\title{
Plant Signaling \\ Molecules
}

Role and Regulation Under

Stressful Environments

Edited by

M. Iqbal R. Khan, Palakolanu Sudhakar Reddy,

Antonio Ferrante, Nafees A. Khan

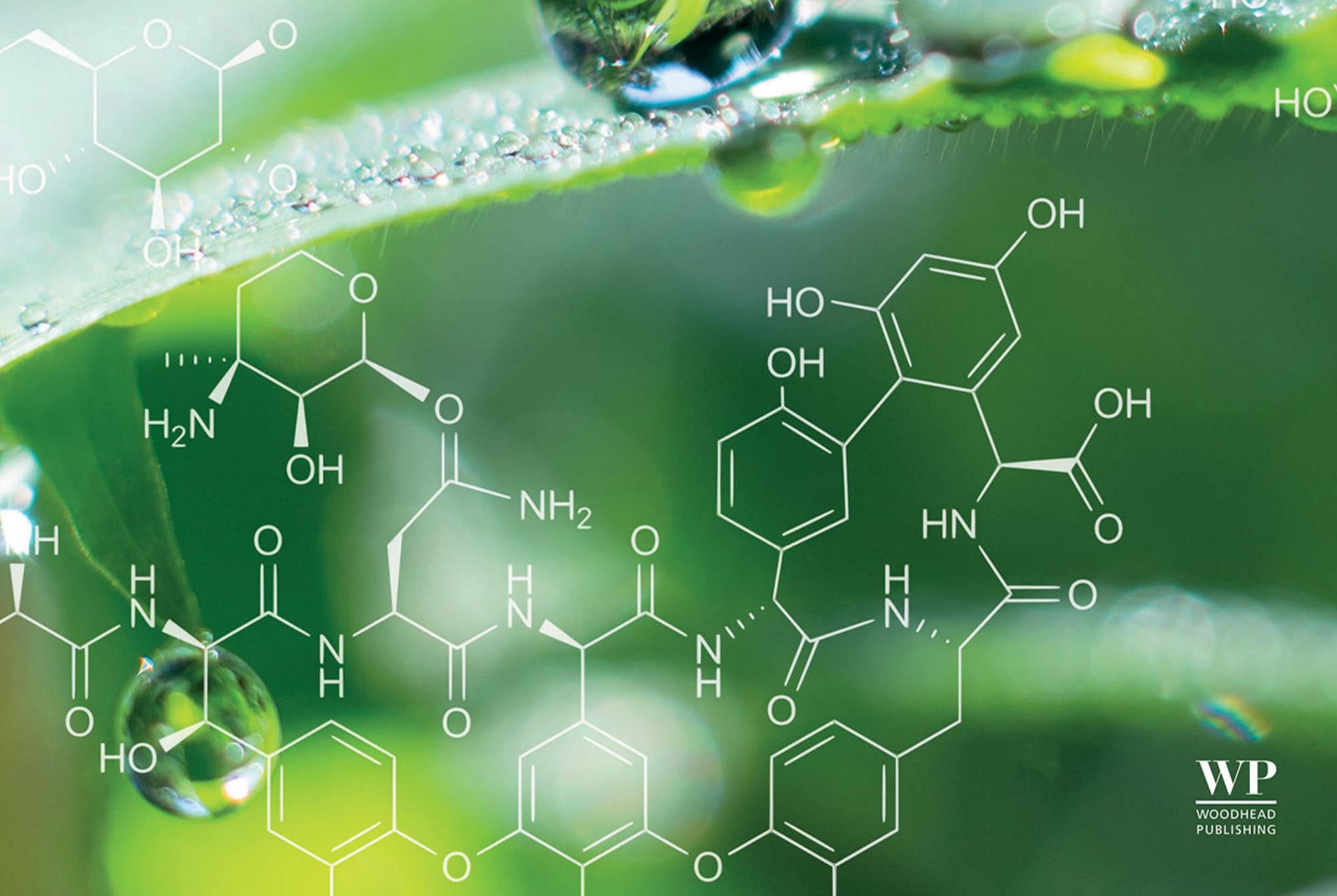




\section{PLANT SIGNALING MOLECULES}




\section{Related Titles}

Genetic and Genomic Resources for Grain Cereal Improvement, (ISBN 978-0-12-802000-5)

Abiotic and Biotic Stresses in Soybean Production (ISBN 978-0-12-801536-0)

Emerging Technologies for Management of Crop Stress Tolerance Volume 1, (ISBN 978-0-12-800876-8)

Emerging Technologies for Management of Crop Stress Tolerance Volume 2, (ISBN 978-0-12-800875-1) 


\section{PLANT SIGNALING MOLECULES \\ Role and Regulation Under Stressful Environments}

Edited by

M. IQBAL R. KHAN

Department of Botany, School of Chemical and Life Sciences, Jamia Hamdard, New Delhi, India

Palakolanu SudhaKar Reddy

Cell, Molecular Biology and Genetic Engineering Group, International Crops Research Institute for the Semi-Arid Tropics (ICRISAT), Telangana, India

Antonio Ferrante

Department of Agricultural and Environmental Sciences, University of Milano, Milano, Italy

NAFEes A. KHAN

Department of Botany, Aligarh Muslim University, Aligarh, India
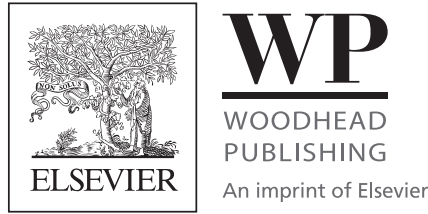
Woodhead Publishing is an imprint of Elsevier

The Officers' Mess Business Centre, Royston Road, Duxford, CB22 4QH, United Kingdom

50 Hampshire Street, 5th Floor, Cambridge, MA 02139, United States

The Boulevard, Langford Lane, Kidlington, OX5 1GB, United Kingdom

Copyright (C) 2019 Elsevier Inc. All rights reserved.

No part of this publication may be reproduced or transmitted in any form or by any means, electronic or mechanical, including photocopying, recording, or any information storage and retrieval system, without permission in writing from the publisher. Details on how to seek permission, further information about the Publisher's permissions policies and our arrangements with organizations such as the Copyright Clearance Center and the Copyright Licensing Agency, can be found at our website: www.elsevier.com/permissions.

This book and the individual contributions contained in it are protected under copyright by the Publisher (other than as may be noted herein).

\section{Notices}

Knowledge and best practice in this field are constantly changing. As new research and experience broaden our understanding, changes in research methods, professional practices, or medical treatment may become necessary.

Practitioners and researchers must always rely on their own experience and knowledge in evaluating and using any information, methods, compounds, or experiments described herein. In using such information or methods they should be mindful of their own safety and the safety of others, including parties for whom they have a professional responsibility.

To the fullest extent of the law, neither the Publisher nor the authors, contributors, or editors, assume any liability for any injury and/or damage to persons or property as a matter of products liability, negligence or otherwise, or from any use or operation of any methods, products, instructions, or ideas contained in the material herein.

British Library Cataloguing-in-Publication Data

A catalogue record for this book is available from the British Library

Library of Congress Cataloging-in-Publication Data

A catalog record for this book is available from the Library of Congress

ISBN: 978-0-12-816451-8 (print)

ISBN: 978-0-12-816452-5 (online)

For information on all Woodhead Publishing publications

visit our website at https://www.elsevier.com/books-and-journals

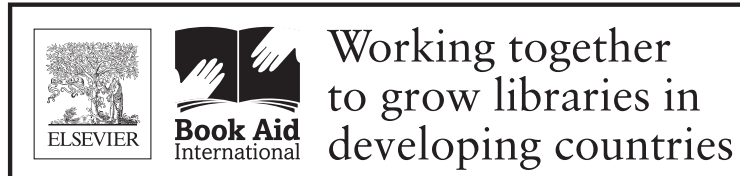

www.elsevier.com • www.bookaid.org

Publisher: Andre G. Wolff

Acquisition Editor: Nancy Maragioglio

Editorial Project Manager: Michael Lutz

Production Project Manager: Debasish Ghosh

Cover Designer: Mark Rogers

Typeset by MPS Limited, Chennai, India 


\section{Contents}

\section{List of Contributors}

1. Physiological Responses and Mechanisms of Signaling Molecules in Plants Stress Tolerance DANIELE MASSA AND SARA MELITO

1.1 Introduction

1.2 Growth and Development

1.3 Leaf Gaseous Exchanges

References

2. Stress Responsive Signaling Molecules and Genes Under Stressful Environments in Plants MURAT DIKILITAS, ERAY SIMSEK AND SEMA KARAKAS

\subsection{Introduction}

2.2 Signaling Molecules Under Stress Conditions

2.3 Signaling Molecules and Plant Responses Under Combined Stress Conditions

2.4 DNA Damage of Plants Under Concurrent of Abiotic and Biotic Stress Combinations

2.5 Genomic and Biochemical Approaches for Plants Under Combined Stresses

2.6 Conclusions and Future Prospects

Acknowledgement

References

3. Engineering Signaling Molecules to Improve Abiotic Stress Tolerance in Crop Plants

KRISHNA KUMAR GUDURU, CHANDRA SEKHAR AKILA

AND CHANDRA OBUL REDDY PULI

3.1 Introduction

3.2 Stress Signal Sensors

3.3 Salt Stress Sensors

3.4 Osmotic Stress Sensors

3.5 ABA Signaling Pathway

3.6 Calcium Sensors and Signaling

3.7 ROS Signaling

3.8 Conclusions and Future Perspectives

Acknowledgement

References

Further Reading

xi 4. Genetic Engineering/Genome Editing Approaches to Modulate Signaling Processes in Abiotic Stress Tolerance

RIWANDAHUN MARWEIN, JOHNI DEBBARMA, YOGITA N. SARKI, INDRANI BARUAH, BANASHREE SAIKIA, H.P.D. BORUAH, NATARAJAN VELMURUGAN

AND CHANNAKESHAVAIAH CHIKKAPUTTAIAH

4.1 Introduction

4.2 Plant Response to Abiotic Stress in Developing Tolerance

4.3 Genetic Engineering Approaches to Modulate Abiotic Stress Signaling Process in Crop Plants

4.4 Genome Editing Approaches to Modulate Abiotic Stress Signaling Processes in Crop Plants

4.5 Conclusions and Future Prospects

References

5. Measurement of Signaling Molecules Calcium , Reactive Sulfur Species, Reactive Carbony Species, Reactive Nitrogen Species, and Reactive Oxygen Species in Plants

33 ZHONG-GUANG LI

5.1 Introduction

5.2 Section 1: $\mathrm{Ca}^{2+}$ Quantification

5.3 Section 2: $\mathrm{H}_{2} \mathrm{~S}$ Quantification

5.4 Section 3: Methylglyoxal Quantification

5.5 Section 4: NO Quantification

5.6 Section 5: $\mathrm{H}_{2} \mathrm{O}_{2}$ Quantification

5.7 Section 6: Superoxide Radical Measurement

5.8 Section 7: HO• Quantification

Acknowledgments

References

Further Reading

43
6. Drought Tolerance in Plants: Molecular Mechanism and Regulation of Signaling Molecules

PRADYUMNA KUMAR SINGH, DIPALI SRIVASTAVA, POONAM TIWARI, MADHU TIWARI, GITI VERMA AND DEBASIS CHAKRABARTY

6.1 Introduction

6.2 Role of Osmoprotectant Regulatory Genes in Drought Stress 
6.3 Redox Regulatory Machinery of Plants During Drought Conditions

6.4 Hormonal Regulation of Drought Tolerance in Plants

6.5 Molecular Mechanism of Regulatory Elements in Drought Stress

6.6 Conclusion and Further Prospects

References

Further Reading

7. Crop Improvement of Cereals Through

Manipulation of Signaling Pathways in Response to Drought Stress

SUNITHA MELLACHERUVU, ASHWINI TALAKAYALA AND

MALLIKARJUNA GARLADINNE

7.1 Introduction

7.2 Transcription Factors Associated With Signaling Mechanism

7.3 Genetic Engineering of Kinases for Drought and Salinity Tolerance

7.4 Modulation of Key Genes Involved in Phytohormone Signaling

7.5 Engineering of Osmoregulatory Genes

7.6 Reactive Oxygen Species Signaling During Drought and Salinity Stress

7.7 Conclusion and Future Prospects

Acknowledgments

References

8. Role and Regulation of ROS and Antioxidants as Signaling Molecules in Response to Abiotic

Stresses

POOJA SHARMA, PRIYANKA SHARMA, PRIYA ARORA, VINOD VERMA, KANIKA KHANNA, POONAM SAINI

AND RENU BHARDWAJ

8.1 Introduction

8.2 Reactive Oxygen Species as Signaling Molecules

8.3 Reactive Oxygen Species Involved in Plant Defense

8.4 Reactive Oxygen Species-Mediated Damage to Macromolecules

8.5 Reactive Oxygen Species Production and Intracellular Protein Oxidation

8.6 Role of Antioxidants and Its Signaling in Abiotic Stress

8.7 Conclusion

References

Further Reading

9. Role and Regulation of Plants Phenolics in Abiotic Stress Tolerance: An Overview

MOHD IRFAN NAIKOO, MUDASIR IRFAN DAR, FARIHA RAGHIB, HASSAN JALEEL, BILAL AHMAD, AAMIR RAINA,

FAREED AHMAD KHAN AND FAUZIA NAUSHIN

9.1 Introduction

9.2 Structure and Classification

9.3 Biosynthesis of Polyphenols
9.4 Phenolics and Abiotic Stress Tolerance 158
109

111

114

117

117

123
9.5 Conclusion and Future Prospects

164

164

Further Reading

168

10. Bioactive Molecules as Regulatory Signals in Plant Responses to Abiotic Stresses

GIULIA FRANZONI, ALICE TRIVELLINI, ROBERTA BULGARI, GIACOMO COCETTA AND ANTONIO FERRANTE

10.1 Introduction

10.2 The Role of Ascorbic Acid, Carotenoids, and Flavonoids in Stress Signaling

Further Reading

11. Biochemical and Molecular

Regulation of Phenylpropanoids Pathway Under Abiotic Stresses

ALESSANDRA FRANCINI, ANDREA GIRO

AND ANTONIO FERRANTE

11.1 Introduction

11.2 Abiotic Stress and Physiological Changes in the Phenylpropanoid Pathway

11.3 Water Stress

11.4 Cold Stress

11.5 Salinity Stress and Phenylpropanoids Accumulation

11.6 Light Stress

186

186

187

11.7 Senescence

11.9 Molecular Changes of the Genes Involved in the Phenylpropanoid Pathways

References

12. Role and Regulation of Glucose as a Signal Molecule to Salt Stress

PÉTER POÓR, ZALÁN CZÉKUS AND ATTILA ÖRDÖG

12.1 Introduction

12.2 Salt Stress Effects on Plants

12.3 Glucose Sensing, Transport, and Signaling in Plants

12.4 Role of Glucose in Salt Stress Responses

12.5 Glucose and Phytohormones Under Salt Stress

12.6 Conclusion and Future Perspectives

References 
13. Role of Sugars in Abiotic Stress Signaling in Plants

IFFAT ZAREEN AHMAD

13.1 Introduction

13.2 Kinases as Enzyme Sensors

13.3 Sugar Signaling at Gene Level

13.4 Sugar Signaling and Plant Metabolism

13.5 Sugar Signaling and Phytohormones

Conclusion

References

Further Reading

14. Methylglyoxal: A Novel Signaling Molecule in Plant Responses to Abiotic Stresses

\section{ZHONG-GUANG LI}

14.1 Introduction

14.2 Generation of MG in Plants

14.3 Elimination of MG in Plants

14.4 MG Signaling Triggered by Environmental Stress in Plants

14.5 Abiotic Stress Tolerance Related to MG in Plants

14.6 Abiotic Stress Tolerance Improved by Overexpressing Glyoxalase Genes

14.7 MG Priming-Induced Abiotic Tolerance

14.8 Signaling Crosstalk Between $\mathrm{MG}$ and $\mathrm{Ca}^{2+}$, ROS, $\mathrm{NO}$, and $\mathrm{H}_{2} \mathrm{~S}$

14.9 Conclusion and Future Prospects

Acknowledgements

References

15. Role of Trehalose and Regulation of its Levels as a Signal Molecule to Abiotic Stresses in Plants

SILVIA MORGUTTI, NOEMI NEGRINI, CHIARA PUCCIARIELLO AND GIAN ATTILIO SACCHI

15.1 Introduction

15.2 Trehalose in Plants

15.3 Trehalose as a Protectant Against Abiotic Stresses

15.4 Trehalose-6-P in Plant Metabolic and Physiological Activities

15.5 Sugar Signaling Systems in Plants

15.6 Trehalose/Trehalose-6-P/SnRK1 System

15.7 Trehalose/Trehalose-6-P/SnRK1 System in Specific Stress Responses: Hypoxia and Cold

15.8 Concluding Remarks

References

Further Reading

\section{Sugar Regulates Plant Growth and}

Development Under In Vitro Conditions

DURDANA SHAH, NASREENA SAJJAD, ROHAYA ALI, NAZISH NAZIR, SUMAYA HASSAN AND SNOBER SHAH

16.1 Introduction
16.2 Role of Sugars in Plant Growth and Development Under In Vitro Conditions

16.3 Sugar Signaling: Physiological, Molecular, and Genetic Approaches in Plants

16.4 Physiological Approaches of Sugar Signaling in Plants

260

16.5 Molecular and Genetic Approaches of Sugar Regulation in Plants

16.6 Importance of Sugar Interaction With Phytohormones in Regulation of Growth and Development Under In Vitro Condition

16.7 Function of Phytohormones Under In Vitro Conditions

16.8 Conclusion and Future Prospects

References

Further Reading

17. Role of Mineral Nutrients in Abiotic Stress Tolerance: Revisiting the Associated Signaling Mechanisms

MOHAMMAD ABASS AHANGER AND PARVAIZ AHMAD

17.1 Introduction

17.2 Mineral Nutrients and Stress Tolerance

17.3 Phosphorous

17.4 Calcium

17.5 Conclusion and Future Prospects

280

Acknowledgments

Further Reading

18. Sulfur Availability Potentiates Phytohormones-Mediated Action in Plants

BADAR JAHAN, ZEBUS SEHAR, ASIM MASOOD, NASER A. ANJUM, M. IQBAL R. KHAN AND NAFEES A. KHAN

18.1 Introduction

18.2 Overview of Sulfur Assimilation and its Role in Plant Metabolism

18.3 Crosstalk Between Sulfur and Phytohormones

References

Further Reading

19. Role and Regulation of Plant Hormones as a Signal Molecule in Response to Abiotic Stresses

PALAK BAKSHI, NEHA HANDA, VANDANA GAUTAM, PARMINDER KAUR, SHELJA SAREEN, BILALAHMAD MIR AND RENU BHARDWAJ

19.1 Introduction

19.2 Perception and Transduction of Signals

19.3 Regulation of Various Abiotic Stresses by Plant Hormones

19.4 Conclusion and Future Prospects 
20. Role and Regulation of Auxin Signaling in Abiotic Stress Tolerance

VEENA PANDEY, INDRA DUTT BHATT AND SHYAMAL KUMAR NANDI

20.1 Introduction

20.2 Conclusion

Acknowledgements

References

21. The Regulatory Signaling of Gibberellin Metabolism and Its Crosstalk With Phytohormones in Response to Plant Abiotic Stresses

ADITYA BANERJEE AND ARYADEEP ROYCHOUDHURY

21.1 Introduction

21.2 Gibberellic Acid Metabolism in Plants

21.3 Regulatory Signaling of Gibberellic Acids During Abiotic Stresses

21.4 The Signaling Crosstalks Between Gibberellic Acids and Related Phytohormones

21.5 Gibberellic Acids in Plant Abiotic Stress: A Recent Update

21.6 Conclusion and Future Perspectives

Acknowledgements

References

22. Abscisic Acid, a Principal Regulator of Plant Abiotic Stress Responses

SUSHMA SAGAR AND AMARJEET SINGH

22.1 Introduction

22.2 ABA Biosynthesis

22.3 Catabolism of ABA

22.4 ABA Signaling Pathway

22.5 ABA Receptors

22.6 Protein Phosphatase 2C

22.7 SNF1-Related Protein Kinase 2 (SnRK2)

22.8 ABA-Dependent Gene Expression

22.9 The Role of ABA in Abiotic Stress Signaling

22.10 Conclusion

Acknowledgment

References

Further Reading

23. Salicylic Acid-Mediated Defense Mechanisms to Abiotic Stress Tolerance FARHAN AHMAD, ANANYA SINGH AND AISHA KAMAL

23.1 Introduction

23.2 Biosynthetic Pathway and Modifications of Salicylic Acid

23.3 Role of Salicylic Acid in Plant Growth and Development

23.4 Salicylic Acid-Mediated Defense Mechanisms to Abiotic Stress

358
23.5 Crosstalk of Salicylic Acid With Other Phytohormones Under Abiotic Stress

23.6 Interaction and Involvement of DELLA With SA-GA Crosstalk Under Stress 363

23.7 Conclusion and Future Prospects 364

Acknowledgements $\quad 365$

References $\quad 365$

Further Reading $\quad 369$

24. Role of Methyl Jasmonates in Salt Stress

Tolerance in Crop Plants

BILAL AHMAD, AAMIR RAINA, MOHD IRFAN NAIKOO AND SAMIULLAH KHAN

24.1 Introduction

24.2 Jasmonate Biosynthesis 372

24.3 Jasmonate Signaling

24.5 Effect of Salt Stress on Plants 376

24.6 Conclusion and Future Perspectives 380

References

380

Further Reading

25. Insights Into the Nitric Oxide Mediated Stress Tolerance in Plants

PARANKUSAM SANTISREE, SRIVANI S. ADIMULAM, KAPIL SHARMA, POOJA BHATNAGAR-MATHUR AND KIRAN K. SHARMA

25.1 Introduction

25.2 NO in Plant Stress Responses

25.3 NO and Phytohormones Crosstalk in Abiotic Stress Tolerance

25.4 Concluding Remarks and Future Perspectives 398

Acknowledgements

References

Further Reading

26. Brassinosteroid Signaling and Complex

Interplay of ROS, NADPH Oxidase, and MAPK

Mediated Biotic and Abiotic Stress Acclimation

in Plants

DEEPESH BHATT, MAYANK SHARMA, MANOJ NATH, MEGHA D. BHATT AND SAURABH BADONI

26.1 Introduction

407

26.2 Brassinosteroids

408

26.3 Brassinosteroid Signaling in Plants

26.4 Transcription Factors Involved in BR Signaling

26.5 Role of RD26 in BR Signaling

409

26.6 BR Mediated Defense Signaling

26.7 BR Mediated ROS Signaling and Its Role in Plant Defense

26.8 Conclusion

References

413

Further Reading 
27. Role and Regulation of Osmolytes and ABA Interaction in Salt and Drought Stress Tolerance

GUDDIMALLI RAJASHEKER, GANDRA JAWAHAR, NARAVULA JALAJA, SOMANABOINA ANIL KUMAR, PALAVALASA HIMA KUMARI, DEVINENI LAKSHMI PUNITA, APPA RAO KARUMANCHI, PALAKOLANU SUDHAKAR REDDY, POLAVARAPU RATHNAGIRI, NESE SREENIVASULU AND POLAVARAPU BILHAN KAVI KISHOR

27.1 Introduction

27.2 Abscisic Acid-Sensing Mechanism of Plants and Downstream Events

27.3 Role of Abscisic Acid in Osmolyte Biosynthesis

27.4 Regulation of Proline Dehydrogenase

27.5 Signaling Molecules and Osmolyte Synthesis

27.6 Functions of Osmolytes During Abiotic Stress

27.7 Osmolytes and Signaling Processes

27.8 Conclusions and Future Prospects

Acknowledgments

References

Further Reading

28. Regulatory Role of Proline in Heat Stress Tolerance: Modulation by Salicylic Acid

NOUSHINA IQBAL, MEHER FATMA, NAFEES A. KHAN AND SHAHID UMAR

28.1 Introduction

28.2 Aftermaths of Heat Stress

28.3 Proline in Heat Tolerance

28.4 Phytohormones in Heat Tolerance

28.5 Role of Salicyclic Acid in Heat Tolerance

28.6 Interaction Between Salicyclic Acid, Ethylene, and Abscisic Acid for Heat Tolerance

28.7 Conclusion and Future Prospects

References

Further Reading

29. Osmolyte Diversity, Distribution, and Their Biosynthetic Pathways

GANDRA JAWAHAR, GUDDIMALLI RAJASHEKER, PARVEDA MAHESHWARI, DEVINENI LAKSHMI PUNITA, NARAVULA JALAJA, PALAVALASA HIMA KUMARI, SOMANABOINA ANIL KUMAR, RUHI AFREEN, APPA RAO KARUMANCHI, POLAVARAPU RATHNAGIRI, NESE SREENIVASULU AND POLAVARAPU BILHAN KAVI KISHOR

29.1 Introduction

29.2 Diversity and Distribution of Osmolytes in Different Plant Species

29.3 Biosynthetic Pathways of Proline and Its Derivatives

29.4 Biosynthetic Pathways of Glycine Betaine and Its Derivatives

29.5 Biosynthetic Pathways of Trehalose and Sugar Alcohols

29.6 Conclusions

Acknowledgments

References

Further Reading
30. Role and Regulation of Osmolytes as Signaling Molecules to Abiotic Stress Tolerance

TITASH DUTTA, NAGESWARA RAO REDDY NEELAPU, SHABIR H. WANI AND CHALLA SUREKHA

30.1 Introduction

30.2 Osmolyte Mediated Abiotic Stress Responses

30.3 Biosynthesis, Accumulation, and Regulation

of Osmolytes in Response to Abiotic Stress

461

30.4 Conclusion and Future Prospects 471

Acknowledgment $\quad 472$

References

472

\section{Proteomics Insights Into Salt Stress}

Signaling in Plants

ARAJMAND FRUKH, ALTAF AHMAD AND TARIQ OMAR SIDDIQI

31.1 Introduction

31.2 Proteomics: Edge Over Other Technologies

31.3 Technical Advances in Proteomics

31.4 Salt Stress in Plants

481

31.5 Response of Plants Towards Salt Stress

31.6 Salt Stress Signaling Pathways

31.7 Proteomics Approach in Understanding Salt Stress Signaling Pathways

31.8 Conclusion and Future Perspectives

References

Further Reading

32. Heat Shock Proteins (Hsps) Mediated Signalling Pathways During Abiotic Stress Conditions

KUMMARI DIVYA, POOJA BHATNAGAR-MATHUR, KIRAN K. SHARMA AND PALAKOLANU SUDHAKAR REDDY

32.1 Introduction 499

32.2 A General Account on Heat Shock Proteins

32.3 Heat Shock Protein Induction Phenomena in Plants

32.4 Abiotic Stresses Induce the Heat Shock Protein-Mediated Signaling Pathways

32.5 Crosstalk Between Reactive Oxygen Species, Mitogen Activated Protein Kinases Cascades, $\mathrm{Ca}^{+2}$ and Heat Shock Factor/Heat Shock Proteins

32.6 Genetic Engineering of Heat Shock Proteins Signaling Molecules

32.7 Conclusions and Future Perspectives
33. $\mathrm{C}_{4} / \mathrm{CAM}$ Facultative Photosynthesis as a Means to Improve Plant Sustainable Productivity Under Abiotic-Stressed Conditions: Regulatory Mechanisms and Biotechnological Implications RENATA CALLEGARI FERRARI AND LUCIANO FRESCHI

33.1 Initial Considerations 
33.2 $\mathrm{C}_{4}$ and Crassulacean Acid Metabolism: Similarities and Differences

33.3 Environmental Cues Controlling $\mathrm{C}_{4}$ and Crassulacean Acid Metabolism

33.4 Stress Signaling Networks Controlling $\mathrm{C}_{4}$ and Crassulacean Acid Metabolism

33.5 $\mathrm{C}_{4} /$ Crassulacean Acid Metabolism Compatibility

33.6 Engineering $\mathrm{C}_{4}$ and Crassulacean Acid Metabolism: Challenges and Possibilities

33.7 Concluding Remarks

Acknowledgments

References

Further Reading

34. Protein Kinases and Phosphatases in Stress Transduction: Role in Crop Improvement

THAMMINENI CHAKRADHAR, RAMESHA A. REDDY AND THUMMALA CHANDRASEKHAR

34.1 Introduction

34.2 Receptors Like Kinases

34.3 Mitogen Activated Protein Kinases

34.4 Calcium Kinases

34.5 Sucrose Nonfermenting 1-Related Protein Kinases

34.6 Diacylglycerol Kinases
34.7 Histidine Kinases

542

51834.8 Genomics Efforts in Plant Protein Kinase Towards Crop Improvement

34.9 Conclusions

543

References

524 Further Reading

543

525

35. Nanoparticles and Abiotic Stress Tolerance in Plants: Synthesis, Action, and Signaling Mechanisms

SYED UZMA JALIL AND MOHAMMAD ISRAIL ANSARI

35.1 Introduction

35.2 Plant and Abiotic Stress

35.3 Mode of Action of Nanomaterials Under Abiotic Stresses

35.4 Signaling Mechanism of Nanoparticles During Abiotic Stress Conditions
35.5 Conclusion

557

Further Reading 


\section{List of Contributors}

Aamir Raina Department of Botany, Mutation Breeding Laboratory, Aligarh Muslim University, Aligarh, Uttar Pradesh, India; Botany Section, Women's College, Aligarh Muslim University, Aligarh, Uttar Pradesh, India

Aditya Banerjee Department of Biotechnology, St. Xavier's College (Autonomous), Kolkata, West Bengal, India

Aisha Kamal Department of Bioengineering, Integral University, Lucknow, Uttar Pradesh, India

Alessandra Francini Institute of Life Sciences, Sant'Anna School of Advanced Studies, Pisa, Italy

Alice Trivellini Institute of Life Sciences, Scuola Superiore Sant'Anna, Pisa, Italy

Altaf Ahmad Department of Botany, Faculty of Life Sciences, Aligarh Muslim University, Aligarh, Uttar Pradesh, India

Amarjeet Singh National Institute of Plant Genome Research, New Delhi, India

Ananya Singh Department of Biosciences, Integral University, Lucknow, Uttar Pradesh, India

Andrea Giro DAFNAE-Department of Agronomy Animals Food Natural Resources and Environment, University of Padua, AGRIPOLIS-University Avenue, Legnaro, Italy

Antonio Ferrante Department of Agricultural and Environmental Sciences, Università degli Studi di Milano, Milano, Italy

Appa Rao Karumanchi Department of Genetics, Osmania University, Hyderabad, Telangana, India; Department of Biotechnology, Acharya Nagarjuna University, Nagarjuna Nagar, Guntur, India

Arajmand Frukh Department of Botany, School of Chemical and Life Sciences, Jamia Hamdard, New Delhi, India

Aryadeep Roychoudhury Department of Biotechnology, St. Xavier's College (Autonomous), Kolkata, West Bengal, India

Ashwini Talakayala Plant Molecular Biology Laboratory, Agri Biotech Foundation, Hyderabad, India

Asim Masood Plant Physiology and Biochemistry Division, Department of Botany, Aligarh Muslim University, Aligarh, Uttar Pradesh, India

Attila Ördög Department of Plant Biology, University of Szeged, Szeged, Hungary

Badar Jahan Plant Physiology and Biochemistry Division, Department of Botany, Aligarh Muslim University, Aligarh, Uttar Pradesh, India
Banashree Saikia Biotechnology Group, Biological Sciences and Technology Division, CSIR-North East Institute of Science and Technology (CSIR-NEIST), Jorhat, Assam, India; Academy of Scientific and Innovative Research (AcSIR), CSIR-NEIST, Jorhat, Assam, India

Bilal Ahmad Plant Physiology Laboratory, Department of Botany, Aligarh Muslim University, Aligarh, Uttar Pradesh, India

BilalAhmad Mir Department of Botany, School of Life Sciences, Satellite campus Kargil, University of Kashmir, Srinagar, Jammu and Kashmir, India

Challa Surekha Department of Biochemistry and Bioinformatics, Institute of Science, Gandhi Institute of Technology and Management (GITAM Deemed to be University), Visakhapatnam, Andhra Pradesh, India

Chandra Obul Reddy Puli Plant Molecular Biology Laboratory, Department of Botany, Yogi Vemana University, Kadapa, Andhra Pradesh, India

Chandra Sekhar Akila Department of Biotechnology, Yogi Vemana University, Kadapa, Andhra Pradesh, India

Channakeshavaiah Chikkaputtaiah Biotechnology Group, Biological Sciences and Technology Division, CSIR-North East Institute of Science and Technology (CSIR-NEIST), Jorhat, Assam, India

Chiara Pucciariello Institute of Life Sciences, Sant'Anna School of Advanced Studies, Pisa, Italy

Daniele Massa CREA, Research Centre for Vegetable and Ornamental Crops, Council for Agricultural Research and Economics, Pescia (PT), Italy

Debasis Chakrabarty Council of Scientific and Industrial Research - National Botanical Research Institute (CSIR-NBRI), Lucknow, India; Academy of Scientific and Innovative Research (AcSIR), New Delhi, India

Deepesh Bhatt Department of Biotechnology, Shree Ramkrishna Institute of Computer Education and Applied Sciences, Veer Narmad South Gujarat University, Surat, Gujarat, India

Devineni Lakshmi Punita Department of Genetics, Osmania University, Hyderabad, Telangana, India

Dipali Srivastava Council of Scientific and Industrial Research - National Botanical Research Institute (CSIR-NBRI), Lucknow, India

Durdana Shah Plant Tissue Culture Laboratory, Centre of Research for Development, University of Kashmir, Srinagar, Jammu and Kashmir, India 
Eray Simsek Department of Plant Protection, Harran University, Şanlıurfa, Turkey

Fareed Ahmad Khan Plant Ecology and Environment Laboratory, Department of Botany, Aligarh Muslim University, Aligarh, Uttar Pradesh, India

Farhan Ahmad Department of Bioengineering, Integral University, Lucknow, Uttar Pradesh, India

Fariha Raghib Plant Ecology and Environment Laboratory, Department of Botany, Aligarh Muslim University, Aligarh, Uttar Pradesh, India

Fauzia Naushin Botany Section, Women's College, Aligarh Muslim University, Aligarh, Uttar Pradesh, India

Gandra Jawahar Department of Genetics, Osmania University, Hyderabad, Telangana, India

Giacomo Cocetta Department of Agricultural and Environmental Sciences, Università degli Studi di Milano, Milano, Italy

Gian Attilio Sacchi Department of Agricultural and Environmental Sciences-Production, Landscape, Agroenergy, University of Milan, Milan, Italy

Giti Verma Council of Scientific and Industrial Research National Botanical Research Institute (CSIR-NBRI), Lucknow, India

Giulia Franzoni Department of Agricultural and Environmental Sciences, Universià degli Studi di Milano, Milano, Italy

Guddimalli Rajasheker Department of Genetics, Osmania University, Hyderabad, Telangana, India

H.P.D. Boruah Biotechnology Group, Biological Sciences and Technology Division, CSIR-North East Institute of Science and Technology (CSIR-NEIST), Jorhat, Assam, India

Hassan Jaleel Plant Physiology Laboratory, Department of Botany, Aligarh Muslim University, Aligarh, Uttar Pradesh, India

Iffat Zareen Ahmad Department of Bioengineering, Integral University, Lucknow, Uttar Pradesh, India

Indra Dutt Bhatt G. B. Pant National Institute of Himalayan Environment and Sustainable Development, Kosi-Katarmal, Almora, Uttarakhand, India

Indrani Baruah Biotechnology Group, Biological Sciences and Technology Division, CSIR-North East Institute of Science and Technology (CSIR-NEIST), Jorhat, Assam, India; Academy of Scientific and Innovative Research (AcSIR), CSIR-NEIST, Jorhat, Assam, India

Johni Debbarma Biotechnology Group, Biological Sciences and Technology Division, CSIR-North East Institute of Science and Technology (CSIR-NEIST), Jorhat, Assam, India; Academy of Scientific and Innovative Research (AcSIR), CSIR-NEIST, Jorhat, Assam, India

Kanika Khanna Department of Botanical and Environmental Sciences, Guru Nanak Dev University, Amritsar, Punjab, India

Kapil Sharma Department of Plant Sciences, Repository of Tomato Genomics Resources, University of Hyderabad, Hyderabad, Telangana, India
Kiran K. Sharma Cell, Molecular Biology \& Genetic Engineering Group, Research Program-Genetic Gains, International Crops Research Institute for the Semi-Arid Tropics (ICRISAT), Patancheru, Hyderabad, Telangana, India

Krishna Kumar Guduru Plant Molecular Biology Laboratory, Department of Botany, Yogi Vemana University, Kadapa, Andhra Pradesh, India

Kummari Divya Cell, Molecular Biology \& Genetic Engineering Group, Research Program-Genetic Gains, International Crops Research Institute for the Semi-Arid Tropics (ICRISAT), Patancheru, Hyderabad, Telangana, India

Luciano Freschi Department of Botany, Institute of Biosciences, University of São Paulo, São Paulo, Brazil

M. Iqbal R. Khan Department of Botany, School of Chemical and Life Sciences, Jamia Hamdard, New Delhi, India

Madhu Tiwari Council of Scientific and Industrial Research - National Botanical Research Institute (CSIR-NBRI), Lucknow, India

Mallikarjuna Garladinne Plant Molecular Biology Laboratory, Agri Biotech Foundation, Hyderabad, India

Manoj Nath Indian Council of Agricultural Research, New Delhi, Uttar Pradesh, India

Mayank Sharma Martin Luther University of HalleWittenberg, Halle, Germany

Megha D. Bhatt GSFC AgroTech Ltd. Gujarat State Fertilizers \& Chemicals Ltd., Vadodara, India

Meher Fatma Plant Molecular Biology, School of life Sciences, Jawaharlal Nehru University, New Delhi, India

Mohammad Abass Ahanger Department of Botany, Govt PG College Rajouri, Rajouri, Jammu and Kashmir, India

Mohammad Israil Ansari Department of Botany, University of Lucknow, Lucknow, India

Mohd Irfan Naikoo Plant Ecology and Environment Laboratory, Department of Botany, Aligarh Muslim University, Aligarh, Uttar Pradesh, India

Mudasir Irfan Dar Plant Ecology and Environment Laboratory, Department of Botany, Aligarh Muslim University, Aligarh, Uttar Pradesh, India

Murat Dikilitas Department of Plant Protection, Harran University, Şanlıurfa, Turkey

Nafees A. Khan Plant Physiology and Biochemistry Division, Department of Botany, Aligarh Muslim University, Aligarh, Uttar Pradesh, India

Nageswara Rao Reddy Neelapu Department of Biochemistry and Bioinformatics, Institute of Science, Gandhi Institute of Technology and Management (GITAM Deemed to be University), Visakhapatnam, Andhra Pradesh, India

Naravula Jalaja Department of Biotechnology, Vignan University, Vadlamudi, Guntur, India 
Naser A. Anjum Plant Physiology and Biochemistry Division, Department of Botany, Aligarh Muslim University, Aligarh, Uttar Pradesh, India

Nasreena Sajjad Department of Biochemistry, University of Kashmir, Srinagar, Jammu and Kashmir, India

Natarajan Velmurugan Branch Laboratory Itanagar, CSIRNorth East Institute of Science and Technology (CSIR-NEIST), Jorhat, Assam, India

Nazish Nazir Plant Tissue Culture Laboratory, Centre of Research for Development, University of Kashmir, Srinagar, Jammu and Kashmir, India

Neha Handa Department of Botanical and Environmental Sciences, Guru Nanak Dev University, Amritsar, Punjab, India

Nese Sreenivasulu Grain Quality and Nutrition Center, Plant Breeding Division, International Rice Research Institute, Metro Manila, Philippines

Noemi Negrini Department of Agricultural and Environmental Sciences-Production, Landscape, Agroenergy, University of Milan, Milan, Italy

Noushina Iqbal Department of Botany, Hamdard University, New Delhi, India

Palak Bakshi Department of Botanical and Environmental Sciences, Guru Nanak Dev University, Amritsar, Punjab, India

Palakolanu Sudhakar Reddy Cell, Molecular Biology \& Genetic Engineering Group, Research Program-Genetic Gains, International Crops Research Institute for the SemiArid Tropics (ICRISAT), Patancheru, Hyderabad, Telangana, India

Palavalasa Hima Kumari Department of Genetics, Osmania University, Hyderabad, Telangana, India

Parankusam Santisree International Crops Research Institute for the Semi-Arid Tropics (ICRISAT), Patancheru, Hyderabad, Telangana, India

Parminder Kaur Department of Botanical and Environmental Sciences, Guru Nanak Dev University, Amritsar, Punjab, India

Parvaiz Ahmad Department of Botany, Govt PG College Rajouri, Rajouri, Jammu and Kashmir, India; Department of Botany and Microbiology, Faculty of Science, King Saud University, Riyadh, Saudi Arabia

Parveda Maheshwari Department of Genetics, Osmania University, Hyderabad, Telangana, India

Péter Poór Department of Plant Biology, University of Szeged, Szeged, Hungary

Polavarapu Bilhan Kavi Kishor Department of Genetics, Osmania University, Hyderabad, Telangana, India

Polavarapu Rathnagiri Genomix CARL Pvt. Ltd., Kadapa, Andhra Pradesh, India

Pooja Bhatnagar- Mathur Cell, Molecular Biology \& Genetic Engineering Group, Research Program-Genetic Gains, International Crops Research Institute for the SemiArid Tropics (ICRISAT), Patancheru, Hyderabad, Telangana, India
Pooja Sharma Department of Botanical and Environmental Sciences, Guru Nanak Dev University, Amritsar, Punjab, India

Poonam Saini Department of Botanical and Environmental Sciences, Guru Nanak Dev University, Amritsar, Punjab, India

Poonam Tiwari Council of Scientific and Industrial Research - National Botanical Research Institute (CSIR-NBRI), Lucknow, India

Pradyumna Kumar Singh Council of Scientific and Industrial Research - National Botanical Research Institute (CSIR-NBRI), Lucknow, India; Academy of Scientific and Innovative Research (AcSIR), New Delhi, India

Priya Arora Department of Botanical and Environmental Sciences, Guru Nanak Dev University, Amritsar, Punjab, India

Priyanka Sharma Department of Biotechnology, Modern College of Arts, Science and Commerce, Ganeshkhind, Pune, Maharashtra, India

Ramesha A. Reddy Seri-Biotech Research Laboratory, Central Silk Board, Bangalore, Karnataka, India

Renata Callegari Ferrari Department of Botany, Institute of Biosciences, University of São Paulo, São Paulo, Brazil

Renu Bhardwaj Department of Botanical and Environmental Sciences, Guru Nanak Dev University, Amritsar, Punjab, India

Riwandahun Marwein Biotechnology Group, Biological Sciences and Technology Division, CSIR-North East Institute of Science and Technology (CSIR-NEIST), Jorhat, Assam, India; Academy of Scientific and Innovative Research (AcSIR), CSIR-NEIST, Jorhat, Assam, India

Roberta Bulgari Department of Agricultural and Environmental Sciences, Universià degli Studi di Milano, Milano, Italy

Rohaya Ali Department of Biochemistry, University of Kashmir, Srinagar, Jammu and Kashmir, India

Ruhi Afreen Department of Genetics, Osmania University, Hyderabad, Telangana, India

Samiullah Khan Department of Botany, Mutation Breeding Laboratory, Aligarh Muslim University, Aligarh, Uttar Pradesh, India

Sara Melito Department of Agriculture, University of Sassari, Sassari, Italy

Saurabh Badoni Plant Breeding Division, International Rice Research Institute, Metro Manila, Philippines

Sema Karakas Department of Soil Science and Plant Nutrition, Harran University, Şanlıurfa, Turkey

Shabir H. Wani Mountain Research Centre for Field Crops, Sher-e-Kashmir University of Agricultural Sciences and Technology, Srinagar, Jammu and Kashmir, India; Department of Plant, Soil and Microbial Sciences, Michigan State University, East Lansing, MI, United States 
Shahid Umar Department of Botany, Hamdard University, New Delhi, India

Shelja Sareen Department of Biotechnology, BBK DAV College for Women, Amritsar, Punjab, India

Shyamal Kumar Nandi G. B. Pant National Institute of Himalayan Environment and Sustainable Development, Kosi-Katarmal, Almora, Uttarakhand, India

Silvia Morgutti Department of Agricultural and Environmental Sciences-Production, Landscape, Agroenergy, University of Milan, Milan, Italy

Snober Shah Cytogenitics and Molecular Biology Laboratory, Centre of Research for Development, University of Kashmir, Srinagar, Jammu and Kashmir, India

Somanaboina Anil Kumar Department of Genetics, Osmania University, Hyderabad, Telangana, India

Srivani S. Adimulam International Crops Research Institute for the Semi-Arid Tropics (ICRISAT), Patancheru, Hyderabad, Telangana, India

Sumaya Hassan Department of Biochemistry, University of Kashmir, Srinagar, Jammu and Kashmir, India

Sunitha Mellacheruvu Plant Molecular Biology Laboratory, Agri Biotech Foundation, Hyderabad, India

Sushma Sagar National Institute of Plant Genome Research, New Delhi, India

Syed Uzma Jalil Amity Institute of Biotechnology, Amity University Uttar Pradesh, Lucknow Campus, Lucknow, Uttar Pradesh, India

Tariq Omar Siddiqi Department of Botany, School of Chemical and Life Sciences, Jamia Hamdard, New Delhi, India

Thammineni Chakradhar Thakora Crop Sciences Pvt. Ltd., Anantapur, Andhra Pradesh, India
Thummala Chandrasekhar Department of Environmental Sciences, Yogi Vemana University, Kadapa, Andhra Pradesh, India

Titash Dutta Department of Biochemistry and Bioinformatics, Institute of Science, Gandhi Institute of Technology and Management (GITAM Deemed to be University), Visakhapatnam, Andhra Pradesh, India

Vandana Gautam Department of Botanical and Environmental Sciences, Guru Nanak Dev University, Amritsar, Punjab, India

Veena Pandey G. B. Pant National Institute of Himalayan Environment and Sustainable Development, Kosi-Katarmal, Almora, Uttarakhand, India

Vinod Verma Department of Botany, DAV University, Jalandhar, Punjab, India

Yogita N. Sarki Biotechnology Group, Biological Sciences and Technology Division, CSIR-North East Institute of Science and Technology (CSIR-NEIST), Jorhat, Assam, India; Academy of Scientific and Innovative Research (AcSIR), CSIR-NEIST, Jorhat, Assam, India

Zalán Czékus Department of Plant Biology, University of Szeged, Szeged, Hungary; Doctoral School in Biology, Faculty of Science and Informatics, University of Szeged, Szeged, Hungary

Zebus Sehar Plant Physiology and Biochemistry Division, Department of Botany, Aligarh Muslim University, Aligarh, Uttar Pradesh, India

Zhong-Guang Li School of Life Sciences, Yunnan Normal University, Kunming, P.R. China; Engineering Research Center of Sustainable Development and Utilization of Biomass Energy, Ministry of Education, Kunming, P.R. China; Key Laboratory of Biomass Energy and Environmental Biotechnology, Yunnan Province, Yunnan Normal University, Kunming, P.R. China 


\section{1 \\ Physiological Responses and Mechanisms of Signaling Molecules in Plants Stress Tolerance \\ Daniele Massa ${ }^{1}$ and Sara Melito ${ }^{2}$}

${ }^{1}$ CREA, Research Centre for Vegetable and Ornamental Crops, Council for Agricultural Research and Economics, Pescia (PT), Italy ${ }^{2}$ Department of Agriculture, University of Sassari, Sassari, Italy

\section{O U T L I N E}

1.1 Introduction

1.2 Growth and Development

1.2.1 Plant Response to Salinity: Signaling Pathway at Tissue and Organ Level

1.2.2 Temporal Signature and Related Cell Response Mechanisms
1.3 Leaf Gaseous Exchanges

1.3.1 Stomatal Conductance and Water Relations

1.3.2 Photosynthesis

References

\subsection{INTRODUCTION}

The signaling pathways involved in the response to salinity are very complex and highly heterogeneous due to the many biological, botanical, and environmental variables that actively interact with one another. Salinity produces several changes in plant physiology that range from osmotic effect to ion toxicity and nutritional disorders (Läuchli and Grattan, 2007; Munns, 2002; Khan et al., 2014a,b) thus causing a number of growth and development alterations from seed germination to plant maturity. It has been observed that saline soils (with at least $4 \mathrm{dS} / \mathrm{m}$ electrical conductivity, roughly equivalent to $40 \mathrm{mM} \mathrm{NaCl}$ ) significantly affect the growth rate of most crops, thus causing significant decreases in produce yield (Shrivastava and Kumar, 2015). The plant response to salinity depends on several factors such as genotypes, plant age, stress duration, salt concentration, and the plant organ involved. Under salinity, plants show typical morphological traits: reduction of leaves numbers, reduction of plant size and of roots length and biomass production (Shannon and Grieve, 1999). These traits are the consequence of the negative effects of high salt concentration on membrane permeability, ions imbalance, stomatal conductance, and lower photosynthesis efficiency (Ivanova et al., 2014). Tolerant species generally show high capacity in sensing saline environments and prompt reaction to change their physiological status. Stomatal closure indeed is one of the first mechanisms to counteract the detrimental effects of osmotic stress (Munns and Tester, 2008; Robinson et al., 1997).

Based on their salt-tolerance capacity, plant species are classified into glycophytes and halophytes. The 
plant tolerance to $\mathrm{Na}^{+}$largely varies among plant species; Flowers (2004) reported a low tolerance to $\mathrm{Na}^{+}$in tomato, citrus, and avocado species, while a high tolerance was found for barley and cotton. In general, most of the crops require water with a very low $\mathrm{Na}^{+}$concentration (micromolar) and are referred to as glycophytes. Growth alteration, lack of fruiting and seed germination, occur for several crops under $100 \mathrm{mM}$ $\mathrm{NaCl}$ (Flowers, 2004; Park et al., 2016; Zhu, 2001). For other plants (i.e., halophytes), such as Atriplex, Salicornia, Rhizophora, and Suaeda, growth and development depend on high $\mathrm{NaCl}$ concentration $(200 \mathrm{mM})$ (Bohnert and Cushman, 2000; Flowers et al., 2010). The $\mathrm{NaCl}$ cellular concentration is the result of the dynamic fluxes in and out of the plant cell. Halophytes show high capacity of controlling $\mathrm{NaCl}$ concentration, because of specific ion-gated channels while glycophytes have a more limited ability to regulate the $\mathrm{NaCl}$ fluxes (Glenn et al., 1999). To control the $\mathrm{Na}^{+}$ level inside the cell, a plasma-membrane antiport $\mathrm{Na}^{+} / \mathrm{H}^{+}$is required (Horie and Schroeder, 2004). Some halophytic species use this antiport system to compartmentalize $\mathrm{Na}^{+}$and $\mathrm{Cl}^{-}$ions in vacuoles within the cell, maintaining a low $\mathrm{NaCl}$ level in the cytosol (Zhang et al., 2010a). Both glycophytes and halophytes are not able to tolerate high salinity in their cytoplasm; consequently they regulate the salt concentration in the cytosol compartmentalizing ions in vacuoles or translocating them in different plant tissues (Turkan and Demiral, 2009).

\subsection{GROWTH AND DEVELOPMENT}

\subsubsection{Plant Response to Salinity: Signaling Pathway at Tissue and Organ Level}

Each phenological stage responds differently to the salinity stress (Munns, 2002; Sairam and Tyagi, 2004). For instance, germination and seedling growth are deeply sensitive to ion imbalance, while the following stage of vegetative growth, in general, shows less sensitivity to salt concentration. Finally, the salinity stress is more damaging during the initial flowering stage as compared with seeds that are already set. Overall, in susceptible plants the shoot growth showed a greater size reduction compared with the root size (Läuchli and Grattan, 2007).

At the germination level, the first signal-molecule received as stress signal is $\mathrm{NaCl}$. High salinity, due to the accumulation of $\mathrm{Na}^{+}$and $\mathrm{Cl}^{-}$, affects the germination because it induces a variation of the osmotic potential that decreases the water uptake, thus leading to a reduction in seed water absorption. In this case, salinity enforces the "dormancy" seed status inhibiting water absorption and the metabolic processes associated with the first stage of germination (Hannachi and Van Labeke, 2018). In addition to the osmotic effect, also the toxic effect of ion accumulation showed to negatively affect plant germination (Bajji et al., 2002). High $\mathrm{Na}^{+}$and $\mathrm{Cl}^{-}$levels inhibit the expanding and division cell process, causing reductions in the germination percentage and further lengthening the time of germination in several plant species such as tomato and eggplant (Cuartero and Fernandez-Munoz, 1999; Hannachi and Van Labeke, 2018). Halophyte and glycophite plant seeds present different germination response. In both groups, salinity induces a reduction of germination: in halophytes the altered osmotic potential is the main cause, while for glycophytes the ion toxicity has an additive role (Bajji et al., 2002). As an adaptive mechanism, seeds of salt-tolerant species have a low osmotic potential inside the seed coat, which ensures adequate water absorption. Two principal strategies are used to create a water potential difference between the environment and the seeds: the use of organic solutes, to keep the osmotic potential, and the $\mathrm{NaCl}$ compartmentalization ( Zhang et al., 2010b).

Osmotic adjustment, ion flux regulation, and $\mathrm{Na}^{+}$ and $\mathrm{Cl}^{-}$compartmentalization are salt tolerance strategies with a high-energy cost that compete with the seed germination and plant growth and development (Zhang et al., 2010b). The metabolic cost for the salt tolerance absorbs carbon reserves and energy otherwise required for the germination and seedling development, inducing a reduction of successful germination and an increase of the germination time. As well as the ion concentration and the quantitative variation of endogenous plant hormone regulates the plant response to salt stress.

One of the principal factors involved in salt stress response is abscisic acid (ABA). ABA is a plant hormone that plays a key role in several plant growth and development aspects under abiotic stress (Devinar et al., 2013; Fahad et al., 2015a; Huang et al., 2012). $\mathrm{ABA}$ is considered as the major internal plant signal molecule allowing the plant survival in adverse environmental conditions (Keskin et al., 2010).

Under salinity, seed germination typically decreases because of the higher level of ABA, which generates a sort of "induced dormancy" (Kang et al., 2015). The effect of salinity in seed germination has been deeply studied in Arabidopsis thaliana. The germination is regulated by $\mathrm{ABA}$ and gibberellins (GA) phytohormones, which have an opposite activity. ABA blocks the seed germination, while GA promote this process (Finkelstein et al., 2008; Piskurewicz et al., 2008; Yamaguchi, 2008). While the ABA biosynthesis is upregulated, at the same time, salt stress induces an 
upregulation of genes involved in GA inactivation and suppression of GA signaling, with the result of a germination reduction (Achard and Genschik, 2009; Colebrook et al., 2014; Kim et al., 2008; Magome et al., 2008; Yuan et al., 2011).

In other plant stages, ABA and GA interactions play crucial roles. The ABA biosynthesis increases in roots as a consequence of osmotic stress and water deficit generated by salinity conditions (Cabot et al., 2009; Cramer and Quarrie, 2002; Gupta and Huang, 2014; Kang et al., 2015). The high level of ABA has been found to induce the activation of the salt tolerance mechanisms in plants that mitigate the negative effects of salinity on plant growth and development, photosynthesis, and assimilated translocation (Yoshida et al., 2004; Zhu et al., 2014). Despite ABA produced in roots and transported to leaves through the xylem as response to salinity stress, which has an important local and systemic effect, Seung et al. (2012) showed that also the new ABA produced in diverse plant tissues can regulate the physiological response in plants.

GA influence as signaling molecule, in plant processes other than germination (leaf expansion, stem elongation, flowering, and fruit development), is also relevant (Yamaguchi, 2008). The regulation of these processes is associated to the role of GA on the photosynthetic enzymes, which is also associated to leaf area index, light interception, and efficiency in nutrient absorption and translocation (Khan et al., 2007; Yamaguchi, 2008). The reduction of plant growth, under salinity stress, is therefore the most important physiological response due to altered hormonal balance in plants. Nonetheless, high level of GA has been associated to an increased activity of the source potential and to an efficient redistribution of photosynthates in the sink sites (Khan et al., 2007) while reduced level of GA highlighted a close correlation between salt induced growth reduction and alteration of GA pathway (Achard and Genschik, 2009).

In $A$. thaliana, a reduction of GA content has been observed as consequence of salt stress. The role of GA in vegetable salt stress tolerance has been elucidated through DELLA mutants, a quadruple mutant defective of the GAI, RGA, RGL1, and RGL2 genes associated to the GA pathway; DELLA mutants were less tolerant to salt stress as compared with the wild type (Achard and Genschik, 2009). In addition, a minor reduction of flowering delay was shown in DELLA mutants, which supports the association between flowering and salt stress. These data were confirmed by the "spy" mutant, in A. thaliana, which showed a higher GA level and an increased salt tolerance compared with the wild type (Olszewski et al., 2002, 2010; Qin et al., 2011).
Despite several studies have been conducted on plant response to salinity in different plant development stages, little exploration has been done on the impact of salinity effect on the young seedlings during the early phases of plant growth. In general, most of the studies showed that annual crops are more salt-tolerant at the germination and less resistant at the following stages such as emergence and early vegetative development (Läuchli and Grattan, 2007). Furthermore, the classification and evaluation of salt tolerance is not the same during all the stages. While for germination and seedling, the tolerance is evaluated as percentage of germinated plant, for the other stages the evaluation is based on growth rating.

Beside plant development stages (seed, seedling, and mature plant), salinity response also shows organspecific trends. Shoot and root are both affected by salinity, but the recovery mechanism is probably different. A reduction of leaves growth under salinity is mainly caused by the osmotic effect nearby the roots. One of the secondary effects of the salt exposure is the osmotic imbalance that leads to water deficit, with a consequent temporary and partial plant loss of turgor. Cells recover the original volume using osmotically active metabolites to properly adjust the osmotic cell conditions (Munns and Tester, 2008). The type of osmolytes is species-specific and beside the tolerance to salt stress, these metabolites are used as active molecules for resistance to other unfavorable environmental conditions, such as freezing, heat, and drought stress. Sugars (glucose, sucrose, fructose, trehalose, and raffinose), polyols (sorbitol, mannitol, glycerol, arabinitol, and pinitol), ions (predominantly $\mathrm{K}^{+}$), specific proteins, and charged metabolites (betaine, proline, glutamate, aspartate, glycine, choline, putrescine, oxalate, and malate) (Cheong and Yun, 2007; Sairam and Tyagi, 2004) are the most common osmolytes used to balance the osmotic stress, and to protect proteins and membranes.

Some of the above-mentioned molecules such as sugars and proteins have shown multiple roles: metabolic resources, structural components, osmolytes, and signaling molecules under salt stress. The role of sugars as signaling molecule in salt stress conditions has been better explained in A. thaliana (Hanson and Smeekens, 2009). In this species, hexokinase 1 (HXK1) is a sugar (glucose) sensor that integrates nutritional and hormonal plant status with plant growth and gene expression under abiotic stress including salt stress (Cho et al., 2006; Moore et al., 2003). HXK1 sugar sensor can be found in cytosol and organelles (chloroplasts and mitochondria), (Rolland et al., 2002; Wiese et al., 1999). Furthermore, HXK1 can be transferred to the nucleus (Aki et al., 2007). When the glucose level increases, the 
HXK1 nuclear complex suppresses the expression of nuclear photosynthetic genes (Cho et al., 2006).

Among proteins and amino acids, the role of proline in plant abiotic stress response has been more deeply explored. Proline accumulation has been observed in several plant species as response to abiotic stress, especially under salt and drought conditions (Mattioli et al., 2009; Szabados and Savoure, 2010; Trovato et al., 2008; Verbruggen and Hermans, 2008). Beside its role as compatible osmolytes, proline accumulation acts as cell redox balancer (Hare and Cress, 1997) and as stress-related growth regulating signal (Khedr et al., 2003; Maggio et al., 2002). In A. thaliana, transcriptomic analysis revealed that the exogenous application of proline activated defense or detoxification genes, and in the meantime repressed genes involved in photosynthesis (Deuschle et al., 2004; Hellmann et al., 2000; Nanjo et al., 2003). The association between proline accumulation and photosynthesis reduction suggests that high concentration of proline in plants may also affect the function of chloroplasts.

$\mathrm{K}^{+}$and $\mathrm{Ca}^{2+}$ are the most important nutrient ions involved in the physiological response of salt-stressed plants. Under salinity stress, indeed $\mathrm{K}^{+}$plays a strategic role in boosting plant growth and development due to its activity in maintaining $\mathrm{Na}^{+} / \mathrm{K}^{+}$homeostasis. The decrease in extracellular $\mathrm{K}^{+}$concentration causes the root epidermal plasma membrane hyperpolarization, thus increasing the accumulation of $\mathrm{Ca}^{2+}$ in the cytosol (Demidchik et al., 2002). The presence of $\mathrm{Ca}^{2+}$ in the cytosol has also been related to improved plant nutrition under salinity (Wilkins et al., 2016). Furthermore there are evidences for possible connections between nitric-nitrogen nutrition and $\mathrm{Ca}^{+}$accumulation in the cytosol and vice versa (Wilkins et al., 2016). The above relationships between $\mathrm{Ca}^{2+}$ and plant nutrition underline the importance of the crosstalk between nutrient ions and stress signaling under salinity.

In conditions of moderate salt stress, several other symptoms of growth and development alteration appear as late plant responses. An inhibition of lateral shoot development, as well as early flowering or a reduced number of florets, is observed over weeks and/or months (Munns and Tester, 2008). In the meantime, older leaves die with a reduction of production of new leaves. These effects are mainly due to the osmotic stress caused by salt outside the roots. Beside the osmotic impact, $\mathrm{NaCl}$ affects plant growth through the toxicity of $\mathrm{Na}^{+}$accumulation in cytosol (Sumer et al., 2004). The mechanism of leaves and shoot development reduction is still to be well understood. As shown by Fricke and Peters (2002), carbohydrates supply and water status only marginally affect the leaves growth. In addition, also the nutrient deficiency and ion toxicity seems to play only a minor role in the shoot development (Hu et al., 2007, 2005; Munns and Tester, 2008). The most probable cause is associated to long distance signals from roots such as hormones that are involved in the plant development alteration and that induce a cascade signal in other plant organs. Shoot and root elongation are both influenced by salinity. The impact of $\mathrm{NaCl}$ exposure on root growth is less important than on leaves and its recovery is faster (a few hours, for a moderate stress, and a few days for a severe stress conditions) (Guo et al., 2015; Munns and Tester, 2008). In contrast to leaves, the root recovery occurs even if the turgor is not completely recovered, suggesting different response mechanisms.

\subsubsection{Temporal Signature and Related Cell Response Mechanisms}

Under salinity conditions, plant growth is reduced due to salt accumulation in shoots or due to processes independent of the shoot salt accumulation. These two phenomena are closely related to the plant timeresponse: the immediate response to salinity (from a few minutes to a few days) and longtime response (from several days to few weeks). In the early response, the plant reacts in a few minutes and a rapid and transient change in growth can be observed (Sirault et al., 2009). These effects can be considered independent of the salt shoot accumulation because of the quick plant response mechanism. The overall effect is the reduction of new leaves and a general reduction of shoot size caused by the "osmotic phase" (Munns and Tester, 2008). Roots are tissue directly involved in the salt stress perception and its signal transduction. From the roots, hormonal signals are generated inducing a general growth reduction during the osmotic phase (Ismail et al., 2014). The second and slower phase causes growth inhibition because of the salt accumulation during a longer period, mainly in older leaves. Salt accumulation in leaves induces cellular damage and necrotic lesion and later cell death. In this case, the cell death and consecutive abscission is an adaptive mechanism of the plant to remove the salt accumulated in older leaves. This phase is called the ionic phase (Bera et al., 2014).

In highly susceptible plants, the rate of dead leaves (for salt accumulation) is higher than the rate of new leaf production. This is a consequence of the photosynthetic activity, which is not sufficient to supply the carbohydrate required for the growth and plant development. The photosynthates produced by the plant are not sufficient to reach the reproductive stage and produce viable seeds (Läuchli and Grattan, 2007). This two-stage response concept implies that the 
osmotic effect of salt detected by roots causes an initial growth reduction, in both resistant and susceptible plant genotypes. However, the differences between tolerant and non-tolerant plant species is mainly associated to the second phase and consequently, to the plant ability to prevent the toxic salt accumulation in transpiring leaves (Munns et al., 2006).

Early and late phases involve specific signal molecules that deeply affect growth and development in plants. Temporal pattern of signal molecules induces adaptation or cell death. High salt level causes a fast increase of $\mathrm{Na}^{+}$in the cytoplasm through the nonselective cation channel (NSCCs) and later, through the $\mathrm{K}^{+}$ transporter called HKT1 (Essah et al., 2003; Ismail et al., 2014) (Fig. 1.1). Two principal types of NSCCs are involved: hyperpolarization-activated (HA) NSCCs and depolarization-activated (DA) NSCCs. HA-NSCCs are activated later than DA-NSCCs and have low selectivity for monovalent cations and are reported to be predominant in salinity susceptible plants; by contrast, DA-NSCCs are mainly detected in tolerant species. The NSCCs have three principal functions: to regulate the $\mathrm{Na}^{+}$flux into the cytosol, to activate the $\mathrm{Ca}^{2+}$ signal, and modulate $\mathrm{K}^{+}$flux in the cell. The quick DANSCCs determines a fast $\mathrm{Na}^{+}$influx with the consequent activation of $\mathrm{Ca}^{2+}$ signal pathway and the rapid inhibition of $\mathrm{K}^{+}$loss thus maintaining the $\mathrm{Na}^{+} / \mathrm{K}^{+}$ homeostasis in the plant (Shabala et al., 2006). In less tolerant plants, the slower response will be less efficient to regulate the water potential with a consequent loss of water. The inhibition of $\mathrm{Na}^{+}$influx is not sufficient to tolerate the stress condition; ions at this point need to be removed from cytosol to prevent accumulation in plant organelles.

The osmotic stress activates the synthesis of $\mathrm{ABA}$, which upregulates the gene expression of the $\mathrm{Na}^{+} / \mathrm{H}^{+}$ antiport for the accumulation of $\mathrm{Na}^{+}$in vacuoles (Shi and Zhu, 2002; Yokoi et al., 2002) (Fig. 1.1). In addition, ABA induces increasing concentration of $\mathrm{Ca}^{2+}$ in the cytosol that is associated to the stomatal closure under exposure to salinity. Turkan and Demiral (2009) reported that one of the early plant responses to salinity associated to osmotic stress consists of the increase of $\mathrm{Ca}^{2+}$ in the cytosol (after $1 \mathrm{~min}$ ). The normal cytoplasmic $\mathrm{Ca}^{2+}$ is between 100 and $200 \mathrm{nM}$, while in plant cell organelles is $1-2 \mathrm{mM}$ (Ismail et al., 2014). $\mathrm{Ca}^{2+}$ channels are distributed on the surface of cytoplasmic membrane and the membranes of different organelles (vacuoles, chloroplasts, and mitochondria). Variation of $\mathrm{Ca}^{2+}$ levels regulated downstream signals for the stress response activation, which includes plant development hormones (Park et al., 2016). The fast increase of $\mathrm{Ca}^{2+}$ activates the antiport ATPase responsible of the $\mathrm{Na}^{+}$vacuole accumulation (Fig. 1.1). $\mathrm{Ca}^{2+}$ is reported to be involved both in the early response and long-term plant response tolerance to salinity (Tuteja, 2007).

The role of $\mathrm{Ca}^{2+}$ as signal molecules for salinity tolerance has been better elucidated in association with the salt overly sensitive (SOS) pathway. Tolerance to salt stress and the maintaining of a favorable ion ratio in plant cytoplasm was associated to the SOS1-SOS3 genes pathway in A. thaliana ( $\mathrm{Zhu}, 2002)$. The study of the SOS pathway function highlighted the role of $\mathrm{Ca}^{2+}$ in the cell ion equilibrium. SOS3 is a $\mathrm{Ca}^{2+}$ binding protein strategic for the salt tolerance; its role as $\mathrm{Ca}^{2+}$ sensor is, in fact, crucial to transduce the salt stress signal in plant. SOS3-SOS2 complex stimulates the $\mathrm{Na}^{+} / \mathrm{H}^{+}$ antiport activity of SOS1 with the purpose to reestablish the cellular ion homeostasis. In addition to the SOS1-SOS3, also two other proteins involved in SOS pathway have been characterized: SOS4 and SOS5 (Mahajan et al., 2008). These two proteins are involved in cell wall structure and integrity under salinity conditions.

In cross-talking with the $\mathrm{Ca}^{2+}$ signal and with the hormonal response, $\mathrm{H}^{+}$also acts as an efficient salt stress signal molecules in plants (Gao et al., 2014; Ismail et al., 2014). Increasing level of $\mathrm{H}^{+}$has been reported as an early plant salt response occurring immediately after $\mathrm{NaCl}$ stress recognition (Ismail et al., 2014) (Fig. 1.1). Protons regulate the plant metabolism acting on the proteins (enzymes) conformation and activity. In $A$. thaliana, the cytosol alkalization, mediated by ABA and methyl jasmonate (MeJa) increasing level, causes stomatal closure (Suhita et al., 2004). The increase in $\mathrm{H}^{+}$influx, often associated with the $\mathrm{Ca}^{2+}$, induces the activation of $\mathrm{Ca}^{2+}$ channels (Ismail et al., 2014) and the $\mathrm{Ca}^{2+}$-mediated signal pathway. In addition, variation of $\mathrm{pH}$ regulates the ratio between inactive and active jasmonic acid (JA), which act as stress signal molecules (Fonseca et al., 2009).

A more rapid and persistent alkalization of the apoplast has been correlated to an increased salt tolerance in Vitis vinifera (Ismail et al., 2014). Furthermore, $\mathrm{H}^{+}$is associated with the $\mathrm{Ca}^{2+}$ fluctuations. Under salinity stress $\mathrm{Ca}^{2+}$ and $\mathrm{H}^{+}$influx can occur simultaneously: the increase of apoplast $\mathrm{pH}$, in fact, is a strong signal for the rapid $\mathrm{Ca}^{2+}$ influx (Felix et al., 1999). Briefly, the alkalization of cytosol provokes a temporary accumulation of $\mathrm{Na}^{+}$and $\mathrm{Ca}^{2+}$, which then represent the early signal molecules of salinity conditions.

After a few minutes of exposure to salinity conditions, salt stress has a general effect on the plant metabolism that involves specific signal molecules. Under salinity, the reduced photosynthesis rate and alternated respiration process generate a general metabolism imbalance in the oxidative processes, which causes an increasing level of reactive oxygen species (ROS) (Fig. 1.1; Khan and Khan, 2017). The 

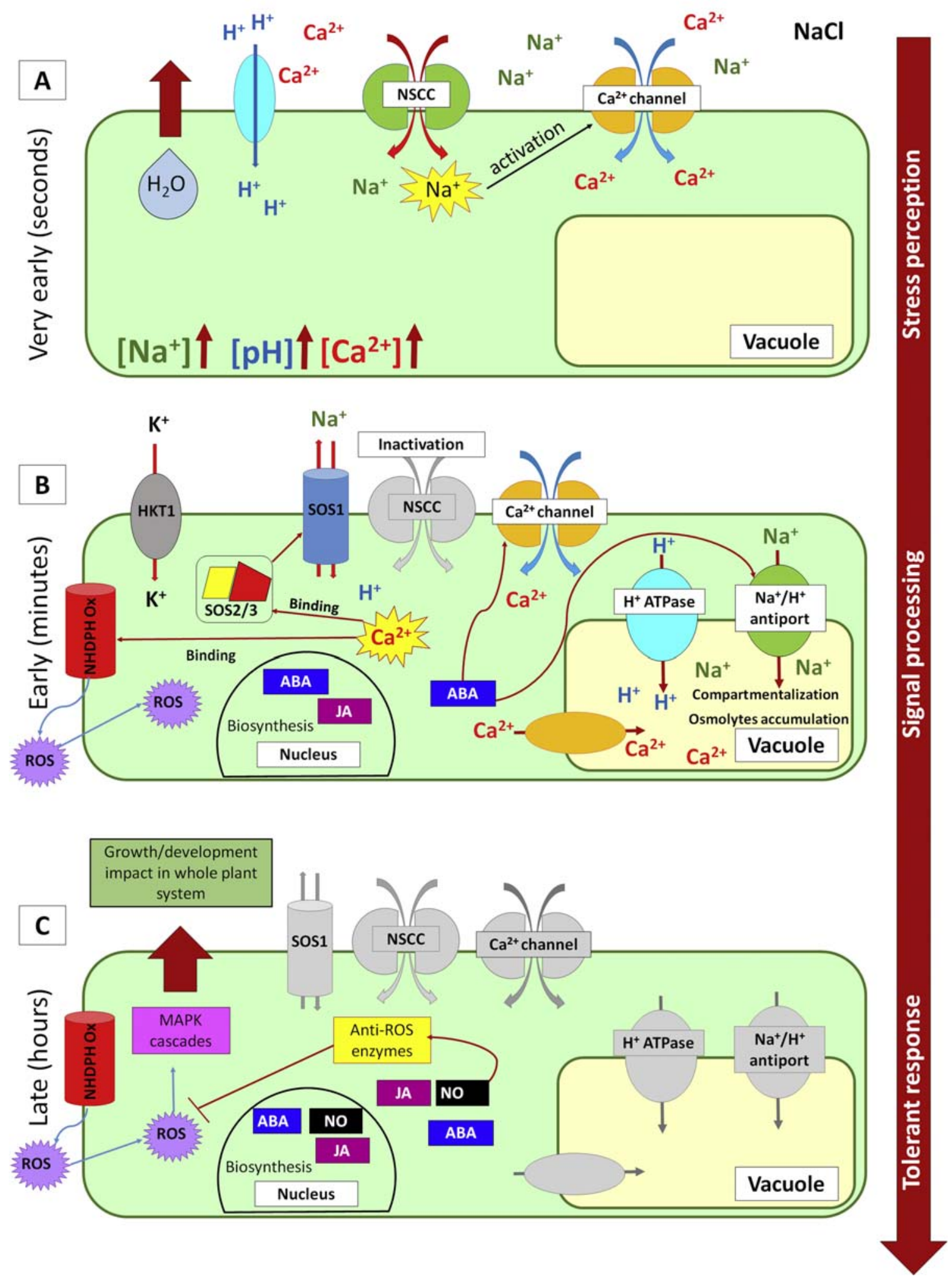

FIGURE 1.1 Plant response signals under salinity stress. (A) During the very early response, tolerant cells are mainly involved in the salt stress perception. High $\mathrm{NaCl}$ levels induced the activation of $\mathrm{Ca}^{2+}, \mathrm{H}^{+}$, and $\mathrm{Na}^{+}$(NSCC) channel. In a few seconds, cytosol alkalization occurs as well as an increasing level of cytosolic $\mathrm{Ca}^{2+}$ and $\mathrm{Na}^{+}$levels. Osmotic potential changes inducing a loss of cell water content. (B) During the early response (minutes), the high $\mathrm{Ca}^{2+}$ level activated the SOS system. $\mathrm{Ca}^{2+}$ binds to SOS2/SOS3 complex, which activates the SOS1 membrane protein. SOS1 is a membrane pump that excretes $\mathrm{Na}^{+}$outside the cell, working on the rebalancing of the $\mathrm{Na}^{+}$level in the cytosol. In the meantime, the $\mathrm{Ca}^{2+}$ binds to the NHDPH oxidase inducing the ROS production. The increased level of ABA, associated to the cell stress condition, induced the compartmentalization of $\mathrm{Na}^{+}, \mathrm{Ca}^{2+}$, and $\mathrm{H}^{+}$into the vacuole through antiport systems. Meanwhile the biosynthesis of ABA, JA, and NO is activated in the nucleus. (C) In the late responses (hours), the influx of $\mathrm{Na}^{+}, \mathrm{Ca}^{2+}$, and $\mathrm{H}^{+}$is blocked and NO activates the production of anti-ROS enzymes for the reduction of ROS level in the cytosol. The initial high level of ROS induced the MAPK cascade with general growth and development consequences in the whole plant system. In a tolerant cell, the osmotic potential at this stage is normalizing and the vacuole compartmentalization is not active anymore. 
high level of apoplastic ROS is caused by the apoplastic depletion of proton and the ROS influx into the cytoplasm. ROS are recognized by the membranelocalized histidine kinase, which activates the mitogen activated protein kinase (MAPK) signaling cascade (Fig. 1.1). The MAPK cascade is a highly conserved signaling pathway in eukaryotes (Ichimura et al., 2002). MAPK cascades, which include a minimum set of three subsequential proteins (MPK, MAPKK, and MKK or MEK), activate downstream targets by phosphorylation system. MAPKs are associated to several plant growth and development aspects (gametogenesis, embryogenesis, morphogenesis, senescence, abscission, flowering, and seed development), ( $\mathrm{Xu}$ and Zhang, 2015). The $A$. thaliana genome analysis revealed 20 different MPKs; 3 of which (MPK3, MPK4, and MPK6) are involved in both stress and developmental responses (Colcombet and Hirt, 2008; Lampard et al., 2009; Liu et al., 2010). Studies conducted on MPK6 loss of function mutants, highlighted alteration of embryo and root development (Bush and Krysan, 2007; Müller et al., 2010; Wang et al., 2010). Primary roots originate from the embryo and generate lateral roots during vegetative growth. A well-developed root system architecture has an important role in plant-environment interaction, especially in case of drought and salinity stress (Casimiro et al., 2003; Dubrovsky and Forde, 2012). Müller et al. (2010) demonstrated a strong relationship between MPK6 activity and cell division. MPK6, in fact, interact with $\gamma$-tubulin and the microtubule plus end protein EB1 associated to the cell division during stress conditions (Kohoutova et al., 2015). Furthermore, Li et al. (2017a,b) have demonstrated that MPK6 is involved in the MAPKs cascade that modulates the ABA response regulating the cell division and elongation in root.

Taj et al. (2010) showed that three MAPKs (MPK4, MPK6, MAPKK1) are triggered under salinity; the overexpression of MAPKK2 in A. thaliana caused a constitutive MAPK4 and MPK6 activity that increased plant salt tolerance. In addition, MPK4 was found to regulate salicylic acid (SA) and ROS production in $A$. thaliana (Gao et al., 2008; Takac et al., 2016). Besides SA and ROS production, MPK4 and MPK6 activate the ACC synthases enzymes responsible for the ethylene biosynthesis, which is involved in several biological processes (from senescence to fruit maturation) (Liu and Zhang, 2004). Considering the cascade of signals generated by the ROS, their production needs to be strongly regulated, otherwise their overproduction could result in a cell death. In addition, a close connection has been demonstrated between ROS and $\mathrm{Ca}^{2+}$ in response to stress, which generated alteration in plant growth (Hu et al., 2007; Jiang et al., 2003; Shoresh et al., 2011). ROS, such as hydrogen peroxide $\left(\mathrm{H}_{2} \mathrm{O}_{2}\right)$, activated the $\mathrm{Ca}^{2+}$ channel increasing its concentration in the cytosol (Pei et al., 2000). The increased level of $\mathrm{Ca}^{2+}$ controls the plant cell expansion. On the other hand, cytosolic $\mathrm{Ca}^{2+}$ regulated the NADPH oxidase, which generates ROS (Fig. 1.1). The ROS, generated by NADPH oxidase, in the plasma membrane, activates the ABA signals cascade. In this intricate signal network, nitric oxide (NO) has an important role for plant salt tolerance. The action of NO is more closely related as signal molecules that cooperate with JA and ethylene. The cross-talking of NO with hormones associated to plant stress response induced alteration of plant growth and development (mainly during seed germination and roots formation). In Lupines liteus, for instance, NO increased the antioxidant activity of "anti-ROS" enzymes superoxide dismutase (SOD), catalase (CAT), and peroxidase (POD), better known as antioxidant enzymes (Kopyra and Gwozdz, 2003). Because of high SOD and CAT activities (NO induced) a decreased ROS level (mainly $\mathrm{O}^{2-}$ in the mitochondria) is reported, which makes it easier to report cells to a redox homeostasis (Lamattina et al., 2003).

In addition to the $\mathrm{ABA}$ and ethylene, ROS also influences JA biosynthesis in roots, which is known to act as an important hormone regulating plant growth and development (Xiong and $\mathrm{Zhu}, 2003$ ). JA is produced as an early signal, occurring between minutes and hours after the $\mathrm{NaCl}$ stress recognition (Ismail et al., 2014). As a hormone, JA is involved in several plant biological processes, determining alteration of seed germinations, primary root growth, flowering, and plant senescence (Fahad et al., 2015b; Riemann et al., 2013; Robson et al., 2010; Tani et al., 2008). The JA biosynthesis is localized in roots' and leaves' peroxisomes and chloroplasts (Cheong and Choi, 2003). The relationship between JA and salinity tolerance has been demonstrated in several species such as Solanum lycopersicum (Pedranzani et al., 2003) and Iris hexagona (Wang et al., 2001) in which high level of JA enhanced the salinity tolerance. Salt-tolerant cultivars of Oryza sativa showed higher JA levels than the susceptible cultivars (Kang et al., 2005); the increase of JA was detected either in roots or in leaf tissues (Tani et al., 2008). It has been shown that salt-tolerant plants at the beginning activate the JA pathway (through a transient pick of ROS), and later JA production is shut off because of the activation of the ABA signaling. ABA and JA signaling are in fact antagonist, mainly for mutual competition for common signaling factors (Anderson et al., 2004). Overaccumulation of JA caused, for instance, the delay of $\mathrm{Ca}^{2+}$ signaling activation thereby leading to membrane damage and cell death (Ismail et al., 2014).

Both salt-tolerant and sensitive plants use the same signal molecules. Timing appears to have a crucial role 
in plant cell adaptation to $\mathrm{NaCl}$ or death. The ability to survive and adapt to salinity is associated to the cell timing of the transient status of salt stress signal pathway. Tolerant plants can efficiently regulate the crosstalk between different signal pathways controlling the temporal intensification of the signal. If the stress signals persist longer, or start later, other plant pathways not associated to the salt tolerance response are activated. A delay in the switching on (and in the turning off) of $\mathrm{Ca}^{2+}$ signal will let increase the ROS production (over the plant cell acceptability) activating the JA pathway, which culminates with plant stress symptoms and eventually cell death.

\subsection{LEAF GASEOUS EXCHANGES}

\subsubsection{Stomatal Conductance and Water Relations}

The reduction of the stomatal activity is one of the most rapid reactions in plants exposed to saline environments. Reduced gaseous exchange rates are associated to limited leaf transpiration (Mittler and Blumwald, 2015), which in turn limits water uptake and fluxes within and between plant tissues, and limited $\mathrm{CO}_{2}$ and $\mathrm{O}_{2}$ exchanges. Such phenomena are known to negatively affect carbon intake and/or sugar respiration (Koyro et al., 2011; Mittler and Blumwald, 2015; Munns and Tester, 2008; Park et al., 2016). Limited stomatal activity causes disorders in primary plant metabolism leading to photoinhibition, oxidative stress, and eventually reduced growth and development in the medium-long period, until cell and plant death (Mittler and Blumwald, 2015; Park et al., 2016).

Salt stress drastically reduces the tolerance to salinity causing significant reduction in both crop quality and yield (Munns and Tester, 2008). Stomatal activity regulation is one of the most important plant defense/ modulation mechanisms to face salinity stress. In fact, stomatal closure occurs both in halophyte and glycophyte plants when exposed to saline conditions (Tester and Davenport, 2003). Indeed, plants that are sensitive to salinity commonly show poor capacity to reduce stomatal conductance (Robinson et al., 1997). Salinity induces plant stress at different levels including osmotic stress, ion toxicity, and nutrient imbalance. Therefore, many different response mechanisms were found to be associated to different botanical families and growth environments (Munns and Tester, 2008). The osmotic stress rapidly influences stomatal conductance that is reduced as a response to the presence of stress-signaling molecules. Yet, it has been demonstrated that lack of molecules signaling saline stress, or the absence of connections between these molecules and adequate ecophysiological plant response, are typical of plants sensitive to salinity (Apel and Hirt, 2004; Basu and Rabara, 2017; Hetherington, 2001; Huang et al., 2012; Munns and Tester, 2008; Zhu, 2002).

Stomatal conductance in salt-stressed plants is primarily reduced, to keep optimal tissue water relations and to maintain cellular turgor, by $\mathrm{ABA}$ as the main signaling molecule (Dar et al., 2017; Mittler and Blumwald, 2015; Park et al., 2016). In addition, the reduced photosynthetic activity induces feedback mechanisms on stomatal closure in which ROS play a crucial role as second messengers (Apel and Hirt, 2004; Koyro et al., 2011; Shi et al., 2015). ROS, therefore, largely contribute to the stomatal reactions to salinity exposure, showing many different functions that are directly or indirectly related to abiotic stresses, including salinity (Mittler and Blumwald, 2015; Tester and Davenport, 2003). Among $\mathrm{ROS}, \mathrm{H}_{2} \mathrm{O}_{2}$ has been found to decrease stomatal conductance both in earlier (McAinsh et al., 1996) and in recent studies conducted on different plant species (An et al., 2008; Li et al., 2017b; Rodrigues et al., 2017; Shi et al., 2015). The effects of $\mathrm{H}_{2} \mathrm{O}_{2}$ on stomata behavior have mainly been related to the role of this molecule in the activation of $\mathrm{Ca}^{2+}$ channels to mediate ABA signaling in guard cells (Basu and Rabara, 2017; Kim et al., 2010; Mittler and Blumwald, 2015; Pei et al., 2000). Nonetheless, recent studies underline the importance of the combined action of ROS and the reactive nitrogen species, NO, as connection point between $\mathrm{Ca}^{2+}$ and ABA signaling cascade in the control of stomatal closure (Li et al., 2017b; Saxena et al., 2016). Possible interactions of the different signaling molecules that play strategic role in the stomata control are summarized in Fig. 1.2.

ABA plays a major role as signaling molecule in salt-stressed plants. Hence, several reports have been produced in the last few decades leading to increased knowledge on this hormone and its role in controlling stomatal behavior in higher plants (Apel and Hirt, 2004; Basu and Rabara, 2017; Danquah et al., 2014; Hetherington, 2001; Huang et al., 2012; Munns and Tester, 2008; Saxena et al., 2016; Taiz et al., 2015; Zhu, 2002). ABA promptly promotes stomatal closure by acting on ion fluxes in guard cells of plants exposed to salinity (Mittler and Blumwald, 2015; Parida and Das, 2005). Its action is explicated through different mechanisms that basically aim to favor the release of $\mathrm{K}^{+}$from guard cells thus leading to $\mathrm{K}^{+}$concentration levels not sufficient to maintain cellular turgor and stomata opening. In sweet pepper exposed to saline environment and different levels of atmospheric $\mathrm{CO}_{2}$, the accumulation of ABA was correlated with stomatal conductance (Piñero et al., 2014). In transgenic cotton, the overexpression of a gene encoding for a bZIP transcription factor, which can improve the ABA signaling 


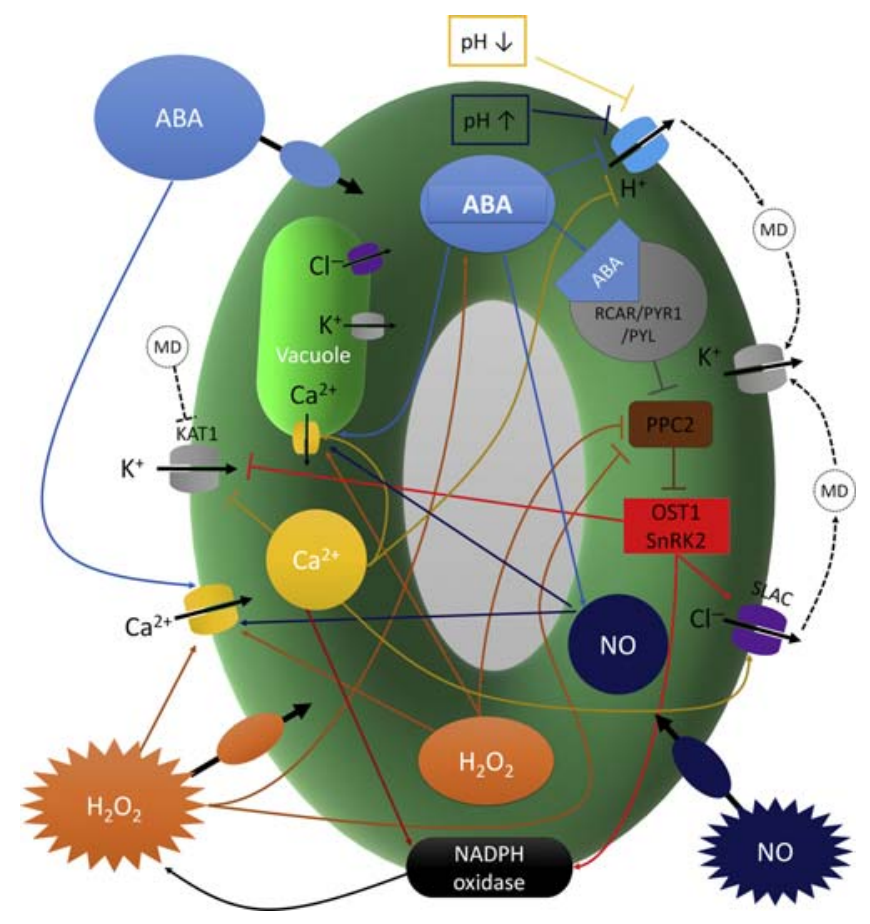

FIGURE 1.2 Stomatal response signals under salinity stress. The main biochemical mechanisms behind the induction of stomata closure are schematically reported in the figure without taking into consideration the real cell guard shape. The possible interactions among the different primary and secondary signaling molecules, which lead to membrane depolarization (MD) and turgor loss, are summarized from various authors (see the text for details). $A B A$, abscisic acid; $\mathrm{H}_{2} \mathrm{O}_{2}$, hydrogen peroxide; KAT1, inward potassium channels; $M D$, membrane depolarization; $N O$, nitric oxide; OST1, open stomata 1; $S n R K 2$ protein kinase; $N A D P H$, reduced form of nicotinamide adenine dinucleotide phosphate; $R C A R / P Y R 1 / P Y L$, ABA-receptor/pyrabactin resistant protein/PYR-like proteins; SLAC, slow anion channels.

pathway, resulted in higher resistance to different stresses, including salinity, showing increased chlorophyll, proline, and soluble sugar content (Wang et al., 2017). The rapid increase in ABA at local (leaf) level, after a few minutes of $\mathrm{NaCl}$ exposure, has been detected in barley (Fricke et al., 2004). However, longer expositions (hours), to the saline environment still induced a reduction in the stomatal conductance, while ABA concentration at leaf level decreased significantly (Fricke et al., 2006, 2004). Therefore, the stomatal response to salinity, in the medium-long period, has mainly been related to signaling mechanisms responding to $\mathrm{ABA}$ produced at the root level (Chaves et al., 2009; Davies et al., 2005). However, many works have highlighted the importance of ABA produced in the various organs during the physiological response of plants exposed to stress conditions (Park et al., 2016). Exogenous ABA has commonly been found to activate genes involved in the response to osmotic stress (Huang et al., 2012) despite that there are genes active against osmotic stress that are not ABAdependent (López-Pérez et al., 2009; Zhu, 2002). Table 1.1 reports a non-exhaustive compendium of recent works that describes the role of $\mathrm{ABA}$ and other signaling molecules involved in the control of stomatal movements under salinity.

ABA-induced stomatal closure can be associated to an ABA-activated protein kinase that controls anion channels in the plasma membrane (Ashraf and Harris, 2013; Danquah et al., 2014; Mittler and Blumwald, 2015; Fig. 1.2). The ABA receptors RCAR/PYR1/PYL (regulatory components of ABA-receptor/pyrabactin resistant protein/PYR-like proteins) binds ABA and forms a complex that inhibits the $2 \mathrm{C}$ protein phosphatases (PP2C) responsible for dephosphorylation, and then, inactivation of the protein kinase open stomata 1 (OST1) in absence of stress. OST1 belongs to a large category of protein kinases (SnRK2) that respond to ABA-dependent and ABA-independent signals under osmotic stress (Yoshida et al., 2014). After phosphorylation, the activated OST1 induces stomatal closure mainly through (1) depolarization of the intracellular plasma, operated by the activation of anion channels, which causes the release of $\mathrm{K}^{+}$; and (2) inhibition of guard cell-inward KAT1 $\mathrm{K}^{+}$channels, which causes higher osmotic potential (Sato et al., 2009). OST1, via a plasma membrane-bound NADPH oxidase (Sirichandra et al., 2009), also promotes the production of $\mathrm{H}_{2} \mathrm{O}_{2}$, which catalyzes the inactivation of PP2C showing a feedback loop on OST1 activity (Mittler and Blumwald, 2015).

In summary (Fig. 1.2), ABA influences stomatal activity following different mechanisms based on the induction of stomatal closure and the inhibition of stomatal opening (Danquah et al., 2014). In this process, many different signaling molecules, which represent powerful second messengers, support ABA actions. ROS play a relevant role in these signaling mechanisms (Gudesblat et al., 2007). The accumulation of ROS in the apoplast can indeed be linked to stomatal closure (An et al., 2008). This was demonstrated through ABI1 and ABI2, which are encoding enzymes involved in stomatal closing. Using the abi1 and abi2 mutants, which show limitation in the phosphatase activities, $A B A$ was found to be unable to generate ROS only in abi1 mutants showing different ROS upstream and downstream action by ABI1 and ABI2, respectively (Murata et al., 2001). Other ABA-dependent mechanisms act through MAPKs, which are involved in stomatal development (Wang et al., 2008). MAPKs were found to be sensitive to the presence of $\mathrm{H}_{2} \mathrm{O}_{2}$ thus enhancing the transduction of $\mathrm{H}_{2} \mathrm{O}_{2}$ signaling at the cellular level (Danquah et al., 2014; Pitzschke and Hirt, 2009). 
TABLE 1.1 Overview of Recent (2010-17) Works Assessing Relationships Between Primary or Secondary Signaling Molecules and Stomatal Activity in Salt-Stressed Plants

\begin{tabular}{|c|c|c|c|c|c|}
\hline Species & $\begin{array}{l}\text { Stomata } \\
\text { measurements }\end{array}$ & Other performance parameters & $\begin{array}{l}\text { Signaling } \\
\text { molecule/s }\end{array}$ & Methodology & References \\
\hline $\begin{array}{l}\text { Dianthus } \\
\text { superbus }\end{array}$ & $\begin{array}{l}\text { Stoma and } \\
\text { chloroplast } \\
\text { development }\end{array}$ & $\begin{array}{l}\text { Growth; photosynthesis; } \\
\text { antioxidant enzyme activity }\end{array}$ & $\begin{array}{l}\mathrm{H}_{2} \mathrm{O}_{2} \\
\mathrm{O}_{2}{ }^{-}\end{array}$ & $\begin{array}{l}\text { Pot experiment; increasing } \mathrm{NaCl} \\
\text { concentrations }\end{array}$ & Ma et al. (2017) \\
\hline $\begin{array}{l}\text { Triticum } \\
\text { aestivum }\end{array}$ & $\begin{array}{l}\text { Stomata } \\
\text { movements and } \\
\text { development }\end{array}$ & $\begin{array}{l}\text { Growth; leaf water loss; proline } \\
\text { and soluble sugar accumulation }\end{array}$ & $\begin{array}{l}\mathrm{ABA} \\
\mathrm{H}_{2} \mathrm{O}_{2}\end{array}$ & $\begin{array}{l}\text { Liquid solution culture; } 200 \mathrm{mM} \\
\mathrm{NaCl}+\text { exogenous } \mathrm{ABA}\end{array}$ & $\begin{array}{l}\text { Yang et al. } \\
\text { (2016) }\end{array}$ \\
\hline $\begin{array}{l}\text { Medicago } \\
\text { truncatula }\end{array}$ & $\begin{array}{l}\text { Stomatal } \\
\text { conductance }\end{array}$ & $\begin{array}{l}\text { Growth; antioxidant enzyme } \\
\text { activity; proline accumulation }\end{array}$ & $\mathrm{H}_{2} \mathrm{O}_{2} ; \mathrm{NO}$ & $\begin{array}{l}\text { Pot experiment (perlite/sand); } \\
\text { growth chamber; } 200 \mathrm{mM} \mathrm{NaCl}\end{array}$ & $\begin{array}{l}\text { Filippou et al. } \\
\text { (2016) }\end{array}$ \\
\hline $\begin{array}{l}\text { Arabidopsis } \\
\text { thaliana }\end{array}$ & $\begin{array}{l}\text { Stomatal } \\
\text { movements }\end{array}$ & Cotyledons characteristics & $\mathrm{ABA}$ & $\begin{array}{l}\text { MS agar medium; } 0,125,150 \text { and } \\
175 \mathrm{mM} \mathrm{NaCl}+\text { exogenous } \mathrm{ABA}\end{array}$ & $\begin{array}{l}\text { Singh et al. } \\
(2015)\end{array}$ \\
\hline $\begin{array}{l}\text { Arabidopsis } \\
\text { thaliana }\end{array}$ & $\begin{array}{l}\text { Leaf osmotic } \\
\text { potential }\end{array}$ & $\begin{array}{l}\text { Biometric parameters; leaf water } \\
\text { loss; enzyme activity }\end{array}$ & $\mathrm{H}_{2} \mathrm{O}_{2}$ & $\begin{array}{l}\text { Hydroponic culture; growth } \\
\text { chamber; } 80 \mathrm{mM} \mathrm{NaCl}+\text { exogenous } \\
\text { tunicamycin }\end{array}$ & $\begin{array}{l}\text { Ozgur et al. } \\
\text { (2014) }\end{array}$ \\
\hline $\begin{array}{l}\text { Ocimum } \\
\text { basilicum }\end{array}$ & $\begin{array}{l}\text { Stomatal } \\
\text { conductance and } \\
\text { density }\end{array}$ & Growth; volatile compounds & ABA & $\begin{array}{l}\text { Greenhouse/growth chamber; 0, 100, } \\
200 \mathrm{mM} \mathrm{NaCl} \text {; two cultivars }\end{array}$ & $\begin{array}{l}\text { Barbieri et al. } \\
\text { (2012) }\end{array}$ \\
\hline Pisum sativum & $\begin{array}{l}\text { Stomatal } \\
\text { conductance }\end{array}$ & $\begin{array}{l}\text { Growth; photosynthesis; } \\
\text { antioxidant enzyme activity; } \\
\text { mineral content }\end{array}$ & $\mathrm{H}_{2} \mathrm{O}_{2}$ & $\begin{array}{l}\text { Hydroponic system; growth } \\
\text { chamber; } 150 \mathrm{mM} \mathrm{NaCl}\end{array}$ & $\begin{array}{l}\text { Martí et al. } \\
(2011)\end{array}$ \\
\hline $\begin{array}{l}\text { Solanum } \\
\text { lycopersicum }\end{array}$ & $\begin{array}{l}\text { Stomatal } \\
\text { conductance }\end{array}$ & $\begin{array}{l}\text { Growth; yield; proline } \\
\text { accumulation }\end{array}$ & $\mathrm{ABA}$ & $\begin{array}{l}\text { Greenhouse experiment; soilless } \\
\text { rockwool; } 0,20,40 \mathrm{mM} \mathrm{NaCl}\end{array}$ & $\begin{array}{l}\text { Orsini et al. } \\
(2010)\end{array}$ \\
\hline
\end{tabular}

The role of ABA in membrane depolarization is relevant for stomatal closure (Fig. 1.2). The phosphorylation of the slow anion channels (SLAC), which release anions outside the guard cells, is a fundamental mechanisms for the depolarization process (Brandt et al., 2012; Lee et al., 2009). In addition, ABA acts through the inhibition of the cell plasma membrane $\mathrm{H}^{+}$-ATPase (Danquah et al., 2014; Hayashi et al., 2011) and the activation of $\mathrm{Ca}^{2+}$ channels in which $\mathrm{H}_{2} \mathrm{O}_{2}$ takes part (Pei et al., 2000; Wang et al., 2016a). Membrane depolarization is also driven by the alkalization of the cytosol (Fig. 1.2) since $\mathrm{pH}$ increases have been observed to precede ROS signaling in stomatal closure (Sobahan et al., 2015). Cytosolic acidification has instead been associated to ROS removal in guard cells. Conversely, in presence of osmotic stress, stromal acidification in chloroplast has been observed at increasing ROS concentration (Wang et al., 2016a) as reversed conditions of optimal photosynthetic activity when stromal pH is typically higher (Taiz et al., 2015). Elevated concentrations of $\mathrm{Ca}^{2+}$ in the cytosol promotes the activation of SLAC1 thus causing the inhibition of inward KAT1 $\mathrm{K}^{+}$channels due to membrane depolarization (Osakabe et al., 2014; Wang et al., 2016b). Yet, $\mathrm{Ca}^{2+}$ would stimulate the activity of $\mathrm{NADPH}$ oxidase (Fig. 1.2) responsible for increased
$\mathrm{H}_{2} \mathrm{O}_{2}$ (Sirichandra et al., 2009) acting in a feedback loop to boost $\mathrm{Ca}^{2+}$ influx and to improve stomatal closure efficiency in general (Mittler and Blumwald, 2015; Munemasa et al., 2013; Saxena et al., 2016). Together with ROS, $\mathrm{Ca}^{2+}$ therefore appears to be a strategic second messenger in the stomatal response of plants exposed to saline stress (Basu and Rabara, 2017). Recently, it has been highlighted that the interaction between $\mathrm{H}_{2} \mathrm{O}_{2}$ and $\mathrm{NO}$ would improve the signaling cascade between $\mathrm{Ca}^{2+}$ and ABA in the control of stomatal movements (Li et al., 2017b; Saxena et al., 2016). Yet, the interaction between $\mathrm{Ca}^{2+}, \mathrm{H}_{2} \mathrm{O}_{2}$, and $\mathrm{NO}$ in the control of stomata closure has been observed in $A$. thaliana during pharmacological experiments (Niu and Liao, 2016). Wang et al. (2016a,b) have proposed a model in which chloroplast $\mathrm{Ca}^{2+}$ firstly, and then ROS, are involved in the achievement of the early state for stomatal closure.

NO takes part in many mechanisms of response to stress conditions thereby resulting in a strategic signaling molecule in the ecophysiology of plants growing under saline environments (Niu and Liao, 2016). Endogenous NO is synthetized by NO synthase but also by nitrate reductase via reduction of nitrite (Neill et al., 2008). The increase in $\mathrm{H}_{2} \mathrm{O}_{2}$ concentration, induced by $\mathrm{ABA}$, brings about $\mathrm{NO}$ generation via 
nitrate reductase and nitrogen oxide synthase activity (Neill et al., 2008). The positive effect of NO in stomatal closure has largely been demonstrated through exogenous NO application in many different plant species (Desikan et al., 2002; Mata and Lamattina, 2001; Neill et al., 2002). However, the production of endogenous $\mathrm{NO}$ has been observed when ABAinduced stomatal closure occurs, while the presence of treatment against NO partially limited the closure mechanism (Neill et al., 2008). In A. thaliana, it has been demonstrated that $\mathrm{NO}$ and $\mathrm{H}_{2} \mathrm{O}_{2}$ are both involved in the stimulation of stomatal closure ( $\mathrm{He}$ et al., 2013). Yet, transgenic plants of $A$. thaliana were modified to overexpress NO synthase activity, which resulted in an increased accumulation of $\mathrm{NO}$ and eventually displayed improved resistance to salt stress (Foresi et al., 2015). Indeed, a strong interaction between $\mathrm{H}_{2} \mathrm{O}_{2}$ and $\mathrm{NO}$, as second messengers in signaling stressful conditions, has been well documented for higher plants growing under saline conditions (Niu and Liao, 2016).

Other than stomatal movements, ABA produced under salinity stress acts on plant water balance also by increasing guard cell permeability to water, and by the activation of water channels. ABA has, in fact, been identified to be a signal hormone for aquaporins influencing their activity through different mechanisms from gene expression to the modification of gene products (Dar et al., 2017). In ABA-deficient barley mutant Az34, the presence of exogenous ABA induced the proliferation of aquaporins, and improved hydraulic conductivity of root cells (Sharipova et al., 2016). Corpas et al. (2017) have supposed that ABA-activated aquaporins could have a relevant role in the translocation of $\mathrm{H}_{2} \mathrm{O}_{2}$ produced by peroxisomes as second messengers of abiotic stress in plants. Yet, aquaporins have also been reported to facilitate the intake of $\mathrm{H}_{2} \mathrm{O}_{2}$ into guard cells during ABA-induced stomatal closure (Rodrigues et al., 2017). Undoubtedly, the abundance of aquaporins can be associated to possible plant strategies of resistance under salinity (López-Pérez et al., 2009).

\subsubsection{Photosynthesis}

The main consequence of stomatal closure is the reduced photosynthesis (Mittler and Blumwald, 2015; Munns and Tester, 2008; Neill et al., 2008; Park et al., 2016) since the photosynthetic machinery is generally inhibited by salinity (Parida and Das, 2005). In higher plants, the photosynthetic activity is one of the most important performance, and then stress, indicators for its implications in the production of primary and secondary metabolites, and signaling molecules as well
(Ashraf and Harris, 2013). Net photosynthesis shows heterogeneous responses when plants undergo saline stress depending on time of exposure, intensity, and plant tolerance. Increase in the assimilation of $\mathrm{CO}_{2}$ per unit area has been observed in salt-stressed wheat (James et al., 2002). This apparently controversial plant response is due to the higher concentration of chloroplast caused by the positive association between salinity and leaf thickness (Munns and Tester, 2008). On the other hand, when photosynthesis is expressed per unit chlorophyll reductions are commonly observed in salt-stressed plants (Munns and Tester, 2008). The lower carbon intake which, in turn, limits plant growth, is therefore a phenomenon that mostly occurs at the whole-plant level and mainly relates to the lower leaf area showed by salt-stressed plants (Parida and Das, 2005). However, the reduction in plant growth causes the accumulation of carbohydrates in plant tissue thereby stimulating feedback signals to control photosynthetic activity (Munns and Tester, 2008).

When photosynthesis is inhibited the production of ROS increases, due to photoxidative stress, as well as the enzyme activity involved in their removal (Apel and Hirt, 2004; Chaves et al., 2009; Foyer and Noctor, 2005; Munns and Tester, 2008; Suo et al., 2017). Photoxidative stress is one of the main metabolic processes producing ROS in plants due to many reactions involved in the photosynthesis and photorespiration process (Foyer and Noctor, 2003), which in presence of reduced stomatal conductance cause the increase of the $\mathrm{O}_{2} / \mathrm{CO}_{2}$ ratio (Koyro et al., 2011). The production of ROS at low level is normal when plants are exposed to optimal growing conditions but it increases dramatically in presence of salinity stress thus causing their abnormal accumulation in the production sites then translocated within plant tissues. The limited photosynthesis activity under salinity is therefore one of the most relevant mechanisms triggering stress signaling related to ROS, especially superoxide and $\mathrm{H}_{2} \mathrm{O}_{2}$, which take part in many plant growth and development processes as second messengers (Apel and Hirt, 2004; Foyer and Noctor, 2005; Koyro et al., 2011; Khan et al., 2013).

ROS in salt-stressed plants are largely produced at the chloroplast and mitochondrial levels other than in the peroxisomes (Apel and Hirt, 2004; Miller et al., 2010). In chloroplasts, oxygen reduction by PSI brings about the production of superoxide, then converted in $\mathrm{H}_{2} \mathrm{O}_{2}$ through $\mathrm{CuZn}$-superoxide dismutase (Asada, 1999; Asada et al., 2000) as an intermediate product of the water-water cycle that, in normal conditions, would eventually end with the production of $\mathrm{H}_{2} \mathrm{O}$ (Miller et al., 2010). Furthermore, when the intracellular concentration of $\mathrm{CO}_{2}$ declines, due to stomatal 
closure, the photorespiratory oxygenation of ribulose 1,5-bisphosphate becomes strategic to preserve the integrity of PSII. Nevertheless, this process leads to the production of 2-phosphoglycolate, which is then converted to glycolate, whose oxidation process causes the accumulation of $\mathrm{H}_{2} \mathrm{O}_{2}$ in plant cells (Apel and Hirt, 2004). Under salinity stress, singlet oxygen is also accumulated in chloroplasts due to PSII activity (Miller et al., 2010). In mitochondria, one of the most relevant mechanisms that generates ROS has been related to the overreduction of the electron transport chain. However, the contribution of these organelles to the total ROS balance is lower than chloroplasts and peroxisomes (Miller et al., 2010). In peroxisomes, $\mathrm{H}_{2} \mathrm{O}_{2}$ and superoxide are both abundantly produced as a result of many metabolic processes (Miller et al., 2010). The oxidation of glycolate to glyoxylic acid would cause the production of $\mathrm{H}_{2} \mathrm{O}_{2}$ in peroxisomes at increasing rates of photorespiration (Mittler et al., 2004). In addition, the dismutation of superoxide, fatty acid b-oxidation, and the flavin oxidase pathway are all processes causing the formation of $\mathrm{H}_{2} \mathrm{O}_{2}$ in these organelles. In salinity-like stress (i.e., drought stress) experiments, polyamine catabolism has also been found to regulate ROS concentration in peroxisomes (Miller et al., 2010).

When plants are able to counteract the detrimental presence of ROS in cells, a steady state between ROS formation and removal is achieved while $\mathrm{H}_{2} \mathrm{O}_{2}$ is kept at a level optimal for cell signaling (Mittler et al., 2004; Munns and Tester, 2008). The production of ROS due to photoxidative stress triggers the activation of many genes involved in antioxidant activities by various enzymes such as superoxide dismutase, glycolate oxidase, catalase, and ascorbate peroxidase, which play a strategic role for the removal of ROS (Apel and Hirt, 2004). The progressive rise of the above enzyme activity has been observed in plants under salinity stress induced in the root zone (Hussain et al., 2015). However, enzymatic ROS scavenging activities are not the only mechanisms in counteracting salinity stress (Miller et al., 2010, 2007) while the complex pathway surrounding ROS as signaling molecules is still not well understood (Mittler et al., 2004).

The above discussed aspects underline the toxicity of ROS, while also highlighting their beneficial effect as powerful molecules improving the transduction of stress-related signals (Miller et al., 2010). Nonetheless, many experiments have been set to prove the role of ROS and NO in the stomatal response as plant strategy to face salinity stress (Desikan et al., 2005; Niu and Liao, 2016). The higher ability of pumpkin-grafted cucumber to counteract the effects of osmotic stress was associated to the increase of $\mathrm{H}_{2} \mathrm{O}_{2}$ in plant tissue that caused earlier stomatal closure relative to self- grafted plants (Niu et al., 2017). Similar findings were obtained using tomato grown under standard and increased concentration of atmospheric $\mathrm{CO}_{2}$ (Yi et al., 2015), and pea as test plants (Desikan et al., 2004; Yi et al., 2015). In Vicia faba, it was observed that NO and ROS act together to mediate the ABA-induced inhibition of stomatal opening (Yan et al., 2007). While the role of ROS and NO in stomatal control was rarely investigated under salinity stress (Filippou et al., 2016), many authors (Table 1.1) report that ABA plays a relevant role in both stomatal closure and opening to control salinity effects with the main aim of reducing water loss (Dar et al., 2017; Kim et al., 2010).

The signaling molecules that regulate gaseous exchange activity of plants exposed to salinity are mostly revealed and the basic action mechanisms are often well understood. Since many processes follow multigenic mechanisms of activation and action, one of the main difficulties lies in the individuation of key genes and proteins. However, the ongoing challenge is to turn the knowledge of those mechanisms and the related physiological responses into tools for boosting cropping systems in areas affected by salinity.

\section{References}

Achard, P., Genschik, P., 2009. Releasing the brakes of plant growth: how GAs shutdown DELLA proteins. J. Exp. Bot. 60, 1085-1092. Available from: https://doi.org/10.1093/jxb/ern301.

Aki, T., Konishi, M., Kikuchi, T., Fujimori, T., Yoneyama, T., Yanagisawa, S., 2007. Distinct modulations of the hexokinase1mediated glucose response and hexokinase1-independent processes by HYS1/CPR5 in Arabidopsis. J. Exp. Bot. 58, 3239-3248. Available from: https://doi.org/10.1093/jxb/erm169.

An, Z., Jing, W., Liu, Y., Zhang, W., 2008. Hydrogen peroxide generated by copper amine oxidase is involved in abscisic acidinduced stomatal closure in Vicia faba. J. Exp. Bot. 59, 815-825. Available from: https://doi.org/10.1093/jxb/erm370.

Anderson, J.P., Badruzsaufari, E., Schenk, P.M., Manners, J.M., Desmond, O.J., Ehlert, C., et al., 2004. Antagonistic interaction between abscisic acid and jasmonate-ethylene signaling pathways modulates defense gene expression and disease resistance in Arabidopsis. Plant Cell 16, 3460-3479. Available from: https://doi. org/10.1105/tpc.104.025833.

Apel, K., Hirt, H., 2004. Reactive oxygen species: metabolism, oxidative stress, and signal transduction. Annu. Rev. Plant Biol. 55, 373-399. Available from: https://doi.org/10.1146/annurev. arplant.55.031903.141701.

Asada, K., 1999. The water-water cycle in chloroplasts: scavenging of active oxygens and dissipation of excess photons. Annu. Rev. Plant Biol. 50, 601-639.

Asada, K., Allen, J., Foyer, C.H., Matthijs, H.C.P., 2000. The waterwater cycle as alternative photon and electron sinks. Philos. Trans. R. Soc. B Biol. Sci. 355, 1419-1431. Available from: https:/ / doi.org/10.1098/rstb.2000.0703.

Ashraf, M., Harris, P.J.C., 2013. Photosynthesis under stressful environments: an overview. Photosynthetica 51, 163-190. Available from: https:/ / doi.org/10.1007/s11099-013-0021-6.

Bajji, M., Kinet, J.M., Lutts, S., 2002. Osmotic and ionic effects of $\mathrm{NaCl}$ on germination, early seedling growth, and ion content of 
Atriplex halimus (Chenopodiaceae). Can. J. Bot. Can. Bot. 80, 297-304. Available from: https://doi.org/10.1139/B02-008.

Barbieri, G., Vallone, S., Orsini, F., Paradiso, R., De Pascale, S., Negre-Zakharov, F., et al., 2012. Stomatal density and metabolic determinants mediate salt stress adaptation and water use efficiency in basil (Ocimum basilicum L.). J. Plant Physiol. 169, 1737-1746. Available from: https://doi.org/10.1016/j. jplph.2012.07.001.

Basu, S., Rabara, R., 2017. Abscisic acid - an enigma in the abiotic stress tolerance of crop plants. Plant Gene 11, 90-98. Available from: https://doi.org/10.1016/j.plgene.2017.04.008.

Bera, B., Siretanu, I., Stuart, M.A.C., Duits, M.H.G., van den Ende, D., Mugele, F., 2014. Ion-adsorption induced wetting transition for increasing efficiency during low salinity waterflooding. Abstr. Pap. Am. Chem. Soc. 247.

Bohnert, H.J., Cushman, J.C., 2000. The ice plant cometh: lessons in abiotic stress tolerance. J. Plant Growth Regul. 19, 334-346. Available from: https://doi.org/10.1007/s003440000033.

Brandt, B., Brodsky, D.E., Xue, S., Negi, J., Iba, K., Kangasjärvi, J., et al., 2012. Reconstitution of abscisic acid activation of SLAC1 anion channel by CPK6 and OST1 kinases and branched ABI1 PP2C phosphatase action. Proc. Natl. Acad. Sci. U. S. A. 109, 10593-10598. Available from: https://doi.org/10.1073/ pnas. 1116590109.

Bush, S.M., Krysan, P.J., 2007. Mutational evidence that the Arabidopsis MAP kinase MPK6 is involved in anther, inflorescence, and embryo development. J. Exp. Bot. 58, 2181-2191. Available from: https://doi.org/10.1093/jxb/erm092.

Cabot, C., Sibole, J.V., Barcelo, J., Poschenrieder, C., 2009. Abscisic acid decreases leaf $\mathrm{Na}^{+}$exclusion in salt-treated Phaseolus vulgaris L. J. Plant Growth Regul. 28, 187-192. Available from: https:// doi.org/10.1007/s00344-009-9088-5.

Casimiro, I., Beeckman, T., Graham, N., Bhalerao, R., Zhang, H.M., Casero, P., et al., 2003. Dissecting Arabidopsis lateral root development. Trends Plant Sci. 8, 165-171. Available from: https://doi. org/10.1016/S1360-1385(03)00051-7.

Chaves, M.M., Flexas, J., Pinheiro, C., 2009. Photosynthesis under drought and salt stress: regulation mechanisms from whole plant to cell. Ann. Bot. 103, 551-560. Available from: https://doi.org/ $10.1093 / \mathrm{aob} / \mathrm{mcn} 125$.

Cheong, J.J., Choi, Y.D., 2003. Methyl jasmonate as a vital substance in plants. Trends Genet. 19, 409-413. Available from: https:// doi.org/10.1016/S0168-9525(03)00138-0.

Cheong, M.S., Yun, D.J., 2007. Salt-stress signaling. J. Plant Biol. 50, 148-155. Available from: https://doi.org/10.1007/Bf03030623.

Cho, Y.H., Yoo, S.D., Sheen, J., 2006. Regulatory functions of nuclear hexokinase1 complex in glucose signaling. Cell 127, 579-589. Available from: https://doi.org/10.1016/j.cell.2006.09.028.

Colcombet, J., Hirt, H., 2008. Arabidopsis MAPKs: a complex signalling network involved in multiple biological processes. Biochem. J. 413, 217-226. Available from: https://doi.org/10.1042/ Bj20080625.

Colebrook, E.H., Thomas, S.G., Phillips, A.L., Hedden, P., 2014. The role of gibberellin signalling in plant responses to abiotic stress. J. Exp. Biol. 217, 67-75. Available from: https://doi.org/10.1242/ jeb.089938.

Corpas, F.J., Barroso, J.B., Palma, J.M., Rodriguez-Ruiz, M., 2017. Plant peroxisomes: a nitro-oxidative cocktail. Redox Biol. 11, 535-542. Available from: https://doi.org/10.1016/j. redox.2016.12.033.

Cramer, G.R., Quarrie, S.A., 2002. Abscisic acid is correlated with the leaf growth inhibition of four genotypes of maize differing in their response to salinity. Funct. Plant Biol. 29, 111-115. Available from: https://doi.org/Unsp1445-4408/02/ 01011110.1071/Pp01131.
Cuartero, J., Fernandez-Munoz, R., 1999. Tomato and salinity. Sci. Hortic. (Amsterdam). 78, 83-125.

Danquah, A., de Zelicourt, A., Colcombet, J., Hirt, H., 2014. The role of ABA and MAPK signaling pathways in plant abiotic stress responses. Biotechnol. Adv. 32, 40-52. Available from: https:// doi.org/10.1016/j.biotechadv.2013.09.006.

Dar, N.A., Amin, I., Wani, W., Wani, S.A., Shikari, A.B., Wani, S.H., et al., 2017. Abscisic acid: a key regulator of abiotic stress tolerance in plants. Plant Gene 11, 106-111. Available from: https:// doi.org/10.1016/j.plgene.2017.07.003.

Davies, W.J., Kudoyarova, G., Hartung, W., 2005. Long-distance ABA signaling and its relation to other signaling pathways in the detection of soil drying and the mediation of the plant's response to drought. J. Plant Growth Regul. 24, 285-295. Available from: https:/ / doi.org/10.1007/s00344-005-0103-1.

Demidchik, V., Bowen, H.C., Maathuis, F.J.M., Shabala, S.N., Tester, M.A., White, P.J., et al., 2002. Arabidopsis thaliana root nonselective cation channels mediate calcium uptake and are involved in growth. Plant J. 32, 799-808. Available from: https:// doi.org/10.1046/j.1365-313X.2002.01467.x.

Desikan, R., Griffiths, R., Hancock, J., Neill, S., 2002. A new role for an old enzyme: nitrate reductase-mediated nitric oxide generation is required for abscisic acid-induced stomatal closure in Arabidopsis thaliana. Proc. Natl. Acad. Sci. U. S. A. 99, 16314-16318. Available from: https://doi.org/10.1073/ pnas.252461999.

Desikan, R., Cheung, M.-K., Clarke, A., Golding, S., Sagi, M., Fluhr, R., et al., 2004. Hydrogen peroxide is a common signal for darkness- and ABA-induced stomatal closure in Pisum sativum. Funct. Plant Biol. 31, 913-920. Available from: https://doi.org/10.1071/ FP04035.

Desikan, R., Hancock, J.T., Bright, J., Harrison, J., Weir, I., Hooley, R., et al., 2005. A role for ETR1 in hydrogen peroxide signaling in stomatal guard cells. Plant Physiol. 137, 831-834. Available from: https://doi.org/10.1104/pp.104.056994.

Deuschle, K., Funck, D., Forlani, G., Stransky, H., Biehl, A., Leister, D., et al., 2004. The role of Delta(1)-Pyrroline-5-carboxylate dehydrogenase in proline degradation. Plant Cell 16, 3413-3425. Available from: https://doi.org/10.1105/tpc.104.023622.

Devinar, G., Llanes, A., Masciarelli, O., Luna, V., 2013. Different relative humidity conditions combined with chloride and sulfate salinity treatments modify abscisic acid and salicylic acid levels in the halophyte Prosopis strombulifera. Plant Growth Regul. 70, 247-256. Available from: https://doi.org/10.1007/s10725-0139796-5.

Dubrovsky, J.G., Forde, B.G., 2012. Quantitative analysis of lateral root development: pitfalls and how to avoid them. Plant Cell 24, 4-14. Available from: https://doi.org/10.1105/ tpc.111.089698.

Essah, P.A., Davenport, R., Tester, M., 2003. Sodium influx and accumulation in Arabidopsis. Plant Physiol. 133, 307-318. Available from: https://doi.org/10.1104/pp.103.022178.

Fahad, S., Hussain, S., Bano, A., Saud, S., Hassan, S., Shan, D., et al., 2015a. Potential role of phytohormones and plant growthpromoting rhizobacteria in abiotic stresses: consequences for changing environment. Environ. Sci. Pollut. Res. 22, 4907-4921. Available from: https://doi.org/10.1007/s11356-014-3754-2.

Fahad, S., Hussain, S., Matloob, A., Khan, F.A., Khaliq, A., Saud, S., et al., 2015b. Phytohormones and plant responses to salinity stress: a review. Plant Growth Regul. 75, 391-404. Available from: https:// doi.org/10.1007/s10725-014-0013-y.

Felix, G., Duran, J.D., Volko, S., Boller, T., 1999. Plants have a sensitive perception system for the most conserved domain of bacterial flagellin. Plant J. 18, 265-276. Available from: https://doi.org/ 10.1046/j.1365-313X.1999.00265.x. 
Filippou, P., Antoniou, C., Obata, T., Van Der Kelen, K., Harokopos, V., Kanetis, L., et al., 2016. Kresoxim-methyl primes Medicago truncatula plants against abiotic stress factors via altered reactive oxygen and nitrogen species signalling leading to downstream transcriptional and metabolic readjustment. J. Exp. Bot. 67, 1259-1274. Available from: https://doi.org/10.1093/jxb/ erv516.

Finkelstein, R., Reeves, W., Ariizumi, T., Steber, C., 2008. Molecular aspects of seed dormancy. Annu. Rev. Plant Biol. 59, 387-415. Available from: https://doi.org/10.1146/annurev. arplant.59.032607.092740.

Flowers, T.J., 2004. Improving crop salt tolerance. J. Exp. Bot. 55, 307-319. Available from: https://doi.org/10.1093/jxb/erh003.

Flowers, T.J., Galal, H.K., Bromham, L., 2010. Evolution of halophytes: multiple origins of salt tolerance in land plants. Funct. Plant Biol. 37, 604-612. Available from: https://doi.org/10.1071/ FP09269.

Fonseca, S., Chico, J.M., Solano, R., 2009. The jasmonate pathway: the ligand, the receptor and the core signalling module. Curr. Opin. Plant Biol. 12, 539-547. Available from: https://doi.org/10.1016/ j.pbi.2009.07.013.

Foresi, N., Mayta, M.L., Lodeyro, A.F., Scuffi, D., Correa-Aragunde, N., García-Mata, C., et al., 2015. Expression of the tetrahydrofolate-dependent nitric oxide synthase from the green alga Ostreococcus tauri increases tolerance to abiotic stresses and influences stomatal development in Arabidopsis. Plant J. 82, 806-821. Available from: https://doi.org/10.1111/tpj.12852.

Foyer, C.H., Noctor, G., 2003. Redox sensing and signalling associated with reactive oxygen in chloroplasts, peroxisomes and mitochondria. Physiol. Plant. 119, 355-364. Available from: https:// doi.org/10.1034/j.1399-3054.2003.00223.x.

Foyer, C.H., Noctor, G., 2005. Oxidant and antioxidant signalling in plants: a re-evaluation of the concept of oxidative stress in a physiological context. Plant, Cell Environ. 28, 1056-1071. Available from: https://doi.org/10.1111/j.13653040.2005.01327.x.

Fricke, W., Peters, W.S., 2002. The biophysics of leaf growth in saltstressed barley. A study at the cell level. Plant Physiol. 129, 374-388. Available from: https://doi.org/10.1104/pp.001164.

Fricke, W., Akhiyarova, G., Veselov, D., Kudoyarova, G., 2004. Rapid and tissue-specific changes in ABA and in growth rate in response to salinity in barley leaves. J. Exp. Bot. 55, 1115-1123. Available from: https://doi.org/10.1093/jxb/erh117.

Fricke, W., Akhiyarova, G., Wei, W., Alexandersson, E., Miller, A., Kjellbom, P.O., et al., 2006. The short-term growth response to salt of the developing barley leaf. J. Exp. Bot. 57, 1079-1095. Available from: https://doi.org/10.1093/jxb/erj095.

Gao, A.L., Wu, Q.Y., Zhang, Y., Miao, Y.C., Song, C.P., 2014. Arabidopsis calcium-dependent protein kinase CPK28 is potentially involved in the response to osmotic stress. Chinese Sci. Bull. 59, 1113-1122. Available from: https://doi.org/10.1007/s11434013-0062-z.

Gao, S., Ouyang, C., Wang, S., Xu, Y., Tang, L., Chen, F., 2008. Effects of salt stress on growth, antioxidant enzyme and phenylalanine ammonia-lyase activities in Jatropha curcas L. seedlings. Plant Soil Environ. 54, 374-381.

Glenn, E.P., Brown, J.J., Blumwald, E., 1999. Salt tolerance and crop potential of halophytes. CRC. Crit. Rev. Plant Sci. 18, 227-255. Available from: https://doi.org/10.1080/ 07352689991309207.

Gudesblat, G.E., Iusem, N.D., Morris, P.C., 2007. Guard cell-specific inhibition of Arabidopsis MPK3 expression causes abnormal stomatal responses to abscisic acid and hydrogen peroxide. New Phytol. 173, 713-721. Available from: https://doi.org/10.1111/ j.1469-8137.2006.01953.x.
Guo, J.R., Suo, S.S., Wang, B.S., 2015. Sodium chloride improves seed vigour of the euhalophyte Suaeda salsa. Seed Sci. Res. 25, 335-344. Available from: https://doi.org/10.1017/S0960258515000239.

Gupta, B., Huang, B.R., 2014. Mechanism of salinity tolerance in plants: physiological, biochemical, and molecular characterization. Int. J. Genomics. Available from: https://doi.org/70159610.1155/ 2014/701596.

Hannachi, S., Van Labeke, M.C., 2018. Salt stress affects germination, seedling growth and physiological responses differentially in eggplant cultivars (Solanum melongena L.). Sci. Hortic. (Amsterdam). 228, 56-65. Available from: https:// doi.org/10.1016/j.scienta.2017.10.002.

Hanson, J., Smeekens, S., 2009. Sugar perception and signaling - an update. Curr. Opin. Plant Biol. 12, 562-567. Available from: https://doi.org/10.1016/j.pbi.2009.07.014.

Hare, P.D., Cress, W.A., 1997. Metabolic implications of stress-induced proline accumulation in plants. Plant Growth Regul. 21, 79-102. Available from: https://doi.org/10.1023/A:1005703923347.

Hayashi, M., Inoue, S.-I., Takahashi, K., Kinoshita, T., 2011. Immunohistochemical detection of blue light-induced phosphorylation of the plasma membrane $\mathrm{H}^{+}$-ATPase in stomatal guard cells. Plant Cell Physiol 52, 1238-1248. Available from: https:// doi.org/10.1093/pcp/pcr072.

He, J.-M., Ma, X.-G., Zhang, Y., Sun, T.-F., Xu, F.-F., Chen, Y.-P., et al., 2013. Role and interrelationship of $\mathrm{G \alpha}$ protein, hydrogen peroxide, and nitric oxide in ultraviolet B-induced stomatal closure in Arabidopsis leaves. Plant Physiol. 161, 1570-1583. Available from: https://doi.org/10.1104/pp.112.211623.

Hellmann, H., Funck, D., Rentsch, D., Frommer, W.B., 2000. Hypersensitivity of an Arabidopsis sugar signaling mutant toward exogenous proline application. Plant Physiol. 123, 779-789.

Hetherington, A.M., 2001. Guard cell signaling. Cell 107, 711-714. Available from: https://doi.org/10.1016/S0092-8674(01)00606-7.

Horie, T., Schroeder, J.I., 2004. Sodium transporters in plants. Diverse genes and physiological functions. Plant Physiol. 136, 2457-2462. Available from: https://doi.org/10.1104/ pp.104.046664.

Hu, G.G., Kamp, A., Linning, R., Naik, S., Bakkeren, G., 2007. Complementation of Ustilago maydis MAPK mutants by a wheat leaf rust, Puccinia triticina homolog: potential for functional analyses of rust genes. Mol. Plant-Microbe Interact. 20, 637-647. Available from: https://doi.org/10.1094/Mpmi-20-60637.

Hu, J.B., Liu, J., Yan, H.B., Xie, C.H., 2005. Histological observations of morphogenesis in petiole derived callus of Amorphophallus rivieri Durieu in vitro. Plant Cell Rep. 24, 642-648. Available from: https: / / doi.org/10.1007/s00299-005-0002-8.

Huang, G.-T., Ma, S.-L., Bai, L.-P., Zhang, L., Ma, H., Jia, P., et al., 2012. Signal transduction during cold, salt, and drought stresses in plants. Mol. Biol. Rep. 39, 969-987. Available from: https:// doi.org/10.1007/s11033-011-0823-1.

Hussain, T., Koyro, H.-W., Huchzermeyer, B., Khan, M.A., 2015. Ecophysiological adaptations of Panicum antidotale to hyperosmotic salinity: water and ion relations and anti-oxidant feedback. Flora - Morphol. Distrib. Funct. Ecol. Plants 212, 30-37. Available from: https:// doi.org/10.1016/j.flora.2015.02.006.

Ichimura, K., Shinozaki, K., Tena, G., Sheen, J., Henry, Y., Champion, A., et al., 2002. Mitogen-activated protein kinase cascades in plants: a new nomenclature. Trends Plant Sci. 7, 301-308.

Ismail, A., Takeda, S., Nick, P., 2014. Life and death under salt stress: same players, different timing? J. Exp. Bot. 65, 2963-2979. Available from: https://doi.org/10.1093/jxb/eru159.

Ivanova, K., Tzvetkova, N., Georgieva, T., Markovska, Y., 2014. Photosynthesis and growth responses of five Paulownia lines to salt stress. Comptes Rendus L Acad. Bulg. Des Sci. 67, $1101-1106$. 
James, R.A., Rivelli, A.R., Munns, R., Von Caemmerer, S., 2002. Factors affecting $\mathrm{CO}_{2}$ assimilation, leaf injury and growth in saltstressed durum wheat. Funct. Plant Biol. 29, 1393-1403. Available from: https://doi.org/10.1071/FP02069.

Jiang, J., An, G.Y., Wang, P.C., Wang, P.T., Han, J.F., Jin, Y.B., et al., 2003. MAP kinase specifically mediates the ABA-induced $\mathrm{H}_{2} \mathrm{O}_{2}$ generation in guard cells of Vicia faba L. Chinese Sci. Bull. 48, 1919-1926. Available from: https://doi.org/10.1360/03wc0155.

Kang, D.J., Seo, Y.J., Lee, J.D., Ishii, R., Kim, K.U., Shin, D.H., et al., 2005. Jasmonic acid differentially affects growth, ion uptake and abscisic acid concentration in salt-tolerant and salt-sensitive rice cultivars. J. Agron. Crop Sci. 191, 273-282. Available from: https://doi.org/10.1111/j.1439-037X.2005.00153.x.

Kang, J., Yim, S., Choi, H., Kim, A., Lee, K.P., Lopez-Molina, L., et al., 2015. Abscisic acid transporters cooperate to control seed germination. Nat. Commun. 6. Available from: https://doi.org/ Artn811310.1038/Ncomms9113.

Keskin, B.C., Sarikaya, A.T., Yuksel, B., Memon, A.R., 2010. Abscisic acid regulated gene expression in bread wheat (Triticum aestivum L.). Aust. J. Crop Sci. 4, 617-625.

Khan, M.I.R., Khan, N., 2017. Reactive Oxygen Species and Antioxidant System in Plants: Role and Regulation Under Abiotic Stress. Springer Nature, Singapore978-981-10-5254-5.

Khan, M.I.R., Asgher, M., Iqbal, N., Khan, N.A., 2013. Potentiality of sulfur-containing compounds in salt tolerance. In: Ahmad, P., Prasad, M.N.V. (Eds.), Ecophysiology and Responses of Plants Under Salt Stress. Springer-Verlag, New York, pp. $443-472$.

Khan, M.I.R., Asgher, M., Khan, N.A., 2014a. Alleviation of saltinduced photosynthesis and growth inhibition by salicylic acid involves glycinebetaine and ethylene in mungbean (Vigna radiata L.). Plant Physiol. Biochem. 80, 67-74.

Khan, N.A., Khan, M.I.R., Asgher, M., Fatma, M., Masood, A., Syeed, S., 2014b. Salinity tolerance in plants: revisiting the role of sulfur metabolites. J. Plant Biochem. Physiol. 2, 2.

Khan, R., Khan, M.M.A., Singh, M., Nasir, S., Naeem, M., Siddiqui, M.H., et al., 2007. Gibberellic acid and triacontanol can ameliorate the opium yield and morphine production in opium poppy (Papaver somniferum L.). Acta Agric. Scand. Sect. B-Soil Plant Sci. 57, 307-312.

Khedr, A.H.A., Abbas, M.A., Wahid, A.A.A., Quick, W.P., Abogadallah, G.M., 2003. Proline induces the expression of saltstress-responsive proteins and may improve the adaptation of Pancratium maritimum L. to salt-stress. J. Exp. Bot. 54, 2553-2562. Available from: https://doi.org/10.1093/jxb/erg277.

Kim, S.T., Kang, S.Y., Wang, W., Kim, S.G., Hwang, D.H., Kang, K. Y., 2008. Analysis of embryonic proteome modulation by GA and ABA from germinating rice seeds. Proteomics 8, 3577-3587. Available from: https:/ /doi.org/10.1002/ pmic.200800183.

Kim, T.-H., Böhmer, M., Hu, H., Nishimura, N., Schroeder, J.I., 2010. Guard cell signal transduction network: advances in understanding abscisic acid, $\mathrm{CO}_{2}$, and $\mathrm{Ca}^{2+}$ Signaling. Annu. Rev. Plant Biol. 61, 561-591. Available from: https://doi.org/10.1146/annurevarplant-042809-112226.

Kohoutova, L., Kourova, H., Nagy, S.K., Volc, J., Halada, P., Meszaros, T., et al., 2015. The Arabidopsis mitogen-activated protein kinase 6 is associated with -tubulin on microtubules, phosphorylates EB1C and maintains spindle orientation under nitrosative stress. New Phytol. 207, 1061-1074. Available from: https://doi.org/10.1111/nph.13501.

Kopyra, M., Gwozdz, E.A., 2003. Nitric oxide stimulates seed germination and counteracts the inhibitory effect of heavy metals and salinity on root growth of Lupinus luteus. Plant Physiol. Biochem. 41, 1011-1017. Available from: https://doi.org/10.1016/j. plaphy.2003.09.003.
Koyro, H.-W., Geissler, N., Seenivasan, R., Huchzermeyer, B., 2011. Plant stress physiology: physiological and biochemical strategies allowing plants/crops to thrive under ionic stress. In: Pessarakli, M. (Ed.), Handbook of Plant and Crop Stress. CRC Press, Boca Raton, FL, p. 1245.

Lamattina, L., Garcia-Mata, C., Graziano, M., Pagnussat, G., 2003. Nitric oxide: the versatility of an extensive signal molecule. Annu. Rev. Plant Biol. 54, 109-136. Available from: https://doi. org/10.1146/annurev.arplant.54.031902.134752.

Lampard, G.R., Lukowitz, W., Ellis, B.E., Bergmann, D.C., 2009. Novel and expanded roles for MAPK signaling in Arabidopsis stomatal cell fate revealed by cell type-specific manipulations. Plant Cell 21, 3506-3517. Available from: https://doi.org/10.1105/ tpc.109.070110.

Läuchli, A., Grattan, S.R., 2007. Plant growth and development under salinity stress. In: Jenks, M.A., Hasegawa, P.M., Jain, S.M. (Eds.), Advances in Molecular Breeding Toward Drought and Salt Tolerant Crops. Springer, Netherlands, Dordrecht, pp. 1-32. Available from: https://doi.org/10.1007/978-1-4020-5578-2_1.

Lee, S.C., Lan, W., Buchanan, B.B., Luan, S., 2009. A protein kinasephosphatase pair interacts with an ion channel to regulate ABA signaling in plant guard cells. Proc. Natl. Acad. Sci. U. S. A. 106, 21419-21424. Available from: https://doi.org/10.1073/ pnas.0910601106.

Li, K., Yang, F.B., Miao, Y.C., Song, C.P., 2017a. Abscisic acid signaling is involved in regulating the mitogen-activated protein kinase cascade module, AIK1-MKK5-MPK6. Plant Signal. Behav. 12. Available from: https://doi.org/ARTNe132118810.1080/ 15592324.2017.1321188.

Li, Q., Wang, Y.-J., Liu, C.-K., Pei, Z.-M., Shi, W.-L., 2017b. The crosstalk between $\mathrm{ABA}$, nitric oxide, hydrogen peroxide, and calcium in stomatal closing of Arabidopsis thaliana. Biol. 72, 1140-1146. Available from: https://doi.org/10.1515/biolog-2017-0126.

Liu, X.M., Kim, K.E., Kim, K.C., Nguyen, X.C., Han, H.J., Jung, M.S., et al., 2010. Cadmium activates Arabidopsis MPK3 and MPK6 via accumulation of reactive oxygen species. Phytochemistry 71, 614-618. Available from: https://doi.org/10.1016/j. phytochem.2010.01.005.

Liu, Y.D., Zhang, S.Q., 2004. Phosphorylation of 1aminocyclopropane-1-carboxylic acid synthase by MPK6, a stressresponsive mitogen-activated protein kinase, induces ethylene biosynthesis in Arabidopsis. Plant Cell 16, 3386-3399. Available from: https: / / doi.org/10.1105/tpc.104.026609.

López-Pérez, L., Martínez-Ballesta, M.D.C., Maurel, C., Carvajal, M., 2009. Changes in plasma membrane lipids, aquaporins and proton pump of broccoli roots, as an adaptation mechanism to salinity. Phytochemistry 70, 492-500. Available from: https://doi. org/10.1016/j.phytochem.2009.01.014.

Ma, X., Zheng, J., Zhang, X., Hu, Q., Qian, R., 2017. Salicylic acid alleviates the adverse effects of salt stress on Dianthus Superbus (Caryophyllaceae) by activating photosynthesis, protecting morphological structure, and enhancing the antioxidant system. Front. Plant Sci. 8, 600. Available from: https://doi.org/10.3389/ fpls.2017.00600.

Maggio, A., Miyazaki, S., Veronese, P., Fujita, T., Ibeas, J.I., Damsz, B., et al., 2002. Does proline accumulation play an active role in stress-induced growth reduction? Plant J. 31, 699-712. Available from: https:/ / doi.org/10.1046/j.1365-313X.2002.01389.x.

Magome, H., Yamaguchi, S., Hanada, A., Kamiya, Y., Oda, K., 2008. The DDF1 transcriptional activator upregulates expression of a gibberellin-deactivating gene, GA2ox7, under high-salinity stress in Arabidopsis. Plant J. 56, 613-626. Available from: https://doi. org/10.1111/j.1365-313X.2008.03627.x.

Mahajan, S., Pandey, G.K., Tuteja, N., 2008. Calcium- and salt-stress signaling in plants: shedding light on SOS pathway. Arch. 
Biochem. Biophys. 471, 146-158. Available from: https://doi. org/10.1016/j.abb.2008.01.010.

Martí, M.C., Florez-Sarasa, I., Camejo, D., Ribas-Carbó, M., Lázaro, J. J., Sevilla, F., et al., 2011. Response of mitochondrial thioredoxin PsTrxo1, antioxidant enzymes, and respiration to salinity in pea (Pisum sativum L.) leaves. J. Exp. Bot. 62, 3863-3874. Available from: https://doi.org/10.1093/jxb/err076.

Mata, C.G., Lamattina, L., 2001. Nitric oxide induces stomatal closure and enhances the adaptive plant responses against drought stress. Plant Physiol. 126, 1196-1204. Available from: https://doi.org/ 10.1104/pp.126.3.1196.

Mattioli, R., Falasca, G., Sabatini, S., Altamura, M.M., Costantino, P., Trovato, M., 2009. The proline biosynthetic genes P5CS1 and P5CS2 play overlapping roles in Arabidopsis flower transition but not in embryo development. Physiol. Plant. 137, 72-85. Available from: https:/ / doi.org/10.1111/j.1399-3054.2009.01261.x.

McAinsh, M.R., Clayton, H., Mansfield, T.A., Hetherington, A.M., 1996. Changes in stomatal behavior and guard cell cytosolic free calcium in response to oxidative stress. Plant Physiol. 111, 1031-1042. Available from: https://doi.org/10.1104/ pp.111.4.1031.

Miller, G., Suzuki, N., Rizhsky, L., Hegie, A., Koussevitzky, S., Mittler, R., 2007. Double mutants deficient in cytosolic and thylakoid ascorbate peroxidase reveal a complex mode of interaction between reactive oxygen species, plant development, and response to abiotic stresses. Plant Physiol. 144, 1777-1785. Available from: https://doi.org/10.1104/pp.107.101436.

Miller, G., Suzuki, N., Ciftci-Yilmaz, S., Mittler, R., 2010. Reactive oxygen species homeostasis and signalling during drought and salinity stresses. Plant, Cell Environ. 33, 453-467. Available from: https://doi.org/10.1111/j.13653040.2009.02041.x.

Mittler, R., Blumwald, E., 2015. The roles of ROS and ABA in systemic acquired acclimation. Plant Cell 27, 64-70. Available from: https:/ / doi.org/10.1105/tpc.114.133090.

Mittler, R., Vanderauwera, S., Gollery, M., Van Breusegem, F., 2004. Reactive oxygen gene network of plants. Trends Plant Sci. 9, 490-498. Available from: https://doi.org/10.1016/j. tplants.2004.08.009.

Moore, B., Zhou, L., Rolland, F., Hall, Q., Cheng, W.H., Liu, Y.X., et al., 2003. Role of the Arabidopsis glucose sensor HXK1 in nutrient, light, and hormonal signaling. Science. 300, 332-336. Available from: https://doi.org/10.1126/science.1080585.

Müller, J., Beck, M., Mettbach, U., Komis, G., Hause, G., Menzel, D., et al., 2010. Arabidopsis MPK6 is involved in cell division plane control during early root development, and localizes to the preprophase band, phragmoplast, trans-Golgi network and plasma membrane. Plant J. 61, 234-248. Available from: https://doi.org/ 10.1111/j.1365-313X.2009.04046.x.

Munemasa, S., Muroyama, D., Nagahashi, H., Nakamura, Y., Mori, I. C., Murata, Y., 2013. Regulation of reactive oxygen speciesmediated abscisic acid signaling in guard cells and drought tolerance by glutathione. Front. Plant Sci. 4. Available from: https:// doi.org/10.3389/fpls.2013.00472.

Munns, R., 2002. Comparative physiology of salt and water stress. Plant Cell Environ. 25, 239-250. Available from: https://doi.org/ 10.1046/j.0016-8025.2001.00808.x.

Munns, R., Tester, M., 2008. Mechanisms of salinity tolerance. Annu. Rev. Plant Biol. 59, 651-681. Available from: https://doi.org/ 10.1146/annurev.arplant.59.032607.092911.

Munns, R., James, R.A., Lauchli, A., 2006. Approaches to increasing the salt tolerance of wheat and other cereals. J. Exp. Bot. 57, 1025-1043. Available from: https://doi.org/10.1093/jxb/erj100.

Murata, Y., Pei, Z.-M., Mori, I.C., Schroeder, J., 2001. Abscisic acid activation of plasma membrane $\mathrm{Ca}^{2+}$ channels in guard cells requires cytosolic $\mathrm{NAD}(\mathrm{P}) \mathrm{H}$ and is differentially disrupted upstream and downstream of reactive oxygen species production in abi1-1 and abi2-1 protein phosphatase $2 \mathrm{C}$ mutants. Plant Cell 13, 2513-2523. Available from: https://doi.org/10.1105/ tpc.13.11.2513.

Nanjo, T., Fujita, M., Seki, M., Kato, T., Tabata, S., Shinozaki, K., 2003. Toxicity of free proline revealed in an Arabidopsis T-DNAtagged mutant deficient in proline dehydrogenase. Plant Cell Physiol. 44, 541-548. Available from: https://doi.org/10.1093/ Pcp/Pcg066.

Neill, S., Barros, R., Bright, J., Desikan, R., Hancock, J., Harrison, J., et al., 2008. Nitric oxide, stomatal closure, and abiotic stress. J. Exp. Bot. 59, 165-176. Available from: https://doi.org/10.1093/ jxb/erm293.

Neill, S.J., Desikan, R., Clarke, A., Hancock, J.T., 2002. Nitric oxide is a novel component of abscisic acid signaling in stomatal guard cells. Plant Physiol. 128, 13-16. Available from: https://doi.org/ 10.1104/pp.128.1.13.

Niu, L., Liao, W., 2016. Hydrogen peroxide signaling in plant development and abiotic responses: crosstalk with nitric oxide and calcium. Front. Plant Sci. 7. Available from: https://doi.org/ 10.3389/fpls.2016.00230.

Niu, M., Huang, Y., Sun, S., Sun, J., Cao, H., Shabala, S., et al., 2017. Root respiratory burst oxidase homologue-dependent $\mathrm{H}_{2} \mathrm{O}_{2}$ production confers salt tolerance on a grafted cucumber by controlling $\mathrm{Na}^{+}$exclusion and stomatal closure. J. Exp. Bot. Available from: https://doi.org/10.1093/jxb/erx386.

Olszewski, N., Sun, T.P., Gubler, F., 2002. Gibberellin signaling: biosynthesis, catabolism, and response pathways. Plant Cell 14, 61-80. Available from: https://doi.org/10.1105/ tpc.010476.

Olszewski, N.E., West, C.M., Sassi, S.O., Hartweck, L.M., 2010. OGlcNAc protein modification in plants: evolution and function. Biochim. Biophys. Acta-General Subj. 1800, 49-56. Available from: https:// doi.org/10.1016/j.bbagen.2009.11.016.

Orsini, F., Cascone, P., De Pascale, S., Barbieri, G., Corrado, G., Rao, R., et al., 2010. Systemin-dependent salinity tolerance in tomato: evidence of specific convergence of abiotic and biotic stress responses. Physiol. Plant. 138, 10-21. Available from: https:// doi.org/10.1111/j.1399-3054.2009.01292.x.

Osakabe, Y., Yamaguchi-Shinozaki, K., Shinozaki, K., Tran, L.-S.P., 2014. ABA control of plant macroelement membrane transport systems in response to water deficit and high salinity. New Phytol. 202, 35-49. Available from: https://doi.org/10.1111/ nph.12613.

Ozgur, R., Turkan, I., Uzilday, B., Sekmen, A.H., 2014. Endoplasmic reticulum stress triggers ROS signalling, changes the redox state, and regulates the antioxidant defence of Arabidopsis thaliana. J. Exp. Bot. 65, 1377-1390. Available from: https://doi.org/ $10.1093 / \mathrm{jxb} / \mathrm{eru} 034$.

Parida, A.K., Das, A.B., 2005. Salt tolerance and salinity effects on plants: a review. Ecotoxicol. Environ. Saf. 60, 324-349. Available from: https://doi.org/10.1016/j.ecoenv.2004.06.010.

Park, H.J., Kim, W.-Y., Yun, D.-J., 2016. A new insight of salt stress signaling in plant. Mol. Cells 39, 447-459. Available from: https:/ / doi.org/10.14348/molcells.2016.0083.

Pedranzani, H., Racagni, G., Alemano, S., Miersch, O., Ramirez, I., Pena-Cortes, H., et al., 2003. Salt tolerant tomato plants show increased levels of jasmonic acid. Plant Growth Regul. 41, 149-158. Available from: https://doi.org/10.1023/ A:1027311319940.

Pei, Z.-M., Murata, Y., Benning, G., Thomine, S., Klüsener, B., Allen, G.J., et al., 2000. Calcium channels activated by hydrogen peroxide mediate abscisic acid signalling in guard cells. Nature 406, 731. 
Piñero, M.C., Houdusse, F., Garcia-Mina, J.M., Garnica, M., del Amor, F.M., 2014. Regulation of hormonal responses of sweet pepper as affected by salinity and elevated $\mathrm{CO}_{2}$ concentration. Physiol. Plant. 151, 375-389. Available from: https://doi.org/ 10.1111/ppl.12119.

Piskurewicz, U., Jikumaru, Y., Kinoshita, N., Nambara, E., Kamiya, Y., Lopez-Molina, L., 2008. The gibberellic acid signaling repressor RGL2 inhibits Arabidopsis seed germination by stimulating abscisic acid synthesis and ABI5 activity. Plant Cell 20, 2729-2745. Available from: https://doi.org/10.1105/ tpc.108.061515.

Pitzschke, A., Hirt, H., 2009. Disentangling the complexity of mitogen-activated protein kinases and reactive oxygen species signaling. Plant Physiol. 149, 606-615. Available from: https:// doi.org/10.1104/pp.108.131557.

Qin, F., Kodaira, K.S., Maruyama, K., Mizoi, J., Tran, L.S.P., Fujita, Y., et al., 2011. SPINDLY, a negative regulator of gibberellic acid signaling, is involved in the plant abiotic stress response. Plant Physiol. 157, 1900-1913. Available from: https://doi.org/ 10.1104/pp.111.187302.

Riemann, B., Sehr, M.A., Schittenhelm, R.S., Rinderknecht, S., 2013. Real Gyroscopic Uncertainties in Robust Control of Flexible Rotors, in: 52nd Annual Conference on Decision and Control (Cdc). pp. 3762-3769.

Robinson, M.F., Véry, A.-A., Sanders, D., Mansfield, T.A., 1997. How can stomata contribute to salt tolerance? Ann. Bot. 80, 387-393. Available from: https://doi.org/10.1006/anbo.1996.0435.

Robson, F., Okamoto, H., Patrick, E., Harris, S.R., Wasternack, C., Brearley, C., et al., 2010. Jasmonate and phytochrome a signaling in Arabidopsis wound and shade responses are integrated through JAZ1 stability. Plant Cell 22, 1143-1160. Available from: https:/ / doi.org/10.1105/tpc.109.067728.

Rodrigues, O., Reshetnyak, G., Grondin, A., Saijo, Y., Leonhardt, N., Maurel, C., et al., 2017. Aquaporins facilitate hydrogen peroxide entry into guard cells to mediate ABA- and pathogentriggered stomatal closure. Proc. Natl. Acad. Sci. U. S. A. 114, 9200-9205. Available from: https://doi.org/10.1073/ pnas. 1704754114.

Rolland, F., Moore, B., Sheen, J., 2002. Sugar sensing and signaling in plants. Plant Cell 14, S185-S205. Available from: https://doi. org/10.1105/tpc.010455.

Sairam, R.K., Tyagi, A., 2004. Physiology and molecular biology of salinity stress tolerance in plants. Curr. Sci. 86, 407-421.

Sato, A., Sato, Y., Fukao, Y., Fujiwara, M., Umezawa, T., Shinozaki, K., et al., 2009. Threonine at position 306 of the KAT1 potassium channel is essential for channel activity and is a target site for ABA-activated SnRK2/OST1/SnRK2.6 protein kinase. Biochem. J. 424, 439-448. Available from: https://doi.org/10.1042/ BJ20091221.

Saxena, I., Srikanth, S., Chen, Z., 2016. Cross talk between $\mathrm{H}_{2} \mathrm{O}_{2}$ and interacting signal molecules under plant stress response. Front. Plant Sci. 7, 570. Available from: https://doi.org/10.3389/ fpls.2016.00570.

Seung, D., Risopatron, J.P.M., Jones, B.J., Marc, J., 2012. Circadian clock-dependent gating in ABA signalling networks. Protoplasma 249, 445-457. Available from: https://doi.org/10.1007/s00709011-0304-3.

Shabala, S., Demidchik, V., Shabala, L., Cuin, T.A., Smith, S.J., Miller, A.J., et al., 2006. Extracellular $\mathrm{Ca}^{2+}$ ameliorates $\mathrm{NaCl}$-induced $\mathrm{K}^{+}$ loss from Arabidopsis root and leaf cells by controlling plasma membrane $\mathrm{K}^{+}$-permeable channels. Plant Physiol. 141, 1653-1665. Available from: https://doi.org/10.1104/ pp.106.082388.

Shannon, M.C., Grieve, C.M., 1999. Tolerance of vegetable crops to salinity. Sci. Hortic. (Amsterdam). 78, 5-38.
Sharipova, G., Veselov, D., Kudoyarova, G., Fricke, W., Dodd, I.C., Katsuhara, M., et al., 2016. Exogenous application of abscisic acid (ABA) increases root and cell hydraulic conductivity and abundance of some aquaporin isoforms in the ABA-deficient barley mutant Az34. Ann. Bot. 118, 777-785. Available from: https:// doi.org/10.1093/aob/mcw117.

Shi, H.Z., Zhu, J.K., 2002. Regulation of expression of the vacuolar $\mathrm{Na}^{+} / \mathrm{H}^{+}$antiporter gene AtNHX1 by salt stress and abscisic acid. Plant Mol. Biol. 50, 543-550. Available from: https://doi.org/ 10.1023/A:1019859319617.

Shi, K., Li, X., Zhang, H., Zhang, G., Liu, Y., Zhou, Y., et al., 2015. Guard cell hydrogen peroxide and nitric oxide mediate elevated $\mathrm{CO}_{2}$-induced stomatal movement in tomato. New Phytol. 208, 342-353. Available from: https://doi.org/10.1111/nph.13621.

Shoresh, M., Spivak, M., Bernstein, N., 2011. Involvement of calciummediated effects on ROS metabolism in the regulation of growth improvement under salinity. Free Radic. Biol. Med. 51, 1221-1234. Available from: https://doi.org/10.1016/j. freeradbiomed.2011.03.036.

Shrivastava, P., Kumar, R., 2015. Soil salinity: a serious environmental issue and plant growth promoting bacteria as one of the tools for its alleviation. Saudi J. Biol. Sci. 22, 123-131. Available from: https:// doi.org/10.1016/j.sjbs.2014.12.001.

Singh, A., Jha, S.K., Bagri, J., Pandey, G.K., 2015. ABA inducible rice protein phosphatase $2 \mathrm{C}$ confers $\mathrm{ABA}$ insensitivity and abiotic stress tolerance in Arabidopsis. PLoS One 10. Available from: https://doi.org/10.1371/journal.pone.0125168.

Sirault, X.R.R., James, R.A., Furbank, R.T., 2009. A new screening method for osmotic component of salinity tolerance in cereals using infrared thermography. Funct. Plant Biol. 36, 970-977. Available from: https://doi.org/10.1071/FP09182.

Sirichandra, C., Gu, D., Hu, H.-C., Davanture, M., Lee, S., Djaoui, M., et al., 2009. Phosphorylation of the Arabidopsis AtrbohF NADPH oxidase by OST1 protein kinase. FEBS Lett. 583, 2982-2986. Available from: https://doi.org/10.1016/j.febslet.2009.08.033.

Sobahan, M.A., Akter, N., Okuma, E., Uraji, M., Ye, W., Mori, I.C., et al., 2015. Allyl isothiocyanate induces stomatal closure in Vicia faba. Biosci. Biotechnol. Biochem. 79, 1737-1742. Available from: https://doi.org/10.1080/09168451.2015.1045827.

Suhita, D., Raghavendra, A.S., Kwak, J.M., Vavasseur, A., 2004. Cytoplasmic alkalization precedes reactive oxygen species production during methyl jasmonate- and abscisic acid-induced stomatal closure. Plant Physiol. 134, 1536-1545. Available from: https:/ / doi.org/10.1104/pp.103.032250.

Sumer, A., Zorb, C., Yan, F., Schubert, S., 2004. Evidence of sodium toxicity for the vegetative growth of maize (Zea mays L.) during the first phase of salt stress. J. Appl. Bot. Food Qual. Bot. 78, $135-139$.

Suo, J., Zhao, Q., David, L., Chen, S., Dai, S., 2017. Salinity response in chloroplasts: insights from gene characterization. Int. J. Mol. Sci. 18. Available from: https://doi.org/10.3390/ijms18051011.

Szabados, L., Savoure, A., 2010. Proline: a multifunctional amino acid. Trends Plant Sci. 15, 89-97. Available from: https://doi. org/10.1016/j.tplants.2009.11.009.

Taiz, L., Zeiger, E., Moller, I.M., Murphy, A., 2015. Plant Physiology and Development, sixth ed Sinauer Associates, Sunderland, MA.

Taj, G., Agarwal, P., Grant, M., Kumar, A., 2010. MAPK machinery in plants. Plant Signal. Behav. 5, 1370-1378. Available from: https://doi.org/10.4161/psb.5.11.13020.

Takac, T., Obert, B., Rolcik, J., Samaj, J., 2016. Improvement of adventitious root formation in flax using hydrogen peroxide. $\mathrm{N}$. Biotechnol. 33, 728-734. Available from: https://doi.org/ 10.1016/j.nbt.2016.02.008.

Tani, T., Sobajima, H., Okada, K., Chujo, T., Arimura, S.I., Tsutsumi, N., et al., 2008. Identification of the OsOPR7 gene encoding 
12-oxophytodienoate reductase involved in the biosynthesis of jasmonic acid in rice. Planta 227, 517-526. Available from: https: / / doi.org/10.1007/s00425-007-0635-7.

Tester, M., Davenport, R., 2003. $\mathrm{Na}^{+}$tolerance and $\mathrm{Na}^{+}$transport in higher plants. Ann. Bot 91, 503-527. Available from: https://doi. org $/ 10.1093 / \mathrm{aob} / \mathrm{mcg} 058$.

Trovato, M., Mattioli, R., Costantino, P., 2008. Multiple roles of proline in plant stress tolerance and development. P. Rend. Fis. Acc. Lincei 19, 325-346. Available from: https://doi.org/10.1007/ s12210-008-0022-8.

Turkan, I., Demiral, T., 2009. Recent developments in understanding salinity tolerance. Environ. Exp. Bot. 67, 2-9. Available from: https://doi.org/10.1016/j.envexpbot.2009.05.008.

Tuteja, N., 2007. Mechanisms of high salinity tolerance in plants. Osmosens. Osmosignal. 428, 419-438. Available from: https:// doi.org/10.1016/S0076-6879(07)28024-3.

Verbruggen, N., Hermans, C., 2008. Proline accumulation in plants: a review. Amino Acids 35, 753-759. Available from: https://doi. org/10.1007/s00726-008-0061-6.

Wang, C., Lu, G., Hao, Y., Guo, H., Guo, Y., Zhao, J., et al., 2017. ABP9, a maize bZIP transcription factor, enhances tolerance to salt and drought in transgenic cotton. Planta 246, 453-469. Available from: https:/ / doi.org/10.1007/s00425-017-2704-x.

Wang, H., Liu, Y., Bruffett, K., Lee, J., Hause, G., Walker, J.C., et al., 2008. Haplo-insufficiency of MPK3 in MPK6 mutant background uncovers a novel function of these two MAPKs in Arabidopsis ovule development. Plant Cell 20, 602-613. Available from: https:/ / doi.org/10.1105/tpc.108.058032.

Wang, P.C., Du, Y.Y., Li, Y.A., Ren, D.T., Song, C.P., 2010. Hydrogen peroxide-mediated activation of MAP kinase 6 modulates nitric oxide biosynthesis and signal transduction in Arabidopsis. Plant Cell 22, 2981-2998. Available from: https://doi.org/10.1105/ tpc.109.072959.

Wang, W.-H., He, E.-M., Guo, Y., Tong, Q.-X., Zheng, H.-L., $2016 a$. Chloroplast calcium and ROS signaling networks potentially facilitate the primed state for stomatal closure under multiple stresses. Environ. Exp. Bot. 122, 85-93. Available from: https://doi.org/ 10.1016/j.envexpbot.2015.09.008.

Wang, Y.-F., Zhang, A., Ren, H.-M., Tan, Y.-Q., Qi, G.-N., Yao, F.-Y., et al., 2016b. S-type anion channels SLAC1 and SLAH3 function as essential negative regulators of inward $\mathrm{K}^{+}$channels and stomatal opening in Arabidopsis. Plant Cell. 28, 949-965. Available from: https:// doi.org/10.1105/tpc.16.01050.

Wang, Y.Y., Mopper, S., Hasenstein, K.H., 2001. Effects of salinity on endogenous ABA, IAA, JA, and SA in Iris hexagona. J. Chem. Ecol. 27, 327-342. Available from: https://doi.org/10.1023/ A:1005632506230.

Wiese, A., Groner, F., Sonnewald, U., Deppner, H., Lerchl, J., Hebbeker, U., et al., 1999. Spinach hexokinase I is located in the outer envelope membrane of plastids. Febs Lett. 461, 13-18. Available from: https://doi.org/10.1016/S0014-5793(99)01417-9.

Wilkins, K.A., Matthus, E., Swarbreck, S.M., Davies, J.M., 2016. Calcium-mediated abiotic stress signaling in roots. Front. Plant Sci. 7, 1296. Available from: https://doi.org/10.3389/ fpls.2016.01296.

Xiong, L.M., Zhu, J.K., 2003. Regulation of abscisic acid biosynthesis. Plant Physiol. 133, 29-36. Available from: https://doi.org/ 10.1104/pp.103.025395.

Xu, J., Zhang, S.Q., 2015. Mitogen-activated protein kinase cascades in signaling plant growth and development. Trends Plant Sci. 20,
56-64. Available from: https://doi.org/10.1016/j. tplants.2014.10.001.

Yamaguchi, S., 2008. Gibberellin metabolism and its regulation. Annu. Rev. Plant Biol. 59, 225-251. Available from: https://doi. org/10.1146/annurev.arplant.59.032607.092804.

Yan, J., Tsuichihara, N., Etoh, T., Iwai, S., 2007. Reactive oxygen species and nitric oxide are involved in ABA inhibition of stomatal opening. Plant, Cell Environ. 30, 1320-1325. Available from: https://doi.org/10.1111/j.1365-3040.2007.01711.x.

Yang, T., Yao, S., Hao, L., Zhao, Y., Lu, W., Xiao, K., 2016. Wheat bHLH-type transcription factor gene TabHLH1 is crucial in mediating osmotic stresses tolerance through modulating largely the ABA-associated pathway. Plant Cell Rep 35, 2309-2323. Available from: https://doi.org/10.1007/s00299-016-2036-5.

Yi, C., Yao, K., Cai, S., Li, H., Zhou, J., Xia, X., et al., 2015. High atmospheric carbon dioxide-dependent alleviation of salt stress is linked to RESPIRATORY BURST OXIDASE 1 (RBOH1)-dependent $\mathrm{H} 2 \mathrm{O} 2$ production in tomato (Solanum lycopersicum). J. Exp. Bot. 66, 7391-7404. Available from: https://doi.org/10.1093/jxb/ erv435.

Yokoi, S., Quintero, F.J., Cubero, B., Ruiz, M.T., Bressan, R.A., Hasegawa, P.M., et al., 2002. Differential expression and function of Arabidopsis thaliana NHX Na$/ / \mathrm{H}^{+}$antiporters in the salt stress response. Plant J. 30, 529-539. Available from: https://doi.org/ 10.1046/j.1365-313X.2002.01309.x.

Yoshida, K., Igarashi, E., Wakatsuki, E., Miyamoto, K., Hirata, K., 2004. Mitigation of osmotic and salt stresses by abscisic acid through reduction of stress-derived oxidative damage in Chlamydomonas reinhardtii. Plant Sci. 167, 1335-1341. Available from: https://doi.org/10.1016/j.plantsci.2004.07.002.

Yoshida, T., Mogami, J., Yamaguchi-Shinozaki, K., 2014. ABAdependent and ABA-independent signaling in response to osmotic stress in plants. Curr. Opin. Plant Biol. 21, 133-139. Available from: https://doi.org/10.1016/j.pbi.2014.07.009.

Yuan, K., Rashotte, A.M., Wysocka-Diller, J.W., 2011. ABA and GA signaling pathways interact and regulate seed germination and seedling development under salt stress. Acta Physiol. Plant. 33, 261-271. Available from: https://doi.org/10.1007/s11738-0100542-6.

Zhang, T., Gong, H.M., Wen, X.G., Lu, C.M., 2010a. Salt stress induces a decrease in excitation energy transfer from phycobilisomes to photosystem II but an increase to photosystem I in the cyanobacterium Spirulina platensis. J. Plant Physiol. 167, 951-958. Available from: https://doi.org/10.1016/j. jplph.2009.12.020.

Zhang, Z.P., Yao, Q.H., Wang, L.J., 2010b. Expression of yeast Hem1 gene controlled by Arabidopsis HemA1 promoter improves salt tolerance in Arabidopsis plants. BMB Rep. 43, 330-336. Available from: https://doi.org/10.5483/ Bmbrep.2010.43.5.330.

Zhu, J.-K., 2002. Salt and drought stress signal transduction in plants. Annu. Rev. Plant Biol. 53, 247-273. Available from: https://doi. org/10.1146/annurev.arplant.53.091401.143329.

Zhu, J.K., 2001. Plant salt tolerance. Trends Plant Sci. 6, 66-71. Available from: https://doi.org/10.1016/S1360-1385(00)01838-0.

Zhu, L., Guo, J.S., Zhu, J., Zhou, C., 2014. Enhanced expression of EsWAX1 improves drought tolerance with increased accumulation of cuticular wax and ascorbic acid in transgenic Arabidopsis. Plant Physiol. Biochem. 75, 24-35. Available from: https://doi. org/10.1016/j.plaphy.2013.11.028. 


\title{
2 \\ Stress Responsive Signaling Molecules and Genes Under Stressful Environments in Plants
}

\author{
Murat Dikilitas ${ }^{1}$ Eray Simsek ${ }^{1}$ and Sema Karakas ${ }^{2}$
}

${ }^{1}$ Department of Plant Protection, Harran University, Şanlıurfa, Turkey ${ }^{2}$ Department of Soil Science and Plant Nutrition, Harran University, Şanlıurfa, Turkey

\section{O U T L I N E}

\subsection{Introduction}

2.2 Signaling Molecules Under Stress Conditions

2.3 Signaling Molecules and Plant Responses Under Combined Stress Conditions

2.4 DNA Damage of Plants Under Concurrent of Abiotic and Biotic Stress Combinations
2.5 Genomic and Biochemical Approaches for Plants Under Combined Stresses 34

2.6 Conclusions and Future Prospects 36

Acknowledgement 36

References $\quad 36$

\subsection{INTRODUCTION}

The changing climatic and environmental conditions and human-oriented pollution increase the possible occurrences of combined stresses of pathogens and abiotic stresses. Evidence suggests that abiotic stresses will affect the distribution and pathogenicity of quite a range of pathogens with increased virulent strain or race development (Garrett et al., 2006; Dikilitas and Karakas, 2012; Ramegowda and Senthil-Kumar, 2015). Although the possible interactions between biotic and abiotic agents in plants were analyzed in the past through the examination of stress parameters via the biochemical and molecular methods, the molecular and biochemical basis of combined interactions between abiotic-abiotic, and abiotic-biotic interactions remain elusive in terms of signaling molecules such as the production of nitric oxide (NO), reactive oxygen species (ROS), and accumulation of plant growth regulators such as salicylic acid (SA), ethylene (ET), jasmonic acid (JA), and abscisic acid (ABA) (Ramegowda et al., 2013; Khan et al., 2015; Khan and Khan 2017; Lai et al., 2018; Lalithya et al., 2017; Per et al., 2018; Zheng et al., 2018). When individual stress agents are examined, their low-level effect could increase the transmission of signaling molecules, which 
induce the defense responses. When the stress is prolonged and becomes severe, these molecules could act as prooxidants and become signals of diseases.

In nature, plants are exposed to the combination of abiotic and biotic stresses either simultaneously or sequentially. Although this type of stress has been recognized for many years, their molecular and biochemical pathways should be evaluated by producing new pathways. The interactions between stresses could be negative, positive, or additive. When plant faces both abiotic and biotic stress factors simultaneously or sequentially, their behaviors in terms of molecular, physiological, and biochemical pathways have potential to modify themselves to the changing conditions. The changing pattern of host plants to the stress could be slow or fast depending on the severity and duration of the stress factors as well as the resistance of crop plants. In most cases, the response of crop plants is slow and therefore, crop plants die quickly due to sudden depletion of biochemical metabolites that have already been prepared for only one type of stress in advance. The response of plants to the simultaneous or sequential actions of several combined stress factors is different from those of plants exposed to a single stress factor. Ramegowda and Senthil-Kumar (2015) stated that concurrent occurrence of drought and pathogen during plants' development provoked complex pathways controlled by various signaling events. Plants tolerating two or more independently occurring stresses need not necessarily tolerate when these stresses occur simultaneously or sequentially (Nostar et al., 2013; Dikilitas et al., 2017). The reaction of plants, in general, depends on the sensitivity of species or cultivars, the intensity of stress factors, duration of exposure, and the mode of action of stress agents on plant metabolism.

In abiotic and biotic stress combinations, the instant simultaneous effect is not very common. One of the stress agents, in general, develops gradually. For example, pathogens can infect plants that are already stressed with abiotic stress agents or abiotic stress gradually develops on already pathogen-infected plants. Under these circumstances, the outcome of these interactions varies depending on the severity of each stress (Xu et al., 2008; Dikilitas et al., 2017). Plants exposed to mild abiotic stress or low pathogenicity of pathogens activate their basal defense systems. On the other hand, severe abiotic stress causes membrane leakage, which fills up the apoplast with cellular nutrients and enhances the sporulation and development of attacking pathogens and as a result of that, it facilitates successful pathogen infection. The same case is also valid for the attacking of virulent pathogens. They could destroy the defense barrier of the host via secretion of cell wall degrading enzymes or toxins, which could stop the many functions of cell metabolites and predispose the plants to abiotic stressors (Dikilitas et al., 2018). For example, a significant positive correlation between spot blotch (caused by Cochliobolus sativus) disease severity in wheat genotypes and average nighttime temperature was evident (Sharma et al., 2007). Again, high-temperature stress in combination with Puccinia striiformis f. sp. tritici (causal agent of stripe rust) increased the disease incidence in spring wheat (Triticum aestivum) (Milus et al., 2009). They stated that temperature-adapted "new" isolates $\left(12-28^{\circ} \mathrm{C}\right)$ were more aggressive than "old" isolates adapted to cool temperatures $\left(10-18^{\circ} \mathrm{C}\right)$ for all cultivars treated. At low-temperature, "new" isolates sporulated earlier, grew faster, and produced more spores per lesion per day compared with those of "old" isolates. Similar patterns were also observed at hightemperature regime. The authors suggested that wheat rust fungi could cause severe disease in unfavorable conditions via adaptation to extreme temperatures. Similar issues were observed with those of salinity, high temperature, and drought stresses. For example, simultaneous exposure of common bean (Phaseolus vulgaris) to drought stress and a fungal pathogen, Macrophomina phaseolina (causal agent of charcoal rot and seedling blight), resulted in high transpiration rate and leaf temperature when compared with plants subjected to only drought stress (Mayek-Pérez et al., 2002). Again, exposure of tomato plants (Lycopersicon esculentum Mill.) to salinity stress either before or after inoculation with Pseudomonas syringae pv. tomato or Phytophthora capsici induced root and crown rot with severe symptoms when compared with nonstressed control or pathogen-inoculated plants (Pye et al., 2013; Bostock et al., 2014). Salt stress significantly reduced fresh weights and increased root and crown necrosis under pathogen infection. Similar findings were also made by Dikilitas (2003) who stated that even very low concentrations of $\mathrm{NaCl}(50 \mathrm{mmol} / \mathrm{L})$ along with the inoculation of wilt pathogen Verticillium albo-atrum on tomato plants resulted in more severe symptoms than those of $\mathrm{NaCl}(50 \mathrm{mmol} / \mathrm{L})$ salinity or $V$. alboatrum infection alone (Fig. 2.1). The pathogen was able to survive in a large range of $\mathrm{NaCl}$ concentrations (Fig. 2.2). Again, under salinity stress, the performance of cell wall degrading enzymes of Rhizoctonia solani and Sclerotium rolfsii and their pathogenicity varied profoundly (El-Abyad et al., 1997). The activities of cell wall degrading enzymes of $R$. solani decreased and those of $S$. rolfsii increased with the increase of salt concentrations when the cell wall material was used as a sole carbon source. Recently, Shankar et al. (2016) reported that the increased $\mathrm{Na}^{+}$uptake and accumulation by stressed plants resulted in increased root permeability in chickpea. The increased severity of plants 

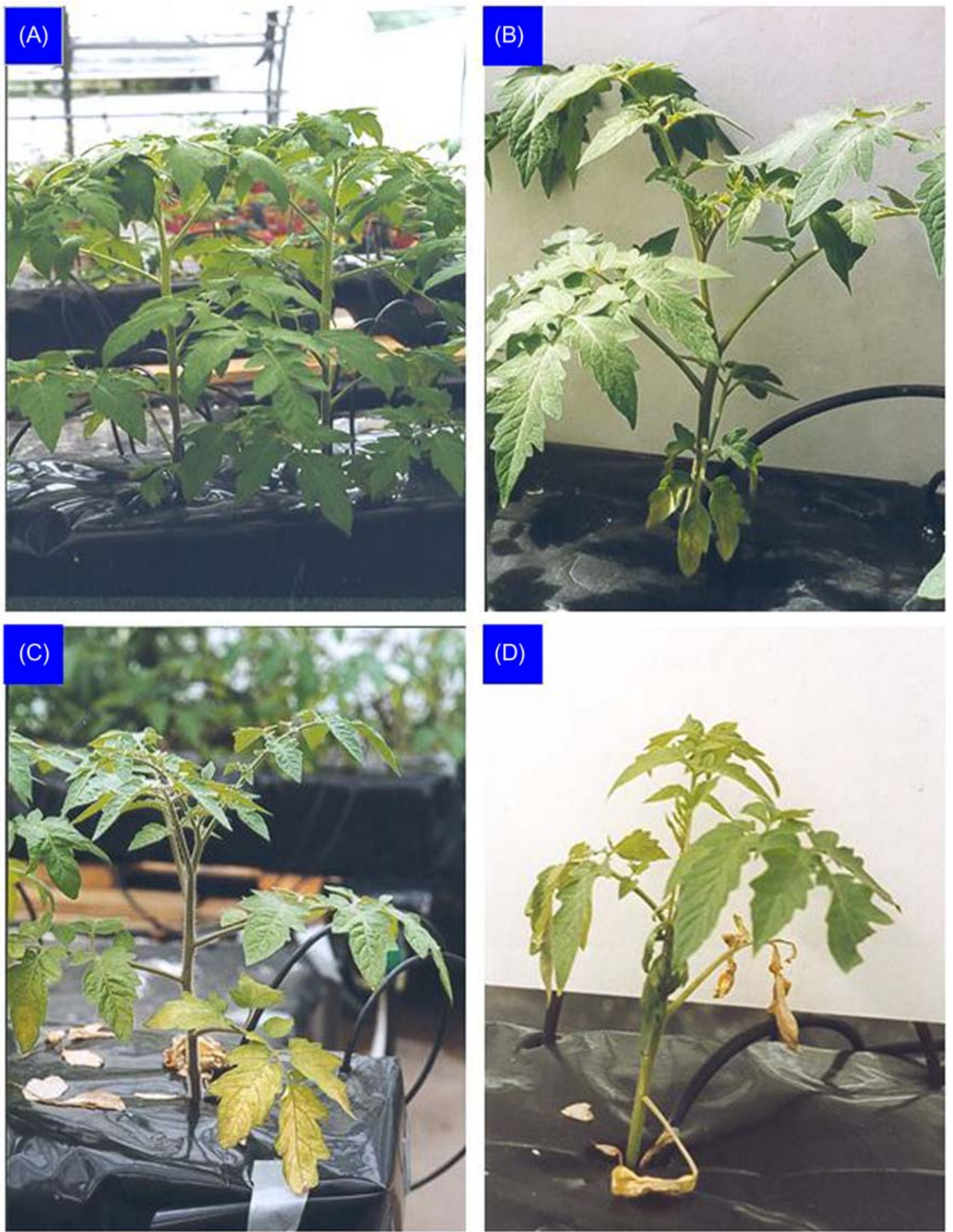

(D)

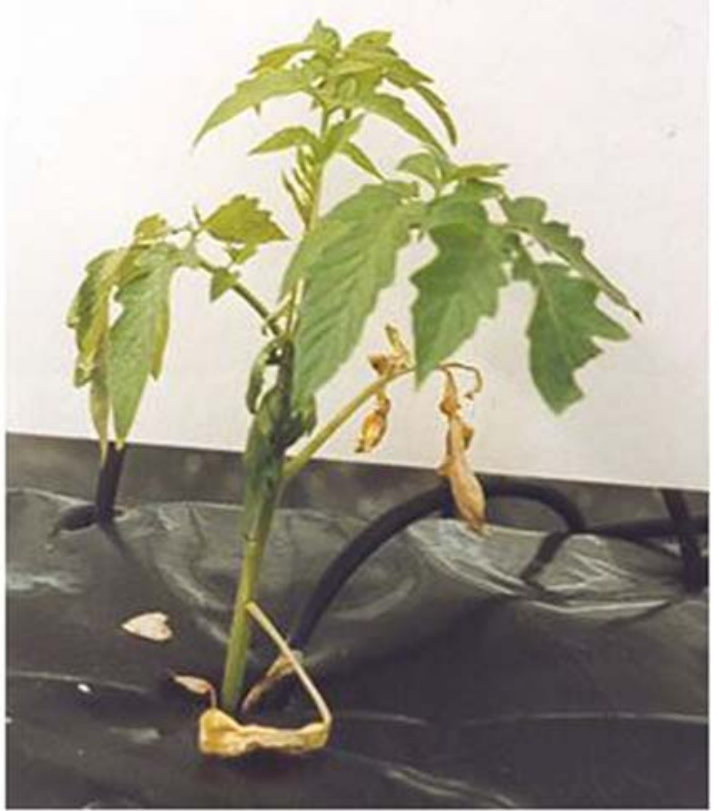

FIGURE 2.1 Tomato plants (A) control; (B) inoculated with Verticillium albo-atrum (isolate V2), growing under hydroponic conditions showing symptoms such as wilting and flaccidity on the lower leaves; (C) treated with $\mathrm{NaCl}$ showing wilting and chlorosis; and (D) inoculated with V. albo-atrum (isolate V2) and treated with $50 \mathrm{mmol} / \mathrm{L} \mathrm{NaCl}$ (Dikilitas, 2003).

under $\mathrm{NaCl}$ stress was also attributed to the stimulatory effect of $\mathrm{Na}^{+}$ions on mycelial growth of fungi as in the case of Botrytis cinerea (Boumaaza et al., 2015; HanumanthaRao et al., 2016). Similarly, Huot et al. (2017) reported that high-temperature stress promoted translocation of bacterial effector proteins into plant cells and caused a loss of ICS1-mediated SA biosynthesis. Therefore, the combined stress of temperature and pathogen prevented the SA-based defense mechanism. 


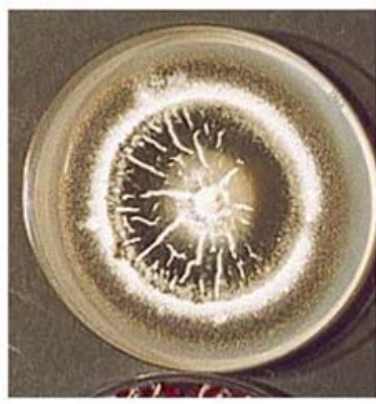

(A)

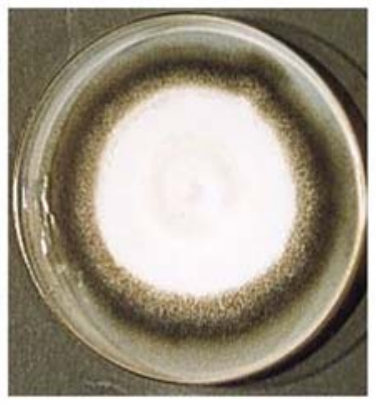

(E)

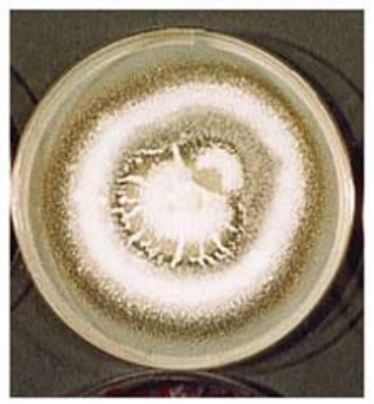

(B)

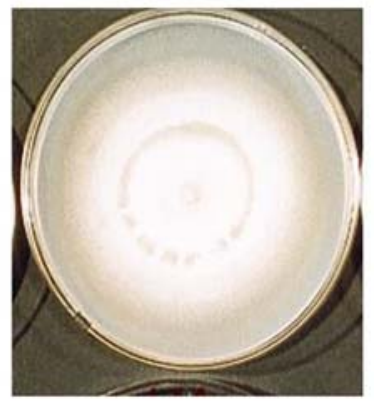

(F)

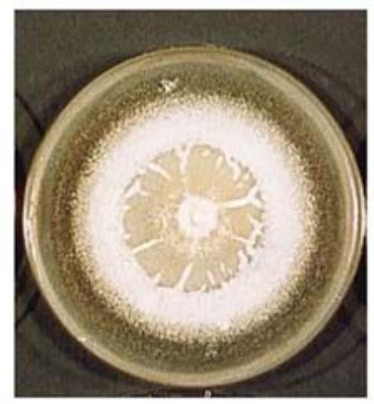

(C)

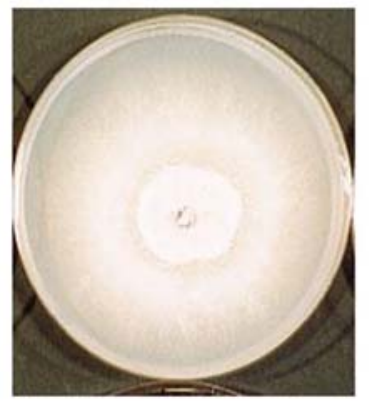

(G)

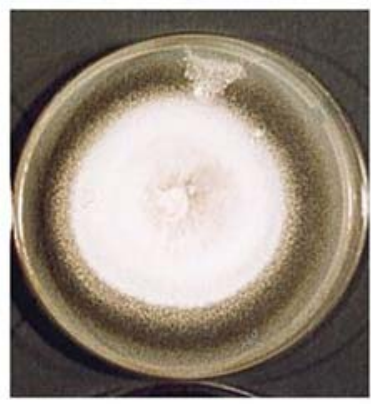

(D)

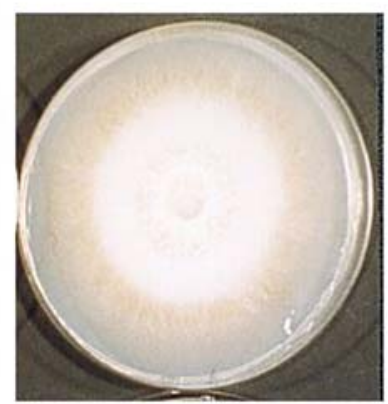

$(\mathrm{H})$

FIGURE 2.2 Development of mycelium of $V$. albo-atrum (isolate V1) on Dox medium containing different concentrations of NaCl. (A) Control, $0 \mathrm{mmol} / \mathrm{L} \mathrm{NaCl}$; (B) $25 \mathrm{mmol} / \mathrm{L}$; (C) $50 \mathrm{mmol} / \mathrm{L}$; (D) $100 \mathrm{mmol} / \mathrm{L}$; (E) $150 \mathrm{mmol} / \mathrm{L}$; (F) $250 \mathrm{mmol} / \mathrm{L}$; (G) $300 \mathrm{mmol} / \mathrm{L}$; and (H) $350 \mathrm{mmol} / \mathrm{L} \mathrm{NaCl}$ (Dikilitas, 2003).

To prevent the crop plants from secondary stress agents, scientists suggested some ideas related to plant nutrition such as application of $\mathrm{CaSO}_{4}$ or $\mathrm{K}_{2} \mathrm{SO}_{4}$ or plant growth substances, etc., which could have adverse effects on growing fungi in leaf surfaces and positive effects on salt tolerance of crop plants (Stockwell et al., 2012; Jabnoun-Khiareddine et al., 2016). However, under abiotic stress conditions such as salinity, drought, temperature, and heavy metal pollution stresses, plants tend to accumulate appreciable amounts of chloride, sulfate, and other toxic ions in tissues that limit the growth and yield potential as well as defense responses. By supplying beneficial substances to stressful plants could be a limited solution. The microorganisms could adapt themselves to the conditions of harsh environments. For example, Abbas and Mandeel (1995) isolated Fusarium oxysporum, F. sambucinum, F. clamidosporum, F. solani, F. moniliforme, etc., from the soil characterized with high salinity. Similar issues were made by Maggi et al. (2013) who stated that macrofungal community of some Italian alpine habitats, in which Inocybe microfastigiata, Laccaria Montana, and Lactarius salicis-herbaceae fungi were found adapted to very cold temperatures. Previously, Hasan (2002) reported that fungal species such as Aspergillus flavus, A. niger, F. oxysporum, Penicillium spp., Rhizopus spp. inhabiting in rhizosphere and rhizoplane of faba bean, sesame, soybean, etc. produced more gibberellin and indole-acetic acid (IAA) at $0.5 \%$ and $1 \% \mathrm{NaCl}$ after 5 days. Therefore, application of fertilizers or chemicals that help crop plants grow better in harsh conditions would have no efficient use due to the adaptation of pathogens. Since virulence was positively correlated with the activities of cell wall degrading enzymes exerted by the pathogens, it is important to note the level of pathogenic enzymes before or after the stress, especially after long-term stress, which could lead to increase of pathogenic enzymes.

Interactions between abiotic stress and virus diseases were evident as well. Simultaneous exposure of Arabidopsis plants to drought and turnip mosaic virus (TuMV) led to a higher reduction in plant weight and leaf numbers compared with those of individual stresses (Prasch and Sonnewald, 2013). Similarly, Moury et al. (1998) and Király et al. (2008) stated that the combined effects of high temperature and tomato spotted wilt virus or tobacco mosaic virus (TMV) suppressed the resistance of pepper (Capsicum annuum and tobacco (Nicotiana tabacum), respectively. Similar findings were also made by Dossa et al. (2017) who stated that plant height and dry shoot biomass of rice plants were remarkably reduced by drought stress treatments. On the other hand, the authors concluded that effect of drought stress response could vary according to rice 
genotype. It is possible that drought stress could interfere with accumulation of ABA over the plant defense response. For example, Mohr and Cahill (2003) stated that both additions of $100 \mu \mathrm{M}$ ABA to plants and drought stress resulted in increased susceptibility of Arabidopsis thaliana to an avirulent isolate of $P$. syringae pv. tomato. In contrast, an ABA-deficient mutant of Arabidopsis exhibited reduced susceptibility to virulent isolates of Peronospora parasitica or $P$. syringae pv. tomato. Similar issues were also made by Sherwood et al. (2015) in which the drought-induced proline accumulation and ROS metabolism invoked susceptibility toward Diplodia pinea in Austrian pine.

There is a synergistic relationship between frost and pathogen occurrences as well. Ferrante and Scortichini (2014) reported that autumn and winter frosts resulted in a sudden outbreak of bacterial canker caused by $P$. syringae pv. actinidiae in Actinidia chinensis and A. deliciosa trees. During thawing stages, bacterial colonization was observed at a 2-cm distance upwards and downwards from the site of inoculation within $3 \mathrm{~min}$ and the leaves were extensively colonized with the pathogen. It appears that the defense mechanisms of plants are weakened, and further damage caused by sequential stress agents result in quality and quantity loss at large. At this point, it is very difficult to assess the level of damage in advance due to changing mechanisms of plant cell response to multiple attacks.

There can also be an antagonistic effect among stressors that the effect of one stress factor is reduced while the effect of other stress factor stays the same as if acting alone. It should be kept in mind that two different stressors would not nullify each other, therefore, plants have to deal with at least one of the stress factors all the time. This antagonistic scenario gets further complicated by a combined stress occurrence. For example, salinity stress increased resistance of barley (Hordeum vulgare) plants to Blumeria graminis (causal agent of powdery mildew) in a concentration-dependent manner (Wiese et al., 2004). They stated that salinity stress exerted both osmotic and ion toxicity on the pathogen growth, which eventually reduced the pathogenicity. Similarly, Kissoudis et al. (2016) stated susceptibility of susceptible or partial resistant lines of tomato increased at mild salt stress $(50 \mathrm{mmol} / \mathrm{L} \mathrm{NaCl})$ toward Oidium neolycopersici (a causal agent of powdery mildew in tomato). However, $\mathrm{NaCl}$ stress over $150 \mathrm{mmol} / \mathrm{L}$ reduced the disease symptoms in resistant cultivars. The authors stated that $\mathrm{Na}^{+}$and $\mathrm{Cl}^{-}$accumulation in the leaves was linearly related to the decreased pathogen symptoms under combined stress. They concluded that increased susceptibility under combined stress was associated with the induction of ET and JA pathway genes. Similarly, drought stress reduced fungal pathogen, B. cinerea (causal agent of gray mold) infection by $50 \%$ in tomato plants and suppressed the spread of another fungal pathogen, $O$. neolycopersici (causal agent of powdery mildew) due to a concomitant increase in endogenous ABA levels (Achuo et al., 2006). Ramegowda et al. (2013) stated that drought acclimation in advance in Nicotiana benthamiana plants imparted tolerance to necrotrophic fungus, Sclerotinia sclerotiorum, and to the hemibiotrophic bacterial pathogen, P. syringae pv. tabaci. Acclimated plants with drought stress led to less disease-induced cell death and reduced disease symptoms compared with nonacclimated plants. They stated that the generation of ROS during drought acclimation resulted in increased disease resistance and primed a defense response that subsequently led to tolerance against pathogens. Ramegowda et al. (2013) also suggested that ABA could change pattern for the attacking pathogens via closing stomata or reduction photosynthesis. Therefore, early exposure of plants to drought stress results in the onset of increased ABA and ROS levels and induction of PR-protein coding genes and contributed to disease resistance under combined stress (Ramegowda and Senthil-Kumar, 2015).

As seen so far, the role of ROS and ABA has been controversial in plant defense systems. The susceptibility of plants could be affected by abiotic stress agents in both ways. Ramegowda and Senthil-Kumar (2015) stated that pathogen infection on already droughtstressed plants can either lead to plant resistance to pathogen infection through drought-induced activation of basal defense or can result in susceptibility due to weakened basal defense. Drought-induced pathogen resistance was attributed to the enhanced expression of antimicrobial and PR-proteins activated by drought. On the other hand, the susceptibility of crop plants was attributed to high levels of ABA under drought stress, which can interfere with pathogen-induced plant defense signaling and thereby reducing the expression of defense-related genes ( $\mathrm{Li}$ and Luan, 2014; Ali et al., 2017). For example, Ali et al. (2017) reported that pretreatment of Brassica juncea with SA and JA stimulators downregulated each other's signature genes suggesting an antagonistic relationship between SA and JA. They also reported that after ABA treatment, SA signatures were downregulated while JA signature genes were upregulated. ABA-SA interaction is two-sided. SA signaling via pathogen inoculation negatively affects ABA responses. However, ABA contributes to the formation of callose, which prevents pathogen penetration. On the other hand, ABA signaling mainly inhibits signals that activate biotic stress signaling. Therefore, combined stress enhances pathogen spread from the initial site of infection. Combined stress, if one of them is not detoxified, is able to affect the nucleus and its contents. Just like ROS, the 
concentration of ABA plays significant roles in resistance to disease. It can either prevent the pathogen infection through stomatal closure or make plants more susceptible to invading pathogens by suppressing JA, SA, or ET-mediated signaling. Here, it is important to note that the level of ROS or ABA should not exceed a certain level that would be characterized as toxic. For example, Gupta et al. (2016) stated that the sporulation of $P$. syringae pv. tomato under drought stress was reduced due to activation of basal defense response through ABA-mediated gene regulation in $A$. thaliana plants. This also reduced ROS generation and cell death and suppressed the effect of pathogen infection (Fujita et al., 2006). Sinha et al. (2016) stated that drought-stressed chickpea plants challenged with $P$. syringae pv. phaseolicola (leaf pathogen) and Ralstonia solanacearum (xylem inhabiting wilt pathogen) exhibited reduced bacterial colonization in chickpea plants as compared with pathogen infection alone. In a similar manner, proline, a stress-related amino acid, could be accumulated upon drought-, salinity- or heavy metal-exposed plants (Per et al., 2017). In this response, one of the stress agents, especially biotic ones, should have low or mild effects. On the other hand, nonvirulent or less virulent biotic stress agents act as elicitors and enable protection for plants to further abiotic stress agents. However, if the stress agents such as drought, salinity, heavy metal pollution, etc. have a continuous effect, then the antagonistic effect of the biotic stress agents would have no beneficial effects after some stage.

The antagonistic interactions were also found between virus and abiotic stress factors as well. $N$. benthamiana plants infected with brome mosaic virus (BMV), cucumber mosaic virus (CMV), and TMV showed delayed the appearance of leaf wilting and stem dehydration under the combination of drought and virus stress when compared with the only drought-stressed plants ( $\mathrm{Xu}$ et al., 2008). BMV- and CMV-inoculated plants exhibited increased accumulation of osmoprotectants such as glucose, fructose, and sucrose (Xu et al., 2008; Tauzin and Giardina, 2014). In addition, virus-infected plants showed lower transpiration rate due to the partial stomatal closure.

Antagonistic effects could be observed among abiotic stress factors as well. For example, mild stress factors could trigger physiological and biochemical defense mechanisms that enable plants to continue growth under stressful conditions. For example, Bandurska et al. (2013) reported that UV-B radiation enhanced the resistance of $A$. thaliana to water stress. They concluded that $\mathrm{H}_{2} \mathrm{O}_{2}, \mathrm{NO}, \mathrm{ABA}, \mathrm{JA}, \mathrm{ET}$, and SA participated in the activation of defense mechanisms. However, if the duration of stress is prolonged or one of them is severe, the stress tolerance cannot be achieved, leading to cell death and tissue necrosis. Similar results were also made by Majsec et al. (2016) who stated that toxic effects of cadmium alone were more prominent on tobacco than that of the combined stress of cadmium and copper. However, mixtures of high concentrations of both cadmium and copper had the most adverse effects. This was directly related to the increase of ROS, malondialdehyde (MDA), and protein carbonyl (PC) contents.

Sometimes antagonistic responses between stresses result in synergistic effects in crop plants. For example, during heat stress, plants open their stomata to cool down their leaves by transpiration. However, heat stress with a combination of drought would not allow plants to open their stomata and their leaf temperature would be higher resulting in more damaging effects on plants (Rizhsky et al., 2002; Mittler, 2006). It is known that the stomatal opening and closing organization is regulated by signaling molecules. However, the sequence of stress would determine the function of stomata. Lu et al. (2017) stated that the combination of $P$. capsici infection and heat caused more severe damages to pepper seedlings than either of the individual stresses. Under heat stress, plant leaves make more transpiration by opening their stomata to cool down the extra heat, however, this predisposes crop plants and makes them more susceptible to invading pathogens (Prasch and Sonnewald, 2015). If plants close their stomata to prevent pathogen infection and spread within leaf tissues, this increases the canopy temperature and leads to heat injury (Atkinson and Urwin, 2012). It is possible that overaccumulation of ROS may cause dysfunction of the cell membrane and results in susceptibility in susceptible and moderately resistant cultivars. When signaling molecules and gene expressions were tested, $C$. annuum heat shock protein (CaHSP) genes were upregulated in resistant (CM334) and susceptible (EC) varieties of pepper plants under stress alone. However, under heat stress, P. capsici infection, the gene expression increased in the resistant cultivar CM334 while decreasing in the susceptible cultivar EC. The defense-related genes were more upregulated under the combined stress than under the individual stresses. On the other hand, the $\mathrm{Ca}^{2+}$ signaling pathway genes were enhanced in susceptible cultivar "EC," they were inhibited in resistant cultivar "CM334." HSP genes were expressed during heat stress alone to alleviate the conditions of plants under heat stress, but these genes were stable under P. capsici inoculation alone in both the susceptible "EC" and resistant "CM334" cultivars (Bokszczanin et al., 2013; Lu et al., 2017). Similarly, pathogenesis-related (PR) proteins play significant roles to prevent further pathogen attack and improve the conditions of plants under pathogenic stresses (Sudisha et al., 2012). Under 
P. capsici inoculation alone, CaPO1 (peroxidase), CaPR1 (pathogenesis-related protein), CaSAR82A (systemic acquired resistance) genes were upregulated in resistant CM334 pepper cultivar, and the response was increased when $P$. capsici was combined with heat stress treatment. However, no significant expression in pathogenesis-related genes was noticed in the susceptible cultivar EC. Since a higher antioxidant capacity along with lower ROS accumulation is associated with plant tolerance/resistance to abiotic or biotic or to both stresses (Suzuki et al., 2014), cultivars that could not express signaling molecules and stress-related genes would not be able to survive under the combined stresses in the long run. Similarly, calcium plays important roles in mediating abiotic and biotic stress signals. Lu et al. (2017) stated that $\mathrm{Ca}^{2+}$-signalingrelated genes under heat stress were suppressed in susceptible pepper cultivar EC but enhanced in resistant cultivar CM334. However, this did not follow a clear pattern between abiotic and biotic stresses. Therefore, the molecular responses could not be predicted in advance by examining the responses of individual stresses.

In this chapter, interactions between abiotic and biotic stressors were evaluated and their combined stressful effects were outlined in crop plants in terms of signaling molecules, gene expression patterns and enzymatic and hormonal responses in the light of new molecular and biochemical findings and approaches. The mechanisms of interactions were briefly outlined in Table 2.1 before detailed evaluation.

\subsection{SIGNALING MOLECULES UNDER STRESS CONDITIONS}

When crop plants get exposed to stress factors, they respond by enhancing production of ROS, which eventually leads to protein-, lipid-, and carbohydrateoxidation and DNA damage (Ahmad et al., 2010). Although ROS have destructive effects on cell metabolism, they also act as signaling molecules in the cellular process. Low concentrations of signaling molecules participate in the activation of defense-related mechanisms involving enzymatic and nonenzymatic antioxidant systems, flavonoid biosynthesis, accumulation of low-molecular weight compounds, vitamin $\mathrm{C}$, anthocyanins, etc. (Liang et al., 2018). Therefore, the signal transduction pathways, in general, lead to activation of biosynthesis of proteins, osmoprotectants, and detoxification enzyme systems (Prasch and Sonnewald, 2015). However, under continuous stress or severe short-term stress, the efficiency of the antioxidant system decreases, and the capacity of the defense system becomes exhausted leading to membrane damage and even cell death (Bandurska et al., 2013). Plants have developed effective stress detection mechanisms and efficient signal transduction pathways to respond to various pathological and environmental stresses quickly (Petrov et al., 2015; Hoque et al., 2016). The signaling molecules include various plant hormones such as SA, JA and ET, ABA, auxin, gibberellin (GA), cytokinin (CK) and brassinosteroid (BL), ROS, lipid, mRNA accumulation, vitamins, ion homeostasis, proteins, sugar, $\mathrm{NO}, \mathrm{Ca}^{2+}$ signaling, etc. They play crucial roles in regulating developmental processes and signaling networks in a coordinated way in a wide range of biotic and abiotic stresses (Robert-Seilaniantz et al., 2007; Baxter et al., 2014; Prasch and Sonnewald, 2015). Signal transduction is the metabolism in which extracellular physiological stimuli are transmitted via signaling cascade into intracellular signals that involve multiple genes/proteins to regulate the expression patterns of the key genes. When these are orchestrated properly, a quick response will be triggered to protect plants from further damages. Signal transduction starts from the receptor activation and simply involve secondary messengers, transcription factors, stressresponsive genes, phosphoprotein cascades (Gong et al., 2013). Therefore, all signaling molecules take part in the regulation of the defense and growth mechanisms. Many scientists have reported that the plasma membrane $(\mathrm{PM})$ is responsible for perceiving and transmitting external stress signals (Gong et al., 2013). Sensors located in membranes perceive the signals. For example, phytohormones magnify the initial signals and trigger a new signaling event either following the same pathway or using other signaling pathways with different components (Huang et al., 2012; Prasch and Sonnewald, 2015). For example, leaf stomata or leaf surfaces are closed under drought stress and cell osmotic potential decreases. As a result, photosynthesis is reduced. Plants, therefore, must constantly adjust stomatal conductance to allow enough $\mathrm{CO}_{2}$ uptake and avoid unnecessary water loss during water stress. In another way, plants must always sense the water stress. ABA, a phytohormone, plays important roles in stress signaling such as transcriptional changes, stomatal closure, etc. (Sreenivasulu et al., 2012; Dar et al., 2017). The accumulation of ABA in roots is one of the fastest responses to water or drought stress. It is loaded to xylem vessels and transported to the leaf cells via the transpiration stream. This hormone plays a crucial role in regulating the expression of many stress-related genes. Crop plants under stress synthesize proteins, enzymes, and nonenzymatic metabolites such as ascorbic acid (vitamin C), glutathione, proline, etc. to remediate the harmful effects of the stresses. All these responses are triggered by ABA-dependent and ABA-independent 
TABLE 2.1 Interactions Between Abiotic-Abiotic; Abiotic-Biotic Stress Agents

\begin{tabular}{|c|c|c|c|c|c|c|}
\hline \multirow[b]{2}{*}{$\begin{array}{l}\text { Stress } \\
\text { interactions }\end{array}$} & \multicolumn{2}{|c|}{ Biotic stress } & \multirow[b]{2}{*}{$\begin{array}{l}\text { Abiotic } \\
\text { stress }\end{array}$} & \multirow[b]{2}{*}{ Host plant(s) } & \multirow[b]{2}{*}{$\begin{array}{l}\text { Physiological, biochemical, } \\
\text { and molecular response }\end{array}$} & \multirow[b]{2}{*}{ References } \\
\hline & Causal agent & $\begin{array}{l}\text { Disease } \\
\text { name }\end{array}$ & & & & \\
\hline \multirow[t]{9}{*}{$\begin{array}{l}\text { Biotic \& Abiotic } \\
\text { stress } \\
\text { combinations } \\
\text { (synergistic or } \\
\text { additive) }\end{array}$} & $\begin{array}{l}\text { Cochliobolus } \\
\text { sativus }\end{array}$ & $\begin{array}{l}\text { A causal } \\
\text { agent of Spot } \\
\text { blotch } \\
\text { disease }\end{array}$ & Temperature & Wheat & Increased disease symptoms & $\begin{array}{l}\text { Sharma et al. } \\
\text { (2007) }\end{array}$ \\
\hline & $\begin{array}{l}\text { Puccinia } \\
\text { striiformis f.sp. } \\
\text { tritici }\end{array}$ & $\begin{array}{l}\text { A causal } \\
\text { agent of } \\
\text { Stripe rust } \\
\text { disease }\end{array}$ & Temperature & Wheat & Increased disease symptoms & $\begin{array}{l}\text { Milus et al. } \\
(2009)\end{array}$ \\
\hline & $\begin{array}{l}\text { Macrophomina } \\
\text { phaseolina }\end{array}$ & $\begin{array}{l}\text { Charcoal rot } \\
\text { and seedling } \\
\text { blight }\end{array}$ & Drought & $\begin{array}{l}\text { Phaseolus } \\
\text { vulgaris }\end{array}$ & Higher transpiration rate & $\begin{array}{l}\text { Mayek-Pérez } \\
\text { et al. (2002) }\end{array}$ \\
\hline & $\begin{array}{l}\text { Phytophthora } \\
\text { parasitica }\end{array}$ & $\begin{array}{l}\text { Root and } \\
\text { crown rot } \\
\text { disease }\end{array}$ & Salinity & Tomato & Severe root rot symptoms & $\begin{array}{l}\text { Swiecki and } \\
\text { MacDonald } \\
\text { (1991) }\end{array}$ \\
\hline & $\begin{array}{l}\text { Verticillium albo- } \\
\text { atrum }\end{array}$ & Wilt disease & Salinity & Vegetables & Increased wilting and root rots & Dikilitas (2003) \\
\hline & $\begin{array}{l}\text { Turnip mosaic } \\
\text { virus }\end{array}$ & $\begin{array}{l}\text { Mosaic } \\
\text { disease }\end{array}$ & Drought & $\begin{array}{l}\text { Arabidopsis } \\
\text { thaliana }\end{array}$ & Higher reduction in plant weight & $\begin{array}{l}\text { Prasch and } \\
\text { Sonnewald } \\
\text { (2013) }\end{array}$ \\
\hline & Rhizoctonia solani & $\begin{array}{l}\text { Root rot } \\
\text { disease }\end{array}$ & Salinity & Sugarbeet & $\begin{array}{l}\text { Synthesis of cell wall degrading enzymes } \\
\text { decreased (salinity caused an } \\
\text { antagonistic effect on the fungus) }\end{array}$ & $\begin{array}{l}\text { El-Abyad et al. } \\
\text { (1997) }\end{array}$ \\
\hline & Blumeria graminis & $\begin{array}{l}\text { Powdery } \\
\text { mildew }\end{array}$ & Salinity & Barley & Salinity caused antagonistic activity & $\begin{array}{l}\text { Wiese et al. } \\
(2004)\end{array}$ \\
\hline & $\begin{array}{l}\text { Botrytis cinerea, } \\
\text { Oidium } \\
\text { neolycopersici }\end{array}$ & $\begin{array}{l}\text { Gray mold, } \\
\text { Powdery } \\
\text { mildew }\end{array}$ & Drought & Tomato & $\begin{array}{l}\text { Infection and the growth of fungal } \\
\text { pathogens were suppressed by drought }\end{array}$ & $\begin{array}{l}\text { Achuo et al. } \\
\text { (2006) }\end{array}$ \\
\hline \multirow[t]{6}{*}{$\begin{array}{l}\text { Biotic and abiotic } \\
\text { stress } \\
\text { combinations } \\
\text { (antagonistic) }\end{array}$} & $\begin{array}{l}\text { Oidium } \\
\text { neolycopersici }\end{array}$ & $\begin{array}{l}\text { A causal } \\
\text { agent of } \\
\text { powdery } \\
\text { mildew in } \\
\text { tomato }\end{array}$ & Salinity & Tomato & $\begin{array}{l}\text { Accumulation of } \mathrm{Na}^{+} \text {and } \mathrm{Cl}^{-} \text {ions in the } \\
\text { leaves was linearly related to the } \\
\text { decreased pathogen symptoms under } \\
\text { combined stress }\end{array}$ & $\begin{array}{l}\text { Kissoudis et al. } \\
\text { (2016) }\end{array}$ \\
\hline & Sclerotium rolfsii & $\begin{array}{l}\text { Root rot } \\
\text { disease }\end{array}$ & Salinity & Sugarbeet & $\begin{array}{l}\text { Synthesis of cell wall degrading enzymes } \\
\text { increased (Salinity caused a synergistic } \\
\text { effect on the fungus) }\end{array}$ & $\begin{array}{l}\text { El-Abyad et al. } \\
\text { (1997) }\end{array}$ \\
\hline & Botrytis cinerea & $\begin{array}{l}\text { Gray mold } \\
\text { disease }\end{array}$ & Salinity & Tomato & $\begin{array}{l}\text { Increased } \mathrm{Na}^{+} \text {ion uptake stimulated the } \\
\text { growth of fungus (salinity caused a } \\
\text { synergistic effect on the fungus) }\end{array}$ & $\begin{array}{l}\text { Boumaaza } \\
\text { et al. (2015) }\end{array}$ \\
\hline & $\begin{array}{l}\text { Tomato spotted } \\
\text { wilt virus, Tobacco } \\
\text { mosaic virus }\end{array}$ & $\begin{array}{l}\text { Mosaic } \\
\text { disease, } \\
\text { Spotted wilt } \\
\text { disease }\end{array}$ & Temperature & $\begin{array}{l}\text { Pepper and } \\
\text { tobacco }\end{array}$ & $\begin{array}{l}\text { Suppressed the resistance of plant, } \\
\text { synergistic effect }\end{array}$ & $\begin{array}{l}\text { Király et al. } \\
(2008)\end{array}$ \\
\hline & $\begin{array}{l}\text { Pseudomonas } \\
\text { syringae pv. } \\
\text { actinidiae }\end{array}$ & $\begin{array}{l}\text { Kiwifruit } \\
\text { canker } \\
\text { disease }\end{array}$ & Frost & $\begin{array}{l}\text { Actinidia } \\
\text { chinensis, } A . \\
\text { deliciosa }\end{array}$ & $\begin{array}{l}\text { Bacterial colonization increased during } \\
\text { thawing, synergistic effect on the } \\
\text { pathogen }\end{array}$ & $\begin{array}{l}\text { Ferrante and } \\
\text { Scortichini } \\
(2014)\end{array}$ \\
\hline & & $\begin{array}{l}\text { Mosaic } \\
\text { disease }\end{array}$ & Drought & Rice, beet & & Xu et al. (2008) \\
\hline
\end{tabular}


TABLE 2.1 (Continued)

\begin{tabular}{|c|c|c|c|c|c|c|}
\hline \multirow[b]{2}{*}{$\begin{array}{l}\text { Stress } \\
\text { interactions }\end{array}$} & \multicolumn{2}{|c|}{ Biotic stress } & \multirow[b]{2}{*}{$\begin{array}{l}\text { Abiotic } \\
\text { stress }\end{array}$} & \multirow[b]{2}{*}{ Host plant(s) } & \multirow[b]{2}{*}{$\begin{array}{l}\text { Physiological, biochemical, } \\
\text { and molecular response }\end{array}$} & \multirow[b]{2}{*}{ References } \\
\hline & Causal agent & $\begin{array}{l}\text { Disease } \\
\text { name }\end{array}$ & & & & \\
\hline & $\begin{array}{l}\text { Brome mosaic } \\
\text { virus Cucumber } \\
\text { mosaic virus }\end{array}$ & & & & $\begin{array}{l}\text { Drought caused an antagonistic effect on } \\
\text { pathogens with delayed disease } \\
\text { symptoms }\end{array}$ & \\
\hline & Blumeria graminis & $\begin{array}{l}\text { Powdery } \\
\text { mildew }\end{array}$ & Ozone & Wheat & $\begin{array}{l}\text { Ozone cause some adverse effects } \\
\text { including necrosis and chlorosis in } \\
\text { plants, however, it can trigger different } \\
\text { defense mechanisms against powdery } \\
\text { mildew }\end{array}$ & $\begin{array}{l}\text { Pazarlar et al. } \\
\text { (2017) }\end{array}$ \\
\hline $\begin{array}{l}\text { Abiotic and } \\
\text { abiotic stress } \\
\text { combinations } \\
\text { (synergistic) }\end{array}$ & - & - & $\begin{array}{l}\text { UV-B } \\
\text { radiation } \\
\text { and water } \\
\text { stress }\end{array}$ & $\begin{array}{l}\text { Arabidopsis } \\
\text { thaliana }\end{array}$ & $\begin{array}{l}\text { UV-B radiation caused a synergistic } \\
\text { effect for Arabidopsis sp. with enhanced } \\
\text { resistance to water stress }\end{array}$ & $\begin{array}{l}\text { Bandurska } \\
\text { et al. (2013) }\end{array}$ \\
\hline \multirow{3}{*}{$\begin{array}{l}\text { Abiotic stress } \\
\text { causing pathogen } \\
\text { adaptation }\end{array}$} & $\begin{array}{l}\text { Fusarium } \\
\text { oxysporum }\end{array}$ & Wilt disease & Salinity & - & Saline-adapted Fusarium oxysporum & $\begin{array}{l}\text { Abbas and } \\
\text { Mandeel (1995) }\end{array}$ \\
\hline & Laccaria montana & - & Cold & - & Cold-adapted Laccaria montana & $\begin{array}{l}\text { Maggi et al. } \\
(2013)\end{array}$ \\
\hline & $\begin{array}{l}\text { Penicillium spp. } \\
\text { Aspergillus niger } \\
\text { Fusarium } \\
\text { oxysporum }\end{array}$ & $\begin{array}{l}\text { Plant-fungal } \\
\text { disease }\end{array}$ & Salinity & $\begin{array}{l}\text { Vicia faba, } \\
\text { Corchorus } \\
\text { olitorius, } \\
\text { Sesamum } \\
\text { indicum }\end{array}$ & $\begin{array}{l}\text { Saline-adapted fungus species produced } \\
\text { high gibberellic indol acetic acid (IAA) at } \\
\text { moderate or high } \mathrm{NaCl} \text { levels }\end{array}$ & Hasan (2002) \\
\hline \multirow[t]{4}{*}{$\begin{array}{l}\text { Abiotic stress } \\
\text { agents }\end{array}$} & - & - & Heat & Wheat & $\begin{array}{l}\text { Heat stress significantly reduced seed } \\
\text { germination and seedling growth, plant } \\
\text { water-use efficiency }\end{array}$ & $\begin{array}{l}\text { Akter and } \\
\text { Islam (2017) }\end{array}$ \\
\hline & - & - & Salinity & Wheat & $\begin{array}{l}\text { Salinity stress caused oxidative stress } \\
\text { resulting in enhancement of lipid } \\
\text { peroxidation }\end{array}$ & $\begin{array}{l}\text { Ahanger and } \\
\text { Agarwal (2017) }\end{array}$ \\
\hline & - & - & Cold & Wheat & $\begin{array}{l}\text { Cold stress caused flower abortion, } \\
\text { pollen and ovule infertility, and affects } \\
\text { seed filling, leading to low seed set and } \\
\text { ultimately low grain yield }\end{array}$ & $\begin{array}{l}\text { Thakur et al. } \\
\text { (2010) }\end{array}$ \\
\hline & - & - & Heavy metal & Wheat & $\begin{array}{l}\text { Heavy metal stress caused inhibition of } \\
\text { root growth, enzyme inactivation, and } \\
\text { plant death }\end{array}$ & $\begin{array}{l}\text { Karataglis et al. } \\
\text { (1991) }\end{array}$ \\
\hline
\end{tabular}

transduction pathways (Shinozaki and YamaguchiShinozaki, 2007). ABA stimulates the activity of NADPH oxidase and $\mathrm{H}_{2} \mathrm{O}_{2}$ generation to enhance the production of $\mathrm{NO}$ that triggers the defense mechanism via synthesis of ET, which leads to stomatal closure (He et al., 2011).

It is important that signaling molecules should be at low concentrations. For example, under low UV-B conditions, the ROS level is controlled through a specific signaling pathway involving UVR8, COP1, and HY5/ HYP transcription factors triggering activation of a gene involved in antioxidant defense system (Bandurska et al., 2013; Rejeb et al., 2014). However, under high UV-B conditions, this may not be achieved due to insufficient antioxidant scavenging capacities after signaling mechanism and gene expression pathway, because overproduction of ROS causes damage to organelles and DNA and disrupts the membrane structure and downregulates the defense-related genes (Brosché and Strid, 2003; Jenkins, 2009). Generation of ROS differs from one stress to another. The sense and expression of ROS are different in abiotic and biotic stress conditions. ROS induces tolerance or resistance by activating stress-related mitogen-activated protein kinases (MAPKs), transcription factors, antioxidant enzymes, and stress-related and pathogen-related proteins (Rejeb et al., 2014). ROS were initially considered as a byproduct of cell metabolism, however, they are 
now regarded as triggering molecules of signal transduction. This means that cells use ROS as biological stimuli and signals to activate and regulate various genetic stress response processes (Gong et al., 2013). ROS contain both free radical $\left({ }^{\circ} \mathrm{O}^{-}{ }_{2}, \mathrm{RO}^{\bullet}, \mathrm{HO}_{2}{ }^{\circ}, \mathrm{OH}^{*}\right)$ and nonradical forms $\left(\mathrm{H}_{2} \mathrm{O}_{2},{ }^{\circ} \mathrm{O}_{2}\right)$. They are highly reactive toxic and harmful byproducts of normal cellular metabolism that damage proteins, lipids, carbohydrates, DNA and cause cell death eventually. ROS are produced at various parts of plants such as chloroplasts, mitochondria, plasma membranes, peroximoses, apoplasts, endoplasmic reticulum and at cell walls in both unstressed and stressed cells (Sharma et al., 2012). Although $\mathrm{H}_{2} \mathrm{O}_{2}$ was recognized as a toxicant, recently, it has been regarded as a signaling molecule and a regulator of the expression of some genes encoding antioxidants and defense proteins and signaling proteins such as kinase and phosphatase (Shu-Hsien et al., 2005).

Under stress, production of ROS is balanced with the production of enzymatic and nonenzymatic antioxidants. However, this equilibrium is perturbed by abiotic and biotic stressors such as salinity, drought, heavy metals, UV light, pathogens, etc. Once the balance is disturbed, various signals are preceded via different pathways to level the imbalance and to protect cells from extra ROS. Whether ROS would act as signaling molecules or could lead to oxidative damage to the tissues or organs, this totally depends on the fine equilibrium between ROS production and their scavenging capacity (Sharma et al., 2012). It has been estimated that $1 \%$ of $\mathrm{O}_{2}$ consumed by plants is diverted to produce ROS (Asada and Takahashi, 1987). From them, oxygen radicals and $\mathrm{H}_{2} \mathrm{O}_{2}$ are produced. $\mathrm{H}_{2} \mathrm{O}_{2}$ is moderately reactive and relatively long-lived molecule. It can readily cross biological membranes and cause cellular damage far from the site of its formation (Torres et al., 2002). At low concentration, $\mathrm{H}_{2} \mathrm{O}_{2}$ can regulate biological processes and trigger tolerance against various environmental and biotic stresses. At high concentration, it can oxidize the cysteine or methionine residues and inactivate enzymes through oxidation of their thiol groups (Halliwell and Gutteridge, 2015).

Reactive nitrogen species (RNS) also play a significant role in signaling. $\mathrm{NO}, \mathrm{NO}_{2}$, as well as nonradical nitrous acid $\left(\mathrm{HNO}_{2}\right)$, act as signaling molecules to trigger different essential pathways involved in plant-stress interactions (Hossain et al., 2018). These molecules are present at very low concentrations at every developmental stage of plants. During stress development, NO quickly diffuses across the plant membranes due to its lipophilic properties (Vaishnav et al., 2018). The NO is mainly produced from agricultural soils. Soil nitrogen contents, $\mathrm{pH}$ level, temperature, and moisture content affect the production of NO. Increase in concentrations of NO plays a crucial role in neutralizing ROS by directly interacting with them or inducing an antioxidant enzyme activity. Therefore, application of NO thorough sodium nitroprusside (SNP) enhances plants resistance against stressors by activating the antioxidant system and plasma membrane transporter, which leads to decrease in ROS accumulation (Fu et al., 2015). As a signaling molecule, $\mathrm{NO}$ is able to change the metabolic activities of plants, bacteria, and fungi. Therefore, it would be appropriate to employ NO-producing plants or microorganisms in polluted soils for sustainable agriculture practices. However, the signaling functions of $\mathrm{NO}$ should be evaluated in detail under considered stress conditions, because combined stress may not allow NO to function as a signaling molecule by decreasing the concentration of it.

Methylglyoxal (MG) has been noticed as an emerging signaling molecule recently in plant stress responses and tolerance (Hoque et al., 2016). It is produced as a byproduct of a number of metabolic reactions. Under natural growth conditions, MG remains low in plants. However, under stress conditions, it can be accumulated in much higher levels. When it reaches high levels, it functions as a toxic molecule and it inhibits growth and development including germination, root growth, photosynthesis, etc. At low levels, like other oxidants, it acts as a signaling molecule (Li, 2016). It regulates many physiological and biochemical events such as cell proliferation and survival, cell homeostasis, and physiological and biochemical functions. It can also modulate stress responses by adjusting stomatal opening and closure, the production of ROS, cytosolic calcium ion concentrations and the expression of many stress-signal transduction and functions for promising adaptation of plants growing under stress conditions. To explore the role of MG in detail, it is important to find out its relationships with other signaling molecules, that is, whether it has synergistic or antagonistic pathways with them. Since this signaling molecule is new, it would be appropriate to find its role under the combined stress conditions to sustain crop plants.

Calcium is one of the important secondary messengers. Calcium ions regulate ion homeostasis thereby enhancing stress tolerance (Huda et al., 2013). It acts as a crucial signaling molecule in both abiotic and biotic stress tolerance. Calcium is bound to different organic molecules including proteins and lipids. In plant cell, calcium ions are placed in endoplasmic reticulum, vacuoles, plastids, and mitochondria and internal concentrations of calcium levels are maintained by various channels, pumps, and transporters (Dixit and Jayabaskaran, 2014). It regulates gene expression and 
defense mechanism. For example, Goswami et al. (2015) reported that exogenous $\mathrm{Ca}^{2+}$ application showed negative correlation with lipid peroxidation and positive correlation with total antioxidant capacity of the cell system under the elevated temperature stress. They also reported that efflux of $\mathrm{Ca}^{2+}$ triggered the activities of kinase and peroxidase enzymes in tolerant $T$. aestivum plants as compared with those of susceptible cultivars.

MAPK cascades are highly conserved signaling modules that transduce extracellular stimuli into intracellular responses (Bitrián et al., 2012). Plant MAPK cascades play important roles in signaling defense mechanism against pathogen attack. Activation of MAPKs is one of the earliest signaling events after sensing pathogen effectors. MAPK is also involved in signaling of multiple defense responses including plant hormones, ROS generation, stomatal closure, gene expression, phytoalexin synthesis, cell wall strengthening. However, pathogens are able to produce effector proteins to suppress plant MAPK activation and downstream defense responses to promote pathogenesis (Bitrián et al., 2012).

Another signaling molecule is lipid and its derivatives. They are one of the major constituents of biological membranes that can sense the stress at first stage. Lipids provide the structural basis for cell membranes and provide energy for cell metabolism. Lyophospholipid, fatty acid, phosphatidic acid, inositol phosphate, and $\mathrm{N}$-acylethanolamine have been proposed to function as signaling lipids (Okazaki and Saito, 2014). Studies have demonstrated that each lipid has class-specific signaling cascades, which activate defense reactions. Lipids can also function as stress mitigators to reduce the intensity of stressors. The increased amount of synthesis of lipids has been noticed under stress conditions (Okazaki and Saito, 2014). The accumulation of oligogalactolipids has recently been found to mitigate freezing and nutritiondepletion stresses. Signaling lipid molecules are usually present in small quantities in tissues and very quickly synthesized from preexisting membrane lipids (Gill and Tuteja, 2010; Markham et al., 2013).

Stress treatment also affected sugar metabolism and sugar signaling (Baena-González and Sheen, 2008). In general, apoplastic accumulation of hexoses is shifted during stress. This leads to increase in sugar concentration to initiate the defense response. In most plant stress relations, a high level of sugars in plant tissues enhances plant resistance. Sugars constitute the primary substrate and provide energy and structural material for defense responses in plants. On the other hand, they also act as signal molecules interacting with the hormonal signaling network, which regulates the prime immune system (Morkunas and Ratajczak, 2014). Sugars enhance oxidative burst at early stages of infection, increase lignification of cell walls, stimulate the synthesis of flavonoids, and induce PR protein. Like other signaling molecules, sugars at low concentrations play important roles for defense mechanisms. They regulate cellular activity at multiple levels, from transcription and translation to protein stability and activity (Rolland et al., 2006). Hexokinase is the bestinvestigated glucose sensor, while this protein also serves in the enzymatic processes, and catalyzes the first step of glycolysis (Smeekens et al., 2010). Apart from glucose, sucrose also functions as a signaling molecule (Wind et al., 2010). It enhances the expression of anthocyanin biosynthesis genes. Control of sugar and energy metabolism in cells is a highly important plant defense mechanism. It should be remembered that in combined stresses, higher accumulation of sugar prior to pathogen attack has a significant role for increasing the virulence and spread of pathogens since sugars are good sources of carbon for attacking pathogens. Santino et al. (2013) showed that phytohormones played a key role in plant defense as well as in plant development. They function as signaling molecules, that is, ET, JA, ABA, SA, etc.

\subsection{SIGNALING MOLECULES AND PLANT RESPONSES UNDER COMBINED STRESS CONDITIONS}

Plants have to respond and cope with various abiotic or biotic stress all the time. To survive in stressed conditions, plants must recognize the stress and rapidly exert multiple defense reactions. Their responses depend on the speed and ability to sense the stress. So far, plant molecular and biochemical responses have been evaluated considering single stress factors. For example, drought, soil flooding, high or low temperatures, pathogens, insects, etc., have now been in interaction with each other more than ever due to changes in climate and environmental conditions. Recently, biochemical or molecular responses of crop plants have been elucidated more commonly in combined interactions (Bonnet et al., 2017). These interactions could be between abiotic and biotic stressors or between abiotic and abiotic or between biotic and biotic stressors. However, interactions between abiotic and abiotic or abiotic and biotic stressors have now been noticed more due to changes in global temperature and environmental pollution (Guerret et al., 2016). These interactions have been modulated by evaluating a complicated network of signaling pathways examining $\mathrm{Ca}^{2+}$ signaling (Seybold et al., 2014; Nguyen et al., 2016), ROS and RNS (Wang et al., 2013; Baxter et al., 2014), and phytohormones (De Vleesschauwer et al., 2014; Kazan, 2015). Plants are often simultaneously or sequentially 
exposed to multiple biotic and abiotic stresses that result in substantial yield loss. Simultaneous occurrence of an abiotic stress with a biotic stress either aggravates or inhibits the effect of the latter, which leads to either enhanced or reduced susceptibility to pathogens. Thus, abiotic stress can change plant behavior in terms of tolerance or susceptibility toward pathogens by different mechanisms. Signaling molecules have been evaluated under single abiotic or biotic stress factors. However, the pathway of these molecules is different at combined stress conditions from those of single stress conditions, Fig. 2.3. The outcome of interactions between biotic and abiotic stress signaling is not easy to predict by evaluating the signaling pathways in single stress responses. More experimental outputs are needed not only in model plants like $A$. thaliana but

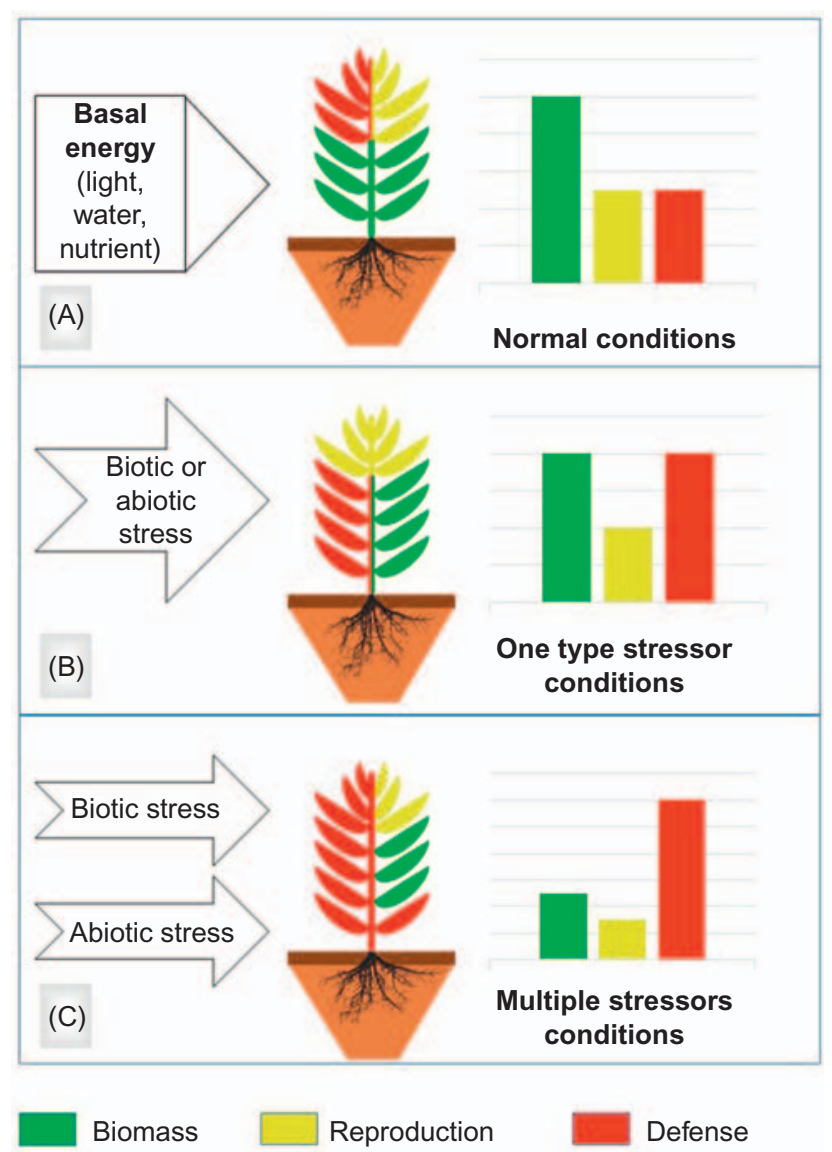

FIGURE 2.3 Energy and metabolic levels of plants under single or multiple stress conditions. The interactions between abiotic and biotic stress agents are not straightforward. Under one type of stress condition, a great amount of energy is diverted into defense. Under the combined stress, most of the energy is diverted into defense, and a very low level of energy is spent for reproduction. Flowering or fruiting stage would be accelerated and fruits would be very small and less compared with those of normal metabolic conditions. In severe cases, early maturity or death of crop plants could be inevitable. also in nonmodel crop plants including grains and vegetables and even trees.

Concentrations of these molecules could change or cross-talk with other signaling molecules. Thereby, new pathways should be produced to understand the complex interactions if we aim to remediate the conditions of crop plants under combined stress conditions (Fig. 2.4). Because, if a stress-related protein or enzyme is to be produced under stress conditions, plants would not produce this metabolite from different pathways, instead it will produce this metabolite using a common or shared pathway to use their energy resources efficiently under the combined stresses. This pathway should be quick and cost-effective as compared with those of pathways under single stress conditions. Therefore, plant adaptation strategy to a combination of two or three stresses consists of both "shared" and "unique" response. Many researchers refer to the "shared" response as the molecular, biochemical, and physiological responses using common pathways by two or three different stresses. They refer to "unique" responses as the specific individual responses in combined stress conditions (Atkinson et al., 2013; Prasch and Sonnewald, 2013). Signaling molecules under combined stress include production and detoxification of ROS, calcium-, phytohormone-, and MAPK-signaling pathway (Atkinson and Urwin, 2012; Suzuki et al., 2012, 2014; Rejeb et al., 2014). Most signaling molecules for abiotic and biotic stresses share common pathway and molecules including ROS (Møller et al., 2007; Wong and Shimamoto, 2009), calcium ions (Galon et al., 2010), transcription factors (Walley and Dehesh, 2010), hormones (Fonseca et al., 2009; Ton et al., 2009), (MAPK) cascades (Pitzschke et al., 2009), etc.

The shared responses play important roles in the adaptation of plants to harsh conditions. Some unique adaptation strategies tailored for stress combinations have been identified in the recent reports (Choi et al., 2013; Prasch and Sonnewald, 2013). For example, a combination of heat stress and virus infection resulted in upregulation of cytosolic invertases instead of cell wall-bound invertases (Prasch and Sonnewald, 2013).

The combination of abiotic and biotic stresses shares the unique or shared pattern (Prasad et al., 2011; Bostock et al., 2014; Rejeb et al., 2014; Pandey et al., 2015; Dikilitas et al., 2017). However, it is not easy to predict the results of stress combination by elucidating each stress alone. Even if they share unique or shared response, it is crucial to test the combination of these stresses in vitro or in vivo conditions. The combination of stresses, in general, is sensed as a new state of stress (Mittler, 2006). Here, the response of plants to stress combination is mainly characterized by the dominant stress factor. This could be described as a kind of a 


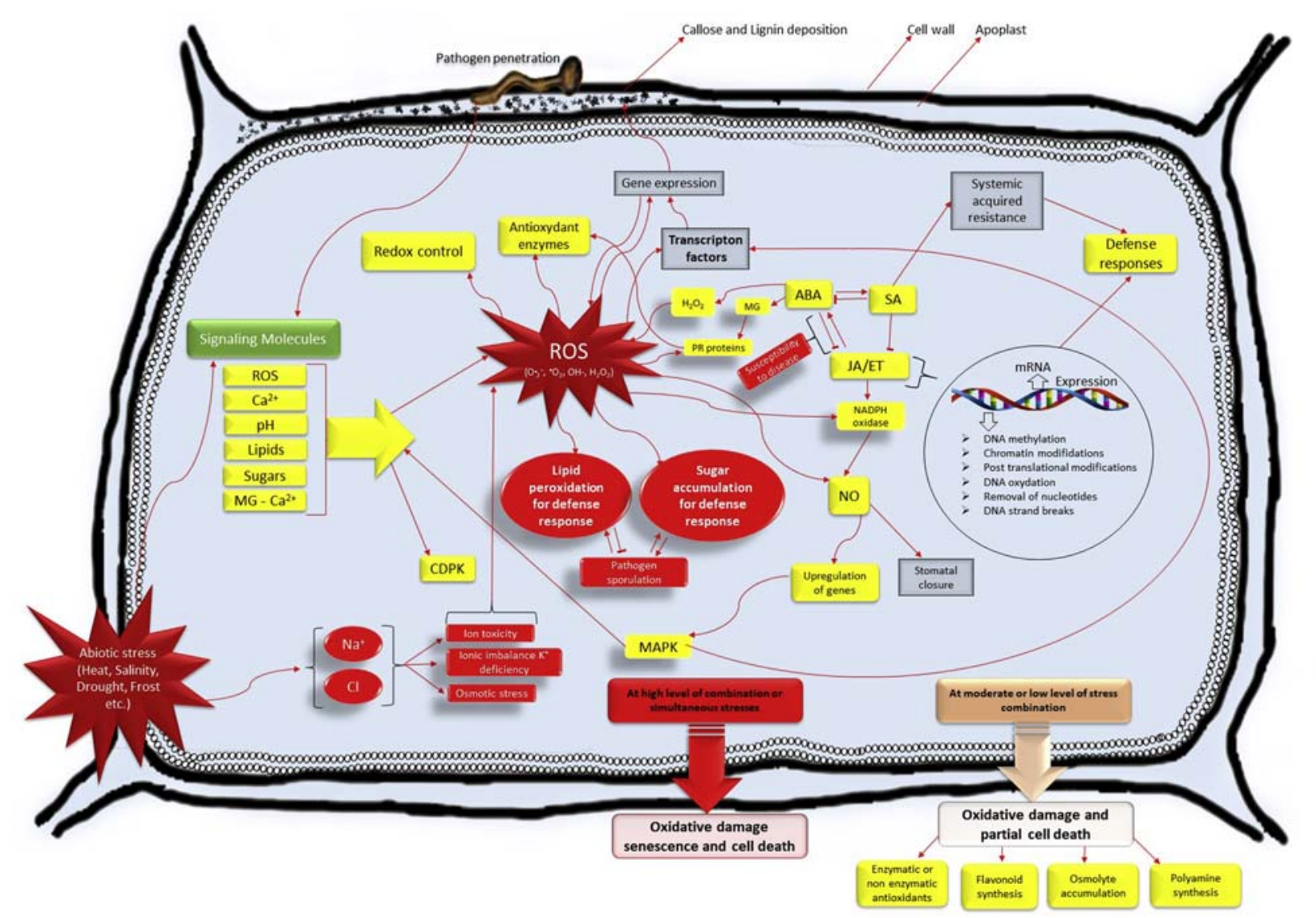

FIGURE 2.4 Signaling pathways of abiotic and biotic stress at the cellular level. Arrows indicate induction and bars indicate inhibition. The combination of stresses could be additive, synergistic, or antagonistic in terms of symptoms or disease progress on crop plants. Abiotic and biotic stress factors affect the homeostasis of chemical signals at the apoplastic space. Abiotic stress disrupts the structure and properties of physical barriers of the cell wall that protects the cell from pathogenic attacks, therefore, abiotic stress predisposes the plants to pathogenic attack. ABA signaling negatively affects hormonal signaling exerted by pathogens. ABA results in closure of stomata, this prevents water loss and pathogen entry, however, overproduction of ABA results in enhancement of pathogen spread and sporulation, because ABA and SA, JA, ET negatively affect each other, and therefore, overproduction of ABA resulted from abiotic stress could suppress the SA-based defense mechanism of plants against pathogenic attack. Therefore, under the combined stress, crop plants might stay defenseless due to cross-talk of signaling molecules. Also, high level of abiotic stress such as toxic ions or high temperatures or drought promote the translocation of effector proteins of pathogens into the host cell (plant). Here, one of the important mechanisms of the combined stress is the order of stresses; the stresses could be sequential or concurrent (simultaneous). Which type of signaling molecules would be prevalent and play a decisive role simply depends on the severity of the stress that would synthesize the signaling molecules. Since detoxification mechanisms would be slow and under capacity, the excess accumulation of ROS could, therefore, damage proteins and lipids and DNA in severe cases. Studies related to genomics, proteomics, transcriptomics, and metabolomics are needed to further elucidate the combined stress interactions.

more severe stress of an individual stress. Although it is not easy to predict the result of stress combination in terms of physiological, molecular, biochemical, or disease progression in advance, we could state whether the different stresses lead to the same or different kinds of changes in plants. The identification of signaling molecules involved in shared and unique response under combined stresses would be an important step to develop resistant crop plants.

Overlap responses between cross-talk of signaling pathways can be a source of potential stress tolerance traits that can be engineered into crops to confer multiple stress resistance into plants (Kissoudis et al., 2016).
For example, different calcium-dependent protein kinase (CDPK) genes in T. aestivum showed that 12 CDPKs were expressed in response to $B$. graminis $\mathrm{pv}$. tritici (causal agent of powdery mildew in wheat) attack, eight of them also responded to abiotic stresses (Li et al., 2008). Similarly, genes involved in ROS scavenging pathway like ascorbate peroxidase (APX) gene have been shown to take part against various abiotic and biotic stresses (Choi and Hwang, 2012). The transcriptomic and proteomic analysis of plants under combined stresses can produce useful information regarding common and unique genes regulated under combined stresses. 
Under combined stress, hormonal signaling interacts with each other. How such interactions affect plant responses under multiple stresses is not well known although such interactions frequently occur in nature. For example, Vos et al. (2013) stated that drought stress enhanced insect resistance due to synergistic interactions between JA and ABA signaling. On the other hand, flooding or waterlogging decreased resistance to chewing herbivores due to its negative cross-talk with JA. It is clear that ABA synthesis and signaling is required to activate defense responses and resistance against abiotic and biotic stresses. However, very low or high concentration of $A B A$ predisposes crop plants to pathogenic attack. For example, Kissoudis et al. (2016) stated that powdery mildew resistance in tomato plants was affected by salt stress in a genotype- and stress intensity-dependent manner. Mild salt stress $(50 \mathrm{mmol} / \mathrm{L} \mathrm{NaCl})$ increased the susceptibility of tomato while $150 \mathrm{mmol} / \mathrm{L} \mathrm{NaCl}$ reduced disease symptoms. They suggested that accumulation of $\mathrm{Na}^{+}$and $\mathrm{Cl}^{-}$ions in the leaves was linearly associated with the decreased pathogen symptoms under severe stress. They concluded that complete resistance was enabled in tomato genotypes carrying $\mathrm{Ol}-2$ and $\mathrm{Ol}-4$ resistance genes. Increased susceptibility and senescence under combined stress was associated with the induction of ET and JA pathway genes. The majority of these studies conclude that there is a negative impact of abiotic stress (mostly drought and salinity stress) on pathogen resistance (Suzuki et al., 2014). ABA is mainly involved in responses to abiotic stresses whereas SA, JA, ET have important roles in biotic stress signaling (Sebastiani et al., 2017). ABA appears to be a central modulator of the regulatory cross-talk, directly impacting biosynthesis of SA, the major regulatory hormone for defense responses against biotrophic pathogens (De Torres Zabala et al., 2009). Therefore, antagonistic relations were evident between $\mathrm{ABA}$ and other signaling molecules. However, under abiotic stress conditions, the role of ABA is significant. For example, enhanced callose deposition to prevent membrane leakage of plants was reported to be regulated by ABA pathway (Cao et al., 2011). Fujita et al. (2006) stated that the ABA-deficient tomato mutant cultivars (deficient in functional enzyme activity at the final step in ABA biosynthesis) had increased resistance to pathogens and application of exogenous ABA restored the susceptibility (Audenaert et al., 2002; Thaler and Bostock, 2004). High ABA concentrations inhibited the SA-dependent defense responses in tomato. ABA and ET are well known antagonistic hormones (Beaudoin et al., 2000; Ghassemian et al., 2000). Additionally, exogenous application of $\mathrm{ABA}$ resulted in the downregulation of JA- or ET-responsive defense gene expression in wild-type plants, whereas higher expression levels of these defense genes were observed in ABA-deficient mutants without any treatments (Anderson et al., 2004). From the above findings, exogenous application of JA and ET cannot restore the defense gene expression of plants suppressed by exogenous ABA application, because the ABA-regulated abiotic stress response is a dominant stress at the severe case when compared with the biotic stress process (Anderson et al., 2004). Verma et al. (2013) reported that ABA was produced during water stress. Various genes induced during drought and cold stress also get induced by application of ABA exogenously, indicating ABAdependent signal transduction pathways. On the other hand, several genes may not be induced by application of $\mathrm{ABA}$ exogenously indicating that the existence of ABA-independent signal transduction is evident as in the case of Arabidopsis ( $\mathrm{Zhu}, 2002$; YamaguchiShinozaki and Shinozaki, 2006). Whenever a plant encounters any kind of stress, this is sensed by the potential sensors, which generate secondary messengers (e.g., ABA, ROS, $\mathrm{Ca}^{2+}$, inositol phosphates, etc.). The secondary messengers can alter the levels of intracellular $\mathrm{Ca}^{2+}$. Any changes in cytoplasmic $\mathrm{Ca}^{2+}$ is sensed by $\mathrm{Ca}^{2+}$ sensors, that is, calcium-binding proteins to regulate stress-related genes. The expression of stress-regulated genes leads to the production of various kinds of regulatory molecules like the phytohormones ABA, ET, and SA and transcription factors, etc. These signaling networks are interconnected at many levels for transferring information to generate firm plant response. A successful signal transduction pathway demands proper coordination of all signaling molecules (Suzuki and Katano, 2018). Fujita et al. (2006) reported that although a few studies dealt with revealing the pathway under combined stress interactions, most of the signaling stress pathways need to be elucidated. However, recent studies have revealed several molecules, including transcription factors and kinases, involved in cross-talk (antagonistic or synergistic actions) between signaling pathways. For example, heavy metal $\left(\mathrm{CuSO}_{4}\right)$ stress with incompatible necrotrophic pathogen infection revealed a significant overlap between responses to biotic and abiotic stresses in terms of gene expression (Fujita et al., 2006). This relationship may simply suggest that plants have developed strategies to avoid simultaneously producing proteins that are involved in abiotic stress and disease resistance responses (Anderson et al., 2004). Suzuki et al. (2014) stated that despite a certain degree of overlap, each combined stress requires a unique mechanism. In addition, the concurrent occurrence of different biotic and abiotic stresses was shown to result in a high degree of complexity in plant responses. Therefore, the mechanisms should be clarified to tackle 
the combined stress. Abiotic stress alters pathogenrelated signaling networks, which could lead to the deactivation of defense responses and the higher susceptibility of plants. Many authors reported that when combined stress triggered the defense mechanism, signaling molecules activated the defense-related genes (Li et al., 2008). For example, increase of cytosolic calcium $\left(\mathrm{Ca}^{2+}\right)$ levels in response to pathogen attack, osmotic stress, water stress, cold, or wounding triggers simultaneous pathways and activates calciuminteracting proteins such as $\mathrm{Ca}^{2+}$-dependent protein kinases (CDPKs) and calmodulin, which are involved in transcription factors. In a combined stress, ET, JA, $\mathrm{SA}$ regulate the defense mechanism regarding biotic stress while $A B$ regulates the abiotic stress responses. In general, the biotic defense signaling networks regulated by phytohormones are mainly dependent on the nature of the pathogen and its mode of action. SA plays an important role against biotrophic and hemibiotrophic pathogens through the establishment of systemic acquired resistance (SAR). By contrast, JA and ET are usually associated with defense against necrotrophic pathogens and insects. JA and ET function synergistically to activate the expression of defense genes as reported by various workers (Thomma et al., 2001; Glazebrook, 2005). For example, Audenaert et al. (2002) stated that tomato mutants (ABA-deficient) showed a reduction in susceptibility to $B$. cinerea and virulent isolates of $P$. syringae $\mathrm{pv}$ tomato DC3000 (Thaler and Bostock, 2004; De Torres-Zabala et al., 2007). Similarly, ABA-deficient Arabidopsis showed reduced susceptibility to the oomycete Hyaloperonospora parasitica (Mohr and Cahill, 2003). In general, it could be stated that $A B A$ is mainly involved in the negative regulation of plant defenses against various biotrophic and necrotrophic pathogens. However, the positive side of ABA was reported in pathogen-involved cases (Mauch-Mani and Mauch, 2005). For example, ABAactivated stomatal closure played an important role in preventing bacterial infection (Melotto et al., 2006). However, there are conflicting results in combined stresses even though these results may come from the same authors. ABA-deficient mutants do not always prevent the disease occurrence. Ton and Mauch-Mani (2004) showed that ABA-deficient mutants showed susceptibility to $P$. syringae pv. tomato while ABA-applied plants showed resistance against Alternaria brassicicola and Plectosphaerella cucumerina. Biochemical and molecular analysis showed that the effects of combined stress were not linear when the effects of single stress value were summed. The increase of ABA under the effects of abiotic stress induces stomatal closure, therefore, virus, bacteria, or fungi like powdery mildew and $B$. cinerea may have faced a barrier before infection. However, if the infection gets started, the progress of the disease gains speed due to cross-talk between $A B A$ and SA pathways. ABA and SA have an antagonistic role in plant defense against stressors (Bowler and Fluhr, 2000; Robert-Seilaniantz et al., 2007).

Massa et al. (2013) stated that gene expressions of Solanum tuberosum in triple combined stress (abiotic stress + hormone + biotic stress) differed from those of individual stress. For example, $40 \%$ of the genes were upregulated under abiotic stress while $19 \%$ and $10 \%$ of the genes were upregulated under biotic and hormonal stress conditions, respectively. Only 6\% of the genes were upregulated under triple combined stress conditions. It could, therefore, be concluded that defense mechanisms of plants are reduced due to decreasing number of defense-related genes under multiple stress conditions. Similarly, Prasch and Sonnewald (2013) stated that double or triple or multiple stress responses (heat, drought, virus) by transcriptome and metabolome analysis revealed that gene expressions were downregulated. The increased cytoplasmic response was also evident; however, this was not observed under single stress conditions. The authors suggested that abiotic stress factors significantly altered virus-specific signaling networks, which led to the deactivation of defense responses and a higher susceptibility of plants. For example, TMV was able to overcome the $\mathrm{M}$ gene-mediated resistance at temperatures above $28^{\circ} \mathrm{C}$ in tobacco ( $N$. tabacum) (Király et al., 2008). Massa et al. (2013) stated that overlap between abiotic, biotic, and hormone stresses showed that hormones (ABA, IAA, $\mathrm{GA}_{3}$ ) and abiotic stresses (heat, salt) shared more differentially expressed genes than each showed with the biotic stress (Phytophthora infestans) agent.

ROS signals after pathogen inoculation may change stomatal conductance and ion uptake transport. Abiotic stress such as salt and drought affect ABA signaling and systemic acquired resistance, therefore, pathogen spread is quickly enhanced and colonizes the whole plant. For example, SA signaling, induced after infection with biotrophic fungi, can attenuate ABA signaling, which takes important roles in adapting plants to abiotic stresses (Kim et al., 2011).

\subsection{DNA DAMAGE OF PLANTS UNDER CONCURRENT OF ABIOTIC AND BIOTIC STRESS COMBINATIONS}

The plant defense mechanism has also been examined in terms of genetic responses in combined stress conditions. Many authors reported that genes and gene products have been expressed under combined stress. Abiotic and biotic stresses affect genome stability and even cause multiple DNA damages. The DNA 
damage could be direct or indirect depending on the source, duration, and level of stress as well as the resistance traits of organisms. During stress, plants are able to recover from the stress, however, this depends on the duration, severity, and source of stress. However, combined stress may not allow plants to recover (Dikilitas et al., 2018). If the concentrations of antioxidant molecules exceed a certain level that the host cannot tolerate, these molecules damage the cell compounds including DNA (Wan-Ibrahim et al., 2010). For example, Čabarkapa et al. (2017) reported that dry olive leaf extract (DOLE) had radical scavenging activity in peripheral blood lymphocytes (PBL), however, the concentrations over $1 \mathrm{mg} / \mathrm{mL}$ led to a severe increase of DNA damage exhibiting prooxidant rather than antioxidant effect.

Although most of these molecules act as signaling molecules at low concentrations, however, overexpression of them is highly toxic and cause protein oxidation, carbohydrate degradation, lipid peroxidation, pigment breakdown, and DNA strand breaks. Although there is no report that the combined stress has made strand breakages in DNA molecules, DNA methylation, a prestep of DNA damage, is evident. This damaging effect could lead to DNA damage and the damage, if not repaired, has an ongoing effect.

The concentration of signaling molecules and DNA damage has not been correlated in plant crop science studies to the best of our knowledge. It should be one of the important steps in identifying the pathway in terms of signaling molecules and DNA damage parameters to generate resistant plants. Plants have to face enormous challenges to maintain their genome integrity due to global warming and environmental pollution, and their immobile character makes this case worse. For example, UV light, ionizing radiation, high soil salinity, drought, heavy metal, chemical mutagens, free radicals, alkalyting agents, pesticides, and even sometimes biological agents and their toxins have the potential to result in DNA methylation and DNA damage (Dikilitas et al., 2015; Georgieva et al., 2017; Silveira et al., 2017). DNA damage generated by abiotic and biotic stress affects plant growth, development, and crop productivity. Therefore, to maintain the stability of genome, plants have developed an extensive array of mechanisms for detection and repair of DNA damage. DNA damage could vary such as DNA base oxidation, single and double strand breaks, DNA intercross-links, DNA methylation, etc. (Roy, 2014). Lesions in the DNA structure as a result of stress agents may lead to biochemical and physical changes in the DNA structures. These lesions have tended to accumulate and have potential to break DNA strands at the end. Under combined stress conditions, the damaging effects of both abiotic and biotic stresses could be more prominent and be able to break the single and double strands of DNA. Individual stress factors, even at low doses, may produce additive or synergistic interactions between them and their negative effects could be visible on DNA. For example, the interactions of ultraviolet lights (UV-B) with different concentrations of cadmium or lead on Bacillus cereus resulted in DNA damage, cytotoxicity, depletion of glutathione, and formation of lipid peroxidation. Although UV-B rays alone enhanced glutathione production, the combined stress exhibited high genotoxic activity due to inhibition of the DNA repair mechanism (El-Sonbaty and El-Hadedy, 2015).

\subsection{GENOMIC AND BIOCHEMICAL APPROACHES FOR PLANTS UNDER COMBINED STRESSES}

If a stress is beyond tolerance level, defense mechanisms are activated at molecular, physiological, and biochemical level. When stress is managed and controlled, the cell signaling returns to normal level. The signals are able to induce expression of specific genes that lead to the assembly of defense reaction (Jaspers and Kangasjärvi, 2010; Ji et al., 2011). Advances in plant genomics research have opened up new perspectives and opportunities to improve crop plants in terms of quantity and quality. For example, many of the key enzymes (peroxidase, catalase, ascorbate peroxidase, and superoxide dismutase) involved in $\mathrm{ABA}$ synthesis have been used in transgenic plants to improve abiotic stress tolerance (Sun et al., 2017). Transgenic plants overexpressing the genes involved in ABA synthesis showed increased tolerance to drought and salinity stress (Ji et al., 2011). Also, the genes involved in the synthesis of osmoprotectants such as proline, glycine betaine, sugars showed increased tolerance to abiotic stresses. These transgenic modifications of biosynthetic and metabolic pathways indicated that higher stress tolerance and the accumulation of compatible solutes may also protect plants against damage by scavenging of ROS and maintain protein structures and functions (Umezawa et al., 2006; $\mathrm{Hu}$ et al., 2016). Low molecular weight compounds, polyamines, were regulated with arginine decarboxylase $(A D C)$. Therefore, transgenic plants overexpressing $A D C$ gene showed an increase in biomass and better performance under salt stress conditions. Genetic engineering in plant signaling pathways is also a valuable option to improve tolerance of crop plants to various stresses (Radwan, 2015).

Although most of the pathways have concentrated on the quick signaling pathway to reduce the effect of stress or stresses, one of the most appropriate ways is 
to eliminate or reduce the impact of stress factors from the very beginning. For example, phytoremediation is one of the most secure and cost-effective ways to reduce the salinity or heavy metals from the vicinity of root area of crop plants. The cultivation of crop plants with halophytes (plants that remove salt or heavy metals from the soil) could reduce the toxic ions that could exert toxicity on crop plants (Karakas et al., 2016). Thus, crop plants would have to deal with at least one stress factor. Also, halophytes would provide plants with minerals, hormones, and other beneficial metabolites during the stress period.

Another approach for the plants under combined stress is the use of organic metabolites. For example, Wani et al. (2017) stated that the effect of foliar spray of 24-epibrassinolide (EBL) on Cicer arietinum L. increased nitrogen metabolism, antioxidant system, photosynthetic characteristics, and chlorophyll fluorescence under cadmium $(\mathrm{Cd} ; 50 \mu \mathrm{M})$ and/or $\mathrm{NaCl}$ $(100 \mathrm{mM})$ stress. In addition to that, EBL application enhanced the activities of enzymes such as nitrogenase, glutamate synthase, glutamine synthase, and glutamate dehydrogenase under both stress and stressfree conditions (Gupta et al., 2017; Wani et al., 2017). It is possible that the remediation effect was due to increase in signaling molecules. Similar findings were made by Derevyanchuk et al. (2017) who stated that the phospholipid signaling was the early steps of brassinosteroids signal transduction. On the other hand, Yusuf et al. (2017) stated that cumulative effort of proline metabolism and enhanced antioxidant system along with increased soluble sugar content, via EBL supply, successfully reduced the negative effects of the combined stress of aluminum and salt. There are other activators that result in similar findings. It is important that these bioactivators should be environmentally friendly and should lead to quick signaling response under the combined stress conditions and should be carefully evaluated for possible antagonistic effects toward other signaling pathways under the stress conditions. Therefore, suitable markers such as SA, NO, $\mathrm{ET}$, and ABA measurement should be employed to test the biochemical signaling pathways.

To sustain the effect of bioactivators naturally, the stress-tolerant plant growth-promoting rhizobacteria (PGPR) and arabuscular mycorrhizal fungi (AMF) have been recently used under stressed conditions (Sinclair et al., 2014; Mishra et al., 2018). The secondary metabolites produced by PGPR are capable of improving crop plants. The secondary metabolites, although they have little or no effect on the primary metabolism, are important for survival and protection of the cell, particularly in stressed conditions. Accumulation of osmoprotectants such as soluble sugars and amino acids help in maintaining the equilibrium across the plasma membranes and reduces cell osmotic potential and increases the turgor pressure in the cell. Recent studies have proved that volatile organic compound (VOC) secreted by B. subtilis GB03 could trigger phytohormone signaling including auxin, cytokinins, SA, and gibberellins in A. thaliana (Liu and Zhang, 2015). Similarly, Orhan (2016) stated that IAA produced by halotolerant and halophilic PGPRs increased the root and shoot length and total fresh weight of the wheat plants under saline conditions. Like other bioactivators, stress-tolerant PGPRs may stimulate plants to selectively take up $\mathrm{Ca}^{2+}$ to maintain a high $\mathrm{Ca}^{+} / \mathrm{Na}^{+}$ ratio (Sharma et al., 2016). They stated PGPR inoculated peanut seedlings accumulated less ROS and exhibited high transcript levels of antioxidant genes. The authors thus concluded that selective ion uptake and redox homeostasis was an important protective mechanism of PGPRs.

Another promising approach to improve the conditions of stress tolerance of plants under both abiotic and biotic stress conditions is to prime the plants with chemical compounds to enable better stress tolerance. This could be achieved with chemicals having high signal transduction traits. Since conventional priming is timeconsuming and others are not acceptable (genetically modified organisms, GMO) in many places of the world, an alternative way is priming or hardening the plants under single or multiple combined stress conditions. Savvides et al. (2016) showed that sodium nitroprusside (SNP), hydrogen peroxide $\left(\mathrm{H}_{2} \mathrm{O}_{2}\right)$, melatonin, and sodium hydrosulfide enhanced tolerance of plants when exposed to multiple stresses. They stated that chemical priming agents shared similar components in their modes of action.

Recent studies have also shown that polyamines (PAs) took a great part in a large number of abiotic and biotic stresses (Alcázar et al., 2010). PAs are low molecular weight polycationic compounds found in most living organisms. Diamine putrescine (Put), triamine spermidine (Spd), tetramines spermine (Spm), and thermospermine (tSpm) can be in free and conjugated forms. Recently, the synthesis and accumulation of PAs were found to be connected to other signaling molecules and took great part in the signaling network. Depletion of PAs in cells is generally detrimental. Therefore, it is necessary to study the interconnections of PA with other signaling molecules especially under combined stress conditions.

Because the exogenous application of PAs might play significant roles to protect plants from further damage even if different stressors use antagonistic pathways (SA or JA pathways), polyamine coding generally engineered plants or PA synthesizing plants or exogenously PA applied plants would protect themselves from stress only when needed without 
significant modifications in biochemical pathways responsible for protein and enzyme synthesis.

\subsection{CONCLUSIONS AND FUTURE PROSPECTS}

Scientists try to generate more resistant or tolerant plant species to get higher crop production in adverse conditions, however, so far only one particular stress agent such as drought, salinity, pathogen, heat, weed, etc. was considered to make plants resistant or tolerant. Now, the problems are more complex and interwoven due to changes in climatic and environmental conditions. Therefore, crop plants have to be generated or bred for multiple stress factors. However, when multiple or combined stresses are involved, we should consider the interactions between abiotic and biotic stress agents. For this reason/matter, stress-responsive signaling molecules including genes, hormones, enzymes, and antioxidant and oxidant molecules should be evaluated in response to multiple stress factors.

Understanding the combined stress involves a fine description of the underlying mechanisms at the cellular and molecular levels. The major challenge is to identify and differentiate the pathways at single or combined stress conditions. Many studies have revealed that the molecular and metabolic responses of plants to combined or multiple stresses is unique when compared with those of single stresses and cannot be predicted from the responses of each individual stress factor. Therefore, any combined stress interactions even if studied with the physiological and biochemical parameters should be identified at the molecular level including signaling pathways to draw a reliable pathway. This is because the majority of pathways produced so far have been composed of the combination of two or more different stress factors, which need to be evaluated in detail to generate resistant crop plants against multiple stress factors occurring simultaneously or sequentially in nature.

Transcriptome analyses and proteomic studies have given rise to rapid progress in the field of plant signal transduction and gene regulation studies. GeneChip and cDNA microarray studies with genome sequencing are useful in identifying novel signaling molecules. Proteomics approach is another useful study in signal transduction studies. To reveal the cross-talk between signaling molecules, more studies, especially in DNA microarray technology, transcriptomic studies, and new generation sequencing techniques, would be very crucial. Nejat and Mantri (2017) stated that when a plant faces one stress, it may tolerate another stress; this phenomenon is called cross-tolerance. Two different stress factors have antagonistic, additive, or synergistic effects, and each stress factor should be verified, and their simultaneous or sequential effects should be considered at first. This is because the sequence of stress plays important roles in the determination of characteristics of stress. Here, each signaling molecule has been discussed and their possible interactions with other signaling molecules have been tried to be evaluated in combined stress issues. A significant gap has been noticed in terms of mechanisms of signaling molecules in combined stress issues. Therefore, mechanisms of signaling molecules should be evaluated individually and possible interactions should be considered. Gene expression studies and signaling molecules would open new pathways and clarify the complex defense mechanisms. Exposure to combined stress can either be more detrimental than a single stress due to synergistic or additive effects or have an attenuating effect due to antagonistic effects.

So far, only a few studies have been conducted on the whole genome responses under multiple stress conditions; this mainly has been performed on Arabidopsis spp. We could simply state that the survival of plants under combined stress conditions depends on the ability to perceive stress signals and transmit the signal into cellular components to biochemical changes to start molecular, biochemical, and physiological defense mechanisms.

\section{Acknowledgement}

The authors apologize to colleagues whose work was not cited due to space limitations.

\section{References}

Abbas, J.A., Mandeel, Q.A., 1995. Survey of Fusarium species in an arid environment of Bahrain III. Abundance and seasonal changes of Fusarium species in the terrestrial habitats of Bahrain Island. Arab Gulf J. Sci. Res. 13 (3), 637-651.

Achuo, E.A., Prinsen, E., Höfte, M., 2006. Influence of drought, salt stress and abscisic acid on the resistance of tomato to Botrytis cinerea and Oidium neolycopersici. Plant Pathol. 55 (2), 178-186. Available from: https://doi.org/10.1111/j.1365-3059.2006.01340.x.

Ahanger, M.A., Agarwal, R.M., 2017. Salinity stress induced alterations in antioxidant metabolism and nitrogen assimilation in wheat (Triticum aestivum L.) as influenced by potassium supplementation. Plant Physiol. Biochem. 115, 449-460. Available from: https://doi.org/10.1016/j.plaphy.2017.04.017.

Ahmad, P., Jaleel, C.A., Salem, M.A., Nabi, G., Sharma, S., 2010. Roles of enzymatic and nonenzymatic antioxidants in plants during abiotic stress. Crit. Rev. Biotechnol. 30 (3), 161-175. Available from: https:/ / doi.org/10.3109/07388550903524243.

Akter, N., Islam, M.R., 2017. Heat stress effects and management in wheat. A review. Agron. Sustain. Dev. 37 (5), 37. Available from: https://doi.org/10.1007/s13593-017-0443-9.

Alcázar, R., Altabella, T., Marco, F., Bortolotti, C., Reymond, M., Koncz, C., et al., 2010. Polyamines: molecules with regulatory functions in plant abiotic stress tolerance. Planta 231 (6), 1237-1249. Available from: https://doi.org/10.1007/s00425-010-1130-0. 
Ali, S., Mir, Z.A., Tyagi, A., Bhat, J.A., Chandrashekar, N., Papolu, P. K., et al., 2017. Identification and comparative analysis of Brassica juncea pathogenesis-related genes in response to hormonal, biotic and abiotic stresses. Acta Physiol. Plant. 39 (12), 268. Available from: https://doi.org/10.1007/s11738-017-2565-8.

Anderson, J.P., Badruzsaufari, E., Schenk, P.M., Manners, J.M., Desmond, O.J., Ehlert, C., et al., 2004. Antagonistic interaction between abscisic acid and jasmonate-ethylene signaling pathways modulates defense gene expression and disease resistance in Arabidopsis. Plant Cell 16 (12), 3460-3479. Available from: https://doi.org/10.1105/tpc.104.025833.

Asada, K., Takahashi, M., 1987. Production and scavenging of active oxygen radicals in photosynthesis. In: Kyle, D.J., Osmond, C.B., Arntzen, C.J. (Eds.), Photoinhibition, vol. 9. Elsivier, Amsterdam, pp. 227-288.

Atkinson, N.J., Lilley, C.J., Urwin, P.E., 2013. Identification of genes involved in the response of Arabidopsis to simultaneous biotic and abiotic stresses. Plant Physiol. 162 (4), 2028-2041. Available from: https:/ / doi.org/10.1104/pp.113.222372.

Atkinson, N.J., Urwin, P.E., 2012. The interaction of plant biotic and abiotic stresses: from genes to the field. J. Exp. Bot. 63 (10), 3523-3543. Available from: https://doi.org/10.1093/jxb/ ers100.

Audenaert, K., De Meyer, G.B., Höfte, M.M., 2002. Abscisic acid determines basal susceptibility of tomato to Botrytis cinerea and suppresses salicylic acid-dependent signaling mechanisms. Plant Physiol. 128 (2), 491-501. Available from: https://doi.org/ $10.1104 /$ pp.010605.

Baena-González, E., Sheen, J., 2008. Convergent energy and stress signaling. Trends Plant Sci. 13 (9), 474-482. Available from: https://doi.org/10.1016/j.tplants.2008.06.006.

Bandurska, H., Niedziela, J., Chadzinikolau, T., 2013. Separate and combined responses to water deficit and UV-B radiation. Plant Sci. 213, 98-105. Available from: https://doi.org/10.1016/j. plantsci.2013.09.003.

Baxter, A., Mittler, R., Suzuki, N., 2014. ROS as key players in plant stress signalling. J. Exp. Bot. 65 (5), 1229-1240. Available from: https://doi.org/10.1093/jxb/ert375.

Beaudoin, N., Serizet, C., Gosti, F., Giraudat, J., 2000. Interactions between abscisic acid and ethylene signaling cascades. Plant Cell 12 (7), 1103-1115. Available from: https://doi.org/10.1105/ tpc.12.7.1103.

Bitrián, M., Zarza, X., Altabella, T., Tiburcio, A.F., Alcázar, R., 2012. Polyamines under abiotic stress: metabolic crossroads and hormonal crosstalks in plants. Metabolites 2 (3), 516-528. Available from: https://doi.org/10.3390/metabo2030516.

Bokszczanin, K.L., SPOT-ITN Consortium, Fragkostefanakis, S., 2013. Perspectives on deciphering mechanisms underlying plant heat stress response and thermotolerance. Front. Plant Sci. 4, 315. Available from: https://doi.org/10.3389/fpls.2013.00315.

Bonnet, C., Lassueur, S., Ponzio, C., Gols, R., Dicke, M., Reymond, P., 2017. Combined biotic stresses trigger similar transcriptomic responses but contrasting resistance against a chewing herbivore in Brassica nigra. BMC Plant Biol. 17 (1), 127. Available from: https:// doi.org/10.1186/s12870-017-1074-7.

Bostock, R.M., Pye, M.F., Roubtsova, T.V., 2014. Predisposition in plant disease: exploiting the nexus in abiotic and biotic stress perception and response. Annu. Rev. Phytopathol. 52, 517-549. Available from: https://doi.org/10.1146/annurev-phyto-081211172902.

Boumaaza, B., Benkhelifa, M., Belkhoudja, M., 2015. Effects of two salts compounds on mycelial growth, sporulation, and spore germination of six isolates of Botrytis cinerea in the Western North of Algeria. Int. J. Microbiol. 2015 (2015), 1-8. Available from: https: / / doi.org/10.1155/2015/572626.
Bowler, C., Fluhr, R., 2000. The role of calcium and activated oxygens as signals for controlling cross-tolerance. Trends Plant Sci. 5 (6), 241-246. Available from: https://doi.org/10.1016/S1360-1385(00) 01628-9.

Brosché, M., Strid, A., 2003. Molecular events following perception of ultraviolet-B radiation by plants. Physiol. Plant. 117 (1), 1-10. Available from: https:/ / doi.org/10.1034/j.1399-3054.2003.1170101.x.

Čabarkapa, A., Dekanski, D., Živković, L., Milanović-Čabarkapa, M., Bajić, V., Topalović, D., et al., 2017. Unexpected effect of dry olive leaf extract on the level of DNA damage in lymphocytes of lead intoxicated workers, before and after CaNa2EDTA chelation therapy. Food Chem. Toxicol. 106, 616-623. Available from: https:// doi.org/10.1016/j.fct.2016.12.023.

Cao, F.Y., Yoshioka, K., Desveaux, D., 2011. The roles of ABA in plant-pathogen interactions. J. Plant Res. 124 (4), 489-499. Available from: https://doi.org/10.1007/s10265-011-0409-y.

Choi, H.W., Hwang, B.K., 2012. The pepper extracellular peroxidase $\mathrm{CaPO}_{2}$ is required for salt, drought and oxidative stress tolerance as well as resistance to fungal pathogens. Planta 235 (6), 1369-1382. Available from: https://doi.org/10.1007/s00425-011-1580-z.

Choi, M.S., Kim, W., Lee, C., Oh, C.S., 2013. Harpins, multifunctional proteins secreted by gram-negative plant-pathogenic bacteria. Mol. Plant Microb. Interact. 26 (10), 1115-1122. Available from: https://doi.org/10.1094/MPMI-02-13-0050-CR.

Dar, N.A., Amin, I., Wani, W., Wani, S.A., Shikari, A.B., Wani, S.H., et al., 2017. Abscisic acid: a key regulator of abiotic stress tolerance in plants. Plant Gene 11, 106-111. Available from: https:// doi.org/10.1016/j.plgene.2017.07.003.

De Torres Zabala, M., Bennett, M.H., Truman, W.H., Grant, M.R., 2009. Antagonism between salicylic and abscisic acid reflects early host-pathogen conflict and moulds plant defence responses. Plant J. 59 (3), 375-386. Available from: https://doi.org/10.1111/ j.1365-313X.2009.03875.x.

De Torres-Zabala, M., Truman, W., Bennett, M.H., Lafforgue, G., Mansfield, J.W., Egea, P.R., et al., 2007. Pseudomonas syringae pv. tomato hijacks the Arabidopsis abscisic acid signalling pathway to cause disease. EMBO J. 26 (5), 1434-1443. Available from: https://doi.org/10.1038/sj.emboj.7601575.

De Vleesschauwer, D., Xu, J., Höfte, M., 2014. Making sense of hormone-mediated defense networking: from rice to Arabidopsis. Front. Plant Sci. 5, 611. Available from: https://doi.org/10.3389/ fpls.2014.00611.

Derevyanchuk, M., Kretynin, S., Iakovenko, O., Litvinovskaya, R., Zhabinskii, V., Martinec, J., et al., 2017. Effect of 24epibrassinolide on Brassica napus alternative respiratory pathway, guard cells movements and phospholipid signaling under salt stress. Steroids 117, 16-24. Available from: https://doi.org/ 10.1016/j.steroids.2016.11.006.

Dikilitas, M., 2003. Effect of salinity, its interactions with Verticillium albo-atrum on the disease development in tomato (Lycopersicon esculentum Mill.) and lucerne (Medicago sativa and M. media) plants. Ph.D. Thesis. University of Wales, Swansea.

Dikilitas, M., Collins, A.R., Kocyigit, A., El-Yamani, N., Karakas, S., 2015. DNA damage in potato plants exposed to high level of $\mathrm{NaCl}$ stress. Frontiers in Genetics Conference Abstract: ICAW 2015-11th International Comet Assay Workshop, Antwerpen, Belgium, September 1-4, 2015. https://doi.org/10.3389/conf. fgene.2015.01.00066.

Dikilitas, M., Karakas, S., 2012. Behaviour of plant pathogens for crops under stress during the determination of physiological, biochemical, and molecular approaches for salt stress tolerance. In: Ashraf, M., Öztürk, M., Ahmad, M.S.A., Aksoy, A. (Eds.), Crop Production for Agricultural Improvement. Springer, Dordrecht, pp. 417-441. Available from: https://doi.org/ 10.1007/978-94-007-4116-4_16. 
Dikilitas, M., Yucel, N., Dervis, S., 2017. Production of antioxidant and oxidant metabolites in tomato plants infected with Verticillium dahliae under saline conditions. In: Khan, M.I.R., Khan, N.A. (Eds.), Reactive Oxygen Species and Antioxidant Systems in Plants: Role and Regulation Under Abiotic Stress. Springer, Singapore, pp. 315-329. Available from: https://doi. org/10.1007/978-981-10-5254-5_13.

Dikilitas, M., Karakas, S., Ahmad, P., 2018. Predisposition of crop plants to stress is directly related to their DNA health. In: Egamberdieva, D., Ahmad, P. (Eds.), Plant Microbiome: Stress Response, Vol. 5. Springer, Singapore, pp. 233-254. Available from: https://doi.org/10.1007/978-981-10-5514-0_11.

Dixit, A.K., Jayabaskaran, C., 2014. Calcium signaling during abiotic stress in plants: roles of calcium dependent protein kinases. In: Hemantaranjan, A. (Ed.), Physiology of Nutrition and Environmental Stresses on Crop Productivity, Vol. 14. Scientific Publishers, India, pp. 348-369.

Dossa, G.S., Torres, R., Henry, A., Oliva, R., Maiss, E., Cruz, C.V., et al., 2017. Rice response to simultaneous bacterial blight and drought stress during compatible and incompatible interactions. Eur. J. Plant Pathol. 147 (1), 115-127. Available from: https:/ / doi. org /10.1007/s10658-016-0985-8.

El-Abyad, M.S., Abu-Taleb, A.M., Abdel-Mawgoud, T., 1997. Response of host cultivar to cell wall-degrading enzymes of the sugarbeet pathogens Rhizoctonia solani Kühn and Sclerotium rolfsii Sacc. under salinity stress. Microbiol. Res. 152 (1), 9-17. Available from: https://doi.org/10.1016/S0944-5013(97)80018-3.

El-Sonbaty, S.M., El-Hadedy, D.E., 2015. Combined effect of cadmium, lead, and UV rays on Bacillus cereus using comet assay and oxidative stress parameters. Environ. Sci. Pollut. Res. 22 (5), 3400-3407. Available from: https:// doi.org/10.1007/s11356-012-1250-0.

Ferrante, P., Scortichini, M., 2014. Frost promotes the pathogenicity of Pseudomonas syringae pv. actinidiae in Actinidia chinensis and A. deliciosa plants. Plant Pathol. 63 (1), 12-19. Available from: https: / / doi.org/10.1111/ppa.12070.

Fonseca, S., Chico, J.M., Solano, R., 2009. The jasmonate pathway: the ligand, the receptor and the core signalling module. Curr. Opin. Plant Biol. 12 (5), 539-547. Available from: https://doi.org/ 10.1016/j.pbi.2009.07.013.

Fu, J., Chu, X., Sun, Y., Miao, Y., Xu, Y., Hu, T., 2015. Nitric oxide mediates 5-aminolevulinic acid-induced antioxidant defense in leaves of Elymus nutans griseb. exposed to chilling stress. PLoS One 10 (7), e0130367. Available from: https://doi.org/10.1371/ journal.pone.0130367.

Fujita, M., Fujita, Y., Noutoshi, Y., Takahashi, F., Narusaka, Y., Yamaguchi-Shinozaki, K., et al., 2006. Crosstalk between abiotic and biotic stress responses: a current view from the points of convergence in the stress signaling networks. Curr. Opin. Plant Biol. 9 (4), 436-442. Available from: https://doi.org/10.1016/j. pbi.2006.05.014.

Galon, Y., Finkler, A., Fromm, H., 2010. Calcium-regulated transcription in plants. Mol. Plant 3 (4), 653-669. Available from: https:// doi.org/10.1093/mp/ssq019.

Garrett, K.A., Dendy, S.P., Frank, E.E., Rouse, M.N., Travers, S.E., 2006. Climate change effects on plant disease: genomes to ecosystems. Annu. Rev. Phytopathol. 44, 489-509. Available from: https:/ / doi.org/10.1146/annurev.phyto.44.070505.143420.

Georgieva, M., Rashydov, N.M., Hajduch, M., 2017. DNA damage, repair monitoring and epigenetic DNA methylation changes in seedlings of Chernobyl soybeans. DNA. Repair. (Amst). 50, 14-21. Available from: https://doi.org/10.1016/j.dnarep.2016.12.002.

Ghassemian, M., Nambara, E., Cutler, S., Kawaide, H., Kamiya, Y., McCourt, P., 2000. Regulation of abscisic acid signaling by the ethylene response pathway in Arabidopsis. Plant Cell 12 (7), 1117-1126. Available from: https://doi.org/10.1105/tpc.12.7.1117.
Gill, S.S., Tuteja, N., 2010. Reactive oxygen species and antioxidant machinery in abiotic stress tolerance in crop plants. Plant Physiol. Biochem. 48 (12), 909-930. Available from: https://doi.org/ 10.1016/j.plaphy.2010.08.016.

Glazebrook, J., 2005. Contrasting mechanisms of defense against biotrophic and necrotrophic pathogens. Annu. Rev. Phytopathol. 43, 205-227. Available from: https://doi.org/10.1146/annurev. phyto.43.040204.135923.

Gong, Y., Rao, L., Yu, D., 2013. Abiotic stress in plants. In: Stoytcheva, M., Zlatev, R. (Eds.), Agricultural Chemistry. InTech, pp. 113-152. Available from: https://doi.org/10.5772/ 55865.

Goswami, S., Kumar, R.R., Sharma, S.K., Kala, Y.K., Singh, K., Gupta, R., et al., 2015. Calcium triggers protein kinases-induced signal transduction for augmenting the thermotolerance of developing wheat (Triticum aestivum) grain under the heat stress. J. Plant Biochem. Biotechnol. 24 (4), 441-452. Available from: https:// doi.org/10.1007/s13562-014-0295-1.

Guerret, M.G., Barbetti, M.J., You, M.P., Jones, R.A., 2016. Effects of temperature on disease severity in plants of subterranean clover infected singly or in mixed infection with Bean yellow mosaic virus and Kabatiella caulivora. J. Phytopathol. 164 (9), 608-619. Available from: https://doi.org/10.1111/jph.12484.

Gupta, A., Dixit, S.K., Senthil-Kumar, M., 2016. Drought stress predominantly endures Arabidopsis thaliana to Pseudomonas syringae infection. Front. Plant Sci. 7, 808. Available from: https://doi. org/10.3389/fpls.2016.00808.

Gupta, P., Srivastava, S., Seth, C.S., 2017. 24-Epibrassinolide and sodium nitroprusside alleviate the salinity stress in Brassica juncea L. cv. Varuna through cross talk among proline, nitrogen metabolism and abscisic acid. Plant Soil 411 (1-2), 483-498. Available from: https://doi.org/10.1007/s11104-016-3043-6.

Halliwell, B., Gutteridge, J.M., 2015. Free Radicals in Biology and Medicine. Oxford University Press, USA.

HanumanthaRao, B., Nair, R.M., Nayyar, H., 2016. Salinity and high temperature tolerance in mungbean [Vigna radiata (L.) Wilczek] from a physiological perspective. Front. Plant Sci. 7, 957. Available from: https://doi.org/10.3389/fpls.2016.00957.

Hasan, H.A.H., 2002. Gibberellin and auxin-indole production by plant root-fungi and their biosynthesis under salinity-calcium interaction. Acta Microbiol. Immunol. Hung. 49 (1), 105-118. Available from: https://doi.org/10.1556/AMicr.49.2002.1.11.

He, J.M., Zhang, Z., Wang, R.B., Chen, Y.P., 2011. UV-B-induced stomatal closure occurs via ethylene-dependent NO generation in Vicia faba. Funct. Plant Biol. 38 (4), 293-302. Available from: https:// doi.org/10.1071/FP10219.

Hoque, T.S., Hossain, M.A., Mostofa, M.G., Burritt, D.J., Fujita, M., Tran, L.S.P., 2016. Methylglyoxal: an emerging signaling molecule in plant abiotic stress responses and tolerance. Front. Plant Sci. 7, 1341. Available from: https://doi.org/10.3389/ fpls.2016.01341.

Hossain, M.A., Li, Z.G., Hoque, T.S., Burritt, D.J., Fujita, M., MunnéBosch, S., 2018. Heat or cold priming-induced cross-tolerance to abiotic stresses in plants: key regulators and possible mechanisms. Protoplasma 255 (1), 399-412. Available from: https://doi. org/10.1007/s00709-017-1150-8.

Hu, T., Zhu, S., Tan, L., Qi, W., He, S., Wang, G., 2016. Overexpression of OsLEA4 enhances drought, high salt and heavy metal stress tolerance in transgenic rice (Oryza sativa L.). Environ. Exp. Bot. 123, 68-77. Available from: https://doi.org/ 10.1016/j.envexpbot.2015.10.002.

Huang, G.T., Ma, S.L., Bai, L.P., Zhang, L., Ma, H., Jia, P., et al., 2012. Signal transduction during cold, salt, and drought stresses in plants. Mol. Biol. Rep. 39, 969-987. Available from: https://doi. org/10.1007/s11033-011-0823-1. 
Huda, K.M.K., Banu, M.S.A., Tuteja, R., Tuteja, N., 2013. Global calcium transducer P-type Ca $2+$-ATPases open new avenues for agriculture by regulating stress signalling. J. Exp. Bot. 64, 3099-3109. Available from: https://doi.org/10.1093/jxb/ert182.

Huot, B., Castroverde, C.D.M., Velásquez, A.C., Hubbard, E., Pulman, J.A., Yao, J., et al., 2017. Dual impact of elevated temperature on plant defence and bacterial virulence in Arabidopsis. Nat. Commun. 8 (1), 1808. Available from: https://doi.org/10.1038/ s41467-017-01674-2.

Jabnoun-Khiareddine, H., Abdallah, R., El-Mohamedy, R., AbdelKareem, F., Gueddes-Chahed, M., Hajlaoui, A., et al., 2016. Comparative efficacy of potassium salts against soil-borne and air-borne fungi and their ability to suppress tomato wilt and fruit rots. J. Microb. Biochem. Technol. 8 (2), 45-55. Available from: https://doi.org/10.4172/1948-5948.1000261.

Jaspers, P., Kangasjärvi, J., 2010. Reactive oxygen species in abiotic stress signaling. Physiol. Plant. 138 (4), 405-413. Available from: https:/ / doi.org/10.1111/j.1399-3054.2009.01321.x.

Jenkins, G.I., 2009. Signal transduction in responses to UV-B radiation. Annu. Rev. Plant Biol. 60, 407-431. Available from: https:// doi.org/10.1146/annurev.arplant.59.032607.092953.

Ji, X., Dong, B., Shiran, B., Talbot, M.J., Edlington, J.E., Hughes, T., et al., 2011. Control of abscisic acid catabolism and abscisic acid homeostasis is important for reproductive stage stress tolerance in cereals. Plant Physiol. 156 (2), 647-662. Available from: https://doi.org/10.1104/pp.111.176164.

Karakas, S., Cullu, M.A., Kaya, C., Dikilitas, M., 2016. Halophytic companion plants improve growth and physiological parameters of tomato plants grown under salinity. Pak. J. Bot. 48 (1), 21-28.

Karataglis, S., Moustakas, M., Symeonidis, L., 1991. Effect of heavy metals on isoperoxidases of wheat. Biol. Plant. 33 (1), 3-9. Available from: https:/ / doi.org/10.1007/BF02873778.

Kazan, K., 2015. Diverse roles of jasmonates and ethylene in abiotic stress tolerance. Trends Plant Sci. 20 (4), 219-229. Available from: https://doi.org/10.1016/j.tplants.2015.02.001.

Kim, T.H., Hauser, F., Ha, T., Xue, S., Böhmer, M., Nishimura, N., et al., 2011. Chemical genetics reveals negative regulation of abscisic acid signaling by a plant immune response pathway. Curr. Biol. 21 (11), 990-997. Available from: https://doi.org/ 10.1016/j.cub.2011.04.045.

Király, L., Hafez, Y.M., Fodor, J., Király, Z., 2008. Suppression of tobacco mosaic virus-induced hypersensitive-type necrotization in tobacco at high temperature is associated with downregulation of NADPH oxidase and superoxide and stimulation of dehydroascorbate reductase. J. Gen. Virol. 89 (3), 799-808. Available from: https://doi.org/10.1099/vir.0.83328-0.

Kissoudis, C., Sunarti, S., Van De Wiel, C., Visser, R.G., van der Linden, C.G., Bai, Y., 2016. Responses to combined abiotic and biotic stress in tomato are governed by stress intensity and resistance mechanism. J. Exp. Bot. 67 (17), 5119-5132. Available from: https://doi.org/10.1093/jxb/erw285.

Khan, M.I.R., Khan, N., 2017. Reactive Oxygen Species and Antioxidant System in Plants: Role and Regulation Under Abiotic Stress. Springer Nature, Singapore,978-981-10-5254-5.

Khan, M.I.R., Fatma, M., Per, T.S., Anjum, N.A., Khan, N.A., 2015. Salicylic acid-induced abiotic stress tolerance and underlying mechanisms in plants. Front. Plant Sci. 6, 462.

Lai, J., Cao, X., Yu, T., Wang, Q., Zhang, Y., Zheng, X., et al., 2018. Effect of Cryptococcus laurentii on inducing disease resistance in cherry tomato fruit with focus on the expression of defenserelated genes. Food. Chem. 254, 208-216. Available from: https:/ / doi.org/10.1016/j.foodchem.2018.01.100.

Lalithya, K.A., Manjunatha, G., Raju, B., Kulkarni, M.S., Lokesh, V., 2017. Plant growth regulators and signal molecules enhance resistance against bacterial blight disease of pomegranate.
J. Phytopathol. 165 (11-12), 727-736. Available from: https://doi. org/10.1111/jph.12612.

Li, F., Vallabhaneni, R., Wurtzel, E.T., 2008. PSY3, a new member of the phytoene synthase gene family conserved in the Poaceae and regulator of abiotic stress-induced root carotenogenesis. Plant Physiol. 146 (3), 1333-1345. Available from: https://doi.org/ 10.1104/pp.107.111120.

Li, J.B., Luan, Y.S., 2014. Molecular cloning and characterization of a pathogen-induced WRKY transcription factor gene from late blight resistant tomato varieties Solanum pimpinellifolium L3708. Physiol. Mol. Plant Pathol. 87, 25-31. Available from: https:// doi.org/10.1016/j.pmpp.2014.05.004.

Li, Z.G., 2016. Methylglyoxal and glyoxalase system in plants: old players, new concepts. Bot. Rev. 82 (2), 183-203. Available from: https:// doi.org/10.1007/s12229-016-9167-9.

Liang, D., Shen, Y., Ni, Z., Wang, Q., Lei, Z., Xu, N., et al., 2018. Exogenous melatonin application delays senescence of kiwifruit leaves by regulating the antioxidant capacity and biosynthesis of flavonoids. Front. Plant Sci. 9, 426. Available from: https://doi. org/10.3389/fpls.2018.00426.

Liu, X.M., Zhang, H., 2015. The effects of bacterial volatile emissions on plant abiotic stress tolerance. Front. Plant Sci. 6, 774. Available from: https://doi.org/10.3389/fpls.2015.00774.

Lu, J., Guo, M., Zhai, Y., Gong, Z., Lu, M., 2017. Differential responses to the combined stress of heat and Phytophthora capsici infection between resistant and susceptible germplasms of pepper (Capsicum annuum L.). J. Plant Growth Regul. 36 (1), 161-173. Available from: https:/ / doi.org/10.1007/s00344-016-9627-9.

Maggi, O., Tosi, S., Angelova, M., Lagostina, E., Fabbri, A.A., Pecoraro, L., et al., 2013. Adaptation of fungi, including yeasts, to cold environments. Plant Biosyst. 147, 247-258. Available from: https:/ / doi.org/10.1080/11263504.2012.753135.

Majsec, K., Cvjetko, P., Tolić, S., Tkalec, M., Balen, B., Pavlica, M., 2016. Integrative approach gives new insights into combined $\mathrm{Cd} /$ $\mathrm{Cu}$ exposure in tobacco. Acta Physiol. Plant. 38 (6), 142. Available from: https: / / doi.org/10.1007/s11738-016-2158-y.

Markham, J.E., Lynch, D.V., Napier, J.A., Dunn, T.M., Cahoon, E.B., 2013. Plant sphingolipids: function follows form. Curr. Opin. Plant Biol. 16 (3), 350-357. Available from: https://doi.org/ 10.1016/j.pbi.2013.02.009.

Massa, A.N., Childs, K.L., Buell, C.R., 2013. Abiotic and biotic stress responses in Solanum tuberosum group Phureja DM1-3 516 R44 as measured through whole transcriptome sequencing. Plant Genome 6 (3), 1-10. Available from: https://doi.org/10.3835/ plantgenome2013.05.0014.

Mauch-Mani, B., Mauch, F., 2005. The role of abscisic acid in plant-pathogen interactions. Curr. Opin. Plant Biol. 8 (4), 409-414. Available from: https://doi.org/10.1016/j.pbi.2005.05.015.

Mayek-Pérez, N., Garć́a-Espinosa, R., LÓpez-CastaÑeda, C., AcostaGallegos, J.A., Simpson, J., 2002. Water relations, histopathology and growth of common bean (Phaseolus vulgaris L.) during pathogenesis of Macrophomina phaseolina under drought stress. Physiol. Mol. Plant Pathol. 60 (4), 185-195. Available from: https://doi. org/10.1006/pmpp.2001.0388.

Melotto, M., Underwood, W., Koczan, J., Nomura, K., He, S.Y., 2006. Plant stomata function in innate immunity against bacterial invasion. Cell 126 (5), 969-980. Available from: https://doi.org/ 10.1016/j.cell.2006.06.054.

Milus, E.A., Kristensen, K., Hovmøller, M.S., 2009. Evidence for increased aggressiveness in a recent widespread strain of Puccinia striiformis f. sp. tritici causing stripe rust of wheat. Phytopathology 99 (1), 89-94. Available from: https://doi.org/ 10.1094/PHYTO-99-1-0089.

Mishra, J., Fatima, T., Arora, N.K., 2018. Role of secondary metabolites from plant growth-promoting rhizobacteria in combating 
salinity stress. In: Egamberdieva, D., Ahmad, P. (Eds.), Plant Microbiome: Stress Response. Microorganisms for Sustainability, vol 5. Springer, Singapore, pp. 127-163. Available from: https:// doi.org/10.1007/978-981-10-5514-0_6.

Mittler, R., 2006. Abiotic stress, the field environment and stress combination. Trends Plant Sci. 11 (1), 15-19. Available from: https:// doi.org/10.1016/j.tplants.2005.11.002.

Mohr, P.G., Cahill, D.M., 2003. Abscisic acid influences the susceptibility of Arabidopsis thaliana to Pseudomonas syringae pv. tomato and Peronospora parasitica. Funct. Plant Biol. 30 (4), 461-469. Available from: https://doi.org/10.1071/FP02231.

Møller, I.M., Jensen, P.E., Hansson, A., 2007. Oxidative modifications to cellular components in plants. Annu. Rev. Plant Biol. 58, 459-481. Available from: https://doi.org/10.1146/annurev. arplant.58.032806.103946.

Morkunas, I., Ratajczak, L., 2014. The role of sugar signaling in plant defense responses against fungal pathogens. Acta Physiol. Plant. 36 (7), 1607-1619. Available from: https://doi.org/10.1007/ s11738-014-1559-z.

Moury, B., Selassie, K.G., Marchoux, G., Daubèze, A.M., Palloix, A., 1998. High temperature effects on hypersensitive resistance to tomato spotted wilt to spovirus (TSWV) in pepper (Capsicum chinense Jacq.). Eur. J. Plant Pathol. 104 (5), 489-498. Available from: https:/ / doi.org/10.1023/A:1008618022144.

Nejat, N., Mantri, N., 2017. Plant Immune System: crosstalk between responses to biotic and abiotic stresses the missing link in understanding plant defence. Curr. Issues Mol. Biol. 23, 1-16. Available from: https://doi.org/10.21775/cimb.023.001.

Nguyen, D., Rieu, I., Mariani, C., Van Dam, N.M., 2016. How plants handle multiple stresses: hormonal interactions underlying responses to abiotic stress and insect herbivory. Plant Mol. Biol. 91 (6), 727-740. Available from: https://doi.org/10.1007/s11103016-0481-8.

Nostar, O., Ozdemir, F., Bor, M., Turkan, I., Tosun, N., 2013. Combined effects of salt stress and cucurbit downy mildew (Pseudoperospora cubensis Berk. and Curt. Rostov.) infection on growth, physiological traits and antioxidant activity in cucumber (Cucumis sativus L.) seedlings. Physiol. Mol. Plant Pathol. 83, 84-92. Available from: https://doi.org/10.1016/j. pmpp.2013.05.004.

Okazaki, Y., Saito, K., 2014. Roles of lipids as signaling molecules and mitigators during stress response in plants. Plant J. 79 (4), 584-596. Available from: https://doi.org/10.1111/tpj.12556.

Orhan, F., 2016. Alleviation of salt stress by halotolerant and halophilic plant growth-promoting bacteria in wheat (Triticum aestivum). Braz. J. Microbiol. 47 (3), 621-627. Available from: https:// doi.org/10.1016/j.bjm.2016.04.001.

Pandey, P., Ramegowda, V., Senthil-Kumar, M., 2015. Shared and unique responses of plants to multiple individual stresses and stress combinations: physiological and molecular mechanisms. Front. Plant Sci. 6, 723. Available from: https://doi.org/10.3389/ fpls.2015.00723.

Pazarlar, S., Cetinkaya, N., Bor, M., Ozdemir, F., 2017. Ozone triggers different defence mechanisms against powdery mildew (Blumeria graminis DC. Speer f. sp. tritici) in susceptible and resistant wheat genotypes. Funct. Plant Biol. 44 (10), 1016-1028. Available from: https:// doi.org/10.1071/FP17038.

Per, T.S., Khan, N.A., Reddy, P.S., Masood, A., Hasanuzzaman, M., Khan, M.I.R., et al., 2017. Approaches in modulating proline metabolism in plants for salt and drought stress tolerance: phytohormones, mineral nutrients and transgenics. Plant Physiol. Biochem. 115, 126-140. Available from: https://doi.org/10.1016/ j.plaphy.2017.03.018.

Petrov, V., Hille, J., Mueller-Roeber, B., Gechev, T.S., 2015. ROSmediated abiotic stress-induced programmed cell death in plants.
Front. Plant Sci. 6, 69. Available from: https://doi.org/10.3389/ fpls.2015.00069.

Pitzschke, A., Schikora, A., Hirt, H., 2009. MAPK cascade signalling networks in plant defence. Curr. Opin. Plant Biol. 12 (4), 421-426. Available from: https://doi.org/10.1016/j.pbi.2009.06.008.

Prasad, P.V.V., Pisipati, S.R., Momčilović, I., Ristic, Z., 2011. Independent and combined effects of high temperature and drought stress during grain filling on plant yield and chloroplast EF-Tu expression in spring wheat. J. Agron. Crop Sci. 197 (6), 430-441. Available from: https://doi.org/10.1111/j.1439-037X.2011.00477.x.

Prasch, C.M., Sonnewald, U., 2013. Simultaneous application of heat, drought, and virus to Arabidopsis plants reveals significant shifts in signaling networks. Plant Physiol. 162 (4), 1849-1866. Available from: https://doi.org/10.1104/pp.113.221044.

Prasch, C.M., Sonnewald, U., 2015. Signaling events in plants: stress factors in combination change the picture. Environ. Exp. Bot. 114, 4-14. Available from: https://doi.org/10.1016/j.envexpbot.2014.06.020.

Pye, M.F., Hakuno, F., MacDonald, J.D., Bostock, R.M., 2013. Induced resistance in tomato by SAR activators during predisposing salinity stress. Front. Plant Sci. 4, 116. Available from: https://doi. org/10.3389/fpls.2013.00116.

Radwan, O., 2015. Genomics improvement of agronomic crops to abiotic stress. J. Invest. Gen. 2 (3), 1-7. Available from: https:// doi.org/10.15406/jig.2015.02.00026.

Ramegowda, V., Senthil-Kumar, M., 2015. The interactive effects of simultaneous biotic and abiotic stresses on plants: mechanistic understanding from drought and pathogen combination. J. Plant Physiol. 176, 47-54. Available from: https://doi.org/10.1016/j. jplph.2014.11.008.

Ramegowda, V., Senthil-Kumar, M., Ishiga, Y., Kaundal, A., Udayakumar, M., Mysore, K.S., 2013. Drought stress acclimation imparts tolerance to Sclerotinia sclerotiorum and Pseudomonas syringae in Nicotiana benthamiana. Int. J. Mol. Sci. 14 (5), 9497-9513. Available from: https://doi.org/10.3390/ijms14059497.

Rejeb, I.B., Pastor, V., Mauch-Mani, B., 2014. Plant responses to simultaneous biotic and abiotic stress: molecular mechanisms. Plants 3 (4), 458-475. Available from: https://doi.org/10.3390/ plants3040458.

Rizhsky, L., Liang, H., Mittler, R., 2002. The combined effect of drought stress and heat shock on gene expression in tobacco. Plant Physiol. 130 (3), 1143-1151. Available from: https://doi. org/10.1104/pp.006858.

Robert-Seilaniantz, A., Navarro, L., Bari, R., Jones, J.D., 2007. Pathological hormone imbalances. Curr. Opin. Plant Biol. 10 (4), 372-379. Available from: https://doi.org/10.1016/j.pbi.2007.06.003.

Rolland, F., Baena-Gonzalez, E., Sheen, J., 2006. Sugar sensing and signaling in plants: conserved and novel mechanisms. Annu. Rev. Plant Biol. 57, 675-709. Available from: https://doi.org/10.1146/ annurev.arplant.57.032905.105441.

Roy, S., 2014. Maintenance of genome stability in plants: repairing DNA double strand breaks and chromatin structure stability. Front. Plant Sci. 5, 487. Available from: https://doi.org/10.3389/ fpls.2014.00487.

Santino, A., Taurino, M., De Domenico, S., Bonsegna, S., Poltronieri, P., Pastor, V., et al., 2013. Jasmonate signaling in plant development and defense response to multiple (a) biotic stresses. Plant Cell Rep. 32 (7), 1085-1098. Available from: https://doi.org/ 10.1007/s00299-013-1441-2.

Savvides, A., Ali, S., Tester, M., Fotopoulos, V., 2016. Chemical priming of plants against multiple abiotic stresses: mission possible? Trends Plant Sci. 21 (4), 329-340. Available from: https://doi. org/10.1016/j.tplants.2015.11.003.

Sebastiani, M.S., Bagnaresi, P., Sestili, S., Biselli, C., Zechini, A., Orrù, L., et al., 2017. Transcriptome analysis of the melon-Fusarium oxysporum f. sp. melonis race 1.2 pathosystem in susceptible and 
resistant plants. Front. Plant Sci. 8, 362. Available from: https:// doi.org/10.3389/fpls.2017.00362.

Seybold, H., Trempel, F., Ranf, S., Scheel, D., Romeis, T., Lee, J., 2014. $\mathrm{Ca}^{2+}$ signalling in plant immune response: from pattern recognition receptors to $\mathrm{Ca}^{2+}$ decoding mechanisms. New Phytol. 204 (4), 782-790. Available from: https://doi.org/ 10.1111/nph.13031.

Shankar, V., Kumar, D., Agrawal, V., 2016. Assessment of antioxidant enzyme activity and mineral nutrients in response to $\mathrm{NaCl}$ stress and its amelioration through glutathione in chickpea. Appl. Biochem. Biotechnol. 178 (2), 267-284. Available from: https:// doi.org/10.1007/s12010-015-1870-1.

Sharma, P., Jha, A.B., Dubey, R.S., Pessarakli, M., 2012. Reactive oxygen species, oxidative damage, and antioxidative defense mechanism in plants under stressful conditions. J. Bot. 2012, 1-26. Available from: https://doi.org/10.1155/2012/217037.

Sharma, R.C., Duveiller, E., Ortiz-Ferrara, G., 2007. Progress and challenge towards reducing wheat spot blotch threat in the Eastern Gangetic Plains of South Asia: is climate change already taking its toll? Field Crops Res. 103 (2), 109-118. Available from: https:/ / doi.org/10.1016/j.fcr.2007.05.004.

Sharma, S., Kulkarni, J., Jha, B., 2016. Halotolerant rhizobacteria promote growth and enhance salinity tolerance in peanut. Front. Microbiol. 7, 1600. Available from: https://doi.org/10.3389/ fmicb.2016.01600.

Sherwood, P., Villari, C., Capretti, P., Bonello, P., 2015. Mechanisms of induced susceptibility to Diplodia tip blight in droughtstressed Austrian pine. Tree Physiol. 35 (5), 549-562. Available from: https://doi.org/10.1093/treephys/tpv026.

Shinozaki, K., Yamaguchi-Shinozaki, K., 2007. Gene networks involved in drought stress response and tolerance. J. Exp. Bot. 58 (2), 221-227. Available from: https://doi.org/10.1093/jxb/erl164.

Shu-Hsien, H., Chih-Wen, Y.U., Lin, C.H., 2005. Hydrogen peroxide functions as a stress signal in plants. Bot. Bullet. Acad. Sin. 46, 1-10.

Silveira, G.L., Lima, M.G.F., dos Reis, G.B., Palmieri, M.J., AndradeVieria, L.F., 2017. Toxic effects of environmental pollutants: comparative investigation using Allium cepa L. and Lactuca sativa L. Chemosphere 178, 359-367. Available from: https://doi.org/ 10.1016/j.chemosphere.2017.03.048.

Sinclair, G., Charest, C., Dalpé, Y., Khanizadeh, S., 2014. Influence of colonization by arbuscular mycorrhizal fungi on three strawberry cultivars under salty conditions. Agric. Food Sci. 23 (2), 146-158.

Sinha, R., Gupta, A., Senthil-Kumar, M., 2016. Understanding the impact of drought on foliar and xylem invading bacterial pathogen stress in chickpea. Front. Plant Sci. 7, 902. Available from: https: / / doi.org/10.3389/fpls.2016.00902.

Smeekens, S., Ma, J., Hanson, J., Rolland, F., 2010. Sugar signals and molecular networks controlling plant growth. Curr. Opin. Plant Biol. 13 (3), 273-278. Available from: https://doi.org/10.1016/j. pbi.2009.12.002.

Sreenivasulu, N., Harshavardhan, V.T., Govind, G., Seiler, C., Kohli, A., 2012. Contrapuntal role of ABA: does it mediate stress tolerance or plant growth retardation under long-term drought stress? Gene 506 (2), 265-273. Available from: https:/ / doi.org/10.1016/j. gene.2012.06.076.

Stockwell, M.P., Clulow, J., Mahony, M.J., 2012. Sodium chloride inhibits the growth and infective capacity of the amphibian chytrid fungus and increases host survival rates. PLoS One 7 (5), e36942. Available from: https://doi.org/10.1371/journal.pone.0036942.

Sudisha, J., Sharathchandra, R.G., Amruthesh, K.N., Kumar, A., Shetty, H.S., 2012. Pathogenesis related proteins in plant defense response. In: Mérillon, J., Ramawat, K. (Eds.), Plant Defence: Biological Control. Progress in Biological Control, vol. 12. Springer, Dordrecht, pp. 379-403. Available from: https://doi.org/10.1007/ 978-94-007-1933-0_17.
Sun, M., Feng, X.X., Gao, J.J., Peng, R.H., Yao, Q.H., Wang, L.J., 2017. VvMYBA6 in the promotion of anthocyanin biosynthesis and salt tolerance in transgenic Arabidopsis. Plant Biotechnol. Rep. 11 (5), 299-314. Available from: https://doi.org/10.1007/s11816-0170452-9.

Suzuki, N., Katano, K., 2018. Coordination between ROS regulatory systems and other pathways under heat stress and pathogen attack. Front. Plant Sci. 9, 490. Available from: https://doi.org/ 10.3389 /fpls.2018.00490.

Suzuki, N., Koussevitzky, S., Mittler, R.O.N., Miller, G.A.D., 2012. ROS and redox signalling in the response of plants to abiotic stress. Plant Cell Environ. 35 (2), 259-270. Available from: https: / / doi.org/10.1111/j.1365-3040.2011.02336.x.

Suzuki, N., Rivero, R.M., Shulaev, V., Blumwald, E., Mittler, R., 2014. Abiotic and biotic stress combinations. New Phytol. 203 (1), 32-43. Available from: https://doi.org/10.1111/nph.12797.

Swiecki, T.J., MacDonald, J.D., 1991. Soil salinity enhances phytophthora root rot of tomato but hinders asexual reproduction by Phytophthora parasitica. J. Amer. Soc. Hort. Sci. 116, $471-477$

Per, T.S., Khan, M.I.R., Anjum, N.A., Masood, A., Hussain, S.J., Khan, N.A., 2018. Jasmonates in plants under abiotic stresses: crosstalk with other phytohormones matters. Environ. Exp. Bot. $145,104-120$.

Tauzin, A.S., Giardina, T., 2014. Sucrose and invertases, a part of the plant defense response to the biotic stresses. Front. Plant Sci. 5, 293. Available from: https://doi.org/10.3389/fpls.2014.00293.

Thakur, P., Kumar, S., Malik, J.A., Berger, J.D., Nayyar, H., 2010. Cold stress effects on reproductive development in grain crops: an overview. Environ. Exp. Bot. 67 (3), 429-443. Available from: https://doi.org/10.1016/j.envexpbot.2009.09.004.

Thaler, J.S., Bostock, R.M., 2004. Interactions between abscisic-acidmediated responses and plant resistance to pathogens and insects. Ecology 85 (1), 48-58. Available from: https://doi.org/ 10.1890/02-0710.

Thomma, B.P., Penninckx, I.A., Cammue, B.P., Broekaert, W.F., 2001. The complexity of disease signaling in Arabidopsis. Curr. Opin. Immunol. 13 (1), 63-68. Available from: https://doi.org/ 10.1016/S0952-7915(00)00183-7.

Ton, J., Flors, V., Mauch-Mani, B., 2009. The multifaceted role of ABA in disease resistance. Trends Plant Sci. 14 (6), 310-317. Available from: https://doi.org/10.1016/j.tplants.2009.03.006.

Ton, J., Mauch-Mani, B., 2004. $\beta$-amino-butyric acid-induced resistance against necrotrophic pathogens is based on ABA-dependent priming for callose. Plant J. 38 (1), 119-130. Available from: https://doi.org/10.1111/j.1365-313X.2004.02028.x.

Torres, M.A., Dangl, J.L., Jones, J.D., 2002. Arabidopsis gp91phox homologues AtrbohD and AtrbohF are required for accumulation of reactive oxygen intermediates in the plant defense response. Proc. Natl. Acad. Sci. 99 (1), 517-522. Available from: https:// doi.org/10.1073/pnas.012452499.

Umezawa, T., Fujita, M., Fujita, Y., Yamaguchi-Shinozaki, K., Shinozaki, K., 2006. Engineering drought tolerance in plants: discovering and tailoring genes to unlock the future. Curr. Opin. Biotechnol. 17 (2), 113-122. Available from: https://doi.org/ 10.1016/j.copbio.2006.02.002.

Vaishnav, A., Sharma, S.K., Choudhary, D.K., Sharma, K.P., Ahmad, E., Sharma, M.P., et al., 2018. Nitric oxide as a signaling molecule in plant-bacterial interactions. In: Egamberdieva, D., Ahmad, P. (Eds.), Plant Microbiome: Stress Response. Springer, Singapore, pp. 183-199. Available from: https://doi.org/10.1007/978-98110-5514-0_8.

Verma, S., Nizam, S., Verma, P.K., 2013. Biotic and abiotic stress signaling in plants. In: Sarwat, M., Ahmad, A., Abdin, M.Z. (Eds.), Stress Signaling in Plants: Genomics and Proteomics Perspective, 
vol. 1. Springer, New York, NY, pp. 25-49. Available from: https://doi.org/10.1007/978-1-4614-6372-6_2.

Vos, I.A., Verhage, A., Schuurink, R.C., Watt, L.G., Pieterse, C.M., Van Wees, S., 2013. Onset of herbivore-induced resistance in systemic tissue primed for jasmonate-dependent defenses is activated by abscisic acid. Front. Plant Sci. 4, 539. Available from: https:/ / doi.org/10.3389/fpls.2013.00539.

Walley, J.W., Dehesh, K., 2010. Molecular mechanisms regulating rapid stress signaling networks in Arabidopsis. J. Integr. Plant Biol. 52 (4), 354-359. Available from: https://doi.org/10.1111/j.17447909.2010.00940.x.

Wang, Y., Loake, G.J., Chu, C., 2013. Cross-talk of nitric oxide and reactive oxygen species in plant programed cell death. Front. Plant Sci. 4, 314. Available from: https://doi.org/10.3389/ fpls.2013.00314.

Wani, A.S., Tahir, I., Ahmad, S.S., Dar, R.A., Nisar, S., 2017. Efficacy of 24-epibrassinolide in improving the nitrogen metabolism and antioxidant system in chickpea cultivars under cadmium and/or $\mathrm{NaCl}$ stress. Sci. Hortic. (Amsterdam) 225, 48-55. Available from: https:/ / doi.org/10.1016/j.scienta.2017.06.063.

Wan-Ibrahim, W.I., Sidik, K., Kuppusamy, U.R., 2010. A high antioxidant level in edible plants is associated with genotoxic properties. Food. Chem. 122 (4), 1139-1144. Available from: https://doi. org/10.1016/j.foodchem.2010.03.101.

Wiese, J., Kranz, T., Schubert, S., 2004. Induction of pathogen resistance in barley by abiotic stress. Plant Biol. 6 (5), 529-536. Available from: https://doi.org/10.1055/s-2004-821176.
Wind, J., Smeekens, S., Hanson, J., 2010. Sucrose: metabolite and signaling molecule. Phytochemistry 71 (14-15), 1610-1614. Available from: https://doi.org/10.1016/j.phytochem.2010.07.007.

Wong, H.L., Shimamoto, K., 2009. Sending ROS on a bullet train. Sci. Signal. 2 (90), pe60. Available from: https://doi.org/10.1126/ scisignal.290pe60.

Xu, P., Chen, F., Mannas, J.P., Feldman, T., Sumner, L.W., Roossinck, M.J., 2008. Virus infection improves drought tolerance. New Phytol. 180 (4), 911-921. Available from: https://doi.org/ 10.1111/j.1469-8137.2008.02627.x.

Yamaguchi-Shinozaki, K., Shinozaki, K., 2006. Transcriptional regulatory networks in cellular responses and tolerance to dehydration and cold stresses. Annu. Rev. Plant Biol. 57, 781-803. Available from: https:/ / doi.org/10.1146/annurev.arplant.57.032905.105444.

Yusuf, M., Fariduddin, Q., Khan, T.A., Hayat, S., 2017. Epibrassinolide reverses the stress generated by combination of excess aluminum and salt in two wheat cultivars through altered proline metabolism and antioxidants. South Afr. J. Bot. 112, 391-398. Available from: https://doi.org/10.1016/j.sajb.2017.06.034.

Zheng, J., Ma, X., Zhang, X., Hu, Q., Qian, R., 2018. Salicylic acid promotes plant growth and salt-related gene expression in Dianthus superbus L. (Caryophyllaceae) grown under different salt stress conditions. Physiol. Mol. Biol. Plants 24 (2), 231-238. Available from: https:/ / doi.org/10.1007/s12298-017-0496-x.

Zhu, J.K., 2002. Salt and drought stress signal transduction in plants. Annu. Rev. Plant Biol. 53 (1), 247-273. Available from: https:// doi.org/10.1146/annurev.arplant.53.091401.143329. 


\section{3}

\section{Engineering Signaling Molecules to Improve Abiotic Stress Tolerance in Crop Plants}

\section{Krishna Kumar Guduru , Chandra Sekhar Akila and Chandra Obul Reddy Puli ${ }^{1}$}

${ }^{1}$ Plant Molecular Biology Laboratory, Department of Botany, Yogi Vemana University, Kadapa, Andhra Pradesh, India

${ }^{2}$ Department of Biotechnology, Yogi Vemana University, Kadapa, Andhra Pradesh, India

\section{O U T L I N E}

3.1 Introduction

3.2 Stress Signal Sensors

3.3 Salt Stress Sensors

3.4 Osmotic Stress Sensors

3.5 ABA Signaling Pathway

3.6 Calcium Sensors and Signaling
$43 \quad 3.7$ ROS Signaling

$44 \quad 3.8$ Conclusions and Future Perspectives 55

45 Acknowledgement $\quad 55$

45 References 56

51 Further Reading 62

53

\subsection{INTRODUCTION}

Plants as sessile organisms come across several biotic and abiotic stresses during their course of growth and development. Global climatic changes impose abiotic stresses such as drought, salt, and extreme temperatures, which majorly affect geographical distribution of plants and agricultural productivity and cause a dearth of food (Fedoroff et al., 2010). It has been estimated that globally $70 \%$ of plants' potential yield is reduced by abiotic stresses (Acquaah, 2007). Rapid changes in global climate are predicted to increase the intensity of abiotic stresses in the near future; at the same time global population is expected to reach 9 billion by 2030 (Husaini and Tuteja, 2013). Plant scientists anticipate a need to improve plants to enhance productivity under adverse climatic conditions to meet the global population demands. Conventional breeding methods proved unsuccessful for complex traits, that is, abiotic stress. Advancements in the field of plant improvement technologies such as transgenic approaches permit to introduce gene(s) from a wide range of organisms into plants and to develop transgenic plants to cope with complex abiotic stresses in a faster way. Identification of candidate genes is the foremost step in the development of transgenic plants. It is of utmost importance to understand how the plants sense abiotic stress signals and transduce at the molecular level for successful 
development of stress tolerant plants (Xiong et al., 2002; Zhu, 2016). Plants respond to this hostile environment, assimilate several molecular and cellular responses, and develop defense mechanisms to overcome the effect of abiotic stresses. Abiotic stress signal perception and transduction at the cellular level are important steps to ignite molecular adaptive responses of plants to extreme environments. The key genes involved in abiotic stress signal sensing, perception, and transduction comprise receptor molecules/osmosensors, phospholipid-cleaving enzymes, reactive oxygen species (ROS), mitogen-activated protein kinases (MAPKs), and $\mathrm{Ca}^{2+}$ sensors. Several signaling molecules have been successfully used for the development of transgenic plants for abiotic stress tolerance in plants. In this chapter we discuss mainly the abiotic stress responsive signaling molecules used for the engineering of plants for abiotic stress tolerance.

\subsection{STRESS SIGNAL SENSORS}

Plant cells apparently display precise gene expression, metabolic, and phenotypic response to different environmental stresses (Zhu, 2016). However, despite a lot of research to date only a few putative stress sensors have been identified. Most of the genes encoding stress sensors display functional redundancy for a variety of environmental stresses. Environmental stresses persuade incursion of transient $\mathrm{Ca}^{2+}$ into the cytoplasm $\left(\mathrm{Ca}^{2+}\right)_{\text {cyt }}$ of the cell (Sanders et al., 1999; Knight, 2000; Xiong et al., 2002). The $\mathrm{Ca}^{2+}$ channels are mainly involved in the transport of $\left(\mathrm{Ca}^{2+}\right)_{\text {cyt. }}$ Osmosensing $\mathrm{Ca}^{2+}$ channels might serve as osmosensors in bacteria, yeast, and animal cells (Kung, 2005; Arnadottir et al., 2010). Recently, a hyperosmotic responsive putative plasma membrane bound $\mathrm{Ca}^{2+}$ sensor gene OSCA1 (reduced hyperosmolality-induced calcium increase 1) was identified in Arabidopsis roots through a calcium imaging-based unbiased forward genetic screen (Yuan et al., 2014a,b). Arabidopsis wild plants accumulated more $\left(\mathrm{Ca}^{2+}\right)_{\text {cyt }}$ displayed drought and salt stress phenotypic symptoms when grown in the media supplemented with sorbitol and mannitol compared with mutant plants with loss-of-function of oscal gene (Yuan et al., 2014a,b). This data suggests that OSCA1 might function as an osmotic sensor in response to environmental stress to induce $\left(\mathrm{Ca}^{2+}\right)_{\text {cyt }}$. Nonetheless, the mechanism of how OSCA1 senses the osmotic stress signals is not known yet; probably it might decrease the turgor pressure and affect the plasma membrane-cell wall interactions (Zhu, 2016) and osmosensors. Several eukaryotic organisms apart from plants have numerous mechanosensitive channels, a subtype of osmosensors including MscS-like, Piezo, K2P, DEG/ENaC, and TRP
(Arnadottir et al., 2010; Hedrich, 2012). Among them, MscS-like family proteins and Piezo mechanosensitive channel proteins were well characterized (Hedrich, 2012). One of the Arabidopsis mechanosensitive ion channel proteins, MSL8, an MscS-like family protein is critical for protection of pollen from hypoosmotic shock during repeated desiccation and rehydration cycles of pollen germination and pollen tube growth (Hamilton et al., 2015). The MSL8 are confined to plasma membrane of tricellular, mature pollen, and pollen tubes, express only during osmotic stress, regulate the movement of ions, and protect the cell membrane from bursting (Hamilton and Haswell, 2017).

Further, plants also encompass a family of glutamate receptor-like (GLR) channels and a large family of nonspecific cation channels cyclic nucleotide-gated channels (CNGCs) to sense environmental signal and modulate cytosolic calcium signals (Finka et al., 2012; Steinhorst and Kudla, 2013; Swarbreck et al., 2013). The CNGCs family consists of 20 members in Arabidopsis, which mainly interact with a self-activated $\alpha$-subunit of heterotrimeric $G$ proteins to activate the $\mathrm{Ca}^{2+}$ channels through GTPase activity (Ma et al., 2015). Recently, Ma et al. (2015) identified a QTL harboring gene, chilling-tolerance divergence 1 (COLD1), which confers cold tolerance to rice. COLD1 belongs to the CNGC family transmembrane protein; it is localized in the plasma membrane and endoplasmic reticulum (ER), acts as a cold-sensing calcium channel protein or regulates calcium channel proteins to sense low temperature stress signal, and might interact with RGA1 of the $\alpha$-subunit of G-protein (Ma et al., 2015). Further, overexpression studies of COLD1 gene in Zhonghua10 of japonica cultivar clearly conferred enhanced cold tolerance nature of the gene; in contrast antisense expression of COLD1 in transgenic plants displayed decreased cold tolerance (Ma et al., 2015).

Another important group of proteins that sense the changes in the fluidity of plasma membrane during environmental stress are membrane bound receptorlike kinases (RLKs) (Sangwan et al., 2002). RLKs are serine/threonine protein kinase gene family proteins consisting of large numbers of genes including 610 in Arabidopsis and 1132 members of rice (Shiu et al., 2004). The RLK protein comprises an extracellular ligand-binding domain (ECLB), a transmembrane domain (TM), and a protein kinase catalytic domain (PKC) (Walker et al., 1995; Greeff et al., 2012). The ECLB domain combines the stress signal and transmits through a TM domain; a further signal is transmitted to the intracellular region by the PKC domain, which activates or represses the gene expression based on phosphorylation and dephosphorylation of protein conformation changes (Ye et al., 2016). Several RLK 
genes have been identified and functionally characterized in several other plant species in response to environmental stimuli (Ye et al., 2016). A drought and ABA-induced LRR-RLK gene of rice, FON1, when overexpressed in transgenic rice, conferred drought tolerance and sensitivity to ABA compared with FON1-RNAi suppressed transgenic rice plants (Feng et al., 2014).

\subsection{SALT STRESS SENSORS}

Salt concentration $\left(\mathrm{Na}^{+}\right)$in the soil significantly influences the growth and development of plants; it also leads to hyperosmotic stress and oxidative stress (Zhu, 2002). Information is extremely limited on how the soil salinity $\left(\mathrm{Na}^{+}\right)$is sensed by the multicellular organisms (Zhu, 2016). Unicellular organisms such as yeast sense the $\mathrm{Na}^{+}$ions through calmodulin and calcineurin. However, plants do not have calcineurin; as a substitute they use calcium-binding protein kinase proteins SOS (salt overly sensitive) (Zhu, 2002). In the root cells, the soil salt concentration educes cytosolic calcium levels, and the calcium sensor protein SOS3 senses the signal and activates a serine/threonine protein kinase protein (SOS2), which phosphorylates and positively regulates the SOS1, a plasma membrane bound sodium/proton antiporter ( $\mathrm{Zhu}, 2002)$. The SOS1 localizes in both the root epidermis as well as in xylem cells; the one that is localized in the root epidermal cells excretes the $\mathrm{Na}^{+}$into the soil media and protects the cells from sodium toxicity, and the one that is localized in the xylem parenchymatic cells excludes the $\mathrm{Na}^{+}$from xylem cells to the apoplastic space of leaf mesophyll cells for long-distance transport (Shi et al., 2002; Zhu, 2016). The SOS3 along with SOS3 homolog protein SCaBBP8/calcineurin B-like 10 (CBL10) augments SOS2 kinase activity and protect the shoots and roots from salt-induced damage (Du et al., 2008). In Arabidopsis several (sucrose nonfermenting 1)-related protein kinase (SnRKs) families whose catalytic domain was similar to SOS2 protein kinases were identified. A total of 37 member SnRKs belonging to 3 subfamilies interacts with SOS3-like calcium-binding proteins (ScaBPs) (Guo et al., 2001). The ScaBP further interacts with PKS/CIPK and inhibits $\mathrm{H}^{+}$-ATPase on the plasma membrane (Fuglsang et al., 2007). Recently, genetic analysis in Arabidopsis revealed that a cellulose synthase like D6 (AtCSLD6)/SOS6 provides osmotic tolerance through regulation of ROS levels (Zhu et al., 2010).

Transgenic overexpression of SOS1 cDNA in the salt sensitive Arabidopsis sos1 mutant cells enhances the salt tolerance (Shi et al., 2000). Transgenic tomato plants overexpressing the SISOS1 revealed that SOS1 is not only important for salt tolerance, but also it is vital for the distribution of $\mathrm{Na}^{+}$between plant organs (Olías et al., 2009). Constitutive overexpression of SOS2 in Arabidopsis (AtSOS2), tomato (SISOS2), apple (MdSOS2), and poplar (PtSOS2) increased the salt tolerance in transgenic plants by enabling optimal ion homeostasis and enhanced antioxidative capacity (Yang et al., 2015). Transgenic overexpression of SOS pathway genes SOS1, SOS2, SOS3 along with AtNHX1 with different combinations moderately improved the salt tolerance similar to the overexpression of the any of the genes alone (Yang et al., 2009). However, in contrast transgenic coexpression of Arabidopsis thaliana SOS1 + SOS2 + SOS3 + CBL10 genes under a stress inducible promoter $r d 29 a$ in tall fescue plants improved the excretion of $\mathrm{Na}^{+}$and uptake of $\mathrm{K}^{+}$in transgenic plant roots and improved the salt tolerance in transgenic tall fescue (Ma et al., 2014). Similarly, stacked overexpression of AtNHX1 and SOS1 in transgenic Arabidopsis plants significantly improved the salt tolerance in transgenic Arabidopsis plants (Pehlivan et al., 2016). This data clearly revealed that genetic manipulation of SOS pathway improves the salt tolerance in plants (Table 3.1).

\subsection{OSMOTIC STRESS SENSORS}

As discussed earlier the calcium channel OSCA1 senses the osmotic stress signal and elicits cytosolic calcium levels (Yuan et al., 2014a,b). The calcium levels activate the CPKs and ScaBP/CBL-PKS/CIPKs (Zhu, 2016). In Arabidopsis several SnRK2 family protein kinases were activated by osmotic stress (Boudsocq et al., 2004). However, it is not clear how the osmotic stress activates the SnRK2s. The genetic studies in Arabidopsis where all 10 members of SnRK2 family members were deactivated revealed that the hyperosmotic stress retards the growth, gene expression, accumulation of compatible osmolyte proline, secondary messenger inositol 1,4,5-trisphosphate (IP3), and abscisic acid (ABA) content in snrk2 mutant plants, however in the absence of osmotic stress they grow like wild-type plants (Fujii et al., 2011). This study clearly disclosed that SnRK2 are important players; they are upstream components of ABA accumulation and are critical for osmotic adjustment during osmotic stress signaling (Fujii et al., 2011). A recent study in the moss Psyscomitrella patens revealed that the ABA and abiotic stress-responsive Raf-like kinase (ARK) play a major role in activation of SnRK2s under both osmotic as well ABA stress (Saruhashi et al., 2015).

Tobacco NtC7 might be involved in sensing of osmotic stress signals. Transient expression of 
TABLE 3.1 Transgenic Plants Overexpressing Signaling Molecules for Abiotic Stress Tolerance

\begin{tabular}{|c|c|c|c|c|c|c|c|}
\hline $\begin{array}{l}\text { Functional } \\
\text { category }\end{array}$ & Genes & Protein function & Origin & $\begin{array}{l}\text { Transformation } \\
\text { receptor }\end{array}$ & Promoter & $\begin{array}{l}\text { Abiotic } \\
\text { stress } \\
\text { regulation }\end{array}$ & References \\
\hline Protein kinase & SOS1 & Salt overly sensitive 1 & Arabidopsis & Arabidopsis & - & $\begin{array}{l}\text { Salt } \\
\text { tolerance }\end{array}$ & $\begin{array}{l}\text { Qiu et al. } \\
\text { (2004) }\end{array}$ \\
\hline Protein kinase & SOS2 & Salt overly sensitive 2 & Arabidopsis & Arabidopsis & - & $\begin{array}{l}\text { Salt } \\
\text { tolerance }\end{array}$ & $\begin{array}{l}\text { Ohta et al. } \\
\text { (2003) }\end{array}$ \\
\hline Protein kinase & SOS2 & Salt overly sensitive 2 & Arabidopsis & Arabidopsis & - & $\begin{array}{l}\text { Salt } \\
\text { tolerance }\end{array}$ & $\begin{array}{l}\text { Liu et al. } \\
\text { (2011) }\end{array}$ \\
\hline Protein kinase & SOS3 & Salt overly sensitive 3 & Arabidopsis & Arabidopsis & - & $\begin{array}{l}\text { Salt } \\
\text { tolerance } \\
\text { Ion } \\
\text { homeostasis }\end{array}$ & $\begin{array}{l}\text { Guo et al. } \\
\text { (2001) }\end{array}$ \\
\hline Protein kinase & SOS3 & Salt overly sensitive 3 & Arabidopsis & Arabidopsis & CaMV35S & $\begin{array}{l}\text { Salt } \\
\text { tolerance }\end{array}$ & $\begin{array}{l}\text { Ishitani et al. } \\
\text { (2000) }\end{array}$ \\
\hline Protein kinase & $\begin{array}{l}\text { SOS2-1 } \\
\text { and } \\
\text { SOS3-1 }\end{array}$ & $\begin{array}{l}\text { Salt overly sensitive 2-1 } \\
\text { and 3-1 }\end{array}$ & Arabidopsis & Arabidopsis & $\begin{array}{l}\mathrm{T} 7 \\
\text { promoter }\end{array}$ & $\begin{array}{l}\text { Salt } \\
\text { tolerance }\end{array}$ & $\begin{array}{l}\text { Kamei et al. } \\
(2005)\end{array}$ \\
\hline Protein kinase & $\begin{array}{l}\text { SOS6/ } \\
\text { AtCSLD5 }\end{array}$ & $\begin{array}{l}\text { Salt overly sensitive/ } \\
\text { Cellulose synthase like } \\
\text { protein }\end{array}$ & Arabidopsis & Arabidopsis & $\mathrm{Rd} 29 \mathrm{~A}$ & $\begin{array}{l}\text { ROS } \\
\text { signaling } \\
\text { Osmotic } \\
\text { tolerance } \\
\text { Salt } \\
\text { tolerance } \\
\text { Drought } \\
\text { tolerance }\end{array}$ & $\begin{array}{l}\text { Zhu et al. } \\
\text { (2010) }\end{array}$ \\
\hline Protein kinase & $\begin{array}{l}\text { SCABP8/ } \\
\text { CBL10 }\end{array}$ & $\begin{array}{l}\text { SOS3-like calcium } \\
\text { binding protein } 8 / \\
\text { Calcineurin B-like-10 }\end{array}$ & Arabidopsis & Arabidopsis & CaMV35S & $\begin{array}{l}\text { Salt } \\
\text { tolerance }\end{array}$ & $\begin{array}{l}\text { Quan et al. } \\
\text { (2007) }\end{array}$ \\
\hline Protein kinase & LKS1 & Low $\mathrm{K}+$ sensitive 1 & Arabidopsis & Arabidopsis & Super & $\begin{array}{l}\text { Ion } \\
\text { homeostasis }\end{array}$ & $\begin{array}{l}\text { Xu et al. } \\
(2006)\end{array}$ \\
\hline Protein kinase & $\begin{array}{l}\text { CBL-CIPK } \\
3 / 9 / 23 / 26\end{array}$ & $\begin{array}{l}\text { Calcineurin B-like-CBL } \\
\text { interacting protein } \\
\text { kinases }\end{array}$ & Arabidopsis & Arabidopsis & CBL2 & $\begin{array}{l}\mathrm{Mg}^{2+} \\
\text { tolerance }\end{array}$ & $\begin{array}{l}\text { Tang et al. } \\
\text { (2015) }\end{array}$ \\
\hline Protein kinase & SnRK2 & $\begin{array}{l}\text { Sucrose nonfermenting } \\
\text { 1-related protein } \\
\text { kinase } 2\end{array}$ & Arabidopsis & Arabidopsis & CaMV35S & $\begin{array}{l}\text { Hyper } \\
\text { osmotic } \\
\text { tolerance } \\
\text { Saline } \\
\text { tolerance }\end{array}$ & $\begin{array}{l}\text { Boudsocq } \\
\text { et al. (2004) }\end{array}$ \\
\hline Protein kinase & SnRK2 & $\begin{array}{l}\text { Sucrose nonfermenting } \\
\text { 1-related protein kinase } \\
2\end{array}$ & Arabidopsis & Arabidopsis & - & $\begin{array}{l}\text { Osmotic } \\
\text { tolerance }\end{array}$ & $\begin{array}{l}\text { Fujii et al. } \\
\text { (2011) }\end{array}$ \\
\hline Protein kinase & $\begin{array}{l}\text { SnRK2.2/ } \\
2.3 / 2.6\end{array}$ & $\begin{array}{l}\text { Sucrose nonfermenting } \\
\text { 1-related protein kinase } \\
2.2 / 2.3 / 2.6\end{array}$ & Arabidopsis & Arabidopsis & - & $\begin{array}{l}\text { ABA } \\
\text { signaling }\end{array}$ & $\begin{array}{l}\text { Fujii et al. } \\
\text { (2009) }\end{array}$ \\
\hline Protein kinase & $\begin{array}{l}\text { SnRK2.2/ } \\
2.3 / 2.6\end{array}$ & $\begin{array}{l}\text { Sucrose nonfermenting } \\
\text { 1-related protein kinase } \\
2.2 / 2.3 / 2.6\end{array}$ & Arabidopsis & Arabidopsis & - & $\begin{array}{l}\text { ABA } \\
\text { signaling }\end{array}$ & $\begin{array}{l}\text { Wang et al. } \\
(2013)\end{array}$ \\
\hline Protein kinase & SnRK2.6 & $\begin{array}{l}\text { Sucrose nonfermenting } \\
\text { 1-related protein } \\
\text { kinase2.6 }\end{array}$ & Arabidopsis & Arabidopsis & $\begin{array}{l}\text { Native } \\
\text { promoter }\end{array}$ & $\begin{array}{l}\text { ABA } \\
\text { signaling }\end{array}$ & $\begin{array}{l}\text { Wang et al. } \\
\text { (2015) }\end{array}$ \\
\hline
\end{tabular}


TABLE 3.1 (Continued)

\begin{tabular}{|c|c|c|c|c|c|c|c|}
\hline $\begin{array}{l}\text { Functional } \\
\text { category }\end{array}$ & Genes & Protein function & Origin & $\begin{array}{l}\text { Transformation } \\
\text { receptor }\end{array}$ & Promoter & $\begin{array}{l}\text { Abiotic } \\
\text { stress } \\
\text { regulation }\end{array}$ & References \\
\hline Protein kinase & SnRK2 & $\begin{array}{l}\text { Sucrose nonfermenting } \\
\text { 1-related protein kinase } \\
2\end{array}$ & Arabidopsis & Arabidopsis & CaMV35S & $\begin{array}{l}\text { Dehydration } \\
\text { tolerance }\end{array}$ & $\begin{array}{l}\text { Yoshida et al. } \\
\text { (2006) }\end{array}$ \\
\hline Protein kinase & ARK & $\begin{array}{l}\text { ABA and abiotic stress } \\
\text { responsive Raf-like } \\
\text { kinase }\end{array}$ & Physcomitrella patens & $\begin{array}{l}\text { Physcomitrella } \\
\text { patens }\end{array}$ & CaMV35S & $\begin{array}{l}\text { Hyper } \\
\text { osmotic } \\
\text { tolerance }\end{array}$ & $\begin{array}{l}\text { Saruhashi } \\
\text { et al. (2015) }\end{array}$ \\
\hline Protein kinase & ROP11 & $\begin{array}{l}\text { Plant specific rho-like } \\
\text { small GTPase }\end{array}$ & Arabidopsis & Arabidopsis & CaMV35S & $\begin{array}{l}\mathrm{ABA} \\
\text { signaling }\end{array}$ & $\begin{array}{l}\text { Li et al. } \\
\text { (2010) }\end{array}$ \\
\hline Protein kinase & $\begin{array}{l}\text { SLAC1/ } \\
\text { OST1 }\end{array}$ & Open stomata 1 & Arabidopsis & Arabidopsis & - & $\begin{array}{l}\text { Drought } \\
\text { stress }\end{array}$ & $\begin{array}{l}\text { Geiger et al. } \\
\text { (2009) }\end{array}$ \\
\hline Protein kinase & OST1 & Open stomata 1 & Arabidopsis & Arabidopsis & - & $\begin{array}{l}\text { ROS } \\
\text { signaling } \\
\text { ABA } \\
\text { signaling }\end{array}$ & $\begin{array}{l}\text { Sirichandra } \\
\text { et al. (2009) }\end{array}$ \\
\hline Protein kinase & OST1 & Open stomata 1 & Arabidopsis & Arabidopsis & CaMV35S & $\begin{array}{l}\mathrm{ABA} \\
\text { signaling }\end{array}$ & $\begin{array}{l}\text { Grondin } \\
\text { et al. (2015) }\end{array}$ \\
\hline Protein kinase & OST1 & Open stomata 1 & Arabidopsis & Arabidopsis & CBF3 & $\begin{array}{l}\text { Freezing } \\
\text { tolerance }\end{array}$ & $\begin{array}{l}\text { Ding et al. } \\
\text { (2015) }\end{array}$ \\
\hline Protein kinase & OST1 & Open stomata 1 & Arabidopsis & Arabidopsis & POST1 & $\begin{array}{l}\text { ABA } \\
\text { signaling } \\
\text { ROS } \\
\text { signaling }\end{array}$ & $\begin{array}{l}\text { Mustilli et al. } \\
\text { (2002) }\end{array}$ \\
\hline Protein kinase & SLAC1 & S-type anion currents 1 & Arabidopsis & Arabidopsis & pUBQ10 & $\begin{array}{l}\text { ABA } \\
\text { signaling }\end{array}$ & $\begin{array}{l}\text { Brandt et al. } \\
\text { (2015) }\end{array}$ \\
\hline Protein kinase & SLAC1 & S-type anion currents 1 & Arabidopsis & Arabidopsis & - & $\begin{array}{l}\mathrm{ABA} \\
\text { signaling }\end{array}$ & $\begin{array}{l}\text { Geiger et al. } \\
\text { (2010) }\end{array}$ \\
\hline Protein kinase & SLAC1 & S-type anion currents 1 & Arabidopsis & Arabidopsis & pUBQ10 & $\begin{array}{l}\mathrm{ABA} \\
\text { signaling }\end{array}$ & $\begin{array}{l}\text { Brandt et al. } \\
\text { (2015) }\end{array}$ \\
\hline Protein kinase & SLAC1 & S-type anion currents 1 & Arabidopsis & Arabidopsis & - & $\begin{array}{l}\mathrm{ABA} \\
\text { signaling }\end{array}$ & $\begin{array}{l}\text { Geiger et al. } \\
\text { (2010) }\end{array}$ \\
\hline Protein kinase & CIPK26 & CBL-interacting PK26 & Arabidopsis & N. benthamiana & $\mathrm{EF} 1 \alpha$ & $\begin{array}{l}\text { ROS } \\
\text { signaling } \\
\mathrm{Ca}^{2+} \\
\text { signaling }\end{array}$ & $\begin{array}{l}\text { Drerup et al. } \\
(2013)\end{array}$ \\
\hline Protein kinase & $\begin{array}{l}\text { Tyr } \\
\text { nitration }\end{array}$ & Tyrosin nitration & Arabidopsis & Arabidopsis & CaMV35S & $\begin{array}{l}\mathrm{ABA} \\
\text { signaling }\end{array}$ & $\begin{array}{l}\text { Castillo et al. } \\
\text { (2015) }\end{array}$ \\
\hline Protein kinase & CBK3 & $\begin{array}{l}\text { CaM binding protein } \\
\text { kinase-3 }\end{array}$ & Arabidopsis & Arabidopsis & CaMV35S & $\begin{array}{l}\text { Heat } \\
\text { tolerance }\end{array}$ & $\begin{array}{l}\text { Liu et al. } \\
\text { (2011) }\end{array}$ \\
\hline Protein kinase & $\begin{array}{l}\text { ABI1 and } \\
\text { PP2CA }\end{array}$ & $\begin{array}{l}\text { Type } 2 \mathrm{C} \text { protein } \\
\text { phosphatase }\end{array}$ & Arabidopsis & Arabidopsis & CaMV35S & $\begin{array}{l}\text { ABA } \\
\text { signaling }\end{array}$ & $\begin{array}{l}\text { Rodrigues } \\
\text { et al. (2013) }\end{array}$ \\
\hline Protein kinase & SrlK & $\begin{array}{l}\text { Leucine-rich repeat RLK } \\
\text { gene }\end{array}$ & Medicagotrancatula & Medicagotrancatula & $\begin{array}{l}\text { SrlK } \\
\text { promoter }\end{array}$ & $\begin{array}{l}\text { Salt } \\
\text { tolerance }\end{array}$ & $\begin{array}{l}\text { de Lorenzo } \\
\text { et al. (2009) }\end{array}$ \\
\hline His kinase & AtHKT1 & Histidine kinase 1 & Arabidopsis & Arabidopsis & HKT1 & $\begin{array}{l}\text { Salt } \\
\text { tolerance }\end{array}$ & $\begin{array}{l}\text { Maser et al. } \\
\text { (2002) }\end{array}$ \\
\hline His kinase & AtHKT1 & Histidine kinase 1 & Arabidopsis & Arabidopsis & HKT1 & $\begin{array}{l}\text { Salt } \\
\text { tolerance }\end{array}$ & $\begin{array}{l}\text { Rus et al. } \\
\text { (2004) }\end{array}$ \\
\hline
\end{tabular}


TABLE $3.1 \quad$ (Continued)

\begin{tabular}{|c|c|c|c|c|c|c|c|}
\hline $\begin{array}{l}\text { Functional } \\
\text { category }\end{array}$ & Genes & Protein function & Origin & $\begin{array}{l}\text { Transformation } \\
\text { receptor }\end{array}$ & Promoter & $\begin{array}{l}\text { Abiotic } \\
\text { stress } \\
\text { regulation }\end{array}$ & References \\
\hline His kinase & AtHKT1 & Histidine kinase 1 & Arabidopsis & Arabidopsis & - & $\begin{array}{l}\text { Salt } \\
\text { tolerance }\end{array}$ & $\begin{array}{l}\text { Rus et al. } \\
\text { (2001) }\end{array}$ \\
\hline His kinase & AtHKT1 & Histidine kinase 1 & Arabidopsis & Arabidopsis & CaMV35S & $\begin{array}{l}\text { Vegetative } \\
\text { osmotic } \\
\text { stress } \\
\text { signaling }\end{array}$ & $\begin{array}{l}\text { Wohlbach } \\
\text { et al. (2008) }\end{array}$ \\
\hline ABA receptor & PYL8 & $\begin{array}{l}\text { Pyrabactin resistance 1- } \\
\text { like protein } 8\end{array}$ & Arabidopsis & Arabidopsis & IAA19 & $\begin{array}{l}\text { Promote } \\
\text { lateral root } \\
\text { growth }\end{array}$ & $\begin{array}{l}\text { Zhao et al. } \\
\text { (2009) }\end{array}$ \\
\hline ABA receptor & $\begin{array}{l}\text { PYL6/ } \\
\text { RCAR9 }\end{array}$ & $\begin{array}{l}\text { Pyrabactin resistance 1- } \\
\text { like protein } 6\end{array}$ & Arabidopsis & N. benthamiana & $\begin{array}{l}\text { Ubiquitin } \\
10\end{array}$ & $\begin{array}{l}\text { ABA and } \\
\text { jasmonic } \\
\text { acid } \\
\text { signaling }\end{array}$ & $\begin{array}{l}\text { Aleman et al. } \\
(2016)\end{array}$ \\
\hline ABA receptor & PYR/PYL & Pyrabactin resistance & Arabidopsis & N. benthamiana & $\operatorname{Rd} 29 A$ & $\begin{array}{l}\mathrm{ABA} \\
\text { signaling }\end{array}$ & $\begin{array}{l}\text { Park et al. } \\
\text { (2009) }\end{array}$ \\
\hline ABA receptor & $\begin{array}{l}\text { PYR1 } \\
\text { PYL1/2/ } \\
4 / 5 / 8\end{array}$ & $\begin{array}{l}\text { Pyrabactin resistance } 1 / \\
\text { PYR1-like } 1 / 2 / 4 / 5 / 8\end{array}$ & Arabidopsis & Arabidopsis & - & $\begin{array}{l}\text { ABA } \\
\text { signaling }\end{array}$ & $\begin{array}{l}\text { Gonzalez- } \\
\text { Guzman } \\
\text { et al. (2012) }\end{array}$ \\
\hline $\mathrm{ABA}$ receptors & $\begin{array}{l}\text { PYR/ } \\
\text { PYL/ } \\
\text { RCAR }\end{array}$ & $\begin{array}{l}\text { Regulating components } \\
\text { of ABA receptors }\end{array}$ & Arabidopsis & N. benthamiana & CaMV35S & $\begin{array}{l}\mathrm{ABA} \\
\text { signaling }\end{array}$ & $\begin{array}{l}\text { Rodriguez } \\
\text { et al. (2014) }\end{array}$ \\
\hline $\begin{array}{l}\text { Transcription } \\
\text { factor }\end{array}$ & AREB1 & $\begin{array}{l}\text { ABA responsive element } \\
\text { binding protein } 1\end{array}$ & Arabidopsis & Arabidopsis & CaMV35S & $\begin{array}{l}\mathrm{ABA} \\
\text { signaling }\end{array}$ & $\begin{array}{l}\text { Furihata } \\
\text { et al. (2006) }\end{array}$ \\
\hline $\begin{array}{l}\text { Transcription } \\
\text { factor }\end{array}$ & $\begin{array}{l}\mathrm{CBF} \text { and } \\
\mathrm{COR}\end{array}$ & $\begin{array}{l}\text { C-repeat binding factors } \\
\text { and cold responsive } \\
\text { genes }\end{array}$ & Arabidopsis & Arabidopsis & CBF3 & $\begin{array}{l}\text { Cold } \\
\text { tolerance }\end{array}$ & $\begin{array}{l}\text { Norén et al. } \\
(2016)\end{array}$ \\
\hline $\begin{array}{l}\text { Transcription } \\
\text { factor }\end{array}$ & $\begin{array}{l}\text { AtMYC2 } \\
\text { and } \\
\text { AtMYB2 }\end{array}$ & $\begin{array}{l}\text { MYB-related } \\
\text { transcription factors }\end{array}$ & Arabidopsis & Arabidopsis & CaMV35S & $\begin{array}{l}\text { ABA } \\
\text { signaling } \\
\text { Drought } \\
\text { tolerance }\end{array}$ & $\begin{array}{l}\text { Abe et al. } \\
\text { (2003) }\end{array}$ \\
\hline $\begin{array}{l}\text { Transcription } \\
\text { factor }\end{array}$ & $\begin{array}{l}\text { CBF2/ } \\
\text { DREB1 }\end{array}$ & $\begin{array}{l}\text { C-repeat binding } \\
\text { factor2/dehydration } \\
\text { responsive element- } \\
\text { binding factor } 1\end{array}$ & Arabidopsis & Arabidopsis & $\mathrm{CBF} 2$ & $\begin{array}{l}\text { Freezing } \\
\text { tolerance }\end{array}$ & $\begin{array}{l}\text { Novillo et al. } \\
\text { (2004) }\end{array}$ \\
\hline $\begin{array}{l}\text { Transcription } \\
\text { factor }\end{array}$ & MBF1C & $\begin{array}{l}\text { Multi-protein binding } \\
\text { factor } 1 \mathrm{c}\end{array}$ & Arabidopsis & Arabidopsis & CaMV35S & $\begin{array}{l}\text { Heat } \\
\text { tolerance }\end{array}$ & $\begin{array}{l}\text { Suzuki et al. } \\
\text { (2011) }\end{array}$ \\
\hline $\begin{array}{l}\text { Transcription } \\
\text { factor }\end{array}$ & WRKY39 & - & Arabidopsis & Arabidopsis & CaMV35S & $\begin{array}{l}\text { Heat } \\
\text { tolerance }\end{array}$ & $\begin{array}{l}\text { Li et al. } \\
\text { (2010) }\end{array}$ \\
\hline $\begin{array}{l}\text { Transcription } \\
\text { factor }\end{array}$ & $\begin{array}{l}\text { HD- } \\
\text { START }\end{array}$ & $\begin{array}{l}\text { Homeodomain- } \\
\text { START-TF }\end{array}$ & Arabidopsis & Arabidopsis & CaMV35S & $\begin{array}{l}\text { Drought } \\
\text { tolerance }\end{array}$ & $\begin{array}{l}\text { Yu et al. } \\
(2008)\end{array}$ \\
\hline MAPK & MPK9\&12 & $\begin{array}{l}\text { MAPK-protein } \\
\text { kinase9\&12 }\end{array}$ & Arabidopsis & Arabidopsis & Actin 2 & $\begin{array}{l}\text { ROS } \\
\text { signaling } \\
\text { ABA } \\
\text { signaling }\end{array}$ & $\begin{array}{l}\text { Jammes et al. } \\
(2009)\end{array}$ \\
\hline MAPK & MKK2 & MAPK kinase kinase 2 & Arabidopsis & Arabidopsis & $\mathrm{ADH}$ & $\begin{array}{l}\text { Cold } \\
\text { tolerance } \\
\text { Salt } \\
\text { tolerance }\end{array}$ & $\begin{array}{l}\text { Teige et al. } \\
\text { (2004) }\end{array}$ \\
\hline
\end{tabular}


TABLE 3.1 (Continued)

\begin{tabular}{|c|c|c|c|c|c|c|c|}
\hline $\begin{array}{l}\text { Functional } \\
\text { category }\end{array}$ & Genes & Protein function & Origin & $\begin{array}{l}\text { Transformation } \\
\text { receptor }\end{array}$ & Promoter & $\begin{array}{l}\text { Abiotic } \\
\text { stress } \\
\text { regulation }\end{array}$ & References \\
\hline MAPK & CRLK1 & $\begin{array}{l}\text { Calcium/Calmodulin- } \\
\text { regulated receptor like } \\
\text { kinase-1 }\end{array}$ & Arabidopsis & Arabidopsis & - & $\begin{array}{l}\text { Cold } \\
\text { tolerance }\end{array}$ & $\begin{array}{l}\text { Yang et al. } \\
\text { (2010) }\end{array}$ \\
\hline MAPK & TPC1 & Two pose channel 1 & Arabidopsis & Arabidopsis & CaMV35S & $\begin{array}{l}\text { ROS } \\
\text { signaling } \\
\text { Salt } \\
\text { tolerance }\end{array}$ & $\begin{array}{l}\text { Choi et al. } \\
\text { (2014) }\end{array}$ \\
\hline MAPK & MKK2 & MAPK-kinase kinase 2 & Arabidopsis & Arabidopsis & CaMV35S & $\begin{array}{l}\text { Cold } \\
\text { tolerance } \\
\text { Salt } \\
\text { tolerance }\end{array}$ & $\begin{array}{l}\text { Teige et al. } \\
\text { (2004) }\end{array}$ \\
\hline MAPK & $\begin{array}{l}\text { OsMEK1 } \\
\text { and } \\
\text { OsMAP } 1\end{array}$ & - & Arabidopsis & Arabidopsis & - & $\begin{array}{l}\text { Low } \\
\text { temperature } \\
\text { tolerance }\end{array}$ & $\begin{array}{l}\text { Wen et al. } \\
\text { (2002) }\end{array}$ \\
\hline HSFs & HSFA1S & A1 heat shock factors & Arabidopsis & Arabidopsis & - & $\begin{array}{l}\text { Heat } \\
\text { tolerance }\end{array}$ & $\begin{array}{l}\text { Liu et al. } \\
\text { (2011) }\end{array}$ \\
\hline HSFs & AtCaM3 & $\begin{array}{l}\text { Arabidopsis Calmodulin } \\
3\end{array}$ & Arabidopsis & Arabidopsis & CaMV35S & $\begin{array}{l}\text { Heat shock } \\
\text { signal } \\
\text { transduction }\end{array}$ & $\begin{array}{l}\text { Zhang et al. } \\
\text { (2009) }\end{array}$ \\
\hline $\begin{array}{l}\text { PMRK (Plasma } \\
\text { membrane } \\
\text { receptor like) }\end{array}$ & GHR1 & $\begin{array}{l}\text { Guard cell hydrogen } \\
\text { peroxide resistant } 1\end{array}$ & Arabidopsis & Arabidopsis & SUPER & $\begin{array}{l}\text { ROS } \\
\text { signaling } \\
\text { ABA } \\
\text { signaling }\end{array}$ & $\begin{array}{l}\text { Hua et al. } \\
\text { (2012) }\end{array}$ \\
\hline $\begin{array}{l}\text { ERK (Extra } \\
\text { cellular signal } \\
\text { regulated kinase) }\end{array}$ & HAMK & $\begin{array}{l}\text { Heat shock activated } \\
\text { MAPK }\end{array}$ & Alfalfa & Alfalfa & - & $\begin{array}{l}\text { Cold } \\
\text { tolerance } \\
\text { Heat } \\
\text { tolerance }\end{array}$ & $\begin{array}{l}\text { Sangwan } \\
\text { et al. (2002) }\end{array}$ \\
\hline CDPK & CPK5 & $\begin{array}{l}\text { Calcium dependent PK } \\
5\end{array}$ & Arabidopsis & Arabidopsis & CaMV35S & $\begin{array}{l}\text { Defense } \\
\text { mechanism }\end{array}$ & $\begin{array}{l}\text { Dubiella } \\
\text { et al. (2013) }\end{array}$ \\
\hline Peroxidases & FBP1 & $\begin{array}{l}\text { French bean peroxidase } \\
\text { type } 1\end{array}$ & Phasiolus vulgaris & Arabidopsis & CaMV35S & $\begin{array}{l}\text { Defense } \\
\text { mechanism }\end{array}$ & $\begin{array}{l}\text { Bindschedler } \\
\text { et al. (2006) }\end{array}$ \\
\hline CBFTPs & ICE1 & $\begin{array}{l}\text { Inducer of CBF } \\
\text { expression } 1\end{array}$ & Arabidopsis & Arabidopsis & CBF3 & $\begin{array}{l}\text { Freezing } \\
\text { tolerance }\end{array}$ & $\begin{array}{l}\text { Chinnusamy } \\
\text { et al. (2003) }\end{array}$ \\
\hline ST gene & ST6-66 & Salt-tilerace 6-66 gene & Thellungiellahalophila & Arabidopsis & CaMV35S & $\begin{array}{l}\text { Salt } \\
\text { tolerance }\end{array}$ & $\begin{array}{l}\text { Du et al. } \\
\text { (2008) }\end{array}$ \\
\hline $\begin{array}{l}\text { Stress inducible } \\
\text { gene }\end{array}$ & VuNCEDI & $\begin{array}{l}\text { 9-cis-epoxy carotenoid } \\
\text { dioxygenase }\end{array}$ & Vignaunguiculata & Vignaunguiculata & CaMV35S & $\begin{array}{l}\text { Drought } \\
\text { tolerance }\end{array}$ & $\begin{array}{l}\text { Iuchi et al. } \\
\text { (2000) }\end{array}$ \\
\hline USP & SpUSP & Universal stress protein & Solanum pennellii & Solanum pennellii & CaMV35S & $\begin{array}{l}\text { Drought } \\
\text { tolerance }\end{array}$ & $\begin{array}{l}\text { Loukehaich } \\
\text { et al. (2012) }\end{array}$ \\
\hline $\begin{array}{l}\text { Protein } \\
\text { phosphatase }\end{array}$ & $\mathrm{PP}_{2} \mathrm{C}$ & $\begin{array}{l}\text { Type } 2 \mathrm{C} \text { protein } \\
\text { phosphatase }\end{array}$ & Arabidopsis & Arabidopsis & - & $\begin{array}{l}\text { ABA } \\
\text { signaling }\end{array}$ & $\begin{array}{l}\text { Ma et al. } \\
\text { (2009) }\end{array}$ \\
\hline G-proteins & $\begin{array}{l}\text { GTG1 and } \\
\text { GTG2 }\end{array}$ & GPCR-type G protein & Arabidopsis & Arabidopsis & - & $\begin{array}{l}\mathrm{ABA} \\
\text { signaling }\end{array}$ & $\begin{array}{l}\text { Pandey et al. } \\
\text { (2009) }\end{array}$ \\
\hline $\begin{array}{l}\text { ABA-binding } \\
\text { START protein }\end{array}$ & PP2C & $\begin{array}{l}\text { Type2c-protein } \\
\text { phosphatases }\end{array}$ & Arabidopsis & Arabidopsis & - & $\begin{array}{l}\mathrm{ABA} \\
\text { signaling }\end{array}$ & $\begin{array}{l}\text { Park et al. } \\
\text { (2009) }\end{array}$ \\
\hline
\end{tabular}


TABLE 3.1 (Continued)

\begin{tabular}{|c|c|c|c|c|c|c|c|}
\hline $\begin{array}{l}\text { Functional } \\
\text { category }\end{array}$ & Genes & Protein function & Origin & $\begin{array}{l}\text { Transformation } \\
\text { receptor }\end{array}$ & Promoter & $\begin{array}{l}\text { Abiotic } \\
\text { stress } \\
\text { regulation }\end{array}$ & References \\
\hline Osmosensors & OSCA1 & $\begin{array}{l}\text { Hyper osmolality } \\
\text { induced }\left(\mathrm{Ca}^{2+}\right) \mathrm{i} \\
\text { increase } 1\end{array}$ & Arabidopsis & Arabidopsis & $\operatorname{Rd} 29 A$ & $\begin{array}{l}\text { Osmotic } \\
\text { tolerance } \\
\text { Drought } \\
\text { tolerance }\end{array}$ & $\begin{array}{l}\text { Yuan et al. } \\
(2014 a, b)\end{array}$ \\
\hline $\begin{array}{l}\text { MscS (Mechano } \\
\text { sensitive channel } \\
\text { of small } \\
\text { conductance) }\end{array}$ & MSL8 & Mechanical stresses & E. coli & Arabidopsis & $\begin{array}{l}\text { LAT52 } \\
\text { (pollen } \\
\text { specific } \\
\text { promoter) }\end{array}$ & $\begin{array}{l}\text { Hypo- } \\
\text { osmotic } \\
\text { tolerance }\end{array}$ & $\begin{array}{l}\text { Hamilton } \\
\text { et al. (2015) }\end{array}$ \\
\hline Stress sensor & COLD1 & $\begin{array}{l}\text { Activates } \mathrm{Ca} 2+\text { ion } \\
\text { channel }\end{array}$ & Rice & Rice & CaMV35S & $\begin{array}{l}\text { Chilling } \\
\text { tolerance }\end{array}$ & $\begin{array}{l}\text { Ma et al. } \\
(2015)\end{array}$ \\
\hline ROS signaling & Chy1 & $\begin{array}{l}\text { Peroxisomal } \\
\beta \text {-hydroxyisobutanyl Co- } \\
\text { A hydrolase }\end{array}$ & Arabidopsis & Arabidopsis & CBF3 & $\begin{array}{l}\text { ROS } \\
\text { signaling } \\
\text { Cold } \\
\text { tolerance }\end{array}$ & $\begin{array}{l}\text { Dong et al. } \\
(2009)\end{array}$ \\
\hline
\end{tabular}

receptor-like protein gene $\mathrm{NtC7}$ in onion epidermal cells confirmed that it is a transmembrane protein and its overexpression in tobacco provides osmotic stress tolerance to seeds induced by mannitol, but not by $\mathrm{NaCl}$ (Tamura et al., 2003). Several genetic and biochemical studies on the salt stress tolerance revealed that MAP kinases are important signal transducers and they were regulated by osmotic and salt stress both at transcriptional and protein levels. In plants such as Arabidopsis the protein AtHK1 (yeast osmosensor SLN1 homolog) senses the osmotic signal and transmits the signal to an MAPK downstream cascade and activates MEKK1, AtMPK3, AtMPK4, and AtMPK6 (Mizoguchi et al., 1996; Ichimura et al., 2000; Droillard et al., 2002; Wohlbach et al., 2008). Transgenic overexpression of AtHK1 in Lycium barbarum exhibited tolerance to salt and water stress by modulating the antioxidative enzymes and osmolytes (Chen et al., 2010). In the MAPK signaling pathway the activated MEKK1 further elicits the activity of MKK4 and MKK5 (Asai et al., 2002). During salt and cold stress the activated MKK2 as an activator subsequently activates MPK4 and MPK6. The transgenic overexpression of the MKK2 gene in Arabidopsis plants upregulates several stress responsive genes and improves cold and salt tolerance (Teige et al., 2004). Under salt stress conditions the MAP6 kinase activates are regulated by phosphatidic acid (PA) and activated MAP6 associates with MYB41 TF, improves the salt tolerance in Arabidopsis plants (Yu et al., 2010; Hoang et al., 2012).

The osmotic stress-induced MKK and MPK members might activate the upstream activators such as $\operatorname{rd} 29 \mathrm{~A}$ and $\mathrm{rd} 29 \mathrm{~B}$ gene promoters under salt stress conditions (Hua et al., 2012). Transgenic overexpression of maize ZmSIMK1 in Arabidopsis plants triggers several known stress responsive genes such as rd29A and P5CS1 under salt stress conditions and improves the salt tolerance of transgenic plants (Gu et al., 2010). In a recent study Kim et al (2012) identified an osmotic regulator gene in Arabidopsis, MKKK20, a new MAP kinase kinase gene, by activating MPK6. Transgenic Arabidopsis plants overexpressing MKKK20 genes displayed osmotic tolerance nature compared with mutant mkkk20 plants (Kim et al., 2012). Earlier findings suggest that Arabidopsis MKK4 improves the osmotic tolerance at cells and seedling stage by activation of MPK3 (Kim et al., 2011). Osmotic stress also activates the MPK17 in Arabidopsis and its homolog ZmMPK17 in maize (Pan et al., 2012). As part of the hypoosmotic stress-signaling pathway Arabidopsis MPK20 interacts with proline dehydrogenase (ProDH) under hypoosmotic stress conditions and provides osmotic tolerance to plant cells (Moustafa et al., 2008). Transgenic tobacco plants overexpressing maize MAP kinase kinase ZmMKK4 improved the osmotic stress tolerance and its transcript levels were modulated by salinity stress (Kong et al., 2011). Several salt-induced osmotic stress MAP kinases were identified in rice, that is, OsMPK44 (Jeong et al., 2006), OsMSRMK3 (Agrawal et al., 2003), OsMSRMK2 (Agrawal et al., 2002), OsEDR1 (Kim et al., 2003), OsMAPK5 (Xiong and Yang, 2003), and OsMAPK4 (Fu et al., 2002). Ectopic overexpression of rice MAP kinase genes, OsMPK5 and OsMPK44, in rice plants improved tolerance to salt stress (Jeong et al., 2006; Xiong and Yang, 2003). In contrast stress responsive overexpression of 
rice OsMAPK33 in transgenic rice plants increases sensitivity to salt stress by altering the expression of several ion transport genes such as efflux pumps and the $\mathrm{K}^{+} / \mathrm{H}^{+}$antiporter. These data clearly suggest that OsMAPK33 could have a negative role in salt stress tolerance (Lee et al., 2011).

Transgenic overexpression of cotton MAP kinase GhMPK2 in cotton plants improved the osmotic adjustment of the transgenic plants and enhanced tolerance to salt stress (Zhang et al., 2011). Osmotic stress responsive MAP kinase of cucumber root CSNMPAK, upon overexpression in tobacco plants, improved the germination rates of seeds under salt stress conditions compared with wild type plants $\mathrm{Xu}$ et al., 2010a,b). Transgenic lines overexpressing Populus trichocarpa MAP kinase PtMAPKK4 displayed tolerance to salt stress by improving oxidative stress tolerance compared with wild type plants (Yang et al., 2017) (Table 3.1).

\subsection{ABA SIGNALING PATHWAY}

The plant hormone ABA plays a crucial role in response to several stresses, regulates the movements of stomata, regulates the gene expression, and modulates various metabolic pathways (Cutler et al., 2010). The concentration of ABA varies in response to abiotic stresses, particularly under drought and salinity (Khan and Hakeem, 2014). The phytohormone ABA synchronizes the complex regulatory network of drought and salt stresses, hence it is vital for the plants to cope with extreme environmental conditions ( $\mathrm{Zhu}, 2002)$. Two plasma membrane proteins such as ATP-binding cassette (ABC) transporters AtABCG40/AtPDR12/ and AtABCG25 were identified in Arabidopsis through genetic and biochemical analysis studies (Kang et al., 2010). Among them AtABCG25 export ABA from ABA biosynthesis cells to apoplastic areas, further AtABCG40 import ABA from apoplastic cells to guard cells to close the stomata (Kuromori and Shinozaki, 2010). Further, to elucidate the genetic network of ABA signaling, comprehensive genetic screening tests and protein-interaction studies have been carried out in several model plant species and have identified pyrabactin (4-bromo-N-[pyridin2-ylmethyl] naphthalene-1-sulfonamide) resistance (PYR)/regulatory component of ABA receptor (RCAR) proteins, also called PYL family proteins, or soluble START domain proteins, as receptor for ABA chemical signal (Park et al., 2009; Ma et al., 2009). The hormone ABA interacts with PYL family receptors through affinity binding; in the presence of co-receptors such as type $2 \mathrm{C}$ protein phosphatases (PP2Cs), ABI1, ABI2, HAB1, and PP2CA. (Ma et al., 2009).

The SnRK2 kinases such as SnRK2.2, 2.3, and SnRK2.6 bind to PP2Cs in the absence of ABA, and inactivate the catalytic domain of PP2Cs by dephosphorylating the activation loop (Soon et al., 2012). The structural studies revealed that PYL/RCAR protein forms a central hydrophobic ligand-binding pocket that plays a vital role as an ABA binding site. ABA enters into this pocket and stimulates the gate and latch loops to close and lock the pocket, and forms the binding surface to PP2Cs (Melcher et al., 2009). In the pocket the tryptophan residue of $\mathrm{PP} 2 \mathrm{C}$ tightly binds to the ABA and stabilizes the ABA-PYLs-PP2C complex, and the ABA-PYL complex inhibits the active site of the PP2C and liberates the SnRK2s (Park et al., 2009). The liberated SnRK2s autophosphorylates and activates, and the activated SnRK2s further modulate several downstream effectors (Furihata et al., 2006; Fujii et al., 2009). The SnRK2 family comprises three subfamilies, SnRK1, SnRK2, and SnRK3 (Hrabak et al., 2003). The members of SnRK2 were activated by both osmotic stress and ABA; however subfamily II members, except SnRK2.2, SnRK2.3, and SnRK2.6, were weakly activated by ABA (Boudsocq et al., 2004). The ABA activated SnRK2 kinase proteins phosphorylate bZIP transcription factors such as ABA responsive element binding factors (ABFs) and AB15 (Furihata et al., 2006); the plasma membrane anion channels SLAC1 (slow anion channel-associated 1$)$ and KAT1 $\left(\mathrm{K}^{+}\right.$channel in Arabidopsis thaliana 1) that are critical for ABA regulation of stomatal movement, and RBOHF (respiratory burst oxidase homolog F) that functions in ROS generation in response to ABA (Sirichandra et al., 2009); miRNA regulation, chromatin regulation, and RNA splicing (Wang et al., 2013).

Calcium is an important molecule within the ABA dependent signaling pathway and plant guard cell regulation. Genetic analysis studies revealed that Arabidopsis mutant plants with four redundant, nonfunction, calcium-dependent protein kinases, CPK5, CPK6, CPK11, and CPK23, are unable to close the stomata in response to ABA (Brandt et al., 2015). The ABA activated CPK simultaneously phosphorylates two key phosphorylation sites in S-type anion channel (SLAC1) and closes the guard cell (Geiger et al., 2010; Brandt et al., 2015). The ABA activated calcium signal activates the CBL-interacting protein kinase (CIPK26)/ calcineurin B-like (CBL), which interacts with the Nterminal of $\mathrm{RBOH}$ protein and phosphorylates it and enhances the production of ROS (the $\mathrm{RBOH} \mathrm{Ca}{ }^{2+}$ sensor proteins together with their interacting kinases (CIPKs) (Drerup et al., 2013). Furthermore, recent genetic analysis studies in mutant plants revealed that 
ABA also induced the production of nitric oxide (NO) and phosphatidic acid (Hou et al., 2016). Further, NO deactivates the SnRK2 and PYLs by modifying the S-nitrosylation of a cystenine residue (Wang et al., 2015; Castillo et al., 2015).

The protein PYRs upon binding to ABA modulates several gene expressions and plays a vital role in plant growth and development. Knockout studies and protein interaction experiments revealed that in the presence of ABA the protein PYL6 strongly interacts with a key basic helix-loop-helix transcription factor MYC2 of jasmonic acid (JA) signaling pathway and modulates the expression of JA pathway genes ZA6 and ZA8 (Aleman et al., 2016). However, it was shown that under stress conditions higher concentrations of ABA limit the growth of primary and lateral roots. It was shown that a higher concentration of ABA limits the growth and development of primary and lateral roots. However, the protein PYL8 at low concentrations of ABA directly interacts with the transcription factors MYB77; and MYB 77 triggers the induction of several auxin-responsive genes as resulted lateral roots growth enters into the recovery phase (Zhao et al., 2014).

Several studies have shown the potential applications of engineering ABA signaling molecules through transgenic overexpression/suppression of signaling pathway genes. Transgenic constitutive overexpression of ABA receptor proteins (clade A PP2C interacting proteins) PYL5 and HAB1 in Arabidopsis, displayed disparity in phenotypes in responses to ABA. The PYL5 OE lines exhibit hypersensitivity to ABA, whereas HAB1 OE lines showed reduced sensitivity to ABA. Simultaneous overexpression of PYL5 and HAB1 (PYL5-OE HAB1-OE) transgenic lines exhibited a phenotype similar to transgenic PYL5-OE lines, which revealed that PYL5 has alienated HAB1 function in the transgenic plants. Further, this study demonstrates PYL5-OE lines displayed enhanced drought tolerance in transgenic plants (Santiago et al., 2009). Constitutive overexpression of another ABA receptor protein PYL2 in Arabidopsis activated the ABA signaling compared with mutant plants (Mosquna et al., 2011). Genetic analysis studies in Arabidopsis mutant plants identified that in the absence of ABA, the receptor molecule PYL4A ${ }^{1947}$ interacts with PP2CA. Transgenic overexpression of PYL4A ${ }^{1947}$ under a constitutive promoter enhances the sensitivity of transgenic Arabidopsis plants to ABA. The transgenic plants also displayed increased tolerance to drought stress compared with nontransformed or transformed 35S:PYL4 plants (Pizzio et al., 2013). Similarly, overexpression of a rice cytosolic ABA receptor OsPYL/RCAR5 in rice plants under a maize ubiquitin promoter trigger the expression of several stress responsive genes such as LEA, dehydrin, and Hsp under normal growth conditions and enhanced the drought and salt tolerance of rice at the vegetative growth stage. However, transgenic plants yielded reduced seed and plant height under field conditions. This study demonstrates that fine regulation of expression OsPYL needed to overcome the detrimental effects in transgenic plants (Kim et al., 2012; Kim et al., 2014). Transgenic overexpression of two rice ABA receptor genes OsPYL3 and OsPYL9 in rice plants demonstrated that in the presence of ABA, OsPYLs expressed lines showed positive seed germination and improved drought and salt tolerance compared with wild type plants (Tian et al., 2015). Likewise, constitutive overexpression of rice OsPYL3 in Arabidopsis plants displayed an ABA hypersensitive phenotype and improved tolerance to cold and drought stress (Lenka et al., 2018). Transgenic overexpression of poplar ABA receptors PtPYRL1 and PtPYRL5 in Arabidopsis plants improved hypersensitivity to ABA and drought stress tolerance (Yu et al., 2016). In a recent work, two different types of transgenic plants were developed in Arabidopsis by transgenic overexpression of two tomato monomeric subgroup ABA receptors, AtPY4-6 and AtPYL7-10, and a tomato dimeric receptor from the subgroup AtPYL1. The Arabidopsis transgenic lines carrying tomato monomeric-type receptors displayed improved drought tolerance, while transgenic Arabidopsis lines carrying a tomato dimeric receptor did not show drought tolerance (Gonzalez-Guzman et al., 2012).

The transcription factors bZIP play vital roles in the ABA signaling pathway. Transgenic overexpression of one of the positive regulators of the ABA and a member of rice bZIP, OsbZIP72, in rice transgenic plants demonstrated hypersensitivity to $A B A$ and modulated the expression of $A B A$ responsive genes such as LEAs and improved drought tolerance in transgenic rice plants compared with wild-type plants (Lu et al., 2009). Rice third subfamily member bZIP transcription factor OsbZIP46 was activated by drought, heat, hydrogen peroxide, and ABA, but not by salt and cold. Transgenic overexpression of OsbZIP46 displayed hypersensitivity to ABA, but no tolerance to drought stress. Mutational modification with a deletion of domain $\mathrm{D}$ created a constitutive active form of OsbZIP46 (OsbZIP46CA1). Transgenic overexpression of the active form in rice seedlings improved tolerance to drought and osmotic stress by modulating several downstream stress responsive genes (Tang et al., 2012). These studies proved that 
engineering ABA signaling pathway genes was an ideal strategy to improve abiotic stress tolerance in plants (Table 3.1).

\subsection{CALCIUM SENSORS AND SIGNALING}

Various extracellular factors such as biotic and abiotic stress and intracellular responses stimulate calcium $\left(\mathrm{Ca}^{2+}\right)$ as a secondary messenger (Snedden and Fromm, 1998, 2001; DeFalco et al., 2010). A number of $\mathrm{Ca}^{2+}$ sensors including calmodulin (CaM), calmodulin-like (CML), calcineurin B-like proteins (CBLs), and CBLinteracting protein kinases (CIPKs)/calcium dependent protein kinases (CDPKs) (Yang and Poovaiah, 2003; Bouche et al., 2005) have been reported in plants. The CaMs are small acidic proteins, unique $\mathrm{Ca}^{2+}$ sensor proteins, which consist of four EF-hand domains located at both $\mathrm{N}$-terminal and C-terminal to bind $\mathrm{Ca}^{2+}$ in plants and animals (Perochon et al., 2011). Osmotic stressinduced CaM-mediated signaling pathway is well documented in several plant species (Bouche et al., 2005) such as AtCML8, an ortholog of GmCaM4 (Park et al., 2010), and AtCML9 (Magnan et al., 2008). Transgenic Arabidopsis plants overexpressing soybean CaM gene GmCaM4 improved drought and salt tolerance by modulating the transcriptional activity of an MYB2 transcription factor, which in turn activated the expression of salt and drought responsive genes (Abe et al., 2003; Yoo et al., 2005). A novel rice calmodulin-like gene OsMSR2 (multistress-responsive gene 2) overexpressed in transgenic Arabidopsis plant exhibited drought and salt tolerance by modulating the known stress responsive genes in an ABA-mediated pathway (Xu et al., 2011). Several CaMBPs involved in the calcium signaling pathway were induced by drought, salt, or osmotic stresses (Zeng et al., 2015). Overexpression of wheat CaMBP TaCCaMK in Arabidopsis plants reduces the ABA sensitivity and acts as a negative regulator of ABA-mediated signaling pathway (Yang et al., 2011). The transgenic expression of one of the $\mathrm{Ca}^{2+} / \mathrm{CBP}$ proteins BjGly-I of Brassica juncea in tobacco plants conferred tolerance to methylglyoxal and high salt stress (Veena et al., 1999). In Arabidopsis drought, low temperature and high salt stress-induced protein AtCaMBP25 reduced the osmotic tolerance nature of transgenic plants by acting as negative regulator, silencing of AtCaMBP25 through antisense approach increased the osmotic tolerance (Perruc et al., 2004). Transgenic overexpression of AtCaM3 in Arabidopsis plants increased the temperature tolerance (Zhang et al., 2009). High temperature responsive signaling pathway genes were AtCBK3, AtPP7, AtHSF, and AtHSP elevated in the transgenic Arabidopsis plants overexpressing rice $\mathrm{OsCaM}_{1-1}$.
Calcineurin B-like protein-interacting protein kinases (CIPKs) are important components of $\mathrm{Ca}^{2+}$ mediated CBL-CIPK network in response to various stresses (Xiang et al., 2007; Zhao et al., 2009). Several CIPK family genes have been identified and a few of their functional roles have been characterized in plant species such as Arabidopsis (Kolukisaoglu), rice (Kolukisaoglu et al., 2004), canola (Zhang et al., 2014a, b), maize (Chen et al., 2011), and wheat (Sun et al., 2015). Numerous CIPK genes involved in the SOS signaling pathway mediated salt tolerance have been well characterized (Qiu et al., 2004; Tang et al., 2011). In Arabidopsis, root salt tolerance and $\mathrm{Na}^{+}$efflux are controlled by the $\mathrm{Na}^{+} / \mathrm{H}^{+}$antiporter; the interaction between AtCBL4 and CIPK24 (SOS3-SOS2) complex mainly regulates NHX activity (Liu et al., 2011), whereas in the shoots AtCBL10-AtCIPK24 complex protects from salt stress (Quan et al., 2007). Transgenic overexpression of maize CIPK21 enhanced salt tolerance of Arabidopsis by activating the downstream stress responsive transactivator dehydration-responsive element-binding (DREB) proteins, decreased accumulation of $\mathrm{Na}+$, and enhanced root length under salt stress conditions (Chen et al., 2014). Ectopic constitutive expression of halophyte Hordeum brevisubulatum kinase HbCIPK2 improved the osmotic stress tolerance in Arabidopsis mutant sos2-1 plants, as well as in wildtype Arabidopsis plants during seed germination under salt stress (Li et al., 2010). Heterologous expression of Populus euphratica CBL1 (PeCBL1) improves the ion homeostasis by interacting with CIPK24, CIPK25, and CIPK26 and enhanced salt tolerance (Zhang et al., 2013). Transgenic tobacco plants overexpressing a wheat TaCIPK2 displayed enhanced tolerance to drought by improving antioxidative enzymes and regulating stomata movements (Wang et al., 2016). A CBL-interacting protein kinase of wheat TaCIPK29 when overexpressed in transgenic tobacco plants expresses throughout the cells and interacts with genes such as TaCBL2, TaCBL3, NtCBL2, NtCBL3, NtCAT1; regulates ROS homeostasis and cations; and improves salt stress tolerance of transgenic plants (Deng et al., 2013).

The CDPKs are known to be involved in abiotic stress-induced $\mathrm{Ca}^{2+}$ signaling pathway. The ion channel proteins and transporters are the substrates for CDPKs. Several CDPKs have been observed to induce drought stress ( $\mathrm{Xu}$ et al., 2010a,b). Genetic analysis revealed that CPK3 and CPK6 regulate ion channels of guard cells. CPK3 localizes in the plasma membrane and vacuole, and regulates abiotic stress signaling independent of MAPK (Mehlmer et al., 2010). However, CPK6 is a functionally redundant positive regulator for abiotic stress, and enhanced constitutive expression of CPK6 in Arabidopsis plants and improved 
the drought and salt tolerance of transgenic plants by modulating expression of several known stress responsive genes (Xu et al., 2010a,b). The Arabidopsis transgenic plants overexpressing CPK10 displayed drought tolerance, in the transgenic plants the CPK10 protein might interact with HSP1 and plays a critical role in regulating $\mathrm{ABA}-$ and $\mathrm{Ca}^{2+}$-mediated stomatal movements (Zou et al., 2010). The CDPK proteins of Arabidopsis phosphorylate the subfamily of bZIP transcription factors, ABA-responsive element binding factors (ABFs). The CPK4 and CPK11 proteins, phosphorylates ABF1 and ABF4 improve the tolerance to salt and drought stress by regulating various physiological process (Zhu et al., 2007). Similarly, CPK32 interacts and phosphorylates $\mathrm{ABF} 4$, the transgenic overexpression of ABF4 regulated expression of several ABA-responsive genes (Choi et al., 2014). In contrast, the closely related CPK21 and CPK23 act as negative regulators in abiotic stress-induced signaling pathway. The mutant lines with loss of function of CPK21 and CPK23 displayed enhanced drought and salt tolerance (Ma and Wu, 2007; Franz et al., 2010). The overexpression of CPK23 in transgenic lines exhibited drought and salt stress sensitivity by increasing stomatal apertures and altered ion homeostasis (Ma and $W u, 2007$ ). Several CDPK genes, OsCDPK7 (Saijo et al., 2000), OsCDPK 13 (Abbasi et al., 2004), and OsCPK21 (Asano et al., 2011), were transformed into rice and the transgenic rice plants displayed enhanced tolerance to drought, salt, and cold, suggesting that all of these CDPKs are involved in ABA and abiotic stress signaling pathways (Table 3.1).

\subsection{ROS SIGNALING}

Aerobic organisms in response to different environmental cues produce ROS, such as hydrogen peroxide $\left(\mathrm{H}_{2} \mathrm{O}_{2}\right)$, superoxide radical $\left(\mathrm{O}_{2}{ }^{-}\right)$, hydroxyl radical $(\mathrm{OH})$, and singlet oxygen $(\mathrm{O})$ from excitation or incomplete reduction of molecular oxygen, which are harmful to organisms. Furthermore, ROS play a crucial role in signaling pathways to regulate plant growth and development under various biotic and abiotic stresses (Apel and Hirt, 2004; Miller et al., 2010; Chakradhar et al., 2017; Khan and Khan, 2017). Genetic analysis studies revealed that ROS molecules such as $\mathrm{O}_{2}{ }^{--}$and $\mathrm{H}_{2} \mathrm{O}_{2}$, which are considered as primary ROS in plants, act as secondary messenger molecules in the signaling pathway to regulate various biological functions in plants (Foyer and Noctor, 2005). Due to high reactive nature ROS they react with various biological molecules such as membrane lipids, carbohydrates, proteins, and DNA. ROS molecules such as $\mathrm{H}_{2} \mathrm{O}_{2}$ rapidly diffuse through biological membranes through aquaporins. Moreover, plants also develop efficient enzymatic and nonenzymatic antioxidative systems to protect from various stresses. ROS scavenging systems including ascorbate peroxidases (APX), catalase (CAT), dehydroascorbate reductase (DHAR), glutathione peroxidases (GPX), glutathione Stransferase (GST), glutathione reductase (GR), monodehydroascorbate reductase (MDHAR), peroxiredoxin (PRX), and superoxide dismutase (SOD) are localized in different parts of the plant to detoxify ROS. Avoiding of excess ROS production is an important process to maintain ROS homeostasis under stress conditions. Maxwell et al. (1999) reported that alternative oxidases (AOX) prevent excess ROS production in mitochondria. Other mechanisms like leaf movements, leaf curling, and rearranging photosynthetic apparatus maintain limited ROS production through balancing absorbed energy levels with availing of $\mathrm{CO}_{2}$ (Mittler, 2002).

Genetic analysis studies in the past few decades identified ROS molecules as important signaling molecules in plants (Pitzschke and Hirt, 2006). The overexpression of OsHK3 in transgenic rice regulated NADPH oxidase expression and $\mathrm{H}_{2} \mathrm{O}_{2}$ production in ABA signaling (Wen et al., 2002). The plant NADPH oxidases also referred to as respiratory burst oxidase homologs (RBOHs) are mostly enzymatic sources to produce ROS as a signaling molecule during abiotic stress. Transgenic overexpression of NADPH oxidase of tomato SIRBOH1 induced the production of $\mathrm{H} 2 \mathrm{O} 2$, ABA accumulation, stomatal closure/opening and oxidative stress tolerance (Xia et al., 2014; Zhou et al., 2014). Transgenic overexpression of pepper receptorlike protein kinase 1 (CaRLK1) improved the expression of $\mathrm{RBOH}$ genes and production of $\mathrm{O}_{2}$; the resulting transgenic plants displayed tolerance to pathogen infection (Yi et al., 2010).

These ROS molecules regulate the expression of several genes as a signaling molecule. The ROS levels vary the gene expression by oxidating the signaling pathway components and activating the redox sensitive transcription factors (Laloi et al., 2004). The overexpression of various families of transcription factors including WRKY, Zat, RAV, GRAS, and Myb resulted in increased ROS signaling in genetically modified plants (Desikan et al., 2001; Vranova et al., 2002; Epple et al., 2003; Pnueli et al., 2003; Rizhsky et al., 2003; Rizhsky et al., 2004). In Arabidopsis, the expression of AtPep1 increased $\mathrm{H}_{2} \mathrm{O}_{2}$ synthesis and ROS signaling defense mechanism (Orozco-Cardenas et al., 2001; Huffaker et al., 2006). Rentel et al. (2004) reported that the overexpression of OXI1 (oxidative signal-inducible 1) gene in Arabidopsis enhanced the $\mathrm{H}_{2} \mathrm{O}_{2}$ signaling. Similarly, transgenic alfalfa expressing $\mathrm{H}_{2} \mathrm{O}_{2}$ induced OMTK1 activates the MAPK pathway downstream genes MMK3 revealed that OMTK1 can be activated not only by $\mathrm{H}_{2} \mathrm{O}_{2}$; but also by ethylene and other elicitors. (Nakagami et al., 2004). 
In rice, h-type thioredoxin (Trx) OsTRXh1, which is involved in the regulation of apoplastic redox state, has been identified and transgenic plants with overexpression of OsTRXh1 induced less concentration of $\mathrm{H}_{2} \mathrm{O}_{2}$ regulated accumulation of ROS in apoplast, which influenced the expression of several developmental and stress responsive genes leading to the salt sensitive phenotype (Zhang and Guo., 2012). A rice NADPH thioredoxin reductase (NTRC) which utilizes the NADPH to reduce the chloroplast 2-Cys PRX BAS1 decreases the amount of $\mathrm{H}_{2} \mathrm{O}_{2}$ and protects the chloroplast against oxidative damage (Perez-Ruiz et al., 2006). The $\mathrm{Ca}^{2+} / \mathrm{CaM}$-dependent protein kinase (CCaMK) of rice OsDMI3 is required for the ABA dependent regulation of ROS by enhancing the expression of NADPH oxidase genes (Shi et al., 2012). A C2H2-type ZFP protein ZFP182 is a component of ABA-induced antioxidant defense system. ABAactivated MPKs induce the expression of ZFP proteins such as ZFP36, which activates the expression of NADPH oxidase, MAPK genes, and ABA-induced production of ABA (Zhang et al., 2014a,b). Transgenic rice plants with overexpression of ZFP36 recorded greater activities of antioxidative enzymes and tolerance to water stress (Zhang et al., 2014a,b).

Several MAPK cascades are crucial players in the ROS signaling pathway (Kovtun et al., 2000; Pitzschke and Hirt, 2006; Pitzschke et al., 2009). Transgenic overexpression of cotton MAPK kinase protein GhMKK1 in tobacco exhibited greater levels of antioxidative enzymes and improved tolerance to salt and drought stresses ( $\mathrm{Lu}$ et al., 2013). Similarly, transgenic overexpression of GhMKK5 in tobacco plants activated several ROS and apoptosis related genes (Zhang et al., 2012). The CDPK proteins are known to be involved in abiotic stressinduced ROS regulation (You and Chan, 2015). Rice transgenic plants overexpressing OsCPK12-OX expressed several ROS scavenging enzyme genes (OsAPx2 and OsAPx8) and recorded lower concentration of $\mathrm{H}_{2} \mathrm{O}_{2}$ in the leaves compared with wild-type plants. Transgenic plants also displayed low levels of NADPH oxidase gene compared with wild-type plants (Asano et al., 2012). Transgenic overexpression of an apple CIPK gene MdSOS2L1 induced the expression of several ROS scavenging proteins and enhanced tolerance to salt stress (Hu et al., 2015).

\subsection{CONCLUSIONS AND FUTURE PERSPECTIVES}

Plants have refined and compactly regulated abiotic stress signal sensing and transduction mechanisms at the cellular or at the whole plant levels to protect them from various environmental cues. Plants exhibit genotypic variation in their signal sensing and transduction. Classical and advanced technologies were applied to develop stress tolerant plants by targeting signal transduction pathways. The classical breeding programs were not successful for abiotic stress tolerance as it is a multigenic trait. Thus, a genetic engineering approach offers a new way towards the development of stress tolerant transgenic plants for multiple abiotic stresses. The most critical step towards the development of an abiotic stress tolerant plant is the identification of candidate stress sensors. So far, forward and reverse genetic analysis in combination with chemical screening methods identified a limited number of sensors involved in the intricate abiotic stress signaling pathway due to gene redundancy problems. Advancement in the technologies such as genome editing and chemical genetic screening approaches would enable to overcome these problems. To date research on the abiotic stress signaling pathway have been carried out in plants grown in the culture media; however in nature, plants growing in a complex environment simultaneously face multiple abiotic and biotic stresses and there would be crosstalk between the abiotic and biotic signaling pathways. Hence, more research focus should be on field grown plants to decipher the signaling molecules involved in the multiple stresses.

The transgenic plants developed using the abiotic stress signaling molecules have significantly improved the stress tolerance under laboratory conditions and greenhouse experiments; however, these transgenic plants need to be evaluated in the field before being integrated in the molecular breeding programs. Furthermore, transgenic plants depend on the foreign genetic source, hence before release these transgenic plants need to undergo risk assessment tests. To overcome these regulatory issues, recently, genome editing technologies such as zinc finger nucleases (ZFNs), a transcription activatorlike effector nucleases (TALENs) and clustered regularly interspaced short palindromic repeats/CRISPR associated 9 (CRISPR/Cas9) have been identified as viable tools and have started being used in several crop species not only for genetic improvement of desired traits, but also for functional characterization of genes.

\section{Acknowledgement}

The authors acknowledge the financial support (No.SR/SO/PS-62/ 08 dated 24/9/2009) from Department of Science and Technology (DST), New Delhi, India and Council of Scientific and Industrial Research (CSIR), New Delhi (No. 38(1212)/09/EMR-II dated March 4 , 2009). KKG acknowledge the financial support by Department of Science and Technology_INSPIRE Fellowship (IF120376). 


\section{References}

Abbasi, F., Onodera, H., Toki, S., Tanaka, H., Komatsu, S., 2004. OsCDPK13, a calcium-dependent protein kinase gene from rice, is induced by cold and gibberellin in rice leaf sheath. Plant Mol. Biol. 55, 541-552.

Abe, H., Urao, T., Ito, T., Seki, M., Shinozaki, K., YamaguchiShinozaki, K., 2003. Arabidopsis AtMYC2 (bHLH) and AtMYB2 (MYB) function as transcriptional activators in abscisic acid signaling. Plant Cell. 15, 63-78. Available from: https://doi.org/ $10.1105 /$ tpc.006130.

Acquaah, G., 2007. Principles of Plant Genetics and Breeding. Blackwell, Oxford, UK.

Agrawal, G.K., Rakwal, R., Iwahashi, H., 2002. Isolation of novel rice (Oryza sativa L.) multiple stress responsive MAP kinase gene, OsMSRMK2, whose mRNA accumulates rapidly in response to environmental cues. Biochem. Biophys. Res. Commun. 294, 1009-1016.

Agrawal, G.K., Agrawal, S.K., Shibato, J., Iwahashi, H., Rakwal, R., 2003. Novel rice MAP kinases OsMSRMK3 and OsWJUMK1 involved in encountering diverse environmental stresses and developmental regulation. Biochem. Biophys. Res. Commun. 300, 775-783.

Aleman, F., et al., 2016. An ABA-increased interaction of the PYL6 ABA receptor with MYC2 Transcription Factor: A putative link of ABA and JA signaling. Sci. Rep 6, 28941. Available from: https:// doi.org/10.1038/srep28941.

Apel, K., Hirt, H., 2004. Reactive oxygen species metabolism, oxidative stress, and signal transduction. Annu. Rev. Plant Biol. 55, 373-399. Available from: https://doi.org/10.1146/annurev. arplant.55.031903.141701.

Arnadottir, J., Chalfi, M., et al., 2010. Eukaryotic mechanosensitive channels. Annu. Rev. Biophys. 39, 111-137.

Asai, T., Tena, G., Plotnikova, J., Willmann, M.R., Chiu, W.L., Gomez-Gomez, L., et al., 2002. MAP kinase signalling cascade in Arabidopsis innate immunity. Nature 415, 977-983. Available from: https://doi.org/10.1038/415977a. PMID: 11875555.

Asano, T., Hakata, M., Nakamura, H., Aoki, N., Komatsu, S., Ichikawa, H., et al., 2011. Functional characterisation of OsCPK21, a calcium-dependent protein kinase that confers salt tolerance in rice. Plant Mol. Biol. 75, 179-191.

Asano, T., Hayashi, N., Kobayashi, M., Aoki, N., Miyao, A., Mitsuhara, I., et al., 2012. A rice calcium-dependent protein kinase OsCPK12 oppositely modulates salt-stress tolerance and blast disease resistance. Plant J. 69, 26-36. Available from: https: / / doi.org/10.1111/j.1365-313X.2011.04766.x.

Bindschedler, L.V., Dewdney, J., Blee, K.A., Stone, J.M., Asai, T., Plotnikov, J., Denoux, C., Hayes, T., Gerrish, C., Davies, D.R., et al., 2006. Peroxidase-dependent apoplastic oxidative burst in Arabidopsis required for pathogen resistance. The Plant J. For Cell and Mol. Bio 47 (6), 851-863. Available from: https://doi. org/10.1111/j.1365-313X.2006.02837.x.

Bouche, N., Yellin, A., Snedden, W.A., Fromm, H., 2005. Plantspecific calmodulin-binding proteins. Annu. Rev. Plant Biol. 56, $435-466$

Boudsocq, M., Barbier-Brygoo, H., Lauriè re, C., 2004. Identification of nine sucrose non fermenting 1-related protein kinases 2 activated by hyper osmotic and saline stresses in Arabidopsis thaliana. J. Biol. Chem. 279, 41758-41766.

Brandt, B., Munemasa, S., Wang, C., Nguyen, D., Yong, T., Yang, P. G., Poretsky, E., Belknap, T.F., Waadt, R., Aleman, F., et al., 2015. Calcium specificity signaling mechanisms in abscisic acid signal transduction in Arabidopsis guard cells. eLife. 4, e03599. Available from: https://doi.org/10.7554/eLife.03599.

Castillo, M.C., Lozano-Juste, J., González-Guzmán, M., Rodriguez, L., Rodriguez, P.L., León, J., 2015. Inactivation of PYR/PYL/RCAR
ABA receptors by tyrosine nitration may enable rapid inhibition of ABA signaling by nitric oxide in plants. Sci. Signal 8, ra89.

Chakradhar, T., Mahanty, S., Reddy, R.A., Divya, K., Reddy, P.S., Reddy, M.K., 2017. Biotechnological perspective of reactive oxygen species (ROS)-mediated stress tolerance in plants. In: Khan, M., Khan, N. (Eds.), Reactive Oxygen Species and Antioxidant Systems in Plants: Role and Regulation under Abiotic Stress. Springer, Singapore.

Chen, L.G., Zhang, L.P., Yu, D.Q., 2010. Wounding-induced WRKY8 is involved in basal defense in Arabidopsis. Mol. Plant Microb. Interact. 23, 558-565.

Chen, X.F., Gu, Z.M., Xin, D.D., Hao, L.A., Liu, C.J., Huang, J., et al., 2011. Identification and characterization of putative CIPK genes in maize. J. Genet. Genom. 38, 77-87. Available from: https:// doi.org/10.1016/j.jcg.2011.01.005.

Chen, Y., Jiang, J., Chang, Q., Gu, C., Song, A., Chen, F., et al., 2014. Cold acclimation induces freezing tolerance via antioxidative enzymes, proline metabolism and gene expression changes in two Chrysanthemum species. Mol. Biol. Rep. 41, 815-822. Available from: https://doi.org/10.1007/s11033-013-2921-8.

Choi, W.-G., Toyota, M., Kim, S.-H., Hilleary, R., Gilroy, S., 2014. Salt stress-induced $\mathrm{Ca}^{2+}$ waves are associated with rapid, long-distance root-to-shoot signaling in plants. Proc. Natl. Acad. Sci. USA 111 (17), 6497-6502. Available from: https://doi.org/10.1073/ pnas.1319955111.

Chinnusamy, V., Ohta, M., Kanrar, S., Lee, B., Hong, X., Agarwal, M., Zhu, J.-K., 2003. ICE1: a regulator of cold-induced transcriptome and freezing tolerance in Arabidopsis. Genes \& Deve 17 (8), 1043-1054. Available from: https://doi.org/10.1101/gad.1077503.

Cutler, S.R., Rodriguez, P.L., Finkelstein, R.R., Abrams, S.R., 2010. Abscisic acid: emergence of a core signaling network. Annu. Rev. Plant Biol. 61, 651-679. Available from: https://doi.org/10.1146/ annurev-arplant-042809-112122.

de Lorenzo, L., Merchan, F., Laporte, P., Thompson, R., Clarke, J., Sousa, C., Crespi, M., 2009. A novel plant leucine-rich repeat receptor kinase regulates the response of Medicago truncatula roots to salt stress. The Plant Cell 21, 668-680. Available from: https://doi.org/10.1105/tpc.108.059576.

DeFalco, T.A., Bender, K.W., Snedden, W.A., 2010. Breaking the code: Ca2 + sensors in plant signalling. Biochem. J. 425, 27-40.

Deng, X., Hu, W., Wei, S., Zhou, S., Zhang, F., Han, J., et al., 2013. TaCIPK29, a CBL-interacting protein kinase gene from wheat, confers salt stress tolerance in transgenic tobacco. PLoS One 8, e69881. Available from: https://doi.org/10.1371/journal.pone.0069881.

Desikan, R., Mackerness, S.A.H., Hancock, J.T., Neill, S.J., 2001. Regulation of the Arabidopsis transcriptome by oxidative stress. Plant Physiol. 127, 159-172.

Dong, C.J., Yang, X.D., Liu, J.Y., 2009. Enzymatic properties of a recombinant phospholipid hydroperoxide glutathione peroxidase from Momordica charantia and its complementation function in yeast. Biochem 74 (5), 502-508.

Drerup, M.M., Schlucking, K., Hashimoto, K., Manishankar, P., Steinhorst, L., Kuchitsu, K., et al., 2013. The calcineurin B-like calcium sensors CBL1 and CBL9 together with their interacting protein kinase CIPK26 regulate the Arabidopsis NADPH oxidase RBOHF. Mol. Plant 6, 559-569. Available from: https://doi.org/ $10.1093 / \mathrm{mp} / \mathrm{sst009}$.

Droillard, M., Boudsocq, M., Barbier-Brygoo, H., Laurière, C., 2002. Different protein kinase families are activated by osmotic stresses in Arabidopsis thaliana cell suspensions. Involvement of the MAP kinases AtMPK3 and AtMPK6. FEBS Lett. 527, 43-50.

Du, J., Huang, Y.P., Xi, J., Cao, M.J., Ni, W.S., et al., 2008. Functional gene-mining for salt-tolerance genes with the power of Arabidopsis. Plant J 56, 653-664. Available from: https://doi. org/10.1111/j.1365-313X.2008.03602.x. 
Dubiella, U., Seybold, H., Durian, G., Komander, E., Lassig, R., Witte, C.-P., Schulze, W.X., Romeis, T., 2013. Calcium-dependent protein kinase/NADPH oxidase activation circuit is required for rapid defense signal propagation. Proc. Natl. Acad. Sci. USA 110 (21), 8744-8749. Available from: https://doi.org/10.1073/ pnas.1221294110.

Epple, P., Mack, A.A., Morris, V.R., Dangl, J.L., 2003. Antagonistic control of oxidative stress-induced cell death in Arabidopsis by two related, plant-specific zinc finger proteins. Proc. Natl. Acad. Sci. USA. 100, 6831-6836.

Fedoroff, N.V., Battisti, D.S., Beachy, R.N., Cooper, P.J., Fischhoff, D. A., Hodges, C.N., et al., 2010. Radically rethinking agriculture for the 21st century. Science. 327, 833-834. Available from: https:// doi.org/10.1126/science.1186834.

Feng, L., Gao, Z.R., Xiao, G.Q., Huang, R.F., Zhang, H.W., 2014. Leucinerich repeat receptor-like kinase FON1 regulates drought stress and seed germination by activating the expression of ABAresponsive genes in Rice. Plant Mol. Biol. Rep. 32 (6), 1-11.

Finka, A., Cuendet, A.F., Maathuis, F.J., Saidi, Y., Goloubinoff, P., et al., 2012. Plasma membrane cyclic nucleotide gated calcium channels control land plant thermal sensing and acquired thermo tolerance. Plant Cell 24, 3333-3348.

Foyer, C.H., Noctor, G., 2005. Redox homeostasis and antioxidant signaling: a metabolic interface between stress perception and physiological responses. Plant Cell. 17, 1866-1875. Available from: https://doi.org/10.1105/tpc.105.033589.

Franz, S., Ehlert, B., Liese, A., Kurth, J., Cazale, A.C., Romeis, T., 2010. Calcium-dependent protein kinase CPK21 functions in abiotic stress response in Arabidopsis thaliana. Mol. Plant. 4, 83-96.

Fu, S.F., Chou, W.C., Huang, D.D., Huang, H.J., 2002. Transcriptional regulation of a rice mitogen-activated protein kinase gene, OsMAPK4, in response to environmental stresses. Plant Cell Physiol. 43, 958-963.

Fuglsang, A.T., Guo, Y., Cuin, T.A., Qiu, Q., Song, C.P., Kristiansen, K.A., et al., 2007. Arabidopsis protein kinase PKS5 inhibits the plasma membrane $\mathrm{H}^{+}$-ATPase by preventing interaction with 14-3-3 protein. Plant Cell 19, 1617-1634.

Fujii, H., Chinnusamy, V., Rodrigues, A., Rubio, S., Antoni, R., Park, S.Y., et al., 2009. In vitro reconstitution of an abscisic acid signalling pathway. Nature 462, 660-664.

Fujii, H., Verslues, P.E., Zhu, J.K., 2011. Arabidopsis decuple mutant reveals the importance of SnRK2 kinases in osmotic stress responses in vivo. Proc. Natl. Acad. Sci. USA. 108, 1717-1722.

Furihata, T., Maruyama, K., Fujita, Y., Umezawa, T., Yoshida, R., Shinozaki, K., et al., 2006. Abscisic acid-dependent multisite phosphorylation regulates the activity of a transcription activator AREB1. Proc. Natl. Acad. Sci. USA. 103, 1988-1993.

Geiger, D., Scherzer, S., Mumm, P., Marten, I., Ache, P., Matschi, S., Liese, A., Wellmann, C., Al-Rasheid, K.A., Grill, E., et al., 2010. Guard cell anion channel SLAC1 is regulated by CDPK protein kinases with distinct $\mathrm{Ca}^{2+}$ affinities. Proc. Natl. Acad. Sci. USA 107 (17), 8023-8028. Available from: https://doi.org/10.1073/ pnas.0912030107.

Geiger, D., Scherzer, S., Mumm, P., Stange, A., Marten, I., Bauer, H., Ache, P., Matschi, S., Liese, A., Al-Rasheid, K.A., et al., 2009. Activity of guard cell anion channel SLAC1 is controlled by drought-stress signaling kinase-phosphatase pair. Proc. Natl. Acad. Sci. USA 106 (50), 21425-21430. Available from: https:// doi.org/10.1073/pnas.0912021106.

Gonzalez-Guzman, M., Pizzio, G.A., Antoni, R., Vera-Sirera, F., Merilo, E., Bassel, G.W., et al., 2012. Arabidopsis PYR/PYL/RCAR Receptors Play a Major Role in Quantitative Regulation of Stomatal Aperture and Transcriptional Response to Abscisic Acid. The Plant Cell 24 (6), 2483-2496. Available from: https:// doi.org/10.1105/tpc.112.098574.
Greeff, C., Roux, M., Mundy, J., Petersen, M., 2012. Receptor-like kinase complexes in plant innate immunity. Front. Plant Sci. 3, 209.

Gu, L., Liu, Y., Zong, X., Liu, L., Li, D.P., Li, D.Q., 2010. Overexpression of maize mitogen-activated protein kinase gene, ZmSIMK1 in Arabidopsis increases tolerance to salt stress. Mol. Biol. Rep. 37, 4067-4073.

Guo, Y., Halfer, U., Ishitani, M., Zhu, J.K., 2001. Molecular characterization of functional domains in the protein kinase SOS2 that is required for plant salt tolerance. Plant Cell. 13, 1383-1399.

Hamilton, E.S., Haswell, E.S., 2017. The tension-sensitive ion transport activity of MSL8 is 690 critical for its function in pollen hydration and germination. Plant Cell Physiol. 1-16. Available from: https://doi.org/10.1093/pcp/pcw230.

Hamilton, E.S., Schlegel, A.M., Haswell, E.S., 2015. United in diversity: mechanosensitive ion channels in plants. Annu. Rev. Plant Biol. 66, 113-137.

Hedrich, R., 2012. Ion channels in plants. Physiol. Rev. 92, 1777-1811. Available from: https://doi.org/10.1152/physrev.00038.2011.

Hoang, M.H., Nguyen, X.C., Lee, K., Kwon, Y.S., Pham, H.T., Park, H.C., et al., 2012. Phosphorylation by ATMPK6 is required for the biological function of AtMYB41 in Arabidopsis. Biochem. Biophys. Res. Commun. 422, 181-186.

Hou, Q.C., Ufer, G., Bartels, D., 2016. Lipid signalling in plant responses to abiotic stress. Plant Cell Environ 39, 1029-1048. Available from: https://doi.org/10.1111/pce.12666.

Hrabak, E.M., Chan, C.W., Gribskov, M., Harper, J.F., Choi, J.H., Halford, N., et al., 2003. The Arabidopsis CDPK-SnRK super family of protein kinases. Plant Physiol. 132, 666-680.

Hu, D.G., Ma, Q.J., Sun, C.H., Sun, M.H., You, C.X., Hao, Y.J., 2015. Over expression of MdSOS2L1, a CIPK protein kinase, increases the antioxidant metabolites to enhance salt tolerance in apple and tomato. Physiol. Plant. Available from: https://doi.org/10.1111/ ppl.12354.

Hua, D., Wang, C., He, J., Liao, H., Duan, Y., Zhu, Z., Guo, Y., Chen, Z., Gong, Z., 2012. A Plasma Membrane Receptor Kinase, GHR1, Mediates Abscisic Acid- and Hydrogen Peroxide-Regulated Stomatal Movement in Arabidopsis. The Plant Cell 24 (6), 2546-2561. Available from: https://doi.org/10.1105/ tpc.112.100107.

Huffaker, A., Pearce, G., Ryan, C.A., 2006. An endogenous peptide signal in Arabidopsis activates components of the innate immune response. Proc Natl. Acad. Sci. USA. 103, 10098-10103.

Husaini, A.M., Tuteja, N., 2013. Biotech crops: imperative for achieving the Millennium Development Goals and sustainability of agriculture in the climate change era. GM Crops Food. 4, 1-9. Available from: https://doi.org/10.4161/gmcr.22748.

Ichimura, K., Mizoguchi, T., Yoshida, R., Yuasa, T., Shinozaki, K., 2000. Various abiotic stresses rapidly activate Arabidopsis MAP kinases ATMPK4 and ATMPK6. Plant J. 24, 655-665. Available from: https:/ / doi.org/10.1046/j.1365-313x.2000.00913.x.

Ishitani, M., Liu, J., Halfter, U., Kim, C.-S., Shi, W., Zhu, J.-K., 2000. SOS3 Function in Plant Salt Tolerance Requires N-Myristoylation and Calcium Binding. The Plant Cell 12 (9), 1667-1678.

Iuchi, S., Kobayashi, M., Yamaguchi-Shinozaki, K., Shinozaki, K., 2000. A Stress-Inducible Gene for 9-cis-Epoxycarotenoid Dioxygenase Involved in Abscisic Acid Biosynthesis under Water Stress in Drought-Tolerant Cowpea. Plant Physiol. 123 (2), $553-562$.

Jammes, F., et al., 2009. MAP kinases MPK9 and MPK12 are preferentially expressed in guard cells and positively regulate ROSmediated ABA signaling. Proc. Natl. Acad. Sci. USA 106, 20520-20525. Available from: https://doi.org/10.1073/ pnas.0907205106.

Jeong, M.J., Lee, S.K., Kim, B.G., Kwon, T.R., Cho, W.S., Park, Y.T., et al., 2006. A rice (Oryza sativa L.) MAP kinase gene, OsMAPK44, 
is involved in response to abiotic stresses. Plant Cell Tissue Organ Cult. 85, 151-160.

Kamei, A., Seki, M., Umezawa, T., Ishida, J., Satou, M., Akiyama, K., et al., 2005. Analysis of gene expression profiles in Arabidopsis salt overly sensitive mutants sos2-1 and sos3-1. Plant Cell Environ 28, 1267-1275. Available from: https://doi.org/10.1111/j.13653040.2005.01363.x.

Kang, J., Hwang, J.U., Lee, M., Kim, Y.Y., Assmann, S.M., Martinoia, E., et al., 2010. PDR-type ABC transporter mediates cellular uptake of the phytohormone abscisic acid. Proc. Natl. Acad. Sci. USA. 107, 2355-2360. Available from: https://doi.org/10.1073/ pnas.0909222107.

Khan, F., Hakeem, K.R., 2014. Cell signaling during drought and salt stress. In: Hakeem, K.R., Rehman, R., Tahir, I. (Eds.), Plant Signaling: Understanding the Molecular Cross Talk. Springer, India, pp. 227-239.

Khan, M.I.R., Khan, N., 2017. Reactive Oxygen Species and Antioxidant System in Plants: Role and Regulation Under Abiotic Stress. Springer Nature 978-981-10-5254-5.

Kim, J.A., Agrawal, G.K., Rakwal, R., Han, K.S., Kim, K.N., Yun, C. H., et al., 2003. Molecular cloning and mRNA expression analysis of a novel rice (Oryza sativa L.) MAPK kinase kinase, OsEDR1, an ortholog of Arabidopsis AtEDR1, reveal its role in defense/stress signalling pathways and development. Biochem. Biophys. Res. Commun. 300, 868-876.

Kim, J.M., Woo, D.H., Kim, S.H., Lee, S.Y., Park, H.Y., Seok, H.Y., et al., 2012. Arabidopsis MKKK20 is involved in osmotic stress response via regulation of MPK6 activity. Plant Cell Rep. 31, 217-224.

Kim, S.H., Woo, D.H., Kim, J.M., Lee, S.Y., Chung, W.S., Moon, Y.H., 2011. Arabidopsis MKK4 mediates osmotic-stress response via its regulation of MPK3 activity. Biochem. Biophys. Res. Commun. 412, 150-154.

Kim, H., Lee, K., Hwang, H., Bhatnagar, N., Kim, D.-Y., Yoon, I.S., et al., 2014. Overexpression of PYL5 in rice enhances drought tolerance, inhibits growth, and modulates gene expression. J. Exp. Bot. 65 (2), 453-464. Available from: https://doi.org/10.1093/ jxb/ert397.

Knight, H., 2000. Calcium signaling during abiotic stress in plants. Int. Rev. Cytol. 195, 269-324.

Kolukisaoglu, U., Weinl, S., Blazevic, D., Batistic, O., Kudal, J., 2004. Calcium sensors and their interacting protein kinases: genomics of the Arabidopsis and rice CBL-CIPK signaling networks. Plant Physiol. 134, 43-58. Available from: https://doi.org/10.1104/ pp.103.033068.

Kong, X., Sun, L., Zhou, Y., Zhang, M., Liu, Y., Pan, J., et al., 2011. ZmMKK4 regulates osmotic stress through reactive oxygen species scavenging in transgenic tobacco. Plant Cell Rep. 30, 2097-2104.

Kovtun, Y., Chiu, W.L., Tena, G., Sheen, J., 2000. Functional analysis of oxidative stress-activated mitogen-activated protein kinase cascade in plants. Proc. Natl. Acad. Sci. USA. 97, 2940-2945. Available from: https://doi.org/10.1073/pnas.97.6.2940.

Kung, C., 2005. A possible unifying principle for mechanosensation. Nature. 436, 647-654.

Kuromori, T., Shinozaki, K., 2010. ABA transport factors found in Arabidopsis $\mathrm{ABC}$ transporters. Plant Signal Behav. 5, 1124-1126.

Laloi, C., Apel, K., Danon, A., 2004. Reactive oxygen signalling: the latest news. Curr. Opin. Plant Biol. 7, 323-328.

Lee, S.K., Kim, B.G., Kwon, T.R., Jeong, M.J., Park, S.R., Lee, J.W., et al., 2011. Overexpression of the mitogen-activated protein kinase gene OsMAPK33 enhances sensitivity to salt stress in rice (Oryza sativa L.). J. Biosci. 36, 139-151.
Lenka, S.K., Muthusamy, S.K., Chinnusamy, V., et al., 2018. Ectopic expression of rice PYL3 enhances cold and drought tolerance in Arabidopsis thaliana. Mol. Biotechnol. 60, 350. Available from: https://doi.org/10.1007/s12033-018-0076-5.

Liu, H.C., Liao, H.T., Charng, Y.Y., 2011. The role of class A1 heat shock factors (HSFA1s) in response to heat and other stresses in Arabidopsis. Plant Cell Environ 34, 738-751. Available from: https://doi.org/10.1111/j.1365-3040.2011.02278.x.

Li, S., Zhou, X., Chen, L., Huang, W., Yu, D., 2010. Functional characterization of Arabidopsis thaliana WRKY39 in heat stress. Mol. Cells. 29, 475-483. Available from: https://doi.org/10.1007/ s10059-010-0059-2.

Loukehaich, R., Wang, T., Ouyang, B., Ziaf, K., Li, H., Zhang, J., et al., 2012. SpUSP, an annexin-interacting universal stress protein, enhances drought tolerance in tomato. J. Exp. Bot 63 (15), 5593-5606. Available from: https://doi.org/10.1093/jxb/ers220.

Lu, G., Gao, C., Zheng, X., Han, B., 2009. Identification of OsbZIP72 as a positive regulator of $\mathrm{ABA}$ response and drought tolerance in rice. Planta. 229, 605-615.

Lu, W., Chu, X., Li, Y., Wang, C., Guo, X., 2013. Cotton GhMKK1 induces the tolerance of salt and drought stress, and mediates defence responses to pathogen infection in transgenic Nicotiana benthamiana. PLoS One 8, e68503. Available from: https://doi. org/10.1371/journal.pone.0068503.

Ma, D.M., Xu, W.R., Li, H.W., Jin, F.X., Guo, L.N., Wang, J., et al., 2014. Co-expression of the Arabidopsis SOS genes enhances salt tolerance in transgenic tall fescue (Festuca arundinacea Schreb.). Protoplasma. 251, 219-231. Available from: https://doi.org/ 10.1007/s00709-013-0540-9.

Ma, S.Y., Wu, W.H., 2007. AtCPK23 functions in Arabidopsis responses to drought and salt stresses. Plant Mol. Biol. 65, $511-518$

Ma, Y., Szostkiewicz, I., Korte, A., Moes, D., Yang, Y., Christmann, A., et al., 2009. Regulators of PP2C phosphatase activity function as abscisic acid sensors. Science. 324, 1064-1068.

Ma, Y., Dai, X., Xu, Y., Luo, W., Zheng, X., Zeng, D., et al., 2015. COLD1 confers chilling tolerance in rice. Cell. 160, 1209-1221.

Magnan, F., Ranty, B., Charpenteau, M., Sotta, B., Galaud, J.P., Aldon, D., 2008. Mutations in AtCML9, a calmodulin-like protein from Arabidopsis thaliana, alter plant responses to abiotic stress and abscisic acid. Plant J. 56, 575-589. Available from: https:// doi.org/10.1111/j.1365-313X.2008.03622.x.

Maxwell, D.P., Wang, Y., Mcintosh, L., 1999. The alternative oxidase lowers mitochondrial reactive oxygen production in plant cells. Proc. Natl. Acad. Sci. USA. 96, 8271-8276. Available from: https://doi.org/10.1073/pnas.96.14.8271.

Mehlmer, N., Wurzinger, B., Stael, S., Hofmann-Rodrigues, D., Csaszar, E., Pfister, B., et al., 2010. The $\mathrm{Ca}^{2+}$-dependent protein kinase CPK3 is required for MAPK-independent salt-stress acclimation in Arabidopsis. Plant J. 63, 484-498.

Melcher, K., Ng, L.M., Zhou, X.E., Soon, F.F., Xu, Y., Suino-Powell, K.M., et al., 2009. A gate-latch-lock mechanism for hormone signalling by abscisic acid receptors. Nature. 462, 602-608.

Miller, G., Suzuki, N., Ciftci-Yilmaz, S., Mittler, R., 2010. Reactive oxygen species homeostasis and signaling during drought and salinity stresses. Plant Cell Environ. 33, 453-467. Available from: https: / / doi.org/10.1111/j.1365-3040.2009.02041.x.

Mittler, R., 2002. Oxidative stress, antioxidants and stress tolerance. Trends Plant Sci. 7, 405-410. Available from: https://doi.org/ 10.1016/S1360-1385(02)02312-9.

Mizoguchi, T., Irie, K., Hirayama, T., Hayashida, N., YamaguchiShinozaki, K., Matsumoto, K., et al., 1996. Gene encoding a mitogen-activated protein kinase kinase kinase is induced 
simultaneously with genes for a mitogen-activated protein kinase and an S6 ribosomal protein kinase by touch, cold, and water stress in Arabidopsis thaliana. Proc. Natl. Acad. Sci. USA. 93, 765-769.

Mosquna, A., Peterson, F.C., Park, S.Y., Lozano-Juste, J., Volkman, B. F., Cutler, S.R., 2011. Potent and selective activation of abscisic acid receptors in vivo by mutational stabilization of their agonistbound conformation. Proc. Natl. Acad. Sci. USA 108 (51), 20838-20843. Available from: https://doi.org/10.1073/ pnas. 1112838108 .

Moustafa, K., Lefebvre-De Vos, D., Leprince, A.S., Savouree, A., Lauriere, C., 2008. Analysis of the Arabidopsis mitogen-activated protein kinase families: organ specificity and transcriptional regulation upon water stresses. Sch. Res. Exch. 12. Available from: https:/ / doi.org/10.3814/2008/143656.

Mustilli, A.-C., Merlot, S., Vavasseur, A., Fenzi, F., Giraudat, J., 2002. Arabidopsis OST1 Protein Kinase Mediates the Regulation of Stomatal Aperture by Abscisic Acid and Acts Upstream of Reactive Oxygen Species Production. The Plant Cell 14 (12), 3089-3099. Available from: https://doi.org/10.1105/tpc.007906.

Nakagami, H., Kiegerl, S., Hirt, H., 2004. OMTK1, a novel MAPKKK, channels oxidative stress signaling through direct MAPK interaction. J. Biol. Chem. 279, 26959-26966.

Norén, L., Kindgren, P., Stachula, P., Rühl, M., Eriksson, M.E., Hurry, V., Strand, A., 2016. Circadian and Plastid Signaling Pathways Are Integrated to Ensure Correct Expression of the CBF and COR Genes during Photoperiodic Growth. Plant Physiol. 171 (2), 1392-1406. Available from: https://doi.org/10.1104/ pp.16.00374.

Novillo, F., Alonso, J.M., Ecker, J.R., Salinas, J., 2004. CBF2/DREB1C is a negative regulator of $C B F 1 / D R E B 1 B$ and $C B F 3 / D R E B 1 A$ expression and plays a central role in stress tolerance in Arabidopsis. Proc. Natl. Acad. Sci. USA 101 (11), 3985-3990. Available from: https://doi.org/10.1073/pnas.0303029101.

Ohta, M., Guo, Y., Halfter, U., Zhu, J.-K., 2003. A novel domain in the protein kinase SOS2 mediates interaction with the protein phosphatase 2C ABI2. Proc. Natl. Acad. Sci. USA 100 (20), 11771-11776. Available from: https://doi.org/10.1073/ pnas. 2034853100.

Olías, R., Eljakaoui, Z., Li, J., De Morales, P.A.Z.A., Marín-Manzano, M.C., Pardo, J.M., et al., 2009. The plasma membrane $\mathrm{Na}+/ \mathrm{H}+$ antiporter SOS1 is essential for salt tolerance in tomato and affects the partitioning of $\mathrm{Na}+$ between plant organs. Plant Cell Environ. 32, 904-916.

Orozco-Cardenas, M.L., Narvaez-Vasquez, J., Ryan, C.A., 2001. Hydrogen peroxide acts as a second messenger for the induction of defense genes in tomato plants in response to wounding, system in, and methyl jasmonate. Plant Cell. 13, 179-191.

Pan, J., Zhang, M., Kong, X., Xing, X., Liu, Y., Zhou, Y., et al., 2012. ZmMPK17, a novel maize group D MAP kinase gene, is involved in multiple stress responses. Planta. 235, 661-676.

Pandey, S., Nelson, D.C., Assmann, S.M., 2009. Two novel GPCRtype $\mathrm{G}$ proteins are abscisic acid receptors in Arabidopsis. Cell. 136, 136-148. Available from: https://doi.org/10.1016/j. cell.2008.12.026.

Park, H.C., Park, C.Y., Koo, S.C., Cheong, M.S., Kim, K.E., Kim, M. C., et al., 2010. AtCML8, a calmodulin-like protein, differentially activating CaM-dependent enzymes in Arabidopsis thaliana. Plant Cell Rep. 29, 1297-1304. Available from: https://doi.org/ 10.1007/s00299-010-0916-7.

Park, S.Y., Fung, P., Nishimura, N., Jensen, D.R., Fujii, H., Zhao, Y., et al., 2009. Abscisic acid inhibits type $2 \mathrm{C}$ protein phosphatases via the PYR/PYL family of START proteins. Science. 324, 1068-1071.
Pehlivan, N., Sun, L., Jarrett, P., Yang, X., Mishra, N., Chen, L., et al., 2016. Co-overexpressing a plasma membrane and a vacuolar membrane sodium/proton antiporter significantly improves salt tolerance in transgenic Arabidopsis plants. Plant Cell Physiol. 57, 1069-1084.

Perez-Ruiz, J.M., Spinola, M.C., Kirchsteiger, K., Moreno, J., Sahrawy, M., Cejudo, F.J., 2006. Rice NTRC is a high-efficiency redox system for chloroplast protection against oxidative damage. Plant Cell. 18, 2356-2368. Available from: https://doi.org/ 10.1105/tpc.106.041541.

Perochon, A., Aldon, D., Galaud, J.P., Ranty, B., 2011. Calmodulin and calmodulin-like proteins in plant calcium signaling. Biochimie. 93, 2048-2053. Available from: https://doi.org/ 10.1016/j.biochi.2011.07.012.

Perruc, E., Charpenteau, M., Ramirez, B.C., Jauneau, A., Galaud, J.P., Ranjeva, R., et al., 2004. A novel calmodulin-binding protein functions as a negative regulator of osmotic stress tolerance in Arabidopsis thaliana seedlings. Plant J. 38, 410-420. Available from: https:/ / doi.org/10.1111/j.1365-313X.2004.02062.x.

Pitzschke, A., Hirt, H., 2006. Mitogen-activated protein kinases and reactive oxygen species signaling in plants. Plant Physiol. 141, 351-356. Available from: https://doi.org/10.1104/pp.106.079160.

Pitzschke, A., Djamei, A., Bitton, F., Hirt, H., 2009. A major role of the MEKK1-MKK1/2-MPK4 path way in ROS signalling. Mol. Plant. 2, 120-137. Available from: https://doi.org/10.1093/mp/ ssn079.

Pizzio, G.A., Rodriguez, L., Antoni, R., Gonzalez-Guzman, M., Yunta, C., Merilo, E., et al., 2013. The PYL4 A194T Mutant Uncovers a Key Role of PYR1-LIKE4/PROTEIN PHOSPHATASE 2CA Interaction for Abscisic Acid Signaling and Plant Drought Resistance. Plant Physiol. 163 (1), 441-455. Available from: https://doi.org/10.1104/pp.113.224162.

Pnueli, L., Liang, H., Rozenberg, M., Mittler, R., 2003. Growth suppression, altered stomatal responses, and augmented induction of heat shock proteins in cytosolic ascorbate peroxidase (Apx1)-deficient Arabidopsis plants. Plant J. 34, 187-203.

Qiu, Q.S., Guo, Y., Quintero, F.J., Pardo, J.M., Schumaker, K.S., Zhu, J.K., 2004. Regulation of vacuolar $\mathrm{Na}^{+} / \mathrm{H}^{+}$exchange in Arabidopsis thaliana by the salt-overly-sensitive (SOS) pathway. J. Biol. Chem. 279, 207-215. Available from: https://doi.org/ 10.1074/jbc.M307982200.

Quan, R., Lin, H.X., Mendoza, I., Zhang, Y.G., Cao, W.H., Yang, Y.Q., et al., 2007. SCABP8/CBL10, a putative calcium sensor, interacts with the protein kinase SOS2 to protect Arabidopsis shoots from salt stress. Plant Cell 19, 1415-1431. Available from: https://doi. org/10.1105/tpc.106.042291.

Rentel, M.C., Lecourieux, D., Ouaked, F., Usher, S.L., Petersen, L., et al., 2004. OXI1 kinase is necessary for oxidative burst-mediated signalling in Arabidopsis. Nature. 427, 858-861.

Rizhsky, L., Liang, H., Mittler, R., 2003. The water-water cycle is essential for chloroplast protection in the absence of stress. J. Biol. Chem. 278, 38921-38925.

Rizhsky, L., Davletova, S., Liang, H., Mittler, R., 2004. The zinc finger protein Zat12 is required for cytosolic ascorbate peroxidase 1 expression during oxidative stress in Arabidopsis. J. Biol. Chem. $279,11736-11743$.

Rodrigues, A., Adamo, M., Crozet, P., Margalha, L., Confraria, A., Martinho, C., et al., 2013. ABI1 and PP2CA Phosphatases Are Negative Regulators of Snf1-Related Protein Kinase1 Signaling in Arabidopsis. The Plant Cell 25 (10), 3871-3884. Available from: https://doi.org/10.1105/tpc.113.114066.

Rodriguez, L., Gonzalez-Guzman, M., Diaz, M., Rodrigues, A., Izquierdo-Garcia, A.C., Peirats-Llobet, M., Fernandez, M.A., 
Antoni, R., Fernandez, D., Marquez, J.A., et al., 2014. C2-Domain Abscisic Acid-Related Proteins Mediate the Interaction of PYR/ PYL/RCAR Abscisic Acid Receptors with the Plasma Membrane and Regulate Abscisic Acid Sensitivity in Arabidopsis. The Plant Cell 26 (12), 4802-4820. Available from: https://doi.org/10.1105/ tpc.114.129973.

Rus, A., Lee, B., Muñoz-Mayor, A., Sharkhuu, A., Miura, K., Zhu, J.K., Bressan, R.A., Hasegawa, P.M., 2004. AtHKT1 Facilitates $\mathrm{Na}^{+}$ Homeostasis and $\mathrm{K}^{+}$Nutrition in Planta. Plant Physiol. 136 (1), 2500-2511. Available from: https://doi.org/10.1104/ pp.104.042234.

Rus, A., Yokoi, S., Sharkhuu, A., Reddy, M., Lee, B.-H., Matsumoto, T.K., Koiwa, H., Zhu, J.-K., Bressan, R.A., Hasegawa, P.M., 2001. AtHKT1 is a salt tolerance determinant that controls $\mathrm{Na}^{+}$entry into plant roots. Proc. Natl. Acad. Sci. USA 98, 14150-14155. Available from: https://doi.org/10.1073/pnas.241501798.

Saijo, Y., Hata, S., Kyozuka, J., Shimamoto, K., Izui, K., 2000. Overexpression of a single $\mathrm{Ca} 2+$-dependent protein kinase confers both cold and salt/drought tolerance on rice plants. Plant J 23, 319-327.

Sanders, D., Brownlee, C., Harper, J.F., 1999. Communicating with calcium. Plant Cell. 11, 691-706.

Sangwan, V., Orvar, B.L., Beyerly, J., Hirt, H., Dhindsa, R.S., 2002. Opposite changes in membrane fluidity mimic cold and heat stress activation of distinct plant MAP kinase pathways. Plant J. 31, 629-638.

Santiago, J., Rodrigues, A., Saez, A., Rubio, S., Antoni, R., Dupeux, F., Park, S.Y., Márquez, J.A., Cutler, S.R., Rodriguez, P.L., 2009. Modulation of drought resistance by the abscisic acid receptor PYL5 through inhibition of clade APP2Cs. Plant J 60, 575-588. Available from: https://doi.org/10.1111/j.1365313X.2009.03981.x.

Saruhashi, M., Kumar Ghosh, T., Arai, K., Ishizaki, Y., Hagiwara, K., Komatsu, K., et al., 2015. Plant Raf-like kinase integrates abscisic acid and hyperosmotic stress signaling upstream of SNF1-related protein kinase2. Proc. Natl. Acad. Sci. USA. 112, E6388-E6396.

Shi, H., Ishitani, M., Kim, C., Zhu, J.K., 2000. The Arabidopsis thaliana salt tolerance gene SOS1 encodes a putative $\mathrm{Na}+/ \mathrm{H}+$ antiporter. Proc. Natl. Acad. Sci. USA. 97, 6896-6901.

Shi, H., Quintero, F.J., Pardo, J.M., Zhu, J.K., 2002. The putative plasma membrane $\mathrm{Na}+/ \mathrm{H}+$ antiporter SOS1 controls long distance $\mathrm{Na}+$ transport in plants. Plant Cell 14, 465-477.

Shiu, S.H., Karlowski, W.M., Pan, R., Tzeng, Y.H., Mayer, K.F.X., Li, W.H., 2004. Comparative analysis of the receptor-like kinase family in Arabidopsis and rice. Plant Cell. 16 (5), 1220-1234.

Sirichandra, C., Gu, D., Hu, H.C., Davanture, M., Lee, S., Djaoui, M., et al., 2009. Phosphorylation of the Arabidopsis AtrbohF NADPH oxidase by OST1 protein kinase. FEBS Lett. 583, 2982-2986.

Snedden, W.A., Fromm, H., 1998. Calmodulin, calmodulin-related proteins and plant response to the environment. Trends Plant Sci. 3, 299-304.

Snedden, W.A., Fromm, H., 2001. Calmodulin as a versatile calcium signal transducer in plants. New Phytol. 151, 35-66.

Soon, F.F., Ng, L.M., Zhou, X.E., West, G.M., Kovach, A., Tan, M.H., et al., 2012. Molecular mimicry regulates ABA signaling by SnRK2 kinases and PP2C phosphatases. Science. 335, 85-88.

Steinhorst, L., Kudla, J., 2013. Calcium and reactive oxygen species rule the waves of signaling. Plant Physiol. 163, 471-485.

Sun, T., Wang, Y., Wang, M., Li, T.T., Zhou, Y., Wang, X.T., et al., 2015. Identification and comprehensive analyses of the $C B L$ and CIPK gene families in wheat (Triticum aestivem L.). BMC Plant Biol. 15, 269. Available from: https://doi.org/10.1186/s12870015-0657-4.
Suzuki, N., Sejima, H., Tam, R., Schlauch, K., Mittler, R., 2011. Identification of the MBF1 heat-response regulon of Arabidopsis thaliana. The Plant J. For Cell and Mol. Bio 66 (5), 844-851. Available from: https://doi.org/10.1111/j.1365-313X.2011.04550. $x$.

Swarbreck, S.M., Colaco, R., Davies, J.M., 2013. Plant calciumpermeable channels. Plant Physiol. 163, 514-522. Available from: https:/ / doi.org/10.1104/pp.113.220855.

Tamura, T., Hara, K., Yamaguchi, Y., Koizumi, N., Sano, H., 2003. Osmotic stress tolerance of transgenic tobacco expressing a gene encoding a membrane-located receptor-like protein from tobacco plants. Plant Physiol 131, 454-462.

Tang, N., Zhang, H., Li, X., Xiao, J., Xiong, L., 2012. Constitutive activation of transcription factor OsbZIP46 improves drought tolerance in rice. Plant Physiol. 158, 1755-1768.

Tang, W., Yuan, M., Wang, R., Yang, Y., Wang, C., Oses-Prieto, J.A., et al., 2011. PP2A activates brassino steroid-responsive gene expression and plant growth by dephosphorylating BZR1. Nat. Cell Biol. 13, 124-131.

Tang, R.-J., Zhao, F.-G., Garcia, V.J., Kleist, T.J., Yang, L., Zhang, H.X., Luan, S., 2015. Tonoplast CBL-CIPK calcium signaling network regulates magnesium homeostasis in Arabidopsis. Proc. Natl. Acad. Sci. USA 112 (10), 3134-3139. Available from: https://doi.org/10.1073/pnas.1420944112.

Teige, M., Scheikl, E., Eulgem, T., Doczi, R., Ichimura, K., Shinozaki, K., et al., 2004. The MKK2 pathway mediates cold and salt stress signaling in Arabidopsis. Mol Cell. 15, 141-152. Available from: https://doi.org/10.1016/j.molcel.2004.06.023. PMID: 15225555.

Tian, X., Wang, Z., Li, X., Lv, T., Liu, H., Wang, L., et al., 2015. Characterization and functional analysis of pyrabactin resistancelike abscisic acid receptor family in rice. Rice. 8, 28. Available from: https://doi.org/10.1186/s12284-015-0061-6.

Veena, Reddy, V.S., Sopory, S.K., 1999. Glyoxalase I from Brassica juncea: molecular cloning, regulation and its over-expression confer tolerance in transgenic tobacco under stress. Plant J. 17385-17395. Available from: https://doi.org/10.1046/j.1365313X.1999.00390.x.

Vranova, E., Atichartpongkul, S., Villarroel, R., Van Montagu, M., Inzé, D., Van Camp, W., 2002. Comprehensive analysis of gene expression in Nicotiana tabacum leaves acclimated to oxidative stress. Proc. Natl. Acad. Sci. USA. 99, 10870-10875.

Walker, S.D., Murray, N.R., Burns, D.J., Fields, A.P., 1995. Protein kinase $C$ chimeras: catalytic domains of alpha and beta II protein kinase C contain determinants for isotype-specific function. Proc. Natl. Acad. Sci. USA. 92, 9156-9160.

Wang, P., Xue, L., Batelli, G., Lee, S., Hou, Y.J., Van Oosten, M.J., et al., 2013. Quantitative phosphoproteomics identifies SnRK2 protein kinase substrates and reveals the effectors of abscisic acid action. Proc. Natl. Acad. Sci. USA. 110, 11205-11210.

Wang, P., Du, Y., Hou, Y.-J., Zhao, Y., Hsu, C.-C., Yuan, F., Zhu, X., Tao, W.A., Song, C.P., Zhu, J.K., 2015. Nitric oxide negatively regulates abscisic acid signaling in guard cells by S-nitrosylation of OST1. Proc. Natl. Acad. Sci. USA 112 (2), 613-618. Available from: https: / / doi.org/10.1073/pnas.1423481112.

Wang, Y., Sun, T., Li, T.T., Wang, M., Yang, G.X., He, G.Y., 2016. A CBL-interacting protein kinase TaCIPK2 confers drought tolerance in transgenic tobacco plants through regulating the stomatal movement. PLoS One 11, e0167962. Available from: https://doi. org/10.1371/journal.pone.0167962.

Wen, J.-Q., Oono, K., Imai, R., 2002. Two Novel Mitogen-Activated Protein Signaling Components, OsMEK1 and OsMAP1, Are Involved in a Moderate Low-Temperature Signaling Pathway in Rice. Plant Physiol 129 (4), 1880-1891. Available from: https:// doi.org/10.1104/pp.006072. 
Wohlbach, D.J., Quirino, B.F., Sussman, M.R., 2008. Analysis of the Arabidopsis histidine kinase ATHK1 reveals a connection between vegetative osmotic stress sensing and seed maturation. Plant Cell. 20 (4), 1101-1117.

Xia, X.J., Gao, C.J., Song, L.X., Zhou, Y.H., Shi, K., Yu, J.Q., 2014. Role of $\mathrm{H} 2 \mathrm{O} 2$ dynamics in brassinosteroid-induced stomatal closure and opening in Solanum lycopersicum. Plant Cell Environ. 37, 2036-2050. Available from: https://doi.org/10.1111/pce.12275.

Xiang, Y., Huang, Y., Xiong, L., 2007. Characterization of stressresponsive CIPK genes in rice for stress tolerance improvement. Plant Physiol. 144, 1416-1428. Available from: https://doi.org/ $10.1104 /$ pp.107.101295.

Xiong, L., Yang, Y., 2003. Disease resistance and abiotic stress tolerance in rice are inversely modulated by an abscisic acid inducible mitogen-activated protein kinase. Plant Cell. 15, 745-759.

Xiong, L., Schumaker, K.S., Zhu, J.K., 2002. Cell signaling during cold, drought, and salt stress. Plant Cell. 14, S165-S183.

Xu, G.Y., Rocha, P.S., Wang, M.L., Xu, M.L., Cui, Y.C., Li, L.Y., et al., 2011. A novel rice calmodulin-like gene, OsMSR2, enhances drought and salt tolerance and increases ABA sensitivity in Arabidopsis. Planta. 234, 47-59. Available from: https://doi.org/ 10.1007 / s00425-011-1386-z.

Xu, H., Li, K., Yang, F., Shi, Q., Wang, X., 2010a. Overexpression of CsNMAPK in tobacco enhanced seed germination under salt and osmotic stresses. Mol. Biol. Rep. 37, 3157-3163.

Xu, J., Tian, Y.S., Peng, R.H., Xiong, A.S., Zhu, B., Jin, X.F., et al., 2010b. AtCPK6, a functionally redundant and positive regulator involved in salt/drought stress tolerance in Arabidopsis. Planta. 231, 1251-1260.

Yang, T., Ali, G.S., Yang, L., Du, L., Reddy, A., Poovaiah, B., 2010. Calcium/calmodulin-regulated receptor-like kinase CRLK1 interacts with MEKK1 in plants. Plant Sig. \& Behav 5 (8), 991-994. Available from: https://doi.org/10.4161/psb.5.8.12225.

Yang, C., Li, A., Zhao, Y., et al., 2011. Overexpression of a wheat CCaMK gene reduces ABA sensitivity of Arabidopsis thaliana during seed germination and seedling growth. Plant Mol. Biol. Rep. 29, 681-692.

Yang, C., Wang, R., Gou, L., Si, Y., Guan, Q., 2017. Overexpression of Populus trichocarpa mitogen-activated protein kinase kinase 4 enhances salt tolerance in tobacco. Int. J. Mol. Sci. 18, 2090. Available from: https://doi.org/10.3390/ijms18102090.

Yang, Q., Chen, Z.Z., Zhou, X.F., Yin, H.B., Li, X., Xin, X.F., et al., 2009. Overexpression of SOS (salt overly sensitive) genes increases salt tolerance in transgenic Arabidopsis. Mol. Plant. 2, 22-31. Available from: https://doi.org/10.1093/mp/ssn058.

Yang, T., Poovaiah, B.W., 2003. Calcium/calmodulin-mediated signal network in plants. Trends Plant Sci. 8, 505-512.

Yang, Y., Tang, R.J., Jiang, C.M., Li, B., Kang, T., Liu, H., et al., 2015. Overexpression of the PtSOS2 gene improves tolerance to salt stress in transgenic poplar plants. Plant Biotechnol. J. 13, 962-973. Available from: https://doi.org/10.1111/pbi.12335.

Ye, Y., Ding, Y., Jiang, Q., Wang, F., Sun, J., Zhu, C., 2016. The role of receptor-like protein kinases (RLKs) in abiotic stress response in plants. Plant Cell Rep. 36, 235-242. Available from: https://doi. org/10.1007/s00299-016-2084-x.

Yi, S.Y., et al., 2010. A novel pepper (Capsicum annuum) receptor-like kinase functions as a negative regulator of plant cell death via accumulation of superoxide anions. New Phytol. 185, 701-715.

Yoo, J.H., Park, C.Y., Kim, J.C., Do Heo, W., Cheong, M.S., Park, H. C., et al., 2005. Direct interaction of a divergent CaM isoform and the transcription factor, MYB2, enhances salt tolerance in Arabidopsis. J. Biol. Chem. 280, 3697-3706. Available from: https:/ / doi.org/10.1074/jbc.M408237200.

Yoshida, R., Umezawa, T., Mizoguchi, T., Takahashi, S., Takahashi, F., Shinozaki, K., 2006. The regulatory domain of SRK2E/OST1/ SnRK2.6 interacts with ABI1 and integrates abscisic acid (ABA) and osmotic stress signals controlling stomatal closure in Arabidopsis. J. Biol. Chem. 281, 5310 8. Available from: https:// doi.org/10.1074/jbc.M509820200. PMID:16365038.

You, J., Chan, Z., 2015. ROS regulation during abiotic stress responses in crop plants. Front. Plant Sci. 6, 1092. Available from: https://doi.org/10.3389/fpls.2015.01092.

Yu, J.L., Yang, L., Liu, X., Tang, R.J., Wang, Y., Ge, H.M., et al., 2016. Overexpression of poplar pyrabactin resistance-like abscisic acid receptors promotes abscisic acid sensitivity and drought resistance in transgenic Arabidopsis. PLoS One 11, e0168040. Available from: https:/ / doi.org/10.1371/journal.pone.0168040.

Yu, L., Nie, J., Cao, C., Jin, Y., Yan, M., Wang, F., et al., 2010. Phosphatidic acid mediates salt stress response by regulation of MPK6 in Arabidopsis thaliana. New Phytol. 188, 762-773.

Yuan, F., et al., 2014a. Nature. 514, 367-371. Available from: https:// doi.org/10.1038/nature13593.

Yuan, F., Yang, H., Xue, Y., Kong, D., Ye, R., Li, C., et al., $2014 b$. OSCA1 mediates osmotic-stress evoked Ca2 + increases vital for osmosensing in Arabidopsis. Nature. 514, 367-371.

Zeng, H., Xu, L., Singh, A., Wang, H., Du, L., Poovaiah, B.W., 2015. Involvement of calmodulin and calmodulin-like proteins in plant responses to abiotic stresses. Front. Plant Sci. 6, 600. Available from: https://doi.org/10.3389/fpls.2015.00600.

Zhang, C.J., Guo, Y., 2012. OsTRXh1 regulates the redox state of the apoplast and influences stress responses in rice. Plant Signal. Behav. 7, 441-443.

Zhang, H., Lv, F., Han, X., Xia, X., Yin, W., 2013. The calcium sensor PeCBL1, interacting with PeCIPK24/25 and PeCIPK26, regulates $\mathrm{Na}+/ \mathrm{K}+$ homeostasis in Populus euphratica. Plant Cell Rep 32, 611-621.

Zhang, H., Liu, Y., Wen, F., Yao, D., Wang, L., Guo, J., et al., 2014b. An ovel rice $\mathrm{C} 2 \mathrm{H} 2$-type zinc finger protein, ZFP36, is a key player involved in abscisic acid-induced antioxidant defence and oxidative stress tolerance in rice. J. Exp. Bot. 65, 5795-5809. Available from: https://doi.org/10.1093/jxb/eru313.

Zhang, H.F., Yang, B., Liu, W.Z., Li, H.W., Wang, L., Wang, B.Y., et al., 2014a. Identification and characterization of $C B L$ and CIPKgene families in canola (Brassica napus L.). BMC Plant Biol. 14, 8. Available from: https:/ / doi.org/10.1186/1471-2229-14-8.

Zhang, L., Xi, D., Li, S., Gao, Z., Zhao, S., Shi, J., et al., 2011. A cotton group C MAP kinase gene, GhMPK2, positively regulates salt and drought tolerance in tobacco. Plant Mol Biol. 77, 17-31.

Zhang, L., Li, Y., Lu, W., Meng, F., Wu, C.A., Guo, X., 2012. Cotton GhMKK5 affects disease resistance, induces HR-like cell death, and reduces the tolerance to salt and drought stress in transgenic Nicotiana benthamiana. J. Exp. Bot. 63, 3935-3951. Available from: https://doi.org/10.1093/jxb/ers086.

Zhang, W., Zhou, R.G., Gao, Y.J., Zheng, S.Z., Xu, P., Zhang, S.Q., et al., 2009. Molecular and genetic evidence for the key role of atcam3 in heat-shock signal transduction in Arabidopsis. Plant Physiol. 149, 1773-1784. Available from: https://doi.org/ $10.1104 /$ pp.108.133744.

Zhao, J., Sun, Z., Zheng, J., Guo, X., et al., 2009. Cloning and characterization of a novel CBL-interacting protein kinase from maize. Plant Mol. Biol. 69, 661-674.

Zhou, J., Wang, J., Li, X., Xia, X.J., Zhou, Y.H., Shi, K., et al., 2014a. $\mathrm{H}_{2} \mathrm{O}_{2}$ mediates the cross talk of brassinosteroid and abscisic acid in tomato responses to heat and oxidative stresses. J. Exp. Bot. 65, 4371-4383. Available from: https://doi.org/10.1093/jxb/eru217.

Zhu, J., Lee, B.H., Dellinger, M., Cui, X., Zhang, C., Wu, S., et al., 2010. A cellulose synthase-like protein is required for osmotic stress tolerance in Arabidopsis. Plant J. 63, 128-140. Available from: https:/ / doi.org/10.1111/j.1365-313X.2010.04227.x.

Zhu, J.K., 2002. Salt and drought stress signal transduction in plants. Ann. Rev. Plant Biol. 53, 247-273. 
Zhu, J.K., 2016. Abiotic stress signaling and responses in plants. Cell. $167,313-324$.

Zhu, S.Y., Yu, X.C., Wang, X.J., Zhao, R., Li, Y., Fan, R.C., et al., 2007. Two calcium-dependent protein kinases, CPK4 and CPK11, regulate abscisic acid signal transduction in Arabidopsis. Plant Cell. 19, 3019-3036.

Zou, J.J., Wei, F.J., Wang, C., Wu, J.J., Ratnasekera, D., Liu, W.X., et al., 2010. Arabidopsis calcium-dependent protein kinase CPK10 functions in abscisic acid- and $\mathrm{Ca}^{2+}$-mediated stomatal regulation in response to drought stress. Plant Physiol. 154, 1232-1243. Available from: https://doi.org/10.1104/pp.110.157545.

\section{Further Reading}

Campo, S., Baldrich, P., Messeguer, J., Lalanne, E., Coca, M., SanSegundo, B., 2014. Over expression of a calcium-dependent protein kinase confers salt and drought tolerance in rice by preventing membrane lipid peroxidation. Plant Physiol. 165, 688-704. Available from: https://doi.org/10.1104/pp.113.230268.
Fukao, T., Yeung, E., Bailey-Serres, J., 2011. The submergence tolerance regulator SUB1A mediates cross talk between submergence and drought tolerance in rice. Plant Cell. 23, 412-427. Available from: https: / / doi.org/10.1105/tpc.110.080325.

Gill, R.A., Ali, B., Cui, P., et al., 2016. Comparative transcriptome profiling of two Brassica napus cultivars under chromium toxicity and its alleviation by reduced glutathione. BMC Genom. 17 (1), 885.

Mittal, D., Madhyastha, D.A., Grover, A., 2012. Genome-wide transcriptional profiles during temperature and oxidative stress reveal coordinated expression patterns and overlapping regulons in rice. PLoS One 7, e40899. Available from: https://doi.org/ 10.1371/journal.pone.0040899.

Wu, L., Zhang, Z., Zhang, H., Wang, X.C., Huang, R., 2008. Transcriptional modulation of ethylene response factor protein JERF3 in the oxidative stress response enhances tolerance of tobacco seedlings to salt, drought, and freezing. Plant Physiol. 148, 1953-1963. Available from: https://doi.org/10.1104/ pp.108.126813. 


\section{4}

\section{Genetic Engineering/Genome Editing Approaches to Modulate Signaling Processes in Abiotic Stress Tolerance}

Riwandahun Marwein ${ }^{1,2}$, Johni Debbarma ${ }^{1,2}$, Yogita N. Sarki ${ }^{1,2}$, Indrani Baruah $^{1,2}$, Banashree Saikia, ${ }^{1,2}$, H.P.D. Boruah ${ }^{1}$, Natarajan Velmurugan ${ }^{3}$ and Channakeshavaiah Chikkaputtaiah ${ }^{1}$

${ }^{1}$ Biotechnology Group, Biological Sciences and Technology Division, CSIR-North East Institute of Science and Technology (CSIR-NEIST), Jorhat, Assam, India ${ }^{2}$ Academy of Scientific and Innovative Research (AcSIR), CSIRNEIST, Jorhat, Assam, India ${ }^{3}$ Branch Laboratory Itanagar, CSIR-North East Institute of Science and Technology (CSIR-NEIST), Jorhat, Assam, India

\section{O U T L I N E}

4.1 Introduction

4.2 Plant Response to Abiotic Stress in Developing Tolerance 4.2.1 Late Embryogenesis Abundant Proteins

4.2.2 Production of Methyglyoxyl Under Abiotic Stress

4.2.3 Hybrid Proline Rich Proteins (HyPRPs) and Abiotic Stress Responses

4.2.4 Role of Protein Kinases in Response to Abiotic Stress

4.2.5 Cross-Talk Signaling of Jasmonate and Ethylene Biosynthesis in Abiotic Stress Tolerance
4.3 Genetic Engineering Approaches to Modulate Abiotic Stress Signaling Process in Crop Plants 4.3.1 Transgenic Approaches

4.4 Genome Editing Approaches to Modulate Abiotic Stress Signaling Processes in Crop Plants $\quad 74$ 4.4.1 Zinc Finger Nucleases $\quad 74$ 4.4.2 Transcription Activator Like Effector Nucleases 74 4.4.3 CRISPR-Cas9 Genome Editing 74 4.4.4 CRISPR-Cpf1 75

4.5 Conclusions and Future Prospects 75

$\begin{array}{ll}\text { References } & 77\end{array}$ 


\subsection{INTRODUCTION}

Global climatic changes and human anthropogenic activities have exhibited detrimental effects of abiotic stresses on crop productivity (Savvides et al., 2016). Rapidly unexpected climate change will challenge world food security by $70 \%$ of global food production to feed up to an additional 9 billion people by 2050 (Commission on Genetic Resources for Food and Agriculture), and the World Bank extrapolated the decrease of $20 \%$ crop yield by 2050 due to climate change (Gill et al., 2014). Climate variation in temperature, precipitation, and ozone concentration is adversely affecting plant growth and development quantitatively and qualitatively (Tripathi et al., 2016; Ramirez-Cabral et al., 2017). Plants being sessile are naturally exposed to both biotic and abiotic stresses, but exceeding a threshold level of these stress deteriorated plant growth and productivity (Meena et al., 2017). Abiotic stresses imposed on plants induce changes in the physiological metabolism affecting growth and yield (Ben Rejeb et al., 2014; Khan et al., 2016; Pasala et al., 2016; Hasanuzzaman et al., 2018). Although plants exhibit an adaptive defense response against abiotic stress, concurrent exposure to these stresses limits their potential, hindering their growth and development (Mohanta et al., 2017). About 50\% of world agricultural yield is affected by abiotic stresses, which include drought, flooding, extreme temperature (heating or freezing), nutrient deficiency, ion toxicity (salinity, heavy metals), excess light, UV radiation, and soil hardness, impacting agricultural productivity (Khan et al., 2012, 2015, 2016; Anjum et al., 2015; Chikkaputtaiah et al., 2017). The combined effects of these stresses have a significantly detrimental effect on plant growth and development compared with the impact they have individually (Prasad et al., 2011; Pandey et al., 2017). Further, interaction between abiotic (mainly drought and salinity) and biotic stresses had a negative impact on the pathogen resistance of the plant (Suzuki et al., 2014; Kissoudis et al., 2016). Environmental stress also leads to the reduction in arable land due to soil degradation, erosion, and salinization, which requires the development of stress tolerant plants adaptable to unfavorable land to feed the growing world population (Koyro et al., 2012). Concerning the future food scenario and global population, rapid development of climate resilient plants is imperative and could be achieved through breeding or by engineering plant tolerance to abiotic stress (Rodziewicz et al., 2013).

Although use of conventional breeding for abiotic stress tolerant plants is achievable, its slow process is limited by the incompatible transfer of traits between species, involvement of thousands of genes, requirement of several back crosses to obtain a desired trait (Ahmad and Mukhtar, 2017), and a suitable gene for breeding (Abdallah et al., 2014; Parmar et al., 2017). Genetic engineering surmounts conventional breeding by its ability to modify a target gene of interest within the same or distantly related species (Singh and Singh, 2014). With the advent of structural and functional genomics approaches, understanding metabolic regulatory pathways of stress responsive genes and identifying valuable genetic traits through the use of modern molecular markers, genome wide association studies, and next generation sequencing approaches, facilitates the incorporation of these traits through genetic engineering in the making of abiotic stress tolerant plant cultivars (Mohanta et al., 2017).

This book chapter describes the plant response to abiotic stress in developing tolerance through stress signaling molecules such as late embryogenesis abundant (LEA) proteins, HSPs, methylglyoxyl, HyPRPs, and protein kinases. Various genetic engineering approaches to modulate abiotic stress signaling process in crop plants were also discussed along with recent case studies. The topic highlights a special emphasis on ubiquitination pathway genes, epigenetic regulation, small RNAs, and helicases in modulating signaling processes in abiotic stress tolerance. The chapter also explains the scope of modern genome editing system (CRISPR-Cas9 and CRISPR-Cpf1) in developing multiple abiotic stress tolerance in crop plants.

\subsection{PLANT RESPONSE TO ABIOTIC STRESS IN DEVELOPING TOLERANCE}

Plants complete their life cycle in one place and have to encounter different parameters of stress, that is, biotic or abiotic. Thus, plants have acquired the capability to respond to sudden change in the environment by activating signals positively or negatively. In response to abiotic stress, plants undergo a series of morphological, biochemical, physiological, and molecular changes thus reprogramming their genetic makeup, which determines their survival. Understanding the adaptive mechanism underlying the response of plants to multiple abiotic stresses causing disturbance in cellular homeostasis is crucial to developing abiotic stress tolerant plants. The response of plants to abiotic stress is highly complex because multiple stress response is governed by complex signaling pathways that either activate or inhibit downstream processes (Suzuki et al., 2014). However, living in an era where omics and bioinformatics technologies are greatly advancing, understanding the molecular mechanisms, interactions, and interconnected signaling 
pathways of stress responsive genes in both model and crop plants gives us an insight into the biological functions, gene expression, and finally key genes regulating the tolerance (Ramesh, 2017), which might alleviate stress tolerance and possibly memory. Upon stress recognition, downstream signal transduction led to the activation of various stress responsive genes. These stress responsive genes have been divided into two classes: (1) genes encoding protective proteins like heat-shock proteins (HSPs) or chaperones, LEA proteins, osmoprotectants, antifreeze protein, detoxification enzymes and free radical scavengers, aquaporins, transporters (sugar and proline), enzymes involved in fatty acid metabolism, inhibitors of proteinase, ferritin and proteins that transfer lipid, ROS and RNS produced during abiotic stress; and (2) genes involved in the downstream signaling processes such as transcription factors, mitogen activated protein kinase (MAPK), calcium dependent protein kinase (CDPK), SOS kinase and phospholipase, and DEAD-box RNA helicases (Ciarmiello et al., 2011; Fang et al., 2016; Baruah et al., 2017). Another important plant product is methylglyoxylase, which is toxic in stress conditions (Hoque et al., 2016). In contrast, plant hormones such as abscisic acid (ABA) play a pivotal role in defense against abiotic stress by triggering the activation of short term responsive genes regulating osmotic balances, and maintaining root and shoot architecture, root hydraulic conductivity, and stomatal closure by regulation of ion channel thus reducing water loss in plants and the upregulation of other downstream stress inducible genes (Lata and Prasad, 2011; Verma et al., 2016) through an ABA dependent or ABA independent pathway (Shao et al., 2015). Fig. 4.1 describes the signaling mechanism modulating the plant response to abiotic stress.

\subsubsection{Late Embryogenesis Abundant Proteins}

LEA proteins are a group of hydrophilic proteins that were first identified in cotton (Gossypium hirsutum) seeds during the late stage of seed development in occurrence of desiccation. Their function in protecting orthodox seeds, pollen, and anhydrobiotic plants from dehydration suggests their protective role in plant tolerance to water scarcity. Their expression is induced by plant stress hormones, ABA during seed maturation, and by various abiotic stresses (Dalal et al., 2009). LEA proteins have been described as intrinsically unstructured proteins characterized by high proportion of charged amino acid residues such as alanine, serine, or threonine enabling them to change conformation to variable cellular environments such as water deficit thus facilitating their interactions with other macromolecules and stabilizing the negative impact caused by abiotic stress (Saucedo et al., 2017). Their functional protective role during desiccation is dedicated to their ability to prevent protein aggregation; to act as a molecular shield and membrane stabilizer, particularly those of dehydrins in cold stress; in ion sequestration; and to prevent inactivation of enzymes such as lactate and malate dehydrogenase 2 (Reddy et al., 2012; Battaglia and Covarrubias, 2013; Amara et al., 2014; Liang et al., 2016; Saucedo et al., 2017). In the model plant Arabidopsis LEA proteins are widely distributed subcellularly in the cytosol, nucleus, plastids, mitochondria, endoplasmic reticulum, and pexophagosomes, signifying their importance in protective mechanisms against desiccation or cold stress (Amara et al., 2014; Candat et al., 2014).

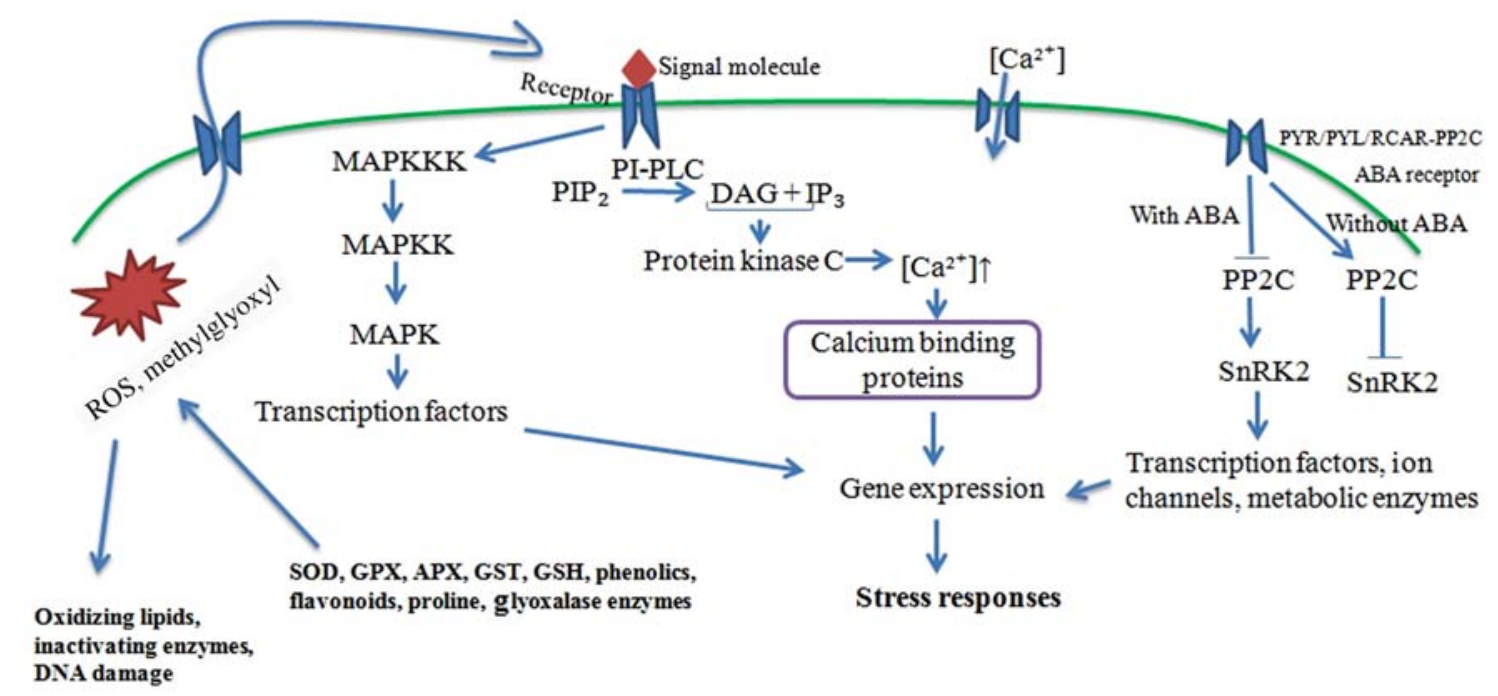

FIGURE 4.1 Overview of signaling mechanism modulating the plant response to abiotic stress. 


\subsubsection{Production of Methyglyoxyl Under Abiotic Stress}

Methylglyoxyl $\left(\mathrm{CH}_{3} \mathrm{C}(\mathrm{O}) \mathrm{CHO}\right)$ is a byproduct form from glycolytic intermediates via enzymatic and nonenzymatic elimination of phosphate from glyceraldehydes3-phosphate (GAP) and dihydroxyacetone phosphate (DHAP) and lipid metabolism, occurring in all living systems, however, in plants it is a 20th century discovery formed spontaneously by nonenzymatic mechanisms under physiological conditions from glycolysis and from photosynthetic intermediate (Hoque et al., 2016). Methyglyoxyl (MG) at low concentration acts as a signaling molecule wherein it regulates functional events such as cell proliferation and survival, maintaining redox reaction and cellular homeostasis (Hossain et al., 2012; Kaur et al., 2014; Hoque et al., 2016). Higher accumulation is toxic to the cell by directly disrupting cellular metabolism causing oxidation of macromolecules leading to formation of reactive oxygen species (ROS), inactivating antioxidant enzymes, photoreduction of $\mathrm{O}_{2}$ to superoxide $\left(\mathrm{O}_{2}{ }^{-}\right)$ in photosystem I increasing oxidative stress, membrane disruption, ion leakage, and DNA strand cleavage leading to death of plant cells. In rice OsDJ-1C converts MG into D-lactate in a single step, acting as a shorter route to MG detoxification (Ghosh et al., 2016). In Arabidopsis DJ1/PfpI superfamily has been reported to exhibit glyoxylase activity against MG (Kwon et al., 2013). With understanding the metabolic pathway of MG and glyoxalase system, several studies have shown improved crop tolerance to abiotic stress by modifying one or two of these pathways (Singla-Pareek et al., 2003). Since both antioxidants and glyoxalase system are involved in the detoxification of ROS, coordination of both pathways can mitigate the accumulation of ROS in the plant cell (Nahar et al., 2015; Hasanuzzaman et al., 2017). In Arabidopsis, the gene encoding for glyoxalase II enzymes, GLX2-1, which is found to be nonfunctional during normal growth, is highly expressed in response to abiotic stress such as hypoxia, drought, and salt stress. The role of MG was also studied in induction of glycolytic enzymes triose phosphate isomerize (TPI) in a concentration dependent manner in rice resulting in decreased DHAP by shifting reactions toward GAP thus decreasing MG via feedback mechanism, which results in survival mechanism under abiotic stress (Sharma et al., 2012). More information on methylglyoxal, a novel signaling molecule in plant responses to abiotic stress, is covered in Chapter 10, Bioactive Molecules as Regulatory Signals in Plant Responses to Abiotic Stresses.

\subsubsection{Hybrid Proline Rich Proteins (HyPRPs) and Abiotic Stress Responses}

HyPRPs represent a group of putative cell wall proteins characterized by their unusual domain structure, which was deduced only from the primary amino acid sequence (Dvořáková et al., 2012). HyPRPs constitute a family of cell wall proteins that is phylogenetically related to nonspecific lipid transfer proteins. Members of the HyPRP family are involved in basic cellular processes and their expression and activity are modulated by environmental factors. Extensins (hydroxyprolinerich glycoproteins), proline-rich proteins (PRPs), and glycine-rich proteins (GRPs) are structural proteins of the primary plant cell wall. According to recent research, expression of a HyPRP1 is suppressed by various abiotic stresses, including drought, high salinity, cold, heat, oxidative stress, and phytohormone ABA in Solanum pennellii ( $\mathrm{Li}$ et al., 2016a). Investigation of transgenic functional analysis and transcriptional level SlHyPRP1 and SpHyPRP1 that were isolated from cultivated tomato Solanum lycopersicum and wild tomato S. pennellii, respectively, demonstrated that HyPRP1 possibly plays a negative role in stress tolerance (Li et al., 2016a). Loss-of-function and gain-of-function studies revealed that overexpression plants showed a significant increase in cold tolerance than the wild type plants, which is conferred by the accumulation of OsPRP3 protein during cold treatment (Gothandam et al., 2010). In a recent study, GhHyPRP4, gene encoding putative HyPRP, was isolated from cotton cDNA library under cold stress. GhHyPRP4 expression was significantly upregulated in leaves of cotton seedlings. GUS (b-glucuronidase) gene driven by GhHyPRP4 promoter was specifically expressed in leaves and cotyledons of the transgenic Arabidopsis thaliana, which was remarkably induced by cold stress (Huang et al., 2011).

\subsubsection{Role of Protein Kinases in Response to Abiotic Stress}

Plants' adaptive tolerance to multiple abiotic stress relies on the coordinated signaling pathway perceived via multiple cell surface receptors and translating it into cellular response by activation of protein kinases such as mitogen activated protein (MAP) kinases, SNF1, CDK, etc. in response to various external and internal stimuli (Agarwal et al., 2010). MAP kinases' multifunctional role in plants involves the growth and development, immune defense system, hormone signaling, and response to biotic and abiotic stresses (Moustafa et al., 2014). These evolutionary conserved signal transduction modules are functionally interlinked through phosphorylation events and include the MAPK kinase kinase (MAPKKK), MAPK kinase (MAPKK), and a MAPK activating each other and other proteins in a sequential manner (Moustafa, 2014). The coordinated signaling is brought about by the activity of MAPK phosphatase that functions as an 
on/off switch signal regulating gene expression of target proteins such as cytoskeletal proteins and transcription factors in response to environmental stresses (Moustafa et al., 2014). Many studies have revealed the functional role of plant MAPK kinase in adaptation to abiotic stresses such as drought, salinity, temperature fluctuations, etc. Because abiotic stress interferes with translational processes, $\mathrm{Xu}$ and Chua (2012) reported the role of MPK6 in promoting mRNA decapping by phosphorylating a decapping protein DCP1 thus getting associated with DCP5 conveying rapid response to dehydration stress in Arabidopsis. In rice, overexpression of DSM1 gene encoding a putative MAPKKK of the Raf family protein kinase increases tolerance to dehydration stress at seedling stage by regulating the scavenging activity of ROS (Ning et al., 2010). Cotton MAPK gene (GhMPK2) conferred drought and salt stress tolerance in transgenic tobacco plant by upregulating various stress responsive genes such as DIN1, Osmotin, and NtLEA5 and proline accumulation (Zhang et al., 2011). Functional role of Arabidopsis transcription factors AtMYB41 in salt tolerance was found to be activated and interact with MPK6 (Hoang et al., 2012). In vitro assay of Arabidopsis MKKK20 was shown to regulate MPK6 activity under salt and cold stress and that MKKK20 overexpressing plant increased tolerance to salt stress (Kim et al., 2012). Rice OsMKK6 and OsMKK6DD were found to phosphorylate OsMPK3 enhancing chilling tolerance, confirming the signaling pathway for moderately induced low temperature (Xie et al., 2012).

CDPKs play a major role as calcium sensors to a changing intracellular calcium ion concentration, as well as transducers, translating signals into downstream phosphorylation events (Singh et al., 2018). Increased cytosolic $\mathrm{Ca}^{2+}$ concentration occurred as a result of exposure to various biotic and abiotic stresses initiating signaling cascades, a feature of an acclimatization response in plants (Tang and Luan, 2017). Realizing their role in abiotic stress response, studies have started exploring functions of CDPKs genes exposed to multiple stresses. The protective role of rice OsCPK4 gene towards salt and drought stress was found to be effective by preventing membrane lipid peroxidation and reduced electrolyte leakage, thus protecting from stress induced oxidative damage (Campo et al., 2014). Similar function was reported by Bundo and Coca (2017) for OsCPK10 gene isoform during drought stress and was also found to positively regulate blast disease resistance by a reduced production of hydrogen peroxide interfering with fungal growth. Reducing water loss and maintaining osmotic potential is crucial under drought conditions; this has been reported for rice CPK gene OsCPK9, which when overexpressed improved drought stress tolerance by enhancing stomatal closure and maintaining osmotic balance in an ABA dependent manner, in addition to increased spikelet fertility (Wei et al., 2014). Transgenic Arabidopsis plants expressing maize CPK genes such as ZmCPK4 enhanced drought tolerance through ABA mediated regulation of stomatal closure (Jiang et al., 2013); ZmCPK12 conferred tolerance to drought and salt stress (Wang and Song, 2013) and ZmCK3 towards drought and heat stress (Wang and Song, 2014). In response to salinity stress overexpression of OsCPK12 gene increases tolerance to salt stress by regulating expression of ROS genes such as OsAPX2 and OsAPX8, however showing ABA sensitivity and susceptibility to blast fungal disease (Asano et al., 2012). Transportation of toxic metal arsenite in Arabidopsis was shown to be negatively regulated by CPK31 protein interacting with the nodulin 26-like intrinsic protein (NIP 1;1), an aquaporin involved in As (III) uptake (Ji et al., 2017). Gene encoding CPK of barley HVCPK $2 a$ was found to be upregulated by drought stress and undergo autophosphorylation; however its overexpression in Arabidopsis reduced tolerance to drought, reduced nitrogen balance index, increased chlorophyll content, and decreased relative water content (Ciesla et al., 2016).

\subsubsection{Cross-Talk Signaling of Jasmonate and Ethylene Biosynthesis in Abiotic Stress Tolerance}

The developmental processes in plants are governed by plant growth regulators such as ethylene (ET), jasmonic acid (JA), auxin, gibberellin, ABA, cytokinin, and salicylic acid (SA) (Khan and Khan, 2014; Khan et al., 2015, 2016; Huang et al., 2017). Under adverse environmental conditions, a plant's metabolic reactions and hormonal signaling pathways are disrupted. To avoid these, plants have set up a modified mechanism by which they can trigger a cascade to develop resilience. Ethylene is an important phytohormone that is involved in maintenance of seed germination, fruit ripening, abscission, and senescence (Abeles et al., 1992; Iqbal et al., 2017). The MAPK cascade pathways have integrated in major signaling systems with ethylene in various plants (Cristina et al., 2010). Jasmonate (JA) acts as a crucial signal to modulate multiple stress development in plants (Per et al., 2018). Recent studies have demonstrated the involvement of JA in leaf senescence and cold stress tolerance. Exogenous JA exhibits the expression of senescence related genes and leaf programmed cell death (PCD) in Arabidopsis. In response to cold stress, exogenous application of JA enhances Arabidopsis cold stress tolerance. Thus, JA also shows interaction with other hormone signaling pathways like ethylene to enhance tolerance upon cold stress (Hu et al., 2017). Phytohormone shows transport 
activity from their sites of synthesis to mediate physiological and molecular responses of plants under stressful conditions. JA has been exercised for its biological role in response to abiotic stresses in growth and development (Clarke et al., 2009; Brossa et al., 2011; Zhao et al., 2013). Despite all environmental cues, JA and ET inducible $A P 2 / E R F$ genes have been reported to confer stress tolerance when overexpressed in diverse species of plants (Chen et al., 2012). It has also been suggested that modification of upstream components may affect plant development. Moreover, it probably can say that downstream elements have a key positive regulatory role for genetic improvement of stress mediated crops. Thus, application of genetic engineering of JA and ET signaling pathways can offer a new platform to improve efficiency of abiotic stress tolerance in crops.

\subsection{GENETIC ENGINEERING APPROACHES TO MODULATE ABIOTIC STRESS SIGNALING PROCESS IN CROP PLANTS}

\subsubsection{Transgenic Approaches}

We are in a situation where a tremendous increase of crop productivity must be achieved with no increase in arable land and in the face of multiple environmental stresses. To overcome this problem, in the past few decades, a great deal of efforts have been devoted to breeding and biotechnology of stress tolerant crops with higher yields and improved qualities against multiple environmental stresses. However, it remains as one of the greatest challenges faced by modern agriculture (Takeda and Matsuoka, 2008; Newton et al., 2011; Liu et al., 2014a). Apart from the conventional breeding approaches that have marginal success due to the complexity of abiotic stress tolerance traits, modern transgenic technology has been in the limelight, which deals with introducing novel exogenous genes or altering the expression levels of endogenous genes to improve stress tolerance. The molecular mechanisms by which plants discern and transduce stress signals to cellular machinery to initiate adaptive responses can be deciphered proving it to be an essential prerequisite for identification of the key genes and pathways to genetically engineer stress-tolerant crop plants (Heidarvand and Maali Amiri, 2010; Ray et al., 2010; Sanchez et al., 2011). Significant progress has been made to elucidate the molecular mechanisms of abiotic stress responses in plants by means of high throughput sequencing and functional genomics tools. Pea DNA Helicase 45 ( $P D H 45)$ has been discovered in combating multiple abiotic stresses in chili (Shivakumara et al.,
2017). Molecular genetics and functional genomics of abiotic stress responsive genes (dehydrins and LEA) in crop plants have been recently reported (Singh et al., 2015; Chikkaputtaiah et al., 2017). A scheme of genetic engineering approaches to develop abiotic stress tolerance in crop plants is given in Fig. 4.2. More information on transgenic approaches to modulate signaling processes in abiotic stress tolerance is covered in Chapter 6, Drought Tolerance in Plants: Role of Signaling Molecules and its Molecular Mechanisms and Regulation.

\subsubsection{Genetic Engineering for Biosynthesis of Osmoprotectants and LEA}

Osmoprotectants are small molecules having low molecular weight, and are electrically neutral, highly soluble, and nontoxic at molar concentrations (Ahn et al., 2011). The accumulation of osmoprotectants is a common plant response under abiotic stresses. They basically stabilize proteins and membranes and contribute to the cell osmotic pressure (Zandalinas et al., 2018). In particular, proline accumulates in many plant species in response to environmental stress. Ailanthus altissima (Miller), when exposed to salinity and drought conditions showed the activity of proline biosynthesis enzyme, 1-pyrroline-5-carboxylate synthetase (Filippou et al., 2014). Maize plants truly suffered with abiotic stress and accumulated more proline to defend salt, dehydration, and heat stresses (Huang et al., 2018).

Thousands of LEA genes have been isolated and cloned to study functional role in abiotic stress responses. A novel gene KvLEA has been isolated and expressed from Kosteletzya virginica, a plant of economic importance that showed tolerance to multiple abiotic stresses (Tang et al., 2016). Thirteen LEA genes from Pinus tabuliformis introduced in Escherichia coli cells showed enhanced resistance under salt and heat stress (Gao and Lan, 2016). A dehydrin LEA gene CsLEA11 was identified and characterized from Cucumis sativus that is induced by heat and cold stress. Overexpression of this gene in E. coli protects cell viability and confers tolerance to heat by preventing inactivation of lactate dehydrogenase enzymes and cold stress (Zhou et al., 2017). Molecular genetic characterization of LEA proteins have shown enhanced drought tolerance in upland cotton Gossypium tomentosum (Magwanga et al., 2018).

\subsubsection{Aquaporin Genes Associated With Abiotic Stress Tolerance}

Aquaporins are intrinsic membrane channel proteins, members of the major intrinsic protein (MIP) family, ubiquitously present in all kingdoms except archaea and intracellular bacteria. They facilitate the 


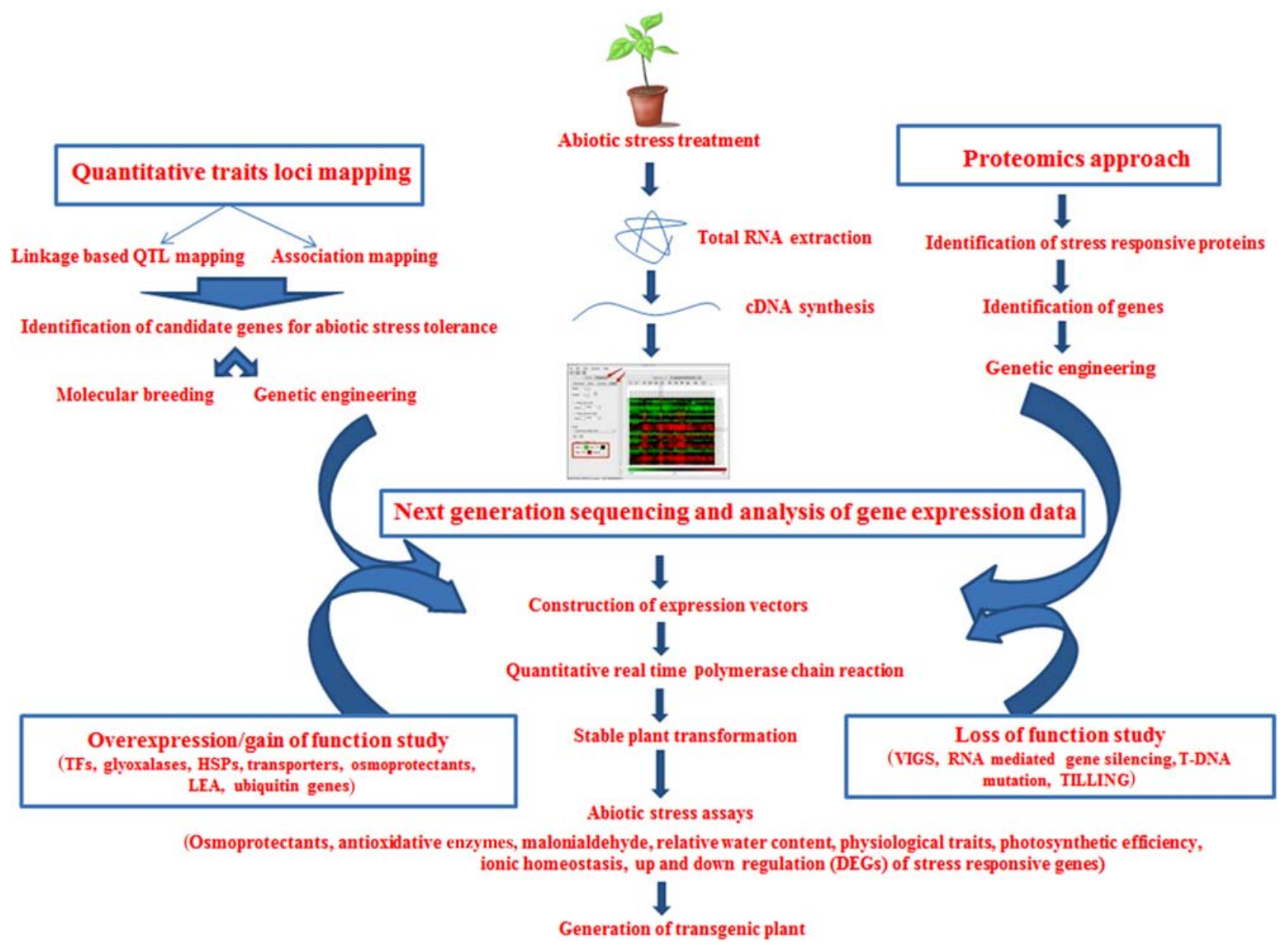

FIGURE 4.2 Scheme of genetic engineering approaches to develop abiotic stress tolerance in crop plants.

bidirectional flow of water across cellular membrane and other solutes such as glycerol, urea, $\mathrm{CO}_{2}, \mathrm{NH}_{3}$, metalloids, and ROS that are important for the growth and development of plants. Abiotic stresses such as drought, water logging, and salinity regulate the expression and activity of aquaporins maintaining water content to short and long term exposures, regulating the hydraulic conductivity in root, stem, and leaf and abiotic stress signaling (Srivastava et al., 2014; Moshelion et al., 2015). A considerable amount of work has been done on aquaporin subfamilies PIP (plasma membrane intrinsic proteins) and TIP (tonoplast intrinsic protein) because of their abundance in plants and their role in plant water transport. Their expression pattern is differentially regulated depending on the isoform, tissue, species, or level of stress (Šrbanovski and Grant, 2014). Overexpression functional studies of aquaporins genes have been carried out in both model and crop plants when imposed with different stresses. Among these, PIP genes from tomato SIPIP2;1, SIPIP2;7, and SIPIP2;5 enhance drought tolerance by improving plant water content and osmotic balance (Li et al., 2016c); wheat aquaporins genes TaAQP8 (Hu et al., 2012) and TaNIP (Gao et al., 2010) enhance salt tolerance in transgenic Arabidopsis plant. Arabidopsis AtTIP5;1 increased tolerance to boron toxicity with a better phenotypic development (Pang et al., 2010). Improved drought tolerance in Solanum tuberosum L. was observed in a constitutively overexpressed StPIP1 gene by improving water use efficiency, increasing nonstructural carbohydrates thus minimizing carbon starvation, and increasing biomass yield (Wang et al., 2017b). Under high salt and osmotic stress, transgenic Arabidopsis plant expressing barley exhibits stress tolerance conditions by minimizing oxidative damage, higher chlorophyll and water retention, and increases expression of osmoprotectant genes (Alavilli et al., 2016). The functions of aquaporins in $\mathrm{CO}_{2}$ conductance were studied in the T-DNA insertion line in Arabidopsis AtPIP1;2 observing a reduced net photosynthesis under drought stress (Boudichevskaia et al., 2015).

However some aquaporin genes display contrasting effects when subjected to abiotic stress, such as the 
wheat TaTIP2;2 that acts as a negative regulator to both drought and salt stress downregulating various stress responsive genes and decreasing proline content in an ABA independent manner ( $\mathrm{Xu}$ et al., 2013a). In a study conducted by Li et al. (2015), Arabidopsis plant expressing GoPIP1 transcript from Galega orientalis increased sensitivity to drought stress as a result of increased transpiration, fluctuated osmolyte accumulation, and changes in ABA signaling, however, no changes were observed in response to salt stress conditions. Negative impact on drought tolerance was also observed when Arabidopsis PIP1b was expressed in tobacco plant causing faster wilting under drought stress with no response under salt stress (Aharon et al., 2003). Further study into the mechanism of aquaporin genes in regulating abiotic stress tolerance is needed to decipher their functional role in different plant species.

\subsubsection{Genetic Engineering of Molecular Chaperones, HSPs, and Plant Transcription Factors for Abiotic Stress Tolerance}

Molecular chaperones are involved in protein folding, assembly, translocation, and degradation in a broad array of normal cellular processes. It has been reported that a wide range of proteins have chaperone activity. However, many molecular chaperones are stress proteins and many of them were originally identified as heat-shock proteins (HSPs) (Wang et al., 2004). HSP gene expression is strongly correlated with heat stress transcription factors (HSFs) that are cytoplasmically located (inactive) regulatory proteins and are activated by stress conditions. They undergo oligomerization and recompartmentalization to the nucleus, under in vivo conditions (Baniwal et al., 2004). HSPs are classified into five major families based on their molecular weight (15-42 kDa): Hsp70 (DnaK), chaperonins (GroEL and Hsp60), Hsp90, Hsp100 (Clp), and small Hsp (sHsp) family (Wang et al., 2004). Under heat stress condition, five Hsp90 genes of Glycine max were overexpressed in $A$. thaliana. This resulted in higher biomass production, pod setting, and reduction in lipid peroxidation and loss of chlorophyll ( $\mathrm{Xu}$ et al., 2013b). In transgenic rice, overexpression of OsHSP18.6 produced crops with increased tolerance to heat, drought, salt, and cold temperatures. The exogenous expression of the Hsp70 gene significantly improved salinity tolerance in transgenic rice at the whole plant level (Hoang et al., 2015). Overexpression of $\mathrm{PgHsc70}$ and $\mathrm{PgHsp} 90$ improves the salt and heat stress tolerance in E. coli cells (Reddy et al., 2010, 2011). Overexpression of EaHSP70 in transgenic sugarcane plants showed enhanced tolerance to water deficiency and high salt concentrations, cell membrane thermostability, enhanced RWC and chlorophyll content, and enhanced photosynthetic efficiency (Augustine et al., 2015). It was reported that a strongly

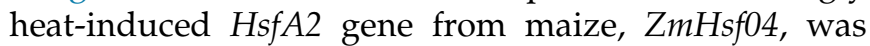
isolated and characterized. The gene has shown its function in the heat/salt-stress response and is localized to the nucleus. Transcription activation data demonstrate that an AHA2 domain protein is necessary for the transcriptional activity of $\mathrm{ZmHsf04}$. The function of ZmHsf04 was characterized in detail in transgenic plants of $A$. thaliana where it was observed that overexpression of $\mathrm{ZmHsf04}$ in Arabidopsis enhanced thermotolerance and positively regulated short-term acquired thermotolerance (Jiang et al., 2017).

\subsubsection{Epigenetic Regulation of Abiotic Stress Tolerance}

Plant gene regulation in response to abiotic stress is also epigenetically controlled without involving changes in the underlying sequence information, in a heritable and reversible manner. This epigenetic changes are brought about by modification in chromatin structure mediated by DNA methylation, histone posttranslational modification, and small noncoding RNAs (miRNA and siRNA) inducing RNA dependent DNA methylation, modulating the transcriptional and posttranscriptional gene expression (Kumar, 2016). These modifications brought about the differential expression of stress responsive genes by altering the binding efficiency of transcription factors (Zhu et al., 2014), and such changes working together or individually enable plants to adapt to unfavorable stresses (Yaish, 2017). Modification of histones occurred in the $\mathrm{N}$ terminal region called the histone tail via methylation, acetylation, phosphorylation, and ubiquitination of lysine and arginine residues altering gene activity that is wrapped around the core histone, and such modification can lead to activation as with acetylation or silencing of abiotic stress related genes (Kim et al., 2015b). Alteration in histone modification has been associated with abiotic stress such as drought, temperature, and salinity maintaining plant physiological status under adverse conditions. Histones, which form the structural unit of chromatin, and any changes, be they through enzymatic modification or incorporation of histone variants, impact chromatin stability and accessibility of transcriptional factors regulating gene expression (Asensi-Fabado et al., 2017). This concept has been explored with several findings, such as that the transcriptional activation of $O s D R E B b$ rice gene in response to cold stress is marked by hyperacetylation of histones H3K9, H3K14ac, and H3K27ac resulting in enhanced tolerance to salinity and cold stress (Scott et al., 2014). DNA methylation has also been shown to differentially regulate miRNA gene expression under temperature stress conditions. Through genetic 
engineering approaches the role of DNA methylation and histone modification in the transcriptional activation or repression of transcription factors MYB, bZIP, and AP2/DREB in soybean tolerance to salinity stress has been experimentally proved (Ci et al., 2015). Studies on the functions of histone modification on various stress responsive genes such as the transcription factors are widely explored on the model plant Arabidopsis by the loss-of-function mutation analysis (Song et al., 2012). Histone deacetylase gene HDA6 is required to survive under cold stress (To et al., 2011), and is involved in salt stress inducing response (Chen and $\mathrm{Wu}, 2010)$. HD2 proteins function in increased tolerance to salt stress (Luo et al., 2012). Histone modification is also correlated with the transcriptional regulation of cell cycle genes during various abiotic stresses. Under abiotic stress in maize seedlings, modifications of histones at specific lysine residue on the cell cycle gene promoter region regulate gene expression by inhibiting cell proliferation, inhibiting plant growth and development. Increased histone acetylation at H3K9ac and H4k5ac was observed with heat stress. Different stress regulates different phase of the cell cycle and reduced expression of cell cycle kinase CDKs during abiotic stress. And because increased acetylation functions in chromatin activation, such studies imply the role of modification in abiotic stress tolerance ( $\mathrm{Zhu}$ et al., 2014). The altered modification might reset to their normal conditions upon relief from stress or persist for future adaptations to recurrent stress, referred to as stress memory (Chinnusamy and Zhu, 2009). This has been observed for several abiotic stress factors such as in the case of drought. Further understanding the epigenetic regulation mechanism for different abiotic stresses through various genetic engineering approaches will facilitate the development of stress tolerant plants by controlling expression of various stress related genes epigenetically modified.

\subsubsection{Functional Role of Ubiquitination Encoding Proteins in Abiotic Stress Tolerance}

Ubiquitination is an intrinsic posttranslational protein modification playing an important role in plant development and environmental stress. Plants adapting to environmental stresses such as drought, salinity, water deficit, and fluctuating temperature undergo changes in their physiological metabolism by modulating the amount and activity of proteins involved in cellular defense (Lyzenga and Stone, 2011). Ubiquitination mediates degradation of a target protein, fine tuning their abundance and stability, regulating gene expression and signaling (Ding et al., 2015). Regulated protein response to abiotic stress involves the regulation of stomatal opening, ROS, hormonal signaling, protein stabilization, and maintaining cell membrane integrity in addition to the degradation of proteins accumulated during abiotic stress (Dametto et al., 2015). The process is mediated by a conserved protein ubiquitin that covalently binds to a target protein on lysine residue and is subjected to $26 \mathrm{~S}$ proteosomal degradation. The degradation is accomplished by sequential action of three enzymes: the ubiquitin activating enzymes (E1), ubiquitin conjugating enzymes (E2), and ubiquitin ligase (E3) (Sharma et al., 2016). The role of ubiquitin proteasome complex has been widely studied with respect to abiotic stress response, mainly on the E3 complex that plays a major role in the system (Stone, 2014). The highlights of a few of the recently identified ubiquitin encoding genes in response to different abiotic stresses through genetic engineering approaches are given in Table 4.1.

\subsubsection{Genetic Engineering of Helicases in Plant Abiotic Stress Tolerance}

Helicases are motor enzymes catalyzing the unwinding of duplex DNA (DNA helicases), RNA secondary structures, or RNPs (RNA helicases) in an ATP dependent manner (Macovei et al., 2012; Tuteja et al., 2012). DNA helicases function in DNA replication, repair, recombination, and transcription whereas RNA helicases are involved in transcription, ribosomes biogenesis, and translation initiation. Most of them are members of the DEAD-box protein subfamily (Sanan-Mishra et al., 2005). Because abiotic stresses interfere with the transcriptional and translation machinery, it is clear that molecules such as helicases involved in maintaining the integrity of nucleic acids are affected, and many studies have reported the positive role of these helicases to counteract with abiotic stresses (Sahoo et al., 2012; Raikwar et al., 2015). DEAD-box RNA helicases are known to be enriched for multiple abiotic stresses like drought, salinity, cold, and oxidative stress in both prokaryotes and eukaryotes as well (Baruah et al., 2017). Overexpression of a DEAD-box pea DNA helicase PDH45 in tobacco exhibited salinity tolerance probably by stabilizing protein synthesis and association with DNA multi subunit complexes regulating gene expression (Sanan-Mishra et al., 2005). OsABP, a DEAD-box RNA helicase ATP binding protein was found to be responsive to multiple abiotic stresses by interacting with proteins involved in RNA metabolism, signal transduction, and stress response (Macovei et al., 2012). Transgenic chili plant overexpressing pea DNA helicase PDH45 was found to alleviate multiple abiotic stresses such as drought, salinity, senescence, and oxidative stress by improving plant physiological parameters, production of antioxidative enzymes, and enhancing expression of 
TABLE 4.1 List of Ubiquitin Encoding Genes in Response to Different Abiotic Stresses Through Genetic Engineering Approaches

\begin{tabular}{|c|c|c|c|c|c|}
\hline Plant & $\begin{array}{l}\text { Ubiquitin } \\
\text { complex }\end{array}$ & Encoding genes & Stress & Functional Expression studies & References \\
\hline Arabidopsis & $\begin{array}{l}\text { U box E3 } \\
\text { ligase }\end{array}$ & PUB46 and PUB48 & Drought & $\begin{array}{l}\text { Negatively regulate plant response to drought stress by } \\
\text { degrading target proteins }\end{array}$ & Adler et al. (2017) \\
\hline Arabidopsis & $\begin{array}{l}\text { RING } \\
\text { finger E3 } \\
\text { ligase }\end{array}$ & SDIR1 & Salt & $\begin{array}{l}\text { SDIRIP1 is a substrate for SDIR1, which negatively } \\
\text { regulates ABA and salt stress response }\end{array}$ & Zhang et al. (2015) \\
\hline Apple & $\begin{array}{l}\text { RING E3 } \\
\text { ligase }\end{array}$ & MdMIEL1 & $\begin{array}{l}\text { Salt and } \\
\text { oxidative } \\
\text { stress }\end{array}$ & $\begin{array}{l}\text { Negatively regulates salt stress and oxidative stress } \\
\text { tolerance by increasing ROS concentration }\end{array}$ & An et al. (2017) \\
\hline Arabidopsis & $\begin{array}{l}\text { F-Box E3 } \\
\text { ligase }\end{array}$ & AtDIF1 & $\begin{array}{l}\text { Salt and } \\
\text { drought }\end{array}$ & $\begin{array}{l}\text { Induced by salinity, drought, ABA but repressed by cold } \\
\text { treatment. Confers salt tolerance by maintaining } \mathrm{Na}^{+} / \mathrm{K}^{+} \\
\text {homeostasis and promote seedlings growth. However, } \\
\text { negatively regulates drought stress tolerance with } \\
\text { retarding seed germination and survival rate }\end{array}$ & Gao et al. (2017) \\
\hline $\begin{array}{l}\text { Grimmia } \\
\text { pilifera }\end{array}$ & $\begin{array}{l}\text { RING E3 } \\
\text { ligase }\end{array}$ & GpDSR7 & Drought & $\begin{array}{l}\text { Induced by water deficit, enhances tolerance to drought } \\
\text { stress pre and post recovery by reducing water loss }\end{array}$ & Li et al. (2016b) \\
\hline Watermelon & $\begin{array}{l}\text { RING E3 } \\
\text { ligase }\end{array}$ & $G d R Z F 1$ & Drought & $\begin{array}{l}\text { Reduced expression enhances tolerance to drought stress } \\
\text { showing reduced leaf wilting, higher relative water } \\
\text { content, lower ion leakage and MDA content, higher } \\
\text { proline content in the antisense lines }\end{array}$ & Chung et al. (2017) \\
\hline Wheat & E3 ligase & TaSAP5 & Drought & $\begin{array}{l}\text { Promotes degradation of DRIP proteins leading to the } \\
\text { accumulation of DREB2A protein enhancing survival } \\
\text { rates of wheat seedlings under drought stress }\end{array}$ & Zhang et al. (2017) \\
\hline Rice & $\begin{array}{l}\text { RING E3 } \\
\text { ligase }\end{array}$ & OsHTAS & Heat & $\begin{array}{l}\text { Enhances rice heat tolerance through an ABA dependent } \\
\text { and DST (drought and salt tolerance) mediated pathway } \\
\text { via an induced } \mathrm{H}_{2} \mathrm{O}_{2} \text { stomatal closure in the leaf blade }\end{array}$ & Liu et al. (2016a) \\
\hline $\begin{array}{l}\text { Brassica } \\
\text { napus }\end{array}$ & $\begin{array}{l}\text { RINGv E3 } \\
\text { ligase }\end{array}$ & $\begin{array}{l}\text { BnTR1 (thermal } \\
\text { resistance) }\end{array}$ & Heat & $\begin{array}{l}\text { Modest expression confers thermal tolerance likely by } \\
\text { regulating calcium channels altering heat-shock factors } \\
\text { and heat-shock proteins expression }\end{array}$ & Liu et al. (2014b) \\
\hline Ryegrass & $\begin{array}{l}\text { Ubiquitin } \\
\text { like protein } \\
\text { family }\end{array}$ & $\begin{array}{l}\text { LpHUB1 (homology to } \\
\text { Ub1) }\end{array}$ & Drought & $\begin{array}{l}\text { Overexpression improves drought tolerance with higher } \\
\text { relative water content, leaf water potential, and } \\
\text { chlorophyll content under drought stress }\end{array}$ & Patel et al. (2015) \\
\hline Maize & $\begin{array}{l}\text { RING } \\
\text { finger E3 } \\
\text { ligase }\end{array}$ & $\begin{array}{l}\text { ZmAIRP4 (Zea mays } \\
\text { abscisic acid insensitive } \\
\text { RING protein) }\end{array}$ & Drought & $\begin{array}{l}\text { Overexpressing transgenic Arabidopsis enhance drought } \\
\text { tolerance by reducing water loss rate and altering other } \\
\text { stress responsive genes }\end{array}$ & Yang et al. (2018) \\
\hline Pepper & $\begin{array}{l}\text { RING type } \\
\text { E3 ligase }\end{array}$ & CaREL1 & Drought & $\begin{array}{l}\text { Overexpressing transgenic Arabidopsis exhibits low } \\
\text { sensitivity to abscisic acid, increased water loss rate, and } \\
\text { impaired stomatal closure, confirming as a negative } \\
\text { regulator to drought stress }\end{array}$ & Lim et al. (2017) \\
\hline Cotton & $\begin{array}{l}\text { RING } \\
\text { finger E3 } \\
\text { ligase }\end{array}$ & $\begin{array}{l}\text { GhSARP1 (salt- } \\
\text { associated ring finger } \\
\text { protein) }\end{array}$ & Salt & $\begin{array}{l}\text { Overexpressing transgenic Arabidopsis reduces tolerance } \\
\text { to salt stress with reduced germination rate }\end{array}$ & Liu et al. (2016b) \\
\hline
\end{tabular}

stress responsive genes (Shivakumara et al., 2017). Many plant DEAD-box RNA helicases have been identified and their active participation in the stress signaling pathway has been reported. Overexpression of Pea p68 transgenic tobacco plants accumulates less $\mathrm{Na}^{+}$and more $\mathrm{K}^{+}$as compared with the WT plants. Higher $\mathrm{K}^{+}$content that controls endonuclease and caspase-like protease activity results in delayed leaf senescence in the transgenic lines under stress conditions (Tuteja et al., 2014). Similarly, SIDEAD30, SIDEAD31, AtRH36, and AtRH9 confer resistance against salinity, drought, cold, and ABA stress conditions, and also regulate the expression of other genes involved in the stress signaling pathway (Zhu et al., 


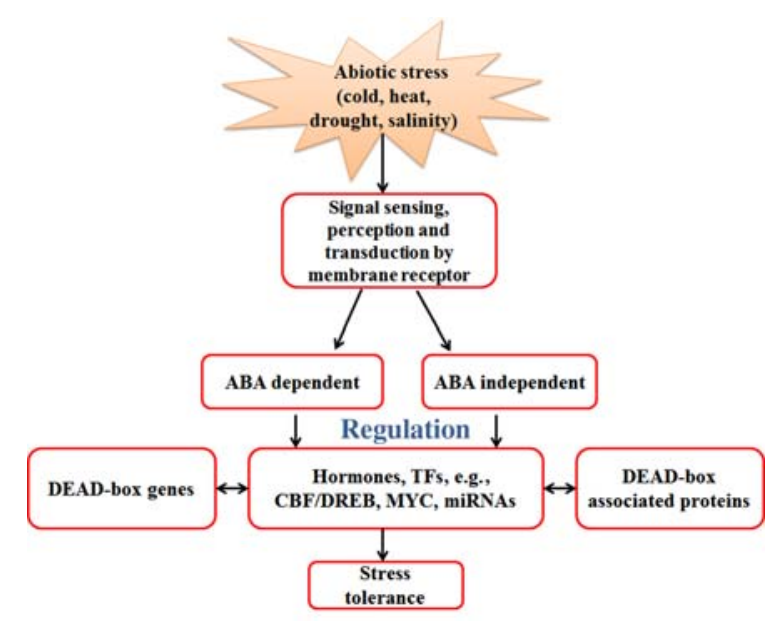

FIGURE 4.3 Schematic presentation of abiotic stress response of DEAD-box genes by interacting with transcription factors (TFs), miRNAs, and DEAD-box associated proteins.

2015). Transgenic groundnut plants expressing DEAD-box RNA helicase gene PgeIF4A were recently developed through genetic engineering that exhibit tolerance to stress conditions like oxidative, salt, and drought stress (Santosh Rama Bhadra Rao et al., 2017). The role of DExD/H-box RNA helicase member of $\mathrm{SF}_{2}$ superfamily in rRNA biogenesis has been attributed during cold stress, where missense mutation of AtRH10 impaired rRNA processing with physiological defects under high temperature; similarly AtRH7 mutants exhibit severe growth retardation in plants exposed to cold stress (Liu and Imai, 2018). The negative role of DEAD-box RNA helicases has been identified for Arabidopsis encoding genes stress response suppressor 1 (STRS1) and STRS2. They are found to be downregulated by multiple abiotic stresses whose mutation increased tolerance by inducing expression of multiple stress responsive genes (Kant et al., 2007). A schematic representation of abiotic stress response of DEAD-box genes by interacting with transcription factors (TFs), miRNAs, and DEADbox associated proteins are given in Fig. 4.3. The role of the DEAD-box RNA helicases in regulating responses to multiple abiotic stresses in plants has been recently reported in detail (Baruah et al., 2017).

\subsubsection{Genetic Engineering of Small RNAs for Abiotic Stress Responses}

MicroRNAs are endogenous small interfering noncoding RNAs of 20-24 nt long that negatively regulate the posttranscriptional expression of specific genes in response to abiotic stresses. Regulation is brought about by mRNA degradation, translational repression, and epigenetic modification. Upregulation of miRNA downregulates stress responsive genes and their inhibition causes expression of functional genes. Identification of these RNAs through various computational approaches could help in engineering plant genes improving plant abiotic stress tolerance (Shriram et al., 2016). miRNA mediated transcriptional regulation was based on the presence of abiotic stress response elements on the promoter region of miRNA (Barciszewska-Pacak et al., 2015). miRNAs are encoded by MIR genes and transcribed by RNA polymerases II forming double stranded stem loop primary miRNA. The duplex is then processed by a micropressor DICER like protein (DCL 1), HYL1, SE protein, and stabilized by HEN1 protein forming mature miRNA-miRNÂ. This is then transported in the cytoplasm, where the miRNA associate with the argonaute protein in the RISC complex while its cognate miRNÂ is degraded. Guided by the argonaute protein miRNA complementary base pair with the target mRNA leading to its degradation and transcriptional inhibition (Gielen et al., 2012). miRNA has been widely accepted to regulate stress responsive genes under abiotic stress and any changes can affect expression conferring adaptation advantages, thus identifying these targets would be beneficial in understanding the regulatory role of miRNA in plant abiotic stress response (Chen et al., 2017). Expression analysis revealed the downregulation of TCP (teosinite branched/cycloidea/proliferating cell factors) and a homolog OsNAC domain in transgenic plant. Overexpression of cold responsive OsmiR156K in rice decreased tolerance to cold stress and suggested the downregulation of cold stress responsive genes 01g22249, OsP5CS, and SPL (Cui et al., 2015). OsSPL2 and OsSPL14 genes were targeted by miR529a in overexpression rice improving plant tolerance to oxidative stress (Yue et al., 2017). Transgenic chickpea overexpressed miR408 increases drought tolerance and it is involved in the expression of transcription factor $D R E B$ genes (Hajyzadeh et al., 2015). Role of soybean miRNA172c for drought and salt stress and MIR394a for drought was investigated in transgenic overexpression lines of the Arabidopsis (Ni et al., 2012; Li et al., 2016d). The overexpression plant improved the plant's physiological metabolism, promoted early flowering under drought stress, and regulated ROS accumulation suggesting the positive role in drought and salt stress tolerance; however it showed hypersensitivity to ABA. The overexpression of two conserved microRNAs miR319a and $m i R 319 b$ in rice improved leaf morphology and enhanced tolerance to cold induced stress (Yang et al., 2013). Suppressing the expression of a gene ESK1 with siRNA together with overexpression of $C B F$ gene in Arabidopsis enhanced drought tolerance. However, suppression of this gene reduced respiration rate during drought stress and reduced seed production in normal watering conditions ( $\mathrm{Xu}$ et al., 2014). 


\subsection{GENOME EDITING APPROACHES TO MODULATE ABIOTIC STRESS SIGNALING PROCESSES IN CROP PLANTS}

Genome or gene editing technology is a new technique of precisely manipulating genome structure in a simple and efficient manner. It involves modifying the known sequence in a genome, targeting only a few nucleotide bases, facilitating functional studies of the gene related to the phenotypic traits. This system encompasses use of site-specific nuclease (SSN) and site-specific recombinase (SSR) to generate the knockout of an undesirable trait or knock-in gene activation in the genome of an organism. In plants this technology has been widely used to study and improve crop nutritional quality and yield, disease management, and improve crop tolerance to abiotic stresses. It is also regarded as a non-GM technology since it does not involve manipulating a large sequence and/or introduction of foreign gene species (Abdallah et al., 2016). Site-specific nuclease generates a double-strand break (DSB) in the targeted gene sequences and uses the cell endogenous DNA repair mechanism, nonhomologous end joining (NHEJ), and homology directed repair (HDR) to repair breaks, creating a new gene function. This includes the zinc finger nuclease (ZFN), transcription activator like effector nucleases (TALENs), and clustered regularly interspaced short palindromic repeats (CRISPR-Cas9) system (Kim et al., 2015a). NHEJ is an error prone repair system resulting in gene knockout by creating an indel mutation causing frameshift mutations in the coding region of a gene or disrupting the cis-regulatory elements of promoters and enhancers, whereas homology dependent repair uses a template with sequence homology flanking the region of interest resulting in insertion of the desired sequence mediated by homologous recombination repair (Kamburova et al., 2017b).

\subsubsection{Zinc Finger Nucleases}

Zinc finger nucleases are engineered restriction nucleases consisting of a sequence specific zinc finger DNA binding protein domain fused to a nuclease domain FokI (dimer) for generating double-strand break cleavage at specific loci (Petolino, 2015). ZFN specifically creates the DNA double-strand breaks on the chromosome, and cause site-specific mutagenesis and base substitution, which can alter the expression of a target gene, preferably gene knockdown (Li et al., 2013). In Arabidopsis, ZFNs have been generated that recognize target DNA to introduce indel mutations (de Pater et al., 2009; Hou et al., 2014). Site-specific mutagenesis and base substitution play a very important role in the plant genetic improvement. Thus, ZFNs are one of the potential genome editing tools to be explored for abiotic stress tolerance in diversified plants.

\subsubsection{Transcription Activator Like Effector Nucleases}

TALENs are the proteins derived from the plant pathogenic bacterium Xanthomonas campestris, which binds to specific DNA sequences (Curtin et al., 2012). TALEN allows site-directed mutagenesis in the target gene of interest (Hou et al., 2014). TALENs are based on fusion of a Fok1 nuclease domain to the DNA binding TALE repeats (Mahfouz et al., 2011). TALENs have been successfully introduced in plants such as Arabidopsis, tobacco, rice, and Brachypodium (Curtin et al., 2012; Shan et al., 2013). Recently, TALEN was shown to induce different heritable mutation in rice (Zhang et al., 2016). Improved cold storage and processing traits in potato have been engineered through TALENs (Clasen et al., 2016). Multiple assembly methods based on golden gate cloning have revolutionized the rapid adoption of TALEN technology for genome editing in plants for several applications including developing abiotic stress tolerant crop plants.

\subsubsection{CRISPR-Cas9 Genome Editing}

The clustered regularly interspaced short palindromic repeat/CRISPR associated protein (Cas9) is a novel genome editing tool that precisely creates a double strand cleavage of target DNA (Khatodia et al., 2016). Discovered from the adaptive defense mechanism of E. coli and archaea against invading foreign DNA, this type II system is commonly used to study the function of a gene by transcriptional activation or repression, altering the metabolic pathway, which can be used to improve crop quality and for drug development because of its simplicity and ability for multiplex genome editing, especially for plant abiotic stress complex trait (Arora and Narula, 2017; Liu et al., 2017). Active Cas9/sgRNA complex cleaves targeted DNA upon binding and the resultant blunt end DSB is then repaired either by NHEJ using DNA ligase IV introducing frameshift or indel mutation leading to gene knockout or by HDR repair pathway causing the insertion of oligotemplate resulting in gene replacement or knock-in of foreign DNA (Song et al., 2016). The basic strategy of the CRISPRCas9/strand-based genome editing system is represented in Fig. 4.4. 


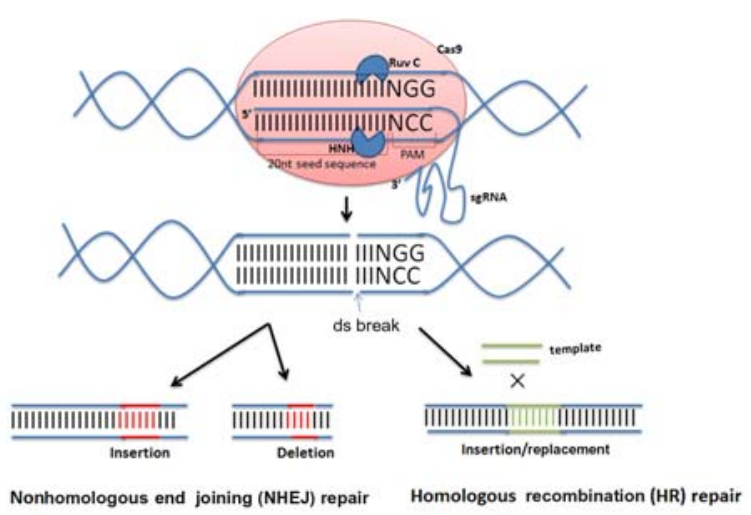

FIGURE 4.4 The basic strategy of the CRISPR-Cas9/sgRNA based genome editing system.

\subsubsection{Application of CRISPR-Cas9 in Plant Abiotic Stress Tolerance}

The role of annexin genes in conferring abiotic stress tolerance such as drought and salinity through overexpression studies has been investigated (Ijaz et al., 2017), however the role in cold tolerance is exploited using CRISPR-Cas9 knockout gene mutation and decreased tolerance to cold stress has been observed (Shen et al., 2017). The CRISPR-Cas9 technology using HRD mediated gene replacement has been used to generate novel ARGOS8 variant in maize, which in its native form has low expression level. Modified ARGOS8 variant increased expression transcript, and was found to increase grain yield by five bushels per acre compared with wild type under drought conditions and no yield loss under well-watered conditions (Shi et al., 2017). MAPKs are important signaling molecules for drought stress. Tomato mutant line SIMAPK3 generated using CRISPR-Cas9 exhibits severe leaf wilting, increased hydrogen peroxide content, reduced antioxidant enzymes, and caused membrane damage under drought conditions, thus confirm the positive role of SIMAPK3 in drought tolerance by maintaining cell membrane structure (Wang et al., 2017a). CRISPRCas9 has been also used to target noncoding regulator element, the promoter region of rice OsRAV2 for its functional role with salt treatment (Duan et al., 2015).

\subsubsection{CRISPR-Cpf1}

While the ease and efficiency of using CRISPR-Cas9 complements other SSNs such as ZFN and TALEN, its limitations in generating off target effects and its uncertain intellectual property landscape reduce its scientific adoption (Begemann et al., 2017). Alternative to CRISPR-Cas9, a recently discovered approach for genome editing is the CRISPR-Cpf1 a type V CRISPRCas system, which is an RNA guided endonuclease
(Xu et al., 2017). This system discovered from Prevotella and Francisella has emerged as a new, efficient tool for target gene modification including DNA free editing in plants, and shows higher efficiency and potential to the CRISPR-Cas9 system, exhibiting fewer off target effects in plants (Zaidi et al., 2017). The CRISPR-Cpf1 uses a single crRNA with approximately 44 nucleotides guiding cleavage by Cpf1 nuclease, in contrast to 100 nucleotide in Cas9, which requires tracrRNA to pair with crRNA for its functional role. Thus a shorter gRNA sequence is required for designing CRISPR-Cpf1 simplifying cloning process. The functional role of Cpf1 nuclease is governed by only RuvC without $\mathrm{HNH}$ domain. Cpf1 recognizes a thymine rich 5'-TTTN-3' PAM site distal to its target DNA at the $5^{\prime}$ end, and generates cohesive sticky ends compared with the blunt end of CRISPR-Cas9, improving efficiency for NHEJ gene insertion (Malzahn et al., 2017; Verwaal et al., 2017). The advantage of multiplexing genome editing using Cpf1 has been demonstrated (Zetsche et al., 2017), where using a single crRNA can target up to four genes simultaneously in mammalian cells, possibly because of the ability of Cpf1 to process its own crRNA, thus minimizing the limits of generating a large expression construct by CRISPR-Cas9. However, further studies on its advantages and disadvantages need to be explored as well as the generation of mutated lines. In rice targeted gene editing using Cpf1 generated precise gene insertion and indels with up to $8 \%$ greater efficiency compared with other nucleases (Begemann et al., 2017), and generated a biallelic mutation of $100 \%$ efficiency using Cpf1 from Lachnospiraceae bacterium (Tang et al., 2017).

Using these approaches genome editing has been widely used for the functional characterization of plant genes and genetic improvement of agricultural crops. A detailed mechanism employed by different genome editing approaches (ZFNs, TALENs, and CRISPRCas9), and their relative comparisons and applications in improving plant tolerance to abiotic stress are briefly explained in Table 4.2. A scheme of the development of multiple abiotic stress tolerance in a crop model using target specific multiplex multigene CRISPR-Cas9 genome editing approach is given in Fig. 4.5.

\subsection{CONCLUSIONS AND FUTURE PROSPECTS}

The impact of rapid global climate change on crop production has emerged as a major research priority during the past decade. The major abiotic stress factors such as drought, salinity, and extreme 
TABLE 4.2 Comparison of Mechanism, Application, Advantages, and Disadvantages of Different Genome Editing Approaches (ZFNs, TALENs, and CRISPR-Cas9)

\begin{tabular}{|c|c|c|c|}
\hline Section & Zinc finger nuclease & TALEN & CRISPR-Cas9 \\
\hline Origin & Zinc finger motifs & Xanthomonas campestris & Streptococcus pyogenes \\
\hline Mechanism & $\begin{array}{l}\text { Cleavage occur by binding of } 4-6 \text { tethered zinc } \\
\text { finger protein domains (Cys2-His } 2 \text { fingers) on } \\
\text { both strands of target DNA fused with a } \\
\text { dimerized nuclease FokI }\end{array}$ & $\begin{array}{l}\text { Cleavage occurs by binding of pairs of } \\
\text { TAL effectors fused to restriction } \\
\text { endonuclease FokI for one target site }\end{array}$ & $\begin{array}{l}\text { Cleavage occurs by binding of a } \\
\text { chimeric sgRNA to the target } \\
\text { sequence mediated by Cas } 9 \\
\text { nuclease }\end{array}$ \\
\hline $\begin{array}{l}\text { Catalytic } \\
\text { domain }\end{array}$ & Required dimerize FokI & Required dimerize FokI & $\begin{array}{l}\text { Only a single Cas9 nuclease } \\
\text { required }\end{array}$ \\
\hline $\begin{array}{l}\text { Sequence } \\
\text { recognition }\end{array}$ & $18-24$ bps & $30-40$ bps & $20-22$ bps \\
\hline Advantage & Can target any sequence & $\begin{array}{l}\text { - } \text { Can target any sequence } \\
\text { - Higher specificity than CRISPR } \\
\text { - } \text { Reduced off target }\end{array}$ & 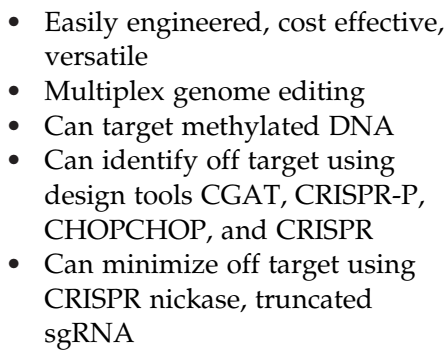 \\
\hline Limitations & $\begin{array}{l}\text { Engineering is difficult because of the context } \\
\text { dependent effects } \\
\text { - Low affinity and specificity } \\
\text { - High off target and toxicity } \\
\text { - Determining off target is difficult due to } \\
\text { context dependence of protein-DNA } \\
\text { interaction }\end{array}$ & $\begin{array}{l}\text { Difficult for engineering nuclease } \\
\text { and TALEN, time consuming and } \\
\text { expensive } \\
\text { Cannot target methylated DNA, } \\
\text { which plays important role in abiotic } \\
\text { stress tolerance } \\
\text { - Determining off target is difficult } \\
\text { due to context dependence of } \\
\text { protein-DNA interaction } \\
\text { Binding site starts with T base }\end{array}$ & $\begin{array}{l}\text { - Higher off target effects than } \\
\text { TALEN } \\
\text { - Required PAM sequence for } \\
\text { recognition } \\
\text { - Suitable for targets containing } \\
\text { high GC content }\end{array}$ \\
\hline References & Kamburova et al. (2017a), Zhao and Wolt (2017) & $\begin{array}{l}\text { Malzahn et al. (2017), Zhao and Wolt } \\
\text { (2017) }\end{array}$ & $\begin{array}{l}\text { Malzahn et al. (2017), Zhao and } \\
\text { Wolt (2017) }\end{array}$ \\
\hline
\end{tabular}

temperatures have negatively impacted the agricultural productivity worldwide. Most plants have developed genetic, biochemical, and physiological mechanisms to cope with different abiotic stresses, which include activation and repression of key signaling pathways; negative and positive regulation of specific stress signaling genes such as DEAD-box RNA helicases, hybrid proline rich proteins, dehydrins, methylglyoxalases, ethylene response factors, and transcription factor genes; and hyper- and hypoaccumulation of compatible salts, and stress proteins such as aquaporins, molecular chaperones, HSPs, kinase proteins, and helicases. With a greater understanding of genetic engineering and functional genomics approaches such as transgenics, overexpression of elite genes, next generation sequencing, lossof-function and gain-of-function mutations, and small RNAs several abiotic stress tolerant crop plants have been developed in the past decade to date. Major genetic engineering has also been extensively used in recent years for development of abiotic stress tolerance in crop plants through epigenetic regulation, ubiquitination, and transcription factor regulation. Very recently the CRISPR-Cas9 and CRISPR-Cpf1 genome editing systems have become the significant discovery of modern day agricultural biotechnology. Widely regarded as a non-GM approach, CRISPR technology has emerged as a breakthrough genome editing system that could be a potential game changer for expanding our research on developing sustainable multiple abiotic stress tolerance in crop plants for the future. Considering the huge loss of crop productivity majorly due to abiotic stress factors, there is an urgent need to direct our research focus to develop sustainable multistress crop tolerance in combination with high yields using genome editing technology to counter the climate change related adverse effects on the productivity of crops. 
I. Genomic target selection a) 20bp target sequence upstream of PAM (NGG)

II. SgRNA design

a) sgRNA is expressed using a small RNA promoter b) Guide sequence should match the target

III. Assembly of Cas9/sgRNA construct a) Golden Gate Cloning

b) GFP reporter in frame for screening

IV. Deliver into target crop plant a) Protoplast transfection

b) Agrobacterium transformation of seedlings/cotyledons

c) Plant transformation through foral dip

V. Regeneration and screening of CRISPR/Cas9 targets

a) $\mathrm{RE}$

b) $\mathrm{SA}$

c) NGS

VI. Selection of GE-specific modifications in target crop edited by CRISPR/Cas9

a) Loss-of-function analysis

b) Genomic fragments

VII. Functional genomic of multiple abiotic stress tolerance

a) Multiple abiotic stress analysis

b) Field evaluation and multi-location trials

VIII. Multiple abiotic stress tolerant cultivar of target crop
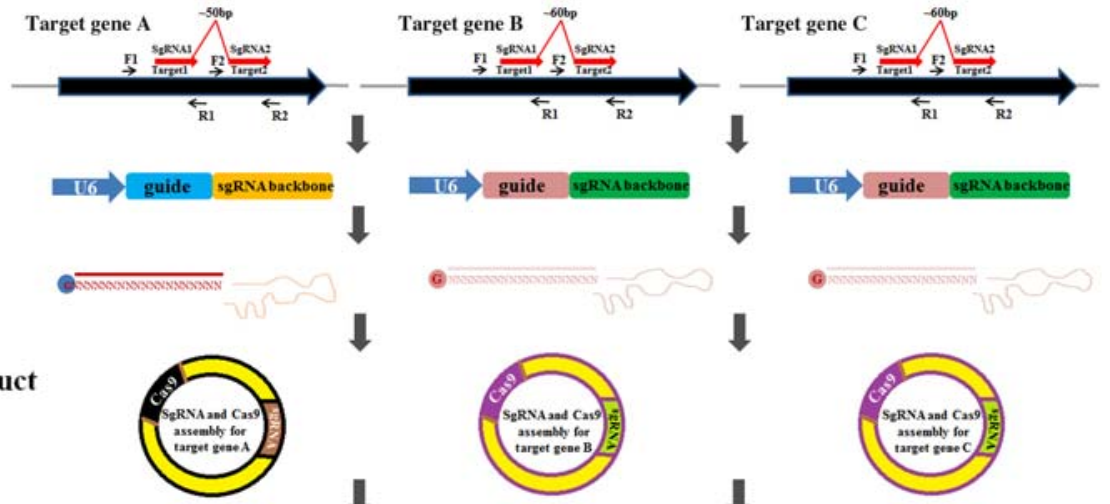

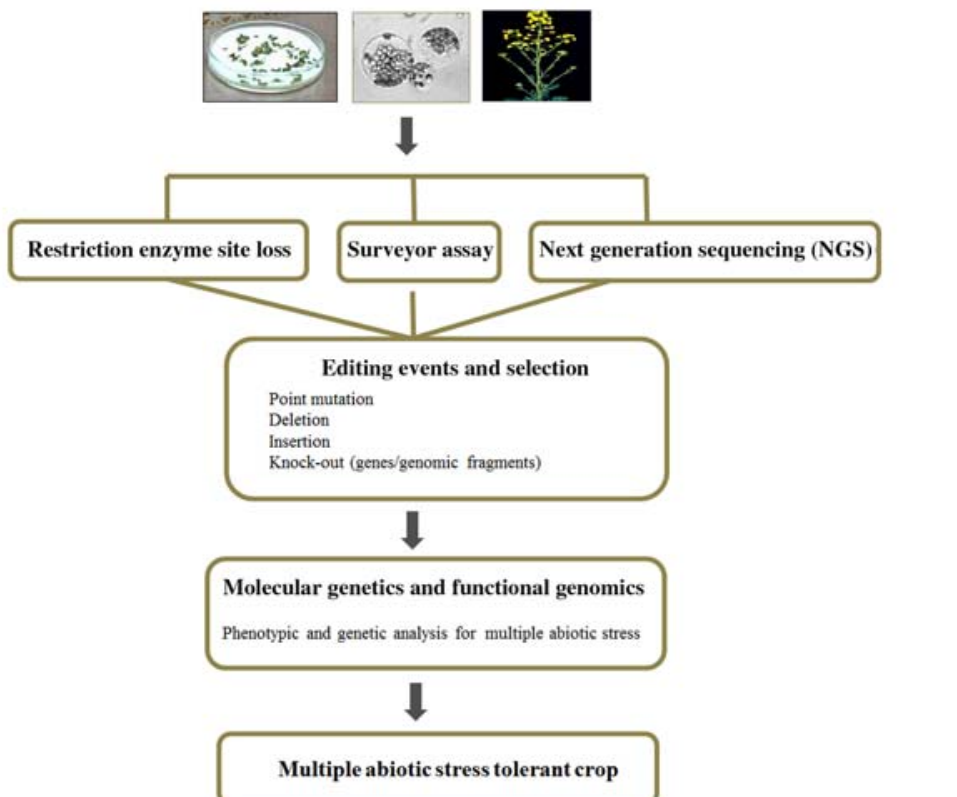

FIGURE 4.5 Scheme of development of multiple abiotic stress tolerance in a crop model using target specific multiplex multigene CRISPRCas9 genome editing approach.

\section{References}

Abdallah, N.A., Moses, V., Prakash, C.S., 2014. The impact of possible climate changes on developing countries. GM Crops Food 5, 77-80.

Abdallah, N.A., Prakash, C.S., McHughen, A.G., 2016. Genome editing for crop improvement: challenges and opportunities. GM Crops Food 6, 183-205.

Abeles, F.B., Morgan, P.W., Saltveit Jr, M.E., 1992. Chapter 3-The Biosynthesis of Ethylene. Ethylene in Plant Biology, second ed. Academic Press, New York, pp. 26-55.

Adler, G., Konrad, Z., Zamir, L., Mishra, A.K., Raveh, D., Bar-Zvi, D., 2017. The Arabidopsis paralogs, PUB46 and PUB48, encoding U-box E3 ubiquitin ligases, are essential for plant response to drought stress. BMC Plant Biol. 17, 8.

Agarwal, P.K., Gupta, K., Jha, B., 2010. Molecular characterization of the Salicornia brachiata SbMAPKK gene and its expression by abiotic stress. Mol. Biol. Rep. 37, 981-986.

Aharon, R., Shahak, Y., Wininger, S., Bendov, R., Kapulnik, Y., Galili, G., 2003. Overexpression of a plasma membrane aquaporin in transgenic tobacco improves plant vigor under favorable growth conditions but not under drought or salt stress. Plant Cell 15, 439-447.

Ahmad, N., Mukhtar, Z., 2017. Genetic manipulations in crops: challenges and opportunities. Genomics 109, 494-505.

Ahn, C., Park, U., Park, P.B., 2011. Increased salt and drought tolerance by D-ononitol production in transgenic Arabidopsis thaliana. Biochem. Biophys. Res. Commun. 415, 669-674.

Alavilli, H., Awasthi, J.P., Rout, G.R., Sahoo, L., Lee, B.-H., Panda, S. K., 2016. Overexpression of a barley aquaporin gene, HvPIP2;5 confers salt and osmotic stress tolerance in yeast and plants. Front. Plant Sci. 7, 1566.

Amara, I., Zaidi, I., Masmoudi, K., Ludevid, M.D., Pagès, M., Goday, A., et al., 2014. Insights into late embryogenesis abundant (LEA) proteins in plants: from structure to the functions. Am. J. Plant Sci. 05, 3440-3455.

An, J.-P., Liu, X., Song, L.-Q., You, C.-X., Wang, X.-F., Hao, Y.-J., 2017. Apple RING finger E3 ubiquitin ligase MdMIEL1 negatively regulates salt and oxidative stresses tolerance. J. Plant Biol. 60, 137-145.

Anjum, N.A., Singh, H.P., Khan, M.I., Masood, A., Per, T.S., Negi, A., et al., 2015. Too much is bad-an appraisal of phytotoxicity of 
elevated plant-beneficial heavy metal ions. Environ. Sci. Pollut. Res. Int. 22, 3361-3382.

Arora, L., Narula, A., 2017. Gene editing and crop improvement using CRISPR-Cas9 system. Front. Plant Sci. 8.

Asano, T., Hayashi, N., Kikuchi, S., Ohsugi, R., 2012. CDPKmediated abiotic stress signaling. Plant Signal. Behav. 7, 817-821.

Asensi-Fabado, M.-A., Amtmann, A., Perrella, G., 2017. Plant responses to abiotic stress: the chromatin context of transcriptional regulation. Biochim. Biophys. Acta (BBA) 1860, 106-122.

Augustine, S.M., Narayan, J.A., Syamaladevi, D.P., Appunu, C., Chakravarthi, M., Ravichandran, V., et al., 2015. Erianthus arundinaceus HSP70 (EaHSP70) overexpression increases drought and salinity tolerance in sugarcane (Saccharum spp. hybrid). Plant Sci. $232,23-34$.

Baniwal, S.K., Bharti, K., Chan, K.Y., Fauth, M., Ganguli, A., Kotak, S., et al., 2004. Heat stress response in plants: a complex game with chaperones and more than twenty heat stress transcription factors. J. Biosci. 29, 471-487.

Barciszewska-Pacak, M., Milanowska, K., Knop, K., Bielewicz, D., Nuc, P., Plewka, P., et al., 2015. Arabidopsis microRNA expression regulation in a wide range of abiotic stress responses. Front. Plant Sci. 6.

Baruah, I., Debbarma, J., Boruah, H.P.D., Keshavaiah, C., 2017. The DEAD-box RNA helicases and multiple abiotic stresses in plants: a systematic review of recent advances and challenges. Plant Omics 10, 252-262.

Battaglia, M., Covarrubias, A., 2013. Late embryogenesis abundant (LEA) proteins in legumes. Front. Plant Sci. 4, 190.

Begemann, M.B., Gray, B.N., January, E., Gordon, G.C., He, Y., Liu, H., et al., 2017. Precise insertion and guided editing of higher plant genomes using Cpf1 CRISPR nucleases. Sci. Rep. 7.

Ben Rejeb, I., Pastor, V., Mauch-Mani, B., 2014. Plant responses to simultaneous biotic and abiotic stress: molecular mechanisms. Plants 3, 458-475.

Boudichevskaia, A., Heckwolf, M., Kaldenhoff, R., 2015. T-DNA insertion in aquaporin gene AtPIP1;2generates transcription profiles reminiscent of a low $\mathrm{CO}_{2}$ response. Plant Cell Environ. 38, 2286-2298.

Brossa, R., López-Carbonell, M., Jubany-Marí, T., Alegre, L., 2011. Interplay between abscisic acid and jasmonic acid and its role in water-oxidative stress in wild-type, ABA-deficient, JA-deficient, and ascorbate-deficient Arabidopsis plants. J. Plant Growth Regul. 30 (3), 322-333.

Bundo, M., Coca, M., 2017. Calcium-dependent protein kinase OsCPK10 mediates both drought tolerance and blast disease resistance in rice plants. J Exp Bot 68, 2963-2975.

Campo, S., Baldrich, P., Messeguer, J., Lalanne, E., Coca, M., San Segundo, B., 2014. Overexpression of a calcium-dependent protein kinase confers salt and drought tolerance in rice by preventing membrane lipid peroxidation. Plant Physiol. 165, 688-704.

Candat, A., Paszkiewicz, G., Neveu, M., Gautier, R., Logan, D.C., Avelange-Macherel, M.- H., et al., 2014. The ubiquitous distribution of late embryogenesis abundant proteins across cell compartments inarabidopsisoffers tailored protection against abiotic stress. Plant Cell 26, 3148-3166.

Chen, L.-T., Wu, K., 2010. Role of histone deacetylases HDA6 and HDA19 in ABA and abiotic stress response. Plant Signal. Behav. 5, 1318-1320.

Chen, T., Yang, Q., Zhang, X., Ding, W., Gruber, M., 2012. An alfalfa (Medicago sativa L.) ethylene response factor gene, MsERF11, enhances salt tolerance in transgenic Arabidopsis. Plant Cell Rep. 31, 1737-1746.

Chen, X.-Y., Yang, Y., Ran, L.-P., Dong, Z.-D., Zhang, E.-J., Yu, X.-R., et al., 2017. Novel insights into mirna regulation of storage protein biosynthesis during wheat caryopsis development under drought stress. Front. Plant Sci. 8.

Chikkaputtaiah, C., Debbarma, J., Baruah, I., Havlickova, L., Deka Boruah, H.P., Curn, V., 2017. Molecular genetics and functional genomics of abiotic stress-responsive genes in oilseed rape (Brassica napus L.): a review of recent advances and future. Plant Biotechnol. Rep. 11, 365-384.

Chinnusamy, V., Zhu, J.-K., 2009. Epigenetic regulation of stress responses in plants. Curr. Opin. Plant. Biol. 12, 133-139.

Chung, J., Park, S., Min, J., Min, K., Lee, S., Lee, K., et al., 2017. Reduced expression of Gongdae Ring Zinc Finger 1 (GdRZF1) enhances drought stress tolerance in watermelon (Citrullus lanatus). Korean J. Horticult. Sci. Technol. 35, 637-646.

Ci, D., Song, Y., Tian, M., Zhang, D., 2015. Methylation of miRNA genes in the response to temperature stress in Populus simonii. Front. Plant Sci. 6.

Ciarmiello, L.F., Woodrow, P., Fuggi, A., Pontecorvo, G., Carillo, P., 2011. Plant genes for abiotic stress. In: Shanker, A., Venkateswarlu, B. (Eds.), Abiotic Stress in Plants-Mechanisms and Adaptations. InTech, Rijeka, chapter 13.

Ciesla, A., Mitula, F., Misztal, L., Fedorowicz-Stronska, O., Janicka, S., Tajdel-Zielinska, M., et al., 2016. A role for barley calciumdependent protein kinase CPK2a in the response to drought. Front. Plant Sci. 7.

Clarke, S.M., Cristescu, S.M., Miersch, O., Harren, F.J.M., Wasternack, C., Mur, L.A.J., 2009. Jasmonates act with salicylic acid to confer basal thermotolerance in Arabidopsis thaliana. New Phytol. 182, 175-187.

Clasen, B.M., Stoddard, T.J., Luo, S., Demorest, Z.L., Li, J., Cedrone, F., et al., 2016. Improving cold storage and processing traits in potato through targeted gene knockout. Plant. Biotechnol. J. 14, 169-176.

Cristina, M., Petersen, M., Mundy, J., 2010. Mitogen-activated protein kinase signaling in plants. Annu. Rev. Plant. Biol. 61, 621-649.

Cui, N., Sun, X., Sun, M., Jia, B., Duanmu, H., Lv, D., et al., 2015. Overexpression of OsmiR156k leads to reduced tolerance to cold stress in rice (Oryza sativa). Mol. Breeding 35, 214.

Curtin, S.J., Voytas, D.F., Stupar, R.M., 2012. Genome engineering of crops with designer nucleases. Plant Genome J. 5, 42.

Dalal, M., Tayal, D., Chinnusamy, V., Bansal, K.C., 2009. Abiotic stress and ABA-inducible Group 4 LEA from Brassica napus plays a key role in salt and drought tolerance. J. Biotechnol. 139, $137-145$

Dametto, A., Buffon, G., dos Reis Blasi, É.A., Sperotto, R.A., 2015. Ubiquitination pathway as a target to develop abiotic stress tolerance in rice. Plant Signal. Behav. 10, e1057369.

de Pater, S., Neuteboom, L.W., Pinas, J.E., Hooykaas, P.J.J., van der Zaal, B.J., 2009. ZFN-induced mutagenesis and gene-targeting in Arabidopsis through Agrobacterium-mediated floral dip transformation. Plant. Biotechnol. J. 7, 821-835.

Ding, Y., Li, H., Zhang, X., Xie, Q., Gong, Z., Yang, S., 2015. OST1 kinase modulates freezing tolerance by enhancing ICE1 stability in Arabidopsis. Dev. Cell. 32, 278-289.

Duan, Y.-B., Li, J., Qin, R.-Y., Xu, R.-F., Li, H., Yang, Y.-C., et al., 2015. Identification of a regulatory element responsible for salt induction of rice OsRAV2 through ex situ and in situ promoter analysis. Plant Mol. Biol. 90, 49-62.

Dvořáková, L., Srba, M., Opatrny, Z., Fischer, L., 2012. Hybrid proline-rich proteins: novel players in plant cell elongation? Ann. Bot. (Lond.) 109, 453-462.

Fang, D.D., Zhou, B., Zhang, L., Ullah, A., Jin, X., Yang, X., et al., 2016. Identification of multiple stress responsive genes by sequencing a normalized cDNA library from sea-land cotton (Gossypium barbadense L.). PLoS One 11, e0152927. 
Filippou, P., Bouchagier, P., Skotti, E., Fotopoulos, V., 2014. Proline and reactive oxygen/nitrogen species metabolism is involved in the tolerant response of the invasive plant species Ailanthus altissima to drought and salinity. Environ. Exp. Bot. 97, 1-10.

Gao, J., Lan, T., 2016. Functional characterization of the late embryogenesis abundant (LEA) protein gene family from Pinus tabuliformis (Pinaceae) in Escherichia coli. Sci. Rep. 6.

Gao, S., Song, J.B., Wang, Y., Yang, Z.M., 2017. An F-box E3 ubiquitin ligase-coding gene AtDIF1 is involved in Arabidopsis salt and drought stress responses in an abscisic acid-dependent manner. Environ. Exp. Bot. 138, 21-35.

Gao, Z., He, X., Zhao, B., Zhou, C., Liang, Y., Ge, R., et al., 2010. Overexpressing a putative aquaporin gene from wheat, TaNIP, enhances salt tolerance in transgenic Arabidopsis. Plant Cell Physiol. 51, 767-775.

Ghosh, A., Kushwaha, H.R., Hasan, M.R., Pareek, A., Sopory, S.K., Singla-Pareek, S.L., 2016. Presence of unique glyoxalase III proteins in plants indicates the existence of shorter route for methylglyoxal detoxification. Sci. Rep. 6.

Gielen, H., Remans, T., Vangronsveld, J., Cuypers, A., 2012. MicroRNAs in metal stress: specific roles or secondary responses? Int. J. Mol. Sci. 13, 15826-15847.

Gill, S.S., Gill, R., Tuteja, R., Tuteja, N., 2014. Genetic engineering of crops: a ray of hope for enhanced food security. Plant Signal. Behav. 9, e28545.

Gothandam, K.M., Nalini, E., Karthikeyan, S., Shin, J.S., 2010. OsPRP3, a flower specific proline-rich protein of rice, determines extracellular matrix structure of floral organs and its overexpression confers cold-tolerance. Plant Mol. Biol. 72, 125-135.

Hajyzadeh, M., Turktas, M., Khawar, K.M., Unver, T., 2015. miR408 overexpression causes increased drought tolerance in chickpea. Gene 555, 186-193.

Hasanuzzaman, M., Nahar, K., Hossain, M., Mahmud, J., Rahman, A., Inafuku, M., et al., 2017. Coordinated actions of glyoxalase and antioxidant defense systems in conferring abiotic stress tolerance in plants. Int. J. Mol. Sci. 18, 200.

Hasanuzzaman, M., Nahar, K., Anee, T.I., Khan, M.I.R., Fujita, M., 2018. Silicon-mediated regulation of antioxidant defense and glyoxalase systems confers drought stress tolerance in Brassica napus L. South Afr. J. Bot. 115, 50-57.

Heidarvand, L., Maali Amiri, R., 2010. What happens in plant molecular responses to cold stress? Acta Physiol. Plant. 32, 419-431.

Hoang, M.H., Nguyen, X.C., Lee, K., Kwon, Y.S., Pham, H.T., Park, H.C., et al., 2012. Phosphorylation by AtMPK6 is required for the biological function of AtMYB41 in Arabidopsis. Biochem. Biophys. Res. Commun. 422, 181-186.

Hoang, T.M.L., Moghaddam, L., Williams, B., Khanna, H., Dale, J., Mundree, S.G., 2015. Development of salinity tolerance in rice by constitutive-overexpression of genes involved in the regulation of programmed cell death. Front. Plant Sci. 6, 175.

Hoque, T.S., Hossain, M.A., Mostofa, M.G., Burritt, D.J., Fujita, M., Tran, L.-S.P., 2016. Methylglyoxal: an emerging signaling molecule in plant abiotic stress responses and tolerance. Front. Plant Sci. 7.

Hossain, M.A., Piyatida, P., da Silva, J.A.T., Fujita, M., 2012. Molecular mechanism of heavy metal toxicity and tolerance in plants: central role of glutathione in detoxification of reactive oxygen species and methylglyoxal and in heavy metal chelation. J. Bot. 2012, 1-37.

Hou, H., Atlihan, N., Lu, Z.-X., 2014. New biotechnology enhances the application of cisgenesis in plant breeding. Front. Plant Sci. 5.

Hu, W., Yuan, Q., Wang, Y., Cai, R., Deng, X., Wang, J., et al., 2012. Overexpression of a wheat aquaporin gene, TaAQP8, enhances salt stress tolerance in transgenic tobacco. Plant Cell Physiol. 53, 2127-2141.
Hu, Y., Jiang, Y., Han, X., Wang, H., Pan, J., Yu, D., 2017. Jasmonate regulates leaf senescence and tolerance to cold stress: crosstalk with other phytohormones. J. Exp. Bot. 68, 1361-1369.

Huang, G., Gong, S., Xu, W., Li, P., Zhang, D., Qin, L., et al., 2011. GhHyPRP4, a cotton gene encoding putative hybrid proline-rich protein, is preferentially expressed in leaves and involved in plant response to cold stress. Acta Biochim. Biophys. Sin. (Shanghai). 43, 519-527.

Huang, H., Liu, B., Liu, L., Song, S., 2017. Jasmonate action in plant growth and development. J. Exp. Bot. 68, 1349-1359.

Huang, Q., Wang, M., Xia, Z., 2018. The SULTR gene family in maize (Zea mays L.): gene cloning and expression analyses under sulfate starvation and abiotic stress. J. Plant. Physiol. 220, 24-33.

Ijaz, R., Ejaz, J., Gao, S., Liu, T., Imtiaz, M., Ye, Z., et al., 2017. Overexpression of annexin gene AnnSp2, enhances drought and salt tolerance through modulation of ABA synthesis and scavenging ROS in tomato. Sci. Rep. 7.

Iqbal, N., Khan, N.A., Ferrante, A., Trivellini, A., Francini, A., Khan, M.I.R., 2017. Ethylene role in plant growth, development and senescence: interaction with other phytohormones. Front. Plant Sci. 8.

Ji, R., Zhou, L., Liu, J., Wang, Y., Yang, L., Zheng, Q., et al., 2017. Calcium-dependent protein kinase CPK31 interacts with arsenic transporter AtNIP1;1 and regulates arsenite uptake in Arabidopsis thaliana. PLoS One 12.

Jiang, S., Zhang, D., Wang, L., Pan, J., Liu, Y., Kong, X., et al., 2013. A maize calcium-dependent protein kinase gene, ZmCPK4, positively regulated abscisic acid signaling and enhanced drought stress tolerance in transgenic Arabidopsis. Plant. Physiol. Biochem. 71, 112-120.

Jiang, Y., Zheng, Q., Chen, L., Liang, Y., Wu, J., 2017. Ectopic overexpression of maize heat shock transcription factor gene $\mathrm{ZmHsf04}$ confers increased thermo and salt-stress tolerance in transgenic Arabidopsis. Acta Physiol. Plant. 40, 9.

Kamburova, V.S., Nikitina, E.V., Shermatov, S.E., Buriev, Z.T., Kumpatla, S.P., Emani, C., et al., 2017a. Genome editing in plants: an overview of tools and applications. Int. J. Agron. 2017, 15.

Kamburova, V.S., Nikitina, E.V., Shermatov, S.E., Buriev, Z.T., Kumpatla, S.P., Emani, C., et al., 2017b. Genome editing in plants: an overview of tools and applications. Int. J. Agron. 2017, 1-15.

Kant, P., Kant, S., Gordon, M., Shaked, R., Barak, S., 2007. Stress Response Suppressor1 and Stress Response Suppressor2, two Dead-box RNA helicases that attenuate Arabidopsis responses to multiple abiotic stresses. Plant Physiol. 145, 814-830.

Kaur, C., Ghosh, A., Pareek, A., Sopory, Sudhir, K., Singla-Pareek, et al., 2014. Glyoxalases and stress tolerance in plants. Biochem. Soc. Trans. 42, 485-490.

Khan, M.I., Khan, N.A., 2014. Ethylene reverses photosynthetic inhibition by nickel and zinc in mustard through changes in PS II activity, photosynthetic nitrogen use efficiency, and antioxidant metabolism. Protoplasma 251, 1007-1019.

Khan, M.I., Khan, N.A., Masood, A., Per, T.S., Asgher, M., 2016. Hydrogen peroxide alleviates nickel-inhibited photosynthetic responses through increase in use-efficiency of nitrogen and sulfur, and glutathione production in mustard. Front. Plant Sci. 7.

Khan, M.I.R., Iqbal, N., Masood, A., Khan, N.A., 2012. Variation in salt tolerance of wheat cultivars: role of glycinebetaine and ethylene. Pedosphere 22, 746-754.

Khan, M.I.R., Fatma, M., Per, T.S., Anjum, N.A., Khan, N.A., 2015. Salicylic acid-induced abiotic stress tolerance and underlying mechanisms in plants. Front. Plant Sci. 6, 462.

Khatodia, S., Bhatotia, K., Passricha, N., Khurana, S.M.P., Tuteja, N., 2016. The CRISPR/cas genome-editing tool: application in improvement of crops. Front. Plant Sci. 7.

Kim, H., Kim, S.-T., Kim, S.-G., Kim, J.-S., 2015a. Targeted genome editing for crop improvement. Plant Breeding Biotechnol. 3, $283-290$. 
Kim, J.-M., Sasaki, T., Ueda, M., Sako, K., Seki, M., 2015b. Chromatin changes in response to drought, salinity, heat, and cold stresses in plants. Front. Plant Sci. 6.

Kim, J.M., Woo, D.H., Kim, S.H., Lee, S.Y., Park, H.Y., Seok, H.Y., et al., 2012. Arabidopsis MKKK20 is involved in osmotic stress response via regulation of MPK6 activity. Plant Cell Rep. 31, 217-224.

Kissoudis, C., Sunarti, S., van de Wiel, C., Visser, R.G.F., van der Linden, C.G., Bai, Y., 2016. Responses to combined abiotic and biotic stress in tomato are governed by stress intensity and resistance mechanism. J. Exp. Bot. 67, 5119-5132.

Koyro, H.-W., Ahmad, P., Geissler, N., 2012. Abiotic stress responses in plants: an overview. In: Ahmad, P., Prasad, M.N.V. (Eds.), Environmental Adaptations and Stress Tolerance of Plants in the Era of Climate Change. Springer, New York, pp. 1-28.

Kumar, S., 2016. Epigenetic regulation of abiotic stress tolerance in plants. Adv. Plants Agric. Res. 5.

Kwon, K., Choi, D., Hyun, J.K., Jung, H.S., Baek, K., Park, C., 2013. Novel glyoxalases from Arabidopsis thaliana. FEBS J. 280, 3328-3339.

Lata, C., Prasad, M., 2011. Role of DREBs in regulation of abiotic stress responses in plants. J. Exp. Bot. 62, 4731-4748.

Li, J., Ban, L., Wen, H., Wang, Z., Dzyubenko, N., Chapurin, V., et al., 2015. An aquaporin protein is associated with drought stress tolerance. Biochem. Biophys. Res. Commun. 459, 208-213.

Li, J., Ouyang, B., Wang, T., Luo, Z., Yang, C., Li, H., et al., 2016 a. HyPRP1 gene suppressed by multiple stresses plays a negative role in abiotic stress tolerance in tomato. Front. Plant Sci. 7.

Li, M., Li, Y., Zhao, J., Liu, H., Jia, S., Li, J., et al., 2016b. GpDSR7, a novel E3 ubiquitin ligase gene in grimmia pilifera is involved in tolerance to drought stress in Arabidopsis. PLoS One 11.

Li, R., Wang, J., Li, S., Zhang, L., Qi, C., Weeda, S., et al., 2016c. Plasma membrane intrinsic proteins SIPIP2;1, SIPIP2;7 and SIPIP2;5 conferring enhanced drought stress tolerance in tomato. Sci. Rep. 6, 31814.

Li, W., Wang, T., Zhang, Y., Li, Y., 2016d. Overexpression of soybean miR172c confers tolerance to water deficit and salt stress, but increases ABA sensitivity in transgenic Arabidopsis thaliana. J. Exp. Bot. 67, 175-194.

Li, W.T., He, M., Wang, J., Wang, Y.P., 2013. Zinc finger protein $(\mathrm{ZFP})$ in plants-A review.

Liang, Y., Xiong, Z., Zheng, J., Xu, D., Zhu, Z., Xiang, J., et al., 2016. Genome-wide identification, structural analysis and new insights into late embryogenesis abundant (LEA) gene family formation pattern in Brassica napus. Sci. Rep. 6.

Lim, C.W., Park, C., Kim, J.-H., Joo, H., Hong, E., Lee, S.C., 2017. Pepper CaREL1, a ubiquitin E3 ligase, regulates drought tolerance via the ABA-signalling pathway. Sci. Rep. 7.

Liu, J., Zhang, C., Wei, C., Liu, X., Wang, M., Yu, F., et al., 2016a. The RING finger ubiquitin E3 ligase OsHTAS enhances heat tolerance by promoting $\mathrm{H}_{2} \mathrm{O}_{2}$-induced stomatal closure in rice. Plant Physiol. 170, 429-443.

Liu, J.-H., Peng, T., Dai, W., 2014a. Critical cis-acting elements and interacting transcription factors: key players associated with abiotic stress responses in plants. Plant. Mol. Biol. Rep. 32, 303-317.

Liu, X., Wu, S., Xu, J., Sui, C., Wei, J., 2017. Application of CRISPR/ Cas9 in plant biology. Acta Pharm. Sin. B 7, 292-302.

Liu, Y., Imai, R., 2018. Function of plant DExD/H-Box RNA helicases associated with ribosomal RNA biogenesis. Front. Plant Sci. 9, 125.

Liu, Y., Zhang, X., Zhu, S., Zhang, H., Li, Y., Zhang, T., et al., 2016b. Overexpression of GhSARP1 encoding a E3 ligase from cotton reduce the tolerance to salt in transgenic Arabidopsis. Biochem. Biophys. Res. Commun. 478, 1491-1496.

Liu, Z.-B., Wang, J.-M., Yang, F.-X., Yang, L., Yue, Y.-F., Xiang, J.-B., et al., 2014b. A novel membrane-bound E3 ubiquitin ligase enhances the thermal resistance in plants. Plant. Biotechnol. J. 12, 93-104.

Luo, M., Wang, Y.Y., Liu, X., Yang, S., Lu, Q., Cui, Y., et al., 2012. HD2C interacts with HDA6 and is involved in ABA and salt stress response in Arabidopsis. J. Exp. Bot. 63, 3297-3306.

Lyzenga, W.J., Stone, S.L., 2011. Abiotic stress tolerance mediated by protein ubiquitination. J. Exp. Bot. 63, 599-616.

Macovei, A., Vaid, N., Tula, S., Tuteja, N., 2012. A new DEAD-box helicase ATP-binding protein (OsABP) from rice is responsive to abiotic stress. Plant Signal. Behav. 7, 1138-1143.

Magwanga, R.O., Lu, P., Kirungu, J.N., Lu, H., Wang, X., Cai, X., et al., 2018. Characterization of the late embryogenesis abundant (LEA) proteins family and their role in drought stress tolerance in upland cotton. BMC Genet. 19, 6.

Mahfouz, M.M., Li, L., Shamimuzzaman, M., Wibowo, A., Fang, X., Zhu, J.K., 2011. De novo-engineered transcription activator-like effector (TALE) hybrid nuclease with novel DNA binding specificity creates double-strand breaks. Proc. Natl. Acad. Sci. U.S.A. 108, 2623-2628.

Malzahn, A., Lowder, L., Qi, Y., 2017. Plant genome editing with TALEN and CRISPR. Cell Biosci. 7.

Meena, K.K., Sorty, A.M., Bitla, U.M., Choudhary, K., Gupta, P., Pareek, A., et al., 2017. Abiotic stress responses and microbemediated mitigation in plants: the omics strategies. Front. Plant Sci. 8.

Mohanta, T.K., Bashir, T., Hashem, A., Abd Allah, E.F., 2017. Systems biology approach in plant abiotic stresses. Plant. Physiol. Biochem. 121, 58-73.

Moshelion, M., Halperin, O., Wallach, R., Oren, R.A.M., Way, D.A., 2015. Role of aquaporins in determining transpiration and photosynthesis in water-stressed plants: crop water-use efficiency, growth and yield. Plant Cell Environ. 38, 1785-1793.

Moustafa, K., 2014. MAPK transgenic circuit to improve plant stresstolerance? Plant Signal. Behav. 9, e970101.

Moustafa, K., AbuQamar, S., Jarrar, M., Al-Rajab, A.J., TrémouillauxGuiller, J., 2014. MAPK cascades and major abiotic stresses. Plant Cell Rep. 33, 1217-1225.

Nahar, K., Hasanuzzaman, M., Alam, M.M., Fujita, M., 2015. Glutathione-induced drought stress tolerance in mung bean: coordinated roles of the antioxidant defence and methylglyoxal detoxification systems. AoB Plants 7, plv069.

Newton, A.C., Johnson, S.N., Gregory, P.J., 2011. Implications of climate change for diseases, crop yields and food security. Euphytica 179, 3-18.

Ni, Z., Hu, Z., Jiang, Q., Zhang, H., 2012. Overexpression of gmaMIR394a confers tolerance to drought in transgenic Arabidopsis thaliana. Biochem. Biophys. Res. Commun. 427, 330-335.

Ning, J., Li, X., Hicks, L.M., Xiong, L., 2010. A raf-like MAPKKK gene mediates drought resistance through reactive oxygen species scavenging in rice. Plant Physiol. 152, 876.

Pandey, P., Irulappan, V., Bagavathiannan, M.V., Senthil-Kumar, M., 2017. Impact of combined abiotic and biotic stresses on plant growth and avenues for crop improvement by exploiting physiomorphological traits. Front. Plant Sci. 8, 537.

Pang, Y., Li, L., Ren, F., Lu, P., Wei, P., Cai, J., et al., 2010. Overexpression of the tonoplast aquaporin AtTIP5;1 conferred tolerance to boron toxicity in Arabidopsis. J. Genet. Genom. 37, 389-397.

Parmar, N., Singh, K.H., Sharma, D., Singh, L., Kumar, P., Nanjundan, J., et al., 2017. Genetic engineering strategies for biotic and abiotic stress tolerance and quality enhancement in horticultural crops: a comprehensive review. 3 Biotech. 7.

Pasala, R.K., Khan, M.I.R., Minhas, P., Per, T., Deokate, P.P., Nafees, A.K., et al., 2016. Can plant bioregulators minimise the crop productivity losses caused by drought, heat stress and salinity. J. Appl. Bot. Food Qual. 89, 113-125. 
Patel, M., Milla-Lewis, S., Zhang, W., Templeton, K., Reynolds, W.C., Richardson, K., et al., 2015. Overexpression of ubiquitin-like LpHUB1gene confers drought tolerance in perennial ryegrass. Plant Biotechnol. J. 13, 689-699.

Per, T.S., Khan, M.I.R., Anjum, N.A., Masood, A., Hussain, S.J., Khan, N.A., 2018. Jasmonates in plants under abiotic stresses: crosstalk with other phytohormones matters. Environ. Exp. Bot. $145,104-120$.

Petolino, J.F., 2015. Genome editing in plants via designed zinc finger nucleases. In Vitro Cell. Dev. Biol. Plant 51, 1-8.

Prasad, P.V.V., Pisipati, S.R., Momčilović, I., Ristic, Z., 2011. Independent and combined effects of high temperature and drought stress during grain filling on plant yield and chloroplast EF-Tu expression in spring wheat. J. Agron. Crop Sci. 197, 430-441.

Raikwar, S., Srivastava, V.K., Gill, S.S., Tuteja, R., Tuteja, N., 2015. Emerging importance of helicases in plant stress tolerance: characterization of Oryza sativa repair helicase XPB2 promoter and its functional validation in tobacco under multiple stresses. Front. Plant Sci. 6, 1094.

Ramesh, M., 2017. Emerging trends on abiotic stress tolerance investigation in crop plants. Adv. Biotechnol. Microbiol. 6.

Ramirez-Cabral, N.Y.Z., Kumar, L., Shabani, F., 2017. Global alterations in areas of suitability for maize production from climate change and using a mechanistic species distribution model (CLIMEX). Sci. Rep. 7, 5910.

Ray, S., Dansana, P.K., Bhaskar, A., Giri, J., Kapoor, S., Khurana, J.P., et al., 2010. Emerging Trends in Functional Genomics for Stress Tolerance in Crop Plants. Plant Stress Biology. Wiley-VCH Verlag GmbH \& Co. KGaA, pp. 37-63.

Reddy, P.S., Mallikarjuna, G., Kaul, T., Chakradhar, T., Mishra, R.N., Sopory, S.K., et al., 2010. Molecular cloning and characterization of gene encoding for cytoplasmic Hsc70 from Pennisetum glaucum may play a protective role against abiotic stresses. Mol. Genet. Genomics 283 (3), 243-254.

Reddy, P.S., Reddy, G.M., Pandey, P., Chandrasekhar, K., Reddy, M. K., 2012. Cloning and molecular characterization of a gene encoding late embryogenesis abundant protein from Pennisetum glaucum: protection against abiotic stresses. Mol. Biol. Rep. 39, 7163-7174.

Reddy, P.S., Thirulogachandar, V., Vaishnavi, C.S., Aakrati, A., Sopory, S.K., Reddy, M.K., 2011. Molecular characterization and expression of a gene encoding cytosolic Hsp90 from Pennisetum glaucum and its role in abiotic stress adaptation. Gene 474 (1), 29-38.

Rodziewicz, P., Swarcewicz, B., Chmielewska, K., Wojakowska, A., Stobiecki, M., 2013. Influence of abiotic stresses on plant proteome and metabolome changes. Acta Physiol. Plant. 36, 1-19.

Sahoo, R.K., Gill, S.S., Tuteja, N., 2012. Pea DNA helicase 45 promotes salinity stress tolerance in IR64 rice with improved yield. Plant Signal. Behav. 7, 1042-1046.

Sanan-Mishra, N., Pham, X.H., Sopory, S.K., Tuteja, N., 2005. Pea DNA helicase 45 overexpression in tobacco confers high salinity tolerance without affecting yield. Proc. Natl. Acad. Sci. U.S.A. 102, 509.

Sanchez, D.H., Pieckenstain, F.L., Szymanski, J., Erban, A., Bromke, M., Hannah, M.A., et al., 2011. Comparative functional genomics of salt stress in related model and cultivated plants identifies and overcomes limitations to translational genomics. PLoS One 6, e17094.

Santosh Rama Bhadra Rao, T., Vijaya Naresh, J., Sudhakar Reddy, P., Reddy, M.K., Mallikarjuna, G., 2017. Expression of pennisetum glaucum eukaryotic translational initiation factor 4A (PgeIF4A) confers improved drought, salinity, and oxidative stress tolerance in groundnut. Front. Plant Sci. 8, 453.

Saucedo, A.L., Hernández-Domínguez, E.E., de Luna-Valdez, L.A., Guevara-García, A.A., Escobedo-Moratilla, A., BojorquézVelázquez, E., et al., 2017. Insights on structure and function of a late embryogenesis abundant protein from amaranthus cruentus: an intrinsically disordered protein involved in protection against desiccation, oxidant conditions, and osmotic stress. Front. Plant Sci. 8, 497.

Savvides, A., Ali, S., Tester, M., Fotopoulos, V., 2016. Chemical priming of plants against multiple abiotic stresses: mission possible? Trends. Plant. Sci. 21, 329-340.

Scott, K.C., Roy, D., Paul, A., Roy, A., Ghosh, R., Ganguly, P., et al., 2014. Differential acetylation of histone H3 at the regulatory region of OsDREB1b promoter facilitates chromatin remodelling and transcription activation during cold stress. PLoS One 9, e100343.

Shan, Q., Wang, Y., Chen, K., Liang, Z., Li, J., Zhang, Y., et al., 2013. Rapid and efficient gene modification in rice and brachypodium using TALENs. Mol. Plant 6, 1365-1368.

Shao, H., Wang, H., Tang, X., 2015. NAC transcription factors in plant multiple abiotic stress responses: progress and prospects. Front. Plant Sci. 6.

Sharma, B., Joshi, D., Yadav, P.K., Gupta, A.K., Bhatt, T.K., 2016. Role of ubiquitin-mediated degradation system in plant biology. Front. Plant Sci. 7.

Sharma, S., Mustafiz, A., Singla-Pareek, S.L., Shankar Srivastava, P., Sopory, S.K., 2012. Characterization of stress and methylglyoxal inducible triose phosphate isomerase (OscTPI) from rice. Plant Signal. Behav. 7, 1337-1345.

Shen, C., Que, Z., Xia, Y., Tang, N., Li, D., He, R., et al., 2017. Knock out of the annexin gene OsAnn3 via CRISPR/Cas9-mediated genome editing decreased cold tolerance in rice. J. Plant Biol. 60, 539-547.

Shi, J., Gao, H., Wang, H., Lafitte, H.R., Archibald, R.L., Yang, M., et al., 2017. ARGOS8 variants generated by CRISPR-Cas9 improve maize under field drought stress conditions. Plant. Biotechnol. J. 15, 207-216.

Shivakumara, T.N., Sreevathsa, R., Dash, P.K., Sheshshayee, M.S., Papolu, P.K., Rao, U., et al., 2017. Overexpression of Pea DNA Helicase 45 (PDH45) imparts tolerance to multiple abiotic stresses in chili (Capsicum annuum L.). Sci. Rep. 7, 2760.

Shriram, V., Kumar, V., Devarumath, R.M., Khare, T.S., Wani, S.H., 2016. MicroRNAs as potential targets for abiotic stress tolerance in plants. Front. Plant Sci. 7.

Singh, A., Sagar, S., Biswas, D.K., 2018. Calcium dependent protein kinase, a versatile player in plant stress management and development. CRC. Crit. Rev. Plant. Sci. 1-17.

Singh, H.P., Singh, B.P., 2014. Genetic engineering of field, industrial and pharmaceutical crops. Am. J. Plant Sci. 05, 3974-3993.

Singh, J., Reddy, P.S., Reddy, C.S., Reddy, M.K., 2015. Molecular cloning and characterization of salt inducible dehydrin gene from the C4 plant Pennisetum glaucum. Plant Gene 4, 55-63.

Singla-Pareek, S.L., Reddy, M.K., Sopory, S.K., 2003. Genetic engineering of the glyoxalase pathway in tobacco leads to enhanced salinity tolerance. Proc. Natl. Acad. Sci. U.S.A. 100, 14672-14677.

Song, G., Jia, M., Chen, K., Kong, X., Khattak, B., Xie, C., et al., 2016. CRISPR/Cas9: a powerful tool for crop genome editing. Crop J. 4, $75-82$.

Song, Y., Ji, D., Li, S., Wang, P., Li, Q., Xiang, F., 2012. The dynamic changes of DNA methylation and histone modifications of salt responsive transcription factor genes in soybean. PLoS One 7, 18.

Srivastava, A.K., Penna, S., Nguyen, D.V., Tran, L.-S.P., 2014. Multifaceted roles of aquaporins as molecular conduits in plant responses to abiotic stresses. Crit. Rev. Biotechnol. 1-10.

Stone, S.L., 2014. The role of ubiquitin and the 26 S proteasome in plant abiotic stress signaling. Front. Plant Sci. 5.

Šurbanovski, N., Grant, O.M., 2014. Chapter 18-The Emerging Role of Aquaporins in Plant Tolerance of Abiotic Stress. Emerging Technologies and Management of Crop Stress Tolerance. Academic Press, San Diego, pp. 431-447.

Suzuki, N., Rivero, R.M., Shulaev, V., Blumwald, E., Mittler, R., 2014. Abiotic and biotic stress combinations. New Phytol. 203, 32-43.

Takeda, S., Matsuoka, M., 2008. Genetic approaches to crop improvement: responding to environmental and population changes. Nat. Rev. Genet. 9, 444-457. 
Tang, R.J., Luan, S., 2017. Regulation of calcium and magnesium homeostasis in plants: from transporters to signaling network. Curr. Opin. Plant. Biol. 39, 97-105.

Tang, X., Wang, H., Chu, L., Shao, H., 2016. KvLEA, a new isolated late embryogenesis abundant protein gene from Kosteletzkya virginica responding to multiabiotic stresses. Biomed. Res. Int. 2016, $1-10$.

Tang, X., Lowder, L.G., Zhang, T., Malzahn, A.A., Zheng, X., Voytas, D.F., et al., 2017. A CRISPR-Cpf1 system for efficient genome editing and transcriptional repression in plants. Nat. Plants 3, 17018.

To, T.K., Kim, J.M., Matsui, A., Kurihara, Y., Morosawa, T., Ishida, J., et al., 2011. Arabidopsis HDA6 regulates locus-directed heterochromatin silencing in cooperation with MET1. PLoS Genet. $7,28$.

Tripathi, A., Tripathi, D.K., Chauhan, D.K., Kumar, N., Singh, G.S., 2016. Paradigms of climate change impacts on some major food sources of the world: a review on current knowledge and future prospects. Agric. Ecosyst. Environ. 216, 356-373.

Tuteja, N., Singh, S., Tuteja, R., 2012. Helicases in Improving Abiotic Stress Tolerance in Crop Plants. Improving Crop Resistance to Abiotic Stress. Wiley-VCH Verlag GmbH \& Co. KGaA, Weinheim, pp. 435-449.

Tuteja, N., Banu, M.S., Huda, K.M., Gill, S.S., Jain, P., Pham, X.H., et al., 2014. Peap68, a DEAD-box helicase, provides salinity stress tolerance in transgenic tobacco by reducing oxidative stress and improving photosynthesis machinery. PLoS One 9.

Verma, V., Ravindran, P., Kumar, P.P., 2016. Plant hormonemediated regulation of stress responses. BMC Plant Biol. 16.

Verwaal, R., Buiting-Wiessenhaan, N., Dalhuijsen, S., Roubos, J.A., 2017. CRISPR/Cpf1 enables fast and simple genome editing of Saccharomyces cerevisiae. Yeast 35 (2), 201-211.

Wang, C.-T., Song, W., 2013. Calcium-dependent protein kinase gene ZmCPK12 from maize confers tolerance to drought and salt stresses in transgenic plants. Acta Physiol. Plant. 35, 1659-1666.

Wang, C.-T., Song, W., 2014. ZmCK3, a maize calcium-dependent protein kinase gene, endows tolerance to drought and heat stresses in transgenic Arabidopsis. J. Plant Biochem. Biotechnol. 23, 249-256.

Wang, L., Chen, L., Li, R., Zhao, R., Yang, M., Sheng, J., et al., 2017a. Reduced drought tolerance by CRISPR/Cas9-mediated SIMAPK3 mutagenesis in tomato plants. J. Agric. Food. Chem. 65, $8674-8682$.

Wang, L., Liu, Y., Feng, S., Yang, J., Li, D., Zhang, J., 2017b. Roles of plasmalemma aquaporin gene stpip1 in enhancing drought tolerance in potato. Front. Plant Sci. 8, 616.

Wang, W., Vinocur, B., Shoseyov, O., Altman, A., 2004. Role of plant heat-shock proteins and molecular chaperones in the abiotic stress response. Trends. Plant. Sci. 9, 244-252.

Wei, S., Hu, W., Deng, X., Zhang, Y., Liu, X., Zhao, X., et al., 2014. A rice calcium-dependent protein kinase OsCPK9 positively regulates drought stress tolerance and spikelet fertility. BMC Plant Biol. 14, 133 .

Xie, G., Kato, H., Imai, R., 2012. Biochemical identification of the OsMKK6-OsMPK3 signalling pathway for chilling stress tolerance in rice. Biochem. J. 443, 95-102.

Xu, C., Wang, M., Zhou, L., Quan, T., Xia, G., 2013a. Heterologous expression of the wheat aquaporin gene TaTIP2;2 compromises the abiotic stress tolerance of Arabidopsis thaliana. PLoS One 8, e79618.

Xu, F., Liu, Z., Xie, H., Zhu, J., Zhang, J., Kraus, J., et al., 2014. Increased drought tolerance through the suppression of ESKMO1 gene and overexpression of CBF-related genes in Arabidopsis. PLoS One 9.
Xu, J., Chua, N.H., 2012. Dehydration stress activates Arabidopsis MPK6 to signal DCP1 phosphorylation. EMBO J. 31, 1975-1984.

Xu, J., Xue, C., Xue, D., Zhao, J., Gai, J., Guo, N., et al., 2013b. Overexpression of GmHsp90s, a heat shock protein 90 (Hsp90) gene family cloning from soybean, decrease damage of abiotic stresses in Arabidopsis thaliana. PLoS One 8.

Xu, R., Qin, R., Li, H., Li, D., Li, L., Wei, P., et al., 2017. Generation of targeted mutant rice using a CRISPR-Cpf1 system. Plant. Biotechnol. J. 15, 713-717.

Yaish, M.W., 2017. Editorial: epigenetic modifications associated with abiotic and biotic stresses in plants: an implication for understanding plant evolution. Front. Plant Sci. 8, 1983.

Yang, C., Li, D., Mao, D., Liu, X.U.E., Ji, C., Li, X., et al., 2013. Overexpression of microRNA319 impacts leaf morphogenesis and leads to enhanced cold tolerance in rice (Oryza sativa L.). Plant Cell Environ. 36, 2207-2218.

Yang, L., Wu, L., Chang, W., Li, Z., Miao, M., Li, Y., et al., 2018. Overexpression of the maize E3 ubiquitin ligase gene ZmAIRP4 enhances drought stress tolerance in Arabidopsis. Plant. Physiol. Biochem. 123, 34-42.

Yue, E., Liu, Z., Li, C., Li, Y., Liu, Q., Xu, J.-H., 2017. Overexpression of miR529a confers enhanced resistance to oxidative stress in rice (Oryza sativa L.). Plant Cell Rep. 36, 1171-1182.

Zaidi, S.S.-E.-A., Mahfouz, M.M., Mansoor, S., 2017. CRISPR-Cpf1: a new tool for plant genome editing. Trends. Plant. Sci. 22, $550-553$.

Zandalinas, S.I., Mittler, R., Balfagon, D., Arbona, V., GomezCadenas, A., 2018. Plant adaptations to the combination of drought and high temperatures. Physiol. Plant. 162, 2-12.

Zetsche, B., Heidenreich, M., Mohanraju, P., Fedorova, I., Kneppers, J., DeGennaro, E.M., et al., 2017. Multiplex gene editing by CRISPRCpf1 using a single crRNA array. Nat. Biotechnol. 35, 31-34.

Zhang, H., Cui, F., Wu, Y., Lou, L., Liu, L., Tian, M., et al., 2015. The RING finger ubiquitin E3 ligase SDIR1 targets SDIR1INTERACTING PROTEIN1 for degradation to modulate the salt stress response and ABA signaling in Arabidopsis. Plant Cell 27, 214-227.

Zhang, H., Gou, F., Zhang, J., Liu, W., Li, Q., Mao, Y., et al., 2016. TALEN-mediated targeted mutagenesis produces a large variety of heritable mutations in rice. Plant. Biotechnol. J. 14, 186-194.

Zhang, L., Xi, D., Li, S., Gao, Z., Zhao, S., Shi, J., et al., 2011. A cotton group C MAP kinase gene, GhMPK2, positively regulates salt and drought tolerance in tobacco. Plant Mol. Biol. 77, 17-31.

Zhang, N., Yin, Y., Liu, X., Tong, S., Xing, J., Zhang, Y., et al., 2017. The E3 ligase TaSAP5 alters drought stress responses by promoting the degradation of DRIP proteins. Plant Physiol. 175, 1878-1892.

Zhao, H., Wolt, J.D., 2017. Risk associated with off-target plant genome editing and methods for its limitation. Emerg. Top. Life Sci. 1, 231.

Zhao, Y., Dong, W., Zhang, N., Ai, X., Wang, M., Huang, Z., et al., 2013. A wheat allene oxide cyclase gene enhances salinity tolerance via jasmonate signaling. Plant Physiol. 164, 1068-1076.

Zhou, Y., He, P., Xu, Y., Liu, Q., Yang, Y., Liu, S., 2017. Overexpression of CsLEA11, a Y3SK2-type dehydrin gene from cucumber (Cucumis sativus), enhances tolerance to heat and cold in Escherichia coli. AMB Express 7, 017-0483.

Zhu, M., Chen, G., Dong, T., Wang, L., Zhang, J., Zhao, Z., et al., 2015. SIDEAD31, a putative DEAD-Box RNA helicase gene, regulates salt and drought tolerance and stress-related genes in tomato. PLoS One 10.

Zhu, W.-G., Zhao, L., Wang, P., Hou, H., Zhang, H., Wang, Y., et al., 2014. Transcriptional regulation of cell cycle genes in response to abiotic stresses correlates with dynamic changes in histone modifications in Maize. PLoS One 9, e106070. 


\section{Measurement of Signaling Molecules Calcium Ion, Reactive Sulfur Species, Reactive Carbonyl Species, Reactive Nitrogen Species, and Reactive Oxygen Species in Plants Zhong-Guang $\mathrm{Li}^{1,2,3}$}

${ }^{1}$ School of Life Sciences, Yunnan Normal University, Kunming, P.R. China ${ }^{2}$ Engineering Research Center of

Sustainable Development and Utilization of Biomass Energy, Ministry of Education, Kunming, P.R. China

${ }^{3}$ Key Laboratory of Biomass Energy and Environmental Biotechnology, Yunnan Province, Yunnan Normal University, Kunming, P.R. China

\section{O U T L I N E}

\subsection{Introduction}

5.2 Section 1: $\mathrm{Ca}^{2+}$ Quantification 5.2.1 Method 1: MTB Method

5.2.2 Method 2: Fura-2 AM Fluorescence Method

5.3 Section 2: $\mathrm{H}_{2} \mathrm{~S}$ Quantification 5.3.1 Method 1: DTNB Method

5.3.2 Method 2: MB Method

5.3.3 Method 3: WSP-1 Fluorescence Method

5.4 Section 3: Methylglyoxal Quantification

5.4.1 Method 1: DAB Method

5.4.2 Method 2: DNP Method

5.4.3 Method 3: NAC Method

5.4.4 Method 4: DAF-2 and DAR-1 Fluorescence

Methods

5.5 Section 4: NO Quantification 5.5.1 Method 1: Griess Reagent Method
84

84

84

85

85

85

86

87

87

87

88

89

\section{9}

90

90
5.5.2 Method 2: DAF-FMDA Fluorescence Method

5.6 Section 5: $\mathrm{H}_{2} \mathrm{O}_{2}$ Quantification 91

5.6.1 Method 1: KI Method 91

5.6.2 Method 2: $\mathrm{Ti}\left(\mathrm{SO}_{4}\right)_{2}$ Method 92

5.6.3 Method 3: XO Method 93

5.6.4 Method 4: ABTS Method 93

5.6.5 Method 5: DCHBS-AAP Method 94

5.6.6 Method 6: DAB Method 95

5.6.7 Method 7: $\mathrm{DCFH}_{2}$-DA and DCF

Fluorescence Methods 95

5.6.8 Method 8: AR and AUR Fluorescence Methods 97

5.6.9 Method 9: BES- $\mathrm{H}_{2} \mathrm{O}_{2}$-Ac and BES- $\mathrm{H}_{2} \mathrm{O}_{2}$ Fluorescence Method

5.7 Section 6: Superoxide Radical Measurement $\quad 98$

5.7.1 Method 1: NBT Method 98

5.7.2 Method 2: XTT Method 99 
5.7.3 Method 3: BESSo-AM and BESSo Fluorescence Methods

5.8 Section 7: HO• Quantification

5.8.1 Method 1: Benzoate Method

5.8.2 Method 2: 2-deoxy-D-Ribose Method
Acknowledgments

References

101

101

Further Reading

\subsection{INTRODUCTION}

Plants commonly perceive, respond, and transform environmental signals by many second messengers, and finally adapt to changing environments (Tardieu and Tuberosa, 2010; Zhu, 2016; Jeandroz and Lamotte et al., 2017). These signaling molecules include calcium messenger systems (mainly calcium ion: $\mathrm{Ca}^{2+}$ ), reactive sulfur species (RSS, mainly hydrogen sulfide: $\mathrm{H}_{2} \mathrm{~S}$ ), reactive carboxyl species (RCS, mainly methylglyoxal: $\mathrm{MG}$ ), reactive nitrogen species (RNS, mainly nitric oxide: $\mathrm{NO}$ ), and reactive oxygen species (ROS, mainly superoxide radical: $\mathrm{O}_{2} \bullet^{-}$; hydrogen peroxide: $\mathrm{H}_{2} \mathrm{O}_{2}$; and hydroxyl radical: $\bullet \mathrm{OH}$ ) (Gill and Tuteja et al., 2010; Li, 2016; Campbell, 2018; Sami et al., 2018). Plants have developed multiple biosynthetic pathways (such as $\mathrm{H}_{2} \mathrm{~S}, \mathrm{MG}, \mathrm{NO}$, and $\mathrm{H}_{2} \mathrm{O}_{2}$ ) or stored pools (such as $\mathrm{Ca}^{2+}$ ) of second messengers and their scavenging mechanisms (Gill and Tuteja et al., 2010; Li, 2016; Campbell, 2018; Sami et al., 2018). These signaling molecules can be rapidly produced when needed, while expediently and efficiently eliminated when not needed by plants (Neill et al., 2002; Dodd et al., 2010; Li et al., 2016; da-Silva and Modolo, 2018). In addition, intracellular and extracellular cues can rapidly trigger an increase in second messengers simultaneously or successively in cytoplasm or other organelles (Neill et al., 2002; Dodd et al., 2010; Li et al., 2016). Therefore, signaling molecules $\left(\mathrm{Ca}^{2+}, \mathrm{H}_{2} \mathrm{~S}, \mathrm{MG}\right.$, $\mathrm{NO}$, and $\mathrm{H}_{2} \mathrm{O}_{2}$ ) play a crucial role in plant metabolism, growth, development, and response to environmental stress including abiotic and biotic stresses. Nowadays, messenger transportation and signal transduction are research hotspots in the field of plant biology, especially in plant stress biology, that are receiving extensive attention. Additionally, qualitative and quantitative analysis of signaling molecules, especially imaging in living cells, is an important fundamental for investigating signaling molecules in plants. In this chapter, based on the current protocols (Mustafiz et al., 2010; Rodriguez and Taleisnik, 2012; Xie and Shen, 2012; Li and Gong, 2014; Li, 2015; Angelini et al., 2018; Antoniou et al., 2018; Campbell, 2018; Park and Roubelakis-Angelakis, 2018) and research papers (Schopfer et al., 2001; Gay and Gebicki, 2000, 2003; Fraisse et al., 2002; Yu et al., 2003; Ashtamker et al., 2007; Maeda et al., 2007; Liu et al., 2011; Wild et al., 2012; Shaheen et al., 2014; Qian et al., 2015), in vivo (including living cell imaging) and in vitro methods, namely histochemical staining method, fluorometry, and spectrophotometry, for determination of $\mathrm{Ca}^{2+}$, $\mathrm{H}_{2} \mathrm{~S}, \mathrm{MG}, \mathrm{NO}, \mathrm{O}_{2} \bullet^{-}, \mathrm{H}_{2} \mathrm{O}_{2}$, and $\bullet \mathrm{OH}$, were investigated. Its aim is to arouse the research and development of signaling molecules in the field of plant biology, especially plant stress biology.

\subsection{SECTION 1: $\mathrm{CA}^{2+}$ QUANTIFICATION}

\subsubsection{Method 1: MTB Method}

\section{Principle}

Free calcium ion $\left(\mathrm{Ca}^{2+}\right)$ can rapidly react with methylthymol blue (MTB) in alkaline conditions and produces a blue dye-Ca ${ }^{2+}$ complex. The complex has a maximal absorbance at $610 \mathrm{~nm}$ (Fig. 5.1; Qian et al., 2015). The light absorption is proportional to the content of $\mathrm{Ca}^{2+}$ in plant samples. Therefore, free $\mathrm{Ca}^{2+}$ content can be measured by spectrophotometry at $610 \mathrm{~nm}$.<smiles>O=S1(=O)OC(P)(P)c2ccccc21</smiles><smiles>[B]c1cc(C(C)C)c(O)c(CN(CC(=O)O[Na])C(=O)O[Na])c1C</smiles>

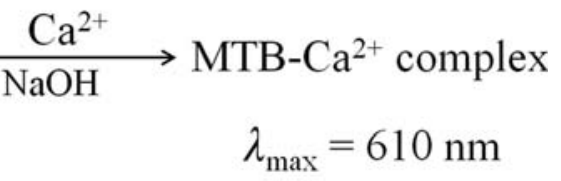

FIGURE 5.1 $\mathrm{Ca}^{2+}$ reacts with MTB under alkaline conditions.

\section{MTB}




\section{Materials}

2.1. $100 \mathrm{~mL}$ of MTB solution: $100 \mathrm{~mL}$ of $0.1 \mathrm{~mol} / \mathrm{L}$ $\mathrm{HCl}$ (mix $0.84 \mathrm{~mL}$ concentrated $\mathrm{HCl}$ with $99.16 \mathrm{~mL}$ of deionized water) containing $0.25 \mathrm{mM}$ MTB (21.1 mg, MW $=844.74)$ and $0.6 \%$ polyvinyl pyrrolidone (PVP, $0.6 \mathrm{~g}$ ).

2.2. $100 \mathrm{~mL}$ of alkaline solution: $100 \mathrm{~mL}$ of solution containing $0.2 \mathrm{M} \mathrm{Na}_{2} \mathrm{SO}_{3}$ (2.53 g, $\left.\mathrm{MW}=126.04\right)$, $0.1 \%(\mathrm{w} / \mathrm{v})$ glycine $(0.1 \mathrm{~g})$, and $0.23 \mathrm{M} \mathrm{NaOH}$ $(0.92 \mathrm{~g}, \mathrm{MW}=40)$.

2.3. Spectrophotometer or microplate reader.

\section{Methods}

3.1. Plant tissue roots or leaves $(0.1 \mathrm{~g} \mathrm{FW})$ are homogenized in $1 \mathrm{~mL}$ deionized water with mortar and pestle.

3.2. Centrifuged at $12,000 \times \mathrm{g}$ for $10 \mathrm{~min}$ at $4^{\circ} \mathrm{C}$, and the supernatant was collected and used for $\mathrm{Ca}^{2+}$ assay.

3.3. Add $50 \mu \mathrm{L}$ of supernatant and $1 \mathrm{~mL}$ of MTB solution to $2 \mathrm{~mL}$ alkaline solution.

3.4. Read absorbance at $610 \mathrm{~nm}$.

3.5. Calculate the amount of $\mathrm{Ca}^{2+}$ from a standard curve prepared using dilutions of a $2.5 \mathrm{mM} \mathrm{Ca}^{2+}$ stock solution and expressed as $\mu \mathrm{mol} / \mathrm{g}$ FW.

\section{Precaution}

Alkaline solution should be freshly prepared.

\subsubsection{Method 2: Fura-2 AM Fluorescence Method}

\section{Principle}

Fluorescent probes Fura-2 AM and Fura-3 AM are not able to bind $\mathrm{Ca}^{2+}$. When they enter freely into the cytosol, Fura-2 AM and Fura-3 AM are converted by esterase into Fura-2 and Fura-3, respectively, in the cytosol. Both can rapidly bind to $\mathrm{Ca}^{2+}$ and produce a green fluorescent substance (Fig. 5.2; Campbell, 2018). The intensity of fluorescence is proportional to the content of $\mathrm{Ca}^{2+}$ in plant samples. The fluorescence can be visualized by fluorescence microscope with excitation at $506 \mathrm{~nm}$ and emission at $525 \mathrm{~nm}$.

\section{Materials}

2.1. $100 \mathrm{~mL}$ of $100 \mathrm{mM}$ Tris- $\mathrm{HCl}$ ( $\mathrm{pH}$ 8.0): Add $50 \mathrm{~mL}$ of $200 \mathrm{mM}$ Tris $(2.42 \mathrm{~g} / 100 \mathrm{~mL})$ to $26.8 \mathrm{~mL}$ of $200 \mathrm{mM} \mathrm{HCl}(1.68 \mathrm{~mL}$ concentrated $\mathrm{HCl} / 100 \mathrm{~mL}$ ). Mix and make up to $100 \mathrm{~mL}$ with deionized water.

2.2. $5 \mu \mathrm{M}$ of Fluo-2 AM: Dissolve $1 \mathrm{mg}$ Fluo-2 AM (MW $=1001.85$ ) in $1 \mathrm{~mL}$ of dimethylsulfoxide (DMSO) to obtain $1 \mathrm{mM}$ Fluo-2 AM. Dilute to final concentration with $100 \mathrm{mM}$ Tris- $\mathrm{HCl}(\mathrm{pH}$ 8.0).

2.3. Fluorescence microscope.

\section{Methods}

3.1. Plant tissues (tissues or cells) are incubated in $100 \mathrm{mM}$ Tris- $\mathrm{HCl}$ (pH 8.0) buffer containing $15 \mu \mathrm{M}$ Fluo-2AM in darkness at $25^{\circ} \mathrm{C}$ for $30 \mathrm{~min}$.

3.2. The fluorescent image was captured using a fluorescence microscope with 506/525 nm (excitation/emission) filter set.

3.3. Take image of tissues or cells using fluorescent microscope.

\section{Precautions}

4.1. Fluo-2 AM should be freshly prepared or stored at $-20^{\circ} \mathrm{C}$ after subpackage of stock solution of $1 \mathrm{mM}$.

4.2. Inverted fluorescence microscope is used for tissues, tissue slices, and cells, while laser confocal fluorescence microscopy for tissues and organs such as roots and leaves.

\subsection{SECTION 2: $\mathrm{H}_{2} \mathrm{~S}$ QUANTIFICATION}

\subsubsection{Method 1: DTNB Method}

\section{Principle}

Under neutral conditions, $\mathrm{H}_{2} \mathrm{~S}$ can react with 5,5'dithiobis(2-nitrobenzoic acid) (DTNB; Ellman's Reagent) to produce a yellow product 5-thio-2nitrobenzoic acid (TNB). The TNB exhibits a maximal light absorption at $412 \mathrm{~nm}$ and the molar extinction coefficient is $1.4 \times 10^{4} \mathrm{M}^{-1} \mathrm{~cm}^{-1}$

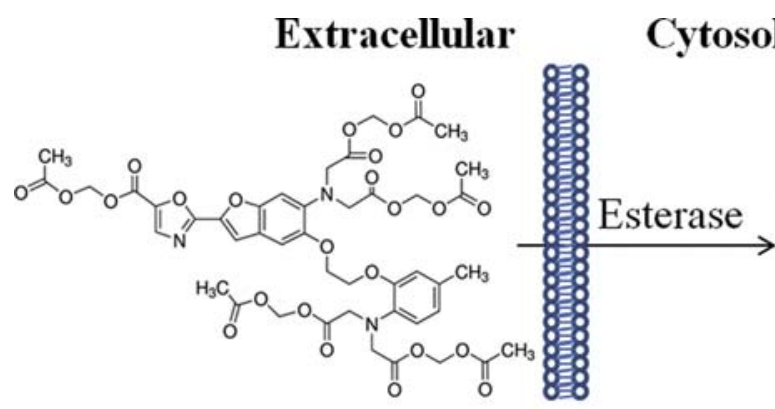

Fura-2 AM

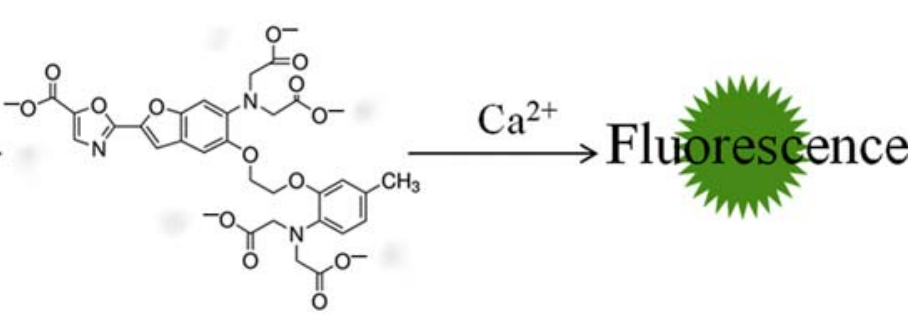

Fura-2 $\left(\lambda_{\text {ex } 506} / \lambda_{\text {em525 nm }}\right)$

FIGURE 5.2 Fura-2 AM, which can freely enter into cytosol, is deacetylated by intracellular esterase to generate Fura-2, which can react with $\mathrm{NO}$ to generate fluorescence. 
DTNB

FIGURE $5.3 \quad \mathrm{H}_{2} \mathrm{~S}$ reacts with DTNB under neutral conditions.

(Fig. 5.3; Li, 2015; Antoniou et al., 2018). The absorbance is in direct proportion to the content of $\mathrm{H}_{2} \mathrm{~S}$ in plant samples. So, $\mathrm{H}_{2} \mathrm{~S}$ content in plant samples can be determined by spectrophotometry.

\section{Materials}

2.1. $100 \mathrm{~mL}$ of extraction buffer: $100 \mathrm{~mL}$ of $100 \mathrm{mM}$ potassium phosphate buffer ( $\mathrm{pH}$ 7.0) containing $10 \mathrm{mM}$ ethylenediaminetetraacetic acid (EDTA, $\mathrm{MW}=292.2$ ). Mix $6.15 \mathrm{~mL}$ of $1 \mathrm{M} \mathrm{K}_{2} \mathrm{HPO}_{4}$ $(17.418 \mathrm{~g} / 100 \mathrm{~mL})$ with $3.85 \mathrm{~mL}$ of $1 \mathrm{M} \mathrm{KH}_{2} \mathrm{PO}_{4}$ $(13.609 \mathrm{~g} / 100 \mathrm{~mL})$, add deionized water to reach a final volume of $100 \mathrm{~mL}$. Then dissolve $0.292 \mathrm{~g}$ EDTA in K-phosphate buffer.

2.2. $20 \mathrm{mM}$ Ellman's reagent: Namely $20 \mathrm{mM}$ DTNB: Dissolve 39.6 mg DTNB (MW = 396.35) in $5 \mathrm{~mL}$ of extraction buffer (see note).

2.3. Spectrophotometer or microplate reader.

\section{Methods}

3.1. Plant roots or leaves $(100 \mathrm{mg})$ are homogenized in liquid nitrogen with mortar and pestle and then add $1 \mathrm{~mL}$ of extraction buffer.

3.2. Centrifuge at $10,000 \times \mathrm{g}$ for $15 \mathrm{~min}$ at $4{ }^{\circ} \mathrm{C}$.

3.3. Add $1880 \mu \mathrm{L}$ of extraction buffer into $100 \mu \mathrm{L}$ of supernatant.

3.4. Add $20 \mu \mathrm{L}$ of $20 \mathrm{mM}$ DTNB (add $1980 \mu \mathrm{L}$ of extraction buffer to $20 \mu \mathrm{L}$ of $20 \mathrm{mM}$ DTNB as the blank).

3.5. Incubate at room temperature for $2 \mathrm{~min}$.

3.6. Transfer $300 \mu \mathrm{L}$ of the reaction mixture to 96 well plate and read absorbance at $412 \mathrm{~nm}$ in the plate reader spectrometer, or read absorbance using spectrophotometer.

3.7. A standard curve is performed with known concentrations of a sodium hydrosulfide hydrate (NaHS, $\mathrm{H}_{2} \mathrm{~S}$ donor) ranging from 0 to $100 \mu \mathrm{M}$.

3.8. The $\mathrm{H}_{2} \mathrm{~S}$ content is calculated using standard curve or the molar extinction coefficient of 1.4 $\times 10^{4} \mathrm{M}^{-1} \mathrm{~cm}^{-1}$ for TNB and expressed in $\mu \mathrm{mol} / \mathrm{g} \mathrm{FW}$.

\section{Precaution}

DTNB should be stored in the dark between $0^{\circ} \mathrm{C}$ and $5^{\circ} \mathrm{C}$ in where it can be stable for 6 months.

\subsubsection{Method 2: MB Method}

\section{Principle}

$\mathrm{H}_{2} \mathrm{~S}$ can react with $N, N$-dimethyl-pphenylenediaminedihydrochloride (DMPD) under

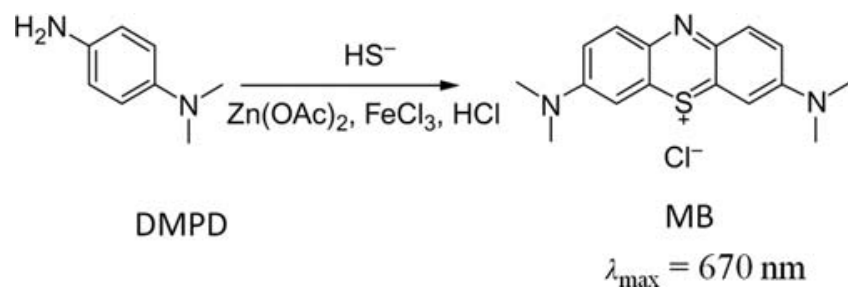

FIGURE 5.4 $\mathrm{H}_{2} \mathrm{~S}$ reacts with DMPD under acidic conditions.

acidic conditions and generates a blue product methylene blue (MB). The MB has a maximal light absorption at $670 \mathrm{~nm}$ and the extinction coefficient of $1.5 \times 10^{7} \mathrm{M}^{-1} \mathrm{~cm}^{-1}$ (Fig. 5.4; Li, 2015). A positive correlation between the light absorption $\left(\mathrm{A}_{670}\right)$ and the $\mathrm{H}_{2} \mathrm{~S}$ content in plant samples can be noted.

\section{Materials}

2.1. $100 \mathrm{mM}$ sodium phosphate buffer ( $\mathrm{pH}$ 7.0): Mix $61 \mathrm{~mL}$ of $200 \mathrm{mM} \mathrm{Na}_{2} \mathrm{HPO}_{4}(53.65 \mathrm{~g}$

$\mathrm{Na}_{2} \mathrm{HPO}_{4} \cdot 7 \mathrm{H}_{2} \mathrm{O}$ or $71.64 \mathrm{~g} \mathrm{Na} \mathrm{HPO}_{4} \cdot 12 \mathrm{H}_{2} \mathrm{O} /$

$1000 \mathrm{~mL}$ ) with $39 \mathrm{~mL}$ of $200 \mathrm{mM} \mathrm{NaH}{ }_{2} \mathrm{PO}_{4}$ (27.6 $\mathrm{g} \mathrm{NaH}_{2} \mathrm{PO}_{4} \cdot \mathrm{H}_{2} \mathrm{O}$ or $31.2 \mathrm{~g}$

$\mathrm{NaH}_{2} \mathrm{PO}_{4} \cdot 2 \mathrm{H}_{2} \mathrm{O} / 1000 \mathrm{~mL}$ ). Mix and make up to $200 \mathrm{~mL}$ with deionized water.

2.2. $100 \mathrm{~mL}$ of extraction buffer: $100 \mathrm{~mL}$ of $100 \mathrm{mM}$ sodium phosphate buffer ( $\mathrm{pH} 7.0$ ) containing $10 \mathrm{mM}$ EDTA (0.292 g, MW $=292.2)$ and $20 \mathrm{mM}$ $\mathrm{Zn}(\mathrm{OAc})_{2} \cdot 2 \mathrm{H}_{2} \mathrm{O}(0.439 \mathrm{~g}, \mathrm{MW}=219.51)$.

2.3. $100 \mathrm{~mL}$ of $30 \mathrm{mM} \mathrm{FeCl}_{3}$ : Dissolve $0.487 \mathrm{~g} \mathrm{FeCl}_{3}$ $(\mathrm{MW}=162.2)$ in $1.2 \mathrm{M} \mathrm{HCl}(10.08 \mathrm{~mL}$ concentrated $\mathrm{HCl} / 100 \mathrm{~mL}$ ).

2.4. $100 \mathrm{~mL}$ of $20 \mathrm{mM}$ DMPD: Dissolve $0.418 \mathrm{~g}$ DMPD (MW $=209.12)$ in $7.2 \mathrm{M} \mathrm{HCl}(60.48 \mathrm{~mL}$ concentrated $\mathrm{HCl} / 100 \mathrm{~mL}$ ).

\section{Methods}

3.1. Plant roots or other tissues ( $2 \mathrm{~g}$ ) are ground into fine powder with a mortar and pestle under liquid nitrogen, and then are homogenized in $2 \mathrm{~mL}$ of extraction buffer.

3.2. Centrifuge at $10,000 \times \mathrm{g}$ for $15 \mathrm{~min}$ at $4^{\circ} \mathrm{C}$.

3.3. Add to $1 \mathrm{~mL}$ of $30 \mathrm{mM} \mathrm{FeCl}_{3}$ and $1 \mathrm{~mL}$ of $20 \mathrm{mM}$ DMPD to $1 \mathrm{~mL}$ of the supernatant.

3.4. Incubate at room temperature for $15 \mathrm{~min}$ and read the absorbance at $670 \mathrm{~nm}$.

3.5. Graph the standard curve as absorbance $\left(\mathrm{A}_{670}\right)$ versus known $\mathrm{NaHS}$ or $\mathrm{Na}_{2} \mathrm{~S}$ concentrations from 0 to $10 \mu \mathrm{M}$. 
3.6. Calculate the $\mathrm{H}_{2} \mathrm{~S}$ content from the graph or the extinction coefficient of $1.5 \times 10^{7} \mathrm{M}^{-1} \mathrm{~cm}^{-1}$ and expressed as nmol/g FW.

\section{Precaution}

$\mathrm{FeCl}_{3}$ and DMPD can be dissolved in 3.5 and $100 \mathrm{mM} \mathrm{H}_{2} \mathrm{SO}_{4}$ to obtain $5 \mathrm{mM}$ DMPD and $50 \mathrm{mM}$ $\mathrm{FeCl}_{3}$, respectively.

\subsubsection{Method 3: WSP-1 Fluorescence Method}

\section{Principle}

WSP-1 (Washington StateProbe-1), 3'-methoxy-3oxo-3H-spiro(isobenzofuran-1, 9'-xanthen)-6'-yl 2(pyridin-2-yldisulfanyl)benzoate, is a fluorescent probe for detecting $\mathrm{H}_{2} \mathrm{~S}$ within living cells or plant extraction with high-sensitivity and selectivity. WSP-1 can freely enter into the cells across membrane and reacts with $\mathrm{H}_{2} \mathrm{~S}$ to generate benzodithiolone and a fluorophore with excitation and emission maxima of 465 and $515 \mathrm{~nm}$, respectively (Fig. 5.5; Liu et al., 2011). The intensity of fluorescence is positively pro rata to the content of $\mathrm{H}_{2} \mathrm{~S}$ in plant samples. The fluorescence is detected by fluorescence microscope or fluorospectrophotometer with excitation at 465 and an emission at $515 \mathrm{~nm}$ via in vivo or in vitro methods.

\section{Materials}

2.1. $100 \mathrm{~mL}$ of $20 \mathrm{mM}$ Hepes- $\mathrm{NaOH}$ (pH 7.5): Dissolve $0.477 \mathrm{~g}$ Hepes $(\mathrm{MW}=238.31)$ in approximately $80 \mathrm{~mL}$ of deionized water. Adjust $\mathrm{pH}$ to 7.5 with $1 \mathrm{M} \mathrm{NaOH}$, and then make up to $100 \mathrm{~mL}$ with deionized water. Store at $4^{\circ} \mathrm{C}$.

2.2. $15 \mu \mathrm{M}$ WSP-1: Dissolve $1 \mathrm{mg}$ WSP-1 $(\mathrm{MW}=561.65)$ in $1 \mathrm{~mL}$ of DMSO to obtain stock solution of $1.78 \mathrm{mM}$, and then dilute to final concentration with $20 \mathrm{mM}$ Hepes- $\mathrm{NaOH}$ ( $\mathrm{pH} 7.5)$.

2.3. Fluorescence microscope or fluorospectrophotometer.

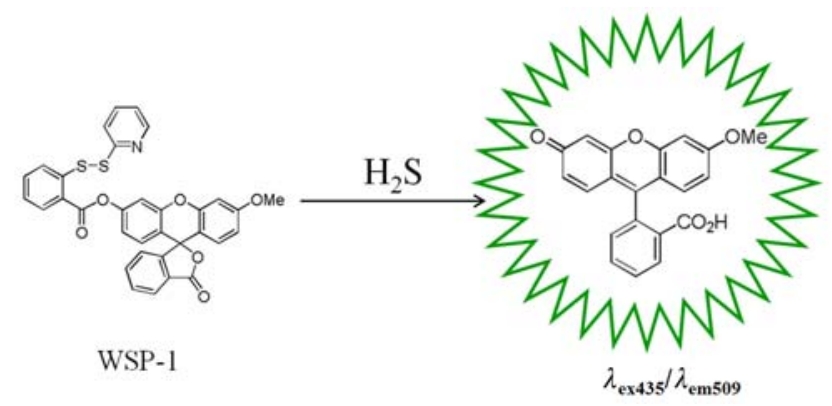

FIGURE 5.5 $\mathrm{H}_{2} \mathrm{~S}$ reacts with WSP-1 and generates green fluorescence.

\section{Methods}

3.1. For in vitro method, $\mathrm{H}_{2} \mathrm{~S}$ extracted from plant samples as DTNB method.

3.2. Plant extraction or tissues is incubated in $20 \mathrm{mM}$ Hepes- $\mathrm{NaOH}$ ( $\mathrm{pH}$ 7.5) containing $15 \mu \mathrm{M}$ WSP-1 for $40 \mathrm{~min}$ (in vitro method needs a short time, approximately $10 \mathrm{~min}$ ).

3.3. The plant tissues were washed with distilled water three times (this step ignored in in vitro method).

3.4. Detect immediately by a fluorescence microscope or fluorospectrophotometer with a 465/515 nm (excitation/emission) filter set.

3.5. Photograph or calculate $\mathrm{H}_{2} \mathrm{~S}$ content using standard curve with known concentrations of $\mathrm{NaHS}$ or $\mathrm{Na}_{2} \mathrm{~S}$ from 0 to $50 \mu \mathrm{M}$.

\section{Precautions}

WSP-1 should be freshly prepared or stored at $-20^{\circ} \mathrm{C}$ after subpackage of stock solution of $1.78 \mathrm{mM}$.

\subsection{SECTION 3: METHYLGLYOXAL QUANTIFICATION}

\subsubsection{Method 1: DAB Method}

\section{Principle}

Methylglyoxal (MG) is produced during normal cellular metabolism, such as glycolysis and photosynthesis. This aldehyde has long been considered as a cytotoxin, which inhibits the growth of cells in all types of organisms, but now is found to be a signaling molecule. MG can react with 1,2-diaminobenzene (DAB) and produces a product hydrazone. The hydrazone has a maximal optical density (OD) at $336 \mathrm{~nm}$ (Fig. 5.6; Mustafiz et al., 2010). The light absorption $\left(\mathrm{OD}_{336}\right)$ is positively proportional to the content of $\mathrm{MG}$ in plant samples.
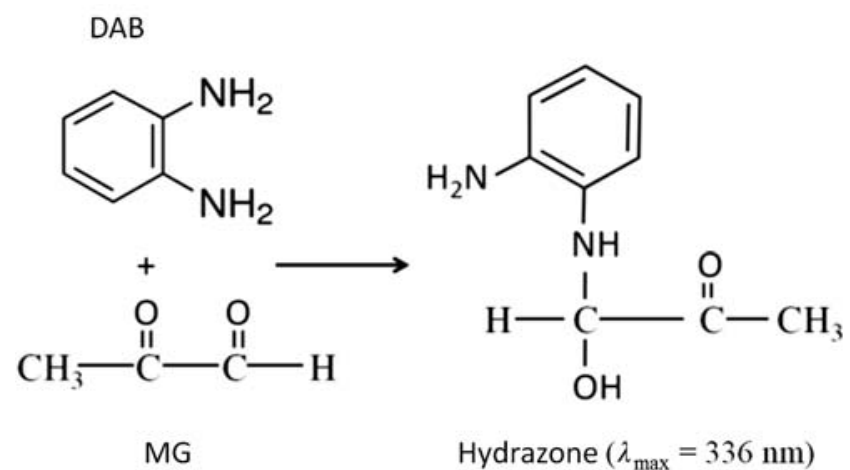

FIGURE 5.6 MG reacts with DAB to produce hydrazone. 


\section{Materials}

2.1. $100 \mathrm{~mL}$ of $0.5 \mathrm{M}$ perchloric acid (PA): Mix $4.17 \mathrm{~mL}$ of PA $(M=12)$ in $100 \mathrm{~mL}$ of deionized water.

2.2. $100 \mathrm{~mL}$ of $5 \mathrm{M}$ PA: Mix $41.7 \mathrm{~mL}$ of PA $(M=12)$ in $100 \mathrm{~mL}$ of deionized water.

2.3. Charcoal.

2.4. Saturated solution of $\mathrm{K}_{2} \mathrm{CO}_{3}$ : Dissolve $\mathrm{K}_{2} \mathrm{CO}_{3}$ in water in excess amount so that some amount of the solute remains undissolved in the solution.

2.5. $100 \mathrm{~mL}$ of $7.2 \mathrm{mM}$, 2-diaminobenzene (DAB): Dissolve $77.9 \mathrm{mg}$ DAB (MW = 108.14) in $100 \mathrm{~mL}$ of deionized water.

2.6. Methylglyoxal $(40 \%, \rho=1.178,6.54 \mathrm{M})$.

2.7. Spectrophotometer or microplate reader.

3. Methods

3.1. Fresh plant tissue $(250 \mathrm{mg})$ is homogenized in liquid nitrogen with mortar and pestle.

3.2. Add $2.5 \mathrm{~mL}$ of $0.5 \mathrm{M} \mathrm{PA}$ and mix well.

3.3. Centrifuge at $11,000 \times \mathrm{g}$ for $10 \mathrm{~min}$ at $4^{\circ} \mathrm{C}$.

3.4. The supernatant is decolorized using charcoal $(10 \mathrm{mg} / \mathrm{mL})$ and kept at room temperature for $15 \mathrm{~min}$. The mixture is centrifuged at $11,000 \times \mathrm{g}$ for $10 \mathrm{~min}$. The clear supernatant is collected and used for MG assay.

3.5. The solution is neutralized using saturated $\mathrm{K}_{2} \mathrm{CO}_{3}$ which should be added gradually (initially $20-30 \mu \mathrm{L}$ should be added, and then one should add $2 \mu \mathrm{L}$ at a time) and the $\mathrm{pH}$ of the supernatant should be checked using a $\mathrm{pH}$ paper time to time. With each addition of $\mathrm{K}_{2} \mathrm{CO}_{3}$, the solution should be mixed properly and the bubbles of $\mathrm{CO}_{2}$ gas should be allowed to come out.

3.6. The neutralized extract is kept at room temperature for $15 \mathrm{~min}$ and centrifuge at $11,000 \times \mathrm{g}$ for $10 \mathrm{~min}$.

3.7. Add $250 \mu \mathrm{L}$ of $7.2 \mathrm{mM}$ DAB and $100 \mu \mathrm{L}$ of $5 \mathrm{M}$ PA to $650 \mu \mathrm{L}$ of neutralized supernatant.

3.8. Incubate at room temperature for $30 \mathrm{~min}$ and take the absorbance at $336 \mathrm{~nm}$ with spectrophotometer or microplate reader.

3.9. A standard curve of different concentrations of MG $(10,25,50$, and $100 \mu \mathrm{M})$ is made using stock solution of MG.

3.10. $\mathrm{MG}$ content is calculated using the standard curve and expressed as $\mu \mathrm{mol} / \mathrm{g}$ FW.

\section{Precautions}

4.1. MG solution should be freshly prepared and stored in the dark due to its sensitivity to light.

4.2. MG should be handled carefully due to its high toxicity.

\subsubsection{Method 2: DNP Method}

1. Principle

MG, similar to other aldehydes, can react with 2,4-dinitrophenylhydrazine (2,4-DNP) in acidic conditions and produces a pink product MG-bis-2,4DNP-hydrazone. The hydrazone has a maximal absorbance at $432 \mathrm{~nm}$ and the molecular absorption coefficient of $3.36 \times 10^{4} \mathrm{M}^{-1} \mathrm{~cm}^{-1}$ (Fig. 5.7; Wild et al., 2012). The absorbance is proportional to the content of MG in plant samples. Therefore, MG can be quantified by spectrophotometry.

\section{Materials}

2.1. $0.2 \mathrm{mM}$ 2,4-dinitrophenylhydrazine (DNP): Prepare first $100 \mathrm{~mL}$ of stock solution of $10 \mathrm{mM}$ 2,4-DNP (0.198 g, MW = 198) in ethanol, and then dilute freshly to $0.2 \mathrm{mM}$ (1:50) using a mixture of $\mathrm{HCl}$ and ethanol $(12: 100, \mathrm{v} / \mathrm{v})$.

2.2. $1 \mathrm{mM}$ MG working solution: Mix $15.3 \mu \mathrm{L}$ of stock solution of MG $(M=6.54 \mathrm{M})$ with $100 \mathrm{~mL}$ deionized water. Freshly prepare from the stock solution every day.

2.3. Spectrophotometer or microplate reader.

2.4. Shaker.

3. Methods

3.1. MG extracted from plant samples as per DTNB method.

3.2. Take $50 \mu \mathrm{L}$ of neutralized supernatant (volume can be adjusted according to the amount of MG in plant samples) to $950 \mu \mathrm{L}$ of $0.2 \mathrm{mM} 2,4-\mathrm{DNP}$.

3.3. Incubate at $42^{\circ} \mathrm{C}$ for $45 \mathrm{~min}$ in a shaker at $600 \mathrm{rpm}$.

3.4. Recovery at room temperature for $5 \mathrm{~min}$.

3.5. Read absorbance at $432 \mathrm{~nm}$.

3.6. Calculate MG content according to the molecular absorption coefficient of $3.36 \times 10^{4} \mathrm{M}^{-1} \mathrm{~cm}^{-1}$ for MG-bis-2,4-DNPhydrazone and expressed as $\mu \mathrm{mol} / \mathrm{g}$ FW.

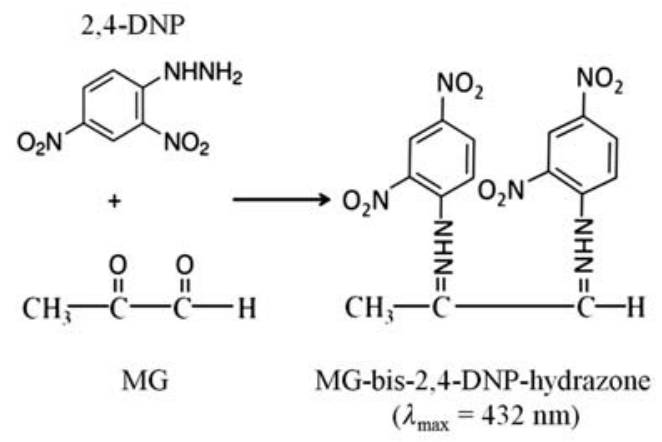

FIGURE 5.7 MG reacts with 2,4-DNP to produce MG-bis-2,4DNP-hydrazone. 


\section{Precautions}

4.1. MG solution should be freshly prepared and stored in the dark due to its sensitivity to light.

4.2. MG should be handled carefully due to its high toxicity.

\subsubsection{Method 3: NAC Method}

\section{Principle}

Under neutral conditions, MG can react with $N$ acetyl-L-cysteine (NAC) to produce $N$ - $\alpha$-acetyl-S-(1hydroxy-2-oxo-prop-1-yl) cysteine (NASC). The NASC exhibits a maximal absorbance at $288 \mathrm{~nm}$ and the molecular absorption coefficient is $248 \mathrm{M}^{-1} \mathrm{~cm}^{-1}$ (Fig. 5.8; Wild et al., 2012). The light absorption $\left(\mathrm{A}_{288}\right)$ is positively related to the content of MG in plant samples. Herein, MG can be detected by spectrophotometer.

\section{Materials}

2.1. $500 \mathrm{mM}$ NAC: Freshly dissolve $81.6 \mathrm{mg}$ NAC $(\mathrm{MW}=163.2)$ in $1 \mathrm{~mL}$ of deionized water.

2.2. $100 \mathrm{mM}$ sodium phosphate buffer ( $\mathrm{pH}$ 7.0): Mix $61 \mathrm{~mL}$ of $200 \mathrm{mM} \mathrm{Na} 2 \mathrm{HPO}_{4}(53.65 \mathrm{~g}$ $\mathrm{Na}_{2} \mathrm{HPO}_{4} \cdot 7 \mathrm{H}_{2} \mathrm{O}$ or $71.64 \mathrm{~g} \mathrm{Na}_{2} \mathrm{HPO}_{4} \cdot 12 \mathrm{H}_{2} \mathrm{O} /$ $1000 \mathrm{~mL}$ ) with $39 \mathrm{~mL}$ of $200 \mathrm{mM} \mathrm{NaH}{ }_{2} \mathrm{PO}_{4}$ (27.6 $\mathrm{g} \mathrm{NaH}_{2} \mathrm{PO}_{4} \cdot \mathrm{H}_{2} \mathrm{O}$ or $31.2 \mathrm{~g}$ $\mathrm{NaH}_{2} \mathrm{PO}_{4} \cdot 2 \mathrm{H}_{2} \mathrm{O} / 1000 \mathrm{~mL}$ ). Mix and make up to $200 \mathrm{~mL}$ with deionized water.
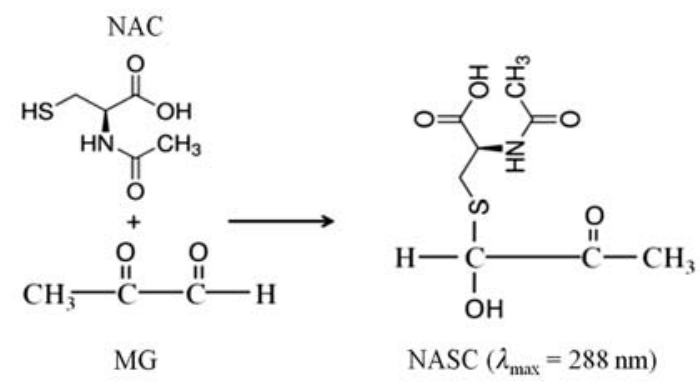

FIGURE 5.8 MG reacts with NAC to generate NASC with maximal absorbance at $288 \mathrm{~nm}$.

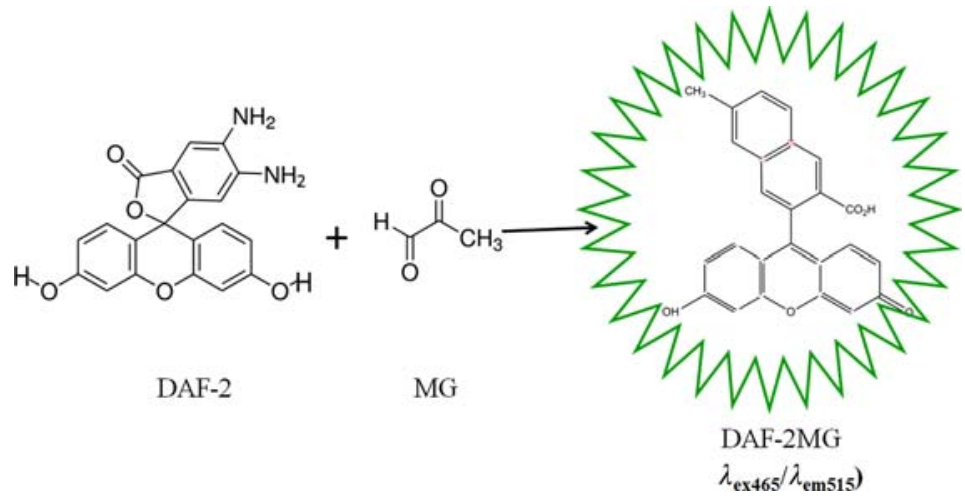

2.3. Spectrophotometer.

\section{Methods}

3.1. MG extracted from plant samples according to DTNB method.

3.2. Pipette $50 \mu \mathrm{L}$ of neutralized supernatant (volume can be adjusted according to MG content in plant samples) into $930 \mu \mathrm{L}$ of $100 \mathrm{mM}$ sodium phosphate buffer ( $\mathrm{pH} 7.0$ ).

3.3. Set to zero with a spectrophotometer.

3.4. Add $20 \mu \mathrm{L}$ of $500 \mathrm{mM}$ NAC (final concentration up to $10 \mathrm{mM}$ ).

3.5. Reaction at room temperature for $10 \mathrm{~min}$.

3.6. Read absorbance at $288 \mathrm{~nm}$.

3.7. Calculate MG content as the molecular absorption coefficient of $248 \mathrm{M}^{-1} \mathrm{~cm}^{-1}$ for NASC and expressed as $\mu \mathrm{mol} / \mathrm{g}$ FW.

\section{Precaution}

NAC should be freshly prepared.

\subsubsection{Method 4: DAF-2 and DAR-1 Fluorescence Methods}

\section{Principle}

Fluorescent probes 4,5-diaminofluorescein (DAF-

2) and 4,5-diamino-rhodamine (DAR-1) can react with MG and produce fluorescence substances DAF-2MG and DAR-1MG, respectively (Fig. 5.9; Shaheen et al., 2014). The intensity of fluorescence is proportional to the amount of MG in plant materials. Therefore, the imaging of DAF-2MG and DAR-1MG can be visualized by fluorescence microscope with excitation at $435 \mathrm{~nm}$ and emission at $509 \mathrm{~nm}$ for DAF-2MG, as well as excitation $545 \mathrm{~nm}$ and emission at $566 \mathrm{~nm}$ for DAR-1MG. The amount of MG in plant samples can be detected by in vivo or in vitro fluorometry.

2. Materials

2.1. $200 \mathrm{~mL}$ of $100 \mathrm{mM}$ sodium phosphate buffer (pH 7.4): Mix $81 \mathrm{~mL}$ of $200 \mathrm{mM} \mathrm{Na}_{2} \mathrm{HPO}_{4}$ (53.65 $\mathrm{g} \mathrm{Na}_{2} \mathrm{HPO}_{4} \cdot 7 \mathrm{H}_{2} \mathrm{O}$ or $71.64 \mathrm{~g}$ $\mathrm{Na}_{2} \mathrm{HPO}_{4} \cdot 12 \mathrm{H}_{2} \mathrm{O} / 1000 \mathrm{~mL}$ ) with $19 \mathrm{~mL}$ of

FIGURE 5.9 MG reacts with DAF-2 to generate a green fluorescence. 
$200 \mathrm{mM} \mathrm{NaH}{ }_{2} \mathrm{PO}_{4}\left(27.6 \mathrm{~g} \mathrm{NaH} \mathrm{PO}_{4} \cdot \mathrm{H}_{2} \mathrm{O}\right.$ or $31.2 \mathrm{~g} \mathrm{NaH}_{2} \mathrm{PO}_{4} \cdot 2 \mathrm{H}_{2} \mathrm{O} / 1000 \mathrm{~mL}$ ). Mix and make up to $200 \mathrm{~mL}$ with deionized water.

2.2. $10 \mu \mathrm{M}$ DAF-2: Dissolve $1 \mathrm{mg}$ DAF-2 (a pack, $\mathrm{MW}=446.4$ ) or DAR-1 (a pack, $\mathrm{MW}=472.6$ ) in $1 \mathrm{~mL}$ of DMSO to obtain $2.24 \mathrm{mM}$ DAF-2 or $2.12 \mathrm{mM}$ DAR-1, and then dilute to final concentration with $100 \mathrm{mM}$ sodium phosphate buffer ( $\mathrm{pH}$ 7.4).

2.3. Fluorescence microscope or fluorospectrophotometer.

\section{Methods}

3.1. For the in vitro assay, MG extracted from plant samples as DTNB method.

3.2. Add $5 \mu \mathrm{L}$ of $2000 \mu \mathrm{M}$ DAF-2 or DAR-1 (final concentration is $10 \mu \mathrm{M}$ ) to $995 \mu \mathrm{L}$ of $100 \mathrm{mM}$ sodium phosphate buffer ( $\mathrm{pH}$ 7.4) and incubate at $37^{\circ} \mathrm{C}$ for $20 \mathrm{~min}$ (depending the amount of $\mathrm{MG})$.

3.3. Develop fluorescence in a fluorospectrophotometer as excitation at $435 \mathrm{~nm}$ and emission at $509 \mathrm{~nm}$ for DAF-2-MG, and 545 and $566 \mathrm{~nm}$ for DAR-1-MG.

3.4. 3.4 Calculate the content of MG in plant samples using standard curve with known concentrations of MG from 0 to $50 \mu \mathrm{M}$.

\section{Precautions}

4.1. This method can detect MG in vivo using $100 \mathrm{mM}$ sodium phosphate buffer ( $\mathrm{pH}$ 7.4) containing $10 \mu \mathrm{M}$ DAF-2 or DAR-1.

4.2. DAF-2 and DAR-1 should be freshly prepared and stored in the dark due to their sensitivity to light.

\subsection{SECTION 4: NO QUANTIFICATION}

\subsubsection{Method 1: Griess Reagent Method}

\section{Principle}

Signaling molecule nitric oxide (NO) can be rapidly converted into $\mathrm{NO}_{2}^{-}$in solution in the presence of oxygen $\left(\mathrm{O}_{2}\right)$. Under acidic conditions, $\mathrm{NO}_{2}{ }^{-}$can react with sulfanilamide (SA) and $\mathrm{N}$ naphthylethylenediamine (NNED) to form a

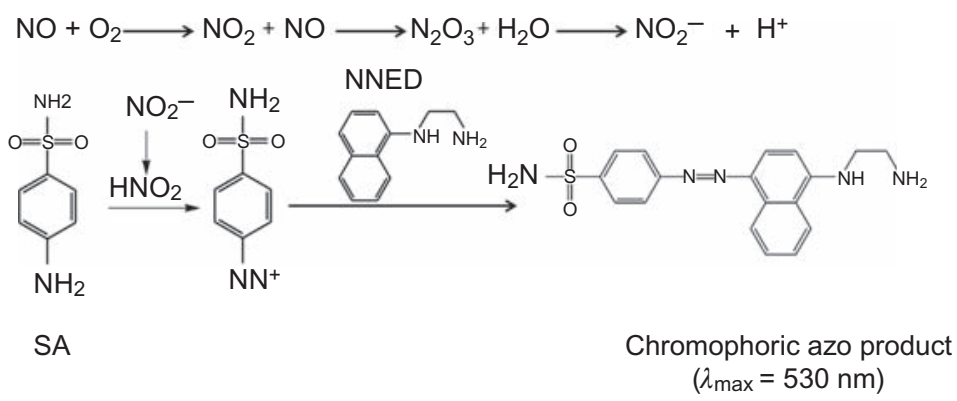

chromophoricazo product. The pink product exhibits a maximal absorbance at $530 \mathrm{~nm}$ (Fig. 5.10; Antoniou et al., 2018). A direct correlation between the absorbance $\left(\mathrm{A}_{530}\right)$ and the concentration of $\mathrm{NO}$ in plant samples can be found.

\section{Materials}

2.1. Extraction buffer: Namely $50 \mathrm{mM}$ acetate buffer $(\mathrm{pH}$ 3.6) containing $4 \%(\mathrm{w} / \mathrm{v})$ zinc acetate: Mix $46.3 \mathrm{~mL}$ of $200 \mathrm{mM}$ acetic acid $(11.55 \mathrm{~mL} /$ $1000 \mathrm{~mL}$ ) with $3.7 \mathrm{~mL}$ of $200 \mathrm{mM} \mathrm{C}_{2} \mathrm{H}_{2} \mathrm{O}_{2} \mathrm{Na}$ (16.4 g C ${ }_{2} \mathrm{H}_{2} \mathrm{O}_{2} \mathrm{Na}$ or $27.2 \mathrm{~g} \mathrm{C}_{2} \mathrm{H}_{2} \mathrm{O}_{2} \mathrm{Na} \cdot 3 \mathrm{H}_{2} \mathrm{O} /$ $1000 \mathrm{~mL}$ ), and then fill it up to $200 \mathrm{~mL}$ with deionized water. Dissolve $4 \mathrm{~g}$ of zinc acetate in $100 \mathrm{~mL}$ buffer.

2.2. Griess reagent: Reagent consisting of $0.5 \%$ $(\mathrm{w} / \mathrm{v}) \mathrm{SA}$ and $0.05 \%(\mathrm{w} / \mathrm{v})$ naphthyl ethylenediamine dihydrochloride (NNED). For SA solution, dissolve $1 \mathrm{~g}$ of SA in $100 \mathrm{~mL}$ of $5 \%$ phosphoric acid (add $5.88 \mathrm{~mL}$ of phosphoric acid to $94.12 \mathrm{~mL}$ deionized water). For NED solution, add $0.1 \mathrm{~g}$ to $100 \mathrm{~mL}$ deionized water. Before measurement, mix NED solution with SA solution in equal volume.

\section{Methods}

3.1. Plant tissue $(100 \mathrm{mg})$ is homogenized in liquid nitrogen with mortar and pestle, and then add $1 \mathrm{~mL}$ extraction buffer.

3.2. Mix well and centrifuge at $15,000 \times \mathrm{g}$ for $15 \mathrm{~min}$ at $4^{\circ} \mathrm{C}$.

3.3. Extract again with $0.5 \mathrm{~mL}$ extraction buffer from pellet and centrifuge.

3.4. Mix supernatant for $\mathrm{NO}$ assay.

3.5. Add $1 \mathrm{~mL}$ of supernatant to $1 \mathrm{~mL}$ of Griess reagent.

3.6. Mix and incubate at room temperature for $30 \mathrm{~min}$.

3.7. Transfer $300 \mu \mathrm{L}$ of the reaction mixture to $96-$ well plate and read absorbance at $530 \mathrm{~nm}$ or read in a spectrophotometer.

3.8. A standard curve is performed with known concentrations of $\mathrm{NaNO}_{2}$ ranging from 0 to $10 \mu \mathrm{M}$.

3.9. NO content is expressed as $\mathrm{nmol} / \mathrm{g} \mathrm{FW}$.

FIGURE 5.10 NO reacts with Griess reagent to generate a chromophoric azo product. 


\section{Precautions}

4.1. All extraction buffers should be kept cool in a container with crushed ice.

4.2. Store the two solutions (NNED and SA) at $4{ }^{\circ} \mathrm{C}$ with NNED covered in aluminum foil due to its sensitivity to light.

4.3. If your supernatant contains chlorophylls (green tissues), they can be remove from the supernatant by adding $\sim 0.1 \mathrm{~g}$ active carbon.

4.4. Griess reagent from the fridge should be equilibrated at room temperature before the reaction takes place.

4.5. Sulfanilamide and NNED solutions also can be separately and orderly added to supernatant without mixing.

\subsubsection{Method 2: DAF-FMDA Fluorescence Method}

\section{Principle}

Fluorescent probes 3-amino,4-aminomethyl-2', $7^{\prime}$ difluorescein, diacetate (DAF-FM DA) can freely enter into the cytosol across the biomembrane where it is deacetylated by intracellular esterase to produce DAF-FM, which is trapped. The DAF-FM can react with $\mathrm{NO}$ to produce a green fluorescent substance (Fig. 5.11; Xie and Shen, 2012), which has a maximal emission at $525 \mathrm{~nm}$ after excitation at $490 \mathrm{~nm}$. The intensity of fluorescence is positively proportional to the amount of NO in plant samples. Therefore, NO in plant can be detected by in vivo or in vitro methods.

\section{Materials}

2.1. $100 \mathrm{~mL}$ of $20 \mathrm{mM}$ of Hepes- $\mathrm{NaOH}$ (pH 7.5): Dissolve $0.477 \mathrm{~g}$ Hepes $(\mathrm{MW}=238.31)$ in approximately $80 \mathrm{~mL}$ of deionized water. Adjust $\mathrm{pH}$ to 7.5 with $1 \mathrm{M} \mathrm{NaOH}$, and then make up to $100 \mathrm{~mL}$ with deionized water. Store at $4^{\circ} \mathrm{C}$.

2.2. $15 \mu \mathrm{M}$ of DAF-FM DA: Dissolve $1 \mathrm{mg}$ DAF-FM DA $(\mathrm{MW}=496.42)$ in $1 \mathrm{~mL}$ of DMSO to obtain the stock solution of $2 \mathrm{mM}$, and then dilute to final concentration with $20 \mathrm{mM}$ of Hepes$\mathrm{NaOH}(\mathrm{pH} 7.5)$.

2.3. Fluorescence microscope.

\section{Methods}

3.1. Plant tissues are incubated in $20 \mathrm{mM}$ Hepes$\mathrm{NaOH}$ ( $\mathrm{pH} 7.5$ ) containing $15 \mu \mathrm{MDAF}-\mathrm{FM}$ DA at $25^{\circ} \mathrm{C}$ for $15 \mathrm{~min}$.

3.2. Wash plant tissues with distilled water three times, and determine the fluorescence using a fluorescence microscope with excitation $490 \mathrm{~nm}$ and emission $525 \mathrm{~nm}$.

3.3. Photo is taken by using fluorescent microscope.

4. Precautions

4.1. DAF-FM DA should be freshly prepared and stored in the dark.

4.2. This method can detect $\mathrm{NO}$ in vitro using DAF FM instead of DAF-FM DA after NO extracted from plant samples following Method 1.

\subsection{SECTION 5: $\mathrm{H}_{2} \mathrm{O}_{2}$ QUANTIFICATION}

\subsubsection{Method 1: KI Method}

\section{Principle}

Iodine ion $\left(\mathrm{I}^{-}\right)$in $\mathrm{KI}$ solution can be reduced to iodine $\left(\mathrm{I}_{2}\right)$ in the present of $\mathrm{H}_{2} \mathrm{O}_{2}$, which in turn forms the yellow product triiodide $\left(\mathrm{I}_{3}{ }^{-}\right)$. Triiodide exhibits a maximal light absorption at $390 \mathrm{~nm}$, the absorbance is positively related to the content of $\mathrm{H}_{2} \mathrm{O}_{2}$ in plant samples (Fig. 5.12; Li and Gong, 2014; Antoniou et al., 2018).

FIGURE 5.11 DAF-FM DA, which can freely permeate into cytosol, is deacetylated by intracellular esterase to produce DAF-FM, which can react with NO to generate fluorescence. 


$$
2 \mathrm{I}^{-}+\mathrm{H}_{2} \mathrm{O}_{2}+2 \mathrm{H}^{+} \longrightarrow \mathrm{I}_{2}+2 \mathrm{H}_{2} \mathrm{O}+\mathrm{I}^{-} \underset{\left(\lambda_{\max }=390 \mathrm{~nm}\right)}{\longrightarrow} \mathrm{I}_{3}^{-}
$$

FIGURE 5.12 $\mathrm{H}_{2} \mathrm{O}_{2}$ reacts with $\mathrm{KI}$ to generate triiodide with maximal absorbance at $390 \mathrm{~nm}$.

\section{Materials}

2.1. Extraction buffer: Namely $0.1 \%(\mathrm{w} / \mathrm{v})$ trichloroacetic acid (TCA). Add $227 \mathrm{~mL}$ deionized water to $500 \mathrm{~g}$ TCA (a pack) and forms $100 \%(\mathrm{w} / \mathrm{v}) \mathrm{TCA}$, and then dilute to final concentration with deionized water. TCA should be stored at $4^{\circ} \mathrm{C}$.

2.2. $10 \mathrm{mM}$ potassium phosphate buffer ( $\mathrm{pH}$ 7.0): Mix $6.15 \mathrm{~mL}$ of $100 \mathrm{mM} \mathrm{K}_{2} \mathrm{HPO}_{4}(17.418 \mathrm{~g} /$ $100 \mathrm{~mL}$ ) with $3.85 \mathrm{~mL}$ of $100 \mathrm{mM} \mathrm{KH}_{2} \mathrm{PO}_{4}$ $(13.609 \mathrm{~g} / 100 \mathrm{~mL})$, and add deionized water to reach a final volume of $100 \mathrm{~mL}$.

2.3. $1 \mathrm{M}$ potassium iodide: Dissolve $16.6 \mathrm{~g} \mathrm{KI}$ $(\mathrm{MW}=166)$ in $100 \mathrm{~mL}$ deionized water. Store at $4^{\circ} \mathrm{C}$.

2.4. Spectrophotometer or microplate reader.

\section{Methods}

3.1. Plant tissue $(100 \mathrm{mg})$ is homogenized in $1 \mathrm{~mL}$ of $0.1 \%(\mathrm{w} / \mathrm{v})$ TCA with mortar and pestle.

3.2. Centrifuge at $15,000 \times \mathrm{g}$ for $15 \mathrm{~min}$ at $4^{\circ} \mathrm{C}$.

3.3. Add $0.5 \mathrm{~mL}$ of the supernatant to $0.5 \mathrm{~mL}$ of $10 \mathrm{mM}$ phosphate buffer (pH 7.0) (use $0.5 \mathrm{~mL}$ of $0.1 \%(\mathrm{w} / \mathrm{v})$ TCA instead of the supernatant as the blank).

3.4. Add $1 \mathrm{~mL}$ of $\mathrm{KI}$ to initiate the reaction as quick as possible.

3.5. Mix gently and incubate in the dark for 2-15 min (depending on plant tissue).

3.6. Transfer $300 \mu \mathrm{L}$ of the reaction mixture to $96-$ well plate and read the absorbance at $390 \mathrm{~nm}$ or read in a spectrophotometer.

3.7. A standard curve is performed using the known concentrations of $\mathrm{H}_{2} \mathrm{O}_{2}$ ranging from 0 to $250 \mu \mathrm{M}$.

3.8. $\mathrm{H}_{2} \mathrm{O}_{2}$ content is calculated following standard curve and expressed as $\mu \mathrm{mol} \mathrm{H}_{2} \mathrm{O}_{2} / \mathrm{g}$ FW.

\section{Precaution}

Because KI is sensitive to light and liberates free iodine, it should be freshly prepared and covered with aluminum foil. Store at $4^{\circ} \mathrm{C}$.

\subsubsection{Method 2: $\mathrm{Ti}\left(\mathrm{SO}_{4}\right)_{2}$ Method}

\section{Principle}

$\mathrm{H}_{2} \mathrm{O}_{2}$ can react with $\mathrm{Ti}^{4+}$ in $\mathrm{Ti}\left(\mathrm{SO}_{4}\right)_{2}$ or $\mathrm{TiCl}_{4}$ solution under acidic conditions to form a yellow titanium-hydrogen peroxide complex $\left(\mathrm{Ti}-\mathrm{H}_{2} \mathrm{O}_{2}\right)$. The complex exhibits a maximal absorbance at $410 \mathrm{~nm}$ and has a molar extinction coefficient of $2.8 \times 10^{5} \mathrm{M}^{-1} \mathrm{~cm}^{-1}$ (Fig. 5.13; Yu et al., 2003; Li

$$
\begin{array}{r}
\mathrm{H}_{2} \mathrm{O}_{2}+\mathrm{Ti}\left(\mathrm{SO}_{4}\right)_{2} \longrightarrow \underset{\text { Ti- }}{\mathrm{H}} \mathrm{H}_{2} \mathrm{O}_{2} \text { complex } \\
\left(\lambda_{\max }=410 \mathrm{~nm}\right)
\end{array}
$$

FIGURE 5.13 $\mathrm{H}_{2} \mathrm{O}_{2}$ reacts with $\mathrm{Ti}\left(\mathrm{SO}_{4}\right)_{2}$ to generate a yellow Ti$\mathrm{H}_{2} \mathrm{O}_{2}$ complex.

and Gong, 2014). The yellow product (the amount of $\mathrm{Ti}-\mathrm{H}_{2} \mathrm{O}_{2}$ complex) is positively proportional to the content of $\mathrm{H}_{2} \mathrm{O}_{2}$ in plant samples.

2. Materials

2.1. Extraction buffer: Namely $50 \mathrm{mMK}$-phosphate buffer (pH 6.5): Mix $31.5 \mathrm{~mL}$ of $100 \mathrm{mM}$ $\mathrm{K}_{2} \mathrm{HPO}_{4}(17.418 \mathrm{~g} / 100 \mathrm{~mL})$ with $68.5 \mathrm{~mL}$ of $100 \mathrm{mM} \mathrm{KH}{ }_{2} \mathrm{PO}_{4}(13.609 \mathrm{~g} / 100 \mathrm{~mL})$, and add deionized water to reach a final volume of $200 \mathrm{~mL}$.

2.2. $0.1 \%(\mathrm{w} / \mathrm{v}) \mathrm{Ti}\left(\mathrm{SO}_{4}\right)_{2}$ : Dissolve $0.1 \mathrm{~g} \mathrm{Ti}\left(\mathrm{SO}_{4}\right)_{2}$ in $100 \mathrm{~mL}$ of $20 \%(\mathrm{v} / \mathrm{v}) \mathrm{H}_{2} \mathrm{SO}_{4}$.

2.3. Centrifuger.

2.4. Spectrophotometer.

\section{Methods}

3.1. $\mathrm{H}_{2} \mathrm{O}_{2}$ is extracted by homogenizing $0.5 \mathrm{~g}$ plant tissues with $3 \mathrm{~mL}$ of $50 \mathrm{mM}$ K-phosphate buffer $(\mathrm{pH} 6.5)$ at $4{ }^{\circ} \mathrm{C}$. The homogenate is centrifuged at $10,000 \times \mathrm{g}$ for $15 \mathrm{~min}$, and supernatant is collected and used for $\mathrm{H}_{2} \mathrm{O}_{2}$ assay.

3.2. A 3-mL sample of supernatant is mixed with $1 \mathrm{~mL}$ of $0.1 \% \mathrm{TiCl}_{4}$, and reacted at room temperature for $5 \mathrm{~min}$.

3.3. The mixture was then centrifuged at $10,000 \times \mathrm{g}$ for $10 \mathrm{~min}$ at room temperature and supernatant was collected.

3.4. The optical density of the supernatant was measured by spectrophotometer at $410 \mathrm{~nm}$.

3.5. $\mathrm{H}_{2} \mathrm{O}_{2}$ content is calculated as the extinction coefficient of $2.8 \times 10^{5} \mathrm{M}^{-1} \mathrm{~cm}^{-1}$ and expressed as $\mu \mathrm{mol} / \mathrm{g}$ FW.

\section{Precautions}

4.1. To avoid the degradation of $\mathrm{H}_{2} \mathrm{O}_{2}$ by catalase, the process of $\mathrm{H}_{2} \mathrm{O}_{2}$ extraction must be carried out at $4{ }^{\circ} \mathrm{C}$, or $1 \mathrm{mM}$ hydroxylamine (final concentration) is added to $50 \mathrm{mMK}$-phosphate buffer ( $\mathrm{pH}$ 6.5) to inhibit catalase.

4.2. $\mathrm{Ti}\left(\mathrm{SO}_{4}\right)_{2}$ can be replaced with $\mathrm{TiCl}_{4}$ (correspondingly dissolved in $20 \% \mathrm{HCl}$ ) in this method.

4.3. If plant sample contains pigment, it should be removed using active carbon from supernatant. 


\subsubsection{Method 3: XO Method}

\section{Principle}

Under acidic conditions, $\mathrm{Fe}^{2+}$ can be oxidized by $\mathrm{H}_{2} \mathrm{O}_{2}$ to $\mathrm{Fe}^{3+}$, which reacts with xylenol orange $(\mathrm{XO})$ to generate a purple complex $\left(\mathrm{Fe}^{3+}-\mathrm{XO}\right)$. The complex has maximal absorbance at $560 \mathrm{~nm}$ and molar extinction coefficient of $2.24 \times 10^{5} \mathrm{M}^{-1} \mathrm{~cm}^{-1}$ (Fig. 5.14; Gay and Gebicki, 2000, 2003; Li and Gong, 2014). The purple product (the amount of $\mathrm{Fe}^{3+}-\mathrm{XO}$ ) is positively correlated to the content of $\mathrm{H}_{2} \mathrm{O}_{2}$ in plant samples and the sensitivity can be improved when proper concentrations of sorbitol are added to the reaction system.

\section{Materials}

2.1. Extraction solution: Namely $200 \mathrm{mM}$ perchloric acid (PA): Add $1.7 \mathrm{~mL}$ of $\mathrm{HClO}_{4}(M=12)$ to $98.3 \mathrm{~mL}$ of deionized water.

2.2. Working reagent: $100 \mathrm{~mL}$ of working reagent containing $500 \mu \mathrm{M}$ ammonium ferrous sulfate (19.6 mg, $\mathrm{MW}=392), 50 \mathrm{mM} \mathrm{H}_{2} \mathrm{SO}_{4}(0.28 \mathrm{~mL}$, $M=18 \mathrm{M}), 200 \mu \mathrm{M}$ xylenol orange (tetrasodium salt, $14.3 \mathrm{mg}$, MW $=716.6$ ), and $200 \mathrm{mM}$ sorbitol (3.64 g, MW = 182).

2.3. Centrifuger.

2.4. Spectrophotometer or microplate reader.

\section{Methods}

3.1. Plant samples roots or leaves $(0.2 \mathrm{~g})$ are extracted in $200 \mathrm{mM}$ PA with mortar and pestle.

3.2. Centrifuge at $4^{\circ} \mathrm{C}, 10,000 \times \mathrm{g}$ for $15 \mathrm{~min}$, collect supernatant and use for $\mathrm{H}_{2} \mathrm{O}_{2}$ assay.

3.3. To $1.5 \mathrm{~mL}$ of supernatant, add $1.5 \mathrm{~mL}$ of working reagent. Develop at $30^{\circ} \mathrm{C}$ for $30 \mathrm{~min}$.

3.4. The optical density $\left(O D_{560}\right)$ of the purple solution was measured by spectrophotometer at $560 \mathrm{~nm}$.

3.5. $\mathrm{H}_{2} \mathrm{O}_{2}$ content is calculated as the extinction coefficient of $2.24 \times 10^{5} \mathrm{M}^{-1} \mathrm{~cm}^{-1}$ and expressed as $\mu \mathrm{mol} / \mathrm{g}$ FW.

\section{Precautions}

4.1. The specificity for $\mathrm{H}_{2} \mathrm{O}_{2}$ was tested by eliminating $\mathrm{H}_{2} \mathrm{O}_{2}$ in the reaction mixture with catalase (CAT) after being neutralized with $1 \mathrm{M}$ $\mathrm{NaOH}$.

4.2. If plant sample contains pigment, it should be removed using active carbon from supernatant.

\subsubsection{Method 4: ABTS Method}

\section{Principle}

$\mathrm{H}_{2} \mathrm{O}_{2}$ can react with 2,2'-azino-bis (3ethylbenzthiazoline-6-sulfonic acid) diammonium salt (ABTS) in the present of the peroxidase such as horseradish peroxidase (HRP) to generate a soluble end product (radical monocation $\mathrm{ABTS}^{+}{ }^{+}$) that is green in color. The ABTS ${ }^{+}$can be detected spectrophotometrically at 405 or $734 \mathrm{~nm}$ (Fig. 5.15; Angelini et al., 2018). The light absorption $\left(\mathrm{A}_{405}\right.$ or $\left.A_{734}\right)$ is positively proportional to the content of $\mathrm{H}_{2} \mathrm{O}_{2}$ in plant samples.

\section{Materials}

2.1. $100 \mathrm{mM} \mathrm{K}$-phosphate buffer ( $\mathrm{pH}$ 7.0): Mix $61 \mathrm{~mL}$ of $100 \mathrm{mM} \mathrm{K}_{2} \mathrm{HPO}_{4}(17.418 \mathrm{~g} / 100 \mathrm{~mL})$ with $39 \mathrm{~mL}$ of $100 \mathrm{mM} \mathrm{KH} \mathrm{PO}_{4}(13.609 \mathrm{~g} /$ $100 \mathrm{~mL}$ ).

2.2. Extraction buffer: $100 \mathrm{~mL}$ of $100 \mathrm{mM} \mathrm{K}$ phosphate buffer ( $\mathrm{pH}$ 7.0) containing 10\% polyvinyl pyrrolidone (PVP, $10 \mathrm{~g}$ ) and $1 \mathrm{mM}$ hydroxylamine $(0.695 \mathrm{~g}, \mathrm{MW}=69.5)$.

2.3. $10 \mathrm{mM} \mathrm{H}_{2} \mathrm{O}_{2}$ : Add $10 \mu \mathrm{L}$ of $30 \% \mathrm{H}_{2} \mathrm{O}_{2}$ solution to $9990 \mu \mathrm{L}$ of $100 \mathrm{mM}$ K-phosphate buffer (pH 7.0) to obtain $10 \mathrm{mMH}_{2} \mathrm{O}_{2}$. Measure the absorbance at $240 \mathrm{~nm}$ to correct its concentration following the extinction coefficient of $43 \mathrm{M}^{-1} \mathrm{~cm}^{-1}$.

2.4. Horseradish peroxidase (HRP) solution: Dissolve $1 \mathrm{mg}$ HRP ( 150 U/mg) in $1 \mathrm{~mL}$ of 100 mM K-phosphate buffer ( $\mathrm{pH}$ 7.0).

$$
\begin{aligned}
& \mathrm{H}_{2} \mathrm{O}_{2}+\mathrm{Fe}^{2+} \longrightarrow \mathrm{Fe}^{3+} \stackrel{\text { xo }}{\longrightarrow} \mathrm{XO}-\mathrm{Fe}^{3+} \text { complex } \\
& \left(\lambda_{\max }=560 \mathrm{~nm}\right)
\end{aligned}
$$

FIGURE $5.14 \mathrm{Fe}^{2+}$ is reduced by $\mathrm{H}_{2} \mathrm{O}_{2}$ to $\mathrm{Fe}^{3+}$ that can react with $\mathrm{XO}$ to produce a purple XO-Fe ${ }^{3+}$ complex.

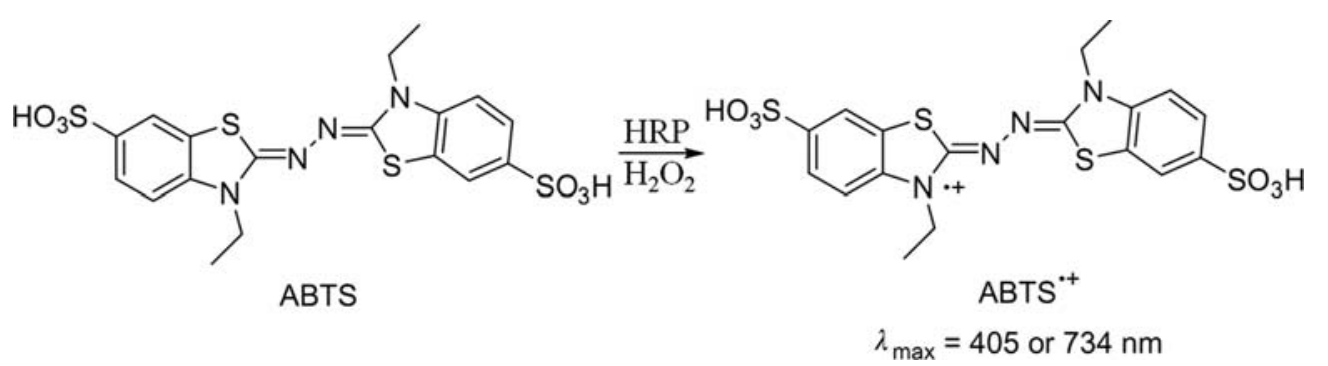

FIGURE 5.15 ABTS reacts with $\mathrm{H}_{2} \mathrm{O}_{2}$ in the present of peroxidase to ABTS ${ }^{+}$. 
2.5. $50 \mathrm{mM}$ ABTS: Dissolve $0.24 \mathrm{~g}$ ABTS $(\mathrm{MW}=548.68)$ in $10 \mathrm{~mL}$ of deionized water.

2.6. Spectrophotometer or microplate reader.

\section{Methods}

3.1. Set up standard curve

1. Dilute the $10 \mathrm{mM} \mathrm{H}_{2} \mathrm{O}_{2}$ solution to $0,50,100$, 150, and $2001 \mu \mathrm{M}$ with $100 \mathrm{mM} \mathrm{K}-$ phosphate buffer ( $\mathrm{pH} 7.0)$.

2. Mix $200 \mu \mathrm{L}$ of the different concentrations of $\mathrm{H}_{2} \mathrm{O}_{2}$ with $10 \mu \mathrm{L}$ of $\mathrm{HRP}$, and then add $10 \mu \mathrm{L}$ of $50 \mathrm{mM}$ ABTS.

3. Incubate for $3 \mathrm{~min}$ at room temperature, and then measure the absorbance at $405 \mathrm{~nm}$.

4. Draw the standard curve with absorbance values on the $Y$-axis and $\mathrm{H}_{2} \mathrm{O}_{2}$ amount (nmol) on $X$-axis or establish regression equation.

3.2. Extraction and measurement of $\mathrm{H}_{2} \mathrm{O}_{2}$

1. Plant tissues $(500 \mathrm{mg}$ ) are homogenized in $1 \mathrm{~mL}$ of extraction buffer with a prechilled mortar and pestle at $4^{\circ} \mathrm{C}$.

2. Centrifuge at $10,000 \times \mathrm{g}, 4^{\circ} \mathrm{C}$ for $10 \mathrm{~min}$, collect supernatant and use for $\mathrm{H}_{2} \mathrm{O}_{2}$ assay.

3. Add $10 \mu \mathrm{L}$ of HRP and $10 \mu \mathrm{L}$ of $50 \mathrm{mM}$ ABTS to $200 \mu \mathrm{L}$ supernatant.

4. Incubate at room temperature for $3 \mathrm{~min}$.

5. Read absorbance at $405 \mathrm{~nm}$.

6. Calculate $\mathrm{H}_{2} \mathrm{O}_{2}$ content using the standard curve and express as $\mu \mathrm{mol} / \mathrm{g}$ FW.

\section{Precautions}

4.1. The molar concentration of $30 \%(\mathrm{w} / \mathrm{w}) \mathrm{H}_{2} \mathrm{O}_{2}$ with a density of $1.11 \mathrm{~g} / \mathrm{mL}$ is $9.8 \mathrm{M}$.

Due to the instability of $\mathrm{H}_{2} \mathrm{O}_{2}$, it should be freshly prepared and immediately corrected using the molar extinction coefficient of $43 \mathrm{M}^{-1} \mathrm{~cm}^{-1}$.

4.2. This method can in vivo detect $\mathrm{H}_{2} \mathrm{O}_{2}$ in plant materials by incubating in $100 \mathrm{mM} \mathrm{K}$-phosphate buffer ( $\mathrm{pH} 7.0)$ containing HRP $(45 \mu \mathrm{g} / \mathrm{mL})$ and $2.5 \mathrm{mM}$ ABTS for the indicated time, and then taking a photo.

\subsubsection{Method 5: DCHBS-AAP Method}

\section{Principle}

The reaction of $\mathrm{H}_{2} \mathrm{O}_{2}$ and 3,5-dichloro-2-hydroxybenzene sulfonic acid (DCHBS) generates a radical DCHBS $\bullet$ in the presence of peroxidase such as horseradish peroxidase (HRP). The DCHBS• further reacts with 4-aminoantipyrine (AAP) to form a nonradical byproduct that is pink in color. The pink product exhibits a maximal absorbance at $515 \mathrm{~nm}$ and the molar extinction coefficient is $2.6 \times 10^{4} \mathrm{M}^{-1} \mathrm{~cm}^{-1}$ (Fig. 5.16; Fraisse et al., 2002; Li and Gong, 2014). The light absorption is proportional to the content of $\mathrm{H}_{2} \mathrm{O}_{2}$ in plant samples. Therefore, the $\mathrm{H}_{2} \mathrm{O}_{2}$ production can be measured as the formation of a pink adduct (nonradical byproduct) via in vivo and in vitro methods.

\subsubsection{In Vivo Method}

\section{Materials}

2.1. $100 \mathrm{mM}$ K-phosphate buffer ( $\mathrm{pH}$ 7.0): Mix $61 \mathrm{~mL}$ of $100 \mathrm{mM} \mathrm{K} \mathrm{HPO}_{4}(17.418 \mathrm{~g} / 100 \mathrm{~mL})$ with $39 \mathrm{~mL}$ of $100 \mathrm{mM} \mathrm{KH}_{2} \mathrm{PO}_{4}(13.609 \mathrm{~g} /$ $100 \mathrm{~mL}$ ).

2.2. $5 \mathrm{mM}$ AAP: Weigh $10 \mathrm{mg} A \mathrm{AP}(\mathrm{MW}=203)$ and transfer to $5 \mathrm{~mL}$ of $100 \mathrm{mM} \mathrm{K}$-phosphate buffer ( $\mathrm{pH}$ 7.0). Mix and make up to $10 \mathrm{~mL}$ with $100 \mathrm{mM}$ K-phosphate buffer ( $\mathrm{pH}$ 7.0).

2.3. Reaction mixture: The solution containing $100 \mu \mathrm{M}$ AAP, $1 \mathrm{mM}$ DCHBS, $60 \mu \mathrm{g} / \mathrm{mL}$ HRP: Weigh $13 \mathrm{mg}$ DCHBS (MW = 265) and $3 \mathrm{mg}$ $\mathrm{HRP}$, respectively, and then add $1 \mathrm{~mL} 5 \mathrm{mM}$ AAP solution. Mix and make up to $50 \mathrm{~mL}$ with $100 \mathrm{mM}$ K-phosphate buffer (pH 7.0).

2.4. Vacuum pump.

2.5. Spectrophotometer.

\section{Methods}

3.1. Submerge the plant material completely in the reaction mixture by vacuum infiltration for $1-3$ times (1 min each time).<smiles>O=S(=O)([O-])c1cc(Cl)cc(Cl)c1O</smiles><smiles>Cc1c(N)c(=O)n(CCN(C)C)n1C</smiles>

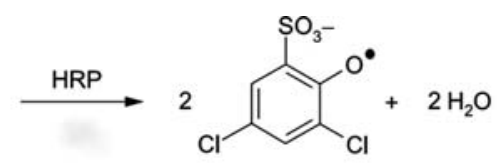

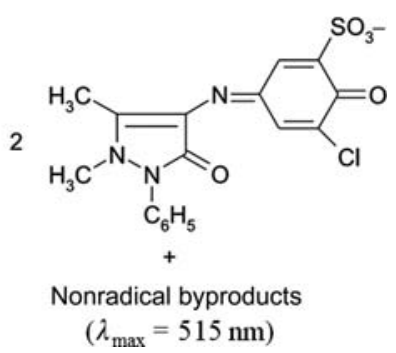

FIGURE 5.16 $\mathrm{H}_{2} \mathrm{O}_{2}$ can react with DCHBS and $\mathrm{AAP}$ in the present of peroxidase to generate a pink product. 
3.2. Incubate for $2 \mathrm{~h}$ at $30^{\circ} \mathrm{C}$ in the dark.

3.3. Collect the incubation medium and centrifuge at $10,000 \times \mathrm{g}$ for $5 \mathrm{~min}$.

3.4. Measure the absorbance at $515 \mathrm{~nm}$.

3.5. Calculate $\mathrm{H}_{2} \mathrm{O}_{2}$ concentration using the molar extinction coefficient $2.6 \times 10^{4} \mathrm{M}^{-1} \mathrm{~cm}^{-1}$.

\section{Precautions}

4.1. To study the effect of environmental stress on $\mathrm{H}_{2} \mathrm{O}_{2}$ production, an appropriate amount of $\mathrm{NaCl}$, sorbitol, and heavy metal is added to reaction mixture to carry out salt, osmotic, and heavy metal stresses. In addition, plant materials incubated in reaction mixture are submitted to high or cold temperature to explore the production of $\mathrm{H}_{2} \mathrm{O}_{2}$.

4.2. For the $\mathrm{H}_{2} \mathrm{O}_{2}$ specificity control, add $1.66 \mathrm{~g} \mathrm{KI}$ to the reaction mixture before adjusting to the final volume.

\subsubsection{In Vitro Method}

\section{Material}

2.1. $100 \mathrm{mM} \mathrm{K}$-phosphate buffer ( $\mathrm{pH}$ 7.0): Mix $61 \mathrm{~mL}$ of $100 \mathrm{mM} \mathrm{K} \mathrm{HPO}_{4}(17.418 \mathrm{~g} / 100 \mathrm{~mL})$ with $39 \mathrm{~mL}$ of $100 \mathrm{mM} \mathrm{KH}{ }_{2} \mathrm{PO}_{4}(13.609 \mathrm{~g} /$ $100 \mathrm{~mL})$.

2.2. HRP $(1 \mathrm{mg} / \mathrm{mL})$ solution: Dissolve $1 \mathrm{mg}$ HRP in $1 \mathrm{~mL}$ of $100 \mathrm{mM} \mathrm{K}$-phosphate buffer $(\mathrm{pH}$ 7.0).

2.3. $10 \mathrm{mM}$ DCHBS stock solution: Dissolve $26.5 \mathrm{mg}$ DCHBS (MW $=265)$ in $10 \mathrm{~mL}$ of water and stored at $4^{\circ} \mathrm{C}$.

2.4. $1 \mathrm{mM}$ AAP stock solution: Dissolve $20.3 \mathrm{mg}$ DCHBS (MW $=203)$ in $100 \mathrm{~mL}$ of water and stored at $4^{\circ} \mathrm{C}$.

2.5. Spectrophotometer.

\section{Methods}

3.1. $\mathrm{H}_{2} \mathrm{O}_{2}$ is extracted from plant materials as in ABTS method.

3.2. Add $50 \mu \mathrm{L}$ of HRP, $100 \mu \mathrm{L}$ of $1 \mathrm{mM}$ APP, and $100 \mu \mathrm{L}$ of $10 \mathrm{mM}$ DCHBS to $200 \mu \mathrm{L}$ of the supernatant.

3.3. Add $100 \mathrm{mM}$ K-phosphate buffer ( $\mathrm{pH}$ 7.0) to final volume of $1 \mathrm{~mL}$.

3.4. Measure absorbance at $515 \mathrm{~nm}$ using a spectrophotometer.

3.5. Calculate $\mathrm{H}_{2} \mathrm{O}_{2}$ content using the extinction coefficient of $2.6 \times 10^{4} \mathrm{M}^{-1} \mathrm{~cm}^{-1}$.

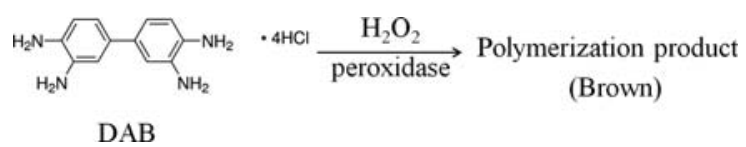

FIGURE 5.17 $\mathrm{H}_{2} \mathrm{O}_{2}$ reacts with DBA in the present of peroxidase to a brown polymerization product.

\section{Precautions}

This system can be used to determine the activity of enzymes involved in $\mathrm{H}_{2} \mathrm{O}_{2}$ production by adding enzymatic substances to reaction mixture, such as copper amine oxidase, glucose oxidase.

\subsubsection{Method 6: DAB Method}

\section{Principle}

$\mathrm{H}_{2} \mathrm{O}_{2}$ is usually detected in plant tissues by using $3,3^{\prime}$-diaminobenzidine tetrahydrochloride (DAB) as substrate in the present of peroxidase. A marked brown and insoluble polymerization product is formed by the reaction of DAB with $\mathrm{H}_{2} \mathrm{O}_{2}$ (Fig. 5.17; Rodriguez and Taleisnik, 2012). The amount of brown product represents the concentration of $\mathrm{H}_{2} \mathrm{O}_{2}$ in plant tissues.

\section{Materials}

2.1. Staining solution: namely $1 \mathrm{mg} / \mathrm{mL} \mathrm{DAB}$ solution: Dissolve $100 \mathrm{mg}$ DAB in $100 \mathrm{~mL}$ of deionized water (adjust $\mathrm{pH}$ to 3.8 with $1 \mathrm{M} \mathrm{HCl}$ ).

2.2. $96 \%(\mathrm{v} / \mathrm{v})$ ethanol: Add $4 \mathrm{~mL}$ of deionized water to $96 \mathrm{~mL}$ of absolute ethanol.

2.3. Fixed solution: ethanol: lactic acid: glycerol (3:1:1).

2.4. Vacuum pump.

2.5. Camera.

3. Methods

3.1. Submerge the interested plant tissues in staining solution by vacuum infiltration $2-3$ times (1-2 min per time) until the plant tissues are completely infiltrated.

3.2. Incubate for $5-6 \mathrm{~h}$ till brown precipitates are observed.

3.3. Chlorophyll can be removed by repeated washes with $96 \%(\mathrm{v} / \mathrm{v})$ ethanol under heating at $40^{\circ} \mathrm{C}$.

3.4. Stained plant tissues can be fixed with fixer solution.

3.5. Photograph will be taken with the help of a camera.

\section{Precautions}

4.1. A low $\mathrm{pH}$ of 3.8 is necessary for proper solubilization of DAB.

4.2. During vacuum infiltration, it is important to release the vacuum gently to enable better infiltration of plant tissues.

\subsubsection{Method 7: $\mathrm{DCFH}_{2}$-DA and DCF Fluorescence Methods}

\section{Principle}

Fluorescence probe $2^{\prime}, 7^{\prime}$-dichlorofluorescein diacetate $\left(\mathrm{DCFH}_{2}-\mathrm{DA}\right)$ is one of the most commonly used 
dyes for determining total ROS (mainly $\mathrm{H}_{2} \mathrm{O}_{2}$ and $\mathrm{O}_{2} \bullet^{-}$). Esterified $\mathrm{DCFH}_{2}$-DA (nonfluorescent) can be permeable to cell membranes and enter freely the cells. Inside the cells, $\mathrm{DCFH}_{2}-\mathrm{DA}$ is deacetylated by intracellular esterases to generate $2^{\prime}, 7^{\prime}$-dichlorofluorescein (DCF) that is trapped. The DCF can be oxidized by ROS molecules to a highly fluorescent substance with excitation at $504 \mathrm{~nm}$ and emission at $524 \mathrm{~nm}$ (Fig. 5.18; Rodriguez and Taleisnik, 2012; Park and RoubelakisAngelakis, 2018). The intensity of fluorescence is proportional to the amount of ROS in plant tissues. Therefore, ROS can be monitored by fluorescence microscopy or fluorospectrophotometer using in vitro and in vivo methods.

\subsubsection{In Vivo Method: (DCFH - -DA Method)}

\section{Materials}

2.1. $20 \mathrm{mM} \mathrm{K-phosphate} \mathrm{buffer} \mathrm{(} \mathrm{pH}$ 6.0): Add $13.2 \mathrm{~mL}$ of $100 \mathrm{mM} \mathrm{K}_{2} \mathrm{HPO}_{4}(1.74 \mathrm{~g} / 100 \mathrm{~mL})$ to $86.8 \mathrm{~mL}$ of $100 \mathrm{mM} \mathrm{KH}{ }_{2} \mathrm{PO}_{4}(1.36 \mathrm{~g} / 100 \mathrm{~mL})$. Autoclave at $121^{\circ} \mathrm{C}$ for $20 \mathrm{~min}$. Store at room temperature. Before use, dilute with water from the concentrated stock to $20 \mathrm{mM} \mathrm{K}$-phosphate buffer.

2.2. $10 \mu \mathrm{M} \mathrm{DCFH} \mathrm{H}_{2}$-DA: Dissolve $1 \mathrm{mg}$ of $\mathrm{DCFH}_{2}$-DA (a pack, MW $=487$ ) in $2 \mathrm{~mL}$ of DMSO to obtain $1 \mathrm{mM} \mathrm{DCFH}_{2}$-DA. Storage in separate tubes at $-20^{\circ} \mathrm{C}$ in a black box. Before experiments, dilute from $1 \mathrm{mM}$ stock solution to $10 \mu \mathrm{M}$ $\mathrm{DCFH}_{2}$-DA with $20 \mathrm{mM} \mathrm{K}$-phosphate buffer.

2.3. Confocal laser scanning microscopy.

\section{Methods}

3.1. Submerge the detach plant tissues (or whole seedlings) in $10 \mu \mathrm{M} \mathrm{DCFH}_{2}$-DA for $30 \mathrm{~min}$.

3.2. Rinse the samples thoroughly with water at least twice to eliminate any remaining fluorescent dye.

3.3. Visualize green fluorescence by confocal laser scanning microscopy with 504/524 nm (excitation/emission).

3.4. Photograph.

\section{Precautions}

4.1. $\mathrm{DCFH}_{2}$-DA is sensitive to light. The stock solution should be covered with aluminum foil to prevent light exposure.

4.2. For the $\mathrm{H}_{2} \mathrm{O}_{2}$ specificity control, add $1.66 \mathrm{~g} \mathrm{KI}$ to $10 \mu \mathrm{M} \mathrm{DCFH}{ }_{2}$-DA.

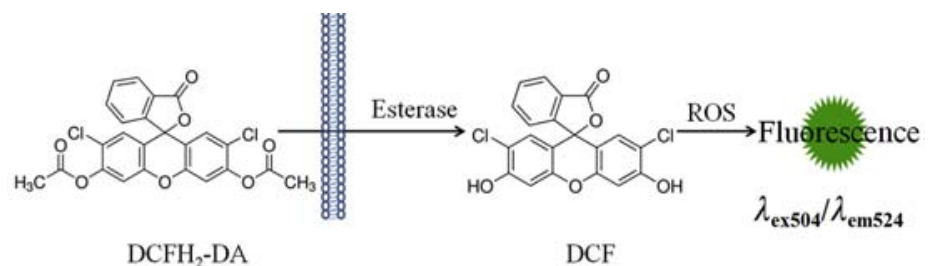

\subsubsection{In Vitro Method: (DCF Method)}

\section{Materials}

2.1. Dimethylsulfoxide (DMSO).

2.2. Extraction buffer: Namely $10 \mathrm{mM}$ Tris $-\mathrm{HCl}(\mathrm{pH}$ 7.2): Mix $50 \mathrm{~mL}$ of $200 \mathrm{mM}$ Tris $(2.42 \mathrm{~g} / 100 \mathrm{~mL})$ with $44.2 \mathrm{~mL}$ of $200 \mathrm{mM} \mathrm{HCl}(1.7 \mathrm{~mL} / 100 \mathrm{~mL})$. Make up to $1000 \mathrm{~mL}$ with deionized water.

2.3. $10 \mu \mathrm{M}$ DCF: Dissolve $1 \mathrm{mg}$ DCF $(\mathrm{MW}=401)$ in $1 \mathrm{~mL}$ of DMSO to obtain $2.5 \mathrm{mM}$ DCF. Dilute to final concentration of $10 \mu \mathrm{M}$ DCF with $10 \mathrm{mM}$ Tris- $\mathrm{HCl}(\mathrm{pH}$ 7.2).

2.4. Bradford reagent (used for protein quantification).

2.5. Refrigerated centrifuge.

2.6. Fluorescence microscopy or fluorospectrophotometer.

\section{Methods}

3.1. Before starting the experiment, turn on the fluorescence microscope to warm up.

3.2. Plant tissues $(100 \mathrm{mg})$ are ground in liquid nitrogen with mortar and pestle.

3.3. Homogenates are mixed with $1 \mathrm{~mL}$ of extraction buffer.

3.4. Centrifuge at $10,000 \times \mathrm{g}$ for $15 \mathrm{~min}$ at $4^{\circ} \mathrm{C}$.

3.5. Add $100 \mu \mathrm{L}$ of supernatant to $890 \mu \mathrm{L}$ of $10 \mathrm{mM}$ Tris $-\mathrm{HCl}$ (pH 7.2) [use $1 \mathrm{~mL}$ of $10 \mathrm{mM}$ Tris- $\mathrm{HCl}(\mathrm{pH} 7.2)$ as the blank].

3.6. Add $10 \mu \mathrm{L}$ of $1 \mathrm{mM}$ DCF (final concentration will be $10 \mu \mathrm{M}$ ) to sample. Vortex and incubate for $10 \mathrm{~min}$ in dark [use the mixture of $100 \mu \mathrm{L}$ of plant extract and $900 \mu \mathrm{L}$ of Tris $-\mathrm{HCl}(\mathrm{pH} 7.2)$ as the control to remove the background fluorescence].

3.7. Visualize by fluorescence microscope or fluorospectrophotometer with excitation at $504 \mathrm{~nm}$ and emission at $524 \mathrm{~nm}$.

3.8. Estimate protein concentration in samples using Bradford method.

3.9. The amount of ROS is expressed as relative fluorescence units $\mathrm{mg}^{-1}$ protein.

\section{Precautions}

4.1. DCF is sensitive to light. The stock solution should be covered with aluminum foil to prevent light exposure.

4.2. To get the specificity for $\mathrm{H}_{2} \mathrm{O}_{2}$, catalase (300 units $/ \mathrm{mL}$ ) should be added to reaction mixture to remove $\mathrm{H}_{2} \mathrm{O}_{2}$ before DCF is added.

FIGURE 5.18 $\mathrm{DCFH}_{2}$-DA, which can freely permeate into cytosol, is deacetylated by intracellular esterases to produce DCF, which can be oxidized by ROS to generate fluorescence. 
4.3. This method can carry out in vitro measurement for $\mathrm{H}_{2} \mathrm{O}_{2}$ using $10 \mu \mathrm{M} \mathrm{DCFH} \mathrm{H}_{2}$-DA instead of $10 \mu \mathrm{M}$ DCF.

\subsubsection{Method 8: AR and AUR Fluorescence Methods}

\section{Principle}

Fluorescence probes10-acetyl-3,7dihydroxyphenoxazine, also known as Ampliflu Red (AR, OxiRed probe) and Amplex UltraRed reagent (AUR), are highly sensitive and stable probes for $\mathrm{H}_{2} \mathrm{O}_{2}$. In the presence of horseradish peroxidase (HRP), the AR and AUR probes react in a 1:1 stoichiometry with $\mathrm{H}_{2} \mathrm{O}_{2}$ to produce highly fluorescent resorufin with excitation at $571 \mathrm{~nm}$ and emission at $585 \mathrm{~nm}$ for AR, as well as excitation at $568 \mathrm{~nm}$ and emission at $581 \mathrm{~nm}$ for AUR (Fig. 5.19; Rodriguez and Taleisnik, 2012; Park and Roubelakis-Angelakis, 2018). Because AUR cannot cross the cell membrane, but AR can, AUR is widely used to detect extracellular $\mathrm{H}_{2} \mathrm{O}_{2}$, while AR is used to detect intracellular $\mathrm{H}_{2} \mathrm{O}_{2}$. The intensity of fluorescence is proportional to the amount of $\mathrm{H}_{2} \mathrm{O}_{2}$. Therefore the content of $\mathrm{H}_{2} \mathrm{O}_{2}$ in plant samples can be assayed by colorimetric or fluorometric methods.

\section{Materials}

2.1. $50 \mathrm{mM}$ sodium phosphate buffer ( $\mathrm{pH}$ 7.4): Add $19 \mathrm{~mL}$ of $100 \mathrm{mM} \mathrm{NaH} \mathrm{PO}_{4} \cdot \mathrm{H}_{2} \mathrm{O}$

$\left(\mathrm{NaH}_{2} \mathrm{PO}_{4} \cdot \mathrm{H}_{2} \mathrm{O}: 1.39 \mathrm{~g} / 100 \mathrm{~mL}\right)$ to $81 \mathrm{~mL}$ of $100 \mathrm{mM} \mathrm{Na} 2 \mathrm{HPO}_{4}\left(\mathrm{Na}_{2} \mathrm{HPO}_{4} \cdot 7 \mathrm{H}_{2} \mathrm{O}_{2}\right)$.

$68 \mathrm{~g} / 100 \mathrm{~mL}$ or $\mathrm{Na}_{2} \mathrm{HPO}_{4} \cdot 12 \mathrm{H}_{2} \mathrm{O}(3.59 \mathrm{~g} /$ $100 \mathrm{~mL}$ ). Autoclave at $121^{\circ} \mathrm{C}$ for $20 \mathrm{~min}$. Store at room temperature. Before use, dilute to $50 \mathrm{mM}$ sodium phosphate buffer with deionized water.

2.2. $50 \mu \mathrm{M} A R$ working reagent: Dissolve $1.29 \mathrm{mg}$ of AR (MW $=257.25)$ in $1 \mathrm{~mL}$ of DMSO to obtain $5 \mathrm{mM}$ AR, which is stored at $-20^{\circ} \mathrm{C}$. Just prior to use, dilute to final concentration $(50 \mu \mathrm{M})$ with $50 \mathrm{mM}$ sodium phosphate buffer ( $\mathrm{pH} 7.4$ ).

2.3. $50 \mu \mathrm{M}$ AUR reagent working solution: Dissolve $1 \mathrm{mg}$ AUR $(\mathrm{MW}=300)$ in $666 \mu \mathrm{L}$ DMSO to obtain $5 \mathrm{mM}$ AUR. Dilute to $1 \mathrm{mM}$ AUR with DMSO and then store at $-20^{\circ} \mathrm{C}$. Just prior to use, dilute $1 \mathrm{mM}$ AUR to final concentration of

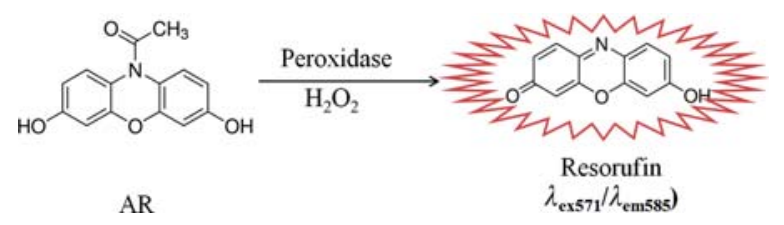

FIGURE 5.19 AR reacts with $\mathrm{H}_{2} \mathrm{O}_{2}$ in the present of peroxidase to generate resorufin.
$50 \mu \mathrm{M}$ with $50 \mathrm{mM}$ sodium phosphate buffer ( $\mathrm{pH} 7.4)$.

2.4. Fluorescence microscopy or fluorospectrophotometer.

\section{Methods}

See "DCFH ${ }_{2}$-DA and DCF Methods." In this experiment, $\mathrm{DCFH}_{2}$-DA and DCF should be replaced with AR and AUR.

3.1. Plant tissues are incubated in $50 \mu \mathrm{M}$ AR or $50 \mu \mathrm{M}$ AUR working solution for 30-60 min at room temperature in the dark.

3.2. Assay fluorescence by fluorescence microscopy with excitation at $571 \mathrm{~nm}$ and emission at $585 \mathrm{~nm}$ for $\mathrm{AR}$, and excitation at $568 \mathrm{~nm}$ and emission at $581 \mathrm{~nm}$ for AUR.

3.3. Photograph or calculate $\mathrm{H}_{2} \mathrm{O}_{2}$ content according to standard curve with known concentrations of $\mathrm{H}_{2} \mathrm{O}_{2}$ from 0 to $50 \mu \mathrm{M}$.

4. Precautions

4.1. DMSO is hazardous. Reagents containing DMSO should be carefully handled. Avoid contact with skin and eyes.

4.2. This method can carry out in vitro measurement for $\mathrm{H}_{2} \mathrm{O}_{2}$ using $50 \mu \mathrm{MAR}$ or AUR instead of $10 \mu \mathrm{M}$ DCF (see DCF method).

4.3. For the $\mathrm{H}_{2} \mathrm{O}_{2}$ specificity control, add $1.66 \mathrm{~g} \mathrm{KI}$ to $50 \mu \mathrm{M}$ AR or $50 \mu \mathrm{M}$ AUR.

\subsubsection{Method 9: BES- $\mathrm{H}_{2} \mathrm{O}_{2}-\mathrm{Ac}$ and BES- $\mathrm{H}_{2} \mathrm{O}_{2}$ Fluorescence Method}

\section{Principle}

Fluorescence probes 3'-O-Acetyl-6'-O-pentafluorobenzenesulfonyl-2'-7'-difluorofluorescein $\left(\mathrm{BES}-\mathrm{H}_{2} \mathrm{O}_{2}\right.$-Ac) and 6'-O-penta-

fluorobenzenesulfonyl-2'-7'-difluorofluorescein $\left(\mathrm{BES}-\mathrm{H}_{2} \mathrm{O}_{2}\right.$ ) are nonfluorescent. When they are oxidized by $\mathrm{H}_{2} \mathrm{O}_{2}$ they form highly fluorescent oxidized products $\left(2^{\prime}, 7^{\prime}\right.$-difluorofluorescein) with excitation at $485 / 530 \mathrm{~nm}$ (excitation/emission) (Fig. 5.20; Maeda et al., 2007; Rodriguez and Taleisnik, 2012; Park and Roubelakis-Angelakis, 2018). The intensity of green fluorescence is a direct correlation to the amount of $\mathrm{H}_{2} \mathrm{O}_{2}$ in plant samples. BES- $\mathrm{H}_{2} \mathrm{O}_{2}$-Ac can freely enter into the cells, which is usually used to explore intracellular $\mathrm{H}_{2} \mathrm{O}_{2}$; while BES- $\mathrm{H}_{2} \mathrm{O}_{2}$ cannot cross the cell membrane, extracellular $\mathrm{H}_{2} \mathrm{O}_{2}$ is commonly detected by fluorescence microscope.

\section{Materials}

2.1. $20 \mathrm{mM} \mathrm{K}$-phosphate buffer ( $\mathrm{pH}$ 6.0): Add $13.2 \mathrm{~mL}$ of $100 \mathrm{mM} \mathrm{K}_{2} \mathrm{HPO}_{4}(1.74 \mathrm{~g} / 100 \mathrm{~mL})$ to $86.8 \mathrm{~mL}$ of $100 \mathrm{mM} \mathrm{KH}_{2} \mathrm{PO}_{4}(1.36 \mathrm{~g} / 100 \mathrm{~mL})$. Autoclave at $121^{\circ} \mathrm{C}$ for $20 \mathrm{~min}$. Store at room 
temperature. Before use, dilute to $20 \mathrm{mM} \mathrm{K}$ phosphate buffer with deionized water.

2.2. $50 \mu \mathrm{M}$ BES- $\mathrm{H}_{2} \mathrm{O}_{2}$-Ac working reagent: Dissolve $1 \mathrm{mg}$ BES- $\mathrm{H}_{2} \mathrm{O}_{2}$-Ac (MW $\left.=640.44\right)$ in $310 \mu \mathrm{L}$ of DMSO to obtain 5 mMBES- $\mathrm{H}_{2} \mathrm{O}_{2}$-Ac, which is stored at $4{ }^{\circ} \mathrm{C}$. Just prior to use, dilute to final concentration $(50 \mu \mathrm{M})$ with $20 \mathrm{mM} \mathrm{K}$-phosphate buffer ( $\mathrm{pH}$ 6.0).

2.3. $50 \mu \mathrm{M}$ BES- $\mathrm{H}_{2} \mathrm{O}_{2}$ reagent working solution: Dissolve $1 \mathrm{mg} \mathrm{BES}-\mathrm{H}_{2} \mathrm{O}_{2}(\mathrm{MW}=598.40)$ in $334 \mu \mathrm{L}$ of DMSO to obtain $5 \mathrm{mM} \mathrm{BES}-\mathrm{H}_{2} \mathrm{O}_{2}$, which is stored at $4{ }^{\circ} \mathrm{C}$. Just prior to use, dilute to final concentration $(50 \mu \mathrm{M})$ with $20 \mathrm{mM} \mathrm{K}$ phosphate buffer ( $\mathrm{pH} 6.0$ ).

2.4. Fluorescence microscopy or fluorospectrophotometer.

\section{Methods}

See "DCFH ${ }_{2}$-DA and DCF Methods." In this experiment, $\mathrm{DCFH}_{2}$-DA and DCF should be replaced with BES- $\mathrm{H}_{2} \mathrm{O}_{2}-\mathrm{Ac}$ and $\mathrm{BES}-\mathrm{H}_{2} \mathrm{O}_{2}$.

3.1. Plant tissues are incubated in $50 \mu \mathrm{M}$ BES- $\mathrm{H}_{2} \mathrm{O}_{2}$ Acor $50 \mu \mathrm{M}$ BES- $\mathrm{H}_{2} \mathrm{O}_{2}$ reagent working solution for indicated time at room temperature in the dark.

3.2. Detect fluorescence by fluorescence microscopy or fluorospectrophotometer with excitation at $485 \mathrm{~nm}$ and emission at $530 \mathrm{~nm}$ for BES- $\mathrm{H}_{2} \mathrm{O}_{2-}$ Ac (for extracellular $\mathrm{H}_{2} \mathrm{O}_{2}$ ) and BES- $\mathrm{H}_{2} \mathrm{O}_{2}$ (for intracellular $\mathrm{H}_{2} \mathrm{O}_{2}$ ).

3.3. Photograph or calculate $\mathrm{H}_{2} \mathrm{O}_{2}$ content according to standard curve with known concentrations of $\mathrm{H}_{2} \mathrm{O}_{2}$ from 0 to $50 \mu \mathrm{M}$.

\section{Precautions}

4.1. DMSO is hazardous. Reagents containing DMSO should be carefully handled. Avoid contact with skin and eyes.

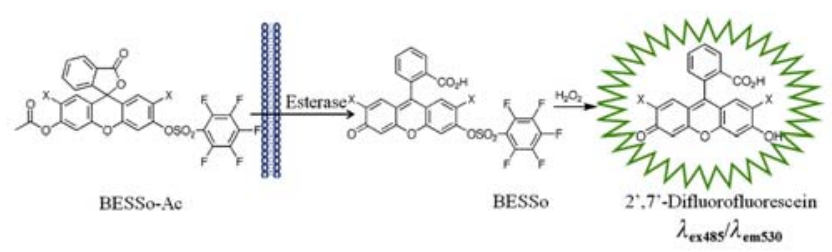

FIGURE 5.20 BESSo, which can freely permeate into cytosol, is deacetylated by intracellular esterases to produce BESSo, which can be oxidized by $\mathrm{H}_{2} \mathrm{O}_{2}$ to generate green fluorescence.

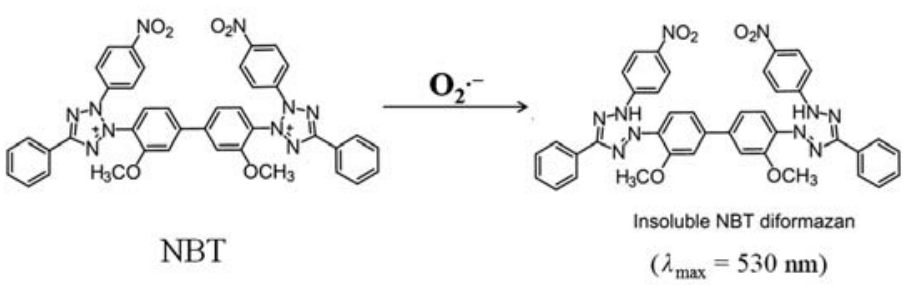

4.2. This method can carry out in vitro measurement for $\mathrm{H}_{2} \mathrm{O}_{2}$ using $50 \mu \mathrm{M}$ BES- $\mathrm{H}_{2} \mathrm{O}_{2-}$ Ac or BES- $\mathrm{H}_{2} \mathrm{O}_{2}$ instead of $10 \mu \mathrm{M}$ DCF (see DCF method).

4.3. For the $\mathrm{H}_{2} \mathrm{O}_{2}$ specificity control, add $1.66 \mathrm{~g} \mathrm{KI}$ to $50 \mu$ MBES- $\mathrm{H}_{2} \mathrm{O}_{2}$-Acor $50 \mu \mathrm{M}$ BES- $\mathrm{H}_{2} \mathrm{O}_{2}$.

\subsection{SECTION 6: SUPEROXIDE RADICAL MEASUREMENT}

\subsubsection{Method 1: NBT Method}

\section{Principle}

The qualitative determination of $\mathrm{O}_{2}{ }^{\bullet}$ with nitrotetrazolium blue chloride depends on the formation of a blue insoluble 1,3,5triphenyltetrazolium formazan precipitate that results from the reaction between $\mathrm{O}_{2}{ }^{-}$and NBT. The formazan has a maximal absorbance at $530 \mathrm{~nm}$ and the extinction coefficient of $1.28 \times 10^{4} \mathrm{M}^{-1} \mathrm{~cm}^{-1}$ (Fig. 5.21; Rodriguez and Taleisnik, 2012; Li and Gong, 2014). The absorbance is positively proportional to the amount of $\mathrm{O}_{2} \bullet^{-}$in plant samples. Therefore, $\mathrm{O}_{2}{ }^{-}$production can be detected by in vivo and in vitro methods.

\section{Materials}

2.1. $10 \mathrm{mM} \mathrm{MnCl}_{2}$ : Dissolve162 $\mathrm{mg} \mathrm{MnCl}_{2}$ $(\mathrm{MW}=161.87)$ in $100 \mathrm{~mL}$ of deionized water.

2.2. $100 \mathrm{mM}$ K-phosphate buffer ( $\mathrm{pH}$ 7.0): Mix $61 \mathrm{~mL}$ of $100 \mathrm{mM} \mathrm{K} \mathrm{HPO}_{4}(17.418 \mathrm{~g} / 100 \mathrm{~mL})$ with $39 \mathrm{~mL}$ of $100 \mathrm{mM} \mathrm{KH}_{2} \mathrm{PO}_{4}$ $(13.609 \mathrm{~g} / 100 \mathrm{~mL})$.

2.3. $0.5 \%$ NBT solution: Dissolve $0.5 \mathrm{~g}$ NBT $(\mathrm{MW}=817.64)$ in $100 \mathrm{~mL}$ of $100 \mathrm{mM} \mathrm{K}-$ phosphate buffer ( $\mathrm{pH}$ 7.0).

2.4. Destaining solution: A mixture of ethanol and glycerin (9:1).

2.5. Vacuum pump.

2.6. Camera.

\subsubsection{Spectrophotometric Method}

\section{Methods}

3.1. Submerge the plant tissues $(200 \mathrm{mg}$ ) in $0.5 \%$ NBT solution by vacuum infiltration $1-3$ times (1 min each time).

FIGURE 5.21 NBT can react with $\mathrm{O}_{2}{ }^{-}$to generate insoluble NBT diformazan. 
3.2. Incubate for indicated time (such as $1 \mathrm{~h}$ ) at room temperature in the dark.

3.3. Centrifuge at $8000 \times \mathrm{g}$ for $5 \mathrm{~min}$ at room temperature.

3.4. The supernatant is heated at $85^{\circ} \mathrm{C}$ for $15 \mathrm{~min}$, and then cooled rapidly on ice.

3.5. Read absorbance at $530 \mathrm{~nm}$ with a spectrophotometer.

3.6. The $\mathrm{O}_{2}{ }^{{ }^{-}-}$content is expressed as the increase in absorbance $\mathrm{g}^{-1} \mathrm{FW}$ or calculated using the extinction coefficient of $1.28 \times 10^{4} \mathrm{M}^{-1} \mathrm{~cm}^{-1}$.

\subsubsection{Histochemical Staining Method}

\section{Methods}

3.1. Submerge the plant tissues ( $200 \mathrm{mg}$ ) in $0.5 \%$ NBT solution by vacuum infiltration $1-3$ times (1 min each time).

3.2. Incubate for $2 \mathrm{~h}$ at $30^{\circ} \mathrm{C}$ in the dark.

3.3. Remove NBT solution and add destaining solution. Boil for $10 \mathrm{~min}$ in water bath until the green color has faded almost completely (this step can be ignored for nongreen tissues).

3.4. Photograph will be taken by using a camera.

\section{Precautions}

4.1. NBT can be replaced with cytochrome C, the extinction coefficient of formazan is $2.1 \times 10^{4} \mathrm{M}^{-1} \mathrm{~cm}^{-1}$ at $550 \mathrm{~nm}$.

4.2. To study the effect of environmental stress on $\mathrm{O}_{2}{ }^{\cdot-}$ production, an appropriate amount of $\mathrm{NaCl}$, sorbitol, and heavy metal is added to NBT solution to carry out salt, osmotic, and heavy metal stresses. In addition, plant materials incubated in NBT solution are submitted to high or cold temperature to explore the production of $\mathrm{H}_{2} \mathrm{O}_{2}$.

4.3. For the $\mathrm{O}_{2}{ }^{\cdot-}$ specificity control, add $0.5 \mathrm{~mL}$ of the $\mathrm{MnCl}_{2}$ solution or SOD $(50 \mu \mathrm{m} / \mathrm{mL})$ before making up to the final volume of NBT.

\subsubsection{Method 2: XTT Method}

\section{Principle}

Tetrazolium compounds that form soluble formazans can be used to quantify $\mathrm{O}_{2}{ }^{-}$ production. The reaction between $\mathrm{O}_{2} \bullet^{-}$and $\mathrm{Na}$, 3'-[1-[(phenylamino)-carbonyl]-3, 4-tetrazolium] (4-methoxy-6-nitro) benzene sulfonic acid hydrate (XTT) produces a soluble formazan, which has a maximal light absorption at $470 \mathrm{~nm}$ and the extinction coefficient of $2.16 \times 10^{4} \mathrm{M}^{-1} \mathrm{~cm}^{-1}$ (Fig. 5.22; Rodriguez and Taleisnik, 2012; Li and Gong, 2014). The absorbance is proportional to the content of $\mathrm{O}_{2}{ }^{-}$ in plant samples.

\section{Materials}

2.1. $10 \mathrm{mM} \mathrm{MnCl}_{2}$ : Dissolve $162 \mathrm{mg} \mathrm{MnCl}_{2}$ $(\mathrm{MW}=161.87)$ in $100 \mathrm{~mL}$ of deionized water.

2.2. $100 \mathrm{mM} \mathrm{K}$-phosphate buffer ( $\mathrm{pH}$ 7.0): Mix $61 \mathrm{~mL}$ of $100 \mathrm{mM} \mathrm{K}_{2} \mathrm{HPO}_{4}(17.418 \mathrm{~g} / 100 \mathrm{~mL})$ with $39 \mathrm{~mL}$ of $100 \mathrm{mM} \mathrm{KH}_{2} \mathrm{PO}_{4}(13.609 \mathrm{~g} /$ $100 \mathrm{~mL}$ ).

2.3. $0.5 \mathrm{mM} X \mathrm{XTT}$ : Dissolve $34 \mathrm{mg} X \mathrm{XTT}$ $(\mathrm{MW}=673.52)$ in $100 \mathrm{~mL}$ of $100 \mathrm{mM} \mathrm{K}-$ phosphate buffer ( $\mathrm{pH} 7.0$ ).

2.4. Vacuum pump.

2.5. Spectrophotometer.

3. Methods

3.1. Submerge the plant material completely in the XTT solution by vacuum infiltration for $1 \mathrm{~min}$.

3.2. Incubate for $5 \mathrm{~h}$ at $30^{\circ} \mathrm{C}$ in the dark.

3.3. Collect the incubation medium and centrifuge at $10,000 \times \mathrm{g}$ for $5 \mathrm{~min}$.

3.4. Measure the absorbance of the incubation medium at $470 \mathrm{~nm}$.

3.5. Calculate the $\mathrm{O}_{2}{ }^{--}$production using molar extinction coefficient of $2.16 \times 10^{4} \mathrm{M}^{-1} \mathrm{~cm}^{-1}$ ).

\section{Precautions}

4.1. To study the effect of environmental stress on $\mathrm{O}_{2}{ }^{\cdot-}$ production, an appropriate amount of $\mathrm{NaCl}$, sorbitol, and heavy metal is added to XTT solution to carry out salt, osmotic, and heavy metal stresses. In addition, plant materials incubated in XTT solution are submitted to high or cold temperature to explore the production of $\mathrm{H}_{2} \mathrm{O}_{2}$.

4.2. For the $\mathrm{O}_{2}{ }^{\cdot-}$ specificity control, add $0.5 \mathrm{~mL}$ of the $\mathrm{MnCl}_{2}$ solution or SOD $(50 \mu \mathrm{m} / \mathrm{mL})$ before making up to the final volume of XTT.

FIGURE 5.22 XTT can react with $\mathrm{O}_{2}^{\cdot-}$ to generate soluble NBT formazan.

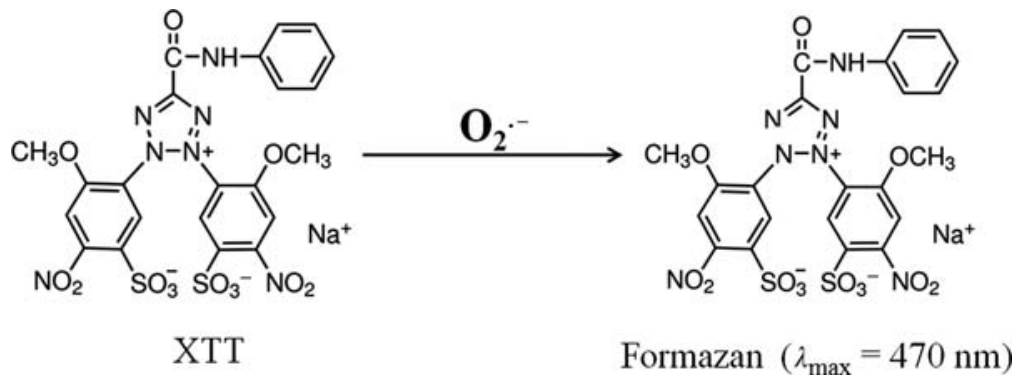




\subsubsection{Method 3: BESSo-AM and BESSo Fluorescence Methods}

\section{Principle}

Superoxide radical $\left(\mathrm{O}_{2} \bullet^{-}\right)$fluorescence probes $3^{\prime}-$ o-(4,5-dimethoxy-2-nitrobenzenesulfonyl)-2', $4^{\prime}, 5^{\prime}, 7^{\prime}-$ tetrafluorofluorescein (BESSo) and acetylated BESSo (BESSo-AM) are nonfluorescent. BES- $\mathrm{H}_{2} \mathrm{O}_{2}$-AM can freely permeate into the cells in where it is deacetylated by intracellular esterase to form BESSo (Maeda et al., 2007; Rodriguez and Taleisnik, 2012; Park and Roubelakis-Angelakis, 2018). In addition, BESSo cannot cross the membrane into the cells. BESSo can be oxidized by $\mathrm{O}_{2}^{\bullet-}$ to form $2^{\prime}, 7^{\prime}-$ difluorofluorescein, which has highly fluorescent oxidized products with excitation at 505/544 nm (excitation/emission) (Fig. 5.23; Maeda et al., 2007; Rodriguez and Taleisnik, 2012; Park and Roubelakis-Angelakis, 2018). Therefore, BESSo is usually used to detect extracellular $\mathrm{O}_{2}{ }^{-}$; while BESSo-AM can determine intracellular $\mathrm{O}_{2}{ }^{-}$in plant materials. Because the intensity of green fluorescence is direct correlation to the amount of $\mathrm{O}_{2} \bullet^{-}$in plant samples, the $\mathrm{O}_{2} \bullet^{-}$content can be detected by fluorescence microscope.

\section{Materials}

2.1. $20 \mathrm{mM}$ K-phosphate buffer ( $\mathrm{pH}$ 6.0): Add $13.2 \mathrm{~mL}$ of $100 \mathrm{mM} \mathrm{K} \mathrm{HPO}_{4}(1.74 \mathrm{~g} / 100 \mathrm{~mL})$ to $86.8 \mathrm{~mL}$ of $100 \mathrm{mM}$ stock $\mathrm{KH}_{2} \mathrm{PO}_{4}(1.36 \mathrm{~g} /$ $100 \mathrm{~mL}$ ). Autoclave at $121^{\circ} \mathrm{C}$ for $20 \mathrm{~min}$. Store at room temperature. Before use, dilute to $20 \mathrm{mM}$ K-phosphate buffer with deionized water.

2.2. $30 \mu \mathrm{M}$ BESSo-AM reagent working solution: Dissolve $1 \mathrm{mg}$ BESSo-AM (MW = 721.54) in $462 \mu \mathrm{L}$ of DMSO to obtain $3 \mathrm{mM}$ BES-So-AM, which is stored at $4{ }^{\circ} \mathrm{C}$ in a black box. Just prior to use, dilute to final concentration $(30 \mu \mathrm{M})$ with $20 \mathrm{mM}$ K-phosphate buffer ( $\mathrm{pH}$ 6.0).

2.3. $30 \mu \mathrm{M}$ BESSo reagent working solution: Dissolve $1 \mathrm{mg}$ BES-So (MW = 649.48) in $195 \mu \mathrm{L}$ of DMSO to get $3 \mathrm{mM}$ BESSo, which is stored at $4^{\circ} \mathrm{C}$ in a black box. Just prior to use, dilute to final concentration $(30 \mu \mathrm{M})$ with $20 \mathrm{mM} \mathrm{K}$ phosphate buffer ( $\mathrm{pH}$ 6.0).

2.4. Confocal laser scanning microscopy or fluorospectrophotometer.

\section{Methods}

See "DCFH ${ }_{2}$ and DCF Method." In this experiment, $\mathrm{DCFH}_{2} \mathrm{DA}$ and DCF should be replaced with BESSo-AM and BESSo.

3.1. Plant tissues are incubated in $30 \mu \mathrm{M}$ BESSo-AM or $30 \mu \mathrm{M}$ BESSo reagent working solution for 30-60 min at room temperature in the dark.

3.2. Detect fluorescence by confocal laser scanning microscopy (for in vivo method) or fluorospectrophotometer (for in vitro method) with excitation at $505 \mathrm{~nm}$ and emission at $544 \mathrm{~nm}$.

3.3. Photograph.

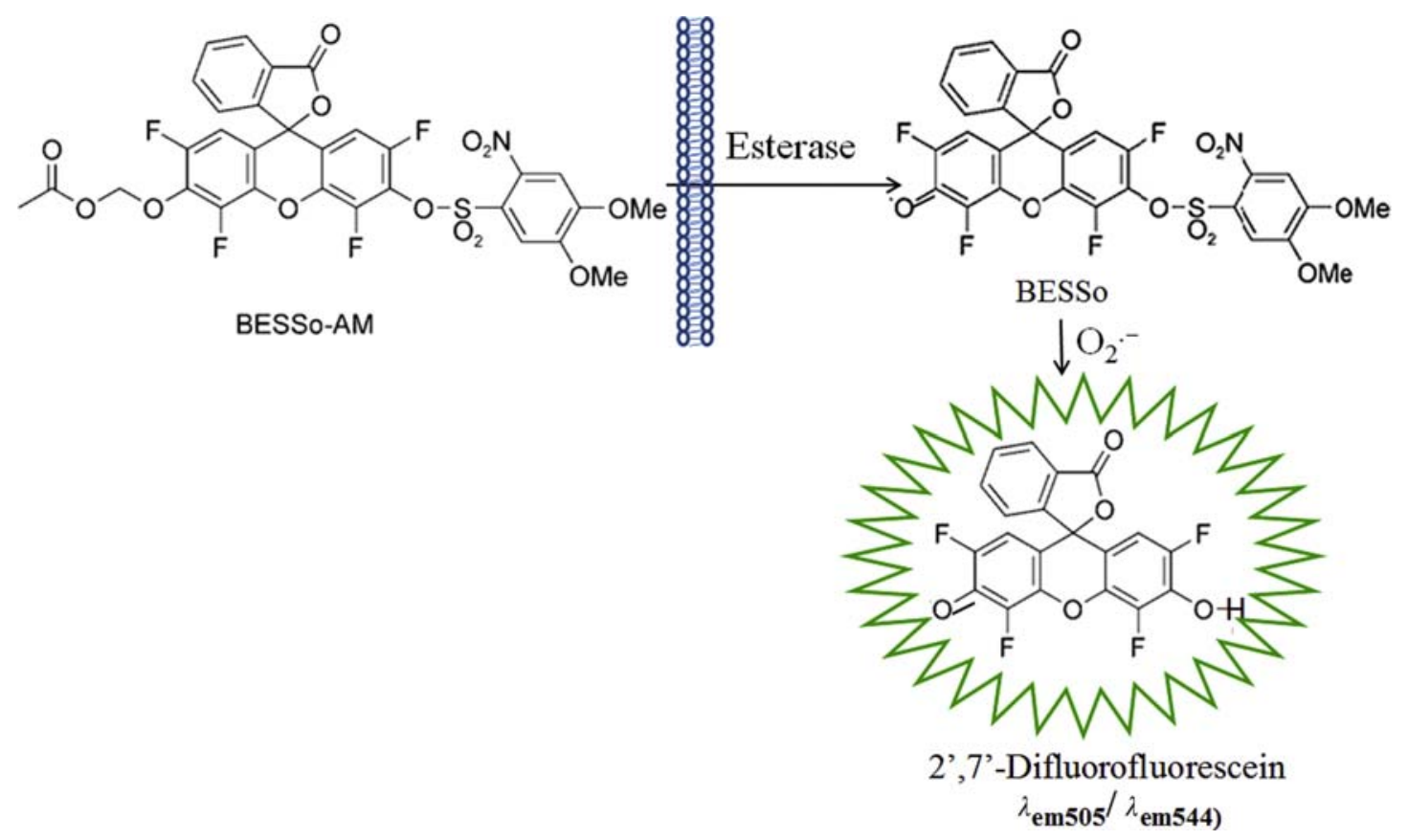

FIGURE 5.23 BESSo-AM, which can freely permeate into cytosol, is deacetylated by intracellular esterases to produce BESSo, which can be oxidized by $\mathrm{O}_{2} \bullet-$ to generate green fluorescence. 


\section{Precautions}

4.1. BESSo-AM and BESSo working reagent should be freshly prepared and stored at $4^{\circ} \mathrm{C}$ in a black box to protect from light.

4.2. This method can carry out in vitro measurement for $\mathrm{O}_{2}{ }^{--}$using $30 \mu \mathrm{M}$ BESSo-AM or $30 \mu \mathrm{M}$ BESSo instead of $10 \mu \mathrm{M}$ DCF (see DCF method).

4.3. DMSO is hazardous. Reagents containing DMSO should be carefully handled. Avoid contact with skin and eyes.

\subsection{SECTION 7: HO• QUANTIFICATION}

\subsubsection{Method 1: Benzoate Method}

\section{Principle}

Hydroxyl radical $(\mathrm{HO} \bullet)$ production can be quantified by spectrofluorometry as the formation of hydroxyl benzoate (fluorescence can be produced at $407 \mathrm{~nm}$ emission after excitation at $305 \mathrm{~nm}$ ) resulting from the reaction of $\mathrm{HO}^{\circ}$ and benzoate (BZ) (Fig. 5.24; Rodriguez and Taleisnik, 2012).

\section{Materials}

2.1. $20 \mathrm{mM} \mathrm{K}$-phosphate buffer ( $\mathrm{pH}$ 6.0): Add $13.2 \mathrm{~mL}$ of $100 \mathrm{mM} \mathrm{K}_{2} \mathrm{HPO}_{4}(1.74 \mathrm{~g} / 100 \mathrm{~mL})$ to $86.8 \mathrm{~mL}$ of $100 \mathrm{mM}$ stock $\mathrm{KH}_{2} \mathrm{PO}_{4}(1.36 \mathrm{~g} /$ $100 \mathrm{~mL}$ ). Dilute to $20 \mathrm{mM}$ K-phosphate buffer with deionized water.

2.2. $2.5 \mathrm{mM}$ BZ: Dissolve $31 \mathrm{mg} B Z(\mathrm{MW}=122)$ in $100 \mathrm{~mL}$ of $20 \mathrm{mM}$ K-phosphate buffer (pH 6.0). Store at $4^{\circ} \mathrm{C}$ in a black bottle to protect from light.

2.3. Vacuum pump.

2.4. Spectrofluorometer.

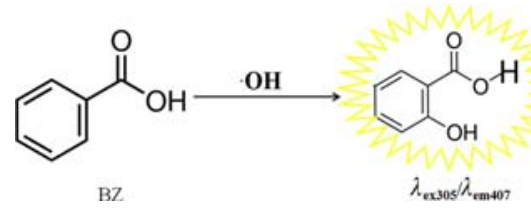

FIGURE 5.24 $\mathrm{HO}$ can react with $\mathrm{BZ}$ to generate a fluorescence product hydroxyl benzoate.

\section{Methods}

3.1. Submerge the plant material completely in the BZ solution by vacuum infiltration for $1 \mathrm{~min}$.

3.2. Incubate for indicated time (such as $7 \mathrm{~h}$ ) at $30^{\circ} \mathrm{C}$ in the dark.

3.3. Collect the incubation medium and centrifuge at $10,000 \times \mathrm{g}$ for $5 \mathrm{~min}$.

3.4. Measure the fluorescence using a spectrofluorometer with $305 / 407 \mathrm{~nm}$ (excitation/emission).

3.5. Run blanks without BZ in parallel to correct for unspecific fluorescence.

3.6. Prepare a BZ calibration curve by measuring a series of BZ dilutions (from 0 to $2.5 \mathrm{mM}$ ).

3.7. Transform the fluorescence values of the biological samples into ${ }^{\circ} \mathrm{OH}$ molar concentration using the calibration curve values.

\section{Precautions}

4.1. To study the effect of environmental stress on - OH production, an appropriate amount of $\mathrm{NaCl}$, sorbitol, and heavy metal is added to $\mathrm{BZ}$ solution to carry out salt, osmotic, and heavy metal stresses. In addition, plant materials incubated in BZ solution are subjected to high or cold temperature to explore the production of $\mathrm{H}_{2} \mathrm{O}_{2}$.

4.2. Due to sensitivity of $\mathrm{BZ}$ to light, $\mathrm{BZ}$ solution should be freshly prepared and stored at $4{ }^{\circ} \mathrm{C}$ in a black bottle to protect from light.

\subsubsection{Method 2: 2-deoxy-D-Ribose Method}

\section{Principle}

Hydroxyl radical $\left(\mathrm{HO}^{\circ}\right)$ can react with 2-deoxy-Dribose (DDR) to generate malondialdehyde (MDA), which in turn reacts with 2-thiobarbituricacid (TBA) and produces a pink product. The product exhibits a maximal light absorption at $532 \mathrm{~nm}$ and the molar extinction coefficient of $1.55 \times 10^{5} \mathrm{M}^{-1} \mathrm{~cm}^{-1}$ (Fig. 5.25; Schopfer et al., 2001). The absorbance is proportional to the content of $\mathrm{HO}^{\circ}$ in plant samples.

\section{Materials}

2.1. $20 \mathrm{mM} \mathrm{K}$-phosphate buffer ( $\mathrm{pH}$ 6.0): Add $13.2 \mathrm{~mL}$ of $100 \mathrm{mM} \mathrm{K}_{2} \mathrm{HPO}_{4}(1.74 \mathrm{~g} / 100 \mathrm{~mL})$ to $86.8 \mathrm{~mL}$ of $100 \mathrm{mM}$ stock $\mathrm{KH}_{2} \mathrm{PO}_{4}(1.36 \mathrm{~g} /$

FIGURE 5.25 DDR is converted by $\bullet \mathrm{OH}$ into MDA, which can react with TBA to generate a pink product.

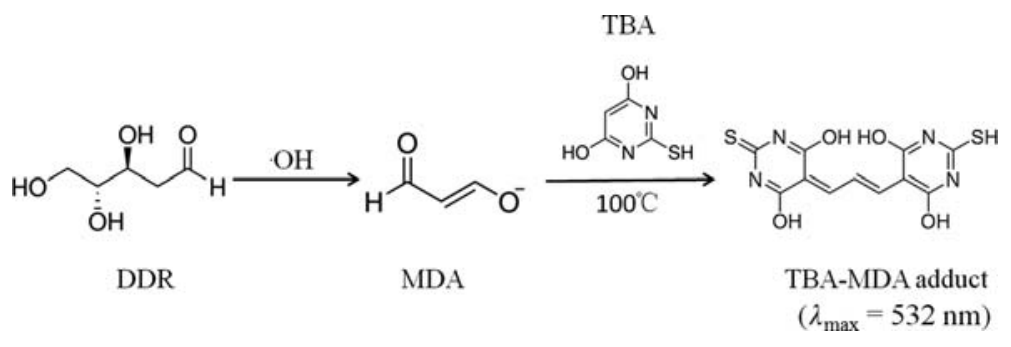


$100 \mathrm{~mL}$ ). Dilute to $20 \mathrm{mM}$ K-phosphate buffer with deionized water.

2.2. $20 \mathrm{mM}$ 2-deoxy-D-ribose: Dissolve $268 \mathrm{mg}$ 2deoxy-D-ribose (MW = 134) in $100 \mathrm{~mL}$ of $20 \mathrm{mM}$ K-phosphate buffer (pH 6.0).

2.3. $1 \%(\mathrm{w} / \mathrm{v})$ TBA: Dissolve $1 \mathrm{~g}$ TBA $(\mathrm{MW}=144)$ in $100 \mathrm{~mL}$ of $50 \mathrm{mM} \mathrm{NaOH}(0.2 \mathrm{~g} / 100 \mathrm{~mL})$.

2.4. $2.8 \%(\mathrm{w} / \mathrm{v})$ trichloroacetic acid (TCA): Add $227 \mathrm{~mL}$ of deionized water to $500 \mathrm{~g}$ TCA (a pack, $\mathrm{MW}=163$ ) to obtain $100 \%(\mathrm{w} / \mathrm{v}) \mathrm{TCT}$, and then dilute to $2.8 \%$ TCA with deionized water.

2.5. Vacuum pump.

2.6. Spectrophotometer or spectrofluorometer.

\section{Methods}

3.1. Submerge the plant tissues completely in $20 \mathrm{mM}$ 2-deoxy-D-ribose by vacuum infiltration for $1 \mathrm{~min}$.

3.2. Incubate for an indicated time (such as $7 \mathrm{~h}$ ) in darkness at $25^{\circ} \mathrm{C}$ on a shaker.

3.3. Collect the incubation medium and centrifuge at $10,000 \times \mathrm{g}$ for $5 \mathrm{~min}$.

3.4. Mix $0.5 \mathrm{~mL}$ of centrifuged incubation medium with $0.5 \mathrm{~mL}$ of $1 \%$ TBA. Mix and then add $0.5 \mathrm{~mL}$ of $2.8 \%$ TCA.

3.5. Boil at $100^{\circ} \mathrm{C}$ for $10 \mathrm{~min}$.

3.6. Measure the fluorescence using a spectrofluorometer (excitation: $532 \mathrm{~nm}$, emission: $553 \mathrm{~nm}$ ) or a spectrophotometer at $532 \mathrm{~nm}$.

3.7. Calculate MDA content using the extinction coefficient of $1.55 \times 10^{5} \mathrm{M}^{-1} \mathrm{~cm}^{-1}$ and convert into $\mathrm{HO}^{\circ}$ content.

\section{Precautions}

4.1. This method also can be used to determine MDA content in plant samples by spectrophotometry.

4.2. $\mathrm{TAB}$ should be freshly prepared.

\section{Acknowledgments}

This research is supported by National Natural Science Foundation of China (31760069; 31360057) and Doctor Startup Foundation of Yunnan Normal University China (01200205020503099).

\section{References}

Angelini, R., Cona, A., Tavladoraki, P., 2018. Determination of copper amine oxidase activity in plant tissues. In: Alcázar, R., Tiburcio, A.F. (Eds.), Polyamines: Methods and Protocols. Springer, New York, pp. 129-140.

Antoniou, C., Savvides, A., Georgiadou, E.C., Fotopoulos, V., 2018. Spectrophotometric quantification of reactive oxygen, nitrogen and sulfur species in plant samples. In: Alcázar, R., Tiburcio, A.F. (Eds.), Polyamines: Methods and Protocols. Springer, New York, pp. $155-162$.
Ashtamker, C., Kiss, V., Sagi, M., Davydov, O., Fluhr, R., 2007. Diverse subcellular locations of cryptogein-induced reactive oxygen species production in tobacco bright yellow-2 cells. Plant Physiol. 143, 1817-1826.

Campbell, A.K., 2018. Fundamentals of Intracellular Calcium. Wiley, Chichester, pp. 89-121.

da-Silva, C.J., Modolo, L.V., 2018. Hydrogen sulfide: a new endogenous player in an old mechanism of plant tolerance to high salinity. Acta Bot. Brasil. 32, 150-160.

Dodd, A.N., Kudla, J., Sanders, D., 2010. The language of calcium signaling. Ann. Rev. Plant Biol. 61, 593-620.

Fraisse, L., Bonnet, M.C., de Farcy, J.P., Agut, C., Dersigny, D., Bayol, A., 2002. A colorimetric 96-well microtiter plate assay for the determination of urate oxidase activity and its kinetic parameters. Anal. Biochem. 309, 173-179.

Gay, C.A., Gebicki, J.M., 2000. A critical evaluation of the effect of sorbitol on the ferric-xylenol orange hydroperoxide assay. Anal. Biochem. 284, 217-220.

Gay, C.A., Gebicki, J.M., 2003. Measurement of protein and lipid hydroperoxides in biological systems by the ferric-xylenol orange method. Anal. Biochem. 315, 29-35.

Gill, S.S., Tuteja, N., 2010. Reactive oxygen species and antioxidant machinery in abiotic stress tolerance in crop plants. Plant Physiol. Biochem. 48, 909-930.

Jeandroz, S., Lamotte, O., 2017. Plant responses to biotic and abiotic stresses: lessons from cell signaling. Front. Plant Sci. 8, 1772.

Li, Z.G., 2015. Quantification of hydrogen sulfide concentration using methylene blue and 5,5'-dithiobis (2-nitrobenzoic acid) methods in plants. Methods Enzymol 554, 101-110.

Li, Z.G., 2016. Methylglyoxal and glyoxalase system in plants: old players, new concepts. Bot. Rev. 82, 183-203.

Li, Z.G., Gong, M., 2014. Comprehensive and Designed Experimental Tutorial of Plant Physiology. Huazhong University of Science and Technology Press, Wuhan.

Li, Z.G., Min, X., Zhou, Z.H., 2016. Hydrogen sulfide: a signal molecule in plant cross-adaptation. Front. Plant Sci. 7, 1621.

Liu, C., Pan, J., Li, S., Zhao, Y., Wu, L.Y., Berkman, C.E., et al., 2011. Capture and visualization of hydrogen sulfide by a fluorescent probe. Angew. Chem. 123, 10511-10513.

Maeda, H., Yamamoto, K., Kohno, I., Hafsi, L., Itoh, N., Nakagawa, S., et al., 2007. Design of a practical fluorescent probe for superoxide based on protection-deprotection chemistry of fluoresceins with benzenesulfonyl protecting groups. Chem. Eur. J. 13, 1946-1954.

Mustafiz, A., Sahoo, K.K., Singla-Pareek, S.L., Sopory, S.K., 2010. Metabolic engineering of glyoxalase pathway for enhancing stress tolerance in plants. In: Sunkar, R. (Ed.), Plant Stress Tolerance., 639. Springer, pp. 95-118.

Neill, N., Desikan, R., Hancock, J., 2002. Hydrogen peroxide signaling. Curr. Opin. Plant Biol. 5, 388-395.

Park, K.Y., Roubelakis-Angelakis, K.A., 2018. Real-time in vivo monitoring of reactive oxygen species in guard cells. In: Alcázar, R., Tiburcio, A.F. (Eds.), Polyamines: Methods and Protocols. Springer, New York, pp. 417-426.

Qian, B., Li, X., Liu, X., Wang, M., 2015. Improved oxidative tolerance in suspension cultured cells of $\mathrm{C}_{4}$-pepc transgenic rice by $\mathrm{H}_{2} \mathrm{O}_{2}$ and $\mathrm{Ca}^{2+}$ under PEG-6000. J. Integr. Plant Biol. 57, 534-549.

Rodriguez, A.A., Taleisnik, E.L., 2012. Determination of reactive oxygen species in salt-stressed plant tissues. In: Shabala, S., Cuin, T. A. (Eds.), Plant Salt Tolerance: Methods and Protocols. Springer, London, pp. 225-236.

Sami, F., Faizan, M., Faraz, A., Siddiqui, H., Yusuf, M., Hayat, S., 2018. Nitric oxide-mediated integrative alterations in plant metabolism to confer abiotic stress tolerance, NO crosstalk with phytohormones and NO-mediated post translational modifications in modulating diverse plant stress. Nitric Oxide 73, 22-38. 
Schopfer, P., Plachy, C., Frahry, G., 2001. Release of reactive oxygen intermediates (superoxide radicals, hydrogen peroxide, and hydroxyl radicals) and peroxidase in germinating radish seeds controlled by light, gibberellin, and abscisic acid. Plant Physiol. 125, 1591-1602.

Shaheen, F., Shmygol, A., Rabbani, N., Thornalley, P.J., 2014. A fluorogenic assay for methylglyoxal. Biochem. Soc. Trans. 42, $548-555$.

Tardieu, F., Tuberosa, R., 2010. Dissection and modelling of abiotic stress tolerance in plants. Curr. Opin. Plant Biol. 13, 206-212.

Wild, R., Ooi, L., Srikanth, V., Münch, G., 2012. A quick, convenient and economical method for the reliable determination of methylglyoxal in millimolar concentrations: the N-acetyl-L-cysteine assay. Anal. Bioanal. Chem. 403, 2577-2581.

Xie, Y.J., Shen, W.B., 2012. In vivo imaging of nitric oxide and reactive oxygen species using laser scanning confocal microscopy.
In: Shabala, S., Cuin, T.A. (Eds.), Plant Salt Tolerance: Methods and Protocols. Springer, London, pp. 191-200.

Yu, C.H., Murphy, T.M., Lin, C.H., 2003. Hydrogen peroxideinduced chilling tolerance in mung beans mediated through ABA-independent glutathione accumulation. Funct. Plant Biol. 30, 955-963.

Zhu, J.K., 2016. Abiotic stress signaling and responses in plants. Cell $167,313-324$.

\section{Further Reading}

Jena, S., Choudhuri, M.A., 1981. Glycolate metabolism of three submerged aquatic angiosperms during aging. Aquat. Bot. 12, $345-354$. 
This page intentionally left blank 
Drought Tolerance in Plants: Molecular Mechanism and Regulation of Signaling Molecules

Pradyumna Kumar Singh $h^{1,2}$, Dipali Srivastava ${ }^{1}$, Poonam Tiwari ${ }^{1}$, Madhu Tiwari ${ }^{1}$, Giti Verma ${ }^{1}$ and Debasis Chakrabarty ${ }^{1,2}$

${ }^{1}$ Council of Scientific and Industrial Research - National Botanical Research Institute (CSIR-NBRI), Lucknow, India ${ }^{2}$ Academy of Scientific and Innovative Research (AcSIR), New Delhi, India

\section{O U T L I N E}

\subsection{Introduction}

6.2 Role of Osmoprotectant Regulatory Genes in Drought Stress
6.2.1 Proline
6.2.2 Polyols/Sugars
6.2.3 Glycine Betaine
6.2.4 Osmotin
6.2.5 Dehydrins

6.3 Redox Regulatory Machinery of Plants

During Drought Conditions

\subsubsection{Enzymatic ROS Regulation}

During Drought

6.3.2 Nonenzymatic ROS Regulation

During Drought

6.4 Hormonal Regulation of Drought

Tolerance in Plants

6.4.1 Auxin

6.4.2 Cytokinins

6.4.3 Gibberellins
106

107

107

108

108

108

108

109

109

110

111

111

112

112
6.4.4 Abscisic Acid

6.4.5 Ethylene

6.4.6 Brassinosteroids

6.4.7 Salicylic Acid

6.4.8 Jasmonic Acid

6.5 Molecular Mechanism of Regulatory

Elements in Drought Stress

6.5.1 MYB Transcription Factor Family

6.5.2 Ethylene Response Element-Binding

Factors (AP2/ERF) Family

6.5.3 Basic Leucine Zipper Transcription

Family

6.5.4 Zn-Finger Transcription Factor Family

6.5.5 NAC Transcription Factor Family

6.6 Conclusion and Further Prospects

112

113

113

114

114

114

115

115

116

116

116

117

References

117

Further Reading

123 


\subsection{INTRODUCTION}

The changing climatic conditions have enhanced disaster events like droughts, floods, and snowstorms. A decrease in the rainy season, but heavy rain in a short time, is observed worldwide, which creates flood conditions at one time and increases the risk of drought at the rest season (Trenberth, 2011). Drought stress negatively affects plant growth and soil fertility and is a major environmental threat to limit crop yield in the agriculture sector (Khan et al., 2015). The severity of the problem may be understood by the fact that nearly $60 \%$ of cereals grain production is dependent on the rainfed regions (Rosegrant et al., 2002), which plays a key role to ensure food security for people. The conditions may be worst for those areas dependent on high water-consuming crop production, for example, rice.

The impact of drought stress on the plant may depend on the duration of water deficit in the soil. Drought stress causes water loss from a cell, which leads to cellular dehydration, osmotic stress, and reactive oxygen species (ROS) production (Hasanuzzaman et al., 2018; Khan and Khan, 2017). Plants have evolved several morphological, physiological, and molecular adaptation mechanisms, such as reduction in water loss, enhancement in antioxidant activity, production of osmolytes, and modulation of hormonal and transcriptional regulation, to mitigate drought stress. However, the plant reduces water loss by limiting leaf surface area and stomatal opening. The increase in plant root surface area by the deep rooting system and lateral root growth is a strategy to maintain water balance and nutrient supply in drought stress (Hund et al., 2009). ROSs such as superoxide, peroxide, and hydroxyl radical damage the plant cell membrane and negatively affect the redox potential and photosynthesis apparatus. Drought stress increases the ROS production, stomatal closure, and dehydration, and disturbs the osmotic homeostasis of the plant. The antioxidant enzymes such as superoxide dismutase, catalase, ascorbate peroxidase, glutathione reductase, and glutathione-s-transferase reduced the ROS content and maintained the reduced glutathione (GSH) pool to balance redox state of a plant cell in drought stress (Gill and Tuteja, 2010; Nahar et al., 2015). In addition, nonenzymatic antioxidants like ascorbic acid and GSH play an important role in detoxification of ROS and act as a photoprotectant in abiotic stress conditions including drought stress (Khan et al., 2012, 2016).

The primary strategy of drought tolerance in the plant is a continuation of osmotic balance to protect cellular structure and function during water deficit conditions (Bhargava and Sawant, 2013; Slama et al.,
2015). Osmolytes are nontoxic, highly soluble, low molecular weight compounds that maintain the cellular protein function, membrane integrity, and physiological function of the plant in drought stress (Slama et al., 2015). A large group of compounds such as amino acid, sugar, and polyhydric alcohols act as osmoprotectants and ROS scavengers. The accumulation of osmolytes (proline, sucrose, trehalose, glycine betaine) in plants enhances drought tolerance (Slama et al., 2015; Masood et al., 2016; Per et al., 2017).

The classical plant hormones such as auxin (IAA), gibberellins (GA), cytokinins (CKs), abscisic acid (ABA), and ethylene regulate the growth and developmental processes in various stresses like drought, heat, cold, and salt (Wani et al., 2016). Besides, other signaling molecules' (nitric oxide, hydrogen peroxide, brassinosteroids, and salicylic acid) role in response to drought stress elucidates their importance in the key physiological processes (Wani et al., 2016; Duan et al., 2017). Plants enhanced the ABA biosynthesis in water deficit conditions, leading to stomatal closures inhibiting the water loss, but at the same time, also decreased the gaseous exchange and hydraulic conductance resulting in slower photosynthesis rate and plant growth (Bhargava and Sawant, 2013). Accumulation of ABA activates the ROS signaling, which leads to nitric oxide (NO) biosynthesis resulting in stomata closing (Yu et al., 2014). A higher level of IAA stimulated the expression of stress-related genes, modulated root architecture, and maintained the water homeostasis in the plant to cope with drought stress (Kazan, 2013). The GA deficient Arabidopsis plant showed higher tolerance to water stress but compromise the growth (Colebrook et al., 2014). Drought stress negatively affects the carbon and nitrogen metabolism. Higher levels of CKs enhance tolerance to drought stress by promoting the carbon and nitrogen metabolism in the plant (Reguera et al., 2013; Zwack and Rashotte, 2015). Plant hormones, NO, and salicylic acid (SA) also modulate drought adaptive physiological responses to water deficit stress. Induced level of SA enhances the ABA-independent stomatal closure and drought tolerance by influencing ROS production and the ascorbate-glutathione cycle (Miura et al., 2013; Miura and Tada, 2014). The SA mediated drought tolerance mechanism is not well known to date.

Drought tolerance response is the result of various molecular pathway cascades including the perception of water deficit stress, activation of signaling network followed by transcriptional, metabolic, and regulatory element response (Liu et al., 2014). Drought-responsive molecular mechanisms are tightly controlled by the regulatory elements such as transcription factors and protein kinases. The $M Y B, N A C, b Z I P, A P 2 / E R F$, and 
$A R E B / A B F$ transcription factor (TF) family genes regulate the stomatal movement and expression of drought-responsive genes acting up- or downstream of a metabolic pathway (Kim et al., 2010; Joshi et al., 2016). Protein kinases, which add a phosphate group to the amino acid residue of the protein, are a key regulator of protein activity via post-translational modification. The role of mitogen-activated protein kinases (MAPKs), including calcium-dependent protein kinase (CDPKs) and CBL-interacting protein kinases (CIPKs) in drought tolerance has been elucidated in the plant by using transgenic approaches (Wang et al., 2016a,b). In response to drought stress plants undergo adaptive mechanisms at a molecular, physiological, and metabolic level, which subsequently activates stressresponsive mechanisms to reestablish homeostasis. Among these mechanisms, one category belongs to those that are directly involved in the protection of important proteins and membranes such as osmoprotectants, late embryogenesis abundant (LEA) proteins like dehydrins, free radical scavengers, and chaperones.

\subsection{ROLE OF OSMOPROTECTANT REGULATORY GENES IN DROUGHT STRESS}

At the metabolic level, plants decrease their cellular osmotic potential by the accumulation of compatible solutes or osmoprotectants. They help in the stabilization of membranes and proteins against abiotic stresses without disrupting plant metabolism (Giri, 2011). Many important crops cannot synthesize osmoprotectants that are present in various stress-tolerant organisms. Subsequently, engineering genes related to biosynthetic pathways of osmoprotectants can enhance tolerance to stress. Fig. 6.1 gives an overview of biosynthetic pathways of the significant osmoprotectants

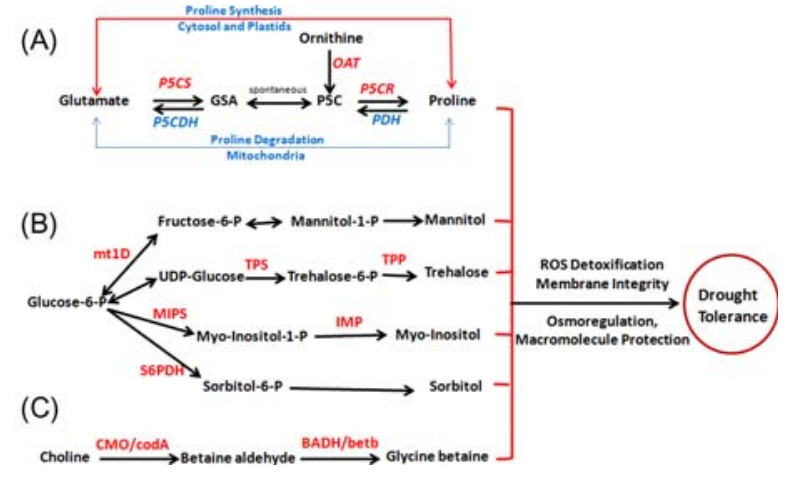

FIGURE 6.1 Overview of osmoprotectants' biosynthesis pathway and their role in drought stress tolerance. discussed in this chapter. This figure also highlights the expression of the transgene for plant genetic engineering, which subsequently resulted in drought and other abiotic stress tolerance. Osmoprotectants include amino acids (e.g., proline), polyol/sugars (e.g., mannitol, trehalose, fructans), and glycine betaine.

\subsubsection{Proline}

In plants, accumulation of proline has been suggested to contribute to stress tolerance by acting as osmoprotectant, molecular chaperone, and as an antioxidant. Proline biosynthesis follows two pathways in plants, that is, glutamate and ornithine pathway. The glutamate is the major proline accumulation pathway, and it is biosynthesized from glutamate by $\Delta 1$-pyrroline-5-carboxylate synthetase (P5CS) further it is reduced to proline by $\Delta 1$-pyrroline-5-carboxylate reductase (P5CR) (Fig. 6.1A). In the alternative pathway, ornithine is transaminated to P5C by ornithine$\delta$-aminotransferase, which further follows the same pathway. As proline biosynthesis follows feedback inhibition, the understanding of proline catabolism becomes equally important. Proline catabolism occurs in mitochondria using the sequential action of proline dehydrogenase or proline oxidase (PDH or POX) on proline to produce $\mathrm{P} 5 \mathrm{C}$ from proline and further $\mathrm{P} 5 \mathrm{C}$ is converted to glutamate by $\mathrm{P} 5 \mathrm{C}$ dehydrogenase (P5CDH). Both P5CS and PDH catalyze the first and the rate limiting steps of proline biosynthesis and degradation, respectively, and their activities could influence proline accumulation and plant growth under stress. Overexpression of moth bean P5CS in tobacco plants resulted in improved salt and drought tolerance (Kishor et al., 1995; Zhu et al., 1998). Overexpression of Arabidopsis and Oryza sativa P5CS conferred drought tolerance in petunia plants (Yamada et al., 2005). However, antisense-AtP5CS transgenic plants showed hypersensitivity to stress (Nanjo et al., 1999). Similarly, insertion mutation of p5cs1 (Székely et al., 2008) also resulted in reduced osmotolerance. Overexpression of Arabidopsis P5CR (AtP5R) gene in soybean improved drought and heat stress (De Ronde et al., 2000, 2004). Overexpression of $P D H$ did not confer osmotolerance in Arabidopsis, except in the presence of exogenously supplied proline. There are several reports that show that the P5CS expression in Arabidopsis and rice plants was upregulated during dehydration and the production of $P D H$ was upregulated during rehydration (Oono et al., 2003; Nanjo et al., 2003). These findings highlighted the role of proline biosynthesis in plants against osmotic stress induced by drought and other abiotic stresses. 


\subsubsection{Polyols/Sugars}

Overall carbon metabolism and the levels of specific sugars are severely affected by drought and other abiotic stress. Polyols such as glycerol, mannitol, D-ononitol, sorbitol, and trehalose are osmoprotectants in algae and certain halophytic plants. Overexpression of mannitol 1-phosphate dehydrogenase $(m t l D)$, the gene for the biosynthesis of mannitol in transgenic tobacco and Arabidopsis, resulted in mannitol production and a salinity-tolerant (Thomas et al., 1995). The expression of $m t l D$ and mannitol accumulation in the chloroplast conferred osmotic stress tolerance. It has also been reported that the ectopic expression of the $m t l D$ gene in wheat improves tolerance to water stress and salinity (Abebe et al., 2003). Tobacco plants were transformed with imtl gene encoding myo-inositolomethyl-transferase of ononitol biosynthetic pathway showed higher tolerance to salt and drought stress than wild-type control (Sheveleva et al., 1997). Several transgenic plants with genes of sorbitol biosynthesis have been reported. Transgenic tobacco plants expressing apple Stpd1 (sorbitol-6-phosphate-dehydrogenase) demonstrated that the plants with lower sorbitol content showed normal growth while the one with higher content exhibited growth retardation (Sheveleva et al., 1998). Another example is of Diospyros kaki transformed with Stpd1, which also accumulated sorbitol but showed higher photosynthetic activity than control plants (Gao et al., 2001).

Trehalose is a non-reducing disaccharide and its pretreatment induces drought tolerance in radish (Raphanus satious L.) plants (Akram et al., 2015). The overexpression of a fusion gene, trehalose-6-phosphate synthase/phosphatase (TPSP), containing the coding region of Escherichia coli trehalose biosynthetic genes (ots $A$ and otsB) (Fig. 6.1B) improved abiotic stress tolerance in rice. The transgenic rice plants accumulated 3-10 times more trehalose than that of the control plants and showed high levels of tolerance to salt, drought, and low-temperature stresses as compared with the non-transformed plant (Garg et al., 2002). Another similar report shows a fusion of the droughtinducible promoter StDS2 to trehalose-6-phosphate synthase (TPS1) in potato. The transgenic lines showed high stomatal conductance and a satisfactory rate of net photosynthesis (Stiller et al., 2008). In another study, Arabidopsis overexpressing AtTPS1 accumulated trehalose only at low levels, but transgenic plants acquired desiccation tolerance. This study has also shown that AtTPS1 has a role in the regulation of glucose and ABA signaling during vegetative development (Avonce et al., 2004). Apart from its known function of chaperone and osmolyte, the role of trehalose and trehalose-6P in signaling is of great importance (Ferndez et al., 2010).

\subsubsection{Glycine Betaine}

Glycine betaine (GB) is a methylated derivative of glycine and an important osmoprotectant in bacteria, animals, and plants. GB accumulation has been reported in plants when exposed to environmental stresses such as salt, drought, and extreme temperatures (Giri, 2011). It can be synthesized in transgenic plants using genes from plants that encode for either of the enzymes: choline monooxygenase $(C M O)$, betaine aldehyde dehydrogenase $(B A D H)$ or alternatively betaine aldehyde dehydrogenase (bet $B$ ) or choline oxidase $(\operatorname{cod} A)$ genes from bacteria (Fig. 6.1C). GB accumulation leads to enhanced tolerance to drought and salt stress (Sakamoto and Murata, 2000). Targeted accumulation of GB in chloroplasts has been achieved by engineering a plastid-expressed $C M O$ gene, leading to higher PSII activity. Thus the protection of PSII can lead to improved drought and salt tolerance in crops (Zhang et al., 2008). Rice, maize, and many crop plants lack the ability to accumulate GB naturally during abiotic stress (Giri, 2011); introduction of GB biosynthesis gene(s) into nonaccumulators by transgenic approach has been successfully used for improved abiotic stress tolerance in diverse plant species, for example, rice (Su et al., 2006), potato (Ahmad et al., 2008), and wheat (Wang et al., 2010).

\subsubsection{Osmotin}

Osmotin and osmotin-like proteins (OLPs) are a group of stress-responsive proteins that belong to the PR-5 pathogenesis-related group of proteins. Several studies reported the dual function of osmotin and osmotin-like proteins in plant defense against abiotic stress (Subramanyam et al., 2012) and osmotic stress (Chowdhury et al., 2017). Although the underlying mechanism still remains unclear, the overexpression of the osmotin gene in tobacco plants improved their tolerance to salinity, wounding, infection, and drought stress (Barthakur et al., 2001). It has been reported in wheat (Noori and Sokhansanj, 2008), tomato (Goel et al., 2010), and soybean (Subramanyam et al., 2012) that the overexpression of the osmotin gene conferred tolerance against various abiotic stresses.

\subsubsection{Dehydrins}

Dehydrins are one of the key components of dehydration tolerance, although the precise mechanism is 
not clear. However, it has been proposed that they may carry out their function through membrane stabilization by acting as chaperones to prevent the aggregation and/or inactivation of proteins under dehydration (Yang et al., 2012). Several transgenic studies revealed that dehydrin gene expression has a positive effect on plant stress tolerance, including cold, drought, and salinity. It has been reported that OsDHN1 overexpressing rice confers high tolerance to drought and salt stress (Kumar et al., 2014). Liu et al. (2015) also reported a positive correlation between ShDHN gene expression and drought tolerance in tomato (Solanum habrochaites L.). At the functional level, OsjDHN genes exhibited subfunctionalization, that is, different genes displaying differential expression profiling during various developmental stages and under PEG-induced drought stress (Verma et al., 2017). In another study, overexpressing dehydrin genes such as ERD10, LTI30, RcDhn5, and DHN-5 in Arabidopsis plants showed tolerance to various abiotic stresses (Puhakainen et al., 2004; Brini et al., 2007; Peng et al., 2008). Similarly, the differential accumulation of $31-$ and $40-\mathrm{kDa}$ dehydrins in the droughtsensitive and tolerant warm-season Bermuda grass indicated their contribution to drought or dehydration tolerance in warm-season Bermuda grass (Hu et al., 2010). A study from Verma et al. (2017) reported evolutionary conservation of dehydrins during the course of domestication/evolution in 11 Oryza species and subspecies, as they play an important role in combating dehydration stress. Recently, we found that rice transgenic lines overexpressing OsDHN gene have improved tolerance against drought, osmotic stress, exogenous $\mathrm{ABA}$, and $\mathrm{ABA}+\mathrm{PEG}$ treatment (unpublished).

\subsection{REDOX REGULATORY MACHINERY OF PLANTS DURING DROUGHT CONDITIONS}

One of the significant reasons for plant growth reduction on exposure to drought is the breakdown of redox homeostasis of the cell (Halliwell, 2006). Production of ROS causes extensive damage to DNA, proteins, membrane lipids, and other cellular components (Foyer and Noctor, 2005). The most common ROS comprise $\mathrm{O}_{2}{ }^{\cdot-}$ (superoxide radical), ${ }^{1} \mathrm{O}_{2}$ (singlet oxygen), ${ }^{\circ} \mathrm{OH}$ (hydroxyl radical), and $\mathrm{H}_{2} \mathrm{O}_{2}$ (hydrogen peroxide). ROS are being produced as a byproduct of electron transport chain in deferent cellular compartments like cytosol, chloroplasts, mitochondria, and peroxisomes under both favorable as well as unfavorable conditions. The antioxidant machinery of the cell plays a vital role in maintaining redox homeostasis by scavenging ROS for normal cellular functioning, and comprises two types of components: enzymatic and nonenzymatic. Enzymatic components include several antioxidant enzymes such as superoxide dismutase (SOD; EC 1.15.1.1), catalase (CAT; EC 1.11.1.6), ascorbate peroxidase (APX; EC 1.11.1.11), guaiacol peroxidase (GPX; EC 1.11.1.7), glutathione peroxidase (GPx; EC 1.11.1.9), monodehydroascorbatereductase (MDHAR; EC 1.6.5.4), dehydroascorbate reductase (DHAR; EC 1.8.5.1), glutathione reductase (GR; GR, EC 1.6.4.2), and glutathione-S-transferase (GST; EC 2.5.1.18) whereas ascorbate (ASC), $\alpha$-tocopherol ( $\alpha$-toc), and GSH fall into the category of nonenzymatic components. Both types of components are equally important and work in a coordinated manner to provide a significant defense from oxidative stress conditions.

\subsubsection{Enzymatic ROS Regulation During Drought}

The role of ROS scavenging enzymes has been elucidated by manipulation of antioxidant components through genetic engineering and can enhance the performance of plants under drought stress leading to increased amounts and activities of antioxidants (Cao et al., 2017). SOD, an antioxidant enzyme, is a metalloenzyme that forms the first line of defense against superoxide-induced damages under stress conditions. Overexpression of the Arachis hypogaea AhCuZnSOD gene in tobacco demonstrated that transgenic lines exhibited improved growth parameters in response to drought-induced osmotic stress (Negi et al., 2015). Likewise, transgenic cotton plants overexpressing Tamarix albiflonum TaMnSOD enhanced drought tolerance through improved plant growth development with reduced oxidative stress (Zhang et al., 2014). Interestingly, co-expression of cytapx (from Pisum sativum) and/or cytsod (from Spinacia oleracea) in tobacco plants not only increased the SOD and APX activity but also enhanced the POX and CAT enzyme activity resulting in a reduction of oxidative stress under drought conditions (Faize et al., 2011). CATs and APXs both are key components of the antioxidative machinery that regulate the $\mathrm{H}_{2} \mathrm{O}_{2}$ content in peroxisomes and in the cytosol, as well as in the chloroplast, respectively. Studies have suggested that catalase gene expression enhanced during drought and ABA treatment ultimately maintains the redox homeostasis in a plant cell by increasing the enzyme activity of CAT (Du et al., 2008; Nie et al., 2015). Moreover, drought tolerant plants were raised by simultaneous homologous overexpression of both cassava $\mathrm{Cu} / \mathrm{Zn}$ superoxide dismutase $(\mathrm{MeCu} / \mathrm{ZnSoD})$ and catalase (MeCat1) 
enzymes (Xu et al., 2013). On the other hand, exposure of drought stress increased the expression of APX gene and altered the cellular metabolism (Koussevitzky et al., 2008). Recently, Cao et al. (2017) reported that on the introduction of cytosolic APX gene from Populus tomentosa into tobacco plants, there were found to be decreased levels of malondialdehyde (MDA) and $\mathrm{H}_{2} \mathrm{O}_{2}$ levels with higher APX activity in the transgenic plants suggesting its role in drought tolerance. A study demonstrated that rice seedlings with loss of function in rice OsAPX2 mutants show susceptibility to drought stress, and exhibit lower APX activity with higher $\mathrm{H}_{2} \mathrm{O}_{2}$ and MDA levels, while constitutive overexpression lines showed drought tolerance (Zhang et al., 2013). MDHAR and DHAR enzymes keep the redox state of the plant cell in drought conditions by retaining the cellular ASC pool by using NADPH and GSH respectively. Polyethylene glycol (PEG) induced osmotic stress increased the expression of MDHAR in the wild-type tomato. Further, a higher status of net photosynthesis rate, lower level of hydrogen peroxide, MDA, and a coordinated higher activity of the antioxidant enzymes served as the major determinants in a sense transgenic line for depicting PEG-induced osmotic stress tolerance compared with antisense lines and WT plants (Li et al., 2012). Similarly, enhanced tolerance to PEG stress has been developed by overexpression of AtMDAR1 in transgenic tobacco (Eltayeb et al., 2007). Moreover, Eltayeb et al. (2006) also found that transgenic tobacco plants carrying cytosolic DHAR gene from Arabidopsis thaliana result in higher enzyme activity, which in turn results in improved photosynthesis protection and increased revival under drought stress.

GR maintains the glutathione pool of the cell by catalyzing the reduction of GSSG to GSH in normal as well as stress conditions. The deviation in cytosolic and dual targeted GR gene expression to drought stress was investigated in cowpea tolerant cultivar and a susceptible cultivar. Cytosolic GR gene was upregulated in both cultivars. However, upregulation of dualtargeted GR was found in the susceptible cultivars only under drought treatment (Contour-Ansel et al., 2006). As ABA is the biomarker of drought stress, the transcription of $B$. campestris $B c g G R 1$ is strongly induced by exogenous application of ABA suggesting its role in drought stress (Lee et al., 2002). Arabidopsis plants overexpressing ApGPX2 and PgGPx (Pennisetum glauccum) genes showed tolerance under drought stress (Gaber et al., 2006; Islam et al., 2015). Plant GSTs are well-known enzymes for detoxification of xenobiotic compounds, but also, they play a role in response to drought-induced oxidative stress (Cicero et al., 2015; Liu et al., 2013). Several studies have revealed that heterologous expression of GST genes improves resistance to drought stress in plants by enhancing their ROS scavenging ability (Yang et al., 2014; Xu et al., 2016) (Table 6.1)

\subsubsection{Nonenzymatic ROS Regulation During Drought}

Apart from enzymatic antioxidants, plant cells possess other non-antioxidant compounds (ASC, GSH, and $\alpha$-toc) that play a fundamental role in plant metabolism. These low-molecular weight antioxidants modulate the cellular redox homeostasis by interacting with ROS and affect the physiological and biochemical processes of plants to ameliorate the adverse effects during drought conditions. Several studies revealed that drought tolerance could be enhanced by the manipulation of key enzymes of biosynthetic pathways of antioxidant compounds and they maintain the intracellular redox environment of plants in stress conditions (Ma et al., 2014; Woo et al., 2014). Ascorbate has been abundantly found in reduced form in all cell compartments and acts as a principal ROS detoxifying compound in response to different types of stress conditions (Ahmad et al., 2010). In a study, an alfalfa GDP-mannose 3, 5-epimerase gene encoding the enzyme involved in ascorbate biosynthetic pathway was overexpressed in Arabidopsis and it was found that higher accumulation of ascorbate in transgenic plants positively correlated with drought stress tolerance (Ma et al., 2014). Similarly, Lim et al. (2012) introduced the rat L-gulono- $\gamma$-lactone oxidase (GLOase) gene into the genome of the tomato plant and developed transgenic plants with higher ascorbate and chlorophyll content as compared with wild-type plants. Arabidopsis ascorbate-deficient vtc1 mutant exhibited droughtsensitive phenotype with reduced activity of antioxidant enzymes and an increased ratio of dehydroascorbate/total ascorbate.

In addition, a lipid-soluble antioxidant $\alpha$-tocopherol is an important component of the plant defense machinery that not only scavenges the harmful radicals but also performs the quenching of singlet oxygen generated in the photosynthesis process (Espinoza et al., 2013). Transgenic plants overexpressing tocopherol cyclase coding gene from Arabidopsis and tobacco respectively showed higher tolerance to drought stress with better growth parameters and antioxidant activity than wild-type plants (Liu et al., 2008; Woo et al., 2014).

Glutathione is a tripeptide thiol that is abundantly found in its reduced form and scavenges reactive oxygen species (Foyer and Noctor, 2005). It synthesized in two steps involving two enzymes: glutamyl-cysteinyl synthetase $(\gamma$-ECS) and glutathione synthetase (GS). 
TABLE 6.1 The Transgenic Intervention of Various Antioxidant Enzymes in Crop Plants to Improve Drought Tolerance

\begin{tabular}{|c|c|c|c|c|}
\hline Antioxidant enzymes & Host & Target & Gene & References \\
\hline \multirow[t]{4}{*}{ APX } & Salicornia brachiata & Nicotiana tabacum & SbpAPX & Singh et al. (2014) \\
\hline & Eleusine coracana & E. coli & Ec-apx1 & Bhatt et al. (2013) \\
\hline & Populus tomentosa & Nicotiana tabacum & PcAPX & Cao et al. (2017) \\
\hline & Oryza sativa & Oryza sativa & OsAPX2 & Zhang et al. (2013) \\
\hline DHAR & Arabidopsis thaliana & Nicotiana tabacum & DHAR & Eltayeb et al. (2006) \\
\hline \multirow[t]{2}{*}{ GPX } & Pennisetum glaucum & Oryza sativa & PgGPx & Islam et al. (2015) \\
\hline & SynechocystisPCC6803 & Arabidopsis thaliana & GPX-2 & Gaber et al. (2006) \\
\hline MDAR & Arabidopsis thaliana & Nicotiana tabacum & AtMDAR1 & Eltayeb et al. (2007) \\
\hline \multirow[t]{3}{*}{ SOD } & Sedum alfredii & Arabidopsis thaliana & $\mathrm{SaCu} / \mathrm{Zn} \mathrm{SOD}$ & Li et al. (2017) \\
\hline & Arachis hypogaea & Nicotiana tabacum & CuZnSOD & Negi et al. (2015) \\
\hline & Tamarix albiflonum & Gossypium hirsutum L.) & TaMnSOD & Zhang et al. (2014) \\
\hline SOD/ APX & Spinacia oleracea/Pisum sativum & Nicotiana tabacum & cytsod/cytapx & Faize et al. (2011) \\
\hline \multirow[t]{5}{*}{ GST } & L. esculentum & Arabidopsis thaliana & LeGSTU2 & Xu et al. (2015) \\
\hline & Pyrus pyrifolia & Nicotiana tabacum & PpGST & Liu et al. (2013) \\
\hline & Arabidopsis thaliana & Arabidopsis thaliana & AtGSTU19 & Xu et al. (2016) \\
\hline & Tamarix hispida & Arabidopsis thaliana & ThGSTZ1 & Yang et al. (2014) \\
\hline & Citrus sinensis & Nicotiana tabacum & CsGSTU & Cicero et al. (2015) \\
\hline SOD/CAT & Manihot esculenta & Manihot esculenta & $\mathrm{MeCu} / \mathrm{ZnSOD}$ and MeCat1 & Xu et al. (2013) \\
\hline
\end{tabular}

Bhatt, D., Saxena, S.C., Jain, S., Dobriyal, A.K., Majee, M. and Arora, S. 2013. Cloning, expression and functional validation of drought inducible ascorbate peroxidase (Ec-apx1) from Eleusine coracana. Mol. Biol. Rep. 40, 1155-1165.

Li, Z., Han, X., Song, X., Zhang, Y., Jiang, J., Han, Q., Liu, M., Qiao, G. and Zhuo, R. 2017. Overexpressing the Sedum alfredii Cu/Zn superoxide dismutase increased resistance to oxidative stress in transgenic Arabidopsis. Front Plant Sci. 8, 1010.

Singh, N., Mishra, A. and Jha, B. 2014. Over-expression of the peroxisomal ascorbate peroxidase (SbpAPX) gene cloned from halophyte Salicornia brachiata confers salt and drought stress tolerance in transgenic tobacco. Mar. Biotechnol. 16, 321-332.

The proportion of GSH to GSSG (oxidized form) plays a central role in maintaining cellular redox state. Exogenous application of glutathione enhanced the antioxidant enzyme activities in drought-treated mung bean seedlings and improved their physiological activity and growth (Nahar et al., 2015). Some studies have revealed the advantage of increasing GSH level through overexpression of $\gamma$-ECS that enhanced resistance to drought and ABA-induced osmotic stresses (Sengupta et al., 2012; Wu et al., 2009).

\subsection{HORMONAL REGULATION OF DROUGHT TOLERANCE IN PLANTS}

Plant endurance during drought is governed by several pathways; among them, plant hormones play an inevitable role. Plant growth regulators, in spite of their biological roles, are central players during abiotic or biotic stresses. Advancement in transcriptome and mutant analysis provides a better lead towards an understanding of plant growth regulators during drought. The two hormones, ABA and ethylene, are well studied to date and proved to be activated and provide tolerance during low water deficit condition. All other major hormones like auxin, CKs, and GA are also involved significantly during drought but the molecular mechanisms related to these hormones are partially understood. Moreover, the other growth regulators such as brassinosteroids, SA, and jasmonic acid (JA) are also significantly involved to cope with drought.

\subsubsection{Auxin}

During drought, soil layers become dry and plant lateral root development and curvature is needed to maintain the effective uptake of water. Auxin is known to be responsible for lateral root development and maintaining the root architecture. YUCCA genes are the rate limiting step of auxin biosynthesis and are drought-induced, although molecular mechanism is 
needed to decipher. iaaM-OX transgenic lines of Arabidopsis with higher endogenous IAA are drought resistant while yuc1yuc2yuc6 triple mutants decreased stress resistance in comparison with WT plants (Shi et al., 2014). The plant overexpressing MIZ1 (MIZUKUSSEI1), a regulator of hydrotropism, has less lateral and more penetrating root and is drought sensitive, showed that it inhibits the auxin response in the pericycle cells. This inhibition is overcome by the exogenous auxin treatment in Arabidopsis (Moriwaki et al., 2011). Auxin-responsive factors (ARFs) are also a key regulator of lateral root development. Microarray analysis shows that drought-stressed plant having upregulated transcripts of ARFs. ARFs are also regulated at the posttranscriptional level via several miRNAs, like miR167 (Kinoshita et al., 2012) and miR164 (Guo et al., 2005) in Arabidopsis, and modulate root architecture, lateral root development. The miR167a is a negative regulator of IAR3. During drought conditions, miR167 is reported to be downregulated results in an increased IAR3 level. Overexpressed IAR3 Arabidopsis plant having freer auxin accumulation and enhanced root architecture leads to drought tolerance impact (Kinoshita et al., 2012). miR393 is an osmotic stress-induced miRNA that degrades TIR and AFB2 transcripts and inhibits the lateral root growth (Chen et al., 2012a,b). This miRNA is responsible for attenuating the auxinmediated response. Auxin also upregulated the function of small auxin-upregulated RNAs (SAURs), which have been shown to be responsible for drought stress tolerance via more root development and upregulation of stress-related genes when overexpressed in Arabidopsis (Guo et al., 2017) but the molecular mechanism of drought tolerance in respect to auxin is still unknown.

\subsubsection{Cytokinins}

Cytokinins (CKs) are the phytohormones involved in cytokinesis and that promote cell division and differentiation. Cytokinin levels were reported to be downregulated during drought stress. This leads to low CK level in roots and leaves (Merewitz et al., 2010). Several plants like bentgrass, tobacco, and rice have been proved to be more drought tolerant when either exogenously supplied with CK compounds or overexpressed adenosine phosphate-isopentyl transferase (IPT) genes (Peleg et al., 2011). It has been suggested that drought mediated signaling hindered the CK production either at CK biosynthesis or at degradation level. Transcript analysis of IPT transgenic plant compared with WT plant was having less upregulated and more downregulated drought-responsive gene (Rivero et al., 2010). It suggested that CK regulates several metabolic processes drought-responsive genes. Interestingly, overexpression of cytokinin degradation enzymes cytokinin oxidase/dehydrogenase1 (CKX1) by the root-specific promoter WRKY6 showed more degradation and low level of CK in root which leads to enlarging the root system required for more water absorption in a water deficit environment (Macková et al., 2013). From these data, it can be hypothesized that in water deficit conditions, low level (WTKY6: CKX1) of cytokinins in roots while high IPT level in shoots (35S: CKX1) have more tolerant effects against drought.

\subsubsection{Gibberellins}

Gibberellins (GAs) are well known as a classical growth hormone. The exact pathway of GA metabolism and signaling during water deficit is yet to be unraveled but GA level has been reported to be downregulated in maize (Wang et al., 2008a,b). The three dioxygenases that participated in the metabolic pathway during GA biosynthesis, GA 20-oxidase, GA 3oxidase, and GA 2-oxidase, have been overexpressed in Arabidopsis under 35S promoter and knockout lines also prepared and studied under drought. The results suggested that high GA concentration leads to drought sensitive response of plants while low GA concentration is drought tolerant (Colebrook et al., 2014). Upregulation of GA 2-oxidase inhibits the GA biosynthesis. The DELLA protein also plays a crucial role in the regulation of GA biosynthesis. DELLA protein, in the absence of GA, binds to GID1, which is a receptor for GA. GA binds to the GID1 and leads to the interaction with an SCF ubiquitin ligase to the DELLA protein. The subsequent $26 \mathrm{~S}$ proteasomal degradation of DELLA protein results in GID1 import to the nucleus and further gene activation in response to GA signaling. Several proteins involved in different hormone signaling pathways interact through DELLA (Bai et al., 2012) suggesting that GA may cross-talk through different hormones. DELLA has also been reported as a positive factor for stress tolerance (Achard et al., 2006). An ethylene responsive factor ERF6 has been reported (Dubois et al., 2013) to participate in both ethylene and GA mediated responses. DELLA gene RGL3 is also transcriptionally upregulated under JA signaling (Wild et al., 2012).

\subsubsection{Abscisic Acid}

The first hormone that comes to mind for drought stress is ABA, which is also thought to be a negative regulator of many developmental processes in plants. The NCED3 enzyme is a rate limiting factor of ABA 
biosynthesis. Mutated nced3 have shown to be less tolerant to drought stress in several plant species while overexpression of NCED3 showed more water use efficiency and drought tolerance (Tung et al., 2008). Stomatal closure is the earliest response to reduce transpirational water loss in water stress and is tightly regulated by $\mathrm{ABA}$-dependent or $\mathrm{ABA}$-independent pathways. ABA bound to the plasma membranebound receptors like PYR, PYL, RCARs present in the guard cell and its downstream signaling involves the generation of secondary signals like $\mathrm{H}_{2} \mathrm{O}_{2}$, which induces $\mathrm{Ca}^{+2}$ release from the plasma membrane, and intrinsic (cytoplasmic) $\mathrm{Ca}^{+2}$ also increases via tonoplast and ultimately leads to stomatal closure via activating outwardly rectifying $\mathrm{K}^{+}$(Ma et al., 2009; Taiz and Zeiger, 2002). Several reports are available that suggest the direct role of ABA to close the inward rectifying $\mathrm{K}^{+}$channels that are responsible for stomatal opening. Biosynthesis and transport of $\mathrm{ABA}$ is enhanced during drought stress. ABA is sensed by receptors like pyrabactin resistance 1 (PYR), pyrabactin resistance1-like (PYL), and the regulatory component of abscisic acid receptor proteins (RCARs). ABA inhibits binding of phosphatase type 2Cs (PP2C) protein to sucrose nonfermenting 1-related protein kinase 2s (SnRK2). In a nonstress environment, SnRK2 is bound with PP2C-like (ABA insensitive-1 and 2, ABI1, and $\mathrm{ABI} 2$ ), which prevented the SnRK2 kinase activity. Once ABA bound to the PP2Cs, SnRK2 was released and free to phosphorylate many ABA-responsive factors (ABFs) (Fujii et al., 2009). ABFs are basically bZIP (basic leucine zipper) proteins and activate many ABA-responsive elements (ABREs) containing genes. These genes involved various transcription factors of stress-related genes, genes of osmolytes production, wax deposition genes, and senescence and dormancy related genes. ABA also inhibits the nonstomatal loss of water from cuticular deposition by enhancing wax gene synthesis.

Senescence is a physiological response that includes programmed cell death (PCD) of mature leaves and nutrient transfer from the senescing organ. ABA induces early senescence genes (premature leaves) during severe drought stress (Volaire and Norton, 2006). The SWEET15 gene is reported to have an enhanced synthesis in senescence leaves having ABA receptor (PYL) overexpressed plants than the WT (Zhao et al., 2016). Another crucial event is dormancy, which is also regulated by ABA. Many bud dormancy related genes possess a CACGTGT motif in their promoters (González-Grandío et al., 2013), which are recognized by ABA-related bZIP TFs (Shen and Ho, 1995). ABA maintains the life of developing tissues (sink) and leads to the death of source tissues, which is crucial during extreme drought conditions.

\subsubsection{Ethylene}

Ethylene has been reported to increase during drought stress in several plant species like French bean, orange, avocado, Vicia faba, and in many other plant species. The 1-aminocyclopropane-1-carboxylic acid synthase (ACS) is a key enzyme of ethylene biosynthesis. C-terminal region of ACS protein has different phosphorylation sites. ACSs are divided into three types on the basis of different phosphorylation site. Type I ACSs have mitogen-activated protein kinase 3 and/or 6 (AtMPK3-6) as well as calcium-dependent protein kinase (AtCDPK2, CDPK, or CPK) phosphorylation domain, type II ACS proteins have only CPK phosphorylation site, and type III do not possess any phosphorylation site in the C-terminal domain. ACSs are not only regulated by phosphorylation but also by phytohormones and secondary messengers like $\mathrm{Ca}^{+2}$ (Arraes et al., 2015). Aminocyclopropane-1-acid carboxylic oxidase (ACO) is a final enzyme that converts ACC to ethylene. ACC moves through the xylem from the root to more distant plant parts and is converted to ethylene wherever it acquires the signal. In maize, it has been shown that ethylene acts as an inhibitor of leaf growth and ACC act as a signal transduction molecule under water deficit conditions (Sairam et al., 2008). RD29A and RD29B genes are upregulated during drought stress. The promoter of RD29A has two cis-acting elements; one is an ABA-responsive element (ABRE), activated via $A B A$, and the other one is a dehydration-responsive element (DRE), activated under osmotic stress. RD29B only has ABA-mediated activation and ABRE domain (Yamaguchi-Shinozaki and Shinozaki, 1994). Ethylene-responsive factor (ERFs) also binds to the DRE elements. Arabidopsis plant with a constitutive promoter, 35S: ERF1, proved to be drought tolerant and ERF protein bound directly to the DRE element of the RD29B promoter (Cheng et al., 2013). DRE element contains the core sequences A/GCCGAC, a cis-acting promoter element that is involved in regulation of gene expression under drought and leads to low yield and higher survival.

\subsubsection{Brassinosteroids}

Polyhydroxysteroids have been recognized as the sixth class of plant hormone, called brassinosteroids, and possess distinct growth-promoting ability (Bishop and Yokota, 2001). Brassinosteroids serve as a positive regulator during drought stress but the molecular mechanism is still unresolved. Brassica napus seedlings treated with epibrassinolide (EBR) during drought stress results in high antioxidant enzyme and reduction in reactive oxygen species leads to tolerance to drought stress (Kagale et al., 2007). A drought sensitive 
variety of wheat coped well during drought via increased water uptake and reduced ion leakage after treatment with brassinosteroids (Sairam, 1994). These results indicate a significant role of brassinosteroids to cope with drought via upregulating different enzymes and antioxidants. Drought stress related marker genes were studied in EBR treated and nontreated Arabidopsis plants. The consistent accumulations of $R D 29 A, E R D 10$, and RD22 transcripts have been identified in EBR treated Arabidopsis plant. RD29A and ERD10 both have a chaperone-like function, and prevent aggregation of proteins during water deficit. There is a possible interaction between ABA and brassinosteroids, although the complete pathway has not yet been deciphered, but ABA-responsive genes like LPT4 and ABA-marker gene RD22 have been identified to be upregulated during $B R$ treatments in the plant (Divi et al., 2010).

\subsubsection{Salicylic Acid}

Salicylic acid (SA), a phenolic phytohormone, mediates systemic acquired resistance during biotic stress and also provides tolerance against abiotic stresses. During water deficit, SA content increased twofold in barley root (Bandurska, 2005). Addition of SA prior to the drought treatment improves drought tolerance in various plants like wheat (Hamada, 2001), muskmelon seedlings (Korkmaz et al., 2007), tomato and bean seeds (Senaratna et al., 2000). Low levels of ROS accumulation have been reported when plants are pretreated with 0.1-0.5 mM of SA (Harfouche et al., 2008). ROS acts as a secondary messenger and leads to ROS mediated enzymatic antioxidant activation such as SOD, GPX, and HSPs (Shi et al., 2006) and many nonenzymatic antioxidants like glutathione, ascorbic acid, and carotenoids (Miyake and Asada, 1994). SA accumulating mutants of Arabidopsis plants like adr1, myb96-1d, siz1, acd6, and cpr5 were drought tolerant when supplemented with SA (Miura and Tada, 2014) LTI29 and LTI30 genes upregulated during drought stress and SA treatment in microarray data. LTI29 and LTI30 overexpressed lines accumulated more dehydrin (Puhakainen et al., 2004). Dehydrin is an important player during drought stress in plants (Brini et al., 2007) so it may also be concluded that SA is involved in dehydrin accumulation and drought tolerance. MAP kinase cascade is also activated through SA and ROS as downstream signaling (Cristina et al., 2010). The phosphorylation of MPK6 in Arabidopsis roots is associated with the application of SA (Mockaitis and Howell, 2000). This led to the conclusion that at low concentration SA acts as a positive regulator of drought tolerance by activating secondary signals like ROS, and by upregulating dehydrins and MPKs.

\subsubsection{Jasmonic Acid}

Jasmonic acid also provides tolerance against drought, however, reports also suggested that JA has a negative impact on plants under drought, so till now, its exact role is under observation (Riemann et al., 2015). The controversial action of JA is also varied due to observations under different experimental setups such as developmental stage of the plant, the concentration of drought agents, etc. (Kim et al., 2009a,b). The plant exposed to drought accumulated more proline and antioxidants (either enzymatic or nonenzymatic). Exogenously supplied JA or MeJA to the plant enhanced antioxidant capacity (Nafie et al., 2011) and proline accumulation (Mahmood et al., 2012). Three ecotypes of $A$. Thaliana have been observed under drought and wound stress. Wounding activated both JA and 12-OPDA (biosynthetic intermediate of JA), but drought stress induced only 12-OPDA, which probably gives an idea that drought mediated signals block the conversion of 12-OPDA to JA, and 12-OPDA is a functional jasmonate during drought. Transgenic plants of Arabidopsis having the same level of ABA but the range of 12-OPDA generation indicated that more 12-OPDA is promising against drought stress with reduced stomatal opening (Savchenko et al., 2014). Arabidopsis seedlings defective functional JA-Ile biosynthesis (jar11) and JA insensitive lines (jai1 and coi1-16) were reported for more proline accumulation after exposure to dehydration showed that more proline accumulation is independent of ABA (De Ollas et al., 2015).

\subsection{MOLECULAR MECHANISM OF REGULATORY ELEMENTS IN DROUGHT STRESS}

Modern genetics and molecular advancement have improved our knowledge in deciphering the role of metabolic and regulatory proteins during water stress utilizing transcriptomics, proteomics, and metabolomics approaches (Hirayama and Shinozaki, 2010). The regulatory genes include TFs, protein kinases, and protein phosphatases, which play the crucial role in abiotic stress synchronizing signal perception and transduction to regulate downstream stress-responsive genes (Wani et al., 2013). Transcription factors act as the key ingredient of gene differential regulation and their expression. As a rule, the transcription factors possess a DNA binding domain and a transcriptional activation/repression domain, to regulate transcription 
process of the targeted gene and vast reports are available emphasizing their role in abiotic stress particularly drought. Plant TFs widely known to play a role in water stress have been categorized into different subfamilies, for example, $M Y B, A P 2 / E R F, b Z I P, \mathrm{Zn}$ finger, and NAC (Riechmann et al., 2000).

\subsubsection{MYB Transcription Factor Family}

The MYB gene family is one of the immensely vast and functionally varied classes of TFs, and plays a central role in the control of plant-specific processes, including primary and secondary metabolism, cell fate and identity, and development response to abiotic and biotic stresses (Dubos et al., 2010). Predominantly, MYB proteins act as transcription factors and characterized by the presence of conserved MYB repeats (R) at $\mathrm{N}$-terminus and the variable region at $\mathrm{C}$-terminal. The conserved $\mathrm{N}$-terminus region is responsible for DNA-binding and protein-protein interactions while the variable region is associated with harmonizing regulatory activity of the protein. In rice, it was reported that $65 \%$ of $M Y B$ genes expressed in seedlings were differentially regulated under drought stress (Katiyar et al., 2012). An even higher percentage was observed in Arabidopsis thaliana (L.) Heynh. Transcriptomic data collected in the GENEVESTIGATOR database (Zimmermann et al., 2004) showed that $51 \%$ of AtMYB genes were upregulated and $41 \%$ were downregulated in drought stress (Katiyar et al., 2012). For example, virus-induced gene silencing (VIGS) of NbPHAN, a member of MYB TFs family, showed altered leaf shapes, impaired tolerance against drought stress, and shallow expression of stress-related genes in Nicotiana benthamiana (Huang et al., 2013). It is also involved in regulation of lateral root growth via the ABA-auxin signaling network to impart drought tolerance. Overexpressing AtMYB96 exhibited drought tolerant and reduced stomatal aperture while myb96-1 mutant plants showed the reverse phenotype under drought and ABA treatment. The MYB60 expression was enhanced by blue and white light while it was reduced in the presence of ABA and desiccation (Newman et al., 2004). Conversely, MYB61 causes reticence of light-induced stomatal opening as its expression was upregulated in the dark (Suzuki et al., 2014). Two other Arabidopsis guard cell expressing genes AtMYB44 (Jung et al., 2008) and AtMYB15 (Ding et al., 2009) showed higher expression upon exposure to ABA and several abiotic stresses. The overexpression lines showed drought tolerant phenotype opposite to the atmyb44 knockout mutant. AtMYB44 acts as a negative regulator of $A B A$ signaling.

$M Y B$ transcription factor gene GbMYB5 conferred drought tolerance in cotton and transgenic tobacco by modulating the efficient ROS scavenging system, polyamine biosynthesis genes ( $A D C 1$ and $S A M D C)$, the LEA abundant protein-encoding gene ERD10D, and drought-responsive genes (NCED3, BG, RD26) (Chen et al., 2015). A unique example of AtMYB21 expression under drought in flowers leads to maintenance of fertility. On the other hand atmyb21 mutant plants' flowers delayed filament elongation recovery after drought treatment as compared with wild-type plants. It suggests that AtMYB21 is a vital target gene that contributes to flower development as well as to drought tolerance possibly by involving cross-talk between JA/GA and ABA in drought response (Su et al., 2013). Thus we could summarize that MYB TFs play an important role in regulating and maintaining plant homeostasis during various abiotic stresses including drought.

\subsubsection{Ethylene Response Element-Binding Factors (AP2/ERF) Family}

Another important class of transcription factor is the APETALA2/ethylene response element-binding factors (AP2/ERF) family, which is involved in development along with biotic and abiotic stress responses (Xu et al., 2011; Sharoni et al., 2010). It has been characterized by the presence of conserved AP2/ERF DNAbinding domain (Song et al., 2013) specific to GCC box involved in ethylene-responsive transcription (Rashid et al., 2012). By number and similarity of the AP2/ERF domains, this family has been divided into four subfamilies: AP2 (Apetala 2), RAV (related to ABI3/VP1), DREB (dehydration-responsive element-binding protein), and ERF (Rashid et al., 2012; Sharoni et al., 2010). Among these four subfamilies, the most studied family of AP2/ERF in abiotic stress response are the AP2 and DREBs proteins.

In rice, six DREB2 family genes had been identified (Srivasta et al., 2010), while DREB2A and DREB2B were highly induced under drought stress (Nakashima et al., 2014) and resulted in improved osmotic tolerance of transgenic plants (Mizoi et al., 2013). A total of eight members of the DREB2 subgroup in Arabidopsis, $D R E B 2 A$, and DREB2B expression were induced by dehydration, high salinity, and heat in an ABAindependent manner (Nakashima et al., 2000; Sakuma et al., 2006). Constitutive expression OsDREB2B in Arabidopsis plant improved tolerance towards water stress by targeting DREB2A target genes (Matsukura et al., 2010). Improved drought tolerance was visible in a variety of species overexpressing DREB1/CBF gene including tomato, soybean (De Paiva Rolla et al., 2014), rice (Nakashima et al., 2014), tobacco (Phuong et al., 2015), and wheat (Shavrukov et al., 2016). Arabidopsis 
DREB1C gene in the transgenic rice plants improved drought stress in upland cultivar (Ishizaki et al., 2013). OsDREB1F homologous constitutive overexpression enhanced dehydration stress in rice via the ABAmediated pathway (Wang et al., 2008a,b). Various other AP2/ERF-type like TFs were functionally characterized in rice to decipher their role in drought tolerance (Abogadallah et al., 2011).

\subsubsection{Basic Leucine Zipper Transcription Family}

The basic leucine zipper family is composed of basic nuclear localizing, DNA binding N-terminal conserved bZIP domain and leucine-rich motif at C-terminus for dimerization (Wang et al., 2015). Function of bZIP TF family is much diversified in plants (Liu et al., 2014; Pourabed et al., 2015). The bZIP role is also explored to understand its role in drought tolerance mechanism in crops (Llorca et al., 2014). Studies would help in portraying its functioning in dehydration stress, such as how SIAREB in tomato (Solanum lycopersicum) improved tolerance to drought and salt stress by regulating the stress-responsive gene in an ABA-dependent manner (Hsieh et al., 2010). Likewise, overexpression of OsbZIP23, OsbZIP46, and OsbZIP16 in rice improved drought tolerance through ABA-mediated signaling (Xiang et al., 2008; Tang et al., 2012; Chen et al., 2012a,b).

A recent study in Arabidopsis revealed that overexpression of TabZIP60 improved plant's tolerance against multiple stresses like drought, salt and freezing along with increased sensitivity to ABA (Zhang et al., 2015). In Arabidopsis, overexpression of ABF4/AREB2, $A R E B 1$, and $A B F 3$ TFs led to enhanced drought tolerance by reducing the transpiration rate of the transgenic line as compared with wild-type ( Fujita et al., 2011). Moreover, areb1 areb2 abf3 triple mutant laid out ABA hyposensitivity and lessened dehydration stress tolerance as compared with single and double AREB/ $A B F$ knockout mutants. This outcome suggested synergistic action between the three bZIP TFs help in shoring up the plants to withstand drought and osmotic stress (Yoshida et al., 2010).

\subsubsection{Zn-Finger Transcription Factor Family}

Zinc-finger proteins (ZFPs) also play a diverse role in plants, and express accordingly to the type and degree of different abiotic stresses (Sun et al., 2010). On the basis of number and sequence order of $C$ and $\mathrm{H}$ residues in the secondary structure, DNA binding $\mathrm{Zn}$ fingers are divided into nine groups: $\mathrm{C} 2 \mathrm{H} 2, \mathrm{C} 8, \mathrm{C} 6$, $\mathrm{C} 3 \mathrm{HC} 4, \mathrm{C} 2 \mathrm{HC}, \mathrm{C} 2 \mathrm{HC} 5, \mathrm{C} 4, \mathrm{C} 4 \mathrm{HC} 3$, and $\mathrm{C} 3 \mathrm{H}$ (Schumann et al., 2007; Gupta et al., 2012). Zinc-finger
TFs are a large family of TFs that consist of 23 subfamilies, and WRKY is one of the most studied TFs in regards to biotic and abiotic stress ( $\mathrm{Li}$ et al., 2014). Here, we emphasize its function in abiotic stress particularly in drought stress. Most of the time, WRKY transcription factors and ABA signaling work hand in hand. Zn finger TF, Zat10 knockout, and RNAi mutants were more tolerant to osmotic and salinity stress in Arabidopsis. ZFP252 (TFIIIA C2H2 ZF) constitutive expression in rice enhanced the drought tolerance by enhancing proline accumulation, sugar content, and drought-responsive OsDREB1A gene expression ( $\mathrm{Xu}$ et al., 2008). Transgenic tobacco, overexpressing GhWRKY41/SpWRKY1, regulated stomatal conductance and ROS levels to impart salt and drought tolerance (Chu et al., 2015; Li et al., 2015). FcWRKY70 in Fortunella crassifolia accumulated a more significant amount of putrescine in comparison with wild-type (WT) and enhanced dehydration stress (Gong et al., 2015). Similarly, SiWRKY066/082 (from Setaria italica) and PgWRKY1 (from Panax ginseng) were involved in stress by regulating hormonal signaling (Muthamilarasan et al., 2015; Nuruzzaman et al., 2016). AtWRKY46 plays an important role in hormonal regulation especially of $\mathrm{ABA}$ and auxin facilitated lateral root development under osmotic and salinity stress (Ding et al., 2015). Transgenic Arabidopsis harboring HaWRKY76 from sunflower provided drought and flood tolerance (Raineri et al., 2015). Zinc-finger TFs help in coping with drought stress through regulation a number of factors like ROS scavenging (Baek et al., 2015) increasing the contents of proline, abscisic acid (ABA), chlorophyll, soluble sugars, and reducing the water loss rate (Luo et al., 2012; Wang et al., 2016a,b). Through detailed study and analysis (TaZFPs) from Triticum aestivum, ZFPs was considered to play an important role in environmental adaptation during drought stress (Cheuk and Houde, 2016).

\subsubsection{NAC Transcription Factor Family}

The NAC gene family is also one of the largest gene families of TFs, performing diverse functions from developmental processes, leaf senescence, and lateral root development to biotic and abiotic stress response (Nuruzzaman et al., 2012). NAM (for no apical meristem), ATAF 1 and 2 (Arabidopsis transcription activation factor), and CUC2 (for cup-shaped cotyledon) collectively form the NAC domain of the NAC gene family. Unlike other TFs, NAC proteins consist of conserved nuclear localizing DNA-binding N-terminal domain and variable C-terminal transcription regulatory (TR) region (Olsen et al., 2005). Multiple reports suggested that NAC genes are involved in drought stress 
responses. Transgenic rice harboring the OsNAC2/6 and OsNAC10 genes improved the drought and salt tolerance (Nakashima et al., 2009; Jeong et al., 2010), and enhanced grain yield of plants overexpressing SNAC1 gene under drought stress ( $\mathrm{Hu}$ et al., 2006). NAC TF genes were induced by hormone application such as auxin, ethylene, and ABA (He et al., 2005; Sperotto et al., 2009). But the role of ABA was prominent in adaptation to drought stress as it regulated the lateral root development for efficient water use. Similarly, rice homologs OsNAC071/OsNAC5/ OsNAC009 and OsNAC6 expression were induced by dehydration, high salinity, and ABA (Takasaki et al., 2010; Nakashima et al., 2007). The mechanism underlying ABA-mediated signaling under stress ensures the expression of drought-responsive genes like LEA protein, MYB, bZIP, and MYC, etc., to protect the vegetative and reproductive tissue from cellular damage on dehydration. ANAC019 and ANAC055 overexpression in Arabidopsis imparts drought tolerance by targeting JA/ABA-mediated pathway (Bu et al., 2008). Heterologous overexpression of AhNAC2 gene in Arabidopsis improved drought and salt tolerance by targeting stress-responsive genes: $R D 29 A, R D 29 B, R A B 18$, ERD1, AtMYB2, AtMYC2, COR47, COR15a, KIN1, $A R E B 1$, and CBF1. Moreover, rice harboring ONAC045 gene enhanced drought and salt tolerance by targeting desiccation tolerant genes OsLEA3-1 and OsPM1 (Zheng et al., 2009). All these evident reports emphasized the major role of NAC TFs in abiotic stresses particularly drought and salinity through hormone regulation.

\subsection{CONCLUSION AND FURTHER PROSPECTS}

Drought stress causes membrane damage, ROS generation, protein dysfunction, and an imbalance in metabolic and redox homeostasis in the plant. Several molecular pathways for tolerance and adaptation to drought stress have been illustrated (Figs. 6.1 and 6.2). The cross-talk between the different signaling molecule and regulatory genes (MYB, WRKY, DREB/ERF, and NAC) suggested that drought tolerance in the plant is a multifaceted phenomenon, and is governed by a complex regulatory mechanism. So, to find a key player in drought stress might be a potential candidate for crop improvement.

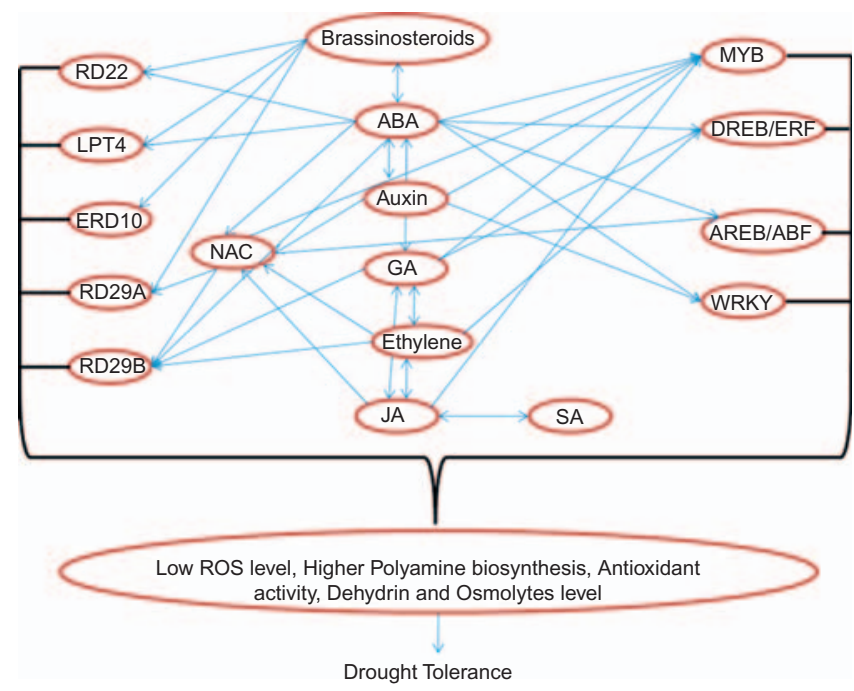

FIGURE 6.2 Cross-talk between the different signaling molecule and regulatory genes that take part in water deficit stress.

\section{References}

Abebe, T., Guenzi, A.C., Martin, B., Cushman, J.C., 2003. Tolerance of mannitol-accumulating transgenic wheat to water stress and salinity. Plant Physiol. 131, 1748-1755.

Abogadallah, G.M., Nada, R.M., Malinowski, R., Quick, P., 2011. Overexpression of HARDY, an AP2/ERF gene from Arabidopsis, improves drought and salt tolerance by reducing transpiration and sodium uptake in transgenic Trifolium alexandrinum L. Planta 233, 1265-1276.

Achard, P., Cheng, H., De Grauwe, L., Decat, J., Schoutteten, H., Moritz, T., et al., 2006. Integration of plant responses to environmentally activated phytohormonal signals. Science 311, 91-94.

Ahmad, P., Jaleel, C.A., Salem, M.A., Nabi, G., Sharma, S., 2010. Roles of enzymatic and nonenzymatic antioxidants in plants during abiotic stress. Crit. Rev. Biotechnol. 30, 161-175.

Ahmad, R., Kim, M.D., Back, K.H., Kim, H.S., Lee, H.S., Kwon, S.Y., et al., 2008. Stress-induced expression of choline oxidase in potato plant chloroplasts confers enhanced tolerance to oxidative, salt, and drought stresses. Plant Cell Rep. 27, 687-698.

Akram, N.A., Noreen, S., Noreen, T., Ashraf, M., 2015. Exogenous application of trehalose alters growth, physiology and nutrient composition in radish (Raphanussativus L.) plants under waterdeficit conditions. Braz. J. Bot. 38, 431-439.

Arraes, F.B.M., Beneventi, M.A., De Sa, M.E.L., Paixao, J.F.R., Albuquerque, E.V.S., Marin, S.R.R., et al., 2015. Implications of ethylene biosynthesis and signaling in soybean drought stress tolerance. BMC Plant Biol. 15, 213.

Avonce, N., Leyman, B., Mascorro-Gallardo, J.O., Van Dijck, P., Thevelein, J.M., Iturriaga, G., 2004. The Arabidopsis trehalose-6-P synthase AtTPS1 gene is a regulator of glucose, abscisic acid, and stress signaling. Plant Physiol. 136, 3649-3659.

Baek, D., Cha, J.Y., Kang, S., Park, B., Lee, H.J., Hong, H., et al., 2015. The Arabidopsis a zinc finger domain protein ARS1 is essential for seed germination and ROS homeostasis in response to ABA and oxidative stress. Front. Plant Sci. 6, 963.

Bai, M.Y., Shang, J.X., Oh, E., Fan, M., Bai, Y., Zentella, R., et al., 2012. Brassinosteroid, gibberellin and phytochrome impinge on a 
common transcription module in Arabidopsis. Nat. Cell Biol. 14, $810-817$.

Bandurska, H., 2005. The effect of salicylic acid on barley response to water deficit. Acta. Physiol. Plant. 27, 379-386.

Barthakur, S.B.V.B., Babu, V., Bansa, K.C., 2001. Over-expression of osmotin induces proline accumulation and confers tolerance to osmotic stress in transgenic tobacco. J. Plant Biochem. Biotechnol. $10,31-37$.

Bhargava, S., Sawant, K., 2013. Drought stress adaptation: metabolic adjustment and regulation of gene expression. Plant Breed. 132, $21-32$.

Bishop, G.J., Yokota, T., 2001. Plants steroid hormones, brassinosteroids: current highlights of molecular aspects on their synthesis/ metabolism, transport, perception and response. Plant Cell Physiol. 42, 114-120.

Brini, F., Hanin, M., Lumbreras, V., Amara, I., Khoudi, H., Hassairi, A., et al., 2007. Overexpression of wheat dehydrin DHN-5 enhances tolerance to salt and osmotic stress in Arabidopsis thaliana. Plant Cell Rep. 26, 2017-2026.

Bu, Q.Y., Jiang, H.L., Li, C.B., Zhai, Q.Z., Zhang, J.Y., Wu, X.Q., et al., 2008. Role of the Arabidopsis thaliana NAC transcription factors ANAC019 and ANAC055 in regulating jasmonic acid-signaled defense responses. Cell Res. 18, 756-767.

Cao, S., Du, X.H., Li, L.H., Liu, Y.D., Zhang, L., Pan, X., et al., 2017. Overexpression of Populus tomentosa cytosolic ascorbate peroxidase enhances abiotic stress tolerance in tobacco plants. Russ. J. Plant Physiol. 64, 224-234.

Chen, H., Li, Z., Xiong, L., 2012a. A plant microRNA regulates the adaptation of roots to drought stress. FEBS Lett. 586, 1742-1747.

Chen, L.G., Song, Y., Li, S.J., Zhang, L.P., Zou, C.S., Yu, D.Q., 2012 b. The role of WRKY transcription factors in plant abiotic stresses. Biochim. Biophys. Acta. 1819, 120-128.

Chen, T.Z., Li, W.J., Hu, X.H., Guo, J.R., Liu, A.M., Zhang, B.L., 2015. A cotton MYB transcription factor, GbMYB5, is positively involved in plant adaptive response to drought stress. Plant Cell Physiol. 56, 917-929.

Cheng, M.C., Liao, P.M., Kuo, W.W., Lin, T.P., 2013. The Arabidopsis Ethylene Response Factor1 regulates abiotic stress-responsive gene expression by binding to different cis-acting elements in response to different stress signals. Plant Physiol. 162, 1566-1582.

Cheuk, A., Houde, M., 2016. Genome wide identification of C1-2i zinc finger proteins and their response to abiotic stress in hexaploid wheat. Mol. Genet. Genomic 291, 873-890.

Chowdhury, S., Basu, A., Kundu, S., 2017. Overexpression of a new osmotin-like protein gene (SindOLP) confers tolerance against biotic and abiotic stresses in sesame. Front. Plant Sci. 8, 410.

Chu, X., Wang, C., Chen, X., Lu, W., Li, H., Wang, X., et al., 2015. The cotton WRKY gene GhWRKY41 positively regulates salt and drought stress tolerance in transgenic Nicotiana benthamiana. PLoS One 10, e0143022.

Cicero, L.L., Madesis, P., Tsaftaris, A., Piero, A.R.L., 2015. Tobacco plants over-expressing the sweet orange tau glutathione transferases (CsGSTUs) acquire tolerance to the diphenyl ether herbicide fluorodifen and to salt \& drought stresses. Phytoche. 116, 69-77.

Colebrook, E.H., Thomas, S.G., Phillips, A.L., Hedden, P., 2014. The role of gibberellin signalling in plant responses to abiotic stress. J. Exp. Biol. 217, 67-75.

Contour-Ansel, D., Torres-Franklin, M.L., Cruz De Carvalho, M.H., D'arcy-Lameta, A., Zuily-Fodil, Y., 2006. Glutathione reductase in leaves of cowpea: cloning of two cDNAs, expression and enzymatic activity under progressive drought stress, desiccation and abscisic acid treatment. Ann. Bot. 98, 1279-1287.

Cristina, M.S., Petersen, M., Mundy, J., 2010. Mitogen-activated protein kinase signaling in plants. Annu. Rev. Plant Biol. 61, 621-649.
De Ollas, C., Arbona, V., Gómez-Cadenas, A., 2015. Jasmonic acid interacts with abscisic acid to regulate plant responses to water stress conditions. Plant Signal. Behav. 10, e1078953.

De Paiva Rolla, A.A., De Fatima Correa Carvalho, J., FugantiPagliarini, R., Engels, C., Do Rio, A., Marin, S.R., et al., 2014. Phenotyping soybean plants transformed with $r d 29 A$ AtDREB1A for drought tolerance in the greenhouse and field. Transgenic Res. 23, 75-87.

De Ronde, J.A., Spreeth, M.H., Cress, W.A., 2000. Effect of antisense L-1-pyrroline-5-carboxylate reductase transgenic soybean plants subjected to osmotic and drought stress. Plant Growth Regul. 32, $13-26$.

De Ronde, J.A., Laurie, R.N., Caetano, T., Greyling, M.M., Kerepesi, I., 2004. Comparative study between transgenic and nontransgenic soybean lines proved transgenic lines to be more drought tolerant. Euphytica 138, 123-132.

Ding, Z.H., Li, S.M., An, X.L., Liu, X.J., Qin, H.M., Wang, D., 2009. Transgenic expression of MYB15 confers enhanced sensitivity to abscisic acid and improved drought tolerance in Arabidopsisthaliana. J. Genet. Genomic. 36, 17-29.

Ding, Z.J., Yan, J.Y., Li, C.X., Li, G.X., Wu, Y.R., Zheng, S.J., 2015. Transcription factor WRKY46 modulates the development of Arabidopsis lateral roots in osmotic/salt stress conditions via regulation of ABA signaling and auxin homeostasis. Plant J. 84, 56-69.

Divi, U.K., Rahman, T., Krishna, P., 2010. Brassinosteroid-mediated stress tolerance in Arabidopsis shows interactions with abscisic acid, ethylene and salicylic acid pathways. BMC Plant Biol. 10, 151.

Du, Y.Y., Wang, P.C., Chen, J., Song, C.P., 2008. Comprehensive functional analysis of the catalase gene family in Arabidopsis thaliana. J. Integr. Plant Biol. 50, 1318-1326.

Duan, F., Ding, J., Lee, D., Lu, X., Feng, Y., Song, W., 2017. Overexpression of SoCYP85A1, a spinach cytochrome p450 gene in transgenic tobacco enhances root development and drought stress tolerance. Front. Plant Sci. 8 (1909).

Dubois, M., Skirycz, A., Claeys, H., Maleux, K., Dhondt, S., De Bodt, S., et al., 2013. Ethylene Response Factor6 acts as a central regulator of leaf growth under water-limiting conditions in Arabidopsis. Plant Physiol. 162, 319-332.

Dubos, C., Stracke, R., Grotewold, E., Weisshaar, B., Martin, C., Lepiniec, L., 2010. MYB transcription factors in Arabidopsis. Trends Plant Sci. 15, 573-581.

Eltayeb, A.E., Kawano, N., Badawi, G.H., Kaminaka, H., Sanekata, T., Morishima, I., et al., 2006. Enhanced tolerance to ozone and drought stresses in transgenic tobacco overexpressing dehydroascorbate reductase in cytosol. Physiol. Plant. 127, 57-65.

Eltayeb, A.E., Kawano, N., Badawi, G.H., Kaminaka, H., Sanekata, T., Shibahara, T., et al., 2007. Overexpression of monodehydroascorbate reductase in transgenic tobacco confers enhanced tolerance to ozone, salt and polyethylene glycol stresses. Planta 225, 1255-1264.

Espinoza, A., San Martín, A., López-Climent, M., Ruiz-Lara, S., Gómez-Cadenas, A., Casaretto, J.A., 2013. Engineered droughtinduced biosynthesis of $\alpha$-tocopherol alleviates stress-induced leaf damage in tobacco. J. Plant Physiol. 170, 1285-1294.

Faize, M., Burgos, L., Faize, L., Piqueras, A., Nicolas, E., Barba-Espin, G., et al., 2011. Involvement of cytosolic ascorbate peroxidase and $\mathrm{Cu} / \mathrm{Zn}$-superoxide dismutase for improved tolerance against drought stress. J. Exp. Bot. 62, 2599-2613.

Ferndez, O., Béthencourt, L., Quero, A., Sangwan, R.S., Clément, C., 2010. Trehalose and plant stress responses: friend or foe? Trends Plant Sci. 15, 409-417.

Foyer, C.H., Noctor, G., 2005. Redox homeostasis and antioxidant signaling: a metabolic interface between stress perception and physiological responses. The Plant Cell 17, 1866-1875. 
Fujii, H., Chinnusamy, V., Rodrigues, A., Rubio, S., Antoni, R., Park, S.Y., et al., 2009. In vitro reconstitution of an abscisic acid signalling pathway. Nat. 462, 660-664.

Fujita, Y., Fujita, M., Shinozaki, K., Yamaguchi-Shinozaki, K., 2011. ABA-mediated transcriptional regulation in response to osmotic stress in plants. J. Plant Res. 124, 509-525.

Gaber, A., Yoshimura, K., Yamamoto, T., Yabuta, Y., Takeda, T., Miyasaka, H., et al., 2006. Glutathione peroxidase-like protein of Synechocystis PCC 6803 confers tolerance to oxidative and environmental stresses in transgenic Arabidopsis. Physiol. Plant. 128, $251-262$.

Gao, M., Tao, R., Miura, K., Dandekar, A.M., Sugiura, A., 2001. Transformation of Japanese persimmon (Diospyros kaki Thunb.) with apple cDNA encoding NADP-dependent sorbitol-6phosphate dehydrogenase. Plant Sci. 160, 837-845.

Garg, A.K., Kim, J.K., Owens, T.G., Ranwala, A.P., Do Choi, Y., Kochian, L.V., et al., 2002. Trehalose accumulation in rice plants confers high tolerance levels to different abiotic stresses. Proc. Natl. Acad. Sci. U.S.A. 99, 15898-15903.

Gill, S.S., Tuteja, N., 2010. Reactive oxygen species and antioxidant machinery in abiotic stress tolerance in crop plants. Plant Physiol. Biochem. 48, 909-930.

Giri, J., 2011. Glycinebetaine and abiotic stress tolerance in plants. Plant Signal. Behav. 6, 1746-1751.

Goel, D., Singh, A.K., Yadav, V., Babbar, S.B., Bansal, K.C., 2010. Overexpression of osmotin gene confers tolerance to salt and drought stresses in transgenic tomato (Solanum lycopersicum L.). Protoplasma 245, 133-141.

Gong, X., Zhang, J., Hu, J., Wang, W., Wu, H., Zhang, Q., et al., 2015. FCWRKY70, a WRKY protein of Fortunella crassifolia, functions in drought tolerance and modulates putrescine synthesis by regulating arginine decarboxylase gene. Plant Cell Environ. 38, 2248-2262.

González-Grandío, E., Poza-Carrión, C., Sorzano, C.O.S., Cubas, P., 2013. Branched 1 promotes axillary bud dormancy in response to shade in Arabidopsis. Plant Cell 25, 834-850.

Guo, H.S., Xie, Q., Fei, J.F., Chua, N.H., 2005. MicroRNA directs mRNA cleavage of the transcription factor NAC1 to downregulate auxin signals for Arabidopsis lateral root development. Plant Cell $17,1376-1386$.

Guo, Y., Jiang, Q., Hu, Z., Sun, X., Fan, S., Zhang, H., 2017. Function of the auxin-responsive gene TaSAUR75 under salt and drought stress. Crop J. Available from: https://doi.org/10.1016/ j.cj.2017.08.005.

Gupta, S.K., Rai, A.K., Kanwar, S.S., Sharma, T.R., 2012. Comparative analysis of zinc finger proteins involved in plant disease resistance. PloS One 7, e42578.

Halliwell, B., 2006. Reactive species and antioxidants. Redox biology is a fundamental theme of aerobic life. Plant Physiol. 141, 312-322.

Hamada, A., 2001. Salicylic acid versus salinity-drought-induced stress on wheat seedlings. Rostlinna Vyroba-UZPI (Czech Republic).

Harfouche, A.L., Rugini, E., Mencarelli, F., Botondi, R., Muleo, R., 2008. Salicylic acid induces $\mathrm{H}_{2} \mathrm{O}_{2}$ production and endochitinase gene expression but not ethylene biosynthesis in Castanea sativa in vitro model system. J. Plant Physiol. 165, 734-744.

Hasanuzzaman, M., Nahar, K., Anee, T.I., Khan, M.I.R., Fujita, M., 2018. Silicon-mediated regulation of antioxidant defense and glyoxalase systems confers drought stress tolerance in Brassica napus L. South Afr. J. Bot. 115, 50-57.

He, X.J., Mu, R.L., Cao, W.H., Zhang, Z.G., Zhang, J.S., Chen, S.Y., 2005. AtNAC2, a transcription factor downstream of ethylene \& auxin signaling pathways, is involved in salt stress response \& lateral root development. Plant J 44, 903-916.

Hirayama, T., Shinozaki, K., 2010. Research on plant abiotic stress responses in the post-genome era: past, present and future. Plant J. 61, 1041-1052.
Hsieh, T.H., Li, C.W., Su, R.C., Cheng, C.P., Tsai, Y.C., Chan, M.T., 2010. A tomato bZIP transcription factor, SIAREB, is involved in water deficit and salt stress response. Planta 231, 1459-1473.

Hu, H.H., Dai, M.Q., Yao, J.L., Xiao, B.Z., Li, X.H., Zhang, Q.F., et al., 2006. Overexpressing a NAM, ATAF, and CUC (NAC) transcription factor enhances drought resistance and salt tolerance in rice. Proc. Natl. Acad. Sci. U.S.A. 103, 12987-12992.

Hu, L., Wang, Z., Du, H., Huang, B., 2010. Differential accumulation of dehydrins in response to water stress for hybrid and common Bermuda grass genotypes differing in drought tolerance. J. Plant Physiol. 167, 103-109.

Huang, C., Hu, G., Li, F., Li, Y., Wu, J., Zhou, X., 2013. NbPHAN, a MYB transcriptional factor, regulates leaf development and affects drought tolerance in Nicotiana benthamiana. Physiol Plant. $149,297-309$.

Hund, A., Ruta, N., Liedgens, M., 2009. Rooting depth and water use efficiency of tropical maize inbred lines, differing in drought tolerance. Plant Soil 318, 311-325.

Ishizaki, T., Maruyama, K., Obara, M., Fukutani, A., YamaguchiShinozaki, K., Ito, Y., et al., 2013. Expression of Arabidopsis DREB1C improves survival, growth, and yield of upland New Rice for Africa (NERICA) under drought. Mol. Breed. 31, 255-264.

Islam, T., Manna, M., Reddy, M.K., 2015. Glutathione peroxidase of Pennisetum glaucum $(P g G P x)$ is a functional $\mathrm{Cd}^{2+}$ dependent peroxiredoxin that enhances tolerance against salinity and drought stress. PloS one 10, e0143344.

Jeong, J.S., Kim, Y.S., Baek, K.H., Jung, H., Ha, S.H., Do Choi, Y., et al., 2010. Root-specific expression of OsNAC10 improves drought tolerance and grain yield in rice under field drought conditions. Plant Physiol. 153, 185-197.

Joshi, R., Wani, S.H., Singh, B., Bohra, A., Dar, Z.A., Lone, A.A., et al., 2016. Transcription factors and plants response to drought stress: current understanding and future directions. Front. Plant Sci. 7, 1029.

Jung, C., Seo, J.S., Han, S.W., Koo, Y.J., Kim, C.H., Song, S.I., et al., 2008. Overexpression of AtMYB44 enhances stomatal closure to confer abiotic stress tolerance in transgenic Arabidopsis. Plant Physiol. 146, 623-635.

Kagale, S., Divi, U.K., Krochko, J.E., Keller, W.A., Krishna, P., 2007. Brassinosteroid confers tolerance in Arabidopsis thaliana and Brassica napus to a range of abiotic stresses. Planta 225, 353-364.

Katiyar, A., Smita, S., Lenka, S.K., Rajwanshi, R., Chinnusamy, V., Bansal, K.C., 2012. Genome-wide classification and expression analysis of MYB transcription factor families in rice and Arabidopsis. BMC Genomics 13, 544.

Kazan, K., 2013. Auxin and the integration of environmental signals into plant root development. Ann. Bot. 112, 1655-1665.

Khan, M.I.R., Khan, N., 2017. Reactive oxygen species and antioxidant system in plants: Role and regulation under abiotic stress. Springer Nature, Singapore978-981-10-5254-5.

Khan, M.I.R., Iqbal, N., Masood, A., Khan, N.A., 2012. Variation in salt tolerance of wheat cultivars: evaluation of the role of glycinebetaine and ethylene. Pedosphere. 22, 746-754.

Khan, M.I.R., Asgher, M., Fatma, M., Per, T.S., Khan, N.A., 2015. Drought stress vis a vis plant functions in the era of climate change. Clim. Change Environ. Sustainability. 3, 13-25.

Khan, M.I.R., Khan, N.A., Masood, A., Per, T.S., Asgher, M., 2016. Hydrogen peroxide alleviates nickel-inhibited photosynthetic responses through increase in use-efficiency of nitrogen and sulfur, and glutathione production in mustard. Front. Plant Sci. 7,44 .

Kim, E.H., Kim, Y.S., Park, S.H., Koo, Y.J., Do Choi, Y., Chung, Y.Y., et al., 2009a. Methyl jasmonate reduces grain yield by mediating stress signals to alter spikelet development in rice. Plant Physiol. $149,1751-1760$. 
Kim, J.H., Woo, H.R., Kim, J., Lim, P.O., Lee, I.C., Choi, S.H., et al., 2009b. Trifurcate feed-forward regulation of age-dependent cell death involving miR164 in Arabidopsis. Sci. 323, 1053-1057.

Kim, T.H., Bohmer, M., Hu, H., Nishimura, N., Schroeder, J.I., 2010. Guard cell signal transduction network: advances in understanding abscisic acid, CO2, and $\mathrm{Ca}^{2+}$ signaling. Annu. Rev. Plant Biol. $61,561-591$.

Kinoshita, N., Wang, H., Kasahara, H., Liu, J., Macpherson, C., Machida, Y., et al., 2012. IAA-Ala Resistant3, an evolutionarily conserved target of miR167, mediates Arabidopsis root architecture changes during high osmotic stress. Plant Cell 24, 3590-3602.

Kishor, P.K., Hong, Z., Miao, G.H., Hu, C.A.A., Verma, D.P.S., 1995. Overexpression of [delta]-pyrroline-5-carboxylate synthetase increases proline production and confers osmotolerance in transgenic plants. Plant Physiol. 108, 1387-1394.

Korkmaz, A., Uzunlu, M., Demirkiran, A.R., 2007. Treatment with acetyl salicylic acid protects muskmelon seedlings against drought stress. Acta. Physiol. Plant. 29, 503-508.

Koussevitzky, S., Suzuki, N., Huntington, S., Armijo, L., Sha, W., Cortes, D., et al., 2008. Ascorbate peroxidase 1 plays a key role in the response of Arabidopsis thaliana to stress combination. J. Biol. Chem. 283, 34197-34203.

Kumar, M., Lee, S.C., Kim, J.Y., Kim, S.J., Kim, S.R., 2014. Overexpression of dehydrin gene, OsDhn1, improves drought and salt stress tolerance through scavenging of reactive oxygen species in rice (Oryza sativa L.). J. Plant Biol. 57, 383-393.

Lee, H., Won, S.H., Lee, B.H., Park, H.D., Chung, W.I., Jo, J., 2002. Genomic cloning and characterization of glutathione reductase gene from Brassica campestris var. Pekinensis. Mol. Cells 13, $245-251$.

Li, F., Wu, Q., Duan, M., Dong, X., Li, B., Meng, Q., 2012. Transgenic tomato plants overexpressing chloroplastic monodehydroascorbate reductase are resistant to salt-and PEG-induced osmotic stress. Photosynthetica 50, 120-128.

Li, J.B., Luan, Y.S., Liu, Z., 2015. Overexpression of SpWRKY1 promotes resistance to Phytophthora nicotianae and tolerance to salt and drought stress in transgenic tobacco. Physiol. Plant. 155, $248-266$.

Li, W.T., Chen, W.L., Yang, C., Wang, J., Yang, L., He, M., et al., 2014. Identification and network construction of zinc finger protein (ZFP) genes involved in the rice-'Magnaporthe oryzae'interaction. Plant Omics. 7, 540.

Lim, M.Y., Pulla, R.K., Park, J.M., Harn, C.H., Jeong, B.R., 2012. Over-expression of l-gulono- $\gamma$-lactone oxidase (GLOase) gene leads to ascorbate accumulation with enhanced abiotic stress tolerance in tomato. In Vitro Cell Dev. Biol. Plant. 48, 453-461.

Liu, D., Liu, Y., Rao, J., Wang, G., Li, H., Ge, F., et al., 2013. Overexpression of the glutathione S-transferase gene from Pyrus pyrifolia fruit improves tolerance to abiotic stress in transgenic tobacco plants. Mol. Biol. 47, 515-523.

Liu, H., Yu, C., Li, H., Ouyang, B., Wang, T., Zhang, J., et al., 2015. Overexpression of ShDHN, a dehydrin gene from Solanumhabrochaites enhances tolerance to multiple abiotic stresses in tomato. Plant Sci. 231, 198-211.

Liu, J., Chen, N., Chen, F., Cai, B., Dal Santo, S., Tornielli, G.B., et al., 2014. Genome-wide analysis and expression profile of the bZIP transcription factor gene family in grapevine (Vitis vinifera). BMC Genomics 15, 281.

Liu, X., Hua, X., Guo, J., Qi, D., Wang, L., Liu, Z., et al., 2008. Enhanced tolerance to drought stress in transgenic tobacco plants overexpressing VTE1 for increased tocopherol production from Arabidopsis thaliana. Biotechnol. Lett. 30, 1275-1280.

Llorca, C.M., Potschin, M., Zentgraf, U., 2014. bZIPs and WRKYs: two large transcription factor families executing two different functional strategies. Front. Plant. Sci. 5, 169.
Luo, X., Bai, X., Zhu, D., Li, Y., Ji, W., Cai, H., et al., 2012. GsZFP1, a new Cys2/His2-type zinc-finger protein, is a positive regulator of plant tolerance to cold and drought stress. Planta 235, 1141-1155.

Ma, L., Wang, Y., Liu, W., Liu, Z., 2014. Overexpression of an alfalfa GDP-mannose 3, 5-epimerase gene enhances acid, drought and salt tolerance in transgenic Arabidopsis by increasing ascorbate accumulation. Biotechnol. Lett. 36, 2331-2341.

Ma, Y., Szostkiewicz, I., Korte, A., Moes, D., Yang, Y., Christmann, A., et al., 2009. Regulators of PP2C phosphatase activity function as abscisic acid sensors. Sci 324, 1064-1068.

Macková, H., Hronková, M., Dobrá, J., Turečková, V., Novák, O., Lubovská, Z., et al., 2013. Enhanced drought and heat stress tolerance of tobacco plants with ectopically enhanced cytokinin oxidase/dehydrogenase gene expression. J. Exp. Bot. 64, 2805-2815.

Mahmood, M., Bidabadi, S.S., Ghobadi, C., Gray, D.J., 2012. Effect of methyl jasmonate treatments on alleviation of polyethylene glycolmediated water stress in banana (Musa acuminata cv.'Berangan', AAA) shoot tip cultures. Plant Growth Regul 68, 161-169.

Masood, A., Per, T.S., Asgher, M., Fatma, M., Khan, M.I.R., Rasheed, F., et al., 2016. Glycine betaine: role in shifting plants toward adaptation under extreme environments. Osmolytes and Plants Acclimation to Changing Environment: Emerging Omics Technologies. Springer, New Delhi, pp. 69-82.

Matsukura, S., Mizoi, J., Yoshida, T., Todaka, D., Ito, Y., Maruyama, K., et al., 2010. Comprehensive analysis of rice DREB2-type genes that encode transcription factors involved in the expression of abiotic stress-responsive genes. Mol. Genet. Genomic 283, 185-196.

Merewitz, E.B., Gianfagna, T., Huang, B., 2010. Effects of SAG12-ipt and HSP18. 2-ipt expression on cytokinin production, root growth, and leaf senescence in creeping bentgrass exposed to drought stress. J. Am. Soc. Hortic. Sci. 135, 230-239.

Miura, K., Tada, Y., 2014. Regulation of water, salinity, and cold stress responses by salicylic acid. Front. Plant Sci. 5, 4.

Miura, K., Okamoto, H., Okuma, E., Shiba, H., Kamada, H., Hasegawa, P.M., et al., 2013. SIZ1 deficiency causes reduced stomatal aperture and enhanced drought tolerance via controlling salicylic acid-induced accumulation of reactive oxygen species in Arabidopsis. Plant J. 73, 91-104.

Miyake, C., Asada, K., 1994. Ferredoxin-dependent photoreduction of the monodehydroascorbate radical in spinach thylakoids. Plant Cell Physiol. 35, 539-549.

Mizoi, J., Ohori, T., Moriwaki, T., Kidokoro, S., Todaka, D., Maruyama, K., et al., 2013. GmDREB2A;2, a canonical dehydration-responsive element-Binding protein2-type transcription factor in soybean, is posttranslationally regulated and mediates dehydration-responsive element-dependent gene expression. Plant Physiol. 161, 346-361.

Mockaitis, K., Howell, S.H., 2000. Auxin induces mitogenic activated protein kinase (MAPK) activation in roots of Arabidopsis seedlings. Plant J. 24, 785-796.

Moriwaki, T., Miyazawa, Y., Kobayashi, A., Uchida, M., Watanabe, C., Fujii, N., et al., 2011. Hormonal regulation of lateral root development in Arabidopsis modulated by MIZ1 and requirement of GNOM activity for MIZ1 function. Plant Physiol. 157, 1209-1220.

Muthamilarasan, M., Bonthala, V.S., Khandelwal, R., Jaishankar, J., Shweta, S., Nawaz, K., et al., 2015. Global analysis of WRKY transcription factor superfamily in Setaria identifies potential candidates involved in abiotic stress signaling. Front. Plant Sci. 6, 910.

Nafie, E., Hathout, T., Mokadem, A., Shyma, A., 2011. Jasmonic acid elicits oxidative defense and detoxification systems in Cucumis melo L. cells. Braz. J. Plant Physiol. 23, 161-174.

Nahar, K., Hasanuzzaman, M., Alam, M., Fujita, M., 2015. Glutathione-induced drought stress tolerance in mung bean: 
coordinated roles of the antioxidant defence and methylglyoxal detoxification systems. AoB Plants 7.

Nakashima, K., Shinwari, Z.K., Sakuma, Y., Seki, M., Miura, S., Shinozaki, K., et al., 2000. Organization and expression of two Arabidopsis DREB2 genes encoding DRE-binding proteins involved in dehydration and high-salinity-responsive gene expression. Plant Mol. Biol. 42, 657-665.

Nakashima, K., Ito, Y., Yamaguchi-Shinozaki, K., 2009. Transcriptional regulatory networks in response to abiotic stresses in Arabidopsis and grasses. Plant Physiol. 149, 88-95.

Nakashima, K., Tran, L.S.P., Van Nguyen, D., Fujita, M., Maruyama, K., Todaka, D., et al., 2007. Functional analysis of a NAC-type transcription factor OsNAC6 involved in abiotic and biotic stressresponsive gene expression in rice. Plant J. 51, 617-630.

Nakashima, K., Yamaguchi-Shinozaki, K., Shinozaki, K., 2014. The transcriptional regulatory network in the drought response and its cross-talk in abiotic stress responses including drought, cold, and heat. Front. Plant Sci. 5, 170.

Nanjo, T., Kobayashi, M., Yoshiba, Y., Sanada, Y., Wada, K., Tsukaya, H., et al., 1999. Biological functions of proline in morphogenesis and osmotolerance revealed in antisense transgenic Arabidopsis thaliana. Plant J. 18, 185-193.

Nanjo, T., Fujita, M., Seki, M., Kato, T., Tabata, S., Shinozaki, K., 2003. Toxicity of free proline revealed in an Arabidopsis T-DNAtagged mutant deficient in proline dehydrogenase. Plant Cell Physiol. 44, 541-548.

Negi, N.P., Shrivastava, D.C., Sharma, V., Sarin, N.B., 2015. Overexpression of $\mathrm{CuZnSOD}$ from Arachis hypogaea alleviates salinity and drought stress in tobacco. Plant Cell Rep. 34, $1109-1126$.

Newman, L.J., Perazza, D.E., Juda, L., Campbell, M.M., 2004. Involvement of the R2R3-MYB, AtMYB61, in the ectopic lignification and dark-photomorphogenic components of the det3 mutant phenotype. Plant J. 37, 239-250.

Nie, Q., Gao, G.L., Fan, Q.J., Qiao, G., Wen, X.P., Liu, T., et al., 2015. Isolation and characterization of a catalase gene "HuCAT3" from pitaya (Hylocereus undatus) and its expression under abiotic stress. Gene 563, 63-71.

Noori, S.S., Sokhansanj, A., 2008. Wheat plants containing an osmotin gene show enhanced ability to produce roots at high $\mathrm{NaCl}$ concentration. Russ. J. Plant Physiol. 55, 256-258.

Nuruzzaman, M., Cao, H., Xiu, H., Luo, T., Li, J., Chen, X., et al., 2016. Transcriptomics-based identification of WRKY genes and characterization of a salt and hormone-responsive PgWRKY1 gene in Panax ginseng. Acta. Biochim. Biophys. Sin. 48, 117-131.

Nuruzzaman, M., Sharoni, A.M., Satoh, K., Kondoh, H., Hosaka, A., Kikuchi, S., 2012. A genome-wide survey of the NAC transcription factor family in monocots and eudicots. Introduction to Genetics-DNA Methylation, Histone Modification and Gene Regulation.

Olsen, A.N., Ernst, H.A., Lo Leggio, L., Skriver, K., 2005. NAC transcription factors: structurally distinct, functionally diverse. Trends Plant Sci. 10, 79-87.

Oono, Y., Seki, M., Nanjo, T., Narusaka, M., Fujita, M., Satoh, R., et al., 2003. Monitoring expression profiles of Arabidopsis gene expression during rehydration process after dehydration using ca. 7000 full-length cDNA microarray. Plant J 34, 868-887.

Peleg, Z., Reguera, M., Tumimbang, E., Walia, H., Blumwald, E., 2011. Cytokinin-mediated source/sink modifications improve drought tolerance and increase grain yield in rice under waterstress. Plant Biotechnol. J 9, 747-758.

Peng, Y., Reyes, J.L., Wei, H., Yang, Y., Karlson, D., Covarrubias, A. A., et al., 2008. RcDhn5, a cold acclimation-responsive dehydrin from Rhododendron catawbiense rescues enzyme activity from dehydration effects in vitro and enhances freezing tolerance in
RcDhn5-overexpressing Arabidopsis plants. Physiol. Plant 134, 583-597.

Per, T.S., Khan, N.A., Reddy, P.S., Masood, A., Hasanuzzaman, M., Khan, M.I.R., et al., 2017. Approaches in modulating proline metabolism in plants for salt and drought stress tolerance: phytohormones, mineral nutrients and transgenics. Plant Physiol. Biochem. 115, 126-140.

Phuong, N.D., Tuteja, N., Nghia, P.T., Hoi, P.X., 2015. Identification and characterization of a stress-inducible gene OsNLI-IF enhancing drought tolerance in transgenic tobacco. Curr. Sci. 109, 541-551.

Pourabed, E., Ghane Golmohamadi, F., Soleymani Monfared, P., Razavi, S.M., Shobbar, Z.S., 2015. Basic leucine zipper family in barley: genome-wide characterization of members and expression analysis. Mol. Biotechnol. 57, 12-26.

Puhakainen, T., Hess, M.W., Mäkelä, P., Svensson, J., Heino, P., Palva, E.T., 2004. Overexpression of multiple dehydrin genes enhances tolerance to freezing stress in Arabidopsis. Plant Mol. Biol. 54, 743-753.

Raineri, J., Ribichich, K.F., Chan, R.L., 2015. The sunflower transcription factor HaWRKY76 confers drought and flood tolerance to Arabidopsisthaliana plants without yield penalty. Plant Cell Rep. 34, 2065-2080.

Rashid, M., He, G.Y., Yang, G.X., Hussain, J., Yan, X., 2012. AP2/ERF Transcription factor in rice: genome-wide canvas and syntenic relationships between monocots and eudicots. Evol. Bioinform. 8, 321-355.

Reguera, M., Peleg, Z., Abdel-Tawab, Y.M., Tumimbang, E.B., Delatorre, C.A., Blumwald, E., 2013. Stress-induced cytokinin synthesis increases drought tolerance through the coordinated regulation of carbon and nitrogen assimilation in rice. Plant Physiol. $163,1609-1622$.

Riechmann, J.L., Heard, J., Martin, G., Reuber, L., Jiang, C.Z., Keddie, J., et al., 2000. Arabidopsis transcription factors: genome-wide comparative analysis among eukaryotes. Sci. 290, 2105-2110.

Riemann, M., Dhakarey, R., Hazman, M., Miro, B., Kohli, A., Nick, P., 2015. Exploring jasmonates in the hormonal network of drought and salinity responses. Front. Plant Sci. 6.

Rivero, R.M., Gimeno, J., Van Deynze, A., Walia, H., Blumwald, E., 2010. Enhanced cytokinin synthesis in tobacco plants expressing PSARK: IPT prevents the degradation of photosynthetic protein complexes during drought. Plant Cell Physiol. 51, 1929-1941.

Rosegrant, M., Cai, X., Cline, S., Nakagawa, N., 2002. The role of rainfed agriculture in the future of global food production. Environment and Production Technology Division Discussion 90.

Sairam, R., 1994. Effects of homobrassinolide application on plant metabolism and grain yield under irrigated and moisture-stress conditions of two wheat varieties. Plant Growth Regul. 14, $173-181$

Sairam, R., Kumutha, D., Ezhilmathi, K., Deshmukh, P., Srivastava, G., 2008. Physiology and biochemistry of waterlogging tolerance in plants. Biol. Plant. 52, 401-412.

Sakamoto, A., Murata, N., 2000. Genetic engineering of glycinebetaine synthesis in plants: current status and implications for enhancement of stress tolerance. J. Exp. Bot. 51, 81-88.

Sakuma, Y., Maruyama, K., Osakabe, Y., Qin, F., Seki, M., Shinozaki, K., et al., 2006. Functional analysis of an Arabidopsis transcription factor, DREB2A, involved in drought-responsive gene expression. Plant Cell 18, 1292-1309.

Savchenko, T., Kolla, V.A., Wang, C.Q., Nasafi, Z., Hicks, D.R., Phadungchob, B., et al., 2014. Functional convergence of oxylipin and abscisic acid pathways controls stomatal closure in response to drought. Plant Physiol. 164, 1151-1160.

Schumann, U., Prestele, J., O'geen, H., Brueggeman, R., Wanner, G., Gietl, C., 2007. Requirement of the C3HC4 zinc RING finger of the Arabidopsis PEX10 for photorespiration and leaf peroxisome 
contact with chloroplasts. Proc. Natl. Acad. Sci. U.S.A. 104, $1069-1074$.

Senaratna, T., Touchell, D., Bunn, E., Dixon, K., 2000. Acetyl salicylic acid (Aspirin) and salicylic acid induce multiple stress tolerance in bean and tomato plants. Plant Growth Regul. 30, 157-161.

Sengupta, D., Ramesh, G., Mudalkar, S., Kumar, K.R.R., Kirti, P.B., Reddy, A.R., 2012. Molecular cloning and characterization of $\gamma$-glutamyl cysteine synthetase ( $\operatorname{rr} r C S)$ from roots of Vigna radiata (L.)Wilczek under progressive drought stress and recovery. Plant Mol. Biol. Rep. 30, 894-903.

Sharoni, A.M., Nuruzzaman, M., Satoh, K., Shimizu, T., Kondoh, H., Sasaya, T., et al., 2010. Gene structures, classification and expression models of the $A P 2 / E R E B P$ transcription factor family in rice. Plant Cell Physiol. 52, 344-360.

Shavrukov, Y., Baho, M., Lopato, S., Langridge, P., 2016. The TaDREB3 transgene transferred by conventional crossings to different genetic backgrounds of bread wheat improves drought tolerance. Plant Biotechnol. J. 14, 313-322.

Shen, Q., Ho, T., 1995. Functional dissection of an abscisic acid (ABA)-inducible gene reveals two independent ABA-responsive complexes each containing a G-box and a novel cis-acting element. Plant Cell 7, 295-307.

Sheveleva, E., Chmara, W., Bohnert, H.J., Jensen, R.G., 1997. Increased salt and drought tolerance by D-ononitol production in transgenic Nicotiana tabacum L. Plant Physiol. 115, 1211-1219.

Sheveleva, E.V., Marquez, S., Chmara, W., Zegeer, A., Jensen, R.G., Bohnert, H.J., 1998. Sorbitol-6-phosphate dehydrogenase expression in transgenic tobacco high amounts of sorbitol lead to necrotic lesions. Plant Physiol. 117, 831-839.

Shi, H., Chen, L., Ye, T., Liu, X., Ding, K., Chan, Z., 2014. Modulation of auxin content in Arabidopsis confers improved drought stress resistance. Plant Physiol. Biochem. 82, 209-217.

Shi, Q., Bao, Z., Zhu, Z., Ying, Q., Qian, Q., 2006. Effects of different treatments of salicylic acid on heat tolerance, chlorophyll fluorescence, and antioxidant enzyme activity in seedlings of Cucumis sativa L. Plant Growth Regul. 48, 127-135.

Slama, I., Abdelly, C., Bouchereau, A., Flowers, T., Savoure, A., 2015. Diversity, distribution and roles of osmoprotective compounds accumulated in halophytes under abiotic stress. Ann. Bot. 115, $433-447$.

Song, X.M., Li, Y., Hou, X.L., 2013. Genome-wide analysis of the AP2/ERF transcription factor superfamily in Chinese cabbage (Brassica rapa ssp pekinensis). BMC Genomics 14.

Sperotto, R.A., Ricachenevsky, F.K., Duarte, G.L., Boff, T., Lopes, K. L., Sperb, E.R., et al., 2009. Identification of up-regulated genes in flag leaves during rice grain filling and characterization of OsNAC5, a new ABA-dependent transcription factor. Planta 230, 985-1002.

Srivasta, A., Mehta, S., Lindlof, A., Bhargava, S., 2010. Overrepresented promoter motifs in abiotic stress-induced DREB genes of rice and sorghum and their probable role in regulation of gene expression. Plant Signal. Behav. 5, 775-784.

Stiller, I., Dulai, S., Kondrák, M., Tarnai, R., Szabó, L., Toldi, O., et al., 2008. Effects of drought on water content and photosynthetic parameters in potato plants expressing the trehalose-6phosphate synthase gene of Saccharomyces cerevisiae. Planta 227, 299.

Su, J., Hirji, R., Zhang, L., He, C., Selvaraj, G., Wu, R., 2006. Evaluation of the stress-inducible production of choline oxidase in transgenic rice as a strategy for producing the stress-protectant glycine betaine. J. Exp. Bot. 57, 1129-1135.

$\mathrm{Su}$, Z., Ma, X., Guo, H.H., Sukiran, N.L., Guo, B., Assmann, S.M., et al., 2013. Flower development under drought stress: morphological and transcriptomic analyses reveal acute responses and long-term acclimation in Arabidopsis. Plant Cell 25, 3785-3807.
Subramanyam, K., Arun, M., Mariashibu, T.S., Theboral, J., Rajesh, M., Singh, N.K., et al., 2012. Overexpression of tobacco osmotin (Tbosm) in soybean conferred resistance to salinity stress and fungal infections. Planta 236, 1909-1925.

Sun, S.J., Guo, S.Q., Yang, X., Bao, Y.M., Tang, H.J., Sun, H., et al., 2010. Functional analysis of a novel Cys2/His2-type zinc finger protein involved in salt tolerance in rice. J. Exp. Bot. 61, $2807-2818$.

Suzuki, N., Rivero, R.M., Shulaev, V., Blumwald, E., Mittler, R., 2014. Abiotic and biotic stress combinations. New Phytol. 203, 32-43.

Székely, G., Ábrahám, E., Cséplő, Á., Rigó, G., Zsigmond, L., Csiszár, J., et al., 2008. Duplicated P5CS genes of Arabidopsis play distinct roles in stress regulation and developmental control of proline biosynthesis. Plant J. 53, 11-28.

Taiz, L., Zeiger, E., 2002. Plant Physiol. 3rd. pp. 720, 21.

Takasaki, H., Maruyama, K., Kidokoro, S., Ito, Y., Fujita, Y., Shinozaki, K., et al., 2010. The abiotic stress-responsive NAC-type transcription factor OsNAC5 regulates stress-inducible genes and stress tolerance in rice. Mol. Genet. Genomic 284, 173-183.

Tang, N., Zhang, H., Li, X.H., Xiao, J.H., Xiong, L.Z., 2012. Constitutive activation of transcription factor OsbZIP46 improves drought tolerance in rice. Plant Physiol. 158, 1755-1768.

Thomas, J.C., Sepahi, M., Arendall, B., Bohnert, H.J., 1995. Enhancement of seed germination in high salinity by engineering mannitol expression in Arabidopsis thaliana. Plant Cell Environ. 18, 801-806.

Trenberth, K.E., 2011. Changes in precipitation with climate change. Clim. Res. 47, 123-138.

Tung, S.A., Smeeton, R., White, C.A., Black, C.R., Taylor, I.B., Hilton, H.W., et al., 2008. Over-expression of LeNCED1 in tomato (Solanum lycopersicum L.) with the rbcS3C promoter allows recovery of lines that accumulate very high levels of abscisic acid and exhibit severe phenotypes. Plant Cell Environ 31, 968-981.

Verma, G., Dhar, Y.V., Srivastava, D., Kidwai, M., Chauhan, P.S., Bag, S.K., et al., 2017. Genome-wide analysis of rice dehydrin gene family: its evolutionary conservedness and expression pattern in response to PEG induced dehydration stress. PloS One 12, e0176399.

Volaire, F., Norton, M., 2006. Summer dormancy in perennial temperate grasses. Ann. Bot. 98, 927-933.

Wang, C., Yang, A., Yin, H., Zhang, J., 2008a. Influence of water stress on endogenous hormone contents and cell damage of maize seedlings. J. Integr. Plant Biol. 50, 427-434.

Wang, C., Lu, W., He, X., Wang, F., Zhou, Y., Guo, X., 2016a. The cotton mitogen-activated protein kinase 3 functions in drought tolerance by regulating stomatal responses and root growth. Plant Cell Physiol. 57, 1629-1642.

Wang, G.P., Li, F., Zhang, J., Zhao, M.R., Hui, Z., Wang, W., 2010. Overaccumulation of glycine betaine enhances tolerance of the photosynthetic apparatus to drought and heat stress in wheat. Photosynthetica 48, 30-41.

Wang, Q., Guan, Y., Wu, Y., Chen, H., Chen, F., Chu, C., 2008b. Overexpression of a rice OsDREB1F gene increases salt, drought, and low temperature tolerance in both Arabidopsis and rice. Plant Mol. Biol. 67, 589-602.

Wang, Y., Sun, T., Li, T., Wang, M., Yang, G., He, G., 2016b. A CBLInteracting Protein Kinase TaCIPK2 confers drought tolerance in transgenic tobacco plants through regulating the stomatal movement. PLoS One 11, e0167962.

Wang, Z., Cheng, K., Wan, L., Yan, L., Jiang, H., Liu, S., et al., 2015. Genome-wide analysis of the basic leucine zipper (bZIP) transcription factor gene family in six legume genomes. BMC Genomics 16, 1053.

Wani, S.H., Singh, N., Devi, T.R., Haribhushan, A., Jeberson, S., Malik, C., 2013. Engineering abiotic stress tolerance in plants: 
extricating regulatory gene complex. Conventional and NonConventional Interventions in Crop Improv. 1-19.

Wani, S.H., Kumar, V., Shriram, V., Sah, S.K., 2016. Phytohormones and their metabolic engineering for abiotic stress tolerance in crop plants. The Crop J. 4, 162-176.

Wild, M., Davière, J.M., Cheminant, S., Regnault, T., Baumberger, N., Heintz, D., et al., 2012. The Arabidopsis DELLA RGA-LIKE3 is a direct target of MYC2 and modulates jasmonate signaling responses. Plant Cell 24, 3307-3319.

Woo, H.J., Sohn, S.I., Shin, K.S., Kim, J.K., Kim, B.G., Lim, M.H., 2014. Expression of tobacco tocopherol cyclase in rice regulates antioxidative defense and drought tolerance. Plant Cell Tiss. Org. $119,257-267$.

Wu, J., Qu, T., Chen, S., Zhao, Z., An, L., 2009. Molecular cloning and characterization of a $\gamma$-glutamylcysteine synthetase gene from Chorispora bungeana. Protoplasma 235, 27-36.

Xiang, Y., Tang, N., Du, H., Ye, H.Y., Xiong, L.Z., 2008. Characterization of OsbZIP23 as a key player of the basic leucine zipper transcription factor family for conferring abscisic acid sensitivity and salinity and drought tolerance in rice. Plant Physiol. $148,1938-1952$.

Xu, D.Q., Huang, J., Guo, S.Q., Yang, X., Bao, Y.M., Tang, H.J., et al., 2008. Overexpression of a TFIIIA-type zinc finger protein gene ZFP252 enhances drought and salt tolerance in rice (Oryza sativa L.). FEBS Lett. 582, 1037-1043.

Xu, J., Duan, X., Yang, J., Beeching, J.R., Zhang, P., 2013. Coupled expression of $\mathrm{Cu} / \mathrm{Zn}$-superoxide dismutase and catalase in cassava improves tolerance against cold and drought stresses. Plant Signal. Behav. 8, e24525.

Xu, J., Xing, X.J., Tian, Y.S., Peng, R.H., Xue, Y., Zhao, W., et al., 2015. Transgenic Arabidopsis plants expressing tomato glutathione S-transferase showed enhanced resistance to salt and drought stress. PLoS One 10, e0136960.

Xu, J., Tian, Y.S., Xing, X.J., Peng, R.H., Zhu, B., Gao, J.J., et al., 2016. Over-expression of AtGSTU19 provides tolerance to salt, drought and methyl viologen stresses in Arabidopsis. Physiol. Plant. 156, 164-175.

Xu, Z.S., Chen, M., Li, L.C., Ma, Y.Z., 2011. Functions and application of the AP2/ERF transcription factor family in crop improvement. J. Int. Plant Biol. 53, 570-585.

Yamada, M., Morishita, H., Urano, K., Shiozaki, N., YamaguchiShinozaki, K., Shinozaki, K., et al., 2005. Effects of free proline accumulation in petunias under drought stress. J. Exp. Bot. 56, 1975-1981.

Yamaguchi-Shinozaki, K., Shinozaki, K., 1994. A novel cis-acting element in an Arabidopsis gene is involved in responsiveness to drought, low-temperature, or high-salt stress. Plant Cell 6, $251-264$.

Yang, G., Wang, Y., Xia, D., Gao, C., Wang, C., Yang, C., 2014. Overexpression of a GSTgene (ThGSTZ1) from Tamarix hispida improves drought and salinity tolerance by enhancing the ability to scavenge reactive oxygen species. Plant Cell Tiss. Org. 117, 99-112.

Yang, Y., He, M., Zhu, Z., Li, S., Xu, Y., Zhang, C., et al., 2012. Identification of the dehydrin gene family from grapevine species and analysis of their responsiveness to various forms of abiotic and biotic stress. BMC Plant Biol 12, 140.

Yoshida, T., Fujita, Y., Sayama, H., Kidokoro, S., Maruyama, K., Mizoi, J., et al., 2010. AREB1, AREB2, and ABF3 are master transcription factors that cooperatively regulate ABRE-dependent ABA signaling involved in drought stress tolerance and require ABA for full activation. Plant J. 61, 672-685.

Yu, M., Lamattina, L., Spoel, S.H., Loake, G.J., 2014. Nitric oxide function in plant biology: a redox cue in deconvolution. New Phytol. 202, 1142-1156.
Zhang, D.Y., Yang, H.L., Li, X.S., Li, H.Y., Wang, Y.C., 2014. Overexpression of Tamarix albiflonum TaMnSOD increases drought tolerance in transgenic cotton. Mol. Breed. 34, 1-11.

Zhang, J., Tan, W., Yang, X.H., Zhang, H.X., 2008. Plastid-expressed choline monooxygenase gene improves salt and drought tolerance through accumulation of glycine betaine in tobacco. Plant Cell Rep. 27, 1113.

Zhang, L., Zhang, L., Xia, C., Zhao, G., Liu, J., Jia, J., et al., 2015. A novel wheat bZIP transcription factor, TabZIP60, confers multiple abiotic stress tolerances in transgenic Arabidopsis. Physiol. Plant. $153,538-554$.

Zhang, Z., Zhang, Q., Wu, J., Zheng, X., Zheng, S., Sun, X., et al., 2013. Gene knockout study reveals that cytosolic ascorbate peroxidase 2 (OsAPX2) plays a critical role in growth and reproduction in rice under drought, salt and cold stresses. PloS One 8, e57472.

Zhao, Y., Chan, Z., Gao, J., Xing, L., Cao, M., Yu, C., et al., 2016. ABA receptor PYL9 promotes drought resistance and leaf senescence. Proc. Natl. Acad. Sci. U.S.A. 113, 1949-1954.

Zheng, X., Chen, B., Lu, G., Han, B., 2009. Overexpression of a NAC transcription factor enhances rice drought and salt tolerance. Biochem. Biophys. Res. Commun. 379, 985-989.

Zhu, B., Su, J., Chang, M., Verma, D.P.S., Fan, Y.L., Wu, R., 1998. Overexpression of a 1-pyrroline-5-carboxylate synthetase gene and analysis of tolerance to water-and salt-stress in transgenic rice. Plant Sci. 139, 41-48.

Zimmermann, P., Hirsch-Hoffmann, M., Hennig, L., Gruissem, W., 2004. Genevestigator. Arabidopsis microarray database and analysis toolbox. Plant Physiol. 136, 2621-2632.

Zwack, P.J., Rashotte, A.M., 2015. Interactions between cytokinin signalling and abiotic stress responses. J. Exp. Bot. 66, 4863-4871.

\section{Further Reading}

Gao, S.Q., Chen, M., Xu, Z.S., Zhao, C.P., Li, L., Xu, H.J., et al., 2011. The soybean GmbZIP1 transcription factor enhances multiple abiotic stress tolerances in transgenic plants. Plant Mol. Biol. 75, 537-553.

Gao, S., Zhang, Y.L., Yang, L., Song, J.B., Yang, Z.M., 2014. AtMYB20 is negatively involved in plant adaptive response to drought stress. Plant Soil 376, 433-443.

Guo, Y., Gan, S., 2006. AtNAP, a NAC family transcription factor, has an important role in leaf senescence. Plant J. 46, 601-612.

Huang, J., Hirji, R., Adam, L., Rozwadowski, K.L., Hammerlindl, J. K., Keller, W.A., et al., 2000. Genetic engineering of glycinebetaine production toward enhancing stress tolerance in plants: metabolic limitations. Plant Physiol. 122, 747-756.

Jiang, C., Gu, J., Chopra, S., Gu, X., Peterson, T., 2004. Ordered origin of the typical two- and three-repeat MYB genes. Gene 326, 13-22.

Liao, Y., Zou, H.F., Wei, W., Hao, Y.J., Tian, A.G., Huang, J., et al., 2008. Soybean GmbZIP44, GmbZIP62 and GmbZIP78 genes function as negative regulator of ABA signaling and confer salt and freezing tolerance in transgenic Arabidopsis. Planta 228, 225-240.

Seo, P.J., Xiang, F., Qiao, M., Park, J.Y., Lee, Y.N., Kim, S.G., et al., 2009. The MYB96 transcription factor mediates abscisic acid signaling during drought stress response in Arabidopsis. Plant Physiol. 151, 275-289.

Wang, J., Zhou, J., Zhang, B., Vanitha, J., Ramachandran, S., Jiang, S. Y., 2011. Genome-wide expansion and expression divergence of the basic leucine zipper transcription factors in higher plants with an emphasis on sorghum. J. Integr. Plant Biol. 53, 212-231.

Woo, H.R., Kim, J.H., Kim, J., Kim, J., Lee, U., Song, I.J., et al., 2010. The RAV1 transcription factor positively regulates leaf senescence in Arabidopsis. J. Exp. Bot. 61, 3947-3957. 
This page intentionally left blank 


\title{
Crop Improvement of Cereals Through Manipulation of Signaling Pathways in Response to Drought Stress
}

\section{Sunitha Mellacheruvu, Ashwini Talakayala and Mallikarjuna Garladinne}

\author{
Plant Molecular Biology Laboratory, Agri Biotech Foundation, Hyderabad, India
}

\section{O U T L I N E}

7.1 Introduction

125

7.2 Transcription Factors Associated With Signaling Mechanism

7.2.1 Dehydration Responsive Element Binding Proteins

7.2.2 MYB Transcription Factor

7.2.3 NAC Transcription Factor

7.2.4 Basic Leucine Zipper Protein Transcription Factor

7.3 Genetic Engineering of Kinases for Drought and Salinity Tolerance 7.3.1 Protein Kinases
7.4 Modulation of Key Genes Involved in Phytohormone Signaling

7.5 Engineering of Osmoregulatory Genes

7.6 Reactive Oxygen Species Signaling During Drought and Salinity Stress

7.7 Conclusion and Future Prospects

Acknowledgments

References

135

\subsection{INTRODUCTION}

Since plants are sessile organisms, they have to adapt continuously under adverse environmental stresses. These unfavorable conditions suppress plant growth and development and ultimately affect crop yield. Abiotic stresses like drought, salinity, cold, heat, and heavy metals are the major abiotic stress factors that impact agriculture productivity and threaten the global food security (Zhu, 2016; Pasala et al., 2016). The scenario of climate change and global warming has led to increase in intensity of extreme weather, alterations in cropping texture in terms of crop life cycle, increased water evaporation, and severe drought and salinity stress coupled with oxidative stress (Fedoroff et al., 2010). However, plants have developed intricate mechanisms to overcome these adverse conditions. Crop plants need to cope with adverse external 
pressure created by environmental and edaphic conditions with their intrinsic biological mechanisms. However, this affects growth and development and ultimately affects productivity (Meena et al., 2017). Plants sense the stress signals and try to adapt under these unfavorable environmental stress conditions through various evolutionary mechanisms like physiological and biochemical alterations connecting various signal transduction pathways and cascades of endogenous metabolic developmental activities (Budak et al., 2015; Khan and Khan, 2017; Phukan et al., 2017) (Fig. 7.1).

The process of signal perception and activation of signaling cascades involves a large number of factors and molecules belonging to diverse classes of gene families. In contrast to animals, plants harbor larger multigene families along with the occurrence of plant-specific ones. In this chapter, various abiotic stress-responsive genes were analyzed and categorized into different classes such as osmoprotectants (glycine betaine, proline), reactive oxygen species (ROS) scavengers (superoxide dismutase, SOD; ascorbate peroxidase, APX; dehydroascorbate reductase,
DHAR; monodehydroascorbate reductase, MDHAR; and glutathione reductase, GR), late abundant (LEA) proteins), heat shock proteins, and transcription factors (TFs) such as C-repeat/dehydration-responsive element binding protein (CBF/DREB), myeloblastosis/ myelocytomatosis (MYB/MYC), basic leucine zipper protein (bZIP), no apical meristem ATAF \& cup shaped cotyledon (NAC), abscisic acid/ABA-responsive element (ABA/ABRE), etc. (Todaka et al., 2015; Per et al., 2017). These gene families are involved in regulation of stress signal transduction pathways in plants.

Improving plant tolerance against environmental stresses by manipulating the signaling pathways is a very important strategy for crop sustainability and enhancing productivity. The availability of several genetic approaches like overexpression of genes, silencing, and genome editing have made it easy to understand the role of signaling molecules and their pathways (Akpinar et al., 2015). Recent approaches like RNA deep sequencing, transcriptomic analysis have been employed at transcription level for identification of the genes involved in various processes

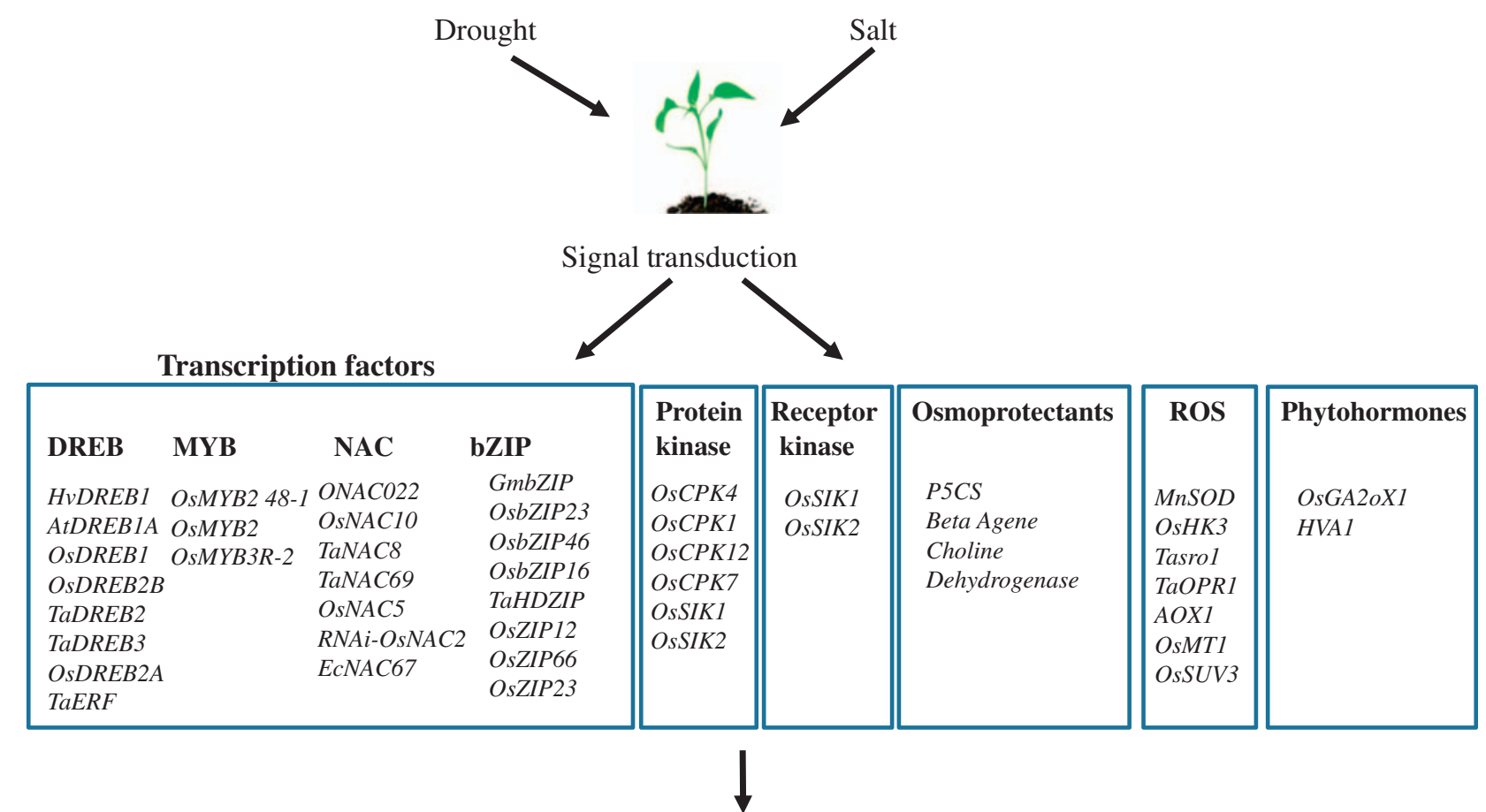

Activation of stress responsive genes

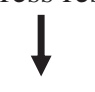

Stress tolerance

FIGURE 7.1 Schematic representation of signaling pathways during abiotic stresses. Upon stress signal perception, different transcription factors and signaling pathways get activated leading to differential gene expression for generating appropriate stress tolerance. 
including signaling. Agrobacterium tumefaciens and particle bombardment methods of transformation have been extensively been used for generation of transgenic plants for achieving stress tolerant cereal crops. Significant progress has been made in the generation of drought and salinity tolerant transgenic crops.

\subsection{TRANSCRIPTION FACTORS ASSOCIATED WITH SIGNALING MECHANISM}

TFs act as master switches and trigger simultaneous expression of a large number of stress-response genes that contribute to the stress tolerance phenotype
(Zandkarimi et al., 2015). TFs are key regulators that function in the upregulation of downstream genes by interacting with ABA, jasmonic acid, and salicylic acid in regulating the signaling pathways during drought stress (Nakashima et al., 2014; Per et al., 2018). Mostly the TFs belong to either ABA dependent or independent signal transduction pathways. These pathways were studied extensively in rice and Arabidopsis systems (Todaka et al., 2012). Different kinds of TFs including $D R E B / C B F, E R F, M Y B, A R E B / A B F, N A C$, zinc fingers, etc. are known to be involved in regulation of signaling mechanism in response to drought and salinity stresses (Yamaguchi-Shinozaki and Shinozaki, 2009). Several research groups have developed transgenic plants by manipulating the TFs; these are listed in Table 7.1.

TABLE 7.1 Expression of Transcription Factors Involved in ABA-Dependent and -Independent Signaling Pathways Through Transgenic Approach

\begin{tabular}{|c|c|c|c|c|c|}
\hline Item & Gene & Promoter & Plant & Phenotype of transgenics & References \\
\hline \multirow[t]{8}{*}{ DREB } & HVDREB1 & CaMV35S & Wheat & Increased salt stress tolerance & Xu et al. (2009) \\
\hline & OsDREB2B & $\begin{array}{l}\text { CaMV35S } \\
\operatorname{rd} 29 A\end{array}$ & Rice & Nonfunctional transcript in rice & Matsukura et al. (2010) \\
\hline & $\begin{array}{l}\text { TaDREB2 and } \\
\text { TaDREB3 }\end{array}$ & $\begin{array}{l}\text { Double 35S, Maize } \\
\text { Rab17 }\end{array}$ & $\begin{array}{l}\text { Wheat } \\
\text { and } \\
\text { barley }\end{array}$ & $\begin{array}{l}\text { Constitutively expressing plants delayed in growth, } \\
\text { flowering and grain yield; drought and frost tolerance }\end{array}$ & Morran et al. (2011) \\
\hline & TaERF & Ubiquitin & Wheat & $\begin{array}{l}\text { Proline was accumulated under drought stress and } \\
\text { increased chlorophyll content in levels }\end{array}$ & Rong et al. (2014) \\
\hline & OsDREB2A & $R d 29 A$ & Rice & $\begin{array}{l}\text { Drought and salinity tolerance with higher number of } \\
\text { panicles and more grain yield }\end{array}$ & Mallikarjuna et al. (2011) \\
\hline & $\begin{array}{l}\text { AtDREB1A/ } \\
\text { CBF3 }\end{array}$ & $R d 29 A$ & Wheat & $\begin{array}{l}\text { Substantial tolerance to water stress and delayed in } \\
\text { wilting under drought stress }\end{array}$ & Pellegrineschi et al. (2004) \\
\hline & $\begin{array}{l}\text { AtDREB1A/ } \\
\text { CBF3 }\end{array}$ & $35 s C A M V$ & Rice & $\begin{array}{l}\text { Tolerance to abiotic stress without displaying stunted } \\
\text { growth }\end{array}$ & Oh et al. (2005) \\
\hline & OsDREB1/CBF & CaMV35S & Rice & Expression of cold responsive genes & Ito et al. (2006) \\
\hline \multirow[t]{3}{*}{ MYB } & OsMYB3R-2 & CaMV35S & Rice & Increased chilling tolerance & Ma et al. (2009) \\
\hline & OsMYB48-1 & CaMV35S & Rice & $\begin{array}{l}\text { Drought and salinity tolerance. Reduced rate of water } \\
\text { loss/lower MDA, higher proline content under stress } \\
\text { conditions }\end{array}$ & Xiong et al. (2014) \\
\hline & OsMYB2 & Ubiquitin & Rice & $\begin{array}{l}\text { Hypersensitive to shoot and root inhibition by } \\
\text { exogenous ABA }\end{array}$ & Zhao et al. (2014) \\
\hline \multirow[t]{5}{*}{ NAC } & OsNAC10 & CaMV35S & Rice & $\begin{array}{l}\text { Drought, salinity and low-temperature stress } \\
\text { toleranceImproved in grain yield }\end{array}$ & Jeong et al. (2010) \\
\hline & TaNAC8 & CaMV35S & Wheat & $\begin{array}{l}\text { Transcriptional gene regulation and showed biotic and } \\
\text { abiotic stress tolerance }\end{array}$ & Xia et al. (2010) \\
\hline & TaNAC69 & $\begin{array}{l}\text { Drought-inducible } \\
\text { promoter (HvDhn4s) }\end{array}$ & Wheat & $\begin{array}{l}\text { Drought tolerance with improvement in water use } \\
\text { efficiency }\end{array}$ & Xue et al. (2011) \\
\hline & OsNAC5 & CaMV35S & Rice & Tolerant to drought and salt & Song et al. (2011) \\
\hline & EcNAC67 & $R d 29 A$ & Rice & $\begin{array}{l}\text { Drought and salt tolerance with increase of high root } \\
\text { and biomass }\end{array}$ & Rahman et al. (2016) \\
\hline
\end{tabular}


TABLE $7.1 \quad$ (Continued)

\begin{tabular}{|c|c|c|c|c|c|}
\hline Item & Gene & Promoter & Plant & Phenotype of transgenics & References \\
\hline & ONAC022 & CaMV35S & Rice & $\begin{array}{l}\text { Accumulation of proline and minimized water loss, } \\
\text { reduced stomatal open, high survival ratio during stress } \\
\text { conditions }\end{array}$ & Hong et al. (2016) \\
\hline & OsNAC2 & CaMV35S & Rice & $\begin{array}{l}\text { Silencing lines showed enhanced tolerance to high } \\
\text { salinity and drought stress at both the vegetative and } \\
\text { flowering stages }\end{array}$ & Shen et al. (2017) \\
\hline \multirow[t]{7}{*}{ bZIP } & OsbZIP23 & Ubiquitin & Rice & $\begin{array}{l}\text { Drought, salt tolerance but sensitivity to exogenous } \\
\text { ABA }\end{array}$ & Xiang et al. (2008) \\
\hline & GmbZIP & CaMV35S, rd29A & Wheat & Drought tolerance without growth retardation & Gao et al. (2011a) \\
\hline & OsbZIP46 & Ubiquitin & Rice & $\begin{array}{l}\text { Drought and salt tolerance. Hypersensitive to shoot } \\
\text { growth inhibition by exogenous ABA }\end{array}$ & Tang et al. (2012) \\
\hline & OsbZIP16 & CaMV35S, rd29A & Rice & $\begin{array}{l}\text { Drought and salt tolerance. Hypersensitive to shoot } \\
\text { growth inhibition by exogenous ABA }\end{array}$ & Todaka et al. (2015) \\
\hline & TaHDZIP & CaMV35S & $\begin{array}{l}\text { Bread } \\
\text { Wheat }\end{array}$ & $\begin{array}{l}\text { Drought and frost tolerance with undesired features like } \\
\text { reduced plant growth, delayed flowering and reduced } \\
\text { biomass and yield }\end{array}$ & Yang et al. (2017) \\
\hline & OsbZIP12 & Cytochrome c & Rice & $\begin{array}{l}\text { Improved drought tolerance and hypersensitivity to } \\
\text { ABA. }\end{array}$ & Joo et al. (2014) \\
\hline & OsbZIP66 & $\begin{array}{l}\text { GOS2 (rice } \\
\text { eukaryotic } \\
\text { translation } \\
\text { initiation factor 1- } \\
\text { like gene) and RCc3 } \\
\text { (rice lipid transfer } \\
\text { protein-like gene) }\end{array}$ & Rice & Enhanced drought tolerance & Yoon et al. (2017) \\
\hline
\end{tabular}

\subsubsection{Dehydration Responsive Element Binding Proteins}

The DREBs are novel TFs, belonging to the AP2 (apetala)/ERF (ethylene responsive element binding factors) family distinctive to plants (Agarwal et al., 2006). There are approximately 124 ERF proteins sharing a conserved 58-59 amino acid conserved region (ERF domain) that binds to two cis-acting elements, that is, C-repeat/dehydration responsive motif (CRT/ DRE) (A/GCCGAC) found in the expression of cold and dehydration responsive genes and GCC box, found in many pathogenesis-related gene promoters conferring ethylene responsiveness (Agarwal et al., 2006; Zhang et al., 2004). The DREB TFs induce a set of downstream and abiotic stress related genes that help in plant survival during adverse environmental conditions. DREB TFs are dichotomized as DREB1 and DREB2, and are involved in signal transduction pathways under low temperature and dehydration, respectively (Agarwal et al., 2006). DREB1/DREB2homologous genes have been isolated from several crop species such as rice, wheat, barley, maize, sorghum, etc. In important cereal crops like wheat and barley, a number of $C B F$ homologs have been mapped to the Fr-2 chromosomal region (Skinner et al., 2005; Miller et al., 2006). A functional Fr-A1/VRN1 allele (winter hardiness/vernalization requirement) plays an important role in regulating the CBF-mediated Cor/Lea gene expression in wheat (Kobayashi et al., 2005).

The DREB TFs are potential genes that have been exploited for generation of transgenic cereals for enhancing drought and salt stress tolerance. The overexpression of AtDREB1A/CBF3 driven by the stressinducible $r d 29 A$ promoter in transgenic wheat improved water stress tolerance, and delayed wilting under greenhouse conditions (Pellegrineschi et al., 2004). Similarly, the constitutive overexpression of DREB1A/CBF3 under $35 S$ promoter in rice increased stress tolerance to drought and high salinity without any growth inhibition or phenotypic aberrations (Oh et al., 2005). Constitutive overexpression of OsDREB1 showed improved tolerance against drought, high salt, and low temperature and also accumulated the osmoprotectants like proline and different soluble sugars in transgenic rice plants. However, stunted growth was observed in transgenic rice plants (Ito et al., 2006). Expression of HvDREB1 gene in barley leaves was significantly 
induced by salt, drought, and low-temperature stress (Xu et al., 2009). Similarly, maize DRE-binding proteins DBF1 and DBF2 were involved in rab17 regulation through the drought-responsive element in an ABAdependent pathway (Kizis and Pages, 2002).

Stress-inducible expression of OsDREB2A conferred drought and salinity stress tolerance in rice and also improved the number of panicles and seed weight in comparison to control plants (Mallikarjuna et al., 2011). The expression of OsDREB2B in transgenic rice and Arabidopsis plants led to transactivation of its target stress adaptive genes (Matsukura et al., 2010). Ectopic expression of cotton DREB leads to enhanced drought, salt, and cold stress tolerance due to soluble sugars and chlorophyll production in wheat (Gao et al., 2009). TaDREB2 and TaDREB3 TFs of wheat were expressed constitutively (CaMV35S) and also inducible expression (Maize Rab17) in wheat and barley showed significant frost tolerance. The constitutively overexpressing plants showed delay in growth and yield, but the plants expressing under inducible condition displayed superior growth performance than untransformed plants. The induced expression of these two genes led to upregulation of 10 other CBF/DREB TFs and also massive number of other stress responses like $L E A /$ $C O R / D H N$ genes involved in protection from desiccation stress (Morran et al., 2011). The transgenic rice expressing SbDREB2 under the control of RD29A promoter exhibited drought tolerance with normal seed set, whereas constitutive expression of the same gene under the regulation of CaMV35S promoter showed abnormal plant growth and seed set (Bihani et al., 2011). Overexpression of wheat TaERF (AP2/ethylene responsive factor) promoted drought tolerance by accumulating the proline and increased levels of chlorophyll levels in wheat (Rong et al., 2014). The heterologous expression of TaCBF14 and TaCBF15 led to elevated transcript of gibberellin catabolic HvGA2ox5 gene through accumulation of DELLA protein, but dwarf phenotype was observed in transgenic barley (Soltész et al., 2013). Overall, the stress-inducible expression of DREB TFs significantly improves the stress tolerant phenotypes without growth penalty despite that constitutive expression possesses the abnormal plant growth and development.

\subsubsection{MYB Transcription Factor}

The MYB family of TFs is a large family and plays a fundamental role in response to biotic and abiotic stresses through ABA-dependent signal transduction pathway. $M Y B$ genes code for TFs with a characteristic of 52 amino acid MYB motifs. These TFs contain varying numbers from one to four MYB domains termed as
R1, R2, R3, and R4 (Du et al., 2009). Each MYB domain has three regularly spaced tryptophan residues that are separated by 18 or 19 amino acid residues and each domain forms a helix-turn-helix fold that is crucial for MYB TF-DNA interaction (Saikumar et al., 1990). The MYB TFs play an important role in biological processes such as cell cycle regulation (Cominelli and Tonelli, 2009), cell proliferation (Xie et al., 2010), developmental processes (Komaki and Sugimoto, 2012), hormone signal transduction (Zhao et al., 2014a, b), and abiotic stress responses (Katiyar et al., 2012). In rice, the MYB-related TF OsMYB48-1 acts in response to water stress by regulating the transcription of genes involved in early and late response to stress and also stress-induced ABA biosynthesis genes (Xiong et al., 2014). Rice R2R3 type MYB, OsMYB2 TF was overexpressed in rice, and conferred abiotic stress tolerance and also accumulated elevated levels of soluble sugars and proline in transgenic plants under salt stress. Moreover, there were improved levels of antioxidant enzymes and highly upregulated stress related genes like OsLEA3, OsRab16A, and OsDREB2A in transgenic rice plants compared with wild type (Yang et al., 2012). In another study, overexpression of OsMYB3R-2 exhibited elevated proline content with enhanced cold tolerance in transgenic rice plants (Ma et al., 2009).

\subsubsection{NAC Transcription Factor}

NAC TFs are highly conserved and play a key role in flower development, formation of secondary walls, cell division, shoot apical meristem formation, and leaf senescence, as well as protective role to biotic and abiotic stresses (Olsen et al., 2005; Nakashima et al., 2012; Nuruzzaman et al., 2013; Banerjee and Roychoudhury, 2015). These proteins share a common structure with a conserved N-terminal NAC domain and a highly variable C-terminal domain. The NAC domain designation is derived from a conserved domain originally associated with the no apical meristem, ATAF1, ATAF2, and cup-shaped cotyledon genes from Arabidopsis (Aida et al., 1997). NAC family TF OsNAP is a transcriptional activator, induced by ABA, drought, salinity, and lowtemperature treatments in transgenic rice plants. Interestingly, the OsNAP stimulated the expression of other stress related TFs like OsDREB1A, OsMYB2, OsAP37, and Osap59 (Chen et al., 2014). The transgenic rice plants constitutively expressing OsNAC022 exhibited enhanced drought tolerance, which was attained by accumulation of proline and soluble sugars. In addition, minimized water loss and reduced stomatal opening were observed in transgenic plants (Hong et al., 2016). Overexpression of OsNAC1 and OsNAC10 TF under control of two different constitutive 
promoters such as GOS2 (rice eukaryotic translation initiation factor 1-like gene) and root specific promoter $R C c 3$ (rice lipid transfer protein-like gene) enhanced drought, salinity, and low-temperature stress tolerance at the vegetative stage. However, root specific expression of OsNAC10 in rice led to improvement in grain yield even under field drought conditions (Jeong et al., 2010, 2013; Redillas et al., 2012). TaNAC8, a novel NAC of wheat, responds to stripe rust pathogen infection and abiotic stresses (Xia et al., 2010). Similarly, overexpression of TaNAC69 in transgenic wheat leads to enhanced dehydration tolerance and improvement of water use efficiency (Xue et al., 2011). Barley HVA1 gene conferred drought and salt tolerance in transgenic maize (Nguyen and Sticklen, 2013). Also transgene pyramiding of the HVA1 and mtlD conferred drought and salt tolerance with enhanced crop biomass in maize (Zea mays L.) (Nguyen et al., 2013).

The role of OsNAC5 was investigated by generating knockdown and overexpressing in rice. The RNAi lines became sensitive to abiotic stresses, whereas overexpressing lines positively correlated with accumulation of higher content of proline, soluble sugars, and reduced levels of malondialdehyde (MDA) and $\mathrm{H}_{2} \mathrm{O}_{2}$. It suggested that the OsNAC5 overexpressing lines possessed more tolerance to oxidative and salt stress (Song et al., 2011). OsNAC2 overexpressing lines negatively correlated with downregulation of several ABA-dependent stress-related genes, whereas silencing of this gene showed elevated tolerance to high salinity and drought stress during vegetative and flowering stage (Shen et al., 2017). A NAC TF from finger millet, EcNAC67 ectopically expressed in rice, exhibited robust root and shoot biomass with higher relative water content in comparison with wild type during drought and salinity stress at the greenhouse level. Moreover, the transgenic rice lines possessed reduced in spikelet sterility and higher grain yield (Rahman et al., 2016). The above results indicated that these NAC TFs are potential candidate genes for generating transgenic lines in other crops also.

\subsubsection{Basic Leucine Zipper Protein Transcription Factor}

bZIPs comprise a large family of TFs that contain a highly conserved bZIP domain with two structural features, that is, a basic domain responsible for sequencespecific DNA binding and an adjacent heptad leucine repeat domain referred to as a leucine zipper (Zhang et al., 2017). bZIPs are present throughout the plant kingdom and are involved in a variety of physiological processes, such as seed maturation, germination, flower development, fertility (Zou et al., 2008), plant senescence (Lee et al., 2006), photomorphogenesis, light signaling
(Mallappa et al., 2006), responses to various abiotic stresses, and/or stress signaling (Hossain et al., 2010; Wang et al., 2010). Some bZIP family members, including OsbZIP23, OsbZIP46, OsbZIP71, and OsbZIP16, were positively upregulated and conferred tolerance to different abiotic stresses (Todaka et al., 2015).

Overexpression of GmbZIP1 in wheat enhanced the responses of transgenic plants to $\mathrm{ABA}$ and improved tolerance to several abiotic stresses without any growth retardation of plants (Gao et al., 2011a). The stress-responsive TaHDZip1-5 gene coding for homeodomain leucine zipper class $1 \mathrm{TF}$ is observed to play a key role in plant tolerance against frost and drought stresses. The constitutive and stress-inducible expression of TaHDZip1-5 gene elevated the frost and drought tolerance in transgenic wheat plants. However, it showed negative impact on phenotypic features and reduced grain yield (Yang et al., 2017). The RING finger containing E3 ligase, that is, salt and drought induced ring finger 1 (OsSDIR1) expressing transgenic rice exhibited strong drought tolerance (Gao et al., 2011b). Another leucine zipper (HD-ZIP) from maize, that is, Oshox 22 acts as a strong transcriptional activator in rice, which is induced by ABA, salt, and polyethylene glycol through ABA-dependent signaling pathway (Zhang et al., 2012).

Expression of Zmhdz10 confers enhanced levels of proline content and reduced electrolyte leakage and lower MDA content in transgenic rice, which contributed to drought and salt stress tolerance through ABAdependent signaling pathway (Zhao et al., 2014). The transgenic rice plants overexpressing OsbZIP23 TF showed elevated levels of drought and salt stress tolerance through ABA signaling mechanism in transgenic rice plants (Xiang et al., 2008). Similarly, overexpression of the OsbZIP66 TF enhances drought tolerance of rice plants in an ABA-dependent manner (Yoon et al., 2017). Microarray analysis of the same plants reported to be involved in regulation of different downstream genes of OsbZIP23 included protein kinases, LEA proteins, and several other stress related TFs (Xiang et al., 2008). OsbZIP12 is an important player in rice for conferring ABA-dependent drought tolerance and has high potential for use in the genetic improvement of stress tolerance (Joo et al., 2014).

\subsection{GENETIC ENGINEERING OF KINASES FOR DROUGHT AND SALINITY TOLERANCE}

\subsubsection{Protein Kinases}

Protein kinases are central integrators of plant abiotic stress signaling that link metabolic and physiological processes through regulation of ionic and osmotic 
homeostasis in plant cells. Mitogen-activated protein kinases (MAPK) and sucrose nonfermenting related protein kinases (SnRK) are well-studied protein kinases in plants. SnRK1 is a subclass of protein kinases playing a key role in abiotic stress adaptation through the Salt Overly Sensitive (SOS) mechanism with regulation of ion homeostasis under salinity stress. Intriguingly, another SnRK2 kinase, SAPK4 in rice, was involved in homeostasis through ionic and oxidative stress signaling pathways (Diédhiou et al., 2008). The SnRK2 family consisting of serine/threonine kinases was first reported to be involved in ABA signaling in wheat (Fujii and Zhu, 2012). Interestingly these proteins are involved in ABA-independent pathway in finger millet (Lata and Prasad, 2011).

Another class of protein kinases, MAPK mediated signal transduction cascades, has provided a pivotal role between physiological and cellular pathways in response to stresses. MAPK are highly conserved in all eukaryotes play an important role in response to biotic and abiotic stress stimuli. Overexpression of MPK5 in rice was attributed a dual role as regulator of resistance against brown spot pathogen Cochliobolus miyabeanus and also as mediator of abiotic stress tolerance (Beckers et al., 2009).

Overexpression of calcium dependent protein kinase (OsCPK4) leads to enhanced drought and salt tolerance in overexpressing rice lines (Campo et al., 2014). In these transgenic lines, the oxidative stressresponsive genes like peroxidases, thioredoxin, glutathione, and laccase gene were upregulated in response to drought stress (Campo et al., 2011, 2014). In other studies, overexpression of OsCDPK7 showed enhanced levels of drought and salinity stresses but not lowtemperature stresses in rice, which indicated involvement in two different distinctive signal transduction pathways in rice (Saijo et al., 2000). Whereas the transgenic rice lines expressing OsCIPK12 have been found to be involved in drought tolerance coupled with accumulation of osmoprotectants like proline and soluble sugars in transgenic plants (Xiang et al., 2007) (Table 7.2).

\subsubsection{Receptor-Like Kinases}

Plant receptor-like kinases (RLKs) are considered to be the most diverse super family of plant protection proteins involved in prevention of self-pollination, pathogen defense, hormone perception, adaptation to abiotic stresses, and increasing the crop yield (Bai et al., 2009). The plant cells often sense the environmental changes with the help of cell surface receptors on the membrane through activated RLK signaling pathways to trigger adaptive changes in the plants. RLKs are transmembrane proteins with amino terminal and carboxyl intracellular kinase domains with protein kinase activity. RLKs initiate the cellular signaling in plants. They are involved in different hormonal signaling pathways, growth, development, and plant pathogen interactions. S-domain receptor-like kinases (SRKs) are a highly polymorphic family of RLKs found in plants, play a key role in control of diseases, and improve the yield parameters in rice. The first plant $R L K$ gene (ZmPK1) was identified and isolated from maize (Walker and Zhang, 1990) and then several RLKs were isolated from different plant species. Overexpression of LRR-RLK gene LRK1 enhanced panicles and spikelets, which led to yield enhancement in rice (Zha et al., 2009). Another receptor-like protein, overexpression of OsSIK2, showed stress tolerance and delayed leaf senescence in transgenic rice plants (Chen et al., 2013). The transgenic expression of SRK, OsLSK1 (large spike-like SRK 1) responded to exogenous application of growth hormones and to abiotic stresses. Overexpression of truncated OsLSK1 increased plant height and number of panicles, which ultimately

TABLE 7.2 Expression of Kinases Involved in Signaling Pathways Through Transgenic Approach

\begin{tabular}{lllllll}
\hline Item & Gene & Promoter & Plant & Phenotype of transgenics & References \\
\hline Protein kinase & OsCPK7 & CaMV35S & Rice & Enhanced drought and salinity tolerance but not cold tolerance & Saijo et al. (2000) \\
& OsCPK12 & Ubiquitin & Rice & $\begin{array}{l}\text { Improved cold, drought and salt tolerance. Osmoprotectant, proline } \\
\text { accumulated }\end{array}$ & Xiang et al. (2007) \\
& OsCPK1 & Ubiquitin & Rice & $\begin{array}{l}\text { Gene was negatively regulated and the silencing lines showed } \\
\text { drought tolerance }\end{array}$ & Ho et al. (2013) \\
OsCPK4 & Ubiquitin & Rice & $\begin{array}{l}\text { Drought, salt and oxidative stress tolerance. Peroxidase, thioredoxin, } \\
\text { glutathione laccase genes upregulated }\end{array}$ & Campo et al. (2014) \\
kinases (RLKs) & OsSIK1 & CaMV35S & Rice & $\begin{array}{l}\text { Elevated drought and salinity tolerance. SOD, peroxidase, and catalase } \\
\text { activity increased }\end{array}$ & Ouyang et al. (2010) \\
& OsSIK2 & CaMV35S & Rice & Enhanced salt and drought stress tolerance & Chen et al. (2013) \\
\hline
\end{tabular}


TABLE 7.3 Expression of Genes Related to Phytohormones, Osmoprotectants, and ROS Signaling Genes

\begin{tabular}{|c|c|c|c|c|c|}
\hline Item & Gene & Promoter & Plant & Phenotype of transgenics & References \\
\hline \multirow[t]{2}{*}{ Phytohormones } & OsGA2ox1 & Actin & Rice & $\begin{array}{l}\text { GA synthesis in shoots during abiotic stress } \\
\text { conditions }\end{array}$ & Sakamoto et al. (2003) \\
\hline & $H V A 1$ & $\begin{array}{l}\text { Maize } \\
\text { Ubiquitin }\end{array}$ & Wheat & $\begin{array}{l}\text { Improved water use efficiency, drought and salinity } \\
\text { stress tolerance continuously for six seasons }\end{array}$ & $\begin{array}{l}\text { Sivamani et al. (2000), } \\
\text { Bahieldin et al. (2005) }\end{array}$ \\
\hline \multirow[t]{4}{*}{ Osmoprotectants } & P5CS & CaMV35S & Wheat & $\begin{array}{l}\text { Abiotic stress tolerance Showed increased levels of } \\
\text { proline accumulation }\end{array}$ & Zhu et al. (1998) \\
\hline & P5CS & CaMV35S & Wheat & Salt stress tolerance & $\begin{array}{l}\text { Sawahel and Hassan } \\
\text { (2002) }\end{array}$ \\
\hline & $\begin{array}{l}\text { Choline } \\
\text { dehydrogenase }\end{array}$ & CaMV35S & Maize & $\begin{array}{l}\text { Drought tolerance, higher grain yield, enhanced } \\
\text { glycine betaine accumulation }\end{array}$ & Quan et al. (2004) \\
\hline & Bet $A$ & CaMV35S & Wheat & Salinity tolerance, higher germination rates & He et al. (2010) \\
\hline \multirow[t]{6}{*}{ ROS } & OsHK3 & CaMV35S & Rice & $\begin{array}{l}\text { Crucial regulator of ABA signaling involved in } \\
\text { antioxidant defense in rice }\end{array}$ & Wen et al. (2015) \\
\hline & Tasro1 & $\begin{array}{l}\text { Ubiquitin } \\
\text { and } \\
\text { CaMV35S }\end{array}$ & $\begin{array}{l}\text { Wheat and } \\
\text { Arabidopsis }\end{array}$ & $\begin{array}{l}\text { Increased salinity tolerance and activated AsA-GSH } \\
\text { signaling pathway }\end{array}$ & Liu et al. (2014) \\
\hline & TaOPR1 & $\begin{array}{l}\text { Ubiquitin } \\
\text { and } \\
\text { CaMV35S }\end{array}$ & $\begin{array}{l}\text { Wheat and } \\
\text { Arabidopsis }\end{array}$ & $\begin{array}{l}\text { Induced salt stress tolerance coupled with ABA } \\
\text { signaling pathway }\end{array}$ & Dong et al. (2013) \\
\hline & $A O X 1$ & CaMV35S & Rice & $\begin{array}{l}\text { Reduced oxidative stress under cold stress. } \\
\text { Scavenging ROS }\end{array}$ & Li et al. (2013b) \\
\hline & OsMT1 & OsActin & Rice & $\begin{array}{l}\text { Enhancement of antioxidative enzymes like catalase, } \\
\text { peroxidase. and ascorbic acid }\end{array}$ & Yang et al. (2009) \\
\hline & OsSUV3 & CaMV35S & Rice & $\begin{array}{l}\text { Reduction in lipid peroxidation and increase in } \\
\text { antioxidative enzymes and salinity stress tolerance }\end{array}$ & Tuteja et al. (2013) \\
\hline
\end{tabular}

produced higher grain yield compared with nontransgenic plants (Zou et al., 2015) (Table 7.3).

\subsection{MODULATION OF KEY GENES INVOLVED IN PHYTOHORMONE SIGNALING}

Phytohormones are signaling molecules that regulate a variety of cellular processes, as well as growth and developmental activities in response to biotic and abiotic stresses in plants (Peleg et al., 2011). They work as chemical messengers to coordinate cellular activities and signal transduction pathways in higher plants (Vob et al., 2014). Although plant response to abiotic stresses depends on various factors, phytohormones are considered to be the most important endogenous substances for modulating physiological and molecular responses, a critical requirement for plant survival as sessile organisms (Fahad et al., 2015). They often rapidly alter gene expression by inducing or preventing the degradation of transcriptional regulators via ubiquitin proteosome system (Santner and Estelle, 2010).
They are also involved in ion homeostasis and enhanced antioxidant defense pathway (Sreenivasulu et al., 2012). Their pivotal roles in promoting plant acclimatization to ever-changing environments by mediating growth, development, source/sink transitions, and nutrient allocation have been well established (Fahad et al., 2015). Phytohormones are auxin (IAA), cytokinins (CKs), ABA, ethylene (ET), gibberellins (GAs), salicylic acid (SA), brassinosteroids (BRs), and jasmonates (JAs). The strigolactones (SL) are relatively new phytohormones. Engineering of key genes of phytohormone signaling could be a perfect platform for biotechnologists to improve biotic/abiotic tolerant crops. Considering that phytohormones are key regulators of plant growth and development as well as mediators of the response to environmental stresses (Sreenivasulu et al., 2012; Khan et al., 2012, 2013, 2014, 2015a,b, 2016, 2017; Khan and Khan, 2014; Per et al., 2017), hormone metabolism and signaling processes are excellent targets of manipulation to obtain enhanced abiotic stress tolerance. However, maintenance of hormonal balance to minimize possible adverse effects on 
growth and development is critical (Cabello et al., 2014; Khan and Khan, 2013).

Among various phytohormones, $\mathrm{ABA}$ is perhaps the most sought-after hormone for engineering abiotic stress tolerance in crop plants owing to its identity as a stress hormone and its vast array of functions under environmental stress conditions, particularly drought. As a result, many of the key ABA biosynthetic pathway enzymes have been investigated through genetic transformation of related genes for improved abiotic stress tolerance (Jewell et al., 2010). Park et al. (2008) overexpressed an $\mathrm{ABA}$ responsive stress related gene in Arabidospsis and the resulting plants exhibited greater osmotic stress tolerance. Constitutively expressing HVA1 under the regulation of maize ubiquitin promoter conferred enhanced water use efficiency, drought, salt, and higher biomass production due to ABA signaling in wheat (Sivamani et al., 2000). Moreover, higher yields were recorded continuously for six seasons at multiple locations. However, it was not released for commercial use (Bahieldin et al., 2005). Overexpression of gene(s) involved in ABA biosynthesis or catabolic pathways resulted in increased drought tolerance, but led to impaired growth due to pleiotropic effects even with the use of inducible promoters (Hwang et al., 2010). To offset these unwanted growth anomalies, Zhang et al. (2013) overexpressed CRK45, a stress-inducible kinase involved in ABA signaling, and the resulting transgenic plants showed enhanced drought tolerance but with tighter control of ABA levels and signaling, indicating the role of CRK45 in fine-tuning of ABA levels. Similarly, IPT was expressed under the control of stress-inducible promoters to avoid pleiotropic effects, leading to increased cytokinin content, antioxidant scavenging, and better root growth with overall improved grain yield under drought conditions in Agrostis stolonifera ( $\mathrm{Xu}$ et al., 2016).

Arabidopsis YUCCA6 gene (a member of the YUCCA family of Flavin monooxygenase-like proteins), involved in tryptophan-dependent IAA biosynthesis pathway, was expressed in poplar under the control of stressinducible SWPA2 promoter and conferred abiotic stress tolerance (Wang et al., 2015). The transgenic lines displayed IAA overproduction morphological phenotypes, including rapid shoot growth and retarded tap root development with increased root hair formation. In addition, the plants had higher levels of free IAA and early expression of the IAA responsive gene transcripts. The transgenic lines showed tolerance to drought stress, associated with reduced levels of ROS (Wang et al., 2015). The rice mutant CONSTITUTIVELY WILTED1 was deficient in the YUCCA homolog (Woo et al., 2007). Drought tolerance was achieved by overexpression of AtYUC6 in potato and the plants were recovered after withholding water for 18 days (Kim et al., 2014). Constitutive overexpression of OsGA2ox1 (GA2 oxidase) under regulation of actin promoter showed dwarf phenotype and failed to set grains during the flowering stage (Sakamoto et al., 2003). Attempts to engineer phytohormones for enhanced abiotic stress tolerance of plants are listed in Table 7.2.

\subsection{ENGINEERING OF OSMOREGULATORY GENES}

Osmoprotectants regulate the cellular osmotic adjustment, mitigate damaging risk caused by ROS, prevent membrane injury, and stabilize proteins and enzymes (Ashraf and Foolad, 2007). Many crop plants lack the ability to synthesize the special osmoprotectants that are naturally accumulated by stress tolerant organisms. Recently has been much emphasis on catalyzing biosynthetic pathways of osmoprotectants in crop plants. Water scarcity is a major abiotic stress that threatens the cereal productivity in subtropical regions. Genes involved in the synthesis of osmoprotectantsorganic compounds such as amino acids (proline), a variety of sugars, sugar alcohols (mannitol, trehalose, and galactinol), quaternary, and other amines (glycine betaine and polyamines)-that accumulate during osmotic adjustment have been used to generate stresstolerant transgenic plants (Vinocur and Altman, 2005; Masood et al., 2016; Per et al., 2017). Naturally, the glycine betaine (GB) accumulates in several plants like sugar beet, maize, spinach, and barley in response to abiotic stresses (Hurkman et al., 1991). Improvement in salt tolerance was observed in transgenic wheat by introducing bet $A$ gene for GB synthesis (He et al., 2010). Expression of GB1 in maize and soybean showed higher level of GB1 content in transgenic lines in comparison with wild type (Castiglioni et al., 2018). Transgenic rice transformed with bacterial choline dehydrogenase gene leads to accumulation of higher levels of glycine betaine, which showed improved tolerance to drought and chilling conditions (Quan et al., 2004). Delta pyroline-5-carboylate synthetase, a bifunctional enzyme involved in proline biosynthesis ( $\mathrm{Hu}$ et al., 1992), was induced by high salt stress and dehydration (Delauney and Verma, 1993). Interestingly, the transgenic wheat plants carrying Vigna aconitifolia pyrroline $T$ carboxylate synthetase (P5CS) gene exhibited the drought stress tolerance even after 15 days of water shortage. Proline was accumulated in these transgenic plants under drought stress conditions (Vendruscolo et al., 2007). Transgenic rice plants expressing P5CS gene showed increased levels of proline (Sawahel and Hassan, 2002). A rice gene, ornithine 
delta-aminotransferase gene OsOAT, was overexpressed in rice conferring increased content of $\delta$-OAT and proline levels coupled with enhanced drought, osmotic, and oxidative stress tolerance (You et al., 2012).

\subsection{REACTIVE OXYGEN SPECIES SIGNALING DURING DROUGHT AND SALINITY STRESS}

ROS are harmful byproducts of physiological changes and environmental stress responses in plants. ROS are known to be signaling molecules and secondary messengers involved in regulation of plant growth and development during biotic and abiotic stresses (Mittler et al., 2004; Khan and Khan, 2017). ROS are generated through incomplete reduction of molecular oxygen in plants (Miller et al., 2010). Excess production of ROS ultimately results in apoptosis of cells (Gill and Tuteja, 2010). ROS includes hydrogen peroxide $\left(\mathrm{H}_{2} \mathrm{O}_{2}\right)$, superoxide anions $\left(\mathrm{O}_{2} \bullet-\right)$, hydroxyl radical $(\mathrm{OH} \bullet-)$, and singlet oxygen. ROS are precisely regulated by enzymatic and nonenzymatic antioxidant defense systems. Scavenging ROS are mediated through enzymatic reactions and regulating signaling pathways in crop plants (Baxter et al., 2013; Chakradhar et al., 2017). The plants have developed coherent antioxidant ROS scavenging machinery through enzymatic (SOD, APX, GR, MDHAR, and DHAR) and nonenzymatic reactions (ascorbic acid, reduced glutathione, tocopherols, carotenoids, flavonoids, etc.) (You and Chan, 2015). There is significant evidence that manipulating ROS provides an opportunity to enhance the abiotic stress tolerance in genetically engineered crop plants.

Several ROS scavenging enzymes have been studied in cereals. Plant NADPH oxidase is known as a homolog of respiratory burst oxidase, key signaling enzymes involved in ROS regulation and several signal transduction pathways (Marino et al., 2012). The activity of NADPH oxidase was induced by treatment with ABA and $\mathrm{Ca}^{2+}$ in leaves of maize seedlings (Jiang and Zhang, 2003). The NADPH oxidase genes were regulated by histidine kinase (OsHK3) in rice, which was involved in the production of $\mathrm{H}_{2} \mathrm{O}_{2}$ in ABA signaling (Wen et al., 2015). Transgenic rice plants overexpressing $M n$-SOD1 exhibited scavenging of mitochondrial peroxide free radicals during stress conditions ( $\mathrm{Li}$ et al., 2013a). The transgenic rice expressing OsDhn1 showed drought, salt, and methyl viologen tolerance coupled with enhancement of chlorophyll content, biomass, and also induced scavenging of free radicals (Kumar et al., 2014) The overexpression of Ta-sro1 (similar to radical induced cell death) gene in wheat and Arabidospsis stimulated the scavenging of ROS and also upregulated the activity of AsA-GSH (ascorbic acid-glutathione reductase) and GPX (glutaredoxin) cycle enzymes, which helps in cellular redox homeostasis (Liu et al., 2014). Another ROS regulating enzyme, TaOPR1 (wheat oxophytodienoate reductase), also exhibited tolerance to salt coupled with ABA signaling pathway in wheat and Arabidopsis (Dong et al., 2013). In another study, the alternative oxidase 1 (AOX1) gene overexpressed in rice conferring reduced the oxidative stress damage under cold stress by ROS scavenging ( $\mathrm{Li}$ et al., 2013b). Overexpression of OsMT1 (metallothionein) gene led to the enhancement of antioxidant enzyme activities of catalase, peroxidase, and ascorbic acid under drought stress and also OsMT1 gene induced the expression of several zinc finger TFs by alteration of $\mathrm{Zn}^{2+}$ homeostasis (Yang et al., 2009). Overexpression of OsSUV3 (a dual helicase) resulted in reduction of lipid peroxidation and $\mathrm{H}_{2} \mathrm{O}_{2}$ production coupled with increase in antioxidative enzymes and also the transgenic plants showed high salinity tolerance in rice (Tuteja et al., 2013). To improve abiotic stress in crop plants by exploiting ROS signaling pathway genes, key regulatory molecules need to be identified. Taken together these pathway genes can be utilized for the genetic transformation of different genetic backgrounds of cereals to enhance the abiotic stress tolerance in field conditions.

\subsection{CONCLUSION AND FUTURE PROSPECTS}

Climate change and severity of abiotic stresses are major threats that will impact basic fundamental processes, growth, and development of plants. These modulations ultimately affect the agriculture production and global food security.

In the present chapter, we highlighted the success stories of crop improvement through genetic engineering approaches by utilizing the genes related to abiotic stress signaling. Genetic engineering of TFs, phytohormones, osmoprotectants, protein kinases, and receptor kinases represents an important platform for abiotic stress tolerance, providing new opportunities to maintain sustainable crop production to feed the world under changing environmental conditions. Rapid development of genomic technology and efforts of many research groups have led to understanding of plant abiotic stress response. Still many challenges lie ahead to uncover and understand the complexity hidden in stress signal-transduction pathways. Among the greatest challenges that remain to be addressed are the development of stable engineered crops that produce staple foods such as rice, wheat, maize and corn. Recently, genome editing has become the most promising tool for facilitating the alterations or silencing the 
gene of a particular locus. The CRISPR-Cas/Cpf tool is widely used for inducing knockin or knockouts of rice, wheat, maize, barley, etc. These applications may pave the way to improve yield and extend crop improvement or trait improvement such as biotic, abiotic, and nutritional value enrichment. It may resolve problems associated with stress response and adaptation pathways. Toward this goal, study should be focused on a combination of stress responses such as those in field environments, because different stresses are most likely to occur simultaneously under field conditions.

\section{Acknowledgments}

The authors are grateful to Agri Biotech Foundation for its support.

\section{References}

Agarwal, M., Hao, Y., Kapoor, A., Dong, C.H., Fujii, H., Zheng, X., et al., 2006. A R2R3 type MYB transcription factor is involved in the cold regulation of $\mathrm{CBF}$ genes and in acquired freezing tolerance. J. Biol. Chem. 281 (49), 37636-37645.

Aida, M., Ishida, T., Fukaki, H., Fujisawa, H., Tasaka, M., 1997. Genes involved in organ separation in Arabidopsis: an analysis of the cup-shaped cotyledon mutant. Plant Cell 9 (6), 841-857.

Akpinar, B.A., Magni, F., Yuce, M., Lucas, S.J., Šimková, H., Šafář, J., et al., 2015. The physical map of wheat chromosome 5DS revealed gene duplications and small rearrangements. BMC Genom. 16 (1), 453.

Ashraf, M., Foolad, M., 2007. Roles of glycine betaine and proline in improving plant abiotic stress resistance. Environ. Exp. Bot. 59 (2), 206-216.

Bai, L., Zhang, G., Zhou, Y., Zhang, Z., Wang, W., Du, Y., et al., 2009. Plasma membrane-associated proline-rich extensin-like receptor kinase 4 , a novel regulator of $\mathrm{Ca} 2+$ signalling, is required for abscisic acid responses in Arabidopsis thaliana. Plant J. 60 (2), 314-327.

Bahieldin, A., Mahfouz, H.T., Eissa, H.F., Saleh, O.M., Ramadan, A. M., Ahmed, I.A., et al., 2005. Field evaluation of transgenic wheat plants stably expressing the HVA1 gene for drought tolerance. Physiol. Plant. 123 (4), 421-427.

Banerjee, A., Roychoudhury, A., 2015. WRKY proteins: signaling and regulation of expression during abiotic stress responses. Sci. World J. 2015.

Baxter, A., Mittler, R., Suzuki, N., 2013. ROS as key players in plant stress signalling. J. Exp. Bot. 65 (5), 1229-1240.

Beckers, G.J., Jaskiewicz, M., Liu, Y., Underwood, W.R., He, S.Y., Zhang, S., et al., 2009. Mitogen-activated protein kinases 3 and 6 are required for full priming of stress responses in Arabidopsis thaliana. Plant Cell 21 (3), 944-953.

Bihani, P., Char, B., Bhargava, S., 2011. Transgenic expression of sorghum DREB2 in rice improves tolerance and yield under water limitation. J. Agric. Sci. 149 (1), 95-101.

Budak, H., Hussain, B., Khan, Z., Ozturk, N.Z., Ullah, N., 2015. From genetics to functional genomics: improvement in drought signaling and tolerance in wheat. Front. Plant Sci. 6, 1012.

Cabello, J.V., Lodeyro, A.F., Zurbriggen, M.D., 2014. Novel perspectives for the engineering of abiotic stress tolerance in plants. Curr. Opin. Biotechnol. 26, 62-70.

Campo, S., Peris-Peris, C., Montesinos, L., Peñas, G., Messeguer, J., San Segundo, B., 2011. Expression of the maize ZmGF14-6 gene in rice confers tolerance to drought stress while enhancing susceptibility to pathogen infection. J. Exp. Bot. 63 (2), 983-999.

Campo, S., Baldrich, P., Messeguer, J., Lalanne, E., Coca, M., San Segundo, B., 2014. Overexpression of a calcium-dependent protein kinase confers salt and drought tolerance in rice by preventing membrane lipid peroxidation. Plant Physiol. 32, 739-747.

Castiglioni, P., Bell, E., Lund, A., Rosenberg, A.F., Galligan, M., Hinchey, B.S., et al., 2018. Identification of GB1, a gene whose constitutive overexpression increases glycinebetaine content in maize and soybean. Plant Direct 2 (2), e00040.

Chakradhar, T., Mahanty, S., Reddy, R.A., Divya, K., Reddy, P.S., Reddy, M.K., 2017. Biotechnological Perspective of Reactive Oxygen Species (ROS)-Mediated Stress Tolerance in Plants. In Reactive Oxygen Species and Antioxidant Systems in Plants: Role and Regulation Under Abiotic Stress. Springer, Singapore, pp. 53-87.

Chen, L.J., Wuriyanghan, H., Zhang, Y.Q., Duan, K.X., Chen, H.W., Li, Q.T., et al., 2013. An S-domain receptor-like kinase, OsSIK2, confers abiotic stress tolerance and delays dark-induced leaf senescence in rice. Plant Physiol. 163 (4), 1752-1765.

Chen, X., Wang, Y., Lv, B., Li, J., Luo, L., Lu, S., et al., 2014. The NAC family transcription factor OsNAP confers abiotic stress response through the ABA pathway. Plant Cell Physiol. 55 (3), $604-619$.

Cominelli, E., Tonelli, C., 2009. A new role for plant R2R3-MYB transcription factors in cell cycle regulation. Cell Res. 19 (11), 1231.

Delauney, A.J., Verma, D.P.S., 1993. Proline biosynthesis and osmoregulation in plants. Plant J. 4 (2), 215-223.

Diédhiou, C.J., Popova, O.V., Dietz, K.J., Golldack, D., 2008. The SNF1type serine-threonine protein kinase SAPK4 regulates stressresponsive gene expression in rice. BMC Plant Biol. 8 (1), 49.

Dong, W., Wang, M., Xu, F., Quan, T., Peng, K., Xiao, L., et al., 2013. Wheat oxophytodienoate reductase gene TaOPR1 confers salinity tolerance via enhancement of abscisic acid signaling and reactive oxygen species scavenging. Plant Physiol. 161 (3), 1217-1228.

Du, H., Zhang, L., Liu, L., Tang, X.F., Yang, W.J., Wu, Y.M., et al., 2009. Biochemical and molecular characterization of plant MYB transcription factor family. Biochemistry (Moscow) 74 (1), 1-11.

Fahad, S., Nie, L., Chen, Y., Wu, C., Xiong, D., Saud, S., et al., 2015. Crop plant hormones and environmental stress. In: Lichtfouse, E. (Ed.), Sustainable Agriculture Reviews. Springer, Cham, pp. 371-400.

Fedoroff, N.V., Battisti, D.S., Beachy, R.N., Cooper, P.J., Fischhoff, D. A., Hodges, C.N., et al., 2010. Radically rethinking agriculture for the $21^{\text {st }}$ century. Science 327 (5967), 833-834.

Fujii, H., Zhu, J.K., 2012. Osmotic stress signaling via protein kinases. Cell. Mol. Life Sci. 69 (19), 3165-3173.

Gao, S.Q., Chen, M., Xia, L.Q., Xiu, H.J., Xu, Z.S., Li, L.C., et al., 2009. A cotton (Gossypium hirsutum) DRE-binding transcription factor gene, GhDREB, confers enhanced tolerance to drought, high salt, and freezing stresses in transgenic wheat. Plant Cell Rep. 28 (2), 301-311.

Gao, S.Q., Chen, M., Xu, Z.S., Zhao, C.P., Li, L., Xu, H.J., et al., 2011a. The soybean GmbZIP1 transcription factor enhances multiple abiotic stress tolerances in transgenic plants. Plant Mol. Biol. 75 (6), 537-553.

Gao, T., Wu, Y., Zhang, Y., Liu, L., Ning, Y., Wang, D., et al., 2011b. OsSDIR1 overexpression greatly improves drought tolerance in transgenic rice. Plant Mol. Biol. 76 (1-2), 145-156.

Gill, S.S., Tuteja, N., 2010. Reactive oxygen species and antioxidant machinery in abiotic stress tolerance in crop plants. Plant. Physiol. Biochem. 48 (12), 909-930.

He, C., Yang, A., Zhang, W., Gao, Q., Zhang, J., 2010. Improved salt tolerance of transgenic wheat by introducing betA gene for glycine betaine synthesis. Plant Cell Tissue Organ Cult. 101 (1), $65-78$. 
Ho, S.L., Huang, L.F., Lu, C.A., He, S.L., Wang, C.C., Yu, S.P., et al., 2013. Sugar starvation-and GA-inducible calcium-dependent protein kinase 1 feedback regulates GA biosynthesis and activates a 14-3-3 protein to confer drought tolerance in rice seedlings. Plant Mol. Biol. 81 (4-5), 347-361.

Hong, Y., Zhang, H., Huang, L., Li, D., Song, F., 2016. Overexpression of a stress-responsive NAC transcription factor gene ONAC022 improves drought and salt tolerance in rice. Front. Plant Sci. 7, 4.

Hossain, M.A., Lee, Y., Cho, J.I., Ahn, C.H., Lee, S.K., Jeon, J.S., et al., 2010. The bZIP transcription factor OsABF1 is an ABA responsive element binding factor that enhances abiotic stress signaling in rice. Plant Mol. Biol. 72 (4-5), 557-566.

Hu, C.A., Delauney, A.J., Verma, D.P., 1992. A bifunctional enzyme (delta 1-pyrroline-5-carboxylate synthetase) catalyzes the first two steps in proline biosynthesis in plants. Proc. Natl. Acad. Sci. 89 (19), 9354-9358.

Hurkman, W.J., Tao, H.P., Tanaka, C.K., 1991. Germin-like polypeptides increase in barley roots during salt stress. Plant Physiol. 97 (1), 366-374.

Hwang, S.G., Chen, H.C., Huang, W.Y., Chu, Y.C., Shii, C.T., Cheng, W.H., 2010. Ectopic expression of rice OsNCED3 in Arabidopsis increases ABA level and alters leaf morphology. Plant Sci. 178 (1), $12-22$.

Ito, Y., Katsura, K., Maruyama, K., Taji, T., Kobayashi, M., Seki, M., et al., 2006. Functional analysis of rice DREB1/CBF-type transcription factors involved in cold-responsive gene expression in transgenic rice. Plant Cell Physiol. 47 (1), 141-153.

Jiang, M., Zhang, J., 2003. Cross-talk between calcium and reactive oxygen species originated from NADPH oxidase in abscisic acidinduced antioxidant defence in leaves of maize seedlings. Plant Cell Environ. 26 (6), 929-939.

Jeong, J.S., Kim, Y.S., Baek, K.H., Jung, H., Ha, S.H., Do Choi, Y., et al., 2010. Root-specific expression of OsNAC10 improves drought tolerance and grain yield in rice under field drought conditions. Plant Physiol. 153 (1), 185-197.

Jeong, J.S., Kim, Y.S., Redillas, M.C., Jang, G., Jung, H., Bang, S.W., et al., 2013. OsNAC5 overexpression enlarges root diameter in rice plants leading to enhanced drought tolerance and increased grain yield in the field. Plant. Biotechnol. J. 11 (1), 101-114.

Jewell, M.C., Campbell, B.C., Godwin, I.D., 2010. Transgenic plants for abiotic stress resistance. Transgenic Crop Plants. Springer, Berlin, Heidelberg, New York, pp. 67-132.

Joo, J., Lee, Y.H., Song, S.I., 2014. Overexpression of the rice basic leucine zipper transcription factor OsbZIP12 confers drought tolerance to rice and makes seedlings hypersensitive to ABA. Plant Biotechnol. Rep. 8 (6), 431-441.

Katiyar, A., Smita, S., Lenka, S.K., Rajwanshi, R., Chinnusamy, V., Bansal, K.C., 2012. Genome-wide classification and expression analysis of MYB transcription factor families in rice and Arabidopsis. BMC Genom. 13 (1), 544.

Kim, H., Lee, K., Hwang, H., Bhatnagar, N., Kim, D.Y., Yoon, I.S., et al., 2014. Overexpression of PYL5 in rice enhances drought tolerance, inhibits growth, and modulates gene expression. J. Exp. Bot. 65 (2), 453-464.

Kizis, D., Pages, M., 2002. Maize DRE-binding proteins DBF1 and DBF2 are involved in rab17 regulation through the droughtresponsive element in an ABA-dependent pathway. Plant J. 30 (6), 679-689.

Khan, M.I.R., Iqbal, N., Masood, A., Khan, N.A., 2012. Variation in salt tolerance of wheat cultivars: evaluation of the role of glycinebetaine and ethylene. Pedosphere 22, 746-754.

Khan, M.I.R., Iqbal, N., Masood, A., Per, T.S., Khan, N.A., 2013. Salicylic acid alleviates adverse effects of heat stress on photosynthesis through changes in proline production and ethylene formation. Plant Signal. Behav. 8, e26374.

Khan, M.I.R., Khan, N.A., 2013. Salicylic acid and jasmonates: approaches in abiotic stress tolerance. Plant Biochem. Physiol. 1, 4.

Khan, M.I.R., Khan, N.A., 2014. Ethylene reverses photosynthetic inhibition by nickel and zinc in mustard through changes in PS II activity, photosynthetic-nitrogen use efficiency and antioxidant metabolism. Protoplasma 251, 1007-1019.

Khan, M.I.R., Khan, N., 2017. Reactive Oxygen Species and Antioxidant System in Plants: Role and Regulation Under Abiotic Stress. Springer Nature, Singapore978-981-10-5254-5.

Khan, M.I.R., Asgher, M., Khan, N.A., 2014. Alleviation of saltinduced photosynthesis and growth inhibition by salicylic acid involves glycinebetaine and ethylene in mungbean (Vigna radiata L.). Plant. Physiol. Biochem. 80, 67-74.

Khan, M.I.R., Fatma, M., Per, T.S., Anjum, N.A., Khan, N.A., 2015a. Salicylic acid-induced abiotic stress tolerance and underlying mechanisms in plants. Front. Plant Sci. 6, 462.

Khan, M.I.R., Khan, N.A., Fatma, M., Asgher, M., Syeed, S., Masood, A., et al., 2015b. Photosynthesis in changing environment: analyzing the role of phytohormones in modulation. In: Khan), N.A. (Ed.), Photosynthesis: Functional Genomics, Physiological Processes and Environmental Issues Chapters Books. Nova Publication, New York, pp. 129-166.

Khan, M.I.R., Khan, N.A., Masood, A., Per, T.S., Asgher, M., 2016. Hydrogen peroxide alleviates nickel-inhibited photosynthetic responses through increase in use-efficiency of nitrogen and sulfur, and glutathione production in mustard. Front. Plant Sci. 7, 44.

Khan, N.A., Khan, M.I.R., Ferrante, A., Poór, P., 2017. Ethylene: a key regulatory molecule in plants. Front. Plant Sci. Available from: https:/ / doi.org/10.3389/fpls.2017.01782.

Kobayashi, F., Takumi, S., Kume, S., Ishibashi, M., Ohno, R., Murai, K., et al., 2005. Regulation by Vrn-1/Fr-1 chromosomal intervals of CBF-mediated Cor/Lea gene expression and freezing tolerance in common wheat. J. Exp. Bot. 56 (413), 887-895.

Komaki, S., Sugimoto, K., 2012. Control of the plant cell cycle by developmental and environmental cues. Plant Cell Physiol. 53 (6), 953-964.

Kumar, M., Lee, S.C., Kim, J.Y., Kim, S.J., Kim, S.R., 2014. Overexpression of dehydrin gene, OsDhn1, improves drought and salt stress tolerance through scavenging of reactive oxygen species in rice (Oryza sativa L.). J. Plant Biol. 57 (6), 383-393.

Lata, C., Prasad, M., 2011. Role of DREBs in regulation of abiotic stress responses in plants. J. Exp. Bot. 62 (14), 4731-4748.

Lee, S.C., Choi, H.W., Hwang, I.S., Choi, D.S., Hwang, B.K., 2006. Functional roles of the pepper pathogen-induced bZIP transcription factor, CAbZIP1, in enhanced resistance to pathogen infection and environmental stresses. Planta 224 (5), 1209-1225.

Li, F., Han, Y., Feng, Y., Xing, S., Zhao, M., Chen, Y., et al., 2013 a. Expression of wheat expansin driven by the RD29 promoter in tobacco confers water-stress tolerance without impacting growth and development. J. Biotechnol. 163 (3), 281-291.

Li, C.R., Liang, D.D., Li, J., Duan, Y.B., Li, H.A.O., Yang, Y.C., et al., 2013b. Unravelling mitochondrial retrograde regulation in the abiotic stress induction of rice ALTERNATIVE OXIDASE 1 genes. Plant Cell Environ. 36 (4), 775-788.

Liu, S., Liu, S., Wang, M., Wei, T., Meng, C., Wang, M., et al., 2014. A wheat SIMILAR TO RCD-ONE gene enhances seedling growth and abiotic stress resistance by modulating redox homeostasis and maintaining genomic integrity. Plant Cell 26 (1), 164-180.

Ma, Q., Dai, X., Xu, Y., Guo, J., Liu, Y., Chen, N., et al., 2009. Enhanced tolerance to chilling stress in OsMYB3R-2 transgenic rice is mediated by alteration in cell cycle and ectopic expression of stress genes. Plant Physiol. 150 (1), 244-256. 
Marino, D., Dunand, C., Puppo, A., Pauly, N., 2012. A burst of plant NADPH oxidases. Trends. Plant. Sci. 17 (1), 9-15.

Mallikarjuna, G., Mallikarjuna, K., Reddy, M.K., Kaul, T., 2011. Expression of OsDREB2A transcription factor confers enhanced dehydration and salt stress tolerance in rice (Oryza sativa L.). Biotechnol. Lett. 33 (8), 1689-1697.

Masood, A., Per, T.S., Asgher, M., Fatma, M., Khan, M.I.R., Rasheed, F., et al., 2016. Glycine betaine: role in shifting plants toward adaptation under extreme environments. In: Iqbal, N., Nazar, R., Khan, N.A. (Eds.), Osmolytes and Plants Acclimation to Changing Environment: Emerging Omics Technologies. Springer, New Delhi, pp. 69-82.

Matsukura, S., Mizoi, J., Yoshida, T., Todaka, D., Ito, Y., Maruyama, K., et al., 2010. Comprehensive analysis of rice DREB2-type genes that encode transcription factors involved in the expression of abiotic stress-responsive genes. Mol. Genet. Genomics. 283 (2), 185-196.

Mallappa, C., Yadav, V., Negi, P., Chattopadhyay, S., 2006. A basic leucine zipper transcription factor, G-box-binding factor 1, regulates blue light-mediated photomorphogenic growth in Arabidopsis. J. Biol. Chem. 281 (31), 22190-22199.

Meena, K.K., Sorty, A.M., Bitla, U.M., Choudhary, K., Gupta, P., Pareek, A., et al., 2017. Abiotic stress responses and microbemediated mitigation in plants: the omics strategies. Front. Plant Sci. $8,172$.

Miller, A.K., Galiba, G., Dubcovsky, J., 2006. A cluster of 11 CBF transcription factors is located at the frost tolerance locus Fr-A m 2 in Triticum monococcum. Mol. Genet. Genomics. 275 (2), 193-203.

Miller, G.A.D., Suzuki, N., Ciftci-Yilmaz, S., Mittler, R.O.N., 2010. Reactive oxygen species homeostasis and signalling during drought and salinity stresses. Plant Cell Environ. 33 (4), 453-467.

Mittler, R., Vanderauwera, S., Gollery, M., Van Breusegem, F., 2004. Reactive oxygen gene network of plants. Trends. Plant. Sci. 9 (10), 490-498.

Morran, S., Eini, O., Pyvovarenko, T., Parent, B., Singh, R., Ismagul, A., et al., 2011. Improvement of stress tolerance of wheat and barley by modulation of expression of DREB/CBF factors. Plant. Biotechnol. J. 9 (2), 230-249.

Nakashima, K., Takasaki, H., Mizoi, J., Shinozaki, K., YamaguchiShinozaki, K., 2012. NAC transcription factors in plant abiotic stress responses. Biochim. Biophys. Acta 1819 (2), 97-103.

Nakashima, K., Yamaguchi-Shinozaki, K., Shinozaki, K., 2014. The transcriptional regulatory network in the drought response and its crosstalk in abiotic stress responses including drought, cold, and heat. Front. Plant Sci. 5, 170.

Nguyen, T.X., Sticklen, M., 2013. Barley HVA1 gene confers drought and salt tolerance in transgenic maize (Zea mays L.). Adv. Crop Sci. Tech. 1 (105), 2.

Nguyen, T.X., Nguyen, T., Alameldin, H., Goheen, B., Loescher, W., Sticklen, M., 2013. Transgene pyramiding of the HVA1 and mtlD in T3 maize (Zea mays L.) plants confers drought and salt tolerance, along with an increase in crop biomass. Int. J. Agron 2.

Nuruzzaman, M., Sharoni, A.M., Kikuchi, S., 2013. Roles of NAC transcription factors in the regulation of biotic and abiotic stress responses in plants. Front. Microbiol. 4, 248.

Oh, S.J., Song, S.I., Kim, Y.S., Jang, H.J., Kim, S.Y., Kim, M., et al., 2005. Arabidopsis CBF3/DREB1A and ABF3 in transgenic rice increased tolerance to abiotic stress without stunting growth. Plant Physiol. 138 (1), 341-351.

Olsen, A.N., Ernst, H.A., Leggio, L.L., Skriver, K., 2005. NAC transcription factors: structurally distinct, functionally diverse. Trends. Plant. Sci. 10 (2), 79-87.

Ouyang, S.Q., Liu, Y.F., Liu, P., Lei, G., He, S.J., Ma, B., et al., 2010. Receptor-like kinase OsSIK1 improves drought and salt stress tolerance in rice (Oryza sativa) plants. Plant J 62 (2), 316-329.
Park, H.Y., Seok, H.Y., Park, B.K., Kim, S.H., Goh, C.H., Lee, B.H., et al., 2008. Overexpression of Arabidopsis ZEP enhances tolerance to osmotic stress. Biochem. Biophys. Res. Commun. 375 (1), 80-85.

Pasala, R.K., Khan, M.I.R., Minhas, P.S., Farooq, M.A., Sultana, R., Per, T.S., et al., 2016. Can plant bio-regulators minimize crop productivity losses caused by drought, heat and salinity stress? An integrated review. J. Appl. Bot. Food. 89, 113-125.

Pellegrineschi, A., Reynolds, M., Pacheco, M., Brito, R.M., Almeraya, R., Yamaguchi-Shinozaki, K., et al., 2004. Stress-induced expression in wheat of the Arabidopsis thaliana DREB1A gene delays water stress symptoms under greenhouse conditions. Genome 47 (3), 493-500.

Peleg, Z., Reguera, M., Tumimbang, E., Walia, H., Blumwald, E., 2011. Cytokinin-mediated source/sink modifications improve drought tolerance and increase grain yield in rice under water stress. Plant. Biotechnol. J. 9 (7), 747-758.

Per, T.S., Khan, N.A., Reddy, P.S., Masood, A., Hasanuzzaman, M., Khan, M.I.R., et al., 2017. Approaches in modulating proline metabolism in plants for salt and drought stress tolerance: phytohormones, mineral nutrients and transgenics. Plant. Physiol. Biochem. 115, 126-140.

Per, T.S., Khan, M.I.R., Anjum, N.A., Masood, A., Hussain, S.J., Khan, N.A., 2018. Jasmonates in plants under abiotic stresses: crosstalk with other phytohormones matters. Environ. Exp. Bot. $145,104-120$.

Phukan, U.J., Jeena, G.S., Tripathi, V., Shukla, R.K., 2017. Regulation of Apetala2/ethylene response factors in plants. Front. Plant Sci. 8,150 .

Quan, R., Shang, M., Zhang, H., Zhao, Y., Zhang, J., 2004. Improved chilling tolerance by transformation with betA gene for the enhancement of glycinebetaine synthesis in maize. Plant Sci. 166 (1), 141-149.

Rahman, H., Ramanathan, V., Nallathambi, J., Duraialagaraja, S., Muthurajan, R., 2016. Over-expression of a NAC 67 transcription factor from finger millet (Eleusine coracana L.) confers tolerance against salinity and drought stress in rice. BMC Biotechnol. 16 (1), 35 .

Redillas, M.C., Jeong, J.S., Kim, Y.S., Jung, H., Bang, S.W., Choi, Y. D., et al., 2012. The overexpression of OsNAC9 alters the root architecture of rice plants enhancing drought resistance and grain yield under field conditions. Plant. Biotechnol. J. 10 (7), 792-805.

Rong, W., Qi, L., Wang, A., Ye, X., Du, L., Liang, H., et al., 2014. The ERF transcription factor TaERF3 promotes tolerance to salt and drought stresses in wheat. Plant. Biotechnol. J. 12 (4), $468-479$.

Saikumar, P., Murali, R., Reddy, E.P., 1990. Role of tryptophan repeats and flanking amino acids in Myb-DNA interactions. Proc. Natl. Acad. Sci. 87 (21), 8452-8456.

Saijo, Y., Hata, S., Kyozuka, J., Shimamoto, K., Izui, K., 2000. Overexpression of a single $\mathrm{Ca} 2+$-dependent protein kinase confers both cold and salt/drought tolerance on rice plants. Plant J. 23 (3), 319-327.

Sakamoto, T., Morinaka, Y., Ishiyama, K., Kobayashi, M., Itoh, H., Kayano, T., et al., 2003. Genetic manipulation of gibberellin metabolism in transgenic rice. Nat. Biotechnol. 21 (8), 909.

Santner, A., Estelle, M., 2010. The ubiquitin-proteasome system regulates plant hormone signaling. Plant J. 61 (6), 1029-1040.

Sawahel, W.A., Hassan, A.H., 2002. Generation of transgenic wheat plants producing high levels of the osmoprotectant proline. Biotechnol. Lett. 24 (9), 721-725.

Shen, J., Lv, B., Luo, L., He, J., Mao, C., Xi, D., et al., 2017. The NACtype transcription factor OsNAC2 regulates ABA-dependent genes and abiotic stress tolerance in rice. Sci. Rep. 7, 40641. 
Sivamani, E., Bahieldin, A., Wraith, J.M., Al-Niemi, T., Dyer, W.E., Ho, T.H.D., et al., 2000. Improved biomass productivity and water use efficiency under water deficit conditions in transgenic wheat constitutively expressing the barley HVA1 gene. Plant Sci. 155 (1), 1-9.

Skinner, J.S., von Zitzewitz, J., Szúcs, P., Marquez-Cedillo, L., Filichkin, T., Amundsen, K., et al., 2005. Structural, functional, and phylogenetic characterization of a large CBF gene family in barley. Plant Mol. Biol. 59 (4), 533-551.

Soltész, A., Smedley, M., Vashegyi, I., Galiba, G., Harwood, W., Vágúffalvi, A., 2013. Transgenic barley lines prove the involvement of TaCBF14 and TaCBF15 in the cold acclimation process and in frost tolerance. J. Exp. Bot. 64 (7), 1849-1862.

Song, S.Y., Chen, Y., Chen, J., Dai, X.Y., Zhang, W.H., 2011. Physiological mechanisms underlying OsNAC5-dependent tolerance of rice plants to abiotic stress. Planta 234 (2), 331-345.

Sreenivasulu, N., Harshavardhan, V.T., Govind, G., Seiler, C., Kohli, A., 2012. Contrapuntal role of ABA: does it mediate stress tolerance or plant growth retardation under long-term drought stress? Gene 506 (2), 265-273.

Tang, N., Zhang, H., Li, X., Xiao, J., Xiong, L., 2012. Constitutive activation of transcription factor OsbZIP46 improves drought tolerance in rice. Plant Physiol. 158 (4), 1755-1768.

Todaka, D., Nakashima, K., Shinozaki, K., Yamaguchi-Shinozaki, K., 2012. Toward understanding transcriptional regulatory networks in abiotic stress responses and tolerance in rice. Rice 5 (1), 6.

Todaka, D., Shinozaki, K., Yamaguchi-Shinozaki, K., 2015. Recent advances in the dissection of drought-stress regulatory networks and strategies for development of drought-tolerant transgenic rice plants. Front. Plant Sci. 6, 84.

Tuteja, N., Sahoo, R.K., Garg, B., Tuteja, R., 2013. OsSUV3 dual helicase functions in salinity stress tolerance by maintaining photosynthesis and antioxidant machinery in rice (Oryza sativa L. cv. IR64). Plant J. 76 (1), 115-127.

Vendruscolo, E.C.G., Schuster, I., Pileggi, M., Scapim, C.A., Molinari, H.B.C., Marur, C.J., et al., 2007. Stress-induced synthesis of proline confers tolerance to water deficit in transgenic wheat. J. Plant. Physiol. 164 (10), 1367-1376.

Vinocur, B., Altman, A., 2005. Recent advances in engineering plant tolerance to abiotic stress: achievements and limitations. Curr. Opin. Biotechnol. 16 (2), 123-132.

Vob, U., Bishopp, A., Farcot, E., Bennett, M.J., 2014. Modelling hormonal response and development. Trends. Plant. Sci. 19 (5), $311-319$.

Walker, J.C., Zhang, R., 1990. Relationship of a putative receptor protein kinase from maize to the S-locus glycoproteins of Brassica. Nature 345 (6277), 743.

Wang, X., Jiang, D., Yang, D., 2015. Fast-tracking determination of homozygous transgenic lines and transgene stacking using a reliable quantitative real-time PCR assay. Appl. Biochem. Biotechnol. 175 (2), 996-1006.

Wang, Y., Gao, C., Liang, Y., Wang, C., Yang, C., Liu, G., 2010. A novel bZIP gene from Tamarix hispida mediates physiological responses to salt stress in tobacco plants. J. Plant. Physiol. 167 (3), 222-230.

Wen, F., Qin, T., Wang, Y., Dong, W., Zhang, A., Tan, M., et al., 2015. OsHK3 is a crucial regulator of abscisic acid signaling involved in antioxidant defense in rice. J. Int. Plant Biol. 57 (2), 213-228.

Woo, Y.M., Park, H.J., Su'udi, M., Yang, J.I., Park, J.J., Back, K., et al., 2007. Constitutively wilted 1 , a member of the rice YUCCA gene family, is required for maintaining water homeostasis and an appropriate root to shoot ratio. Plant Mol. Biol. 65 (1-2), 125-136.

Xia, N., Zhang, G., Sun, Y.F., Zhu, L., Xu, L.S., Chen, X.M., et al., 2010. TaNAC8, a novel NAC transcription factor gene in wheat, responds to stripe rust pathogen infection and abiotic stresses. Physiol. Mol. Plant Pathol. 74 (5-6), 394-402.
Xiang, Y., Huang, Y., Xiong, L., 2007. Characterization of stressresponsive CIPK genes in rice for stress tolerance improvement. Plant Physiol. 144 (3), 1416-1428.

Xiang, Y., Tang, N., Du, H., Ye, H., Xiong, L., 2008. Characterization of OsbZIP23 as a key player of the basic leucine zipper transcription factor family for conferring abscisic acid sensitivity and salinity and drought tolerance in rice. Plant Physiol. 148 (4), 1938-1952.

Xie, Z., Lee, E., Lucas, J.R., Morohashi, K., Li, D., Murray, J.A., et al., 2010. Regulation of cell proliferation in the stomatal lineage by the Arabidopsis MYB FOUR LIPS via direct targeting of core cell cycle genes. Plant Cell 22 (7), 2306-2321.

Xiong, H., Li, J., Liu, P., Duan, J., Zhao, Y., Guo, X., et al., 2014. Overexpression of OsMYB48-1, a novel MYB-related transcription factor, enhances drought and salinity tolerance in rice. PLoS One 9 (3), e92913.

Xu, Z.S., Ni, Z.Y., Li, Z.Y., Li, L.C., Chen, M., Gao, D.Y., et al., 2009. Isolation and functional characterization of HvDREB1-a gene encoding a dehydration-responsive element binding protein in Hordeum vulgare. J. Plant. Res. 122 (1), 121-130.

Xu, Q., Burgess, P., Xu, J., Meyer, W., Huang, B., 2016. Osmotic stress-and salt stress-inhibition and gibberellin-mitigation of leaf elongation associated with up-regulation of genes controlling cell expansion. Environ. Exp. Bot. 131, 101-109.

Xue, G.P., Way, H.M., Richardson, T., Drenth, J., Joyce, P.A., McIntyre, C.L., 2011. Overexpression of TaNAC69 leads to enhanced transcript levels of stress up-regulated genes and dehydration tolerance in bread wheat. Mol. Plant 4 (4), 697-712.

Yamaguchi-Shinozaki, K., Shinozaki, K., 2009. DREB regulons in abiotic-stress-responsive gene expression in plants. In Molecular Breeding of Forage and Turf. Springer, New York, pp. 15-28.

Yang, A., Dai, X., Zhang, W.H., 2012. A R2R3-type MYB gene, OsMYB2, is involved in salt, cold, and dehydration tolerance in rice. J. Exp. Bot. 63 (7), 2541-2556.

Yang, Y., Luang, S., Harris, J., Riboni, M., Li, Y., Bazanova, N., et al., 2017. Overexpression of the class I homeodomain transcription factor TaHDZipI-5 increases drought and frost tolerance in transgenic wheat. Plant. Biotechnol. J. 16 (6), 1227-1240.

Yang, Z., Wu, Y., Li, Y., Ling, H.Q., Chu, C., 2009. OsMT1a, a type 1 metallothionein, plays the pivotal role in zinc homeostasis and drought tolerance in rice. Plant Mol. Biol. 70 (1-2), 219-229.

You, J., Hu, H., Xiong, L., 2012. An ornithine $\delta$-aminotransferase gene OsOAT confers drought and oxidative stress tolerance in rice. Plant Sci. 197, 59-69.

You, J., Chan, Z., 2015. ROS regulation during abiotic stress responses in crop plants. Front. Plant Sci. 6, 1092.

Yoon, S., Lee, D.K., Yu, I.J., Kim, Y.S., Do Choi, Y., Kim, J.K., 2017. Overexpression of the OsbZIP66 transcription factor enhances drought tolerance of rice plants. Plant Biotechnol. Rep. 11 (1), $53-62$.

Zha, X., Luo, X., Qian, X., He, G., Yang, M., Li, Y., et al., 2009. Overexpression of the rice LRK1 gene improves quantitative yield components. Plant. Biotechnol. J. 7 (7), 611-620.

Zandkarimi, H., Ebadi, A., Salami, S.A., Alizade, H., Baisakh, N., 2015. Analyzing the expression profile of AREB/ABF and DREB/ $\mathrm{CBF}$ genes under drought and salinity stresses in grape (Vitis vinifera L.). PLoS One 10 (7), e0134288.

Zhang, C., Li, C., Liu, J., Lv, Y., Yu, C., Li, H., et al., 2017. The OsABF1 transcription factor improves drought tolerance by activating the transcription of COR413-TM1 in rice. J. Exp. Bot. 68 (16), 4695-4707.

Zhang, H., Huang, Z., Xie, B., Chen, Q., Tian, X., Zhang, X., et al., 2004. The ethylene-, jasmonate-, abscisic acid-and NaCl-responsive tomato transcription factor JERF1 modulates expression of 
GCC box-containing genes and salt tolerance in tobacco. Planta 220 (2), 262-270.

Zhang, S., Haider, I., Kohlen, W., Jiang, L., Bouwmeester, H., Meijer, A.H., et al., 2012. Function of the HD-Zip I gene Oshox22 in ABA-mediated drought and salt tolerances in rice. Plant Mol. Biol. 80 (6), 571-585.

Zhang, X., Yang, G., Shi, R., Han, X., Qi, L., Wang, R., et al., 2013. Arabidopsis cysteine-rich receptor-like kinase 45 functions in the responses to abscisic acid and abiotic stresses. Plant. Physiol. Biochem. 67, 189-198.

Zhao, Y., Xing, L., Wang, X., Hou, Y.J., Gao, J., Wang, P., et al., 2014a. The ABA receptor PYL8 promotes lateral root growth by enhancing MYB77-dependent transcription of auxin-responsive genes. Sci. Signal. 7 (328), ra53. pp.

Zhao, Y., Ma, Q., Jin, X., Peng, X., Liu, J., Deng, L., et al., 2014b. A novel maize homeodomain-leucine zipper (HD-Zip) I gene,
Zmhdz10, positively regulates drought and salt tolerance in both rice and Arabidopsis. Plant Cell Physiol. 55 (6), $1142-1156$.

Zhu, J.K., 2016. Abiotic stress signaling and responses in plants. Cell 167 (2), 313-324.

Zhu, B., Su, J., Chang, M., Verma, D.P.S., Fan, Y.L., Wu, R., 1998. Overexpression of a $\Delta 1$-pyrroline-5-carboxylate synthetase gene and analysis of tolerance to water-and salt-stress in transgenic rice. Plant Sci. 139 (1), 41-48.

Zou, M., Guan, Y., Ren, H., Zhang, F., Chen, F., 2008. A bZIP transcription factor, OsABI5, is involved in rice fertility and stress tolerance. Plant Mol. Biol. 66 (6), 675-683.

Zou, X., Qin, Z., Zhang, C., Liu, B., Liu, J., Zhang, C., et al., 2015. Over-expression of an S-domain receptor-like kinase extracellular domain improves panicle architecture and grain yield in rice. J. Exp. Bot. 66 (22), 7197-7209. 
This page intentionally left blank 


\title{
8
}

\section{Role and Regulation of ROS and Antioxidants as Signaling Molecules in Response to Abiotic Stresses}

\author{
Pooja Sharma ${ }^{1}$, Priyanka Sharma ${ }^{2}$, Priya Arora ${ }^{1}$, Vinod Verma ${ }^{3}$, Kanika \\ Khanna ${ }^{1}$, Poonam Saini ${ }^{1}$ and Renu Bhardwaj ${ }^{1}$ \\ ${ }^{1}$ Department of Botanical and Environmental Sciences, Guru Nanak Dev University, Amritsar, Punjab, India \\ ${ }^{2}$ Department of Biotechnology, Modern College of Arts, Science and Commerce, Ganeshkhind, Pune, Maharashtra, \\ India ${ }^{3}$ Department of Botany, DAV University, Jalandhar, Punjab, India
}

\section{O U T L I N E}

8.1 Introduction

8.2 Reactive Oxygen Species as Signaling Molecules

8.3 Reactive Oxygen Species Involved in Plant

Defense

8.3.1 Process of Reactive Oxygen Species Signaling in Plants

8.4 Reactive Oxygen Species-Mediated Damage to Macromolecules

8.4.1 Lipids

8.4.2 Proteins

8.4.3 DNA
141

142

144

144

145

145

146

146
8.5 Reactive Oxygen Species Production and Intracellular Protein Oxidation

8.5.1 Reactive Oxygen Species-Induced Posttranslational Modifications

8.6 Role of Antioxidants and Its Signaling in Abiotic Stress

8.7 Conclusion

152

References

152

Further Reading

156

\subsection{INTRODUCTION}

Plants normally come across a combination of many abiotic stresses in their natural habitats. Abiotic stresses like drought, salt, cold, heat, and heavy metal cause a series of morphological, physiological, biochemical, and molecular changes that unfavorably affect plant growth, development, and productivity 
(Asgher et al., 2015; Khan et al., 2012, 2013, 2014, 2015, 2016; Pasala et al., 2016). These abiotic stresses modulate pathways of metabolism and boost expression level of transcription factors (TFs), which activate stress responsive genes. During plant stress response, reactive oxygen species (ROS) are produced and act as important molecules that activate downstream metabolic pathways (Chan et al., 2016). ROS interact with numerous metabolites and cause severe damage to proteins, DNA, and lipids and affect normal functioning of the cell (Apel and Hirt, 2004; Foyer and Noctor, 2005; Ashraf, 2009; Khan and Khan, 2017). ROS mainly includes singlet oxygen, hydrogen peroxide, superoxide radical, and hydroxyl radical. The excitation of $\mathrm{O}_{2}$ results in the formation of singlet oxygen $\left({ }^{1} \mathrm{O}_{2}\right)$ (Triantaphylidès and Havaux, 2009). The transfer of one, two, or three electrons to $\mathrm{O}_{2}$ forms a superoxide radical $\left(\mathrm{O}_{2}^{--}\right), \mathrm{H}_{2} \mathrm{O}_{2}$ or a hydroxyl radical $\left(\mathrm{OH}^{*}\right)$, respectively (Mittler, 2002). These are mainly synthesized by involvement of NADPH oxidases (termed respiratory burst oxidase homologs; $\mathrm{RBOHs}$ ) and some oxidases and peroxidases, and in chloroplast, mitochondria, peroxisome, and other cellular compartments, via different pathways (Suzuki et al., 2011; Vaahtera et al., 2014; Gilroy et al., 2016; MignoletSpruyt et al., 2016). ROS generation (metabolically or for signaling purposes) and ROS scavenging is a continuous process and occurs in all cellular compartments of the cells. This process is controlled by the ROS gene network (Mittler et al., 2004). Overproduction of ROS causes oxidative stress. During oxidative stress the capacity of cellular defenses to remove these toxic species is lowered in comparison with their production. During stress, ROS generation is higher than ROS consumption (Ahmad et al., 2008, 2010a,b, 2011; Koyro et al., 2012). ROS also changes gene expression by modifying TFs. At lower or optimal concentrations, ROS function as secondary messengers in signaling cascades. Redox homeostasis in plants during stressful conditions is regulated by two mechanisms involving the enzymatic components comprising the superoxide dismutase (SOD), ascorbate peroxidase (APX), glutathione-S-transferase, peroxidase (POX) and catalase (CAT), and the nonenzymatic low molecular compounds like ascorbic acid (AA), reduced glutathione (GSH), $\alpha$-tocopherol, carotenoids, flavonoids, and proline (Gill and Tuteja, 2010; Miller et al., 2010).

\subsection{REACTIVE OXYGEN SPECIES AS SIGNALING MOLECULES}

ROS are accepted as central regulators of plant development and studies on tip growing systems indicated a main function for the NADPH oxidases in forming such developmentally significant ROS. Tip growing cells also revealed the role of cytosolic ROS in regulation of ion channel gating and are directly associated with ROS generation in the apoplast, where they act to alter the properties of the cell wall. The organization of ROS generation and their activities between compartments is budding as an imperative theme in understanding how growth and development programs are coordinated (Swanson and Gilroy, 2010). ROS plays an important function in the acclimation process of plants to abiotic stress conditions. They mainly function as signaling molecules that regulate various pathways during plant acclimation to stress (Choudhury et al., 2017). Abiotic stresses are coupled with the formation of chemical entities called ROS, that is, $\mathrm{H}_{2} \mathrm{O}_{2}, \mathrm{O}_{2}{ }^{-}, \mathrm{OH}^{-}$, etc. They are able to induce cellular damages by breakdown of proteins, enzyme inactivation, changes in the gene, and inhibition in different pathways of metabolic significance. ROS behave as secondary messengers that signal important cellular roles, that is, cell proliferation, necrosis, and apoptosis (Choudhury et al., 2013). Under abiotic stress conditions, ROS production occurs that causes harm to normal functioning of plants. It also plays an important function in cellular damage and abiotic stress signaling. Plants contain various antioxidative mechanisms that deal with high ROS in the cells (Gautam et al., 2017). The participation of ROS in signal transduction indicates that there is a coordinated role of regulation networks to sustain ROS at nontoxic levels in a fragile balancing act between ROS generation and ROSscavenging pathways, and to adjust ROS responses and following downstream processes (Mittler et al., 2004). Many studies from various plant species showed the ROS production and activity of different antioxidant enzymes enhanced during abiotic stresses (Damanik et al., 2010; Selote and Chopra, 2010; Tang et al., 2010; Turan and Ekmekci, 2011).

The ABA is the main regulator of abiotic stress resistance in plants, and regulates various stress responsive genes by a complex regulatory network so as to present tolerance to the environmental stresses (Cutler et al., 2010; Raghavendra et al., 2010). The induction of ABA stress tolerance is partly associated with activation of antioxidant defense systems, that is, enzymatic and nonenzymatic components, which provide protection to the plant cells against oxidative damage (Huang et al., 2012; Zhang et al., 2012, 2014a, b). Water stress-induced ABA enhancement and exogenous application of ABA inhibit the enhanced production of ROS, then leads to antioxidant system activation in crops (Jiang and Zhang, 2002; Ye et al., 2011). Various studies have showed that brassinosteroids (BRs) can activate antioxidant defense to enhance 
stress tolerance in crop plants (Özdemir et al., 2004; Xia et al., 2009). Gibberellins (GAs) take part in the reaction of plants to abiotic stress and their action is linked with the management of growth by controlling cell elongation and division (Colebrook et al., 2014). GAs play their role through DELLA protein regulation, negative regulators of GAs signaling (Achard et al., 2006). The GAs binding with the nuclear receptor GID1 induces conformational variations in the proteins and favoring associations with DELLA proteins. As a result of this, DELLA is ubiquitinated and targeted for breakdown through $26 \mathrm{~S}$ proteasome (Colebrook et al., 2014). The signaling of GA regulates stress tolerance by controlling the cellular redox homeostasis. Under water-deficit conditions the GA concentration was decreased in the maize and as a result there was enhancement in the activity of DELLA proteins that resulted in enhanced ROS quenching capacity and enhanced endurance (Wang and Li, 2008). Nitric oxide (NO) is the most important ROS and linked with various physiological processes in the plants (Niu and Liao, 2016). NO mediates the posttranslational amendments of marked proteins through S-nitrosylation and nitration. ABA induces the synthesis of $\mathrm{NO}$ and ROS under water-deficit conditions. Both $\mathrm{NO}$ and ROS form 8-nitro-cGMP and induce the closure of stomata (Joudoi et al., 2013). Auxin also induces the synthesis of ROS and NO, and both ROS and NO act in auxininduced signaling (Yadav and David Bhatla, 2011; Farnese et al., 2016). In Arabidopsis Col-0 and gsnor1-3 (a mutant defective in protein denitrosylation), auxin signaling and auxin transport were correlated and Shi et al. (2015) reported that auxin signaling and polar auxin transport was decreased indicating the function of S-nitrosylation in auxin signaling. NO and ROS were studied to take part in osmotic tolerance of wheat seedlings by enhancing the biosynthesis of ABA and NO by protecting against oxidative damage (Misra et al., 2010).

ROS plays a significant function in development of plants and ROS that are formed by NADPH oxidases produce the $\mathrm{O}_{2}{ }^{-2}$ (Segal and Abo, 1993). Application of inhibitors, that is, diphenylene iodonium (DPI), indicated that NADPH oxidases obtained by ROS controls development of cells in roots of maize showing that ROS helps in growth. DPI is a flavin enzyme inhibitor and when treated with DPI may also have influence in other protein activities in count to NADPH oxidases (Moulton et al., 2000; Liszkay et al., 2004). NADPH oxidases also play an important role in controlling the cell growth and there are some indications that these also participate in apical dominance and leaf shape (Sagi et al., 2004). The cell wall has an important function in the expansion of the cell, wall loosening, which allows the cells to enlarge, whereas cross-linking of wall reduces the expansion. There are facts that ROS take part in such processes. They are concerned with cell wall loosening in growing tissues and as the cell growth ceases and cells get differentiated the cell wall becomes hard (Gapper and Dolan, 2006).

ROS scavenging enzymes, that is, SOD, CAT, and APX play a significant role in the ROS-scavenging pathway. The existence of antioxidant enzymes and compounds in every cellular part indicates the significance of ROS detoxification for defense against stress conditions (Mittler et al., 2004). Small molecules, that is, $\mathrm{Ca}^{2+}$ and calmodulin $(\mathrm{CaM}), \mathrm{NO}$, and ROS have been observed to take part in the ABA-induced antioxidant defense system (Jiang and Zhang, 2003; Hu et al., 2007). In rice $\mathrm{Ca}^{2+} / \mathrm{CaM}$-dependent protein kinase, OsDMI3 is significant for ABA-induced enhancement in the expression and SOD and CAT activities. ABAinduced $\mathrm{H}_{2} \mathrm{O}_{2}$ generation activates OsDMI3 and their activation also increased $\mathrm{H}_{2} \mathrm{O}_{2}$ generation by enhancing the expression of NADPH oxidase genes (Shi et al., 2014). Moreover, it was also observed that OsDMI3 plays a role upstream of OsDMI1, to regulate the antioxidant enzyme activities and in the generation of $\mathrm{H}_{2} \mathrm{O}_{2}$ in rice plants (Shi et al., 2014). ABA-induced $\mathrm{H}_{2} \mathrm{O}_{2}$ generation and ABA-induced activation of OsMPKs promotes the ZFP36 and ZFP36 expression and regulates the NADPH oxidase expression and MAPK genes and the $\mathrm{H}_{2} \mathrm{O}_{2}$ generation in ABA signaling (Zhang et al., 2014a,b). $\mathrm{H}_{2} \mathrm{O}_{2}$ molecules formed in the chloroplast can disperse outside the organelles and act as a signaling molecule in the cytoplasm, switching MAP-kinase cascade activation that activates nuclear genes, mainly the cytoplasmic APX encoding gene (Yabuta et al., 2004; Mubarakshina et al., 2010). It also participates in inducing the expression of light responsive genes. Therefore, treatment of plants with $\mathrm{H}_{2} \mathrm{O}_{2}$ enhanced expression of APX2 (which encodes APX), and ZAT10 and ZAT12 (genes encoding TFs). However addition of CAT resulted in decreased APX2 and ZAT10 expression (Davletova et al., 2005).

Apart from chloroplasts, plant mitochondria are the major site for generation of ROS such as $\mathrm{H}_{2} \mathrm{O}_{2}$ and also the target of ROS (Rasmusson et al., 2004). Plant mitochondria have particular electron transport chain (ETC) components and roles in processes such as photorespiration. The mitochondrial ETC harbors electrons with high free energy for direct reduction of $\mathrm{O}_{2}$, which is the inevitable main source of mitochondrial ROS production in aerobic respiration (Rhoads et al., 2006). Moreover, generation of ROS in mitochondria occurs under normal respiratory conditions but may be increased due to biotic and abiotic stress conditions. Complexes I and II are recognized as the site of $\mathrm{O}_{2}^{-}$ generation and in aqueous solution $\mathrm{O}_{2}^{-}$is fairly reactive but it is reduced to $\mathrm{H}_{2} \mathrm{O}_{2}$ by SOD dismutation (Quan, 
2008; Möller, 2001; Grene, 2002). Further $\mathrm{H}_{2} \mathrm{O}_{2}$ reacts with iron and copper to form highly toxic $\mathrm{HO}$ and this uncharged $\mathrm{HO}$ can enter through membranes and leaves the mitochondria (Rhoads et al., 2006). Abstraction of hydrogen atom by ROS, mainly by $\mathrm{HO}$, starts peroxidation of mitochondrial membrane polyunsaturated fatty acid. The significance of this is the generation of cytotoxic lipid alkenals, aldehydes, hydroxyalkenals, etc. Nevertheless, plant mitochondria control production of ROS through energy dissipating systems and as result of these mitochondria play a key function in the adaptation of plants to abiotic stress conditions. Scarpeci et al. (2008) reported that methyl viologen induced production of $\mathrm{O}_{2}^{-}$in Arabidopsis thaliana chloroplasts during active photosynthesis and indicate that $\mathrm{O}_{2}^{-}$produced in photosynthetically active chloroplast leads to activation of genes that take part in the signaling pathways. $C_{3}$ and $C_{4}$ photosynthesis under salt stress conditions was reported and it was observed that amaranth plants unlike wheat plants were able to detoxify the $\mathrm{O}_{2}^{-}$by SOD and amaranthine antioxidant and decreased the LPO intensity (Gambarova and Gins, 2008). At low concentrations $\mathrm{H}_{2} \mathrm{O}_{2}$ acts as a signaling molecule that takes part in acclamatory signaling inhibiting tolerance to biotic and abiotic stress conditions, and at high concentrations leads to programmed cell death (Quan, 2008). $\mathrm{H}_{2} \mathrm{O}_{2}$ also has been revealed to act as an important regulator in various physiological processes, that is, senescence, photorespiration, photosynthesis, stomatal movements, cell cycle, growth, and development (Bright et al., 2006; Peng et al., 2005; Mittler et al., 2004; Foreman et al., 2003; Noctor and Foyer, 1998). It is also accepted as a secondary messenger for the signals produced through ROS because of its comparatively long life and high permeability across the membranes (Quan, 2008).

\subsection{REACTIVE OXYGEN SPECIES INVOLVED IN PLANT DEFENSE}

Different types of biotic or abiotic stresses enhance the levels of ROS production, which further inactivates different antioxidants and enzymatic systems in plants. This causes oxidative stress in plants that illustrates the perquisite for initiation of the signaling response in plants for acclimation mechanisms (Jaspers and Kandasjarvi, 2010; Miller et al., 2010). ROS are defined as the group of different free radicals, ions, and molecules that play a crucial role as oxidation signaling molecules and are mainly derived from $\mathrm{O}_{2}$ (Foyer and Noctor, 2009). On an estimate, around $1 \%$ of $\mathrm{O}_{2}$ utilized by plants is directed for ROS production in different cell organelles such as peroxisomes, chloroplasts, and mitochondria (Sharma et al., 2012).
Plants mainly possess three mechanisms for ROS production: (1) in ETC in mitochondria and chloroplasts; (2) different oxidases and peroxidases such as amine oxidase, lipoxygenase, NADH oxidase, xanthine oxidase, glycolate oxidase, and NADPH oxidase; and (3) chlorophyll containing molecules (Blokhina et al., 2003). In chloroplasts, photosynthetic products are the predominant ROS producers. For example, Rubisco enzyme catalyzing carboxylase-oxygenase reactions leads to both utilization and production of oxygen. Therefore, ETC in chloroplasts produces higher levels of oxygen, which leads to electron leakage that generates ROS. Moreover, chlorophyll coupled with photosensitizers also generates ROS by sunlight (Mittler, 2002). However, the principal producers of ROS are the mitochondria, which generate molecules such as $\mathrm{H}_{2} \mathrm{O}_{2}$ by reducing oxygen in aerobic respiration (Koyro et al., 2012; Ahmad et al., 2013). The signals initiated by ROS in these organelles promote transcriptional changes and reprogramming of cells, which either undergo cell death or cell protection (Foyer and Noctor, 2005; Fig. 8.1). Different types of ROS produced in the cells are hydrogen peroxide $\left(\mathrm{H}_{2} \mathrm{O}_{2}\right)$, hydroxyl radical $\left(\mathrm{OH}^{*}\right)$, superoxide radical $\left(\mathrm{O}_{2}{ }^{-}\right)$, and singlet oxygen $\left({ }^{1} \mathrm{O}_{2}\right)$, which are nontoxic until they react with the organic molecules to get activated (Apel and Hirt, 2004).

$\mathrm{O}_{2}$ activation can occur by two different ways: (1) energy absorption to reverse the spin of unpaired electrons, or (2) reduction of monovalent ions in stepwise manner. By this act, the formation of ${ }^{1} \mathrm{O}_{2}$ takes place followed by its reduction to $\mathrm{H}_{2} \mathrm{O}_{2}, \mathrm{OH}$ and $\mathrm{O} \bullet-_{2}$ (Apel and Hirt, 2004).

\subsubsection{Process of Reactive Oxygen Species Signaling in Plants}

As a consequence of abiotic stress a flux of calcium results in the cytosol of the plant cell. Calcium acts as a secondary messenger and directly activates $\mathrm{RBOHs}$, and/or triggers a cascade of events that activate calcium-dependent protein kinases. These kinases phosphorylate and further activate $\mathrm{RBOHs}$ (Miller et al., 2009; Mittler et al., 2011; Dubiella et al., 2013; Gilroy et al., 2014). The activated RBOHs produce ROS at the apoplast, which is sensed by neighboring cells. It triggers the flux of calcium in these cells and results in the activation of their own RBOHs. ROS-derived calcium flux, coupled with activation of $\mathrm{RBOHs}$ by calcium, is then autopropagated from cell to neighboring cell throughout the entire plant, and triggers systemic responses to abiotic stress (Miller et al., 2009).

ROS generated as a result of environmental stress also results in the activation of mitogen activated protein kinases (MAPKs), which are highly conserved signaling pathway proteins that play an important role in 


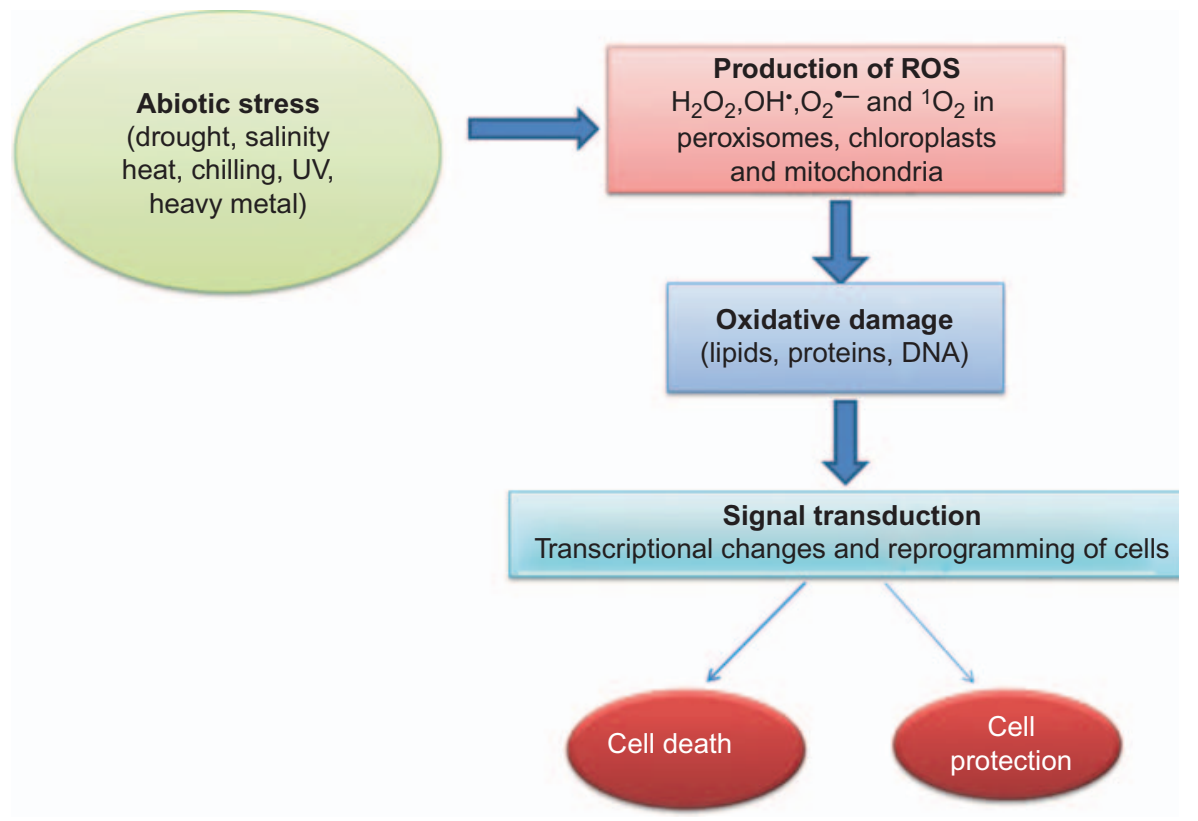

FIGURE 8.1 Abiotic stress induced ROS production and reprogramming of cells.

signal transduction under stress conditions. These are activated in response to abiotic stress such as drought, salt, and osmotic stress. MAPKs phosphorylate specific serine/threonine residues to regulate various cellular functions and activities (Fig. 8.2). In the MAPKs pathway, the MAPK cascade consists of three functionally interlinked protein kinases, MAPK kinase kinase [MAPKKK], MAPK kinase [MAPKK], and MAPK (Ho, 2015). The activated MAPKKK phosphorylates and activates MAPKK, which further phosphorylates and activates a MAPK, and results in the activation of specific signaling molecules such as TFs to induce cellular responses from the nucleus of a cell. These phosphorylation cascades work either upstream or downstream of ROS (Asai et al., 2002)

\subsection{REACTIVE OXYGEN SPECIES- MEDIATED DAMAGE TO MACROMOLECULES}

When the level of ROS exceeds the defense mechanisms, a cell is said to be in a state of "oxidative stress." High levels of ROS can cause damage to lipids, proteins, and DNA (Valko et al., 2006) leading to aging and cell death (Ashraf, 2009).

\subsubsection{Lipids}

On exceeding threshold limit, the ROS increases lipid peroxidation in cell membranes ultimately affecting normal cellular function. Lipid peroxidation produces lipid-derived radicals leading to enhanced oxidative stress, which eventually damages proteins and nucleic acids. Plants growing under environmental stress reveal increased lipid peroxidation consecutively corresponding to aggravated levels of ROS. One of the final products of lipid peroxidation is malondialdehyde (MDA). Two common sites of ROS attack on the phospholipid molecules are the ester linkage between glycerol and the fatty acid and the unsaturated (double) bond between two carbon atoms. The polyunsaturated fatty acids (PUFAs) present in membrane phospholipids are predominantly susceptible to attack by ROS. A single $\bullet \mathrm{OH}$ can result in peroxidation of many polyunsaturated fatty acids as the reactions involved in this process are part of a cyclic chain reaction (Sharma et al., 2012).

In general, the process of lipid peroxidation involves three discrete stages: (1) initiation, (2) progression, and (3) termination. The initial phase comprises activation of $\mathrm{O}_{2}$, which is a rate limiting step. $\mathrm{O}_{2}^{--}$and $\bullet \mathrm{OH}$ can react with methylene groups of PUFA forming lipid peroxy radicals, conjugated dienes, and hydroperoxides (Smirnoff, 1995).

1. Initiation step:

$$
\begin{aligned}
& \text { PUFA }-\mathrm{H}+\mathrm{X}^{\bullet} \rightarrow \mathrm{PUFA}+\mathrm{X}-\mathrm{H} \\
& \text { PUFA }+\mathrm{O}_{2} \rightarrow \text { PUFA }-\mathrm{OO}^{\bullet}
\end{aligned}
$$

2. Progression step: The peroxy radical formed is highly reactive and propagates the chain reaction.

$$
\text { PUFA - } \mathrm{OO}^{\bullet}+\text { PUFA - } \mathrm{OOH} \rightarrow \text { PUFA }-\mathrm{OOH}+\mathrm{PUFA}^{\bullet}
$$

3. Termination step: The formation of conjugated diene occurs when free radicals attack the 


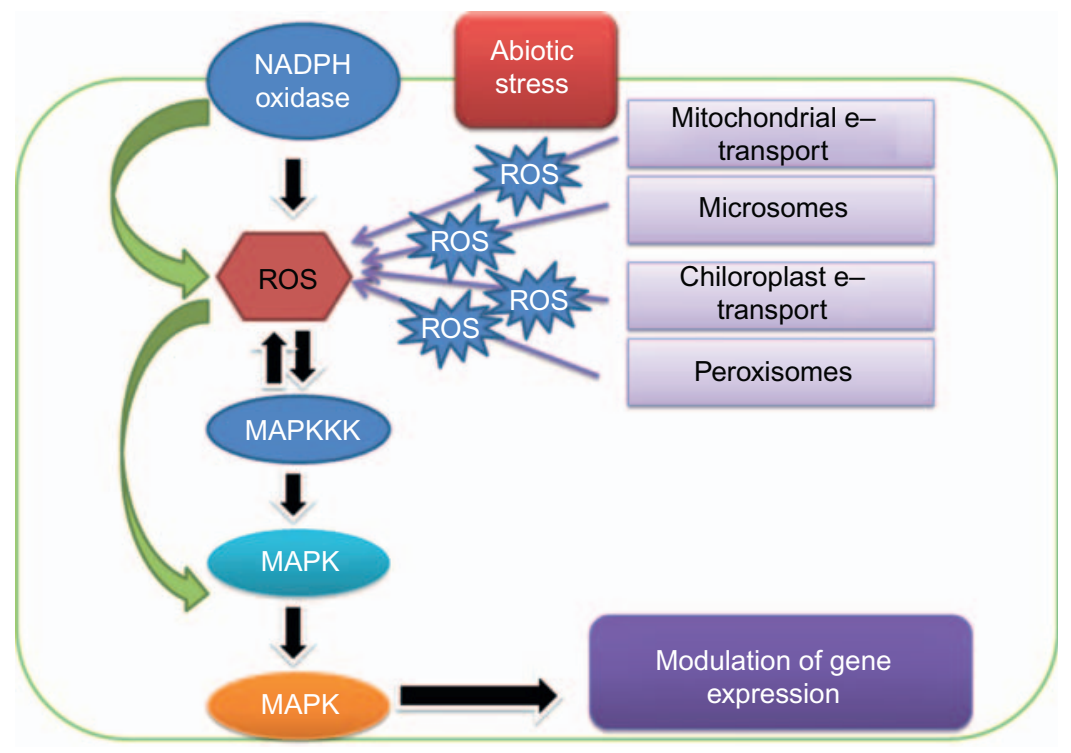

FIGURE 8.2 Mitogen activated protein kinases (MAPK) signaling pathway abiotic stresses. Source: Adapted from Jalmi and Sinha (2015).

hydrogens of methylene groups separating double bonds thereby, rearranging the bonds (Recknagal and Glende, 1984). The lipid hydroperoxides produced (PUFA-OOH) can undergo reductive cleavage by reduced metals (e.g., $\mathrm{Fe}^{2+}$ ) as follows:

$$
\begin{aligned}
& \mathrm{Fe}^{2+} \text { complex }+ \text { PUFA }-\mathrm{OOH} \rightarrow \mathrm{Fe} 3+\text { complex } \\
& \quad+\text { PUFA }-\mathrm{O}^{\bullet}
\end{aligned}
$$

Numerous reactive species such as lipid alkoxyl radicals, malonyldialdehyde, crotonaldehyde, acrolein, alkanes, lipid epoxides, and alcohols can be simply formed by the decomposition of lipid hydroperoxide (Davies, 2000). The lipid alkoxy radical produced (PUFA-O•) can initiate additional chain reactions (Buettner, 1993).

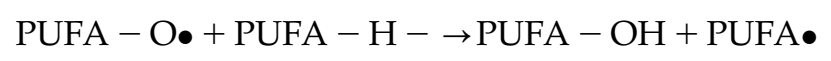

Peroxidation of polyunsaturated fatty acid by ROS can cause chain breakage leading to increased membrane permeability and fluidity.

\subsubsection{Proteins}

ROS can aggravate reversible or irreversible alterations of proteins, consecutively causing modifications in the regulation of plant metabolism, as well as the activation of transcriptional regulatory networks. Due to augmented ROS production, fragmentation of the peptide chain, aggregation of cross-linked reaction products, site-specific amino acid modification, and increased susceptibility of proteins to proteolysis occur. Tissues damaged by oxidative stress generally contain increased concentrations of carbonylated proteins, which are extensively used as a marker of protein oxidation (Moller and Kristensen, 2004). Diverse stresses in plants cause enhanced modification of proteins (Tanoua et al., 2009a,b). The amino acids in a peptide differ in their propensity to attack by ROS. The most vulnerable residues to oxidation are the sulfur containing methionine and cysteine. The thiol of cysteine may be oxidized by hydroxyl radicals, superoxide, and hydrogen peroxide to a disulfide that can be readily reversible. Oxidation of methionine in numerous proteins has a minute effect on protein structure and function. An example of the reversible oxidation of methionine is the inactivation of the small heat shock protein in chloroplasts that is reactivated by thioredoxin in reaction catalyzed by methionine sulfoxide reductase (Gustavsson et al., 2002). Tyrosine oxidation can modify residue hydrophobicity with subsequent effect on protein structure. Oxidation of tryptophan is an irreversible protein modification (Rinalducci et al., 2008). Another example of irreversible protein modification is oxidation of iron-sulfur centers by $\mathrm{O}_{2}^{--}$. It has been suggested that protein oxidation could predispose it to ubiquitination consequently making it a target for proteasomal degradation (Cabiscol et al., 2000).

\subsubsection{DNA}

ROS induces severe damage to DNA, including cross-links, base deletion, deoxyribose oxidation, strand breaks, pyrimidine dimers, and base modifications such as oxidation and alkylation (Tuteja et al., 2001). ROS can cause oxidative damage to nuclear, chloroplastic, and mitochondrial DNA. Moreover, changes in the nucleotides of one strand can cause mismatches with the nucleotides in the other strand 
causing subsequent mutations. Any damage to the DNA can lead to changes in the encoded proteins, which may result in malfunctions or complete inactivation of the encoded proteins. Both the sugar and base moieties of DNA are susceptible to oxidation by ROS. It has been reported that $\mathrm{OH}^{\bullet}$ is most reactive and can cause damage to all components of the DNA molecule, damaging both the purine and pyrimidine bases and also the deoxyribose sugar backbone (Halliwell and Gutteridge, 1999). ${ }^{1} \mathrm{O}_{2}$ primarily attacks guanine; $\mathrm{H}_{2} \mathrm{O}_{2}$ and $\mathrm{O}_{2}{ }^{-}$do not react at all (Wiseman and Halliwell, 1996). DNA damage results in various physiological effects, such as cell membrane destruction, reduced protein synthesis, and damage to photosynthetic proteins, ultimately affecting growth and development of the whole organism (Britt, 1999). DNA damage can result either in arrest or induction of transcription, stimulation of signal transduction pathways, replication errors, and genomic instability (Cooke et al., 2003). The major type of DNA damage caused by exposure to UV-B is the formation of dimers between neighboring pyrimidines. UV photoproducts consist primarily of 6-4PPs dimers and cyclobutane pyrimidine dimers (Tuteja et al., 2009). Excessive changes caused by ROS lead to permanent damage to the DNA with potentially detrimental effects for the cell.

\subsection{REACTIVE OXYGEN SPECIES PRODUCTION AND INTRACELLULAR PROTEIN OXIDATION}

During photosynthesis and respiration process, ROS lead to protein oxidation of carbonyl (CO) groups. Among the 20 amino acids of the protein, several amino acids can be directly modified by means of side-chain reactions with ROS. Most susceptible amino acids are those having sulfhydryl groups and those with aromatic side-chain groups. The condition of aromatic side-chain amino acids, including tryptophan, phenylalanine, ROS-induced oxidation starts during an array of intermediates (Moller et al., 2007; Foyer and Noctor, 2009). For example, the oxidation of phenylalanine residues leads to the development of mono- and dihydroxy derivatives but tryptophan residues are transformed into numerous hydroxy derivatives. Furthermore, histidine residues can be oxidized to 2oxohistidine and 4-OH-glutamate, however tyrosine residues are converted to a dihydroxy derivative, dopamine (DOPA), nitrotyrosine, chlorotyrosin, and a dityrosine derivative. The carbonyl groups (aldehydes and ketones) are also produced on protein side chains (especially of proline, arginine, lysine, and threonine) where they are oxidized. Protein carbonyl derivatives can also be generated through oxidative cleavage of proteins either by the $\alpha$-amidation pathway or by oxidation of the glutamyl side chain. The nucleophilic side chains of Cys, His, and Lys residues may be formed when the CO group is introduced into proteins. The carbonyl group can further react with $\alpha$-amino group of lysine residues, which leads to the formation of intra- or intermolecular cross-links promoting protein aggregation (Davies, 2000; Dietz, 2014; Waszczak et al., 2015).

Both Met and Cys play crucial roles in cell metabolism. Met is mostly found in globular protein in the interior membrane-spanning protein domains. These are liable to oxidation to MetO residues (Waszczak et al., 2015, Miki and Funaro, 2012). The Cys residues provide a lot of functions, such as catalysis, stabilization of protein arrangement during metal binding, disulfides, and regulation of protein role. Cys residues also focus on many posttranslational modifications (Fig. 8.3). Reversible oxidation of Cys thiols is known to participate in redox regulation of proteins by means of the formation of sulfenic acid intermediates (RSOH), inter- and intramolecular disulfide bonds ( $R-S-S-R)$, diverse disulfide bond with glutathione (R-S-SG), and overoxidation to sulfinic acids $\left(\mathrm{R}-\mathrm{SO}_{2} \mathrm{H}\right)$ (Miki and Funaro, 2012). These redox-derived changes in protein function can affect transcription, phosphorylation, and other important signaling events, and/or alter metabolic fluxes and reactions in the cell by altering enzymatic properties (Mittler, 2017). The studies of Gulyas et al. (2017) also provided evidence about the redox control of free amino acid profile in wild-type and ascorbate or glutathione deficient mutant $A$. thaliana plants before and after hydroponic treatment with various redox agents. As a consequence of excessive ROS production, site-specific amino acid modification, fragmentation of the peptide chain, aggregation of crosslinked reaction products, altered electric charge, and increased susceptibility of proteins to proteolysis occur. Tissues injured by oxidative stress generally contain increased concentrations of carbonylated proteins, which are a widely used marker of protein oxidation. Enhanced modification of proteins has been reported in plants under various stresses (Niu and Liao, 2016; Liu and He, 2017; Mittler, 2017).

\subsubsection{Reactive Oxygen Species-Induced Posttranslational Modifications}

Redox reactions on metabolic processes can alter a wide variety of downstream protein targets by influencing key regulators of distinct PTMs, such as phosphorylation, acetylation, and ubiquitination. Such modifications include components that control various metabolic rates, that is, AMP-activated protein kinase, 


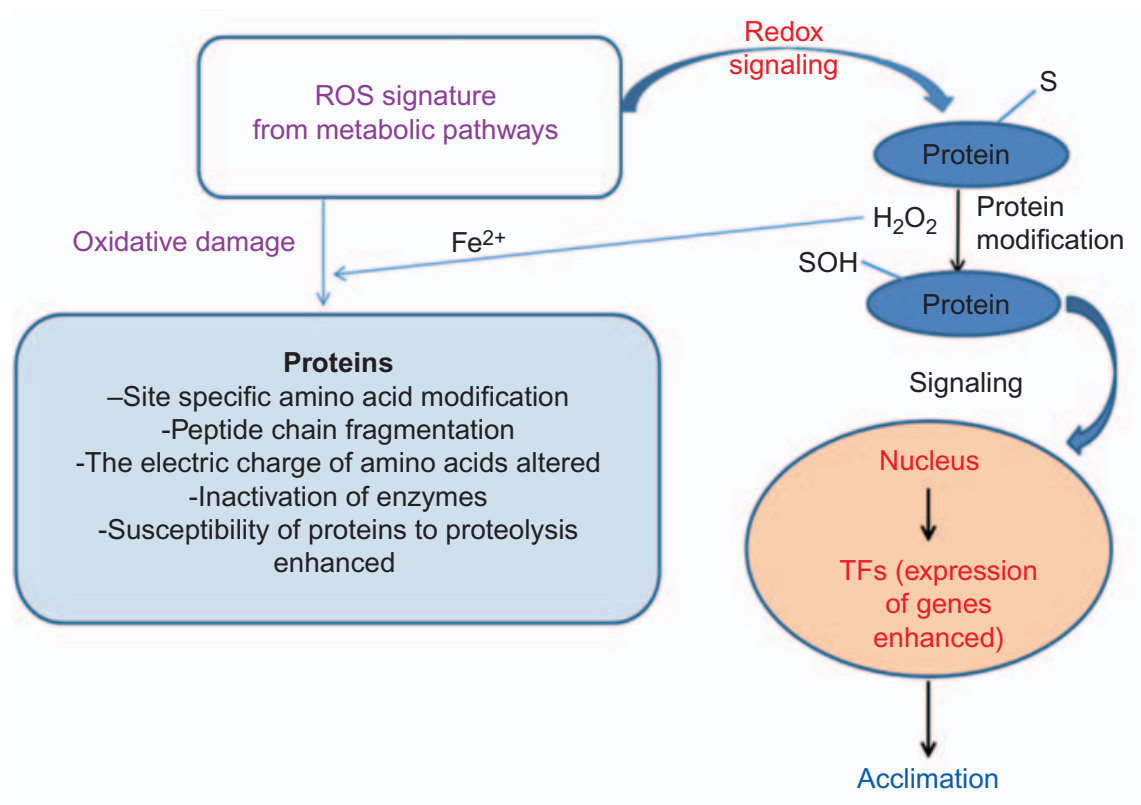

FIGURE 8.3 Oxidation of cysteine residues on proteins by $\mathrm{H}_{2} \mathrm{O}_{2}$, affecting their structure and function and triggering/regulating cellular signaling pathways (Mittler, 2017).

protein kinase $C$, adenylate kinase, and pyruvate kinase (Corcoran and Cotter, 2013). In particular redox modifications of cysteine (thiol) residues have been extensively studied and can result in reversible and irreversible modifications with effects on protein function (Fig. 8.3). Fortunately, a lot of developments have been made in the area of redox proteomics to enhance the determination of alterations to proteins under conditions of stress. At the same time the oxidized products can be important secondary signaling molecules, and in such cases damage and signaling are two sides of the same story, which can either coexist or cause severe imbalances (Fig. 8.3). The stress of ROS also causes modification of proteins in a variety of ways, that is, direct and indirect. In direct modifications, modulation of a protein's activity occurs through Snitrosylations, carbonylation, disulfide bond formation (sulfonylation), and glutathionylation. However, by indirect process, proteins can be modified by interaction of conjugation with byproducts of fatty acid peroxidation (Choudhury et al., 2017).

\subsubsection{S-Nitrosylation}

The effects of nitric oxide (NO) on signaling and wide metabolic pathways are recognized as Snitrosylation (or nitrosation) on key cysteine residues proteins (McDonagh, 2017). The formation of Snitrosylation is the process when the covalent binding of NO to thiol groups of Cys protein occurs through $\mathrm{ONOO}-$ and there are also reports of transfer of nitrosyl or transnitrosylation by the actions of proteins, such as thioredoxins (Benhar, 2015). S-nitrosylation on target proteins is considered an important mechanism for NO signaling transduction, posttranslational modification that can regulate the function of some proteins during stress; however as this PTM is reversible and highly labile PTM, it has been suggested as an intermediate in the formation of disulfide bonds (Wolhuter and Eaton, 2017). Camejo et al. (2013) showed that different enzymes involved in respiration, antioxidation, and photorespiration were Snitrosylated during salinity stress. In plants subjected to low temperatures, the main S-nitrosylated proteins were those related to $C$ metabolism (Puyaubert et al., 2014). S-Nitrosylation of proteins is essential for metabolic reprogramming that is necessary to keep homeostasis under stress conditions. S-Nitrosylation also induces changes in some TFs, which affect their binding to DNA, as well as inactivate $\mathrm{RBOH}$ (Yun et al., 2011). For example, S-nitrosylation can act as a negative regulator of MYB TFs, which are essential regulators of abiotic stress responses (Tavares et al., 2014).

\subsubsection{Protein Carbonylation}

Protein carbonylation is an irreversible PTM associated with the oxidation of residues, such as Arg, His, Lys, Pro, and Thr (Choudhury et al., 2017). Carbonylation of proteins can also be induced by indirect reactions of lipoperoxidation products with Cys and His residues of proteins (Madian and Regnier, 2010) and even with glycation/glycoxidation (for Lys) process. A broad range of carbonylation proteins in plants and other organisms have been studied, and various quantification techniques developed in recent years are extensively reviewed (Fedorova et al., 2014; Rogowska-Wrzesinska et al., 2014). Choudhury et al. (2017) highlighted that mitochondrial enzymes such as aconitase, pyruvate dehydrogenase, and glycine 
decarboxylase are sensitive to inactivation by oxidation and carbonylation, and these enzymes might be inhibited by an increase in ROS production that results in slowing down the Tricarboxylic acid cycle (TCA) cycle and consequently decreasing the energy status of the cell (Schwarzlander and Finkemeier, 2013; Camejo et al., 2015). However, some studies suggested that the pattern of (chloroplast) protein carbonylation in Arabidopsis is distinct from that in nonphotosynthetic eukaryotes, due to the mechanism that carbonylation first increases with age but drops abruptly prior to the vegetative-to-reproductive transition and does not coincide with senescence (Johansson et al., 2004). These findings propose that old leaves rid themselves of oxidized proteins prior to bolting. We speculate that this is due to the targeted removal of organellar content through a combination of chlorophagy, Rubiscocontaining bodies, and senescence-associated vacuoles (Wang and Blumwald, 2014; Van Wijk, 2015; Xie et al., 2015). There are multiple protein carbonylation studies investigated with various plant species mainly focusing on chloroplasts and mitochondria in leaves as well as seed biology and fruit ripening (Lounifi et al., 2013). Carbonylated proteins have been detected especially in cytosol and mitochondria (Smakowska et al., 2014), and chloroplasts are involved in various metabolic pathways, protein folding, and other processes. However, within these pathways, carbonylation is not evenly distributed, and specific proteins appear preferentially targeted for this PTM. It was also proposed that there is cross-talk between ROS-induced carbonylation and reactive nitrogen species-induced protein nitrosylation, particularly under biotic and abiotic stress (Lounifi et al., 2013).

\subsubsection{Sulhydryl Oxidations of Met and Cys (Sulfonylation)}

Sulfonylation is one of the main mechanisms that cause the oxidation of sulfhydryl groups by $\mathrm{H}_{2} \mathrm{O}_{2}$ generating sulfenic acid (R-SOH) (Fig. 8.3). This process can initiate to the formation of disulfide $(S-S)$ bonds between cysteine residues, which in turn results in conformational changes and protein/enzyme activity alteration. The "recovery" of a protein from this oxidized state is mainly mediated via thioredoxins (Trxs), peroxiredoxin (PRXs), and the glutathione (GSH) system in response to stress. The previous studies indicated that several enzymes of the Calvin cycle [Fru-1,6bisphosphatase (FBPase)] are regulated, reduced, and are active in the light whereas they are oxidized and inactive in dark conditions (Scheibe et al., 2005; Balsera et al., 2014; Dietz and Hell, 2015). Choudhury et al. (2017) reviewed that regulation via reduced Trx prevents a waste of energy by activation of enzymes such as FBPase and seduheptulose-bisphosphatase in the reductive cycle and a parallel inactivation of the Glc-6$\mathrm{P}$ dehydrogenase found in the oxidative cycle in the light. However, in the dark, Trx becomes oxidized and the opposite situation becomes predominant. The reduction state of $\operatorname{Trx}$ creates a conditional separation of metabolic fluxes within the same compartment.

In addition to the nonenzymatic oxidation of Cys, a small set of plant Cys oxidases (PCOs) is enzymatically oxidizing N-terminal Cys in the case of several ethylene response factor (ERF)-VII TFs, generating a sulfenic acid PTM (Weits et al., 2014). The PTM turns these TFs into a substrate for arginyl transferases, ultimately leading to polyubiquitination and proteasomal degradation (Van Dongen and Licausi, 2015). In contrast to Cys oxidation, another type of sufonylation is known for Met oxidation and its impact on plants. Nevertheless, a study in Arabidopsis identified and quantified hundreds of Met oxidation sites induced by $\mathrm{H}_{2} \mathrm{O}_{2}$ generated in situ in the peroxisomes through enhanced photorespiration and in a peroxisomal catalase mutant (Jacques et al., 2015). A family of Met sulfoxide reductase enzymes reduced the oxidation of Met by Trx, thus repairing these oxidized (damaged) proteins.

\subsubsection{Sulfur Glutathionylation}

Tripeptide glutathione ( $\gamma$-glutamylcysteinyln-glycine, GSH) is one of the crucial low molecular weight nonprotein thiols that plays an important role in intracellular defense against ROS-induced oxidative damage. It gains a physiological importance in buffering the GSSG/GSH pool as well as having additional regulatory functions against oxidative stress and maintaining thiol homeostasis (Zaffagnini et al., 2012a; Rouhier et al., 2015). Due to its reducing power, GSH plays an important role in diverse biological processes. GSH can form a disulfide bridge with available free thiol on a protein and forms a protein named S-glutathionylation. Several plant enzymes have been shown to undergo glutathionylation, in particular in mitochondria and chloroplasts but also in the cytosol (Zaffagnini et al., 2012b). The most extensive glutathionylation proteomics study in photosynthetic organisms was done for Chlamydomonas reinhardtii, showing that many metabolic pathways (e.g., nucleotides, thiamine, chlorophyll, fatty acid, and respiration) are targets for this PTM (Zaffagnini et al., 2012a). Protein glutathionylation can be regarded a posttranslational modification mechanism with significant effects on the various enzyme activities and TFs. However, even with lot of these recent findings, protein glutathionylation in plants still remains as one of the poorly studied aspects in abiotic stress response. 


\subsubsection{Acetylation}

Protein acetylation is a posttranslational regulatory mechanism that regulates gene expression under redox state. The previous studies revealed that protein acetylation occurs in two diverse forms: Nterminal acetylation and Lys acetylation. N-terminal acetylation is a nonreversible $\mathrm{N} \alpha$-terminal modification that results in the loss of a positive charge and is carried out by $\mathrm{N}$-terminal acetyl transferases (the NAT family). It emerges that these NATs can also acetylate the $\epsilon$-amine of Lys (Starheim et al., 2012). It was proved that $\mathrm{N}$-terminal acetylation is positively correlated to the levels of acetyl-CoA and involves cellular metabolic state to this PTM. The majority of proteins are $\mathrm{N}$-acetylated and it was proved by Ferrandez-Ayela et al. (2013) that a NAT-A loss-offunction in Arabidopsis mutant showed pleiotropic developmental and growth defects in Arabidopsis, however a loss of function of NAT-C illustrated reduced plant growth and photosynthetic capacity (Pesaresi et al., 2011). Recently, Dinh et al. (2015) identified a chloroplast NAT enzyme (AtNAA70) in Arabidopsis and showed N-a-acetylation when expressed in Escherichia coli, in particular for Met, Ala, Thr, and Ser. However, Lys acetylation is reversible $\varepsilon$-amino group modification of lysine $(\mathrm{K})$ by $\mathrm{K}$ acetyl transferases (KATs) and $\mathrm{K}$ deacetylases (KDACs) (Friso and Van Wijk, 2015). Histones are one of the target proteins of KATs and KDACs. Studies of crop acetylome were performed in rice, wheat, and soybeans (Nallamilli et al., 2014; SmithHammond et al., 2014; Zhang et al., 2016). It was highlighted that a wide range of cellular processes were affected by $\mathrm{K}$ acetylation from metabolic processes, signal transduction, RNA processing, protein translation, and stability. Proteins related to cell death were included among 44 proteins identified as acetylated proteins in rice. This was followed by a study in wheat, another important cereal crop (Zhang et al., 2016). As described above, protein acetylation controls the metabolic adaptations via modifying nonhistone metabolic enzymes. Furthermore, it participates in the shift of energy production via changing gene expression.

PTMs is the dynamic nature of the modifications in cell signaling and potential cross-talk between redox and nonredox dependent PTMs that regulate protein activity. Including an increased number of potential PTMs combined with targeted postanalysis would increase sensitivity and provide a comprehensive overview on the role of ROS-induced protein modifications and their role in stress acclimation in cellular signaling (Friso and van Wink, 2015; McDonagh, 2017; Choudhury et al., 2017).

\subsection{ROLE OF ANTIOXIDANTS AND ITS SIGNALING IN ABIOTIC STRESS}

Plants have a variety of constitutively expressed antioxidant defense mechanisms to scavenge the ROS generated during abiotic stress. The importance of the cellular antioxidant machinery in protection against various stresses is emphasized by many researchers (Dalton et al., 1999, Tuteja, 2007, 2009). Plants produce antioxidants, which can scavenge ROS. Antioxidants are of two types: enzymatic and nonenzymatic antioxidants. Enzymatic antioxidants include catalase (CAT), SOD, peroxidase (POX), APX, monodehydroascorbate reductase (MDHAR or MDAR), dehydroascorbate reductase (DHAR or DAR), and glutathione reductase (GR) (Ahmad et al., 2008, 2010a,b, 2011). The nonenzymatic antioxidants are glutathione (GSH), ascorbate (AsA), carotenoids, tocopherols, flavones, and anthocyanins (Gupta et al., 2005). These enzymes are located in different plant cells and work together to detoxify ROS. Enzymatic antioxidants containing SOD, CAT, APX, POX, GR, and MDAR decrease the levels of $\mathrm{O}_{2}$ and $\mathrm{H}_{2} \mathrm{O}_{2}$ in plants (Ahmad et al., 2013; Rasool et al., 2013). Under stress conditions, SOD forms the first line of defense against ROS-induced damages. The SOD removes $\mathrm{O}^{\bullet-}$ by catalyzing its dismutation into $\mathrm{O}_{2}$ and $\mathrm{H}_{2} \mathrm{O}_{2}$. APX reduces $\mathrm{H}_{2} \mathrm{O}_{2}$ to $\mathrm{H}_{2} \mathrm{O}$ and (dehydroascorbate) DHA, using AA as a reducing agent. DHAR reduces dehydroascorbate (DHA) to AA by using reduced glutathione (GSH) as an electron donor and GSH is converted to its oxidized form (GSSG) (Eltayeb

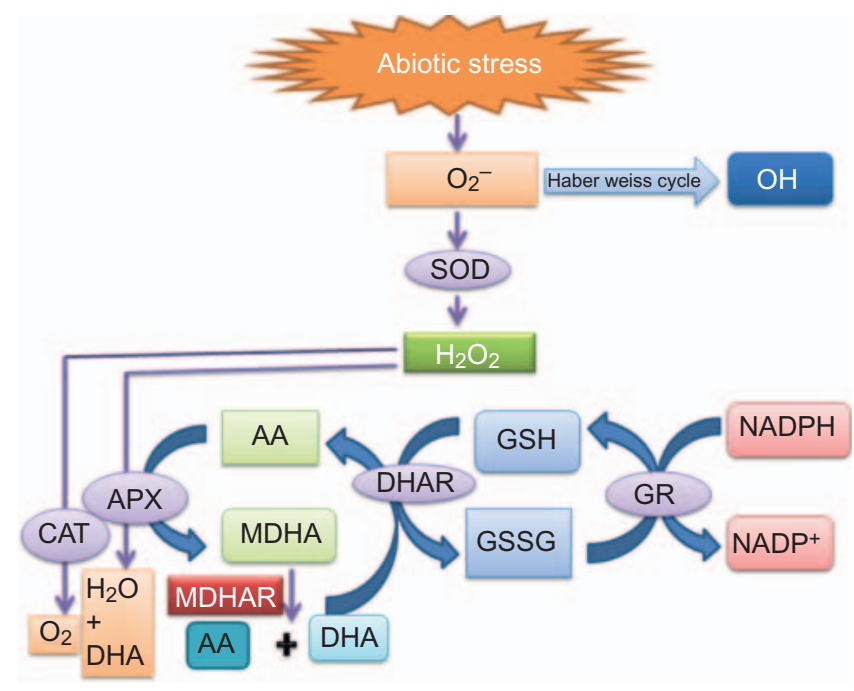

FIGURE 8.4 Antioxidant defense mechanism. Source: Adapted from Gill, S.S., Tuteja, N., 2010. Reactive oxygen species and antioxidant machinery in abiotic stress tolerance in crop plants. Plant Physiol. Biochem. 48, 909-930. doi:10.1016/j.plaphy.2010.08.016 and Choudhury, S., Panda, P., Sahoo, L., Kumar, S., Panda, S.K., 2013. Reactive oxygen species signalling in plants under abiotic stress. Plant Signal Behav. (8)4, e23681. 
et al., 2007). The organellar redox state is maintained by different enzymatic antioxidants like GR and MDHAR. MDHAR regenerates AA from the shortlived monodehydroascorbate (MDHA) and ultimately replenishes the cellular AA pool. GR reduces GSSG to GSH by using NADPH as a reducing agent (Fig. 8.4). The role of various antioxidants in plants during abiotic stress is given in Table 8.1.

TABLE 8.1 Antioxidant Defense Mechanism in Various Plant Species During Abiotic Stress

\begin{tabular}{|c|c|c|c|c|}
\hline Plant & Stress & Stress imposition & Antioxidant activity & References \\
\hline $\begin{array}{l}\text { Solanum } \\
\text { lycopersicum }\end{array}$ & Low temperature & $\begin{array}{l}15^{\circ} \mathrm{C} \text {-Day } \\
8^{\circ} \mathrm{C} \text {-Night }\end{array}$ & $\begin{array}{l}\text { Enhanced activities of SOD, APX, } \\
\text { MDHAR, DHAR }\end{array}$ & Liu et al. (2018) \\
\hline $\begin{array}{l}\text { Passiflora } \\
\text { edulis }\end{array}$ & Aluminum & $\begin{array}{l}0.2 \mathrm{mM} \mathrm{Al}^{3+} \\
0.2 \mathrm{mM} \mathrm{Al}^{3+} \\
\text { For } 10 \text { days }\end{array}$ & SOD activity increased & Preeti et al. (2018) \\
\hline Zea mays & Drought & $\begin{array}{l}80 \% \mathrm{FC} \\
60 \% \mathrm{FC} \\
40 \% \mathrm{FC}\end{array}$ & $\begin{array}{l}\text { SOD, CAT, and POX activity decreased } \\
\text { with severity of drought }\end{array}$ & Anjum et al. (2017) \\
\hline $\begin{array}{l}\text { Nicotiana } \\
\text { tobacum }\end{array}$ & $\begin{array}{l}\mathrm{H}_{2} \mathrm{O}_{2} \text {, salt, and } \\
\text { drought }\end{array}$ & $\begin{array}{l}1 \% \mathrm{H}_{2} \mathrm{O}_{2} \\
300 \mathrm{mM} \mathrm{NaCl} \\
30 \% \text { PEG }\end{array}$ & Overexpression of DHAR & Chang et al. (2017) \\
\hline $\begin{array}{l}\text { Citrullus } \\
\text { lanatus }\end{array}$ & Drought & $30 \%$ FC for 12 days & SOD, CAT, APX, GR, and MDHAR & Mo et al. (2016) \\
\hline $\begin{array}{l}\text { Cicer } \\
\text { arietinum }\end{array}$ & Salt & $\begin{array}{l}50-100 \mathrm{mM} \mathrm{NaCl} 25 \text {-day-old plant to } \\
70 \text {-day-old plant }\end{array}$ & SOD, APX, CAT, GR activities increased & Ahmad et al. (2016) \\
\hline $\begin{array}{l}\text { Lycopersicon } \\
\text { esculentum }\end{array}$ & Low temperature & $\begin{array}{l}10^{\circ} \mathrm{C} \text {-Day } \\
3^{\circ} \mathrm{C} \text {-Night } \\
12^{\circ} \mathrm{C} \text {-Day } \\
7^{\circ} \mathrm{C} \text {-Night } \\
20^{\circ} \mathrm{C} \text {-Day } \\
14^{\circ} \mathrm{C} \text {-Night } \\
25^{\circ} \mathrm{C} \text {-Day } \\
18^{\circ} \mathrm{C} \text {-Night }\end{array}$ & $\begin{array}{l}\text { SOD, CAT activity increased. Proline } \\
\text { content also increased in leaves with } \\
\text { decreasing temperature }\end{array}$ & Khan et al. (2015) \\
\hline $\begin{array}{l}\text { Triticium } \\
\text { aestivum }\end{array}$ & Osmotic & PEG-6000 for 5 days & SOD, GR, APX activity increased & Naderi et al. (2014) \\
\hline $\begin{array}{l}\text { Limonium } \\
\text { sinense }\end{array}$ & Salt & $\begin{array}{l}50 \mathrm{~mL} \text { of } 500 \mathrm{mM} / \mathrm{L} \mathrm{NaCl} \text { solution for } 7 \\
\text { days }\end{array}$ & $\begin{array}{l}\text { Increased activity of SOD, POX, CAT. } \\
\text { MDA content increased in seedlings }\end{array}$ & $\begin{array}{l}\text { Zhang et al. (2014a, } \\
\text { b) }\end{array}$ \\
\hline $\begin{array}{l}\text { Triticum } \\
\text { aestivum }\end{array}$ & Cold & $\begin{array}{l}5^{\circ} \mathrm{C} \text {-Day } \\
2^{\circ} \mathrm{C} \text {-Night for } 3 \text { days }\end{array}$ & Increased activity of SOD, POX, GR, APX & Turk et al. (2014) \\
\hline $\begin{array}{l}\text { Eichhornia } \\
\text { crassipes }\end{array}$ & Lead & $\begin{array}{l}\text { Seedlings were treated with different } \\
\text { concentrations of } \mathrm{Pb}\left(\mathrm{NO}_{3}\right)_{2}(100,200 \text {, } \\
400,600,800,1000 \mathrm{mg} / \mathrm{L}) \text { for } 10 \text { days }\end{array}$ & $\begin{array}{l}\text { Increased activity of SOD, APX, POX. } \\
\text { MDA content increased with increasing } \\
\text { concentration of lead }\end{array}$ & Malar et al. (2014) \\
\hline $\begin{array}{l}\text { Vigna } \\
\text { radiate }\end{array}$ & Lead and salt & $\begin{array}{l}\text { Lead chloride } 70 \mathrm{mM} \\
\text { Lead nitrate } 150 \mathrm{mM} \\
\mathrm{NaCl} 225 \mathrm{mM}\end{array}$ & SOD, APX, GPX, GR activity increased & Siddiqui (2013) \\
\hline Oryza sativa & Copper & $8 \mu \mathrm{M}$ copper for 3 days & Upregulation POX, APX, and DHAR & Song et al. (2013) \\
\hline
\end{tabular}


TABLE 8.1 (Continued)

\begin{tabular}{|c|c|c|c|c|}
\hline Plant & Stress & Stress imposition & Antioxidant activity & References \\
\hline Canna edulis & Drought & Drought stress for 35 days & $\begin{array}{l}\text { MDA content increased, SOD, POX, CAT } \\
\text { activities first increased and then } \\
\text { decreased }\end{array}$ & Zhang et al. (2013) \\
\hline $\begin{array}{l}\text { Solanum } \\
\text { tuberosum }\end{array}$ & Drought & $\begin{array}{l}\text { Drought conditions maintained for } 2 \\
\text { weeks }\end{array}$ & Increased activity of POX and SOD & $\begin{array}{l}\text { Boguszewska et al. } \\
\text { (2010) }\end{array}$ \\
\hline $\begin{array}{l}\text { Cassia } \\
\text { auriculata }\end{array}$ & UV-B & $\begin{array}{l}50 \text { and } 100 \mathrm{~min} \text { exposure to UV-B } \\
\text { source }\end{array}$ & Enhanced SOD, CAT activity & Agarwal (2007) \\
\hline T. aestivum & Cadmium & $100 \mathrm{mg} \mathrm{Cd} / \mathrm{kg}$ soil & Increased GR activity & Khan et al. (2007) \\
\hline
\end{tabular}

\subsection{CONCLUSION}

Various abiotic stresses lead to the overproduction of ROS in plants, which are highly reactive and result in oxidative stress. These species affect cell membrane properties and cause oxidative damage to nucleic acids, lipids, and proteins and make them nonfunctional. The plant cell and its organelles like chloroplast, mitochondria, and peroxisomes have antioxidant defense systems to overcome ROS-induced oxidative stress. The antioxidant defense mechanisms include enzymatic antioxidants and nonenzymatic antioxidants. ROS also acts as a signaling molecule and has capacity to regulate the downstream signaling pathway components and to impart a specific response toward a particular stress. Persistent research is required to understand the mechanisms regulating ROS signaling pathways and their interplay during abiotic stresses.

\section{References}

Achard, P., Cheng, H., De Grauwe, L., Decat, J., Schoutteten, H., Moritz, T., et al., 2006. Integration of plant responses to environmentally activated phytohormonal signals. Science 311, 91-94.

Agarwal, S., 2007. Increased antioxidant activity in Cassia seedlings under UV-B radiation. Biol. Plant. 51, 157-160.

Ahmad, P., Sarwat, M., Sharma, S., 2008. Reactive oxygen species, antioxidants and signalling in plants. J. Plant. Biol. 167-173.

Ahmad, P., Jaleel, C.A., Salem, M.A., Nabi, G., Sharma, S., 2010a. Roles of enzymatic and non-enzymatic antioxidants in plants during abiotic stress. Crit. Rev. Biotechnol. 30, 161-175.

Ahmad, P., Umar, S., Sharma, S., 2010b. Mechanism of free radical scavenging and role of phytohormones during abiotic stress in plants. In: Ashraf, M., Ozturk, M., Ahmad, M.S.A. (Eds.), Plant Adaptation and Phytoremediation. Springer, pp. 99-108.

Ahmad, P., Nabi, G., Jaleel, C.A., Umar, S., 2011. Free radical production, oxidative damage and antioxidant defense mechanisms in plants under abiotic stress. In: Ahmad, P., Umar, S., (Eds.), Oxidative Stress: Role of Antioxidants in Plants, pp. 19-53.

Ahmad, P., Ashraf, M., Azooz, M.M., Rasool, S., Akram, N.A., 2013. Potassium starvation induced oxidative stress and antioxidant defense responses in Brassica juncea. J. Plant Interact . Available from: https:/ / doi.org/10.1080/17429145.2012.747629.
Ahmad, P., Abdel Latef, A.A., Hashem, A., Abd_Allah, E.F., Gucel, S., Tran, L.S.P., 2016. Nitric oxide mitigates salt stress by regulating levels of osmolytes and antioxidant enzymes in chickpea. Front. Plant Sci. 7, 347.

Anjum, S.A., Ashraf, U., Tanveer, M., Khan, I., Hussain, S., Shahzad, B., et al., 2017. Drought induced changes in growth, osmolyte accumulation and antioxidant metabolism of three maize hybrids. Front Plant Sci. 8, 69. Available from: https://doi.org/10.3389/fpls.2017.00069.

Apel, K., Hirt, H., 2004. Reactive oxygen species: metabolism, oxidative stress, and signal transduction. Annu. Rev. Plant Biol. 55, 373-399.

Asai, T., Tena, G., Plotnikova, J., Willmann, M.R., Chiu, W.L., Gomez-Gomez, L., et al., 2002. MAP kinase signalling cascade in Arabidopsis innateimmunity. Nature 415, 977-983. Available from: https://doi.org/10.1038/415977a.

Asgher, M., Khan, I.R., Anjum, N.A., Khan, N.A., 2015. Minimising toxicity of cadmium in plants-role of plant growth regulators. Protoplasma 252 (2), 399-413.

Ashraf, M., 2009. Biotechnological approach of improving plant salt tolerance using antioxidants as markers. Bio. Adv. 27, 84-93.

Balsera, M., Uberegui, E., Schurmann, P., Buchanan, B.B., 2014. Evolutionary development of redox regulation in chloroplasts. Antioxid. Redox. Signal 21, 1327-1355.

Benhar, M., 2015. Nitric oxide and the thioredoxin system: a complex interplay in redox regulation. Biochim. Biophy. Acta 1850, 2476-2484.

Blokhina, O., Virolainen, E.O., Fagerstedt, K.V., 2003. Antioxidants, oxidative damage and oxygen deprivation stress: a review. Ann. Bot. 91, 179-194.

Boguszewska, D., Grudkowska, M., Zagdańska, B., 2010. ) Droughtresponsive antioxidant enzymes in Potato (Solanum tuberosum L.). Potato Res. 53, 373-382.

Bright, J., Desikan, R., Hancock, J.T., Weir, I.S., Neill, S.J., 2006. ABAinduced $\mathrm{NO}$ generation and stomatal closure in Arabidopsis are dependent on $\mathrm{H}_{2} \mathrm{O}_{2}$ synthesis. Plant J. 45, 113-122.

Britt, A.B., 1999. Molecular genetics of DNA repair in higher plants. Trends Plant. Sci 4, 20-25.

Buettner, G.R., 1993. The pecking order of free radicals and antioxidants: lipid peroxidation, $\alpha$-tocopherol, and ascorbate. Arch. Biochim. Biophys. 300 (2), 535-543.

Cabiscol, E., Piulats, E., Echave, P., Herrero, E., Ros, J., 2000. Oxidative stress promotes specific protein damage in Saccharomyces cerevisiae. J. Biol. Chem. 275 (35), 27393-27398.

Camejo, D., Romero-Puertas Mdel, C., Rodriguez-Serrano, M., Sandalio, L.M., Lazaro, J.J., 2013. Salinity-induced changes in S-nitrosylation of pea mitochondrial proteins. J. Proteomics 79, 87-99.

Camejo, D., Jimenez, A., Palma, J.M., Sevilla, F., 2015. Proteomic identification of mitochondrial carbonylated proteins in two maturation stages of pepper fruits. Proteomics 15, 2634-2642. 
Chan, K.X., Phua, S.Y., Crisp, P., McQuinn, R., Pogson, B.J., 2016. Learning the languages of the chloroplast: retrograde signalling and beyond. Annu. Rev. Plant Biol. 67, 25-53.

Chang, L., Sun, H., Yang, H., Wang, X., Su, Z., Chen, F., et al., 2017. Over-expression of dehydroascorbate reductase enhances oxidative stress tolerance in tobacco. Electronic J. Biotechnol. 25, 1-8.

Choudhury, F.K., Rivero, R.M., Blumwald, E., Mittler, R., 2017. Reactive oxygen species, abiotic stress and stress combination. Plant J. 90, 856-867. Available from: https://doi.org/10.1111/tpj.13299.

Choudhury, S., Panda, P., Sahoo, L., Kumar, S., Panda, S.K., 2013. Reactive oxygen species signalling in plants under abiotic stress. Plant Signal Behav 8 (4), e23681.

Colebrook, E.H., Thomas, S.G., Phillips, A.L., Hedden, P., 2014. The role of gibberellin signalling in plant responses to abiotic stress. J. Exp. Biol. 217, 67-75.

Cooke, M.S., Evans, M.D., Dizdaroglu, M., Lunec, J., 2003. Oxidative DNA damage: mechanisms, mutation, and disease. FASEB J. 17, 1195-1214.

Corcoran, A., Cotter, T.G., 2013. Redox regulation of protein kinases. FEBS J 280, 1944-1965. Available from: https://doi.org/10.1111/ febs.12224.

Cutler, S.R., Rodriguez, P.L., Finkelstein, R.R., Abrams, S.R., 2010. Abscisic acid:emergence of a core signalling network. Annu. Rev. Plant Bio. 61, 651-679. Available from: https://doi.org/10.1146/ annurev-arplant-042809-112122.

Dalton, T.H., Shertzer, A., Puga, A., 1999. Regulation of gene expression by reactive oxygen. Annual Review Pharmacol. Toxicol. 39, 67-101.

Damanik, R.I., Maziah, M., Ismail, M.R., Ahmad, S., Zain, A., 2010. Responses of the antioxidative enzymes in Malaysian rice (Oryzasativa L.) cultivars under submergence condition. Acta Physiol. Plant32 739-747. Available from: https://doi.org/ $10.1007 /$ s11738-009-0456.

Davies, K.J.A., 2000. Oxidative stress, antioxidant defenses and damage removal, repair, and replacement systems. IUBMB Life. 50 (45), 279-289.

Davletova, S., Schlauch, K., Coutu, J., Mittler, R., 2005. The zincfinger protein ZAT12 plays a central role in reactive oxygen and abiotic stress signalling in Arabidopsis. Plant Physiol. 139, 847-856.

Dietz, K.J., 2014. Redox regulation of transcription factors in plant stress acclimation and development. Antioxid. Redox Signal 21, 1356-1372. Available from: https://doi.org/10.1089/ ars.2013.5672.

Dietz, K.J., Hell, R., 2015. Thiol switches in redox regulation of chloroplasts: balancing redox state, metabolism and oxidative stress. Biol. Chem. 396, 483-494.

Dinh, T.V., Bienvenut, W.V., Linster, E., Feldman-Salit, A., Jung, V. A., et al., 2015. Molecular identification and functional characterization of the first Na-acetyltransferase in plastids by global acetylome profiling. Proteomics 15, 2426-2435. Available from: https://doi.org/10.1016/j.bbagen.2015.09.010.

Dubiella, U., Seybold, H., Durian, G., Komander, E., Lassig, R., Witte, C.P., et al., 2013. Calcium-dependent protein kinase/ NADPH oxidase activation circuit is required for rapid defense signal propagation. Proc. Natl Acad. Sci. USA 110, 8744-8749.

Eltayeb, A.E., Kawano, N., Badawi, G.H., Kaminaka, H., Sanekata, T., Shibahara, T., et al., 2007. Overexpression of monodehydroascorbate reductase in transgenic tobacco confers enhanced tolerance to ozone, salt and polyethylene glycol stresses. Planta. 225, $1255-1264$.

Farnese, F.S., Menezes-Silva, P.E., Gusman, G.S., Oliveira, J.A., 2016. When bad guys become good ones: the key role of reactive oxygen species and nitric oxide in the plant responses to abiotic stress. Front. Plant Sci. 7, 471.
Fedorova, M., Bollineni, R.C., Hoffmann, R., 2014. Protein carbonylation as a major hallmark of oxidative damage: update of analytical strategies. Mass Spectrom. Rev 33, 79-97. Available from: https://doi.org/10.1002/mas.21381.

Ferrandez-Ayela, A., Micol-Ponce, R., Sánchez-García, A.B., AlonsoPeral, M.M., Micol, J.L., Ponce, M.R., 2013. Mutation of an Arabidopsis NatB N-alpha-terminal acetylation complex component causes pleiotropic developmental defects. PLoS One 8, e80697.

Foreman, J., Demidchik, V., Bothwell, J.H., Mylona, P., Miedema, H., et al., 2003. Reactive oxygen species produced by NADPH oxidase regulate plant cell growth. Nature 422, 442-446.

Foyer, C.H., Noctor, G., 2005. Redox homeostasis and antioxidant signalling: a metabolic interface between stress perception and physiological responses. Plant Cell 17, 1866-1875.

Foyer, C.H., Noctor, G.D., 2009. Redox regulation in photosynthetic organisms: signalling, acclimation, and practical implications. Antiox. Redox. Signal 11, 861-905.

Friso, G., Van Wijk, K.J., 2015. Posttranslational protein modifications in plant metabolism. Plant Physiol. 169, 1469-1487.

Gambarova, N.G., Gins, M.S., 2008. Characteristics of oxidative stress of plants with C3 and C4 photosynthesis during salinization. Russ. Agric. Sci. 34, 77-80.

Gapper, C., Dolan, L., 2006. Control of plant development by reactive oxygen species. Plant Physiol. 141, 341-345.

Gautam, V., Kaur, R., Kohli, S.K., Verma, V., Kaur, P., et al., 2017. ROS compartmentalization in plant cells under abiotic stress condition In: Khan, M.I.R., Khan, N.A. (Eds.), Reactive Oxygen Species and Antioxidant Systems in Plants: Role and Regulation under Abiotic Stress, pp. 89-114. <https://doi.org/10.1007/978981-10-5254-5-4>.

Gill, S.S., Tuteja, N., 2010. Reactive oxygen species and antioxidant machinery in abiotic stress tolerance in crop plants. Plant Physiol. Biochem. 48, 909-930. Available from: https://doi.org/10.1016/j. plaphy.2010.08.016.

Gilroy, S., Suzuki, N., Miller, G., Choi, W.G., Toyota, M., Devireddy, A.R., et al., 2014. A tidal wave of signals: calcium and ROS at the forefront of rapid systemic signaling. Trends Plant Sci. 19, $623-630$.

Gilroy, S., Bialasek, M., Suzuki, N., Gorecka, M., Devireddy, A., et al., 2016. ROS, calcium and electric signals: key mediators of rapid systemic signalling in plants. Plant Physiol. 171, 1606-1615.

Grene, R., 2002. Oxidative stress and acclimation mechanisms in plants. In: Somerville, C.R., Myerowitz, E.M. (Eds.), The Arabidopsis Book. American Society of Plant Biologists, Rockville, MD, pp. 1-19.

Gulyas, Z., Simon-Sarkadi, L., Badics, E., Novak, A., Mednyanszky, Z., et al., 2017. Redox regulation of free amino acid levels in Arabidopsis thaliana. Physiol Plant 159, 264-276. Available from: https:// doi.org/10.1111/ppl.12510.

Gupta, K.J., Stoimenova, M., Kaiser, W.M., 2005. In higher plants, only root mitochondria, but not leaf mitochondria reduce nitrite to NO, in vitro and in situ. J. Exp. Bot. 56, 2601-2609.

Gustavsson, N., Kokke, B.P., Harndahl, U., Silow, M., Bechtold, U., Poghosyan, Z., et al., 2002. A peptide methionine sulfoxide reductase highly expressed in photosynthetic tissue in Arabidopsis thaliana can protect the chaperone-like activity of a chloroplastlocalized small heat shock protein. Plant J. 29, 545-553.

Halliwell, B., Gutteridge, J.M.C., 1999. Free radicals in biology and medicine. In: Halliwell, B., Gutteridge, J.M.C. (Eds.), Free Radicals in Biology and Medicine, third ed. Oxford University Press, Oxford, pp. 1-25.

Ho, H.L., 2015. Functional roles of plant protein kinases in signal transduction pathways during abiotic and biotic stress. J Biodivers Biopros Dev. 2 (2), 147. Available from: https://doi. org/10.4172/2376-0214.1000147. 
Hu, X., Jiang, M., Zhang, J., Zhang, A., Lin, F., Tan, M., 2007. Calcium calmodulin is required for abscisic acid-induced antioxidant defense and functions both upstream and downstream of $\mathrm{H}_{2} \mathrm{O}_{2}$ production in leaves of maize (Zea mays) plants. New Phytol. 173, 27-38. Available from: https://doi.org/10.1111/ j.1469-8137.2006.01888.

Huang, J., Sun, S., Xu, D., Lan, H., Sun, H., Wang, Z., et al., 2012. A TFIIIA type zinc finger protein confers multiple abiotic stress tolerances in transgenic rice (Oryzasativa L.). Plant Mol. Biol. 80, 337-350. Available from: https://doi.org/10.1007/s11103-012-9955-5.

Jacques, S., Ghesquière, B., De Bock, P.J., Demol, H., Wahni, K., Willems, P., et al., 2015. Protein methionine sulfoxide dynamics in Arabidopsis thaliana under oxidative stress. Mol Cell Proteomics $14,1217-1229$.

Jalmi, S.K., Sinha, A.K., 2015. ROS mediated MAPK signaling in abiotic and biotic stress-striking similarities and differences. Front. Plant Sci. 6, 769 .

Jaspers, P., Kandasjarvi, J., 2010. Reactive oxygen species in abiotic stress signalling. Physiol. Plant 138, 405-413.

Jiang, M., Zhang, J., 2002. Involvement of plasma-membrane NADPH oxidase in abscisic acid-and water stress-induced antioxidant defense in leaves of maize seedlings. Planta 215, 1022-1030. Available from: https:/ / doi.org/10.1007/s00425-002-0829-y.

Jiang, M., Zhang, J., 2003. Cross talk between calcium and reactive oxygen species originated from NADPH oxidase in abscisic acid - induced antioxidant defence in leaves of maize seedlings. Plant Cell Environ. 26, 929-939. Available from: https://doi.org/ 10.1046/j.13653040.2003.01025.x.

Johansson, E., Olsson, O., Nystrom, T., 2004. Progression and specificity of protein oxidation in the life cycle of Arabidopsis thaliana. J. Biol. Chem. 279, 22204-22208.

Joudoi, T., Shichiri, Y., Kamizono, N., Akaike, T., Sawa, T., et al., 2013. Nitrated cyclic GMP modulates guard cell signalling in Arabidopsis. Plant Cell. 25, 558-571.

Khan, M.I.R., Asgher, M., Khan, N.A., 2013. Rising temperature in the changing environment: a serious threat to plants. Climate Change Environ. Sustain. 1, 25-36. Available from: https://doi. org /10.5958/j.2320-6411.1.1.004.

Khan, M.I.R., Asgher, M., Khan, N.A., 2014. Alleviation of saltinduced photosynthesis and growth inhibition by salicylic acid involves glycinebetaine and ethylene in mungbean (Vigna radiata L.). Plant Physiol. Biochem. 80, 67-74. Available from: https:// doi.org/10.1016/j.plaphy.2014.03.026.

Khan, M.I.R., Fatma, M., Per, S.T., Anjum, N.A., 2015. Salicylic acidinduced abiotic stress tolerance and underlying mechanisms in plants. Front. Plant Sci. 6, 462. Available from: https://doi.org/ 10.3389/fpls.2015.00462.

Khan, M.I.R., Iqbal, N., Masood, A., Mobin, M., Anjum, N.A., Khan, N.A., 2016. Modulation and significance of nitrogen and sulfur metabolism in cadmium challenged plants. Plant Growth Regul. 78 (1), 1-11.

Khan, M.L.R., Khan, N.A. (Eds.), 2017. Reactive Oxygen Species and Antioxidant Systems in Plants: Role and Regulation Under Abiotic Stress. Springer, New York.

Khan, N.A., Samiullah, S.S., Nazar, R., 2007. Activities of antioxidative enzymes, sulphur assimilation, photosynthetic activity and growth of wheat (Triticum aestivum) cultivars differing in yield potential under cadmium stress. J. Agro. Crop. Sci. 193, 435-444.

Khan, N.A., Nazar, R., Iqbal, N., Anjum, N.A., 2012. Phytohormones and Abiotic Stress Tolerance in Plants. Springer, Berlin. Available from: http:/ / doi.org/10.1007/978-3-642-25829-9.

Khan, T.A., Fariduddin, Q., Yusuf, M., 2015. Lycopersicon esculentum under low temperature stress: an approach toward enhanced antioxidants and yield. Environ. Sci. Pollut. Res. Int. 22 (18), 78-88. Available from: https://doi.org/10.1007/s11356-015-4658-5. 141.
Koyro, H.W., Ahmad, P., Geissler, N., 2012. Abiotic stress responses in plants: an overview. In: Ahmad, P., Prasad, M.N.V. (Eds.), Environmental Adaptations and Stress Tolerance of Plants in the Era of Climate Change, pp. 1-28.

Liszkay, A., Van der Zalm, E., Schopfer, P., 2004. Production of reactive oxygen intermediates $\mathrm{O}_{2}{ }^{2}, \mathrm{H}_{2} \mathrm{O}_{2}$, and $\mathrm{OH}$ by maize roots and their role in wall loosening and elongation growth. Plant Physiol. 136, 3114-3123.

Liu, T., Hu, X., Zhang, J., Zhang, J., Du, Q., et al., 2018. $\mathrm{H}_{2} \mathrm{O}_{2}$ mediates ALA-induced glutathione and ascorbate accumulation in the perception and resistance to oxidative stress in Solanum lycopersicum at low temperatures. BMC Plant Biol 18, 34. Available from: https://doi.org/10.1186/s12870-018-1254-0.

Liu, Y., He, C., 2017. A review of redox signalling and the control of MAP kinase pathway in plants. Redox. Biol. 11, 192-204.

Lounifi, I., Arc, E., Molassiotis, A., Job, D., Rajjou, L., Tanou, G., 2013. Interplay between protein carbonylation and nitrosylation in plants. Proteomics 13, 568-578.

Madian, A.G., Regnier, F.E., 2010. Proteomic identification of carbonylated proteins and their oxidation sites. J. Proteome Res. 9, 3766-3780.

Malar, S., Vikram, S.S., Favas, P.J.C., Perumal, V., 2014. Lead heavy metal toxicity induced changes ongrowth and antioxidative enzymes level in water hyacinths [Eichhornia crassipes (Mart.)]. Bot. Stud. 55, 54-65.

McDonagh, B., 2017. Detection of ROS induced proteomic signatures by mass spectrometry. Front Physiol. 8, 470. Available from: https://doi.org/10.3389/fphys.2017.00470.

Mignolet-Spruyt, L., Xu, E., Idanheimo, N., Hoeberichts, F.A., Muhlenbock, P., Brosche, M., et al., 2016. Spreading the news: subcellular and organellar reactive oxygen species production and signalling. J. Exp. Bot. 67, 3831-3844.

Miki, H., Funaro, Y.J., 2012. Regulation of intracellular signalling through cysteine oxidation by reactive oxygen species. Biochim 151 (3), 255-261. Available from: https://doi.org/10.1093/JB/ mvs006.

Miller, G., Schlauch, K., Tam, R., Cortes, D., Torres, M.A., Shulaev, V., et al., 2009. The plant NADPH oxidase RBOHD mediates rapid systemic signaling in response to diverse stimuli. Sci. Signal. 2, ra45.

Miller, G., Suzuki, N., Ciftci-Yilmaz, S., Mittler, R., 2010. Reactive oxygen species homeostasis and signalling during drought and salinity stresses. Plant Cell Environ. 33, 453-467.

Misra, A.N., Misra, M., Singh, R., 2010. Nitric oxide: a ubiquitous signalling molecule with diverse role in plants. Afric. J. Plant Sci. 5, $57-74$.

Mittler, R., 2002. Oxidative stress, antioxidants and stress tolerance. Trends Plant Sci. 7, 405-410.

Mittler, R., 2017. ROS are good. Trends Plant Sci. 22, 11-19. Available from: https://doi.org/10.1016/j.tplants.2016.08.002.

Mittler, R., Vanderauwera, S., Gollery, M., Van, B.F., 2004. Reactive oxygen gene network of plants. Trends Plant Sci. 9, 490-498.

Mittler, R., Vanderauwera, S., Suzuki, N., Miller, G., Tognetti, V.B., Vandepoele, K., et al., 2011. ROS signaling: the new wave? Trends Plant Sci. 16, 300-309.

Mo, Y., Wang, Y., Yang, R., Zheng, J., Liu, C., Li, H., et al., 2016. Regulation of plant growth, photosynthesis, antioxidation and osmosis by an arbuscular mycorrhizal fungus in watermelon seedlings under well-watered and drought conditions. Front Plant Sci. 7, 644. Available from: https://doi.org/10.3389/ fpls.2016.00644.

Möller, I.M., 2001. Plant mitochondria and oxidative stress: electron transport NADPH turnover, and metabolism of reactive oxygen species. Annu. Rev. Plant Physiol. Mol. Biol. 52, 561-591.

Moller, I.M., Kristensen, B.K., 2004. Protein oxidation in plant mitochondria as a stress indicator. Photochem. Photobiol. Sci. 3, 730-735. 
Moller, I.M., Jensen, P.E., Hansson, A., 2007. Oxidative modifications to cellular components in plants. Annu. Rev. Plant Biol. 58, 459-481. Available from: https://doi.org/10.1146/annurev. arplant.58.032806.103946.

Moulton, P., Martin, H., Ainger, A., Cross, A., Hoare, C., Doel, J., et al., 2000. The inhibition of flavoproteins by phenoxaiodonium, a new iodonium analogue. Eur. J. Pharmacol. 401, 115-120.

Mubarakshina, M.M., Ivanov, B.N., Naidov, I.A., Hillier, W., Badger, M.R., et al., 2010. Production and diffusion of chloroplastic $\mathrm{H}_{2} \mathrm{O}_{2}$ and its implication to signalling. J. Exp. Bot. 61, 3577-3587.

Naderi, R., Valizadeh, M., Toorchi, M., Shakiba, M.R., 2014. Antioxidant enzyme changes in response to osmotic stress in wheat ( Triticum aestivum L.) seedling. Acta Biologica. Szegediensis 58 (2), 95-101.

Nallamilli, B.R., Edelmann, M.J., Zhong, X., Tan, F., Mujahid, H., 2014. Global analysis of lysine acetylation suggests the involvement of protein acetylation in diverse biological processes in rice (Oryza sativa). PLoS One 9, e89283.

Niu, L., Liao, W., 2016. Hydrogen peroxide signalling in plant development and abiotic responses: crosstalk with nitric oxide and calcium. Front Plant Sci. 7, 230.

Noctor, G., Foyer, C.H., 1998. A re-evaluation of the ATP: NADPH budget during C3 photosynthesis. A contribution from nitrate assimilation and its associated respiratory activity. J. Exp. Bot. 49, 1895-1908.

Özdemir, F., Bor, M., Demiral, T., Türkan, I., 2004. Effects of 24epibrassinolideon seed germination, seedling growth, lipid peroxidation, proline content and antioxidative system of rice (Oryza sativa L.) under salinity stress. Plant Growth Regul. 42, 203-211. Available from: https://doi.org/10.1023/b:grow.0000026509.25995.13.

Pasala, R., Khan, M.I.R., Minhas, P.S., Farooq, M.A., Sultana, R., Per, T.S., et al., 2016. Can plant bio-regulators minimize crop productivity losses caused by drought, salinity and heat stress? An integrated review. J. Appl. Bot. Food Qual. 89, 113-125. Available from: https:/ / doi.org/10.5073/JABFQ.2016.089.014.

Peng, C.L., Ou, Z.Y., Liu, N., Lin, G.Z., 2005. Response to high temperature in flag leaves of super high-yielding rice Pei'ai 64S/E32 and Liangyoupeijiu. Rice Sci. 12, 179-186.

Pesaresi, P., Pribil, M., Wunder, T., Leister, D., 2011. Dynamics of reversible protein phosphorylation in thylakoids of flowering plants: the roles of STN7, STN8 and TAP38. Biochim. Biophys. Acta 1807, 887-896.

Preeti, I.R., Luz, A.C., Perdigao, T.L., Araujo, R.A., Batitucci, M.C.P., 2018. Gene expression and antioxidant enzymatic activity in passion fruit exposed to aluminum. Afr. J. Agric. Res. 13, 115-120. Available from: https://doi.org/10.5897/AJAR2017.12834.

Puyaubert, J., Fares, A., Reze, N., Peltier, J.B., Baudouin, E., 2014. Identification of endogenously S-nitrosylated proteins in Arabidopsis plantlets: effect of cold stress on cysteine nitrosylation level. Plant Sci. 216, 150-156.

Quan, L.J., 2008. Hydrogen peroxide in plants a versatile molecule of reactive oxygen species network. J. Integr. Plant Biol. 50, 2-18.

Raghavendra, A.S., Gonugunta, V.K., Christmann, A., Grill, E., 2010. ABA perception and signalling. Trends Plant Sci. 15, 395-401. Available from: https://doi.org/10.1016/j.tplants.2010.04.006.

Rasmusson, A.G., Soole, K.L., Elthon, T.E., 2004. Alternative NAD(P) $\mathrm{H}$ dehydrogenases of plant mitochondria. Annu Rev. Plant Biol. $55,23-39$.

Rasool, S., Ahmad, A., Siddiqi, T.O., Ahmad, P., 2013. Changes in growth, lipid peroxidation and some key antioxidant enzymes in chickpea genotypes under salt stress. Acta Physiol. Plant 35, $1039-1050$.

Recknagal, R.O., Glende, E.A., 1984. Oxygen radicals in biological systems. Method Enzymol 105, 331-337.

Rhoads, D.M., et al., 2006. Mitochondrial reactive oxygen species. Contribution to oxidative stress and inter organellar signalling. Plant Physiol. 141, 125-134.
Rinalducci, S., Murgiano, L., Zolla, L., 2008. Redox proteomics: basic principles and future perspectives for the detection of protein oxidation in plants. J. Exp. Bot. 59, 3781-3801.

Rogowska-Wrzesinska, A., Wojdyla, K., Nedić, O., Baron, C.P., Griffiths, H.R., 2014. Analysis of protein carbonylation-pitfalls and promise in commonly used methods. Free Radic. Res. 48, 1145-1162.

Rouhier, N., Cerveau, D., Couturier, J., Reichheld, J.P., Rey, P., 2015. Involvement of thiol-based mechanisms in plant development. Biochim. Biophys. Acta 1850, 1479-1496.

Sagi, M., Davydov, O., Orazova, S., Yesbergenova, Z., Ophir, R., Stratmann, J.W., et al., 2004. Plant respiratory burst oxidase homologs impinge on wound responsiveness and development in Lycopersicon esculentum. Plant Cell 16, 616-628.

Scarpeci, T.E., Zanor, M.I., Carrillo, N., Mueller-Roeber, B., Valle, E. M., 2008. Generation of superoxide anion in chloroplasts of Arabidopsis thaliana during active photosynthesis: a focus on rapidly induced genes. Plant Mol. Biol. 66, 361-378.

Scheibe, R., Backhausen, J.E., Emmerlich, V., Holtgrefe, S., 2005. Strategies to maintain redox homeostasis during photosynthesis under changing conditions. J. Exp. Bot. 56, 1481-1489.

Schwarzlander, M., Finkemeier, I., 2013. Mitochondrial energy and redox signalling in plants. Antioxid. Redox Signal 18, 2122-2144.

Segal, A.W., Abo, A., 1993. The biochemical basis of the NADPH oxidase of phagocytes. Trends Biochem. Sci. 18, 43-47.

Selote, D.S., Chopra, R.K., 2010. Antioxidant response of wheat roots to drought acclimation. Protoplasma 245, 153-163. Available from: https:// doi.org/10.1007/s00709-010-0169-x.

Sharma, P., Jha, A.B., Dubey, R.S., Pessarakli, M., 2012. Reactive oxygen species, oxidative damage, and antioxidative defense mechanism in plants under stressful conditions. J. Bot. Article ID 217037, 26 pages.

Shi, Y.F., Wang, D.L., Wang, C., Culler, A.H., Kreiser, M.A., et al., 2015. Loss of GSNOR1 function leads to compromised auxin signalling and polar auxin transport. Mol. Plant. 8, 1350-1365.

Shi, B., Ni, L., Liu, Y., Zhang, A., Tan, M., Jiang, M., 2014. OsDMI3mediated activation of OsMPK1 regulates the activities of antioxidant enzymes in abscisic acid signalling in rice. Plant Cell Environ. 37, 341-352. Available from: https://doi.org/10.1111/ pce.12154.

Siddiqui, Z.S., 2013. Effects of double stress on antioxidant enzyme activity in Vigna radiata (L.) Wilczek. Acta Bot. Croat. 72 (1), 145-156.

Smakowska, E., Czarna, M., Janska, H., 2014. Mitochondrial ATPdependent proteases in protection against accumulation of carbonylated proteins. Mitochondrion 19, 245-251.

Smirnoff, N., 1995. Antioxidant systems and plant response to the environment. In: Smirnoff, V. (Ed.), Environment and Plant Metabolism: Flexibility and Acclimation. BIOS Scientific Publishers, Oxford, pp. 217-243.

Smith-Hammond, C.L., Swatek, K.N., Johnston, M.L., Thelen, J.J., Miernyk, J.A., 2014. Initial description of the developing soybean seed protein Lys-N( $(\varepsilon)$-acetylome. J. Proteomics 96, 56-66.

Song, Y., Cui, J., Zhang, H., Wang, G., Zhao, F.J., Shen, Z., 2013. Proteomics analysis of copper stress responses in the roots of two rice (Oryza sativa L.) varieties. Plant Soil 366, 647-358.

Starheim, K.K., Gevaert, K., Arnesen, T., 2012. Protein N-terminal acetyltransferases: when the start matters. Trends Biochem. Sci. 37, 152-161.

Suzuki, N., Miller, G., Morales, J., Shulaev, V., Torres, M.A., Mittler, R., et al., 2011. Respiratory burst oxidases: the engines of ROS signalling. Curr. Opin. Plant Biol. 14, 691-699.

Swanson, S., Gilroy, S., 2010. ROS in plant development. Physiol. Plant 138 (4), 384-392. Available from: https://doi.org/10.1111/ j.1399-3054.2009.01313.x.

Tang, B., Xu, S.Z., Zou, X.L., Zheng, Y.L., Qiu, F.Z., 2010. Changes of antioxidative enzymes and lipid peroxidation in leaves and roots of water logging-tolerant and water logging sensitive maize 
genotype sat seedling stage. Agric. Sci. China 9, 651-661. Available from: https://doi.org/10.1016/S1671-2927(09)60140-1.

Tanoua, G., Molassiotis, A., Diamantidis, G., 2009a. Hydrogen peroxide-and nitric oxide-induced systemic antioxidant primelike activity under $\mathrm{NaCl}$-stress and stress-free conditions in citrus plants. J. Plant Physiol. 166, 1904-1913.

Tanoua, G., Molassiotis, A., Diamantidis, G., 2009b. Induction of reactive oxygen species and necrotic death-like destruction in strawberry leaves by salinity. Environ. Exp. Bot. 6, 270-281.

Tavares, C.P., Vernal, J., Delena, R.A., Lamattina, L., Cassia, R., Terenzi, H., et al., 2014. S-nitrosylation influences the structure and DNA binding activity of AtMYB30 transcription factor from Arabidopsis thaliana. Biochim. Biophys. Acta 1844, 810-817.

Triantaphylidès, C., Havaux, M., 2009. Singlet oxygen in plants: production, detoxification and signalling. Trends Plant Sci. 14 (4), 19-28. Available from: https://doi.org/10.1016/j.tplants.2009.01.008.

Turan, O., Ekmekci, Y., 2011. Activities of photosystem II and antioxidant enzymes in chickpea (Cicerarietinum L.) cultivars exposed to chilling temperatures. Acta Physiol. Plant 33, 67-78. Available from: https:/ / doi.org/10.1007/s11738-010-0517-7.

Turk, H., Erdal, S., Genisel, M., Atici, O., Demir, Y., Yanmis, D., 2014. The regulatory effect of melatonin on physiological, biochemical and molecular parameters in cold-stressed wheat seedlings. Plant Growth Regul. 74, 139-152.

Tuteja, N., 2007. Mechanisms of high salinity tolerance in plants. Meth. Enzymol 428, 419-438.

Tuteja, N., 2009. Cold, salinity, and drought stress. In: Hirt, H. (Ed.), Plant Stress Biology. Wiley VCH Verlag $\mathrm{GmbH}$, Weinheim, pp. 137-159.

Tuteja, N., Singh, M.B., Misra, M.K., Bhalla, P.L., Tuteja, R., 2001. Molecular mechanisms of DNA damage and repair: progress in plants. Crit. Rev. Biochem. Mol. Biol. 36, 337-397.

Tuteja, N., Ahmad, P., Panda, B.B., Tuteja, R., 2009. Genotoxic stress in plants: shedding light on DNA damage, repair and DNA repair helicases. Mutat. Res. 681, 134-149.

Vaahtera, L., Brosche, M., Wrzaczek, M., Kangasjarvi, J., 2014. Specificity in ROS signalling and transcript signatures. Antioxid. Redox Signal. 21, 1422-1441.

Valko, M., Rhodes, C.J., Moncol, J., Izakovic, M., Mazur, M., 2006. Free radicals, metals and antioxidants in oxidative stress-induced cancer. Chem. Biol. Interac. 160, 1-40.

Van Dongen, J.T., Licausi, F., 2015. Oxygen sensing and signalling. Annu. Rev. Plant Biol. 66, 345-367.

Van Wijk, K.J., 2015. Protein maturation and proteolysis in plant plastids, mitochondria, and peroxisomes. Annu. Rev. Plant Biol. 66, 75-111.

Wang, C.Q., Li, R.C., 2008. Enhancement of superoxide dismutase activity in the leaves of white clover (Trifolium repens L.) in response to polyethylene glycol-induced water stress. Acta Physiol. Plant 30, 841-847.

Wang, S., Blumwald, E., 2014. Stress-induced chloroplast degradation in Arabidopsis is regulated via a process independent of autophagy and senescence-associated vacuoles. Plant Cell 114.

Waszczak, C., Akter, S., Jacques, S., Huang, J., Messens, J., Van Breusegem, F., et al., 2015. Oxidative post-translational modifications of cysteine residues in plant signal transduction. J Exp. Bot. 66, 2923-2934. Available from: https://doi.org/10.1093/jxb/erv084.

Weits, D.A., Giuntoli, B., Kosmacz, M., Parlanti, S., Hubberten, H.M., Riegler, H., et al., 2014. Plant cysteine oxidases control the oxygen-dependent branch of the N-end-rule pathway. Nat. Commun. 5, 3425.

Wiseman, H., Halliwell, B., 1996. Damage to DNA by reactive oxygen and nitrogen species: role in inflammatory disease and progression to cancer. Biochem 313, 17-29.
Wolhuter, K., Eaton, P., 2017. How widespread is stable protein Snitrosylation as an end-effector of protein regulation? Free Radic. Biol. Med. 109, 156-166. Available from: https://doi.org/ 10.1016/j.freeradbiomed.2017.02.013.

Xia, X.J., Wang, Y.J., Zhou, Y.H., Tao, Y., Mao, W.H., Shi, K., et al., 2009. Reactive oxygen species are involved in brassinosteroid induced stress tolerance in cucumber. Plant Physiol. 150, 801-814. Available from: https://doi.org/10.1104/pp.109.138230.

Xie, Q., Michaeli, S., Peled-Zehavi, H., Galili, G., 2015. Chloroplast degradation: one organelle, multiple degradation pathways. Trends Plant Sci. 20, 264-265.

Yabuta, Y., Maruta, T., Yoshimura, K., Ishikawa, T., Shigeoka, S., 2004. Two distinct redox signalling pathways for cytosolic APX induction under photo oxidative stress. Plant Cell Physiol. 45, 1586-1594.

Yadav, S., David Bhatla, S.C., 2011. Nitric oxide accumulation and actin distribution during auxin-induced adventitious root development in sunflower. Sci. Hort. 129, 159-166.

Ye, N., Zhu, G., Liu, Y., Li, Y., Zhang, J., 2011. ABA controls $\mathrm{H}_{2} \mathrm{O}_{2}$ accumulation through the induction of OsCATB in rice leaves underwater stress. Plant Cell Physiol. 52, 689-698. Available from: https://doi.org/10.1093/pcp/pcr028.

Yun, B.W., Feechan, A., Yin, M., Saidi, N.B., Le Bihan, T., et al., 2011. S-nitrosylation of NADPH oxidase regulates cell death in plant immunity. Nature 478, 264-268.

Zaffagnini, M., Bedhomme, M., Groni, H., Marchand, C.H., Puppo, C., Gontero, B., et al., 2012a. Glutathionylation in the photosynthetic model organism Chlamydomonas reinhardtii: a proteomic survey. Mol. Cell Proteomics 11, M111.014142.

Zaffagnini, M., Bedhomme, M., Marchand, C.H., Morisse, S., Trost, P., Lemaire, S.D., et al., 2012b. Redox regulation in photosynthetic organisms: focus on glutathionylation. Antioxid. Redox Signal 16, 567-586.

Zhang, H., Ni, L., Liu, Y., Wang, Y., Zhang, A., Tan, M., et al., 2012. The $\mathrm{C}_{2} \mathrm{H}_{2}$-type zinc finger protein ZFP182 is involved in abscisic acidinduced antioxidant defense in rice. J. Integr. Plant Biol. 54, 500-510. Available from: https://doi.org/10.1111/j.1744-7909.2012.01135.x.

Zhang, H., Liu, Y., Wen, F., Yao, D., Wang, L., Guo, J., et al., 2014a. A novel rice $\mathrm{C}_{2} \mathrm{H}_{2}$ type zinc finger protein, ZFP36, is a key player involved in abscisic acid- induced antioxidant defence and oxidative stress tolerance in rice. J. Exp. Bot. 65, 5795-5809. Available from: https://doi.org/10.1093/jxb/eru313.

Zhang, W., Tian, Z., Pan, X., Zhao, X., Wang, F., 2013. Oxidative stress and non-enzymatic antioxidants in leaves of three edible canna cultivars under drought stress. Hortic. Environ. Biotechnol. 54, 1-8.

Zhang, X., Yin, H., Chen, S., He, J., Guo, S.L., 2014b. Changes in antioxidant enzyme activity and transcript levels of related genes in Limonium sinense Kuntze seedlings under $\mathrm{NaCl}$ Stress. J. Chem 749047, 6 pages.

Zhang, Y., Song, L., Liang, W., Mu, P., Wang, S., Lin, Q., 2016. Comprehensive profiling of lysine acetylproteome analysis reveals diverse functions of lysine acetylation in common wheat. Sci. Rep. 6, 21069. Available from: https://doi.org/10.1038/ srep21069.

\section{Further Reading}

Wang, C., Wen, D., Sun, A., Han, X., Zhang, J., Wang, Z., et al., 2014. Changes antioxidant enzyme activity and gene expression in response to high temperature stress in wheat seedlings. J. Cereal Sci. 60, 653-659.

Yang, Y., Han, C., Liu, Q., Lin, B., Wang, J., 2008. Effect of drought and low light on growth and enzymatic antioxidant system of Picea asperata seedlings. Acta Physiol. Plant. 30, 433-440. 


\title{
Role and Regulation of Plants Phenolics in Abiotic Stress Tolerance: An Overview
}

\author{
Mohd Irfan Naikoo ${ }^{1}$, Mudasir Irfan Dar ${ }^{1}$, Fariha Raghib ${ }^{1}$, Hassan Jaleel ${ }^{2}$, \\ Bilal Ahmad', Aamir Raina ${ }^{3,4}$, Fareed Ahmad Khan ${ }^{1}$ and Fauzia Naushin ${ }^{3}$ \\ ${ }^{1}$ Plant Ecology and Environment Laboratory, Department of Botany, Aligarh Muslim University, Aligarh, \\ Uttar Pradesh, India ${ }^{2}$ Plant Physiology Laboratory, Department of Botany, Aligarh Muslim University, Aligarh, \\ Uttar Pradesh, India ${ }^{3}$ Botany Section, Women's College, Aligarh Muslim University, Aligarh, Uttar Pradesh, India \\ ${ }^{4}$ Mutation Breeding Laboratory, Department of Botany, Aligarh Muslim University, Aligarh, Uttar Pradesh, India
}

\section{O U T L I N E}

9.1 Introduction

9.2 Structure and Classification

9.3 Biosynthesis of Polyphenols

9.4 Phenolics and Abiotic Stress Tolerance

9.4.1 Phenolics as Ultraviolet Sunscreens

9.4.2 Plant Phenolics and Their Role in Heavy Metal Stress

9.4.3 Plant Phenolics and Their Role in Drought Stress
157

158

158

158

161

162

163

\subsection{INTRODUCTION}

Plants are exposed to multifarious abiotic stresses in constantly changing environments that are unfavorable for growth and development (Zhu, 2016). These abiotic stresses include water (drought and flooding), heavy metals, salinity, excess or deficiency of nutrients, high and low temperatures (chilling and freezing), extreme levels of light (high and low), radiation
(UV-B and UV-A; ultraviolet, UV), ozone, sulfur dioxide, mechanical factors, and other less frequently occurring stressors (Pereira, 2016). Plants being rooted in the environment they grow in have to adapt with the changing conditions due to abiotic stresses and the accumulation of phenolics in plant tissues are considered as an adaptive response of plants to these adverse environmental conditions (Pereira, 2016; Lattanzio, 2013). 
Plants synthesize an enormous number of chemicals categorized as primary and secondary metabolites. The primary metabolites, that is, sugars, fatty acids, amino, and nucleic acids being essential for plant growth and development are ubiquitously distributed in all plants (Fiehn, 2002; Wu and Chappell, 2008). Secondary metabolites being much more diverse than the primary metabolites structurally as well as chemically are the specialized compounds that are not directly essential for basic plant metabolism but are required by plants for survival in the environment.

Plant phenolics or polyphenols are the most widely occurring groups of secondary metabolites with substantial physiological and morphological importance in plants. They are aromatic compounds with one or more hydroxyl groups and emerge from shikimate/ phenylpropanoid pathway or polyketide acetate/malonate pathway, producing monomeric and polymeric phenols and polyphenols (Randhir et al., 2004). Plant phenolics play an important role in plant growth, development, and reproduction, and a key role as defense compounds against abiotic stresses, such as high light, low temperatures, UV-B radiations, heavy metals and nutrient deficiency (Lattanzio, 2013), protection against pathogens and predators (Bravo, 1998), producing color and sensory characteristics of fruits and vegetables (Alasalvar et al., 2001), besides exhibiting other essential properties like antiallergenic, antimicrobial, and antioxidant activity (Balasundram et al., 2006). Plant phenolics or polyphenols are the most widely distributed secondary metabolites and predominate in the plant kingdom. Bacteria, fungi, and algae produce peculiar phenolic compounds whereas bryophytes are regular producers of polyphenols like flavonoids, but in the vascular plants a full range of phenolic compounds or polyphenols are found (Swain, 1975; Harborne, 1980). An estimation of about $2 \%$ of all the carbon photosynthesized by plants is converted into phenolic compounds (Robards and Antolovich, 1997). Several thousand phenolic compounds are known to be synthesized by higher plants and the characterization of these compounds is continuously increasing. Plant leaves contain esters; amides and glycosides of hydroxycinnamic acids (HCAs); glycosylated flavonoids, particularly flavonols; and proanthocyanidins and their derivatives. Lignin, suberin, and pollen sporopollenin are some other polyphenolic polymers. Some soluble phenolics like chlorogenic acid are widely distributed, whereas some are restricted to specific genera or families, thereby are handy biomarkers for taxonomic studies.

\subsection{STRUCTURE AND CLASSIFICATION}

Structurally, phenolic compounds contain an aromatic ring with one or more hydroxyl substituents attached to it, ranging from simple phenolic molecules to highly polymerized compounds, thereby showing great structural diversity, and are often referred to as polyphenols (Bravo, 1998). Most phenolic compounds naturally appear as conjugates with mono- and polysaccharides, coupled with one or more phenolic groups, and may also exist as functional derivatives like esters and methyl esters (Harborne, 1989; Harborne et al., 1999; Shahidi and Naczk, 1995).

Phenolics though a very large and diverse group of chemical compounds can be classified in a number of ways. Harborne and Simmonds (1964) classified them into different groups based on the number of carbons in the molecule (Table 9.1).

\subsection{BIOSYNTHESIS OF POLYPHENOLS}

Plant phenolics are biosynthesized in plants from a biosynthetic intermediate, phenylalanine and shikimic acid through the shikimic acid pathway (Fig. 9.1). The starting metabolites of the pathway are erythose-4phosphate and phosphoenolpyruvate (PEP), which are intermediates of pentose phosphate pathway (PPP) and glycolysis, respectively.

The first step involves the conversion of glucose in the PPP to glucose-6-phosphate and then irreversibly to ribulose-5-phosphate by the aid of glucose-6-phosphate dehydrogenase (G6PDH). The PPP advances to produce erythrose-4-phosphate. Similarly from glycolysis, phosphoenolpyruvate is generated, which is then used together with erythrose-4-phosphate through the phenylpropanoid pathway to generate phenolic compounds after being channeled to the shikimic acid pathway to produce phenylalanine (Fig. 9.1).

\subsection{PHENOLICS AND ABIOTIC STRESS TOLERANCE}

The great degree of interactions between plants and their changing environments has been a major driving force behind the emergence of specific natural products (Lattanzio, 2013). In this regard, the accumulation of phenolics in plant tissues is considered as an adaptive response of plants to adverse environmental conditions, thereby expanding evolutionary fitness. This accumulation resulted due to the activity of phenylalanine ammonia lyase (PAL), chalcone synthase (CHS), and other enzymes. Activity of PEPcarboxylase also increases, indicating a shift from sucrose production to the processes of defense and repair. Plant phenolics confer various physiological functions for survival and adaptation to environmental disturbances (Landolt et al., 1997; Andersen, 2003; 
TABLE 9.1 Classification of Phenolic Compounds

\begin{tabular}{|c|c|c|c|}
\hline Class & No. of C-atoms & Structure & Occurrence \\
\hline Simple phenolics, benzoquinones & 6 & C6 & Rare to common \\
\hline Phenolic acids and related compounds & 7 & $\mathrm{C} 6-\mathrm{C} 1$ & Common \\
\hline Acethophenones, phenylacetic acids & 8 & $\mathrm{C} 6-\mathrm{C} 2$ & Rare \\
\hline $\begin{array}{l}\text { HCAs, phenylpropanoids (coumarins, isocoumarins, } \\
\text { chromones, chromenes) }\end{array}$ & 9 & $\mathrm{C} 6-\mathrm{C} 3$ & Rare to common \\
\hline Napthoquinones & 10 & $\mathrm{C} 6-\mathrm{C} 4$ & \\
\hline Xanthones & 13 & $\mathrm{C} 6-\mathrm{C} 1-\mathrm{C} 6$ & Rare \\
\hline Stilbenes, anthraquinones & 14 & $\mathrm{C} 6-\mathrm{C} 2-\mathrm{C} 6$ & Rare \\
\hline Flavonoids, isoflavonoids & 15 & $\mathrm{C} 6-\mathrm{C} 2-\mathrm{C} 6$ & Common \\
\hline Betacyanins & 18 & & Rare \\
\hline Lignans, neolignans & 18 & $(\mathrm{C} 6-\mathrm{C} 3)_{2}$ & \\
\hline Biflavonoids & 30 & $(\mathrm{C} 6-\mathrm{C} 3-\mathrm{C} 6)_{2}$ & Rare \\
\hline Lignin & & $(\mathrm{C} 6-\mathrm{C} 3)_{n}$ & Common \\
\hline Melanins & $\mathrm{N}$ & $(\mathrm{C} 6)_{n}$ & \\
\hline Condensed tannins (proanthocyanidins flavolans) & & $(\mathrm{C} 6-\mathrm{C} 3-\mathrm{C} 6)_{n}$ & \\
\hline
\end{tabular}
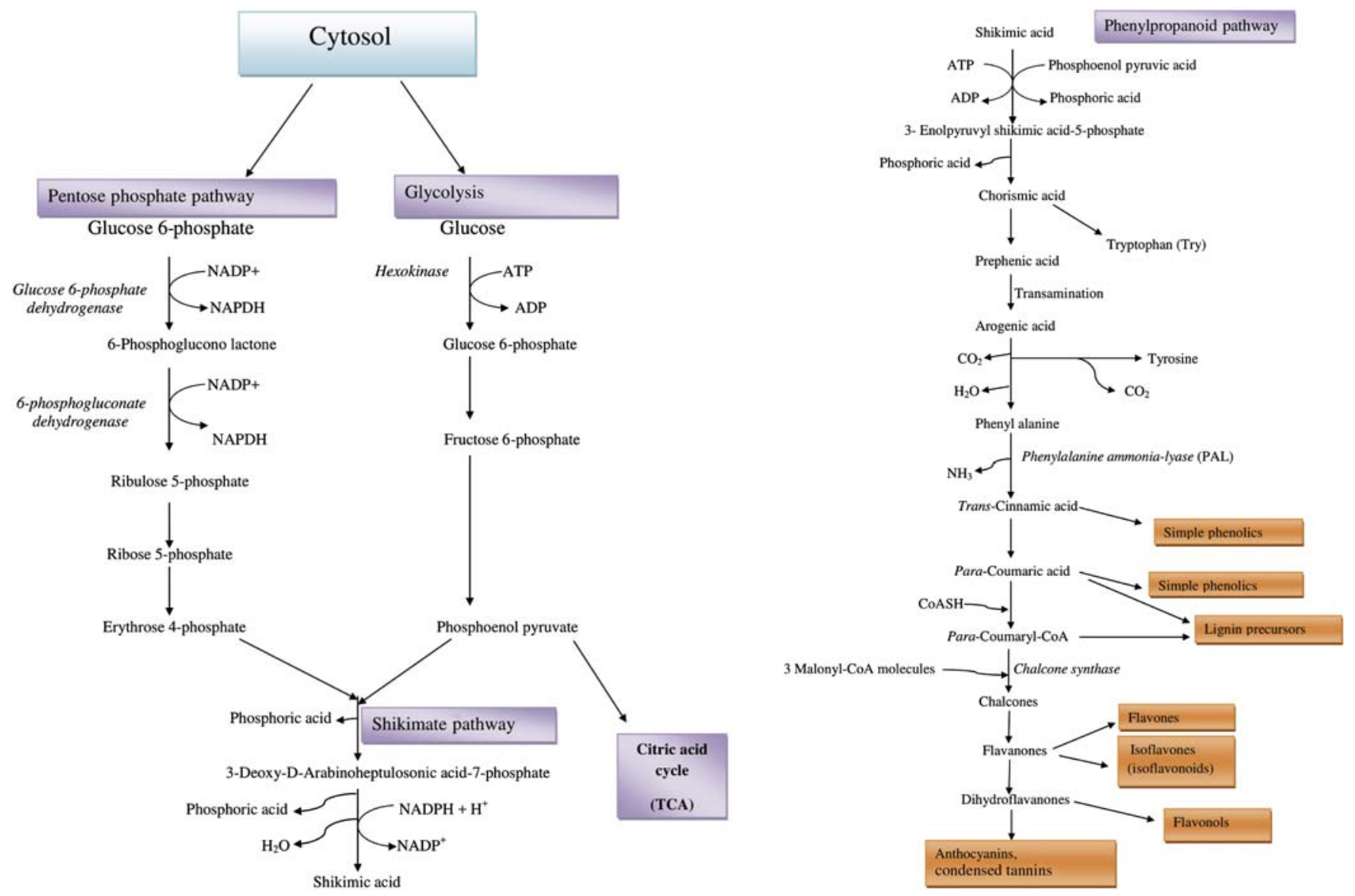

FIGURE 9.1 The phenolic compound biosynthesis pathway. A schematic representation of the biosynthesis of phenolic compounds in the pentose phosphate, shikimate, and phenylpropanoid pathways in plants. Source: Redrawn from Lattanzio, V., 2013. Phenolic compounds: introduction. In: Ramawat, K.G., Mérillon, J.M. (Eds.), Natural Products, Springer-Verlag: Berlin, Heidelberg. http://doi.org/10.1007/978-3-642-22144-6_57 and Lin et al. (2016). 
Lattanzio et al., 2009). Plant phenolics are generally considered pivotal defense compounds when environmental stresses, such as high light or UV radiation, low temperatures, pathogen infection, herbivores, heavy metals, and nutrient deficiency, lead to increased production of free radicals and other oxidative species in plants. Plants respond to these biotic and abiotic stress factors by increasing their capacity to scavenge reactive oxygen species (ROS; Khan and Khan, 2017). The induction of secondary metabolism gene expression by the abovementioned environmental stresses is often mediated by integrating signaling molecules such as salicylic acid, jasmonic acid, and their derivatives (WinkelShirley, 2002; Gould and Lister, 2006; Nascimento and Fett-Neto, 2010; Khan and Khan, 2013; Khan et al., 2013, 2014, 2015; Per et al., 2018).

Plants are exposed to various abiotic stresses during their life cycle. When a plant is subjected to abiotic stress, a number of genes are turned on or off, resulting in increasing levels of several metabolites and proteins, some of which may be responsible for conferring a certain degree of defense against these stresses (Ahmad et al., 2008; Jaleel et al., 2009; Tuteja et al., 2009). Abiotic stress promotes the production of damaging active oxygen species within the cells (Dar et al., 2017). Phenolics are varied secondary metabolites (flavonoids, tannins, hydroxycinnamate esters, and lignin) found abundantly in plant tissues and are actively involved in defense mechanisms against biotic and abiotic stress. As compared with nonstressed conditions, plants often produce a higher quantity of phenolic compounds under certain stress conditions (Selmar, 2008). Phenolic compounds performing as antioxidants terminate free radical chains and chelate redox-active metal ions that are capable of catalyzing lipid peroxidation (Schroeter et al., 2002). Phytophenolics, particularly polyphenols, function as antioxidants to support the primary ascorbatedependent detoxification system as a backup defense mechanism of vascular plants contrasting to monophenols (Yamasaki et al., 1995, 1999). Polyphenols are more effective antioxidants in vitro than ascorbate and tocopherols and have an ideal structural chemistry for free radical-scavenging activity. Antioxidative features of polyphenols arise from the ability of the polyphenol-derived radical to steady and delocalize the unpaired electron (chain-breaking function), their high reactivity as hydrogen or electron donors and from their capability to chelate transition metal ions (termination of the Fenton reaction) (Rice-Evans et al., 1997). Kagan and Tyurina (1997) reported that phenolics are univalently oxidized to their respective phenoxyl radicals when they function as antioxidants either by enzymatic or direct radical-scavenging mechanisms. Plants synthesize phenolic compounds to survive in stress conditions (UV radiation, drought, salt, metal, and low temperature stress). Most plants constitutively synthesize phenylpropanoids including flavonoids and HCAs. However, accumulation of phenolics in plants can be induced by abiotic and biotic stresses, for example, UV radiation, high light illumination, low temperatures, wounding, low nutrients, and pathogen attack (Dixon and Paiva, 1995; Yamasaki et al., 1995).

Certain secondary metabolic compounds are intensively synthesized under conditions of abiotic stress like drought where these act as antioxidants (Nascimento and Fett-Neto, 2010). Phenolic compounds accumulation in plant tissues is regarded as a distinctive plant stress characteristic. One among the largest three groups of secondary metabolites produced in plants, phenols have been alienated into five subgroups (coumarins, flavonoids, lignins, phenolic acids, and tannins) (Gumul et al., 2007), and are synthesized in plants via shikimic acid and chorismic acid pathways. Phenolic compounds have been regarded as metabolic alteration byproducts (Solecka, 1997). These not only serve a vital function of defense in plants but are also known to influence animals and humans that consume these phenol-enriched plant products (Franca et al., 2001; Amarowicz and Weidner, 2009). The expression of phenolic compounds has been, however, observed to be upregulated (Wróbel et al., 2005; Weidner et al., 2009a) or downregulated (Weidner et al., 2007, 2009b) in response to diverse environmental stresses, thus leading to the increased or decreased content of the phenolic compounds. A number of studies have demonstrated the increased production of phenols in different plant tissues under abiotic stress conditions (Dixon and Paiva, 1995; Wróbel et al., 2005; Weidner et al., 2009a). During water deficit and chilling stress conditions, Chung et al. (2006) have reported increased content of total phenolic compounds in Rehmannia glutinosa. Further confirmation has come from the studies of Posmyk et al. (2005) in soybean subjected to chilling stress. This accumulation is due to enhanced activities of enzymes, PAL, CHS, and other enzymes involved in their biosynthesis. Additionally, phosphoenolpyruvate (PEP)-carboxylase activity also increases, which suggests a shift from the production of sucrose to metabolic processes supporting defense and repair. Phenolics confer a variety of physiological functions to plants to survive and adapt to various environmental disturbances (Landolt et al., 1997; Andersen, 2003; Lattanzio et al., 2009). Phenolic acids are synthesized in response to abiotic stress through hydration, dehydration, and methylation of cinnamic acid (Dixon and Paiva, 1995). Many 
secondary metabolism products in plants that exhibit antioxidant properties belong to this class of compounds (Oszman'ski, 1995). As antioxidants, these phenolic compounds scavenge ROS (Amarowicz et al., 2004, 2010; Caillet et al., 2006; Amarowicz and Weidner, 2009), catalyze oxygenation reactions by forming complexes with some metals, and hinder the activities of certain oxidizing enzymes (Elavarthi and Martin, 2010). As stated earlier, accumulation of phenols in plant tissues is a characteristic feature of several environmental stresses that divert considerable quantity of substrates from primary metabolism to the formation of secondary products leading to significant perturbations in the cellular homeostasis. Strong stimulation of mRNAs encoding G6PDH, a carbohydrate metabolism enzyme that provides shikimate pathway substrates, and 3-deoxyarabinoheptulosonate 7-phosphate synthase, which is a shikimate pathway enzyme required for phenylalanine biosynthesis has been observed in response to stress (Cheynier et al., 2013). Furthermore, accumulation of free proline in plants as a result of various biotic and abiotic stresses has been reported. Researchers have anticipated that a stress-stimulated enhancement in the shift of reducing equivalents into proline biosynthesis (cytosolic) as well as degradation (mitochondrial) cycle might be responsible for enabling sensitive regulation of redox potential in the cytosol (Logemann et al., 2000; Lattanzio et al., 2009; Verslues and Sharma, 2010). The abovementioned points imply that different environmental perturbations selectively induce the primary as well as secondary metabolic activities, which are directly and indirectly involved in the accretion of phenolic compounds (Cheynier et al., 2013; Lattanzio et al., 2009). A likely series of biochemical reactions occurring inside the cell, which convey a signal from the outside cell environment into the inside of the plant cell, leading to an effective physiological response, may thus be envisaged (Fig. 9.2) (Hare and Cress, 1997; Lattanzio et al., 2009). This signaling pathway proposes a connection between primary and secondary metabolism, which couples the accretion of proline (a stress metabolite) with the energy transfer toward the biosynthesis of phenylpropanoid through the oxidative PPP (Cheynier et al., 2013). Under various stress conditions, the plant undergoes forceful accumulation of ample amounts of free proline. It can be synthesized de novo or can be released by the protein degradation and is accompanied by NADPH oxidation. Enhanced $\mathrm{NADP}^{+} / \mathrm{NADPH}$ ratio leads to increased activity of the oxidative PPP, which in turn provides precursors for biosynthesis of phenolic compounds via the shikimic acid pathway (Cheynier et al., 2013; Lattanzio et al., 2009).

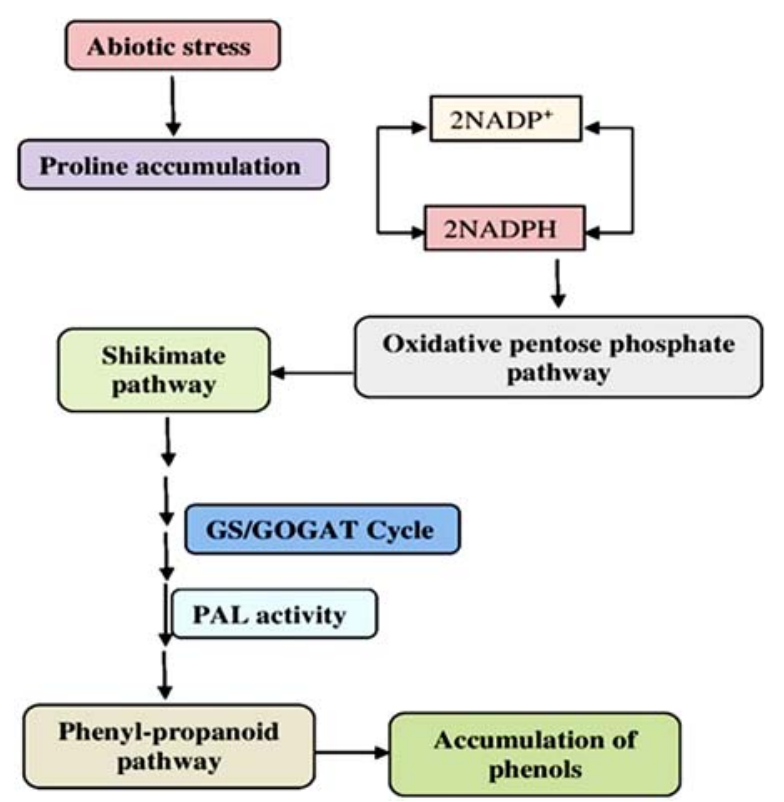

FIGURE 9.2 Showing the abiotic stress-mediated phenol biosynthesis in plants. Source: Redrawn from Cheynier et al. (2013); Lattanzio V., Cardinali, A., Ruta, C., Morone Fortunato, I., Lattanzio, V.M.T., Linsalata, $V$., et al., 2009. Relationship of secondary metabolism to growth in oregano (Origanum vulgare L.) shoot cultures under nutritional stress. Environ. Exp. Bot. 65, 54-62.

\subsubsection{Phenolics as Ultraviolet Sunscreens}

Light is a well-known physical factor that can affect the synthesis of metabolites in plants. Exposure of ambient solar UV-B radiation $(280-320 \mathrm{~nm})$ to plants in open fields adversely affects DNA, proteins, and membranes and alters metabolism through the generation of ROS. Plants synthesize phenolic compounds, which act as a screen inside the epidermal cell layer to defend themselves from this damaging radiation and by adjusting the antioxidant systems at both the cell and whole organism level thereby intercepting mutagenesis and cell death by dimerization of thymine units in the DNA, and possible photo destruction of coenzymes NAD or NADP (Daayf and Lattanzio, 2008). Flavonoids with their high absorptivity at $250-270$ and $335-360 \mathrm{~nm}$ act as good UV screens (Lattanzio, 2013; Winkel-Shirley, 2002; Carletti et al., 2003). Flavonoids and other phenolic compounds play a significant role in UV protection ( $\mathrm{Li}$ et al., 1993). Increased flavonoid synthesis was observed in plants radiated with UV light, which confirms the ability of flavonoids to absorb radiation of high energy with the maximum absorption at 250-270 and 335-360 nm (Michalak, 2006; Falcone Ferreyra et al., 2012; Winkel-Shirley, 2002; Liang et al., 2006). It is an observed fact that tropical and high-altitude plants have a higher percentage of flavonoids than temperate plants. The change in the 
proportion of flavonoids composition of plant leaves is due to excess of light or UV radiation largely due the activation of flavonoid biosynthetic genes (Kolb et al., 2001). Several studies have confirmed that the excess of light or UV radiation changes the flavonoid composition of plant leaves (Olsson et al., 1998; Kolb et al., 2001). Plant defense mechanisms of polyphenols against UV radiation as direct shields has been regarded as a key biological function by several authors (Rozema et al., 2002; Burchard et al., 2000). Larcher (1995) revealed that phenolics, particularly anthocyanin, which accumulate in the epidermis can act as a darkening filter and protect the mesophyll from extreme radiation. Flavonoids (especially kaempferol derivatives), phenolic acid esters, or isoflavonoids and psoralens accumulate during stress and prevent UV-B from reaching the mesophyll (Stapleton, 1992). Flavones and flavonols, the two important groups of flavonoids found in flowers, accumulate in epidermal layers of leaves and stems and absorb light strongly in the UV-B region without interrupting visible (PAR) wavelengths, thus function to shield cells from UV-B radiation (Lake et al., 2009). Ryan et al. (2001) proved that flavonoids are essential in UV protection using mutants of Arabidopsis, UV-hypersensitive phenotypes that have a block in flavonoid production. Anthocyanin synthesis was stimulated by UV light from 280 to $320 \mathrm{~nm}$ synergistically when combined with red light in apples (Arakawa et al., 1985). Liu et al. (1995) revealed that flavonoid improved in barley and Kramer et al. (1991) also found increased content of polyamines in cucumber by UV-B radiation. Flavonols concentration also improved in Norway spruce (Picea abies) on UV-B exposure (Fischbach et al., 1999). Along similar lines, the increased production of the important anticancerous phenolic compounds, that is, vinblastine and vincristine, due to UV-B exposure in Catharanthus roseus has been reported by Bernard et al. (2009). Shiozaki et al. (1999) also revealed that flavonoids in the roots of pea plants was enhanced on UV (300-400 nm) exposure. Flavonols production was stimulated by UV-B in silver birch and grape leaves (Tegelberg et al., 2004). Moreover, photosynthetic pigments, condensed tannins were accumulated under six different daily doses of UV radiation (UV-A and UV-B), whereas its precursor, (+)-catechin, significantly decreased (Lavola et al., 2003). In a UV-tolerant rice cultivar, C-glycosylflavone contents increasingly appeared but were not found in a susceptible cultivar when exposed to different UV-B light levels (Markham et al., 1998). Furthermore, in several plant species, enhanced flavonoid levels have been measured at higher altitudes (Bachereau et al., 1998; Zidorn et al., 2005; Rieger et al., 2008; Spitaler et al.,
2008; Murai et al., 2009). It has been proven in several plant species that the expression of $\mathrm{CHS}$ is transcriptionally activated by UV light, which is the first enzyme in the flavonoid biosynthesis pathway (Koes et al., 1989; Schulze-Lefert et al., 1989).

\subsubsection{Plant Phenolics and Their Role in Heavy Metal Stress}

Heavy metal toxicity is one of the important abiotic stresses that alter physiological and metabolic processes, thus leading to harmful effects in plants (Villiers et al., 2011). It has been reported that certain flavonoids exhibit the ability to provide heavy metal stress protection by transition metals chelation (e.g., $\mathrm{Fe}, \mathrm{Cu}, \mathrm{Ni}, \mathrm{Zn}$ ), which generates hydroxyl radical via Fenton's reaction (Mira et al., 2002;Williams et al., 2004). Kidd et al. (2001) revealed that the chelation of these metals in the soil may be an effective form of defense against the effects of high metals concentration toxicity. Michalak (2006) observed that the biosynthesis of phenolic compounds that are precursors of lignin intensifies under stress conditions, for example, in plants subjected to heavy metal stress. Research on corn plants (Zea mays L.) confirmed this further when grown on soil contaminated with aluminum ions and root exudates were found with high levels of catechin and quercetin. Winkel-Shirley (2001) reported that flavonoids are involved in plants' defense, growing in soils that are rich in toxic metals such as aluminum. The production of betalains in Beta vulgaris is stimulated by $\mathrm{Cu}^{2+}$ (Trejo-Tapia et al., 2001). The hairy roots were exposed to metal ions to improve betalaines production (Thimmaraju and Ravishankar, 2004). Red cabbage seedlings accumulated phenolic compounds, total antioxidant capacity, and increased PAL activity when treated with copper (Posmyk et al., 2009). Accumulation of betacyanins in callus cultures of Amaranthus caudatus is stimulated by $\mathrm{Cu}^{2+}$ (Obrenovic, 1990). Flavonoids accumulation was also observed in cell cultures of Ginkgo biloba treated with $\mathrm{CuSO}_{4}$ as compared with untreated cells (Kim et al., 1999). Similarly, association between concentration of $\mathrm{CuSO}_{4}$ and flavonoid level in cell cultures of Digitalis lanata was reported (Bota and Deliu, 2011). Nickel stress leads to significant decrease in anthocyanin levels as observed by Hawrylak et al. (2007). Michalak (2006) observed that plants with high content of tannins, such as tea, are able to tolerate high concentrations of manganese in a soil, as they are protected by the direct chelation of these ions. Lavid et al. (2001) reported the heavy metal ions binding with polyphenols in Nympheae where heavy metals $(\mathrm{Hg}, \mathrm{Pb}, \mathrm{Cr})$ were chelating by the polyphenols rich methanol extract. 


\subsubsection{Plant Phenolics and Their Role in Drought Stress}

Drought is the major abiotic stress that affects plant growth and development and causes losses in agricultural production. As has been reported by several studies, phenolics content increased in plants under water scarcity. Flavonoid accumulation is important to improve drought tolerance in wild-type and Arabidopsis thaliana mutants revealed by transcriptomic and metabolomic approaches (Nakabayashi et al., 2014). Ballizany et al. (2012) revealed that the quercetin (a flavonol) contents enhanced significantly in white clover under drought conditions, which was higher in the more drought-resistant genotypes. Kirakosyan et al. (2003) reported that under drought conditions, flavonols have been increased in other species also, such as Crataegus laevigata and Crataegus monogyna. Similarly, in Cistus clusii plants upon controlled drought treatments, and in plants collected from the field in summer, characterized by high temperatures and prolonged lack of rain in the Mediterranean climate, an increase in flavanol levels has been reported (Hernandez et al., 2004). Akula and Ravishankar (2011) reported that the defense mechanism against drought stress is triggered by bioactivity of leaf phenolic molecules. Phenolic acids and flavonoids as antioxidant accumulation and sunshields are involved in plants' response to drought stress (Nichols et al., 2015). Larson (1988) also reported the increased level of flavonoids and phenolic acids in willow leaves under drought conditions causes oxidative stress. In droughtresistant tomato cultivars kaempferol and quercetin (flavonoids) were enhanced while reduced in drought sensitive cultivars reported by Sánchez-Rodríguez et al. (2011). In red-hulled and black-hulled rice the radical-scavenging ability depended on the concentrations of proanthocyanidins and anthocyanins, respectively (Oki et al., 2002). Flavonoids and phenolic acids were synthesized in a large amount in wheat leaves and cell-damaging oxidants also generated under drought stress (Ma et al., 2014). Chalker-Scott (1999) reported that plant tissues containing anthocyanins are usually rather resistant to drought. For example, a purple cultivar of chili resists water stress better than a green cultivar revealed by Bahler et al. (1991). In Chenopodium quinoa saponins content decreased from $0.46 \%$ to $0.38 \%$ dry weight $(\mathrm{dw})$ in plants growing under low water deficit and in high water deficit conditions, respectively (Soliz-Guerrero et al., 2002). Phenolic compounds concentration was $10 \%$ improved in Hypericum brasiliense grown under drought stress as compared with control plants (De Abreu and Mazzafera, 2005). Similarly, phenolic compound were also increased in pea plants (Pisum sativum) when grown under drought conditions (Nogue's et al., 1998). In both leaves and flowers of Tridax procumbens significant increases of total phenolic content were observed under drought stress (Gnanasekaran and Kalavathy, 2017). Antioxidant capacity of phenolic acids was reflected by change in their contents during the process of finger millet malting (Subba Rao and Muralikrishna, 2002). Nichols et al. (2015) reported that the high levels of flavonols, quercetin, and kaempferol contents were related with improved stress tolerance capacity of white clover under drought conditions.

\subsubsection{Plant Phenolics and Their Role in Cold Stress}

It has been observed that nonfreezing low temperatures enhance phenolic metabolism in plants (Akula and Ravishankar, 2011). Phenolic metabolism is stimulated at a critical low temperature, which is the threshold temperature at which chilling injury is also induced (Janska et al., 2010). This low temperature results in cold-induced stimulation of the PAL activity (EC 4.3.1.5) as well as other enzymes necessary for phenolic biosynthesis, leading to increased phenolic production and modified plant development either independently or by interaction with known plant growth promoters, especially ethylene (Lattanzio et al., 1994, 2001). Cold stress increases phenolic production into the cell wall either as suberin or lignin (Griffith and Yaish, 2004). Lignification and suberin deposition increase resistance to cold stress. These cell wall thickenings protect the plant from freezing stress. An increase in cell wall thickening could reduce cell collapse during freezing-induced dehydration and mechanical stress, thus providing freezing resistance of the plant (Chalker-Scott and Fuchigami, 1989). Apple trees are found to be associated with high levels of chlorogenic acid as an adaptive measure to cold climate (Perez-Ilzarbe et al., 1997). Christie et al. (1994) reported the anthocyanins accumulation during cold stress and Pedranzani et al. (2003) reported that cold and water stresses initiated changes in endogenous jasmonates in Pinus.

\subsubsection{Plant Phenolics and Their Role in Nutrient Stress}

Plant growth depends on the supply of recycled nutrients. External nutrient supply and nutrient mineralization by soil microorganisms contribute the nutrient requirements. The factors regulating nutrient cycle include climate, substrate (litter) quality, and decomposer organisms. Polyphenols influence the supply 
and flow of inorganic and organic soil nutrients available for plants and/or microbes. Phenolic compounds find their way to the soil as leachates from the aboveas well as below-ground parts of plants and/or within above and belowground plant litter (Hättenschwiler and Vitousek, 2000). Polyphenols affect the composition and activity of decomposers thus influencing the rates of decomposition and nutrient cycling (Lattanzio et al., 2006).

Phenolic compounds show a sensitive response to nutrient deficiency, thus providing a method for diagnosing nutrient disorders prior to the appearance of visible symptoms. Deficiencies of $\mathrm{N}, \mathrm{P}, \mathrm{K}$, and $\mathrm{S}$ usually result in increased concentrations of phenolic compounds, and abundant $\mathrm{N}$ generally inhibits phenolic accumulation (Gershenzon, 1913; McClue, 1977). Visual symptoms of $\mathrm{N}$ or P deficiency are red or purple tints of the leaves due to accumulation of anthocyanins (Hewitt, 1963). Nutrient stress increased threefold anthocyanidins level and doubled quercetin-3-Oglucoside in tomato (Bongue-Bartelsman Phillips, 1995). Osmotic stress by sucrose and other agents regulate anthocyanin production in Vitis vinifera cultures (Tuteja and Mahajan, 2007).

\subsection{CONCLUSION AND FUTURE PROSPECTS}

Plant phenolics are the most common and widespread secondary metabolites, comprising a large reservoir of natural chemical diversity with a huge range of compounds and enzymes and a wide spectrum of mechanisms of gene regulation, and transport of metabolites and enzymes. Plants accumulate phenolic compounds in their tissues as an adaptive response to adverse environmental stresses including wounding, pathogen attack, mineral deficiencies, and temperature stress. Polyphenols modify the developmental status of the plant independently or by interacting with plant growth promoters like ethylene. Furthermore, these compounds, the precursors for lignin and suberin, are polymerized into the cell wall. These cell wall thickenings protect the plant from freezing stress. An increase in cell wall thickening could reduce cell collapse during freezing-induced dehydration and mechanical stress, thus providing freezing resistance of the plant (Chalker-Scott and Fuchigami, 1989). Polyphenols influence the supply and flow of inorganic and organic soil nutrients available for plants and/or microbes. They also show a sensitive response to nutrient deficiency, thus providing a method for diagnosing nutrient disorders prior to the appearance of visible symptoms.
Despite a handful of studies on biosynthesis of phenolic compounds and their accumulation as an adaptive response against abiotic stresses, studies on the proper mechanism of their accumulation and their interactions with other cell metabolites are desperately needed to develop a complete understanding of their increased expression and imparting tolerance under such circumstances.

\section{References}

Ahmad, P., Sarwat, M., Sharma, S., 2008. Reactive oxygen species, antioxidants and signaling in plants. J. Plant Biol. 51 (3), 167-173.

Akula, R., Ravishankar, G.A., 2011. Influence of abiotic stress signals on secondary metabolites in plants. Plant Signal. Behav. 6, 1720-1731.

Alasalvar, C., Grigor, J.M., Zhang, D., Quantick, P.C., Shahidi, F., 2001. Comparison of volatiles, phenolics, sugars, antioxidant vitamins, and sensory quality of different colored carrot varieties. J. Agric. Food Chem. 49, 1410-1416.

Amarowicz, R., Weidner, S., 2009. Biological activity of grapevine phenolic compounds. In: Roubelakis-Angelakis, K.A. (Ed.), Grapevine Molecular Physiology and Biotechnology, second ed Springer, New York, pp. 389-405.

Amarowicz, R., Pegg, R.B., Rahimi-Moghaddam, P., Barl, B., Weil, J.A., 2004. Free-radical scavenging capacity and antioxidant activity of selected plant species from the Canadian prairies. Food Chem. 84, 551-562.

Amarowicz, R., Weidner, S., Wojtowicz, I., Karmac, M., Kosin'ska, A., Rybarczyk, A., 2010. Influence of low-temperature stress on changes in the composition of grapevine leaf phenolic compounds and their antioxidant properties. Funct. Plant Sci. Biotechnol. 4, 90-96.

Andersen, C., 2003. Source-sink balance and carbon allocation below ground in plants exposed to ozone. New Phytol. 157, 213-228.

Arakawa, O., Hori, Y., Ogata, R., 1985. Relative effectiveness and interaction of ultraviolet-B, red and blue light in anthocyanin synthesis of apple fruit. Physiol. Plant. 64, 323-327.

Bachereau, F., Marigo, G., Asta, J., 1998. Effect of solar radiation (UV and visible) at high altitude on CAM-cycling and phenolic compounds biosynthesis in Sedum album. Physiol. Plant. 104, 203-210.

Bahler, B.D., Steffen, K.L., Orzolek, M.D., 1991. Morphological and biochemical comparison of a purple-leafed and a green-leafed pepper cultivar. HortScience. 26, 736 .

Balasundram, N., Sundram, K., Samman, S., 2006. Phenolic compounds in plants and agri-industrial by-products: antioxidant activity, occurrence, and potential uses. Food Chem. 99, 191-203.

Ballizany, W.L., Hofmann, R.V., Jahufer, M.Z.Z., Barrett, B.B., 2012. Multivariate associations of flavonoid and biomass accumulation in white clover (Trifolium repens) under drought. Funct. Plant Biol. 39, 167-177.

Bernard, Y.K., Binder Christie, A.M., Peebles Jacqueline, V., Shanks, K.-Y.S., 2009. The effects of UV-B stress on the production of terpenoid indole alkaloids in Catharanthus roseus hairy roots. Biotechnol. Prog. 25, 8615.

Bongue-Bartelsman, M., Phillips, D.A., 1995. Nitrogen stress regulates gene expression of enzymes in the flavonoids biosynthetic pathway of tomato. Plant Physiol. Biochem. 33, 539-546.

Bota, C., Deliu, C., 2011. The effect of copper sulphate on the production of flavonoids in Digitalis lanata cell cultures. Farmacia 59, $113-118$

Bravo, L., 1998. Polyphenols: chemistry, dietary sources, metabolism, and nutritional significance. Nutr. Rev. 56, 317-333. 
Burchard, P., Bilger, W., Weissenbock, G., 2000. Contribution of hydroxycinnamates and flavonoids to epidermal shielding of UVA and UV-B radiation in developing rye primary leaves as assessed by ultraviolet-induced chlorophyll fluorescence measurements. Plant Cell Environ. 23, 1373-1380.

Caillet, S., Salmieri, S., Lacriox, M., 2006. Evaluation of free radicalscavenging properties of commercial grape phenol extracts by a fast colorimetric method. Food Chem. 95, 1-8.

Carletti, P., Masi, A., Wonisch, A., Grill, D., Tausz, M., Ferretti, M., 2003. Changes in antioxidant and pigment pool dimensions in UV-B irradiated maize seedlings. Environ. Exp. Bot. 50, 149-157.

Chalker-Scott, L., 1999. Environmental significance of anthocyanins in plant stress responses. Photochem. Photobiol. 70, 1-9.

Chalker-Scott, L., Fuchigami, 1989. The role of phenolic compounds in plant stress responses. In: Li, P.H. (Ed.), Low Temperature Stress Physiology in Crops. CRC Press, Boca Raton, FL, pp. 67-76.

Cheynier, V., Comte, G., Davies, K.M., Lattanzio, V., Martens, S., 2013. Plant phenolics: recent advances on their biosynthesis, genetics, and ecophysiology. Plant Physiol. Biochem. 72, 1-20.

Christie, P.J., Alfenito, M.R., Walbot, V., 1994. Impact of low temperature stress on general phenylpropanoid and anthocyanin pathways: enhancement of transcript abundance and anthocyanin pigmentation in maize seedlings. Planta 194, 541-549.

Chung, I.M., Kim, J.J., Lim, J.D., Yu, C.Y., Kim, S.H., Hahn, S.J., 2006. Comparison of resveratrol, SOD activity, phenolic compounds and free amino acid in Rehmannia glutinose under temperature and water stress. Environ. Exp. Bot. 56, 44-53.

Daayf, F., Lattanzio, V. (Eds.), 2009. Recent advances in polyphenol research. John Wiley \& Sons, West Sussex, UK.

Dar, M.I., Naikoo, M.I., Khan, F.A., Rehman, F., Green, I.D., Naushin, F., et al., 2017. An introduction to reactive oxygen species metabolism under changing climate in plants. In: Khan, M.I. R., Khan, N.A. (Eds.), Reactive Oxygen Species and Antioxidant Systems in Plants: Role and Regulation under Abiotic Stress. Springer, Berlin, Germany, pp. 25-52.

De Abreu, I.N., Mazzafera, P., 2005. Effect of water and temperature stress on the content of active constituents of Hypericum brasiliense Choisy. Plant Physiol. Biochem. 43 (3), 241-248.

Dixon, R.A., Paiva, N., 1995. Stress-induced phenylpropanoid metabolism. Plant Cell. 7, 1085-1097.

Elavarthi, S., Martin, B., 2010. Spectrophotometric assays for antioxidant enzymes in plants. Methods Mol. Biol. 639, 273-281.

Falcone Ferreyra, M.L., Rius, S.P., Casati, P., 2012. Flavonoids: biosynthesis, biological functions, and biotechnological applications. Front Plant. Available from: https://doi.org/10.3389/ fpls.2012.00222.

Fiehn, O., 2002. Metabolomics - the link between genotypes and phenotypes. Plant Mol. Biol. 48, 155-171.

Fischbach, R.J., Kossmann, B., Panten, H., Steinbrecher, R., Heller, W., Seidlitz, H.K., 1999. Seasonal accumulation of ultraviolet-B screening pigments in needles of Norway spruce (Picea abies (L.) Karst). Plant Cell Environ. 22, 27-37.

Franca, S.C., Roberto, P.G., Marins, M.A., Puga, R.D., Rodrigeuz, A., Pereira, J.O., 2001. Biosynthesis of secondary metabolites in sugarcane. Gen. Mol. Biol. 24, 243-250.

Gershenzon, J., 1913. Changes in levels of plant secondary metabolites under water and nutrient stress. Recent Adv. Phytochem. 18, $273-320$.

Gnanasekaran, N., Kalavathy, S., 2017. Drought stress signal promote the synthesis of more reduced phenolic compounds (chloroform insoluble fraction) in Tridax procumbens. Free Rad. Antioxid. 7 (1), 128-136.

Gould, K.S., Lister, C., 2006. Flavonoid functions in plants. In: Andersen, Ø.M., Markham, K.R. (Eds.), Flavonoids Chemistry, Biochemistry and Applications. CRC Taylor \& Francis, Boca Raton, FL, pp. 397-411.
Griffith, M., Yaish, M.W.F., 2004. Antifreeze proteins in overwintering plants: a tale of two activities. Trends Plant Sci. 9, 399-405. Available from: https://doi.org/10.1016/j.tplants.2004.06.007. PMID:15358271.

Gumul, D., Korus, J., Achremowicz, B., 2007. The influence of extrusion on the content of polyphenols and antioxidant/antiradical activity of rye grains (Secale cereal L.). Acta Sci. Pol. 6, 103-111.

Harborne, J.B., 1980. Plant phenolics. In: Bell, E.A., Charlwood, B.V. (Eds.), Encyclopedia of Plant Physiology, vol. 8, Secondary Plant Products. Springer, Berlin, pp. 329-402.

Harborne, J.B., 1989. General procedures and measurement of total phenolics. In: Harborne, J.B. (Ed.), Methods in Plant Biochemistry: Volume 1 Plant Phenolics. Academic Press, London, pp. 1-28.

Harborne, J.B., Simmonds, N.W., 1964. Natural distribution of the phenolic aglycones. In: Harborne, J.B. (Ed.), Biochemistry of Phenolic Compounds. Academic Press, London, pp. 77-128.

Harborne, J.B., Baxter, H., Moss, G.P. (Eds.), 1999. Phytochemical Dictionary: Handbook of Bioactive Compounds from Plants. second ed Taylor \& Francis, London.

Hare, P.D., Cress, W.A., 1997. Metabolic implications of stressinduced proline accumulation in plants. Plant Growth Regul. 21, 79-102.

Hawrylak, B., Matraszek, R., Szymanska, M., 2007. Response of lettuce (Lactuca sativa L.) to selenium in nutrient solution contaminated with nickel. Veg. Crop Res. Bull. 67, 63-70.

Hernandez, I., Alegre, L., Munne-Bosch, S., 2004. Drought-induced changes in flavonoids and other low molecular weight antioxidants in Cistus clusii grown under Mediterranean field conditions. Tree Physiol. 24, 1303-1311.

Hewitt, E.J., 1963. The essential nutrients: requirements and interactions in plants. In: Steward, F.C. (Ed.), Plant Physiology. Academic Press, New York \& London, pp. 137-360.

Hättenschwiler, S., Vitousek, P.M., 2000. The role of polyphenols in terrestrial ecosystem nutrient cycling. Trends Ecol. Evol. 15, 238-243.

Jaleel, C.A., Gopi, R., Manivannan, P., Gomathinayagam, M., Riadh, K., Inès, J., et al., 2009. Antioxidant defense responses: physiological plasticity in higher plants under abiotic constraints. Acta Physiol. Plant 31 (3), 427-436.

Janska, A., Marsik, P., Zelenkova, S., Ovesna, J., 2010. Cold stress and acclimation-what is important for metabolic adjustment? Plant Biol. 12, 395-405.

Kagan, V.E., Tyurina, Y.Y., 1997. Recycling and redox cycling of phenolic antioxidants. Ann. N.Y. Acad. Sci. 854, 425-434.

Khan, M.I.R., Khan, N., 2017. Reactive Oxygen Species and Antioxidant System in Plants: Role and Regulation Under Abiotic Stress. Springer Nature, Singapore978-981-10-5254-5.

Khan, M.I.R., Khan, N.A., 2013. Salicylic acid and jasmonates: approaches in abiotic stress tolerance. Plant Biochem. Physiol. 1, 4.

Khan, M.I.R., Iqbal, N., Masood, A., Per, T.S., Khan, N.A., 2013. Salicylic acid alleviates adverse effects of heat stress on photosynthesis through changes in proline production and ethylene formation. Plant Signal. Behav. 8, e26374.

Khan, M.I.R., Asgher, M., Khan, N.A., 2014. Alleviation of saltinduced photosynthesis and growth inhibition by salicylic acid involves glycinebetaine and ethylene in mungbean (Vigna radiata L.). Plant Physiol. Biochem. 80, 67-74.

Khan, M.I.R., Fatma, M., Per, T.S., Anjum, N.A., Khan, N.A., 2015. Salicylic acid-induced abiotic stress tolerance and underlying mechanisms in plants. Front. Plant Sci. 6, 462.

Kidd, P.S., Llugany, M., Poschenrieder, C., Gunsé, B., Barceló, J., 2001. The role of root exudates in aluminium resistance and silicon-induced amelioration of aluminium toxicity in three varieties of maize (Zea mays L.). J. Exp. Bot. 52, 1339-1352. 
Kim, M.S., Kim, C., Jo, D.H., Ryu, Y.W., 1999. Effect of fungal elicitor and heavy metals on the production of flavonol glycosides in cell cultures of Ginkgo biloba. J. Microbiol. Biotechnol. 9, 661-667.

Kirakosyan, A., Seymour, E., Kaufman, P.B., Warber, S., Bolling, S., Chang, S.C., 2003. Antioxidant capacity of polyphenolic extracts from leaves of Crataegus laevigata and Crataegus monogyna (Hawthorn) subjected to drought and cold stress. J. Agric. Food Chem. 51, 3973-3976.

Koes, R.E., Spelt, C.E., Mol, J.N.M., 1989. The chalcone synthase multigene family of Petunia hybrida (V30): differential, light regulated expression during flower development and UV light induction. Plant Mol. Biol. 12, 213-225.

Kolb, C.A., Käser, M.A., Kopecký, J., Zotz, G., Riederer, M., Pfündel, E.E., 2001. Effects of natural intensities of visible and ultraviolet radiation on epidermal ultraviolet screening and photosynthesis in grape leaves. Plant Physiol. 127, 863-875.

Kramer, G.F., Norman, H.A., Krizek, D.T., Mirecki, R.M., 1991. Influence of UV-B radiation on polyamines, lipid peroxidation and membrane lipids in cucumber. Phytochemistry 30, 2101-2108.

Lake, J.A., Field, K.J., Davey, M.P., Beerling, D.J., Lomax, B.H., 2009. Metabolomic and physiological responses reveal multi-phasic acclimation of Arabidopsis thaliana to chronic UV radiation. Plant Cell Environ. 32 (10), 1377-1389.

Landolt, W., Gunthardt-Georg, M.S., Pfenninger, I., Einig, W., Hampp, R., Maurer, S., et al., 1997. Effect of fertilization on ozone-induced changes in the metabolism of birch (Betula pendula) leaves. New Phytol. 137, 389-397.

Larcher, W., 1995. Physiological Plant Ecology. Springer, Berlin, Heidelberg.

Larson, R.A., 1988. The antioxidants of higher plants. Phytochemistry 27, 969-978

Lattanzio, V., 2013. Phenolic compounds: introduction. In: Ramawat, K.G., Mérillon, J.M. (Eds.), Natural Products. Springer-Verlag, Berlin, Heidelberg. Available from: http://doi.org/10.1007/9783-642-22144-6_57.

Lattanzio, V., Cardinali, A., Di Venere, D., Linsalata, V., Palmieri, S., 1994. Browning phenomena in stored artichoke (Cynara scolymus L.) heads: enzymic or chemical reactions? Food Chem. 50, $1-7$.

Lattanzio, V., Di Venere, D., Linsalata, V., Bertolini, P., Ippolito, A., Salerno, M., 2001. Low temperature metabolism of apple phenolics and quiescence of Phlyctaena vagabonda. J. Agric. Food Chem. $49,5817-5821$.

Lattanzio, V., Lattanzio, V.M.T., Cardinali, A., 2006. Role of phenolics in the resistance mechanisms of plants against fungal pathogens and insects. In: Imperato, F. (Ed.), Phytochemistry: Advances in Research. Research Signpost, Trivandrum, pp. 23-67.

Lattanzio, V., Cardinali, A., Ruta, C., Morone Fortunato, I., Lattanzio, V.M.T., Linsalata, V., et al., 2009. Relationship of secondary metabolism to growth in oregano (Origanum vulgare L.) shoot cultures under nutritional stress. Environ. Exp. Bot. 65, 54-62.

Lavid, N., Schwartz, A., Yarden, O., Tel-Or, E., 2001. The involvement of polyphenols and peroxidase activities in heavy metal accumulation by epidermal glands of waterlily (Nymphaeceaea). Planta. 212, 323-331.

Lavola, A., Aphalo, P.J., Lahti, M., Julkunen-Tiitto, R., 2003. Nutrient availability and the effect of increasing UV-B radiation on secondary plant compounds in Scots pine. Environ. Exp. Bot. 49, 49-60.

Li, J., Ou-Lee, T.M., Raba, R., Amundson, R.G., Last, R.L., 1993. Arabidopsis flavonoid mutants are hypersensitive to UV-B irradiation. Plant Cell 5, 171-179.

Lin, D., Xiao, M., Zhao, J., Li, Z., Xing, B., Li, X., et al., 2016. An overview of plant phenolic compounds and their importance in human nutrition and management of type 2 diabetes. Molecules 21, 1374 .
Liang, B., Huang, X., Zhang, G., Zhang, F., Zhou, Q., 2006. Effect of lanthanum on plants under supplementary ultraviolet-B radiation: effect of lanthanum on flavonoid contents in Soybean seedlings exposed to supplementary ultraviolet-B radiation. J. Rare Earths. 24, 613-616.

Liu, L., Gitz III, D.C., McClure, J.,W., 1995. Effects of UV-B on flavonoids, ferulic acid, growth and photosynthesis in barley primary leaves. Physiol. Plant. 93, 734-738.

Logemann, E., Tavernaro, A., Schulz, W., Somssich, I.E., Hahlbrock, K., 2000. UV light selectively coinduces supply pathways from primary metabolism and flavonoid secondary product formation in parsley. Proc. Natl. Acad. Sci. USA 97, 1903-1907.

Ma, D., Sun, D., Wang, C., Li, Y., Guo, T., 2014. Expression of flavonoid biosynthesis genes and accumulation of flavonoid in wheat leaves in response to drought stress. Plant Physiol. Biochem. 80, $60-66$.

Markham, K.R., Tanner, G.J., Caasi-Lit, M., Whitecross, M.I., Nayudu, M., Mitchell, K.A., 1998. Possible protective role for 31, 41-dihydroxyflavones induced by enhanced UV-B in a UVtolerant rice cultivar. Phytochemistry 49, 1913-1919.

McClue, J.W., 1977. The physiology of phenolic compounds in plants. Recent Adv. Phytochem. 12, 525-556.

Michalak, A., 2006. Phenolic compounds and their antioxidant activity in plants growing under heavy metal stress. Pol. J. Environ. Stud. 15, 523-530.

Mira, L., Fernandez, M.T., Santos, M., Rocha, R., Florêncio, M.H., Jennings, K.R., 2002. Interactions of flavonoids with iron and copper ions: a mechanism for their antioxidant activity. Free Radic. Res. 36, 1199-1208.

Murai, Y., Takemura, S., Takeda, K., Kitajima, K., Iwashina, T., 2009. Altitudinal variation of UV-absorbing compounds in Plantago asiatica. Biochem. Syst. Ecol. 37, 378-384.

Nakabayashi, R., Yonekura-Sakakibara, K., Urano, K., Suzuki, M., Yamada, Y., Nishizawa, T., et al., 2014. Enhancement of oxidative and drought tolerance in Arabidopsis by overaccumulation of antioxidant flavonoids. Plant J. 77, 367-379.

Nascimento, N.C., Fett-Neto, A.G., 2010. Plant secondary metabolism and challenges in modifying its operation: an overview. In: FettNeto, A.G. (Ed.), Plant Secondary Metabolism Engineering Methods and Application, Methods in Molecular Biology, vol. 643. Humana Press, New York, pp. 1-13.

Nichols, S.N., Hofmann, R.W., Williams, W.M., 2015. Physiological drought resistance and accumulation of leaf phenolics in white clover interspecific hybrids. Environ. Exp. Bot. 119, 40-47.

Nogue's, S., Allen, D.J., Morison, J.I.L., Baker, N.R., 1998. Ultraviolet$B$ radiation effects on water relations, leaf development, and photosynthesis in droughted pea plants. Plant Physiol. 117 (1), $173-181$.

Obrenovic, S., 1990. Effect of Cu (11) D-penicillanine on phytochrome mediated betacyanin formation in Amaranthus caudatus seedlings. Plant Physiol. Biochem. 28, 639-646.

Oki, T., Masuda, M., Kobayashi, M., Nishiba, Y., Furuta, S., Suda, L., et al., 2002. Polymeric procyanidins as radical-scavenging components in red-hulled rice. J. Agric. Food Chem. 50, $7524-7529$.

Olsson, L.C., Veit, M., Weissenböck, G., Bornman, J.F., 1998. Differential flavonoid response to enhanced UV-B radiation in Brassica napus. Phytochemistry 49, 1021-1028.

Oszman'ski, J., 1995. Polyphenols as antioxidants in food. Przem Spo_z 3, 94-96. in Polish.

Pedranzani, H., Sierra-de-Grado, R., Vigliocco, A., Miersch, O., Abdala, G., 2003. Cold and water stresses produce changes in endogenous jasmonates in two populations of Pinus pinaster Ait. Plant Growth Regul. 52, 111-116. 
Per, T.S., Khan, M.I.R., Anjum, N.A., Masood, A., Hussain, S.J., Khan, N.A., 2018. Jasmonates in plants under abiotic stresses: crosstalk with other phytohormones matters. Environ. Exp. Bot. $145,104-120$.

Pereira, A., 2016. Plant abiotic stress challenges from the changing environment. Front. Plant Sci. 7, 1123.

Perez-Ilzarbe, J., Hernandez, T., Estrella, I., Vendrell, M., 1997. Cold storage of apples (cv. Granny Smith) and changes in phenolic compounds. Z Lebensm Unters Forsch. 204, 52-55.

Posmyk, M.M., Bailly, C., Szafran'ska, K., Jasan, K.M., Corbineau, F., 2005. Antioxidant enzymes and isoflavonoids in chilled soybean [Glycine max (L.) Merr.] seedlings. J. Plant Physiol. 162, 403-412.

Posmyk, M.M., Balabusta, M., Wieczorek, M., Sliwinska, E., Janas, K. M., 2009. Melatonin applied to cucumber (Cucumis satious L.) seeds improves germination during chilling stress. J. Pineal Res. (46), 214-223.

Randhir, R., Lin, Y.-T., Shetty, K., 2004. Phenolics, their antioxidant and antimicrobial activity in dark germinated fenugreek sprouts in response to peptide and phytochemical elicitors. Asia Pac. J. Clin. Nutr. 13, 295-307.

Rice-Evans, C.A., Miller, N.J., Paganga, G., 1997. Antioxidant properties of phenolic compounds. Trends Plant Sci. 2, 152-159.

Rieger, G., Muller, M., Guttenberger, H., Bucar, F., 2008. Influence of altitudinal variation on the content of phenolic compounds in wild populations of Calluna vulgaris, Sambucus nigra, and Vaccinium myrtillus. J. Agric. Food Chem. 58, 9080-9086.

Robards, R., Antolovich, M., 1997. Analytical chemistry of fruit bioflavonoids. A review. Analyst 122, 11R-34R.

Rozema, J., Bjorn, L.O., Bornman, J.F., Gaberscik, A., Hader, D.P., Trost, T., et al., 2002. The role of UV-B radiation in aquatic and terrestrial ecosystems - an experimental and functional analysis of the evolution of UV-absorbing compounds. J. Photochem. Photobiol. B. 66, 2-12.

Ryan, K.G., Swinny, E.E., Winefield, C., Markham, K.R., 2001. Flavonoids and UV photoprotection in Arabidopsis mutants. Z. Naturforsch. 56, 745-754.

Sánchez-Rodríguez, E., Moreno, D.A., Ferreres, F., del Mar RubioWilhelmi, M., Ruiz, J.M., 2011. Differential responses of five cherry tomato varieties to water stress: changes on phenolic metabolites and related enzymes. Phytochemistry 72, 723-729.

Schroeter, H., Boyd, C., Spencer, J.P., Williams, R.J., Cadenas, E., Rice-Evans, C., 2002. MAPK signaling in neurodegeneration: influences of flavonoids and of nitric oxide. Neurobiol. Aging. 23, 861-880.

Schulze-Lefert, P., Dangl, J.L., Becker-Andre, M., Hahlbrock, K., Schulz, W., 1989. Inducible in vivo DNA footprints define sequences necessary for UV light activation of the parsley chalcone synthase gene. EMBO J. 8, 651-656.

Selmar, D., 2008. Potential of salt and drought stress to increase pharmaceutical significant secondary compounds in plants. Landbauforsch. Volkenrode. 58, 139-144.

Shahidi, F., Naczk, M., 1995. Food Phenolics: Sources, Chemistry, Effects, Applications. Technomic Publishing Company Inc, Lancaster, PA.

Shiozaki, N., Hattori, I., Gojo, R., Tezuka, T., 1999. Activation of growth and nodulation in symbiotic system between pea plants and leguminous bacteria by near UV radiation. J. Photochem. Photob. B. Biol. 50, 33-37.

Solecka, D., 1997. Role of phenylpropanoid compounds in plant responses to different stress factors. Acta Physiol. Plant. 19, 257-268.

Soliz-Guerrero, J.B., de Rodriguez, D.J., Rodriguez-Garcia, R., Angulo-Sanchez, J.L., Mendez-Padilla, G., 2002. Quinoasaponins: concentration and composition analysis. In: Janick, J., Whipkey,
A. (Eds.), Trends in New Crops and New Uses. ASHS Press, Alexandria, p. 110.

Spitaler, R., Winkler, A., Lins, I., Yanar, S., Stuppner, H., Zidorn, C., 2008. Altitudinal variation of phenolic contents in flowering heads of Arnica montana cv. ARBO: a 3-year comparison. J. Chem. Ecol. 34, 369-375.

Stapleton, A.E., 1992. Ultraviolet radiation and plants: burning questions. Plant Cell. 4, 1353-1358.

Subba Rao, M.V., Muralikrishna, G., 2002. Evaluation of the antioxidant properties of free and bound phenolic acids from native and malted finger millet (Ragi, Eleusine, coracana, Indaf-15). J. Agric. Food Chem. 50, 889-892.

Swain, T., 1975. Evolution of flavonoid compounds. In: Harborne, J.B., Mabry, T.J., Mabry, H. (Eds.), The Flavonoids. Chapman \& Hall, London, pp. 1096-1138.

Tegelberg, R., Julkunen-Tiitto, R., Aphalo, P.J., 2004. Red: farred light ratio and UV-B radiation: their effects on leaf phenolics and growth of silver birch seedlings. Plant Cell Environ. 27, 1005-1013.

Thimmaraju, B.N., Ravishankar, G.A., 2004. In situ and ex situ adsorption and recovery of betalains from hairy root cultures of Beta vulgaris. Biotechnol. Prog. 20, 777-785.

Trejo-Tapia, G., Jimenez-Aparicio, A., Rodriguez-Monroy, M., De Jesus-Sanchez, A., Gutierrez-Lopez, G., 2001. Influence of cobalt and other microelements on the production of betalains and the growth of suspension cultures of Beta vulgaris. Plant Cell Tissue Organ. Cult. 67, 19-23.

Tuteja, N., Mahajan, S., 2007. Calcium signaling network in plants: an overview. Plant Signal. Behav. 2, 79-85. PMID: 19516972.

Tuteja, N., Ahmad, P., Panda, B.B., Tuteja, R., 2009. Genotoxic stress in plants: shedding light on DNA damage, repair and DNA repair helicases. Mutat. Res. 681, 134-149.

Verslues, P.E., Sharma, S., 2010. Proline metabolism and its implications for plant environment interaction. Arabidopsis Book 8, 0140. Available from: https://doi.org/10.1199/tab.0140.

Villiers, F., Ducruix, C., Hugouvieux, V., Jarno, N., Ezan, E., Garin, J., et al., 2011. Investigating the plant response to cadmium exposure by proteomic and metabolomic approaches. Proteomics 11 (9), 1650-1663.

Weidner, S., Karamać, M., Amarowicz, R., Szypulska, E., Golgowska, A., 2007. Changes in composition of phenolic compounds and antioxidant properties of Vitis amurensis seeds germinated under osmotic stress. Acta Physiol. Plant 29, 238-290.

Weidner, S., Karolak, M., Karamać, M., Kosińska, A., Amarowicz, R., 2009a. Phenolic compounds and properties of antioxidants in grapevine roots (Vitis vinifera) under drought stress followed by regeneration. Acta Soc. Bot. Pol. 78, 97-103.

Weidner, S., Kordala, E., Brosowska-Arend, T.W., Karama, M., Kosińska, A., Amarowicz, R., 2009b. Phenolic compounds and properties of antioxidants in grapevine roots followed by recovery. Acta Soc. Bot. Pol. 78, 279-286.

Williams, R.J., Spencer, J.P., Rice-Evans, C., 2004. Flavonoids: antioxidants or signaling molecules? Free Radic. Biol. Med. 36, $838-849$.

Winkel-Shirley, B., 2001. Flavonoid biosynthesis, a colorful model for genetics, biochemistry, cell biology and biotechnology. Plant Physiol. 26, 485-493.

Winkel-Shirley, B., 2002. Biosynthesis of flavonoids and effects of stress. Curr. Opin. Plant Biol. 5, 218-223.

Wróbel, M., Karmać, M., Amarowicz, R., Frączek, E., Weidner, S., 2005. Metabolism of phenolic compounds in Vitis riparia seeds during stratification and during germination under optimal and low temperature stress conditions. Acta Physiol. Plant 27 (3A), 313-320. 
Wu, S., Chappell, J., 2008. Metabolic engineering of natural products in plants; tools of the trade and challenges for the future. Curr. Opin. Biotechnol. 19, 145-152.

Yamasaki, H., Heshiki, R., Ikehara, N., 1995. Leaf-goldening induced by high light in Ficus microcarpa L. f., a tropical fig. J. Plant Res. $108,171-180$.

Yamasaki, H., Takahashi, S., Heshiki, R., 1999. The tropical fig Ficus microcarpa L. f. cv. Golden Leaves lacks heat-stable dehydroascorbate reductase activity. Plant Cell Physiol.

Zhu, J.K., 2016. Abiotic stress signalling and responses in plants. Cell 167 (2), 313-324.

Zidorn, C., Schubert, B., Stuppner, H., 2005. Altitudinal differences in the contents of phenolics in flowering heads of three members of the tribe Lactuceae (Asteraceae) occurring as introduced species in New Zealand. Biochem. Syst. Ecol. 33, 855-872.

\section{Further Reading}

Alscher, R.G., Donahue, H.L., Cramer, C.L., 1997. Reactive oxygen species and antioxidants: relationships in green cells. Physiol. Plant 100, 224-233.

Blumthaler, M., Ambach, M., Ellinger, R., 1997. Increase in solar UV radiation with altitude. J. Photochem. Photobiol. B 39, 130-134.

Draper, J., 1997. Salicylate, superoxide synthesis and cell suicide in plant defence. Trends Plant Sci. 2, 162-165.

Lattanzio, V., Linsalata, V., Palmieri, S., Van Sumere, C.F., 1989. The beneficial effect of citric and ascorbic acid on the phenolic browning reaction in stored artichoke (Cynara scolymus L.) heads. Food Chem. 33, 93-106. 


\section{0}

\section{Bioactive Molecules as Regulatory Signals in Plant Responses to Abiotic Stresses}

\section{Giulia Franzoni ${ }^{1}$, Alice Trivellini ${ }^{2}$, Roberta Bulgari ${ }^{1}$, Giacomo Cocetta ${ }^{1}$ and Antonio Ferrante ${ }^{1}$}

${ }^{1}$ Department of Agricultural and Environmental Sciences, Università degli Studi di Milano, Milano, Italy ${ }^{2}$ Institute of Life Sciences, Scuola Superiore Sant'Anna, Pisa, Italy

\section{O U T L I N E}

10.1 Introduction

10.2 The Role of Ascorbic Acid, Carotenoids, and Flavonoids in Stress Signaling

10.3 Amino Acids and Derivates Under Stress

10.4 Poly- and Oligosaccharides and Plant Responses

10.5 Nitrate Accumulation and Stress Regulation
10.6 Melatonin Bioactive Molecule in the Regulation of Abiotic Stress

10.6.1 Melatonin Biosynthesis and Its Level Under Stressful Conditions

10.6.2 Priming Plants With Melatonin Against Abiotic Stresses

Further Reading

\subsection{INTRODUCTION}

The bioactive compounds in plants are a wide range of molecules that are produced under suboptimal growing conditions. The biosynthesis of these molecules is aimed to enhance crop tolerance to abiotic and biotic stresses and overcome stressful conditions or to avoid attack from animal and pathogens. Most bioactive compounds in plants belong to the secondary metabolites, but there are also compounds coming from primary metabolism. These molecules have also several beneficial effects in humans and animals, if eaten, and they are of great interest in the diet. The primary role of these compounds is played in plants because they produce them for their benefit in certain environmental conditions. Plants have modified their structure and metabolism for adaptation to different environments. Their adaptation ability has an energy cost that represents part of the energy invested in their defense strategies. Plants based on their adaptation strategies have colonized stressful environments characterized by drought (predesert and desert zones), 
salinity (coastal areas), high or low temperatures, high or low light intensities, polluted areas, etc.

The physiological, biochemical, and molecular changes behind the plant's adaptation ability are extremely important to exploit in the agricultural systems and extend the arable lands in poor environments.

\subsection{THE ROLE OF ASCORBIC ACID, CAROTENOIDS, AND FLAVONOIDS IN STRESS SIGNALING}

Being sessile organisms, plants have developed several strategies to counteract stressful environmental conditions. Most of these strategies are promptly activated and can involve different classes of molecules, which play specific roles in the signaling network. Moreover, different species can respond to the same stimulus in a different way and the molecules involved in the response and signaling can vary even for a specific variety within a species. The stress response can involve a large array of different compounds that are constitutively synthetized by plants or specifically produced in response to an external stimulus or environmental condition. These last compounds are classified as plant secondary metabolites, they can belong to various chemical classes of molecules and can be involved in different metabolic pathways. Many studies have been conducted on the role of plants' secondary metabolites in defense responses and signaling related to biotic stress factors (Akula and Ravishankar, 2011; Nakabayashi and Saito, 2015). However, more recently new functions in plant signaling in response to abiotic stresses have been also discovered, especially for ascorbic acid, flavonoids, carotenoids, and their derivatives.

Ascorbic acid (AsA) is a very important molecule in plants, being involved in several physiological processes tightly connected to both primary and secondary metabolism (Davey et al., 2000), including stress responses. Its biosynthesis can occur via different independent pathways (Smirnoff et al., 2001). Ascorbate is not often considered as a signaling molecule in plants and for several years, its importance as an antioxidant has been largely underlined. AsA can, in fact, actively quench the oxidative cascade and the consequent accumulation of reactive oxygen species (ROS), being oxidized to monodehydroascorbic acid (MDHA) and to dehydroascorbic acid (DHA). Also, its antioxidant activity can be efficiently mediated by the combined action of a series of enzymatic reactions, known as the ascorbate-glutathione cycle (or Halliwell-Asada cycle) (Chew et al., 2003; Khan and Khan, 2017). There is a large plethora of studies produced in the last few years on this topic and on the practical implications in both plant stress metabolism (Cocetta et al., 2014; Pandey et al., 2017) and development (Cocetta et al., 2012; Alós et al., 2014; Wang et al., 2015). It should be considered that ROS are among the key players in plant stress signaling (Suzuki et al., 2012; Baxter et al., 2013), thus the ability of AsA in maintaining ROS homeostasis could indicate an active role in signaling for this molecule too. However, the importance of AsA is due to several other biological functions in which it is actively involved. A protective role of melatonin has been proposed in tomato subjected to heat and salt stress. The authors showed that melatonin treatment altered the expression of genes encoding for the enzymes involved in AsA recycling (Martinez et al., 2018). More recently, it has been suggested that AsA can have a role in cytosolic $\mathrm{Ca}^{2+}$ signals, by activating $\mathrm{Ca}^{2+}$ influx in Arabidopsis roots (Makavitskaya et al., 2018). AsA is also involved in hormone signaling. Arabidopsis mutants with low levels of AsA showed an increment in ethylene production and reduced biomass accumulation. The authors observed also that low AsA altered the expression of genes involved in the hormone pathways that control growth (Caviglia et al., 2018).

Carotenoids are a large group of molecules covering several roles in plants. They act as antioxidants, pigments involved in photoassimilation and in plant-animal interactions (Nisar et al., 2015), and they are the precursors of abscisic acid, one of the most important plant hormones (Walter and Strack, 2011). Recently, new functions for carotenoids have been discovered, including the role in modulating gene expression in response to stress. In fact, ROS-induced oxidation of certain carotenoids (mainly $\beta$-carotene) determines the accumulation of carotenoid derivatives, which are potential signal molecules (Havaux, 2014). Among those molecules, $\beta$-cyclocitral or $\beta$-ionone were reported to increase in response to photooxidative stress and to stimulate the expression of genes related to cellular defense against stress in Arabidopsis (Ramel et al., 2012). Isoprene, which is the constituent unit of carotenoids, could also have a role in stress signaling (Havaux, 2014). In fact, different abiotic stressful conditions have been reported to stimulate the emission of large amounts of isoprene-oxidation products (Jardine et al., 2013). Also, in a previous study, volatile isoprenoids have been proposed as important actors able to modulate oxidative stress induced by various factors (Vickers et al., 2009). It always important to consider that different metabolic routes or responses can be activated at once and no one of the considered responses act separately from the others. For example, it has been shown that an impairment in carotenoids and porphyrins (chlorophyll precursors) biosynthesis obtained by exogenous treatments with chemical 
inhibitors, can be sensed by stress signaling molecules, such as hydrogen peroxide, ascorbate, glutathione, and salicylic acid, and can in turn activate enzymemediated antioxidant responses in rice (Khan et al., 2016; Park and Jung, 2018).

Flavonoids are a subgroup of phenolic compounds that have been reported to have several biological activities in both plants and animals. The efficacy of flavonoids as antioxidants is well known and has been largely documented, also in relation to response to biotic and abiotic stresses (Brunetti et al., 2013; Rejeb et al., 2014). However, it is also known that these molecules can have other important biological functions, being involved in transport and signaling transduction pathways (Peer and Murphy, 2006). The presence of flavonoids in the nucleus has been demonstrated in different plant species, and the potential target of flavonoid regulation are transcription factors, kinases, and ABC (ATP-binding cassette) transporters. However, most of these interactions are thought to be related to developmental processes more than stress response metabolism. For example, heavy metal stress has been reported to induce an increment in both ROS and flavonoids (Khan and Khan, 2014). In this sense flavonoids can alter the ROS-mediated signal transduction by acting as antioxidant and metal chelators (Skórzyńska-Polit et al., 2010; Brown et al., 1998), but the presence of a specific signaling mechanism involving them has not been demonstrated. More recently, it has proposed that the interaction of flavonoids with protein kinases could also suggest an important role for flavonoids in photoprotection other than in plant development. The authors concluded that flavonoids could affect MPAK signaling by directly binding to the active sites of the proteins or by modulating their activation through ROS-scavenging activity (Brunetti et al., 2013). Important changes in flavonoids accumulation were observed in Arabidopsis in response to drought stress, but the exact mechanisms of signaling and regulation are still unclear (Nakabayashi et al., 2014).

Glucosinolates are a class of sulfur-containing secondary metabolites, typically from the Brassicaceae family. These compounds are particularly appreciated as components of functional foods and, at the same time, they have an important role in plant stress responses, together with their degradation products (such as isothiocyanates and nitriles) (Halkier and Gershenzon, 2006). Recently, great attention has been paid to the cross-talk between some of the physiological processes in stress response and glucosinolate metabolism, and it has been proposed that glucosinolates may act as signals themselves (Martínez-Ballesta et al., 2013). However, this aspect need to be further explored.
In the near future, the application of innovative techniques of investigation like transcriptome analysis, gene silencing, proteomics, metabolomics, as well as the use of mutants, will help to better understand the mechanisms of stress sensing, signaling, and regulation and perhaps to discover novel functions or molecules involved in this complex network.

\subsection{AMINO ACIDS AND DERIVATES UNDER STRESS}

Amino acids are molecules made up of two functional groups: amine $\left(-\mathrm{NH}_{2}\right)$ and carboxylic acid $(-\mathrm{COOH})$, and a specific side-chain $(\mathrm{R})$, which is responsible for the differences in chemical properties. They can be classified in many ways depending on their polarity, $\mathrm{pH}$ level, or functional groups location. According to the structure and the chemical characteristics of the $\mathrm{R}$ group, amino acids are arranged in six classes: aliphatic, hydroxyl or sulfur containing, cyclic, aromatic, basic, acidic, and their amides (Anjum et al., 2014).

Amino acids have commonly been considered as precursors and constituents of proteins, but they are also building blocks for several other nitrogen containing compounds such as nucleic acids or numerous metabolites such as hormones, cell wall components, chlorophyll, and secondary metabolites, and are present in the cell in a free form too. Moreover, they act as regulatory and signaling molecules improving plant stress tolerance and regulating the timing and developmental progression of leaf senescence (Less and Galili, 2008; Watanabe et al., 2013; Araújo et al., 2015; Planchet et al., 2015). Amino acids also have an important role in plant nutrition, for example, glutamine synthetase is a key enzyme involved in the assimilation and transport across the cell membrane of nitrogen (Moe, 2013; Anjum et al., 2014). Due to this biological significance amino acids are commonly used as fertilizers or components in several products such as biostimulants.

Plants are often exposed to suboptimal environmental conditions that are unfavorable for growth, development, and can reduce agronomic yield. The prevalent abiotic stresses limiting crop productivity are usually related to water amount (drought, flooding), salinity, temperature (cold, heat), nutrient deficiency, chemical toxicity, and pollutants. Defense and adaptation mechanisms of plants are dynamic, complex, and affect several metabolic pathways according to the nature of the stress, plant species, tissue, or developmental stage (Planchet et al., 2015; Araújo et al., 2015). However, plants exhibit shared responses that are in common to different stresses. Some of these 
lead to an alteration both the amino acid spectra and their derivates (Rai, 2002; Less and Galili, 2008; Gill and Tuteja, 2010; Hussain et al., 2011; Araújo et al., 2015). Moreover, a bunch of studies suggested that exogenous application of amino acids and polyamines such as putrescine, spermine, and spermidine results in abiotic stress tolerance in various plants (Gill and Tuteja, 2010).

It is widely assumed that the amino acid pool is much induced during abiotic stress. Indeed, an overall increase of several defense molecules commonly referred to as osmoprotectants or compatible osmolytes (peptides, amines, amino acids, proteins, betaines, sugars) is a part of the plant mechanism to cope with osmotic stress, in particular due to drought, salinity, and flooding (Joshi et al., 2010), allowing the maintenance of cell turgor. Among these, specific amino acids such as proline, alanine, arginine, glycine, amides such as glutamine and asparagine, nonprotein amino acids like $\gamma$-aminobutyric acid, pipecolic acid, citrulline, and ornithine have been reported for their enhancement in plants under these conditions (Skirycz et al., 2010; Obata and Fernie, 2012; Anjum et al., 2014).

An important increase in the branched-chain amino acids (isoleucine, valine, and leucine) and aromatic amino acids (tryptophan, tyrosine, phenylalanine) has been observed also under less severe stress conditions, acting as alternative respiratory substrates (Araújo et al., 2011).

To cope with flooding and anaerobiosis, cytosolic pH commonly decreases and amino acids level changes. In those stressful conditions it has been observed that $\gamma$-aminobutyric acid, alanine, glycine, serine, and proline increase to limit cytoplasmic acidosis, while aspartic acid and glutamine decrease (Reggiani et al., 1988; Roberts et al., 1992).

Several studies have attributed a nonenzymatic antioxidant activity to proline and polyamines, suggesting ROS scavenging role and singlet oxygen quencher (Smirnoff and Cumbes, 1989; Mohanty et al., 2001; Matysik et al., 2002). Proline can also have an indirect action in protecting and stabilizing ROS scavenging enzymes such as peroxidase, glutathion-S-transferase, superoxide dismutase, and catalase, activating alternative detoxification pathways (Hare and Cress, 1997). However, there is contrasting evidence about this role. Indeed, Signorelli et al. (2013) demonstrated that proline does not play any role in quenching singlet oxygen.

Polyamines are also involved in these kinds of responses, working as antioxidants, free radical scavengers, or as membrane stabilizers (Velikova et al., 2000).

Amino acids such as asparagine and proline, but mostly polyamines bind to metals such as zinc, cadmium, and copper by forming a metal complex useful for protecting the activity of some enzymes against the effects of toxic heavy metals (Rai, 2002; Planchet et al., 2015).

There is a large amount of information on amino acids involvement in plant stress responses as reported above; otherwise currently very little is known about their signaling function especially under unfavorable conditions. Indeed, whereas in the past only hormones were considered as signal molecules, data of recent years suggest that this function may be performed also by other compounds.

Proline is one of the most important amino acids and plays several roles in plants both in stressed and unstressed conditions. Different studies have suggested an extra role as a signaling molecule involved in regulation of gene expression under stress (Szabados and Savoure, 2010; Hayat et al., 2012).

Maggio et al. (2002) proposed that proline may act as regulatory molecule in salt-induced vacuolation. This cellular strategy has been reported in yeast (Bone et al., 1998) and plants (Chang et al., 1996). It has been observed that signal transduction system involved with perception and adaptation to osmotic stress is associated with accumulation of increased intracellular proline. Some branched-chain amino acids (BCAAs) such as serine, proline, and leucine have been shown to have a direct activity as signaling molecules and others are the precursors for a variety of secondary metabolites or phytohormones with signaling function (Nambara et al., 1998; Urano et al., 2009; Hildebrandt et al., 2015). Examples of regulation of transduction signal induced by amino acids involve target of rapamycin (TOR) kinase. Through this pathway, functioning as regulatory integrators of environmental signals, BCAAs, in particular leucine, act to modulate global RNA (Kimball and Jefferson, 2006).

Hannah et al. (2010) designed a large experiment to investigate the response of Arabidopsis to different conditions. They applied transcriptomics and metabolomic analysis to monitor the changes induced by environmental perturbation. Interesting variation in leucine has been observed suggesting a possible role as regulator of gene expression. Based on the results obtained from the experiment they attributed to leucine an important role as mediator molecule. Indeed, a high correlation between gene expression analysis and transgenic Arabidopsis plant overproducing leucine has been observed. This leucine function has not been described in plants but in other organisms where leucine probably activates the TOR pathway stimulating protein synthesis (Dann and Thomas, 2006). It is very likely that a similar mechanism exists also in vegetable tissues (Dinkeloo et al., 2017). 
Furthermore, Häusler et al. (2014) recently focused on some amino acids' role as metabolic intermediates / end-products and as signal molecules, comparing medical and plants fields. They suggested that the amino acid serine has a role in signaling. Both L-serine and D-serine act as metabolic signal in the communication between male gametophytes and pistil by regulating a glutamate receptor-like $\mathrm{Ca}^{2+}$ channel in pollen tubes. This mechanism looks like what happens in other organisms, where serine functions as neurotransmitter and regulates the activity of a nonselective ion channel. As well as leucine, serine may be also related to the TOR pathway even if there is no direct evidence to prove it.

Gamma-aminobutyric acid (GABA), a four-carbon nonprotein amino acid, is a significant component of the free amino acid pool. It accumulates rapidly in plant tissues as a response to biotic and abiotic stresses, but its physiological significance and the specificity of the response are largely unclear. Different roles have been proposed, such as the regulation of cellular $\mathrm{pH}$ and osmolarity, maintaining $\mathrm{C} / \mathrm{N}$ balance, scavenging of ROS and defense against insect attack (Shelp et al., 1999; Bouche and Fromm, 2004; Mekonnen et al., 2016). Its fast increase may be related with the glutamate decarboxylase (GAD) activity and the interaction with $\mathrm{Ca}^{2+} /$ calmodulin. Indeed, different stress conditions trigger a transduction pathway that leads to an increase in cytosolic $\mathrm{Ca}^{2+}$, which activates GAD resulting in GABA synthesis from glutamate (Shelp et al., 1999).

Different studies have also reported a strong link between the TCA cycle and GABA shunt, the pathway involving GABA synthesis and breakdown (Shelp et al., 1999; Bouche and Fromm, 2004). It has been reported that changes in the TCA cycle upstream of succinate production enhanced the flux through the GABA shunt, and vice versa, a mutation of the GABA pathways impact the accumulation of TCA cycle intermediates, since GABA is a significant source of succinate in this network (Gilliham and Tyerman, 2016).

Furthermore, Mekonnen et al. (2016) proposed that GABA acts as a signal molecule suggesting that its accumulation under drought conditions is a stressspecific response and this phenomenon induces the regulation of stomatal opening thereby preventing loss of water. The control of stomatal movement may be regulated by the interaction between GABA and ALMT (aluminum-activated malate transporter) channel and through the modulation of the activity of $\mathrm{H}^{+}$ATPase via 14-3-3 proteins. Under drought stress GABA concentration increases leading to a deactivation of the flux of anions into vacuole binding to ALMT6 protein. This implicates ALMT proteins as GABA receptors in plants acting as metabolic sensors that are able to translate environmental changes into physiological outputs. GABA has been proposed to be able to affect $\mathrm{Ca}^{2+}$ channel activity via provoking changes in membrane potential (Ramesh et al., 2015). The regulation of membrane potential is known to constitute a signal in several cell types.

Additionally, GABA represses the expression of 143-3 proteins, which are involved in light-induced stomata opening (Lancien and Roberts, 2006). All this speculation on GABA's signaling role results also from the similarities between its mechanisms in plant and mammalian cells where GABA is described as a neurotransmitter (Chebib and Johnston, 1999).

Recent studies have suggested an interplay between the GABA shunt and the TCA cycle through various bypasses (Fait et al., 2008). Although further analysis of these metabolic pathways is required, our metabolome analysis allows us to speculate that reduction of proline and agmatine as members of the glutamate family, and methionine, lysine, and saccharopine as members of the aspartate family, caused activation of the GABA shunt and the TCA cycle in nc3-2 under dehydration.

Polyamines are critical for plant growth and development, and play an important role in controlling downstream stress responses by modulating gene expression and regulating different transporters (Tuteja and Sopory, 2008). Transcriptomic analysis shows that spermine and spermidine act as anabolic regulators, while putrescine acts as a catabolic regulator in tomato fruit. Moreover it is supposed that there is a connection between TOR and polyamines due to their linkage with anabolic processes and C:N signaling in tomato (Handa and Mattoo, 2010). Spermine is also involved in defense signaling against plant pathogens by regulating a subset of defense-related genes and leading to hypersensitive response and to programmed cell death (Takahashi and Kakehi, 2009).

Polyamines have been positively correlated with plant responses to abiotic stresses for a long time. They accumulate under several unfavorable conditions such as salinity, drought, chilling, heat, hypoxia, heavy metals, and UV radiation (Alcázar et al., 2010; Gill and Tuteja, 2010; Gupta et al., 2013; Mattoo et al., 2015). Polyamines act in different ways to help plant tolerance as free radical scavengers and keep cellular ionic balance affecting $\mathrm{K}^{+} / \mathrm{Na}^{+}$homeostasis and regulating ion channels (Zhao et al., 2007). Several studies have reported that polyamines act as signal molecules in the ABA-regulated stress response pathway and through the production of $\mathrm{H}_{2} \mathrm{O}_{2}$. Indeed, has been shown that salt stress and treatment with $\mathrm{ABA}$ induce polyamine transport in the apoplast (Gupta et al., 2013). Metabolome and transcriptome analysis have also revealed that polyamines and $\mathrm{ABA}$ modulate each 
other's biosynthesis under stress to increase plant adaptive potential (Urano et al., 2009; Pal et al., 2015). Moreover, $\mathrm{H}_{2} \mathrm{O}_{2}$ produced during polyamines catabolism leads to the synthesis of ROS, which triggers a downstream signal cascade upregulating various stress responses proteins. Yamasaki and Cohen (2006) reported evidence of cross-talk of polyamine also with NO, a small gaseous molecule functioning as an intraand intercellular messenger, but the mechanism for this action is still not understood and more experiments are needed. NO accumulation has been reported after spermidine and spermine treatments in Arabidopsis. This linkage between NO and polyamines could explain several physiological effects of polyamines under stress conditions (Pal et al., 2015). Polyamines play an important role in the regulation of ion channels during stress through the induction of signaling molecules like ROS and NO. Several studies also reported a role in the regulation of the vacuolar $\mathrm{Ca}^{2+}$ concentration and during the sequestration of $\mathrm{Na}^{+}$in salt stress conditions (Yamaguchi et al., 2006; Kusano et al., 2007; Liu et al., 2014). A lot of experiments have shown that amino acids and polyamines act as an elicitor of plant defense responses under stress. Much progress has been made by developing amino acids or polyamine deficient mutants, or by a multidisciplinary approach. However, the exact mechanism of action is not fully understood.

\subsection{POLY- AND OLIGOSACCHARIDES AND PLANT RESPONSES}

Oligosaccharides and polysaccharides are carbohydrate polymers made up of units of simple sugars called monosaccharides interconnected by glycosidic linkages. Generally, the first contain three to ten sugar residues; the latter are a long chain of monosaccharides that can reach up to a molecular weight of 100,000 daltons and comprise much of the biomass on the planet.

Carbohydrates have a pivotal role as sources of energy and carbon skeleton for organic compounds and as storage components. Cellulose, chitin, and pectin are the most abundant carbohydrates present in plant cells. High sugar level generally stimulates expression of genes involved in growth, storage of protein, and biosynthesis of starch and fructans (Gupta and Kaur, 2005). Their concentration and metabolism alter both during development and in response to different environmental signals. For example, sugar accumulation is stimulated by nitrogen limitation and high-intensity light. Under unfavorable conditions, soluble sugars, sugar alcohols, cyclic carbohydrates, and raffinose family oligosaccharides (RFOs) function as osmolytes to maintain cell turgor.

In addition, an important regulatory function as signaling molecules involved in plant growth and stress resistance has been recently observed. Studies on carbohydrates revealed that fragments from plant cell walls might act as signaling molecules to activate plant defensive genes and change plant growth and development (Ryan, 1987). In general, this metabolic network is linked with plant hormones. Indeed, several experiments have shown that a cross-talk occurs between glucose and ABA signaling (Rook and Bevan, 2003; Rolland et al., 2006; Bolouri-Moghaddam et al., 2010; Elsayed et al., 2014). Moreover, stress-induced ABA can upregulate different transcription factors that activate both galactinol synthase and raffinose synthase (Valluru and Van Den Ende, 2011). Interaction between sugars and ABA signaling might control the induction of leaves' senescence during drought stress (Wingler and Roitsch, 2008). This evidence suggests an involvement of carbohydrates in a process called "sweet immunity," stimulating the immune system of plants and abiotic stress responses (Zhang et al., 2009; Bolouri Moghaddam and Van Den Ende, 2013). This concept is commonly related to small sugars (mono-, di-, and some small oligosaccharides) such as sucrose, raffinose, or galactinol, that are able to activate plant defenses against pathogen attacks. Several observations suggested that also fructans (fructose-based oligo- and polysaccharides) may be involved in sweet priming processes, counteracting biotic and abiotic stresses.

Kafi et al. (2003) observed that levels of fructans increased in salt-stress wheat reaching higher levels in tolerant cultivars compared with sensitive ones. These molecules are the major storage sugars in cereals and cool zone grasses and are involved also in freezing and drought tolerance mechanisms. Indeed, they accumulate during stress conditions because they are highly soluble in water and resistant to crystallization at subzero temperatures (Kerepesi et al., 2002; Kawakami et al., 2008; Joudi et al., 2012; Van Den Ende and El-Esawe, 2014). Fructans have also an important role stabilizing membranes by inserting into the lipid headgroup region. This helps prevent leakage when water is removed from the system both during freezing and drought (Livingston et al., 2009). Their role is also confirmed by the higher expression of both defense-related TFs and fructans biosynthetic genes (Gaudet et al., 2011). Moreover, the introduction of fructan biosynthesis genes in nonfructan accumulator cultivars confers abiotic stress tolerance (Bie et al., 2012; Van Arkel et al., 2013). Wang et al. (2009) observed that after spraying a preparation containing fructan on tobacco leaves, the resistance against 
tobacco mosaic virus was increased. They suggested an involvement of salicylic acid-dependent signaling pathways. This preparation increased the content of lignin and enhanced the activity of defense-related enzymes such as peroxidase or superoxide dismutase in the same leaves. Similar results have been obtained in tomato fruit treated with the same preparation in postharvest diseases (Sun et al., 2013). This treatment increased also the mRNA level of genes encoding various pathogenesis-related proteins and phenylanaline ammonialyase (PAL) gene.

Nishizawa et al. (2008) obtained similar results after overexpression of genes involved in biosynthesis pathway of RFOs. Van den Ende and Valluru (2008) hypothesized that RFOs, galactinol, and fructans might function as transportable stress signals mediating different stress responses. It's known that galactinol acts as a signal molecule in pathogen-induced resistance (Kim et al., 2008); however, the signal role of these molecules under abiotic stress is still unclear (Elsayed et al., 2014).

\subsection{NITRATE ACCUMULATION AND STRESS REGULATION}

Nitrogen $(\mathrm{N})$ is an essential macronutrient required for the synthesis of vital molecules, such as proteins, nucleic acids, and chlorophyll, and it is a fundamental nutrient for cellular metabolism (Parker and Newstead, 2014). Its absorption at the root level determines plant growth and consequently crop productivity (Krapp et al., 2014; O’Brien et al., 2016).

In plants, several processes, including $\mathrm{N}$ uptake and assimilation, are known to be adversely affected by abiotic stresses, such as salinity, drought, and extreme temperatures (Goel and Singh, 2015). It is a wellestablished fact that high salt concentration inhibits nitrate reductase (NR) activity; this enzyme catalyzes the first enzymatic step in nitrate assimilation in plants, involving reduction of nitrate to nitrite. Several metabolic and environmental signals regulate NR activity (Kaiser et al., 1999). The reduced activity of the enzyme in the leaves of salt-stressed plants is attributed to salinity inhibited nitrate transport to the shoot, which in turn is due to interference with nitrate uptake and xylem loading (Cramer et al., 1995). The uptake of nitrogen, its translocation, and also the assimilation has been found to be affected by high salinity in cowpea plants, as reported by da Silveira et al. (1999). Nitrogen use efficiency was also reported to be reduced significantly with increased salinity levels $\left(1.5,4.5\right.$, and $6.5 \mathrm{dS} \mathrm{m}^{-1}$ ) in chile pepper (Huez López et al., 2011). The high salinity has been shown to inhibit the activity of many of the key enzymes involved in nitrogen assimilation in maize plants, Brassica juncea, and tomato seedlings (Khan and Srivastava, 1998; Debouba et al., 2007; Goel and Singh, 2015). Likewise, under drought stress conditions, the activity of nitrate reductase and glutamine synthetase were found to be reduced in barley plants, as reported by Robredo et al. (2011). Effect of drought stress on Brassica juncea was reported by Singh et al. (2009) in a work that showed that photosynthesis, transpiration, water use efficiency, and other morphophysiological characters were adversely affected by this stressful condition (Goel and Singh, 2015). Increasing concentration of $\mathrm{NaCl}$, polyethylene glycol, and metal salts on rice seedlings showed a marked inhibitory effect on the activity of NR (Sharma and Dubey, 2005). An important signaling molecule with diverse physiological functions in plants is nitric oxide (NO), which leads to the expression of stress response genes under various abiotic/biotic stressful conditions (Arasimowicz and Floryszak-Wieczorek, 2007 and references therein; Qiao and Fan, 2008). An enzymatic source of NO in plants is NR. Production of NO, dependent on NR activity, was recorded in several plant species, like cucumber (de la Haba et al., 2001), sunflower, spinach, maize (Rockel et al., 2002), Arabidopsis (Desikan et al., 2002), wheat, and tobacco (Planchet et al., 2005, 2006), and its production was demonstrated in vitro (Yamasaki, 2000) and in vivo (Rockel et al., 2002). This synthesis was strictly dependent on nitrite and nitrate content in the plant tissue (Yamasaki et al., 1999; Kaiser, 2001).

\subsection{MELATONIN BIOACTIVE MOLECULE IN THE REGULATION OF ABIOTIC STRESS}

Melatonin is a tryptophan derivative compound ( $N$ acetyl-5-methoxytryptamine) that was discovered in the late 1950s in bovine pineal tissue (Lerner et al., 1958). Initially, melatonin was considered only to occur in animals, but further evidence emerged confirming that this compound is ubiquitously present in almost all organisms from primitive photosynthetic bacteria, red and green algae, fungi, and plants to humans (Poeggeler et al., 1991; Tilden et al., 1997; Yilmaz et al., 2014; Hattori et al., 1995; Dubbels et al., 1995).

In animals this signaling molecule is considered a hormone, acting as a neurotransmitter and influencing sleep, body temperature, mood, the retina, and sexual behavior through the circadian clock present in animals (Reiter et al., 2010). Anyway, this wide range of melatonin's functions in animals were acquired later during evolution (Reiter et al., 2010; Tan et al., 2010). 
Several studies in unicellular organisms, plants and animals have confirmed the primary function of this phylogenetically ancient molecule, as a free radical scavenger and antioxidant to protect organisms from a variety of environmental and internal oxidative stresses (Galano et al., 2011; Manchester et al., 2015; Shi et al., 2015; Arnao and Hernandez-Ruiz, 2015; Zhou et al., 2015). Since the discovery of melatonin in plants around two decades ago (Hattori et al., 1995), several aspects have been investigated and driven by what is known in animals. These studies can be summarized in three main focus areas: (1) modulation of circadian rhythms and photoperiod-dependent processes; (2) medicinal and nutritional features (as nutraceutical value), with the aim of identifying plants species containing particularly high levels of melatonin; and (3) as a scavenger of free radicals thus preserving the integrity of plants under a variety of stressful conditions.

Thus, the data summarized below primarily consider the roles of melatonin related with diverse aspects linked to its protective function against abiotic stressors in plants. In particular, the melatonin levels in plants under stress conditions and the possibility of melatonin priming against multiple abiotic stresses are discussed.

\subsubsection{Melatonin Biosynthesis and Its Level Under Stressful Conditions}

Plant melatonin is synthesized via similar biosynthetic pathways to those in animals and comprises four consecutive enzymatic steps (Kang et al., 2011). The first step is the decarboxylation of tryptophan into tryptamine by tryptophan decarboxylase (TDC). The second step is the hydroxylation of the amine by the cytochrome P450 enzyme tryptamine 5-hydroxylase (T5H) to form serotonin (Fujiwara et al., 2010). Serotonin, in both plants and animals, is converted to $\mathrm{N}$-acetyl serotonin in an enzymatic reaction catalyzed by serotonin $\mathrm{N}$-acetyltransferase (SNAT), which is then O-methylated by hydroxyindole-O-methyltransferase (ASMT, formerly known as hydroxymethyl O methyltransferase, HIOMT) resulting in the formation of melatonin (Nawaz et al., 2016).

The rate limiting enzyme in the biosynthetic pathway of melatonin is ASMT (Liu and Borjigin, 2005). Interesting, recent works on subcellular localization of the final enzyme in this pathway (ASMT) show that this enzyme is localized in both mitochondria (Wang et al., 2017) and chloroplast (Zheng et al., 2017), thus representing the primary sites for melatonin biosynthesis. In fact, these plant organelles are major sources of free radical generation, such as ROS and RNS, and thus, they require strong protection against oxidative stress. Melatonin, produced in high level in plants by both chloroplast and mitochondria (Arnao and Hernández-Ruiz, 2014), is a potent antioxidant and free radical scavenger that provides onsite protection against the byproducts of energy metabolism and preserves the physiological functions of these important organelles, working as a powerhouse at photosynthetic sites (Tan et al., 2013).

Many studies show that endogenously produced melatonin increases significantly when plants are exposed to rapid change in the environmental conditions perceived by the plants as stress clues. These include hot or cold temperatures, salinity, drought, overwatering, ultraviolet radiation, high irradiation, and chemical or metal pollutants in water and soils. In barley roots (Arnao and Hernández-Ruiz, 2009) and lupin plants (Arnao and Hernández-Ruiz, 2013) the changes in endogenous levels of melatonin under natural or artificially perturbations were measured. A remarkable observation from these studies was that nearly all stress factors, such as drought, anaerobic, $\mathrm{pH}$, and cold stress as well as using $\mathrm{ZnSO}_{4}, \mathrm{NaCl}$, and $\mathrm{H}_{2} \mathrm{O}_{2}$ as chemical stress agents, upregulated melatonin biosynthesis (Arnao and Hernández-Ruiz, 2009, 2013). The elevated melatonin production associated to different forms of environmental perturbation may serve as an adaptive reaction of plants to tolerate adverse environmental conditions due to its well-known antioxidant properties. Moreover, exposure to heavy metals is associated with toxic effects on biological macromolecules leading to oxidative damage. The significant rise in melatonin content observed in green microalgae Ulva sp. exposed to cadmium, lead, and zinc (Tal et al., 2011), and the mitigation of heavy metals-induced stress by melatonin exogenously applied, suggest the involvement of melatonin in adaptation to environmental stress.

Increases of melatonin were also reported to be a consequence of light intensity and spectral quality. Comparing plants grown indoors with plants grown under field conditions subjected to more variable conditions and sunlight showed that melatonin concentrations were increased more than threefold in plants cultivated in the field (Tan et al., 2007). Pepper nonshaded fruits contained more melatonin content than that of shaded fruit (Riga et al., 2014). In addition, Conti et al. (2002) reported that Alpine and Mediterranean species that normally are exposed to high natural light and UV intensities contained enhanced levels of melatonin compared with the same or related species from other areas. Similar results were obtained in Glycyrrhyza uralensis grown under high intensity UV-B radiation compared with those grown under low intensity radiation, showing increase melatonin levels in roots for plants exposed to high 
UVB radiation (Afreen et al., 2006). Therefore, the upregulation of melatonin biosynthesis may protect the plant from large quantities of free radicals generated during photosynthesis, especially under stressful conditions such as exposure to excessive light intensity and UV radiations.

\subsubsection{Priming Plants With Melatonin Against Abiotic Stresses}

A key role for melatonin as a potent antioxidant appears to be a fundamental stress resistance mechanism in plants, supporting their resistance to different forms of stress (Zhang et al., 2015; Wang et al., 2013). As in the above-discussed studies, there are also many recent investigations that reveal a causal relationship between environmental stresses and induction of oxidative stress and damage, which are mitigated by exogenous treatments of melatonin. Melatonin pretreatment significantly increases the tolerance of both drought-tolerant Malus prunifolia and drought-sensitive M. hupehensis plants (Li et al., 2015) by the downregulation of genes involved in abscisic acid (ABA) catabolic route leading to a lower ABA content, and the enhancement of antioxidant enzyme activities, which directly scavenge $\mathrm{H}_{2} \mathrm{O}_{2}$ ( $\mathrm{Li}$ et al., 2015). In Bermuda grass pretreated with melatonin and then exposed to different cold stress treatments a positive effect was observed of this indoleamine molecule toward cold resistance by maintaining cell membrane stability, increasing antioxidant enzymes activities, and improving the photosynthetic process (Fan et al., 2015). The role of melatonin in the alleviation of photoinhibition in tomato seedlings exposed to moderate light during chilling was recently reported to be dependent on its dual mode of action, as a direct scavenger of ROS and as a trigger of antioxidant responses, both actions leading to reduced level of ROS (Ding et al., 2017). Using exogenously applied melatonin Arora and Bhatla (2017) reported an interaction between this molecule and nitric oxide signaling pathways that is involved in the regulation of seedling growth leading to an attenuation in both oxidative and nitrosative stress under salt stress in sunflower. Furthermore, melatonin has been shown to contribute to extending leaf longevity while enhancing stress tolerance. Long-term pretreatment with melatonin significantly reduced chlorophyll degradation and suppressed the upregulation of senescence-associated gene 12 (SAG12) and helped maintain photosystem function under drought and dark-induced leaf senescence in apple (Wang et al., 2013). Similar results were obtained in rice where treatments with melatonin significantly reduced chlorophyll degradation, suppressed the transcripts of senescence-associated genes, delayed the leaf senescence, and enhanced salt stress tolerance (Liang et al., 2015). These authors, using a high-throughput RNA sequencing, reported a complex molecular network under the control of transcriptional activators and repressors (such as bZIP, NAC, MYB TFs, DREBs, and HSFs transcription factors), which consequently modulates the expression levels of genes involved in oxidation-reduction, chlorophyll biosynthesis, stress responses, nutrient metabolism and remobilization processes, and finally delays leaf senescence and enhances salt stress tolerance (Liang et al., 2015). These results indicate also that melatonin is an essential feature closely related to the delay of plant senescence.

\subsection{CONCLUSION}

Crop plants are subjected to multiple abiotic and artificial-induced stresses during their life span that greatly reduce productivity and also the quality of these commodities during postharvest. Recent research reviewed in Savvides et al. (2016) suggests that plants can be primed by chemical compounds to better tolerate different environmental perturbations. The evidence indicates that melatonin exogenously applied as a priming agent can prepare plant stress response due to its multifaceted action (Arnao and Hernández-Ruiz, 2014; Zhang et al., 2015; Wang et al., 2013). Given the promising potential of melatonin as a direct antioxidant molecule, as a trigger of antioxidant responses in plants, and its beneficial effects on senescence and cell death processes, it could represent an effective strategy to employ in crop management to both enhance tolerance against multiple abiotic stressors and to prolong shelf life of harvested leafy vegetables and flowers.

\section{References}

Afreen, F., Zobayed, S., Kozai, T., 2006. Melatonin in Glycyrrhiza uralensis: response of plant roots to spectral quality of light and UVB radiation. J. Pineal Res. 41, 108-115.

Akula, R., Ravishankar, G.A., 2011. Influence of abiotic stress signals on secondary metabolites in plants. Plant Signal. Behav. 6 (11), 1720-1731

Alcázar, R., et al., 2010. Polyamines: molecules with regulatory functions in plant abiotic stress tolerance. Planta 231 (6), 1237-1249.

Alós, E., Rodrigo, M.J., Zacarías, L., 2014. Differential transcriptional regulation of L-ascorbic acid content in peel and pulp of citrus fruits during development and maturation. Planta 239 (5), 1113-1128.

Anjum, N.A., Gill, S.S., Gill, R. (Eds.), 2014. Plant Adaptation to Environmental Change: Significance of Amino Acids and Their Derivatives. CABI, UK.

Arasimowicz, M., Floryszak-Wieczorek, J., 2007. Nitric oxide as a bioactive signalling molecule in plant stress responses. Plant Sci. 172 (5), 876-887. 
Araújo, W.L., et al., 2011. Protein degradation-an alternative respiratory substrate for stressed plants. Trends Plant Sci. 16 (9), 489-498.

Araújo, S.S., Beebe, S., Crespi, M., Delbreil, B., Gonzalez, E.M., Gruber, V., et al., 2015. Abiotic stress responses in legumes: strategies used to cope with environmental challenges. Crit. Rev. Plant Sci. 34 (1-3), 237-280.

Arnao, M.B., Hernández-Ruiz, J., 2009. Chemical stress by different agents affects the melatonin content of barley roots. J. Pineal Res. 46, 295-299.

Arnao, M.B., Hernández-Ruiz, J., 2013. Growth conditions determine different melatonin levels in Lupinus albus L. J. Pineal Res. 55, 149-155.

Arnao, M.B., Hernández-Ruiz, J., 2014. Melatonin: plant growth regulator and/or biostimulator during stress? Trends Plant Sci. 19, 789-797.

Arnao, M.B., Hernandez-Ruiz, J., 2015. Functions of melatonin in plants: a review. J. Pineal Res. 59, 133-150.

Arora, D., Bhatla, S.C., 2017. Melatonin and nitric oxide regulate sunflower seedling growth under salt stress accompanying differential expression of $\mathrm{Cu} / \mathrm{Zn}$ SOD and Mn SOD. Free Rad. Biol. Med. 106, 315-328.

Baxter, A., Mittler, R., Suzuki, N., 2013. ROS as key players in plant stress signalling. J. Exp. Bot. 65 (5), 1229-1240.

Bie, X., et al., 2012. Combinational transformation of three wheat genes encoding fructan biosynthesis enzymes confers increased fructan content and tolerance to abiotic stresses in tobacco. Plant Cell Rep. 31 (12), 2229-2238.

Bolouri Moghaddam, M.R., Van Den Ende, W., 2013. Sweet immunity in the plant circadian regulatory network. J. Exp. Bot. 64 (6), 1439-1449.

Bolouri-Moghaddam, M.R., et al., 2010. Sugar signalling and antioxidant network connections in plant cells. FEBS J. 277 (9), 2022-2037.

Bone, N., et al., 1998. Regulated vacuole fusion and fission in Schizosaccharomyces pombe: an osmotic response dependent on MAP kinases. Curr. Biol. 8 (3), 135-144.

Bouche, N., Fromm, H., 2004. GABA in plants: just a metabolite? Trends Plant Sci. 9 (3), 110-115.

Brown, J.E., Rice-Evans, C.A., 1998. Luteolin-rich artichoke extract protects low density lipoprotein from oxidation in vitro. Free Radic. Res. 29 (3), 247-255.

Brunetti, C., Di Ferdinando, M., Fini, A., Pollastri, S., Tattini, M., 2013. Flavonoids as antioxidants and developmental regulators: relative significance in plants and humans. Int. J. Mol. Sci. 14 (2), 3540-3555.

Caviglia, M., Morales, L.M., Concellón, A., Grozeff, G.G., Wilson, M., Foyer, C.H., et al., 2018. Ethylene signaling triggered by low concentrations of ascorbic acid regulates biomass accumulation in Arabidopsis thaliana. Free Rad. Biol. Med.

Chang, P.-F.L., et al., 1996. Alterations in cell membrane structure and expression of a membrane-associated protein after adaptation to osmotic stress. Physiol. Plant. 98 (3), 505-516.

Chebib, M., Johnston, G.A.R., 1999. The "ABC" of GABA receptors: a brief review. Clin. Exp. Pharmacol. Physiol. 26 (11), 937-940.

Chew, O., Whelan, J., Millar, A.H., 2003. Molecular definition of the ascorbate-glutathione cycle in Arabidopsis mitochondria reveals dual targeting of antioxidant defenses in plants. J. Biol. Chem. 278 (47), 46869-46877.

Cocetta, G., Karppinen, K., Suokas, M., Hohtola, A., Häggman, H., Spinardi, A., et al., 2012. Ascorbic acid metabolism during bilberry (Vaccinium myrtillus L.) fruit development. J. Plant Physiol. 169 (11), 1059-1065.

Cocetta, G., Baldassarre, V., Spinardi, A., Ferrante, A., 2014. Effect of cutting on ascorbic acid oxidation and recycling in fresh-cut baby spinach (Spinacia oleracea L.) leaves. Postharvest. Biol. Technol. 88, 8-16.

Conti, A., Tettamanti, C., Singaravel, M., Haldar, C., Pandi-Perumal, R.S., Maestroni, G.J.M., 2002. Melatonin: an ubiquitous and evolutionary hormone. In: Haldar, C., Singaravel, M., Maitra, S.K. (Eds.), Treatise on Pineal Gland and Melatonin. Science Publishers, Enfield, NH, pp. 105-143.

Cramer, M.D., Schierholt, A., Wang, Y.Z., Lips, S.H., 1995. The influence of salinity on the utilization of root anaplerotic carbon and nitrogen metabolism in tomato seedlings. J. Exp. Bot. 46 (10), 1569-1577.

da Silveira, J.A.G., de Melo, A.R.B., Viégas, R.A., 1999. Salt-induced decrease in nitrate uptake and assimilation in cowpea plants. Rev. Brasil. Fisiol. Vegetal.

Dann, S.G., Thomas, G., 2006. The amino acid sensitive TOR pathway from yeast to mammals. FEBS Lett. 580 (12), 2821-2829.

Davey, M.W., Montagu, M.V., Inze, D., Sanmartin, M., Kanellis, A., Smirnoff, N., et al., 2000. Plant L-ascorbic acid: chemistry, function, metabolism, bioavailability and effects of processing. J. Sci. Food. Agric. 80 (7), 825-860.

de la Haba, P., Agüera, E., Benitez, J.M., 2001. Maldonado Modulation of nitrite reductase activity in cucumber (Cucumis sativus) roots. Plant Sci. 161, 231-237.

Debouba, M., Maâroufi-Dghimi, H., Suzuki, A., Ghorbel, M.H., Gouia, H., 2007. Changes in growth and activity of enzymes involved in nitrate reduction and ammonium assimilation in tomato seedlings in response to $\mathrm{NaCl}$ stress. Ann. Bot. (Lond.) 99 (6), 1143-1151.

Desikan, R., Griffiths, R., Hancock, J., Neill, S., 2002. A new role for an old enzyme: nitrate reductase-mediated nitric oxide generation is required for abscisic acid-induced stomatal closure in Arabidopsis thaliana. Proc. Natl. Acad. Sci. USA 99, 16314-16318.

Ding, F., Wang, M., Liu, B., Zhang, S., 2017. Exogenous melatonin mitigates photoinhibition by accelerating non-photochemical quenching in tomato seedlings exposed to moderate light during chilling. Front. Plant Sci. 8, 244.

Dinkeloo, K., Boyd, S., Pilot, G., 2017. Update on amino acid transporter functions and on possible amino acid sensing mechanisms in plants. Seminars in Cell \& Developmental Biology. Academic Press.

Dubbels, R., Reiter, R.J., Klenke, E., et al., 1995. Melatonin in edible plants identified by radioimmunoassay and by high performance liquid chromatography-mass spectrometry. J. Pineal Res. 18, $28-31$.

Elsayed, A.I., Rafudeen, M.S., Golldack, D., 2014. Physiological aspects of raffinose family oligosaccharides in plants: protection against abiotic stress. Plant Biol. 16 (1), 1-8.

Fan, J., Hu, Z., Xie, Y., Chan, Z., Chen, K., Amombo, E., et al., 2015. Alleviation of cold damage to photosystem II and metabolisms by melatonin in Bermudagrass. Front. Plant Sci. 6, 925.

Fait, A., Fromm, H., Walter, D., Galili, G., Fernie, A., 2008. Highway or byway: the metabolic role of the GABA shunt in plants. Trends Plant Sci 13 (1), 14-19.

Fujiwara, T., Maisonneuve, S., Isshiki, M., Mizutani, M., Chen, L., Wong, H.L., et al., 2010. Sekiguchi lesion gene encodes a cytochrome P450 monooxygenase that catalyzes conversion of tryptamine to serotonin in rice. J. Biol. Chem. 285, 11308-11313.

Galano, A., Tan, D.X., Reiter, R.J., 2011. Melatonin as a natural ally against oxidative stress: a physicochemical examination. J. Pineal Res. 51, 1-16.

Gaudet, D.A., et al., 2011. Low temperature induced defence gene expression in winter wheat in relation to resistance to snow moulds and other wheat diseases. Plant Sci. 180 (1), 99-110. 
Gill, S.S., Tuteja, N., 2010. Polyamines and abiotic stress tolerance in plants. Plant Signal. Behav. 5 (1), 26-33.

Gilliham, M., Tyerman, S.D., 2016. Linking metabolism to membrane signaling: the GABA-malate connection. Trends Plant Sci. 21 (4), 295-301.

Goel, P., Singh, A.K., 2015. Abiotic stresses downregulate key genes involved in nitrogen uptake and assimilation in Brassica juncea L. PLoS One 10 (11), e0143645.

Gupta, A.K., Kaur, N., 2005. Sugar signalling and gene expression in relation to carbohydrate metabolism under abiotic stresses in plants. J. Biosci. 30 (5), 761-776.

Gupta, K., Dey, A., Gupta, B., 2013. Plant polyamines in abiotic stress responses. Acta Physiol. Plant. 35 (7), 2015-2036.

Halkier, B.A., Gershenzon, J., 2006. Biology and biochemistry of glucosinolates. Annu. Rev. Plant Biol. 57, 303-333.

Handa, A.K., Mattoo, A.K., 2010. Differential and functional interactions emphasize the multiple roles of polyamines in plants. Plant Physiol. Biochem. 48 (7), 540-546.

Hannah, M.A., et al., 2010. Combined transcript and metabolite profiling of Arabidopsis grown under widely variant growth conditions facilitates the identification of novel metabolite-mediated regulation of gene expression. Plant Physiol. 152 (4), 2120-2129.

Hare, P.D., Cress, W.A., 1997. Metabolic implications of stressinduced proline accumulation in plants. Plant Growth Regul. 21 (2), $79-102$

Hattori, A., Migitaka, H., Iigo, M., et al., 1995. Identification of melatonin in plants and its effects on plasma melatonin levels and binding to melatonin receptors in vertebrates. Biochem. Mol. Biol. Int. 35, 627-634.

Häusler, R.E., Ludewig, F., Krueger, S., 2014. Amino acids-a life between metabolism and signaling. Plant Sci. 229, 225-237.

Havaux, M., 2014. Carotenoid oxidation products as stress signals in plants. Plant J. 79 (4), 597-606.

Hayat, S., et al., 2012. Role of proline under changing environments: a review. Plant Signal. Behav. 7 (11), 1456-1466.

Hildebrandt, T.M., et al., 2015. Amino acid catabolism in plants. Mol. Plant 8 (11), 1563-1579.

Huez López, M.A., Ulery, A.L., Samani, Z., Picchioni, G., Flynn, R.P., 2011. Response of chile pepper (Capsicum annuum L.) to salt stress and organic and inorganic nitrogen sources: I. Growth and yield. Trop. Subtrop. Agroecosyst. 14 (1).

Hussain, S.S., et al., 2011. Polyamines: natural and engineered abiotic and biotic stress tolerance in plants. Biotechnol. Adv. 29 (3), $300-311$

Jardine, K.J., Meyers, K., Abrell, L., Alves, E.G., Serrano, A.M.Y., Kesselmeier, J., et al., 2013. Emissions of putative isoprene oxidation products from mango branches under abiotic stress. J. Exp. Bot. 64 (12), 3669.

Joshi, V., et al., 2010. Interdependence of threonine, methionine and isoleucine metabolism in plants: accumulation and transcriptional regulation under abiotic stress. Amino Acids 39 (4), 933-947.

Joudi, M., et al., 2012. Comparison of fructan dynamics in two wheat cultivars with different capacities of accumulation and remobilization under drought stress. Physiol. Plant. 144 (1), 1-12.

Kafi, M., Stewart, W.S., Borland, A.M., 2003. Carbohydrate and proline contents in leaves, roots, and apices of salt-tolerant and saltsensitive wheat cultivars1. Russian J. Plant Physiol. 50 (2), $155-162$

Kaiser, S.C., 2001. Huber Post-translational regulation of nitrate reductase: mechanisms, physiological relevance and environmental triggers. J. Exp. Bot. 52, 1981-1989.

Kaiser, W.M., Weiner, H., Huber, S.C., 1999. Nitrate reductase in higher plants: a case study for transduction of environmental stimuli into control of catalytic activity. Physiol. Plant. 105 (2), $384-389$
Kang, K., Kong, K., Park, S., Natsagdorj, U., Kim, Y., Back, K., 2011. Molecular cloning of a plant $\mathrm{N}$-acetylserotonin methyltransferase and its expression characteristics in rice. J. Pineal Res. 50, 304-309.

Kawakami, A., Sato, Y., Yoshida, M., 2008. Genetic engineering of rice capable of synthesizing fructans and enhancing chilling tolerance. J. Exp. Bot. 59 (4), 793-802.

Kerepesi, I., Banyai-Stevanovits, E., Galiba, G., 2002. Fructans in wheat under stress conditions. Acta Biol. Szeged. 46 (3-4), $101-102$.

Khan, M.I.R., Khan, N.A., 2014. Ethylene reverses photosynthetic inhibition by nickel and zinc in mustard through changes in PS II activity, photosynthetic-nitrogen use efficiency and antioxidant metabolism. Protoplasma 251, 1007-1019.

Khan, M.I.R., Khan, N.A., Masood, A., Per, T.S., Asgher, M., 2016. Hydrogen peroxide alleviates nickel-inhibited photosynthetic responses through increase in use-efficiency of nitrogen and sulfur, and glutathione production in mustard. Front. Plant Sci. 7, 44.

Khan, M.I.R., Khan, N., 2017. Reactive Oxygen Species and Antioxidant System in Plants: Role and Regulation Under Abiotic Stress. Springer Nature, 978-981-10-5254-5.

Khan, M.G., Srivastava, H.S., 1998. Changes in growth and nitrogen assimilation in maize plants induced by $\mathrm{NaCl}$ and growth regulators. Biol. Plant. 41 (1), 93-99.

Kim, M.S., et al., 2008. Galactinol is a signaling component of the induced systemic resistance caused by Pseudomonas chlororaphis O6 root colonization. Mol. Plant Microbe. Interact. 21 (12), 1643-1653.

Kimball, S.R., Jefferson, L.S., 2006. New functions for amino acids: effects on gene transcription and translation. Am. J. Clin. Nutr. 83 (2), 500S-507S.

Krapp, A., David, L.C., Chardin, C., Girin, T., Marmagne, A., Leprince, A.S., et al., 2014. Nitrate transport and signalling in Arabidopsis. J. Exp. Bot. 65 (3), 78.

Kusano, T., et al., 2007. Advances in polyamine research in 2007. J. Plant Res. 120 (3), 345-350.

Lancien, M., Roberts, M.R., 2006. Regulation of Arabidopsis thaliana 14-3-3 gene expression by $\gamma$-aminobutyric acid. Plant Cell Environ. 29 (7), 1430-1436.

Lerner, A.B., Case, J.D., Takahashi, Y., 1958. Isolation of melatonin, a pineal factor that lightens melanocytes. J. Am. Chem. Soc. 80, 2587.

Less, H., Galili, G., 2008. Principal transcriptional programs regulating plant amino acid metabolism in response to abiotic stresses. Plant Physiol. 147 (1), 316-330.

Li, C., Liang, D., Chang, C., Jia, D., Ma, F., 2015. Melatonin mediates the regulation of ABA metabolism, free-radical scavenging, and stomatal behavior in two Malus species under drought stress. J. Exp. Bot. 66, 669-680.

Liang, C., Zheng, G., Li, W., et al., 2015. Melatonin delays leaf senescence and enhances salt stress tolerance in rice. J. Pineal Res. 59, 91-101.

Liu, T., Borjigin, J., 2005. N-acetyltransferase is not the rate-limiting enzyme of melatonin synthesis at night. J. Pineal Res. 2005 (39), 91-96.

Liu, T., et al., 2014. Oryza sativa polyamine oxidase 1 back-converts tetraamines, spermine and thermospermine, to spermidine. Plant Cell Rep. 33 (1), 143-151.

Livingston, D.P., Hincha, D.K., Heyer, A.G., 2009. Fructan and its relationship to abiotic stress tolerance in plants. Cell. Mol. Life Sci. 66 (13), 2007-2023.

Maggio, A., Miyazaki, S., Veronese, P., Fujita, T., Ibeas, J.I., Damsz, B., et al., 2002. Does proline accumulation play an active role in stress-induced growth reduction? Plant J. 31 (6), 699-712. 
Makavitskaya, M., Svistunenko, D., Navaselsky, I., Hryvusevich, P., Mackievic, V., Rabadanova, C., et al., 2018. Novel roles of ascorbate in plants: induction of cytosolic $\mathrm{Ca} 2+$ signals and efflux from cells via anion channels. J. Exp. Bot.

Manchester, L.C., Coto-Montes, A., Boga, J.A., et al., 2015. Melatonin: an ancient molecule that makes oxygen metabolically tolerable. J. Pineal Res. 59, 403-419.

Martinez, V., Nieves-Cordones, M., Lopez-Delacalle, M., Rodenas, R., Mestre, T.C., Garcia-Sanchez, F., et al., 2018. Tolerance to stress combination in tomato plants: new insights in the protective role of melatonin. Molecules 23 (3), 535.

Martínez-Ballesta, M., Moreno, D.A., Carvajal, M., 2013. The physiological importance of glucosinolates on plant response to abiotic stress in Brassica. Int. J. Mol. Sci. 14 (6), 11607-11625.

Mattoo, A.K., Upadhyay, R.K., Rudrabhatla, S., 2015. Abiotic stress in crops: candidate genes, osmolytes, polyamines, and biotechnological intervention, Elucidation of Abiotic Stress Signaling in Plants, 2015. Springer, New York, NY, pp. 415-437.

Matysik, J., et al., 2002. Molecular mechanisms of quenching of reactive oxygen species by proline under stress in plants. Curr. Sci. $525-532$.

Mekonnen, D.W., Flügge, U.I., Ludewig, F., 2016. Gammaaminobutyric acid depletion affects stomata closure and drought tolerance of Arabidopsis thaliana. Plant Sci. 245, 25-34.

Moe, L.A., 2013. Amino acids in the rhizosphere: from plants to microbes. Am. J. Bot. 100 (9), 1692-1705.

Mohanty, P., et al., 2001. Effect of proline on the production of singlet oxygen. Amino Acids 21 (2), 195-200.

Nakabayashi, R., Mori, T., Saito, K., 2014. Alternation of flavonoid accumulation under drought stress in Arabidopsis thaliana. Plant Signal. Behav. 9 (8), e29518.

Nakabayashi, R., Saito, K., 2015. Integrated metabolomics for abiotic stress responses in plants. Curr. Opin. Plant Biol. 24, $10-16$.

Nambara, E., et al., 1998. Characterization of an Arabidopsis thaliana mutant that has a defect in ABA accumulation: ABA-dependent and $\mathrm{ABA}$-independent accumulation of free amino acids during dehydration. Plant Cell Physiol. 39 (8), 853-858.

Nawaz, M.A., Huang, Y., Bie, Z., Reiter, R.J., Niu, M., Hameed, S., 2016. Melatonin: current status and future perspectives in plant science. Front. Plant Sci. 6, 1230.

Nisar, N., Li, L., Lu, S., Khin, N.C., Pogson, B.J., 2015. Carotenoid metabolism in plants. Mol. Plant 8 (1), 68-82.

Nishizawa, A., Yabuta, Y., Shigeoka, S., 2008. Galactinol and raffinose constitute a novel function to protect plants from oxidative damage. Plant Physiol. 147 (3), 1251-1263.

Obata, T., Fernie, A.R., 2012. The use of metabolomics to dissect plant responses to abiotic stresses. Cell. Mol. Life Sci. 69 (19), 3225-3243.

O'Brien, J.A., Vega, A., Bouguyon, E., Krouk, G., Gojon, A., Coruzzi, G., et al., 2016. Nitrate transport, sensing, and responses in plants. Mol. Plant 9 (6), 837-856.9-798.

Pal, M., Szalai, G., Janda, T., 2015. Speculation: polyamines are important in abiotic stress signaling. Plant Sci. 237, 16-23.

Pandey, S., Fartyal, D., Agarwal, A., Shukla, T., James, D., Kaul, T., et al., 2017. Abiotic stress tolerance in plants: myriad roles of ascorbate peroxidase. Front. Plant Sci. 8, 581.

Park, J.H., Jung, S., 2018. Perturbations in carotenoid and porphyrin status result in differential photooxidative stress signaling and antioxidant responses. Biochem. Biophys. Res. Commun. 496 (3), 840-845.

Peer, Murphy, 2006. In: Grotewold, E. (Ed.), The Science of Flavonoids. The Ohio State University, Columbus, Ohio, USA.

Planchet, E., Gupta, K.J., Sonoda, M., Kaiser, W.M., 2005. Nitric oxide emission from tobacco leaves and cell suspensions: rate limiting factors and evidence for the involvement of mitochondrial electron transport. Plant J. 41, 732-743.

Planchet, E., Limami, A.M., D'Mello, J.P., 2015. Amino acid synthesis under abiotic stress. Amino acids in higher plants. $\mathrm{CAB}$ International, Wallingford, CT, US, 978-1-78064-263-5pp. 262-276.

Planchet, M., Sonoda, M., Zeier, J., Kaiser, W.M., 2006. Nitric oxide (NO) as an intermediate in the cryptogein-induced hypersensitive responses: a critical re-evalution. Plant Cell Environ. 29, 59-69.

Poeggeler, B., Balzer, I., Hardeland, R., et al., 1991. Pineal hormone melatonin oscillates also in the dinoflagellate Gonyaulax polyedra. Naturwissenschaften. 78, 268-269.

Qiao, W., Fan, L.M., 2008. Nitric oxide signaling in plant responses to abiotic stresses. J. Integr. Plant Biol. 50 (10), 1238-1246.

Rai, V.K., 2002. Role of amino acids in plant responses to stresses. Biol. Plant. 45 (4), 481-487.

Ramesh, S.A., Tyerman, S.D., Xu, B., Bose, J., Kaur, S., Conn, V., et al., 2015. GABA signalling modulates plant growth by directly regulating the activity of plant-specific anion transporters. Nat. Commun. 6, 7879 .

Ramel, F., Birtic, S., Ginies, C., Soubigou-Taconnat, L., Triantaphylidès, C., Havaux, M., 2012. Carotenoid oxidation products are stress signals that mediate gene responses to singlet oxygen in plants. Proc. Natl. Acad. Sci. 109 (14), 5535-5540.

Reggiani, R., et al., 1988. Accumulation and interconversion of amino acids in rice roots under anoxia. Plant Cell Physiol. 29 (6), 981-987.

Reiter, R.J., Tan, D.X., Fuentes-Broto, L., 2010. Melatonin: a multitasking molecule. Prog. Brain. Res. 181, 127-151.

Rejeb, I.B., Pastor, V., Mauch-Mani, B., 2014. Plant responses to simultaneous biotic and abiotic stress: molecular mechanisms. Plants 3 (4), 458-475.

Riga, P., Medina, S., Garcia-Flores, L.A., Gil-Izquierdo, A., 2014. Melatonin content of pepper and tomato fruits: effects of cultivar and solar radiation. Food. Chem. 156, 347-352.

Roberts, J.K.M., et al., 1992. Contribution of malate and amino acid metabolism to cytoplasmic $\mathrm{pH}$ regulation in hypoxic maize root tips studied using nuclear magnetic resonance spectroscopy. Plant Physiol. 98 (2), 480-487.

Robredo, A., Pérez-López, U., Miranda-Apodaca, J., Lacuesta, M., Mena-Petite, A., Muñoz-Rueda, A., 2011. Elevated CO2 reduces the drought effect on nitrogen metabolism in barley plants during drought and subsequent recovery. Environ. Exp. Bot. 71 (3), 399-408.

Rockel, P., Strube, F., Rockel, A., Wildt, J., Kaiser, W.M., 2002. Regulation of nitric oxide (NO) production by plant nitrate reductase in vivo and in vitro. J. Exp. Bot. 53, 103-110.

Rolland, F., Baena-Gonzalez, E., Sheen, J., 2006. Sugar sensing and signaling in plants: conserved and novel mechanisms. Annu. Rev. Plant Biol. 57, 675-709.

Rook, F., Bevan, M.W., 2003. Genetic approaches to understanding sugar-response pathways. J. Exp. Bot. 54 (382), 495-501.

Ryan, C.A., 1987. Oligosaccharide signalling in plants. Annu. Rev. Cell. Biol. 3 (1), 295-317.

Savvides, A., Ali, S., Tester, M., Fotopoulos, V., 2016. Chemical priming of plants against multiple abiotic stresses: mission possible? Trends Plant Sci. 21, 329-340.

Sharma, P., Dubey, R.S., 2005. Modulation of nitrate reductase activity in rice seedlings under aluminium toxicity and water stress: role of osmolytes as enzyme protectant. J. Plant Physiol. 162 (8), $854-864$

Shelp, B.J., Bown, A.W., Mclean, M.D., 1999. Metabolism and functions of gamma-aminobutyric acid. Trends Plant Sci. 4 (11), $446-452$.

Shi, H., Wang, X., Tan, D.X., et al., 2015. Comparative physiological and proteomic analyses reveal the actions of melatonin in the 
reduction of oxidative stress in Bermuda grass (Cynodon dactylon L.). Pers.). J. Pineal Res. 59, 120-131.

Signorelli, S., et al., 2013. Proline does not quench singlet oxygen: evidence to reconsider its protective role in plants. Plant Physiol. Biochem. 64, 80-83.

Singh, M., Chauhan, J.S., Meena, S.S., 2009. Drought induce changes in water use efficiency and other morpho-physiological characters in Indian mustard (Brassica juncea L.). In: 16th Australian Research Assembly on Brassicas, Ballarat Victoria; 2009. pp. 1-5.

Skirycz, A., et al., 2010. Developmental stage specificity and the role of mitochondrial metabolism in the response of Arabidopsis leaves to prolonged mild osmotic stress. Plant Physiol. 152 (1), $226-244$.

Skórzyńska-Polit, E., Drążkiewicz, M., Krupa, Z., 2010. Lipid peroxidation and antioxidative response in Arabidopsis thaliana exposed to cadmium and copper. Acta Physiol. Plant. 32 (1), 169.

Smirnoff, N., Cumbes, Q.J., 1989. Hydroxyl radical scavenging activity of compatible solutes. Phytochemistry 28 (4), 1057-1060.

Smirnoff, N., Conklin, P.L., Loewus, F.A., 2001. Biosynthesis of ascorbic acid in plants: a renaissance. Annu. Rev. Plant Biol. 52 (1), 437-467.

Sun, F., et al., 2013. Burdock fructooligosaccharide induces fungal resistance in postharvest Kyoho grapes by activating the salicylic acid-dependent pathway and inhibiting browning. Food. Chem. 138 (1), 539-546.

Suzuki, N., Koussevitzky, S., Mittler, R.O.N., Miller, G.A.D., 2012. ROS and redox signalling in the response of plants to abiotic stress. Plant Cell Environ. 35 (2), 259-270.

Szabados, L., Savoure, A., 2010. Proline: a multifunctional amino acid. Trends Plant Sci. 15 (2), 89-97.

Takahashi, T., Kakehi, J.I., 2009. Polyamines: ubiquitous polycations with unique roles in growth and stress responses. Ann. Bot., 105 (1)), 1-6.

Tal, O., Haim, A., Harel, O., Gerchman, Y., 2011. Melatonin as an antioxidant and its semi-lunar rhythm in green macroalga Ulva sp. J. Exp. Bot. 62, 1903-1910.

Tan, D.X., Hardeland, R., Manchester, L.C., et al., 2010. The changing biological roles of melatonin during evolution: from an antioxidant to signals of darkness, sexual selection and fitness. Biol. Rev. Camb. Philos. Soc. 85, 607-623.

Tan, D.X., Manchester, L.C., Di Mascio, P., Martinez, G.R., Prado, F. M., Reiter, R.J., 2007. Novel rhythms of N-1-acetyl-N-2-formyl-5methoxykynuramine and its precursor melatonin in water hyacinth: importance for phytoremediation. FASEB J. 21, 1724-1729.

Tan, D.X., Manchester, L.C., Liu, X.Y., Rosales-Corral, S.A., AcunaCastroviejo, D., Reiter, R.J., 2013. Mitochondria and chloroplasts as the original sites of melatonin synthesis: a hypothesis related to melatonin's primary function and evolution in eukaryotes. J. Pineal Res. 54, 127-138.

Tilden, A.R., Becker, M.A., Amma, L.L., et al., 1997. Melatonin production in an aerobic photosynthetic bacterium: an evolutionarily early association with darkness. J. Pineal Res. 22, 102-106.

Tuteja, N., Sopory, S.K., 2008. Chemical signaling under abiotic stress environment in plants. Plant Signal. Behav. 3 (8), 525-536.

Urano, K., et al., 2009. Characterization of the ABA-regulated global responses to dehydration in Arabidopsis by metabolomics. Plant J. 57 (6), 1065-1078.

Valluru, R., Van Den Ende, W., 2011. Myo-inositol and beyond-emerging networks under stress. Plant Sci. 181 (4), 387-400.

Van Arkel, J., et al., 2013. Tailor-made fructan synthesis in plants: a review. Carbohydr. Polym. 93 (1), 48-56.
Van Den Ende, W., El-Esawe, S.K., 2014. Sucrose signaling pathways leading to fructan and anthocyanin accumulation: a dual function in abiotic and biotic stress responses. Environ. Exp. Bot. 108, $4-13$.

Van Den Ende, W., Valluru, R., 2008. Sucrose, sucrosyl oligosaccharides, and oxidative stress: scavenging and salvaging. J. Exp. Bot. 60 (1), 9-18.

Velikova, V., Yordanov, I., Edreva, A., 2000. Oxidative stress and some antioxidant systems in acid rain-treated bean plants: protective role of exogenous polyamines. Plant Sci. 151 (1), 59-66.

Vickers, C.E., Gershenzon, J., Lerdau, M.T., Loreto, F., 2009. A unified mechanism of action for volatile isoprenoids in plant abiotic stress. Nat. Chem. Biol. 5 (5), 283.

Walter, M.H., Strack, D., 2011. Carotenoids and their cleavage products: biosynthesis and functions. Nat. Prod. Rep. 28 (4), 663-692.

Wang, F., Feng, G., Chen, K., 2009. Defense responses of harvested tomato fruit to burdock fructooligosaccharide, a novel potential elicitor. Postharvest. Biol. Technol. 52 (1), 110-116.

Wang, G.L., Xu, Z.S., Wang, F., Li, M.Y., Tan, G.F., Xiong, A.S., 2015. ). Regulation of ascorbic acid biosynthesis and recycling during root development in carrot (Daucus carota L.). Plant Physiol. Biochem. 94, 10-18.

Wang, L., Feng, L., Zheng, X., Guo, Y., Zhou, F., Shan, D., et al., 2017. Plant mitochondria synthesize melatonin and enhance the tolerance of plants to drought stress. J. Pineal Res. 69, e12429.

Wang, P., Sun, X., Li, C., Wei, Z., Liang, D., Ma, F., 2013. Long-term exogenous application of melatonin delays drought-induced leaf senescence in apple. J. Pineal Res. 54, 292-302.

Watanabe, M., et al., 2013. Comprehensive dissection of spatiotemporal metabolic shifts in primary, secondary, and lipid metabolism during developmental senescence in Arabidopsis. Plant Physiol. 162 (3), 1290-1310.

Wingler, A., Roitsch, T., 2008. Metabolic regulation of leaf senescence: interactions of sugar signalling with biotic and abiotic stress responses. Plant Biol. 10 (s1), 50-62.

Yamaguchi, K., et al., 2006. The polyamine spermine protects against high salt stress in Arabidopsis thaliana. FEBS Lett. 580 (30), 6783-6788.

Yamasaki, H., Cohen, M.F., 2006. NO signal at the crossroads: polyamine-induced nitric oxide synthesis in plants? Trends Plant Sci. 11 (11), 522-524.

Yamasaki, H., Sakihama, Y., Takahashi, S., 1999. An alternative pathway for nitric oxide production in plants: new featured of an old enzyme. Trends Plant Sci. 4, 128-129.

Yamasaki, Y.S., 2000. Simultaneous production of nitric oxide and peroxynitrite by plant nitrate reductase: in vitro evidence for the NR-dependent formation of active nitrogen species. FEBS Lett. $468,89-92$.

Yilmaz, C., Kocadagli, T., Gokmen, V., 2014. Formation of melatonin and its isomer during bread dough fermentation and effect of baking. J. Agric. Food. Chem. 62, 2900-2905.

Zhang, N., Sun, Q., Zhang, H., Cao, Y., Weeda, S., Guo, Y.-D., 2015. Roles of melatonin in abiotic stress resistance in plants. J. Exp. Bot. 66, 647-656.

Zhang, P.Y., et al., 2009. A novel burdock fructooligosaccharide induces changes in the production of salicylates, activates defence enzymes and induces systemic acquired resistance to Colletotrichum orbiculare in cucumber seedlings. J. Phytopathol. 157 (4), 201-207. 
Zhao, F., et al., 2007. Polyamines improve $\mathrm{K}+/ \mathrm{Na}+$ homeostasis in barley seedlings by regulating root ion channel activities. Plant Physiol. 145 (3), 1061-1072.

Zheng, X., Tan, D.X., Allan, A.C., et al., 2017. Chloroplastic biosynthesis of melatonin and its involvement in protection of plants from salt stress. Sci. Rep. 7, 41236.

Zhou, L., Chen, X., Liu, T., et al., 2015. Melatonin reverses $\mathrm{H}_{2} \mathrm{O}_{2}$ induced premature senescence in mesenchymal stem cells via the SIRT1-dependent pathway. J. Pineal Res. 59, 190-205.

\section{Further Reading}

Cramer, G.R., Urano, K., Delrot, S., Pezzotti, M., Shinozaki, K., 2011. Effects of abiotic stress on plants: a systems biology perspective. BMC Plant Biol. 11 (1), 163.

Corpas, F.J., Chaki, M., Fernández-Ocaña, A., Valderrama, R., Palma, J.M., Carreras, A., et al., 2008. Metabolism of reactive nitrogen species in pea plants under abiotic stress conditions. Plant Cell Physiol. 49 (11), 1711-1722. Available from: https://doi.org/ $10.1093 / \mathrm{pcp} / \mathrm{pcn} 144$. 


\title{
11
}

\section{Biochemical and Molecular Regulation of Phenylpropanoids Pathway Under Abiotic Stresses}

\author{
Alessandra Francini ${ }^{1}$, Andrea Giro ${ }^{2}$ and Antonio Ferrante ${ }^{3}$ \\ ${ }^{1}$ Institute of Life Sciences, Sant'Anna School of Advanced Studies, Pisa, Italy ${ }^{2}$ DAFNAE-Department of Agronomy \\ Animals Food Natural Resources and Environment, University of Padua, AGRIPOLIS—University Avenue, Legnaro, \\ Italy ${ }^{3}$ Department of Agricultural and Environmental Sciences, Universià degli Studi di Milanot, Milano, Italy
}

\section{O U T L I N E}

11.1 Introduction

11.2 Abiotic Stress and Physiological Changes in the Phenylpropanoid Pathway

11.3 Water Stress

11.4 Cold Stress

186

11.5 Salinity Stress and Phenylpropanoids Accumulation

11.6 Light Stress

11.7 Senescence

11.8 Nitrogen Deficiency and Phenylpropanoids
11.9 Molecular Changes of the Genes Involved in the Phenylpropanoid Pathways

11.9.1 Phenylalanine Ammonia-Lyase and Chalcone Synthase Transcriptional Induction

11.9.2 Anthocyanidin Synthase Transcriptional Changes

11.10 Regulation and Phenylpropanoids Pathway 190

11.11 Conclusion

References

Further Reading

\subsection{INTRODUCTION}

The phenylpropanoids pathway is very important in plants, since leads to the biosynthesis of important molecules with signaling and protection functions. The phenylpropanoids are ubiquitous compounds in plants and their biosynthesis is enhanced under stress conditions (Weaver and Herrmann, 1997). Indeed, plants have to cope with adverse environmental conditions by increase their tolerance against the different biotic and abiotic stresses, thus the potential and diversification ability of plants to produce functional molecules 
is essential for plant adaptation (Dixon et al., 2002). In agriculture, the understanding of plant adaptation is very important for reducing the quality and yield losses in crops during the adverse seasons, because the yield losses due to abiotic stress conditions can reach $70 \%$ of the total (Boyer, 1982; Mariani and Ferrante, 2017). Therefore, the biochemical and molecular studies of the biosynthesis of phenylpropanoids pathway can provide useful information for improving the yield in different agricultural areas in the world and leading the crop breeding and selection toward cultivars with different tolerance.

Furthermore, the phenylpropanoids pathway is the main sources of secondary metabolites in plants that are important because they have also antioxidant skills and the accumulation of these molecules inside fruits and vegetables can provide beneficial effects in the human diet.

The term "secondary" metabolites means that these compounds do not participate in the essential vital processes of the plant. However, contrary to this first definition these compounds play a very important role in the defense mechanism. In the past, these were considered as excretion products or final products of metabolism because they were not always present in the plants. Therefore, they were considered not essential. However, the phenylpropanoids are still classified as secondary metabolites as they are less important than compounds belonging to the primary metabolism.

The regulation of the biosynthesis of the different branches of this pathway can be exploited for improving either the nutraceutical value of food or the plant tolerance against the environmental stresses. Horticultural crops rich in flavonoids and anthocyanins are appreciated for their beneficial effect in counteracting stress and age-related diseases. The accumulation of phenolic compounds in plants under stress is well known, even if the phenolics composition of plant tissues under different abiotic stresses conditions has not easy to study. Indeed, the phenylpropanoids pathway is the main stream of thousands of molecules and the biosynthesis of them is regulated at several molecular and biochemical levels. Changes of the gene expression or biochemical activation of different enzymes are often tightly correlated with environment and stress factors. The defense mechanism of most phenylpropanoids is due to irritant, toxic, or repellent properties. Phenols are compounds with at least an aromatic ring with one or more hydroxy groups. The main classes of phenols in plants are reported in Table 11.1 (Balasundram et al., 2006; Pereira et al., 2009).

Phenylpropanoids derive from cinnamic acid and the main compounds are reported in Fig. 11.1.
TABLE 11.1 Classes of Phenolic Compounds in Plants and Carbon Number

\begin{tabular}{lll}
\hline Class & Structure & $\begin{array}{l}\text { C } \\
\text { number }\end{array}$ \\
\hline Simple phenolics, benzoquinones & C6 & 6 \\
Hydroxybenzoic acids, phenolic acids & C6-C1 & 7 \\
Acethophenones, phenylacetic acids & C6-C2 & 8 \\
$\begin{array}{l}\text { Hydroxycinnamic acids, } \\
\text { phenylpropanoids (coumarins, } \\
\text { isocoumarins, chromones, chromenes) }\end{array}$ & C6-C3 & 9 \\
$\begin{array}{l}\text { Napthoquinones } \\
\text { Xanthones }\end{array}$ & C6-C4 & 10 \\
Stilbenes, anthraquinones & $\mathrm{C} 6-\mathrm{C} 1-\mathrm{C} 6$ & 13 \\
Flavonoids, isoflavonoids & $\mathrm{C} 6-\mathrm{C} 2-\mathrm{C} 6$ & 14 \\
Lignans, neolignans & $\mathrm{C} 6-\mathrm{C} 3-\mathrm{C} 6$ & \\
$\begin{array}{l}\text { Biflavonoids } \\
\text { Lignins }\end{array}$ & $(\mathrm{C} 6-\mathrm{C} 3)_{2}$ & 18 \\
$\begin{array}{l}\text { Condensed tannins (proanthocyanidins } \\
\text { or flavolans) }\end{array}$ & $(\mathrm{C} 6-\mathrm{C} 3-\mathrm{C} 6)_{2}$ & 22 \\
\hline & $(\mathrm{C} 6-\mathrm{C} 3)_{n}$ & $\mathrm{n}$ \\
& $(\mathrm{C} 6-\mathrm{C} 3-\mathrm{C} 6)$ & \\
\hline
\end{tabular}

\subsection{ABIOTIC STRESS AND PHYSIOLOGICAL CHANGES IN THE PHENYLPROPANOID PATHWAY}

The phenylpropanoids pathway includes several enzymes such as phenylalanine ammonia-lyase (PAL, E.C. 4.3.1.5), chalcone synthase (CHS, E.C. 2.3.1.74), chalcone isomerase (E.C. 5.5.1.6), dihydroflavonol reductase (E.C. 1.1.1.219), and anthocyanidin synthase (ANS, E.C. 1.14.11.19). These enzymes induce the accumulation of purple pigments in the plants. The ANS is involved in anthocyanidins biosynthesis and last step of biosynthesis is catalyzed by flavonoid glycosyltransferases. Anthocyanins are water-soluble flavonoids that are produced in the cytoplasm and transferred inside the vacuole. These enzymes are responsible for the flavonoid biosynthesis before the accumulation in the vacuole (Holton and Cornish, 1995; Tanaka et al., 1995; Winkel-Shirley, 2001; Vogt and Jones, 2000).

The phenylpropanoids structure can be simple with C6-C3 carbon skeleton compounds and are biosynthesized from cinnamic acid. These compounds can have origin from the phenylalanine and undergo acylation, condensation, cyclization, glycosylation, hydroxylation, methylation, prenylation, and dehydration reactions. These include caffeic, ferulic, $p$-coumaric, and sinapic acids. The free acids are not accumulated in the cells and are often conjugated with organic acids and 


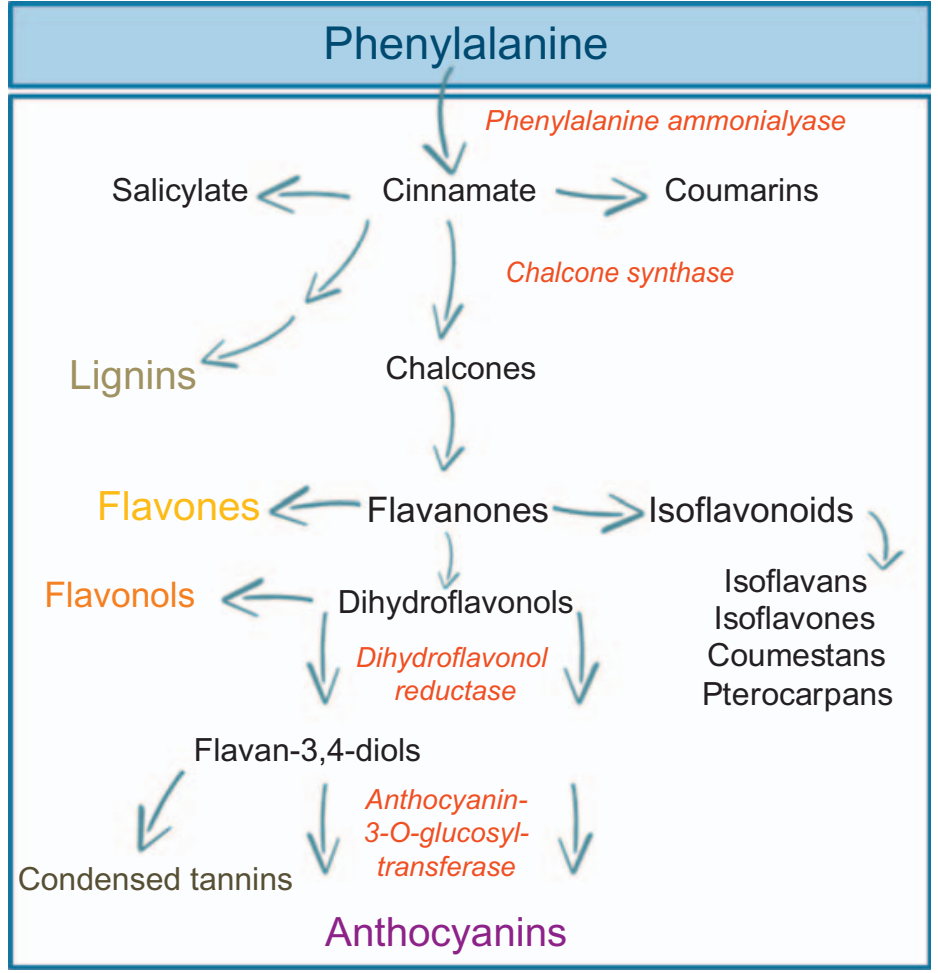

FIGURE 11.1 Phenylpropanoids pathway key enzymes involved in the biosynthesis of the main phenolic compounds. Most of these compounds are accumulated under stress conditions and provide defense mechanism. carbohydrates. However, compounds with higher carbon skeleton with $\mathrm{C} 15$ are flavonoids that are biosynthesized from chalcone synthetase (Table 11.1). This enzyme condenses $p$-cumaryl-CoA and three molecules of malonyl-CoA. These C15 flavonoid skeleton compounds are the most stress-induced phenylpropanoids and lead to flavones, flavanones, anthocyanins, and 3-deoxyanthocyanins (Dixon and Paiva, 1995).

Several studies have reported that the large number of phenolic compounds can change the spectra in relation to the copigments, $\mathrm{pH}$, and cell shape (Forkman, 1991). The pH usually is responsible for the color formation. Different species are not able to accumulate specific phenolic compounds indicating mutations or variation of the biosynthetic pathway. Molecular biology tools and plant transformation allowed obtaining plants with a modified the phenylpropanoids biosynthetic pathway. This strategy can be also developed in the plant's biofactory. A review highlights studies on transgenic plants with pigment pathways altered, including petunia, torenia, and carnation by the overexpression of heterologous flavonoid biosynthetic genes and/or the downregulation of endogenous genes (Tanaka, 2006).

Phenylpropanoids have an antioxidant activity and they can scavenge the reactive oxygen species (ROS) increasing tolerance to stressful conditions (Grace and Logan, 2000). ROS play a dual role as toxic byproducts of normal cell metabolism and as regulatory molecules in biotic and abiotic stress perception and signal transduction. Therefore, crops that are able to increase the biosynthesis and the accumulation of these compounds usually had better tolerance to the stress conditions.

\subsection{WATER STRESS}

The water stress usually increases the phyenylpropanoids biosynthesis in different plant species (Nogués et al., 1998; Parida et al., 2007). Water stress or drought reduces the plant metabolism, lowering the transpiration and photosynthesis activity. All physiological processes that consume water are drastically reduced. Unfortunately, the reduction of water has as consequence an increase of plant temperature with increase of respiration and the reduction of photosynthesis leading leaves to stress conditions for an excess of light. These conditions lead to accumulation of ROS and cell damage. The phenylpropanoids are involved in the detoxification and protection mechanisms. The induction of phenylpropanoids biosynthesis and accumulation has been observed in different plant species and different plant organs under water stress conditions.

In Scrophularia striata seedlings the exposure of seedling to water stress increased PAL and tyrosine ammonia-lyase (EC 4.3.1.23) enzymes activities and were correlated with the biosynthesis of secondary metabolites (Falahi et al., 2018). In stressed roots of 
S. striata the main phenolic acids and flavonoids accumulated were salicylic acid, ferulic acid, cinnamic acid, coumaric acid, caffeic acid (phenolic acids), genistein, myricetin (flavonoids), and resveratrol (stilbenoid).

The accumulation of total phenols under water stress occurs in all plants and in both shoots and roots. Experiments carried out on soybean under water stress demonstrated that at the apical region of roots in the elongation zone an increase of isoflavone content was observed (Fig. 11.2). CHS proteins were highly accumulated in this root region (Yamaguchi et al., 2010). Hypericum brasiliense plants exposed to water stress showed an increase of phenols but with difference in terms of compounds between shoots and roots. Rutin and quercetin increased in shoots while these were not detected in roots (de Abreu and Mazzafera, 2005), indicating a different phenolics composition and distribution in the plant. The increase of quercetin and rutin under water stress has been also observed in other wood plants such as Ligustrum vulgare (Guidi et al., 2008) and Crataegus monogyna (Kirakosyan et al., 2004). These compounds can be associated to water stress and can be used as a marker for screening plant's tolerance to this abiotic stress.

In cherry tomatoes, it has been demonstrated that cultivars with higher biosynthesis of phenylpropanoids and flavonoids had higher tolerance to water stress. Cherry tomatoes under moderate water stress in

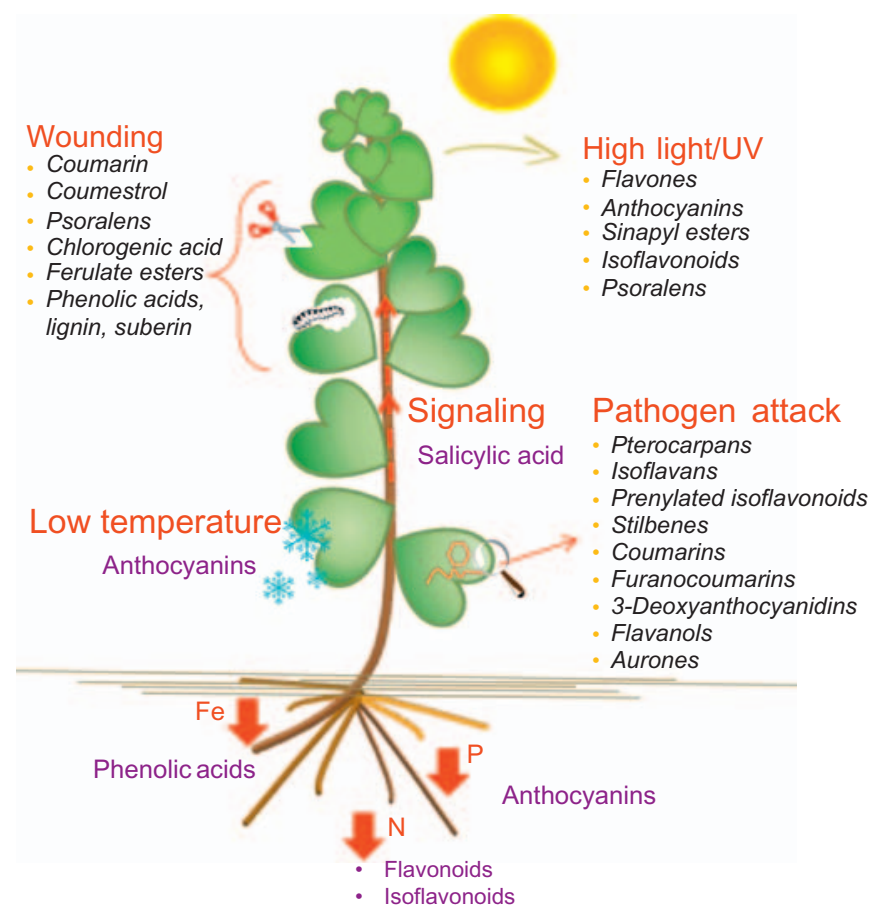

FIGURE 11.2 Environmental stresses affecting the biosynthesis of bioactive molecules able to protect the plants and induce adaptation strategies for coping with adverse conditions. the most tolerant cultivar significant changes were observed among phenolic acids and in quercetin-3-apirut, kaempferol-3-api-rut, and kaempferol-3-rut (Sánchez-Rodríguez, et al., 2011). Medicinal plants that are usually rich in bioactive compounds exposed to water stress accumulated higher amounts of phenols. In Echinacea purpurea, the drought can induce an increase of accumulation of total phenol up to $67 \%$ (Gray et al., 2003).

\subsection{COLD STRESS}

The low temperatures can induce damage to different plant organs acting at plasma membrane level. The cold injury usually acts by reducing the membrane stability and can disrupt the phospholipids. The tissue damage can be monitored by measuring the lipid peroxidation or electrolyte leakage (Campos et al., 2003; Ferrante et al., 2008). Chilling injury can also induce an increased respiration with accumulation of ROS (Suzuki and Mittler, 2006), which can be an accumulation from the stress, but they can also act as signaling molecules. The plants in response to cold can induce the biosynthesis of several compounds such as soluble sugars, sugar alcohol, and compounds derived from nitrogen such as proline. Among the defense mechanism, also the phenylpropanoid compounds play a role as scavengers of ROS (Sharma et al., 2012). Low environmental temperatures increase the PAL activities with a consequent accumulation of phenols. This behavior has been found in tomato and watermelon plants grown at $15^{\circ} \mathrm{C}$ compared with $25^{\circ} \mathrm{C}$ (Rivero et al., 2001). Results confirm that the accumulation of phenolic compounds can be a strategy of plants to increase tolerance and improve adaptation to suboptimal environmental temperature conditions.

\subsection{SALINITY STRESS AND PHENYLPROPANOIDS ACCUMULATION}

Plants subjected to stress conditions such as salinity respond by activating tolerance mechanisms at multiple levels of organization (anatomical, morphological, and molecular). The salt-stressed plants usually undergo damages from an excess of sodium accumulation in the cells. Most sodium is translocated and accumulated in the vacuoles, but when increased in the cytoplasm many physiological processes are compromised. From a biochemical point of view, the complex way of the phenylpropanoids pathway is usually activated in response to salt stress. This pathway is a key point for production of many secondary metabolites inducing scientists to investigate the mechanism of 
regulation of their biosynthesis under salt stress conditions. In fact, phenylpropanoid pathway is, considered in the same way, a model for understanding metabolites flux control and a target for biotechnological manipulation to improve molecule accumulation.

Regarding salinity effects on phenolic compounds in olive plants, Wiesman et al. (2004) showed that the high polyphenol contents recorded in oils of saline irrigated plants can be explained by the acceleration of maturation of the olives, which could account for the higher levels of phenols.

The use of saline water in Chemlali olive plants irrigation induces phenol accumulation in oil produced (Behn et al., 2009). Rossi et al. (2016) demonstrated that phenolic compounds in Frantoio leaves increased under salinity stress in new and old leaves. On the other hand, in Leccino, where the translocation of sodium to leaf was even higher than in Frantoio, the phenolic compounds remained stable under salinity stress.

The phenylpropanoids pathway in cumin seems to be stimulated under salinity (Bettaieb Rebey et al., 2017). The total polyphenol content was higher in treated seeds and salinity improved the amount of individual phenolic compounds. The accumulation of phenolic compounds in response to $\mathrm{NaCl}$ treatment was primarily caused by an increase in the concentrations of the two major compounds, $p$-coumaric acid and luteolin, despite the fact that the profile of minor compounds was changed (Bettaieb Rebey et al., 2017). Mild salt stress $(80 \mathrm{mmol} / \mathrm{L} \mathrm{NaCl})$ improves strawberry fruit quality by increasing the phenylpropanoids content. The increase of total phenolic content in strawberry was most related to the increase in the content of $(+)$-catechin. Since catechins and epicatechins have strong antioxidant properties, the increase in these phenolic acids implies an improvement on the functional properties of strawberry fruit.

\subsection{LIGHT STRESS}

Light is an essential factor for plant growth and development and each species has specific requirements to complete their physiologic life cycle. Light quantity (intensity and duration) and quality (spectral composition) affect fundamental plant physiological, morphological, and anatomical processes (Devlin et al., 2007). Furthermore, the quality and intensity of light is essential for the photosynthesis, the development, and reproduction. Leaves are organs that have the light harvesting complexes (chlorophylls, carotenoids) and the photoreceptors. However, the excess of light compared with the photosynthetic capacity of the plants can damage leaves' cells. The excess of light can be dissipated as fluorescence and heat by photosystems, but the excitation energy and electrons can generate ROS and accumulation in tissues, leading to cell damage. Plants for defense activate several detoxification enzymes and metabolites such as anthocyanins that are able to scavenge the ROS to reduce the damages of these radicals (Niyogi, 1999). This explains why phenols and in particular anthocyanins are accumulated under high intensity light stress. Anthocyanins accumulation under high-light conditions has been proven to act as a photoprotection strategy of the plants (Chalker-Scott, 1999; Field et al., 2001). The accumulation usually occurs in the upper part of the leaf epidermal layer, protecting the leaf pigments from photodamage. The protection function of anthocyanins has been proved by treating the plants with an inhibitor of PAL enzyme, the as 2-amino-indan-2-phosphonic acid (AIP). The treated plants showed higher leaf damage after the exposure to UV-B than the control, demonstrating the importance of phenylpropanoids in photoprotection (Gitz et al., 1998). The UV light induces in plants the biosynthesis of phenylpropanoids as a protection strategy. In fact, UV-A and UV-B can induce the activation of phenylpropanoids pathway (Mariz-Ponte et al., 2018). In particular, the short exposure to UV-A/B lights increased the phenolic compounds, without increasing the antioxidant systems. This means that the phenolic compounds are able to counteract the light damage without inducing any further protection systems.

\subsection{SENESCENCE}

Senescence is a genetically and highly programmed process that represents the last stage of organ or organism development (Rogers, 2013; Iqbal et al., 2017; Khan et al., 2017; Zentgraf et al., 2018). Leaves and flowers are plant organs that undergo senescence for specific function, such as the renewal of the leaves or after pollination in flowers. The leaf senescence in many deciduous plants during the autumn season is characterized by anthocyanins biosynthesis (Hoch et al., 2001), turning the leaf color dark purple. The accumulation of anthocyanins seems to be correlated with leaf protection from excessive light when during senescence other protecting pigments decline. This strategy can be associated with nutrient recycling of deciduous plants and the anthocyanins are responsible for the leaf functionality in this last stage of development (Field et al., 2001). The accumulation of anthocyanins occurs before the chlorophyll degradation and they should act as scavengers for ROS that increase during leaf and flower senescence (Chalker-Scott, 1999; Trivellini et al., 2017). 
The anthocyanins have been considered to protect senescing leaves from excessive irradiance, substituting for the carotenoids' function. However, another hypothesis is the antioxidant function for removing the ROS formed during senescence. It can represent the last chance for avoiding cell death. The accumulation of anthocyanins prior to senescence has been observed in several plant species. In Nicotiana mutabilis the petal senescence is associated with the accumulation of anthocyanins (Macnish et al., 2010). The application of flower senescence inhibitors such as ethylene inhibitor delayed the anthocyanins accumulation, demonstrating the relationship between senescence and anthocyanins. In petunia, the higher anthocyanins content in different cultivars was also associated with longer flower life and treatments with AOA, an inhibitor of ethylene biosynthesis, reduces the anthocyanins biosynthesis (Ferrante et al., 2006).

\subsection{NITROGEN DEFICIENCY AND PHENYLPROPANOIDS}

Nutrient deficiency reduces growth and induces physiological disorders in plants. The most stressful nutrient deficiency can be observed in plants under nitrogen starvation. Nitrogen $(\mathrm{N})$ is an essential element for plant growth and yield (Rubio-Wilhelmi et al., 2011). $\mathrm{N}$ is one of the main elements involved in amino acids biosynthesis (Fukushima and Kusano, 2014). Many metabolic changes occur in plants under low availability of nitrogen because its assimilation is strictly related into a complex network with primary metabolites (Fritz et al., 2006). Furthermore, the flux of primary metabolites, such as sugars and amino acids, is transferred into secondary metabolism by PAL inside the phenylpropanoids pathway. Lignin, flavonoids, and anthocyanins are biosynthesized through this pathway, so their accumulation in plants is related to PAL activity. PAL regulates the initial step of phenylpropanoids synthesis removing nitrogen from phenylalanine (Olsen et al., 2008). Therefore, low N content triggers the biosynthesis of phenols, because in response to the lack of nitrogen, plants increase PAL activity, to recycle amine from phenylalanine to counteract the reduced availability of free amine for the synthesis of new amino acids. As a consequence of the removal, ammonium ions released by PAL can be assimilated in different ways, for example, by GS/GOGAT system; hence the resulting nitrogen-free carbon skeletons of $t$-cinnamate can be shunted into different pathways of secondary metabolism. However, removing the amine group from the phenylalanine is a futile cycle that is transiently activated by plants. Structural genes such as PAL, chalcone synthase (CHS), and flavanone 3-hydroxylase $(\mathrm{F} 3 \mathrm{H})$ are overexpressed in response to low-N availability (Olsen et al., 2008; Sun et al., 2017). Moreover, other enzymes involved in the subsequent pathway of anthocyanins biosynthesis such as ANS are positively and negatively regulated by MYB transcription factors triggers by $\mathrm{N}$-deficiency (Soubeyrand et al., 2014) (Sun et al., 2017). Therefore, using a subdeficient fertilization it is possible to enhance accumulation of phenols and anthocyanins in plants, improving their nutraceutical content.

\subsection{MOLECULAR CHANGES OF THE GENES INVOLVED IN THE PHENYLPROPANOID PATHWAYS}

The amount and the type of phenylpropanoidderived compounds vary among the different plant species and also depend on the stress sensitivity of plants and the biosynthetic ability of the tissues. In Arabidopsis model plants, phenylpropanoids biosynthesis and metabolism have been widely studied and confirmed in different agricultural crops. Rocket plants exposed to preharvest (salinity, heat, and nitrogen starvation) and postharvest stresses (cold, dehydration, dark, wounding) showed several differentially expressed genes belonging to phenylalanine metabolism (Cavaiuolo et al., 2017).

A transcriptome approach analysis carried out on Begonia semperflorens studied the differential expressed genes and the phenylpropanoid biosynthesis genes under high-light and low-temperature stress conditions (Table 11.2). Results indicated that begonia activates the same defence strategy at both high-light and low temperature conditions, inducing the same phenylpropanoids related genes. Although, the phenilpropanoid metabolites were different under the two stressful conditions. Anthocyanins were biosynthesized under high-light conditions, while under low temperature lignin, proanthocyanidins, and anthocyanins were mainly accumulated (Dong et al., 2018).

\subsubsection{Phenylalanine Ammonia-Lyase and Chalcone Synthase Transcriptional Induction}

Among the abiotic stresses, the suboptimal environmental temperatures induce the expression of genes encoding for enzymes involved in the phenylpropanoids biosynthetic pathway. Low temperatures in correlation with light intensity regulate the phenylpropanoids pathway and particularly the key enzymes PAL and CHS (Leyva et al., 1995). Differently, microarray studies of Arabidopsis exposed to water stress revealed among the differential expressed genes the phenylpropanoid enzymes (Bray, 2002). 
TABLE 11.2 Genes Encoding for Phenylpropanoid Enzymes Involved in the Stress Response in Plants

\begin{tabular}{|c|c|c|c|}
\hline Species & Gene & Stress & References \\
\hline $\begin{array}{l}\text { Antirrhinum } \\
\text { majus, } \\
\text { Medicago sativa }\end{array}$ & CHS & $\mathrm{CuCl}_{2}$, Wounding, UV & $\begin{array}{l}\text { Junghans et al. (1993), } \\
\text { Lipphardt et al. (1988) }\end{array}$ \\
\hline $\begin{array}{l}\text { Arabidopsis } \\
\text { thaliana }\end{array}$ & PAL, CHS, ANS & $\begin{array}{l}\text { Low temperature, UV-B, UV-A, blue } \\
\text { light, high-lights intensity, salicylic } \\
\text { acid, ethylene, MeJ }\end{array}$ & $\begin{array}{l}\text { Leyva et al. (1995), Fuglevand et al. (1996), } \\
\text { Hartmann et al. (1998), Wade et al. (2001), } \\
\text { Feinbaum and Ausubel (1988) }\end{array}$ \\
\hline Brassica rapa & ANS & Cold, freezing & Ahmed et al. (2015) \\
\hline $\begin{array}{l}\text { Petroselinum } \\
\text { crispum }\end{array}$ & CHS & UV & $\begin{array}{l}\text { Schmelzer et al. (1988), } \\
\text { Schulze-Lefert et al. (1989) }\end{array}$ \\
\hline Petunia hybrida & CHS & UV, low temperature & Koes et al. (1989), Shvarts et al. (1997) \\
\hline Picea glauca & CHS & Wounding, JA, MeJ & Richard et al. (2000) \\
\hline Pinus sylvestris & $\mathrm{CHS}$ & UV-B & Schnitzler et al. (1997) \\
\hline $\begin{array}{l}\text { Solanum } \\
\text { tuberosum }\end{array}$ & $\begin{array}{l}\text { PAL, CHS, flavanoid } \\
\text { monooxygenase, } \\
\text { flavone } 3 \beta \text {-hydroxylase, } \\
\text { chalcone-flavone isomerases, } \\
\text { and dihydroflavonol } \\
\text { 4-reductase }\end{array}$ & Drought & Schafleitner et al. (2007) \\
\hline Vitis vinifera & $\mathrm{CHS}$ & Wounding, UV-C & Vannozzi et al. (2012) \\
\hline
\end{tabular}

At the molecular level, the stress conditions induce always the gene expression of PAL that is the key enzyme of phenylpropanoids. The lettuce plants treated with AIP, a specific inhibitor of PAL, showed lower tolerance to water stress and slower growth $(\mathrm{Oh}$ et al., 2009).

However, in cold stressed plants or lowtemperature adapted plants activate the biosynthesis of many others phenylpropanoids compounds, in particular anthocyanin. The biosynthesis of these molecules has been reported in several plants in young, mature, or senescing leaves exposed to cold temperature. Moreover, maize seedlings, exposed to low temperature, showed as well as the increase of mRNA abundance of PAL, CHS, and ANS leading to anthocyanins accumulation (Christie et al., 1994). The CHS is regulated by different abiotic stress such as UV light, bacterial, or fungal infection (Dao et al., 2011) and the increase of this enhances the accumulation of flavonoids for reducing the stress of plants.

Water stress analogously as observed for other stress induces the gene expression of phenylpropanoid genes in different tissues. In roots of maize under water stress two cinnamoyl-CoA reductase 1 and 2 genes were enhanced the lignin biosynthesis as a defense mechanism strategy (Fan et al., 2006).

Salinity stress also affects the phenylpropanoids pathway. Lotus japonicus exposed to salt stress reduced PAL activities in roots, but in the tissue the quercetin glycosides increased while others phenylpropanoids decreased (Mrázová et al., 2017). Microarray analysis in Vitis vinifera showed that $V v C H S$ gene family was upregulated by wounding and UV-C (Vannozzi et al., 2012).

\subsubsection{Anthocyanidin Synthase Transcriptional Changes}

Maize seedlings were exposed to low temperature for studying the molecular chances of the phenylpropanoid genes. The exposure of the seedlings at $10^{\circ} \mathrm{C}$ for $24 \mathrm{~h}$ induced the expression of genes involved in the anthocyanins accumulation. The anthocyanins genes were upregulated by 7 - to 10-fold (Christie et al., 1994). Crops with high accumulation of anthocyanins during fruit ripening, such as grapevines, show earlier ripening when exposed to water stress. The water limitation increases the expression of the phenylpropanoid genes leading to higher accumulation and anticipated fruit ripening (Castellarin et al., 2007).

Brassica rapa plants exposed to cold or freezing temperature respond by activating four SNS genes (Ahmed et al., 2015). The expression of ANS genes was variable among species and the highest mRNA abundance was observed after $8 \mathrm{~h}$ of cold stress.

In potato plants after 23 days of drought upregulation $P A L, C H S$, flavanoid monooxygenase, flavone $3 \beta$-hydroxylase, chalcone-flavone isomerases, and dihydroflavonol 4-reductase genes were observed (Schafleitner et al., 2007). This induction can be a defense strategy to control the oxidative stress induced by the drought conditions. 


\subsection{REGULATION AND PHENYLPROPANOIDS PATHWAY}

MicroRNAs (miRNAs) are gene products composed by 20-21 nucleotides that are able to regulate the transcription of target genes (Lee et al., 2002). The regulation of phenylpropanoids by microRNAs (miRNA) under abiotic stresses has been demonstrated in Arabidopsis. In particular, the miRNA156 seems to play an important role in the regulation of plant tolerance (Cui et al., 2014). The overexpression of miRNA156 induced an increase of stress tolerance to salt and drought, while the reduction of the expression increased the sensitivity. This study demonstrated the active role of miRNAs in plant adaptation under environmental stress conditions. In a study carried out on poplar and Arabidopsis a miRNA, the ptr miR408, was found to be associated with the tissue lignification as response to pathogen attack ( $\mathrm{Lu}$ et al., 2005). Since, miRNAs are short sequences of RNA they can be synthetized and used for function studies. The application of artificial miRNAs can be exploited for the regulation of phenylpropanoids pathway and enhance the secondary metabolites accumulation and increase the crop tolerance (Sreekumar and Soniya, 2018).

\subsection{CONCLUSION}

The phenylpropanoids pathway has a pivotal role in the regulation of plant physiology and metabolism under stress conditions. These compounds induce crop tolerance by reducing the oxidative stress of tissues and increasing the plant recovery poststress events. All tissues under stress can induce the accumulation of phenylpropanoids under stress conditions. The most important organs of the plants, such as leaves, flowers, and roots, are able to accumulate high amount of phenylpropanoids. The identification of plant with high tolerance to abiotic stresses has to be searched among those that have high accumulation of phenylpropanoids. Further studies can be useful for leading genetic improvements with the aim to increase plant adaptation or in agricultural crops leading to higher yields even under stressful conditions.

\section{References}

Ahmed, N.U., Park, J.I., Jung, H.J., Hur, Y., Nou, I.S., 2015. Anthocyanin biosynthesis for cold and freezing stress tolerance and desirable color in Brassica rapa. Funct. Integr. Genomics. 15 (4), 383-394.

Balasundram, N., Sundram, K., Samman, S., 2006. Phenolic compounds in plants and agri-industrial by-products: antioxidant activity, occurrence, and potential uses. Food. Chem. 99 (1), 191-203.
Ben, A.C., Ben, R.B., Sensoy, S., Boukhriss, M., Ben, A.F., 2009. Saline water irrigation effects on antioxidant defense system and proline accumulation in leaves and roots of field-grown olive. J. Agric. Food Chem. 57 (24), 11484-11490.

Bettaieb Rebey, I., Bourgou, S., Rahali, F.Z., Msaada, K., Ksouri, R., Marzouk, B., 2017. Relation between salt tolerance and biochemical changes in cumin (Cuminum cyminum L.) seeds. J. Food drug Anal. 25 (391), e402.

Boyer, J.S., 1982. Plant productivity and environment. Science 218, $443-448$.

Bray, E.A., 2002. Classification of genes differentially expressed during water-deficit stress in Arabidopsis thaliana: an analysis using microarray and differential expression data. Ann. Bot. (Lond.) 89 (7), 803-811.

Campos, P.S., Nia Quartin, V., Chicho Ramalho, J., Nunes, M.A., 2003. Electrolyte leakage and lipid degradation account for cold sensitivity in leaves of Coffea sp. plants. J. Plant. Physiol. 160 (3), 283-292.

Castellarin, S.D., Pfeiffer, A., Sivilotti, P., Degan, M., Peterlunger, E., Di Gaspero, G., 2007. Transcriptional regulation of anthocyanin biosynthesis in ripening fruits of grapevine under seasonal water deficit. Plant Cell Environ. 30 (11), 1381-1399.

Cavaiuolo, M., Cocetta, G., Spadafora, N.D., Müller, C.T., Rogers, H. J., Ferrante, A., 2017. Gene expression analysis of rocket salad under pre-harvest and postharvest stresses: a transcriptomic resource for Diplotaxis tenuifolia. PLoS One 12 (5), e0178119.

Chalker-Scott, L., 1999. Environmental significance of anthocyanins in plant stress responses. Photochem. Photobiol. 70 (1), 1-9.

Christie, P.J., Alfenito, M.R., Walbot, V., 1994. Impact of lowtemperature stress on general phenylpropanoid and anthocyanin pathways: enhancement of transcript abundance and anthocyanin pigmentation in maize seedlings. Planta 194 (4), 541-549.

Cui, L.G., Shan, J.X., Shi, M., Gao, J.P., Lin, H.X., 2014. The miR156-SPL9DFR pathway coordinates the relationship between development and abiotic stress tolerance in plants. Plant J. 80 (6), 1108-1117.

Dao, T.T.H., Linthorst, H.J.M., Verpoorte, R., 2011. Chalcone synthase and its functions in plant resistance. Phytochem. Rev. 10 (3), 397.

de Abreu, I.N., Mazzafera, P., 2005. Effect of water and temperature stress on the content of active constituents of Hypericum brasiliense Choisy. Plant. Physiol. Biochem. 43 (3), 241-248.

Devlin, P.F., Christie, J.M., Terry, M.J., 2007. Many hands make light work. J. Expt. Bot. 58, 3071-3077.

Dixon, R.A., Paiva, N.L., 1995. Stress-induced phenylpropanoid metabolism. Plant Cell 7 (7), 1085

Dixon, R.A., Achnine, L., Kota, P., Liu, C.J., Reddy, M.S., Wang, L., 2002. The phenylpropanoid pathway and plant defence-a genomics perspective. Mol. Plant Pathol. 3 (5), 371-390.

Dong, Y., Qu, Y., Qi, R., Bai, X., Tian, G., Wang, Y., et al., 2018. Transcriptome analysis of the biosynthesis of anthocyanins in Begonia semperflorens under low-temperature and high-light conditions. Forests 9 (2), 87.

Falahi, H., Sharifi, M., Maivan, H.Z., Chashmi, N.A., 2018. Phenylethanoid glycosides accumulation in roots of Scrophularia striata as a response to water stress. Environ. Exp. Bot. 147, 13-21.

Fan, L., Linker, R., Gepstein, S., Tanimoto, E., Yamamoto, R., Neumann, P.M., 2006. Progressive inhibition by water deficit of cell wall extensibility and growth along the elongation zone of maize roots is related to increased lignin metabolism and progressive stelar accumulation of wall phenolics. Plant Physiol. 140 (2), 603-612.

Feinbaum, R.L., Ausubel, F.M., 1988. Transcriptional regulation of the Arabidopsis thaliana chalcone synthase gene. Mol. Cell. Biol. 8 (5), 1985-1992.

Ferrante, A., Incrocci, L., Serra, G., 2008. Quality changes during storage of fresh-cut or intact Swiss chard leafy vegetables. J. Food Agric. Environ. 6 (3\&4), 60-62. 
Ferrante, A., Vernieri, P., Tognoni, F., Serra, G., 2006. Changes in abscisic acid and flower pigments during floral senescence of petunia. Biol. Plant. 50 (4), 581.

Field, T.S., Lee, D.W., Holbrook, N.M., 2001. Why leaves turn red in autumn. The role of anthocyanins in senescing leaves of red-osier dogwood. Plant Physiol. 127, 566-574.

Fritz, C., Palacios-Rojas, N., Feil, R., Stitt, M., 2006. Regulation of secondary metabolism by the carbon-nitrogen status in tobacco: nitrate inhibits large sectors of phenylpropanoid metabolism. Plant J. 46 (4), 533-548. Available from: https://doi.org/10.1111/ j.1365-313X.2006.02715.x.

Forkman, G., 1991. Flavonoids as flower pigments: the formation of the natural spectrum and its extension by genetic engineering. Plant Breeding 106, 1-26.

Fuglevand, G., Jackson, J.A., Jenkins, G.I., 1996. UV-B, UV-A, and blue light signal transduction pathways interact synergistically to regulate chalcone synthase gene expression in Arabidopsis. Plant Cell 8 (12), 2347-2357.

Fukushima, A., Kusano, M., 2014. A network perspective on nitrogen metabolism from model to crop plants using integrated "omics" approaches. J. Exp. Bot. 65 (19), 5619-5630. Available from: https://doi.org/10.1093/jxb/eru322.

Gitz III, D.C., Liu, L., McClure, J.W., 1998. Phenolic metabolism, growth, and UV-B tolerance in phenylalanine ammonia-lyaseinhibited red cabbage seedlings. Phytochemistry 49 (2), $377-386$.

Grace, S.C., Logan, B.A., 2000. Energy dissipation and radical scavenging by the plant phenylpropanoid pathway. Philos. Trans. Royal Soc. Lond. B: Biol. Sci. 355 (1402), 1499-1510.

Gray, D.E., Pallardy, S.G., Garrett, H.E., Rottinghaus, G., 2003. Acute drought stress and plant age effects on alkamide and phenolic acid content in purple coneflower roots. Planta Med. 69, $50-55$.

Guidi, L., Degl'Innocenti, E., Remorini, D., Massai, R., Tattini, M., 2008. Interactions of water stress and solar irradiance on the physiology and biochemistry of Ligustrum vulgare. Tree. Physiol. 28 (6), 873-883.

Hartmann, U., Valentine, W.J., Christie, J.M., Hays, J., Jenkins, G.I., Weisshaar, B., 1998. Identification of UV/blue light-response elements in the Arabidopsis thaliana chalcone synthase promoter using a homologous protoplast transient expression system. Plant Mol. Biol 36 (5), 741-754.

Hoch, W.A., Zeldin, E.L., McCown, B.H., 2001. Physiological significance of anthocyanins during autumnal leaf senescence. Tree. Physiol. 21 (1), 1-8.

Holton, T.A., Cornish, E.C., 1995. Genetic and biochemistry of anthocyanin biosynthesis. Plant Cell 7, 1071-1083.

Iqbal, N., Khan, N.A., Ferrante, A., Trivellini, A., Francini, A., Khan, M.I.R., 2017. Ethylene role in plant growth, development and senescence: interaction with other phytohormones. Front. Plant Sci. 8,475 .

Junghans, H., Dalkin, K., Dixon, R.A., 1993. Stress responses in alfalfa (Medicago sativa L.). 15. Characterization and expression patterns of members of a subset of the chalcone synthase multigene family. Plant Mol. Biol. 22 (2), 239-253.

Khan, N.A., Khan, M.I.R., Ferrante, A., Poor, P., 2017. Ethylene: a key regulatory molecule in plants. Front. Plant Sci. 8, 1782.

Kirakosyan, A., Kaufman, P., Warber, S., Zick, S., Aaronson, K., Bolling, S., et al., 2004. Applied environmental stresses to enhance the levels of polyphenolics in leaves of hawthorn plants. Physiol. Plant. 121, 182-186.

Koes, R.E., Spelt, C.E., van den Elzen, P.J., Mol, J.N., 1989. Cloning and molecular characterization of the chalcone synthase multigene family of Petunia hybrida. Gene 81 (2), 245-257.
Lee, Y., Jeon, K., Lee, J.T., Kim, S., Kim, V.N., 2002. MicroRNA maturation: stepwise processing and subcellular localization. EMBO J 21 (17), 4663-4670.

Leyva, A., Jarillo, J.A., Salinas, J., Martinez-Zapater, J.M., 1995. Low temperature induces the accumulation of phenylalanine ammonialyase and chalcone synthase mRNAs of Arabidopsis thaliana in a light-dependent manner. Plant Physiol. 108 (1), 39-46.

Lipphardt, S., Brettschneider, R., Kreuzaler, F., Schell, J., Dangl, J.L., 1998. UV-inducible transient expression in parsley protoplasts identifies regulatory cis-elements of a chimeric Antirrhinum majus chalcone synthase gene. EMBO J. 7 (13), 4027-4033.

Lu, S., Sun, Y.H., Shi, R., Clark, C., Li, L., Chiang, V.L., 2005. Novel and mechanical stress-responsive microRNAs in Populus trichocarpa that are absent from Arabidopsis. Plant Cell 17 (8), 2186-2203.

Macnish, A.J., Jiang, C.Z., Negre-Zakharov, F., Reid, M.S., 2010. Physiological and molecular changes during opening and senescence of Nicotiana mutabilis flowers. Plant Sci. 179 (3), 267-272.

Mariani, L., Ferrante, A., 2017. Agronomic management for enhancing plant tolerance to abiotic stresses-drought, salinity, hypoxia, and lodging. Horticulturae 3, 52. Available from: https://doi. org/10.3390/horticulturae3040052.

Mariz-Ponte, N., Mendes, R.J., Sario, S., de Oliveira, J.F., Melo, P., Santos, C., 2018. Tomato plants use non-enzymatic antioxidant pathways to cope with moderate UV-A/B irradiation: a contribution to the use of UV-A/B in horticulture. J. Plant. Physiol. 221, $32-42$.

Mrázová, A., Belay, S., Eliášová, A., Perez-Delgado, C., Kaducová, M., Betti, M., et al., 2017. Expression, activity of phenylalanineammonia-lyase and accumulation of phenolic compounds in Lotus japonicus under salt stress. Biologia 72 (1), 36-42. Available from: https://doi.org/10.1515/biolog-2017-0001.

Niyogi, K.K., 1999. Photoprotection revisited. Annu. Rev. Plant. Physiol. Plant. Mol. Biol. 50, 333-359.

Nogués, S., Allen, D.J., Morison, J.I.L., Baker, N.R., 1998. Ultraviolet-B radiation effects on water relations, leaf development, and photosynthesis in droughted pea plants. Plant Physiol. 117, 173-181.

Oh, M.M., Trick, H.N., Rajashekar, C.B., 2009. Secondary metabolism and antioxidants are involved in environmental adaptation and stress tolerance in lettuce. J. Plant. Physiol. 166 (2), 180-191.

Olsen, K.M., Lea, U.S., Slimestad, R., Verheul, M., Lillo, C., 2008. Differential expression of four Arabidopsis PAL genes; PAL1 and PAL2 have functional specialization in abiotic environmentaltriggered flavonoid synthesis. J. Plant. Physiol. 165 (14), 1491-1499. Available from: https://doi.org/10.1016/j. jplph.2007.11.005.

Parida, A.K., Dagaonkar, V.S., Phalak, M.S., Umalkar, G.V., Aurangabadkar, L.P., 2007. Alterations in photosynthetic pigments, protein and osmotic components in cotton genotypes subjected to short-term drought stress followed by recovery. Plant Biotechnol. Rep. 1 (1), 37-48.

Pereira, D.M., Valentão, P., Pereira, J.A., Andrade, P.B., 2009. Phenolics: from chemistry to biology. Molecules 14, 2202-2211.

Richard, S., Morency, M.J., Drevet, C., Jouanin, L., Séguin, A., 2000. Isolation and characterization of a dehydrin gene from white spruce induced upon wounding, drought and cold stresses. Plant Mol. Biol 43 (1), 1-10.

Rivero, R.M., Ruiz, J.M., Garcia, P.C., Lopez-Lefebre, L.R., Sánchez, E., Romero, L., 2001. Resistance to cold and heat stress: accumulation of phenolic compounds in tomato and watermelon plants. Plant Sci. 160 (2), 315-321.

Rogers, H.J., 2013. From models to ornamentals: how is flower senescence regulated? Plant Mol. Biol. 82 (6), 563-574. 
Rossi, L., Borghi, M., Francini, A., Lin, X., Xie, D.Y., Sebastiani, L., 2016. Salt stress induces differential regulation of the phenylpropanoid pathway in Olea europaea cultivars Frantoio (salt-tolerant) and Leccino (salt-sensitive). J. Plant. Physiol. 204, 8-15.

Rubio-Wilhelmi, M.M., Sanchez-Rodriguez, E., Rosales, M.A., Begoña, B., Rios, J.J., Romero, L., et al., 2011. Effect of cytokinins on oxidative stress in tobacco plants under nitrogen deficiency. Environ. Exp. Bot. 72 (2), 167-173. Available from: https://doi. org/10.1016/j.envexpbot.2011.03.005.

Sánchez-Rodríguez, E., Moreno, D.A., Ferreres, F., del Mar RubioWilhelmi, M., Ruiz, J.M., 2011. Differential responses of five cherry tomato varieties to water stress: changes on phenolic metabolites and related enzymes. Phytochemistry 72 (8), 723-729.

Schafleitner, R., Rosales, R.O.G., Gaudin, A., Aliaga, C.A.A., Martinez, G.N., Marca, L.R.T., et al., 2007. Capturing candidate drought tolerance traits in two native Andean potato clones by transcription profiling of field grown plants under water stress. Plant. Physiol. Biochem. 45 (9), 673-690.

Schnitzler, J.P., Jungblut, T.P., Feicht, C., Köfferlein, M., Langebartels, C., Heller, W., et al., 1997. UV-B induction of flavonoid biosynthesis in Scots pine (Pinus sylvestris L.) seedlings. Trees 11 (3), $162-168$.

Schulze-Lefert, P., Dangl, J.L., Becker-André, M., Hahlbrock, K., Schulz, W., 1989. Inducible in vivo DNA footprints define sequences necessary for UV light activation of the parsley chalcone synthase gene. EMBO J. 8 (3), 651-656.

Schmelzer, E., Jahnen, W., Hahlbrock, K., 1988. In situ localization of light-induced chalcone synthase mRNA, chalcone synthase, and flavonoid end products in epidermal cells of parsley Leaves. Proc. Natl. Acad. Sci. 85 (9), 2989-2993.

Sharma, P., Jha, A.B., Dubey, R.S., Pessarakli, M., 2012. Reactive oxygen species, oxidative damage, and antioxidative defense mechanism in plants under stressful conditions. J. Bot. 217037.

Shvarts, M., Borochov, A., Weiss, D., 1997. Low temperature enhances petunia flower pigmentation and induces chalcone synthase gene expression. Physiol. Plantarum 99 (1), 67-72.

Soubeyrand, E., Basteau, C., Hilbert, G., Van Leeuwen, C., Delrot, S., Gomès, E., 2014. Nitrogen supply affects anthocyanin biosynthetic and regulatory genes in grapevine cv. Cabernet-Sauvignon berries. Phytochemistry 103, 38-49. Available from: https://doi. org/10.1016/j.phytochem.2014.03.024.

Sreekumar, S., Soniya, E.V., 2018. Artificial MicroRNAs promote highlevel production of biomolecules through metabolic engineering of phenylpropanoid pathway. CRC. Crit. Rev. Plant. Sci. 1-14.

Sun, X., Jia, X., Huo, L., Che, R., Gong, X., Wang, P., et al., 2017. MdATG18a overexpression improves tolerance to nitrogen deficiency and regulates anthocyanin accumulation through increased autophagy in transgenic apple. Plant Cell Environ. 469-480. Available from: https://doi.org/10.1111/pce.13110.

Suzuki, N., Mittler, R., 2006. Reactive oxygen species and temperature stresses: a delicate balance between signaling and destruction. Physiol. Plant. 126 (1), 45-51.

Tanaka, Y., 2006. Flower colour and cytochromes P450. Phyochem. Rev. 5, 283-291.

Tanaka, Y., Fukui, Y., Fukuchi-Mizutani, M., Holton, T.A., Higgins, E., Kusumi, T., 1995. Molecular cloning and characterization of
Rosa hybrida dihydroflavonol 4-reductase gene. Plant Cell Physiol. 36, 1023-1031.

Trivellini, A., Cocetta, G., Francini, A., Ferrante, A., 2017. Reactive oxygen species production and detoxification during leaf senescence. Reactive Oxygen Species and Antioxidant Systems in Plants: Role and Regulation Under Abiotic Stress. Springer, Singapore, pp. 115-128.

Vannozzi, A., Dry, I.B., Fasoli, M., Zenoni, S., Lucchin, M., 2012. Genome-wide analysis of the grapevine stilbene synthase multigenic family: genomic organization and expression profiles upon biotic and abiotic stresses. BMC Plant Biol. 12 (1), 130.

Vogt, T., Jones, P., 2000. Glycosyltransferases in plant natural product synthesis: characterization of a supergene family. Trends. Plant. Sci. 5, 380-386.

Wade, H.K., Bibikova, T.N., Valentine, W.J., Jenkins, G.I., 2001. Interactions within a network of phytochrome, cryptochrome and UV-B phototransduction pathways regulate chalcone synthase gene expression in Arabidopsis leaf tissue. Plant J 25 (6), $675-685$.

Weaver, L.M., Herrmann, K.M., 1997. Dynamics of the shikimate pathway in plants. Trends. Plant. Sci. 2 (9), 346-351.

Wiesman, Z., Itzhakb, D., Ben, D.N., 2004. Optimization of saline water level for sustainable Barnea olive and oil production in desert conditions. Sci. Hortic. 100, 257-266.

Winkel-Shirley, B., 2001. Flavonoid biosynthesis. A colorful model for genetics, biochemistry, cell biology, and biotechnology. Plant Physiol. 126, 485-493.

Yamaguchi, M., Valliyodan, B., Zhang, J., Lenoble, M.E., Yu, O., Rogers, E.E., et al., 2010. Regulation of growth response to water stress in the soybean primary root. I. Proteomic analysis reveals region-specific regulation of phenylpropanoid metabolism and control of free iron in the elongation zone. Plant Cell Environ. 33 (2), 223-243.

Zentgraf, U., Doll, J., Riester, L., 2018. Live and let die: the core circadian oscillator coordinates plant life history and pilots leaf senescence. Mol. Plant 11 (3), 351-353.

\section{Further Reading}

Dixon, R.A., Paiva, N.L., Dixonl, R.A., Roberts, S., Foundation, N., 1995. Metabolism phenylpropanoid. Plant Cell 7 (7), 1085-1097. Retrieved from < http://www.jstor.org/stable/3870059>.

Feild, T.S., Lee, D.W., Holbrook, N.M., 2001. Why leaves turn red in autumn. The role of anthocyanins in senescing leaves of red-osier dogwood. Plant Physiol. 127 (2), 566-574.

Galli, V.R., da Silva Messias, Perin, E.C., Borowski, J.M., Bamberg, A. L., Rombaldi, C.V., 2016. Mild salt stress improves strawberry fruit quality. Food Sci. Technol. 73, 693e699.

Mosadegh, H., Trivellini, A., Ferrante, A., Lucchesini, M., Vernieri, P., Mensuali, A., 2018. Applications of UV-B lighting to enhance phenolic accumulation of sweet basil. Sci. Hortic. (Amsterdam) 229, 107-116.

Seigler, D.S., 1998. Phenylpropanoids. Plant Secondary Metabolism. Springer, Boston, MA.

Yordanov, I., Velikova, V., Tsonev, T., 2000. Plant responses to drought, acclimation, and stress tolerance. Photosynthetica 38 (2), 171-186. 


\title{
12
}

\section{Role and Regulation of Glucose as a Signal Molecule to Salt Stress}

\author{
Péter Poór ${ }^{1}$, Zalán Czékus ${ }^{1,2}$ and Attila Ördög ${ }^{1}$ \\ ${ }^{1}$ Department of Plant Biology, University of Szeged, Szeged, Hungary ${ }^{2}$ Doctoral School in Biology, Faculty of \\ Science and Informatics, University of Szeged, Szeged, Hungary
}

\section{O U T L I N E}

12.1 Introduction

12.2 Salt Stress Effects on Plants

12.3 Glucose Sensing, Transport, and Signaling in Plants

12.4 Role of Glucose in Salt Stress Responses
12.5 Glucose and Phytohormones Under Salt Stress

12.6 Conclusion and Future Perspectives

Acknowledgments

\subsection{INTRODUCTION}

Environmental stresses can disrupt cellular structures and impair physiological functions of plants leading to growth perturbation, reduced fertility, premature senescence, and yield loss (Khan and Khan, 2013; Pasala et al., 2016). To counteract stressful changes and grow successfully, plants launch resistance mechanisms by reprogramming metabolism and gene expression to the stressful conditions, and by acquiring a new equilibrium between growth and development or defense (Morkunas and Ratajczak, 2014). Soluble sugars, the products of photosynthesis (glucose, fructose, and sucrose) interacting with phytohormones play an important role in maintaining the overall cell structure, in growth and development and in regulating stress responses in a complex manner (Couée et al., 2006; Chaves et al., 2009; Rosa et al., 2009; Wind et al., 2010; Per et al., 2018). Salt stress is one of the most harmful environmental stresses in arid and semiarid regions, which can also disrupt the homeostasis of plant cells (Khan et al., 2012, 2014), that is, accompanied by changes in sugar metabolism (Ashraf and Harris, 2004; Sami et al., 2016). Moreover, there is a strong correlation between changes in soluble sugar concentration and development of successful stress tolerance in plants. In this review we summarize the current knowledge about the effects of salt stress on glucose concentration, as well as the physiological, and the molecular aspects of glucose metabolism. Cross-talk between sugars and several phytohormones under salt stress has also been discussed (Fig. 12.1). 


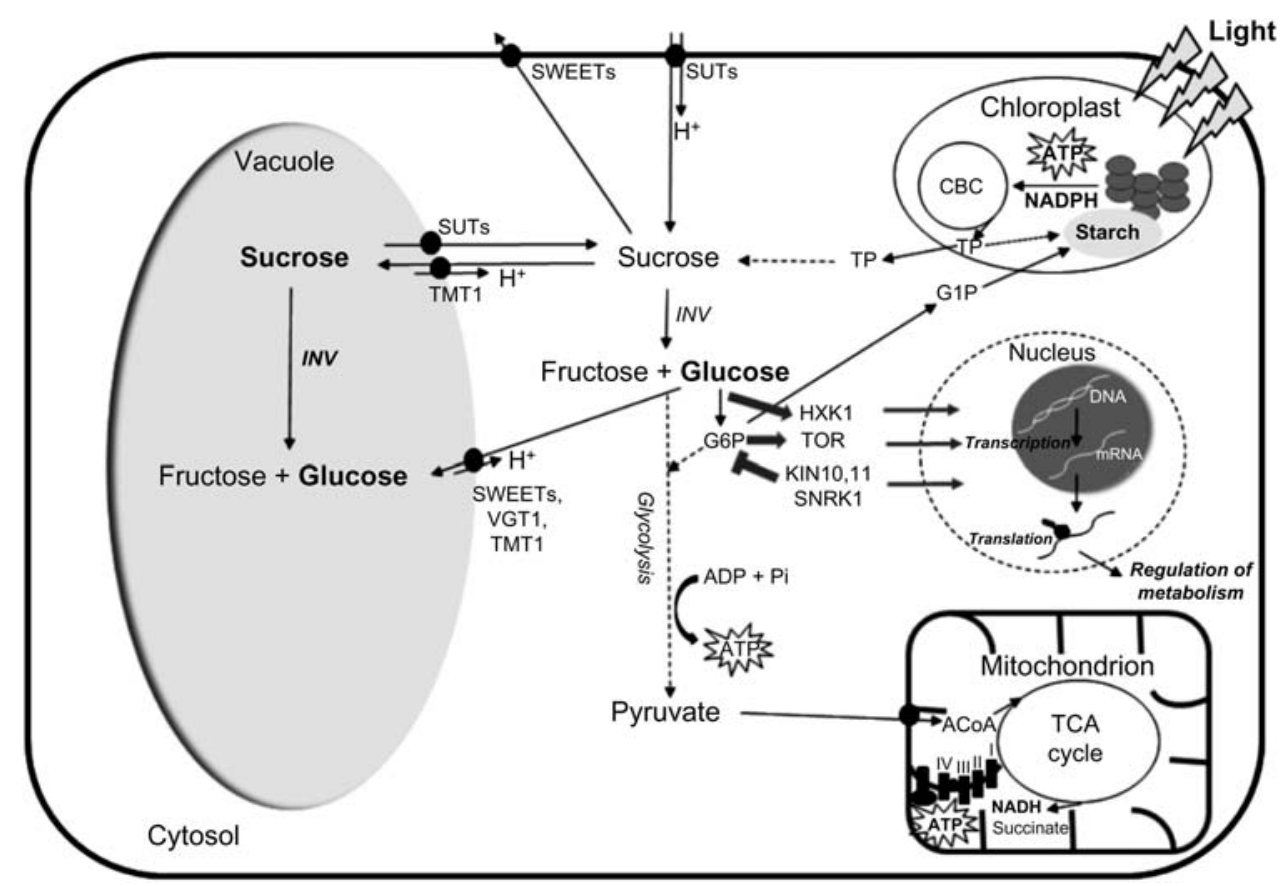

FIGURE 12.1 Schematic representation of glucose metabolism, transport, storage, and signaling in plant cells. $A C o A$, Acetyl coenzyme A; $C B C$, Calvin-Benson cycle; G1P, glucose 1-phosphate; G6P, glucose 6-phosphate; HXK1, hexokinase 1; INV, invertase; KIN10,11, Arabidopsis protein kinase 10,11; SUTs, sucrose transporters; SWEETs, SUGARS WILL EVENTUALLY BE EXPORTED sugar facilitators; TCA cycle, tricarboxylic acid cycle; TMT1, glucose, fructose, sucrose- $\mathrm{H}^{+}$exchanger; TOR, target of rapamycin; TP, triose-phosphate; VGT1, vacuolar glucose transporter 1.

\subsection{SALT STRESS EFFECTS ON PLANTS}

Salt stress is one of the most important environmental stress factors that decreases the yield and productivity of crops (Munns, 2002; Munns and Tester, 2008; Pasala et al., 2016). Munns and Tester (2008) defined three distinct responses of plants to high salinity. Firstly, it induces osmotic stress in plants and reduces water uptake by the roots leading to a reduction in relative water content of the tissues and to decreased water potential, which results in growth inhibition, stomatal closure, and a decrease in photosynthesis and transpiration. Secondly, salt-specific ionic stress disrupts the optimal $\mathrm{K}^{+} / \mathrm{Na}^{+}$ratio in the cells, where the high $\mathrm{K}^{+}$ and low $\mathrm{Na}^{+}$concentrations in the cytoplasm play a primary role in the protection of cellular functions (Zhu, 2002). The supraoptimal concentration of $\mathrm{Na}^{+}$in the leaf tissues results in premature senescence of older leaves, decreased biomass production, or it may induce programmed cell death of plants (Shabala, 2009). Finally, salt stress can cause deregulation, overflow or even disruption of the electron transport chain in mitochondria and chloroplasts and thus induces high and rapid production of reactive oxygen (ROS) and nitrogen species in plant tissues, which can destroy membranes, proteins, and macromolecules (Gémes et al., 2011; Khan and Khan, 2017; Poór et al., 2015a).
Plant responses under salt stress depend not only on the concentration of $\mathrm{NaCl}$ but also on the duration of the stress and on the age and sensitivity of the plants (Munns and Gilliham, 2015). Plants have evolved two strategies to vary the harmful degree of salt stress: excluding $\mathrm{Na}^{+}$and $\mathrm{Cl}^{-}$, particularly from photosynthesizing leaves, and synthetizing organic compounds for osmotic adjustment ("ion exclusion"); or accumulating $\mathrm{Na}^{+}$and $\mathrm{Cl}^{-}$, but having strict ionic regulation in various cell compartments ("tissue tolerance") (Roy et al., 2014; Munns and Gilliham, 2015). Firstly, osmotic adjustment can be achieved by the accumulation of compatible solutes such as proline, glycine betaine, mannitol, or soluble sugars (glucose, fructose, and sucrose) (Sakamoto and Murata, 2002; Mahajan and Tuteja, 2005; Krasensky and Jonak, 2012; Khan and Khan, 2013; Khan et al., 2014; Sami et al., 2016; Takács et al., 2017; Per et al., 2017). Secondly, the maintenance of the optimal $\mathrm{K}^{+} / \mathrm{Na}^{+}$ratio, the high cytosolic $\mathrm{K}^{+}$, and low $\mathrm{Na}^{+}$concentrations are of primary importance to protect cellular functions ( $\mathrm{Zhu}$, 2003). The precise control of $\mathrm{K}^{+} / \mathrm{Na}^{+}$is mediated by several $\mathrm{K}^{+}$and $\mathrm{Na}^{+}$transporters and $\mathrm{H}^{+}$pumps $\left(\mathrm{H}^{+}\right.$-ATPases, $\mathrm{H}^{+}$-PPases, SOS1, HKTs, and NHXs) in shoot and root tissues (Rodríguez-Rosales et al., 2009; Yamaguchi et al., 2013; Shabala, 2013; Hamamoto et al., 2015; Almeida et al., 2017). Both osmotic and 
ionic effects of salt stress induce the accumulation of ROS, which negatively affects cellular structures and metabolism. Thus, the generation and detoxification of ROS by various antioxidant systems can also determinate the survival of plants under salinity stress (Miller et al., 2010; Kocsy et al., 2013; Poór et al., 2015b; Tari et al., 2015). However, the role of glucose in the regulation of ROS scavenging in plant tissues under salt stress has not been examined in detail (Hu et al., 2012).

\subsection{GLUCOSE SENSING, TRANSPORT, AND SIGNALING IN PLANTS}

Sugars, interacting with phytohormones, play a pivotal role in the life cycle of plants, not only as the main carbon and energy source but also as signaling molecules. The photosynthetic tissues as a source can produce their own sugars while the nonphotosynthesizing tissues act as only a sink. Thus, the control of production, transport, storage, and utilization is crucial for controlling growth, development, and defense of plants (Sheen, 2014; Daloso et al., 2016; AguileraAlvarado and Sánchez-Nieto, 2017). Glucose is one of the hydrolytic products of sucrose by invertase (INV) and it acts as a major sugar signaling metabolite (Rolland et al., 2006; Sheen, 2014). Namely, it regulates cellular activity at multiple levels, from transcription and translation to protein stability and activity (Rolland et al., 2006). There were found three glucosemodulated master regulators in Arabidopsis (Sheen, 2014; Li and Sheen, 2016). Hexokinase (HXK1) is the most characterized direct glucose sensor mediating multiple functions in the glucose repression and promotion of gene transcription (Moore et al., 2003; Cho et al., 2006). At the same time, HXK catalyzes the first step of glycolysis, the conversion of glucose to glucose 6-phosphate (Granot et al., 2013). Control of glucose abundance and signaling by HXK contributes not only to the regulation of plant development and growth, but also to the defense mechanisms in accordance with several phytohormones, like ethylene or abscisic acid (ABA) (Claeyssen and Rivoal, 2007; Cho et al., 2010; Granot et al., 2013; Li and Sheen, 2016; AguileraAlvarado and Sánchez-Nieto, 2017). Besides the direct sensing of glucose by HXK1, intracellular sugar levels can be perceived as metabolic input by other energy sensing regulators thus coordinating plant metabolism and stress signaling ( $\mathrm{Li}$ and Sheen, 2016). The evolutionarily conserved energy sensor TOR (target of rapamycin) protein kinase is also activated by glucose (Xiong and Sheen, 2014). While KIN10/11 protein kinases are repressed by glucose, which provides catalytic activity in the evolutionarily conserved energy sensor complex SNRK1 (Baena-González et al., 2007).
Not only sensing, but also carbohydrate partitioning, the process of assimilating, transporting and distributing sugars from source to sink tissues, is fundamental to plant growth and development, as well as to acclimatization under environmental stress stimuli (Rosa et al., 2009; Lemoine et al., 2013; Julius et al., 2017). Sucrose (SUTs) and monosaccharide (MSTs) transporters are the main components of this process and can mediate long-distance transport of sugars from source to sink organs. Two families of transporters are involved in the loading and unloading of plant monosaccharides across the plasma membrane. The first family members are MSTs, which are distributed to other seven subfamilies according to their substrate specificity, like the glucose transporting sugar transport protein (STP) and vacuolar glucose transporter (VGT). The second family are the SWEETs, which were initially characterized also as glucose uniporters (Chen et al., 2010; Julius et al., 2017; Martinoia, 2018).

It is well known that glucose plays role in controlling photosynthesis by repressing of chlorophyll $a / b$ binding protein (CAB) and Rubisco small subunit (RBCS) genes (Krapp et al., 1993). Several experiments suggested that increasing sugar levels delayed seed germination and stimulated the induction of flowering or senescence in various plant species (Gibson, 2005). It was also observed that the senescence-associated gene 12 (SAG12), whose expression is highly senescence-specific, was induced several hundred-fold by growth on glucose in combination with low nitrogen supply (Wingler et al., 2005). These results suggest the important role of the timing, the amount, and tissue-specific accumulation of glucose during stress stimuli in plants.

\subsection{ROLE OF GLUCOSE IN SALT STRESS RESPONSES}

Various salt treatments can influence sugar production, transportation, or accumulation in many plant species and plant organs in a time- and concentrationdependent manner (Table 12.1). Glucose accumulation was observed in roots (Hartzendorf and Rolletschek, 2001; Anjum, 2008; Mišić et al., 2012; Siahpoosh et al., 2012; Gashaw et al., 2014; Swain et al., 2017), root exudate (Sacchi et al., 2000), stem (Hu et al., 2000; Kerepesi and Galiba, 2000), leaves (Hassine and Lutts, 2010; Rubio et al., 2010; Cao et al., 2011; Theerawitaya et al., 2012; Grzesiak et al., 2013; Lee et al., 2016), fruits (Navarro et al., 2005; Rubio et al., 2009; Yin et al., 2009; Kataoka et al., 2017), cotyledon (Ruffino et al., 2010), seedling (Morsy et al., 2007), calli (dos Santos and Caldeira, 1999; Liu and Van Staden, 2001), and cell suspension cultures (Mahasal et al., 2011) after various 
TABLE 12.1 Effects of Salt Stress on Glucose Contents

\begin{tabular}{|c|c|c|c|c|c|}
\hline Plants & Organs & $\begin{array}{l}\text { Dose of } \mathrm{NaCl} \\
\text { exposure }\end{array}$ & $\begin{array}{l}\text { Duration of } \\
\mathrm{NaCl} \text { exposure }\end{array}$ & Plant responses & References \\
\hline Atriplex halimus & Leaf & $160 \mathrm{mM}$ & 10 days & $\begin{array}{l}\mathrm{NaCl} \text { induced the accumulation of nonreducing } \\
\text { sucrose, glucose, and fructose. Exogenous abscisic } \\
\text { acid (ABA) treatment }(50 \mu \mathrm{M}) \text { had no impact on } \\
\text { glucose and fructose levels under salinity stress }\end{array}$ & $\begin{array}{l}\text { Hassine and } \\
\text { Lutts (2010) }\end{array}$ \\
\hline $\begin{array}{l}\text { Bruguiera } \\
\text { sexangula }\end{array}$ & $\begin{array}{l}\text { Cell } \\
\text { suspension }\end{array}$ & $150 \mathrm{mM}$ & $24 \mathrm{~h}$ & $\begin{array}{l}\text { Glucose level was significantly reduced in salt-treated } \\
\text { cells. Together with the reduced glucose level, the } \\
\text { pool sizes of glucose-6-phosphate and fructose-6- } \\
\text { phosphate were also reduced in salt-stressed cells }\end{array}$ & $\begin{array}{l}\text { Suzuki et al. } \\
\text { (2005) }\end{array}$ \\
\hline Capsicum annuum & Leaf & $30 \mathrm{mM}$ & 3 days & $\begin{array}{l}\text { Salinity increased the fructose, glucose, and sucrose } \\
\text { levels. Water-soluble carbohydrates increased with } \\
\text { decreasing } \mathrm{K}^{+} \text {concentration }\end{array}$ & $\begin{array}{l}\text { Rubio et al. } \\
\text { (2010) }\end{array}$ \\
\hline Capsicum annuum & Fruit & $30 \mathrm{mM}$ & 184 days & $\begin{array}{l}\text { Glucose and fructose increased in green and red fruits } \\
\text { of pepper plants under saline condition }\end{array}$ & $\begin{array}{l}\text { Rubio et al. } \\
\text { (2009) }\end{array}$ \\
\hline $\begin{array}{l}\text { Catharanthus } \\
\text { roseus }\end{array}$ & Leaf & $250 \mathrm{mM}$ & 3 weeks & $\begin{array}{l}\text { Treatment with } \mathrm{NaCl} \text { led to a significant decrease in } \\
\text { the contents of fructose and glucose during the } 3 \text { - } \\
\text { week course observed. In this case, sucrose content } \\
\text { increased at the second and third week of treatment. } \\
\text { Exogenous trehalose treatment }(10 \mathrm{mM}) \text { elevated the } \\
\text { contents of these sugars under salinity stress }\end{array}$ & $\begin{array}{l}\text { Chang et al. } \\
\text { (2014) }\end{array}$ \\
\hline $\begin{array}{l}\text { Chenopodium } \\
\text { quinoa }\end{array}$ & $\begin{array}{l}\text { Seed } \\
\text { (embryonic } \\
\text { axes, } \\
\text { cotyledon) }\end{array}$ & $400 \mathrm{mM}$ & $4,6,10$, and $14 \mathrm{~h}$ & $\begin{array}{l}\text { Reduced glucose and fructose contents were found in } \\
\text { embryonic axes in the presence of } \mathrm{NaCl} \text {. However, } \\
\text { the glucose and fructose contents did not differ } \\
\text { significantly in the cotyledons. Levels of sucrose were } \\
\text { higher in } \mathrm{NaCl} \text {-treated cotyledons than in untreated } \\
\text { seeds }\end{array}$ & $\begin{array}{l}\text { Prado et al. } \\
\text { (2000) }\end{array}$ \\
\hline $\begin{array}{l}\text { Chenopodium } \\
\text { quinoa }\end{array}$ & Cotyledon & $250 \mathrm{mM}$ & $\begin{array}{l}6,12, \text { and } 21 \\
\text { days }\end{array}$ & $\begin{array}{l}\text { The concentration of total soluble sugars, sucrose, and } \\
\text { glucose were higher but the level of fructose was } \\
\text { lower in salt-treated than in control cotyledons }\end{array}$ & $\begin{array}{l}\text { Ruffino et al. } \\
\text { (2010) }\end{array}$ \\
\hline Citrus limon & Leaf & $80 \mathrm{mM} \mathrm{NaCl}$ & 60 days & $\begin{array}{l}\text { Sucrose was dramatically reduced, while leaf fructose } \\
\text { concentration was significantly increased and glucose } \\
\text { content did not change under salt stress }\end{array}$ & $\begin{array}{l}\text { Tsabarducas } \\
\text { et al. (2015) }\end{array}$ \\
\hline $\begin{array}{l}\text { Citrus reshni, } \\
\text { Poncirus trifoliata } \\
x \text { Citrus sinensis }\end{array}$ & Root, leaf & 40 and $80 \mathrm{mM}$ & 12 weeks & $\begin{array}{l}\text { The concentrations of fructose, glucose, and sucrose } \\
\text { in the leaves of Cleopatra mandarin and both in the } \\
\text { leaves and roots of Troyer citrange decreased with the } \\
\text { increase of salinity level. However, the concentration } \\
\text { of sugars in the roots of Cleopatra mandarin } \\
\text { increased with the increase in salinity level }\end{array}$ & Anjum (2008) \\
\hline Glycine max & Calli & $100 \mathrm{mM}$ & $1,4,8,12$ days & $\begin{array}{l}\text { Glucose and fructose concentrations increased in the } \\
\text { callus after } \mathrm{NaCl} \text { treatments }\end{array}$ & $\begin{array}{l}\text { Liu and Van } \\
\text { Staden (2001) }\end{array}$ \\
\hline $\begin{array}{l}\text { Gossypium } \\
\text { hirsutum }\end{array}$ & $\begin{array}{l}\text { Root } \\
\text { exudate }\end{array}$ & $100 \mathrm{mM}$ & 9 days & $\begin{array}{l}\text { Higher net exudation of glucose and fructose was } \\
\text { observed from roots of cotton plants grown under } \\
\text { saline condition }\end{array}$ & $\begin{array}{l}\text { Sacchi et al. } \\
(2000)\end{array}$ \\
\hline Helianthus annus & Calli & 50 and $100 \mathrm{mM}$ & 30 days & $\begin{array}{l}\text { Higher concentration of } \mathrm{NaCl} \text { increased glucose and } \\
\text { fructose contents, but the moderate concentration of } \\
\mathrm{NaCl} \text { did not change significantly glucose and sucrose } \\
\text { contents in calli }\end{array}$ & $\begin{array}{l}\text { Dos Santos } \\
\text { and Caldeira } \\
(1999)\end{array}$ \\
\hline Lolium perenne & $\begin{array}{l}\text { Leaf } \\
\text { protoplast }\end{array}$ & $250 \mathrm{mM}$ & $6 \mathrm{~h}$ & $\begin{array}{l}\text { The survival rate of the protoplasts increased when } \\
\text { xylitol, cellobiose, 1-kestose, maltose, maltotriose, } \\
\text { raffinose and trehalose were introduced, while no } \\
\text { changes occurred when fructose, fucose, galactose, } \\
\text { glucose, inositol, mannitol, mannose, rhamnose, } \\
\text { sorbitol, sorbose, fructobiose, lactose, and sucrose } \\
\text { were introduced }\end{array}$ & $\begin{array}{l}\text { Unno and } \\
\text { Maeda (2008) }\end{array}$ \\
\hline
\end{tabular}


TABLE 12.1 (Continued)

\begin{tabular}{|c|c|c|c|c|}
\hline Plants & Organs & $\begin{array}{l}\text { Dose of } \mathrm{NaCl} \\
\text { exposure }\end{array}$ & $\begin{array}{l}\text { Duration of } \\
\mathrm{NaCl} \text { exposure }\end{array}$ & Plant responses \\
\hline Lotus japonicus & Nodule & $50 \mathrm{mM}$ & 10 weeks & $\begin{array}{l}\text { Sucrose and glucose decreased in nodules exposed to } \\
\mathrm{NaCl} \text { stress. Salt and trehalase inhibitor validamycin } \\
\mathrm{A}(30 \mu \mathrm{M}) \text { increased total soluble carbohydrates of } \\
\text { nodules but not those such as starch, sucrose, and } \\
\text { glucose }\end{array}$ \\
\hline Lupinus albus & Leaf & 50 and $150 \mathrm{mM}$ & 12 days & $\begin{array}{l}\text { Glucose content decreased with salt stress, sucrose } \\
\text { content was almost three times higher in plants } \\
\text { treated with } 150 \mathrm{mM} \mathrm{NaCl} \text { and fructose content did } \\
\text { not change significantly }\end{array}$ \\
\hline Olea europaea & Leaf & 75 and $125 \mathrm{mM}$ & 5 months & $\begin{array}{l}\text { Glucose content increased after } 75 \mathrm{mM} \text {, but decreased } \\
\text { after } 125 \mathrm{mM} \mathrm{NaCl} \text { treatment. There is a close } \\
\text { relationship between oleuropein concentration and } \\
\text { glucose, which is characterized by a significant } \\
\text { negative correlation and an opposite response of both } \\
\text { substances as salinity increased. Oleuropein acts as a } \\
\text { glucose-reservoir for osmoregulation or high energy- } \\
\text { consuming processes required for plant adaptation to } \\
\text { salinity }\end{array}$ \\
\hline
\end{tabular}

Oryza sativa $\quad$ Seedling $\quad 100 \mathrm{mM} \quad 4$ days

In chilling-sensitive rice genotype, galactose decreased or was unchanged while glucose and fructose increased in response to salt stress. In tolerant seedlings, these parameters did not change significantly, but sucrose decreased

Oryza sativa $\quad$ Leaf $\quad 150 \mathrm{mM} \quad 24 \mathrm{~h}$

Salt stress induced the accumulation of glucose, fructose, and saccharose in the leaves of wild-type References

López et al. (2006)

Fernandes et al. (2004)

Petridis et al. (2012) rice. In OsGMST1 knockdown rice seedlings, a mutant in the Golgi-localized monosaccharide transporter is hypersensitive to $\mathrm{NaCl}$ stress, as the $\mathrm{NaCl}$-induced accumulation of glucose and fructose is impaired in knockdown plants, and the accumulation of sucrose was also different from the wild-type plants

\begin{tabular}{|c|c|c|c|c|}
\hline Oryza sativa & $\begin{array}{l}\text { Cell } \\
\text { suspension }\end{array}$ & $100 \mathrm{mM}$ & $\begin{array}{l}1,3,6,10 \text { and } \\
14 \text { days }\end{array}$ & $\begin{array}{l}\text { Increased expression level of OsMST3 (Oryza sativa } \mathrm{L} \text {. } \\
\text { monosaccharide transporter } 3 \text { ) and high glucose } \\
\text { concentration at the early period of } \mathrm{NaCl} \text { treatment } \\
\text { was observed in suspension cells }\end{array}$ \\
\hline Oryza sativa & Leaf & $150 \mathrm{mM}$ & 3 and 7 days & $\begin{array}{l}\text { Glucose, fructose, and total soluble sugar contents in } \\
\text { the flag leaf tissues of salt stressed Pathumthani } 1 \\
\text { (PT1; salt-sensitive) rice were significantly enhanced } \\
\text { especially after } 3 \text { days of salt exposure. In Homjan } \\
\text { (HJ; salt-tolerant) rice, the soluble sugar level in the } \\
\text { flag leaf was unchanged }\end{array}$ \\
\hline Oryza sativa & Root & $100 \mathrm{mM}$ & 13 days & $\begin{array}{l}\text { Decreased pool sizes (glucose) were found in the } \\
\text { sensitive rice cultivars upon exposure to salt stress. } \\
\text { The salt-tolerant Pokkali cultivar showed increased } \\
\text { pools of glucose, fructose, inositol, galactinol, and } \\
\text { raffinose in the root. Leaves accumulated these } \\
\text { carbohydrates after salt treatment }\end{array}$ \\
\hline Oryza sativa & Leaf & $200 \mathrm{mM}$ & $\begin{array}{l}1,4 \text { and } \\
8 \text { days }\end{array}$ & $\begin{array}{l}\text { Glucose, fructose, and total sugar content increased as } \\
\text { a function of time in both salt-tolerant (Pokkali) and } \\
\text { salt-sensitive (IR29) genotypes of rice }\end{array}$ \\
\hline Oryza sativa & Root, leaf & $16.6 \mathrm{dS} \mathrm{m}^{-1}$ & 10 and 15 days & $\begin{array}{l}\text { Soluble sugars, including sucrose, glucose, and } \\
\text { fructose in leaf blade and leaf sheath were enriched in } \\
0.1 \mathrm{mM} \mathrm{N}-2 \text {-(chloro- } 4 \text {-pyridyl)- } \mathrm{N} \text {-phenyl urea (CPPU) } \\
\text { pretreated plants subsequently exposed to } \mathrm{NaCl} \text {. The } \\
\text { patterns of accumulation of glucose and fructose in } \\
\text { the root tissues were similar }\end{array}$ \\
\hline
\end{tabular}

Mahasal et al. (2011)

Boriboonkaset et al. (2012)

Morsy et al. (2007)

Cao et al. (2011) 
TABLE 12.1 (Continued)

\begin{tabular}{lllll}
\hline Plants & Organs & $\begin{array}{l}\text { Dose of } \mathrm{NaCl} \\
\text { exposure }\end{array}$ & $\begin{array}{l}\text { Duration of } \\
\mathrm{NaCl} \text { exposure }\end{array}$ & Plant responses \\
\hline Oryza sativa & Root, shoot & $200 \mathrm{mM}$ & $24 \mathrm{~h}$ & $\begin{array}{l}\text { The rice G-protein } \gamma \text { subunit }\left(\mathrm{T}_{2} \text { RGG1) overexpressing }\right. \\
\text { transgenic plants accumulated almost twice the } \\
\text { amount of glucose and 3.5-fold more fructose in both } \\
\text { their roots and shoots as weighed against the WT } \\
\text { plants during salinity stress }\end{array}$
\end{tabular}

$\begin{array}{lll}\begin{array}{l}\text { Pennisetum } \\ \text { clandestinum }\end{array} \quad \text { Leaf } & \begin{array}{l}50,100,150 \text { and } 15 \text { days } \\ 200 \mathrm{mM}\end{array}\end{array}$

clandestinum

$200 \mathrm{mM}$

$\begin{array}{lll}\begin{array}{l}\text { Phragmites } \\ \text { australis }\end{array} & \begin{array}{l}\text { Root, } \\ \text { rhizome, }\end{array} & 1.5 \text { and } 10 \% \text { days } \\ & \text { leaf } & \end{array}$

\begin{tabular}{|c|c|c|c|}
\hline Schenkia spicata & $\begin{array}{l}\text { Root } \\
\text { cultures }\end{array}$ & $\begin{array}{l}50,100, \text { and } \\
200 \mathrm{mM}\end{array}$ & 4 weeks \\
\hline
\end{tabular}

Solanum $\quad$ Fruit $\quad 60 \mathrm{mM}$

lycopersicum

$\begin{array}{lll}\begin{array}{l}\text { Solanum } \\ \text { lycopersicum }\end{array} & \text { Fruit } & 50 \mathrm{mM} \\ \begin{array}{l}\text { Solanum } \\ \text { lycopersicum }\end{array} & \text { Fruit } & 160 \mathrm{mM}\end{array}$

lycopersicum
The sucrose content was increased at 50 and $100 \mathrm{mM}$ $\mathrm{NaCl}$, but decreased at 150 and $200 \mathrm{mM} \mathrm{NaCl}$ in kikuyu grass. Accumulation of hexoses (glucose and fructose) was observed after treatments with 150 and $200 \mathrm{mM} \mathrm{NaCl}$ and a lower activity of glucokinase (GK), phosphoglucoisomerase (GPI), pyruvate kinase (PK), and glucose-6-phosphate dehydrogenase $(\mathrm{G} 6 \mathrm{PDH})$ was also detected

The contents of sucrose, glucose, and fructose were higher in leaves $>$ rhizomes $>$ roots. Sugar contents increased up to 3.5 -fold from $1.5 \%$ o to $10 \%$ salinity level, but were lower at $1.5 \%$ versus the control in the rhizome. Sugar contents were the lowest in the roots and the highest in the leaves at $1.5 \%$ salinity. The sugar composition did not vary significantly except for leaves where the fraction of sucrose decreased, but glucose and fructose increased with increasing salinity level

In salt tolerant roots of S. spicata, glucose and fructose contents significantly increased with increasing salt concentration up to $100 \mathrm{mM} \mathrm{NaCl}$, and a decrease was observed under severe salinity. In the roots of the salt sensitive genotype, sugar levels did not change

During the Spring-Summer growing season

$\mathrm{NaCl}$ treatment increased glucose and fructose contents in tomato fruit juice. Treatments with $\mathrm{Ca}^{2+}$ and/or $\mathrm{NH}_{4}{ }^{+}$or $\mathrm{HCO}_{3}$ in combination with $\mathrm{NaCl}$ increased sugar concentrations compared to the control

$21,28,35,42$, and 49 days after flowering

Salinity stress strongly promoted the accumulation of sucrose, but did not elevate significantly glucose and fructose contents in tomato fruits

$10,18,26,34$, and 42 days after flowering

Sugar levels of salinity-stressed tomato fruits were unchanged at 34 days after flowering, but had increased substantially by 42 days. Only sucrose had been kept at a similar level during this period even under saline conditions. Salinity stress enhanced the accumulation of glucose by 2.43 times, fructose by 2.05 times, sucrose by 7.87 times, and total sugars by 2.27 times at 42 days after flowering compared with those of the control. In contrast, starch content decreased during this period

Salt-stressed tomato plants showed lower concentration of glucose in roots and leaves, but no

difference between $\mathrm{NaCl}$ treatments could be detected
Muscolo et al. (2003)

Hartzendorf and Rolletschek (2001)

Mišić et al. (2012)

Navarro et al. (2005)

Saito et al. (2008)

Yin et al. (2009)

Hunsche et al. (2010) lycopersicum Solanum pennellii
Root, old 70,140 , and 8 weeks leaf, young $210 \mathrm{mM}$ leaf 
TABLE 12.1 (Continued)

\begin{tabular}{|c|c|c|c|c|c|}
\hline Plants & Organs & $\begin{array}{l}\text { Dose of } \mathrm{NaCl} \\
\text { exposure }\end{array}$ & $\begin{array}{l}\text { Duration of } \\
\mathrm{NaCl} \text { exposure }\end{array}$ & Plant responses & References \\
\hline $\begin{array}{l}\text { Solanum } \\
\text { lycopersicum }\end{array}$ & Root, leaf & $100 \mathrm{~mm}$ & 1 week & $\begin{array}{l}\text { Salt stress decreased sucrose and glucose levels in the } \\
\text { leaves and roots of tomato plants. Growth in } 10^{-4} \mathrm{M} \\
\text { salicylic acid (SA)-containing solution led to } \\
\text { accumulation of soluble sugars in both leaf and root } \\
\text { tissues, which remained higher in both plant parts } \\
\text { during salt stress at } 10^{-4} \mathrm{M} \text { SA. The activity of } \\
\text { hexokinase (HXK) with glucose, but not fructose, as } \\
\text { substrate was reduced by SA treatment in leaf and } \\
\text { root samples, leading to accumulation of glucose and } \\
\text { fructose in leaf tissues. HXK activity decreased } \\
\text { further under high salinity in both plant organs }\end{array}$ & $\begin{array}{l}\text { Poór et al. } \\
(2011)\end{array}$ \\
\hline $\begin{array}{l}\text { Solanum } \\
\text { lycopersicum }\end{array}$ & Fruit & $50 \mathrm{mM}$ & 21 days & $\begin{array}{l}\text { Salinity treatment caused a significant increase in } \\
\text { sucrose, glucose, and fructose contents in tomato } \\
\text { fruits. There was no effect from chemical or } \\
\text { organomineral fertilizer on the increasing sugar } \\
\text { concentration under the salinity condition, except for } \\
\text { sucrose and glucose in the summer cropping, when } \\
\text { accumulation of these sugars was higher }\end{array}$ & $\begin{array}{l}\text { Kataoka et al. } \\
\text { (2017) }\end{array}$ \\
\hline Triticum aestivum & Stem & $200 \mathrm{mM}$ & 4 days & $\begin{array}{l}\text { Drought- and salt-tolerant wheat genotypes (Sakha-8; } \\
\text { Kobomugi) accumulated more soluble carbohydrates } \\
\text { (glucose and sucrose) than did the sensitive ones } \\
\text { (Chinese spring; Regina) after the } \mathrm{NaCl} \text { treatment }\end{array}$ & $\begin{array}{l}\text { Kerepesi and } \\
\text { Galiba (2000) }\end{array}$ \\
\hline Triticum aestivum & Stem & $120 \mathrm{mM}$ & $\begin{array}{l}\text { Continuously } 3 \\
\text { d after } \\
\text { emergence of } \\
\text { leaf } 4^{\text {th }}\end{array}$ & $\begin{array}{l}\text { The distribution of carbohydrates along the leaf axis } \\
\text { showed distinct patterns that were altered by salinity } \\
\text { and time in the photoperiod. Glucose and fructose } \\
\text { concentrations were low at the base of the elongation } \\
\text { zone and increased sharply up to the end of the leaf } \\
\text { elongation zone after the salt treatment }\end{array}$ & $\begin{array}{l}\text { Hu et al. } \\
(2000)\end{array}$ \\
\hline Triticum aestioum & Leaf & $0.4 \mathrm{~mol} \mathrm{dm}^{-3}$ & 2 days & $\begin{array}{l}\mathrm{NaCl} \text { stimulated the accumulation of glucose and } \\
\text { saccharose in leaves of different wheat genotypes }\end{array}$ & $\begin{array}{l}\text { Grzesiak et al. } \\
\text { (2013) }\end{array}$ \\
\hline $\begin{array}{l}\text { Eutremal } \\
\text { Thellungiella spp. } \\
\text { accessions }\end{array}$ & Leaf & $\begin{array}{l}25,50,100,200 \\
300,400,500,600 \\
\text { and } 700 \mathrm{mM}\end{array}$ & 26 days & $\begin{array}{l}\text { The accumulation of glucose, fructose, and saccharose } \\
\text { was positively correlated with the increasing salt } \\
\text { stress levels }\end{array}$ & $\begin{array}{l}\text { Lee et al. } \\
(2016)\end{array}$ \\
\hline Vicia faba & Pod & 50 and $100 \mathrm{mg} / \mathrm{L}$ & $\begin{array}{l}5 \text { times at } \\
10 \text { day intervals } \\
\text { (foliar-sprayed) }\end{array}$ & $\begin{array}{l}\text { Under normal watering the two } \mathrm{NaCl} \text { concentrations } \\
\text { induced loss of sugars (glucose, fructose, and sucrose) } \\
\text { both to immature (pods harvested } 10 \text { days before the } \\
\text { end of the experiment) and mature pods }\end{array}$ & $\begin{array}{l}\text { Ouzounidou } \\
\text { et al. (2014) }\end{array}$ \\
\hline
\end{tabular}

$\mathrm{NaCl}$ treatments. In contrast, glucose content did not change or decreased after $\mathrm{NaCl}$ treatments in leaves (Muscolo et al., 2003; Fernandes et al., 2004; Hunsche et al., 2010; Poór et al., 2011; Boriboonkaset et al., 2012; Petridis et al., 2012; Chang et al., 2014; Tsabarducas et al., 2015), fruits (Saito et al., 2008; Ouzounidou et al., 2014), seed (Prado et al., 2000), nodule (López et al., 2006), callus (dos Santos and Caldeira, 1999), cell suspension culture (Suzuki et al., 2005), and leaf protoplasts (Unno and Maeda, 2008) of various plant species (Table 12.1). These results suggested that in not only plant organs but also plant species/genotypes, the concentration and the duration of salt exposure could determine the intracellular glucose levels.
First of all, the genotypes of the given crops seemed to be crucial in salt stress responses. There were several important investigations with rice plants (Oryza sativa L.) to detect the effects of salt stress on different plant genotypes. Boriboonkaset et al. (2012) observed that glucose, fructose, and total soluble sugar contents in the flag leaf of salt stressed Pathumthani 1 (PT1; salt-sensitive) rice were significantly enhanced, especially on the third day of the salt exposure. In contrast, soluble sugar levels did not change in the flag leaf of Homjan (HJ; salt-tolerant) rice after treatment with $150 \mathrm{mM} \mathrm{NaCl}$. At the same time, glucose, fructose, and total sugar contents increased as a function of time in leaves of both salt-sensitive (IR29) and salt-tolerant 
(Pokkali) rice genotypes (Theerawitaya et al., 2012). In contrast, in the roots, glucose decreased in the sensitive cultivars, but it increased in the salt-tolerant Pokkali cultivar after $100 \mathrm{mM} \mathrm{NaCl}$ treatment (Siahpoosh et al., 2012). In roots of other plant species, glucose concentration also increased upon salinity stress. Glucose and fructose contents significantly elevated in salt-tolerant root culture of Schenkia spicata (L.) after $100 \mathrm{mM} \mathrm{NaCl}$ treatment, but sugar levels did not change in the salt-sensitive genotype (Mišić et al., 2012). Similar tendencies were observed in wheat (Triticum aestioum L.) cultivars. Drought- and salttolerant wheat genotypes (Sakha-8; Kobomugi) accumulated more soluble carbohydrates (glucose and sucrose) after 4 days in the stem than the sensitive ones (Chinese spring; Regina) after $200 \mathrm{mM} \mathrm{NaCl}$ treatment (Kerepesi and Galiba, 2000). In contrast, Morsy et al. (2007) investigated chilling-sensitive and chilling-tolerant rice genotypes after salt stress and they found that glucose and fructose concentrations increased in response to $100 \mathrm{mM} \mathrm{NaCl}$, but only in the chilling-sensitive rice genotype. However, sugar concentration did not change significantly (only sucrose decreased) in the chilling-tolerant seedlings after the 4day-long salt exposure. Based on these investigations we can conclude that glucose accumulation is common phenomenon in salt or drought (but not in chilling) tolerant crop genotypes in various plant organs (leaf, stem, and root).

Nevertheless, there can be significant differences in the glucose levels in different organs or in different parts of the organs. The concentrations of fructose, glucose, and sucrose decreased in the leaves of Cleopatra mandarin (Citrus reshni Hort. ex Tan), but at the same time the concentration of these sugars elevated in the roots after treatment with $80 \mathrm{mM} \mathrm{NaCl}$ (Anjum, 2008). In contrast, sucrose, glucose, and fructose increased in leaf blade and leaf sheath of rice and similar patterns of the accumulation of glucose and fructose were measured in roots after the salt exposure (Gashaw et al., 2014; Swain et al., 2017). However, $100 \mathrm{mM} \mathrm{NaCl}$ decreased sucrose and glucose levels both in leaves and roots of tomato (Solanum lycopersicum L.) plants (Poór et al., 2011). In addition, dynamic source-sink relations can be found between the various plant organs (fruit-leaf-root) or between differently aged organs. Hunsche et al. (2010) investigated the concentration of soluble sugars in old as well as in young leaves of different tomato genotypes. They found lower concentration of glucose in roots and leaves in salt-stressed plants upon 70, 140, and $210 \mathrm{mM} \mathrm{NaCl}$ treatments (Hunsche et al., 2010). Glucose levels can be different in fruits depending on their ripening stages. Rubio et al. (2009) observed elevated glucose and fructose levels in green and red fruits of pepper plants under saline condition. Moreover, contents of sucrose, glucose, and fructose were higher in leaves $>$ rhizomes $>$ roots of Phragmites australis (Cav.) Trin. ex Steud after different salt exposures (Hartzendorf and Rolletschek, 2001). In addition, the distribution of carbohydrates along the leaf axis can show distinct patterns, which can be altered by salinity and time in the photoperiod. It was provided that glucose and fructose concentrations were low at the base and increased sharply up to the end of the leaf elongation zone of wheat stem after salt treatment with $120 \mathrm{mM} \mathrm{NaCl}$ (Hu et al., 2000). It can be concluded that distribution of glucose between the different plant organs or inside the organ can be crucial in responses against high salinity. From this aspect, not only the production and accumulation of glucose but also the regulation of glucose transport can be crucial in case of the salinity stress. There are only a few investigations in rice plants, where the role of monosaccharide transporters was examined upon salt stress. Cao et al. (2011) found that in OsGMST1 knockdown rice seedlings, a mutant in the Golgi-localized monosaccharide transporter is hypersensitive to $150 \mathrm{mM} \mathrm{NaCl}$, as the $\mathrm{NaCl}$-induced accumulation of glucose and fructose is impaired in knockdown rice leaves. Moreover, the potential role of monosaccharide transporters was confirmed in rice suspension cells, where $100 \mathrm{mM} \mathrm{NaCl}$ caused significant increase in the expression level of OsMST3 $(O$. sativa L. monosaccharide transporter 3) and induced high glucose concentration at the early period of the salt treatment (Mahasal et al., 2011).

Changes in glucose metabolism can be dependent on the dose of $\mathrm{NaCl}$ treatments. Fortunately, many authors investigated effects of different concentration of $\mathrm{NaCl}$ on the selected plant species. Based on these observations, it can be concluded that glucose contents can significantly elevate with the increasing amount of salt up to the lethal salt concentration and a decrease can be observed under severe salinity. This tendency was confirmed by dos Santos and Caldeira (1999) in sunflower (Helianthus annus L.) calli after 50 and $100 \mathrm{mM} \mathrm{NaCl}$ treatments, by Muscolo et al. (2003) in leaves of kikuyu grass (Pennisetum clandestinum Hochst) after 50, 100, 150, and $200 \mathrm{mM} \mathrm{NaCl}$ treatments, by Petridis et al. (2012) in leaves of olive (Olea europaea L.) after 75 and $125 \mathrm{mM} \mathrm{NaCl}$ treatments, by Mišić et al. (2012) in S. spicata root cultures after 50, 100 , and $200 \mathrm{mM} \mathrm{NaCl}$ treatments and by Lee et al. (2016) in leaves of Eutrema/Thellungiella spp. accessions after 25, 50, 100, 200, 300, 400, 500, 600, and $700 \mathrm{mM}$ $\mathrm{NaCl}$ treatments. However, lower concentration of glucose was detected in roots and leaves of tomato plants after 70,140 , and $210 \mathrm{mM} \mathrm{NaCl}$ treatments but there were no differences between the glucose levels after 8 weeks (Hunsche et al., 2010). Nevertheless, others 
found that higher concentration of $\mathrm{NaCl}(>250 \mathrm{mM})$ can induce cell death in tomato plants (Poór et al., 2012, 2013). $400 \mathrm{mM}$ of $\mathrm{NaCl}$ decreased also glucose and fructose contents in seeds of Chenopodium quinoa (Prado et al., 2000) and $100 \mathrm{mM} \mathrm{NaCl}$ caused similar effects on glucose levels in pods of Vicia faba (Ouzounidou et al., 2014). Interestingly, both 50 and $150 \mathrm{mM}$ concentrations of $\mathrm{NaCl}$ also decreased glucose content in leaves of Lupinus albus (L.) after 12 days (Fernandes et al., 2004).

The time and duration of salt exposure can be also crucial in glucose metabolism and plant stress responses. High glucose concentration was observed at the early period of $100 \mathrm{mM} \mathrm{NaCl}$ treatment after few days in rice suspension cells (Mahasal et al., 2011) and in soybean (Glycine max L.) calli (Liu and Van Staden, 2001). Increase in glucose content can be observed as a function of days in leaves of rice (Theerawitaya et al., 2012) and in cotyledons of C. quinoa (Ruffino et al., 2010), but it can be detected after several hours in $C$. quinoa seeds (Prado et al., 2000). In contrast, glucose levels elevated only several weeks after the salt exposure in fruits of tomato (Yin et al., 2009).

The accumulated glucose can play a role in several signaling and metabolic processes in the salt stressed tissues. Application of exogenous glucose attenuated the effects of salt stress in a dose-dependent manner of glucose $(0.1,0.5$, and $50 \mathrm{mM})$ in leaves of wheat plants. Pretreatment with glucose showed significant reversal of salt stress caused by $200 \mathrm{mM} \mathrm{NaCl}$ in chlorophyll decay, loss of water and dry weight, shortening of root length, and accumulation of proline. The glucoseinduced salt stress resistance was associated with enhanced intracellular $\mathrm{K}^{+}$and higher $\mathrm{K}^{+} / \mathrm{Na}^{+}$ratio in wheat leaves. Moreover, pretreatment with glucose activated antioxidant enzyme activities (superoxide dismutase, peroxidase, catalase), thus decreasing lipid peroxidation in wheat seedlings based on thiobarbituric acid reactive substances (TBARS) and malondialdehyde (MDA) contents (Hu et al., 2012). In contrast, others found that in in vitro cell culture, treatment with $25 \mathrm{mM}$ glucose together with $80 \mathrm{mM} \mathrm{NaCl}$ increased the ion leakage, TBARS, superoxide production, superoxide dismutase activity, and hydrogen peroxide $\left(\mathrm{H}_{2} \mathrm{O}_{2}\right)$ contents after 2 days in rice suspension cells. However, these changes were lower or not significant after 4 days compared with the $\mathrm{NaCl}$ treated samples (Zhang et al., 2013). These findings suggested that glucose can modulate key antioxidant enzymes, thus can decrease the lipid peroxidation, reduce the membrane permeability and maintain the optimal $\mathrm{K}^{+}$/ $\mathrm{Na}^{+}$ratio. Rubio et al. (2010) also observed that watersoluble carbohydrates increased with decreasing $\mathrm{K}^{+}$ concentration in the nutrient solution induced by salinity stress. Thus, the loss of $\mathrm{K}^{+}$can be an important signal to sugar accumulation and induction of defense mechanism.

Interestingly, several chemicals can induce glucose accumulation under salt stress. Chang et al. (2014) found that exogenous trehalose treatment $(10 \mathrm{mM})$ elevated the contents of soluble sugars in leaves of Catharanthus roseus under salinity stress, but trehalase inhibitor validamycin A $(30 \mu \mathrm{M})$ increased the total soluble carbohydrates of nodules in Lotus japonicus, but had no effect on starch, sucrose, and glucose contents (López et al., 2006). Chemical or organomineral fertilizer was also effective in the accumulation of glucose in fruits of tomato under salt stress (Kataoka et al., 2017) and treatments with $\mathrm{Ca}^{2+}$ and/or $\mathrm{NH}_{4}{ }^{+}$ or $\mathrm{HCO}_{3}$ in combination with $\mathrm{NaCl}$ increased the sugar concentrations of tomato fruits (Navarro et al., 2005). Accumulated glucose can contribute to higher energy consumption in the stressed plant organs and can play a crucial role in osmoregulation (Hunsche et al., 2010; Petridis et al., 2012). Unfortunately, the enzymatic regulation of carbohydrate metabolism and in parallel the analysis of the coding sequences of these enzymes under salt stress was detected only in a few studies in more detail such as in case of the glycolysis (Muscolo et al., 2003), the role of hexokinases (Poór et al., 2011), and starch metabolism (Boriboonkaset et al., 2012).

\subsection{GLUCOSE AND PHYTOHORMONES UNDER SALT STRESS}

Glucose is a potent modulator of synthesis and the actions of several phytohormones (Sheen, 2014). Price et al. (2003) observed that exogenously applied glucose increased the expression of genes $(A B A 2, A B I 1$, and $A B I 4)$ involved in the biosynthesis of $A B A$ in Arabidopsis seedlings. In addition, it was found that the antagonistic interaction between glucose and ethylene is mediated partly through ABA biosynthesis and signaling (León and Sheen, 2003). Moreover, glucose enhances the degradation of ETHYLENE-INSENSITIVE3 (EIN3), a key transcriptional regulator of ethylene signaling, through HXK1 (Yanagisawa et al., 2003).

Thus, regulation of glucose levels can be mediated by several phytohormones under salt stress. Hassine and Lutts (2010) observed that exogenous ABA treatment $(50 \mu \mathrm{M})$ had no impact on glucose and fructose levels under salt stress in the leaves of Atriplex halimus. In addition, Yin et al. (2009) observed that salinity induced carbohydrate accumulation was also independent of ABA in tomato fruits. In contrast, exogenous pretreatment with salicylic acid (SA) led to the accumulation of soluble sugars in both leaf and root tissues of tomato plants under salinity stress (Poór et al., 2011). 
Effects of other phytohormones, like ethylene in glucose metabolism under salt stress, will likely lead to new discoveries.

\subsection{CONCLUSION AND FUTURE PERSPECTIVES}

Despite the fact that the role of glucose has been intensively studied in the past 20 years, there are many gaps in comprehending the production, transport, storage, and utilization of glucose under salt stress. In this review, we highlighted the current state of the physiological and molecular aspects of $\mathrm{NaCl}-$ modulated glucose levels in different plant species, genotypes, and organs. Moreover, the regulation of glucose levels by phytohormones under salt stress were also mentioned.

Based on the revised works we can conclude that glucose accumulation is a common phenomenon of salt tolerant genotypes in various plant organs (leaf, stem, fruits and root). At the same time, there can be significant differences in the glucose levels in the different plant species, organs, or parts of the organs. Namely, distribution of glucose along the leaf axis can show distinct patterns, which can be altered by salinity in a time-dependent manner. Moreover, not only the production and accumulation of glucose but also the regulation of glucose transport can be crucial in the case of salt stress. The changes in glucose levels in the roots or in fruits can be also crucial to survive the severe salinity stress. Changes in glucose metabolism can be dependent on the dose and duration of $\mathrm{NaCl}$ treatments. Unfortunately, only a few authors have investigated the effects of different concentrations of $\mathrm{NaCl}$ on selected plant species. Based on other studies, glucose contents were increased up to lethal salt concentration and decreased under severe salinity. Moreover, most of the authors determined glucose accumulation only at one time-point after the salt exposure, although the rise may vary from some hours to a few days or weeks. The accumulated glucose can play a role in several signaling and metabolic processes mediated by various phytohormones in the salt stressed tissues, in which salicylic acid can be a significant component. Accumulated glucose can contribute to higher energy consumption in the salt stressed plant organs and can play a role in osmoregulation.

In the future, accurate description of the role of the key enzymes in the glucose metabolism and the role of glucose transport and glucose utilization mediated by various phytohormones would provide new insights into converging and diverging signaling pathways under different salt conditions. Understanding the mechanism that can regulate glucose levels at the cellular, tissue, organ or whole plant levels is an important problem in current plant biology as well as in agriculture. Precise investigation of salt tolerant genotypes may be used to increase the yield and salt stress tolerance under today's changing environment.

\section{Acknowledgments}

We apologize to those colleagues whose work was not reviewed here. This work was supported by grants from the Hungarian Scientific Research Fund (OTKA FK 124871) and by the EU-funded Hungarian Grant EFOP-3.6.1-16-2016-00008. No conflict of interest is declared.

\section{References}

Aguilera-Alvarado, G., Sánchez-Nieto, S., 2017. Plant hexokinases are multifaceted proteins. Plant Cell Physiol. 58 (7), 1151-1160.

Almeida, D.M., Oliveira, M.M., Saibo, N.J., 2017. Regulation of $\mathrm{Na}^{+}$ and $\mathrm{K}^{+}$homeostasis in plants: towards improved salt stress tolerance in crop plants. Genet. Mol. Biol. 40 (1 suppl 1), 326-345.

Anjum, M.A., 2008. Effect of $\mathrm{NaCl}$ concentrations in irrigation water on growth and polyamine metabolism in two citrus rootstocks with different levels of salinity tolerance. Acta Physiol. Plant. 30 (1), 43-52.

Ashraf, M.J.C., Harris, J.C., 2004. Potential biochemical indicators of salinity tolerance in plants. Plant Sci. 166 (1), 3-16.

Baena-González, E., Rolland, F., Thevelein, J.M., Sheen, J., 2007. A central integrator of transcription networks in plant stress and energy signalling. Nature 448 (7156), 938-943.

Boriboonkaset, T., Theerawitaya, C., Pichakum, A., Cha-um, S., Takabe, T., Kirdmanee, C., 2012. Expression levels of some starch metabolism related genes in flag leaf of two contrasting rice genotypes exposed to salt stress. Aust. J. Crop Sci. 6 (11), 1579.

Cao, H., Guo, S., Xu, Y., Jiang, K., Jones, A.M., Chong, K., 2011. Reduced expression of a gene encoding a Golgi localized monosaccharide transporter (OsGMST1) confers hypersensitivity to salt in rice (Oryza sativa). J. Exp. Bot. 62 (13), 4595-4604.

Chang, B., Yang, L., Cong, W., Zu, Y., Tang, Z., 2014. The improved resistance to high salinity induced by trehalose is associated with ionic regulation and osmotic adjustment in Catharanthus roseus. Plant. Physiol. Biochem. 77, 140-148.

Chaves, M.M., Flexas, J., Pinheiro, C., 2009. Photosynthesis under drought and salt stress: regulation mechanisms from whole plant to cell. Ann. Bot. (Lond.) 103 (4), 551-560.

Chen, L.Q., Hou, B.H., Lalonde, S., Takanaga, H., Hartung, M.L., Qu, X.Q., et al., 2010. Sugar transporters for intercellular exchange and nutrition of pathogens. Nature 468 (7323), 527-532.

Cho, Y.H., Sheen, J., Yoo, S.D., 2010. Low glucose uncoupleshexokinase1-dependent sugar signaling from stress and defense hormone abscisic acid and $\mathrm{C}_{\mathrm{H}} 4$ responses in Arabidopsis. Plant Physiol. 152 (3), 1180-1182.

Cho, Y.H., Yoo, S.D., Sheen, J., 2006. Regulatory functions of nuclear hexokinase1 complex in glucose signaling. Cell 127 (3), 579-589.

Claeyssen, É., Rivoal, J., 2007. Isozymes of plant hexokinase: occurrence, properties and functions. Phytochemistry 68 (6), 709-731.

Couée, I., Sulmon, C., Gouesbet, G., El Amrani, A., 2006. Involvement of soluble sugars in reactive oxygen species balance and responses to oxidative stress in plants. J. Exp. Bot. 57 (3), 449-459.

Daloso, D.M., Anjos, L., Fernie, A.R., 2016. Roles of sucrose in guard cell regulation. New Phytol. 211 (3), 809-818. 
dos Santos, C.V., Caldeira, G., 1999. Comparative responses of Helianthus annuus plants and calli exposed to $\mathrm{NaCl}$ : I. Growth rate and osmotic regulation in intact plants and calli. J. Plant. Physiol. 155 (6), 769-777.

Fernandes, F.M., Arrabaça, M.C., Carvalho, L.M.M., 2004. Sucrose metabolism in Lupinus albus L. under salt stress. Biol. Plant. 48 (2), 317-319.

Gashaw, A., Theerawitaya, C., Samphumphuang, T., Cha-um, S., Supaibulwatana, K., 2014. CPPU elevates photosynthetic abilities, growth performances and yield traits in salt stressed rice (Oryza sativa L. s indica) via free proline and sugar accumulation. Pestic. Biochem. Physiol. 108, 27-33.

Gémes, K., Poór, P., Horváth, E., Kolbert, Z., Szopkó, D., Szepesi, Á., et al., 2011. Cross-talk between salicylic acid and NaCl-generated reactive oxygen species and nitric oxide in tomato during acclimation to high salinity. Physiol. Plant. 142 (2), 179-192.

Gibson, S.I., 2005. Control of plant development and gene expression by sugar signaling. Curr. Opin. Plant. Biol. 8 (1), 93-102.

Granot, D., David-Schwartz, R., Kelly, G., 2013. Hexose kinases and their role in sugar-sensing and plant development. Front. Plant Sci. 4,44 .

Grzesiak, M., Filek, M., Barbasz, A., Kreczmer, B., Hartikainen, H., 2013. Relationships between polyamines, ethylene, osmoprotectants and antioxidant enzymes activities in wheat seedlings after short-term PEG- and NaCl-induced stresses. Plant Growth Regul. 69 (2), 177-189.

Hamamoto, S., Horie, T., Hauser, F., Deinlein, U., Schroeder, J.I., Uozumi, N., 2015. HKT transporters mediate salt stress resistance in plants: from structure and function to the field. Curr. Opin. Biotechnol. 32, 113-120.

Hartzendorf, T., Rolletschek, H., 2001. Effects of NaCl-salinity on amino acid and carbohydrate contents of Phragmites australis. Aquat. Bot. 69 (2-4), 195-208.

Hassine, A.B., Lutts, S., 2010. Differential responses of saltbush Atriplex halimus L. exposed to salinity and water stress in relation to senescing hormones abscisic acid and ethylene. J. Plant. Physiol. 167 (17), 1448-1456.

Hu, M., Shi, Z., Zhang, Z., Zhang, Y., Li, H., 2012. Effects of exogenous glucose on seed germination and antioxidant capacity in wheat seedlings under salt stress. Plant Growth Regul. 68 (2), 177-188.

Hu, Y., Schnyder, H., Schmidhalter, U., 2000. Carbohydrate deposition and partitioning in elongating leaves of wheat under saline soil conditions. Funct. Plant Biol. 27 (4), 363-370.

Hunsche, M., Lankes, C., Hoffstall, H., Noga, G., 2010. Vegetative performance, leaf water potential, and partitioning of minerals and soluble sugars: traits for ranking the NaCl-tolerance of tomato genotypes? Plant Growth Regul. 62 (2), 151-162.

Julius, B.T., Leach, K.A., Tran, T.M., Mertz, R.A., Braun, D.M., 2017. Sugar transporters in plants: new insights and discoveries. Plant Cell Physiol. 58 (9), 1442-1460.

Kataoka, K., Sugimoto, K., Ohashi, H., Yamada, H., 2017. Effect of organo-mineral fertilizer on tomato fruit production and incidence of blossom-end rot under salinity. Horticult. J. 86 (3), 357-364.

Kerepesi, I., Galiba, G., 2000. Osmotic and salt stress-induced alteration in soluble carbohydrate content in wheat seedlings. Crop Sci. 40 (2), 482-487.

Khan, M.I.R., Khan, N.A., 2013. Salicylic acid and jasmonates: approaches in abiotic stress tolerance. Plant Biochem. Physiol. 1, 4.

Khan, M.I.R., Khan, N.A., 2017. Reactive Oxygen Species and Antioxidant System in Plants: Role and Regulation Under Abiotic Stress. Springer Nature, Singapore978-981-10-5254-5.

Khan, M.I.R., Asgher, M., Khan, N.A., 2014. Alleviation of saltinduced photosynthesis and growth inhibition by salicylic acid involves glycinebetaine and ethylene in mungbean (Vigna radiata L.). Plant. Physiol. Biochem. 80, 67-74.
Khan, M.I.R., Iqbal, N., Masood, A., Khan, N.A., 2012. Variation in salt tolerance of wheat cultivars: evaluation of the role of glycinebetaine and ethylene. Pedosphere 22, 746-754.

Kocsy, G., Tari, I., Vanková, R., Zechmann, B., Gulyás, Z., Poór, P., et al., 2013. Redox control of plant growth and development. Plant Sci. 211, 77-91.

Krapp, A., Hofmann, B., Schäfer, C., Stitt, M., 1993. Regulation of the expression of $r b c S$ and other photosynthetic genes by carbohydrates: a mechanism for the 'sink regulation' of photosynthesis? Plant J. 3 (6), 817-828.

Krasensky, J., Jonak, C., 2012. Drought, salt, and temperature stressinduced metabolic rearrangements and regulatory networks. J. Exp. Bot. 63 (4), 1593-1608.

Lee, Y., Funk, C., Erban, A., Kopka, J., Köhl, K.I., Zuther, E., et al., 2016. Salt stress responses in a geographically diverse collection of Eutrema/Thellungiella s accessions. Funct. Plant Biol. 43 (7), $590-606$.

Lemoine, R., La Camera, S., Atanassova, R., Dédaldéchamp, F., Allario, T., Pourtau, N., et al., 2013. Source-to-sink transport of sugar and regulation by environmental factors. Front. Plant Sci. 4, 272.

León, P., Sheen, J., 2003. Sugar and hormone connections. Trends. Plant. Sci. 8 (3), 110-116.

Li, L., Sheen, J., 2016. Dynamic and diverse sugar signaling. Curr. Opin. Plant. Biol. 33, 116-125.

Liu, T., Van Staden, J., 2001. Partitioning of carbohydrates in saltsensitive and salt-tolerant soybean callus cultures under salinity stress and its subsequent relief. Plant Growth Regul. 33 (1), 13-17.

López, M., Herrera-Cervera, J.A., Lluch, C., Tejera, N.A., 2006. Trehalose metabolism in root nodules of the model legume Lotus japonicus in response to salt stress. Physiol. Plant. 128 (4), 701-709.

Mahajan, S., Tuteja, N., 2005. Cold, salinity and drought stresses: an overview. Arch. Biochem. Biophys. 444 (2), 139-158.

Mahasal, K., Chaopaknam, A., Ngampanya, B., 2011. Expression analysis of $\mathrm{Na}^{+} / \mathrm{H}^{+}$exchanger and monosaccharide transporter genes in rice suspension cells under salt stress. Thai J. Agric. Sci. 44 (5), 125-128.

Martinoia, E., 2018. Vacuolar transporters - companions on a longtime journey. Plant Physiol. 176, 1384-1407.

Miller, G.A.D., Suzuki, N., Ciftci-Yilmaz, S., Mittler, R., 2010. Reactive oxygen species homeostasis and signalling during drought and salinity stresses. Plant Cell Environ. 33 (4), 453-467.

Mišić, D., Dragićević, M., Šiler, B., Živković, J.N., Maksimović, V., Momčilović, I., et al., 2012. Sugars and acid invertase mediate the physiological response of Schenkia spicata root cultures to salt stress. J. Plant. Physiol. 169 (13), 1281-1289.

Moore, B., Zhou, L., Rolland, F., Hall, Q., Cheng, W.H., Liu, Y.X., et al., 2003. Role of the Arabidopsis glucose sensor HXK1 in nutrient, light, and hormonal signaling. Science 300 (5617), 332-336.

Morkunas, I., Ratajczak, L., 2014. The role of sugar signaling in plant defense responses against fungal pathogens. Acta Physiol. Plant. 36 (7), 1607-1619.

Morsy, M.R., Jouve, L., Hausman, J.F., Hoffmann, L., Stewart, J.M., 2007. Alteration of oxidative and carbohydrate metabolism under abiotic stress in two rice (Oryza sativa L.) genotypes contrasting in chilling tolerance. J. Plant. Physiol. 164 (2), 157-167.

Munns, R., 2002. Comparative physiology of salt and water stress. Plant Cell Environ. 25 (2), 239-250.

Munns, R., Gilliham, M., 2015. Salinity tolerance of crops - what is the cost? New Phytol. 208 (3), 668-673.

Munns, R., Tester, M., 2008. Mechanisms of salinity tolerance. Annu. Rev. Plant. Biol. 59, 651-681.

Muscolo, A., Panuccio, M.R., Sidari, M., 2003. Effects of salinity on growth, carbohydrate metabolism and nutritive properties of kikuyu grass (Pennisetum clandestinum Hochst). Plant Sci. 164 (6), 1103-1110. 
Navarro, J.M., Flores, P., Carvajal, M., Martinez, V., 2005. Changes in quality and yield of tomato fruit with ammonium, bicarbonate and calcium fertilisation under saline conditions. J. Horticult. Sci. Biotechnol. 80 (3), 351-357.

Ouzounidou, G., Ilias, I., Giannakoula, A., Theoharidou, I., 2014. Effect of water stress and $\mathrm{NaCl}$ triggered changes on yield, physiology, biochemistry of broad bean (Vicia faba) plants and on quality of harvested pods. Biologia 69 (8), 1010-1017.

Pasala, R.K., Khan, M.I.R., Minhas, S., Farooq, M.A., Sultana, R., Per, T.S., et al., 2016. Can plant bio-regulators minimize crop productivity losses caused by drought, heat and salinity stress? An integrated review. J. Appl. Bot. Food 89, 113-125.

Per, T.S., Khan, N.A., Reddy, S., Masood, A., Hasanuzzaman, M., Khan, M.I.R., et al., 2017. Approaches in modulating proline metabolism in plants for salt and drought stress tolerance: phytohormones, mineral nutrients and transgenics. Plant. Physiol. Biochem. 115, 126-140.

Per, T.S., Khan, M.I.R., Anjum, N.A., Masood, A., Hussain, S.J., Khan, N.A., 2018. Jasmonates in plants under abiotic stresses: crosstalk with other phytohormones matters. Environ. Exp. Bot. 145, 104-120.

Petridis, A., Therios, I., Samouris, G., Tananaki, C., 2012. Salinity-induced changes in phenolic compounds in leaves and roots of four olive cultivars (Olea europaea L.) and their relationship to antioxidant activity. Environ. Exp. Bot. 79, 37-43.

Poór, P., Gémes, K., Horváth, F., Szepesi, A., Simon, M.L., Tari, I., 2011. Salicylic acid treatment via the rooting medium interferes with stomatal response, $\mathrm{CO}_{2}$ fixation rate and carbohydrate metabolism in tomato, and decreases harmful effects of subsequent salt stress. Plant Biol. 13 (1), 105-114.

Poór, P., Kovács, J., Szopkó, D., Tari, I., 2013. Ethylene signaling in salt stress-and salicylic acid-induced programmed cell death in tomato suspension cells. Protoplasma 250 (1), 273-284.

Poór, P., Laskay, G., Tari, I., 2015a. Role of nitric oxide in salt stress-induced programmed cell death and defense mechanisms. In: Khan, M.N., Mobin, M., Mohammad, F., Corpas, F.J. (Eds.), Nitric Oxide Action in Abiotic Stress Responses in Plants. Springer, pp. 193-219.

Poór, P., Kovács, J., Borbély, P., Takács, Z., Szepesi, Á., Tari, I., 2015b. Salt stress-induced production of reactive oxygen-and nitrogen species and cell death in the ethylene receptor mutant Never ripe and wild type tomato roots. Plant. Physiol. Biochem. 97, 313-322.

Poór, P., Szopkó, D., Tari, I., 2012. Ionic homeostasis disturbance is involved in tomato cell death induced by $\mathrm{NaCl}$ and salicylic acid. In Vitro Cell. Dev. Biol. Plant 48 (3), 377-382.

Prado, F.E., Boero, C., Gallardo, M., Gonzalez, J.A., 2000. Effect of $\mathrm{NaCl}$ on germination, growth, and soluble sugar content in Chenopodium quinoa Willd. seeds. Bot. Bullet. Acad. Sinica 41, 27-34.

Price, J., Li, T.C., Kang, S.G., Na, J.K., Jang, J.C., 2003. Mechanisms of glucose signaling during germination of Arabidopsis. Plant Physiol. 132 (3), 1424-1438.

Rodríguez-Rosales, M.P., Gálvez, F.J., Huertas, R., Aranda, M.N., Baghour, M., Cagnac, O., et al., 2009. Plant NHX cation/proton antiporters. Plant Signal. Behav. 4 (4), 265-276.

Rolland, F., Baena-Gonzalez, E., Sheen, J., 2006. Sugar sensing and signaling in plants: conserved and novel mechanisms. Annu. Rev. Plant. Biol. 57, 675-709.

Rosa, M., Prado, C., Podazza, G., Interdonato, R., González, J.A., Hilal, M., et al., 2009. Soluble sugars: metabolism, sensing and abiotic stress: a complex network in the life of plants. Plant Signal. Behav. 4 (5), 388-393.

Roy, S.J., Negrão, S., Tester, M., 2014. Salt resistant crop plants. Curr. Opin. Biotechnol. 26, 115-124.
Rubio, J.S., Garcia-Sanchez, F., Rubio, F., Martinez, V., 2009. Yield, blossom-end rot incidence, and fruit quality in pepper plants under moderate salinity are affected by $\mathrm{K}^{+}$and $\mathrm{Ca}^{2+}$ fertilization. Sci. Hortic. (Amsterdam) 119 (2), 79-87.

Rubio, J.S., García-Sánchez, F., Rubio, F., García, A.L., Martínez, V., 2010. The importance of $\mathrm{K}^{+}$in ameliorating the negative effects of salt stress on the growth of pepper plants. Europ. J. Horticult. Sci. $75,33-41$.

Ruffino, A.M.C., Rosa, M., Hilal, M., González, J.A., Prado, F.E., 2010. The role of cotyledon metabolism in the establishment of quinoa (Chenopodium quinoa) seedlings growing under salinity. Plant Soil 326 (1-2), 213-224.

Sacchi, G.A., Abruzzese, A., Lucchini, G., Fiorani, F., Cocucci, S., 2000. Efflux and active re-absorption of glucose in roots of cotton plants grown under saline conditions. Plant Soil 220 (1-2), 1-11.

Saito, T., Matsukura, C., Ban, Y., Shoji, K., Sugiyama, M., Fukuda, N., et al., 2008. Salinity stress affects assimilate metabolism at the gene-expression level during fruit development and improves fruit quality in tomato (Solanum lycopersicum L.). J. Jap. Soc. Horticult. Sci. 77 (1), 61-68.

Sakamoto, A., Murata, N., 2002. The role of glycine betaine in the protection of plants from stress: clues from transgenic plants. Plant Cell Environ. 25 (2), 163-171.

Sami, F., Yusuf, M., Faizan, M., Faraz, A., Hayat, S., 2016. Role of sugars under abiotic stress. Plant. Physiol. Biochem. 109, 54-61.

Shabala, S., 2009. Salinity and programmed cell death: unravelling mechanisms for ion specific signalling. J. Exp. Bot. 60 (3), $709-712$.

Shabala, S., 2013. Learning from halophytes: physiological basis and strategies to improve abiotic stress tolerance in crops. Ann. Bot. (Lond.) 112 (7), 1209-1221.

Sheen, J., 2014. Master regulators in plant glucose signaling networks. J. Plant Biol. 57 (2), 67-79.

Siahpoosh, M.R., Sanchez, D.H., Schlereth, A., Scofield, G.N., Furbank, R.T., van Dongen, J.T., et al., 2012. Modification of OsSUT1 gene expression modulates the salt response of rice Oryza sativa cv. Taipei 309. Plant Sci. 182, 101-111.

Suzuki, M., Hashioka, A., Mimura, T., Ashihara, H., 2005. Salt stress and glycolytic regulation in suspension-cultured cells of the mangrove tree, Bruguiera sexangula. Physiol. Plant. 123 (3), 246-253.

Swain, D.M., Sahoo, R.K., Srivastava, V.K., Tripathy, B.C., Tuteja, R., Tuteja, N., 2017. Function of heterotrimeric G-protein $\gamma$ subunit RGG1 in providing salinity stress tolerance in rice by elevating detoxification of ROS. Planta 245 (2), 367-383.

Takács, Z., Poór, P., Szepesi, Á., Tari, I., 2017. In vivo inhibition of polyamine oxidase by a spermine analogue, MDL-72527, in tomato exposed to sublethal and lethal salt stress. Funct. Plant Biol. 44 (5), 480-492.

Tari, I., Csiszár, J., Horváth, E., Poór, P., Takács, Z., Szepesi, Á., 2015. The alleviation of the adverse effects of salt stress in the tomato plant by salicylic acid shows a time- and organ-specific antioxidant response. Acta Biol. Cracov. s. Bot. 57 (1), 21-30.

Theerawitaya, C., Boriboonkaset, T., Cha-um, S., Supaibulwatana, K., Kirdmanee, C., 2012. Transcriptional regulations of the genes of starch metabolism and physiological changes in response to salt stress rice (Oryza sativa L.) seedlings. Physiol. Mol. Biol. Plants 18 (3), 197-208.

Tsabarducas, V., Chatzistathis, T., Therios, I., Koukourikou-Petridou, M., Tananaki, C., 2015. Differential tolerance of 3 self-rooted Citrus limon cultivars to $\mathrm{NaCl}$ stress. Plant. Physiol. Biochem. 97, 196-206.

Unno, H., Maeda, Y., 2008. Effect of exogenous application of sugars on the salt tolerance of perennial ryegrass protoplasts. Biologia 63 (2), 204-206. 
Wind, J., Smeekens, S., Hanson, J., 2010. Sucrose: metabolite and signaling molecule. Phytochemistry 71 (14-15), 1610-1614.

Wingler, A., Purdy, S., MacLean, J.A., Pourtau, N., 2005. The role of sugars in integrating environmental signals during the regulation of leaf senescence. J. Exp. Bot. 57 (2), 391-399.

Xiong, Y., Sheen, J., 2014. The role of target of rapamycin signaling networks in plant growth and metabolism. Plant Physiol. 164 (2), 499-512.

Yamaguchi, T., Hamamoto, S., Uozumi, N., 2013. Sodium transport system in plant cells. Front. Plant Sci. 4, 410.

Yanagisawa, S., Yoo, S.D., Sheen, J., 2003. Differential regulation of EIN3 stability by glucose and ethylene signalling in plants. Nature 425 (6957), 521-525.
Yin, Y.G., Kobayashi, Y., Sanuki, A., Kondo, S., Fukuda, N., Ezura, H., et al., 2009. Salinity induces carbohydrate accumulation and sugar-regulated starch biosynthetic genes in tomato (Solanum lycopersicum L. cv.'Micro-Tom') fruits in an ABA- and osmotic stress-independent manner. J. Exp. Bot. 61 (2), 563-574.

Zhang, L., Liu, J., Wang, X., Bi, Y., 2013. Glucose-6-phosphate dehydrogenase acts as a regulator of cell redox balance in rice suspension cells under salt stress. Plant Growth Regul. 69 (2), 139-148.

Zhu, J.K., 2002. Salt and drought stress signal transduction in plants. Annu. Rev. Plant. Biol. 53 (1), 247-273.

Zhu, J.K., 2003. Regulation of ion homeostasis under salt stress. Curr. Opin. Plant. Biol. 6 (5), 441-445. 
This page intentionally left blank 


\section{3}

\section{Role of Sugars in Abiotic Stress Signaling in Plants \\ Iffat Zareen Ahmad}

Department of Bioengineering, Integral University, Lucknow, Uttar Pradesh, India

\section{O U T L I N E}

13.1 Introduction 13.1.1 Sugars as Signaling Molecules

13.2 Kinases as Enzyme Sensors

13.3 Sugar Signaling at Gene Level

13.4 Sugar Signaling and Plant Metabolism

\subsection{INTRODUCTION}

Sugars are the most vital biomolecules since they are present in every cell of the body, and are rich in energy. They play an important role in primary as well as secondary metabolism of plants and animals (Lloyd and Zakhleniuk, 2004; Zhang et al., 2014). In plants, they are the product of photosynthesis and are a component of most of the biologically active compounds (Pego et al., 2000). Recently, the function of sugars as critical signaling molecules in relation to both the metabolic status in cells and response to different abiotic stresses has been shown (Lastdrager et al., 2014; Rolland et al., 2006). The diverse and complex networks of sugars warrant a detailed comprehension of their impact on regulatory and metabolic processes at the cellular and the whole plant levels (Lastdrager et al., 2014). Sugar signaling research will remain an exciting area of investigations for many years to come (Smeekens et al., 2014).

Sugars can be classified as monosaccharides, disaccharides, and polysaccharides on the basis of the number of monomers present. The functional groups they possess include aldoses and ketoses. Monosaccharides, mainly glucose, play the role of an important sensory metabolite. Disaccharides (sucrose, trehalose), raffinose, and fructans are three important categories of water-soluble sugars that primarily play a role in plant stress responses and might interact with reactive oxygen species (ROS) signaling pathways. Besides, oligosaccharides and some kinases have also been reported to contribute significantly to signaling at the cellular level (Fig. 13.1). Regardless of the expected complexity of sugar sensing and signaling in photosynthetic 


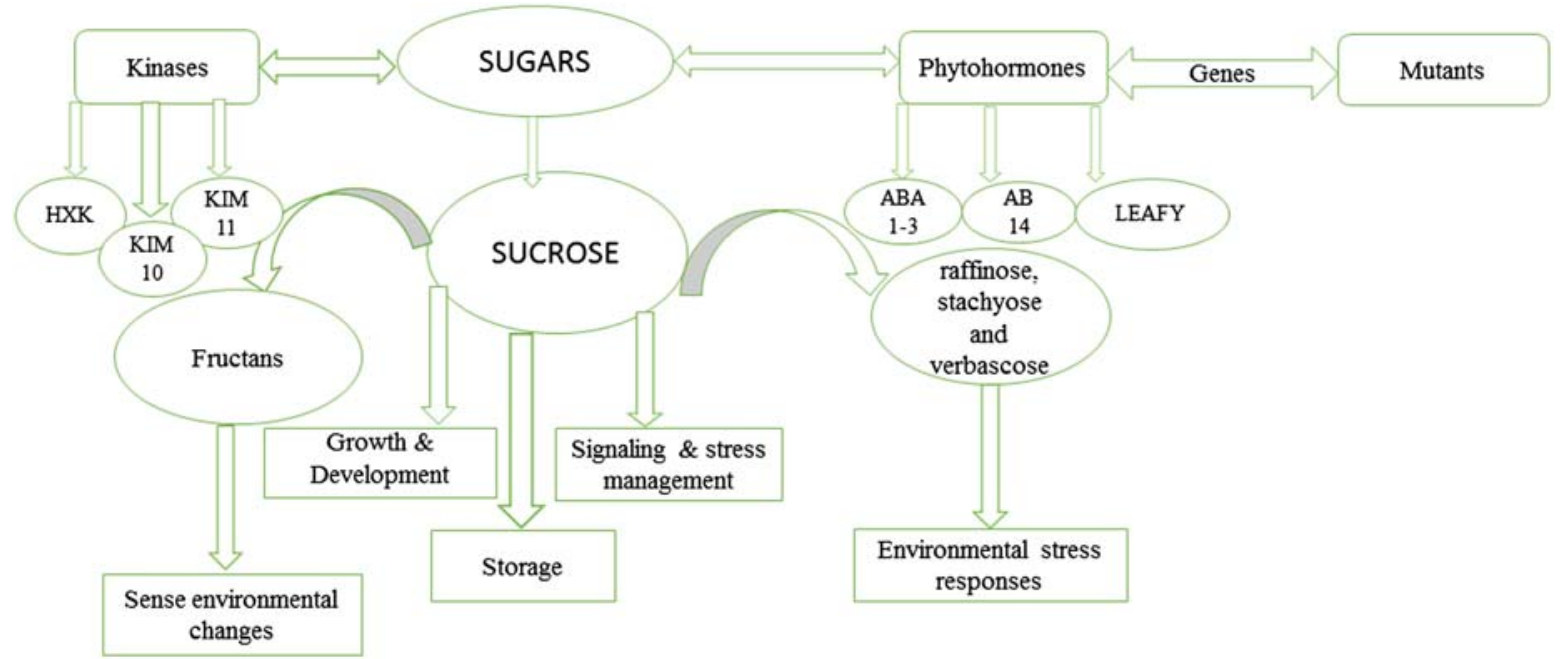

FIGURE 13.1 An overview of sugar signaling in plants in response to stress.

plants, stimulating progress has been made in the past 2 years. Some of the important sugars as signaling molecules are listed below:

1. Sucrose is the most commonly studied sugar in gene regulation development (Gibson and Graham, 1999; Halford et al., 1999; Jang et al., 1997; Koch, 1996; Lalond et al., 1999; Roitsch, 1999; Smeekens and Rook, 1997). It is the major form of translocated sugars in plants. Its role in growth, development, storage, signaling, and stress acclimation makes it the most studied molecule (Salerno and Curatti, 2003).

2. Sucrose combines with galactosyl moieties to give rise to raffinose, stachyose, and verbascose in the cytosol (Schneider and Keller, 2009). Their accumulation, gene expression, and metabolism are greatly associated with environmental stress responses (Nishizawa et al., 2008).

3. Fructans, fructose polymers derived from sucrose, are present in around 15\% of angiosperms. There are many plants for which fructans are the main storage form of carbohydrates (Suzuki et al., 1993). These plants are very important parts of ecosystems in which there are frequent environmental changes (Albrecht et al., 1997).

4. Oligogalacturonides (OGs) (Ferrari et al., 2013) illustrate an excellent example of a sugar being both a metabolite and a signaling molecule.

5. Hexokinase $(\mathrm{HXK})$ is a double-function enzyme and glucose sensor, the first to be documented in plants (Graham et al., 1994; Jang et al., 1997;

Pego et al., 1999; Smeekens and Rook, 1997;

Umemura et al., 1998).

Sugar signaling can be thrust area of research, and a compilation of the present data on this also can be very useful for those researchers who are working on this topic (Table 13.1). The present chapter aims at providing insight into the known information on sugars as signaling molecules under stressful and optimal environments.

\subsubsection{Sugars as Signaling Molecules}

The growth and development of the plant must be in accordance with its metabolism, particularly with the rate of photosynthesis and the nutrient uptake. This synchronization involves local association between hormonal response and metabolic state, as well as long-distance networks between shoot and root tissues. Recently, numerous mechanisms at the molecular level have been given to elucidate the association of sugar signaling with hormone pathways. In a study, DELLA and PIF proteins were developed as hubs in sugar-hormone cross-regulation networks (Ljung et al., 2015). The studies on mutants stimpy or ramosa3 have proposed the role of sugars as signaling molecules for controlling various facets of plant development. In the developmental activities, it was shown that mutant phenotypes are modulated by sugars and they also play a putative role (e.g., trehalose-6-phosphate) in inflorescence development. Because of the plasticity in nature of plant growth and development they are greatly influenced by environmental and dietary conditions (Eveland and Jackson, 2012).

Sugars may control expression of hundreds of genes in plants. A novel biological system was developed that was based on stem cell-like Arabidopsis suspension culture. The cells were maintained in a hormone-free medium and were provided xylose as 
TABLE 13.1 The Effects of Sugars and Enzymes on Different Physiological Parameters and Signaling

\begin{tabular}{|c|c|c|c|c|}
\hline S. no. & Sugar/enzyme & Effects & Metabolites/genes affected & References \\
\hline 1. & Sucrose & Gene regulation & $\begin{array}{l}\text { Maize sucrose synthase and soluble } \\
\text { invertase }\end{array}$ & Koch (1996) \\
\hline 2. & Hexokinase & Gene regulation & Antisense hexokinase (AtHXK) genes & Jang et al. (1997) \\
\hline 3. & Sucrose \& glucose & $\begin{array}{l}\text { Gene regulation } \\
\text { metabolism }\end{array}$ & $\begin{array}{l}\text { Sucrose phosphorylase, enzymes of } \\
\text { glycolysis }\end{array}$ & Smeekens and Rook (1997) \\
\hline 4. & Sucrose & Signaling & Source sink regulation & Roitsch (1999) \\
\hline 5. & Hexokinase & Sugar sensor & Sugars & $\begin{array}{l}\text { Halford, Purcell, and Hardie (1999), } \\
\text { Jang and Sheen (1997), Smeekens and } \\
\text { Rook (1997) }\end{array}$ \\
\hline 6. & Galactinol and raffinose & Gene expression & GolS1 or GolS2 & Nishizawa et al. (2008) \\
\hline 7. & Fructans & $\begin{array}{l}\text { Sugar accumulation in } \\
\text { hypoxia, flood }\end{array}$ & Sugars & Albrecht et al. (1997) \\
\hline 8. & Sucrose & Gene expression & $\begin{array}{l}\text { Cucumber malate synthase (MS) and } \\
\text { isocitrate lyase (ICL) }\end{array}$ & Graham et al. (1994) \\
\hline 9. & Sugars & $\begin{array}{l}\text { Sugar-mediated plant } \\
\text { growth regulation }\end{array}$ & $\begin{array}{l}\text { Trehalose-6-phosphate, target of } \\
\text { rapamycin kinase, and Snf1-related } \\
\text { kinase } 1 \text { regulatory systems }\end{array}$ & Lastdrager et al. (2014) \\
\hline 10. & Mannose & $\begin{array}{l}\text { Germination of } \\
\text { Arabidopsis seeds }\end{array}$ & Hexokinase expression & Pego et al. (1999) \\
\hline 11. & Trehalose-6-phosphate & Inflorescence & & Eveland and Jackson (2012) \\
\hline 12. & Sugars & Fungal pathogen & Increased resistance & Morkunas (2014) \\
\hline 13. & Sucrose & $\begin{array}{l}\text { Sugar signaling, } \\
\text { phosphate deficiency }\end{array}$ & Increased size of roots & Hammond and White (2011) \\
\hline 14. & $\begin{array}{l}\text { Raffinose, sucrose, and } \\
\text { starch }\end{array}$ & Gene expression & TOR kinase & Dobrenel et al. (2013) \\
\hline 15. & Invertase & Plant development & Phenotypic alterations in carrot embryo & Tang et al. (1999) \\
\hline 16. & Sucrose & Sugar signaling & Circadian rhythms & $\begin{array}{l}\text { Moghaddam and Van den Ende } \\
\text { (2013) }\end{array}$ \\
\hline 17. & $\begin{array}{l}\text { Suc itself, glucose (Glc) } \\
\text { and trehalose-6- } \\
\text { phosphate (T6P) }\end{array}$ & $\begin{array}{l}\text { Sugar signaling, growth } \\
\text { and development }\end{array}$ & Sugar-mediated plant growth regulation & Dahiya et al. (2017) \\
\hline 18. & $\begin{array}{l}\text { Oligogalacturonides } \\
\text { (OGs) }\end{array}$ & $\begin{array}{l}\text { Defense response and } \\
\text { development }\end{array}$ & Auxin & Ferrari et al. (2013) \\
\hline 19. & Hexokinase & $\begin{array}{l}\text { Sugar sensor and } \\
\text { metabolism }\end{array}$ & $\begin{array}{l}\text { Snf1-related kinases (SnRKs), } \\
\text { extracellular sugar sensors, and trehalose } \\
\text { metabolism }\end{array}$ & Rolland et al. (2006) \\
\hline 20. & Hexokinase & $\begin{array}{l}\text { Sugar sensing and } \\
\text { signaling, physiology, } \\
\text { and development }\end{array}$ & $\begin{array}{l}\text { KIN10 and KIN11 protein kinases, bZIP } \\
\text { transcription factors }\end{array}$ & Hanson and Smeekens (2009) \\
\hline
\end{tabular}

the only carbon source. Using functional genomics 290 sugar-responsive genes were identified, responding rapidly (within $1 \mathrm{~h}$ ) and specifically to low concentration $(1 \mathrm{mM})$ of glucose, fructose, and/or sucrose. The exact nature of the signaling sugar molecules and sites of their perception were elaborated using nonmetabolizable sugar analogs for selected genes. The expression of selected sugar-responsive genes was not restricted to a specific tissue or cell type, as shown in transgenic and wild-type Arabidopsis thaliana seedlings. It was further proved that response was associated with photoperiod-related changes in sugar availability (Kunz et al., 2014). Studies indicated that sugars can act as signaling molecules and can control gene expression and developmental processes in plants in a manner similar to plant hormones. 
Uncoupling of glucose signaling from its metabolism provided substantial proof in favor of this. Glucose sensors have been identified and isolation and characterization of mutants and other regulatory components have been done in plant sugar signal transduction pathways (Sheen et al., 1999).

Sugars play osmoprotective roles and their levels increase to avoid degradation of enzymes and to decrease the amount of ROS. In the presence of drought stress, synthesis of different sugar molecules, such as mono-, di-, and oligosaccharides, accelerates to give osmoregulation. Another approach to osmoregulation is by balancing the low molecular sugars by phloem transport and sucrose metabolism. Other than osmoregulation, soluble sugars also act as signaling molecules to modulate the sensitivity of plants and thus help in cell responses. The sensor proteins are needed for sensing the level of sugars in cells and lead to signal transduction cascades. The exogenous application of various sugar molecules (trehalose, mannitol, sorbitol, etc.) has been found to be an effective approach to increase the resistance against various abiotic stresses including drought stress. Plants that are grown under abiotic stress are under distress, which results in the increased accumulation of ROS (Khan and Khan, 2014). This buildup of ROS invokes both injurious as well as signaling effects at the organellar and cellular levels. The consequence of an environmental trial is mostly dependent on the subtle equilibrium between the synthesis of ROS and their scavenging by both enzymatic and nonenzymatic antioxidants (Choudhry et al., 2016; Khan and Khan, 2014; Khan et al., 2014; You and Chan, 2015). Nevertheless, this classical knowledge needs greater and renewed understanding, since it has become gradually more clear that soluble sugars such as disaccharides, raffinose family oligosaccharides, and fructans-next to their associated metabolic enzymes-are intensely associated with stress-induced collection of ROS in plants. There are several examples of such signaling sugar molecules that are based on their location within the cell, as distinct organelles seem to use different mechanisms. Interestingly, the vacuole seems to be a most significant component in the network involved in ROS signaling of plants. The explicit understanding of the connection between the mechanisms regulating ROS signaling under abiotic stress will help in clarifying and taking up mechanistic approaches to improve crop tolerance to different environmental conditions due to abiotic stresses and the mitigation of ROS produced due to this (Choudhry et al., 2016; Keunen et al., 2013; You and Chan, 2015). The role of exogenous application of sugars under drought stress is also studied. Drought has significant effects on plants at the cellular and metabolic levels. The amount of osmoprotectants increase, which prevents degradation of enzymes and scavenges ROS.
One of these protective compounds is sugar. To provide osmoregulation under drought conditions, the levels of mono-, di-, and oloigosaccharides is enhanced. Sugars also act as signaling molecules and initiate signal transduction cascades. Even the exogenous application of some sugars like trehalose, mannitol, sorbitol, etc. has been shown to improve drought resistance (Bhardwaj et al., 2016). This book chapter reviews the metabolism of sugar as well as its role in stress signaling. Considerable information has been presented in the last 2 years on important groups in sugar-mediated plant growth regulation, with particular stress on trehalose-6phosphate, target of rapamycin kinase, and Snf1-related kinase 1 regulatory systems. The synthesis of protein has been regulated by sugars and this aspect of metabolism is fundamental in controlling the growth of the plant, and many research efforts to understand this regulation of translation by sugars have been carried out (Lastdrager et al., 2014).

Sugars are involved in many metabolic and signaling pathways in plants. Sugar signals may also contribute to immune responses against pathogens and probably function as priming molecules leading to pathogen-associated molecular patterns-triggered immunity and effector-triggered immunity in plants. These putative roles also depend greatly on coordinated relationships with hormones and the light status in an intricate network. Although evidence in favor of sugar-mediated plant immunity is accumulating, more in-depth fundamental research is required to unravel the sugar signaling pathways involved. This might pave the way for the use of biodegradable sugar-(like) compounds to counteract plant diseases as cheaper and safer alternatives for toxic agrochemicals (Moghaddam and den Ende, 2012). In most cases in which fungus affects a plant, an increased amount of sugars in plant tissues improves the resistance of plant. Many different theories have been suggested by various researchers working in this area over time to explore the mechanistic approach of this "high-sugar resistance." Sugars act as an important substrate that releases energy and also provides the building blocks for defense responses in plants, while they may also play a role as signal molecules in networking with the hormonal signaling systems, which regulate the immune system of the plant. Sugars increase the oxidative burst during the initial phases of infection, resulting in increased lignification of cell walls, stimulation of the flavonoid synthesis, and induction of some proteins. Some sugars act as grooming agents that stimulate and induce greater resistance of the plant to pathogens (Morkunas, 2014).

The studies carried out during the last decade have revealed the understanding behind the sensing signal by plants and their response towards the availability 
soil phosphorus (P) (Amtmann et al., 2006; George et al., 2011; Nilsson et al., 2010; Vance, 2010; White and Hammond, 2008; Yang and Finnegan, 2010). Earlier studies have shown the potential of shoot-derived carbohydrate signals to induce acclimatory responses in roots in low $\mathrm{P}$ condition. In this perspective, these carbohydrates act as systemic plant growth regulators (Hammond and White, 2011). Photosynthate is transported mainly to sink tissues as sucrose via the phloem. In low $\mathrm{P}$ availability, plants store sugars and starch in their leaves. Enhanced loading of sucrose to the phloem in conditions of $\mathrm{P}$ starvation mostly works to relocate carbon resources to the roots, which results in the increase in their size in comparison with the shoot (Hermans et al., 2006). The translocation of sugars via the phloem also has the ability to initiate sugar signaling cascades that modify the expression of genes responsible for plant responses to low $\mathrm{P}$ availability. These comprises of improving biochemical changes in root to acquire soil $\mathrm{P}$, by increasing expression and activity of inorganic phosphate $(\mathrm{Pi})$ transporters, the secretion of acid phosphatases and organic acids to release $\mathrm{P}$ from the soil, and the optimization of internal P use (Hammond and White, 2011).

\subsubsection{Glucose}

Glucose has been shown to be a positive regulator of TOR kinase enzyme and the explanation is given about its effect on different processes including biosynthesis of the stress-related sugar raffinose, sucrose, and starch as well as glycolysis (Dobrenel et al., 2013).

Glucose has the characteristics of a plant hormone and its specific effects on the level of gene expression and developmental plans are a feature of plant hormone actions (Koch, 1996; Lalond et al., 1999). Moreover, a study showing that HXK acts as a specific glucose sensor, and that the action of nonmetabolizable glucose signals is mediated through unknown sensors/receptors, further proved the behavior of glucose as a plant hormone with dual functions of a signaling molecule and a metabolite. The latest research on transgenic plants (Herbers et al., 1996; Jang et al., 1997; Tang, Luscher \& Sturm, 1999) and sugar response mutants (Dijkwel et al., 1997; Mita et al., 1997; Zhou et al., 1998) has revealed close cross-talk between glucose and other plant hormone signaling pathways. The characterization of transgenic carrot plants recently with antisense cell wall invertase or vacuolar invertase constructs showed malformed embryos and bushy shoots that can be corrected by hexoses (Tang et al., 1999). These phenotypes resulted due to lack of hexose signaling, which might be responsible for maintaining the balance between the plant hormones auxin and cytokinin. In gin2 mutant, which has been isolated recently, the lack of AtHXK1 resulted in changed sensitivity to auxin and cytokinin.

\subsubsection{Sucrose}

A different characteristic regarding sugars as signaling molecules has been given in articles reporting the assimilation of sucrose-mediated signaling pathways in cellular networks (Moghaddam and Van den Ende, 2013). This explained the interaction of sugar signals with other critical signaling systems of the cell including the circadian clock and phytohormones, in monitoring defense responses and developmental activities such as flowering (Moghaddam and Van den Ende, 2013). In the same way, the regulatory steps that combine diurnal signals with downstream cellular responses may occur at the sugar uptake step. The work has been reported on the sucrose transporter 4 (SUT4) from potato (Chincinska et al., 2013). Another instance where sugars function as regulatory molecules has been done on RAPAMYCIN (TOR) kinase complexes (Dobrenel et al., 2013). These complexes combine with additional partner proteins to bring about and involve a broad range of cellular responses, including metabolism, mRNA processing, and autophagy, frequently along with nutrient signaling.

Two aspects of sugar biochemistry that have been extensively studied as the characteristic features of many sugars, mainly sucrose, are their controlled distribution within the cell and transport over long distances from sinks to sources. The metabolism of sucrose at the cellular level is dependent on, and regulated by, the activities of sucrose synthase and sucrosephosphate synthase (SPS). Researchers have provided understanding on two rice SPS paralogs, OsSPS1 and OsSPS11, and their particular expressions in response to diurnal factors and carbohydrate availability (Yonekura et al., 2013). The long-distance transport of sucrose is assisted by the activities of specialized transport proteins. Scientists explored the functioning of these transporters as checkpoints to forward information on metabolic fluxes to initiate cellular responses (Chincinska et al., 2013).

Sucrose metabolism plays crucial roles in plant development mainly by generating a range of sugar signaling molecules such as Suc itself, glucose, and trehalose-6-phosphate. Sugars not only fuel cellular carbon and energy metabolism but also play pivotal roles as signaling molecules. Sugars have a central regulatory function in steering plant growth.

\subsubsection{Oligogalacturonides}

The information given by a researcher in a review article on OGs demonstrated an outstanding example of a sugar being both a metabolic and a signaling 
molecule (Ferrari et al., 2013). OGs comprise of $\alpha-1,4-$ linked galacturonosyl residues and are essential constituents of the cell wall. Biotic stress conditions lead to the release of hydrolytic enzymes by the cell wall, which is either stimulated by fungal growth or by mechanical damage imposed by herbivores. The OGs released due to this stimulus then work as signaling molecules to produce a defense response in the particular plant cell and adjacent tissues (Ferrari et al., 2013).

An improved understanding of the regulation of developmental processes and allelopathic and defenseinducing characteristics of "oligosaccharin" signaling carbohydrates gives opportunity not only for better knowledge of plant biology, but also offers better opportunities for commercial exploitation.

\subsection{KINASES AS ENZYME SENSORS}

The latest evidence suggested that even a millimolar range of signaling molecules can stimulate sensing and signaling by applying sugar binding enzymes, proteins, or transporters. Even though multiple sugar sensors/receptors probably exist, HXK as a dual-function enzyme and glucose sensor was the first to be reported in plants (Graham et al., 1994; Jang and Sheen, 1997; Jang et al., 1997; Smeekens and Rook, 1997; Umemura et al., 1998). Sucrose is the main form of translocated sugars in plants and is the most commonly studied plant sugar with respect to gene regulation and development (Gibson and Graham, 1999; Halford et al., 1999; Jang et al., 1997; Koch, 1996; Lalond et al., 1999; Roitsch, 1999; Smeekens and Rook, 1997).

HXK acts in a dual manner and it functions both as enzyme and sensor. HXK is a dimeric cytosolic enzyme that is required for glycolysis. It is a matter of debate whether HXK is a glucose sensor and it senses the intracellular glucose signals, whether the catalytic and regulatory functions of HXK are separable, and whether the ATP/AMP (adenosine triphosphate and adenosine monophosphate) ratio is the actual signal. Manipulation of glucose metabolites in a leaf cell showed that sugar phosphates, the changes in ATP levels, phosphate depletion, and other metabolites cannot substitute for the glucose signal (Jang and Sheen, 1997). The inhibition of Arabidopsis seed germination by mannose could occur without affecting ATP or inorganic phosphate levels in seeds, and could be overcome by a HXK inhibitor (Pego et al., 1999). These data gave support in favor of the uncoupling of glucose signaling and glucose metabolism. In genetically modified Arabidopsis plants and yeast, different regulatory functions in glucose repression but similar catalytic activities were exhibited by plant and yeast HXK. Therefore, glucose metabolism alone cannot explain many of the glucose responses. The function of HXK as a glucose sensor seems to be conserved in plants, as overexpression of the Arabidopsis HXK1 gene stimulated glucose hypersensitivity in transgenic tomato and in transformed maize leaf cells (Sheen, unpublished data). Arabidopsis HXK1 mutants lacking glucose repression have been identified recently, with no corresponding effects on sugar metabolism.

Sugars act as an important signaling molecule in plants. Different sugar signals are being generated to monitor growth, development, and stress response. The study at the gene level showed extensive interactions between sugar and plant hormone signaling, and a main role for HXK as a conserved glucose sensor. Varied sugar signals stimulate multiple HXK-dependent and HXK-independent pathways and utilize diverse molecular mechanisms to regulate transcription, translation, protein stability, and enzymatic activity. Significant and complicated roles for Snf1-related kinases (SnRKs), extracellular sugar sensors, and trehalose metabolism in plant sugar signaling are now also developing (Rolland et al., 2006). Sugar sensors have been documented and proposed that include the most studied glucose sensor HXK1, besides sucrose and trehalose-6-phosphate. The diversity and complexity of sugar sensing and signaling and their effects on many physiological and developmental processes and integration with other signaling pathways make their study quite interesting. Notably, KIN10 and KIN11 protein kinases are fundamental in coordinating several of the responses to sugars and stress. bZIP transcription factors play an important role and facilitate effects of sugar signaling on gene expression and metabolite content (Hanson and Smeekens, 2009).

The perception of sugars by the cell is another very significant aspect in sugar research. The most significant indication on cellular sugar sensing systems presently comes from hexose kinases, which phosphorylate glucose (hexokinase) and fructose (fructokinase). HXKI from Arabidopsis has been involved in the early steps (Jang et al., 1997; Moore et al., 2003), and two related review articles provided comprehensive information on HXK and fructokinases in plants (Granot et al., 2013; Tiessen and Padilla-Chacon, 2013), along with other sugar metabolizing enzymes such as invertases, sucrose synthases, and SPS (Tiessen and PadillaChacon, 2013). Literature that has been produced on various proteins with respect to their gene families, their subcellular localization, and particular metabolic activities, as well as effects on developmental activities and association with signal transduction events. Further, regulatory steps involved in sugar and stress-related signal transduction are dependent primarily on the activity of SnRK1-protein kinases. These kinases are multienzyme complexes and 
cystathionine- $\beta$-synthase (CBS) domain-containing proteins belong to this group. The two subunits of these complexes, AtPV42a and AtPV42b, are misregulated in histone acetyltransferase 1 (hac1) mutants (Heisel et al., 2013). The hac1 mutants showed abnormal sugar responses and fertility defects, which could be partly clarified by the altered levels of AtPV42a and AtPV42b expression. It was strongly shown that there is the participation of microRNAs in SnRK1-protein kinasedependent processes (Confraria et al., 2013). Sugar response regulation also requires mRNA processing steps, as shown by Funck et al. (2012). They were identified by map-based cloning of a sugar response mutation as ESP1, a CstF64-like putative RNA processing factor. There is a role of ESP1 in mRNA $3^{\prime}$-end formation, and the work involves RNA maturation as a crucial factor for normal sugar response (Funck et al., 2012).

\subsection{SUGAR SIGNALING AT GENE LEVEL}

Sugars can behave as signaling molecules as they have the tendency to be global regulators of gene expression. For example, mimicking hormones for transforming nutrient status to regulate growth and the floral development process (Koch, 1996, 2004; Ohto et al., 2001; Price et al., 2004; Rolland et al., 2002, 2006; Smeekens, 2000; Smeekens et al., 2010; Wobus and Weber, 1999). In this manner, sugar-dependent regulation of the gene reveals an excess of carbohydrate or its exhaustion (Koch et al., 1996; Koch, 2004; Rolland et al., 2002). The interplay between nutrient status and regulation of transcription permits the plant to regulate growth, both at the level of the complete plant and at the cellular level, which is dependent on a particular tissue or cell so as to strongly manage developmental processes with existing carbohydrate. In conditions of low sugar levels, genes involved in photosynthesis, carbohydrate transport and storage, and nitrogen metabolism are upregulated. On the other hand, the excess of sugar stimulates the usual sink organ activities including import of carbohydrate, consumption, and storage, and the biosynthesis of starch and anthocyanin.

Several genes that are involved in sugar sensing and signaling have been recognized in mutants for transformed responses to exogenous sugars during germination of seed and early growth of seedling in Arabidopsis (Gibson, 2005; Rolland et al., 2002, 2006; Smeekens, 2000). For example, glucose insensitive (gin) mutants don't undergo growth arrest under inhibitory concentration of glc, demonstrating usual hypocotyl elongation, cotyledon greening, and expansion. Screening of mutants related to other sugar response phenotypes showed that certain sucrose uncoupled (sun), sugar insensitive (sis), and / or impaired sucrose induction (isi) mutations were allelic to gin loci, giving an idea that these genes may function at the border of various sugar signaling pathways (Gibson, 2005; Rolland et al., 2002; Smeekens, 2000; Zhou et al., 1998). Such mutants could lead to secondary responses to hexose products produced from sucrose-dependent stimulation of sucrose-splitting enzymes, for instance extracellular invertases. Moreover, the disaccharide trehalose, and its intermediary metabolites, trehalose-6phosphate (T6P), are involved in regulating some growth responses (Eastmond and Graham, 2003; Paul, 2008; Smeekens et al., 2010)

\subsection{SUGAR SIGNALING AND PLANT METABOLISM}

The regulation of metabolism and specific growth responses tend to be triggered and/or moderated on the basis of the nature of the sugar signal. For example, the transport form of sugar in plants, that is, sucrose, can be recognized as a signal directly (Chiou and Bush, 1998) or, otherwise, a signal can arise through its hexose cleavage products, glucose (glc) or UDP-glc and fructose (Li et al., 2011; Price et al., 2004; Rolland et al., 2002).

As reported, the generation of sugar signals depends on either the concentration or the relative ratios to other metabolites, for example, C:N (Coruzzi and Bush, 2001; Palenchar et al., 2004), or through sugar-specific sensors and/or transporters (Buttner, 2010; Lalonde et al., 1999, 2004; Vaughn et al., 2002; Williams et al., 2000). The latest addition to this is the possible role of metabolic enzymes as dominant members of transcriptional regulatory complexes; HXK represent the best example. The abovementioned study is an example of remarkable cross-talk between metabolic pathways and/or sensors, and gene regulation. Further, one more sensing mechanism has been reported involving cell surface receptors, such as RGS1, which negatively regulate G-protein signaling (Chen et al., 2003; Chen and Jones, 2004).

In general, signaling potential of hexoses tends to have greater effect on promoting organ growth and cell proliferation, whereas sucrose is normally associated with differentiation and maturation (Borisjuk et al., 2002; Koch, 2004). Relative ratios of hexoses to sucrose are sensed and retained by sucrose metabolic enzymes, which function in a spatiotemporal manner to synchronize growth during important developmental phases (Koch, 2004; Xu et al., 1996). The role of sugar metabolic enzymes and transporters is to establish sugar gradients within tissues (Weschke et al., 2000, 2003). Earlier work on developing legume embryos revealed that differential glc concentrations along a spatial gradient can 
be related to enhanced mitosis (Borisjuk et al., 1998, 2003), proposing a connection between hexoses and the cell cycle. In accordance with this, sugars have been reported to control cell division through modulation of cyclinD (CycD) gene expression (Gaudin et al., 2000; Riou-Khamlichi et al., 1999, 2000).

\subsection{SUGAR SIGNALING AND PHYTOHORMONES}

Sugars can also cross-talk with existing phytohormone signaling networks to regulate important growth processes like embryo development, seed germination, and seedling and tuber growth (Gazzarrini and McCourt, 2001; Gibson, 2004, 2005; Leon and Sheen, 2003; Rolland et al., 2002, 2006). There is also evidence to suggest that sugars can regulate meristem maintenance and identity at the genetic level (SatohNagasawa et al., 2006; Wu et al., 2005). Indeed, Pien et al. (2001) observed spatiotemporal expression of genes of carbohydrate metabolism in the tomato shoot apical meristem (SAM) and developing leaf primordia. In Arabidopsis, misexpression of a specific extracellular invertase, in the SAM resulted into alterations in flowering time and inflorescence structure (Heyer et al., 2004). In addition, sucrose can salvage flowering time mutant phenotypes, by controlling meristem identity genes, such as LEAFY (Ohto et al., 2001). The latest research has also revealed that exogenous sucrose can compensate for regulators of meristem maintenance in the shoot (Wu et al., 2005) and root (Wahl et al., 2010).

It has been shown that plants defective in abscisic acid (ABA) and/or ethylene sensitivity and signaling display changed sugar response phenotypes. Therefore, research has revealed a huge overlap between sugar, ABA, and ethylene signals in regulating processes such as seed germination and seedling growth (Gazzarrini and McCourt, 2001; Gibson, 2004, 2005; Leon and Sheen, 2003; Rolland et al., 2002, 2006). A number of mutants identified in sugar response screens also showed defect in ABA metabolism, which proved a cross-talk between them. For example, certain ABA biosynthesis $(a b a)$ and ABA-insensitive (abi) mutants are insensitive to high glc (Arenas-Huertero et al., 2000; Brocard et al., 2002; Dekkers et al., 2008; Leon and Sheen, 2003). Rigorous cross-talk between sugar and ABA signaling pathways has been described for various aspects of plant development and metabolism (Gazzarrini and McCourt, 2001; Finkelstein and Gibson, 2002; Gibson, 2004, 2005); for example, embryo growth, transition in phase from rapid cell division to cell enlargement and storage of reserves (Finkelstein and Gibson, 2002; Wobus and Weber, 1999a,b). ABA increases sucrose stimulation of starch biosynthetic genes (Rook et al., 2001). On the other hand, ABA and glc act antagonistically during seed germination and initial growth of seedlings, where exogenous glc enables wild-type Arabidopsis seeds for germination on otherwise inhibitory ABA levels (Leon and Sheen, 2003). A direct association between sugar signaling and hormone biosynthesis was shown by characterization of ABA biosynthetic genes, $A B A 1-A B A 3$, which have been also individually isolated as gin mutants (Arenas-Huertero et al., 2000; Laby et al., 2000; Rook et al., 2001). Exogenous glc can enhance both expression of these ABA synthesis genes and, subsequently, endogenous ABA levels (Cheng et al., 2002). An important association between sugars and ABA perception is demonstrated by $A B I 4$, which encodes an AP2 domain transcription factor involved in sugar response during germination and seedling growth (Arenas-Huertero et al., 2000; Huijser et al., 2000; Laby et al., 2000; Rook et al., 2001). In maize seeds, $A B I 4$ is controlled by sugar in the developing embryo, and binds regulatory elements for both $\mathrm{ABA}$ and sugar (Niu et al., 2002). Further, a recent study recognized a splicing factor, $S R 45$, as a negative regulator of sugar signaling during early seedling growth. SR45 is involved in the repression of glc-induced ABA accumulation, and downregulation of genes for ABA biosynthesis and signaling (Carvalho et al., 2010).

Ethylene signaling pathways are also closely connected with sugar and ABA sense networks (Gazzarrini and McCourt, 2001; Leon and Sheen, 2003). For example, ethylene mutants, ethylene receptor 1 (etr1) and ethylene insensitive 2 and 3 (ein2 and ein3), are glc hypersensitive, while constitutive triple response 1(ctr1), a negative regulator of ethylene signaling, is glc insensitive (Gibson et al., 2001; Yanagisawa et al., 2003; Zhou et al., 1998). ABA levels are improved in the ein 2 mutant, and wildtype seedlings treated with the ethylene precursor 1-aminocyclopropane-1-carboxylic acid (ACC) phenocopy gin mutants (Ghassemian et al., 2000).

\section{CONCLUSION}

Sugars are known to have enormous and diversified roles in the organism. Besides being an important energy-giving primary metabolite, now the focus of research has shifted towards sugars' function as regulatory and signaling molecules. The literature in this particular area of research emphasizes the variety of processes related to sugars and their requirement in the cell, and also the regulatory networks to which they are associated. The role of sugar signaling in circadian rhythm, and particular developmental programs has also been discussed. Interestingly, sugar signaling works at the gene level and also in 
association with various phytohormones. The function of sugars as signaling molecules becomes obvious when the plant is under biotic or abiotic stress. In such conditions, plants produce osmoprotectants, mainly sugars, for the maintenance of homeostasis and to scavenge ROS. Sugars provide protection to enzymes from degradation and also initiate the signaling pathways to combat stress. Despite the existing research on this topic, there remains a dearth of knowledge, as many major issues would be addressed if we could understand the regulatory complexity and the components involved in sugar homeostasis, (sub)cellular allocation, and long-distance transport. Sugar signaling study will continue to be a fascinating area of research for many years to come.

\section{References}

Albrecht, G., Biemelt, S., Baumgartner, S., 1997. Accumulation of fructans following oxygen deficiency stress in related plant species with different flooding tolerances. New Phytol. 136, 137-144.

Amtmann, A., Hammond, J.P., Armengaud, P., White, P.J., 2006. Nutrient sensing and signalling in plants: potassium and phosphorus. Adv. Bot. Res. 43, 209-257.

Arenas-Huertero, F., Arroyo, A., Zhou, L., Sheen, J., Leon, P., 2000. Analysis of Arabidopsis glucose insensitive mutants, gin5 and gin6, reveals a central role of the plant hormone $\mathrm{ABA}$ in the regulation of plant vegetative development by sugar. Genes Dev. 14, 2085-2096.

Bhardwaj, P.R., Handa, N., Kaur, H., Rattan, A., Bali, S., Gautam, V., et al., 2016. Sugar signaling in plants: a novel mechanism for drought stress management. In: Ahmad, P. (Ed.), Water Stress and Crop Plants: A Sustainable Approach. John Wiley \& Sons, Ltd, Chichester, pp. 287-302.

Borisjuk, L., Walenta, S., Weber, H., Mueller-Klieser, W., Wobus, U., 1998. High-resolution histographical mapping of glucose concentrations in developing cotyledons of Vicia faba in relation to mitotic activity and storage processes: glucose as a possible developmental trigger. Plant J. 15, 583-591.

Borisjuk, L., Walenta, S., Rolletschek, H., Mueller-Klieser, W., Wobus, U., Weber, H., 2002. Spatial analysis of plant metabolism: sucrose imaging within Vicia faba cotyledons reveals specific developmental patterns. Plant J. 29, 521-530.

Borisjuk, L., Rolletschek, H., Wobus, U., Weber, H., 2003. Differentiation of legume cotyledons as related to metabolic gradients and assimilate transport into seeds. J. Exp. Bot. 54, 503-512.

Brocard, I.M., Lynch, T.J., Finkelstein, R.R., 2002. Regulation and role of the Arabidopsis abscisic acid-insensitive 5 gene in abscisic acid, sugar, and stress response. Plant Physiol. 129, 1533-1543.

Buttner, M., 2010. The Arabidopsis sugar transporter (AtSTP) family: an update. Plant Biol. 12, 35-41.

Carvalho, R.F., Carvalho, S.D., Duque, P., 2010. The plant-specific SR45 protein negatively regulates glucose and ABA signaling during early seedling development in Arabidopsis. Plant Physiol. 154, 772-783.

Chen, J.G., Jones, A.M., 2004. AtRGS1 function in Arabidopsis thaliana. Methods Enzymol. 389, 338-350.

Chen, J.G., Willard, F.S., Huang, J., Liang, J., Chasse, S.A., Jones, A. M., et al., 2003. A seven-transmembrane RGS protein that modulates plant cell proliferation. Science 301, 1728-1731.

Cheng, W.H., Endo, A., Zhou, L., et al., 2002. A unique short-chain dehydrogenase/reductase in Arabidopsis glucose signaling and abscisic acid biosynthesis and functions. Plant Cell 14, 2723-2743.
Chincinska, I., Gier, K., Krügel, U., Liesche, J., He, H., Grimm, B., et al., 2013. Photoperiodic regulation of the sucrose transporter StSUT4 affects the expression of circadian-regulated genes and ethylene production. Front. Plant. Sci. 4, 26.

Chiou, T.J., Bush, D.R., 1998. Sucrose is a signal molecule in assimilate partitioning. Proc. Natl Acad. Sci. 95, 4784-4788.

Choudhry, F.K., Rivero, R.M., Blumwald, E., Mittler, R., 2016. Reactive oxygen species, abiotic stress and stress combination. Plant J. 90, 856-867.

Confraria, A., Martinho, C., Elias, A., Rubio-Somoza, I., BaenaGonzález, E., 2013. miRNAs mediate SnRK1-dependent energy signaling in Arabidopsis. Front. Plant. Sci. 4, 197.

Coruzzi, G., Bush, D.R., 2001. Nitrogen and carbon nutrient and metabolite signaling in plants. Plant Physiol. 125, 61-64.

Dahiya, A., Saini, R., Saini, H.S., Devi, A., 2017. Sucrose metabolism: controls the sugar sensing and generation of signaling molecules in plants. J. Pharmacogn. Phytochem. 6, 1563-1572.

Dekkers, B.J., Schuurmans, J.A., Smeekens, S.C., 2008. Interaction between sugar and abscisic acid signaling during early seedling development in Arabidopsis. Plant Mol. Biol. 67, 151-167.

Dijkwel, P.P., Huijser, C., Weisbeeek, P.J., Chua, N.H., Smeekens, S., 1997. Sucrose control of phytochrome A signaling in Arabidopsis. Plant Cell 9, 583-595.

Dobrenel, T., Marchive, C., Azzopardi, M., Clément, G., Moreau, M., Sormani, R., et al., 2013. Sugar metabolism and the plant target of rapamycin kinase: a sweet operator? Front. Plant. Sci. 4, 93.

Eastmond, P.J., Graham, I.A., 2003. Trehalose metabolism: a regulatory role for trehalose-6-phosphate? Curr. Opin. Plant Biol. 6, 231-235.

Eveland, A.L., Jackson, D.P., 2012. Sugars, signaling, and plant development. J. Exp. Bot. 63, 3367-3377.

Ferrari, S., Savatin, D.V., Sicilia, F., Gramegna, G., Cervone, F., Lorenzo, G.D., 2013. Oligogalacturonides: plant damageassociated molecular patterns and regulators of growth and development. Front. Plant. Sci. 4, 49.

Finkelstein, R.R., Gibson, S.I., 2002. ABA and sugar interactions regulating development: cross-talk or voices in a crowd? Curr. Opin. in Plant Biol. 5, 26-32.

Funck, D., Clauß, K., Frommer, W.B., Hellmann, H.A., 2012. The Arabidopsis CstF64-like RSR1/ESP1 protein participates in glucose signaling and flowering time control. Front. Plant. Sci. 3, 80.

Gaudin, V., Lunness, P.A., Fobert, P.R., Towers, M., Riou-Khamlichi, C., Murray, J.A.H., et al., 2000. The expression of D-cyclin genes defines distinct developmental zones in snapdragon apical meristems and is locally regulated by the cycloidea gene. Plant Physiol. $122,1137-1148$.

Gazzarrini, S., McCourt, P., 2001. Genetic interactions between ABA, ethylene and sugar signaling pathways. Curr. Opin. Plant Biol. 4, 387-391.

George, T.S., Fransson, A.M., Hammond, J.P., White, P.J., 2011. Phosphorus nutrition: rhizosphere processes, plant response and adaptations. In: Bunemann, E., Oberson, A., Frossard, E. (Eds.), Phosphorus in Action: Soil Biology. Springer, Dordrecht, The Netherlands, pp. 245-271.

Ghassemian, M., Nambara, E., Cutler, S., Kawaide, H., Kamiya, Y., McCourt, P., 2000. Regulation of abscisic acid signaling by the ethylene response pathway in Arabidopsis. Plant Cell 12, 1117-1126.

Gibson, S.I., 2004. Sugar and phytohormone response pathways: navigating a signaling network. J. Exp. Bot. 55, 253-264.

Gibson, S.I., 2005. Control of plant development and gene expression by sugar signaling. Curr. Opin. in Plant Biol. 8, 93-102.

Gibson, S.I., Graham, I.A., 1999. Commentary: another player joins the complex field of sugar-regulated gene expression in plants. Proc. Natl Acad. Sci. 96, 4746-4748.

Gibson, S.I., Laby, R.J., Kim, D.G., 2001. The sugar-insensitive1 (sis1) mutant of Arabidopsis is allelic to ctr1. Biochem. Biophys. Res. Commun. 280, 196-203. 
Graham, I.A., Denby, K.J., Leaver, C.J., 1994. Carbon catabolite repression regulates glyoxylate cycle gene expression in cucumber. Plant Cell 6, 761-772.

Granot, D., David-Schwartz, R., Kelly, G., 2013. Hexose kinases and their role in sugar-sensing and plant development. Front. Plant. Sci. 4, 44.

Halford, N.G., Purcell, P.C., Hardie, D.G., 1999. Is hexokinase really a sugar sensor in plants? Trends Plant Sci. 4, 117-120.

Hammond, J.P., White, P.J., 2011. Sugar signaling in root responses to low phosphorus availability. Plant Pysiol. 156, 1033-1040.

Hanson, J., Smeekens, S., 2009. Sugar perception and signaling-an update. Curr. Opin. Plant Biol. 12, 562-567.

Heisel, T.J., Li, C.Y., Grey, K.M., Gibson, S.I., 2013. Mutations in histone acetyltransferase1 affect sugar response and gene expression in Arabidopsis. Front. Plant. Sci. 4 (245).

Herbers, K., Meuwly, P., Frommer, W.B., Metraux, J.P., Sonnewald, U., 1996. Systemic acquired resistance mediated by the ectopic expression of invertase: possible hexose sensing in the secretory pathway. Plant Cell 8, 793-803.

Hermans, C., Hammond, J.P., White, P.J., Verbruggen, N., 2006. How do plants respond to nutrient shortage by biomass allocation? Trends Plant Sci 11, 610-617.

Heyer, A.G., Raap, M., Schroeer, B., Marty, B., Willmitzer, L., 2004. Cell wall invertase expression at the apical meristem alters floral, architectural, and reproductive traits in Arabidopsis thaliana. Plant J. 39, 161-169.

Huijser, C., Kortstee, A., Pego, J., Weisbeek, P., Wisman, E., Smeekens, S., 2000. The Arabidopsis SUCROSE UNCOUPLED6 gene is identical to ABSCISIC ACID INSENSITIVE-4: involvement of abscisic acid in sugar responses. Plant J. 23, $577-585$.

Jang, J.C., Sheen, J., 1997. Sugar sensing in higher plants. Trends Plant Sci. 2, 208-214.

Jang, J.C., León, P., Zhou, L., Sheen, J., 1997. Hexokinase as a sugar sensor in higher plants. Plant Cell 9, 5-19.

Keunen, E., Peshev, D., Vangronsveld, J., Ende, W.V.D., Cuypers, A., 2013. Plant sugars are crucial players in the oxidative challenge during abiotic stress: extending the traditional concept. Plant Cell Environ. 36, 1242-1255.

Khan, M.I.R., Khan, N.A., 2014. Ethylene reverses photosynthetic inhibition by nickel and zinc in mustard through changes in PS II activity, photosynthetic nitrogen use efficiency, and antioxidant metabolism. Protoplasma 251, 1007-1019.

Khan, M.I.R., Asghar, M., Khan, N.A., 2014. Alleviation of saltinduced photosynthesis and growth inhibition by salicylic acid involves glycinebetaine and ethylene in mungbean (L.). Plant Physiol. Biochem. 80, 67-74.

Koch, K., 2004. Sucrose metabolism: regulatory mechanisms and pivotal roles in sugar sensing and plant development. Curr. Opin. Plant Biol. 7, 235-246.

Koch, K.E., 1996. Carbohydrate modulated gene expression in plants. Annu. Rev. Plant Physiol. Plant Mol. Biol. 47, 509-540.

Koch, K.E., Wu, Y., Xu, J., 1996. Sugar and metabolic regulation of genes for sucrose metabolism: potential influence of maize sucrose synthase and soluble invertase responses on carbon partitioning and sugar sensing. J. Exp. Bot. 47, 1179-1185.

Kunz, S., Pesquet, E., Kleczkowski, L.A., 2014. Functional dissection of sugar signals affecting gene expression in Arabidopsis thaliana. PLoS One 9, e100312.

Laby, R.J., Kincaid, M.S., Kim, D., Gibson, S.I., 2000. The Arabidopsis sugar-insensitive mutants sis4 and sis5 are defective in abscisic acid synthesis and response. Plant J. 23, 587-596.

Lalond, S., Boles, E., Hellmann, H., Barker, L., Patrick, J.W., Frommer, W.B., et al., 1999. The dual function of sugar carriers: transport and sugar sensing. Plant Cell 11, 707-726.
Lalonde, S., Wipf, D., Frommer, W.B., 2004. Transport mechanisms for organic forms of carbon and nitrogen between source and sink. Annu. Rev. Plant Biol. 55, 341-372.

Lastdrager, J., Hanson, J., Smeekens, S., 2014. Sugar signals and the control of plant growth and development. J. Exp. Bot. 65, 799-807.

Leon, P., Sheen, J., 2003. Sugar and hormone connections. Trends Plant Sci. 8, 110-116.

Li, P., Wind, J.J., Shi, X., Zhang, H., Hanson, J., Smeekens, S.C., et al., 2011. Fructose sensitivity is suppressed in Arabidopsis by the transcription factor ANAC089 lacking the membrane-bound domain. Proc. Natl Acad. Sci. 108, 3436-3441.

Ljung, K., Jennifer, L.N., Perata, P., 2015. New mechanistic links between sugar and hormone signaling networks. Curr. Opin. Plant Biol. 25, 130-137.

Lloyd, J.C., Zakhleniuk, O.V., 2004. Responses of primary and secondary metabolism to sugar accumulation revealed by microarray expression analysis of the Arabidopsis mutant, pho3. J. Exp. Bot. 55, 1221-1230.

Mita, S., Murano, N., Akaike, M., Nakamura, K., 1997. Mutants of Arabidopsis thaliana with pleiotropic effects on the expression of the gene for b-amylase and on the accumulation of anthocyanin that are inducible by sugars. Plant 11, 841-851.

Moghaddam, B.M.R., Van den Ende, W., 2013. Sugars, the clock and transition to flowering. Front. Plant. Sci. 4, 22.

Moghaddam, M.R.B., den Ende, W.V., 2012. Sugars and plant innate immunity. J. Exp. Bot. 63, 3989-3998.

Moore, B., Zhou, L., Rolland, F., Hall, Q., Cheng, W.H., Liu, Y.X., 2003. Role of the Arabidopsis glucose sensor HXK1 in nutrient, light and hormonal signaling. Science 300, 332-336.

Morkunas, I., 2014. The role of sugar signaling in plant defense responses against fungal pathogens. Acta Physiol. Plant 36, 1607-1619.

Nilsson, L., Muller, R., Nielsen, T.H., 2010. Dissecting the plant transcriptome and the regulatory responses to phosphate deprivation. Physiol. Plant 139, 129-143.

Nishizawa, A., Yabuta, Y., Shigeoka, S., 2008. Galactinol and raffinose constitute a novel function to protect plants from oxidative damage. Plant Physiol. 147, 1251-1263.

Niu, X., Helentjaris, T., Bate, N.J., 2002. Maize ABI4 binds coupling element1 in abscisic acid and sugar response genes. Plant Cell 14, 2565-2575.

Ohto, M., Onai, K., Furukawa, Y., Aoki, E., Araki, T., Nakamura, K., 2001. Effects of sugar on vegetative development and floral transition in Arabidopsis. Plant Physiol. 127, 252-261.

Palenchar, P.M., Kouranov, A., Lejay, L.V., Coruzzi, G.M., 2004. Genome-wide patterns of carbon and nitrogen regulation of gene expression validate the combined carbon and nitrogen (CN)-signaling hypothesis in plants. Genome Biol. 5, R91.

Paul, M.J., 2008. Trehalose 6-phosphate: a signal of sucrose status. Biochem. J. 412, e1-e2.

Pego, J.V., Weisbeek, P.J., Smeekens, S.C.M., 1999. Mannose inhibits Arabidopsis germination via a hexokinase-mediated step. Plant Physiol. 119, 1017-1023.

Pego, J.V., Kortstee, A.J., Huijser, C., Smeekens, S.C.M., 2000. Photosynthesis, sugars and the regulation of gene expression. J. Exp. Bot. 51, 407-416.

Pien, S., Wyrzykowska, J., Fleming, A.J., 2001. Novel marker genes for early leaf development indicate spatial regulation of carbohydrate metabolism within the apical meristem. Plant J. 25, 663-674.

Price, J., Laxmi, A., St Martin, S.K., Jang, J.C., 2004. Global transcription profiling reveals multiple sugar signal transduction mechanisms in Arabidopsis. Plant Cell 2128-2150.

Roitsch, T., 1999. Source-sink regulation by sugar and stress. Curr. Opin. Plant Biol. 2, 198-206.

Rolland, F., Moore, B., Sheen, J., 2002. Sugar sensing and signaling in plants. Plant Cell 14, S185-S205. 
Rolland, F., Baena-Gonzalez, E., Sheen, J., 2006. Sugar sensing and signaling in plants: conserved and novel mechanisms. Annu. Rev. Plant Biol. 57, 675-709.

Rook, F., Corke, F., Card, R., Munz, G., Smith, C., Bevan, M.W., 2001. Impaired sucrose-induction mutants reveal the modulation of sugar-induced starch biosynthetic gene expression by abscisic acid signaling. Plant J. 26, 421-433.

Salerno, G.L., Curatti, L., 2003. Origin of sucrose metabolism in higher plants: when, how and why? Trends Plant Sci. 8, 63-69.

Satoh-Nagasawa, N., Nagasawa, N., Malcomber, S., Sakai, H., Jackson, D., 2006. A trehalose metabolic enzyme controls inflorescence architecture in maize. Nature 441, 227-230.

Schneider, T., Keller, F., 2009. Raffinose in chloroplasts is synthesized in the cytosol and transported across the chloroplast envelope. Plant Cell Physiol. 50, 2174-2182.

Sheen, J., Zhou, L., Jang, J.C., 1999. Sugars as signaling molecules. Curr. Opin. Plant Biol. 2, 410-418.

Smeekens, S., 2000. Sugar-induced signal transduction in plants. Annu. Rev. Plant Physiol. Plant Mol Biol. 51, 49-81.

Smeekens, S., Rook, F., 1997. Sugar sensing and sugar-mediated signal transduction in plants. Plant Physiol. 115, 7-13.

Smeekens, S., Ma, J., Hanson, J., Rolland, F., 2010. Sugar signals and molecular networks controlling plant growth. Curr. Opin. Plant Biol. 13, 274-279.

Smeekens, S., Hellmann, H.A., 2014. Sugar sensing and signaling in plants. Front. Plant Sci. 5, 113.

Suzuki, M., Chatterton, N.J., Hendry, G.A.F., Wallace, R.K., 1993. The origin, distribution, and evolutionary significance of fructans. In: Suzuki, M., Chatterton, N.J. (Eds.), Science and Technology of Fructans. CRC Press, Boca Raton, FL, pp. 119-139.

Tang, G.Q., Luscher, M., Sturm, A., 1999. Antisense repression of vacuolar and cell wall invertase in transgenic carrot alters early plant development and sucrose partitioning. Plant Cell 11, 177-189.

Tiessen, A., Padilla-Chacon, D., 2013. Subcellular compartmentation of sugar signaling: links among carbon cellular status, route of sucrolysis, sink-source allocation, and metabolic partitioning. Front. Plant. Sci. 3, 306.

Umemura, T.A., Perata, P., Futsuhara, Y., Yamaguchi, J., 1998. Sugar sensing and a-amylase gene repression in rice embryos. Planta 204, 420-428.

Vance, C.P., 2010. Quantitative trait loci, epigenetics, sugars, and micro- RNAs: quaternaries in phosphate acquisition and use. Plant Physiol. 154, 582-588.

Vaughn, M.W., Harrington, G.N., Bush, D.R., 2002. Sucrose-mediated transcriptional regulation of sucrose symporter activity in the phloem. Proc. Natl. Acad. Sci. 99, 10876-10880.

Wahl, V., Brand, L.H., Guo, Y.L., Schmid, M., 2010. The FANTASTIC FOUR proteins influence shoot meristem size in Arabidopsis thaliana. BMC Plant Biol. 10, 285.

Weschke, W., Panitz, R., Sauer, N., Wang, Q., Neubohn, B., Weber, H., et al., 2000. Sucrose transport into barley seeds: molecular characterization of two transporters and implications for seed development and starch accumulation. Plant J. 21, 455-467.

Weschke, W., Panitz, R., Gubatz, S., Wang, Q., Radchuk, R., Weber, H., et al., 2003. The role of invertases and hexose transporters in controlling sugar ratios in maternal and filial tissues of barley caryopses during early development. Plant J. 33, 395-411.

White, P.J., Hammond, J.P., 2008. Phosphorus nutrition of terrestrial plants. In: White, P.J., Hammond, J.P. (Eds.), The Ecophysiology of Plant-Phosphorus Interactions. Springer, Dordrecht, The Netherlands, pp. 51-81.

Williams, L.E., Lemoine, R., Sauer, N., 2000. Sugar transporters in higher plants-a diversity of roles and complex regulation. Trends Plant Sci. 5, 283-290.
Wobus, U., Weber, H., 1999a. Seed maturation: genetic programmes and control signals. Curr. Opin. Plant Biol. 2, 33-38.

Wobus, U., Weber, H., 1999b. Sugars as signal molecules in plant seed development. Biol. Chem. 937-944.

Wu, X., Dabi, T., Weigel, D., 2005. Requirement of homeobox gene STIMPY/WOX9 for Arabidopsis meristem growth and maintenance. Curr. Biol. 15, 436-440.

Xu, J., Avigne, W.T., McCarty, D.R., Koch, K.E., 1996. A similar dichotomy of sugar modulation and developmental expression affects both paths of sucrose metabolism: evidence from a maize invertase gene family. Plant Cell 8, 1209-1220.

Yanagisawa, S., Yoo, S.D., Sheen, J., 2003. Differential regulation of EIN3 stability by glucose and ethylene signaling in plants. Nature $425,521-525$.

Yang, X.J., Finnegan, P.M., 2010. Regulation of phosphate starvation responses in higher plants. Ann. Bot 105, 513-526.

Yonekura, M., Aoki, N., Hirose, T., Onai, K., Ishiura, M., Okamura, M., et al., 2013. The promoter activities of sucrose phosphate synthase genes in rice, OsSPS1 and OsSPS11, are controlled by light and circadian clock, but not by sucrose. Front. Plant. Sci. 4, 31.

You, J., Chan, Z., 2015. ROS Regulation during abiotic stress responses in crop plants. Front. Plant Sci. 6, 1092.

Zhang, Y., Xu, D., Huang, H., Chen, S., Wang, L., Zhu, L., et al., 2014. Regulation of glucose homeostasis and lipid metabolism by PPP1R3Gmediated hepatic glycogenesis. Mol. Endocrinol. 28, 116-126.

Zhou, L., Jang, J.C., Jones, T.L., Sheen, J., 1998. Glucose and ethylene signal transduction crosstalk revealed by an Arabidopsis glucoseinsensitive mutant. Proc. Natl Acad. Sci. U.S.A. 95, 10294-10299.

\section{Further Reading}

Ahmad, I.Z., Ahmad, A., Mabood, A., Tabassum, H., 2017. Effects of different metal stresses on the antioxidant defense systems of medicinal plants. In: Khan, M.I.R., Khan, N.A. (Eds.), Reactive Oxygen Species and Antioxidant System in Plants: Role and Regulation Under Abiotic Stress. Springer nature, Singapore, pp. 215-256.

Cheng, P.C., Greyson, R.I., Walden, D.B., 1983. Organ initiation and the development of unisexual flowers in the tassel and ear of Zea mays. Am. J. Bot. 70, 450-462.

Eastmond, P.J., van Dijken, A.J., Spielman, M., Kerr, A., Tissier, A.F., Dickinson, H.G., et al., 2002. Trehalose-6-phosphate synthase 1, which catalyses the first step in trehalose synthesis, is essential for Arabidopsis embryo maturation. Plant J. 29, 225-235.

Field, R.A., 2009. Oligosaccharide signaling molecules. In: Osbourn, A., Lanzotti, V. (Eds.), Plant-Derived Natural Products. Springer, New York, pp. 349-359.

Goddijn, O.J., Van Dun, K., 1999. Trehalose metabolism in plants. Trends Plant Sci. 4, 315-319.

Li, Y., Lee, K.K., Walsh, S., Smith, C., Hadingham, S., Sorefan, K., et al., 2006. Establishing glucose- and ABA-regulated transcription networks in Arabidopsis by microarray analysis and promoter classification using a Relevance Vector Machine. Genome Res. 16, 414-427.

Ohto, M.A., Hayashi, S., Sawa, S., Hashimoto-Ohta, A., Nakamura, K., 2006. Involvement of HLS1 in sugar and auxin signaling in Arabidopsis leaves. Plant Cell Physiol. 47, 1603-1611.

Paul, M., Pellny, T., Goddijn, O., 2001. Enhancing photosynthesis with sugar signals. Trends Plant Sci. 6, 197-200.

Paul, M.J., Primavesi, L.F., Jhurreea, D., Zhang, Y., 2008. Trehalose metabolism and signaling. Annu. Rev. Plant Biol. 59, 417-441.

Rolland, F., Winderickx, J., Thevelein, J.M., 2001. Glucose-sensing mechanisms in eukaryotic cells. Trends Biochem. Sci. 26, 310-317. 
This page intentionally left blank 


\section{4}

\section{Methylglyoxal: A Novel Signaling Molecule in Plant Responses to Abiotic Stresses}

$$
\text { Zhong-Guang } L i^{1,2,3}
$$

${ }^{1}$ School of Life Sciences, Yunnan Normal University, Kunming, P.R. China ${ }^{2}$ Engineering Research Center of Sustainable Development and Utilization of Biomass Energy, Ministry of Education, Kunming, P.R. China

${ }^{3}$ Key Laboratory of Biomass Energy and Environmental Biotechnology, Yunnan Province, Yunnan

Normal University, Kunming, P.R. China

\section{O U T L I N E}

14.1 Introduction

14.2 Generation of MG in Plants

14.2.1 Nonenzymatic Pathway: A Key Player

14.2.2 Enzymatic Pathways: A Secondary Way

14.3 Elimination of MG in Plants

14.3.1 Glyoxalase System: The First Defense Line

14.3.2 Nonglyoxalase System: A Minor Route

14.4 MG Signaling Triggered by Environmental Stress in Plants

14.5 Abiotic Stress Tolerance Related to MG in Plants

14.5.1 Salt Tolerance

\subsection{INTRODUCTION}

Oxygenated short aldehyde methylglyoxal (MG) containing $\alpha, \beta$-dicarbonyl is a side-product of many
14.5.2 Drought Tolerance 224

14.5.3 Heavy Metal Stress Tolerance 225

14.5.4 Heat Tolerance 226

14.5.5 Chilling Tolerance 226

14.6 Abiotic Stress Tolerance Improved by Overexpressing Glyoxalase Genes

14.7 MG Priming-Induced Abiotic Tolerance 228

14.8 Signaling Crosstalk Between $\mathrm{MG}$ and $\mathrm{Ca}^{2+}$, ROS, NO, and $\mathrm{H}_{2} \mathrm{~S}$

14.9 Conclusion and Future Prospects

Acknowledgements

References metabolic processes, such as glycolysis, photosynthesis, fatty acid degradation, and the oxidation of amino acids and proteins. The generation of MG is spontaneous and unavoidable in the life activity of all 
organisms (Kaur et al., 2014a,b; Hossain et al., 2011, 2016; Singh and Dhaka, 2016). Due to high reactivity of MG with protein, DNA, RNA, and lipid, for a long time, it has been considered to be a toxic metabolite (Kaur et al., 2014a,b; Hossain et al., 2011, 2016; Singh and Dhaka, 2016). Nowadays, MG is emerging as a novel signaling molecule at low concentration, which takes part in the regulation of seed germination, plant growth, development, reproduction, and response and adaptation to adverse environment (including biotic and abiotic stress), similar to other signaling molecules such as $\mathrm{Ca}^{2+}$, hydrogen peroxide $\left(\mathrm{H}_{2} \mathrm{O}_{2}\right)$, nitric oxide (NO), and hydrogen sulfide $\left(\mathrm{H}_{2} \mathrm{~S}\right)$ (Kaur et al., 2015a,b; Hossain et al., 2011, 2016; Khan et al., 2016; Li, 2016; Singh and Dhaka, 2016). Under normal physiological conditions, MG in plant cells can be maintained a low and nontoxic physiological level, approximately $25-75 \mu \mathrm{M}$ in many plant species such as (Yadav et al., 2005a,b; Hossain et al., 2009; Khan et al., 2016; Li, 2016; Singh and Dhaka, 2016). Under stress conditions, endogenous MG levels in plant cells can be dramatically triggered and produce a MG "burst" or "wave." This burst has become a common stress signaling or marker, followed by regulating the formation of plant abiotic stress tolerance or inducing MG stress (Hossain et al., 2016, 2017; Hoque et al., 2016). MG stress, also known as carbonyl stress, an analogue to oxidative stress due to overaccumulation of reactive oxygen species (ROS), refers to the rapid reaction of MG with protein, nucleic acid, and lipid (Kaur et al., 2014a,b; Hossain et al., 2011, 2016; Khan and Khan, 2017; Singh and Dhaka, 2016). In plants, enzymatic and nonenzymatic pathways all can produce MG during the process of cellular metabolism, but the latter is the major contributor, especially under stress conditions (Kaur et al., 2014a,b; Hossain et al., 2016; Li, 2016; Singh and Dhaka, 2016). In general, approximately $0.05 \%-0.3 \%$ glucose can be spontaneously converted into MG in glycolysis and this ratio increases under stress conditions (Kaur et al., 2014a,b; Hossain et al., 2016; Li, 2016; Singh and Dhaka, 2016).

To maintain MG homeostasis in plant cells, plants have evolved a unique and effective detoxification system composed of a glyoxalase system and nonglyoxalase system. The former refers to glyoxalase I (Gly I), glyoxalase II (Gly II), and glyoxalase III (Gly III), which is the key player in MG scavenging, eliminating $99 \%$ MG. Nonglyoxalase system mainly includes aldose/ aldehyde reductase (ALR), aldo-keto reductase (AKR), MG dehydrogenase (MGDH), MG reductase (MDR), and aldehyde dehydrogenase (ADH). They convert MG into lactaldehyde or pyruvate using reduced nicotinamide adenine dinucleotide (NADH) from glycolysis or tricarboxylic acid cycle as cofactor (Kaur et al., 2014a,b; Hossain et al., 2016; Singh and Dhaka, 2016;
Hasanuzzaman et al., 2018). Nonglyoxalase system only eliminates less than $1 \% \mathrm{MG}$, it is a minor route under normal conditions unless the glyoxalase system is blocked (Kaur et al., 2014a,b; Hossain et al., 2016; Singh and Dhaka, 2016).

As a novel signaling molecule, the MG metabolic pathway and its physiological function has been extensively studied in the field of plant biology and huge progress has been made (Kaur et al., 2014a,b; Hossain et al., 2016; Li, 2016; Singh and Dhaka, 2016). In this chapter, based on the current knowledge on MG and its detoxification system, the generation and elimination of MG, MG signaling triggered by adverse environments, environment and chemical priming-induced abiotic stress tolerance related to $M G$, and signaling crosstalk of MG with other signaling molecules such as $\mathrm{Ca}^{2+}, \mathrm{H}_{2} \mathrm{O}_{2}, \mathrm{NO}$, and $\mathrm{H}_{2} \mathrm{~S}$ have been highlighted. This chapter helps researchers to further understand MG homeostasis in plant cells and its physiological functions, laying a foundation for crop improvement related to abiotic stress tolerance.

\subsection{GENERATION OF MG IN PLANTS}

Like signaling molecules $\mathrm{Ca}^{2+}, \mathrm{H}_{2} \mathrm{O}_{2}, \mathrm{NO}$, and $\mathrm{H}_{2} \mathrm{~S}$, multiple pathways of anabolism and catabolism are the important criteria of signaling molecules (Neill et al., 2002; Dodd et al., 2010; Li et al., 2016; da-Silva and Modolo, 2018). Multiple metabolic pathways can rapidly produce signaling molecules when needed, but are effectively eliminated when not needed by plants (Neill et al., 2002; Dodd et al., 2010; Li et al., 2016; daSilva and Modolo, 2018). In plants, MG is commonly synthesized in cytoplasm, mitochondria, chloroplast, and nucleus and maintains homeostasis in plant cells via multiple metabolic pathways, discussed as follows.

\subsubsection{Nonenzymatic Pathway: A Key Player}

Glyceraldehyde-3-phosphate (G3P) and dihydroxyacetone phosphate (DHAP), collectively known as triosephosphate (TP), are important intermediate products of glycolysis and photosynthesis. Due to its instability, the phosphoryl group of TP, especially DHAP, is easy to remove and produce MG (Kaur et al., 2014a,b; Hossain et al., 2016; Min et al., 2016; Singh and Dhaka, 2016; Fig. 14.1). G3P and DHAP can interconvert via the catalyzation of isomerase (TPI). Therefore, DHAP is the major contributor of MG production by nonenzymatic pathway, and TPI plays a key role (Kaur et al., 2014a,b; Hossain et al., 2016; Min et al., 2016; Singh and Dhaka, 2016; Fig. 14.1). In general, during glycolysis, about $0.05 \%-0.3 \%$ glucose can be spontaneously 


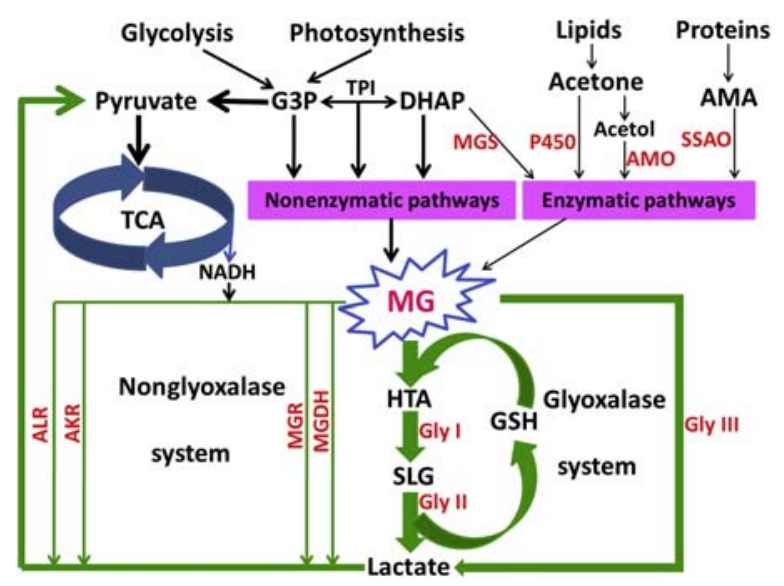

FIGURE 14.1 MG homeostasis in plants. MG can maintain homeostasis in plant cells by generating from enzymatic and nonenzymatic pathways as well as eliminating via glyoxalase and nonglyoxalase systems in plants. Source: Adopted from Wang, Y., Zhou, Z. H., Ye, X.Y., Li, Z.G., 2018. Methylglyoxal: a new signaling molecule in plants. Plant Physiol. J. 54, 10-18 (Wang et al, 2018).

converted into MG by nonenzymatic pathway, which is enhanced under stress conditions (Kaur et al., 2014a, b; Hossain et al., 2016; Li, 2016; Singh and Dhaka, 2016). Therefore, the nonenzymatic pathway is a key player of MG biosynthesis in plants.

\subsubsection{Enzymatic Pathways: A Secondary Way}

In addition to nonenzymatic pathways, plants can also actively synthesize MG by the following four enzymatic pathways. Firstly, DHAP can be converted into MG by the catalyzation of MG synthase (MGS). Secondly, cytochrome P450 can catalyze the conversion of acetone produced from fatty acid metabolism to MG. Thirdly, acetol from acetone can be transformed into MG by the catalyzer acetol monooxygenase (AMO). Fourthly, aminoacetone (AMA) originated from protein metabolism is able to convert into MG via the catalysis of semicarbazide-sensitive amine oxidase (SSAO) (Kaur et al., 2014a,b; Hossain et al., 2016; Li, 2016; Singh and Dhaka, 2016; Fig. 14.1). Enzymatic pathways have been found in many organisms including animals, yeast, and fungus, but they are not completely clear in plants (Kaur et al., 2014a,b; Hossain et al., 2016; Li, 2016; Min et al., 2016; Singh and Dhaka, 2016).

\subsection{ELIMINATION OF MG IN PLANTS}

Due to its toxic effect at high concentrations, plant cells maintain homeostasis of MG via production and scavenging system. Similar to its generation, MG has both glyoxalase system and nonglyoxalase system to scavenge excessive MG in plants, listed as follows.

\subsubsection{Glyoxalase System: The First Defense Line}

The glyoxalase system, also known as the MG detoxification system, is composed of glyoxalase I (Gly I), glyoxalase I (Gly II), and glyoxalase III (Gly III) (Kaur et al., 2014a,b, 2016; Hossain et al., 2016; Singh and Dhaka, 2016; Fig. 14.1). During the process of detoxification, MG spontaneously reacts with reduced glutathione (GSH) and forms hemithioacetal (HTA), which is then converted to S-D-lactoylglutahione (SLG) by Gly I. Afterwards, Gly II catalyzes the conversion of SLG to D-lactate and regenerates GSH, which is recycled back into the glyoxalase system or regulates the redox homeostasis in plant cells (Kaur et al., 2014a, b; Hossain et al., 2016; Singh and Dhaka, 2016; Fig. 14.1). For Gly III, it can directly convert MG into D-lactate in a single step without the help of any cofactor including GSH, referred to as the shorter route (Kaur et al., 2014a,b; Hossain et al., 2016; Singh and Dhaka, 2016; Fig. 14.1). Commonly, more than 99\% MG produced from enzymatic and nonenzymatic pathways are eliminated by the glyoxalase system (Kaur et al., 2014a,b; Hossain et al., 2016; Singh and Dhaka, 2016; Fig. 14.1). Therefore, the glyoxalase system is the first line of defense against MG in plants.

\subsubsection{Nonglyoxalase System: A Minor Route}

In addition to the glyoxalase system, plants can scavenge MG by the nonglyoxalase system. The nonglyoxalase system mainly includes aldose/aldehyde reductase (ALR), aldo-keto reductase (AKR), MG reductase (MDR), and MG dehydrogenase (MGDH) (Kaur et al., 2014a,b; Hossain et al., 2016; Singh and Dhaka, 2016; Fig. 14.1). These enzymes use reduced nicotinamide adenine dinucleotide (NADH) produced from glycolysis and/or tricarboxylic acid cycle (TCA) as cofactor to convert MG into lactaldehyde and pyruvate; the latter goes back to TCA, which in turn regulates energy metabolism and redox homeostasis (Kaur et al., 2014a,b; Hossain et al., 2016; Singh and Dhaka, 2016; Fig. 14.1). As the elimination of MG by nonglyoxalase system is less than $1 \%$, this pathway is a minor route for scavenging MG in plants (Kaur et al., 2014a,b; Hossain et al., 2016; Li, 2016; Singh and Dhaka, 2016).

\subsection{MG SIGNALING TRIGGERED BY ENVIRONMENTAL STRESS IN PLANTS}

As mentioned above, under normal physiological conditions plant cells contain a small amount of MG, from 25 to $75 \mu \mathrm{M}$, varying in concentration in different organs and plant species (Kaur et al., 2014a,b; Hossain 
et al., 2016; Li, 2016; Singh and Dhaka, 2016). However, under abiotic stress such as salt, drought, heavy metal, extreme temperature, and so forth, MG can be rapidly produced and increases two to sixfold, reaching 150-450 $\mu \mathrm{M}$, triggering MG signaling "burst" (Kaur et al., 2014a,b; Hossain et al., 2016; Li, 2016; Singh and Dhaka, 2016; Fig. 14.2). This burst has become a common response and/or adaptation signaling of plant to abiotic stress, followed by inducing the formation of plant abiotic stress tolerance or leading to MG stress.

In rice (Oryza sativa) seedlings, $\mathrm{NaCl}$ stress with different concentrations (150 and $200 \mathrm{mM}$ ) increased endogenous MG level to $160 \%$ and $290 \%$ respectively, and this increase was enhanced with increasing stress strength and duration (Mostofa et al., 2015a,b; Rahman et al., 2016d). Similar results were observed in mustard (Brassica campestris) seedling by Hossain et al. (2013a). Our previous results also showed that $\mathrm{NaCl}$ stress could rapidly induce an increase in endogenous MG level in wheat (Triticum aestivum) seedlings, increasing 1.6-fold compared with the control ( $\mathrm{Li}$ et al., 2017a,b). In addition, under moderate and severe drought stress simulated with $5 \%$ and 25\% PEG-6000, MG content rapidly increased in mung bean (Vigna radiata L.) seedlings and severe stress seedlings accumulated more MG $(39 \mu \mathrm{mol} / \mathrm{g} \mathrm{FW})$ than that of moderate stress seedlings $(15 \mu \mathrm{mol} / \mathrm{g}$ FW (Nahar et al., 2015b, 2017a). In Brassica juncea seedlings, a significant accumulation of endogenous MG was recorded under drought stress (Reddy and Sopory, 1999). Also, under heavy metal stress such as $0.1 \mathrm{mM}$ copper $(\mathrm{Cu}), 0.5$ and $1.0 \mathrm{mM}$ arsenic (As), as well as $0.25,0.30$, and $0.5 \mathrm{mM}$ cadmium $(\mathrm{Cd})$, the

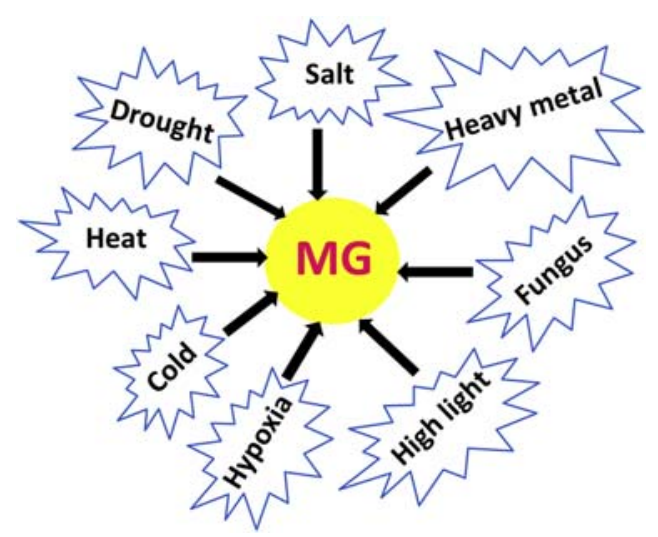

FIGURE 14.2 Adverse environments can trigger MG signaling in plants. MG signaling can be induced by adverse environments such as salt, drought, heavy metal, heat, cold, high light, and hypoxia in plants. Source: Adopted from Wang, Y., Zhou, Z.H., Ye, X.Y., Li, Z.G., 2018. Methylglyoxal: a new signaling molecule in plants. Plant Physiol. J. 54, 10-18. content of endogenous MG was markedly increased in stress-rice seedlings (Mostofa et al., 2015c; Rahman et al., 2015, 2016a, c). In mung bean seedlings, Cd stress $(1.0$ and $1.5 \mathrm{mM})$ more significantly increased the accumulation of endogenous MG (Nahar et al., 2016c, 2017a). In addition to these abiotic stresses, extreme temperature can also induce an increase in endogenous MG level in several plant species. For example, when Ficus concinna seedlings were subjected to high temperature stress at $35^{\circ} \mathrm{C}$ and $40^{\circ} \mathrm{C}$, the accumulation of endogenous MG was quickly activated (Jin et al., 2015). Similar results were reported in mung bean seedlings under heat stress by Nahar et al. (2017a). Furthermore, cold stress at $6^{\circ} \mathrm{C}$ also increased the level of endogenous MG in mung bean seedlings from 14 (control) to $26 \mu \mathrm{mol} / \mathrm{g}$ FW (cold stress for 2 days), and this increase was strengthened with increasing cold stress duration from 26 ( 2 days) to $35 \mu \mathrm{mol} / \mathrm{g}$ FW (3 days) (Nahar et al., 2015d). Similarly, in tea (Camellia sinensis) seedlings, a rapid increase in MG was observed under cold stress at $4{ }^{\circ} \mathrm{C}$, rising from 170 (cold stress for $4 \mathrm{~h}$ ) to $350 \mu \mathrm{mol} / \mathrm{g}$ FW (cold stress for $142 \mathrm{~h}$ ) (Kumar and Yadav, 2009).

\subsection{ABIOTIC STRESS TOLERANCE RELATED TO MG IN PLANTS}

In general, protein (including enzyme) denaturation, loss of biomembrane integrity, and oxidative, osmotic, and MG stresses are the common traits of abiotic stress such as salt, drought, heavy metal, and extreme temperature in plants (Golldack et al., 2014; Iqbal et al., 2016; Li et al., 2016; Min et al., 2016; Hasanuzzaman et al., 2013, 2017d). Correspondingly, the acquisition of plant abiotic stress tolerance is usually implicated in synthesis of stress proteins, repair and reestablishment of biomembrane integrity, enhancement of antioxidant system, osmotic adjustment, and activation of glyoxalase system (Golldack et al., 2014; Li et al., 2016; Hasanuzzaman et al., 2013, 2017d). Environmental stresses can trigger second messengers including MG signaling, which in turn stimulates the glyoxalase system, followed by inducing the formation of plant abiotic stress tolerance (Fig. 14.2; Table 14.1).

\subsubsection{Salt Tolerance}

Overaccumulation of salt such as $\mathrm{NaCl}, \mathrm{Na}_{2} \mathrm{SO}_{4}$, $\mathrm{NaCO}_{3}$, and $\mathrm{NaHCO}_{3}$ in soil leads to salt stress. Salt stress commonly results in MG, oxidative, osmotic, and ion stresses (Zhu, 2002; Céccoli et al., 2015; Hoque 
TABLE 14.1 Chemical Priming-Induced Abiotic Stress Tolerance and Its Relation to Glyoxalase System in Plants

\begin{tabular}{|c|c|c|c|c|}
\hline Priming reagents & Glyoxalase & Abiotic tolerance & Plant species & References \\
\hline Put, Sp, and Spd & Gly I $\uparrow$, Gly II $\uparrow$ & Salt & $V$. radiata & Nahar et al. (2016a) \\
\hline Pro and GB & Gly I $\uparrow$, Gly II $\uparrow$ & Salt & $V$. radiata & Hossain and Fujita (2010) \\
\hline $\mathrm{Ca}^{2+}$ & Gly I $\uparrow$, Gly II $\uparrow$ & Salt & O. sativa & Rahman et al. (2016d) \\
\hline Tre & Gly I $\uparrow$, Gly II $\uparrow$ & Salt & O. sativa & Mostofa et al. (2015a) \\
\hline $\mathrm{H}_{2} \mathrm{~S}$ & Gly I $\uparrow$, Gly II $\uparrow$ & Salt & O. sativa & Mostofa et al. (2015b) \\
\hline MG & Gly I $\uparrow$, Gly II $\uparrow$ & Salt & T. aestivum & Li et al. (2017a) \\
\hline HS & Gly I $\uparrow$, Gly II $\uparrow$ & Salt and drought & B. campestris & Hossain et al. (2013a, 2013b) \\
\hline Se & Gly I $\uparrow$, Gly II $\uparrow$ & drought & B. napus & Hasanuzzaman and Fujita (2011) \\
\hline SNP & Gly I $\uparrow$, Gly II $\uparrow$ & PEG & B. napus & Hasanuzzaman et al. (2017a) \\
\hline MG & Gly I $\uparrow$, Gly II $\uparrow$ & $\mathrm{Cd}$ & T. aestivum & Li et al. (2017b) \\
\hline Spd & Gly I $\uparrow$, Gly II $\uparrow$ & $\mathrm{Al}$ & $V$. radiata & Nahar et al. (2017b) \\
\hline Spm & Gly I $\uparrow$, Gly II $\uparrow$ & $\mathrm{Cd}$ & $V$. radiata & Nahar et al. (2016b) \\
\hline Put and NO & Gly I $\uparrow$, Gly II $\uparrow$ & $\mathrm{Cd}$ & $V$. radiata & Nahar et al. (2016c) \\
\hline $\mathrm{Ca}^{2+}$ & Gly I $\uparrow$, Gly II $\uparrow$ & $\mathrm{Cd}$, As & O. sativa & Rahman et al. $(2015,2016 a)$ \\
\hline $\mathrm{Si}$ & Gly I $\uparrow$, Gly II $\uparrow$ & $\mathrm{Cd}$ & B. napus & Hasanuzzaman et al. (2017b) \\
\hline $\mathrm{H}_{2} \mathrm{O}_{2}$ & Gly I $\uparrow$, Gly II $\uparrow$ & $\mathrm{Cd}$ & B. napus & Hasanuzzaman et al. (2017c) \\
\hline GABA & Gly I $\uparrow$, Gly II $\uparrow$ & $\mathrm{Cr}$ & B. campestris & Mahmud et al. (2017a) \\
\hline MA & Gly I $\uparrow$, Gly II $\uparrow$ & $\mathrm{Cr}$ & B. campestris & Mahmud et al. (2017b) \\
\hline CA & Gly I $\uparrow$, Gly II $\uparrow$ & $\mathrm{Cd}$ & B. campestris & Mahmud et al. (2018) \\
\hline SA and SNP & Gly I $\uparrow$, Gly II $\uparrow$ & $\mathrm{Zn}$ & C. tinctorius & Namdjoyan et al. (2017) \\
\hline $\mathrm{Mn}$ & Gly I $\uparrow$, Gly II $\uparrow$ & Drought and Cd & O. sativa & Rahman et al. $(2016 b, c)$ \\
\hline Spm & Gly I $\uparrow$, Gly II $\uparrow$ & Heat and drought & $V$. radiata & Nahar et al. (2017a) \\
\hline GSH & Gly I $\uparrow$, Gly II $\uparrow$ & Heat, drought, and salt & $V$. radiata & Nahar et al. $(2015 a, b, c)$ \\
\hline Se & Gly I $\uparrow$, Gly II $\uparrow$ & Heat & B. napus & Hasanuzzaman et al. (2014) \\
\hline Pro and GB & Gly I $\uparrow$, Gly II $\uparrow$ & Chilling & C. sinensis & Kumar and Yadav (2009) \\
\hline Spm & Gly I $\uparrow$, Gly II $\uparrow$ & Chilling & $V$. radiata & Nahar et al. (2015d) \\
\hline
\end{tabular}

CA, citric acid; $\mathrm{Ca}^{2+}$, calcium ion; GABA, gamma-aminobutyric acid; $\mathrm{GB}$, glycine betaine; $\mathrm{GSH}$, glutathione; $\mathrm{H}_{2} \mathrm{O}_{2}$, hydrogen peroxide; $\mathrm{H}_{2} \mathrm{~S}$, hydrogen sulfide; $\mathrm{HS}$, heat shock; MA, maleic acid; MG, methylglyoxal; Mn, manganese; NO, nitric oxide; Pro, proline; Put, putrescine; SA, salicylic acid; Se, selenium; Si, silicon; Sp, spermidine; Spd, spermine; Tre, trehalose; SNP, sodium nitroprusside. Arrow $(\uparrow)$ indicates increase.

et al., 2016; Singh and Dhaka, 2016). Therefore, the improvement of salt tolerance in plants is closely associated with the induction of the glyoxalase system and antioxidant system, synthesis of osmolytes (also known as compatible solutes), and ion homeostasis (Zhu, 2002; Céccoli et al., 2015; Hoque et al., 2016; Singh and Dhaka, 2016).

In mung bean $(V$. radiata $L$.$) seedlings, salt stress$ led to the toxicity of $\mathrm{Na}$, a decrease in mineral nutrient uptake such as $\mathrm{K}, \mathrm{Ca}, \mathrm{Mg}$, and $\mathrm{Zn}$ in roots and shoots; oxidative stress (as reflected in increase in lipid peroxidation, $\mathrm{H}_{2} \mathrm{O}_{2}$, and $\mathrm{O}_{2}{ }^{-}$); and $\mathrm{MG}$ stress (as indicated in an increase in MG level) (Nahar et al., 2016a). Pretreatment of bean seedlings with putrescine (Put), spermidine (Spd), and spermine (Spm) alone increased the activities of antioxidant enzymes (dehydroascorbate reductase, DHAR; glutathione reductase, GR; catalase, CAT; and glutathione peroxidase, GPX) and glyoxalase enzyme (Gly I and Gly II), and the contents of GSH and ascorbate (AsA), which in turn weakened salt-induced oxidative and MG stresses (Nahar et al., 2016a). In addition, combination of exogenous Put, Spd, and Spm could increase their endogenous levels, followed by reducing cellular $\mathrm{Na}$ 
accumulation and maintaining nutrient homeostasis (Nahar et al., 2016a). Similarly, under salt stress, exogenously applied proline (Pro) and glycine betaine (GB) to mung bean seedlings resulted in an increase in GSH content, which in turn maintained a high GSH redox state and higher activities of Gly I, Gly II, GPX, glutathione-S-transferase (GST), and GR, followed by decreasing the accumulation of $\mathrm{MG}, \mathrm{H}_{2} \mathrm{O}_{2}$, and $\mathrm{O}_{2}{ }^{\circ-}$ compared with the control seedlings (Hossain and Fujita, 2010). In addition, salt stress significantly improved the contents of endogenous $\mathrm{MG}$, malondialdehyde (MDA), $\mathrm{H}_{2} \mathrm{O}_{2}, \mathrm{O}_{2}{ }^{--}$, Pro, $\mathrm{GSH}$, and oxidized glutathione (GSSG), and reduced AsA content and the GSH/GSSG ratio in mung bean seedlings (Nahar et al., 2015a). In addition, the activities of Gly I, Gly II, monodehydroascorbate reductase (MDHAR), DHAR, and CAT declined in salt-stressed seedlings; while the activities of APX, GR, SOD, GST, and GPX increased (Nahar et al., 2015a). Interestingly, saltstressed mung bean seedlings treated with exogenous GSH improved the activities of Gly I, Gly II, APX, MDHAR, DHAR, GR, SOD, CAT, GPX, and GST, as well as AsA and GSH content and GSH/GSSG ratio, compared with treatment with $\mathrm{NaCl}$ alone, which in turn decreased the MG, MDA, $\mathrm{H}_{2} \mathrm{O}_{2}$, and $\mathrm{O}_{2}^{\cdot-}$ contents, followed by improving the resistance of the mung bean seedlings to the salt stress (Nahar et al., 2015a). These data suggest that polyamines (PAs), Pro, GB, and GSH have a protective action against salt-induced MG and oxidative stresses by enhancing MG detoxification and antioxidant defense systems in mung bean seedlings.

In rice $(O$. sativa) seedlings, salt stress resulted in oxidative stress (an increase in lipoxygenase activity and the accumulation of $\mathrm{O}_{2}{ }^{-}, \mathrm{H}_{2} \mathrm{O}_{2}$, and MDA), MG stress (MG accumulation), and osmotic stress (an increase in Pro and a decrease in relative water content), which in turn led to stunted growth, severe yellowing and leaf rolling (Mostofa et al., 2015a,b). On the other hand, pretreatment with trehalose (Tre) and $\mathrm{NaHS}\left(\mathrm{H}_{2} \mathrm{~S}\right.$ donor) alone dramatically depressed the activity of lipoxygenase (LOX) and the accumulation of $\mathrm{O}_{2}{ }^{\circ-}, \mathrm{H}_{2} \mathrm{O}_{2}$, MDA, and Pro; considerably increased the contents of AsA, GSH, relative water content (RWC), and Chl, as well as redox status; and remarkedly stimulated the activities of Gly I, Gly II, SOD, GPX, APX, GST, MDHAR, DHAR, and GR in saltstressed rice seedlings, which in turn significantly addressed salt-induced toxicity symptoms (Mostofa et al., 2015a,b). In the same way, salt stress led to destruction of ion homeostasis (through $\mathrm{Na}^{+}$influx and $\mathrm{K}^{+}$efflux), loss of mineral nutrient uptake, and oxidative, and MG stresses, and finally caused growth inhibition, chlorosis, and water shortage in the saltstressed rice seedlings (Rahman et al., 2016d).
Adversely, the salt-stressed seedlings supplemented with exogenous $\mathrm{Ca}^{2+}$ improved ion homeostasis by inhibition of $\mathrm{Na}^{+}$influx and $\mathrm{K}^{+}$leakage, and increased the capacity of ROS and MG detoxification by activating the antioxidant defense and glyoxalase systems, followed by recovering from water loss, chlorosis, and growth inhibition (Rahman et al., 2016d).

\subsubsection{Drought Tolerance}

The primary effects of drought stress are osmotic, oxidative, and MG stresses. The strategies that can alleviate these stresses can improve plant drought stress tolerance. Chemical priming is a very important and effective approach that increases plant abiotic stress tolerance including drought tolerance (Antoniou et al., 2016; Hossain et al., 2017). Chemical priming commonly induces the enhancement of the antioxidant system and glyoxalase system and the accumulation of osmolytes such as Pro, GB, Tre, soluble sugar, and $\gamma$-aminobutyric acid (GABA), which in turn improve plant drought tolerance (Antoniou et al., 2016; Hossain et al., 2017).

In rapeseed (Brassica napus) seedlings, drought stress simulated using polyethylene glycol (PEG) resulted in reduction in RWC and the activities of Gly I and Gly II, while it increased Pro, $\mathrm{H}_{2} \mathrm{O}_{2}$, and MDA contents (Hasanuzzaman et al., 2017a). In contrast, exogenous NO (using sodium nitroprusside (SNP) as donor) improved the levels of nonenzymatic antioxidant pool (GSH and AsA) and the activities of antioxidant enzymes (APX, MDHAR, DHAR, GR, GPX, GST, and CAT) and glyoxalase system (Gly I and Gly II), which in turn successfully alleviated oxidative stress (as reflected by a decrease in $\mathrm{H}_{2} \mathrm{O}_{2}$ and MDA) and MG accumulation (Hasanuzzaman et al., 2017a). Likewise, drought stress mimicked by PEG caused a significant increase in the contents of $\mathrm{H}_{2} \mathrm{O}_{2}, \mathrm{MDA}, \mathrm{GSH}$, GSSG, and AsA, as well as the activities of MDHAR, GR, GST, GPX, and Gly I; a decrease in Gly II and CAT; but was unchanged in APX in rapeseed seedlings (Hasanuzzaman and Fujita, 2011). On the other hand, further increase in the contents of AsA and GSH and the activities of APX, DHAR, MDHAR, GR, GST, GPX, CAT, Gly I, and Gly II was observed in selenium (Se)pretreated seedlings under drought stress, which in turn maintained a higher GSH/GSSG ratio and lower ROS and MG levels, and ultimately increased the tolerance of the rapeseed plants to drought stress (Hasanuzzaman and Fujita, 2011).

In mung bean seedlings, Nahar et al. (2015b) reported that drought stress resulted in oxidative stress (as evidenced by $\mathrm{H}_{2} \mathrm{O}_{2}, \mathrm{O}_{2}{ }^{--}$, and $\mathrm{MDA}$ ); 
increased the MG, GSSG, GSH, and Pro levels and the activities of APX, GST, Gly I, and Gly II; and decreased leaf succulence, leaf chlorophyll (Chl), RWC, AsA, and GSH/GSSG ratio. In contrast to drought stress alone, exogenous GSH further enhanced the components of the antioxidant and glyoxalase systems in droughtaffected mung bean seedlings except for AsA, Pro, Gly I, and DHAR. Similarly, in Brassica species seedlings, drought stress significantly increased the LOX activity and the levels of MDA, $\mathrm{H}_{2} \mathrm{O}_{2}$, Pro, oxidized ascorbate (DHA), and GSSG, which in turn reduced seedling biomass, Chl content, and RWC. In contrast, spraying drought-stressed seedlings with JA increased Gly I, Gly II, DHAR, GR, and GPX activities (Alam et al., 2014).

Interestingly, heat shock (HS) as environment priming can induce cross-tolerance in plants (Hossain et al., 2017). In mustard seedlings, as compared with the control, HS $\left(42^{\circ} \mathrm{C}, 5 \mathrm{~h}\right)$ positively modulates the activities of APX, DHAR, GR, GST, GPX, CAT, Gly I, and Gly II, and maintained lower levels of GSSG, $\mathrm{H}_{2} \mathrm{O}_{2}, \mathrm{MDA}$, and MG (Hossain et al., 2013a). These results showed that HS (previous stress exposure) protects the mustard plants from salt- and drought-induced oxidative stress by a coordination of antioxidant and glyoxalase systems.

\subsubsection{Heavy Metal Stress Tolerance}

With the advance of industrialization, urbanization, and agriculturalization, heavy metal pollutants are more and more severe, which significantly affects crop productivity and human heath (Hossain et al., 2012). The excessive accumulation of MG and ROS is a common consequence of heavy metal toxicity in plants, which leads to MG and oxidative stresses, that is, lipid peroxidation, protein oxidation, enzyme inactivation, and DNA damage (Hossain et al., 2012). Plants can acquire the tolerance against heavy metal stress by sophisticated glyoxalase and antioxidant defense systems to scavenge excessive MG and ROS (Hossain et al., 2012).

In B. juncea seedlings, Cd stress led to its endogenous accumulation in the roots and shoots in a dose-dependent manner. This accumulation resulted in oxidative damage (as reflected in elevated MDA, $\mathrm{H}_{2} \mathrm{O}_{2}, \mathrm{O}_{2}{ }^{--}$, and LOX activity), MG stress (as indicated in accumulated $\mathrm{MG}$ ), and downregulating of the antioxidant defense and glyoxalase systems, which in turn reduced growth, biomass, water status, and Chl content (Khan and Khan, 2014; Mahmud et al., 2018). However, treatment with citric acid (CA) enhanced the pool of AsA and GSH and the activities of the antioxidant enzymes (APX, MDHAR, DHAR, GR, GPX, SOD, and $\mathrm{CAT}$ ) and the glyoxalase system (Gly I and Gly II), and the phytochelatin (PC) content. Therefore, $\mathrm{CA}$ reduced oxidative damage, increased leaf $\mathrm{RWC}, \mathrm{Chl}$, and the content of $\mathrm{Cd}$ in the root and shoot, as well as $\mathrm{Cd}$ translocation from the roots to the shoots in a dose-dependent manner (Mahmud et al., 2018). These data suggest for the first time that CA plays a dual role in mustard seedlings by increasing phytoremediation and upregulating the antioxidant defense and glyoxalase systems (Mahmud et al., 2018). Similarly, accumulation of chromium $(\mathrm{Cr})$ increased in a dose-dependent manner, which in turn disrupted antioxidant defense and glyoxalase systems and led to an increase in $\mathrm{H}_{2} \mathrm{O}_{2}, \mathrm{O}_{2}{ }^{--}, \mathrm{MG}$, and $\mathrm{Chl}$ contents, RWC, and LOX activity, followed by reducing mustard seedling growth (Mahmud et al., 2017a,b). Furthermore, pretreatment with GABA and maleic acid (MA) alone reduced $\mathrm{Cr}$ uptake and upregulated the contents of AsA and GSH and the activities of APX, MDHAR, DHAR, GR, GPX, SOD, CAT, Gly I, and Gly II, which in turn reduced oxidative damage and MG stress. In addition, GABA increased leaf RWC and Chl content, but decreased Pro and PC levels, and finally restored mustard seedling growth (Mahmud et al., 2017a,b).

In rice seedlings, $\mathrm{Cd}$ stress increased the levels of MG and ROS, while $\mathrm{Ca}^{2+}$ treatment significantly increased the content of AsA and the activities of Gly I, Gly II, SOD, CAT, GST, MDHAR, and DHAR; and reduced $\mathrm{Cd}$ uptake, which in turn reversed overproduced MG and ROS, thereby reducing Cd toxicity (Rahman et al., 2016a). Likewise, exogenously applied $\mathrm{Ca}^{2+}$ significantly decreased As and ROS levels, increased AsA content and the activities of Gly I, Gly II, MDHAR, DHAR, CAT, GPX, and SOD, restoring water loss and plant growth, compared with rice seedlings exposed to As only (Rahman et al., 2015). In rapeseed (B. napus L.) seedlings, $\mathrm{Cd}$ stress increased the contents of $\mathrm{H}_{2} \mathrm{O}_{2}$, MDA, and GSSG, as well as the activities of LOX, APX, and GST; and decreased the contents of AsA and GSH, GSH/GSSG ratio, as well as their redox pool. In addition, $\mathrm{Cd}$ declined the activities of MDHAR, DHAR, and CAT, but was unchanged in Gly I and Gly II. Inversely, exogenous application of $\mathrm{Si}$ and $\mathrm{H}_{2} \mathrm{O}_{2}$ alone reduced $\mathrm{H}_{2} \mathrm{O}_{2}$ and MDA contents and increased the AsA and GSH pools and activities of APX, MDHAR, DHAR, GR, CAT, Gly I, and Gly II (Hasanuzzaman et al., 2017b,c). Therefore, the enhancement of the nonenzymatic and enzymatic antioxidants and glyoxalase system helped to decrease the oxidative damage and MG stress, followed by improving the resistance of rapeseed seedlings to Cd toxicity. 


\subsubsection{Heat Tolerance}

High temperature deleteriously affects the whole plant life cycle from seed germination, seedling establishment, growth, development, to senescence, and even survival (Wahid et al., 2007; Khan et al., 2013a,b; Hemmati et al., 2015). High temperature commonly leads to direct injury, namely loss of biomembrane integrity and protein denaturation and indirect injury also known as secondary injury, that is, oxidative, osmotic, and MG stresses. These injuries result in physiological disorder, metabolic inordinance, and loss of crop productivity (Wahid et al., 2007; Hemmati et al., 2015). Plants have correspondingly developed a series of complex strategies to perception, response and adaptation to high temperature at the morphological, anatomical, physiological, biochemical, and molecular levels (Wahid et al., 2007; Hemmati et al., 2015). Understanding the mechanism of damage and adaptation of plants to high temperature stress is fundamental for the development of tolerant plant species.

Application of PAs plays a key role in conferring abiotic stress tolerance in plants. In mung bean seedlings, pretreatment with Spm upregulated the activities of antioxidant enzymes (SOD, CAT, GPX, DHAR, and GR) and glyoxalase system (Gly I and Gly II); and maintained a higher levels of AsA and GSH, which in turn reduced oxidative stress (a decrease in $\mathrm{H}_{2} \mathrm{O}_{2}$, $\mathrm{O}_{2}{ }^{\cdot-}$, LOX activity, and MDA) and MG stress (a lower MG accumulation) under high temperature stress (Nahar et al., 2017a). In addition, exogenous PAs could increase its endogenous content, as osmolyte, which carried out osmoregulation and restoration of plant water status under high temperature stress, followed by preventing the degradation of photosynthetic pigments and improving seedling growth parameters (Nahar et al., 2017a). Similarly, high temperature stress markedly reduced the activities of MDHAR, DHAR, GPX, CAT, and Gly I, followed by triggering oxidative stress, as reflected in an increase in $\mathrm{H}_{2} \mathrm{O}_{2}, \mathrm{O}_{2}{ }^{\bullet-}$, and MDA, but increased Gly II (Nahar et al., 2015c). However, pretreatment of mung bean seedlings with exogenous GSH increased the activities of APX, MDHAR, DHAR, GR, GPX, GST, CAT, Gly I, and Gly II; improved endogenous GSH content and the GSH/ GSSG ratio; and reduced GSSG content, compared with the control seedlings (Nahar et al., 2015c). Therefore, GSH improved the glyoxalase and antioxidant systems, which in turn reduced MG and oxidative stresses, followed by exhibiting better physiological performance under high temperature stress (Nahar et al., 2015c).

Selenium, a minor element, plays a crucial role in the acquisition of plant abiotic stress tolerance (Hasanuzzaman and Fujita, 2011; Hasanuzzaman et al., 2014; Khan et al., 2015). Exogenously applied Se to rapeseed seedlings improved the activities of MDHAR, DHAR, GR, GPX, CAT, Gly I, and Gly II, and the contents of Pro, AsA, and GSH, as well as the GSH/GSSG ratio under high temperature stress (Hasanuzzaman et al., 2014). The activation of antioxidant and glyoxalase systems by Se further alleviated oxidative damage and MG toxicity, as indicated by a decrease in $\mathrm{MDA}, \mathrm{H}_{2} \mathrm{O}_{2}$, and $\mathrm{MG}$, as compared with heat-treated seedlings without $\mathrm{Se}$ supplementation (Hasanuzzaman et al., 2014). Similarly, in rice seedlings, high temperature stress led to oxidative stress, as indicated by an increase in LOX activity, MDA, and $\mathrm{H}_{2} \mathrm{O}_{2}$, which in turn decreased fresh weight (FW) and Chl content (Mostofa et al., 2014). In addition, high temperature stress also significantly increased the activities of Gly I and Gly II (Mostofa et al., 2014). In contrast, foliar spray with Spm alleviated an increase in MDA and $\mathrm{H}_{2} \mathrm{O}_{2}$, but improved the contents of AsA, GSH, FW, Chl and AsA, as well as GSH redox status (Mostofa et al., 2014).

\subsubsection{Chilling Tolerance}

Chilling injury refers to the damage of chillingsensitive plants from tropic and subtropic regions by low temperature (above ice point) but below the temperature that they normally experience (Ruelland et al., 2009; Zhu, 2016). Chilling stress commonly triggers membrane transition, that is, conversion of liquid-crystal state to solid state, which leads to loss of biomembrane integrity (Ruelland et al., 2009; Zhu, 2016). In addition, oxidative, osmotic, and MG stresses also can be induced by chilling stress in many plant species (Ruelland et al., 2009; Zhu, 2016). Therefore, the strategies, including environment or chemical priming, that can maintain liquid-crystal state of biomembrane and remit oxidative, osmotic, and MG stresses all can improve the tolerance of chilling-sensitive plants to chilling stress. In Jatropha curcas seedlings, chilling shock $\left(5^{\circ} \mathrm{C}, 4 \mathrm{~h}\right)$ or chilling hardening $\left(12^{\circ} \mathrm{C}, 2 \mathrm{~h}\right)$ could improve the chilling tolerance by a coordination of osmolytes and antioxidant system (Ao et al, 2013a,b; Li et al., 2013). Similarly, in mustard seedlings, salt, and drought stress abruptly increased $\mathrm{H}_{2} \mathrm{O}_{2}$ and MDA levels, producing oxidative stress (Hossain et al., 2013b). In addition, both stresses significantly improved the contents of GSH and GSSG, and the activities of MDHAR and Gly I; but declined CAT and Gly II activities. However, chilling shock $\left(6^{\circ} \mathrm{C}, 5.5 \mathrm{~h}\right)$ maintained a higher level of AsA and GSH contents and GSH/GSSG ratio; higher activities of APX, DHAR, GR, GST, GPX, CAT, Gly I, and Gly II; and lowered the levels of GSSG, $\mathrm{H}_{2} \mathrm{O}_{2}$, and 
MDA in mustard seedlings, as compared with the control (Hossain et al., 2013b). These adaptive changes in the antioxidant system and glyoxalase system induced by chilling shock are the basis for the acquisition of abiotic stress tolerance in chillingsensitive plants.

In mung bean seedlings, chilling stress led to oxidative stress, as indicated in an increase in $\mathrm{H}_{2} \mathrm{O}_{2}$ and MDA contents, by modulating the nonenzymatic (AsA and GSH) and enzymatic components (APX, GR, DHAR, and MDHAR) of AsA-GSH (Nahar et al., 2015d). Also, chilling stress reduced RWC, Chl content, and seedling growth. On the other hand, exogenous pretreatment of mung bean seedlings with Spd significantly increased the contents of AsA and GSH, and the ratios of AsA/DHA and GSH/GSSG; and decreased DHA and GSSG contents under chilling stress (Nahar et al., 2015d). Similarly, Spd pretreatment also increased APX, MDHAR, DHAR, and GR activities in mung bean seedlings under chilling stress, which in turn reduced the oxidative stress and MG toxicity, followed by improving seedling growth (Nahar et al., 2015d). In the same way, chilling stress led to MG stress (MG accumulation) and oxidative stress (an increase in MDA) in tea bud (Kumar and Yadav, 2009). However, exogenous application of Pro and GB alleviated a decrease in Gly I and Gly II activities and an increase in MG level under chilling stress (Kumar and Yadav, 2009). In addition, exogenous Pro activated GST and GR activities, but GB only increased GR under chilling stress (Kumar and Yadav, 2009). Interestingly, involvement of Gly I and Gly II in the acquisition of chilling tolerance was identified using proteomics protocol in arabidopsis (Arabidopsis thaliana), rice, and onion (Allium cepa) (Goulas et al., 2006; Lee et al., 2009; Chen et al., 2013).

\subsection{ABIOTIC STRESS TOLERANCE IMPROVED BY OVEREXPRESSING GLYOXALASE GENES}

As mentioned above, plant abiotic stress tolerance is closely connected with the glyoxalase system. A number of studies have found that transgenic plants overexpressing glyoxalase genes (Gly I, Gly II, or Gly II) alone or in combination can improve multiple abiotic stress tolerance such as salt, drought, heavy metal, heat, and chilling tolerance (Li, 2016; Hasanuzzaman et al., 2017d; Hossain et al., 2017). For example, transgenic rice plants overexpressing GLY I gene (from Brassica) and GLY II gene (from O. sativa) increased the activities of Gly I, Gly II, and TPI; and reduced the levels of G3P, DHAP, pyruvate, GSH, and MAD under salt, drought, and heat stresses (Gupta et al., 2018). In addition, transgenic plants alleviated the oxidative damage of chloroplast and mitochondrial ultrastructure by ROS and maintained photosynthetic efficiency under these stresses (Gupta et al., 2018). Finally, plants overexpressing GLY I combined with GLY II imparted tolerance to abiotic stresses like salinity, drought heat, and provided resistance to the sheath blight fungus (Gupta et al., 2018).

Similarly, under normal conditions, in rice seedlings, a gene OsGly I encoding Gly I was ubiquitously expressed in different organs such as root, stem, leaf, leaf sheath, and spikelet with varying abundance (Zeng et al., 2016). Under $\mathrm{NaCl}, \mathrm{ZnCl}_{2}$, and mannitol stresses, OsGly I was markedly upregulated in different organs of rice seedlings (Zeng et al., 2016). In addition, transgenic rice seedlings overexpressing OsGly $I$ increased Gly I activity, which in turn reduced the accumulation of MG and MDA, followed by improving the tolerance of transgenic seedlings to $\mathrm{NaCl}, \mathrm{ZnCl}_{2}$, and mannitol stresses, compared with wild-type plants (Zeng et al., 2016). Also, transgenic plants performed higher seed setting rate and yield (Zeng et al., 2016). Both Gly I and Gly II genes, known as JcGLYI and JcGLYII from J. curcas, were heterologously expressed in Escherichia coli and yeast individually, which conferred the resistance to PEG (5\%), NaCl (200 mM), and MG (5 mM) stresses (Mudalkar et al., 2017). Furthermore, in B. juncea seedlings, overexpression of Gly I improved the tolerance to several stresses such as salinity, heavy metal, and drought stress as compared with untransformed control plants, indicating that the overexpression of the gly I gene is a better option for improving salt, drought, and heavy metal stress tolerance in transgenic plants (Rajwanshi et al., 2016).

In addition, the transgenic tobacco overexpressing Gly I and Gly II alone or in combination increased the activities of GR, GST, GPX, and APX, GSH level, and GSG/GSSG ratio; and reduced the accumulation of endogenous MG and MDA, which in turn improved the growth of transgenic plants under salt stress as compared with the nontransgenic plants (Yadav et al., 2005b). In addition to glyoxalase, AKR is another enzyme involved in MG detoxification in plants. Its expression level can be greatly induced by abscisic acid (ABA), $\mathrm{H}_{2} \mathrm{O}_{2}, \mathrm{NaCl}$, and mannitol treatments (Turoczy et al., 2011). Furthermore, the transgenic tobacco overexpressing OsAKR1 from rice exhibited higher AKR activity and less accumulation of MG in leaves than the wild-type plants under normal and heat stress conditions, which in turn increased the resistance to high temperature stress at $44^{\circ} \mathrm{C}$ (Turoczy et al., 2011). 


\subsection{MG PRIMING-INDUCED ABIOTIC TOLERANCE}

Many signaling molecules such as $\mathrm{Ca}^{2+}, \mathrm{H}_{2} \mathrm{O}_{2}, \mathrm{NO}$, and $\mathrm{H}_{2} \mathrm{~S}$ priming can improve the abiotic stress tolerance in various plant species; the acquisition of plant abiotic stress tolerance is involved in MG and ROS detoxification systems, osmotic adjustment, synthesis of stress proteins, and so forth (Antoniou et al., 2016; Li et al., 2016; Zhou et al., 2017; Hossain et al., 2017). A lot of studies have reported that MG priming can increase the resistance of plants to a wide range of abiotic stresses, further supporting the signaling role of MG. For example, in Brassica rapa L. seedlings, MG priming could stimulate the activities of MG detoxification system (Gly I and Gly II) and ROS detoxification enzymes (SOD, APX, CAT, GR, and APX), reduced the oxidative damage and MG toxicity, as reflected in a decrease in the accumulation of $\mathrm{H}_{2} \mathrm{O}_{2}, \mathrm{O}_{2}{ }^{-}$, and $\mathrm{MG}$, which in turn promoted seed germination and subsequent seedling growth of seedlings under zirconium stress (Bless et al., 2017). In addition, $\mathrm{NaCl}$ stress reduced seed germination and seedling growth, as indicated in seedling height, root length, fresh weight, and dry weight ( $\mathrm{Li}$ et al., 2017a). However, the toxic effects of $\mathrm{NaCl}$ were remitted by exogenous MG treatment, but worsen by MG scavenger $N$-acetyl-L-cysteine (NAC), illustrating that MG could improve the tolerance of wheat to salt stress ( $\mathrm{Li}$ et al., 2017a). Further experiments showed that MG activated Gly I, Gly II, SOD, CAT, APX GR; increased GSH and AsA levels; and decreased oxidative stress indicated by decrease in $\mathrm{O}_{2}{ }^{--}, \mathrm{H}_{2} \mathrm{O}_{2}$, and MDA levels in wheat seedlings under $\mathrm{NaCl}$ stress ( $\mathrm{Li}$ et al., 2017a). In addition, MG also accumulated osmolytes such as Pro and GB soluble sugar, thereby enhancing the capacity of osmotic adjustment in wheat seedlings under $\mathrm{NaCl}$ stress. The above positive effects of MG were weakened by NAC, further verifying the signaling role of MG ( $\mathrm{Li}$ et al., 2017a).

Similarly, under Cd stress, wheat seedlings showed reduced seedling height, root length, FW, and DW in a concentration dependent manner ( $\mathrm{Li}$ et al., 2017b). Interestingly, the negative effects of $\mathrm{Cd}$ on wheat seedlings were alleviated by exogenous MG, suggesting that MG could improve $\mathrm{Cd}$ toxicity in wheat ( $\mathrm{Li}$ et al., 2017b). Further investigations have illustrated that MG increased the activities of GR and gammaglutamylcysteine synthetase $(\gamma$-ECS), a key enzyme in GSH biosynthesis, and GSH level in Cd-stress wheat seedlings compared with the control seedlings without MG treatment ( $\mathrm{Li}$ et al., 2018). In contrast, $\gamma$-ECS and GR activities and GSH level were weakened by NAC, DHBA (2,4-dihydroxy-benzylamine), and
BCNU (1,3-bischloroethyl-nitrosourea), specific inhibitors of GR, and BSO (buthionine sulfoximine), a specific inhibitor of GSH biosynthesis (Li et al., 2018). Moreover, MG increased the activities of Gly I and Gly II and declined endogenous MG level in Cdtreated seedlings ( $\mathrm{Li}$ et al., 2018). On the other hand, an increase in Gly I and Gly II activities and a decrease in MG level were reversed by NAC, IAS (isoascorbate), and SA (squaric acid), specific inhibitors of Gly I (Li et al., 2018). Furthermore, MG alleviated an increase in $\mathrm{H}_{2} \mathrm{O}_{2}$ and MDA in Cd-treated wheat seedlings (Li et al., 2018).

\subsection{SIGNALING CROSSTALK BETWEEN MG AND CA ${ }^{2+}$, ROS, NO, AND $\mathrm{H}_{2} \mathrm{~S}$}

In general, the acquisition of plant abiotic stress tolerance can be classified into four steps: stress perception by sensors, signal transduction and signal network formation, gene expression, and physiological and biochemical change (Antoniou et al., 2016; Li et al., 2016; Hossain et al., 2017). As mentioned above, environmental stress can trigger second messengers such as $\mathrm{Ca}^{2+}, \mathrm{H}_{2} \mathrm{O}_{2}, \mathrm{NO}, \mathrm{H}_{2} \mathrm{~S}$ and $\mathrm{MG}$, while exogenous signaling molecule priming can increase their endogenous levels, which in turn switches on plant abiotic stress tolerance via signaling crosstalk (Antoniou et al., 2016; Li et al., 2016; Hossain et al., 2017).

In prokaryote E. coli, $\left[\mathrm{Ca}^{2+}\right]_{\mathrm{cyt}}$ also was induced by exogenous MG in a concentration dependent manner, and this induction was blocked by $\mathrm{la}^{3+}$, a plasma membrane $\mathrm{Ca}^{2+}$ channel blocker (Campbell et al., 2007; Hoque et al., 2016). Similarly, in eukaryote Saccharomyces cerevisiae, exogenous MG treatment could activate a high osmolarity glycerol-mitogenactivated protein kinase cascade, which in turn increased cytosolic free $\mathrm{Ca}^{2+}\left(\left[\mathrm{Ca}^{2+}\right]_{\text {cyt }}\right)$ through stimulating $\mathrm{Ca}^{2+}$ channels, followed by triggering the calcineurin-mediated $\mathrm{Ca}^{2+}$ signaling pathway (Maeta et al., 2005; Hoque et al., 2016). These results suggest that $\mathrm{Ca}^{2+}$ transient requires the entry of extracellular $\mathrm{Ca}^{2+}$ into cells across the plasma membrane. In Brassica oleracea, calmodulin (CaM) inhibitors, trifluoperazine (TFP), and chlorpromazine (CPZ), alone inhibited the activity of Gly I (Bagga et al., 1987; Hoque et al., 2016). Likewise, Gly I activity in B. juncea was activated by $\mathrm{Ca}^{2+} / \mathrm{CaM}$, but blocked by TFP (Deswal and Sopory, 1999; Hoque et al., 2016). These data indicate that the calcium messenger system (mainly $\mathrm{Ca}^{2+}$ and CaM) may activate Gly I, which in turn regulates $\mathrm{MG}$ homeostasis in cells, further verifying the interaction of $\mathrm{MG}$ and $\mathrm{Ca}^{2+} / \mathrm{CaM}$. 


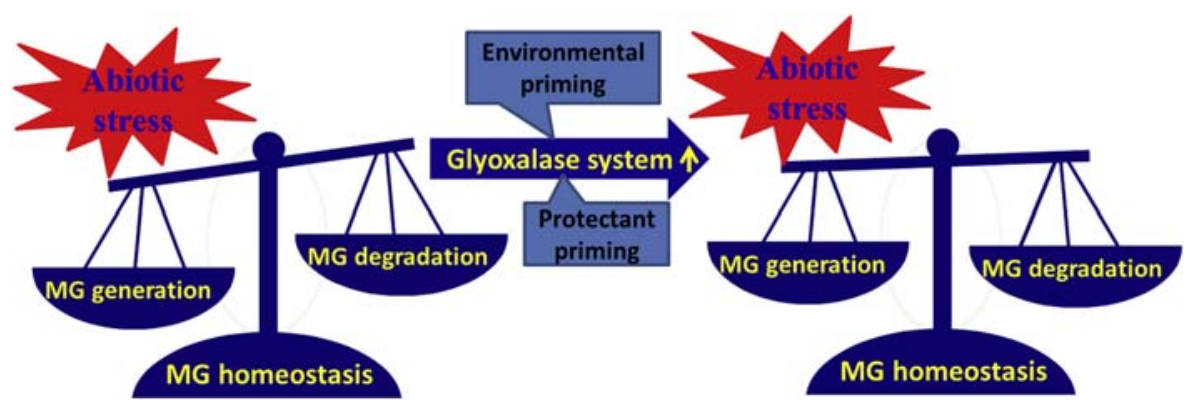

FIGURE 14.3 Key role of glyoxalase system in MG homeostasis in plants. Adverse environments induce MG excessive accumulation, while environment (hardening) or chemical (protectant) priming restores MG homeostasis, followed by switching on abiotic stress tolerance in plants.
In guard cells of Arabidopsis, MG could increase the activity of salicylhydroxamic acid (SHAM)-sensitive peroxidases (POD), which in turn induced extracellular $\operatorname{ROS}\left(\mathrm{H}_{2} \mathrm{O}_{2}\right.$ and $\left.\mathrm{O}_{2}{ }^{-}\right)$production, followed by inducing $\left[\mathrm{Ca}^{2+}\right]_{\mathrm{cyt}}$ oscillations in guard cells, and ultimately led to stomatal closure (Hoque et al., 2012c). Similarly, MG also could inhibit inward-rectifying potassium channels, reducing the accumulation of $\mathrm{K}^{+}$ in guard cells, followed by inhibiting light-induced stomatal opening in Arabidopsis (Hoque et al., 2012a). In addition, MG could catalyze the photoreduction of $\mathrm{O}_{2}$ at photosystem I (PS I), leading to $\mathrm{O}_{2}{ }^{--}$production, which is converted into $\mathrm{H}_{2} \mathrm{O}_{2}$ by CAT or APX, further exerting the signaling role of $\mathrm{H}_{2} \mathrm{O}_{2}$ and $\mathrm{O}_{2}{ }^{--}$(Saito et al., 2011; Li, 2016). Many studies also found that MG could induce the accumulation of $\mathrm{H}_{2} \mathrm{O}_{2}$ and $\mathrm{O}_{2}{ }^{-}$by inhibiting mitochondrial electron transfer chain, complex III and antioxidant enzymes (Chang et al., 2005; Wang et al., 2009; Desai et al., 2010; Hoque et al., 2016).

Interestingly, in animal cells, many studies have found crosstalk between MG and NO (Su et al., 2013), ROS (Chang et al., 2005), and $\mathrm{H}_{2} \mathrm{~S}$ (Yang et al., 2014; Chang et al., 2010).

\subsection{CONCLUSION AND FUTURE PROSPECTS}

Signaling molecule $\mathrm{MG}$, similar to other second messengers such as $\mathrm{Ca}^{2+}, \mathrm{H}_{2} \mathrm{O}_{2}, \mathrm{NO}$, and $\mathrm{H}_{2} \mathrm{~S}$, can be rapidly triggered by adverse environments (including salt, drought, heavy metal, heat, chilling, and high light stresses) or chemicals (like $\mathrm{ABA}, \mathrm{H}_{2} \mathrm{O}_{2}$, and 2,4dichlorophenoxyacetic) via coordinating action of its production (mainly nonenzymatic pathways) and scavenging (principally glyoxalase system) systems in plants (Hossain et al., 2009; Fig. 14.2). Owing to the dual role of $M G$, excessive MG has to be eliminated by glyoxalase and nonglyoxalase systems (Fig. 14.1) and maintain a low and nontoxic physiological level to exert its signaling role in seed germination, plant growth, development, reproduction, and response to abiotic stress. The acquisition of plant abiotic stress tolerance (such as salt, drought, heavy metal, heat, and chilling tolerance) induced by environment or chemical (protectant) priming is closely related to MG and its detoxification system, especially glyoxalase system plays a key role (Table 14.1 and Fig. 14.3).

Recently, the research on MG and its detoxification system has extensively attracted attention and obtained great progress in seed germination, plant growth, development, reproduction, and adaptation to abiotic stress (Hasanuzzaman et al., 2017d; Hossain et al., 2017; Sankaranarayanan et al., 2017). However, some open questions have to be settled urgently:

1. With the development of genomics, transcriptomics, proteomics, and metabolomics, an in-depth understanding of MG homeostasis and its signaling roles will be expounded in various plant species using omics approach in the future.

2. MG priming-induced abiotic stress tolerance and its mechanisms are waiting for answer at the physiological, biochemical, and molecular level.

3. In animal cells, signaling crosstalk between MG and $\mathrm{Ca}^{2+}, \mathrm{ROS}, \mathrm{NO}$, and $\mathrm{H}_{2} \mathrm{~S}$ has gotten much attention (Maeta et al., 2005; Campbell et al., 2007; Chang et al., 2005, 2010; Su et al., 2013), but their crosstalk is not completely clear in plants.

4. MG can lead to glycation of proteins, which in turn regulates protein secondary structure and activity (Gomord and Faye, 2004). However, whether glycated proteins are the receptors of MG needs to be further investigated.

5. MG as a novel signaling molecule, its concentration in cells, or compartmentalization requires precise and timely quantitative determination in vivo and in vitro. Shaheen et al. (2014) used fluorogenic probes, DAF-2 (4,5-diaminofluorescein) and DAR-1 (4,5-diaminorhodamine), to detect the content of endogenous MG in living animal cells, while these probes have nonspecificity. Therefore, specific fluorescence methods must be further explored in the response of plants to abiotic stress and signaling crosstalk in plants in future. 


\section{Acknowledgements}

This research is supported by National Natural Science Foundation of China $(31760069 ; 31360057)$ and Doctor Startup Foundation of Yunnan Normal University China (01200205020503099).

\section{References}

Alam, M.M., Nahar, K., Hasanuzzaman, M., Fujita, M., 2014. Exogenous jasmonic acid modulates the physiology, antioxidant defense and glyoxalase systems in imparting drought stress tolerance in different brassica, species. Plant Biotechnol. Rep. 8, 279-293.

Antoniou, C., Savvides, A., Christou, A., Fotopoulos, V., 2016. Unravelling chemical priming machinery in plants: the role of reactive oxygen-nitrogen-sulfur species in abiotic stress tolerance enhancement. Curr. Opin. Plant Biol. 33, 101-107.

Ao, P.X., Li, Z.G., Gong, M., 2013a. Involvement of compatible solutes in chill hardening-induced chilling tolerance in Jatropha curcas seedlings. Acta Physiol. Plant. 35, 3457-3464.

Ao, P.X., Li, Z.G., Fan, D.M., Gong, M., 2013b. Involvement of antioxidant defense system in chill hardening-induced chilling tolerance in Jatropha curcas seedlings. Acta Physiol. Plant. 35, 153-160.

Bagga, S., Das, R., Sopory, S., 1987. Inhibition of cell proliferation and glyoxalase-I activity by calmodulin inhibitors and lithium in Brassica oleracea. J. Plant Physiol. 129, 149-153.

Bless, Y., Ndlovu, L., Gokul, A., Keyster, M., 2017. Exogenous methylglyoxal alleviates zirconium toxicity in Brassica rapa $\mathrm{L}$. seedling shoots. South Afr. J. Bot. 109, 327.

Campbell, A.K., Naseem, R., Holland, I.B., Matthews, S.B., Wann, K. T., 2007. Methylglyoxal and other carbohydrate metabolites induce lanthanum-sensitive $\mathrm{Ca}^{2+}$ transients and inhibit growth in E. coli. Arch. Biochem. Biophys. 468, 107-113.

Céccoli, G., Ramos, J., Pilatti, V., Dellaferrera, I., Tivano, J.C., Taleisnik, E., et al., 2015. Salt glands in the poaceae family and their relationship to salinity tolerance. Bot. Rev. 81, 162-178.

Chang, T., Wang, R., Wu, L., 2005. Methylglyoxal-induced nitric oxide and peroxynitrite production in vascular smooth muscle cells. Free Rad. Biol. Med. 38, 286-293.

Chang, T., Untereiner, A., Liu, J., Wu, L., 2010. Interaction of methylglyoxal and hydrogen sulfide in rat vascular smooth muscle cells. Antioxid. Redox Signal. 12, 1093-1100.

Chen, K., Renaut, J., Sergeant, K., Wei, H., Arora, R., 2013. Proteomic changes associated with freeze-thaw injury and post-thaw recovery in onion (Allium cepa L.) scales. Plant Cell Environ. 36, 892-905.

da-Silva, C.J., Modolo, L.V., 2018. Hydrogen sulfide: a new endogenous player in an old mechanism of plant tolerance to high salinity. Acta Bot. Brasil. 32, 150-160.

Desai, K.M., Chang, T., Wang, H., Banigesh, A., Dhar, A., Liu, J.H., et al., 2010. Oxidative stress and aging: is methylglyoxal the hidden enemy? Can. J. Physiol. Pharmacol. 88, 273-284.

Deswal, R., Sopory, S.K., 1999. Glyoxalase I from Brassica juncea is a calmodulin stimulated protein. Biochim. Biophys. Acta 1450, 460-467.

Dodd, A.N., Kudla, J., Sanders, D., 2010. The language of calcium signaling. Ann. Rev. Plant Biol. 61, 593-620.

Golldack, D., Li, C., Mohan, H., Probst, N., 2014. Tolerance to drought and salt stress in plants: unraveling the signaling networks. Front. Plant Sci. 5, 151.

Gomord, V., Faye, L., 2004. Posttranslational modification of therapeutic proteins in plants. Curr. Opin. Plant Biol. 7, 171-181.

Goulas, E., Schubert, M., Kieselbach, T., Kleczkowski, L.A., Gardestrom, P., Schroder, W., et al., 2006. The chloroplast lumen and stromal proteomes of Arabidopsis thaliana show differential sensitivity to short-and long-term exposure to low temperature. Plant J. 47, 720-734.
Gupta, B.K., Sahoo, K.K., Ghosh, A., Tripathi, A.K., Anwar, K., Das, P., et al., 2018. Manipulation of glyoxalase pathway confers tolerance to multiple stresses in rice. Plant Cell Environ. 41, $1186-1200$.

Hasanuzzaman, M., Fujita, M., 2011. Selenium pretreatment upregulates the antioxidant defense and methylglyoxal detoxification system and confers enhanced tolerance to drought stress in rapeseed seedlings. Biol. Trace Elem. Res. 143, $1758-1776$.

Hasanuzzaman, M., Nahar, K., Alam, M.M., Roychowdhury, R., Fujita, M., 2013. Physiological, biochemical, and molecular mechanisms of heat stress tolerance in plants. Inter. J. Mol. Sci. $14,9643-9684$.

Hasanuzzaman, M., Nahar, K., Alam, M.M., Fujita, M., 2014. Modulation of antioxidant machinery and the methylglyoxal detoxification system in selenium-supplemented Brassica napus seedlings confers tolerance to high temperature stress. Biol. Trace Elem. Res. 161, 297-307.

Hasanuzzaman, M., Nahar, K., Hossain, M.S., Anee, T.I., Parvin, K., Fujita, M., 2017a. Nitric oxide pretreatment enhances antioxidant defense and glyoxalase systems to confer PEG-induced oxidative stress in rapeseed. J. Plant Interact. 12, 323-331.

Hasanuzzaman, M., Nahar, K., Anee, T.I., Fujita, M., 2017b. Exogenous silicon attenuates cadmium-induced oxidative stress in Brassica napus L. by modulating AsA-GSH pathway and glyoxalase system. Front. Plant Sci. 8, 1061.

Hasanuzzaman, M., Nahar, K., Gill, S.S., Alharby, H.F., Razafindrabe, B.H.N., Fujita, M., 2017c. Hydrogen peroxide pretreatment mitigates cadmium-induced oxidative stress in Brassica napus L.: an intrinsic study on antioxidant defense and glyoxalase systems. Front. Plant Sci. 8, 115.

Hasanuzzaman, M., Nahar, K., Hossain, M.S., Mahmud, J.A., Rahman, A., Inafuku, M., et al., 2017d. Coordinated actions of glyoxalase and antioxidant defense aystems in conferring abiotic stress tolerance in plants. Inter. J. Mol Sci. 18, 200.

Hasanuzzaman, M., Nahar, K., Anee, T.I., Khan, M.I.R., Fujita, M., 2018. Silicon-mediated regulation of antioxidant defense and glyoxalase systems confers drought stress tolerance in Brassica napus L. South Afr. J. Bot. 115, 50-57.

Hemmati, H., Gupta, D., Basu, C., 2015. Molecular physiology of heat stress responses in plants. In: Pandey, G.K. (Ed.), Elucidation of Abiotic Stress Signaling in Plants: Functional Genomics Perspectives. Springer, New York, pp. 109-142.

Hoque, T.S., Okuma, E., Uraji, M., Furuichi, T., Sasaki, T., Hoque, M. A., 2012a. Inhibitory effects of methylglyoxal on light-induced stomatal opening and inward $\mathrm{K}^{+}$channel activity in Arabidopsis. Biosci. Biotechnol. Biochem. 76, 617-619.

Hoque, T.S., Uraji, M., Ye, W., Hossain, M.A., Nakamura, Y., Murata, Y., 2012c. Methylglyoxal-induced stomatal closure accompanied by peroxidase-mediated ROS production in Arabidopsis. J. Plant Physiol. 169, 979-986.

Hoque, T.S., Hossain, M.A., Mostofa, M.G., Burritt, D.J., Masayuki, F., Tran, L.P., 2016. Methylglyoxal: an emerging signaling molecule in plant abiotic stress responses and tolerance. Front. Plant Sci. 7, 1341.

Hossain, M.A., Fujita, M., 2010. Evidence for a role of exogenous glycinebetaine and proline in antioxidant defense and methylglyoxal detoxification systems in mung bean seedlings under salt stress. Physiol. Mol. Biol. Plants 16, 19-29.

Hossain, M.A., Hossain, M.Z., Fujita, M., 2009. Stress-induced changes of methylglyoxal level and glyoxalase I activity in pumpkin seedlings and cDNA cloning of glyoxalase I gene. Austr. J. Crop Sci. 3, 53-64.

Hossain, M.A., da Silva, J.A.T., Fujita, M., 2011. Glyoxalase system and reactive oxygen apecies detoxification aystem in plant abiotic 
stress response and tolerance: an intimate relationship. In: Shanker, A. (Ed.), Abiotic Stress in Plants-Mechanisms and Adaptations. InTech, Rijeka, pp. 235-266.

Hossain, M.A., Piyatida, P., da Silva, J.A.T., Fujita, M., 2012. Molecular mechanism of heavy metal toxicity and tolerance in plants: central role of glutathione in detoxification of reactive oxygen species and methylglyoxal and in heavy metal chelation. J. Bot. 2012, 872875

Hossain, M.A., Mostofa, M.G., Fujita, M., 2013a. Heat-shock positively modulates oxidative protection of salt and drought-stressed mustard (Brassica campestris L.) seedlings. J. Plant Sci. Mol. Breed. $2,1-14$.

Hossain, M.A., Mostofa, M.G., Fujita, M., 2013b. Cross protection by cold-shock to salinity and drought stress-induced oxidative stress in mustard (Brassica campestris L.) seedlings. Mol. Plant Breed. 4, 50-70.

Hossain, M.A., Burritt, D.J., Fujita, M., 2016. Cross-stress tolerance in plants: molecular mechanisms and possible involvement of reactive oxygen species and methylglyoxal detoxification systems. In: Tuteja, N., Gill, S.S. (Eds.), Abiotic Stress Response in Plants. Wiley, New York, pp. 323-375.

Hossain, M.A., Li, Z.G., Hoque, T.S., Burritt, D.J., Fujita, M., MunnéBosch, M., 2017. Heat or cold priming-induced cross-tolerance to abiotic stresses in plants: key regulators and possible mechanisms. Protoplasma 255, 399-412.

Iqbal, N., Nazar, R., Khan, N.A., 2016. Osmolytes and Plants Acclimation to Changing Environment: Emerging Omics Technologies. Springer, London.

Jin, S., Li, X.Q., Wang, G.G., Zhu, X.T., 2015. Brassinosteroids alleviate high-temperature injury in Ficus concinna seedlings via maintaining higher antioxidant defence and glyoxalase systems. AoB Plants 7, plv009.

Kaur, C., Ghosh, A., Pareek, A., Sopory, S.K., Singla-Pareek, S.L., 2014a. Glyoxalases and stress tolerance in plants. Biochem. Soc. Trans. 42, 485-490.

Kaur, C., Singla-Pareek, S.L., Sopory, S.K., 2014b. Glyoxalase and methylglyoxal as biomarkers for plant stress tolerance. Crit. Rev. Plant Sci. 33, 429-456.

Kaur, C., Kushwaha, H.R., Mustafiz, A., Pareek, A., Sopory, S.K., Singlapareek, S.L., 2015a. Analysis of global gene expression profile of rice in response to methylglyoxal indicates its possible role as a stress signal molecule. Front. Plant Sci. 6, 682.

Kaur, C., Sharma, S., Singla-Pareek, S.L., Sopory, S.K., 2015 b. Methylglyoxal, triose phosphate isomerase, and glyoxalase pathway: implications in abiotic stress and signaling in plants. In: Pandey, G.K. (Ed.), Elucidation of Abiotic Stress Signaling in Plants. Springer, New York, pp. 347-366.

Kaur, C., Sharma, S., Singla-Pareek, S.L., Sopory, S.K., 2016. Methylglyoxal detoxification in plants: role of glyoxalase pathway. Indian J. Plant Physiol. 21, 377-390.

Khan, M.I.R., Khan, N.A., 2014. Ethylene reverses photosynthetic inhibition by nickel and zinc in mustard through changes in PS II activity, photosynthetic-nitrogen use efficiency and antioxidant metabolism. Protoplasma 251, 1007-1019.

Khan, M.I.R., Khan, N., 2017. Reactive Oxygen Species and Antioxidant System in Plants: Role and Regulation Under Abiotic Stress. Springer Nature 978-981-10-5254-5.

Khan, M.I.R., Iqbal, N., Masood, A., Per, T.S., Khan, N.A., 2013a. Salicylic acid alleviates adverse effects of heat stress on photosynthesis through changes in proline production and ethylene formation. Plant Signal. Behav. 8, e26374.

Khan, M.I.R., Asgher, M., Khan, N.A., 2013b. Rising temperature in the changing environment: a serious threat to plants. Clim. Change Environ. Sustain. 1, 25-36.
Khan, M.I.R., Nazir, F., Asgher, M., Per, T.S., Khan, N.A., et al., 2015. Selenium and sulfur influence ethylene formation and alleviate cadmium-induced oxidative stress by improving proline and glutathione production in wheat. J Plant Physiol. 173, 9-18.

Khan, M.I.R., Khan, N.A., Masood, A., Per, T.S., Asgher, M., 2016. Hydrogen peroxide alleviates nickel-inhibited photosynthetic responses through increase in use-efficiency of nitrogen and sulfur, and glutathione production in mustard. Front. Plant Sci. 7, 44.

Kumar, V., Yadav, S.K., 2009. Proline and betaine provide protection to antioxidant and methylglyoxal detoxification systems during cold stress in Camellia sinensis (L.) O. Kuntze. Acta Physiol. Plant. 31, 261-269.

Lee, D.G., Ahsan, N., Lee, S.H., Lee, J.J., Bahk, J.D., Kang, K.Y., et al., 2009. Chilling stress-induced proteomic changes in rice roots. J. Plant Physiol. 166, 1-11.

Li, Z.G., 2016. Methylglyoxal and glyoxalase system in plants: old players, new concepts. Bot. Rev. 82, 183-203.

Li, Z.G., Yuan, L.X., Wang, Q.L., Ding, Z.L., Dong, C.Y., 2013. Combined action of antioxidant defense system and osmolytes in chilling shock-induced chilling tolerance in Jatropha curcas seedlings. Acta Physiol. Plant. 35, 2127-2136.

Li, Z.G., Min, X., Zhou, Z.H., 2016. Hydrogen sulfide: a signal molecule in plant cross-adaptation. Front. Plant Sci. 7, 1621.

Li, Z.G., Duan, X.Q., Min, X., Zhou, Z.H., 2017a. Methylglyoxal as a novel signal molecule induces the salt tolerance of wheat by regulating the glyoxalase system, the antioxidant system, and osmolytes. Protoplasma 254, 1995-2006.

Li, Z.G., Duan, X.Q., Xia, Y.M., Wang, Y., Zhou, Z.H., Min, X., 2017b. Methylglyoxal alleviates cadmium toxicity in wheat (Triticum aestivum L). Plant Cell Rep. 36, 367-370.

Li, Z.G., Nie, Q., Wang, Y., Zhou, Z.H., 2018. Signal molecule methylglyoxal ameliorates cadmium injury in wheat (Triticum aestivum L) by a coordinated induction of glutathione pool and glyoxalase system. Ecotoxicol. Environ. Saf. 149, 101-107.

Maeta, K., Izawa, S., Inoue, Y., 2005. Methylgyoxal, a metabolite derived from glycolysis, functions as a signal initiator of the high osmolarity glycerol-mitogen-activated protein kinase cascade and calcineurin/Crz1- mediated pathway in Saccharomyces cerevisiae. J. Biol. Chem. 280, 253-260.

Mahmud, J.A.L., Hasanuzzaman, M., Nahar, K., Rahman, A., Hossain, M.S., Fujita, M., 2017a. $\gamma$-aminobutyric acid (GABA) confers chromium stress tolerance in Brassica juncea L. by modulating the antioxidant defense and glyoxalase systems. Ecotoxicology 26, $675-690$.

Mahmud, J.A., Hasanuzzaman, M., Nahar, K., Rahman, A., Hossain, M.A., Fujita, M., 2017b. Maleic acid assisted improvement of metal chelation and antioxidant metabolism confers chromium tolerance in Brassica juncea L. Ecotoxicol. Environ. Safe. 144, $216-226$.

Mahmud, J.A., Hasanuzzaman, M., Nahar, K., Bhuyan, M.H.M.B., Fujita, M., 2018. Insights into citric acid-induced cadmium tolerance and phytoremediation in Brassica juncea L.: coordinated functions of metal chelation, antioxidant defense and glyoxalase systems. Ecotoxicol. Environ. Safe. 147, 990-1001.

Min, X., Zhou, Z.H., Li, Z.G., 2016. The metabolism of signal molecule hydrogen sulfide and its role in the acquisition of heat tolerance in plants. Plant Physiol. J. 52 (1), 37-46.

Mostofa, M.G., Yoshida, N., Fujita, M., 2014. Spermidine pretreatment enhances heat tolerance in rice seedlings through modulating antioxidative and glyoxalase systems. Plant Growth Regul. $73,31-44$.

Mostofa, M.G., Hossain, M.A., Fujita, M., 2015a. Trehalose pretreatment induces salt tolerance in rice (Oryza sativa L.) seedlings: 
oxidative damage and co-induction of antioxidant defense and glyoxalase systems. Protoplasma 252, 461-475.

Mostofa, M.G., Saegusa, D., Fujita, M., Tran, L.P., 2015b. Hydrogen sulfide regulates salt tolerance in rice by maintaining $\mathrm{Na}^{+} / \mathrm{K}^{+}$ balance, mineral homeostasis and oxidative metabolism under excessive salt stress. Front. Plant Sci. 6, 1055.

Mostofa, M.G., Hossain, M.A., Fujita, M., Tran, L.S., 2015c. Physiological and biochemical mechanisms associated with trehalose-induced copper-stress tolerance in rice. Sci. Rep. 5, 11433.

Mudalkar, S., Sreeharsha, R.V., Reddy, A.R., 2017. Involvement of glyoxalases and glutathione reductase in conferring abiotic stress tolerance to Jatropha curcas L. Environ. Exp. Bot. 134, 141-150.

Nahar, K., Hasanuzzaman, M., Alam, M.M., Fujita, M., 2015a. Roles of exogenous glutathione in antioxidant defense system and methylglyoxal detoxification during salt stress in mung bean. Biol. Plant. 59, 745-756.

Nahar, K., Hasanuzzaman, M., Alam, M.M., Fujita, M., $2015 b$. Glutathione-induced drought stress tolerance in mung bean: coordinated roles of the antioxidant defense and methylglyoxal detoxification systems. AoB Plants 7, plv069.

Nahar, K., Hasanuzzaman, M., Alam, M.M., Fujita, M., 2015c. Exogenous glutathione confers high temperature stress tolerance in mung bean (Vigna radiata, L.) by modulating antioxidant defense and methylglyoxal detoxification system. Environ. Exp. Bot. 112, 44-54.

Nahar, K., Hasanuzzaman, M., Alam, M.M., Fujita, M., 2015d. Exogenous spermidine alleviates low temperature injury in mung bean (Vigna radiata L.) seedlings bymodulating ascorbateglutathione and glyoxalase pathway. Int. J. Mol. Sci. 16, 30117-30132.

Nahar, K., Hasanuzzaman, M., Rahman, A., Alam, M.M., Mahmud, J.A., Suzuki, T., et al., 2016a. Polyamines confer salt tolerance in Mung Bean (Vigna radiata L.) by reducing sodium uptake, improving nutrient homeostasis, antioxidant defense, and methylglyoxal detoxification systems. Front. Plant Sci. 7, 1104.

Nahar, K., Rahman, M., Hasanuzzaman, M., Alam, M.M., Rahman, A., Suzuki, T., et al., 2016b. Physiological and biochemical mechanisms of spermine-induced cadmium stress tolerance in mung bean (Vigna radiata L.) seedlings. Environ. Sci. Pollut. Res. 23, 21206-21218.

Nahar, K., Hasanuzzaman, M., Alam, M.M., Rahman, A., Suzuki, T., Fujita, M., 2016c. Polyamine and nitric oxide crosstalk: antagonistic effects on cadmium toxicity in mung bean plants through upregulating the metal detoxification, antioxidant defense and methylglyoxal detoxification systems. Ecotoxicol. Environ. Safe. 126, 245-255.

Nahar, K., Hasanuzzaman, M., Alam, M.M., Rahman, A., Mahmud, J.A., Suzuki, T., et al., 2017a. Insights into spermine-induced combined high temperature and drought tolerance in mung bean: osmoregulation and roles of antioxidant and glyoxalase system. Protoplasma 254, 445-460.

Nahar, K., Hasanuzzaman, M., Suzuki, T., Fujita, M., 2017b. Polyamines-induced aluminum tolerance in mung bean: a study on antioxidant defense and methylglyoxal detoxification systems. Ecotoxicology 26, 58-73.

Namdjoyan, S., Kermanian, H., Sooirk, A.A., Tabatabaei, S.M., Elyasi, N., 2017. Interactive effects of Salicylic acid and nitric oxide in alleviating zinc toxicity of Safflower (Carthamus tinctorius L.). Ecotoxicology 26, 752-761.

Neill, N., Desikan, R., Hancock, J., 2002. Hydrogen peroxide signaling. Curr. Opin. Plant Biol. 5, 388-395.

Rahman, A., Alam, M.M., Nahar, K., Hasanuzzaman, M., Fujita, M., 2015. Calcium mitigates arsenic toxicity in rice seedlings by reducing arsenic uptake and modulating the antioxidant defense and glyoxalase systems and stress markers. BioMed Res. Intern. 2015, 1-12.

Rahman, A., Mostofa, M.G., Nahar, K., Hasanuzzaman, M., Fujita, M., 2016a. Exogenous calcium alleviates cadmium-induced oxidative stress in rice (Oryza sativa L.) seedlings by regulating the antioxidant defense and glyoxalase systems. Braz. J. Bot. 39, 393-407.

Rahman, A., Hossain, M.S., Mahmud, J.A., Nahar, K., Hasanuzzaman, M., Fujita, M., 2016b. Manganese-induced salt stress tolerance in rice seedlings: regulation of ion homeostasis, antioxidant defense and glyoxalase systems. Physiol. Mol. Biol. Plants 22, 291-306.

Rahman, A., Nahar, K., Hasanuzzaman, M., Fujita, M., 2016c. Manganese-induced cadmium stress tolerance in rice seedlings: coordinated action of antioxidant defense, glyoxalase system and nutrient homeostasis. Comp. Rend. Biol. 339, 462-474.

Rahman, A., Nahar, K., Hasanuzzaman, M., Fujita, M., 2016 d. Calcium supplementation improves $\mathrm{Na}^{+} / \mathrm{K}^{+}$ratio, antioxidant defense and glyoxalase systems in salt-stressed rice seedlings. Front. Plant Sci. 7, 609.

Rajwanshi, R., Kumar, K., Yusuf, M.A., DebRoy, S., Sarin, N.B., 2016. Stress-inducible overexpression of glyoxalase I is preferable to its constitutive overexpression for abiotic stress tolerance in transgenic Brassica juncea. Mol. Breed. 36, 76.

Reddy, V.S., Sopory, S.K., 1999. Glyoxalase I from Brassica juncea: molecular cloning, regulation and its over-expression confer tolerance in transgenic tobacco under stress. Plant J. 17, 385-395.

Ruelland, E., Vaultier, M.N., Zachowski, A., Hurry, V., 2009. Cold signalling and cold acclimation in plants. Adv. Bot. Res. 49, 35-150.

Saito, R., Yamamoto, H., Makino, A., Sugimoto, T., Miyake, C., 2011. Methylglyoxal functions as Hill oxidant and stimulates the photoreduction of $\mathrm{O}_{2}$ at photosystem I: a symptom of plant diabetes. Plant Cell Environ. 34, 1454-1464.

Sankaranarayanan, S., Jamshed, M., Kumar, A., Skori, L., Scandola, S., Wang, T., et al., 2017. Glyoxalase goes green: the expanding roles of glyoxalase in plants. Int. J. Mol. Sci. 18, 898.

Shaheen, F., Shmygol, A., Rabbani, N., Thornalley, P.J., 2014. A fluorogenic assay for methylglyoxal. Biochem. Soc. Trans. 42, $548-555$.

Singh, P., Dhaka, N., 2016. Glyoxalase system and salinity stress in plants. In: Wani, S.H., Hossain, M.A. (Eds.), Managing Salt Tolerance in Plants: Molecular and Genomic Perspectives. CRC Press, New York, pp. 173-185.

Su, Y., Qadri, S.M., Wu, L., Liu, L., 2013. Methylglyoxal modulates endothelial nitric oxide synthase-associated functions in EA. hy926 endothelial cells. Cardiovas. Diabetol. 12 (134).

Turoczy, Z., Kis, P., Torok, K., Cserhati, M., Lendvai, A., Dudits, D., et al., 2011. Overproduction of a rice aldo-keto reductase increases oxidative and heat stress tolerance by malondialdehyde and methylglyoxal detoxification. Plant Mol. Biol. 75, 399-412.

Wahid, A., Gelani, S., Ashraf, M., Foolad, M.R., 2007. Heat tolerance in plants: an overview. Environ. Exp. Bot. 61, 199-223.

Wang, H., Liu, J., Wu, L., 2009. Methylglyoxal-induced mitochondrial dysfunction in vascular smooth muscle cells. Biochem. Pharmacol. 77, 1709-1716.

Wang, Y., Zhou, Z.H., Ye, X.Y., Li, Z.G., 2018. Methylglyoxal: a new signaling molecule in plants. Plant Physiol. J. 54, 10-18.

Yadav, S.K., Singla-Pareek, S.L., Ray, M., Reddy, M.K., Sopory, S.K., 2005a. Methylglyoxal levels in plants under salinity stress are dependent on glyoxalase I and glutathione. Biochem. Biophys. Res. Commun. 337, 61-67.

Yadav, S.K., Singla-Pareek, S.L., Ray, M., Reddy, M.K., Sopory, S.K., 2005b. Transgenic tobacco plants overexpressing glyoxalase 
enzymes resist an increase in methylglyoxal and maintain higher reduced glutathione levels under salinity stress. FEBS Lett. 579, 6265-6271.

Yang, C., Zhao, Y., Xian, M., Li, J., Dong, Q., Bai, H., et al., 2014. A novel controllable hydrogen sulfide-releasing molecule rrotects human skin keratinocytes against methylglyoxal-induced injury and dysfunction. Cell Physiol. Biochem. 34, 1304-1317.

Zeng, Z.M., Xiong, F.J., Yu, X.H., Gong, X.P., Luo, J.T., Jiang, Y.D., et al., 2016. Overexpression of a glyoxalase gene, OsGly I, improves abiotic stress tolerance and grain yield in rice (Oryza sativa L.). Plant Physiol. Biochem. 109, 62-71.

Zhou, Z.H., Wang, Y., Min, X., Li, Z.G., 2017. Crosstalk between hydrogen sulfide signal and other signal regulates drought tolerance of plant. Biotechnol. Bull. 33 (6), 1-9.

Zhu, J.K., 2002. Salt and drought stress signal transduction in plants. Ann. Rev. Plant Biol. 53, 247-273.

Zhu, J.K., 2016. Abiotic stress signaling and responses in plants. Cell $167,313-324$. 
This page intentionally left blank 


\title{
15
}

\section{Role of Trehalose and Regulation of its Levels as a Signal Molecule to Abiotic Stresses in Plants}

\author{
Silvia Morgutti ${ }^{1}$, Noemi Negrini ${ }^{1}$, Chiara Pucciariello ${ }^{2}$ and \\ Gian Attilio Sacchi ${ }^{1}$
}

${ }^{1}$ Department of Agricultural and Environmental Sciences-Production, Landscape, Agroenergy, University of Milan, Milan, Italy ${ }^{2}$ Institute of Life Sciences, Sant'Anna School of Advanced Studies, Pisa, Italy

\section{O U T L I N E}

15.1 Introduction

15.2 Trehalose in Plants

15.3 Trehalose as a Protectant Against Abiotic Stresses

15.4 Trehalose-6-P in Plant Metabolic and Physiological Activities

15.5 Sugar Signaling Systems in Plants

15.6 Trehalose/Trehalose-6-P/SnRK1 System
235

236

236

239

240

240
15.7 Trehalose/Trehalose-6-P/SnRK1 System in Specific Stress Responses: Hypoxia and Cold

15.7.1 Hypoxia 243

15.7.2 Cold 245

15.8 Concluding Remarks 247

References 250

Further Reading 255

and thus control the energy status is crucial for the cell survival. In plants, sugar sensing varies during life in relation to developmental and environmental factors. Several studies on the role of sugar signaling in plant development have been conducted that mostly focus on specific developmental processes (O'Hara et al., 2013). The cellular levels of the disaccharide sucrose (Suc) and the hexoses glucose (Glu) and fructose (Fru) indirectly regulate the expression of different genes involved in 
the control of plant growth and development, as well as in the responses to stresses. This chapter focuses on the nonreducing disaccharide trehalose (Tre) and its phosphorylated precursor, trehalose-6-P (T6P). For these sugars roles in signaling the cell energy status, including the response to abiotic stresses, are to date emerging.

\subsection{TREHALOSE IN PLANTS}

Tre high solubility and chemical nonreactivity allow the accumulation of this sugar at high cellular concentrations in all major groups of organisms except vertebrates without significant interference with metabolism (Figueroa and Lunn, 2016). Due to these properties, Tre has been reported to play roles as osmolyte, osmoprotectant, and C storage. In higher plants, this role was attributed to Suc, since for a long time it was supposed that in angiosperms only some resurrection plants were able to synthesize Tre that at high concentrations was thought to contribute to cell membrane stabilization in the desiccated state. Several studies demonstrated that the Tre biosynthetic pathway is instead widespread in the plant kingdom and that the intermediate T6P plays unexpected functional roles, including the regulation of plant development and growth, relationships with other organisms, and plant responses to several stresses (Fernandez et al., 2010).

Tre is formed by an $\alpha, \alpha-1,1$-glucoside bond between two $\alpha$-Glu units. In plants, Tre biosynthesis is a twostep process that occurs in the cytosol and involves the enzymes T6P synthase (TPS) and T6P phosphatase (TPP). These enzymes catalyze the condensation reaction of uridine diphosphate glucose (UDPG) and Glu6P to T6P and the subsequent dephosphorylation of T6P to Tre, respectively. Finally, Tre is hydrolyzed by trehalase (TRE) into two Glu molecules (Fig. 15.1).

In Arabidopsis, 11 TPS genes, four homolog to yeast TPS1 (AtTPS1-AtTPS4, grouped as class I) and seven homolog to yeast TPS2 (AtTPS5-AtTPS11, grouped as class II), were identified. Recently, it was demonstrated that, together with AtTPS1, other TPS isoforms have TPS activity. The successful complementation of tps1 yeast mutants with AtTPS1, AtTPS2, and AtTPS3 demonstrates the catalytic activity of these isoforms (Delorge et al., 2015). For the other proteins of the family, and in particular for those of class II, which possess both synthase and phosphatase domains but are devoid of TPS activity (John et al., 2017), the function still needs to be clarified and currently a regulatory activity as sensors for the level of T6P has been suggested (Vandesteene et al., 2010; Zang et al., 2011). Ten TPP genes encoding active enzymes when expressed in yeast or in Escherichia coli have been identified in Arabidopsis thaliana (Vandesteene et al., 2012).
Excluding the TPP catalytic phosphatase box domains, they do not show sequence homology with the related microbial genes. The presence of multiple TPP genes strongly suggests the need for a fine regulation, at different histological and/or cellular levels, of the substrate (T6P) and/or the product (Tre) of the encoded activities. The presence of active TPSs and TPPs is not limited to A. thaliana, but is widespread among all the major plant taxa including monocots (Avonce et al., 2006; Lunn, 2007; Pramanik and Imai, 2005; Shima et al., 2007; Zang et al., 2011).

Only one TRE-encoding gene has been identified in A. thaliana, as well as in other higher plants among which are rice and soybean (Frison et al., 2007; O'Hara et al., 2013; Paul et al., 2008).

The regulation of TPS and TPP activities in plants is poorly understood. TPS1 proteins possess an autoinhibitory $\mathrm{N}$-terminal domain, which regulates the catalytic activity; upon removal of that domain, the enzyme catalytic activity is increased. The region responsible for the inhibition corresponds to the $\mathrm{R} / \mathrm{L}$ rich region located at the N-terminal site of the protein, which is present in several orthologs from different plant genera and species. The autoinhibitory domain is absent in the AtTPS2-isoforms. AtTPS1 can be phosphorylated on $\mathrm{S}^{252}$ by $\mathrm{Ca}^{2+}$-dependent protein kinases (Lunn et al., 2014) even if, to our best knowledge, no report on the effect of this regulation on activity is available. In the resurrection plant Selaginella lepidophylla, SITPS1 is activated by $\mathrm{K}^{+}, \mathrm{Mg}^{2+}$, and $\mathrm{Ca}^{2+}$ but is insensitive to Tre or Suc (Valenzuela-Soto et al., 2004); nevertheless, it should be considered that SITPS1 kinetic properties may be peculiar for this species. Class II AtTPSs may also be phosphorylated by a sugar-dependent mechanism, possibly also involved in protecting them from ubiquitin (UQ)-proteasome system degradation (Lunn et al., 2014).

Other regulatory mechanisms of this pathway likely exist, possibly involved in the fine tuning of the Tre/ T6P ratio (see later, Section 15.3). Moreover, Tre levels have been suggested to regulate activity of TRE (John et al., 2017).

\subsection{TREHALOSE AS A PROTECTANT AGAINST ABIOTIC STRESSES}

Similarly to other sugars and, more in general, to compatible solutes, direct roles in tackling the effects of stressful environmental conditions (drought, salt, extreme temperatures, chemicals, flooding) have for a long time been assumed for Tre when it accumulates inside the cell. This hypothesis has been largely investigated in higher plants by experimentally modifying the cellular levels of Tre through exogenous treatments 
$\underbrace{\mathrm{O}_{\mathrm{O}}^{\mathrm{O}=\mathrm{O}^{-}}}_{\mathrm{OH}}$

G1P

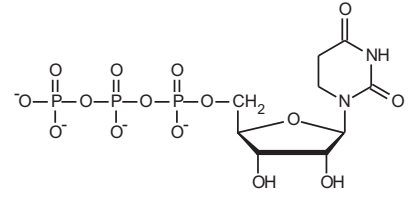

UTP
FIGURE 15.1 The trehalose (Tre) metabolic pathway in plants and its possible regulation. Red or green lines indicate negative or positive regulation, respectively. Dashed lines summarize the existence of multiple-step processes.<smiles>O=C1CCN(C2OC(COP(=O)([O-])OP(=O)([O-])OC3OC(CO)C4OC3C(O)C4O)C(O)CC2O)C(=O)N1</smiles>

UDPG

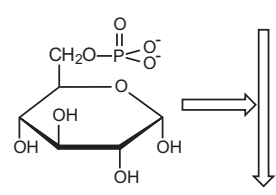

G6P

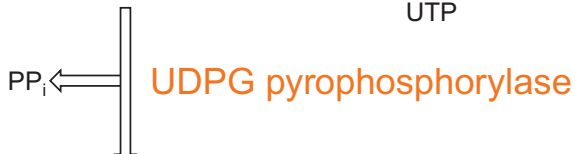<smiles>O=[PH](=O)([O-])OC1C(O)C2OC(C(O)C(CO)C(O)C2O)C1O</smiles>

(1)

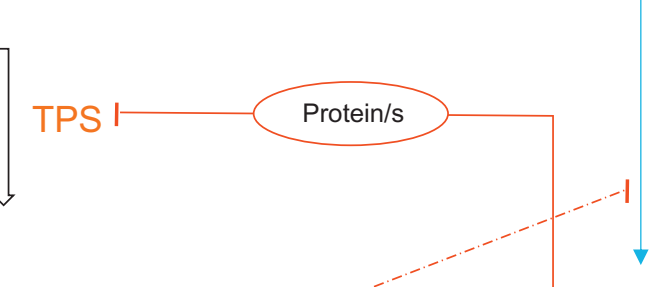

$\alpha, \alpha-1,1$-glucoside bond
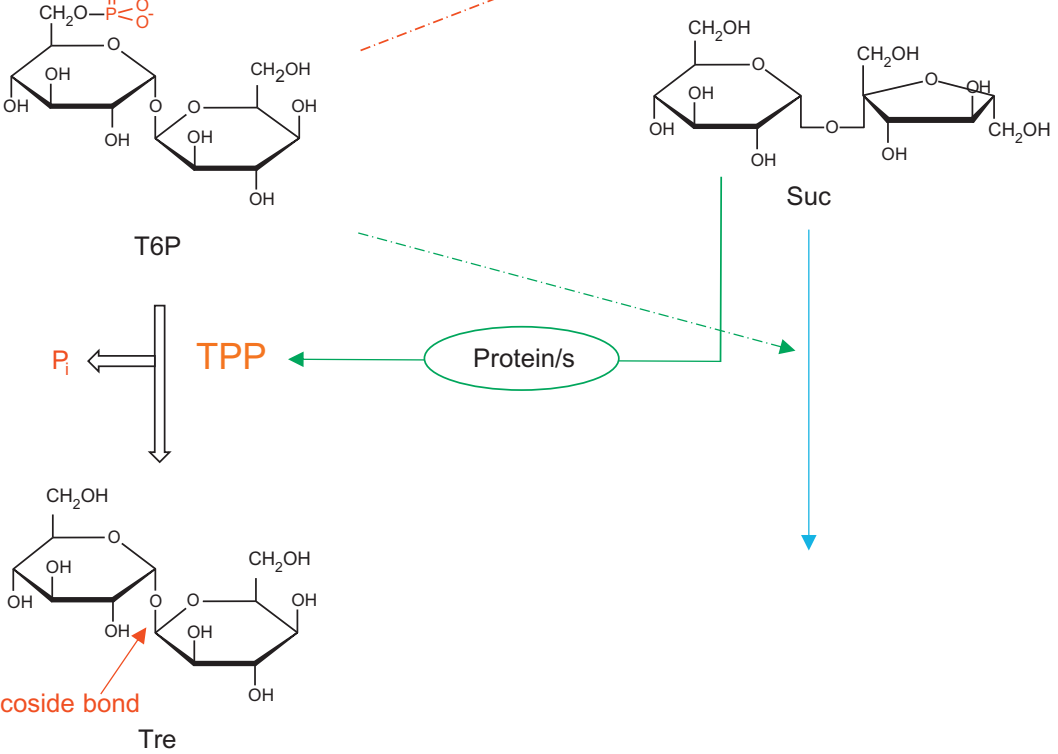

TRE

2<smiles>OCC1OC(O)C(O)C(O)C1O</smiles>

Glu

and/or manipulation of its metabolism using chemical inhibitors or transgenic approaches.

On the other hand, plants may face Tre-dependent effects upon contact with pathogens like bacteria and fungi, and larvae and adults of herbivorous insects that contain high Tre amounts. In these cases, Tre can be perceived as an elicitor of the plant response to biotic stress (Tayeh et al., 2014). To our best 
knowledge, to date specific Tre transporters have not yet been described in plant tissues (Griffiths et al., 2016). Nevertheless, in A. thaliana an ERD6 sugar transporter, homolog to the TRET1 Tre transporter of insect cells (Kikawada et al., 2007), has been identified (Schluepmann and Paul, 2009).

Some evidence has shown that Tre treatment enhances the plant's tolerance to different abiotic stresses since it directly or indirectly induces the plant antioxidant system(s), scavenging the overproduction and accumulation of reactive oxygen species (ROS) generated as a secondary stress factor by virtually all environmental primary stresses (Aldesuquy and Ghanem, 2015; Sharma et al., 2012).

Intracellular ROS levels are kept within the range of tolerability by a vast array of antioxidant enzymes and chemical compounds (Hasanuzzaman et al., 2012). Sugars (Suc, raffinose family oligosaccharides, and fructans) also contribute to abiotic stress tolerance being involved in the cellular redox status balance, although high sugar levels may correspond to both activation and decrease in specific ROS-producing pathways, and both high and low sugar levels may result in an enhancement of ROS antioxidant/signaling responses (Keunen et al., 2013). Tre has been proposed to act against the damage produced by oxygen radicals (Elbein et al., 2003), scavenging ROS in vitro (Stoyanova et al., 2011) and in vivo (Nery et al., 2008). In both monocots and dicots, the effects of Tre treatment are often accompanied by upregulation of genes encoding antioxidant enzymes and accumulation of antioxidants eventually leading to the enhancement of enzymatic and nonenzymatic antioxidant defense mechanisms (Ali and Ashraf, 2011; Nounjan et al., 2012; Sadak, 2016). Cluster analysis of expression profiling data in Tre-fed A. thaliana seedlings showed induction of genes encoding activities that suggest a link between Tre metabolism and oxidative stress responses (Bae et al., 2005a; Schluepmann et al., 2004). Nevertheless, an opposite effect of the Tre treatment on antioxidant systems has been observed concerning the expression of an APR1 gene (Bae et al., 2005a) that encodes a 5'-adenylylsulfate reductase of the plant $\mathrm{SO}_{4}{ }^{2-}$ assimilation pathway involved in the synthesis of antioxidant thiols (Leustek et al., 2000). Tre treatment induced in A. thaliana cells the expression of detoxification and stress response proteins (Bae et al., 2005b), like a cytosolic dehydroascorbate reductase1 (DHAR1) concomitant with an increase in the Tre levels in the cell. Consistently, in A. thaliana seedlings, exogenous Tre counteracted the increased ROS levels induced by salt treatment (Yang et al., 2014).

Tre ameliorates the effects of oxidative stress independently from the primary stressing cause. Indeed, in winter wheat under heat stress, low amounts of Tre supplied in the growth medium increase the internal levels of Tre and diminish electrolyte leakage (a parameter linked to membrane damage), levels of malondialdehyde (MDA), the product of lipid peroxidation, superoxide $\left(\mathrm{O}_{2}{ }^{-}\right)$, hydrogen peroxide $\left(\mathrm{H}_{2} \mathrm{O}_{2}\right)$, and lipoxygenase activity (Luo et al., 2010). In droughtaffected wheat, Tre treatment significantly increases the activities of the detoxifying enzymes ascorbate peroxidase and peroxidase, and of phenylalanine ammonia lyase, with consistent effects also on the levels of nonenzymatic antioxidant compounds (Aldesuquy and Ghanem, 2015; Ibrahim and Abdellatif, 2016). In drought-stressed maize, fenugreek, Brassica juncea, and radish seedlings, application of Tre increases compounds and enzymatic activities related to oxidative stress resistance, and decreases the levels of oxidative stress-related parameters, with positive effects also on growth, photosynthetic pigments, and proteins (Akram et al., 2016; Alam et al., 2014; Ali and Ashraf, 2011; Sadak, 2016; Shafiq et al., 2015). When stress is induced in barley by acid rain, Tre treatment limited membrane damage at the root level (reduction in MDA levels and increase in $\mathrm{H}^{+}$extrusion) and also increases catalase and peroxidase activities and general antioxidant capacity (Ding et al., 2018). Tre application is also effective in reducing oxidative stress in wheat callus, where the beneficial effect is ascribed to nonenzymatic mechanisms (Ma et al., 2013), and in salt-stressed rice (Mostofa et al., 2015a; Nounjan et al., 2012).

Heavy metals pollution can also generate ROS (Jalmi et al., 2018). In rice, Tre treatment prior to plant exposure to excessive $\mathrm{Cu}$ amounts improves ascorbic acid content, redox status, and the activities of major antioxidant enzymes (Mostofa et al., 2015b). In the aquatic plant Lemna gibba, Tre increases upon Cd exposure, and application of external Tre increases the enzymatic and nonenzymatic antioxidant activity-related parameters whereas it decreases the levels of oxidative stressrelated compounds (Duman et al., 2011).

Changes induced in Tre metabolism may also interfere with oxidative stress effects and responses. Transgenic tobacco overproducing AtTPS1 shows lesser oxidative damage when exposed to $\mathrm{Cd}$ and excess $\mathrm{Cu}$ (Martins et al., 2014). Al, which induces oxidative stress and oxidative stress-related genes in $A$. thaliana (Richards et al., 1998) and inhibits energy supply in plants, increases Tre levels and reduces TRE activity in mycorrhized roots of Pinus massioniana (Tan et al., 2005).

Cell ROS levels increase dramatically upon ultraviolet (UV) radiation exposure (Saini et al., 2018). Although the existing literature on a possible relationship between UV stress and Tre metabolism in plants is scanty, in silico expression profiling of all AtTPS and AtTPP genes revealed that AtTPS4 is specifically induced by UV-B treatment, whereas AtTPS3, AtTPPB, and AtTPPI are repressed (Iordachescu and Imai, 2008). 
ZAT10 is a zinc finger of $A$. thaliana transcription factor (TF) whose mRNA abundance is regulated by several stimuli, among which are ROS. Its constitutive expression results in the increase of different ROSresponse transcripts (Mittler et al., 2006). Arabidopsis plants overexpressing ZAT10 show enhanced tolerance to both high light- or $\mathrm{H}_{2} \mathrm{O}_{2}$-induced oxidative stress. A TPS gene has been identified among those upregulated in ZAT10-overexpressing plants (Rossel et al., 2007). It may then be hypothesized that there is a possible connection between ZAT10 expression and Tre metabolism in the framework of the responses to oxidative stress.

Photosynthesis and photorespiration are major sources of ROS (Foyer and Noctor, 2005). Exposure to excess light increases ROS generation, resulting in photooxidative damage. In vitro, Tre prevents the inactivation of isolated photosystem II (PSII) complexes probably affecting the extent of hydration, favoring optimal protein conformation and cyclic electron flow around PSII against photo- and heat-induced inactivation (Mamedov et al., 2015). In vivo, in Tre-treated A. thaliana seedlings, the levels of DHAR1 were increased (Bae et al., 2005b), increasing the possibility to protect PSII from photooxidative damage.

High temperatures (HT) induce ROS synthesis (Ruelland and Zachowski, 2010). Wheat seedlings treated with Tre prior to exposure to HT showed enhanced PSII activity and higher levels of D1 protein (Luo et al., 2018). In vitro, Tre scavenges heat stress-generated free radicals, and protects superoxide dismutase (SOD) activity from heat inactivation (Luo et al., 2008).

Most of the effects cited earlier, rather than to significant Tre accumulation, appear correlated to changes in the ratio between Tre and its precursor T6P. The exact mechanism underlying the Tre-induced increase in T6P has not yet been clarified (Yadav et al., 2014), although it has been suggested that Tre inhibits TPP activity (Delatte et al., 2011). Moreover, the protective effect should be carefully evaluated because of the presence of the apoplastic-oriented TRE that may degrade Tre (Frison et al., 2007). Tre may thus function essentially as an elicitor and induce specific signal transduction pathways (Delorge et al., 2014).

\subsection{TREHALOSE-6-P IN PLANT METABOLIC AND PHYSIOLOGICAL ACTIVITIES}

Alterations in the Tre biosynthetic pathway causing fluctuations in the level of T6P lead to a plethora of effects on plant metabolic, physiological, and developmental processes, like photosynthesis, sucrose utilization, starch metabolism, cell division, cell wall synthesis, inflorescence architecture, embryo and leaf development, seedling and adult plant biomass, and tolerance/sensitivity to different stresses (Griffiths et al., 2016; O'Hara et al., 2013; Paul et al., 2008; Ponnu et al., 2011).

T6P is a low abundance molecule whose levels are determined by the relative rates of biosynthesis (TPS) and dephosphorylation (TPP), controlled by transcriptional and posttranslational mechanisms, and related to the cell Suc status (Lunn et al., 2006; Yadav et al., 2014). A few elements are recognized toward the feasibility of T6P as a signaling molecule (Paul, 2008; Paul et al., 2008), with particular regard to its low amounts, lack of participation in major metabolic fluxes, and observation that the genes involved in its metabolic pathway converge on the regulation of T6P levels.

It is now largely accepted that T6P contents are correlated to those of Suc: T6P accumulates when Suc in the cell is high, acting as a specific signal for Suc availability. On the other hand, C starvation depletes T6P levels (Figueroa and Lunn, 2016; Nunes et al., 2013a). This conclusion is supported by evidence from several experiments: (1) in C-starved $A$. thaliana seedlings the level of T6P collapses, but when they are treated with exogenous Suc, in a very short time the level of T6P dramatically increases, more than those of other soluble sugars (Lunn et al., 2006); (2) the rapid described restoration of T6P levels is not observed when other sugars that cannot be metabolically converted to Suc are supplied as C-source (Yadav et al., 2014); (3) the diurnal phase shifts of T6P overlap with those of Suc in Arabidopsis shoots (Martins et al., 2013); (4) in P- or S-starved plants no response concerning the T6P levels is observed, whereas in the case of N-starvation fluctuations in T6P levels do occur, but they are concomitant with the fluctuations of Suc consequent to changes in the N-metabolism (Yadav et al., 2014), reinforcing the idea that T6P specifically monitors cell Cavailability.

These observations, originally obtained in the model plant $A$. thaliana, have been confirmed in other plant species including wheat, maize, tomato, and cucumber (for extensive references, see Figueroa et al., 2016).

The exact mechanism(s) by which Suc fluctuations are transduced in those of T6P still have to be completely elucidated. Any mass action effect of the T6P precursors G6P and UDPG has been experimentally excluded (Yadav et al., 2014), whereas little knowledge is to date available about the existence of direct kinetic effects of Suc on TPS and TPP activities. When Suc is supplied to C-starved plants in the presence of the transcriptional inhibitor cordycepin, the Suc-dependent T6P increase is observed, whereas it does not take place if protein synthesis is inhibited by cycloheximide, indicating dependence of the process from de novo protein biosynthesis (Yadav et al., 2014). 
The link between Suc and T6P is mediated by one or more not yet identified protein/s in turn acting stimulating the activity of TPS and/or reducing that of TPP (Fig. 15.1). Nevertheless, recent results obtained by immunoblotting experiments and polysome-loading analyses appear to exclude that the target is the TPS enzyme. Overexpression in $A$. thaliana of $E$. coli ots $A$ or ots $B$ genes encoding TPS and TPP, respectively, suggests the existence also of a feedback mechanism by which T6P is able to control, directly or indirectly, the Suc level reducing its synthesis and/or stimulating its metabolic consumption (Yadav at al., 2014; Fig. 15.1).

The magnitude of the T6P/Suc ratio values seems critical, contributing, in specific cell types and plant developmental stages, to the regulation of Suc availability (Yadav et al., 2014). In plants, developmental transitions and growth are supported by time- and organ-specific metabolic changes that reprogram the source-sink relationships. The sensing of adequate availability of $C$ sources, and the consequent trigger of signaling cascades that lead to specific metabolic changes, must be highly coordinated to ensure that the C sink is capable to sustain a developmental program (Wingler, 2018).

Adverse environmental conditions result in a decline in the cell energy levels. Stress response strategies adopted by plants include perception of unbalanced $C$ metabolism induced by the stress condition and the onset of metabolic changes aimed at restoring an adequate energy level even at the cost of a growth slowdown.

\subsection{SUGAR SIGNALING SYSTEMS IN PLANTS}

In plants, two regulatory networks related to the sugar signaling system are involved in the response to changes in nutrient and energy status (Wingler, 2018): the high-C- and the low-C-availability signaling pathways. In the former one, accumulation of hexose is sensed by hexokinase-1 (Granot et al., 2014) and the protein kinase target of rapamycin (TOR; Lastdrager et al., 2014), acting through two independent pathways (Xiong et al., 2013). Accumulation of Suc is reflected in a consequent increase in T6P levels, triggering changes in the energy metabolism ( $\mathrm{O}^{\prime}$ Hara et al., 2013). In plants the latter one is centered around the activities of (1) the Snf1-related protein kinase 1 (SnRK1), homolog of animal AMP-activated protein kinase (AMPK) and yeast sucrose nonfermenting 1 (SNF1) kinase (Wurzinger et al., 2018); (2) the C/S1 group of the bZIP (basic leucine zipper) TFs; and (3) the miRNAs dependent repression of specific genes (Wingler, 2018).
T6P represents a cross-point between the high- $\mathrm{C}$ and the low-C signaling pathways. Indeed, although the regulation of SnRK1 is complex, it is important to stress that its activity is downregulated when T6P is high, and the contrary happens when the level of T6P is low and a starvation response (energy saving program) is triggered (Lastdrager et al., 2014; Nunes et al., 2013a; Zhang et al., 2009). Despite their common function in promoting growth in response to C-availability, no direct interaction between T6P and TOR signaling has been identified (Figueroa and Lunn, 2016).

In Arabidopsis leaves T6P also acts on plastidial metabolism. It promotes the redox activation of ADPglucose pyrophosphorylase (AGPase) in response to cytosolic sugar levels enhancing starch synthesis (Kolbe et al., 2005). T6P also affects the SnRK1mediated transcriptional regulation of genes encoding enzymes involved in starch biosynthesis and degradation (Zhang et al., 2009).

As shown in A. thaliana plants expressing the E. coli T6P synthase gene (ots $A$ ), increased T6P levels may also determine a transient decrease in Suc as a consequence of posttranslational activation of nitrate reductase (NR) and phosphoenolpyruvate carboxylase. The final effect would be that of diverting photoassimilates from Suc and generate $\mathrm{C}$ skeletons and fixed $\mathrm{N}$ for amino acid synthesis (Figueroa et al., 2016).

\subsection{TREHALOSE/TREHALOSE-6-P/ SNRK1 SYSTEM}

The evolutionarily conserved SnRK1 is the plant homolog of yeast SNF1 and animal AMPK (Wurzinger et al., 2018). These kinases, altogether known as the SNF1/AMPK/SnRK1 family, regulate the cell energy homeostasis activating catabolic energy-producing pathways and inhibiting the anabolic energyconsuming ones when intrinsic or environmental factors impact with the overall cell energy availability. The mechanisms by which these orthologous enzymes sense the cell energetic status in different organisms, thus triggering the adaptive responses aimed at reestablishing the energy homeostasis, are quite different. In this section details about the plant SnRK1 system will be furnished, whereas for SNF1 and AMPK updated information can be found in dedicated reviews (Margalha et al., 2016).

The SNRK1 complex, like its orthologs, is a heterotrimeric protein consisting of one catalytic and two regulatory subunits. In the different plant species several isoforms have been identified for each subunit type, suggesting the existence of different isoformspecific functions and/or tissue/subcellular localizations (Emanuelle et al., 2016). 
The SnRK1 system has been most largely investigated in the model plant $A$. thaliana, but increasing evidence is available also for several other plant species (Margalha et al., 2016). In A thaliana two isoforms of the catalytic subunit ( $\alpha 1$ and $\alpha 2$, also known as KIN10 and KIN11 or SnRK1.1 and SnRK1.2), three ( $\beta 1, \beta 2$, and $\beta 3$ ) of the first noncatalytic subunit, and one $(\beta \gamma)$ of the second one have been identified as constituents of the SnRK1 complexes. In Arabidopsis the existence of a further gene has been reported, encoding a third type of catalytic subunit ( $\alpha 3$, KIN12 or SnRK1.3), but it is poorly expressed in most plant tissues (Margalha et al., 2016). Finally, a subunit $(\gamma)$ whose ortholog is present and active in SNF1 and AMPK complexes has been observed in plants, but it does not seem to be part of the SnRK1 complex and no functional role has been clearly demonstrated (Emanuelle et al., 2015). Different combinations of all subunits result in the presence, in A. thaliana, of six different SnRK1 isoenzymes; hormones and metabolic and environmental factors differently modulate the expression of the specific SnRK1 subunits. All the $\alpha$ subunits present an activation T-loop containing a specific $\mathrm{Thr}\left(\mathrm{T}^{175}\right.$ and $\mathrm{T}^{176}$ in $\alpha 1$ and $\alpha 2$, respectively) whose phosphorylation by three possible kinases (geminivirus kinase 1 and 2 (GRIK1 and GRIK2), and $\mathrm{Ca}^{2+}$-activated calcineurin B-like interaction protein kinase15 (CIPK15)), is decisive for activity. Dephosphorylation of $\mathrm{T}^{175 / 176}$ by two alternative upstream phosphatases, abscisic acid (ABA) insensitive1 (ABI1) and protein phosphatase 2CA (PP2CA), inactivates SnRK1 (Emanuelle et al., 2015). The function of the $\beta$ subunits is probably to act as a scaffold between the $\alpha$ and the $\beta \gamma$ subunits. Sequence analyses show that the $\beta 1$ and $\beta 2$ subunits of SnRK1 contain a carbohydrate-binding motif (CBM) that, nevertheless, does not seem to be decisive for activity; activity persists even when the complex includes the $\beta 3$ subunit that lacks CBM. Interestingly, the plant typical $\beta \gamma$ subunit harbors a CBM domain fused with the $\mathrm{N}$-terminal sequence of the $\gamma$ subunit that shares with other eukaryotes (Viana et al., 2007). This peculiarity makes $\beta \gamma$ able to complement the snf4 yeast mutant, carrying a modified CBM sequence on the $\beta$ subunit that makes it unable to bind carbohydrates (Emanuelle et al., 2015). Other than phosphorylation/dephosphorylation of $\mathrm{T}^{175 / 176}$ in the $\alpha$ subunit, several other posttranslational modifications control the activity of SnRK1, among which are ubiquitination of the different subunits, sumoylation of the $\alpha$ subunits and successive degradation by the UQ-proteasome system, and myristoylation of the $\beta 1$ and $\beta 2$ subunits with consequent changes in their subcellular localization (for a review, see Margalha et al., 2016). SnRK1 activity is regulated also by its redox status: a simulated oxidative burst leads to changes in $\alpha 1$ activity involving a $C$ residue. This seems a novel regulatory mechanism for modulation of SnRK1 activity regardless of phosphorylation state (Wurzinger et al., 2017). Differently from SNF1 and AMPK, the activity of SnRK1 is not allosterically regulated by the cellular AMP/ATP and ADP/ATP ratios, due to lack, in the $\beta \gamma$ subunit, of the cystathionine beta-synthase domain that binds the adenosyl groups (Emanuelle et al., 2015).

Several experiments have shown that sugars indirectly and/or directly finely modulate the activity of SnRK1, supporting the idea of its involvement in sugar sensing/signaling. This hypothesis has been progressively corroborated demonstrating that activation of SnRK1 upon exposure to several stresses inducing cellular energy deficiencies can be removed by addition of Glu or Suc (Baena-González and Hanson, 2017). Moreover, it is now increasingly clear that the transcript, protein, and metabolite profiles observed in plants under starvation largely overlap with those induced by the activation of the SnRK1 pathway, and are widely different from those observed in Glu- or Suc-fed plants (Cookson et al., 2016). Under lowenergy conditions, SnRK1 becomes active triggering a cascade of regulative events that repress anabolic pathways limiting developmental and growth processes. Therefore, the activation of SnRK1 regulates transcription and metabolism in response to energy deprivation and, by slowing down energy-consuming processes, allows the cell to survive $C$ shortage/stress conditions through the modulation of the activity of key enzymes in N, C, or fatty acid metabolism, and/ or massive transcriptional reprogramming (Harthill et al., 2006; Kulma et al., 2004; Mair et al., 2015; Tomé et al., 2014).

Using SnRK1 complexes extracted from A. thaliana leaves (Zhang et al., 2009), wheat grain (MartínezBarajas et al., 2011), or potato tubers (Debast et al., 2011), it has been shown that T6P, Glu1P, and Glu6P have inhibitory effects on this kinase. The Ki values of the different phosphorylated sugars are consistent with their physiological concentrations in the cell (Nunes et al., 2013a).

Microarray data showed opposite effects of T6P on SnRK1-regulated genes: genes downregulated by SnRK1 and involved in reactions of anabolic importance (amino acid, protein, and nucleotide synthesis) are upregulated by T6P, whereas genes upregulated by SnRK1 and involved in degradation processes are downregulated by T6P. This result further supports the inhibitory effect of T6P on SnRK1 in growing tissues, likely requiring a protein factor not present in mature tissues (Zhang et al., 2009). Also ribose-5-P (R5P), an intermediate of the oxidative pentose-5-P (OPP) pathway, strongly allosterically inhibits SnRK1 
in wheat grain (Piattoni et al., 2011). R5P may indicate the operativeness of the anabolic OPP pathway: therefore, the R5P inhibition of SnRK1 appears functionally consistent with the C status of the cell (Nunes et al., 2013b).

From the earlier statements it becomes clear that SnRK1 is a regulator that confers to the cell the ability to adequate and integrate, at multiple levels, the metabolism to the needs imposed by internal (developmental) processes and/or external (environmental) conditions. The effects of SnRK1 activity on the cell transcriptional profiles are realized through (1) direct regulation of TFs (Mair et al., 2015; Puranik et al., 2012); (2) SnRK1 direct binding to the chromatin; and (3) miRNAs and transcript turnover rates. Translational regulation involves ribosome biogenesis and the initiation factor of protein synthesis. Targets of posttranslational SnRK1 protein phosphorylation are key metabolic enzymes, actors of mitogen-activated protein kinase (MAPK) cascades and, interestingly, class II TPS proteins suggesting a feedback regulatory loop on the Tre metabolic pathway (Broeckx et al., 2016; Margalha et al., 2016).

The S1/C group of bZIP TFs plays a key role within the SnRK1 signaling pathway in relation to C-availability. In fact, SnRK $1 \alpha 1 /$ SnRK $1 \alpha 2$ and Suc exert opposite effects on the transcription/translation

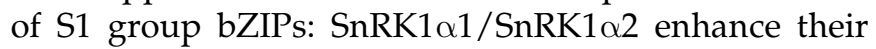
transcription, whereas Suc inhibits translation of their mRNAs (Baena-González, 2010; Baena-González et al., 2007; Kang et al., 2010; Lastdrager et al., 2014; Weltmeier et al., 2009). The specific components bZIP1, bZIP11, and bZIP53 participate to the metabolic response to low energy supply (Dietrich et al., 2011; Ma et al., 2011). Specifically, overexpression of bZIP11, while inducing growth inhibition, is positively related to the levels of TPP5/TPP6 expression and TRE activity, with diminished T6P levels (Ma et al., 2011). The Arabidopsis bZIP63 also plays a key role in regulating the starvation response (Baena-González et al., 2007). Its phosphorylation by SnRK1 is suggested to induce an altered interaction with other bZIP proteins, eventually leading to changes in gene expression (Mair et al., 2015; Nukarinen et al., 2016). Moreover, bZIP63 seems to integrate the sugar and ABA signals, since repression of its translation requires the $\mathrm{ABA}$ biosynthetic pathway, suggesting an interesting crosstalk with stress conditions (Matiolli et al., 2011). This hypothesis is supported by the observation that other bZIPs, like AREBP/ABF (ABA response element-binding protein/ factor) contain highly conserved SnRK1 target sites and are phosphorylated by SnRK1 (Broeckx et al., 2016). Some TFs belonging to NAC (NAM (nonapical meristem)-ATAF-CUC (cup-shaped cotyledon)) families can also interact with SnRK1 by regulating the expression of downstream genes in response to physiological/developmental processes and abiotic stresses (Liu et al., 2014; Pinheiro et al., 2009). In particular, the Arabidopsis ATAF1 (Arabidopsis transcription activation factor) binds the $\alpha 1$ and $\alpha 2$ catalytic subunits, possibly functioning as a component of complexes regulating the transcriptional activity (Kleinow et al., 2009). TRE1 expression is enhanced by overexpression of ATAF1, parallel to reduced T6P levels and sugar starvation metabolome (Garapati et al., 2015). ATAF1 and closely related NACs are upregulated by different stresses and plants overexpressing ATAF1 are more tolerant to drought (Wu et al., 2009). Overall, the action of bZIP/ NAC TFs appears to mimic that of starvation/stress conditions by mechanism(s) at different steps of the regulatory Tre/T6P/SnRK1 system.

Several other TFs interact with SnRK1 and are involved in different physiological processes (Broeckx et al., 2016; Margalha et al., 2016). SnRK1 has been reported to control, through protein complexes involving other TFs, the expression of specific genes. In Oryza sativa and A. thaliana, under submergenceinduced hypoxia, $\alpha 1$ enhances the expression of genes encoding the two enzymes of anaerobic fermentation, alcohol dehydrogenase1 and pyruvate decarboxylase1, through chromatin binding (Cho et al., 2012, 2016).

In Arabidopsis, SnRK1 $1 / 2$ affect the expression of more than 1000 genes, repressing the ribosomal protein genes and eventually inhibiting the translation process (Baena-González, 2010; Baena-González et al., 2007, 2008). Genes upregulated by SnRK $1 \alpha 1$ or repressed by addition of exogenous sugars seem thus involved in the switch into catabolic pathways that provide metabolic energy. Conversely, those involved in biosynthetic pathways are, with few exceptions, repressed by SnRK1 1 and starvation (BaenaGonzález, 2010; Baena-González et al., 2008).

SnRK1 appears to be involved in an antagonistic crosstalk between the Glu and the ethylene signaling pathways: in Glu-fed A. thaliana protoplasts, over-

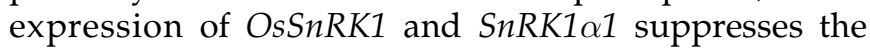
expression of the ethylene responsive factor1 (ERF1), a marker gene of the ethylene transduction pathway (Cho et al., 2012).

MicroRNAs, which posttranscriptionally control specific cell processes also involving stress responses, are involved in the SnRK1 signaling cascade. In A. thaliana, upon dark-induced starvation or SnRK1 $\alpha 1$ overexpression, the transcript levels of genes encoding miRNAs precursors decrease (Confraria et al., 2013). In wheat, the grain development-associated miR1211 has been suggested to target the regulatory subunit of SnRK1 (Meng et al., 2013).

Phosphorylation by SnRK1 may reversibly inactivate enzymes involved in biosynthetic/assimilatory 
pathways, like NR and sucrose phosphate synthase. SnRK1 also phosphorylates 6-phospho-fructo-2kinase/fructose 2,6-bisphosphatase, decreasing its activity (Nukarinen et al., 2016) and altering the levels of the important regulator Fru 2,6-bisphosphate. Phosphorylation by SnRK1 of the key glycolytic enzyme cytoplasmic pyruvate kinase (Beczner et al., 2010) seems to target the enzyme to the UQ/26S proteasome pathway for degradation (Tang et al., 2003). As a whole, these results appear to strengthen the view of a SnRK1-mediated redirection of C flux toward a starvation program (Cho et al., 2016).

Phosphoproteomic analyses in SnRK1-mutant Arabidopsis plants under extended darkness and hypoxia linked specific phosphorylation events and T6P metabolism. SnRK1 was confirmed to phosphorylate in vivo, and inactivate, the class II TPSs 5/7/8 (Cho et al., 2016; Nukarinen et al., 2016).

The T6P/SnRK1 system may impact on the levels of expression of genes including components of other signaling cascades (like $\mathrm{Ca}^{2+}$ modulators) capable to further amplify its signal (Baena-González et al., 2008). In Arabidopsis plants with increased T6P levels increased expression of a gene encoding a calmodulin-like calcium binding protein was detected, suggesting a crosstalk with $\mathrm{Ca}^{2+}$ signaling/transduction pathway(s) (Schluepmann et al., 2004). Moreover, in rice, the regulation of the SnRK1-dependent pathway by OsCIPK15 suggests a possible relationship of $\mathrm{Ca}^{2+}$-dependent regulation mechanism(s) with the energy-sensing network (Lee et al., 2009) (Fig. 15.2).

\subsection{TREHALOSE/TREHALOSE-6-P/ SNRK1 SYSTEM IN SPECIFIC STRESS RESPONSES: HYPOXIA AND COLD}

\subsubsection{Hypoxia}

Changes in $\mathrm{O}_{2}$ availability often occur during the plant life due to environmental conditions and developmental processes. Several worldwide areas are susceptible to water flooding events, when located close to rivers or exposed to heavy rains. Plants can experience low $\mathrm{O}_{2}$ conditions in organs and tissues characterized by steep $\mathrm{O}_{2}$ gradients due to low gas diffusion rate or high metabolic activity (van Dongen and Licausi, 2015).

Plants cannot survive low $\mathrm{O}_{2}$ conditions for a long period. Oxygen is required in the electron transport chain in mitochondria to produce ATP, and $\mathrm{O}_{2}$ shortage eventually leads to an energy crisis that can culminate with death (Perata and Alpi, 1993). A metabolic reconfiguration is the primary hallmark of $\mathrm{O}_{2}$ limitation. This includes the shift from aerobic respiration to degradation of carbohydrates via glycolysis and subsequent fermentation, which partially compensate the drop in ATP availability.

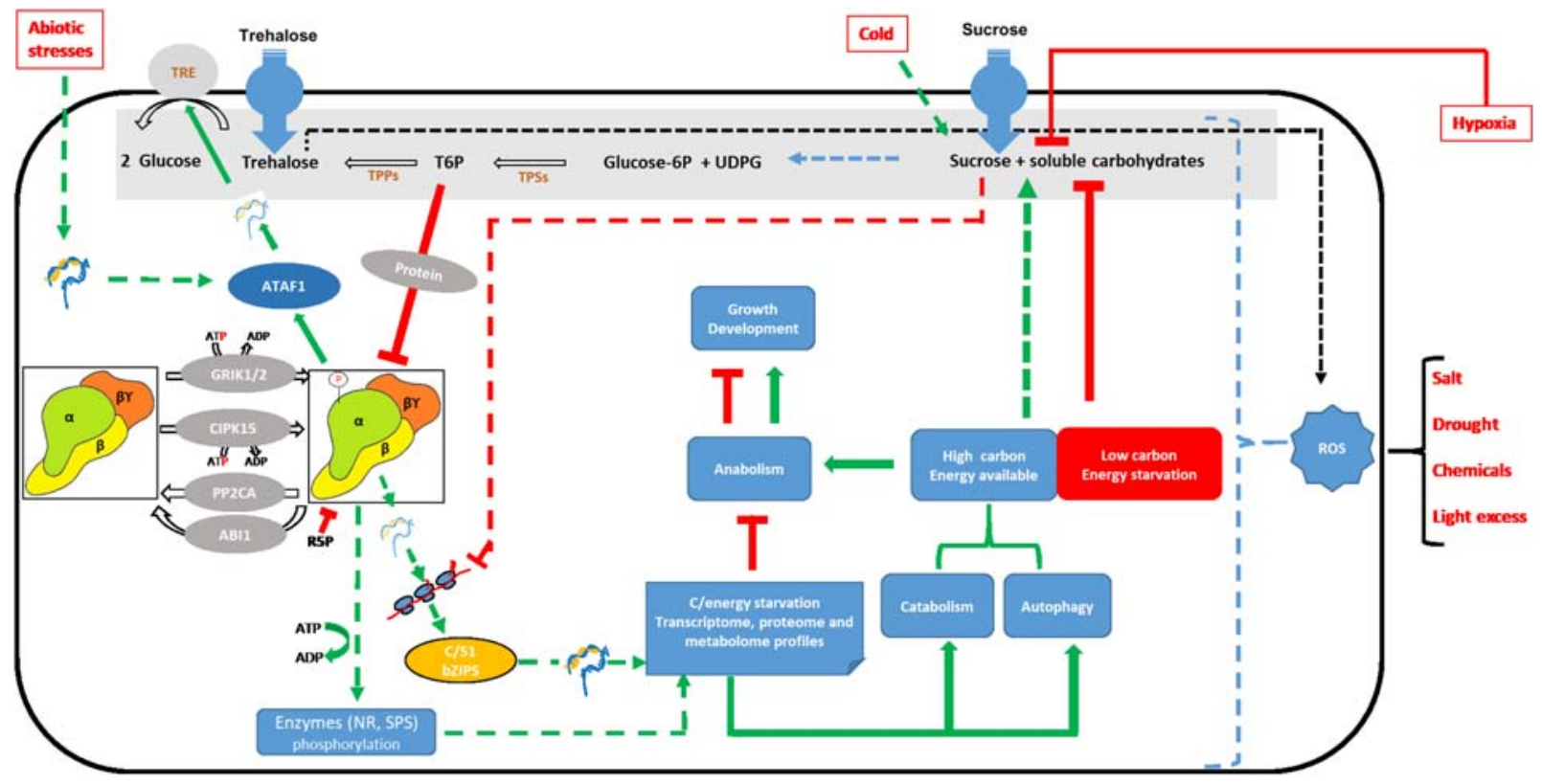

FIGURE 15.2 Proposed model of the Tre/T6P/SnRK1 system and its regulation. Red or green lines indicate negative or positive regulation, respectively. Dashed lines summarize the existence of multiple-step processes. ABI1, ABA insensitive1; CIPK15, calcineurin B-like interaction protein kinase15; GRIK, geminivirus kinase; NR, nitrate reductase; PP2CA, protein phosphatase 2CA; R5P, ribose-5-P; ROS, reactive oxygen species; SPS, sucrose phosphate synthase; TPS, T6P synthase; TPP, T6P phosphatase; TOR, target of rapamycin; Tre, trehalose; T6P, trehalose-6-P; UDPG, uridine diphosphate glucose. 
Under energy starvation, sugar mobilization for a proper reallocation of consumption is crucial, and communication between source and sink organs has a pivotal role in determining the plant survival. In this context, rice (O. sativa L.) is one of the few crops that can cope with the energetically critical low $\mathrm{O}_{2}$ condition. This semiaquatic crop has been the focus of many studies, since it is well adapted to different types of flooding and displays several mechanisms of adaptation (Singh et al., 2017).

Germination and seedling establishment are critical phases for rice survival under low $\mathrm{O}_{2}$ (Miro and Ismail, 2013). At this stage, source to sink communication through signaling has a major role in the mobilization of nutrients from the endosperm (source) to the elongating embryonic axis (sink; Yu et al., 2015). In cereal grains, starch is the major reserve in providing a C-source to generate energy and metabolites for coleoptile and radicle growth until the achievement of the photoautotrophic stage. Therefore, the regulation of $\alpha$-amylases hydrolytic enzymes gene expression and activity to degrade starch in the endosperm is of primary importance. Among major cereals, only rice harbors the $\alpha$-amylase subfamily of enzymes necessary to the endosperm starch mobilization to readily fermentable sugars under $\mathrm{O}_{2}$ deprivation (Perata et al., 1992, 1993; Guglielminetti et al., 1995). In cereal grains, SnRK1 has a key role in source to sink communication at germination and seedling developmental stage, since it converges the crosstalk among sugar and nutrient demand, and hormone and abiotic stress signal to regulate nutrient mobilization (Yu et al., 2015).

Recently, the major quantitative trait locus qAG-9-2 for rice anaerobic germination capability has been identified in the landrace Khao Hlan On (KHO; Kretzschmar et al., 2015). The genetic determinant for the low $\mathrm{O}_{2}$ tolerance was recognized to be the OsTPP7 gene, whose product converts T6P into Tre. In KHO rice, TPP7 activity likely modifies the T6P/Suc homeostasis to reduce the repressive action of T6P on SnRK1. In rice, the SnRK1 family members SnRK1A and SnRK1B are analogous to the mammalian and yeast counterparts. During anaerobic rice germination, SnRK1A acts through the sugar starvation-responsive MYB1 (MYBS1) complex to activate the expression of the starvation-induced $\alpha$-amylase Ramy $3 D$ gene and the $\alpha$-amylase activity for the endosperm hydrolysis of starch. The MYBS1 TF interacts with the key TA-box sugar responsive element located on the sugar responsive complex of the $\alpha$-amylase promoter region ( $\mathrm{Lu}$ et al., 2002, 2007). As consequence, rice varieties that do harbor the OsTPP7 gene are suggested to be more effective in activating starch hydrolysis in a fast and continuous way, therefore increasing the ability to elongate the coleoptile and establish the plantlet under $\mathrm{O}_{2}$ shortage. In this context, CIPK15 has been found to play a critical role in the SnRK1-dependent pathway under low $\mathrm{O}_{2}$. In fact, a cipk15 knockout rice mutant is impaired in anaerobic germination and has a reduced activity of $\alpha$-amylases hydrolytic enzymes, probably because of a posttranscriptional regulation of SnRK1A by CIPK15 phosphorylation (Lee et al., 2009). CIPK15 was identified as the hub of both low $\mathrm{O}_{2}$ and sugar starvation signals, where the former involves the presence of $\mathrm{a} \mathrm{Ca}^{2+}$ signal likely perceived by the $\mathrm{Ca}^{2+}$ sensing calcineurin B-like CBL4 (Ho et al., 2017) and relayed through CIPK15. Notably, MYBS1 and CIPK15 transcripts have been found to be upregulated in AG-1 rice plants expressing OsTPP7 under the control of its native promoter in the IR64 variety, which lacks the native OsTPP7 gene (Kretzschmar et al., 2015).

Only a few investigations have focused on the T6Prelated signaling in adult rice plants under submergence. In deepwater rice, the striking internode elongation under water, finalized to reach the air surface to restore aerobic respiration, is positively regulated by the ERFs SNORKEL1 and SNORKEL2 (Hattori et al., 2009). A recent transcriptomic analysis of shoots from those plants revealed that the expression of genes related to Tre biosynthesis, OsTPS12-OsTPS14 and OsTPP1 and OsTPP11 (corresponding to OsTPP7), is strongly upregulated under submergence in the deepwater cv C9285 in comparison with to the nondeepwater one Taichung 65 . This suggests that T6P and Tre accumulate in deepwater rice and are possibly involved in the adaptive mechanism (Minami et al., 2018).

Similarly, a fine regulation of $\mathrm{C}$ metabolism is a key element in the rice quiescence strategy regulated by the ERF-VII Submergence1A (Sub1A) gene under submergence (Fukao et al., 2006). Sub1A possibly acts repressing $\alpha$-amylases under low $\mathrm{O}_{2}$ to preserve $C$ for the recovery phase. A detailed transcriptome and metabolome comparison between the M202 cv and its near-isogenic Sub1A introgressed line M202(Sub1A) revealed that Sub1A presence steadily dampens CIPK15 mRNA under submergence, consistent with the observed modest increase in $\alpha$-amylases transcripts (Locke et al., 2018). However, Sub1A presence does not clearly affect the T6P content, despite the strong differences observed in the regulation of transcripts related to the T6P metabolism. The levels of T6P dramatically decline in rice shoots during submergence in both M202 and M202(Sub1A), suggesting a possible release of SnRK1 activation to signal sucrose limitation.

In rice seedlings under abiotic stresses, the SnRK1Ainteractive negative regulators SKINs dampen SnRK1A 
and MYBS1 migration to the nucleus where they activate enzyme expression for nutrient mobilization (Lin et al., 2014). Under submergence, the overexpression of SKIN1 and SKIN2 results in an arrest in shoot elongation that is recovered by a sugar supply. However, it is not yet known whether the T6P pathway is involved in this additional mechanism of SnRK1 regulation.

Under low $\mathrm{O}_{2}$ the activation of Tre metabolism is suggested to be a common mechanism among different organisms. Indeed, TPS and TPP genes have been found to be expressed upon $\mathrm{O}_{2}$ shortage in some bacteria, fungi, and plants other than rice, among which is Arabidopsis (Mustroph et al., 2010). However, in Arabidopsis little is known about the regulation of this pathway under $\mathrm{O}_{2}$ shortage. In this plant, the presence of a molecular mechanism that senses the $\mathrm{O}_{2}$ variations, and which relies on a direct sensor of $\mathrm{O}_{2}$, is suggested to be at the basis of the plant acclimation. Studies on A. thaliana showed that ERF-VII TFs are stabilized under hypoxia and can drive the transcription of hypoxia-related genes, including those involved in fermentative metabolism which can provide a certain level of ATP to preserve the plant basal metabolism (Gibbs et al., 2011; Licausi et al., 2011). Recent results showed that limited carbohydrate sources under $\mathrm{O}_{2}$ shortage dampen the induction of anaerobic genes. This likely occurs independent of the stabilization of ERF-VII but upstream of their interaction with the anaerobic genes promoters, suggesting a further mechanism of regulation (Loreti et al., 2018). However, when testing the role of the SnRK1.1 protein, the use of SnRK1.1-overexpressing plants and of a SnRK1.1 dominant negative mutant ruled out a possible involvement of a Suc sensing mechanism activated through this way (Baena-González et al., 2007).

Investigations on key components in the regulation of plants' source to sink communication via the T6P/ Suc balance under low $\mathrm{O}_{2}$ are only relatively recent. Indeed, a full understanding of the molecular mechanism(s) that regulate(s) sugar mobilization through that way may suggest key strategies for crop improvement and enhanced tolerance to flooding.

\subsubsection{Cold}

To date, in Europe rice is increasingly sown on dry soil since this water-saving practice also requires lower labor costs compared with the traditional sowing on flooded soil (Mazza et al., 2016). In these conditions, however, rice seeds and plantlets face some risks concerning, amongst others, cold stress, due to the low or drastically dropped temperatures during early sowing in the absence of the thermal buffer effect of water.
Exposure to extreme temperatures is one of the most common risks for plant growth and crop yield (Yadav, 2010). Concerning low temperatures, in the term "cold stress" are included two distinct stresses, chilling and freezing, encountered by plants species originating in temperate areas when exposed to temperatures below $20^{\circ} \mathrm{C}$ or $0^{\circ} \mathrm{C}$, respectively (Miura and Furumoto, 2013).

Plants are generally most sensitive to low temperature at the seedling and reproductive stages. However, at different developmental phases cold induces evident phenotypic symptoms of suffering like poor germination, stunted seedling establishment, growth retardation, reduced leaf expansion, chlorosis and necrosis, and pollen sterility. In a relatively short time, chilling induces alterations of membrane fluidity and integrity, and a general functional decline of transport and intracellular compartmentalization with consequent negative effects on metabolic activities. Ultrastructural changes in a wide range of cell components are observed, followed by severe effects on reduction and impairment of photosynthesis, protein synthesis, and general metabolic processes. Freezing is first of all characterized by the onset of structural injuries at the cell and tissue levels due to the early formation of ice crystals in the apoplast (Ruelland and Zachowski, 2010; Takahashi et al., 2013; Theocharis et al., 2012; Yadav, 2010).

Following the perception of low temperatures, plants react by adjusting cellular metabolism to enhance tolerance mechanisms according to acclimation programs. In some cases, whenever freezing conditions are preceded by progressive chilling periods, the acclimation processes can lead the plant to the acquisition of freezing tolerance (Miura and Furumoto, 2013; Rihan et al., 2017).

In major detail, membrane rigidification/fluidification and/or cytoskeleton depolymerization could be responsible for sensing the altered temperature and trigger signaling responses that involve altered levels of intracellular free $\mathrm{Ca}^{2+}$ and activation of phospholipase $\mathrm{D}$ and consequent phospholipid degradation (Ruelland and Zachowski, 2010). Changes of enzyme activities have an impact on energy metabolism. In particular, concerning the photosynthetic machinery, cold reduces the release of Pi and determines the accumulation of phosphorylated intermediates with consequent low regeneration of ribulose bisphosphate and overall photosynthesis inhibition (Ruelland and Zachowski, 2010). The slowdown of metabolic reactions alters the balance between photochemistry (light energy input) and metabolic reactions and growth (energy use), and of the plastoquinone redox state, 
inducing the production of ROS, whose accumulation at low temperatures is also favored by reduced activities of ROS-scavenging enzymes (Ruelland and Zachowski, 2010). Plant metabolism is thus redirected toward the synthesis of osmo- and cryoprotectant molecules like soluble sugars (Suc, raffinose, stachyose, Tre), sugar alcohols (sorbitol, inositol), and lowmolecular weight $\mathrm{N}$ compounds (proline, glycine betaine). These compounds, together with dehydrins, cold-regulated (COR) proteins, and others, contribute to stabilization of membrane components and cytoplasmic proteins, maintenance of hydrophobic interactions and ion homeostasis, and ROS scavenging. Vacuolar fructans are released by a vesicle-mediated transport into the apoplast, where they stabilize membranes and indirectly contribute to osmotic adjustment upon freezing and dehydration by the release of hexose sugars. Therefore, both symplastic and apoplastic soluble sugars contribute to membrane stabilization (Krasensky and Jonak, 2012). Cold acclimation is also accompanied by increases in the levels of antioxidant compounds and in the activity of ROS-scavenging enzymes. The increase in the proportion of unsaturated fatty acids in phospholipids and the suppression of phospholipase $\mathrm{D}$ help to maintain membrane functionality and reduce phospholipid degradation (Janská et al., 2010).

All these events are under hormonal (particularly ABA) control, and are mediated by $\mathrm{Ca}^{2+}$ and some metabolites, including sugars, that act as signals (Janská et al., 2010; Yadav, 2010; Zhang et al., 2014). The different signal transduction pathways converge toward expression of target genes encoding CORs, in turn responsible for the accumulation of regulatory and functional proteins and metabolites leading to stress tolerance. Many intermediates, like the components of the complex systems of protein kinases $\left(\mathrm{Ca}^{2+}\right.$-dependent protein kinases, MAPKs cascades), as well as different TFs, like ICEs (inducer of C-repeat binding factor (CBF) expression), CBF/DREBs (dehydration-responsive-element-binding), MYC/MYBs, are involved (Janská et al., 2010; Miura and Furumoto, 2013; Rihan et al., 2017; Theocharis et al., 2012).

In E. coli a cold shock (rapid decrease from $37^{\circ} \mathrm{C}$ to $16^{\circ} \mathrm{C}$ ) induces an increase in the levels of both ost $A$ and ostB mRNAs as well as of Tre (up to eightfold) accompanied by enhanced cell viability at the lowest temperature, suggesting that Tre metabolism is involved in chilling tolerance in this organism (Kandror et al., 2002).

Also in plants increasing evidence reports on the effects of cold stress in enhancing Tre/T6P metabolism. A metabolomic study in A. thaliana aimed at identifying metabolite temporal dynamics associated with acclimation-induced freezing tolerance indicates that the Tre levels increase by eightfold 4 days after cold exposure (Kaplan et al., 2004). In both japonica and indica rice, OsTPP1 and OsTPP2 genes have been found to be differentially expressed under chilling other than drought and salt; the OSTPP1 transcripts are induced very early $(1-2 \mathrm{~h})$ by chilling (Ge et al., 2008; Nakamura et al., 2011; Pramanik and Imai, 2005; Shima et al., 2007). The accumulation of OsTPP1 transcripts is accompanied by increases in TPP activity and Tre contents; however, the increase in Tre is transient and too low to account for a function as stress protectant, suggesting again the possible role of Tre in the regulation of metabolism, with particular regard to the C one, under cold stress (Pramanik and Imai, 2005). Moreover, OsTPP1 overexpression enhances cold tolerance and is accompanied by upregulation of OSTPS1 and of a few cold stress-induced genes, like the lowtemperature-induced protein genes Lip5 and Lip9, and the TF DREB1B (Ge et al., 2008). OsTPS1 is constitutively expressed and not upregulated upon abiotic stress. Nevertheless, its overexpression induces cold tolerance and Tre accumulation associated to upregulation of genes involved in Tre/T6P metabolism (OsTPP1, OsTPP2, and OsTRE1). Transformed lines overexpressing individually class II OsTPSs genes also show improved tolerance to cold (Li et al., 2011). As reported in Section 15.2, the role of class II TPSs is not yet completely clarified, even if it has been proposed that they modulate TPS1 activity through the formation of protein complexes (Zang et al., 2011).

In a chilling-tolerant rice CT6748-8-CA-17 cv the levels of Tre are constitutively higher than those reported in the sensitive one, INIAP12. Upon cold stress, Tre contents decrease in the tolerant cultivar that accumulates higher levels of sugars (Suc, raffinose, galactose) and shows strongly reduced levels of oxidative products (MDA and $\mathrm{H}_{2} \mathrm{O}_{2}$ ) compared with the sensitive one. In the tolerant cultivar, Tre may contribute to membrane stabilization and protection from stress-generated ROS. However, it cannot be excluded that the constitutive presence of higher Tre levels may act in advance as a priming factor in response to the stress (Morsy et al., 2007). A similar behavior was recently reported in $A$. thaliana (Nunes et al., 2013a).

In grapevine (Vitis vinifera L.), exposure to low temperature induces Tre accumulation; in parallel, the levels of expression of a VvTPP gene and of VvTRE are up- and downregulated, respectively, whereas the levels of VvTPS1 transcripts remain essentially unchanged. In this system, the increase in T6P levels 
upon cold exposure is parallel to the observed chilling-induced accumulation of Suc, reinforcing the hypothesis of a possible action of $\mathrm{T} 6 \mathrm{P}$ as a signal molecule possibly involving SnRK1 also in response to cold (Fernandez et al., 2012). A comprehensive transcriptomic profiling with meta-analysis of data to discriminate the transcriptomic changes related to general or specific cold-responses was conducted concerning the combined effects of severe cold and circadian rhythm variations in maize seedlings (Jończyk et al., 2017). Alteration of circadian regulation of gene expression appears to be one of the main targets of severe cold and the transcriptome changes largely precede the physiological response. Of particular interest is the link between the massive repression of photosynthesis-related genes during the day and photoinhibition, and the participation of Tre and stachyose in low-temperature signaling as suggested by the transcript profiles of several genes related to stachyose and Tre biosynthesis/degradation, that appear to be dramatically and specifically altered by cold.

A few studies focused on the interplay between Tre metabolism and signaling pathways activated by cold. In particular, in rice a few components (ICEs, MYBS3, NAC5) of TF systems appear to be involved in a complex crosstalk with the Tre/T6P system related to the upregulation of OSTPP1 and OsTPP2 (Nakamura et al., 2011; Song et al., 2011; Su et al., 2010). Moreover, an OsmiRNA319 gene encoding a miRNA that responds to cold stress has been individuated. Its overexpression enhances cold tolerance through the downregulation of two plant-specific TFs (OsPCF6 and OsTCP21, involved in the control of cell proliferation in growing tissues), and induces upregulation of a few cold stress-responding genes among which is OsTPP1/2 (Wang et al., 2014). This evidence suggests the possible involvement of a different mechanism in the modulation of cold stress response in relation to the Tre/T6P/SnRK1 system (Confraria et al., 2013; Tomé et al., 2014).

Chilling stress induces a condition of limited growth due to a reduced sink capacity even in the presence of high sugar availability. In Arabidopsis seedlings fed with Suc or grown under sink-limited conditions induced by low temperature, the T6P levels always correlate closely with Suc and both compounds increase under low temperature (in vitro SnRK1 activity is poorly affected by temperature). As expected, the increase in T6P levels in the sinklimited condition upregulates a few marker genes (in particular TPS5) normally downregulated by SnRK1, and downregulates the SnRK1-upregulated marker genes. Interestingly, plants with reduced T6P contents for overexpression of $E$. coli ots $B$, or overexpressing SnRK1 $\alpha 1$, when transferred from cold (characterized by high Suc levels) to warm (characterized by low Suc), recover growth less promptly than their isogenic lines, suggesting that T6P is necessary to allow growth restart when the stress origin is removed, independent of overall Suc availability. Under sinklimited conditions T6P seems not directly related to growth rate but changes in gene expression induced by T6P are related to the plant recovery of growth when stress is removed (Nunes et al., 2013a). Moreover, at low temperature the relationship between endogenous Suc levels and expression of TPS1 is not linear, suggesting that factors other than Suc may regulate TPS1 expression and T6P content under low temperature (Nunes et al., 2013c).

\subsection{CONCLUDING REMARKS}

Global climate change, which is to date exacerbating extreme events in frequency and intensity, has potentially dramatic consequences for crops, with a negative impact on food availability on a worldwide scale. Water shortage, temperature-related stress, flooding, and salinization of lands are all factors capable to jeopardize overall crop yield. In this context, studies on the plant's capability to tolerate such abiotic stresses in harsh environments and/or marginal lands, and the development of tolerant crop varieties will surely, in the long term, have positive consequences on the wellbeing of a large part of the world's population. The understanding of the basis mechanisms that regulate the energy metabolism in the context of the sink-source relationships both during plant development and in response to stress conditions is of paramount importance. The finely tuned Tre metabolic pathway is suggested to play a key role in the regulation, together with the SnRK1 system, of such plant processes. This network involves a plethora of actors at several cell/tissue/organ levels therefore appearing extremely complex during both normal and stress conditions.

Although further studies are necessary to elucidate all the steps (at the molecular, biochemical, and physiological levels) of this network, promising perspectives can be foreseen for the possibility to exploit this knowledge with the aim of obtaining crop species and cultivars with improved characteristics of stress tolerance and resistance in the field (Table 15.1). 
TABLE 15.1 Trehalose Metabolism and Abiotic Stresses

\begin{tabular}{l}
$\begin{array}{l}\text { Stress } \\
\text { type } \quad \text { Plant material }\end{array}$ \\
\hline TREATMENT WITH EXOGENOUS TREHALOSE
\end{tabular}

None Arabidopsis thaliana (L.) Heynh., Columbia ecotype (Col-0); seedling liquid culture

Treatment/condition

Response/effect

References

30 or $100 \mathrm{mM}$ Tre
supplemented in liquid
culture

Drought A. thaliana, Col-0 ecotype, seedlings and $0.5,1,5 \mathrm{mM}$ Tre protoplasts supplemented to hydroponic medium

Maize (Zea mays L., cvs Agaiti-2002 and Foliar spray (30 mM Tre) EV-1098) leaves

Wheat (Triticum aestivum L., cvs Gemmieza-7 and Sahel-1) leaves

Foliar spray $(1.5 \mathrm{mM}$ Tre)

Wheat (T. aestioum, cv Giza 168) leaves

Foliar spray (10 mM Tre

Wheat (T. aestivum, cv Zhoumai 18) callus

$50 \mathrm{mM}$ Tre

supplemented to callus growing medium

Brassica spp. (B. napus L., cv BARI Sharisha 13; B. campestris L., cv BARI Sharisha 9; B. juncea L., cv BARI Sharisha 11) seedlings and leaves

Fenugreek (Trigonella foenum graecum, cv Giza 30) leaves and seeds

Radish (Raphanus satious L., cvs Manu and 40-day) leaves

Radish ( $R$. satious, cvs Manu and 40day) roots

Salt

A. thaliana Heynh., Col-0 ecotype, seedlings and protoplasts

Rice (O. sativa, cv KDML105) seedling leaves

Rice (O. sativa, cv BR 11) leaves

Acid rain

Heavy metals

Induction of different stress response genes and proteins; repression of an APR1 gene of thiol synthesis

Enhanced SOD and peroxidase (POD) activity

Enhanced enzymatic and nonenzymatic antioxidant systems; decreased levels of stress-related compounds

Enhanced enzymatic and nonenzymatic antioxidant systems; decreased levels of stress related compounds

Increase in nonenzymatic antioxidants and APX activity

Decreased levels of stress related compounds; increased levels of nonenzymatic antioxidants and SOD levels/activity; decreased POD, CAT levels/activity

$5 \mathrm{mM}$ Tre supplemented to seedling growing medium

Enhancement of enzymatic and nonenzymatic antioxidant systems; decreased levels of stress related compounds and enzymatic activities

Foliar spray (500 $\mu \mathrm{M}$ Tre) Enhanced growth, photosynthetic pigments, antioxidant compounds and total antioxidant activity

Seed presoaking (25 mM Increased growth, chlorophyll $a$ content, Tre)

Seed presoaking and foliar spray (25 and $50 \mathrm{mM}$ Tre)

0.5, 1, $5 \mathrm{mM}$ Tre supplemented to hydroponic medium

$10 \mathrm{mM}$ Tre supplemented to hydroponic medium

$10 \mathrm{mM}$ Tre supplemented to hydroponic medium

Barley (Hordeum vulgare L. cv Gairdner) Seed soaking (5, 10, roots and leaves

15 mM Tre)

$10 \mathrm{mM}$ Tre supplemented to hydroponic medium prior to exposure to $\mathrm{Cu}$ water-use efficiency, SOD activity

Enhancement of enzymatic and nonenzymatic antioxidant systems; decreased levels of stress related compounds

Enhanced SOD and POD activity

Upregulation of genes encoding antioxidant enzymes, increased APX activity

Increased nonenzymatic and enzymatic antioxidant systems; reduced ROS accumulation,

Enhanced enzymatic and nonenzymatic antioxidant systems; decreased levels of stress related compounds; increased $\mathrm{H}^{+}$ATPase activity

Enhanced ascorbic acid content, redox status, activities of major antioxidant enzymes
Yang et al. (2014)

Bae et al. (2005a,b), Schluepmann et al. (2004)

Yang et al. (2014)

Ali and Ashraf

(2011)

Aldesuquy and Ghanem (2015)

Ibrahim and Abdellatif (2016)

Ma et al. (2013)

Alam et al. (2014)

Sadak (2016)

Akram et al. (2016) Shafiq et al. (2015)

Nounjan et al. (2012)

Mostofa et al. (2015a)

Ding et al. (2018)

Mostofa et al. (2015b) 
TABLE 15.1 (Continued)

\begin{tabular}{|c|c|c|c|c|}
\hline $\begin{array}{l}\text { Stress } \\
\text { type }\end{array}$ & Plant material & Treatment/condition & Response/effect & References \\
\hline & Duckweed (Lemna gibba L.) fronds & $\begin{array}{l}0.5,1,2,5 \mathrm{mM} \text { Tre } \\
\text { supplemented to } \\
\text { hydroponic medium } \\
\text { together with Cd }\end{array}$ & $\begin{array}{l}\text { Enhanced enzymatic and nonenzymatic } \\
\text { antioxidant systems; decreased levels of } \\
\text { stress-related compounds }\end{array}$ & Duman et al. (2011) \\
\hline Heat & Wheat (T. aestivum) seedlings & $\begin{array}{l}1.5 \mathrm{mM} \text { Tre } \\
\text { supplemented to } \\
\text { hydroponic medium }\end{array}$ & $\begin{array}{l}\text { Increased levels of enzymatic and } \\
\text { nonenzymatic antioxidant systems and } \\
\text { related genes; decreased levels of } \\
\text { oxidative stress-related compounds and } \\
\text { enzymes; increased membrane } \\
\text { functionality; enhanced PSII activity; } \\
\text { higher levels of D1 protein }\end{array}$ & $\begin{array}{l}\text { Luo et al. (2010, } \\
\text { 2018) }\end{array}$ \\
\hline
\end{tabular}

\section{EFFECT OF STRESS CONDITIONS ON TREHALOSE METABOLISM}

Hypoxia Rice (O. sativa, landrace Khao Hlan On) Submersion in water for embryos and coleoptiles

Rice (O. sativa, deepwater cv C9285; nondeepwater cv Taichung 65, ssp. japonica) shoots and leaves

Cold A. thaliana, Columbia ecotype, seedlings

A. thaliana, Col-0 ecotype, seedlings

Rice (O. sativa, ssp. japonica, cv Yukihikari) roots and shoots

Rice (O. sativa, ssp. japonica, cv Yukihikari) roots and shoots

Rice (O. sativa, cvs Nona Bokra (ssp. indica) and Nipponbare (ssp. japonica)) seedlings

Rice (O. sativa, cvs CT6748-8-CA-17, chilling-tolerant, and INIAP12, chillingsensitive) seedlings

Maize (Z. mays, spp. indentata, inbred line CM109) leaves

Grape (Vitis vinifera L., cv Chardonnay clone 7535) roots, stems and leaves from in vitro cultured plants up to 21 days

Submersion in water for up to $24 \mathrm{~h}$ of seedlings at the leaf stages $4 \mathrm{LS}$ and $6 \mathrm{LS}$

Exposure of 2-week-old plants to $4^{\circ} \mathrm{C}$ up to 8 days

Exposure of 7-day-old seedlings to $10^{\circ} \mathrm{C}$ up to $72 \mathrm{~h}$

Exposure of 7-day-old seedlings to $12^{\circ} \mathrm{C}$ and $4^{\circ} \mathrm{C}$ up to $48 \mathrm{~h}$

Exposure of 7-day-old seedlings to $12^{\circ} \mathrm{C}$ up to $48 \mathrm{~h}$

Exposure of 2-week-old seedlings to $8-6^{\circ} \mathrm{C}$ up to $72 \mathrm{~h}$ or to $4^{\circ} \mathrm{C}$ up to $24 \mathrm{~h}$

Exposure of S3 (prophyll emergence stage) plantlets to $13-10^{\circ} \mathrm{C}$ for 4 days

Exposure of V3 (fully developed third leaf) stage plantlets to $8-6^{\circ} \mathrm{C}$ up to $24 \mathrm{~h}$

Exposure of 6-week-old plants to $4^{\circ} \mathrm{C}$ up to $24 \mathrm{~h}$
Activated starch hydrolysis, modified T6P/Suc homeostasis

Increased expression of OsTPS12-

OsTPS14 and OsTPP1 and OsTPP11 in

deepwater rice

Increased Tre levels

Kretzschmar et al. (2015)

Minami et al. (2018)

Kaplan et al. (2004)

Increased T6P levels and enhanced TPS5 Nunes et al. (2013a) expression

Early induction of OsTPP1 transcription; enhanced TPP activity and increased Tre levels

Early induction of OsTPP1 and OsTPP2 transcription

Early induction of OsTPP1 transcription

Constitutive presence of higher Tre

levels and decreased levels of oxidative stress-related compounds in the chillingtolerant $\mathrm{cv}$

Increased levels of TPS, TPP, and TRE transcripts

Jończyk et al. (2017)

Enhanced Tre accumulation and expression of VvTPPA gene; reduced expression of $V v T R E$

Pramanik and Imai (2005)

Shima et al. (2007)

Ge et al. (2008), Nakamura et al. (2011)

Morsy et al. (2007)

\author{
Morsy et al. (2007)
}

BIOTECHNOLOGICAL INTERVENTIONS ON TREHALOSE METABOLISM IN RELATION TO THE RESPONSE TO STRESS CONDITIONS

Hypoxia OsTPP7-overexpressing rice (O. sativa, cv IR64) seedlings

Cold OsTPP1-overexpressing rice (O. sativa, cv Nipponbare) seedlings, shoots
Submersion in water for up to 21 days

Exposure of 2-week-old seedlings to $8-6^{\circ} \mathrm{C}$ for 18 days
Upregulation of hallmark genes (MYBS1 and CIPK15) for anaerobic germination in plants expressing OsTPP7

(2015)

Enhanced cold tolerance; upregulation

Ge et al. (2008)

of OsTPS1 and of cold stress-induced genes (Lip5, Lip9, DREB1B TF) 
TABLE 15.1 (Continued)

\begin{tabular}{|c|c|c|c|c|}
\hline $\begin{array}{l}\text { Stress } \\
\text { type }\end{array}$ & Plant material & Treatment/condition & Response/effect & References \\
\hline & $\begin{array}{l}\text { Class I OsTPS1- and class II OsTPSs- } \\
\text { overexpressing rice (O. sativa, cvs } \\
\text { Nipponbare and ZH11) seedlings, } \\
\text { shoots }\end{array}$ & $\begin{array}{l}\text { Exposure of 2-week-old } \\
\text { seedlings to } 4^{\circ} \mathrm{C} \text { for } 5 \\
\text { days }\end{array}$ & $\begin{array}{l}\text { Increased Tre levels, upregulation of } \\
\text { OsTPP1, OsTPP2, OsTRE1; enhanced } \\
\text { cold tolerance }\end{array}$ & Li et al. (2011) \\
\hline & $\begin{array}{l}\text { OsNAC5-overexpressing rice (O. sativa, } \\
\text { ssp. japonica, cv Zhonghua 10) seedlings }\end{array}$ & $\begin{array}{l}\text { Exposure of } 4 \text {-week-old } \\
\text { seedlings to } 4{ }^{\circ} \mathrm{C} \text { for } 6 \\
\text { days }\end{array}$ & $\begin{array}{l}\text { Enhanced expression of OsTPP1 and } \\
\text { OsTPP2 }\end{array}$ & Song et al. (2011) \\
\hline & $\begin{array}{l}\text { MYBS3-overexpressing rice (O. sativa, } \mathrm{cv} \\
\text { Tainung 67) seedlings }\end{array}$ & $\begin{array}{l}\text { Exposure of } 10 \text {-day-old } \\
\text { seedlings to } 4^{\circ} \mathrm{C} \text { for } 24 \mathrm{~h}\end{array}$ & $\begin{array}{l}\text { Enhanced expression of OsTPP1 and } \\
\text { OsTPP2 }\end{array}$ & Su et al. (2010) \\
\hline & $\begin{array}{l}\text { Osa-miR319b-overexpressing rice ( } O \text {. } \\
\text { sativa, cv Kong Yu 131) seedlings }\end{array}$ & $\begin{array}{l}\text { Exposure of V3 (fully } \\
\text { developed third leaf) } \\
\text { stage seedlings to } 4^{\circ} \mathrm{C} \text { for } \\
7 \text { days }\end{array}$ & $\begin{array}{l}\text { Enhanced expression of OsTPP1 and } \\
\text { OsTPP2 }\end{array}$ & Wang et al. (2014) \\
\hline Drought & $\begin{array}{l}\text { ATAF1-overexpressing } A \text {. thaliana (Col-0 } \\
\text { ecotype) seedlings }\end{array}$ & $\begin{array}{l}\text { Exposure of 17-day-old } \\
\text { seedlings to irrigation } \\
\text { interruption up to } \\
\text { symptom appearance }\end{array}$ & $\begin{array}{l}\text { Increased drought tolerance; enhanced } \\
\text { TRE1 expression and TRE activity; } \\
\text { reduced T6P levels and sugar starvation } \\
\text { metabolome. }\end{array}$ & $\begin{array}{l}\text { Garapati et al. } \\
\text { (2015), Wu et al. } \\
\text { (2009) }\end{array}$ \\
\hline $\begin{array}{l}\text { Heavy } \\
\text { metals }\end{array}$ & $\begin{array}{l}\text { AtTPS1-overexpressing tobacco } \\
\text { (Nicotiana tabacum L., cv Petite Havana } \\
\text { SR1) plants, leaves }\end{array}$ & $\begin{array}{l}\text { Exposure to Cd/excess } \\
\mathrm{Cu}\end{array}$ & $\begin{array}{l}\text { Decreased levels of oxidative stress- } \\
\text { related compounds; increased activities } \\
\text { of detoxifying enzymes }\end{array}$ & Martins et al. (2014) \\
\hline
\end{tabular}

\section{References}

Akram, N.A., Waseem, M., Ameen, R., Ashraf, M., 2016. Trehalose pretreatment induces drought tolerance in radish (Raphanus sativus L.) plants: some key physio-biochemical traits. Acta Physiol. Plant. 38, 3. Available from: https://doi.org/10.1007/s11738-0152018-1.

Alam, M.M., Nahar, K., Hasanuzzaman, M., Fujita, M., 2014. Trehalose-induced drought stress tolerance: a comparative study among different Brassica species. Plant Omics J. 7, 271-283.

Aldesuquy, H., Ghanem, H., 2015. Exogenous salicylic acid and trehalose ameliorate short term drought stress in wheat cultivars by up-regulating membrane characteristics and antioxidant defense system. J. Hortic. 2, 2-10. Available from: https://doi.org/ 10.4172/2376-0354.1000139.

Ali, Q., Ashraf, M., 2011. Induction of drought tolerance in maize (Zea mays L.) due to exogenous application of trehalose: growth, photosynthesis, water relations and oxidative defence mechanism. J. Agron. Crop Sci. 197, 258-271. Available from: https:// doi.org/10.1111/j.1439-037X.2010.00463.x.

Avonce, N., Mendoza-Vargas, A., Morett, E., Iturriaga, G., 2006. Insights on the evolution of trehalose biosynthesis. BMC Evol. Biol. 6, 109. Available from: https://doi.org/10.1186/1471-2148-6109.

Bae, H., Herman, E., Bailey, B., Bae, H.-J., Sicher, R., 2005a. Exogenous trehalose alters Arabidopsis transcripts involved in cell wall modification, abiotic stress, nitrogen metabolism, and plant defense. Physiol. Plant. 125, 114-126. Available from: https:// doi.org/10.1111/j.1399-3054.2005.00537.x.

Bae, H., Herman, E., Sicher, R., 2005b. Exogenous trehalose promotes non-structural carbohydrate accumulation and induces chemical detoxification and stress response proteins in Arabidopsis thaliana grown in liquid culture. Plant Sci. 168, 1293-1301. Available from: https://doi.org/10.1016/j.plantsci.2005.01.006.
Baena-González, E., 2010. Energy signaling in the regulation of gene expression during stress. Mol. Plant 3, 300-313. Available from: https://doi.org/10.1093/mp/ssp113.

Baena-González, E., Hanson, J., 2017. Shaping plant development through the SnRK1-TOR metabolic regulators. Curr. Opin. Plant Biol. 35, 152-157. Available from: https://doi.org/10.1016/j. pbi.2016.12.004.

Baena-González, E., Rolland, F., Sheen, J., 2008. KIN10/11 are master regulators of the convergent stress transcriptome. In: Allen, J.F., Gantt, E., Golbeck, J.H., Osmond, B. (Eds.), Photosynthesis. Energy from the Sun. Proceedings of the 14th International Congress on Photosynthesis. 22-27 July 2007. Springer, Dordrecht, Glasgow, Scotland, UK, pp. 1331-1337.

Baena-González, E., Rolland, F., Thevelein, J.M., Sheen, J., 2007. A central integrator of transcription networks in plant stress and energy signalling. Nature 448, 938-943. Available from: https:// doi.org/10.1038/nature06069.

Beczner, F., Dancs, G., Sós-Hegedűs, A., Antal, F., Bánfalvi, Z., 2010. Interaction between SNF1-related kinases and a cytosolic pyruvate kinase of potato. J. Plant Physiol. 167, 1046-1051. Available from: https:/ / doi.org/10.1016/j.jplph.2010.03.010.

Broeckx, T., Hulsmans, S., Rolland, F., 2016. The plant energy sensor: evolutionary conservation and divergence of SnRK1 structure, regulation, and function. J. Exp. Bot. 67, 6215-6252. Available from: https://doi.org/10.1093/jxb/erw416.

Cho, H.Y., Wen, T.N., Wang, Y.T., Shih, M.C., 2016. Quantitative phosphoproteomics of protein kinase SnRK1 regulated protein phosphorylation in Arabidopsis under submergence. J. Exp. Bot. 67, 2745-2760. Available from: https://doi.org/10.1093/jxb/ erw107.

Cho, Y.H., Hong, J.W., Kim, E.C., Yoo, S.D., 2012. Regulatory functions of SnRK1 in stress-responsive gene expression and in plant growth and development. Plant Physiol. 158, 1955-1964. Available from: https://doi.org/10.1104/pp.111.189829. 
Confraria, A., Martinho, C., Elias, A., Rubio-Somoza, I., BaenaGonzalez, E., 2013. miRNAs mediate SnRK1-dependent energy signaling in Arabidopsis. Front. Plant Sci. 4, 197. Available from: https:/ /doi.org/10.3389/fpls.2013.00197.

Cookson, S.J., Yadav, U.P., Klie, S., Morcuende, R., Usadel, B., Lunn, J.E., et al., 2016. Temporal kinetics of the transcriptional response to carbon depletion and sucrose readdition in Arabidopsis seedlings. Plant Cell Env. 39, 768-786. Available from: https://doi. org /10.1111/pce.12642.

Debast, S., Nunes-Nesi, A., Hajirezaei, M.R., Hofmann, J., Sonnewald, U., Fernie, A.R., et al., 2011. Altering trehalose-6phosphate content in transgenic potato tubers affects tuber growth and alters responsiveness to hormones during sprouting. Plant Physiol. 156, 1754-1771. Available from: https://doi.org/ 10.1104/pp.111.179903.

Delatte, T.L., Sedijani, P., Kondou, Y., Matsui, M., de Jong, G.J., Somsen, G.W., et al., 2011. Growth arrest by trehalose-6-phosphate: an astonishing case of primary metabolite control over growth by way of the SnRK1 signaling pathway. Plant Physiol. 157, 160-174. Available from: https://doi.org/10.1104/ pp.111.180422.

Delorge, I., Janiak, M., Carpentier, S., Van Dijck, P., 2014. Fine tuning of trehalose biosynthesis and hydrolysis as novel tools for the generation of abiotic stress tolerant plants. Front. Plant Sci. 5, 147. Available from: https://doi.org/10.3389/fpls.2014.00147.

Delorge, I., Figueroa, C.M., Feil, R., Lunn, J.E., Van Dijck, P., 2015. Trehalose-6-phosphate synthase 1 is not the only active TPS in Arabidopsis thaliana. Biochem. J. 466, 283-290. Available from: https://doi.org/10.1042/BJ20141322.

Dietrich, K., Weltmeier, F., Ehlert, A., Weiste, C., Stahl, M., Harter, K., et al., 2011. Heterodimers of the Arabidopsis transcription factors bZIP1 and bZIP53 reprogram amino acid metabolism during low energy stress. Plant Cell 23, 381-395. Available from: https://doi.org/10.1105/tpc.110.075390.

Ding, F., Wang, R., Wang, T., 2018. Enhancement of germination, seedling growth, and oxidative metabolism of barley under simulated acid rain stress by exogenous trehalose. Crop Sci. 58, 783-791. Available from: https://doi.org/10.2135/ cropsci2017.08.0491.

Duman, F., Aksoy, A., Aydin, Z., Temizgul, R., 2011. Effects of exogenous glycinebetaine and trehalose on cadmium accumulation and biological responses of an aquatic plant (Lemna gibba L.). Water Air Soil Pollut. 217, 545-556. Available from: https://doi. org/10.1007/s11270-010-0608-5.

Elbein, A.D., Pan, Y.T., Pastuszak, I., Carrol, D., 2003. New insights on trehalose: a multifunctional molecule. Glycobiology 13, 17R-27R. Available from: https://doi.org/10.1093/glycob/ cwg047.

Emanuelle, S., Hossain, M.I., Moller, I.E., Pedersen, H.L., van de Meene, A.M., Doblin, M.S., et al., 2015. SnRK1 from Arabidopsis thaliana is an atypical AMPK. Plant J. 82, 183-192. Available from: https://doi.org/10.1111/tpj.12813.

Emanuelle, S., Doblin, M.S., Stapleton, D.I., Bacic, A., Gooley, P.R., 2016. Molecular insights into the enigmatic metabolic regulator, SnRK1. Trends Plant Sci. 21, 341-353. Available from: https:// doi.org/10.1016/j.tplants.2015.11.001.

Fernandez, O., Béthencourt, L., Quero, A., Sangwan, R.S., Clément, C., 2010. Trehalose and plant stress responses: friend or foe? Trends Plant Sci. 15, 409-417. Available from: https://doi.org/ 10.1016/j.tplants.2010.04.004.

Fernandez, O., Vandesteene, L., Feil, R., Baillieul, F., Lunn, J.E., Clément, C., 2012. Trehalose metabolism is activated upon chilling in grapevine and might participate in Burkholderia phytofirmans induced chilling tolerance. Planta 236, 355-369. Available from: https://doi.org/10.1007/s00425-012-1611-4.
Figueroa, C.M., Feil, R., Ishihara, H., Watanabe, M., Kölling, K., Krause, U., et al., 2016. Trehalose 6-phosphate coordinates organic and amino acid metabolism with carbon availability. Plant J. 85, 410-423. Available from: https://doi.org/10.1111/ tpj.13114.

Figueroa, C.M., Lunn, J.E., 2016. A tale of two sugars: trehalose 6phosphate and sucrose. Plant Physiol. 172, 7-27. Available from: https:// doi.org/10.1104/pp.16.00417.

Foyer, C., Noctor, G., 2005. Oxidant and antioxidant signalling in plants: a re-evaluation of the concept of oxidative stress in a physiological context. Plant Cell Environ. 28, 1056-1071.

Frison, M., Parrou, J.L., Guillaumot, D., Masquelier, D., François, J., Chaumont, F., et al., 2007. The Arabidopsis thaliana trehalase is a plasma membrane-bound enzyme with extracellular activity. FEBS Lett. 581, 4010-4016. Available from: https://doi.org/ 10.1016/j.febslet.2007.07.036.

Fukao, T., Xu, K., Ronald, P.C., Bailey-Serres, J., 2006. A variable cluster of ethylene response factor-like genes regulates metabolic and developmental acclimation responses to submergence in rice. Plant Cell 18, 2021-2034. Available from: https://doi.org/ 10.1105 /tpc.106.043000.

Garapati, P., Feil, R., Lunn, J.E., Van Dijck, P., Balazadeh, S., MuellerRoeber, B., 2015. Transcription factor Arabidopsis activating factor1 integrates carbon starvation responses with trehalose metabolism. Plant Physiol. 169, 379-390. Available from: https://doi.org/ $10.1104 /$ pp.15.00917.

Ge, L.F., Chao, D.Y., Shi, M., Zhu, M.Z., Gao, J.P., Lin, H.X., 2008. Overexpression of the trehalose-6-phosphate phosphatase gene OsTPP1 confers stress tolerance in rice and results in the activation of stress responsive genes. Planta 228, 191-201. Available from: https:/ / doi.org/10.1007/s00425-008-0729-x.

Gibbs, D.J., Lee, S.C., Isa, N.M., Gramuglia, S., Fukao, T., Bassel, G.W., et al., 2011. Homeostatic response to hypoxia is regulated by the N-end rule pathway in plants. Nature 479, 415-418. Available from: https:// doi.org/10.1038/nature10534.

Granot, D., Kelly, G., Stein, O., David-Schwartz, R., 2014. Substantial roles of hexokinase and fructokinase in the effects of sugars on plant physiology and development. J. Exp. Bot. 65, 809-819. Available from: https:// doi.org/10.1093/jxb/ert400.

Griffiths, C.A., Paul, M.J., Foyer, C.H., 2016. Metabolite transport and associated sugar signalling systems underpinning source/sink interactions. Biochim. Biophys. Acta-Bioenergetics 1857, 1715-1725. Available from: https://doi.org/10.1016/j. bbabio.2016.07.007.

Guglielminetti, L., Yamaguchi, J., Perata, P., Alpi, A., 1995. Amylolytic activities in cereal seeds under aerobic and anaerobic conditions. Plant Physiol. 109, 1069-1076. Available from: https:/ / doi.org/10.1104/pp.109.3.1069.

Harthill, J., Meek, S.E., Morrice, N., Peggie, M.W., Borch, J., Wong, B.H.C., et al., 2006. Phosphorylation and 14-3-3 binding of Arabidopsis trehalose-phosphate synthase 5 in response to 2deoxyglucose. Plant J. 47, 211-223. Available from: https://doi. org/10.1111/j.1365-313X.2006.02780.x.

Hasanuzzaman, M., Hossain, M.A., da Silva, J.A.T., Fujita, M., 2012. Plant response and tolerance to abiotic oxidative stress: antioxidant defense is a key factor. In: Venkateswarlu, B., Shanker, A.K., Shanker, C., Mahewari, M. (Eds.), Crop Stress and Its Management: Perspectives and Strategies. Springer, Dordrecht, pp. 261-315. Available from: https://doi.org/10.1007/978-94007-2220-0_8.

Hattori, Y., Nagai, K., Furukawa, S., Song, X.J., Kawano, R., Sakakibara, H., et al., 2009. The ethylene response factors SNORKEL1 and SNORKEL2 allow rice to adapt to deep water. Nature 460, 1026-1030. Available from: https:/ / doi.org/10.1038/ nature08258. 
Ho, V.T., Tran, A.N., Cardarelli, F., Perata, P., Pucciariello, C., 2017. A calcineurin B-like protein participates in low oxygen signalling in rice. Funct. Plant Biol. 44, 917-928. Available from: https:// doi.org/10.1071/FP16376.

Ibrahim, H.A., Abdellatif, Y.M., 2016. Effect of maltose and trehalose on growth, yield and some biochemical components of wheat plant under water stress. Ann. Agric. Sci. 61, 267-274. Available from: https://doi.org/10.1016/j.aoas.2016.05.002.

Iordachescu, M., Imai, R., 2008. Trehalose biosynthesis in response to abiotic stresses. J. Integr. Plant Biol. 50, 1223-1229. Available from: https:/ / doi.org/10.1111/j.1744-7909.2008.00736.x.

Jalmi, S.K., Bhagat, P.K., Verma, D., Noryang, S., Tayyeba, S., Singh, K., et al., 2018. Traversing the links between heavy metal stress and plant signaling. Front. Plant Sci. 9, 12. Available from: https: / / doi.org/10.3389/ fpls.2018.00012.

Janská, A., Maršík, P., Zelenková, S., Ovesná, J., 2010. Cold stress and acclimation-what is important for metabolic adjustment? Plant Biol. 12, 395-405. Available from: https://doi.org/10.1111/ j.1438-8677.2009.00299.x.

John, R., Raja, V., Ahmad, M., Jan, N., Majeed, U., Ahmad, S., et al., 2017. Trehalose: metabolism and role in stress signaling in plants. In: Sarwat, M., et al., (Eds.), Stress Signaling in Plants: Genomics and Proteomics Perspective, Volume 2. Springer, Cham, pp. 261-275. Available from: https://doi.org/10.1007/978-3-31942183-4_11.

Jończyk, M., Sobkowiak, A., Trzcinska-Danielewicz, J., Skoneczny, M., Solecka, D., Fronk, J., et al., 2017. Global analysis of gene expression in maize leaves treated with low temperature. II. Combined effect of severe cold $\left(8^{\circ} \mathrm{C}\right)$ and circadian rhythm. Plant Mol. Biol. 95, 279-302. Available from: https://doi.org/10.1007/ s11103-017-0651-3.

Kandror, O., DeLeon, A., Goldberg, A.L., 2002. Trehalose synthesis is induced upon exposure of Escherichia coli to cold and is essential for viability at low temperatures. Proc. Natl. Acad. Sci. U.S.A. 99, 9727-9732. Available from: https://doi.org/10.1073/ pnas.142314099.

Kang, S.G., Price, J., Lin, P.C., Hong, J.C., Jang, J.C., 2010. The Arabidopsis bZIP1 transcription factor is involved in sugar signaling, protein networking, and DNA binding. Mol. Plant. 3, 361-373. Available from: https://doi.org/10.1093/mp/ssp115.

Kaplan, F., Kopka, J., Haskell, D.W., Zhao, W., Schiller, K.C., Gatzke, N., et al., 2004. Exploring the temperature-stress metabolome of Arabidopsis. Plant Physiol. 136, 4159-4168. Available from: https://doi.org/10.1104/pp.104.052142.

Keunen, E., Peshev, D., Vangronsveld, J., van Den Ende, W., Cuypers, A., 2013. Plant sugars are crucial players in the oxidative challenge during abiotic stress: extending the traditional concept. Plant Cell Environ. 36, 1242-1255. Available from: https:// doi.org/10.1111/pce.12061.

Kikawada, T., Saito, A., Kanamori, Y., Nakahara, Y., Iwata, K.-I., Tanaka, D., et al., 2007. Trehalose transporter 1, a facilitated and high-capacity trehalose transporter, allows exogenous trehalose uptake into cells. Proc. Natl. Acad. Sci. U.S.A. 104, 11587-11590. Available from: https://doi.org/10.1073/pnas.0702538104.

Kleinow, T., Himbert, S., Krenz, B., Jeske, H., Koncz, C., 2009. NAC domain transcription factor ATAF1 interacts with SNF1-related kinases and silencing of its subfamily causes severe developmental defects in Arabidopsis. Plant Sci. 177, 360-370. Available from: https: / / doi.org/10.1016/j.plantsci.2009.06.011.

Kolbe, A., Tiessen, A., Schluepmann, H., Paul, M., Ulrich, S., Geigenberger, P., 2005. Trehalose 6-phosphate regulates starch synthesis via posttranslational redox activation of ADP-glucose pyrophosphorylase. Proc. Natl. Acad. Sci. U.S.A. 102, 11118-11123. Available from: https://doi.org/10.1073/ pnas.0503410102.
Krasensky, J., Jonak, C., 2012. Drought, salt, and temperature stressinduced metabolic rearrangements and regulatory networks. J. Exp. Bot. 63, 1593-1608. Available from: https://doi.org/ 10.1093/jxb/err460.

Kretzschmar, T., Pelayo, M.A.F., Trijatmiko, K.R., Gabunada, L.F.M., Alam, R., Jimenez, R., et al., 2015. A trehalose-6-phosphate phosphatase enhances anaerobic germination tolerance in rice. Nature Plants 1, 15124. Available from: https://doi.org/10.1038/ NPLANTS.2015.124.

Kulma, A., Villadsen, D., Campbell, D.G., Meek, S.E.M., Harthill, J.E., Nielsen, T.H., et al., 2004. Phosphorylation and 14-3-3 binding of Arabidopsis 6-phosphofructo-2-kinase/fructose-2,6-bisphosphatase. Plant J. 37, 654-667. Available from: https://doi.org/ $10.1111 /$ j.1365-313X.

Lastdrager, J., Hanson, J., Smeekens, S., 2014. Sugar signals and the control of plant growth and development. J. Exp. Bot. 65, 799-807. Available from: https://doi.org/10.1093/jxb/ert474.

Lee, K.W., Chen, P.W., Lu, C.A., Chen, S., Ho, T.H., Yu, S.M., 2009. Coordinated responses to oxygen and sugar deficiency allow rice seedlings to tolerate flooding. Sci. Signal. 2, ra61. Available from: https:/ / doi.org/10.1126/scisignal.2000333.

Leustek, T., Martin, M.N., Bick, J.A., Davies, J.P., 2000. Pathways and regulation of sulfur metabolism revealed through molecular and genetic studies. Ann. Rev. Plant Physiol. Plant Mol. Biol. 51, 141-165. Available from: https://doi.org/10.1146/annurev. arplant.51.1.141.

Li, H.W., Zang, B.S., Deng, X.W., Wang, X.P., 2011. Overexpression of the trehalose-6-phosphate synthase gene OsTPS1 enhances abiotic stress tolerance in rice. Planta 234, 1007-1018. Available from: https:/ / doi.org/10.1007/s00425-011-1458-0.

Licausi, F., Kosmacz, M., Weits, D.A., Giuntoli, B., Giorgi, F.M., Voesenek, L.A.C.J., et al., 2011. Oxygen sensing in plants is mediated by an N-end rule pathway for protein destabilization. Nature 479, 419-422. Available from: https://doi.org/10.1038/ nature10536.

Lin, C.R., Lee, K.W., Chen, C.Y., Hong, Y.F., Chen, J.L., Lu, C.A., et al., 2014. SnRK1A-interacting negative regulators modulate the nutrient starvation signaling sensor SnRK1 in source-sink communication in cereal seedlings under abiotic stress. Plant Cell 26, 808-827. Available from: https://doi.org/10.1105/ tpc.113.121939.

Liu, J.H., Peng, T., Dai, W., 2014. Critical cis-acting elements and interacting transcription factors: key players associated with abiotic stress responses in plants. Plant Mol. Biol. Rep. 32, 303-317. Available from: https://doi.org/10.1007/s11105-013-0667-z.

Locke, A.M., Barding Jr, G.A., Sathnur, S., Larive, C.K., Bailey-Serres, J., 2018. Rice SUB1A constrains remodelling of the transcriptome and metabolome during submergence to facilitate postsubmergence recovery. Plant Cell Environ. 41, 721-736. Available from: https:// doi.org/10.1111/pce.13094.

Loreti, E., Valeri, M.C., Novi, G., Perata, P., 2018. Carbon-starvation represses hypoxia responses. Plant Physiol. 176, 1286-1298. Available from: https://doi.org/10.1104/pp.17.01002.

Lu, C.A., Ho, T.H., Ho, S.L., Yu, S.M., 2002. Three novel MYB proteins with one DNA binding repeat mediate sugar and hormone regulation of alpha-amylase gene expression. Plant Cell 14, 1963-1980. Available from: https://doi.org/10.1105/tpc.001735.

Lu, C.-A., Lin, C.-C., Lee, K.-W., Chen, J.-L., Huang, L.-F., Ho, S.-L., et al., 2007. The SnRK1A protein kinase plays a key role in sugar signaling during germination and seedling growth of rice. Plant Cell 19, 2484-2499. Available from: https://doi.org/10.1105/ tpc.105.037887.

Lunn, J.E., 2007. Gene families and evolution of trehalose metabolism in plants. Funct. Plant Biol. 34, 550-563. Available from: https:// doi.org/10.1071/FP06315. 
Lunn, J.E., Delorge, I., Figueroa, C.M., Van Dijck, P., Stitt, M., 2014. Trehalose metabolism in plants. Plant J. 79, 544-567. Available from: https://doi.org/10.1111/tpj.12509.

Lunn, J.E., Feil, R., Hendriks, J.H.M., Gibon, Y., Morcuende, R., Osuna, D., et al., 2006. Sugar-induced increases in trehalose 6phosphate are correlated with redox activation of ADP glucose pyrophosphorylase and higher rates of starch synthesis in Arabidopsis thaliana. Biochem. J. 397, 139-148. Available from: https://doi.org/10.1042/BJ20060083.

Luo, Y., Li, W.M., Wang, W., 2008. Trehalose: protector of antioxidant enzymes or reactive oxygen species scavenger under heat stress? Env. Exp. Bot. 63, 378-384. Available from: https://doi. org/10.1016/j.envexpbot.2007.11.016.

Luo, Y., Li, F., Wang, G.P., Yang, X.H., Wang, W., 2010. Exogenously-supplied trehalose protects thylakoid membranes of winter wheat from heat-induced damage. Biol. Plant. 54, 495-501. Available from: https://doi.org/10.1007/s10535-0100087-y.

Luo, Y., Wang, W., Fan, Y.Z., Gao, Y.M., Wang, D., 2018. Exogenously-supplied trehalose provides better protection for D1 protein in winter wheat under heat stress. Russ. J. Plant Physiol. 65, 115-122. Available from: https://doi.org/10.1134/ S1021443718010168.

Ma, C., Wang, Z., Kong, B., Lin, T., 2013. Exogenous trehalose differentially modulate antioxidant defense system in wheat callus during water deficit and subsequent recovery. Plant Growth Regul. 70, 275-285. Available from: https://doi.org/10.1007/s10725013-9799-2.

Ma, J., Hanssen, M., Lundgren, K., Hernández, L., Delatte, T., Ehlert, A., et al., 2011. The sucrose-regulated Arabidopsis transcription factor bZIP11 reprograms metabolism and regulates trehalose metabolism. New Phytol. 191, 733-745. Available from: https:// doi.org/10.1111/j.1469-8137.2011.03735.x.

Mair, A., Pedrotti, L., Wurzinger, B., Anrather, D., Simeunovic, A., Weiste, C., et al., 2015. SnRK1-triggered switch of bZIP63 dimerization mediates the low-energy response in plants. Elife 4, e05828. Available from: https://doi.org/ 10.7554 /eLife.05828.

Mamedov, M.D., Petrova, I.O., Yanykin, D.V., Zaspa, A.A., Semenov, A.Y., 2015. Effect of trehalose on oxygen evolution and electron transfer in photosystem 2 complexes. Biochemistry (Moscow) 80, 61-66. Available from: https://doi.org/10.1134/ S0006297915010071.

Margalha, L., Valerio, C., Baeña-González, E., 2016. Plant SnRK1 kinases: structure, regulation, and function. In: Cordero, M., Viollet, B. (Eds.), AMP-Activated Protein Kinase. Experientia Supplementum, vol 107. Springer, Cham. Available from: https: / / doi.org/10.1007/978-3-319-43589-3_17.

Martínez-Barajas, E., Delatte, T., Schluepmann, H., de Jong, G.J., Somsen, G.W., Nunes, C., et al., 2011. Wheat grain development is characterized by remarkable trehalose 6-phosphate accumulation pregrain filling: tissue distribution and relationship to SNF1related protein kinase1 activity. Plant Physiol. 156, 373-381. Available from: https://doi.org/10.1104/pp.111.174524.

Martins, M.C.M., Hejazi, M., Fettke, J., Steup, M., Feil, R., Krause, U., et al., 2013. Feedback inhibition of starch degradation in Arabidopsis leaves mediated by trehalose 6-phosphate. Plant Physiol. 163, 1142-1163. Available from: https://doi.org/ 10.1104/pp.113.226787.

Martins, L.L., Mourato, M.P., Baptista, S., Reis, R., Carvalheiro, F., Almeida, A.M., et al., 2014. Response to oxidative stress induced by cadmium and copper in tobacco plants (Nicotiana tabacum) engineered with the trehalose-6-phosphate synthase gene (AtTPS1). Acta Physiol. Plant. 36, 755-765. Available from: https://doi.org/10.1007/s11738-013-1453-0.
Matiolli, C.C., Tomaz, J.P., Duarte, G.T., Prado, F.M., Del Bem, L.E. V., Silveira, A.B., et al., 2011. The Arabidopsis bZIP gene AtbZIP63 is a sensitive integrator of transient abscisic acid and glucose signals. Plant Physiol. 157, 692-705. Available from: https://doi. org/10.1104/pp.111.181743.

Mazza, G., Agnelli, A.E., Orasen, G., Gennaro, M., Valè, G., Lagomarsino, A., 2016. Reduction of global warming potential from rice under alternate wetting and drying practice in a sandy soil of northern Italy. Ital. J. Agrometeorol. 21, 35-44. Available from: https://doi.org/10.19199/2016.2.2038-5625.035.

Meng, F., Liu, H., Wang, K., Liu, L., Wang, S., Zhao, Y., et al., 2013. Development-associated microRNAs in grains of wheat (Triticum aestivum L.). BMC Plant Biol. 13, 140. Available from: https://doi. org/10.1186/1471-2229-13-140.

Minami, A., Yano, K., Gamuyao, R., Nagai, K., Kuroha, T., Ayano, M., et al., 2018. Time-course transcriptomics analysis reveals key responses of submerged deepwater rice to flooding. Plant Physiol. 176, 3081-3102. Available from: https://doi.org/ 10.1104/pp.17.00858.

Miro, B., Ismail, A.M., 2013. Tolerance of anaerobic conditions caused by flooding during germination and early growth in rice (Oryza sativa L.). Front. Plant Sci. 4, 269. Available from: https://doi. org/10.3389/fpls.2013.00269.

Mittler, R., Kim, Y., Song, L., Coutu, J., Coutu, A., Ciftci-Yilmaz, et al., 2006. Gain- and loss-of-function mutations in Zat10 enhance the tolerance of plants to abiotic stress. FEBS Lett. 580, 6537-6542. Available from: https://doi.org/10.1016/j. febslet.2006.11.002.

Miura, K., Furumoto, T., 2013. Cold signaling and cold response in plants. Int. J. Mol. Sci. 14, 5312-5337. Available from: https:// doi.org/10.3390/ijms14035312.

Morsy, M.R., Jouve, L., Hausman, J.-F., Hoffmann, L., Stewart, J.M., 2007. Alteration of oxidative and carbohydrate metabolism under abiotic stress in two rice (Oryza sativa L.) genotypes contrasting in chilling tolerance. J. Plant Physiol. 164, 157-167. Available from: https://doi.org/10.1016/j.jplph.2005.12.004.

Mostofa, M.G., Hossain, M.A., Fujita, M., 2015a. Trehalose pretreatment induces salt tolerance in rice (Oryza sativa L.) seedlings: oxidative damage and co-induction of antioxidant defense and glyoxalase systems. Protoplasma 252, 461-475. Available from: https: / / doi.org/10.1007/s00709-014-0691-3.

Mostofa, M.G., Hossain, M.A., Fujita, M., Tran, L.-S.P., 2015b. Physiological and biochemical mechanisms associated with trehalose-induced copper-stress tolerance in rice. Sci. Rep. 5, 11433. Available from: https://doi.org/10.1038/srep11433.

Mustroph, A., Lee, S.C., Oosumi, T., Zanetti, M.E., Yang, H., Ma, K., et al., 2010. Cross-kingdom comparison of transcriptomic adjustments to low-oxygen stress highlights conserved and plantspecific responses. Plant Physiol. 152, 1484-1500. Available from: https:/ / doi.org/10.1104/pp.109.151845.

Nakamura, J., Yuasa, T., Huong, T.T., Harano, K., Tanaka, S., Iwata, T., et al., 2011. Rice homologs of inducer of CBF expression (OsICE) are involved in cold acclimation. Plant Biotechnol. 28, 303-309. Available from: https://doi.org/10.5511/ plantbiotechnology.11.0421a.

Nery, D.D.C.M., da Silva, C.G., Mariani, D., Fernandes, P.N., Pereira, M.D., Panek, A.D., et al., 2008. The role of trehalose and its transporter in protection against reactive oxygen species. Biochim. Biophys. Acta-General Subjects 1780, 1408-1411. Available from: https: / / doi.org/10.1016/j.bbagen.2008.05.011.

Nounjan, N., Nghia, P.T., Theerakulpisut, P., 2012. Exogenous proline and trehalose promote recovery of rice seedlings from saltstress and differentially modulate antioxidant enzymes and expression of related genes. J. Plant Physiol. 169, 596-604. Available from: https:/ / doi.org/10.1016/j.jplph.2012.01.004. 
Nukarinen, E., Nagele, T., Pedrotti, L., Wurzinger, B., Mair, A., Landgraf, R., et al., 2016. Quantitative phosphoproteomics reveals the role of the AMPK plant ortholog SnRK1 as a metabolic master regulator under energy deprivation. Sci. Rep. 6, 31697. Available from: https:/ / doi.org/10.1038/srep31697.

Nunes, C., O'Hara, L.E., Primavesi, L.F., Delatte, T.L., Schluepmann, H., Somsen, G.W., et al., 2013a. The trehalose 6-phosphate/SnRK1 signaling pathway primes growth recovery following relief of sink limitation. Plant Physiol. 162, 1720-1732. Available from: https://doi.org/10.1104/pp.113.220657.

Nunes, C., Primavesi, L.F., Patel, M.K., Martinez-Barajas, E., Powers, S.J., Sagar, R., et al., 2013b. Inhibition of SnRK1 by metabolites: tissue-dependent effects and cooperative inhibition by glucose 1-phosphate in combination with trehalose 6-phosphate. Plant Physiol. Biochem. 63, 89-98. Available from: https://doi.org/ 10.1016/j.plaphy.2012.11.011.

Nunes, C., Schluepmann, H., Delatte, T.L., Wingler, A., Silva, A.B., Fevereiro, P.S., et al., 2013c. Regulation of growth by the trehalose pathway: relationship to temperature and sucrose. Plant Signal. Behav. 8, e26626. Available from: https://doi.org/10.4161/ psb.26626.

O'Hara, L.E., Paul, M.J., Wingler, A., 2013. How do sugars regulate plant growth and development? New insight into the role of trehalose-6-phosphate. Mol. Plant 6, 261-274. Available from: https://doi.org/10.1093/mp/sss120.

Paul, M.J., 2008. Trehalose 6-phosphate: a signal of sucrose status. Biochem. J. 412, e1-e2. Available from: https://doi.org/10.1042/ BJ20080598.

Paul, M.J., Primavesi, L.F., Jhurreea, D., Zhang, Y., 2008. Trehalose metabolism and signaling. Ann. Rev. Plant Biol. 59, 417-441. Available from: https://doi.org/10.1146/annurev. arplant.59.032607.092945.

Perata, P., Alpi, A., 1993. Plant responses to anaerobiosis. Plant Sci. 93, 1-17. Available from: https://doi.org/10.1016/0168-9452(93) 90029-Y.

Perata, P., Geshi, N., Yamaguchi, J., Akazawa, T., 1993. Effect of anoxia on the induction of $\alpha$-amylase in cereal seeds. Planta 191, 402-408.

Perata, P., Pozueta-Romero, J., Akazawa, T., Yamaguchi, J., 1992. Effect of anoxia on starch breakdown in rice and wheat seeds. Planta 188, 611-618.

Piattoni, C.V., Bustos, D.M., Guerrero, S.A., Iglesias, A., 2011. Nonphosphorylating glyceraldehyde 3-phosphate dehydrogenase is phosphorylated in wheat endosperm at serine-404 by an SNF1related protein kinase allosterically inhibited by ribose 5phosphate. Plant Physiol. 156, 1337-1350. Available from: https://doi.org/10.1104/pp.111.177261.

Pinheiro, G.L., Marques, C.S., Costa, M.D., Reis, P.A., Alves, M.S., Carvalho, C.M., et al., 2009. Complete inventory of soybean NAC transcription factors: sequence conservation and expression analysis uncover their distinct roles in stress response. Gene 444, 10-23. Available from: https://doi.org/10.1016/j. gene.2009.05.012.

Ponnu, J., Wahl, V., Schmid, M., 2011. Trehalose-6-phosphate: connecting plant metabolism and development. Front. Plant Sci. 2, 70. Available from: https://doi.org/10.3389/fpls.2011.00070.

Pramanik, M.H., Imai, R., 2005. Functional identification of a trehalose 6-phosphate phosphatase gene that is involved in transient induction of trehalose biosynthesis during chilling stress in rice. Plant Mol. Biol. 58, 751-762. Available from: https://doi.org/ 10.1007 /s11103-005-7404-4.

Puranik, S., Sahu, P.P., Srivastava, P.S., Prasad, M., 2012. NAC proteins: regulation and role in stress tolerance. Trends Plant Sci. 17, 369-381. Available from: https://doi.org/10.1016/j. tplants.2012.02.004.
Richards, K.D., Schott, E.J., Sharma, Y.K., Davis, K.R., Gardner, R.C., 1998. Aluminum induces oxidative stress genes in Arabidopsis thaliana. Plant Physiol. 116, 409-418. Available from: https://doi. org/10.1104/pp.116.1.409.

Rihan, H.Z., Al-Issawi, M., Fuller, M.P., 2017. Advances in physiological and molecular aspects of plant cold tolerance. J. Plant Interact. 12, 143-157. Available from: https://doi.org/10.1080/ 17429145.2017.1308568.

Rossel, J.B., Wilson, P.B., Hussain, D., Woo, N.S., Gordon, M.J., Mewett, O.P., et al., 2007. Systemic and intracellular responses to photooxidative stress in Arabidopsis. Plant Cell 19, 4091-4110. Available from: https://doi.org/10.1105/tpc.106.045898.

Ruelland, E., Zachowski, A., 2010. How plants sense temperature. Environ. Exp. Bot. 69, 225-232. Available from: https://doi.org/ 10.1016/j.envexpbot.2010.05.011.

Sadak, M.S., 2016. Mitigation of drought stress on fenugreek plant by foliar application of trehalose. Int. J. Chem. Tech. Res. 9, 147-155.

Saini, P., Gani, M., Kaur, J.J., Godara, L.C., Singh, C., Chauhan, S.S., et al., 2018. Reactive Oxygen Species (ROS): a way to stress survival in plants. In: Zargar, S., Zargar, M. (Eds.), Abiotic StressMediated Sensing and Signaling in Plants: An Omics Perspective. Springer, Singapore, pp. 127-153. Available from: https://doi. org/10.1007/978-981-10-7479-0_4.

Schluepmann, H., Paul, M., 2009. Trehalose metabolites in Arabidopsis-elusive, active and central. The Arabidopsis Book 7, e0122. Available from: https://doi.org/10.1199/tab.0122.

Schluepmann, H., Van Dijken, A., Aghdasi, M., Wobbes, B., Paul, M., Smeekens, S., 2004. Trehalose mediated growth inhibition of Arabidopsis seedlings is due to trehalose-6-phosphate accumulation. Plant Physiol. 135, 879-890. Available from: https://doi. org/10.1104/pp.104.039503.

Shafiq, S., Akram, N.A., Ashraf, M., 2015. Does exogenously-applied trehalose alter oxidative defense system in the edible part of radish (Raphanus sativus L.) under water-deficit conditions? Sci. Hort. 185, 68-75. Available from: https://doi.org/10.1016/j. scienta.2015.01.010.

Sharma, P., Jha, A.B., Dubey, R.S., Pessarakli, M., 2012. Reactive oxygen species, oxidative damage, and antioxidative defense mechanism in plants under stressful conditions. J. Bot. 2012, 217037. Available from: https://doi.org/10.1155/2012/217037.

Shima, S., Matsui, H., Tahara, S., Imai, R., 2007. Biochemical characterization of rice trehalose-6-phosphate phosphatases supports distinctive functions of these plant enzymes. FEBS J. 274, 1192-1201. Available from: https://doi.org/10.1111/j.17424658.2007.05658.x.

Singh, A., Septiningsih, E.M., Balyan, H.S., Singh, N.K., Rai, V., 2017. Genetics, physiological mechanisms and breeding of floodtolerant rice (Oryza sativa L.). Plant Cell Physiol. 58, 185-197. Available from: https://doi.org/10.1093/pcp/pcw206.

Song, S.Y., Chen, Y., Chen, J., Dai, X.Y., Zhang, W.H., 2011. Physiological mechanisms underlying OsNAC5-dependent tolerance of rice plants to abiotic stress. Planta 234, 331-345. Available from: https://doi.org/10.1007/s00425-011-1403-2.

Stoyanova, S., Geuns, J., Hideg, E., Van den Ende, W., 2011. The food additives inulin and stevioside counteract oxidative stress. Int. J. Food Sci. Nutr. 62, 207-214. Available from: https://doi.org/ 10.3109/09637486.2010.523416.

Su, C.F., Wang, Y.C., Hsieh, T.H., Lu, C.A., Tseng, T.H., Yu, S.M., 2010. A novel MYBS3-dependent pathway confers cold tolerance in rice. Plant Physiol. 153, 145-158. Available from: https://doi. org/10.1104/pp.110.153015.

Takahashi, D., Li, B., Nakayama, T., Kawamura, Y., Uemura, M., 2013. Plant plasma membrane proteomics for improving cold tolerance. Front. Plant Sci. 4, 90. Available from: https://doi.org/ 10.3389/fpls.2013.00090. 
Tan, J.K., Kong, F.X., Cao, H.S., Yu, Y., Han, X.B., 2005. Effects of acid precipitation and aluminum on carbohydrate metabolism in mycorrhizae of Pinus massioniana. Bull. Environ. Contam. Toxicol. 74, 614-622. Available from: https://doi.org/10.1007/s00128005-0628-9.

Tang, G.-Q., Hardin, S.C., Dewey, R., Huber, S.C., 2003. A novel Cterminal proteolytic processing of cytosolic pyruvate kinase, its phosphorylation and degradation by the proteasome in developing soybean seeds. Plant J. 34, 77-93. Available from: https:// doi.org/10.1046/j.1365-313X.2003.01711.x.

Tayeh, C., Randoux, B., Vincent, D., Bourdon, N., Reignault, P., 2014. Exogenous trehalose induces defenses in wheat before and during a biotic stress caused by powdery mildew. Phytopathology 104, 293-305. Available from: https://doi.org/10.1094/PHYTO07-13-0191-R.

Theocharis, A., Clément, C., Barka, E.A., 2012. Physiological and molecular changes in plants grown at low temperatures. Planta 235, 1091-1105. Available from: https://doi.org/10.1007/s00425012-1641-y.

Tomé, F., Nägele, T., Adamo, M., Garg, A., Marco-llorca, C., Nukarinen, E., et al., 2014. The low energy signaling network. Front. Plant Sci. 5, 353. Available from: https://doi.org/10.3389/ fpls.2014.00353.

Valenzuela-Soto, E.M., Marquez-Escalante, J.A., Iturriaga, G., Figueroa-Soto, C.G., 2004. Trehalose 6-phosphate synthase from Selaginella lepidophylla: purification and properties. Biochem. Biophys. Res. Commun. 313, 314-319. Available from: https:// doi.org/10.1016/j.bbrc.2003.11.128.

van Dongen, J.T., Licausi, F., 2015. Oxygen sensing and signaling. Ann. Rev. Plant Biol. 66, 345-367. Available from: https://doi. org/10.1146/annurev-arplant-043014-114813.

Vandesteene, L., Ramon, M., Le Roy, K., Van Dijck, P., Rolland, F., 2010. A single active trehalose-6-P synthase (TPS) and a family of putative regulatory TPS-like proteins in Arabidopsis. Mol. Plant 3, 406-419. Available from: https://doi.org/10.1093/mp/ssp114.

Vandesteene, L., López-Galvis, L., Vanneste, K., Feil, R., Maere, S., Lammens, W., et al., 2012. Expansive evolution of the TREHALOSE-6-PHOSPHATE PHOSPHATASE gene family in Arabidopsis. Plant Physiol. 160, 884-896. Available from: https:// doi.org/10.1104/pp.112.201400.

Viana, R., Towler, M.C., Pan, D.A., Carling, D., Viollet, B., Hardie, D. G., et al., 2007. A conserved sequence immediately N-terminal to the Bateman domains in AMP-activated protein kinase $\gamma$ subunits is required for the interaction with the $\beta$ subunits. J. Biol. Chem. 282, 16117-16125. Available from: https://doi.org/10.1074/jbc. M611804200.

Wang, S.-t, Sun, X.-l, Hoshino, Y., Yu, Y., Jia, B., Sun, Z.-W., et al., 2014. MicroRNA319 positively regulates cold tolerance by targeting OsPCF6 and OsTCP21 in rice (Oryza sativa L.). PLoS One 9, e91357. Available from: https://doi.org/10.1371/journal. pone.0091357.

Weltmeier, F., Rahmani, F., Ehlert, A., Dietrich, K., Schütze, K., Wang, X., et al., 2009. Expression patterns within the Arabidopsis C/S1 bZIP transcription factor network: availability of heterodimerization partners controls gene expression during stress response and development. Plant Mol. Biol. 69, 107-119. Available from: https://doi.org/10.1007/s11103-008-9410-9.
Wingler, A., 2018. Transitioning to the next phase: the role of sugar signaling throughout the plant life cycle. Plant Physiol. 176, 1075-1084. Available from: https://doi.org/10.1104/pp.17.01229.

Wu, Y., Deng, Z., Lai, J., Zhang, Y., Yang, C., Yin, B., et al., 2009. Dual function of Arabidopsis ATAF1 in abiotic and biotic stress responses. Cell Res. 19, 1279-1290. Available from: https://doi. org/10.1038/cr.2009.108.

Wurzinger, B., Mair, A., Fischer-Schrader, K., Nukarinen, E., Roustan, V., Weckwerth, W., et al., 2017. Redox state-dependent modulation of plant SnRK1 kinase activity differs from AMPK regulation in animals. FEBS Lett. 591, 3625-3636. Available from: https: / / doi.org/10.1002/1873-3468.12852.

Wurzinger, B., Nukarinen, E., Nägele, T., Weckwerth, W., Teige, M., 2018. The SnRK1 kinase as central mediator of energy signaling between different organelles. Plant Physiol. 176, 1085-1094. Available from: https://doi.org/10.1104/pp.17.01404.

Xiong, Y., McCormack, M., Li, L., Hall, Q., Xiang, C., Sheen, J., 2013. Glucose-TOR signalling reprograms the transcriptome and activates meristems. Nature 496, 181-187. Available from: https:// doi.org/10.1038/nature12030.

Yadav, S.K., 2010. Cold stress tolerance mechanisms in plants. A review. Agron. Sustain. Dev. 30, 515-527. Available from: https: / / doi.org/10.1051/agro/2009050.

Yadav, U.P., Ivakov, A., Feil, R., Duan, G.Y., Walther, D., Giavalisco, P., et al., 2014. The sucrose-trehalose 6-phosphate (Tre6P) nexus: specificity and mechanisms of sucrose signalling by Tre6P. J. Exp. Bot. 65, 1051-1068. Available from: https://doi.org/10.1093/jxb/ ert457.

Yang, L., Zhao, X., Zhu, H., Paul, M., Zu, Y., Tang, Z., 2014. Exogenous trehalose largely alleviates ionic unbalance, ROS burst, and PCD occurrence induced by high salinity in Arabidopsis seedlings. Front. Plant Sci. 5, 570. Available from: https://doi.org/10.3389/fpls.2014.00570.

Yu, S.M., Lo, S.F., Ho, T.D., 2015. Source-sink communication: regulated by hormone, nutrient, and stress cross-signaling. Trends Plant Sci. 20, 844-857. Available from: https://doi.org/10.1016/j. tplants.2015.10.009.

Zang, B., Li, H., Li, W., Deng, X.W., Wang, X., 2011. Analysis of trehalose-6-phosphate synthase (TPS) gene family suggests the formation of TPS complexes in rice. Plant Mol. Biol. 76, 507-522. Available from: https://doi.org/10.1007/s11103-011-9781-1.

Zhang, Q., Chen, Q., Wang, S., Hong, Y., Wang, Z., 2014. Rice and cold stress: methods for its evaluation and summary of cold tolerance-related quantitative trait loci. Rice 7, 24. Available from: https:// doi.org/10.1186/s12284-014-0024-3.

Zhang, Y., Primavesi, L.F., Jhurreea, D., Andralojc, P.J., Mitchell, R.A. C., Powers, S.J., et al., 2009. Inhibition of SNF1-related protein kinase1 activity and regulation of metabolic pathways by trehalose-6-phosphate. Plant Physiol. 149, 1860-1871. Available from: https:/ / doi.org/10.1104/pp.108.133934.

\section{Further Reading}

Karim, S., Aronsson, H., Ericson, H., Pirhonen, M., Leyman, B., Welin, B., 2007. Improved drought tolerance without undesired side effects in transgenic plants producing trehalose. Plant Mol. Biol. 64, 371-386. 
This page intentionally left blank 


\title{
16
}

\section{Sugar Regulates Plant Growth and Development Under In Vitro Conditions}

\section{Durdana Shah ${ }^{1}$, Nasreena Sajjad ${ }^{2}$, Rohaya Ali ${ }^{2}$, Nazish Nazir ${ }^{1}$, Sumaya Hassan ${ }^{2}$ and Snober Shah ${ }^{3}$}

\begin{abstract}
${ }^{1}$ Plant Tissue Culture Laboratory, Centre of Research for Development, University of Kashmir, Srinagar, Jammu and Kashmir, India ${ }^{2}$ Department of Biochemistry, University of Kashmir, Srinagar, Jammu and Kashmir, India ${ }^{3}$ Cytogenitics and Molecular Biology Laboratory, Centre of Research for Development, University of Kashmir, Srinagar, Jammu and Kashmir, India
\end{abstract}

\section{O U T L I N E}

16.1 Introduction

16.2 Role of Sugars in Plant Growth and Development Under In Vitro Conditions

16.3 Sugar Signaling: Physiological, Molecular, and Genetic Approaches in Plants

16.4 Physiological Approaches of Sugar Signaling in Plants

16.5 Molecular and Genetic Approaches of Sugar Regulation in Plants
16.6 Importance of Sugar Interaction With Phytohormones in Regulation of Growth and Development Under In Vitro Condition

16.7 Function of Phytohormones Under In Vitro Conditions

16.8 Conclusion and Future Prospects 266

References 266

Further Reading $\quad 268$

\subsection{INTRODUCTION}

Plants as photoautotrophic organisms are able to produce the carbohydrates they require and have developed mechanisms to coordinate carbohydrate production and its metabolism (Rook et al., 2003) Carbohydrate-derived signals regulate the expression of genes involved in both photosynthesis and metabolism, and control carbohydrate partitioning. Genetic screens for sugar-response mutants have shown the close interaction between sugar and hormonesignaling pathways (Rook et al., 2003). Sugars produced from plant photosynthesis play a central role to support and integrate the functions and actions of 
internal and external regulatory signals in driving diverse biological processes from embryogenesis to senescence. Although the knowledge on how plants produce, transport, metabolize, store, and sense diverse sugar signals has been significantly advanced, the spectrum of sugar signals, sensors, and molecular mechanisms mediating primary signaling remain to be fully explored (Granot et al., 2014). Many informative review articles presented recent progress on broad aspects of sugar-related research in plant biology, encompassing source-sink communication; sugar-hormone interactions; new sugar transporters and their functions; sugar regulation of plant development; chloroplast-nuclear signaling; sucrose, starch, and trehalose metabolism and signaling; clock-sugar connections; as well as sugar and stress. The accumulated knowledge will provide an excellent and comprehensive platform for future research, especially on elucidating the molecular, cellular, and biochemical basis of sugar sensing and signaling underlying the plasticity and potential in plant growth and development. Emphasis in this review is placed on the emerging understanding of the dynamic, primary, and integrated sugar signaling mechanisms and transcriptional networks triggered by direct and indirect sugar signals via sugar, energy, and metabolite sensors.

Sugars play a very essential role in growth and developmental processes. An adequate supply of carbohydrates is needed both to energize metabolism and provide the "building blocks" to make cellular structures and cell walls. Plant hormones may integrate metabolism with developmental programs by directing the carbon and determining its use. The pivotal role of sugars as signaling molecules is well illustrated by the variety of sugar sensing and signaling mechanisms discovered in free-living microorganisms such as bacteria and yeast (Ramon et al., 2008). For such unicellular organisms, nutrient availability is the main extracellular factor controlling growth and metabolism. The role of nutrients as regulatory molecules has come to be appreciated only recently in mammals despite extensive previous research on glucose homeostasis and diabetes. In plants, sugar production through photosynthesis is a vital process, and sugar status modulates and coordinates internal regulators and environmental cues that govern growth and development. Although the regulatory effect of sugars on photosynthetic activity and plant metabolism has long been recognized, the concept of sugars as central signaling molecules is relatively novel. Recent progress has begun to reveal the molecular mechanisms underlying sugar sensing and signaling in plants, including the demonstration of hexokinase (HXK) as a glucose sensor that modulates gene expression and multiple plant hormone-signaling pathways (Rolland et al., 2002a,b,c). Analyses of HXK mutants will provide new evidence for distinct signaling and metabolic activities. Diverse roles of Snf1-related protein kinases (SnRKs) in carbon metabolism and sugar signaling also are emerging. In addition, Suc, trehalose, and other HXKindependent sugar sensing and signaling pathways add more complexity in plants (Rolland et al., 2006). Biochemical, molecular, and genetic experiments have supported a central role of sugars in the control of plant metabolism, growth, and development and have revealed interactions that integrate light, stress, and hormone signaling and coordinate carbon and nitrogen metabolism (Ramon et al., 2008). A number of reviews have appeared in the past few years emphasizing different aspects of sugar signaling and its interactions with other plant signal transduction pathways (Cho et al., 2010; Ehlert et al., 2006; Rolland et al., 2002a,b,c; Gazzarrini and McCourt, 2001).

\subsection{ROLE OF SUGARS IN PLANT GROWTH AND DEVELOPMENT UNDER IN VITRO CONDITIONS}

In plants, growth usually is a complex molecular network and irreversible change in size involving cell division and cell elongation. These networks must continuously adapt to an ever-changing environment (Gonzalez et al., 2012; Powell and Lenhard, 2012). Plants have played a crucial role in the evolution of life on earth through the making of energy-rich sugar molecules and oxygen by photosynthetic carbon fixation. Sugars are the most important carbon and energy source to cells, and also have important regulatory functions in controlling metabolism, growth, and development of plants. Sugars operate both as immediate substrates for intermediary metabolism and as effective signaling molecules. Plant sugar regulation is mediated by sugar signals, which are generated at different locations depending on environmental conditions and developmental stage. Sucrose (Suc) transport and hydrolysis play key regulatory roles in sugar signal generation (Ruan, 2014) In plants, sensing and signaling pathways have been described for different sugars (Hanson and Smeekens, 2009), but only for glucose is detailed information on sensing and signaling mechanisms available (Ramon et al., 2008). Over the past decade, various cellular, chemical, genetic, proteomic and genomic approaches in the reference plant Arabidopsis thaliana have begun to unravel the surprisingly broad range of functions and actions of three glucose-modulated master regulators, HXK1,KIN10/11, and TOR, which control the expression of thousands of plant genes involved in a wide spectrum of cellular functions (Dobrenel et al., 2016). 
In the recent years important progress has also been made in identifying the dominant plant growth controlling regulatory systems that receive input from sugars and sugar derived metabolic signals. These systems are either growth promoting or growth inhibiting. Systems that have a promoting role on growth are the hexokinase glucose sensor, the trehalose-6phosphate (T6P) signal, and the Target of rapamycin (TOR) kinase system in response to high sugar levels. Growth and floral transition halted in the absence of either T6P or TOR kinase. Systems that have an inhibitory effect on growth are the plant SNF1-related protein kinase1 (SnRK1), homolog of the animal AMP-activated protein kinase (AMPK) and yeast sucrose nonfermenting 1 (SNF1) kinase, and C/S1 bZIP transcription factor network in response to low sugar level. Therefore, all phases of plant life cycle from seed germination to hypocotyl elongation, cotyledon expansion, adventitious root formation, true leaf formation, flowering, and senescence are dependent on sugar (Rolland et al., 2006). The environmental conditions affect the availability of photosynthetic carbon in the form of sucrose, which in turn controls plant growth and development. The most common sugar translocated in plants is sucrose and out of all translocated materials in plants sucrose represents over $95 \%$ of the dry weight (Zimmermann and Ziegler, 1975). In plant cell, tissue, and organ culture the most common carbon source used is sucrose. In tissue culture medium sucrose is the most widely used carbohydrate in most of the plants, like Curcuma, the Phaseolus, and Hyoscyamus genus. MS media (Murashige and Skoog, 1962) is the most commonly used media in plant tissue culture, which constitutes about $3 \%$ of sucrose. However, the photosynthetic efficiency of cultured plants in the presence of high sucrose concentration in the media is restricted by reducing the levels of chlorophyll, key enzymes for photosynthesis, and epicuticular waxes promoting the formation of structurally and physiologically abnormal stomata. The light conditions, carbon in the media, and their interaction have an important effect on growth and development of plants. For sustaining photomixotrophic metabolism, ensuring optimal development in the plant tissue culture sucrose acts as a fuel source, it has more recently been emphasized that sucrose has other significant roles such as carbon precursor or signaling metabolite (Müller, 2011). Sucrose is metabolized in plant cell and tissue culture for every metabolic process that the cell will conduct to provide energy and carbon skeletons. The maintenance of osmotic potential and the conservation of water in cells are also supported by sucrose. Maltose is one of the other carbon sources used in culture media, in cereal anther culture, and it has resulted in significant enhancement of callus and plant regeneration frequencies, including wheat (Orsinky et al., 1990), barley (Karsai et al., 1994), and rye (Deimling et al., 1992).

Every aspect of plant growth and development including seed germination, cell proliferation and death, cell expansion and elongation, primary root length, seedling growth and development, root gravitropism, lateral roots, carbon and nitrogen metabolism and stress responses, root hairs, shoot meristem maintenance, reproduction, senescence, photosynthetic gene expression, crop yield and product quality are influenced by glucose, which acts as signaling molecule (Eveland and Jackson, 2012).

Trehalose-6-phosphate (T6P), which is a significant signaling metabolite, is the precursor of trehalose in the biosynthetic pathway and is tangled in the regulation of plant growth and development in response to carbon availability. The essential aspects of carbon consumption and metabolism in cell and tissue cultures have yet to be fully understood even though carbohydrates are of chief importance for cell growth, maintenance, and differentiation under in vitro conditions. In cell suspension cultures, where sucrose is promptly broken down for energy and carbon skeleton, most sucrose metabolism research has been performed by using tissue from plants growing in environmental conditions where the synthesized sucrose is for translocation and storage. For the enzymatic cleavage of sucrose there are two pathways. First, in what is called the alternative pathway, sucrose synthase conserves the energy of the glycosidic bond by cleaving sucrose into fructose and UDP glucose. UDP and pyrophosphate are components on which this pathway is dependent (Xu et al., 1998) and in 1986 it was first proposed to operate in plants. In this pathway the reaction is catalyzed by UDP glucose pyrophosphorylase, and generated UDP glucose feeds glucose 1-phosphate directly into glycolysis. Second, in what is called the classic pathway, with the loss of the glycosidic energy bond the invertase reaction produces glucose and fructose. Prior to subsequent metabolization these products must be phosphorylated by glucokinase and fructokinase. One alternative that is described as an adaptative pathway is catalyzed by a readily reversible pyrophosphatedependent phosphofructokinase (PFP) and through the utilization and synthesis of pyrophosphate an equilibrium reaction conserves energy. The second, which is described as maintenance reaction, is catalyzed by the irreversible nucleotide triphosphatedependent phosphofructokinase (PFK). So through UDP glucose pyrophosphorylase PFP when working in the gluconeogenic direction could produce PPi to drive sucrose breakdown (Xu et al., 1998). 


\subsection{SUGAR SIGNALING: PHYSIOLOGICAL, MOLECULAR, AND GENETIC APPROACHES IN PLANTS}

Sugars play a central role in metabolism but they can also act as signaling molecules. During various stresses, that is, biotic or abiotic, the sugar concentration changes dramatically (Smeekens and Hellmann, 2014). The main target of sugar signaling is carbon metabolism and regulation of photosynthesis. As we now know sucrose is the main photosynthetic product, therefore, there are many sugar signaling effects on growth and metabolism. Sugars act as regulatory signals that control the expression of various genes involved in many processes. There are various sugar signaling pathways that regulate gene expression. Discovery of unique and global repression of photosynthetic genes by glucose in Arabidopsis led to identification of HXK1 as the first plant glucose sensor, which mediates glucose repression or promotion of gene transcription and plant growth. Following various signal transduction pathways which occur in plants, for example, HXK1-dependent pathways,that is related with the HXK1-mediated signaling function and the major effect exerted by this pathway is repression of photosynthetic gene expression signal transduction pathways occurring in plants, for example, HXK1-dependent pathways, gene expression, which is related with the HXK1-mediated signaling function and the major effect exerted by this pathway is repression of photosynthetic gene expression (Xiao et al., 2000). Another pathway is glycolysis-dependent, that is, glucose induction of PR1 and PR5 gene expression. Finally, there are HXK1-independent signaling pathways (Fig. 16.1). The presence of sugar is sensed by

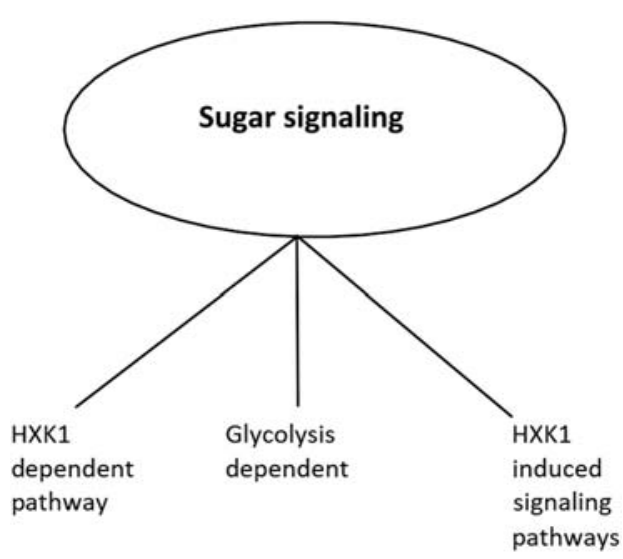

FIGURE 16.1 Various sugar signaling pathways that regulate gene expression in plants. HXK1-dependent pathways gene expression, which is related with the HXK1-mediated signaling function. HXK1-independent signaling pathways and glycolysis-dependent, that is, glucose induction of PR1 and PR5 gene expression. sugar receptors, which then results in downstream signaling events (Jang and Jen Sheen, 1997). The group of bZIP transcription factors that link sugar availability to growth. bZIP transcription factors are transnationally regulated in response to sugar signals. Various numbers of physiological, biochemical, and molecular approaches have been used to study sugar responses in plants (Kang et al., 2010). Arabidopsis is the genetic model system of choice for the isolation of sugarresponse mutants. The sugar status of plant cells is sensed by sensor proteins (Rook et al., 2003). The signaling pathways involve mitogen-activated protein kinases, protein phosphatases, $\mathrm{Ca}^{2+}$ and calmodulins, resulting in appropriate gene expression. Depending on the status of soluble sugars a number of genes are either induced or repressed. Further various stresses like abiotic stresses to plants result in alterations in sugar status and therefore affect the expression of various genes by down- and upregulating their expression.

\subsection{PHYSIOLOGICAL APPROACHES OF SUGAR SIGNALING IN PLANTS}

For all living organisms on this planet survival is dependent on photosynthesis, that is, fixation of carbon and light energy from the sun. As the sugar molecule is the prime carbon and energy source, sugars have acquired important regulatory functions in different organisms early on in evolution. Plants are photosynthetic, sugar-producing, and sessile, where maintaining homeostasis requires a complex and flexible regulatory machinery. Hence, sugar signaling plays an important role in controlling metabolism, stress resistance, growth, and development (Rolland et al., 2006). The regulation is controlled at numerous levels in plants, for example, by allosteric regulation of metabolic enzymes, and tissue-specific or temporalspecific expression of genes (Ehlert et al., 2006) It has been reported previously that expression of genes involved in starch and fructan metabolism, among other pathways, is regulated by sugars (Sun et al., 2003). Generally, in higher plants, high sugar levels stimulate expression of genes involved in sink function, such as growth, storage of proteins, and biosynthesis of starch and other carbohydrates, whereas low sugar levels promote photosynthesis and mobilization of energy reserves, such as breakdown of storage starch or lipids. Sugar signaling can be dissected into three steps, sugar sensing, signal transduction, and target gene expression. Further complicating matters is the dual function of sugars as nutrients and signaling molecules and also the interaction between sugar signaling and hormonal networks. Hexoses, sucrose, and 
trehalose might serve as elicitors of plant sugar signaling (Smeekens and Rook, 1998; Müller et al., 2001).

Sugars are essential to the fundamental processes required for plant growth. Therefore, carbohydrate production, metabolism, and use must be carefully coordinated with photosynthate availability, environmental cues, and timing of key developmental programs. Sugars in general can act as signaling molecules and as global regulators of gene expression, for example, acting like hormones and translating nutrient status to regulation of growth and the floral transition. Therefore, sugar-responsive gene regulation reflects carbohydrate abundance or depletion. The translation of nutrient status to transcriptional regulation allows the plant to modulate growth, both at the whole plant level and locally, in tissue- or cell-specific patterns, potentially to coordinate developmental programs with available carbohydrate. In response to sugar depletion, genes involved in photosynthesis, carbohydrate remobilization and export, and nitrogen $(\mathrm{N})$ metabolism tend to be upregulated. Alternatively, sugar abundance induces typical sink organ activities such as carbohydrate import, utilization, and storage, and starch and anthocyanin biosynthesis. Specific growth and metabolic responses tend to be activated and modulated based on the nature of the sugar signal. For example, sucrose, the primary transport sugar in plants, can be sensed as a signal directly (Chiou and Bush, 1998) or, alternatively, a signal can arise via its hexose cleavage products, glucose or UDP-glc and fructose.

Sugar signaling has been involved in carbon and nitrogen assimilation and transport. Regarding carbon metabolism, one case that has been long studied is the induction of fructan (polymers of Fru) synthesis in grasses. Although in nature fructan metabolism is mainly induced during periods of low temperature, the effect of cold is not direct but through its role in increasing cell Suc concentration due to lower carbon utilization. It has been shown that, at warm temperature, light induces fructan accumulation in detached leaves of different grass species, and that Suc mimics the light effect. Although glucose supply (and other sugars as well) can also induce fructan synthesis, the efficiency of these sugars is much lower than that of Suc. This fact, together with results from the application of various sugar analogs, led to the conclusion that in nature, Suc is most likely the molecule that initiates the signaling cascade leading to the induction of fructan synthesizing enzymes (Wiese et al., 1999). Sucrose also appears to act as a signaling molecule that initiates/activates starch synthesis. Sucrose regulates nitrogen and respiratory metabolism after feeding tobacco with different sugars. Nitrogen transport also appears to be regulated by Suc. A case in which the ammonium transporter gene,
CitAMT1, is specifically induced by Suc has been reported for citrus plants. Besides, it has been shown that the A. thaliana nitrate and ammonium transporter genes are induced after the addition of Suc; however, it is uncertain whether Suc is acting as a signal molecule in this response since hexoses are effective as well. A particularly important protein in carbon and nitrogen metabolisms is PII, which coordinates the regulation of nitrogen assimilation in response to nitrogen, carbon, and energy availability. The expression of the gene (GLB1) that encodes PII protein is induced by light and Suc in darkadapted $A$. thaliana plants. This effect is not triggered by mannitol or nonmetabolizable carbon source. Regarding other mineral nutrients, the expression of genes encoding for ion transporters for phosphate, sulfate, and potassium may be upregulated by Suc. Suc appears to modify the expression of a number of genes related to P starvation, which leads to an altered root physiology. Sucrose also plays an important role in control of copper homeostasis through sugar-responsive miRNAs in $A$. thaliana (Smeekens, 2000).

\subsection{MOLECULAR AND GENETIC APPROACHES OF SUGAR REGULATION IN PLANTS}

Sugar signaling transduction pathways are categorized in three distinct types in plants, based on the role of HXK1. In the first HXK1-dependent pathway, gene expression is correlated with the HXK1-mediated signaling function (Loreti et al., 2008).A major effect of this pathway is the repression of photosynthetic gene expression. Target genes in this pathway can now be defined genetically by the gin2 mutants and catalytically inactive alleles. A second pathway is glycolysisdependent and can also be sustained by heterologous yeast Hxk2 activity. An example is the glucose induction of PR1 and PR5 gene expression. Finally, there is evidence for HXK1-independent signaling pathways. Glucose induction of CHS, PAL1 and genes encoding AGPase as well as glucose repression of ASN1 are observed independent of sense and antisense overexpression of Arabidopsis HXK1 or overexpression of yeast Hxk2 (Cho et al., 2010). Transcriptional responses to nonphosphorylated glucose analogs have been observed in Chenopodium cell suspension cultures, Chlorella, and transgenic Arabidopsis, although one should be cautious about possible nonspecific effects of such chemicals. The glucose analog 3-OMG, not perceived as a sugar signal, is still phosphorylated by HXK with low catalytic efficiency, and the product, 3-OMG-6-phosphate, accumulates in these cells; see Fig. 16.2. Conversely, none of the 200 glucose-responsive Arabidopsis genes 


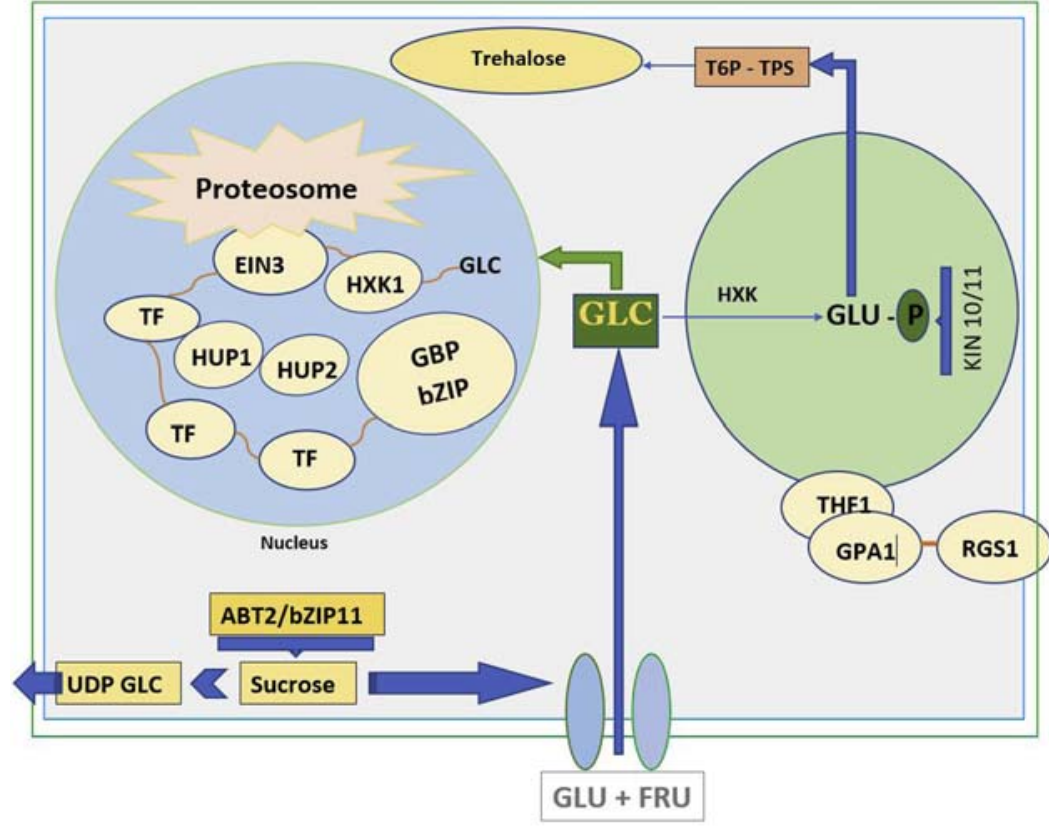

FIGURE 16.2 Glc and Fru are transported or mobilized from cytosolic and vacuolar Suc and plastid starch. Glc then enters metabolism after HXKcatalyzed phosphorylation. The HXK sugar sensor, as a cytosolic protein or associated with mitochondria or other organelles, then could activate a signaling cascade through HXK-interacting proteins (HIPs) or affect transcription directly after nuclear translocation.

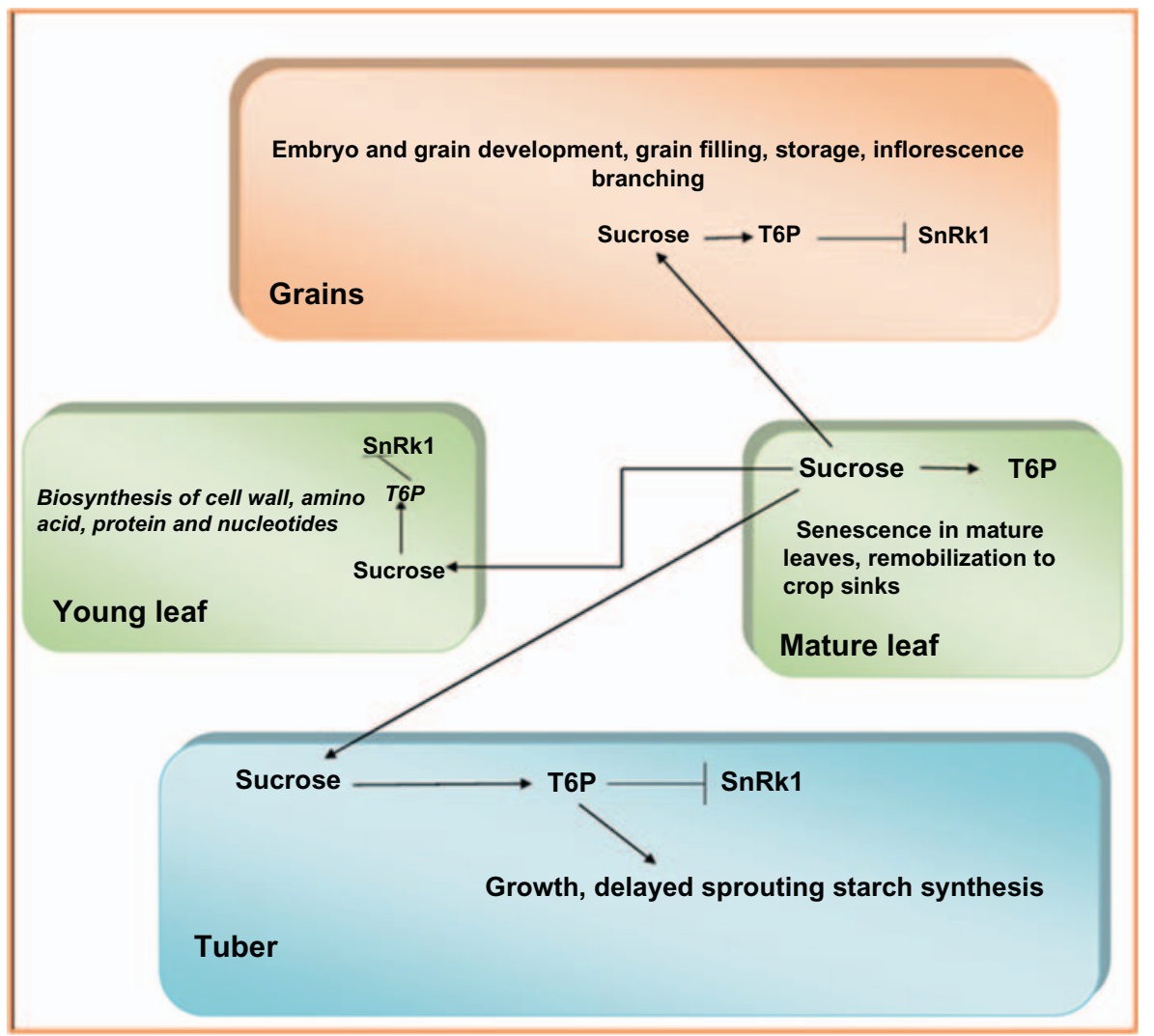

FIGURE 16.3 Sucrose metabolism in various tissues of plants.

identified in a recent study responds to 3-OMG or 6DOG (Dekkers et al., 2004).

There are a number of selected genes, for example, a gene that encodes a sugar beet proton sucrose symporter, whose expression is regulated by sucrose (see Fig. 16.3) but not glucose or fructose; this observation points to an HXK-independent, sucrose-specific signaling pathway. Interestingly, nonmetabolizable 
sucrose analogs such as palatinose and turanose can also affect carbohydrate metabolism and gene expression. This observation suggests the existence of a disaccharide sensing system at the plasma membrane. However, such analogs again have no physiological relevance and can elicit distinct responses consistent with their perception as stress-related stimuli.

Over the years, a large number of plant genes have been found to be transcriptionally regulated by sugars, consistent with the coordinated regulation of source and sink activities. Importantly, several genes that encode metabolic proteins involved in sugar signal generation undergo transcriptional feedback regulation by their own products. Repression of photosynthesis gene promoters, for example, has been studied in mesophyll protoplasts and transgenic seedlings. As well as photosynthesis genes, the INV and SUS genes are also extensively regulated by sugar availability. Also, when sugar levels are high, carbohydrate storage through starch synthesis is upregulated by the induction of genes that encode AGPase (Riou-Khamlichi et al., 2000).

Many sugar-regulated genes and promoters have been used to screen for Arabidopsis mutants with potential defects in transcription control. A screen using the regulatory sequences of the sugar-inducible AGPase large subunit (APL3) gene fused to a negative selection marker has identified several impaired sucrose induction (isi) mutants. Another screen based on the activity of a luciferase (LUC) reporter gene under the control of the APL3 promoter yielded high sugar-response (hsr) mutants that exhibited elevated LUC activity and APL3 expression in response to low sugar concentrations. The screen using sugar-regulated expression of an Arabidopsis $\beta$-amylase generated low beta amylase (lba) and high beta amylase (hba) mutants with altered sugar regulation of a subset of genes. Arabidopsis reduced sugar response (rsr) mutants were selected using the sucrose activated promoter of patatin, a potato tuber storage protein. Molecular analysis of the mutants will bring new information on the mechanisms underlying sugarmediated transcription control. The new microarray technologies now enable genome-wide expression analyses of Arabidopsis sugar and starvation responses. Arabidopsis phosphorus-deficient3 (pho3) mutant plants are used specifically to study the genomic response to sugar accumulation. This mutant is affected in the sucrose transporter2 (SUC2), and therefore accumulates soluble sugars, starch, and anthocyanins. High expression levels of genes that encode sucrose phosphate synthases (SPS), the plastid glucose $\mathrm{G} 6 \mathrm{P} /$ phosphate translocator (characteristically expressed only in heterotrophic tissues), and the AGPase large subunits are consistent with the starch accumulation in the mutant. Also consistent with the phenotype, there is a large increase in the expression of TFs and enzymes involved in anthocyanin biosynthesis. Apparently, secondary metabolism is also an important target for transcription regulation by sugars. Using a more comprehensive approach, the short term effects of glucose and nitrogen in global gene expression in the dark have been studied in liquid-grown Arabidopsis seedlings. The use of the protein synthesis inhibitor cycloheximide shows that glucose repression is a more direct process than glucose induction, which often requires de novo protein synthesis (Moore et al., 2003). TFs with sugar-regulated expression profiles are likely regulators of the broad transcriptional response to sugars. Several global gene expression studies have been published on sugar starvation responses. Using cDNA microarrays and seedlings grown in the presence or absence of sucrose, a small number of (mostly carbohydrate and amino acid metabolism) genes were shown to be upregulated in concert during sugar depletion. A more detailed analysis of nutrient mobilization in response to sucrose starvation in Arabidopsis cells cultured in suspension has been carried out using the ATH1 gene chip. Consistent with extensive nutrient recycling for cell survival, genes that were upregulated are involved in carbohydrate, amino acid, protein and lipid catabolism, and autophagy. Although these cultures were nonphotosynthetic, several photosynthesis associated genes were also upregulated upon starvation. Genes that were downregulated are involved in metabolism (biosynthesis), protein synthesis, and cell division. Similar expression profiles were observed in the responses of Arabidopsis rosettes to an extended night period and a starchless pgm mutant at the end of the night. These studies also introduce the use of MAPMAN, a practical and informative tool to display complex genomic data in diagrams of metabolic and regulatory pathways. Interestingly, the molecular events in dark-induced senescence of Arabidopsis leaves (analyzed using a combination of cDNA microarray and biochemical analyses) exhibited extensive similarities with the sugar starvation response. Many TF genes were identified as putative regulators. However, a comparative microarray study reveals significant differences in gene expression and signaling pathways between developmental and dark/starvation-induced senescence. Extended dark treatment causes a starvation condition that overrides the transcriptional regulation by circadian rhythm. However, in addition to energizing sugar production and (re)setting the clock, light can also directly affect gene expression through light-specific mechanisms. In a recent study, the effects of both light and sugar were examined. The results reveal that the majority of affected genes are coregulated by both stimuli. More extensive time-course gene expression analyses using wild-type and the pgm mutant plants under a $12 \mathrm{~h}$ photoperiod 
provide a clear picture of the essential roles of sugar signals for a large set of circadian regulated genes (Price et al., 2004).

The large genomic datasets generated in microarray experiments provide an excellent opportunity to identify conserved DNA elements in the promoters of coregulated genes. Currently, most information on regulatory cis-elements involved in sugar signaling comes from a few selected genes, encoding sweet potato tuber and cereal seed proteins, and proteins involved in maize photosynthesis (Ohto et al., 2001). Studies on sugar activation of sweet potato tuber class I patatin, SUS, sporamin, and $\beta$-amylase promoters identified several sucrose-responsive cis-elements, including the sucrose-responsive element (SURE), A and B-boxes, the TGGACGG element, an SP8 motif, and an SP8-binding protein, SPF1. SPF1 is a WRKYtype sucrose-repressed negative regulator with putative orthologs in other species, including Arabidopsis. These factors typically bind to (T) TGAC (C/T) Wboxes, also found in defense-related gene promoters. A sugar-induced WRKY-type TF, SUSIBA2, that is, expressed in barley endosperm binds to the SURE and W-box, but not the SP8a element, to activate the barley isoamylase1 (iso1) promoter. In addition, a novel DNA-binding protein, designated STOREKEEPER (STK), specifically recognizes the B-box motif to control sucrose induced patatin expression in potato tubers. A more recent dissection analysis involved the sugar/ABA-induced sweet potato sporamin. A promoter in transgenic tobacco has yielded a minimal promoter (Spomin) that contains negatively acting regions and two carbohydrate metabolite signal responsive elements (CMSRE), CMSRE-1 (TGGACGG) and CMSRE-2, in addition to the SP8a motif. The most recent and fruitful studies of transcription control have been obtained by analyzing the sugar-inducible promoter of a sporamin gene that encodes the most abundant protein in sweet potato storage roots. Two putative TFs, WRI1 (activator of Spomin::LUC1; ASML1) and a novel CCT domain protein (ASML2), were isolated recently by enhancer activation-tagging of an Arabidopsis line carrying the LUC reporter under control of a short, minimal sugar/ABA inducible sporamin promoter. Several sugar-regulated genes, including $\beta A M Y$ and, in the case of ASML2, APL3, are activated in the transgenic lines. Both TF genes are also specifically induced by high sugar concentrations. Apparently, the WRI1 TF plays an important role in directing the carbon flow toward storage when sugar levels are high. The his 2 mutant displays high Spomin: LUC1 reporter activity even in noninducing conditions and is deficient in a novel B3 domain transcriptional repressor (Gomez et al., 2006).
Sugars also modulate hormone signaling at the transcriptional level (Riou-Khamlichi et al., 2000). Most obviously, glucose induces $\mathrm{ABA}$ and $\mathrm{ABI}$ gene expression as a core mechanism of its signal transduction. A detailed analysis of three factors involved in sugar signaling, ABI4, ABI5, and CTR1, documents their specific and developmental stage. Glucose repression of several ethylene biosynthesis and signal transduction genes suggests that interactions between sugar and ethylene signaling take place in part at the transcriptional level. Studies with several maize photosynthetic gene promoters suggest the involvement of different regulatory elements in sugar repression and negative control of positive cis-elements. Extensive studies of sugar repression and starvation induction of transcription have also been carried out on the promoters of rice genes that encode $\alpha$-amylases ( $\alpha \mathrm{AMY})$, involved in seed starch degradation. In a study with a minimal $\alpha$ AMY3 promoter, a sugar-response sequence (SRS) was identified with three essential elements for high sugar starvationinduced expression: the GC-box, the G-box, and the TATCCA element. Interestingly, three novel MYB proteins with a single DNA-binding domain (OsMYBS1-3) specifically bind to the TATCCA element to regulate aAMY expression. The identification of G-box cis-elements provides a link between nutrient stress and other environmental stress responses. The G-box motif (CACGTG) is, for example, involved in phytochromemediated light control of gene expression and is very similar to ABRE (CCACGTGG). The ABRE-binding factors $\mathrm{ABF} 2, \mathrm{ABF} 3$, and $\mathrm{ABF} 4$ have also been implicated in sugar signaling. Analysis of a conserved minimal light-responsive module (CMA5) recently revealed an ABI4 dependent sugar and ABA repression mechanism involving a novel element conserved in several RBCS promoters. This S-box element (CACCTCCA) is an ABI4-binding site and is typically closely associated with the G-box in light-regulated promoters. Novel bioinformatics and experimental approaches will be required to use fully the large number of publicly available microarray data to uncover new regulatory elements and TF functions in sugar regulation (Paul and Foyer, 2001; Schluepmann et al., 2003).

\subsection{IMPORTANCE OF SUGAR INTERACTION WITH PHYTOHORMONES IN REGULATION OF GROWTH AND DEVELOPMENT UNDER IN VITRO CONDITION}

Plant growth regulators include naturally occurring plant hormones such as indole acetic acid (IAA), gibberellins, zeatin, abscisic acid (ABA), and ethylene, and also a number of synthetic chemicals that affect or 
control growth and development in plants (Khan et al., 2012, 2013, 2014, 2015, 2016; Khan and Khan 2014; 2017). These compounds are important regulators of plant growth and mediate responses to both biotic and abiotic stresses. In the future, a major challenge will be to understand how the information conveyed by these simple compounds is integrated during plant growth (Table 16.1). Plant biologists have been fascinated by the regulatory capacity of phytohormones since the time of their discovery, and the notion that hormone levels or responses could be manipulated to improve desired plant traits has long been an area of intense interest (Sasaki et al., 2002).

Each type of plant growth regulator has a wide range of physiological effects in different plants. Phytohormones have been shown to increase growth and yield of plants (Nazar et al., 2014). These effects are determined by the kind of growth regulator, its concentration, the presence or absence of other growth regulators, and by the genetic makeup and the physiological status of the target tissue. The same physiological response in different tissues even of the same plant may require different growth regulator(s) or different combinations of growth regulators. The growth and development of plants under varied environmental conditions determine agricultural production. The growth, development, and senescence of plant's organs can influence crop production by modulating photosynthesis, nutrient remobilization efficiency, and harvest index (Jing et al., 2005; Khan et al., 2012). Every stage of the plant's life cycle is regulated by plant hormones. In general, plant biological activity is manipulated by more than one hormone, thus the biological phenomenon often reflects the combined interplay of several different hormones. Meanwhile, unlike animals, which can escape from harsh environments, plants can only survive through adjusting various biological activities when encountering biotic and abiotic stresses. During these situations, plant hormones also cooperate to modify biological responses for the formation and maintenance of plant stress tolerance.

Sugar production and distribution has been found to play vital role in plant physiology. Plant growth and development in plants is modulated through sugar production by photosynthesis and also organizes internal regulators and environmental signals (Smeekens, 2000). The effect of carbon allocation on organ and on whole plant architecture is illustrated most dramatically by carbohydrate storage and the concomitant cell expansion in reserve organs such as roots, fruit, seed, and tubers. Sugars are the most important carbon and energy source to cells, and also have important regulatory functions in controlling metabolism, growth, and development of plants under in vitro conditions (Ramon et al., 2008). Sugars operate both as immediate substrates for intermediary metabolism and as effective signaling molecules. Sugars as signaling compounds have intense effects in all stages of the plant's life cycle from germination and vegetative growth to reproductive development and seed

TABLE 16.1 Phytohormones and Their Functions in Growth and Development Under In Vitro Conditions

- Auxins
coleoptile sections. Usually the transport is unidirectional, called polar transport, but recently it has been reported that it has
been transported acropetally in phloem
- IAA, IBA are naturally occurring auxins
Functions under in vitro conditions
- Elongates cell
- Regulates apical dominance
- Forms lateral and adventitious roots
- Delays the onset of leaf abscission
- Regulates lateral floral bud development
- Promotes fruit development
- Cytokinins move to the leaves from roots, keeping root and shoot growth in balance
- Occur in both free and bound forms
- in higher plants and bacteria
Foot tip is important for its synthesis; however, cambial tissue and developing seed are also site synthesis
- Proms under in vitro conditions
- Promotes cell division
- Promotes cell expansion in leaves and regulates growth of stem and roots
- Modifies apical dominance and promotes lateral bud growth

The auxin and cytokinin ratio regulates morphogenesis and callus formation under in vitro conditions. 
formation (Smeekens, 2000). Plant sugar regulation is mediated by sugar signals, which are generated at different locations depending on environmental conditions and developmental stage. Sucrose (Suc) transport and hydrolysis play key regulatory roles in sugar signal generation (Ruan, 2014). In plants, sensing and signaling pathways have been described for different sugars (Hanson and Smeekens, 2009) but only for glucose detailed information on sensing and signaling mechanisms is available (Grigston et al., 2008).

\subsection{FUNCTION OF PHYTOHORMONES UNDER IN VITRO CONDITIONS}

Significant evidence of associations between sugar and phytohormones response and other metabolic pathways have also been provided by recent studies (Gibson, 2005). However, no systematic study has been done to explore the molecular basis of interaction between the two signaling molecules, that is, sugar and auxin, although both regulate similar processes and are so fundamental to plants (Mishra et al., 2009). Resistance toward exogenous auxin application is exhibited by glucose insensitive mutant gin2, which is mutated in glucose sensor HXK gene (Moore et al., 2003). Sugar and auxin regulate a number of common responses vis-à-vis root and shoot development, vascular morphogenesis, cell division and expansion, embryogenesis, immunity, chloroplast and anthocyanin biogenesis, de novo organogenesis, lateral root formation, leaf morphology and senescence, stress responses, immunity, nodule organogenesis, and photomorphogenesis (Kieber and Schaller 2010; Müller 2011; Brenner et al., 2012; Gupta and Rashotte 2012; Hwang, Sheen and Müller 2012; Shi and Rashotte 2012; Spíchal 2012). Sugars and cytokinin (CK) can control similar responses, through a two-component signaling cascade. Cytokinin (CK) signaling modulates sugarinduced anthocyanin biosynthesis via regulating sugar-inducible structural and regulatory genes (Das et al., 2012). Cyclin D3 (CycD3) expression is induced by sucrose alone and in combination with cytokinin (Kushwah and Laxmi, 2014). However, CK does not induce $\mathrm{CycD} 3$ expression in the absence of sucrose (Riou-Khamlichi et al., 1999). In cytokinin (CK) metabolism and signaling, the several genes involved can be regulated by glucose (Kushwah and Laxmi, 2014). Physiologically, hypocotyl length in dark could be regulated by both glucose and cytokinin. CK-regulated gene expression may also be affected by glucose via nontranscriptional pathways. Sugar and CK signaling is linked in antagonistic manner in the delayed leaf senescence phenotype found in glucose receptor gin2 mutant (Moore et al., 2003).

\subsection{CONCLUSION AND FUTURE PROSPECTS}

Sugar plays an important role in the metabolism of plants. They are now recognized as regulatory molecules with signaling functions in plants and other organisms. Yeast genetics has enabled the rapid and detailed elucidation of diverse sugar sensing and signaling pathways, plant sugar signaling has proven more difficult to study due to the complexity of source-sink interactions, responses to diverse sugar signals and metabolites, and the intimate integration of a web-like signaling network governed by plant hormones, nutrients, and environmental conditions (Rolland et al., 2006). The use of different experimental systems, including isolated cells, excised tissues, cell cultures, whole plants, and mutants under different environmental and nutrient conditions at various developmental stages is critical in dissecting the plethora of sugar responses and their connections in plants. Microarray and clustering analysis are new, powerful genomic tools to provide a global view on the transcript dynamics controlled by different sugar responses and identify novel regulatory components and target genes. The sharing of massive datasets is beginning to provide new insights into the extent and mechanisms of sugar-regulated gene expression and interactions with other signals. The molecular details of signal transduction pathways and their crosstalk with other pathways will be revealed by using a combination of genomic proteomic and genetic approaches. Current technology limits the ability to visualize and quantify the precise location and concentration of various sugar molecules and metabolites in living cells. Novel molecular sensors and fluorescence resonance energy transfer-based imaging will hopefully circumvent this limitation and provide critical information to facilitate the elucidation of intracellular sugar signal transduction pathways.

\section{References}

Brenner, W.G., Ramireddy, E., Hey, A., Schmülling, T., 2012. Gene regulation by cytokinin in Arabidopsis. Front. Plant Sci. 3, 8.

Chiou, T.J., Bush, D.R., 1998. Sucrose is a signal molecule in assimilate partitioning. Proc. Natl. Acad. Sci. 95, 4784-4788.

Cho, Y.H., Sheen, J., Yoo, S.D., 2010. Low glucose uncouples HXK1dependent sugar signaling from stress and defense hormone ABA and C2H4 responses in Arabidopsis. Plant Physiol. 152, 1180-1182.

Das, P.K., Shin, D.H., Choi, S.-B., Yoo, S.-D., Choi, G., Park, Y.-I., 2012. Cytokinins enhance sugar-induced anthocyanin biosynthesis in Arabidopsis. Mol. Cells 34, 93-101.

Dekkers, B.J., Schuurmans, J.A., Smeekens, S.C., 2004. Glucose delays seed germination in Arabidopsis thaliana. Planta 218, 579-588. 
Dobrenel, T., Caldana, C., Hanson, J., Robaglia, C., Vincentz, M., 2016. TOR signaling and nutrient sensing. Annu. Rev. Plant. Biol. 67, 261-285.

Ehlert, A., Weltmeier, F., Wang, X., Mayer, C.S., Smeekens, S., Vicente Carbajosa, J., et al., 2006. Two-hybrid protein-protein interaction analysis in Arabidopsis protoplasts: establishment of a heterodimerization map of group $\mathrm{C}$ and group $\mathrm{S}$ bZIP transcription factors. Plant J. 46, 890-900.

Eveland, A.L., Jackson, D.P., 2012. Sugars, signalling, and plant development. J. Exp. Bot. 63, 3367-3377.

Gazzarrini, S., McCourt, P., 2001. Genetic interactions between ABA, ethylene and sugar signaling pathways. Curr. Opin. Plant. Biol. 4, 387-391.

Gibson, S.I., 2005. Control of plant development and gene expression by sugar signaling. Curr. Opin. Pant Biol. 8, 93-102.

Gomez, L.D., Baud, S., Gilday, A., Li, Y., Graham, I.A., 2006. Delayed embryo development in the arabidopsis trehalose-6-phosphate synthase 1 mutant is associated with altered cell wall structure, decreased cell division and starch accumulation. Plant J. 46, $69-84$.

Gonzalez, N., Vanhaeren, H., Inzé, D., 2012. Leaf size control: complex coordination of cell division and expansion. Trends. Plant. Sci. (6), 332-340.

Granot, D., Kelly, G., Stein, O., David-Schwartz, R., 2014. Substantial roles of hexokinase and fructokinase in the effects of sugars on plant physiology and development. J. Exp. Bot. 65, 809-819.

Grigston, J.C., Osunac, D., Scheiblec, W.-R., Liua, C., Stittc, M., Jones, A.M., 2008. d-glucose sensing by a plasma membrane regulator of G signaling protein, AtRGS1. FEBS Lett. 582, 3577-3584.

Gupta, S., Rashotte, A.M., 2012. Down-stream components of cytokinin signaling and the role of cytokinin throughout the plant. Plant Cell Rep. 31, 801-812.

Hanson, J., Smeekens, S., 2009. Sugar perception and signaling - an update. Curr. Opin. Plant. Biol. 12 (2), 562-567.

Hwang, I., Sheen, J., Müller, B., 2012. Cytokinin signaling networks. Annu. Rev. Plant. Biol. 63, 353-380.

Jang, J.C., Jen Sheen, J., 1997. Sugar sensing in higher plants. Trends. Plant. Sci. (2), 208-214.

Jing, H.C., Schippers, J.H., Hille, J., Dijkwel, P.P., 2005. Ethyleneinduced leaf senescence depends on age-related changes and OLD genes in Arabidopsis. J. Exp. Bot. 56, 2915-2923.

Kang, S.G., Price, J., Lin, P.C., Hong, J.C., Jang, J.C., 2010. The Arabidopsis bZIP1 transcription factor is involved in sugar signaling, protein networking, and DNA binding. Mol. Plant 3 (2), 361-373.

Karsai, I., Vedo, Z., Hayes, P.M., 1994. Effect of induction medium, $\mathrm{pH}$, and maltose concentration on in vitro androgenesis of hexaploid winter triticale and wheat. Plant Cell Tiss. Organ Cult. 39, 49-53.

Khan, M.I.R., Khan, N.A., 2014. Ethylene reverses photosynthetic inhibition by nickel and zinc in mustard through changes in PS II activity, photosynthetic-nitrogen use efficiency and antioxidant metabolism. Protoplasma 251, 1007-1019.

Khan, M.I.R., Iqbal, N., Masood, A., Per, T.S., Khan, N.A., 2013. Salicylic acid alleviates adverse effects of heat stress on photosynthesis through changes in proline production and ethylene formation. Plant Signal. Behav. 8, e26374.

Khan, M.I.R., Asgher, M., Khan, N.A., 2014. Alleviation of saltinduced photosynthesis and growth inhibition by salicylic acid involves glycine betaine and ethylene in mungbean (Vigna radiata L.). Plant. Physiol. Biochem. 80, 67-74.

Khan, M.I.R., Khan, N.A., Masood, A., Per, T.S., Asgher, M., 2016. Hydrogen peroxide alleviates nickel-inhibited photosynthetic responses through increase in use-efficiency of nitrogen and sulfur, and glutathione production in mustard. Front. Plant Sci. 7, 44.
Khan, M.I.R., Khan, N., 2017. Reactive Oxygen Species and Antioxidant System in Plants: Role and Regulation Under Abiotic Stress. Springer Nature, Singapore978-981-10-5254-5.

Khan, M.I.R., Syeed, S., Nazar, R., Anjum, N.A., 2012. An insight into the role of salicylic acid and jasmonic acid in salt stress tolerance. Phytohormones and Abiotic Stress Tolerance in Plants. SpringerVerlag, Berlin, pp. 277-300.

Khan, M.I.R., Fatma, M., Per, T.S., Anjum, N.A., Khan, N.A., 2015. Salicylic acid-induced abiotic stress tolerance and underlying mechanisms in plants. Front. Plant Sci. 6, 462.

Kieber, J.J., Schaller, G.E., 2010. The perception of cytokinin: a story 50 years in the making. Plant Physiol. 154, 487-492.

Kushwah, S., Laxmi, A., 2014. The interaction between glucose and cytokinin signal transduction pathway in Arabidopsis thaliana. Plant Cell Environ. 37, 235-253.

Loreti, E., Povero, G., Novi, G., Solfanelli, C., Alpi, A., Perat, P., 2008. Gibberellins, jasmonate and abscisic acid modulate the sucroseinduced expression of anthocyanin biosynthetic genes in Arabidopsis. New Phytol. 179, 1004-1016.

Mishra, B.S., Singh, M., Aggrawal, P., Laxmi, A., 2009. Glucose and auxin signaling interaction in controlling Arabidopsis thaliana seedlings root growth and development. PLoS One 4, e4502.

Moore, B., Zhou, L., Rolland, F., Hall, Q., Cheng, W.H., Liu, Y.X., et al., 2003. Role of the Arabidopsis glucose sensor HXK1 in nutrient, light, and hormonal signaling. Science 300, 332-336.

Müller, B., 2011. Generic signal-specific responses: cytokinin and context dependent cellular responses. J. Exp. Bot. 62, 3273-3288.

Müller, J., Aeschbacher, R.A., Wingler, A., Boller, T., Wiemken, A., 2001. Trehalose and trehalase in Arabidopsis. Plant Physiol. 125, 1086-1093.

Murashige, T., Skoog, F., 1962. A revised medium for rapid growth and bioassays with tobacco tissue cultures. Physiol. Plant. 15, 473-497.

Nazar, R., Khan, M.I.R., Iqbal, N., Masood, A., Khan, N.A., 2014. Involvement of ethylene in reversal of salt-inhibited photosynthesis by sulfur in mustard. Physiol. Plant. 152, 331-344.

Ohto, M., Onai, K., Furukawa, Y., Aoki, E., 2001. Effects of sugar on vegetative development and floral transition in Arabidopsis. Plant Physiol. 127, 252-261.

Orsinky, B.L., McGregor, G.I., Johnson, G.I., Kartha, K.K., 1990. Improved embryoid induction and green shoot regeneration from wheat anther cultures with medium with maltose. Plant Cell Rep. 9, 365-369.

Paul, M.J., Foyer, C.H., 2001. Sink regulation of photosynthesis. J. Exp. Bot. 526 (1), 1383-1400.

Powell, A.E., Lenhard, M., 2012. Control of organ size in plants. Curr. Biol. 22, R360-R367.

Price, J., Laxmi, A., St Martin, S.K., Jang, J.C., 2004. Global transcription profiling reveals multiple sugar signal transduction mechanisms in Arabidopsis. Plant Cell 16, 2128-2150.

Ramon, M., Rolland, F., Sheena, J., 2008. Sugar sensing and signaling. Arabidopsis Book 2008 (6), e0117.

Riou-Khamlichi, C., Huntley, R., Jacqmard, A., Murray, J.A.H., 1999. Cytokinin activation of Arabidopsis cell division through a dtype cyclin. Science 283, 1541-1544.

Riou-Khamlichi, C., Menges, M., Healy, J.M.S., Murray, J.A.H., 2000. Sugar control of the plant cell cycle: differential regulation of Arabidopsis d-type cyclin gene expression. Mol. Cell. Biol. 20, $4513-4521$.

Rolland, F., Moore, B., Sheen, J., 2002a. Sugar sensing and signaling in plants. Plant Cell (Suppl. 14), S185-S205.

Rolland, F., Moore, B., Sheen, J., 2002b. Sugar sensing and signaling in plants. Plant Cell 185-204.

Rolland, F., Winderickx, J., Thevelein, J.M., 2002c. Glucose-sensing and -signalling mechanisms in yeast. FEMS. Yeast. Res. (2), 183-201. 
Rolland, F., Baena-Gonzalez, E., Sheen, J., 2006. Sugar sensing and signaling in plants: conserved and novel mechanisms. Annu. Rev. Plant. Biol. 57, 675-709.

Rook, F., Bevan, M.B.M., 2003. Genetic approaches to understanding sugar-response pathways. J. Exp. Bot. 54, 495-501.

Ruan, Y.P., 2014. Sucrose Metabolism: gateway to diverse carbon use and sugar signaling. Annu. Rev. Plant. Biol. 65, 33-67.

Sasaki, A., Ashikari, M., Ueguchi-Tanaka, M., Itoh, H., Nishimura, A., et al., 2002. Green revolution: a mutant gibberellin-synthesis gene in rice. Nature 416, 701-702.

Schluepmann, H., Pellny, T., van Dijken, A., Smeekens, S., Paul, M., 2003. Trehalose 6-phosphate is indispensable for carbohydrate utilization and growth in Arabidopsis thaliana. Proc. Natl. Acad. Sci. U.S.A. 100, 6849-6854.

Shi, X., Rashotte, A.M., 2012. Advances in upstream players of cytokinin phosphorelay: receptors and histidine phosphotransfer proteins. Plant Cell Rep. 31, 789-799.

Smeekens, S., Hellmann, H.A., 2014. Sugar sensing and signaling in plants. Front. Plant Sci. 5, 113.

Smeekens, S., 2000. Sugar-induced signal transduction in plants. Annu. Rev. Plant. Physiol. Plant. Mol. Biol. 51, 49-81.

Smeekens, S., Rook, F., 1998. Sugar sensing and sugar-mediated signal transduction in plants. Plant Physiol. 115, 7-13.

Spíchal, L., 2012. Cytokinins-recent news and views of evolutionally old molecules. Funct. Plant Biol. 39, 267-284.

Sun, C., Ahlandsberg, S., Palmqvist, S., Ohlsson, H., Borén, M., Jansson, C., 2003. A novel WRKY transcription factor, SUSIBA2, participates in sugar signaling in barley by binding to the SURE element of the iso1 promoter. Plant Cell 15, 2076-2092.

Wiese, A., Groner, F., Sonnewald, U., Deppner, H., Lerchl, J., Hebbeker, U., et al., 1999. Spinach hexokinase I is located in the outer envelope membrane of plastids. FEBS Lett. 461, 13-18.

Xiao, W., Sheen, J., Chyun, J., 2000. The role of hexokinase in plant sugar signal transduction and growth and development. Plant. Mol. Biol. 44, 451-461.
Xu, X., Van Lammeren, A.A.M., Vermeer, E., Vreugdenhil, D., 1998. The role of gibberellin, abscisic acid, and sucrose in the regulation of potato tuber formation in vitro. Plant Physiol. 117, $575-584$.

\section{Further Reading}

Iqbal, N., Khan, N.A., Nazar, R., Teixeira da Silva, J.A., 2012. Ethylene stimulated photosynthesis results from increased nitrogen and sulfur. Environ. Exp. Bot. 78, 84-90.

Joseph, D., Clarke, T.Z., 2006. Microarray analysis of the transcriptome as a stepping stone towards understanding biological systems: practical considerations and perspectives. Plant J. 45, $630-650$.

Li, L., Sheen, J., 2016. Dynamic and diverse sugar signaling. Sheen Curr. Opin. Plant Biol. 33, 116-125.

Liu, Y., Bassham, D.C., 2010. TOR is a negative regulator of autophagy in Arabidopsis thaliana. PLoS One 5, 27-30.

Moore, B., Zhou, L., Rolland, F., Hall, Q., Cheng, W.H., Liu, Y.X., et al., 2006. Role of the Arabidopsis glucose sensor HXK1 in nutrient, light, and hormonal signaling. Science 1, 332-336.

Paul, M.J., Primavesi, L.F., Jhurreea, D., Zhang, Y., 2008. Trehalose metabolism and signaling. Annu. Rev. Plant. Biol. 596 (1), 417-441.

Rook, F., Bevan, M.W., 2003. Genetic approaches to understanding sugar-response pathways. J. Exp. Bot. 54, 495-501.

Singh, A., Virdi, Singh, S., Singh, P., 2015. Abiotic stress responses in plants: roles of calmodulin-regulated proteins. Front. Plant Sci. 6, 809.

Smeekens, S., Rook, F., 1997. Sugar sensing and sugar-mediated signal transduction in plants. Plant Physiol. 115, 7-13.

Yongqiang, Z., Junxian, H.E., 2015. Sugar-induced plant growth is dependent on brassinosteroids. Plant Signal. Behav. 10 (12), e1082700. 


\title{
17
}

\section{Role of Mineral Nutrients in Abiotic Stress Tolerance: Revisiting the Associated Signaling Mechanisms}

\author{
Mohammad Abass Ahanger ${ }^{1}$ and Parvaiz Ahmad ${ }^{1,2}$
}

${ }^{1}$ Department of Botany, Govt PG College Rajouri, Rajouri, Jammu and Kashmir, India

${ }^{2}$ Department of Botany and Microbiology, Faculty of Science, King Saud University, Riyadh, Saudi Arabia

\section{O U T L I N E}

17.1 Introduction

17.2 Mineral Nutrients and Stress Tolerance

17.2.1 Nitrogen

17.2.2 Sulfur

17.2.3 Potassium

17.3 Phosphorous

17.4 Calcium
17.5 Conclusion and Future Prospects

Acknowledgments

References

Further Reading
276

278

\subsection{INTRODUCTION}

On one hand, world population is expected to cross 8.3 billion in the next two decades (FAO, 2010) and rapid urbanization and industrialization, on the other hand, have resulted in considerable shrinkage of the arable land area all over the globe, thereby raising the grave issue of sustainable food security. No doubt, it has been widely accepted that most of the food crops have shown apparent increase in productivity during the past few years. Therefore maintaining such positive effects for meeting the needs of the increasing population in future needs the integration of scientific methods with the conventionally employed agricultural practices (Fan et al., 2012; Ahmad and Wani, 2014; Ahanger et al., 2017a). In nature normal growth and development of plants is regulated by several environmental factors affecting the sustainability of crop productivity. Sustainable crop production is primarily a function of several biotic and abiotic environmental stress factors, and also is directly linked with the mineral status of soil (Sogbedi et al., 2006). It is interesting to point out that both biotic as well as abiotic stresses adversely affect the soil mineral status 
and the access of plant roots to the existing minerals in the rhizosphere, therefore affecting growth and development at the plant level (Turkan and Demiral, 2009; Khan et al., 2015; Ahanger and Agarwal, 2017a,b). Increasing magnitude of stresses in future is expected to impose considerable loss of fertile land, for example, high soil salinity impedes plant productivity by triggering ionic and osmotic effects leading to restricted mineral uptake and altered assimilation (Fatma et al., 2014; Ahanger and Agarwal, 2017a). Contrary to this, water deficit impedes nutrient access to plant roots (Osakabe et al., 2014) while metal(loid)s restrict the uptake of mineral ions by potentially reducing the activity of ion transporters (Tamas et al., 2014). Individually stresses are not so lethal to plants when compared with their combined effects, and cumulative exposure of crops to biotic and abiotic stresses can reduce productivity by 75\% (Noman et al., 2017). Loss in productivity is due to the negative effects of the stress-triggered alterations in the key metabolic pathways leading to anomalies in the developmental progression. Among the key negative regulators are included the reactive oxygen species (ROS), which are continuously generated in cellular organelles like chloroplast, mitochondria, peroxisomes, etc. Among the key consequences of ROS are included the inhibition of photosynthetic and mitochondrial electron transport systems, and metabolic dysfunction leading to damage to cellular structures and offering premature senescence (Petrov et al., 2015; Rogers and Munne-Bosch, 2016). However, reports are available that advocate the beneficial role of ROS via their involvement in the stress signaling (Camejo et al., 2016; Mittler, 2017; Khan and Khan, 2017). In addition there are other adversaries triggered by stress exposures that are more or less regulated by the availability of sufficient concentrations of different mineral elements. Availability of mineral elements controls the growth and protects metabolism under extreme conditions (Ahanger et al., $2017 \mathrm{~b}$ ) and has been observed to bring integration of several signaling events either through their direct involvement or by inducing improved synthesis of key signaling molecules (Iqbal et al., 2015; Khan et al., 2015; Ahanger et al., 2015). Besides this having effects on the expression of genes controlling growth and yield, mineral elements strongly affect the distribution of plant species (John et al., 2007; Barbosa et al., 2014). Since environmental stresses are potential threats for sustainable agricultural productivity, the present review focuses on the current understandings about the stress-triggered deleterious effects on the uptake, transport, and assimilation of key mineral elements making interpolation of relevant reports and identifying the key future targets for overcoming such effects. In addition the role of nutrients in mediating signaling individually and combinedly for elicitation of stress tolerance mechanisms has also been discussed.

\subsection{MINERAL NUTRIENTS AND STRESS TOLERANCE}

\subsubsection{Nitrogen}

Nitrogen $(\mathrm{N})$ is a vital plant nutrient considered as a major determining factor required for growth and yield productivity of major crops. It makes up nearly $4 \%$ of total dry matter and is a key component of important molecules like proteins and nucleic acids. Sufficient availability of $\mathrm{N}$ throughout the plant life cycle or growing season is important for attaining the optimum growth and yield. Nitrogen availability has been reported to affect proteins and many other compounds essential for plant growth processes including chlorophyll and enzyme activities (Iqbal et al., 2015). Besides imparting growth promotion through its own effects, $\mathrm{N}$ availability also affects the uptake and utilization of other key elements like phosphorous, potassium, sulfur, zinc, boron, etc. causing significant enhancement in the yield and yield associated attributes (Dash et al., 2015). Therefore, it can be inferred that $\mathrm{N}$ availability has direct influence on the uptake as well as assimilation of other key minerals. However lack of sufficient quantities of these nutrients also influences $\mathrm{N}$ uptake.

Nitrogen availability profusely contributes to biomass accumulation by influencing photosynthesis and synthesis of proteins and nucleic acids, and impeded uptake and assimilation affects growth at the whole plant level (Masclaux-Daubresse et al., 2010). Ammonium $\left(\mathrm{NH}_{4}^{+}\right)$and nitrate $\left(\mathrm{NO}_{3}^{-}\right)$are the abundant forms of $\mathrm{N}$ taken up by plants and $\mathrm{NH}_{4}^{+}$is released from some metabolic processes including amino acid deamination during seed germination, biosynthesis of amino acids, and photorespiration. This $\mathrm{NH}_{4}^{+}$must be reassimilated to meet the cellular nitrogen needs so that any possibility of $\mathrm{NH}_{4}^{+}$toxicity can be avoided. For maintaining the beneficial consequences of $\mathrm{NH}_{4}^{+}$the plant must maintain optimal activity of glutamine synthetase (GS, EC: 6.3.1.2), glutamate synthase (GOGAT, EC: 1.4.1.14), and glutamate dehydrogenase (GDH, EC: 1.4.1.2) enzymes. Contrary to this for $\mathrm{NO}_{3}^{-}$assimilation nitrate reductase (NR, EC: 1.6.6.1) and nitrite reductase (NiR, EC: 1.7.2.1) form key mediators to contribute to the formation of $\mathrm{NH}_{4}^{+}$. The assimilated nitrogen moiety is fixed in the form of organic acids by aminotransferases resulting in biosynthesis of amino acids (Masclaux-Daubresse et al., 2010; Ahanger et al., 2017b). It has been proved that plant species maintaining optimal functioning of nitrogen 
metabolizing enzymes exhibit apparent increase in amino acids involved in growth regulation and stress tolerance (Pandey et al., 2004; Iqbal et al., 2015; Ahanger et al., 2017b). At root plasma membrane levels the transport proteins functioning for the nitrate uptake have been identified as low affinity (LA) and high affinity transport system (HATS) and their expression has been observed to show considerable variation with respect to available nitrate concentration, that is, LATS and HATS act when external nitrate concentration is high and low respectively (MasclauxDaubresse et al., 2010). Several such transport proteins have been isolated from plants like Arabidopsis and their functional characterization has been done, and their role in long distance transport of nitrate has been studied and confirmed using mutant lines (Tsay et al., 1993; Huang et al., 1999; Filleur et al., 2001; Chiu et al., 2004; Almagro et al., 2008). Chopin et al. (2007) have demonstrated that overexpressing AtNRT2.7 accumulates greater nitrate, making the seeds less dormant. The availability of nitrate induces the development of lateral roots in Arabidopsis (Zhang and Forde, 1998). Exposure of Brassica juncea to abiotic (osmotic, salinity, high and low temperatures) stresses downregulates the expression of key genes coding for nitrate and ammonium transport proteins and their assimilatory enzymes (Goel and Singh, 2015).

It has been shown that the availability of nitrogen affects the synthesis of nitrogen containing metabolites leading to regulation of several signaling events by modulating the expression of key molecules like transcription factors (TFs) in addition to their involvement in such processes. $\mathrm{N}$ availability affected the synthesis of glutamine in rice and supplementation of exogenous glutamine reversed the ill effects by affecting the expression important TFs including LBD37-like genes involved in the regulation of nitrogen metabolism and $D R E B 1 A, I R O 2$, and NAC5 TF genes mediating regulation of stress responses (Kan et al., 2015), reflecting in the involvement of glutamine in amplification and subsequent modification of signals, and crosstalk with key molecules for bringing growth regulation under stress. Iqbal et al. (2011; 2015) have demonstrated that increasing $\mathrm{N}$ application optimizes the photosynthetic efficiency of Brassica juncea and the involvement of exogenously applied ethylene was shown to regulate $\mathrm{N}$-use efficiency.

Nitrogen is primarily transported by amino acids and therefore identifying the QTL preferably controlling the $\mathrm{N}$ uptake and the subsequent incorporation into amino acids can be worthwhile for improving the nutrient use efficiency (NUE) in plants. For achieving this integrating plant physiology and genetics to reflect in clear understanding of plant performance under optimal and suboptimal $\mathrm{N}$ supplementation and the identification of several QTL controlling transport, assimilation, and metabolism of $\mathrm{N}$ have been identified (Han et al., 2016). Among these identified genes some have been worked out for their role in $\mathrm{N}$ metabolism, for example, transgenic Pisumsativum overexpressing amino acid transporter AMINO ACID PERMEASE1 (AAP1) showed greater allocation of $\mathrm{N}$ via the vasculature to the shoot and seeds thereby producing greater biomass and yield (Perchlik and Tegeder, 2017).

It has been demonstrated that Saccharomyces cerevisiae Snf1 protein kinase of the Snf1/AMP is directly involved in nitrogen signaling. Its involvement in pseudohyphal differentiation depends on the stimulatory phosphorylation of Snf1 at Thr210 and N limitation induces improved Thr210 phosphorylation besides the negative regulation of Snf1 by rapamycin-sensitive TOR (target of rapamycin) kinase actively involved in signaling nitrogen and amino acid availability (Orlova et al., 2006). In yeast cells, the TOR pathway has been implicated in regulating cellular responses to nutrients, including proliferation, translation, transcription, autophagy, and ribosome biogenesis. It has been reported that overexpressing Tap42-Sit4, a protein phosphatase regulatory subunit, restores the pseudohyphal growth in cells exposed to rapamycin. Possible involvement of Tor protein kinases and Tap42-Sit4 protein phosphatase interactions have been proposed to regulate $\mathrm{N}$ nutrient-sensing pathway involving the activation of the MAP kinase or cAMP pathways (Cutler et al., 2001). In addition, of the molecular regulation the biochemical regulation of $\mathrm{N}$ assimilation has been worked out. Nitrate has been confirmed as the main primary signal molecule triggering activation of the transcription of key genes controlling nitrate assimilation and related genes, which further directly affects the processes like photosynthesis, cell cycle control, and other related translational events (Takei et al., 2002).

\subsubsection{Sulfur}

Sulfur (S) is another key nutrient actively regulating the plant growth and development. Fourth in importance after N, P, and K, S forms an integral part of several metabolically important compounds like vitamins, phytohormones, coenzymes, etc., which affect plant growth and vigor considerably. $\mathrm{S}$ is considered to potentially regulate the growth and development of plant under normal and stress environments (Asgher et al., 2014; Fatma et al., 2016). Limited S availability restricts yield and quality of product, and adequate $S$ nutrition enhances photosynthesis and growth in plants (Fatma et al., 2014). In addition, S shows a growth regulatory interaction with other minerals like 
$\mathrm{N}$ assimilation (Scherer, 2008). Deficiency of S hampers the process of chlorophyll biosynthesis, $\mathrm{N}$ assimilation, photosynthesis, protein synthesis (Lunde et al., 2008). Once taken up by a plant, $\mathrm{S}$ gets incorporated into some key organic molecules, for example, thiol $(-\mathrm{SH})$ groups in cysteine residues or nonprotein thiols like glutathione. Among these S containing compounds, mostly reduced glutathione is sensitive to oxidized environment and has been recognized for its potential role in modulating the plant responses to a range of stresses (Tausz et al., 2004; Szalai et al., 2009; Khan et al., 2015). These $S$ containing compounds like glutathione are involved in the removal of excess ROS thereby preventing the oxidative damage to cells, and maintaining the concentration of ROS for integration of several beneficial roles (Rausch and Bucher, 2002). In addition, optimal availability and assimilation of $S$ regulates the biosynthesis of a nonprotein thiol leading to maintenance of the homeostasis between the reduced (GSH) and oxidized (GSSG) forms of glutathione thereby maintaining the signaling of stress response proteins and hence the oxidative stress amelioration. Since stresses pose deleterious effects on the metabolism of minerals including $S$, therefore a prerequisite requirement for mitigating the ill effects on growth and productivity will be the understanding of the involvement of $S$ in regulation of different signaling pathways under stress.

Sulfur supplementation regulates growth of plants under a range of environmental stresses. It has been observed that $S$ supplementation restricts the generation of excess ROS thereby preventing the oxidative damage (Fatma et al., 2014; Asgher et al., 2014; Khan et al., 2015). Recent reports have suggested the positive influence of applied $\mathrm{S}$ on the regulation of endogenous levels of phytohormones like ABA, JA, NO, and ethylene (Khan et al., 2014a,b; Fatma et al., 2016; Per et al., 2017, 2018). Sulfur is involved in the maintenance of $\mathrm{K} / \mathrm{Na}$ ratio (Abdelhamid et al., 2013) and restricting the accumulation of toxic ions like cadmium (Matraszek et al., 2016). In addition to restricting the generation of ROS, S supplementation improves the synthesis of Rubisco thereby enhancing photosynthetic efficiency and the yield productivity (Lunde et al., 2008), however the detailed mechanisms at the physiological and molecular levels are largely unknown.

Several S containing compounds have been demonstrated to protect plants from the stress induced deleterious effects, however, very little is known about the molecular mechanisms involved in regulating the assimilation of $S$ by stresses. Though reports are available, conferring the demand driven assimilation of $S$ for promoting its greater incorporation in protective compounds (Fatma et al., 2014; Khan et al., 2015) nevertheless it should be noted that excess $\mathrm{S}$ can prove repressive in regulation of growth and development of plants. For mediating scavenging of ROS plants upregulate $S$ assimilation by improving rate of transport as well as reduction. A reduction in the concentrations of GSH induces the activity of 5-phosphosulfate reductase reflecting in the demand driven synthesis of $S$ containing compounds for stress amelioration. $\mathrm{S}$ transport mediated by ATP-sulfurylase and subsequent reduction by 5-phosphosulfate reductase are the key regulatory steps in $S$ metabolism and increased levels of mRNA coding for $S$ transporters like ATPsulfurylase and 5-phosphosulfate reductase have been observed reflecting in the availability dependent regulation at the transpirational level (Nikiforova et al., 2003). Many researchers are of the opinion that Oacetyl serine accumulation during stressful conditions may probably serve as signal for inducing the regulation at transcriptional levels (Ohkama et al., 2002; Hubberten et al., 2012). In Arabidopsis thaliana, Hubberten et al. (2012) have identified six different genes whose expression was correlated with the accumulation of OAS through the OAS mediated changes at transcriptional levels of their gene expression. Under $S$ deficiency the expression of $S$ responsive genes is also regulated by the availability of growth hormones, for example, in Arabidopsis thaliana exogenously supplied cytokinins, trans-zeatin, and trans-zeatin riboside upregulated the expression of sulfur-deficiency responsive element (beta SR), a beta subunit gene of betaconglycinin of seed storage protein (Ohkama et al., 2002). Additionally products of $S$ assimilation like GSH have been proposed to act as long distance signal in bringing coordination between the synthetic precursors and their assimilatory pathways (Lappartient et al., 1999). A kinase, GCN2, has been identified to act as a sensor of the carbon/nitrogen precursor availability, and $S$ limitation precursor, and is transduced to TOR by downregulation of the glucose metabolism and downregulation of TOR activity causes reduction in translation and meristematic activity, while elevating autophagy (Dong et al., 2017). The role of S in regulation of MAPK signaling cascades is not available and the identification of key signaling events triggered by $S$ deficiency or availability needs to be evaluated. Assimilation of $S$ is proved to be under direct control of phytohormones including SA, ethylene, auxins, etc. The involvement of three sulfur-starvation responsive TFs, IAA13, IAA28, and ARF-2 (ARF1-binding protein), related to auxin signaling has been observed to regulate developmental events in plants. Overexpressing or loss of function of $I A A 28$ imparted no major visible morphological changes, whereas IAA13- and ARF1$B P$-overexpressing lines exhibited much slower growth than the wild ones and it was confirmed that the maintenance of steady-state metabolite levels and 
expression of pathway-relevant genes under normal and sulfate-deficient conditions has no exclusive link with $S$ availability. Instead, it has been observed that the up- or downregulation of the specific TFs mediate metabolic changes, which in turn affect sulfur metabolism (Faljenberg et al., 2008).

Decreased S inputs have increased the incidence of S-deficiency in crops, causing decline in yield and yield associated attributes. Ability of crops to respond to S-deficiency stress shows considerable variation between crops and such gaps can be eliminated by introducing the genetic improvement of S-utilization efficiency. Focus of any genetic or molecular study should be improvement in the capture of available $S$ resources followed by subsequent accumulation of $S$ reserves and the remobilization of accumulated reserves. Inability of plant species to overaccumulate S and remobilize S-reserves restricts S-use efficiency. For improving this genetic manipulation of the transporters and their expression can be a key factor (Hawkesford, 2000; Lee et al., 2016).

Howarth et al. (2009) have identified a sulfate deficiency-induced gene, sdi1, in Triticum aestivum L. and it has been demonstrated that knocking down of sdi1 in Arabidopsis thaliana improved the endogenous sulfate concentrations under $\mathrm{S}$ deficient conditions. Another high-affinity sulfate transporter SULT1;1 has been seen to be highly regulated in the epidermal and cortical cells in Arabidopsis roots under $S$ deficiency, and its induction under the control of promoter sulfur responsive element (SURE) has been demonstrated to be regulated by improving external S supplementation like GSH and cysteine (Maruyama-Nakashita et al., 2005).

Controlled gene expression leads to limited uptake of excess $S$ and optimizes subsequent assimilatory pathways with endogenous expression of sulfate transporters being regulated by applied $\mathrm{S}$ where reduced S-containing compounds act as negative regulators and $O$-acetylserine as positive regulators. Contrarily constitutive expression of the transporters will eliminate this regulation paving the accumulation of sulfate reserves so that the accumulated sulfate in the vacuoles and other reduced sulfur pools like glutathione or protein may be remobilized under S-limiting conditions. Targeting the remobilization of vacuolar sulfate can revert the deleterious changes induced by $S$ deficiency ultimately affecting uptake from the soil solution and subsequent delivery to the site of reduction in chloroplast or plastids (Hawkesford, 2000; Kataoka et al., 2004). For further insights about this, the identification of gene family and their phylogenetic relationships and differences in spatial expression to unlock their functional roles remains to be evaluated.

\subsubsection{Potassium}

Potassium is a third important element for plants and is mostly absorbed in the form of ions as $\mathrm{K}^{+}$. Most of the available $\mathrm{K}$ in soil is either dehydrated or coordinated to oxygen atoms, and thereby is rendered unavailable for absorption (Ahanger et al., 2017b,c; Tittal et al., 2017). K availability to plants is sternly affected by the soil nutrient dynamics, soil $\mathrm{K}$ pool, physiochemical properties, and the type of the existing soil. Depending on its availability and accessibility to plants $\mathrm{K}$ has been identified to exist in four different pools, that is, (1) water soluble $\mathrm{K}$, (2) exchangeable K, (3) nonexchangeable K, and (4) structural K. Water soluble $\mathrm{K}$ is easily available to plants in addition to the exchangeable $\mathrm{K}$ pool that is represented by the electrostatically bound $\mathrm{K}$ to clay minerals and organic or humic substances. Both these available $\mathrm{K}$ pools are replenished by crops for their optimal physiological performance, even though they form a very small fraction of the whole $\mathrm{K}$ soil pool. In comparison, nonexchangeable $\mathrm{K}$ obtained from the weathering of mica rich rocks is not easily available to plants while the structural pool existing as micas, muscovite, biotite, and other feldspars constituting a major part of soil $\mathrm{K}$ is totally unavailable for plants (see Agarwal et al., 2009; Zorb et al., 2014). However it shall be noted here that the available $\mathrm{K}$ pool (water soluble $\mathrm{K}$ ) is often leached if unabsorbed by the plant roots. $\mathrm{K}$ application has a direct effect on the structure and properties of soil. Enhancement in the water holding capacity of soil and maintenance of the structural stability of soil particularly the sandy soils has been reported (Holthusen et al., 2012). K or mineral induced soil stability may be ascribed to increased shear resistance contributing to enhanced water retention. Mineral induced stability in soil structure is ascribed to increase in electrolyte concentration causing flocculation subsequently resulting in precipitation in the form of salt crystals (Van Olphen, 1977). In addition to $\mathrm{K}, \mathrm{Mg}^{2+}$ and $\mathrm{Ca}^{2+}$ are also effective cations for mediating the stabilization of soil structure because of their high relative flocculating power (Rengasamy and Sumner, 1998). Increasing water retention potential or water holding capacity can be very beneficial for crop plants in areas with limited water availability.

As an abundant inorganic constituent of cells and cation in the cytosol, $\mathrm{K}$ has a significant role in regulation of several physiological functions including protein synthesis, membrane polarization, and osmotic potential hence controlling osmotically driven functions like cell movements, stomatal functioning, and phloem transport (Zorb et al., 2014; Ahanger et al., 2017c). Therefore, enough quantity of K must be present in soil solution to ensure significant absorption, 
transport, and allocation to various organs. However K in soils displays considerable fluctuations making the fulfillment of demand driven needs of existing crops difficult. Plants have developed certain mechanisms to show efficient uptake of $\mathrm{K}$ so as to ensure maintained growth under limited $\mathrm{K}$ availability. Improved capacity for $\mathrm{K}$ uptake, efficient redistribution of absorbed $\mathrm{K}$ between cytosol and the vacuole for cellular homeostasis, and modification of root system are the key strategies. Such processes require precisely regulated and controlled signaling cascades and mechanisms (Ashley et al., 2006; Cherel et al., 2014). In addition to the complex soil dynamics, $\mathrm{K}$ availability is also influenced by the root-soil interactions. Expression of high affinity $\mathrm{K}$ transporters and the associated signaling cascades are initiated under low $\mathrm{K}$ availability and in this connection ROS and certain phytohormones including auxin, ethylene, JA are believed to mediate the sensing of $\mathrm{K}$ deficiency (Ashley et al., 2006; Agarwal et al., 2009; Ahanger et al., 2017c). Through molecular and electrophysiological studies, certain putative transport proteins are identified for controlling the uptake as well as compartmentalization of $\mathrm{K}$ ions (Osakabe et al., 2013). Transporter proteins, mainly operating at the soil root, xylem, phloem, and tonoplast levels, can be seen elsewhere, however it shall be noted that $\mathrm{K}$ starvation leads to release of vacuolar K (Nieves-Cordones et al., 2014) and it has been shown that expression of transporter genes is increased for ensuring homeostasis in plant tissues under water stress and K deficiency (Song et al., 2015).

Deficient $\mathrm{K}$ leads to growth retardation, accumulation of simple carbohydrates, low chlorophyll content, altered photosynthetic activity, and eventually to reduction in growth and yield (Sharma et al., 2006; Jatav et al., 2014; Ahanger et al., 2015), and can increase sensitivity of plants to biotic as well as abiotic stresses (Zorb et al., 2014) in comparison with sufficient $\mathrm{K}$ that has been reported to maintain growth and yield of most crop plants by inducing positive changes in metabolism (Tiwari et al., 1998; Sharma and Agarwal, 2002; Sharma et al., 2006; Tomar and Agarwal, 2013; Jatav et al., 2012, 2014; Ahanger et al., 2015, 2017b; Ahanger and Agarwal, 2017a,b). Presence of high $\mathrm{K}$ reduces pest and disease incidence through improved synthesis of high molecular weight compounds including proteins, starch, and cellulose (Perrenoud, 1990; Marschner, 1995). Any minor variation in $\mathrm{K}$ induced regulation of biotic infestation reflects the alterations in $\mathrm{K}$ nutritional status at a whole plant level and the form of $\mathrm{K}$ salt applied (Amtmann et al., 2008; Wang et al., 2013). Additionally $\mathrm{K}$ has a critical role as inorganic osmotica as well as the synthesis of other compatible osmolytes affecting functioning like stomatal movements and water relations. $\mathrm{K}$ maintains photoassimilate loading and the activity of key metabolic enzymes (Ahanger et al., 2014; Erel et al., 2015; Ahanger et al., 2015, 2017b). Sufficient availability of $\mathrm{K}$ enhances carboxylation and overall photosynthetic efficiency leading to optimal yield production under normal as well as stressed conditions (Tiwari et al., 1998). It has been observed that under $\mathrm{K}$ deficiency mediated reduction in photosynthetic rates are mainly due to the limited access to the $\mathrm{CO}_{2}$ due to stomatal closure (Jin et al., 2011) and osmotic regulation by $\mathrm{K}$ brings the expansion of leaves and cells. Such reduction in $\mathrm{CO}_{2}$ assimilation causes increase in excited energy dissipations through nonphotochemical quenching (Erel et al., 2015). Improved photosynthetic performance due to $K$ is related to the alterations in stomatal and gas exchange characteristics, and regulation of enzymes like Rubisco (Erel et al., 2015). In addition, $K$ deficient plants show increased photodamage. Photosynthetic arrest under stressful conditions mediated by nonstomatal limitations includes inhibition of chlorophyll biosynthesizing system (Xiao-guang et al., 2015) and K application induces positive influence on the chlorophyll synthesis (Tiwari et al., 1998), chloroplastic $\mathrm{CO}_{2}$ concentrations, and ETS (Xiao-guang et al., 2015).

Due to heterogeneous distribution of the developmental resources, plant growth gets affected, and sensing these changes for optimizing growth is very important. Therefore, it is important to elicit shoot to root or root to shoot signaling processes for coordinating the different root and shoot developmental events for maintaining the overall plant growth. Active involvement of $\mathrm{K}$ in regulating the key physiological responses like stomatal movements together with the hydraulic conductivity can be monitored with the timely examination of the available $\mathrm{K}$ and its subsequent flow through xylem and phloem tissues (Kudoyarova et al., 2015).

Additionally, reduced ion concentrations in xylem can change volume of pectin pit membranes leading to alteration of hydraulic conductivity (Zwieniecki et al., 2001). Cations like $\mathrm{K}^{+}$bring modifications in the pore dimensions of pit membranes thereby modulating the resistance to water flow through the xylem (Wheeler et al., 2005). K supplementation increases hydraulic conductivity as well as leaf specific conductivity due to its interaction with the pectic matrix of vessels. However, K-mediated enhancement in hydraulic conductivity benefits the plant by maintaining cell turgor, stomatal functioning, and gas exchange parameters (Oddo et al., 2012). Under nutrient deficient conditions, biosynthesis of major energy reductants is reduced due to reduced photosynthesis and hence results in perturbed carbon and energy metabolism. Moreover specific tolerance proteins are differentially 
expressed in plants exposed to nutrient starvation including the proteins having a role in protecting the important plant cellular processes like primary and secondary metabolism, energy metabolism and defense, signal transduction, transcription, synthesis, targeting and storage of protein (Deng et al., 2014).

The dominant role of $\mathrm{K}$ ions in maintenance of turgor and water homeostasis is obvious from its evident role in the processes like pressure driven solute transport in xylem and phloem, vacuolar accumulation of $\mathrm{K}^{+}$and $\mathrm{K}^{+}$flux that mediate plant movement. For example, K-induced changes in stomatal movements through its uptake and release greatly affects plant water relations and photosynthetic efficiency (Mahouachi et al., 2006). Adequate K concentrations improve the oxidative damage-averting potential of plants by regulating stomatal functioning, osmoregulation, and water use efficiency (Shabala and Pottosin, 2014). Under field conditions, reports communicating the beneficial role of supplementing relatively higher doses of $\mathrm{K}$ under water stress conditions are well available (Tiwari et al., 1998; Sharma and Agarwal, 2002; Jatav et al., 2012, 2014; Ahanger and Agarwal, 2017a,b).

In stress-exposed plants, efflux of $\mathrm{K}$ and calcium (Ca) occurs from the leaf mesophyll cells, which ultimately alters the stomatal characteristics and the photosynthetic efficiency. Drought induced efflux of K and $\mathrm{Ca}$ from the leaf mesophyll has been correlated with other growth parameters including height, biomass accumulation, and chlorophyll contents as well as photosynthetic attributes like intracellular $\mathrm{CO}_{2}$ concentration, net $\mathrm{CO}_{2}$ assimilation, stomatal conductance, and transpiration rate. Effluxed $\mathrm{K}$ and $\mathrm{Ca}$ from the mesophyll cells sense the intensity of stress (Britto et al., 2010). Stress induced K leakage is because of the increased expression of $\mathrm{K}$ efflux channels caused by the overproduction of ROS, and K starvation further aggravates this situation by reducing the activity of root aquaporins and the hydraulic conductivity (Kanai et al., 2011). Stresses generate excessive ROS leading to lipid peroxidation and intensifying the $\mathrm{K}$ leakage.

Addition of $\mathrm{K}$ can enhance tissue $\mathrm{K}^{+} / \mathrm{Na}^{+}$ratio and hence the stress tolerance of plants. Supplying sufficient $\mathrm{K}$ reduces the oxidative damage by enhancing the activities of antioxidant enzymes (Soleimanzadeh et al., 2010; Soledad et al., 2015; Ahanger et al., 2015; Ahanger and Agarwal, 2017a,b). Under salinity stress, adequate $\mathrm{K}^{+}$in plant tissues is maintained through selective uptake and efficient compartmentalization of $\mathrm{K}^{+}$and $\mathrm{Na}^{+}$ions (Munns et al., 2006) leading to maintenance of structural and functional integrity of the cell. It is believed that cytosolic $\mathrm{K}^{+} / \mathrm{Na}^{+}$ratio not the tissue ratio is the key determinant of saline stress withstanding potential (Shabala and Cuin, 2007). However, identifying the key targets for improving ion sequestration capability of crop plants shall prove very promising in improving stress tolerance.

Plant species showing greater discrimination between $\mathrm{K}^{+}$and $\mathrm{Na}^{+}$during absorption from the soil solution as well as transport to the upper tissue shoot preferably accumulate $\mathrm{K}^{+}$and sequester $\mathrm{Na}^{+}$into the vacuole or apoplast (Wu et al., 2013). Selectivity in $\mathrm{K}^{+}$ and $\mathrm{Na}^{+}$during xylem loading and the potentiality of plants to redirect $\mathrm{Na}^{+}$back from leaves to roots impart greater tolerance to saline stress (Attia et al., 2009). The $\mathrm{Na}^{+}$concentrations are maintained well below the toxic levels and selective uptake of $\mathrm{Na}$ to upper plant parts has been reported quite often (Sharma et al., 2006; Attia et al., 2009, 2011; Tomar and Agarwal, 2013; Jatav et al., 2012, 2014; Iqbal et al., 2015; Ahanger and Agarwal, 2017a). Root tissues sense and transduce K deficiency to cell cytosol leading to initiation of several biochemical and physiological events for short as well as long term responses. It has been suggested that factors including membrane potential, concentration of ROS and phytohormones regulate short term responses to K deficiency (Wang and Wu, 2013; Wang et al., 2013). Studies have reported the induction of genes like TF and mitogen activated protein (MAP) kinase after one hour of mineral starvation (Wang et al., 2002). At transcriptional levels, $K$ deficiency induces regulation of gene expression of proteins coding for metabolic processes, cation binding, ion transporters, and the genes associated with jasmonic acid, defense response, and $\mathrm{K}$ transporters (Ruan et al., 2015). Low $K$ status triggers high affinity $K$ transporters and activates signaling events similar to other stresses like wounding and molecules like ROS and phytohormones like ethylene, auxin, and jasmonic acid. In addition, several developmental responses in root tissues are initiated due to $\mathrm{K}$ deficiency (Ashley et al., 2006). It has been reported that $K$ starvation mediated induction of various genes varies with the plant species and the genotype, for example, genes coding for jasmonic acid, $\mathrm{ROS}, \mathrm{Ca}^{+}$, and receptor-like signaling, lignin synthesis etc., exhibited different pattern of expression in two cultivars of watermelon suggesting that gene repression in response to stress can lead to less energy consumption for better root growth mediation for improving $\mathrm{K}$ uptake and tolerance to K deficiency (Fan et al., 2014).

Transcriptomic studies have revealed that $\mathrm{K}$ starvation reduces growth in rice by triggering the downregulation of TFs like MYB, zinc finger, helix lope helix and bZIP, and transporters involved in metal, lipid, and ions like $\mathrm{K}, \mathrm{Na}, \mathrm{P}$, etc., and several signaling associated molecules like MAPK, phosphatases, and calcium sensors (Shankar et al., 2013). So it could be suggested that $\mathrm{K}$ deprivation mediated activation of 
genes and gene networks acting as concert sensors to external $\mathrm{K}$ availability and its subsequent distribution as well as adaptation. Therefore interplay between both up- and downregulated genes in response to $\mathrm{K}$ concentration variations can be a determining factor for averting the alteration in developmental and physiological stages. It is believed that calcium-mediated CBL-CIPK signaling is involved in regulating the shaker family $\mathrm{K}^{+}$channels $A K T 1$ and $A K T 2$, however exact mechanisms mediating $\mathrm{K}$ deficiency are largely not known (Luan et al., 2009; Tokas et al., 2013; Fan et al., 2014). Research confirming the molecular mechanisms of $\mathrm{K}^{+}$sensing, uptake, distribution, and homeostasis in crop plants is in its infancy therefore extensive work is needed to understand the exact mechanisms underlying $\mathrm{K}$ nutrition and signaling. Nath and Tuteja (2016) have suggested the involvement of microRNAs in sensing and signaling nutrient deficiency in plants. Recently, Song et al. (2017) have demonstrated the involvement of nitric oxide in $\mathrm{K}$ starvation sensing and signaling in tobacco; however further studies are required to unravel the exact mechanisms.

\subsection{PHOSPHOROUS}

Phosphorus $(\mathrm{P})$ is one of the abundant macronutrients in plant tissues; however its low availability in the soil is often a growth-limiting factor. $\mathrm{P}$ ranges from $0.1 \%$ to $1 \%$ of the total plant dry matter thereby making it among the three most abundant and much needed mineral nutrients for plants (Marschner, 2012). $P$ is actively implemented in several growth and developmental aspects of plants, for example, formation of membrane phospholipids and nucleic acids, ATP generation, and its subsequent exchange, in the regulation of cellular processes through mediation of phosphorylation and dephosphorylation of key metabolites, etc. In plants, specifically, some processes like photosynthesis, photorespiration, or the growth patterns like the complex relations between the source and sink of photoassimilates resulting in modular growth makes studying the effect of available $\mathrm{P}$ on plant physiology of special interest. Chiefly acquired as inorganic phosphate $(\mathrm{Pi})$, it remains in equilibrium as $\mathrm{H}_{3} \mathrm{PO}_{4}$, $\mathrm{H}_{2} \mathrm{PO}_{4}{ }^{-}, \mathrm{HPO}_{4}{ }^{2-}$, and $\mathrm{PO}_{4}{ }^{3-}$ anions (Hernandez and Munne-Bosch, 2015). However it should be noted here that under physiological $\mathrm{pH}$, that is, $5-6, \mathrm{H}_{2} \mathrm{PO}_{4}{ }^{-}$is the chief form of $\mathrm{Pi}$ absorbed by roots compared with other forms thereby contributing enormously to the whole P content of the plant (Schachtman et al., 1998). Pi can easily be immobilized in the soil after the formation of insoluble complexes with organic matter or several other mineral cations including $\mathrm{Al}^{3+}$ or $\mathrm{Fe}^{3+}$ and $\mathrm{Mg}^{2+}$ or $\mathrm{Ca}^{2+}$ in acidic and calcareous soils respectively (Manning, 2008). Instead of having abundant occurrence in soils the availability of $\mathrm{Pi}$ is rather scanty for absorption to plants therefore it is emerging as the one of the most important mineral nutrients limiting normal plant growth, development, and yield (Hernandez and Munne-Bosch, 2015). Most Pi is obtained exclusively from mining, however only $20 \%$ of the Pi supplemented to plants is absorbed, making the need for P fertilization to be kept in check with the productivity rate (Mishima et al., 2003). Additionally the leached Pi causes serious environmental pollution leading to eutrophication after entering into fresh water bodies (Carpenter, 2005). The demand for increased crop productivity for serving the world's population and the advent of bioenergetic crops often force agriculturalists to use excessive $\mathrm{P}$ rather to cut down its annual fertilization rates. Therefore in this context it has been suggested that in the future Pi sources may get exhausted or dwindle. Therefore it becomes important to understand the responses of crop plants to fluctuating $\mathrm{Pi}$ status or sources for improved productivity so that the genetic potential of crops can be exploited.

Uptake and transport of Pi are precisely regulated so as to ensure optimal phosphorous use efficiency (PUE). For piercing the plant root $\mathrm{P}$ may prefer either symplastic or apoplastic pathway to reach the vascular bundle (Rausch and Bucher, 2002). Pi moves actively into the plants against gradient and its incorporation into the symplast or apoplast involves several key transport proteins like high affinity $\mathrm{P} / \mathrm{H}+$ cotransporters phosphate transporter 1 (PHT1), which acts as symporter and is responsible for $\mathrm{P}$ uptake in angiosperms. Contrarily another protein, phosphate transporter B (PTB), is hypothesized as a $\mathrm{Na}+/ \mathrm{Pi}$ symporter catalyzing $\mathrm{Pi}$ uptake in chlorophytes (Lopez-Arredondo et al., 2014; Bonnot et al., 2017). Transport mediated by PHT1 is coupled to extrusion of $\mathrm{H}^{+}$by ATPases pumping leading to prevention of alkalinization of extracellular medium, however, small concentration of $\mathrm{Pi}$ is maintained in the root cells to meet the $\mathrm{Pi}$ requirements whilst the remainder is loaded into the xylem for long-distance transport. Within the xylem, loading has been observed to be regulated by another phosphate transporter protein PHO1 in Arabidopsis thaliana L (Nussaume et al., 2011). Under $\mathrm{Pi}$ deficiency its redistribution is mediated by the phloem transport. It has been reported that the PHT1 family is localized mainly in root epidermal cells; however other members like PHT2, PHT3, and PHT4 are localized in the inner membrane of plastids, inner mitochondrial membrane, and Golgi apparatus respectively (Chen et al., 2011; Lopez-Arredondo et al., 2014). It has been postulated that differential distribution of 
PHT transporter families is responsible for controlling the distribution of $\mathrm{Pi}$ among organelles, cells, and tissues (Poirier and Bucher, 2002; Raghothama and Karthikeyan, 2005; Nussaume et al., 2011; Gu et al., 2016). However, little is known about the genetic manipulation of $\mathrm{Pi}$ transport proteins for improving PUE and subsequently understanding the regulatory mechanisms at transcriptional, posttranscriptional, translational, and posttranslational levels (Gu et al., 2016). Most PHT1 members are involved in direct Pi uptake, which might be dominated by arbuscular mycorrhizal Pi uptake pathway solely relying on the AM-inducible PHT1 genes (Smith et al., 2011). However under P starvation several P starvation genes are induced and most of them are regulated at transcriptional levels thereby pointing to their regulation via $P$ starvation signaling as well as the $P$ starvation response transcription factors (PHR TFs) (Bustos et al., 2010; Goel and Singh, 2015). These PHF TFs belong to the MYB-CC family and have been observed to regulate the expression of genes after binding with palindromic sequences available at the proximal promoter regions (Bustos et al., 2010; Wu et al., 2013). In addition the TFs from belonging to other families have also been reported to regulate the $\mathrm{P}$ transport and Pi starvation signaling (Jain et al., 2012).

Genes encoding the signal molecules like AtIPS1/ At4, miRNAs, SPXs, biosynthetic genes of sulfolipids and galactolipids, $\mathrm{Pi}$ transporters and purple acid phosphatases acting downstream of PHR1 are directly or indirectly involved in regulating Pi transport ( $\mathrm{Wu}$ et al., 2013; Gu et al., 2016). Among these molecules SPXs have been widely reported to be involved in sensing and transport of $\mathrm{Pi}$ in both plants as well as yeasts (Secco et al., 2012). Based on the type as well as presence or absence of secondary domain at $C$ terminus major SPXs have been identified as EXS (ERD1/ XPR1/SYG1), MFS (major facilitator superfamily), or RING (really interesting new gene) domain (Secco et al., 2012). Arabidopsis PHO1 encoding a protein of the SPX-EXS subfamily localized to the endomembranes of Golgi and the trans-Golgi network in root pericycle cells has been reported to control loading of Pi into xylem and transport to shoot tissue (Arpat et al., 2012). Further, it has been suggested that AtPHO1 acts as Pi exporter effluxing Pi out of cells into the xylem vessel (Arpat et al., 2012). Nevertheless PHO1:GFP fusion has been reported to get relocated to the plasma membrane in leaves infiltrated high Pi concentrations (Arpat et al., 2012). Therefore further investigating the probable roles of AtPHO1-localized organelles on Pi transport and distribution will be interesting, exploiting the data recently generated describing its topology and domain-specific functions (Wege et al., 2015). It has been revealed that it is the membrane-spanning EXS domain not the SPX domain of AtPHO1 that regulates the Pi export activity, specifying the localization to the Golgi and trans-Golgi network (Wege et al., 2015; Gu et al., 2016). Stefanovic et al. (2007) have observed that as many 10 genes in Arabidopsis encode the close homologs of AtPHO1, and AtPHO1;H1 but not others (AtPHO:H2-H10) partially rescue the phenotype of the pho1 mutant. Khan et al. $(2014 a, b)$ have demonstrated that a SPX-EXS member in Arabidopsis, AtPHO1;H3, is involved in suppression of root-to-shoot Pi transfer and in rice only three SPXEXS genes have been found to be phylogenetically associated with AtPHO1 (Secco et al., 2010).

Additionally the vacuole is a key reservoir of many ions including $\mathrm{Pi}$, however, the proteins responsible for the transport of $\mathrm{Pi}$ across the tonoplast and the underlying regulatory mechanisms are largely unidentified. Wang et al. (2012) have identified the involvement of two rice genes belonging to the SPX-MFS subfamily, OsSPX-MFS1 and OsSPX-MFS2, that can serve as key targets in Pi starvation-induced miRNA, osa-miR827. All these members of SPX-MFS are localized to the tonoplast (Wang et al., 2015; Gu et al., 2016; Ham et al., 2018) where OsSPX MFS1 and 2 are involved respectively in the facilitation of import and export of Pi across the tonoplast, while OsSPX-MFS3 has been shown to mediate Pi efflux from vacuole into cytosol (Wang et al., 2015). By and large the actual roles of OsSPX-MFSs and their protein products in $\mathrm{Pi}$ transport are still not fully understood. Liu et al. (2015) have shown that vacuolar phosphate transporter 1 (VPT1), an ortholog of OsSPX-MFS3 in Arabidopsis, mediates the transport of $\mathrm{Pi}$ into the vacuole (Liu et al., 2015). Under Pi-sufficient and -deficient conditions, the phosphorylation and dephosphorylation of PHT1 regulates its interaction with PHF1 therefore resulting in regulation of $\mathrm{Pi}$ concentration in various cellular compartments (Ham et al., 2018).

During P deficiency plants display mechanisms for improving the uptake and distribution among aboveground tissues so that use efficiency is increased, however, focus has been mainly on the roots for unraveling the role of mechanisms increasing Pi availability. It has been observed that Pi availability to roots is increased by secreting the acid phosphatases, nucleases, or organic acids, therefore modifying the root architecture either through biotechnological approaches or exploitation of beneficial microbes like plant growth promoting rhizobacteria (PGPR) or arbuscular mycorrhizal fungi (AMF), and fine tuning it with optimized uptake to its aboveground parts by enhancing the expression of transport proteins can be a worthwhile future strategy (Cabugao et al., 2017; Battini et al., 2017). Molecular and genetic studies have shown the prime mechanisms regulating uptake 
and utilization of $\mathrm{P}$, and the influence of transporters, regulators, root architecture, metabolic adaptations, quantitative trait loci, hormonal signaling, and microRNAs. In connection to this, for acquiring improved PUE transition from the molecular mechanisms and plant architecture, modification to practical strategies is needed. Therefore, it can be proposed that introducing PGPR and AMF symbioses for efficient solubilization and uptake of $\mathrm{P}$, intercropping with suitable crop species for maintaining $\mathrm{P}$ activation and its subsequent mobilization in the soil and improving the expression of homologous genes with advantageous agronomic properties for attaining greater PUE in plants under normal as well stressful conditions. In addition breeding for phosphorus-efficient varieties and introgression of key quantitative trait loci can be handy in achieving improved crop PUE (Veneklaas et al., 2012; van deWiel et al., 2016; Hasan et al., 2016). Several microRNA molecules have been identified to regulate the sensing and signaling of $\mathrm{P}$ deficiency under stress conditions, and their involvement in triggering mechanisms for controlling $\mathrm{P}$ assimilation needs to be studied (Kumar et al., 2017).

Low P uptake and redistribution leads to imbalance in the ability of plants to process light energy intensifying the chances of photooxidative stress. Xing and $\mathrm{Wu}$ (2014) reported declined carbon assimilation due to $P$ deficiency. In chloroplasts, $\mathrm{Pi}$ is essential for photosynthesis and several reports advocate that Pi deficiency leads to reduced net $\mathrm{CO}_{2}$ assimilation by largely affecting stomatal functioning (Singh et al., 2014; Zhang et al., 2014). Additionally biochemical limitations including reduced generation as well as protection of ribulose-1, 5-bisphosphate or Rubisco or mesophyll limitations to $\mathrm{CO}_{2}$ diffusion are key effects of reduced $\mathrm{P}$ (Rao and Terry, 1995; Singh et al., 2014; Zhang et al., 2014). Besides this, the activity of phosphoenolpyruvate carboxylase, the enzyme responsible for shuttling $\mathrm{CO}_{2}$ from the mesophyll into the bundle sheath, is reduced under low Pi availability (Schlüter et al., 2013). Research is needed for comprehensive understanding of the implication of antioxidants and other defense molecules in the protection of the photosynthetic apparatus under Pi starvation. In Pi starved plants, Okazaki et al. (2013) has observed the replacement of phospholipids by galactolipids in biomembranes and the implication of new plant lipid (glucuronosyldiacylglycerol) has been reported. Moreover roots are the main organs involved in sensing and signaling $\mathrm{P}$ homeostasis in leaves; however, exact mechanisms are not fully known and involvement of $\mathrm{Ca}^{2+}$, ROS, sucrose, membrane proteins, etc. have been proposed (Lei et al., 2011). In addition understanding the modulation of phytohormone concentrations with response to changing $\mathrm{P}$ homeostasis and the subsequent integration of signaling cascades in bringing tolerance at the whole plant level can be interesting.

\subsection{CALCIUM}

$\mathrm{Ca}$ is involved in processes like cell division, cell elongation, cell differentiation, cell polarity, cytoplasmic streaming, gravitropism, photomorphogenesis, plant defense, and stress responses. As a divalent cation $\left(\mathrm{Ca}^{2+}\right)$ acts as an intracellular messenger in the cytosol and has a key role in maintaining the structural stability of cell wall and cell membranes, and in addition acts as a counter cation for anions in the vacuoles (White and Broadley, 2003). Ca provides structural rigidity by forming cross-links within the pectin polysaccharides (Easterwood, 2002). Mostly existing as calcium oxalate crystals in plastids the structural integrity as well as the quality of fruit produced is strongly coupled to $\mathrm{Ca}^{2+}$ availability. It has been reported to activate several enzymes like ATPase, phospholipases, amylase, succinate dehydrogenase, and antioxidants (Pliethand Vollbehr, 2012; Ahanger et al., 2014; Ahmad et al., 2015; He et al., 2015). Ca availability has been observed to affect the stomatal closure, hydraulic conductivity, sap flow, and uptake of ions like $\mathrm{K}^{+}, \mathrm{Mg}^{2+}$, etc. (Cabot et al., 2009; Ahmad et al., 2015). It is believed that in the absence of an external stimulus plant cells tend to maintain low cytosolic $\mathrm{Ca}^{2+}$ concentration, however, a rapid increase in cytosolic concentrations is achieved when exposed to external stimuli like light, touch, hormones, and biotic and abiotic stresses resulting mainly due to $\mathrm{Ca}^{2+} / \mathrm{H}^{+}$antiporter and $\mathrm{Ca}^{2+}$ pumps mediated efflux of Ca (Bush, 1995). Among the common signaling pathways elevating the concentration of cytosolic $\mathrm{Ca}$ is the phospholipase $\mathrm{C}$ (PLC) pathway, which is regulated by cell surface receptors, including $G$ protein coupled receptors and receptor tyrosine kinases activating PLC enzyme leading to hydrolysis of the membrane phospholipid PIP2 resulting in the generation of two secondary messengers, that is, 1,4,5-trisphosphate (IP3) and diacylglycerol (DAG). DAG leads to activation of protein kinase C while IP3 diffuses into the endoplasmic reticulum binding to its receptor (IP3 receptor, which is a $\mathrm{Ca}^{2+}$ channel) thereby leading to release of $\mathrm{Ca}^{2+}$ from the endoplasmic reticulum. Such stimulus mediated increase of Ca in cytoplasm is called Ca signature and it is believed that in addition to IP3, cyclic ADP ribose also has significant influence on $\mathrm{Ca}^{2+}$ channels therefore it could be said that increased Ca concentration in cytosol is due to the cumulative effect of different stimulatory molecules on the activity of Ca channels (Guse et al., 1999; Pottosin and Schonknecht, 2007; Noh et al., 2015). More importantly the transduction of Ca 
signatures into the biochemical and morphological responses are rather complex and a number of factors have been identified to regulate the specificity of $\mathrm{Ca}$ towards a particular response (Guse et al., 1999; Zhang et al., 2014). In addition to this the internal Ca concentration and Ca signature integrate the external stimuli with key intracellular responses, and spatial and temporal changes in $\mathrm{Ca}^{2+}$ determine the fate of a particular physiological response and its dynamics and amplitude (Allen et al., 1999; Zhu, 2016). Ca binding proteins have a critical role in decoding and subsequently transducing the Ca signatures and the activation of target as well as metabolic pathway (Bagur and Hajnoczky, 2017).

Increased concentration of cytosolic Ca leads to the activation of several $\mathrm{Ca}^{2+}$ sensor proteins thereby converting the signals into a biochemical change. Among these sensors are included calmodulin (CaM), calcium dependent protein kinases (CDPK), and calcineurin B-like (CBL) having a crucial role in the abiotic stress signaling in plants (Das and Pandey, 2010). Conformational changes are triggered in the sensor molecule due to binding of $\mathrm{Ca}^{2+}$ and hydrophobic pockets get exposed leading to interactions of a sensor and target proteins (Wilkins et al., 2016). Kinases inhibit autophosphorylation thereby increasing substrate phosphorylation. Ca and its sensors like calmodulin $(\mathrm{CaM})$ regulate the expression of structural and regulatory genes by acting as TFs or increased $\mathrm{Ca}^{2+}$ may directly bind TFs modulating their activity hence regulating the gene expression (Yael et al., 2010). Usually $\mathrm{Ca}^{2+} / \mathrm{CaM}$ complex interacts with $\mathrm{TFs}$ and modulates either their DNA binding or transcriptional activities or indirectly regulates transcription by associating with the multicomponent transcriptional machinery consisting of $\mathrm{Ca}^{2+} / \mathrm{CaM}$ complex, transcription factor binding protein (TFBP), and TFs. Finally, $\mathrm{Ca}^{2+} /$ CaM complex leads to regulation of gene expression by modulating the phosphorylation status of TFs. This indirect regulation is achieved by a CaM binding protein kinase and a CaM binding protein phosphatase (Kim et al., 2009). It has been reported that plants exhibiting overexpression of these protein kinases show greater stress tolerance, for example, transgenic rice overexpressing OsCDPK7 increases drought and salinity tolerance by improving the induction of stress responsive genes (Saijo et al., 2000). Overexpression of CBL5 protein in Arabidopsis enhanced the tolerance to salt and drought stress (Cheong et al., 2010).

$\mathrm{Ca}^{2+}$ addition under drought stress has been reported to enhance water conservation and improve the hydrophobicity of cellular membranes while lowering its permeability through its interaction with phosphates, carboxyl of phosphatides, and proteins in cellular membranes, thus strengthening their stability
(Shao et al., 2008). $\mathrm{Ca}^{2+}$ alters the hydration status of membranes and improves the cohesive property of cell walls leading to enhancement of the protoplasm viscosity and hence imparting more resistance to cellular dehydration. It can be concluded that $\mathrm{Ca}^{2+}$ brings stabilization of plant cells by direct positive effects on the structural components or basis of drought resistance (Ma et al., 2009; Ahanger et al., 2014). Addition of $\mathrm{Ca}$ has been reported to increase contents of several amino acids like alanine and $\gamma$-aminobutyric acid, polyamines including putrescine and spermidine, in addition to chlorophyll content Picearubens exposed to low temperature stress (Schaberg et al., 2011). Ca supplementation significantly mitigated the negative effects of drought stress on fresh and dry weight, chlorophyll, and relative water content concomitant with reduced membrane leakage in Vicia faba (Abdel-Basset, 1998). Ca mediated heat stress tolerance has been attributed to increased antioxidant potential and the maintenance of tissue water content (Jiang and Huang, 2001). Xu et al. (2013) has demonstrated Ca-induced upregulation of antioxidant system to influence photosynthetic efficiency of plants under water stress. Such effects of $\mathrm{Ca}$ on metabolism have been shown to involve the Ca-mediated regulation of ABA signaling and antioxidant system (Wang et al., 2017). Upregulation of the antioxidant system by Ca protects the functioning of PSII and associated photosynthetic components by checking the ROS concentrations (Sakhonwasee and Phinkasan, 2017).

Similar to other macroelements, $\mathrm{Ca}^{2+}$ as secondary signal plays an important role in integrating extracellular signals and environmental cues including light and stress factors eliciting changes in the cellular Ca levels, termed as calcium signatures. The concentration of $\mathrm{Ca}^{2+}$ is precisely maintained by the $\mathrm{Ca}^{2+}$ stores like vacuoles, endoplasmic reticulum, mitochondria, and cell wall. $\mathrm{Ca}^{2+}$ is present in $\mathrm{mM}$ concentrations in the cell wall and vacuoles and is released whenever required by the cell. It has been demonstrated that organelles including mitochondria, chloroplasts, and nuclei have the potential to generate calcium signals on their own (Xiong et al., 2006).

$\mathrm{Ca}^{2+}$ is an important secondary signaling molecule and makes a convergence point for several signaling pathways. Plant cells tend to reprogram the cellular setup by initiating a network of signaling events from perception to response. It shall be noted here that only cytosolic $\mathrm{Ca}^{2+}$ is involved in diverse signaling pathways and responds to numerous stimuli. Stress mediated increase of $\mathrm{Ca}$ concentration activates calmodulin, which in turn regulates the expression of several TFs and kinase and phosphatase proteins thereby integrating the signal perception with the expression of stress-specific genes in the nucleus 
(Virdi et al., 2015). Therefore, identification of stress specific TFs and their subsequent manipulations through genetic and molecular approaches can prove helpful in unraveling the hidden mechanisms of $\mathrm{Ca}$ mediated stress tolerance.

$\mathrm{Ca}^{2+}$ signaling pathway also regulates a $\mathrm{K}^{+}$channel for low-K response in Arabidopsis. Ca forms an essential component of the sucrose signaling pathway that leads to the induction of fructan synthesis (Martinez-Noel et al., 2006), in addition also to regulating cell cycle progression in abiotic stress exposed plants. $\mathrm{Ca}^{2+}$ competes with other cations both for these sites and for the uptake from the soil. High levels of $\mathrm{Ca}^{2+}$ ameliorates the uptake and negative effects of toxic ions ( $\mathrm{Cd}, \mathrm{Al}$ and $\mathrm{Na}$ ) while maintaining the higher levels of other cations like $\mathrm{K}, \mathrm{Mg}$, $\mathrm{P}$, etc., and in certain cases it has been observed that uptake of $\mathrm{Ca}$ is affected by the presence of essential elements in the soil solution (Sanders et al., 2002; Sakhonwasee and Phinkasan, 2017). Ca deficiency occurs due to low base saturation of soils or greater acidification or competition with other cations or restricted transpiration bringing down the xylem flow mediated supplementation of Ca to growing tissues (Zhang et al., 2014). Calcium deficiency results in stunted root growth and altered leaf appearances (Ahmad et al., 2015). Under drastic deficiency there are symptoms like reduced cell membrane integrity and appearance of bitter pit, blossom end rot, and tip burn in apple fruit, tomato, and lettuce respectively.

\subsection{CONCLUSION AND FUTURE PROSPECTS}

Stresses alter the uptake as well as metabolism of key nutrient elements by affecting the expression of transport and metabolism proteins. Resource variability due to the heterogeneous distribution intensifies the effects on plant development. Therefore, sensing the change in mineral concentrations may be beneficial for optimizing growth via the integrative mechanisms at root and shoot levels. Responses of plants elicited in response to limited nutrient availability may have some similarity with some stresses, and identification of genes and gene products that are up- or downregulated in response to the availability of a particular nutrient and the subsequent modulations through various biotechnological interventions can be helpful in understanding the actual mechanisms involved. Focus should be on dissecting the molecules regulating the root-to-shoot and shoot-to-root signaling events for bringing coordination between root and shoot under optimal, suboptimal, and supraoptimal availability of nutrients. Moreover, identification and studying the possible involvement of phytohormones, TFs, metabolites, etc. in sensing the nutrient availability and triggering the downstream events for bringing the modulations in metabolism is required. Developing a defined set of markers for enhancing the uptake, allocation, and metabolism of nutrients and hence the use-efficiency can help in understanding the genus or species specific responses to nutrient availability. Using "omics" techniques the identification of key signaling components regulating physiological responses at the whole plant level and the associated tolerance adaptation.

\section{Acknowledgments}

Facilities provided by Higher Education Department, Government of Jammu and Kashmir are gratefully acknowledged.

\section{References}

Abdel-Basset, R., 1998. Calcium channels and membrane disorders induced by drought stress in Vicia faba plants supplemented with calcium. Acta Physiol. Plant. 2 (2), 149-153.

Abdelhamid, M., Eldardiry, E., El-Hady, M.A., 2013. Ameliorate salinity effect through sulphur application and its effect on some soil and plant characters under different water quantities. Agric. Sci. 4 (1), 39-47.

Agarwal, R.M., Tomar, N.S., Singh, K., Sharma, G.L., 2009. Potassium induced changes in flowering plants. In: Vimala, Y. (Ed.), Flower Retrospect and Prospect (ProfesssorVishwambharPuri Birth Centenary Volume). SR Scientific Publication, Delhi, pp. 158-186.

Ahanger, M.A., Agarwal, R.M., 2017a. Salinity stress induced alterations in antioxidant metabolism and nitrogen assimilation in wheat (Triticumaestioum L) as influenced by potassium supplementation. Plant Physiol. Biochem. 115, 449-460.

Ahanger, M.A., Agarwal, R.M., 2017b. Potassium improves antioxidant metabolism and alleviates growth inhibition under water and osmotic stress in wheat (Triticumaestivum L). Protoplasma. 254 (4), 1471-1486.

Ahanger, M.A., Tyagi, S.R., Wani, M.R., Ahmad, P., 2014. Drought tolerance: Roles of organic osmolytes, growth regulators and mineral nutrients. In: Ahmad, P., Wani, M.R. (Eds.), Physiological Mechanisms and Adaptation Strategies in Plants Under Changing Environment, vol. 1. Springer Science + Business Media, Inc., New York, pp. 25-56.

Ahanger, M.A., Agarwal, R.M., Tomar, N.S., Shrivastava, M., 2015. Potassium induces positive changes in nitrogen metabolism and antioxidant system of oat (Avenasativa L cultivar Kent). J. Plant Inter. 10 (1), 211-223

Ahanger, M.A., Akram, N.A., Ashraf, M., Alyemni, M.N., Wijaya, L., Ahmad, P., 2017a. Plant responses to environmental stresses from gene to biotechnology. Ann. Bot. (AoB) Plants. 9 (4), PLX025. Available from: https://doi.org/10.1093/aobpla/plx025.

Ahanger, M.A., Tittal, M., Mir, R.A., Agarwal, R.M., 2017b. Alleviation of water and osmotic stress-induced changes in nitrogen metabolizing enzymes in Triticumaestivum L. cultivars by potassium. Protoplasma 254 (5), 1953-1963.

Ahanger, M.A., Tomar, N.S., Tittal, M., Argal, S., Agarwal, R.M., 2017c. Plant growth under water/ salt stress: ROS production; antioxidants and significance of added potassium under such conditions. Physiol. Mol. Bio. Plants 23 (4), 731-744. 
Ahmad, P., Sarwat, M., Bhat, N.A., Wani, M.R., Kazi, A.G., Tran, L.S., 2015. Alleviation of cadmium toxicity in Brassica juncea L. (Czern. \&Coss.) by calcium application involves various physiological and biochemical strategies. PLoS One 10 (1), e0114571. Available from: https:/ / doi.org/10.1371/journal.pone.0114571.

Ahmad, P., Wani, M.R., 2014. Mechanisms and Adaptation Strategies in Plants Under Changing Environment, vol. 1. Springer Science + Business Media, Inc, New York.

Allen, G.A., Kwak, J.M., Chu, S.P., Llopis, J., Tsien, R.Y., Harper, J.F., et al., 1999. Cameleon calcium indicator reports cytoplasmic calcium dynamics in Arabidopsis guard cells. Plant J. 19, 735-747.

Almagro, A., Lin, S., Tsay, Y., 2008. Characterization of the Arabidopsis nitrate transporter NRT1.6 reveals a role of nitrate in early embryo development. Plant Cell. 20, 3289-3299.

Amtmann, A., Troufflard, S., Armengaud, P., 2008. The effect of potassium nutrition on pest and disease resistance in plants. Physiol. Plant. 133, 682-691.

Arpat, A.B., Magliano, P., Wege, S., Rouached, H., Stefanovic, A., Poirier, Y., 2012. Functional expression of PHO1 to the Golgi and trans-Golgi network and its role in export of inorganic phosphate. Plant J. 71, 479-491.

Asgher, M., Khan, N.A., Khan, M.I.R., Fatma, M., Masood, A., 2014. Ethylene production is associated with alleviation of cadmiuminduced oxidative stress by sulfur in mustard types differing in ethylene sensitivity. Ecotox. Environ. Safety 106, 54-61.

Ashley, M.K., Grant, M., Grabov, A., 2006. Plant responses to potassium deficiencies: a role for potassium transport proteins. J. Exp. Bot. 57 (2), 425-436.

Attia, H., Karray, N., Ellili, A., Msilini, N., Lachaal, M., 2009. Sodium transport in basil. Acta Physiol. Plant. 31, 1045-1051.

Attia, H., Ouhibi, C., Ellili, A., Msilini, N., Bouzaen, G., Karray, N., et al., 2011. Analysis of salinity effects on basil leaf surface area, photosynthetic activity and growth. Acta Physiol. Plant. 33, 823-833.

Bagur, R., Hajnoczky, G., 2017. Intracellular $\mathrm{Ca}^{2+}$ sensing: its role in calcium homeostasis and signalling. Mol. Cell. 66 (6), 780-788.

Barbosa, E.R.M., Tomlinson, K.W., Carvalheiro, L.G., Kirkman, K., de Bie, S., Prins, H.H.T., et al., 2014. Short-term effect of nutrient availability and rainfall distribution on biomass production and leaf nutrient content of savanna tree species. PLoS One 9 (3), e92619. Available from: https://doi.org/10.1371/journal.pone.0092619.

Battini, F., Gronlund, M., Agnolucci, M., Jakobsen, I., 2017. Facilitation of phosphorus uptake in maize plants by mycorrhizosphere bacteria. Sci. Rep. 7, 4686. Available from: https://doi. org/10.1038/s41598-017-04959-0.

Bonnot, C., Proust, H., Pinson, B., Colbalchini, F.P.L., Lesly-Veillard, A., Breuninger, H., et al., 2017. Functional PTB phosphate transporters are present in streptophyte algae and early diverging land plants. New Phytol. 214, 1158-1171.

Britto, D.T., Ebrahimi-Ardebili, S., Hamam, A.M., Coskun, D., Kronzucker, H.J., 2010. 42K analysis of sodium-induced potassium efflux in barley: mechanism and relevance to salt tolerance. New Phytol. 186, 373-384.

Bush, D.S., 1995. Calcium regulation in plant cells and its role in signalling. Annu. Rev. Plant Physiol. Plant Mol. Biol. 46, 95-122.

Bustos, R., Castrillo, G., Linhares, F., Puga, M.I., Rubio, V., PerezPerez, J., et al., 2010. A central regulatory system largely controls transcriptional activation and repression responses to phosphate starvation in Arabidopsis. PLoS One 9. Available from: https:// doi.org/10.1371/journal.pgen.1001102.

Cabot, C., SiboleBarcelo, J.V., Poschenrieder, C., 2009. Sodiumcalcium interactions with growth, water, and photosynthetic parameters in salt treated beans. J. Plant Nutr. Soil Sci. 172, 637-643.

Cabugao, K.G., Timm, C.M., Carrell, A.A., Childs, J., Lu, T.Y.S., Pelletier, D.A., et al., 2017. Root and rhizosphere bacterial phosphatase activity varies with tree species and soil phosphorus availability in puertorico tropical forest. Front Plant Sci. 8, 1834. Available from: https://doi.org/10.3389/fpls.2017.01834.

Camejo, D., Guzman Cedeno, A., Moreno, A., 2016. Reactive oxygen species, essential molecules, during plantepathogen interactions. Plant Physiol. Biochem. 103, 10-23.

Carpenter, S.R., 2005. Eutrophication of aquatic ecosystems: bistability and soil phosphorus. Proc. Nat. Acad. Sci. 102 (29), 10002-10005.

Chen, J., Liu, Y., Ni, J., Wang, Y., Bai, Y., Shi, J., et al., 2011. OsPHF1 regulates the plasma membrane localization of low- and highaffinity inorganic phosphate transporters and determines inorganic phosphate uptake and translocation in rice. Plant Physiol. 157 (1), 269-278.

Cheong, Y.H., Sung, S.J., Kim, B.G., Pandey, G.K., Cho, J.S., Kim, K.N., et al., 2010. Constitutive Over expression of the calcium sensor CBL5 Confers osmotic or drought stresstolerance in Arabidopsis. Mol. Cells. 29, 159-165.

Cherel, I., Lefoulon, C., Boeglin, M., Sentenac, H., 2014. Molecular mechanisms involved in plant adaptation to low $\mathrm{K}^{+}$availability. J. Exp. Bot. 65 (3), 833-848.

Chiu, C.C., Lin, C.S., Hsia, A.P., Su, R.C., Lin, H.L., Tsay, Y.F., 2004. Mutation of a nitrate transporter, AtNRT1:4, results in a reduced petiole nitrate content and altered leaf development. Plant Cell Physiol. 45, 1139-1148.

Chopin, F., Orsel, M., Dorbe, M.F., Chardon, F., Truong, H.N., Miller, A.J., et al., 2007. The Arabidopsis ATNRT2.7 nitrate transporter controls nitrate content in seeds. Plant Cell 19, 1590-1602.

Cutler, N.S., Pan, X., Heitman, J., Cardenas, M.E., 2001. The TOR signal transduction cascade controls cellular differentiation in response to nutrients. Mol. Biol. Cell. 12 (12), 4103-4113.

Das, R., Pandey, G.K., 2010. Expressional analysis and role of calcium regulated kinases in abiotic stress signalling. Curr. Genom. $11,2-13$.

Dash, A.K., Singh, H.K., Mahakud, T., Pradhan, K.C., Jena, D., 2015. Interaction effect of nitrogen, phosphorus, potassium with sulphur, boron and zinc on yield and nutrient uptake by rice under rice - rice cropping system in inceptisol of coastal odisha. Int. Res. J Agri. Sci. Soil Sci. 5 (1), 14-21.

Deng, G., Liu, L.J., Zhong, X.Y., Lao, C.Y., Wang, H.Y., Wang, B., et al., 2014. Comparative proteome analysis of the response of ramie under N, P and K deficiency. Planta. 239, 1175-1186.

Dong, Y., Silbermann, M., Speiser, A., Forieri, I., Linster, E., Poschet, G., et al., 2017. Sulfur availability regulates plant growth via glucose-TOR signalling. Nat. Comm. 8, 1174. Available from: https: / / doi.org/10.1038/s41467-017-01224-w.

Easterwood, G.W., 2002. Calcium's role in plant nutrition. Fluid J. 1, $1-3$.

Erel, R., Yermiyahu, U., Ben-Gal, A., Dag, A., Shapira, O., Schwartz, A., 2015. Modification of non-stomatal limitation and photoprotection due to $\mathrm{K}$ and Na nutrition of olive trees. J. Plant Physiol. $177,1-10$.

Faljenberg, B., Witt, I., Zanor, M.I., Steinhauser, D., Mueller-Roeber, B., Hesse, H., et al., 2008. Transcription factors relevant to auxin signalling coordinate broad-spectrum metabolic shifts including sulphur metabolism. J. Exp. Bot. 59 (10), 2831-2846.

Fan, M., Huang, Y., Zhong, Y., Kong, Q., Xie, J., Niu, M., et al., 2014. Comparative transcriptome profiling of potassium starvation responsiveness in two contrasting watermelon genotypes. Plants 239 (2), 397-410. Available from: https://doi.org/10.1007/ s00425-013-1976-z. Epub 2013 Nov 2.

Fan, M., Shen, J., Yuan, L., Jiang, R., Chen, X., Davies, W.J., et al., 2012. Improving crop productivity and resource use efficiency to ensure food security and environmental quality in China. J. Exp. Bot. 63 (1), 13-24. 
FAO, 2010. FAO Land and plant nutrition management service. http://www.fao.org

Fatma, M., Asgher, M., Masood, A., Khan, N.A., 2014. Excess sulfur supplementation improves photosynthesis and growth in mustard under salt stress through increased production of glutathione. Environ. Exp. Bot. 107, 55-63.

Fatma, M., Masood, A., Per, T.S., Khan, N.A., 2016. Nitric oxide alleviates salt stress inhibited photosynthetic performance by interacting with sulfur assimilation in mustard. Front. Plant Sci. 7, 521. Available from: https://doi.org/10.3389/fpls.2016.00521.

Filleur, S., Dorbe, M., Cerezo, M., Orsel, M., Granier, F., Gojon, A., et al., 2001. An arabidopsis T-DNA mutant affected in Nrt2 genes is impaired in nitrate uptake. FEBS Lett. 489, 220-224.

Goel, P., Singh, A.K., 2015. Abiotic stresses down-regulate key genes involved in nitrogen uptake and assimilation in Brassica juncea L. PLoS One 10 (11), e0143645. Available from: https://doi.org/ 10.1371 / journal.pone. 0143645 .

Gu, M., Chen, A., Sun, S., Xu, G., 2016. Complex regulation of plant phosphate transporters and the gap between molecular mechanisms and practical application: what is missing? Mol Plant 9, 396-416.

Guse, A.H., de Silva, C.P., Berg, I., Skapenko, A.L., Weber, K., Heyer, P., et al., 1999. Regulation of calcium signalling in T lymphocytes by the second messenger cyclic ADP-ribose. Nature. 398 (6722), $70-73$.

Ham, B.K., Chen, J., Yan, Y., Lucas, W.J., 2018. Insights into plant phosphate sensing and signalling. Curr. Opin. Biotech. 49, 1-9.

Han, M., Wong, J., Tao, S., Beatty, P.H., Good, A.G., 2016. Identification of nitrogen use efficiency genes in barley: searching for QTLs controlling complex physiological traits. Front. Plant Sci. 7, 1587. Available from: https://doi.org/10.3389/fpls.2016.01587.

Hasan, M.M., Hasan, M.M., da Silva, J.A.T., Li, X., 2016. Regulation of phosphorus uptake and utilization: transitioning from current knowledge to practical strategies. Cell Mol. Biol. Lett. 21, 7. Available from: https:/ / doi.org/10.1186/s11658-016-0008-y.

Hawkesford, M.J., 2000. Plant responses to sulphur deficiency and the genetic manipulation of sulphate transporters to improve Sutilization efficiency. J. Exp. Bot. 51 (342), 131-138. Available from: https://doi.org/10.1093/jexbot/51.342.131.

He, L., Li, B., Lu, X., Yuan, L., Yang, Y., Yuan, Y., et al., 2015. The effect of exogenous calcium on mitochondria, respiratory metabolism enzymes and ion transport in cucumber roots under hypoxia. Sci. Rep. 5, 11391. Available from: https://doi.org/10.1038/ srep11391.

Hernandez, I., Munne-Bosch, S., 2015. Linking phosphorus availability with photo-oxidative stress in plants. J. Exp. Bot. 66 (10), 2889-2900.

Holthusen, D., Reeb, D., Horn, R., 2012. Influence of potassium fertilization, water and salt stress, and their interference on rheological soil parameters in planted containers. Soil Tillage Res. 125, 72-79.

Howarth, J.R., Parmar, S., Barraclough, P.B., Hawkesford, M.J., 2009. A sulphur deficiency-induced gene, sdi1, involved in the utilization of stored sulphate pools under sulphur-limiting conditions has potential as a diagnostic indicator of sulphur nutritional status. Plant Biotech J. 7, 200-209.

Huang, N.C., Liu, K.H., Lo, H.J., Tsay, Y.F., 1999. Cloning and functional characterization of an Arabidopsis nitrate transporter gene that encodes a constitutive component of low-affinity uptake. Plant Cell. 11, 1381-1392.

Hubberten, H.M., Klie, S., Caldana, C., Degenkolbe, T., Willmitzer, L., Hoefgen, R., 2012. Additional role of O-acetylserine as a sulfur status-independent regulator during plant growth. Plant J. 70 (4), 666-677.

Iqbal, N., Nazar, R., Syeed, S., Massod, A., Khan, N.A., 2011. Exogenously-sourced ethylene increases stomatal conductance, photosynthesis, and growth under optimal and deficient nitrogen fertilization in mustard. J. Exp. Bot. 62 (14), 4955-4963.

Iqbal, N., Umar, S., Khan, N.A., 2015. Nitrogen availability regulates proline and ethylene production and alleviates salinity stress in mustard (Brassica juncea). J. Plant Physiol. 178, 84-91.

Jain, A., Nagarajan, V.K., Raghothama, K.G., 2012. Transcriptional regulation of phosphate acquisition by higher plants. Cell Mol. Life Sci. 69, 3207-3224.

Jatav, K.S., Agarwal, R.M., Tomar, N.S., Tyagi, S.R., 2014. Nitrogen metabolism, growth and yield responses of wheat (Triticumaestivum $\mathrm{L}$ ) to restricted water supply and varying potassium treatments. J. Indian Bot. Soc. 93, 177-189.

Jiang, Y., Huang, B., 2001. Effect of calcium on antioxidant activities and water relations associated with heat tolerance in two coolseason grasses. J. Exp. Bot. 52 (355), 31-349.

Jin, S.H., Huang, J.Q., Li, X.Q., Zheng, B.S., Wu, J.S., Wang, Z.J., et al., 2011. Effects of potassium supply on limitations of photosynthesis by mesophyll diffusion conductance in Caryacathayensis. Tree Physiol. 31, 1142-1151.

John, R., Dalling, J.W., Harms, K.E., Yavitt, J.B., Stallard, R.F., Mirabello, M., et al., 2007. Soil nutrients influence spatial distributions of tropical tree species. PNAS. 104 (3), 864-869.

Kan, C.C., Chung, T.Y., Juo, Y.A., Hsieh, M.H., 2015. Glutamine rapidly induces the expression of key transcription factor genes involved in nitrogen and stress responses in rice roots. BMC Genom. 16, 731. Available from: https://doi.org/10.1186/s12864-015-1892-7.

Kanai, S., Moghaieb, R.E., El-Shemy, H.A., Panigrahid, R., Mohapatra, P.K., Ito, J., et al., 2011. Potassium deficiency affects water status and photosynthetic rate of the vegetative sink in green house tomato prior to its effects on source activity. Plant Sci. 180, 368-374.

Kataoka, T., Watanabe-Takahashi, A., Hayashi, N., Ohnishi, M., Mimura, T., Buchner, P., et al., 2004. Vacuolarsulfate transporters are essential determinants controlling internal distribution of sulfate in Arabidopsis. Plant Cell. 16, 2693-2704.

Khan, G.A., Bouraine, S., Wege, S., Li, Y.Y., de Carbonnel, M., Berthomieu, P., et al., 2014a. Coordination between zinc and phosphate homeostasis involves the transcription factor PHR1, the phosphate exporter PHO1, and its homologue $\mathrm{PHO}$; $\mathrm{H} 3$ in Arabidopsis. J. Exp. Bot. 65, 871-884.

Khan, M.I.R., Asgher, M., Khan, N.A., 2014b. Alleviation of saltinduced photosynthesis and growth inhibition by salicylic acid involves glycine betaine and ethylene in mungbean (Vignaradiata L.). Plant Physiol. Biochem. 80, 67-74.

Khan, M.I.R., Khan, N.A., 2017. Reactive Oxygen Species and Antioxidant Systems in Plants: Role and Regulation under Abiotic Stress.

Khan, M.I.R., Nazir, F., Asgher, M., Per, T.S., Khan, N.A., 2015. Selenium and sulfur influence ethylene formation and alleviate cadmium-induced oxidative stress by improving proline and glutathione production in wheat. J. Plant Physiol. 173, 9-18.

Kim, M.C., Chung, W.S., Yun, D.J., Cho, M.J., 2009. Calcium and calmodulin-mediated regulation of gene expression in plants. Mol. Plant 2, 13-21.

Kudoyarova, G.R., Dodd, I.C., Veselov, D.S., Rothwell, S.A., Veselov, S.Y., 2015. Common and specific responses to availability of mineral nutrients and water. J. Exp. Bot. 66 (8), 2133-2144.

Kumar, S., Verma, S., Trivedi, P.K., 2017. Involvement of small rnas in phosphorus and sulfur sensing, signalling and stress: current update. Front. Plant Sci. 10. Available from: https://doi.org/ 10.3389/fpls.2017.00285.

Lappartient, A.G., Vidmar, J.J., Leustek, T., Glass, A.D.M., Touraine, B., 1999. Interorgan signalling in plant: regulation of ATP sulfurylase and sulfate transporter genes expression in roots mediated by phloem-translocated compounds. Planta 18, 89-95. 
Lee, B.R., Zaman, R., Avice, J.C., Ourry, A., Kim, T.H., 2016. Sulfur use efficiency is a significant determinant of drought stress tolerance in relation to photosynthetic activity in Brassica napuscultivars. Front. Plant Sci. 7, 459. Available from: https://doi.org/10.3389/ fpls.2016.00459.

Lei, M., Liu, Y., Zhang, B., Zhao, Y., Wang, X., Zhou, Y., et al., 2011. Genetic and genomic evidence that sucrose is a global regulator of plant responses to phosphate starvation in Arabidopsis. Plant Physiol. 156, 1116-1130.

Liu, J.H., Yang, L., Luan, M.D., Wang, Y., Zhang, C., Zhang, B., et al., 2015. A vacuolar phosphate transporter essential for phosphate homeostasis in Arabidopsis. Proc. Natl Acad. Sci. USA. 112 (47), $6571-6578$.

Lopez-Arredondo, D., Leyva-Gonzalez, M., Gonzalez-Morales, S., Lopez-Bucio, J., Herrera-Estrella, L., 2014. Phosphate nutrition: improving low-phosphate tolerance in crops. Ann. Rev. Plant Bio. $65,95-123$.

Luan, S., Lan, W., Chul Lee, S., 2009. Potassium nutrition, sodium toxicity, and calcium signalling: connections through the CBLCIPK network. Curr. Opin. Plant Biol. 12, 339-346.

Lunde, C., Zygadlo, A., Simonsen, H.T., Nielsen, P.L., Blennow, A., Haldrup, A., 2008. Sulfur starvation in rice: the effect on photosynthesis, carbohydrate metabolism, and oxidative stress protective pathways. Physiol. Plant. 134, 508-521.

Ma, Y.Y., Song, W.Y., Liu, Z.H., Zhang, H.M., Guo, X.L., Shao, H.B., et al., 2009. The dynamic changing of $\mathrm{Ca}^{2+}$ cellular localization in maize leaflets under drought stress. Cell Biol 332, 35-362.

Mahouachi, J., Socorro, A.R., Talon, M., 2006. Responses of papaya seedlings (Carica papaya L.) to water stress and re-hydration: growth, photosynthesis and mineral nutrient imbalance. Plant Soil. 281, 137-146.

Marschner, H., 1995. Mineral Nutrition of Higher Plants, second ed Academic Press, New York.

Marschner, H., 2012. Marschner's Mineral Nutrition of Higher Plants, third ed. Academic Press, New York.

Martinez-Noel, G., Tognetti, J., Nagaraj, V., Wiemken, A., Pontis, H., 2006. Calcium is essential for fructan synthesis induction mediated by sucrose in wheat. Planta 225, 183-191.

Maruyama-Nakashita, A., Nakamura, Y., Watanabe-Takahashi, A., Inoue, E., Yamaya, T., Takahashi, H., 2005. Identification of a novel cis-acting element conferring sulfur deficiency response in Arabidopsis roots. Plant J. 42 (3), 305-314.

Masclaux-Daubresse, C., Daniel-Vedele, F., Dechorgnat, J., Chardon, F., Gaufichon, L., Suzuki, A., 2010. Nitrogen uptake, assimilation and remobilization in plants: challenges for sustainable and productive agriculture. Ann. Bot. 105 (7), 1141-1157.

Matraszek, R., Hawrylak-Nowak, B., Chwil, S., Chwil, M., 2016. Interaction between cadmium stress and sulphur nutrition level on macronutrient status of Sinapisalba L. Water Air Soil Pollut. 227 (9), 355.

Mishima, S., Itahashi, S., Kimura, R., Inoue, T., 2003. Trends of phosphate fertilizer demand and phosphate balance in farmland soils in Japan. Soil Sci. Plant Nutr. 49, 39-45.

Mittler, R., 2017. ROS are good. Trends Plant Sci. 22 (1), 11-19.

Munns, R., James, R.A., Lauchli, A., 2006. Approaches to increasing the salt tolerance of wheat and other cereals. J. Exp. Bot. 57 (5), 1025-1043.

Nath, M., Tuteja, N., 2016. NPKS uptake, sensing, and signalling and miRNAs in plant nutrient stress. Protoplasma 253 (3), $767-786$

Nieves-Cordones, M., Aleman, F., Martinez, V., Rubio, F., 2014. K ${ }^{+}$ uptake in plant roots. The systems involved, their regulation and parallels in other organisms. J. Plant Physiol 171, 688-695.

Nikiforova, V., Freitag, J., Kempa, S., Adamik, M., Hesse, H., Hoefgen, R., 2003. Transcriptome analysis of sulfur depletion in
Arabidopsis thaliana: interlacing of biosynthetic pathways provides response specificity. Plant J. 33, 633-650.

Noh, S., Lee, S.R., Jeong, Y.J., Ko, K.S., Rhee, B.D., Kim, N., et al., 2015. The direct modulatory activity of zinc toward ion channels. Integ. Med. Res. 4 (3), 142-146.

Noman, A., Fahad, S., Aqeel, M., Ali, U., Amanullah, Anwar, S., et al., 2017. miRNAs: major modulators for crop growth and development under abiotic stresses. Biotech. Lett. 39 (5), 85-700.

Nussaume, L., Kanno, S., Javot, H., Marin, E., Pochon, N., Ayadi, A., et al., 2011. Phosphate import in plants: focus on the PHT1 transporters. Front. Plant Sci. Available from: https://doi.org/ 10.3389/fpls.2011.00083.

Oddo, E., Inzerillo, S., Bella, F.L., Grisafi, F., Salleo, S., Nardini, A., 2012. Short-term effects of potassium fertilization on the hydraulic conductance of LaurusnobilisL. Tree Physiol. 31, 131-138.

Ohkama, N., Takei, K., Sakakibara, H., Hayashi, H., Yoneyama, T., Fujiwara, T., 2002. Regulation of sulfur-responsive gene expression by exogenously applied cytokinins in Arabidopsis thaliana. Plant Cell Physiol. 43 (12), 1493-1501.

Okazaki, Y., Otsuki, H., Narisawa, T., Kobayashi, M., Sawai, S., Kamide, Y., et al., 2013. A new class of plant lipid is essential for protection against phosphorus depletion. Nat. Commun. 4, 1510. Available from: https://doi.org/10.1038/ncomms2512.

Orlova, M., Kanter, E., Krakovich, D., Kuchin, S., 2006. Nitrogen availability and TOR regulate the SNF1 protein kinase in Saccharomyces cerevisiae. Eukayot. Cell. 5 (11), 1831-1837.

Osakabe, Y., Arinaga, N., Umezawa, T., Katsura, S., Nagamachi, K., Tanaka, H., et al., 2013. Osmotic stress responses and plant growth controlled by potassium transporters in Arabidopsis. Plant Cell. 25, 609-624.

Osakabe, Y., Osakabe, K., Shinozaki, K., Tran, L.S.P., 2014. Response of plants to water stress. Front. Plant Sci. 5 (1). Available from: https://doi.org/10.3389/fpls.2014.00086.

Pandey, R., Agarwal, R.M., Jeevaratnam, K., Shrama, G.L., 2004. Osmotic stress - induced alterations in rice (Oryzasativa L.) and recovery on stress release. Plant Growth Reg. 42, 79-87.

Perchlik, M., Tegeder, M., 2017. Improving plant nitrogen use efficiency through alteration of amino acid transport processes. Plant Physiol. 175, 235-247.

Perrenoud, S., 1990. Potassium and Plant Health, second ed. International Potash Institute, Bern, Switzerland.

Petrov, V., Hille, J., Mueller-Roeber, B., Gechev, T.S., 2015. ROSmediated abiotic stress-induced programmed cell death in plants. Front. Plant Sci. 6, 69. Available from: https://doi.org/10.3389/ fpls.2015.00069.

Plieth, C., Vollbehr, S., 2012. Calcium promotes activity and confers heat stability on plant peroxidases. Plant Sig. Beh. 7 (6), 650-660. Available from: https://doi.org/10.4161/psb.20065.

Poirier, Y., Bucher, M., 2002. Phosphate transport and homeostasis in Arabidopsis. Arabidopsis Book. 1, e0024. Available from: https:// doi.org/10.1199/tab.0024.

Pottosin, I.I., Schonknecht, G., 2007. Vacuolar calcium channels. J. Exp. Bot. 58 (7), 1559-1569.

Raghothama, K.G., Karthikeyan, A.S., 2005. Phosphate acquisition. Plant Soil. 274, 37-49.

Rao, I.M., Terry, N., 1995. Leaf phosphate status, photosynthesis, and carbon partitioning in sugar beet. IV. Changes with time following increased supply of phosphate to low-phosphate plants. Plant Physiol. 107, 1313-1321.

Rausch, C., Bucher, M., 2002. Molecular mechanisms of phosphate transport in plants. Planta 216 (1), 23-37.

Rengasamy, P., Sumner, M.E., 1998. Processes involved in sodic behavior. In: Sumner, E., Naidu, R. (Eds.), Sodic Soils Distribution, Properties, Management and Environmental Consequences. Oxford Press, New York. 
Rogers, H., Munne-Bosch, S., 2016. Production and scavenging of reactive oxygen species and redox signalling during leaf and flower senescence: similar but different. Plant Physiol. 171, 1560-1568.

Ruan, L., Zhang, J., Xin, X., Zhang, C., Ma, D., Chen, L., et al., 2015. Comparative analysis of potassium deficiency-responsive transcriptomes in low potassium susceptible and tolerant wheat (Triticumaestivum L.). Sci. Rep. 5, 10090. Available from: https:// doi.org/10.1038/srep10090.

Saijo, Y., Hata, S., Kyozuka, J., Shimamoto, K., Izui, K., 2000. Over expression of single calcium dependent protein kinase confers both cold and salt/drought tolerance on rice plants. Plant J. 28 (3), 319-327.

Sakhonwasee, S., Phinkasan, W., 2017. Effects of the foliar application of calcium on photosynthesis, reactive oxygen species production, and changes in water relations in tomato seedlings under heat stress. Hort. Environ. Biotech. 58 (2), 119-126.

Sanders, D., Pelloux, J., Brownlee, C., Harper, J.F., 2002. Calcium at the crossroads of signalling. Plant Cell. 14, S401-S417.

Schaberg, P.G., Minocha, R., Long, S., Halman, J.M., Hawley, G.J., Eagar, C., 2011. Calcium addition at the Hubbard Brook Experimental Forest increases the capacity for stress tolerance and carbon capture in red spruce (Picearubens) trees during the cold season. Trees. 25, 1053-1061.

Schachtman, D.P., Reid, R.J., Ayling, S.M., 1998. Phosphorus uptake by plants: from soil to cell. Plant Physiol. 116, 447-453.

Scherer, H.W., 2008. Impact of sulphur on N2 fixation of legumes. In: Khan, N.A., Singh, S., Umar, S. (Eds.), Sulfur Assimilation and Abiotic Stresses in Plants. Springer-Verlag, New York, pp. 43-54.

Schlüter, U., Colmsee, C., Scholz, U., Bräutigam, A., Weber, A.P.M., Zellerhoff, N., et al., 2013. Adaptation of maize source leaf metabolism to stress related disturbances in carbon, nitrogen and phosphorus balance. BMC Genom. 201314, 442. Available from: https://doi.org/10.1186/1471-2164-14-442.

Secco, D., Baumann, A., Poirier, Y., 2010. Characterization of the rice PHO1 gene family reveals a key role for OsPHO1;2 in phosphate homeostasis and the evolution of a distinct clade in dicotyledons. Plant Physiol. 152, 1693-1704.

Secco, D., Wang, C., Shou, H.X., Whelan, J., 2012. Phosphate homeostasis in the yeast Saccharomyces cerevisiae, the key role of the SPX domain-containing proteins. FEBS Lett. 586, 289-295.

Shabala, S., Cuin, T.A., 2007. Potassium transport and plant salt tolerance. Physiol. Plant. 133, 651-669.

Shabala, S., Pottosin, I., 2014. Regulation of potassium transport in plants under hostile conditions: implications for abiotic and biotic stress tolerance. Physiol. Plant. 151, 257-279.

Shankar, A., Singh, A., Kanwar, P., Srivastava, A.K., Pandey, A., Suprasanna, P., et al., 2013. Gene expression analysis of rice seedling under potassium deprivation reveals major changes in metabolism and signalling components. PLoS One 8 (7), e70321. Available from: https://doi.org/10.1371/journal. pone.0070321.

Shao, H.B., Chu, L.Y., Shao, M.A., 2008. Calcium as a versatile plant signal transducer under soil water stress. Bio Essays 30, 634-641.

Sharma, G.L., Agarwal, R.M., 2002. Potassium-induced changes in nitrate reductase activity in Cicer arietinum L. Ind. J. Plant Physiol. 7, 221-226.

Sharma, G.L., Agarwal, R.M., Singh, R.P., 2006. Potassium induced changes in certain aspects of nitrogen metabolism in chickpea (Cicer arietinum L). Physiol. Mol. Biol. Plants. 12, 157-162.

Singh, S.K., Reddy, V.R., Fleisher, D.H., Timlin, D.J., 2014. Growth, nutrient dynamics, and efficiency responses to carbon dioxide and phosphorus nutrition in soybean. J. Plant Int. 9, 838-849.

Smith, S.E., Jakobsen, I., Grønlund, M., Smith, A.F., 2011. Roles of arbuscular mycorrhizas in plant phosphorus nutrition: interactions between pathways of phosphorus uptake in arbuscular mycorrhizal roots have important implications for understanding and manipulating plant phosphorus acquisition. Plant Physiol. 156, 1050-1057.

Sogbedi, J.M., Vaves, H.M., Agbeko, K.L., 2006. Cover cropping and nutrient management strategies for maize production in western Africa. Agron. J. 98, 883-889.

Soledad, O.N., Florencia, M.M., Laura, F.M., Raul, D.G., Balbina, A.A., Pia, O.F., 2015. Potassium phosphite increases tolerance to UV-B in potato. Plant Physiol. Biochem. 88, 1-8.

Soleimanzadeh, H., Habibi, D., Ardakani, M.R., Paknejad, F., Rejali, F., 2010. Effect of potassium levels on antioxidant enzymes and malondialdehyde content under drought stress in sunflower (Helianthus annuusL.) American. J. Agri. Bio. Sci. 5 (1), 56-61.

Song, W., Xue, R., Song, Y., Bi, Y., Liang, Z., Meng, L., et al., 2017. Differential response of first-order lateral root elongation to low potassium involves nitric oxide in two tobacco cultivars. J. Plant Growth Regul. Available from: https://doi.org/10.1007/s00344017-9711-9.

Song, Z.Z., Yang, Y., Ma, R.J., Xu, J.L., Yu, M.L., 2015. Transcription of potassium transporter genes of KT/HAK/KUP family in peach seedlings and responses to abiotic stresses. Biol. Plant. 59 (1), $65-73$.

Stefanovic, A., Ribot, C., Rouached, H., Wang, Y., Chong, J., Belbahri, L., et al., 2007. Members of the PHO1 gene family show limited functional redundancy in phosphate transfer to the shoot, and are regulated by phosphate deficiency via distinct pathways. Plant J. 50, 982-994

Szalai, G., Kellos, T., Galiba, G., Kocsy, G., 2009. Glutathione as an antioxidant and regulatory molecule in plants under abiotic stress conditions. J. Plant Growth Regul. 28, 66-80.

Takei, K., Takahashi, T., Sugiyama, T., Yamaya, T., Sakakibara, H., 2002. Multiple routes communicating nitrogen availability from roots to shoots: a signal transduction pathway mediated by cytokinin. J. Exp. Bot. 53 (370), 971-977.

Tamas, M.J., Sharma, S.K., Ibstedt, S., Jacobson, T., Christen, P., 2014. Heavy metals and metalloids as a cause for protein misfolding and aggregation. Biomol. 4 (1), 252-267.

Tausz, M., Sircelj, H., Grill, D., 2004. The glutathione system as a stress marker in plant ecophysiology: is a stress-response concept valid? J. Exp. Bot. 55, 1955-1962.

Tittal, M., Ahanger, M.A., Agarwal, R.M., 2017. Role of potassium in disease resistance. In: Singh, R.P., Tomar, V.S. (Eds.), Recent Trends in Environmental Science and Technology. Educationist Press, Write and Print Publication, New Delhi, p. 110015.

Tiwari, H.S., Agarwal, R.M., Bhatt, R.K., 1998. Photosynthesis, stomatal resistance and related characteristics, as influenced by potassium under normal water supply and water stress conditions in rice (OryzasativaL.). Indian J. Plant Physiol. 3 (4), 314-316.

Tokas, I., Pandey, A., Pandey, G.K., 2013. Role of calcium-mediated CBL-CIPK network in plant mineral nutrition and abiotic stress. In: Rout, D. (Ed.), Molecular Stress Physiology of Plants. Springer, India. Available from: https://doi.org/10.1007/978-81322-0807-5_10.

Tomar, N.S., Agarwal, R.M., 2013. Influence of treatment of Jatropha curcas L. leachates and potassium on growth and phytochemical constituents of wheat (Triticumaestivum L). Amer. J. Plant Sci. 4, 1134-1150.

Tsay, Y.F., Schroeder, J.I., Feldmann, K.A., Crawford, N.M., 1993. The herbicide sensitivity gene CHL1 of Arabidopsis encodes a nitrate-inducible nitrate transporter. Cell 72, 705-713.

Turkan, I., Demiral, T., 2009. Recent developments in understanding salinity tolerance. Environ. Exp. Bot. 67, 2-9.

van de Wiel, C.C.M., van der Linden, C.G., Scholten, O.E., 2016. Improving phosphorus use efficiency in agriculture: opportunities for breeding. Euphytica. 207 (1), 1-22. 
Van Olphen, H., 1977. An Introduction to Clay Colloid Chemistry, second ed Wiley, New York, NY.

Veneklaas, E.J., Lambers, H., Bragg, J., Finnegan, P.M., Lovelock, C.E., Plaxton, W.C., et al., 2012. Opportunities for improving phosphorus-use efficiency in crop plants. New Phytol. 195 (2), 306-320.

Virdi, A.S., Singh, S., Singh, P., 2015. Abiotic stress responses in plants: roles of calmodulin-regulated proteins. Front. Plant Sci. 6, 809. Available from: https://doi.org/10.3389/fpls.2015.00809.

Wang, C., Huang, W., Ying, Y.H., Li, S., Secco, D., Tyerman, S., et al., 2012. Functional characterization of the rice SPX-MFS family reveals a key role of OsSPX-MFS1 in controlling phosphate homeostasis in leaves. New Phytol. 196 (33), 139-148.

Wang, C., Yue, W.H., Ying, Y.H., Wang, S.D., Secco, D., Liu, Y., et al., 2015. Rice SPX-Major Facility Superfamily, a vacuolar phosphate 4 efflux transporter, is involved in maintaining phosphate homeostasis in rice. Plant Physiol. 169, 2822-2831.

Wang, D.F., Pang, X.J., Yang, F., Kou, L.S., Zhang, X., Yu, P.X., et al., 2017. Antioxidative enzymes, calcium, and ABA signalling pathway are required for the stress tolerance of transgenic wheat plant by the ectopic expression of harpin protein fragment Hpa1 $1_{10-42}$ under heat stress. Russ. J. Plant Physiol. 64 (6), 899-905.

Wang, M., Zheng, Q., Shen, Q., Guo, S., 2013. The critical role of potassium in plant stress response. Int. J. Mol. Sci. 14, 7370-7390.

Wang, Y., Wu, W.H., 2013. Potassium transport and signalling in higher plants. Annu. Rev. Plant Biol. 64, 451-476.

Wang, Y.H., Garvin, D.F., Kochian, L.V., 2002. Rapid induction of regulatory and transporter genes in response to phosphorus, potassium, and iron deficiencies in tomato roots. Evidence for cross talk and root/rhizosphere-mediated signals. Plant Physiol. 130, 1361-1370.

Wege, S., Khan, G.A., Jung, J.Y., Vogiatzaki, E., Pradervand, S., Aller, I., et al., 2015. The EXS domain of PHO1 participates in the response of shoots to phosphate deficiency via a root-to-shoot signal. Plant Physiol. 170, 385-400.

Wheeler, J.K., Sperry, J.S., Hacke, U.G., Hoang, N., 2005. Inter-vessel pitting and cavitation in woody Rosaceae and other vesselled plants: a basis for a safety versus efficiency trade-off in xylem transport. Plant Cell Environ. 28, 800-812.

White, P.J., Broadley, M.R., 2003. Calcium in plants. Ann. Bot. 92, 487-511.

Wilkins, K.A., Matthus, E., Swarbreck, S.M., Davies, J.M., 2016. Calcium-mediated abiotic stress signalling in roots. Front Plant Sci. 7, 1296.

Wu, P., Shou, H.X., Xu, G.H., Lian, X.M., 2013. Improvement of phosphorus efficiency in rice on the basis of understanding phosphate signalling and homeostasis. Curr. Opin. Plant Biol. $16,205-212$.
Xiao-guang, W., Xin-hua, Z., Chun-ji, J., Chun-hong, L., Shan, C., Di, W., et al., 2015. Effects of potassium deficiency on photosynthesis and photoprotection mechanisms in soybean (Glycine max L Merr). J. Integ. Agri. 14, 856-863.

Xing, D., Wu, Y., 2014. Effect of phosphorus deficiency on photosynthetic inorganic carbon assimilation of three climber plant species. Bot Stud. 55, . Available from: https://doi.org/10.1186/s40529014-0060-860.1.

Xiong, T.C., Bourque, S., Lecourieux, D., Amelot, N., Grat, S., Briere, C., et al., 2006. Calcium signalling in plant cell organelles delimited by a double membrane. BiochimBiophysActa. 1763, 1209-1215.

Xu, C., Li, X., Zhang, L., 2013. The effect of calcium chloride on growth, photosynthesis, and antioxidant responses of Zoysia japonica under drought conditions. PLoS One 8 (7), e68214. Available from: https://doi.org/10.1371/journal.pone.0068214.

Yael, G., Aliza, F., Hillel, F., 2010. Calcium-regulated transcription in plants. Mol. Plant. 3 (4), 653-669.

Zhang, L., Du, L., Poovaiah, B.W., 2014. Calcium signalling and biotic defense responses in plants. Plant Sig. Behav. 9 (11), e973818.

Zhang, H., Forde, B.G., 1998. AnArabidopsis MADS box gene that controls nutrient-induced changes in root architecture. Science 279, 407-409.

Zhu, J.K., 2016. Abiotic stress signalling and responses in plants. Cell 167, 313-324.

Zorb, C., Senbayram, M., Peiter, E., 2014. Potassium in agriculture status and perspectives. J. Plant Physiol. 171, 656-669.

Zwieniecki, M.A., Melcher, P.J., Holbrook, N.M., 2001. Hydrogel control of xylem hydraulic resistance in plants. Science 291, 1059-1062.

\section{Further Reading}

Gao, J.T., Guo, L., Zhong, Y.W., Ren, H., Guo, Z.C., 2016. Removal of phosphorus-rich phase from high-phosphorous iron ore by melt separation at $1573 \mathrm{~K}$ in a super-gravity field. Int. J. Min. Met. Mat. 23 (7), 743-750.

Per, T.S., Khan, N.A., Masood, A., Fatma, M., 2016. Methyl jasmonate alleviates cadmium-induced photosynthetic damages through increased S-assimilation and glutathione production in mustard. Front. Plant Sci. 7, 1933. Available from: https://doi.org/ 10.3389/fpls.2016.01933.

Anchutz, U., Becker, D., Shabala, S., 2014. Going beyond nutrition: regulation of potassium homoeostasis as a common denominator of plant adaptive responses to environment. J. Plant Physiol. 171 (9), 670-687.

Munns, R., 2002. Comparative physiology of salt and water stress. Plant. Cell Environ. 25 (2), 239-250. 
This page intentionally left blank 


\title{
18
}

\section{Sulfur Availability Potentiates Phytohormones-Mediated Action in Plants}

\author{
Badar Jahan ${ }^{1}$, Zebus Sehar ${ }^{1}$, Asim Masood ${ }^{1}$, Naser A. Anjum ${ }^{1}$, \\ M. Iqbal R. Khan ${ }^{2}$ and Nafees A. Khan ${ }^{1}$
}

${ }^{1}$ Plant Physiology and Biochemistry Division, Department of Botany, Aligarh Muslim University, Aligarh, Uttar Pradesh, India ${ }^{2}$ Department of Botany, School of Chemical and Life Sciences, Jamia Hamdard, New Delhi, India

\section{O U T L I N E}

18.1 Introduction

18.2 Overview of Sulfur Assimilation and its Role in Plant Metabolism

18.2.1 Sulfur Assimilation

18.2.2 Sulfur Transport Mechanism in Plants

18.2.3 Role of Sulfur and its Compounds in Plant Metabolism

18.3 Crosstalk Between Sulfur and Phytohormones 18.3.1 Auxin 18.3.2 Cytokinin
287
18.3.3 Abscisic Acid

18.3.4 Gibberellic Acid

18.3.5 Ethylene

18.3.6 Jasmonic Acid

18.3.7 Salicylic Acid

18.3.8 Nitric Oxide

18.3.9 Brassinosteroids

18.4 Conclusion

References

Further Reading

\subsection{INTRODUCTION}

Macroelements are ubiquitously present in soil and serve as a major source of mineral nutrients for plants. Amongst these elements, sulfur (S) is one of the foremost indispensable plant nutrient elements like nitrogen $(\mathrm{N})$, phosphorus $(\mathrm{P})$, and potassium $(\mathrm{K})$
(Marschner, 1995). Deficiency of any of the elements in soil significantly impacts plant metabolism, which in turn affects nutritional quality, plant susceptibility to pests, and decreases protein biosynthesis and photosynthetic functions resulting in diminished crop yield (Mazid et al., 2011; Capaldi et al., 2015). In particular, $\mathrm{S}$ serves as a key nutrient universally found in plants 
to mediate plant growth and development and plant metabolism. The importance of $S$ as a plant nutrient has been accepted for a long time, but dynamic research started when extensive $S$ deficiencies were reported. The deficiency of $S$ was reported to impair plant metabolism (Honsel et al., 2012), decrease chlorophyll content and photosynthesis (Kastori et al., 2000), and affect photosynthetic efficiency and the activity of ribulose1, 5 bisphosphate carboxylase (Rubisco) (Lunde et al., 2008). These aspects have been discussed in recent reports in plants under both optimal as well as stressful environments (Dubousset et al., 2010; Iqbal et al., 2013; Khan et al., 2013; Nazar et al., 2014a,b). Sulfur is present in nature both in inorganic and organic forms (Takahashi et al., 2011). The inorganic S is converted to S-containing compounds such as cysteine (Cys) and methionine (Met) and to secondary products such as sulfoxides and glucosinolates through a cascade of enzymatic steps (Leustek et al., 2000; Saito, 2004; Koprivova et al., 2008; Abdallah et al., 2010). These compounds alter several physiological processes and alleviate salt tolerance (Khan et al., 2014a,b). Reports indicate that $S$ is also found in vitamins (biotin and thiamine), active ingredients of a large range of imperative metabolites, cofactors [CoA and S-adenosyl-methionine (SAM)], peptides (glutathione and phytochelatins), many coenzymes, and prosthetic groups (Mazid et al., 2011; Takahashi et al., 2011; Iqbal et al., 2012; Khan et al., 2012a,b, 2013, 2014a,b; Nazar et al., 2012, 2013). Notably, several studies reveal that photosynthetic organisms synthesize a wide variety of S-compounds, using sulfate as a primary S source (Leustek et al., 2000; Saito, 2004). Furthermore, $S$ is used in the form of sulfate by root (Fatma et al., 2016). Sulfate assimilation is an elementary biological pathway of primary metabolism that provides the plant with S (Koprivova and Kopriva, 2016). Sulfur assimilation pathway is also associated with the synthesis of ethylene through Cys and Met. ATP-sulfurylase (ATP-S) and serine acetyl transferase (SAT), the two enzymes of S-assimilation, play a crucial role in Cys synthesis. In addition, ATP-S catalyzes the activation of sulfate, and SAT is dependable for the entry step from serine metabolism to Cys biosynthesis (Fatma et al., 2013). Consequently, the accessibility of sulfate and its uptake and assimilation are vital for plant growth and development, cellular metabolism, and response to a range of biotic and abiotic stresses (Leustek et al., 2000; Saito, 2004; Rausch and Wachter, 2005). In fact, a plant's exposure is inevitable to varied stress factors including salinity, drought, temperature, ozone, soil acidification, and heavy metal toxicity (Bulbovas et al., 2014; Medici et al., 2014; Nogueirol et al., 2015). Nevertheless, plants possess very proficient resistance pathways that tolerate the scavenging of reactive oxygen species (ROS), shielding the cells from oxidative damage (Gratão et al., 2005; Khan and Khan, 2017). Sulfur-containing compounds are involved in ROS metabolism and reduction of oxidative stress (Fatma et al., 2013; reviewed by Anjum et al., 2015). S-assimilation and cellular level of Cys, Met, and reduced glutathione (GSH) can be controlled to improve plant's stress tolerance capacity (Khan et al., 2008; Anjum et al., 2010, 2015). A vast amount of literature is available on the involvement of phytohormones in plant growth and development and also in plant responses to varied stress factors (Khan et al., 2012a,b). Additionally, the involvement of phytohormones such as ethylene in S-mediated improved stress tolerance has been reported in abiotic stressed plants (Asgher et al., 2014; Iqbal et al., 2013; Masood et al., 2012). Further, a large array of phytohormones such as auxins (AU), gibberellins (GA), cytokinins (CK), ethylene (ET), abscisic acid (ABA), brassinosteroids (BR), salicylic acid (SA), and nitric oxide (NO) were shown in crosstalk with $S$ in maintaining plant metabolism (Ohkama et al., 2002; MaruyamaNakashita et al., 2003; Khan et al., 2015a,b). Herein, based on recent reports on the subject, S-assimilation and its role in plant metabolism are overviewed, involvement of S-assimilation in the synthesis of major hormones is highlighted, a crosstalk is presented between $S$ and other phytohormones, and major aspects so far least explored in the current context are listed.

\subsection{OVERVIEW OF SULFUR ASSIMILATION AND ITS ROLE IN PLANT METABOLISM}

\subsubsection{Sulfur Assimilation}

Pathways involved in S uptake and assimilation have been extensively studied (Saito, 2000, 2004; Rausch and Wachter, 2005; Kopriva, 2006). S-assimilation is highly regulated in a demand-driven way (Lappartient and Touraine, 1996; Leustek et al., 2000; Kopriva and Rennenberg, 2004; Kopriva, 2006; Davidian and Kopriva, 2010; Nazar et al., 2011a,b). For the proper growth and development of plants S uptake- and assimilationpathway is generally induced (Kopriva and Rennenberg, 2004). $\mathrm{S}$ is available in the form of sulfate in roots to uptake (Davidian and Kopriva, 2010); whereas under limited S supply, plants are adapted to use foliar absorbed $\mathrm{H}_{2} \mathrm{~S}$ as $\mathrm{S}$ source for the growth (Koralewska et al., 2008). The sulfate uptake by roots and its transport to shoots are stringently restricted and worked as prime points of regulation of S-assimilation. In addition, sulfate reduction occurs in leaf chloroplasts and produces sulfide. The regulatory steps of sulfate assimilation include the transport of sulfate into the cells using ATP-S and the reduction of adenosine 5 '-phosphosulfate (APS) to sulfite by APR (Vauclare et al., 2002). Additionally, with the help of ferredoxin-dependent sulfite reductase (SiR), $S$ is further reduced to sulfide and sulfide is then 


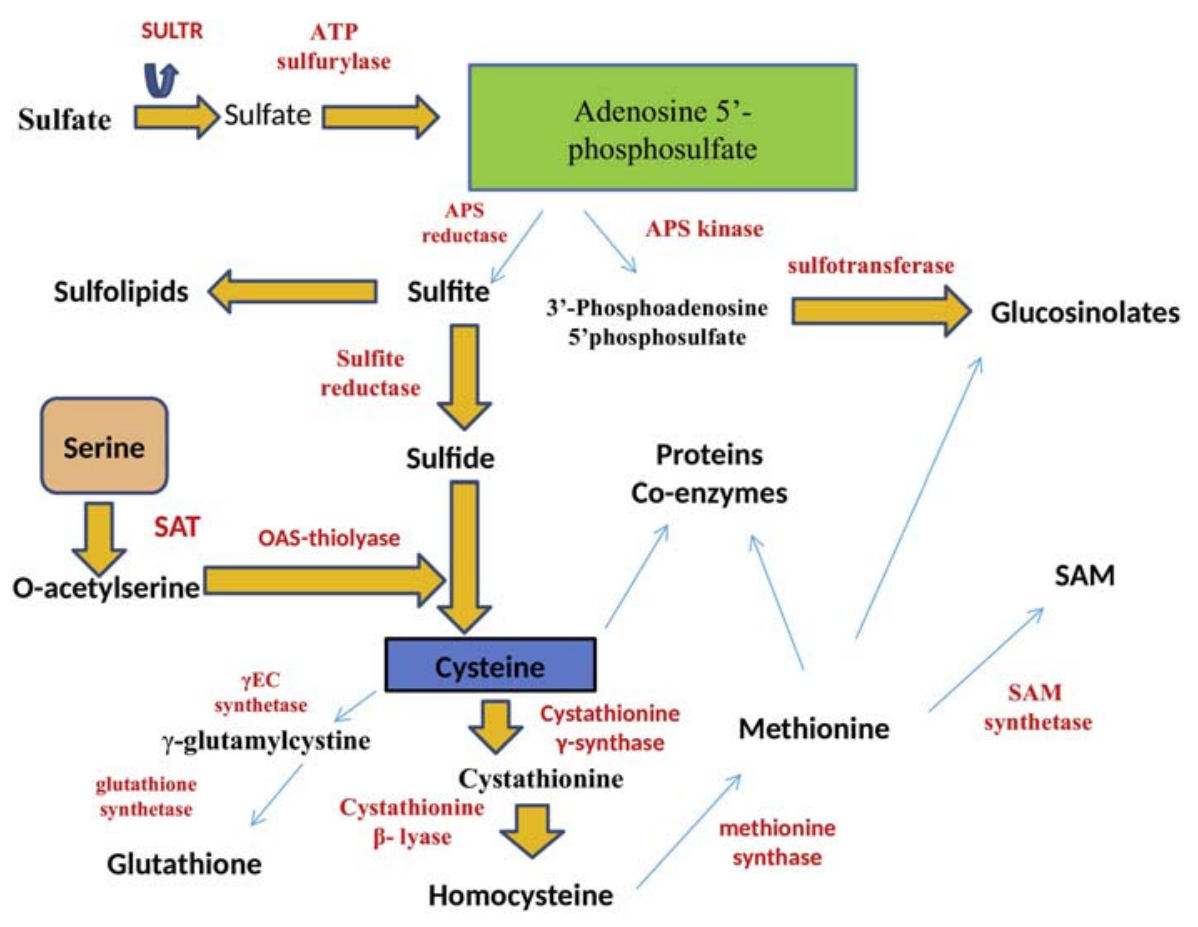

FIGURE 18.1 Schematic demonstration of sulfate assimilation. APS is a branching point as it can enter a reductive assimilation via APS reductase and be phosphorylated by APS kinase. In the reductive branch of sulfate assimilation, a key enzyme is amino acid skeleton of O-acetylserine (OAS) to form cysteine, which can be incorporated into peptides and proteins or serve as a donor of reduced sulfur for methionine and coenzyme syntheses. SULTR, sulfate transporter; APS, adenosine-5'-phosphosulfate; SAT, serine acetyl transferase; $\gamma$-EC, $\gamma$-glutamylcysteine; OAS, O-acetylserine; CoA, acetyl coenzyme A; SAM, S-adenosylmethionine (S-AdoMet). integrated into the amino acid frame of $O$-acetylserine (OAS) to form Cys. However, APS can also be phosphorylated by APS kinase (APK) to form 3-phosphoadenosine 5-phosphosulfate (PAPS), a donor of activated sulfate for many sulfation reactions. Both plants and microorganisms are able to reduce sulfate into sulfide and incorporate it into organic metabolites (Takahashi et al., 2011). The S-assimilation pathway involves the enzymes, ATP-S, APS kinase, PAPS reductase or APS reductase, and SiR to synthesize cysteine (Krueger et al., 2010; Khan et al., 2013; Herrmann et al., 2014;Anjum et al., 2015; Fig. 18.1). Moreover, in sulfide assimilation, two different enzymes are also involved, that is, SAT, which produces OAS from Ser and acetylCoA, and O-acetylserine/O-acetylhomoserine sulfhydrylase, which is involved in the transfers of sulfide at the $\beta$-position of OAS. Both of these enzymes are constrained in the cytosol, chloroplast, and mitochondria, contrasting to those of the reductive phase (Saito, 2000, 2004; Hell et al., 2002). Notably, S homeostasis and response to trace element exposure are linked to plants. Treatment of plants with trace elements leads to increases in both sulfate uptake (Nocito et al., 2002, 2006; Van Hoewyk et al., 2008) and induction in the activities of enzymes involved in S-assimilation (Cobbett, 2000; Lafaye et al., 2005; Herbette et al., 2006; Weber et al., 2006). In contrast, S-assimilation is too stoutly consistent with the assimilation of nitrate as well as carbon (reviewed by Kopriva and Rennenberg, 2004; Nero et al., 2009).

\subsubsection{Sulfur Transport Mechanism in Plants}

$S$ is present in plants chiefly in the oxidized form of inorganic sulfate. $S$ is taken up by the roots as sulfate into plants cells via sulfate transporters (SULTRs) SULTR 1;2 (sulfate- $\mathrm{H}^{+}$co-transporters $\mathrm{M}$ ) and is motivated by the electrochemical gradient established by proton motive force created by ATPase (Leustek et al., 2000; Saito, 2000; Takahashi et al., 2000; Hawkesford, 2000, 2003). A number of genes encoding sulfate transporters were reported to be cloned and characterized in various plant species (Smith et al., 1997; Takahashi et al., 1997, 2000; Bolchi et al., 1999; Vidmar et al., 1999, 2000; Rae and Smith, 2002; Shibagaki et al., 2002; Yoshimoto et al., 2002, 2003; Howarth et al., 2003; Hopkins et al., 2004). Approximately 12-16 genes are reported to encode SULTR in plant species. According to their protein sequence similarities SULTR proteins can be classified into SULTR 1-5 (for a review: Buchner et al., 2004; Davidian and Kopriva, 2010; Hawkesford, 2003). Furthermore, 12 SULTRs, classified into four different groups (SULTR1, SULTR2, SULTR3, and SULTR4) were reported from Arabidopsis thaliana (Takahashi et al., 2012). Their functions in plants and tissue localization have been widely studied (Davidian and Kopriva, 2010; Takahashi et al., 2011). Besides this, the high-affinity transporters SULTR1;1, SULTR1;2, and SULTR1;3 consist of groups 1 sulfate transporters. SULTR1;1 and SULTR1;2 were reported to be expressed in the epidermis and cortex of roots and facilitating the initial uptake of sulfate from the soil (Takahashi et al., 2000; Shibagaki et al., 2002; Yoshimoto et al., 2002). Additionally, it was stated by Yoshimoto et al. (2003) that SULTR1;3 is limited to a small area in the phloem and mediates source-to-sink translocation of sulfate; besides this the group 4 sulfate transporters SULTR4;1 and SULTR4;2 are constrained to the tonoplast and are concerned in remobilization of vacuolar sulfate collection (Kataoka et al., 2004a). There are two ATP-S 
isoforms present in plants. A minor ATP-S form is localized in the cytoplasm and a major ATP-S form is localized in plastids. Moreover, the enhanced in steady-state levels of mRNAs for high-affinity sulfate transporters, ATP-S, and APR upon S undernourishment is detected by Northern analysis (Takahashi et al., 1997; Yamaguchi et al., 1999) or cDNA arrays (Maruyama-Nakashita et al., 2003; Nikiforova et al., 2003).

\subsubsection{Role of Sulfur and its Compounds in Plant Metabolism}

Sulfur metabolism has been described both at the biochemical and molecular levels, and was shown to play crucial roles in plant metabolism and physiology (Schmidt and Jager, 1992; Leustek et al., 2000; Hell et al., 2002; Hesse and Hoefgen, 2003) (Fig. 18.2). S-metabolism and its metabolites play a significant role in modulating plant stress responses (Rausch and Wachter, 2005; Khan et al., 2013). Furthermore, S-metabolism in plants is a highly regulated mechanism that adjusts the production of S-containing metabolites on demand, availability of $S$, and also changes in the environmental conditions and in response to prevent accumulation of toxic substances (Takahashi et al., 2011). Sulfur may up- or downregulate mechanism of plant growth and development through its control on diverse metabolic activities; glutathione (GSH) acts as an important antioxidant under stress and is also the main nonprotein source of $S$ to the plants (Kopriva et al., 2004; Ghelfi et al., 2011; Rennenberg and Herschbach, 2012; Seth et al., 2012). Furthermore, GSH is a universal molecule, which acts as an imperative part of plants related to cellular defense, signal transduction, redox status, and detoxification processes
(Noctor et al., 2012). GSH controls S-assimilation and plays crucial roles in various plant processes including cell differentiation, cell death, balance in redox status, defense against biotic and abiotic stresses, protein folding, as the precursor of phytochelatins, and in detoxification of xenobiotics (Foyer et al., 2001; Mullineaux and Rausch, 2005). The key enzyme in S-metabolism is the OAS-TL that catalyzes the formation of Cys via OAS (Youssefian et al., 2001). The production of GSH against biotic and abiotic stresses is the key preventive step in Cys biosynthesis that can be regarded as the elite function of S reduction in plants (Youssefian et al., 2001; Mera et al., 2014). In GSH biosynthesis, the availability of Cys is an additional vital thing, but a supportable supply of glutamate and glycine is also important (Kopriva and Rennenberg, 2004).

Szalai et al. (2009) reported that GSH maintains Cys, homocysteine, enzymatic proteins, ascorbate (AsA) in active form and may standardize the thiol/disulfide ratio in proteins and defend cell membrane against $\mathrm{H}_{2} \mathrm{O}_{2}$ and free radicals. Additionally, in plant metabolism, SAM is one of the most imperative S-compounds (Azevedo et al., 2006). It is mainly a methyl donor involved in transmethylation of nucleic acids, proteins, polysaccharides, and fatty acids (Ma et al., 2003). However, glucosinolates (GS) are secondary metabolites derived from amino acids, consisting of a thioglucose moiety, a sulfonated aldoxime, and a side chain obtained from aliphatic or aromatic amino acids (Halkier and Gershenzon, 2006).

\subsection{CROSSTALK BETWEEN SULFUR AND PHYTOHORMONES}

Evidence from recent literature reveals that phytohormones are vital for regulation of $\mathrm{S}$ nutrition (Ohkama

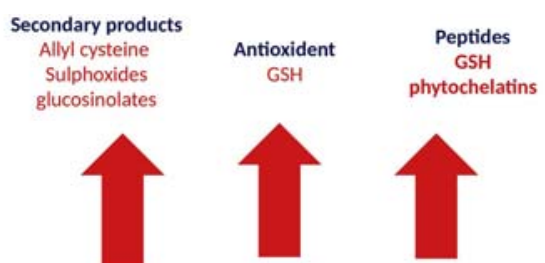

FIGURE 18.2 Diagram represents the involvement of $\mathrm{S}$ in the biosynthesis of plant metabolites or physiological functions. 
et al., 2002; Maruyama-Nakashita et al., 2003). Besides this, some studies have reviewed the significance of the association between S-assimilation and phytohormones (Maruyama-Nakashita et al., 2004, 2005; Kopriva, 2006). According to Peleg and Blumwald (2011) phytohormones are crucial for plant acclimation and adaptation to environmental changes. Moreover, in the plant developmental processes and metabolism and plant defense pathways, efficient nutrient use is linked to signaling pathway of phytohormones (Fatma et al., 2013).

Additionally, phytohormones can interrelate with mineral nutrients under both stress and normal conditions, performing a vital role in salt stress control and disturbing plant growth recovery, germination, cell division, and seed production, still when applied exogenously (Fatma et al., 2013; Khan et al., 2015b; Per et al., 2018). At the same time as phytohormones have a large impact on S-metabolism, some of them require these pathways for their synthesis. A good example is ET; according to Sauter et al. (2013) the synthesis of ET is securely linked with Met metabolism. The substrate for ET biosynthesis, SAM, the activated form of Met is capable of synthesizing the 1-aminocyclopropane carboxylate (ACC). In a Yang cycle the coproduct of ACC synthesis, 5 methylthioadenosine, is cast-off to Met and at the same time as it serves plants as a single source of $\mathrm{S}$ it also inhibits ET production (Sauter et al., 2004). Undoubtedly, among plant hormones, ET is most closely connected with S-metabolism. Studies have shown (Brenner et al., 2005; Kopriva, 2006) CK is well known to be connected to the numerous processes of micronutrients, such as $\mathrm{N}$ cycle and its metabolism, and $\mathrm{P}$ assimilation. It seems that $\mathrm{CK}$ has a common role in the assimilation of the above nutrients, including sulfur. In addition, CKs were reported to decrease the expression of genes encoding macronutrient transporters, that is, nitrate, and phosphate and sulfate transporters. Besides this, CKs were reported to reduce the expression of SULTR1;1 and SULTR1;2, resulting in a reduction of sulfate uptake in roots (MaruyamaNakashita et al., 2004). In general, polyamines are not considered as phytohormones, but are required to be present in higher concentrations for their action. Besides this, there is another group of growth promoting factors, called small signaling peptides, that act more like phytohormones (Czyzewicz et al., 2013).

For the synthesis of one more phytohormone, ABA, $\mathrm{S}$ is indirectly involved and is consequently affected by $S$ deficient conditions. In comparison with ET, the relationship between S-assimilation and ABA synthesis is not quite direct; for the metabolic reactions $S$ is not required (Cao et al., 2014). According to Cao et al. (2014), sulfate deficiency leads to reduced rate of Cys synthesis, resulting in lower tolerance to abiotic stress and steady-state levels of ABA. GA was reported to enhance the effects of salt stress by regulating the availability of other phytohormones in soybean plants Capaldi et al. (2015). Phytohormones, for instance, JA, ABA, SA, and ET play vital roles in the accumulation of GSH under stressful conditions. On the other hand, ET and SA regulate S-metabolism to induce salt tolerance (Kopriva and Rennenberg, 2004; Iqbal et al., 2013; Nazar et al., 2013; Asgher et al., 2014). Additionally, both SA and NO were reported to require GSH for their action. S-nitrosoglutathione (formed when $\mathrm{NO}$ is conjugated to GSH) on other hand is indispensable for its transport and to facilitate nitrosylation of proteins (Stamler, 1994). However, evidence for direct regulation of S-metabolism is insufficient. Therefore, we can say that sulfate assimilation pathway is not only required by phytohormones for their actions, it is also crucial for the synthesis of at least some.

\subsubsection{Auxin}

Auxin is among the most versatile plant hormones and is found to play vital roles in growth and development of plants, and was reported to act as a signaling molecule under sulfate limitation (Nikiforova et al., 2003). Even as AU interacts with diverse aspects of S-metabolism, direct regulation of the pathway by this hormone is not well understood. Additionally, the first link is the production of indole 3-acetic acid (IAA), and indole- 3 acetaldoxime, indolic glucosinolates are common intermediates between AU and S (Mikkelsen et al., 2004). On the other hand, alterations in indolic glucosinolates synthesis are frequently associated with interruption of AU homeostasis (Barlier et al., 2000; Douglas Grubb et al., 2004; Reintanz et al., 2001). Interestingly, the next chief link between $\mathrm{AU}$ and $\mathrm{S}$ is directly associated with the function of $\mathrm{AU}$ in control of root growth (Koprivova and Kopriva, 2016). Several studies reveal S starvation upregulated those genes that are expressed of AU inducible such as IAA18, At1g51950, tryptophan synthase ßchain, At5g38530, putative AU-regulated protein, At2g33830 (Nikiforova et al., 2003; Hirai and Saito, 2004), and indole-3acetonitrile to IAA is converted by NIT3 nitrilase, and powerfully increased by $\mathrm{S}$ starvation (Kutz et al., 2002). According to López-Bucio et al. (2003) AU production may result in an increased in lateral root density in Arabidopsis under sulfate-limited conditions. Furthermore, in wild-type, Arabidopsis suppresses lateral root development by $\mathrm{S}$ deficiency, whereas this response is compromised in the axr1-3 mutant (Dan et al., 2007). In sulfate starvation, microarray results revealed the expression of tryptophan metabolism and some AU responsive genes (Nikiforova et al., 2003; Koprivova and Kopriva, 2016). Based on the 
transcriptome and metabolomic facts AU influx and the IAA28-mediated AU signaling circuit play vital role in modulating $S$ response (Nikiforova et al., $2005 a, b)$. Under the exogenous application of AU, S deficiency-activated BGLU28 ( $\beta$-glucosidase 28) expression is downregulated. In several of the studies, it is hypothesized that BGLU28 acts by releasing $S$ from glucosinolate, which is possibly a major $\mathrm{S}$ derived compound in plant cell vacuoles (MaruyamaNakashita et al., 2003, 2006; Dan et al., 2007). AU signaling component AXR1, a component of the $26 \mathrm{~S}$ proteasome, was also found to be involved in the $S$ starvation (Dan et al., 2007).

\subsubsection{Cytokinin}

CKs are recognized to use authority on the gaining of several macronutrients, such as N, P, and S. Particularly, CKs decreased the expression of genes encoding multiple macronutrient transporters, such as nitrate transporters, sulfate transporters, and phosphate transporters (Brenner et al., 2005). Whereas jasmonate, ET, and ABA can be considered as stress hormones, the participation of CKs in the regulation of S-metabolism is probably the most observable. Additionally, CKs are well known to act together with mineral nutrient plants status (Collier et al., 2003; Franco-Zorrilla et al., 2005; Nam et al., 2012; Sakakibara et al., 2006). Studies have shown (Leustek et al., 2000) that APR1 is a fundamental enzyme in S-metabolism and its upregulation by CKs also suggests that $\mathrm{S}$-assimilation is also regulated by CKs. However, the direct link of CKs and $S$ has been recognized when CKs were initiated to apply a negative effect on expression of SULTR1;1 and SULTR1;2, resulting in sulfate uptake by roots being reduced (Maruyama-Nakashita et al., 2004). Generally, the reduction in transcript levels was accompanied by a reduction in sulfate uptake and was alleviated in cre1 mutants (Maruyama-Nakashita et al., 2004). Apart from this, CKs are also involved in upregulation of APR and SULTR2;1 genes S limitation in Arabidopsis (Ohkama et al., 2002).

\subsubsection{Abscisic Acid}

ABA appears to be the phytohormone that responds more quickly and has the biggest impact on sulfate assimilation. In plants under drought stress, ABA biosynthesis and expression of genes induced by $A B A$ resulted in stomatal closure (Peleg and Blumwald, 2011). Under exogenous ABA application the upregulation of SULTR3;1 transcription in roots indicates that it is essential for a significant coregulation of plastid sulfate uptake (Cao et al., 2013). Sulfate has been reported to affect $\mathrm{ABA}$ root-to-shoot signaling that affects stomatal closure during early stages of water stress in maize plants (Ernst et al., 2010). Conversely, in some circumstances, mostly in salt and drought stress, $\mathrm{ABA}$ is important for signaling and has a great impact on sulfate assimilation as these conditions are accompanied by increased production of ROS. Hence, they reduced $S$ and form a higher demand for GSH. Most enzymes of sulfate assimilation such as APS reductase, ATP-S, SAT, sulfite reductase, cytosolic isoform of OASTL, and sulfate transporters SULTR1;2, 3;1, 3;4 and 4;1 are induced by salts (Barroso et al., 1999; Ruiz and Blumwald, 2002; Koprivova et al., 2008; Cao et al., 2014). According to Zhu (2002), salt stress signaling includes both ABA-dependent and ABA-independent pathways. ABA was reported to suppress the growth of lateral root to enable the growth of primary root to access water deep inside the ground during drought (Xiong and Fei, 2006). Calderwood and Kopriva (2014) showed that many studies were conducted on the role of hydrogen sulfide as a new gas signal in plants during the last few years, both in humans and animals. The well-known function of $\mathrm{H}_{2} \mathrm{~S}$ is in the regulation of stomata opening (García-Mata and Lamattina, 2010; Lisjak et al., 2010; Scuffi et al., 2014). The regulation of stomata opening is intermittent in mutants in the enzyme producing $\mathrm{H}_{2} \mathrm{~S}$ (Scuffi et al., 2014), Furthermore, stomata are closed in the cad2 mutant having high Cys level and low GSH level (Okuma et al., 2011; Noctor et al., 2012), therefore for correct ABA function a steady supply of Cys is essential. It seems that for ABA there is an interconnection between other phytohormones and sulfate assimilation rather than a unidirectional regulation. However, in the $\mathrm{ABA}$ biosynthesis pathway, the conversion of an abscisic aldehyde to ABA is catalyzed by abscisic aldehyde oxidase (EC 1.2.3.1, AAO3), which requires the sulfurated molybdenum cofactor (Mendel and Hänsch, 2002; Schwarz and Mendel, 2006). Sulfuration of Moco is essential for ABA biosynthesis and has been demonstrated by mutating ABA3 (AT1G16540) resulting in ABA deficiency (Xiong et al., 2001). In response to environmental stresses, ABA induces mRNA accumulation of cytosolic OASTL. Accumulation of ABA induces negative role of AtGSTUI7 in stress tolerance by affecting GSH pool (Chen et al., 2012a,b) and indicating that it has more profound effect on control of S-metabolism under stress conditions (Kopriva, 2006). In Arabidopsis, ABA plays an important role in maintaining the redox state by increasing GSH level (Jiang et al., 2010).

\subsubsection{Gibberellic Acid}

The exogenous application of GA enhances photosynthesis, growth, and utilization of $\mathrm{N}$ in mustard. However there is a high requirement of $\mathrm{S}$ in mustard 
and the assimilatory pathway of $\mathrm{S}$ is synchronized with the $\mathrm{N}$. It has been studied that the application of $\mathrm{GA}_{3}$ to mustard enhances the photosynthetic production with the higher efficiency of $\mathrm{N}$ utilization, which in turn increases the S-use efficiency (SUE) of crops (Khan and Mobin, 2005).

It has been studied that the application of GA upregulates the transcript level of APR1 and APR2 in Arabidopsis (Koprivova et al., 2008). The $\mathrm{GA}_{3}$ application to plants accelerates the uptake of $\mathrm{N}$ and $\mathrm{S}$, however, their utilization is interlinked (Khan et al., 2007). It has been suggested that the utilization of $\mathrm{N}$ is impossible in mustard grown in $\mathrm{S}$ deficient soil (Khan and Mobin, 2005).

\subsubsection{Ethylene}

The crosstalk between S-assimilation and ET signaling in plants is considered important because of the close link between ET synthesis and S-metabolism through the Met, a S-containing amino acid that is a substrate for S-adenosyl methionine synthase (SAMS) dependable for the production of SAM or AdoMet (Fontecave et al., 2004; Roje, 2006; Khan et al., 2017). Met and SAM used for polyamines (PA), nicotianamine (NA), and ET biosynthesis are recycled in the Met salvage cycle (known also as a Yang cycle). Significantly, soluble Met seems to be a rate-limiting metabolite of ET biosynthesis (Katz et al., 2006; Bürstenbinder et al., 2007). Noteworthily, sulfate limitation 1 (SLIM1) is the key regulator of sulfate deficiency responses, which is the considerable tie between S and ET signaling. Maruyama-Nakashita et al. (2006) have reported that SLIM1 is constituent of a family of five ethylene insensitive 3-like (EIL) transcription factors. EIN3 also acts as a transcription factor that controls a bulky number of ET-responsive genes (Chao et al., 1997). Additionally, EIN3 regulated the many S related genes (Chang et al., 2013). Interestingly, in Brassica napus investigation for genes' fundamental variation in nutrient homeostasis two genes of ET signaling, EIN2 and EIN5, were shown by associative transcriptomics to be associated with a deviation in sulfate and phosphate levels (Koprivova and Kopriva, 2014). ET signaling is crucial not only for the SLIM1 facilitated response of the sulfate assimilation pathway and managing $S$ metabolite pools, but also for nutrient homeostasis. The interconnection of ET and S has been very freshly reviewed in great detail (Wawrzynska et al., 2015). Recent reports suggested that the combined application of ethephon and $\mathrm{S}$ to cadmium (Cd) treated mustard synergistically enhanced the photosynthetic performance in comparison with Cd-treated plants by reduction in oxidative stress and diminishing the glucose and ET sensitivity with an increase in Met, Cys, GSH (Khan et al., 2016). In Arabidopsis, the application of ET leads to increase in mRNA level of APR1 and APR3 (adenosine 5'-phosphosulfate reductase), which plays a pivotal role in S-assimilation (Koprivova et al., 2008).

\subsubsection{Jasmonic Acid}

The primary and secondary S-metabolism are coordinately affected by jasmonates and during sulfate deficiency jasmonate synthesis is induced (Jost et al., 2005; Nikiforova et al., 2003). JA is an endogenous growth regulator reported to induce a range of physiological and developmental responses in many plant species (Engelberth et al., 2001) and may also act as a stress modulator by enhancing or suppressing the stress responses of plants (Agrawal et al., 2002). Additionally, several studies have revealed that jasmonate or methyl jasmonates (MeJA) regulated those genes that are involved in sulfate reduction, such as Cys content, GSH synthesis, and glucosinolate metabolism at the transcriptional stage (Xiang and Oliver, 1998; Harada et al., 2000; Jost et al., 2005; SasakiSekimoto et al., 2005; Shan and Liang, 2010; Gfeller et al., 2011). In Arabidopsis thaliana treated with MeJA, the levels of mRNA increased consequently to many genes concerned in S-assimilation and GSH synthesis, without affecting the $\mathrm{SO}_{4}^{-2}$ transporters with associated mRNA levels and content of $S$ metabolites (Kopriva, 2006). Hence, JA has a signaling role for suggesting S-assimilation below S shortage (SasakiSekimoto et al., 2005; Srivastava et al., 2012). Analysis using cDNA macroarrays of jasmonate regulated metabolic pathways in Arabidopsis showed that jasmonates activate the expression of factors involved in nine metabolic pathways belonging to two functionally associated groups: (1) biosynthesis of indole glucosinolate, a defense compound occurring in the Brassicaceae family; and (2) glutathione and ascorbate metabolic pathways, which are important in defense responses to oxidative stress (Sasaki-Sekimoto et al., 2005). Again the $S$ deficiency responsive APR1 gene is also reported induced under JA treatment (SasakiSekimoto et al., 2003).

\subsubsection{Salicylic Acid}

While acting as an endogenous signaling molecule SA was found to play crucial roles in plant defense mechanisms, by regulating both physiological and biochemical processes (Gunes et al., 2007; Joseph et al., 2010). SA indirectly assimilates the inorganic $S$ by activating the $S$ assimilatory pathway enzymes' SAT and 
GSH content and increased the heavy metal resistance (Iqbal et al., 2015). It has been found that the SA accelerates the $\mathrm{S}$ uptake and metabolism by regulating the biosynthesis of GSH in ozone exposed Arabidopsis (Yoshida et al., 2009). It has been suggested that various phytohormones like JA, ABA, and SA upregulate the expression of genes that are related to $\mathrm{S}$ metabolism, and hence play a pivotal role in plant stress defense by increasing cellular S-containing compounds like Cys, GSH, thiols, phytochelatins, glucosinolates, etc. (Kutz et al., 2002; Rausch and Wachter, 2005). It has been found that the various S-containing secondary metabolites like thionines, glucosinolates, and GSH were increased by the exogenous application of SA under various abiotic stresses in Brassica napus (Kumar et al., 2015; Kiddle et al.,1994). Nazar et al. (2011b) have shown that the application of $0.5 \mathrm{mM}$ SA increases the inorganic $S$ content in salt-treated mung bean and this inorganic $S$ is easily incorporated into the amino acid Cys through the cascade of enzymatic process. However, the Cys acts as a key regulatory amino acid in the C-terminal region of the transactivation domain of SA receptor NPR1 (Rochon et al., 2006).

\subsubsection{Nitric Oxide}

$\mathrm{NO}$ is a gaseous, signaling, small reactive molecule that interacts with diverse cellular compounds, including other radical species (Correa-Aragunde et al., 2015), and participates in multiple biological processes in plants (Neill et al., 2003; Wendehenne et al., 2004; Crawford and Guo, 2005). It has been suggested that NO triggers the induction of seed germination and reduction of seed dormancy (Beligni and Lamattina, 2000; Libourel et al., 2006; Zhang et al., 2009), induction in cell death (Pedroso and Durzan, 2000), and regulation of plant metabolism and senescence (Ya'acov et al., 1998; Guo and Crawford, 2005). Furthermore, it is also involved in responses to abiotic and biotic stresses, and apoptosis (Delledonne et al., 1998; Garcia-Mata and Lamattina, 2002; Zhao et al., 2004; Zhang et al., 2006).

$\mathrm{NO}$ requires GSH for its action. GSH reacts with $\mathrm{NO}$ and NO-derived peroxynitrite to produce nitrosoglutathione (GSNO) (Barroso et al., 2006), which is crucial for enhanced $S$ requirement of plants for better survival (Wang et al., 2015). In addition, this metabolite can be converted by the enzyme GSNO reductase (GSNOR) into oxidized glutathione (GSSG) and $\mathrm{NH}_{3}$ (Leterrier et al., 2012). Besides this GSNOR regulates the cellular levels of GSNO maintaining NO homeostasis, which is fundamental for transient cell signaling (Malik et al., 2011). Moreover, according to Frungillo et al. (2014) GSNO also regulates the NO concentration in the cell via inhibition of the $\mathrm{N}$ assimilation pathways. In the process of nitration, NO also activates diverse biochemical pathways and interacts with metals to fabricate metal proteins, and with sulfhydryl groups and nitro groups to provide resistance against salt stress (Leterrier et al., 2012). Feng et al. (2013) proposed that NO negatively regulates $\mathrm{CK}$ signaling by restrictive phosphorelay activity via S-nitrosylation. Furthermore, NO acts in a chief role for a salt-induced increase in APR whereas expression of all three APR isoforms and the enzyme activity are induced by salicylate (Koprivova et al., 2008). Additionally, NO regulate stomata aperture dependent on hydrogen sulfide (Scuffi et al., 2014). Noticeably more research is desired to discover how NO is involved in S-metabolism.

\subsubsection{Brassinosteroids}

BRs are the ubiquitous steroidal component considered as a new phytohormone in the plant kingdom; they have pleiotropic effects, which impact various developmental processes like rhizogenesis, seed germination, flowering, abscission, and maturation (Rae and Smith, 2002). In addition to the developmental process, BRs play an important role in abiotic stress (Kapoor et al., 2014). Foliar spray of 24-epiBL has shown a steep rise in enzymatic and nonenzymatic components of the defense system during abiotic stress (Ramakrishna and Rao, 2013; Kapoor et al., 2014). However, there is no interplay link found between S-metabolism and BR hormones.

\subsection{CONCLUSION}

Sulfur has emerged as a requisite nutrient for plant growth and survival owing to the role of $S$ assimilates in structural components of essential cellular molecules and signaling networks. Sulfur-containing compounds (amino acids, vitamins, thioredoxin system, glutathione, lipoic acid, and glucosinolates), play significant roles in plant metabolism and stress response. It has become evident from the literature that $S$ regulates various plant processes and also is the requirement for the activity of almost all the phytohormones-mediated responses.

The involvement of phytohormones in plant growth and development is well accepted. These phytohormonemediated responses are influenced by several factors. Physiological and biochemical insights are available on the outcomes of the crosstalk of S-assimilation with phytohormones such as auxin, gibberellins, cytokinin ethylene, abscisic acid jasmonates, salicylic acid, and nitric oxide. However, it has not been emphasized 
how the availability of sulfur regulates the action of phytohormones. The present chapter has emphasized the importance of $\mathrm{S}$ in regulation of phytohormonesmediated responses. It appears that $S$ is the essential requirement for phytohormones to deliver their best. It would be interesting to get more insights into the molecular genetics of the aforesaid aspects. If done, the outcomes of these studies can help us in sustainably improving plant health and productivity under abiotic stress conditions.

\section{References}

Abdallah, M., Dubousset, L., Meuriot, F., Etienne, P., Avice, J.C., Ourry, A., 2010. Effect of mineral sulphur availability on nitrogen and sulphur uptake and remobilization during the vegetative growth of Brassica napus L. J. Exp. Bot. 61, 2335-2346. Available from: https://doi.org/10.1093/jxb/erq096.

Agrawal, G.K., Rakwal, R., Tamogami, S., Yonekura, M., Kubo, A., Saji, H., 2002. Chitosan activates defense/stress response (s) in the leaves of Oryza sativa seedlings. Plant. Physiol. Biochem. 40, 1061-1069. Available from: https://doi.org/10.1016/S0981-9428 (02)01471-7.

Anjum, N.A., Umar, S., Chan, M.T. (Eds.), 2010. AscorbateGlutathione Pathway and Stress Tolerance in Plants. Springer Science \& Business Media, New York.

Anjum, N.A., Gill, R., Kaushik, M., Hasanuzzaman, M., Pereira, E., Ahmad, I., et al., 2015. ATP-sulfurylase, sulfur-compounds, and plant stress tolerance. Front. Plant Sci. 6, 210. Available from: https:/ / doi.org/10.3389/fpls.2015.00210.

Asgher, M., Khan, N.A., Khan, M.I.R., Fatma, M., Masood, A., 2014. Ethylene production is associated with alleviation of cadmiuminduced oxidative stress by sulfur in mustard types differing in ethylene sensitivity. Ecot. Environ. Safety. 106, 54-61. Available from: https://doi.org/10.1016/j.ecoenv.2014.04.017.

Azevedo, R.A., Lancien, M., Lea, P.J., 2006. The aspartic acid metabolic pathway, an exciting and essential pathway in plants. Amino Acids. 30, 143-162. Available from: https://doi.org/ 10.1007/s00726-005-0245-2.

Barlier, I., Kowalczyk, M., Marchant, A., Ljung, K., Bhalerao, R., Bennett, M., et al., 2000. The SUR2 gene of Arabidopsis thaliana encodes the cytochrome P450 CYP83B1, a modulator of auxin homeostasis. Proc. Nat. Acad. Sci. 97, 14819-14824. Available from: https://doi.org/10.1073/pnas.260502697.

Barroso, C., Romero, L.C., Cejudo, F.J., Vega, J.M., Gotor, C., 1999. Salt-specific regulation of the cytosolic O-acetylserine (thiol) lyase gene from Arabidopsis thaliana is dependent on abscisic acid. Plant. Mol. Biol. 40, 729-736. Available from: https://doi.org/ 10.1023/A:1006285016296.

Barroso, J.B., Corpas, F.J., Carreras, A., Rodríguez-Serrano, M., Esteban, F.J., Fernández-Ocana, A., et al., 2006. Localization of S-nitrosoglutathione and expression of S-nitrosoglutathione reductase in pea plants under cadmium stress. J. Exp. Bot. 57, 1785-1793. Available from: https://doi.org/10.1093/jxb/erj175.

Beligni, M.V., Lamattina, L., 2000. Nitric oxide stimulates seed germination and de-etiolation, and inhibits hypocotyl elongation, three light-inducible responses in plants. Planta. 210, 215-221. Available from: https://doi.org/10.1007/PL00008128.

Bolchi, A., Petrucco, S., Tenca, P.L., Foroni, C., Ottonello, S., 1999. Coordinate modulation of maize sulfate permease and ATP sulfurylase mRNAs in response to variations in sulfur nutritional status: stereospecific down-regulation by L-cysteine. Plant. Mol.
Biol. 39, 527-537. Available from: https://doi.org/10.1023/ A:1006148815106.

Brenner, W.G., Romanov, G.A., Köllmer, I., Bürkle, L., Schmülling, $\mathrm{T} ., 2005$. Immediate-early and delayed cytokinin response genes of Arabidopsis thaliana identified by genome-wide expression profiling reveal novel cytokinin-sensitive processes and suggest cytokinin action through transcriptional cascades. Plant. J. 44, 314-333. Available from: https://doi.org/10.1111/j.1365313X.2005.02530.x.

Buchner, P., Stuiver, C.E.E., Westerman, S., Wirtz, M., Hell, R., Hawkesford, M.J., et al., 2004. Regulation of sulfate uptake and expression of sulfate transporter genes in Brassica oleracea as affected by atmospheric H2S and pedospheric sulfate nutrition. Plant. Physiol. 3396-3408. Available from: https://doi.org/ 10.1104/pp.104.046441.

Bulbovas, P., Souza, S.R., Esposito, J.B.N., Moraes, R.M., Alves, E.S., Domingos, M., et al., 2014. Assessment of the ozone tolerance of two soybean cultivars (Glycine max cv. Sambaíba and Tracajá) cultivated in Amazonian areas. Environ. Sci. Poll. Res. 21, 10514-10524. Available from: https://doi.org/10.1007/s11356014-2934-4.

Bürstenbinder, K., Rzewuski, G., Wirtz, M., Hell, R., Sauter, M., 2007. The role of methionine recycling for ethylene synthesis in Arabidopsis. Plant. J. 49, 238-249. Available from: https://doi. org/10.1111/j.1365-313X.2006.02942.x.

Calderwood, A., Kopriva, S., 2014. Hydrogen sulfide in plants: from dissipation of excess sulfur to signaling molecule. Nitric Oxide 41, 72-78. Available from: https:/ / doi.org/10.1016/j.niox.2014.02.005.

Cao, M., Liu, X., Zhang, Y., Xue, X., Zhou, X.E., Melcher, K., et al., 2013. An ABA-mimicking ligand that reduces water loss and promotes drought resistance in plants. Cell Res. 23, 1043. Available from: https://doi.org/10.1038/cr.2013.95.

Cao, M.J., Wang, Z., Zhao, Q., Mao, J.L., Speiser, A., Wirtz, M., et al., 2014. Sulfate availability affects $A B A$ levels and germination response to ABA and salt stress in Arabidopsis thaliana. Plant. J. 77, 604-615. Available from: https://doi.org/10.1111/tpj.12407.

Capaldi, F.R., Gratão, P.L., Reis, A.R., Lima, L.W., Azevedo, R.A., 2015. Sulfur metabolism and stress defense responses in plants. Tropical. plant. Biol. 8, 60-73. Available from: https://doi.org/ 10.1007/s12042-015-9152-1.

Chang, K.N., Zhong, S., Weirauch, M.T., Hon, G., Pelizzola, M., Li, H., et al., 2013. Temporal transcriptional response to ethylene gas drives growth hormone cross-regulation in Arabidopsis. Elife 2. Available from: https:/ / doi.org/10.7554/eLife.00675.

Chao, Q., Rothenberg, M., Solano, R., Roman, G., Terzaghi, W., Ecker, J.R., 1997. Activation of the ethylene gas response pathway in Arabidopsis by the nuclear protein ETHYLENEINSENSITIVE3 and related proteins. Cell 89, 1133-1144. Available from: https://doi.org/10.1016/S0092-8674(00)80300-1.

Chen, J.H., Jiang, H.W., Hsieh, E.J., Chen, H.Y., Chien, C.T., Hsieh, H.L., et al., 2012a. Drought and salt stress tolerance of an Arabidopsis glutathione S-transferase U17 knockout mutant are attributed to the combined effect of glutathione and abscisic acid. Plant. Physiol. 158, 340-351. Available from: https://doi.org/ 10.1104/pp.111.181875.

Chen, T., Yang, Q., Zhang, X., Ding, W., Gruber, M., 2012b. An alfalfa (Medicago sativa L.) ethylene response factor gene, MsERF11, enhances salt tolerance in transgenic Arabidopsis. Plant. Cell. Rep. 31, 1737-1746. Available from: https://doi.org/ 10.1007/s00299-012-1287-z.

Cobbett, C.S., 2000. Phytochelatins and their roles in heavy metal detoxification. Plant Physiol. 123, 825-832. Available from: https://doi.org/10.1104/pp.123.3.825.

Collier, M.D., Fotelli, M.N., Nahm, M., Kopriva, S., Rennenberg, H., Hanke, D.E., et al., 2003. Regulation of nitrogen uptake by Fagus 
sylvatica on a whole plant level-interactions between cytokinins and soluble N compounds. Plant. Cell. Environ. 26, 1549-1560. Available from: https:/ / doi.org/10.1046/j.1365-3040.2003.01079.x.

Correa-Aragunde, N., Foresi, N., Lamattina, L., 2015. Nitric oxide is a ubiquitous signal for maintaining redox balance in plant cells: regulation of ascorbate peroxidase as a case study. J. Exp. Bot. 66, 2913-2921. Available from: https://doi.org/10.1093/jxb/erv073.

Crawford, N.M., Guo, F.Q., 2005. New insights into nitric oxide metabolism and regulatory functions. Trends. Plant. Sci 10, 5-200. Available from: https://doi.org/10.1016/j.tplants.2005.02.008.

Czyzewicz, N., Yue, K., Beeckman, T., Smet, I.D., 2013. Message in a bottle: small signalling peptide outputs during growth and development. J. Exp. Bot. 64, 5281-5296. Available from: https://doi. org /10.1093/jxb/ert283.

Dan, H., Yang, G., Zheng, Z.L., 2007. A negative regulatory role for auxin in sulphate deficiency response in Arabidopsis thaliana. Plant Mol. Biol. 63, 221-235. Available from: https://doi.org/10.1007/ s11103-006-9084-0.

Davidian, J.C., Kopriva, S., 2010. Regulation of sulfate uptake and assimilation-the same or not the same? Mol. Plant 3, 314-325. Available from: https://doi.org/10.1093/mp/ssq001.

Delledonne, M., Xia, Y., Dixon, R.A., Lamb, C., 1998. Nitric oxide functions as a signal in plant disease resistance. Nature 394 (6693), 585. Available from: https://doi.org/10.1038/29087.

Douglas Grubb, C., Zipp, B.J., Ludwig-Müller, J., Masuno, M.N., Molinski, T.F., Abel, S., 2004. Arabidopsis glucosyltransferase UGT74B1 functions in glucosinolate biosynthesis and auxin homeostasis. Plant J. 40, 893-908. Available from: https://doi. org/10.1111/j.1365-313X.2004.02261.x.

Dubousset, L., Etienne, P., Avice, J.C., 2010. Is the remobilization of S and $\mathrm{N}$ reserves for seed filling of winter oilseed rape modulated by sulfate restrictions occurring at different growth stages? J. Exp. Bot. 61, 4313-4324. Available from: https://doi.org/10.1093/jxb/ erq233.

Engelberth, J., Koch, T., Schüler, G., Bachmann, N., Rechtenbach, J., Boland, W., 2001. Ion channel-forming alamethicin is a potent elicitor of volatile biosynthesis and tendril coiling. Cross talk between jasmonate and salicylate signaling in lima bean. Plant. Physiol. 125, 369-377. Available from: https://doi.org/10.1104/ pp.125.1.369.

Ernst, L., Goodger, J.Q., Alvarez, S., Marsh, E.L., Berla, B., Lockhart, E., et al., 2010. Sulphate as a xylem-borne chemical signal precedes the expression of ABA biosynthetic genes in maize roots. J. Exp. Bot. 61, 3395-3405. Available from: https://doi.org/ $10.1093 / j x b$ / erq160.

Fatma, M., Khan, M.I.R., Masood, A., Khan, N.A., 2013. Coordinate Changes in Assimilatory Sulfate Reduction are Correlated to Salt Tolerance: Involvement of Phytohormones.

Fatma, M., Masood, A., Per, T.S., Rasheed, F., Khan, N.A., 2016. Interplay between nitric oxide and sulfur assimilation in salt tolerance in plants. Crop. J. 4, 153-161. Available from: https://doi. org/10.1016/j.cj.2016.01.009.

Feng, J., Wang, C., Chen, Q., Chen, H., Ren, B., Li, X., et al., 2013. Snitrosylation of phosphotransfer proteins represses cytokinin signaling. Nat. Commun. 4, 1529. Available from: https://doi.org/ $10.1038 /$ ncomms 2541 .

Fontecave, M., Atta, M., Mulliez, E., 2004. S-adenosylmethionine: nothing goes to waste. Trend. Biochem. Sci. 29, 243-249. Available from: https://doi.org/10.1016/j.tibs.2004.03.007.

Foyer, C.H., Theodoulou, F.L., Delrot, S., 2001. The functions of interand intracellular glutathione transport systems in plants. Trends. Plant Sci. 6, 486-492. Available from: https://doi.org/10.1016/ S1360-1385(01)02086-6.

Franco-Zorrilla, J.M., Martín, A.C., Leyva, A., Paz-Ares, J., 2005. Interaction between phosphate-starvation, sugar, and cytokinin signaling in Arabidopsis and the roles of cytokinin receptors CRE1/AHK4 and AHK3. Plant Physiol. 138, 847-857. Available from: https://doi.org/10.1104/pp.105.060517.

Frungillo, L., Skelly, M.J., Loake, G.J., Spoel, S.H., Salgado, I., 2014. S-nitrosothiols regulate nitric oxide production and storage in plants through the nitrogen assimilation pathway. Nat. Communicat. 5, 5401. Available from: https://doi.org/10.1038/ ncomms6401.

Garcia-Mata, C., Lamattina, L., 2002. Nitric oxide and abscisic acid cross talk in guard cells. Plant Physiol. 128, 790-792. Available from: https:/ / doi.org/10.1104/pp.011020.

García-Mata, C., Lamattina, L., 2010. Hydrogen sulphide, a novel gasotransmitter involved in guard cell signalling. New Phytol. 188, 977-984. Available from: https://doi.org/10.1111/j.14698137.2010.03465.x.

Gfeller, A., Baerenfaller, K., Loscos, J., Chételat, A., Baginsky, S., Farmer, E.E., 2011. Jasmonate controls polypeptide patterning in undamaged tissue in wounded Arabidopsis leaves. Plant Physiol. 156, 1797-1807. Available from: https://doi.org/ 10.1104/pp.111.181008.

Ghelfi, A., Gaziola, S.A., Cia, M.C., Chabregas, S.M., Falco, M.C., Kuser-Falcão, P.R., et al., 2011. Cloning, expression, molecular modelling and docking analysis of glutathione transferase from Saccharumofficinarum. Ann. Appl. Biol. 159, 267-280. Available from: https:/ / doi.org/10.1111/j.1744-7348.2011.00491.x.

Gratão, P.L., Polle, A., Lea, P.J., Azevedo, R.A., 2005. Making the life of heavy metal-stressed plants a little easier. Funct. Plant Biol. 32, 481-494. Available from: https://doi.org/10.1071/FP05016.

Gunes, A., Inal, A., Alpaslan, M., Eraslan, F., Bagci, E.G., Cicek, N., 2007. Salicylic acid induced changes on some physiological parameters symptomatic for oxidative stress and mineral nutrition in maize (Zea mays L.) grown under salinity. J. Plant Physiol. 164, 728-736. Available from: https:/ / doi.org/10.1016/j.jplph.2005.12.009.

Guo, F.Q., Crawford, N.M., 2005. Arabidopsis nitric oxide synthase1 is targeted to mitochondria and protects against oxidative damage and dark-induced senescence. Plant Cell 17 (12), 3436-3450. Available from: https://doi.org/10.1105/tpc.105.037770.

Halkier, B.A., Gershenzon, J., 2006. Biology and biochemistry of glucosinolates. Ann. Rev. Plant. Biol. 57, 303-333. Available from: https://doi.org/10.1146/annurev.arplant.57.032905.105228.

Harada, E., Kusano, T., Sano, H., 2000. Differential expression of genes encoding enzymes involved in sulfur assimilation pathways in response to wounding and jasmonate in Arabidopsis thaliana. J. Plant. Physio. 156, 272-276. Available from: https:// doi.org/10.1016/S0176-1617(00)80317-X.

Hawkesford, M.J., 2000. Plant responses to sulphur deficiency and the genetic manipulation of sulphate transporters to improve S-utilization efficiency. J. Exp. Bot. 51, 131-138. Available from: https://doi.org/10.1093/jexbot/51.342.131.

Hawkesford, M.J., 2003. Transporter gene families in plants: the sulfate transporter gene family redundancy or specialization? Physiol. Plant. 117, 155-163. Available from: https://doi.org/ 10.1034/j.1399-3054.2003.00034.x.

Hell, R., Jost, R., Berkowitz, O., Wirtz, M., 2002. Molecular and biochemical analysis of the enzymes of cysteine biosynthesis in the plant Arabidopsis thaliana. Amino Acids 22, 245-257. Available from: https:/ / doi.org/10.1007/s007260200012.

Herbette, S., Taconnat, L., Hugouvieux, V., Piette, L., Magniette, M.L., Cuine, S., et al., 2006. Genome-wide transcriptome profiling of the early cadmium response of Arabidopsis roots and shoots. Biochimie 88 (11), 1751-1765. Available from: https://doi.org/ 10.1016/j.biochi.2006.04.018.

Herrmann, J., Ravilious, G.E., McKinney, S.E., Westfall, C.S., Lee, S.G., Baraniecka, P., et al., 2014. Structure and mechanism of soybean ATP sulfurylase and the committed step in plant sulfur 
assimilation. J. Biol. Chem. 289, 10919-10929. Available from: https://doi.org/10.1074/jbc.M113.540401.

Hesse, H., Hoefgen, R., 2003. Molecular aspects of methionine biosynthesis. Trends Plant Sci. 8, 259-262. Available from: https:// doi.org/10.1016/S1360-1385(03)00107-9.

Hirai, M.Y., Saito, K., 2004. Post-genomics approaches for the elucidation of plant adaptive mechanisms to sulphur deficiency. J. Exp. Bot. 55, 1871-1879. Available from: https://doi.org/ $10.1093 / j x b /$ erh184.

Honsel, A., Kojima, M., Haas, R., Frank, W., Sakakibara, H., Herschbach, C., et al., 2012. Sulphur limitation and early sulphur deficiency responses in poplar: significance of gene expression, metabolites, and plant hormones. J. Exp. Bot. 63, 1873-1893. Available from: https://doi.org/10.1093/jxb/err365.

Hopkins, L., Parmar, S., Bouranis, D.L., Howarth, J.R., Hawkesford, M.J., 2004. Coordinated expression of sulfate uptake and components of the sulfate assimilatory pathway in maize. Plant. Biol. 6, 408-414. Available from: https://doi.org/10.1055/s-2004-820872.

Howarth, J.R., Fourcroy, P., Davidian, J.C., Smith, F.W., Hawkesford, M.J., 2003. Cloning of two contrasting high-affinity sulfate transporters from tomato induced by low sulfate and infection by the vascular pathogen Verticillium dahliae. Planta. 218, 58-64. Available from: https:/ / doi.org/10.1007/s00425-003-1085-5.

Iqbal, N., Nazar, R., Khan, M.I.R., Khan, N.A., 2012. Variation in photosynthesis and growth of mustard cultivars: role of ethylene sensitivity. Sci. Hort. 135, 1-6. Available from: https://doi.org/ 10.1016/j.scienta.2011.12.005.

Iqbal, N., Masood, A., Khan, M.I.R., Asgher, M., Fatma, M., Khan, N. A., 2013. Cross-talk between sulfur assimilation and ethylene signaling in plants. Plant Signal. Behav. 8, 22478. Available from: https: / / doi.org/10.4161/psb.22478.

Iqbal, N., Umar, S., Khan, N.A., 2015. Nitrogen availability regulates proline and ethylene production and alleviates salinity stress in mustard (Brassica juncea). J. Plant Physiol. 178, 84-91. Available from: https://doi.org/10.1016/j.jplph.2015.02.006.

Jiang, H.W., Liu, M.J., Chen, C., Huang, C.H., Chao, L.Y., Hsieh, H. L., 2010. A glutathione S-transferase regulated by light and hormones participates in the modulation of Arabidopsis seedling development. Plant. Physiol. 154, 1646-1658. Available from: https://doi.org/10.1104/pp.110.159152.

Joseph, B., Jini, D., Sujatha, S., 2010. Biological and physiological perspectives of specificity in abiotic salt stress response from various rice plants. Asian J. Agric. Sci 2, 99-105.

Jost, R., Altschmied, L., Bloem, E., Bogs, J., Gershenzon, J., Hähnel, U., et al., 2005. Expression profiling of metabolic genes in response to methyl jasmonate reveals regulation of genes of primary and secondary sulfur-related pathways in Arabidopsis thaliana. Phot. Res. 86, 491-508. Available from: https://doi.org/ $10.1007 / \mathrm{s} 11120-005-7386-8$.

Kapoor, D., Rattan, A., Gautam, V., Kapoor, N., Bharadwaj, R., 2014. 24- Epibrassinolide mediated photosynthetic pigments and antioxidative defense systems of radish seedling under cadmium and mercury stress. J. Stress Physiol. Biochem. 10, 110-121.

Kastori, R., Plesnicar, M., Arsenijevic-Maksimovic, I., Petrovic, N., Pankovic, D., Saka, Z., 2000. Photosynthesis, chlorophyll fluorescence, and water relations in youngsugar beet plants as affected by sulfur supply. J. Plant. Nutr. 23, 1037-1049. Available from: https:/ / doi.org/10.1080/01904160009382080.

Kataoka, T., Watanabe-Takahashi, A., Hayashi, N., Ohnishi, M., Mimura, T., Buchner, P., et al., 2004a. Vacuolar sulfate transporters are essential determinants controlling internal distribution of sulfate in Arabidopsis. Plant. Cell. 16, 2693-2704. Available from: https:// doi.org/10.1105/tpc.104.023960.

Katz, Y.S., Galili, G., Amir, R., 2006. Regulatory role of cystathioninegamma-synthase and denovo synthesis of methionine in ethylene production during tomato fruit ripening. Plant. Mol. Biol. 61, 255-268. Available from: https://doi.org/10.1007/s11103-006-0009-8. Khan, M.I.R., Khan, N., 2017. Reactive Oxygen Species and Antioxidant System in Plants: Role and Regulation Under Abiotic Stress. Springer Nature, New York978-981-10-5254-5.

Khan, M.I.R., Iqbal, N., Masood, A., Khan, N.A., 2012a. Variation in salt tolerance of wheat cultivars: role of glycinebetaine and ethylene. Pedosphere 22, 746-754. Available from: https://doi.org/ 10.1016/S1002-0160(12)60060-5.

Khan, M.I.R., Syeed, S., Nazar, R., Anjum, N.A., 2012b. An insight into the role of salicylic acid and jasmonic acid in salt stress tolerance. Phytohorm. Abiotic Stress Tolerance Plants 277-300. Available from: https://doi.org/10.1007/978-3-642-25829-9.

Khan, M.I.R., Asgher, M., Iqbal, N., Khan, N.A., 2013. Potentiality of sulphur-containing compounds in salt stress tolerance. Ecophysiology and Responses of Plants Under Salt Stress. Springer, New York, NY, pp. 443-472. Available from: https:// doi.org/10.1007/978-1-4614-4747-4_17.

Khan, M.I.R., Fatma, M., Per, T.S., Anjum, N.A., Khan, N.A., 2015b. Salicylic acid-induced abiotic stress tolerance and underlying mechanisms in plants. Front. Plant Sci. 6, 462.

Khan, M.I.R., Nazir, F., Asgher, M., Per, T.S., Khan, N.A., 2015a. Selenium and sulfur influence ethylene formation and alleviate cadmium-induced oxidative stress by improving proline and glutathione production in wheat. J. Plant Physiol. 173, 9-18.

Khan, N.A., Mobin, M., 2005. The influence of gibberellic acid and sulfur fertilization rate on growth and S-use efficiency of mustard (Brassica juncea). Plant. Soil 270, 269-274. Available from: https://doi.org/10.1007/s11104-004-1606-4.

Khan, N.A., Samiullah, Singh, S., Nazar, R., 2007. Activities of antioxidative enzymes, sulphur assimilation, photosynthetic activity and growth of wheat (Triticum aestivum) cultivars differing in yield potential under cadmium stress. J. Agron. Crop Sci. 193, 435-444. Available from: https:// doi.org/10.1111/j.1439-037X.2007.00272.x.

Khan, N.A., Mir, M.R., Nazar, R., Singh, S., 2008. The application of ethephon (an ethylene releaser) increases growth, photosynthesis and nitrogen accumulation in mustard (Brassica juncea L.) under high nitrogen levels. Plant. Biol. 10, 534-538. Available from: https:/ / doi.org/10.1111/j.1438-8677.2008.00054.x.

Khan, N.A., Khan, M.I.R., Asgher, M., Fatma, M., Masood, A., Syeed, S., 2014a. Salinity tolerance in plants: revisiting the role of sulfur metabolites. J. Plant. Biochem. Physiol. 2, 2. Available from: https: / / doi.org/10.4172/2329-9029.1000120.

Khan, N.A., Khan, M.I.R., Asgher, M., Fatma, M., Masood, A., Syeed, S., 2014b. Salinitytolerance in plants: revisiting the role of sulfur metabolites. J. Plant Biochem. Physiol. Available from: https:// doi.org/10.4172/2329-9029.1000120.

Khan, N.A., Asgher, M., Per, T.S., Masood, A., Fatma, M., Khan, M.I. R., 2016. Ethylene potentiates sulfur-mediated reversal of cadmium inhibited photosynthetic responses in mustard. Front. Plant. Sci. 7, 1628. Available from: https://doi.org/10.3389/ fpls.2016.01628.

Khan, N.A., Khan, M.I.R., Ferrante, A., Poór, P., 2017. Ethylene: a key regulatory molecule in plants. Front. Plant Sci. 8, 1782.

Kiddle, G.A., Doughty, K.J., Wallsgrove, R.M., 1994. Salicylic acidinduced accumulation of glucosinolates in oilseed rape (Brassica napus L.) leaves. J. Exp. Bot. 45, 1343-1346. Available from: https:// doi.org/10.1093/jxb/45.9.1343.

Kopriva, S., 2006. Regulation of sulfate assimilation in Arabidopsis and beyond. Ann. Bot. 97, 479-495. Available from: https://doi. org/10.1093/aob/mcl006.

Kopriva, S., Rennenberg, H., 2004. Control of sulfate assimilation and glutathione synthesis: interaction with $\mathrm{N}$ and $\mathrm{C}$ metabolism. J. Exp. Bot. 55, 1831-1842. Available from: https://doi.org/ $10.1093 / \mathrm{jxb} / \mathrm{erh} 203$. 
Koprivova, A., Kopriva, S., 2014. Molecular mechanisms of regulation of sulfate assimilation: first steps on a long road. Front. Plant Sci. 5, 589. Available from: https://doi.org/10.3389/ fpls.2014.00589.

Koprivova, A., Kopriva, S., 2016. Hormonal control of sulfate uptake and assimilation. Plant. Mole. Biol. 9, 617-627. Available from: https: / / doi.org/10.1007/s11103-016-0438-y.

Koprivova, A., North, K.A., Kopriva, S., 2008. Complex signaling network in regulation of adenosine $5^{\prime}$-phosphosulfate reductase by salt stress in Arabidopsis roots. Plant. Physiol. 146, 1408-1420. Available from: https://doi.org/10.1104/pp.107.113175.

Koralewska, A., Stuiver, C.E.E., Posthumus, F.S., Kopriva, S., Hawkesford, M.J., De Kok, L.J., 2008. Regulation of sulfate uptake, expression of the sulfate transporters Sultr1; 1 and Sultr1; 2, and APS reductase in Chinese cabbage (Brassica pekinensis) as affected by atmospheric H2S nutrition and sulfate deprivation. Funct. Plant. Biol. 35, 318-327. Available from: https://doi.org/10.1071/FP07283.

Krueger, S., Donath, A., Lopez-Martin, M.C., Hoefgen, R., Gotor, C., Hesse, H., 2010. Impact of sulfur starvation on cysteine biosynthesis in T-DNA mutants deficient for compartment-specific serineacetyltransferase. Amino Acids. 39, 1029-1042. Available from: https:// doi.org/10.1007/s00726-010-0580-9.

Kumar, A., Irchhaiya, R., Yadav, A., Gupta, N., Kumar, S., Gupta, N., et al., 2015. Metabolites in plants and its classification. World J. Pharm. Pharm. Sci. 4, 287-305.

Kutz, A., Müller, A., Hennig, P., Kaiser, W.M., Piotrowski, M., Weiler, E.W., 2002. A role for nitrilase 3 in the regulation of root morphology in sulphur-starving Arabidopsis thaliana. Plant. J. 30, 95-106. Available from: https://doi.org/10.1046/j.1365313X.2002.01271.x.

Lafaye, A., Junot, C., Pereira, Y., Lagniel, G., Tabet, J.C., Ezan, E., et al., 2005. Combined proteome and metabolite-profiling analyses reveal surprising insights into yeast sulfur metabolism. J. Biol. Chem. 280, 24723-24730. Available from: https://doi.org/ 10.1074 /jbc.M502285200.

Lappartient, A.G., Touraine, B., 1996. Demand-driven control of root ATP sulfurylase activity and SO42-uptake in intact canola (the role of phloem-translocated glutathione). Plant. Physiol. 111, 147-157. Available from: https://doi.org/10.1104/pp.111.1.147.

Leterrier, M., Airaki, M., Palma, J.M., Chaki, M., Barroso, J.B., Corpas, F.J., 2012. Arsenic triggers the nitric oxide (NO) and Snitrosoglutathione (GSNO) metabolism in Arabidopsis. Environ. Pollut. 166, 136-143. Available from: https://doi.org/10.1016/j. envpol.2012.03.012.

Leustek, T., Martin, M.N., Bick, J.A., Davies, J.P., 2000. Pathways and regulation of sulfur metabolism revealed through molecular and genetic studies. Annu. Rev. Plant. Biol. 51, 141-165. Available from: https:/ / doi.org/10.1146/annurev.arplant.51.1.141.

Libourel, I.G., Bethke, P.C., De Michele, R., Jones, R.L., 2006. Nitric oxide gas stimulates germination of dormant Arabidopsis seeds: use of a flow-through apparatus for delivery of nitric oxide. Planta. 223, 813-820. Available from: https://doi.org/10.1007/ s00425-005-0117-8.

Lisjak, M., Srivastava, N., Teklic, T., Civale, L., Lewandowski, K., Wilson, I., et al., 2010. A novel hydrogen sulfide donor causes stomatal opening and reduces nitric oxide accumulation. Plant. Physiol. Biochem. 48, 931-935. Available from: https://doi.org/ 10.1016/j.plaphy.2010.09.016.

López-Bucio, J., Cruz-Ramırez, A., Herrera-Estrella, L., 2003. The role of nutrient availability in regulating root architecture. Curr. Opin. Plant. Biol. 6, 280-287. Available from: https://doi.org/10.1016/ S1369-5266(03)00035-9.

Lunde, C., Zygadlo, A., Simonsen, H.T., Nielsen, P.L., Blennow, A., Haldrup, A., 2008. Sulfur starvation in rice: the effect on photosynthesis, carbohydrate metabolism, and oxidative stress protective pathways. Physiol. Plant. 134, 508-521. Available from: https:// doi.org/10.1111/j.1399-3054.2008.01159.x.

Ma, X., Wang, Z., Qi, Y., Zhao, Y., Zhang, H., 2003. Isolation of S-adenosylmethionine synthetase gene from Suaeda salsa and its differential expression under $\mathrm{NaCl}$ stress. Acta Bot $\mathrm{Sin} 45$ (11), 1359-1365.

Malik, S.I., Hussain, A., Yun, B.-W., Spoel, S.H., Loake, G.J., 2011. GSNOR-mediated de-nitrosylation in the plant defence response. Plant. Sci. 181, 540-544. Available from: https://doi.org/ 10.1016/j.plantsci.2011.04.004.

Marschner, H., 1995. Mineral Nutrition of Higher Plants. second ed. Academic Press, London.

Maruyama-Nakashita, A., Inoue, E., Watanabe-Takahashi, A., Yamaya, T., Takahashi, H., 2003. Transcriptome profiling of sulfur-responsive genes in Arabidopsis reveals global effects of sulfur nutrition on multiple metabolic pathways. Plant. Physiol. 132, 597-605. Available from: https://doi.org/10.1104/pp.102. 019802.

Maruyama-Nakashita, A., Nakamura, Y., Yamaya, T., Takahashi, H., 2004. A novel regulatory pathway of sulfate uptake in Arabidopsis roots: implication of CRE1/WOL/AHK4-mediated cytokinin-dependent regulation. Plant. J 38, 779-789. Available from: https:// doi.org/10.1111/j.1365-313X.2004.02079.x.

Maruyama-Nakashita, A., Nakamura, Y., Watanabe-Takahashi, A., Inoue, E., Yamaya, T., Takahashi, H., 2005. Identification of a novel cis-acting element conferring sulfur deficiency response in Arabidopsis roots. Plant. J 42, 305-314. Available from: https:// doi.org/10.1111/j.1365-313X.2005.02363.x.

Maruyama-Nakashita, A., Nakamura, Y., Tohge, T., Saito, K., Takahashi, H., 2006. Arabidopsis SLIM1 is a central transcriptional regulator of plant sulfur response and metabolism. Plant. Cell. 18, 3235-3251. Available from: https://doi.org/10.1105/ tpc.106.046458.

Masood, A., Iqbal, N., Khan, M.I.R., Khan, N.A., 2012. The coordinated role of ethylene and glucose in sulfur-mediated protection of photosynthetic inhibition by cadmium. Plant Signal. Behav. 7, 1420-1422. Available from: https://doi.org/10.4161/psb.22079.

Mazid, M., Khan, T.M., Mohammad, F., 2011. Response of crop plants under sulphur stress tolerance. J. Stress Physiol. Biochem. 7, 25-57.

Medici, L.O., Reinert, F., Carvalho, D.F., Kozak, M., Azevedo, R.A., 2014. What about keeping plants well watered? Environ. Exp. Bot. 99, 38-42. Available from: https://doi.org/10.1016/j. envexpbot.2013.10.019.

Mendel, R.R., Hänsch, R., 2002. Molybdoenzymes and molybdenum cofactor in plants. J. Exp. Bot. 53, 1689-1698. Available from: https://doi.org/10.1093/jxb/erf038.

Mera, R., Torres, E., Abalde, J., 2014. Sulphate, more than a nutrient, protects the microalga Chlamydomonas moewusii from cadmium toxicity. Aquatic. Toxicol. 148, 92-103. Available from: https:// doi.org/10.1016/j.aquatox.2013.12.034.

Mikkelsen, M.D., Naur, P., Halkier, B.A., 2004. Arabidopsis mutants in the C-S lyase of glucosinolate biosynthesis establish a critical role for indole-3-acetaldoxime in auxin homeostasis. Plan. J. 37, 770-777. Available from: https://doi.org/10.1111/j.1365313X.2004.02002.x.

Mullineaux, P.M., Rausch, T., 2005. Glutathione, photosynthesis and the redox regulation of stress-responsive gene expression. Phot. Res. 86, 459-474. Available from: https://doi.org/10.1007/ s11120-005-8811-8.

Nam, Y.J., Tran, L.S.P., Kojima, M., Sakakibara, H., Nishiyama, R., Shin, R., 2012. Regulatory roles of cytokinins and cytokinin signaling in response to potassium deficiency in Arabidopsis. PLoS One 7, 47797. Available from: https://doi.org/10.1371/journal. pone.0047797. 
Nazar, R., Umar, S., Khan, N.A., 2013. Involvement of salicylic acid in sulfur induced salinity tolerance: a role of glutathione. Ann. Res. Rev. Biol. 4, 3875-3893.

Nazar, R., Iqbal, N., Masood, A., Syeed, S., Khan, N.A., 2011a. Understanding the significance of sulfur in improving salinity tolerance in plants. Environ. Exp. Bot. 70, 80-87. Available from: https://doi.org/10.1016/j.envexpbot.2010.09.011.

Nazar, R., Iqbal, N., Syeed, S., Khan, N.A., 2011b. Salicylic acid alleviates decreases in photosynthesis under salt stress by enhancing nitrogen and sulfur assimilation and antioxidant metabolism differentially in two mungbean cultivars. J. Plant. Physiol. 168, 807-815. Available from: https://doi.org/ 10.1016/j.jplph.2010.11.001.

Nazar, R., Iqbal, N., Masood, A., Khan, M.I.R., Syeed, S., Khan, N.A., 2012. Cadmium toxicity in plants and role of mineral nutrients in its alleviation. Amer. J. Plant Sci. 3, 1476. Available from: https:// doi.org/10.4236/ajps.2012.310178.

Nazar, R., Khan, M.I.R., Iqbal, N., Masood, A., Khan, N.A., 2014a. Involvement of ethylene in reversal of salt-inhibited photosynthesis by sulfur in mustard. Physiol. Plant. 152, 331-344. Available from: https://doi.org/10.1111/ppl.12173.

Nazar, R., Umar, S., Khan, N.A., 2014b. Involvement of salicylic acid in sulfur induced salinity tolerance: a role of glutathione. Ann. Res. Reandview. Biol. 4, 3875.

Neill, S.J., Desikan, R., Hancock, J.T., 2003. Nitric oxide signaling in plants. New. Phytol. 159, 11-35. Available from: https://doi.org/ 10.1046/j.1469-8137.2003.00804.x.

Nero, D., Krouk, G., Tranchina, D., Coruzzi, G.M., 2009. A system biology approach highlights a hormonal enhancer effect on regulation of genes in a nitrate responsive "biomodule". BMC Syst. Biol. 3, 59. Available from: https://doi.org/10.1186/1752-05093-59.

Nikiforova, V., Freitag, J., Kempa, S., Adamik, M., Hesse, H., Hoefgen, R., 2003. Transcriptome analysis of sulfur depletion in Arabidopsis thaliana: interlacing of biosynthetic pathways provides response specificity. Plant. J. 33, 633-650. Available from: https:/ / doi.org/10.1046/j.1365-313X.2003.01657.x.

Nikiforova, V.J., Daub, C.O., Hesse, H., Willmitzer, L., Hoefgen, R., 2005a. Integrative gene-metabolite network with implemented causality deciphers informational fluxes of sulphur stress response. J. Exp. Bot. 56, 1887-1896. Available from: https://doi. org/10.1093/jxb/eri179.

Nikiforova, V.J., Kopka, J., Tolstikov, V., Fiehn, O., Hopkins, L., Hawkesford, M.J., et al., 2005b. Systems rebalancing of metabolism in response to sulfur deprivation, as revealed by metabolome analysis of Arabidopsis plants. Plant Physiol. 138, 304-318. Available from: https://doi.org/10.1104/pp.104.053793.

Nocito, F.F., Pirovano, L., Cocucci, M., Sacchi, G.A., 2002. Cadmiuminduced sulfate uptake in maize roots. Plant. Physiol. 129, 1872-1879. Available from: https://doi.org/10.1104/pp.002659.

Nocito, F.F., Lancilli, C., Crema, B., Fourcroy, P., Davidian, J.C., Sacchi, G.A., 2006. Heavy metal stress and sulfate uptake in maize roots. Plant. Physiol. 141, 1138-1148. Available from: https:/ / doi.org/10.1104/pp.105.076240.

Noctor, G., Mhamdi, A., Chaouch, S., Han, Y., Neukermans, J., Marquez- Garcia, B., et al., 2012. Glutathione in plants: an integrated overview. Plant. Cell. Environ. 35, 454-484. Available from: https:/ / doi.org/10.1111/j.1365-3040.2011.02400.x.

Nogueirol, R.C., Monteiro, F.A., Gratão, P.L., Borgo, L., Azevedo, R.A., 2015. Tropical soils with high aluminum concentrations cause oxidative stress in two tomato genotypes. Environ. Monitor. Assess. 187, 73. Available from: https://doi.org/ 10.1007/s10661-015-4282-3.

Ohkama, N., Goto, D.B., Fujiwara, T., Naito, S., 2002. Differential tissue specific response to sulfate and methionine of a soybean seed storage protein promoter region in transgenic Arabidopsis. Plant. Cell. Physiol. 43, 1266-1275. Available from: https://doi.org/ $10.1093 /$ pcp/pcf149.

Okuma, E., Jahan, M.S., Munemasa, S., Hossain, M.A., Muroyama, D., Islam, M.M., et al., 2011. Negative regulation of abscisic acidinduced stomatal closure by glutathione in Arabidopsis. J. Plant. Physiol. 168, 2048-2055. Available from: https://doi.org/ 10.1016/j.jplph.2011.06.002.

Pedroso, M.C., Durzan, D.J., 2000. Effect of different gravity environments on DNA fragmentation and cell death in Kalanchoe leaves. Annals. Bot. 86, 983-994. Available from: https://doi.org/ 10.1006/anbo.2000.1260.

Peleg, Z., Blumwald, E., 2011. Hormone balance and abiotic stress tolerance in crop plants. Curr. Opin. Plant Boil. 14, 290-295. Available from: https://doi.org/10.1016/j.pbi.2011.02.001.

Per, T.S., Khan, M.I.R., Anjum, N.A., Masood, A., Hussain, S.J., Khan, N.A., 2018. Jasmonates in plants under abiotic stresses: crosstalk with other phytohormones matters. Environ. Exp. Bot. 145, 104-120.

Rae, A.L., Smith, F.W., 2002. Localisation of expression of a highaffinity sulfate transporter in barley roots. Planta. 215, 565-568. Available from: https:// doi.org/10.1007/s00425-002-0777-6.

Ramakrishna, B., Rao, S.S.R., 2013. Preliminary studies on the involvement of glutathione metabolism and redox status against zinc toxicity in radish seedlings by28-homobrassinolide. Environ. Exp. Bot. 96, 52-58. Available from: https://doi.org/10.1016/j. envexpbot.2013.08.003.

Rausch, T., Wachter, A., 2005. Sulfur metabolism: a versatile platform for launching defence operations. Trends. Plant. Sci. 10, 503-509. Available from: https://doi.org/10.1016/j. tplants.2005.08.006.

Reintanz, B., Lehnen, M., Reichelt, M., Gershenzon, J., Kowalczyk, M., Sandberg, G., et al., 2001. Bus, a bushy Arabidopsis CYP79F1 knockout mutant with abolished synthesis of short-chain aliphatic glucosinolates. Plant. Cell. 13, 351-367. Available from: https:// doi.org/10.1105/tpc.13.2.351.

Rennenberg, H., Herschbach, C., 2012. Sulfur compounds in multiple compensation reactions of abiotic stress responses. Sulfur. Metabolism. Plants. Springer, Dordrecht, pp. 203-215. Available from: https: / / doi.org/10.1007/978-94-007-4450-9_25.

Rochon, A., Boyle, P., Wignes, T., Fobert, P.R., Després, C., 2006. The coactivator function of Arabidopsis NPR1 requires the core of its $\mathrm{BTB} / \mathrm{POZ}$ domain and the oxidation of C-terminal cysteines. Plant. Cell. 18, 3670-3685. Available from: https://doi.org/ $10.1105 /$ tpc. 106.046953.

Roje, S., 2006. S-Adenosyl-L-methionine: beyond the universal methyl group donor. Phytochem. 67, 1686-1698. Available from: https:/ / doi.org/10.1016/j.phytochem.2006.04.019.

Ruiz, J., Blumwald, E., 2002. Salinity-induced glutathione synthesis in Brassica napus. Planta. 214, 965-969. Available from: https:// doi.org/10.1007/s00425-002-0748-y.

Saito, K., 2000. Regulation of sulphate transport and synthesis of sulphur-containing amino acids. Curr. Opin. Plant. Biol. 3, 188-195. Available from: https://doi.org/10.1016/S1369-5266(00) 80064-3.

Saito, K., 2004. Sulfur assimilatory metabolism. The long and smelling road. Plant. Physiol. 136, 2443-2450. Available from: https:// doi.org/10.1104/pp.104.046755.

Sakakibara, H., Takei, K., Hirose, N., 2006. Interactions between nitrogen and cytokinin in the regulation of metabolism and development. Trends. Plant. Sci. 11, 440-448. Available from: https:// doi.org/10.1016/j.tplants.2006.07.004.

Sasaki-Sekimoto, Y., Obayashi, T., Matsuumi, M., Kobayashi, Y., Asamizu, E., Shibata, D., et al., 2003. Monitoring of 12-oxophytodienoic acid (OPDA)-induced expression changes in 
Arabidopsis by cDNA macroarray. Advanced Research Plant Lipids. Springer, Dordrecht, pp. 335-338. Available from: https:/ / doi.org/10.1007/978-94-017-0159-4_78.

Sasaki-Sekimoto, Y., Taki, N., Obayashi, T., Aono, M., Matsumoto, F., Sakurai, N., et al., 2005. Coordinated activation of metabolic pathways for antioxidants and defence compounds by jasmonates and their roles in stress tolerance in Arabidopsis. Plant. J. 44, 653-668. Available from: https:/ / doi.org/10.1111/j.1365-313X.2005.02560.x.

Sauter, M., Cornell, K.A., Beszteri, S., Rzewuski, G., 2004. Functional analysis of methylthioribose kinase genes in plants. Plant. physiol. 136, 4061-4071. Available from: https://doi.org/10.1104/ pp.104.053587.

Sauter, M., Moffatt, B., Saechao, M.C., Hell, R., Wirtz, M., 2013. Methionine salvage and S-adenosyl methionine: essential links between sulfur, ethylene and polyamine biosynthesis. Biochem. J. 451, 145-154. Available from: https://doi.org/10.1042/BJ20121744.

Schmidt, A., Jager, K., 1992. Open questions about sulphur metabolism in plants. Ann. Rev. Plant Physiol. Plant Mol. Biol. 43, 325-349.

Schwarz, G., Mendel, R.R., 2006. Molybdenum cofactor biosynthesis and molybdenum enzymes. Annu. Rev. Plant. Biol. 57, 623-647. Available from: https://doi.org/10.1146/annurev.arplant.57. 032905.105437.

Scuffi, D., Alvarez, C., Laspina, N., Gotor, C., Lamattina, L., Garcia-Mata, C., 2014. Hydrogen sulfide generated by L-cysteine desulfhydrase acts upstream of nitric oxide to modulate abscisic acid-dependent stomatal closure. Plant. Physiol. 166, 2065-2076. Available from: https://doi.org/10.1104/pp.114.245373.

Seth, C.S., Remans, T., Keunen, E., Jozefczak, M., Gielen, H., Opdenakker, K., et al., 2012. Phytoextraction of toxic metals: a central role for glutathione. Plant. Cell. Environ. 35, 334-346. Available from: https://doi.org/10.1111/j.1365-3040.2011.02338.x.

Shan, C., Liang, Z., 2010. Jasmonic acid regulates ascorbate and glutathione metabolism in Agropyron cristatum leaves under water stress. Plant. Sci. 178, 130-139. Available from: https://doi.org/ 10.1016/j.plantsci.2009.11.002.

Shibagaki, N., Rose, A., McDermott, J.P., Fujiwara, T., Hayashi, H., Yoneyama, T., et al., 2002. Selenate-resistant mutants of Arabidopsis thaliana identify Sultr1;2, a sulfate transporter required for efficient transport of sulfate into roots. Plant. J. 29, 475-486. Available from: https://doi.org/10.1046/j.0960-7412.2001.01232.x.

Smith, F.W., Hawkesford, M.J., Ealing, P.M., Clarkson, D.T., Berg, P. J., Belcher, A.R., et al., 1997. Regulation of expression of a cDNA from barley roots encoding a high affinity sulfate transporter. Plant J. 12, 875-884. Available from: https://doi.org/10.1046/ j.1365-313X.1997.12040875.x.

Srivastava, S., Srivastava, A.K., Suprasanna, P., D'souza, S.F., 2012. Identification and profiling of arsenic stress-induced microRNAs in Brassica juncea. J. Exp. Bot. 64 (1), 303-315. Available from: https://doi.org/10.1093/jxb/ers333.

Stamler, J.S., 1994. Redox signaling: nitrosylation and related target interactions of nitric oxide. Cell. 78, 931-936. Available from: https: / / doi.org/10.1016/0092-8674(94)90269-0.

Szalai, G., Kellös, T., Galiba, G., Kocsy, G., 2009. Glutathione as an antioxidant and regulatory molecule in plants under abiotic stress conditions. J. Plant. Growth. Regul. 28, 66-80. Available from: https:/ / doi.org/10.1007/s00344-008-9075-2.

Takahashi, H., Yamazaki, M., Sasakura, N., Watanabe, A., Leustek, T., de Almeida Engler, J., et al., 1997. Regulation of sulfur assimilation in higher plants: a sulfate transporter induced in sulfatestarved roots plays a central role in Arabidopsis thaliana. Proc. Natl. Acad. Sci. 94, 11102-11107. Available from: https://doi. org/10.1073/pnas.94.20.11102.

Takahashi, H., Watanabe-Takahashi, A., Smith, F.W., Blake-Kalff, M., Hawkesford, M.J., Saito, K., 2000. The roles of three functional sulfate transporters involved in uptake and translocation of sulfate in Arabidopsis thaliana. Plant. J. 23, 171-182. Available from: https:/ / doi.org/10.1046/j.1365-313x.2000.00768.x.

Takahashi, H., Kopriva, S., Giordano, M., Saito, K., Hell, R., 2011. Sulfur assimilation in photosynthetic organisms: molecular functions and regulations of transporters and assimilatory enzymes. Annu. Rev. Plant. Biol. 62, 157-184.

Takahashi, H., Buchner, P., Yoshimoto, N., Hawkesford, M.J., Shiu, S.H., 2012. Evolutionary relationships and functional diversity of plant sulfate transporters. Front. Plant. Sci. 2, 119. Available from: https://doi.org/10.3389/fpls.2011.00119.

Van Hoewyk, D., Takahashi, H., Inoue, E., Hess, A., Tamaoki, M., Pilon-Smits, E.A., 2008. Transcriptome analyses give insights into selenium-stress responses and selenium tolerance mechanisms in Arabidopsis. Physiol. Plant. 132, 236-253. Available from: https://doi.org/10.1111/j.1399-3054.2007.01002.x.

Vauclare, P., Kopriva, S., Fell, D., Suter, M., Sticher, L., Von Ballmoos, P., et al., 2002. Flux control of sulphate assimilation in Arabidopsis thaliana: adenosine $5^{\prime}$-phosphosulphate reductase is more susceptible than ATP sulphurylase to negative control by thiols. Plant. J. 31, 729-740. Available from: https://doi.org/ 10.1046/j.1365-313X.2002.01391.x.

Vidmar, J.J., Schjoerring, J.K., Touraine, B., Glass, A.D., 1999. Regulation of the hvst1 gene encoding a high-affinity sulfate transporter from Hordeum vulgare. Plant Mol. Biol. 40, 883-892. Available from: https://doi.org/10.1023/A:1006230131841.

Vidmar, J.J., Tagmount, A., Cathala, N., Touraine, B., Davidian, J.C.E., 2000. Cloning and characterization of a root specific high-affinity sulfate transporter from Arabidopsis thaliana. FEBS Lett. 475, 65-69. Available from: https: / / doi.org/10.1016/S0014-5793(00)01615-X.

Wang, D., Liu, Y., Tan, X., Liu, H., Zeng, G., Hu, X., et al., 2015. Effect of exogenous nitric oxide on antioxidative system and S-nitrosylation in leaves of Boehmeria nivea (L.) Gaud under cadmium stress. Environ. Sci. Pollution. Res. 22, 3489-3497. Available from: https://doi.org/10.1007/s11356-014-3581-5.

Wawrzynska, A., Moniuszko, G., Sirko, A., 2015. Links between ethylene and sulfur nutrition-a regulatory interplay or just metabolite association? Front. Plant. Sci. 6, 1053. Available from: https:// doi.org/10.3389/fpls.2015.01053.

Weber, M., Trampczynska, A., Clemens, S., 2006. Comparative transcriptome analysis of toxic metal responses in Arabidopsis thaliana and the $\mathrm{Cd}(2+)$-hypertolerant facultative metallophyte Arabidopsis halleri. Plant. Cell. Environ. 29, 950-963. Available from: https:/ / doi.org/10.1111/j.1365-3040.2005.01479.x.

Wendehenne, D., Durner, J., Klessig, D.F., 2004. Nitric oxide: a new player in plant signalling and defence responses. Curr. Opin. Plant. Biol. 7, 449-455. Available from: https://doi.org/10.1016/ j.pbi.2004.04.002.

Xiang, C., Oliver, D.J., 1998. Glutathione metabolic genes coordinately respond to heavy metals and jasmonic acid in Arabidopsis. Plant. Cell. 10, 1539-1550. Available from: https://doi.org/ 10.1105/tpc.10.9.1539.

Xiong, L., Lee, B.H., Ishitani, M., Lee, H., Zhang, C., Zhu, J.K., 2001. FIERY1 encoding an inositol polyphosphate 1-phosphatase is a negative regulator of abscisic acid and stress signaling in Arabidopsis. Genes Dev. 15 (15), 1971-1984.

Xiong, Y., Fei, S.Z., 2006. Functional and phylogenetic analysis of a DREB/CBF-like gene in perennial ryegrass (Lolium perenne L.). Planta. 224, 878-888. Available from: https://doi.org/10.1007/ s00425-006-0273-5.

Ya'acov, Y.L., Wills, R.B., Ku, V.V.V., 1998. Evidence for the function of the free radical gas-nitric oxide $(\mathrm{NO} \bullet$ ) - as an endogenous maturation and senescence regulating factor in higher plants. Plant Physiol. Biochem. 36 (11), 825-833. Available from: https:/ / doi.org/10.1016/S0981-9428(99)80020-5. 
Yamaguchi, Y., Nakamura, T., Harada, E., Koizumi, N., Sano, H., 1999. Differential accumulation of transcripts encoding sulfur assimilation enzymes upon sulfur and/or nitrogen deprivation in Arabidopsis thaliana. Biosci. Biotechnol. Biochem. 63, 762-766. Available from: https://doi.org/10.1271/bbb.63.762.

Yoshida, S., Tamaoki, M., Ioki, M., Ogawa, D., Sato, Y., Aono, M., et al., 2009. Ethylene and salicylic acid control glutathione biosynthesis in ozoneexposed Arabidopsis thaliana. Physiol. Plant. 136, 284-298. Available from: https://doi.org/10.1111/j.1399-3054.2009.01220.x.

Yoshimoto, N., Takahashi, H., Smith, F.W., Yamaya, T., Saito, K., 2002. Two distinct high-affinity sulfate transporters with different inducibilities mediate uptake of sulfate in Arabidopsis roots. Plant J. 29, 465-473. Available from: https://doi.org/10.1046/ j.0960-7412.2001.01231.x.

Yoshimoto, N., Inoue, E., Saito, K., Yamaya, T., Takahashi, H., 2003. Phloem-localizing sulfate transporter, Sultr1;3, mediates redistribution of sulfur from source to sink organs in Arabidopsis. Plant. Physiol. 131, 1511-1517. Available from: https://doi.org/ 10.1104/pp.014712.

Youssefian, S., Nakamura, M., Orudgev, E., Kondo, N., 2001. Increased cysteine biosynthesis capacity of transgenic tobacco overexpressing an O-acetylserine (thiol) lyase modifies plant responses to oxidative stress. Plant. Physiol. 126, 1001-1011. Available from: https://doi.org/10.1104/pp.126.3.1001.

Zhang, Y., Wang, L., Liu, Y., Zhang, Q., Wei, Q., Zhang, W., 2006. Nitric oxide enhances salt tolerance in maize seedlings through increasing activities of proton-pump and $\mathrm{Na}+/ \mathrm{H}+$ antiport in the tonoplast. Planta. 224, 545-555. Available from: https://doi. org/10.1007/s00425-006-0242-z.

Zhang, Y., Han, X., Chen, X., Jin, H., Cui, X., 2009. Exogenous nitric oxide on antioxidative system and ATPase activities from tomato seedlings under copper stress. Scientia Hort. 123, 217-223. Available from: https://doi.org/10.1016/j.scienta.2009.08.015.

Zhao, L., Zhang, F., Guo, J., Yang, Y., Li, B., Zhang, L., 2004. Nitric oxide functions as a signal in salt resistance in the calluses from two ecotypes of reed. Plant. Physiol. 134, 849-857. Available from: https:// doi.org/10.1104/pp.103.030023.

Zhu, J.K., 2002. Salt and drought stress signal transduction in plants. Annu. Rev. Plant Biol. 53, 247-273. Available from: https://doi. org/10.1146/annurev.arplant.53.091401.14332.

\section{Further Reading}

Azevedo, R.A., Alas, R.M., Smith, R.J., Lea, P.J., 1998. Response of antioxidant enzymes to transfer from elevated carbon dioxide to air and ozone fumigation, in the leaves and roots of wild-type and a catalase-deficient mutant of barley. Physiol. Plant. 104, 280-292. Available from: https://doi.org/10.1034/j.1399-3054.1998.1040217.x.

Bethke, P.C., Libourel, I.G., Jones, R.L., 2005. Nitric oxide reduces seed dormancy in Arabidopsis. J. Exp. Bot. 57, 517-526. Available from: https://doi.org/10.1093/jxb/erj060.

Bethke, P.C., Libourel, I.G., Jones, R.L., 2018. Nitric oxide in seed dormancy and germination. Ann. Plant. Rev. 153-175. Available from: https:/ / doi.org/10.1002/9780470988848.ch7.

Bittner, F., Mendel, R.R., 2010. Cell biology of molybdenum. Cell Biology of Metals and Nutrients. Springer, Berlin, Heidelberg, pp. 119-143. Available from: https://doi.org/10.1007/978-3-64210613-2_6.
Bittner, F., Oreb, M., Mendel, R.R., 2001. ABA3 is a molybdenum cofactor sulfurase required for activation of aldehyde oxidase and xanthine dehydrogenase in Arabidopsis thaliana. J. Biol. Chem. 276, 40381-40384. Available from: https://doi.org/10.1074/jbc. C100472200.

Boerjan, W., Cervera, M.T., Delarue, M., Beeckman, T., Dewitte, W., Bellini, C., et al., 1995. Superroot, a recessive mutation in Arabidopsis, confers auxin overproduction. Plant. Cell 7, 1405-1419. Available from: https://doi.org/10.1105/ tpc.7.9.1405.

Chassot, C., Buchala, A., Schoonbeek, H.J., Métraux, J.P., Lamotte, O., 2008. Wounding of Arabidopsis leaves causes a powerful but transient protection against Botrytis infection. Plant J. 55, 555-567. Available from: https://doi.org/10.1111/j.1365313X.2008.03540.x.

Durner, J., Wendehenne, D., Klessig, D.F., 1998. Defense gene induction in tobacco by nitric oxide, cyclic GMP, and cyclic ADPribose. Proc. Natl. Acad. Sci. 95, 10328-10333. Available from: https:// doi.org/10.1073/pnas.95.17.10328.

Gotor, C., Laureano-Marín, A.M., Moreno, I., Aroca, Á., García, I., Romero, L.C., 2015. Signaling in the plant cytosol: cysteine or sulfide? Amino Acids 47, 2155-2164. Available from: https://doi. org/10.1007/s00726-014-1786-Z.

Huang, P.M., Chen, J.Y., Wang, S.J., 2009. Tissue-specific regulation of rice molybdenum cofactor sulfurase gene in response to salt stress and ABA. Acta. Physiol. Plant. 31, 545-551. Available from: https://doi.org/10.1007/s11738-008-0264-1.

Mhamdi, A., Hager, J., Chaouch, S., 2010. Arabidopsis glutathione reductase 1 plays a crucial role in leaf responses to intracellular $\mathrm{H}_{2} \mathrm{O}_{2}$ and in ensuring appropriate gene expression through both salicylic acid and jasmonic acid signaling pathways. Plant. Physiol. 153, 1144-1160. Available from: https://doi.org/ 10.1104/pp.110.153767.

Mueller, S., Hilbert, B., Dueckershoff, K., Roitsch, T., Krischke, M., Mueller, M.J., et al., 2008. General detoxification and stress responses are mediated by oxidized lipids through TGA transcription factors in Arabidopsis. Plant. Cell. 20, 768-785. Available from: https:// doi.org/10.1105/tpc.107.054809.

Pootakham, W., Gonzalez-Ballester, D., Grossman, A.R., 2010. Identification and regulation of plasma membrane sulfate transporters in Chlamydomonas. Plant. Physiol. 153, 1653-1668. Available from: https://doi.org/10.1104/pp.110.157875.

Rao, S.S.R., Vardhini, B.V., Sujatha, E., Anuradha, S., 2002. Brassinosteroids-a new class of phytohormones. Curr. Sci. $1239-1245$.

Sasaki, Y., Asamizu, D., Shibata, Y., Nakamura, T., Kaneko, K., Awai, T., et al., 2001. Monitoring of methyl jasmonate-responsive genes in Arabidopsis by cDNAmacroarray: self-activation of jasmonic acid biosynthesis and crosstalk with other phytohormone signaling pathways. DNA. Res. 8, 153-161. Available from: https:// doi.org/10.1093/dnares/8.4.153.

Xiang, C., Werner, B.L., Christensen, E.M., Oliver, D.J., 2001. The biological Yamaguchi Y, Nakamura T, Harada E, Koizumi N, Sano H. Differential accumulation of transcripts encoding sulfur assimilation enzymes upon sulfur and/or nitrogen deprivation in Arabidopsis thaliana. Biosci. Biotechnol. Biochem. 1999 (63), 762-766. Available from: https://doi.org/10.1271/bbb.63.762. PMID:10361691. 
This page intentionally left blank 


\section{9}

\section{Role and Regulation of Plant Hormones} as a Signal Molecule in Response to Abiotic Stresses

\section{Palak Bakshi ${ }^{1}$, Neha Handa ${ }^{1}$, Vandana Gautam ${ }^{1}$, Parminder Kaur ${ }^{1}$, Shelja Sareen ${ }^{2}$, BilalAhmad Mir ${ }^{3}$ and Renu Bhardwaj ${ }^{1}$}

${ }^{1}$ Department of Botanical and Environmental Sciences, Guru Nanak Dev University, Amritsar, Punjab, India

${ }^{2}$ Department of Biotechnology, BBK DAV College for Women, Amritsar, Punjab, India

${ }^{3}$ Department of Botany, School of Life Sciences, Satellite campus Kargil, University of Kashmir, Srinagar, Jammu and Kashmir, India

\section{O U T L I N E}

19.1 Introduction

19.2 Perception and Transduction of Signals

19.3 Regulation of Various Abiotic Stresses

by Plant Hormones

19.3.1 Water Stress (Deficit or Flooding)

19.3.2 Temperature Stress

19.3.3 Salinity Stress
303

304

305

305

307

307
19.3.4 Metal Stress

19.3.5 UV Radiation

19.3.6 Crosstalk of Different Hormones

19.4 Conclusion and Future Prospects

References

Further Reading

\subsection{INTRODUCTION}

Plants are multicellular organisms that sense the variations in environment and communicate and coordinate with various signaling cues. Various environmental factors that affect plant growth and productivity include drought, temperature, salinity, flooding, UV radiation, heavy metal (HM) toxicity, etc. Due to anthropogenic activities the pace of plants to get exposed to various abiotic stresses during their complete life cycle has been increased. However, plants adapt themselves to ever-changing environmental conditions by rapidly detecting and responding to external changes, which shows the plasticity of phenotype and represents their dynamic nature. They effectively evaluate and regulate internal as well as external signals and respond accordingly.

Plant growth regulators (PGRs) are synthesized at very low concentrations and act as effective chemical messengers, communicating and coordinating the 
cellular activities in plants. They include auxin (AUX), cytokinin (CK), abscisic acid (ABA), gibberellins (GA), ethylene, jasmonic acid (JA), salicylic acid (SA), nitric oxide (NO), and brassinosteroid (BR). These plant growth regulators are derived from plant biosynthetic pathways and manage to play a dual function in plants. They not only play an essential role in growth and development of plants but also act as signal molecules in response to various biotic and abiotic stress conditions (Zhao et al., 2016). ABA, a 15-carbon compound synthesized by mevalonic acid pathway in chloroplast and other plastids is considered as an effective signal molecule in plant defense against various abiotic stress conditions (Keskin et al., 2010). SA, a phenolic nature plant growth regulator synthesized from shikimate pathway has a well-established role in abiotic stress by redox regulation, synthesis of heat shock proteins, and osmotic balance (Khan et al., 2015). In plants, SA enhances the resistance against salt, drought, and temperature stress (Yuan and Lin, 2008). CKs are adenosine substituted compound synthesized by two distinct pathways, the de novo biosynthetic pathway and $t$-RNA pathway, playing an effective role in various plant developmental processes as well as in stress responses by interacting with other PGRs (Zwack and Rashotte, 2015). GAs are terpenoid compounds synthesized by methyl erythritol phosphate pathway in plastids and also play an important role in various developmental process of plants, that is, growth, germination, dormancy, flowering, senescence, as well as in defense against abiotic stress conditions by enhancing or reducing expression of GA genes such as GA2ox and DELLA (Sharan et al., 2017). JA, an organic compound synthesized by octadecanoid pathway from linolenic acid known to have role in various physiological processes as well as significant role in plants to withstand stress conditions. ABA, ethylene, SA acts as important mediator along with mitogen activated protein kinase (MAPKs) in JA signaling and synthesis (Ahmad et al., 2016). Brassinosteroids (BRs), a class of steroidal PGR, play an essential role in enhancing stress resistance by upregulating the expression of stress marker genes along with plant growth and development (Krishna et al., 2017). Ethylene, olefinic gaseous PGR synthesized from methionine, shows increase in its biosynthesis in plants under various abiotic stress conditions, which shows its pivotal role in stressed conditions as well (Thao et al., 2015; Khan and Khan, 2014; Khan et al., 2014). These plant growth regulators act as effector molecules that perceive the signals, respond downstream by cascading effects, and are involved in ubiquitin proteasomal processes, which trigger the stress response by altering the gene expression (Santner and Estelle, 2010). Expression of different antioxidative enzymes like superoxide dismutase (SOD), peroxidase (POD), ascorbate peroxidase (APX), glutathione peroxidase (GPX), dehydroascorbatereductase (DHAR), and monodehydroascorbate reductase (MDHAR) get modulated in response to various abiotic stress conditions (Khan and Khan, 2017). However, these PGRs also modulate synthesis or response of various other plant hormones, which show they are interrelated with each other either synergistically or antagonistically (Peleg and Blumwald et al., 2011).

In the present chapter, an attempt has been made to understand the recent advances on PGRs as defense responsive molecules. It also covers signal transduction of PGRs, their crosstalk, and defensive responses under various abiotic stresses.

\subsection{PERCEPTION AND TRANSDUCTION OF SIGNALS}

Perception of external changes and internal signaling helps the plant to respond accordingly leading to diverse signaling pathways, which initiates various stress responses. However, the process of the signal transduction pathway is regulated by a sensitive network in which each component, such as sensors, secondary messengers, phosphoprotein cascades, transcription factors, phytohormones, and stress-responsive genes plays an essential role. Eventually the sudden and quick response will be triggered to defend plants from damages in a large sense. Signal transduction depends on various cellular activities and their coordination, which is a complicated process as most of the steps take place in a time- and space-dependent manner (Todaka et al., 2017).

Plant hormones, besides playing an important role in plant growth and development, are also considered as phytomolecules of prime importance in intercellular regulation and transduction of signals (Busch and Benfey, 2010). The generation of signal transduction response through phytohormones and secondary messengers is generated after the primary response like increase in cytosolic calcium. In recent years, regulation and functions of various phytohormones like auxin, cytokinin, ABA, jasmonic acid, ethylene, and brassinosteroid have been reported (Fig. 19.1). Production, accumulation, and gene upregulation of these phytohormones like synthesis of $\mathrm{ABA}$ in response to stress have been well reported.

Phytohormones act as systemic signal molecules and transfer information to different parts, for example, ABA can be transported at various distant sites away from where it is produced and plays important physiological roles (Sauter et al., 2001). Various plant cells respond differently even for the similar hormone signals and even different hormones interact with each 


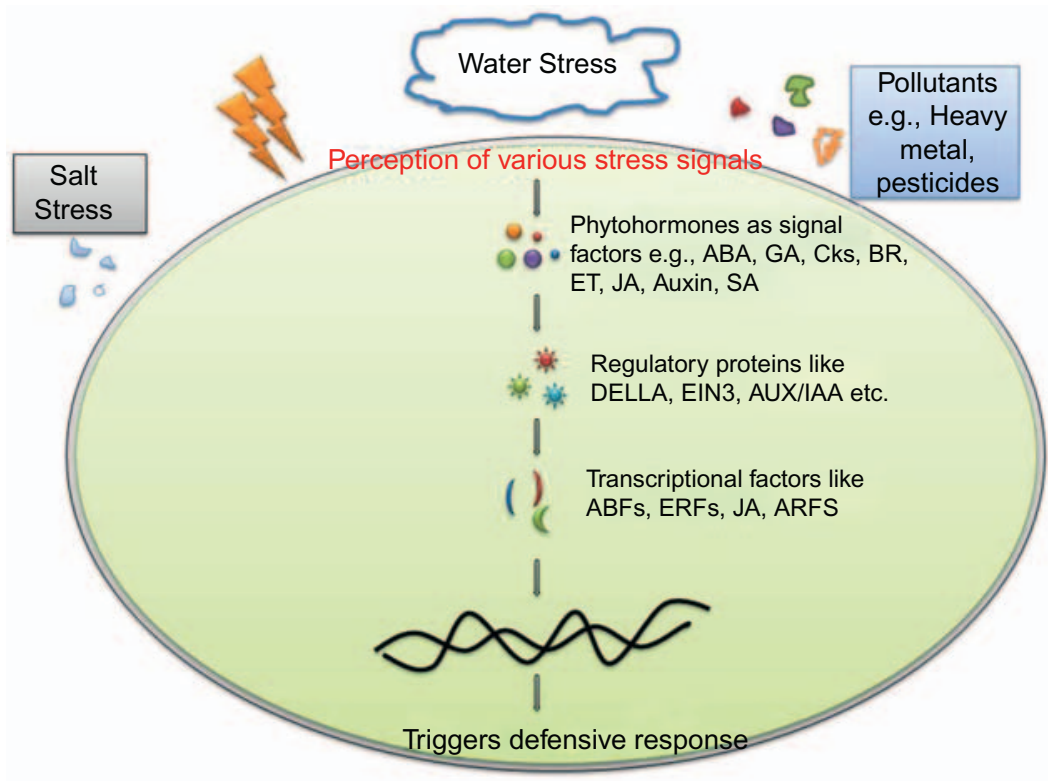

FIGURE 19.1 Signal transduction of plant growth regulators in plants.

other for coherent response to cells. The response to these signals occurs at the molecular level in the form of gene regulation, which could be perceived and studied using transcription (Goda et al., 2008).

\subsection{REGULATION OF VARIOUS ABIOTIC STRESSES BY PLANT HORMONES}

\subsubsection{Water Stress (Deficit or Flooding)}

Water stress causes severe loss in plant development. Both water deficit and excess water conditions end up in decreased crop production. Excess water leads to flooding, which directly affects the supply of oxygen to plant root cells. Inhibition of oxygen supply results in distortion of root functions such as nutrient absorption and uptake. However, under deficit conditions shortage of water availability results in damage of membranes. This is due to the increased porosity in the cell membrane, which disrupts the bilayer structure of the cell wall. Water logging and deficit conditions cause various anatomical, physiological, and molecular changes in plants. Plants face stunned growth due to the deficit supply of oxygen under waterlogged conditions (Sairam et al., 2008). Decline in photosynthesis, respiration due to hindrance in gas diffusion in flooding situation were observed (Amador et al., 2012). Similarly water deficit conditions result in decreased crop and fruit production, reduction in growth, shoot length, and area of leaf as well as decreased gas exchange (Deblonde and
Ledent, 2001; Bourtsoukidis et al., 2014). Various factors control the plant response to water stress, such as opening and closing of stomata, which is mainly controlled by ethylene and ABA (Shinozaki and Yamaguchi Shinozaki, 2000). Response to dehydration in the content of plant hormones in Arabidopsis wildtype (WT) plants and genes regulating biosynthesis of ABA given dehydration treatment was observed. Increase in the content of ABA was observed in early phases of water deficit conditions (Urano et al., 2017).

\subsubsection{Role of Abscisic Acid Under Water Stress}

ABA has been reported to have an important role in water stress by stimulating sudden responses in plants like closing and opening of stomata and control of ion homeostasis (Pandey et al., 2017). ABA also affects the long term effects in plants due to water stress by regulating the genes responsible for stress. Water scarcity or flooding induces $A B A$ production in leaves of the various plant species (Jiang and Zhang, 2002; Nayyar et al., 2005; Satisha et al., 2005). Destressing of plants resulted in reversed levels of ABA content in plants. Enhanced ABA levels restrict plant water loss via transpiration through stomata by inducing closure of stomata, which increases osmotic balance of plants and results in increased root hydraulic conductivity (Thompson et al., 2007; Zandalinas et al., 2018).

The amount of ABA content accumulated in guard cells depends upon the various factors like plant species, leaf age, climatic conditions, plant nutrient status, etc. ABA also upregulates the expression of various genes under water stress such as ZEAXANTHIN 
EPOXIDASE gene (ZEP), LOW EXPRESSION OF OSMOTIC STRESS-RESPONSIVE gene (LOS6), and ALDEHYDE OXIDASE gene (AAO3). Under water deficit conditions, the concentration of $\mathrm{ABA}$ increases abruptly to a level that induces efflux of ion and disruption of uptake of sugars and reduction in aperture size of the stomata. Reports of enhancement of ABA content by 30 times under drought stress have been recorded (Outlaw, 2003).

Exogenous application of ABA has been reported to enhance plant tolerance to water stress (Marcinska et al., 2013; Javid et al., 2011). The increase in the production of ABA during drought is due to the upregulation of genes encoding enzyme NCED3 (9cis-epoxycarotenoid dioxygenase). It was reported by Thompson et al. (2000) that plants given dehydration conditions result in instant increase in NCED3 level significantly after $15-30 \mathrm{~min}$. Induction of genes AtNCED3, AtZEP (Zeaxan thin epoxidase), and AtAA03 (ABA-aldehyde oxidase) in Arabidopsis and overexpression of OsNCED3 in rice was observed after dehydration (Cheng et al., 2002; Ye et al., 2011).

During drought, the key role of ABA in plants is the ion homeostasis and inhibition of intracellular water loss by stimulating root-leaf signaling and ultimately inducing stomatal closure (Wilkinson and Davies, 2002; Tuteja, 2007). ABA accumulation induces the efflux of $\mathrm{K}^{+}$ion by decreasing the turgor pressure in the guard cells (Anjum et al., 2011). The ion channel responsible for exchange of various anions and $\mathrm{K}^{+}$ions, that is, S-type anion channels and $\mathrm{K}^{+}$channels (SLAC1 and KAT1), is regulated by the sucrose nonfermenting-1-related-2-protein kinases (SnRK2). The kinase SnRK2 increases the activity of S-type anion channels and restricts the KAT1. Activation of S-type anion channels in guard cells by $\mathrm{Ca}^{2+}$ and ABA in different plant species has been reported by Raschke et al. (2003), Roelfsema et al. (2004), and Mori et al. (2006).

Enhanced activity of SLAC1 causes efflux of anions, which leads to depolarization of the membrane as a result of phosphorylation by SnRK. The activation of membrane depolarization results in efflux of $\mathrm{K}^{+}$cations from the cell via guard cell outward-rectifying $\mathrm{K}^{+}$channel (GORK), also known as $\mathrm{K}^{+}$efflux channel (Jeanguenin et al., 2008). Under water deficient conditions the accumulation of ABA as well as the S-type anion channel and KAT1 is deactivated by the SnRKs.

Inhibition of KAT1 activity due to accumulation of ABA and $\mathrm{Ca}^{2+}$ ion in the cytoplasm has been reported (Grabov and Blatt, 1999) through phosphorylation regulated by SnRK, which in turn results in the restricted entry of $\mathrm{K}^{+}$ions into the guard cells (Hubbard et al., 2010). Efflux of $\mathrm{K}^{+}$and other anions from the guard cells cause dehydration in cells, which leads to loss of turgor pressure and closure of stomata in response to ABA.

\subsubsection{Role of Jasmonic Acid Under Water Stress}

Jasmonate biosynthesis is activated during stress conditions (Wasternack, 2007) and many of the genes involved in JA signaling are controlled by drought stress (Huang et al., 2008). The significant role of JA during drought stress in closure of stomata has been reported by Suhita et al. (2003, 2004) and Munemasa et al. (2007). Exogenous application of JA or methyl JA (MeJA) results in its conversion to JA-Ile (iso-Jasmonyl-L-isoleucine), a bioactive form of JA. It binds to the ${ }^{\mathrm{SCF}} \mathrm{COI}$ complex, which comprises the coronatine insensitive 1 (COI 1) F-box protein (Sheard et al., 2010).

The coordination of components in a complex results in degradation of the JAZ repressor protein (Jasmonate ZIM-domain) proteasome. The CPK6 $\left(\mathrm{Ca}^{+}\right.$ dependent protein kinase 6) is identified as a positive regulator of MeJA and assist in ABA signaling in the guard cells. Similar to ABA, MeJA activates the S-type anion channels and interacts by increasing the entry of $\mathrm{Ca}^{+}$ions, which activates the CDPK and results in series of events eventually leading to stomatal closure during drought conditions (Munemasa et al., 2007).

\subsubsection{Role of Ethylene Under Water Stress}

Increased biosynthesis of ET under drought stress has been reported by Upreti et al. (1998). The induction of synthesis of ethylene is mainly done by enhancing the synthesis of ACC synthase and ACC oxidase (enzyme responsible for synthesis of ethylene). Accumulation of ethylene helps plants to reduce water loss by increasing plant senescence and inhibited growth. The content of ET accumulation is controlled by various factors and high dose of drought stress tends to downregulate the activity of ACC synthatase enzyme.

Under flooding conditions ET promotes adventitious root formation, which helps plants in growth by regulating nutrient and water uptake (Sairam et al., 2008; Pistelli et al., 2012). During drought direct relationship between ACC expression and biosynthesis of ethylene has been reported in citrus (Gómez-Cadenas et al., 1996). The action of ethylene in repression of ABA induced stomatal closure. The Arabidopsis plants exposed to gaseous ethylene tend to inhibit the closure of stomata even after application of ABA (Tanaka et al., 2005). ABA accumulation reduces the synthesis of ethylene, which results in sudden increase of ABA concentration during water stress and leads to reduced content of ethylene. 


\subsubsection{Role of Auxin and Cytokinin Under Water Stress}

These phytohormones, when present inside plants during water stress, induce opening of stomata whereas when at higher concentrations they result in stomatal closure. Auxin plays a major role in controlling the movements $\left(\mathrm{H}^{+}\right.$-ATPase) across the plasma membrane (PM) in the guard cells. Efflux of $\mathrm{H}^{+}$from the guard cells promotes the influx of $\mathrm{K}^{+}$, which is regulated by the hyperpolarization of the membrane. The exit of $\mathrm{K}^{+}$ ion results in opening of stomata when auxin is present in lower content and high concentration of it leads to efflux of $\mathrm{K}^{+}$ions and restricts the influx leading to stomatal closure (Daszkowska-Golec and Szarejko, 2013). CKs work in association with ABA and mostly inhibit the closing of stomata (Tanaka et al., 2006).

\subsubsection{Role of Brassinosteroids Under Water Stress}

BRs work in association with $\mathrm{ABA}$ and promote closing of stomata to inhibit water loss through transpiration. The same have been studied in Vicia faba where application of EBL (epibrassinolide) indicates the stomatal closure and restricts their opening in epidermal cells (Haubrick et al., 2006). Resistance to water scarcity in Arabidopsis thaliana and Brassica napus given exogenous application of EBL causes drought resistance (Fariduddin et al., 2014). Similarly 24-EBR has been reported to promote closing of stomata in $A$. thaliana through ethylene signaling transduction pathway by activation of $\mathrm{G} \alpha$ protein, hydrogen peroxide $\left(\mathrm{H}_{2} \mathrm{O}_{2}\right)$, and nitric oxide (NO) production (Shi et al., 2015).

\subsubsection{Temperature Stress}

Temperature stress is one of the most hazardous stresses amongst abiotic stresses and an increase by $0.2^{\circ} \mathrm{C}$ in temperature per decade has been predicted leading to global temperature rise by $1.8-4.0^{\circ} \mathrm{C}$ by the year 2100 (IPCC, 2007). Plants, in particular, are at a primary risk and face several adverse effects of stress caused due to temperatures beyond optimum levels. Heat stress especially alters growth and development, various physiochemical processes, and crop quality and yield in plants (Hasanuzzaman et al., 2013; Khan et al., 2013; Khan and Khan, 2013). Similarly, low temperatures lead to poor reproductive development, slow growth, low yield, and have adverse effects on the macromolecules present in the cells, which in turn slows down metabolic pathways and impairs membrane functions due to solidification (Smith and Stitt, 2007; Jewell et al., 2010). Many methods have been employed to enhance the tolerance of plants against temperature stress and the role of plant hormones in enhancing tolerance against temperature extremes is proving highly beneficial. Table 19.1 highlights the various hormones that, when supplied exogenously, aid in improving resistance of plants against temperature stress by modulating various physiological processes and important biomolecules.

\subsubsection{Salinity Stress}

Salinity is high concentration of soluble salt in the water body; it is one of the abiotic stress factors and a primary cause of concern for the human population, as it affects overall agricultural practices (Shrivastava and Kumar, 2015). Out of total land surface about $10 \%$ of the global area is affected by salt stress (Pessaraki and Szabolcs, 2010). FAO's database Aquastat shows that in some countries the severity of damage caused by salinity can be as high as $50 \%$ of the areas fully equipped for irrigation (Food and Agriculture Organization, UN, 2018 http:/ / www.fao.org/soils-portal/soil-management/ management-of-some-problem-soils/salt-affected-soils / more-information-on-salt-affected-soils/en/ (accessed on 6.04.18)) (Table 19.2).

\subsubsection{Metal Stress}

HMs, natural constituents of Earth's crust, lately due to prominent accumulation have become a major problem in terms of both nutrition and environment. HMs are nonbiodegradable chemical species that have genotoxic, cytogenic, and mutagenic effects on living beings (Emamverdian et al., 2015). They can enter the food chain as a result of uptake by plants (Gangwar et al., 2014). Their accumulation in plants also induces the formation of ROS. Plants have hormone regulators that regulate growth and development of plants. From time to time various research studies have shown that exogeneous applications of these plant regulators induce protection against metal induced stress and improve overall yield and biomass (Krishnamurthy and Rathinasabapathi, 2013; Srivastava et al., 2013) (Table 19.3).

\subsubsection{UV Radiation}

Radiation falling between the wavelength spectrums of $10-400 \mathrm{~nm}$ is called ultraviolet (UV) radiation. It is a component of solar radiation, shorter than visible radiation but longer than X-rays; electromagnetic in nature, and about 10 percent of solar radiation is comprised of UV radiation (Andrady et al., 2006). Ultraviolet radiation is further divided into different types according to their wavelength ranges. In recent times, as a consequence of the stratospheric ozone 
TABLE 19.1 Effect of Various Hormones in Regulation of Temperature Stress

\begin{tabular}{|c|c|c|c|c|c|}
\hline $\begin{array}{l}\text { S. } \\
\text { no. }\end{array}$ & $\begin{array}{l}\text { Plant } \\
\text { species }\end{array}$ & Stress & Hormone treatment & Response & References \\
\hline 1. & $\begin{array}{l}\text { Dendrobium } \\
\text { officinale }\end{array}$ & $\begin{array}{l}\text { Chilling stress }\left(0^{\circ} \mathrm{C} \text { for } 6 \&\right. \\
12 \mathrm{~h})\end{array}$ & $\begin{array}{l}\text { SA ( } 1.5 \mathrm{mM} / \mathrm{L} \text { sprayed after } 7 \text { days } \\
\text { of chilling pretreatment) }\end{array}$ & $\begin{array}{l}\text { Improved chlorophyll content, photosynthetic rate, photochemical efficiency, and } \\
\text { superoxide dismutase (SOD) activity; reduced content of malondialdehyde (MDA) }\end{array}$ & $\begin{array}{l}\text { Huang et al. } \\
\text { (2016) }\end{array}$ \\
\hline 2. & $\begin{array}{l}\text { Brassica } \\
\text { juncea }\end{array}$ & $\begin{array}{l}\text { High temperature }\left(44^{\circ} \mathrm{C} \text { for }\right. \\
3 \text { days) }\end{array}$ & $\begin{array}{l}\text { HBL (28-homobrassinolide) } \\
\text { presoaking for } 8 \mathrm{~h} \text { in } 10^{-9} \mathrm{M}\end{array}$ & $\begin{array}{l}\text { Reduced contents of MDA, } \mathrm{H}_{2} \mathrm{O}_{2} \text {, and NO; enhanced activities of SOD, catalase (CAT), } \\
\text { glutathione peroxidase (GPOX), and ascorbate peroxidase (APOX) }\end{array}$ & $\begin{array}{l}\text { Sirhindi et al. } \\
\text { (2017) }\end{array}$ \\
\hline 3. & $\begin{array}{l}\text { Cucumis } \\
\text { sativus }\end{array}$ & $\begin{array}{l}\text { Chilling stress }\left(8^{\circ} \mathrm{C} \text { for } 3\right. \\
\text { days) }\end{array}$ & SA (50 $\mu \mathrm{M}$ foliar spraying after $4 \mathrm{~h})$ & $\begin{array}{l}\text { Enhanced expression of phenylalanine ammonia lyase and benzoic acid-2- hydroxylase; } \\
\text { reduced content of } \mathrm{H}_{2} \mathrm{O}_{2}\end{array}$ & $\begin{array}{l}\text { Dong et al. } \\
\text { (2014) }\end{array}$ \\
\hline 4. & $\begin{array}{l}\text { Cynara } \\
\text { cardunculus }\end{array}$ & High temperature $\left(30^{\circ} \mathrm{C}\right)$ & $\mathrm{ET}(30 \mu / \mathrm{ML})$ & Increase in early root growth, root hair density, root area, and lateral roots & $\begin{array}{l}\text { Shinohara et al. } \\
\text { (2017) }\end{array}$ \\
\hline 5. & $\begin{array}{l}\text { Ficus } \\
\text { concinna }\end{array}$ & $\begin{array}{l}\text { High temperature }\left(35^{\circ} \mathrm{C} \text { and }\right. \\
\left.40^{\circ} \mathrm{C} \text { for } 48 \mathrm{~h}\right)\end{array}$ & $\begin{array}{l}\text { EBR (24-epibrassinolide) }(0.25 \mu \mathrm{M} \\
\text { EBR conc. at the rate } 15 \mathrm{~mL} \text { per } \\
\text { plant) }\end{array}$ & $\begin{array}{l}\text { Enhanced activities of superoxide dismutase (SOD), glutathione-S-transferase (GST), GPX, } \\
\text { APOX, monodehydroascorbate reductase (MDHAR), glutathione reductase (GR), } \\
\text { dehydroascorbate reductase (DHAR), glyoxalase I (Gly I) and glyoxalase II (Gly II); } \\
\text { increase in contents of reduced glutathione (GSH) and ascorbate (AsA); reduced contents of } \\
\text { reactive oxygen species (ROS), malondialdehyde (MDA), and methylglyoxal (MG) }\end{array}$ & Jin et al. (2015) \\
\hline 6. & $\begin{array}{l}\text { Hordeum } \\
\text { vulgare }\end{array}$ & $\begin{array}{l}\text { Low temperature } \\
\left(7 / 5^{\circ} \mathrm{C} \text { day/night }\right. \\
\text { temperature for } 3 \text { days })\end{array}$ & $\begin{array}{l}\text { SA ( } 0.1 \mathrm{mM} \text { applied to } 7 \text {-day-old } \\
\text { plant) }\end{array}$ & $\begin{array}{l}\text { Reduced MDA with SA in both cultivars; } \mathrm{H}_{2} \mathrm{O}_{2} \text { decreased in cv. Akhisar. enhanced } \\
\text { activities of POD and SOD in both cultivars; CAT activity enhanced in Tokak cultivar }\end{array}$ & $\begin{array}{l}\text { Mutlu et al. } \\
\text { (2016) }\end{array}$ \\
\hline 7. & $\begin{array}{l}\text { Lycopersicon } \\
\text { esculentum }\end{array}$ & $\begin{array}{l}\text { High temperature }\left(40 / 30^{\circ} \mathrm{C}\right. \\
\text { day/night temperature for } \\
8 \text { days) }\end{array}$ & $\begin{array}{l}\text { EBR }(0.01,0.1,1.0 \mathrm{mg} / \mathrm{L} \text { applied } 6 \\
\text { weeks after germination) }\end{array}$ & $\begin{array}{l}\text { Net photosynthetic rate, stomatal conductance, maximum carboxylation rate of RuBisCo } \\
\text { enhanced with hormone treatment; activities of SOD, APOX, CAT, and guaiacol peroxidase } \\
\text { (GPOD) increased; reduction in } \mathrm{H}_{2} \mathrm{O}_{2} \text { and MDA production }\end{array}$ & $\begin{array}{l}\text { Ogweno et al. } \\
(2008)\end{array}$ \\
\hline 8. & $\begin{array}{l}\text { Lycopersicon } \\
\text { esculentum }\end{array}$ & $\begin{array}{l}\text { High temperature }\left(38 / 30^{\circ} \mathrm{C}\right. \\
\text { day/night temperature })\end{array}$ & $\begin{array}{l}\text { Plants produced } 1.7-2.4 \text { fold high } \\
\text { spermidine and spermine }\end{array}$ & $\begin{array}{l}\text { Reduced MDA, improved activities of APOX, CAT, GPOD, and SOD; improved } \mathrm{CO}_{2} \\
\text { assimilation }\end{array}$ & $\begin{array}{l}\text { Cheng et al. } \\
\text { (2009) }\end{array}$ \\
\hline 9. & $\begin{array}{l}\text { Lycopersicon } \\
\text { esculentum }\end{array}$ & $\begin{array}{l}\text { Low temperature }\left(4^{\circ} \mathrm{C} \text { for }\right. \\
28 \text { days })\end{array}$ & SA (1 mM for $15 \mathrm{~min}$ before storage) & $\begin{array}{l}\text { Reduced chilling injury; increased expression of GA biosynthetic gene (GA3ox1), increased } \\
\text { GA }_{3} \text { levels and DELLA proteins degradation enhanced; enhanced activities of antioxidative } \\
\text { enzymes and reduced ROS accumulation }\end{array}$ & $\begin{array}{l}\text { Ding et al. } \\
(2016)\end{array}$ \\
\hline 10. & $\begin{array}{l}\text { Malus } \\
\text { domestica }\end{array}$ & Sun exposed, not exposed & $\begin{array}{l}\text { Endogenous levels of IAA, ABA, JA, } \\
\text { SA, and ET were estimated }\end{array}$ & $\begin{array}{l}\text { ABA, SA, and JA increased significantly in exposed tissues indicating the role of these } \\
\text { hormones in modulating defense responses to photooxidative damage; IAA was not found } \\
\text { to be related to injury development }\end{array}$ & $\begin{array}{l}\text { Torres et al. } \\
\text { (2017) }\end{array}$ \\
\hline 11. & $\begin{array}{l}\text { Solanum } \\
\text { melongena }\end{array}$ & $\begin{array}{l}\text { High temperature }\left(43 / 38^{\circ} \mathrm{C}\right. \\
\text { day/night temperature for } \\
8 \text { days) }\end{array}$ & $\begin{array}{l}\text { EBR }(0.1 \mu \mathrm{M} \text { conc. to } 6 \text {-week-old } \\
\text { plant })\end{array}$ & $\begin{array}{l}\text { Enhanced growth, chlorophyll content, net photosynthetic rate, stomatal conductance, } \\
\text { transpiration rate, maximum quantum efficiency of PSII, potential photochemical } \\
\text { efficiency, the quantum efficiency of PSII; increased activities of SOD, POD, CAT, and } \\
\text { APOX; enhanced levels of GSH, ascorbate (AsA), proline, soluble sugar, and proteins; } \\
\text { reduced levels of superoxide anions, } \mathrm{H}_{2} \mathrm{O}_{2} \text { and MDA }\end{array}$ & Wu et al. (2014) \\
\hline 13. & Vitis vinifera & High temperature $\left(140^{\circ} \mathrm{C}\right)$ & SA $(150 \mu \mathrm{M})$ & Accumulation of $P A L$ mRNA and phenolics & $\begin{array}{l}\text { Wen et al. } \\
\text { (2008) }\end{array}$ \\
\hline 14. & Vitis vinifera & $\begin{array}{l}\text { High temperature }\left(43^{\circ} \mathrm{C} \text { for }\right. \\
5 \mathrm{~h})\end{array}$ & SA $(100 \mu \mathrm{M})$ & $\begin{array}{l}\text { Improved net photosynthesis rate and ribulose-1,5-bisphosphate carboxylase (RuBisCo); } \\
\text { heat shock protein } 21 \text { (HSP) immune signals increased in SA treated plants subjected to } \\
\text { heat stress }\end{array}$ & $\begin{array}{l}\text { Wang et al. } \\
\text { (2010) }\end{array}$ \\
\hline
\end{tabular}


TABLE 19.2 Effect of Various Hormones in Regulation of Salinity Stress

\begin{tabular}{|c|c|c|c|c|c|}
\hline $\begin{array}{l}\text { S. } \\
\text { no. }\end{array}$ & $\begin{array}{l}\text { Plant } \\
\text { species }\end{array}$ & Stress & Hormone treatment & Response & References \\
\hline 1. & $\begin{array}{l}\text { Brassica } \\
\text { juncea } L .\end{array}$ & $\begin{array}{l}\mathrm{NaCl}(100 \text { and } \\
150 \mathrm{mM})\end{array}$ & $\begin{array}{l}\mathrm{GA}_{3}(75 \mathrm{mg} / \text { Lonce a week for } \\
45 \text { days) }\end{array}$ & $\begin{array}{l}\text { Exogenous application of } \mathrm{GA}_{3} \text { found to enhance growth and yield } \\
\text { The significant rise in activity of SOD, CAT, POD, GR, and APX was observed }\end{array}$ & Ahmad (2010) \\
\hline 2. & $\begin{array}{l}\text { Medicago } \\
\text { sativa }\end{array}$ & $\mathrm{NaCl}(200 \mathrm{mM})$ & $\begin{array}{l}\text { SA ( } 0.1 \& 0.5 \mathrm{mM} \text { applied } 49 \text { days } \\
\text { after sowing) }\end{array}$ & $\begin{array}{l}\text { The heightened activity of POX, SOD, APX, DHAR, and GR was reported } \\
\text { CAT activity was reduced by } 40 \% \\
\text { The improved photosynthetic efficiency and nitrogen fixation capacity was observed }\end{array}$ & $\begin{array}{l}\text { Palma et al. } \\
\text { (2013) }\end{array}$ \\
\hline 3. & $\begin{array}{l}\text { Medicago } \\
\text { sativa }\end{array}$ & $200 \mathrm{mM} \mathrm{NaCl}$ & $\begin{array}{l}\text { ABA ( } 1 \& 10 \mu \mathrm{M} \text { conc. applied } 2 \text { days } \\
\text { before salt treatment) }\end{array}$ & The significant rise in SOD, CAT, and GR was reported; reduced MDA was also observed & $\begin{array}{l}\text { Palma et al. } \\
\text { (2014) }\end{array}$ \\
\hline 4. & $\begin{array}{l}\text { Medicago } \\
\text { sativa L. }\end{array}$ & $\begin{array}{l}13.6 \mathrm{dS} / \mathrm{m} \mathrm{NaCl} \\
\text { solution }\end{array}$ & Brassinolide $(5 \mu \mathrm{M} / \mathrm{L})$ & $\begin{array}{l}\text { Significant increase in shoot dry weight, fresh weight, and root dry weight in Victoria and golden } \\
\text { empress and increase in root fresh weight in Victoria and Victor } \\
\text { Enhanced activities of SOD, POD \& CAT observed in Victoria \& Victor seedlings. } \\
\text { Significant reduction in MDA accumulation was noticed. }\end{array}$ & $\begin{array}{l}\text { Zhang et al. } \\
\text { (2007) }\end{array}$ \\
\hline 5. & $\begin{array}{l}\text { Pistacia } \\
\text { vera } \mathrm{L} \text {. }\end{array}$ & $\begin{array}{l}(0,30,60, \text { or } \\
90 \mathrm{mM}) \mathrm{NaCl}\end{array}$ & $\begin{array}{l}\text { SA }(0,0.10,0.50 \text {, or } 1.00 \mathrm{mM} \text { applied } 3 \\
\text { weeks after emergence) }\end{array}$ & $\begin{array}{l}\text { Various physiological parameters like water content, leaf chlorophyll content, photosynthetic efficiency, } \\
\text { and chlorophyll fluorescence ratio were found to be increased; SA applications at } 0.50 \text { and } 1.00 \mathrm{mM} \\
\text { were effective in reducing proline content and electrolyte leakage }\end{array}$ & $\begin{array}{l}\text { Bastam et al. } \\
\text { (2013) }\end{array}$ \\
\hline 6. & $\begin{array}{l}\text { Pusa } \\
\text { Basmati-1 }\end{array}$ & $\begin{array}{l}\mathrm{NaCl}(75,100, \\
125 \mathrm{mM})\end{array}$ & $\begin{array}{l}\text { EBL }\left(10^{-11}, 10^{-9}, 10^{-7} \mathrm{M} \text { presoaking }\right. \\
\text { for } 8 \mathrm{~h})\end{array}$ & $\begin{array}{l}\text { Enhanced growth was observed in rice seedlings; there was a significant rise in proline and protein content } \\
\text { Reduced content of MDA was recorded }\end{array}$ & $\begin{array}{l}\text { Sharma et al. } \\
(2013)\end{array}$ \\
\hline 7. & $\begin{array}{l}\text { Ricinus } \\
\text { communis } \\
\text { L. }\end{array}$ & $\mathrm{NaCl}(100 \mathrm{Mm})$ & $\mathrm{GA}_{3}$ (seed presoaking in $250 \mu \mathrm{M}$ ) & $\begin{array}{l}\text { The activity of SOD, POD, and CAT and proline content was increased } \\
\text { Plant height, leaf area, root, stem, leaf weight was also increased with application of } \mathrm{GA}_{3}\end{array}$ & $\begin{array}{l}\text { Zhou et al. } \\
\text { (2014) }\end{array}$ \\
\hline 8. & $\begin{array}{l}\text { Solanum } \\
\text { melongena } \\
\text { L. }\end{array}$ & $\mathrm{NaCl}(90 \mathrm{mM})$ & $\begin{array}{l}\text { EBR }(0,0.025,0.05,0.10 \text {, and } 0.20 \mathrm{mg} / \\
\mathrm{dm}^{3} \text { during fourth or fifth true leaf } \\
\text { stage })\end{array}$ & $\begin{array}{l}\text { The enhanced activity of APX, SOD, CAT, POD, and reduced content of GSH was observed } \\
\text { The reduction in production of MDA and } \mathrm{H}_{2} \mathrm{O}_{2} \text { was significant }\end{array}$ & $\begin{array}{l}\text { Ding et al. } \\
\text { (2012) }\end{array}$ \\
\hline 9. & $\begin{array}{l}\text { Triticum } \\
\text { aestivum } \\
\text { L. }\end{array}$ & $\mathrm{NaCl}(150 \mathrm{mM})$ & $\begin{array}{l}\text { EBR }(0,0.052,0.104,0.156 \mathrm{~L} / \mathrm{M} \text { applied } \\
\text { on 14-day stage) }\end{array}$ & $\begin{array}{l}\text { The improvement in growth parameters were observed only in S- } 24 \\
\text { The enhanced photosystem II efficiency in both the cultivars was reported } \\
\text { There was no change in SOD activity, whilst POD and CAT activities were increased in S-24 only }\end{array}$ & Ali et al. (2008) \\
\hline 10. & $\begin{array}{l}\text { Vigna } \\
\text { radiata L. }\end{array}$ & $\mathrm{NaCl}(50 \mathrm{mM})$ & $\begin{array}{l}\text { SA }(0.5 \mathrm{mM} \text { sprayed } 15 \text { days after } \\
\text { sowing) }\end{array}$ & $\begin{array}{l}\text { The remarkable increase in nitrogen, sulfur assimilation, GSH content, and increase in photosynthesis } \\
\text { was observed } \\
\text { The activity of APX and GR was higher in Pusavishal than T44; whilst activity of SOD was higher in T44 } \\
\text { Overall, the effect of SA was prominent in Pusavishal as compared with T44 }\end{array}$ & $\begin{array}{l}\text { Nazar et al. } \\
(2011)\end{array}$ \\
\hline 11. & $\begin{array}{l}\text { Vigna } \\
\text { sinensis }\end{array}$ & $\begin{array}{l}\mathrm{NaCl}(25,50, \\
100 \text { and } \\
150 \mathrm{mM})\end{array}$ & $\begin{array}{l}\text { Brassinolide }(0.05 \mathrm{ppm} \text { after } 25 \text { and } \\
32 \text { days from sowing })\end{array}$ & $\begin{array}{l}\text { High saline conditions significantly affected the morphological features like shoot and root length, } \\
\text { number of leaves, leaf area, etc.; elevated levels of TBARS content was reported, which was decreased } \\
\text { with exogenous applications of brassinolide } \\
\text { There was a significant increase in soluble protein content, tocopherol content, and GSH } \\
\text { The enhanced activity of antioxidant enzymes PPO, POX, and SOD was also reported }\end{array}$ & $\begin{array}{l}\text { El-Mashad and } \\
\text { Mohamed } \\
(2012)\end{array}$ \\
\hline 12. & Zea mays & $\begin{array}{l}\mathrm{NaCl}(25,50,75 \\
\text { and } 100 \mathrm{mM})\end{array}$ & $\operatorname{HBL}\left(10^{-8}, 10^{-6}, 10^{-4} \mathrm{mM}\right.$ for $\left.12 \mathrm{~h}\right)$ & $\begin{array}{l}\text { The enhanced activities of SOD, POD, CAT, and APOX was observed } \\
\text { Oxidative damage was reduced with the application of } 28 \text {-homoBL. }\end{array}$ & $\begin{array}{l}\text { Arora et al. } \\
(2008)\end{array}$ \\
\hline
\end{tabular}


TABLE 19.3 Effect of Various Hormones in Regulation of Metal Stress

\begin{tabular}{llll}
\hline $\begin{array}{l}\text { S. } \\
\text { no. }\end{array}$ & $\begin{array}{l}\text { Plant } \\
\text { species }\end{array}$ & Stress & Hormone tre \\
\hline 1. & $\begin{array}{l}\text { Solanum } \\
\text { lycoperscium }\end{array}$ & $\begin{array}{l}\text { Cadmium }(\mathrm{Cd})(3, \\
9 \mathrm{mg} / \mathrm{kg})\end{array}$ & $\begin{array}{l}\text { 6-furfurylam } \\
\text { (artificial cy } \\
(10 \mu \mathrm{M})\end{array}$ \\
& & Lead $(\mathrm{Pb})(0.25,0.50$ & $\mathrm{EBL}$ \\
2. & $\begin{array}{l}\text { Brassica } \\
\text { juncea }\end{array}$ & $\& 0.75 \mathrm{mM})$ & $10-7 \mathrm{M}$ an \\
& & $\mathrm{mM}$ for 8 \\
& & & \\
3. & $\begin{array}{l}\text { Brassica } \\
\text { juncea }\end{array}$ & $\begin{array}{l}\text { Manganese }(\mathrm{Mn})(0, \\
3,6, \text { or } 9 \mathrm{mM})\end{array}$ &
\end{tabular}

juncea 3,6 , or $9 \mathrm{mM}$ )

4. Brassica juncea

5. Poa pratensis

6. Pisum sativum

Chromium VI (Cr VI) $(50,100, \&$ $250 \mu \mathrm{M})$

7. Triticum aestivum

$\mathrm{Cd}\left(\mathrm{CH}_{3} \mathrm{COO}\right)_{2}$ (1 mM)

8. Triticum aestivum

9. Vigna radiata
10. Vigna radiata

Nickel (Ni) (0, 20, 40 , and $60 \mathrm{mg} / \mathrm{kg}$ )

Cadmium chloride $\left(\mathrm{CdCl}_{2}\right)(500$ or $1000 \mu \mathrm{M} \mathrm{Cd}$ )

Indole-3-acetic acid (IAA) $(500 \mu \mathrm{M})$ or SA $(500 \mu \mathrm{M})$

Aluminum (Al) (0.0 1.0 , or $10.0 \mathrm{mM}$ )

11. Brassica juncea

\section{$\mathrm{Cd}$}

(1)

( $\mathrm{Cu})$,

Cd $(0,5,10$, or

SA (500 $\mu \mathrm{M}$ for 7 days)

HBL $\left(10^{-10}, 10^{-8}\right.$, and $10^{-6}$ $\mathrm{M}$ for $8 \mathrm{~h}$ )

SA (500 $\mathrm{MM}$ for 7 days)

IAA $(10 \& 100 \mu \mathrm{M})$

SA $(50 \mu \mathrm{M})$

24 28-homobrassinolide
(HBL) $\left(10^{-8} \mathrm{M}\right)$

$\mathrm{GA}_{3}\left(10^{-4} \mathrm{M}\right)$ at 15,30 , and 45 days after germination

Castasterone $\left(10^{-11} \mathrm{M}\right.$, $10^{-9} \mathrm{M}$, and $10^{-7} \mathrm{M}$ for $8 \mathrm{~h}$ )
Response

References

Increase in ascorbate peroxidae (APX), glutathione reductase (GR)

Singh et al.

(2018)

Proline, trehalose, glycine betaine, glutathiones, ascorbic acid, and tocopherol content was increased

Kohli et al. (2018)

The enhanced activities of guaiacol peroxidase, catalase, glutathione reductase, and glutathione-s-transferase were observed

The metal induced stress effects were reversed with the application of SA; improved growth, better photosynthetic traits, proline accumulation, heightened activity of antioxidant enzymes, which is believed to be a contributing factor in stress tolerance in mustard plants

Parashar et al. (2014)

Fariduddin rise in proline content was observed; significant improvement in growth characteristics and photosynthetic parameters were also recorded after 28-HBL applications

Enhanced activities of APX and SOD were observed; CAT activity was decreased; chlorophyll content and mineral nutrients content, for example, $\mathrm{K}, \mathrm{Ca}, \mathrm{Mg}$, and Fe were also increased

Reduction in $\mathrm{H}_{2} \mathrm{O}_{2}$ and MDA was reported

IAA applications protect pea plants against $\mathrm{Cr}$ by regulating oxidative stress and $\mathrm{Cr}$ deposition There is noticeable decrease in nitrate reductase, nitrite reductase, glutamine synthetase, and glutamate synthase (GOGAT) activities, and increase in glutamate dehydrogenase activity was observed

The improved growth characteristics and poststress recovery was observed after SA applications; PAL, the enzyme involved in lignin biosynthesis, was activated and contributed in carrier functioning of cell walls; significant decline in MDA accumulation and electrolyte leakage were also observed

Significant rise in antioxidant activities of SOD, POX, and CAT attenuated the $\mathrm{Cd}$ induced stress in wheat seedlings

Agami and Mohamed (2013)

Ali et al.

The increased activities of antioxidative enzymes CAT, POX, and COD was reported in aluminum stressed plants, (2008) which were further enhanced after 24- EBL and 28-HBL applications; various morphological and physiological parameters were also improved

Enhanced growth parameters pertaining to length, shoot, and root dry weight as well as overall yield were recorded; reduced nickel concentration was also reported

Ali et al. (2015)

Partial regain of biomass as well as enhanced ascorbate peroxidase (APOX), dehydroascorbate reductase (DHAR), and glutathione reductase (GR), glutathione peroxidase (GPOX), and glutathione-s-transferase 


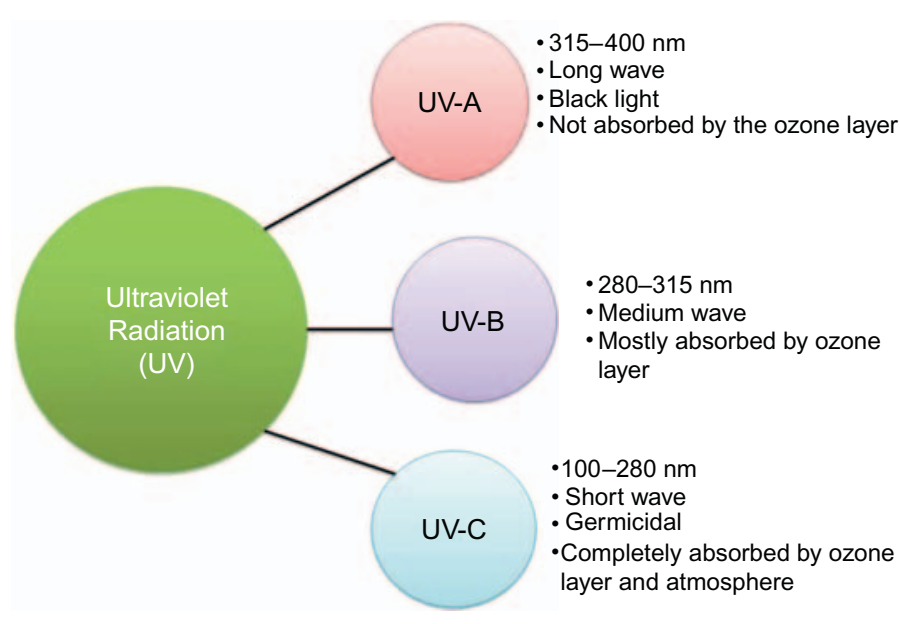

FIGURE 19.2 Different types of ultraviolet radiation and their wavelength range. Source: Modified after Mainster, M.A., 2006. Violet and blue light blocking intraocular lenses: photoprotection versus photoreception. BJO. 90(6), 784-792 (Mainster, 2006).

layer depletion by anthropogenic activities, increased concentrations of UV light has entered into the atmosphere (Gill et al., 2015; Khan et al., 2015). Different types of ultraviolet radiation and their wavelength ranges are given in (Fig. 19.2).

Being nonmotile and needy for sunlight, it is impossible for plants to avoid contact with ultraviolet light. Increase in the UV-B radiation's intensity disturbs the electron transport in PS II; inhibits the normal production and functioning of nucleic acids, photosynthetic pigments, and other metabolites; and injures the membranes, DNA, and proteins, and therefore, causes considerable damage to the crops in terms of productivity (Mohammed and Tarpley, 2011). Plants protect themselves from UV stress by producing UV shielding secondary metabolites. The efficiency of the process depends upon the proper organization and utilization of those metabolites, which is managed by the different plant hormones.

Exogenous application of salicylic acid controlled the UV-B stress impacts in Glycine max plants by increasing the water use efficiency and improving photosynthetic function (Li et al., 2014). Ranceliene and Vyšniauskiene (2012) reported that salicylic acid is capable of decreasing the chromosome aberrations caused by UV-B. Improvement in oil yield and growth of Thymus daenensis Celak and Thymus vulgaris L. plants under UV stress was observed after the foliar spray of salicylic acid (Yadegari, 2017). In the UV-B stressed plants of Oriza sativa, salicylic acid mediated the stress amelioration by increasing the PS II activity, total phenolic content in leaves, pollen viability, and yield (Mohammed and Tarpley, 2013). Auxin plays a vital signaling role in preventing UV-B radiation from causing downward curling of $A$. thaliana leaves (Fierro et al., 2015). Auxin also regulates the ultraviolet radiation induced morphogenesis and flavonoid accumulation (Hectors et al., 2012).

ABA activates the plant's guard against UV radiation stress (Berli et al., 2010). It also increases the concentrations of UV-B radiation absorbing metabolites like kaempferol, flavonols, and quercetin in the plants (Mazid et al., 2011; Tossi et al., 2012). ABA enhances $\mathrm{Ca}^{2+}$ concentration in cytosol; causes alkalinization of the cytosol; increases hydroxycinnamic acid, ferulic acid, and caffeic acid concentrations; and modifies membrane properties, thereby, bringing on the radiation stress tolerance in plants (Berli et al., 2010).

\subsubsection{Crosstalk of Different Hormones}

The defense responses against stresses are regulated by interaction between signaling pathways of several hormones like ABA, SA, JA, and ethylene (Verma et al., 2016). Also, studies have shown that growth promoting hormones, that is, auxins, cytokinins, and gibberellins too have a stress tolerance role (Verma et al., 2016). A study on rice seedlings has shown that the gene TLD1/OsGH3.13 for indole-3-acetic acid (IAA)amidosynthetase was involved in imparting drought tolerance by upregulating the expression of late embryogenesis abundant ( $L E A$ ) genes (Zhang et al., 2009). In addition to this, it was also found that ethylene was involved in regulating the many genes for auxin synthesis, perception, and action (Stepanova and Alonso, 2009). Enzymes for auxin biosynthesis (ASA1/ WEI2/TIR7, ASB1/WEI7, TAA1/SAV3/WEI8), auxin responsive factors (ARF2, ARF1), and auxin transporters (PIN1, PIN2, PIN4, AUX1) are the genes that are specifically regulated by ethylene ( $\mathrm{Li}$ et al., 2004a; Stepanova et al., 2005; Ruzicka et al., 2007; Stepanova et al., 2008). However, in lateral root development, a negative regulation of auxin transport via ethylene was reported (Negi et al., 2010). Also, in Arabidopsis, an analog of SA called benzothiadiazole-S-methyl ester was reported to downregulate the expression of TRANSPORT INHIBITOR RESISTANT 1 (TIR1)/ AUXIN SIGNALING F-BOX (AFB) genes (Wang et al., 2007). This response increased the stability of auxin repression protein (AUX/IAA), which further led to inhibition of auxin responses (Wang et al., 2007). Therefore, it was suggested that suppression of auxin signaling during stress resistance becomes necessary because onset of systemic acquired resistance (SAR) led to reduced auxin responsive gene expression and also, auxins promote susceptibility (Verma et al., 2016).

Cytokinins have also been reported to regulate defense responses synergistically with SA. It was reported that interaction between two transcription factors, that is, 


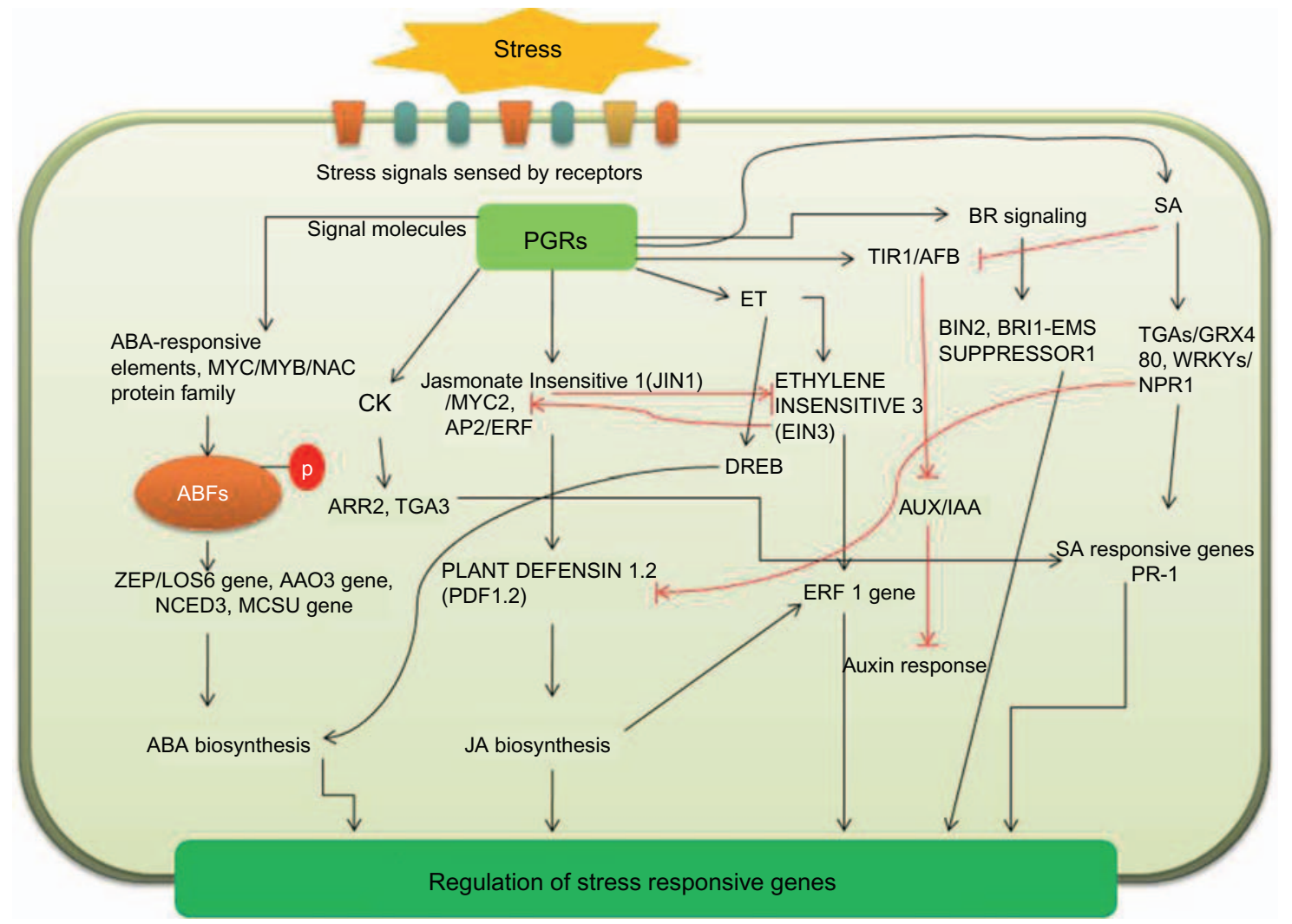

FIGURE 19.3 Plant growth regulators' signaling and their crosstalk.

ARABIDOPSIS RESPONSE REGULATOR 2 (ARR2), which gets activated by cytokinins, and TGA3, a bZIPtype transcription factor, triggers SA dependent defense response (Choi et al., 2010). In rice, the interaction between OsNPR1 and WRKY45 indicated synergistic behaviors of cytokinins and SA leading to resistance against Magnaporthe oryzae (Jiang et al., 2013). ABA has been known to suppress cytokinin biosynthesis (Nishiyama et al., 2011), but studies on cytokinin receptors like Arabidopsis histidine kinases (AHK1, AHK2, AHK3) suggested that AHK1 aided in ABA signaling and provided resistance against drought and salinity stresses. AHK2 and AHK3 were reported to negatively regulate both $\mathrm{ABA}$ signaling and osmotic stress (Tran et al., 2007).

An antagonistic interaction between SA and JA has been reported in case of biotic stresses (Bari and Jones, 2009). It was established by Doherty et al. (1988) that aspirin, an acetyl derivative of SA, inhibited the JAmediated response to wounding. The NON-EXPRESSOR OF PR (pathogen related) GENE 1 (NPR1) is regulated by SA, and this NPR1 suppresses LIPOXYGENASE 2 (LOX2), VEGETATIVE STORAGE PROTEIN (VSP), and PDF1.2; these genes are responsive to JA. Therefore, NPR1 is chiefly responsible for SA-JA antagonism (Spoel et al., 2003). Also, a transcription factor called WRKY70 increased the expression of SA responsive genes (PR genes), while suppressing the expression of JA responsive genes (PDF1.2), thus playing a role in SA-JA antagonism (Li et al., 2004b). Ethylene and JA, however, have been reported to act synergistically in defense responses against biotic stresses (Verma et al., 2016). Ethylene as well as JA aid in either inducing or stabilizing a transcription factor ETHYLENE INSENSITIVE3 (EIN3) that leads to improved tolerance to necrotrophs and root hair formation (Zhu et al., 2011). PR genes were also reported to be activated by ETHYLENE-RESPONSIVE FACTOR1 (ERF1) for which both ethylene and JA are required (Lorenzo et al., 2003). Ethylene induces another transcription factor, dehydration responsive element (DRE)BINDING PROTEIN (DREB), that interacts with ABA when plants are exposed to abiotic stresses (Arc et al., 2013). ABA induces seed dormancy and ethylene helps in breaking dormancy and inducing seed germination (Arc et al., 2013) (Fig. 19.3).

\subsection{CONCLUSION AND FUTURE PROSPECTS}

The present study emphasizes the role of phytohormones in ever-changing environmental conditions. 
Molecular analysis of signal cascade of phytohormones and their interactions play an effective role in understanding the key role of phytohormones in defense. Crosstalk among different hormones also opens new insight in the research field. Molecular level understanding of different pathways helps to form transgenic plants that can withstand extreme stress conditions, and opens up an important aspect in a different area of research.

\section{References}

Agami, R.A., Mohamed, G.F., 2013. Exogenous treatment with indole-3-acetic acid and salicylic acid alleviates cadmium toxicity in wheat seedlings. Ecotoxicol. Environ. Saf. 94, 164-171.

Ahmad, P., 2010. Growth and antioxidant responses in mustard (Brassica juncea L.) plants subjected to combined effect of gibberellic acid and salinity. Arch. Agron. Soil Sci. 56, 575-588.

Ahmad, P., Rasool, S., Gul, A., Sheikh, S.A., Akram, N.A., Ashraf, M., et al., 2016. Jasmonates: multifunctional roles in stress tolerance. Fronti. Plant Sci. 7, 813.

Ali, B., Hasan, S.A., Hayat, S., Hayat, Q., Yadav, S., et al., 2008. A role for brassinosteroids in the amelioration of aluminium stress through antioxidant system in mung bean (Vigna radiata L. Wilczek). Environ. Exper. Bot. 62, 153-159.

Ali, M.A., Asghar, H.N., Khan, M.Y., Saleem, M., Naveed, M., et al., 2015. Alleviation of nickel-induced stress in mungbean through application of gibberellic acid. Int. J. Agric. Biol. 17, 990-994.

Amador, M.L., Sancho, S., Bielsa, B., Gomez-Aparisi, J., RubioCabetas, M.J., 2012. Physiological and biochemical parameters controlling waterlogging stress tolerance in Prunus before and after drainage. Physiol. plant 144, 357-368.

Andrady, A., Aucamp, P.J., Bais, A.F., Ballaré, C.L., Bjorn, L., et al., 2006. Environmental effects of ozone depletion and its interactions with climate change: progress report, 2005. Photochem. Photobiol. Sci. 5, 13-24.

Anjum, S.A., Xie, X.Y., Wang, L.C., Saleem, M.F., Man, C., et al., 2011. Morphological, physiological and biochemical responses of plants to drought stress. Afr. J. Agri. Res. 6, 2026-2032.

Arc, E., Sechet, J., Corbineau, F., Rajjou, L., Marion-Poll, A., 2013. ABA crosstalk with ethylene and nitric oxide in seed dormancy and germination. Front Plant Sci. 4, 63.

Arora, N., Bhardwaj, R., Sharma, P., Arora, H.K., 2008. 28Homobrassinolide alleviates oxidative stress in salt-treated maize (Zea mays L.) plants. Braz. J. Plant Physiol. 20, 153-157.

Bari, R., Jones, J.D., 2009. Role of plant hormones in plant defence responses. Plant Mol. Biol. 69, 473-488.

Bastam, N., Baninasab, B., Ghobadi, C., 2013. Improving salt tolerance by exogenous application of salicylic acid in seedlings of pistachio. Plant Growth Regul. 69, 275-284.

Berli, F.J., Moreno, D., Piccolo, P., Hespanhol-Viana, L., Silva, M.F., et al., 2010. Abscisis acid is involved in the response of grape (Vitis vinifera L.) cv. Malbec leaf tissues to ultraviolet-B radiation by enhancing ultraviolet absorbing compounds, antioxidant enzymes and membrane sterols. Plant Cell Environ. 33, 1-10.

Bourtsoukidis, E., Kawaletz, H., Radacki, D., Schütz, S., Hakola, H., et al., 2014. Impact of flooding and drought conditions on the emission of volatile organic compounds of Quercus robur and Prunus serotina. Trees 28, 193-204.

Busch, W., Benfey, P.N., 2010. Information processing without brains-the power of intercellular regulators in plants. Dev. Cell $137,1215-1226$.
Cheng, L., Zou, Y., Ding, S., Zhang, J., Yu, X., Cao, J., et al., 2009. Polyamine accumulation in transgenic tomato enhances the tolerance to high temperature stress. J. Integr. Plant Biol. 51, 489-499.

Cheng, W.H., Endo, A., Zhou, L., Penney, J., Chen, H.C., Arroyo, A., et al., 2002. A unique short-chain dehydrogenase/reductase in Arabidopsis glucose signaling and abscisic acid biosynthesis and functions. Plant Cell 14, 2723-2743.

Choi, J., Huh, S.U., Kojima, M., Sakakibara, H., Paek, K.H., Hwang, I., 2010. The cytokinin-activated transcription factor ARR2 promotes plant immunity via TGA3/NPR1-dependent salicylic acid signalling in Arabidopsis. Dev. Cell 19, 284-295.

Daszkowska-Golec, A., Szarejko, I., 2013. The molecular basis of ABA-mediated plant response to drought. In: Vahdati, K., Leslie, C. (Eds.), Abiotic Stress - Plant Responses and Applications in Agriculture. Intech. Publ, Rijeka, pp. 103-134.

Deblonde, P.M., Ledent, J.F., 2001. Effects of moderate drought conditions on green leaf number, stem height, leaf length and tuber yield of potato cultivars. Eur. J. Agron. 14, 31-41.

Ding, H.D., Zhu, X.H., Zhu, Z.W., Yang, S.J., Zha, D.S., Zhang, Y., et al., 2012. Amelioration of salt-induced oxidative stress in eggplant by application of 24-epibrassinolide. Biol. Plant 56, 767-770.

Ding, Y., Zhao, J., Nie, Y., Fan, B., Wu, S., et al., 2016. Salicylicacid-induced chilling-and oxidative-stress tolerance in relation to gibberellin homeostasis, C-repeat/dehydration-responsive element binding factor pathway, and antioxidant enzyme systems in cold-stored tomato fruit. J. Agric. Food Chem. 64, $8200-8206$.

Doherty, H.M., Selvendran, R.R., Bowles, D.J., 1988. The wound response of tomato plants can be inhibited by aspirin and related hydroxy-benzoic acids. Physiol. Mol. Plant Pathol. 33, 377-384.

Dong, C.J., Li, L., Shang, Q.M., Liu, X.Y., Zhang, Z.G., 2014. Endogenous salicylic acid accumulation is required for chilling tolerance in cucumber (Cucumis sativus L.) seedlings. Planta 240, $687-700$

El-Mashad, A.A., Mohamed, H.I., 2012. Brassinolide alleviates salt stress and increases antioxidant activity of cowpea plants (Vigna sinensis). Protoplasma 249, 625-635.

Emamverdian, A., Ding, Y., Mokhberdoran, F., Xie, Y., 2015. Heavy metal stress and some mechanisms of plant defense response. Sci. World J. 1-18. Available from: https://doi.org/10.1155/2015/ 756120.

Esim, N., Atici, Ö., 2015. Effects of exogenous nitric oxide and salicylic acid on chilling-induced oxidative stress in wheat (Triticum aestivum). Fronti. Life Sci. 8, 124-130.

Fariduddin, Q., Yusuf, M., Hayat, S., Ahmad, A., 2009. Effect of 28homobrassinolide on antioxidant capacity and photosynthesis in Brassica juncea plants exposed to different levels of copper. Environ. Exper. Bot. 66, 418-424.

Fariduddin, Q., Yusuf, M., Ahmad, I., Ahmad, A., 2014. Brassinosteroids and their role in response of plants to abiotic stresses. Biol. Plant 58, 9-17.

Fierro, A.C., Leroux, O., Coninck, B., Cammue, B., Marchal, K., Prinsen, E., et al., 2015. Ultraviolet-B radiation stimulates downward leaf curling in Arabidopsis thaliana. Plant Physiol. Biochem. 93, 9-17.

Gangwar, S., Singh, V.P., 2011. Indole acetic acid differently changes growth and nitrogen metabolism in Pisum sativum L. seedlings under chromium (VI) phytotoxicity: implication of oxidative stress. Sci. Hortic. 129, 321-328.

Gangwar, S., Singh, V.P., Tripathi, D.K., Chauhan, D.K., Prasad, S.M., Maurya, J.N., 2014. Plant responses to metal stress: the emerging role of plant growth hormones in toxicity alleviation. Emerging Technologies and Management of Crop Stress Tolerance 2, 215-248. 
Gill, S.S., Anjum, N.A., Gill, R., Tuteja, N., 2015. DNA damage and repair in plants under ultraviolet and ionizing radiations. Sci. World J. Available from: https://doi.org/10.1155/2015/250158.

Goda, H., Sasaki, E., Akiyama, K., Maruyama-Nakashita, A., Nakabayashi, K., Ogawa, M., et al., 2008. The AtGenExpress hormone and chemical treatment data set: experimental design, data evaluation, model data analysis and data access. Plant J. 55, $526-542$.

Gómez-Cadenas, A., Tadeo, F.R., Talon, M., Primo-Millo, E., 1996. Leaf abscission induced by ethylene in water-stressed intact seedlings of Cleopatra mandarin requires previous abscisic acid accumulation in roots. Plant Physiol. 112, 401-408.

Grabov, A., Blatt, M.R., 1999. A steep dependence of inwardrectifying potassium channels on cytosolic free calcium concentration increase evoked by hyperpolarization in guard cells. Plant Physiol. 119, 277-288.

Guo, Q., Meng, L., Mao, P.C., Jia, Y.,Q., Shi, Y.J., 2013. Role of exogenous salicylic acid in alleviating cadmium-induced toxicity in Kentucky bluegrass. Biochem. Syst. Ecol. 50, 269-276.

Hasanuzzaman, M., Nahar, K., Alam, M.M., Roychowdhury, R., Fujita, M., 2013. Physiological, biochemical, and molecular mechanisms of heat stress tolerance in plants. Intl. J. Mol. Sci. 14, 9643-9684.

Haubrick, L.L., Torsethaugen, G., Assmann, S.M., 2006. Effect of brassinolide, alone and in concert with abscisic acid, on control of stomatal aperture and potassium currents of Vicia faba guard cell protoplasts. Physiol. Planta. 128, 134-143.

Hectors, K., van Oevelen, S., Guisez, Y., Prinsen, E., Jansen, M.A., 2012. The phytohormone auxin is a component of the regulatory system that controls UV-mediated accumulation of flavonoids and UV-induced morphogenesis. Physiol. Plant 145, 594-603.

Huang, C., Wang, D., Sun, L., Wei, L., 2016. Effects of exogenous salicylic acid on the physiological characteristics of Dendrobium officinale under chilling stress. Plant Growth Regul. 79, 199-208.

Huang, D., Wu, W., Abrams, S.R., Cutler, A.J., 2008. The relationship of drought-related gene expression in Arabidopsis thaliana to hormonal and environmental factors. J. Exp. Bot. 59, 2991-3007.

Hubbard, K.E., Nishimura, N., Hitomi, K., Getzoff, E.D., Schroeder, J.I., 2010. Early abscisic acid signal transduction mechanisms: newly discovered components and newly emerging questions. Genes Dev. 24, 1695-1708.

Intergovernmental Panel on Climate Change (IPCC), 2007. Climate change 2007-The physical science basis. Contribution of Working Group I to the Fourth Assessment Report of the Intergovernmental Panel on Climate Change. Cambridge University Press, Cambridge, UK

Javid, M.G., Sorooshzadeh, A., Moradi, F., ModarresSanavy, S.A.M., Allahdadi, I., 2011. The role of phytohormones in alleviating salt stress in crop plants. Aust. J. Crop Sci. 5, 726-734.

Jeanguenin, L., Lebaudy, A., Xicluna, J., Alcon, C., Hosy, E., Duby, G., et al., 2008. Heteromerization of Arabidopsis $\mathrm{Kv}$ channel $\alpha$-subunits: data and prospects. Plant Signal Behav. 3, 622-625.

Jewell, M.C., Campbell, B.C., Godwin, I.D., 2010. Transgenic plants for abiotic stress resistance. Transgenic Crop Plants. Springer, Berlin Heidelberg, pp. 67-132.

Jiang, C.J., Shimono, M., Sugano, S., Kojima, M., Liu, X., Inoue, H., et al., 2013. Cytokinins act synergistically with salicylic acid to activate defense gene expression in rice. Mol. Plant-Microbe Interact. 26, 287-296.

Jiang, M., Zhang, J., 2002. Water stress-induced abscisic acid accumulation triggers the increased generation of reactive oxygen species and up-regulates the activities of antioxidant enzymes in maize leaves. J. Exp. Bot. 53, 2401-2410.

Jin, S.H., Li, X.Q., Wang, G.G., Zhu, X.T., 2015. Brassinosteroids alleviate high-temperature injury in Ficus concinna seedlings via maintaining higher antioxidant defence and glyoxalase systems. AoB Plants 7, 1-12.

Keskin, B.C., Saikaya, A.T., Yuksel, B., Memoh, A.R., 2010. Abscisic acid regulated gene expression in bread wheat. Aust. J. Crop Sci. 4, 617-625

Khan, M.I.R., Khan, N.A., 2013. Salicylic acid and jasmonates: approaches in abiotic stress. J. Plant Biochem. Physiol. 1, e113. Available from: https://doi.org/10.4172/2329-9029.1000e113.

Khan, M.I.R., Khan, N.A., 2017. Reactive Oxygen Species and Antioxidant System in Plants: Role and Regulation Under Abiotic Stress. Springer Nature, Singapore978-981-10-5254-5.

Khan, M.I.R., Khan, N.A., 2014. Ethylene reverses photosynthetic inhibition by nickel and zinc in mustard through changes in PS II activity, photosynthetic-nitrogen use efficiency and antioxidant metabolism. Protoplasma 251, 1007-1019.

Khan, M.I.R., Iqbal, N., Masood, A., Per, T.S., Khan, N.A., 2013. Salicylic acid alleviates adverse effects of heat stress on photosynthesis through changes in proline production and ethylene formation. Plant Signal. Behav. 8 (11), e26374.

Khan, M.I.R., Asgher, M., Khan, N.A., 2014. Alleviation of saltinduced photosynthesis and growth inhibition by salicylic acid involves glycinebetaine and ethylene in mungbean (Vigna radiata L.). Plant Physiol. Biochem. 80, 67-74.

Khan, M.I.R., Fatma, M., Per, T.S., Anjum, N.A., Khan, N.A., 2015. Salicylic acid-induced abiotic stress tolerance and underlying mechanisms in plants. Front. Plant Sci. 6, 1-17.

Kohli, S.K., Handa, N., Bali, S., Arora, S., Sharma, A., Kaur, R., et al., 2018. Modulation of antioxidativedefense expression and osmolyte content by co-application of 24-epibrassinolide and salicylic acid in $\mathrm{Pb}$ exposed Indian mustard plants. Ecotoxicol. Environ. Saf. 147, 382-393.

Krishna, P., Prasad, B.D., Rahman, T., 2017. Brassinosteroid action in plant abiotic stress tolerance. In: Russinova, E., Caño-Delgado, A.I. (Eds.), Brassinosteroids. Humana Press, New York, NY, pp. 193-202.

Krishnamurthy, A., Rathinasabapathi, B., 2013. Auxin and its transport play a role in plant tolerance to arsenite-induced oxidative stress in Arabidopsis thaliana. Plant Cell Environ. 36, 1838-1849.

Li, H., Johnson, P., Stepanova, A., Alonso, J.M., Ecker, J.R., 2004a. Convergence of signalling pathways in the control of differential cell growth in Arabidopsis. Dev. Cell 7, 193-204.

Li, J., Brader, G., Palva, E.T., 2004b. The WRKY70 transcription factor: a node of convergence for jasmonate-mediated and salicylatemediated signals in plant defense. Plant Cell 16, 319-331.

Li, T., Hu, Y., Du, X., Tang, H., Shen, C., Wu, J., et al., 2014. Salicylic acid alleviates the adverse effects of salt stress in Torreya grandis $\mathrm{cv}$. merrillii seedlings by activating photosynthesis and enhancing antioxidant systems. PLoS One 9, e109492. Available from: https://doi.org/10.1371/journal.pone.0109492.

Lorenzo, O., Piqueras, R., Sánchez-Serrano, J.J., Solano, R., 2003. ETHYLENE RESPONSE FACTOR1 integrates signals from ethylene and jasmonate pathways in plant defense. Plant Cell 15, 165-178.

Mainster, M.A., 2006. Violet and blue light blocking intraocular lenses: photoprotection versus photoreception. BJO. 90 (6), 784-792.

Marcinska, I., Czyczyło-Mysza, E., Skrzypek, M., Grzesiak, F., Janowiak, F., Filek, M., et al., 2013. Alleviation of osmotic stress effects by exogenous application of salicylic or abscisic acid on wheat seedlings. Int. J. Mol. Sci. 14, 13171-13193.

Mazid, M., Khan, T.A., Mohammad, F., 2011. Role of secondary metabolites in defense mechanisms of plants. Biol. Med. 3, 232-249.

Mohammed, A.R., Tarpley, L., 2011. Morphological and physiological responses of nine southern U.S. rice cultivars differing in their 
tolerance to enhanced ultraviolet-B radiation. Environ. Exp. Bot. 70, 174-184. Available from: https://doi.org/10.1016/j. envexpbot.2010.09.001.

Mohammed, A.R., Tarpley, L., 2013. Effects of enhanced ultraviolet-B (UV-B) radiation and antioxidative-type plant growth regulators on Rice (Oryza sativa L.) leaf photosynthetic rate, photochemistry and physiology. J. Agric. Sci. 5, 115-128. Available from: https:// doi.org/10.5539/jas.v5n5p115.

Mori, I.C., Murata, Y., Yang, Y., Munemasa, S., Wang, Y.F., et al., 2006. CDPKs CPK6 and CPK3 function in ABA regulation of guard cell S-type anion-and Ca2 + -permeable channels and stomatal closure. PLoS Boil 4, e327.

Munemasa, S., Oda, K., Watanabe-Sugimoto, M., Nakamura, Y., Shimoishi, Y., et al., 2007. The coronatine-insensitive 1 mutation reveals the hormonal signalling interaction between abscisic acid and methyl jasmonate in Arabidopsis guard cells. Specific impairment of ion channel activation and second messenger production. Plant Physiol. 143, 1398-1407.

Mutlu, S., Atıcı, Ö., Nalbantoğlu, B., Mete, E., 2016. Exogenous salicylic acid alleviates cold damage by regulating antioxidative system in two barley (Hordeum vulgare L.) cultivars. Front. Life Sci. 9, 99-109.

Nayyar, H., Kaur, S., Smita, S.K.J., Dhir, K.K., Bains, T., 2005. Water-stress induced injury to reproductive phase in chick pea: evaluation of stress sensitivity in wild and cultivated species in relation to abscisic acid and polyamines. Agron. Crop Sci. 191, $450-457$.

Nazar, R., Iqbal, N., Syeed, S., Khan, N.A., 2011. Salicylic acid alleviates decreases in photosynthesis under salt stress by enhancing nitrogen and sulfur assimilation and antioxidant metabolism differentially in two mungbean cultivars. J. Plant Physiol. 168, $807-815$.

Negi, S., Sukumar, P., Liu, X., Cohen, J.D., Muday, G.K., 2010. Genetic dissection of the role of ethylene in regulating auxindependent lateral and adventitious root formation in tomato. Plant J. 61, 3-15.

Nishiyama, R., Watanabe, Y., Fujita, Y., Le, D.T., Kojima, M., Werner, T., et al., 2011. Analysis of cytokinin mutants and regulation of cytokinin metabolic genes reveals important regulatory roles of cytokinins in drought, salt and abscisic acid responses, and abscisic acid biosynthesis. Plant Cell 23, 2169-2183.

Ogweno, J.O., Song, X.S., Shi, K., Hu, W.H., Mao, W.H., Zhou, Y.H., et al., 2008. Brassinosteroids alleviate heat induced inhibition of photosynthesis by increasing carboxylation efficiency and enhancing antioxidant systems in Lycopersicon esculentum. J. Plant Growth Regul. 27, 49-57.

Outlaw Jr, W.H., 2003. Integration of cellular and physiological functions of guard cells. Critical Rev. Plant Sci. 22, 503-529.

Palma, F., López-Gómez, M., Tejera, N.A., Lluch, C., 2013. Salicylic acid improves the salinity tolerance of Medicagosativa in symbiosis with Sinorhizobium meliloti by preventing nitrogen fixation inhibition. Plant sci. 208, 75-82.

Palma, F., López-Gómez, M., Tejera, N.A., Lluch, C., 2014. Involvement of abscisic acid in the response of Medicago sativa plants in symbiosis with Sinorhizobium meliloti to salinity. Plant Sci. 223, 16-24.

Pandey, N., Iqbal, Z., Pandey, B.K., Sawant, S.V., 2017. Phytohormones and drought stress: plant responses to transcriptional regulation. In: Pandey, G.K. (ed.), Mechanism of Plant Hormone Signalling under Stress, pp. 477-504.

Parashar, A., Yusuf, M., Fariduddin, Q., Ahmad, A., 2014. Salicylic acid enhances antioxidant system in Brassica juncea grown under different levels of manganese. Int. J. Biol. Macromol. 70, 551-558.

Peleg, Z., Blumwald, E., 2011. Hormone balance and abiotic stress tolerance in crop plants. Curr. Opin. plant 14, 290-295.
Pessaraki, M., Szabolcs, I., 2010. Soil salinity and sodicity as particular plant/crop stress factors. In: Pessaraki, M. (Ed.), Handbook of Plant and Crop stress, third ed CRC Press, Boca Raton, FL.

Pistelli, L., Iacona, C., Miano, D., Cirilli, M., Colao, M.C., Mensuali-Sodi, A., et al., 2012. Novel Prunus rootstock somaclonal variants with divergent ability to tolerate waterlogging. Tree Physiol. 32, 355-368.

Ranceliene, V., Vyšniauskiene, R., 2012. Modification of UV-B radiation effect on Crepis capillarisby antioxidant and environmental conditions. Emirates J. Food Agric. 24, 614-620. Available from: https:/ / doi.org/10.9755/ ejfa.v24i6.614620.

Raschke, K., Shabahang, M., Wolf, R., 2003. The slow and the quick anion conductance in whole guard cells: their voltage-dependent alternation, and the modulation of their activities by abscisic acid and CO2. Planta. 217, 639-650.

Roelfsema, M.R., Levchenko, V., Hedrich, R., 2004. ABA depolarizes guard cells in intact plants, through a transient activation of R-and S-type anion channels. Plant J. 37, 578-588.

Ruzicka, K., Ljung, K., Vanneste, S., Podhorská, R., Beeckman, T., Friml, R., et al., 2007. Ethylene regulates root growth through effects on auxin biosynthesis and transport-dependent auxin distribution. Plant Cell 19, 2197-2212.

Sairam, R.K., Kumutha, D., Ezhilmathi, K., Deshmukh, P.S., Srivastava, G.C., 2008. Physiology and biochemistry of waterlogging tolerance in plants. Biol. Plant 52, 401-412.

Santner, A., Estelle, M., 2010. The ubiquitin-proteasome system regulates plant hormone signalling. Plant J. 61, 1029-1040.

Satisha, J., Prakash, G.S., Murti, G.S.R., Upreti, K.K., 2005. Response of grape genotypes to water deficit: root, shoot growth and endogenous hormones. Indian J. Plant Physiol. 10, 225-230.

Sauter, A., Davies, W.J., Hartung, W., 2001. The long-distance abscisic acid signal in the droughted plant: the fate of the hormone on its way from root to shoot. J. Exp. Bot 52, 1991-1997.

Shakirova, F.M., Allagulova, C.R., Maslennikova, D.R., Klyuchnikova, E.O., Avalbaev, A.M., Bezrukova, M.V., 2016. Salicylic acid-induced protection against cadmium toxicity in wheat plants. Environ. Exper. Bot. 122, 19-28.

Sharan, A., Dkhar, J., Singla-Pareek, S.L., Pareek, A., 2017. Crosstalk between gibberellins and abiotic stress tolerance machinery in plants. In: G.K. Pandey (ed.), Mechanism of Plant Hormone Signaling under Stress, pp. 101-126.

Sharma, I., Ching, E., Saini, S., Bhardwaj, R., Pati, P.K., 2013. Exogenous application of brassinosteroid offers tolerance to salinity by altering stress responses in rice variety Pusa Basmati-1. Plant Physiol. Biochem. 69, 17-26.

Sheard, L.B., Tan, X., Mao, H., Withers, J., Ben-Nissan, G., Hinds, T.R., et al., 2010. Jasmonate perception by inositol-phosphatepotentiated COI1-JAZ co-receptor. Nature 468, 400.

Shi, C., Qi, C., Ren, H., Huang, A., Hei, S., She, X., 2015. Ethylene mediates brassinosteroid-induced stomatal closure via $\mathrm{G \alpha}$ protein-activated hydrogen peroxide and nitric oxide production in Arabidopsis. Plant J. 82, 280-301.

Shinohara, T., Martin, E.A., Leskovar, D.I., 2017. Ethylene regulators influence germination and root growth of globe artichoke seedlings exposed to heat stress conditions. Seed Sci. Technol. 45, $167-178$.

Shinozaki, K., Yamaguchi Shinozaki, K., 2000. Molecular responses to dehydration and low temperature: differences and cross talk between two stress signalling pathways. Curropin. Plant Sci. 3, 217-223.

Shrivastava, P., Kumar, R., 2015. Soil salinity: a serious environmental issue and plant growth promoting bacteria as one of the tools for its alleviation. Saudi. J. Biol. Sci. 22, 123-131.

Singh, S., Singh, A., Srivastava, P.K., Prasad, S.M., 2018. Cadmium toxicity and its amelioration by kinetin in tomato seedlings vis-à-vis ascorbate glutathione cycle. J. Photochem. Photobiol. B. 178, 76-84. 
Sirhindi, G., Kaur, H., Bhardwaj, R., Sharma, P., Mushtaq, R., 2017. 28 Homobrassinolide potential for oxidative interface in Brassica juncea under temperature stress. Acta Physiol. Plant 39, 228.

Smith, A.M., Stitt, M., 2007. Coordination of carbon supply and plant growth. Plant Cell Environ. 30, 1126-1149.

Spoel, S.H., Koornneef, A., Claessens, S.M., Korzelius, J.P., Van Pelt, J.A., Mueller, M.J., et al., 2003. NPR1 modulates cross-talk between salicylate-and jasmonate-dependent defense pathways through a novel function in the cytosol. Plant Cell 15, 760-770.

Srivastava, S., Srivastava, A.K., Suprasanna, P., D'Souza, S.E., 2013. Identification and profiling of arsenic stress-induced microRNAs in Brassica juncea. J. Exp. Bot. 64, 303-315.

Stepanova, A.N., Alonso, J.M., 2009. Ethylene signalling and response: where different regulatory modules meet. Curr. Opin. Plant Biol. 12, 548-555.

Stepanova, A.N., Hoyt, J.M., Hamilton, A.A., Alonso, J.M., 2005. A link between ethylene and auxin uncovered by the characterization of two root-specific ethylene-insensitive mutants in Arabidopsis. Plant Cell 17, 2230-2242.

Stepanova, A.N., Robertson-Hoyt, J., Yun, J., Benavente, L.M., Xie, D. Y., Dolezal, K., et al., 2008. TAA1-mediated auxin biosynthesis is essential for hormone crosstalk and plant development. Cell 133, 177-191.

Suhita, D., Kolla, V.A., Vavasseur, A., Raghavendra, A.S., 2003. Different signalling pathways involved during the suppression of stomatal opening by methyl jasmonate or abscisic acid. Plant Sci. 164, 481-488.

Suhita, D., Raghavendra, A.S., Kwak, J.M., Vavasseur, A., 2004. Cytoplasmic alkalization precedes reactive oxygen species production during methyl jasmonate-and abscisic acid-induced stomatal closure. Plant Physiol. 134, 1536-1545.

Tanaka, Y., Sano, T., Tamaoki, M., Nakajima, N., Kondo, N., Hasezawa, S., 2005. Ethylene inhibits abscisic acid-induced stomatal closure in Arabidopsis. Plant Physiol. 138, 2337-2343.

Tanaka, Y., Sano, T., Tamaoki, M., Nakajima, N., Kondo, N., Hasezawa, S., 2006. Cytokinin and auxin inhibit abscisic acidinduced stomatal closure by enhancing ethylene production in Arabidopsis. J. Exp. Bot. 57, 2259-2266.

Thao, N.P., Khan, M.I.R., Thu, N.B.A., Hoang, X.L.T., Asgher, M., Khan, N.A., et al., 2015. Role of ethylene and its cross talk with other signaling molecules in plant responses to heavy metal stress. Plant physiol. 169, 73-84.

Thompson, A.J., Jackson, A.C., Parker, R.A., Morpeth, D.R., Burbidge, A., 2000. Abscisic acid biosynthesis in tomato: regulation of zeaxanthin epoxidase and 9-cis-epoxycarotenoid dioxygenase mRNAs by light/dark cycles, water stress and abscisic acid. Plant Mol. Biol. 42, 33-845.

Thompson, A.J., Andrews, J., Mulholland, B.J., McKee, J.M., Hilton, H.W., Horridge, J.S., et al., 2007. Overproduction of abscisic acid in tomato increases transpiration efficiency and root hydraulic conductivity and influences leaf expansion. Plant Physiol. 4, 1905-1917.

Todaka, D., Zhao, Y., Yoshida, T., Kudo, M., Kidokoro, S., Mizoi, J., et al., 2017. Temporal and spatial changes in gene expression, metabolite accumulation and phytohormone content in rice seedlings grown under drought stress conditions. Plant J. 90, 61-78.

Torres, C.A., Sepulveda, G., Kahlaoui, B., 2017. Phytohormone interaction modulating fruit responses to photooxidative and heat stress on apple (Malus domestica Borkh.). Front. Plant Sci. 8, 2129.

Tossi, V., Cassia, R., Bruzzone, S., Zocchi, E., Lamattina, L., 2012. ABA says NO to UV-B: a universal response? Trends Plant Sci. $17,510-517$

Tran, L.S., Urao, T., Qin, F., Maruyama, K., Kakimoto, T., Shinozaki, K., et al., 2007. Functional analysis of AHK1/ATHK1 and cytokinin receptor histidine kinases in response to abscisic acid, drought, and salt stress in Arabidopsis. Proc. Natl. Acad. Sci. USA 104, 20623-20628.

Tuteja, N., 2007. Review abscisic acid and abiotic stress signalling. Plant Signal. Behav. 2, 135-138.

Upreti, K.K., Murti, G.S.R., Bhatt, R.M., 1998. Response of French bean cultivars to water deficits: changes in endogenous hormones, proline and chlorophyll. Biol. Plant 40, 381-388.

Urano, K., Maruyama, K., Jikumaru, Y., Kamiya, Y., YamaguchiShinozaki, K., Shinozaki, K., 2017. Analysis of plant hormone profiles in response to moderate dehydration stress. Plant J. 90, $17-36$

Verma, V., Ravindran, P., Kumar, P.P., 2016. Plant hormonemediated regulation of stress responses. BMC Plant Biol. 16, 86. Available from: https://doi.org/10.1186/s12870-016-0771-y.

Wang, D., Pajerowska-Mukhtar, K., Culler, A.H., Dong, X., 2007. Salicylic acid inhibits pathogen growth in plants through repression of the auxin signalling pathway. Curr. Biol. 17, 1784-1790.

Wang, L.J., Fan, L., Loescher, W., Duan, W., Liu, G.J., Luo, H.B., 2010. Salicylic acid alleviates decreases in photosynthesis under heat stress and accelerates recovery in grapevine leaves. BMC Plant Biol. 10, 34.

Wasternack, C., 2007. Jasmonates: an update on biosynthesis, signal transduction and action in plant stress response, growth and development. Ann. Bot. 100, 681-697.

Wen, P.F., Chen, J.Y., Wan, S.B., Kong, W.F., Zhang, P., Wang, W., 2008. Salicylic acid activates phenylalanine ammonia-lyase in grape berry in response to high temperature stress. Plant Growth Regul. 55, 1-10.

Wilkinson, S., Davies, W.J., 2002. ABA-based chemical signalling: the co-ordination of responses to stress in plants. Plant Cell Environ. 25, 195-210.

Wu, X., Yao, X., Chen, J., Zhu, Z., Zhang, H., Zha, D., 2014. Brassinosteroids protect photosynthesis and antioxidant system of eggplant seedlings from high-temperature stress. Acta Physiol. Plant 36, 251-261.

Yadav, P., Kaur, R., Kanwar, M.K., Sharma, A., Verma, V., Sirhindi, G., et al., 2018. Castasterone confers copper stress tolerance by regulating antioxidant enzyme responses, antioxidants, and amino acid balance in B. juncea seedlings. Ecotoxicol. Environ. Saf. 147, 725-734.

Yadegari, M., 2017. Study of phytohormones effects on UV-B stress seeds of thyme species. J. Herbal Drugs 8, 109-115.

Ye, N., Zhu, G., Liu, Y., Li, Y., Zhang, J., 2011. ABA controls $\mathrm{H}_{2} \mathrm{O}_{2}$ accumulation through the induction of OsCATB in rice leaves under water stress. Plant Cell Physiol. 52, 689-698.

Yuan, S., Lin, H.H., 2008. Minireview: role of salicylic acid in plant abiotic stress. Zeitschriftfür Naturforschung C 63 (5-6), 313-320.

Zandalinas, S.I., Mittler, R., Balfagón, D., Arbona, V., GómezCadenas, A., 2018. Plant adaptations to the combination of drought and high temperatures. Physiol. Plant 162, 2-12.

Zhang, S., Hu, J., Zhang, Y., Xie, X.J., Knapp, A., 2007. Seed priming with brassinolide improves lucerne (Medicago sativa L.) seed germination and seedling growth in relation to physiological changes under salinity stress. Aust. J. Agric. Res. 58, 811-815.

Zhang, S.W., Li, C.H., Cao, J., Zhang, Y.C., Zhang, S.Q., et al., 2009. Altered architecture and enhanced drought tolerance in rice via the down-regulation of indole-3-acetic acid by TLD1/OsGH3. 13 activation. Plant Physiol. 151, 1889-1901.

Zhao, Y., Chan, Z., Gao, J., Xing, L., Cao, M., et al., 2016. ABA receptor PYL9 promotes drought resistance and leaf senescence. Proc. Natl. Acad. Sci. 113, 1949-1954.

Zhou, G., Nimir, N., Lu, S., Zhai, F., Wang, Y., 2014. Gibberellic acid and salinity affected growth and antioxidant enzyme activities in castor bean plants at early growth stage. Agron. J. 106, $1340-1348$. 
Zhu, Z., An, F., Feng, Y., Li, P., Xue, L., A, M., et al., 2011. Derepression of ethylene-stabilized transcription factors (EIN3/ EIL1) mediates jasmonate and ethylene signalling synergy in Arabidopsis. Proc. Natl. Acad. Sci. USA 108, 12539-12544.

Zwack, P.J., Rashotte, A.M., 2015. Interactions between cytokinin signalling and abiotic stress responses. J. Exp. Bot. 66 (16), 4863-4871.

\section{Further Reading}

Ashraf, M., Foolad, M., 2007. Roles of glycine betaine and proline in improving plant abiotic stress resistance. Environ. Exp. Bot. 59, 206-216.

Behringer, C., Bartsch, K., Schaller, A., 2011. Safeners recruit multiple signalling pathways for the orchestrated induction of the cellular xenobiotic detoxification machinery in Arabidopsis. Plant Cell Environ. 34, 1970-1985.

Gleason, C., Foley, R.C., Singh, K.B., 2011. Mutant analysis in Arabidopsis provides insight into the molecular mode of action of auxinic herbicide dicamba. PLoS One 6, e17245.

Kummerova, M., Zezulka, V.L., Fišerová, H., 2012. Effect of organic pollutant treatment on the growth of pea and maize seedlings. Cent. Eur. J. Biol. 7, 159-166.

Rowe, J.H., Topping, J.F., Liu, J., Lindsey, K., 2016. Abscisic acid regulates root growth under osmotic stress conditions via an interacting hormonal network with cytokinin, ethylene and auxin. New Phytol. 211 (1), 225-239.

<http://www.fao.org/soils-portal/soil-management/managementof-some-problem-soils / salt-affected-soils / more-information-onsalt-affected-soils/en/>. 
This page intentionally left blank 


\title{
20
}

\section{Role and Regulation of Auxin Signaling in Abiotic Stress Tolerance}

\author{
Veena Pandey, Indra Dutt Bhatt and Shyamal Kumar Nandi
}

G. B. Pant National Institute of Himalayan Environment and Sustainable Development, Kosi-Katarmal, Almora, Uttarakhand, India

\begin{tabular}{lrll} 
& O U T L I N E \\
& 319 & 20.2 Conclusion & 326 \\
20.1 Introduction & 320 & Acknowledgements & 327 \\
20.1.1 Auxin Signaling in Plants & 320 & \\
20.1.2 Auxin Signaling Under Abiotic Stresses & & References \\
Hormones & 326 & \\
& & \\
\hline
\end{tabular}

\subsection{INTRODUCTION}

Plant hormones play a crucial role in regulating growth and development. Among all hormones, auxin (indole-3-acetic acid (IAA)) has a special place since it was the first growth hormone discovered in plants. Auxin, along with cytokinins, differs from other plant hormones in the fact that they are required for viability. No mutants lacking either auxin or cytokinin have been found, suggesting that mutations that eliminate them are lethal. IAA biosynthesis in plants occurs by tryptophan-dependent and tryptophan-independent manner. Four pathways for tryptophan-dependent IAA biosynthesis include IAM (indole-3-acetamide) pathway, IPA (indole-3-pyruvic acid) pathway, TAM (tryptamine) pathway, and IAOX (indole-3-acetaldoxime) pathway (Mano and Nemoto, 2012). Although free IAA is the biologically active form, most of the auxins in plants are found in a conjugated form, covalently bonded to glucose or amino acid. The primary sites of IAA biosynthesis include shoot apical meristems, young leaves, and developing fruits or seeds. Auxin synthesized in the aerial part is transported to other plant parts through polar transport. It is the only plant hormone known to be transported polarly (Taiz and Zeiger, 2002). Polar auxin transport (PAT) includes influx carriers such as AUX1/LAX1 (Auxin Resistant 1/Like Aux1) and auxin efflux carriers such as the PIN (PIN-FORMED), ATP binding cassette type $\mathrm{B}(\mathrm{ABCB})$, and the multidrug resistant proteins or P-glycoproteins (MDR-PGPs) (Kazan, 2013). More recently another class of auxin carriers, called PILS (PIN-LIKES), has been identified that regulates intracellular auxin transport and compartmentalization in plants (Barbez et al., 2012).

The physiological role of auxin in many plant developmental processes like phototropism, gravitropism, apical dominance, cell elongation, vascular differentiation, 
parthenocarpy, fruit development, abscission, and adventitious root formation is well known (Taiz and Zeiger, 2002; Zhao, 2010). In recent years, the role of auxin under various abiotic stresses has also been studied. This chapter provides an overview of the role of auxin signaling in various abiotic stress responses of plants.

\subsubsection{Auxin Signaling in Plants}

Auxin signaling involves auxin perception by receptors like ABP1 (auxin-binding protein) (Taiz and Zeiger, 2002), TIR1 (transport inhibitor response 1), and AFB (Auxin F-Box) proteins (Dharmasiri et al., 2005; Kepinski and Leyser, 2005; Kazan, 2013). Auxin binding to its receptor leads to degradation of Aux/ IAAs (Auxin/IAA) repressor by the $26 \mathrm{~S}$ proteasome. Aux/IAA proteins repress auxin response factors (ARFs), a class of transcription factors (TFs) that bind to auxin responsive elements (ARE) found in the promoter region of auxin-induced genes (Kim et al., 1997). Degradation of Aux/IAAs releases ARFs from suppression, which then bind to the ARE and regulate auxin dependent gene expression (Kazan, 2013).

The two large TF families, that is, the ARFs and the Aux/IAAs, are mainly involved in transcriptional regulation of auxin responsive genes. The expression of these genes responds to environmental signals (Guilfoyle and Hagen, 2007). Due to the potential applications of ARF TFs for the development of improved stress tolerant transgenic crop plants, many ARF families have been identified in a number of crop species like Oryza sativa (Song et al., 2009; Jain and Khurana, 2009; Shen et al., 2010), Sorghum bicolor (Wang et al., 2010), Solanum lycopersicum (Wu et al., 2011), Zea mays (Xing et al., 2011; Wang et al., 2012), Brassica rapa (Mun et al., 2012), and Glycine max (Van Ha et al., 2013). Likewise, transcriptional control of Aux/IAA genes plays a central role in the establishment of the auxin-signaling pathways that regulate plant response to environmental variables. Various TFs that regulate the Aux/IAA genes belong to DREB/ CBF family (which functions in stress tolerance) and directly promote transcription of these genes in response to abiotic stress (Shani et al., 2017).

Recent findings suggest that auxin biosynthesis pathway involving YUCCA gene (encoding a flavin monooxygenase, and belonging to the tryptophandependent auxin biosynthetic pathway) may be exploited to alter plant responses to the environment (Kim et al., 2013; Lee et al., 2012; Park et al., 2013). Plant response to high temperature was found to be mediated through the expression of YUC2 and YUC6 genes in Arabidopsis thaliana and Hordeum vulgare (Cheng, 2006; Sakata et al., 2010). Moreover, Arabidopsis plants overexpressing YUC6 or transgenic poplar expressing Arabidopsis YUC6 under control of stressinducible SWPA2 promoter exhibited improved drought and oxidative stress resistance (Cha et al., 2015; Ke et al., 2015). Elevated auxin positively modulated the expression levels of multiple abiotic stress-related genes ( $R A B 18, R D 22, R D 29 A, R D 29 B$, $D R E B 2 A$ and DREB2B) and increased antioxidant enzyme activities resulting in higher tolerance to the variable environment (Shi et al., 2014).

\subsubsection{Auxin Signaling Under Abiotic Stresses}

The role of auxin in plant development is well known; however, its possible function in response to various stresses is poorly understood. Several recent studies demonstrate a novel role of auxin signaling and transport in plant tolerance to abiotic stress (Krishnamurthy and Rathinasabapathi, 2013). Various abiotic stresses that involve auxin-mediated response in plants are represented in Table 20.1. The following section summarizes the role and signaling of auxin under different abiotic stresses.

\subsubsection{Drought Stress}

Auxin plays an important role in plant responses to drought stress. Auxin biosynthesis, distribution via changes in auxin transport, or conjugation with amino acids or sugar molecules all are regulated under abiotic stresses (Shibasaki et al., 2009). Members of an auxinresponsive GH3 gene family, encoding auxin conjugating enzymes (IAA-amido synthetases) have been shown to be also involved in drought-stress responses. OsGH3.13 has been reported to enhance the expression of LEA (late embryogenesis abundant) genes, which increased the drought tolerance of rice seedlings (Zhang et al., 2009a). Overexpression of OsGH3-2 in rice resulted in IAA-deficient phenotype with reduced free IAA, carotene, and ABA, which caused greater stomatal apertures causing increased water loss and more hypersensitivity to drought ( $\mathrm{Du}$ et al., 2012). In rice, IAA level was reduced to $72 \%$ after 3 days of drought stress (Du et al., 2013a). Auxin positively modulates root biomass and branching, which might improve water uptake efficiency and hence, provide drought stress resistance (Shi et al., 2014).

Transcriptome analysis indicated that under dehydration, the expression levels of many auxin-related genes are changed. Furthermore, auxin partly participates in the positive regulation of drought stress resistance through the regulation of root architecture (Shi et al., 2014). In Arabidopsis, MYB96 TF regulates lateral root (LR) meristem activation under drought conditions, possibly through an ABA-auxin signaling crosstalk, and the MYB96-knockout mutant was more susceptible to drought stress (Seo et al., 2009). 
TABLE 20.1 Abiotic Stresses Which Encompass Auxin-Mediated Signaling Response in Plants

\begin{tabular}{lll}
\hline $\begin{array}{l}\text { Abiotic } \\
\text { stress }\end{array}$ & Crops & Major findings \\
\hline Drought & Arabidopsis, Oryza sativa & Auxin level decrease under drought stress; however, higher auxin
\end{tabular}

stress

Salinity Arabidopsis

stress

Arabidopsis, Phaseolus vulgaris,

$\begin{array}{ll}\text { Temperature } & \text { Arabidopsis, Phase } \\ \text { stress } & \text { Tomato, pepper }\end{array}$

Nitrogen Arabidopsis

deficiency

Phosphorus Arabidopsis, Lupinus albus

deficiency

Potassium

deficiency

Arabidopsis, cotton, rice, tobacco, maize

Sulfur

deficiency

Arabidopsis

Iron

deficiency

Arabidopsis, Phaseolus vulgaris,

Cucumis satious, Trifolium pratens,

Malus xiaojinensis, Pyrus betulifolia

Aluminum Arabidopsis, maize, Triticum

toxicity aestivum

Lead toxicity Arabidopsis, Oryza sativa, Triticum aestivum

Cadmium Arabidopsis, Pisum sativum

toxicity

Copper

toxicity

Arsenic

toxicity
Auxin level decrease under drought stress; however, higher auxin
concentration improves drought tolerance by increasing compatible solutes, antioxidants, and root branching

Regulation of auxin level helps to maintain ion homeostasis under salt stress; plants reduce auxin concentration and signaling to cope with salinity stress

High/low temperature stress reduces auxin concentration by converting active IAA to inactive conjugated form; however, application of exogenous auxin might help to reduce stress-induced plant injury

Many nitrogen uptake transporters also mediate auxin transport in plants and they help plants to alter root architecture under nitrogen deficiency

Phosphorus deficiency induces auxin-mediated lateral root formation in plants and increases plant sensitivity to auxin

Many potassium transporters are crucial for auxin transport and severe potassium deficiency reduces auxin concentration/transport and root growth; however, exogenous auxin application may increase lateral root formation and elongation under potassium deficiency

Plants increase auxin accumulation under sulfur deficiency, so as to induce more root growth and to gain more access to sulfur

Fe-deficiency increases auxin level in roots and induces formation of branched root hairs; auxin mediated response under Fe deficiency involves the interplay of ethylene and nitric oxide

Aluminum toxicity causes reduction in cell expansion and inhibition of root growth by disruption of auxin transport

Lead-induced plant injury is associated with increased IAA-oxidase activity and reduction in IAA levels

Cadmium stress causes enhanced IAA-oxidase activity, auxin degradation, and reduction in plant growth

Copper toxicity inhibits primary root elongation by mediating auxin redistribution in plants

Exogenous supply of IAA can improve arsenic tolerance in plants

\section{References}

Shibasaki et al. (2009), Zhang et al. (2009a), Seo et al. (2009), Du et al (2012), Lee and Luan (2012), Du et al (2013a), Shi et al. (2014), Sharma et al. (2015)

Bao and Li (2002), He et al. (2005), Park et al. (2007), Sun et al. (2008), Popko et al. (2010), Ding et al. (2009), Wang et al. (2009), Iglesias et al. (2010, 2014), Jung and Park (2011), Liu et al. (2015)

Morris (1979), Kuo and Tsai (1984), Franco et al. (1990), El-Abd et al. (1986), Ofir et al. (1993), Fukaki et al. (1996), Huberman et al. (1997), Wyatt et al. (2002), Shibasaki et al. (2009), Sakata et al. (2010), Oshino et al. (2007, 2011), Du et al. (2013b)

Zhang et al. (2007), Gifford et al. (2008), Krouk et al. (2010), Vidal et al. (2010), Kiba et al. (2011), Gojon et al. (2011), Bouguyon et al. (2012)

Gilbert et al. (1998, 2000), Neumann et al. (2000), Lopez-Bucio et al (2002), Hammond et al. (2003), Nacry et al. (2005)

Cao et al. (1993), Philippar et al. (1999), Vicente-Agullo et al. (2004), Armengaud et al. (2004), Ashley et al. (2005), Zhang et al. (2009b), Ma et al. (2012), Song et al. (2015)

Kutz et al. (2002), Nikiforova et al. (2003), Kopriva and Rennenberg (2004), Nikiforova et al. (2005), Maruyama-Nakashita et al. (2005), Kasajima et al. (2007), Falkenberg et al. (2008), Lewandowska and Sirko (2008), Frerigmann and Gigolashvili (2014)

Landsberg (1984), Romheld and Marschner (1986), Kim et al. (1992), Schmidt et al. (2000), Li et al. (2000), Schmidt and Schikora (2001), Zheng et al. (2003), Muller and Schmidt (2004), Li and Li (2004), Lombardo et al. (2006), Chen et al. (2010), Bacaicoa et al. (2011), Romera et al. (2011), Giehl et al. (2012), Wu et al. (2012), Li et al. (2016)

Hasenstein et al. (1988), Kollmeier et al. (2000), Shen et al. (2008), Sun et al. (2010), Yang et al. (2011)

Mukherji and Maitra (1977), Lane et al. (1978), Liu et al. (2009)

Hasenstein et al. (1988), Hagen et al. (1988), Moons (2003), Chaoui and El Ferjani (2005), Ganesan (2008), Mei et al. (2009), Ding and Zhu (2009)

Chaoui and El Ferjani (2005), Yuan et al. (2013)

Krishnamurthy and Rathinasabapathi (2013), Pandey and Gupta (2015) 
Activation of the YUCCA7 gene of auxin biosynthesis resulted in elevated endogenous auxin levels and enhanced drought resistance in Arabidopsis (Lee and Luan, 2012). Auxin also positively modulated the accumulations of compatible solutes such as multiple sugars and sugar alcohols. Additionally, auxin positively regulated the activities of four enzymatic antioxidants (superoxide dismutase, catalase, peroxidase, glutathione reductase) under drought stress condition, thus conferring effective ROS (reactive oxygen species) detoxification to improve drought stress resistance (Shi et al., 2014).

A crosstalk between IAA and other hormones is thought to mediate drought responses of plants. Abscisic acid (ABA) mediated inhibition of LR growth is controlled by reducing PAT. ABA insensitive3 ( $A B I 3$ ) interacts with ARF or Aux/IAA proteins and mediates LR growth (Shkolnik-Inbar and Bar-Zvi, 2010). ABA can alter auxin transport to mediate proton secretion at root tips and control root growth under moderate water deficit (Sharma et al., 2015). Auxin also plays an important synergistic and antagonistic role in the biosynthesis of other stress related hormones like ethylene (Li et al., 2004; Stepanova and Alonso, 2005; Ruzicka et al., 2007; Stepanova et al., 2007) and their crosstalk might be responsible for mediating auxin mediated response under stress.

\subsubsection{Salinity Stress}

Regulation of auxin biosynthesis and transport is required to mediate the response of plants to salt stress. Salt stress in Arabidopsis induced the expression of IAA biosynthesis genes (nitrilase 1 and nitrilase 2), suggesting that the level of IAA might increase in response to salinity stress (Bao and Li, 2002). Salinityinduced expression of NAC-type TF (AtNAC2) mediates the process of plant LR development under salinity stress through auxin signaling pathways ( $\mathrm{He}$ et al., 2005). Overexpression of AtNAC2 in transgenic Arabidopsis plants resulted in the promotion of LR development. Jung and Park (2011) found that a membrane-bound NAC TF (NTM2) mediates the signaling crosstalk between auxin and salt stress via the IAA30 gene during seed germination in Arabidopsis. Germination of the NTM2-deficient ntm2-1 mutant seeds exhibited enhanced resistance to high salinity, while ntm2-1 mutant overexpressing the IAA30 gene reduced salt resistance.

SOS pathway (which maintains ion homeostasis under salt stress) modulates root response by regulating PIN2 protein and auxin asymmetric distribution (Sun et al., 2008). The different levels of salt stress determine the degree of auxin biosynthesis in the shoot apex and the subsequent gradients of auxin in roots, resulting in changes in the number of elongated
LRs and the rates of elongation (Wang et al., 2009). High salinity induced the expression of AtGH3.5 gene, which encodes IAA amido-conjugate synthases. Overexpression of AtGH3.5 resulted in decreased free auxin concentration and increase in auxin conjugate like IAA-Asp (Park et al., 2007). Free auxin content in the developing xylem of Populus declines during abiotic stress, while auxin conjugates increase in response to plant adaptation to stress (Popko et al., 2010).

Adaptation to salinity in Arabidopsis is mediated in part by an auxin/redox interaction. Auxin receptor double mutant (tir1 afb2) showed increased tolerance against salinity as measured by chlorophyll content, germination rate, root elongation, and enhanced antioxidant enzymes compared with wild-type plants (Iglesias et al., 2010). miRNA microarray hybridization analysis revealed that salt stress-induced differential expression of miRNAs in maize roots, some of which were involved in auxin signaling (Ding et al., 2009). Salt stress triggers miR393 expression, which leads to a reduction in the levels of the TIR1 and AFB2 auxin receptors. Salt stress also stabilized Aux/IAA repressors, which lead to downregulation of auxin signaling. It was also reported that miR393 is involved in repression of LR initiation, emergence, and elongation during salinity, since the mir393ab mutant showed reduced inhibition of $\mathrm{LR}$ growth upon $\mathrm{NaCl}$ treatment (Iglesias et al., 2014). Nitric oxide (NO) is involved in repressing PIN expression (thereby reducing auxin levels), stabilizing IAA17 (thereby repressing auxin signaling) and mediating salt-induced inhibition of root meristem growth (Liu et al., 2015). All these findings suggest that the downregulation of auxin signaling might be a plant strategy to enhance tolerance to salinity stress.

\subsubsection{Temperature Stress}

Increasing evidence suggests a potential link between low- and high-temperature stress and auxin signaling. Morris (1979) showed that temperature affects the velocity of exogenous auxin transport in plants. High and low day/night temperature $\left(35 / 24^{\circ} \mathrm{C}\right.$ and $10 / 5^{\circ} \mathrm{C}$ ) was found to reduce fruit set, pollen grain viability, and IAA levels in tomato (El-Abd et al., 1986). Also, a short exposure to $38^{\circ} \mathrm{C}$ to young fruits of tomato caused reduction in auxin-like substances (Kuo and Tsai, 1984). Similarly, in bean (Phaseolus vulgaris), heat stress significantly reduced the diffusion of endogenous IAA from reproductive organs into agar, possibly due to the direct inhibitory effect of the high temperatures $\left(32 / 27^{\circ} \mathrm{C}\right)$ on the transport capacity of related tissues (Ofir et al., 1993). High temperatures (day/night regimes of $32 / 27^{\circ} \mathrm{C}, 40 / 27^{\circ} \mathrm{C}$ ) reduced IAA levels and particularly auxin transport capacity in the reproductive organ in pepper (Capsicum annuum). 
In addition, the reduction of auxin transport capacity was found to be the major mechanism of high temperature induced abscission of the reproductive organ (Huberman et al., 1997). Basipetal auxin transport in wild-type Arabidopsis was stopped at $4^{\circ} \mathrm{C}$; but auxin transport was restored when plants were reintroduced to room temperature (Wyatt et al., 2002). However, $\mathrm{Du}$ et al. (2013b) reported that under the cold stress of 3 days, IAA level increased 1.6-fold, while it was increased by 1.3 -fold after $6 \mathrm{~h}$ of heat stress, as compared with the control.

Cold stress inhibits the inflorescence gravity response in Arabidopsis, which is regulated by an asymmetric distribution of auxin (Fukaki et al., 1996; Wyatt et al., 2002). Cold stress affected the polar transport of auxin by selectively inhibiting the intracellular trafficking of auxin efflux carriers PIN2 and PIN3 (Shibasaki et al., 2009). For transport of auxin towards the shoot, the polar deployment of PIN2 protein and the constitutive cycling of this protein from the membrane to endosome are required for its functionality. Thus, reduced intracellular cycling functionality affects the PINs resulting in decreased shootward transport of auxin and inhibits the capability of roots to form an auxin gradient (Paciorek et al., 2005; Sukumar et al., 2009).

In Arabidopsis, many auxin-responsive genes respond to cold stress (Jain and Khurana, 2009). Likewise, many OsGH3 genes (OsGH3-2, OsGH3-5, OsGH3-6, OsGH3-7, OsGH3-9, OsGH3-11, OsGH3-13), which convert active IAA to an inactive form, were found to be downregulated by heat stress in rice (Franco et al., 1990). High temperature reduced endogenous auxin in developing anthers by reducing expression of the YUCCA auxin biosynthesis and caused male sterility in barley and Arabidopsis. However, application of auxin completely reversed male sterility in both plant species (Sakata et al., 2010). Compromised auxin biosynthesis/IAA level under high temperature condition resulted in nuclear and organellar DNA proliferation arrest due to cotranscriptional alteration, however, exogenous auxin application promoted the expression of DNA replication-related genes, which induce anther cell proliferation under heat stress (Oshino et al., 2007, 2011). This suggests that auxin reduction is the primary cause of high temperature induced injury, and the exogenous auxin application may help to reduce plant injury and to sustain steady yields of crops.

\subsubsection{Nutrient Deficiency Stress}

Plants in nature encounter deficiency of a wide variety of essential mineral elements, which suppresses plant growth. The ability of plants to sense and respond appropriately to the soil nutrient availability is of fundamental importance for their adaptation. Auxin signaling and transport play important roles in regulating plant developmental responses to deficiency of various soil nutrients. Kazan (2013) suggested that a rapid alteration in plant root architecture in response to nutrient deficiency is achieved through auxin signaling.

Nitrogen: Auxin mediates plant response to nitrogen deficiency. Arabidopsis seedlings grown in low nitrogen conditions contain higher levels of auxin in roots as compared with seedlings grown in high nitrogen conditions (Kiba et al., 2011). However, an early report reveals that the inhibitory effect of high nitrate on LR growth of Arabidopsis is not alleviated by exogenous application of auxin, indicating that the auxin content is not the only factor regulating LR development (Zhang et al., 2007).

Using a cell sorting technique, Gifford et al. (2008) identified a nitrogen-inducible auxin response factor (ARF8), which is expressed in pericycle cells. They provided genetic evidence that the nitrogen signal regulates auxin signaling through the action of microRNA (miR167a) to control LR initiation. Recently, the auxin receptor gene $A F B 3$ was also found to be induced by nitrate (Vidal et al., 2010). Another link between nitrate and auxin signaling is provided by Krouk et al. (2010), who showed that NRT1.1/CHL1, which is a nitrate transporter and sensor (Ho et al., 2009), also facilitates cell-tocell auxin transport. Under low $\mathrm{NO}_{3}{ }^{-}$concentrations, nitrate uptake transporter (NITRATE TRANSPORTER1.1 or NRT1.1) promotes basipetal auxin transport (from the LR tip towards shoot) to inhibit auxin accumulation in LR initials. At high $\mathrm{NO}_{3}{ }^{-}$levels, NRT1.1-dependent auxin transport out of the LRs is inhibited, leading to the accumulation of auxin in LR initials and promotion of LR growth (Krouk et al., 2010; Gojon et al., 2011; Bouguyon et al., 2012).

Phosphorus: Phosphorus starvation-induced cluster root formation in Lupinus albus involved the signaling of endogenous phytohormones like auxins and cytokinins (Gilbert et al., 1998, 2000; Neumann et al., 2000). The auxin-transport inhibitors 2,3,5-triiodobenzoic acid (TIBA) and N-(1-napthyl) phtalamic acid (NPA) were found to inhibit the formation of LRs under phosphorus deficiency in Lupinus albus (Gilbert et al., 2000). Phosphorus starvation in Arabidopsis potentially downregulated expression of some auxin regulated genes, suggesting a link between phosphate deficiency and auxin (Hammond et al., 2003). Arabidopsis plants growing under limiting $(1 \mathrm{mM})$ phosphorus concentration were more sensitive to auxins in terms of the inhibition of primary root elongation and increase of LR density, suggesting that changes in auxin sensitivity play an important role in the effect of phosphorus deprivation on root architecture (Lopez-Bucio et al., 2002). Moreover, phosphorus deficiency was found to change root system architecture via modifying local auxin 
concentrations within the root system, through changes in auxin transport rather than auxin synthesis (Nacry et al., 2005).

Potassium: Several reports suggesting links between potassium and auxin transport/signaling are available (Cao et al., 1993; Philippar et al., 1999; Vicente-Agullo et al., 2004; Ashley et al., 2005; Zhang et al., 2009b). The growth of maize coleoptiles was found to be mediated via auxin-regulated expression of ZMK1, an inwardly rectifying potassium channel (Philippar et al., 1999). TRH1 potassium transporter, which regulates root hair development and root gravitropic behavior in Arabidopsis, was found crucial for auxin transport (Vicente-Agullo et al., 2004). Evidence for the role of auxin-dependent processes in acclimation to $\mathrm{K}^{+}$deficiency was provided by demonstrating that the CYP79B2 and CYP79B3 genes involved in the tryptophan-dependent auxin biosynthesis were downregulated upon $\mathrm{K}^{+}$resupply to $\mathrm{K}^{+}$-starved roots (Armengaud et al., 2004). Recent transcriptome analyses of rice roots subjected to $\mathrm{K}^{+}$deficiency have identified a large number of auxin-related genes (Ma et al., 2012), which is further evidence for auxin regulation of root responses to $\mathrm{K}^{+}$deficiency. More recently, it was found that potassium deficiency reduced root growth, auxin concentration, $\left[{ }^{3} \mathrm{H}\right]$ IAA transport, and the expression levels of PIN genes in tobacco plants. However, application of exogenous auxin (NAA) under potassium deficiency increased LR formation and elongation (Song et al., 2015).

Sulfur: Sulfur deficiency increased accumulation of nitrilase (NIT3) gene, which encodes an enzyme that catalyzes the transformation of indole-3-acetonitrile to IAA in Arabidopsis. High NIT3 expression leads to high auxin production and increased root growth, thus allowing the root system to penetrate more to gain access to sulfur (Kutz et al., 2002). Upregulation of genes involved in auxin biosynthesis (including myrosinase and nitrilase) and auxin response under sulfur deficiency was also reported by Nikiforova et al. (2003). Induction of genes involved in auxin synthesis upon S-starvation was also reported by Kopriva and Rennenberg (2004) and Lewandowska and Sirko (2008). Sequences named sulfur-responsive elements (SURE), which are very similar to the auxin response factor (ARF) binding sites, were identified in the promoter of many sulfur-responsive genes (MaruyamaNakashita et al., 2005). Nikiforova et al. (2005) suggested that auxin signaling under sulfur deficiency is highly regulated. Sulfur deficit causes a surplus increase of auxin and the activation of auxin-induced genes. The altered auxin content triggers changes in free calcium levels in plant cells, which are sensed by calmodulin. Activated calmodulin influences the expression of the IAA28 gene, which in turn represses the transcription of auxin-induced genes, thus providing the feedback control of the auxin signaling pathway (Nikiforova et al., 2005).

A genomic study showed that the BIG gene, which encodes a protein necessary for the polar transport of auxin, is involved in regulation of sulfur deficiency-responsive genes in Arabidopsis thaliana (Kasajima et al., 2007). Many auxin-relevant TFs like IAA13, IAA28, and ARF-2 were found to serve as coordinators of the metabolic shifts driving sulfur homeostasis (Falkenberg et al., 2008). The role of various MYBs TFs in the regulation of sulfur assimilation enzymes, and their involvement in mobilization of auxin and LR formation under S deficiency, was confirmed by Frerigmann and Gigolashvili (2014).

Iron: Auxin plays a key role in altering root system architecture under variable iron availability. The induction of Fe deficiency stress responses has been linked to an increase of auxin in root tips (Landsberg, 1984; Romheld and Marschner, 1986). Possibly, the effect of auxin could be through ethylene, since high levels of auxin promote ethylene production by inducing the synthesis of ACC synthase (Kim et al., 1992). Low $\mathrm{Fe}$ availability frequently leads to the formation of branched root hairs (Muller and Schmidt, 2004) through a signaling cascade that involves auxin and ethylene (Schmidt et al., 2000; Schmidt and Schikora, 2001).

Several studies revealed that application of exogenous auxin analogs promoted the induction of root ferric chelate reductase (FCR) activity in Phaseolus vulgaris (Li et al., 2000), Cucumis sativus ( $\mathrm{Li}$ and Li, 2004), and Trifolium pratense (Zheng et al., 2003), which enhanced $\mathrm{Fe}$ uptake under $\mathrm{Fe}$ deficient condition. Chen et al. (2010) concluded that NO acts downstream of auxin to activate root FCR activity under Fe deficiency. NO-mediated root hair development in Arabidopsis and lettuce in response to auxins were also reported by Lombardo et al. (2006). Fe-deficiency increased auxin and NO levels in Arabidopsis and the increase was greater when exogenous auxin was applied, but suppressed by an inhibitor of PAT (Chen et al., 2010). It was concluded that Fe deficiency responses involve the interplay of auxin, ethylene, and NO; however, auxin acts upstream of ethylene and NO (Romera et al., 2011).

Localized availability of iron upregulated AUX1 gene to accumulate auxin in LR apices and induced LR elongation (Giehl et al., 2012). Removal of shoot apex in sunflower, bean plants, and Malus xiaojinensis arrested the rhizospheric acidification induced by $\mathrm{Fe}$ deficiency (Landsberg, 1981, 1984; Wu et al., 2012). However, exogenous application of auxin to the detopped shoots of Pyrus betulifolia recovered the Fe deficiency response (upregulation of citrate synthase ( $P b C S 1)$ expression, which is involved in the synthesis 
of citric acid that acts as a chelate substance for the long-distance transmission of Fe in the root system). In contrast, NPA (auxin transport inhibitor) application to the shoot tips arrested upregulation of the PbCS1 expression. Thus, it was suggested that auxin is involved in mediating Fe-deficient response through regulation of the PbCS1 expression (Li et al., 2016).

\subsubsection{Heavy Metal Stress}

Heavy metals are required in trace amount for the proper growth of the plant, but in excess concentration, they adversely affect the plant normal metabolic and developmental functions (Gill and Tuteja, 2010; Khan and Khan, 2014, 2017; Pandey and Gupta, 2015; Khan et al., 2016). Response of plants to heavy metal stress is mediated in part via auxin signaling. Heavy metal-induced decrease of cell expansion growth in wheat (Lane et al., 1978) and maize (Hasenstein et al., 1988) is attributed to alteration of auxin metabolism and transport. Auxin-mediated inhibition of cell elongation arises either from direct effects of heavy metals on auxin or due to their effect on proton extrusion (Barceló and Poschenrieder, 1990). Auxin signaling was found to be involved in heavy metal induced curvature of the root (away from metal ions) in Zea mays. Treatment of maize roots with auxin transport inhibitors such as 2,3,5-triiodobenzoic acid (TIBA) or naphthylphthalamic acid (NPA) prevented curvature in response to unilateral application of these ions, indicating the significance of auxin transport and distribution (Hasenstein et al., 1988). Link between auxin signaling and heavy metal stress has been studied by many researchers using different metal ions.

Aluminum: Aluminum toxicity is a major problem in acidic soil (Foy, 1988), which causes rapid inhibition of root growth (Doncheva et al., 2005). This aluminuminduced inhibition of root growth is associated with disruption of auxin transport and/or signaling in Zea mays (Kollmeier et al., 2000) and Arabidopsis (Sun et al., 2010). It was reported that $\mathrm{Al}^{3+}$ upregulated the expression of PIN2 (a key component for mediating basipetal auxin transport) and inhibited transport of PIN2 vesicles from plasma membranes to endosomes in Arabidopsis, leading to reductions in auxin concentration in root apical cells (Shen et al., 2008). Likewise, transcriptional levels of AtAUX1 (gene encoding auxin influx protein) were also enhanced when exposed to $\mathrm{Al}^{3+}$, thus indicating that $\mathrm{Al}^{3+}$ may target the PIN2and AUX1-mediated auxin transport system, leading to disruption of auxin distribution in roots and inhibition of root elongation (Sun et al., 2010). Yang et al. (2011) showed that wheat plants cope with aluminum toxicity through the efflux of organic acids (malic acid), which is mediated through auxin.
Lead: Mukherji and Maitra (1977) reported that leadinduced reduction in germination and growth of rice is associated with increased IAA-oxidase activity. Moreover, the application of IAA was found to be partially capable of relieving lead toxicity. Lane et al. (1978) reported that high lead concentration caused reduction in the rate of cell division and elongation in Triticum aestivum, which was mediated through the action of auxin. Microarray study in Arabidopsis showed that lead treatment activated many genes involved in IAA biosynthesis, suggesting a possible role of auxin in heavy metal induced responses (Liu et al., 2009).

Cadmium: Crosstalk between cadmium stress signaling and auxin signaling pathway has been suggested by many researchers (Hagen et al., 1988; Moons, 2003; Ganesan, 2008). Cadmium (20 and $100 \mu \mathrm{M})$ caused oxidative stress, lipoperoxidation, and reduced growth in Pisum sativum seedlings, which is ascribed due to an elevation in the activities of IAA-oxidase and lignifying peroxidases. Enhanced activity of IAAoxidase contributed to auxin degradation and reduced plant growth under heavy metal stress (Chaoui and El Ferjani, 2005). At the molecular level, cadmium stress was found to induce a number of miRNAs in rice (Huang et al., 2009) that function in auxin signaling (Ding and $\mathrm{Zhu}$, 2009). Cation exchanger $\mathrm{H}^{+}$/ cation antiporter is required to induce auxin-mediated root growth in Arabidopsis under cadmium stress (Mei et al., 2009).

Copper: Copper toxicity $(100 \mu \mathrm{M})$ induced a reduction in the growth of Pisum sativum seedlings and this reduction was found to be mediated through the enhanced activity of IAA-oxidase (Chaoui and El Ferjani, 2005). Yuan et al. (2013) reported that coppermediated inhibition of primary root elongation is due to copper-mediated auxin redistribution in Arabidopsis seedlings. Genetic and physiological analysis demonstrated that PIN1, but not PIN2 or AUX1, regulated this process (Yuan et al., 2013).

Arsenic: Auxin also plays an important role in plant tolerance to arsenic-induced oxidative stress (Krishnamurthy and Rathinasabapathi, 2013). Auxin transporter mutants aux1, pin1, and pin2 were significantly more sensitive to As(III) than the wild-type plants. However, exogenous supply of IAA improved As(III) tolerance of aux1 mutant via reactive oxygen species (ROS)-mediated signaling (Krishnamurthy and Rathinasabapathi, 2013). Pandey and Gupta (2015) concluded that application of selenium and auxin alone or in combination were very effective in lowering the As (III) induced stress in rice. Improved arsenic tolerance in the presence of auxin involves physiological, biochemical, and molecular interaction in a synergistic or additive way. 


\subsubsection{Crosstalk of Auxin With Other Hormones}

Auxin interacts with other phytohormones to mediate various signaling responses under stress conditions. One such response includes changes in root architecture, which is mediated by interaction of auxin with other hormones like ABA (Saini et al., 2013), ethylene, and cytokinins (Harrison, 2012). Several miRNAs that are associated with auxin signaling (miR167 and miR168) and are positive regulators of adventitious root development in Arabidopsis contain ABREs, indicating that they are regulated by ABA signaling (Liu et al., 2008).

Arbona and Gómez-Cadenas (2008) found that under prolonged soil waterlogging, plants increased their IAA levels and reduced ABA levels; thereby promoted LR growth. $\mathrm{Xu}$ et al. (2013) found that under moderate drought stress, endogenous ABA accumulation in root tips of Arabidopsis and rice stimulated transport of auxin into the root apex and induced root growth. The transcript levels of AUX1 and PIN2 increased in the root tips under ABA stimuli, suggesting the interaction of auxin and ABA during stressinduced root growth (Xu et al., 2013). Similarly, the increased tolerance to cold stress is due to the combined effects of IAA and ABA. The change in auxin homeostasis affected ABA synthesis under drought and cold stress in rice, and the resulting balance of auxin and ABA homeostasis played a crucial role in stress responses (Du et al., 2012; Du et al., 2013b). Taniguchi et al. (2010) suggested the involvement of ABA in hydrotropic response of roots through modulation in auxin.

Stress response in plants is also dependent on interaction of auxin with ethylene. In a study, adventitious root development in response to flooding was found to be regulated by auxin mediated ethylene production (Vidoz et al., 2010; Muday et al., 2012). Flooded tomato plants treated with the aminoethoxyvinylglycine (ethylene biosynthesis inhibitor) and 1-naphthylphthalamic acid (auxin transport inhibitor) were unable to form adventitious roots. Auxin accumulation in stem upon waterlogging triggers de novo ethylene synthesis, which stimulates the transport of auxins towards the flooded parts of the plant and induced the root development under stress (Vidoz et al., 2010). Ethylene also stimulates enhanced sensitivity to auxins under temporary submergence (Park et al., 2011).

O'Brien and Benková (2013) suggested the possible antagonistic role of cytokinin and auxin in plant defense responses. Dynamic and complementary actions of auxin and cytokinins pathways, along with their crosstalk, regulate a plethora of developmental processes under stress (Bielach et al., 2017). Rowe et al. (2016) suggested that root growth under osmotic stress is regulated by a hormonal network of auxin, cytokinin, $\mathrm{ABA}$, and ethylene. It was shown that PIN1 levels were reduced under osmotic stress in an ABA-dependent manner, overriding ethylene effects. Recently, Liu et al. (2017) found that hormonal crosstalk between auxin, cytokinin, and ethylene regulate root development in Arabidopsis.

\subsection{CONCLUSION}

Auxin is an important phytohormone that plays a crucial role in regulating many developmental processes in plants. Its amount is modulated in plants in

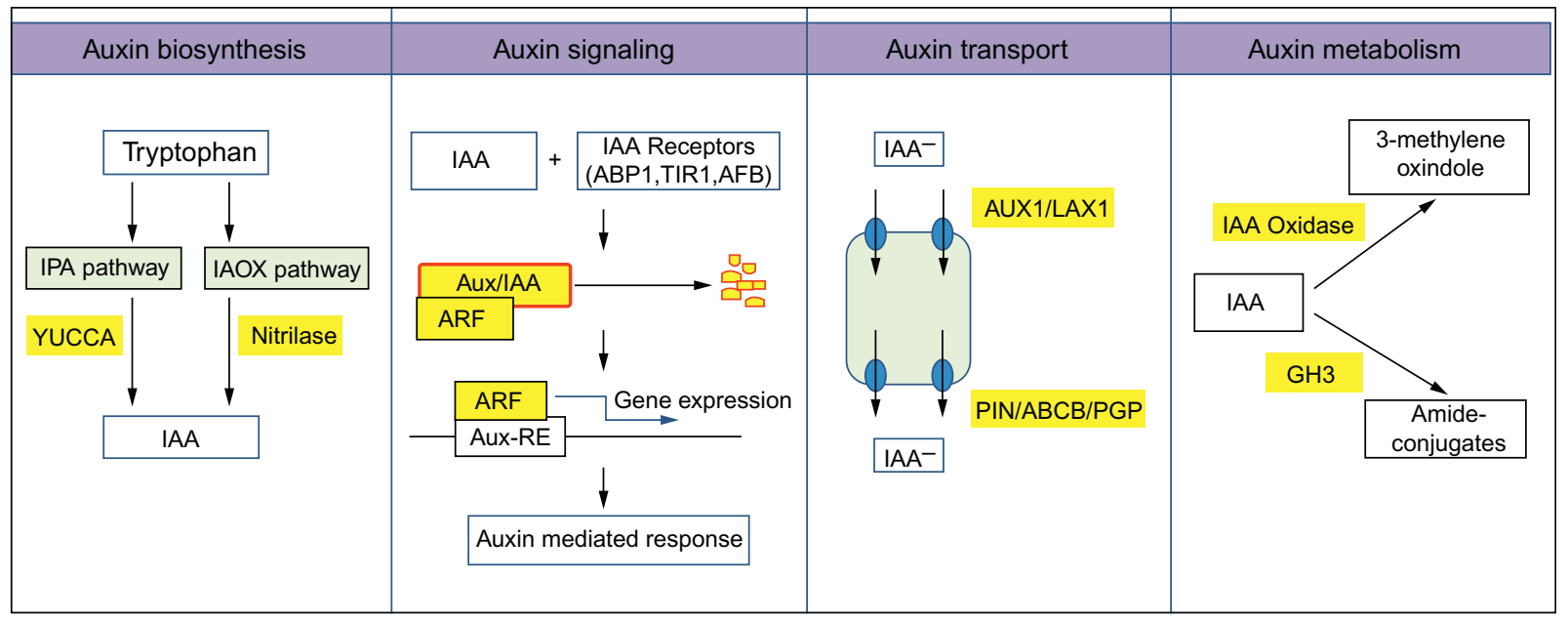

FIGURE 20.1 Components (shown in yellow) of auxin biosynthesis, signaling, transport, and metabolism, which can be targeted for enhancing abiotic stress tolerance in plants. $A B P 1$, auxin-binding protein; $A F B$, auxin F-Box; $A R F$, auxin response factor; $A u x-R E$, auxin responsive elements; IAOX, indole-3-acetaldoxime; IPA, indole-3-pyruvic acid; TIR1, transport inhibitor response 1. 
response to different environmental signals and provides developmental plasticity to plants. It is an essential signaling hormone and regulates plant growth and development under various abiotic stresses like heavy metal stress, nutrient deficiency, drought, salinity, and temperature stress. Roots, which are a key component for water and nutrient absorption, are also involved in sensing environmental signals. The role of auxin in mediating LR development is quite critical under stress conditions. Thus, modulation of auxin concentration in tissue specific manner can be a potential strategy to change root system architecture and to induce stress tolerance. Various auxin biosynthesis (YUCCA gene), metabolism (IAA-oxidase), conjugation (IAA-amidosynthetases), transport (PIN, AUX1/LAX1, $\mathrm{ABCB}$ carriers), or signaling (Aux/IAA, ARF) components have been identified in plants, which can be good candidate genes for improving abiotic stress tolerance in plants (Fig. 20.1). However, many auxinsignaling components and mechanisms are yet to be unraveled to increase auxin mediated tolerance in plants. A major challenge that remains to be addressed is the development of stable auxin-engineered crops that perform well under stressed as well as unstressed conditions. Towards this goal, study should be focused on development of transgenic crop plants that exhibit enhanced stress tolerance with no penalty on yield under unstressed conditions.

\section{Acknowledgements}

First author wants to thank Late Prof. Alok Shukla, Department of Plant Physiology, GBPUAT, Pantnagar, for his initial guidance, motivation and encouragement. Dr. Sandeep Rawat is acknowledged for critically reading the article. Authors also acknowledge Himalayan RA scheme and Mountain Division ( $5^{\text {th }}$ Unit of GBPNIHESD) for providing necessary facilities and infrastructural support.

\section{References}

Arbona, V., Gómez-Cadenas, A., 2008. Hormonal modulation of citrus responses to flooding. J. Plant Growth Regul. 27, 241-250.

Armengaud, P., Breitling, R., Amtmann, A., 2004. The potassiumdependent transcriptome of Arabidopsis reveals a prominent role of jasmonic acid in nutrient signalling. Plant Physiol. 136, 2556-2576.

Ashley, M.K., Grant, M., Grabov, A., 2005. Plant responses to potassium deficiencies: a role for potassium transport proteins. J. Exp. Bot. 57 (2), 425-436.

Bacaicoa, E., Mora, V., Zamarreño, A.M., Fuentes, M., Casanova, E., García-Mina, J.M., 2011. Auxin: a major player in the shoot-toroot regulation of root Fe-stress physiological responses to Fe deficiency in cucumber plants. Plant Physiol. Biochem. 49 (5), 545-556.

Bao, F., Li, J.Y., 2002. Evidence that the auxin signaling pathway interacts with plant stress response. Acta Botanica Sinica 44, $532-536$.
Barbez, E., Kubes, M., Rolcík, J., Beziat, C., Pencík, A., Wang, B., et al., 2012. A novel putative auxin carrier family regulates intracellular auxin homeostasis in plants. Nature 485, 119-122.

Barceló, J.U.A.N., Poschenrieder, C., 1990. Plant water relations as affected by heavy metal stress: a review. J. Plant. Nutr. 13 (1), $1-37$.

Bielach, A., Hrtyan, M., Tognetti, V.B., 2017. Plants under stress: involvement of auxin and cytokinin. Int. J. Mol. Sci. 18, 1427.

Bouguyon, E., Gojon, A., Nacry, P., 2012. Nitrate sensing and signaling in plants. Sem. Cell. Dev. Biol. 23, 648-654.

Cao, Y., Glass, A.D., Crawford, N.M., 1993. Ammonium inhibition of Arabidopsis root growth can be reversed by potassium and by auxin resistance mutationsaux1, axr1, and axr2. Plant Physiol. 102, 983-989.

Cha, J.Y., Kim, W.Y., Kang, S.B., Kim, J.I., Baek, D., Jung, I.J., et al., 2015. A novel thiol-reductase activity of Arabidopsis YUC6 confers drought tolerance independently of auxin biosynthesis. Nat. Commun. 6, 8041.

Chaoui, A., El Ferjani, E., 2005. Effects of cadmium and copper on antioxidant capacities, lignification and auxin degradation in leaves of pea (Pisum sativum L.) seedlings. C. R. Biol. 328 (1), 23-31.

Chen, W.W., Yang, J.L., Qin, C., Jin, C.W., Mo, J.H., Ye, T., et al., 2010. Nitric oxide acts downstream of auxin to trigger root ferricchelate reductase activity in response to iron deficiency in Arabidopsis thaliana. Plant Physiol. 154 (2), 810-819.

Cheng, Y., 2006. Auxin biosynthesis by the YUCCA flavin monooxygenases controls the formation of floral organs and vascular tissues in Arabidopsis. Genes Dev. 20, 1790-1799.

Dharmasiri, N., Dharmasiri, S., Estelle, M., 2005. The F-box protein TIR1 is an auxin receptor. Nature $435,441-445$.

Ding, D., Zhang, L., Wang, H., Liu, Z., Zhang, Z., Zheng, Y., 2009. Differential expression of miRNAs in response to salt stress in maize roots. Ann. Bot. (Lond.) 103 (1), 29-38.

Ding, Y.F., Zhu, C., 2009. The role of microRNAs in copper and cadmium homeostasis. Biochem. Biophys. Res. Commun. 386 (1), 6-10.

Doncheva, S., Amenos, M., Poschenrieder, C., Barcelo, J., 2005. Root cell patterning: a primary target for aluminium toxicity in maize. J. Exp. Bot. 56, 1213-1220.

Du, H., Wu, N., Fu, J., Wang, S., Li, X., Xiao, J., et al., 2012. A GH3 family member, OsGH3-2, modulates auxin and abscisic acid levels and differentially affects drought and cold tolerance in rice. J. Exp. Bot. 63, 6467-6480.

Du, H., Liu, H., Xiong, L., 2013a. Endogenous auxin and jasmonic acid levels are differentially modulated by abiotic stresses in rice. Front. Plant Sci. 4, 397.

Du, H., Wu, N., Chang, Y., Li, X., Xiao, J., Xiong, L., 2013b. Carotenoid deficiency impairs ABA and IAA biosynthesis and differentially affects drought and cold tolerance in rice. Plant Mol. Biol. 83, 475-488.

El-Abd, S.O., El-Beltagy, A.S., Hall, M.A., 1986. Physiological studies on flowering and fruit set in tomatoes. ActaHorticulturae 190, 389-396.

Falkenberg, B., Witt, I., Zanor, M.I., Steinhauser, D., Mueller-Roeber, B., Hesse, H., 2008. Transcription factors relevant to auxin signalling coordinate broad-spectrum metabolic shifts including sulphur metabolism. J. Exp. Bot. 59 (10), 2831-2846.

Foy, C.D., 1988. Plant adaptation to acid, aluminum-toxic soils. Commun. Soil. Sci. Plant. Anal. 19, 959-987.

Franco, A.R., Gee, M.A., Guilfoyle, T.J., 1990. Induction and superinduction of auxin-responsive mRNAs with auxin and protein synthesis inhibitors. J. Biol. Chem. 265, 15845-15849.

Frerigmann, H., Gigolashvili, T., 2014. Update on the role of R2R3$M Y B s$ in the regulation of glucosinolates upon sulfur deficiency. Front. Plant Sci. 5, 626. 
Fukaki, H., Fujisawa, H., Tasaka, M., 1996. Gravitropic response of inflorescence stems in Arabidopsis thaliana. Plant Physiol. 110, 933-943.

Ganesan, V., 2008. Rhizoremediation of cadmium soil using a cadmium-resistant plant growth-promoting rhizopseudomonad. Curr. Microbiol. 56, 403-407.

Giehl, R.F., Lima, J.E., vonWiren, N., 2012. Localized iron supply triggers lateral root elongation in Arabidopsis by altering the AUX1-mediated auxin distribution. Plant Cell 24, 33-49.

Gifford, M.L., Dean, A., Gutierrez, R.A., Coruzzi, G.M., Birnbaum, K.D., 2008. Cell-specific nitrogen responses mediate developmental plasticity. Proc. Natl. Acad. Sci. USA 105 (2), 803-808.

Gilbert, G.A., Vance, C.P., Allan, D.L., 1998. Regulation of white lupin root metabolism by phosphorus availability. Curr. Top. Plant Physiol. 19, 157-167.

Gilbert, G.A., Knight, J.D., Vance, C.P., Allan, D.L., 2000. Proteoid root development of phosphorus deficient lupin is mimicked by auxin and phosphonate. Ann. Bot. (Lond.) 85 (6), 921-928

Gill, S.S., Tuteja, N., 2010. Reactive oxygen species and antioxidant machinery in abiotic stress tolerance in crop plants. Plant. Physiol. Biochem. 48, 909-930.

Gojon, A., Krouk, G., Perrine-Walker, F., Laugier, E., 2011. Nitrate transceptor(s) in plants. J. Exp. Bot. 62, 2299-2308.

Guilfoyle, T.J., Hagen, G., 2007. Auxin response factors. Curr. Opin. Plant. Biol. 10, 453-460.

Hagen, G., Uhrhammer, N., Guilfoyle, T.J., 1988. Regulation of expression of an auxin-induced soybean sequence by cadmium. J. Biol. Chem. 263, 6442-6446.

Hammond, J.P., Bennett, M.J., Bowen, H.C., Broadley, M.R., Eastwood, D.C., May, S.T., et al., 2003. Changes in gene expression in Arabidopsis shoots during phosphate starvation and the potential for developing smart plants. Plant Physiol. 132 (2), 578-596.

Harrison, M.A., 2012. Cross-talk between phytohormones signaling pathways under both optimal and stressful environmental conditions. Phytohormones and Abiotic Stress Tolerance in Plants. Springer, Berlin, Heidelberg, pp. 49-76.

Hasenstein, K.H., Evans, M.L., Stinemetz, C.L., Moore, R., Fondren, W.M., Koon, E.C., et al., 1988. Comparative effectiveness of metal ions in inducing curvature of primary roots of Zea mays. Plant Physiol. 86, 885-889.

He, X.J., Mu, R.L., Cao, W.H., Zhang, Z.G., Zhang, J.S., Chen, S.Y., 2005. AtNAC2, a transcription factor downstream of ethylene and auxin signaling pathways, is involved in salt stress response and lateral root development. Plant J. 44 (6), 903-916.

Ho, C.H., Lin, S.H., Hu, H.C., Tsay, Y.F., 2009. CHL1 functions as a nitrate sensor in plants. Cell 138, 1184-1194.

Huang, S.Q., Peng, J., Qiu, C.X., Yang, Z.M., 2009. Heavy metalregulated new microRNAs from rice. J. Inorg. Biochem. 103 (2), 282-287.

Huberman, M., Riov, J., Aloni, B., Goren, R., 1997. Role of ethylene biosynthesis and auxin content and transport in high temperature-induced abscission of pepper reproductive organs. J. Plant. Growth Regul. 16 (3), 129-135.

Iglesias, M.J., Terrile, M.C., Bartoli, C.G., D’Ippólito, S., Casalongué, C.A., 2010. Auxin signaling participates in the adaptative response against oxidative stress and salinity by interacting with redox metabolism in Arabidopsis. Plant Mol. Biol. 74 (3), 215-222

Iglesias, M.J., Terrile, M.C., Windels, D., Lombardo, M.C., Bartoli, C. G., Vazquez, F., et al., 2014. MiR393 regulation of auxin signaling and redox-related components during acclimation to salinity in Arabidopsis. PLoS. ONE. 9 (9), e107678.
Jain, M., Khurana, J.P., 2009. Transcript profiling reveals diverse roles of auxin-responsive genes during reproductive development and abiotic stress in rice. FEBS J. 276, 3148-3162.

Jung, J.H., Park, C.M., 2011. Auxin modulation of salt stress signaling in Arabidopsis seed germination. Plant Signal. Behav. 6 (8), $1198-1200$

Kasajima, I., Ohkama-Ohtsu, N., Ide, Y., Hayashi, H., Yoneyama, T., Suzuki, et al., 2007. The BIG gene is involved in regulation of sulfur deficiency-responsive genes in Arabidopsis thaliana. Physiol. Plant. 129 (2), 351-363.

Kazan, K., 2013. Auxin and the integration of environmental signals into plant root development. Ann. Bot. (Lond.) 112, 1655-1665.

Ke, Q., Wang, Z., Ji, C.Y., Jeong, J.C., Lee, H.S., Li, H., et al., 2015. Transgenic poplar expressing Arabidopsis YUCCA6 exhibits auxinoverproduction phenotypes and increased tolerance to abiotic stress. Plant. Physiol. Biochem. 94, 19-27.

Kepinski, S., Leyser, O., 2005. The Arabidopsis F-box protein TIR1 is an auxin receptor. Nature 435, 446-451.

Khan, M.I.R., Khan, N., 2017. Reactive Oxygen Species and Antioxidant System in Plants: Role and Regulation Under Abiotic Stress. Springer Nature, Singapore978-981-10-5254-5.

Khan, M.I.R., Khan, N.A., 2014. Ethylene reverses photosynthetic inhibition by nickel and zinc in mustard through changes in PS II activity, photosynthetic-nitrogen use efficiency and antioxidant metabolism. Protoplasma 251, 1007-1019.

Khan, M.I.R., Khan, N.A., Masood, A., Per, T.S., Asgher, M., 2016. Hydrogen peroxide alleviates nickel-inhibited photosynthetic responses through increase in use-efficiency of nitrogen and sulfur, and glutathione production in mustard. Front. Plant Sci. 7, 44.

Kiba, T., Kudo, T., Kojima, M., Sakakibara, H., 2011. Hormonal control of nitrogen acquisition: roles of auxin, abscisic acid, and cytokinin. J. Exp. Bot. 62 (4), 1399-1409.

Kim, J., Harter, K., Theologis, A., 1997. Protein-protein interactions among the Aux/IAA proteins. Proc. Natl. Acad. Sci. U.S.A. 94, 11786-11791.

Kim, J.I., Baek, D., Park, H.C., Chun, H.J., Oh, D.H., Lee, M.K., et al., 2013. Over-expression of Arabidopsis YUCCA6 in potato results in high-auxin developmental phenotypes and enhanced resistance to water deficit. Mol. Plant 6, 337-349.

Kim, W.T., Silberstone, A., Yip, W.K., Dong, J.G., Yang, S.F., 1992. Induction of 1-aminocyclopropane-1-carboxylate synthase mRNA by auxin in mung bean hypocotyls and cultured apple shoots. Plant Physiol. 98, 465-471.

Kollmeier, M., Felle, H.H., Horst, W.J., 2000. Genotypical differences in aluminum resistance of maize are expressed in the distal part of the transition zone. Is reduced basipetal auxin flow involved in inhibition of root elongation by aluminum? Plant Physiol. 122 (3), 945-956.

Kopriva, S., Rennenberg, H., 2004. Control of sulphate assimilation and glutathione synthesis: interaction with $\mathrm{N}$ and $\mathrm{C}$ metabolism. J. Exp. Bot. 55 (404), 1831-1842.

Krishnamurthy, A., Rathinasabapathi, B., 2013. Auxin and its transport play a role in plant tolerance to arsenite-induced oxidative stress in Arabidopsis thaliana. Plant Cell Environ. 36 (10), 1838-1849.

Krouk, G., Lacombe, B., Bielach, A., Perrine-Walker, F., Malinska, K. Mounier, E., et al., 2010. Nitrate-regulated auxin transport by NRT1.1 defines a mechanism for nutrient sensing in plants. Dev. Cell. 18, 927-937.

Kuo, C.G., Tsai, C.T., 1984. Alternation by high temperature of auxin and gibberellin concentrations in the flower buds, flower and young fruit of tomato. HortScience 19, 870-872.

Kutz, A., Muller, A., Hennig, P., Kaiser, W.M., Piotrowski, M., Weiler, E.W., 2002. A role for nitrilase 3 in the regulation of root morphology in sulphur-starving Arabidopsis thaliana. Plant J. 30, 95-106. 
Landsberg, E.C., 1981. Energy driven H + efflux pump in sunflower roots: activated by Fe-deficiency stress. Plant Physiol. 67, 702.

Landsberg, E.C., 1984. Regulation of iron-stress-response by wholeplant activity. J. Plant. Nutr. 7, 609-621.

Lane, S.D., Martin, E.S., Garrod, J.P., 1978. Lead toxicity effect on indole-3-acetic-induced cell elongation. Planta 144, 79-84.

Lee, M., Jung, J.H., Han, D.Y., Seo, P.J., Park, W.J., Park, C.M., 2012. Activation of a flavin monooxygenase gene YUCCA7 enhances drought resistance in Arabidopsis. Planta. 235, 923-938.

Lee, S.C., Luan, S., 2012. ABA signal transduction at the crossroad of biotic and abiotic stress responses. Plant Cell Environ. 35, 53-60.

Lewandowska, M., Sirko, A., 2008. Recent advances in understanding plant response to sulfur-deficiency stress. Acta Biochim. Pol. 55 (3), 457-471.

Li, C., Zhu, X., Zhang, F., 2000. Role of shoot in regulation of iron deficiency responses in cucumber and bean plants. J. Plant. Nutr. 23, 1809-1818.

Li, H., Johnson, P., Stepanova, A., Alonso, J.M., Ecker, J.R., 2004. Convergence of signaling pathways in the control of differential cell growth in Arabidopsis. Dev. Cell. 7, 193-204.

Li, X., Gao, Y., Li, Y., Yan, S., Zhang, J., Zhang, J., 2016. Characterization and expression analysis of $P b C S 1$ gene regulated by iron deficiency and involved in response to auxin in Pyrus betulifolia. Hort. Plant J. 2 (5), 253-260.

$\mathrm{Li}, \mathrm{X} ., \mathrm{Li}, \mathrm{C} ., 2$ 2004. Is ethylene involved in regulation of root ferric reductase activity of dicotyledonous species under iron deficiency. Plant Soil 261, 147-153.

Liu, H.H., Tian, X., Li, Y.J., Wu, C.A., Zheng, C.C., 2008. Microarraybased analysis of stress-regulated microRNAs in Arabidopsis thaliana. RNA $14,836-843$

Liu, J., Moore, S., Chen, C., Lindsey, K., 2017. Crosstalk complexities between auxin, cytokinin and ethylene in Arabidopsis root development: from experiments to systems modelling, and back again. Mol. Plant 10 (12), 1480-1496.

Liu, T., Liu, S., Guan, H., Ma, L., Chen, Z., Gu, H., et al., 2009. Transcriptional profiling of Arabidopsis seedlings in response to heavy metal lead (Pb). Environ. Exp. Bot. 67 (2), 377-386.

Liu, W., Li, R.J., Han, T.T., Cai, W., Fu, Z.W., Lu, Y.T., 2015. Salt stress reduces root meristem size by nitric oxide-mediated modulation of auxin accumulation and signaling in Arabidopsis. Plant Physiol. 168 (1), 343-356.

Lombardo, M.C., Graziano, M., Polacco, J.C., Lamattina, L., 2006. Nitric oxide functions as a positive regulator of root hair development. Plant Signal. Behav. 1, 28-33.

Lopez-Bucio, J., Hernandez-Abreu, E., Sanchez-Calderon, L., Nieto Jacobo, M.F., Simpson, J., Herrera-Estrella, L., 2002. Phosphate availability alters architecture and causes changes in hormone sensitivity in the Arabidopsis root system. Plant Physiol. 129, 244-256.

Ma, L., Wu, W.H., Wang, Y., 2012. Transcriptome analysis of rice root responses to potassium deficiency. BMC Plant Biol. 12, 161.

Mano, Y., Nemoto, K., 2012. The pathway of auxin biosynthesis in plants. J. Exp. Bot. 63 (8), 2853-2872.

Maruyama-Nakashita, A., Nakamura, Y., Watanabe-Takahashi, A., Inoue, E., Yamaya, T., Takahashi, H., 2005. Identification of a novel cis-acting element conferring sulfur deficiency response in Arabidopsis roots. Plant J. 42, 305-314.

Mei, H., Cheng, N.H., Zhao, J., Park, S., Escareno, R.A., Pittman, J.K., et al., 2009. Root development under metal stress in Arabidopsis thaliana requires the $\mathrm{H}^{+}$/cation antiporter CAX4. New Phytol. 183 (1), 95-105.

Moons, A., 2003. Ospdr9, which encodes a PDR-type ABC transporter, is induced by heavy metals, hypoxic stress and redox perturbations in rice roots. FEBS Lett. 553 (3), 370-376.
Morris, D.A., 1979. The effect of temperature on the velocity of exogenous auxin transport in intact chilling-sensitive and chillingresistant plants. Planta 146 (5), 603-605.

Muday, G.K., Rahman, A., Binder, B.M., 2012. Auxin and ethylene: collaborators or competitors? Trends. Plant. Sci. 17, 181-195.

Mukherji, S., Maitra, P., 1977. Growth and metabolism of germinating rice (Oryzasativa L.) seeds as influenced by toxic concentrations of lead. Int. J. Plant Physiol. 81, 26-33.

Muller, M., Schmidt, W., 2004. Environmentally induced plasticity of root hair development in Arabidopsis. Plant Physiol. 134, 409-419.

Mun, J.H., Yu, H.J., Shin, J.Y., Oh, M., Hwang, H.J., Chung, H., 2012. Auxin response factor gene family in Brassica rapa: genomic organization, divergence, expression, and evolution. Mol. Genet. Genomics. 287, 765-784.

Nacry, P., Canivenc, G., Muller, B., Azmi, A., Van Onckelen, H., Rossignol, M., et al., 2005. A role for auxin redistribution in the responses of the root system architecture to phosphate starvation in Arabidopsis. Plant Physiol. 138 (4), 2061-2074.

Neumann, G., Massonneau, A., Langlade, N., Dinkelaker, B., Hengeler, C., Römheld, V., et al., 2000. Physiological aspects of cluster root function and development in phosphorus-deficient white lupin (Lupinus albus L.). Ann. Bot. (Lond.) 85 (6), 909-919.

Nikiforova, V., Freitag, J., Kempa, S., Adamik, M., Hesse, H., Hoefgen, R., 2003. Transcriptome analysis of sulphur depletion in Arabidopsis thaliana: interlacing of biosynthetic pathways provides response specificity. Plant J. 33, 633-650.

Nikiforova, V.J., Daub, C.O., Hesse, H., Willmitzer, L., Hoefgen, R., 2005. Integrative gene-metabolite network with implemented causality deciphers informational fluxes of sulphur stress response. J. Exp. Bot. 56 (417), 1887-1896.

O’Brien, J.A., Benková, E., 2013. Cytokinin cross-talking during biotic and abiotic stress responses. Front. Plant Sci. 4, 451.

Ofir, M., Gross, Y., Bangerth, F., Kigel, J., 1993. High temperature effects on pod and seed production as related to hormone levels and abscission of reproductive structures in common bean (Phaseolus vulgaris L.). Sci. Hortic. (Amsterdam) 55, 201-211.

Oshino, T., Abiko, M., Saito, R., Ichiishi, E., Endo, M., KawagishiKobayashi, M., et al., 2007. Premature progression of anther early developmental programs accompanied by comprehensive alterations in transcription during high-temperature injury in barley plants. Mol. Genet. Genomics. 278, 31-42.

Oshino, T., Miura, S., Kikuchi, S., Hamada, K., Yano, K., Watanabe, M., et al., 2011. Auxin depletion in barley plants under hightemperature conditions represses DNA proliferation in organelles and nuclei via transcriptional alterations. Plant Cell Environ. 34, 284-290.

Paciorek, T., Zazimalova, E., Ruthardt, N., Petrasek, J., Stierhof, Y.D., Kleine-Vehn, J., et al., 2005. Auxin inhibits endocytosis and promotes its own efflux from cells. Nature 435, 1251-1256.

Pandey, C., Gupta, M., 2015. Selenium and auxin mitigates arsenic stress in rice (Oryza sativaL.) by combining the role of stress indicators, modulators and genotoxicity assay. J. Hazard. Mater. 287, 384-391.

Park, H.C., Cha, J.Y., Yun, D.J., 2013. Roles of YUCCAs in auxin biosynthesis and drought stress responses in plants. Plant Signal. Behav. 8, e24495.

Park, J.E., Park, J.Y., Kim, Y.S., Staswick, P.E., Jeon, J., Yun, J., et al., 2007. GH3-mediated auxin homeostasis links growth regulation with stress adaptation response in Arabidopsis. J. Biol. Chem. 282, 10036-10046.

Park, W.J., Hertel, R., Kang, B.G., 2011. Enhancement of auxin sensitivity in Ranunculussceleratusby ethylene: a mechanism to escape from hypoxia under temporary submergence. Environ. Exp. Bot. $72,266-271$. 
Philippar, K., Fuchs, I., Lüthen, H., Hoth, S., Bauer, C.S., Haga, K., et al., 1999. Auxin-induced $\mathrm{K}^{+}$channel expression represents an essential step in coleoptile growth and gravitropism. Proc. Natl. Acad. Sci. USA 96 (21), 12186-12191.

Popko, J., Hänsch, R., Mendel, R.R., Polle, A., Teichmann, T., 2010. The role of abscisic acid and auxin in the response of poplar to abiotic stress. Plant Biol. 12 (2), 242-258.

Romera, F.J., García, M.J., Alcántara, E., Pérez-Vicente, R., 2011. Latest findings about the interplay of auxin, ethylene and nitric oxide in the regulation of Fe deficiency responses by Strategy I plants. Plant Signal. Behav. 6 (1), 167-170.

Romheld, V., Marschner, H., 1986. Mobilization of iron in the rhizosphere of different plant species. Adv. Plant Nutr. 2, 155-204.

Rowe, J.H., Topping, J.F., Liu, J., Lindsey, K., 2016. Abscisic acid regulates root growth under osmotic stress conditions via an interacting hormonal network with cytokinin, ethylene and auxin. New Phytol. 211, 225-239.

Ruzicka, K., Ljung, K., Vanneste, S., Podhorska, R., Beeckman, T., Friml, J., et al., 2007. Ethylene regulates root growth through effects on auxin biosynthesis and transport-dependent auxin distribution. Plant Cell 19, 2197-2212.

Saini, S., Sharma, I., Kaur, N., Pati, P.K., 2013. Auxin: a master regulator in plant root development. Plant Cell Rep. 32, 741-757.

Sakata, T., Oshino, T., Miura, S., Tomabechi, M., Tsunaga, Y., Higashitani, N., et al., 2010. Auxins reverse plant male sterility caused by high temperatures. Proc. Natl. Acad. Sci. USA 107, 8569-8574.

Schmidt, W., Schikora, A., 2001. Different pathways are involved in phosphate and iron stress-induced alterations of root epidermal cell development. Plant Physiol. 125, 2078-2084.

Schmidt, W., Tittel, J., Schikora, A., 2000. Role of hormones in the induction of iron deficiency responses in Arabidopsis roots. Plant Physiol. 122, 1109-1118.

Seo, P.J., Xiang, F., Qiao, M., Park, J.Y., Lee, Y.N., Kim, S.G., et al., 2009. The MYB96 transcription factor mediates abscisic acid signaling during drought stress response in Arabidopsis. Plant Physiol. 151, 275-289.

Shani, E., Salehin, M., Zhang, Y., Sanchez, S.E., Doherty, C., Wang, R., et al., 2017. Plant stress tolerance requires auxin-sensitive Aux/IAA transcriptional repressors. Curr. Biol. 27 (3), 437-444.

Sharma, E., Sharma, R., Borah, P., Jain, M., Khurana, J.P., 2015. Emerging roles of auxin in abiotic stress responses. Elucidation of Abiotic Stress Signaling in Plants. Springer, New York, pp. 299-328.

Shen, C., Wang, S., Bai, Y., Wu, Y., Zhang, S., Chen, M., et al., 2010. Functional analysis of the structural domain of ARF proteins in rice (Oryza sativa L.). J. Exp. Bot. 61, 3971-3981.

Shen, H., Hou, N.Y., Schlicht, M., Wan, Y.L., Baluska, F., 2008. Aluminum toxicity targets PIN2 in Arabidopsis root apices: effects on PIN2 endocytosis vesicular recycling, and polar auxin transport. Chinese Sci. Bull. 53, 2480-2487.

Shi, H., Chen, L., Ye, T., Liu, X., Ding, K., Chan, Z., 2014. Modulation of auxin content in Arabidopsis confers improved drought stress resistance. Plant. Physiol. Biochem. 82, 209-217.

Shibasaki, K., Uemura, M., Tsurumi, S., Rahman, A., 2009. Auxin response in Arabidopsis under cold stress: underlying molecular mechanisms. Plant Cell 21, 3823-3838.

Shkolnik-Inbar, D., Bar-Zvi, D., 2010. ABI4 mediates abscisic acid and cytokinin inhibition of lateral root formation by reducing polar auxin transport in Arabidopsis. Plant Cell 22, 3560-3573.

Song, W., Liu, S., Meng, L., Xue, R., Wang, C., Liu, G., et al., 2015. Potassium deficiency inhibits lateral root development in tobacco seedlings by changing auxin distribution. Plant Soil 396 (2), $163-173$.
Song, Y., Wang, L., Xiong, L., 2009. Comprehensive expression profiling analysis of OsIAA gene family in developmental processes and in response to phytohormone and stress treatments. Planta 229, 577-591.

Stepanova, A.N., Alonso, J.M., 2005. Ethylene signaling and response pathway: a unique signaling cascade with a multitude of inputs and outputs. Physiol. Plant. 123, 195-206.

Stepanova, A.N., Yun, J., Likhacheva, A.V., Alonso, J.M., 2007. Multilevel interactions between ethylene and auxin in Arabidopsis roots. Plant Cell 19 (7), 2169-2185.

Sukumar, P., Edwards, K.S., Rahman, A., Delong, A., Muday, G.K., 2009. PINOID kinase regulates root gravitropism through modulation of PIN2-dependent basipetal auxin transport in Arabidopsis. Plant Physiol. 150, 722-735.

Sun, F.F., Zhang, W.S., Hu, H.Z., Li, B., Wang, Y.N., Zhao, Y.K., et al., 2008. Salt modulates gravity signaling pathway to regulate growth direction of primary roots in Arabidopsis thaliana. Plant Physiol. 146, 178-188.

Sun, P., Tian, Q.Y., Chen, J., Zhang, W.H., 2010. Aluminium-induced inhibition of root elongation in Arabidopsis is mediated by ethylene and auxin. J. Exp. Bot. 61, 347-356.

Taiz, L., Zeiger, E., 2002. Plant Physiology, third ed. Sinauer Associates, Inc. Publishers, Sunderland, USA, pp. 424-460.

Taniguchi, Y.Y., Taniguchi, M., Tsuge, T., Oka, A., Aoyama, T., 2010. Involvement of Arabidopsis thaliana phospholipase Dzeta 2 in root hydrotropism through the suppression of root gravitropism. Planta 231, 491-497.

Van Ha, C., Le, D.T., Nishiyama, R., Watanabe, Y., Sulieman, S., Tran, U.T., et al., 2013. The auxin response factor transcription factor family in soybean: genome-wide identification and expression analyses during development and water stress. DNA Res. 20 (5), 511-524.

Vicente-Agullo, F., Rigas, S., Desbrosses, G., Dolan, L., Hatzopoulos, P., Grabov, A., 2004. Potassium carrier TRH1 is required for auxin transport in Arabidopsis roots. Plant J. 40 (4), 523-535.

Vidal, E.A., Araus, V., Lu, C., Parry, G., Green, P.J., Coruzzi, G.M., et al., 2010. Nitrate-responsive miR393/AFB3 regulatory module controls root system architecture in Arabidopsis thaliana. Proc. Natl. Acad. Sci. USA 107 (9), 4477-4482.

Vidoz, M.L., Loreti, E., Mensuali, A., Alpi, A., Perata, P., 2010. Hormonal interplay during adventitious root formation in flooded tomato plants. Plant J. 63, 551-562.

Wang, S., Bai, Y., Shen, C., Shen, C., Wu, Y., Zhang, S., et al., 2010. Auxin-related gene families in abiotic stress response in Sorghum bicolour. Funct. Integrat. Genom. 10, 533-546.

Wang, Y., Li, K., Li, X., 2009. Auxin redistribution modulates plastic development of root system architecture under salt stress in Arabidopsis thaliana. J. Plant. Physiol. 166 (15), 1637-1645.

Wang, Y., Deng, D., Shi, Y., Miao, N., Bian, Y., Yin, Z., 2012. Diversification, phylogeny and evolution of auxin response factor (ARF) family: insights gained from analyzing maize ARF genes. Mol. Biol. Rep. 39, 2401-2415.

Wu, J., Wang, F., Cheng, L., Kong, F., Peng, Z., Liu, S., et al., 2011. Identification, isolation and expression analysis of auxin response factor (ARF) genes in Solanum lycopersicum. Plant Cell Rep. 30, 2059-2073.

Wu, T., Zhang, H.T., Wang, Y., Jia, W.S., Xu, X.F., Zhang, X.Z., et al., 2012. Induction of root $\mathrm{Fe}$ (III) reductase activity and proton extrusion by iron deficiency is mediated by auxin-based systemic signalling in Malus xiaojinensis. J. Exp. Bot. 63, 859-870.

Wyatt, S.E., Rashotte, A.M., Shipp, M.J., Robertson, D., Muday, G.K., 2002. Mutations in the gravity persistence signal loci in Arabidopsis disrupt the perception and/or signal transduction of gravitropic stimuli. Plant Physiol. 130, 1426-1435. 
Xing, H., Pudake, R.N., Guo, G., Xing, G., Hu, Z., Zhang, Y., et al., 2011. Genome-wide identification and expression profiling of auxin response factor (ARF) gene family in maize. BMC Genomics 12, 178.

Xu, W., Jia, L., Shi, W., Liang, J., Zhou, F., Li, Q., et al., 2013. Abscisic acid accumulation modulates auxin transport in the root tip to enhance proton secretion for maintaining root growth under moderate water stress. New Phytol. 197, 139-150.

Yang, Y., Wang, Q.L., Geng, M.J., Guo, Z.H., Zhao, Z., 2011. Effect of indole-3-acetic acid on aluminum-induced efflux of malic acid from wheat (Triticum aestivum L.). Plant Soil 346 (1), 215-230.

Yuan, H.M., Xu, H.H., Liu, W.C., Lu, Y.T., 2013. Copper regulates primary root elongation through PIN1-mediated auxin redistribution. Plant Cell Physiol. 54 (5), 766-778.

Zhang, H., Rong, H., Pilbeam, D., 2007. Signalling mechanisms underlying the morphological responses of the root system to nitrogen in Arabidopsis thaliana. J. Exp. Bot. 58, 2329-2338.
Zhang, S.W., Li, C.H., Cao, J., Zhang, Y.C., Zhang, S.Q., Xia, Y.F., et al., 2009a. Altered architecture and enhanced drought tolerance in rice via the down-regulation of indole-3-acetic acid by TLD1/OsGH3.13 activation. Plant Physiol. 151, 1889-1901.

Zhang, Z., Yang, F., Li, B., Egrinya Eneji, A., Li, J., Duan, L., et al., 2009b. Coronatine-induced lateral-root formation in cotton (Gossypium hirsutum) seedlings under potassium-sufficient anddeficient conditions in relation to auxin. J. Plant Nutr. Soil Sci. 172 (3), 435-444.

Zhao, Y., 2010. Auxin biosynthesis and its role in plant development. Annu. Rev. Plant. Biol. 61, 49-64.

Zheng, S.J., Tang, C.X., Arakama, Y., Masaoka, Y., 2003. The responses of red clover (Trifolium pratense L.) to iron deficiency: a root Fe(III) chelate reductase. Plant Sci. 164, 679-687. 
This page intentionally left blank 


\title{
21
}

\section{The Regulatory Signaling of Gibberellin Metabolism and Its Crosstalk With Phytohormones in Response to Plant Abiotic Stresses}

\author{
Aditya Banerjee and Aryadeep Roychoudhury
}

Department of Biotechnology, St. Xavier's College (Autonomous), Kolkata, West Bengal, India

\section{O U T L I N E}

\subsection{Introduction}

21.2 Gibberellic Acid Metabolism in Plants

21.3 Regulatory Signaling of Gibberellic Acids During Abiotic Stresses

21.4 The Signaling Crosstalks Between Gibberellic Acids and Related Phytohormones
333

334

21.5 Gibberellic Acids in Plant Abiotic Stress: A Recent Update

21.6 Conclusion and Future Perspectives

Acknowledgements

References

\subsection{INTRODUCTION}

The rapidly expanding human population demands a substantial increase in the global agricultural productivity. However, a major part of crop production is negatively affected by hostile environmental conditions. Such abiotic stresses manifested by salinity, drought, cold, heat, heavy metal toxicity, etc. induce physiological, biochemical, and molecular damages in plants (Tilman et al., 2011; Khan et al., 2012, 2013, 2014, 2015, 2016; Per et al., 2017). In spite of such constraints, global productivity has to be increased by almost $70 \%$ to feed an additional 2.3 billion people by 2050 (Tilman et al., 2011). Thus, novel strategies should be planned for generating the stress tolerant phenotype in susceptible plant species to boost crop yields even under suboptimal conditions.

Phytohormones are diverse signaling molecules with the ability to act as chemical messengers and promote cellular communication even at infinitesimally low concentrations (Roychoudhury and Banerjee, 2015). Phytohormones like auxins, cytokinin (CK), gibberellic acids (GAs), abscisic acid (ABA), and ethylene act as traditional phytohormones. Among these, 
the universal stress hormone ABA performs several important stress responsive functions by suppressing the amounts of GAs (germination and internodal growth promoting phytohormone) (Roychoudhury et al., 2015). The regulation of GA signaling and metabolism is a crucial decisive factor for the stress susceptible/tolerant phenotype. GAs modify the physiological metabolism in plants via their effects on photosynthesis and sink formation (Iqbal et al., 2011). These plant growth regulators promote phloem loading by inducing the activities of fructose-1,6-bisphosphatase and sucrose phosphate synthase. GA-mediated signaling stimulates the maintenance of an efficient source and sink. Such integration of source-sink relations is required for tolerance towards suboptimal conditions and poststress recovery (Iqbal et al., 2011). Hence, it is necessary to understand the functional significance of GAs in plants exposed to unfavorable environmental conditions. The current chapter presents an overview of the frontiers of GA research in plant abiotic stress responses.

\subsection{GIBBERELLIC ACID METABOLISM IN PLANTS}

GA constitute a large group of tetracyclic diterpenoid carboxylic acids, of which mainly $\mathrm{GA}_{1}$ and $\mathrm{GA}_{4}$ predominantly function as PGRs (Colebrook et al., 2014). GAs are positive regulators of germination, leaf expansion, stem elongation, initiation of flowering and trichome and reproductive developments (Claeys et al., 2012). GAs trigger growth-stimulatory functions and facilitate developmental phase transitions. It has been observed that Arabidopsis seedlings exposed to osmotic stress require the interaction of GAs with other phytohormones to exhibit stress responses (Munteanu et al., 2014).

The methylerythritol phosphate pathway operating in the plastids synthesizes GAs from trans-geranylgeranyl diphosphate. Thus, GAs share the common precursor of ABA (Banerjee et al., 2016; Banerjee and Roychoudhury, 2016a). The biosynthesis is facilitated by the sequential catalysis of two plastid localized terpene cyclases. The formed products are oxidized on the endoplasmic reticulum by cytochrome P450 monooxygenase and then by soluble 2-oxoglutarate-dependent dioxygenases (Hedden and Thomas, 2012). The GA-20oxidase (GA20ox), GA3ox isozymes, and GA2ox comprises the family of dioxygenases. Abiotic stresses induce the expression of GA-catabolic GA2ox genes. These along with specific biosynthetic paralogues of GA20ox and GA3ox maintain cellular homeostasis by reducing GA accumulation (Wani et al., 2016).

The pleiotropic action of GAs during plant development is mediated by the degradation of DELLA protein (belonging to the GRAS family of proteins) (Colebrook et al., 2014). The conformational change in the nuclear receptor, GID1, is induced upon association with GA. As a result, the receptor interacts with the conserved $\mathrm{N}$-terminal domain of the DELLA protein and promotes their association with an SKP, CULLIN, F-BOX CONTAINING (SCF) ubiquitin ligase. Thus, DELLA is ubiquitinylated and degraded by the $26 \mathrm{~S}$ proteasomal machinery (Banerjee and Roychoudhury, 2016b) (Fig. 21.1). It has been opined that the DELLAs might activate or suppress gene expression by acting as transcriptional activators or in complexes with other transcription factors (TFs) (Hirano et al., 2012). Similarly they also act as inhibitors by sequestering gene

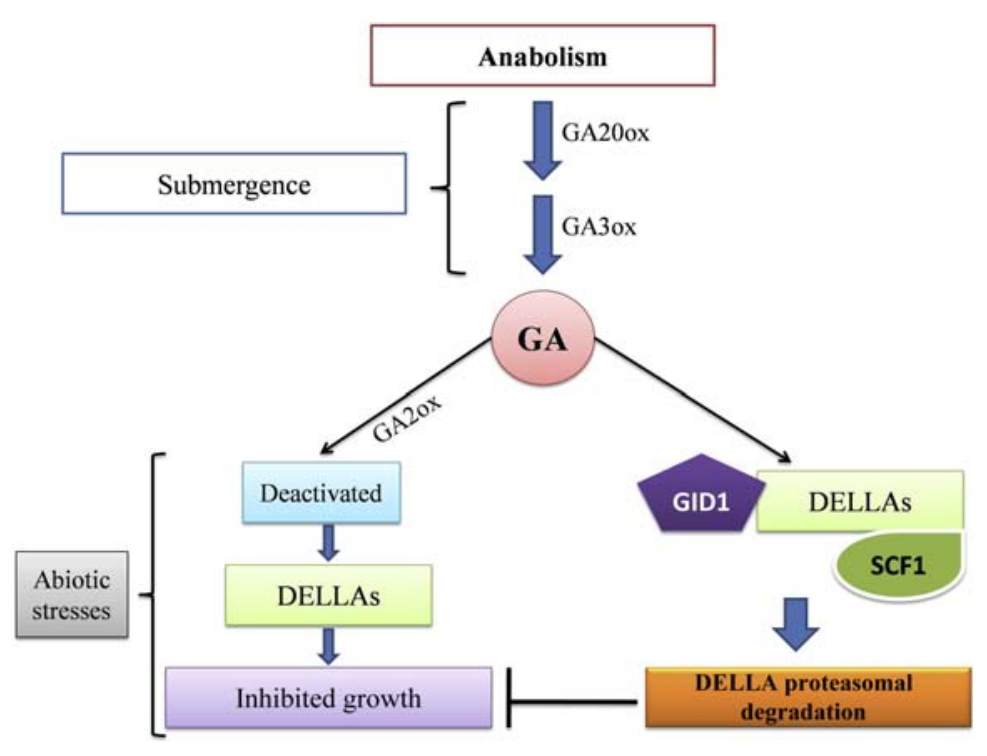

FIGURE 21.1 Gibberellic acid (GA) biosynthesis and its associated equilibrium during abiotic stress responses. Submergence promotes the expression of GA anabolic genes, GA20 oxidase (GA20ox) and GA3ox. However, under other abiotic stresses like salinity, drought, cold etc., the catabolic gene GA2Ox is activated, which degrades GA and activates DELLAs. Thus, plant developmental growth is suppressed. Under natural conditions, GA stimulates the proteasomal degradation of DELLAs via polyubiquitinylation and regulates shoot and root growth (Colebrook et al., 2014). 
activating TFs (Feng et al., 2008). This opens up potential avenues of GA signaling crosstalk with other pathways. DELLAs have been observed to interact with the molecular components of other phytohormonal cascades (Bai et al., 2012). Exploring the putative associations of DELLAs with basic leucine zipper (bZIP) or WRKY TFs can be adopted as future perspectives (Banerjee and Roychoudhury, 2017a; Banerjee and Roychoudhury, 2015).

\subsection{REGULATORY SIGNALING OF GIBBERELLIC ACIDS DURING ABIOTIC STRESSES}

The TF encoding gene, Submergence 1A (Sub1A) [family: APETALA2/Ethylene Response Factor (AP2/ ERF); subfamily: ERF] regulates GA metabolism in Arabidopsis plants exposed to abiotic stress (Dubois et al., 2013). Salt and cold stresses in these plants suppress GA biosynthesis via two TFs of the DEHYDRATION-RESPONSIVE ELEMENT BINDING 1/C-REPEAT BINDING FACTOR (DREB1/CBF) family and one TF of a subfamily of the AP2/ERF family. The DWARF AND DELAYED FLOWERING 1 (DDF1) TF upregulated the expression of AtGA20x7 during salt stress (Magome et al., 2008). Mutation of AtGA2ox7 slightly increased primary root length compared with the wild-type seedlings under saline treatment. However, no change in the extent of salt tolerance could be determined in the ga2ox7 or ddf1 mutants (Colebrook et al., 2014). Plants exposed to cold stress exhibit reduced accumulation of bioactive GAs via the DREB1B/CBF1 mediated upregulation of AtGA2ox3 and $A t G A 20 x 6$. Lowering of cellular GA content resulted in the DELLA-dependent root growth inhibition during low temperatures (Achard et al., 2008a). Dubois et al. (2013) showed that ERF6 controls the expression of GA20x6 during mild osmotic stress. Interestingly, only RGL3 (out of the five DELLA genes in Arabidopsis) was upregulated by stress. The other homologues had to be stabilized only by a low cellular GA content (Colebrook et al., 2014). It is well known that the ABA-independent pathway usually regulates the DREB1/CBFs (Roychoudhury et al., 2013). Association of the ethylene-dependent TF, ETHYLENE INSENSITIVE 3(EIN3) with the DREB1/CBF promoters might integrate these transcriptional regulators in the GA signaling pathway (Shi et al., 2012).

The DELLAs have been implicated in regulating the antioxidant system in Arabidopsis plants experiencing stress conditions (Achard et al., 2008b). The reactive oxygen species (ROS) are usually generated in uncontrolled amounts in almost all kinds of abiotic stresses. They trigger lipid peroxidation in membranes and degrade essential proteins within the cell. ROS can even oxidize nucleic acids and promote mutational effects that are cytotoxic (Banerjee and Roychoudhury, 2017b). DELLAs delay ROS-induced necrosis by inhibiting their accumulation during salt stress (Achard et al., 2008b). ROS controls the GA-mediated root growth. Thus, ROS might be the key regulatory component in the growth and stress tolerance effects exhibited by DELLAs (Achard et al., 2008b). In rice, the DELLA protein, SLENDER LIKE-1 (SLR1) ameliorated oxidative stress in the Sub1A lines separately exposed to drought and to dehydration after flooding (Fukao et al., 2011). Distinct roles of DELLA have been observed in Arabidopsis plants under control and stressed environments. DELLA regulated cellular proliferation under normal conditions. However, when the plants were exposed to osmotic stress, high accumulation of DELLA triggered mitotic exit and reduced the overall cell number compared with the control sets (Claeys et al., 2012). This partly illustrates the basis of DELLA-mediated growth arrest in plants under suboptimal environments.

\subsection{THE SIGNALING CROSSTALKS BETWEEN GIBBERELLIC ACIDS AND RELATED PHYTOHORMONES}

GA signaling is closely integrated with the traditional PGRs, ABA, and ethylene (Roychoudhury and Banerjee, 2017). Exogenous application of ABA resulted in inhibited root growth and corresponding accumulation of DELLAs in the Arabidopsis seedlings. The inhibition was however significantly reduced in the quadruple della mutants (Achard et al., 2006). The ABA-mediated accumulation of DELLA is related to decreased levels of bioactive GAs. Plants with mutated $\mathrm{ABA}$ receptor, $A B A$ Insensitive 1-1 (ABI1-1) did not exhibit the accumulation of DELLA (Colebrook et al., 2014). This shows that DELLA-associated GA signaling operates downstream to the ABI1 mediated cascade. ABI5 is a bZIP TF regulating seed germination and early seedling development by participating in the ABA signaling pathway (Banerjee and Roychoudhury, 2017a). It has been observed that ABI5 acts as an ABA analogue and integrates the components of GA, auxin, $\mathrm{CK}$, jasmonate, and brassinosteroid (BR) signaling cascades (Skubacz et al., 2016). Liu et al. (2016) observed that three NUCLEAR FACTOR-Y C (NF-YC) homologues, NFYC3, NF-YC4, and NF-YC9, regulate the GA and ABAmediated germination of seeds. The NF-YCs associate with the DELLA protein RGL2. The module binds to the CCAAT cis elements in the ABI5 promoter. ABI5 in turn regulates the expression of crucial GA- and ABAresponsive genes. Thus, the NF-YC-RGL2-ABI5 module 


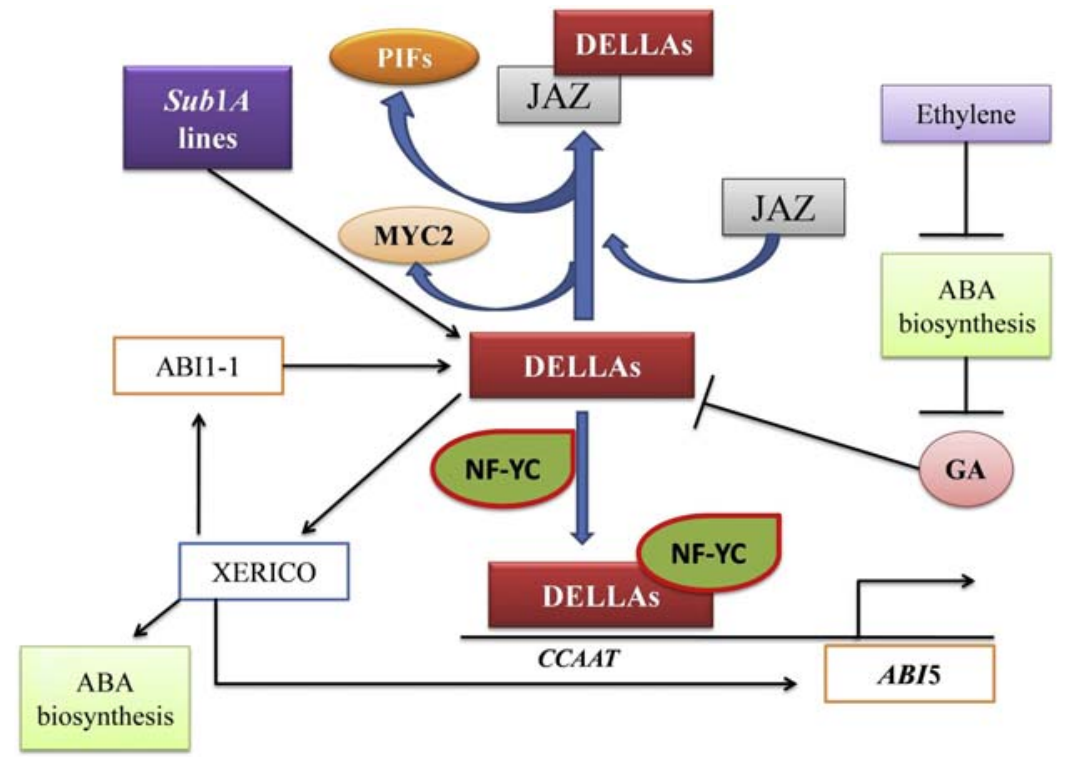

FIGURE 21.2 DELLA interaction with diverse signaling components mediates gibberellic acid crosstalks with other phytohormone signaling pathways. Signal transduction through ABSCISIC ACID INSENSITIVE1-1 (ABI1-1) stimulates the accumulation of DELLAs, which in turn promote abscisic acid biosynthesis by inducing the expression of XERICO. DELLAs also associate with NUCLEAR FACTOR-Y Cs to bind to the cis acting element, CCAAT in the $A B I 5$ promoter. This transcriptionally activates $A B I 5$. The Submergence1A lines also maintain elevated levels of DELLA proteins. DELLAs often outcompete MYC2 to interact with JASMONIC ACID ZIMdomain proteins, which in turn compete with PHYTOCHROME INTERACTING FACTORs to bind with DELLAs. Elevated levels of ethylene during submergence triggers GA accumulation by repressing ABA synthesis. High cellular GA content degrades DELLA proteins and promotes escape responses during submergence. mediates a GA-ABA crosstalk during germination (Liu et al., 2016) (Fig. 21.2). Zentella et al. (2007) showed that DELLA triggers the accumulation of XERICO, which represses a negative regulator of $\mathrm{ABA}$ biosynthesis (Fig. 21.2).

Analysis of the constitutive triple response 1 (ctr1), gibberellic acid insensitive-t6(gai-t6) and repressor of gibberellic acid (rga-24) mutants revealed reduced salt tolerant phenotype, which indicates a possible integration of the ethylene signaling with DELLA (Achard et al., 2006). Ren and Wang (2016) identified two GA-responsive elements and an auxinresponsive element in the promoter of the auxin receptor gene TIR1 in Solanum lycopersicum. Whereas naphthalene acetic acid (NAA) and ABA downregulated SITIR1, exogenous application of GA upregulated the gene. It was observed that plants overexpressing SITIR1 failed to form reproductive seeds as SITIR1 promoted stenospermocarpy (abnormal seed development) (Ren and Wang, 2016). Ethylene accumulation promotes leaf hyponastic responses to submergence; whereas the redistribution of auxin, indole-3-acetic acid (IAA) from the leaf blade dictates the differential growth of the petiole in Rumex palustris (Cox et al., 2006). The petiolar ABA content is reduced in the presence of ethylene, which induces the expression of GA3ox so that the GA content is increased (Benschop et al., 2006). GA promotes escape response by bending the leaves upwards and stimulating petiole and leaf blade elongation to avoid anoxia during submergence (Polko et al., 2011).

Ethylene accumulation in submerged tissues of rice plants stimulates ABA catabolism via increased ABA8 '-hydroxylase activity (Jackson, 2008). The Sub1 locus regulates the quiescence strategy of rice varieties acclimatized to short-lived, deep floods. Jung et al. (2010) highlighted that the ERF subfamily protein, SUB1A, controlled the expression of a variety of transcripts regulated by GA, ABA, ethylene, and CK. Under submergence, the Sub1A lines maintained elevated expression of GA2ox to restrict GA levels, stabilize the DELLA protein SLR1, and promote quiescence (Fukao et al., 2011). As a result of low GA content, the ABA levels were high, which effectively ameliorated the dehydration stress during desubmergence by inducing the expression of ABA-dependent drought-responsive transcripts. The plants thus maintained high relative water potential and exhibited low accumulation of ROS (Colebrook et al., 2014).

Jasmonate or jasmonic acid (JA) actively synthesized during cold stress interacts with phytohormones like GA, auxin, and ethylene to regulate leaf senescence and abiotic stress tolerance ( $\mathrm{Hu}$ et al., 2017; Per et al., 2018). The DELLA proteins compete with MYC2 TF to bind to the JA ZIM-domain (JAZ) proteins (Hou et al., 2010). Yang et al. (2012) reported that even the JAZ proteins compete with the growth promoting PHYTOCHROME INTERACTING FACTORs (PIFs) to bind to DELLAs (Fig. 21.2). JA upregulated the DELLA gene RGA-like 3 (RGL3) and its promoter was targeted by MYC2. RGL3 was found to interact with JAZ1 and JAZ8, of which JAZ8 is resistant to JA-mediated degradation (Wild et al., 2012). Thus, MYC2 released from JAZ1 (degraded by JA) in presence of JA triggers the expression of RGL3. Association of RGL3 with non-JA-degradable JAZ8 further promotes MYC2-dependent JA responses (Wild et al., 2012). 


\subsection{GIBBERELLIC ACIDS IN PLANT ABIOTIC STRESS: A RECENT UPDATE}

In the rainfed lowland areas, submergence stress is considered to be a limiting factor for the growth of rice plants. GA is regarded as a negative regulator of submergence tolerance in rice. Treatment of plants separately with GA and its inhibitor paclobutrazol (PB) led to the identification of 3936 differentially expressed genes (DEGs) among the treated sets and the control. The PB treated plants exhibited submergence tolerance by maintaining photosynthetic capacity and reducing nutrient metabolism (Xiang et al., 2017). Liu et al. (2017) showed that GRAS40 in S. lycopersicum was upregulated by multiple abiotic stress treatments including D-mannitol, $\mathrm{NaCl}$, and $\mathrm{H}_{2} \mathrm{O}_{2}$. It was found that SIGRAS40 interacted with GA and auxin signaling pathways during the vegetative and reproductive growth phases in tomato plants. The transgenics overexpressing SIGRAS40 exhibited increased salt and drought tolerance compared with the control plants (Liu et al., 2017). Genes involved in JA, salicylic acid (SA) synthesis, and SA and BR signaling were downregulated, whereas the genes associated with GA degradation were upregulated in the transgenic plants overexpressing the $C$-repeat binding factor 2 (CBF2) and $C B F 3$, both involved in generating cold tolerance (Li et al., 2017).

In a recent study, several tissue specific GA dioxygenases have been identified as CsGA20ox2, CsGA3ox2, CsGA3ox3, CsGA2ox1, CsGA2ox2, and CsGA2ox4 in Camellia sinensis. These genes have been proposed as candidate marker genes for abiotic stress tolerance breeding in tea plants (Pan et al., 2017). An AP2/ERF family protein, DREB2, from physic nut was found to be induced by ABA, but repressed by GA and salt exposures (Tang et al., 2017). Overexpression of the $D R E B 2$ gene in rice resulted in dwarf and GA-deficient phenotype. Normal growth could however be restored by exogenous treatment with $\mathrm{GA}_{3}$. Expression of biosynthetic genes like OsGA20ox1, OsGA20ox2, OsGA20ox4, and OsGA3ox2 was significantly reduced in the plants that also showed elevated sensitivity to salt stress (Tang et al., 2017). Khalloufi et al. (2017) reported that exogenous application of $10^{-6} \mathrm{M} \mathrm{GA}_{3}$ supplemented by inoculation with arbuscular mycorrhyzal fungi alleviated salt-induced damages in tomato plants by elevating the levels of CK, IAA, and ABA. The plants also maintained a high $\mathrm{K}^{+} / \mathrm{Na}^{+}$ratio during stress (Khalloufi et al., 2017). Casein kinase II (CKII) is an evolutionarily conserved and crucial Ser/ Thr kinase involved in regulating GA signaling during abiotic stress response in plants (Yuan et al., 2017). Analysis of the $\beta$-subunit of CKII (CKB1) was performed by creating Arabidopsis T-DNA mutants (ckb1-1 and ckb1-2) and CKB1 overexpressing plants. The mutants exhibited low sensitivity to ABA during germination and initial growth along with increased stomatal aperture, leaf water loss, and proline (compatible solute) accumulation. However, the CKB1 overexpressing plants showed opposite results via alteration in a number of $\mathrm{ABA}$ and GA-regulatory genes. The research proposed CKB1 to be an ABA signaling-associated protein that positively regulates ABA signaling by establishing a parallel influence on GA metabolism (Yuan et al., 2017).

GA accumulation negatively regulates stress tolerance. Hence, attenuation of endogenous GA level by ectopic expression of GA20x6 increased the yield and promoted drought tolerance in rice (Lo et al., 2017). GA2ox6 regulates the architecture and function of rice plants. The transgenics exhibited a thorough reprogramming in their transcriptomes along with reduced shoot height, more productive tillers, expanded root system, higher water use efficiency, and increased photosynthetic rate. Field trials using the transgenics increased the yield by 10\%-30\% compared with the wild-type plants under drought stress (Lo et al., 2017). GA reportedly regulates redox homeostasis by eliciting electron mobilization in the living aleurone layers of Hordeum vulgare cv. Himalaya grains (Mark et al., 2016). Maintenance of such redox equilibrium is essential for acclimatizing with suboptimal environments.

\subsection{CONCLUSION AND FUTURE PERSPECTIVES}

GA is a germination-promoting phytohormone that also stimulates internodal growth during seedling development. On evidential grounds, the current belief is that abiotic stress represses GA accumulation and its associated signaling cascades. Under such circumstances, cellular content of DELLA proteins is increased and these establish regulatory crosstalks with multiple phytohormones (Fig. 21.2). GA regulates the ROS levels in the cell. Furthermore, essential TFs like MYCs, DREBs, CBFs, JAZ-domain proteins, and PIFs participate in the GA signaling pathway during plant exposure to stress. Such rapid yet intricate interaction among phytohormones complicates the ultimate GA-mediated physiology under stress.

Identification of the epigenomic landscape in plants is an important aspect of understanding the contextdependent molecular physiology (Banerjee and Roychoudhury, 2017c). Epigenetic alterations at the global scale have been reported in abiotic stresses like salt, drought, and cold (Banerjee and Roychoudhury, 2017d; Banerjee et al., 2017). There is no literature available regarding GA-induced epigenetic dynamism 
in plants. Thus, identifying such alterations in the epigenomic landscape under stress can be a novel endeavor. Transgenic rice plants overexpressing the GA catabolic gene, GA2ox, have succeeded in producing high yielding phenotypes with enhanced stress tolerance (Lo et al., 2017). Further, genome-wide studies must be conducted to identify more of such GA catabolic loci, which can be effectively mapped for stringent screening of high yielding, stress tolerant cultivars. Successful field trials followed by cultivation of such transgenic plants will ensure global food safety and a significant surge in the agricultural economy.

\section{Acknowledgements}

Financial support from Council of Scientific and Industrial Research (CSIR), Government of India through the major grant [38(1387)/14/ EMR-II] to Dr. Aryadeep Roychoudhury is gratefully acknowledged. The University Grants Commission, Government of India is acknowledged for providing Junior Research Fellowship to Mr. Aditya Banerjee.

\section{References}

Achard, P., Cheng, H., De Grauwe, L., et al., 2006. Integration of plantresponses to environmentally activated phytohormonal signals. Science 311, 91-94.

Achard, P., Gong, F., Cheminant, S., et al., 2008a. The cold-inducible CBF1 factor-dependent signalling pathway modulates the accumulation of the growth-repressing DELLA proteins via its effect on gibberellin metabolism. Plant Cell 20, 2117-2129.

Achard, P., Renou, J.-P., Berthomé, R., et al., 2008b. Plant DELLAs restrain growth and promote survival of adversity by reducing the levels of reactive oxygen species. Curr. Biol. 18, 656-660.

Bai, M.-Y., Shang, J.-X., Oh, E., et al., 2012. Brassinosteroid, gibberellin and phytochrome impinge on a common transcription module in Arabidopsis. Nat. Cell. Biol. 14, 810-817.

Banerjee, A., Roychoudhury, A., 2015. WRKY proteins: Signalling and regulation of expression during abiotic stress responses. Sci. World J. 2015, 807560.

Banerjee, A., Roychoudhury, A., 2016a. Group II late embryogenesis abundant (LEA) proteins: structural and functional aspects in plant abiotic stress. Plant Growth Regul. 79, 1-17.

Banerjee, A., Roychoudhury, A., 2016b. Plant responses to light stress: Oxidative damages, photoprotection and role of phytohormones. In: Ahammed, G.J., Yu, J.-Q. (Eds.), Plant Hormones Under Challenging Environmental Factors. Springer, Netherlands, pp. 181-213.

Banerjee, A., Roychoudhury, A., 2017a. Abscisic-acid-dependent basic leucine zipper (bZIP) transcription factors in plant abiotic stress. Protoplasma 254, 3-16.

Banerjee, A., Roychoudhury, A., 2017b. Abiotic stress, generation of reactive oxygen species, and their consequences: An overview. In: Singh, V.P., Singh, S., Tripathi, D., Mohan Prasad, S., Chauhan, D.K. (Eds.), Revisiting the Role of Reactive Oxygen Species (ROS) in Plants: ROS Boon or Bane for Plants? John Wiley \& Sons, Inc, USA, pp. 23-50.

Banerjee, A., Roychoudhury, A., 2017c. The gymnastics of epigenomics in rice. Plant Cell Rep. Available from: https://doi.org/ 10.1007/s00299-017-2192-2.
Banerjee, A., Roychoudhury, A., 2017d. Epigenetic regulation during salinity and drought stress in plants: Histone modifications and DNA methylation. Plant Gene 11, 199-204.

Banerjee, A., Wani, S.H., Roychoudhury, A., 2017. Epigenetic control of plant cold responses. Front. Plant Sci. 8, 1643.

Banerjee, A., Roychoudhury, A., Krishnamoorthi, S., 2016. Emerging techniques to decipher microRNAs (miRNAs) and their regulatory role in conferring abiotic stress tolerance in plants. Plant Biotechnol. Rep. 10, 185-205.

Benschop, J.J., Bou, J., Peeters, A.J.M., et al., 2006. Long-term submergence induced elongation in Rumex palustris requires abscisic acid-dependent biosynthesis ofgibberellin1. Plant Physiol. 141, 1644-1652.

Claeys, H., Skirycz, A., Maleux, K., Inze, D., 2012. DELLA signalling mediates stress-induced cell differentiation in Arabidopsis leaves through modulation of anaphase-promoting complex/cyclosome activity. Plant Physiol. 159, 739-747.

Colebrook, E.H., Thomas, S.G., Phillips, A.L., Hedden, P., 2014. The role of gibberellin signalling in plant responses to abiotic stress. J. Exp. Biol. 217, 67-75.

Cox, M.C.H., Peeters, A.J.M., Voesenek, L.A., 2006. The stimulating effects of ethylene and auxin on petiole elongation and on hyponastic curvature are independent processes in submerged Rumex palustris. Plant Cell Environ. 29, 282-290.

Dubois, M., Skirycz, A., Claeys, H., et al., 2013. The ETHYLENERESPONSE FACTOR 6 acts as central regulator of leaf growth under water limiting conditions in Arabidopsis thaliana. Plant Physiol. 162, 319-332.

Feng, S.H., Martinez, C., Gusmaroli, G., et al., 2008. Coordinated regulation of Arabidopsis thaliana development by light and gibberellins. Nature 451, 475-479.

Fukao, T., Yeung, E., Bailey-Serres, J., 2011. The submergence tolerance regulator SUB1A mediates crosstalk between submergence and drought tolerance in rice. Plant Cell 23, 412-427.

Hedden, P., Thomas, S.G., 2012. Gibberellin biosynthesis and its regulation. Biochem. J444, 11-25.

Hirano, K., Kouketu, E., Katoh, H., et al., 2012. The suppressive function of the rice DELLA protein SLR1 is dependent on its transcriptional activation activity. Plant J71, 443-453.

Hou, X., Lee, L.Y.C., Xia, K., et al., 2010. DELLAs modulate jasmonate signalling via competitive binding to JAZs. Dev. Cell 19, 884-894.

Hu, Y., Jiang, Y., Han, X., et al., 2017. Jasmonate regulates leaf senescence and tolerance to cold stress: crosstalk with other phytohormones. J. Exp. Bot. 68, 1361-1369.

Iqbal, N., Nazar, R., Khan, M.I.R., Masood, A., Khan, N., 2011. Role of gibberellins in regulation of source-sink relations under optimal and limiting environmental conditions. Curr. Sci. 100, 998-1007.

Jackson, M.B., 2008. Ethylene-promoted elongation: an adaptation to submergence stress. Ann. Bot. 101, 229-248.

Jung, K.-H., Seo, Y.-S., Walia, H., et al., 2010. The submergence tolerance regulatorSub1A mediates stress-responsive expression of AP2/ERF transcription factors. Plant Physiol. 152, 1674-1692.

Khalloufi, M., Martínez-Andújar, C., Lachaâl, M., et al., 2017. The interaction between foliar GA3 application and arbuscular mycorrhizal fungi inoculation improves growth in salinized tomato (Solanum lycopersicum L.) plants by modifying the hormonal balance. J. Plant Physiol. 214, 134-144.

Khan, M.I.R., Iqbal, N., Masood, A., Khan, N.A., 2012. Variation in salt tolerance of wheat cultivars: Evaluation of the role of glycinebetaine and ethylene. Pedosphere 22, 746-754.

Khan, M.I.R., Asgher, M., Khan, N.A., 2013. Rising temperature in the changing environment: A serious threat to plants. Clim. Change Environ. Sust. 1, 25-36. 
Khan, M.I.R., Asgher, M., Khan, N.A., 2014. Alleviation of saltinduced photosynthesis and growth inhibition by salicylic acid involves glycinebetaine and ethylene in mungbean (Vigna radiata L.). Plant Physiol. Biochem. 80, 67-74.

Khan, M.I.R., Asgher, M., Fatma, M., Per, T.S., Khan, N.A., 2015. Drought stress vis a vis plant functions in the era of climate change. Clim. Change Environ. Sust. 3, 13-25.

Khan, M.I.R., Khan, N.A., Masood, A., Per, T.S., Asgher, M., 2016. Hydrogen peroxide alleviates nickel-inhibited photosynthetic responses through increase in use-efficiency of nitrogen and sulfur, and glutathione production in mustard. Front. Plant Sci. 7, 44.

Li, A., Zhou, M., Wei, D., et al., 2017. Transcriptome profiling reveals the negative regulation of multiple plant hormone signalling pathways elicited by overexpression of C-Repeat Binding Factors. Front. Plant Sci. 8, 1647.

Liu, B., De Storme, N., Geelen, D., 2017. Gibberellin induces diploid pollen formation by interfering with meiotic cytokinesis. Plant Physiol. 173, 338-353.

Liu, X., Hu, P., Huang, M., et al., 2016. The NF-YC-RGL2 module integrates $\mathrm{GA}$ and $\mathrm{ABA}$ signalling to regulate seed germination in Arabidopsis. Nat. Commun. 7, 12768.

Liu, Y., Huang, W., Xian, Z., et al., 2017. Overexpression of SIGRAS40 in tomato enhances tolerance to abiotic stresses and influences auxin and gibberellin signalling. Front. Plant Sci. 8, 1659.

Lo, S.F., Ho, T.D., Liu, Y.L., et al., 2017. Ectopic expression of specific GA2 oxidase mutants promotes yield and stress tolerance in rice. Plant Biotechnol. J. 15, 850-864.

Magome, H., Yamaguchi, S., Hanada, A., et al., 2008. The DDF1transcriptional activator upregulates expression of a gibberellin-deactivating gene, GA2ox7, under high-salinity stress in Arabidopsis. Plant J. 56, 613-626.

Mark, C., Zor, K., Heiskanen, A., et al., 2016. Monitoring intra- and extracellular redox capacity of intact barley aleurone layers responding to phytohormones. Anal. Biochem. 515, 1-8.

Munteanu, V., Gordeev, V., Martea, R., Duca, M., 2014. Effect of gibberellin cross talk with other phytohormones on cellular growth and mitosis to endoreduplication transition. Int. J. Adv. Res. Biol. Sci. 1, 136-153.

Pan, C., Tian, K., Ban, Q., et al., 2017. Genome-wide analysis of the biosynthesis and deactivation of gibberellin-dioxygenases gene family in Camellia sinensis (L.) O. Kuntze. Genes (Basel) 8, piiE235.

Per, T.S., Khan, N.A., Reddy, P.S., Masood, A., Hasanuzzaman, M., Khan, M.I.R., et al., 2017. Approaches in modulating proline metabolism in plants for salt and drought stress tolerance: Phytohormones, mineral nutrients and transgenics. Plant Physiol. Biochem. 115, 126-140.

Per, T.S., Khan, M.I.R., Anjum, N.A., Masood, A., Hussain, S.J., Khan, N.A., 2018. Jasmonates in plants under abiotic stresses: crosstalk with other phytohormones matters. Environ. Exp. Bot. $145,104-120$.
Polko, J.K., Voesenek, L.A.C.J., Peeters, A.J.M., Pierik, R., 2011. Petiole hyponasty: an ethylene-driven, adaptive response to changes in the environment. AoB Plants 2011, plr031.

Ren, Z., Wang, X., 2016. SITIR1 is involved in crosstalk of phytohormones, regulates auxin-induced root growth and stimulates stenospermocarpic fruit formation in tomato. Plant Sci. 253, 13-20.

Roychoudhury, A., Banerjee, A., 2015. Transcriptome analysis of abiotic stress response in plants. Transcriptomics 3, 2.

Roychoudhury, A., Banerjee, A., 2017. Abscisic acid signalling and involvement of mitogen activated protein kinases and calciumdependent protein kinases during plant abiotic stress. In: Pandey, G. (Ed.), Mechanism of Plant Hormone Signalling under Stress., vol. 1. John Wiley \& Sons, Inc, USA, pp. 197-241.

Roychoudhury, A., Paul, S., Basu, S., 2013. Cross-talk between abscisic acid-dependent and abscisic acid-independent pathways during abiotic stress. Plant Cell Rep. 32, 985-1006.

Roychoudhury, A., Banerjee, A., Lahiri, V., 2015. Metabolic and molecular-genetic regulation of proline signalling and its crosstalk with major effectors mediates abiotic stress tolerance in plants. Turk J. Bot. 39, 887-910.

Shi, Y., Tian, S., Hou, L., et al., 2012. Ethylene signalling negatively regulates freezing tolerance by repressing expression of CBF and type-A ARR genes in Arabidopsis. Plant Cell 24, 2578-2595.

Skubacz, A., Daszkowska-Golec, A., Szarejko, I., 2016. The role and regulation of ABI5 (ABA-Insensitive 5) in plant development, abiotic stress responses and phytohormone crosstalk. Front. Plant Sci. 7, 1884.

Tang, Y., Liu, K., Zhang, J., et al., 2017. JcDREB2, a physic nut AP2/ ERF gene, alters plant growth and salinity stress responses in transgenic rice. Front. Plant Sci. 8, 306.

Tilman, D., Balzer, C., Hill, J., Belfort, B.L., 2011. Global food demand and the sustainable intensification of agriculture. Proc. Natl. Acad. Sci. USA 108, 20260-20264.

Wani, S.H., Kumar, V., Shriram, V., Sah, S.K., 2016. Phytohormones and their metabolic engineering for abiotic stress tolerance in crop plants. Crop J. 4, 162-176.

Wild, M., Davière, J.-M., Cheminant, S., et al., 2012. The Arabidopsis DELLA RGA-LIKE3is a direct target of MYC2 and modulates jasmonate signalling responses. Plant Cell 24, 3307-3319.

Xiang, J., Wu, H., Zhang, Y., et al., 2017. Transcriptomic analysis of gibberellin- and paclobutrazol-treated rice seedlings under submergence. Int. J. Mol. Sci. 18, piiE2225.

Yang, D.-L., Yao, J., Mei, C.-S., et al., 2012. Plant hormone jasmonate prioritizes defense over growth by interfering with gibberellin signalling cascade. Proc. Natl. Acad. Sci. USA 109, E1192-E1200.

Yuan, C., Ai, J., Chang, H., et al., 2017. CKB1 is involved in abscisic acid and gibberellic acid signalling to regulate stress responses in Arabidopsis thaliana. J. Plant Res. 130, 587-598.

Zentella, R., Zhang, Z.-L., Park, M., et al., 2007. Global analysis of DELLA direct targets in early gibberellin signalling in Arabidopsis. Plant Cell 19, 3037-3057. 
This page intentionally left blank 


\section{2}

\section{Abscisic Acid, a Principal Regulator of Plant Abiotic Stress Responses}

Sushma Sagar and Amarjeet Singh

National Institute of Plant Genome Research, New Delhi, India

\begin{tabular}{llll} 
& O U T L I N E \\
22.1 Introduction & 341 & 22.8 ABA-Dependent Gene Expression & 346 \\
22.2 ABA Biosynthesis & 342 & 22.9 The Role of ABA in Abiotic Stress & 347 \\
22.3 Catabolism of ABA & 343 & Signaling & 349 \\
22.4 ABA Signaling Pathway & 344 & 22.10 Conclusion & 349 \\
22.5 ABA Receptors & 344 & Acknowledgment & 349 \\
22.6 Protein Phosphatase 2C & 345 & References & 353 \\
22.7 SNF1-Related Protein Kinase 2(SnRK2) & 346 & Further Reading & \\
\hline
\end{tabular}

\subsection{INTRODUCTION}

Plants are routinely troubled by various abiotic stresses such as high salinity, dehydration, and low temperature during their life span. These abiotic stresses have detrimental effect on plant development, longevity, and productivity. Plants have evolved with a sessile nature, and unlike animals, they cannot move away from adverse growth conditions. Rather, they are destined to combat these stress conditions in their stationary habitat. Therefore, plants have devised an adaptive mechanism that entails the activation of several signal transduction pathways, leading to diverse molecular, cellular, and physiological changes (Singh et al., 2016, 2018). Most signal transduction pathways triggered in response to biotic or abiotic stresses are mediated by one or more plant hormones. Therefore, plant hormones are a crucial player in regulating plants' response to various environmental cues (Iqbal et al., 2017; Khan et al., 2015a,b; Khan and Khan, 2014; Kazan, 2015; Per et al., 2018). Generally, phytohormones like salicylic acid (SA), jasmonic acid (JAs), and ethylene (ET) are implicated in plant response to pathogens, wounding, and other biotic stresses, whereas gibberellins (GAs), auxins (IAAs), brassinosteroids (BRs), and cytokinins are known to regulate plant development. However, recent advancements in plant stress related research have shown that all plant hormones could control multiple plant processes and are involved in crosstalk of signaling pathways. 
For example, SA, JA, and ET, apart from biotic stresses, are also involved in plant development and responses to abiotic stresses. Similarly, auxins and GA are crucial in abiotic and biotic stress responses (Colebrook et al., 2014; Kazan, 2013; Khan and Khan, 2013; Santino et al., 2013).

Abscisic acid (ABA) is the key hormone that primarily regulates plants' responses to various abiotic stresses; however, like other phytohormones ABA is also known to regulate plants' response to biotic stress and development (Singh et al., 2016). The discovery of the vital phytohormone ABA dates way back to the 1960s. Several independent and convergent experiments carried out by various research groups led to the discovery of ABA (Cracker and Abeles, 1969). However, the earliest and most convincing was the discovery of ABA in cotton, where it was involved in fruit abscission and dormancy ( $\mathrm{Li}$ et al., 2017). As time and research progressed, newer functions of ABA were unveiled, including adaptation to various stresses, stomatal closure, sugar accumulation, seed development, etc. Due to its crucial role in abiotic and biotic stresses, ABA is known as a "stress hormone". Plenty of research on ABA accumulated ample information on its biosynthesis, storage, catabolism, site of action, and its possible targets. During the last decade, the ABA receptors and their crystal structures have been elucidated (Ma et al., 2009; Park et al., 2009). This information has provided a clear cut paradigm of the ABA signal transduction pathway. Moreover, recruiting the combinations of different key players such as PP2C phosphatase and SnRK2 kinases has helped to understand the signal transduction pathway. Recent studies have provided newer insights into the functional roles of the ABA signaling cascade in various aspects of plant growth and development. In this chapter, we discuss different facets of $\mathrm{ABA}$ in plants, including its biosynthesis, catabolism, ABA signaling pathway and various signaling components, and the role of $\mathrm{ABA}$ in abiotic stresses and plant development.

\subsection{ABA BIOSYNTHESIS}

ABA is synthesized via direct and indirect pathways. In fungi, ABA is synthesized directly from farnesyl diphosphate; on the other hand, in plants ABA is synthesized indirectly from carotenoids ( $\mathrm{Li}$ et al., 2017). ABA synthesis in plants occurs in plastids and cytosol. A five carbon biological isoprene unit, isopentenyl diphosphate (IPP), is the precursor for the formation of ABA. Three molecules of IPP combine with dimethylallyl diphosphate to form a $\mathrm{C}_{20}$ molecule geranylgeranyl diphosphate (GGPP). In the next step, phytoene synthase catalyzes the condensation of two
GGPP molecules together to produce a $\mathrm{C}_{40}$ molecule, phytoene (Kirby and Keasling, 2009). This is the first committed step for the biosynthesis of carotenoids. Further, desaturation and isomerization reactions lead to the formation of intermediates, such as lycopene. Lycopene is cyclized and hydroxylated to produce the first oxygenated carotenoid called zeaxanthin, by the enzyme $\beta$ carotene hydroxylases. In the next step, zeaxanthin by the action of the enzyme zeaxanthin epoxidase (ZEP) is converted to all-trans-violaxanthin, which is further converted to 9'-cis-neoxanthin or 9-cisviolaxanthin by isomerization reaction with the help of the enzyme cis-isomerase. Subsequently, 9'-cis-neoxanthin or 9-cis-violaxanthin is oxidatively cleaved to produce the first C15 intermediate, xanthoxin (Li et al., 2017). This is the first committed step in the ABA biosynthetic pathway. All the reactions mentioned till now occur in the plastid. Xanthoxin is then transported to the cytosol by some unknown transporter where it is acted upon by short chain dehydrogenase reductaselike enzyme (SDR) to produce abscisic aldehyde. Lastly, abscisic aldehyde oxidase (AAO) encoded by Arabidopsis $A B A 2$ gene converts abscisic aldehyde to ABA (Fig. 22.1). AAO requires molybdenum as a cofactor for its activity. The $A B A 3$ gene in Arabidopsis encodes a sulfurase, which is capable of producing a cofactor MoCo. This cofactor is pivotal for ABA synthesis since $a b a 3$ mutants are defective in ABA synthesis due to faulty AAO activity (Bittner et al., 2001). Hormones act optimally within certain concentration range. Too high or too low concentration poses a problem and can have severe consequences, therefore, the amount of hormone should be effectively regulated. The amount of active ABA available in a particular cell is determined by the rate of its biosynthesis, catabolism, and transport. The sensitivity of the plant cell towards ABA concentration is critical for ABA mediated response. During stress conditions there is a need for excess of $\mathrm{ABA}$, thus, genes involved in $\mathrm{ABA}$ biosynthesis and various transcription factors are upregulated and activated (Banerjee and Roychoudhury, 2017). It is well known that zeaxanthin epoxidase (ZEP) has a regulatory role in ABA biosynthesis. Lower level of ZEP expression in etiolated tissue led to reduction in carotenoids, whereas overexpression of ZEP in tobacco resulted in elevated seed dormancy (Frey et al., 1999). Interestingly, the Arabidopsis genome encodes a single ZEP gene, however, even the most severe ZEP mutants do not completely lack ABA, suggesting the existence of an alternate minor pathway for ABA biosynthesis (Barrero et al., 2005). Moreover, the zep mutants are deficient in ABA and showed wilty phenotype and produced nondormant seeds (Finkelstein, 2013). Several reports have shown the upregulation of AtZEP gene in both shoot and root 


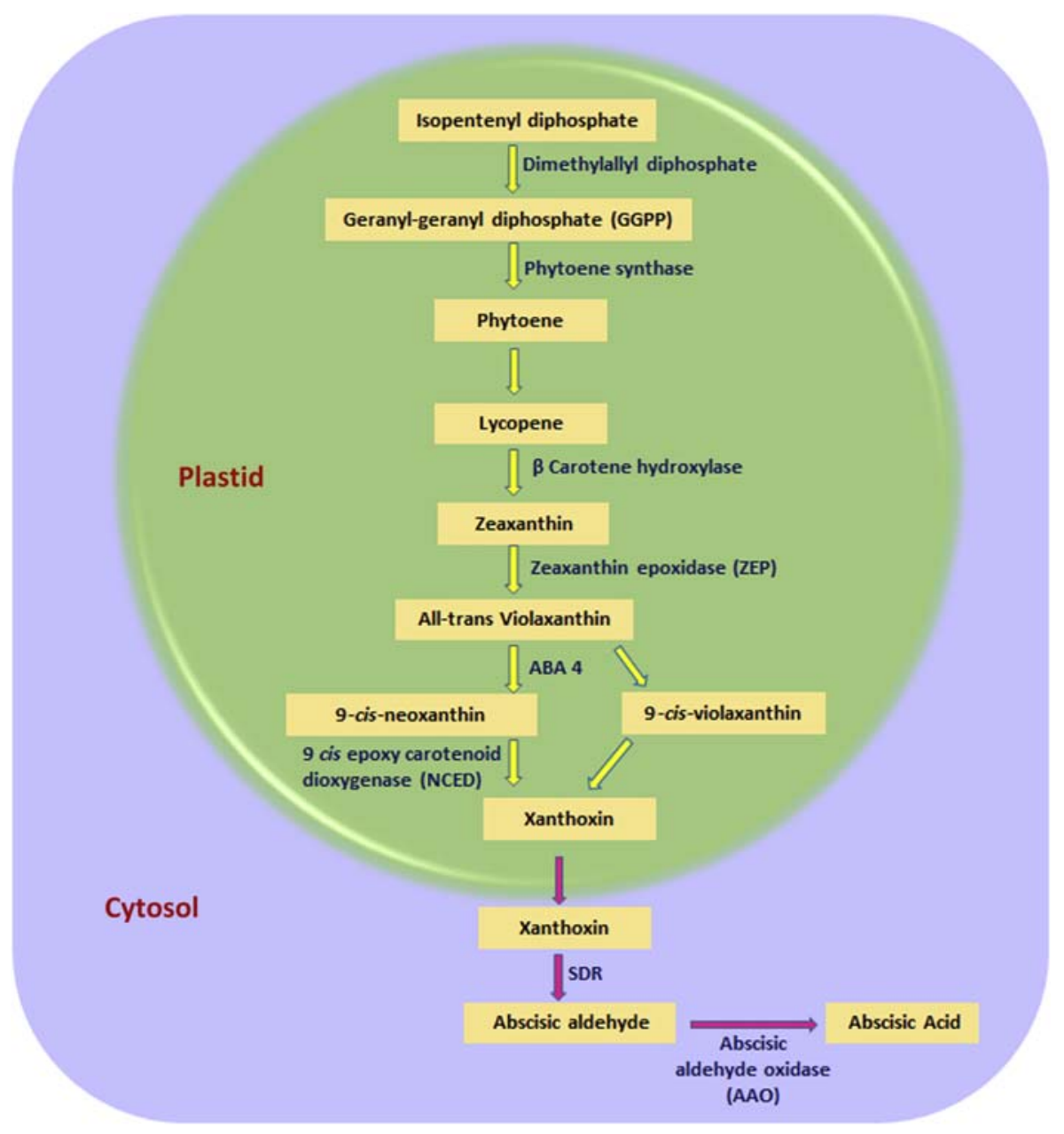

FIGURE 22.1 Schematic representation of abscisic acid biosynthesis pathway. Most of the reactions of ABA biosynthesis occur in the plastid. In the first step isopentenyl diphosphate is converted to a $\mathrm{C}_{20}$ molecule geranylgeranyl diphosphate (GGPP) by the enzyme dimethylallyl diphosphate. Through a series of reactions GGPP is converted to xanthoxin in the plastid. Xanthoxin is transported to the cytosol by an unknown mechanism where it is converted to abscisic aldehyde by the enzyme short chain dehydrogenase/reductase-like (SDR1). Finally, abscisic aldehyde is converted to abscisic acid by the action of the enzyme abscisic aldehyde oxidase (AAO).

when subjected to osmotic stress conditions ( $\mathrm{Li}$ et al., 2017). Upregulation of ZEP gene also occurs on onset of drought stress in Nicotiana plumbaginifolia, Arabidopsis, and Lycopersicon roots (Audran et al., 1998, 2001). Hence, these findings support the key role of ZEP in regulating ABA synthesis in plants. Another crucial gene, 9-cis-epoxy-carotenoid dioxygenase (NCED), has a key role in ABA biosynthesis regulation. It has been observed that with the onset of stress, increase in level of ABA accompanies upsurge in NCED mRNA and protein, and whenever ABA level decreases, there is a fall in the level of NCED expression (Finkelstein, 2013). Rapid conversion of xanthophyll to ABA has been observed, especially during drought stress and xanthophyll is oxidatively cleaved to form ABA (Vishwakarma et al., 2017). This was reported in Phaseolus vulgaris where PvNCED1 accumulates under water deficit conditions, prior to ABA accumulation (Qin and Zeevaart, 1999). In avocado (Persea americana), higher expression of PaNCED1 and $P a N C E D 3$ were correlated with high ABA level during fruit ripening (Chernys and Zeevaart, 2000). Several independent researchers have witnessed such an increase in ABA level upon overexpression of NCED gene, for example; AtNCED3 in Arabidopsis (Audran et al., 2001), LeNCED1 in tomato and PvNCED1 in tobacco (Schwartz and Zeevaart, 2010). Additionally, enzymes involved in the final step of ABA synthesis have a crucial role in regulating ABA level. However, lack of studies limits our knowledge on the role of this gene in the regulation mechanism of ABA synthesis.

\subsection{CATABOLISM OF ABA}

Under normal (unstressed) conditions, the amount of $\mathrm{ABA}$ in the cell must be reduced, thus, excess amount of ABA present needs to be neutralized. Catabolism and conjugation are important mechanisms for regulating the level of ABA (Nambara and MarionPoll, 2005). Hydroxylation is a common reaction to catabolize $\mathrm{ABA}$, and there exist three different ways of ABA hydroxylation in plants. It can be either hydroxylated at the C-7', C-8, or at C-9' position. However, hydroxylation at the $\mathrm{C}-8^{\prime}$ position is the predominant pathway (Li et al., 2017). ABA is inactivated through 
hydroxylation at the $8^{\prime}$ position by the action of the enzyme ABA- $8^{\prime}$ hydroxylase, which is a cytochrome P450 (Okamoto et al., 2011). Since 8'ABA is unstable, it is rapidly converted to phaseic acid, which is further converted to dihydrophaseic acid. In another mechanism, ABA is converted to $9^{\prime}$ hydroxyl ABA by $9^{\prime}$ hydroxylation. Whether both $8^{\prime}$ and $9^{\prime}$ hydroxylation are carried out by the same enzyme is not known and demands investigation. In planta regulation of ABA levels by hydroxylation has been displayed in Arabidopsis during water stress, salinity, and osmotic stress conditions, where abundance of multiple CYP707A transcripts and proteins were observed (Kushiro et al., 2004). In adzuki bean (Vigna angularis), ABA-glucosyltransferase $(A O G)$ gene expression was rapidly increased under drought conditions ( $\mathrm{Xu}$ et al., 2002). AOG catalyzes the transfer of a glycosyl group from a donor to an acceptor. Another way of checking and catabolizing higher level of ABA is by conjugating it with glucose ester to form ABA-GE, which is eventually stored in the vacuole. This form of ABA is inactive and can be considered as a storage form. Low permeability of biomembranes for ABA-GE suggests that it may be involved in long distance transport. ABA may be transported as ABA-GE and upon reaching the destined organ or tissue it may be cleaved to produce a functionally active form.

\subsection{ABA SIGNALING PATHWAY}

Generally, a signal transduction pathway involves initiation of signal by a stimulus, perception by the receptors, and transduction of signal by effectors and secondary messengers, leading to activation or inactivation of downstream components to generate the desired response. The core components of ABA signal transduction pathway include ABA receptors (ABAR), a group of START proteins called Pyrabactin resistance/ Pyrabactin resistance like/Regulatory component of ABA receptor (PYR/PYL/RCAR), protein phosphatase 2C (PP2C), and sucrose non-fermenting 1 (SNF1) related kinase 2 (SnRK2) group of serine/threonine kinases (Singh et al., 2016). In the absence of ABA, ABARs remain unbound and inactive, whereas $\mathrm{PP} 2 \mathrm{C}$ physically interacts with the SnRK2 and masks its kinase activation loop, thereby blocking the downstream signaling cascade. In the presence of $A B A$, as in case of stress and developmental trigger, $\mathrm{ABAR}$ binds to $\mathrm{ABA}$, undergoes a conformational change and exposes its surface to interact with PP2C. Upon binding of PP2C with the receptors, inhibition of SnRK2 activity is removed. After unmasking, SnRK2 undergoes autophosphorylation and becomes active. Activated SnRK2 phosphorylates and regulates the activity of set of downstream genes including transcription factors, ion transport channel and ROS generating enzyme (Fig. 22.2). Function of activated or repressed downstream components ultimately lead to adaptive response (Sheard and Zheng, 2009; Sirichandra et al., 2009; Umezawa et al., 2010). A few of the identified targets of SnRK2s include inward rectifying $\mathrm{K}^{+}$CHANNEL IN Arabidopsis thaliana (KAT1) (Sato et al., 2009), SLOW ANION CHANNEL ASSOCIATED1 (SLAC1) (Geiger et al., 2009; Lee et al., 2009a), ROS generating enzyme RESPIRATORY BURST OXIDASE HOMOLOG F (RBOHF) (Sirichandra et al., 2009) and bZIP transcription factor (Nakashima et al., 2009).

\subsection{ABA RECEPTORS}

ABARs are the key components in ABA perception and were discovered recently by screening for mutants that were unable to grow in the presence of an $\mathrm{ABA}$ agonist Pyrabactin (Park et al., 2009). PYR/PYL/RCAR proteins are the members of soluble ligand binding START-domain protein superfamily (Ma et al., 2009; Park et al., 2009). The proteins of this family are comprised of a characteristic helix-grip fold and form a ligand binding pocket in the center. PYR/PYL/RCAR family proteins contain a START domain or Bet $\mathrm{v}$ fold, named after a major allergen in pollen of white birch (Betula verrucosa) (Radauer et al., 2008). PYR/PYL/ RCARs contain an N-terminal alpha helical segment that is absent in Bet $\mathrm{v}$ fold. Also, they have a helix-grip fold consisting of seven stranded beta sheets flanked by two alpha helices. Crystal structures of PYL1 (Melcher et al., 2009) and PYL2 (Melcher et al., 2009; Yuan et al., 2010) have been delineated, both in free and ABA bound form. The structures of these receptors are highly similar, however there are some dissimilarities in the receptor binding pocket. Pyrabactin is an agonist of PYR1 and PYL1, however, it is an antagonist of PYL2 (Melcher et al., 2009; Peterson et al., 2010). Generally, the ABA binding pocket is buried deep within the receptor. The chemical structure of various functional groups of the ABA molecule complement with the walls of this pocket, thus allowing ABA to fit well inside the pocket. The hydroxyl, carboxylic, and ketone groups of ABA molecule interact with the polar side chain of PYR1. Whereas, the isoprene moiety and the cyclohexane ring of ABA molecule form hydrophobic interaction with apolar side chain of the PYR1 molecule (Santiago et al., 2009). A total of 14 genes encoding 159-211 amino acid-long PYR/PYL/RCAR protein are identified in the Arabidopsis genome (Santiago et al., 2012). PYR/PYL/RCAR proteins are highly conserved at the level of amino acid sequence. The function of PYR/PYL/RCAR proteins as ABA receptors has been validated in plants. The triple 


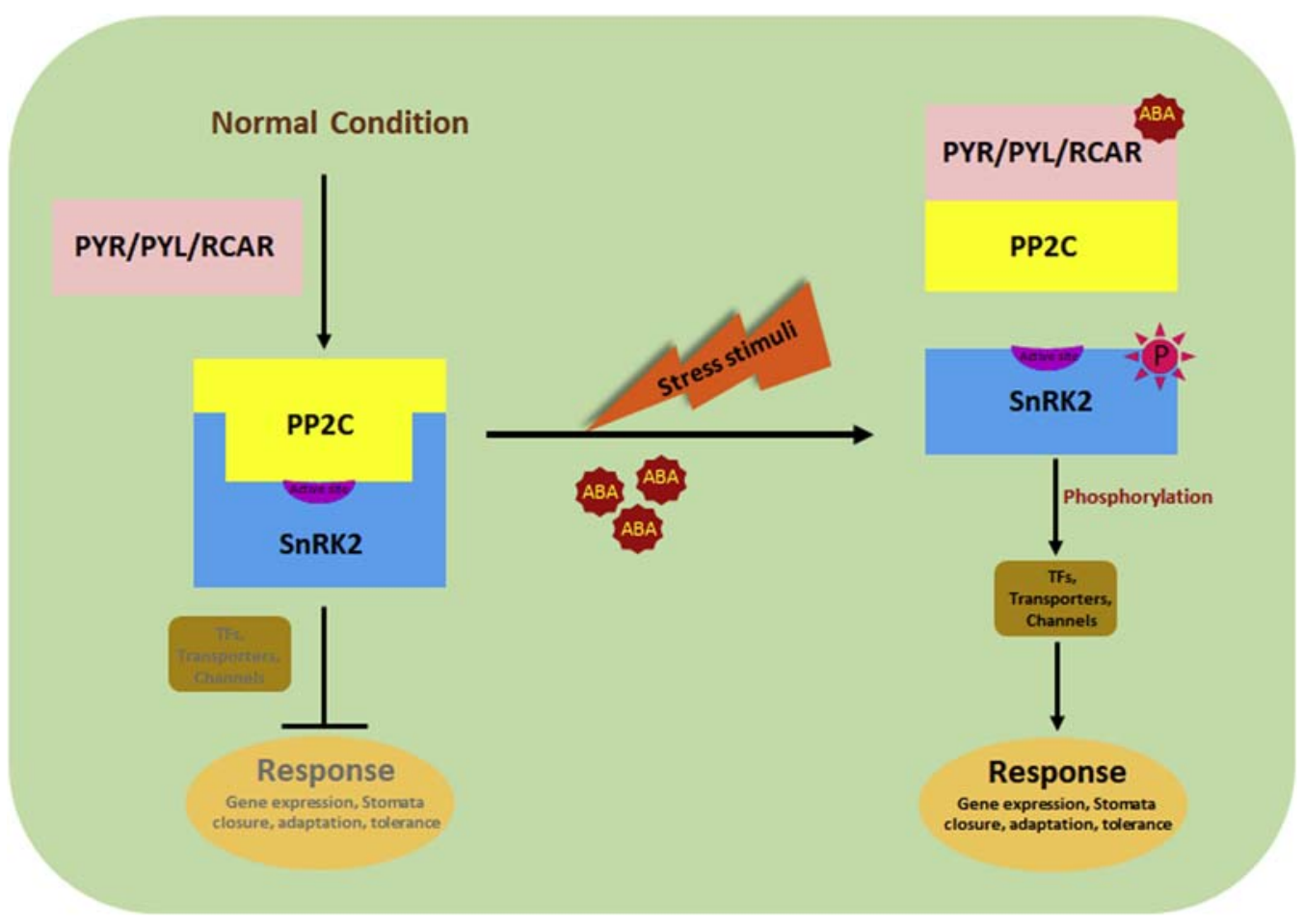

FIGURE 22.2 ABA signal transduction pathway in plants. In normal (unstressed) conditions due to absence or low level of ABA, ABAR (PYR/PYL/RCAR) remains unbound. Whereas, PP2C physically interacts with the SnRK2 and masks its kinase activation loop, thereby blocking the downstream signaling cascade. Perception of stress stimuli results in accumulation of ABA in the cell. In the presence of ABA, ABAR binds to $\mathrm{ABA}$ and undergoes a conformational change and interacts with PP2C. Interaction of ABAR with PP2C unmasks the inhibition from SnRK2, and the free SnRK2 undergoes autophosphorylation and becomes active. Activated SnRK2 phosphorylates and regulates the activity of a set of downstream genes including transcription factors, ion transport channel and ROS generating enzyme to generate an adaptive response, leading to stress tolerance.

mutant pyr1/pyrl1/pyrl4 and the quadruple mutant pyr1/pyrl1/pyrl2/pyrl4 are less sensitive to ABA and have abnormal root growth and germination (Gonzalez-Guzman et al., 2012). Moreover, quadruple mutants showed abnormal stomata closure even in presence of ABA (Nishimura et al., 2009; Santiago et al., 2009). On the other hand, overexpression of PYL8 and PYL9 enhanced transpiration rate in Arabidopsis (Ma et al., 2009; Saavedra et al., 2010). The rice and the poplar (Populus trichocarpa) genomes also encode for 14 members of the PYR/PYL/RCAR family (Tian et al., 2015; Yu et al., 2017a). These ABA receptors have been shown to regulate abiotic stress, particularly drought stress tolerance in rice, Arabidopsis, and tomato (González-Guzmán et al., 2014; Kim et al., 2014; Zhao et al., 2016). In a recent study, overexpression of poplar PtPYL1 and PtPYL5 in Arabidopsis conferred hypersensitivity to ABA and drought tolerance (Yu et al., 2016). Thereafter, overexpression of PtPYL1 and PtPYL5 also enhanced drought tolerance in hybrid poplar (Populus davidiana X Populus bolleana). Moreover, overexpression of PtPYL1 and PtPYL5 in hybrid poplar resulted in increased leaf size, which could be a strategy of the plant to retain more water under drought stress (Yu et al., 2017b). This genetic evidence confirmed the role of PYR/PYL/RCAR as $\mathrm{ABA}$ receptor, their importance in $\mathrm{ABA}$ signaling and their key role in mediating stress tolerance in plants.

\subsection{PROTEIN PHOSPHATASE 2C}

Protein phosphatases 2C (PP2C) are the largest group of serine/threonine protein phosphatase in plants and comprised of 80,90, and 91 members in Arabidopsis, rice, and tomato, respectively (Singh et al., 2016). In rice and Arabidopsis, the PP2C family is subdivided into 11 subclades, from $\mathrm{A}$ to $\mathrm{K}$ (Singh et al., 2010). PP2Cs consist of a catalytic domain, which can be located either at the N-terminus or at the C-terminus (Schweighofer et al., 2004). This contributes to their structural variability and functional diversity. PP2Cs are the monomeric enzymes and they require metal ions such as $\mathrm{Mn}^{2+}$ or $\mathrm{Mg}^{2+}$ as cofactors for their activity (Singh and Pandey, 2012). Recent identification of novel ABA receptors in plants have placed PP2C at the center stage of the entire ABA signaling pathway. Several studies have shown that group A PP2Cs are 
majorly involved in ABA related function, and act as negative regulators of ABA signaling (Bhaskara et al., 2012; Komatsu et al., 2009; Merlot et al., 2001; Saez et al., 2004; Singh et al., 2015). PP2Cs localize both in the cytosol and the nucleus, however they form a complex with ABARs mainly in the nucleus (Moes et al., 2008). Arabidopsis PP2C group A is comprised of nine members including ABI1, ABI2, HAB1, HAB2, AHG1, AHG3, HAI1, HAI2, and HAI3 (Singh et al., 2015) ABI1 is the first PP2C to be cloned (Meyer et al., 1994) followed by its closest homolog ABI2. Group A PP2Cs of rice, Arabidopsis, maize, and tomato are highly inducible under ABA, drought, salinity, and cold stress (Singh et al., 2010, 2015; Sun et al., 2011; Wei and Pan, 2014). In Arabidopsis, group A PP2Cs are critical in regulating $\mathrm{ABA}$ signaling under abiotic stresses. Arabidopsis ABI1 and ABI2 are the most extensively studied PP2Cs in plants and established as the major player of ABA signaling under abiotic stresses and during development (Fuchs et al., 2013; Singh and Pandey, 2012). Elimination of group A PP2Cs allows survival of mosses in complete desiccation conditions, suggesting that group A PP2Cs are important regulators of desiccation tolerance (Komatsu et al., 2013). Besides regulating function of SnRK2s in ABA signaling, PP2Cs also control abiotic stress triggered MAPK signaling (Danquah et al., 2014). PP2Cs dephosphorylate and deactivate MAPKs, thereby blocking the downstream signaling cascade (Smékalová et al., 2014). PP2Cs are also recognized as important regulators of plant development. They control and regulate plant development at various stages, including panicle stage, leaves, roots, inflorescence, etc. (Kamenetsky et al., 2015; Xue et al., 2008). Changes in the transcript abundance of various PP2C genes during reproductive development (seed and panicle) were observed in rice (Singh et al., 2010).

\subsection{SNF1-RELATED PROTEIN KINASE 2 (SNRK2)}

In Arabidopsis, 39 SnRK protein kinases are present that are divided into three different families: SnRK1 (3 members), SnRK2 (10 members), and SnRK3 (26 members) (Hrabak et al., 2003; Yan and Chen, 2017), based on the sequence similarity. SnRK2s have been recognized as the key players of ABA signaling pathway. Several members of SnRK2 family, such as SnRK2.1, 2.2, 2.3, and 2.6 are implicated in ABA related function in Arabidopsis (Fernando and Schroeder, 2016; Nakashima et al., 2009). The first SnRK2 shown to be involved in ABA signaling pathway was PKABA1, which is transcriptionally upregulated by $A B A$ and suppresses gibberellic acid induced gene expression in aleurone layers of barley (Gómez-Cadenas et al., 1999). Later on, in-gel kinase assay showed that AAPK (ABA activated serine-threonine protein kinase) regulates ABA induced stomata closure in Vicia faba (Kwak et al., 2006). OST1 (Open stomata 1; SnRK2.6) is an ortholog of AAPK in Arabidopsis and SnRK 2.2, 2.3 are other members of the kinase family that are closely related to OST1. Double mutant snrk2.2/2.3 exhibited ABA insensitive phenotype, in terms of inhibition of seed generation and root growth, and reduction in expression of ABA inducible genes (Fujii et al., 2007). Furthermore, triple mutant snrk2.2/2.3/2.6 showed severe defects in ABA signaling and related responses, suggesting that SnRK2s are the positive regulators of ABA signaling (Nakashima et al., 2009). Interestingly, all SnRK2s were activated by osmotic stress except SnRK2.9, and Arabidopsis mutants defective in all 10 SnRK2s show defect in gene regulation and ABA accumulation under osmotic stress (Fujii et al., 2011). Hence, SnRK2s are indispensable for ABA dependent osmotic stress response in plants. Recently, overexpression of poplar PtSnRK2.5 and PtSnRK2.7 in Arabidopsis led to enhanced salt stress tolerance (Song et al., 2016). Furthermore, PtSnRK2s exhibited differential expression pattern in response to $\mathrm{ABA}$ treatment and different stress conditions, in an organ specific manner (Yu et al., 2017a). Interestingly, heterologous expression of Arabidopsis SnRK2C resulted in enhanced osmotic and salt stress tolerance in poplar (Yu et al., 2017b). Apart from SnRK2, members of the SnRK3 family are also implicated in ABA signaling. For instance, PKS3/CIPK15 interacted with calcium binding protein $\mathrm{SCaBP} 5 / \mathrm{CBL} 1$, leading to insensitivity in seed germination and stomata movement, thereby negatively regulating the $\mathrm{ABA}$ signaling pathway (Yu et al., 2006). The cipk23 null mutants showed decreased transpirational water loss and stomata opening, thus CIPK23 was recognized as a negative regulator of ABA signaling and response (Cheong et al., 2007; Zhang et al., 2014).

\subsection{ABA-DEPENDENT GENE EXPRESSION}

ABA induces the expression of various genes, especially transcription factors. About $10 \%$ of the protein coding genes are regulated by ABA in Arabidopsis (Fujita et al., 2011). Essentially, ABA dependent gene expression is regulated by two main families of bZIP transcription factors, $\mathrm{AREB} / \mathrm{ABF}$ during vegetative stages and ABI5 in the seeds. During osmotic stress when the plant is still in the vegetative stage, the wide range of the ABA-mediated gene expression is governed by the AREB/ABF pathway (Bartels and Souer, 
2004; Shinozaki and Yamaguchi-Shinozaki, 2007). One essential feature of genes regulated by $\mathrm{ABA}$ is that they contain G-box (CACGTG), ABA responsive ciselement (ABRE: PyAC GTGG/TC) in their promoters (Gómez-Porras et al., 2007). These elements have an ACGT central core that is recognized by the bZIP transcription factors in the plants (Choi et al., 2000). Transcription factors of the AREB family are another major group of genes that mediate ABA-dependent gene expression. Analysis of transgenic Arabidopsis plants overexpressing $A R E B 1 / A B F 2, A R E B 2 / A B F 4$, or $A B F 3$ showed involvement of these transcription factors in stress responsive ABA signaling (Kim et al., 2004). Further, the ectopic expression of $A B F 2$ in transgenic Arabidopsis plants could not activate target genes such as $R D 29 B$ (Fujita et al., 2005) and ABF2 requires ABA for full activation (Yoshida et al., 2010). These transcription factors are considered as the master regulators of ABA-mediated gene expression under osmotic stress. This is supported by the observation that areb1/areb2/areb3 triple mutants are ABA insensitive and showed significantly lower drought tolerance as compared with the single and double mutants (Yoshida et al., 2010). AP2/ERFs are another important group of transcription factors controlling ABAmediated gene expression (Nakano et al., 2006). DREB is the most widely studied AP2/ERF and DRE/CRT binding protein namely, DREB1, has an important role in abiotic stress signaling. These transcription factors bind to the DRE/CRT cis-acting motif (A/GCCGAC), which is crucial for induction of many stress related genes (Agarwal et al., 2006). Recently, overexpression of AtERF15 and AtERF19 conferred drought tolerance in the transgenic Arabidopsis plants (Lee et al., 2015; Scarpeci et al., 2016). RAP2.1 and RAP2.6, the two $\mathrm{AP2} / \mathrm{ERF}$ transcription factors that are identified as subregulons of DREB/CBF (Fowler and Thomashow, 2002). RAP2.6 interacts with cis-element CE1 (TGCCACCGG) or GCC (AGCCGCC) and acts as a positive regulator of osmotic stress in an ABA dependent manner (Zhu et al., 2010). Whereas, RAP2.1 acts as a negative regulator in ABA signaling pathway during cold and drought stress (Dong and Liu, 2010). Another AP2, ADAP (ARIA-interacting double AP2 domain protein) is considered a positive regulator of ABA response. The adap knockout mutant plants showed reduced drought tolerance and partial insensitivity to ABA (Lee et al., 2009b). Plant MYB transcription factors belong to the R2R3-type MYB family. In Arabidopsis, 126 R2R3-MYB genes are present and few of them act as mediators of ABA-dependent gene expression (Dubos et al., 2010). Transgenic Arabidopsis plants overexpressing $M Y B 2$ and $M Y C 2$ exhibited enhanced osmotic stress tolerance and sensitivity to ABA as compared with the wild type plants
(Bhattacharjee and Jain, 2013). MYB 96 mediates ABAdependent expression of $R D 22$ gene to confer drought stress tolerance via auxin signaling pathway (Seo et al., 2009). Hence, it can be inferred that MYB96 converges the ABA and auxin signaling pathway at some point. Furthermore, transcript level of MYB transcription factors, like MYB108 and MYB44, was upregulated upon treatment with various hormones and stress conditions like drought and salinity (Jung et al., 2007, 2008). All these findings by and large confirm that R2R3 MYB transcription factors control ABA-mediated gene expression in various stress conditions by targeting different signaling pathways. In Arabidopsis, 74 WRKY TFs are present and form a large gene family (Rushton et al., 2010). The major role of WRKY transcription factors is the transcriptional reprogramming in diverse cellular processes. WRKY63 controls the expression of stress related genes, such as $A B F 2$ and $R D 29 A$, thereby mediating plants responses to ABA, drought, and salt stress (Jiang et al., 2014; Ren et al., 2010). The expression of AtWRKY40, AtWRKY18, AtWRKY60, and AtWRKY63 was increased upon ABA treatment (Rushton et al., 2012). Overexpression of wheat (Triticum aestivum) TaWRKY1 and TaWRKY33 in Arabidopsis resulted in heat and drought stress tolerance (He et al., 2016). Other important regulators of transcription in plants are NAC transcription factors. They play an important role in biotic and abiotic stress signaling in plants (Nuruzzaman et al., 2013). The expression of NAC transcription factors is induced under drought conditions in vital crop plants like rice, soybean, maize, and wheat (Hao et al., 2011; Hong et al., 2016; Huang et al., 2015; Lu et al., 2012; Rabbani et al., 2003). Several NAC transcription factors have been demonstrated in improving drought stress tolerance through ABA signaling pathway in various plants like rice, wheat, tobacco, and Arabidopsis (Christiansen et al., 2011; Jeong et al., 2010; Ramegowda et al., 2012; Xue et al., 2011). Recently, expression of OsNACO22 was increased fourfold after ABA treatment and OsNAC022 overexpressing plants showed tolerance to drought and salt stress in rice (Hong et al., 2016). These findings prove that the transcription factors have multiplex roles in controlling ABA-responsive gene expressions in plants.

\subsection{THE ROLE OF ABA IN ABIOTIC STRESS SIGNALING}

Plants are sessile and are often exposed to various stresses in their natural habitat. Adverse environmental conditions disrupt the normal cellular functioning, thus decreasing the plants' productivity significantly. However, plants have devised adaptive mechanisms to combat adverse growth conditions. Triggering an array 
of signaling networks is one of the major adaptive responses. ABA signaling has been established as an important signaling network triggered in response to abiotic stresses (Cutler et al., 2010; Singh and Pandey, 2012). ABA mediates drought, salinity, and cold stress responses and tolerance in plants (Huang et al., 2012; Qin et al., 2011). One of the crucial functions of ABA is to regulate stomatal movement under osmotic/ drought stress. Higher levels of ABA promote stomata closure and inhibit the transport of positively charged ions across the guard cell membrane to regulate the rate of transpiration under water deficit conditions (Albert et al., 2017; Haworth et al., 2018). The onset of stress leads to elevated ABA concentration, which instigates an increase in cytosolic calcium concentration. An increase in cytosolic calcium concentrations results in activation of $S$ type (slow activating channel) and R type (rapid transient) channels (Schroeder et al., 2001). SLAC1 anion channel allows release of anionic organic acid from vacuole to the cytoplasm (Sirichandra et al., 2009). Also, an increase in the guard cell ABA level activates the $\mathrm{K}^{+}$outward rectifying channel and inhibits the $\mathrm{K}^{+}$inward rectifying channel (KAT1, KAT2). Regulation of these channels leads to anion efflux from the guard cell, causing membrane depolarization, which further leads to the efflux of $\mathrm{K}^{+}$ from the guard cell (Kim et al., 2010). This reduces the guard cell turgor leading to stomata closure. OST1 is an important protein kinase involved in regulating stomata aperture through ABA signaling (Sirichandra et al., 2009). OST1 is known to be induced under osmotic stress and targets inward rectifying potassium channel KAT1 to control its channel activity through phosphorylation (Sato et al., 2009). Thus, OST1 positively regulates stomata closure by negative regulation of KAT1 activity in an ABA-dependent manner (Umezawa et al., 2010). During water deficit conditions, ABA inhibits shoot growth and promotes root growth, thereby enhancing the root:shoot ratio ( $\mathrm{Li}$ et al., 2017). Increasing the root growth enables the plant to efficiently absorb available water and hence prevents the plant from dehydrating. $\mathrm{ABA}$ is known to influence the activity of various aquaporins by regulating their gene expression or via posttranslational modification of aquaporins (Sharipova et al., 2016). During water scarcity, dephosphorylation of various aquaporins occur at the $\mathrm{N}$-terminus in an ABA dependent manner. The posttranslational modification of aquaporins helps to reduce the water loss (Kline et al., 2010; Shatil-Cohen et al., 2011). Several studies of overexpression transgenic plants have unearthed the important roles of ABA in drought and salinity stress tolerance in various plants species. OsbZIP overexpression in rice plants conferred drought tolerance through ABA signaling pathway (Tang et al., 2012). The overexpression of $\mathrm{ABA}$ responsive transcription factor $A R E B / A B F$ or BIS/AtDPBF led to induction of stressresponsive gene like LEA (late embryogenesis abundant protein) (Bartels and Sunkar, 2005). LEA proteins act as molecular chaperones and protect the cell membrane integrity during stress. During osmotic stress, these proteins protect mRNA, lipid, and various enzymes from dehydration (Yamaguchi-Shinozaki and Shinozaki, 2006). Interestingly, exogenous application of ABA conferred drought tolerance in various flowering plants like Salvia divinorum, Impatiens walleriana, Tagetes erecta, and Viola tricolor (Harris et al., 2011). Recently, dehydrin genes are found to confer osmotic stress tolerance through ABA dependent pathway, in various plant species, including sorghum, maize, and Prunus mume (Bao et al., 2017; Halder et al., 2016; Zamora-Briseño and de Jiménez, 2016). Salt stress is detrimental to the plants as it hampers their photosynthetic efficiency and metabolic processes (Fernando and Schroeder, 2016). ABA is also known to mediate salinity stress response and tolerance in plants. Salinity stress has an inhibitory effect on the primary and lateral root growth. During salinity stress, lateral roots undergo a quiescent stage during which they develop thick casparian strips in the endodermis, a process supported by high ABA concentration. The thick casparian strips act as a barrier to salt diffusion (Duan et al., 2013). The HISTONE DEACETYLASE6 (HDAC6) played a crucial role in salt stress tolerance and seed germination in an ABA-dependent manner (Chen et al., 2010). Also, an important role of cuticle in stress signaling and ABA biosynthesis was elucidated. CED1 (9CIS EPOXYCAROTENOID DIOXYGENASE DEFECTIVE 1) is an essential protein in the cuticle formation and ced1 mutants are defective in ABA production, thereby showing sensitivity towards the osmotic stress (Wang et al., 2011). Recently, Reaumuria trigyna $R t W R K Y$ gene was induced by $\mathrm{ABA}$ and $\mathrm{NaCl}$ treatments and overexpression of RtWRKY in Arabidopsis plants showed enhanced salt tolerance (Du et al., 2017). The AtDIF1 (Arabidopsis thaliana Drought induced F Box1) is induced upon ABA, drought, and salinity treatment. The AtDIF1 overexpression contributes to salt tolerance by reducing $\mathrm{Na}^{+}$content and promoting seedling growth in transgenic plants through $\mathrm{ABA}$ signaling. However AtDIF1 had antagonistic effect under drought stress as transgenic plants showed reduced seedling growth (Gao et al., 2017). These results showed that AtDIF1 positively regulates salinity stress tolerance and negative regulates drought stress response.

In the recent past, various researchers have shown the role of ABA in cold stress tolerance as well. Coldinducible dehydrin (WCS120) and ABA accumulation was reported to confer cold stress tolerance in wheat 
(Kosová et al., 2012). Moreover, ABA and dehydrin accumulation results in increased cold acclimation induced frost tolerance in Triticum monococcum (Vanková et al., 2014). In prior studies, overexpression of wheat WCOR410 (wheat cold regulated gene) in cucumber and potato, and DHN24 (dehydrin 24) in strawberry, resulted in enhanced frost tolerance in transgenic plants (Yin et al., 2006). Overall, these findings advocate that ABA is an important stress hormone and enables the plants to stand against various stress conditions.

\subsection{CONCLUSION}

ABA is an important hormone involved in diverse plant processes and signaling networks. The recent breakthrough discovery of $\mathrm{ABA}$ receptors in plants has enabled researchers to understand the ABA signaling pathway comprehensively. Before understanding the role of $\mathrm{ABA}$ in different conditions, it is important to understand the biosynthesis of ABA in plants. ABA biosynthesis mainly occurs in the plastid and partly in the cytosol, where a number of intermediate products are formed with the help of several enzymes. Genetic manipulation of these enzymes, especially like that of NCED and AAO, would be vital to regulate the ABA level. Thus, a balance of ABA concentration could be achieved in stressed and unstressed conditions. Several groups of transcription factors, such as bZIP, AP2, WRKY, MYB and others, have been the major effectors of ABA signaling under stress and developmental events. These transcription factors receive the signal from upstream and perform transcriptional reprogramming at the downstream for adaptive response. Targeting transcription factors that are involved in ABA response for genetic manipulation has helped researchers to generate plants that show better tolerance to various abiotic stress. Several transgenic based studies have established the crucial role of ABA in osmotic, drought, salinity and cold in a spectrum of plant species. Therefore, ABA is a vital phytohormone required for plant adaptation to different stresses in the environment. In future, analysis of interactions of ABA signaling with other phytohormones such as cytokinin and ethylene, and crosstalk of signaling pathways, may provide the new paradigm in plant abiotic stress signaling.

\section{Acknowledgment}

The authors are thankful to National Institute of Plant Genome Research (NIPGR), India for providing core research grant and DBT (Department of Biotechnology)-eLibrary Consortium (DeLCON), India for providing access to e-resources. SS is thankful to the Council of Scientific and Industrial Research (CSIR), India for research fellowships.

\section{References}

Agarwal, P.K., Agarwal, P., Reddy, M., Sopory, S.K., 2006. Role of DREB transcription factors in abiotic and biotic stress tolerance in plants. Plant Cell Rep. 25 (12), 1263-1274.

Albert, R., Acharya, B.R., Jeon, B.W., Zañudo, J.G., Zhu, M., Osman, K., et al., 2017. A new discrete dynamic model of ABA-induced stomatal closure predicts key feedback loops. PLoS Biol. 15 (9), e2003451.

Audran, C., Borel, C., Frey, A., Sotta, B., Meyer, C., Simonneau, T., et al., 1998. Expression studies of the zeaxanthin epoxidase gene in Nicotiana plumbaginifolia. Plant Physiol. 118 (3), 1021-1028.

Audran, C., Liotenberg, S., Gonneau, M., North, H., Frey, A., Tap-Waksman, K., et al., 2001. Localisation and expression of zeaxanthin epoxidase mRNA in Arabidopsis in response to drought stress and during seed development. Funct. Plant Biol. 28 (12), 1161-1173.

Banerjee, A., Roychoudhury, A., 2017. Abscisic-aciddependent basic leucine zipper (bZIP) transcription factors in plant abiotic stress. Protoplasma 254 (1), 3-16.

Bao, F., Du, D., An, Y., Yang, W., Wang, J., Cheng, T., et al., 2017. Overexpression of Prunus mume dehydrin genes in tobacco enhances tolerance to cold and drought. Front. Plant Sci. 8, 151.

Barrero, J.M., Piqueras, P., González-Guzmán, M., Serrano, R., Rodríguez, P.L., Ponce, M.R., et al., 2005. A mutational analysis of the ABA1 gene of Arabidopsis thaliana highlights the involvement of ABA in vegetative development. J. Exp. Bot. 56 (418), 2071-2083.

Bartels, D., Souer, E., 2004. Molecular responses of higher plants to dehydration. In: Hirt, H., Shinozaki, K. (Eds.), Plant Responses to Abiotic Stress. Topics in Current Genetics. Springer, Berlin, Heidelberg, pp. 9-38.

Bartels, D., Sunkar, R., 2005. Drought and salt tolerance in plants. CRC. Crit. Rev. Plant. Sci. 24 (1), 23-58.

Bhaskara, G.B., Nguyen, T.T., Verslues, P.E., 2012. Unique drought resistance functions of the highly ABA-induced clade A protein phosphatase 2Cs. Plant Physiol. 160 (1), 379-395.

Bhattacharjee, A., Jain, M., 2013. Transcription factor mediated abiotic stress signaling in rice. In: Pandey, G.K. (Ed.), Stress Mediated Signaling in Plants. Plant Stress 7. Global Science Books, pp. 16-25.

Bittner, F., Oreb, M., Mendel, R.R., 2001. ABA3 is a molybdenum cofactor sulfurase required for activation of aldehyde oxidase and xanthine dehydrogenase in Arabidopsis thaliana. J. Biol. Chem. 276 (44), 40381-40384.

Chen, L.T., Luo, M., Wang, Y.Y., Wu, K., 2010. Involvement of Arabidopsis histone deacetylase HDA6 in ABA and salt stress response. J. Exp. Bot. 61 (12), 3345-3353.

Cheong, Y.H., Pandey, G.K., Grant, J.J., Batistic, O., Li, L., Kim, B.G., et al., 2007. Two calcineurin B-like calcium sensors, interacting with protein kinase CIPK23, regulate leaf transpiration and root potassium uptake in Arabidopsis. Plant J. 52 (2), 223-239.

Chernys, J.T., Zeevaart, J.A., 2000. Characterization of the 9-cisepoxycarotenoid dioxygenase gene family and the regulation of abscisic acid biosynthesis in avocado. Plant Physiol. 124 (1), $343-354$.

Choi, H., Hong, J., Ha, J., Kang, J., Kim, S.Y., 2000. ABFs, a family of ABA-responsive element binding factors. J. Biol. Chem. 275 (3), 1723-1730.

Christiansen, M.W., Holm, P.B., Gregersen, P.L., 2011. Characterization of barley (Hordeum vulgare L.) NAC transcription factors suggests conserved functions compared to both monocots and dicots. BMC Res. Notes 4 (1), 302.

Colebrook, E.H., Thomas, S.G., Phillips, A.L., Hedden, P., 2014. The role of gibberellin signaling in plant responses to abiotic stress. J. Exp. Biol. 217, 67-75. 
Cracker, L., Abeles, F.B., 1969. Abscission: role of abscisic acid. Plant Physiol. 44 (8), 1144-1149.

Cutler, S.R., Rodriguez, P.L., Finkelstein, R.R., Abrams, S.R., 2010. Abscisic acid: emergence of a core signaling network. Annu. Rev. Plant. Biol. 61, 651-679.

Danquah, A., de Zelicourt, A., Colcombet, J., Hirt, H., 2014. The role of $\mathrm{ABA}$ and MAPK signaling pathways in plant abiotic stress responses. Biotechnol. Adv. 32 (1), 40-52.

Dong, C.J., Liu, J.Y., 2010. The Arabidopsis EAR-motif-containing protein RAP2. 1 functions as an active transcriptional repressor to keep stress responses under tight control. BMC Plant Biol. 10 (1), 47.

Du, C., Zhao, P., Zhang, H., Li, N., Zheng, L., Wang, Y., 2017. The Reaumuria trigyna transcription factor RtWRKY1 confers tolerance to salt stress in transgenic Arabidopsis. J. Plant. Physiol. 215, 48-58.

Duan, L., Dietrich, D., Ng, C.H., Chan, P.M.Y., Bhalerao, R., Bennett, M.J., et al., 2013. Endodermal ABA signaling promotes lateral root quiescence during salt stress in Arabidopsis seedlings. Plant Cell 25 (1), 324-341.

Dubos, C., Stracke, R., Grotewold, E., Weisshaar, B., Martin, C., Lepiniec, L., 2010. MYB transcription factors in Arabidopsis. Trends. Plant. Sci. 15 (10), 573-581.

Fernando, V.D., Schroeder, D.F., 2016. Role of ABA in arabidopsis salt, drought, and desiccation tolerance. In: Shankar, A. (Ed.), Abiotic and Biotic Stress in Plants-Recent Advances and Future Perspectives. InTech, pp. 507-524.

Finkelstein, R., 2013. Abscisic acid synthesis and response. Arabidopsis Book 11, e0166.

Fowler, S., Thomashow, M.F., 2002. Arabidopsis transcriptome profiling indicates that multiple regulatory pathways are activated during cold acclimation in addition to the CBF cold response pathway. Plant Cell 14 (8), 1675-1690.

Frey, A., Audran, C., Marin, E., Sotta, B., Marion-Poll, A., 1999. Engineering seed dormancy by the modification of zeaxanthin epoxidase gene expression. Plant Mol. Biol. 39 (6), 1267-1274.

Fuchs, S., Grill, E., Meskiene, I., Schweighofer, A., 2013. Type 2C protein phosphatases in plants. FEBS. J. 280 (2), 681-693.

Fujii, H., Verslues, P.E., Zhu, J.-K., 2007. Identification of two protein kinases required for abscisic acid regulation of seed germination, root growth, and gene expression in Arabidopsis. Plant Cell 19 (2), 485-494.

Fujii, H., Verslues, P.E., Zhu, J.-K., 2011. Arabidopsis decuple mutant reveals the importance of SnRK2 kinases in osmotic stress responses in vivo. Proc. Natl. Acad. Sci. 108 (4), 1717-1722.

Fujita, Y., Fujita, M., Satoh, R., Maruyama, K., Parvez, M.M., Seki, M., et al., 2005. AREB1 is a transcription activator of novel ABREdependent ABA signaling that enhances drought stress tolerance in Arabidopsis. Plant Cell 17 (12), 3470-3488.

Fujita, Y., Fujita, M., Shinozaki, K., Yamaguchi-Shinozaki, K., 2011. ABA-mediated transcriptional regulation in response to osmotic stress in plants. J. Plant. Res. 124 (4), 509-525.

Gao, S., Song, J.B., Wang, Y., Yang, Z.M., 2017. An F-box E3 ubiquitin ligase-coding gene AtDIF1 is involved in Arabidopsis salt and drought stress responses in an abscisic acid-dependent manner. Environ. Exp. Bot. 138, 21-35.

Geiger, D., Scherzer, S., Mumm, P., Stange, A., Marten, I., Bauer, H., et al., 2009. Activity of guard cell anion channel SLAC1 is controlled by drought-stress signaling kinase-phosphatase pair. Proc. Natl. Acad. Sci. 106 (50), 21425-21430.

Gómez-Cadenas, A., Verhey, S.D., Holappa, L.D., Shen, Q., Ho, T.-H. D., Walker-Simmons, M., 1999. An abscisic acid-induced protein kinase, PKABA1, mediates abscisic acid-suppressed gene expression in barley aleurone layers. Proc. Natl. Acad. Sci. 96 (4), 1767-1772.

Gómez-Porras, J.L., Riaño-Pachón, D.M., Dreyer, I., Mayer, J.E., Mueller-Roeber, B., 2007. Genome-wide analysis of ABA-responsive elements ABRE and CE3 reveals divergent patterns in Arabidopsis and rice. BMC Genom. 8 (1), 260.

Gonzalez-Guzman, M., Pizzio, G.A., Antoni, R., Vera-Sirera, F., Merilo, E., Bassel, G.W., et al., 2012. Arabidopsis PYR/PYL/ RCAR receptors play a major role in quantitative regulation of stomatal aperture and transcriptional response to abscisic acid. Plant Cell 24 (6), 2483-2496.

González-Guzmán, M., Rodríguez, L., Lorenzo-Orts, L., Pons, C., Sarrión-Perdigones, A., Fernández, M.A., et al., 2014. Tomato PYR/PYL/RCAR abscisic acid receptors show high expression in root, differential sensitivity to the abscisic acid agonist quinabactin, and the capability to enhance plant drought resistance. J. Exp. Bot. 65 (15), 4451-4464.

Halder, T., Agarwal, T., Ray, S., 2016. Isolation, cloning, and characterization of a novel Sorghum dehydrin (SbDhn2) protein. Protoplasma 253 (6), 1475-1488.

Hao, Y.J., Wei, W., Song, Q.X., Chen, H.W., Zhang, Y.Q., Wang, F., et al., 2011. Soybean NAC transcription factors promote abiotic stress tolerance and lateral root formation in transgenic plants. Plant J. 68 (2), 302-313.

Harris, J.C., Hrmova, M., Lopato, S., Langridge, P., 2011. Modulation of plant growth by HD-Zip class I and II transcription factors in response to environmental stimuli. New Phytol. 190 (4), 823-837.

Haworth, M., Marino, G., Cosentino, S.L., Brunetti, C., De Carlo, A., Avola, G., et al., 2018. Increased free abscisic acid during drought enhances stomatal sensitivity and modifies stomatal behaviour in fast growing giant reed (Arundo donax L.). Environ. Exp. Bot. 147, $116-124$

He, G.-H., Xu, J.-Y., Wang, Y.-X., Liu, J.-M., Li, P.-S., Chen, M., et al., 2016. Drought-responsive WRKY transcription factor genes TaWRKY1 and TaWRKY33 from wheat confer drought and/or heat resistance in Arabidopsis. BMC Plant Biol. 16 (1), 116.

Hong, Y., Zhang, H., Huang, L., Li, D., Song, F., 2016. Overexpression of a stress-responsive NAC transcription factor gene ONAC022 improves drought and salt tolerance in rice. Front. Plant Sci. 7, 4.

Hrabak, E.M., Chan, C.W., Gribskov, M., Harper, J.F., Choi, J.H., Halford, N., et al., 2003. The Arabidopsis CDPK-SnRK superfamily of protein kinases. Plant Physiol. 132 (2), 666-680.

Huang, G.-T., Ma, S.-L., Bai, L.-P., Zhang, L., Ma, H., Jia, P., et al., 2012. Signal transduction during cold, salt, and drought stresses in plants. Mol. Biol. Rep. 39 (2), 969-987.

Huang, Q., Wang, Y., Li, B., Chang, J., Chen, M., Li, K., et al., 2015. TaNAC29, a NAC transcription factor from wheat, enhances salt and drought tolerance in transgenic Arabidopsis. BMC Plant Biol. 15 (1), 268.

Iqbal, N., Khan, N.A., Ferrante, A., Trivellini, A., Francini, A., Khan, M.I.R., 2017. Ethylene role in plant growth, development and senescence: interaction with other phytohormones. Front. Plant Sci. 8,475 .

Jeong, J.S., Kim, Y.S., Baek, K.H., Jung, H., Ha, S.-H., Do Choi, Y., et al., 2010. Root-specific expression of OsNAC10 improves drought tolerance and grain yield in rice under field drought conditions. Plant Physiol. 153 (1), 185-197.

Jiang, Y., Duan, Y., Yin, J., Ye, S., Zhu, J., Zhang, F., et al., 2014. Genomewide identification and characterization of the Populus WRKY transcription factor family and analysis of their expression in response to biotic and abiotic stresses. J. Exp. Bot. 65 (22), 6629-6644.

Jung, C., Lyou, S.H., Yeu, S., Kim, M.A., Rhee, S., Kim, M., et al., 2007. Microarray-based screening of jasmonate-responsive genes in Arabidopsis thaliana. Plant Cell Rep. 26 (7), 1053-1063.

Jung, C., Seo, J.S., Han, S.W., Koo, Y.J., Kim, C.H., Song, S.I., et al., 2008. Overexpression of AtMYB44 enhances stomatal closure to confer abiotic stress tolerance in transgenic Arabidopsis. Plant Physiol. 146 (2), 623-635. 
Kamenetsky, R., Faigenboim, A., Mayer, E.S., Michael, T.B., Gershberg, C., Kimhi, S., et al., 2015. Integrated transcriptome catalogue and organ-specific profiling of gene expression in fertile garlic (Allium sativum L.). BMC Genom. 16 (1), 12.

Kazan, K., 2013. Auxin and the integration of environmental signals into plant root development. Ann. Bot. (Lond.) 112, 1655-1665.

Kazan, K., 2015. Diverse roles of jasmonates and ethylene in abiotic stress tolerance. Trends Plant Sci. 20 (4), 219-229.

Khan, M.I.R., Khan, N.A., 2013. Salicylic Acid and Jasmonates: approaches in Abiotic Stress Tolerance. Plant Biochem. Physiol. 1, 4.

Khan, M.I.R., Khan, N.A., 2014. Ethylene reverses photosynthetic inhibition by nickel and zinc in mustard through changes in PS II activity, photosynthetic-nitrogen use efficiency and antioxidant metabolism. Protoplasma 251, 1007-1019.

Khan, M.I.R., Khan, N.A., Fatma, M., Asgher, M., Syeed, S., Masood, A., et al., 2015b. Photosynthesis in changing environment: analyzing the role of phytohormones in modulation. In: Khan, N.A. (Ed.), Photosynthesis: Functional Genomics, Physiological Processes and Environmental Issues Chapters Books. Nova Publication, pp. 129-166.

Khan, M.I.R., Fatma, M., Per, T.S., Anjum, N.A., Khan, N.A., 2015a. Salicylic acid-induced abiotic stress tolerance and underlying mechanisms in plants. Front. Plant Sci. 6, 462.

Kim, H., Lee, K., Hwang, H., Bhatnagar, N., Kim, D.-Y., Yoon, I.S., et al., 2014. Overexpression of PYL5 in rice enhances drought tolerance, inhibits growth, and modulates gene expression. J. Exp. Bot. 65 (2), 453-464.

Kim, J.M., To, T.K., Nishioka, T., Seki, M., 2010. Chromatin regulation functions in plant abiotic stress responses. Plant Cell Environ. 33 (4), 604-611.

Kim, S., Kang, J.Y., Cho, D.I., Park, J.H., Kim, S.Y., 2004. ABF2, an ABRE-binding bZIP factor, is an essential component of glucose signaling and its overexpression affects multiple stress tolerance. Plant J. 40 (1), 75-87.

Kirby, J., Keasling, J.D., 2009. Biosynthesis of plant isoprenoids: perspectives for microbial engineering. Annu. Rev. Plant. Biol. 60, 335-355.

Kline, K.G., Barrett-Wilt, G.A., Sussman, M.R., 2010. In planta changes in protein phosphorylation induced by the plant hormone abscisic acid. Proc. Natl. Acad. Sci. 107 (36), 15986-15991.

Komatsu, K., Nishikawa, Y., Ohtsuka, T., Taji, T., Quatrano, R.S., Tanaka, S., et al., 2009. Functional analyses of the ABI1-related protein phosphatase type $2 \mathrm{C}$ reveal evolutionarily conserved regulation of abscisic acid signaling between Arabidopsis and the moss Physcomitrella patens. Plant Mol. Biol. 70 (3), 327-340.

Komatsu, K., Suzuki, N., Kuwamura, M., Nishikawa, Y., Nakatani, M., Ohtawa, H., et al., 2013. Group A PP2Cs evolved in land plants as key regulators of intrinsic desiccation tolerance. Nat. Commun. 4, 2219.

Kosová, K., Prášil, I.T., Vítámvás, P., Dobrev, P., Motyka, V., Floková, K., et al., 2012. Complex phytohormone responses during the cold acclimation of two wheat cultivars differing in cold tolerance, winter Samanta and spring Sandra. J. Plant. Physiol. 169 (6), 567-576.

Kushiro, T., Okamoto, M., Nakabayashi, K., Yamagishi, K., Kitamura, S., Asami, T., et al., 2004. The Arabidopsis cytochrome P450 CYP707A encodes ABA 8'-hydroxylases: key enzymes in ABA catabolism. EMBO J. 23 (7), 1647-1656.

Kwak, J.M., Nguyen, V., Schroeder, J.I., 2006. The role of reactive oxygen species in hormonal responses. Plant Physiol. 141 (2), 323-329.

Lee, S., Kang, J., Kim, S.Y., 2009b. An ARIA-interacting AP2 domain protein is a novel component of ABA signaling. Mol. Cells 27 (4), $409-416$

Lee, S., Lee, S., Kim, S.Y., 2015. AtERF15 is a positive regulator of ABA response. Plant Cell Rep. 34 (1), 71-81.
Lee, S.C., Lan, W., Buchanan, B.B., Luan, S., 2009a. A protein kinasephosphatase pair interacts with an ion channel to regulate ABA signaling in plant guard cells. Proc. Natl. Acad. Sci. 106 (50), 21419-21424.

Li, X., Chen, L., Forde, B.G., Davies, W.J., 2017. The biphasic root growth response to abscisic acid in Arabidopsis involves interaction with ethylene and auxin signalling pathways. Front. Plant Sci. 8, 1493.

Lu, M., Ying, S., Zhang, D.-F., Shi, Y.-S., Song, Y.-C., Wang, T.-Y., et al., 2012. A maize stress-responsive NAC transcription factor, ZmSNAC1, confers enhanced tolerance to dehydration in transgenic Arabidopsis. Plant Cell Rep. 31 (9), 1701-1711.

Ma, Y., Szostkiewicz, I., Korte, A., Moes, D., Yang, Y., Christmann, A., et al., 2009. Regulators of PP2C phosphatase activity function as abscisic acid sensors. Science 324 (5930), 1064-1068.

Melcher, K., Ng, L.-M., Zhou, X.E., Soon, F.-F., Xu, Y., Suino-Powell, K.M., et al., 2009. A gate-latch-lock mechanism for hormone signaling by abscisic acid receptors. Nature 462 (7273), 602-608.

Merlot, S., Gosti, F., Guerrier, D., Vavasseur, A., Giraudat, J., 2001. The ABI1 and ABI2 protein phosphatases $2 \mathrm{C}$ act in a negative feedback regulatory loop of the abscisic acid signaling pathway. Plant J. 25 (3), 295-303.

Meyer, K., Leube, M.P., Grill, E., 1994. A protein phosphatase 2C involved in ABA signal transduction in Arabidopsis thaliana. Science 264 (5164), 1452-1455.

Moes, D., Himmelbach, A., Korte, A., Haberer, G., Grill, E., 2008. Nuclear localization of the mutant protein phosphatase abil is required for insensitivity towards $\mathrm{ABA}$ responses in Arabidopsis. Plant J. 54 (5), 806-819.

Nakano, T., Suzuki, K., Fujimura, T., Shinshi, H., 2006. Genome-wide analysis of the ERF gene family in Arabidopsis and rice. Plant Physiol. 140 (2), 411-432.

Nakashima, K., Ito, Y., Yamaguchi-Shinozaki, K., 2009. Transcriptional regulatory networks in response to abiotic stresses in Arabidopsis and grasses. Plant Physiol. 149 (1), 88-95.

Nambara, E., Marion-Poll, A., 2005. Abscisic acid biosynthesis and catabolism. Annu. Rev. Plant. Biol. 56, 165-185.

Nishimura, N., Hitomi, K., Arvai, A.S., Rambo, R.P., Hitomi, C., Cutler, S.R., et al., 2009. Structural mechanism of abscisic acid binding and signaling by dimeric PYR1. Science 326 (5958), 1373-1379.

Nuruzzaman, M., Sharoni, A.M., Kikuchi, S., 2013. Roles of NAC transcription factors in the regulation of biotic and abiotic stress responses in plants. Front. Microbiol. 4, 248.

Okamoto, M., Kushiro, T., Jikumaru, Y., Abrams, S.R., Kamiya, Y., Seki, M., et al., 2011. ABA 9'-hydroxylation is catalyzed by CYP707A in Arabidopsis. Phytochemistry 72 (8), 717-722.

Park, S.Y., Fung, P., Nishimura, N., Jensen, D.R., Fujii, H., Zhao, Y., et al., 2009. Abscisic acid inhibits type $2 \mathrm{C}$ protein phosphatases via the PYR/PYL family of START proteins. Science 324 (5930), $1068-1071$.

Per, T.S., Khan, M.I.R., Anjum, N.A., Masood, A., Hussain, S.J., Khan, N.A., 2018. Jasmonates in plants under abiotic stresses: crosstalk with other phytohormones matters. Environ. Exp. Bot. 145, 104-120.

Peterson, F.C., Burgie, E.S., Park, S.-Y., Jensen, D.R., Weiner, J.J., Bingman, C.A., et al., 2010. Structural basis for selective activation of ABA receptors. Nat. Struct. Mol. Biol. 17 (99), 1109-1113.

Qin, F., Shinozaki, K., Yamaguchi-Shinozaki, K., 2011. Achievements and challenges in understanding plant abiotic stress responses and tolerance. Plant Cell Physiol. 52 (9), 1569-1582.

Qin, X., Zeevaart, J.A., 1999. The 9-cis-epoxycarotenoid cleavage reaction is the key regulatory step of abscisic acid biosynthesis in water-stressed bean. Proc. Natl. Acad. Sci. 96 (26), 15354-15361. 
Rabbani, M.A., Maruyama, K., Abe, H., Khan, M.A., Katsura, K., Ito, Y., et al., 2003. Monitoring expression profiles of rice genes under cold, drought, and high-salinity stresses and abscisic acid application using cDNA microarray and RNA gel-blot analyses. Plant Physiol. 133 (4), 1755-1767.

Radauer, C., Lackner, P., Breiteneder, H., 2008. The Bet v 1 fold: an ancient, versatile scaffold for binding of large, hydrophobic ligands. BMC Evol. Biol. 8 (1), 286.

Ramegowda, V., Senthil-Kumar, M., Nataraja, K.N., Reddy, M.K., Mysore, K.S., Udayakumar, M., 2012. Expression of a finger millet transcription factor, EcNAC1, in tobacco confers abiotic stresstolerance. PLoS. ONE. 7 (7), e40397.

Ren, X., Chen, Z., Liu, Y., Zhang, H., Zhang, M., Liu, Q., et al., 2010. $\mathrm{ABO}$, a WRKY transcription factor, mediates plant responses to abscisic acid and drought tolerance in Arabidopsis. Plant J. 63 (3), 417-429.

Rushton, D.L., Tripathi, P., Rabara, R.C., Lin, J., Ringler, P., Boken, A.K., et al., 2012. WRKY transcription factors: key components in abscisic acid signalling. Plant. Biotechnol. J. 10 (1), 2-11.

Rushton, P.J., Somssich, I.E., Ringler, P., Shen, Q.J., 2010. WRKY transcription factors. Trends. Plant. Sci. 15 (5), 247-258.

Saavedra, X., Modrego, A., Rodríguez, D., González-García, M.P., Sanz, L., Nicolás, G., et al., 2010. The nuclear interactor PYL8/ RCAR3 of Fagus sylvatica FsP2C1 is a positive regulator of abscisic acid signaling in seeds and stress. Plant Physiol. 152 (1), $133-150$.

Saez, A., Apostolova, N., Gonzalez-Guzman, M., Gonzalez-Garcia, M.P., Nicolas, C., Lorenzo, O., et al., 2004. Gain-of-function and loss-of-function phenotypes of the protein phosphatase 2C HAB1 reveal its role as a negative regulator of abscisic acid signalling. Plant J. 37 (3), 354-369.

Santiago, J., Dupeux, F., Round, A., Antoni, R., Park, S.-Y., Jamin, M., et al., 2009. The abscisic acid receptor PYR1 in complex with abscisic acid. Nature 462 (7273), 665-668.

Santiago, J., Dupeux, F., Betz, K., Antoni, R., Gonzalez-Guzman, M., Rodriguez, L., et al., 2012. Structural insights into PYR/PYL/ RCAR ABA receptors and PP2Cs. Plant Sci. 182, 3-11.

Santino, A., Taurino, M., De Domenico, S., Bonsegna, S., Poltronieri, P., Pastor, V., et al., 2013. Jasmonate signaling in plant development and defense response to multiple (a)biotic stresses. Plant Cell Rep. 32 (7), 1085-1098.

Sato, A., Sato, Y., Fukao, Y., Fujiwara, M., Umezawa, T., Shinozaki, K., et al., 2009. Threonine at position 306 of the KAT1 potassium channel is essential for channel activity and is a target site for ABA-activated SnRK2/OST1/SnRK2. 6 protein kinase. Biochem. J. 424 (3), 439-448.

Scarpeci, T.E., Frea, V.S., Zanor, M.I., Valle, E.M., 2016. Overexpression of AtERF019 delays plant growth and senescence, and improves drought tolerance in Arabidopsis. J. Exp. Bot. 68 (3), 673-685.

Schroeder, J.I., Kwak, J.M., Allen, G.J., 2001. Guard cell abscisic acid signalling and engineering drought hardiness in plants. Nature 410 (6826), 327-330.

Schwartz, S.H., Zeevaart, J.A., 2010. Abscisic acid biosynthesis and metabolism. In: Davies, P.J. (Ed.), Plant Hormones - Biosynthesis, Signal Transduction, Action, third ed. Springer, Netherland, pp. $137-155$.

Schweighofer, A., Hirt, H., Meskiene, I., 2004. Plant PP2C phosphatases: emerging functions in stress signaling. Trends. Plant. Sci. 9 (5), 236-243.

Seo, P.J., Xiang, F., Qiao, M., Park, J.-Y., Lee, Y.N., Kim, S.-G., et al., 2009. The MYB96 transcription factor mediates abscisic acid signaling during drought stress response in Arabidopsis. Plant Physiol. 151 (1), 275-289.

Sharipova, G., Veselov, D., Kudoyarova, G., Fricke, W., Dodd, I.C., Katsuhara, M., et al., 2016. Exogenous application of abscisic acid
(ABA) increases root and cell hydraulic conductivity and abundance of some aquaporin isoforms in the ABA-deficient barley mutant Az34. Ann. Bot. (Lond.) 118 (4), 777-785.

Shatil-Cohen, A., Attia, Z., Moshelion, M., 2011. Bundle-sheath cell regulation of xylem-mesophyll water transport via aquaporins under drought stress: a target of xylem-borne ABA? Plant J. 67 (1), 72-80.

Sheard, L.B., Zheng, N., 2009. Plant biology: signal advance for abscisic acid. Nature 462 (7273), 575-576.

Shinozaki, K., Yamaguchi-Shinozaki, K., 2007. Gene networks involved in drought stress response and tolerance. J. Exp. Bot. 58 (2), 221-227.

Singh, A., Giri, J., Kapoor, S., Tyagi, A.K., Pandey, G.K., 2010. Protein phosphatase complement in rice: genome-wide identification and transcriptional analysis under abiotic stress conditions and reproductive development. BMC Genom. 11 (1), 435.

Singh, A., Jha, S.K., Bagri, J., Pandey, G.K., 2015. ABA inducible rice protein phosphatase $2 \mathrm{C}$ confers $\mathrm{ABA}$ insensitivity and abiotic stress tolerance in Arabidopsis. PLoS One 10 (4), e0125168.

Singh, A., Pandey, A., Srivastava, A.K., Tran, L.-S.P., Pandey, G.K., 2016. Plant protein phosphatases 2C: from genomic diversity to functional multiplicity and importance in stress management. Crit. Rev. Biotechnol. 36 (6), 1023-1035.

Singh, A., Pandey, G.K., 2012. Protein phosphatases: a genomic outlook to understand their function in plants. J. Plant Biochem. Biotechnol. 21 (1), 100-107.

Singh, A., Sagar, S., Biswas, D.K., 2018. Calcium Dependent Protein Kinase, a Versatile Player in Plant Stress Management and Development. CRC. Crit. Rev. Plant. Sci. Available from: https://doi. org/10.1080/07352689.2018.1428438.

Sirichandra, C., Wasilewska, A., Vlad, F., Valon, C., Leung, J., 2009. The guard cell as a single-cell model towards understanding drought tolerance and abscisic acid action. J. Exp. Bot. 60 (5), 1439-1463.

Smékalová, V., Doskocilová, A., Komis, G., Šamaj, J., 2014. Crosstalk between secondary messengers, hormones and MAPK modules during abiotic stress signalling in plants. Biotechnol. Adv. 32 (1), $2-11$.

Song, X., Yu, X., Hori, C., Demura, T., Ohtani, M., Zhuge, Q., 2016. Heterologous overexpression of poplar SnRK2 genes enhanced salt stress tolerance in Arabidopsis thaliana. Front. Plant Sci. 7, 612.

Sun, L., Wang, Y.-P., Chen, P., Ren, J., Ji, K., Li, Q., et al., 2011. Transcriptional regulation of SIPYL, SIPP2C, and SISnRK2 gene families encoding ABA signal core components during tomato fruit development and drought stress. J. Exp. Bot. 62 (15), 5659-5669.

Tang, N., Zhang, H., Li, X., Xiao, J., Xiong, L., 2012. Constitutive activation of transcription factor OsbZIP46 improves drought tolerance in rice. Plant Physiol. 158 (4), 1755-1768.

Tian, X., Wang, Z., Li, X., Lv, T., Liu, H., Wang, L., et al., 2015. Characterization and functional analysis of pyrabactin resistancelike abscisic acid receptor family in rice. Rice 8 (1), 28.

Umezawa, T., Nakashima, K., Miyakawa, T., Kuromori, T., Tanokura, M., Shinozaki, K., et al., 2010. Molecular basis of the core regulatory network in ABA responses: sensing, signaling and transport. Plant Cell Physiol. 51 (11), 1821-1839.

Vanková, R., Kosová, K., Dobrev, P., Vítámvás, P., Trávníčková, A., Cvikrová, M., et al., 2014. Dynamics of cold acclimation and complex phytohormone responses in Triticum monococcum lines G3116 and DV92 differing in vernalization and frost tolerance level. Environ. Exp. Bot. 101, 12-25.

Vishwakarma, K., Upadhyay, N., Kumar, N., Yadav, G., Singh, J., Mishra, R.K., et al., 2017. Abscisic acid signaling and abiotic stress tolerance in plants: a review on current knowledge and future prospects. Front. Plant Sci. 8, 161. 
Wang, Z.-Y., Xiong, L., Li, W., Zhu, J.-K., Zhu, J., 2011. The plant cuticle is required for osmotic stress regulation of abscisic acid biosynthesis and osmotic stress tolerance in Arabidopsis. Plant Cell 23 (5), 1971-1984.

Wei, K., Pan, S., 2014. Maize protein phosphatase gene family: identification and molecular characterization. BMC Genomics 15 (1), 773.

Xu, Z.-J., Nakajima, M., Suzuki, Y., Yamaguchi, I., 2002. Cloning and characterization of the abscisic acid-specific glucosyltransferase gene from adzuki bean seedlings. Plant Physiol. 129 (3), 1285-1295.

Xue, G.-P., Way, H.M., Richardson, T., Drenth, J., Joyce, P.A., McIntyre, C.L., 2011. Overexpression of TaNAC69 leads to enhanced transcript levels of stress up-regulated genes and dehydration tolerance in bread wheat. Mol. Plant 4 (4), 697-712.

Xue, T., Wang, D., Zhang, S., Ehlting, J., Ni, F., Jakab, S., et al., 2008. Genome-wide and expression analysis of protein phosphatase $2 \mathrm{C}$ in rice and Arabidopsis. BMC Genomics 9 (1), 550.

Yamaguchi-Shinozaki, K., Shinozaki, K., 2006. Transcriptional regulatory networks in cellular responses and tolerance to dehydration and cold stresses. Ann. Rev. Plant Biol. 57, 781-803.

Yan, A., Chen, Z., 2017. The pivotal role of abscisic acid signaling during transition from seed maturation to germination. Plant Cell Rep. 36 (5), 689-703.

Yin, Z., Rorat, T., Szabala, B.M., Ziólkowska, A., Malepszy, S., 2006. Expression of a Solanum sogarandinum SK 3-type dehydrin enhances cold tolerance in transgenic cucumber seedlings. Plant Sci. 170 (6), 1164-1172.

Yoshida, T., Fujita, Y., Sayama, H., Kidokoro, S., Maruyama, K., Mizoi, J., et al., 2010. AREB1, AREB2, and ABF3 are master transcription factors that cooperatively regulate ABRE-dependent $\mathrm{ABA}$ signaling involved in drought stress tolerance and require ABA for full activation. Plant J. 61 (4), 672-685.

Yu, J., Ge, H., Wang, X., Tang, R., Wang, Y., Zhao, F., et al., 2017b. Overexpression of Pyrabactin Resistance-Like Abscisic Acid Receptors Enhances Drought, Osmotic, and Cold Tolerance in Transgenic Poplars. Front. Plant Sci. 8, 1752.
Yu, J., Yang, L., Liu, X., Tang, R., Wang, Y., Ge, H., et al., 2016. Overexpression of Poplar Pyrabactin Resistance-Like Abscisic Acid Receptors Promotes Abscisic Acid Sensitivity and Drought Resistance in Transgenic Arabidopsis. PLoS. ONE. 11 (12), e0168040.

Yu, X., Takebayashi, A., Demura, T., Ohtani, M., 2017a. Differential expression of poplar sucrose nonfermenting1-related protein kinase 2 genes in response to abiotic stress and abscisic acid. J. Plant. Res. 130 (5), 929-940.

Yu, X.-C., Li, M.-J., Gao, G.-F., Feng, H.-Z., Geng, X.-Q., Peng, C.-C., et al., 2006. Abscisic acid stimulates a calcium-dependent protein kinase in grape berry. Plant Physiol. 140, 558-579.

Yuan, G.-F., Jia, C.-G., Li, Z., Sun, B., Zhang, L.-P., Liu, N., et al., 2010. Effect of brassinosteroids on drought resistance and abscisic acid concentration in tomato under water stress. Sci. Hortic. (Amsterdam) 126 (2), 103-108.

Zamora-Briseño, J.A., de Jiménez, E.S., 2016. A LEA 4 protein upregulated by $\mathrm{ABA}$ is involved in drought response in maize roots. Mol. Biol. Rep. 43 (4), 221-228.

Zhang, T., Chen, S., Harmon, A.C., 2014. Protein phosphorylation in stomatal movement. Plant Signal. Behav. 9 (11), e972845.

Zhao, Y., Chan, Z., Gao, J., Xing, L., Cao, M., Yu, C., et al., 2016. ABA receptor PYL9 promotes drought resistance and leaf senescence. Proc. Natl. Acad. Sci. 113 (7), 1949-1954.

Zhu, Q., Zhang, J., Gao, X., Tong, J., Xiao, L., Li, W., et al., 2010. The Arabidopsis AP2/ERF transcription factor RAP2. 6 participates in ABA, salt and osmotic stress responses. Gene 457 (1), $1-12$.

\section{Further Reading}

Lee, S.-J., Cho, D.-I., Kang, J.-Y., Kim, M.-D., Kim, S.Y., 2010. AtNEK6 interacts with ARIA and is involved in ABA response during seed germination. Mol. Cells 29 (6), 559-566. 
This page intentionally left blank 


\title{
23
}

\section{Salicylic Acid-Mediated Defense Mechanisms to Abiotic Stress Tolerance}

\author{
Farhan Ahmad ${ }^{1}$, Ananya Singh ${ }^{2}$ and Aisha Kamal ${ }^{1}$ \\ ${ }^{1}$ Department of Bioengineering, Integral University, Lucknow, Uttar Pradesh, India ${ }^{2}$ Department of Biosciences, \\ Integral University, Lucknow, Uttar Pradesh, India
}

\section{O U T L I N E}

\subsection{Introduction}

23.2 Biosynthetic Pathway and Modifications of Salicylic Acid

23.3 Role of Salicylic Acid in Plant Growth and Development

356

23.4 Salicylic Acid-Mediated Defense Mechanisms to Abiotic Stress

23.4.1 Salicylic Acid Involvement in Modulation of Redox Homeostasis

23.4.2 Salicylic Acid Interaction With Heat Shock Proteins, Reactive Oxygen Species and Mitogen-Activated Protein Kinase

23.4.3 Interaction of Salicylic Acid With Mineral Nutrients and

Osmoprotectant
23.5 Crosstalk of Salicylic Acid With Other Phytohormones Under Abiotic Stress

23.5.1 Salicylic Acid and Auxins 23.5.2 Salicylic Acid and Abscisic Acid 23.5.3 Salicylic Acid and Gibberellic Acid

23.6 Interaction and Involvement of DELLA With SA-GA Crosstalk Under Stress

23.6.1 Salicylic Acid and Ethylene 23.6.2 Salicylic Acid and Jasmonic Acid 363 23.6.3 Salicylic Acid and Brassinosteroids 364 23.6.4 Salicylic Acid and Nitric Oxide

23.7 Conclusion and Future Prospects

Acknowledgements

References

Further Reading

\subsection{INTRODUCTION}

Plant overall growth and development metabolism is well-coordinated and regulated by low molecular weight compounds that work as chemical messengers to perform a precise role in plants known as phytohormones (Voß et al., 2014). The active site of phytohormones may be close to the place of synthesis or may be transported to different location wherever prerequisite for the regulation of biological functioning either in normal or stressful environment (Khan and Khan, 2014; Per et al., 2018). The beneficial effect of 
phytohormones directly linked with plant growth and development and involving engineering for premeditated stress resistance has been well-established (Wani et al., 2016). The positive growth regulators of plants can be listed as auxins (AUX), gibberellic acid (GA), and cytokinins (CK). The growth regulator primarily involved in dormant and stress conditions, for example, salicylic acid (SA), abscisic acid (ABA), ethylene (ET), and recently identified compounds such as, jasmonates (JA) derived compound, brassinosteroids (BRs), and nitric oxide (NO), achieve much consideration to improve plant growth through stress tolerance. Among all, SA, chemically known as 2-hydroxybenzoic acid, is a phenolic watchdog, involved directly or indirectly in growth and development, thermogenesis, and enhanced protection against disease (Khan and Khan, 2013; Khan et al., 2012, 2015a,b; Klessig, 2017). SA is fully dedicated to alleviating lifethreatening consequences of abiotic stresses such as salinity tolerance (Cao et al., 2009; Ahmad et al., 2017), heat stress (Wang and Li, 2006; Khan et al., 2013a,b), metal stress (Nazar et al., 2015), etc. It was reported that exogenous spraying of SA enhanced photosynthetic capability; production of secondary metabolites such as proline, flavonoid, and saponin content; and promoted LOX inhibitory action, thus improving the antioxidant defense system of the plant (Khan et al., 2013a,b; Ibrahim et al., 2017). SA, either in the developmental stage or environmentally adverse conditions prompted the expression of stress-specific genes and proteins, the activation of molecular chaperone, mitogen activated protein kinases (MAPKs), and maintains balance between ROS formation and detoxification, to enhance tolerance against abiotic stress (Xu and Brosché, 2014). Further, the beneficial interactions of SA with other phytohormones in both biotic as well as abiotic response have also been well known with jasmonic acid (Khan et al., 2013a,b; Per et al., 2018), ethylene (Tirani et al., 2013; Nguyen et al., 2016), and brassinosteroids (Saini et al., 2015; Khatoon et al., 2017). However, the detailed mechanism of SA crosstalk with other phytohormones under stress is not well known. In this review, the crosstalk of SA with other phytohormones in boosting plant strength under ideal and adverse environments is deliberated with emphasis on the most suitable and justifiable mechanism, and other unresolved, unfamiliar perspectives have also been explored.

\subsection{BIOSYNTHETIC PATHWAY AND MODIFICATIONS OF SALICYLIC ACID}

The biosynthetic pathway for synthesis of SA adopted by plants encompasses two enzymatic pathways, that is, ICS (isochorismate synthase) and IPL (isochorismate pyruvate lyase), from a common precursor, chorismic acid (Strawn et al., 2007). The conversion of shikimic acid to SA is a multistep, enzyme catalyzed biochemical reaction via intermediates such as phenylalanine and cinnamic acid. The conversion of phenylalanine to SA accomplished by two routes in which transformation of orthocoumaric acid or benzoic acid by enzymatic action of BA 2-hydroxylase (BA2H) in PAL biosynthetic. The conversion of phenylalanine to cinnamic acid required essential phenylalanine ammonialyase enzyme and hence known as PAL biosynthetic pathway (Verberne et al., 1999; Hara et al., 2012). The IC pathway fundamentally is based on the assumption that conversion of chorismate to isochorismate is taking place through the action of IPL and is finally converted to SA. In another pathway, SA biosynthesis in plants has been reported from shikimic acid via chorismic acid and coumaric acid but the detailed mechanism is under question (An and Mou, 2011). The PAL and ICS pathways act independently, and it is well known that the PAL pathway is mainly responsible for the synthesis of SA. Thus the question arises whether the existence of PAL pathway is solely restricted to pathogen-prompted SA production (Chen et al., 2009). Further, the importance of the PAL pathway lends credibility that SA production is governed by intermediates from both the ISC and PAL pathways (Chen et al., 2009).

Once synthesized in the chloroplast, plant MATE proteins facilitate the transportation of SA from chloroplast to cytosol through EDS5 channel (Dempsey et al., 2011). EDS5 is localized to the envelope membranes of chloroplasts and is essential for SA biosynthesis and transportation (Feys and Parker, 2000). However, detailed transporter actions and the mechanism involved in the transportation of SA from chloroplasts is unknown. After transportation, SA undergoes various biochemical modifications such as glucosylation, methylation (MET), and amino acid (AA) conjugation that activates SA into an active growth regulator (Dempsey et al., 2011; Gao et al., 2015). Reversely, glucosylation and methylation also inactivates SA and facilitates the accumulation in vacuoles by making tonoplast more permeable to cross (Dempsey et al., 2011). The attachment of glucose molecules at the hydroxyl group resulted in synthesis of SA 2-O- $\beta-D-$ glucoside (SAG) while at the carboxyl group salicylate glucose ester (SGE) was produced (Klessig, 2017). Whenever required, the SAG release free SA through hydroxylation reaction catalyzed by $\beta$-D-glucan glucohydrolases but the underlying mechanism of conversion is still not clear. Moreover attachment of methyl group to SA transformed it into methyl SA (MeSA), which respond in SAR response originating from an infected leaf to systemic tissues and stimulate conversion of MeSA to SA again (Park et al., 2007). Salicyloyl-aspartate has also been identified 
in the plant, synthesized by WES1 belong to acyl adenylase family that increases the accumulation of SA in the cytosol by suppressing SA-Asp synthesis (Chen et al., 2013). The role and mechanism of amino acid conjugation is also not well-studied but may be associated with catabolic pathway of SA. The schematic representations of possible modes of SA biosynthesis, regulation, and modification have been shown in Fig. 23.1. Before discussing the significant role of SA in various abiotic stresses, an attempt to establish the importance of SA in plant growth and development has been made in the next section of the chapter.

\subsection{ROLE OF SALICYLIC ACID IN PLANT GROWTH AND DEVELOPMENT}

Plants usually secrete a wide range of secondary metabolites; among them, SA is a well-known phenolic compound that greatly influences germination of seeds, adventitious root initiation, transpiration, photosynthesis, flowering, thermogenesis, and membrane permeability (Vlot et al., 2009; Boatwright and Pajerowska-Mukhtar, 2013; Khan et al., 2015a,b; Verma and Agrawal, 2017). In recent years, extensive research on the ameliorative effect of SA in plants exposed to various abiotic stresses has been well documented, such as extreme temperature (high, low, chilling) (He and Liu, 2002; Wang et al., 2010; Khan et al., 2013a,b). Reports are available on the importance of SA and related compounds in plants facing extreme drought conditions (Kareem et al., 2017), salinity (Munns and Tester, 2008; Khan and Khan, 2014), and metals/metalloids (Chen et al., 2007; Zhang et al., 2015). Both endogenous production and exogenous application of SA have been recounted as determining factors in plant metabolism and have authoritarian rheostat over cellular ROS (Herrera-Vásquez et al., 2015). Seed germination is a basic agronomic trait that has impact on

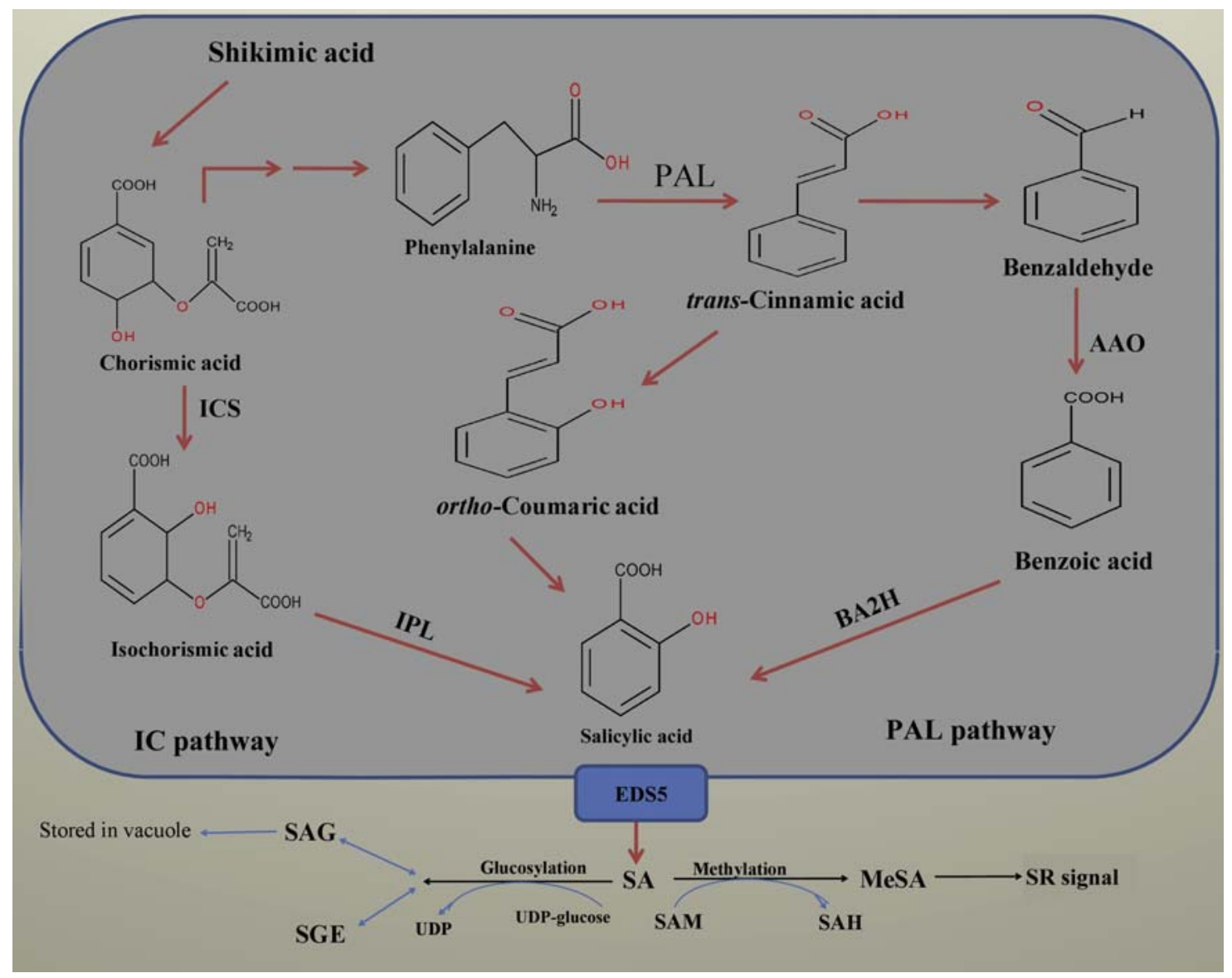

FIGURE 23.1 Steps involved in the biosynthesis and modification of salicylic acid from different pathways. Glucosylation process involved UDP-glucosyltransferases that glucosylate SA whereas methylation process was catalyzed by BA/SA carboxyl methyltransferase. SA has been shown to be sulfonated in vitro by members of the SOT family of sulfotransferases. Conversion back to SA is shown where evidence exists. MeSA is converted to SA by the methyl esterase activity of NtSABP2; the resultant increase in SA triggers systemic defenses. SAH, S-adenosyl homocysteine; $S A M$, S-adenosyl methionine; UDP, uridine diphosphate. 
plant development and growth. It was observed that presoaking of seeds with SA at $1 \mathrm{mM}$ concentration increased germination percentage, index, and mean germination time (MGT) in Pisum sativum exposed to high salinity $(150 \mathrm{mM})$ through retaining relative water content and increased accumulation of proline (Ahmad et al., 2017). In another study, seed priming before sowing with low dose of SA $(0.1 \mathrm{mM})$ showed better germination percentage in Vicia faba under salinity (Anaya et al., 2015). The dose-dependent role of SA in the germination process is still contradictory. For example, in Arabidopsis higher concentration of (1 mM) $\mathrm{SA}$ reduced the germination percentage under $150 \mathrm{mM}$ $\mathrm{NaCl}$ while low concentration $(0.1 \mathrm{mM}) \mathrm{SA}$ facilitated germination percentage (Lee et al., 2010). Besides soaking with SA, foliar applications to stressed plants are a promising approach in making plants tolerant. For instance, foliar application of SA $(0.1 \%$ and $0.2 \%)$ improved plant height, anatomical leaf structure, stem length and dry weight, phenols and proline content, and nitrogen uptake in wheat plants exposed to salinity (El-Sayed and Merwad, 2015). In addition to this, foliarapplied SA improved growth, gas-exchange characteristics, and chlorophyll fluorescence in citrus under saline conditions (Khoshbakht and Asgharei, 2015). The foliar application of $0.5 \mathrm{mM} \mathrm{SA}$ on wheat plat plant alleviated the adverse effect of heat stress by increasing proline and ethylene formation (Khan et al., 2013a,b). Further evidence of foliar application of SA in combination of L-tryptophan enhanced drought tolerance by improving higher relative water content, leaf membrane stability index, chlorophyll, and potassium content (Rao et al., 2002). Reports are also available to foliar application in mitigating other abiotic stress such as metal toxicity, soil acidity, gaseous pollutants, extreme levels of light (Sulmon et al., 2015; Pereira, 2016), etc.

The SA biosynthetic pathway helps in maintaining the architectural structure of roots and associated meristem activity in Oryza sativa (Xu et al., 2017). In addition to this, SA has also been involved in regulating photosynthetic pigments and the antioxidant system, reducing superoxide formation, and ensuring better activity of photosynthetic enzymes such as Rubisco and carbonic anhydrase (Hayat et al., 2012; Khan et al., 2013a,b; Ma et al., 2017). Leaf senescence is a gradual process of cell death taking into account excess production of reactive oxygen species, decreased photosynthetic efficiency, the disintegration of cellular components, and degradation of biomolecules and nucleic acid (Khan and Khan, 2017; Thomas, 2013; Zhao et al., 2016). Advanced proteomic studies confirmed that SA-mediated expression of PAD4, PR1, and SID2 induce leaf senescence in PAT14 mutants (Zhao et al., 2016). The progression from vegetative phase to reproductive phase has been influenced by natural conditions in coordination with hormonal regulations (Denay et al., 2017). The promising role of SA to induce flowering has been extensively explored for a couple of decades. Lee and Skoog (1965) observed that application of $4 \mu \mathrm{M}$ SA to tobacco callus initiates flowering at very early stage. The mechanistic approach of SA to facilitate flowering comprises regulatory action of PAL (phenylalanine ammonia-lyase) enzyme, an important precursor of SA biosynthesis (discussed above) in Pharbitis nil (Wada et al., 2014). Controversially, negative regulation of SA by repressing floral gene such as FLC and flowering pathway cycles have also been reported. Plant senescence is the process of aging, either stress-induced or developmental aging, morphophysiologically described by discoloration of leaves, degradation of chlorophyll pigments, and initiation of necrosis (Vogelmann et al., 2012). The impact of SA to the senescence process has been long established through expression of several senescenceassociated genes (SAGs), such as SAG12 $\alpha \mathrm{VPE}, \gamma \mathrm{VPE}$ (vacuolar processing enzymes), WRKY6, WRKY53 (transcription factors), and SEN1 (protease), which are undetectable in Arabidopsis plants (Morris et al., 2000; Rivas-San Vicente and Plasencia, 2011). However, SA's role in senescence still needs extensive scientific research at the molecular and physiological levels.

Modern agronomy practices face numerous abiotic stresses, for example, high salt accumulation, drought stress, chilling and high temperature stress, and metal stress, which have adverse impact on crops yield (Saud et al., 2013). Approximately, $25 \%$ of the worldwide agricultural land is plagued by drought and about 5\%-7\% land is polluted with salt accumulation (Ismail and Horie, 2017). According to the US Environmental Action Group, heavy metals toxicity has the severe impact on about 10 million people around the world and also affect the food quality of human and animals (ENS, 2006; Jaishankar et al., 2014). It has also been estimated that emission of greenhouse gases $\left(\mathrm{CH}_{4}, \mathrm{CO}_{2}\right.$, and $\left.\mathrm{CFC}\right)$ by $500-1000 \mathrm{ppm}$ will cause an irregular high temperature by $3^{\circ} \mathrm{C}$ by the late twenty-first century. Therefore, the back-to-back deleterious effects of salinity, metal toxicity, drought, water stress, nutritional inadequacy, and frequent climate change have become more challenging.

\subsection{SALICYLIC ACID-MEDIATED DEFENSE MECHANISMS TO ABIOTIC STRESS}

\subsubsection{Salicylic Acid Involvement in Modulation of Redox Homeostasis}

Usually, generation and detoxification of ROS take place in plants in normal physiological conditions. 
Plants thus use ROS as signaling molecules to develop steadfast pathways to protect themselves from ROS toxicity (Foyer and Noctor, 2013). Under stress conditions, disturbance in the equilibrium of ROS production and scavenging activity results in oxidative damages to membrane protein and degradation of nucleic acid (Anjum et al., 2012; Choudhury et al., 2017). Plants have innovative and multifaceted metabolic controls to ensure survival (Fahad and Bano, 2012). Tolerance stimulation in stress conditions demands the stimulation of the antioxidative defense system and scavenging systems under abiotic stress conditions (Khan and Khan, 2017; Khan et al., 2012, 2015a,b; Pasala et al., 2016; El-Mashad and Mohamed, 2012). The involvement of SA in regulating the antioxidant system to enhance tolerance under stress conditions has been extensively reviewed (Khan et al., 2015a,b; Sharma et al., 2017). Ma et al. (2017) showed that pretreatment of SA diminished injurious effects of salinity on growth and improved net photosynthesis rate by enhancing the antioxidant enzymes SOD, CAT, and POD activity in Dianthus superbus. Pretreatment of SA (0.05 mM) improved tolerance in Brassica juncea exposed to salinity stress due to enhanced activity of ascorbate glutathione (AsA-GSH) pathway (Nazar et al., 2015). It was also demonstrated that the hydrogen peroxide processing enzymes (SOD, CAT, and POD) were modulated by SA application on exposure to cold and drought stress (Mutlu et al., 2013; Saruhan et al., 2012). Heavy metal stress, for example, aluminum toxicity, increased the endogenous level of SA by regulating $\mathrm{PAL}$ and $\mathrm{BA} 2 \mathrm{H}$ enzymatic pathway and increased expression of GmNPR1 gene in soybean plants (Liu et al., 2013). The amelioration of oxidative damage caused by $A l$ toxicity was directly linked to SA application by inducing $\mathrm{H}_{2} \mathrm{O}_{2}$ level, which acts as signaling molecule to activate the antioxidant system (Liu et al., 2017). In another study, Triticum aestivum exposed to Cd stress revealed SA biosynthetic pathway correlation with glutathione cycle and attained a degree of Cd tolerance (Kovács et al., 2014). Alleviating effects of SA ( 0.01 and $0.1 \mathrm{mM})$ by improving ASA (ascorbate), GSH (reduced glutathione), and redox ratios ASC/DHA (dihydroxyascorbate) and (GSH/GSSG) under drought stress caused by polyethylene glycol (PEG)in Artemisia aucheri was also observed (Abbaspour and Ehsanpour, 2016).

\subsubsection{Salicylic Acid Interaction With Heat Shock Proteins, Reactive Oxygen Species and Mitogen-Activated Protein Kinase}

The leading role of heat shock proteins (HSP) is to drive molecular chaperones and have a central role in stress tolerance. In response to stress, activation of HSP implicates a multistep pathway of homotrimer formation associated with transcriptional factor for stimulation of stress gene (Liu et al., 2013). Major plant reactions to stress encompasses activation of HSFs/ HSPs associated with ROS shifting capabilities. The interaction between ROS and HSP is accompanied by mitogen-activated protein kinases (MAPK) and SA/ ROS signaling reaction in MAK cascade activation (Cutler et al., 2010). In Arabidopsis, a model has been proposed by researchers to activate different mitogenactivated protein kinase (MPK3, MPK4, and MPK6) in response to a number of abiotic stresses (Ichimura et al., 2000; Teige et al., 2004). Thus, a model has been developed to demonstrate that MAP kinase kinase kinases (MEKKs) are stimulated as a consequences of phosphorylation of MAP kinase kinases (MKKs) that ultimately terminate in activation of MPKs (Jalmi and Sinha, 2015). In Arabidopsis roots, Mockaitis and Howell (2000), showed that phosphorylation of MPK was induced by SA. Profoundly, these cascades linked with SA-mediated downregulation signaling either lack the functionality of MEKK1 or MPK4 that induces the accumulation of SA (Suarez-Rodriguez et al., 2007). It is well understood that ROS generation is a basic factor for activating MAP kinase cascades and ANP1 (Arabidopsis NPK1-like protein kinase1) also activated by $\mathrm{H}_{2} \mathrm{O}_{2}$ and terminated by phosphorylation of MPK3/MPK6 in Arabidopsis plants (Kovtun et al., 2000). MEKK1-MPK4 cascade also has a commanding feature during ROS metabolism (Nakagami et al., 2006). However, these MAPK cascades are critically controlled by both SA and ROS levels. Since oxidative stress plays a central role in response to biotic and abiotic stresses, the redox homeostasis may be concluded to be an indicator for monitoring stress in plants. The interactions of SA and ROS with other important factors involved in various abiotic stresses have been demonstrated in Fig. 23.2.

\subsubsection{Interaction of Salicylic Acid With Mineral Nutrients and Osmoprotectant}

To perform normal physiological and biochemical functions, plants fundamentally need an adequate supply of macro- and microelements. It was estimated that about $60 \%$ of fertile soils have problems related with nutrient deficits thus reduced crop productivity (Bagheri et al., 2017). Nazar et al. (2015) found that SA increased sulfur absorption and nitrogen absorption, which is associated with antioxidant coordination in plants, and offered tolerance to salinity by provoking various physiological reactions. Foliar application of SA (200 ppm) on Gossypium barbadense with potassium 


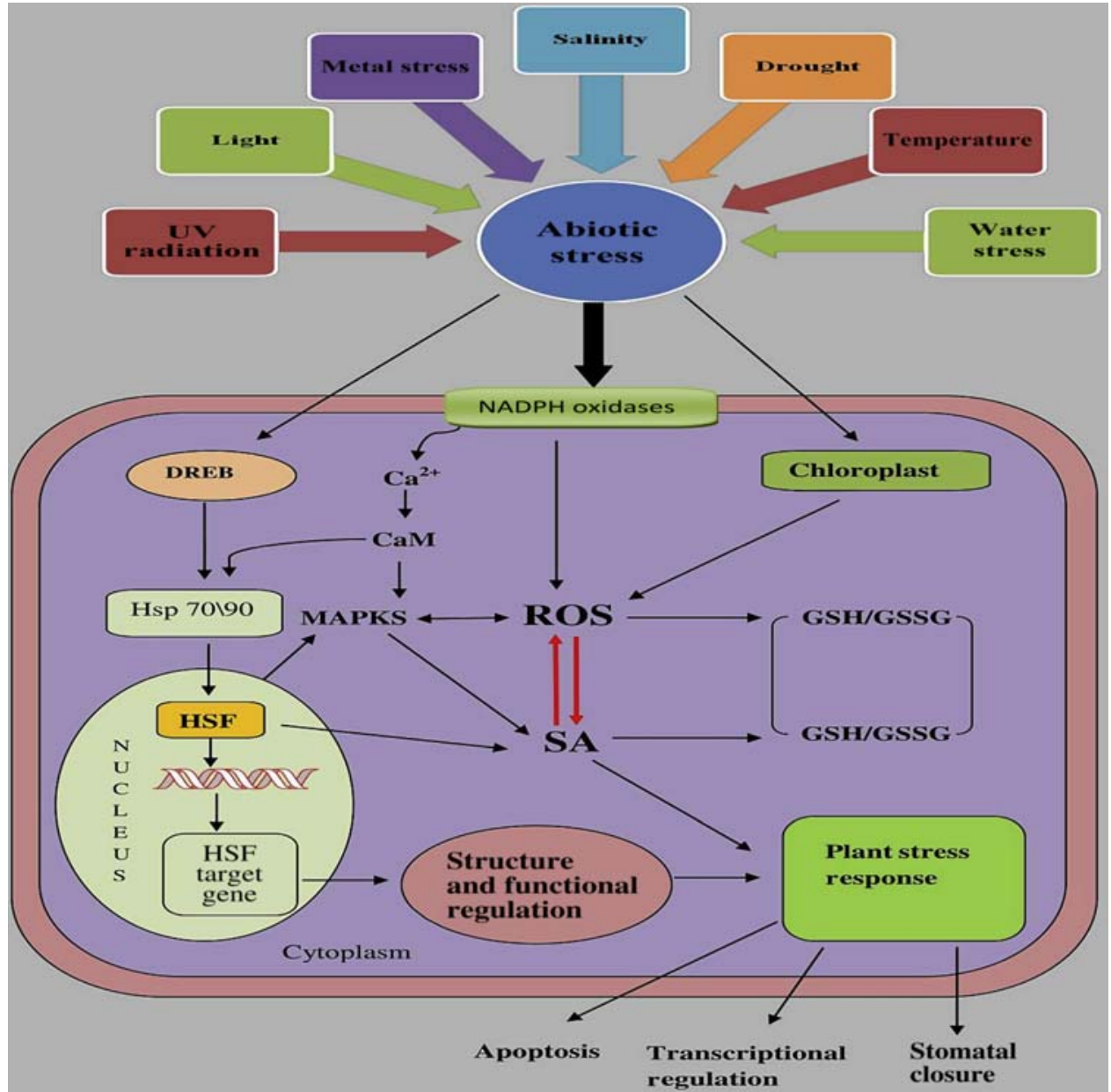

FIGURE 23.2 Interaction of salicylic acid and reactive oxygen species (ROS) with transcriptional factor, heat shock protein under abiotic stress. Reactive oxygen species (ROS) and $\mathrm{Ca}^{++}$are known among others to play a prominent role as transducers (messengers) and mitogenactivated protein kinase (MAPK) cascades have been shown to be used by both types of stresses. Upregulation of transcription factors (TFs), pathogenesis related (PR) and defense genes, heat shock protein (HSP) genes, and further genes involved in protection against stress.

citrate $(2.5 \mathrm{~g} / \mathrm{L})$ enhanced growth and yield and leaf chemical composition under salinity stress (El-Beltagi et al., 2017). Recent outcomes delivered that SA and $\mathrm{Ca}^{2+}$ improved the adverse effect of oxidative trauma by activating the antioxidant system and enhancing photosynthetic efficiency in Triticum aestivum (Yücel and Heybet, 2016). The mutual application of SA and S caused noteworthy diminution in $\mathrm{Na} / \mathrm{K}$ proportions by modulating enzymatic and nonenzymatic antioxidant coordination in Glycine max exposed to salinity stress (Razmi et al., 2017).

Under extreme osmotic stress, there is accumulation of organic compounds such as glycine betaine (GB), proline, soluble sugars, and amines, which act as osmolytes to ensure survival of plants (Wani et al., 2016). These osmolytes are neutral and nontoxic molecules that maintain osmotic equilibrium between the cell's surroundings and the cytosol without affecting the metabolic process (Shabala et al., 2006). GB, chemically known as $\mathrm{N}, \mathrm{N}, \mathrm{N}$-trimethylglycine, is an effective osmolyte ubiquitously found in plants for osmotic balance. GB at physiological $\mathrm{pH}$ is a dipolar but electrically neutral molecule that can hypothetically play a decisive role in operative guard against salt, drought, and extreme temperature stress (Wani et al., 2016; Masood et al., 2016). Plants achieved systematic acquired resistance (SAR) through SA-induced GB accumulation under drought and salinity stress (Jagendorf and Takabe, 2001). Further, $0.5 \mathrm{mM} \mathrm{SA}$ application to Vigna radiate overpowered ethylene formation and increased amino acid content, confirmed GA accumulation, and increased tolerance of plant under salinity stress (Khan and Khan, 2014). Another important osmolyte, proline, also gets accumulated in plants and activates ROS scavenging activity, regulates osmotic equilibrium, and maintains membrane stability under stress conditions (Iqbal et al., 2014). SA $(1 \mathrm{mM})$ significantly increased proline content under 
high level of $\mathrm{NaCl}$ in Pisum sativum plant; this might be due to enhanced activity of pyrroline-5-carboxylate reductase and $\gamma$-glutamyl kinase enzyme involved in proline biosynthesis (Misra and Misra, 2012; Ahmad et al., 2017). Pretreatment of SA (1 mM) was also reported to improve the level of total soluble sugars and protein content in pepper leaves during germination phase (Qados, 2015). Authors conclude from these findings that these molecules in association with SA help in reducing and prevent oxidative stress and maintain structural conformation of proteins in water scarcity.

\subsection{CROSSTALK OF SALICYLIC ACID WITH OTHER PHYTOHORMONES UNDER ABIOTIC STRESS}

Phytohormones showed multitude impact on plant health performance to numerous ecological cues (Griesser et al., 2015). To uphold a harmony amongst development and defense programming in plant systems, crosstalk between various phytohormones has been a striking assessment for plant researchers. The interactive multifarious signaling offered them ideal contenders for assigning defense reactions. Phytohormones act either self-sufficiently or through pathways interconnected with other growth regulators mutually or antagonistically (Kazan, 2015). Notably, hormonal crosstalk involved positive or destructive influence on synthesis, transport, and signaling of other stress hormones ( $\mathrm{Hu}$ et al., 2017). The positive and negative crosstalk of SA with other growth regulators has been summarized in Table 23.1.

\subsubsection{Salicylic Acid and Auxins}

Auxins act as a key player in growth and development of plants by regulating plant metabolic processes as well as root differentiation and also in abiotic stress tolerance (Agtuca et al., 2014; Fahad and Bano, 2012). The antagonistic interaction of indole acetic acid (IAA) and SA signaling pathways hinder functionality of each other in maize plants, where exogenous application of IAA enhanced lateral root formation rather than primary roots development, and SA improved total root biomass (Agtuca et al., 2014). Overexpression of PR1 gene via SA application in auxin TIR1, AFB2 mutants provides better understanding of SA-induced auxin signaling in salinity stressed plants (Iglesias et al., 2011). One of the common approaches of plants to deal with stress conditions is SA-mediated suppression of auxins studied in Arabidopsis (Atkinson et al., 2013). MicroRNA (miR393), the main regulator of
TIR1, AFB2, and AFB (auxin receptors) was also stimulated by cold and salinity stress (Sunkar et al., 2007). Exogenous supplementation of SA caused repression of auxin-related genes and resulted in modulation of Aux/IAA proteins and inhibition of auxin responses in Arabidopsis (Wang et al., 2017). SA elicited the accumulation of abscisic acid (ABA) under both favorable and stress conditions, which thus helped in the osmotic adjustment, enhancement of photosynthetic pigments, and development traits in Solanum lycopersicum (Szepesi et al., 2009).

\subsubsection{Salicylic Acid and Abscisic Acid}

Abscisic acid (ABA) is usually known as a stress hormone mainly associated with abiotic stresses mitigation (Cramer et al., 2011; Vishwakarma et al., 2017). The ABA-dependent signaling pathway countersigns defense genes removing stress protection via transcriptional reprogramming of plant cell metabolism (Bali et al., 2017; Vishwakarma et al., 2017). The regulatory element of ABA receptor (PYR/PYL/RCAR) and protein phosphatase (PP2C) stress-protein subfamily was a major breakthrough in explaining $\mathrm{ABA}$ signaling pathway under stress (Fujii and Zhu, 2009; Soon et al., 2012). SA and ABA act provocatively to regulate cell cycle-related genes in rice plants (Meguro and Sato, 2014; Manohar et al., 2017). In Agrostis stolonifera, coapplication of abscisic acid, $\gamma$-aminobutyric acid (GABA), and SA enhanced drought resistance by protecting membrane strength and water retention ( $\mathrm{Li}$ et al., 2017). Metabolic profiling studies proved that ABA, GABA, and SA have inclined metabolic route and also caused the differential alteration in metabolite accumulation under drought (Li et al., 2017).

The antagonistic performance of $\mathrm{ABA}$ and $\mathrm{SA}$ revealed that ABA kept up the SA-mediated protective mechanism by accumulation of SA, though this defense was perceived to be hindered when ABA was applied exogenously (Yasuda et al., 2008). Further, the endogenous SA levels in the Cd-stressed Zea mays were altered by ABA treatment (Szalai et al, 2013).

\subsubsection{Salicylic Acid and Gibberellic Acid}

Gibberellins (GAs) are naturally occurring tetracyclic diterpenoid carboxylic acids responsible for important process, that is, seed germination, shoot elongation, flowering and fruit development, etc. (Yamaguchi, 2008). They empower development and advance stage changes and are effectively involved in abiotic stress tolerances and adjustment (Colebrook et al., 2014). It has been reported that GAs improved seed germination under salinity (Kaya et al., 2009), as well as increased 
TABLE 23.1 Interaction of SA With Other Plant Growth Regulator Under Optimal/Stress Condition

\begin{tabular}{|c|c|c|c|c|c|c|}
\hline Phytohormones & $\begin{array}{l}\text { Plant } \\
\text { species }\end{array}$ & $\begin{array}{l}\text { Interaction } \\
\text { with SA }\end{array}$ & $\begin{array}{l}\text { Stress/ } \\
\text { optimal } \\
\text { condition }\end{array}$ & Responses & Effect & References \\
\hline \multirow[t]{4}{*}{ Auxin } & A. esculentus & Syn & Salinity & $\begin{array}{l}\text { DPPH (2,2-diphenyl radical scavenging capacity), } \\
\text { antioxidant activities }\end{array}$ & + & $\begin{array}{l}\text { Esan et al. } \\
\text { (2017) }\end{array}$ \\
\hline & \multirow[t]{2}{*}{ H. vulgare } & \multirow[t]{2}{*}{ Syn } & \multirow{2}{*}{$\begin{array}{l}\text { Heavy } \\
\text { metals }\end{array}$} & Cd-induced IAA mediated ROS & - & \multirow{2}{*}{$\begin{array}{l}\text { Tamás et al. } \\
\text { (2015) }\end{array}$} \\
\hline & & & & $\begin{array}{l}\text { Lipoxygenase or glutathione peroxidases along with } \\
\text { stimulated root growth inhibition }\end{array}$ & + & \\
\hline & Zea mays & Syn & Salinity & $\begin{array}{l}\text { Micronutrients } \mathrm{CO} 3+, \mathrm{Mn} 2+, \mathrm{Cu}^{3+}, \mathrm{Fe}^{2+} \text { in roots, chl a, } \\
\text { chl b, carotenoids, sugars, proline, and protein content }\end{array}$ & + & $\begin{array}{l}\text { Fahad and } \\
\text { Bano (2012) }\end{array}$ \\
\hline \multirow[t]{4}{*}{ Gibberellins } & $\begin{array}{l}\text { Pisum } \\
\text { sativum }\end{array}$ & Syn & Salinity & $\begin{array}{l}\text { Seed germination, protein and sugar content, antioxidant } \\
\text { activity }\end{array}$ & + & $\begin{array}{l}\text { Ahmad et al. } \\
\text { (In press) }\end{array}$ \\
\hline & $\begin{array}{l}\text { Sorghum } \\
\text { bicolor }\end{array}$ & Syn & Drought & $\begin{array}{l}\text { Catalase, APX activities, germination percentage, and } \\
\text { germination index }\end{array}$ & + & $\begin{array}{l}\text { Sheykhbaglou } \\
\text { et al. (2014) }\end{array}$ \\
\hline & A. thaliana & Syn & Salinity & $\begin{array}{l}\text { Modulation of biochemical and molecular GA-SA } \\
\text { signaling mechanism, seed germination }\end{array}$ & + & $\begin{array}{l}\text { Lee et al. } \\
(2010)\end{array}$ \\
\hline & Glycine max & Syn/Ant & Salinity & $\begin{array}{l}\text { Upregulation of SA biosynthesis/decreased SA content, } \\
\text { under increased GA3 and elevated SA under } \mathrm{NaCl} \text { stress }\end{array}$ & $+/-$ & $\begin{array}{l}\text { Hamayun } \\
\text { et al. (2010) }\end{array}$ \\
\hline \multirow[t]{5}{*}{ ABA } & A. thaliana & Syn & Water stress & Promote stomatal closure, induce leaf senescence & + & Qi et al. (2015) \\
\hline & $\begin{array}{l}\text { S. } \\
\text { lycopersicum }\end{array}$ & Syn & $\begin{array}{l}\text { Salinity/ } \\
\text { optimal }\end{array}$ & $\begin{array}{l}\text { Osmotic adaptation, photosynthetic pigments, and } \\
\text { growth attributes }\end{array}$ & + & $\begin{array}{l}\text { Szepesi et al. } \\
\text { (2009). }\end{array}$ \\
\hline & T. aestivum & Syn & Drought & $\begin{array}{l}\text { Soluble protein and soluble sugar content, photosynthetic } \\
\text { efficiency }\end{array}$ & + & $\begin{array}{l}\text { Sami et al. } \\
\text { (2016) }\end{array}$ \\
\hline & \multirow[t]{2}{*}{ O. sativa } & \multirow[t]{2}{*}{ Ant } & \multirow[t]{2}{*}{ Optimal } & Cell cycle progression & + & \multirow{2}{*}{$\begin{array}{l}\text { Meguro and } \\
\text { Sato (2014) }\end{array}$} \\
\hline & & & & $\begin{array}{l}\text { DNA replication, thymidine incorporation in the shoot } \\
\text { apical meristem }\end{array}$ & - & \\
\hline \multirow[t]{5}{*}{ Jasmonic acid } & $\begin{array}{l}\text { G. } \\
\text { lemaneiformis }\end{array}$ & Syn & Heat & Hsp70, Hsp60, signaling transduction & + & $\begin{array}{l}\text { Wang et al. } \\
\text { (2017) }\end{array}$ \\
\hline & Citrus limon & Syn & Chilling & Total phenolics and phenylalanine ammonia lyase (PAL) & + & $\begin{array}{l}\text { Siboza et al. } \\
\text { (2014) }\end{array}$ \\
\hline & O. sativa & Ant & $\begin{array}{l}\text { Drought/ } \\
\text { salinity }\end{array}$ & Transcripts and RSOs PR10 proteins & + & $\begin{array}{l}\text { Clarke et al. } \\
\text { (2009) }\end{array}$ \\
\hline & A. thaliana & Syn & Heat stress & Electrolyte leakage & + & $\begin{array}{l}\text { Schenk et al. } \\
\text { (2000) }\end{array}$ \\
\hline & $\begin{array}{l}\text { Lycopersicon } \\
\text { esculentum }\end{array}$ & Ant & Optimal & Pathogen related $(\mathrm{PR})$ protein genes & + & $\begin{array}{l}\text { Thaler et al. } \\
\text { (1999) }\end{array}$ \\
\hline \multirow[t]{3}{*}{ Brassinosteroids } & B. juncea & Syn & $\begin{array}{l}\text { Metal stress } \\
(\mathrm{Pb})\end{array}$ & Photosynthetic efficiency, gene expression & + & $\begin{array}{l}\text { Kohli et al } \\
\text { (2017) }\end{array}$ \\
\hline & A. thaliana & Syn & Salinity & $\begin{array}{l}\text { Transcription factor WRKY70 and WAK1, NPR1 defense } \\
\text { gene expression }\end{array}$ & + & $\begin{array}{l}\text { Divi et al. } \\
\text { (2010) }\end{array}$ \\
\hline & $\begin{array}{l}\text { Arachis } \\
\text { hypogaea }\end{array}$ & Syn & $\begin{array}{l}\text { Metal stress } \\
(\mathrm{Cd})\end{array}$ & Growth and metabolism & + & $\begin{array}{l}\text { Khatoon et al. } \\
\text { (2017) }\end{array}$ \\
\hline \multirow[t]{2}{*}{ NO } & Glycine max & Syn & Salinity & CAT, APX, GPX, accumulation of proline & + & $\begin{array}{l}\text { Simaei et al. } \\
\text { (2012) }\end{array}$ \\
\hline & T. aestivum & Syn & Chilling & Antioxidant activities SOD, POD, CAT & + & $\begin{array}{l}\text { Esim and } \\
\text { Atici (2015) }\end{array}$ \\
\hline
\end{tabular}

Syn, synergistic; Ant, antagonistic; + (positive response), - (negative response). 
pigments, hill activity, and water holding capacity (Shah, 2007). GA signaling enables plants to adapt to adverse environmental conditions, and this can be mediated through SA regulation. GA and SA act in an organized manner to modulate pathogenesis associated protein expression (Miura and Tada, 2014) and activate defense system against abiotic stresses (Fayez and Bazaid, 2014). Inhibition of seed germination by SA occurs due to GA suppressible WRKY genes that block GA-induced $\alpha$-amylase expression (Xie et al., 2007). The antagonistic association between SA and GA was also observed in Arabidopsis plant in trichome development under drought stress. Exogenous application of SA suppresses GA-initiated trichome development through downregulation of Npr1/Nim2 gene (Traw and Bergelson, 2003).

\subsection{INTERACTION AND INVOLVEMENT OF DELLA WITH SA-GA CROSSTALK UNDER STRESS}

DELLA proteins stimulate ROS-detoxifying enzymes by altering ROS strength in abiotic/biotic stress (Achard et al., 2008). GAs activated in both salt and cold stress by cunning DELLA proteins and established noteworthy crosstalk with SA-ET signaling (Verma et al., 2016). Furthermore, upregulation of GA biosynthetic gene expressed itself after SA treatment followed by DELLA protein destruction degradation (Ding et al., 2015). SA and/or GA enhanced prominent CBF1 expression in tomato under cold stress (Zhao et al., 2016). It was reported that in Arabidopsis thaliana, GA stimulate isocitratelyase (ICL), an enzyme essential in lipid assimilation during seed germination also stimulated by SA (Bali et al., 2017).

\subsubsection{Salicylic Acid and Ethylene}

Ethylene (ET) is a self-effacing gaseous hormone, having manifold regulation in growth and development and acts as modulator between plant response to environmental stresses to attain maximum performance (Khan and Khan, 2014). It was shown that SA improved tolerance in Arabidopsis by inducing the expression of NPR1 gene (Jayakannan et al., 2015). A well-coordinated upregulation of ethylene TF genes such as GmERF3, AtERF6, and CarERF116 (Sewelam et al., 2013; Deokar et al., 2015; Liu et al., 2017), while downexpression of SodERF3 (Trujillo et al., 2008) was noticed upon SA application. During high temperature stress, exogenous spraying of SA improves plant growth by limiting ethylene production and enhancing proline metabolism to such a point that modulatory action of ACC synthase (1-aminocyclopropanel-carboxylic acid synthase) enzyme is held back (Khan et al., 2013a,b). According to Poór et al. (2013), ethylene is accountable for cell death under high salt concentration in Lycopersicon esculentum due to the excessive generation of ROS, whereas SA-induced reactive oxygen species production was not reported to be straightforwardly linked with ethylene. The chlorophyll as well as anthocyanin content was reduced noticeably by ethylene application, but SA ameliorates negative effects of salinity (Tirani et al., 2013). Further, SA facilitated the ethylene-dependent hypersensitive reaction in Madia sativa through reducing formation of lipid peroxidation and polyamines (Palma et al., 2013).

\subsubsection{Salicylic Acid and Jasmonic Acid}

Jasmonic acid is a naturally occurring lipid that performs as a regulator in germination and seedling growth and root development, induces tuber formation and gravitropism, embryo formation, determination and development of flowers, fruit ripening, and leaf senescence (Zheng et al.,2017). Additionally, JA plays a vital role in abiotic stress and is regarded as a stress modulator, and enhances stress tolerance by improving photosynthetic efficiency and antioxidant metabolism via modulating proteome profiling (Rincón-Pérez et al., 2016). Proteomic and transcriptomics analysis explored the upregulation of specific stress proteins modulated by JA and/or SA under abiotic stress (Van der Does et al., 2013). Usually, SA and JA signaling pathways interact antagonistically at the level of mitogen activated protein kinases (MAPK) under normal or stress conditions (Khan et al., 2013a,b; Bali et al., 2017).

Caarls et al. (2015) explained that SA inhibits JA action by downregulating the complex COI1-JAZ by altering GCC-box motifs in JA-responsive promoters. The downregulation of the JA pathway comprises MAPK, redox balances, and transcription factors such as WRKY regulated by SA (Pieterse et al., 2012). The inhibitory action of SA in NPR1 suppressing the expression of LOX2 and VSP gene, which are upregulated by JA (Spoel et al., 2003). It is interesting to note that SA-induced NPR1 can activate GRX480, which in turn, can form a complex with TGA factors, and suppress the expression of JA-responsive genes (Backer et al., 2015).

Synergistic interaction between JA and SA are also evidenced; however, the reports are scanty in this context. In Citrus, coapplication of MeJA and SA enhanced synthesis and accumulation of total phenols and activity of phenylalanine ammonia lyase (PAL) and simultaneously deterring the action of polyphenol 
oxidase (PPO) and peroxidase (POD) to chilling stress (Siboza et al., 2014). Moreover, the involvement of the transcription factor and ROS mechanism engaged by both SA and JA suggests feasible crosstalk connecting the JA and SA signal transduction pathway to cold stress (Sharma et al., 2017).

\subsubsection{Salicylic Acid and Brassinosteroids}

Brassinosteroids (BRs) are plant steroidal hormones that have gained much attention recently for their beneficial role to induce tolerance to plants exposed to a wide range of abiotic stress. BRs protect plants from a range of ecological distress, including extreme temperatures, drought, salinity, and pathogen attack, apart from playing a developmental role (Vardhini and Anjum, 2015; Ahmad et al., 2018). The collaborations of BR with SA reported to enhance tolerance to plants have been extensively studied under abiotic stress (Divi et al., 2010; Handa et al., 2017). BR is involved in upregulation of nonexpression of pathogenesis-related genes (NPR1) coupled with SA-mediated tolerance under salinity stress. In addition to this, expression of SA-induced PR genes has been upregulated by BR by modulating transcription factor WRKY70 (Divi et al., 2010). Coapplication of SA and BR was found efficient to adjust under severe condition of salinity (Ding et al., 2012). However, BR mediated resistance does not require involvement of SA and further exploration by quantifying SA accumulation and expression analysis of NahG transgenic tobacco confirmed the independent mode of defense against pathogen attack (Nakashita et al., 2003; Saini et al., 2015). Further, crosstalk of SA and BR pathways negatively suppress immune response provoked by BR. For example, Brassinazole (Bz) reduced the susceptibility of Pythium graminicola observed in rice plants by downregulating of NPR1 and OsWRKY45 that directly linked with SA defense regulators pathway. Moreover, bioformulation of SA and 24-EBL and 24-epicastasterone enhanced the growth of millet plantlet by reducing lipid peroxidation under heat and salt stress (Litvinovskaya et al., 2016).

\subsubsection{Salicylic Acid and Nitric Oxide}

Nitric oxide (NO), a signaling molecule, alters gene expression thus regulating numerous physiological and metabolic pathways in a dose-dependent manner (Fancy et al., 2017). During the last two decades there has been a tremendous outburst in deciphering the role of NO in the germination process, photosynthesis, leaf senescence, and pollen growth (Esim and Atici, 2015). The stomata closure during stress begins with inactivation of $\mathrm{Kin}^{+}$pumps associated with $\mathrm{NO}$ and
ROS interaction in guard cells induced by SA (Khokon et al., 2011). The reduction in oxidative stress and retaining equilibrium of ion-homeostasis thus protects the plant and promotes growth and photosynthetic efficiency when treated with sodium nitroprusside (SNP) as NO source (Zhang et al., 2015; Fatma et al., 2016). Under salinity stress, NO has been stimulated downstream of SA signaling in diminishing oxidative injury in osmotic stresses in Triticum aestivum seedlings (Naser Alavi et al., 2014). The combined application of SA $(1 \mathrm{mM})$ and $\mathrm{NO}(0.1 \mathrm{mM})$ reduced the proportions of malondialdehyde (MDA), $\mathrm{H}_{2} \mathrm{O}_{2}$ and $\mathrm{O}_{2}{ }^{-}$amplified the activity of SOD, CAT, and POX, which subsidized to ROS generation in wheat under chilling stress (Esim and Atici, 2015). Exposure of Ni stress, pretreatment of SA plus SNP mitigates the detrimental effect of heavy metal by dropping root-to-shoot translocation of $\mathrm{Ni}$, enhancing the chlorophyll content, and decreasing lipid peroxidation, $\mathrm{H}_{2} \mathrm{O}_{2}$, and proline accumulation in leaves of Brassica napus (Kazemi et al., 2010). The mutual accomplishment of SA and NO enriched Fe uptake and a smaller amount of leaf interveinal chlorosis in Arachis seedlings rather than individual application of SA or SNP under Fe-deficiency (Kong et al., 2014). Furthermore, synergistic consequence of SA + SNP enhanced activity of SOD, POD, APX, and GR exposed to UV-B stress. Under salinity, SA inhibited NO biosynthesis, demonstrating a conflicting relationship between $\mathrm{NO}$ and SA and showing dual response (Gémes et al., 2011).

\subsection{CONCLUSION AND FUTURE PROSPECTS}

SA is a multidimensional hormone used in physiological metabolic processes and defense mechanism tolerance to abiotic stresses. Studies on the physiological and molecular levels of plant tolerance to numerous stresses will be critical for improved management with prospective ecological alteration. It will be fascinating to investigate the additional roles of SA in the clash between plants and ecological stresses. However, some conflicting consequences are obtained in exploring exogenous SA application and endogenous SA levels depending upon the concentration applied. It is ambiguous if exogenous SA application directly or indirectly enhances endogenous SA levels and whether the effect of SA is coupled with ROS generation. Indepth study must be used to expose an advanced system of upregulation of SA regulated genes under the influence of abiotic stress, and the unambiguous network coordination with other phytohormones to search out precise information regarding plant reaction to abiotic stress. 


\section{Acknowledgements}

The authors are grateful to Dean, R\&D, Section, Integral University, Lucknow, for quick and crisp revision of manuscript and for allotting manuscript number (Yet to be allotted).

\section{References}

Abbaspour, J., Ehsanpour, A.A., 2016. Physiological targets of salicylic acid on Artemisia aucheri BOISS as a medicinal and aromatic plant grown under in vitro drought stress. Bot. Stud. 57 (1), 39.

Achard, P., Gong, F., Cheminant, S., Alioua, M., Hedden, P., Genschik, P., 2008. The cold-inducible CBF1 factor-dependent signaling pathway modulates the accumulation of the growthrepressing DELLA proteins via its effect on gibberellin metabolism. Plant Cell 20 (8), 2117-2129.

Agtuca, B., Rieger, E., Hilger, K., Song, L., Robert, C.A., Erb, M., et al., 2014. Carbon-11 reveals opposing roles of auxin and salicylic acid in regulating leaf physiology, Leaf metabolism, and resource allocation patterns that impact root growth in Zea mays. J. Plant. Growth Regul. 33 (2), 328-339.

Ahmad, F., Singh, A., Kamal, A., 2017. Ameliorative effect of salicylic acid in salinity stressed Pisum sativum by improving growth parameters, activating photosynthesis and enhancing antioxidant defense system. Biosci. Biotechnol. Res. Communicat. 10 (3), 481-489.

Ahmad, F., Singh, A., Kamal, A., 2018. Crosstalk of brassinosteroids with other phytohormones under various abiotic stresses. J. Appl. Biol. Biotechnol. 6 (1), 56-62.

An, C., Mou, Z., 2011. Salicylic acid and its function in plant immunity. J. Integrat. Plant Biol. 53 (6), 412-428.

Anaya, F., Fghire, R., Wahbi, S., Loutfi, K., 2015. Influence of salicylic acid on seed germination of Vicia faba L. under salt stress. J. Saudi Soc. Agric. Sci. 12, 1-8.

Anjum, N.A., Umar, S., Ahmad, A. (Eds.), 2012. Oxidative Stress in Plants: Causes, Consequences and Tolerance. IK International Publishing, New Delhi.

Atkinson, N.J., Lilley, C.J., Urwin, P.E., 2013. Identification of genes involved in the response of Arabidopsis to simultaneous biotic and abiotic stresses. Plant Physiol. 162 (4), 2028-2041.

Backer, R., Mahomed, W., Reeksting, B.J., Engelbrecht, J., IbarraLaclette, E., van den Berg, N., 2015. Phylogenetic and expression analysis of the NPR1-like gene family from Persea americana (Mill.). Front. Plant Sci. 6.

Bagheri, R., Ahmad, J., Bashir, H., Iqbal, M., Qureshi, M.I., 2017. Changes in rubisco, cysteine-rich proteins and antioxidant system of spinach (Spinacia oleracea L.) due to sulphur deficiency, cadmium stress and their combination. Protoplasma 254 (2), 1031-1043.

Bali, S., Gautam, V., Kaur, P., Khanna, K., Kaur, R., Vig, A.P., et al., 2017. Interaction of Salicylic Acid with Plant Hormones in Plants Under Abiotic Stress. Salicylic Acid: A Multifaceted Hormone. Springer, Singapore, pp. 201-219.

Boatwright, J.L., Pajerowska-Mukhtar, K., 2013. Salicylic acid: an old hormone up to new tricks. Mol. Plant Pathol. 14 (6), 623-634.

Caarls, L., Pieterse, C.M., Van Wees, S.C., 2015. How salicylic acid takes transcriptional control over jasmonic acid signaling. Front. Plant Sci. 6.

Cao, Y., Zhang, Z.W., Xue, L.W., Du, J.B., Shang, J., Xu, F., et al., 2009. Lack of salicylic acid in Arabidopsis protects plants against moderate salt stress. Z. Naturforsch. C 64, 231-238.

Chen, J., Zhu, C., Li, L.P., Sun, Z.Y., 2007. Effects of exogenous salicylic acid on growth and $\mathrm{H}_{2} \mathrm{O}_{2}-$ metabolizing enzymes in rice seedlings under lead stress. J. Environ. Sci. 19, 44-49.
Chen, H., Xue, L., Chintamanani, S., Germain, H., Lin, H., Cui, H., et al., 2009. ETHYLENE INSENSITIVE3 and ETHYLENE INSENSITIVE3-LIKE1 repress SALICYLIC ACID INDUCTION DEFICIENT2 expression to negatively regulate plant innate immunity in Arabidopsis. Plant Cell 21 (8), 2527-2540.

Chen, Y., Shen, H., Wang, M., Li, Q., He, Z., 2013. Salicyloylaspartate synthesized by the acetyl-amidosynthetase GH3. 5 is a potential activator of plant immunity in Arabidopsis. Acta Biochim. Biophys. Sin. 45 (10), 827-836.

Choudhury, F.K., Rivero, R.M., Blumwald, E., Mittler, R., 2017. Reactive oxygen species, abiotic stress and stress combination. Plant J. 90 (5), 856-867.

Clarke, S.M., Cristescu, S.M., Miersch, O., Harren, F.J., Wasternack, C., Mur, L.A., 2009. Jasmonates act with salicylic acid to confer basal thermotolerance in Arabidopsis thaliana. New Phytol. 182 (1), 175-187.

Colebrook, E.H., Thomas, S.G., Phillips, A.L., Hedden, P., 2014. The role of gibberellin signalling in plant responses to abiotic stress. J. Exp. Biol. 217 (1), 67-75.

Cramer, G.R., Urano, K., Delrot, S., Pezzotti, M., Shinozaki, K., 2011. Effects of abiotic stress on plants: a systems biology perspective. BMC Plant Biol. 11 (1), 163.

Cutler, S.R., Rodriguez, P.L., Finkelstein, R.R., Abrams, S.R., 2010. Abscisic acid: emergence of a core signaling network. Annu. Rev. Plant. Biol. 61, 651-679.

Dempsey, D.M.A., Vlot, A.C., Wildermuth, M.C., Klessig, D.F., 2011. Salicylic acid biosynthesis and metabolism. The Arabidopsis Book. p. e0156.

Denay, G., Chahtane, H., Tichtinsky, G., Parcy, F., 2017. A flower is born: an update on Arabidopsis floral meristem formation. Curr. Opin. Plant. Biol. 35, 15-22.

Deokar, A.A., Kondawar, V., Kohli, D., Aslam, M., Jain, P.K., Karuppayil, S.M., et al., 2015. The CarERF genes in chickpea (Cicer arietinum L.) and the identification of CarERF116 as abiotic stress responsive transcription factor. Funct. Integr. Genomics. 15 (1), 27-46.

Ding, H.D., Zhu, X.H., Zhu, Z.W., Yang, S.J., Zha, D.S., Wu, X.X., 2012. Amelioration of salt-induced oxidative stress in eggplant by application of 24-epibrassinolide. Biol. Plant. 56 (4), 767-770.

Ding, Y., Sheng, J., Li, S., Nie, Y., Zhao, J., Zhu, Z., et al., 2015. The role of gibberellins in the mitigation of chilling injury in cherry tomato (Solanum lycopersicum L.) fruit. Postharvest. Biol. Technol. 101, 88-95.

Divi, U.K., Rahman, T., Krishna, P., 2010. Brassinosteroid-mediated stress tolerance in Arabidopsis shows interactions with abscisic acid, ethylene and salicylic acid pathways. BMC Plant Biol. 10 (1), 151.

El-Beltagi, H.S., Ahmed, S.H., Namich, A.A.M., Abdel-Sattar, R.R., 2017. Effect of salicylic acid and potassium citrate on cotton plant under salt stress. Fresenius Environ. Bull. 26 (1 A), 1091-1100.

El-Mashad, A.A.A., Mohamed, H.I., 2012. Brassinolide alleviates salt stress and increases antioxidant activity of cowpea plants (Vigna sinensis). Protoplasma 249 (3), 625-635.

ENS, 2006. Environment News Service: Report List World's 10 Worst Pollution Available at: http://www.google.pt/url? $\mathrm{sa}=\mathrm{t} \& \mathrm{rct}=\mathrm{j} \& \mathrm{q}=\& \mathrm{esrc}=\mathrm{s} \&$ source $=\mathrm{web} \& \mathrm{~cd}=2 \& \mathrm{cad}=$ rja\&uac $\mathrm{t}=8 \& \mathrm{ved}=0 \mathrm{CCgQFjAB \& url}=\mathrm{http} \% 3 \mathrm{~A} \% 2 \mathrm{~F} \% 2 \mathrm{Fwww}$.chem.unep. ch $\% 2$ Fpb_and_cd $\% 2$ FDocuments $\% 2$ FNews $\% 2$ F10 $\% 2520$ most $\%$ 2520polluted $\%$ 2520cities.pdf\&ei $=1 \mathrm{zZgVb2iOKKR7AaMoIAo \& usg}=$ AFQjCNHOAyar_69TBPA143KXTt-cAvkP3g\&sig2 =_1TZpa2Y QnsF0slt0Xm7ew\&bvm = bv.93990622,d.ZGU

Esan, A.M., Masisi, K., Dada, F.A., Olaiya, C.O., 2017. Comparative effects of indole acetic acid and salicylic acid on oxidative stress marker and antioxidant potential of okra (Abelmoschus esculentus) fruit under salinity stress. Sci. Hortic. (Amsterdam) 216, 278-283. 
Esim, N., Atici, Ö., 2015. Effects of exogenous nitric oxide and salicylic acid on chilling-induced oxidative stress in wheat (Triticum aestivum). Front. Life Sci. 8 (2), 124-130.

Fahad, S., Bano, A., 2012. Effect of salicylic acid on physiological and biochemical characterization of maize grown in saline area. Pak. J. Bot. 44 (4), 1433-1438.

Fancy, N.N., Bahlmann, A.K., Loake, G.J., 2017. Nitric oxide function in plant abiotic stress. Plant Cell Environ. 40 (4), 462-472.

Fatma, M., Masood, A., Per, T.S., Khan, N.A., 2016. Nitric oxide alleviates salt stress inhibited photosynthetic performance by interacting with sulfur assimilation in mustard. Front. Plant Sci. 7, 521.

Fayez, K.A., Bazaid, S.A., 2014. Improving drought and salinity tolerance in barley by application of salicylic acid and potassium nitrate. J. Saudi Soc. Agric. Sci. 13 (1), 45-55.

Feys, B.J., Parker, J.E., 2000. Interplay of signaling pathways in plant disease resistance. Trends Genet. 16 (10), 449-455.

Foyer, C.H., Noctor, G., 2013. Redox signaling in plants.

Fujii, H., Zhu, J.K., 2009. Arabidopsis mutant deficient in 3 abscisic acid-activated protein kinases reveals critical roles in growth, reproduction, and stress. Proc, Natl. Acad. Sci. 106 (20), $8380-8385$.

Gao, Q.M., Zhu, S., Kachroo, P., Kachroo, A., 2015. Signal regulators of systemic acquired resistance. Front. Plant Sci. 6, 228.

Gémes, K., Poór, P., Horváth, E., Kolbert, Z., Szopkó, D., Szepesi, Á., et al., 2011. Cross-talk between salicylic acid and NaCl-generated reactive oxygen species and nitric oxide in tomato during acclimation to high salinity. Physiol. Plant 142 (2), 179-192.

Griesser, M., Weingart, G., Schoedl-Hummel, K., Neumann, N., Becker, M., Varmuza, K., et al., 2015. Severe drought stress is affecting selected primary metabolites, polyphenols, and volatile metabolites in grapevine leaves (Vitis vinifera cv. Pinot noir). Plant Physiol. Biochem. 88, 17-26.

Hamayun, M., Khan, S.A., Shinwari, Z.K., Khan, A.L., Ahmad, N., Lee, I.J., 2010. Effect of polyethylene glycol induced drought stress on physio-hormonal attributes of soybean. Pak. J. Bot 42 (2), 977-986

Handa, N., Kohli, S.K., Kaur, R., Khanna, K., Bakshi, P., Thukral, A. K., et al., 2017. Emerging Trends in physiological and biochemical responses of salicylic acid. Salicylic Acid: A Multifaceted Hormone. Springer, Singapore, pp. 47-75.

Hara, M., Furukawa, J., Sato, A., Mizoguchi, T., Miura, K., 2012. Abiotic stress and role of salicylic acid in plants. Abiotic Stress Responses in Plants. Springer, New York, pp. 235-251.

Hayat, Q., Hayat, S., Alyemeni, M.N., Ahmad, A., 2012. Salicylic acid mediated changes in growth, photosynthesis, nitrogen metabolism and antioxidant defense system in Cicer arietinum L. Plant Soil Environ. 58 (9), 417-423.

He, Y.L., Liu, Q., 2002. Thermotolerance related to antioxidation induced by salicylic acid and heat acclimation in tall fescue seedlings. J. Plant Physiol. Mol. 28, 89-95.

Herrera-Vásquez, A., Salinas, P., Holuigue, L., 2015. Salicylic acid and reactive oxygen species interplay in the transcriptional control of defense genes expression. Front. Plant Sci. 6, 171.

Hu, Y., Jiang, Y., Han, X., Wang, H., Pan, J., Yu, D., 2017. Jasmonate regulates leaf senescence and tolerance to cold stress: crosstalk with other phytohormones. J. Exp. Bot. 68 (6), 1361-1369.

Ibrahim, M.H., Omar, H., Zain, N.A.M., 2017. Salicylic acid enhanced photosynthesis, secondary metabolites, antioxidant and lipoxygenase inhibitory activity (LOX) in Centella asiatica.

Ichimura, K., Mizoguchi, T., Yoshida, R., Yuasa, T., Shinozaki, K., 2000. Various abiotic stresses rapidly activate Arabidopsis MAP kinases ATMPK4 and ATMPK6. Plant J. 24 (5), 655-665.

Iglesias, M.J., Terrile, M.C., Casalongué, C.A., 2011. Auxin and salicylic acid signalings counteract the regulation of adaptive responses to stress. Plant Signal. Behav. 6 (3), 452-454.
Iqbal, N., Umar, S., Khan, N.A., Khan, M.I.R., 2014. A new perspective of phytohormones in salinity tolerance: regulation of proline metabolism. Environ. Exp. Bot. 100, 34-42.

Ismail, A.M., Horie, T., 2017. Genomics, physiology, and molecular breeding approaches for improving salt tolerance. Annu. Rev. Plant. Biol. 68, 405-434.

Jagendorf, A.T., Takabe, T., 2001. Inducers of glycinebetaine synthesis in barley. Plant Physiol. 127 (4), 1827-1835.

Jaishankar, M., Tseten, T., Anbalagan, N., Mathew, B.B., Beeregowda, K.N., 2014. Toxicity, mechanism and health effects of some heavy metals. Interdiscip. Toxicol. 7 (2), 60-72.

Jalmi, S.K., Sinha, A.K., 2015. ROS mediated MAPK signaling in abiotic and biotic stress-striking similarities and differences. Front. Plant Sci. 6, 769

Jayakannan, M., Bose, J., Babourina, O., Shabala, S., Massart, A., Poschenrieder, C., et al., 2015. The NPR1-dependent salicylic acid signalling pathway is pivotal for enhanced salt and oxidative stress tolerance in Arabidopsis. J. Exp. Bot. 66 (7), 1865-1875.

Kareem, F., Rihan, H., Fuller, M.P., 2017. The effect of exogenous applications of salicylic acid and molybdenum on the tolerance of drought in wheat. Stress 17, 20.

Kaya, C., Tuna, A.L., Yokaş, I., 2009. The role of plant hormones in plants under salinity stress. Salinity and Water Stress. Springer, Netherlands, pp. 45-50.

Kazan, K., 2015. Diverse roles of jasmonates and ethylene in abiotic stress tolerance. Trends. Plant. Sci. 20 (4), 219-229.

Kazemi, N., Khavari-Nejad, R.A., Fahimi, H., Saadatmand, S., NejadSattari, T., 2010. Effects of exogenous salicylic acid and nitric oxide on lipid peroxidation and antioxidant enzyme activities in leaves of Brassica napus L. under nickel stress. Sci. Hortic. (Amsterdam) 126 (3), 402-407.

Khan, M.I.R., Khan, N.A., 2013. Salicylic acid and jasmonates: approaches in abiotic stress tolerance. Plant Biochem. Physiol. 1, 4.

Khan, M.I.R., Khan, N., 2017. Reactive Oxygen Species and Antioxidant System in Plants: Role and Regulation Under Abiotic Stress. Springer Nature, Singapore978-981-10-5254-5.

Khan, M.I.R., Syeed, S., Nazar, R., Anjum, N.A., 2012. An insight into the role of salicylic acid and jasmonic acid in salt stress tolerance. In: Nafees, A., Khan, Nazar, R., Iqbal, N., Anjum, N.A. (Eds.), Phytohormones and Abiotic Stress Tolerance in Plants. SpringerVerlag, pp. 277-300.

Khan, M.I.R., Asgher, M., Khan, N.A., 2013a. Rising temperature in the changing environment: a serious threat to plants. Clim. Change Environ. Sust. 1, 25-36.

Khan, M.I.R., Iqbal, N., Masood, A., Per, T.S., Khan, N.A., 2013 b. Salicylic acid alleviates adverse effects of heat stress on photosynthesis through changes in proline production and ethylene formation. Plant Signal. Behav. 8 (11), e26374.

Khan, M.I.R., Asgher, M., Fatma, M., Per, T.S., Khan, N.A., 2015a. Drought stress vis a vis plant functions in the era of climate change. Clim. Change Environ. Sust. 3, 13-25.

Khan, M.I.R., Fatma, M., Per, T.S., Anjum, N.A., Khan, N.A., 2015b. Salicylic acid-induced abiotic stress tolerance and underlying mechanisms in plants. Front. Plant Sci. 6, 462.

Khan, N.A., Khan, M.I.R., 2014. The ethylene: from senescence hormone to key player in plant metabolism. J. Plant Biochem. Physiol. 2, e124.

Khatoon, H., Singh, A., Ahmad, F., Kamal, A., 2017. Biosciences and Plant Biology. Int. J. Curr. Res. Biosci. Plant Biol. 4 (7), 88-96.

Khokon, M.D., Okuma, E.I.J.I., Hossain, M.A., Munemasa, S., Uraji, M., Nakamura, Y., et al., 2011. Involvement of extracellular oxidative burst in salicylic acid-induced stomatal closure in Arabidopsis. Plant Cell Environ. 34 (3), 434-443.

Khoshbakht, D., Asgharei, M.R., 2015. Influence of foliar-applied salicylic acid on growth, gas-exchange characteristics, and chlorophyll 
fluorescence in citrus under saline conditions. Photosynthetica 53 (3), 410-418.

Klessig, D.F., 2017. How does the multifaceted plant hormone salicylic acid combat disease in plants and are similar mechanisms utilized in humans? BMC Biol. 15 (1), 23.

Kohli, S.K., Handa, N., Sharma, A., Kumar, V., Kaur, P., Bhardwaj, R., 2017. Synergistic effect of 24-epibrassinolide and salicylic acid on photosynthetic efficiency and gene expression in Brassica juncea L. under Pb stress. Turk. J. Biol. 41 (6), 943-953.

Kong, J., Dong, Y., Xu, L., Liu, S., Bai, X., 2014. Effects of foliar application of salicylic acid and nitric oxide in alleviating iron deficiency induced chlorosis of Arachis hypogaea L. Bot. Stud. 55 (1), 9.

Kovács, V., Gondor, O.K., Szalai, G., Darkó, É., Majláth, I., Janda, T., et al., 2014. Synthesis and role of salicylic acid in wheat varieties with different levels of cadmium tolerance. J. Hazard. Mater. 280, 12-19.

Kovtun, Y., Chiu, W.L., Tena, G., Sheen, J., 2000. Functional analysis of oxidative stress-activated mitogen-activated protein kinase cascade in plants. Proc. Natl. Acad. Sci. 97 (6), 2940-2945.

Lee, I., Ambaru, B., Thakkar, P., Marcotte, E.M., Rhee, S.Y., 2010. Rational association of genes with traits using a genome-scale gene network for Arabidopsis thaliana. Nat. Biotechnol. 28 (2), 149-156.

Lee, T.T., Skoog, F., 1965. Effects of substituted phenols on bud formation and growth of tobacco tissue cultures. Physiol. Plant. 18 (2), 386-402.

Li, Z., Yu, J., Peng, Y., Huang, B., 2017. Metabolic pathways regulated by abscisic acid, salicylic acid and $\gamma$-aminobutyric acid in association with improved drought tolerance in creeping bentgrass (Agrostis stolonifera). Physiol. Plant. 159 (1), 42-58.

Litvinovskaya, R.P., Vayner, A.A., Zhylitskaya, H.A., Kolupaev, Y.E., Savachka, A.P., Khripach, V.A., 2016. Synthesis and stressprotective action on plants of Brassinosteroid conjugates with salicylic acid. Chem. Nat. Comp. 52 (3), 452-457.

Liu, P., Xu, Z.S., Pan-Pan, L., Hu, D., Chen, M., Li, L.C., et al., 2013. A wheat PI4K gene whose product possesses threonine autophophorylation activity confers tolerance to drought and salt in Arabidopsis. J. Exp. Bot. 64, 2915-2927.

Liu, N., Song, F., Zhu, X., You, J., Yang, Z., Li, X., 2017. Salicylic acid alleviates aluminum toxicity in soybean roots through modulation of reactive oxygen species metabolism. Front. Chem. 5, 96.

Ma, X., Zheng, J., Zhang, X., Hu, Q., Qian, R., 2017. Salicylic acid alleviates the adverse effects of salt stress on dianthus superbus (Caryophyllaceae) by activating photosynthesis, protecting morphologicalstructure, and enhancing the antioxidant system. Front. Plant Sci. 8.

Manohar, M., Wang, D., Manosalva, P.M., Choi, H.W., Kombrink, E., Klessig, D.F., 2017. Members of the abscisic acid co-receptor PP2C protein family mediate salicylic acid-abscisic acid crosstalk. Plant Direct 1 (5).

Masood, A., Per, T.S., Asgher, M., Fatma, M., Khan, M.I.R., Rasheed, F., et al., 2016. Glycine betaine: role in shifting plants toward adaptation under extreme environments. Osmolytes and Plants Acclimation to Changing Environment: Emerging Omics Technologies. Springer, New Delhi, pp. 69-82.

Meguro, A., Sato, Y., 2014. Salicylic acid antagonizes abscisic acid inhibition of shoot growth and cell cycle progression in rice. Sci. Rep. 4.

Misra, N., Misra, R., 2012. Salicylic acid changes plant growth parameters and proline metabolism in Rauwolfia serpentina leaves grown under salinity stress. Am. Eurasian J. Agri. Environ. Sci 12, 1601-1609.

Miura, K., Tada, Y., 2014. Regulation of water, salinity, and cold stress responses by salicylic acid. Front. Plant Sci. 5.
Mockaitis, K., Howell, S.H., 2000. Auxin induces mitogenic activated protein kinase (MAPK) activation in roots of Arabidopsis seedlings. Plant J. 24 (6), 785-796.

Morris, K., Mackerness, S.A.H., Page, T., John, C.F., Murphy, A.M., Carr, J.P., et al., 2000. Salicylic acid has a role in regulating gene expression during leaf senescence. Plant J. 23 (5), 677-685.

Munns, R., Tester, M., 2008. Mechanisms of salinity tolerance. Annu. Rev. Plant Biol. 59, 651-681.

Mutlu, S., Karadağoğlu, Ö., Atici, Ö., Nalbantoğlu, B., 2013. Protective role of salicylic acid applied before cold stress on antioxidative system and protein patterns in barley apoplast. Biol. Plant. 57 (3), 507-513.

Nakagami, H., Soukupová, H., Schikora, A., Zárský, V., Hirt, H., 2006. A mitogen-activated protein kinase mediates reactive oxygen species homeostasis in Arabidopsis. J. Biol. Chem. 281 (50), 38697-38704.

Nakashita, H., Yasuda, M., Nitta, T., Asami, T., Fujioka, S., Arai, Y., et al., 2003. Brassinosteroid functions in a broad range of disease resistance in tobacco and rice. Plant J. 33 (5), 887-898.

Naser Alavi, S.M., Arvin, M.J., Manoochehri Kalantari, K., 2014. Salicylic acid and nitric oxide alleviate osmotic stress in wheat (Triticum aestivum L.) seedlings. J. Plant Interact. 9 (1), 683-688.

Nazar, R., Umar, S., Khan, N.A., 2015. Exogenous salicylic acid improves photosynthesis and growth through increase in ascorbate-glutathione metabolism and $\mathrm{S}$ assimilation in mustard under salt stress. Plant Signal. Behav. 10 (3), e1003751.

Nguyen, D., Rieu, I., Mariani, C., van Dam, N.M., 2016. How plants handle multiple stresses: hormonal interactions underlying responses to abiotic stress and insect herbivory. Plant Mol. Biol. 91 (6), 727-740.

Palma, F., López-Gómez, M., Tejera, N.A., Lluch, C., 2013. Salicylic acid improves the salinity tolerance of Medicago sativa in symbiosis with Sinorhizobium meliloti by preventing nitrogen fixation inhibition. Plant Sci. 208, 75-82.

Park, S.W., Kaimoyo, E., Kumar, D., Mosher, S., Klessig, D.F., 2007. Methyl salicylate is a critical mobile signal for plant systemic acquired resistance. Science 318 (5847), 113-116.

Pasala, R.K., Khan, M.I.R., Minhas, P.S., Farooq, M.A., Sultana, R., Per, T.S., et al., 2016. Can plant bio-regulators minimize crop productivity losses caused by drought, heat and salinity stress? An integrated review. J. Appl. Bot. Food. 89.

Per, T.S., Khan, M.I.R., Anjum, N.A., Masood, A., Hussain, S.J., Khan, N.A., 2018. Jasmonates in plants under abiotic stresses: crosstalk with other phytohormones matters. Environ. Exp. Bot. $145,104-120$.

Pereira, A., 2016. Plant abiotic stress challenges from the changing environment. Front. Plant Sci. 7, 1123.

Pieterse, C.M., Van der Does, D., Zamioudis, C., Leon-Reyes, A., Van Wees, S.C., 2012. Hormonal modulation of plant immunity. Annu. Rev. Cell. Dev. Biol. 28, 489-521.

Poór, P., Kovács, J., Szopkó, D., Tari, I., 2013. Ethylene signaling in salt stress-and salicylic acid-induced programmed cell death in tomato suspension cells. Protoplasma 250 (1), 273-284.

Rao, M.V., Lee, H.I., Davis, K.R., 2002. Ozone-induced ethylene production is dependent on salicylic acid, and both salicylic acid and ethylene act in concert to regulate ozone-induced cell death. Plant J. 32 (4), 447-456.

Qados, A.M.A., 2015. Effects of salicylic acid on growth, yield and chemical contents of pepper (Capsicum annuum L) plants grown under salt stress conditions. Int. J. Agric. Crop Sci. 8 (2), 107.

Qi, T., Wang, J., Huang, H., Liu, B., Gao, H., Liu, Y., et al., 2015. Regulation of jasmonate-induced leaf senescence by antagonism between bHLH subgroup IIIe and IIId factors in Arabidopsis. Plant Cell 27 (6), 1634-1649. 
Razmi, N., Ebadi, A., Daneshian, J., Jahanbakhsh, S., 2017. Salicylic acid induced changes on antioxidant capacity, pigments and grain yield of soybean genotypes in water deficit condition. J. Plant Interact. 12 (1), 457-464.

Rincón-Pérez, J., Rodríguez-Hernández, L., Ruíz-Valdiviezo, V.M., Abud-Archila, M., Luján-Hidalgo, M.C., Ruiz-Lau, N., et al., 2016. Fatty acids profile, phenolic compounds and antioxidant capacity in elicited callus of thevetiaperuviana (Pers.) K. schum. J. Oleo. Sci. 65 (4), 311-318.

Rivas-San Vicente, M., Plasencia, J., 2011. Salicylic acid beyond defence: its role in plant growth and development. J. Exp. Bot. 62 (10), 3321-3338.

Saini, S., Sharma, I., Pati, P.K., 2015. Versatile roles of brassinosteroid in plants in the context of its homoeostasis, signaling and crosstalks. Front. Plant Sci. 6.

Sami, F., Yusuf, M., Faizan, M., Faraz, A., Hayat, S., 2016. Role of sugars under abiotic stress. Plant. Physiol. Biochem. 109, 54-61.

Saruhan, N., Saglam, A., Kadioglu, A., 2012. Salicylic acid pretreatment induces drought tolerance and delays leaf rolling by inducing antioxidant systems in maize genotypes. Acta Physiol. Plant. 34 (1), 97-106.

Saud, S.H.A.H., Chen, Y., Baowen, L., Fahad, S.H.A.H., Arooj, S., 2013. The different impact on the growth of cool season turf grass under the various conditions on salinity and drought stress. Int. J. Agric. Sci. Res. 3 (4), 77-84.

Schenk, P.M., Kazan, K., Wilson, I., Anderson, J.P., Richmond, T., Somerville, S.C., et al., 2000. Coordinated plant defense responses in Arabidopsis revealed by microarray analysis. Proc. Natl. Acad. Sci. 97 (21), 11655-11660.

Sewelam, N., Kazan, K., Thomas-Hall, S.R., Kidd, B.N., Manners, J.M., Schenk, P.M., 2013. Ethylene response factor 6 is a regulator of reactive oxygen species signaling in Arabidopsis. PLoS One 8 (8), e70289.

Shabala, S., Demidchik, V., Shabala, L., Cuin, T.A., Smith, S.J., Miller, A.J., et al., 2006. Extracellular $\mathrm{Ca} 2+$ ameliorates $\mathrm{NaCl}$-induced $\mathrm{K}+$ loss from Arabidopsis root and leaf cells by controlling plasma membrane $\mathrm{K}+$-permeable channels. Plant Physiol. 141 (4), 1653-1665.

Shah, S.H., 2007. Effects of salt stress on mustard as affected by gibberellic acid application. Gen. Appl. Plant Physiol. 33 (1-2), 97-106.

Sharma, M., Gupta, S.K., Majumder, B., Maurya, V.K., Deeba, F., Alam, A., et al., 2017. Salicylic acid mediated growth, physiological and proteomic responses in two wheat varieties under drought stress. J. Proteomics. 163, 28-51.

Sheykhbaglou, R., Rahimzadeh, S., Ansari, O., Sedghi, M., 2014. The effect of salicylic acid and gibberellin on seed reserve utilization, germination and enzyme activity of sorghum (Sorghum bicolor L.) seeds under drought stress. J. Stress Physiol. Biochem. 10 (1).

Siboza, X.I., Bertling, I., Odindo, A.O., 2014. Salicylic acid and methyl jasmonate improve chilling tolerance in cold-stored lemon fruit (Citrus limon). J. Plant. Physiol. 171 (18), 1722-1731.

Simaei, M., Khavari-Nejad, R.A., Bernard, F., 2012. Exogenous application of salicylic acid and nitric oxide on the ionic contents and enzymatic activities in NaCl-stressed soybean plants. Am. J. Plant Sci. 3 (10), 1495.

Soon, F.F., Ng, L.M., Zhou, X.E., West, G.M., Kovach, A., Tan, M.E., et al., 2012. Molecular mimicry regulates ABA signaling by SnRK2 kinases and PP2C phosphatases. Science 335 (6064), $85-88$.

Spoel, S.H., Koornneef, A., Claessens, S.M., Korzelius, J.P., Van Pelt, J.A., Mueller, M.J., et al., 2003. NPR1 modulates cross-talk between salicylate-and jasmonate-dependent defense pathways through a novel function in the cytosol. Plant Cell 15 (3), $760-770$.
Strawn, M.A., Marr, S.K., Inoue, K., Inada, N., Zubieta, C., Wildermuth, M.C., 2007. Arabidopsis isochorismate synthase functional in pathogen-induced salicylate biosynthesis exhibits properties consistent with a role in diverse stress responses. J. Biol. Chem. 282 (8), 5919-5933.

Suarez-Rodriguez, M.C., Adams-Phillips, L., Liu, Y., Wang, H., Su, S. H., Jester, P.J., et al., 2007. MEKK1 is required for flg22-induced MPK4 activation in Arabidopsis plants. Plant Physiol. 143 (2), $661-669$.

Sulmon, C., Van Baaren, J., Cabello-Hurtado, F., Gouesbet, G., Hennion, F., Mony, C., et al., 2015. Abiotic stressors and stress responses: what commonalities appear between species across biological organization levels? Environ. Poll. 202, 66-77.

Sunkar, R., Chinnusamy, V., Zhu, J., Zhu, J.K., 2007. Small RNAs as big players in plant abiotic stress responses and nutrient deprivation. Trends. Plant. Sci. 12 (7), 301-309.

Szalai, G., Krantev, A., Yordanova, R., Popova, L.P., Janda, T., 2013. Influence of salicylic acid on phytochelatin synthesis in Zea mays during Cd stress. Turkish J. Bot. 37 (4), 708-714.

Szepesi, Á., Csiszár, J., Gémes, K., Horváth, E., Horváth, F., Simon, M.L., et al., 2009. Salicylic acid improves acclimation to salt stress by stimulating abscisic aldehyde oxidase activity and abscisic acid accumulation, and increases $\mathrm{Na}+$ content in leaves without toxicity symptoms in Solanum lycopersicum L. J. Plant. Physiol. 166 (9), 914-925.

Tamás, L., Mistrík, I., Alemayehu, A., Zelinová, V., Bočová, B., Huttová, J., 2015. Salicylic acid alleviates cadmium-induced stress responses through the inhibition of $\mathrm{Cd}$-induced auxin-mediated reactive oxygen species production in barley root tips. J. Plant. Physiol. 173, 1-8.

Teige, M., Scheikl, E., Eulgem, T., Dóczi, R., Ichimura, K., Shinozaki, K., et al., 2004. The MKK2 pathway mediates cold and salt stress signaling in Arabidopsis. Mol. Cell 15 (1), 141-152.

Thaler, J.S., Fidantsef, A.L., Duffey, S.S., Bostock, R.M., 1999. Tradeoffs in plant defense against pathogens and herbivores: a field demonstration of chemical elicitors of induced resistance. J. Chem. Ecol. 25 (7), 1597-1609.

Thomas, H., 2013. Senescence, ageing and death of the whole plant. New Phytol. 197 (3), 696-711.

Tirani, M.M., Nasibi, F., Kalantari, K.M., 2013. Interaction of salicylic acid and ethylene and their effects on some physiological and biochemical parameters in canola plants (Brassica napus L.). Photosynthetica 51 (3), 411-418.

Traw, M.B., Bergelson, J., 2003. Interactive effects of jasmonic acid, salicylic acid, and gibberellin on induction of trichomes in Arabidopsis. Plant Physiol. 133 (3), 1367-1375.

Trujillo, L.E., Sotolongo, M., Menendez, C., Ochogavia, M.E., Coll, Y., Hernandez, I., et al., 2008. SodERF3, a novel sugarcane ethylene responsive factor (ERF), enhances salt and drought tolerance when overexpressed in tobacco plants. Plant Cell Physiol. 49 (4), 512-525.

Van der Does, D., Leon-Reyes, A., Koornneef, A., Van Verk, M.C., Rodenburg, N., Pauwels, L., et al., 2013. Salicylic acid suppresses jasmonic acid signaling downstream of SCFCOI1-JAZ by targeting GCC promoter motifs via transcription factor ORA59. Plant Cell 25 (2), 744-761.

Vardhini, B.V., Anjum, N.A., 2015. Brassinosteroids make plant life easier under abiotic stresses mainly by modulating major components of antioxidant defense system. Front. Environ. Sci. 2, 67.

Verberne, M.C., Muljono, R.A.B., Verpoorte, R., 1999. Salicylic acid biosynthesis, New Comprehensive Biochemistry, Vol. 33. Elsevier, pp. 295-312.

Verma, K., Agrawal, S.B., 2017. Salicylic acid-mediated defence signalling in respect to its perception, alteration and transduction. Salicylic Acid: A Multifaceted Hormone. Springer, Singapore, pp. 97-122. 
Verma, V., Ravindran, P., Kumar, P.P., 2016. Plant hormone-mediated regulation of stress responses. BMC Plant Biol. 16 (1), 86.

Vishwakarma, K., Upadhyay, N., Kumar, N., Yadav, G., Singh, J., Mishra, R.K., et al., 2017. Abscisic acid signaling and abiotic stress tolerance in plants: a review on current knowledge and future prospects. Front. Plant Sci. 8.

Vlot, A.C., Dempsey, D.M.A., Klessig, D.F., 2009. Salicylic acid, a multifaceted hormone to combat disease. Annu. Rev. Phytopathol. 47, 177-206.

Vogelmann, K., Drechsel, G., Bergler, J., Subert, C., Philippar, K., Soll, J., et al., 2012. Early senescence and cell death in Arabidopsis saul1 mutants involves the PAD4-dependent salicylic acid pathway. Plant Physiol. 159 (4), 1477-1487.

Voß, U., Bishopp, A., Farcot, E., Bennett, M.J., 2014. Modelling hormonal response and development. Trends. Plant. Sci. 19 (5), 311-319.

Wada, K.C., Mizuuchi, K., Koshio, A., Kaneko, K., Mitsui, T., Takeno, K., 2014. Stress enhances the gene expression and enzyme activity of phenylalanine ammonia-lyase and the endogenous content of salicylic acid to induce flowering in pharbitis. J. Plant. Physiol. 171 (11), 895-902.

Wang, F., Wang, C., Zou, T., Xu, N., Sun, X., 2017. Comparative transcriptional profiling of Gracilariopsis lemaneiformis in response to salicylic acid-and methyl jasmonate-mediated heat resistance. PLoS. ONE. 12 (5), e0176531.

Wang, L.J., Li, S.H., 2006. Salicylic acid-induced heat or cold tolerance in relation to $\mathrm{Ca}_{2}{ }^{+}$homeostasis and antioxidant systems in young grape plants. Plant Sci. 170 (4), 685-694.

Wang, L.J., Fan, L., Loescher, W., Duan, W., Liu, G.J., Cheng, J.S., et al., 2010. Salicylic acid alleviates decreases in photosynthesis under heat stress and accelerates recovery in grapevine leaves. BMC Plant Biol. 10 (1), 34.

Wani, S.H., Kumar, V., Shriram, V., Sah, S.K., 2016. Phytohormones and their metabolic engineering for abiotic stress tolerance in crop plants. Crop J. 4 (3), 162-176.

Xie, Z., Zhang, Z.L., Hanzlik, S., Cook, E., Shen, Q.J., 2007. Salicylic acid inhibits gibberellin-induced alpha-amylase expression and seed germination via a pathway involving an abscisic-acidinducible WRKY gene. Plant Mol. Biol. 64 (3), 293-303.

Xu, E., Brosché, M., 2014. Salicylic acid signaling inhibits apoplastic reactive oxygen species signaling. BMC Plant Biol. 14 (1), 155.

Xu, L., Zhao, H., Ruan, W., Deng, M., Wang, F., Peng, J., et al., 2017. Abnormal inflorescence meristem 1 functions in salicylic acid biosynthesis to maintain proper reactive oxygen species levels for root meristem activity in rice. Plant Cell 29 (3), 560-574.

Yamaguchi, S., 2008. Gibberellin metabolism and its regulation. Annu. Rev. Plant Biol. 59, 225-251.

Yasuda, M., Ishikawa, A., Jikumaru, Y., Seki, M., Umezawa, T., Asami, T., et al., 2008. Antagonistic interaction between systemic acquired resistance and the abscisic acid-mediated abiotic stress response in Arabidopsis. Plant Cell 20 (6), $1678-1692$.

Yücel, N.C., Heybet, E., 2016. Salicylic acid and calcium treatments improves wheat vigor, lipids and phenolics under high salinity. Acta Chimica Slovenica 63 (4), 738-746.

Zhang, Y., Xu, S., Yang, S., Chen, Y., 2015. ). Salicylic acid alleviates cadmium-induced inhibition of growth and photosynthesis through upregulating antioxidant defense system in two melon cultivars (Cucumis melo L.). Protoplasma 252, 911-924.

Zhao, X.Y., Wang, J.G., Song, S.J., Wang, Q., Kang, H., Zhang, Y., et al., 2016. Precocious leaf senescence by functional loss of PROTEIN S-ACYL TRANSFERASE14 involves the NPR1dependent salicylic acid signaling. Sci. Rep. 6, 20309.

Zheng, Y., Cui, X., Su, L., Fang, S., Chu, J., Gong, Q., et al., 2017. Jasmonate inhibits COP1 activity to suppress hypocotyl elongation and promote cotyledon opening in etiolated Arabidopsis seedlings. Plant J. 90 (6), 1144-1155.

\section{Further Reading}

Asgher, M., Khan, M.I.R., Anjum, N.A., Khan, N.A., 2015. Minimising toxicity of cadmium in plants-role of plant growth regulators. Protoplasma 252 (2), 399-413.

Desoky, E.S.M., Merwad, A.R.M., 2015. Improving the salinity tolerance in wheat plants using salicylic and ascorbic acids. J. Agric. Sci. 7 (10), 203.

Hussain Wani, S., Brajendra Singh, N., Haribhushan, A., Iqbal Mir, J., 2013. Compatible solute engineering in plants for abiotic stress tolerance-role of glycine betaine. Curr. Genomics 14 (3), 157-165.

Wada, K.C., Yamada, M., Shiraya, T., Takeno, K., 2010. Salicylic acid and the flowering gene FLOWERING LOCUS $\mathrm{T}$ homolog are involved in poor-nutrition stress-induced flowering of Pharbitis nil. J. Plant. Physiol. 167 (6), 447-452. 
This page intentionally left blank 


\title{
24
}

\section{Role of Methyl Jasmonates in Salt Stress Tolerance in Crop Plants}

\author{
Bilal Ahmad ${ }^{1}$, Aamir Raina ${ }^{2,3}$, Mohd Irfan Naikoo ${ }^{4}$ and Samiullah Khan ${ }^{2}$ \\ ${ }^{1}$ Plant Physiology Laboratory, Department of Botany, Aligarh Muslim University, Aligarh, Uttar Pradesh, India \\ ${ }^{2}$ Department of Botany, Mutation Breeding Laboratory, Aligarh Muslim University, Aligarh, Uttar Pradesh, India \\ ${ }^{3}$ Botany Section, Women's College, Aligarh Muslim University, Aligarh, Uttar Pradesh, India ${ }^{4}$ Plant Ecology and \\ Environment Laboratory, Department of Botany, Aligarh Muslim University, Aligarh, Uttar Pradesh, India
}

\section{O U T L I N E}

24.1 Introduction

24.2 Jasmonate Biosynthesis

24.3 Jasmonate Signaling

24.4 Methyl Jasmonates: Multifunctional Roles in Abiotic Stress Tolerance

24.5 Effect of Salt Stress on Plants
24.5.1 Jasmonates Counteract Salinity Stress 24.5.2 Salt Stress Response Mediated by JA Signaling

24.6 Conclusion and Future Perspectives

References

Further Reading

\subsection{INTRODUCTION}

Globally salinity is considered as one of the most serious problems of agriculture and allied sectors, especially in arid and semiarid regions. Increased soil salinity may be the main factor in making arable land unfavorable for a wide range of crop species (Epstein et al., 1980). The factors that lead to increased salinity are the usage of saline water for irrigation and application of fertilizer (Epstein et al., 1980). Saline soils are characterized with high levels of sodium and chloride content and thus exert severe abiotic stress on the growth and development of plants by inducing changes in most physiological and biochemical processes. Multiple factors are involved in the alleviation of salinity stress but phytohormones are thought to be among the most essential endogenous substances involved in the underlying mechanisms of tolerance or susceptibility of various plant species (Velitchkova and Fedina, 1998). Plants act in response to abiotic stresses by inducing the expression of different sets of genes whose products play a pivotal role in alleviating a wide range of stresses (Martinez et al., 2018). Phytohormones play an essential role in the plant's ability to acclimatize to environmental flux such as abscisic acid (ABA), a stress hormone known to be 
involved in abiotic stress response and tolerance (Peleg and Blumwald, 2011). Different plant hormones, such as jasmonic acid (JA) and its derivatives, are implicated in regulating plant defense mechanisms in response to environmental stresses including drought, low temperature, and salinity by inducing changes in gene expression (Wasternack and Parthier, 1997; Khan et al., 2012). Acting as stress modulator JAs can repress or augment plant stress responses (Agrawal et al., 2003). JAs have been well documented for their vital roles in plant responses to a wide range of abiotic stresses including drought (Brossa et al., 2011), salt (Dong et al., 2013; Qiu et al., 2014; Zhao et al., 2014), heavy metals (Maksymiec et al., 2005), and heat stress (Clarke et al., 2009). In the plant kingdom jasmonic acid (JA) and its methyl ester, methyl jasmonate (MeJA), are cosmopolitan in distribution (Meyer et al., 1984; Schaller, 2011; Pirbalouti et al., 2014). The initial isolation of JA was reported from the culture filtrate of the fungus Lasiodiplodia theobromae (Aldridge et al., 1971). MeJA, one of the conjugates of JA, is an aromatic volatile compound originally known in flowers of Jasminum grandiflorum and is also known as a primary source to isolate MeJA (Demole et al., 1962; Avanci et al., 2010). MeJA can also considerably direct photosynthesis and antioxidant metabolism by modulating set of proteins. Apart from JA and MeJA, other JAs such as jasmonoyl ACC (JA-ACC), cis-jasmone, and jasmonoyl isoleucine (JA-Ile) with numerous biological functions have been documented (Rohwer and Erwin, 2008; Avanci et al., 2010; Wasternack and Kombrink, 2010). However, literature is very meager on JA crosstalk with other major phytohormones in plants under salt stress. Keeping in view the recent literature, this chapter dissects the role and underlying mechanisms of JA-regulated plant growth and development in response to salt stress, and presents crosstalk on and interaction-outcomes of JA.

\subsection{JASMONATE BIOSYNTHESIS}

Jasmonates, fatty acid derived cyclopentanones ubiquitously found in the kingdom Plantae, are vital regulators found in plants predominantly during various abiotic and biotic stress responses (Reinbothe et al., 2009). An array of metabolic events involving multiple signal transduction cascades regulates the Jasmonate biosynthesis and altered levels have been observed in stressed as well as unstressed plant tissues (Browse, 2009; Reinbothe et al., 2009). Characterized by a pentacyclic ring structure, jasmonates are the products of the oxidative metabolism of polyunsaturated fatty acids and belong to class of compounds called oxylipins, a family of oxygenated fatty acid derivatives. The biosynthesis of jasmonates takes place through the octadecanoid pathway analogous to the biogenesis of animal antiinflammatory prostaglandins (Wasternack and Hause, 2002), involving translocation of chloroplast membrane lipid intermediates to the peroxisomes through cytoplasm (León, 2013). Phospholipases belonging to the class D (PLDs), after activation through certain stimuli, act on the chloroplast membrane lipids releasing an 18 carbon phospholipid $\alpha$-linolenic acid (C18:3) through oxidation (Fig. 24.1). This $\alpha$-linolenic acid leads to the

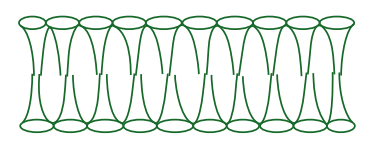

(Lipid membrane)

Lipase

$\alpha$-Linolenic acid

13LOX

13(S)HPOT

AOS

12, 13S-epoxylinolenic acid
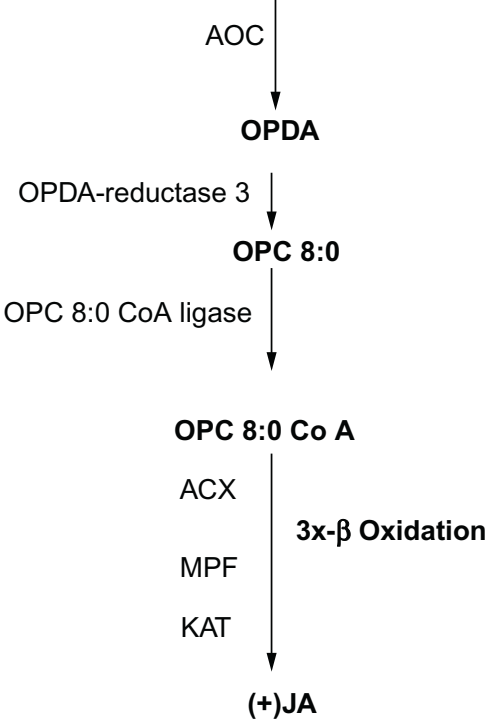

FIGURE 24.1 Pathway intermediates are abbreviated as 13HPOT for (9Z11E15Z13S)-13-hydroperoxy-9,11,15-octadecatrienoic acid (i.e., 13(S)-hydroperoxy linolenic acid), allene oxide for (12,13 (S)-epoxy-9(Z)), 11,15(Z)-octadecatrienoic acid, cis-(+)-OPDA for cis(+)- 12-oxophytodienoic acid, and OPC8:0 for 3-oxo-2(2_(Z)- pentenyl)-cyclopentane-1-octanoic acid. The enzymes are indicated as 13LOX for 13-lipoxygenase, AOS for allene oxide synthase, AOC for allene oxide cyclase, and OPR3 for 12-oxophytodienoate reductase 3. Source: Adapted from Wasternack, C., Hause, B., 2002. Jasmonates and octadecanoids: signals in plant stress. responses and development. Prog. Nucl. Acid Res. Mol. Biol. 72, 165-221. 
production of 12-oxophytodienoic acid (OPDA) involving three steps. First a chloroplastic lipoxygenase (LOX) oxygenates $\alpha$-linolenic acid to generate 13(S)-hydroperoxy linolenic acid derivatives (13-HPOT) (namely $13 S$ (13S-HPODE) and 9S-hydroperoxy derivative of LA hydroperoxy octadecadienoic acids (9S-HPODE) (Vick and Zimmerman, 1984). This oxygenation is followed by cyclization leading to the generation of 12oxophytodienoic acid (OPDA) (Bannenberg et al., 2009) by the help of allene oxide synthase (AOS; EC 4.2.1.92) and allene oxide cyclase (AOC) enzymes. AOS dehydrates the 13S-HPODE producing 12-oxophytodienoic acid (12-OPDA), which is recognized as allene oxide. Further biosynthesis of jasmonic acid from OPDA involves reduction and $\beta$-oxidation after which JA carboxyl methyltransferase (JMT) enzyme comes into play leading to the catabolization of $\beta$-oxidation product into its volatile equivalent methyl jasmonate (MeJA) (Song et al., 2000; Seo et al., 2011). OPDA is exported to the peroxisome where it is oxidized to 3-oxo-2-2'(Z)-pentenyl)-cyclopentane-1-octanoic acid (OPC:8) by the help of the enzyme OPDA reductase (OPR; EC 1.3.1.4). Finally, shortening of the precursor molecule side chains brought about through three cycles of peroxisomal $\beta$-oxidation (Bannenberg et al., 2009; Lyons et al., 2013). The parent compound (octadecatrienoic acid, an 18:3 compound), from which jasmonic acid is derived through a series of enzyme catalyzed reactions, gives the pathway its name as the octadecanoid pathway. Occurrence of three of LOXs (LOX2, LOX3, and LOX4) has been observed in the genome of the Arabidopsis thaliana (Caldelari et al., 2011). AOS and AOC are reported to be chloroplastic enzymes (Ziegler et al., 2000; Hause et al., 2003). Moreover, OPDA is exported by the help of a carrier namely COMATOSE1/PEROXIMAL 1/PEROXISOME ABC TRANSPORTER (ABC CTS1/ PXA1/PED3) from chloroplast to peroxisome (Zolman et al., 2000; Theodoulou et al., 2005; Footitt et al., 2007). Furthermore, the existence of a novel C16 cyclopentenone namely $d n$-OPDA has been revealed from the analysis of the oxylipin content of Arabidopsis leaves (Weber, 2002). Besides, an array of products are derived from JA after its biosynthesis, noteworthy among which are methyl ester of JA (MeJA) and certain conjugates like conjugate with hydroxylated didehydro derivatives and certain derivatives of sugars as well as amide conjugates with certain amino acids (JA-Ile) (Staswick, 2008). Certain proteins and enzymes necessary for the biosynthesis and perception of JA have been reproduced by the help of different plant species and a handful of like AOC, 13-AOS, ACYL-CoA-OXIDASE1, 13-LOX, JAR1, OPR3, and the SCFCOI1-JAZ-coreceptor complex have also been crystallized (Kombrink, 2012; Wasternack and Hause, 2013).

\subsection{JASMONATE SIGNALING}

Jasmonates, lipid-derived signals derived from the lipid constituents of some particular membranes by the help of some oxygenases, are perceived by protein receptors resulting in the activation of certain specific signal transduction cascades in plants (Wasternack and Hause, 2013). JAs mediate the plant responses to various types of biotic and abiotic stresses and in plant development. Quite a few molecules that operate as activators, repressors, and corepressors have been recognized in plants that polish up the gene expression regulated by JA itself and its various conjugates, and insights into the vital components of JA signaling and perception have been gained in addition to the elucidation of each and every step in their biosynthesis in the recent past due to the exhaustive efforts of researchers, which has increased our understanding regarding the functioning of the plant hormone (Wasternack and Strnad, 2016). JA actively participates in numerous metabolic reactions, notably conjugation with various amino acids, carboxylation, glucosylation, hydroxylation, methylation, and sulfation leading to several compounds with varied biological activities. The activity of these metabolites varies from being highly active, partly active, or inactive in specific processes. Carboxylation, hydroxylation, and sulfation lead to inactivation of JA signaling. 12-oxophytodienoic acid (OPDA), the JA-biosynthesis precursor, has been recognized as a JA-independent signaling compound (Wasternack and Strnad, 2016).

Among the chief regulators of JA signaling; JASMONATE ZIM DOMAIN (JAZ) proteins (ZIMdomain protein), Skp/Cullin/F-box complex $\left(\mathrm{SCF}^{\mathrm{CO} 11}\right)$, a type of E3 ubiquitin ligase, and also the $26 \mathrm{~S}$ proteasome and F-box protein CORONATINE INSENSITIVE 1 (COI1), are the most noteworthy (Deshaies, 1999; Turner et al., 2002; Pauwels and Goossens, 2011). The discovery of the ubiquitin-proteasome system in plant signaling was an important breakthrough and it was observed to act as a central regulator in the sensing and signaling of plant hormone including JA. It is comprised of a protein complex consisting of three types of proteins (Skp1/ Cullin/F-box proteins) called the SCF complex, which serves as an E3 ubiquitin ligase. At this complex, a target protein to be ubiquitinated is recognized by the F-box protein and consequently proteasomal degradation is carried out. COI1 serves as an F-box protein during perception and signaling of JA (Xie et al., 1998). The JAZ proteins play a pivotal role in the signaling cascade of JA (Thines et al., 2007). These proteins, which were discovered by chance and named as JASMONATE ZIM DOMAIN (JAZ) proteins (Yan et al., 2007), were found to be upregulated early by JA treatment or wounding. 
These operate as repressors of JA signaling and consequently act as negative regulators of the gene expression. During the JA-induced gene expression, degradation of these repressors is brought about through the $\mathrm{SCF}^{\mathrm{CO}}$ dependent 26S proteasome pathway. Pauwels et al. (2010) elucidated the molecular mechanisms through which JAZ proteins repress the expression of genes. It was shown that in Arabidopsis, the JAZ proteins conscript a type of corepressor called as Groucho/Tup1-type TOPLESS (TPL) and TPL-related proteins (TPRs) through a formerly uncharacterized adaptor protein, named as Novel Interactor of JAZ (NINJA). JAZ degradation allows the liberation of positively acting TFs like MYC2, which are then able to bind to the promoter sequences in the responsive elements of JA-responsive genes, leading to the initiation of transcription. During the resting state JA-Ile levels being very low, JAZ protein represses the attachment of the transcription factor MYC2 to the Gbox. Enhanced endogenous levels of JA-Ile induced by any environmental or developmental signal results in the formation of $\mathrm{SCF}^{\mathrm{COI}}$-JAZ coreceptor complex, which requires JA-Ile, one of the most bioactive compounds acting as the ligand during the formation of $\mathrm{SCF}^{\mathrm{COI1}}-\mathrm{JAZ}$ coreceptor complex. JA-Ile is active as an epimer (+)-7iso-JA-Ile, synthesized by the enzyme jasmonoylisoleucine synthetase, which is encoded by JAR1, a member of the GH3 gene family (Fonseca et al., 2009; Staswick and Tiryaki, 2004; Westfall et al., 2012) JA-Ile level is very important as it plays a regulatory role and its level is primarily determined by the activity and hydroxylation of JAR1 as well as its cleavage by certain amidohydrolases (Koo et al., 2011; Heitz et al., 2012; Aubert et al., 2015). During the scarcity of other plant proteins, the JA-Ile conjugate promotes interaction between COI1 and JAZ1 proteins specifically (Thines et al., 2007). Besides, JA-Ile conjugate-induced destruction of JAZ transcriptional repressors is responsible for the stimulation of downstream response genes (Staswick, 2008). Degradation of JAZ protein repressors can also be facilitated by their binding with F-box protein COI1, resulting in the release of transcription factors which are otherwise repressed (Thines et al., 2007; Pauwels and Goossens, 2011).

MYC2, a very essential member of the MYC-TFfamily, is a master regulator of the signal transduction pathway of JA and this TF belongs to the basic helix-loop-helix (bHLH)-type (Kazan and Manners, 2013). Its chief role has already been established in a number of signal transduction pathways noteworthy among which are synthesis of auxin, ethylene, glucosinolates, tryptophan, and JA in addition to responses to wounding/insects, pathogens, oxidative stress, and ABA-dependent drought stress (Dombrecht et al., 2007; Kazan and Manners, 2008). MYC2 is also known to play a diverse part in various signaling pathways involved in different aspects of defense responses and development in Arabidopsis mediated through JA (Kazan and Manners, 2013). Worth noting is the MYC2-mediated regulation of crosstalk between different plant hormones like ABA, GAs, SA, and auxin, and also the establishment of a link between different signaling pathways such as phytochromes, light, circadian clock, and JA. Investigations at the molecular level have helped to gain insights into the regulation of transcription of JA-responsive elements through MYC2, which has improved our current understanding about the signal transduction pathway of JA in plants a great deal (Chen et al., 2011; Zhai et al., 2013a, b). MYC2 has been reportedly observed to involve distinct molecular mechanisms to differentially regulate the transcription of distinct JA-responsive genes thereby causing the transcriptional activation of various downstream target genes (Katsir et al., 2008; Chen et al., 2011; Zhai et al., 2013a,b). Reports regarding the occurrence of JAZ repressors with high affinity for various related TFs that belong to the bHLH family including MYC2, MYC3 and MYC4, GLABRA3 (GL3), ENHANCER OF GLABRA3 1 (EGL1), and TRANSPARENT TESTA 8 (TT8) are well documented, which actively participate in specific as well as overlapping JA signaling aspects (Cheng et al., 2011; Fernández-Calvo et al., 2011; Niu and Figueroa, 2011; Qi et al., 2011). MYC2 activity has been reported to be blocked due to the interaction of the JAZ repressors and the adaptor protein NINJA with the MYC2 in the absence of functional JAs and also due to the recruitment of the corepressors TOPLESS (TPL) and TPLrelated proteins (reviewed by Pauwels and Goossens, 2011). However, reports regarding the mechanism of differential regulation of various JA-responsive genes at the protein level mediated through MYC2 are scanty. Furthermore, regulation of the target gene transcription can be regulated by MYC2-proteolysis. This has been confirmed by the discovery of a peptide stretch, 12 amino acids long, in the MYC2 transcription activation domain that has been assigned for both transcriptional activity of MYC2 as well as its proteolysis. In addition, phosphorylation of a threonine (Thr-328) residue has also been observed to be an important event in the MYC2-mediated regulation of transcription (Zhai et al., $2013 a, b)$. One more breakthrough in the JA signaling was the discovery of a new regulating element called as JASMONOYL-L-ISOLEUCINE HYDROLASE 1 (JIH1), which has been observed to encode a novel homeostatic step in the signaling of the hormone along with additional JA-Ile inactivation steps like hydroxylation and carboxylation (Woldemariam et al., 2012). JIH1 has been shown to contribute in the defense responses of plants and to reduce the JA-Ile burst (Woldemariam et al., 2012). A number of new 
endogenous bioactive conjugates mimicking the amino acid conjugates of JA have been revealed, which are designed by the conjugation of coronafacic acid (CFA) conjugates with the other amino acids, for example, (+)-7-iso-JA-Ala, (+)-7-iso-JA-Leu, (+)-7-iso-JAMet, and (+)-7-iso-JA-Val (Yan et al., 2007).

Recent studies have revealed the regulatory role of JAs in salt tolerance (Dong, et al., 2013; Qiu, et al., 2014; Zhao et al., 2014). Expression of Triticum aestivumTaAOC1 gene, encoding allene oxide cyclase (AOC) enzyme, in Arabidopsis raises JA levels and increases the tolerance against salt stress, signifying the positive regulatory effect of JAs against salt tolerance. This salt tolerance imparted by the expression of TaAOC1 occurs independent of the ABA as the transgenic expression of TaAOC1 in aba2 (ABA-deficient mutant) did not compromise the salt tolerance. Contrary to this, TaAOC1 expression in the myc2 mutant background impedes salt tolerance. It is therefore apparent that the branch of the JA biosynthetic pathway catalyzed by AOC imparts salt tolerance, which is MYC2-dependent but ABA-independent (Zhao et al., 2014). Likewise, the wheat gene OPR1, which encodes 12-oxophytodienoate reductase 1 enzyme, has been observed to mediate salt tolerance in Arabidopsis when overexpressed (Dong et al., 2013). But, in comparison with the ABA-independent TaAOC1-conferred salt tolerance, TaOPR1-induced salt tolerance is ABA-dependent. The possible mechanism underlying the TaOPR1-mediated salt tolerance appears to be the increased ability of TaOPR1 overexpressing plants to detoxify ROS in an ABA-dependent manner (Dong et al., 2013). Certainly, JAs are known to increase the salt tolerance in wheat as well as the antioxidant enzyme activities (Qiu et al., 2014); however, further study needs to be conducted regarding the JAs-induced TaOPR1-mediated salt tolerance as no instantaneous effect of TaOPR1 expression on the signaling of JA was observed (Dong et al., 2013).

JA-mediated salt tolerance is not limited to Arabidopsis only. In rice, a nuclear protein, namely RICE SALT SENSITIVE3 (RSS3), expressed in the root tip and known to promote cell elongation, is essential for root growth under salt stress. The promotion of root growth is caused by the suppression of root JA responses. Consequently, inhibition of root growth under salinity stress due to the loss of RSS3 function causes is endorsed to the stimulation of JA-responsive genes. RSS3 is known to interact with the components of JA signaling pathway, OsbHLH089 and OsbHLH094, as well as with OsJAZ9 and OsJAZ11, leading to the formation of a stable complex with OsbHLH089 and OsJAZ9, which is required for the repression of the OsbHLH094 transcriptional activity (Toda et al., 2013). Moreover, OsJAZ9, which is a negative regulator of salt tolerance, interacts with OsbHLH062 and suppresses it (Ye et al., 2009). OsbHLH062 is known to regulate the expression of important genes involved in ion homeostasis like OsSKC1, OsHAK21, and OsHAK27 (Wu et al., 2015). A tomato mutant res (restored cell structure by salinity) was observed to accumulate JAs in its roots. This mutant revealed certain developmental alterations in the absence of salinity stress. Fascinatingly, however, on exposure to the salinity stress, the mutant was observed to restore the developmental alterations (Garcia-Abellan et al., 2015). Lastly, systemin, a recently discovered plant hormone that promotes JA production in tomato, is observed to promote salinity tolerance in an ABA-dependent manner. Transgenic tomato in which prosystemin is constitutively expressed have been observed to produce higher biomass enhanced stomatal conductance when exposed to salt stress in comparison with wild-type plants (Orsini et al., 2010). Jointly, all the above findings support that the positive role of JA in the regulation of salt tolerance in plants.

\subsection{METHYL JASMONATES: MULTIFUNCTIONAL ROLES IN ABIOTIC STRESS TOLERANCE}

Jasmonate and MeJA are supposed to play a dynamic role in alleviating biotic and abiotic stress tolerance especially salt stress. Apart from their role in stress tolerance, methyl jasmonates are involved in diverse developmental, growth, and physiological processes such as plant fertility, sex determination, reproductive processes, storage organ formation, root elongation, fruit ripening and senescence, oxidative resistance, and interaction with other phytohormones (Browse, 2009; Moreno et al., 2009; Avanci et al., 2010; Cipollini, 2010; Nafie et al., 2011; Khan et al., 2012; Khan and Khan, 2013; Per et al., 2018). Other physiological functions related to JA are stimulation of seed germination (Creelman and Mullet, 1997), chlorosis (Creelman and Mullet, 1997), buildup of storage proteins (Pelacho and Mingo-Castel, 1991), upregulation of antioxidant enzymes (Soares et al., 2010), seed and flower development (Wasternack et al., 2012), systemic resistance (Pieterse et al., 2002; 2012), elicitors of plant secondary metabolism (De Geyter et al., 2012), herbivory and wounding (Chung et al., 2008; Howe and Jander, 2008; Ballare, 2011; Erb et al., 2012), allelopathy (Baldwin, 2010), and senescence (Seltmann et al., 2010), etc. In addition, methyl jasmonates trigger plant defense mechanisms in response to insect-driven wounding, pathogens, and environmental stresses, such as low temperature, drought, and salinity (Cheong and Do Choi, 2003). Many researchers have reported the role of 
methyl jasmonates in regulating gene expression; this has been documented in a wide range of crops such as Arabidopsis (Sasaki et al., 2001), grapevines (Marchive et al., 2013), rice (Liu et al., 2012), tomato (Boter et al., 2004) etc., and eventually leads to defense against abiotic stresses (Gfeller et al., 2010; Ballare, 2011; Su et al., 2011).

\subsection{EFFECT OF SALT STRESS ON PLANTS}

As per the reports of FAO (2011) soil salinity incurs loss of more than US $\$ 11,000$ million annually and can affect more than $10 \%$ of the world's arable land, which greatly influences agricultural productivity (Tanji, 2002). Salt stress is one of the main abiotic stresses known to affect plant growth and development by inducing ionic, osmotic, and oxidative stress, with detrimental effects on plant yield (Golldack et al., 2014). The stress posed by high salt content in the soil is twofold. Firstly the high concentration of salt ions is toxic to plants and secondly, high salt content leads to water deficiency by lowering the water potential in the soil (Dar et al., 2015). Salinity is known to reduce the growth and development of glycophytes. Soil with electrical conductivity more than $4 \mathrm{dS} / \mathrm{m}$ and high content of salts such as sodium chloride and sodium sulfate imposes osmotic stress and ion-induced injury in a wide range of crops (Munns, 2002; Zhang and $\mathrm{Mu}, 2009)$. The extent of this injury is correlated to the concentration of $\mathrm{NaCl}$, genotype, and the environment. Salt stress influences mineral distribution, carbon and nitrogen metabolism (Kim et al., 2004; Hakeem et al., 2012), membrane permeability (Gupta et al., 2002), chlorophyll biosynthesis (Khan and Abdullah, 2003), and leads to augmented ion toxicity and enhanced respiration rate (Sudhir and Murthy, 2004). High soil salinity leads to the generation of free radicals such as reactive oxygen species (ROS), which plays a vital role in nucleic acid damage, enzyme inactivation, and lipid peroxidation (Smirnoff, 1993; Khan and Khan, 2017). Salt stress also results in the increased ABA biosynthesis, which reduces stomatal aperture and eventually affects photosynthesis rate (Dar et al., 2015). Salinity induced drop in photosynthetic rate may be attributed to the decrease in the carboxylation rate at which RuBPC fixes $\mathrm{CO}_{2}$. This may be caused by decline in either $\mathrm{CO}_{2}$ concentration or in the activity of the enzymes that produce ATP and other reducing equivalents. Many researchers have advocated that the reduction in photosynthetic rate may be credited to two main factors: (1) an indirect effect mediated by stomatal closure causing a drop in $\mathrm{CO}_{2}$ supply, or (2) a direct effect on the photosynthetic machinery independent of altered diffusion limitations. Essa (2002) have documented in soybean that salinity stress induces seed germination inhibition and reduces nodulation and seedling height, and decreases biomass accumulation and total plant yield. In a wide range of crop species salt stress alters diverse developmental processes, downregulates plant growth, and upregulates senescence and apoptosis during prolonged exposure. It is postulated that amongst diverse sources, irrigation and poor drainage are the main sources of soil salinity.

\subsubsection{Jasmonates Counteract Salinity Stress}

Phytohormone application is one of the strategies to counter the salinity stress in plants and the main ones involved are ABA, SA, BRs, and JAs. There is growing evidence that jasmonates alleviate the salt stress in plants (Table 24.1). Exogenous applications of MeJA can significantly alleviate salinity stress symptoms in soybean seedlings (Yoon et al., 2009). Moons et al. (1997a,b) in rice have documented that jasmonates induce salt stress-responsive protein in roots. Exogenous spray of JAs enhances dry mass production in the rice cultivars and the use of JA partially recovered the salt stress in them (Dar et al., 2015). Similarly Tsonev et al. (1998) and Velitchkova and Fedina (1998) have reported that MeJA pretreatment alleviates the salt stress and recovered the rate of $\mathrm{CO}_{2}$ fixation in Pisum sativum. It has been pointed out that the treatment of barley seedlings with the jasmonates improved salt stress tolerance (Tsonev et al., 1998). In response to salt stress, the JA level increases in the leaves of Iris hexagona and roots of rice (Wang et al., 2001).

Increasing evidence supports the idea that JA can play a significant role in alleviating abiotic stress response (Kazan, 2015; Riemann et al., 2015). The JAmediated responses are dependent on COI1, an F-box protein member of the SCFCOI1 ubiquitin-ligase complex (Xie et al., 1998). Chini et al. (2007) and Fonseca et al. (2009) have documented that the presence of the biologically active JA-Ile facilitate degradation of JAZ proteins by SCFCOI1 complex in an ubiquitindependent manner. JAZ proteins play a vital role in the repression of JA induced genes by binding to bHLH transcription factors such as MYC2, MYC3, MYC4, and MYC5 that are activators of JA responses (Chini et al., 2007; Cheng et al., 2011; Fernández-Calvo et al., 2011; Niu et al., 2011; Figueroa and Browse, 2012, 2015; Qi et al., 2015; Zhang et al., 2015). This inhibition of JAZ proteins is released by JA-lle mediated destabilization of JAZ by action of MYC2, MYC3, and MYC4 thereby activating the JA responses (FernándezCalvo et al., 2011; Niu et al., 2011; Zhang et al., 2015). The JA responses include an inhibitory effect on primary root growth. Xie et al. (1998), Chen et al. (2011), 
TABLE 24.1 Role of Methyl Jasmonates in Alleviating Salt Stress in Different Crop Species

\begin{tabular}{|c|c|c|c|c|}
\hline Crop & $\begin{array}{l}\text { Salt stress } \\
\text { concentration }\end{array}$ & Studied parameters & Consequences on plants & Reference \\
\hline Glycine max & $60 \mathrm{mM} \mathrm{NaCl}$ & $\begin{array}{l}\text { Plant growth, endogenous bioactive } \\
\text { gibberellin (GA4), photosynthesis and } \\
\text { transpiration rate }\end{array}$ & $\begin{array}{l}\text { MeJA counteracted the negative effects of } \\
\mathrm{NaCl} \text { stress on plant growth, chlorophyll } \\
\text { content, leaf photosynthetic rate, leaf } \\
\text { transpiration rate, and proline content }\end{array}$ & $\begin{array}{l}\text { Yoon et al. } \\
\text { (2009) }\end{array}$ \\
\hline Oryza sativa & $\begin{array}{l}50 \text { and } 75 \mathrm{mM} \\
\mathrm{NaCl}\end{array}$ & $\begin{array}{l}\text { Molecular and physiological effects of } \\
\text { jasmonic acid (JA) }(<\text { or }=10 \mu \mathrm{M}), \mathrm{ABA} \text {, and } \\
\text { salt stress in roots of rice }\end{array}$ & $\begin{array}{l}\text { JA markedly induced a cationic } \\
\text { peroxidase, two novel } 32-\text { and } 28-\mathrm{kD} \\
\text { proteins, acidic PR-1 and PR-10 } \\
\text { pathogenesis-related proteins, and the } \\
\text { salt stress-responsive SalT protein in } \\
\text { roots }\end{array}$ & $\begin{array}{l}\text { Moons et al. } \\
(1997 a, b)\end{array}$ \\
\hline Iris hexagona & $\begin{array}{l}0,100,200, \text { and } \\
400 \mathrm{mM} \mathrm{NaCl}\end{array}$ & $\begin{array}{l}\text { Effects of salinity on abscisic acid (ABA), } \\
\text { indole-3-acetic acid (IAA), salicylic acid (SA), } \\
\text { and jasmonic acid (JA) in leaves, stalks, fruits, } \\
\text { and seeds of Iris hexagona }\end{array}$ & $\begin{array}{l}\text { ABA and JA generally increased and IAA } \\
\text { and SA declined in response to salinity; } \\
\text { salinity significantly increased JA content } \\
\text { in the apical parts of flower stalks }\end{array}$ & $\begin{array}{l}\text { Wang et al. } \\
\text { (2001) }\end{array}$ \\
\hline Pisum sativum & $30 \mathrm{mM} \mathrm{NaCl}$ & $\begin{array}{l}\mathrm{CO} 2 \text { fixation and relative water content, and } \\
\text { proline content }\end{array}$ & $\begin{array}{l}\text { Pretreatment with JA-Me for } 3 \text { days } \\
\text { before salt treatment diminished the } \\
\text { inhibitory effect of } \mathrm{NaCl} \text { on the rate of } \\
\text { 14CO2 fixation, protein content, and } \\
\text { activity and content of ribulose-1,5- } \\
\text { bisophosphate carboxylase/oxygenase; } \\
\text { the Na }+ \text { and } \mathrm{Cl} \text { - contents in leaves } \\
\text { decreased in JA-Me pretreated plants }\end{array}$ & $\begin{array}{l}\text { Velitchkova } \\
\text { and Fedina } \\
\text { (1998) }\end{array}$ \\
\hline Hordeum vulgare & $100 \mathrm{~mm} \mathrm{NaCl}$ & Growth and photosynthesis & $\begin{array}{l}\text { Both } 100 \mathrm{mM} \mathrm{NaCl} \text { and } 25 \mu \mathrm{M} \mathrm{JA} \\
\text { treatment led to a noticeable decrease in } \\
\text { net photosynthetic rate vs. intercellular } \\
\mathrm{CO} 2 \text { concentration and the maximal rate } \\
\text { of photosynthesis }\end{array}$ & $\begin{array}{l}\text { Tsonev } \\
\text { et al. (1998) }\end{array}$ \\
\hline Oryza sativa & $200 \mathrm{mM} \mathrm{NaCl}$ & Plant growth, root, and shoot length & $\begin{array}{l}\text { Suppression of OsJAZ9, member of the } \\
\text { JAZ subfamily, resulted in reduced salt } \\
\text { tolerance, which was mainly due to the } \\
\text { changes in } \mathrm{K}+\text { homeostasis } \\
\text { OsJAZ9 acts as a transcriptional regulator } \\
\text { by forming a transcriptional regulation } \\
\text { complex with OsNINJA and OsbHLH to } \\
\text { fine tune the expression of JA-responsive } \\
\text { genes involved in salt stress tolerance in } \\
\text { rice }\end{array}$ & $\begin{array}{l}\text { Wu et al. } \\
(2015)\end{array}$ \\
\hline Tamarix hispida & $0.4 \mathrm{M} \mathrm{NaCl}$ & $\begin{array}{l}\text { Adaptation to salty environments in roots of } \\
\text { Tamarix hispida }\end{array}$ & $\begin{array}{l}\text { Ninety redundant unique transcripts } \\
\text { responsive to } \mathrm{NaCl} \text { treatment were } \\
\text { identified. Of them, } 21 \text { genes were novel } \\
\text { or of unknown function while others } \\
\text { were involved in the functional activities, } \\
\text { such as ROS scavenging, lipid } \\
\text { metabolism, osmolyte biosynthesis, signal } \\
\text { transduction, transport, lignin synthesis, } \\
\text { and homeostasis }\end{array}$ & $\begin{array}{l}\text { Li et al. } \\
\text { (2009) }\end{array}$ \\
\hline $\begin{array}{l}\text { Scenedesmus } \\
\text { incrassatulus }\end{array}$ & $175 \mathrm{mM} \mathrm{NaCl}$ & $\begin{array}{l}\text { 14CO2 fixation, free proline and } \\
\text { malondialdehyde }\end{array}$ & $\begin{array}{l}\text { Salt stress resulted in a reduction of } \\
\text { growth and } 14 \mathrm{CO} 2 \text { fixation and in an } \\
\text { increase of accumulation of free proline } \\
\text { and malondialdehyde; exogenously } \\
\text { supplied methyl jasmonate did not } \\
\text { considerably change the } 14 \mathrm{CO} 2 \text { fixation, } \\
\text { but increased proline and MDA } \\
\text { accumulation in the cells }\end{array}$ & $\begin{array}{l}\text { Fedina and } \\
\text { Benderliev } \\
(2000)\end{array}$ \\
\hline
\end{tabular}


TABLE $24.1 \quad$ (Continued)

\begin{tabular}{lll}
\hline Crop & $\begin{array}{l}\text { Salt stress } \\
\text { concentration }\end{array}$ & Studied parameters \\
\hline $\begin{array}{l}\text { Mesembryanthemum } \\
\text { crystallinum }\end{array}$ & $200-500 \mathrm{mM}$ & $\begin{array}{l}\text { Plant growth, photosynthesis, and chlorophyl } \\
\text { content }\end{array}$ \\
& $100 \mathrm{mM} \mathrm{NaCl}$ & $\begin{array}{l}\text { Lipoxygenase protein accumulation, } \\
\text { transcripts of allene oxide synthase and } \\
\text { proteinase inhibitor II, and activities of } \\
\text { esculentum }\end{array}$ \\
& $\begin{array}{l}\text { diacylglycerol kinase and phosphatidate } \\
\text { kinase (enzymes involved in the hosphatidic } \\
\text { acid and diacylglycerol pyrophosphate } \\
\text { metabolism) }\end{array}$
\end{tabular}

Glycine max

$100 \mathrm{mM} \mathrm{NaCl}$ Shoot length, plant fresh weight and dry weight

Triticum aestioum

$150 \mathrm{mM} \mathrm{NaCl}$

Plant height, root length, shoot dry weight, root dry weight

$100,200,300$

and $400 \mathrm{mM}$

Effects of salinity on abscisic acid (ABA), indole-3-acetic acid (IAA), salicylic acid (SA), and jasmonic acid (JA) in leaves, stalks, fruits, and seeds

Hordeum vulgare

$150 \mathrm{mM} \mathrm{NaCl}$

Physiological parameters

Net photosynthetic rate per unit area, stomatal conductance to $\mathrm{CO}_{2}\left(g_{\mathrm{c}}\right)$, and transpiration rate
Consequences on plants

Reference

When plants are grown at high salt Schmitt concentrations, salt is accumulated in the leaves and leaf osmotic pressure increases drastically; in the presence of high salt, the mode of primary carbon fixation switches from $\mathrm{C} 3$ to CAM

Steady-state levels of JA and related compounds were higher in the salttolerant cv. Pera than in cv. Hellfrucht Fruhstamm (HF) and JA levels in both cultivars changed in response to salt stress

It was noted that JA content increased in plants treated with PEG and $\mathrm{NaCl}$, as maximum JA contents were found in plants treated with PEG followed by $\mathrm{NaCl}$

Jasmonic acid aids in regulating stress responses, plant growth, and development; JA could effectively protect wheat seedlings from salt stress damage

Treatments with exogenous JA for 3 days significantly enhanced salt stress tolerance in wheat seedlings by decreasing the concentration of MDA and $\mathrm{H} 2 \mathrm{O} 2$, the production rate of and increasing the transcript levels and activities of SOD, POD, CAT, and APX and the contents of GSH, Chl b and Car, which, in turn, enhanced the growth of salt-stressed seedlings

Leaves of plants exposed to $400 \mathrm{mM}$ salt wilted and eventually died but lower salinities were tolerated; in young leaves, JA content increased in response to salinity concentration

Transcripts that showed significant upregulation under salinity stress are exemplified by jasmonate-responsive, metallothionein-like, late embryogenesisabundant (LEA) and ABA-responsive proteins; downregulation in a category was observed for photosynthesis-related functions

Photosynthetic and sodium ion

Walia et al. accumulation responses were compared after salinity stress, JA treatment, and JA pretreatment followed by salinity stress; the JA-pretreated salt-stressed plants accumulated strikingly low levels of $\mathrm{Na}+$ in the shoot tissue compared with untreated salt-stressed plants after several days of exposure to stress
Pedranzani et al. (2003)

Hamayun et al. (2010)

Qiu et al.

(2014)

Wang et al. (2001)

Ozturk et al. (2002)

(2007) 
TABLE 24.1 (Continued)

\begin{tabular}{|c|c|c|c|c|}
\hline Crop & $\begin{array}{l}\text { Salt stress } \\
\text { concentration }\end{array}$ & Studied parameters & Consequences on plants & Reference \\
\hline Vitis vinifera & $\begin{array}{l}10,20,30,40 \\
50,85,120 \\
155,200, \text { and } \\
300 \mathrm{mM}\end{array}$ & $\begin{array}{l}\text { Using two grapevine cell lines differing in salt } \\
\text { tolerance, the response of jasmonate proteins } \\
\text { a marker for salt adaptation, and markers for } \\
\text { biotic defense were analyzed }\end{array}$ & $\begin{array}{l}\text { JA in the absence of salt stress reduced } \\
\text { growth by } \sim 20 \% \text { in both cell lines as } \\
\text { compared with the control; salt stress } \\
\text { signaling shares several events with } \\
\text { biotic defense including activity of a } \\
\text { gadolinium-sensitive calcium influx } \\
\text { channel and transient induction of JAZ/ } \\
\text { TIFY transcripts; exogenous jasmonate } \\
\text { can rescue growth in the salt-sensitive } \\
\text { cell line }\end{array}$ & $\begin{array}{l}\text { Ismail et al. } \\
\text { (2012) }\end{array}$ \\
\hline Oryza sativa & $\begin{array}{l}20,40, \text { and } \\
80 \mathrm{~mm} \mathrm{NaCl}\end{array}$ & $\begin{array}{l}\text { Plant growth, net photosynthetic rate, leaf } \\
\text { water potential, and dry mass production }\end{array}$ & $\begin{array}{l}\text { The decrease of JA concentrations in salt- } \\
\text { tolerant cultivar was lesser than in the } \\
\text { salt-sensitive cultivar plants in the shoot } \\
\text { Postapplication in the stressed plants } \\
\text { with } 30 \mu \mathrm{m} \text { JA at } 24 \text { and } 48 \mathrm{~h} \text { after } \mathrm{NaCl} \\
\text { treatment recovered salt inhibition on dry } \\
\text { mass production more effectively than } \\
\text { application of JA at } 48 \text { and } 24 \mathrm{~h} \text { before } \\
\text { salt stress, and during salt stress } \\
\text { simultaneously }\end{array}$ & $\begin{array}{l}\text { Kang et al. } \\
(2005)\end{array}$ \\
\hline
\end{tabular}

and Fernández-Calvo et al. (2011) have reported that Arabidopsis null mutants that do not respond to JA signaling possess longer roots in the presence of JA. This was also supported by the results of Galvan-Ampudia and Testerink (2011) that plants develop shorter primary roots and alter the root system architecture in high soil with high salt concentration. The inhibition of root growth has been attributed to the cell division and elongation arrest (West et al., 2004; Geng et al., 2013). Hence, salt stress along with the activation of the JA pathway may have an inhibitory effect on primary root growth. As reported in recent literature, the activation of JA signaling pathway is correlated to salt stress response (Kazan, 2015; Riemann et al., 2015). According to Dong et al. (2013) attenuation of salt stress induced root growth inhibition in Arabidopsis by the overexpression of the wheat JA-biosynthesis gene OPR1. In contrast Toda et al. (2013) in rice (Oryza sativa) have documented that the interaction of OsbHLH089 and OsbHLH094 transcription factors with the salt-sensitive 3, a nuclear localized JAZ-interacting protein lacking a DNA binding domain, results in the formation of a ternary complex that regulates salt induced root cell elongation. However, Kurotani et al. (2015) in rice have reported that salt tolerance may be attributed to the conversion of JA-Ile to an inactive form as a result of overexpression of the $\mathrm{OsCYP94C2b}$ gene.

All these findings reveal that JA signaling may act as either a positive or negative regulator of the salt stress response in a conditional manner (Riemann et al., 2015). However, as per the reports of early workers such as Jiang and Deyholos (2006), Ma et al. (2006), Kilian et al. (2007), and Geng et al. (2013) have advocated salinity induces upregulation of JAbiosynthesis genes like AOC1, AOC2, AOS, LOX3, and OPR3 in root cells. These results provide the basis the notion of salt stress mediated activation of the JA signaling pathway with wide impact on diverse developmental and physiological processes in plants. Additionally, in jai3-1, a JA-resistant mutant allele that encodes stabilized JAZ3 in Arabidopsis, showed an enhanced in root growth rate when compared with wild-type plants under salt stress on a temporal basis (Geng et al., 2013). This finding has reconfirmed that salt stress triggers activation of the JA signaling pathway in the roots leading to growth. Valenzuela et al. (2016) have shown that in the meristematic region and stele of the differentiation region of the Arabidopsis root, salinity stimulated activation of the JA signaling pathway in a JAR1-, COI1-, and proteasomedependent manner. This activation is likely to occur with the participation of core components of the JA signaling pathway, such as COI1, JAZ3 and MYC2, MYC3 and MYC4, leading to arrest of cell elongation in the primary root. All these findings reveal that the salt stress response involves activation of the JA signaling pathway, resulting in inhibition of root growth in Arabidopsis.

\subsubsection{Salt Stress Response Mediated by JA Signaling}

Earlier expression studies showed that some genes implicated in JA biosynthesis were induced in the 
roots by salt stress, signifying that the biosynthesis of this hormone is induced in roots by salt stress (Jiang and Deyholos, 2006; Ma et al., 2006; Kilian et al., 2007). Additionally Valenzuela et al. (2016) while studying the early JA-responsive gene activation in the roots during the salt stress response quantified JAZ expression profile, reported that eight out of nine analyzed $J A Z$ genes are upregulated by salt stress. Together, these gene expression results strongly suggest that JA signaling is activated in the roots at early stages of the salt stress response. Chini et al. (2007), Thines et al. (2007), and Chung et al. (2008) have reviewed that wounding or exogenous spray of JA causes upregulation of JAZ that eventually turns off hormone signaling once the signal has been transmitted by employing negative-feedback mechanism. This JAZ upregulation has also been observed in the roots after $3 \mathrm{~h}$ of salt treatment (Valenzuela et al., 2016) thereby suggesting that JAZ proteins play a crucial role in the downregulation of the JA pathway. Xiao et al. (2004) have reported that coi1 mutants reflected reduced JAZ expression levels when compared with wild-type plants under salt stress. This result confirms that COI1, a core component of the JA-Ile coreceptor (Sheard et al., 2010), is imperative for JAZ transcript upregulation in the roots during the response to salt stress.

The salt stress mediated upregulation of $J A Z$ genes in a COI1-dependent manner observed in the roots is likely to follow the canonical JA signaling pathway (Wasternack and Hause, 2013), with proteasomedependent degradation of JAZ proteins. In Arabidopsis, Valenzuela et al. (2016) have reported that considerable activation of JA signaling after salt or JA treatment, mainly in the stele and lower part of the merismatic zone, involves the proteasome-mediated destabilization of JAZ1. The underlying mechanism of signal transduction induced by salt stress and integration from several upstream signals into upregulation of JA-Ile biosynthesis or downregulation of hormone catabolism is not fully understood yet and is under speculation. Detailed studies are required to reveal early events of JA-Ile homeostasis.

\subsection{CONCLUSION AND FUTURE PERSPECTIVES}

JAs have attained much attention in recent years owing to their considerable involvement in plant growth and development and in alleviating wide range of stresses. It is now clear that JAs are involved in a diversity of functions. JA induced responses are in harmony with diverse complex signals. There exists a complex interplay between JAs with other hormones' signaling pathways for regulating plant responses under salt stress conditions. JAs avert the deteriorating impact of salt stress on diverse developmental and physiological processes. The numerous jasmonate compounds and their different modes of action allow plants to respond specifically and flexibly to environmental flux. However, JA signaling and its roles in signaling crosstalk at the organ, tissue, or cell levels remains under speculation. As research on the biosynthesis and interaction of JA with other phytohormonal activity progresses, a large diversity of metabolites related to different phytohormones would play a role in plant defense and plant-environment interactions. Much work is to be done in the near future to find out the proper answers of the questions like action of JA metabolites, and identification of universal JA receptors, etc. Complete signaling pathways involving MAPKs, CDPK, TGA, SIPK, WIPK, and WRKY TFs are yet to be studied to understand the complete mechanism of action of JA.

\section{References}

Agrawal, G.K., Tamogami, S., Iwahashi, H., Agrawal, V.P., Rakwal, R., 2003. Transient regulation of jasmonic acid-inducible rice MAP kinase gene (OsBWMK1) by diverse biotic and abiotic stresses. Plant Physiol. Biochem. 41, 355-361.

Aldridge, D.C., Galt, S., Giles, D., Turner, W.B., 1971. Metabolites of Lasiodiplodia theobromae. J. Chem. Soc. C: Organic 1623-1627.

Aubert, Y., Widemann, E., Miesch, L., Pinot, F., Heitz, T., 2015. CYP94-mediated jasmonoyl-isoleucine hormone oxidation shapes jasmonate profiles and attenuates defence responses to Botrytis cinerea infection. J. Exp. Bot. 66, 3879-3892.

Avanci, N.C., Luche, D.D., Goldman, G.H., Goldman, M.H.S., 2010. Jasmonates are phytohormones with multiple functions, including plant defense and reproduction. Genet. Mol. Res. 9, 484-505.

Baldwin, I.T., 2010. Plant volatiles. Curr. Biol. 11 (20), R392-R397.

Ballare, C.L., 2011. Jasmonate-induced defenses: a tale of intelligence, collaborators and rascals. Trends Plant Sci. 16, 249-257.

Bannenberg, G., Martínez, M., Hamberg, M., Castresana, C., 2009. Diversity of the enzymatic activity in the lipoxygenase gene family of Arabidopsis thaliana. Lipids 44 (2), 85.

Boter, M., Ruíz-Rivero, O., Abdeen, A., Prat, S., 2004. Conserved MYC transcription factors play a key role in jasmonate signalling both in tomato and Arabidopsis. Genes Dev. 18, 1577-1591.

Brossa, R., López-Carbonell, M., Jubany-Marí, T., Alegre, L., 2011. Interplay between abscisic acid and jasmonic acid and its role in water-oxidative stress in wild-type, ABA-deficient, JA-deficient, and ascorbate-deficient Arabidopsis plants. J. Plant Growth Regul. $30,322-333$.

Browse, J., 2009. Jasmonate passes muster: a receptor and targets for the defense hormone. Annu. Rev. Plant Biol. 60, 183-205.

Caldelari, D., Wang, G., Farmer, E., Dong, X., 2011. Arabidopsis lox3 lox4 double mutants are male sterile and defective in global proliferative arrest. Plant Mol. Biol. 75, 25-33.

Chen, Q., Sun, J., Zhai, Q., Zhou, W., Qi, L., Xu, L., et al., 2011. The basic helix-loop-helix transcription factor MYC2 directly represses PLETHORA expression during jasmonate-mediated modulation of the root stem cell niche in Arabidopsis. Plant Cell 23, 3335-3352.

Cheng, Z., Sun, L., Qi, T., Zhang, B., Peng, W., Liu, Y., et al., 2011. The bHLH transcription factor MYC3 interacts with the jasmonate 
ZIM-domain proteins to mediate jasmonate response in Arabidopsis. Mol. Plant 4, 279-288.

Cheong, J.J., Do Choi, Y., 2003. Methyl jasmonate as a vital substance in plants. Trends Genet. 19, 409-413.

Chini, A., Fonseca, S., Fernandez, G., Adie, B., Chico, J.M., Lorenzo, O., et al., 2007. The JAZ family of repressors is the missing link in jasmonate signalling. Nat. 448, 666 .

Chung, H.S., Koo, A.J., Gao, X., Jayanty, S., Thines, B., Jones, A.D., et al., 2008. Regulation and function of Arabidopsis JASMONATE ZIM-domain genes in response to wounding and herbivory. Plant Physiol. 146, 952-964.

Cipollini, D., 2010. Constitutive expression of methyl jasmonateinducible responses delays reproduction and constrains fitness responses to nutrients in Arabidopsis thaliana. Evol. Ecol. 24, $59-68$.

Clarke, S.M., Cristescu, S.M., Miersch, O., Harren, F.J., Wasternack, C., Mur, L.A., 2009. Jasmonates act with salicylic acid to confer basal thermotolerance in Arabidopsis thaliana. New Phytol. 182, 175-187.

Creelman, R.A., Mullet, J.E., 1997. Biosynthesis and action of jasmonates in plants. Annu. Rev. Plant Biol. 48, 355-381.

Dar, T.A., Uddin, M., Khan, M.M.A., Hakeem, K.R., Jaleel, H., 2015. Jasmonates counter plant stress: a review. Environ. Exp. Bot. 115, $49-57$.

De Geyter, N., Gholami, A., Goormachtig, S., Goossens, A., 2012. Transcriptional machineries in jasmonate-elicited plant secondary metabolism. Trends Plant Sci. 17, 349-359.

Demole, E., Lederer, E., Mercier, D., 1962. Isolement et détermination de la structure du jasmonate de méthyle, constituant odorant caractéristique de l'essence de jasmin. Helvet. Chim. Acta 45, 675-685.

Deshaies, R.J., 1999. SCF and Cullin/Ring H2-based ubiquitin ligases. Annu. Rev. Cell Dev. Biol. 15, 435-467.

Dombrecht, B., Xue, G.P., Sprague, S.J., Kirkegaard, J.A., Ross, J.J., Reid, J.B., et al., 2007. MYC2 differentially modulates diverse jasmonatedependent functions in Arabidopsis. Plant Cell 19, 2225-2245.

Dong, W., Wang, M., Xu, F., Quan, T., Peng, K., Xiao, L., et al., 2013. Wheat oxophytodienoate reductase gene TaOPR1 confers salinity tolerance via enhancement of abscisic acid signalling and reactive oxygen species scavenging. Plant Physiol. 161, 1217-1228.

Epstein, E., Norlyn, J.D., Rush, D.W., Kingsbury, R.W., Kelley, D.B., Cunningham, G.A., et al., 1980. Saline culture of crops: a genetic approach. Science 210, 399-404.

Erb, M., Meldau, S., Howe, G.A., 2012. Role of phytohormones in insect-specific plant reactions. Trends Plant Sci. 17, 250-259.

Essa, T.A., 2002. Effect of salinity stress on growth and nutrient composition of three soybean (Glycine $\max$ L. Merrill) cultivars. J. Agron. Crop. Sci. 188, 86-93.

FAO, 2011. FAO Land and Plant Nutrition Management Service. http:/ /www.fao.org/ag/agl/agll/spush.

Fedina, I.S., Benderliev, K.M., 2000. Response of Scenedesmus incrassatulus to salt stress as affected by methyl jasmonate. Biol. Plant. 43, 625-627.

Fernández-Calvo, P., Chini, A., Fernández-Barbero, G., Chico, J.M., Gimenez-Ibanez, S., Geerinck, J., et al., 2011. The Arabidopsis bHLH transcription factors MYC3 and MYC4 are targets of JAZ repressors and act additively with MYC2 in the activation of jasmonate responses. Plant Cell 23, 701-715.

Figueroa, P., Browse, J., 2012. The Arabidopsis JAZ2 promoter contains a G-Box and thymidine-rich module that are necessary and sufficient for jasmonate-dependent activation by MYC transcription factors and repression by JAZ proteins. Plant Cell Physiol. $53,330-343$.

Figueroa, P., Browse, J., 2015. Male sterility in Arabidopsis induced by overexpression of a MYC5 SRDX chimeric repressor. Plant J. 81, $849-860$.
Fonseca, S., Chini, A., Hamberg, M., Adie, B., Porzel, A., Kramell, R., et al., 2009. (+)-7-iso-Jasmonoyl-L-isoleucine is the endogenous bioactive jasmonate. Nat. Chem. Biol. 5, 344 .

Footitt, S., Dietrich, D., Fait, A., Fernie, A.R., Holdsworth, M.J., Baker, A., et al., 2007. The COMATOSE ATP-binding cassette transporter is required for full fertility in Arabidopsis. Plant Physiol. 144, 1467-1480.

Galvan-Ampudia, C.S., Testerink, C., 2011. Salt stress signals shape the plant root. Curr. Opin. Plant Boil. 14 (3), 296-302.

Garcia-Abellan, J.O., Fernandez-Garcia, N., Lopez-Berenguer, C., Egea, I., Flores, F.B., Angosto, T., et al., 2015. The tomato res mutant which accumulates JA in roots in non-stressed conditions restores cell structure alterations under salinity. Physiol. Plant. $155,296-314$.

Geng, Y., Wu, R., Wee, C.W., Xie, F., Wei, X., Chan, P.M.Y., et al., 2013. A spatio-temporal understanding of growth regulation during the salt stress response in Arabidopsis. Plant Cell 25, 2132-2154.

Gfeller, A., Dubugnon, L., Liechti, R., Farmer, E.E., 2010. Jasmonate biochemical pathway. Sci. Signal. 16, 3 .

Golldack, D., Li, C., Mohan, H., Probst, N., 2014. Tolerance to drought and salt stress in plants: unraveling the signalling networks. Front. Plant Sci. 5, 151.

Gupta, N.K., Meena, S.K., Gupta, S., Khandelwal, S.K., 2002. Gas exchange, membrane permeability, and ion uptake in two species of Indian jujube differing in salt tolerance. Photosynthetica 40, $535-539$.

Hakeem, K.R., Khan, F., Chandna, R., Siddiqui, T.O., Iqbal, M., 2012. Genotypic variability among soybean genotypes under $\mathrm{NaCl}$ stress and proteome analysis of salt-tolerant genotype. Biotechnol. Appl. Biochem. 168, 2309-2329.

Hamayun, M., Sohn, E.Y., Khan, S.A., Shinwari, Z.K., Khan, A.L., Lee, I.J., 2010. Silicon alleviates the adverse effects of salinity and drought stress on growth and endogenous plant growth hormones of soybean (Glycine max L.). Pak. J. Bot 42, 1713-1722.

Hause, B., Hause, G., Kutter, C., Miersch, O., Wasternack, C., 2003. Enzymes of jasmonate biosynthesis occur in tomato sieve elements. Plant Cell Physiol. 44, 643-648.

Heitz, T., Widemann, E., Lugan, R., Miesch, L., Ullmann, P., Désaubry, L., et al., 2012. Cytochromes P450 CYP94C1 and CYP94B3 catalyze two successive oxidation steps of plant hormone jasmonoylisoleucine for catabolic turnover. J. Biol. Chem. 287, 6296-6306.

Howe, G.A., Jander, G., 2008. Plant immunity to insect herbivores. Annu. Rev. Plant Biol. 59, 41-66.

Ismail, A., Riemann, M., Nick, P., 2012. The jasmonate pathway mediates salt tolerance in grapevines. J. Exp. Bot. 63, 2127-2139.

Jiang, Y., Deyholos, M.K., 2006. Comprehensive transcriptional profiling of NaCl-stressed Arabidopsis roots reveals novel classes of responsive genes. BMC Plant Biol. 6, 25.

Kang, D.J., Seo, Y.J., Lee, J.D., Ishii, R., Kim, K.U., Shin, D.H., et al., 2005. Jasmonic acid differentially affects growth, ion uptake and abscisic acid concentration in salt tolerant and salt sensitive rice cultivars. J. Agro. Crop. Sci. 191, 273-282.

Katsir, L., Chung, H.S., Koo, A.J., Howe, G.A., 2008. Jasmonate signalling: a conserved mechanism of hormone sensing. Curr. Opin. Plant Biol. 11, 428-435.

Kazan, K., 2015. Diverse roles of jasmonates and ethylene in abiotic stress tolerance. Trends Plant Sci. 20, 219-229.

Kazan, K., Manners, J.M., 2008. Jasmonate signalling: toward an integrated view. Plant Physiol. 146, 1459-1468.

Kazan, K., Manners, J.M., 2013. MYC2: the master in action. Mol. Plant 6, 686-703.

Khan, M.I.R., Khan, N., 2017. Reactive Oxygen Species and Antioxidant System in Plants: Role and Regulation Under Abiotic Stress. Springer Nature, Singapore978-981-10-5254-5. 
Khan, M.A., Abdullah, Z., 2003. Reproductive physiology of two wheat cultivars differing in salinity tolerance under dense salinesodic soil. J. Food Agric. Environ. 1, 185-189.

Khan, M.I.R., Khan, N.A., 2013. Salicylic acid and jasmonates: approaches in abiotic stress tolerance. J. Plant Biochem. Physiol. 1 (4).

Khan, M.I.R., Syeed, S., Nazar, R., Anjum, N.A., 2012. An insight into the role of salicylic acid and jasmonic acid in salt stress tolerance. Phytohormones and Abiotic Stress Tolerance in Plants. Springer, Berlin Heidelberg, pp. 277-300.

Kilian, J., Whitehead, D., Horak, J., Wanke, D., Weinl, S., Batistic, O., et al., 2007. The AtGenExpress global stress expression data set: protocols, evaluation and model data analysis of UV B light, drought and cold stress responses. Plant J. 50, 347-363.

Kim, Y., Arihara, J., Nakayama, T., Nakayama, N., Shimada, S., Usui, K., 2004. Antioxidative responses and their relation to salt tolerance in Echinochloa oryzicola Vasing and Setaria virdis (L.) Beauv. Plant Growth Regul. 44, 87-92.

Kombrink, E., 2012. Chemical and genetic exploration of jasmonate biosynthesis and signalling paths. Planta 236, 1351-1366.

Koo, A.J., Cooke, T.F., Howe, G.A., 2011. Cytochrome P450 CYP94B3 mediates catabolism and inactivation of the plant hormone jasmonoyl-L-isoleucine. Proc. Nat. Acad. Sci. 108, 9298-9303.

Kurotani, K.I., Hayashi, K., Hatanaka, S., Toda, Y., Ogawa, D., Ichikawa, H., et al., 2015. Elevated levels of CYP94 family gene expression alleviate the jasmonate response and enhance salt tolerance in rice. Plant Cell Physiol. 56, 779-789.

León, J., 2013. Role of plant peroxisome in the production of jasmonic acid based signals. Peroxisomes and Their Key Role in Cellular Signalling and Metabolism. Springer, Netherlands, pp. 299-313.

Li, H., Wang, Y., Jiang, J., Liu, G., Gao, C., Yang, C., 2009. Identification of genes responsive to salt stress on Tamarix hispida roots. Gene 433, 65-71.

Liu, H., Li, X., Xiao, J., Wang, S., 2012. A convenient method for simultaneous quantification of multiple phytohormones and metabolites: application in study of rice-bacterium interaction. Plant Methods 8, 2.

Lyons, R., Manners, J.M., Kazan, K., 2013. Jasmonate biosynthesis and signalling in monocots: a comparative overview. Plant Cell Rep. 32, 815-827.

Ma, S., Gong, Q., Bohnert, H.J., 2006. Dissecting salt stress pathways. J. Exp. Bot. 57, 1097-1107.

Maksymiec, W., Wianowska, D., Dawidowicz, A.L., Radkiewicz, S., Mardarowicz, M., Krupa, Z., 2005. The level of jasmonic acid in Arabidopsis thaliana and Phaseolus coccineus plants under heavy metal stress. J. Plant Physiol. 162, 1338-1346.

Marchive, C., Léon, C., Kappel, C., Coutos-Thévenot, P., CorioCostet, M.F., Delrot, S., et al., 2013. Over-expression of VvWRKY1 in grapevines induces expression of jasmonic acid pathwayrelated genes and confers higher tolerance to the downy mildew. PLoS One 8, e54185.

Martinez, V., Nieves-Cordones, M., Lopez-Delacalle, M., Rodenas, R., Mestre, T.C., Garcia-Sanchez, F., et al., 2018. Tolerance to stress combination in tomato plants: new insights in the protective role of melatonin. Molecules 23, 535. Available from: https://doi.org/ 10.3390 / molecules23030535.

Meyer, A., Miersch, O., Büttner, C., Dathe, W., Sembdner, G., 1984. Occurrence of the plant growth regulator jasmonic acid in plants. J. Plant Growth Regul. 3 (1-4), 1.

Moons, A., De Keyser, A., Van Montagu, M., 1997a. A group 3 LEA cDNA of rice, responsive to abscisic acid, but not to jasmonic acid, shows variety-specific differences in salt stress response. Gene 191, 197-204.

Moons, A., Prinsen, E., Bauw, G., Van Montagu, M., 1997b. Antagonistic effects of abscisic acid and jasmonates on salt stressinducible transcripts in rice roots. Plant Cell 9, 2243-2259.
Moreno, J.E., Tao, Y., Chory, J., Ballaré, C.L., 2009. Ecological modulation of plant defense via phytochrome control of jasmonate sensitivity. Proc. Nat. Acad. Sci. 106, 4935-4940.

Munns, R., 2002. Comparative physiology of salt and water stress. Plant Cell Environ. 25, 239-250.

Nafie, E., Hathout, T., Mokadem, A., Shyma, A., 2011. Jasmonic acid elicits oxidative defense and detoxification systems in Cucumis melo L. cells. Braz. J. Plant Physiol. 23, 161-174.

Niu, Y., Figueroa, P., Browse, J., 2011. Characterization of JAZinteracting bHLH transcription factors that regulate jasmonate responses in Arabidopsis. J. Exp. Bot. 62, 2143-2154.

Niu, Y., Figueroa, P., 2011. Characterization of JAZ-interacting bHLH transcription factors that regulate jasmonate responses in Arabidopsis. J. Exp. Bot. 62, 2143-2154.

Orsini, F., Cascone, P., De Pascale, S., Barbieri, G., Corrado, G., Rao, R., et al., 2010. Systemin-dependent salinity tolerance in tomato: evidence of specific convergence of abiotic and biotic stress responses. Physiol. Plant Arum 138, 10-21.

Ozturk, Z.N., Talamé, V., Deyholos, M., Michalowski, C.B., Galbraith, D.W., Gozukirmizi, N., et al., 2002. Monitoring large-scale changes in transcript abundance in drought-and salt-stressed barley. Plant Mol. Boil. 48, 551-573.

Pauwels, L., Barbero, G.F., Geerinck, J., Tilleman, S., Grunewald, W., Pérez, A.C., et al., 2010. NINJA connects the co-repressor TOPLESS to jasmonate signalling. Nature 464, 788-791.

Pauwels, L., Goossens, A., 2011. The JAZ proteins: a crucial interface in the jasmonate signalling cascade. Plant Cell 23, 3089-3100.

Pedranzani, H., Racagni, G., Alemano, S., Miersch, O., Ramírez, I., Peña-Cortés, H., et al., 2003. Salt tolerant tomato plants show increased levels of jasmonic acid. Plant Growth Regul. 41, 149-158.

Pelacho, A.M., Mingo-Castel, A.M., 1991. Jasmonic acid induces tuberization of potato stolons cultured in vitro. Plant Physiol. 97, 1253-1255.

Peleg, Z., Blumwald, E., 2011. Hormone balance and abiotic stress tolerance in crop plants. Curr. Opin. Plant Biol. 14, 290-295.

Per, T.S., Khan, M.I.R., Anjum, N.A., Masood, A., Hussain, S.J., Khan, N.A., 2018. Jasmonates in plants under abiotic stresses: crosstalk with other phytohormones matters. Environ. Exp. Bot. $145,104-120$.

Pieterse, C.M., Van der Does, D., Zamioudis, C., Leon-Reyes, A., Van Wees, S.C., 2012. Hormonal modulation of plant immunity. Annu. Rev. Cell Dev. Biol. 28.

Pieterse, C.M.J., Van Wees, S.C.M., Ton, J., Van Pelt, J.A., Van Loon, L.C., 2002. Signalling in rhizobacteria-induced systemic resistance in Arabidopsis thaliana. Plant Biol. 4, 535-544.

Pirbalouti, A.G., Mirbagheri, H., Hamedi, B., Rahimi, E., 2014. Antibacterial activity of the essential oils of myrtle leaves against Erysipelothrix rhusiopathiae. Asian Pac. J. Trop. Biomed. 4, S505-S509.

Qi, T., Song, S., Ren, Q., Wu, D., Huang, H., Chen, Y., et al., 2011. The Jasmonate-ZIM-domain proteins interact with the WDRepeat/bHLH/MYB complexes to regulate Jasmonate-mediated anthocyanin accumulation and trichome initiation in Arabidopsis thaliana. Plant Cell 23, 1795-1814.

Qi, T., Huang, H., Song, S., Xie, D., 2015. Regulation of jasmonatemediated stamen development and seed production by a bHLHMYB complex in Arabidopsis. Plant Cell 27, 1620-1633.

Qiu, Z., Guo, J., Zhu, A., Zhang, L., Zhang, M., 2014. Exogenous jasmonic acid can enhance tolerance of wheat seedlings to salt stress. Ecotoxicol. Environ. Saf. 104, 202-208.

Reinbothe, C., Springer, A., Samol, I., Reinbothe, S., 2009. Plant oxylipins: role of jasmonic acid during programmed cell death, defence and leaf senescence. F.E.B.S. J. 276, 4666-4681.

Riemann, M., Dhakarey, R., Hazman, M., Miro, B., Kohli, A., Nick, P., 2015. Exploring jasmonates in the hormonal network of drought and salinity responses. Front. Plant Sci. 6, 1077. 
Rohwer, C.L., Erwin, J.E., 2008. Horticultural applications of jasmonates. J. Hort. Sci. Biotechnol. 83, 283-304.

Sasaki, Y., Asamizu, E., Shibata, D., Nakamura, Y., Kaneko, T., Awai, K., et al., 2001. Monitoring of methyl jasmonate-responsive genes in Arabidopsis by cDNA macroarray: self-activation of jasmonic acid biosynthesis and crosstalk with other phytohormone signalling pathways. DNA Res. 8, 153-161.

Schaller, M., 2011. The behavioural immune system and the psychology of human sociality. Philosop. Trans. Royal Soc. B: Biol. Sci. 366, 3418-3426.

Schmitt, J.M., Fißlthaler, B., Sheriff, A., Lenz, B., Bäßler, M., Meyer, G., 1996. Environmental control of CAM induction in Mesembryanthemum crystallinum-a role for cytokinin, abscisic acid and jasmonate? Crassulacean Acid Metabolism. Springer, Berlin, Heidelberg, pp. 159-175.

Seltmann, M.A., Stingl, N.E., Lautenschlaeger, J.K., Krischke, M., Mueller, M.J., Berger, S., 2010. Differential impact of lipoxygenase 2 and jasmonates on natural and stress-induced senescence in Arabidopsis. Plant Physiol. 152, 1940-1950.

Seo, J.S., Joo, J., Kim, M.J., Kim, Y.K., Nahm, B.H., Song, S.I., et al., 2011. OsbHLH148, a basic helix loop helix protein, interacts with OsJAZ proteins in a jasmonate signalling pathway leading to drought tolerance in rice. Plant J. 65, 907-921.

Sheard, L.B., Tan, X., Mao, H., Withers, J., Ben-Nissan, G., Hinds, T. R., et al., 2010. Jasmonate perception by inositol-phosphatepotentiated COI1-JAZ co-receptor. Nat. 468, 400.

Smirnoff, N., 1993. The role of active oxygen in the response of plants to water deficit and desiccation. New Phytol. 125, 27-58.

Soares, A.M.D.S., Souza, T.F.D., Jacinto, T., Machado, O.L.T., 2010. Effect of methyl jasmonate on antioxidative enzyme activities and on the contents of ROS and $\mathrm{H} 2 \mathrm{O} 2$ in Ricinus communis leaves. Braz. J. Plant Physiol. 22, 151-158.

Song, S., Choi, Y., Moon, Y.H., Kim, S., Choi, Y.D., Lee, J.S., 2000. Systemic induction of a Phytolacca insularis antiviral protein gene by mechanical wounding jasmonic acid, and abscisic acid. Plant Mol. Biol. 43, 439-450.

Staswick, P.E., Tiryaki, I., 2004. The oxylipin signal jasmonic acid is activated by an enzyme that conjugates it to isoleucine in Arabidopsis. Plant Cell 16, 2117-2127.

Staswick, P.E., 2008. JAZing up jasmonate signalling. Trends Plant Sci. 13, 66-71.

Su, M., Li, X.F., Ma, X.Y., Peng, X.J., Zhao, A.G., Cheng, L.Q., et al., 2011. Cloning two P5CS genes from bioenergy sorghum and their expression profiles under abiotic stresses and MeJA treatment. Plant Sci. 181, 652-659.

Sudhir, P., Murthy, S.D.S., 2004. Effects of salt stress on basic processes of photosynthesis. Photosynthetica 42, 481-486.

Tanji, K.K., 2002. Salinity in the soil environment. In: Lauchli, A., Luttge, U. (Eds.), Salinity: Environment-Plants-Molecules. Kluwer Academic Publishers, The Netherlands, pp. 21-51.

Theodoulou, F.L., Job, K., Slocombe, S.P., Footitt, S., Holdsworth, M., Baker, A., et al., 2005. Jasmonic acid levels are reduced in COMATOSE ATP binding cassette transporter mutants: implications for transport of jasmonate precursors into peroxisomes. Plant Physiol. 137, 835-840.

Thines, B., Katsir, L., Melotto, M., Niu, Y., Mandaokar, A., Liu, G., et al., 2007. JAZ repressor proteins are targets of the SCF COI1 complex during jasmonate signalling. Nature 448, 661.

Toda, Y., Tanaka, M., Ogawa, D., Kurata, K., Kurotani, K.I., Habu, Y., et al., 2013. RICE SALT SENSITIVE3 forms a ternary complex with JAZ and class-C bHLH factors and regulates jasmonate-induced gene expression and root cell elongation. Plant Cell. 25, 1709-1725.

Tsonev, T.D., Lazova, G.N., Stoinova, Z.G., Popova, L.P., 1998. A possible role for jasmonic acid in adaptation of barley seedlings to salinity stress. J. Plant Growth Regul. 17, 153-159.
Turner, J.G., Ellis, C., Devoto, A., 2002. The jasmonate signal pathway. Plant Cell 14, 153-164.

Valenzuela, C.E., Acevedo-Acevedo, O., Miranda, G.S., VergaraBarros, P., Holuigue, L., Figueroa, C.R., et al., 2016. Salt stress response triggers activation of the jasmonate signalling pathway leading to inhibition of cell elongation in Arabidopsis primary root. J. Exp. Bot. 67, 4209-4220.

Velitchkova, M., Fedina, I., 1998. Response of photosynthesis of Pisum sativum to salt stress as affected by methyl jasmonate. Photosynthetica 35, 89-97.

Vick, B.A., Zimmerman, D.C., 1984. Biosynthesis of jasmonic acid by several plant species. Plant Physiol. 75, 458-461.

Walia, H., Wilson, C., Condamine, P., Liu, X., Ismail, A.M., Close, T. J., 2007. Large scale expression profiling and physiological characterization of jasmonic acid mediated adaptation of barley to salinity stress. Plant Cell Environ. 30, 410-421.

Wang, Y., Mopper, S., Hasenstein, K.H., 2001. Effects of salinity on endogenous ABA, IAA, JA, and SA in Iris hexagona. J. Chem. Ecol. 27, 327-342.

Wasternack, C., Kombrink, E., 2010. Jasmonates: structural requirements for lipid-derived signals active in plant stress responses and development. ACS Chem. Biol. 5, 63-77.

Wasternack, C., Hause, B., 2013. Jasmonates: biosynthesis, perception, signal transduction and action in plant stress response, growth and development. An update to the 2007 review in Annals of Botany. Annal. Bot. 111, 1021-1058.

Wasternack, C., Parthier, B., 1997. Jasmonate-signalled plant gene expression. Trends Plant Sci. 2, 302-307.

Wasternack, C., Strnad, M., 2016. Jasmonate signalling in plant stress responses and development-active and inactive compounds. $\mathrm{N}$ Biotechnol. 33, 604-613.

Wasternack, C., Goetz, S., Hellwege, A., Forner, S., Strnad, M., Hause, B., 2012. Another JA/COI1-independent role of OPDA detected in tomato embryo development. Plant Signal. Behav. 7, 1349-1353.

Wasternack, C., Hause, B., 2002. Jasmonates and octadecanoids: signals in plant stress. responses and development. Prog. Nucl. Acid Res. Mol. Biol. 72, 165-221.

Weber, H., 2002. Fatty acid-derived signals in plants. Trends plant Sci. 7 (5), 217-224.

West, G., Inzé, D., Beemster, G.T., 2004. Cell cycle modulation in the response of the primary root of Arabidopsis to salt stress. Plant Physiol. 135, 1050-1058.

Westfall, C.S., Zubieta, C., Herrmann, J., Kapp, U., Nanao, M.H., Jez, J.M., 2012. Structural basis for prereceptor modulation of plant hormones by GH3 proteins. Science 336, 1708-1711.

Woldemariam, M.G., Onkokesung, N., Baldwin, I.T., Galis, I., 2012. Jasmonoyl-1-isoleucine hydrolase 1 (JIH1) regulates jasmonoyl-1isoleucine levels and attenuates plant defenses against herbivores. Plant J. 72, 758-767.

Wu, H., Ye, H., Yao, R., Zhang, T., Xiong, L., 2015. OsJAZ9 acts as a transcriptional regulator in jasmonate signalling and modulates salt stress tolerance in rice. Plant Sci. 232, 1-12.

Xiao, S., Dai, L., Liu, F., Wang, Z., Peng, W., Xie, D., 2004. COS1: an Arabidopsis coronatine insensitive1 suppressor essential for regulation of jasmonate-mediated plant defense and senescence. Plant Cell 16, 1132-1142.

Xie, D.X., Feys, B.F., James, S., Nieto-Rostro, M., Turner, J.G., 1998. COI1: an Arabidopsis gene required for jasmonate-regulated defense and fertility. Sci. 280, 1091-1094.

Yan, Y., Stolz, S., Chételat, A., Reymond, P., Pagni, M., Dubugnon, L., et al., 2007. A downstream mediator in the growth repression limb of the jasmonate pathway. Plant Cell 19, 2470-2483.

Ye, H., Du, H., Tang, N., Li, X., Xiong, L., 2009. Identification and expression profiling analysis of TIFY family genes involved in 
stress and phytohormone responses in rice. Plant Mol. Biol. 71, 291-305.

Yoon, J.Y., Hamayun, M., Lee, S.K., Lee, I.J., 2009. Methyl jasmonate alleviated salinity stress in soybean. J. Crop. Sci. Biotechnol. 12, $63-68$.

Zhai, Q., Yan, L., Tan, D., Chen, R., Sun, J., Gao, L., et al., 2013a. Phosphorylation-coupled proteolysis of the transcription factor MYC2 is important for jasmonate-signaled plant immunity. PLoS Genet. 9, e1003422.

Zhai, Y., Wang, Y., Li, Y., Lei, T., Yan, F., Su, L., et al., 2013 b. Isolation and molecular characterization of GmERF7, a soybean ethylene-response factor that increases salt stress tolerance in tobacco. Gene 513, 174-183.

Zhang, F., Yao, J., Ke, J., Zhang, L., Lam, V.Q., Xin, X.F., et al., 2015. Structural basis of JAZ repression of MYC transcription factors in jasmonate signalling. Nat. 525, 269.

Zhang, J.T., Mu, C.S., 2009. Effects of saline and alkaline stresses on the germination, growth, photosynthesis, ionic balance and antioxidant system in an alkali tolerant leguminous forage Lathyrus quinquenervius. Soil. Sci. Plant. Nutr. 55, 685-697.
Zhao, Y., Dong, W., Zhang, N., Ai, X., Wang, M., Huang, Z., et al., 2014. A wheat allene oxide cyclase gene enhances salinity tolerance via jasmonate signalling. Plant Physiol. 164, 1068-1076.

Ziegler, J., Stenzel, I., Hause, B., Maucher, H., Hamberg, M., Grimm, R., et al., 2000. Molecular cloning of allene oxide cyclase. The enzyme establishing the stereochemistry of octadecanoids and jasmonates. J. Biol. Chem. 275, 19132-19138.

Zolman, B.K., Yoder, A., Bartel, B., 2000. Genetic analysis of indole-3butyric acid responses Arabidopsis thaliana reveals four mutant classes. Genetics 156, 1323-1337.

\section{Further Reading}

Cheng, M.C., Liao, P.M., Kuo, W.W., Lin, T.P., 2013. The Arabidopsis ETHYLENE RESPONSE FACTOR1 regulates abiotic stressresponsive gene expression by binding to different cis-acting elements in response to different stress signals. Plant Physiol. 162, 1566-1582.

Kazan, K., Manners, J.M., 2012. JAZ repressors and the orchestration of phytohormone crosstalk. Trends Plant Sci. 17, 22-31. 


\title{
25
}

\section{Insights Into the Nitric Oxide Mediated Stress Tolerance in Plants}

\author{
Parankusam Santisree ${ }^{1}$, Srivani S. Adimulam ${ }^{1}$, Kapil Sharma ${ }^{2}$, Pooja \\ Bhatnagar-Mathur ${ }^{1}$ and Kiran K. Sharma ${ }^{1}$
}

${ }^{1}$ International Crops Research Institute for the Semi-Arid Tropics (ICRISAT), Patancheru, Hyderabad, Telangana, India ${ }^{2}$ Department of Plant Sciences, Repository of Tomato Genomics Resources,

University of Hyderabad, Hyderabad, Telangana, India

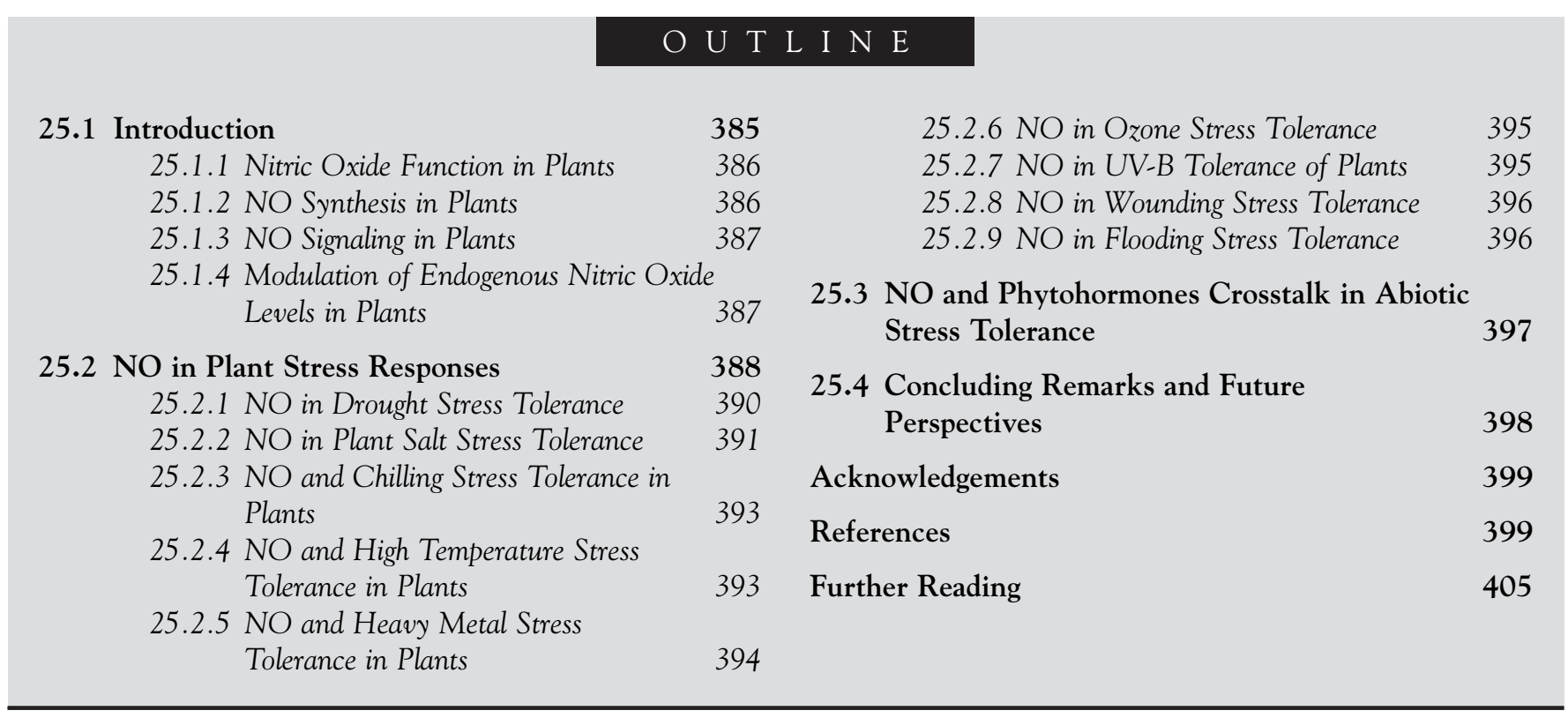

\subsection{INTRODUCTION}

Nitric oxide (NO) was first discovered by Joseph Priestley in 1772 as a colorless and odorless gas named "nitrous air"; just a couple of years later came his discovery of oxygen $\left(\mathrm{O}_{2}\right)$. NO was considered as a toxic gas for next two centuries, until it was shown to emit by air purging of herbicide-treated Glycine max leaves (Klepper, 1979). Thenceforward, NO has long been of major interest in both plant and animal research (Santisree et al., 2015), and in 1992, Science magazine crowned NO as "Molecule of the Year". A joint 
discovery that NO is produced by the mammalian species as a signaling molecule by Robert F. Furchgott, Louis J. Ignarro, and Ferid Murad secured the Nobel Prize in Physiology or Medicine for 1998. During the last two decades, several other discoveries have revealed the biological significance of $\mathrm{NO}$ in both animals and plants. Despite rapid research in animals, NO research in plants has been gradual with increasing excitement.

\subsubsection{Nitric Oxide Function in Plants}

Although initial discoveries in plants recognized $\mathrm{NO}$ as an atmospheric toxic pollutant for plant foliage, it was eventually considered as a modulator of plant defense during pathogen attacks. The increasing number of reports demonstrated the role of NO in a plethora of plant development processes including seed germination (Arc et al., 2013), root formation, different stages of the seed development, gravitropism, stomatal movements, photosynthesis, mitochondrial functionality, senescence, plant maturation (Sun et al., 2017; Patel et al., 2017; Hasanuzzaman et al., 2018; Locato et al., 2016; Mostofa et al., 2015; Asgher et al., 2017), multiple abiotic (Fancy et al., 2017; Santisree et al., 2015; Parankusam et al., 2017; Adimulam et al., 2017; Tossi et al., 2012; Sehrawat et al., 2013; Ziogas et al., 2013) and biotic stress responses in plants (Vaishnav et al., 2018). In addition, a number of studies focused on describing the crucial role of NO in moderating various plant hormone-mediated development and stress responses (Asgher et al., 2017). Further, accumulation of NO has been shown to induce gene expression of defense proteins during stress conditions and recovery (Romero-Puertas et al., 2013; Fancy et al., 2017). Mounting evidence suggests the role of NO in maintaining cellular homeostasis by acting as an antioxidant and negating the intensity of oxidative damage caused by various stress treatments (Hasanuzzaman et al., 2012; Groß et al., 2013). Despite the emergent knowledge about NO-mediated plant stress responses such as decreasing reactive oxygen species (ROS) levels, protecting membranes from damage, osmolyte accumulation, and regulation of various hormonemediated signaling events, its functional status has been far from clear. However, the short life and complex chemistry of this diffusible free radical makes NO research in living systems extremely challenging.

\subsubsection{NO Synthesis in Plants}

\subsubsection{Nitric Oxide Synthase (NOS)}

In animal tissues, NO generation occurs through at least three different nitric oxide synthase (NOS) genes namely neuronal NOS (nNOS), an endothelial enzyme (eNOS), and an inducible enzyme (iNOS). Though NO is known to exist functionally in plants, the origin and signaling of $\mathrm{NO}$ is inconclusive to date. The isolation of NOS enzymes in animals is really rapid compared with the decades of research in plants. It is speculated that $\mathrm{NO}$ is derived from four major sources in plants: NO synthase pathway, which is similar to that of animals (Negi et al., 2010), nitrate reductase (NR) pathway, and other enzymatic and nonenzymatic pathways (Sahay and Gupta, 2017). The generation of NO has also been suggested through reductive pathways including the mitochondrial electron transport system and from xanthine dehydrogenase/oxidase (Sahay and Gupta, 2017). Despite several possible NO generating pathways proposed in plants (Sahay and Gupta, 2017), identification of a definitive enzymatic pathway generating $\mathrm{NO}$ in plants is still awaiting. The first molecular evidence for gene encoding NOS like activity potentially involved in NO synthesis in plants came from Arabidopsis thaliana T-DNA insertion mutant (atnos1) based on the homology to a snail protein (Guo et al., 2014). However, later studies failed to detect any NOS activity in purified recombinant AtNOS1 protein or in similar recombinant proteins encoded by orthologous genes from Zea mays and Oryza sativa. Moreover, this mutant also failed to induce NO synthesis in guard cells in response to abscisic acid (ABA) and other stimuli. These studies concluded AtNOS1 as a regulator of NO levels rather than the molecule of synthesis. Eventually it was renamed as Arabidopsis thaliana nitric oxide associated (atnos1) mutant (Parankusam et al., 2017). Even though a few other recent pharmacological studies in various plant species also suggested the existence of NOS-like enzyme in plants, the attempts of purifying the gene or protein are still underway (Negi et al., 2010; Fröhlich and Durner, 2011). Nonetheless, two genes with NOS like activity and approximately $40 \%$ homology to animal NOS genes have been reported in green algae Ostreococcus tauri and Ostreococcus lucimarinus (Foresi et al., 2015). Though we now have the first NOS genes identified in a green alga, identification of NO synthase gene in higher plants is still awaited. The divergence of this gene to a new nonanimal homologous during the course of evolution might have posed a challenge to isolate NO synthase genes from higher plants.

\subsubsection{Nitrate Reductase (NR)}

Apart from NOS, the other well-known NO-generating enzyme in plants is nitrate reductase (NR). It converts nitrite to $\mathrm{NO}$ in a $\mathrm{NAD}(\mathrm{P}) \mathrm{H}$ dependent manner. In Arabidopsis, NR is encoded by two genes, NIA1 and NIA2. The double mutant of NIA genes accumulate much less NO in guard cells in response to ABA. Eventually the NO generation by NR has been 
confirmed in many other species, like Helianthus annus, Spinacea oleracea, Zea mays, Cucumis sativus, Chlamydomonas reinhardtii, Triticum aestivum, Malaxis monophyllos, and Aloe vera (Sakihama et al., 2002, Xu and Zhao, 2003). Additionally, NO can be generated nonenzymatically under low $\mathrm{pH}$ by the interaction of two nitrous acid $\left(\mathrm{HNO}_{2}\right)$ molecules derived from protonated nitrite, by reduction of $\mathrm{NO}_{2}$ to $\mathrm{NO}$ by carotenoids under light or by oxidation of hydroxylamine, salicylhydroxamate in plant cell cultures, and also during electron transport processes in mitochondria and chloroplasts (Jasid et al., 2006). NO besides being endogenous in origin is also taken up by plants from the external environment. Endogenous NO is synthesized in parallel to ROS accumulation in various organelles like mitochondria, chloroplast, peroxisomes, and apoplast. Recently Gibbs et al. (2014) identified a unifying mechanism for $\mathrm{NO}$ sensing in plants based on targeted proteolysis of plant-specific group VII ERF transcription factors. They proposed $\mathrm{N}$-end rule pathway proteolysis as an essential NO sensing mechanism throughout the plant life cycle. Though the research on $\mathrm{NO}$ is gaining popularity in recent years, the following controversial questions about $\mathrm{NO}$ are around the corner: (1) the major source of NO generation and biosynthesis mechanism, (2) the sensing mechanism of NO by different plant tissues, and (3) the degradation mechanism.

\subsubsection{NO Signaling in Plants}

As the sources of $\mathrm{NO}$ generation have begun to establish, the mechanisms of NO signaling are also increasingly being resolved in parallel. Being lipophilic and highly diffusible in nature, NO is involved in several signaling processes in plants (Baudouin, 2011). Invariably, it has been shown that NO modifies the activity of enzymes and some key signaling components, possibly through posttranslational protein modifications (PTMs). The major PTMs that are integral to NO signaling include protein $S$-nitrosylation and tyrosine nitration (Fancy et al., 2017). A number of S-nitrosylated proteins were identified in Arabidopsis thaliana, Brassica juncea, Citrus aurantium, and Zea mays after stress treatment (Mengel et al., 2013; Fan et al., 2014; Fancy et al., 2017). NO-mediated PTMs including nitrosylation, nitration, and carbonylation have been shown to influence ascorbate peroxidase activity under drought (Santisree et al., 2015). NO mediated S-nitrosylation prevented the inactivation of the antioxidant enzymes in Antiaris toxicaria thereby providing a way to mitigate the $\mathrm{H}_{2} \mathrm{O}_{2}$ concentration in plant cells and increasing desiccation tolerance of seeds (Fan et al., 2014; Santisree et al., 2015). Many target signaling components such as redox-associated proteins, the $\mathrm{K}^{+}$channel at the guard cell plasma membrane, AtRhobD, salicylic acid-binding protein 3, NPR1, TGA1, and auxin signaling TIR1 (Romero-Puertas et al., 2013; Lindermayr et al., 2010; Terrile et al., 2012) are known to be engaged in NO-mediated PTMs. Additionally, NO acts as a signaling molecule at the transcription level by enhancing the expression of stress related kinases and by its interaction with other signaling molecules and phytohormones (Lozano-Juste and León, 2011). NO stimulates myosin activated protein kinase (MAPK), which in turn causes the activation of stress related genes ( $\mathrm{Li}$ and Xue, 2010). Another well-established method by which $\mathrm{NO}$ exerts its effect is by influencing the redox status of the cell. Modulation of guanylate cyclase represents an NOspecific mechanism of signaling and there are many reports showing that cyclic guanosine monophosphate (cGMP) is a facet of NO effects in plants (Mulaudzi et al., 2011). Recently, a novel guanylate cyclase that generates cGMP and binds NO has been described in Arabidopsis thaliana (Mulaudzi et al., 2011). Certain other molecules like $\mathrm{Ca}^{2+}$ and cyclic ADP-ribose (cADPR) are found to be involved in the downstream signaling of $\mathrm{NO}$ in plant stress responses (Mioto and Mercier, 2013). Moreover, the emerging high throughput omics platforms have been very useful in identifying various candidate genes associated with $\mathrm{NO}$ and NO-mediated PTMs (nitrosylation, nitration, and carbonylation) influencing plant stress conditions (Astier and Lindermayr, 2012; Shi et al., 2013; Begara-Morales et al., 2014). It is now imperative to integrate all these molecules and events into our existing knowledge of NO networks.

\subsubsection{Modulation of Endogenous Nitric Oxide Levels in Plants}

Given that NO is an important signal in plant stress responses, there is increasing interest in understanding the impact of altered NO levels in plants. Notwithstanding, the in vivo level of NO appears to be regulated by mutations in diverse genes. Previous studies have reported few mutants of Arabidopsis thaliana including nia1nia2, noa1, and nox1, which failed to exhibit certain developmental and stress responses due to defective $\mathrm{NO}$ accumulation, while noe1 mutation in Oryza sativa resulted in higher NO accumulation (Desikan et al., 2002). The mutation in CUE1 gene encoding a chloroplast phosphoenolpyruvate/ phosphate translocator led to an increase in NO content and delayed flowering in the mutant (He et al., 2004). Meanwhile, the null alleles of Arabidopsis thaliana HOT5 locus encoding S-nitrosoglutathione reductase (GSNOR), showed decreased tolerance to temperature stress due to an increase in levels of nitrate, NO, and 
nitroso species (Santisree et al., 2017). Similarly, enhanced lateral root formation in arginase-negative mutant is associated with an increased NO levels (Santisree et al., 2015). Similarly mutation in Arabidopsis prohibitin (PHB3) gene leads to reduction in absicic acid (ABA)-mediated NO accumulation and auxininduced lateral root formation (Wang et al., 2010a,b).

Although there is no NOS sequence identified in higher plants, few transgenic efforts have constitutively expressed rat and mammalian neural nitric oxide synthase (nNOS) genes in plants (Shi et al., 2014; Santisree et al., 2015). 35S::nNOS transgenic lines of Arabidopsis thaliana displayed improved abiotic and biotic tolerance (Shi et al., 2011). Similarly, overexpression of rat $n N O S$ increased NOS activity and endogenous NO level in transgenic Oryza sativa lines, that led to higher tolerance under both drought and salt stresses (Cai et al., 2015). Further, transgenic plants expressing OtNOS also displayed increased stomatal development and enhanced abiotic stress tolerance due to higher accumulation of NO (Santisree et al., 2015). The fragmentary molecular identities related to NO synthesis and signaling in plants makes the transgenic and genetic studies challenging.

Due to the inadequate molecular information, most of the current NO research in plants bank on exogenous application of NO-donors and inhibitors/scavengers (Table 25.1). So far this pharmacological approach has been used either to mimic an endogenous NO-related response or as a substitute for an endogenous NO deficiency. Exogenous NO donors or inhibitors have been combined with high throughput technologies to study the ability of NO in modulating plant stress responses at the genomic, proteomic, and postproteomic levels. A pioneering proteomic study in Gossypium hirsutum leaves treated with sodium nitroprusside (SNP) identified 166 differentially expressed proteins belonging to diverse pathways, followed by the identification of 167 phosphoproteins to be differentially phosphorylated in response to SNP (Meng et al., 2011). Similarly, proteome profiling revealed 172 downregulated and 76 upregulated proteins in Cicer aritinum leaves (Santisree et al., 2017). Few other studies attempted to understand the proteomic basis of NO mediated stress tolerance (Bai et al., 2011; Sehrawat et al., 2013; Yang et al., 2013; Fan et al., 2014). Nevertheless, high-throughput genomic and proteomic signatures of NO still need to be unfolded to further explore the complexity involved in its signaling under plant stress.

\subsection{NO IN PLANT STRESS RESPONSES}

High temperature and drought are perhaps the two major environmental factors limiting crop growth and yield worldwide (Prasad et al., 2011; Vile et al., 2012). Plants respond at the molecular, cellular, and physiological level by perception and transmission of stress signals followed by a series of responses (Fancy et al., 2017). Longer and severe stress episodes result in production of redox active molecules including reactive oxygen and reactive nitrogen species (RNS), respectively (Astier et al., 2016), which leads to abnormalities at the cellular level due to oxidation of proteins, lipids, and nucleic acids (Hayat et al., 2012). On the other hand, it was demonstrated that abiotic stress often induced NO generation that led to the activation of cellular processes for protection against oxidative stress. $\mathrm{NO}$ protects the plants from oxidative damage by enhancing the $\mathrm{H}_{2} \mathrm{O}_{2}$-scavenging enzymes activities thereby maintaining cellular redox homeostasis (Shi et al., 2014; Zheng et al., 2009). Moreover, exogenous NO donors have often been deployed successfully as priming agents to ward off abiotic stress induced losses in plants (Uchida et al., 2002; Hasanuzzaman et al., 2012; Santisree et al., 2015; Savvides et al., 2016). Although accumulation of NO during various stress conditions appears to be a general response in diverse plant species and tissues, its specificity has been established by using various inhibitors/scavengers such as 2-(4-Carboxyphenyl)-4,4,5,5-tetramethylimidazoline-1-

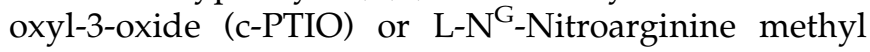
ester; $\mathrm{N}(\mathrm{G})$-Nitro-L-arginine methyl ester (L-NAME), which reversed these NO-mediated effects in many such studies (Santisree et al., 2015). NO plays a critical role in mitigating oxidative stress caused by unfavorable climate (Siddiqui et al., 2017; Table 25.2). Most of the studies reinforce the role of $\mathrm{NO}$ in detoxifying $\mathrm{ROS}$ either directly interacting with $\mathrm{O}^{-2}$ or indirectly by enhancing function of the antioxidant system (Tewari et al., 2008). Interaction of $\mathrm{NO}$ with $\mathrm{O}^{-2}$ forms peroxynitrite, which serves as a nitrating agent in regulating protein activity under stress conditions (Baudouin, 2011). Further, NO upregulated the activity and transcription of ascorbate peroxidase (APX) and glutathione reductase (GR), the two key enzymes in the ascorbic acid-glutathione (AsA-GSH) cycle in Nicotiana tabacum and Cucumis satious leaves (Cui et al., 2011) and conferred resistance to abiotic stress. NO also rapidly reacts with oxygen species, hemes, thiols, and proteins to produce biochemical signals that directly and indirectly regulate enzymatic activity. However, the information available is sometimes contradictory, depending on the plant species, severity, and duration of the stress treatments (Begara-Morales et al., 2014). Despite an increasing number of reports on the role of $\mathrm{NO}$ as an endogenous signaling molecule in plants, there is still a large knowledge gap about underlying molecular mechanisms of its action that can sense and transduce NO signals. 
TABLE 25.1 Various Forms of Nitric Oxide Modulators Used in Plants to Demonstrate the Role of NO Under Plant Abiotic Stress

\begin{tabular}{ll}
\hline & Plant species \\
\hline NO donors & Medicago sativa; \\
& \\
Sodium nitroprusside (SNP) & Triticum aestivum; Zea mays; Vicia faba; Salpichora
\end{tabular}
organifolia; Tagetes erecta; Lycopersicon esculentum

\section{Triticum aestivum}

Chrysanthemum morifolium; zea mays; Phaseolus vulgaris; phragmitis communis; Oryza sativa; Phaseolus radiates; Phragmitis communis; Gingiber officinale; Festuca anundinacea; Solanum lycopersicon

Citrus grandis; Hibiscus moscheutos; Artemisia annua; Lolium perene; Triticum aestivum; Vigna unguiculata

Brassica junica; Arachis hypogeae; Trifolium repens; Typha angustifolia

Triticum aestivum; Pistia stratiotes

Phaseolus vulgaris; Helianthus annuus; Zea mays; Glycene max

\section{Cicer aritinum}

Glycene max

Medicago sativa

Salpichora organifolia; Tradescantia sp; Medicago sativa;

Lactuca sativa; Zea mays

Carboxy-2-phenyl-4,4,5,5, -tetramethylimidazoline-1oxyl 3-oxide (cPTIO)

Nicotiana tabacam; Arabidopsis thaliana; Betula pendula

\section{Mutants}

Transgenics
Atnoa1, nia1nia2, gsnor1-3, respiratory burst oxidase homolog mutants of Arabidopsis thaliana

nia1nia2 mutants of Arabidopsis thaliana

atgsnor1-3/hot5-2 mutants of Arabidopsis thaliana

Homozygous mutants deficient of atips1 of Arabidopsis thaliana

Arabidopsis thaliana and Oryza sativa transgenic plants overexpressing oxide synthase gene (nNOS), OtNOS transgenic lines; 35S::nNOS transgenic lines of Arabidopsis thaliana

\section{Arabidopsis thalianatGLB3}

H7 plants overexpressing Arabidopsis hemoglobin 1 $(A H b 1)$, transgenic cucumber plants overexpressing CsNOA1

Stress imposed References

High Yu et al. (2014)
temperature
stress

Drought stress

Tian and Lei (2007); Hao et al. (2008); Gracia-Mata and Lamattina (2001); Gupta et al. (2013)

Chilling stress Esim et al. (2014)

High temperature Yang et al. (2011a,b); Li et al. (2013b); Song et al. (2008); Li et al. (2013); stress

Chen et al. (2013); Siddiqui et al. (2017)

$\mathrm{Al}^{\mathrm{a}}$ stress

Yang et al. (2016); Aftab et al. (2012); Bai et al. (2011); Sun et al. (2014); Sadeghipour (2016)

$\mathrm{Cd}^{\mathrm{a}}$ stress Dong et al. (2014); Zhao et al. (2016)

$\mathrm{As}^{\mathrm{a}}$ stress Hasanuzzaman et al. (2013)

UV-B stress Shi et al. (2005); Tossi et al. (2012)

Salinity stress

Ahmad et al. (2016)

Salinity stress

Egbichi et al. (2014)

Drought stress

Garcia-Mata and Lamattina (2001); Tian and Lei (2007); Hao et al. (2008)

High temperature stress

Deng and Song (2012); Li et al. (2013b)

Ozone stress

Ederli et al. (2008); Ahlfors et al. (2009a)

Drought stress

Lozano-Juste and León (2011); She et al. (2004)

Chilling stress

Zhao et al. (2009); Liu et al. (2016)

Heat stress

Santisree et al. (2017)

UV-B stress

Lytvyn et al. (2016)

Drought stress

Cai et al. (2015); Shi et al. (2014) Salt stress

Heat stress

Chilling stress
Hossain et al. (2011)

Zhao et al. (2009); Cantrel et al. (2011); Bai et al. (2011); Xu et al. (2012a,b); Liu et al. (2016)

${ }^{a} A l$, aluminum; $C d$, cadmium; $A s$, arsenic. 


\subsubsection{NO in Drought Stress Tolerance}

Prevailing water deficit conditions limit crop yields worldwide (Simontacchi et al., 2015). NO, which is known to involve in various physiological processes of the plants, also plays a vital role in mitigating drought stress conditions in plants (Santisree et al., 2015). Many studies have reported an increased production of NO in drought-stressed plants depending on the duration and severity of the given drought stress (Santisree et al., 2015). Roots of Cucumis sativus when subjected to a mild water deficit for less than $10 \mathrm{~h}$ had slightly enhanced NO synthesis, while it increased to a greater extent with prolonged drought imposition for $17 \mathrm{~h}$ (Arasimowicz-Jelonek et al., 2011). Moreover, accumulation of $\mathrm{NO}$ as a result of application of exogenous donors in many reported studies also correlated well with the amelioration of drought stress, while the use of NO scavengers/inhibitors reversed this effect (Arasimowicz-Jelonek et al., 2011; Planchet et al., 2014).

The increase in NO production under drought stress has been correlated significantly to the decrease in stomatal conductance in Vitis vinifera (Patakas et al., 2010). Given the ability of NO to induce dark-induced stomatal closure in Vicia faba epidermal strips (She et al., 2004), a good number of studies confirmed the generation of $\mathrm{NO}$ in guard cells in response to drought and ABA by using a NO-sensitive fluorescent dye 4,5Diaminofluorescein diacetate (DAF-2DA) (Planchet et al., 2014; Zimmer-Prados et al., 2014). Exogenous NO induces ABA synthesis by upregulating the expression of a key genes including 9-cis-epoxycarotenoid dioxygenase and also negatively regulates the ABA sensitivity thereby enhancing plant tolerance to drought stress (Santisree et al., 2015). While, ABA failed to induce stomatal closure in Atnoa1and nia1nia2 mutants of Arabidopsis thaliana having reduced endogenous NO levels, NO-deficient triple mutant nia1nia2noa1-2 plants were hypersensitive to dehydration and ABA treatment in stomatal closure providing genetic evidence for the involvement of $\mathrm{NO}$ in ABA-mediated stomatal closure (Planchet et al., 2014; Lozano-Juste and León, 2011). Furthermore, the overaccumulation of $\mathrm{NO}$ in guard cells of Arabidopsis thaliana GSNO reductase mutant (gsnor1-3) has led to the defective ABA-induced stomatal closure due to the constitutive S-nitrosylation of open stomata 1 (OST1) reinforcing NO as a key intermediate in ABA-induced stomatal closure (Adimulam et al., 2017). Moreover, reduced NO accumulation and stomatal closure were observed in respiratory burst oxidase homolog mutant of Arabidopsis thaliana having a mutation in nicotinamide adenine dinucleotide phosphate (NADPH) oxidase suggesting a link between $\mathrm{H}_{2} \mathrm{O}_{2}$ and NO accumulation (She et al., 2004). Broadly, $\mathrm{NO}$ enhances antioxidant enzyme activity and induce stomatal closure through ambiguous signaling pathways that require MAPKs, cGMP, and $\mathrm{Ca}^{2+}$. In addition to MAPK, NO also activates other protein kinases such as osmotic stress-activated kinase, NtOSAK in Nicociana tabacum (Baudouin and Hancock, 2014). NO alters protein phosphorylation through the regulation of these kinases and also alters calcium flux to regain normal NO responses to occur in the guard cells under drought. NO increases the cGMP level, which acts as the secondary messenger in promoting the expression of stress related genes and secondary metabolites during water deficit conditions (Santisree et al., 2015).

It was reported that exogenous $\mathrm{NO}$ decreased drought-induced reduction in photochemical quenching in Tagetes erecta (Liao et al., 2012), while enhancing $\mathrm{CO}_{2}$ assimilation and photosynthetic rate in Rumex leaves under osmotic stress ( $\mathrm{Li}$ et al., 2013). In Lycopersicon esculentum, SNP treatment promoted the activity of carbonic anhydrase that catalyzes the interconversion of $\mathrm{CO}_{2}$ and $\mathrm{HCO}_{3}$, and thus indirectly maintain constant supply of $\mathrm{CO}_{2}$ to ribulose-1,5-bisphosphate carboxylase/oxygenase (RuBisCo) in a concentration dependent manner. Additionally, NO ameliorates the stress effects on chloroplasts and combats drought-induced leaf senescence by antagonizing the effects of ethylene (Manjunatha et al., 2012). NO application has been shown to negate the drought-induced decrease in transcription of $p s b A$ gene encoding for D1 protein of PSII complex, thus protecting PSII functionality during grain filling (Wang et al., 2011; Procházková et al., 2013). Similarly, photochemical efficiency of photosystem II (PSII) increased by exogenous NO treatment in drought stressed Populus przewalskii, but decreased under prolonged drought stress conditions (Simontacchi et al., 2013). Conversely, thylakoids isolated from NO-treated Spinacia oleracea exhibited less photosynthetic activity indicating that NO can bind reversibly to PSII and inhibit electron transport (Misra et al., 2014).

Exogenous NO treatment under drought stress often results in reduced $\mathrm{H}_{2} \mathrm{O}_{2}$ content and lipid peroxidation in plants while high $\mathrm{NO}$ causes nitrosative stress (Farooq et al., 2009; Liao et al., 2012; Li et al., 2013). For instance, SNP treatment maintained higher relative water content and reduced ion leakage during drought stress in two turf grass species (Hatamzadeh et al., 2015). Similarly, NO-treated plants maintained high levels of antioxidant enzyme activities and less lipid peroxidation under drought stress in Dendrobium huoshanense and Oryza sativa (Farooq et al., 2009; Fan et al., 2014). Despite reducing the level of oxidative stress, NO also help in maintaining high concentrations of osmotically active solutes and amino acids. NO promoted drought-induced free proline accumulation in many plants (Farooq et al., 2009; Wang et al., 2011). NO also mediates the accumulation of glycine 
betaine by stimulating the activity of betaine aldehyde dehydrogenase in the leaves of drought stressed Zea Mays (Hao et al., 2008). Conversely, neither depleting endogenous NO by its scavenger nor inducing by NO donor had significant effect on the accumulation of proline in Medicago seedlings. Meanwhile, drought stress decreased the DNA methylation levels in Dendrobium huoshanense, while NO increased the methylated sites clearly suggesting the ability of NO to alter gene expression under drought (Fan et al., 2014). Transgenic plants overexpressing the rat neural nitric oxide synthase gene ( $n N O S$ ) in Arabidopsis thaliana and Oryza sativa exhibit enhanced drought tolerance than their untransformed controls (Cai et al., 2015; Shi et al., 2014). Moreover, OtNOS transgenic lines also exhibited better stomatal development compared with control plants. Although our knowledge on the role of NO in drought stress is still emerging, we find enormous potential of $\mathrm{NO}$ in mitigating drought induced adversaries in plants.

\subsubsection{NO in Plant Salt Stress Tolerance}

Soil salinity is one of the major abiotic stress factors for crop production impacting more than 45 million hectares of cultivated land (Slinger and Tenison, 2009; Fatma et al., 2016). With continuous increase in the demand for food, farmlands are being artificially irrigated in greater amounts, leading to increased salt accumulation in the soil. Excess accumulation of $\mathrm{NaCl}$ in soil limits the plant water and mineral uptake (Khan et al., 2012). In addition, the excess salt intake into the cytosol leads to osmotic imbalance and imposes toxic effects on cell membranes (Abeer et al., 2014). Higher salt concentrations cause oxidative stress due to excess production of ROS and thus hinder several metabolic processes (Fatma et al., 2016; Naser Alavi et al., 2014). In the past decade, function of NO in salt stress tolerance has gained a lot of attention among plant researchers (Yang et al., 2011a,b; Mostofa et al., 2015). It was reported that endogenous NO generation has increased in Nicotiana tabacum plants in response to salinity stress. External application of NO donor, $S$-nitroso- $N$-acetylpenicillamine (SNAP), to salinized plants enhanced the growth parameters, leaf relative water content, photosynthetic pigment production, levels of osmolytes, as well as the antioxidant enzyme activities and gene expression in Cicer aritinum (Ahmad et al., 2016). Moreover, exogenous NO enhanced salt tolerance by mitigating the oxidative damage, stimulating proton-pump and $\mathrm{Na}^{+} / \mathrm{H}^{+}$antiport activity in the tonoplast thus promoting $\mathrm{K}^{+} / \mathrm{Na}^{+}$ ratio (Santisree et al., 2015). NO influences salinity tolerance by regulating plasma membrane $\mathrm{H}^{+}$-ATPase and $\mathrm{Na}^{+} / \mathrm{K}^{+}$ratio thereby generating a $\mathrm{H}^{+}$gradient that offers the force for $\mathrm{Na}^{+} / \mathrm{H}^{+}$exchange (Zhang et al., 2006). For instance, NO has been implicated in enhancing $\mathrm{K}^{+}, \mathrm{Ca}^{2+}$, and $\mathrm{Mg}^{2+}$ content in salt-treated Gossypium hirsutum plants (Dong et al., 2014). Moreover, NO reacts with lipid radicals thus preventing salt stress induced lipid oxidation and decreasing membrane permeability (Kopyra, 2004; Fatma and Khan, 2014; Xu et al., 2010b). NO enhances the antioxidant defense systems in plants subjected to salinity by inducing the expression of redox-related genes (Zheng et al., 2009). The application of DiethylenetriamineNONOate (DETA/NO) ameliorated long term salinity effects in Glycene max via the induction of antioxidant enzymes (Egbichi et al., 2014). Studies have provided evidence that NO mediated detoxification is partly by its ability to regulate ascorbate-glutathione cycle through S-nitrosylation (Camejo et al., 2013; Ziogas et al., 2013). It is evident from the studies that salt stress induces an increase in total S-nitrosylation especially S-nitrosylation of the glycine dehydrogenase $\mathrm{P}$ subunit, F1 ATPase $\beta$ subunit, and isocitrate dehydrogenase (ICDH) implying the role of NO-mediated posttranslational modifications in controlling respiratory/ photorespiratory pathways (Fares et al., 2011; Camejo et al., 2013; Abat and Deswal, 2009; Begara-Morales et al., 2015). Conversely, salt stress induced $\mathrm{O}_{2}$ might also reduce the S-nitrosylation level by interacting with S-nitrosoglutathione (GSNO/RSNO) (Fancy et al., 2017). However, the enhanced antioxidant enzyme activity due to foliar spray of $\mathrm{NO}$ in various crop plants has also resulted in increased plant growth under salt stress (Wu et al., 2011; Fatma and Khan, 2014). Similarly, exogenous application of NO was also proved beneficial in enhancing plant dry weight, shoot, and root length of salt-stressed wheat plants (Kausar and Shahbaz, 2013). Not only that, NO participates in enhancement of photosynthesis by inducing the photosynthetic pigments, adenosine triphosphate (ATP) synthesis, and two respiratory electron transport pathways in mitochondria under salt stress (Ruan et al., 2002). This was further ascertained by an improved photosynthesis by quenching excess energy and increasing in quantum yield of PSII by using exogenous NO in Solanum melongena seedlings (Wu et al., 2013). The exogenous application of NO has been reported to control the activity of phosphoenolpyruvate carboxylase kinase in Sorghum bicolor under salt stress (Monreal et al., 2013). NO application enhanced the photosynthetic potential of Brassica juncea under salt stress (Fatma and Khan, 2014). The sustained photosynthesis after application of $\mathrm{NO}$ under salt stress in Lycopersicon esculentum has been attributed to improved gas exchange parameters and chlorophyll fluorescence (Wu et al., 2011). A recent study in 
TABLE 25.2 Various Studies Describing the Involvement of Nitric Oxide (NO) in Plant Abiotic Stress Tolerance

\begin{tabular}{|c|c|}
\hline $\begin{array}{l}\text { Stress } \\
\text { imposed }\end{array}$ & Plant species \\
\hline $\begin{array}{l}\text { Salinity } \\
\text { stress }\end{array}$ & $\begin{array}{l}\text { Brassica nigra; Brassica juncea; Arabidopsis } \\
\text { thaliana; Triticum aestivum; Lycopersicon } \\
\text { esculentum; Sorghum bicoclor; Hordium } \\
\text { vulgare; Cicer aritinum; Glycene max; Zea } \\
\text { mays; Linum ustiatissimum; morua alba; } \\
\text { Gossypium hirsutum }\end{array}$ \\
\hline $\begin{array}{l}\text { Drought } \\
\text { stress }\end{array}$ & $\begin{array}{l}\text { Triticum aestivum; Zea mays; Hordeum } \\
\text { vulgare; Oryza sativa; Vicia faba; Cucumis } \\
\text { sativus; Salpicora organifolia; Tagetes erecta; } \\
\text { Malus hupensis; Spinacea oleracia; Populus } \\
\text { przewalskii; Lycopersecon esculentum }\end{array}$ \\
\hline $\begin{array}{l}\text { Chilling } \\
\text { stress }\end{array}$ & $\begin{array}{l}\text { Helianthus annus; Capsicum annum; } \\
\text { Arabidopsis thaliana; Chirospora bugeana; } \\
\text { Baccaurea ramiflora; Brassica juncea; } \\
\text { Camallia sinensis; Lycopersicon esculentum; } \\
\text { Triticum aestivum }\end{array}$ \\
\hline
\end{tabular}

Response

Lipid peroxidation,

ROS $^{\text {a }}$ scavenging

Activation of antioxidant enzymes

Altered gene transcription,

enhanced photosynthesis

Stomatal closure, enhanced antioxidant defense, increased adventitious root length, reduced lipid peroxidation, mediating $\mathrm{ABA}^{\mathrm{a}}$ signaling

Synthesis of osmolytes, reprogramming of lipid signaling, negatively regulates sphingolipid phosphorylation, increases spermidine and spermine levels, increases antioxidant enzyme activity
References

Khan et al. (2012); Fatma et al. (2016); Fatma and Khan (2014); Monreal et al. (2013); Ahmad et al. (2016); Egbichi et al. (2014); Dong et al. (2014)

Santisree et al. (2015); Garcra-Mata and Lamattina (2002); Tian and Lei (2007); Hao et al. (2008); Cheong et al. (2002); Simontacchi et al. (2013); Gupta et al. (2013)

Cantrel et al. (2011); Ashraf and Foolad (2007); Zhao et al. (2009); Chaki et al. (2011); Airaki et al. (2012); Zhao et al. (2009); Liu et al. (2016); Zhao et al. (2009); Bai et al. (2011); Xu et al. (2012a,b); Abat and Deswal (2009); Wang et al. (2012); Diao et al. (2016); Esim et al. (2014); Baudouin and Jeandroz (2015)

High Medicago sativa; Pisum sativum; temperature Chrysanthemeum morifolium; Nicotiana stress gluca; Helianthus annus; Triticum aestivum zea mays; Phaseolus vulgaris; phragmitis communis; Oryza sativa; Phaseolus radiates; Gingiber officinale; Festuca anundinacea; Solanum lycopersicon; Cicer aritinum

Heavy metal Oryza sativa; Arabidopsis thaliana; stress Nicotiana tabacum; Solanum lycopersicon; Panax ginseng; Brassica junica; Pisum sativum; Triticum aestivum; Citrus grandis; Hibiscus moscheutos; Artemisia annua; Phaseolus vulgaris; Secale cereal; Lolium perene; Vigna unguiculata; Antiaris toxicaria; Glycene max; Lupinus luteus; Arachis hypogeae; Pistia stratiotes

Ozone stress Arabidopsis thaliana; Nicotiana tabacam; Ginkgo biloba

\section{UV-B Zea mays; Betula pendula; Pisum sativum; Solanum tuberosum; Helianthus annuus; Glycene max}

Wounding Arabidopsis thaliana; Pisum sativum; stress Nicotiana tabacum; Helianthus annus; Vicia faba; Triticum aestivum $\begin{array}{ll}\begin{array}{l}\text { Flooding } \\ \text { stress }\end{array} & \begin{array}{l}\text { Arabidopsis thaliana; Hordeum vulgare; } \\ \text { Brassica japonicum }\end{array}\end{array}$ stress Brassica japonicum
ROS scavenging enzymes, alleviated the expression of HSPs,

$\mathrm{NO}$ acts as signal molecule for the stress response, protects the plant from heat stress induced oxidative stress, plays an important role in $\mathrm{H}_{2} \mathrm{O}_{2}{ }^{a}$ metabolism

NO helps challenge heavy metal stress by chelating the heavy metal at the root zone and preventing their accumulation in plant, regulating gene transcription level of $\mathrm{APX}^{\mathrm{a}} \mathrm{GR}^{\mathrm{a}}$ and $\mathrm{DHA},{ }^{\mathrm{a}}$ increases the plasma membrane transport activity, and GSNOR ${ }^{\mathrm{a}}$ activity

Exogenous application or endogenous synthesis of NO reduces the damaging effects of ozone by activating active oxygen scavenging enzymes

NO functions as a secondary messenger under UV-B stress, reduces UV induced photomorphogenic responses

$\mathrm{Ca}^{2+}$ influx and ROS ${ }^{\mathrm{a}}$ production in $\mathrm{NO}^{\mathrm{a}}$ Huang et al. (2004); Chaki et al. (2011); Si dependent pathway, acts as downstream et al. (2017) transduction

Induces ethylene biosynthesis, maintains Wang et al. (2000) $\mathrm{ATP}^{\mathrm{a}}$ levels to prevent cell death signal molecule in wounding signal

${ }^{a} A B A$, abscisic acid; $A P X$, ascorbate peroxidase; ATP, adenosine triphosphate; DHAR, dehydroascorbate reductase; GR, glutathione reductase; GSNOR, S-Nitrasoglutathione reductase; $\mathrm{H}_{2} \mathrm{O}_{2}$, hydrogen peroxide; $H S P$ s, heat shock proteins; $N O$, nitric oxide; $R O S$, reactive oxygen species. 
mustard has suggested that NO improves photosynthetic performance under salt stress more effectively by enhancing sulfur assimilation (Fatma et al., 2016). Besides, exogenous NO induced the accumulation of protecting molecules including proline, GB, total soluble proteins and total soluble sugars in Cicer arietinum, Linum ustiatissimum, and Morua alba, thus confirming its role in mitigating the adverse effects of salinity stress (Khan et al., 2012).

\subsubsection{NO and Chilling Stress Tolerance in Plants}

Exposure of plants to chilling stress severely limits the crop yields due to its deleterious effects including poor seed germination, stunted growth, delayed flowering, and altered gene expression (Minami et al., 2005). Previous studies have documented that an increase in NO synthesis was associated with cold acclimation in a number of plant species including Arabidopsis thaliana, Helianthus annus, and Capsicum annum (Singh et al., 2009; Zhao et al., 2009; Chaki et al., 2011; Airaki et al., 2012). Although, the temperature range for NO generation has not been clearly defined, its formation has been observed at $8 \mathrm{oC}$ in pea leaves, at $4 \mathrm{oC}$ in Arabidopsis thaliana, and at $0 \mathrm{oC}$ in Chorispora bugeana (Zhao et al., 2009; Liu et al., 2011; Cantrel et al., 2011). Moreover, both NO-sensitive fluorescent probe and chemiluminescence confirmed the accumulation of $\mathrm{NO}$ after $1-4 \mathrm{~h}$ of chilling treatment in Arabidopsis thaliana (Cantrel et al., 2011). This chilling induced NO production was impaired in the nia1nia2 nitrate reductase mutant and $\mathrm{H} 7$ plants overexpressing Arabidopsis hemoglobin 1 ( $A H b 1$ ) further supporting its role in chilling tolerance. While NR has been implicated in NO synthesis in Arabidopsis thaliana, loquat fruit, and Baccaurea ramiflora embryos under chilling stress (Zhao et al., 2009; Cantrel et al., 2011; Bai et al., 2011; Xu et al., 2012a,b), NO synthase inhibitors blocked NO accumulation in Chorispora bungeana suggesting the involvement of both NR and NOS like enzymes for NO synthesis during chilling stress (Baudouin and Jeandroz, 2015). Eventually, a number of other studies also confirmed the role of NO in alleviating cold injury (Zhao et al., 2009; Liu et al., 2011; Yang et al., 2011a,b; Tan et al., 2013; Diao et al., 2016). Recently, transgenic cucumber plants overexpressing CsNOA1 constitutively had greater accumulation of soluble sugars, starch, and a lower chilling damage index, while suppression of CsNOA1 expression resulted in opposite effects. Furthermore, ectopic expression of cucumber CSNOA1 in the A. thaliana noa1 mutant enhanced chilling tolerance and rescued the mutant phenotype due to an increase in endogenous NO levels (Liu et al., 2016). Consistent with this, exogenous NO donor has been shown to induce cold acclimation through the synthesis of osmolytes such as glycine betaine and proline (Ashraf and Foolad, 2007; Zhao et al., 2009; Wang et al., 2012) and reprogramming of lipid signaling and composition. Moreover, NO negated the chilling induced transient synthesis of phytosphingosine phosphate and ceramide phosphate in Arabidopsis thaliana (Cantrel et al., 2011; Lynch and Steponkus, 1987; Kawaguchi et al., 2000). Not only that, SNP treatment alleviated chilling injury in Cynodon dactylon by decreasing malondialdehyde (MDA) content and electrolyte leakage due to the stimulation of superoxide dismutase (SOD) and peroxidase (POD) activities (Fan et al., 2015). Similarly, application of exogenous SNP to cold stressed Triticum aestivum seedlings increased the tolerance by enhancing the synthesis of antioxidant enzymes (Esim et al., 2014).

It was documented that cold stress resulted in highest S-nitrosothiol formation followed by drought, high temperature, and salinity in Brassica juncia. Besides, 20 proteins predominantly associated with plant defense, photosynthesis, glycolysis, and signaling were found to be potentially S-nitrosylated after cold stress treatment in Brassica juncea (Abat and Deswal, 2009; Zhao et al., 2009). Importantly, Rubisco carboxylase is one among those enzymes shown to be inactivated by cold stress induced S-nitrosylation (Abat and Deswal, 2009). Collectively, the emerging picture suggests that NO regulates cold stress signaling by cell wall remodeling and promoting ROS detoxification in plants.

\subsubsection{NO and High Temperature Stress Tolerance in Plants}

Heat stress is defined as temperature above the optimal temperature that causes an irreversible damage to the growth and metabolism of plants (Johkan et al., 2011; Yamori et al., 2014; Awasthi et al., 2016; Santisree et al., 2017). Heat stress promoted NOS and GSNOR activities thereby increasing the accumulation of $\mathrm{NO}$ and S-nitrosothiols in plants suggesting a role for $\mathrm{NO}$ in heat stress mitigation (Yu et al., 2014). While exogenous application of NO donors has been able to reduce heat-induced cellular damage, depleting endogenous NO levels by cPTIO reversed these effects, establishing the functional specificity of $\mathrm{NO}$ in plant heat stress amelioration (Hasanuzzaman et al., 2013). The importance of NO homeostasis in heat stress tolerance has been highlighted by a null mutation in atgsnor1-3/hot5-2 locus or RNAi line of Arabidopsis thaliana, where overaccumulation of $\mathrm{NO}$ correlated with high heat sensitivity (Parankusam et al., 2017). Further, NO scavenger could able to rescue heat sensitivity of these mutant lines of Arabidopsis thaliana (Parankusam et al., 2017). 
Another major challenge under heat stress is the maintenance of membrane integrity. Exogenous application of SNP reduced electrolyte leakage and MDA content that substantially enhanced the survival percentage of Zea mays seedlings ( $\mathrm{Li}$ et al., 2013b). In another study, SNP treatment recovered RWC, chlorophyll content, electrolyte leakage in heat stressed Gingiber officinale leaves ( $\mathrm{Li}$ et al., 2013a). Heat stress reduced chlorophyll (chl) biosynthesis and caused great damage to photosynthetic apparatus followed by reduced yield (Parankusam et al., 2017). NO has been shown to negate this heat induced chlorophyll loss and also maintain the activity of photosystem II thereby sustaining photosynthesis in plants (Pospísil, 2016). For example, pretreatment of SNP resulted in enhanced photosynthetic electron transport in heat stressed Festuca arundinacea (Chen et al., 2013). Similarly, SNP application reduced the rate of nonphotochemical quenching in heat shocked Triticum aestivum leaf discs and diverted more energy to PSII (Hossain et al., 2011). However, excess NO has been shown to inhibit electron transport by reversibly binding to thylakoid membrane complexes of Pisum sativum (Ziogas et al., 2013). Furthermore, heat-induced structural and functional changes in the thylakoid membrane often result in ROS formation (Pospísil, 2016). Several studies evident the ability of NO in maintaining the cellular redox homeostasis by neutralizing harmful ROS produced by heat stress (Ziogas et al., 2013). Pretreatment with SNP enhanced ascorbate and glutathione contents and activities of antioxidant enzymes including monodehydroascorbate reductase, dehydroascorbate reductase, and glyoxalase I and II in heat stressed Triticum aestivum seedlings (Hasanuzzaman et al., 2012). Additionally, foliar application of SNP enhanced carotenoid levels thereby protecting against photooxidative damage caused by heat stress in Chrysanthemum morifolium (Yang et al., 2011a,b). However, prolonged heat stress was found to induce nitrosative stress in Pisum sativum due to increased S-nitrosylation (SNO) content (Parankusam et al., 2017). SNP pretreatment also helped in osmotic adjustment under heat stress by upregulating the P5CS gene in Oryza sativa seedlings and reducing putricine (PUT)/polyamine (PAs) ratio in Gingiber officinale (Uchida et al., 2002). It was shown that nearly 13 tyrosine-nitrated proteins including enzymes like ferredoxin-NADP oxidoreductase and carbonic anhydrase got induced by heat stress in Chrysanthemum morifolium seedlings (Chaki et al., 2011). However, detailed global molecular profiling by omics approaches are needed to increase our knowledge on the effect of $\mathrm{NO}$ and NO-mediated PTMs at subcellular level to mitigate nitrosative stress impacts induced by high heat.

\subsubsection{NO and Heavy Metal Stress Tolerance in Plants}

Heavy metals are a group of metals and metalloids that can alter the normal metabolic processes and become toxic to the growth of the plant beyond permissible concentrations (Sahay and Gupta, 2017; Oz et al., 2015). Copper $(\mathrm{Cu})$, cadmium (Cd), aluminum (Al), and arsenic (Ar) are the most commonly reported heavy metals toxic to plants (Sahay and Gupta, 2017). They accumulate in the soil due to the application of fertilizers, mining, agricultural wastes, application of sewage sludge, and industrial activities. Once they enter into the plant cells through plasma membrane transporters, they affect the plants by direct interaction with DNA, proteins, and binding to atoms such as oxygen, sulfur, etc. (Choudhury et al., 2013; ChmielowskaBak et al., 2014). NO mitigate heavy metal stresses in plants mainly by two strategies. One, NO helps plants to challenge metal stress conditions by upregulation of antioxidant enzyme activity or by upregulation of defensive gene expression (Ortega-Galisteo et al., 2012). Two, NO regulates cellular free metal concentration either by excluding the heavy metal entry into the root or by preventing their cellular accumulation to a toxic level (Oz et al., 2015). However, the results are sometimes contradictory, where on one hand several reports evidence the protective role of NO in alleviating heavy metal stress adversaries, but on the other hand, NO together with heavy metals has been shown to contribute to the toxicity and growth inhibition of plants (Wang et al., 2014; Yun et al., 2016). Hence, more detailed studies will facilitate understanding of the exact roles of $\mathrm{NO}$ in regulating cellular responses to heavy metals (Xiong et al., 2010).

Studies suggested an increased NO production in the $\mathrm{Cu}$-treated plants mainly in the root tissues (Tewari et al., 2008). Moreover, application of SNP effectively reduced Cu-induced toxicity and $\mathrm{NH}_{4}{ }^{+}$ accumulation in the leaves of Oryza sativa as well as in the adventitious roots of Panax ginseng (Tewari et al., 2008). Experiments showed the ability of $\mathrm{NO}$ in alleviating the growth inhibition induced by $\mathrm{CuCl}_{2}$ due to the induction of $\mathrm{H}^{+}$-ATPase activity in plasma membrane and also by enhancing the antioxidant system through regulating GSH/GSSG ratio (Yu et al., 2014; Wang et al., 2015; Xu et al., 2010a,b). In addition to regulation of antioxidant defense, $\mathrm{NO}$ is also shown to increase the RuBisCo activity and mineral uptake in $\mathrm{Cu}$-stressed Nicotiana tabacum and Loleumk perenne respectively (Petó et al., 2011; Khairy et al., 2016).

Al affects crop growth and yield in acidic soils mainly by inhibiting uptake of minerals and nutrients. Exogenous application of SNP alleviated the effects of $\mathrm{Al}$ on root elongation in Hibiscus moscheutos (He et al., 
2012a,b). Moreover, SNP treatment promoted Al immobilization in roots by improving the root secretion of malate and citrate there by preventing $\mathrm{Al}$ accumulation inside the cells in Artemisia annua (He et al., 2012a,b; Aftab et al., 2012). Not only that, NO also enhances $\mathrm{Al}$ tolerance by regulating hormonal equilibrium in root apices of Secale cereale and Triticum aestivum (Sun et al., 2014). Besides, SNP treatment improved photosynthetic efficiency, gene transcription, and activity of major antioxidant enzymes of $\mathrm{Al}-$ stressed plants (Aftab et al., 2012; Sun et al., 2014; Bai et al., 2011; Sadeghipour, 2016). While increase in endogenous NO level has been observed by Cd-stress in soybean cell suspension cultures (Kopyra, 2004) and in roots of Lupinus luteus seedlings (ArasimowiczJelonek et al., 2011), a severe decline in the NOSdependent NO production was observed under $\mathrm{Cd}$ stress in Pisum sativum leaves (Rodríguez-Serrano et al., 2009). Studies indicate that NO protects chlorophyll against cadmium stress in Helianthus annuus (Laspina et al., 2005; Groppa et al., 2008) and Cu stress in Lolium perenne (Dong et al., 2014). In another study, NO-induced Cd tolerance in Oryza sativa has been attributed to the ability of $\mathrm{NO}$ to restrict $\mathrm{Cd}$ entry by increasing pectin and hemicellulose content in the cell wall of roots, promoting phosphotic acid accumulation and phospholipase D activity in the plasma membrane (Xiong et al., 2009; Jhanji et al., 2012; Yang et al., 2016). Moreover, SNP treatment significantly decreased the level of ROS and lipid peroxidation due to the increased activities of antioxidant enzymes in $\mathrm{Cd}$ exposed Lolium perenne seedlings (ArasimowiczJelonek et al., 2011; Bai et al., 2011). Interestingly, SNP decreased the translocation of $\mathrm{Cd}$ metal in roots and stems of Arachis hypogeae followed by an increase in photosynthetic activity (Dong et al., 2014). Similarly, SNP treatment increased antioxidant enzyme activities in As stressed Triticum aestivum (Hasanuzzaman et al., 2013). Exogenous application of SNP also decreased As accumulation, which resulted in improved seed germination in mung bean (Ismail, 2012; Leterrier et al., 2012).

Zinc $(\mathrm{Zn})$ is another essential micronutrient that becomes toxic to plants at higher concentration (Sahay and Gupta, 2017). Roots of Triticum aestivum display enhanced NOS activity followed by NO generation when exposed to Zn (Duan et al., 2007). Besides, exogenous $\mathrm{NO}$ also impacts $\mathrm{Zn}$ translocation from root to shoot, increase ascorbate content in Triticum aestivum plants (Buet et al., 2014). Meanwhile, NO treated Triticum aestivum plants show a decrease in $\mathrm{H}_{2} \mathrm{O}_{2}$ and nonprotein thiol content under Fe deficient conditions (Buet et al., 2014). Reports suggest that NO increased uptake of Fe and magnesium that subsequently resulted in improved chlorophyll synthesis, photosynthesis, and transpiration (Kong et al., 2016). Similarly, Nickel (Ni) induced oxidative stress was alleviated by exogenous application of NO in Brassica junica, Solanum lycopersicum, and Triticum aestivum (Mihailovic and Drazic, 2011; Kazemi, 2012; Wang et al., 2010a,b). Another study points to the reduced manganese $(\mathrm{Mn})$ toxicity due to NO donor treatment in excised rice leaves (Srivastava and Dubey, 2012). Collectively, the available data on the involvement of NO in metal stress suggests that $\mathrm{NO}$ alleviates heavy metal toxicity by attenuating oxidative stress and enhancing the activity of antioxidant enzymes.

\subsubsection{NO in Ozone Stress Tolerance}

Ozone $\left(\mathrm{O}_{3}\right)$ is an oxidant gaseous molecule present in the stratosphere region protecting Earth from harmful UV-B radiation. The rising surface $\mathrm{O}_{3}$ levels due to urbanization and the industrial revolution has become a serious concern for both for human health and vegetation (Monks et al., 2015). Increased accumulation of NO was observed in Nicotiana tabacam and Arabidopsis thaliana upon ozone exposure, which disappeared following the addition of $100 \mu \mathrm{M} \mathrm{CPTIO} \mathrm{(Ederli} \mathrm{et} \mathrm{al.,} \mathrm{2008;} \mathrm{Ahlfors}$ et al., 2009; Xua et al., 2012). Moreover, Atnoa1/rif1 mutant with decreased $\mathrm{NO}$ accumulation was found to be $\mathrm{O}_{3}$ sensitive. Conversely, the $\mathrm{O}_{3}$-sensitive $r c d 1$ mutant was found to be an NO overproducer suggesting the significance of $\mathrm{NO}$ generation during $\mathrm{O}_{3}$ stress response. $\mathrm{NO}$ is required to modify hormone biosynthesis and signaling, and associated gene expression in plants during $\mathrm{O}_{3}$ exposure (Ahlfors et al., 2009). Although both NO and ethylene dependent pathways are required for the $\mathrm{O}_{3}$-induced upregulation of alternative oxidase $A O X 1 a$, only $\mathrm{NO}$ is indispensable for the activation of $A O X 1 a$ gene expression. Activating alternative oxidase pathway is essential to reducing the formation of reactive oxygen species and thus allowing increased carbon flux through the tricarboxylic acid cycle to provide carbon skeletons for other cellular processes under limited ADP supply (Ederli et al., 2008). Interestingly, acute ozone fumigation of poplar has changed the total nitrite and nitrosothiol contents of leaves and altered the homeostasis of 32 S-nitrosylated proteins (Vanzo et al., 2014). Together, a total of 172 proteins have been shown to be Snitrosylated in poplar callus and leaf due to ozone fumigation. Besides, ozone fumigation enhanced the activity of phenylalanine ammonia-lyase (PAL) by denitrosylation in poplar (Vanzo et al., 2014).

\subsubsection{NO in UV-B Tolerance of Plants}

In recent years the depleting ozone layer is allowing more UV-B radiation $(280-320 \mathrm{~nm})$ to reach Earth's 
surface. Exposure to high doses of UV-B radiation leads to numerous harmful consequences in plant cells including dimers formation in DNA, genomic instability, overproduction of ROS, lipid peroxidation, followed by damage to the photosynthetic apparatus and membranes, and ultimately to PCD (Lytvyn et al., 2016; Krasylenko et al., 2013). Plants initiate various defense reactions including the activation of ROS scavenging systems, synthesis of UV-absorbing phenolic compounds, activation of DNA repair mechanisms, and upregulation of UV-B sensitive oxidative defense genes (Hideg et al., 2013). Studies indicate that upregulation of chalcone synthase gene responsible for flavonoid production by UV-B requires NO in Arabidopsis thaliana (Mackerness et al., 2001), while NO scavengers counteract this upregulation further asserting $\mathrm{NO}^{\prime} \mathrm{s}$ role in flavonoid accumulation. A recent study suggests that treatment of Arabidopsis thaliana with SNP donor recovered the plant from UV-B inhibited root growth and altered morphology as well as had damaging effects on microtubular organization (Krasylenko et al., 2013). Similarly, a role of NO in UV-B induced stem elongation has been suggested for Pisum sativum. Although NOS-like source for NO generation during UV-B stress has been suggested in Ginkgo biloba (Tossi et al., 2012), the mammalian NOS inhibitors had no effect on UV-B-induced NO generation (Zhang et al., 2011). Conversely, a direct correlation between NR activity, $\mathrm{NO}$ emission, and nitrite $\left(\mathrm{NO}_{2}{ }^{-}\right)$has been shown to be essential for flavonoid accumulation under UV-B radiation, while cPTIO abolished this response in Betula pendula (Zhang et al., 2011).

Homozygous mutants of Arabidopsis thaliana deficient for inositol-3-phosphate synthase 1 (atips1), a key enzyme for biosynthesis of myo-inositol and its derivatives, displayed greater tissue-specific resistance to the action of UV-B. Moreover, pretreatment with low doses of SNP before UV-B exposure resulted in a tissue-specific protective effect in wild type, which was further enhanced in atips1 (Lytvyn et al., 2016). Exogenous NO partially alleviated the UV-B effect by a decrease in chlorophyll content and oxidative damage to the thylakoid membrane in bean seedlings. In addition, the enhanced NO levels also help in maintaining cellular redox homeostasis under UV-B stress by activating antioxidants and secondary metabolite production. Consistent with this, SNP pretreatment prevented the oxidative stress progression in UV-B exposed Phaseolus vulgaris seedlings by decreasing $\mathrm{H}_{2} \mathrm{O}_{2}$ content, increasing the thiol group content and upregulation of active oxygen scavenging genes. Moreover, NO scavenger or inhibitor or cGMP inhibitor partially prevented the negative impact of UV-B on pollen germination and pollen tube growth suggesting the operation of cGMP mediated NO pathway during
UV-B responses of Paulownia tomentosa. However, cataloguing of NO responsive genes, proteins, and other signaling partners under UV-B stress would decipher NO-dependent mechanisms of UV-B stress tolerance in plants.

\subsubsection{NO in Wounding Stress Tolerance}

Wounding stress in plants represents a range of mechanical injuries caused by both biotic (herbivory and infect feeding) and abiotic stresses (raining, wind, touching, hailing, etc.) (Gilroy et al., 2016). In cultivated crops, wounding may also occur during manual manipulation such as harvesting, handling, and storage. In Pisum satioum leaves, NO accumulated $4 \mathrm{~h}$ after wounding followed by an increase in the content of SNOs due to the induction of NOS and GSNOR activities (Gilroy et al., 2016). Interestingly, wounding triggered accumulation of GSNO and some other SNOs due to the downregulation of GSNOR activity, but without affecting the $\mathrm{NO}$ content in Helianthus annus hypocotyls (Chaki et al., 2011). The NO released under wounding in turn acts as a signal to cause a transient increase in the cytoplasmic $\mathrm{Ca}^{2+}$ concentration at the site of injury in Vicia faba and Nicotiana tabacum cell suspensions (Gilroy et al., 2016). More recently, extracellular ATP leaking from the injured cells was also suggested to act as a signal to carry stress response to surrounding healthy cells in plants as well as in animals (Casalongué et al., 2015). Intriguingly, extracellular ATP induced activation of $\mathrm{Ca}^{2+}$ influx, ROS production, and altered gene expression was also mediated through NO-dependent pathway (Chivasa et al., 2005; Song et al., 2008). Another study revealed that systemic accumulation of $\mathrm{H}_{2} \mathrm{O}_{2}$ and $\mathrm{NO}$ at local wound site enhanced cross tolerance to freezing in untreated systemic leaves in Triticum aestivum (Si et al., 2017). The mechanical stress induced NO burst has also been implicated in DNA fragmentation in Arabidopsis thaliana (Garcês et al., 2001). Although these results strongly support the critical role played by NO in wounding stress in plants, additional work is required to understand the detailed mechanism of its signaling in plants.

\subsubsection{NO in Flooding Stress Tolerance}

Flooding is a major limiting factor for crops growing on as much as $12 \%$ of the world's soils that suffer excess water (Bailey-Serres et al., 2012). In general, plants respond to flooding by forming root aerenchyma, initiating a hyponastic response and by initiating a quiescence phase to conserve bioenergy. The decline in mitochondrial respiration under hypoxia 
leads to an increase in NADH and a drop in ATP levels. Flooding causes anaerobic conditions leading to intracellular $\mathrm{NO}_{2}$ accumulation as an alternative source of $\mathrm{NAD}^{+}$to maintain a high glycolytic rate, which is often converted to NO by cytosolic NR in Hordeum vulgare roots (Dongen and Licausi, 2015). Besides NR, hypoxic stress-induced hemoglobin $(\mathrm{Hb})$ also regulates NO levels in Zea mays cell cultures and Medicago sativa root cultures (Igamberdiev et al., 2005). The regulation of NO levels was also shown to be critical by maintaining high nitrate $\left(\mathrm{NO}^{3-}\right)$ concentrations and stress-induced $\mathrm{Hb}$ in Arabidopsis thaliana (Wang et al., 2000). Both increased $\mathrm{Hb}$ gene expression and activation of NR enzyme induce the generation of NO in order to maintain ATP levels and to prevent cell death under flooding (Igamberdiev et al., 2005). Detoxification of NO and nitrite produced due to denitrification in soybean nodules after flooding requires a functional plant oxygen carrier leghemoglobin (Sánchez et al., 2010). Hence, the reduction of nitrate by Brassica japonicum bacteroids leads to legheamoglobin-NO production within Glycene max nodules in response to hypoxia (Meakin et al., 2007). Moreover, flooding caused a decrease in nifH expression and nitrogenase activity in wild-type soybean nodules (Sánchez et al., 2010). Nevertheless, uncertainties not only on the source of $\mathrm{NO}$, but also the complete NO-mediated mechanism under flooding, need to be thoroughly addressed.

\subsection{NO AND PHYTOHORMONES CROSSTALK IN ABIOTIC STRESS TOLERANCE}

Owing to its rich chemistry, NO involves interactions with a number of signaling molecules and phytohormones during abiotic stress responses in plants (Sami et al., 2017). A series of experimental reports established that NO modulates the biosynthesis, distribution, and degradation of phytohormones. The past decade has witnessed the interaction of NO with almost all the plant hormones mostly as a second messenger in the signaling cascades of various plant developmental and stress responses. For example, NO has been intensively involved with hormones such as GA, JA, ET, CK, and AUX during the regulation of stomata under environmental stress conditions (Sami et al., 2017; Nawaz et al., 2017). A majority of the research publications highlighted the interaction between ABA and NO under drought stress. Interestingly, both synergistic and antagonistic crosstalks have been described between NO and ABA depending on the physiological response and tissue during stress (Santisree et al., 2015). For example, NO is involved in the $\mathrm{ABA}$ induced stomatal closure by selectively activating intracellular $\mathrm{Ca}^{2+}$ channels through a cGMP/cADPR-dependent signaling pathway in Vicia faba guard cells (Mioto and Mercier, 2013). ABA induced NOS activity and NO levels have been shown to improve the thermotolerance of Phragmites communis calluses (Song et al., 2008). Guo et al. (2014) found that coordinated action between $\mathrm{NO}$ and ABA upregulated cold-induced MfSAMS1 expression, resulting in enhanced acclimation against cold stress. Similarly, the study using vp14 maize mutant defective in ABA synthesis suggests that the requirement of $A B A$ accumulation after UV-B perception is critical to trigger the elevation of cytosolic $\mathrm{Ca}^{2+}$ concentration resulting in enhanced NOS-mediated NO production (Tossi et al., 2012). NO and Aux display an extensive signaling crosstalk during the development and remodeling of root architecture for the extraction of more water under drought stress Simontacchi et al., 2013. Additionally, several developmental studies indicate the involvement of $\mathrm{NO}$ in auxin mediated lateral and adventitious roots formation (Simontacchi et al., 2013). In agreement with this, interplay of NO-AUX was also evident by significant reduction in root meristem size in salt treated Arabidopsis thaliana. Interestingly, supplementation of NO reduces AUX degradation by downregulating IAA oxidase activity in Medicago truncatula under Cd stress as well as under Al toxicity in Triticum aestivum and Secale cereale (Xu et al., 2010a,b; He et al., 2012a,b). In another study, $\mathrm{Cd}$ induced $\mathrm{NO}$ accumulation promoted the stabilization of AUX repressor protein IAA17 in Arabidopsis through suppression of AUX carriers PIN1/ 3/7 (Kovacs and Lindermayr, 2013). Further, a positive correlation between AUX and NO has been suggested in enhancing ferric-chelate reductase activity in a Fedeficient Arabidopsis thaliana plants (Chen et al., 2010). Similar to auxin, a positive interaction between NO and CK under drought was reported, wherein the treatment with CK regulated photosynthetic machinery by promoting NO signaling in Zea mays (Shao et al., 2010). Conversely, CK reduced NO levels to trigger stomatal opening in dark grown Vicia faba seedlings (Santisree et al., 2015). Foliar application of SNP delayed saltinduced leaf senescence by upregulating the expression of isopentenyl transferase (IPT) in Gossypium hirsutum seedlings (Kong et al., 2016). However, more studies are required to provide strong evidence for $\mathrm{NO}$ and $\mathrm{CK}$ interaction under abiotic stress. Previous studies have reported the participation of NO in SA-induced stomatal closure in Arabidopsis thaliana (Sun et al., 2010). A coordinated action of NO and SA was found to mitigate the damaging effects of osmotic stress in Triticum aestivum seedlings (Naser alavi et al., 2014). In another study, combined application of $\mathrm{NO}$ and SA improved $\mathrm{Ca} 2+/$ $\mathrm{Mg} 2+$ absorption, increased proline accumulation while mitigating the salt stress adversaries in Glycene max 
seedlings. Similarly, combination of NO and SA has been shown to alleviate the toxic effects of $\mathrm{Ni}$ in Brassica napus and Cd in Arachis hypogaea (Kazemi et al., 2010; Xu et al., 2015).

The critical balance between NO and ethylene seems to be essential to prevent cold-induced injury during postharvest fruit ripening and seed conservation (Bai et al., 2011; Xu et al., 2012a,b). Akin to SA, ET induced by Cd stress reduced NO levels in Pisum sativum and Glycine max seedlings. Besides, both $\mathrm{NO}$ and ET are required to upregulate the plasma membrane $\mathrm{H}^{+}$-ATPase and alternative respiratory pathway to modulate ion homeostasis for improved salt tolerance (Wang et al., 2010a,b). A few studies indicate that NO treatment induces production of ethylene to regulate a few $\mathrm{O}_{3}$ induced genes (Ahlfors et al., 2009). Additionally, NO plays a key role in programmed cell death (PCD) and the hyponastic responses by inducing ethylene biosynthesis during flooding stress (Pasqualini et al., 2012). It is well known from the literature that coordinated action between $\mathrm{NO}$ and plant hormones including ABA, JA, GA, and CK induce thermotolerance by activating the antioxidant machinery and upregulating the heat shock protein expression in plants (Zandalinas et al., 2016). Further, pretreatment of Zea mays seedlings with $\mathrm{H}_{2} \mathrm{O}_{2}$ rapidly induced endogenous $\mathrm{H}_{2} \mathrm{O}_{2}, \mathrm{NO}$, and $\mathrm{H}_{2} \mathrm{~S}$ accumulation under heat stress, which was reversed by $\mathrm{H}_{2} \mathrm{O}_{2}$ scavenger dimethylthiourea and $\mathrm{NO}$ scavenger cPTIO, indicating that $\mathrm{H}_{2} \mathrm{O}_{2}$ induced heat stress tolerance was involved in the crosstalk between downstream components $\mathrm{NO}$ and $\mathrm{H}_{2} \mathrm{~S}$ ( $\mathrm{Li}$ et al., 2013b). An interlink between NO and GA has also observed in promoting apical root growth in Al-stressed Triticum aestivum roots (He et al., 2012a,b). Additionally, NO induced reduction in total free polyamines, free put, spermidine ( $\mathrm{Spd}$ ), and polyamine oxidase activity was reported in salt stressed

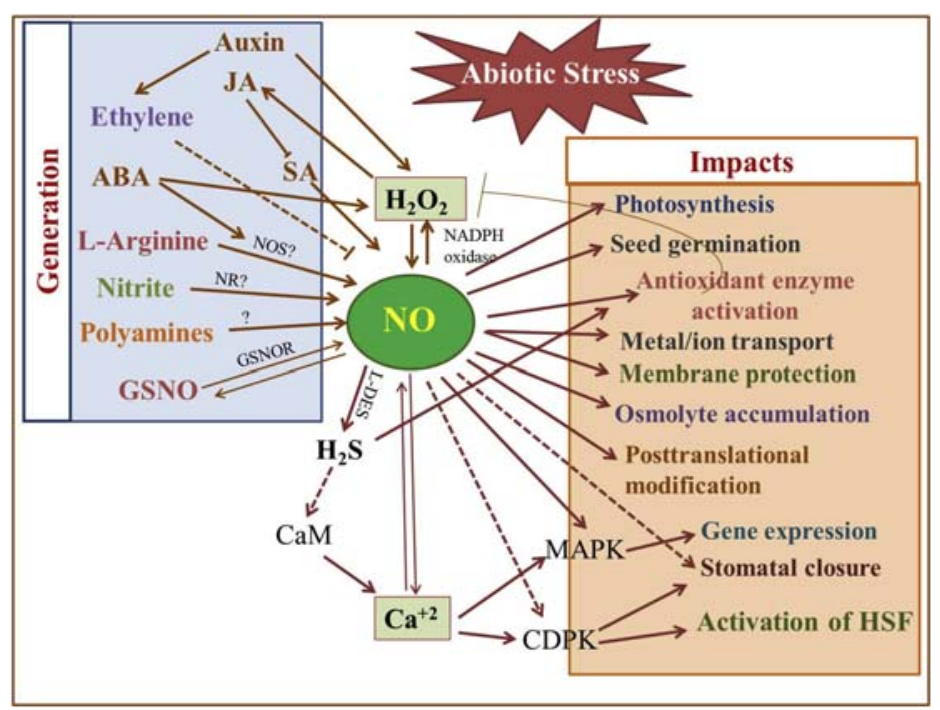

cucumber seedlings (Fan et al., 2013). Despite this evidence, the complete understanding of mechanisms underlying the intersection of NO signaling with other signaling molecules requires further study to explain how NO concomitantly interacts with hormone-related proteins at the posttranscriptional or translational level.

\subsection{CONCLUDING REMARKS AND FUTURE PERSPECTIVES}

As climate change continues, our appreciation of how plants respond to stress is becoming increasingly significant. Accumulating evidence demonstrated a reversible accumulation of $\mathrm{NO}$ under a range of abiotic stresses (Fig. 25.1). Several studies indicate that the NO accumulation potentially enhances plant survival under stress conditions mostly by increasing cellular antioxidant defense. However, the functionality of $\mathrm{NO}$ accumulation depends on its concentration, location, duration, plant growth, and developmental stage and many internal and external factors. Although $\mathrm{NO}$ is recognized as a multitasking molecule with innumerable functions in plant stress responses, many questions remain unanswered. Most importantly, how does the stress-specific NO accumulation translate into a biological function that helps in stress amelioration? Another pertinent question is how the small NO molecule can influence modification of a massive number of molecules that enhance plants tolerance to stress. Since the sensitivity to stress varies from species to species, the stress ameliorating effects of $\mathrm{NO}$ in the given species under given stress may not be extrapolated to other species with different sensitivity. In some of the experiments, in vitro stress treatments used may not be comparable with the natural stress conditions that occur at

FIGURE 25.1 Schematic illustration for synthesis and functional roles of nitric oxide (NO) in plant responses to abiotic stress.Abiotic stress factors in plants led to the generation of reactive oxygen species (ROS) and nitric oxide (NO), which can effect gene expression. NO participates actively in signal transduction by altering the expression of a number of genes, such as protein kinases and transcription factors (TFs), triggering various physiobiochemical responses, including changes in general metabolism and ion/metabolite transport, stress responses, as well as protein degradation. NO either stimulates (normal end arrow) or inhibits (blunt end arrow) plant processes in coordination with various other signaling molecules under abiotic stress. The dotted line denotes the pathways not studied clearly. The double-sided arrow indicates the mutual regulation of molecules. $A B A$, abscisic acid; $J A$, jasmonic acid; $M A P K$, mitogen activated protein kinase; NOS, nitric oxide synthase; $N R$, nitrite reductase; $R O S$, reactive oxygen species; $S A$, salicylic acid; $T F$, transcription factor. 
the field level. Therefore, how the NO signaling pathways function in an open environment can only be addressed by field level studies. Further, detailed studies that are more directly linked to yield, such as the role of NO in increasing protection to photosynthesis and osmolyte accumulation under stress, will add more value to the established antioxidant role of NO.

Despite growing knowledge about NO-mediated plant functions, detailed information on its origin and signaling under individual stress conditions has so far been elusive. Hence, the genetic screens used to explore the consequences of NO deficiency may not be conclusive unless multiple NO generating sources are disabled simultaneously. Clearly, blocking the NO generating sources without complete elucidation of the responsible molecular identities presents a big challenge for genetic and transgenic researchers. Although the use of exogenous donors/inhibitors offers an alternative until the molecular identities of $\mathrm{NO}$ generation in plants are better deciphered, it is critical to consider the stress treatment and the tissue type used to critically analyze the results to avoid ambiguous conclusions. Moreover, the method of NO application needs to be validated considering the cost, impact, and ease of use under large scale applications. In this context, encapsulation of NO donors in nanomaterials looks promising and has several advantages such as improved efficiency and controlled release over direct exogenous applications (Savvides et al., 2016).

Although there is sufficient evidence on the potential involvement of $\mathrm{NO}$ in various hormone-mediated plant growth processes, the crosstalk studies remain fragmental under individual stress conditions. Moreover, the high throughput -omics analysis of NO-mediated stress responses remain largely untapped, but more research in this direction will facilitate research aimed at the identification of NO targets in stress conditions. Together, exploration of NO metabolism and its interacting partners in plants and their physiological relevance under stress will be helpful to enrich our knowledge on NO functions.

\section{Acknowledgements}

This work was supported by a financial grant to PS through the INSPIRE Faculty Award (IFA12-LSPA-08) from the Department of Science and Technology, Government of India, and partial funding from the CGIAR Research Program on Grain Legumes.

\section{References}

Abat, J.K., Deswal, R., 2009. Differential modulation of S-nitrosoproteome of Brassica juncea by low temperature: change in S-nitrosylation of Rubisco is responsible for the inactivation of its carboxylase activity. Proteomics. 9, 4368-4380. Available from: https://doi.org/10.1002/pmic.200800985.

Abeer, H., Abd_Allah, E.F., Alqarawi, A.A., El-Didamony, G., Alwhibi, M., Egamberdieva, D., et al., 2014. Alleviation of adverse impact of salinity on faba bean (Vicia faba L.) by arbuscular mycorrhizal fungi. Pak. J. Bot. 46, 2003-2013.

Adimulam, S.S., Bhatnagar-Mathur, P., Santisree, P., 2017. Interaction of nitric oxide with phytohormones under drought stress. J. Plant Stud. 6, 58-61.

Aftab, T., Khan, M.M.A., Naeem, M., Idrees, M., da Silva, J.A.T., Ram, M., 2012. Exogenous nitric oxide donor protects Artemisia annua from oxidative stress generated by boron and aluminium toxicity. Ecotoxicol. Environ. Saf. 80, 60-68. Available from: https: / / doi.org/10.1016/j.ecoenv.2012.02.007.

Ahlfors, R., Brosché, M., Kollist, H., Kangasjärvi, J., 2009. Nitric oxide modulates ozone-induced cell death, hormone biosynthesis and gene expression in Arabidopsis thaliana. Plant J. 58, 1-12.

Ahmad, P., Abdel Latef, A.A., Hashem, A., Abd_Allah, E.F., Gucel, S., Tran, L.S.P., 2016. Nitric oxide mitigates salt stress by regulating levels of osmolytes and antioxidant enzymes in chickpea. Front. Plant Sci. 7, 347. Available from: https://doi.org/10.3389/ fpls.2016.00347.

Airaki, M., Leterrier, M., Mateos, R.M., Valderrama, R., Chaki, M., Barroso, J.B., et al., 2012. Metabolism of reactive oxygen species and reactive nitrogen species in pepper (Capsicum annuum L.) plants under low temperature stress. Plant Cell Environ. 35, 281-295. Available from: https://doi.org/10.1111/j.1365-3040. 2011.02310.x.

Arasimowicz-Jelonek, M., Floryszak-Wieczorek, J., Gwóźdź, E.A., 2011. The message of nitric oxide in cadmium challenged plants. Plant Sci. 181, 612-620.

Arc, E., Galland, M., Godin, B., Cueff, G., Rajjou, L., 2013. Nitric oxide implication in the control of seed dormancy and germination. Front. Plant Sci. 4, 346.

Asgher, M., Per, T.S., Masood, A., Fatma, M., Freschi, L., Corpas, F.J., et al., 2017. Nitric oxide signaling and its crosstalk with other plant growth regulators in plant responses to abiotic stress. Environ. Sci. Poll. Res. 24, 2273-2285. Available from: https:// doi.org/10.1007/s11356-016-7947-8.

Ashraf, M., Foolad, M., 2007. Roles of glycine betaine and proline in improving plant abiotic stress resistance. Environ. Exp. Bot. 59, 206-216. Available from: https://doi.org/10.1016/j.envexpbot. 2005.12.006.

Astier, J., Lindermayr, C., 2012. Nitric oxide-dependent posttranslational modification in plants: an update. Int. J. Mol. Sci. 13, 15193-15208. Available from: https://doi.org/10.3390/ijms131115193.

Astier, J., Loake, G., Velikova, V., Gaupels, F., 2016. Interplay between NO Signaling, ROS, and the Antioxidant System inPlants. Front. Plant Sci. 7, 1731.

Awasthi, R., Bhandari, K., Nayyar, H., 2016. Temperature stress and redox homeostasis in agricultural crops. Redox homeostasis managers in plants under environmental stresses. Front. Environ. Sci. 3, 11. Available from: https://doi.org/10.3389/fenvs.2015.00011.

Bai, X., Yang, L., Yang, Y., Ahmad, P., Yang, Y., Hu, X., 2011. Deciphering the protective role of nitric oxide against salt stress at the physiological and proteomic levels in maize. J. Proteome. Res. 10, 4349-4364. Available from: https://doi.org/10.1021/ pr200333f.

Bailey-Serres, J., Lee, S.C., Brinton, E., 2012. Waterproofing crops: effective flooding survival strategies. Plant Physiol. 160, 1698-1709. Available from: https://doi.org/10.1104/pp.112.208173.

Baudouin, E., 2011. The language of nitric oxide signalling. Plant Biol. 13, 233-242. Available from: https://doi.org/10.1111/ j.1438-8677.2010.00403.x.

Baudouin, E., Hancock, J., 2014. Nitric oxide signaling in plants. Front. Plant Sci. 4, 553. Available from: https://doi.org/10.3389/ fpls.2013.00553.

Baudouin, E., Jeandroz, S., 2015. Nitric Oxide as a Mediator of Cold Stress Response: A Transcriptional Point of View. In: Khan, M., Mobin, M., Mohammad, F., Corpas, F. (Eds.), Nitric Oxide Action in Abiotic Stress Responses in Plants. Springer, Cham. 
Begara-Morales, J.C., Sánchez-Calvo, B., Luque, F., Leyva-Pérez, M.O., Leterrier, M., Corpas, F.J., et al., 2014. Differential transcriptomic analysis by RNA-Seq of GSNO-responsive genes between Arabidopsis roots and leaves. Plant Cell Physiol. 55, 1080-1095. Available from: https://doi.org/10.1093/pcp/pcu044.

Begara-Morales, J.C., Sánchez-Calvo, B., Chaki, M., Mata-Pérez, C., Valderrama, R., Padilla, M.N., et al., 2015. Differential molecular response of monodehydroascorbate reductase and glutathione reductase by nitration and S-nitrosylation. J. Exp. Bot. 66, 5983-5996. Available from: https://doi.org/10.1093/jxb/erv306.

Buet, A., Moriconi, J.I., Santa-María, G.E., Simontacchi, M., 2014. An exogenous source of nitric oxide modulates zinc nutritional status in wheat plants. Plant. Physiol. Biochem. 83, 337-345.

Cai, W., Liu, W., Wang, W.S., Fu, Z.W., Han, T.T., Lu, Y.T., 2015. Overexpression of rat neurons nitric oxide synthase in rice enhances drought and salt tolerance. PLoS. ONE. 10, e0131599. Available from: https://doi.org/10.1371/journal.pone.0131599.

Camejo, D., del Carmen Romero-Puertas, M., Rodríguez-Serrano, M., Sandalio, L.M., Lázaro, J.J., Jiménez, A., et al., 2013. Salinityinduced changes in S-nitrosylation of pea mitochondrial proteins. J. Proteomics. 79, 87-99. Available from: https://doi.org/ 10.1016/j.jprot.2012.12.003.

Cantrel, C., Vazquez, T., Puyaubert, J., Rezé, N., Lesch, M., Kaiser, W.M., et al., 2011. Nitric oxide participates in cold-responsive phosphosphingolipid formation and gene expression in Arabidopsis thaliana. New Phytol. 189, 415-427. Available from: https: / / doi.org/10.1111/j.1469-8137.2010.03500.x.

Casalongué, C.A., Fiol, D.F., D’Ippólito, S., Tonón, C., París, R., 2015. Insights into the participation of nitric oxide and extra cellular ATP in wounding. Nitric Oxide Action in Abiotic Stress Responses in Plants. Springer, Cham.

Chaki, M., Valderrama, R., Fernández-Ocaña, A.M., Carreras, A., Gómez-Rodríguez, M.V., Pedrajas, J.R., et al., 2011. Mechanical wounding induces a nitrosative stress by down-regulation of GSNO reductase and an increase in S-nitrosothiols in sunflower (Helianthus annuus) seedlings. J. Exp. Bot. 62, 1803-1813. Available from: https://doi.org/10.1093/jxb/erq358.

Chen, K., Chen, L., Fan, J., Fu, J., 2013. Alleviation of heat damage to photosystem II by nitric oxide in tall fescue. Photosynth. Res. 116, 21-31. Available from: https:// doi.org/10.1007/s11120-013-9883-5.

Chen, W.W., Yang, J.L., Qin, C., Jin, C.W., Mo, J.H., Ye, T., et al., 2010. Nitric oxide acts downstream of auxin to trigger root ferricchelate reductase activity in response to iron deficiency in Arabidopsis thaliana. Plant Physiol. 110.

Cheong, Y.H., Chang, H.S., Gupta, R., Wang, X., Zhu, T., Luan, S., 2002. Transcriptional profiling reveals novel interactions between wounding, pathogen, abiotic stress, and hormonal responses in Arabidopsis. Plant Physiol. 129, 661-677. Available from: https://doi.org/10.1104/pp.002857.

Chivasa, S., Ndimba, B.K., Simon, W.J., Lindsey, K., Slabas, A.R., 2005. Extracellular ATP functions as an endogenous external metabolite regulating plant cell viability. Plant Cell 17, 3019-3034. Available from: https://doi.org/10.1105/tpc.105.036806.

Chmielowska-Bạk, J., Gzyl, J., Rucińska-Sobkowiak, R., ArasimowiczJelonek, M., Deckert, J., 2014. The new insights into cadmium sensing. Front. Plant Sci. 5, 245. Available from: https://doi.org/ 10.3389/fpls.2014.00245.

Choudhury, S., Panda, P., Sahoo, L., Panda, S.K., 2013. Reactive oxygen species signaling in plants under abiotic stress. Plant Signal. Behav. 8, e23681. Available from: https://doi.org/10.4161/ psb.23681.

Cui, J.X., Zhou, Y.H., Ding, J.G., Xia, X.J., Shi, K.A.I., Chen, S.C., et al., 2011. Role of nitric oxide in hydrogen peroxide-dependent induction of abiotic stress tolerance by brassinosteroids in cucumber. Plant Cell Environ. 34 (2), 347-358.
Deng, Z., Song, S., 2012. Sodium nitroprusside, ferricyanide, nitrite and nitrate decrease the thermo-dormancy of lettuce seed germination in a nitric oxide-dependent manner in light. South Afr. J. Bot. 78, 139-146. Available from: https://doi.org/10.1016/j. sajb.2011.06.009.

Desikan, R., Griffiths, R., Hancock, J., Neill, S., 2002. A new role for an old enzyme: nitrate reductase-mediated nitric oxide generation is required for abscisic acid-induced stomatal closure in Arabidopsis thaliana. Proc. Natl. Acad. Sci. 16314-16318. Available from: https://doi.org/10.1073/pnas.252461999.

Diao, Q.N., Song, Y.J., Shi, D.M., Qi, H.Y., 2016. Nitric oxide induced by polyamines involves antioxidant systems against chilling stress in tomato (Lycopersicon esculentum Mill.) seedling. J. Zhejiang Univ.-SCIENCE B 17, 916-930. Available from: https:// doi.org/10.1631/jzus.B1600102.

Dong, Y.J., Jinc, S.S., Liu, S., Xu, L.L., Kong, J., 2014. Effects of exogenous nitric oxide on growth of cotton seedlings under $\mathrm{NaCl}$ stress. J. Soil Sci. Plant Nutr. 14, 1-13. Available from: https:// doi.org/10.4067/S0718-95162014005000001.

Dongen, J.T., Licausi, F., 2015. Oxygen sensing and signaling. Annu. Rev. Plant. Biol. 66, 345-367.

Duan, X., Su, X., You, Y., Qu, H., Li, Y., Jiang, Y., 2007. Effect of nitric oxide on pericarp browning of harvested longan fruit in relation to phenolic metabolism. Food. Chem. 104 (2), 571-576. Available from: https:/ / doi.org/10.1016/j.foodchem.2006.12.007.

Ederli, L., Meier, S., Borgogni, A., Reale, L., Ferranti, F., Gehring, C., et al., 2008. cGMP in ozone and NO dependent responses. Plant Signal. Behav. 3, 36-37.

Egbichi, I., Keyster, M., Ludidi, N., 2014. Effect of exogenous application of nitric oxide on salt stress responses of soybean. South Afr. J. Bot. 90, 131-136. Available from: https://doi.org/10.1016/j. sajb.2013.11.002.

Esim, N., Atici, O., Mutlu, S., 2014. Effects of exogenous nitric oxide in wheat seedlings under chilling stress. Toxicol. Ind. Health 30, 268-274. Available from: https:/ / doi.org/10.1177/0748233712457444.

Fan, H.F., Du, C.X., Guo, S.R., 2013. Nitric oxide enhances salt tolerance in cucumber seedlings by regulating free polyamine content. Environ. Exp. Bot. 86, 52-59.

Fan, J., Chen, K., Amombo, E., Hu, Z., Chen, L., Fu, J., 2015. Physiological and molecular mechanism of nitric oxide (NO) involved in Bermudagrass response to cold stress. PLoS. ONE. 10, e0132991. Available from: https://doi.org/10.1371/journal. pone.0132991.

Fan, S., Meng, Y., Song, M., Pang, C., Wei, H., Liu, J., et al., 2014. Quantitative phosphoproteomics analysis of nitric oxide-responsive phosphoproteins in cotton leaf. PLoS. ONE. 9, e94261. Available from: https://doi.org/10.1371/journal.pone.0094261.

Fancy, N.N., Bahlmann, A.K., Loake, G.J., 2017. Nitric oxide function in plant abiotic stress. Plant, Cell Environ. 40, 462-472. Available from: https:// doi.org/10.1111/pce.12707.

Fares, A., Rossignol, M., Peltier, J.B., 2011. Proteomics investigation of endogenous S-nitrosylation in Arabidopsis. Biochem. Biophys. Res. Commun. 416, 331-336. Available from: https://doi.org/ 10.1016/j.bbrc.2011.11.036.

Farooq, M., Basra, S.M.A., Wahid, A., Rehman, H., 2009. Exogenously applied nitric oxide enhances the drought tolerance in fine grain aromatic rice (Oryza sativa L.). J Agron Crop Sci 195, 254-261. Available from: https:// doi.org/10.1111/j.1439-037X.2009.00367.x.

Fatma, M., Khan, N.A., 2014. Nitric oxide protects photosynthetic capacity inhibition by salinity in Indian mustard. J. Funct. Environ. Bot. 4, 106-116.

Fatma, M., Masood, A., Per, T.S., Khan, N.A., 2016. Nitric oxide alleviates salt stress inhibited photosynthetic performance by interacting with sulfur assimilation in mustard. Front. Plant Sci. 7, 521. Available from: https:// doi.org/10.3389/fpls.2016.00521. 
Foresi, N., Mayta, M.L., Lodeyro, A.F., Scuffi, D., Correa-Aragunde, N., García-Mata, C., et al., 2015. Expression of the tetrahydrofolate-dependent nitric oxide synthase from the green alga Ostreococcus tauri increases tolerance to abiotic stresses and influences stomatal development in Arabidopsis. Plant J. 82, 806-821. Available from: https://doi.org/10.1111/tpj.12852.

Fröhlich, A., Durner, J., 2011. The hunt for plant nitric oxide synthase (NOS): is one really needed? Plant Sci. 181, 401-404. Available from: https:/ / doi.org/10.1016/j.plantsci.2011.07.014.

Garcia-Mata, Lamattina, L., 2001. Nitric Oxide Induces Stomatal Closure and Enhances the Adaptive Plant Responses against Drought Stress. Plant Physiol. 126, 1196-1204. Available from: http://dx.doi.org/10.1104/pp.126.3.1196.

Garcês, H., Durzan, D., Pedroso, M.C., 2001. Mechanical stress elicits nitric oxide formation and DNA fragmentation in Arabidopsis thaliana. Ann. Bot. (Lond.) 87, 567-574.

Gibbs, D.J., Isa, N.M., Movahedi, M., Lozano-Juste, J., Mendiondo, G.M., Berckhan, S., et al., 2014. Nitric oxide sensing in plants is mediated by proteolytic control of group VII ERF transcription factors. Mol. Cell 53, 369-379. Available from: https://doi.org/ 10.1016/j.molcel.2013.12.020.

Gilroy, S., Białasek, M., Suzuki, N., Górecka, M., Devireddy, A.R., Karpiński, S., et al., 2016. ROS, calcium, and electric signals: key mediators of rapid systemic signaling in plants. Plant Physiol. 171 (3), 1606-1615.

Groppa, M.D., Rosales, E.P., Iannone, M.F., Benavides, M.P., 2008. Nitric oxide, polyamines and Cd-induced phytotoxicity in wheat roots. Phytochemistry 69, 2609-2615. Available from: https:// doi.org/10.1016/j.phytochem.2008.07.016.

Groß, F., Durner, J., Gaupels, F., 2013. Nitric oxide, antioxidants and prooxidants in plant defence responses. Front. Plant Sci. 4, 419. Available from: https://doi.org/10.3389/fpls.2013.00419.

Guo, Q., Wu, B., Chen, W., Zhang, Y., Wang, J., Li, X., 2014. Effects of nitric oxide treatment on the cell wall softening related enzymes and several hormones of papaya fruit during storage. Food Sci. Technol. Int. 20 (4), 309-317.

Gupta, D.K., Inouhe, M., Rodríguez-Serrano, M., Romero-Puertas, M.C., Sandalio, L.M., 2013. Oxidative stress and arsenic toxicity: role of NADPH oxidases. Chemosphere 90, 1987-1996.

Hao, G.P., Xing, Y., Zhang, J.H., 2008. Role of nitric oxide dependence on nitric oxide synthase-like activity in the water stress signaling of maize seedling. J. Integrat. Plant Biol. 50, 435-442. Available from: https: / / doi.org/10.1016/10.1111/j.1744-7909.2008.00637.x.

Hasanuzzaman, M., Nahar, K., Alam, M.M., Fujita, M., 2012. Exogenous nitric oxide alleviates high temperature induced oxidative stress in wheat ('Triticum aestivum'L.) seedlings by modulating the antioxidant defense and glyoxalase system. Austr. J. Crop Sci. 6, 1314.

Hasanuzzaman, M., Nahar, K., Alam, M.M., Roychowdhury, R., Fujita, M., 2013. Physiological, biochemical, and molecular mechanisms of heat stress tolerance in plants. Int. J. Mol. Sci. 14, 9643-9684. Available from: https://doi.org/10.3390/ijms14059643.

Hasanuzzaman, M., Oku, H., Nahar, K., Bhuyan, M.B., Al Mahmud, J., Baluska, F., et al., 2018. Nitric oxide-induced salt stress tolerance in plants: ROS metabolism, signaling, and molecular interactions. Plant Biotechnol. Rep. 1-16.

Hatamzadeh, A., Molaahmad Nalousi, A., Ghasemnezhad, M., Biglouei, M.H., 2015. The potential of nitric oxide for reducing oxidative damage induced by drought stress in two turfgrass species, creeping bentgrass and tall fescue. Grass forage Sci. 70, 538-548.

Hayat, S., Khalique, G., Irfan, M., Wani, A.S., Tripathi, B.N., Ahmad, A., 2012. Physiological changes induced by chromium stress in plants: an overview. Protoplasma 249 (3), 599-611.

He, H., Zhan, J., He, L., Gu, M., 2012a. Nitric oxide signaling in aluminum stress in plants. Protoplasma 249, 483-492. Available from: https:/ / doi.org/10.1007/s00709-011-0310-5.
He, H.Y., He, L.F., Gu, M.H., Li, X.F., 2012b. Nitric oxide improves aluminum tolerance by regulating hormonal equilibrium in the root apices of rye and wheat. Plant Sci. 183, 123-130.

He, Y., Tang, R.H., Hao, Y., Stevens, R.D., Cook, C.W., Ahn, S.M., et al., 2004. Nitric oxide represses the Arabidopsis floral transition. Science 305, 1968-1971. Available from: https://doi.org/ 10.1016/10.1126/science.1098837.

Hideg, É., Jansen, M.A., Strid, Å., 2013. UV-B exposure, ROS, and stress: inseparable companions or loosely linked associates? Trends. Plant. Sci. 18, 107-115. Available from: https://doi.org/ 10.1016/j.tplants.2012.09.003.

Hossain, K.K., Nakamura, T., Yamasaki, H., 2011. Effect of nitric oxide on leaf non-photochemical quenching of fluorescence under heat-stress conditions. Russian J. Plant Physiol. 58, 629-633. Available from: https://doi.org/10.1134/S1021443711030046.

Huang, X., Stettmaier, K., Michel, C., Hutzler, P., Mueller, M.J., Durner, J., 2004. Nitric oxide is induced by wounding and influences jasmonic acid signaling in Arabidopsis thaliana. Planta 218, 938-946. Available from: https://doi.org/10.1007/s00425-0031178-1.

Igamberdiev, A.U., Baron, K., Manac'H-Little, N., Stoimenova, M., Hill, R.D., 2005. The haemoglobin/nitric oxide cycle: involvement in flooding stress and effects on hormone signalling. Ann. Bot. (Lond.) 96, 557-564.

Ismail, G.S.M., 2012. Protective role of nitric oxide against arsenicinduced damages in germinating mung bean seeds. Acta Physiol. Plant. 34, 1303-1311.

Jasid, S., Simontacchi, M., Bartoli, C.G., Puntarulo, S., 2006. Chloroplasts as a nitric oxide cellular source. Effect of reactive nitrogen species on chloroplastic lipids and proteins. Plant Physiol. 142, 1246-1255. Available from: https://doi.org/ 10.1104 /pp.106.086918.

Jhanji, S., Setia, R.C., Kaur, N., Kaur, P., Setia, N., 2012. Role of nitric oxide in cadmium-induced stress on growth, photosynthetic components and yield of Brassica napus L. J. Environ. Biol. 33, 1027.

Johkan, M., Oda, M., Maruo, T., Shinohara, Y., 2011. Crop production and global warming. In: Casalegno, S. (Ed.), Global Warming Impacts-Case Studies on the Economy, Human Health, and on Urban and Natural Environments. InTech.

Kausar, F., Shahbaz, M., 2013. Interactive effect of foliar application of nitric oxide (NO) and salinity on wheat (Triticum aestioum L.). Pak. J. Bot. 45 (SI), 67-73.

Kazemi, N., 2012. Effect of exogenous nitric oxide on alleviating nickel-induced oxidative stress in leaves of tomato plants. Int. J. Agric. Sci. 2, 799-809.

Kazemi, N., Khavari-Nejad, R.A., Fahimi, H., Saadatmand, S., NejadSattari, T., 2010. Effects of exogenous salicylic acid and nitric oxide on lipid peroxidation and antioxidant enzyme activities in leaves of Brassica napus L. under nickel stress. Sci. Hortic. (Amsterdam) 126 (3), 402-407.

Khairy, A.I.H., Oh, M.J., Lee, S.M., Roh, K.S., 2016. Nitric oxide overcomes $\mathrm{Cd}$ and $\mathrm{Cu}$ toxicity in in vitro-grown tobacco plants through increasing contents and activities of rubisco and rubisco activase. Biochim. Open 2, 41-51.

Khan, M.N., Siddiqui, M.H., Mohammad, F., Naeem, M., 2012. Interactive role of nitric oxide and calcium chloride in enhancing tolerance to salt stress. Nitric Oxide 27, 210-218. Available from: https://doi.org/10.1016/j.niox.2012.07.005.

Klepper, L., 1979. Nitric oxide (NO) and nitrogen dioxide (NO2) emissions from herbicide-treated soybean plants. Atmos. Environ. 13 (4), 537-542 (1967).

Kong, X., Wang, T., Li, W., Tang, W., Zhang, D., Dong, H., 2016. Exogenous nitric oxide delays salt-induced leaf senescence in cotton (Gossypium hirsutum L.). Acta Physiol. Plant. 38 (3), 61. 
Kopyra, M., 2004. The role of nitric oxide in plant growth regulation and responses to abiotic stresses. Acta Physiol. Plant. 26, 459-473. Available from: https:/ / doi.org/10.1007/s11738-004-0037-4.

Kovacs, I., Lindermayr, C., 2013. Nitric oxide-based protein modification: formation and site-specificity of protein S-nitrosylation. Front. Plant Sci. 4, 137.

Krasylenko, Y.A., Yemets, A.I., Blume, Y.B., 2013. Plant microtubules reorganization under the indirect UV-B exposure and during UVB-induced programmed cell death. Plant Signal. Behav. 8, e24031. Available from: https://doi.org/10.4161/psb.24031.

Laspina, N.V., Groppa, M.D., Tomaro, M.L., Benavides, M.P., 2005. Nitric oxide protects sunflower leaves against Cd-induced oxidative stress. Plant Sci. 169, 323-330. Available from: https://doi. org/10.1016/j.plantsci.2005.02.007.

Leterrier, M., Valderrama, R., Chaki, M., Airaki, M., Palma, J.M., Barroso, J.B., et al., 2012. Function of nitric oxide under environmental stress conditions. In: Khan, N.A., Nazar, R., Iqbal, N., Anjum, N.A. (Eds.), Phytohormones and abiotic Stress tolerance in plants. Springer, Berlin, pp. 99-113.

Li, H.D., Wang, W.B., Li, P.M., Xu, K., Gao, H.Y., Xiao, J., 2013. Effects of addition of external nitric oxide on the allocation of photosynthetic electron flux in Rumex K-1 leaves under osmotic shock. Photosynthetica 51 (4), 509-516. Available from: https:// doi.org/10.1007/s11099-013-0049-7.

Li, S.W., Xue, L., 2010. The interaction between $\mathrm{H}_{2} \mathrm{O}_{2}$ and NO, $\mathrm{Ca}^{2+}$, cGMP, and MAPKs during adventitious rooting in mung bean seedlings. In Vitro Cell. Dev. Biol. Plant 46, 142-148. Available from: https:/ / doi.org/10.1007/s11627-009-9275-x.

Li, X., Baio, G., Yun, W., 2013a. Heat stress mitigation by exogenous nitric oxide application involves polyamine metabolism and psII physiological strategies in ginger leaves. Agric. Sci. China 47, 1171-1179.

Li, Z.G., Yang, S.Z., Long, W.B., Yang, G.X., Shen, Z.Z., 2013 b. Hydrogen sulphide may be a novel downstream signal molecule in nitric oxide-induced heat tolerance of maize (Zea mays L.) seedlings. Plant, Cell Environ. 36, 1564-1572. Available from: https:/ / doi.org/10.1111/pce.12092.

Liao, W.B., Huang, G.B., Yu, J.H., Zhang, M.L., 2012. Nitric oxide and hydrogen peroxide alleviate drought stress in marigold explants and promote its adventitious root development. Plant. Physiol. Biochem. 58, 6-15.

Lindermayr, C., Sell, S., Müller, B., Leister, D., Durner, J., 2010. Redox regulation of the NPR1-TGA1 system of Arabidopsis thaliana by nitric oxide. Plant Cell 22, 2894-2907. Available from: https:/ / doi.org/10.1105/tpc.109.066464.

Liu, X., Liu, B., Xue, S., Cai, Y., Qi, W., Jian, C., et al., 2016. Cucumber (Cucumis sativus L.) nitric oxide synthase associatedgene1 (CsNOA1) plays a role in chilling stress. Front. Plant Sci. 7 (1652). Available from: https:/ / doi.org/10.3389/fpls.2016.01652.

Liu, X., Wang, L., Liu, L., Guo, Y., Ren, H., 2011. Alleviating effect of exogenous nitric oxide in cucumber seedling against chilling stress. Afr. J. Biotechnol. 10, 4380-4386.

Locato, V., Paradiso, A., Sabetta, W., De Gara, L., de Pinto, M.C., 2016. Nitric oxide and reactive oxygen species in PCD signaling, Advances in BotanicalResearch, Vol. 77. Academic Press, pp. $165-192$.

Lozano-Juste, J., León, J., 2011. Nitric oxide regulates DELLA content and PIF expression to promote photomorphogenesis in Arabidopsis. Plant Physiol. 156, 1410-1423. Available from: https://doi.org/10.1104/pp.111.177741.

Lynch, D.V., Steponkus, P.L., 1987. Plasma membrane lipid alterations associated with cold acclimation of winter rye seedlings (Secale cereale L. cv Puma). Plant Physiol. 83, 761-767.

Lytvyn, D.I., Raynaud, C., Yemets, A.I., Bergounioux, C., Blume, Y. B., 2016. Involvement of inositol biosynthesis and nitric oxide in the mediation of UV-B induced oxidative stress. Front. Plant Sci. 7,430 .

Mackerness, S., John, C.F., Jordan, B.R., Thomas, B., 2001. Early signalling componets in ultraviolet-B responses: distinct roles for different reactive oxygen species and nitric oxide. FEBS Lett. 489, $237-242$.

Manjunatha, G., Gupta, K.J., Lokesh, V., Mur, L.A., Neelwarne, B., 2012. Nitric oxide counters ethylene effects on ripening fruits. Plant Signal. Behav. 7 (4), 476-483.

Meakin, G.E., Bueno, E., Jepson, B., Bedmar, E.J., Richardson, D.J., Delgado, M.J., 2007. The contribution of bacteroidal nitrate and nitrite reduction to the formation of nitrosylleghaemoglobin complexes in soybean root nodules. Microbiology 153, 411-419. Available from: https://doi.org/10.1099/mic.0.2006/000059-0.

Meng, Y., Liu, F., Pang, C., Fan, S., Song, M., Wang, D., et al., 2011. Label-free quantitative proteomics analysis of cotton leaf response to nitric oxide. J. Proteome. Res. 10, 5416-5432. Available from: https:/ / doi.org/10.1021/pr200671d.

Mengel, A., Chaki, M., Shekariesfahlan, A., Lindermayr, C., 2013. Effect of nitric oxide on gene transcription-S-nitrosylation of nuclear proteins. Front. Plant Sci. 4, 293. Available from: https:// doi.org/10.3389/fpls.2013.00293.

Mihailovic, N., Drazic, G., 2011. Incomplete alleviation of nickel toxicity in bean by nitric oxide supplementation. Plant Soil Environ. 57, 396-401.

Minami, A., Nagao, M., Ikegami, K., Koshiba, T., Arakawa, K., Fujikawa, S., et al., 2005. Cold acclimation in bryophytes: lowtemperature-induced freezing tolerance in Physcomitrella patens is associated with increases in expression levels of stress-related genes but not with increase in level of endogenous abscisic acid. Planta 220 (3), 414-423.

Mioto, P.T., Mercier, H., 2013. Abscisic acid and nitric oxide signaling in two different portions of detached leaves of Guzmania monostachia with CAM up-regulated by drought. J. Plant. Physiol. 170 (11), 996-1002.

Misra, A.N., Vladkova, R., Singh, R., Misra, M., Dobrikova, A.G., Apostolova, E.L., 2014. Action and target sites of nitric oxide in chloroplasts. Nitric Oxide 39, 35-45. Available from: https:// doi. org/10.1016/j.niox.2014.04.003.

Monks, P.S., Archibald, A.T., Colette, A., Cooper, O., Coyle, M., Derwent, R., et al., 2015. Tropospheric ozone and its precursors from the urban to the global scale from air quality to short-lived climate forcer. Atmosph. Chem. Phys. 15 (15), 8889-8973.

Monreal, J.A., Arias-Baldrich, C., Pérez-Montaño, F., Gandullo, J., Echevarría, C., García-Mauriño, S., 2013. Factors involved in the rise of phosphoenolpyruvate carboxylase-kinase activity caused by salinity in sorghum leaves. Planta 237, 1401-1413. Available from: https://doi.org/10.1007/s00425-013-.

Mostofa, M.G., Rahman, A., Ansary, M.M.U., Watanabe, A., Fujita, M., Tran, L.S.P., 2015. Hydrogen sulfide modulates cadmiuminduced physiological and biochemical responses to alleviate cadmium toxicity in rice. Sci. Rep. 5, 14078. Available from: https:// doi.org/10.1038/srep14078.

Mulaudzi, T., Ludidi, N., Ruzvidzo, O., Morse, M., Hendricks, N., Iwuoha, E., et al., 2011. Identification of a novel Arabidopsis thaliana nitric oxide-binding molecule with guanylate cyclase activity in vitro. FEBS Lett. 585, 2693-2697. Available from: https://doi. org/10.1016/j.febslet.2011.07.023.

Naser Alavi, S.M., Arvin, M.J., Manoochehri Kalantari, K., 2014. Salicylic acid and nitric oxide alleviate osmotic stress in wheat (Triticum aestivum L.) seedlings. J. Plant Interact. 9 (1), 683-688.

Nawaz, F., Shabbir, R.N., Shahbaz, M., Majeed, S., Raheel, M., Hassan, W., et al., 2017. Cross Talk between Nitric Oxide and Phytohormones Regulate Plant Development during Abiotic Stresses. In: El-Esawi, M. (Ed.), Phytohormones-Signaling 
Mechanisms and Crosstalk in Plant Development and Stress Responses. InTech.

Negi, S., Santisree, P., Kharshiing, E.V., Sharma, R., 2010. Inhibition of the ubiquitin-proteasome pathway alters cellular levels of nitric oxide in tomato seedlings. Mol. Plant 3, 854-869. Available from: https://doi.org/10.1093/mp/ssq033.

Ortega-Galisteo, A.P., Rodríguez-Serrano, M., Pazmiño, D.M., Gupta, D.K., Sandalio, L.M., Romero-Puertas, M.C., 2012. S-Nitrosylated proteins in pea (Pisum sativum L.) leaf peroxisomes: changes under abiotic stress. J. Exp. Bot. 63, 2089-2103. Available from: https://doi.org/10.1093/jxb/err414.

Oz, M.T., Eyidogan, F., Yucel, M., Öktem, H.A., 2015. Functional role of nitric oxide under abiotic stress conditions. In: Khan, M., Mobin, M., Mohammad, F., Corpas, F. (Eds.), Nitric Oxide Action in Abiotic Stress Responses in Plants. Springer, Cham, pp. 21-41. Available from: https://doi.org/10.1007/978-3-319-17804-2_2.

Parankusam, S., Bhatnagar-Mathur, P., Sharma, K.K., 2017. Heat responsive proteome changes reveal molecular mechanisms underlying heat tolerance in chickpea. Environ. Exp. Bot. 141, 132-144. Available from: https://doi.org/10.1016/j.envexpbot.2017.07.007.

Pasqualini, S., Reale, L., Calderini, O., Pagiotti, R., Ederli, L., 2012. Involvement of protein kinases and calcium in the NO-signalling cascade for defence-gene induction in ozonated tobacco plants. J. Exp. Bot. 63, 4485-4496.

Patakas, A.A., Zotos, A., Beis, A.S., 2010. Production, localisation and possible roles of nitric oxide in drought-stressed grapevines. Austr. J. Grape Wine Res. 16, 203-209. Available from: https:// doi.org/10.1111/j.1755-0238.2009.00064.x.

Patel, P., Kadur Narayanaswamy, G., Kataria, S., Baghel, L., 2017. Involvement of nitric oxide in enhanced germination and seedling growth of magnetoprimed maize seeds. Plant Signal. Behav. 12 (12), e1293217.

Petó, A., Lehotai, N., Lozano-Juste, J., León, J., Tari, I., Erdei, L., et al., 2011. Involvement of nitric oxide and auxin in signal transduction of copper-induced morphological responses in Arabidopsis seedlings. Ann. Bot. (Lond.) 108, 449-457. Available from: https:/ / doi.org/10.1093/aob/mcr176.

Planchet, E., Verdu, I., Delahaie, J., Cukier, C., Girard, C., Morère-Le Paven, M.C., et al., 2014. Abscisic acid-induced nitric oxide and proline accumulation in independent pathways under waterdeficit stress during seedling establishment in Medicago truncatula. J. Exp. Bot. 65 (8), 2161-2170.

Pospísill, P., 2016. Production of reactive oxygen species by photosystem II as a response to light and temperature stress. Front. Plant Sci. 7, 1950. Available from: https://doi.org/10.3389/fpls.2016.01950.

Prasad, P.V.V., Pisipati, S.R., Momčilović, I., Ristic, Z., 2011. Independent and combined effects of high temperature and drought stress during grain filling on plant yield and chloroplast EF-Tu expression in spring wheat. J Agron Crop Sci 197, 430-441. Available from: https:/ / doi.org/10.1111/j.1439-037X.2011.00477.x.

Procházková, D., Haisel, D., Wilhelmová, N., Pavlíková, D., Száková, J., 2013. Effects of exogenous nitric oxide on photosynthesis. Photosynthetica 51, 483-489. Available from: https://doi.org/ 10.1007/s11099-013-0053-y.

Rodríguez-Serrano, M., Romero-Puertas, M.C., Pazmino, D.M., Testillano, P.S., Risueño, M.C., Luis, A., et al., 2009. Cellular response of pea plants to cadmium toxicity: cross talk between reactive oxygen species, nitric oxide, and calcium. Plant Physiol. 150, 229-243. Available from: https://doi.org/10.1104/pp.108.131524.

Romero-Puertas, M.C., Rodríguez-Serrano, M., Sandalio, L.M., 2013. Protein S-nitrosylation in plants under abiotic stress: an overview. Front. Plant Sci. 4, 373. Available from: https://doi.org/10.3389/ fpls.2013.00373.

Ruan, H., Shen, W., Ye, M., Xu, L., 2002. Protective effects of nitric oxide on salt stress-induced oxidative damage to wheat (Triticum aestivum L.) leaves. Chinese Sci. Bull. 47, 677. Available from: https: / / doi.org/10.1360/02tb9154.

Sadeghipour, O., 2016. Pretreatment with nitric oxide reduces lead toxicity in cowpea (Vigna unguiculata [L.] walp.). Arch. Biol. Sci. $68,165-175$.

Sahay, S., Gupta, M., 2017. An update on nitric oxide and its benign role in plant responses under metal stress. Nitric Oxide 67, 39-52. Available from: https://doi.org/10.1016/j.niox.2017.04.011.

Sakihama, Y., Nakamura, S., Yamasaki, H., 2002. Nitric oxide production mediated by nitrate reductase in the green alga Chlamydomonas reinhardtii: an alternative NO production pathway in photosynthetic organisms. Plant Cell Physiol. 43, 290-297. Available from: https://doi.org/10.1093/pcp/pcf034.

Sami, F., Faizan, M., Faraz, A., Siddiqui, H., Yusuf, M., Hayat, S., 2017. Nitric oxide-mediated integrative alterations in plant metabolism to confer abiotic stress tolerance, NO crosstalk with phytohormones and NO-mediated post translational modifications in modulating diverse plant stress. Nitric Oxide

Sánchez, C., Gates, A.J., Meakin, G.E., Uchiumi, T., Girard, L., Richardson, D.J., et al., 2010. Production of nitric oxide and nitrosylleghemoglobin complexes in soybean nodules in response to flooding. Mol. Plant Microbe. Interact. 23, 702-711. Available from: https://doi.org/10.1094/MPMI-23-5-0702.

Santisree, P., Bhatnagar-Mathur, P., Sharma, K.K., 2015. NO to drought-multifunctional role of nitric oxide in plant drought: do we have all the answers? Plant Sci. 239, 44-55. Available from: https:/ / doi.org/10.1016/j.plantsci.2015.07.012.

Santisree, P., Bhatnagar-Mathur, P., Sharma, K.K., 2017. Molecular insights into the functional role of nitric oxide (NO) as a signal for plant responses in chickpea. Funct. Plant Biol. 45, 267-283. Available from: https://doi.org/10.1071/FP16324.

Savvides, A., Ali, S., Tester, M., Fotopoulos, V., 2016. Chemical priming of plants against multiple abiotic stresses: mission possible? Trends. Plant. Sci. 21, 329-340. Available from: https://doi.org/ 10.1016/j.tplants.2015.11.003.

Sehrawat, A., Abat, J.K., Deswal, R., 2013. RuBisCO depletion improved proteome coverage of cold responsive S-nitrosylated targets in Brassica juncea. Front. Plant Sci. 4, 342. Available from: https:/ / doi.org/10.3389/fpls.2013.00342.

Shao, R., Wang, K., Shangguan, Z., 2010. Cytokinin-induced photosynthetic adaptability of Zea mays L. to drought stress associated with nitric oxide signal: probed by ESR spectroscopy and fast OJIP fluorescence rise. J. Plant. Physiol. 167 (6), 472-479.

She, X.P., Song, X.G., He, J.M., 2004. Role and relationship of nitric oxide and hydrogen peroxide in light/dark-regulated stomatal movement in Vicia faba. Acta Botanica Sinica-English Edition 46, $1292-1300$

Shi, H., Ye, T., Chen, F., Cheng, Z., Wang, Y., Yang, P., et al., 2013. Manipulation of arginase expression modulates abiotic stress tolerance in Arabidopsis: effect on arginine metabolism and ROS accumulation. J. Exp. Bot. 64, 1367-1379. Available from: https://doi.org/10.1093/jxb/ers400.

Shi, H., Ye, T., Zhu, J.K., Chan, Z., 2014. Constitutive production of nitric oxide leads to enhanced drought stress resistance and extensive transcriptional reprogramming in Arabidopsis. J. Exp. Bot. 65, 4119-4131. Available from: https://doi.org/10.1093/jxb/eru184.

Shi, H.T., Li, R.J., Cai, W., Liu, W., Wang, C.L., Lu, Y.T., 2011. Increasing nitric oxide content in Arabidopsis thaliana by expressing rat neuronal nitric oxide synthase resulted in enhanced stress tolerance. Plant Cell Physiol. 53, 344-357. Available from: https://doi.org/10.1093/pcp/pcr181.

Si, T., Wang, X., Wu, L., Zhao, C., Zhang, L., Huang, M., et al., 2017. Nitric oxide and hydrogen peroxide mediate wounding-induced freezing tolerance through modifications in photosystem and antioxidant system in wheat. Front. Plant Sci. 8, 1284. 
Siddiqui, M., Alamri, S.A., Mutahhar, Y.Y., Al-Khaishany, M.A., AlQutami, H.M., Nasir Khan, M.A., 2017. Nitric Oxide and calcium induced physiobiochemical changes in tomato (Solanum Lycopersicum) plant under heat stress. Fresenius Environ. Bull. 26, $1663-1672$.

Simontacchi, M., Galatro, A., Ramos-Artuso, F., Santa-María, G.E., 2015. Plant survival in a changing environment: the role of nitric oxide in plant responses to abiotic stress. Front. Plant Sci. 6, 977. Available from: https://doi.org/10.3389/fpls.2015.00977.

Simontacchi, M., Garcia-Mata, C., Bartoli, C.G., Santa-María, G.E., Lamattina, L., 2013. Nitric Oxide as a Key Component in Hormone-Regulated Processes. Plant Cell Rep. 32, 853-866. Available from: http://dx.doi.org/10.1007/s00299-013-1434-1.

Singh, S.P., Singh, Z., Swinny, E.E., 2009. Postharvest nitric oxide fumigation delays fruit ripening and alleviates chilling injury during cold storage of Japanese plums (Prunus salicina Lindell). Postharvest. Biol. Technol. 53, 101-108. Available from: https:// doi.org/10.1016/j.postharvbio.2009.04.007.

Slinger, D., Tenison, K., 2009. Salinity Glove Box Guide. Industry \& Investment NSW, Tasmania.

Song, L., Ding, W., Shen, J., Zhang, Z., Bi, Y., Zhang, L., 2008. Nitric oxide mediates abscisic acid induced thermotolerance in the calluses from two ecotypes of reed under heat stress. Plant Sci. 175, 826-832.

Srivastava, S., Dubey, R.S., 2012. Nitric oxide alleviates manganese toxicity by preventing oxidative stress in excised rice leaves. Acta Physiol. Plant. 34 (2), 819-825.

Sun, C., Lu, L., Liu, L., Liu, W., Yu, Y., Liu, X., et al., 2014. Nitrate reductase-mediated early nitric oxide burst alleviates oxidative damage induced by aluminum through enhancement of antioxidant defenses in roots of wheat (Triticum aestivum). New Phytol. 201, 1240-1250.

Sun, H., Tao, J., Zhao, Q., Xu, G., Zhang, Y., 2017. Multiple roles of nitric oxide in root development and nitrogen uptake. Plant Signal. Behav. 12 (1), e1274480.

Sun, L.R., Hao, F.S., Lu, B.S., Ma, L.Y., 2010. AtNOA1 modulates nitric oxide accumulation and stomatal closure induced by salicylic acid in Arabidopsis. Plant Signal. Behav. 5 (8), 1022-1024.

Tan, J., Zhuo, C., Guo, Z., 2013. Nitric oxide mediates cold- and dehydration-induced expression of a novel MfHyPRP that confers tolerance to abiotic stress. Physiol. Plant 149, 310-320.

Terrile, M.C., París, R., Calderón-Villalobos, L.I., Iglesias, M.J., Lamattina, L., Estelle, M., et al., 2012. Nitric oxide influences auxin signaling through S-nitrosylation of the Arabidopsis TRANSPORT INHIBITOR RESPONSE 1 auxin receptor. Plant J. 70, 492-500. Available from: https://doi.org/10.1111/j.1365313X.2011.04885.x.

Tewari, R.K., Hahn, E.J., Paek, K.Y., 2008. Modulation of copper toxicity-induced oxidative damage by nitric oxide supply in the adventitious roots of Panax ginseng. Plant Cell Rep. 27, 171-181. Available from: https://doi.org/10.1007/s00299-007-0423-7.

Tian, X.R., Lei, Y.B., 2007. Physiological responses of wheat seedlings to drought and UV-B radiation. Effect of exogenous sodium nitroprusside application. Russian J. Plant Physiol. 54, 676-682.

Tossi, V., Lombardo, C., Cassia, R., Lamattina, L., 2012. Nitric oxide and flavonoids are systemically induced by UV-B in maize leaves. Plant Sci. 193, 103-109. Available from: https://doi.org/10.1016/ j.plantsci.2012.05.012.

Uchida, A., Jagendorf, A.T., Hibino, T., Takabe, T., Takabe, T., 2002. Effects of hydrogen peroxide and nitric oxide on both salt and heat stress tolerance in rice. Plant Sci. 163, 515-523.

Vaishnav, A., et al., 2018. Nitric Oxide as a Signaling Molecule in Plant-Bacterial Interactions. In: Egamberdieva, D., Ahmad, P. (Eds.), Plant Microbiome: Stress Response. Microorganisms for Sustainability, vol 5. Springer, Singapore.
Vanzo, E., Ghirardo, A., Merl-Pham, J., Lindermayr, C., Heller, W., Hauck, S.M., et al., 2014. S-nitroso-proteome in poplar leaves in response to acute ozone stress. PLoS. ONE. 9 (9), e106886.

Vile, D., Pervent, M., Belluau, M., Vasseur, F., Bresson, J., Muller, B., et al., 2012. Arabidopsis growth under prolonged high temperature and water deficit: independent or interactive effects? Plant, Cell Environ. 35, 702-718. Available from: https://doi.org/ 10.1111/j.1365-3040.2011.02445.x.

Wang, L., Su, H., Han, L., Wang, C., Sun, Y., Liu, F., 2014. Differential expression profiles of poplar MAP kinase kinases in response to abiotic stresses and plant hormones, and overexpression of PtMKK4 improves the drought tolerance of poplar. Gene 545, 141-148. Available from: https://doi.org/10.1016/j.gene.2014.04.058.

Wang, P., Du, Y., Hou, Y.J., Zhao, Y., Hsu, C.C., Yuan, F., et al., 2015. Nitric oxide negatively regulates abscisic acid signaling in guard cells by S-nitrosylation of OST1. Proc. Natl. Acad. Sci. 112, 613-618. Available from: https://doi.org/10.1073/pnas.1423481112.

Wang, Q.F., Zhao, Y., Yi, Q., Li, K.Z., Yu, Y.X., Chen, L.M., 2010a. Overexpression of malate dehydrogenase in transgenic tobacco leaves: enhanced malate synthesis and augmented Al-resistance. Acta Physiol. Plant. 32, 1209-1220. Available from: https://doi. org/10.1007/s11738-010-0522-x.

Wang, R., Guegler, K., LaBrie, S.T., Crawford, N.M., 2000. Genomic analysis of a nutrient response in Arabidopsis reveals diverse expression patterns and novel metabolic and potential regulatory genes induced by nitrate. Plant Cell 12, 1491-1509. Available from: https://doi.org/10.1105/tpc.12.8.1491.

Wang, Y., Ries, A., Wu, K., Yang, A., Crawford, N.M., 2010b. The Arabidopsis prohibitin gene PHB3 functions in nitric oxide-mediated responses and in hydrogen peroxide-induced nitric oxide accumulation. Plant Cell 22, 249-259. Available from: https:/ / doi.org/10.1105/tpc.109.072066.

Wang, Y., Suo, B., Zhao, T., Qu, X., Yuan, L., Zhao, X., et al., 2011. Effect of nitric oxide treatment on antioxidant responses and psbA gene expression in two wheat cultivars during grain filling stage under drought stress and rewatering. Acta Physiol. Plant. 33, 1923. Available from: https:// doi.org/10.1007/s11738-011-0740-x.

Wang, Y.H., Li, X.C., Zhu-Ge, Q., Jiang, X., Wang, W.D., Fang, W.P., et al., 2012. Nitric oxide participates in cold-inhibited Camellia sinensis pollen germination and tube growth partly via cGMP in vitro. PLoS. ONE. 7, e52436.

Wu, X., Zhu, W., Zhang, H., Ding, H., Zhang, H.J., 2011. Exogenous nitric oxide protects against salt-induced oxidative stress in the leaves from two genotypes of tomato (Lycopersicom esculentum Mill.). Acta Physiol. Plant. 33, 1199-1209. Available from: https:/ / doi.org/10.1007/s11738-010-0648-x.

Wu, X.X., Zhu, X.H., Chen, J.L., Yang, S.J., Ding, H.D., Zha, D.S., 2013. Nitric oxide alleviates adverse salt-induced effects by improving the photosynthetic performance and increasing the anti-oxidant capacity of eggplant (Solanum melongena L.). J. Hort. Sci. Biotechnol. 88, 352-360. Available from: https://doi.org/ 10.1080/14620316.2013.11512976.

Xiong, J., An, L., Lu, H., Zhu, C., 2009. Exogenous nitric oxide enhances cadmium tolerance of rice by increasing pectin and hemicellulose contents in root cell wall. Planta 230, 755-765.

Xiong, J., Fu, G., Tao, L., Zhu, C., 2010. Roles of nitric oxide in alleviating heavy metal toxicity in plants. Arch. Biochem. Biophys. 497, 13-20. Available from: https://doi.org/10.1016/j.abb.2010. 02.014 .

Xu, J., Wang, W., Yin, H., Liu, X., Sun, H., Mi, Q., 2010a. Exogenous nitric oxide improves antioxidative capacity and reduces auxin degradation in roots of Medicago truncatula seedlings under cadmium stress. Plant Soil 326, 321.

Xu, J., Yin, H., Liu, X., Li, X., 2010b. Salt affects plant Cd-stress responses by modulating growth and $\mathrm{Cd}$ accumulation. Planta 
231, 449-459. Available from: https://doi.org/10.1007/s00425009-1070-8.

Xu, L.L., Fan, Z.Y., Dong, Y.J., Kong, J., Bai, X.Y., 2015. Effects of exogenous salicylic acid and nitric oxide on physiological characteristics of two peanut cultivars under cadmium stress. Biol. Plant. 59 (1), 171-182.

Xu, M., Dong, J., Zhang, M., Xu, X., Sun, L., 2012a. Cold-induced endogenous nitric oxide generation plays a role in chilling tolerance of loquat fruit during postharvest storage. Postharvest. Biol. Technol. 65, 5-12. Available from: https://doi.org/10.1016/j. postharvbio.2011.10.008.

Xu, M., Zhu, Y., Dong, J., Jin, H., Sun, L., Wang, Z., et al., 2012b. Ozone induces flavonol production of Ginkgo biloba cells dependently on nitrate reductase-mediated nitric oxide signaling. Environ. Exp. Bot. 75, 114-119. Available from: https://doi.org/ 10.1016/j.envexpbot.2011.09.005.

Xu, Y.C., Zhao, B.L., 2003. The main origin of endogenous NO in higher non-leguminous plants. Plant. Physiol. Biochem. 41, 833-838. Available from: https://doi.org/10.1016/S0981-9428(03) 00116-5.

Yamori, W., Hikosaka, K., Way, D.A., 2014. Temperature response of photosynthesis in C3, C4, and CAM plants: temperature acclimation and temperature adaptation. Photosynth. Res. 119, 101-117. Available from: https://doi.org/10.1007/s11120-013-9874-6.

Yang, H., Wu, F., Cheng, J., 2011a. Reduced chilling injury in cucumber by nitric oxide and the antioxidant response. Food. Chem. 127, 1237-1242. Available from: https://doi.org/10.1016/j. foodchem.2011.02.011.

Yang, L., Ji, J., Harris-Shultz, K.R., Wang, H., Wang, H., Abd-Allah, E.F., et al., 2016. The dynamic changes of the plasma membrane proteins and the protective roles of nitric oxide in rice subjected to heavy metal cadmium stress. Front. Plant Sci. 7, 190.

Yang, L., Tian, D., Todd, C.D., Luo, Y., Hu, X., 2013. Comparative proteome analyses reveal that nitric oxide is an important signal molecule in the response of rice to aluminum toxicity. J. Proteome. Res. 12, 1316-1330. Available from: https://doi.org/ $10.1021 /$ pr300971n.

Yang, W., Sun, Y., Chen, S., Jiang, J., Chen, F., Fang, W., et al., 2011b. The effect of exogenously applied nitric oxide on photosynthesis and antioxidant activity in heat stressed chrysanthemum. Biol. Plant. 55, 737.

Yu, M., Lamattina, L., Spoel, S.H., Loake, G.J., 2014. Nitric oxide function in plant biology: a redox cue in deconvolution. New Phytol. 202, 1142-1156. Available from: https://doi.org/10.1111/ nph.12739.

Yun, B.W., Skelly, M.J., Yin, M., Yu, M., Mun, B.G., Lee, S.U., et al., 2016. Nitric oxide and S-nitrosoglutathione function additively during plant immunity. New Phytol. 211, 516-526. Available from: https://doi.org/10.1111/nph.13903.

Zandalinas, S.I., Balfagón, D., Arbona, V., Gómez-Cadenas, A., Inupakutika, M.A., Mittler, R., 2016. ABA is required for the accumulation of APX1 and MBF1c during a combination of water deficit and heat stress. J. Exp. Bot. 67 (18), 5381-5390.

Zhang, M., Dong, J.F., Jin, H.H., Sun, L.N., Xu, M.J., 2011. Ultraviolet-B-induced flavonoid accumulation in Betula pendula leaves is dependent upon nitrate reductase-mediated nitric oxide signaling. Tree. Physiol. 31, 798-807.

Zhao, M.G., Chen, L., Zhang, L.L., Zhang, W.H., 2009. Nitric reductase-dependent nitric oxide production is involved in cold acclimation and freezing tolerance in Arabidopsis. Plant Physiol. 151, 755-767. Available from: https://doi.org/ 10.1104/pp.109.140996.

Zhao, H., Jin, Q., Wang, Y., Chu, L., Li, X., Xu, Y., 2016. Effects of nitric oxide on alleviating cadmium stress in Typha angustifolia. Plant Growth Regul. 78, 243-251.
Zheng, C., Jiang, D., Liu, F., Dai, T., Liu, W., Jing, Q., et al., 2009. Exogenous nitric oxide improves seed germination in wheat against mitochondrial oxidative damage induced by high salinity. Environ. Exp. Bot. 67, 222-227. Available from: https://doi.org/ 10.1016/j.envexpbot.2009.05.002.

Zimmer-Prados, L.M., Moreira, A.S.F.P., Magalhaes, J.R., França, M. G.C., 2014. Nitric oxide increases tolerance responses to moderate water deficit in leaves of Phaseolus vulgaris and Vigna unguiculata bean species. Physiol. Mol. Biol. Plants 20, 295-301. Available from: https://doi.org/10.1007/s12298-014-0239-1.

Ziogas, V., Tanou, G., Filippou, P., Diamantidis, G., Vasilakakis, M., Fotopoulos, V., et al., 2013. Nitrosative responses in citrus plants exposed to six abiotic stress conditions. Plant. Physiol. Biochem. 68, 118-126. Available from: https://doi.org/10.1016/j. plaphy.2013.04.004.

\section{Further Reading}

Allakhverdiev, S.I., Kreslavski, V.D., Klimov, V.V., Los, D.A., Carpentier, R., Mohanty, P., 2008. Heat stress: an overview of molecular responses in photosynthesis. Photosynth. Res. 98, 541. Available from: https:/ / doi.org/10.1007/s11120-008-9331-0.

An, L., Liu, Y., Zhang, M., Chen, T., Wang, X., 2005. Effects of nitric oxide on growth of maize seedling leaves in the presence or absence of ultraviolet-B radiation. J. Plant. Physiol. 162, 317-326.

Besson-Bard, A., Astier, J., Rasul, S., Wawer, I., Dubreuil-Maurizi, C., Jeandroz, S., et al., 2009. Current view of nitric oxide-responsive genes in plants. Plant Sci. 177, 302-309. Available from: https:// doi.org/10.1016/j.plantsci.2009.06.006.

Bethke, P.C., Libourel, I.G., Aoyama, N., Chung, Y.Y., Still, D.W., Jones, R.L., 2007. The Arabidopsis aleurone layer responds to nitric oxide, gibberellin, and abscisic acid and is sufficient and necessary for seed dormancy. Plant Physiol. 143, 1173-1188. Available from: https://doi.org/10.1104/pp.106.093435.

Casati, P., Walbot, V., 2004. Rapid transcriptome responses of maize (Zea mays) to UV-B in irradiated and shielded tissues. Genome. Biol. 5, R16.

Chen, K., Feng, H., Zhang, M., Wang, X., 2003. Nitric oxide alleviates oxidative damage in the green alga Chlorella pyrenoidosa caused by UV-B radiation. Folia. Microbiol. (Praha). 48, 389. Available from: https:/ / doi.org/10.1007/BF02931372.

Domingos, P., Prado, A.M., Wong, A., Gehring, C., Feijo, J.A., 2015. Nitric oxide: a multitasked signaling gas in plants. Mol. Plant 8, 506-520. Available from: https:/ / doi.org/10.1016/j.molp.2014.12.010.

FAO Food Agricultural Organization, 2009. How to Feed the World in 2050. Food and Agriculture Organization, Rome. Available online.

Gayatri, G., Agurla, S., Raghavendra, A.S., 2013. Nitric oxide in guard cells as an important secondary messenger during stomatal closure. Front. Plant Sci. 4, 425.

Gill, S.S., Peter Singh, L., Gill, R., Tuteja, N., 2012. Generation and Scavenging of reactive oxygen species in plants under stress. In: Tuteja, N., Gill, S.S., Tiburcio, A.F., Tuteja, R. (Eds.), Improving Crop Resistance to Abiotic Stress. Wiley-VCH Verlag GmbH \& Co. KGaA, Weinheim, Germany, pp. 49-70.

Guillas, I., Zachowski, A., Baudouin, E., 2011. A matter of fat: interaction between nitric oxide and sphingolipid signaling in plant cold response. Plant Signal. Behav. 6, 140-142. Available from: https://doi.org/10.4161/psb.6.1.14280.

Hao, G.P., Du, X.H., Hai, R.J., 2007. Exogenous nitric oxide accelerates soluble sugar, proline and secondary metabolite synthesis in Ginkgo biloba under drought stress. J. Plant Physiol. Mol. Biol. 33, 499-506.

He, J.M., Xu, H., She, X.P., Song, X.G., Zhao, W.M., 2005. The role and the interrelationship of hydrogen peroxide and nitric oxide 
in the UV-B-induced stomatal closure in broad bean. Funct. Plant Biol. 32, 237-247. Available from: https://doi.org/10.1071/ FP04185.

Lin, D.I., Lur, H.S., Chu, C., 2001. Effects of abscisic acid on ozone tolerance of rice (Oryza sativa L.) seedlings. Plant Growth Regul. 35, 295-300. Available from: https://doi.org/10.1023/A:1014427505353.

Maurice, N., Ping, C.Y., Miaomiao, Q., Constantine, U., Bo, Y., Qi, K. Y., 2016. Effects of exogenous nitric oxide on germination and carbohydrates mobilization in alfalfa seedlings under cadmium stress. Int. J. Environ. Sci. Technol. 4, 2337-2350.

Nazar, R., Khan, M.I.R., Iqbal, N., Masood, A., Khan, N.A., 2014. Involvement of ethylene in reversal of salt-inhibited photosynthesis by sulfur in mustard. Physiol. Plant. 152, 331-344. Available from: https://doi.org/10.1111/ppl.12173.

Parankusam, S., Adimulam, S.S., Bhatnagar-Mathur, P., Sharma, K.K., 2017b. Nitric oxide (NO) in plant heat stress tolerance: current knowledge and perspectives. Front. Plant Sci. 8, 1582.

Pasqualini, S., Meier, S., Gehring, C., Madeo, L., Fornaciari, M., Romano, B., et al., 2009. Ozone and nitric oxide induce cGMPdependent and-independent transcription of defence genes in tobacco. New Phytol. 181, 860-870. Available from: https://doi. org/10.1111/j.1469-8137.2008.02711.x.
Popova, L., Tuan, T., 2010. Nitric oxide in plants: properties, biosynthesis and physiological functions. Iranian J. Sci. Technol. (Sci.) 34, 173-183.

Rao, K.P., Vani, G., Kumar, K., Wankhede, D.P., Misra, M., Gupta, M., et al., 2011. Arsenic stress activates MAP kinase in rice roots and leaves. Arch. Biochem. Biophys. 506, 73-82. Available from: https://doi.org/10.1016/j.abb.2010.11.006.

Tian, Q.Y., Sun, D.H., Zhao, M.G., Zhang, W.H., 2007. Inhibition of nitric oxide synthase (NOS) underlies aluminum-induced inhibition of root elongation in Hibiscus moscheutos. New Phytol. 174, 322-331. Available from: https://doi.org/10.1111/j.1469-8137.2007.02005.x.

Wang, H., Liang, X., Wan, Q., Wang, X., Bi, Y., 2009. Ethylene and nitric oxide are involved in maintaining ion homeostasis in Arabidopsis callus under salt stress. Planta 230 (2), 293-307.

Wang, M., Li, Q., Fu, S., Xiao, D., Dong, B., 2005. Effects of exogenous nitric oxide on drought-resistance of poplar. Ying yong sheng tai xue bao = The J. Appl. Ecol. 16, 805-810.

Zhang, H., Shen, W.B., Zhang, W., Xu, L.L., 2005. A rapid response of $\beta$-amylase to nitric oxide but not gibberellin in wheat seeds during the early stage of germination. Planta 220, 708-716. Available from: https://doi.org/10.1007/s00425-004-1390-7. 


\title{
26
}

\section{Brassinosteroid Signaling and Complex Interplay of ROS, NADPH Oxidase, and MAPK Mediated Biotic and Abiotic Stress Acclimation in Plants}

\author{
Deepesh Bhatt ${ }^{1, *}$, Mayank Sharma ${ }^{2, *}$, Manoj $\mathrm{Nath}^{3}$, \\ Megha D. Bhatt ${ }^{4}$ and Saurabh Badoni ${ }^{5}$
}

${ }^{1}$ Department of Biotechnology, Shree Ramkrishna Institute of Computer Education and Applied Sciences, Veer Narmad South Gujarat University, Surat, Gujarat, India ${ }^{2}$ Martin Luther University of Halle-Wittenberg, Halle, Germany ${ }^{3}$ Indian Council of Agricultural Research, New Delhi, Uttar Pradesh, India ${ }^{4}$ GSFC AgroTech Ltd. Gujarat State Fertilizers \& Chemicals Ltd., Vadodara, India ${ }^{5}$ Plant Breeding Division, International Rice Research Institute, Metro Manila, Philippines

\section{O U T L I N E}

26.1 Introduction

26.2 Brassinosteroids

26.3 Brassinosteroid Signaling in Plants

26.4 Transcription Factors Involved in BR Signaling

26.5 Role of RD26 in BR Signaling
407

26.6 BR Mediated Defense Signaling

$408 \quad 26.7$ BR Mediated ROS Signaling and Its

408

Role in Plant Defense

26.8 Conclusion

409 References

$410 \quad$ Further Reading

\subsection{INTRODUCTION}

Plants are constantly exposed to multiple biotic and abiotic stresses during their entire life, which include pathogen attacks and insect herbivory, high or low temperatures stress, drought and salinity stress, etc. Therefore, to survive, plants have evolved a range of intricate signaling mechanisms thus adapting towards these fluctuating environments. Plant growth regulators, also termed as phytohormones, are increasingly

*The authors (Deepesh Bhatt and Mayank Sharma) contributed equally to this work. 
recognized to play vital roles in plant stress adaptations. Several studies indicated that biological processes in plants are influenced by stressful conditions, which are regulated through different hormonal signaling pathways in plants (Teale et al., 2008). Phytohormones, like salicylic acid (SA), jasmonic acid (JA), ethylene (ET), and abscisic acid (ABA), are primary signals that regulate responses to mitigate biotic and abiotic stresses in plants (Lorenzo and Solano, 2005; Mauch-Mani and Mauch, 2005). These stresses, if not properly regulated, may further lead to the generation of a key intermediate termed as reactive oxygen species (ROS). ROS are generated in response to stress as well as to regulate normal metabolic processes as a signaling agent (Mittler et al., 2011; Sewelam et al., 2016). Additionally, respiratory burst oxidase homologs (RBOHs) and NADPH oxidases are also known to be major components of ROS production system in plants (Suzuki et al., 2011; Kadota et al., 2015). Furthermore several other studies also propose that ROS plays a critical role in enhancing stress tolerance by activating mitogenactivated protein kinases (MAPKs), antioxidant enzymes, and other related transcription factors (Gechev et al., 2006). The generation of ROS has been shown to be a key process that is shared among many transcriptional pathways related to stress signaling. Thus at the molecular level, the perception of external stimuli results in the subsequent activation of stress signaling through some intermediate signal molecules. Brassinosteroids are a group of steroidal hormones and play a major role in various developmental pathways and are involved in stress response (Choudhary et al., 2012). Here in this chapter we will mainly emphasize how brassinosteroids are able to influence and regulate concomitant generation of ROS, NADPH oxidase, and MAPK, which act as intermediate components in the signaling cascade to regulate biotic and abiotic stress acclimation in plants.

\subsection{BRASSINOSTEROIDS}

Mitchell and coworkers explored a sixth class of plant hormones named brassinosteroids (BRs) in 1970 (Mitchell et al., 1970). BR was first isolated from the pollen of Brassica napus. BRs are polyhydroxysteroids similar to steroid hormones from animals (Clouse, 2011). Furthermore they play an important role in regulating various aspects of plant growth and development (Wei and Li, 2016). This includes cell division and elongation, tissue morphogenesis, plant defense, seed germination, and reproduction (Krishna, 2003; Ashraf et al., 2010). They also play a direct or indirect role in stress signaling in plants (Bajguz and Hayat, 2009). Approximately 70 different kinds of analogue of BRs have been identified to date from various plant species and tissue samples. Brassinolide has
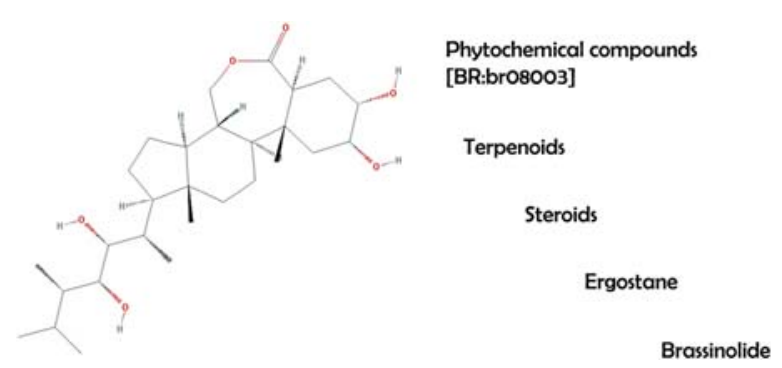

FIGURE 26.1 Chemical structure of the most active brassinosteroid 'brassinolide' $\mathrm{C}_{28} \mathrm{H}_{48} \mathrm{O}_{6}$ (Image from PubChem) and its phytochemical compounds tree.

been reported as a most active BR identifies hitherto (Grove et al., 1979, Tang et al., 2016) (Fig. 26.1). BRs are continuously biosynthesized, mostly in pollens, immature seeds, roots, and flower (Takatsuto, 1994; Kutschera and Wang, 2012). Excess of BRs can be metabolized by plants via different chemical reactions to render them into inactive form. However, they can be converted into the active form whenever needed to maintain the BR homeostasis (Bishop and Yokota, 2001; Bajguz, 2007). Plant cell consists of various receptors for BRs, which recognize them and stimulate the downstream signaling processes. Characterization of BR-insensitive (bri1) mutants led to the discovery of the first BR receptor in Arabidopsis thaliana (Clouse et al., 1996). BRI1 encodes a leucine-rich repeat-receptor like kinase (LRR-RLK) composing of an intracellular kinase domain and extracellular LRR domain (Li and Chory, 1997). The bril mutants were shown to be male sterile with several developmental and tissue deformities. Three BRI1 homologs namely BRL1, BRL2, and BRL3 (BRI1 like 1, 2, and 3) were also reported to recognize BRs in Arabidopsis (Cano-Delgado et al., 2004), though they display a weak phenotype in respective knockout plants when compared with BRI1. A further molecular dissection, by two different scientific groups, contributed in identification of interacting partners of BRI1 named BRI1 associated kinase 1 (BAK1) or somatic embryogenesis receptor-like kinase (SERK3) (Hecht et al., 2001; Nam and Li, 2002). BAK1 interacts with BRI1 to persuade the BR signaling. The SERK family of proteins with five members seems to play an important role in BR signaling. SERK1 and SERK4 have a similar role as of BAK1/SERK3 in BR signaling. Just to avoid confusion it should be noted that BAK1 and SERK3 were discovered and named separately, however later on it came out that BAK1 and SERK3 are the same protein.

\subsection{BRASSINOSTEROID SIGNALING IN PLANTS}

BR signaling is a phosphorylation and dephosphorylation relay system among BR receptors, coreceptors, 
and downstream transcription factors. In absence of BR, a BRI1-KINASE INHIBITOR 1 (BKI1) remains bound to BRI1 (Wang et al., 2005; Wang and Chory, 2006). The binding of BR to BRI1 and coreceptor BAK1 releases BKI1 and makes it available for 14-3-3 proteins that phosphorylate it and thus inhibit its binding to BRI1 (Gampala et al., 2007). Furthermore the mutual phosphorylation of BRI1 and BAK1 leads to activation of downstream signaling. The activation of BRI1 and BAK1 also requires the TWISTED DWARF 1 (TWD1/ FKBP42) protein (Zhao et al., 2016). An activated BRI1 phosphorylates the downstream signaling proteins namely BR-SIGNALING KINASE (BSK1) and CONSTITUTIVE DIFFERENTIAL GROWTH 1 (CDG1) kinase (Tang et al., 2008; Kim et al., 2009, 2011; Sreeramulu et al., 2013). After phosphorylation, this in turn leads to the activation of BRI1-SUPPRESSOR 1 (BSU1). BSU1 is a PP1-type phosphatase (Kim et al., 2011). The activation of BRI1 and BAK1 also requires the TWISTED DWARF 1 (TWD1/FKBP42) protein. The BSU1 further dephosphorylates and inactivates BRASSINOSTEROID INSENSITIVE 2 (BIN2). BIN2 is a G11SK3-like kinase and proposed to be degraded via its ubiquitinylation by a ubiquitin ligase KINK SUPPRESSED IN BZR1-1D (KIB1) (Zhu et al., 2017). Another protein, BES1/BZR1 (BRI1 EMS SUPPRESSOR
1/BRASSINAZOLE RESISTANT 1), a BR responsive transcription factor, plays a key role in activation of BRinduced genes. In absence of BR, the BIN2 phosphorylates BES1/BZR1 leading to its inactivation. But in the presence of BR, BIN2 is degraded and PROTEIN PHOSPHATASE 2A (PP2A) dephosphorylates BES1/ BZR1 (He et al., 2001). The BES1/BZR1 later enters in the nucleus, binds to DNA and activates the BR-induced genes with the help of some additional transcription factors (Ryu et al., 2007). For a schematic view, refer to Fig. 26.2A-B.

\subsection{TRANSCRIPTION FACTORS INVOLVED IN BR SIGNALING}

Genome-wide analysis of genes regulated by BES1/ BZR1 revealed that the expression of several thousand genes is influenced in BR signaling (Yu et al., 2011; Sun et al., 2010). The downstream BR signaling genes are either directly or indirectly regulated by BES1/BZR1. Several BES1/BZR1 interacting proteins and transcription factors have been identified so far. The complex interplay with these transcription factors in turn regulates the expression of BR signaling genes (Li, 2010). This include PHYTOCHROME-INTERACTING FACTORS
(A)

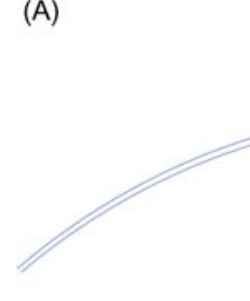

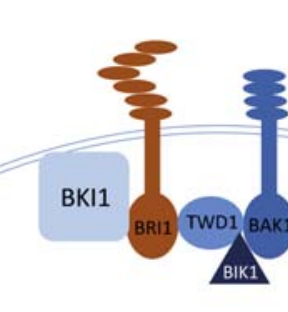

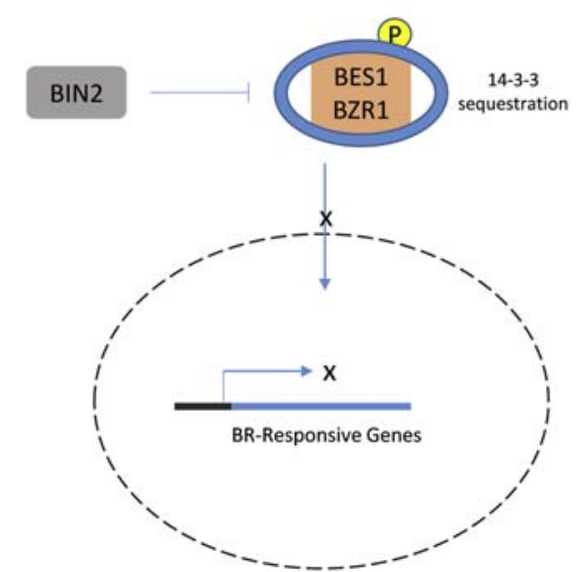

(B)
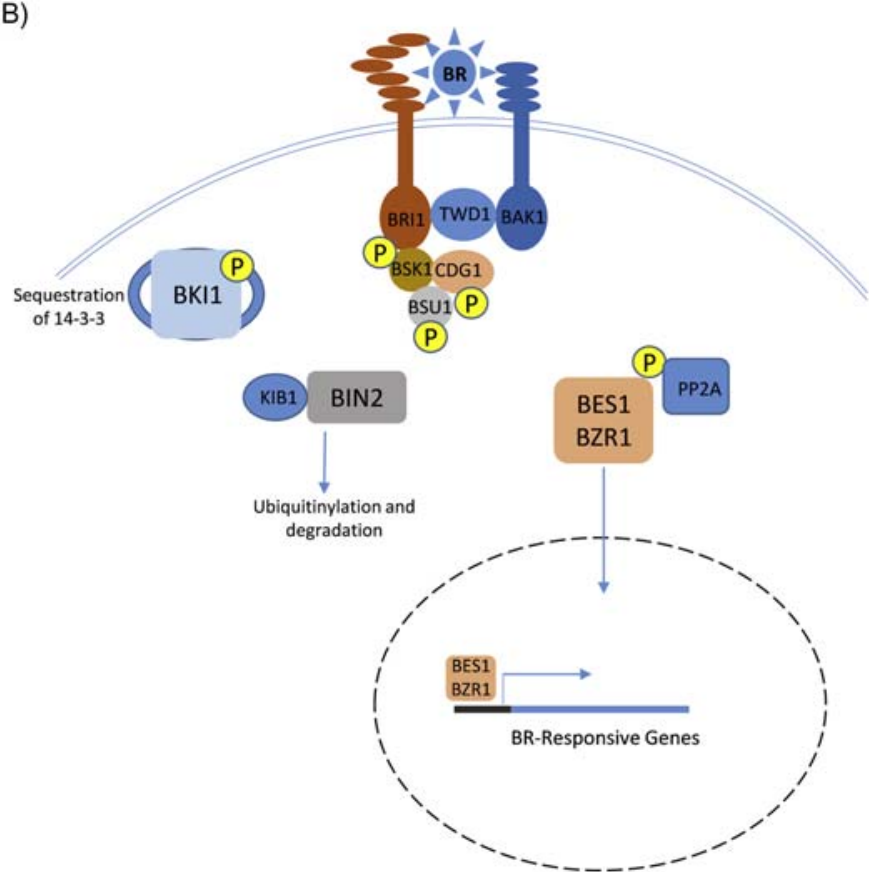

FIGURE 26.2 (A) A schematic view of BR-signaling in plant cell. A BR-regulated transcription factor BES1/BZR1 is the main player in BRsignaling. In absence of BR, it remains phosphorylated and cannot bind to BR responsive gene in the nucleus. 14-3-3 protein sequesters BES1/ BZR1 and keeps it phosphorylated. (See Section 26.3 for details.) (B) A schematic view of BR-signaling in plant cell. When BR is available and binds to BR receptors and coreceptors, this phosphorylates several interacting proteins. A protein, BKI1, when phosphorylated, sequesters 143-3 protein thus keeping BES1/BZR1 free. PP2A dephosphorylates BES1/BZR1 and later it enters in the nucleus to activates the BR responsive genes. (See Section 26.3 for details.) Source: Model adapted from Nolan, T.M., Brennan, B., Yang, M., Chen, J., Zhang, M., Li, Z., et al., 2017. Selective autophagy of BES1 mediated by DSK2 balances plant growth and survival. Dev. Cell 41, 33-46. 
TABLE 26.1 List of Proteins That Directly Bind to BES1 and or BZR1 and Regulate BR Responsive Gene Expression or Protein Stability

\begin{tabular}{|c|c|c|c|}
\hline Gene name & Molecular activity & $\begin{array}{l}\text { Binding } \\
\text { partner }\end{array}$ & References \\
\hline $\begin{array}{l}\text { PIFs (PHYTOCHROME-INTERACTING } \\
\text { FACTORS) }\end{array}$ & Regulation of BR responsive genes & BES1/BZR1 & $\begin{array}{l}\text { De Lucas and Prat } \\
\text { (2014) }\end{array}$ \\
\hline $\begin{array}{l}\text { ARF6/8 (AUXIN RESPONSIVE TRANSCRIPTION } \\
\text { FACTORS) }\end{array}$ & Regulation of growth and defense related genes & $\begin{array}{l}\text { PIF4 and } \\
\text { BZR1 }\end{array}$ & Oh et al. (2012) \\
\hline MYB30 & $\begin{array}{l}\text { Regulation of BR responsive gene induction and } \\
\text { ABA response }\end{array}$ & BES1 & Li et al. (2009) \\
\hline $\begin{array}{l}\text { HAT1 (HOMEODOMAIN-LEUCINE ZIPPER } \\
\text { PROTEIN 1) }\end{array}$ & Control of BR responsive genes & BES1 & Li et al. (2009) \\
\hline $\begin{array}{l}\text { BRAVO(BRs AT VASCULAR AND ORGANIZING } \\
\text { CENTER) }\end{array}$ & Regulation of cell division in quiescent center & BES1 & $\begin{array}{l}\text { Vilarrasa-Blasi et al. } \\
\text { (2014) }\end{array}$ \\
\hline BIM1 (BES1-INTERACTING MYC-LIKE1) & Control of BR responsive genes & BES1 & Yin et al. (2005) \\
\hline TOPLESS and TOPLESS Related (TPR) & Antagonist of ABA signaling & BES1 & $\begin{array}{l}\text { Espinosa-Ruiz et al. } \\
\text { (2017) }\end{array}$ \\
\hline DSK2(DOMINANT SUPPRESSOR OF KAR2) & BES1 degradation during stress condition & BES1 & Nolan et al. (2017) \\
\hline
\end{tabular}

(PIFs), BES1-INTERACTING MYC-LIKE1 (BIM1), the auxin responsive transcription factors (ARFS), $A T B S 1 /$ PRE (ACTIVATION-TAGGED bri1 SUPPRESSOR1/ PACLOBUTRAZOL-RESISTANCE), AIFS/IBH1 (ATBS1INTERACTING FACTORS/INCREASED LAMINA INCLINATION INTERACTING bHLH1) bHLH transcription factors, etc. (Wang et al., 2009; Zhang et al., 2009; de Lucas and Prat, 2014; Oh et al., 2012; Wang et al., 2012). PIFs are the transcription factors regulated by light and circadian clock. PIF4 has been validated to interact with BZR1 and forms a heterodimer, which together binds to the G-BOX (CACGTG) element present in the upstream promoter region of $\mathrm{BR}$ responsive genes. Similarly BIM1 interacts with BES1 and binds together with E-Box (CANNTG) element. The ARF6 and $A R F 8$ also shown to interact with $B Z R 1$ and later on bind to ARF motif (TGTCTC) present in the promoter region of BR signaling genes. BZR1, PIFs, and ARFs together activate the downstream basic HELIX LOOP HELIX (bHLH) transcription factors including ATBS1/PRE family protein, which works as antagonist to AIFs/IBH1 proteins and activate proteins, which promotes the cell elongation. Similarly, a number of other transcription factors are involved in BR signaling that are directly regulated by BES1/BZR1. A list of such proteins is included in Table 26.1.

\subsection{ROLE OF RD26 IN BR SIGNALING}

BR plays an important role in stress acclimation. Plants, being sessile in nature, try to reduce its growth and development to expense the resources in stress management (Claeys and Inze, 2013). BES1 is known to be involved in reduction of plant growth under stress conditions especially when the drought stress is encountered. RESPONSIVE TO DESICCATION 26 $(R D 26)$ is a NAC transcription factor, which has been shown to play a crucial role in abiotic stress signaling in plants. The expression of RD26 gene is regulated by $B E S 1$. BES1 binds to the BRRE-binding site present in the promoter region of RD26 gene (Tran et al., 2004). $B R$ response was found to be aberrated in plants overexpressing RD26 gene. The plants showed a strong phenotype of reduced growth and development. RD26 is induced under stress conditions thus providing a hint for RD26 as a connecting link between stress signaling and BR responses (Fujita et al., 2004). Genomewide analysis of these RD26OX plants revealed the antagonist expression of RD26 and BR-responsive genes. The output provided a hint that $R D 26$ might inhibit BR responsive signaling under stress conditions (Chung et al., 2014). BES1 and RD26 share their target genes, however one is an inducer and another is a repressor or vice versa. These two transcription factors are known to bind to E-Box element and BR-response element (BRRE) respectively. Both BES1 and RD26 were experimentally validated to be the interacting partner and together bind on to the DNA elements in an antagonistic fashion, thus neutralizing the gene regulation under normal plant growth conditions (Ye et al., 2017). While under stress conditions the expression of RD26 increases this result in increased expression of BR-repressed genes and reduced expression of 

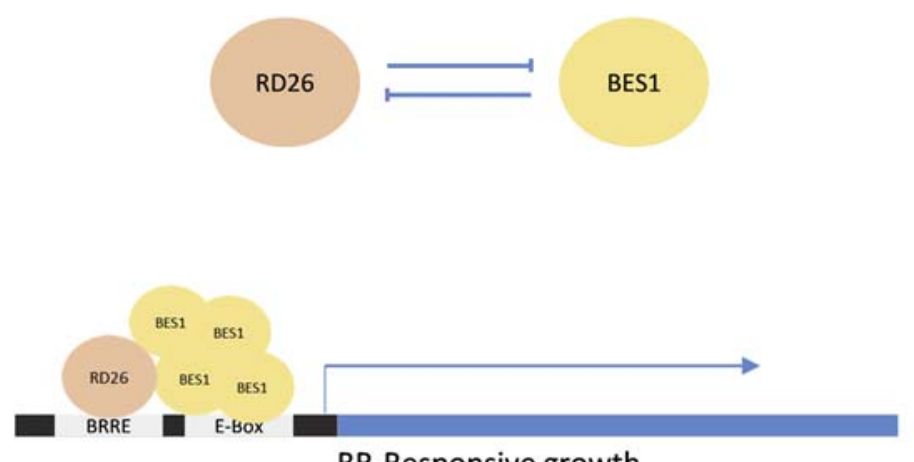

BR-Responsive growth

related genes

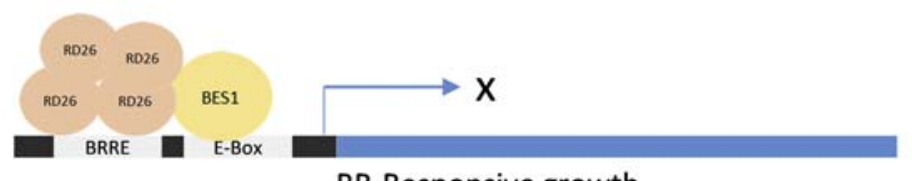

BR-Responsive growth

related genes

BES1 targeted or BR-induced growth related genes. Extensive gene expression analysis of BR-responsive genes and RD26 target gene under drought stress conditions (with external application of ABA) indicated that $R D 26, B E S 1$ and their target genes fluctuate in terms of expression to mitigate changing environmental conditions and sustain the plant growth (Fujita et al., 2004 ) (Fig. 26.3).

\subsection{BR MEDIATED DEFENSE SIGNALING}

The relationship of BR signaling and plant defense is similar to that of the BR signaling- drought crosstalk (Lozano-Durán and Zipfel, 2015). Plants need to utilize their resources carefully under stress conditions and this sometimes leads to a temporary inhibition of the growth and development while making the plant ready to tolerate the stress. As many BR responsive genes are involved in regulating plant growth and development, they are the first target to be suppressed to reduce the resource utilization and hence the crosstalk between BR-signaling genes and defense related genes needs to be established. Studies on the influence of BR signaling and defense indicated that PAMP triggered immunity or PTI in plants is somehow influenced by BR-receptors or BR-responsive genes. PTI is a defense mechanism in plants, which recognizes the pathogen associated molecular patterns (PAMPs) and activates the downstream defense signaling cascade. PAMPs are the chemical signature moiety derived from the pathogens and recognized by specific receptors present on the plasma membrane. Two main components of the BR signaling cascade, namely BAK1
FIGURE 26.3 Antagonistic regulation of RD26 and BES1 during drought stress.RD26 and BES1 are known to compete with each other for binding to BR-responsive gene promoters. When plants experience drought stress, the expression of RD26 is elevated and this inhibits binding of BES1 to BR-responsive genes thus inhibiting their expression. (See Section 26.5 for details.)
Drought

Condition and BRI1, have been shown to play an important role in PTI (Albrecht et al., 2012; Goddard et al., 2014). BAK1, a coreceptor for BR, also serves as coreceptor for bacterial PAMPs. It has been proved that bacterial flagellin (a PAMP) binds to its receptor FLAGELLIN SENSING 2 (FLS2) together with coreceptor BAK1 and hence it is proposed that bacterial PAMPs and BR compete with each other for BAK1 (Chinchilla et al., 2007). So in the case when the PTI is activated, the BR pathway is inhibited up to a certain extent and vice versa. Although several studies proposed different hypothesis for the mechanism of BAK1 action in PTI, the conclusion remains the same (Albrecht et al., 2012; Belkhadir et al., 2014). PTI in plants activates the ROS signaling, which leads to an oxidative burst in the cell, resulting in several defense related phenotypes like stomatal movement and callose deposition on the cell wall (Ingle et al., 2006; Nürnberger et al., 2004). An elevated BR signaling has been shown to reduce the oxidative burst in the plant cell and made plants more susceptible to the pathogen. For example, the overexpression of a BR biosynthetic gene has been shown to limit the plant's response against a bacterial PAMP, flg22 (Belkhadir et al., 2012). Moreover, the plants overexpressing BRI1 (BR receptor) resulted in similar phenotype indicating the antagonistic relationship between PTI and BR-signaling. A list of different experiments carried out to understand the effect of manipulation of BR-signaling genes on plants' pathogen defense has been summarized in Table 26.2 (also reviewed by Nolan et al., 2017). Another protein, BSK1, involved in BR-signaling, also plays a direct role in PTI via its interaction with FLS2 and positively regulates ROS production in the cell (Shi et al., 2013a,b). Two other members of BR-signaling related genes, 
TABLE 26.2 Effect of Genetic Manipulation of BR-Signaling Genes on Disease Susceptibility

\begin{tabular}{|c|c|c|c|c|}
\hline Host & Pathogen/PAMP & Genetic manipulation & Phenotype & References \\
\hline $\begin{array}{l}\text { Arabidopsis } \\
\text { thaliana }\end{array}$ & flg22, elf19 & BRI1 Overexprssion & $\begin{array}{l}\text { Suppression of PTI, reduced ROS } \\
\text { production }\end{array}$ & $\begin{array}{l}\text { Belkhadir et al. } \\
\text { (2012) }\end{array}$ \\
\hline $\begin{array}{l}\text { Arabidopsis } \\
\text { thaliana }\end{array}$ & $\begin{array}{l}\text { Pseudomonas syringae pv. } \\
\text { tomatoDC } 3000\end{array}$ & bik1 mutant & Enhanced Susceptibility & Lu et al. (2010) \\
\hline $\begin{array}{l}\text { Arabidopsis } \\
\text { thaliana }\end{array}$ & flg22 & $\operatorname{bin} 2$ & $\begin{array}{l}\text { Suppression of PTI, reduced ROS } \\
\text { production }\end{array}$ & $\begin{array}{l}\text { Lozano-Durán et al. } \\
\text { (2013) }\end{array}$ \\
\hline $\begin{array}{l}\text { Arabidopsis } \\
\text { thaliana }\end{array}$ & Thrips tabaci & bzr1-D mutant & Enhanced tolerance & Miyaji et al. (2014) \\
\hline $\begin{array}{l}\text { Arabidopsis } \\
\text { thaliana }\end{array}$ & $\begin{array}{l}\text { Pseudomonas syringae pv. } \\
\text { tomatoDC } 3000\end{array}$ & JUB1 Overexpression & Enhanced susceptibility & $\begin{array}{l}\text { Shahnejat-Bushehri } \\
\text { et al. (2016) }\end{array}$ \\
\hline $\begin{array}{l}\text { Arabidopsis } \\
\text { thaliana }\end{array}$ & $\begin{array}{l}\text { Pseudomonas syringae pv. } \\
\text { tomatoDC } 3000\end{array}$ & bes1 mutant & Enhanced susceptibility & Kang et al. (2015) \\
\hline Hordeum vulgare & Fusarium culmorum & $\begin{array}{l}\text { BRI1 mutation in kinase } \\
\text { domain }\end{array}$ & Enhanced tolerance & Ali et al. (2014) \\
\hline Brassica napus & $\begin{array}{l}\text { Sclerotinia sclerotiorum, } \\
\text { Leptosphaeria maculans }\end{array}$ & AtDWF4 Overexpression & Enhanced tolerance & Sahni et al. (2016) \\
\hline $\begin{array}{l}\text { Brachypodium } \\
\text { distachyon }\end{array}$ & Necrotrophic fungus & bri1 mutation & Enhanced tolerance & Goddard et al. (2014) \\
\hline
\end{tabular}

namely BIN2 and BZR1, were also reported to enhance tolerance against pathogen attack when inhibited or mutated (Lozano-Durán et al., 2013; Miyaji et al., 2014). Hence, providing supporting evidence for the hypothesis stating that the suppression of BR signaling decreases the susceptibility against pathogen attack. However this does not hold true for every experiment carried out and for different kinds of pathogen, indicating that the mechanism and interplay of BRsignaling is complex and the response might vary according to experimental setup. A study on BES1 is one such example where the mutants were shown to behave differently for a fungal and a bacterial pathogen. BES1 was shown to be a potential candidate connecting the MAPK (mitogen-activated protein kinase) signaling pathway of plant defense to BR-signaling (Kang et al., 2015). Another example comes from Brassica napus, where the overexpression of a BR biosynthetic gene, AtDWF4, leads to enhance disease tolerance against fungal pathogens. While plants from Belkhadir et al. (2012), where bacterial PAMP were used, displayed compromised tolerance.

\subsection{BR MEDIATED ROS SIGNALING AND ITS ROLE IN PLANT DEFENSE}

ROS are often considered as major determinant of stress and serve as a key player in various signaling pathways in plant cells, entailing in photosynthetic regulation, perception of abiotic or pathogen response and hormonal action, programmed cell death (PCD), and other important growth and developmental pathways (Dat et al., 2000; Mittler, 2002; Mullineaux and Karpinski, 2002; Apel and Hirt, 2004; Khan and Khan, 2017). ROS consist of free radicals corresponding to superoxide $\left(\cdot \mathrm{O}_{2}^{-}\right)$and hydroxyl radicals $(\cdot \mathrm{OH})$, and nonfree radicals such as hydrogen peroxide $\left(\mathrm{H}_{2} \mathrm{O}_{2}\right)$ and singlet oxygen $\left(\mathrm{O}_{2}\right)$. In plants, ROS are mainly generated during the process of respiration, photosynthesis, and $\mathrm{N}_{2}$ fixation in the chloroplast, mitochondria, peroxisome, cytosol, plasma membrane, and the apoplastic space. Furthermore, generation of ROS in cell wall bound peroxidases takes place in the apoplastic space. While, in the plasma membrane, an NADPH oxidase complex functions as the ROSproducing system (Bolwell, 1999). Suzuki and Mittler (2006) interpreted that $\mathrm{ROS}$, like superoxide $\left(. \mathrm{O}_{2^{-}}\right)$, are produced by NADPH oxidases during stress and trigger the downstream stress-response pathways and thereby induce several underlying defense pathways. Besides this, ROS lead to oxidative burst by initiating membrane depolarization and influx of calcium ions inside the cell, which evoke several pathways required for combating both biotic and abiotic stresses. This includes defense related pathways, production of antioxidant enzymes, dehydrins and heat shock proteins, synthesis of pathogenesis-related proteins (Gechev et al., 2006), MAPKs signaling (Apel and Hirt, 2004), etc. 
$\mathrm{H}_{2} \mathrm{O}_{2}$ is one of the most stable ROS having the ability to behave both as an oxidant and as a reductant (Salin, 1991). Being a strong oxidant it may trigger localized oxidative damage leading to disruption of vital metabolic function and can also diffuse. Conversely, to mediate ROS signaling, $\mathrm{H}_{2} \mathrm{O}_{2}$ plays multiple roles by behaving as a signal, a mediator, and an effector molecule (Levine et al., 1994) and was found to accumulate in response to both biotic and abiotic stresses. Additionally, to reinforce the cell wall, $\mathrm{H}_{2} \mathrm{O}_{2}$ is utilized by apoplastic peroxidases that catalyze the cross-linking between and polysaccharides and cell wall extension, hampering the pathogen penetration (Passardi et al., 2004). Moreover, being as a secondary messenger in intracellular signaling pathways, it induces expression of genes involved in pathogen response and chilling acclimation (Chen et al., 1993; Prasad et al., 1994). It is also identified as one of the earliest factors involved in the transcriptional activation of defense related genes in Birch (Pellinen et al., 2002). Owing to stresses, sudden and transient increase of $\mathrm{H}_{2} \mathrm{O}_{2}$ is considered as a general alarm signal for subsequent eliciting the durable form of defenses that are helpful in facilitating protection against all these stresses (Mittler and Berkowitz, 2001). Additionally, $\mathrm{H}_{2} \mathrm{O}_{2}$ may also contribute in induction of systemic acquired acclimation (SAA), which is raised due to exposure of one part of the plant to high light that renders the unexposed parts resilient to high light (Karpinski et al., 1999). BR-induced $\mathrm{H}_{2} \mathrm{O}_{2}$ accumulation is caused by increased activity of NADPH oxidase as confirmed by measuring the activity of plasma membrane NADPH oxidase in plants treated with 24epibrassinolide (EBR), which is a bioactive brassinosteroid derivative (Xia et al., 2009). Additionally, EBR was also shown to be effective in restoring the NADPH oxidase activity of brassinazole (BRZ, a specific inhibitor of BR biosynthesis) treated plants. Similarly exogenously applied $\mathrm{H}_{2} \mathrm{O}_{2}$ showed a similar pattern to that of EBR (Deng et al., 2016). Studies suggest that apart from BAK1 (for BRI1-associated receptor kinase 1), BIN2 (a GSK3/SHAGGY-like kinase), BSU1 (for BRI1 suppressor 1, a phosphatase) and, three other regulatory genes, $\mathrm{RBOH}$ (respiratory burst oxidase homolog), MAPK1 (mitogen-activated protein kinases), and MAPK3, were upregulated upon treatment with EBR but downregulated after BRZ treatment emphasizing their vital role (Xia et al., 2009).

Exogenous application of BR on the leaves of Niotiana benthamiana were shown to enhance the plant tolerance against several different pathogens (Nakashita et al., 2003). This is accompanied by accumulation of BR-induced MAPK and RBOHB (an NADPH oxidase B) genes and subsequent ROS burst. Eventually, the end products of the target genes directly take part into the cellular protection. Nevertheless, further studies are warranted to provide genetic evidence for NADPH oxidase's role in BR-induced ROS generation (Deng et al., 2016). This would pave the way to unveil the critical signaling components in BR signal perception and downstream stress responses, and to elucidate the molecular mechanisms involved in the crosstalk between BR and other hormones.

\subsection{CONCLUSION}

Elucidation of BR signaling pathways is still a challenging task for scientists worldwide. Its multifaceted nature and crosstalk with different growth and developmental pathways in plants make it a complex system to study. As far as stress signaling and $B R$ crosstalk is concerned, a clear role of BR has been established. However, the availability of different model systems and different experimental approaches make it more complicated to untangle the exact pathway. The role of BR signaling in both biotic and abiotic stresses seems to be connected with ROS signaling and potential of an extensive crosstalk between two pathways can be visualized but the BR signaling research is still in its juvenile phase and many more factors are needed to be uncovered to have a clear view of this complex machinery in the plant cell.

\section{References}

Albrecht, C., Boutrot, F., Segonzac, C., Schwessinger, B., GimenezIbanez, S., Chinchilla, D., et al., 2012. Brassinosteroids inhibit pathogen-associated molecular pattern-triggered immune signalling independent of the receptor kinase BAK1. Proc. Natl Acad. Sci. U.S.A. 109, 303-308.

Ali, S.S., Gunupuru, L.R., Kumar, G.B.S., Khan, M., Scofield, S., Nicholson, P., et al., 2014. Plant disease resistance is augmented in uzu barley lines modified in the brassinosteroid receptor BRI1. BMC Plant Biol. 14, 227.

Apel, K., Hirt, H., 2004. Reactive oxygen species: metabolism, oxidative stress, and signal transduction. Annu. Rev. Plant Biol. 55, 373-399.

Ashraf, M., Akram, N.A., Arteca, R.N., Foolad, M.R., 2010. The physiological, biochemical and molecular roles of brassinosteroids and salicylic acid in plant processes and salt tolerance. Crit Rev. Plant Sci. 29, 162-190.

Bajguz, A., 2007. Metabolism of brassinosteroids in plants. Plant Physiol Biochem. 45, 95-107.

Bajguz, A., Hayat, S., 2009. Effects of brassinosteroids on the plant responses to environmental stresses. Plant Physiol Biochem. 47, $1-8$.

Belkhadir, Y., Jaillais, Y., Epple, P., Balsemao-Pires, E., Dangl, J.L., Chory, J., 2012. Brassinosteroids modulate the efficiency of plant immune responses to microbe-associated molecular patterns. Proc. Natl Acad. Sci. U.S.A. 109, 297-302.

Belkhadir, Y., Yang, L., Hetzel, J., Dangl, J.L., Chory, J., 2014. The growth-defence pivot: crisis management in plants mediated by LRR-RK surface receptors. Trends Biochem. Sci. 39, 447-456. 
Bishop, G.J., Yokota, T., 2001. Plants steroid hormones, brassinosteroids: current highlights of molecular aspects on their synthesis / metabolism, transport, perception and response. Plant Cell Physiol. 42, 114-120.

Bolwell, G.P., 1999. Role of reactive oxygen species and NO in plant defence responses. Curr. Opin. Plant Biol. 2, 287-294.

Cano-Delgado, A., Yin, Y., Yu, C., Vafeados, D., Mora-Garcia, S., Cheng, J.C., et al., 2004. BRL1 and BRL3 are novel brassinosteroid receptors that function in vascular differentiation in Arabidopsis. Development 131, 5341-5351.

Chen, Z., Silva, H., Klessig, D.F., 1993. Chilling-induced oxidative stress in maize seedlings and a regulatory role for hydrogen peroxide. Plant Cell 6, 65-74.

Chinchilla, D., Zipfel, C., Robatzek, S., Kemmerling, B., Nürnberger, T., Jones, J.D.G., et al., 2007. A flagellin-induced complex of the receptor FLS2 and BAK1 initiates plant defence. Nature 448, 497-500.

Choudhary, S.P., Yu, J.Q., Yamaguchi-Shinozaki, K., Shinozaki, K., Tran, L.S.P., 2012. Benefits of brassinosteroid crosstalk. Trends in Plant Sci. 17 (10), 594-605.

Chung, Y., Kwon, S.I., Choe, S., 2014. Antagonistic regulation of Arabidopsis growth by brassinosteroids and abiotic stresses. Mol. Cells 37, 795-803.

Claeys, H., Inze, D., 2013. The agony of choice: how plants balance growth and survival under water-limiting conditions. Plant Physiol. 162, 1768-1779.

Clouse, S.D., 2011. Brassinosteroids. The Arabidopsis Book 011 (9), e0151.

Clouse, S.D., Langford, M., McMorris, T.C., 1996. A brassinosteroidinsensitive mutant in Arabidopsis thaliana exhibits multiple defects in growth and development. Plant Physiol. 111, 671-678.

Dat, J., Vandenabeele, S., Vranova, E., Van Montagu, M., Inze, D., Van Breusegem, F., 2000. Dual action of the active oxygen species during plant stress responses. Cell. Mol. Life Sci. 57, 779-795.

de Lucas, M., Prat, S., 2014. PIFs get BR right: phytochrome interacting factors as integrators of light and hormonal signals. New Phytol. 202, 1126-1141.

Deng, X.G., Zhu, T., Zou, L.J., Han, X.Y., Zhou, X., Xi, D.H., et al., 2016. Orchestration of hydrogen peroxide and nitric oxide in brassinosteroid-mediated systemic virus resistance in Nicotiana benthamiana. The Plant Journal 85, 478-493.

Espinosa-Ruiz, A., Martínez, C., de Lucas, M., Fàbregas, N., Bosch, N., Caño-Delgado, A.I., et al., 2017. TOPLESS mediates brassinosteroid control of shoot boundaries and root meristem development in Arabidopsis thaliana. Development 144, 1619-1628.

Fujita, M., Fujita, Y., Maruyama, K., Seki, M., Hiratsu, K., OhmeTakagi, M., et al., 2004. A dehydration-induced NAC protein, RD26, is involved in a novel ABA-dependent stress-signalling pathway. Plant J. 39, 863-876.

Gampala, S.S., Kim, T.-W., He, J.-X., Tang, W., Deng, Z., Bai, M.-Y., et al., 2007. An essential role for 14-3-3 proteins in brassinosteroid signal transduction in Arabidopsis. Dev. Cell 13, 177-189.

Gechev, T.S., Van Breusegem, F., Stone, J.M., Denev, I., Laloi, C., 2006. Reactive oxygen species as signals that modulate plant stress responses and programmed cell death. Bioessays 28 (11), 1091-1101.

Goddard, R., Peraldi, A., Ridout, C., Nicholson, P., 2014. Enhanced disease resistance caused by BRI1 mutation is conserved between Brachypodium distachyon and barley (Hordeum vulgare). Mol. Plant Microbe Interact. 27, 1095-1106.

Grove, M.D., Spencer, G.F., Rohwedder, W.K., Mandava, N., Worley, J.F., Warthen, J.D., et al., 1979. Brassinolide, a plant growth promoting steroid isolated from Brassica napus pollen. Nature 281, 216-217.

Hecht, V., Vielle-Calzada, J.P., Hartog, M.V., Schmidt, E.D., Boutilier, K., Grossniklaus, U., et al., 2001. The Arabidopsis SOMATIC
EMBRYOGENESIS RECEPTOR KINASE 1gene is expressed in developing ovules and embryos and enhancesembryogenic competence in culture. Plant Physiol. 127, 803-816.

Ingle, R.A., Carstens, M., Denby, K.J., 2006. PAMP recognition and the plant-pathogen arms race. Bioessays 28, 880-889.

Kadota, Y., Shirasu, K., Zipfel, C., 2015. Regulation of the NADPH oxidase RBOHD during plant immunity. Plant Cell Physiol. 56 (8), 1472-1480.

Kang, S., Yang, F., Li, L., Chen, H., Chen, S., Zhang, J., 2015. The Arabidopsis transcription factor BRASSINOSTEROID INSENSITIVE 1-ETHYL METHANESULFONATE-SUPPRESSOR 1 is a direct substrate of MITOGEN-ACTIVATED PROTEIN KINASE 6 and regulates immunity. Plant Physiol. 167, 1076-1086.

Karpinski, S., Reynolds, H., Karpinska, B., Wingsle, G., Creissen, G., Mullineaux, P., 1999. Systemic signalling and acclimation in response to excess excitation energy in Arabidopsis. Science 284, $654-657$.

Khan, M.I.R., Khan, N., 2017. Reactive Oxygen Species and Antioxidant System in Plants: Role and Regulation Under Abiotic Stress. Springer Nature 978-981-10-5254-5.

Kim, T.-W., Guan, S., Sun, Y., Deng, Z., Tang, W., Shang, J.-X., et al., 2009. Brassinosteroid signal transduction from cell-surface receptor kinases to nuclear transcription factors. Nat. Cell Biol. 11, $1254-1260$.

Kim, T.-W., Guan, S., Burlingame, A.L., Wang, Z.-Y., 2011. The CDG1 kinase mediates brassinosteroid signal transduction from BRI1 receptor kinase to BSU1 phosphatase and GSK3-like kinase BIN2. Mol. Cell 43, 561-571.

Krishna, P., 2003. Brassinosteroid-mediated stress responses. J. Plant Growth Regul. 22, 289-297.

Kutschera, U., Wang, Z.Y., 2012. Brassinosteroid action in flowering plants: a Darwinian perspective. J. Exp. Bot. 63, 3511-3522.

Levine, A., Tenhaken, R., Dixon, R., Lamb, C., 1994. H2O2 from the oxidative burst orchestrates the plant hypersensitive disease resistance response. Cell 79, 583-593.

Li, J., 2010. Regulation of the nuclear activities of brassinosteroid signalling. Curr. Opin. Plant Biol. 13, 540-547.

Li, J., Chory, J., 1997. A putative leucine-rich repeat receptor kinase involved in brassinosteroid signal transduction. Cell 90, 929-938.

Li, L., Yu, X., Thompson, A., Guo, M., Yoshida, S., Asami, T., et al., 2009. Arabidopsis MYB30 is a direct target of BES1 and cooperates with BES1 to regulate brassinosteroid-induced gene expression. Plant J. 58 (2), 275-286.

Lorenzo, O., Solano, R., 2005. Molecular players regulating the jasmonate signalling network. Curr. Opin. Plant Biol. 8, 532-540.

Lozano-Durán, R., Zipfel, C., 2015. Trade-off between growth and immunity: role of brassinosteroids. Trends Plant Sci. 20, 12-19.

Lozano-Durán, R., Macho, A.P., Boutrot, F., Segonzac, C., Somssich, I.E., Zipfel, C., 2013. The transcriptional regulator BZR1 mediates trade-off between plant innate immunity and growth. eLife 2, e00983.

Lu, D., Wu, S., Gao, X., Zhang, Y., Shan, L., He, P., 2010. A receptorlike cytoplasmic kinase, BIK1, associates with a flagellin receptor complex to initiate plant innate immunity. Proc. Natl Acad. Sci. U.S.A. 107, 496-501.

Mauch-Mani, B., Mauch, F., 2005. The role of abscisic acid in plant-pathogen interactions. Curr. Opin. Plant Biol. 8, 409-414.

Mitchell, J., Mandava, N., Worley, J., Plimmer, J., Smith, M., 1970. Brassins-a new family of plant hormones from rape pollen. Nature 225, 1065-1066.

Mittler, R., 2002. Oxidative stress, antioxidants and stress tolerance. Trends Plant Sci. 7, 405-410.

Mittler, R., Berkowitz, B., 2001. Hydrogen peroxide, a messenger with too many roles? Redox Rep. 6, 69-72. 
Mittler, R., Vanderauwera, S., Suzuki, N., Miller, G., Tognetti, V.B., Vandepoele, K., et al., 2011. ROS signalling: the new wave? Trends Plant Sci. 16, 300-910.

Miyaji, T., Yamagami, A., Kume, N., Sakuta, M., Osada, H., Asami, T., et al., 2014. Brassinosteroid-related transcription factor BIL1/BZR1 increases plant resistance to insect feeding. Biosci. Biotechnol. Biochem. 78, 960-968.

Mullineaux, P., Karpinski, S., 2002. Signal transduction in response to excess light: getting out of the chloroplast. Curr. Opin. Plant Biol. 5, 43-48.

Nakashita, H., Nitta, T., Asami, T., Fujioka, S., Arai, Y., Sekimata, K., et al., 2003. Brassinosteroid functions in a broad range of disease resistance in tobacco and rice. Plant J. 33, 887-898.

Nam, K.H., Li, J., 2002. BRI1/BAK1, a receptor kinase pair mediating brassinosteroid signalling. Cell 110, 203-212.

Nolan, T.M., Brennan, B., Yang, M., Chen, J., Zhang, M., Li, Z., et al., 2017. Selective autophagy of BES1 mediated by DSK2 balances plant growth and survival. Dev. Cell 41, 33-46.

Nürnberger, T., Brunner, F., Kemmerling, B., Piater, L., 2004. Innate immunity in plants and animals: striking similarities and obvious differences. Immunol. Rev. 198, 249-266.

Oh, E., Zhu, J.-Y., Wang, Z.-Y., 2012. Interaction between BZR1 and PIF4 integrates brassinosteroid and environmental responses. Nat. Cell Biol. 14, 802-809.

Passardi, F., Penel, C., Dunand, C., 2004. Performing the paradoxical: how plant peroxidases modify the cell wall. Trends Plant Sci. 9 (11), 534-540.

Pellinen, R.I., Korhonen, M.-S., Tauriainen, A.A., Palva, T., Kangasjärvi, J., 2002. Hydrogen peroxide activates cell death and defence gene expression in birch. Plant Physiol. 130, 549-560.

Prasad, T.K., Anderson, M.D., Stewart, C.R., 1994. Acclimation, Hydrogen peroxide, and Abscisic acid protect mitochondria against irreversible chilling injury in maize seedlings. Plant Physiol. 105, 619-627.

Ryu, H., Kim, K., Cho, H., Park, J., Choe, S., Hwang, I., 2007. Nucleocytoplasmic shuttling of BZR1 mediated by phosphorylation is essential in Arabidopsis brassinosteroid signalling. Plant Cell 19, 2749-2762.

Sahni, S., Prasad, B.D., Liu, Q., Grbic, V., Sharpe, A., Singh, S.P., et al., 2016. Overexpression of the brassinosteroid biosynthetic gene DWF4 in Brassica napus simultaneously increases seed yield and stress tolerance. Sci. Rep. 6, 28298.

Salin, M.L., 1991. Chloroplast and mitochondrial mechanisms for protection against oxygen toxicity. Free Rad. Res. Commun. 12, 851-858.

Sewelam, N., Kazan, K., Schenk, P.M., 2016. Global plant stress signalling: reactive oxygen species at the cross-road. Front. Plant Sci.

Shahnejat-Bushehri, S., Nobmann, B., Devi Allu, A., Balazadeh, S., 2016. JUB1 suppresses Pseudomonas syringae-induced defence responses through accumulation of DELLA proteins. Plant Signal. Behav. 11, e1181245.

Shi, H., Shen, Q., Qi, Y., Yan, H., Nie, H., Chen, Y., et al., 2013a. BRSIGNALLING KINASE 1 physically associates with FLAGELLIN SENSING 2 and regulates plant innate immunity in Arabidopsis. Plant Cell 25, 1143-1157.

Shi, H., Yan, H., Li, J., Tang, D., 2013b. BSK1, a receptor-like cytoplasmic kinase, involved in both BR signalling and innate immunity in Arabidopsis. Plant Signal. Behav. 8, e24996.

Sun, Y., Fan, X.-Y., Cao, D.-M., Tang, W., He, K., Zhu, J.-Y., et al., 2010. Integration of brassinosteroid signal transduction with the transcription network for plant growth regulation in Arabidopsis. Dev. Cell 19, 765-777.

Suzuki, N., Mittler, R., 2006. Reactive oxygen species and temperature stresses: a delicate balance between signalling and destruction. Physiol Plant. 126, 45.
Suzuki, N., Miller, G., Morales, J., Shulaev, V., Torres, M.A., Mittler, R., 2011. Respiratory burst oxidases: the engines of ROS signalling. Curr. Opin. Plant Biol. 14 (6), 691-699.

Takatsuto, S., 1994. Brassinosteroids: distribution in plants, bioassays and microanalysts by gas chromatography-mass spectrometry. J Chromatogr. A 658, 3-15.

Tang, J., Han, Z., Chai, J., 2016. Q\&A: what are brassinosteroids and how do they act in plants? BMC biology 14, 113.

Tang, W., Kim, T.-W., Oses-Prieto, J.A., Sun, Y., Deng, Z., Zhu, S., et al., 2008. BSKs mediate signal transduction from the receptor kinase BRI1 in Arabidopsis. Science 321, 557-560.

Teale, W.D., Ditengou, F.A., Dovzhenko, A.D., Li, X., Molendijk, A.M., Ruperti, B., et al., 2008. Auxin as a model for the integration of hormonal signalprocessing and transduction. Mol. Plant 1, 229-237.

Tran, L.S.P., Nakashima, K., Sakuma, Y., Simpson, S.D., Fujita, Y., Maruyama, K., et al., 2004. Isolation and functional analysis of Arabidopsis stress-inducible NAC transcription factors that bind to a drought-responsive cis-element in the early responsive to dehydration stress 1 promoter. Plant Cell 16, 2481-2498.

Vilarrasa-Blasi, J., González-García, M.-P., Frigola, D., Fábregas, N., Alexiou, K.G., López-Bigas, N., et al., 2014. Regulation of plant stem cell quiescence by a brassinosteroid signalling module. Dev. Cell 30, 36-47.

Wang, H., Zhu, Y., Fujioka, S., Asami, T., Li, J., Li, J., 2009. Regulation of Arabidopsis brassinosteroid signalling by atypical basic helix-loop-helix proteins. Plant Cell 21, 3781-3791.

Wang, X., Chory, J., 2006. Brassinosteroids regulate dissociation of BKI1, a negative regulator of BRI1 signalling, from the plasma membrane. Science 313, 1118-1122.

Wang, X., Goshe, M.B., Soderblom, E.J., Phinney, B.S., Kuchar, J.A., $\mathrm{Li}$, J., et al., 2005. Identification and functional analysis of in vivo phosphorylation sites of the Arabidopsis BRASSINOSTEROIDINSENSITIVE1 receptor kinase. Plant Cell 17, 1685-1703.

Wang, Z.-Y., Bai, M.-Y., Oh, E., Zhu, J.-Y., 2012. Brassinosteroid signalling network and regulation of photomorphogenesis. Ann. Rev. Genet. 46, 701-724.

Wei, Z., Li, J., 2016. Brassinosteroids regulate root growth, development, and symbiosis. Mol Plant. 9, 86-100.

Xia, X.J., Wang, Y.J., Zhou, Y.H., Tao, Y., Mao, W.H., Shi, K., et al., 2009. Reactive oxygen species are involved in brassinosteroids induced stress tolerance in Cucumis sativus. Plant Physiol. 150, 801-814.

Ye, H., Liu, S., Tang, B., Chen, J., Xie, Z., Nolan, T.M., et al., 2017. RD26 mediates crosstalk between drought and brassinosteroidsignalling pathways. Nat. Commun. 8, 14573.

Yin, Y., Vafeados, D., Tao, Y., Yoshida, S., Asami, T., Chory, J., 2005. A new class of transcription factors mediates brassinosteroidregulated gene expression in Arabidopsis. Cell 120 (2), 249-259.

Yu, X., Li, L., Zola, J., Aluru, M., Ye, H., Foudree, A., et al., 2011. A brassinosteroid transcriptional network revealed by genomewide identification of BESI target genes in Arabidopsis thaliana. Plant J. 65, 634-646.

Zhang, L.-Y., Bai, M.-Y., Wu, J., Zhu, J.-Y., Wang, H., Zhang, Z., et al., 2009. Antagonistic HLH/bHLH transcription factors mediate brassinosteroid regulation of cell elongation and plant development in rice and Arabidopsis. Plant Cell 21, 3767-3780.

Zhao, B., Lv, M., Feng, Z., Campbell, T., Liscum, E., Li, J., 2016. TWISTED DWARF 1 associates with BRASSINOSTEROIDINSENSITIVE 1 to regulate early events of the brassinosteroid signalling pathway. Mol. Plant 9, 582-592.

Zhu, J.-Y., Li, Y., Cao, D.-M., Yang, H., Oh, E., Bi, Y., et al., 2017. The F-box protein KIB1 mediates brassinosteroid-induced inactivation and degradation of GSK3-like kinases in Arabidopsis. Mol. Cell $66,648-657$. 


\section{Further Reading}

He, J.-X., Gendron, J.M., Yang, Y., Li, J., Wang, Z.-Y., 2002. The GSK3-like kinase BIN2 phosphorylates and destabilizes BZR1, a positive regulator of the brassinosteroid signalling pathway in Arabidopsis. Proc. Natl Acad. Sci. U.S.A. 99, 10185-10190.

Lin, F., Ding, H.D., Wang, J.X., Zhang, H., Zhang, A.Y., Zhang, Y., et al., 2009. Positive feedback regulation of maize NADPH oxidase by mitogen-activated protein kinase cascade in abscisic acid signalling. J. Exp. Bot. 60, 3221-3238.

Mittler, R., Vanderauwera, S., Gollery, M., Van Breusegem, F., 2004. Reactive oxygen gene network of plants. Trends Plant Sci. 9, 490-498.
Xia, X.-J., Gao, C.-J., Song, L.-X., Zhou, Y.-H., Shi, K., Yu, J.-Q., 2014. Role of $\mathrm{H} 2 \mathrm{O} 2$ dynamics in brassinosteroid-induced stomatal closure and opening in Solanum lycopersicum. Plant Cell Environ. 37, 2036-2050.

Zhang, A.Y., Jiang, M.Y., Zhang, J.H., Tan, M.P., Hu, X.L., 2006. Mitogenactivatedprotein kinase is involved in abscisic acidinduced antioxidant defence and acts downstream of reactive oxygen species production in leaves of maize plants. Plant Physiol. 141, 475-487.

Zhou, J., Wang, J., Li, X., Xia, X.-J., Zhou, Y.-H., Shi, K., et al., 2014. $\mathrm{H} 2 \mathrm{O} 2$ mediates the crosstalk of brassinosteroid and abscisic acid in tomato responses to heat and oxidative stresses. J. Exp. Bot. 65, $4371-4383$ 


\section{7}

\section{Role and Regulation of Osmolytes and ABA Interaction in Salt and Drought Stress Tolerance}

Guddimalli Rajasheker ${ }^{1}$, Gandra Jawahar ${ }^{1}$, Naravula Jalaja ${ }^{2}$, Somanaboina Anil Kumar ${ }^{1}$, Palavalasa Hima Kumari ${ }^{1}$, Devineni Lakshmi Punita ${ }^{1}$, Appa Rao Karumanchi, Palakolanu Sudhakar Reddy, Polavarapu Rathnagiri, Nese Sreenivasulu ${ }^{6}$ and Polavarapu Bilhan Kavi Kishor ${ }^{1}$

${ }^{1}$ Department of Genetics, Osmania University, Hyderabad, Telangana, India ${ }^{2}$ Department of Biotechnology, Vignan University, Vadlamudi, Guntur, India ${ }^{3}$ Department of Biotechnology, Acharya Nagarjuna University, Nagarjuna Nagar, Guntur, India ${ }^{4}$ International Crops Research Institute for the Semi-Arid Tropics (ICRISAT), Patancheru, Hyderabad, Telangana, India ${ }^{5}$ Genomix CARL Pvt. Ltd., Kadapa, Andhra Pradesh, India ${ }^{6}$ Grain Quality and Nutrition Center, Plant Breeding Division, International Rice Research Institute, Metro Manila, Philippines

\section{O U T L I N E}

27.1 Introduction

27.2 Abscisic Acid-Sensing Mechanism of Plants and Downstream Events

27.3 Role of Abscisic Acid in Osmolyte Biosynthesis

27.3.1 Abscisic Acid-Dependent and -Independent Signaling Pathways and Proline Biosynthesis

27.3.2 Role of Hormones in the Regulation of P5CS and Proline Synthesis

27.4 Regulation of Proline Dehydrogenase 27.4.1 Glycine Betaine Biosynthesis and Its Modulation by Abscisic Acid

27.5 Signaling Molecules and Osmolyte Synthesis
41827.6 Functions of Osmolytes During Abiotic Stress 27.6.1 Osmolyte Accumulation and Osmotic Adjustment During Stress

27.6.2 Osmolytes and Protection of Photosynthetic Machinery During Abiotic Stress

27.6.3 Osmolyte Accumulation and Oxidative Stress

27.6.4 Osmolytes and Amelioration of $\mathrm{NaCl}$ - and Metal-Induced $\mathrm{K}^{+}$Efflux Under Stress 426

27.6.5 Osmolytes and Their Metal Chelation Properties During Metal Stress 426

27.6.6 Role of Osmolytes in Membrane and Native Protein Structure Stabilizations 428

27.6.7 Osmolytes as Sources of Energy and Carbon Reserve During and After the Release of Stress 
27.7 Osmolytes and Signaling Processes

27.7.1 Proline and Signaling Processes

27.7.2 Proline Metabolism and Signaling Pathways in Plant Senescence

27.7.3 Osmolytes as Sensing Compounds and/or Growth Regulators
429

429

429

430
27.8 Conclusions and Future Prospects

Acknowledgments

References

Further Reading

\subsection{INTRODUCTION}

Plants are constantly exposed to diverse abiotic stresses like salinity, drought, cold, high temperature, and high light intensity (photooxidative stress) during their life cycle. Such stresses initiate oxidative stress releasing reactive oxygen species (ROS) that comprise free radicals $\left(\mathrm{O}_{2}{ }^{\bullet},{ }^{1} \mathrm{O}_{2}, . \mathrm{OH}, \mathrm{HO}_{2}\right.$ • $)$ and nonradical forms $\left(\mathrm{H}_{2} \mathrm{O}_{2}\right)$. Production of ROS damages cellular fabric, disrupts metabolism, and causes functional loss of cell organelles. This leads to death of plants in the majority of the cases (Blokhina et al., 2003; Khan and Khan, 2017). To minimize the deleterious effect of ROS, plants develop mechanisms such as scavenging them with antioxidative enzyme systems like ascorbate peroxidase (APX), catalase (CAT), glutathione $S$-transferase, superoxide dismutase (SOD), etc., or quenching them with the help of nonenzymatic molecules such as ascorbic acid, reduced glutathione, $\alpha$-tocopherol, carotenoids, flavonoids, and osmolytes such proline, glycine betaine (GB), mannitol, and trehalose (Khan and Khan, 2014; Khan et al., 2014, 2015; Per et al., 2018). Several compatible solutes or osmolytes are synthesized in bacteria as well as in plant systems when exposed to abiotic stresses. They protect protein structure stability and also scavenge the ROS as mentioned. Biosynthesis of certain osmolytes like proline and signaling contribute to the redox balance of cells under abiotic stress as well devoid of stress as has been pointed out by Per et al. (2017). However, multiple pathways exist for osmolyte biosynthesis in bacteria as well as in plants and the gene regulation is complex. The synthesis and accumulation of osmolytes is certainly controlled by phytohormones besides many other factors like mineral nutrients (Per et al., 2017). By interacting synergistically with proline and GB metabolism, phytohormones bring about stress tolerance in plants (Iqbal et al., 2014). But, the comprehensive role of hormones in modulating many osmolyte biosynthesis leading to salt and drought stress tolerance is not totally explored. Further, several signaling molecules like nitric oxide (NO), carbon monoxide (CO), and hydrogen sulfide $\left(\mathrm{H}_{2} \mathrm{~S}\right)$ play a pivotal role in the regulation of osmolyte biosynthesis ( $\mathrm{He}$ and $\mathrm{He}, 2017$ ).
Accordingly, it is vital to understand the role of these molecules on osmolyte accumulation and metabolism to resolve the adaptive roles played by plants to avoid abiotic stresses. This review focuses on two such osmolyte molecules, proline and GB, which are well studied and modulated by ABA and other phytohormones and the associated signaling pathways. The regulation of other osmolytes by hormones is not well known, and hence not included here. Myriad functions that are performed by osmolytes during stress, plant growth, and development are also emphasized. This review may provide new insights and opportunities in modulating osmolyte metabolism to impart salt and drought stress tolerance to crop plants, thereby contributing to sustainable agricultural yields in future.

\subsection{ABSCISIC ACID-SENSING MECHANISM OF PLANTS AND DOWNSTREAM EVENTS}

Abscisic acid (ABA) plays an important role in seed development, dormancy, and acclimation of plants to abiotic stresses. When plants are exposed to salt or drought stresses, rapid de novo synthesis of ABA was observed in leaves. This leads to the closure of stomata, which helps in protecting the plants against evaporation of water (Schwartz and Zeevaart, 2010). Henson (1984) and Mohapatra et al. (1988) reported enhanced levels of ABA in tissues exposed to abiotic stresses. Further, ABA plays a pivotal role during drought stress by modulating many physiological responses that lead to plant adaptations to unfavorable conditions. Exogenous application of ABA can also induce several genes in plants that are not subjected to stress (Mundy and Chua, 1988). This indicates that $\mathrm{ABA}$ is associated with functions other than abiotic stress. ABA signaling pathway perceives and transmits the hormone stimulus to activate several of the downstream events in the plants (Fujii et al., 2009; Ma et al., 2009; Park et al., 2009). This comprises three protein classes, namely pyrabactin resistance (PYR)/pyrabactin resistance-like (PYL)/regulatory component of ABA receptor (RCAR), which regulate protein phosphatase 2C (PP2C) negatively (Fig. 27.1A), and the positive 
(A)

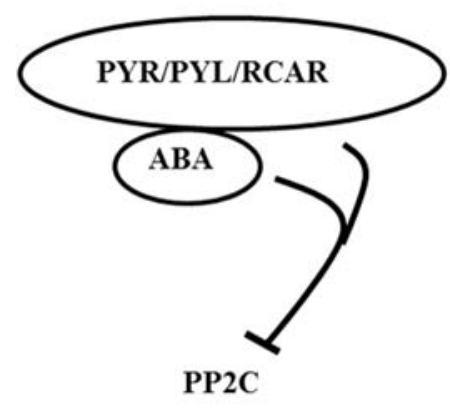

(B)

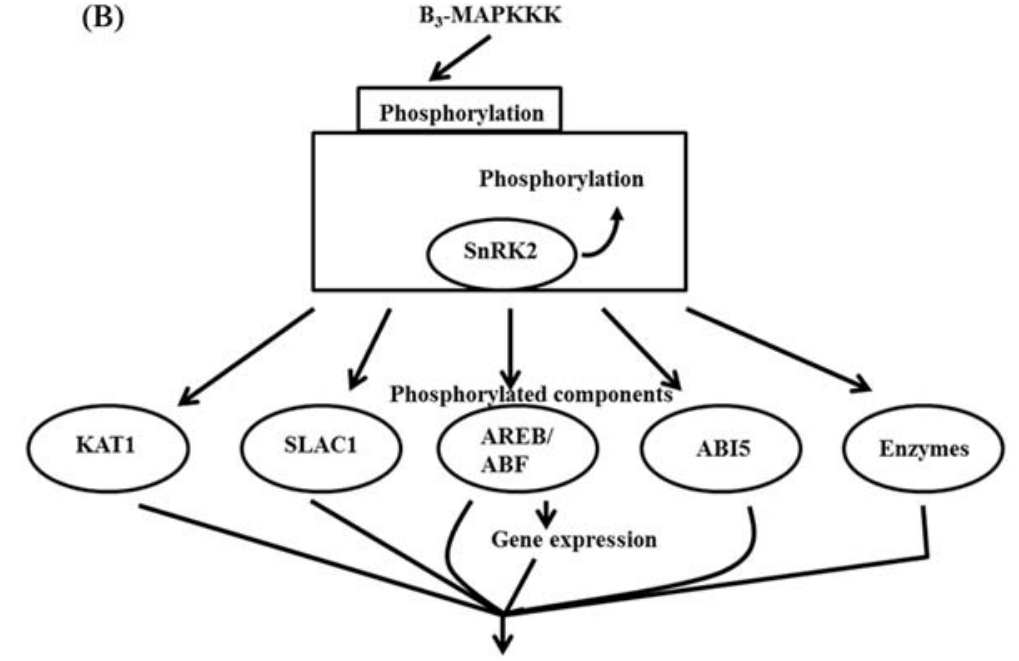

ABA-induced responses such as abiotic stress response, stomatal closure, seed maturation are observed

FIGURE 27.1 Abscisic acid signaling module. (A) An abscisic acid signaling module involves the presence of ABA, ABA receptors PYR/ PYL/RCAR which bind to ABA and inhibit protein phosphatase 2C (PP2C). (B) PP2C inhibition activates SnRK2s through autophosphorylation. SnRK2 then phosphorylates the downstream targets initiating abiotic stress responses.

regulators sucrose nonfermenting 1-related protein kinase type 2 (SnRK2s) (Fig. 27.1B). When enough concentrations of ABA are not present in the plant systems, SnRK2s are inactivated by PP2Cs (Umezawa et al., 2010). Once ABA binds to PYR/PYL/RCAR receptors, they undergo conformational change that allows the binding of PP2C (Cutler et al., 2010; Seiler et al., 2014). Therefore, ABA-induced inhibition of PP2Cs leads to phosphorylation of SnRK2 and its activation (Boudsocq et al., 2007). It has been found that SnRK2 activity is sensitive to staurosporine, but not to hyperosmolarity or ABA. This infers that SnRK2 activation by phosphorylation is mediated by staurosporine-insensitive kinase. SnRK2s then can phosphorylate downstream proteins like ion channels, NADPH oxidases, and others (Sah et al., 2016). While a Raf-like kinase (B-MAPKKK) activates SnRK2, a casein kinase 2 phosphorylates SnRK2's carboxyl-terminal serine residues. This enhances SnRK2PP2C interaction and thus, ABA can bring about activation of several of the downstream events.

\subsection{ROLE OF ABSCISIC ACID IN OSMOLYTE BIOSYNTHESIS}

\subsubsection{Abscisic Acid-Dependent and - Independent Signaling Pathways and Proline Biosynthesis}

Proline accumulation is a primary response to stress and is dependent on de novo synthesis (Verbruggen et al., 1993; Per et al., 2017). Therefore, the upstream signaling cascade must be in place for controlling proline biosynthesis as has been pointed out earlier (Hare et al., 1999). But, the signal transduction events controlling P5CS1 are not completely known. However, it has been shown that different signaling pathways regulate P5CS1 during cold and osmotic stress (Yoshiba et al., 1995; Igarashi et al., 2000; Hare et al., 1999). While AtP5CS1 is strongly ABAresponsive, AtP5CS2 is moderately responsive (Strizhov et al., 1997; Abrahám et al., 2003). Induction of AtP5CS transcript in salt-treated seedlings is associated with the early transcriptional response regulated by ABA signaling, but not observed by the deficiency of ABA biosynthesis in the abal Arabidopsis mutant (Strizhov et al., 1997). On the other hand, AtP5CS2 mRNA level is inhibited by cycloheximide. Mutations abi1 and axr2 affecting ABA-perception in Arabidopsis decrease the accumulation of both AtP5CS mRNAs during salt stress. At the same time, ABA signaling functions defined by the abi2 and abi3 mutations have no effect on salt induction of the P5CS genes (Strizhov et al., 1997). Verslues and Bray (2006) pointed out that low water potential-induced proline accumulation requires $A B A$ levels like those of wild-type levels. Estimations of ABA and proline levels in ABAinsensitive mutants, abi1-1, abi2-1, abi3, abi4, and abi5 revealed that abi4 had higher accumulation of proline at low water potential, but a reduced response to exogenous ABA. They found out that these responses could be modified by sucrose treatment. Also, while abi1 had reduced accumulation of proline in response to low water potential and ABA application, abi1-1 and abi2-1 had enhanced ABA accumulation. ABA-insensitive mutants are impaired in their response to sugars also 
(Gibson, 2005). It is known that sucrose inhibits ABAinduced proline accumulation (Verslues and Bray, 2006). It appears therefore, that ABI4 is connected to $\mathrm{ABA}$ and sugar-signaling in the regulation of proline accumulation (Verslues and Bray, 2006). Also, ABAdependent induction of P5CS1 expression is prevented by pretreatment with the hormone brassinosteroid (Abrahám et al., 2003). Thus, their analysis suggests the existence of both ABA-dependent and ABAindependent signaling pathways. Further, analysis of the P5CS1 and P5CS2 promoters showed the presence of cis-acting ABA-responsive elements only in P5CS1, but not P5CS2. Also, no dehydration-responsive element could be identified in the promoter sequences (Hare et al., 1999). In contrast, P5CS2 is weakly induced by ABA. It has been discovered that MYB-type of transcription factors PHOSPHATE STARVATION RESPONSE1 (PHR1) and PHR1-LIKE1 (PHL1) bind to P5CS1 regulatory sequences in the first intron, which contains a conserved PHR1-binding site motif (Aleksza et al., 2017). Phosphate starvation of Arabidopsis seedlings led to the activation of P5CS1, proline dehydrogenase 2 (ProDH2), as well as an increase in proline content. Such an accumulation was not noticed in the ABA-deficient aba1-3 and ABA-insensitive abi4-1 mutants. They also noticed that ABA is implicated in growth retardation in such nutritional stress. These results point out that proline biosynthesis is modulated by a crosstalk between ABA signaling and regulation of phosphate homeostasis through PHR1- and PHL1mediated transcriptional activation of the P5CS1 gene (Aleksza et al., 2017). Factors affecting P5CS and proline dehydrogenase (ProDH), enzymes associated in proline metabolism, are shown in the Table 27.1.

\subsubsection{Role of Hormones in the Regulation of P5CS and Proline Synthesis}

The results of You et al. (2012) indicated that ornithine $\delta$-aminotransferase $(O A T)$ is strongly induced by ABA and indole-3-acetic acid, and is slightly induced by brassinosteroids and jasmonic acid (JA) indicating that $O A T$ is responsive to multiple stresses. You et al. (2012) found out that the drought-induced expression of OsOAT is contributed by both ABA-dependent and ABA-independent pathways. Auxin upregulates the expression levels of both P5CS1 and P5CS2 genes, while cytokinin downregulates P5CS1, but enhances the expression of P5CS2 (Yoshiba et al., 1995; Strizhov et al., 1997; Hare et al., 1999; Abrahám et al., 2003). Induction of P5CS1 is light-dependent. Also, both ABA and salt stress strongly activate P5CS1 gene in Arabidopsis. At the same time, ABA and salt stress weakly stimulate P5CS2 gene and downregulate PDH gene expression in light-grown Arabidopsis plants
(Abrahám et al., 2003). Thus, their experiments proved that proline accumulation is strongly dependent on light, salt stress, and ABA, which is due to the activation of P5CS1 gene. At the same time, ABA and lightdependent activation of P5CS1 gene is inhibited in dark-grown plants. Proline accumulation in response to ABA and salt stress is mostly controlled by lightdependent activation of P5CS1 gene, but is inhibited by brassinosteroid signaling in Arabidopsis thaliana (Abrahám et al., 2003). In Arabidopsis, dark conditions downregulated P5CS2. Induction of P5CS1 is increased in the ABA-hypersensitive pleiotropic regulatory locus1 (prl1) and brassinosteroid-deficient deetiolated 2 (det2) mutants. On the other hand, both ABA and salt stress increased the P5CS2 gene induction only in det2 mutants. Thus, proline accumulation is certainly controlled by ABA as well as salt stress via P5CS1 gene. In the S6P5CS promoter region, a methyl jasmonate (MeJA)-responsive motif (TGACG-motif) was predicted, inferring that MeJA activates SbP5CS expression (Su et al., 2011). Salicylic acid (SA) positively regulated proline metabolism and helped in proline accumulation in several plants (Kanade, 2008; Misra and Saxena, 2009). SA induced proline accumulation might be due to increased P5CR activity and the stress protective effect of SA is perhaps controlled due to proline accumulation. SA treatment enhanced the accumulation of proline in barley shoots but not in roots (El-Tayeb, 2005). SA signaling is also associated with the expression of P5CS2 after infection with avirulent Pseudomonas (Fabro et al., 2004). Indeed, SAresponsive element was noticed in the promoter region of SbP5CS (Su et al., 2011). Likewise, a gibberellin (GA)-responsive element, GARE, was predicted in the upstream region of SbP5CS gene. But, no experiment was conducted to show that GA activates P5CS expression. Brassinosteroids inhibited the expression of both P5CS1 and P5CS2, and they could not stimulate ProDH expression (Abrahám et al., 2003). Thus, regulation of P5CS1 appears to be rather complex. During stress conditions, other secondary messengers or hormones like $\mathrm{NO}$ and ROS such as hydrogen peroxide $\left(\mathrm{H}_{2} \mathrm{O}_{2}\right)$ are known to mediate $\mathrm{ABA}$ signals and affect proline metabolism as has been shown by Desikan et al. (2002), Neill et al. (2008), and Yang et al. (2009). NO has been shown to be involved in the ABA-induced proline accumulation in wheat seedlings (Hai-Hua et al., 2004). While NO treatment enhanced the copperinduced proline accumulation (Zhang et al., 2008), the same was not noticed in Brassica rapa under salt stress conditions (Lopez-Carrion et al., 2008). It increased the activity of P5CS1, but downregulated the activity of ProDH in wheat (Hai-Hua et al., 2004). NO stimulated P5CS1 expression in Arabidopsis, but inhibited the expression of ProDH1 (Zhao et al., 2009). They also noticed that nitrate reductase rather than NO synthase 
TABLE 27.1 Factors Affecting P5CS1, ProDH, and Proline Accumulation in Plants

\begin{tabular}{|c|c|c|}
\hline Factor & Gene/expression & References \\
\hline Phosphate starvation & ProDH2 upregulation in A. thaliana & Aleksza et al. (2017) \\
\hline Light & P5CS1 upregulation in A. thaliana & Feng et al. (2016) \\
\hline Heat/temperature & P5CS1 upregulation in A. thaliana and Prunus persica & Shin et al. (2016), Wei-Tao et al. (2011) \\
\hline Hydrogen peroxide & P5CS1 upregulation in A. thaliana & Ben Rejeb et al. (2015) \\
\hline Diphenylene iodonium & P5CS1 downregulation in A. thaliana & Ben Rejeb et al. (2015) \\
\hline Menadione sodium bisulfite & P5CS1 upregulation in A. thaliana & Jiménez-Arias et al. (2015) \\
\hline Salt stress & ProT upregulation in Kosteletzkya virginica & Wang et al. (2015) \\
\hline Salt stress & OAT upregulation in Kosteletzkya virginica & Wang et al. (2015) \\
\hline Phosphatidylinositol 3-kinase & ProDH1 upregulation in A. thaliana & Leprince et al. (2015) \\
\hline $\mathrm{H} 2 \mathrm{~S}$ & Downregulation of ProDH1 in Musa paradisica & Luo et al. (2015) \\
\hline Salt stress & ProDH1 downregulation Saccharomyces cerevisiae and Helianthus tuberosus & Huang et al. (2013) \\
\hline Methyl jasmonate & P5CS2 upregulation in Sorghum bicolor & Su et al. (2011) \\
\hline Nitric oxide & P5CS1 upregulation in A. thaliana & Zhao et al. (2009) \\
\hline Carbon monoxide (CO) & P5CS1 upregulation in Triticum aestivum & Yuan et al. (2009) \\
\hline Phospholipase C & P5CS1 upregulation in A. thaliana & Parre et al. (2007) \\
\hline Phospholipase D & P5CS1 downregulation under nonstress conditions in A. thaliana & Thiery et al. (2004) \\
\hline Salicylic acid & P5CS2 upregulation in A. thaliana & Fabro et al. (2004) \\
\hline Pathogens & P5CS2 upregulation in A. thaliana & Fabro et al. (2004) \\
\hline Rehydration & ProDH1 upregulation in A. thaliana & Satoh et al. (2002) \\
\hline Brassinosteroids & P5CS2 downregulation in A. thaliana & Abrahám et al. (2003) \\
\hline Metal & P5CS1 upregulation/overexpression in Chlamydomonas reinhardtii & Siripornadulsil et al. (2002) \\
\hline Light & P5CS1 upregulation in A. thaliana & Hayashi et al. (2000) \\
\hline Dark conditions & ProDH1 upregulation in A. thaliana & Hayashi et al. (2000) \\
\hline Cold/Low temperature & P5CS1 upregulation in Oryza sativa & Igarashi et al. (1997) \\
\hline Abscisic acid & P5CS1 upregulation in A. thaliana & Strizhov et al. (1997) \\
\hline Indole-3-acetic acid & P5CS1 upregulation in A. thaliana & Strizhov et al. (1997) \\
\hline Salt stress & ProDH1 downregulation Saccharomyces cerevisiae and Helianthus tuberosus & Peng et al. (1996) \\
\hline Water stress & ProDH1 downregulation in A. thaliana & Kiyosue et al. (1996) \\
\hline Salt stress & P5CS1 upregulation in A. thaliana & Yoshiba et al. (1995) \\
\hline Drought & P5CS1 upregulation in A. thaliana & Savouré et al. (1995) \\
\hline Low nitrogen & P5CS1 upregulation in Vigna aconitifolia & Delauney et al. (1993) \\
\hline
\end{tabular}

is responsible for NO-mediated regulation of proline accumulation and also freezing tolerance.

Experiments to unravel the upstream signaling pathway of P5CS gene resulted in the identification of phospholipase $\mathrm{D}$, involved in water stress response and ABA signal transduction (Hallouin et al., 2002). But, phospholipase D downregulated P5CS1 activity under normal and also abiotic stress conditions (Thiery et al., 2004). It is known that calcium plays a pivotal role in proline accumulation under salt stress conditions. But, $\mathrm{CaCl}_{2}$ and phospholipase $\mathrm{D}$ treatment resulted in the upregulation of P5CS1 gene. They suggested that calcium regulates phospholipase $\mathrm{D}$ as a downstream signal messenger. However, there are many gaps in our understanding of the activation of some of the enzymes involved in proline metabolism 
and signaling pathways. Also, how some of these hormones regulatedP5CS and ProDH expressions at the molecular level and controlled the fine tuning of proline accumulation or degradation during stress, plant growth, and development is still to be discovered and perhaps pivotal for a comprehensive understanding of this osmolyte. Nevertheless, these experiments revealed a link between proline, hormonal signal, and downstream stress responses in plants.

\subsection{REGULATION OF PROLINE DEHYDROGENASE}

Dark conditions upregulated ProDH gene in shoots. ProDH was inhibited by both ABA and salt stress in shoots and roots of light-grown plants unlike that of P5CS1. In Arabidopsis, while prl1 mutation reduced the basal level of $P D H$ gene expression, the det2 mutation enhanced the inhibition of $P D H$ by ABA (Abrahám et al., 2003). Thus, it appears that $P D H$ expression is also regulated by $\mathrm{ABA}$. In plants, the receptors histidine kinases (AHKs) and elements of the twocomponent system have been proposed to function in water stress responses by regulating various stress-responsive genes. But, not much information is available concerning AHK phosphorelay-mediated downstream signaling (Veerabagu et al., 2014). ProDH is associated with the catabolic process and converts proline to pyrroline-5-carboxylate (P5C). It is known that ProDH1 expression undergoes extensive regulation by exogenous and endogenous signals, but the mechanism of its transcriptional and posttranscriptional regulation is not known completely. Accumulation of ProDH1 is controlled by the ACTCAT cis-acting element (ACT-box) in the ProDH1 promoter (Satoh et al., 2002) via basic region leucine zipper (bZIP) transcriptional activators from the S1group (Satoh et al., 2004). Analysis of ProDH1 regulation revealed that the S1-group members of TFs like bZIP1 and bZIP53 bind to the promoter of ProDH (Dietrich et al., 2011). But, not much is known about the functioning of C-group bZIP factors except perhaps AtbZIP63, which is a sensitive integrator of transient ABA and glucose signals under water stress (Matiolli et al., 2011). Veerabagu et al. (2014) have shown that the Arabidopsis type-B response regulator 18 (ARR18) acts as an osmotic stress response regulator in the seeds of Arabidopsis. This regulator affects the activity of the ProDH1 promoter, controlled by C-group bZIP transcription factors. They showed that ARR18 interaction negatively interferes with the bZIP63 on the ProDH1 promoter. Thus, regulation of ProDH via response regulators appears to be crucial for osmotic stress tolerance. However, such response regulators have not yet been discovered for other genes involved in the biosynthesis of other osmolytes.

\subsubsection{Glycine Betaine Biosynthesis and Its Modulation by Abscisic Acid}

GB interacts with plant hormones including ABA. Drought stress induced ABA accumulation was noticed first in corn, followed by GB accumulation (Zhang et al., 2012a,b). Thus, ABA and GB are positively associated with stress tolerance in plants. Both in wheat and pears, water stress has increased GB accumulation (Nayyar and Walia, 2004; Gao et al., 2004b). Zhang et al. (2012a,b) noticed enhanced GB accumulation with the application of ABA and also leaf relative water content and shoot dry matter production in two maize cultivars during water stress. They concluded that endogenous $\mathrm{ABA}$ is involved in modulating GB accumulation. Kurepin et al. (2015) reported upregulation of the genes associated with the GB biosynthetic pathway by increases in ABA and SA contents. An interplay between these hormones and GB appeared necessary for protection of photosynthesis under abiotic stress conditions. Synergistic effects of $\mathrm{ABA}$ and GB have been shown to protect photosynthetic apparatus in the cold acclimation process in higher plants (Kurepin et al., 2015). Transgenic plants overexpressing GB biosynthetic pathway genes produced better biomass in comparison with untransformed plants under stress (Hayashi et al., 1998). But, many researchers (Kurepin et al., 2013; Hüner et al., 2014) are of the opinion that GB in nonstressed plants can modify the production of endogenous phytohormones (ABA, ethylene, SA) associated with plant stress responses. It is not only drought stress conditions that increased the betaine aldehyde dehydrogenase (BADH) mRNA levels, even exogenously supplied ABA upregulated the BADH mRNA in leaves and roots of barley (Ishitani et al., 1995; Jagendorf and Takabe, 2001). Besides ABA and SA, JA also enhanced the GB accumulation in higher plants (Jagendorf and Takabe, 2001; Gao et al., 2004a). This implied that GB biosynthesis is under the hormonal control like that of proline. Taken together, it is suggested that a close interaction and synergistic effect of $A B A$ and $G B$ are necessary for effective acclimation of freezing and abiotic stress tolerance in plants. The above results also indicated that the hormone ABA transduces the signal for the biosynthesis of GB. But, how the signal is transduced and what are the different components associated in the pathway are not known. While proline biosynthesis is mediated by both ABA-dependent and ABA-independent signaling pathways (Hare et al., 1999), in contrast, GB biosynthesis appeared upregulated directly by ABA. 
But, not much is known about the regulation of the genes associated with the biosynthesis of other osmolytes like proline derivatives, GB derivatives, trehalose, and sugar alcohols by ABA and other hormones.

\subsection{SIGNALING MOLECULES AND OSMOLYTE SYNTHESIS}

Environmental stresses result in the production of signaling molecules like $\mathrm{NO}, \mathrm{CO}$, and $\mathrm{H}_{2} \mathrm{~S}$ in plants (He and He, 2017). However, the molecular mechanisms associated with the induction of osmolyte synthesis are not totally known. Evidence exists that NO induces one of the important osmolytes, proline, in wheat (Hai-Hua et al., 2004), Chinese cabbage (LopezCarrion et al., 2008), and rye grass (Liu et al., 2010) by upregulating P5CS and downregulating ProDH genes. Ke et al. (2014) reported that NO is associated with salt tolerance by regulating proline metabolism in tobacco, thus indicating the importance of $\mathrm{NO}$ in proline synthesis during abiotic stress. Yuan et al. (2009), Zhang et al. $(2012 a, b)$ reported CO stimulated P5CS and suppression of ProDH in wheat and Cassia obtusifolia seedlings as well as salt stress alleviation. Luo et al. (2015) and Chen et al. (2016) reported chilling injury and drought stress alleviation respectively with $\mathrm{H}_{2} \mathrm{~S}$ treatment. Further, Tian et al. (2016) reported $\mathrm{H}_{2} \mathrm{~S}$ and proline ameliorated metal $(\mathrm{Cd})$ stress in foxtail millet inferring a complex regulatory mechanism for proline synthesis and its relation to abiotic stress alleviation. However, the molecular mechanisms underlying activation of the biosynthetic pathway genes of proline metabolism and also the effects of these signal molecules on the biosynthesis of other osmolytes have not been understood so far.

\subsection{FUNCTIONS OF OSMOLYTES DURING ABIOTIC STRESS}

\subsubsection{Osmolyte Accumulation and Osmotic Adjustment During Stress}

Compatible solute accumulation, known for its osmotic adjustment, has long been recognized (Brown and Simpson, 1972; Borowitzka and Brown, 1974). Further, a correlation has also been found between the quantity of compatible solute and stress tolerance levels in plants (Storey and Jones, 1977; Flowers and Hall, 1978; Kishor et al., 1995). Amino acids play a vital role in protein biosynthesis. However, certain amino acids like proline accumulate under stressful environment and impart stress tolerance by maintaining cell turgor or osmotic balance, stabilizing membranes thereby preventing electrolyte leakage (Szabados and Savoure, 2010). When proline and GB were supplied exogenously, plants displayed salt stress tolerance. Both of them mediate upregulation of genes associated with antioxidant defense and glyoxalase systems and thus protect seedlings of rice from salt-induced oxidative damage (Hasanuzzaman et al., 2014). A large number of plants were genetically modified with the biosynthetic pathway genes associated with proline (reviewed in Kumar et al., 2014) and GB (reviewed in Khan et al., 2009), which displayed higher tolerance to salt stress. Higher accumulation of proline and GB in leaves of transgenic lines has been noticed in comparison with untransformed plants. This indicated that introduced genes are properly integrated and expressed in the host genome. It appeared that accumulation in leaves of stressed plants is regulated, at least in part, via the changes in the expression of biosynthetic pathway genes. But, the signals that provoke these changes in gene expressions have not been clearly identified in higher plants. It is not clear how osmolytes like proline and GB affect the cell turgor in plants and what signals are associated with them. It seems that in yeast, Synthetic Lethal of $N$-end rule $1(S \ln 1)$ osmosensor histidine kinase monitors the changes in turgor pressure as demonstrated by Saito and Tatebayashi (2004). It has been shown that reduction in turgor pressure caused by hyperosmotic stress activates the mitogen activated protein kinase high osmolarity glycerol 1 (HOG1) through SLN1 branch of the glycerol pathway (Reiser et al., 2003). In higher plants, activity of the plant histidine kinase Cre1 (cytokinin response 1) is regulated by changes in turgor pressure like that of $\operatorname{Sln} 1$ in yeast. It is known that Cre1 complemented the deficient Hog1 response in $\operatorname{Sln} 1$ mutant yeast cells (Reiser et al., 2003). These authors proposed that Cre1 has dual functions in plants acting both as a cytokinin receptor and also as an osmosensor. In addition to the possible role of osmolytes in osmotic adjustment and in stabilizing membranes upon salt/water stress, they play several other important regulatory functions in stressed plants (Lokhande and Suprasanna, 2012). Sugars not only sustained the growth of sink tissues, but also affected sugar-sensing systems that regulated the expression, either positively or negatively, of a variety of genes involved in photosynthesis, respiration, and the synthesis and degradation of starch and sucrose (Hare et al., 1998). Accumulation of sugar alcohols such as mannitol, sorbitol, pinitol, and others might serve dual functions: facilitating osmotic adjustment and supporting redox control (Tarczynski et al., 1993; Shen et al., 1999). Similarly, other osmolytes exhibited multiple functions in plants (Bohnert and Shen, 1998; Shen et al., 1999; Elbein et al., 2003; Livingston et al., 2009). 


\subsubsection{Osmolytes and Protection of Photosynthetic Machinery During Abiotic Stress}

Light including ultraviolet light-B (UV-B) radiation stress affects plant productivity drastically by inhibiting photosynthetic activity. Therefore, plants accumulate proline besides several other antioxidative and flavonoid molecules (Saradhi et al., 1995). As shown by Arora and Saradhi (2002), proline might protect the plants by scavenging the singlet oxygen or free radicals generated during light or UV-B radiation stress. A combination of $\mathrm{NaCl}$ and UV-B radiation showed an additive effect on most of the parameters studied in barley. UV-B treatment decreased the chlorophyll/ carotenoid ratio in barley seedlings and also photochemical efficiency of PSII (Fedina et al., 2003). Fedina et al. (2003) pointed out that $\mathrm{NaCl}$ preexposure decreased $\mathrm{H}_{2} \mathrm{O}_{2}$ generation and alleviated the inhibitory effect of UV-B on PSII. Proline accumulated during $\mathrm{NaCl}$ preexposure might be one of the reasons for the observed tolerance of barley seedlings to UV-B radiation. Pretreatment of Senedesmus (algal member) with proline decreased lipid peroxidation and UV-B induced malondialdehyde (MDA) generation (Tripathi and Gaur, 2004). Thus far, the exact mechanism of light-dependent stimulation of proline biosynthetic pathway genes and proline accumulation are not known. It was shown by Uchida et al. (2002) that $\mathrm{H}_{2} \mathrm{O}_{2}$ pretreatment induces increased ROS scavenging enzyme activities and enhanced expression of P5CS, sucrose phosphate synthase, and the small heat shock protein 26 in rice. These experiments indicated that $\mathrm{NO}$ and $\mathrm{H}_{2} \mathrm{O}_{2}$ act as signaling molecules that modulate both salt and heat stress tolerance by regulating the gene expression associated with it. Likewise, transgenic Arabidopsis leaves that expressed choline oxidase (COD) gene for the accumulation of GB displayed enhanced levels of $\mathrm{H}_{2} \mathrm{O}_{2}$ in comparison with untransformed plants (Alia et al., 1999). Further, activities of the enzymes such as APX and CAT were higher in transgenics than in the wild-type plants. These results indicated that the $\mathrm{H}_{2} \mathrm{O}_{2}$ generated by overexpression of $C O D$ gene might have stimulated the expression of scavenging enzymes (Sakamoto and Murata, 2002). Thus, $\mathrm{H}_{2} \mathrm{O}_{2}$ generated during stress might play as a signal transducer in stimulating proline biosynthetic pathway gene P5CS as well as ROS scavenging enzymes under the influence of $C O D$.

Photosynthesis is a major target of high-temperature as well as other abiotic stresses in plants and PSII is the most temperature-sensitive component. It is known that GB enhances the tolerance of photosynthetic machinery to photoinhibition (Sakamoto and Murata, 2002). Gorham (1995) indicated that GB protects enzymes and protein complexes against heat-induced inactivation. Photoinhibition involves photoinduced damage to PSII and the light-dependent repair of PSII complex (Aro et al., 1993). Abiotic stresses especially high temperature impair the activity of Rubisco enzyme also in several species (Haldimann and Feller, 2005). While D1 protein, one of the constituents of PSII, is damaged, steps are taken immediately by the plant to ensure the removal and replacement of the damaged D1 protein. GB plays a dual function of repairing the PSII complex during photoinhibition as well as protecting the complex proteins (Allakhverdiev et al., 2007). Alia et al. (1998) reported protection of transgenic Arabidopsis lines overexpressing GB biosynthetic pathway genes. This could be because of the protection of Rubisco activase by GB, which was later supported by Yang et al. (2005) in transgenic tobacco. Alia et al. (1999) found out that $\operatorname{cod} A$ overexpression in Arabidopsis resulted in light stress tolerance. They also found that GB had no effect on photodamage, but participated in repair of the PSII complex. Holmström et al. (2000) revealed that overexpression of choline dehydrogenase $(\mathrm{CDH})$ in tobacco resulted in the removal of photodamaged D1 protein and reconstitution of the functional PSII complex. It is believed that increased $\mathrm{CO}_{2}$ assimilation rate in transgenic lines is associated with the Rubisco activase-mediated activation of Rubisco by GB (Yang et al., 2005). Proline has been found to reduce the inhibitory effects of $\mathrm{NaCl}$ on the activity of enzymes like Rubisco in vitro in Tamarix jordanis (Solomon et al., 1994). Papageorgiou and Murata (1995) showed that osmolytes prevented dissociation of the oxygen-evolving complex of photosystems II. Yang et al. (2005) also reported improved thermostability of the oxygen-evolving complex and the reaction center of PSII (Yang et al., 2007) when GB biosynthetic pathway genes are overexpressed. However, the mechanistic explanation of how exactly osmolytes, including GB, protect PSII under stress is not clear to date.

\subsubsection{Osmolyte Accumulation and Oxidative Stress}

Chloroplasts and mitochondria are the two powerhouses of plant systems. The redox state of these two cell organelles is maintained by a delicate balance between energy production and consumption. These organelles need to avoid always the excess production of ROS, especially under abiotic stress conditions. While optimal levels of ROS are useful for signal transduction and several developmental activities, excess amounts cause damage to the nucleic acids, oxidation of proteins and lipids, and degradation of chlorophyll molecules (Davies, 1987; Imlay and Linn, 1988). Plants need to utilize the redox cues that are generated both in chloroplasts and mitochondria not only for maintaining metabolic fluxes, but also for coping with 
environmental changes via a complex network (Suzuki et al., 2012). The degradation pathway of proline is downregulated during osmotic stress, allowing free proline to accumulate. Miller et al. (2009) showed that overexpression of MsProDH in tobacco and Arabidopsis or impairment of $\mathrm{P} 5 \mathrm{C}$ oxidation in the Arabidopsis $p 5 c d h$ mutant did not change the cellular proline to $\mathrm{P} 5 \mathrm{C}$ ratio under ambient and osmotic stress conditions. This reveals that excess $\mathrm{P} 5 \mathrm{C}$ is reduced to proline in a mitochondrial-cytosolic cycle. This cycle involves conversion of proline by ProDH to P5C and back to proline by P5CR enzyme and is known to exist in animal cells (P5C-proline cycle). Miller et al. (2009) demonstrated that when an excess of exogenous L-proline is provided, it generates mitochondrial ROS by delivering electrons to $\mathrm{O}_{2}$. This was demonstrated by them using mitochondria specific MitoSox staining of superoxide ions. When there is a lack of P5CDH enzyme activity, it has led to higher ROS production in the presence of excess proline (Miller et al., 2009). It appears therefore, balancing not only chloroplastic but also mitochondrial ROS production during enhanced proline oxidation is critical for avoiding proline related toxic effects. To avoid the generation of ROS production by P5C-proline cycling, plants must oxidize P5C back to glutamate by $\mathrm{P} 5 \mathrm{CDH}$.

Regulation of ROS is coordinated by both enzymatic and nonenzymatic mechanisms. Further, exogenous application of proline or genetic manipulation of its synthesis or degradation has amply demonstrated its role in plant responses to abiotic stresses in different species like tobacco, sugarcane, grapevine, and sorghum (Smirnoff and Cumbes, 1989; Okuma et al., 2004; Molinari et al., 2007; Ozden et al., 2009; Reddy et al., 2015). Proline has enhanced the primary photochemical activities in isolated thylakoid membranes of Brassica juncea by arresting photoinhibitory damage (Alia and Saradhi, 1991). They suggested that proline protects the components involved in water oxidation capacity by reducing the production of free radicals and/or scavenging the free radicals thereby reducing thylakoid lipid peroxidation. Alia et al. (1997) also proposed that proline produced a considerable reduction in the lipid peroxidation-linked formation of both conjugated dienes and MDA in the thylakoids during exposure to strong light. They demonstrated that proline is involved in reducing the photodamage in the thylakoid membranes by scavenging and/or reducing the production of singlet oxygen. Alia et al. (2001) utilized spin trapping electron paramagnetic resonance (EPR) spectroscopy for analyzing the singlet quenching action of proline. Their results show that proline is very effective in reducing the production of singlet oxygen $\left({ }^{1} \mathrm{O}_{2}\right)$. Quenching of ${ }^{1} \mathrm{O}_{2}$ by proline seems to be based on its capability to form a charge-transfer complex due to low ionization potential. Proline is also a scavenger of hydroxyl radicals $(\mathrm{OH})$ as shown by Smirnoff and Cumbes (1989). But, proline does not interact with superoxide radicals. Kaul et al. (2008) showed the free radical scavenging potential of Lproline using in vitro assay system. However, it is difficult to explain the exact mechanism of quenching of ${ }^{1} \mathrm{O}_{2}$ or $\mathrm{OH}$ by proline. Overall, it appears that proline can stabilize proteins, DNA, as well as membranes under stress conditions as has also been pointed out by Matysik et al. (2002). High proline producing genotypes of niger (Guizotia abyssinica) exhibited higher antioxidative enzymes compared with low proline producing lines (Sarvesh et al., 1996). Exogenous supply of proline alleviated the oxidative stress and increased the vase life of Rosa hybrida flowers (Kumar et al., 2010). This increase in vase life coincided with higher levels of endogenous proline, lower levels of superoxide radicals, and higher activity of $\mathrm{PDH}$ in proline treated flowers. Exogenous application of proline has also been shown to associate with antioxidative enzyme activities (Hoque et al., 2007a,b). Proline ameliorated the enzymatic inactivation of APX and peroxidase, while SOD and CAT activities were reduced in grapevine (Ozden et al., 2009). In transgenic sugarcane overexpressing P5CS, a negative correlation between proline and lipid peroxidation were observed. This suggested that proline might protect against osmotic stress by increasing antioxidant systems. De Campos et al. (2011) demonstrated that transgenic citrumelo plants were able to cope with water deficit better than untransformed controls. Since these transgenics expressing VignaP5CSF129A are able produce high endogenous proline levels, proline must have contributed to gas exchange parameters and elevated levels of antioxidative enzymes (APX, SOD) but not CAT, and thereby ameliorated the deleterious effects of drought-induced oxidative stress. Transgenic Sorghum bicolor plants overexpressing P5CSF129A also displayed higher proline and higher antioxidative enzyme activities under salt stress (Reddy et al., 2015). Kaushal et al. (2011) demonstrated that proline has induced heat tolerance in Cicer arietinum plants by protecting vital enzymes of carbon and antioxidative metabolism. Posmyk and Janas (2007) noticed a positive correlation between endogenous levels of proline content in seeds of Vigna radiata and germination upon exposure to chilling stress. When seeds of $V$. radiata were pretreated with proline, it had a stimulatory effect on germination. This increase in seed germination by exogenously supplied proline under chilling temperatures is attributed to its potential to stabilize cell membrane by quenching both ${ }^{1} \mathrm{O}_{2}$ and $\mathrm{OH}$. Heat stress induced $\mathrm{H}_{2} \mathrm{O}_{2}$ in Saccharum species, but pretreatment with proline and GB has substantially reduced the $\mathrm{H}_{2} \mathrm{O}_{2}$ production, improved the accumulation of soluble sugars, and protected the developing tissues from heat stress effects in sprouting sugarcane buds (Rasheed 
et al., 2011). Ben Rejeb et al. (2015) investigated the role of NADPH oxidases, respiratory burst oxidase homologues (Rboh) in the induction of proline accumulation under $\mathrm{NaCl}$ and mannitol stress conditions. Both salt and mannitol stresses have increased $\mathrm{H}_{2} \mathrm{O}_{2}$ accompanied by accumulation of proline. They also found out that dimethylthiourea (a scavenger of $\mathrm{H}_{2} \mathrm{O}_{2}$ ) and diphenylene iodonium (an inhibitor of $\mathrm{H}_{2} \mathrm{O}_{2}$ production by NADPH oxidase) inhibited P5CS activity and proline accumulation under these stresses. Supporting this phenomenon, evidence was also presented in Arabidopsis thaliana knockout mutants lacking either AtRboHD or AtRbohF. Wild-type plants accumulated more proline than these mutants (Ben Rejeb et al., 2015). These results suggest that Rbohs contribute to $\mathrm{H}_{2} \mathrm{O}_{2}$ production in response to salt and mannitol stresses and help in proline accumulation in Arabidopsis.

Some of the sugars like trehalose (a nonreducing disaccharide) also protect the plants against oxygen radicals. Cell lines defective in trehalose synthesis were more sensitive to oxygen radicals than the wild-type indicating that trehalose protected the plants against oxidative stress. Oxygen radicals damage the amino acids in cellular proteins, but trehalose in the cells prevented this damage indicating that trehalose acts as a free radical scavenger. Hincha et al. (2002) also pointed out that when trehalose is present in high concentrations in cells, plants show resistance to heat, dehydration, and oxygen stress. Galactose as well as mannitol protected the cells, but not sucrose since sucrose does not have the ability to quench oxygen radicals (Benaroudj et al., 2001). Depending upon the species, many plants display high plasticity to accumulate various kinds of sugars (levels may vary in cells depending on the stage of the growth, nutritional status, and environmental conditions prevailing at that time) like raffinose series (Gala-1-6-sucrose and higher), stachyose, and other sucrose oligosaccharides that give protection against different stress conditions. While mannitol may function to shield susceptible thiol-regulated enzymes (such as phosphoribulokinase) from inactivation by hydroxyl radicals in plants, GB is not effective as a hydroxy radical scavenger (Shen et al., 1997; Smirnoff and Cumbes, 1989). Thus, several lines of research clearly indicate the ROS scavenging functions of osmolytes, but the exact molecular mechanisms are not yet completely known.

\subsubsection{Osmolytes and Amelioration of $\mathrm{NaCl}$ - and Metal-Induced $\mathrm{K}^{+}$Efflux Under Stress}

Potassium $\left(\mathrm{K}^{+}\right)$homeostasis plays a central role during salt stress tolerance in the plant systems (Tester and Davenport, 2003). When plants are exposed to $\mathrm{NaCl}$ stress, a massive efflux of $\mathrm{K}^{+}$from plant cells is observed (Shabala et al., 2003; Chen et al., 2005). Prevention or mitigation of $\mathrm{K}^{+}$efflux is well correlated to salt stress tolerance in barley (Carden et al., 2003; Chen et al., 2005). It was not known until recently that compatible solutes are implicated with the $\mathrm{K}^{+}$transport under salt stress. Cuin and Shabala (2005) hypothesized that osmoprotectants may maintain cytosolic $\mathrm{K}^{+}$homeostasis by preventing $\mathrm{NaCl}$ induced $\mathrm{K}^{+}$leakage from the barley cells. They showed that either proline or GB at a concentration of $0.5-5 \mathrm{mM}$, when supplied exogenously, instantaneously reduced the $\mathrm{NaCl}$-induced $\mathrm{K}^{+}$efflux from barley roots in a dose dependent manner. Proline at $5-\mathrm{mM}$ concentrations reduced the hydroxyl-radical induced $\mathrm{K}^{+}$efflux in barley (Cuin and Shabala, 2007a). They also measured membrane potentials in addition to $\mathrm{K}^{+}$and $\mathrm{Na}^{+}$concentrations, which are consistent with the concept that cytosolic $\mathrm{K}^{+}$homeostasis is maintained by proline by preventing $\mathrm{NaCl}-$ induced leakage of $\mathrm{K}^{+}$from the cells. Proline may possibly control this through the increased activity of $\mathrm{H}^{+}$-ATPase, controlling voltage-dependent outward-rectifying $\mathrm{K}^{+}$channels and creating the electrochemical gradient that is essential for ion transport processes (Cuin and Shabala, 2005). Thus, evidence has been provided for the first time for the regulation of ion fluxes across the plasma membrane by addition of proline/osmolytes. Cuin and Shabala (2007b) further showed that 21 out of 26 amino acids tested caused a significant mitigation of the $\mathrm{NaCl}$-induced $\mathrm{K}^{+}$efflux. Surprisingly, both valine and ornithine prevented the $\mathrm{NaCl}$-induced $\mathrm{K}^{+}$efflux significantly along with proline and maintained $\mathrm{K}^{+}$homeostasis. They pointed out that physiologically relevant concentrations of amino acids might contribute to salt stress adaptation by regulating $\mathrm{K}^{+}$transport across the plasma membrane. This might perhaps help the plants to maintain optimal $\mathrm{K}^{+} / \mathrm{Na}^{+}$ratio, which is vital during salt stress. But, the exact mechanism underlying proline prevented $\mathrm{K}^{+}$efflux under stress is not known. Still though it is thought that free radicals can mediate this. Heavy metals such as $\mathrm{Cu}^{2+}$ when added to lower plants (algal members) also cause leakage of $\mathrm{K}^{+}$from the cells. Whether proline or other osmolytes are associated with $\mathrm{Cu}^{2+} /$ metal-induced $\mathrm{K}^{+}$leakage prevention in metal tolerant plants is not known.

\subsubsection{Osmolytes and Their Metal Chelation Properties During Metal Stress}

Several crop plants (Cajanus cajan, Vigna mungo, Triticum aestivum) accumulate proline in response to heavy metal stress (Alia and Saradhi, 1991; Bassi and Sharma, 1993). In seedlings of Oryza sativa, exogenous 
supply of proline reduced the copper uptake (Chen et al., 2004). They noticed that proline supplement accompanied by $\mathrm{Cu}^{2+}$ exposure induce a barrier of $\mathrm{Cu}^{2+}$ influx and efflux in rice roots. It appeared that excess $\mathrm{Cu}^{2+}$ leads to inadequate proline in rice roots and results in the malfunction of copper transport barrier. An increase up to $>20$-fold in the proline content was noticed in leaves of metal nontolerant Silene vulgaris (Schat et al., 1997). The shoot proline content is higher than that in roots in Silene. On the other hand, root proline levels increased in Lactuca sativa with an increase in cadmium concentration (Costa and Morel, 1994). Not only nonmetal-tolerant plants, but also several metal-tolerant species like Armeria maritima, Deschampsia cespitosa, and Silene vulgaris have been reported to contain substantially higher constitutive proline levels when compared with nontolerant relatives (Farago and Mullen, 1979; Smirnoff and Stewart, 1987; Schat et al., 1997). But A. maritima plants, when grown in a noncopper site did not exhibit higher proline content (Farago and Mullen, 1979). Proline also accumulated in lower plants like algal members (Anacystis, Chlorella, Scendesmus, etc.) when exposed to heavy metal stress (Wu et al., 1995, 1998; Tripathi and Gaur, 2004). Thus, a large body of information existed with regard to proline accumulation under metal stress. Costa and Morel (1994) suggested that inhibition of proline oxidation is the reason for higher root proline levels under metal stress. Chen et al. (2001) pointed out that increased P5CR or OAT activities are responsible for higher proline accumulation in rice. Since water balance is disturbed under heavy metal stress, it is reasonable to speculate an increased proline synthesis and accumulation (Barceló and Poschenrieder, 1990). Chen et al. (2001) also suggested ABA mediated $\mathrm{Cu}$-induced proline accumulation in rice leaves. Later, it has been reported that copperinduced proline synthesis in the green algal member Chlamydomonas reinhardtii is associated with NO generation. They further investigated the effect of $\mathrm{Cu}^{2+}$ and NO on the activity and transcript amount of P5CS, and observed that application of sodium nitroprusside (NO specific donor) is able to stimulate the P5CS activity in the Cu-treated algae (Zhang et al., 2008). Their results indicated that $\mathrm{Cu}$-responsive proline synthesis is related to $\mathrm{NO}$ generation in C. reinhardtii.

Based on the existing information, the following speculations can be drawn for the possible metal stress mitigation by proline/osmolytes in plants. Proline may be acting as a metal chelator as demonstrated by Farago and Mullen (1979). They showed that $\mathrm{Cu}^{2+}$ in the roots of A. maritima existed as $\mathrm{Cu}$-proline complex. Proline protected glucose-6-phosphate dehydrogenase and nitrate reductase activities in vitro against zinc and copper-induced inhibition due to the formation of a metal-proline complex (Sharma et al., 1998). Experiments conducted by Siripornadulsil et al. (2002) contradict these results. Their experiments revealed that transgenic Chlamydomonas reinhardtii expressing the mothbean P5CS gene exhibits tolerance to $100 \mu \mathrm{M}$ cadmium and has $80 \%$ higher proline levels than the wild-type cells. They observed that cadmium does not bind to proline in transgenic algae but is coordinated tetrahedrally by sulfur of phytochelatin. In contrast to P5CS-expressing cells, in wild-type cells, cadmium is coordinated tetrahedrally by two oxygen and two sulfur atoms. These results suggested that free proline acts as an antioxidant in cadmium-stressed cells with higher reduced glutathione (GSH) levels. Enhanced GSH levels in turn facilitate phytochelatin synthesis and sequestration of cadmium, since GSH-heavy metal adducts are the substrates for phytochelatin synthase, as pointed out by Siripornadulsil et al. (2002). The above fact that cadmium is coordinated by oxygen and sulfur atoms supported the findings of Adhiya et al. (2002). Proline chelation of cadmium does not seem to be important since cadmium induces phytochelatins that can chelate the metal (de Knecht et al., 1994). Therefore, it is of interest to find out the role of proline or other osmolytes in binding metal ions that do not form complexes with phytochelatins as has been also pointed out by Sharma and Dietz (2006). The second possibility is that proline may be acting as an antioxidant during metal stress. Free radicals are generated under heavy metal stress, which could lead to oxidative stress. As mentioned in the earlier sections, both ${ }^{1} \mathrm{O}_{2}$ and $\mathrm{OH}^{-}$can be scavenged by proline. Heavy metal exposure causes lipid peroxidation as well as $\mathrm{K}^{+}$ efflux in algal members. Wu et al. (1995) observed that when Anacystis nidulans (Cyanobacteria) was exposed to $\mathrm{Cu}^{2+}, \mathrm{K}^{+}$is effluxed out, but exogenously supplied proline reduced the leakage. Wu et al. (1998) also noticed that when proline was supplied exogenously to Chlorella species prior to copper treatment, it resulted in desorption of the adsorbed $\mathrm{Cu}^{2+}$ immediately after the addition of proline. These results indicate that one function of accumulated proline is to reduce the uptake of metal ions. Mehta and Gaur (1999) reported that Chlorella vulgaris accumulates proline within few hours of exposure to a wide range of heavy metals. Their experiments demonstrate that pretreatment of $C$. vulgaris with proline counteract metalinduced lipid peroxidation as well as $\mathrm{K}^{+}$efflux. Even in lichens Trebouxia erici (Bačkor et al., 2004), proline content is positively correlated to $\mathrm{Cu}^{2+}$ tolerance. Taken together, it is unlikely that proline binds to metals and chelates them during metal stress, but preferentially acts as an antioxidant molecule and detoxifies the ROS. 


\subsubsection{Role of Osmolytes in Membrane and Native Protein Structure Stabilizations}

Galinski (1993) noticed thermal stability of enzymes in the presence of osmolytes. Several other lines of evidence show that osmolytes effectively protect plant enzymes against stress induced denaturation (Solomon et al., 1994; Yang et al., 2007). Arguments and counterarguments were raised against and in favor of the effective compatible solute concentrations that are needed for protection of enzymes in vitro. While $500 \mathrm{mM}$ has been suggested as an effective concentration for membrane stability, such high concentrations are often not detected in vivo (Bohnert and Shen, 1998). It appears that the osmolyte concentration may not be important since Zhao et al. (1992) have shown protection of thylakoid and plasma membranes against freezing damage under high as well as low concentrations. Experiments conducted to date indicate that the local concentration of membrane or protein surfaces is vital rather than the absolute concentrations for membrane stabilization or enzyme protection in plants undergoing stress.

Fructans are a class of polysaccharides known for their protective effects on liposomes during conditions of drying. Both bacteria (levan produced from Bacillus subtilis) and plants (inulin synthesized from chicory roots) have been found to protect liposomes from leakage during freeze-drying or air-drying (Hincha et al., 2000; Vereyken et al., 2003). Besides chicory, many grass species also accumulate osmolytes like fructans (Livingston et al., 2009). Differences exist between fruc$\tan$ molecules in their size, structure, and also tissue localization, which is vital for the survival of the whole plants under cold stress conditions (Livingston et al., 2005, 2006). Hincha et al. (2000) showed that inulin is a mixture of polysaccharides with a degree of polymerization (DP) between 10 and 30 (Hincha et al., 2000) and molecular masses 1600 and 5000. These researchers pointed out that during freeze-drying, inulin in phosphatidylcholine liposome preparations reduces the degree of leakage after rehydration by establishing $\mathrm{H}$-bonds to the lipid $\mathrm{P}=\mathrm{O}$. But, high-DP fructans from oat and rye are not able to prevent leakage or fusion in liposomes during drying (Hincha et al., 2007). On the other hand, inulins and fructans from the same species with 7-10 degrees of polymerization (more soluble) do not precipitate during air-drying and provide protection to liposomes (Hincha et al., 2002, 2007; Vereyken et al., 2003). It appears that fructans are transported in the phloem of Agave deserti leaf tissues (Wang and Nobel, 1998). Similarly, it has been found that fructan DP3 is transported via the apoplast (phloem) in transgenic potato inferring that fructans in the apoplast protect the tissues from freezing/dehydration injury besides serving as a hexose reserve (Zuther et al., 2004). Levan from Bacillus subtilis has a DP of about 125, found to have higher solubility, but protects liposomes from leakage and fusion (Vereyken et al., 2003). Taken together, it appears that specific structural features of oligosaccharides determine their efficacy as membrane stabilizers during drying/ freezing stress.

Some plants, especially anhydrobiotic organisms produce very high concentrations of trehalose, and other di- and oligosaccharides under abiotic stress conditions (Zentella et al., 1999; Elbein et al., 2003). In plants, sucrose also plays a similar role to that of trehalose in yeast (Anandarajah and McKersie, 1990). A large body of evidence suggests that trehalose, due to its structure and stereochemistry, depresses the phase transition temperature of the dry lipids, which maintains them in the liquid crystalline phase in the absence of water (Crowe and Crowe, 1988). Trehalose appears to preserve labile proteins during drying probably by interacting directly with the dry protein by hydrogen bonding between its hydroxyl groups and polar residues in the protein (Carpenter and Crowe, 1989). Two contrasting models that have been proposed to explain protective or stabilizing effects of compatible solutes on membrane/protein structures are (1) the preferential exclusion model (Arakawa and Timasheff, 1985) and (2) the preferential interaction model. In the first model, compatible solutes are excluded from the hydration shell of proteins that stabilize protein structure or promote protein/protein interaction under stress. But, Schobert (1977) is of the opinion that interactions between the compatible solutes and proteins are necessary, and protein's hydration shell is crucial for its structural stability. It appears that osmolytes interact with the hydrophobic domains of proteins and prevent their destabilization. The molecular mechanism for osmolyte-induced protein stability has been elucidated by Street et al. (2006). They pointed out that in the equilibrium protein folding reaction, unfolded $(\mathrm{U}) \leftrightarrow$ native $(\mathrm{N})$, high concentrations of protecting osmolytes push the equilibrium of protein folding towards $\mathrm{N}$, while denaturing osmolytes push it toward the unfolded form (U). It appears that the configuration of the protein backbone is the most important determinant of stabilization or denaturation. As yet, there is no universal molecular theory that explains the mechanism by which osmolytes interact with the protein to alter its stability in higher plants under abiotic stress conditions. However, more experiments will be necessary to gain a better insight into the membrane/protein stabilizing effects of osmolytes. 


\subsubsection{Osmolytes as Sources of Energy and Carbon Reserve During and After the Release of Stress}

It is interesting to note that drought, salt, and flooding stresses increase generally soluble sugar concentrations in plants, but high light intensity, heavy metals, and ozone decrease sugar accumulation depending on the genotype (Gill et al., 2001; Morsy et al., 2007). It is possible that not all soluble sugars play identical roles during stress (Almodares et al., 2008). If sugars are accumulated in high concentrations, that can lead to downregulation of further energy synthesis (Koch, 2004; Chen, 2007). While low sugar content in the tissues increases photosynthesis, high sugar level promotes carbohydrate storage. Thus, changes in $\mathrm{CO}_{2}$ assimilation are possible by sugar accumulation under stress. This is one of the ways perhaps to maintain energy homeostasis in plants during abiotic stress conditions as has been pointed out by Rosa et al. (2009a). Gupta and Kaur (2005) described that both glucose and sucrose act as sources of carbon and energy, but also as osmolytes (but not fructose) to maintain cell homeostasis. Oxidation of sugars via glycolytic and other pathways leads to the production of ATP, NADPH, and erythrose-4-phosphate, which can be utilized once the stress is released. Osmolytes like proline are involved in the alleviation of cytoplasmic acidosis and sustaining $\mathrm{NADP}^{+} / \mathrm{NADPH}$ ratios at the levels required for metabolism (Hare and Cress, 1997). The functions of proline are dependent on spatial and temporal control of its synthesis as well as its catabolism. In turn, this helps the plants either to take up or release reductant and energy at a site/tissue location where it is necessary for metabolic functions (Sharma et al., 2011). Plants prefer NADPH over NADH as an electron donor for the biosynthesis of proline (Murahama et al., 2001). This helps the plants to regenerate $\mathrm{NADP}^{+}$in the chloroplast and thus prevents ROS production and photoinhibition as has been pointed out by Szabados and Savoure (2010). The work of Sharma et al. (2011) demonstrated that both p5cs1 (involved in proline synthesis) and pdh1 mutants (blocked in proline catabolism) are required for optimal growth at low water potential. Many lines of evidence suggest that the generation of $\mathrm{NADP}^{+}$and $\mathrm{NADPH}$ during proline synthesis and degradation respectively and maintaining a favorable ratio of $\mathrm{NADP}^{+}$/NADPH are also critical for the survival of plants under stress. Once the stress is relieved, accumulated proline is oxidized in mitochondria and energy is released. Both proline and trehalose (Becker et al., 1996) are the major sugars and are consumed during flight in insects. Thornburg (2007) analyzed the content of proline in ornamental tobacco (LxS8 line) flowers, which was $2020 \mu \mathrm{M}$, while the concentration of other amino acids are in the range of only $114-547 \mu \mathrm{M}$. Bertazzini et al. (2010) found that artificial nectar containing proline is preferred by forager honeybees. Trehalose is stored in fungal spores and its hydrolysis helps in spore germination and is a source of carbon for synthesis of glucose (Thevelein, 1984). Thus, osmolytes act as both as a source of carbon and energy during and after release from the stress conditions.

\subsection{OSMOLYTES AND SIGNALING PROCESSES}

\subsubsection{Proline and Signaling Processes}

Proline propels two major signaling events like cellular survival as well as apoptosis. During proline oxidation, ROS are formed in the mitochondria, which have been implicated in the hypersensitive response in plants. Further, ROS leads to induction of intrinsic and extrinsic apoptotic cell death pathways in animals (Liu et al., 2006; Hu et al., 2007). Thus, ROS appear to be the main signal transducers for downstream responses during proline oxidation. However, several critical issues remain elusive in our understanding of these events. First, is there any threshold level of proline that is required for metabolic switchover from inducing survival pathways to cellular apoptosis? Second, it is not known if there are any other mediators or components that are associated with signaling phenomena during proline metabolism. Existing evidence suggests that proline biosynthetic pathway enzymes interact with redox proteins like thioredoxin (Liang et al., 2013). It would be interesting to find out if there are any other interacting partners of proline metabolic enzymes that play a role in cellular signaling networks leading to the triggering of downstream events.

\subsubsection{Proline Metabolism and Signaling Pathways in Plant Senescence}

Proline is also associated with plant senescence. Nearly 14-fold increase in proline content was recorded in petals of cut roses during the process of senescence. Enhanced activities of P5CS and ProDH were also noticed during the course of senescence (Kumar et al., 2009). While the expression of ProDH2 in the vascular tissue and abscission zone of petals is regulated by the transcription factor bZIP11 (Hanson et al., 2008), ProDH1 expression is modulated by bZIP1 and bZIP53 (Dietrich et al., 2011) in Arabidopsis thaliana. This infers that proline is catabolized rapidly 
whenever sucrose levels are low in plants (Funck et al., 2010; Llorca et al., 2014). During senescence, proline metabolism also influences ROS signaling pathways that delay the process of senescence (Zhang and Becker, 2015). But, more studies are needed on the regulation of proline metabolic shifts that occur during senescence. Such studies may provide novel insights that rescue crop plants undergoing abiotic stress and also preserve postharvest agricultural products.

\subsubsection{Osmolytes as Sensing Compounds and/or Growth Regulators}

Sugars such as glucose and sucrose may act as sources of carbon and energy. But, fructose plays a different role from that of glucose and sucrose. Hilal et al. (2004) demonstrated that fructose acts as a precursor for the synthesis of lignin and several phenolic compounds, thus inferring that sugar accumulation under stress performs diverse roles. Sugars can act as primary messengers and regulate signals that control the expressions of genes (Gupta and Kaur, 2005; Gibson, 2005; Chen, 2007). Since sugars are rapidly metabolized and also interconverted depending upon the environmental stresses (Rosa et al., 2009b), it is difficult to pinpoint if sensing of soluble sugars depends upon their metabolism. But, it is known that sugar levels modulate differential expression of genes (Koch et al., 1992). Rook et al. (1998) demonstrated that sucrose-specific signaling pathways to be responsible for repression of ATB2bZIP transcription factor. Many genes are negatively regulated by sugars (sucrose, glucose, and fructose) at the transcription level (Yamaguchi-Shinozaki and Shinozaki, 2006). When $30 \mathrm{mM}$ proline was applied exogenously, it ameliorated the salt stress effects in rice, but $40-50 \mathrm{mM}$ levels resulted in poor growth (Roy et al., 1993). Overexpression of microbial genes for trehalose biosynthesis caused dwarfism and aberrant root development (Vogel et al., 1998). Müller et al. (1999) found such growth defects in transgenic rice producing trehalose. These findings have led to postulate that osmolytes might function as plant growth regulators. The plausible explanation that has been given is that small amounts of trehalose or trehalose-phosphate might be toxic to the plants. Else, trehalose metabolism may act as a signal in sugar sensing and partitioning of assimilates like other sugars (Müller et al., 1999).

\subsection{CONCLUSIONS AND FUTURE PROSPECTS}

ABA is central to the signal perception and subsequent transduction events during abiotic stress. The core signaling module regulates several downstream events including osmolyte biosynthesis and subsequently abiotic stress tolerance. Diverse osmolytes accumulated during abiotic stress conditions are regulated by many phytohormones. Osmolytes perform many vital functions such as osmotic adjustment, scavenging ROS, controlling the redox state, and cell survival and apoptosis during stress. However, the precise molecular mechanisms underlying the triggering of genes associated with several of the osmolyte biosyntheses and catabolisms (barring a few) are not completely known. Therefore, it is of prime importance to unravel the intricate networks, molecular mechanisms, and the signaling events leading to the better survival of crop plants exposed to different abiotic stress conditions. Such a comprehensive knowledge about molecular mechanisms and signaling events leading to the regulation of osmolyte biosynthesis and effective scavenging of ROS will enable us to develop strategies to genetically modify crop plants and use them for sustainable agricultural yields.

\section{Acknowledgments}

PBK is thankful for sanctioning CSIR-Emeritus Scientist Fellowship through the Grant Number 38(1325)/12/EMR-II) by the Council for Scientifics and Industrial Research, New Delhi.

\section{References}

Abrahám, E., Rigó, G., Székely, G., Nagy, R., Koncz, C., Szabados, L., 2003. Light-dependent induction of proline biosynthesis by abscisic acid and salt stress is inhibited by brassinosteroid in Arabidopsis. Plant Mol. Biol. 51 (3), 363-372.

Adhiya, J., Cai, X., Sayre, R.T., Traina, S.J., 2002. Binding of aqueous cadmium by the lyophilized biomass of Chlamydomonas reinhardtii. Colloids Surfaces A: Physicochem. Eng. Aspects 210 (1), $1-11$.

Aleksza, D., Horváth, G.V., Sándor, G., Szabados, L., 2017. Proline accumulation is regulated by transcription factors associated with phosphate starvation. Plant Physiol. 175 (1), 555-567.

Alia, Hayashi, H., Sakamoto, A., Murata, N., 1998. Enhancement of the tolerance of Arabidopsis to high temperatures by genetic engineering of the synthesis of glycinebetaine. Plant J. 16 (2), $155-161$.

Alia, Kondo, Y., Sakamoto, A., Nonaka, H., Hayashi, H., Saradhi, P. P., et al., 1999. Enhanced tolerance to light stress of transgenic Arabidopsis plants that express the codA gene for a bacterial choline oxidase. Plant Mol. Biol. 40 (2), 279-288.

Alia, Mohanty, P., Matysik, J., 2001. Effect of proline on the production of singlet oxygen. Amino Acids 21 (2), 195-200.

Alia, Saradhi, P.P., 1991. Proline accumulation under heavy metal stress. J. Plant. Physiol. 138 (5), 554-558.

Alia, Saradhi, P.P., Mohanty, P., 1997. Involvement of proline in protecting thylakoid membranes against free radical-induced photodamage. J. Photochem. Photobiol. B: Biol. 38 (2-3), 253-257.

Allakhverdiev, S.I., Los, D.A., Mohanty, P., Nishiyama, Y., Murata, N., 2007. Glycinebetaine alleviates the inhibitory effect of moderate heat stress on the repair of photosystem II during 
photoinhibition. Biochim. Biophys. Acta (BBA)-Bioenerg. 1767 (12), 1363-1371.

Almodares, A., Taheri, R., Chung, M., Fathi, M., 2008. The effect of nitrogen and potassium fertilizers on growth parameters and carbohydrate contents of sweet sorghum cultivars. J. Environ. Biol. 29 (6), 849-852.

Anandarajah, K., McKersie, B.D., 1990. Manipulating the desiccation tolerance and vigor of dry somatic embryos of Medicago sativa L. with sucrose, heat shock and abscisic acid. Plant Cell Rep. 9 (8), $451-455$.

Arakawa, T., Timasheff, S.N., 1985. The stabilization of proteins by osmolytes. Biophys. J. 47 (3), 411-414.

Aro, E.M., Virgin, I., Andersson, B., 1993. Photoinhibition of photosystem II. Inactivation, protein damage and turnover. Biochim. Biophys. Acta (BBA)-Bioenerg. 1143 (2), 113-134.

Arora, S., Saradhi, P.P., 2002. Light induced enhancement in proline levels under stress is regulated by non-photosynthetic events. Biol. Plant. 45 (4), 629-632.

Bačkor, M., Fahselt, D., Wu, C.T., 2004. Free proline content is positively correlated with copper tolerance of the lichen photobiont Trebouxia erici (Chlorophyta). Plant Sci. 167 (1), 151-157.

Barceló, J.U.A.N., Poschenrieder, C., 1990. Plant water relations as affected by heavy metal stress: a review. J. Plant. Nutr. 13 (1), $1-37$.

Bassi, R., Sharma, S.S., 1993. Proline accumulation in wheat seedlings exposed to zinc and copper. Phytochemistry 33 (6), 1339-1342.

Becker, A., Schlöder, P., Steele, J.E., Wegener, G., 1996. The regulation of trehalose metabolism in insects. Experientia 52 (5), 433-439.

Ben Rejeb, K., Vos, L.D., Le Disquet, I., Leprince, A.S., Bordenave, M., Maldiney, R., et al., 2015. Hydrogen peroxide produced by NADPH oxidases increases proline accumulation during salt or mannitol stress in Arabidopsis thaliana. New Phytol. 208 (4), $1138-1148$.

Benaroudj, N., Lee, D.H., Goldberg, A.L., 2001. Trehalose accumulation during cellular stress protects cells and cellular proteins from damage by oxygen radicals. J. Biol. Chem. 276 (26), 24261-24267.

Bertazzini, M., Medrzycki, P., Bortolotti, L., Maistrello, L., Forlani, G., 2010. Amino acid content and nectar choice by forager honeybees (Apis mellifera L.). Amino Acids 39 (1), 315-318.

Blokhina, O., Virolainen, E., Fagerstedt, K.V., 2003. Antioxidants, oxidative damage and oxygen deprivation stress: a review. Ann. Bot. (Lond.) 91 (2), 179-194.

Bohnert, H.J., Shen, B.O., 1998. Transformation and compatible solutes. Sci. Hortic. (Amsterdam) 78 (1-4), 237-260.

Borowitzka, L.J., Brown, A.D., 1974. The salt relations of marine and halophilic species of the unicellular green alga. Dunaliella. Arch. Microbiol. 96 (1), 37-52.

Boudsocq, M., Droillard, M.J., Barbier-Brygoo, H., Laurière, C., 2007. Different phosphorylation mechanisms are involved in the activation of sucrose non-fermenting 1 related protein kinases 2 by osmotic stresses and abscisic acid. Plant Mol. Biol. 63 (4), 491-503.

Brown, A.D., Simpson, J.R., 1972. Water relations of sugar-tolerant yeasts: the role of intracellular polyols. Microbiology 72 (3), 589-591.

Carden, D.E., Walker, D.J., Flowers, T.J., Miller, A.J., 2003. Single-cell measurements of the contributions of cytosolic $\mathrm{Na}^{+}$and $\mathrm{K}^{+}$to salt tolerance. Plant Physiol. 131 (2), 676-683.

Carpenter, J.F., Crowe, J.H., 1989. An infrared spectroscopic study of the interactions of carbohydrates with dried proteins. Biochemistry 28 (9), 3916-3922.

Chen, C.T., Chen, L.M., Lin, C.C., Kao, C.H., 2001. Regulation of proline accumulation in detached rice leaves exposed to excess copper. Plant Sci. 160 (2), 283-290.
Chen, C.T., Chen, T.H., Lo, K.F., Chiu, C.Y., 2004. Effects of proline on copper transport in rice seedlings under excess copper stress. Plant Sci. 166 (1), 103-111.

Chen, J.G., 2007. Sweet sensor, surprising partners. Sci. STKE 2007 (373), pp.pe7-pe7.

Chen, Z., Newman, I., Zhou, M., Mendham, N., Zhang, G., Shabala, S., 2005. Screening plants for salt tolerance by measuring $\mathrm{K}+$ flux: a case study for barley. Plant Cell Environ. 28 (10), 1230-1246.

Chen, J., Shang, Y.T., Wang, W.H., Chen, X.Y., He, E.M., Zheng, H. L., et al., 2016. Hydrogen sulfide-mediated polyamines and sugar changes are involved in hydrogen sulfide-induced drought tolerance in Spinacia oleracea seedlings. Front. Plant Sci. 7, 1173.

Costa, G., Morel, J.L., 1994. Water relations, gas exchange and amino acid content in Cd-treated lettuce. Plant. Physiol. Biochem. (France).

Crowe, L.M., Crowe, J.H., 1988. Trehalose and dry dipalmitoylphosphatidylcholine revisited. Biochim. Biophys. Acta (BBA)-Biomembr. 946 (2), 193-201.

Cuin, T.A., Shabala, S., 2005. Exogenously supplied compatible solutes rapidly ameliorate $\mathrm{NaCl}$-induced potassium efflux from barley roots. Plant Cell Physiol. 46 (12), 1924-1933.

Cuin, T.A., Shabala, S., 2007a. Compatible solutes reduce ROSinduced potassium efflux in Arabidopsis roots. Plant Cell Environ. 30 (7), 875-885.

Cuin, T.A., Shabala, S., 2007b. Amino acids regulate salinity-induced potassium efflux in barley root epidermis. Planta 225 (3), $753-761$.

Cutler, S.R., Rodriguez, P.L., Finkelstein, R.R., Abrams, S.R., 2010. Abscisic acid: emergence of a core signaling network. Annu. Rev. Plant. Biol. 61, 651-679.

Davies, K.J., 1987. Protein damage and degradation by oxygen radicals. I. general aspects. J. Biol. Chem. 262 (20), 9895-9901.

de Campos, M.K.F., de Carvalho, K., de Souza, F.S., Marur, C.J., Pereira, L.F.P., Bespalhok Filho, J.C., et al., 2011. Drought tolerance and antioxidant enzymatic activity in transgenic 'Swingle'citrumelo plants over-accumulating proline. Environ. Exp. Bot. 72 (2), 242-250.

de Knecht, J.A., van Dillen, M., Koevoets, P.L., Schat, H., Verkleij, J. A., Ernst, W.H., 1994. Phytochelatins in cadmium-sensitive and cadmium-tolerant Silene vulgaris (chain length distribution and sulfide incorporation). Plant Physiol. 104 (1), 255-261.

Delauney, A.J., Hu, C.A., Kishor, P.B., Verma, D.P., 1993. Cloning of ornithine delta-aminotransferase cDNA from Vigna aconitifolia by trans-complementation in Escherichia coli and regulation of proline biosynthesis. J. Biol. Chem. 268 (25), 18673-18678.

Desikan, R., Griffiths, R., Hancock, J., Neill, S., 2002. A new role for an old enzyme: nitrate reductase-mediated nitric oxide generation is required for abscisic acid-induced stomatal closure in Arabidopsis thaliana. Proc. Natl. Acad. Sci. 99 (25), 16314-16318.

Dietrich, K., Weltmeier, F., Ehlert, A., Weiste, C., Stahl, M., Harter, K., et al., 2011. Heterodimers of the Arabidopsis transcription factors bZIP1 and bZIP53 reprogram amino acid metabolism during low energy stress. Plant Cell 23 (1), 381-395.

Elbein, A.D., Pan, Y.T., Pastuszak, I., Carroll, D., 2003. New insights on trehalose: a multifunctional molecule. Glycobiology 13 (4), 17R-27R.

El-Tayeb, M.A., 2005. Response of barley grains to the interactive effect of salinity and salicylic acid. Plant Growth Regul. 45 (3), $215-224$.

Fabro, G., Kovacs, I., Pavet, V., Szabados, L., Alvarez, M.E., 2004. Proline accumulation and AtP5CS2 gene activation are induced by plant-pathogen incompatible interactions in Arabidopsis. Mol. Plant-Microbe Interact. 17, 343-350.

Farago, M.E., Mullen, W.A., 1979. Plants which accumulate metals. Part IV. A possible copper-proline complex from the roots of Armeria maritima. Inorganica. Chim. Acta 32, L93-L94. 
Fedina, I.S., Grigorova, I.D., Georgieva, K.M., 2003. Response of barley seedlings to UV-B radiation as affected by $\mathrm{NaCl}$. J. Plant. Physiol. 160 (2), 205-208.

Feng, X.J., Li, J.R., Qi, S.L., Lin, Q.F., Jin, J.B., Hua, X.J., 2016. Light affects salt stress-induced transcriptional memory of P5CS1 in Arabidopsis. Proc. Natl. Acad. Sci. 113 (51), E8335-E8343.

Flowers, T.J., Hall, J.L., 1978. Salt tolerance in the halophyte, Suaeda maritima (L.) Dum.: the influence of the salinity of the culture solution on the content of various organic compounds. Ann. Bot. (Lond.) 42 (5), 1057-1063.

Fujii, H., Chinnusamy, V., Rodrigues, A., Rubio, S., Antoni, R., Park, S.Y., et al., 2009. In vitro reconstitution of an abscisic acid signalling pathway. Nature 462 (7273), 660-664.

Funck, D., Eckard, S., Müller, G., 2010. Non-redundant functions of two proline dehydrogenase isoforms in Arabidopsis. BMC Plant Biol. 10 (1), 70.

Galinski, E.A., 1993. Compatible solutes of halophilic eubacteria: molecular principles, water-solute interaction, stress protection. Experientia 49 (6-7), 487-496.

Gao, X.P., Pan, Q.H., Li, M.J., Zhang, L.Y., Wang, X.F., Shen, Y.Y., et al., 2004b. Abscisic acid is involved in the water stress-induced betaine accumulation in pear leaves. Plant Cell Physiol. 45 (6), $742-750$.

Gao, X.P., Wang, X.F., Lu, Y.F., Zhang, L.Y., Shen, Y.Y., Liang, Z., et al., 2004a. Jasmonic acid is involved in the water-stress-induced betaine accumulation in pear leaves. Plant Cell Environ. 27 (4), 497-507.

Gibson, S.I., 2005. Control of plant development and gene expression by sugar signaling. Curr. Opin. Plant. Biol. 8 (1), 93-102.

Gill, P.K., Sharma, A.D., Singh, P., Bhullar, S.S., 2001. Effect of various abiotic stresses on the growth, soluble sugars and water relations of sorghum seedlings grown in light and darkness. Bulg. J. Plant Physiol 27 (1-2), 72-84.

Gorham, J., 1995. Betaine in higher plants- biosynthesis and role in stress metabolism. In: Wallsgrove, R.M. (Ed.), Amino Acids in Higher Plants. Cambridge University Press, Cambridge, pp. 171-203.

Gupta, A.K., Kaur, N., 2005. Sugar signalling and gene expression in relation to carbohydrate metabolism under abiotic stresses in plants. J. Biosci. 30 (5), 761-776.

Hai-Hua, R., Wen-Biao, S., Lang-Lai, X., 2004. Nitric oxide involved in the abscisic acid induced proline accumulation in wheat seedling leaves under salt stress. Acta Botanica Sinica 46 (11), 1307-1315.

Haldimann, P., Feller, U., 2005. Growth at moderately elevated temperature alters the physiological response of the photosynthetic apparatus to heat stress in pea (Pisum sativum L.) leaves. Plant Cell Environ. 28 (3), 302-317.

Hallouin, M., Ghelis, T., Brault, M., Bardat, F., Cornel, D., Miginiac, E., et al., 2002. Plasmalemma abscisic acid perception leads to RAB18 expression via phospholipase D activation in Arabidopsis suspension cells. Plant Physiol. 130 (1), 265-272.

Hanson, J., Hanssen, M., Wiese, A., Hendriks, M.M., Smeekens, S., 2008. The sucrose regulated transcription factor bZIP11 affects amino acid metabolism by regulating the expression of Asparagine synthetase 1 and Proline dehydrogenase2. Plant J. 53 (6), 935-949.

Hare, P.D., Cress, W.A., 1997. Metabolic implications of stressinduced proline accumulation in plants. Plant Growth Regul. 21 (2), 79-102.

Hare, P.D., Cress, W.A., Van Staden, J., 1998. Dissecting the roles of osmolyte accumulation during stress. Plant Cell Environ. 21 (6), 535-553.

Hare, P.D., Cress, W.A., Van Staden, J., 1999. Proline synthesis and degradation: a model system for elucidating stress-related signal transduction. J. Exp. Bot. 50 (333), 413-434.
Hasanuzzaman, M., Alam, M., Rahman, A., Hasanuzzaman, M., Nahar, K., Fujita, M., 2014. Exogenous proline and glycine betaine mediated upregulation of antioxidant defense and glyoxalase systems provides better protection against salt-induced oxidative stress in two rice (Oryza sativa L.) varieties. Biomed. Res. Int. 2014.

Hayashi, H., Sakamoto, A., Murata, N., 1998. Enhancement of the tolerance of Arabidopsis to high temperatures by genetic engineering of the synthesis of glycinebetaine. Plant J. 16 (2), 155-161.

Hayashi, F., Ichino, T., Osanai, M., Wada, K., 2000. Oscillation and regulation of proline content by P5CS and ProDH gene expressions in the light/dark cycles in Arabidopsis thaliana L. Plant Cell Physiol. 41 (10), 1096-1101.

He, H., He, L.F., 2017. Regulation of gaseous signaling molecules on proline metabolism in plants. Plant Cell Rep. 37 (3), 387-392.

Henson, I.E., 1984. Effects of atmospheric humidity on abscisic acid accumulation and water status in leaves of rice (Oryza sativa L.). Ann. Bot. (Lond.) 54 (4), 569-582.

Hilal, M., Parrado, M.F., Rosa, M., Gallardo, M., Orce, L., Massa, E. M., et al., 2004. Epidermal lignin deposition in quinoa cotyledons in response to UV-B radiation. Photochem. Photobiol. 79 (2), 205-210

Hincha, D.K., Hellwege, E.M., Heyer, A.G., Crowe, J.H., 2000. Plant fructans stabilize phosphatidylcholine liposomes during freezedrying. FEBS. J. 267 (2), 535-540.

Hincha, D.K., Zuther, E., Hellwege, E.M., Heyer, A.G., 2002. Specific effects of fructo-and gluco-oligosaccharides in the preservation of liposomes during drying. Glycobiology 12 (2), 103-110.

Hincha, D.K., Livingston, D.P., Premakumar, R., Zuther, E., Obel, N., Cacela, C., et al., 2007. Fructans from oat and rye: composition and effects on membrane stability during drying. Biochim. Biophys. Acta (BBA)-Biomembr. 1768 (6), 1611-1619.

Holmström, K.O., Somersalo, S., Mandal, A., Palva, T.E., Welin, B., 2000. Improved tolerance to salinity and low temperature in transgenic tobacco producing glycine betaine. J. Exp. Bot. 51 (343), 177-185.

Hoque, M.A., Banu, M.N.A., Okuma, E., Amako, K., Nakamura, Y., Shimoishi, Y., et al., 2007b. Exogenous proline and glycinebetaine increase $\mathrm{NaCl}$-induced ascorbate-glutathione cycle enzyme activities, and proline improves salt tolerance more than glycinebetaine in tobacco bright yellow-2 suspension-cultured cells. J. Plant. Physiol. 164 (11), 1457-1468.

Hoque, M.A., Okuma, E., Banu, M.N.A., Nakamura, Y., Shimoishi, Y., Murata, Y., 2007a. Exogenous proline mitigates the detrimental effects of salt stress more than exogenous betaine by increasing antioxidant enzyme activities. J. Plant. Physiol. 164 (5), $553-561$.

Hu, C.A., Donald, S.P., Yu, J., Lin, W.W., Liu, Z., Steel, G., et al., 2007. Overexpression of proline oxidase induces prolinedependent and mitochondria-mediated apoptosis. Mol. Cell. Biochem. 295 (1-2), 85-92.

Huang, Z., Zhao, L., Chen, D., Liang, M., Liu, Z., Shao, H., et al., 2013. Salt stress encourages proline accumulation by regulating proline biosynthesis and degradation in Jerusalem artichoke plantlets. PLoS One 8 (4), e62085.

Hüner, N., Dahal, K., Kurepin, L.V., Savitch, L., Singh, J., Ivanov, A. G., et al., 2014. Potential for increased photosynthetic performance and crop productivity in response to climate change: role of CBFs and gibberellic acid. Front. Chem. 2, 18.

Igarashi, Y., Yoshiba, Y., Sanada, Y., Yamaguchi-Shinozaki, K., Wada, K., Shinozaki, K., 1997. Characterization of the gene for $\Delta$ 1-pyrroline-5-carboxylate synthetase and correlation between the expression of the gene and salt tolerance in Oryza sativa L. Plant Mol. Biol. 33 (5), 857-865. 
Igarashi, Y., Yoshiba, Y., Takeshita, T., Nomura, S., Otomo, J., Yamaguchi-Shinozaki, K., et al., 2000. Molecular cloning and characterization of a cDNA encoding proline transporter in rice. Plant Cell Physiol. 41 (6), 750-756.

Imlay, J.A., Linn, S., 1988. DNA damage and oxygen radical toxicity. Science 240 (4857), 1302-1309.

Iqbal, N., Umar, S., Khan, N.A., Khan, M.I.R., 2014. A new perspective of phytohormones in salinity tolerance: regulation of proline metabolism. Environ. Exp. Bot. 100, 34-42.

Ishitani, M., Nakamura, T., Han, S.Y., Takabe, T., 1995. Expression of the betaine aldehyde dehydrogenase gene in barley in response to osmotic stress and abscisic acid. Plant Mol. Biol. 27 (2), 307-315.

Jagendorf, A.T., Takabe, T., 2001. Inducers of glycinebetaine synthesis in barley. Plant Physiol. 127 (4), 1827-1835.

Jiménez-Arias, D., Borges, A.A., Luis, J.C., Valdés, F., Sandalio, L.M., Pérez, J.A., 2015. Priming effect of menadione sodium bisulphite against salinity stress in Arabidopsis involves epigenetic changes in genes controlling proline metabolism. Environ. Exp. Bot. 120, $23-30$.

Kanade, M.B., 2008. Effect of foliar application of salicylic acid on polyphenol, proline and carbohydrates content in wheat and sorghum. Adv. Plant Sci. 21 (1), 321-322.

Kaul, S., Sharma, S.S., Mehta, I.K., 2008. Free radical scavenging potential of L-proline: evidence from in vitro assays. Amino Acids 34 (2), 315-320.

Kaushal, N., Gupta, K., Bhandhari, K., Kumar, S., Thakur, P., Nayyar, H., 2011. Proline induces heat tolerance in chickpea (Cicer arietinum L.) plants by protecting vital enzymes of carbon and antioxidative metabolism. Physiol. Mol. Biol. Plants 17 (3), 203-213.

Ke, X., Cheng, Z., Ma, W., Gong, M., 2014. Effects of nitric oxide on smoregulation of tobacco cells under salt stress. Acta Bot. Boreal Occident. Sin. 34, 1596-1607.

Khan, M.S., Yu, X., Kikuchi, A., Asahina, M., Watanabe, K.N., 2009. Genetic engineering of glycine betaine biosynthesis to enhance abiotic stress tolerance in plants. Plant Biotechnol. 26 (1), 125-134.

Khan, M.I.R., Khan, N.A., 2014. Ethylene reverses photosynthetic inhibition by nickel and zinc in mustard through changes in PS II activity, photosynthetic-nitrogen use efficiency and antioxidant metabolism. Protoplasma 251, 1007-1019.

Khan, M.I.R., Khan, N., 2017. Reactive Oxygen Species and Antioxidant System in Plants: Role and Regulation Under Abiotic Stress. Springer Nature, New York978-981-10-5254-5.

Khan, M.I.R., Asgher, M., Khan, N.A., 2014. Alleviation of saltinduced photosynthesis and growth inhibition by salicylic acid involves glycinebetaine and ethylene in mungbean (Vigna radiata L.). Plant. Physiol. Biochem. 80, 67-74.

Khan, M.I.R., Fatma, M., Per, T.S., Anjum, N.A., Khan, N.A., 2015. Salicylic acid-induced abiotic stress tolerance and underlying mechanisms in plants. Front. Plant Sci. 6, 462.

Kishor, P.B.K., Hong, Z., Miao, G.H., Hu, C.A.A., Verma, D.P.S., 1995. Overexpression of [delta]-pyrroline-5-carboxylate synthetase increases proline production and confers osmotolerance in transgenic plants. Plant Physiol. 108 (4), 1387-1394.

Kiyosue, T., Yoshiba, Y., Yamaguchi-Shinozaki, K., Shinozaki, K., 1996. A nuclear gene encoding mitochondrial proline dehydrogenase, an enzyme involved in proline metabolism, is upregulated by proline but downregulated by dehydration in Arabidopsis. Plant Cell 8 (8), 1323-1335.

Koch, K., 2004. Sucrose metabolism: regulatory mechanisms and pivotal roles in sugar sensing and plant development. Curr. Opin. Plant. Biol. 7 (3), 235-246.

Koch, K.E., Nolte, K.D., Duke, E.R., McCarty, D.R., Avigne, W.T., 1992. Sugar levels modulate differential expression of maize sucrose synthase genes. Plant Cell 4 (1), 59-69.
Kumar, N., Pal, M., Srivastava, G.C., 2009. Proline metabolism in senescing rose petals (Rosa hybrida L. 'First Red'). J. Hort. Sci. Biotechnol. 84 (1), 536-540.

Kumar, N., Pal, M., Singh, A., SaiRam, R.K., Srivastava, G.C., 2010. Exogenous proline alleviates oxidative stress and increase vase life in rose (Rosa hybrida L.'Grand Gala'). Sci. Hortic. (Amsterdam) 127 (1), 79-85.

Kumar, R., Zhao, S., Vetting, M.W., Wood, B.M., Sakai, A., Cho, K., et al., 2014. Prediction and biochemical demonstration of a catabolic pathway for the osmoprotectant proline betaine. mBio 5 (1), e00933-13.

Kurepin, L.V., Dahal, K.P., Savitch, L.V., Singh, J., Bode, R., Ivanov, A.G., et al., 2013. Role of CBFs as integrators of chloroplast redox, phytochrome and plant hormone signaling during cold acclimation. Int. J. Mol. Sci. 14 (6), 12729-12763.

Kurepin, L.V., Ivanov, A.G., Zaman, M., Pharis, R.P., Allakhverdiev, S.I., Hurry, V., et al., 2015. Stress-related hormones and glycinebetaine interplay in protection of photosynthesis under abiotic stress conditions. Photosynth. Res. 126 (2-3), 221-235.

Leprince, A.S., Magalhaes, N., De Vos, D., Bordenave, M., Crilat, E., Clément, G., et al., 2015. Involvement of phosphatidylinositol 3kinase in the regulation of proline catabolism in Arabidopsis thaliana. Front. Plant Sci. 5, 772.

Liang, X., Zhang, L., Natarajan, S.K., Becker, D.F., 2013. Proline mechanisms of stress survival. Antioxid. Redox. Signal. 19 (9), 998-1011.

Liu, J., Hu, H., Wang, X., Li, B., 2010. Effect of nitric oxide on proline accumulation in ryegrass seedlings subjected to salt stress. Acta Agrestia Sinica 18 (6), 786-791.

Liu, Y., Borchert, G.L., Surazynski, A., Hu, C.A., Phang, J.M., 2006. Proline oxidase activates both intrinsic and extrinsic pathways for apoptosis: the role of ROS/superoxides, NFAT and MEK/ERK signaling. Oncogene 25 (41), 5640.

Livingston, D.P., Tallury, S.P., Premkumar, R., Owens, S.A., Olien, C. R., 2005. Changes in the histology of cold-hardened oat crowns during recovery from freezing. Crop Sci. 45 (4), 1545-1558.

Livingston, D.P., Premakumar, R., Tallury, S.P., 2006. Carbohydrate partitioning between upper and lower regions of the crown in oat and rye during cold acclimation and freezing. Cryobiology 52 (2), 200-208.

Livingston, D.P., Hincha, D.K., Heyer, A.G., 2009. Fructan and its relationship to abiotic stress tolerance in plants. Cell. Mol. Life Sci. 66 (13), 2007-2023.

Llorca, C.M., Potschin, M., Zentgraf, U., 2014. bZIPs and WRKYs: two large transcription factor families executing two different functional strategies. Front. Plant Sci. 5, 169.

Lokhande, V.H., Suprasanna, P., 2012. Prospects of halophytes in understanding and managing abiotic stress tolerance. In: Parvaiz, A., Prasad, M.N.V. (Eds.), Environmental Adaptations to Changing Climate: Metabolism, Productivity and Sustainability. Springer, New York, pp. 29-56.

Lopez-Carrion, A.I., Castellano, R., Rosales, M.A., Ruiz, J.M., Romero, L., 2008. Role of nitric oxide under saline stress: implications on proline metabolism. Biol. Plant. 52 (3), 587.

Luo, Z., Li, D., Du, R., Mou, W., 2015. Hydrogen sulfide alleviates chilling injury of banana fruit by enhanced antioxidant system and proline content. Sci. Hortic. (Amsterdam) 183, 144-151.

Ma, Y., Szostkiewicz, I., Korte, A., Moes, D., Yang, Y., Christmann, A., et al., 2009. Regulators of PP2C phosphatase activity function as abscisic acid sensors. Science 324 (5930), 1064-1068.

Matiolli, C.C., Tomaz, J.P., Duarte, G.T., Prado, F.M., Del Bem, L.E. V., Silveira, A.B., et al., 2011. The Arabidopsis bZIP gene AtbZIP63 is a sensitive integrator of transient abscisic acid and glucose signals. Plant Physiol. 157 (2), 692-705. 
Matysik, J., Alia, Bhalu, B., Mohanty, P., 2002. Molecular mechanisms of quenching of reactive oxygen species by proline under stress in plants. Curr. Sci. 525-532.

Mehta, S.K., Gaur, J.P., 1999. Heavy-metal-induced proline accumulation and its role in ameliorating metal toxicity in Chlorella vulgaris. New Phytol. 143 (2), 253-259.

Miller, G., Honig, A., Stein, H., Suzuki, N., Mittler, R., Zilberstein, A., 2009. Unraveling $\Delta 1$-pyrroline-5-carboxylate-proline cycle in plants by uncoupled expression of proline oxidation enzymes. J. Biol. Chem. 284 (39), 26482-26492.

Misra, N., Saxena, P., 2009. Effect of salicylic acid on proline metabolism in lentil grown under salinity stress. Plant Sci. 177 (3), 181-189.

Mohapatra, S.S., Poole, R.J., Dhindsa, R.S., 1988. Abscisic acidregulated gene expression in relation to freezing tolerance in Alfalfa. Plant Physiol. 87 (2), 468-473.

Molinari, H.B.C., Marur, C.J., Daros, E., De Campos, M.K.F., De Carvalho, J.F.R.P., Pereira, L.F.P., et al., 2007. Evaluation of the stress-inducible production of proline in transgenic sugarcane (Saccharum spp.): osmotic adjustment, chlorophyll fluorescence and oxidative stress. Physiol. Plant. 130 (2), 218-229.

Morsy, M.R., Jouve, L., Hausman, J.F., Hoffmann, L., Stewart, J.M., 2007. Alteration of oxidative and carbohydrate metabolism under abiotic stress in two rice (Oryza sativa L.) genotypes contrasting in chilling tolerance. J. Plant. Physiol. 164 (2), 157-167.

Müller, J., Wiemken, A., Aeschbacher, R., 1999. Trehalose metabolism in sugar sensing and plant development. Plant Sci. 147 (1), 37-47.

Mundy, J., Chua, N.H., 1988. Abscisic acid and water-stress induce the expression of a novel rice gene. EMBO J. 7 (8), 2279-2286.

Murahama, M., Yoshida, T., Hayashi, F., Ichino, T., Sanada, Y., Wada, K., 2001. Purification and characterization of 81 -pyrroline5 -carboxylate reductase isoenzymes, indicating differential distribution in spinach (Spinacia oleracea L.) leaves. Plant Cell Physiol. 42 (7), 742-750.

Nayyar, H., Walia, D.P., 2004. Genotypic variation in wheat in response to water stress and abscisic acid-induced accumulation of osmolytes in developing grains. J Agron Crop Sci 190 (1), 39-45.

Neill, S., Barros, R., Bright, J., Desikan, R., Hancock, J., Harrison, J., et al., 2008. Nitric oxide, stomatal closure, and abiotic stress. J. Exp. Bot. 59 (2), 165-176.

Okuma, E., Murakami, Y., Shimoishi, Y., Tada, M., Murata, Y., 2004. Effects of exogenous application of proline and betaine on the growth of tobacco cultured cells under saline conditions. Soil Sci. Plant Nutr. 50 (8), 1301-1305.

Ozden, M., Demirel, U., Kahraman, A., 2009. Effects of proline on antioxidant system in leaves of grapevine (Vitis vinifera L.) exposed to oxidative stress by $\mathrm{H}_{2} \mathrm{O}_{2}$. Sci. Hortic. (Amsterdam) 119 (2), 163-168.

Papageorgiou, G.C., Murata, N., 1995. The unusually strong stabilizing effects of glycine betaine on the structure and function of the oxygen-evolving photosystem II complex. Photosynth. Res. 44 (3), 243-252.

Park, S.Y., Fung, P., Nishimura, N., Jensen, D.R., Fujii, H., Zhao, Y., et al., 2009. Abscisic acid inhibits PP2Cs via the PYR/PYL family of ABA-binding START proteins. Science (New York, NY) 324 (5930), 1068-1071.

Parre, E., Ghars, M.A., Leprince, A.S., Thiery, L., Lefebvre, D., Bordenave, M., et al., 2007. Calcium signaling via phospholipase $\mathrm{C}$ is essential for proline accumulation upon ionic but not nonionic hyperosmotic stresses in Arabidopsis. Plant Physiol. 144 (1), 503-512.

Peng, Z., Lu, Q., Verma, D.P.S., 1996. Reciprocal regulation of $\Delta^{1}$ pyrroline-5-carboxylate synthetase and proline dehydrogenase genes controls proline levels during and after osmotic stress in plants. Mol. Gen. Genet. 253 (3), 334-341.
Per, T.S., Khan, N.A., Reddy, P.S., Masood, A., Hasanuzzaman, M., Khan, M.I.R., et al., 2017. Approaches in modulating proline metabolism in plants for salt and drought stress tolerance: phytohormones, mineral nutrients and transgenics. Plant. Physiol. Biochem. 115, 126-140.

Per, T.S., Khan, M.I.R., Anjum, N.A., Masood, A., Hussain, S.J., Khan, N.A., 2018. Jasmonates in plants under abiotic stresses: crosstalk with other phytohormones matters. Environ. Exp. Bot. $145,104-120$.

Posmyk, M.M., Janas, K.M., 2007. Effects of seed hydropriming in presence of exogenous proline on chilling injury limitation in Vigna radiata L. seedlings. Acta Physiol. Plant. 29 (6), 509-517.

Rasheed, R., Wahid, A., Farooq, M., Hussain, I., Basra, S.M., 2011. Role of proline and glycinebetaine pretreatments in improving heat tolerance of sprouting sugarcane (Saccharum sp.) buds. Plant Growth Regul. 65 (1), 35-45.

Reddy, P.S., Jogeswar, G., Rasineni, G.K., Maheswari, M., Reddy, A. R., Varshney, R.K., et al., 2015. Proline over-accumulation alleviates salt stress and protects photosynthetic and antioxidant enzyme activities in transgenic sorghum [Sorghum bicolor (L.) Moench]. Plant. Physiol. Biochem. 94, 104-113.

Reiser, V., Raitt, D.C., Saito, H., 2003. Yeast osmosensor Sln1 and plant cytokinin receptor Cre1 respond to changes in turgor pressure. J. Cell. Biol. 161 (6), 1035-1040.

Rook, F., Gerrits, N., Kortstee, A., Van Kampen, M., Borrias, M., Weisbeek, P., et al., 1998. Sucrose-specific signalling represses translation of the Arabidopsis ATB2 bZIP transcription factor gene. Plant J. 15 (2), 253-263.

Rosa, M., Hilal, M., Gonzalez, J.A., Prado, F.E., 2009b. Lowtemperature effect on enzyme activities involved in sucrose-starch partitioning in salt-stressed and salt-acclimated cotyledons of quinoa (Chenopodium quinoa Willd.) seedlings. Plant. Physiol. Biochem. 47 (4), 300-307.

Rosa, M., Prado, C., Podazza, G., Interdonato, R., González, J.A., Hilal, M., et al., 2009a. Soluble sugars: metabolism, sensing and abiotic stress: a complex network in the life of plants. Plant Signal. Behav. 4 (5), 388-393.

Roy, D., Basu, N., Bhunia, A., Banerjee, S.K., 1993. Counteraction of exogenous L-proline with $\mathrm{NaCl}$ in salt-sensitive cultivar of rice. Biol. Plant. 35 (1), 69.

Sah, S.K., Reddy, K.R., Li, J., 2016. Abscisic acid and abiotic stress tolerance in crop plants. Front. Plant Sci. 7, 571.

Saito, H., Tatebayashi, K., 2004. Regulation of the osmoregulatory HOG MAPK cascade in yeast. J. Biochem. 136 (3), 267-272.

Sakamoto, A., Murata, N., 2002. The role of glycine betaine in the protection of plants from stress: clues from transgenic plants. Plant Cell Environ. 25 (2), 163-171.

Saradhi, P.P., AliaArora, S., Prasad, K.V.S.K., 1995. Proline accumulates in plants exposed to UV radiation and protects them against UV-induced peroxidation. Biochem. Biophys. Res. Commun. 209 (1), $1-5$.

Sarvesh, A., Anuradha, M., Pulliah, T., Reddy, T.P., Kavi Kishor, P. B., 1996. Salt stress and antioxidant response in high and low proline producing cultivars of niger, Guizotia abyssinica (Lf) Cass. Indian J. Exp. Biol. 34 (3), 252-256.

Satoh, R., Fujita, Y., Nakashima, K., Shinozaki, K., YamaguchiShinozaki, K., 2004. A novel subgroup of bZIP proteins functions as transcriptional activators in hypoosmolarity-responsive expression of the ProDH gene in Arabidopsis. Plant Cell Physiol. 45 (3), 309-317.

Satoh, R., Nakashima, K., Seki, M., Shinozaki, K., YamaguchiShinozaki, K., 2002. ACTCAT, a novel cis-acting element for proline-and hypoosmolarity-responsive expression of the ProDH gene encoding proline dehydrogenase in Arabidopsis. Plant Physiol. 130 (2), 709-719. 
Savouré, A., Jaoua, S., Hua, X.J., Ardiles, W., Van Montagu, M., Verbruggen, N., 1995. Isolation, characterization, and chromosomal location of a gene encoding the $\Delta 1$-pyrroline-5-carboxylate synthetase in Arabidopsis thaliana. FEBS Lett. 372 (1), 13-19.

Schat, H., Sharma, S.S., Vooijs, R., 1997. Heavy metal-induced accumulation of free proline in a metal-tolerant and a nontolerant ecotype of Silene vulgaris. Physiol. Plant. 101 (3), 477-482.

Schobert, B., 1977. Is there an osmotic regulatory mechanism in algae and higher plants? J. Theor. Biol. 68 (1), 17-26.

Schwartz, S.H., Zeevaart, J.A.D., 2010. Abscisic acid biosynthesis and metabolism. In: Davies, P.J. (Ed.), Plant Hormones: Biosynthesis, Signal Transduction and Action, third (revised) ed. Springer, Dordrecht, pp. 137-155.

Seiler, C., Harshavardhan, V.T., Reddy, P.S., Hensel, G., Kumlehn, J., Eschen-Lippold, L., et al., 2014. Abscisic acid flux alterations result in differential abscisic acid signaling responses and impact assimilation efficiency in barley under terminal drought stress. Plant Physiol. 164 (4), 1677-1696.

Shabala, S., Shabala, L., Van Volkenburgh, E., 2003. Effect of calcium on root development and root ion fluxes in salinised barley seedlings. Funct. Plant Biol. 30 (5), 507-514.

Sharma, S., Villamor, J.G., Verslues, P.E., 2011. Essential role of tissue-specific proline synthesis and catabolism in growth and redox balance at low water potential. Plant Physiol. 157 (1), 292-304.

Sharma, S.S., Dietz, K.J., 2006. The significance of amino acids and amino acid-derived molecules in plant responses and adaptation to heavy metal stress. J. Exp. Bot. 57 (4), 711-726.

Sharma, S.S., Schat, H., Vooijs, R., 1998. In vitro alleviation of heavy metal-induced enzyme inhibition by proline. Phytochemistry 49 (6), 1531-1535.

Shen, B., Jensen, R.G., Bohnert, H.J., 1997. Mannitol protects against oxidation by hydroxyl radicals. Plant Physiol. 115 (2), 527-532.

Shen, B., Hohmann, S., Jensen, R.G., Bohnert, H.J., 1999. Roles of sugar alcohols in osmotic stress adaptation. Replacement of glycerol by mannitol and sorbitol in yeast. Plant Physiol. 121 (1), $45-52$.

Shin, H., Oh, S., Kim, K., Kim, D., 2016. Proline accumulates in response to higher temperatures during dehardening in peach shoot tissues. Hort. J. 85 (1), 37-45.

Siripornadulsil, S., Traina, S., Verma, D.P.S., Sayre, R.T., 2002. Molecular mechanisms of proline-mediated tolerance to toxic heavy metals in transgenic microalgae. Plant Cell 14 (11), 2837-2847.

Smirnoff, N., Cumbes, Q.J., 1989. Hydroxyl radical scavenging activity of compatible solutes. Phytochemistry 28 (4), 1057-1060.

Smirnoff, N., Stewart, G.R., 1987. Nitrogen assimilation and zinc toxicity to zinc-tolerant and non-tolerant clones of Deschampsia cespitosa (L.) Beauv. New Phytol. 107 (4), 671-680.

Solomon, A., Beer, S., Waisel, Y., Jones, G.P., Paleg, L.G., 1994. Effects of $\mathrm{NaCl}$ on the carboxylating activity of Rubisco from Tamarix jordanis in the presence and absence of proline-related compatible solutes. Physiol. Plant. 90 (1), 198-204.

Storey, R., Jones, R.W., 1977. Quaternary ammonium compounds in plants in relation to salt resistance. Phytochemistry 16 (4), $447-453$.

Street, T.O., Bolen, D.W., Rose, G.D., 2006. A molecular mechanism for osmolyte-induced protein stability. Proc. Natl. Acad. Sci. 103 (38), 13997-14002.

Strizhov, N., Ábrahám, E., Ökrész, L., Blickling, S., Zilberstein, A., Schell, J., et al., 1997. Differential expression of two P5CS genes controlling proline accumulation during salt-stress requires ABA and is regulated by ABA1, ABI1 and AXR2 in Arabidopsis. Plant J. 12 (3), 557-569.

Su, M., Li, X.F., Ma, X.Y., Peng, X.J., Zhao, A.G., Cheng, L.Q., et al., 2011. Cloning two P5CS genes from bioenergy sorghum and their expression profiles under abiotic stresses and MeJA treatment. Plant Sci. 181 (6), 652-659.

Suzuki, N., Koussevitzky, S., Mittler, R.O.N., Miller, G.A.D., 2012. ROS and redox signalling in the response of plants to abiotic stress. Plant Cell Environ. 35 (2), 259-270.

Szabados, L., Savoure, A., 2010. Proline: a multifunctional amino acid. Trends. Plant. Sci. 15 (2), 89-97.

Tarczynski, M.C., Jensen, R.G., Bohnert, H.J., 1993. Stress protection of transgenic tobacco by production of the osmolyte mannitol. Science 259 (5094), 508-510.

Tester, M., Davenport, R., 2003. $\mathrm{Na}^{+}$tolerance and $\mathrm{Na}^{+}$transport in higher plants. Ann. Bot. (Lond.) 91 (5), 503-527.

Thevelein, J.M., 1984. Regulation of trehalose mobilization in fungi. Microbiol. Rev. 48 (1), 42.

Thiery, L., Leprince, A.S., Lefebvre, D., Ghars, M.A., Debarbieux, E., Savouré, A., 2004. Phospholipase D is a negative regulator of proline biosynthesis in Arabidopsis thaliana. J. Biol. Chem. 279 (15), 14812-14818.

Thornburg, R.W., 2007. Molecular biology of the Nicotiana floral nectary. In: Nicolson, S. (Ed.), Nectaries and Nectar. Springer-Verlag, Berlin, pp. 265-287.

Tian, B., Qiao, Z., Zhang, L., Li, H., Pei, Y., 2016. Hydrogen sulfide and proline cooperate to alleviate cadmium stress in foxtail millet seedlings. Plant. Physiol. Biochem. 109, 293-299.

Tripathi, B.N., Gaur, J.P., 2004. Relationship between copper-and zinc-induced oxidative stress and proline accumulation in Scenedesmus sp. Planta 219 (3), 397-404.

Uchida, A., Jagendorf, A.T., Hibino, T., Takabe, T., Takabe, T., 2002. Effects of hydrogen peroxide and nitric oxide on both salt and heat stress tolerance in rice. Plant Sci. 163 (3), 515-523.

Umezawa, T., Nakashima, K., Miyakawa, T., Kuromori, T., Tanokura, M., Shinozaki, K., et al., 2010. Molecular basis of the core regulatory network in ABA responses: sensing, signaling and transport. Plant Cell Physiol. 51 (11), 1821-1839.

Veerabagu, M., Kirchler, T., Elgass, K., Stadelhofer, B., Stahl, M., Harter, K., et al., 2014. The interaction of the Arabidopsis response regulator ARR18 with bZIP63 mediates the regulation of Proline dehydrogenase expression. Mol. Plant 7 (10), 1560-1577.

Verbruggen, N., Villarroel, R., Van Montagu, M., 1993. Osmoregulation of a pyrroline-5-carboxylate reductase gene in Arabidopsis thaliana. Plant Physiol. 103 (3), 771-781.

Vereyken, I.J., Chupin, V., Hoekstra, F.A., Smeekens, S.C., de Kruijff, B., 2003. The effect of fructan on membrane lipid organization and dynamics in the dry state. Biophys. J. 84 (6), 3759-3766.

Verslues, P.E., Bray, E.A., 2006. Role of abscisic acid (ABA) and Arabidopsis thaliana ABA-insensitive loci in low water potentialinduced $\mathrm{ABA}$ and proline accumulation. J. Exp. Bot. 57 (1), 201-212.

Vogel, G., Aeschbacher, R.A., Müller, J., Boller, T., Wiemken, A., 1998. Trehalose-6-phosphate phosphatases from Arabidopsis thaliana: identification by functional complementation of the yeast tps2 mutant. Plant J. 13 (5), 673-683.

Wang, H., Tang, X., Wang, H., Shao, H.B., 2015. Proline accumulation and metabolism-related genes expression profiles in Kosteletzkya virginica seedlings under salt stress. Front. Plant Sci. 6, 792.

Wang, N., Nobel, P.S., 1998. Phloem transport of fructans in the crassulacean acid metabolism species Agave deserti. Plant Physiol. 116 (2), 709-714.

Wei-Tao, L., Lin, B., Zhang, M., Hua, X.J., 2011. Proline accumulation is inhibitory to Arabidopsis seedlings during heat stress. Plant Physiol. 156 (4), 1921-1933.

Wu, J.T., Chang, S.C., Chen, K.S., 1995. Enhancement of intracellular proline level in cells of Anacystis nidulans (cyanobacteria) exposed to deleterious concentrations of copper. J. Phycol. 31 (3), $376-379$. 
Wu, J.T., Hsieh, M.T., Kow, L.C., 1998. Role of proline accumulation in response to toxic copper in Chlorella sp. (Chlorophyceae) cells. J. Phycol. 34 (1), 113-117.

Yamaguchi-Shinozaki, K., Shinozaki, K., 2006. Transcriptional regulatory networks in cellular responses and tolerance to dehydration and cold stresses. Annu. Rev. Plant. Biol. 57, 781-803.

Yang, S.L., Lan, S.S., Gong, M., 2009. Hydrogen peroxide-induced proline and metabolic pathway of its accumulation in maize seedlings. J. Plant. Physiol. 166 (15), 1694-1699.

Yang, X., Liang, Z., Lu, C., 2005. Genetic engineering of the biosynthesis of glycinebetaine enhances photosynthesis against high temperature stress in transgenic tobacco plants. Plant Physiol. 138 (4), 2299-2309.

Yang, X., Wen, X., Gong, H., Lu, Q., Yang, Z., Tang, Y., et al., 2007. Genetic engineering of the biosynthesis of glycinebetaine enhances thermotolerance of photosystem II in tobacco plants. Planta 225 (3), 719-733.

Yoshiba, Y., Kiyosue, T., Katagiri, T., Ueda, H., Mizoguchi, T., Yamaguchi-Shinozaki, K., et al., 1995. Correlation between the induction of a gene for $\Delta 1$-pyrroline-5-carboxylate synthetase and the accumulation of proline in Arabidopsis thaliana under osmotic stress. Plant J. 7 (5), 751-760.

You, J., Hu, H., Xiong, L., 2012. An ornithine $\delta$-aminotransferase gene OsOAT confers drought and oxidative stress tolerance in rice. Plant Sci. 197, 59-69.

Yuan, X., Wang, J., Xie, Y., Shen, W., 2009. Effect of carbon monoxide on salt tolerance and proline contents in root of wheat seedlings. Plant Physiol. Communicat. 45 (6), 567-570.

Zentella, R., Mascorro-Gallardo, J.O., Van Dijck, P., Folch-Mallol, J., Bonini, B., Van Vaeck, C., et al., 1999. A Selaginella lepidophylla trehalose-6-phosphate synthase complements growth and stresstolerance defects in a yeasttps1 mutant. Plant Physiol. 119 (4), 1473-1482.
Zhang, L., Becker, D., 2015. Connecting proline metabolism and signaling pathways in plant senescence. Front. Plant Sci. 6, 552.

Zhang, L., Gao, M., Hu, J., Zhang, X., Wang, K., Ashraf, M., 2012a. Modulation role of abscisic acid (ABA) on growth, water relations and glycinebetaine metabolism in two maize (Zea mays L.) cultivars under drought stress. Int. J. Mol. Sci. 13 (3), 3189-3202.

Zhang, L.P., Mehta, S.K., Liu, Z.P., Yang, Z.M., 2008. Copperinduced proline synthesis is associated with nitric oxide generation in Chlamydomonas reinhardtii. Plant Cell Physiol. 49 (3), $411-419$.

Zhang, C., Li, Y., Yuan, F., Hu, S., He, P., 2012b. Effects of hematin and carbon monoxide on the salinity stress responses of Cassia obtusifolia L. seeds and seedlings. Plant Soil 359 (1-2), 85-105.

Zhao, M.G., Chen, L., Zhang, L.L., Zhang, W.H., 2009. Nitric reductase-dependent nitric oxide production is involved in cold acclimation and freezing tolerance in Arabidopsis. Plant Physiol. 151 (2), 755-767.

Zhao, Y., Aspinall, D., Paleg, L.G., 1992. Protection of membrane integrity in Medicago sativa L. by glycinebetaine against the effects of freezing. J. Plant. Physiol. 140 (5), 541-543.

Zuther, E., Kwart, M., Willmitzer, L., Heyer, A.G., 2004. Expression of a yeast-derived invertase in companion cells results in longdistance transport of a trisaccharide in an apoplastic loader and influences sucrose transport. Planta 218 (5), 759-766.

\section{Further Reading}

Ruan, H.H., Shen, W.B., Xu, L.L., 2004. Nitric oxide involved in the abscisic acid induced proline accumulation in wheat seedling leaves under salt stress. Acta Botanica Sinica-English Edition 46 (11), 1307-1315. 


\section{8}

\section{Regulatory Role of Proline in Heat Stress Tolerance: Modulation by Salicylic Acid}

\section{Noushina Iqbal ${ }^{1}$, Meher Fatma ${ }^{2}$, Nafees A. Khan ${ }^{3}$ and Shahid Umar ${ }^{1}$}

${ }^{1}$ Department of Botany, Hamdard University, New Delhi, India ${ }^{2}$ Plant Molecular Biology, School of life Sciences, Jawaharlal Nehru University, New Delhi, India ${ }^{3}$ Plant Physiology and Biochemistry Division, Department of Botany,

Aligarh Muslim University, Aligarh, Uttar Pradesh, India

\section{O U T L I N E}

28.1 Introduction

28.2 Aftermaths of Heat Stress

28.3 Proline in Heat Tolerance

28.4 Phytohormones in Heat Tolerance

28.5 Role of Salicyclic Acid in Heat Tolerance
28.6 Interaction Between Salicyclic Acid, Ethylene, and Abscisic Acid for Heat Tolerance

28.7 Conclusion and Future Prospects

References

Further Reading

\subsection{INTRODUCTION}

Heat stress is the continual rise in temperature for a longer period of time affecting plant growth and productivity (Fahad et al., 2017; Hatfield and Prueger, 2011; Khan et al., 2013a,b). With continual rise in temperature and global warming there is a need to develop heat-tolerant plants to protect food production and ensure crop safety. The complexity of global warming is of concern as it affects food security and risks extending famine if suitable tolerance strategies for plants are not well worked out. Plants, being immobile, cannot migrate and escape the extremities of temperature change and therefore have to develop avoidance or tolerance mechanisms. Avoidance or tolerance might decrease their production potential. According to the FAO Land and Nutrition Management Service (2009), the global agricultural aim for increasing the production of food by $70 \%$ by the year 2050 for approximately 2.3 billion additional people is facing dire obstruction in the context of poverty, hunger, and minor change with the environmental modification and exploitation of rare natural resources more economically. It is predicated that temperature increases of $1.5^{\circ} \mathrm{C}-5.8^{\circ} \mathrm{C}$ by 2100 will cause heat stress and create threats to agricultural production (Rosenzweig et al., 2001). Atmospheric $\mathrm{CO}_{2}$ will rise with an increase in global temperature ranging from $1.1^{\circ} \mathrm{C}$ to $6.4^{\circ} \mathrm{C}$ depending on the basis of global emissions scenarios. 
High temperature affects plant physiology, biochemistry, and genetics, and protein structure and functions are affected in the whole development of the plant. The adverse effect of heat stress has been discussed in many studies explaining the famine and malnutrition conditions due to decreased crop nutritional values besides the physiological and biochemical changes (Bita and Gerats, 2013; Kazemi-Shahandashti et al., 2014; Larkindale and Knight, 2002; Siboza et al., 2014). Development of plants that can tolerate high temperature requires understanding the mechanism plants employ under heat stress.

Plants either employ antioxidants, proteins, ion transporters or osmoprotectants to cope with heat stress. Upon exposure to heat stress plants activate their signaling cascades and transcriptional machinery to reduce physiological and biochemical alterations (Hasanuzzaman et al., 2013). The reactive oxygen species (ROS) generated during stress are scavenged through the antioxidants or osmoprotectants (Khan and Khan, 2017). The heat shock proteins (HSPs) are just another heat tolerance strategy (Wang et al., 2014). Bita and Gerats (2013) reported that high temperature is sensed by sensors in different cellular compartments. These sensors send signals that modifications have to be done to incorporate changing temperature with increased membrane fluidity. As a result of temperature change the lipid based signaling cascade in the membrane sends a signal for increase in $\mathrm{Ca}^{2+}$ influx and cytoskeletal reorganization. These signal for the production of osmolytes and antioxidants. Phytohormone levels are also regulated during heat stress (Abeles, 1986; Khan et al., 2013,a,b; Lv et al., 2011). To produce heat-tolerant plants one strategy could be the regulation of osmolytes like proline by salicylic acid (SA). In the present chapter we will focus on the role of proline in heat tolerance together with its regulation by SA. Further, since hormonal regulation cannot be governed by a single hormone, the interaction between them should instead be dealt with to get better insight. We will also consider the relationship of SA with abscisic acid (ABA) and ethylene in regulating each other and proline for heat tolerance.

\subsection{AFTERMATHS OF HEAT STRESS}

Heat stress influences nearly every aspect of plants' growth and development at the physiological and molecular level and there have been major limitations to crop productivity (Bita and Gerats, 2013; Pasala et al., 2016; Wahid et al., 2007). It causes oxidative stress, membrane injury, protein degradation, enzyme inactivation, and DNA damage. Negative impact of heat stress on major world food crop yields is increasingly robust including reducing leaf photosynthesis and enhancing leaf senescence rate (Mathur and Jajoo, 2014; Sharma and Sharma, 2017; Sita et al., 2017). Moreover, extreme heat stress affects plant photosynthetic and transpiration efficiencies and negatively impacts on plant development, collectively affecting plant yield. The decreases in photosynthetic rate under heat stress attributes to lower internal plant $\mathrm{CO}_{2}$, inhibition of photosynthetic enzymes, and synthesis of ATP, which produces chemical energy that is required for plant biochemical reactions. Heat stress also inhibited photosynthesis (Salvucci, 2008; Sage and Kubien, 2007). The inhibition of net photosynthesis by heat stress had been featured to an inability of rubisco activase to maintain rubisco in an active form. Hemantaranjan et al. (2014) reported that nonphotochemical quenching of chlorophyll fluorescence, the effective quantum yield of photochemical energy conversion, and the maximum yield of PSII were sensitive to temperature in Antarctic hairgrass and spinach. Moreover, Tripathy and Rebeiz (1986) and Porra (1997) elucidated in detail the biosynthesis of porphyrins and particularly chlorophyll during early greening stages of seedlings. In cucumber seedlings, biosynthesis of chlorophyll under chill and heat stress was affected by $90 \%$ and $60 \%$, respectively. Inhibition of chlorophyll biosynthesis was partly due to impairment of 5-aminolevulinic acid biosynthesis both in chill and heat stress states. Also, protochlorophyllide synthesis was inhibited by $90 \%$ and $70 \%$ in chill and heat stress, respectively. Heat stress increases the rate of reproductive development, which shortens the time for photosynthesis to contribute to fruit or seed production and reduces total fruit or grain yield (Hall, 2001). Lobell et al. (2012) and Pradhan et al. (2012) noted that heat stress increased the supply of assimilate during grain filling in wheat plant, which was unable to fully compensate for the shorter duration of the grain filling period. Heat stress altered the regulation of senescence processes, such as reduced photosynthesis and leaf chlorophyll content, resulting in accelerated senescence (Harding et al., 1990; Yang et al., 2002; Zhao et al., 2007). Pradhan et al. (2012) reported decrease in leaf chlorophyll, individual grain weight, and grain yield in an increasing magnitude of drought or high temperature and with combined stress. Hays et al. (2007) reported grain abortion by $25 \%$ in one wheat cultivar and no response in another in response to a heat shock event 10 days after pollination. Those differences were attributed to genetic variation in heat tolerance. Tashiro and Wardlaw (1990) reported that heat shock also produced wheat kernels that are small, notched, and split that also significantly affect crop yield and quality. High temperatures had also been shown to reduce the grain filling period by $45 \%-60 \%$ by Shah and Paulsen (2003). 


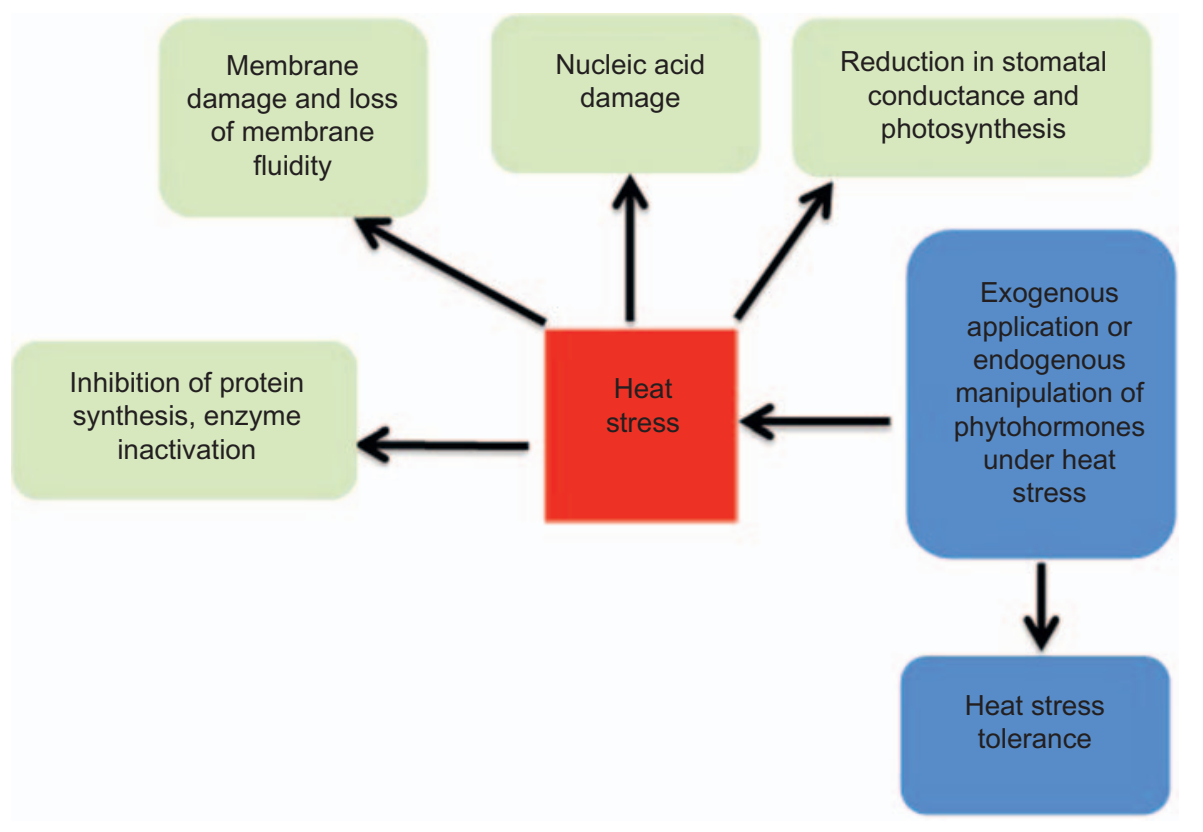

FIGURE 28.1 Major impacts of heat stress on plants and phytohormone coping mechanisms.

Each plant species has a specific temperature range characterized by a minimum, maximum, and optimum that affects the rate of plant growth and development. Those values were reviewed by Hatfield et al. (2008, 2011) for different species typical of grain and fruit production. Barlow et al. (2015) reported that extreme temperature, frost, and heat caused sterility and abortion of formed grains during frost and reduction in grain number and reduced duration of the grain filling period during heat stress in wheat plant. The report of Meehl et al. (2007) exposed that daily minimum temperatures would increase more rapidly than daily maximum temperatures leading to the increase in the daily mean temperatures and those changes had negative effects on grain yield. The effect of heat stress is increased under a high vapor pressure deficit because pollen viability depends on pollen moisture content which strongly depends on vapor pressure deficit (Fonseca and Westgate, 2005).

Under heat stress, Uchida et al. (2002) reported that pretreatment of $\mathrm{H}_{2} \mathrm{O}_{2}$ or $\mathrm{NO}$ induced not only ROS scavenging enzyme activities but also expression of oxidative stress-related genes in rice seedlings. Likewise, Larkindale and Knight (2002) reported that heat produced $\mathrm{H}_{2} \mathrm{O}_{2}$ and induced oxidative stress in plant cells and established that the production of $\mathrm{H}_{2} \mathrm{O}_{2}$ contributes to the transduction of the heat signal into the expression of HSPs and their downstream proteins (Königshofer et al., 2008). Likewise, Sanmiya et al. (2004) in tobacco plants and Xin et al. (2011) in wheat plant reported significant modifications in protein pattern. Sanmiya et al. (2004) and Reddy et al. (2016) noted that high temperature stress induced the synthesis of polypeptides and accumulated the different members of HSP families (96, 80, 70, 35, and $24 \mathrm{kDa}$ ). Heat stress or direct high temperature injuries caused protein denaturation and aggregation and increased fluidity of membrane lipids whereas slower heat injuries or indirect effect caused inactivation of enzymes in chloroplast and mitochondria, inhibition of protein synthesis, protein degradation, and loss of membrane integrity (Howarth et al., 2005). Heat stress also affected the organization of microtubules by splitting and/or elongation of spindles, microtubule asters formation in mitotic cells, and elongation of phragmoplast microtubules (Smertenkon et al., 1997). Fig. 28.1 shows the major impact of heat stress on plants.

\subsection{PROLINE IN HEAT TOLERANCE}

Proline accumulates under heat stress and helps in osmotic adjustment and plays a protective role (Szabados and Savouré, 2010; Kaur and Asthir, 2015; Per et al., 2017). Its synthesis mainly occurs from glutamate and proline metabolizing enzyme, pyrroline-5carboxylate synthetase (P5CS), which reduces glutamate to glutamate-semialdehyde (GSA) and spontaneously to pyrroline-5-carboxylate (P5C). Finally proline formation occurs from the reduction of $\mathrm{P} 5 \mathrm{C}$ by $\mathrm{P} 5 \mathrm{C}$ reductase (P5CR). Proline catabolism occurs by the sequential action of proline dehydrogenase or proline oxidase (PDH or POX) resulting in P5C from proline, and P5C dehydrogenase $(\mathrm{P} 5 \mathrm{CDH})$, which regenerates glutamate from 5C. Alternatively, ornithine is transaminated by ornithine-delta-aminotransferase (OAT) producing GSA and $\mathrm{P} 5 \mathrm{C}$ and then converted to proline. Besides glutamate this is another method of proline synthesis in 
plants (Iqbal et al., 2014). Thus, proline level should increase with increase in P5CS, P5C, and OAT. However, increase in proline under heat stress is not ubiquitous since the increase under heat stress varies depending on different conditions. While proline was reported to increase in the leaves of tomato (Rivero et al., 2004) and tobacco (Cvikrová et al., 2012), it decreased in the germinating wheat seeds (Song et al., 2005) and remain unaltered in Arabidopsis plants (Hua et al., 2001; Rizhsky et al., 2004). This differential proline accumulation was due to altered expression of proline biosynthetic genes. Manipulation of proline could therefore be one potential heat tolerance mechanism. In Arabidopsis overexpression of pyrroline-5-carboxylate reductase gene (AtP5CR) in soybean improved heat tolerance (de Ronde et al., 2004). In heat stressed exposed leaves of sugarcane proline was responsible for increased pressure potential (Wahid et al., 2007). Proline helps in osmotic adjustment, increases the concentration of cell protoplasm to maintain normal membrane function under heat stress, and increases plants adaptability. Its capability to act as an osmoprotectant helps in stabilizing the antioxidant system and thus the deleterious effect of ROS (Reddy et al., 2015; Szabados and Savouré, 2010). Increased proline content was observed in leaf of lettuce seedlings under heat stress but the increase in heat-tolerant varieties was significantly higher than the nonheat-tolerant varieties (Han et al., 2013). Dobra et al. (2010), through the use of transgenic plant constitutively overexpressing a gene for the proline biosynthetic enzyme $\Delta 2$-pyrroline-5-carboxylate synthetase (P5CSF129A; EC 2.7.2.11/1.2.1.41) in tobacco, showed that the transformant with enhanced proline production had a more negative leaf osmotic potential and enhanced xanthophyll cycle pigments that provide protection to plants. In transgenic sugarcane plants with heterologous P5CS gene under control of stress inducible promoter, ABA-inducible promoter complex, Molinari et al. (2007) found that under stress, proline production increased more in the transgenic sugarcane leading to higher protection of chlorophyll and the PSII together with high Malondialdehyde (MDA) content responsible for enhanced antioxidant system. However, osmotic potential in the transgenic sugarcane with higher proline accumulation decreased, not supporting its role in osmotic adjustment. Thus, the role of proline depends upon species; however, we cannot ignore its role in heat tolerance. Rivero et al. (2004) suggested using $\mathrm{NH}_{4}{ }^{+}$as $\mathrm{N}$ source rather than $\mathrm{NO}_{3}{ }^{-}$for heat tolerance. The tolerance was related to higher accumulation of proline with $\mathrm{NH}_{4}{ }^{+}$than $\mathrm{NO}_{3}{ }^{-}$. Kocsy et al. (2005) reported that the genetic manipulation of proline in Glycine max caused enhanced activity of antioxidative enzymes and glutathione content under simultaneous drought and heat stress treatment. The role of proline in the protection of plants against simultaneous drought and heat stress was confirmed by de Ronde et al. (2000). Plants with higher proline content were less damaged compared with wild-type plants with lower proline. However, the effect of proline is not independent but relies on many factors, one among them being phytohormones. In the next section we are going to discuss the role of phytohormones in stress tolerance.

\subsection{PHYTOHORMONES IN HEAT TOLERANCE}

Hormones control a variety of functions in plants and their level determines plants' fate under stress situations. Together with proline, phytohormones also play a key role under heat stress. Per et al. (2017) reviewed that phytohormones play a role in modulating proline metabolism. Arabidopsis plants ectopically expressing the $\Delta$ (1)-pyrroline-5-carboxylate synthetase 1 gene (AtP5CS1) under the control of the HSP17.6II gene promoter had enhanced proline biosynthesis but a decreased thermotolerance, most likely due to higher ROS production and inhibition of $A B A$ and ethylene biosynthesis ( $\mathrm{Lv}$ et al., 2011). Increased level of ABA, $\mathrm{SA}$, and ethylene were observed under heat stress whereas, cytokinin (CK), auxin (AUX), and gibberellic acids (GAs) decreased causing premature plant senescence (Talanova et al., 2003; Larkindale and Huang, 2004; Larkindale et al., 2005). Binder and Patterson (2009) reported that the abscission of reproductive organs was caused by increased $\mathrm{ABA}$ and ethylene levels and reduced levels and transport of AUX. Hormones' association with heat stress tolerance may be fast to immediately provide adaptability or slow to enhance their defense pathway. Dobra et al. (2010) reported that under stress conditions the increased level of ABA is associated both with providing immediate protection through stomatal closure and slower metabolic changes coinciding with activation of defense pathways. Hays et al. (2007), in studying wheat genotypes, reported the role of ethylene in abortion of kernel and reduction in the weight of kernel in the heat susceptible "Karl 92" plants under heat stress. Inhibition of ethylene action in "Karl 92" by methylcyclopropane (1-MCP) before applying heat stress resulted in inhibition of both kernel abortion and reduction in kernel weight confirming the role of ethylene in heat stress induced damages. Heat stress evolved ethylene in such cases served as a signal for developmental arrest and senescence. Phytohormones work by reducing the heat induced oxidative damage as reported by Larkindale and Knight (2002). They applied SA, 1-aminocyclopropane-1-carboxylic acid (a precursor to ethylene), and $\mathrm{ABA}$ to plants and 
observed not only protection from oxidative damage but also the insensitive mutants of ethylene; etr-1, the $A B A ; a b i-1$, and a transgenic line expressing nah $G$ (consequently inhibited in SA production) showed increased susceptibility to heat and thus the role of these hormones in heat tolerance. In lettuce seeds exposed to high temperature, ethylene regulated thermoinhibition and the seeds' ability to germinate at high temperature was positively correlated to seed ethylene (Abeles, 1986).

Phytohormones are signal molecules that regulate plant growth and interact with proline metabolism to increase stress tolerance in plants. Reports are available for the synergistic interaction of phytohormones with proline metabolism for stress tolerance (Khan et al., 2013a,b; Iqbal et al., 2014; Iqbal et al., 2015; Per et al., 2017). However, not much is known about the interaction of proline with ethylene, $\mathrm{ABA}$, and $\mathrm{SA}$ in respect to heat tolerance. Although, the relationship of nitrogen $(\mathrm{N})$ with proline and regulation of $\mathrm{N}$ by phytohormones has been discussed (Iqbal et al., 2015) and the regulation of proline by different phytohormones under salt stress (Iqbal et al., 2014). However, not much is reported about regulation of proline by SA through interaction between the three hormones under heat stress.

Kumar et al. (2012) found that ABA induces tolerance to heat in seedlings of Cicer arietinum L. by accumulating osmoprotectants like proline, glycine betaine, and trehalose. ABA induces heat shock transcription factor 3, which acts synergistically with chimeric genes with a small HSP promoter (Rojas et al., 1999). Its role in induced thermotolerance has been supported (Jiang and Huang, 2001; Larkindale and Knight, 2002; Larkindale and Huang, 2004). Larkindale and Knight (2002) reported the induced thermotolerance in ethylene and ABA treated plants was through increased activity of antioxidative enzymes that reduced heat stress induced oxidative stress. They further showed that those mutants and transgenic plants that do not signal ABA and ethylene are more heat sensitive than wild-type plants. Lv et al. (2011) however, provided a completely different picture to what we usually see. He reported that proline overproducing mutants inhibit ethylene and ABA synthesis and increased oxidative stress via the proline/P5C cycle, decreasing thermotolerance in Arabidopsis seedlings.

SA treatment was reported to increase proline content in chickpea (Cicer arietinum) and increased heat tolerance compared with the control plants not receiving SA (Chakraborty and Tongden, 2005). Khan et al. $(2013 a, b)$ reported the role of SA in heat tolerance, showing that SA at $0.5 \mathrm{mM}$ resulted in tolerating heat induced stress through increased proline production, which helped in maintaining osmotic and water potential necessary for photosynthesis. The increase in proline content was followed by the increase in proline metabolizing enzyme $\gamma$-glutamyl kinase (GK) and decrease in POX activity. Ethylene increased proline accumulation under salt stress to provide tolerance in mustard through the increase in proline metabolizing enzymes (Iqbal et al., 2015).

\subsection{ROLE OF SALICYCLIC ACID IN HEAT TOLERANCE}

Recently, SA has received great attention in the regulation of numerous plant developmental processes under abiotic stresses including heat stress (Khan et al., 2012; Khan and Khan, 2013; Khan et al., 2015). The mechanism of thermotolerance in plants by SA interaction gives an insight into some scientific approaches to modulate plants' responses for heat stress. The SA mechanism was reported by Oata (1975) and Pieterse and Muller (1977). They reported that SA-induced flowering by acting as a chelating agent. Their view was supported by Raskin et al. (1987), who confirmed that SA functioned as endogenous growth regulators of flowering and florigenic effects. It induces flowering, increases flower life, retards senescence, and increases cell metabolic rate. Furthermore, SA is a natural phenolic compound that acts as an endogenous signal molecule that plays a role in the regulation of plant growth, development, and responses to environmental stress. The positive effect of SA offered protection against a number of abiotic stresses, mainly heat stress. Exogenous application of SA is an important way to promote economic utilization by providing thermotolerance in many major crops, for example, in potato (Dat et al., 1998a), tomato (Shaheen et al., 2017; Senaratna et al., 2000), wheat (Sadak and Orabi, 2015), and Arabidopsis (Clarke et al., 2004; Larkindale and Knight, 2002) respectively. SA-mediated improved plant tolerance to heat stress has also been reported by He et al. (2002a); Wang et al. (2010); Khan et al. (2013a,b).

SA has been attributed to an increased $\mathrm{CO}_{2}$ assimilation and photosynthetic rate and increased mineral uptake by the stressed plant (Vazirimehr and Rigi, 2014). Analysis of SA-mediated change in physiological, biochemical, molecular alternations under high temperature stress was proven by the application of SA in improving the phenology, photosynthesis, remobilization of carbohydrates, and yield under normal and late sown high temperature conditions. Larkindale and Knight (2002) reported that the transgenic Arabidopsis seedlings showing a bacterial SA-decomposing salicylate hydroxylase were less tolerant to heat stress. Cold/ heat treated grape plant had shown cold or heat tolerance through the supplementation of SA (Wang and 
Li, 2006). After both heat stress and recovery, SA declined electrolyte leakage and oxidative stress and improved maximum yield of PSII, Fv/Fm, and the quantum yield of the PSII electron transport in cucumber seedlings (Shi et al., 2006). Khan et al. (2013a,b) reported that SA treatment alleviated heat stress in wheat by increasing the production of proline and restriction of the stress ethylene formation. SA derivative acetyl SA application enhanced thermotolerance in potato microplants that simplified the production of virus free potato plants through tissue culture (LopezDelgado et al., 1998). Several other studies reported that exogenous application of SA or acetylsalicylate had been shown to enhance thermotolerance in Arabidopsis (Dat et al., 1998b; Clarke et al., 2004). SA marked differential antioxidant response by upregulating the Halliwell-Asada pathway in roots and attaining high peroxidase activity in both seedlings of maize under high temperature stress (Khanna et al., 2016).

SA pretreatment alleviated the heat stress through inducing decrease in net photosynthesis through maintaining higher Rubisco activation state and it accelerated the net photosynthesis recovery principally through effects on PSII function (Wang et al., 2010). Wang and Li (2007) reported that SA treatment maintained at higher net photosynthesis in grape leaves under heat stress. Wang and $\mathrm{Li}$ (2006) reported that spraying with a $0.1 \mathrm{mM}$ solution of SA decreased thiobarbituric acid-reactive substances and relative electrolyte leakage in young grape leaves under heat stress, signifying that it induced intrinsic heat tolerance in grapevines by upregulating the antioxidant system. Wang et al. (2010) noted the effect of SA on photosynthesis of grape leaves before, during, and after heat stress. Heat stress led to decline in maximum yield of PSII, FPSII, and photochemical quenching and increased of Non-photochemical quenching (NPQ) in relation to the treatment of SA. Maximum quantum yield of PSII, FPSII, and photochemical quenching gradually rise and SA-treated leaves were always greater than control leaves during recovery. SA-treated and control leaves had much lower FPSII under heat stress and had greater thermal dissipation of excitation energy as measured by increased NPQ and with the recovery from heat stress, FPSII of SA-treated and control plants increased, and this was accompanied by increases in maximum yield of PSII and photochemical quenching and a rapid decline of nonphotochemical quenching. However, heat stress significantly reduced the crop productivity whereas in supplementation of SA played substantial role in combating the negative effect of it.

SA caused changes in net photosynthesis and changes in proline under heat stress suggesting that its application protects photosynthesis through alleviating the negative effects of heat stress on proline accumulation and ethylene formation (Khan et al., 2013a,b). Chakraborty and Tongden (2005) reported that SA induces heat tolerance via increase in antioxidative enzymes, protein, and proline in Cicer arietinum $\mathrm{L}$. Sakhabutdinova et al. (2003) found that SA treatment to water stressed plants resulted in increase in ABA and development of antistress reactions like proline accumulation for stress tolerance. In tall fescue seedlings, SA increased the antioxidative enzymes to bring about salt tolerance under abiotic stress (He et al., 2002b). Krantev et al. (2008) reported cadmium tolerance in Zea mays through the increase in proline content.

In lentil, SA alleviated the negativity of salt stress by increasing P5CS, GK, and proline and thus maintaining turgor under salt stress (Misra and Saxena, 2006). SA treatment increased the proline content under stress (Szepsi et al., 2005). Similar proline increase after SA treatment was also reported in Triticum aestivum, Avena sativa, Phaseolus vulgaris, and Lycopersicum plants under oxidative stress (Tasgin et al., 2006). As SA increases proline so was proline associated with induction of SA signaling in tobacco (Chen et al., 2011). It was found that SA increases proline formation through regulating $\mathrm{N}$-assimilation and was dependent on $\mathrm{N}$ source and application method (Iqbal et al., 2014; Tarighaleslami et al., 2012). Application of $\mathrm{N}$ and sulfur individually or in combination increased nitrate reductase activity (NRA), $\mathrm{N}$ content, proline accumulation in Brassica juncea to reduce salt stress (Rais et al., 2013). SA was reported to increase NR activity and activity of glutamine synthase in Cucumis sativa under drought stress (Jing-hong et al., 2012). Under salt stress, SA was responsible for increase in activity of $\mathrm{NR}$ and $\mathrm{NiR}$ in Vigna radiata (Akhtar et al., 2013). Application of SA increased proline accumulation through increased NRA in water stressed seedlings of Amaranthus hybridus (Umebese et al., 2009). However, this increase was found to be concentration dependent in Vigna mungo where low SA-induced NRA while high concentration of SA decreased it (Ramanujam et al., 1998). Thus SA might be responsible for increasing proline for heat tolerance via regulating $\mathrm{N}$ metabolism. Table 28.1 shows the effect of SA on proline content under different stresses.

\subsection{INTERACTION BETWEEN SALICYCLIC ACID, ETHYLENE, AND ABSCISIC ACID FOR HEAT TOLERANCE}

Now let us focus on the interaction between the three hormones to find out how they can regulate proline under heat stress to induce tolerance. The interaction between SA and ethylene was reported by 
TABLE 28.1 Effect of Salicylic Acid on Proline Content Under Different Stresses

\begin{tabular}{llll}
\hline Plant species & Effect on proline & Stresses & References \\
\hline Triticum aestivum & GK increases PROX decreases & Heat & Khan et al. (2013a,b) \\
Lentil & P5CS, GK, proline increases & Salt & Misra and Saxena (2006) \\
Hordeum vulgare cv Gerbel & Proline increases & Salt & El-Tayeb (2005) \\
Triticum aestivum & Proline increases & Salt & Shakirova et al. (2003) \\
Zea mays & Proline increases & Salt & Hussein et al. (2007) \\
Glycine max & SbPRP increase & Salt & He et al. (2002b) \\
& & Drought & Calicylic acid \\
Cicer arietinum L. & Proline increases & Heat & Chakraborty and Tongden (2005) \\
Triticum aestivum & Proline increases & Salicylic acid/heat & Azooz and Youssef (2010) \\
Zea mays & Proline decreases & Cadmium & Krantev et al. (2008) \\
Lycopersicum esculentum & Proline level decreases at higher salt level & Salinity & Zahra et al. (2010) \\
\hline
\end{tabular}

Khan et al. (2013a,b). Under heat stress SA worked as an inhibitor of ethylene synthesis inhibiting activity of ethylene synthesis enzyme 1-aminocyclopropane carboxylic acid (ACC) synthase (ACS) and brought ethylene to an optimum level from stress ethylene level. Optimal ethylene resulted in increased proline metabolism, N-assimilation and photosynthesis. Rao et al. (2002) found that SA is required for stress ethylene production and under ozone stress SA treatment increased ethylene production and ozone sensitivity to induce cell death. In canola plants ethylene treatment reduced chlorophyll and carotenoid content and increased lipid peroxidation while SA treatment after ethylene treatment reversed the ethylene induced oxidative damage and increased chlorophyll and carotenoid contents (Tirani et al., 2013). Lee et al. (1999) found inhibitory effect of SA on AUX-induced ethylene through inhibiting 1-aminocyclopropane-1-carboxylic acid oxidase (ACO) enzyme activity probably through reversible interaction with $\mathrm{Fe}^{2+}$, an essential cofactor of the enzyme. Nazar et al. (2015) reported restricted ethylene formation in drought stressed Brassica juncea upon SA treatment. SA inhibited the ACS activity to influence proline metabolism and photosynthesis.

Similar interaction between ABA and SA is also recorded. Both $\mathrm{ABA}$ and $\mathrm{SA}$ production increases in stress situations. Genes involved in ABA synthesis (AtNCED genes encoding 9-cisepoxycarotenoid dioxygenases) are expressed when Arabidopsis seeds are imbibed at high temperature. Li et al. (2017) reported that the actions of ABA and SA are different under stress. While ABA promotes the accumulation of organic acid, SA promoted the accumulation of amino acids, one among them being proline. ABA was found to suppress the SA signaling pathway in rice by repressing the transcriptional upregulation of WRKY45 and OsNPR1, the two key components of the SA signaling pathway in rice. ABA biosynthesis mutants (aba1, aba2, and aba3), and SA deficient mutant (NahG transgenic lines) showed weaker defects. The role of ABA in thermoinhibition was confirmed by Tamura et al. (2006) when they showed that Arabidopsis seeds both ABA-deficient and ABA-insensitive (aba1 and abi3) germinated at supraoptimal temperatures. The first biochemical step of ABA biosynthesis is catalyzed by AtNCED9 and it plays a major role in thermoinhibition (Toh et al., 2008). Both SA and ABA decreased oxidative stress and increased antioxidative enzymes activity and thereby increased chlorophyll and carotenoid contents, relative water content, membrane stability index, leaf area, and total biomass over control plants in water stressed wheat genotypes.

Xie et al. (2007) reported that for the suppression of GA-induced seed germination by $\mathrm{SA}$ and ABA, HvWRKY38 gene in barley serves as a converging node of signal pathways for $\mathrm{SA}$ and $\mathrm{ABA}$ interaction. Audenaert et al. (2002) found that endogenous ABA level in wild-type plants increased plants' susceptibility to $B$. cinerea infection and they suggested ABA negatively regulates SA-dependent defense signaling, which in turn appears to be an effective plant defense mechanism against $B$. cinerea. The antagonistic interaction between ABA and SA was found in Lycopersicon esculentum where ABA was found to increase the susceptibility of tomato to necrotrophic fungus Botrytis cinerea. However, SA was found to provide protection against the fungus. Mutants with reduced ABA level were more resistant to the fungus and showed increase 
in phenylalanine ammonia lyase activity (pathway for $\mathrm{SA}$ synthesis) and thus suggest the role of ABA in SA-dependent defense pathway in tomato.

Szepesi et al. (2009) found positive interaction between SA and ABA and SA was reported to increase ABA accumulation under salt stress; however, such an increase was dose dependent. At $10^{-4} \mathrm{M}$ concentration SA led to ABA accumulation and enhanced activity of aldehyde oxidase responsible for conversion of ABAaldehyde to ABA. Shakirova et al. (2003) reported high ABA level in SA-treated wheat seedlings that was responsible for proline accumulation under stress, which resulted in preadaptation of plants to stress. $\mathrm{ABA}$ is known to have a key role in the induction of the synthesis of a range of stress proteins (Rock, 2000) and its increased level under stress suggests a significant role of $\mathrm{ABA}$ in protection through changes in proline, in SA-induced preadaptation of wheat plants to stress. SA increases ABA content and proline accumulation under salt stress in wheat (Shakirova et al., 2003). Liu et al. (2006) reported that $100 \mu \mathrm{M}$ ABA biosynthesis inhibitor inhibited not only increase in ABA content but also in SA formation during heat stress. This implies that increase in ABA content under heat stress is also responsible for increasing free SA level focusing on the relationship between the two hormones under heat stress. Both ethylene and ABA are stress hormones involved in germination, leaf abscission, flower senescence, fruit ripening, and in biotic and abiotic stress responses. Some functions of ethylene overlap with ABA throwing light on the interaction between $\mathrm{ABA}$ and ethylene pathway under normal or stress conditions. The signaling pathway of $\mathrm{ABA}$ and ethylene are generally antagonistic (Beaudoin et al., 2000; Anderson et al., 2004). They studied that ethylene insensitive mutant (etr1, ein2, ein3) and ABA-insensitive mutant (aba1, aba2, abi1, abi2) antagonistically affect the expression of stressrelated genes and therefore differently modulate plants' abiotic stress responses. Both ethylene and ABA limit the formation of each other. In Arabidopsis higher level of endogenous ABA was found in ethylene insensitive mutants ein $2 /$ era 3 and etr 1 in comparison with higher ethylene level in ABA-deficient mutant aba2 (LeNoble et al., 2004; Chiwocha et al., 2005). This clearly shows that both ethylene and ABA action are opposite to each other restricting the formation of one another. However, we cannot say that such an antagonistic interaction is ubiquitously present in all plants and in all species. Generally plants' responses are species specific or depend on growth processes and conditions. Therefore, the synergistic interaction between ethylene and ABA cannot be ignored in regulating plants' development and stress responses. Ma et al. (2014) studied synergistic interaction between ethylene and ABA in inhibiting root growth. Ethylene-deficient mutants showed resistance to both ethylene and ABA while in ABA-deficient mutant only $A B A$ resistance was present. In such case ethylene signaling was responsible for ABA-inhibited root growth (Beaudoin et al., 2000; Ghassemian et al., 2000). Thus ABA and ethylene signaling pathways have a close interplay in plant growth, development, and stress response. Hormone signals act either antagonistically or synergistically. The synergistic interaction was found when mutation in ethylene signaling

TABLE 28.2 Effect of Salicylic Acid on Ethylene and Abscisic Acid Under Different Abiotic Stresses

\begin{tabular}{|c|c|c|c|c|}
\hline & Type of stress/condition & Plant species & Response & References \\
\hline \multirow[t]{7}{*}{ Ethylene } & Heat stress & Triticum aestioum & Decreases stress ethylene & Khan et al. $(2013 a, b)$ \\
\hline & Wounding & Lycopersicon esculentum & $\begin{array}{l}\text { Ethylene decreased by preventing accumulation } \\
\text { of ACS transcript }\end{array}$ & Li et al. (1992) \\
\hline & Salt stress & Vigna radiata & Ethylene decreases & Khan et al. (2014) \\
\hline & Cold stress & Zea mays & ACC decrease & Szalai et al. (2000) \\
\hline & Ozone stress & $\begin{array}{l}\text { Arabidopsis mutants, } \\
\text { eto1 and eto3 }\end{array}$ & Ethylene increases & Rao et al. (2002) \\
\hline & Hypocotyl & Vigna mungo & Inhibits ACO enzyme activity & Lee et al. (1999) \\
\hline & Drought stress & Brassica juncea & $\begin{array}{l}\text { Restrict ethylene formation by restricting } \\
\text { ACS enzyme activity }\end{array}$ & Nazar et al. (2015) \\
\hline \multirow[t]{4}{*}{ Abscisic acid } & Salinity & Solanum lycopersicum & Increase & Szepesi et al. (2009) \\
\hline & Salinity & Triticum aestivum & Increase & Shakirova et al. (2003) \\
\hline & Shoot growth and cell cycle & Rice & Antagonizes ABA action & Meguro and Sato (2014) \\
\hline & Seed germination & Arabidopsis & increased synthesis of ABA-regulated proteins & Rajjou et al. (2006) \\
\hline
\end{tabular}


enhanced seed dormancy by altering the sensitivities of germinating seeds to exogenous ABA (Beaudoin et al., 2000; Matilla and Matilla-Vazquez, 2008).

Larkindale et al. (2005) reported that root growth and seedling survival in ABA signaling mutants (abi1 and abi2) were more defective in acquired thermotolerance than basal thermotolerance while ethylene signaling mutants (ein 2 and etr1) were more defective in basal than acquired thermotolerance, especially under high light. Table 28.2 shows the effect of SA on ABA and ethylene production under different conditions and the responses thereof.

\subsection{CONCLUSION AND FUTURE PROSPECTS}

Thus we can see that SA interacts with both ABA and ethylene and ethylene and $\mathrm{ABA}$ also interact with each other. Individually all of them have a role in heat tolerance and affect proline. Although it may be assumed that SA decreases ethylene content and decreased ethylene might increase ABA signaling to increase proline content for heat tolerance, however, this is not always true in all circumstances. Incidence of positive $\mathrm{ABA}$-ethylene and $\mathrm{ABA}-\mathrm{SA}$ interactions are also available. If we can figure out how these three hormones are interacting with each other under stress conditions to regulate proline metabolism, then mutations in hormones or mechanisms for enhancing proline accumulation through regulating the hormones to breed stress tolerant crop will be a promising strategy to combat heat stress in future and develop heat resistant plants. However, one thing is clear-that proline is normally involved in heat tolerance and SA, ABA, and ethylene affect proline under abiotic stresses. So, to develop heat resistant varieties manipulation of proline is an important technique.

\section{References}

Abeles, F.B., 1986. Role of ethylene in Lactuca sativa cv 'Grand Rapids' seed germination. Plant Physiol. 81, 780-787.

Akhtar, J., Ahmad, R., Ashraf, M.Y., Tanveer, A., Waraich, E.A., Oraby, H., 2013. Influence of exogenous application of salicylic acid on salt stressed mungbean (Vigna radiata): growth and nitrogen metabolism. Pak. J. Bot. 45, 119-125.

Anderson, J.P., Badruzsaufari, E., Schenk, P.M., Manners, J.M., Desmond, O.J., Ehlert, C., et al., 2004. Antagonistic interaction between abscisic acid and jasmonate-ethylene signaling pathways modulates defense gene expression and disease resistance in Arabidopsis. Plant Cell 16, 3460-3479.

Audenaert, K., De Meyer, G.B., Höfte, M.M., 2002. Abscisic acid determines basal susceptibility of tomato to Botrytis cinerea and suppresses salicylic acid-dependent signaling mechanisms. Plant Physiol. 128, 491-501.
Azooz, M.M., Youssef, M.M., 2010. Evaluation of heat shock and salicylic acid treatments as inducer of drought stress tolerance in Hassawi wheat. Am. J. Plant Physiol. 5, 56-70.

Barlow, K.M., Christy, B.P., O'Leary, G.J., Riffkin, P.A., Nuttall, J.G., 2015. Simulating the impact of extreme heat and frost events on wheat crop production: a review. Field Crops Res. 171, 109-119.

Beaudoin, N., Serizet, C., Gosti, F., Giraudat, J., 2000. Interactions between abscisic acid and ethylene signaling cascades. Plant Cell 12, 1103-1115.

Binder, B.M., Patterson, S.E., 2009. Ethylene-dependent and -independent regulation of abscission. Stewart Postharvest Rev. 5, 1-10.

Bita, C.E., Gerats, T., 2013. Plant tolerance to high temperature in a changing environment: scientific, fundamentals and production of heat stress-tolerant crops. Front. Plant Sci. 4, 273.

Chakraborty, U., Tongden, C., 2005. Evaluation of heat acclimation and salicylic acid treatments as potent inducers of thermotolerance in Cicer arietinum L. Curr. Sci. 89, 384-389.

Chen, J., Zhang, Y., Wang, C., Lü, W., Jin, J.B., Hua, X., 2011. Proline induces calcium-mediated oxidative burst and salicylic acid signaling. Amino Acids. 40, 1473-1484.

Chiwocha, S.D., Cutler, A.J., Abrams, S.R., Ambrose, S.J., Yang, J., Ross, A.R., et al., 2005. The etr1-2 mutation in Arabidopsis thaliana affects the abscisic acid, auxin, cytokinin and gibberellin metabolic pathways during maintenance of seed dormancy, moistchilling and germination. Plant J. 42, 35-48.

Clarke, S.M., Mur, L.A., Wood, J.E., Scott, I.M., 2004. Salicylic acid dependent signaling promotes basal thermotolerance but is not essential for acquired thermotolerance in Arabidopsis thaliana. The Plant J. 38, 432-447.

Cvikrová, M., Gemperlová, L., Dobrá, J., Martincová, O., Prásil, I.T., Gubis, J., et al., 2012. Effect of heat stress on polyamine metabolism in proline-over-producing tobacco plants. Plant Sci. 182, 49-58.

Dat, J.F., Lopez-Delgado, H., Foyer, C.H., Scott, I.M., 1998b. Parallel changes in $\mathrm{H}_{2} \mathrm{O}_{2}$ and catalase during thermotolerance induced by salicylic acid or heat acclimation in mustard plants. Plant Physiol. 116, 1351-1357.

Dat, J.F., Foyer, C.H., Scote, I.M., 1998a. Changes in salicylic acid and antioxidants during induced thermotolerance in Mustard seedlings. Plant Physiol. 118, 1455-1461.

de Ronde, J.A., Cress, W.A., Kruger, G.H.J., Strasser, R.J., Van Staden, J., 2004. Photosynthetic response of transgenic soybean plants, containing an Arabidopsis P5CR gene, during heat and drought stress. J. Plant Physiol. 161, 1211-1224.

de Ronde, J.A., Cress, W.A., Van Staden, J., 2000. Interaction of osmotic and temperature stress on transgenic soybean. South Afr. J. Bot. 67, 655-660.

Dobra, J., Motyka, V., Dobrev, P., Malbeck, J., Prasil, I.T., Haisel, D., et al., 2010. Comparison of hormonal responses to heat, drought and combined stress in tobacco plants with elevated proline content. J. Plant Physiol. 167, 1360-1370.

El-Tayeb, M.A., 2005. Response of barley grains to the interactive effect of salinity and salicylic acid. Plant Growth Regul. 45, $215-224$.

Fahad, S., Bajwa, A.A., Nazir, U., Anjum, S.A., Farooq, A., Zohaib, A., et al., 2017. Crop production under drought and heat stress: plant responses and management options. Front. Plant Sci. Available from: https://doi.org/10.3389/fpls.2017.01147.

FAO, Food and Agricultural Organization, 2009. Available from: <http://www.fao.org/fileadmin/templates/wsfs/docs/expert_ paper/How_to_Feed_the_World_in_2050.pdf $>$.

Fonseca, A.E., Westgate, M.E., 2005. Relationship between desiccation and viability of maize pollen. Field Crops Res. 94, 114-125.

Ghassemian, M., Nambara, E., Cutler, S., Kawaide, Y., Kamiya, Y., et al., 2000. Regulation of abscisic acid signaling by the ethylene response pathway in Arabidopsis. Plant Cell 12, 1117-1126. 
Hall, A.E., 2001. Crop Responses to Environment. CRC Press LLC, Boca Raton, FL.

Han, Y., Fan, S., Zhang, Q., Wang, Y., 2013. Increased proline content was observed in leaf of lettuce seedlings under heat stress but the increase in heat-tolerant varieties was significantly higher than the non-heat tolerant varieties. Agric. Sci. 4, 112-115.

Harding, S.A., Guikema, J.A., Paulsen, G.M., 1990. Photosynthetic decline from high temperature stress during maturation of wheat: I. Interaction with senescence processes. Plant Physiol. 92, $648-653$.

Hasanuzzaman, M., Nahar, K., Alam, M.M., Roychowdhury, R., Fujita, M., 2013. Physiological, biochemical, and molecular mechanisms of heat stress tolerance in plants. Int. J. Mol. Sci. 14, 9643-9684.

Hatfield, J., Boote K., Fay P., Hahn L., Izaurralde C., Kimball B.A., et al., 2008. Agriculture. In: The Effects of Climate Change on Agriculture, Land resources, Water resources, and Biodiversity. A Report by the U.S. Climate Change Science Program and the Subcommittee on Global Change Research. Washington, DC., USA, 2008, 362 pp.

Hatfield, J.L., Prueger, J.H., 2011. In: Yadav, S.S., Redden, R.J., Hatfield, J.L., Lotze-Campen, H., Hall, A.E. (Eds.), Agroecology: Implications for Plant Response to Climate Change. WileyBlackwell, West Sussex, UK, pp. 27-43.

Hays, D.B., Do, J.H., Mason, R.E., Finlayson, S.A., 2007. Heat stress induced ethylene production in developing wheat grains induces kernel abortion and increased maturation in a susceptible cultivar. Plant Sci. 172, 1113-1123.

He, C.Y., Zhang, J.S., Chen, S.Y., 2002b. A soybean gene encoding a proline-rich protein is regulated by salicylic acid, an endogenous circadian rhythm and by various stresses. Theor. Appl. Genet. $104,1125-1131$.

He, Y.L., Liu, Y.L., Chen, Q., Bian, A.H., 2002a. Thermotolerance related to antioxidation induced by salicylic acid and heat hardening in tall fescue seedlings. J. Plant Physiol. Mol. Biol. 28, 89-95.

Hemantaranjan, A., Nishant Bhanu, A., Singh, M.N., Yadav, D.K., Patel, P.K., et al., 2014. Heat stress reponses and thermotolerance. Adv. Plants Agricul. Res. 1, 00012.

Howarth, C.J., Ashraf, M., Harris, P.J.C., 2005. Genetic improvements of tolerance to high temperature. In: Ashraf, M., Harris, P.J.C. (Eds.), Abiotic Stresses: Plant Resistance Through Breeding and Molecular Approaches. Haworth Press Inc., New York, pp. 277-300.

Hua, X.J., Van de Cotte, B., Van Montagu, M., Verbruggen, N., 2001. The $5^{\prime}$ untranslated region of the At-P5R gene is involved in both transcriptional and post-transcriptional regulation. Plant J. 26, 157-169.

Hussein, M.M., Balbaa, L.K., Gaballah, M.S., 2007. Salicylic acid and salinity effects on growth of maize plants. Res. J. Agric. Biol. Sci. 3, 321-328.

Iqbal, N., Umar, N., Khan, N.A., Khan, M.I.R., 2014. A new perspective of phytohormones in salinity tolerance: regulation of proline metabolism. Environ. Exp. Bot. 100, 34-42.

Iqbal, N., Umar, S., Khan, N.A., 2015. Nitrogen availability regulates proline and ethylene production and alleviates salinity stress in mustard (Brassica juncea). J. Plant Physiol. 178, 84-91.

Jiang, Y., Huang, B., 2001. Drought and heat stress injury to two cool season turfgrasses in relation to antioxidant metabolism and lipid peroxidation. Crop Sci. 41, 436-442.

Jing-hong, H., Yang, Y., Qing-mao, S., Chun-juan, D., Zhi-gang, Z., 2012. Effect ofexogenous salicylic acid on nitrogen assimilation of cucumber seedling underdrought stress. Acta Hort. Sinice 39, 81-90.

Kaur, G., Asthir, B., 2015. Proline: a key player in plant abiotic stress tolerance. Biol. Plant. 59, 609-619.
Kazemi-Shahandashti, S.S., Maali-Amiri, R., Zeinali, H., Khazaei, M., Talei, A., Ramezanpour, S.S., 2014. Effect of short-term cold stress on oxidative damage and transcript accumulation of defenserelated genes in chickpea seedlings. J. Plant Physiol. 171, 1106-1116.

Khan, M.I.R., Asgher, M., Khan, N.A., 2013a. Rising temperature in the changing environment: a serious threat to plants. Clim. Change Environ. Sust. 1, 25-36.

Khan, M.I.R., Khan, N., 2017. Reactive Oxygen Species and Antioxidant System in Plants: Role and regulation Under Abiotic Stress. Springer Nature, New York978-981-10-5254-5.

Khan, M.I.R., Syeed, S., Nazar, R., Anjum, N.A., 2012. An insight into the role of salicylic acid and jasmonic acid in salt stress tolerance. In: Khan, N.A., Nazar, R., Iqbal, N., Anjum, N.A. (Eds.), Phytohormones and Abiotic Stress Tolerance in Plants. SpringerVerlag, New York, pp. 277-300.

Khan, M.I.R., Khan, N.A., 2013. Salicylic acid and jasmonates: approaches in abiotic stress tolerance. Plant Biochem. Physiol. 1, 4.

Khan, M.I.R., Asgher, M., Khan, N.A., 2014. ). Alleviation of saltinduced photosynthesis and growth inhibition by salicylic acid involves glycinebetaine and ethylene in mungbean (Vigna radiata L.). Plant Physiol. Biochem. 80, 67-74.

Khan, M.I.R., Iqbal, N., Masood, A., Per, T.S., Khan, N.A., 2013 b. Salicylic acid alleviates adverse effects of heat stress on photosynthesis through changes in proline production and ethylene formation. Plant Signal. Behav. 8, e26374.

Khan, M.I.R., Fatma, M., Per, T.S., Anjum, N.A., Khan, N.A., 2015. Salicylic acid-induced abiotic stress tolerance and underlying mechanisms in plants. Front. Plant Sci. 6, 462.

Khanna, P., Kaur, K., Gupta, A.K., 2016. Salicylic acid induces differential antioxidant response in spring maize under high temperature stress. Indian J. Exp. Biol. 54, 386-393.

Kocsy, G., Laurie, R., Szalai, G., Szilágyi, V., Simon-Sarkadi, L., Galiba, G., et al., 2005. Genetic manipulation of proline levels affects antioxidants in soybean subjected to simultaneous drought and heat stresses. Physiol. Plant. 124, 227-235.

Königshofer, H., Tromballa, H.W., Löppert, H.G., 2008. Early events in signalling high-temperature stress in tobacco BY2 cells involve alterations in membrane fluidity and enhanced hydrogen peroxide production. Plant Cell Environ. 31, 1771-1780.

Krantev, A., Yordanova, R., Janda, T., Szalai, G., Popova, L., 2008. Treatment with salicylic acid decreases the effect of cadmium on photosynthesis in maize plants. J. Plant physiol. 165, 920-931.

Kumar, S., Kaushal, N., Nayyar, H., 2012. Abscisic acid induces heat tolerance in chickpea (Cicer arietinum L.) seedlings by facilitated accumulation of osmoprotectants. Acta Physiol. Plant. 34, 1651-1658.

Larkindale, J., Hall, J.D., Knight, M.R., Vierling, E., 2005. Heat stress phenotypes of Arabidopsis mutants implicate multiple signaling pathways in the acquisition of thermotolerance. Plant Physiol. $138,882-897$.

Larkindale, J., Huang, B., 2004. Thermotolerance and antioxidant systems in Agrostis stolonifera: involvement of salicylic acid, abscisic acid, calcium, hydrogen peroxide, and ethylene. J. Plant Physiol. 161, 405-413.

Larkindale, J., Knight, M.R., 2002. Protection against heat stressinduced oxidative damage in Arabidopsis involves calcium, abscisic acid, ethylene, and salicylic acid. Plant Physiol. 128, 682-695.

Lee, J.H., Jin, E.S., Kim, W.T., 1999. Inhibition of auxin-induced ethylene production by salicylic acid in mung bean hypocotyls. J. Plant Biol. 42, 1-7.

LeNoble, M.E., Spollen, W.G., Sharp, R.E., 2004. Maintenance of shoot growth by endogenous $\mathrm{ABA}$ : genetic assessment of the involvement of ethylene suppression. J. Exp. Bot. 55, 237-245.

Li, N., Parsons, B.L., Liu, D., Mattoo, A.K., 1992. Accumulation of wound-inducible ACC synthase transcript in tomato fruit is 
inhibited by salicylic acid and polyamines. Plant Mol. Biol. 18, 477-487.

Li, Z., Yuc, J., Penga, Y., Huang, B., 2017. Metabolic pathways regulated by abscisic acid, salicylic acid, and $\gamma$-aminobutyric acid in association with improved drought tolerance in creeping bentgrass (Agrostis stolonifera). Physiol. Plant. 159, 42-58.

Liu, H.T., Liu, Y.Y., Pan, Q.H., Yang, H.R., Zhan, J.C., Huang, W.D., 2006. Novel interrelationship between salicylic acid, abscisic acid, and PIP2-specific phospholipase $\mathrm{C}$ in heat acclimation-induced thermotolerance in pea leaves. J. Exp. Bot. 57, 3337-3347.

Lobell, D.B., Sibley, A., Ortiz-Monasterio, J.I., 2012. Extreme heat effects on wheat senescence in India. Nat. Clim. Chan. 2, 186-189.

Lopez-Delgado, H., Dat, J.F., Foyer, C.H., Scott, I.M., 1998. Induction of thermo-tolerance in potato micro-plants by acetyl salicylic acid and $\mathrm{H}_{2} \mathrm{O}_{2}$. J. Exp. Bot. 49, 713-720.

Lv, W.T., Lin, B., Zhang, M., Hua, X.J., 2011. Proline accumulation is inhibitory to Arabidopsis seedlings during heat stress. Plant Physiol. 156, 1921-1933.

Ma, B., Yin, C.C., He, S.J., Lu, X., Zhang, W.K., Lu, T.G., et al., 2014. Ethylene-induced inhibition of root growth requires abscisic acid function in rice (Oryza sativa L.) seedlings. PLoS Gen. 10, e1004701.

Mathur, S., Jajoo, A., 2014. Effects of heat stress on growth and crop yield of wheat (Triticum aestivum). In: Ahmad, P., Wani, M.R. (Eds.), Physiological Mechanisms and Adaptation Strategies in Plants Under Changing Environment, vol. 1. Springer, New York, pp. 163-191.

Matilla, A.J., Matilla-Vazquez, M.A., 2008. Involvement of ethylene in seed physiology. Plant Sci. 175, 87-97.

Meehl, G.A., Stocker, T.F., Collins, W.D., Gaye, A.J., Gregory, J.M., Kitoh, A., et al., 2007. In: Solomon, S., Qin, D., Manning, M., Chen, Z., Marquis, M., Averyt, K.B., et al.,Global Climate Projections. Cambridge University Press, Cambridge, UK and NY.

Meguro, A., Sato, Y., 2014. Salicylic acid antagonizes abscisic acid inhibition of shoot growth and cell cycle progression in rice. Sci. Rep. 4, 4555.

Misra, N., Saxena, P., 2006. Effect of salicylic acid on proline metabolism in lentil grown under salinity stress. Plant Sci. 177, 181-189.

Molinari, H.B.C., Daros, E., Marur, C.J., Vieira, L.G.E., 2007. Evaluation of the stress-inducible production of proline in transgenic sugarcane (Saccharum spp.): osmotic adjustment, chlorophyll fluorescence and oxidative stress. Physiol. Plant. 130, 218-229.

Nazar, R., Umar, S., Khan, N.A., Sareer, O., 2015. Salicylic acid supplementation improves photosynthesis and growth in mustard through changes in proline accumulation and ethylene formation under drought stress. South Afr. J. Bot. 98, 84-94.

Oata, Y., 1975. Short day flowering of Lemna gibba $\mathrm{G}_{3}$ induced by salicylic acid. Plant Cell Physiol. 16, 1131-1135.

Pasala, R.K., Khan, M.I.R., Minhas, P.S., Farooq, M.A., Sultana, R., Per, T.S., et al., 2016. Can plant bio-regulators minimize crop productivity losses caused by drought, heat and salinity stress? An integrated review. J. Appl. Bot. Food. 89, 243-248.

Per, T.S., Khan, N.A., Reddy, P.S.B., Masood, A., Hasanuzzaman, M., Khan, M.I.R., et al., 2017. Approaches in modulating proline metabolism in plants for salt and drought stress tolerance: phytohormones, mineral nutrients and transgenics. Plant Physiol Biochem. 115, 126-140.

Pieterse, A.H., Muller, L.J., 1977. Induction of flowering in Lemna gibba $G_{3}$ under short day conditions. Plant Cell Physiol. 18, 45-53.

Porra, R.J., 1997. Recent progress in porphyrin and chlorophyll biosynthesis. Photochem. Photobiol. 65, 492-516.

Pradhan, G.P., Prasad, P.V.V., Fritz, A.K., Kirkham, M.B., Gill, B.S., 2012. Effects of drought and high temperature stress on synthetic hexaploid wheat. Funct. Plant Biol. 39, 190-198.
Rais, L., Masood, A., Inam, A., Khan, N., 2013. Sulfur and nitrogen co-ordinately improve photosynthetic efficiency, growth and proline accumulation in two cultivars of mustard under salt stress. J. Plant Biochem. Physiol. 1, 1.

Rajjou, L., Belghazi, M., Huguet, R., Robin, C., Moreau, A., Job, C., et al., 2006. Proteomic investigation of the effect of salicylic acid on Arabidopsis seed germination and establishment of early defense mechanisms. Dev. Hormone Action. 141, 910-923.

Ramanujam, M.P., Jaleel, V.A., Kumaravelu, G., 1998. Effect of salicylic acid on nodulation, nitrogenous compounds and related enzymes of Vigna mungo. Biol. Plant. 41, 307-311.

Rao, M.V., Lee, Hi, Dav, K.R., 2002. Ozone-induced ethylene production is dependent on salicylic acid, and both salicylic acid and ethylene act in concert to regulate ozone-induced cell death. Plant J. 32, 447-456.

Raskin, I., Ehmann, A., Melander, W.R., Meeuse, B.J.D., 1987. Salicylic acid-a natural inducer of heat production in Arum lilies. Science. 237, 1545-125.

Reddy, P.S., Jogeswar, G., Rasineni, G.K., Maheswari, M., Reddy, A. R., Varshney, R.K., et al., 2015. Proline over-accumulation alleviates salt stress and protects photosynthetic and antioxidant enzyme activities in transgenic sorghum [Sorghum bicolor (L.) Moench], Plant Physiol. Biochem., 94. pp. 104-113.

Reddy, P.S., Chakradhar, T., Reddy, R.A., Nitnavare, R.B., Mahanty, S., Reddy, M.K., 2016. Role of heat shock proteins in improving heat stress tolerance in crop plants. Heat Shock Proteins and Plants. Springer, Cham, pp. 283-307.

Rivero, R.M., Ruiz, J.M., Romero, L., 2004. Importance of N source on heat stress tolerance due to the accumulation of proline and quaternary ammonium compounds in tomato plants. Plant Biol. 6, 702-707.

Rizhsky, L., Liang, H.J., Shuman, J., Shulaev, V., Davletova, S., Mittler, R., 2004. When defense pathways collide: the response of Arabidopsis to a combination of drought and heat stress. Plant Physiol. 134, 1683-1696.

Rock, C.D., 2000. Pathways to abscisic acid-regulated gene expression. New Phytol. 148, 357-396.

Rojas, A., Almoguera, C., Jordan, J., 1999. Transcriptional activation of a heat shock gene promoter in sunflower embryos: synergism between ABI3 and heat shock factors. The Plant J. 20, 601-610.

Rosenzweig, C., Iglesias, A., Yang, X.B., Epstein, P.R., Chivian, E., 2001. Climate change and extreme weather events. Implications for food production, plant diseases and pests. Global Change Human Health 2, 90-104.

Sadak, M.S., Orabi, S.A., 2015. Improving thermo tolerance of wheat plant by foliar application of citric acid or oxalic acid. Int. J. Chem. Technol. Res. 8, 333-345.

Sage, R.F., Kubien, D.S., 2007. The temperature response of $C_{3}$ and $\mathrm{C}_{4}$ photosynthesis. Plant Cell Environ. 30, 1086-1106.

Sakhabutdinova, A.R., Fatkhutdinova, D.R., Bezrukova, M.V., Shakirova, F.M., 2003. Salicylic acid prevents the damaging action of stress factors on wheat plants. Bulg J. Plant Physiol 314-319. Special issue 21.

Salvucci, M.E., 2008. Association of Rubisco activase with chaperonin-60beta: a possible mechanism for protecting photosynthesis during heat stress. J. Exp. Bot. 59, 1923-1933.

Sanmiya, K., Suzuki, K., Egawa, Y., Shono, M., 2004. Mitochondrial small heat-shock protein enhances thermotolerance in tobacco plants. FEBS Lett. 557, 265-268.

Senaratna, T., Touchell, D., Bunn, E., Dixon, K., 2000. Acetyl salicylic acid (Aspirin) and salicylic acid induce multiple stress tolerance in bean and tomato plants. Plant Growth Regul. 30, 157-161.

Shah, N., Paulsen, G., 2003. Interaction of drought and high temperature on photosynthesis and grain-filling of wheat. Plant Soil. 257, 219-226. 
Shaheen, M.R., Ayyub, C.M., Yaqub, M.S., Sarwar, N., Yaseen, R., et al., 2017. Salicylic acid upsurges the heat tolerance of tomato by improving plant water relations and antioxidants activity. Int. J. Biosci. 10, 120-130.

Shakirova, F.M., Sakhabutdinova, A.R., Bezrukova, M.V., Falkhutdinova, R.A., Fatkhutdinova, D.R., 2003. Changes in the hormonal status of wheat seedlings induced by salicylic acid and salinity. Plant Sci. 164, 317-322.

Sharma, R., Sharma, R., 2017. Terminal heat stress in bread wheat: a critical study. Int. J. Eng. Technol. Sci. Res. 4, 12.

Shi, Q., Bao, Z., Zhu, Z., Ying, Q., Qian, Q., 2006. Effects of different treatments of salicylic acid on heat tolerance, chlorophyll fluorescence, and antioxidant enzyme activity in seedlings of Cucumis sativa L. Plant Growth Regul. 48, 127-135.

Siboza, X.I., Bertling, I., Odindo, A.O., 2014. Salicylic acid and methyl jasmonate improve chilling tolerance in cold-stored lemon fruit (Citrus limon). J. Plant Physiol. 171, 1722-1731.

Sita, K., Sehgal, A., Rao, B.H., Nair, R.M., Prasad, P.V., Kumar, S., et al., 2017. Food legumes and rising temperatures: effects, adaptive functional mechanisms specific to reproductive growth stage and strategies to improve heat tolerance. Front. Plant Sci. Available from: https://doi.org/10.3389/fpls.2017.01658.

Smertenkon, A., Draber, P., Viklicky, V., Opatrny, Z., 1997. Heat stress affects the organization of microtubules and cell division in Nicotiana tabacum cells. Plant Cell Environ. 20, 1534-1542.

Song, S.Q., Lei, Y.B., Tian, X.R., 2005. Proline metabolism and crosstolerance to salinity and heat stress in germinating wheat seeds. Russ. J. Plant Physiol. 52, 793-800.

Szabados, L., Savouré, A., 2010. Proline: a multifunctional amino acid. Trends Plant Sci. 15, 89-97.

Szalai, G., Tari, I., Janda, T., Pestenácz, A., Páldi, E., 2000. Effects of cold acclimation and salicylic acid on changes in ACC and MACC contents in maize during chilling. Biol. Plant. 43, 637-640.

Szepesi, A., Csiszár, J., Gèmes, K., Horváth, E., Horváth, F., Simon, L. M., et al., 2009. Salicylic acid improves acclimation to salt stress by stimulating abscisic aldehyde oxidase activity and abscisic acid accumulation, and increases $\mathrm{Na}^{+}$content in leaves without toxicity symptoms in Lycopersicum esculentum Mill. L. J. Plant Physiol. 166, 914-925.

Szepsi, A., Csiszar, J., Bajkan, S.Z., Gemes, K., Horvath, F., Erdei, L., et al., 2005. Role of salicyclic acid pre-treatment on the acclimatization of tomato plant to salt and osmotic stress. Acta Biologica Szegediensis 49, 123-125.

Talanova, V., Akimova, T., Titov, A., 2003. Effect of whole plant and local heating on the ABA content in cucumber seedling leaves and roots and on their heat tolerance. Russ. J. Plant Physiol. 50, 90-94.

Tamura, K., Yoshida, T., Tanaka, A., Sasaki, R., Bando, A., Toh, S., et al., 2006. Isolation and characterization of high temperatureresistant germination mutants of Arabidopsis thaliana. Plant Cell Physiol. 47, 1081-1094.

Tarighaleslami, M., Zarghami, R., Boojar, M.M.A., Oveysi, M., 2012. Effects of drought stress and different nitrogen levels on morphological traits of proline in leaf and protein of corn seed (Zea mays L.). Am-Eur. J. Agricul. Environ. Sci. 12, 49-56.

Tasgin, E., Atici, O., Nulbantoglu, B., Popova, L.P., 2006. Effects of salicylic acid and cold treatment on protein levels and on the activities of antioxidant enzymes in the apoplast of winter wheat leaves. Phytochemistry 67, 710-715.

Tashiro, T., Wardlaw, I., 1990. The Response to high temperature shock and humidity changes prior to and during the early stages of grain development in wheat. Funct. Plant Biol. 17, 551-561.

Tirani, M.M., Nasibi, F., Kalantari, K.M., 2013. ). Interaction of salicylic acid and ethylene and their effects on some physiological and biochemical parameters in canola plants (Brassica napus L.). Photosynthetica 51, 411-418.
Toh, S., Imamura, A., Watanabe, A., Nakabayashi, K., Okamoto, M., Jikumaru, Y., et al., 2008. High temperature-induced abscisic acid biosynthesis and its role in the inhibition of gibberellin action in Arabidopsis seeds. Plant Physiol. 146, 1368-1385.

Tripathy, B.C., Rebeiz, C.A., 1986. Chloroplast biogenesis: demonstration of monovinyl and divinyl monocarboxylic routes of chlorophyll biosynthesis in higher plants. J. Biol. Chem. 261, 13556-13564.

Uchida, A., Jagendorf, A.T., Hibino, T., Takabe, T., Takabe, T., 2002. Effects of hydrogen peroxide and nitric oxide on both salt and heat stress tolerance in rice. Plant Sci. 163, 515-523.

Umebese, C.E., Olatimilehin, T.O., Ogunsusi, T.A., 2009. Salicylic acid protects nitrate reductase activity, growth and proline in Amaranth and tomato plants during water deficit. Am. J. Agric. Biol. Sci. 4, 224-229.

Vazirimehr, M.R., Rigi, K., 2014. Effect of salicylic acid in agriculture. Int. J. Plant Animal Environ. Sci. 4, 291-296.

Wahid, A., Gelani, S., Ashraf, M., Foolad, M.R., 2007. Heat tolerance in plants: an overview. Environ. Exp. Bot. 61, 199-223.

Wang, K., Zhang, X., Goatley, M., Ervin, E., 2014. Heat shock proteins in relation to heat stress tolerance of creeping bentgrass at different N Levels. PLoS One. 9, e102914.

Wang, L.J., Li, S.H., 2006. Salicylic acid-induced heat or cold tolerance in relation to $\mathrm{Ca}^{2+}$ homeostasis and antioxidant systems in young grape plants. Plant Sci. 170, 685-694.

Wang, L.J., Li, S.H., 2007. The effects of salicylic acid on distribution of ${ }^{14} \mathrm{C}$ assimilation and photosynthesis in young grape plants under heat stress. Acta Hort. 738, 779-7851.

Wang, L.-J., Fan, L., Loesher, W., Duan, W., Liu, G.-J., Cheng, J.-S., et al., 2010. Salicylic acid alleviates decreases in photosynthesis under heat stress and accelerates recovery in grapevine leaves. BMC Plant Biol. 10, 34.

Xie, Z., Zhang, Z.L., Hanzlik, S., Cook, E., Shen, Q.J., 2007. Salicylic acid inhibits gibberellin-induced alpha-amylase expression and seed germination via a pathway involving an abscisic-acidinducible WRKY gene. Plant Mol. Biol. 64, 293-303.

Xin, M., Wang, Y., Yao, Y., Song, N., Hu, Z., Qin, D., et al., 2011. Identification and characterization of wheat long non-protein coding RNAs responsive to powdery mildew infection and heat stress by using microarray analysis and SBS sequencing. BMC Plant Biol. 11, 61.

Yang, J., Sears, R.G., Gill, B.S., Paulsen, G.M., 2002. Growth and senescence characteristics associated with tolerance of wheatalien amphiploids to high temperature under controlled conditions. Euphytica. 126, 185-193.

Zahra, S., Amin, B., Ali, V.S.M., Ali, Y., Mehdi, Y., 2010. ). The salicylic acid effect on the tomato (Lycopersicon esculentum Mill.) sugar, protein and proline contents under salinity stress $(\mathrm{NaCl})$. J. Biophys. Struct. Biol. 2, 35-41.

Zhao, H., Dai, T., Jing, Q., Jiang, D., Cao, W., 2007. Leaf senescence and grain filling affected by post-anthesis high temperatures in two different wheat cultivars. Plant Growth Regul. 51, 149-158.

\section{Further Reading}

Kavi Kishor, P.B., Hong, Z., Miao, G.H., Hu, C.A.A., Verma, D.P.S., 1995. Overexpression of $\Delta 1$-pyrroline-5-carboxylate synthetase increases proline production and confers osmotolerance in transgenic plants. Plant Physiol. 108, 1387-1394.

Leung, J., Giraudat, J., 1998. Abscisic acid signal transduction. Ann. Rev. Plant Mol. Biol. 49, 199-222.

Roosens, N.H., Al Bitar, F., Loenders, K., Angenon, G., Jacobs, M., 2002. Overexpression of ornithine- $\delta$-aminotransferase increases proline biosynthesis and confers osmotolerance in transgenic plants. Mol. Breed. 9, 73-80. 


\section{9}

\section{Osmolyte Diversity, Distribution, and Their Biosynthetic Pathways}

Gandra Jawahar ${ }^{1}$, Guddimalli Rajasheker ${ }^{1}$, Parveda Maheshwari ${ }^{1}$, Devineni Lakshmi Punita ${ }^{1}$, Naravula Jalaja ${ }^{2}$, Palavalasa Hima Kumari ${ }^{1}$, Somanaboina Anil Kumar ${ }^{1}$, Ruhi Afreen ${ }^{1}$, Appa Rao Karumanchi ${ }^{1}$, Polavarapu Rathnagiri ${ }^{3}$, Nese Sreenivasulu ${ }^{4}$ and Polavarapu Bilhan Kavi Kishor

${ }^{1}$ Department of Genetics, Osmania University, Hyderabad, Telangana, India ${ }^{2}$ Department of Biotechnology, Vignan University, Vadlamudi, Guntur, India ${ }^{3}$ Genomix CARL Pvt. Ltd., Kadapa, Andhra Pradesh, India ${ }^{4}$ Grain Quality and Nutrition Center, Plant Breeding Division, International Rice Research Institute, Metro Manila, Philippines

\section{O U T L I N E}

29.1 Introduction

29.2 Diversity and Distribution of Osmolytes in Different Plant Species

29.2.1 What Are Osmolytes or Osmoprotectants

29.2.2 Why Are Osmolytes Compatible?

29.2.3 Diversity and Distribution of Osmolytes

29.3 Biosynthetic Pathways of Proline and Its Derivatives

29.3.1 Biosynthesis of Proline

29.3.2 Biosynthesis of Proline Derivatives
450

450

450

450

451

452

452

452
29.4.1 Biosynthesis of Glycine Betaine

29.4.2 Biosynthesis of Glycine Betaine Derivatives
453

454

29.5 Biosynthetic Pathways of Trehalose and Sugar Alcohols

29.5.1 Biosynthesis of Trehalose (Sugar) 29.5.2 Biosynthesis of Sugar Alcohols

29.6 Conclusions

Acknowledgments

456

References

456

Further Reading

29.4 Biosynthetic Pathways of Glycine Betaine and Its Derivatives 


\subsection{INTRODUCTION}

Salt and water stresses, nutrient/mineral deficiencies, and extreme temperatures are the major environmental perturbations that adversely affect both growth and development and generate a threat to sustainable agriculture. Since abiotic stress is a complex phenomenon, it is necessary to understand the anatomical, physiological, biochemical, and molecular mechanisms associated with it. Development of crop plants that resist adverse environmental factors is an important approach since they can mitigate the problem of global food shortage in future. Environmental perturbations evoke multiple responses in the plant systems (Cheeseman, 2013; Khan et al., 2013a,b, 2017). While water deficit leads to osmotic stress, salt causes both ionic and osmotic stresses. Osmotic stress also leads to buildup of salt levels in and around the roots and inhibition of water uptake, cell expansion, and lateral root development (Munns and Tester, 2008). On the other hand, when $\mathrm{Na}^{+}$and $\mathrm{Cl}^{-}$ accumulate in plants under salt stress, it leads to a decline in photosynthesis with a simultaneous increase in chlorosis and cell death (Glenn et al., 1999). But, plants adapt a number of mechanisms that can help them to cope with such adverse conditions. Accumulation of both energetically cheap inorganic ions like $\mathrm{Na}^{+}$and $\mathrm{Cl}^{-}$ and low-molecular weight organic solutes such as proline and glycine betaine $\left(N, N^{\prime}, N^{\prime \prime}\right.$-trimethylglycine) helps the plants in osmotic adjustment (Glenn et al., 1999). While the inorganic ions $\left(\mathrm{Na}^{+}\right.$and $\left.\mathrm{Cl}^{-}\right)$accumulate in the vacuoles through complex biochemical mechanisms, osmolytes (amino acids like proline, tertiary sulfonium compounds (e.g., 3-dimethylsulfoniopropionate (DMSP)), quaternary ammonium compounds (e.g., glycine betaine, proline betaine, $\beta$-alanine betaine, and choline- $O$-sulfate), sugars (e.g., trehalose and others), and sugar alcohols or polyhydric alcohols) accumulation takes place primarily in the cytoplasm and to some extent in chloroplasts (proline and glycine betaine). The quaternary ammonium compounds possess a fully methyl substituted nitrogen atom and a negatively charged carboxyl group (in the case of betaines) or sulfate group (in the case of cholineO-sulfate) (Rhodes and Hanson, 1993; Gorham, 1995). While glycine betaine, $\beta$-alanine betaine, and proline betaine act as osmotic agents (based on growth assays), choline, an alcohol, without a negatively charged group is not an osmoprotectant per se (Kishor, 1988; Hanson et al., 1994). This review discusses the diversity, distribution, and biosynthesis of osmolytes that occur in different taxa, and the factors that regulate their accumulation. Multiple biosynthetic pathways; the interaction of hormones, especially abscisic acid in osmolyte regulation; and the wide array of osmolytes functions during salt and drought stress tolerance are discussed.

\subsection{DIVERSITY AND DISTRIBUTION OF OSMOLYTES IN DIFFERENT PLANT SPECIES}

\subsubsection{What Are Osmolytes or Osmoprotectants}

In plants, biochemical reactions require specific inorganic ions. But under stress, such as high $\mathrm{NaCl}$ conditions, the concentrations of the inorganic ions increase above the threshold levels that are typically found in plant cells. Such high concentrations generally perturb the protein functions. Contrarily, the effect of organic osmolytes on proteins is much less, in other words, compatible. Firstly, $\mathrm{NaCl}$ stress causes osmotic flux of water out of cells, and then elevates the concentration of all cellular constituents and inorganic ions (Burg et al., 2007). The perturbing inorganic ions are slowly replaced by organic osmolytes. Under different abiotic stress conditions, plants accumulate in high concentrations, a wide array of organic compounds, also known as compatible solutes or osmolytes. These compounds are highly soluble in water and do not interfere with normal physiological or metabolic activities in plants (Yancey, 2005; Kishor et al., 2005). They are nontoxic at high cellular concentrations and protect cellular components from dehydration. Hence, osmolytes are also commonly referred as osmoprotectants.

\subsubsection{Why Are Osmolytes Compatible?}

It has long been known that cells use osmolytes for osmotic adjustment under stress conditions. Osmolytes are not charged (or zwitterionic such as glycine betaine) at a neutral $\mathrm{pH}$ and are highly soluble in water (Bellantyne and Chamberlin, 1994). They modify the solvent properties of water and stabilize the internal osmotic potential (Yancey et al., 1982; Yancey, 2005). Many cells respond to hyperosmotic stress by accumulating nontoxic organic compatible solutes. Many different types of compatible solutes accumulate and have been classified as (1) water structure-makers, (2) promoters of protein association, (3) protein stabilizers, and (4) salting-out solutes and enzyme activators (Bellantyne and Chamberlin, 1994). Nonspecific binding of solutes to proteins (other than catalytic site) denatures proteins. On the other hand, solutes that are excluded from the surface of the proteins will stabilize folded protein structures (due to entropically unfavorable situation). Thus, osmolytes are excluded from the hydration sphere of proteins and protect the stability of folded proteins, and cellular components from dehydration injury (Low, 1985). Precisely for these reasons, osmolytes are also named as osmoprotectants. 


\subsubsection{Diversity and Distribution of Osmolytes}

Organic osmolytes fall into few major chemical categories in all: small carbohydrates including mono-, di-, oligo-, and polysaccharides (e.g., glucose, fructose, sucrose, trehalose, raffinose, and fructans), sugar alcohols or polyols (e.g., glycerol, inositol, sorbitol, mannitol etc.), and inositol derivatives or methylated inositol like o-methyl-inositol, amino acids (alanine, arginine, glycine, proline, methylated proline-related compounds such as methyl-proline, proline betaine, and hydroxyproline betaine, other betaines such as glycine betaine, $\beta$-alanine betaine, choline- $O$-sulfate, taurine (observed among marine animals, but not in plants), etc.), amides such as glutamine, asparagine, nonprotein amino acids like $\gamma$-aminobutyric acid (GABA), pipecolic acid, citrulline, ornithine and amino acid derivatives (e.g., ectoine), methylammonium ( $N$-trimethylamine oxide), methylsulfonium, tertiary sulfonium compounds and DMSP (Rhodes et al., 2002; Ashraf and Foolad, 2007). Ectoine is not discussed in this chapter since it is of bacterial origin. The above common osmolytes are synthesized from primary metabolic pathways and accumulated under conditions of abiotic stress in a wide range of plant families (Ahmad et al., 1981; Mansour, 2000; Slama et al., 2015). Being a proteogenic amino acid, proline occurs in all plant species. Glycine betaine is also the most common quaternary ammonium compound among plants exposed to a range of abiotic stresses (Guo et al., 2009).

Lower as well as higher plants synthesize and accumulate either one type or many types of osmolytes when exposed to one or multiple stresses simultaneously. But, all plants do not accumulate all kinds of osmolytes at the same level in the same tissues within the same or different families. Depending on the species and the type of environmental stress, the type and quantity or the level of accumulation (some species synthesize and accumulate low or high or may not accumulate) also varies to a large extent (Ashraf and Foolad, 2007; Szabados et al., 2011). Osmolyte synthesis in the cellular organelles is also important for low or high accumulation of glycine betaine as shown by Nuccio et al. (2000). Both glycophytes (e.g., Citrus) and halophytes accumulate several types of osmolytes at the same time (Gagneul et al., 2007). This indicates their synergistic mode of action when plants are exposed to abiotic stress conditions. Further, seasonal pattern, developmental stage of the plant, age of plant, type of tissue or organ and environmental parameters like temperature, moisture, nutritional status (including nitrogen) of the soil, $\mathrm{pH}$ of the medium greatly influence the accumulation (Hare et al., 1998; Murakeözy et al., 2003). Subcellular partitioning of several osmolytes is also reported depending on the growing conditions (Aubert et al., 1999; Kishor et al., 2005). While proline accumulates and also degrades rapidly, glycine betaine accumulates for a longer period (Gagneul et al., 2007) in comparison with proline. Accumulation of proline and glycine betaine can be cultivar or genotype specific. In Guizotia abyssinica (niger), both high and low proline accumulating cultivars have been found (Sarvesh et al., 1996). Similarly, some genotypes of Sorghum bicolor and corn accumulate glycine betaine, while others do not (Grote et al., 1994; Saneoka et al., 1995) inferring the occurrence of stress-tolerant and stress-susceptible genotypes within the species. Generally, accumulations of osmolytes are higher under stress due to increased biosynthesis or decreased degradation or both (Kishor et al., 2005; Khan et al., 2013, 2014; Per et al., 2017) indicating regulation of genes associated with such metabolic fluctuations and also homeostasis of biological reductants (Szabados and Savoure, 2010).

\subsubsection{Quaternary Ammonium Compounds}

Glycine betaine, $\beta$-alanine betaine, proline betaine, hydroxyproline betaine, choline-O-sulfate, and pipecolate betaine are the quaternary ammonium compounds found mostly in halophytic plant species (Ashraf and Harris, 2004). They are common among the members of the families Amaranthaceae (former Chenopodiaceae) and Plumbaginaceae (Slama et al., 2015). Among the many quaternary ammonium compounds, glycine betaine is the most commonly accumulated osmolyte when exposed to diverse abiotic stresses in plants (Hanson et al., 1991; Guo et al., 2009; Türkan and Demiral, 2009). Glycine betaine is accumulated in many halophytes with the exception of Chenopodium quinoa, Noaea mucronata, and others (Ruffino et al., 2010; Tipirdamaz et al., 2006). Nuccio et al. (1998) reported that endogenous choline supply limits glycine betaine synthesis in transgenic tobacco expressing choline monooxygenase (CMO). Proline betaine and pipecolate betaine accumulate together in species like Medicago sativa and Achillea (Wood et al., 1991; Bonham et al., 1995). Stewart and Larher (1980) and Rosenthal (1982) reported that in Fabaceae, pipecolic acid is accumulated in high concentrations, which are derived from lysine. Biosynthesis of pipecolate betaine and hydroxypipecolate betaine is discussed under the biosynthesis part of this chapter.

\subsubsection{Tertiary Sulfonium Compounds}

Tertiary sulfonium compounds like DMSP are distributed in algae, in grasses like Spartina alterniflora, and in Wollastonia biflora (Otte et al., 2004). Methionine is the precursor for the synthesis of DMSP in both algae and higher plants (Kocsis and Hanson, 2000). While methionine is transaminated to form 
4-methylthio-2-oxobutyrate in algae, it is methylated first to form $S$-methyl methionine and then converted to dimethylsulfoniopropionaldehyde (DMSP-ald) by some mechanism. It is believed that DMSP is a sulfur detoxifier and an osmoregulator and accumulates both in cytoplasm and vacuoles (Otte et al., 2004). Nakajima et al. (2014) isolated DMSP from a green sea alga and found that it suppresses Ehrlich ascites carcinoma.

\subsubsection{Sugars and Sugar Alcohols}

Several plants accumulate simple sugars such as glucose, fructose, sucrose, trehalose, and fructans under salt and drought stress conditions (Briens and Larher, 1982; Yuanyuan et al., 2009) in families like Amaranthaceae, Brassicaceae, Cyperaceae, Juncaceae, Plumbaginaceae, Poaceae, etc. (Slama et al., 2015). Both sugars and polyols are the dominant solutes in many plants, highly soluble in water and hence act as compatible solutes. The nonreducing disaccharide trehalose was first discovered in resurrection plants, but not widely distributed in higher plants (Lunn et al., 2014). Several cyclic polyols (e.g., pinitol) and noncyclic (e.g., mannitol, sorbitol) sugar alcohols accumulate both in halophytic and nonhalophytic species. While pinitol is common in the members of the families Aizoaceae and Fabaceae, mannitol occurs mostly in Combretaceae. Pinitol is widely reported in halophytic species and is derived from the methylation of myo-inositol. Epimerization of ononitol also leads to the production of pinitol (Sengupta et al., 2008). But, the biosynthesis of these osmolytes is an energy consuming process, requiring many molecules of ATP (Flowers and Colmer, 2008). Then, what makes plants use organic osmotica or osmolytes under stress in place of cheaper inorganic osmotica such as $\mathrm{Na}^{+}$and $\mathrm{Cl}^{-}$is not known. Probably, glycophytes do not have the ability for tissue tolerance of $\mathrm{Na}^{+} / \mathrm{Cl}^{-}$, and hence depend on the accumulation of compatible organic solutes for osmotic adjustment under stress.

\subsection{BIOSYNTHETIC PATHWAYS OF PROLINE AND ITS DERIVATIVES}

\subsubsection{Biosynthesis of Proline}

Different routes of proline biosynthesis from glutamate and ornithine are shown in the Fig. 29.1. Also, proline derivatives like proline betaine are shown in Fig. 29.1. The differences that exist in the proline anabolic and catabolic pathways and the genes that encode the enzymes involved both in bacteria and plants, their localizations in different cellular compartments have been reviewed earlier (Kishor et al., 2005; Szabados and Savoure, 2010; Per et al., 2017). But, proline accumulation under stress conditions is not a universal phenomenon.

\subsubsection{Biosynthesis of Proline Derivatives}

Proline is converted to hydroxyproline by hydroxylation. Proline betaine is seen in members of Plumbaginaceae, Capparidaceae, Rutaceae, Labiatae, Compositae, and Leguminosae (Wyn Jones and Storey, 1981; Hanson et al., 1994). It has been pointed out that proline betaine catabolism contributes to rhizobial colonization of seedling roots (Phillips et al., 1998). Both proline and hydroxyproline can be converted to L-proline betaine (also called $\mathrm{N}, \mathrm{N}$-dimethyl-L-proline or stachydrine) and hydroxyproline betaine (known as trans-4-hydroxy-L-proline betaine or betonicine) respectively. Proline is first converted to $\mathrm{N}$-methylproline by a methyl transferase and later to proline betaine by methylation again (Fig. 29.1). Likewise, hydroxyproline is converted first to $\mathrm{N}$-methyl hydroxyproline by a methyl transferase enzyme. $N$-Methyl hydroxyproline is then converted to hydroxyproline betaine by methylation again (Fig. 29.1). Isotope-labeling studies by Trinchant et al. (2004) in alfalfa indicate that

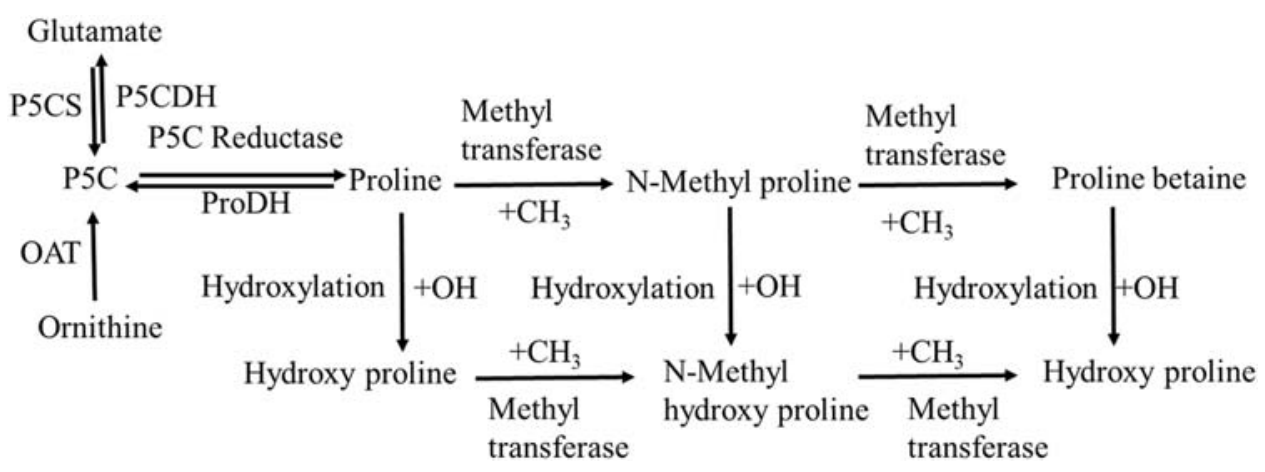

FIGURE 29.1 Biosynthesis of proline, proline betaine (stachydrine) and hydroxyproline betaine (betonicine) in bacteria and plants. P5CS, $\Delta^{1}$-pyrroline-5-carboxylate synthetase; $P 5 \mathrm{C}$, pyrroline-5-carboxylate; $P 5 C R, \mathrm{P} 5 \mathrm{C}$ reductase; $P$ ro $D H$, proline dehydrogenase; $P 5 C D H, P 5 C$ dehydrogenase; $\mathrm{OAT}$, ornithine $\delta$-aminotransferase; $\mathrm{CH}_{3}$, methyl group; $\mathrm{OH}$, hydroxyl group. 


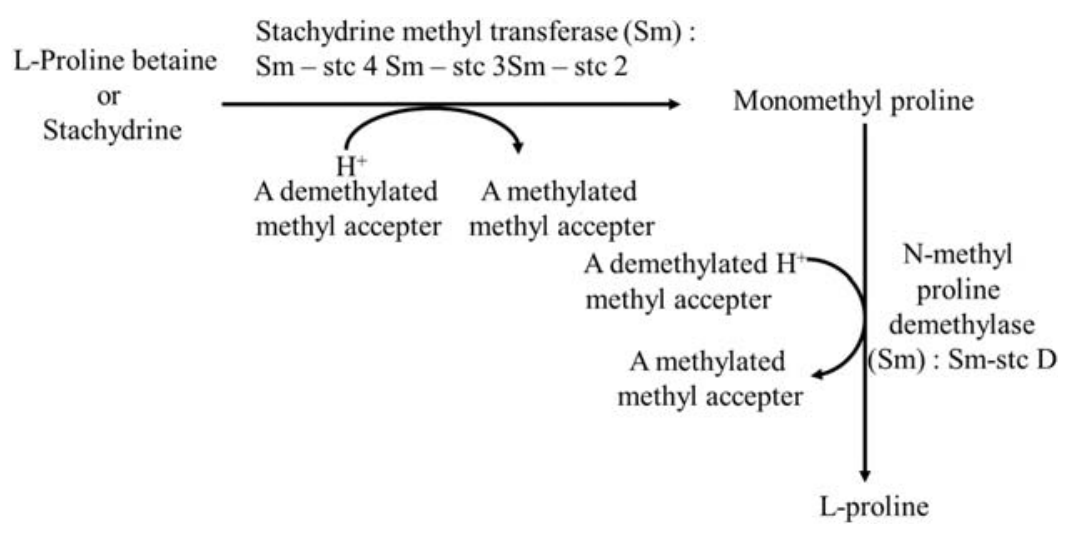

FIGURE 29.2 Catabolism of L-proline betaine to L-proline in Sinorhizobium meliloti.

$\left[{ }^{14} \mathrm{C}\right]$ proline betaine is synthesized from $\mathrm{L}-\left[{ }^{14} \mathrm{C}\right]$ proline. Nolte et al. (1997) and Trinchant et al. (2004) reported biosynthesis of proline betaine (Fig. 29.1) as a long-term response to salinity in Citrus and Medicago species respectively. Both proline betaine and hydroxyproline betaine (albeit to a lesser extent) are simultaneously produced in species like Citrus that grow in chronically dry areas (Nolte et al., 1997). Proline betaine and hydroxyproline betaine protected bacteria against salt stress, but not $N$-methyl-L-proline and trans-4-Lhydroxyproline (Bashir et al., 2014). Hydroxyproline betaine is synthesized from hydroxyproline by a methyl transferase enzyme (Kishor et al., 2005). In species like Citrus, methylated derivatives of proline such as $N$-methylproline (hygric acid), 4-hydroxy-L-proline betaine, and $\mathrm{N}, \mathrm{N}$-dimethyl-L-proline accumulate to ameliorate abiotic stress (Servillo et al., 2011a). Both proline betaine and hydroxyproline betaine have been believed to be metabolically inert as cell protectants against salt, drought, and temperature stresses. But, Kumar et al. (2014) reported that several of the hydroxyproline betaine degradation pathway enzymes also function in proline betaine degradation in bacteria such as Paracoccus denitrificans and Rhodobacter sphaeroides. Proline betaine is ultimately converted back to glutamate in bacteria. Proline betaine catabolic pathway was repressed by both osmotic and cold stresses in these species. However, it is not known if such a catabolic pathway operates in plants also. Trinchant et al. (2004) showed that proline betaine is catabolized through sequential demethylations via $\mathrm{N}$-methylproline and proline (Fig. 29.2). L-proline betaine is used by Sinorhizobium meliloti as a carbon and nitrogen source and as an osmoprotectant (Goldmann et al., 1991). Devoid of any stress, this organism degrades proline betaine. But, in osmotically stressed cells, it is strongly reduced resulting in significant amounts of accumulation (Gloux and Le Rudulier, 1989). Boscari et al. (2002) identified a DNA segment encoding a protein named as BetS in Sinorhizobium meliloti. This gene displays significant sequence identities to the choline transporter
BetT of Escherichia coli and to the glycine betaine transporter OpuD of Bacillus subtilis. Thus, BetS appears as a major glycine betaine/proline betaine transporter needed for early osmotic adjustment in Sinorhizobium meliloti. L-proline betaine is degraded like glycine betaine that involves two sequential demethylations as shown in Fig. 29.2 and produces monomethyl glycine in bacteria (Smith et al., 1988). While the first demethylation reaction is catalyzed by a Rieske type iron-sulfur monooxygenase enzyme (Burnet et al., 2000), the second is carried out by oxidoreductase flavoproteins (Phillips et al., 1998). Using radioisotope $\left[{ }^{14} \mathrm{C}\right]$, Trinchant et al. (2004) reported that proline betaine is catabolized through sequential demethylations via $\mathrm{N}$ methylproline and proline in alfalfa. The compartmentalization of proline betaine and proline within root nodules revealed that salt stress induced an increase in cytosol and bacteroids (Trinchant et al., 2004). But, the existence of proline betaine degrading enzymes in higher plants (other than nodules) has not been reported yet. Also, Kim et al. (2017) discovered that trans-4-L-hydroxyproline also acts as a compatible solute in Halobacillus halophilus. This species accumulates glycine betaine, glutamine, proline, and ectoine to cope with salt stress conditions. It appears that trans-4hydroxy-L-proline (Hyp) is accumulated in H. halophilus in response to salinity. This suggests that Hyp may be a vital compatible solute in this species. They have also identified the candidate genes associated with the biosynthetic pathway of Hyp.

\subsection{BIOSYNTHETIC PATHWAYS OF GLYCINE BETAINE AND ITS DERIVATIVES}

\subsubsection{Biosynthesis of Glycine Betaine}

Multiple pathways exist for the biosynthesis of glycine betaine (Fig. 29.3A-C). It is synthesized from choline (through a series of reactions, L-serine is converted 
to choline in plants), which is converted to betaine aldehyde by CMO in higher plants (Fig. 29.3A). Ethanolamine is the precursor of choline, which is converted to monomethyl ethanolamine by the addition of a methyl group from $S$-adenosylmethionine (SAM). While choline is formed in cytosol, it is transported to the chloroplasts where glycine betaine is synthesized (Weretilnyk et al., 1989). Once it is synthesized, it is transported to different plant parts via phloem (Hattori et al., 2009) through proline porters in plants (common for both proline and glycine betaine). However, it is not known if proline porters also transport proline betaine and hydroxyproline betaine in higher plants. Like proline, younger leaves accumulated more glycine betaine than the older ones under stress (Chen and Murata, 2011; Khan et al., 2012; Masood et al., 2016). In barley, glycine betaine mostly accumulated in vascular tissues of leaves and the pericycle of roots. In most of the glycine betaine accumulating species, it is synthesized from betaine aldehyde by the action of the enzyme betaine aldehyde dehydrogenase (BADH) (Ashraf and Foolad, 2007; Fitzgerald et al., 2009). Nuccio et al. (1998) inserted a chloroplastic glycine betaine synthesizing pathway gene into tobacco and the transformants produced very little amounts. Contrary to this, a cytosolic glycine betaine synthesis pathway accumulated more glycine betaine (Nuccio et al., 2000). This suggested that subcellular localization influences the glycine betaine pathway flux. But in bacteria, three pathways exist. In soil bacterium

(A)

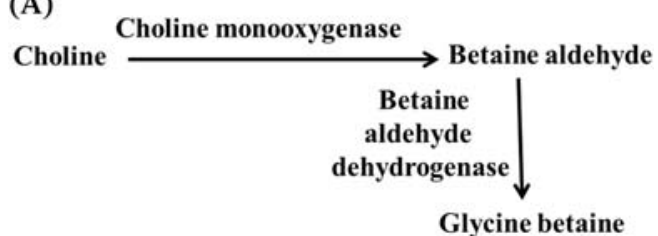

FIGURE 29.3A Biosynthesis of glycine betaine in E. coli.

(B)

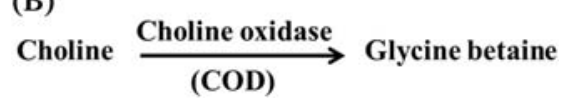

FIGURE 29.3B Biosynthesis of glycine betaine in higher plants.

(C)

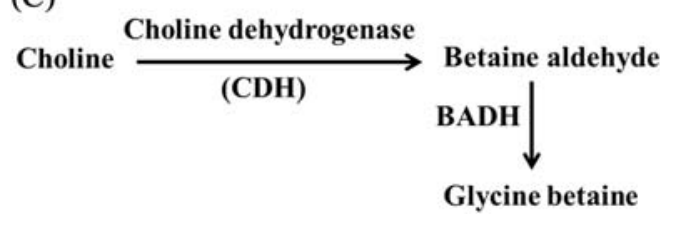

FIGURE 29.3C Biosynthesis of glycine betaine in Arthrobacter globiformis. $B A D H=$ Betaine aldehyde dehydrogenase.
Arthrobacter globiformis, choline is converted to glycine betaine by a single-enzyme choline oxidase (Fig. 29.3B), an $\mathrm{H}_{2} \mathrm{O}_{2}$-generating oxidase (Ikuta et al., 1977). In E. coli, choline is catalyzed by choline dehydrogenase (Fig. 29.3C) into betaine aldehyde. In extreme halophiles like Ectothiorhodospira halochloris and Actinopolyspora halophila glycine betaine is formed by a novel pathway from glycine through three $N$-methylations with SAM as a methyl donor, sarcosine and dimethylglycine as intermediates. The reactions are catalyzed by glycine sarcosine methyltransferase (GSMT) and sarcosine dimethylglycine methyltransferase (SDMT). GSMT first converts glycine to sarcosine and also sarcosine to dimethylglycine (with overlapping functions). Again SDMT catalyzes the methylation of sarcosine or dimethylglycine to dimethylglycine or glycine betaine respectively (Nyyssölä et al., 2000; Kimura et al., 2010). Similar pathway also exists in Myxococcus xanthus (Kimura et al., 2010). The enzyme BADH is common for both plants and animals that can convert betaine aldehyde (formed from choline) to glycine betaine. Thus, differences in glycine betaine pathway exist between bacteria and higher plants.

\subsubsection{Biosynthesis of Glycine Betaine Derivatives}

Rathinasabapathi et al. (2001) reported biosynthesis of $\beta$-alaline betaine by the catalysis of SAM-dependent $\mathrm{N}$ methylation of $\beta$-alanine via $N$-methyl $\beta$-alanine and $N$, $N$-dimethyl $\beta$-alanine. Glycine betaine requires oxygen for its biosynthesis, but not $\beta$-alanine betaine. $\beta$-Alanine betaine may be a more suitable osmoprotectant in halophytic species (e.g., Limonium latifolium and others) than glycine betaine under saline hypoxic conditions since it can avoid the requirement of oxygen for its biosynthesis (Hanson et al., 1991, 1994). The genus Citrus contains osmolytes like trigonelline (nicotinic acid betaine) and choline, but not GABA betaine (Servillo et al., 2011b). Nicotinic acid is converted to nicotinic acid betaine by nicotinic acid N-methyltransferase. Interestingly, Citrus bergamia and Medicago sativa produce pipecolic acid betaine or pipecolate betaine, also known as homostachydrine (Servillo et al., 2012). Accumulation of pipecolic acid betaine is reported under stress conditions in vegetables, Citrus species, Medicago, and Achillea (Wood et al., 1991; Moulin et al., 2006; Servillo et al., 2012). Pipecolate betaine is synthesized from pipecolic acid via $\mathrm{N}$-methylpipecolate with the addition of methyl groups in each step (Fig. 29.4). Pipecolic acid is also converted to hydroxypipecolate by the addition of $\mathrm{OH}$ group. Hydroxypipecolate is first converted to $\mathrm{N}$-methylhydroxypipecolate and then to hydroxypipecolate betaine with the addition of methyl groups 


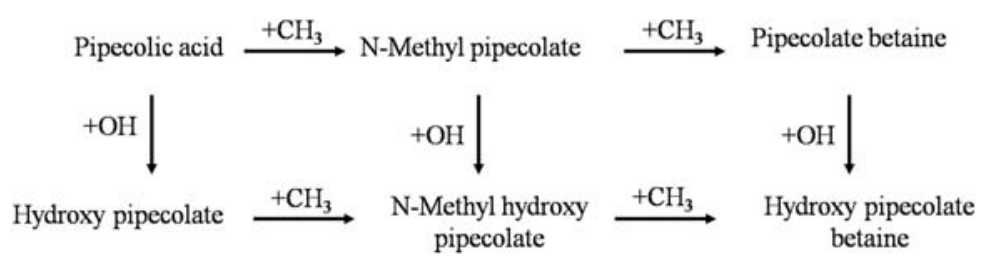

FIGURE 29.4 Biosynthetic pathway of pipecolate betaine and hydroxyl pipecolate betaine.

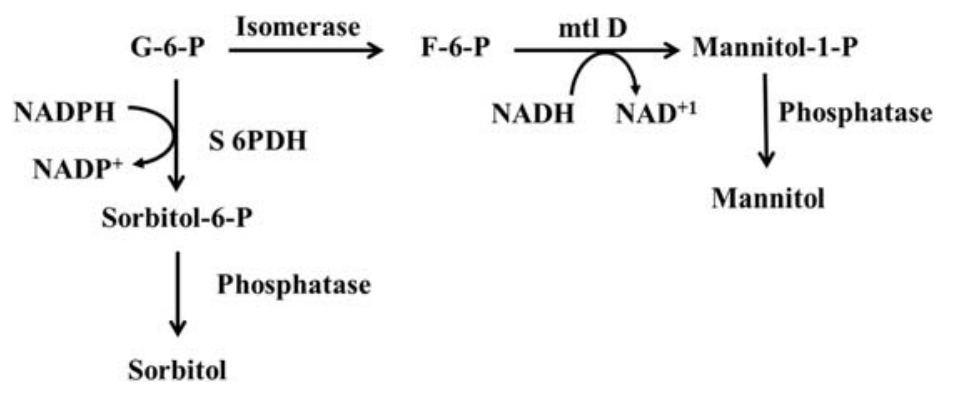

FIGURE 29.5 Biosynthesis of mannitol and sorbitol in plants. G-6-P, glucose-6-phospahate; $m t l D$, mannitol-1-phospahate; $S 6 P D H$, sorbitol-6-phosphate dehydrogenase; mannitol-1$P$, mannitol-1-phosphate.
(Fig. 29.4). However, the enzymes that catalyze the pipecolic acid to the betaines are not exactly known. Presence of pipecolic acid in Citrus and Medicago species led to the postulation that it serves as a homostachydrine precursor through direct methylation. Thus, the pathways for the synthesis of proline and glycine betaine vary depending upon the species but all the pathways are not well characterized in higher plants.

\subsection{BIOSYNTHETIC PATHWAYS OF TREHALOSE AND SUGAR ALCOHOLS}

\subsubsection{Biosynthesis of Trehalose (Sugar)}

Biosynthesis of trehalose is complicated and three different pathways have been noticed to date. OtsA in E. coli and the enzyme trehalose phosphate synthase catalyze the transfer of glucose from UDP-glucose to glucose-6-phosphate (G-6-P) to produce trehalose-6phosphate and uridine diphosphate (Cabib and Leloir, 1958; Roth and Sussman, 1966). Trehalose-6-phosphate is then converted to trehalose by dephosphorylation by phosphatase enzyme. Organisms that use OtsA pathway have a trehalose-P phosphatase or OtsB in E. coli to produce trehalose (Elbein et al., 2003). The second pathway for trehalose biosynthesis (e.g., Pimelobacter species) involves intramolecular rearrangement of maltose (glucosyl-a1-4-glucopyranoside) to convert the 1,4-linkage to the 1,1-bond of trehalose by the enzyme trehalose synthase (Nishimoto et al., 1996). Trehalose synthesis was also reported from some bacteria (e.g., Sulfolobus acidocaldarius) involving the conversion of maltooligosaccharides or starch to trehalose. Maltooligosyltrehalose synthase (TreY) catalyzes the conversion of maltodextrin to maltooligosyltrehalose and then maltooligosyltrehalose trehalohydrolase
(TreZ) hydrolyzes this to trehalose (Maruta et al., 1996). In the third pathway (like in mushrooms), glucose is rearranged at the reducing end of a glycogen chain to convert the alpha 1,4-linkage to an a, a1,1bond. A second enzyme then produces the disaccharide trehalose (Elbein et al., 2003).

\subsubsection{Biosynthesis of Sugar Alcohols}

Both mannitol and sorbitol accumulate in large quantities and are widespread in higher plants. Mannitol has been detected in more than 50 families. But, other sugar alcohols like dulcitol and ribitol are less represented in plants (Lewis and Smith, 1967). Glucose-6-phosphate is the precursor for the synthesis of sugar alcohols such as mannitol and sorbitol. G-6-P is first converted to fructose-6-phosphate (F-6-P) by an isomerase enzyme. F-6-P is then converted to mannitol and the reaction is catalyzed by mannitol-1-phosphate dehydrogenase (mtlD). Mannitol-1-phosphate is converted to mannitol by a phosphatase enzyme (Fig. 29.5). Mannitol not only acts a compatible solute but it also protects the plants against abiotic stress. Besides, it has a possible role in plant responses to pathogen attack (Stoop et al., 1996). Sorbitol-6-phosphate dehydrogenase (S6PDH) catalyzes the conversion of G-6-P to sorbitol-6phosphate $(\mathrm{S} 6 \mathrm{P})$, releasing $\mathrm{NADP}^{+}$. S6P produces sorbitol (Fig. 29.5) by dephosphorylation (Shen et al., 1999). Mesembryanthemum crystallinum, a facultative halophyte, produces rare sugar alcohols like pinitol (ID-3-O-methyl chiro-inositol) from inositol (a polyol and a 6-carbon compound). Pinitol is accumulated during salt and drought stress in this halophyte and also in many plants. In the pathway, a methylation step is catalyzed by a position specific SAM-dependent O-methyl transferase. Ononitol is then converted to pinitol by an 
epimerase reaction that requires $\mathrm{NAD}^{+}$and/or NADPH (Loewus and Dickinson, 1982). But tracer studies with labeled inositols revealed that the biosynthesis of D-pinitol in several leguminous plants like Medicago sativa, Trifolium incarnatum, Simmondsia chinensis does not occur by epimerization of sequoyitol. It only occurs via D-ononitol (Dittrich and Brandl, 1987). Thus, rare sugar alcohols like pinitol and ononitol are produced not only in halophytic species, but also in glycophytes like in leguminous plants.

\subsection{CONCLUSIONS}

Diverse osmolytes have been identified in a wide range of microbial and plant species with multiple biosynthetic pathways. Unfortunately, the biosynthetic pathway enzymes and the corresponding genes have not yet been isolated in higher plants. However, it has been recognized that osmolytes perform multifarious functions during abiotic stress conditions though the exact molecular mechanisms are not completely known. Thus, several gaps exist in our understanding of the osmolyte biosynthesis and their precise regulation at the cellular level.

\section{Acknowledgments}

PBK is thankful for sanctioning CSIR-Emeritus Scientist Fellowship through the Grant Number 38(1325)/12/EMR-II by the Council for Scientific and Industrial Research, New Delhi.

\section{References}

Ahmad, I., Larher, F., Stewart, G., 1981. The accumulation of $\Delta^{\prime}$-acetylornithine and other solutes in the salt marsh grass Puccinellia maritima. Phytochemistry 20 (7), 1501-1504.

Ashraf, M., Foolad, M., 2007. Roles of glycine betaine and proline in improving plant abiotic stress resistance. Environ. Exp. Bot. 59 (2), 206-216.

Ashraf, M.P.J.C., Harris, P.J.C., 2004. Potential biochemical indicators of salinity tolerance in plants. Plant Sci. 166 (1), 3-16.

Aubert, S., Hennion, F., Bouchereau, A., Gout, E., Bligny, R., Dorne, A.J., 1999. Subcellular compartmentation of proline in the leaves of the subantarctic Kerguelen cabbage Pringlea antiscorbutica R. Br. In vivo13C-NMR study. Plant Cell Environ. 22 (3), 255-259.

Bashir, A., Hoffmann, T., Kempf, B., Xie, X., Smits, S.H., Bremer, E., 2014. Plant-derived compatible solutes proline betaine and betonicine confer enhanced osmotic and temperature stress tolerance to Bacillus subtilis. Microbiology 160 (10), 2283-2294.

Bellantyne, J.S., Chamberlin, M.E., 1994. Regulation of cellular amino acid levels. In: Strange, K. (Ed.), Cellular and Molecular Physiology of Cell Volume Regulation. CRC Press, Boca Raton, FL, pp. 111-122.

Bonham, C.C., Wood, K.V., Yang, W.J., Nadolska-Orczyk, A., Samaras, Y., Gage, D.A., et al., 1995. Identification of quaternary ammonium and tertiary sulfonium compounds by plasma desorption mass spectrometry. J. Mass. Spectrom. 30 (8), 1187-1194.

Boscari, A., Mandon, K., Dupont, L., Poggi, M.C., Le Rudulier, D., 2002. BetS is a major glycine betaine/proline betaine transporter required for early osmotic adjustment in Sinorhizobium meliloti. J. Bacteriol. 184 (10), 2654-2663.

Briens, M., Larher, F., 1982. Osmoregulation in halophytic higher plants: a comparative study of soluble carbohydrates, polyols, betaines and free proline. Plant Cell Environ. 5 (4), 287-292.

Burg, M.B., Ferraris, J.D., Dmitrieva, N.I., 2007. Cellular response to hyperosmotic stresses. Physiol. Rev. 87 (4), 1441-1474.

Burnet, M.W., Goldmann, A., Drong, R., El Amrani, A., Loreau, O., Slightom, J., et al., 2000. The stachydrine catabolism region in Sinorhizobium meliloti encodes a multi-enzyme complex similar to the xenobiotic degrading systems in other bacteria. Gene 244 (1), 151-161.

Cabib, E., Leloir, L.F., 1958. The biosynthesis of trehalose phosphate. J. Biol. Chem. 231 (1), 259-275.

Cheeseman, J.M., 2013. The integration of activity in saline environments: problems and perspectives. Funct. Plant Biol. 40 (9), $759-774$.

Chen, T.H., Murata, N., 2011. Glycinebetaine protects plants against abiotic stress: mechanisms and biotechnological applications. Plant Cell Environ. 34 (1), 1-20.

Dittrich, P., Brandl, A., 1987. Revision of the pathway of D-pinitol formation in Leguminosae. Phytochemistry 26 (7), 1925-1926.

Elbein, A.D., Pan, Y.T., Pastuszak, I., Carroll, D., 2003. New insights on trehalose: a multifunctional molecule. Glycobiology 13 (4), 17R-27R.

Fitzgerald, T.L., Waters, D.L., Henry, R.J., 2009. Betaine aldehyde dehydrogenase in plants. Plant Biol. 11 (2), 119-130.

Flowers, T.J., Colmer, T.D., 2008. Salinity tolerance in halophytes. New Phytol. 179 (4), 945-963.

Gagneul, D., Aïnouche, A., Duhazé, C., Lugan, R., Larher, F.R., Bouchereau, A., 2007. A reassessment of the function of the socalled compatible solutes in the halophytic Plumbaginaceae Limonium latifolium. Plant Physiol. 144 (3), 1598-1611.

Glenn, E.P., Brown, J.J., Blumwald, E., 1999. Salt tolerance and crop potential of halophytes. CRC. Crit. Rev. Plant. Sci. 18 (2), $227-255$

Gloux, K., Le Rudulier, D., 1989. Transport and catabolism of proline betaine in salt-stressed Rhizobium meliloti. Arch. Microbiol. 151 (2), 143-148.

Goldmann, A., Boivin, C., Fleury, V., Lecoeur, L., Maille, M., Tepfer, D., 1991. Betaine use by rhizosphere bacteria: genes essential for trigonelline, stachydrine, and carnitine catabolism in Rhizobium meliloti are located on pSym in the symbiotic region. Mol. PlantMicrobe Int. MPMI 4 (6), 571-578.

Gorham, J., 1995. Betaine in higher plants- biosynthesis and role in stress metabolism. In: Wallsgrove, R.M. (Ed.), Amino Acids in Higher Plants. Cambridge University Press, Cambridge, pp. 171-203.

Grote, E.M., Ejeta, G., Rhodes, D., 1994. Inheritance of glycinebetaine deficiency in sorghum. Crop Sci. 34 (5), 1217-1220.

Guo, P., Baum, M., Grando, S., Ceccarelli, S., Bai, G., Li, R., et al., 2009. Differentially expressed genes between drought-tolerant and drought-sensitive barley genotypes in response to drought stress during the reproductive stage. J. Exp. Bot. 60 (12), 3531-3544.

Hanson, A.D., Rathinasabapathi, B., Chamberlin, B., Gage, D.A., 1991. Comparative physiological evidence that $\beta$-alanine betaine and choline-O-sulfate act as compatible osmolytes in halophytic Limonium species. Plant Physiol. 97 (3), 1199-1205.

Hanson, A.D., Rathinasabapathi, B., Rivoal, J., Burnet, M., Dillon, M. O., Gage, D.A., 1994. Osmoprotective compounds in the Plumbaginaceae: a natural experiment in metabolic engineering of stress tolerance. Proc. Natl. Acad. Sci. 91 (1), 306-310.

Hare, P.D., Cress, W.A., Van Staden, J., 1998. Dissecting the roles of osmolyte accumulation during stress. Plant Cell Environ. 21 (6), $535-553$.

Hattori, T., Mitsuya, S., Fujiwara, T., Jagendorf, A.T., Takabe, T., 2009. Tissue specificity of glycinebetaine synthesis in barley. Plant Sci. 176 (1), 112-118. 
Ikuta, S., Imamura, S., Misaki, H., Horiuti, Y., 1977. Purification and characterization of choline oxidase from Arthrobacter globiformis. J. Biochem. 82 (6), 1741-1749.

Kishor, P.B.K., 1988. Effect of salt stress on callus cultures of Oryza sativa L. J. Exp. Bot. 39 (2), 235-240.

Kishor, P.B.K., Sangam, S., Amrutha, R.N., Laxmi, P.S., Naidu, K. R., Rao, K.R.S.S., et al., 2005. Regulation of proline biosynthesis, degradation, uptake and transport in higher plants: its implications in plant growth and abiotic stress tolerance. Curr. Sci. 424-438.

Kim, K.H., Jia, B., Jeon, C.O., 2017. Identification of Trans-4-hydroxyL-proline as a Compatible Solute and Its Biosynthesis and Molecular Characterization in Halobacillus halophilus. Front. Microbiol. 8, 2054.

Kimura, Y., Kawasaki, S., Yoshimoto, H., Takegawa, K., 2010. Glycine betaine biosynthesized from glycine provides an osmolyte for cell growth and spore germination during osmotic stress in Myxococcus xanthus. J. Bacteriol. 192 (5), 1467-1470.

Khan, M.I.R., Iqbal, N., Masood, A., Khan, N.A., 2012. Variation in salt tolerance of wheat cultivars: role of glycinebetaine and ethylene. Pedosphere 22 (6), 746-754.

Khan, M.I.R., Asgher, M., Khan, N.A., 2014. Alleviation of saltinduced photosynthesis and growth inhibition by salicylic acid involves glycinebetaine and ethylene in mungbean (Vigna radiata L.). Plant. Physiol. Biochem. 80, 67-74.

Khan, M.I.R., Iqbal, N., Masood, A., Per, T.S., Khan, N.A., $2013 a$. Salicylic acid alleviates adverse effects of heat stress on photosynthesis through changes in proline production and ethylene formation. Plant Signal. Behav. 8 (11), e26374.

Khan, M.I.R., Asgher, M., Khan, N.A., 2013b. Rising temperature in the changing environment: a serious threat to plants. Clim. Change Environ. Sustain. 1, 25-36.

Khan, N.A., Khan, M.I.R., Ferrante, A., Poór, P., 2017. Ethylene: a key regulatory molecule in plants. Front. Plant Sci. 8, 1782.

Kocsis, M.G., Hanson, A.D., 2000. Biochemical evidence for two novel enzymes in the biosynthesis of 3-dimethylsulfoniopropionate in Spartina alterniflora. Plant Physiol. 123 (3), 1153-1162.

Kumar, R., Zhao, S., Vetting, M.W., Wood, B.M., Sakai, A., Cho, K., et al., 2014. Prediction and biochemical demonstration of a catabolic pathway for the osmoprotectant proline betaine. mBio 5 (1), e00933-13.

Lewis, D.H., Smith, D.C., 1967. Sugar alcohols (polyols) in fungi and green plants. New Phytol. 66 (2), 143-184.

Loewus, F.A., Dickinson, D.B., 1982. In: Tanner, W. (Ed.), Encyclopedia of plant Physiology: Plant Carbohydrates I: Intracellular Carbohydrates, 13. Springer-Verlag, Berlin, pp. 193-206.

Low, P.S., 1985. Molecular basis of the biological compatibility of nature's osmolytes. In: Gilles, R., Gilles, B.M. (Eds.), Transport Processes, Iono-and Osmoregulation. Springer, Berlin: Heidelberg, pp. $469-477$.

Lunn, J.E., Delorge, I., Figueroa, C.M., Van Dijck, P., Stitt, M., 2014. Trehalose metabolism in plants. Plant J. 79 (4), 544-567.

Mansour, M.M.F., 2000. Nitrogen containing compounds and adaptation of plants to salinity stress. Biol. Plant. 43 (4), 491-500.

Maruta, K., Mitsuzumi, H., Nakada, T., Kubota, M., Chaen, H., Fukuda, S., et al., 1996. Cloning and sequencing of a cluster of genes encoding novel enzymes of trehalose biosynthesis from thermophilic archaebacterium Sulfolobus acidocaldarius. Biochimica et Biophysica Acta (BBA)-General Subjects 1291 (3), 177-181.

Masood, A., Per, T.S., Asgher, M., Fatma, M., Khan, M.I.R., Rasheed, F., et al., 2016. Glycine betaine: role in shifting plants toward adaptation under extreme environments. Osmolytes and Plants Acclimation to Changing Environment: Emerging Omics Technologies. Springer, New Delhi, pp. 69-82.
Moulin, M., Deleu, C., Larher, F., Bouchereau, A., 2006. The lysineketoglutarate reductase-saccharopine dehydrogenase is involved in the osmo-induced synthesis of pipecolic acid in rapeseed leaf tissues. Plant. Physiol. Biochem. 44 (7-9), 474-482.

Munns, R., Tester, M., 2008. Mechanisms of salinity tolerance. Annu. Rev. Plant. Biol. 59, 651-681.

Murakeözy, É.P., Nagy, Z., Duhazé, C., Bouchereau, A., Tuba, Z., 2003. Seasonal changes in the levels of compatible osmolytes in three halophytic species of inland saline vegetation in Hungary. J. Plant. Physiol. 160 (4), 395-401.

Nakajima, K., Tsujiwaki, S., Nakajima, Y., 2014. A tertiary sulfonium compound, dimethylsulfoniopropionate in green sea algae, completely suppresses crucial Ehrlich ascites carcinoma in mice. Anticancer Res. 34 (8), 4045-4050.

Nishimoto, T., Nakano, M., Nakada, T., Chaen, H., Fukuda, S., Sugimoto, T., et al., 1996. Purification and properties of a novel enzyme, trehalose synthase, from Pimelobacter sp. R48. Biosci. Biotechnol. Biochem. 60 (4), 640-644.

Nolte, K.D., Hanson, A.D., Gage, D.A., 1997. Proline accumulation and methylation to proline betaine in Citrus: implications for genetic engineering of stress resistance. J. Am. Soc. Hortic. Sci. 122 (1), 8-13.

Nuccio, M.L., Russell, B.L., Nolte, K.D., Rathinasabapathi, B., Gage, D.A., Hanson, A.D., 1998. The endogenous choline supply limits glycine betaine synthesis in transgenic tobacco expressing choline monooxygenase. Plant J. 16 (4), 487-496.

Nuccio, M.L., McNeil, S.D., Ziemak, M.J., Hanson, A.D., Jain, R.K., Selvaraj, G., 2000. Choline import into chloroplasts limits glycine betaine synthesis in tobacco: analysis of plants engineered with a chloroplastic or a cytosolic pathway. Metab. Eng. 2 (4), 300-311.

Nyyssölä, A., Kerovuo, J., Kaukinen, P., von Weymarn, N., Reinikainen, T., 2000. Extreme halophiles synthesize betaine from glycine by methylation. J. Biol. Chem. 275 (29), 22196-22201.

Otte, M.L., Wilson, G., Morris, J.T., Moran, B.M., 2004. Dimethylsulphoniopropionate (DMSP) and related compounds in higher plants. J. Exp. Bot. 55 (404), 1919-1925.

Per, T.S., Khan, N.A., Reddy, P.S., Masood, A., Hasanuzzaman, M., Khan, M.I.R., et al., 2017. Approaches in modulating proline metabolism in plants for salt and drought stress tolerance: phytohormones, mineral nutrients and transgenics. Plant. Physiol. Biochem. 115, 126-140.

Phillips, D.A., Sande, E.S., Vriezen, J.A.C., de Bruijn, F.J., Le Rudulier, D., Joseph, C.M., 1998. A new genetic locus in Sinorhizobium meliloti is involved in stachydrine utilization. Appl. Environ. Microbiol. 64 (10), 3954-3960.

Rathinasabapathi, B., Fouad, W.M., Sigua, C.A., 2001. $\beta$-Alanine betaine synthesis in the plumbaginaceae. Purification and characterization of a trifunctional, S-adenosyl-l-methionine-dependent $\mathrm{N}$ methyltransferase from Limonium latifolium leaves. Plant Physiol. 126 (3), 1241-1249.

Rhodes, D., Hanson, A.D., 1993. Quaternary ammonium and tertiary sulfonium compounds in higher plants. Annu. Rev. Plant. Biol. 44 (1), 357-384.

Rhodes, D., Nadolska-Orczyk, A., Rich, P.J., 2002. Salinity, osmolytes and compatible solutes. In: Lauchli, A., Luttge, U. (Eds.), Salinity: Environment-Plants-Molecules. Springer, Dordrecht, pp. 181-204.

Rosenthal, G., 1982. Plant Nonprotein Amino and Imino Acids: Biological, Biochemical, and Toxicological Properties. Elsevier, Amsterdam.

Roth, R., Sussman, M., 1966. Trehalose synthesis in the cellular slime mold Dictyostelium discoideum. Biochimica et Biophysica Acta (BBA)-Enzymology and Biological Oxidation 122 (2), 225-231.

Ruffino, A.M.C., Rosa, M., Hilal, M., González, J.A., Prado, F.E., 2010. The role of cotyledon metabolism in the establishment of quinoa (Chenopodium quinoa) seedlings growing under salinity. Plant Soil 326 (1-2), 213-224. 
Saneoka, H., Nagasaka, C., Hahn, D.T., Yang, W.J., Premachandra, G.S., Joly, R.J., et al., 1995. Salt tolerance of glycinebetainedeficient and-containing maize lines. Plant Physiol. 107 (2), 631-638.

Sarvesh, A., Anuradha, M., Pulliah, T., Reddy, T.P., Kavi Kishor, P. B., 1996. Salt stress and antioxidant response in high and low proline producing cultivars of niger, Guizotia abyssinica (Lf) Cass. Indian J. Exp. Biol. 34 (3), 252-256.

Sengupta, S., Patra, B., Ray, S., Majumder, A.L., 2008. Inositol methyl transferase from a halophytic wild rice, Porteresia coarctata Roxb. (Tateoka): regulation of pinitol synthesis under abiotic stress. Plant Cell Environ. 31 (10), 1442-1459.

Servillo, L., Giovane, A., Balestrieri, M.L., Bata-Csere, A., Cautela, D., Castaldo, D., 2011a. Betaines in fruits of Citrus genus plants. J. Agric. Food. Chem. 59 (17), 9410-9416.

Servillo, L., Giovane, A., Balestrieri, M.L., Ferrari, G., Cautela, D., Castaldo, D., 2011b. Occurrence of pipecolic acid and pipecolic acid betaine (homostachydrine) in Citrus genus plants. J. Agric. Food. Chem. 60 (1), 315-321.

Servillo, L., Giovane, A., Balestrieri, M.L., Cautela, D., Castaldo, D., 2012. N-Methylated tryptamine derivatives in Citrus genus plants: identification of $N, N, N$-trimethyltryptamine in bergamot. J. Agric. Food. Chem. 60 (37), 9512-9518.

Shen, B., Hohmann, S., Jensen, R.G., Bohnert, H.J., 1999. Roles of sugar alcohols in osmotic stress adaptation. Replacement of glycerol by mannitol and sorbitol in yeast. Plant Physiol. 121 (1), $45-52$.

Slama, I., Abdelly, C., Bouchereau, A., Flowers, T., Savouré, A., 2015. Diversity, distribution and roles of osmoprotective compounds accumulated in halophytes under abiotic stress. Ann. Bot. (Lond.) 115 (3), 433-447.

Smith, L.T., Pocard, J.A., Bernard, T., Le Rudulier, D., 1988. Osmotic control of glycine betaine biosynthesis and degradation in Rhizobium meliloti. J. Bacteriol. 170 (7), 3142-3149.

Stewart, G.R., Larher, F., 1980. Accumulation of amino acids and related compounds in relation to environmental stress. In: Miflin, B.J. (Ed.), Amino Acids and Derivatives, 5. pp. 609-635.

Stoop, J.M.H., Williamson, J.D., Pharr, D.M., 1996. Mannitol metabolism in plants: a method for coping with stress. Trends. Plant. Sci. 1 (5), 139-144.

Szabados, L., Savoure, A., 2010. Proline: a multifunctional amino acid. Trends. Plant. Sci. 15 (2), 89-97.
Szabados, L., Kovács, H., Zilberstein, A., Bouchereau, A., 2011. Plants in extreme environments: importance of protective compounds in stress tolerance. Adv. Botan. Res. 57, 105-150.

Tipirdamaz, R., Gagneul, D., Duhazé, C., Aïnouche, A., Monnier, C., Özkum, D., et al., 2006. Clustering of halophytes from an inland salt marsh in Turkey according to their ability to accumulate sodium and nitrogenous osmolytes. Environ. Exp. Bot. 57 (1-2), 139-153.

Trinchant, J.C., Boscari, A., Spennato, G., Van de Sype, G., Le Rudulier, D., 2004. Proline betaine accumulation and metabolism in alfalfa plants under sodium chloride stress. Exploring its compartmentalization in nodules. Plant Physiol. 135 (3), 1583-1594.

Türkan, I., Demiral, T., 2009. Recent developments in understanding salinity tolerance. Environ. Exp. Bot. 67 (1), 2-9.

Weretilnyk, E.A., Bednarek, S., McCue, K.F., Rhodes, D., Hanson, A. D., 1989. Comparative biochemical and immunological studies of the glycine betaine synthesis pathway in diverse families of dicotyledons. Planta 178 (3), 342-352.

Wood, K.V., Stringham, K.J., Smith, D.L., Volenec, J.J., Hendershot, K.L., Jackson, K.A., et al., 1991. Betaines of alfalfa: characterization by fast atom bombardment and desorption chemical ionization mass spectrometry. Plant Physiol. 96 (3), 892-897.

Wyn Jones, R.G., Storey, R., 1981. Betaines. In: Paleg, L.G., Aspinall, D. (Eds.), The Physiology and Biochemistry of Drought Resistance in Plants. Academic Press, Sydney, pp. 171-204.

Yancey, P.H., 2005. Organic osmolytes as compatible, metabolic and counteracting cytoprotectants in high osmolarity and other stresses. J. Exp. Biol. 208 (15), 2819-2830.

Yancey, P.H., Clark, M.E., Hand, S.C., Bowlus, R.D., Somero, G.N., 1982. Living with water stress: evolution of osmolyte systems. Science 217 (4566), 1214-1222.

Yuanyuan, M., Yali, Z., Jiang, L., Hongbo, S., 2009. Roles of plant soluble sugars and their responses to plant cold stress. Afr. J. Biotechnol. 8 (10).

\section{Further Reading}

Servillo, L., Giovane, A., Balestrieri, M.L., Cautela, D., Castaldo, D., 2010. Proline derivatives in fruits of bergamot (Citrus bergamia Risso et Poit): presence of $\mathrm{N}$-methyl-L-proline and 4-hydroxy-Lprolinebetaine. J. Agric. Food. Chem. 59 (1), 274-281. 


\title{
30
}

\section{Role and Regulation of Osmolytes as Signaling Molecules to Abiotic Stress Tolerance}

\author{
Titash Dutta ${ }^{1}$, Nageswara Rao Reddy Neelapu ${ }^{1}$, \\ Shabir H. Wani ${ }^{2,3}$ and Challa Surekha ${ }^{1}$
}

${ }^{1}$ Department of Biochemistry and Bioinformatics, Institute of Science, Gandhi Institute of Technology and Management (GITAM Deemed to be University), Visakhapatnam, Andhra Pradesh, India ${ }^{2}$ Mountain Research

Centre for Field Crops, Sher-e-Kashmir University of Agricultural Sciences and Technology, Srinagar, Jammu and Kashmir, India ${ }^{3}$ Department of Plant, Soil and Microbial Sciences, Michigan State University, East Lansing, MI, United States

\section{O U T L I N E}

30.1 Introduction

30.2 Osmolyte Mediated Abiotic Stress Responses

30.3 Biosynthesis, Accumulation, and Regulation of

Osmolytes in Response to Abiotic Stress

30.3.1 Proline

30.3.2 Glycine Betaine
459

460

461

461

463
30.3.3 Polyamines

30.3.4 Sugars and Sugar Alcohols

30.4 Conclusion and Future Prospects

Acknowledgment

References

\subsection{INTRODUCTION}

Abiotic stresses, predominantly salinity, drought, flooding, metals, and extreme temperatures contribute to significant loss in global crop production. The scenario is further aggravated by the growing population, which is expected to cross 9.1 billion in 2050 (Sah et al., 2016). According to a survey, it was estimated that $60 \%$ increase in production of important crops such as cereals and legumes is necessary to meet the food supply of the growing population (FAO, 2010).

Abiotic stress factors are considered detrimental for crops as they minimize photosynthesis, photosynthetic pigments, mineral use efficiencies, pollen viability, seed quality, and yield (Khan et al., 2012, 2013, 2014, 2015, 2016; Nath et al., 2017; Sade et al., 2017). 
Acquaah (2007) reported that exposure to abiotic stresses lead to $70 \%$ reduction in crop yield annually. Plants are unable to avoid stress exposure due to their sessile nature. Abiotic stresses severely impair the cellular homeostasis and increases reactive oxygen species (ROS) accumulation, eventually leading to plant death (Khan and Khan, 2017; Zandalinas et al., 2018). Subsequently changes in global climate pattern and diminished rainfall have increased the severity and frequency of the abiotic stress conditions. Thus, the world food security is under constant threat as yield loss of agriculturally important crops (rice, maize, wheat, and barley) has been associated with climate change and abiotic stresses (Jaleel et al., 2009; Khan and Khan, 2013).

Plants at the molecular level trigger a sequence of biochemical events in response to the abiotic stress conditions. Stress tolerance is conferred by transcriptionally regulating specific gene families (Joshi et al., 2018). These genes families are classified into three categories based on their function. The first category contains genes involved in osmoprotection such as osmoprotectants, antioxidant enzymes, late embryogenesis abundant (LEA) proteins, and heat shock proteins. The second category comprises the genes coding ion transporters such as $\mathrm{Na}^{+} / \mathrm{H}^{+}$channels and aquaporins (facilitates water uptake). The third category includes the genes responsible for signal perception and transcriptional regulation such as mitogenactivated protein kinases (MAPKs), salt overly sensitive kinases (Ji et al., 2013), ethylene-responsive element binding proteins, heat shock transcriptional factors, CBF/DREB (C-repeat-binding / dehydration-responsive element), bZIP (basic-domain leucine zipper), NAC (NAM, ATAF, and CUC), MYC (myelocytomatosis oncogene)/MYB (myeloblastosis oncogene), Cys2/His2 zinc-finger motifs, and WRKY protein domains (Umezawa et al., 2006). During the last decade, plant breeders focused on developing potential stress tolerant cultivars by incorporating transgenic approaches in conventional breeding techniques. The transgenic cultivars exhibit enhanced qualitative and quantitative traits and induce stress tolerance (Wani et al., 2016; BhatnagarMathur et al., 2008).

Salt and drought stress are responsible for cellular dehydration. Loss of intracellular water to denaturation of cellular proteins alters the cellular homeostasis. To minimize the water loss, maintain cellular integrity, and protect the proteins, plants have evolved strategies to accumulate specific organic molecules classified as osmolytes or compatible solutes. The hallmark of these osmolytes includes low molecular weight and high solubility. Proline, glycine betaine (GB), polyols, sugar alcohols, and soluble sugars are the important osmolytes that accumulate in plants in response to abiotic stresses. These osmolytes are also involved in scavenging of ROS, maintaining cellular redox potential and $\mathrm{pH}$, osmotic adjustments, and stabilizing proteins structures and membranes.

Thus, osmolyte biosynthesis and their accumulation in plants contribute towards osmoprotection thereby conferring abiotic as well as secondary (osmotic and ionic) stress conditions. Genome-wide screening and transcriptome analysis of major cereals, pulses, and in A. thaliana exposed to abiotic stresses led to identification of a set of genes involved in the biosynthetic pathway of these osmolytes (Suprasanna et al., 2016; Chakraborty and Sairam, 2017). Thus, the focus lies in developing transgenic cultivars by introducing the candidate genes of these osmolytes to enhance stress tolerance and crop productivity (Surekha et al., 2015). Several transgenic cultivars have been developed successfully by targeting osmoprotection. The transgenic plants exhibited improved grain yield, biomass, and abiotic stress tolerance. These include rice (Garg et al., 2002; Su and $\mathrm{Wu}, 2004)$, potato (Zhang et al., 2011b), wheat (Sawahel and Hassan, 2002), tomato (Park et al., 2007), maize (Quan et al., 2004), tobacco (Szabados and Savouré, 2010), soyabean (Qin et al., 2017) and pigeonpea (Surekha et al., 2014).

The present review article focuses on identifying the various osmolytes that plants accumulate, and their specific role and regulation as signaling molecules in response to abiotic stresses. Extensive research on their biosynthetic pathways highlighted the candidate genes essential for their synthesis. The review also features the transgenic plants developed bearing the candidate biosynthetic genes thereby enhancing osmoprotection and abiotic stress tolerance.

\subsection{OSMOLYTE MEDIATED ABIOTIC STRESS RESPONSES}

Exposure to abiotic stress initiates morphological, physiological, metabolic, and molecular changes that significantly hamper plant development and survival. Prolonged exposure to salinity inhibits plant growth as observed in the biphasic response model: initial phase (disruption of cellular homeostasis) and the final phase (disruption of ionic homeostasis) (Munns, 2002; Adem et al., 2014; Wani et al., 2017). To counter these stress conditions, plants have evolved three strategies, namely, ion exclusion, $\mathrm{Na}^{+}$compartmentalization into vacuoles, and osmoprotection (Munns, 2002; Wani et al., 2017).

In stressed plants, osmoprotection is achieved by increasing the synthesis and accumulation of specific osmolytes such as GB and proline (Ashfaque et al., 2014; Masood et al., 2016; Per et al., 2017). The osmolytes are generally characterized by their low 
molecular weight, high polarity, solubility, and hydrophilic nature. These features allow the solutes to protect the membrane protein structures during stress conditions. The osmolytes accumulated in plants are grouped into three classes: (1) free amino acids (proline), (2) quaternary amines (GB and polyamines (PAs)), and (3) sugars and sugar alcohols (trehalose, fructans, mannitol, and sorbitol) (Yancey, 2005; Roychoudhury et al., 2015). GB and trehalose serve as osmoprotectants by stabilizing the membranes and the quaternary structures of proteins. Mannitol acts as a free-radical scavenger. Proline functions as a vital storage sink for carbon and nitrogen as well as a freeradical scavenger (Kaur and Asthir, 2015). Moreover it also lends stability to subcellular structures (membranes and proteins), and maintains the cellular redox potential under abiotic stress. Due to their role in osmoprotection, these organic osmolytes are termed as osmoprotectants (Chen and Murata, 2002; Surekha et al., 2014; Roychoudhury et al., 2015; Blum, 2017).

Osmolyte mediated abiotic stress response involves compartmentalization of osmolytes at the subcellular level, thereby reducing the water potential and enhancing osmoregulation. Niu et al. (1995) reported reduced water potential is associated with regulation of tissue water content in saline stressed soils. They also function as molecular chaperones by reducing protein misfolding, stabilizing integral protein structures and membrane. Osmolytes form strong hydrogen bonding with these protein structures, which prevents their denaturation and simultaneously enhances their stability and integrity (Kumar, 2009; Slama et al., 2015). Moreover they also scavenge hydroxyl radicals (Ozgur et al., 2013). Oxidative damage is the major detrimental effect of abiotic stress characterized by elevated levels of ROS affecting the organelles due to cellular toxicity. Osmolytes scavenge hydroxyl radicals, singlet oxygen species (Roychoudhury and Chakraborty, 2013) thus lowering the lipid peroxidation level, which serves as plant ROS status indicator. The importance of osmolytes has been thoroughly exploited by researchers towards development of transgenic cultivars able to express the candidate biosynthetic genes leading to enhanced osmolyte accumulation and abiotic stress tolerance (Singh et al., 2015; Wani et al., 2017).

Osmolyte accumulation is triggered by the onset of abiotic stresses (salt, drought and cold). The stress stimuli is first sensed by the primary sensors (histidine kinase) and is passed onto specific transcriptional factors (MYC/MYB, AREB/ABF, NAC/ZF-HD, DREB, etc.) that regulate the expression of specific genes involved in the biosynthesis of osmolytes (Zhou et al., 2016). The entire stress perception and signaling is mediated by the MAPK pathway (Fig. 30.1). Stress

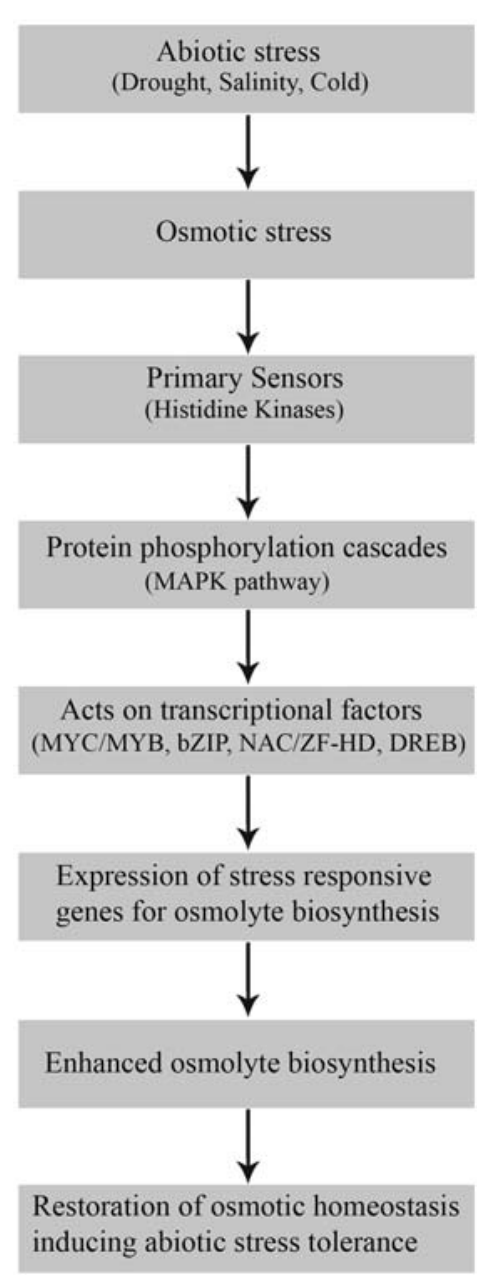

FIGURE 30.1 Abiotic stress signal perception, transduction, and response.

stimuli perception is carried out via a protein phosphorylation cascade. The signals of the plasma membrane receptors activate MAP kinase kinase kinases (MAPKKK), which subsequently activates MAP kinase kinases (MAPKK) and MAPK via reversible phosphorylation (Rodriguez et al., 2010). The MAPK regulates the transcription factors (TFs) that in turn regulate the osmolyte biosynthetic genes.

\subsection{BIOSYNTHESIS, ACCUMULATION, AND REGULATION OF OSMOLYTES IN RESPONSE TO ABIOTIC STRESS}

\subsubsection{Proline}

Proline is structurally an $\alpha$-amino acid derivative, and plays a key role in regulating plant growth and development under stressed environment. The high solubility of proline in water $(1.54 \mathrm{~kg} / \mathrm{L})$ makes it an automatic choice for an ideal solute (Huang et al., 2008). 
Proline synthesis is common in innumerable plant species both under stressed and nonstressed environments. Under nonstressed circumstances proline is involved in regulation of seed formations and embryo development (Mattioli et al., 2009) and it functions as precursor for enzyme and protein synthesis (Nanjo et al., 1999; VivesPeris et al., 2017). Moreover proline enhances the stability of protein secondary structures due to its superior conformational rigidity (Lehmann et al., 2010; Funck et al., 2012) and also serves as a reservoir for storing cellular carbon and nitrogen during the plant recovery phase (Kavi Kishor et al., 2005).

A high level of proline accumulation is frequently observed when plants are exposed to salt and drought stressed environments (Per et al., 2017; Mansour and Ali, 2017). Under such conditions, it serves as an osmoprotectant by restoring the adverse effects of the secondary stress factors (oxidative and osmotic). As plants are exposed to salinity over a prolonged period, high levels of proline get accumulated in the cytoplasm, which regulates the cellular homeostasis and redox potential (Heuer, 2003) and protein secondary structural stability (Suprasanna et al., 2014), and scavenges free radicals thereby suppressing oxidative stress (Kavi Kishor et al., 2014; Matysik et al., 2002).

Endogenous proline production in all plant species at an optimum level is essential for its use as an osmoprotectant or compatible solute. However, studies reveal that important cereals including wheat, rice, and maize are not able to synthesize adequate levels of proline necessary to negate the deleterious effects of salinity and drought and promote plant revival (Slama et al., 2015). Therefore, to enhance the endogenous levels of proline, the plants were treated with exogenous supply of proline (Ashraf and Foolad, 2007). Exogenous application of proline in alfalfa (Ehsanpour and Fatahian, 2003), ice plant (Shevyakova et al., 2009), A. thaliana (Hare et al., 2003), rice (Bhusan et al., 2016), maize (Alam et al., 2017), Brassica juncea (Arif et al., 2017), and soybean (Sabagh et al., 2017) improved the endogenous proline accumulation. However in some plants, such as spinach and rapeseed, exogenous application was associated with adverse side effects due to noncompatibility of the accumulated proline with intracellular environments (Sulpice et al., 1998). Such drawbacks persuaded scientists to elucidate the machinery of biosynthetic pathways of these solutes and identify enzymes that play a key role in their synthesis. These results have been incorporated to develop transgenic plants so as to upregulate the endogenous level of osmolytes thereby inducing abiotic stress tolerance.

In plants, cytosol is the site of proline synthesis and can be accomplished by any of the two pathways: glutamate or ornithine pathway (Kavi Kishor et al., 2005;

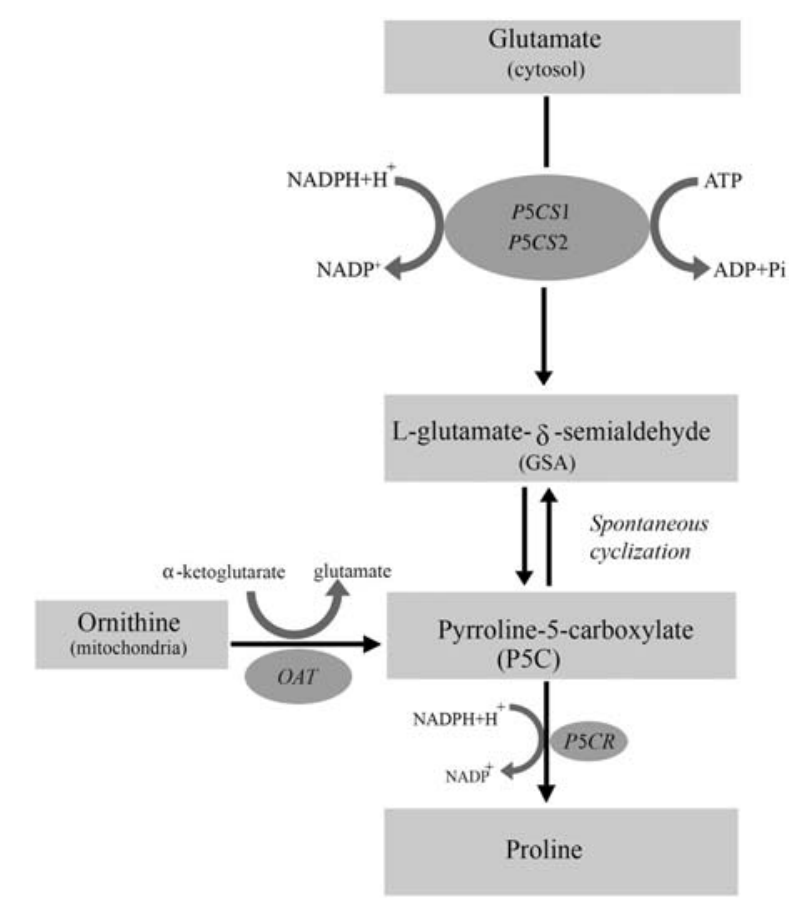

FIGURE 30.2 Proline biosynthesis pathway. P5CS1/P5CS2, pyrroline 5-carboxylate synthetase; $P 5 C R$, pyrroline 5-carboxylate reductase; $O A T$, ornithine D-aminotransferase.

Suprasanna et al., 2014; Zarattini and Forlani, 2017) (Fig. 30.2). The glutamate pathway is completed in two enzyme dependent steps and requires two NADPH molecules. The first step involves ATP dependent phosphorylation of glutamate thereby activating it. This is followed by reduction of the activated glutamate to glutamatic- - -glutamyl kinase (GSA) and its subsequent cyclization to pyrroline 5-carboxylate (P5C). The cyclization of GSA to P5C is catalyzed by pyrroline 5-carboxylate synthetase (P5CS) enzyme. The final enzyme catalyzed step of the glutamate pathway involves reduction of the P5C to proline by pyrroline 5-carboxylate reductase (P5CR) enzyme. The alternative ornithine pathway uses ornithine as the precursor for proline biosynthesis instead of glutamate. The steps involve transamination of ornithine to P5C catalyzed by Orn-D-aminotransferase and reduction of P5C to proline by the enzyme P5CR.

The mechanism regulating proline biosynthesis has been studied in the model plant $A$. thaliana (Yoshiba et al., 1995), tobacco (Delauney and Verma, 1993), and rice (Lutts et al., 1999; Joseph et al., 2015). The findings identified the two enzymes P5CS and P5CR as potential candidate genes capable of regulating proline production under abiotic stressed environments. Two genes, P5CS1 and P5CS2, code for the enzyme P5CS whereas a single gene codes for P5CR enzyme. These two genes have been incorporated in many economically important crops to develop their transgenic counterparts 
capable of increased proline accumulation under stress thus negating the adverse effects of osmotic stress induced by salt and drought stress.

Kishor et al. (1995) developed transgenic cultivars overexpressing the P5CS gene from Vigna aconitifolia (moth bean). The transformed cultivars showed increased proline accumulation compared with the nontransformed (wild) plants by $10-$ to 18 -fold. The enhanced levels of proline contributed to flower development and increased root and plant dry biomass. There was one major concern: the negative feedback inhibition of the P5CS enzyme due to increased proline accumulation. This glitch was taken care of when Hong et al. (2000) developed the P5CS129A gene, which was a mutated version of the $V$. acontifolia P5CS enzyme. They substituted the phenylalanine (Phe) residue at the 129th position with an alanine (Ala) residue. The mutated version of the enzyme did not exhibit negative feedback inhibition and increased proline accumulation by 36 -fold in the transformed tobacco. Kumar et al. (2010) achieved similar results when they developed transgenic rice overexpressing the mutated P5CS129A gene. Moreover, the transgenic lines showed lower lipid peroxidation level when exposed to $150 \mathrm{mM} \mathrm{NaCl}$ stress.

The same $V$. acontifolia P5CSF129A gene increased fourfold more proline accumulation in first generation of transgenic Cajanus cajan lines under $200 \mathrm{mM} \mathrm{NaCl}$ stress thereby inducing salt tolerance (Surekha et al., 2014). The transgenic lines showed increased chlorophyll content and lower lipid peroxidation levels, which resulted in better growth and development. Similarly, Gleeson et al. (2005) overexpressed the same gene in Larix leptoeuropaea (forest tree), proline content increased significantly by 30 -fold in the transgenic plants in comparison with its wild type counterparts. The transformed cultivars exhibited remarkable growth and recovery when subjected to salt $(200 \mathrm{mM}$ $\mathrm{NaCl})$ and low temperature $\left(4^{\circ} \mathrm{C}\right)$ stress.

The rice OsP5CS1 and OsP5CS2 genes were expressed together in tobacco by Zhang et al. (2014). The transgenic cultivars of the second generation $\left(T_{2}\right)$ recorded 3.2-fold increased proline accumulation along with improved root length and mean fresh weight in comparison with its wild type counterparts treated with $200 \mathrm{mM} \mathrm{NaCl}$. Similarly Chen et al. (2013) coexpressed the Phaseolus vulgarisP5CS (PvP5CS1 and PvP5CS2) genes in the model plant, A. thaliana. The transgenic lines were subjected to different salt concentrations $(0,100$, and $200 \mathrm{mM} \mathrm{NaCl})$ and they accumulated 1.6 times and 1.9 times more proline at 100 and $200 \mathrm{mM} \mathrm{NaCl}$ concentration respectively.

The other enzymes involved in proline biosynthesis and degradation (P5CS, P5CR, ornithine aminotransferase (OAT) and $\mathrm{P} 5 \mathrm{CDH}$ ) have also been exploited to develop transgenic rice (Su and $\mathrm{Wu}, 2004$; Karthikeyan et al., 2011), sugarcane (Guerzoni et al., 2014), wheat (Vendruscolo et al., 2007), olive (Behelgardy et al., 2012) carrot (Han and Hwang, 2003), Jerusalem artichoke (Huang et al., 2013), Kosteletzkya virginica (Wang et al., 2015), and sweet potato (Liu et al., 2014) with enhanced abiotic stress tolerance (Table 30.1). All these experiments aimed at improving proline accumulation in transgenic plants highlight the function of these enzymes in regulating the osmolyte levels depending on the environmental conditions. Moreover proline also restores cellular homeostasis by reducing oxidative damage scavenging free radicals.

\subsubsection{Glycine Betaine}

GB represents quaternary amines and is a derivative of $N$-trimethyl glycine. The properties of GB that make it a suitable osmolyte includes its low molar mass, water solubility, amphoteric nature, and nontoxicity even in high concentrations. Its amphoteric nature allows its interaction with hydrophilic and hydrophobic side chains of macromolecules (Gupta and Huang, 2014). In plants, GB serves as an effective compatible solute by regulating cellular osmotic homeostasis, shielding photosystem II and thylakoid membranes, maintaining protein stability and reducing oxidative stress (Khan et al., 2009; Allakhverdiev et al., 2003; Kurepin et al., 2017). But very few plants, namely sugar beet, maize, spinach, and barley are natural GB accumulators (Kishitani et al., 1994; Chen and Murata, 2008). However, the amount of GB accumulated is negligible and is triggered when plants are exposed to abiotic stress (Wani et al., 2013). The major agricultural plants such as potato, rice, eggplant, and tomato are naturally nonaccumulators of GB (De Zwart et al., 2003; Park et al., 2004). The cause can be attributed to loss of functional domains, premature stop codons, and shortened transcripts of the genes involved in the GB biosynthetic pathway (Rasheed et al., 2017). To overcome the shortcomings, researchers aimed at developing transgenic lines of these nonaccumulators harboring candidate GB biosynthetic genes from other species to increase the endogenous GB levels (Khan et al., 2009; Chen and Murata, 2011).

In plants GB biosynthetic pathway takes place in the stroma of the chloroplast and involves the precursor choline molecule (Sakamoto and Murata, 2002). The two steps involve oxidation of choline to betaine aldehyde catalyzed by choline monooxygenase (CMO) followed by its oxidation to GB. The final step is catalyzed by betaine aldehyde dehydrogenase (BADH) and involves the cofactor NAD $^{+}$(Wani et al., 2013; 
TABLE 30.1 List of Transgenic Plants Overexpressing Candidate Genes for Proline Accumulation

\begin{tabular}{|c|c|c|c|c|c|}
\hline $\begin{array}{l}\text { S. } \\
\text { no. }\end{array}$ & Transgene & Host & $\begin{array}{l}\text { Target crop } \\
\text { plants/trees }\end{array}$ & Remarks & References \\
\hline 1 & P5CS & $\begin{array}{l}\text { Vigna } \\
\text { aconitifolia }\end{array}$ & $\begin{array}{l}\text { Nicotiana } \\
\text { tabacum }\end{array}$ & $\begin{array}{l}\text { Enhanced } 10-18 \text { fold proline accumulation and induced salt } \\
\text { tolerance }\end{array}$ & $\begin{array}{l}\text { Kavi Kishor et al. } \\
\text { (1995) }\end{array}$ \\
\hline 2 & P5CSF129A & V. aconitifolia & O. sativa & Enhanced proline accumulation and salt tolerance & $\begin{array}{l}\text { Kumar et al. } \\
(2010)\end{array}$ \\
\hline 3 & P5CS & V. aconitifolia & $\begin{array}{l}\text { Larix } \\
\text { leptoeuropaea }\end{array}$ & $\begin{array}{l}\text { Alleviates oxidative stress, high chlorophyll content and 30-fold } \\
\text { enhanced proline accumulation }\end{array}$ & $\begin{array}{l}\text { Gleeson et al. } \\
\text { (2005) }\end{array}$ \\
\hline 4 & P5CS & V. aconitifolia & $\begin{array}{l}\text { Saccharum } \\
\text { officinarum }\end{array}$ & $\begin{array}{l}\text { Enhanced proline content, biomass production, low lipid } \\
\text { peroxidation level, and oxidative stress protection; }\end{array}$ & $\begin{array}{l}\text { Guerzoni et al. } \\
\text { (2014) }\end{array}$ \\
\hline 5 & P5CSF129A & $\begin{array}{l}\text { Vigna } \\
\text { acontifolia }\end{array}$ & Cajanus cajan & $\begin{array}{l}\text { enhanced proline accumulation, seed germination rate, chlorophyll } \\
\text { content, low lipid peroxidation level }\end{array}$ & $\begin{array}{l}\text { Surekha et al., } \\
2014\end{array}$ \\
\hline 6 & P5CS & $\begin{array}{l}\text { Vigna } \\
\text { aconitifolia }\end{array}$ & Daucus carota & $\begin{array}{l}\text { Enhanced proline accumulation, biomass production, and low lipid } \\
\text { peroxidation level }\end{array}$ & $\begin{array}{l}\text { Han and Hwang } \\
\text { (2003) }\end{array}$ \\
\hline 7 & P5CS & $\begin{array}{l}\text { Vigna } \\
\text { aconitifolia }\end{array}$ & T. aestivum & $\begin{array}{l}\text { Enhanced proline accumulation, biomass production, and low lipid } \\
\text { peroxidation level }\end{array}$ & $\begin{array}{l}\text { Sawahel and } \\
\text { Hassan (2002) }\end{array}$ \\
\hline 9 & $\begin{array}{l}\text { PvP5CS1, } \\
\text { PvP5CS2 }\end{array}$ & P. vulgaris & A. thaliana & Increased 1.9 times proline content, flower and seed development & Chen et al. (2013) \\
\hline 10 & $P 5 C R$ & A. thaliana & Glycine max L. & $\begin{array}{l}\text { Increased proline accumulation, RWC and WUE content, high } \\
\text { spikelet fertility }\end{array}$ & $\begin{array}{l}\text { De Ronde et al. } \\
\text { (2000) }\end{array}$ \\
\hline 11 & P5CS & A. thaliana & Olea europaea & Increased proline accumulation, ionic homeostasis & $\begin{array}{l}\text { Behelgardy et al. } \\
\text { (2012) }\end{array}$ \\
\hline 12 & P5CS & A. thaliana & S. tuberosum & $\begin{array}{l}\text { Enhanced proline accumulation, tuber yield, and biomass } \\
\text { production }\end{array}$ & $\begin{array}{l}\text { Hmida-Sayari } \\
\text { et al. (2005) }\end{array}$ \\
\hline 13 & $\begin{array}{l}\text { OsP5CS1 and } \\
\text { OsP5CS2 }\end{array}$ & O. sativa & N. tabacum & $\begin{array}{l}\text { Enhanced } 3.2 \text { times proline content, biomass production, oxidative } \\
\text { stress protection }\end{array}$ & $\begin{array}{l}\text { Zhang et al. } \\
\text { (2014) }\end{array}$ \\
\hline 14 & $O A T$ & O sativa & O sativa & $\begin{array}{l}\text { Proline accumulation, water retention, spikelet fertility, and } \\
\text { increased biomass }\end{array}$ & You et al. (2012) \\
\hline 15 & HtP5CS & $\begin{array}{l}\text { Helianthus } \\
\text { tuberosus L. }\end{array}$ & $\begin{array}{l}\text { Helianthus } \\
\text { tuberosus L. }\end{array}$ & Enhanced proline accumulation and salt tolerance & $\begin{array}{l}\text { Huang et al. } \\
\text { (2013) }\end{array}$ \\
\hline 16 & $I b P 5 C R$ & $\begin{array}{l}\text { Ipomoea } \\
\text { batatas (L.) }\end{array}$ & $\begin{array}{l}\text { Ipomoea batatas } \\
\text { (L.) }\end{array}$ & Enhanced proline accumulation and salt tolerance. & Liu et al. (2014) \\
\hline 17 & P5CS & $\begin{array}{l}\text { Vigna } \\
\text { aconitifolia }\end{array}$ & $\begin{array}{l}\text { Medicago } \\
\text { truncatula }\end{array}$ & Enhanced proline accumulation and salt tolerance & $\begin{array}{l}\text { Verdoy et al. } \\
\text { (2006) }\end{array}$ \\
\hline 18 & KvP5CS1 & $\begin{array}{l}\text { Kosteletzkya } \\
\text { virginica }\end{array}$ & $\begin{array}{l}\text { Kosteletzkya } \\
\text { virginica }\end{array}$ & Enhanced 6.83 times proline content and salt tolerance & $\begin{array}{l}\text { Wang et al. } \\
\text { (2015) }\end{array}$ \\
\hline 19 & $\begin{array}{l}\operatorname{LrP5CS1} \\
\operatorname{LrP5CS} 2\end{array}$ & Lilium regale & A. thaliana & $\begin{array}{l}\text { Enhanced proline accumulation and tolerance to salt, drought, and } \\
\text { osmotic stress }\end{array}$ & Wei et al. (2016) \\
\hline
\end{tabular}

Chen and Murata, 2002) (Fig. 30.3). Scientists exploited the genes involved in the GB biosynthetic pathway (bacterial $\operatorname{cod} A, \mathrm{CMO}$, and $\mathrm{BADH}$ ) to develop transgenic varieties of agriculturally important crops such as rice, potato, soybean, groundnut, and maize, among other plants (Sawahel,2003; Ranganayakulu et al., 2013). Significant increase in yield and growth parameters was observed in barley, soybean, wheat, maize, tobacco, beans, and sunflower exposed to abiotic stresses (Ashraf and Foolad, 2007). The $\operatorname{codA}$ gene of Arthobacter spp. has been introduced in many plant species such as A. thaliana, Solanum tuberosum, Zea mays, S. lycopersicum, and Lycopersicon esculentum (Giri,2011; Quan et al., 2004; Wei et al., 2017; Yu et al., 2017). The transgenic lines showed increased GB accumulation, which enhanced photosynthetic activity, plant development, and crop yield under abiotic stress conditions.

Sakamato and Murata (1998) overexpressed the $A$. globiformis codA gene in rice (non-GB accumulator) and observed that the transgenic varieties accumulated $5.3 \mathrm{mmol} / \mathrm{g}$ fresh weight of GB. Park et al. (2004) 


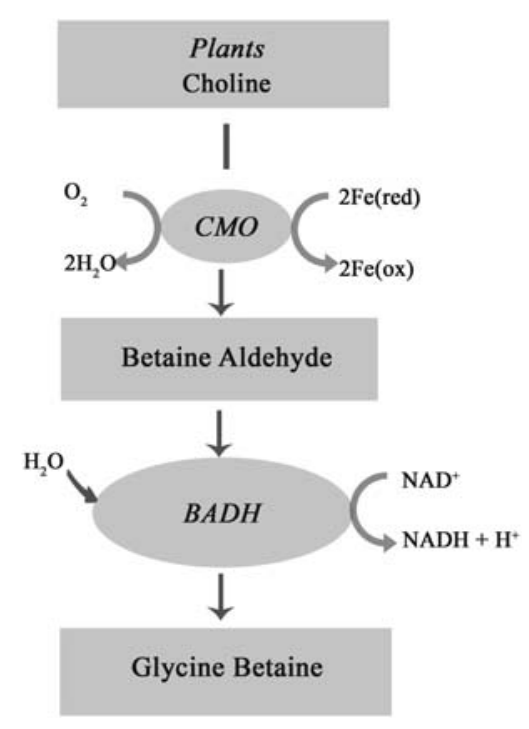

FIGURE 30.3 Glycine betaine biosynthesis pathway. CMO, choline monooxygenase; $C D H$, choline dehydrogenase; $C O D$, choline oxidase; $B A D H$, betaine aldehyde dehydrogenase.

developed transgenic tomato harboring the same $\operatorname{cod} A$ gene and exposed it to salt $(200 \mathrm{mM} \mathrm{NaCl})$ and low temperature $\left(4^{\circ} \mathrm{C}\right)$ stress. Under salt and low temperature stress, the transgenic tomato plants accumulated 3 times and 5 times more GB respectively than the nontransformed plants and exhibited 10\%-30\% increase in fruit formation. Enhanced GB accumulation in the transgenic varieties contributed towards improving the net photosynthetic rate, activity of antioxidant enzyme, and lowering the lipid peroxidation levels in the transgenic lines.

Similarly, Ahmad et al. (2014) overexpressed the A. globiformis $\operatorname{codA}$ gene in potato and exposed the plants to salt $(100 \mathrm{mM} \mathrm{NaCl})$ and low temperature $\left(4^{\circ} \mathrm{C}\right)$ stress. The transformed varieties were characterized by normal growth, improved plant biomass, and high tuber yield both under salt and low temperature stress. The transgenic potato tuber yield was reported to be $44 \mathrm{~g}$ /plant while it was only $33.6 \mathrm{~g} /$ plant in the wild type (WT) plants. Wei et al. (2017) also transformed tomato with the same $A$. globiformis codA gene. The transgenic varieties exhibited increased GB accumulation in comparison with the nonaccumulator wild types. Moreover there was significant improvement in photosynthetic rates due to restoration of chlorophyll content, and activity of antioxidant enzymes in lowering ROS content.

The $B A D H$ gene coding for BADH enzyme that regulates the oxidation of betaine aldehyde to GB has also been exploited to enhance GB accumulation in sweet potato. Fan et al. (2012) developed transgenic sweet potato harboring the $S o B A D H$ gene isolated from Spinacia oleracea. The amount of GB accumulation in the transgenic plants doubled conferring tolerance to salt, cold, and oxidative stress. Enhanced GB accumulation resulted in improved integrity of cellular membranes, net photosynthetic activity, and ROS scavenging. Transgenic tomato developed by $\mathrm{Li}$ et al. (2014) carrying the SoBADH gene exhibited high photosynthetic capacity even at $42^{\circ} \mathrm{C}$. Enhanced GB accumulation increased the $D_{1}$ protein content thereby preventing photosystem II from denaturation. Moreover, there was significant decrease in $\mathrm{H}_{2} \mathrm{O}_{2}$ content and superoxide radical in the transgenic tomato plants. Wang et al. (2010) reported $170.7 \mu \mathrm{mol} / \mathrm{g}$ dry weight GB accumulation in chloroplast of transgenic wheat lines harboring the BADH gene isolated from cyanobacteria under salt and drought stress. These experiments highlight the regulatory role of GB in improving plant growth and development under abiotic stress conditions. Similar transgenic lines have been developed with improved GB accumulation potential and have been listed in Table 30.2.

\subsubsection{Polyamines}

PAs belong to the class of aliphatic amines and are present in bacteria, animals, and plants (Hussain et al., 2011; Wimalasekera et al., 2011; Zhang et al., 2011a,b). The PAs found in plants include putrescine (Put), spermidine (Spd), and spermine (Spm) (Tiburcio et al., 2014; Sharma et al., 2017). PAs have been found to significantly regulate plant stress and development physiology during the stages of elongation stage, floral development, fruit ripening, and cellular apoptosis (Alcázar et al., 2010; Feng et al., 2011; Alet et al., 2012).

In plants, arginine (Arg) serves as the precursor for diamine putrescine (Put) synthesis and involves the sequential action of three enzymes: arginine decarboxylase (ADC), agmatine iminohydrolase (AIH), and $N$-carbamoyl Put amidohydrolase. Putrescine biosynthesis can be alternatively carried out in a single step by converting mitochondrial ornithine to Put catalyzed by the enzyme ornithine decarboxylase (ODC). The second PA, spermidine, is synthesized from Put by the action of spermidine synthase (SPDS) while spermine (tetramine) is formed from spermidine. This reaction is catalyzed by spermine synthase (SPMS) and decarboxylated S-adenosylmethionine (dcSAM) provides the aminopropyl group essential for the synthesis of spermidine and spermine (Fig. 30.4). The aminopropyl donor group is obtained from methionine via two enzyme catalyzed reactions by $S$-adenosylmethionine (SAM) synthase and SAM decarboxylase (SAMDC) (Takahashi et al., 2010; Moschou et al., 2008).

Transcriptome analysis of PAs revealed their accumulation in plants subjected to salt, drought, low and high temperature, and oxidative stresses (Romero et al., 2018; 
TABLE 30.2 List of Transgenic Plants Overexpressing Candidate Genes for Glycine Betaine Accumulation

\begin{tabular}{|c|c|c|c|c|c|}
\hline $\begin{array}{l}\text { S. } \\
\text { no. }\end{array}$ & Transgene & Host & $\begin{array}{l}\text { Target crop } \\
\text { plants/trees }\end{array}$ & Remarks & References \\
\hline 1 & $\operatorname{cod} A$ & $\begin{array}{l}\text { Arthrobacter } \\
\text { globiformis }\end{array}$ & Oryza sativa L. & Enhanced 5.3-fold GB accumulation and induced salt tolerance & $\begin{array}{l}\text { Sakamoto and } \\
\text { Murata (1998) }\end{array}$ \\
\hline 2 & $\operatorname{cod} A$ & $\begin{array}{l}\text { Arthrobacter } \\
\text { globiformis }\end{array}$ & $\begin{array}{l}\text { Solanum } \\
\text { lycopersicum }\end{array}$ & Enhanced fivefold GB accumulation and induced salt tolerance & Park et al. (2004) \\
\hline 3 & $\operatorname{cod} A$ & $\begin{array}{l}\text { Arthrobacter } \\
\text { globiformis }\end{array}$ & $\begin{array}{l}\text { Solanum } \\
\text { tuberosum }\end{array}$ & Enhanced GB content and tolerance to salt and chilling stress & Ahmad et al. (2014) \\
\hline 4 & SoBADH & Spinacia oleracea & $\begin{array}{l}\text { Ipomoea batatas } \\
\text { (L.) }\end{array}$ & $\begin{array}{l}\text { Enhanced GB accumulation and tolerance to salinity, low } \\
\text { temperature and oxidative stress }\end{array}$ & Fan et al. (2012) \\
\hline 5 & $\operatorname{cod} A$ & $\begin{array}{l}\text { Arthrobacter } \\
\text { globiformis }\end{array}$ & $\begin{array}{l}\text { Solanum } \\
\text { lycopersicum }\end{array}$ & $\begin{array}{l}\text { Induced GB accumulation, antioxidant enzyme activity, and } \\
\text { salt tolerance }\end{array}$ & Wei et al. (2017) \\
\hline 6 & SoBADH & Spinacia oleracea & $\begin{array}{l}\text { Solanum } \\
\text { lycopersicum }\end{array}$ & Enhanced GB accumulation and heat stress & Li et al. (2014) \\
\hline 7 & $B A D H$ & Cyanobacteria & Triticum aestivum & Induced $170.7 \mu \mathrm{M} / \mathrm{g}$ GB accumulation and salt tolerance & Wang et al. (2010) \\
\hline 9 & $\mathrm{BvCMO}$ & Beta vulgaris & $\begin{array}{l}\text { Nicotiana } \\
\text { tobacum }\end{array}$ & Enhanced fivefold GB accumulation and salt tolerance & Zhang et al. (2008) \\
\hline 10 & $\begin{array}{l}\text { GSMT and } \\
\text { DMT } a\end{array}$ & $\begin{array}{l}\text { Aphanothece } \\
\text { halophytica }\end{array}$ & O. sativa & Enhanced GB biosynthesis, salt and cold stress tolerance & Niu et al. (2014) \\
\hline 11 & $B A D H$ & E. coli & M. sativa & Enhanced GB accumulation and salt tolerance & Yan et al. (2012) \\
\hline 12 & OsBADH1 & O. sativa & N. tabacum & Salinity stress tolerance & $\begin{array}{l}\text { Hasthanasombut } \\
\text { et al. (2010) }\end{array}$ \\
\hline 13 & SIBADH & $\begin{array}{l}\text { Suaeda } \\
\text { liaotungensis }\end{array}$ & $\begin{array}{l}\text { Solanum } \\
\text { lycopersicum }\end{array}$ & Enhanced GB accumulation under salt stress & Wang et al. (2013) \\
\hline 14 & $\operatorname{cod} A$ & $\begin{array}{l}\text { Arthrobacter } \\
\text { globiformis }\end{array}$ & $\begin{array}{l}\text { Lycopersicon } \\
\text { esculentum }\end{array}$ & $\begin{array}{l}\text { Enhanced seed germination rate, GB accumulation, and } \\
\text { tolerance to salt and drought. }\end{array}$ & Goel et al. (2011) \\
\hline 15 & $\operatorname{cod} A$ & $\begin{array}{l}\text { Arthrobacter } \\
\text { globiformis }\end{array}$ & N. tabacum & $\begin{array}{l}\text { Enhanced antioxidant enzyme activity by } 50 \% \text { and salt } \\
\text { tolerance. }\end{array}$ & Jing et al. (2013) \\
\hline 16 & bet $A$ & Escherichia coli & Zea mays & Enhanced GB, seed germination rate, and tolerance to drought & Quan et al. (2004) \\
\hline 17 & $\operatorname{cod} A$ & $\begin{array}{l}\text { Arthrobacter } \\
\text { globiformis }\end{array}$ & $\begin{array}{l}\text { Transgenic } \\
\text { populus spp. }\end{array}$ & $\begin{array}{l}\text { Enhanced GB accumulation, photosystem II integrity, and } \\
\text { multiple stress tolerance }\end{array}$ & Ke et al. (2016) \\
\hline
\end{tabular}

Ma et al., 2017; Tavladoraki et al., 2012). To gain more insights in their role in inducing stress tolerance, exogenous application of these PAs was carried out for plants growing in stressed environments.

Exogenous application of PAs under abiotic stress has been extensively studied in plants species such as tomato, rice, wheat, barley, cucumber, soybean, Welsh onion, etc. (Yiu et al., 2009; Li et al., 2013; Sagor et al., 2013; Sequera-Mutiozabal et al., 2017). Nahar et al. (2016) exogenously applied spermine $(0.2 \mathrm{mM})$ on mung bean species growing under high temperature $\left(40^{\circ} \mathrm{C}\right)$, salt $(200 \mathrm{~mm} \mathrm{NaCl})$, and drought stress. The plants exhibited lowered ROS status depicted by less $\mathrm{H}_{2} \mathrm{O}_{2}$ content, lipoxygenase activity, and malondialdehyde activity (marker for membrane peroxidation) in comparison with the controls (nontreated). Moreover, activity of important antioxidant enzymes such as glutathione (GSH), superoxide dismutase (SOD), catalase, and glutathione peroxidase was significantly enhanced. Sánchez-Rodríguez et al. (2016) also reported similar findings when they treated the drought-tolerant cultivar (Zarina) and drought-sensitive cultivar (Josefina) and subjected them to water stress. However, in both studies, it was observed that the effect of the exogenously supplied Pas reduced drastically as the duration of stress was increased. This drawback prompted scientists to enhance the endogenous PAs levels by developing transgenic varieties harboring genes encoding the PA biosynthesis enzymes (ADC, ODC, SPDS, and SAM) (Gill and Tuteja, 2010; Gupta et al.,2013; Shukla and Mattoo, 2013).

Kasukabe et al. (2004) overexpressed the Cucurbita ficifolia spermidine synthase gene (CSSPDS) in the model plant $A$. thaliana under the regulation of a 


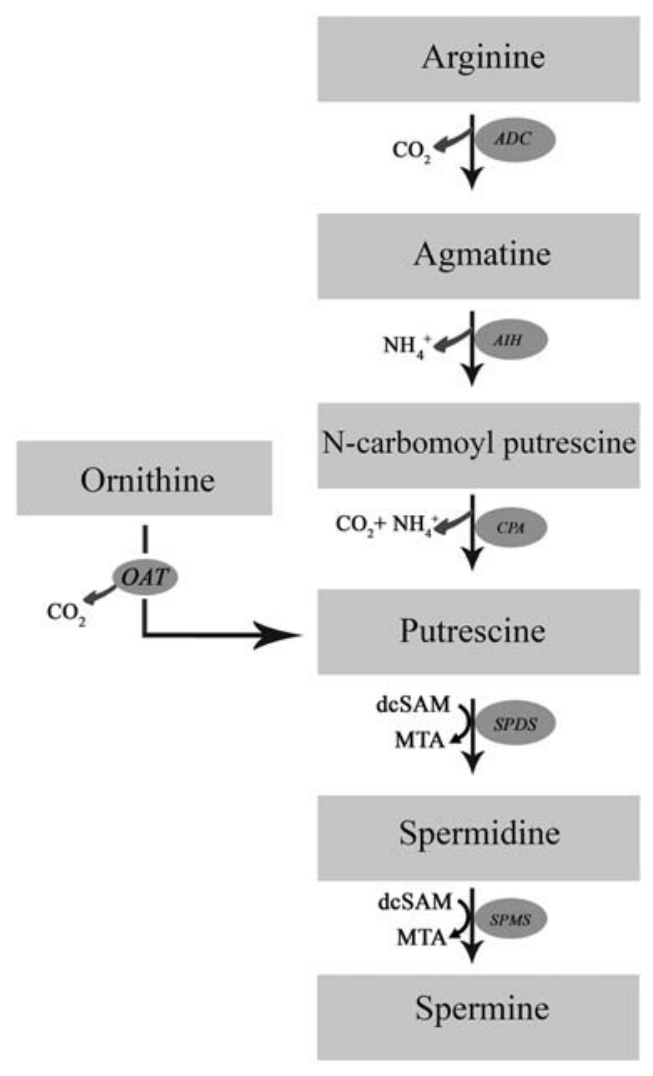

FIGURE 30.4 Polyamines biosynthesis pathway. $A D C$, arginine decarboxylase; $A I H$, agmatine iminohydrolase; $C P A, N$-carbamoylPut amidohydrolase; SPDS, spermidine synthase; SPMS, spermine synthase; dcSAM, decarboxylated S-adenosylmethionine; MTA, 5methylthioasdenosine. constitutive cauliflower mosaic virus 35S (CaMV 35S) promoter. The transformed $A$.thaliana lines exhibited twofold more accumulation of spermidine under salt $(200 \mathrm{mM} \mathrm{NaCl})$, low temperature $\left(4^{\circ} \mathrm{C}\right)$, and drought stress when compared with its wild types. Capell et al. (2004) engineered rice with the Datura stramonium ADC gene $(D s A D C)$ and expressed it under the inducible monocot Ubi-1 promoter. The transformed rice varieties showed threefold increase in endogenous putrescine levels and also facilitated spermidine and spermine synthesis under drought stress. In another study, Franceschetti et al. (2004) developed transgenic tobacco expressing the D. stramonium SPDS gene (DsSPDS) and reported high spermidine accumulation, restoration of tissue water, and chlorophyll content facilitating plant growth and development under $200 \mathrm{mM} \mathrm{NaCl}$ stress.

He et al. (2008) studied the enzymatic and nonenzymatic antioxidant capacity of Pyrus communis harboring the SPDS gene from apple exposed to $200 \mathrm{mM} \mathrm{NaCl}$ stress. The transformed pear varieties showed enhanced spermidine accumulation and antioxidant enzyme activity, and low levels of malondialdehyde and hydrogen peroxide levels (markers of ROS status). The transgenic lines exhibited high ROS scavenging that helped in shielding membrane and protein structures from denaturation. Similar studies led to the development of many transgenic plants (Table 30.3) expressing the different PA biosynthetic genes and were associated with enhanced endogenous PA accumulation inducing abiotic stress tolerance.

TABLE 30.3 List of Transgenic Plants Overexpressing Candidate Genes for Polyamines Accumulation

\begin{tabular}{|c|c|c|c|c|c|}
\hline S. no. & Transgene & Host & $\begin{array}{l}\text { Target crop plants/ } \\
\text { trees }\end{array}$ & Remarks & References \\
\hline 1 & CsSPDS & Cucurbita ficifolia & A. thaliana & $\begin{array}{l}\text { Enhanced twofold accumulation of spermidine, inducing abiotic stress } \\
\text { tolerance }\end{array}$ & Kasukabe et al. (2004) \\
\hline 2 & $D s A D C$ & Daturastramonium & O. sativa $\mathrm{L}$. & Enhanced threefold putrescine accumulation inducing drought tolerance & Capell et al. (2004) \\
\hline 3 & DsSPDS & D. stramonium & N. tobacum & $\begin{array}{l}\text { Enhanced spermidine accumulation, chlorophyll content, and plant } \\
\text { growth }\end{array}$ & $\begin{array}{l}\text { Franceschetti et al. } \\
\text { (2004) }\end{array}$ \\
\hline 4 & SPDS & Maluspumila & Pyruscommunis & $\begin{array}{l}\text { Enhanced spermidine content and ROS scavenging, inducing salinity } \\
\text { tolerance }\end{array}$ & He et al. (2008) \\
\hline 5 & $S A M D C$ & $\begin{array}{l}\text { Saccharomyces } \\
\text { cerevisiae }\end{array}$ & $\begin{array}{l}\text { Lycopersicon } \\
\text { esculentum }\end{array}$ & $\begin{array}{l}\text { 1.7- to 2.4-fold higher levels of spermidine and spermine, heat stress } \\
\text { tolerance, } \mathrm{CO}_{2} \text { assimilation }\end{array}$ & Cheng et al. (2009) \\
\hline 6 & AsADC & Avena sativa $\mathrm{L}$. & O. sativa $L$. & Enhanced polyamine accumulation inducing abiotic stress tolerance & Roy and Wu (2001) \\
\hline 7 & $S A M D C$ & Tritordeum & O. sativa $L$. & $\begin{array}{l}\text { three and fourfold increase of spermidine and spermine, enhanced seed } \\
\text { germination, salt tolerance }\end{array}$ & Roy and Wu (2002) \\
\hline 8 & MdSPDS1 & Malusdomestica & Pyrus communis L. & $\begin{array}{l}\text { Enhanced spermidine accumulation leading to salt and osmotic stress } \\
\text { tolerance }\end{array}$ & Wen et al. (2008) \\
\hline 9 & LcSAMDC1 & Leymuschinensis & A. thaliana & $\begin{array}{l}\text { Enhanced spermine, proline, and chlorophyll content under salt and cold } \\
\text { stress }\end{array}$ & Liu et al. (2017) \\
\hline 10 & $S A M D C$ & Homo sapiens & Solanumlycopersicum & $\begin{array}{l}\text { Enhanced polyamine accumulation, delayed ripening, and improved } \\
\text { postharvest storage }\end{array}$ & $\begin{array}{l}\text { Madhulatha et al. } \\
\text { (2014) }\end{array}$ \\
\hline 12 & $A v A D C$ & Avena sativa $\mathrm{L}$ & Medicago truncatula & Enhanced polyamine content, seed yield, and desiccation stress tolerance & Duque et al. (2016) \\
\hline
\end{tabular}




\subsubsection{Sugars and Sugar Alcohols}

Prolonged exposure to abiotic stress interferes with the cellular metabolism of carbon and alters the cellular levels of certain sugars and polyols (alcohol derivatives of sugars). Sugars (trehalose and fructose) and polyols (mannitol, sorbitol, and ononitol) have been studied in relation to abiotic stress and it has been observed that plants accumulate high levels of these osmolytes (Wani et al., 2016; O'Hara et al., 2013). These low molecular weight molecules regulate cellular osmolarity, ROS scavenging, and stability of protein and membrane structures. Moreover they also serve as molecular chaperones and carbon reservoir (Gupta and Huang, 2014; Parvaiz and Satyawati, 2008). This section highlights the biosynthetic pathways and the regulatory function of the various sugars and their alcohol derivatives in connection to abiotic stress tolerance. Moreover, the successfully engineered transgenic plants harboring potential biosynthetic genes of these osmolytes have also been enlisted in Table 30.4.

TABLE 30.4 List of Transgenic Plants Overexpressing Candidate Genes for Sugar and Sugar Alcohols

\begin{tabular}{|c|c|c|c|c|c|}
\hline S. no. & Transgene & Host & $\begin{array}{l}\text { Target crop } \\
\text { plants/trees }\end{array}$ & Remarks & References \\
\hline 1 & ScTPS1 & $\begin{array}{l}\text { Saccharomyces } \\
\text { cervisiae }\end{array}$ & N. tabacum & $\begin{array}{l}0.17 \mathrm{mg} / \mathrm{g} \mathrm{FW} \text { in leaves trehalose content, shunted growth, } \\
\text { drought tolerance }\end{array}$ & Romero et al. (1997) \\
\hline 2 & ScTPS1 & $\begin{array}{l}\text { Saccharomyces } \\
\text { cervisiae }\end{array}$ & $\begin{array}{l}\text { Solanum } \\
\text { tuberosum }\end{array}$ & Twofold trehalose accumulation, salt and drought tolerance & Yeo et al. (2000) \\
\hline 3 & ScTPS1 & $\begin{array}{l}\text { Saccharomyces } \\
\text { cervisiae }\end{array}$ & $\begin{array}{l}\text { Solanum } \\
\text { lycopersicum }\end{array}$ & 2.5-fold trehalose accumulation, salt, and drought tolerance & $\begin{array}{l}\text { Cortina and } \\
\text { Culiáñez-Macià } \\
\text { (2005) }\end{array}$ \\
\hline 4 & $\begin{array}{l}\text { ots } A \text { and } \\
\text { ots } B\end{array}$ & E. coli & Oryza sativa L. & $\begin{array}{l}3-10 \text { fold trehalose accumulation, salt, drought, and low } \\
\text { temperature tolerance }\end{array}$ & Garg et al. (2002) \\
\hline 5 & PyTPS & Porphyrayezoensis & $\begin{array}{l}\text { Oryza sativa } \mathrm{L} \\
\text { TP309 }\end{array}$ & Enhanced trehalose accumulation, seed germination, and yield & Guo et al. (2014) \\
\hline 6 & GfTPS & Grifolafrondosa & N. tabacum & 12-fold more trehalose content, abiotic stress tolerance & Zhang et al. (2005) \\
\hline 7 & $\begin{array}{l}\text { CvTPS1 }+ \\
\text { CvTPS2 }\end{array}$ & $\begin{array}{l}\text { Saccharomyces } \\
\text { cervisiae }\end{array}$ & $\begin{array}{l}\text { Arabidopsis } \\
\text { thaliana }\end{array}$ & $\begin{array}{l}\text { Enhanced plant growth and abiotic stress tolerance, twofold } \\
\text { trehalose content }\end{array}$ & Miranda et al. (2007) \\
\hline 9 & $\begin{array}{l}\text { CvTPS1 }+ \\
\text { CvTPS2 }\end{array}$ & $\begin{array}{l}\text { Saccharomyces } \\
\text { cervisiae }\end{array}$ & M. sativa & $\begin{array}{l}\text { Enhanced plant growth and abiotic stress tolerance, enhanced } \\
\text { trehalose content }\end{array}$ & Suárez et al. (2009) \\
\hline 10 & $S a c B$ & Bacillus subtilis & N. tabacum & $\begin{array}{l}0.35 \mathrm{mg} / \mathrm{g} \text { fructans accumulation, } 55 \% \text { enhanced plant growth } \\
\text { under drought stress }\end{array}$ & $\begin{array}{l}\text { Pilon-Smits et al. } \\
\text { (1995) }\end{array}$ \\
\hline 11 & $S a c B$ & Bacillus subtilis & Beta vulgaris L & $\begin{array}{l}\text { Accumulated } 0.5 \% \text { more fructans, inducing drought stress } \\
\text { tolerance }\end{array}$ & $\begin{array}{l}\text { Pilon-Smits et al. } \\
\text { (1999) }\end{array}$ \\
\hline 12 & $1-S S T$ & Lactuca sativa & N. tabacum & $\begin{array}{l}\text { High soluble carbohydrate, fructose, fructan content inducing } \\
\text { cold stress tolerance }\end{array}$ & Li et al. (2007) \\
\hline 13 & $m t l D$ & E. coli & $\begin{array}{l}\text { Arabidopsis } \\
\text { thaliana }\end{array}$ & $\begin{array}{l}\text { Enhanced mannitol }(3 \mu \mathrm{mol} / \mathrm{g} \text { FW }) \text {, germination rate, optimum } \\
\text { shoot and root growth under salt stress }\end{array}$ & Thomas et al. (1995) \\
\hline 14 & $m t l D$ & E. coli & N. tabacum & $6 \mu \mathrm{mol} / \mathrm{g}$ FW of mannitol inducing salt tolerance & $\begin{array}{l}\text { Tarczynski et al. } \\
\text { (1992) }\end{array}$ \\
\hline 15 & $m t l D$ & E. coli & $\begin{array}{l}\text { Triticum } \\
\text { aestioum }\end{array}$ & $\begin{array}{l}\text { Enhanced mannitol content, plant height, dry and fresh weight } \\
\text { under salt stress. }\end{array}$ & Abebe et al. (2003) \\
\hline 16 & $m t l D$ & E. coli & $\begin{array}{l}\text { Solanum } \\
\text { tuberosum }\end{array}$ & $\begin{array}{l}\text { Enhanced mannitol level leading to } 65 \% \text { plant survival under } \\
\text { salt stress. }\end{array}$ & $\begin{array}{l}\text { Rahnama et al. } \\
\text { (2011) }\end{array}$ \\
\hline 17 & $\begin{array}{l}\text { HVA1 + } \\
m t l D\end{array}$ & $\begin{array}{l}\text { Hordeumoulgare } \\
\text { and E. coli }\end{array}$ & Zea mays & $\begin{array}{l}\text { Improved rate of plant survival, shoot and root biomass under } \\
\text { multiple abiotic stress conditions }\end{array}$ & Nguyen et al. (2013) \\
\hline 18 & $M p S 6 P D H$ & Maluspumila & N. tabacum & $0.2-130 \mu \mathrm{mol} / \mathrm{g} \mathrm{FW}$ sorbitol content in response to salt stress & $\begin{array}{l}\text { Sheveleva et al. } \\
\text { (1998) }\end{array}$ \\
\hline 19 & $M p S 6 P D H$ & Maluspumila & Diospyros kaki & $\begin{array}{l}14.5-61.5 \mu \mathrm{mol} / \mathrm{g} \text { FW sorbitol content and high chlorophyll } \\
\text { content under salt stress }\end{array}$ & Gao et al. (2001) \\
\hline
\end{tabular}




\subsubsection{Trehalose}

Trehalose, a nonreducing disaccharide is composed of two glucose units and has been found in bacteria, fungi, and higher plants (Djilianov et al., 2005; Elbein et al., 2003). Trehalose biosynthesis in plants is accomplished in two enzyme catalyzed reactions. In the first step, uridine diphosphate glucose (UDP-glucose) and glucose-6-phosphate are catalyzed by the enzyme trehalose-6-phosphate synthase (TPS) forming trehalose6-phosphate (T6P) intermediate. In the final step, the intermediate T6P is converted to trehalose via a dephosphorylation reaction catalyzed by trehalose-6-phosphate phosphatase (TPP) (Iordachescu and Imai, 2011; Paul et al., 2008; John et al., 2017) (Fig. 30.5A).

Trehalose has been associated with protection of protein and membrane structures of plants exposed to abiotic stress (Garg et al., 2002; Wingler, 2002). It shields the structures against denaturation and is also involved in regulation of cell proliferation, cell differentiation, and cellular homeostasis (Wani et al., 2016). Transcriptome analysis of Arabidopsis revealed the presence of 11 TPS and 10 TPP enzymes respectively while nine TPS and nine TPP enzymes have been found in the case of rice. To suppress the deleterious effects of abiotic stress, many scientists focused on exploiting the role of trehalose either by treating plants with trehalose supplements or by developing transgenic plants overexpressing the candidate biosynthetic genes.

Sadak (2016) studied the effect of exogenous effect of $500 \mathrm{mM}$ trehalose on fenugreek plants under drought stress. They reported that the plants exhibited

(A)

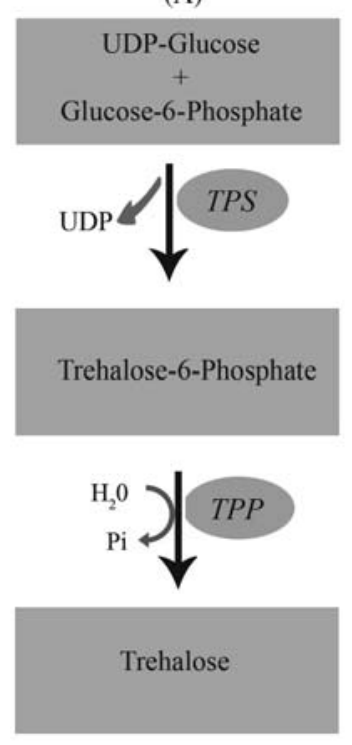

FIGURE 30.5A Trehalose biosynthetic pathway.

TPS: trehalose-6-phosphate synthase; TPP: trehalose-6-phosphate phosphatase; UDP: uridine diphosphate. increased chlorophyll content, protein, and flavonoid content when compared with their WT counterparts. In radish a very small trehalose supplement $(25 \mathrm{mM})$ increased root dry biomass, antioxidant enzyme activity (SOD and peroxidase), chlorophyll a content, total soluble sugar, and free proline content after 45 days exposure to drought stress (Akram et al., 2016).

The rapid progresses in the field of genetic engineering has enabled researchers to harness the potential trehalose biosynthetic genes (TPP and TPS) from plants as well as prokaryotes and develop transgenic varieties. These transgenic lines are designed to enhance endogenous trehalose accumulation, which in turn protects the plants from the deleterious effects of abiotic stress (Iordachescu and Imai, 2008; Sah et al., 2016). The ScTPS1 gene was isolated from yeast and used to develop the first transgenic tobacco plants expressing the TPS enzyme. The trehalose accumulation in the transgenic cultivars was recorded as $0.17 \mathrm{mg} / \mathrm{g}$ fresh weight in leaves. Though the plants exhibited stunted growth and lancet-shaped leaves they were tolerant to prolonged drought exposure (Romero et al., 1997). The yeast (ScTPS1) gene was overexpressed in transgenic potato (Yeo et al., 2000) and tomato (Cortina and Culiáñez-Macià, 2005) respectively under the regulation of a $35 \mathrm{~S}$ CaMV promoter and exposed to salt, drought, and cold stress. Both the transgenic plants showed enhanced trehalose accumulation leading to abiotic stress tolerance.

Garg et al. (2002) developed transgenic rice varieties expressing the E. coli trehalose biosynthetic genes (ots $A$

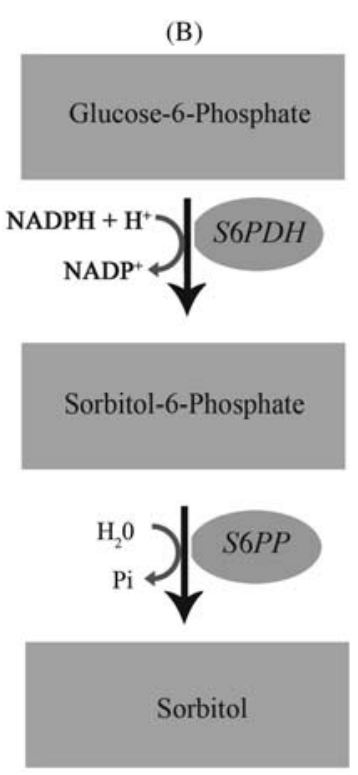

FIGURE 30.5B Sorbitol biosynthesis pathway. S6PDH: sorbitol-6-phosphate dehydrogenase; S6PP: sorbitol-6pyrophosphatase. 
and $o t s B$ ) under the control of a stress responsive promoter. The transformed plants accumulated 3- to 10-fold more trehalose resulting in improved growth, less photooxidative damage, and maintenance of mineral homeostasis under salt, drought, and low temperature. Leaf rolling and wilting were common in nontransformed plants whereas the transformed plants exhibited significant shoot development. Guo et al. (2014) expressed Porphyra yezoensis (PyTPS) gene in a particular rice variety (TP309). The transgenic plants were characterized with improved seed germination rate and yield parameters due to increased trehalose accumulation.

Zhang et al. (2005) reported significant trehalose (2.1-2.5 mg/g fresh weight) accumulation in transgenic tobacco plants expressing the Grifola frondosa trehalose synthase gene. The transgenic plants accumulated trehalose up to $2.1-2.5 \mathrm{mg} / \mathrm{g}$ fresh weight while the level of trehalose remained untraced in the controls (nontransformed). High levels of trehalose accumulation were reported in transgenic lines harboring the $A$. thaliana (AtTPS1) gene, which increased net photosynthesis, improved antioxidant enzyme activity, and lowered peroxidation levels (Vinocur and Altman, 2005). Miranda et al. (2007) coexpressed the yeast TPS1 and TPS2 gene in Arabidopsis and the same gene construct was later introduced in Alfalfa (Suárez et al., 2009). In both the experiments, the transformed varieties were able to thrive in drought, heat, freezing, and salt stress conditions and showed normal growth and development.

\subsubsection{Fructans}

Fructose polymers are referred as fructans and are synthesized from sucrose molecule characterized by transfer of a fructosyl group in two steps catalyzed by sucrose:sucrose 1-fructosyltransferase (1-SST) and sucrose:fructan 6-fructosyltransferase(6-SFT) respectively (Fig. 30.5C). Under abiotic stress condition, plants accumulate fructan in vacuoles and they serve as carbohydrate sinks that are utilized during the nutrient recovery phase (Vijn and Smeekens, 1999; Konstantinova et al., 2002)

The genes involved in fructan biosynthesis have been used to engineer transgenic tobacco, potato (Van Der Meer et al., 1994), rice (Kawakami et al., 2008), and sugar beet varieties tolerant to abiotic stresses. Pilon-Smits et al. (1995) developed transgenic tobacco expressing the Bacillus subtilis gene $(\mathrm{SacB})$ under the constitutive (CaMV 35S) promoter. The transgenic plants accumulated higher fructan levels $(0.35 \mathrm{mg} / \mathrm{g}$ fresh weight) under drought stress and growth rate was enhanced by $55 \%$. Moreover the transgenic lines recorded $33 \%$ and $59 \%$ increase in fresh and dry weight when compared with their wild type. The same gene when expressed in sugar beet accumulated sevenfold more trehalose predominantly in roots

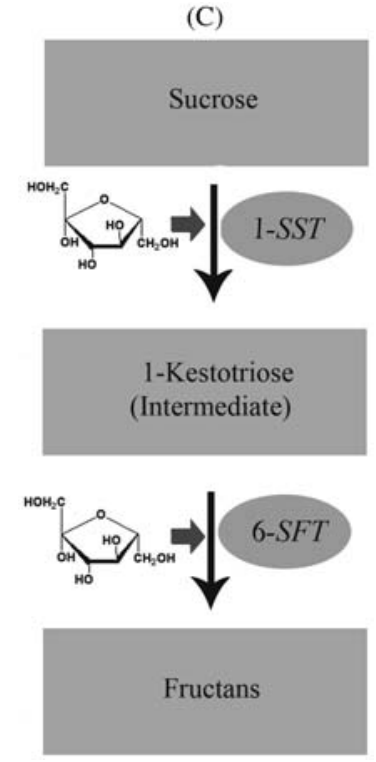

FIGURE 30.5C Fructan biosynthesis pathway.

1-SST: sucrose:sucrose 1-fructosyltransferase; 6-SFT: sucrose:fructan 6-fructosyltransferase.

under drought stress in comparison to its WT (Pilon-Smits et al., 1999).

Li et al. (2007) also focused on transgenic tobacco expressing the sucrose 1-fructosyltransferase (1-SST) gene isolated from Lactuca sativa. The transgenic lines exhibited increase in soluble carbohydrate content, fructan, and fructose content and net photosynthesis but similar antioxidant enzyme activity (SOD) under low temperature stress $\left(4^{\circ} \mathrm{C}\right)$ in comparison with wild types.

\subsubsection{Mannitol}

Mannitol belongs to the class of sugar alcohols and is a 6-carbon molecule. Three enzymes, namely, mannose-6-phosphate isomerase, mannose-6-phosphate reductase, and mannose-1-phosphate phosphatase sequentially catalyze the synthesis of mannitol from fructose-6-phosphate (Loescher et al., 1992) (Fig. 30.5D). In plants, enhanced mannitol accumulation is triggered when exposed to abiotic stress and regulates stability of macromolecules and ROS scavenging (Llanes et al., 2013). Thomas et al. (1995) introduced the E. colimtlD gene into Arabidopsis (nonmannitol accumulator). Mannitol dehydrogenase is the major rate limiting enzyme involved in conversion of fructose-6-phosphate to mannitol-1-phosphate. The transgenic plants were shown to accumulate increased mannitol levels ( $3 \mu \mathrm{mol} / \mathrm{g}$ fresh weight), which resulted in improved biomass and germination rate, and normal shoot and root development even when exposed under $400 \mathrm{mM}$ $\mathrm{NaCl}$ stress. The same E. colimtlD gene was inserted in 
(D)

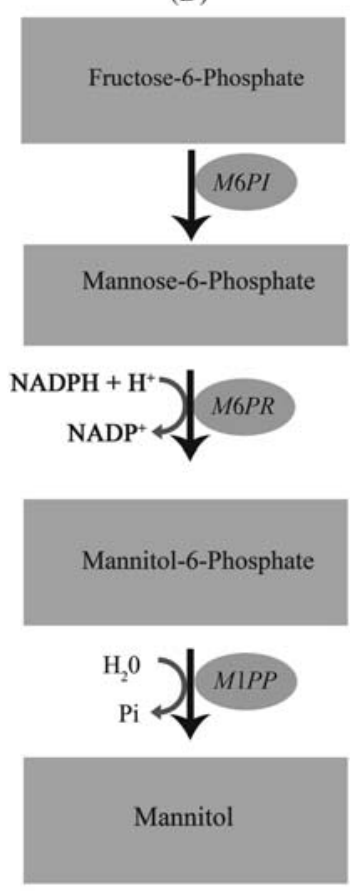

FIGURE 30.5D Mannitol biosynthesis pathway.

M6PI: mannose-6-phosphate isomerase; M6PR: mannose-6phosphate reductase; M1PP: mannose-1-phosphate phosphatase.

tobacco to develop its transgenic lines (Tarczynski et al., 1992). The transgenic tobacco lines accumulated mannitol $(6 \mu \mathrm{mol} / \mathrm{g}$ fresh weight) unlike the wild types. Moreover the transgenic lines thrived in $150 \mathrm{mM} \mathrm{NaCl}$ stress and did not show reduction in dry weight as in the case of wild types, which showed $44 \%$ reduction in dry weight.

Rahnama et al. (2011) introduced the E. coli(mtlD) gene in potato and exposed both the transformed and the control varieties to $100 \mathrm{mM} \mathrm{NaCl}$ stress. The transformed plants only observed $17.3 \%$ reduction in shoot fresh weight while the reduction was $76.5 \%$ weight in the nontransformed potato lines. In another study, Nguyen et al. (2013) designed a gene construct of Hordeum vulgare HVA1 (a group 3 LEA protein) and E. coli $(m t l D)$ genes under the control of rice actine promoter (Act1). This construct was expressed in maize exposed to high salinity $(300 \mathrm{mM} \mathrm{NaCl})$ and drought stress. The successfully transformed varieties expressing the HVA1 + mtlD construct exhibited better shoot and root biomass and sustained throughout the stress period while their wild type counterparts failed to survive.

\subsubsection{Sorbitol}

Sorbitol also belongs to the category of sugar alcohol and is produced from glucose-6-phosphate by the action of sorbitol-6-phosphate dehydrogenase (S6PDH) and sorbitol-6-pyrophosphatase (S6PP) enzymes (Fig. 30.5B). The genes regulating the sorbitol biosynthetic pathway have been exploited to engineer some transgenic plants aimed for developing abiotic stress tolerance. Sheveleva et al. (1998) expressed the sorbitol6-phosphate dehydrogenase $(S 6 P D H)$ gene from apple in tobacco. The transformed varieties accumulated sorbitol $(2-130 \mu \mathrm{mol} / \mathrm{g}$ fresh weight) when subjected to $200 \mathrm{mM}$ salt stress and were more stress tolerant in comparison with its WT plants. Gao et al. (2001) also expressed the same S6PDH gene in Japanese persimmon and exposed them to $200 \mathrm{mM} \mathrm{NaCl}$ stress. The transgenic lines accumulated sorbitol $(14.5-61.5 \mu \mathrm{mol} / \mathrm{g}$ fresh weight) in comparison with its wild types (naturally nonaccumulator). The accumulation of sorbitol was attributed to enhanced photosynthetic activity and chlorophyll content increased in the transgenic lines thereby conferring salt stress tolerance.

\subsection{CONCLUSION AND FUTURE PROSPECTS}

Abiotic stresses are responsible for significant damage to crop productivity annually and the situation is further aggravated due to changes in world climatic pattern and global warming. The major research conducted these days focuses on evolving new techniques aimed at enhancing plant stress physiology. The major concern remains in ensuring sustainable crop productivity for the ever-growing population. Under such a scenario, the diverse role of osmolytes in conferring abiotic stress tolerance can be harnessed to develop potential transgenic varieties of agriculturally important plants. Extensive studies have been conducted to elucidate the biosynthetic pathways of these osmolytes and the valuable inputs have translated to the identification of many candidate genes regulating the biosynthetic pathways of the above discussed osmolytes. The transgenic cultivars expressing the candidate genes in plants improved osmolyte accumulation significantly and contributed to specific stress tolerance. However all the developed transgenic plants discussed so far have not been subjected to field trials and all responses are only true to laboratory conditions. Hence, special focus should be made to develop transgenic plants that can successfully thrive in field conditions. Moreover plants being sessile are exposed to multiple abiotic stresses simultaneously, hence it is of utmost importance in developing transgenic plants capable of expressing multigenic traits (simultaneous expression of osmolyte biosynthesis genes and other genes involved in stress tolerance like ion transporters, aquaporins, transcriptional factors, LEA proteins, etc.). 


\section{Acknowledgment}

CS, NNR, and TD are grateful to GITAM (Deemed to be University) for providing necessary facilities to carry out the research work and for extending constant support in writing this chapter. TD is thankful for financial support in the form of DST Inspire Fellowship (IF 160964), Department of Science and Technology, New Delhi.

\section{References}

Acquaah, G., 2007. Principles of Plant Genetics and Breeding. Blackwell, Oxford.

Adem, G.D., Roy, S.J., Zhou, M., Bowman, J.P., Shabala, S., 2014. Evaluating contribution of ionic, osmotic and oxidative stress components towards salinity tolerance in barley. BMC Plant Biol. $14(1), 113$

Ahmad, R., Hussain, J., Jamil, M., Kim, M.D., Kwak, S.S., Shah, M. M., et al., 2014. Glycinebetaine synthesizing transgenic potato plants exhibit enhanced tolerance to salt and cold stresses. Pak. J. Bot. 46, 1987-1993.

Akram, N.A., Waseem, M., Ameen, R., Ashraf, M., 2016. Trehalose pretreatment induces drought tolerance in radish (Raphanus sativus L.) plants: some key physio-biochemical traits. Acta Physiol. Plant. 38 (1), 3.

Alam, R., Das, D.K., Islam, M.R., Murata, Y., Hoque, M.A., 2017. Exogenous proline enhances nutrient uptake and confers tolerance to salt stress in maize (Zea mays L.). Prog. Agric. 27 (4), 409-417.

Alcázar, R., Altabella, T., Marco, F., Bortolotti, C., Reymond, M., Koncz, C., et al., 2010. Polyamines: molecules with regulatory functions in plant abiotic stress tolerance. Planta 231 (6), 1237-1249.

Alet, A.I., Sánchez, D.H., Cuevas, J.C., Marina, M., Carrasco, P., Altabella, T., et al., 2012. New insights into the role of spermine in Arabidopsis thaliana under long-term salt stress. Plant Sci. 182, 94-100.

Allakhverdiev, S.I., Hayashi, H., Nishiyama, Y., Ivanov, A.G., Aliev, J.A., Klimov, V.V., et al., 2003. Glycinebetaine protects the D1/ D2/Cytb559 complex of photosystem II against photo-induced and heat-induced inactivation. J. Plant. Physiol. 160 (1), 41-49.

Arif, S.W., Faraz, A., Faizan, M., Ahmad, A., Hayat, S., Tahir, I., 2017. Foliar Spray of Proline Enhanced the Photosynthetic Efficiency and Antioxidant System in Brassica juncea. Notulae Botanicae Horti Agrobotanici Cluj-Napoca 45 (1).

Ashfaque, F., Khan, M.I.R., Khan, N.A., 2014. Exogenously applied $\mathrm{H} 2 \mathrm{O} 2$ promotesproline accumulation, water relations, photosynthetic efficiency and growth of wheat (Triticumaestivum L.) under salt stress. Annu. Res. Rev. Biol 4, 105-120.

Ashraf, M., Foolad, M., 2007. Roles of glycine betaine and proline in improving plant abiotic stress resistance. Environ. Exp. Bot. 59 (2), 206-216.

Behelgardy, M.F., Motamed, N., Jazii, F.R., 2012. Expression of the P5CS gene in transgenic versus nontransgenic olive (Olea europaea) under salinity stress. World Appl. Sci. J. 18 (4), 580-583.

Bhatnagar-Mathur, P., Vadez, V., Sharma, K.K., 2008. Transgenic approaches for abiotic stress tolerance in plants: retrospect and prospects. Plant Cell Rep. 27 (3), 411-424.

Bhusan, D., Das, D.K., Hossain, M., Murata, Y., Hoque, M.A., 2016. Improvement of salt tolerance in rice ('Oryza sativa' L.) by increasing antioxidant defense systems using exogenous application of proline. Aust. J. Crop Sci. 10 (1), 50.

Blum, A., 2017. Osmotic adjustment is a prime drought stress adaptive engine in support of plant production. Plant Cell Environ. 40 (1), 4-10.
Capell, T., Bassie, L., Christou, P., 2004. Modulation of the polyamine biosynthetic pathway in transgenic rice confers tolerance to drought stress. Proc. Natl. Acad. Sci. U.S.A. 101 (26), 9909-9914.

Chakraborty, K., Sairam, R.K., 2017. Induced-expression of osmolyte biosynthesis pathway genes improves salt and oxidative stress tolerance in Brassica species. Int. J. Exp. Bio. 55 (10), 711-721.

Chen, J.B., Yang, J.W., Zhang, Z.Y., Feng, X.F., Wang, S.M., 2013. Two P5CS genes from common bean exhibiting different tolerance to salt stress in transgenic Arabidopsis. J. Genet. 92 (3), 461-469.

Chen, T.H., Murata, N., 2002. Enhancement of tolerance of abiotic stress by metabolic engineering of betaines and other compatible solutes. Curr. Opin. Plant. Biol. 5 (3), 250-257.

Chen, T.H., Murata, N., 2008. Glycinebetaine: an effective protectant against abiotic stress in plants. Trends. Plant. Sci. 13 (9), 499-505.

Chen, T.H., Murata, N., 2011. Glycinebetaine protects plants against abiotic stress: mechanisms and biotechnological applications. Plant Cell Environ. 34 (1), 1-20.

Cheng, L., Zou, Y., Ding, S., Zhang, J., Yu, X., Cao, J., et al., 2009. Polyamine accumulation in transgenic tomato enhances the tolerance to high temperature stress. J. Integr. Plant Biol. 51 (5), $489-499$.

Cortina, C., Culiáñez-Macià, F.A., 2005. Tomato abiotic stress enhanced tolerance by trehalose biosynthesis. Plant Sci. 169 (1), 75-82.

De Ronde, J.A., Van Der Mescht, A., Steyn, H.S.F., 2000. Proline accumulation in response to drought and heat stress in cotton. Afr. Crop Sci. J. 8, 85-92.

De Zwart, F.J., Slow, S., Payne, R.J., Lever, M., George, P.M., Gerrard, J.A., et al., 2003. Glycine betaine and glycine betaine analogues in common foods. Food. Chem. 83 (2), 197-204.

Delauney, A.J., Verma, D.P.S., 1993. Proline biosynthesis and osmoregulation in plants. Plant J. 4 (2), 215-223.

Djilianov, D., Georgieva, T., Moyankova, D., Atanassov, A., Shinozaki, K., Smeeken, S.C.M., et al., 2005. Improved abiotic stress tolerance in plants by accumulation.

Duque, A.S., López-Gómez, M., Kráčmarová, J., Gomes, C.N., Araújo, S.S., Lluch, C., et al., 2016. Genetic engineering of polyamine metabolism changes Medicago truncatula responses to water deficit. Plant Cell, Tissue Organ Cult. (PCTOC) 127 (3), 681-690.

Ehsanpour, A.A., Fatahian, N., 2003. Effects of salt and proline on Medicago sativa callus. Plant Cell Tissue Organ Cult. 73 (1), $53-56$.

Elbein, A.D., Pan, Y.T., Pastuszak, I., Carroll, D., 2003. New insights on trehalose: a multifunctional molecule. Glycobiology 13 (4), 17R-27R.

Fan, W., Zhang, M., Zhang, H., Zhang, P., 2012. Improved tolerance to various abiotic stresses in transgenic sweet potato (Ipomoea batatas) expressing spinach betaine aldehyde dehydrogenase. PLoS One 7 (5), e37344.

FAO, 2010. FAO Land and plant nutrition management service. Retrieved from http://www.fao.org/tempref/agl/agll/docs/ sudan.pdf.

Feng, H.Y., Wang, Z.M., Kong, F.N., Zhang, M.J., Zhou, S.L., 2011. Roles of carbohydrate supply and ethylene, polyamines in maize kernel set. J. Integr. Plant Biol. 53 (5), 388-398.

Franceschetti, M., Fornalè, S., Tassoni, A., Zuccherelli, K., Mayer, M. J., Bagni, N., 2004. Effects of spermidine synthase overexpression on polyamine biosynthetic pathway in tobacco plants. J. Plant. Physiol. 161 (9), 989-1001.

Funck, D., Winter, G., Baumgarten, L., Forlani, G., 2012. Requirement of proline synthesis during Arabidopsis reproductive development. BMC Plant Biol. 12 (1), 191.

Gao, M., Tao, R., Miura, K., Dandekar, A.M., Sugiura, A., 2001. Transformation of Japanese persimmon (Diospyros kaki Thunb.) with apple cDNA encoding NADP-dependent sorbitol-6phosphate dehydrogenase. Plant Sci. 160 (5), 837-845. 
Garg, A.K., Kim, J.K., Owens, T.G., Ranwala, A.P., Do Choi, Y., Kochian, L.V., et al., 2002. Trehalose accumulation in rice plants confers high tolerance levels to different abiotic stresses. Proc. Natl. Acad. Sci. 99 (25), 15898-15903.

Gill, S.S., Tuteja, N., 2010. Polyamines and abiotic stress tolerance in plants. Plant Signal. Behav. 5 (1), 26-33.

Giri, J., 2011. Glycinebetaine and abiotic stress tolerance in plants. Plant Signal. Behav. 6 (11), 1746-1751.

Gleeson, D., Lelu-Walter, M.A., Parkinson, M., 2005. Overproduction of proline in transgenic hybrid larch (Larix $x$ leptoeuropaea (Dengler)) cultures renders them tolerant to cold, salt and frost. Mol. Breed. 15 (1), 21-29.

Goel, D., Singh, A.K., Yadav, V., Babbar, S.B., Murata, N., Bansal, K. C., 2011. Transformation of tomato with a bacterial $\operatorname{cod} A$ gene enhances tolerance to salt and water stresses. J. Plant. Physiol. 168 (11), 1286-1294.

Guerzoni, J.T.S., Belintani, N.G., Moreira, R.M.P., Hoshino, A.A., Domingues, D.S., Bespalhok Filho, J.C., et al., 2014. Stressinduced $\Delta 1$-pyrroline-5-carboxylate synthetase (P5CS) gene confers tolerance to salt stress in transgenic sugarcane. Acta Physiol. Plant. 36 (9), 2309-2319.

Guo, B.T., Wang, B., Weng, M.L., Qiao, L.X., Feng, Y.B., Wang, L., et al., 2014. Expression of porphyra yezoensis (tps) gene in transgenic rice enhanced the salt tolerance. J. Plant Breed. Genet. 2 (1), 45-55.

Gupta, B., Huang, B., 2014. Mechanism of salinity tolerance in plants: physiological, biochemical, and molecular characterization. Int. J. Genomics 2014, 701596.

Gupta, K., Dey, A., Gupta, B., 2013. Plant polyamines in abiotic stress responses. Acta Physiol. Plant. 35 (7), 2015-2036.

Han, K.H., Hwang, C.H., 2003. Salt tolerance enhanced by transformation of a P5CS gene in carrot. J. Plant Biotechnol. 5, 149-153.

Hare, P.D., Cress, W.A., Van Staden, J., 2003. A regulatory role for proline metabolism in stimulating Arabidopsis thaliana seed germination. Plant Growth Regul. 39 (1), 41-50.

Hasthanasombut, S., Ntui, V., Supaibulwatana, K., Mii, M., Nakamura, I., 2010. Expression of Indica rice OsBADH1 gene under salinity stress in transgenic tobacco. Plant Biotechnol. Rep. 4 (1), 75-83.

He, L., Ban, Y., Inoue, H., Matsuda, N., Liu, J., Moriguchi, T., 2008. Enhancement of spermidine content and antioxidant capacity in transgenic pear shoots overexpressing apple spermidine synthase in response to salinity and hyperosmosis. Phytochemistry 69 (11), 2133-2141.

Heuer, B., 2003. Influence of exogenous application of proline and glycine betaine on growth of salt-stressed tomato plants. Plant Sci. 165 (4), 693-699.

Hmida-Sayari, A., Gargouri-Bouzid, R., Bidani, A., Jaoua, L., Savouré, A., Jaoua, S., 2005. Overexpression of $\Delta$ 1-pyrroline-5carboxylate synthetase increases proline production and confers salt tolerance in transgenic potato plants. Plant Sci. 169 (4), $746-752$.

Hong, Z., Lakkineni, K., Zhang, Z., Verma, D.P.S., 2000. Removal of feedback inhibition of $\Delta 1$-pyrroline-5-carboxylate synthetase results in increased proline accumulation and protection of plants from osmotic stress. Plant Physiol. 122 (4), 1129-1136.

Huang, B., Jin, L., Liu, J.Y., 2008. Identification and characterization of the novel gene GhDBP2 encoding a DRE-binding protein from cotton (Gossypium hirsutum). J. Plant. Physiol. 165 (2), 214-223.

Huang, Z., Zhao, L., Chen, D., Liang, M., Liu, Z., Shao, H., et al., 2013. Salt stress encourages proline accumulation by regulating proline biosynthesis and degradation in Jerusalem artichoke plantlets. PLoS. ONE. 8 (4), e62085.

Hussain, S.S., Ali, M., Ahmad, M., Siddique, K.H., 2011. Polyamines: natural and engineered abiotic and biotic stress tolerance in plants. Biotechnol. Adv. 29 (3), 300-311.
Iordachescu, M., Imai, R., 2008. Trehalose biosynthesis in response to abiotic stresses. J. Integr. Plant Biol. 50 (10), 1223-1229.

Iordachescu, M., Imai, R., 2011. Trehalose and abiotic stress in biological systems. Abiotic Stress in Plants-Mechanisms and Adaptations. InTech.

Jaleel, C.A., Manivannan, P., Wahid, A., Farooq, M., Al-Juburi, H.J., Somasundaram, R., et al., 2009. Drought stress in plants: a review on morphological characteristics and pigments composition. Int. J. Agric. Biol. 11 (1), 100-105.

Ji, H., Pardo, J.M., Batelli, G., Van Oosten, M.J., Bressan, R.A., Li, X., 2013. The Salt Overly Sensitive (SOS) pathway: established and emerging roles. Mol. Plant 6 (2), 275-286.

Jing, J., Li, H., He, G., Yin, Y., LiuM, Liu, B., et al., 2013. Overexpression of the $\operatorname{codA}$ gene by Rd29Apromoter improves salt tolerance in Nicotiana tabacum. Pak. J. Bot. 45, 821-827.

John, R., Raja, V., Ahmad, M., Jan, N., Majeed, U., Ahmad, S., et al., 2017. Trehalose: metabolism and role in stress signalling in plants, Stress Signalling in Plants: Genomics and Proteomics Perspective, Vol. 2. Springer International Publishing, pp. 261-275.

Joseph, E.A., Radhakrishnan, V.V., Mohanan, K.V., 2015. A Study on the accumulation of Proline-an osmoprotectant amino acid under salt stress in some native rice cultivars of north Kerala, India. Univ. J. Agric. Res. 3 (1), 15-22.

Joshi, R., Singla-Pareek, S.L., Pareek, A., 2018. Engineering abiotic stress response in plants for biomass production. J. Biol. Chem. pp. jbc-TM117.

Karthikeyan, A., Pandian, S.K., Ramesh, M., 2011. Transgenic indica rice cv. ADT 43 expressing a $\Delta 1$-pyrroline-5-carboxylate synthetase (P5CS) gene from Vigna aconitifolia demonstrates salt tolerance. Plant Cell, Tissue Organ Culture (PCTOC) 107 (3), 383-395.

Kasukabe, Y., He, L., Nada, K., Misawa, S., Ihara, I., Tachibana, S., 2004. Overexpression of spermidine synthase enhances tolerance to multiple environmental stresses and up-regulates the expression of various stress-regulated genes in transgenic Arabidopsis thaliana. Plant Cell Physiol. 45 (6), 712-722.

Kaur, G., Asthir, B., 2015. Proline: a key player in plant abiotic stress tolerance. Biol. Plant. 59 (4), 609-619.

Kawakami, A., Sato, Y., Yoshida, M., 2008. Genetic engineering of rice capable of synthesizing fructans and enhancing chilling tolerance. J. Exp. Bot. 59 (4), 793-802.

Ke, Q., Wang, Z., Ji, C.Y., Jeong, J.C., Lee, H.S., Li, H., et al., 2016. Transgenic poplar expressing codA exhibits enhanced growth and abiotic stress tolerance. Plant. Physiol. Biochem. 100, 75-84.

Khan, M.I.R., Khan, N.A., 2013. Salicylic acid and jasmonates: approaches in abiotic stress tolerance. Plant Biochem. Physiol. 1, 4.

Khan, M.I.R., Khan, N., 2017. Reactive Oxygen Species and Antioxidant System in Plants: Role and Regulation Under Abiotic Stress. Springer Nature, New York978-981-10-5254-5.

Khan, M.I.R., Asgher, M., Khan, N.A., 2014. Alleviation of saltinduced photosynthesis and growth inhibition by salicylic acid involves glycine betaine and ethylene in mungbean (Vignaradiata L.). Plant. Physiol. Biochem. 80, 67-74.

Khan, M.I.R., Iqbal, N., Masood, A., Khan, N.A., 2012. Variation in salt tolerance of wheat cultivars: evaluation of the role of glycine betaine and ethylene. Pedosphere 22, 746-754.

Khan, M.I.R., Iqbal, N., Masood, A., Per, T.S., Khan, N.A., 2013. Salicylic acid alleviates adverse effects of heat stress on photosynthesis through changes in proline production and ethylene formation. Plant Signal. Behav. 8, 263-274. e.

Khan, M.I.R., Fatma, M., Per, T.S., Anjum, N.A., Khan, N.A., 2015. Salicylic acid-induced abiotic stress tolerance and underlying mechanisms in plants. Front. Plant Sci. 6, 462.

Khan, M.I.R., Khan, N.A., Masood, A., Per, T.S., Asgher, M., 2016. Hydrogen peroxide alleviates nickel-inhibited photosynthetic 
responses through increase in use-efficiency of nitrogen and sulfur, and glutathione production in mustard. Front. Plant Sci. 7,44 .

Khan, M.S., Yu, X., Kikuchi, A., Asahina, M., Watanabe, K.N., 2009. Genetic engineering of glycine betaine biosynthesis to enhance abiotic stress tolerance in plants. Plant Biotechnol. 26 (1), 125-134.

Kishitani, S., Watanabe, K., Yasuda, S., Arakawa, K., Takabe, T., 1994. Accumulation of glycinebetaine during cold acclimation and freezing tolerance in leaves of winter and spring barley plants. Plant Cell Environ. 17 (1), 89-95.

Kishor, P.K., Hong, Z., Miao, G.H., Hu, C.A.A., Verma, D.P.S., 1995. Overexpression of [delta]-pyrroline-5-carboxylate synthetase increases proline production and confers osmotolerance in transgenic plants. Plant Physiol. 108 (4), 1387-1394.

Kishor, P.K., Sangam, S., Amrutha, R.N., Laxmi, P.S., Naidu, K.R., Rao, K.R.S.S., et al., 2005. Regulation of proline biosynthesis, degradation, uptake and transport in higher plants: its implications in plant growth and abiotic stress tolerance. Curr. Sci. 424-438.

Kishor, P.K., Polavarapu, B., Sreenivasulu, N., 2014. Is proline accumulation per se correlated with stress tolerance or is proline homeostasis a more critical issue? Plant Cell Environ. 37 (2), $300-311$.

Konstantinova, T., Parvanova, D., Atanassov, A., Djilianov, D., 2002. Freezing tolerant tobacco, transformed to accumulate osmoprotectants. Plant Sci. 163 (1), 157-164.

Kumar, R., 2009. Role of naturally occurring osmolytes in protein folding and stability. Arch. Biochem. Biophys. 491 (1), 1-6.

Kumar, V., Shriram, V., Kishor, P.K., Jawali, N., Shitole, M.G., 2010. Enhanced proline accumulation and salt stress tolerance of transgenic indica rice by over-expressing P5CSF129A gene. Plant Biotechnol. Rep. 4 (1), 37-48.

Kurepin, L.V., Ivanov, A.G., Zaman, M., Pharis, R.P., Hurry, V., Hüner, N.P., 2017. Interaction of glycine betaine and plant hormones: protection of the photosynthetic apparatus during abiotic stress. Photosynthesis: Structures, Mechanisms, and Applications. Springer, Cham, pp. 185-202.

Lehmann, S., Funck, D., Szabados, L., Rentsch, D., 2010. Proline metabolism and transport in plant development. Amino Acids 39 (4), 949-962.

Li, B., He, L., Guo, S., Li, J., Yang, Y., Yan, B., et al., 2013. Proteomics reveal cucumber Spd-responses under normal condition and salt stress. Plant. Physiol. Biochem. 67, 7-14.

Li, H.J., Yang, A.F., Zhang, X.C., Gao, F., Zhang, J.R., 2007. Improving freezing tolerance of transgenic tobacco expressing sucrose: sucrose 1-fructosyltransferase gene from Lactuca sativa. Plant Cell Tissue Organ Cult. 89 (1), 37-48.

Li, M., Li, Z., Li, S., Guo, S., Meng, Q., Li, G., et al., 2014. Genetic engineering of glycine betaine biosynthesis reduces heatenhanced photoinhibition by enhancing antioxidative defense and alleviating lipid peroxidation in tomato. Plant. Mol. Biol. Rep. 32 (1), 42-51.

Liu, D., He, S., Zhai, H., Wang, L., Zhao, Y., Wang, B., et al., 2014. Overexpression of $\mathrm{IbP} 5 \mathrm{CR}$ enhances salt tolerance in transgenic sweet potato. Plant Cell, Tissue Organ Culture (PCTOC) 117 (1), $1-16$.

Liu, N., Lin, S., Huang, B., 2017. Differential Effects of Glycine Betaine and Spermidine on Osmotic Adjustment and Antioxidant Defense Contributing to Improved Drought Tolerance in Creeping Bentgrass. J. Am. Soc. Hortic. Sci. 142 (1), 20-26.

Llanes, A., Bertazza, G., Palacio, G., Luna, V., 2013. Different sodium salts cause different solute accumulation in the halophyte Prosopis strombulifera. Plant Biol. 15 (s1), 118-125.

Loescher, W.H., Tyson, R.H., Everard, J.D., Redgwell, R.J., Bieleski, R.L., 1992. Mannitol Synthesis in Higher Plants Evidence for the
Role and Characterization of a NADPH-Dependent Mannose 6Phosphate Reductase. Plant Physiol. 98 (4), 1396-1402.

Lutts, S., Majerus, V., Kinet, J.M., 1999. $\mathrm{NaCl}$ effects on proline metabolism in rice (Oryza sativa) seedlings. Physiol. Plant. 105 (3), $450-458$.

Ma, Y., Shukla, V., Merewitz, E.B., 2017. Transcriptome analysis of creeping bentgrass exposed to drought stress and polyamine treatment. PLoS. ONE. 12 (4), e0175848.

Madhulatha, P., Gupta, A., Gupta, S., Kumar, A., Pal, R.K., Rajam, M.V., 2014. Fruit-specific over-expression of human Sadenosylmethionine decarboxylase gene results in polyamine accumulation and affects diverse aspects of tomato fruit development and quality. J. Plant Biochem. Biotechnol. 23 (2), 151-160.

Mansour, M.M.F., Ali, E.F., 2017. Evaluation of proline functions in saline conditions. Phytochemistry 140, 52-56.

Masood, A., Per, T.S., Asgher, M., Fatma, M., Khan, M.I.R., Rasheed, F., et al., 2016. Glycine betaine: role in shifting plants toward adaptation under extreme environments. Osmolytes and Plants Acclimation to Changing Environment: Emerging Omics Technologies. Springer, New Delhi, pp. 69-82.

Mattioli, R., Costantino, P., Trovato, M., 2009. Proline accumulation in plants: not only stress. Plant Signal. Behav. 4 (11), 1016-1018.

Matysik, J., Alia, Bhalu, B., Mohanty, P., 2002. Molecular mechanisms of quenching of reactive oxygen species by proline under stress in plants. Curr. Sci. 525-532.

Miranda, J.A., Avonce, N., Suárez, R., Thevelein, J.M., Van Dijck, P., Iturriaga, G., 2007. A bifunctional TPS-TPP enzyme from yeast confers tolerance to multiple and extreme abiotic-stress conditions in transgenic Arabidopsis. Planta 226 (6), 1411-1421.

Moschou, P.N., Sanmartin, M., Andriopoulou, A.H., Rojo, E., Sanchez-Serrano, J.J., Roubelakis-Angelakis, K.A., 2008. Bridging the gap between plant and mammalian polyamine catabolism: a novel peroxisomal polyamine oxidase responsible for a full back-conversion pathway in Arabidopsis. Plant Physiol. 147 (4), $1845-1857$.

Munns, R., 2002. Comparative physiology of salt and water stress. Plant Cell Environ. 25, 239-250.

Nahar, K., Hasanuzzaman, M., Rahman, A., Alam, M.M., Mahmud, J.A., Suzuki, T., et al., 2016. Polyamines confer salt tolerance in mung bean (Vigna radiata L.) by reducing sodium uptake, improving nutrient homeostasis, antioxidant defense, and methylglyoxal detoxification systems. Front. Plant Sci. 7.

Nanjo, T., Kobayashi, M., Yoshiba, Y., Sanada, Y., Wada, K., Tsukaya, H., et al., 1999. Biological functions of proline in morphogenesis and osmotolerance revealed in antisense transgenic Arabidopsis thaliana. Plant J. 18 (2), 185-193.

Nath, M., Bhatt, D., Prasad, R., Tuteja, N., 2017. Reactive Oxygen Species (ROS) Metabolism and Signalling in Plant-Mycorrhizal Association Under Biotic and Abiotic Stress Conditions. In Mycorrhiza-Eco-Physiology, Secondary Metabolites, Nanomaterials. Springer, Cham, pp. 223-232.

Nguyen, V.L., Ribot, S.A., Dolstra, O., Niks, R.E., Visser, R.G., van der Linden, C.G., 2013. Identification of quantitative trait loci for ion homeostasis and salt tolerance in barley (Hordeum vulgare L.). Mol. Breed. 31 (1), 137-152.

Niu, X., Bressan, R.A., Hasegawa, P.M., Pardo, J.M., 1995. Ion homeostasis in $\mathrm{NaCl}$ stress environments. Plant Physiol. 109 (3), 735.

Niu, X., Xiong, F., Liu, J., Sui, Y., Zeng, Z., Lu, B.R., et al., 2014. Coexpression of ApGSMT and ApDMT promotes biosynthesis of glycine betaine in rice (Oryza sativa L.) and enhances salt and cold tolerance. Environ. Exp. Bot. 104, 16-25.

O'Hara, L.E., Paul, M.J., Wingler, A., 2013. How do sugars regulate plant growth and development? New insight into the role of trehalose-6-phosphate. Mol. Plant 6 (2), 261-274. 
Ozgur, R., Uzilday, B., Sekmen, A.H., Turkan, I., 2013. Reactive oxygen species regulation and antioxidant defence in halophytes. Funct. Plant Biol. 40 (9), 832-847.

Park, E.J., Jeknić, Z., Sakamoto, A., DeNoma, J., Yuwansiri, R., Murata, N., et al., 2004. Genetic engineering of glycinebetaine synthesis in tomato protects seeds, plants, and flowers from chilling damage. Plant J. 40 (4), 474-487.

Park, E.J., Jeknić, Z., Pino, M.T., Murata, N., Chen, T.H.H., 2007. Glycinebetaine accumulation is more effective in chloroplasts than in the cytosol for protecting transgenic tomato plants against abiotic stress. Plant Cell Environ. 30 (8), 994-1005.

Parvaiz, A., Satyawati, S., 2008. Salt stress and phyto-biochemical responses of plants-a review. Plant Soil Environ. 54 (3), 89.

Paul, M.J., Primavesi, L.F., Jhurreea, D., Zhang, Y., 2008. Trehalose metabolism and signalling. Annu. Rev. Plant. Biol. 59, 417-441.

Per, T.S., Khan, N.A., Reddy, P.S., Masood, A., Hasanuzzaman, M., Khan, M.I.R., et al., 2017. Approaches in modulating proline metabolism in plants for salt and drought stress tolerance: phytohormones, mineral nutrients and transgenics. Plant. Physiol. Biochem. 115, 126-140.

Pilon-Smits, E.A., Ebskamp, M.J., Paul, M.J., Jeuken, M.J., Weisbeek, P.J., Smeekens, S.C., 1995. Improved performance of transgenic fructan-accumulating tobacco under drought stress. Plant Physiol. 107 (1), 125-130.

Pilon-Smits, E.A., Terry, N., Sears, T., van Dun, K., 1999. Enhanced drought resistance in fructan-producing sugar beet. Plant. Physiol. Biochem. 37 (4), 313-317.

Qin, D., Zhao, C.L., Liu, X.Y., Wang, P.W., 2017. Transgenic soybeans expressing betaine aldehyde dehydrogenase from Atriplex canescens show increased drought tolerance. Plant Breed. 136 (5), 699-709.

Quan, R., Shang, M., Zhang, H., Zhao, Y., Zhang, J., 2004. Engineering of enhanced glycine betaine synthesis improves drought tolerance in maize. Plant. Biotechnol. J. 2 (6), 477-486.

Rahnama, H., Vakilian, H., Fahimi, H., Ghareyazie, B., 2011. Enhanced salt stress tolerance in transgenic potato plants (Solanum tuberosum L.) expressing a bacterial $m t l D$ gene. Acta Physiol. Plant. 33 (4), 1521-1532.

Ranganayakulu, G.S., Veeranagamallaiah, G., Sudhakar, C., 2013. Effect of salt stress on osmolyte accumulation in two groundnut cultivars (Arachis hypogaea L.) with contrasting salt tolerance. Afr. J. Plant Sci. 7 (12), 586-592.

Rasheed, R., Iqbal, M., Ashraf, M.A., Hussain, I., Shafiq, F., Yousaf, A., et al., 2017. Glycine betaine counteracts the inhibitory effects of waterlogging on growth, photosynthetic pigments, oxidative defence system, nutrient composition, and fruit quality in tomato. J. Hort. Sci. Biotechnol. 1-7.

Rodriguez, M.C., Petersen, M., Mundy, J., 2010. Mitogen-activated protein kinase signalling in plants. Annu. Rev. Plant. Biol. 61, 621-649.

Romero, C., Bellés, J.M., Vayá, J.L., Serrano, R., Culiáñez-Macià, F.A., 1997. Expression of the yeast trehalose-6-phosphate synthase gene in transgenic tobacco plants: pleiotropic phenotypes include drought tolerance. Planta 201 (3), 293-297.

Romero, F.M., Maiale, S.J., Rossi, F.R., Marina, M., Ruíz, O.A., Gárriz, A., 2018. Polyamine Metabolism Responses to Biotic and Abiotic Stress. Polyamines. Humana Press, New York, NY, pp. 37-49.

Roy, M., Wu, R., 2001. Arginine decarboxylase transgene expression and analysis of environmental stress tolerance in transgenic rice. Plant Sci. 160 (5), 869-875.

Roy, M., Wu, R., 2002. Overexpression of S-adenosylmethionine decarboxylase gene in rice increases polyamine level and enhances sodium chloride-stress tolerance. Plant Sci. 163 (5), 987-992.
Roychoudhury, A., Chakraborty, M., 2013. Biochemical and molecular basis of varietal difference in plant salt tolerance. Annu. Rev. Res. Biol. 3 (4), 422-454.

Roychoudhury, A., Banerjee, A., Lahiri, V., 2015. Metabolic and molecular-genetic regulation of proline signalling and its crosstalk with major effectors mediates abiotic stress tolerance in plants. Turkish J. Bot. 39 (6), 887-910.

Sabagh, A.E., Sorour, S., Ragab, A., Saneoka, H., Islam, M.S., 2017. The effect of exogenous application of proline and glycine betaine on the nodule activity of soybean under saline condition. J. Agric. Biotechnol. 2 (1).

Sadak, M.S., 2016. Mitigation of drought stress on Fenugreek plant by foliar application of trehalose. Inter. J. Chem Tech Res. 9, 147-155.

Sade, N., del Mar Rubio-Wilhelmi, M., Umnajkitikorn, K., Blumwald, E., 2017. Stress-induced senescence and plant tolerance to abiotic stress. J. Exp. Bot.

Sagor, G.H.M., Berberich, T., Takahashi, Y., Niitsu, M., Kusano, T., 2013. The polyamine spermine protects Arabidopsis from heat stress-induced damage by increasing expression of heat shockrelated genes. Transgenic res. 22 (3), 595-605.

Sah, S.K., Kaur, G., Wani, S.H., 2016. Metabolic Engineering of Compatible Solute Trehalose for Abiotic Stress Tolerance in Plants. Osmolytes and Plants Acclimation to Changing Environment: Emerging Omics Technologies. Springer, India, pp. 83-96.

Sakamoto, A., Murata, A.N., 1998. Metabolic engineering of rice leading to biosynthesis of glycinebetaine and tolerance to salt and cold. Plant Mol. Biol. 38 (6), 1011-1019.

Sakamoto, A., Murata, N., 2002. The role of glycine betaine in the protection of plants from stress: clues from transgenic plants. Plant Cell Environ. 25 (2), 163-171.

Sánchez-Rodríguez, E., Romero, L., Ruiz, J.M., 2016. Accumulation of free polyamines enhances the antioxidant response in fruits of grafted tomato plants under water stress. J. Plant. Physiol. 190, 72-78.

Sawahel, W., 2003. Improved performance of transgenic glycinebetaine-accumulating rice plants under drought stress. Biol. Plant. 47 (1), 39-44.

Sawahel, W.A., Hassan, A.H., 2002. Generation of transgenic wheat plants producing high levels of the osmoprotectant proline. Biotechnol. Lett. 24 (9), 721-725.

Sequera-Mutiozabal, M., Antoniou, C., Tiburcio, A.F., Alcázar, R., Fotopoulos, V., 2017. Polyamines: emerging hubs promoting drought and salt stress tolerance in plants. Curr. Mol. Biol. Rep. 3 (1), 28-36.

Sharma, S., Pareek, S., Sagar, N.A., Valero, D., Serrano, M., 2017. Modulatory effects of exogenously applied polyamines on postharvest physiology, antioxidant system and shelf life of fruits: a review. Int. J. Mol. Sci. 18 (8), 1789.

Sheveleva, E.V., Marquez, S., Chmara, W., Zegeer, A., Jensen, R.G., Bohnert, H.J., 1998. Sorbitol-6-phosphate dehydrogenase expression in transgenic tobacco high amounts of sorbitol lead to necrotic lesions. Plant Physiol. 117 (3), 831-839.

Shevyakova, N.I., Bakulina, E.A., Kuznetsov, V.V., 2009. Proline antioxidant role in the common ice plant subjected to salinity and paraquat treatment inducing oxidative stress. Russian J. Plant Physiol. 56 (5), 663-669.

Shukla, V., Mattoo, A.K., 2013. Developing robust crop plants for sustaining growth and yield under adverse climatic changes. Climate Change Plant Abiotic Stress Tolerance 27-56.

Singh, M., Kumar, J., Singh, S., Singh, V.P., Prasad, S.M., 2015. Roles of osmoprotectants in improving salinity and drought tolerance in plants: a review. Rev. Environ. Sci. Bio/Technol. 14 (3), 407-426.

Slama, I., Abdelly, C., Bouchereau, A., Flowers, T., Savouré, A., 2015. Diversity, distribution and roles of osmoprotective compounds accumulated in halophytes under abiotic stress. Ann. Bot. (Lond.) 115 (3), 433-447. 
$\mathrm{Su}, \mathrm{J} ., \mathrm{Wu}, \mathrm{R} ., 2004$. Stress-inducible synthesis of proline in transgenic rice confers faster growth under stress conditions than that with constitutive synthesis. Plant Sci. 166 (4), 941-948.

Suárez, R., Calderón, C., Iturriaga, G., 2009. Enhanced tolerance to multiple abiotic stresses in transgenic alfalfa accumulating trehalose. Crop Sci. 49 (5), 1791-1799.

Sulpice, R., Gibon, Y., Bouchereau, A., Larher, F., 1998. Exogenously supplied glycine betaine in spinach and rapeseed leaf discs: compatibility or noncompatibility? Plant Cell Environ. 21 (12), 1285-1292.

Suprasanna, P., Nikalje, G.C., Rai, A.N., 2016. Osmolyte accumulation and implications in plant abiotic stress tolerance. Osmolytes and Plants Acclimation to Changing Environment: Emerging Omics Technologies. Springer, India, pp. 1-12.

Suprasanna, P., Rai, A.N., HimaKumari, P., Kumar, S.A., Kavi Kishor, P.B., 2014. Modulation of proline: implications in plant stress tolerance and development. Plant Adaptation to Environmental Change. CAB International, Oxfordshire, pp. 68-96.

Surekha, C., Aruna, L., Hossain, M.A., Wani, S.H., Neelapu, N.R.R., 2015. Present status and future prospects of transgenic approaches for salt tolerance in plants/crop plants. In: Wani, S. H., Hossain, M.A. (Eds.), Managing Salt Tolerance in Plants, Molecular and Genomic Perspectives. Taylor \& Francis 1 (18), pp. 329-352.

Surekha, C., Kumari, K.N., Aruna, L.V., Suneetha, G., Arundhati, A., Kishor, P.K., 2014. Expression of the Vigna aconitifolia P5CSF129A gene in transgenic pigeonpea enhances proline accumulation and salt tolerance. Plant Cell, Tissue Organ Culture (PCTOC) 116 (1), $27-36$

Szabados, L., Savouré, A., 2010. Proline: a multifunctional amino acid. Trends. Plant. Sci. 15 (2), 89-97.

Takahashi, Y., Cong, R., Sagor, G.H.M., Niitsu, M., Berberich, T., Kusano, T., 2010. Characterization of five polyamine oxidase isoforms in Arabidopsis thaliana. Plant Cell Rep. 29 (9), 955-965.

Tarczynski, M.C., Jensen, R.G., Bohnert, H.J., 1992. Expression of a bacterial $m t l D$ gene in transgenic tobacco leads to production and accumulation of mannitol. Proc. Natl. Acad. Sci. 89 (7), 2600-2604.

Tavladoraki, P., Cona, A., Federico, R., Tempera, G., Viceconte, N., Saccoccio, S., et al., 2012. Polyamine catabolism: target for antiproliferative therapies in animals and stress tolerance strategies in plants. Amino Acids 42 (2-3), 411-426.

Thomas, J.C., Sepahi, M., Arendall, B., Bohnert, H.J., 1995. Enhancement of seed germination in high salinity by engineering mannitol expression in Arabidopsis thaliana. Plant Cell Environ. 18 (7), 801-806

Tiburcio, A.F., Altabella, T., Bitrián, M., Alcázar, R., 2014. The roles of polyamines during the lifespan of plants: from development to stress. Planta 240 (1), 1-18.

Umezawa, T., Fujita, M., Fujita, Y., Yamaguchi-Shinozaki, K., Shinozaki, K., 2006. Engineering drought tolerance in plants: discovering and tailoring genes to unlock the future. Curr. Opin. Biotechnol. 17 (2), 113-122.

Van Der Meer, I.M., Ebskamp, M.J., Visser, R.G., Weisbeek, P.J., Smeekens, S.C., 1994. Fructan as a new carbohydrate sink in transgenic potato plants. Plant Cell 6 (4), 561-570.

Vendruscolo, E.C.G., Schuster, I., Pileggi, M., Scapim, C.A., Molinari, H.B.C., Marur, C.J., et al., 2007. Stress-induced synthesis of proline confers tolerance to water deficit in transgenic wheat. J. Plant. Physiol. 164 (10), 1367-1376.

Verdoy, D., Coba De La Peña, T., Redondo, F.J., Lucas, M.M., Pueyo, J.J., 2006. Transgenic Medicago truncatula plants that accumulate proline display nitrogen-fixing activity with enhanced tolerance to osmotic stress. Plant Cell Environ. 29 (10), 1913-1923.
Vijn, I., Smeekens, S., 1999. Fructan: more than a reserve carbohydrate? Plant Physiol. 120 (2), 351-360.

Vinocur, B., Altman, A., 2005. Recent advances in engineering plant tolerance to abiotic stress: achievements and limitations. Curr. Opin. Biotechnol. 16 (2), 123-132.

Vives-Peris, V., Gómez-Cadenas, A., Pérez-Clemente, R.M., 2017. Citrus plants exude proline and phytohormones under abiotic stress conditions. Plant Cell Rep. 36 (12), 1971-1984.

Wang, G.P., Li, F., Zhang, J., Zhao, M.R., Hui, Z., Wang, W., 2010. Overaccumulation of glycine betaine enhances tolerance of the photosynthetic apparatus to drought and heat stress in wheat. Photosynthetica 48 (1), 30-41.

Wang, H., Tang, X., Wang, H., Shao, H.B., 2015. Proline accumulation and metabolism-related genes expression profiles in Kosteletzkya virginica seedlings under salt stress. Front. Plant Sci. 6

Wang, J.Y., Tong, S.M., Li, Q.L., 2013. Constitutive and salt-inducible expression of SlBADH gene in transgenic tomato (Solanum lycopersicum L. cv. Micro-Tom) enhances salt tolerance. Biochem. Biophys. Res. Commun. 432 (2), 262-267.

Wani, S.H., Singh, N.B., Haribhushan, A., Mir, J.I., 2013. Compatible Solute Engineering in Plants for Abiotic Stress Tolerance - Role of Glycine Betaine. Curr. Genomics 14 (3), 157-165.

Wani, S.H., Dutta, T., Neelapu, N.R.R., Surekha, C., 2017. Transgenic approaches to enhance salt and drought tolerance in plants. Plant Gene.

Wani, S.H., Sah, S.K., Hossain, M.A., Kumar, V., Balachandran, S.M., 2016. Transgenic approaches for abiotic stress tolerance in crop plants. Advances in Plant Breeding Strategies: Agronomic, Abiotic and Biotic Stress Traits. Springer International Publishing, pp. 345-396.

Wei, C., Cui, Q., Zhang, X.Q., Zhao, Y.Q., Jia, G.X., 2016. Three P5CS genes including a novel one from Lilium regale play distinct roles in osmotic, drought and salt stress tolerance. J. Plant Biol. 59 (5), $456-466$.

Wei, D., Zhang, W., Wang, C., Meng, Q., Li, G., Chen, T.H., et al., 2017. Genetic engineering of the biosynthesis of glycinebetaine leads to alleviate salt-induced potassium efflux and enhances salt tolerance in tomato plants. Plant Sci. 257, 74-83.

Wen, X.P., Pang, X.M., Matsuda, N., Kita, M., Inoue, H., Hao, Y.J., et al., 2008. Over-expression of the apple spermidine synthase gene in pear confers multiple abiotic stress tolerance by altering polyamine titers. Transgenic. Res. 17 (2), 251-263.

Wimalasekera, R., Tebartz, F., Scherer, G.F., 2011. Polyamines, polyamine oxidases and nitric oxide in development, abiotic and biotic stresses. Plant Sci. 181 (5), 593-603.

Wingler, A., 2002. The function of trehalose biosynthesis in plants. Phytochemistry 60 (5), 437-440.

Yan, L.P., Liu, C.L., Liang, H.M., Mao, X.H., Wang, F., Pang, C.H., et al., 2012. Physiological responses to salt stress of T2 alfalfa progenies carrying a transgene for betaine aldehyde dehydrogenase. Plant Cell Tissue Organ Culture (PCTOC) 108 (2), 191-199.

Yancey, P.H., 2005. Organic osmolytes as compatible, metabolic and counteracting cytoprotectants in high osmolarity and other stresses. J. Exp. Biol. 208 (15), 2819-2830.

Yeo, E.T., Kwon, H.B., Han, S.E., Lee, J.T., Ryu, J.C., Byu, M.O., 2000. Genetic engineering of drought resistant potato plants by introduction of the trehalose-6-phosphate synthase (TPS1) gene from Saccharomyces cerevisiae. Mol. Cells 10 (3), 263-268.

Yiu, J.C., Juang, L.D., Fang, D.Y.T., Liu, C.W., Wu, S.J., 2009. Exogenous putrescine reduces flooding-induced oxidative damage by increasing the antioxidant properties of Welsh onion. Sci. Hortic. (Amsterdam) 120 (3), 306-314.

Yoshiba, Y., Kiyosue, T., Katagiri, T., Ueda, H., Mizoguchi, T., Yamaguchi-Shinozaki, K., et al., 1995. Correlation between the 
induction of a gene for $\Delta 1$-pyrroline-5-carboxylate synthetase and the accumulation of proline in Arabidopsis thaliana under osmotic stress. Plant J. 7 (5), 751-760.

You, J., Hu, H., Xiong, L., 2012. An ornithine $\delta$-aminotransferase gene OsOAT confers drought and oxidative stress tolerance in rice. Plant Sci. 197, 59-69.

Yu, H.Q., Zhou, X.Y., Wang, Y.G., Zhou, S.F., Fu, F.L., Li, W.C., 2017. A betaine aldehyde dehydrogenase gene from Ammopiptanthus nanus enhances tolerance of Arabidopsis to high salt and drought stresses. Plant Growth Regul. 83 (2), 265-276.

Zandalinas, S.I., Mittler, R., Balfagón, D., Arbona, V., Gómez Cadenas, A., 2018. Plant adaptations to the combination of drought and high temperatures. Physiol. Plant. 162 (1), pp2-12.

Zarattini, M., Forlani, G., 2017. Toward unveiling the mechanisms for transcriptional regulation of proline biosynthesis in the plant cell response to biotic and abiotic stress conditions. Front. Plant Sci. 8, 927.

Zhang, J., Tan, W., Yang, X.H., Zhang, H.X., 2008. Plastid-expressed choline monooxygenase gene improves salt and drought tolerance through accumulation of glycine betaine in tobacco. Plant Cell Rep. 27 (6), 1113.
Zhang, N., Si, H.J., Wen, G., Du, H.H., Liu, B.L., Wang, D., 2011 a. Enhanced drought and salinity tolerance in transgenic potato plants with a BADH gene from spinach. Plant Biotechnol. Rep. 5 (1), 71-77.

Zhang, S.Z., Yang, B.P., Feng, C.L., Tang, H.L., 2005. Genetic transformation of tobacco with the trehalose synthase gene from Grifola frondosa Fr. enhances the resistance to drought and salt in tobacco. J. Integr. Plant Biol. 47 (5), 579-587.

Zhang, X., Tang, W., Lui, J., 2014. Co-expression of rice OsP5CS1 and OsP5CS2 genes in transgenic tobacco resulted in elevated proline biosynthesis and enhanced abiotic stress tolerance. Chin. J. Appl. Environ. Biol. 20 (4), 717-722.

Zhang, Y., Wu, R., Qin, G., Chen, Z., Gu, H., Qu, L.J., 2011 b. Overexpression of WOX1 leads to defects in meristem development and polyamine homeostasis in Arabidopsis. J. Integr. Plant Biol. 53 (6), 493-506.

Zhou, X., Naguro, I., Ichijo, H., Watanabe, K., 2016. Mitogenactivated protein kinases as key players in osmotic stress signalling. Biochimica et Biophysica Acta (BBA)-General Subjects 1860 (9), 2037-2052. 
This page intentionally left blank 


\title{
31
}

\section{Proteomics Insights Into Salt Stress Signaling in Plants}

\author{
Arajmand Frukh ${ }^{1}$, Altaf Ahmad ${ }^{2}$ and Tariq Omar Siddiqi ${ }^{1}$ \\ ${ }^{1}$ Department of Botany, School of Chemical and Life Sciences, Jamia Hamdard, New Delhi, India \\ ${ }^{2}$ Department of Botany, Faculty of Life Sciences, Aligarh Muslim University, Aligarh, Uttar Pradesh, India
}

\section{O U T L I N E}

31.1 Introduction

31.2 Proteomics: Edge Over Other Technologies

31.3 Technical Advances in Proteomics 31.3.1 Global Proteome Analysis

31.3.2 Targeted Proteome Analysis

31.4 Salt Stress in Plants

31.5 Response of Plants Towards Salt Stress

31.5.1 Osmotic Stress

31.5.2 Ionic Stress

31.6 Salt Stress Signaling Pathways

31.6.1 Salt Overly Sensitive (SOS) Signaling Pathway

31.6.2 Mitogen-Activated Protein Kinase

(MAPK) Signaling Pathway
479

480

481

481

483

485

485

485

485

485

485

486
31.6.3 ABA-Signaling Pathway

31.6.4 $\mathrm{Ca}^{2+} /$ Calmodulin (CaM) Signaling Pathway

31.6.5 Phospholipid Signaling Pathway

31.6.6 ROS Signaling Pathway

31.6.7 Jasmonic Acid (JA), Ethylene (ET), and Salicylic Acid (SA) Signaling Pathways

31.7 Proteomics Approach in Understanding Salt Stress Signaling Pathways

31.8 Conclusion and Future Perspectives 493

References

493

Further Reading

497

\subsection{INTRODUCTION}

Plants are continuously being subjected to a number of environmental stresses that diminish the productivity of various economically important crops (Sewelam et al., 2016; Khan and Khan, 2017). Environmental stress conditions include biotic stress, such as pathogen infection and herbivore attack, and abiotic stress, such as excess salt, drought, heat, cold, etc. ( $\mathrm{Zhu}, 2016)$. Among all, salt stress is a major environmental constraint to agricultural productivity (Ji et al., 2013). It is estimated that more than $20 \%$ of the total irrigated land on Earth has been affected by salinity, which is expected to continue to increase over time 
(Ji et al., 2016). This increase in salinity on the other hand is the major cause of decrease in crop productivity throughout the world. The increase in the salt concentration in soil occurs either naturally by means of rock weathering, salt deposited from saline wind, rain near the coast, and soil evaporation or by anthropogenic activities, which include intense irrigation, land clearing, poor agricultural practices, etc. (Hafiz CheOthman et al., 2017). Salinity imposes both osmotic as well as ionic stress in plants (Munns and Tester, 2008). In the early phase, inhibition of water uptake, cell elongation, root development, formation of new leaves occurs, while in the later phase, salt ions accumulate and cause premature senescence, disruption in enzyme functionality, and inhibition of photosynthesis (Khan et al., 2012, 2014; Roy et al., 2014; Shelke et al., 2017). It affects most of the developmental stages of plants from seed germination to reproductive stage. Plants survive against these different environmental challenges by a complicated protection mechanism. One such mechanism is the ability to sense complex stress factors to activate complex signaling pathways to respond appropriately (Sewelam et al., 2016). To improve the adaptability of plants, a better understanding of plant signaling pathways is the need of the hour.

Plants have evolved a complex signaling system to enable cell-to-cell communication during growth and development. This communication is highly responsible for plants to adjust their metabolism, growth, and development to a highly dynamic environment especially during stress conditions. Plants are unable to relocate during unfavorable environmental conditions and instead rely on intricate signaling pathways that work at the local and systemic level to withstand stress (Carella et al., 2016). The crucial part of the biological research is to study the nature and mechanism of signaling events. Stress signaling pathways in plants are complex and they don't involve only the components that transfer a "signal" from receptors to TFs or other intracellular effector proteins (Kaufmann et al., 2011). The signaling mechanism also relies on intricate networks, which encompass feedback loops, crosstalk with other signaling components, and the integration of information related to the internal state of the cell (Choudhary and Mann, 2010). Although genomics and transcriptomics have conferred the role of various genes that activate multiple signal transduction pathways they have been far less successful in identifying the signaling components involved. This may be because of the fact that there is redundancy in pathways and in components of pathways, or lethality by mutated genes encoding the proteins (Peck et al., 2001).

Genomics can offer clues regarding signaling molecules. However, the genome of an organism is static and does not necessarily reflect the pool of genes that are actually transcribed or translated into proteins in a given individual in a given environment (Song and Lin, 2017). The transcriptome analyses of gene expression at the mRNA level have contributed greatly to understand the complex signaling mechanism. However, the level of mRNA does not always correlate well with the level of protein. This is mainly due to posttranscriptional modifications. Therefore, it is insufficient to predict protein expression level from quantitative mRNA data (Yan et al., 2006). Thus, studies via alternative methods are required to complement existing genetic studies to elucidate the complex pattern of signaling. Proteome analysis aims at the complete set of proteins encoded by the genome and thus complements the transcriptome studies.

\subsection{PROTEOMICS: EDGE OVER OTHER TECHNOLOGIES}

Proteomics is evolving phenomenally as one of the major tools of "omics" to identify proteins. Proteomics has many applications. Among them signaling is one of the components. The term proteomics was coined in 1997 in analogy with genomics, the study of the genome. The word proteome is actually a combination of protein and genome and was coined by Mark Wilkins in 1994. The proteome is the entire set of proteins produced by a living organism and proteomics deals with the study of proteome. Proteomics has advanced over time to enrich the understanding of salt stress mechanism in plants. While the other two fields of "omics," that is, genomics and transcriptomics, deal with the analysis of genes, regulatory elements, and their transcripts, the field of proteomics deals with the analysis of proteins (Ashwin et al., 2017). Proteomic based technologies have been widely used in several crop species to understand the changes in the cellular activities at the protein level under salt stress.

Proteins are the important macromolecules exhibiting diverse functions in plant stress tolerance. They act as enzymes, exhibit protective functions, interact with other proteins and other biomolecules, and scavenge ROS (reactive oxygen species) either directly via chemical reactions or indirectly via metal cofactors. It is therefore highly important to study changes in proteome composition under stress to uncover key proteins involved in mechanisms underlying plant acclimation to stress. However, it is quite evident that the diverse functionality of one protein depends on its subcellular localization, PTMs, and interacting partners. Therefore, studies of particulate protein, PTMs, as well as protein-protein interactions are extremely important. The major focus of proteomics studies in the future would probably shift from a mere identification of 
differentially expressed proteins to a proper characterization of protein function in plant stress response (Kosová et al., 2013). 2-DE (2-dimensional electrophoresis) coupled with mass spectrometry are the widely used quantitative proteomics methods. Nonetheless, the defects of low rate of protein detection, low reproducibility, and difficult isolation of hydrophobic proteins restricted the full potential of 2-DE in systematic analysis of proteomic changes (Sun et al., 2017). This technical disadvantage of 2-DE gave rise to the gel-free based protein quantitative approach, which is also regarded as shotgun proteomics.

\subsection{TECHNICAL ADVANCES IN PROTEOMICS}

There are ideally three critical stages in proteomic approaches, that is, sample preparation, gel/columnbased protein/peptide separation, and identification of proteins using MS (mass spectrometry) (Ashwin et al., 2017). In case of model systems including human, yeast, and bacterial proteomes advanced proteomic technologies are being developed but they may not be directly applied to plant tissues (Timperio et al., 2008). In the case of plant tissue the main critical step for proteome analysis is sample preparation because of rigid cell wall, presence of secondary metabolites like phenolic compounds, polysaccharides, etc. These secondary metabolites usually cause protein precipitation during the disruption of tissues. Therefore, it is difficult to obtain high quality protein in case of plants. Moreover, roots and fruits have got the least amount of protein content and high amount of interfering substances, which makes it challenging to extract protein for proteome analysis. There are many techniques of extracting proteins from plant tissues for proteomic studies but phenol and trichloroacetic acid (TCA)based extraction methods have been found to give superior protein yield and good quality 2-DE gels for certain plant tissues (Wang et al., 2016). Further, the sample preparation methodology should be compatible with the downstream proteomic strategies for separation, identification/quantification, and analysis (Agrawal et al., 2011).

MS has emerged as an indispensable tool for proteomics over the years as the technology has advanced in its robustness, accuracy, sensitivity, and selectiveness in identification and quantification of proteins. Besides this, increased speed and accuracy in matching proteins/peptides from vast database searches in in silico tools for MS data analysis have been rapidly refined. Also de novo assisted database search for highly reliable protein/peptide predictions and quantifications of labeled or label-free proteins/peptides, the algorithms for de novo sequencing for organisms that do not have reference genome/proteome database, are evolving continuously. Many proteomic approaches have emerged owing to these technological advances, which can be broadly classified into global and targeted profiling of proteomes (Liebler and Zimmerman, 2013).

\subsubsection{Global Proteome Analysis}

Global proteome analysis is the most common approach for proteome analysis. This approach is generally preferred whenever there is comparative analysis of two or more proteomes or to establish a reference proteome map. The global proteome profiling approach can be further classified into two broad categories, that is, gel-based and gel-free/shotgun approaches as represented in Fig. 31.1. Gel-based approaches include 1DGE (one-dimensional gel electrophoresis), 2DGE, 2DE-DIGE (two-dimensional-differential in gel electrophoresis), and 3DGE. These are the preferred techniques used in amalgamation with MS (Görg et al., 2009). On the other hand, gel-free or shotgun approaches include isotope-coded affinity tags (ICAT), isobaric tags for relative and absolute quantitation (iTRAQ), stable isotope labeling by amino acids in cell culture (SILAC), multidimensional protein identification technology (MudPIT), and deep proteome analysis approaches. In the gel-free proteomics approach, the extracted protein mixture is directly subjected to trypsin digestion and the digested peptides are chromatographically separated and analyzed by MS. Shotgun or gel-free proteomics approach can raise the number of different proteins that can be recognized from complex samples, contrasted to more traditional gel-based approaches (Hakeem et al., 2013).

\subsubsection{Two-Dimensional Gel Electrophoresis (2DGE)}

In plant proteomics, 2DE remains one of the main methods of choice for the reproducible separation of proteins and their isoforms in complex extracts (Ngara and Ndimba, 2014). The standard approach for 2DE in the analysis of plant proteins uses immobilized $\mathrm{pH}$ gradient (IPG) gels in the first dimension for charge separation and then an orthogonal separation, in the presence of SDS (sodium dodecyl sulfate), to resolve the proteins according to their molecular mass (Cash and Argo, 2009). Using this technique, different biological samples are compared and the proteins that show differential expression are revealed in the form of spots individual gels following silver or Coomassie Blue staining. Those proteins that are of our interest are then identified by MS. The limitation of $2 \mathrm{DE}$ is the lack of reproducibility and quantitation. 


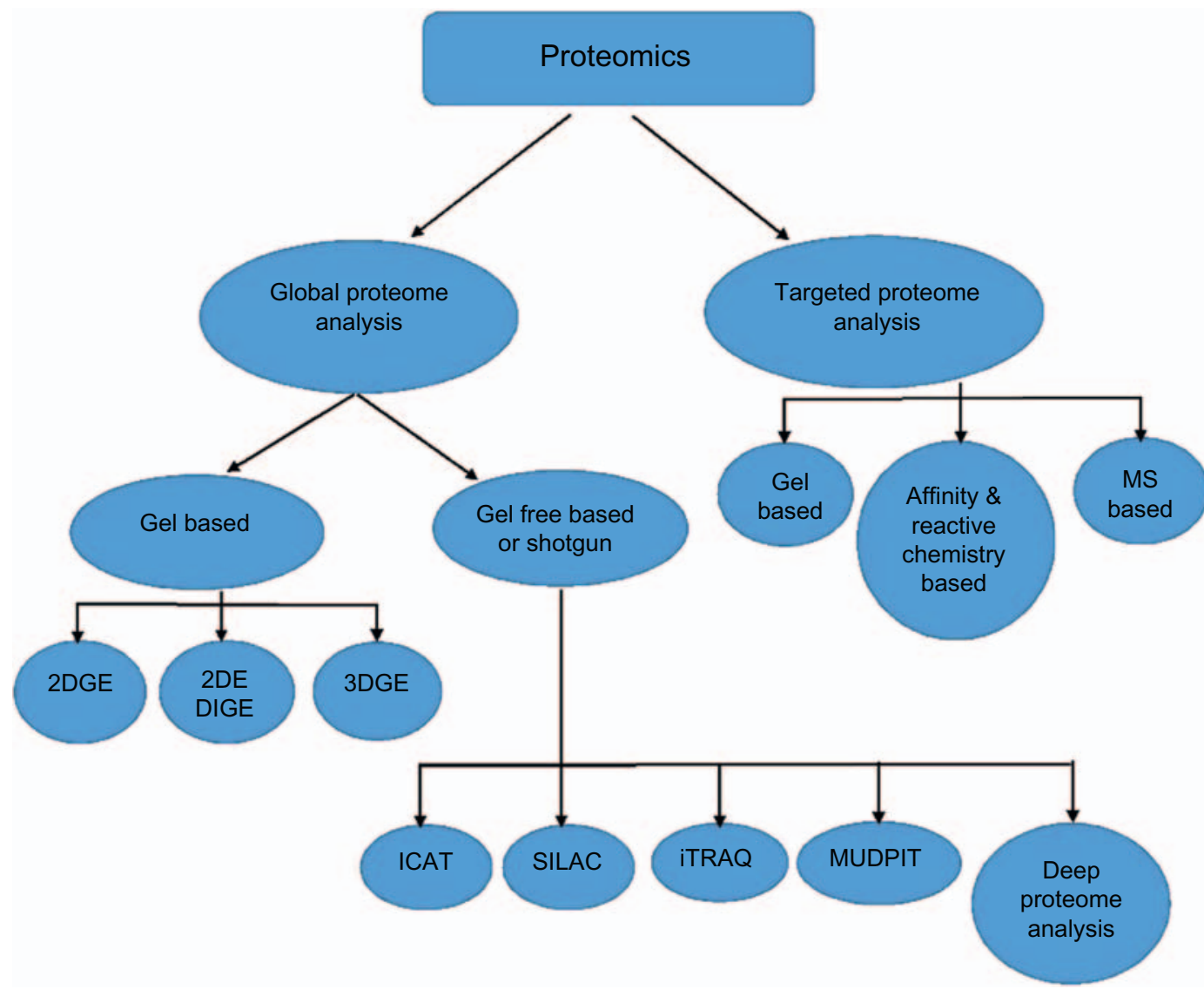

FIGURE 31.1 Schematic representation of types of proteomic techniques used for the analysis, identification, and quantification of proteins. 2DGE two-dimensional gel electrophoresis, 2DE-DIGE two-dimensional-differential in gel electrophoresis, 3DGE three-dimensional gel electrophoresis, ICAT isotope coded affinity tags, SILAC stable isotope labeling with amino acids in cell culture, iTRAQ isobaric tag for relative and absolute quantification, MudPIT multidimensional protein identification technology.

\subsubsection{Three-Dimensional Gel Electrophoresis (3DGE)}

Three-dimensional (3D)-gel electrophoresis has advancement over 2DE because the protein analysis is carried out in a separation medium that extends substantially in all three spatial dimensions. 3DGE was developed to overcome some of the limitations of 2DGE such as lack of accuracy in protein identification and variance in relative quantification of spots that occur mainly due to comigration of proteins. In this method the analysis of the proteins occurs according to three independent separation parameters, that is, native size, $\mathrm{pI}$, and molecular mass (MM) (Ventzki and Stegemann, 2010). Another advantage of this method is that it aids in unambiguous identification of posttranslational modification (PTM) of proteins (Rabilloud, 2013). Despite its high accuracy than 2DE this principle is yet to be employed in unraveling the signaling pathway related proteome.

\subsubsection{Isotope-Coded Affinity Tag (ICAT)}

ICAT is the first in vitro method that permits tagging of proteins and peptides of all types of biological samples using stable isotopes. It was developed by Gygi and his associates in 1999 (Gygi et al., 1999). This technique employs ICAT reagent, which comprises of mainly three structures, that is, iodo-acetamide group or N-ethymaleimide, a spacer or linker arm, and biotin. Iodo-acetamide group or N-ethymaleimide is highly reactive group that binds with the thiol groups (cysteines) of proteins and leads to its alkylation. A spacer or linker arm is meant for introduction of mass shift by incorporation of different isotopes in different samples. Isotopes introduce mass difference of in the labeled or unlabeled amino acid residues producing light and heavy tags. Biotin is an affinity tag that captures all the cysteine containing peptides from the mixture and in this way assists purification of labeled peptides (Zargar et al., 2016). This technique adopts the strategy of chromatographic fractionation of ICAT labeled tryptic peptides, followed by identification and quantification of proteins using tandem MS (Shiio and Aebersold, 2006). No doubt, this technique offers accuracy as samples are similarly treated by protease and in this way experimental variations are prevented. 
Moreover, sample complexity is also reduced due to tagging of only cysteine residues but it is also associated with loss of information leading to lower proteome coverage. Also the expensive tags have made the ICAT approach inapt for use.

\subsubsection{Stable Isotope Labeling by Amino Acids in Cell Culture (SILAC)}

SILAC is a simple in vivo technique that was first developed in 2002 (Ong et al., 2002). Isotopic lysine $\left(\mathrm{C}_{6} \mathrm{H}_{14} \mathrm{~N}_{2} \mathrm{O}_{2}\right)$ and arginine $\left(\mathrm{C}_{6} \mathrm{H}_{14} \mathrm{~N}_{4} \mathrm{O}_{2}\right)$ amino acid tags are mainly used in this strategy. This technique works on the principle of incorporation of nonradioactive heavy isotopes in either amino acids or metabolites in the culture medium, which can be easily identified by tandem MS (Geiger et al., 2011). However, in the case of plant cells only partial labeling of plant proteins with selected stable isotope-coded amino acids appears to be feasible due to the autotrophic nature of plant cells allowing the synthesis of amino acids from inorganic nitrogen (Oeljeklaus et al., 2009). This is the main reason that the strengths of the SILAC strategy could not be fully exploited for the analysis of plant proteomes. Moreover, the approach is relatively more expensive and cumbersome compared with other shotgun proteomic approaches.

\subsubsection{Isobaric Tag for Relative and Absolute Quantification (iTRAQ)}

The strategy of iTRAQ was introduced by Ross and his associates in 2004, and involves isobaric labeling of peptides (Ross et al., 2004). This strategy has been utilized efficiently to explore the diverse molecular mechanisms occurring in plants. Among the shotgun approaches iTRAQ is the most preferred and relatively less sophisticated method for proteomic studies. The iTRAQ reagents usually consist of an $\mathrm{N}$-methyl piperazine reporter group that introduces mass shifts, a balance group that is required to maintain the overall mass of isobaric tag, and an N-hydroxy succinimide ester group that is reactive with the primary amines of peptides. The balance groups, which are mainly carbonyl group, function to make the labeled peptides from each sample isobaric and the quantification is facilitated through analysis of reporter groups that are generated upon fragmentation in the mass spectrometer. Reporter groups have a mass range varying from 114 to $117 \mathrm{Da}$ and 113 to $121 \mathrm{Da}$, which is compensated by balance group having a range from 28 to $31 \mathrm{Da}$ and 184 to 192 Da (Rauniyar and Yates, 2014). There are currently two mainly used reagents: 4-plex (mass $\operatorname{tag}=145 \mathrm{Da}$ ) and 8-plex (mass tag $=305 \mathrm{Da}$ ), which can be used to label all peptides from different samples/treatments. These samples are then pooled and usually fractionated by nano liquid chromatography and analyzed by tandem mass spectrometry (MS/MS). The sensitivity of detecting peptides is higher in iTRAQ than other shotgun approaches because the labels are isobaric and uniform to all peptides (Evans et al., 2012).

\subsubsection{Multidimensional Protein Identification Technology (MudPIT)}

MudPIT is the first gel-free approach developed for complex proteome analysis (Washburn et al., 2001). In case of multidimensional protein identification technology (MudPIT) biphasic or triphasic microcapillary columns are coupled to high-performance liquid chromatography, tandem mass spectrometry, and database searching. This multidimensional separation results in the increase in the sensitivity and identification of the low abundant peptides increases due to which an exhaustive list of proteins present in a sample is generated (Florens and Washburn, 2006). MudPIT has been proven to be an excellent tool for both qualitative and quantitative proteomic analysis. Although this technique is less complex and cheaper than iTRAQ, quantitation accuracy is relatively less and largely dependent on sample preparation.

\subsubsection{Deep Proteome Analysis}

Deep proteome analysis, also known as deep proteomics, is one of the approaches used to get the maximum coverage of proteins present in a complex protein mixture. The basic workflow of this technique relies on three protein separation techniques, that is, 1D PAGE, in-gel IEF (iso-electric focusing) and reverse phase LC-MS (liquid chromatography-mass spectrometry). The enhancement of the resolving power is achieved by gel fractionation and trypsin digestion steps after 1D-PAGE (Atanassov and Urlaub, 2013). To obtain the maximum and deeper coverage of peptides sometimes MudPIT approach is followed after trypsin digestion of fractionated gel slices. All these combined approaches coupled with Orbitrap MS enhance the sensitivity and peptide coverage by many folds, which in turn improves the identification of LAPs (linear azole-containing peptides) and posttranslationally modified proteins. Due to these advantages this is the most often used approach in recent years, often used for nonmodel plants.

\subsubsection{Targeted Proteome Analysis}

Targeted proteome analysis, as the name indicates, is the selective analysis in which only a few selective or targeted peptides are quantified. Earlier the targeted protein analysis was carried out by ELISA (enzyme linked immunosorbent assay) but it requires specific 
antibodies and complete information of the proteins to be analyzed and thus it is not suitable for analysis of novel proteins. Targeted proteome analysis is mainly used for identification of interacting proteins or proteins of interest (immunoprecipitation) and identification of posttranslationally modified proteins. The profiling or identification of specific protein or selective group of proteins in this case is carried out by the use of motif or PTM-specific stains, antibodies (immunoassay) or targeted MS assays. Among these methods MS-based approaches are gaining more attention and utility over others because of the advantage of multiplexing many target peptides with quantitative information in a single experiment. Moreover, it has got the ability to systematically configure any protein or protein modifications of interest (Liebler and Zimmerman, 2013).

\subsubsection{Gel-Based Targeted Proteomics}

In case of gel based targeted proteomics proteinspecific or PTM-specific staining procedures are followed after global profiling of proteins using 2DGE. PTM specific stains like ProQ-Diamond used for the detection of phosphoproteins, Lissamine Rhodamine B sulfonyl hydrazine (LRSH) used for the detection of glycoproteins are used for visualizing the proteins of interest. Another way of detection of phosphoproteins is by comparative analysis between phosphatase treated proteome profiles and untreated profiles, which would aid in mapping differential migration of proteins (Yamagata et al., 2002). Some web-based tools are also there that aid in putative identification of modified proteins in in silico gels. The limitation of this approach is that it can only detect certain PTMs like phosphorylation, glycosylation, acetylation, and ubiquitination after MS analysis (Mann and Jensen, 2003). These limitations are the main reason that the gel based targeted proteome analysis is not followed nowadays.

\subsubsection{Affinity and Reactive Chemistry-Based Proteomics}

For the large scale study of proteins or peptides of interest in a complex sample enrichment or purification is required. Immobilized metal affinity chromatography (IMAC) using transition metals such as $\mathrm{Fe}^{3+}$ or $\mathrm{Ga}^{3+}$ (Ficarro et al., 2002) as well as titanium dioxide $\left(\mathrm{TiO}_{2}\right)\left(\right.$ Macek et al., 2009) or zirconium dioxide $\left(\mathrm{ZrO}_{2}\right)$ (Kweon and Håkansson, 2006) chromatography, often used in combination with strong cation exchange (SCX) chromatography, are the most common enrichment strategies used to date. Immunoprecipitation using phosphotyrosine-specific antibodies is another enrichment method frequently employed. This technique is usually employed for those low abundant peptides / proteins that tend to elude MS detection following other enrichment strategies (Kaboord and Perr, 2008).

\subsubsection{Mass Spectrometry-Based Targeted Proteomics}

There are a number of technologies that can be used to study proteomes, but arguably none is more powerful than mass spectrometry. The goal of a targeted proteomics experiment is to monitor or select a few proteins of interest with high sensitivity, reproducibility, and quantitative accuracy. Although various mass spectrometric platforms have been used for targeted proteomics analysis only a few tools like triple quadrupole, quadrupole trap (Q-Trap), and linear trap quadrupole-orbitrap (LTQ-Orbitrap) are widely employed. The triple quadrupole mass spectrometer operates as a dual mass filter that allows molecular ions of predetermined masses to be selected for fragmentation in the instrument. Peptide ions travel into the first mass filter, which is programmed to select specific "precursor" ions on the basis of their $\mathrm{m} / \mathrm{z}$ ratio for fragmentation. The second mass filter then selects the target "product" ions, which are guided to the detector for quantification, resulting in a trace of signal intensity versus retention time for each precursor ion. The process of detecting specific signals or modifications in triple quadrupole is called selected reaction monitoring (SRM). Similarly, the process of detecting multiple modifications is called multiple reaction monitoring (MRM) (Liebler and Zimmerman, 2013). SRM is the most mature mass spectrometry-based technology but as it is laborious new methodologies are on the horizon. Sequential window acquisition of all theoretical spectra (SWATH) is one such approach in which complex mass spectra generated by data-independent acquisition are queried for the presence of specific peptides using libraries of qualified peptide fragment spectra. Another new approach called parallel reaction monitoring (PRM) provides increased speed, sensitivity, and selectivity because all transitions are monitored in parallel in a single analysis (Gallien et al., 2014). Despite many advances in detection and processing algorithms, MSbased label-free quantification methods suffer from light precision errors between samples. For this reason the strategy of isotope dilution is implemented with SRM/MRM/PRM techniques. After isotope labeling, the true and precise abundance of each protein or peptide is determined by standards such as absolute quantification (AQUA), quantification concatamers (QconCAT), and protein standard absolute quantification (PSAQ). QconCAT, PSAQ, and AQUA methods are highly specific and sophisticated, and find a better scope in clinical applications. 


\subsection{SALT STRESS IN PLANTS}

Salinity imposes two kinds of stresses to plants; osmotic stress arising due to increased osmotic pressure, and ionic stress due to the increase in the levels of toxic ions like $\mathrm{Na}^{+}$and $\mathrm{Cl}^{-}$leading to ionic imbalance (Flowers and Colmer, 2008). Osmotic stress causes cell membrane disruption in plants. Plants respond to osmotic stress, with sensors and signal transduction networks providing information to the cell about the osmolarity of its surroundings. These signals activate responses to deal with extreme conditions. Ionic stress on the other hand disrupts the mineral relations of plants. $\mathrm{Na}^{+}$induce $\mathrm{K}^{+}$deficiency and $\mathrm{Cl}^{-}$inhibit $\mathrm{NO}^{3-}$ absorption, which eventually generates imbalances and nutritional disorders and finally may confront the structure and composition of plant cells, injure macromolecules, and consequently lead to senescence of the leaves (Hakeem et al., 2013). Although the general effects of salinity stresses on plant growth and development have been studied, their influence at the physiological and biochemical levels is not well understood (Manuchehri and Salehi, 2014).

\subsection{RESPONSE OF PLANTS TOWARDS SALT STRESS}

\subsubsection{Osmotic Stress}

Osmotic stress occurs due to the lower osmotic potential of the soil because of the higher concentration of soluble salts in saline soils. Whenever plants are exposed to osmotic stress, they exhibit a wide range of responses at the molecular, cellular, and whole-plant levels. These include morphological and developmental changes, adjustment in ion transport, and metabolic changes (Xiong and Zhu, 2002). Plants tend to lose water to the external environment during osmotic stress, which in turn causes decrease in the turgor pressure. As a result of loss of turgor pressure the main processes of cell division and cell expansion are highly affected. This indirectly inhibits the plant growth. The decreased osmotic potential induces specific signaling events in plants, which include MAPK signaling, calcium signaling, phospholipid signaling, and ABA signaling (Kosová et al., 2013).

\subsubsection{Ionic Stress}

Ionic effect is considered as a continuous, long-term effect of an accumulative nature since it usually depends on the intracellular salt ion levels, which increase with the duration of salinity stress and also with aging processes of a given plant tissue. Salinity stress leads to an increase in the activity of salt ions in soil water surrounding plant root cells. This results in the entry of $\mathrm{Na}^{+}$ions into the cytoplasm passively via nonselective cation channels because of the negative electrical potential of plant cell cytoplasm. The excessive $\mathrm{Na}^{+}$ions harm plant growth and metabolism. To diminish these harmful effects sequestration of excessive $\mathrm{Na}^{+}$ions is required for which the SOS pathway paves the way.

\subsection{SALT STRESS SIGNALING PATHWAYS}

\subsubsection{Salt Overly Sensitive (SOS) Signaling Pathway}

Salt stress leads to ionic stress, which is crucial for normal plant growth. Hence, ion homeostasis by compartmentalization is an essential process for growth of plants under stress conditions. Glycophytes and halophytes both cannot tolerate high $\mathrm{NaCl}$ concentration. Therefore, salt is either transported or sequestered in older tissues to protect plants from salt stress. Although a number of possible salt signaling pathways for ionic homeostasis have been proposed, none is established in terms of signaling proteins and inputs and outputs. One exception is the SOS pathway, which emerged recently as a result of combinatorial approaches of genetic, molecular, and biochemical analysis. The SOS signaling transduction pathway is a major regulatory mechanism for ion homeostasis.

The $\mathrm{Na}^{+}$ions that enter the cytoplasm are transported to vacuoles via $\mathrm{Na}^{+} / \mathrm{H}^{+}$antiporter. There are usually two types of $\mathrm{H}^{+}$pumps present in the vacuolar membrane: vacuolar type $\mathrm{H}^{+}$-ATPase (V-ATPase) and the vacuolar pyrophosphatase (V-PPase) (Chinnusamy et al., 2005). Between the two pumps V-ATPase is the most dominant one and plays an important role in ion homeostasis. The SOS signaling pathway consists of three major proteins, SOS1, SOS2, and SOS3. SOS1 encodes plasma membrane $\mathrm{Na}^{+} / \mathrm{H}^{+}$antiporter, which is one of the possible $\mathrm{Na}$ sensors and is essential in regulating $\mathrm{Na}^{+}$efflux at cellular level. $\mathrm{Na}^{+} / \mathrm{H}^{+}$antiporter also plays a critical role in controlling long distance $\mathrm{Na}^{+}$transport from root to shoot. SOS1 protein has 10-12 transmembrane domains and a long tail (of more than 700 amino acids) to sense $\mathrm{Na}^{+}$and is supposed to exist in the cytoplasm. Another protein, that is, SOS5 containing two putative AGP-like (arabinogalactanproteins-like) domains and two alternatively organized fasciclin-like domains residing in the outer surface of the plasma membrane also seems as a candidate for being a $\mathrm{Na}^{+}$sensor, although limited data is available about the function of this protein 
(Türkan and Demiral, 2009). SOS2 gene encodes serine/ threonine kinase, which is activated by $\mathrm{Ca}^{+}$signals elicited by salt stress. The serine/threonine kinase protein has got $\mathrm{N}$-terminal catalytic domain and C-terminal regulatory domain (Liu et al., 2000). The regulatory domain contains 21 amino acid long FISL motif (also called NAF domain), which serves as a site of interaction for $\mathrm{Ca}^{+}$binding SOS3 binding protein. SOS3 encodes EFhand $\mathrm{Ca}^{2+}$ binding protein, also called myristolylated $\mathrm{Ca}^{+}$binding protein. This protein has an N-terminus myristolylation site, which plays an important role in conferring salt stress (Ishitani et al., 2000).

Whenever there is increase in the $\mathrm{Na}^{+}$level, intracellular $\mathrm{Ca}^{+}$levels get elicited. The cytosolic $\mathrm{Ca}^{+}$perturbations are decoded by $\mathrm{Ca}^{2+}$ sensing proteins such as calcineurin B-like proteins (CBLs) and their cooperative partners CBL interacting protein kinases (CIPKs) (Knight and Knight, 2001). This sharp increase in the $\mathrm{Ca}^{+}$levels facilitates the binding of SOS3 protein with SOS2. The SOS3 protein activates SOS2 protein by releasing its self-inhibition. The SOS3-SOS2 complex is then loaded onto the plasma membrane and their interaction results in the activation of kinases (Guo et al., 2004). Activated kinase phosphorylates SOS1 protein thereby increasing its transport activity. The phosphorylation of SOS1 results in increased $\mathrm{Na}^{+}$ efflux reducing the $\mathrm{Na}^{+}$toxicity (Gupta and Huang, 2014). SOS1 transcript present in Arabidopsis plants was found to be upregulated by $\mathrm{NaCl}$ treatment ( $\mathrm{Zhu}$, 2001). Moreover, molecular studies have also shown that SOS1 gene expression is regulated by SOS2 and SOS3 (Huang et al., 2012). SOS3-SOS2 protein complex also downregulates the activity of low affinity $\mathrm{Na}^{+}$ transporter HKT1 under salt stress so as to regulate the entry of $\mathrm{Na}^{+}$ions into cells (Mahajan et al., 2008). SOS2 also influences vacuolar $\mathrm{Na}^{+} / \mathrm{H}^{+}$exchanger activity to sequestrate $\mathrm{Na}^{+}$ions into vacuolar compartment and thus facilitates the process of ion homeostasis (Fig. 31.2). SOS2 also regulates vacuolar $\mathrm{H}^{+} / \mathrm{Ca}^{+}$ antiporter CAX1, which is independent of SOS3 and thus maintains the $\mathrm{Ca}^{+}$homeostasis. It also interacts with $\mathrm{AB} 12$, which restores the homeostasis after stress condition by dephosphorylating the proteins that are phosphorylated by SOS2 (Ohta et al., 2003). Phosphorylation of SOS1 by the SOS2-SOS3 kinase complex activates SOS1 and results in increased tolerance to $\mathrm{NaCl}$ and enhanced $\mathrm{Na}$ exclusion in yeast (Quintero et al., 2002).

\subsubsection{Mitogen-Activated Protein Kinase (MAPK) Signaling Pathway}

The MAPK kinase pathways are intracellular signal modules that mediate signal transduction from the cell

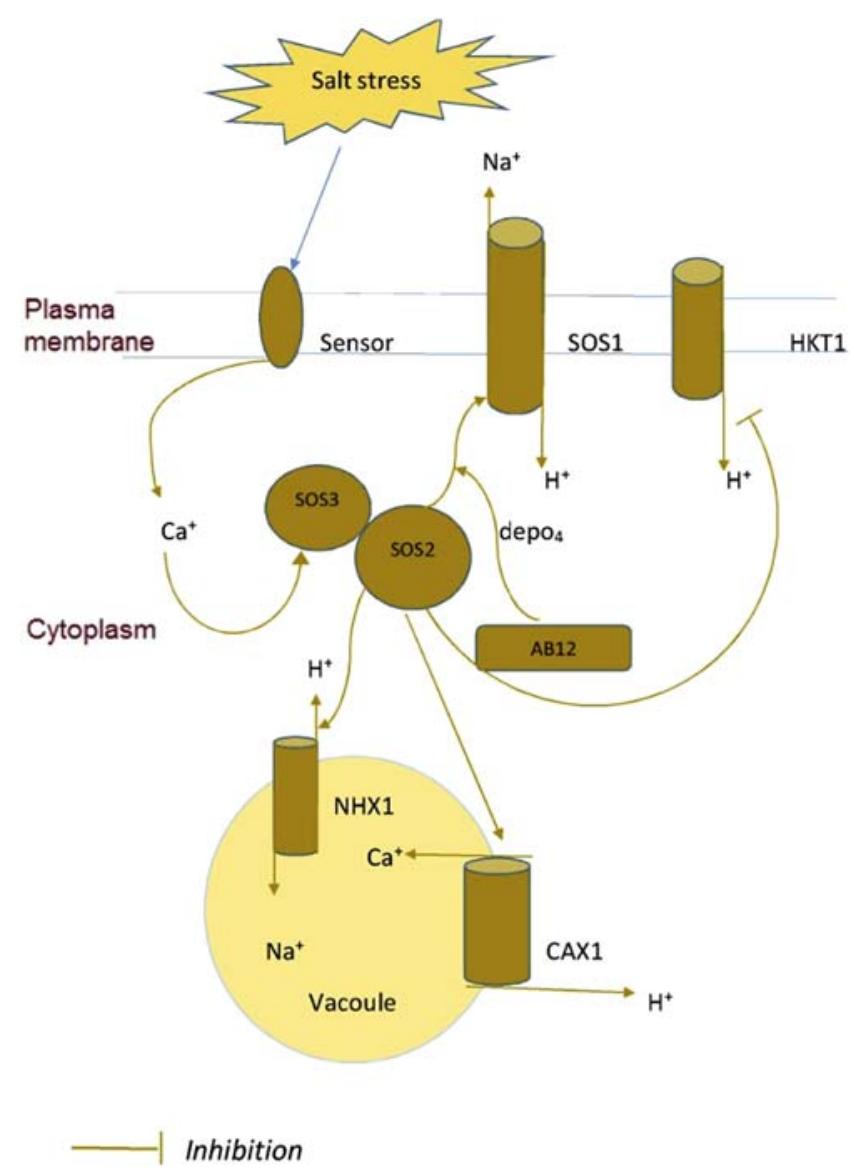

FIGURE 31.2 SOS (salt overly sensitive) pathway for the regulation of ion homeostasis during salt stress. Excessive $\mathrm{Na}^{+}$ions are sensed by unknown sensors at the plasma membrane, which leads to elicitation of cytosolic $\mathrm{Ca}^{2+}$ ions. $\mathrm{Ca}^{2+}$ signals are perceived by SOS3, which activates the SOS2 kinase. The SOS3-SOS2 complex phosphorylates plasma membrane $\mathrm{Na}^{+} / \mathrm{H}^{+}$antiporter SOS1 to efflux $\mathrm{Na}^{+}$out of the cytoplasm. Further, it inhibits HKT1 activity so as to restrict $\mathrm{Na}^{+}$ions into the cytoplasm thereby regulating ion homeostasis. SOS2, independent of SOS3, regulates NHX1 activity to transport $\mathrm{Na}^{+}$from cytosol to vacuole so as to regulate cellular $\mathrm{Na}^{+}$levels. SOS2 also regulates vacuolar $\mathrm{H}^{+} / \mathrm{Ca}^{+}$antiporter CAX1 to maintain the $\mathrm{Ca}^{+}$homeostasis. It also interacts with $\mathrm{AB} 12$, which restores the homeostasis after stress conditions by dephosphorylating the proteins that are phosphorylated by SOS2.

surface to nucleus. These are usually osmolarity signaling modules as they respond to the osmotic stress caused by various abiotic stress components (Kaur and Gupta, 2005). The MAP kinase family belongs to the CMGC group of the eukaryotic protein kinase superfamily. All MAPKs are characterized by the unique signature motif that distinguishes them from other eukaryotic protein kinases. This signature motif consists of part of the phosphorylation lip and the "P +1 " substrate-binding pocket, both of which are located on loop 12 of the three-dimensional structure of the MAP kinase and are critical for MAPK activity (Kültz and Burg, 1998). MAPK is one of the most studied 
signaling mechanisms in plants. MAPK cascade is composed of 3 protein kinases: MAP3K, MAP2K, and MAPK. MAPK cascade has been mainly studied in Arabidopsis, which contains more than 80 MAPKs, 10 MAP2Ks, and 20 MAP3Ks. It has been observed that under salt stress MAPKs respond to the stress stimuli. The salt stress activated MAPKs are activated by secondary signals rather than primary osmotic stress signals. The rapid activation of multiple MAPKs, including MAPK3, 4, and 6, has long been observed in plants in response to biotic as well as abiotic stimuli (deZelicourt et al., 2016). These MAPKs are activated by MAP2Ks, which in turn are activated by MAP3Ks in a sequential manner via phosphorylation (Colcombet and Hirt, 2008) (Fig. 31.3). An activated MAPKKK (MAP3K) first phosphorylates two serine and/or threonine residues $\left(\mathrm{S} / \mathrm{T}-\mathrm{X}_{3-5}-\mathrm{S} / \mathrm{T}\right)$ located in the activation loop of MAPKK (MAP2K), which in turn phosphorylates the T-X-Y motif present in the activation loop of MAPK and in this way triggers the activation of MAPK (Hamel et al., 2012; Danquah et al., 2014). Activated

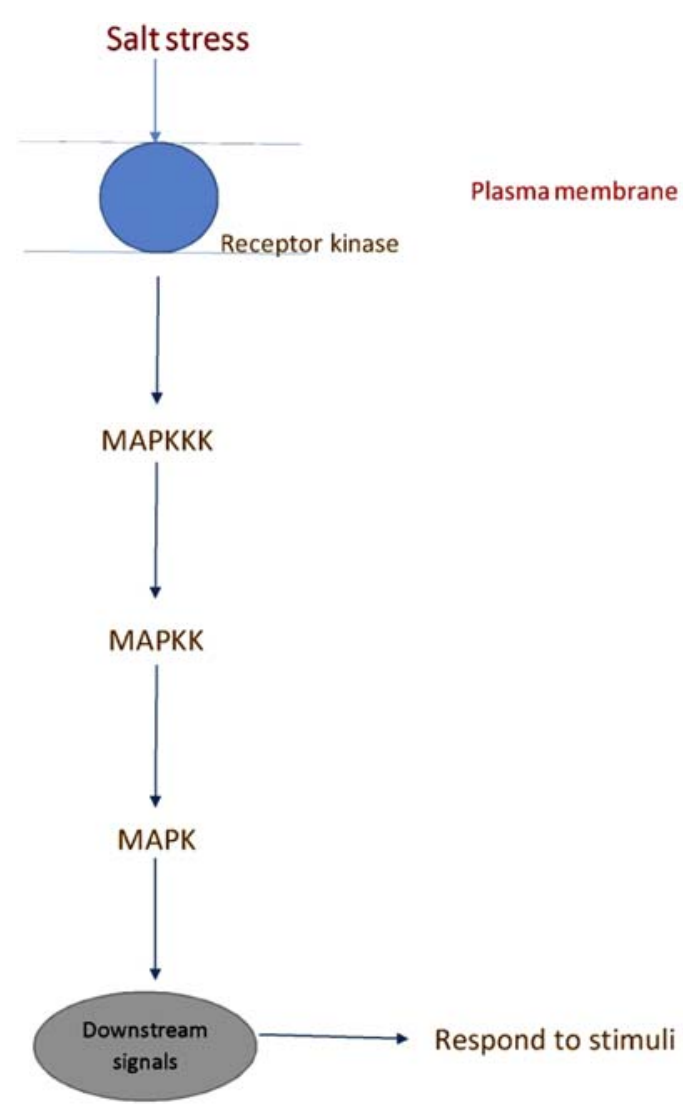

FIGURE 31.3 MAPK (mitogen activated protein kinase) cascade. In this pathway the salt stress induces receptor kinases present in the plasma membrane, which in turn causes the phosphorylation of MAPKKK. The MAPKKK causes the downstream signals by the activation of MAPKK and MAPK via sequential phosphorylation, ultimately leading to response to salt stress stimuli.
MAPK is imported into the nucleus, where it phosphorylates and activates specific downstream signaling components. The sequential activation of MAPK cascade eventually results in the activation of transcription factors, phospholipases, cytoskeletal, and microtubule associated proteins and also results in the expression of number of genes that respond to the stress stimuli (Taj et al., 2010). In case of Arabidopsis it was observed that MKK2 acts as a key signal transducer for salt stress (Teige et al., 2004). The activity of GMK1 (Glycine max MAP kinase 1) was found to be induced and regulated by posttranslational modification with increasing salt concentrations (Im et al., 2012). Ectopic expression of ZmSIMK1 (Zea mays salt-induced mitogen-activated protein kinase 1) in Arabidopsis resulted in increased resistance against salt stress suggesting that ZmSIMK1 plays an important role in salt stress (Gu et al., 2010). In Arabidopsis it was found that MKK5 is involved in $\mathrm{NaCl}$-induced salt stress via regulating the expression of iron superoxide dismutase gene (Xing et al., 2015).

\subsubsection{ABA-Signaling Pathway}

ABA belongs to class of isoprenoids (terpenoids) and also belongs to the most important phytohormones involved in the plant growth, development, and adaptation to various stress conditions. ABA plays a vital role in controlling downstreaming response essential for adaptation to stress (Raghavendra et al., 2010). ABA signaling cascades, synchronized with the plant growth stage and other hormone levels regulate important abiotic stress responses (Park et al., 2016). One of the most important advances in the past few decades has been the identification of $\mathrm{ABA}$ receptors and the elucidation of the core ABA-signaling pathway. Stress induced biosynthesis of ABA primarily occurs in vascular tissue but it exerts its response in various cells, including distant guard cells (Kuromori et al., 2010). ABA signaling transduction occurs via central signaling module made up of proteins belonging to three classes: pyracbactin resistance/pyracbactin resistance like/regulatory component of ABA receptors (PYR/PYL/RCARs), which act as ABA receptors; protein phosphatase 2Cs (PP2Cs), which act as negative regulators; and SNF1-related protein kinase 2s (SnRKs), which act as positive regulators (Danquah et al., 2014). PYR/PYL/RCAR, a family of soluble START domain proteins, plays a central role in ABA perception (Melcher et al., 2010). Members of this family like PYR1, PYL1, and PYL2 were biochemically shown to directly bind to ABA. The entire family of PYR/PYL/RCAR is capable of activating ABA signaling response, which indicates that nearly all members can function as ABA receptors (Fujii and Zhu, 2009). 


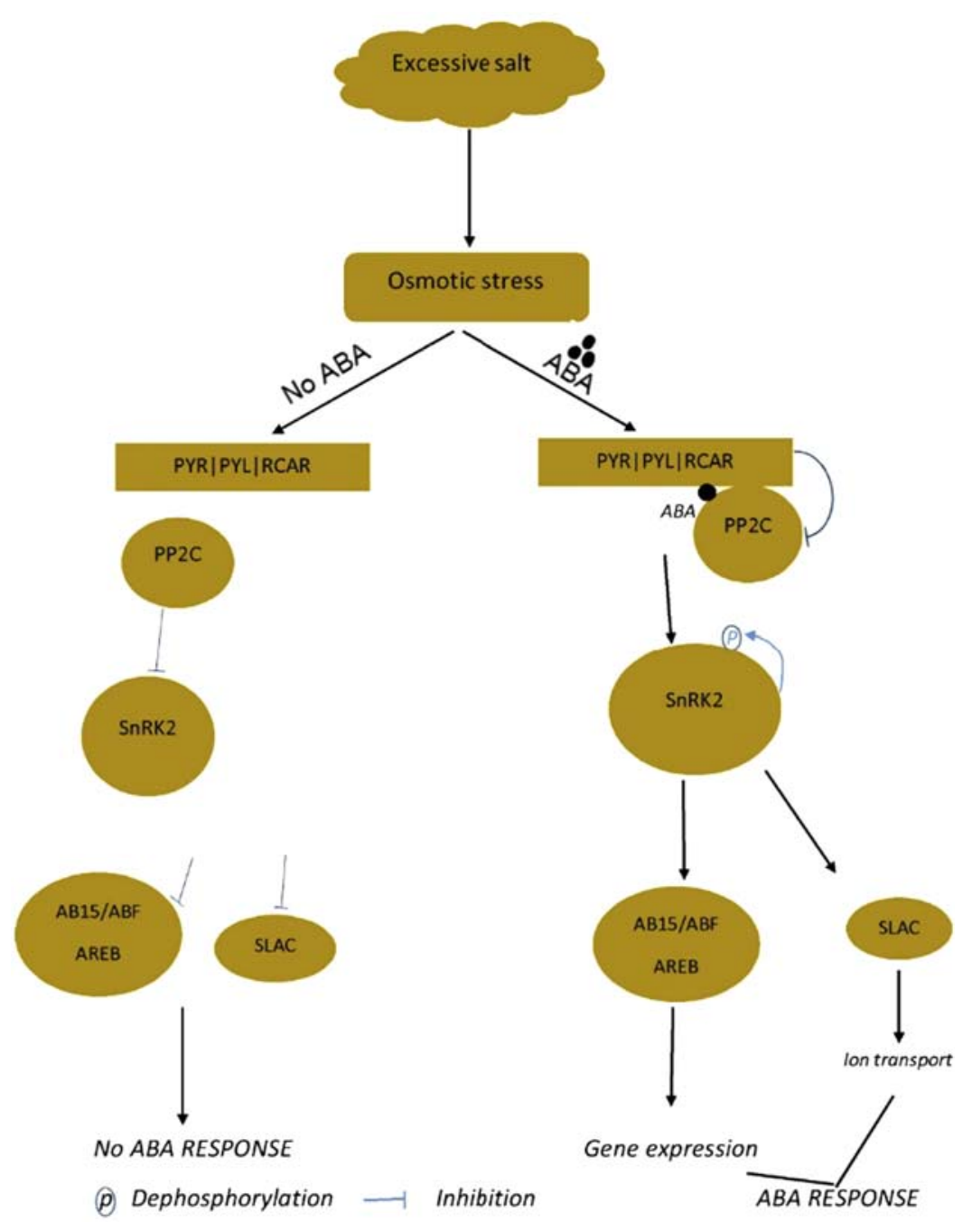

FIGURE 31.4 ABA signaling events during salt stress. Salt stress causes osmotic stress in plants, which is responded to by $A B A$. Whenever there is presence of ABA, the ABA receptors PYR/PIL/ RCAR proteins bind to $A B A$ and in turn inhibit PP2C activity, allowing the activation of SnRK2 through autophosphorylation. Active SnRK2 kinases phosphorylate downstream target proteins to induce $\mathrm{ABA}$ response. In absence of $\mathrm{ABA}, \mathrm{PP} 2 \mathrm{C}$ is active because of the inhibition of $\mathrm{ABA}$ receptor proteins and hence it inhibits the activity of SnRK2. Due to inhibition of SnRK2 there is no downstream signals and hence no ABA response.
PYLs bind ABA in presence of clade A PP2Cs, such as ABI1 (ABA-INSENSITIVE 1), ABI2, HAB1 (Homology to ABI1), and PP2CA. This is the main reason these PP2Cs are considered as coreceptors. In the absence of ABA, PP2Cs dephosphorylate SnRK2 kinases including SnRK2.1, 2.3, and SnRK2.6 in a serine residue in the kinase activation loop. This phosphorylation is necessary for kinase activity (Soon et al., 2012). There is docking of kinase activation loop of SnRK2 into the active site of PP2Cs, while the insertion of the conserved ABA-sensing tryptophan of PP2Cs into the kinase catalytic cleft occurs. In this way the kinases are kept inactive by blocking their catalytic cleft and by dephosphorylating the activation loop ultimately leading to the inhibition of enzyme activity (Miyazono et al., 2009; Melcher et al., 2010). In the presence of ABA, the ABA receptors PYR/PYL/RCAR proteins bind to ABA and in turn inhibit PP2C activity, allowing the activation of SnRK2s through autophosphorylation. This allows the SnRK2s to rely upon the ABA signal to downstream effectors (Boudsocq et al.,
2007) (Fig 31.4). 10 SnRK2 were activated by hyperosmolarity induced by $\mathrm{NaCl}$ in Arabidopsis, indicating an important role of the SnRK2 family in osmotic signaling (Boudsocq et al., 2004). In rice it was found that overexpression of OsPP1 enhanced tolerance to high salt treatment (Liao et al., 2016).

\subsection{4 $\mathrm{Ca}^{2+} /$ Calmodulin (CaM) Signaling Pathway}

Calcium was first described as an essential macronutrient element in plants, which is taken by roots and then delivered to shoot via xylem. $\mathrm{Ca}^{2+}$ ions represent an important signaling molecule and a convergence point of many disparate signaling pathways. The cytosolic $\mathrm{Ca}^{2+}$ in plant cells increases in response to various abiotic stresses. Salt stress-induced $\mathrm{Ca}^{2+}$ dependent signaling network has been reported to mediate $\mathrm{Na}^{+}$homeostasis and salt resistance (Mahajan et al., 2008). An elevation in cytosolic free calcium due 
to opening of stretch-dependent or voltage-gated calcium channels is the secondary signal caused by many different environmental stresses. Cytosolic calcium waves are produced within the cytoplasm by successive recruitment of particular $\mathrm{Ca}^{2+}$ channels to coordinate cellular response. The local elevation of cytosolic $\mathrm{Ca}^{2+}$ generates secondary messengers such as IP3 (inositol trisphosphate) or cADPR that activate a relay of spatially separate $\mathrm{Ca}^{2+}$ channels (Drøbak and Watkins, 2000). The information encoded in transient $\mathrm{Ca}^{2+}$ changes is decoded by an array of $\mathrm{Ca}^{2+}$-binding proteins, which fall into two main classes, referred to as sensor relays and sensor responders. Sensor relays, which include calmodulin (CaM), CaM-related proteins and calcineurin B-like proteins (CBLs), function through biomolecular interaction. Sensor responders such as the $\mathrm{Ca}^{2+}$-dependent protein kinases (CDPK) function at first through intramolecular interactions and undergo a $\mathrm{Ca}^{2+}$-induced conformational change that alters the protein's own activity or structure (Lecourieux et al., 2006). Calmodulin (CaM) is a small $(17 \mathrm{KDa})$, highly conserved, acidic protein. It has two globular domains each containing two EF hands connected by a flexible $\alpha$-helical linker (Luan et al., 2002). $\mathrm{CaM}$ appears to be regulatory protein and induces large changes in interhelical angles as $\mathrm{Ca}^{2+}$ is bound. There is influence of particular target proteins for affinity of CaM for $\mathrm{Ca}^{2+}$. CaM-like proteins differ from the CaM in containing more than 148 amino acid residues and have between one and six EF hand motifs. CDPKs are serine/threonine protein kinases with a Cterminal calmodulin-like domain with up to 4 EF-hand motifs that can directly bind $\mathrm{Ca}^{2+}$. Some CDPKs have an N-terminal myristoylation motif suggesting potential association with membranes. Calcineurin B-like proteins (CBLs) are relatively a new class of calcium sensors that are essential in the SOS signaling pathway to reinstate cellular ion homeostasis. There are several other proteins that bind $\mathrm{Ca}^{2+}$ but do not contain EF hand motifs. These include the phospholipase D (PLD), annexins, calreticulin, and Pistil-expressed $\mathrm{Ca}^{2+}$ binding protein (PCP) (Tuteja and Mahajan, 2007). The information that is decoded by calcium binding proteins during stress gives rise to a cascade of downstream effects, including altered protein phosphorylation and gene expression patterns (Sanders et al., 2002). Studies on Arabidopsis revealed that expression of OsMSR2 (Oryza sativa L. Multistress-Responsive gene 2), a novel calmodulin-like protein gene conferred enhanced tolerance in it under high salinity ( $\mathrm{Xu}$ et al., 2011). Similarly, in another study of Arabidopsis, overexpression of a salt-induced CaM gene from soybean, GmCaM4, conferred salt stress tolerance through the upregulation of DNA-binding activity of a MYB transcription factor MYB2 (Zeng et al., 2015). Studies also provided the information that CaM methylation plays a regulatory role in Arabidopsis under salt stress (Banerjee et al., 2013). In another study the mRNA of CaM-4 was significantly induced in response to salt stress in soybean (Park et al., 2004).

\subsubsection{Phospholipid Signaling Pathway}

Membrane lipids give rise to various signaling messengers, such as phosphatidic acid (PA), diacylglycerol (DAG), DAG-pyrophosphate (DAG-PP), lysophospholipids, free fatty acids (FFAs), oxylipins, phosphoinositides, and inositol polyphosphates (Wang et al., 2007). Phosphatidic acid acts as a messenger in osmotic stress. Signaling PA is generated by two principal routes in plants. One is the direct PA production by phospholipase D (PLD)-catalyzed hydrolysis of common membrane phospholipids. Another is phospholipase C (PLC) hydrolysis of phosphatidylinositol $(4,5)$ bisphosphate PI(4, 5)P2 followed by phosphorylation of DAG by DAG kinase (DGK). PLD and PA have been suggested to affect osmotic stress-induced production of proline (Thiery et al., 2004). Moreover, it has been found that 1-butanol, which is an inhibitor of PA production by $\mathrm{PLD}$, reduces $\mathrm{NaCl}$-induced $\mathrm{H}^{+}$ATPase activation, whereas applied PA stimulated $\mathrm{H}^{+}$-ATPase activity (Zhang et al., 2006). These results suggest that PLD and PA play an important role in salt stress. Under salt and hyperosmotic conditions, PLC-produced DAG is phosphorylated to PA by DGK and it has been found that the PLD and PLC/DGK reactions are activated differentially in response to different stimuli (Zonia and Munnik, 2004). PA regulates ABI1 function by inhibiting its phosphatase activity and by sequestering it in the plasma membrane thereby leading to the closure of stomata. Phosphoinositides (PIs) are phosphorylated phosphatidylinositols and they include three monophosphorylated PI3P, PI4P, and PI5P; three bisphosphorylated PI $(4,5) \mathrm{P} 2, \operatorname{PI}(3,4)$ $\mathrm{P} 2$, and $\mathrm{PI}(3,5) \mathrm{P} 2$; and one trisphosphorylated $\mathrm{PI}(3,4,5) \mathrm{P3}$. PIs are important signaling molecules that regulate actin organization, membrane trafficking, endo/exocytosis, and ion channels. Increase in PA and $\mathrm{PIP}_{2}$ with respect to time and dose was observed in rice under the effect of salinity (Darwish et al., 2009). In another study rapidly increased PI(4, 5)P2 synthesis in response to $\mathrm{NaCl}$ treatment was observed in Arabidopsis suggesting its important role in salt tolerance (DeWald et al., 2001).

\subsubsection{ROS Signaling Pathway}

ROS are produced during abiotic stresses and it has been hypothesized that ROS production can be the primary symptom of phytotoxicity. ROS are capable of 
inducing cellular damage by degradation of proteins, inactivation of enzymes, alterations in the gene, and interfering in various pathways of metabolic importance. ROS includes hydrogen peroxide $\left(\mathrm{H}_{2} \mathrm{O}_{2}\right)$, superoxide radical $\left(\mathrm{O}^{\bullet}{ }^{-}\right)$, hydroxyl radical $\left(\mathrm{OH}^{\bullet}\right)$, and singlet oxygen $\left({ }^{1} \mathrm{O}_{2}\right)$ etc. (Khan and Khan, 2013, 2017). In plants, NADPH oxidases, respiratory burst oxidase homologs (RBOHs), play a key role in the network of ROS production. In recent years, several studies have revealed that plant $\mathrm{RBOHs}$ are involved in a multitude of different signaling pathways including stomatal closure, plant defense, and acclimation to different abiotic stresses (Suzuki et al., 2011). Plant RBOHs have cytosolic FAD- and NADPH-binding domains in the C-terminal region, and six conserved transmembranespanning domains. RBOHs have a cytosolic N-terminal extension comprised of two $\mathrm{Ca}^{2+}$-binding EF-hand motifs and phosphorylation target sites that are important for their activity (Kimura et al., 2012). Salt stress induces elevation in cytosolic $\mathrm{Ca}^{2+}$ ions leading to the activation of Rboh (Lecourieux et al., 2006). Once activated, superoxide $\left(\mathrm{O}_{2}{ }^{-}\right)$is produced at the apoplast via the function of $\mathrm{RBOH}$ proteins, which is then dismutated to $\mathrm{H}_{2} \mathrm{O}_{2}$ by SOD. O2 ${ }^{\bullet-}$ and $\mathrm{H}_{2} \mathrm{O}_{2}$ act as secondary messengers in plants by regulating diverse function of growth and development. It is quite evident that ROS act as intracellular signaling molecules, but the controversy lies in the fact that how they can set specific signaling duties. This controversy arises because the specificity in signaling pathways is generally mediated via the noncovalent binding of a ligand to its cognate receptor through a shapecomplementary fit between macromolecules but ROS deliver signaling events via chemical reactions that lead to protein modifications (Nathan, 2003). The chemical characteristics and the biological activities of each ROS reveal an answer to this question. $\mathrm{O}_{2}{ }^{--}$is unstable and is not able to diffuse through membranes because of its negative charge, which makes this ROS a relatively poor signaling molecule. On the other hand $\mathrm{H}_{2} \mathrm{O}_{2}$ is relatively stable and can diffuse through biological membranes because it is not charged, which makes it fit for signaling. The intracellular signal transduction is mediated by $\mathrm{H}_{2} \mathrm{O}_{2}$ through chemoselective oxidation of Cys residues in signaling proteins, such as glutathione, thioredoxins, and peroxiredoxins (Paulsen and Carroll, 2009). High indiscriminate reactivity of $\mathrm{OH}^{\cdot}$ limits its diffusion to sites of production. ${ }^{1} \mathrm{O}_{2}$ could deliver specific signaling events mainly through spatial aspects of ROS production because the half-life time of ${ }^{1} \mathrm{O}_{2}$ is very short and as a result it can react directly only with molecules in close proximity to its production location. In recent years rapid progress has been made in defining ROS as a major signal in diverse biological processes in plants. These are considered as highly controlled signaling molecules, which are able to transfer the environmental signals, with other signaling intermediates, to the genetic machinery (Polidoros et al., 2005).

In plant cells, the ROS production is strictly regulated by ROS scavenging pathways involving enzymatic and nonenzymatic antioxidants. The major antioxidants that play crucial role in ROS detoxification include ascorbic acid (AA), $\alpha$-tocopherol, glutathione, catalase (CAT), peroxidases (POX), superoxide dismutase (SOD), glutathione reductase (GR), etc. SOD is considered as a first-line defense against ROS (Khan and Khan, 2014; Khan et al., 2015, 2017). It converts superoxide to $\mathrm{H}_{2} \mathrm{O}_{2}$ while APX, GPX, and CAT detoxify $\mathrm{H}_{2} \mathrm{O}_{2}$. AA is used as a substrate by ascorbate peroxidase (APX) to reduce $\mathrm{H}_{2} \mathrm{O}_{2}$ to $\mathrm{H}_{2} \mathrm{O}$ in the ascorbate-glutathione cycle and generate monodehydroascorbate, which further dissociates to AA and dehydroascorbate (Choudhury et al., 2013). The ROS signaling pathway can regulate additional pathways that are physically nonadjacent to the pathway in which it was formed (Nathan, 2003). One such example is the activation of a secondary messenger such as a MAPK or a plant hormone by ROS, which in turn activate remote signaling pathways.

\subsubsection{Jasmonic Acid (JA), Ethylene (ET), and Salicylic Acid (SA) Signaling Pathways}

JA is one of the important signaling phytohormones that play an essential role in plant development as well as respond to biotic and abiotic stresses (Khan et al., 2012; Per et al., 2018). JA synthesis usually occurs from the $\alpha$-linolenic acid precursor through the octadecanoid pathway. JA is activated by enzymatic coupling to isoleucine amino acid. The resulting JA-Ile functions as a ligand promoting assembly of a coreceptor complex between the F-box protein COI1 and JA ZIM-domain (JAZ) proteins (Thines et al., 2007). JAZ proteins bind to bHLH (basic helix-loop-helix) transcription factors (e.g., MYC2, MYC3, MYC4, and MYC5) that are activators of JA responses repressing their transcriptional activity and turning off the expression of the early JA-responsive genes (Qi et al., 2015; Zhang et al., 2015). JAR1 (jasmonate resistant 1) is a JA-amidosynthetase that catalyzes the conversion of JA to JA-Ile, which is in turn recognized by the COI1-JAZ coreceptor (Sheard et al., 2010). After destabilizing JAZ by action of JA-lle, transcription factors are released from repression activating early JA responses. This switching on of JA responses leads to the attenuation of hormone signaling by induction of the JA-responsive JAZ genes to avoid the inhibitory effect that overactivation of JA responses has on plant 
growth (Zhang and Turner, 2008). JAZ proteins are transcriptional repressors that prevent the transcription of target genes under low JA-Ile levels, and are specifically ubiquitinated when JA-Ile accumulates under biotic stress. JA-Ile is therefore a master switch controlling various aspects of plant immunity/adaptation. Exogenous supply of JA confirmed that it acts as a positive regulator of salt tolerance in wheat (Qiu et al., 2014). In Arabidopsis elevated JA levels promoted salt tolerance, suggesting that JAs positively regulate salt tolerance (Zhao et al., 2014). Although role for jasmonates for the adaptation to salt stress has been suggested (Fujita et al., 2006), molecular mechanisms of the role of jasmonates for salt stress-signaling are still mostly unclear.

Ethylene is a simple gaseous hormone synthesized through three enzymatic reaction steps: in the first reaction methionine is converted to $S$-adenosyl-methionine (S-AdoMet) by $S$-adometsynthetase, after this the direct precursor of ethylene ACC(1-aminocyclopropane-1-carboxylic) is synthesized from S-AdoMet by ACS (aminocyclopropane-1-carboxylic acid synthase), and finally ethylene is produced through the oxidation of ACC by ACO (ACC oxidase) (Lin et al., 2009; Khan et al., 2017). The main components of ethylene signal transduction model include five ethylene receptors, a negative regulator CTR1 (CONSTITUTIVE TRIPLE RESPONSE1), a key positive regulator EIN2 (ETHYLENE INSENSITIVE2), primary transcription factors EIN3/EILs (ETHYLENE-INSENSITIVE-LIKE), and many downstream ethylene-response factors. The main ethylene signaling components that pave the way to downstream the signal of EIN3 are ERFs (ethylene-responsive element binding factors). During normal conditions, ethylene receptors interact with CTR1 and activate it, which in turn phosphorylates EIN2 and prevents its translocation into nucleus, therefore inhibits ethylene signal transduction. But whenever ethylene production is induced by environmental signals, it binds with receptors to inhibit their interaction with CTR1. This leads to the inactivation of CTR1, causing the dephosphorylation and cleavage of EIN2 (Tao et al., 2015). There is translocation of C-terminus of EIN2 into nucleus to stabilize downstream transcription factors EIN3/EILs (Wen et al., 2012). The whole ethylene signaling pathway possibly mediates the stress signal transduction as the whole pathway is involved in salinity stress response in plants. Ethylene receptors have been found to have contrasting roles in seed germination in Arabidopsis during salt stress. ETR1 and EIN4 stimulate ABA biosynthesis to inhibit germination. On the other hand ETR2 suppresses ETR1 and EIN4, reduces ET levels, and improves germination under salt stress (Wilson et al., 2014). Also the lack of ETO1 (ETHYLENE OVERPRODUCER1) function in Arabidopsis conferred soil-salinity tolerance through improved shoot $\mathrm{Na} / \mathrm{K}$ homeostasis, effected via the ETHYLENE RESISTANT1-CONSTITUTIVE TRIPLE RESPONSE1 ethylene signaling pathway (Jiang et al., 2013).

Salicylic acid (SA) is a phenolic compound and it is involved in the regulation of some of the important plant physiological processes such as photosynthesis, nitrogen metabolism, proline (Pro) metabolism, production of glycinebetaine (GB), antioxidant defense system, and plant-water relations under stress conditions and thereby provides protection in plants against abiotic stresses (Nazar et al., 2011; Miura and Tada, 2014; Khan et al., 2015, 2012). Salicylic acid is synthesized via SID2-dependent and SID2-independent biosynthesis. To induce defense signaling, SA binds to some specific receptors including SA methyl transferase 1 (SAMT1) and SA-binding protein 2 (SABP2). Transcriptomic-profiling study on tomato revealed that SABP2 was induced by salinity suggesting involvement of SABP2 in the salt tolerance mechanisms (Sun et al., 2010). Another SA receptor, NPR1 (nonexpresser of PR proteins 1) emerged as a master regulatory protein of SA-dependent defense responses (Wu et al., 2012). Arabidopsis npr1 mutant showed enhanced growth during salt stress (Hao et al., 2012). On the other hand NPR1-hyper accumulating Arabidopsis double mutant (npr3npr4) failed to undergo programmed cell death (Fu et al., 2012). This controversial role of NPR1 during salt stress suggests that salt tolerance in plants can be controlled by both NPR1-independent and NPR1dependent mechanisms (Jayakannan et al., 2013). One of the major actions of SA is the inhibition of catalase, a major $\mathrm{H}_{2} \mathrm{O}_{2}$ enzyme scavenging, thereby increasing cellular concentrations of $\mathrm{H}_{2} \mathrm{O}_{2}$, which acts as a second messenger and activates defense-related genes (Ananieva et al., 2002).

\subsection{PROTEOMICS APPROACH IN UNDERSTANDING SALT STRESS SIGNALING PATHWAYS}

Plants' response to salinity at the proteomic level has been investigated in both halophytes as well as glycophytes but there are only few reports on differential protein abundance of some salinity signaling involved protein. The main reason for this is that the signaling involved proteins are mostly low abundant proteins, which are hardly detectable on the 2DE gels and for proteome analysis $2 \mathrm{DE}$ is the most preferred technique. However, the gel free based techniques have more or less overcome this problem.

Proteomic studies of rice (Li et al., 2010) and S. salsa (Li et al., 2011) revealed that the calcium binding 
proteins are regulated by salinity while studying the salt stress induced plasma membrane-associated proteome of rice shoots. This observation was further confirmed through the study of salt stress induced differential proteome response in the shoots of Aeluropus lagopoides (Sobhanian et al., 2010) and proteomic changes in maize roots under saline conditions (Zörb et al., 2010). Among all calcium binding proteins the most studied ones are calreticulin and calmodulin. During the study of root proteome of barley under salt stress, annexin, a calcium dependent membrane protein that mediates osmotic stress and ABA signaling transduction, was found to be upregulated in salt tolerant barley line. Annexins sense $\mathrm{Ca}^{+}$signals induced by $\mathrm{ABA}$ signaling and transmit them to downstream signaling pathways. This study also confirmed the role of JA during salt stress because the most important components involved in the biosynthesis of oxylipins (JA), that is, linolate 9S-lipoxygenase 1 and lipoxygenase 2, were found to be increased under salt stress (Mostek et al., 2015). Some other researchers also found that the proteins involved in JA biosynthesis are regulated by salinity. Among these proteins two 12oxophytodienoate reductase proteins involved in JA biosynthesis were found to be reduced by salt stress in sensitive cucumber plants (Fan et al., 2015); and the enzymes such as allene oxide synthase, 4-coumarateCoA ligase-like 4, lipoxygenase 2.2 were found to be upregulated under higher salt stress level in durum wheat (Capriotti et al., 2014). One of the proteins, that is, 14-3-3, which is ubiquitous and multifunctional, interacts with calcium dependent protein kinases

TABLE 31.1 List of Proteins Involved in Salt Stress Signal Transduction Identified by Proteome Analysis

\begin{tabular}{llll}
\hline S. NO & Signaling proteins identified & Proteomic technique used & Plant species \\
\hline 1 & $\begin{array}{l}\text { I. } \text { Upregulation of serine/threonine protein phosphatase, PP1 catalytic subunit } \\
\text { (PPP1C), and serine/threonine protein phosphatase 2B regulatory subunit } \\
\text { (PPP3R) }\end{array}$ & $\begin{array}{l}\text { iTRAQ coupled with } \\
\text { LCMS/MS }\end{array}$ & Carex rigescens \\
& L &
\end{tabular}

II. Downregulation of COI-1 protein, which is involved in JA response

III. CTR-1 protein, involved in ethylene signaling, was found to be downregulated

2 I. Calmodulin-1/11/16, a $\mathrm{Ca}^{2+}$ binding protein was found to be downregulated in salt tolerant variety

II. 14-3-3 like protein $\mathrm{E}$ was found to be regulated by salt stress

3 I. LysM domain receptor like kinase-4 involved in signal transduction was found to be differentially regulated

4 I. Calmodulin 490 proteins (F2E7M2 and A0MMD0) showed significant decrease under salt stress

5 I. Probable mannitol dehydrogenase involved in signaling was differentially expressed

II. Histone deactylase signaling protein was regulated under salt stress iTRAQ coupled with SCX $\quad$ Cotton $^{\text {a }}$
and LCMS/MS

iTRAQ coupled with $\quad$ Maize $^{c}$
LCMS/MS

iTRAQ coupled with nano- $\quad$ Barley $^{\mathrm{d}}$ HPLC-MS/MS

2D-PAGE coupled with

MALDI-TOF/TOF-MS

Azolla

microphylla

2D-PAGE coupled with MALDI-TOF/TOF-MS

Mustard $^{\text {g }}$ to be upregulated under salt stress

II. SNF-1 related kinase was upregulated under salt stress

III. SOS2 was found to be upregulated under salt stress

IV. Remonin family protein and zinc finger protein, putative were also upregulated

$7 \quad$ I. Serine/threonine protein kinase was found to undergo posttranslational modifications

II. PI3-kinase was also found to be modified

III. Acid phosphatase was also found to be modified under salt stress

${ }^{a}$ Gong, W., Xu, F., Sun, J., Peng, Z., He, S., Pan, Z. and Du, X., 2017. iTRAQ-based comparative proteomic analysis of seedling leaves of two upland cotton genotypes differing in salt tolerance. Frontiers in plant science, $8,2113$.

${ }^{b}$ Li, M., Zhang, K., Long, R., Sun, Y., Kang, J., Zhang, T. and Cao, S., 2017. iTRAQ-based comparative proteomic analysis reveals tissue-specific and novel early-stage molecular mechanisms of salt stress response in Carex rigescens. Environ. Exp. Bot., 143, 99-114.

${ }^{c}$ Luo, M., Zhao, Y., Wang, Y., Shi, Z., Zhang, P., Zhang, Y., Song, W. and Zhao, J., 2017. Comparative proteomics of contrasting maize genotypes provides insights into saltstress tolerance mechanisms. J. Proteome Res., 17(1), 141-153.

${ }^{d}$ Shen, Q., Yu, J., Fu, L., Wu, L., Dai, F., Jiang, L., Wu, D. and Zhang, G., 2017. Ionomic, metabolomic and proteomic analyses reveal molecular mechanisms of root adaption to salt stress in Tibetan wild barley. Plant Physiol. Biochem.

${ }^{e}$ Tanou, G., Filippou, P., Belghazi, M., Job, D., Diamantidis, G., Fotopoulos, V. and Molassiotis, A., 2012. Oxidative and nitrosative-based signaling and associated posttranslational modifications orchestrate the acclimation of citrus plants to salinity stress. Plant J., 72(4), 585-599.

${ }^{f}$ Thagela, P., Yadav, R.K., Mishra, V., Dahuja, A., Ahmad, A., Singh, P.K., Tiwari, B.S. and Abraham, G., 2017. Salinity-induced inhibition of growth in the aquatic pteridophyte Azolla microphylla primarily involves inhibition of photosynthetic components and signaling molecules as revealed by proteome analysis. Protoplasma, 254(1), 303-313.

'Yousuf, P.Y., Ahmad, A., Ganie, A.H., Sareer, O., Krishnapriya, V., Aref, I.M. and Iqbal, M., 2017. Antioxidant response and proteomic modulations in Indian mustard grown under salt stress. Plant Growth Regul., 81(1), 31-50. 
during stress conditions and is hence involved in many cellular signaling pathways. This protein has been found to be involved in salt signaling transduction through proteomic studies of rice (Malakshah et al., 2007), P. patens (Wang et al., 2008), and mangrove (Wang et al., 2014). Proteomic study of bread wheat revealed that the ethylene receptor (ETR) and three isoforms of receptor protein kinase (RPK) were stimulated by salinity, which suggests the possible role of ethylene and ABA signaling pathways in salt response (Peng et al., 2009). Another protein involved in the synthesis of ethylene, that is, ACC oxidase, was found to be downregulated under salt stress in Tangut nitraria, which validates the earlier reports of the role of ethylene signaling in response to salt stress (Cheng et al., 2015). The role of ABA signaling was also confirmed while studying Brachypodium distachyon in which protein PP2C 70 and serine/threonine protein kinase SAPK8 were found to be phosphorylated under salt stress, which indicates that these proteins function in the phosphorylation status in response to salt stress (Lv et al., 2014). The number of signal transduction proteins was found to be regulated while studying proteomic changes in salt stressed radish. These proteins include annexin 1 and annexin E1, calcium dependent protein kinase 2 and calcium dependent protein kinase 21 , calmodulin 5, calmodulin like protein 13 and calmodulin like protein 20, mitogenactivated protein kinase 3 , phospholipase $\mathrm{C}$ and phospholipase D (Sun et al., 2017). Reportedly, one of the members of mitogen-activated protein kinase pathway, that is, mitogen-activated protein kinase 4, was found to be modulated under saline conditions, which further signifies the implication of this pathway in salt stress signaling (Cheng et al., 2015). Phosphorylation of polyphosphoinositide phosphatase 1016 under salt stress implies that the phospholipid signaling pathway has its role to play during salt stress ( $\mathrm{Lv}$ et al., 2014). Moreover, the increased level of iniostol1;3,4-triphosphate 5/6- kinase in A. thaliana (Ndimba et al., 2005) and $S$ europaea (Wang et al., 2009) in the earlier reports also provides insights of phospholipid signaling pathway under salt stress. Some of the salt stress induced proteins related to signaling are enlisted in Table 31.1.

\subsection{CONCLUSION AND FUTURE PERSPECTIVES}

2-DE is considered as the most preferred technique for comparative studies of dynamic protein profiling during developmental or stress responses. However, the poor resolution of the low abundant proteins has led to introduction of shotgun proteomics as a complementary approach to address these technical limitations. Besides this the major challenge in the field of abiotic stress proteomics research is to unravel the process of PTMs and interactomics (protein-protein interactions) so that the major components involved in signaling pathways can be explored. Also the crosstalk between signaling pathways needs to be understood to find out how plants interact with and adapt to the stress conditions. Hopefully, in the coming years the integration of plant proteomics will deliver the raw information to predict which protein forms, PTMs, and protein complexes are present at a specific moment in a given tissue upon stress so that the information can be used to reveal the mechanism of salt tolerance and further implemented to generate tolerance in those crops that fail to survive under saline conditions.

\section{References}

Agrawal, G.K., Bourguignon, J., Rolland, N., Ephritikhine, G., Ferro, M., Jaquinod, M., et al., 2011. Plant organelle proteomics: collaborating for optimal cell function. Mass. Spectrom. Rev. 30 (5), $772-853$.

Ananieva, E.A., Alexieva, V.S., Popova, L.P., 2002. Treatment with salicylic acid decreases the effects of paraquat on photosynthesis. J. Plant. Physiol. 159 (7), 685-693.

Ashwin, N.M.R., Barnabas, L., Sundar, A.R., Malathi, P., Viswanathan, R., Masi, A., et al., 2017. Advances in proteomic technologies and their scope of application in understanding plant-pathogen interactions. J. Plant Biochem. Biotechnol. 26 (4), 371-386.

Atanassov, I., Urlaub, H., 2013. Increased proteome coverage by combining PAGE and peptide isoelectric focusing: comparative study of gel-based separation approaches. Proteomics. 13 (20), 2947-2955.

Banerjee, J., Magnani, R., Nair, M., Dirk, L.M., DeBolt, S., Maiti, I.B., et al., 2013. Calmodulin-mediated signal transduction pathways in Arabidopsis are fine-tuned by methylation. Plant Cell 25 (11), 4493-4511.

Boudsocq, M., Barbier-Brygoo, H., Laurière, C., 2004. Identification of nine sucrose nonfermenting 1-related protein kinases 2 activated by hyperosmotic and saline stresses in Arabidopsis thaliana. J. Biol. Chem. 279 (40), 41758-41766.

Boudsocq, M., Droillard, M.J., Barbier-Brygoo, H., Laurière, C., 2007. Different phosphorylation mechanisms are involved in the activation of sucrose non-fermenting 1 related protein kinases 2 by osmotic stresses and abscisic acid. Plant Mol. Biol. 63 (4), 491-503.

Capriotti, A.L., Borrelli, G.M., Colapicchioni, V., Papa, R., Piovesana, S., Samperi, R., et al., 2014. Proteomic study of a tolerant genotype of durum wheat under salt-stress conditions. Anal. Bioanal. Chem. 406 (5), 1423-1435.

Carella, P., Wilson, D.C., Kempthorne, C.J., Cameron, R.K., 2016. Vascular sap proteomics: providing insight into long-distance signalling during stress. Front. Plant Sci. 7, 651.

Cash, P., Argo, E., 2009. Analysis of bacterial proteins by 2DE. Two-Dimensional Electrophoresis Protocols. Humana Press, pp. 131-144.

Cheng, T., Chen, J., Zhang, J., Shi, S., Zhou, Y., Lu, L., et al., 2015. Physiological and proteomic analyses of leaves from the halophyte Tangut Nitraria reveals diverse response pathways critical for high salinity tolerance. Front. Plant Sci. 6, 30. 
Chinnusamy, V., Jagendorf, A., Zhu, J.K., 2005. Understanding and improving salt tolerance in plants. Crop Sci. 45 (2), 437-448.

Choudhary, C., Mann, M., 2010. Decoding signalling networks by mass spectrometry-based proteomics. Nat. Rev. Mol. Cell Biol. 11 (6), 427.

Choudhury, S., Panda, P., Sahoo, L., Panda, S.K., 2013. Reactive oxygen species signalling in plants under abiotic stress. Plant Signal. Behav. 8 (4), e23681.

Colcombet, J., Hirt, H., 2008. Arabidopsis MAPKs: a complex signalling network involved in multiple biological processes. Biochem. J. 413 (2), 217-226.

Danquah, A., de Zelicourt, A., Colcombet, J., Hirt, H., 2014. The role of $\mathrm{ABA}$ and MAPK signalling pathways in plant abiotic stress responses. Biotechnol. Adv. 32 (1), 40-52.

Darwish, E., Testerink, C., Khalil, M., El-Shihy, O., Munnik, T., 2009. Phospholipid signaling responses in salt-stressed rice leaves. Plant Cell Physiol. 50 (5), 986-997.

DeWald, D.B., Torabinejad, J., Jones, C.A., Shope, J.C., Cangelosi, A. R., Thompson, J.E., et al., 2001. Rapid accumulation of phosphatidylinositol 4, 5-bisphosphate and inositol 1, 4, 5-trisphosphate correlates with calcium mobilization in salt-stressed Arabidopsis. Plant Physiol. 126 (2), 759-769.

deZelicourt, A., Colcombet, J., Hirt, H., 2016. The role of MAPK modules and ABA during abiotic stress signalling. Trends. Plant. Sci. 21 (8), 677-685.

Drøbak, B.K., Watkins, P.A., 2000. Inositol $(1,4,5)$ trisphosphate production in plant cells: an early response to salinity and hyperosmotic stress. FEBS Lett. 481 (3), 240-244.

Evans, C., Noirel, J., Ow, S.Y., Salim, M., Pereira-Medrano, A.G., Couto, N., et al., 2012. An insight into iTRAQ: where do we stand now? Anal. Bioanal. Chem. 404 (4), 1011-1027.

Fan, H., Xu, Y., Du, C., Wu, X., 2015. Phloem sap proteome studied by ITRAQ provides integrated insight into salinity response mechanisms in cucumber plants. J. Proteomics. 125, 54-67.

Ficarro, S.B., McCleland, M.L., Stukenberg, P.T., Burke, D.J., Ross, M. M., Shabanowitz, J., et al., 2002. Phosphoproteome analysis by mass spectrometry and its application to Saccharomyces cerevisiae. Nat. Biotechnol. 20 (3), 301.

Florens, L., Washburn, M.P., 2006. Proteomic analysis by multidimensional protein identification technology. New and Emerging Proteomic Techniques. Humana Press, pp. 159-175.

Flowers, T.J., Colmer, T.D., 2008. Salinity tolerance in halophytes. New Phytol. 179 (4), 945-963.

Fu, Z.Q., Yan, S., Saleh, A., Wang, W., Ruble, J., Oka, N., et al., 2012. NPR3 and NPR4 are receptors for the immune signal salicylic acid in plants. Nature 486 (7402), 228.

Fujii, H., Zhu, J.K., 2009. Arabidopsis mutant deficient in 3 abscisic acid-activated protein kinases reveals critical roles in growth, reproduction, and stress. Proc. Natl. Acad. Sci. 106 (20), 8380-8385.

Fujita, M., Fujita, Y., Noutoshi, Y., Takahashi, F., Narusaka, Y., Yamaguchi-Shinozaki, K., et al., 2006. Crosstalk between abiotic and biotic stress responses: a current view from the points of convergence in the stress signalling networks. Curr. Opin. Plant. Biol. 9 (4), 436-442.

Gallien, S., Bourmaud, A., Kim, S.Y., Domon, B., 2014. Technical considerations for large-scale parallel reaction monitoring analysis. J. Proteomics. 100, 147-159.

Geiger, T., Wisniewski, J.R., Cox, J., Zanivan, S., Kruger, M., Ishihama, Y., et al., 2011. Use of stable isotope labeling by amino acids in cell culture as a spike-in standard in quantitative proteomics. Nat. Protoc. 6 (2), 147.

Görg, A., Drews, O., Lück, C., Weiland, F., Weiss, W., 2009. 2-DE with IPGs. Electrophoresis. 30 (S1).

Guo, Y., Qiu, Q.S., Quintero, F.J., Pardo, J.M., Ohta, M., Zhang, C., et al., 2004. Transgenic evaluation of activated mutant alleles of
SOS2 reveals a critical requirement for its kinase activity and Cterminal regulatory domain for salt tolerance in Arabidopsis thaliana. Plant Cell 16 (2), 435-449.

Gu, L., Liu, Y., Zong, X., Liu, L., Li, D.P., Li, D.Q., 2010. Overexpression of maize mitogen-activated protein kinase gene, ZmSIMK1 in Arabidopsis increases tolerance to salt stress. Mol. Biol. Rep. 37 (8), 4067-4073.

Gupta, B., Huang, B., 2014. Mechanism of salinity tolerance in plants: physiological, biochemical, and molecular characterization. Int. J. Genomics 2014, 1-18.

Gygi, S.P., Rochon, Y., Franza, B.R., Aebersold, R., 1999. Correlation between protein and mRNA abundance in yeast. Mol. Cell. Biol. 19 (3), 1720-1730.

Hafiz Che-Othman, M., Harvey Millar, A., Taylor, N.L., 2017. Connecting salt stress signalling pathways with salinity induced changes in mitochondrial metabolic processes in C3 plants. Plant, Cell Environ. 40 (12), 2875-2905.

Hakeem, K.R., Chandna, R., ulRehman, R., Tahir, I., Sabir, M., Iqbal, M., 2013. Unravelling Salt Stress in Plants Through Proteomics. Salt Stress in Plants. Springer, New York, NY, pp. 47-61.

Hamel, L.P., Nicole, M.C., Duplessis, S., Ellis, B.E., 2012. Mitogenactivated protein kinase signalling in plant-interacting fungi: distinct messages from conserved messengers. Plant Cell 24 (4), 1327-1351.

Hao, L., Zhao, Y., Jin, D., Zhang, L., Bi, X., Chen, H., et al., 2012. Salicylic acid-altering Arabidopsis mutants response to salt stress. Plant Soil 354 (1-2), 81-95.

Huang, G.T., Ma, S.L., Bai, L.P., Zhang, L., Ma, H., Jia, P., et al., 2012. Signal transduction during cold, salt, and drought stresses in plants. Mol. Biol. Rep. 39 (2), 969-987.

Im, J.H., Lee, H., Kim, J., Kim, H.B., Seyoung, K., Kim, B.M., et al., 2012. A salt stress-activated mitogen-activated protein kinase in soybean is regulated by phosphatidic acid in early stages of the stress response. J. Plant Biol. 55 (4), 303-309.

Ishitani, M., Liu, J., Halfter, U., Kim, C.S., Shi, W., Zhu, J.K., 2000. SOS3 function in plant salt tolerance requires $\mathrm{N}$-myristoylation and calcium binding. Plant Cell 12 (9), 1667-1677.

Jayakannan, M., Bose, J., Babourina, O., Rengel, Z., Shabala, S., 2013. Salicylic acid improves salinity tolerance in Arabidopsis by restoring membrane potential and preventing salt-induced $\mathrm{K}+$ loss via a GORK channel. J. Exp. Bot. 64 (8), 2255-2268.

Ji, H., Pardo, J.M., Batelli, G., Van Oosten, M.J., Bressan, R.A., Li, X., 2013. The Salt Overly Sensitive (SOS) pathway: established and emerging roles. Mol. Plant 6 (2), 275-286.

Ji, W., Koh, J., Li, S., Zhu, N., Dufresne, C.P., Zhao, X., et al., 2016. Quantitative proteomics reveals an important role of GsCBRLK in salt stress response of soybean. Plant Soil 402 (1-2), 159-178.

Jiang, C., Belfield, E.J., Cao, Y., Smith, J.A.C., Harberd, N.P., 2013. An Arabidopsis soil-salinity-tolerance mutation confers ethylenemediated enhancement of sodium/potassium homeostasis. Plant Cell 25 (9), 3535-3552.

Kaboord, B., Perr, M., 2008. Isolation of proteins and protein complexes by immunoprecipitation. 2D PAGE: Sample Preparation and Fractionation. Humana Press, pp. 349-364.

Kaufmann, K., Smaczniak, C., de Vries, S., Angenent, G.C., Karlova, R., 2011. Proteomics insights into plant signalling and development. Proteomics. 11 (4), 744-755.

Kaur, N., Gupta, A.K., 2005. Signal transduction pathways under abiotic stresses in plants. Curr. Sci. 1771-1780.

Kimura, S., Kaya, H., Kawarazaki, T., Hiraoka, G., Senzaki, E., Michikawa, M., et al., 2012. Protein phosphorylation is a prerequisite for the $\mathrm{Ca}^{2+}$-dependent activation of Arabidopsis NADPH oxidases and may function as a trigger for the positive feedback regulation of $\mathrm{Ca}^{2+}$ and reactive oxygen species. Biochimicae tBiophysica Acta (BBA)-Mol. Cell Res. 1823 (2), 398-405. 
Khan, M.I.R., Khan, N.A., 2013. Salicylic acid and jasmonates: approaches in abiotic stress tolerance. Plant Biochem. Physiol. 1, 4.

Khan, M.I.R., Khan, N.A., 2014. Ethylene reverses photosynthetic inhibition by nickel and zinc in mustard through changes in PS II activity, photosynthetic-nitrogen use efficiency and antioxidant metabolism. Protoplasma 251, 1007-1019.

Khan, M.I.R., Khan, N., 2017. Reactive Oxygen Species and Antioxidant System in Plants: Role and Regulation Under Abiotic Stress. Springer Nature978-981-10-5254-5.

Khan, M.I.R., Syeed, S., Nazar, R., Anjum, N.A., 2012. An Insight into the Role of Salicylic Acid and Jasmonic Acid in Salt Stress Tolerance. In: Khan, N.A., Nazar, R., Iqbal, N., Anjum, N.A. (Eds.), Phytohormones and Abiotic Stress Tolerance in Plants. Springer-Verlag, pp. 277-300.

Khan, M.I.R., Asgher, M., Khan, N.A., 2014. Alleviation of saltinduced photosynthesis and growth inhibition by salicylic acid involves glycinebetaine and ethylene in mungbean (Vigna radiata L.). Plant. Physiol. Biochem. 80, 67-74.

Khan, M.I.R., Fatma, M., Per, T.S., Anjum, N.A., Khan, N.A., 2015. Salicylic acid-induced abiotic stress tolerance and underlying mechanisms in plants. Front. Plant Sci. 6, 462.

Khan, N.A., Khan, M.I.R., Ferrante, A., Poór, P., 2017. Ethylene: a key regulatory molecule in plants. Front. Plant Sci. 8, 1782.

Knight, H., Knight, M.R., 2001. Abiotic stress signalling pathways: specificity and cross-talk. Trends. Plant. Sci. 6 (6), 262-267.

Kosová, K., Prášil, I.T., Vítámvás, P., 2013. Protein contribution to plant salinity response and tolerance acquisition. Int. J. Mol. Sci. 14 (4), 6757-6789.

Kültz, D., Burg, M., 1998. Evolution of osmotic stress signalling via MAP kinase cascades. J. Exp. Biol. 201 (22), 3015-3021.

Kuromori, T., Miyaji, T., Yabuuchi, H., Shimizu, H., Sugimoto, E., Kamiya, A., et al., 2010. ABC transporter AtABCG25 is involved in abscisic acid transport and responses. Proc. Natl. Acad. Sci. 107 (5), 2361-2366.

Kweon, H.K., Håkansson, K., 2006. Selective zirconium dioxidebased enrichment of phosphorylated peptides for mass spectrometric analysis. Anal. Chem. 78 (6), 1743-1749.

Lecourieux, D., Ranjeva, R., Pugin, A., 2006. Calcium in plant defence-signalling pathways. New Phytol. 171 (2), 249-269.

Li, W., Zhang, C., Lu, Q., Wen, X., Lu, C., 2011. The combined effect of salt stress and heat shock on proteome profiling in Suaeda salsa. J. Plant. Physiol. 168 (15), 1743-1752.

Li, X.J., Yang, M.F., Chen, H., Qu, L.Q., Chen, F., Shen, S.H., 2010. Abscisic acid pretreatment enhances salt tolerance of rice seedlings: proteomic evidence. Biochimicaet Biophysica Acta (BBA)Proteins Proteomics 1804 (4), 929-940.

Liao, Y.D., Lin, K.H., Chen, C.C., Chiang, C.M., 2016. Oryza sativa protein phosphatase 1a (OsPP1a) involved in salt stress tolerance in transgenic rice. Mol. Breed. 36 (3), 22.

Liebler, D.C., Zimmerman, L.J., 2013. Targeted quantitation of proteins by mass spectrometry. Biochemistry 52 (22), 3797-3806.

Lin, Z., Zhong, S., Grierson, D., 2009. Recent advances in ethylene research. J. Exp. Bot. 60 (12), 3311-3336.

Liu, J., Ishitani, M., Halfter, U., Kim, C.S., Zhu, J.K., 2000. The Arabidopsis thaliana SOS2 gene encodes a protein kinase that is required for salt tolerance. Proc. Natl. Acad. Sci. 97 (7), $3730-3734$.

Luan, S., Kudla, J., Rodriguez-Concepcion, M., Yalovsky, S., Gruissem, W., 2002. Calmodulins and calcineurin B-like proteins: calcium sensors for specific signal response coupling in plants. Plant Cell 14 (suppl 1), S389-S400.

Lv, D.W., Subburaj, S., Cao, M., Yan, X., Li, X., Appels, R., et al., 2014. Proteome and phosphoproteome characterization reveals new response and defense mechanisms of Brachypodiumdistachyon leaves under salt stress. Mol. Cell. Proteomics. 13 (2), 632-652.
Macek, B., Mann, M., Olsen, J.V., 2009. Global and site-specific quantitative phosphoproteomics: principles and applications. Annu. Rev. Pharmacol. Toxicol. 49, 199-221.

Mahajan, S., Pandey, G.K., Tuteja, N., 2008. Calcium-and salt-stress signaling in plants: shedding light on SOS pathway. Arch. Biochem. Biophys. 471 (2), 146-158.

Malakshah, S.N., Rezaei, M.H., Heidari, M., Salekdeh, G.H., 2007. Proteomics reveals new salt responsive proteins associated with rice plasma membrane. Biosci. Biotechnol. Biochem. 71 (9), 2144-2154.

Mann, M., Jensen, O.N., 2003. Proteomic analysis of posttranslational modifications. Nat. Biotechnol. 21 (3), 255.

Manuchehri, R., Salehi, H., 2014. Physiological and biochemical changes of common bermudagrass (Cynodondactylon [L.] Pers.) under combined salinity and deficit irrigation stresses. South Afr. J. Bot. 92, 83-88.

Melcher, K., Zhou, X.E., Xu, H.E., 2010. Thirsty plants and beyond: structural mechanisms of abscisic acid perception and signaling. Curr. Opin. Struct. Biol. 20 (6), 722-729.

Miura, K., Tada, Y., 2014. Regulation of water, salinity, and cold stress responses by salicylic acid. Front. Plant Sci. 5, 4.

Miyazono, K.I., Miyakawa, T., Sawano, Y., Kubota, K., Kang, H.J., Asano, A., et al., 2009. Structural basis of abscisic acid signalling. Nature 462 (7273), 609.

Mostek, A., Börner, A., Badowiec, A., Weidner, S., 2015. Alterations in root proteome of salt-sensitive and tolerant barley lines under salt stress conditions. J. Plant. Physiol. 174, 166-176.

Munns, R., Tester, M., 2008. Mechanisms of salinity tolerance. Annu. Rev. Plant. Biol. 59, 651-681.

Nathan, C., 2003. Specificity of a third kind: reactive oxygen and nitrogen intermediates in cell signaling. J. Clin. Invest. 111 (6), 769-778.

Nazar, R., Iqbal, N., Syeed, S., Khan, N.A., 2011. Salicylic acid alleviates decreases in photosynthesis under salt stress by enhancing nitrogen and sulfur assimilation and antioxidant metabolism differentially in two mungbean cultivars. J. Plant. Physiol. 168 (8), 807-815.

Ndimba, B.K., Chivasa, S., Simon, W.J., Slabas, A.R., 2005. Identification of Arabidopsis salt and osmotic stress responsive proteins using two-dimensional difference gel electrophoresis and mass spectrometry. Proteomics. 5 (16), 4185-4196.

Ngara, R., Ndimba, B.K., 2014. Understanding the complex nature of salinity and drought-stress response in cereals using proteomics technologies. Proteomics. 14 (4-5), 611-621.

Oeljeklaus, S., Meyer, H.E., Warscheid, B., 2009. Advancements in plant proteomics using quantitative mass spectrometry. J. Proteomics. 72 (3), 545-554.

Ohta, M., Guo, Y., Halfter, U., Zhu, J.K., 2003. A novel domain in the protein kinase SOS2 mediates interaction with the protein phosphatase 2C ABI2. Proc. Natl. Acad. Sci. 100 (20), 11771-11776.

Ong, S.E., Blagoev, B., Kratchmarova, I., Kristensen, D.B., Steen, H., Pandey, A., et al., 2002. Stable isotope labeling by amino acids in cell culture, SILAC, as a simple and accurate approach to expression proteomics. Mol. Cell. Proteomics. 1 (5), 376-386.

Park, H.C., Kim, M.L., Kang, Y.H., Jeon, J.M., Yoo, J.H., Kim, M.C., et al., 2004. Pathogen-and NaCl-induced expression of the SCaM4 promoter is mediated in part by a GT- 1 box that interacts with a GT-1-like transcription factor. Plant Physiol. 135 (4), 2150-2161.

Park, H.J., Kim, W.Y., Yun, D.J., 2016. A new insight of salt stress signaling in plant. Mol. Cells 39 (6), 447.

Paulsen, C.E., Carroll, K.S., 2009. Orchestrating redox signaling networks through regulatory cysteine switches. ACS. Chem. Biol. 5 (1), 47-62.

Peck, S.C., Nühse, T.S., Hess, D., Iglesias, A., Meins, F., Boller, T., 2001. Directed proteomics identifies a plant-specific protein 
rapidly phosphorylated in response to bacterial and fungal elicitors. Plant Cell 13 (6), 1467-1475.

Peng, Z., Wang, M., Li, F., Lv, H., Li, C., Xia, G., 2009. A proteomic study of the response to salinity and drought stress in an introgression strain of bread wheat. Mol. Cell. Proteomics. 8 (12), 2676-2686.

Per, T.S., Khan, M.I.R., Anjum, N.A., Masood, A., Hussain, S.J., Khan, N.A., 2018. Jasmonates in plants under abiotic stresses: crosstalk with other phytohormones matters. Environ. Exp. Bot. $145,104-120$.

Polidoros, A.N., Mylona, P.V., Pasentsis, K., Scandalios, J.G., Tsaftaris, A.S., 2005. The maize alternative oxidase 1a (Aox1a) gene is regulated by signals related to oxidative stress. Redox. Rep. 10 (2), 71-78.

Qi, T., Huang, H., Song, S., Xie, D., 2015. Regulation of jasmonatemediated stamen development and seed production by a bHLHMYB complex in Arabidopsis. Plant Cell 27 (6), 1620-1633.

Qiu, Z., Guo, J., Zhu, A., Zhang, L., Zhang, M., 2014. Exogenous jasmonic acid can enhance tolerance of wheat seedlings to salt stress. Ecotoxicol. Environ. Saf. 104, 202-208.

Quintero, F.J., Ohta, M., Shi, H., Zhu, J.K., Pardo, J.M., 2002. Reconstitution in yeast of the Arabidopsis SOS signaling pathway for $\mathrm{Na}+$ homeostasis. Proc. Natl. Acad. Sci. 99 (13), 9061-9066.

Rabilloud, T., 2013. When 2D is not enough, go for an extra dimension. Proteomics. 13 (14), 2065-2068.

Rauniyar, N., Yates III, J.R., 2014. Isobaric labeling-based relative quantification in shotgun proteomics. J. Proteome. Res. 13 (12), $5293-5309$.

Raghavendra, A.S., Gonugunta, V.K., Christmann, A., Grill, E., 2010. ABA perception and signalling. Trends. Plant. Sci. 15 (7), 395-401.

Ross, P.L., Huang, Y.N., Marchese, J.N., Williamson, B., Parker, K., Hattan, S., et al., 2004. Multiplexed protein quantitation in Saccharomyces cerevisiae using amine-reactive isobaric tagging reagents. Mol. Cell. Proteomics. 3 (12), 1154-1169.

Roy, S.J., Negrão, S., Tester, M., 2014. Salt resistant crop plants. Curr. Opin. Biotechnol. 26, 115-124.

Sanders, D., Pelloux, J., Brownlee, C., Harper, J.F., 2002. Calcium at the crossroads of signaling. Plant Cell 14 (suppl 1), S401-S417.

Sewelam, N., Kazan, K., Schenk, P.M., 2016. Global plant stress signaling: reactive oxygen species at the cross-road. Front. Plant Sci. $7,187$.

Sheard, L.B., Tan, X., Mao, H., Withers, J., Ben-Nissan, G., Hinds, T. R., et al., 2010. Jasmonate perception by inositol-phosphatepotentiated COI1-JAZ co-receptor. Nature 468 (7322), 400.

Shelke, D.B., Pandey, M., Nikalje, G.C., Zaware, B.N., Suprasanna, P., Nikam, T.D., 2017. Salt responsive physiological, photosynthetic and biochemical attributes at early seedling stage for screening soybean genotypes. Plant. Physiol. Biochem. 118, 519-528.

Shiio, Y., Aebersold, R., 2006. Quantitative proteome analysis using isotope-coded affinity tags and mass spectrometry. Nat. Protoc. 1 (1), 139.

Sobhanian, H., Motamed, N., Jazii, F.R., Nakamura, T., Komatsu, S., 2010. Salt stress induced differential proteome and metabolome response in the shoots of Aeluropuslagopoides (Poaceae), a halophyte C4 plant. J. Proteome. Res. 9 (6), 2882-2897.

Song, X., Lin, Q., 2017. Genomics, transcriptomics and proteomics to elucidate the pathogenesis of rheumatoid arthritis. Rheumatol. Int. 37 (8), 1257-1265.

Soon, F.F., Ng, L.M., Zhou, X.E., West, G.M., Kovach, A., Tan, M.E., et al., 2012. Molecular mimicry regulates ABA signaling by SnRK2 kinases and PP2C phosphatases. Science 335 (6064), 85-88.

Sun, W., Xu, X., Zhu, H., Liu, A., Liu, L., Li, J., et al., 2010. Comparative transcriptomic profiling of a salt-tolerant wild tomato species and a salt-sensitive tomato cultivar. Plant Cell Physiol. 51 (6), 997-1006.
Sun, X., Wang, Y., Xu, L., Li, C., Zhang, W., Luo, X., et al., 2017. Unraveling the Root Proteome Changes and Its Relationship to Molecular Mechanism Underlying Salt Stress Response in Radish (Raphanus sativus L.). Front. Plant Sci. 8, 1192.

Suzuki, N., Miller, G., Morales, J., Shulaev, V., Torres, M.A., Mittler, R., 2011. Respiratory burst oxidases: the engines of ROS signaling. Curr. Opin. Plant. Biol. 14 (6), 691-699.

Taj, G., Agarwal, P., Grant, M., Kumar, A., 2010. MAPK machinery in plants: recognition and response to different stresses through multiple signal transduction pathways. Plant Signal. Behav. 5 (11), 1370-1378.

Tao, J.J., Chen, H.W., Ma, B., Zhang, W.K., Chen, S.Y., Zhang, J.S., 2015. The role of ethylene in plants under salinity stress. Front. Plant Sci. 6, 1059.

Teige, M., Scheikl, E., Eulgem, T., Dóczi, R., Ichimura, K., Shinozaki, K., et al., 2004. The MKK2 pathway mediates cold and salt stress signaling in Arabidopsis. Mol. Cell 15 (1), 141-152.

Thiery, L., Leprince, A.S., Lefebvre, D., Ghars, M.A., Debarbieux, E., Savouré, A., 2004. Phospholipase D is a negative regulator of proline biosynthesis in Arabidopsis thaliana. J. Biol. Chem. 279 (15), 14812-14818.

Thines, B., Katsir, L., Melotto, M., Niu, Y., Mandaokar, A., Liu, G., et al., 2007. JAZ repressor proteins are targets of the SCF COI1 complex during jasmonate signalling. Nature 448 (7154), 661.

Timperio, A.M., Egidi, M.G., Zolla, L., 2008. Proteomics applied on plant abiotic stresses: role of heat shock proteins (HSP). J. Proteomics. 71 (4), 391-411.

Türkan, I., Demiral, T., 2009. Recent developments in understanding salinity tolerance. Environ. Exp. Bot. 67 (1), 2-9.

Tuteja, N., Mahajan, S., 2007. Calcium signaling network in plants: an overview. Plant Signal. Behav. 2 (2), 79-85.

Ventzki, R., Stegemann, J., 2010. 3D-Gel Electrophoresis-A New Development in Protein Analysis. Mass Spectro. Microb. Proteom. 205-221.

Wang, L., Liu, X., Liang, M., Tan, F., Liang, W., Chen, Y., et al., 2014. Proteomic analysis of salt-responsive proteins in the leaves of mangrove Kandelia candel during short-term stress. PLoS. ONE. 9 (1), e83141.

Wang, N., Wu, X., Ku, L., Chen, Y., Wang, W., 2016. Evaluation of three protein-extraction methods for proteome analysis of maize leaf midrib, a compound tissue rich in sclerenchyma cells. Front. Plant Sci. 7, 856.

Wang, X., Zhang, W., Li, W., Mishra, G., 2007. Phospholipid signaling in plant response to drought and salt stress. Advances in Molecular Breeding Toward Drought and Salt Tolerant Crops. Springer, Dordrecht, pp. 183-192.

Wang, X., Yang, P., Gao, Q., Liu, X., Kuang, T., Shen, S., et al., 2008. Proteomic analysis of the response to high-salinity stress in Physcomitrella patens. Planta 228 (1), 167-177.

Wang, X., Fan, P., Song, H., Chen, X., Li, X., Li, Y., 2009. Comparative proteomic analysis of differentially expressed proteins in shoots of Salicornia europaea under different salinity. J. Proteome. Res. 8 (7), 3331-3345.

Washburn, M.P., Wolters, D., Yates III, J.R., 2001. Large-scale analysis of the yeast proteome by multidimensional protein identification technology. Nat. Biotechnol. 19 (3), 242.

Wen, X., Zhang, C., Ji, Y., Zhao, Q., He, W., An, F., et al., 2012. Activation of ethylene signaling is mediated by nuclear translocation of the cleaved EIN2 carboxyl terminus. Cell Res. 22 (11), 1613.

Wilson, R.L., Kim, H., Bakshi, A., Binder, B.M., 2014. The ethylene receptors ETHYLENE RESPONSE1 and ETHYLENE RESPONSE2 have contrasting roles in seed germination of Arabidopsis during salt stress. Plant Physiol. 165 (3), 1353-1366. 
Wu, Y., Zhang, D., Chu, J.Y., Boyle, P., Wang, Y., Brindle, I.D., et al., 2012. The Arabidopsis NPR1 protein is a receptor for the plant defense hormone salicylic acid. Cell Rep. 1 (6), 639-647.

Xing, Y., Chen, W.H., Jia, W., Zhang, J., 2015. Mitogen-activated protein kinase kinase 5 (MKK5)-mediated signalling cascade regulates expression of iron superoxide dismutase gene in Arabidopsis under salinity stress. J. Exp. Bot. 66 (19), 5971-5981.

Xiong, L., Zhu, J.K., 2002. Molecular and genetic aspects of plant responses to osmotic stress. Plant, Cell Environ. 25 (2), 131-139.

Xu, G.Y., Rocha, P.S., Wang, M.L., Xu, M.L., Cui, Y.C., Li, L.Y., et al., 2011. A novel rice calmodulin-like gene, OsMSR2, enhances drought and salt tolerance and increases ABA sensitivity in Arabidopsis. Planta 234 (1), 47-59.

Yamagata, A., Kristensen, D.B., Takeda, Y., Miyamoto, Y., Okada, K., Inamatsu, M., et al., 2002. Mapping of phosphorylated proteins on two-dimensional polyacrylamide gels using protein phosphatase. Proteomics. 2 (9), 1267-1276.

Yan, S.P., Zhang, Q.Y., Tang, Z.C., Su, W.A., Sun, W.N., 2006. Comparative proteomic analysis provides new insights into chilling stress responses in rice. Mol. Cell. Proteomics. 5 (3), 484-496.

Zargar, S.M., Gupta, N., Mir, R.A., Rai, V., 2016. Shift from gel based to gel free proteomics to unlock unknown regulatory network in plants: a comprehensive review. J. Adv. Res. Biotech 1, 19.

Zeng, H., Xu, L., Singh, A., Wang, H., Du, L., Poovaiah, B.W., 2015. Involvement of calmodulin and calmodulin-like proteins in plant responses to abiotic stresses. Front. Plant Sci. 6, 600.

Zhang, F., Yao, J., Ke, J., Zhang, L., Lam, V.Q., Xin, X.F., et al., 2015. Structural basis of JAZ repression of MYC transcription factors in jasmonate signalling. Nature 525 (7568), 269.
Zhang, Y., Wang, L., Liu, Y., Zhang, Q., Wei, Q., Zhang, W., 2006. Nitric oxide enhances salt tolerance in maize seedlings through increasing activities of proton-pump and $\mathrm{Na}+/ \mathrm{H}+$ antiport in the tonoplast. Planta 224 (3), 545-555.

Zhang, Y.I., Turner, J.G., 2008. Wound-induced endogenous jasmonates stunt plant growth by inhibiting mitosis. PLoS. ONE. 3 (11), e3699.

Zhao, Y., Dong, W., Zhang, N., Ai, X., Wang, M., Huang, Z., et al., 2014. A wheat allene oxide cyclase gene enhances salinity tolerance via jasmonate signaling. Plant Physiol. 164 (2), 1068-1076.

Zhu, J.K., 2001. Cell signaling under salt, water and cold stresses. Curr. Opin. Plant. Biol. 4 (5), 401-406.

Zhu, J.K., 2016. Abiotic stress signaling and responses in plants. Cell 167 (2), 313-324.

Zonia, L., Munnik, T., 2004. Osmotically induced cell swelling versus cell shrinking elicits specific changes in phospholipid signals in tobacco pollen tubes. Plant Physiol. 134 (2), 813-823.

Zörb, C., Schmitt, S., Mühling, K.H., 2010. Proteomic changes in maize roots after short-term adjustment to saline growth conditions. Proteomics. 10 (24), 4441-4449.

\section{Further Reading}

Kosová, K., Vítámvás, P., Prášil, I.T., Renaut, J., 2011. Plant proteome changes under abiotic stress-contribution of proteomics studies to understanding plant stress response. J. Proteomics. 74 (8), $1301-1322$.

Mahajan, S., Tuteja, N., 2005. Cold, salinity and drought stresses: an overview. Arch. Biochem. Biophys. 444 (2), 139-158. 
This page intentionally left blank 


\title{
32
}

\section{Heat Shock Proteins (Hsps) Mediated Signalling Pathways During Abiotic Stress Conditions}

\author{
Kummari Divya, Pooja Bhatnagar-Mathur, \\ Kiran K. Sharma and Palakolanu Sudhakar Reddy
}

Cell, Molecular Biology \& Genetic Engineering Group, Research Program-Genetic Gains, International Crops Research Institute for the Semi-Arid Tropics (ICRISAT), Patancheru, Hyderabad, Telangana, India

\section{O U T L I N E}

32.1 Introduction

32.2 A General Account on Heat Shock Proteins 32.2.1 Small Heat Shock Proteins

32.3 Heat Shock Protein Induction Phenomena in Plants

32.4 Abiotic Stresses Induce the Heat Shock Protein-Mediated Signaling Pathways

32.4.1 Reactive Oxygen Species

32.4.2 Mitogen Activated Protein Kinases

32.4.3 Calcium and Calcium-Regulated

Proteins
499

501

501
32.5 Crosstalk Between Reactive Oxygen Species, Mitogen Activated Protein Kinases Cascades, $\mathrm{Ca}^{+2}$ and Heat Shock Factor/Heat Shock Proteins

32.6 Genetic Engineering of Heat Shock Proteins Signaling Molecules

32.7 Conclusions and Future Perspectives

Acknowledgments

References

\subsection{INTRODUCTION}

During their entire life span plants encounter several abiotic stresses and also experience various complex environmental interactions comprising multiple factors. Plants have evolved with specific adaptive mechanisms to cope with stress conditions (Meena et al., 2017; Khan and Khan, 2017). When plants are exposed to various stresses plant metabolism is disturbed (Massad et al., 2012; Khan et al., 2012, 2013; Per et al., 2017, 2018), which eventually affects the plants' growth and productivity up to $>50 \%$ (Wang et al., 2003; Shao et al., 2008). A crucial step in plants' stress tolerance mechanism to abiotic stress is the timely recognition of the stress signals and activation of the composite signaling cascades of defense in a specific and efficient manner (Chinnusamy et al., 2004; AbouQamar et al., 2009; Andreasson and Ellis, 2010; Khan et al., 2016). To protect against different stresses, plants activate several specific stress-responsive mechanisms by perceiving 
the stress signals, then producing molecules through signal transduction by up/down regulation of the many sets of mechanisms and their genes (Wang et al., 2003; Per et al., 2017, 2018). Products of stress-induced genes are classified into two major groups: (1) proteins, which directly protect against stresses include chaperones, Lea proteins, antifreeze proteins, and detoxification enzymes; and (2) some of which regulate gene expression and signal transduction pathways include transcription factors (TFs), protein kinases, and enzymes (Basu and Roychoudhury, 2014). Among them, Hsps come under the umbrella of chaperones, which show important stress-related chaperone functions in plants under abiotic stress conditions (Hendrick and Hartl, 1993; Bartels et al., 2007; Reddy et al., 2010, 2011, 2014a,b, 2016). Chaperones include specific stress-related proteins and are involved in protein synthesis, targeting, maturation, degradation, membrane stabilization, and protein renaturation (Reddy et al., 2014, 2016; Díaz-Villanueva et al., 2015). In the presence of abiotic stresses, there is an assembly of some chemical messengers that positively affects a plant's stimulus to the stresses and hence protects it from different aggressors (Pastori and Foyer, 2002; Rasmussen et al., 2013; Khan et al., 2015, 2016). Plants' reflex in adverse stress conditions is by altering the expression of a complex array of genes and elucidation of the biochemical and molecular pathways (Fig. 32.1). Genes associated with these pathways have been the foremost focus of research over the last two to three decades and the mechanisms by which these genes and their products interact remain relatively less focused.

Commonly, Hsps shield cells from injury and assist in revival and endurance after a return to normal growth conditions (Al-Whaibi, 2011). Under stress, by default Hsps play as molecular chaperones whereas under nonthermal stress their function can be changed (Timperio et al., 2008). It is implied that Hsps play a consistent role as molecular chaperones by regulating the enfolding, aggregation, transport, and degradation of the proteins in the plants ( $\mathrm{Hu}$ et al., 2009; Tripp et al., 2009; Gupta et al., 2010; Al-Whaibi, 2011; Reddy et al., 2010, 2011, 2016). Based on their protein size, Hsps can be divided into five families that have been shown to have important stress-related chaperone functions in the plants (Török et al., 2001; Reddy et al., 2016). Each Hsp family has a distinctive mechanism and the role of each family is summarized in the subsequent sections (Table 32.1). Hsps' function in signal transduction has been established from experimental results that Hsp90 and Hsp70 proteins are associated with a number of signaling molecules, including v-Src, Raf1, Akt, and steroid receptor protein kinases like MAPK and $\mathrm{Ca}^{+2}$ (Sato et al., 2000; Nollen and Morimoto, 2002; Wang and Huang, 2017). The components or signaling molecules and cochaperones of both ABA-dependent and independent signaling pathways that activate the TFs needs to be defined genetically for assessing whether a signaling pathway is specific to a particular stress or is involved in crosstalk with

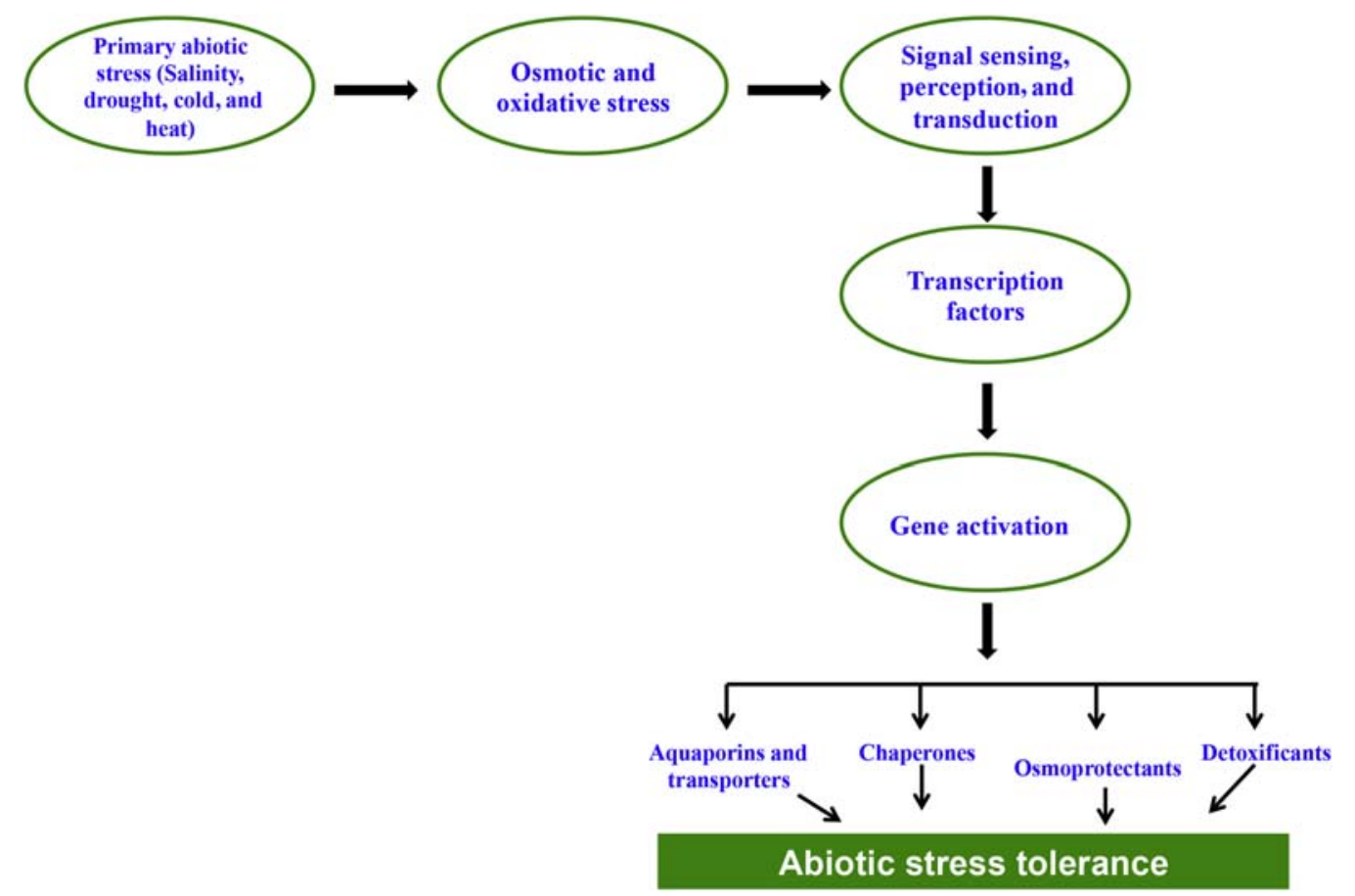

FIGURE 32.1 Complex signaling network of the plant response against different abiotic stress. 
TABLE 32.1 Different Classes of Heat Shock Protein Families, Their Location and Functions

\begin{tabular}{|c|c|c|c|}
\hline Classes & $\begin{array}{l}\text { Representative } \\
\text { members }\end{array}$ & Location & Major functions \\
\hline Hsp100/Clp & Hsp100 & $\begin{array}{l}\text { Cytosol, } \\
\text { mitochondria }\end{array}$ & $\begin{array}{l}\text { Disaggregation, } \\
\text { unfolding }\end{array}$ \\
\hline Subfamily: & & Chloroplast & \\
\hline \multirow[t]{2}{*}{ Class I } & $\mathrm{ClpB}, \mathrm{ClpA} / \mathrm{C}$ & Chloroplast & \\
\hline & ClpD & & \\
\hline \multirow[t]{2}{*}{ Class II } & ClpM, ClpN & & \\
\hline & ClpX, ClpY & & \\
\hline \multirow[t]{4}{*}{ Hsp90 } & Hsp90 & Cytosol & \multirow{4}{*}{$\begin{array}{l}\text { Facilitating } \\
\text { maturation of } \\
\text { signaling molecules, } \\
\text { genetic buffering }\end{array}$} \\
\hline & & Chloroplast & \\
\hline & & Mitochondria & \\
\hline & & $\begin{array}{l}\text { Endoplasmic } \\
\text { reticulum }\end{array}$ & \\
\hline Hsp70 & Hsp/Hsc70 & Cytosol & \multirow{4}{*}{$\begin{array}{l}\text { Preventing } \\
\text { aggregation, assisting } \\
\text { refolding, protein } \\
\text { import and } \\
\text { translocation, signal } \\
\text { transduction, and } \\
\text { transcriptional } \\
\text { activation }\end{array}$} \\
\hline Subfamily: & Hsp70 & $\begin{array}{l}\text { Chloroplast, } \\
\text { mitochondria }\end{array}$ & \\
\hline \multirow[t]{2}{*}{ DnaK } & Bip & $\begin{array}{l}\text { Endoplasmic } \\
\text { reticulum }\end{array}$ & \\
\hline & Hsp91 & Cytosol & \\
\hline \multicolumn{4}{|l|}{ Hsp110/SSE } \\
\hline \multirow[t]{2}{*}{$\begin{array}{l}\text { Chaperonin/ } \\
\text { Hsp60 }\end{array}$} & Cpn60 & $\begin{array}{l}\text { Chloroplast, } \\
\text { mitochondria }\end{array}$ & \multirow[t]{2}{*}{$\begin{array}{l}\text { Folding and assisting } \\
\text { refolding }\end{array}$} \\
\hline & CCT & Cytosol & \\
\hline \multicolumn{4}{|l|}{ Subfamily: } \\
\hline \multicolumn{4}{|l|}{ Group I } \\
\hline \multicolumn{4}{|l|}{ Group II } \\
\hline sHsp & & Cytosol & \multirow{9}{*}{$\begin{array}{l}\text { Preventing } \\
\text { aggregation, } \\
\text { stabilizing nonnative } \\
\text { proteins }\end{array}$} \\
\hline Subfamily: & & Cytosol & \\
\hline I & Hsp17.6 & Chloroplast & \\
\hline II & Hsp17.9 & $\begin{array}{l}\text { Endoplasmic } \\
\text { reticulum }\end{array}$ & \\
\hline \multirow[t]{2}{*}{ III } & Hsp21 & Mitochondria & \\
\hline & Hsp26.2 & Membrane & \\
\hline IV & Hsp22 & & \\
\hline V & Hsp23 & & \\
\hline VI & Hsp22.3 & & \\
\hline
\end{tabular}

other pathways (Yoshida et al., 2014). Signal transduction in plants under various environmental stresses have been divided into three major types, that is, (1) osmotic/oxidative stress signaling through MAP kinases, (2) $\mathrm{Ca}^{+2}$-dependent signaling, and (3) reactive oxygen species (ROS) signaling (Xiong et al., 2002). Resolving the mechanisms that control $\mathrm{Ca}^{+2}$, MAPK, and ROS signaling in cells during abiotic stress can provide an efficient way to increase plant tolerance to the environmental stresses. These signaling mechanisms are emphasized more in the following sections pertaining to Hsps as well as the functionality of different Hsps and their crosstalk in different signaling mechanisms.

\subsection{A GENERAL ACCOUNT ON HEAT SHOCK PROTEINS}

Abiotic stresses induce a set of special class of proteins called heat shock proteins (Hsps) or stressinduced proteins (Reddy et al., 2016). Based on their protein size, Hsps were classified into five subfamilies including small Hsp (sHsp), Chaperonins, Hsp70, Hsp90, and Hsp100 (Wang et al., 2004; Kotak et al., 2007; Gupta et al., 2010; Reddy et al., 2016). Hsps are involved in maintaining the cellular equilibrium, protein conformation, preventing aggregation, and hence preserving the nonnative protein in a competent state for further remodeling, which is attained by other Hsps/chaperones (Lindquist, 1986; Wang et al., 2004). The denatured or incorrectly folded proteins make aggregates, and they can be resolubilized by Hsp100/ Clp followed by refolding, or degraded by proteases (Mishra et al., 2016). Some Hsps/chaperones (e.g., Hsp70, Hsp90) accompany the signal transduction and transcription switch on the synthesis of Hsps (Wang et al., 2004; Al-Whaibi, 2011). The detailed role of each Hsp family is emphasized in the subsequent sections of this chapter.

\subsubsection{Small Heat Shock Proteins}

Small heat shock proteins (sHsps) have a unique alpha-crystallin domain (ACD) containing 80-100 amino acid residues located in the C-terminal region (Seo et al., 2006). sHsps have several characteristic features including the degradation of the proteins that cannot fold back and ATP independent chaperonic function (Miernyk, 1999; Reddy et al., 2014, 2015). The sHsps cannot refold nonnative proteins, but they can bind to partly folded or denatured substrate proteins and prevent permanent unfolding or incorrect protein aggregation (Sun et al., 2002). Neoteric study revealed that under in vitro conditions, sHsp18.1 from Pisum sativum as well as the sHsp16.6 from Synechocystis sp. PCC6803 binds to unfolded proteins and allows further refolding by Hsp70/Hsp100 complexes (Mogk et al., 2003). Studies in apple indicated that the 
mitochondrial sHsp protects the NADDH:ubiquinone oxidoreductase (complex I) during heat stress (Downs and Heckathorn, 1998). AsHsp17 modulates the expression of photosynthetic genes through ABAdependent and independent signaling pathways (Sun et al., 2016). In addition to abiotic stress, sHsps also play a role in biotic stress by associating with Hsp70/ Hsp40 chaperone complex (Rousch et al., 2004). Another sHsp20 is known to specifically interact with I-2, which confers resistance to Fusarium oxysporum (Simons et al., 1998).

\subsubsection{Chaperonins}

Hsp60 comes under chaperonins and is involved in facilitating the vast range of newly synthesized proteins, and translocates to organelles such as chloroplasts and mitochondria (Bukau and Horwich, 1998; Wang et al., 2004). Plant chaperonins commonly play a major role in assisting the plastid proteins such as Rubisco (Tompa and Kovacs, 2010). Hsp60 family proteins bind to different types of proteins after their transcription and prior to folding to avoid their aggregation (Parsell and Lindquist, 1993). Arabidopsis chlCpn60a mutated species was shown to exhibit defects in chloroplast development and subsequently in the plant development (Apuya et al., 2001). Antisense Cpn60b in transgenic tobacco plants showed drastic phenotypic alterations, including slow growth, delayed flowering, stunting, and leaf chlorosis (Zabaleta et al., 1994). Hsp10 is a cochaperone that functions together with Hsp60 in an ATP-dependent manner and helps in folding, assembly, and sorting of proteins (Bukau and Horwich, 1998). The Hsp60-Hsp10 complex enhances the osmotic as well as salt stress tolerance in transformed E. coli and yeast cells (Wang et al., 2004).

\subsubsection{Heat Shock Protein 70}

The abundant Hsps present in eukaryotic cell is Hsp70 proteins. Hsp70 proteins play a role as chaperones for newly synthesized proteins to stop their accumulations as aggregates, and assist in accurate folding and help in translocation (Su and Li, 2008; Wang et al., 2014). Hsp70 protein binds in an ATP-dependent manner to hydrophobic patches of moderate unfolded proteins and prevent protein aggregation (Mayer and Bukau, 2005; Reddy et al., 2010). Hsp70 along with other Hsps behave as molecular chaperones and play an important role in protecting plant cells from the detrimental effects of abiotic stresses including heat stress (Rousch et al., 2004). Hsp70-sHsp17.6 chaperone complex may play a role in cross adaptation to temperature stress induced by heat or cold pretreatment in grape plants (Zhang et al., 2008). Under heat stress, Hsc70 cognates are essential for general cellular functions due to their involvement in the control of protein homeostasis, categorizing of proteins by interaction with mitochondrial and chloroplast protein import complexes, and making a connection to the proteasomal degradation pathway through ubiquitin (Hartl and Hayer-Hartl, 2002; Mirus and Schleiff, 2009). Chaperone complex (SGT1b-Hsp70-Hsp90) with other client protein (COI1) helps in hormone signaling by stabilizing the client protein. The Came/AtBAG5/ Hsc70 signaling complex plays a pivotal role in modulating the plant senescence (Li et al., 2016). Salicylic acid (SA) is a crucial molecule in signaling, which activates several plant defense mechanisms, and enhances the expression of Hsp70, which further helps in modulation of the heat shock response (HSR) in tomato seedlings (Cronjé et al., 2004), tobacco protoplasts (Cronjé et al., 2004), and seedlings of Arabidopsis. Another receptor that could straight away bind to mycobacterial Hsp70 is the chemokine receptor CCRS, which has a considerable significance for signaling cascades induced by Hsps (Floto et al., 2006). Hsp70 protein forms guidance complex with other proteins and directs import bound to the protein precursor to be transferred through the membranes into the organelles such as chloroplast and mitochondria (JacksonConstan et al., 2001). Other studies indicate that Hsp70B found in the stroma of chloroplasts takes part in photoprotection and the repairing of photosystem II during and after the photoinhibition (Schroda et al., 1999). Study on A. thaliana also indicated the necessity of Hsp70 found in the stroma of chloroplast for the differentiation of germinating seeds and its tolerance of heat stress (Su and Li, 2008).

\subsubsection{Heat Shock Protein 90}

Hsp90 class proteins are the most abundant class of Hsps with $\sim 1 \%$ of total proteins in prokaryotic and eukaryotic cells. Hsp90 increases up to $4 \%-6 \%$ of total protein under elevated heat stress conditions (Wegele et al., 2004). Hsp90 proteins majorly reside in the cytoplasm, and are rarely seen in the other organelles like endoplasmic reticulum (ER), mitochondria, and chloroplasts (Hao et al., 2010; Reddy et al., 2011). Hsp90 proteins are seen to interact and bind with Hsp70 in many chaperone complexes in signaling and trafficking (Pratt and Toft, 2003). Hsp90 proteins are involved indirectly along with other proteins in the process of signal transduction. Hence, losing the activity may lead to different disturbances in cells. Hsp90 interacting proteins are categorized into three types, which include auxiliary proteins (cochaperones), regulatory factors (regulators), and substrate proteins (substrates) (Xu et al., 2012). Accessory proteins are involved in the modulation of ATP enzymatic function of Hsp90s in cytoplasm and help in interactions between Hsp90s and 
the substrates (Johnson and Brown, 2009; Zuehlke and Johnson, 2010). All Hsp90 proteins have the TPR-binding site in their structure and help in interaction of the TPR domain-containing proteins (Aviezer-Hagai et al., 2007). Under control conditions, Arabidopsis ROF1 interacts with Hsp90 through the TPR domain and targets into the cytoplasm (Aviezer-Hagai et al., 2007) but under heat stress, ROF1-Hsp90 makes a complex with HsfA2 and migrates to the nucleus and regulates sHsp transcription to increase heat tolerance (Meiri and Breiman, 2009). In control state, A. thaliana Hsp90 negatively inhibited Hsf, but under heat stress modulated positively (Yamada et al., 2007). The Hsp90-TIR1 module integrates the temperature and auxin signaling to regulate plant development (Wang et al., 2016). Hsp90s chaperonic activity mainly depends on phosphorylation and stimulates heme to modulate heme-regulated inhibitor (HRI) through casein kinase II or secondary phosphorylation (Szyszka Kramer and Hardesty, 1989). Hsp90s affect the folding and activation of a wide variety of substrate proteins, most of which are kinases and TFs involved in signal transduction and regulatory processes (Pratt et al., 2004; Krukenberg et al., 2011). Hsp90 has also been seen to function in brassinosteroid (BR) signaling (Samakovli et al., 2014). This highlights the importance of the Hsp90s' role in signaling of hormones in plants and animals. Gene silencing of RPS4 results in cell death, which is dependent on the three plant signaling components, EDS1, SGT1, and Hsp90 (Zhang et al., 2004). These studies substantiate that Hsp90 is an important signaling component under abiotic stress conditions.

\subsubsection{Heat Shock Protein 100}

The Hsp100 family belongs to the AAA ATPase super family with a wide range of functional properties (Agarwal et al., 2001). Besides playing a role in protein aggregation and misfolding, the Hsp100 families are seen to be crucial for disaggregation and/or degradation of proteins (Singh and Grover, 2010). Removal of degraded polypeptides, which are harmful due to misfolding, accumulation, or denaturation is crucial for maintaining equilibrium of the cells. A characteristic activity of Hsp100 is a reactivation of accumulated proteins by resolubilization of nonfunctional protein aggregates as well as in assisting to degrade damaged and irreparable polypeptides (Parsell and Lindquist, 1993; Bösl et al., 2006; Kim et al., 2007). Hsp101 is also seen to be playing a crucial role in high temperature stress tolerance, which can be used in genetic engineering of plants. This may improve sustenance during situations of acute environmental stress (Queitsch et al., 2000).

\subsubsection{Heat Shock Transcription Factors}

In the plant genome, $\sim 7 \%$ of the coding sequences constitute TFs and among them, the majority are of large gene families in comparison with their counterparts in animals and yeasts, such as Hsfs (Udvardi et al., 2007). To survive under adverse conditions, plants have greater number of Hsfs in comparison with other systems like mammalian cells. Hsfs belong to a multigenic family with more than 21 in Arabidopsis (Scharf et al., 2012), 24 in tomato (Fragkostefanakis et al., 2015), 52 in soybean (Scharf et al., 2012), and at least 56 in wheat (Xue et al., 2014) are anticipated and may be involved in stress responsiveness. Hsfs are seen to be involved in plant response to various abiotic stress conditions. Hsfs specifically bind to the heat stress elements (HSEs) present in the promoters of target genes, which further activate the genes (Baniwal et al., 2004; Sakurai and Enoki, 2010; Scharf et al., 2012). In tomato, only two Hsfs, HsfA2 and HsfB1, are induced by heat (Scharf et al., 1990), whose expression is modulated by HsfA1 (Mishra et al., 2002). In fact, the interaction of HsfA2 with HsfA1 is required for the colocalization of HsfA2 into the nucleus (Scharf et al., 1998). HsfA2 is the most predominant Hsf during heat stress conditions (Mishra et al., 2002). Under heat stress, AtHsfA1d and AtHsfA1e together bind to the HSE cis-element of the AtHsfA2 gene and activate the AtHsfA2 expression (Nishizawa-Yokoi et al., 2011). Hsfs playing a role in drought and salinity stresses are modulated by ABA-dependent and ABA-independent signaling pathways (Yoshida et al., 2008). At high temperature, the expression of the Hsps is modulated by Hsfs (Saidi et al., 2011; Scharf et al., 2012). Further, Hsfs translocate to the nucleus in heat stress where they bind to HSEs of respective promoter regions of Hsp genes (Voellmy and Boellmann, 2007; Scharf et al., 2012). The alternative regulatory systems for the activation of different Hsp expression are seen to be operated in plants by modulating WRKY, DREB2A, and DREB2C TFs (Scharf et al., 2012). Besides WRKY, there are more than 90 TFs in Arabidopsis that have the binding sites for CAM that comprise the Calmodulinbinding transcription activator (CAMTA). Expression of the genes coding to these proteins increases in response to heat conditions (Reddy et al., 2011), but it is unknown if this increase is important for Hsp expression or not. HsfA2, HsfA3, and HsfA7a of Arabidopsis are considered as the strongest activators of HS transcription during recovery and are responsible for the heat-acclimation phenotype (Charng et al., 2007; Nishizawa et al., 2006; Schramm et al., 2008). Some studies have also concluded that Hsfs play a role in the sensing of ROS. Mittler and Zilinskas (1992) and Storozhenko et al. (1998) have revealed the presence of 
a HSF-binding sequence in the 5 ' region of the gene that codes for $\mathrm{H}_{2} \mathrm{O}_{2}$-scavenging enzyme Apx1. In addition, Hsf and HSE (PgApx1) specificity to each other and their expression profile shows a critical interlink in heat and oxidative stress signaling pathways, which are known to play an important part in understanding the mechanisms involved in plant abiotic stress tolerance (Reddy et al., 2009). Furthermore, HsfA4A carries out the function of Arabidopsis $\mathrm{H}_{2} \mathrm{O}_{2}$ under oxidative stress (Miller and Mittler, 2006). HvHsfB2c is coexpressed in the main hub of sHsps and hence it may be modulating the expression levels of different sHsps in barley (Reddy et al., 2014). DREB2A signaling is mediated by HsfA3, which again induces the expression of Hsfs (Schramm et al., 2008; Yoshida et al., 2008). It has been shown that calmodulin is an essential component in Arabidopsis signal transduction, and with the help of a yeast two-hybrid assay, it has been demonstrated that calmodulin-binding protein kinase 3 interacts with heat shock factor (Hsf) AtHsfAla (Liu et al., 2008). Wang and Huang (2017) also demonstrated that transcriptional regulation of HsfA2c-mediated heat tolerance involving lipid and calcium signaling pathways in tall fescue.

\subsection{HEAT SHOCK PROTEIN INDUCTION PHENOMENA IN PLANTS}

Hsp synthesis in plants, including rice, Medicago and tomato was qualitatively and quantitatively dependent on the type of condition and nature of tissue (Hernandez and Vierling, 1993; Pareek et al., 1998; $\mathrm{Hu}$ et al., 2009; Fragkostefanakis et al., 2015; Zhou et al., 2016). A further study on expression of cytoplasmic class of proteins in leaves, flowers, and developing seed pods in Medicago sativa was carried out. Results indicated the recurrent formation of these proteins in flowers and buds, but no expression in the leaves (Hernandez and Vierling, 1993). Heat stress is an increase in temperature for a short term at $37-38^{\circ} \mathrm{C}$, which has no negative impact, but enhances the ability of plants to sustain the subsequent damaging heat treatment, termed heat shock; this phenomenon is known as induced or acquired thermotolerance (Saidi et al., 2011). The induced thermotolerance of plants is seen to be dependent on the expression of Hsps. Plant Hsp101 is seen to play the main role in induced thermotolerance (Queitsch et al., 2000). Expression of Hsp90 in rice plant indicated that the Hsp90 was present after $2 \mathrm{~h}$ of heat stress (from $28^{\circ} \mathrm{C}$ to $45^{\circ} \mathrm{C}$ ), and its quantity was high and stable even after heat stress $(4 \mathrm{~h})$ and after return to normal conditions. It was also found that Hsp90 (Hsp85 and Hsp87) could be induced by other than heat stress, such as salinity, drought, and cold (Pareek et al., 1998). Hsp100/ClpB proteins tend to interact tightly with
sHsps that are incorporated into the protein aggregates under heat shock, thus promoting the disaggregation activity of Hsp100/ClpB (Rikhvanov et al., 2007). Hu et al. (2009) examined a global expression profiling with heat stressed rice seedling, and then compared the results with the previous rice data under cold, drought, and salt stresses. They found that Hsps and Hsfs might be important molecules in crosstalk of different stress signal transduction networks. The expression of Hsps and its factors' Hsfs was induced largely by heat, cold, salinity, and osmotic stresses. The response to other stress factors depended on protein class and tissue. For example, under stress, high expression response for class Hsp20 was recorded with high similarity of their information. Wounding the roots of the plant stimulated (after $12 \mathrm{~h}$ ) the expression of several genes, including Hsp20, Hsp70, and Hsp100. High expression of Hsps and Hsfs was observed under UV-B stress in aerial tissues (shoot), but in nonaerial tissues (root system) there was no expression (Swindell et al., 2007).

\subsection{ABIOTIC STRESSES INDUCE THE HEAT SHOCK PROTEIN-MEDIATED SIGNALING PATHWAYS}

Abiotic stress response of plants is governed through the signaling pathways knitted at the cellular as well as molecular levels (Knight and Knight, 2001). Perception of abiotic stress initiates the signals that activate downstream signaling cascades and transcriptional controls and simultaneous pathways (Wang et al., 2003; Vij and Tyagi, 2007). The first step in the signal transduction pathway is the perception of the signal, which is carried out by receptors/sensors as phytochromes, histidine kinases and receptor-like kinases, G-protein-coupled receptors, and hormones. And then, secondary signaling molecules such as inositol phosphatase, ROS, and ABA are produced. Eventually, the secondary moleculemediated modulation of intracellular $\mathrm{Ca}^{2+}$ level activates protein phosphorylation cascades (i.e., MAP kinases, CDP kinases, protein phosphatase, SOS3/protein kinase, etc.), TFs, and stress-responsive genes (Boguszewska and Zagdańska, 2012; Gong et al., 2013). For genetically engineering stress tolerance traits in plants, proper insights of both endpoints and the precession are required for signaling pathways (Chinnusamy et al., 2004; Akpinar et al., 2012). Taking into account the first principle concepts and the developments of late, succeeding sections briefly give an insight into the significance of ROS, calcium and calcium-regulated proteins, and MAPK cascades in signaling pathways in plants under different abiotic stresses. The different signaling molecules and varied Hsps and Hsfs activated in response to elevated temperatures are given in Table 32.2. 
TABLE 32.2 Summary of the Key Signaling Mechanisms Modulating the Expression of Heat Shock Proteins in Plants

\begin{tabular}{|c|c|c|c|c|}
\hline $\begin{array}{l}\text { Signaling } \\
\text { component }\end{array}$ & Plant & $\begin{array}{l}\text { Treatment } \\
\left({ }^{\circ} \mathrm{C}\right)\end{array}$ & Remarks & References \\
\hline \multirow[t]{7}{*}{$\begin{array}{l}\text { Membrane and } \\
\mathrm{Ca}^{2+}\end{array}$} & Arabidopsis & $\begin{array}{l}22 \\
37\end{array}$ & $\begin{array}{l}\text { Induction of AtHsp } 18.2 \text { triggered by } \mathrm{Ca}^{2+} \text { and inhibited by Ca channel } \\
\text { blockers and EGTA }\end{array}$ & Liu et al. (2005) \\
\hline & \multirow[t]{2}{*}{ Tobacco } & 25 & $\begin{array}{l}\text { Hsp70 induced by membrane fluidizers and prevented by membrane } \\
\text { rigidifiers }\end{array}$ & \multirow[t]{2}{*}{$\begin{array}{l}\text { Suri and Dhindsa } \\
\text { (2008) }\end{array}$} \\
\hline & & $>38$ & Acquired thermotolerance diminished by EGTA and enhanced by $\mathrm{Ca}^{2+}$ & \\
\hline & Alfalfa & 37 & $\mathrm{Ca}^{2+}$ require for MAPK induction & $\begin{array}{l}\text { Sangwan et al. } \\
\text { (2002) }\end{array}$ \\
\hline & Maize & 27 & DNA-binding activity of Hsfs induced by $\mathrm{Ca}^{2+}$ & Li et al. (2004) \\
\hline & \multirow[t]{2}{*}{ Physcomitrella } & 22 & $\begin{array}{l}\text { Membrane fluidizers induced } \mathrm{Ca}^{2+} \text { influx, triggered HSR and enhanced } \\
\text { thermotolerance }\end{array}$ & \multirow[t]{2}{*}{ Saidi et al. (2009) } \\
\hline & & $>27$ & $\begin{array}{l}\text { Elicited } \mathrm{Ca}^{2+} \text { influx. Blocking } \mathrm{Ca}^{2+} \text { entry abolished the expression of } \\
\text { Hsps and reduced thermotolerance }\end{array}$ & \\
\hline \multirow[t]{6}{*}{$\begin{array}{l}\text { Calmodulin and } \\
\text { kinase }\end{array}$} & \multirow[t]{2}{*}{ Arabidopsis } & 37 & $\begin{array}{l}\text { AtCaM3 induced by elevated temperature. DNA-binding activity of } \\
\text { Hsfs reduced in cam3 mutant }\end{array}$ & Zhang et al. (2009) \\
\hline & & 37 & $\begin{array}{l}\text { At HsfA1 a specifically phosphorylated by At CBK3in the presence of } \\
\mathrm{CaM} \text { and } \mathrm{Ca}^{2+} \text {; binding activity of Hsfs to HSEs impaired in AtCBK3 } \\
\text { mutants and improved in the overexpressors; accumulation of Hsps } \\
\text { reduced in AtCBK3 mutants and enhanced in the overexpressors }\end{array}$ & Liu et al. (2008) \\
\hline & Tobacco & 37 & Hsp70 accumulation repressed by MAPKK inhibitors & \\
\hline & Maize & 27 & $\begin{array}{l}\text { DNA-binding activity of Hsfs induced by CaM and reduced by CaM } \\
\text { antagonist }\end{array}$ & Li et al. (2004) \\
\hline & Wheat & 37 & CaM antagonists decreased Hsp26 and Hsp70 expression & Liu et al. (2003) \\
\hline & Physcomitrella & 36 & $\begin{array}{l}\text { Pretreatment with kinase inhibitor reduced HSR and negatively affected } \\
\text { thermotolerance }\end{array}$ & Saidi et al. (2009) \\
\hline \multirow[t]{7}{*}{$\mathrm{H}_{2} \mathrm{O}_{2}$ and $\mathrm{NO}$} & \multirow[t]{5}{*}{ Arabidopsis } & 20 & $\begin{array}{l}\text { DNA-binding activity of Hsfs occurred in protein extracts from } \mathrm{H}_{2} \mathrm{O}_{2}- \\
\text { treated cells }\end{array}$ & $\begin{array}{l}\text { Volkov et al. } \\
\text { (2006) }\end{array}$ \\
\hline & & 23 & HsfA2 and Hsp25 were induced by treatment with $\mathrm{H}_{2} \mathrm{O}_{2}$ & \multirow[t]{2}{*}{ Banti et al. (2010) } \\
\hline & & 20 & Exposure to $\mathrm{H}_{2} \mathrm{O}_{2}$ induced expression of hsp17.6 and hsp18.2 & \\
\hline & & 37 & $\begin{array}{l}\text { Heat increased endogenous } \mathrm{H}_{2} \mathrm{O}_{2} \text { levels. } \\
\text { Hsp expression is reduced by peroxide scavenger; peroxide scavenger } \\
\text { blocked Hsf DNA-binding activity }\end{array}$ & $\begin{array}{l}\text { Volkov et al. } \\
\text { (2006) }\end{array}$ \\
\hline & & 37 & $\begin{array}{l}\text { DNA-binding activity of Hsfs and Hsp18.2 expression were reduced in } \\
\text { noa1 mutant and rescued by the addition of an NO donor }\end{array}$ & Xuan et al. (2010) \\
\hline & \multirow[t]{2}{*}{ Tobacco } & 45 & Endogenous NO levels increased during heat shock & Gould et al. (2003) \\
\hline & & 36 & Small Hsp induction reduced by inhibitor of $\mathrm{H}_{2} \mathrm{O}_{2}$ generation & $\begin{array}{l}\text { Königshofer et al. } \\
\text { (2008) }\end{array}$ \\
\hline \multirow[t]{3}{*}{$\begin{array}{l}\text { Cytoskeletonand } \\
\text { protein } \\
\text { denaturation }\end{array}$} & Arabidopsis & 20 & $\begin{array}{l}\text { Expression of HsfA2, Hsp70A, and small Hsps induced by chemical } \\
\text { generation of misfolded AZC-mediated Hsp70A expression reduced in } \\
\text { hsfA2 mutant }\end{array}$ & Sugio et al. (2009) \\
\hline & \multirow[t]{2}{*}{ Tobacco } & 25 & $\begin{array}{l}\text { Microfilament and microtubule destabilizers induced Hsp70 } \\
\text { accumulations }\end{array}$ & \multirow[t]{2}{*}{$\begin{array}{l}\text { Suri and Dhindsa } \\
\text { (2008) }\end{array}$} \\
\hline & & 37 & $\begin{array}{l}\text { Hsp70 accumulation repressed by microfilament and microtubule } \\
\text { stabilizers }\end{array}$ & \\
\hline
\end{tabular}


TABLE 32.2 (Continued)

\begin{tabular}{|c|c|c|c|c|}
\hline $\begin{array}{l}\text { Signaling } \\
\text { component }\end{array}$ & Plant & $\begin{array}{l}\text { Treatment } \\
\left({ }^{\circ} \mathrm{C}\right)\end{array}$ & Remarks & References \\
\hline & Rice & 28 & Activation of Oshsp17.3 promoter induced by AZC & Guan et al. (2010) \\
\hline & Physcomitrella & 40 & $\begin{array}{l}\text { Heat-denatured luciferase was not sufficient to induce Hsps when } \\
\text { extracellular } \mathrm{Ca}^{2+} \text { was immobilized }\end{array}$ & Saidi et al. (2009) \\
\hline \multirow[t]{2}{*}{ Hsp90 inhibition } & Arabidopsis & 22 & $\begin{array}{l}\text { Hsp90 inhibitors activated the transcription of Hsps and increased } \\
\text { thermotolerance }\end{array}$ & $\begin{array}{l}\text { Yamada et al. } \\
\text { (2007) }\end{array}$ \\
\hline & & 37 & $\begin{array}{l}\text { Hsp90.2 binds HsfA1d in the absence of heat; heat treatment inhibited } \\
\text { Hsp90 activity }\end{array}$ & \\
\hline \multirow[t]{2}{*}{ Hsp90 inhibition } & Physcomitrella & 37 & $\begin{array}{l}\text { ROF1-Hsp90 complex translocates to the nucleus by heat via interaction } \\
\text { with HsfA2 }\end{array}$ & $\begin{array}{l}\text { Meiri and } \\
\text { Breiman (2009) }\end{array}$ \\
\hline & & 22 & Hsp90 inhibitors induced a $\mathrm{Ca}^{2+}$-dependent HSR & Saidi et al. (2009) \\
\hline
\end{tabular}

\subsubsection{Reactive Oxygen Species}

ROS and its reaction products are the most significant second messengers that actively participate in stress signal transduction (Gong et al., 2013; Chakradhar et al., 2017; Khan and Khan, 2017). ROS have been credibly evidenced to play a significant role in signaling, redox-sensing mechanisms, and plant survival under abiotic stresses (Mittler, 2002; Mittler et al., 2004, 2008, 2010; Gill and Tuteja, 2010). A number of researchers have considered $\mathrm{H}_{2} \mathrm{O}_{2}$ as an active signaling molecule in plants, where a variety of cellular responses are accomplished due to redox-sensingmediated $\mathrm{H}_{2} \mathrm{O}_{2}$ accumulation (Bhattacharjee, 2005; Del Rio and Velez-Pardo, 2006; Halliwell, 2006; Kovalchuk, 2010; Ashfaque et al., 2014; Khan et al., 2016). Though little information is available on ROS-mediated induction of the redox-sensing mechanisms and the associated signaling pathways, the contribution of ROS-induced signaling in the activation of defense genes and subsequent stress tolerance/specific acclamatory responses has been widely accepted (Aslund et al., 1999; Goyer et al., 2002; Locato et al., 2009). Researchers provided the clues in support of $\mathrm{H}_{2} \mathrm{O}_{2}$ as a central metabolite and diffusible signal that has the capacity to induce a number of defense related genes (Chen et al., 1993; Prasad et al., 1994). Chloroplastic ROS contributing to the signaling cascade of the $\mathrm{Hsp}$ genes were not affected by apoplastic $\mathrm{H}_{2} \mathrm{O}_{2}$ or $\mathrm{H}_{2} \mathrm{O}_{2}$ produced by the plasma membrane (Scarpeci et al., 2008). Increase in temperature would enhance the mitochondrial production of ROS in plant cells (Pucciariello et al., 2012; Suzuki et al., 2012; Kreslavski et al., 2012). An increase in $\mathrm{mt} \Delta \psi$ (potential difference across the inner mitochondrial membrane) (Rikhvanov et al., 2007; Pyatrikas et al., 2014; Pavlova et al., 2009; Pulyaevskaya et al., 2011) and ROS production (Saidi et al., 2011;Volkov et al., 2006; Miller and Mittler, 2006) is essential for the activation of Hsp expression under heat stress. Inhibitors and uncouplers of mitochondria suppress the increase in $\mathrm{mt} \Delta \psi$ and inhibit the activation of the Hsp expression at elevated temperatures, even though these agents sometimes activate Hsp expression in the absence of heat stress (Rikhvanov et al., 2007; Pyatrikas et al., 2014). Likely, the addition of antioxidants inhibits Hsp expression under heat stress (Volkov et al., 2006; Saidi et al., 2011; Mittler et al., 2012). Production of ROS under heat stress is probably associated with changes in $\left[\mathrm{Ca}^{2+}\right]$ cyt and with hyperpolarization of the mitochondrial membrane. On the other hand, the rate of ROS generation in mammalian mitochondria is known to enhance with an increase in $\mathrm{mt} \Delta \psi$ (Korshunov et al., 1997). Similar concepts can be applied to plant cells. The increase in $\mathrm{mt} \Delta \psi$ induced by extracellular ATP (Sun et al., 2012) and camptothecin (Weir et al., 2003) stimulated ROS production, whereas the protonophore CCCP known to diminish $\mathrm{mt} \Delta \psi$ inhibited this process. Hence, $\mathrm{mt} \Delta \psi$ level can be among the factors determining ROS formation under heat stress. Apparently, plant cell mitochondria participate in the regulation of Hsp expression by controlling not only the $\left[\mathrm{Ca}^{2+}\right]$ cyt level but also ROS generation. This also shows the crosstalk between different signaling pathways in the regulation of Hsps.

\subsubsection{Mitogen Activated Protein Kinases}

Mitogen activated protein kinases (MAPKs) are the best-studied plant protein kinases that connect different receptors/sensors to cellular and nuclear responses (Tena et al., 2001; Sinha et al., 2011). The MAP kinase pathways facilitate signal transduction from the surface of the cell to the nucleus and are extensively used as osmolarity signaling modules. The environmental 
signals are initially perceived by specific receptors, which on being activated initiate a cascade to transmit the signal intracellularly and activate nuclear TFs to induce expression of specific target genes. Evidence shows that plants quickly activate MAPK when exposed to multiple abiotic stress stimuli (Kiegerl et al., 2000; Ligterink and Hirt, 2001). MAPKs are dual function kinases that are stimulated through phosphorylation of their two amino acids in sequential order, starting from the MAPK kinase kinase (MAPKKK) to MAPK kinase (MAPKK) to MAPK (Fig. 32.2). A series of subfamilies (i.e., MAP4K, MAP3K, MAP2K, MAPK) are chronologically activated in this MAPK cascade as a result of various environmental stimuli that in turn activate TFs like Hsf, phospholipases, or microtubuleassociated proteins, and the expression of Hsps (Wang et al., 2003; Sasabe et al., 2006) (Fig. 32.2). MAPK modules in plant tolerance to abiotic stress is illustrated, in which a tobacco MAPKKK ANP orthologue, NPK1, was expressed in an active form in Arabidopsis thaliana. NPK1 mediates $\mathrm{H}_{2} \mathrm{O}_{2}$ regulated gene expression in plants (Kovtun et al., 2000). In Arabidopsis, MAPK6 targets AtHsfA2, phosphorylates it on T249, and changes its intracellular localization under HS conditions (Evrard et al., 2013). AtHsfA4A interacts with the MAP kinases MPK3 and MPK6 and is phosphorylated in vitro on three distinct sites, with Ser-309 being the major phosphorylation site (Pérez-Salamó et al., 2014).
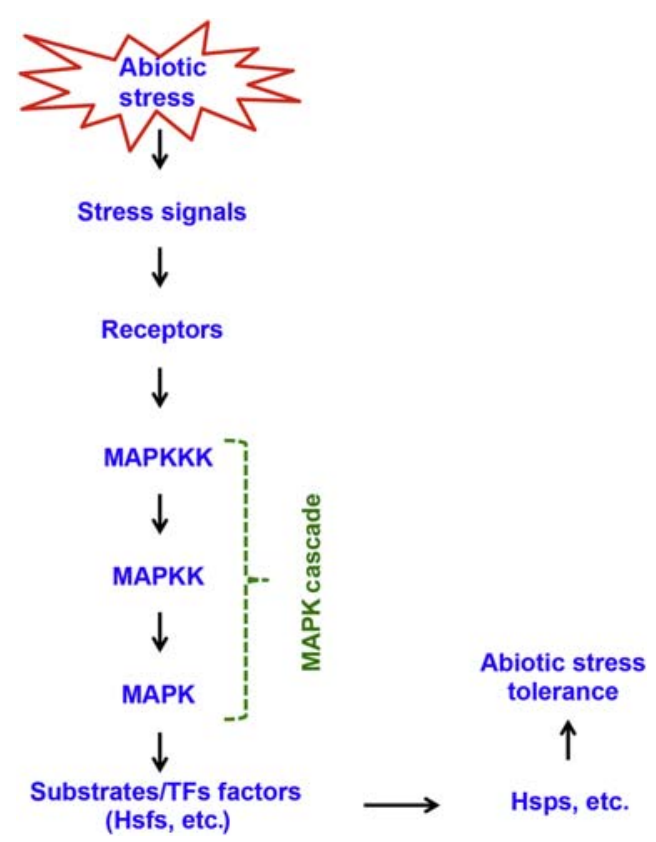

FIGURE 32.2 Predicted model of MAP kinase cascade pathway represents the cascade mechanism of MAPK phosphorylation system that serves as a link between receptors, stress signals, and downstream signaling components such as TFs to activate genes and induce abiotic stress tolerance.
Nishizawa-Yokoi et al. (2010) described that AtHsfA2 was regulated by the accumulation of polyubiquitinated proteins generated by the inhibition of $26 \mathrm{~S}$ proteasome and AtHsp90.

\subsubsection{Calcium and Calcium-Regulated Proteins}

In plants, $\mathrm{Ca}^{2+}$ serves as an important, ubiquitous second messenger and regulates many physiological processes (Tuteja and Sopory, 2008; Tuteja, 2009; Boudsocq et al., 2010). $\mathrm{Ca}^{2+}$ channels, pumps, and exchangers (carriers) control the plant $\mathrm{Ca}^{2+}$ homeostasis maintenance under a variety of stimuli through the regulation of diverse $\mathrm{Ca}^{2+}$ transport systems (Gong et al., 2013; Boudsocq et al., 2010; Kudla et al., 2010). In addition, reduction in $\mathrm{Ca}^{2+}$ mobility, localization, and spatial concentration elevations are facilitated by the abundance of buffering $\mathrm{Ca}^{2+}$ sensors (Dodd et al., 2010). $\mathrm{Ca}^{2+}$ sensor groups, namely, sensor relays [calmodulin (CaMs), calcineurin B-like (CBL)], and sensor responders, that is, sensor protein kinases [CDPKs, calcium and calmodulindependent protein kinases (CCaMKs)] lack any intrinsic enzymatic activity, directly activated upon $\mathrm{Ca}^{2+}$ binding, decode cellular $\mathrm{Ca}^{2+}$ signals, and transmit the $\mathrm{Ca}^{2+}$ induced modification to target proteins (Fig. 32.3).

Activation and inactivation of Hsf are determined by the degree of its phosphorylation and dephosphorylation, because of which it should be clear that $\mathrm{Ca}^{2+}$ activates Hsf indirectly, by modulating the activity of protein kinases and phosphatases (Voellmy and Boellmann, 2007). Serine residue phosphorylation at position 230 leads to transcriptional activity of a human Hsf1. This process is carried out by $\mathrm{Ca}^{2+} / \mathrm{CaM}$ dependent kinase II (CaMK II) (Holmberg et al., 2001). It is evident that $\mathrm{Ca}^{2+} / \mathrm{CaM}$ binding protein kinases $(\mathrm{CBK})$ and $\mathrm{Ca}^{2+}$ dependent protein kinases (CPK) in plants perform a similar function (Fig. 32.3). It is seen that CBK3 phosphorylated HsfA1a (Liu et al., 2008), while CPK3 and CPK13 phosphorylated HsfB2a (Kanchiswamy et al., 2010a; Kanchiswamy et al., 2010b), which activated Hsp expression in heat stressed $A$. thaliana. MAP kinase (HAMK), which is activated by heat, may also be involved in Hsf phosphorylation. HAMK was shown to be a $\mathrm{Ca}^{2+}$ dependent kinase and activated under heat stress in cultured tobacco cells, which is necessary for the expression of Hsp70 (Suri and Dhindsa, 2008). Dephosphorylation of certain serine residues in Hsf also leads to its activation. In A. thaliana dephosphorylation is performed by serine/threonine phosphatase PP7. It was shown that PP7 interacts with both CaM and Hsf. A mutation in PP7 gene inhibited the expression of Hsps under heat stress (Liu et al., 2007). It is not excluded that the ability of Hsp70 and Hsp90 to regulate Hsf activity 

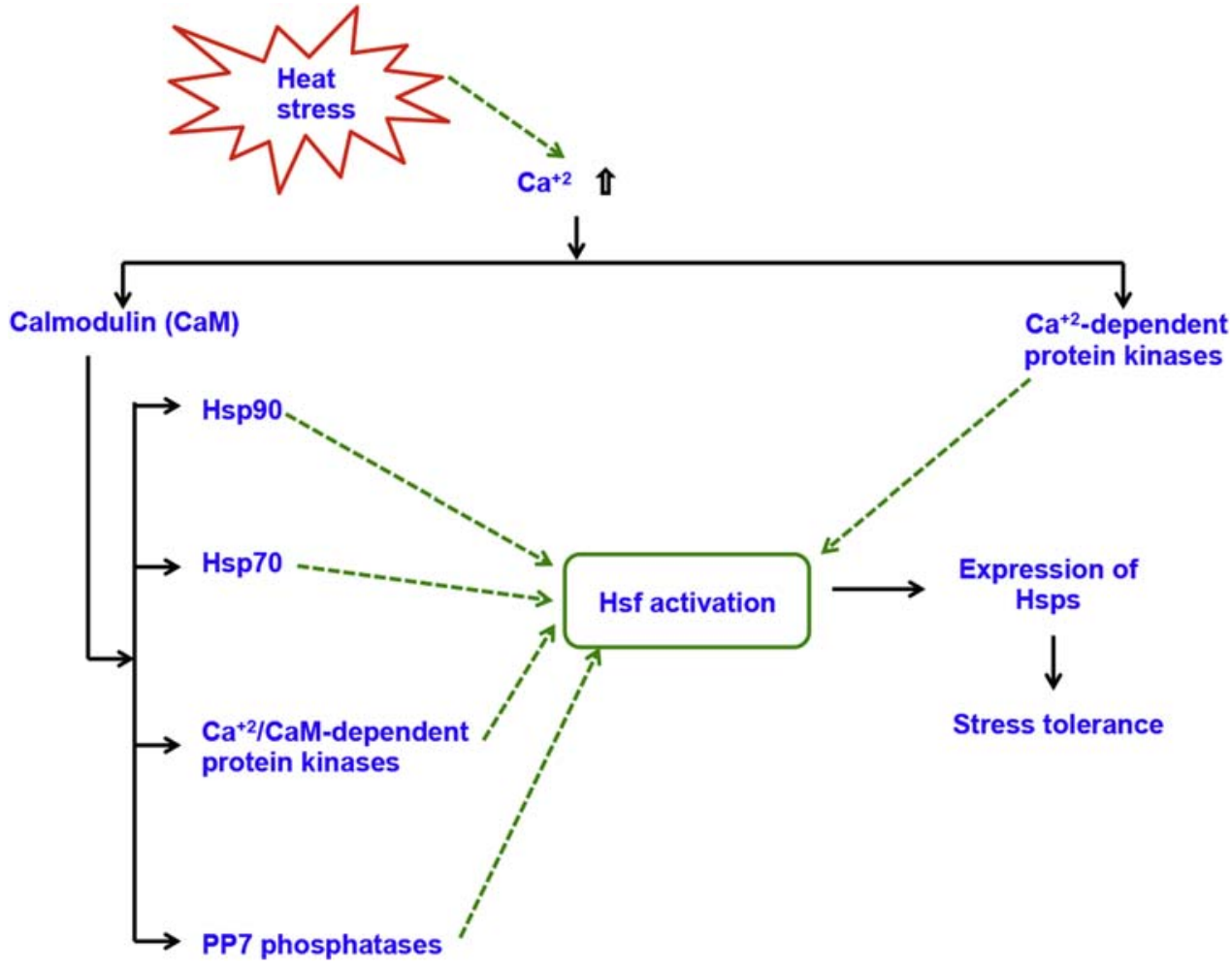

FIGURE 32.3 Mechanism depicting Ca-dependent activation of Hsf and Hsps in heat stressed plants.

depends on $\left[\mathrm{Ca}^{2+}\right]$ cyt. As seen, Hsp70 contains the CaM binding site (Sun et al., 2000). Although Hsp90 is devoid of such site, its activity can be modulated by adapter proteins possessing the CaM binding site (Meiri and Breiman, 2009; Saidi et al., 2011; Mittler et al., 2012). Activation of Hsps through calcium ion in heat stress conditions sustains the plants in heat stress conditions (Fig. 32.3).

\subsection{CROSSTALK BETWEEN REACTIVE OXYGEN SPECIES, MITOGEN ACTIVATED PROTEIN KINASES CASCADES, $\mathrm{CA}^{+2}$ AND HEAT SHOCK FACTOR/HEAT SHOCK PROTEINS}

Abiotic stresses are perceived by different signaling mechanisms, among which few are specific, and some may have crosstalk at different stages. Intricate teamwork and interaction of signaling mechanism is adopted by abiotic-stressed plants to perceive transducer stress signals and finally to transduce stress responses. ROS, TFs, $\mathrm{Ca}^{2+}$ and $\mathrm{Ca}^{2+}$-regulated proteins, and MAPK cascades are credibly being evidenced to sport a significant role in plant abiotic stress signaling cascades. Induction of changes in $\mathrm{Ca}^{2+}$ in the cytosol is observed with hormonal signals (Poovaiah et al., 1993). The initiation of MAPK in plants has also been reported to be evoked by $\mathrm{H}_{2} \mathrm{O}_{2}$ (Desikan et al., 2001; Grant et al., 2000; Neill et al., 2002; Song et al., 2008; Palavan-Unsal and Arisan, 2009). Involvement of $\mathrm{Ca}^{2+}$-dependent MAPK pathways in signaling of abiotic stress in plant cells is also known (Wurzinger et al., 2011). However, although much has been attained in the area of plant abiotic stress signaling pathways, efforts should be made with the assistance of powerful molecular tools, including transcriptome and proteome analysis, to understand more about molecular mechanism(s) underlying $\mathrm{ROS}$ and $\mathrm{Ca}^{2+}$ sensing and signal transduction.

Hsp as well as Hsfs are the most important factors in crosstalk between various stress signaling transduction networks as understood through the comparative study of global expression profiling in heat, salt, and drought stresses in rice seedlings ( $\mathrm{Hu}$ et al., 2009). A number of recent studies have shown proofs in context to interrelations among Hsfs, Hsps, ROS, and ROS scavengers under heat stress conditions. To activate Hsfs directly, elevated temperatures enhance the accumulation of ROS, which again activates Hsfs, either straight away or indirectly through triggering the MAPK pathway. The Hsfs tend to bind to HSE in the 5 upstream region of different genes like Hsf, Hsp, miRNA398, and ROS scavenging genes. The miRNA398-dependent repression of SOD scavengers may have a role in the quick ROS accumulation upon exposure to heat. 
This supports the triggering of Hsfs, which then boosts induction of the heat stress response in a lower duration. sHsp protein plays a crucial part in safeguarding PSII and PSI during chilling stress in low illumination in tobacco (Guo et al., 2007). These findings suggest that Hsps are not active only in heat stress, but are also active in other stresses by activating a cascade of signaling reactions. In the longer term, the induction and stabilization of other scavengers would start to suppress ROS levels to avoid excessive cellular damage.

\subsection{GENETIC ENGINEERING OF HEAT SHOCK PROTEINS SIGNALING MOLECULES}

Different approaches have been made to produce stress resistant plants for tackling global warming. The molecular mechanism of abiotic stress tolerance depends on activation and modulation of genes that are related to specific stress conditions. Abiotic stress sustenance in plants can be achieved by the combination of molecular and traditional plant breeding (Wang et al., 2003; Vinocur and Altman, 2005) and alternatively through forward and reverse genetic approaches (Lavania et al., 2015; Driedonks et al., 2015; Usman et al., 2014; Reddy et al., 2016). There is much evidence that shows that a broad conservation of the Hsp/ chaperone web as a multiple stress defense mechanism admits all land plants. Indeed, plant alteration was shown to be strongly dependent on Hsps as shown from the diversification of the Hsp families. Proteomic and genomic studies have also revealed a positive correlation between the expression of Hsps and tolerance to different abiotic stress conditions. Transgenic plants that are overexpressing either of Hsps or Hsfs possess enhanced antioxidant activities, higher osmolyte levels, and higher expression of the stress inducible genes, which is indicative of the activation of different stressresponsive mechanisms by Hsps and their role in the cross stress tolerance of the plants ( $\mathrm{Li}$ et al., 2012; Lee et al., 2012; Zhou et al., 2012b; Zou et al., 2012; Mu et al., 2013; Song et al., 2014; Wang et al., 2015; Masand and Yadav, 2016; Wan et al., 2016). Overexpression and knockout studies showed Hsp70 plays a protective role during dehydration stress in tobacco, soybean, and citrus (Yu et al., 2015). OsAhl1 of rice was shown to directly induce Hsp101 and Hsp90 expression leading to drought resistance (Zhou et al., 2016). OsHsp90-2 and OsHsp90-4 were also seen to be upregulated under drought, cold, heat, and salt stresses (Zhang et al., 2016). OsHsp90-2, when introduced in E. coli, was sufficient to induce resistance to heat, huge salinity conditions, and drought (Reddy et al., 2011; Zhang et al., 2016). Similar regulations of these Hsfs and Hsps were seen in tomato in stimuli to heat, drought, and salinity (Fragkostefanakis et al., 2015). In wheat, overexpression of Triticum HsfA6f was demonstrated to direct the expression of several Hsps, leading to thermotolerance (Xue et al., 2014). Increased expression of soybean GmHsp90s decreases the destruction of abiotic stresses in Arabidopsis thaliana ( $\mathrm{Xu}$ et al., 2013). The expression of $\mathrm{ZmHsf06}$ (Zea mays) is sufficient to confer heat and drought stress resistance in Arabidopsis. Identification of Hsf signaling from monocots to dicots definitely shows an intense preservation of Hsp-based multiple stress responses in crops.

Hsps repair the structure of the protein and the target at incorrectly aggregated and nonnative proteins for removing it out of the cells (Cho and Hong, 2006). It was found that both the overexpression of Hsc70 and the use of a dominant negative $(\mathrm{DN})$ form of Hsp90 disrupted ABA-mediated stomata closure, thereby negatively affecting water loss in stress conditions. The targets of Hsp90 and Hsc70 are not known yet, but must be downstream of SnRK2 as it was fully activated after ABA treatment, despite the use of an Hsp90 inhibitor (Clément et al., 2011). NtHsp70-1 is one among such proteins, which is constitutively overexpressed in tobacco to find out its role in plant drought response and tolerance. The tolerance of transgenic seedlings to drought was enhanced and their optimum water content was sustained after drought stress (Cho and Hong, 2006). Hsp24 from Trichederma was seen to develop significantly higher resistance to salt, drought, and heat stress in yeast (Liming et al., 2008). Expression of the CaHsp26 gene in transgenic tobacco showed that the mRNA accumulation of CaHsp26 was triggered by heat stress (Guo et al., 2007). Overexpression of soybean GmHsfA1 can increase the thermotolerance of transgenic soybeans because of the activation under HS of downstream genes, such as GmHsp70, GmHsp22, and other GmHsps (Zhu et al., 2006). HsfA2 has been identified to be the dominant Hsf in tomato and Arabidopsis based on its high activator potential for transcription of $H s p$ genes and the strong accumulation under conditions of long-term HS or repeated cycles of HS and recovery (Mishra et al., 2002; von Koskull-Döring et al., 2007). Studies on tomato HsfB1 with a variant of lysine residue that was replaced by its Arabidopsis counterpart showed that $H s f B 1$ and $H s f B 2 b$ may promote the activity of $H s f A 1$ under HS conditions by repressing Hsps that interfere with the nuclear migration of HsfA1s, an activator of the early HS response (Ikeda et al., 2011). In Arabidopsis, overexpression of RcHsp17.8 enhanced the SOD activity (Jiang et al., 2009) whereas overexpression of ZmHSP16.9 in tobacco enhanced POD, CAT, and SOD activity (Sun et al., 2012). Overexpression of the LeCDJ1 DnaJ protein-coding gene also known as 
J-protein or Hsp40 (Qiu et al., 2006) in tomato resulted in improved thermotolerance, accompanied by increased Apx and SOD activity after heat stress and reduced accumulation of $\mathrm{O}_{2}{ }^{-}$and $\mathrm{H}_{2} \mathrm{O}_{2}$. Also, tomato plants overexpressing the DnaJ/Hsp40 LeCDJ1 showed both higher heat and chilling tolerance (Kong et al., 2013, 2014a) and overexpression of BRZ-INSENSITIVE-LONG HYPOCOTYLS 2 (BIL2), a mitochondrial-localized DnaJ/Hsp40 family member, enhanced resistance against salinity and high light stress (Bekh-Ochir et al., 2013). Zhang et al. (2009) could demonstrate that AtCaM3 knockout mutant plants in Arabidopsis were more sensitive to heat stress, whereas high expression of CaM3 showed enhanced thermotolerance. Moreover, CaM3 is supposed to be involved in the activation of Hsfs shown by electrophoretic mobility-shift assays, real-time quantitative reverse transcription-polymerase chain reaction, and Western-blot analyses (Zhang et al., 2009). HsfA3 helps in DREB2A signaling inducing the expression of Hsfs (Schramm et al., 2008; Yoshida et al., 2008). The multiprotein bridging factor1c (MBF1c) modulates several genes, including $D R E B 2 A$ and two classes of B Hsfs. MBF1c overexpression in plants revealed increased tolerance to heat, osmotic, and biotic stress (Bechtold et al., 2010; Suzuki et al., 2005, 2011).

Overexpression of Hsf3 in Arabidopsis has shown enhanced functionality of $A p x$ at the time of postheatstress recovery and expressed solid induction of $A p x 2$ in comparison with the wild-type Arabidopsis (Panchuk et al., 2002). In a knockout Apx1, crops accumulated $\mathrm{H}_{2} \mathrm{O}_{2}$ in mild light stress in which $H s f 21$ was seen to be increased in light stress initial stages (Davletova et al., 2005a, Pnueli et al., 2003). When wild-type cells are put on with $\mathrm{H}_{2} \mathrm{O}_{2}$, accumulation of transcripts that encode for Hsf21 are seen to be aggregated (Davletova et al., 2005b). A variant of $H s f 21$ showed reduced expression of Zat12, which is a $\mathrm{H}_{2} \mathrm{O}_{2}$-responsive zinc finger protein wanted for expression of Apx1 in transgenic plants (Davletova et al., 2005a). From these studies, the critical function of Hsfs in initial sensing of $\mathrm{H}_{2} \mathrm{O}_{2}$ and Apx1, Apx2, and Zat12 expression is revealed.

\subsection{CONCLUSIONS AND FUTURE PERSPECTIVES}

The ubiquitous role of Hsps is seen in stabilization of proteins and maintaining the homeostasis in the cell. Heat stress muddles cellular homeostasis, which leads to developmental aberrations, growth retardation, and eventually plant death in some cases. Heat stress acts initially upon the quaternary structure of the folding protein, activity of which is lost due to exposure to elevated temperatures, which have deleterious effects on plant metabolism. This results in the removal of connections between the signaling pathways. In this context, further elucidation is required to understand how plants better respond to heat and other major abiotic stresses from the physiological and molecular perspectives. Still, we need to understand precisely how Hsps/chaperones participate in sensing, translocation of immune receptors, signal transduction, and transcriptional activation of several stress genes. Hence, first what needs to be focused upon is understanding the way plants respond to heat stress and utilizing this knowledge in the development of thermotolerant crops.

Many abiotic stress signaling components have been identified from mutants or functional genomic studies. Still, there is only a fragmentary view of understanding the abiotic stress signaling pathways. Functional genomics approaches by forward and reverse genetics will continue to be imperative to break through these complex pathways. Conventional genetic screens have yielded immensely important insight into stress signal transduction; this approach may ultimately be limited due to functional redundancy of components within the signaling pathways. Molecular screens based on reporter genes are a better way to identify upstream signaling components that control subsets of responses that may not manifest as visible tolerance phenotypes. Detailed characterization of mutant phenotypes will provide an indication if a signaling component function is in a specific pathway or is involved in multiple pathways. Analysis of spatial and temporal expression patterns, in combination with biochemical analysis, will be required to firmly establish specificity or crosstalk of the signaling mechanisms and their pathways.

More elucidation is required to explore opportunities to better understand how plants respond to major abiotic stresses and their cross connectivity. Multiple genes affected under abiotic stresses imply that there could not be a single marker for protection against stress. There is a lot of crosstalk taking place between various hormonal pathways, and the exact nature of this crosstalk during simultaneous biotic and abiotic stress is yet to be investigated. Researchers should look forward for defined set of markers to predict tolerance towards a particular stress with a definite degree of affirmation. Though much has been achieved in the context of plant abiotic stress signaling pathways, efforts should be made with the aid of powerful modern molecular tools, including transcriptomic and proteomics technologies, to get more insights into molecular mechanism(s) underlying various signaling cascades, that is, ROS and $\mathrm{Ca}^{2+}$ sensing signal transduction pathways. Mutations or edits enhancing Hsf/ Hsps expression or activity undoubtedly are valuable targets to engineer multi-stress resistant crops through 
recently discovered CRISPR/Cas9 (clustered regularly interspaced short palindromic repeat-CRISPR associated 9) system and TILLING (targeted induced local lesion in genomes), which would allow a rapid technology transfer in crops.

\section{Acknowledgments}

PSR acknowledges the Department of Science and Technology, Govt. of India for the fellowship and research grant through the INSPIRE Faculty Award No. IFALSPA-06. This work was undertaken as part of the CGIAR Research Program on Dry land Cereals.

\section{References}

AbouQamar, S., Luo, H., Laluk, K., Mickelbart, M.V., Mengiste, T., 2009. Crosstalk between biotic and abiotic stress responses in tomato is mediated by the AIM1 transcription factor. Plant J. 58 (2), 347-360.

Agarwal, M., Katiyar-Agarwal, S., Sahi, C., Gallie, D.R., Grover, A., 2001. Arabidopsis thaliana Hsp100 proteins: kith and kin. Cell Stress Chap. 6 (3), 219-224.

Akpinar, B.A., Avsar, B., Lucas, S.J., Budak, H., 2012. Plant abiotic stress signalling. Plant Signal. Behav. 7 (11), 1450-1455.

Al-Whaibi, M.H., 2011. Plant heat-shock proteins: a mini review. J. King Saud Univ. Sci. 23 (2), 139-150.

Andreasson, E., Ellis, B., 2010. Convergence and specificity in the Arabidopsis MAPK nexus. Trends. Plant. Sci. 15 (2), 106-113.

Apuya, N.R., Yadegari, R., Fischer, R.L., Harada, J.J., Zimmerman, J. L., Goldberg, R.B., 2001. The Arabidopsis embryo mutant schlepperless has a defect in the chaperonin- $60 \alpha$ gene. Plant Physiol. 126 (2), 717-730.

Åslund, F., Zheng, M., Beckwith, J., Storz, G., 1999. Regulation of the OxyR transcription factor by hydrogen peroxide and the cellular thiol-disulfide status. Proc. Natl. Acad. Sci. 96 (11), 6161-6165.

Ashfaque, F., Khan, M.I.R., Khan, N.A., 2014. Exogenously applied $\mathrm{H} 2 \mathrm{O} 2$ promotes proline accumulation, water relations, photosynthetic efficiency and growth of wheat (Triticum aestivum L.) under salt stress. Annu. Res. Rev. Biol. 4, 105-120.

Aviezer-Hagai, K., Skovorodnikova, J., Galigniana, M., FarchiPisanty, O., Maayan, E., Bocovza, S., et al., 2007. Arabidopsis immunophilins ROF1 (AtFKBP62) and ROF2 (AtFKBP65) exhibit tissue specificity, are heat-stress induced, and bind HSP90. Plant Mol. Biol. 63 (2), 237-255.

Baniwal, S.K., Bharti, K., Chan, K.Y., Fauth, M., Ganguli, A., Kotak, S., et al., 2004. Heat stress response in plants: a complex game with chaperones and more than twenty heat stress transcription factors. J. Biosci. 29 (4), 471-487.

Banti, V., Mafessoni, F., Loreti, E., Alpi, A., Perata, P., 2010. The heatinducible transcription factor HsfA2 enhances anoxia tolerance in Arabidopsis. Plant Physiol. 152, 1471-1483.

Bartels, D., Phillips, J., Chandler, J., 2007. Desiccation tolerance: gene expression, pathways, and regulation of gene expression. Plant Desiccat. Toler. 35, 115-137.

Basu, S., Roychoudhury, A., 2014. Expression profiling of abiotic stress-inducible genes in response to multiple stresses in rice (Oryza sativa L.) varieties with contrasting level of stress tolerance. Biomed. Res. Int. 2014.

Bechtold, U., Lawson, T., MEJIA-CARRANZA, J.A.I.M.E., Meyer, R. C., Brown, I.R., Altmann, T., et al., 2010. Constitutive salicylic acid defences do not compromise seed yield, drought tolerance and water productivity in the Arabidopsis accession C24. Plant Cell Environ. 33 (11), 1959-1973.

Bekh-Ochir, D., Shimada, S., Yamagami, A., Kanda, S., Ogawa, K., Nakazawa, M., et al., 2013. A novel mitochondrial DnaJ/Hsp40 family protein BIL2 promotes plant growth and resistance against environmental stress in brassinosteroid signaling. Planta 237 (6), 1509-1525.

Bhattacharjee, S., 2005. Reactive oxygen species and oxidative burst: roles in stress, senescence and signal transduction in plants. Curr. Sci. 1113-1121.

Boguszewska, D., Zagdańska, B., 2012. ROS as signalling molecules and enzymes of plant response to unfavorable environmental conditions. Oxidative Stress-Molecular Mechanisms and Biological Effects. InTech, Rijeka, Croatia, pp. 341-362.

Bösl, B., Grimminger, V., Walter, S., 2006. The molecular chaperone Hsp104-a molecular machine for protein disaggregation. J. Struct. Biol. 156 (1), 139-148.

Boudsocq, M., Willmann, M.R., McCormack, M., Lee, H., Shan, L., $\mathrm{He}, \mathrm{P}$. , et al., 2010. Differential innate immune signalling via Ca $2+$ sensor protein kinases. Nature 464 (7287), 418.

Bukau, B., Horwich, A.L., 1998. The Hsp70 and Hsp60 chaperone machines. Cell 92 (3), 351-366.

Chakradhar, T., Mahanty, S., Reddy, R.A., Divya, K., Reddy, P.S., Reddy, M.K., 2017. Biotechnological perspective of reactive oxygen species (ROS)-mediated stress tolerance in plants. Reactive Oxygen Species and Antioxidant Systems in Plants: Role and Regulation under Abiotic Stress. Springer, Singapore, pp. 53-87.

Charng, Y.Y., Liu, H.C., Liu, N.Y., Chi, W.T., Wang, C.N., Chang, S. H., et al., 2007. A heat-inducible transcription factor, HsfA2, is required for extension of acquired thermotolerance in Arabidopsis. Plant Physiol. 143 (1), 251-262.

Chen, Z., Silva, H., Klessig, D.F., 1993. Active oxygen species in the induction of plant systemic acquired resistance by salicylic acid. Science 262 (5141), 1883-1886.

Chinnusamy, V., Schumaker, K., Zhu, J.K., 2004. Molecular genetic perspectives on cross-talk and specificity in abiotic stress signalling in plants. J. Exp. Bot. 55 (395), 225-236.

Cho, E.K., Hong, C.B., 2006. Over-expression of tobacco NtHSP70-1 contributes to drought-stress tolerance in plants. Plant Cell Rep. 25 (4), 349-358.

Clément, M., Leonhardt, N., Droillard, M.J., Reiter, I., Montillet, J.L., Genty, B., et al., 2011. The cytosolic/nuclear HSC70 and HSP90 molecular chaperones are important for stomatal closure and modulate abscisic acid-dependent physiological responses in Arabidopsis. Plant Physiol. 156 (3), 1481-1492.

Cronjé, M.J., Weir, I.E., Bornman, L., 2004. Salicylic acid-mediated potentiation of Hsp70 induction correlates with reduced apoptosis in tobacco protoplasts. Cytom. Part A 61 (1), 76-87.

Davletova, S., Schlauch, K., Coutu, J., Mittler, R., 2005a. The zincfinger protein Zat12 plays a central role in reactive oxygen and abiotic stress signaling in Arabidopsis. Plant Physiol. 139 (2), $847-856$

Davletova, S., Rizhsky, L., Liang, H., Shengqiang, Z., Oliver, D.J., Coutu, J., et al., 2005b. Cytosolic ascorbate peroxidase 1 is a central component of the reactive oxygen gene network of Arabidopsis. Plant Cell 17 (1), 268-281.

Del Rio, M.J., Velez-Pardo, C., 2006. Insulin-like growth factor-1 prevents $\mathrm{Ab}[25-35] /(\mathrm{H} 2 \mathrm{O} 2)$-induced apoptosis in lymphocytes by reciprocal NF-kB activation and p53 inhibition via PI3Kdependent pathway. Growth Factors 24 (1), 67-78.

Desikan, R., Hancock, J.T., Ichimura, K., Shinozaki, K., Neill, S.J., 2001. Harpin Induces Activation of the Arabidopsis MitogenActivated Protein Kinases AtMPK4 and AtMPK6. Plant Physiol. 126 (4), 1579-1587. 
Díaz-Villanueva, J.F., Díaz-Molina, R., García-González, V., 2015. Protein folding and mechanisms of proteostasis. Int. J. Mol. Sci. 16 (8), 17193-17230.

Dodd, A.N., Kudla, J., Sanders, D., 2010. The language of calcium signalling. Annu. Rev. Plant. Biol. 61, 593-620.

Downs, C.A., Heckathorn, S.A., 1998. The mitochondrial small heatshock protein protects NADH: ubiquinone oxidoreductase of the electron transport chain during heat stress in plants. FEBS Lett. 430 (3), 246-250.

Driedonks, N., Xu, J., Peters, J.L., Park, S., Rieu, I., 2015. Multi-level interactions between heat shock factors, heat shock proteins, and the redox system regulate acclimation to heat. Front. Plant Sci. 6, 999.

Evrard, A., Kumar, M., Lecourieux, D., Lucks, J., von KoskullDöring, P., Hirt, H., 2013. Regulation of the heat stress response in Arabidopsis by MPK6-targeted phosphorylation of the heat stress factor HsfA2. PeerJ 1, e59.

Floto, R.A., MacAry, P.A., Boname, J.M., Mien, T.S., Kampmann, B., Hair, J.R., et al., 2006. Dendritic cell stimulation by mycobacterial Hsp70 is mediated through CCR5. Science 314 (5798), 454-458.

Fragkostefanakis, S., Simm, S., Paul, P., Bublak, D., Scharf, K.D., Schleiff, E., 2015. Chaperone network composition in Solanum lycopersicum explored by transcriptome profiling and microarray meta-analysis. Plant Cell Environ. 38 (4), 693-709.

Gill, S.S., Tuteja, N., 2010. Reactive oxygen species and antioxidant machinery in abiotic stress tolerance in crop plants. Plant. Physiol. Biochem. 48 (12), 909-930.

Gong, P., Xu, X., Shi, J., Ni, L., Huang, Q., Xia, L., et al., 2013. Phosphorylation of mitogen-and stress-activated protein kinase-1 in astrocytic inflammation: a possible role in inhibiting production of inflammatory cytokines. PLoS ONE 8 (12), e81747.

Gould, K.S., Lamotte, O., Klinguer, A., Pugin, A., Wendehenne, D., 2003. Nitric oxide production in tobacco leaf cells: a generalized stress response? Plant Cell Environ. 26 (11), 1851-1862.

Goyer, A., Haslekås, C., Miginiac-Maslow, M., Klein, U., Le Marechal, P., Jacquot, J.P., et al., 2002. Isolation and characterization of a thioredoxin-dependent peroxidase from Chlamydomonas reinhardtii. FEBS. J. 269 (1), 272-282.

Grant, M., Brown, I., Adams, S., Knight, M., Ainslie, A., Mansfield, J., 2000. The RPM1 plant disease resistance gene facilitates a rapid and sustained increase in cytosolic calcium that is necessary for the oxidative burst and hypersensitive cell death. Plant J. 23 (4), $441-450$.

Guan, J.C., Yeh, C.H., Lin, Y.P., Ke, Y.T., Chen, M.T., You, J.W., et al., 2010. A 9 bp cis-element in the promoters of class I small heat shock protein genes on chromosome 3 in rice mediates Lazetidine-2-carboxylic acid and heat shock responses. J. Exp. Bot. 61, 4249-4261.

Guo, S.J., Zhou, H.Y., Zhang, X.S., Li, X.G., Meng, Q.W., 2007. Overexpression of CaHSP26 in transgenic tobacco alleviates photoinhibition of PSII and PSI during chilling stress under low irradiance. J. Plant. Physiol. 164 (2), 126-136.

Gupta, S.C., Sharma, A., Mishra, M., Mishra, R.K., Chowdhuri, D.K., 2010. Heat shock proteins in toxicology: how close and how far? Life. Sci. 86 (11-12), 377-384.

Halliwell, B., 2006. Reactive species and antioxidants. Redox biology is a fundamental theme of aerobic life. Plant Physiol. 141 (2), $312-322$.

Hao, H., Naomoto, Y., Bao, X., Watanabe, N., Sakurama, K., Noma, K., et al., 2010. HSP90 and its inhibitors. Oncol. Rep. 23 (6), 1483-1492.

Hartl, F.U., Hayer-Hartl, M., 2002. Molecular chaperones in the cytosol: from nascent chain to folded protein. Science 295 (5561), 1852-1858.

Hendrick, J.P., Hartl, F.U., 1993. Molecular chaperone functions of heat-shock proteins. Annu. Rev. Biochem. 62 (1), 349-384.
Hernandez, L.D., Vierling, E., 1993. Expression of low molecular weight heat-shock proteins under field conditions. Plant Physiol. 101 (4), 1209-1216.

Holmberg, C.I., Hietakangas, V., Mikhailov, A., Rantanen, J.O., Kallio, M., Meinander, A., et al., 2001. Phosphorylation of serine 230 promotes inducible transcriptional activity of heat shock factor 1. EMBO J. 20 (14), 3800-3810.

Hu, W., Hu, G., Han, B., 2009. Genome-wide survey and expression profiling of heat shock proteins and heat shock factors revealed overlapped and stress specific response under abiotic stresses in rice. Plant Sci. 176 (4), 583-590.

Ikeda, M., Mitsuda, N., Ohme-Takagi, M., 2011. Arabidopsis HsfB1 and HsfB2b act as repressors of the expression of heat-inducible Hsfs but positively regulate the acquired thermotolerance. Plant Physiol. 157 (3), 1243-1254.

Jackson-Constan, D., Akita, M., Keegstra, K., 2001. Molecular chaperones involved in chloroplast protein import. Biochimica et Biophysica Acta (BBA)-Mol. Cell Res. 1541 (1-2), 102-113.

Jiang, C., Xu, J., Zhang, H.A.O., Zhang, X., Shi, J., Li, M.I.N., et al., 2009. A cytosolic class I small heat shock protein, RcHSP17. 8, of Rosa chinensis confers resistance to a variety of stresses to Escherichia coli, yeast and Arabidopsis thaliana. Plant Cell Environ. 32 (8), 1046-1059.

Johnson, J.L., Brown, C., 2009. Plasticity of the Hsp90 chaperone machine in divergent eukaryotic organisms. Cell Stress Chap. 14 (1), 83-94.

Kanchiswamy, C.N., Takahashi, H., Quadro, S., Maffei, M.E., Bossi, S., Bertea, C., et al., 2010a. Regulation of Arabidopsis defense responses against Spodoptera littoralis by CPK-mediated calcium signalling. BMC Plant Biol. 10 (1), 97.

Kanchiswamy, C.N., Muroi, A., Maffei, M.E., Yoshioka, H., Sawasaki, T., Arimura, G.I., 2010b. Ca2 +-dependent protein kinases and their substrate HsfB2a are differently involved in the heat response signaling pathway in Arabidopsis. Plant Biotechnol. 27 (5), 469-473.

Khan, M.I.R., Khan, N., 2017. Reactive Oxygen Species and Antioxidant System in Plants: Role and Regulation Under Abiotic Stress. Springer Nature, New York, ISBN: 978-981-10-5254.

Khan, M.I.R., Iqbal, N., Masood, A., Khan, N.A., 2012. Variation in salt tolerance of wheat cultivars: evaluation of the role of glycinebetaine and ethylene. Pedosphere 22, 746-754.

Khan, M.I.R., Asgher, M., Khan, N.A., 2013. Rising temperature in the changing environment: a serious threat to plants. Clim. Change Environ. Sust. 1, 25-36.

Khan, M.I.R., Fatma, M., Per, T.S., Anjum, N.A., Khan, N.A., 2015. Salicylic acid-induced abiotic stress tolerance and underlying mechanisms in plants. Front. Plant Sci. 6, 462.

Khan, M.I.R., Khan, N.A., Masood, A., Per, T.S., Asgher, M., 2016. Hydrogen peroxide alleviates nickel-inhibited photosynthetic responses through increase in use-efficiency of nitrogen and sulfur, and glutathione production in mustard. Front. Plant Sci. 7, 44.

Kiegerl, S., Cardinale, F., Siligan, C., Gross, A., Baudouin, E., Liwosz, A., et al., 2000. SIMKK, a mitogen-activated protein kinase (MAPK) kinase, is a specific activator of the salt stress-induced MAPK, SIMK. Plant Cell 12 (11), 2247-2258.

Kim, H.J., Hwang, N.R., Lee, K.J., 2007. Heat shock responses for understanding diseases of protein denaturation, Molecules \& Cells, 23. Springer Science \& Business Media BV (2).

Knight, H., Knight, M.R., 2001. Abiotic stress signalling pathways: specificity and cross-talk. Trends. Plant. Sci. 6 (6), 262-267.

Kong, F., Deng, Y., Zhou, B., Wang, G., Wang, Y., Meng, Q., 2013. A chloroplast-targeted DnaJ protein contributes to maintenance of photosystem II under chilling stress. J. Exp. Bot. 65 (1), 143-158.

Kong, F., Deng, Y., Wang, G., Wang, J., Liang, X., Meng, Q., 2014a. LeCDJ1, a chloroplast DnaJ protein, facilitates heat tolerance in transgenic tomatoes. J. Integr. Plant Biol. 56 (1), 63-74. 
Königshofer, H., Tromballa, H.W., Löppert, H.G., 2008. Early events in signalling high-temperature stress in tobacco BY2 cells involve alterations in membrane fluidity and enhanced hydrogen peroxide production. Plant Cell Environ. 31 (12), 1771-1780.

Korshunov, S.S., Skulachev, V.P., Starkov, A.A., 1997. High protonic potential actuates a mechanism of production of reactive oxygen species in mitochondria. FEBS Lett. 416 (1), 15-18.

Kotak, S., Larkindale, J., Lee, U., von Koskull-Döring, P., Vierling, E., Scharf, K.D., 2007. Complexity of the heat stress response in plants. Curr. Opin. Plant. Biol. 10 (3), 310-316.

Kovalchuk, I., 2010. Multiple roles of radicals in plants. Reactive Oxygen Species and Antioxidants in Higher Plants. CRC Press, New York, NY, pp. 31-44.

Kovtun, Y., Chiu, W.L., Tena, G., Sheen, J., 2000. Functional analysis of oxidative stress-activated mitogen-activated protein kinase cascade in plants. Proc. Natl. Acad. Sci. 97 (6), 2940-2945.

Kreslavski, V.D., Los, D.A., Allakhverdiev, S.I., Kuznetsov, V.V., 2012. Signalling role of reactive oxygen species in plants under stress. Russian J. Plant Physiol. 59 (2), 141-154.

Krukenberg, K.A., Street, T.O., Lavery, L.A., Agard, D.A., 2011. Conformational dynamics of the molecular chaperone Hsp90. Q. Rev. Biophys. 44 (2), 229-255.

Kudla, J., Batistič, O., Hashimoto, K., 2010. Calcium signals: the lead currency of plant information processing. Plant Cell 22 (3), $541-563$.

Lavania, D., Siddiqui, M.H., Al-Whaibi, M.H., Singh, A.K., Kumar, R., Grover, A., 2015. Genetic approaches for breeding heat stress tolerance in faba bean (Vicia faba L.). Acta Physiol. Plant. 37 (1), 1737.

Lee, K.W., Cha, J.Y., Kim, K.H., Kim, Y.G., Lee, B.H., Lee, S.H., 2012. Overexpression of alfalfa mitochondrial HSP23 in prokaryotic and eukaryotic model systems confers enhanced tolerance to salinity and arsenic stress. Biotechnol. Lett. 34 (1), 167-174.

Li, B., Liu, H.T., Sun, D.Y., Zhou, R.G., 2004. Ca2 + and calmodulin modulate DNA-binding activity of maize heat shock transcription factor in vitro. Plant Cell Physiol. 45, 627-634.

Li, L., Xing, Y., Chang, D., Fang, S., Cui, B., Li, Q., et al., 2016. CaM/ BAG5/Hsc70 signalling complex dynamically regulates leaf senescence. Sci. Rep. 6, 31889.

Li, M., Ji, L., Yang, X., Meng, Q., Guo, S., 2012. The protective mechanisms of CaHSP26 in transgenic tobacco to alleviate photoinhibition of PSII during chilling stress. Plant Cell Rep. 31 (11), 1969-1979.

Ligterink, W., Hirt, H., 2001. Mitogen-activated protein (MAP) kinase pathways in plants: versatile signalling tools. Int. Rev. Cytol. 201, 209-275.

Liming, Y., Qian, Y., Pigang, L., Sen, L., 2008. Expression of the HSP24 gene from Trichoderma harzianum in Saccharomyces cerevisiae. J. Therm. Biol. 33 (1), 1-6.

Lindquist, S., 1986. The heat-shock response. Annu. Rev. Biochem. 55 (1), 1151-1191.

Liu, H.T., Li, B., Shang, Z.L., Li, X.Z., Mu, R.L., Sun, D.Y., et al., 2003. Calmodulin is involved in heat shock signal transduction in wheat. Plant Physiol. 132 (3), 1186-1195.

Liu, H.T., Li, G.L., Chang, H.U.I., Sun, D.Y., Zhou, R.G., Li, B., 2007. Calmodulin-binding protein phosphatase PP7 is involved in thermotolerance in Arabidopsis. Plant Cell Environ. 30 (2), 156-164.

Liu, H.T., Sun, D.Y., Zhou, R.G., 2005. Ca2 + and AtCaM3 are involved in the expression of heat shock protein gene in Arabidopsis. Plant Cell Environ. 28 (10), 1276-1284.

Liu, H.T., Gao, F., Li, G.L., Han, J.L., Liu, D.L., Sun, D.Y., et al., 2008. The calmodulin-binding protein kinase 3 is part of heat-shock signal transduction in Arabidopsis thaliana. Plant J. 55 (5), 760-773.

Locato, V., De Pinto, M.C., De Gara, L., 2009. Different involvement of the mitochondrial, plastidial and cytosolic ascorbate- glutathione redox enzymes in heat shock responses. Physiol. Plant. 135 (3), 296-306.

Masand, S., Yadav, S.K., 2016. Overexpression of MuHSP70 gene from Macrotyloma uniflorum confers multiple abiotic stress tolerance in transgenic Arabidopsis thaliana. Mol. Biol. Rep. 43 (2), 53-64.

Massad, T.J., Dyer, L.A., Vega, G., 2012. Costs of defense and a test of the carbon-nutrient balance and growth-differentiation balance hypotheses for two co-occurring classes of plant defense. PLoS ONE 7 (10), e47554.

Mayer, M., Bukau, B., 2005. Hsp70 chaperones: cellular functions and molecular mechanism. Cell. Mol. Life Sci. 62, 670-684.

Meena, K.K., Sorty, A.M., Bitla, U.M., Choudhary, K., Gupta, P., Pareek, A., et al., 2017. Abiotic stress responses and microbemediated mitigation in plants: the omics strategies. Front. Plant Sci. 8, 172.

Meiri, D., Breiman, A., 2009. Arabidopsis ROF1 (FKBP62) modulates thermotolerance by interacting with HSP90. 1 and affecting the accumulation of HsfA2-regulated sHSPs. Plant J. 59 (3), 387-399.

Miernyk, J.A., 1999. Protein folding in the plant cell. Plant Physiol. 121 (3), 695-703.

Miller, G.A.D., Mittler, R.O.N., 2006. Could heat shock transcription factors function as hydrogen peroxide sensors in plants? Ann. Bot. (Lond.) 98 (2), 279-288.

Miller, G.A.D., Suzuki, N., Ciftci-Yilmaz, S., Mittler, R.O.N., 2010. Reactive oxygen species homeostasis and signalling during drought and salinity stresses. Plant Cell Environ. 33 (4), 453-467.

Miller, G., Shulaev, V., Mittler, R., 2008. Reactive oxygen signalling and abiotic stress. Physiol. Plant. 133 (3), 481-489.

Mirus, O., Schleiff, E., 2009. The evolution of tetratricopeptide repeat domain containing receptors involved in protein translocationreview. Endocytob. Cell Res. (19).

Mishra, R.C., Singh, A., Tiwari, L.D., Grover, A., 2016. Characterization of $5^{\prime}$ UTR of rice ClpB-C/Hsp100 gene: evidence of its involvement in post-transcriptional regulation. Cell Stress Chap. 21 (2), 271-283.

Mishra, S.K., Tripp, J., Winkelhaus, S., Tschiersch, B., Theres, K., Nover, L., et al., 2002. In the complex family of heat stress transcription factors, HsfA1 has a unique role as master regulator of thermotolerance in tomato. Genes Dev. 16 (12), 1555-1567.

Mittler, R., 2002. Oxidative stress, antioxidants and stress tolerance. Trends. Plant. Sci. 7 (9), 405-410.

Mittler, R., Zilinskas, B.A., 1992. Molecular cloning and characterization of a gene encoding pea cytosolic ascorbate peroxidase. J. Biol. Chem. 267 (30), 21802-21807.

Mittler, R., Finka, A., Goloubinoff, P., 2012. How do plants feel the heat? Trends. Biochem. Sci. 37 (3), 118-125.

Mittler, R., Vanderauwera, S., Gollery, M., Van Breusegem, F., 2004. Reactive oxygen gene network of plants. Trends. Plant. Sci. 9 (10), 490-498.

Mogk, A., Schlieker, C., Friedrich, K.L., Schönfeld, H.J., Vierling, E., Bukau, B., 2003. Refolding of substrates bound to small Hsps relies on a disaggregation reaction mediated most efficiently by ClpB/DnaK. J. Biol. Chem. 278 (33), 31033-31042.

Mu, C., Zhang, S., Yu, G., Chen, N., Li, X., Liu, H., 2013. Overexpression of small heat shock protein LimHSP16. 45 in Arabidopsis enhances tolerance to abiotic stresses. PLoS ONE 8 (12), e82264.

Neill, S.J., Desikan, R., Clarke, A., Hurst, R.D., Hancock, J.T., 2002. Hydrogen peroxide and nitric oxide as signalling molecules in plants. J. Exp. Bot. 53 (372), 1237-1247.

Nishizawa, A., Yabuta, Y., Yoshida, E., Maruta, T., Yoshimura, K., Shigeoka, S., 2006. Arabidopsis heat shock transcription factor A2 as a key regulator in response to several types of environmental stress. Plant J. 48 (4), 535-547. 
Nishizawa-Yokoi, A., Tainaka, H., Yoshida, E., Tamoi, M., Yabuta, Y., Shigeoka, S., 2010. The $26 \mathrm{~S}$ proteasome function and Hsp90 activity involved in the regulation of HsfA2 expression in response to oxidative stress. Plant Cell Physiol. 51 (3), 486-496.

Nishizawa-Yokoi, A., Nosaka, R., Hayashi, H., Tainaka, H., Maruta, T., Tamoi, M., et al., 2011. HsfA1d and HsfAle involved in the transcriptional regulation of HsfA2 function as key regulators for the Hsf signalling network in response to environmental stress. Plant Cell Physiol. 52 (5), 933-945.

Nollen, E.A., Morimoto, R.I., 2002. Chaperoning signalling pathways: molecular chaperones as stress-sensing heat shock proteins. J. Cell. Sci. 115 (14), 2809-2816.

Palavan-Unsal, N., Arisan, D., 2009. Nitric oxide signalling in plants. Bot. Rev. 75 (2), 203-229.

Panchuk, I.I., Volkov, R.A., Schöffl, F., 2002. Heat stress-and heat shock transcription factor-dependent expression and activity of ascorbate peroxidase in Arabidopsis. Plant Physiol. 129 (2), 838-853.

Pareek, A., Singla, S.L., Grover, A., 1998. Plant Hsp90 family with special reference to rice. J. Biosci. 23 (4), 361-367.

Parsell, D.A., Lindquist, S., 1993. The function of heat-shock proteins in stress tolerance: degradation and reactivation of damaged proteins. Annu. Rev. Genet. 27 (1), 437-496.

Pastori, G.M., Foyer, C.H., 2002. Common components, networks, and pathways of cross-tolerance to stress. The central role of "redox" and abscisic acid-mediated controls. Plant Physiol. 129 (2), 460-468.

Pavlova, E.L., Rikhvanov, E.G., Tauson, E.L., Varakina, N.N., Gamburg, K.Z., Rusaleva, T.M., et al., 2009. Effect of salicylic acid on the development of induced thermotolerance and induction of heat shock protein synthesis in the Arabidopsis thaliana cell culture. Russian J. Plant Physiol. 56 (1), 68-73.

Per, T.S., Khan, N.A., Reddy, P.S., Masood, A., Hasanuzzaman, M., Khan, M.I.R., et al., 2017. Approaches in modulating proline metabolism in plants for salt and drought stress tolerance: phytohormones, mineral nutrients and transgenics. Plant. Physiol. Biochem. 115, 126-140.

Per, T.S., Khan, M.I.R., Anjum, N.A., Masood, A., Hussain, S.J., Khan, N.A., 2018. Jasmonates in plants under abiotic stresses: crosstalk with other phytohormones matters. Environ. Exp. Bot. 145, 104-120.

Pérez-Salamó, I., Papdi, C., Rigó, G., Zsigmond, L., Vilela, B., Lumbreras, V., et al., 2014. The heat shock factor A4A confers salt tolerance and is regulated by oxidative stress and the mitogenactivated protein kinases MPK3 and MPK6. Plant Physiol. 165 (1), 319-334.

Pnueli, L., Liang, H., Rozenberg, M., Mittler, R., 2003. Growth suppression, altered stomatal responses, and augmented induction of heat shock proteins in cytosolic ascorbate peroxidase (Apx1)-deficient Arabidopsis plants. Plant J. 34 (2), 187-203.

Poovaiah, B.W., Reddy, A.S.N., Feldman, L., 1993. Calcium and signal transduction in plants. CRC. Crit. Rev. Plant. Sci. 12 (3), 185-211.

Prasad, T.K., Anderson, M.D., Martin, B.A., Stewart, C.R., 1994. Evidence for chilling-induced oxidative stress in maize seedlings and a regulatory role for hydrogen peroxide. Plant Cell 6 (1), 65-74.

Pratt, W.B., Toft, D.O., 2003. Regulation of signalling protein function and trafficking by the hsp $90 /$ hsp70-based chaperone machinery. Exp. Biol. Med. 228 (2), 111-133.

Pratt, W.B., Galigniana, M.D., Harrell, J.M., DeFranco, D.B., 2004. Role of hsp90 and the hsp90-binding immunophilins in signalling protein movement. Cell. Signal. 16 (8), 857-872.

Pucciariello, C., Banti, V., Perata, P., 2012. ROS signalling as common element in low oxygen and heat stresses. Plant. Physiol. Biochem. 59, 3-10.

Pulyaevskaya, M.A., Varakina, N.N., Gamburg, K.Z., Rusaleva, T.M., Stepanov, A.V., Voinikov, V.K., et al., 2011. Sodium fluoride inhibits HSP synthesis in heat-stressed cultured cells of Arabidopsis thaliana. Russian J. Plant Physiol. 58 (4), 589-596.

Pyatrikas, D.V., Rikhvanov, E.G., Fedoseeva, I.V., Varakina, N.N., Rusaleva, T.M., Tauson, E.L., et al., 2014. Mitochondrial retrograde regulation of HSP101 expression in Arabidopsis thaliana under heat stress and amiodarone action. Russian J. Plant Physiol. 61 (1), 80-89.

Qiu, X.B., Shao, Y.M., Miao, S., Wang, L., 2006. The diversity of the DnaJ/Hsp40 family, the crucial partners for Hsp70 chaperones. Cell. Mol. Life Sci. CMLS 63 (22), 2560-2570.

Queitsch, C., Hong, S.W., Vierling, E., Lindquist, S., 2000. Heat shock protein 101 plays a crucial role in thermotolerance in Arabidopsis. Plant Cell 12 (4), 479-492.

Rasmussen, S., Barah, P., Suarez-Rodriguez, M.C., Bressendorff, S., Friis, P., Costantino, P., et al., 2013. Transcriptome responses to combinations of stresses in Arabidopsis. Plant Physiol. 161 (4), 1783-1794.

Reddy, A.S., Ali, G.S., Celesnik, H., Day, I.S., 2011. Coping with stresses: roles of calcium-and calcium/calmodulin-regulated gene expression. Plant Cell 23 (6), 2010-2032.

Reddy P.S., Chakradhar T., Reddy R.A., Nitnavare R.B., Mahanty S., Reddy M.K., 2016. Role of Heat Shock Proteins in Improving Heat Stress Tolerance in Crop Plants. In Heat Shock Proteins and Plants, Springer, Cham, 283-307.

Reddy, P.S., Mallikarjuna, G., Kaul, T., Chakradhar, T., Mishra, R.N., Sopory, S.K., et al., 2010. Molecular cloning and characterization of gene encoding for cytoplasmic Hsc70 from Pennisetum glaucum may play a protective role against abiotic stresses. Mol. Genet. Genomics. 283 (3), 243-254.

Reddy, P.S., Thirulogachandar, V., Vaishnavi, C.S., Aakrati, A., Sopory, S.K., Reddy, M.K., 2011. Molecular characterization and expression of a gene encoding cytosolic Hsp90 from Pennisetum glaucum and its role in abiotic stress adaptation. Gene 474 (1), 29-38.

Reddy, P.S., Kishor, P.B.K., Seiler, C., Kuhlmann, M., EschenLippold, L., Lee, J., et al., 2014a. Unraveling regulation of the small heat shock proteins by the heat shock factor $\mathrm{HvHsfB} 2 \mathrm{c}$ in barley: its implications in drought stress response and seed development. PLoS ONE 9 (3), e89125.

Reddy, P.S., Vadez, V., Nese, S., Kishor, P.K., 2014b. Tackling the heat-stress tolerance in crop plants: a bioinformatics approach. Agricultural Bioinformatics. Springer, India, pp. 33-57.

Reddy, P.S., Sharma, K.K., Vadez, V., Reddy, M.K., 2015. Molecular cloning and differential expression of cytosolic class I small Hsp gene family in Pennisetum glaucum (L.). Appl. Biochem. Biotechnol. 176 (2), 598-612.

Reddy, R.A., Kumar, B., Reddy, P.S., Mishra, R.N., Mahanty, S., Kaul, T., et al., 2009. Molecular cloning and characterization of genes encoding Pennisetum glaucum ascorbate peroxidase and heat-shock factor: interlinking oxidative and heat-stress responses. J. Plant. Physiol. 166 (15), 1646-1659.

Rikhvanov, E.G., Gamburg, K.Z., Varakina, N.N., Rusaleva, T.M., Fedoseeva, I.V., Tauson, E.L., et al., 2007. Nuclear-mitochondrial cross-talk during heat shock in Arabidopsis cell culture. Plant J. 52 (4), 763-778.

Rousch, J.M., Bingham, S.E., Sommerfeld, M.R., 2004. Protein expression during heat stress in thermo-intolerant and thermo-tolerant diatoms. J. Exp. Mar. Bio. Ecol. 306 (2), 231-243.

Saidi, Y., Finka, A., Muriset, M., Bromberg, Z., Weiss, Y.G., Maathuis, F.J., et al., 2009. The heat shock response in moss plants is regulated by specific calcium-permeable channels in the plasma membrane. Plant Cell 21, 2829-2843.

Saidi, Y., Finka, A., Goloubinoff, P., 2011. Heat perception and signalling in plants: a tortuous path to thermotolerance. New Phytol. 190 (3), 556-565. 
Sakurai, H., Enoki, Y., 2010. Novel aspects of heat shock factors: DNA recognition, chromatin modulation and gene expression. FEBS. J. 277 (20), 4140-4149.

Samakovli, D., Margaritopoulou, T., Prassinos, C., Milioni, D., Hatzopoulos, P., 2014. Brassinosteroid nuclear signalling recruits HSP90 activity. New Phytol. 203 (3), 743-757.

Sangwan, V., Örvar, B.L., Beyerly, J., Hirt, H., Dhindsa, R.S., 2002. Opposite changes in membrane fluidity mimic cold and heat stress activation of distinct plant MAP kinase pathways. Plant J. $31(5), 629-638$.

Sasabe, M., Soyano, T., Takahashi, Y., Sonobe, S., Igarashi, H., Itoh, T.J., et al., 2006. Phosphorylation of NtMAP65-1 by a MAP kinase down-regulates its activity of microtubule bundling and stimulates progression of cytokinesis of tobacco cells. Genes Dev. 20 (8), 1004-1014.

Sato, S., Fujita, N., Tsuruo, T., 2000. Modulation of Akt kinase activity by binding to Hsp90. Proc. Natl. Acad. Sci. 97 (20), 10832-10837.

Scarpeci, T.E., Zanor, M.I., Carrillo, N., Mueller-Roeber, B., Valle, E. M., 2008. Generation of superoxide anion in chloroplasts of Arabidopsis thaliana during active photosynthesis: a focus on rapidly induced genes. Plant Mol. Biol. 66 (4), 361-378.

Scharf, K.D., Rose, S., Zott, W., Schöffl, F., Nover, L., Schöff, F., 1990. Three tomato genes code for heat stress transcription factors with a region of remarkable homology to the DNA-binding domain of the yeast HSF. EMBO J. 9 (13), 4495-4501.

Scharf, K.D., Höhfeld, I., Nover, L., 1998. Heat stress response and heat stress transcription factors. J. Biosci. 23 (4), 313.

Scharf, K.D., Berberich, T., Ebersberger, I., Nover, L., 2012. The plant heat stress transcription factor (Hsf) family: structure, function and evolution. Biochimica et Biophysica Acta (BBA)-Gene Regul. Mech. 1819 (2), 104-119.

Schramm, F., Larkindale, J., Kiehlmann, E., Ganguli, A., Englich, G., Vierling, E., et al., 2008. A cascade of transcription factor DREB2A and heat stress transcription factor HsfA3 regulates the heat stress response of Arabidopsis. Plant J. 53 (2), 264-274.

Schroda, M., Vallon, O., Wollman, F.A., Beck, C.F., 1999. A chloroplast-targeted heat shock protein 70 (HSP70) contributes to the photoprotection and repair of photosystem II during and after photoinhibition. Plant Cell 11 (6), 1165-1178.

Seo, H.R., Chung, D.Y., Lee, Y.J., Lee, D.H., Kim, J.I., Bae, S., et al., 2006. Heat shock protein 25 or inducible heat shock protein 70 activates heat shock factor 1 dephosphorylation on serine 307 through inhibition of ERK1/2 phosphorylation. J. Biol. Chem. 281 (25), 17220-17227.

Shao, H.B., Chu, L.Y., Jaleel, C.A., Zhao, C.X., 2008. Water-deficit stress-induced anatomical changes in higher plants. C. R. Biol. 331 (3), 215-225.

Simons, G., Groenendijk, J., Wijbrandi, J., Reijans, M., Groenen, J., Diergaarde, P., et al., 1998. Dissection of the Fusarium I2 gene cluster in tomato reveals six homologs and one active gene copy. Plant Cell 10 (6), 1055-1068.

Singh, A., Grover, A., 2010. Plant Hsp100/ClpB-like proteins: poorlyanalyzed cousins of yeast ClpB machine. Plant Mol. Biol. 74 (4-5), 395-404.

Sinha, A.K., Jaggi, M., Raghuram, B., Tuteja, N., 2011. Mitogenactivated protein kinase signalling in plants under abiotic stress. Plant Signal. Behav. 6 (2), 196-203.

Song, A., Zhu, X., Chen, F., Gao, H., Jiang, J., Chen, S., 2014. A chrysanthemum heat shock protein confers tolerance to abiotic stress. Int. J. Mol. Sci. 15 (3), 5063-5078.

Song, X., Xu, A., Pan, W., Wallin, B., Kivlin, R., Lu, S., et al., 2008. Minocycline protects melanocytes against $\mathrm{H} 2 \mathrm{O} 2$-inducedcell death via JNK and p38 MAPK pathways. Int. J. Mol. Med. 22 (1), 9-16.

Storozhenko, S., De Pauw, P., Van Montagu, M., Inzé, D., Kushnir, S., 1998. The heat-shock element is a functional component of the
Arabidopsis APX1 gene promoter. Plant Physiol. 118 (3), 1005-1014.

Su, P.H., Li, H.M., 2008. Arabidopsis stromal 70-kD heat shock proteins are essential for plant development and important for thermotolerance of germinating seeds. Plant Physiol. 146 (3), 1231-1241.

Sugio, A., Dreos, R., Aparicio, F., Maule, A.J., 2009. The cytosolic protein response as a subcomponent of the wider heat shock response in Arabidopsis. Plant Cell 21 (2), 642-654.

Sun, J., Zhang, C.L., Deng, S.R., Lu, C.F., Shen, X., Zhou, X.Y., et al., 2012. An ATP signalling pathway in plant cells: extracellular ATP triggers programmed cell death in Populus euphratica. Plant Cell Environ. 35 (5), 893-916.

Sun, W., Van Montagu, M., Verbruggen, N., 2002. Small heat shock proteins and stress tolerance in plants. Biochimica et Biophysica Acta (BBA)-Gene Struct. Exp. 1577 (1), 1-9.

Sun, X.T., Li, B., Zhou, G.M., Tang, W.Q., Bai, J., Sun, D.Y., et al., 2000. Binding of the maize cytosolic Hsp70 to calmodulin, and identification of calmodulin-binding site in Hsp70. Plant Cell Physiol. 41 (6), 804-810.

Sun, X., Sun, C., Li, Z., Hu, Q., Han, L., Luo, H., 2016. AsHSP17, a creeping bentgrass small heat shock protein modulates plant photosynthesis and ABA-dependent and independent signalling to attenuate plant response to abiotic stress. Plant Cell Environ. 39 (6), 1320-1337.

Suri, S.S., Dhindsa, R.S., 2008. A heat-activated MAP kinase (HAMK) as a mediator of heat shock response in tobacco cells. Plant Cell Environ. 31 (2), 218-226.

Suzuki, N., Rizhsky, L., Liang, H., Shuman, J., Shulaev, V., Mittler, R., 2005. Enhanced tolerance to environmental stress in transgenic plants expressing the transcriptional coactivator multiprotein bridging factor 1c. Plant Physiol. 139 (3), 1313-1322.

Suzuki, N., Sejima, H., Tam, R., Schlauch, K., Mittler, R., 2011. Identification of the MBF1 heat-response regulon of Arabidopsis thaliana. Plant J. 66 (5), 844-851.

Suzuki, N., Koussevitzky, S., Mittler, R.O.N., Miller, G.A.D., 2012. ROS and redox signalling in the response of plants to abiotic stress. Plant Cell Environ. 35 (2), 259-270.

Swindell, W.R., Huebner, M., Weber, A.P., 2007. Transcriptional profiling of Arabidopsis heat shock proteins and transcription factors reveals extensive overlap between heat and non-heat stress response pathways. BMC Genomics 8 (1), 125.

Szyszka, R., Kramer, G., Hardesty, B., 1989. The phosphorylation state of the reticulocyte $90-\mathrm{kDa}$ heat shock protein affects its ability to increase phosphorylation of peptide initiation factor 2 . alpha. subunit by the heme-sensitive kinase. Biochemistry 28 (4), 1435-1438.

Tena, G., Asai, T., Chiu, W.L., Sheen, J., 2001. Plant mitogenactivated protein kinase signalling cascades. Curr. Opin. Plant. Biol. 4 (5), 392-400.

Timperio, A.M., Egidi, M.G., Zolla, L., 2008. Proteomics applied on plant abiotic stresses: role of heat shock proteins (HSP). J. Proteomics. 71 (4), 391-411.

Tompa, P., Kovacs, D., 2010. Intrinsically disordered chaperones in plants and animals. Biochem. Cell. Biol. 88 (2), 167-174.

Török, Z., Goloubinoff, P., Horváth, I., Tsvetkova, N.M., Glatz, A., Balogh, G., et al., 2001. Synechocystis HSP17 is an amphitropic protein that stabilizes heat-stressed membranes and binds denatured proteins for subsequent chaperone-mediated refolding. Proc. Natl. Acad. Sci. 98 (6), 3098-3103.

Tripp, J., Mishra, S.K., Scharf, K.D., 2009. Functional dissection of the cytosolic chaperone network in tomato mesophyll protoplasts. Plant Cell Environ. 32 (2), 123-133.

Tuteja, N., 2009. Integrated calcium signalling in plants. Signalling in plants. Springer Berlin Heidelberg, pp. 29-49. 
Tuteja, N., Sopory, S.K., 2008. Chemical signalling under abiotic stress environment in plants. Plant Signal. Behav. 3 (8), 525-536.

Udvardi, M.K., Kakar, K., Wandrey, M., Montanari, O., Murray, J., Andriankaja, A., et al., 2007. Legume transcription factors: global regulators of plant development and response to the environment. Plant Physiol. 144 (2), 538-549.

Usman, M.G., Rafii, M.Y., Ismail, M.R., Malek, M.A., Latif, M.A., Oladosu, Y., 2014. Heat shock proteins: functions and response against heat stress in plants. Int. J. Sci. Technol. Res 3 (11), 204-218.

Vij, S., Tyagi, A.K., 2007. Emerging trends in the functional genomics of the abiotic stress response in crop plants. Plant. Biotechnol. J. 5 (3), 361-380.

Vinocur, B., Altman, A., 2005. Recent advances in engineering plant tolerance to abiotic stress: achievements and limitations. Curr. Opin. Biotechnol. 16 (2), 123-132.

Voellmy, R., Boellmann, F., 2007. Chaperone regulation of the heat shock protein response. Molecular Aspects of the Stress Response: Chaperones, Membranes and Networks. Springer, New York, NY, pp. 89-99.

Volkov, R.A., Panchuk, I.I., Mullineaux, P.M., Schöffl, F., 2006. Heat stress-induced $\mathrm{H} 2 \mathrm{O} 2$ is required for effective expression of heat shock genes in Arabidopsis. Plant Mol. Biol. 61 (4-5), 733-746.

von Koskull-Döring, P., Scharf, K.D., Nover, L., 2007. The diversity of plant heat stress transcription factors. Trends. Plant. Sci. 12 (10), 452-457.

Wan, X.L., Yang, J., Li, X.B., Zhou, Q., Guo, C., Bao, M.Z., et al., 2016. Over-expression of PmHSP17. 9 in transgenic Arabidopsis thaliana confers thermotolerance. Plant. Mol. Biol. Rep. 34 (5), 899-908.

Wang, A., Yu, X., Mao, Y., Liu, Y., Liu, G., Liu, Y., et al., 2015. Overexpression of a small heat-shock-protein gene enhances tolerance to abiotic stresses in rice. Plant Breeding 134 (4), 384-393.

Wang, R., Zhang, Y., Kieffer, M., Yu, H., Kepinski, S., Estelle, M., 2016. HSP90 regulates temperature-dependent seedling growth in Arabidopsis by stabilizing the auxin co-receptor F-box protein TIR1. Nat. Commun. 7, 10269.

Wang, W., Vinocur, B., Altman, A., 2003. Plant responses to drought, salinity and extreme temperatures: towards genetic engineering for stress tolerance. Planta 218 (1), 1-14.

Wang, W., Vinocur, B., Shoseyov, O., Altman, A., 2004. Role of plant heat-shock proteins and molecular chaperones in the abiotic stress response. Trends. Plant. Sci. 9 (5), 244-252.

Wang, X., Huang, B., 2017. Lipid-and calcium-signalling regulation of HsfA2c-mediated heat tolerance in tall fescue. Environ. Exp. Bot. 136, 59-67.

Wang, Y., Lin, S., Song, Q., Li, K., Tao, H., Huang, J., et al., 2014. Genome-wide identification of heat shock proteins (Hsps) and Hsp interactors in rice: Hsp70s as a case study. BMC Genomics 15 (1), 344

Wegele, H., Müller, L., Buchner, J., 2004. Hsp70 and Hsp90-a relay team for protein folding. Reviews of physiology, biochemistry and pharmacology. Springer Berlin Heidelberg, pp. 1-44.

Weir, I.E., Pham, N.A., Hedley, D.W., 2003. Oxidative stress is generated via the mitochondrial respiratory chain during plant cell apoptosis. Cytom. Part A 54 (2), 109-117.

Wurzinger, B., Mair, A., Pfister, B., Teige, M., 2011. Cross-talk of calcium-dependent protein kinase and MAP kinase signalling. Plant Signal. Behav. 6 (1), 8-12.

Xiong, L., Schumaker, K.S., Zhu, J.K., 2002. Cell signaling during cold, drought, and salt stress. Plant Cell 14 (Suppl. 1), S165-S183.

Xu, J., Xue, C., Xue, D., Zhao, J., Gai, J., Guo, N., et al., 2013. Overexpression of GmHsp90s, a heat shock protein 90 (Hsp90) gene family cloning from soybean, decrease damage of abiotic stresses in Arabidopsis thaliana. PLoS ONE 8 (7), e69810.
Xu, Q., Metzler, B., Jahangiri, M., Mandal, K., 2012. Molecular chaperones and heat shock proteins in atherosclerosis. Am. J. Physiol. Heart Circul. Physiol. 302 (3), H506-H514.

Xuan, Y., Zhou, S., Wang, L., Cheng, Y., Zhao, L., 2010. Nitric oxide functions as a signal and acts upstream of AtCaM3 in thermotolerance in Arabidopsis seedlings. Plant Physiol. 153 (4), 1895-1906.

Xue, G.P., Drenth, J., McIntyre, C.L., 2014. TaHsfA6f is a transcriptional activator that regulates a suite of heat stress protection genes in wheat (Triticum aestivum L.) including previously unknown Hsf targets. J. Exp. Bot. 66 (3), 1025-1039.

Yamada, K., Fukao, Y., Hayashi, M., Fukazawa, M., Suzuki, I., Nishimura, M., 2007. Cytosolic HSP90 regulates the heat shock response that is responsible for heat acclimation in Arabidopsis thaliana. J. Biol. Chem. 282 (52), 37794-37804.

Yoshida, T., Mogami, J., Yamaguchi-Shinozaki, K., 2014. ABAdependent and ABA-independent signalling in response to osmotic stress in plants. Curr. Opin. Plant. Biol. 21, 133-139.

Yoshida, T., Sakuma, Y., Todaka, D., Maruyama, K., Qin, F., Mizoi, J., et al., 2008. Functional analysis of an Arabidopsis heat-shock transcription factor HsfA3 in the transcriptional cascade downstream of the DREB2A stress-regulatory system. Biochem. Biophys. Res. Commun. 368 (3), 515-521.

Yu, A., Li, P., Tang, T., Wang, J., Chen, Y., Liu, L., 2015. Roles of Hsp70s in stress responses of microorganisms, plants, and animals. Biomed. Res. Int. 2015.

Zabaleta, E., Oropeza, A., Assad, N., Mandel, A., Salerno, G., Herrera-Estrella, L., 1994. Antisense expression of chaperonin 603 in transgenic tobacco plants leads to abnormal phenotypes and altered distribution of photoassimilates. Plant J. 6 (3), 425-432.

Zhang, H., Li, L., Ye, T., Chen, R., Gao, X., Xu, Z., 2016. Molecular characterization, expression pattern and function analysis of the OsHSP90 family in rice. Biotechnol. Biotechnol. Equip. 30 (4), 669-676.

Zhang, J.H., Wang, L.J., Pan, Q.H., Wang, Y.Z., Zhan, J.C., Huang, W.D., 2008. Accumulation and subcellular localization of heat shock proteins in young grape leaves during cross-adaptation to temperature stresses. Sci. Hortic. (Amsterdam) 117 (3), $231-240$.

Zhang, W., Zhou, R.G., Gao, Y.J., Zheng, S.Z., Xu, P., Zhang, S.Q., et al., 2009. Molecular and genetic evidence for the key role of AtCaM3 in heat-shock signal transduction in Arabidopsis. Plant Physiol. 149 (4), 1773-1784.

Zhang, Y., Dorey, S., Swiderski, M., Jones, J.D., 2004. Expression of RPS4 in tobacco induces an AvrRps4-independent HR that requires EDS1, SGT1 and HSP90. Plant J. 40 (2), 213-224.

Zhou, L., Liu, Z., Liu, Y., Kong, D., Li, T., Yu, S., et al., 2016. A novel gene OsAHL1 improves both drought avoidance and drought tolerance in rice. Sci. Rep. 6, 30264.

Zhou, Y., Chen, H., Chu, P., Li, Y., Tan, B., Ding, Y., et al., 2012b. NnHSP17.5, a cytosolic class II small heat shock protein gene from Nelumbo nucifera, contributes to seed germination vigor and seedling thermotolerance in transgenic Arabidopsis. Plant Cell Rep. 31 (2), 379-389.

Zou, J., Liu, C., Liu, A., Zou, D., Chen, X., 2012. Overexpression of OsHsp17. 0 and OsHsp23. 7 enhances drought and salt tolerance in rice. J. Plant. Physiol. 169 (6), 628-635.

Zhu, B., Ye, C., Lü, H., Chen, X., Chai, G., Chen, J., et al., 2006. Identification and characterization of a novel heat shock transcription factor gene, GmHsfA1, in soybeans (Glycine max). J. Plant. Res. 119 (3), 247-256.

Zuehlke, A., Johnson, J.L., 2010. Hsp90 and co-chaperones twist the functions of diverse client proteins. Biopolymers 93 (3), 211-217. 


\section{3}

\section{$\mathrm{C}_{4} / \mathrm{CAM}$ Facultative Photosynthesis as a Means to Improve Plant Sustainable Productivity Under Abiotic-Stressed Conditions: Regulatory Mechanisms and Biotechnological Implications}

Renata Callegari Ferrari and Luciano Freschi

Department of Botany, Institute of Biosciences, University of São Paulo, São Paulo, Brazil

\section{O U T L I N E}

\subsection{Initial Considerations}

33.2 $\mathrm{C}_{4}$ and Crassulacean Acid Metabolism:

Similarities and Differences

33.2.1 Defining $\mathrm{C}_{4}$ and Crassulacean Acid Metabolism

33.2.2 Modules Common to Both $\mathrm{C}_{4}$ and Crassulacean Acid Metabolism Plants

33.2.3 Exclusive Modules: Transfer Acid Generation and Stomatal Control

33.3 Environmental Cues Controlling $\mathrm{C}_{4}$ and Crassulacean Acid Metabolism

33.3.1 Modulation of $\mathrm{C}_{4}$ by Environmental Cues

33.3.2 Modulation of Crassulacean Acid Metabolism by Environmental Cues
518

33.4 Stress Signaling Networks Controlling $\mathrm{C}_{4}$ and Crassulacean Acid Metabolism

$518 \quad 33.5 \mathrm{C}_{4} /$ Crassulacean Acid Metabolism Compatibility

33.7 Concluding Remarks

Acknowledgments

References

523 Further Reading 


\subsection{INITIAL CONSIDERATIONS}

When referring to carbon concentrating mechanisms (CCMs), it is important take first a brief overview of the selective pressures that lead to their evolution. Under low $\mathrm{CO}_{2}$ availability, the oxygenase activity of ribulose1,5-biphosphate-carboxylase oxygenase (Rubisco) is favored, resulting in the production of 3-phosphoglyceric acid (PGA) and phosphoglycolate (PG), a toxic molecule capable of inhibiting photosynthesis, instead of PGA alone (Erb and Zarzycki, 2018; Miziorko and Lorimer, 1983). As a result, all plant lineages evolved the photorespiratory pathway, a complex mechanism that involves coordinated chemical reactions in chloroplasts, peroxisomes, and mitochondria. Photorespiration leads to the conversion of PG into less toxic products and yields back part of the $\mathrm{CO}_{2}$ that would have been lost due to the formation of PG (Sage et al., 2012). For this reason, although energetically expensive, photorespiration is essential to photosynthetic organisms (Eisenhut et al., 2008).

Different methods for reconstructing atmospheric history converge in estimating that, for the last 40 million years until present date, atmospheric $\mathrm{CO}_{2}$ concentration has decreased from over $800 \mathrm{ppm}$ to the current 390 ppm (Beerling and Royer, 2011; Zhang et al., 2013). In this scenario, photorespiration limits carbon fixation in plants performing $\mathrm{C}_{3}$ photosynthesis, being intensified by high temperatures or stress conditions that promote stomatal closure and consequently lead to a decline in intercellular $\mathrm{CO}_{2}$ levels (Keeley and Rundel, 2003). As atmospheric $\mathrm{CO}_{2}$ levels dropped significantly, some photosynthetic organisms evolved CCMs, which are mechanisms capable of increasing inorganic carbon availability inside cells (Moroney et al., 2013; Raven et al., 2008). CCMs are responsible for a significant portion of Earth's inorganic carbon assimilation since they occur in a vast diversity of phylogenetic groups (Raven et al., 2008). These pathways have diversified among cyanobacteria (Badger et al., 1980, 2002), eukaryotic algae (Giordano et al., 2005; Meyer and Griffiths, 2013), basal embryophyte lineages (Raven et al., 2008), and flowering plants, either terrestrial or aquatic (Keeley and Rundel, 2003; Maberly and Madsen, 2002; Raven et al., 2008; Sage, 2004; Silvera et al., 2010). Among the distinct CCMs currently known, the $\mathrm{C}_{4}$ photosynthesis and the Crassulacean acid metabolism (CAM) are particularly relevant for flowering plants; therefore, they will be the focus of this chapter. We do not intend to exhaustively review the biochemical, anatomical, regulatory, and evolutionary similarities and differences between the $\mathrm{C}_{4}$ and CAM systems, but instead we aim to discuss the compatibility between these two photosynthetic adaptations, the environmental regulation and signaling events controlling both syndromes, and the potential biotechnological implications of engineering $\mathrm{C}_{4}$ and CAM together into one crop species.

\section{2 $\mathrm{C}_{4}$ AND CRASSULACEAN ACID METABOLISM: SIMILARITIES AND DIFFERENCES}

In the low $\mathrm{CO}_{2}$ availability scenario, when increasing $\mathrm{CO}_{2}$ assimilation involved higher transpiration losses, $\mathrm{C}_{4}$ and CAM lineages evolved independently and irradiated, resulting in increased fitness under specific environmental conditions (Edwards and Ogburn, 2012; Ehleringer and Monson, 1993). These two pathways recruited existing genes, formerly involved in anaplerotic functions, to perform photosynthetic-related roles (Edwards and Ogburn, 2012). On their evolutionary trajectories, $\mathrm{C}_{4}$ and CAM evolved from $\mathrm{C}_{3}$ ancestors, and probably the first few characteristics opted lead to their final divergent phenotypes (Sage, 2002). CAM is considered to have arisen first, since it is present in basal lineages such as lycophytes, ferns, isoetids, and cycads, whereas $\mathrm{C}_{4}$ probably evolved more recently (Edwards and Ogburn, 2012; Ehleringer and Monson, 1993; Keeley and Rundel, 2003; Silvera et al., 2010). $C_{4}$ is present in 19 angiosperm families, mostly grasses, representing roughly 3\% of known species (Sage, 2017), while CAM is more widespread, occurring in 35 families (about 6\% of total species number) (Silvera et al., 2010). $C_{4}$ and CAM can be considered as photosynthetic syndromes, since both comprise a whole set of morphoanatomical and biochemical attributes, and may be associated with specific ecological niches.

\subsubsection{Defining $\mathrm{C}_{4}$ and Crassulacean Acid Metabolism}

Both pathways utilize organic four carbon (4-C) acids as temporary reservoirs of $\mathrm{CO}_{2}$. $\mathrm{C}_{4}$ photosynthesis, or dual-cell $\mathrm{C}_{4}$, represents a spatial specialization, where $\mathrm{CO}_{2}$ is prefixed into 4-C acids in mesophyll cells (MC) and transported to bundle sheath cells (BSC), the latter being the only cells containing Rubisco and performing the photosynthetic carbon reductive (PCR) cycle (Monson, 1999). On the other hand, CAM is a temporal specialization, with carbon prefixation into 4-C acids occurring at night, followed by daytime remobilization of acids to release $\mathrm{CO}_{2}$ when the PCR cycle is active (Winter and Smith, 1996).

The steps involved in each mechanism can be described in a comparative manner, as similar biochemical modules are required in both these photosynthetic adaptations. As highlighted in recent next-generation sequencing and biodesign studies (Borland et al., 2014; 
Schluter et al., 2016; Yang et al., 2015), both $C_{4}$ and CAM pathways require a coordinated series of reactions comprising distinct modules (e.g., carboxylation, decarboxylation, $\mathrm{CO}_{2}$ acceptor regeneration, transfer acid generation, anatomy, stomatal control), for which the participating enzymes and regulatory proteins have been identified over the years (Table 33.1). Despite efforts in dissecting the components of these modules, uncertainties remain, particularly regarding the role played by many transporters in both syndromes (Hibberd and Covshoff, 2010; Schluter et al., 2016).

\subsubsection{Modules Common to Both $\mathrm{C}_{4}$ and Crassulacean Acid Metabolism Plants}

\subsubsection{Carboxylation and Decarboxylation Modules}

A common set of enzymes and regulatory proteins are responsible for the carboxylation and decarboxylation modules in both syndromes (Fig. 33.1, Table 33.1). In $\mathrm{C}_{4}$, the carboxylation module is restricted to $\mathrm{MC}$ whereas the decarboxylation module is confined to BSC, and both take place during the daytime (Monson, 1999). In CAM, the carboxylation and decarboxylation modules occur in the same cells (Fig. 33.1), but during four distinct phases: carboxylation occurs at night when stomata are open (phase I); decarboxylation occurs during the day when stomata are closed (phase III); dawn and dusk are transition moments (phases II and IV, respectively), when phosphoenolpyruvate carboxylase (PEPC) activity declines and Rubisco initiates $\mathrm{CO}_{2}$ assimilation (phase II), or vice versa (phase IV) (Winter and Smith, 1996).

The first carboxylation by PEPC is one of the key steps for both the $\mathrm{C}_{4}$ and CAM photosynthesis (Fig. 33.1, Table 33.1). Because of its fundamental role, PEPC regulation has been widely studied and extensively reviewed (e.g., Chollet et al., 1996; Izui et al., 2004; Lepiniec et al., 1994; Nimmo, 2000). Allosteric regulation of PEPC involves effector molecules, being glucose-6-P (G-6-P) and triose-P positive effectors, and malate and aspartate negative regulators (Chollet et al., 1996). Different isoforms of PEPC are encountered in higher plants, involved both in photosynthetic and anaplerotic reactions. According to Christin et al. (2014), there are two major groups of PEPC genes in plants: PPC-1 and PPC-2. The PPC-1 group comprises all $C A M-$ and $\mathrm{C}_{4}$-related genes and was duplicated many types in different plant lineages, while $P P C-2$ genes are related to $C_{3}$ housekeeping functions and are present in a single copy in plant genomes. The PPC-1 lineage is divided in different groups: $P P C-1 E 2$ and PPC-1E1, the latter containing the CAM and $\mathrm{C}_{4}$ specific genes (Christin et al., 2014).
The most significant difference between PEPC isoforms is the substitution of an alanine for a serine residue near the N-terminal domain (Chollet et al., 1996). This serine can be phosphorylated, which is a critical step in the regulation of PEPC activity. A PEPC kinase is responsible for phosphorylating this serine residue, thus activating PEPC, whereas a typical mammalian-type protein phosphatase 2A (PP2A) converts PEPC back to its inactive form by dephosphorylating this residue (Nimmo, 2000). The activation of PEPC occurs in opposite moments of the diel cycle for $\mathrm{C}_{4}$ and CAM (daytime and nighttime, respectively) and results in lower sensitivity to malate inhibition and higher sensitivity to G-6-P activation in both cases (Chollet et al., 1996).

Compared with the conserved role played by PEPC in all $\mathrm{C}_{4}$ and CAM plants, distinct decarboxylation enzymes can be found among representatives of both syndromes. In fact, three types of $\mathrm{C}_{4}$ photosynthesis can be defined according to the decarboxylation enzyme employed (i.e., NAD-ME, NADP-ME, and PEPCK types), which are accompanied by ultrastructural differences involving chloroplast position in the BSC (Kanai and Edwards, 1999). In the NADP-ME type, chloroplasts are arranged centrifugally and have reduced grana stackings; most oxaloacetate (OAA) is converted to malate, which is metabolized in MC and BSC chloroplasts. In NAD-ME type, chloroplasts and mitochondria are arranged centripetally and show well-developed grana stackings; most OAA is converted to aspartate in the cytoplasm, which is transferred to BSC mitochondria to be decarboxylated. While grana-rich chloroplasts are related to NADP and ATP production due to higher photosystem II activities and linear electron flow, lower grana content is related to ATP production and photosystem I-mediated cyclic electron flow (Edwards et al., 2004). In the PEPCKtype, chloroplasts also have developed grana stackings, and there may be a $30 \%$ contribution or more from NAD-ME decarboxylation (Bräutigam et al., 2014; Kanai and Edwards, 1999). Recently, this type has been questioned due to metabolite and energetic specific requirements that may be hard to maintain in BSC (Wang et al., 2014). Overall, it is more likely that NADor NADP-ME act as the main decarboxylating enzyme and PEPCK acts as a second decarboxylating enzyme instead of an exclusive PEPCK type (Bräutigam et al., 2014; Muhaidat et al., 2007; Wang et al., 2014).

The decarboxylation enzyme employed may also vary in CAM according to the species, and options are the same as employed by $\mathrm{C}_{4}$. In addition, the transitory carbohydrate pool converted into phosphoenolpyruvate for $\mathrm{CO}_{2}$ assimilation can be starch or soluble sugars (Borland et al., 2016; Holtum et al., 2005), resulting in an array of combinations of transitory carbohydrate pool and decarboxylation system. 
TABLE 33.1 Core Candidate Genes for $\mathrm{C}_{4}$ and CAM Syndromes Based on Recent Literature

\begin{tabular}{|c|c|c|c|c|}
\hline Module C & Gene & Type & Protein name & Reaction \\
\hline \multicolumn{2}{|c|}{ Carboxylation } & $\mathrm{C}_{4} / \mathrm{CAM}$ & & \\
\hline & CA & & Beta carbonic anhydrase & $\mathrm{H}_{2} \mathrm{CO}_{3}<=>\mathrm{CO}_{2}+\mathrm{H}_{2} \mathrm{O}$ \\
\hline & PEPC & & Phosphoenolpyruvate carboxylase & $\begin{array}{l}\mathrm{H}_{2} \mathrm{O}+\text { phosphoenolpyruvate }+\mathrm{HCO}_{3}^{-}<=> \\
\text {phosphate }+ \text { oxaloacetate }\end{array}$ \\
\hline & V-ATPases & & V-type proton ATPase & $\mathrm{ATP}+\mathrm{H}_{2} \mathrm{O}+\mathrm{H}^{+}$(in) $=\mathrm{ADP}+$ phosphate $+\mathrm{H}^{+}$(out) \\
\hline & ALMT & & Aluminum-activated malate transporter & $\begin{array}{l}\text { Presumably controlling malate influx and efflux in and } \\
\text { out of the tonoplast }\end{array}$ \\
\hline & VPPases & & $\begin{array}{l}\text { Pyrophosphate-energized vacuolar } \\
\text { membrane proton pump }\end{array}$ & $\mathrm{PP}=>2 \mathrm{P}$ \\
\hline \multicolumn{2}{|c|}{ Decarboxylation } & $\mathrm{C}_{4} / \mathrm{CAM}$ & & \\
\hline & NADP-ME & & NADP-dependent malic enzyme & Malate $+\mathrm{NADP}^{+}<=>$pyruvate $+\mathrm{CO}_{2}+\mathrm{NADPH}$ \\
\hline & NAD-ME & & NAD-dependent malic enzyme & Malate $+\mathrm{NAD}^{+}<=>$pyruvate $+\mathrm{CO}_{2}+\mathrm{NADH}$ \\
\hline & PEPCK & & Phosphoenolpyruvate carboxykinase & $\begin{array}{l}\text { ATP }+ \text { oxaloacetate }<=>\text { ADP }+ \text { phosphoenolpyruvate } \\
+\mathrm{CO}_{2}\end{array}$ \\
\hline & DiT2/DCT & & Dicarboxylate transporter & Malate (out) $+X=>$ malate (in) $+X$ \\
\hline & DIC & & Dicarboxylate carrier & 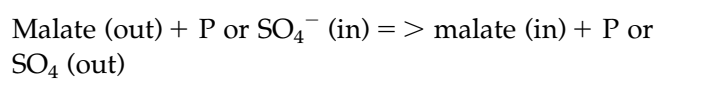 \\
\hline & Pyruvate exporter & & $\begin{array}{l}\text { Unknown pyruvate exporter in } \\
\text { mitochondria and chloroplasts }\end{array}$ & Pyruvate (in) $+X=>$ Pyruvate (out) $+X$ \\
\hline \multicolumn{2}{|c|}{$\mathrm{CO}_{2}$ acceptor regeneration ${ }^{\mathrm{a}}$} & $\mathrm{C}_{4} / \mathrm{CAM}$ & & \\
\hline & PPDK & & Pyruvate, phosphate dikinase & $\begin{array}{l}\text { ATP }+ \text { pyruvate }+ \text { phosphate }<=>\text { AMP }+ \\
\text { phosphoenolpyruvate }+ \text { diphosphate }\end{array}$ \\
\hline & AMK & & AMP kinase & $\mathrm{AMP}+\mathrm{ATP}=>2 \mathrm{ADP}$ \\
\hline & PPase & & Pyrophosphorylase & $\mathrm{PP}=>2 \mathrm{P}$ \\
\hline & BASS2 & & Sodium/pyruvate cotransporter BASS2 & $\begin{array}{l}\text { Pyruvate (out) }+\mathrm{Na}^{+} \text {(out) }=>\text { pyruvate (in) }+\mathrm{Na}^{+} \\
\text {(in) }\end{array}$ \\
\hline & NHD & & $\mathrm{Na} / \mathrm{H}$ antiporter & $\mathrm{H}^{+}$(out) $+\mathrm{Na}^{+}$(in) $=>\mathrm{H}^{+}$(in) $+\mathrm{Na}^{+}$(out) \\
\hline & PPT & & Phosphoenolpyruvate transporter & $\begin{array}{l}\mathrm{PEP}(\text { in })+\mathrm{H}^{+}(\text {in })+\mathrm{P}(\text { out })=>\mathrm{PEP}(\text { out })+\mathrm{H}^{+} \text {(out) }+ \\
\mathrm{P} \text { (in) }\end{array}$ \\
\hline & $\begin{array}{l}\text { Proton pyruvate } \\
\text { transporter }\end{array}$ & & Unknown chloroplast pyruvate importer & Pyruvate (out) $+\mathrm{H}^{+}($out $)=>$pyruvate $($in $)+\mathrm{H}^{+}$(in) \\
\hline \multicolumn{2}{|c|}{ Transfer acid generation ${ }^{\mathrm{b}}$} & $\mathrm{C}_{4}$ & & \\
\hline & $\mathrm{MDH}$ & & Malate dehydrogenase & Malate $+\mathrm{NAD}(\mathrm{P})^{+}<=>$oxaloacetate $+\mathrm{NAD}(\mathrm{P}) \mathrm{H}$ \\
\hline & AlaAT & & Alanine transaminase & Alanine +2 -oxoglutarate $<=>$ pyruvate + glutamate \\
\hline & AspAT & & Aspartate transaminase & $\begin{array}{l}\text { Aspartate }+2 \text {-oxoglutarate }<=>\text { oxaloacetate }+ \\
\text { glutamate }\end{array}$ \\
\hline & DiT1/OMT & & Dicarboxylate transporter 1 & OAA $($ out $)+$ malate $($ in $)=>$ malate $($ out $)+$ OAA (in) \\
\hline & DiT2/DCT & & Dicarboxylate transporter 2 & $\begin{array}{l}\text { OAA (out) }+ \text { aspartate }(\text { in })=>\text { aspartate }(\text { out })+\text { OAA } \\
\text { (in) }\end{array}$ \\
\hline
\end{tabular}

${ }^{a}$ Although a PEP regeneration module has only been named separately for $\mathrm{C}_{4}$, the reactions involved also occur in CAM and, for this work, it was considered here as part of the $\mathrm{CO}_{2}$ acceptor regeneration module.

${ }^{b}$ The role played by many transporters is still unclear for both syndromes; therefore, we summarize the most probable candidate reported in the literature (Schluter et al., 2016). 

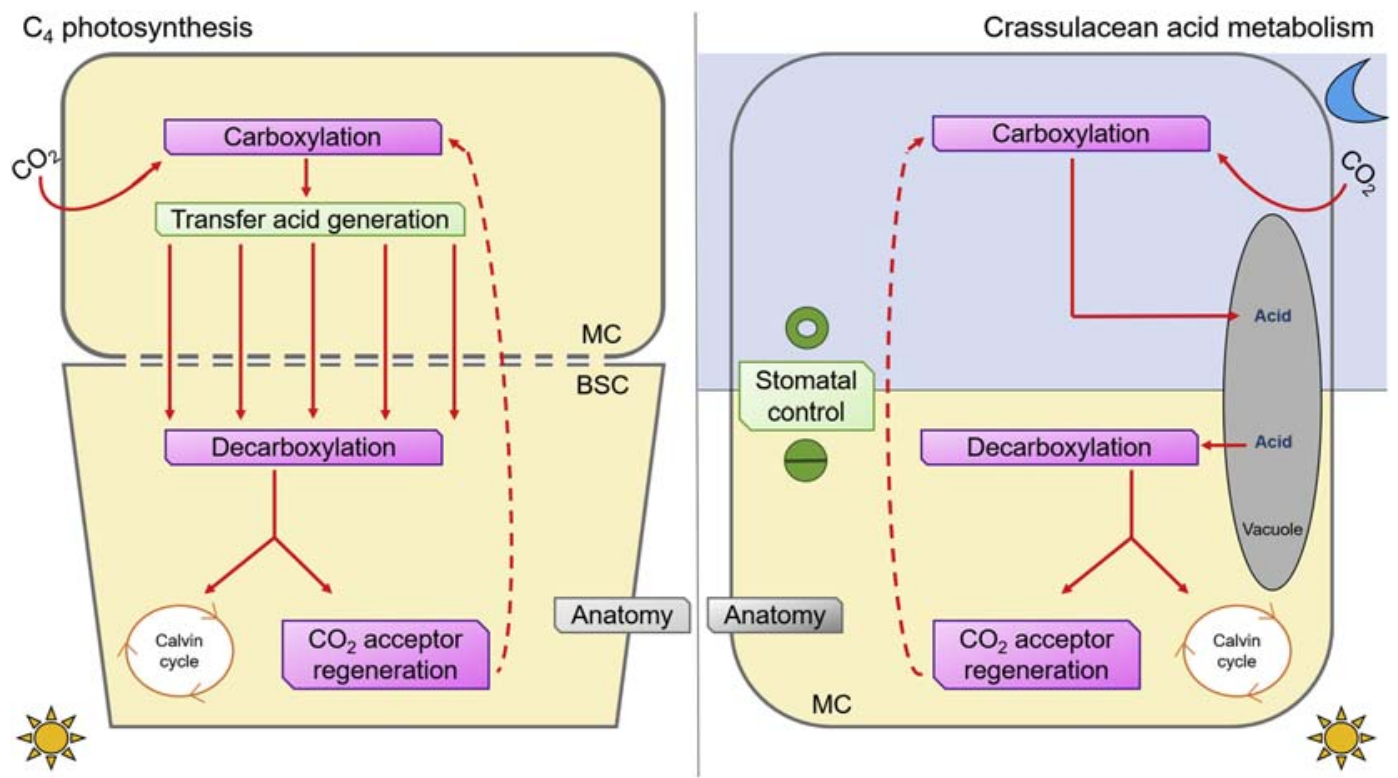

FIGURE 33.1 Comparative scheme between $\mathrm{C}_{4}$ photosynthesis and Crassulacean acid metabolism. Boxes indicate modules composed of a set of genes. Exclusive modules are indicated in green boxes. Common modules are indicated in purple boxes. Gray boxes indicate modules that are different in composition but share a common theme. Red arrows indicate the carbon pathway inside the cells. BSC, Bundle sheath cell; $M C$, mesophyll cell.

\subsubsection{Anatomy Module}

Anatomical traits are encompassed in an anatomy module, but although the theme is common, different attributes are involved in each syndrome (Fig. 33.1, Table 33.1). Most $C_{4}$ plants present Kranz anatomy, named after the well-developed layer of BSC surrounding vascular bundles and containing all Rubisco and glycine decarboxylase activity of the leaf (Hibberd and Covshoff, 2010; Sage, 2004). However, there are also a few examples of single-cell terrestrial $\mathrm{C}_{4}$ photosynthesis, indicating that the presence of Kranz anatomy is not mandatory (Voznesenskaya et al., 2001). In these cases, dimorphic chloroplasts can be found inside a single $\mathrm{MC}$, where there is asymmetrical distribution of $\mathrm{C}_{4}$-related enzymes and organelles between the distal and proximal regions of the cytoplasm, as in Borszczowia aralocaspica, or between internal and external regions, as in Bienertia cycloptera (Edwards et al., 2004; Voznesenskaya et al., 2001, 2002).

Different types of Kranz anatomy can be found among dual-cell $\mathrm{C}_{4}$ representatives based on the position of MC, BSC, vascular bundles, and other tissues, also varying between eudicots and monocots (Dengler and Nelson, 1999; Edwards et al., 2004; Muhaidat et al., 2007). However, there is no well-specified correlation between specific anatomical and biochemical types (Muhaidat et al., 2007). Other important traits associated to Kranz anatomy, all aiming to provide short distances for metabolite diffusion but at the same time avoid $\mathrm{CO}_{2}$ leakage, are (1) low M:BSC ratio with numerous plasmodesmata connecting these two cell types, (2) increased vein density, (3) thin leaves, and (4) a diffusion barrier in or around BSC, with either suberization of cell walls or high concentration of organelles at peripheric regions (Dengler and Nelson, 1999; Edwards et al., 2004; Sage, 2004). Specific mesophyll and bundle sheath trans- and cis-acting elements for core $\mathrm{C}_{4}$ enzymes have been identified and some have already been tested successfully (Wang et al., 2017). Moreover, tissue specificity in $\mathrm{C}_{4}$ leaves is guaranteed via epigenetic, transcriptional and posttranscriptional, translational and posttranslational regulation (Fig. 33.2; Reeves et al., 2016).

In line with the need of accumulating large quantities of 4-C acids in vacuoles of MC overnight, the most frequent anatomical features related to CAM are (1) increased MC size, (2) reduced intercellular air space (IAS), (3) reduced mesophyll surface exposed to IAS $\left(\mathrm{L}_{\text {mes }}\right)$ per unit area, and (4) increased leaf thickness compared with $\mathrm{C}_{3}$ and $\mathrm{C}_{4}$ species (Nelson et al., 2005). This results in more succulent leaves and prevents $\mathrm{CO}_{2}$ efflux during the day, although an increase in succulence itself is not related to higher CAM expression (Nelson and Sage, 2008).

\subsubsection{Exclusive Modules: Transfer Acid Generation and Stomatal Control}

Lastly, there are two exclusive modules, one related to each syndrome alone. The transfer acid generation 
module (Table 33.1) is very relevant for dual-cell $\mathrm{C}_{4}$ representatives since metabolites must flow between two different cell types (i.e., MC and BSC) (Schluter et al., 2016). As the type of transfer acid formed in MC and the place of decarboxylation in BSC may vary, this module involves a variable number of genes depending on the species. Essentially, it comprises proteins responsible for transporting or generating metabolites in different cell compartments such as mitochondria, chloroplasts, and the cytosol (Kanai and Edwards, 1999; Schluter et al., 2016).

On the other hand, the so-called stomatal control module is particularly relevant in CAM research. In $\mathrm{C}_{3}$ and $\mathrm{C}_{4}$, stomata remain open during the day and closed during the night. In contrast, in most CAM plants stomata remain closed during the day (phase III) probably due to the increase in partial $\mathrm{CO}_{2}$ pressure $\left(\mathrm{pCO}_{2}\right)$ from daytime acid decarboxylation, followed by nocturnal stomata opening (Borland and Taybi, 2004; Hartwell, 2005). Stomata may also remain open during transitional phase II in some species, promoting a burst in $\mathrm{CO}_{2}$ assimilation at the beginning of the light period (Winter and Holtum, 2014). In the CAM species Agave americana, several protein kinase-encoding genes, including HIGH LEAF TEMPERATURE 1 (HT1), OPEN STOMATA 1 (OST), and $S n R K 2.10$, presented rescheduled expression compared with their orthologs in Arabidopsis thaliana (Abraham et al., 2016). Furthermore, PHOTOTROPIN 2 (PHOT2), which encodes a blue light receptor, showed an inverted pattern of expression in the CAM plant model Kalanchöe fedtschenkoi compared with that observed in $C_{3}$ species (Yang et al., 2017). Also, genes related to ion channels in guard cells, such as POTASSIUM TRANSPORTER 2/3 (AKT2/3) and CHLORIDE CHANNEL FAMILY (CLC-C), showed reciprocal expression behavior in A. americana compared with that described in Arabidopsis (Abraham et al., 2016). Therefore, these genes exemplify some of the promising candidates associated with the inverted stomatal control typically found in CAM plants (Abraham et al., 2016; Yang et al., 2017).

\subsection{ENVIRONMENTAL CUES CONTROLLING $\mathrm{C}_{4}$ AND CRASSULACEAN ACID METABOLISM}

As stated by Lüttge (2004), environmental cues are defined as an input act upon organisms, referred to as receivers, and this interacting network will result in an output at community and ecosystems level. The input affects genotypes and phenotypes of receivers, the latter including morphotype and physiotype. $\mathrm{C}_{4}$ and CAM, both including genotype and phenotype components, represent two cases of parallel convergent evolution, where unrelated taxa from various phylogenetic backgrounds have evolved similar mechanisms in two independent ways (Christin et al., 2013, 2014; Muhaidat et al., 2007; Sage, 2017; Silvera et al., 2010). This makes the study of how representatives from both syndromes respond to the environment particularly interesting since a diverse array of strategies and different magnitudes of response has been observed.

In $C_{4}$ species such as the traditionally studied monocots maize (Zea mays) and sugarcane (Saccharum officinarum) or in CAM crops such as Agave americana or Opuntia ficus-indica, the CCM is constitutively expressed. Among CAM plants, there are also facultative species, whose CAM expression is highly influenced by environmental cues, thereby representing valuable tools for dissecting the regulatory mechanisms responsible for adjusting the CCM expression in response to the surrounding environmental conditions (Winter and Holtum, 2007; Winter and Holtum, 2014). In addition, increasing attention has been dedicated to the so-called $\mathrm{C}_{3}-\mathrm{C}_{4}$ intermediates, which may shed light on evolutionary paths leading to full $\mathrm{C}_{4}$ expression (Christin et al., 2011).

An interesting strategy to understand how external cues affect organisms is to study these intermediate and facultative phenotypes, because the first may help to understand evolutionary paths, and in the latter, the timing and inducing factors can be controlled without developmental interference (Christin et al., 2011; Winter and Holtum, 2014; Winter and Holtum, 2007).

\subsubsection{Modulation of $\mathrm{C}_{4}$ by Environmental Cues}

The relationship between dual-cell $\mathrm{C}_{4}$ and environmental cues can be traced in an evolutionary panorama or considering phenotypically plastic responses. Regarding the latter, a great description of environmental variables-such as light, water, nitrogen, and temperature-and how they modulate photosynthetic aspects of $C_{4}$ plants was made by Long (1999). When referring to the biochemical types of $\mathrm{C}_{4}$, NAD-type grasses were able to enhance water use efficiency (WUE) at leaf level in a higher proportion than NADPME type grasses, which was probably related to stomatal adjustments (Ghannoum et al., 2002).

Dual-cell $\mathrm{C}_{4}$ requires more specialized structural arrangements when compared with single-cell $\mathrm{C}_{4}, \mathrm{C}_{3}$, and $\mathrm{CAM}$, and presents more restricted geographic distribution than the latter two types. This can be correlated to a reduced potential for phenotypic plasticity when compared with $C_{3}$ plants, as stated by Sage and McKown (2006). These authors highlight that 
acclimation processes must involve coordinated changes in the different tissues involved in $\mathrm{C}_{4}$ to keep functional stoichiometries. Besides, when compared with $C_{3}$ plants, dual-cell $C_{4}$ representatives display restricted potential to modulate leaf thickness, due to the enlarged BSC, the costs associated with changes in vein density, and a limited capacity to modulate Rubisco content. However, there are exceptions to this assumption, since Flaveria bidentis and maize have been shown to exhibit plastic acclimation responses to low-light conditions (Pengelly et al., 2010; Bellasio and Griffiths, 2014), indicating that this subject needs further investigation. Plants exhibiting single-cell $\mathrm{C}_{4}$ do not face such structural constraints since they can more easily modulate photosynthesis-related processes within a single photosynthetic cell (Edwards et al., 2004; Voznesenskaya et al., 2001). Perhaps, in this case, more plastic responses could be observed, but no experimental evidence has been reported yet.

Similarly, little is known about the phenotypic plasticity within $\mathrm{C}_{3}-\mathrm{C}_{4}$ intermediate species. This photosynthetic type is considered a transitional stage between ancestral $C_{3}$ and fully expressed $C_{4}$, as it presents biochemical and anatomical features of both photosynthetic types (Christin et al., 2011; Monson and Moore, 1989). In a different point of view, $C_{3}-C_{4}$ plants can also be treated as a successful adaptive strategy, not merely as a transitional stage, as is the case for Mollugo verticillata and M. nudicaulis. These two species are weeds that show a cosmopolitan distribution, suggesting that the intermediate photosynthetic type may have contributed to their wide geographical distribution (Christin et al., 2011).

Differently from most $\mathrm{C}_{4}$ and $\mathrm{C}_{3}-\mathrm{C}_{4}$ intermediates, the aquatic species Eleocharis vivipara stands out as a remarkable example of $\mathrm{C}_{4}$ expression controlled by environmental circumstances (Ueno et al., 1988). E. vivipara produces $C_{3}$ photosynthetically active culms when underwater, but emerged $C_{3}$ culms wither away and new $\mathrm{C}_{4}$ culms develop after a few days (Ueno et al., 1988; Ueno, 2001). The $\mathrm{C}_{4}$ culms have already been characterized biochemically and anatomically (Ueno, 2001), and $\mathrm{C}_{4}$-related gene expression is not strictly dependent on Kranz anatomy (Uchino et al., 1998).

\subsubsection{Modulation of Crassulacean Acid Metabolism by Environmental Cues}

It is increasingly accepted that a higher plasticity can be found between and within CAM plants than in most $C_{4}$ species (Edwards and Ogburn, 2012). Compared with the rare examples of $\mathrm{C}_{3}-\mathrm{C}_{4}$ facultative plants (e.g., E. vivipara, E. baldwinii), a significantly higher number of $\mathrm{C}_{3}$-CAM facultative species are described in the literature (Silvera et al., 2010; Winter et al., 2015). Moreover, even obligate CAM species display some plasticity in CAM expression during early developmental stages (Freschi et al., 2010; Winter and Holtum, 2007). In this sense, CAM has been considered a trait with continuous distribution oppositely to an all-or-nothing adaptive response (Silvera et al., 2010; Winter et al., 2015).

Besides the variation in plasticity (i.e., constitutive versus facultative CAM), differences in the magnitude of nighttime acid accumulation and the diel pattern of stomata opening also greatly vary among CAM, giving rise to the following CAM modes: (1) CAM cycling, in which gas exchange is limited to daytime, with nocturnal acid production due to respiratory $\mathrm{CO}_{2}$ recycling; and (2) CAM-idling, which is an emergency process triggered during periods of prolonged drought and heat. The latter state is characterized by the complete closure of stomata over the diel cycle associated with a small nocturnal acid production from respiratory $\mathrm{CO}_{2}$ recycling (Cushman, 2001). Both these modes are considered weak versions of CAM as the amount of acids accumulated overnight is usually lower than in plants performing classical CAM.

Facultative plants frequently perform weak CAM for variable portions of their life cycles; therefore, the adaptive advantages of facultative CAM have been questioned (Herrera, 2009). However, even if a low level of CAM is induced, this may improve plant survival by increasing WUE, promoting a positive carbon balance, intensifying the photoprotection of the photosynthetic machinery, and, consequently, improving reproductive success (Herrera, 2009; Winter and Holtum, 2014).

Due to the absence of clear anatomical traits characterizing CAM and the fact that $\delta^{13} \mathrm{C}$ values in facultative CAM species tend to be closer to $C_{3}$ plants, detailed physiological studies are required to properly identify facultative CAM (Silvera et al., 2010; Winter et al., 2015). This may contribute to an underestimation of the total number of facultative $\mathrm{C}_{3}$-CAM species (Silvera et al., 2010). Nevertheless, confirmed facultative CAM species have been described in Bromeliaceae, Crassulaceae, Montiaceae, Piperaceae, Portulacaceae, Talinaceae, and Clusiaceae families (Winter and Holtum, 2014). In most facultative species, CAM expression can be induced or intensified by drought either alone or in combination with other environmental stresses such as nutrient availability and changes in temperature, light intensity, and photoperiod (Silvera et al., 2010). In the halophyte Mesembryanthemum crystallinum, either drought or salt stress strongly induce CAM (Cushman et al., 1990; Herppich et al., 1992; Winter and Holtum, 2007), and although a great deal of physiological, signaling, and molecular studies have 
been performed in this facultative model (Bohnert et al., 1988; Cushman et al., 1990, 2008; Herppich et al., 1992; Holtum and Winter, 1982; Winter and Holtum, 2007), much still remains to be elucidated.

Besides M. crystallinum, constitutive and facultative CAM species belonging the tropical genus Clusia have also been intensively studied over the years (Lüttge, 1996, 2006). CAM induction may be under developmental control as in C. rosea, C. alata, and C. hilariana; under environmental control in a fully reversible manner as in C. pratensis; or even exhibit intermediary influences as in C. minor (Winter and Holtum, 2007). Given the rapid, intense, and completely reversible induction of CAM observed in C. pratensis in response to drought, this species has emerged as an interesting model for future studies on the regulation of facultative CAM (Winter and Holtum, 2014).

\subsection{STRESS SIGNALING NETWORKS CONTROLLING $\mathrm{C}_{4}$ AND CRASSULACEAN ACID METABOLISM}

As presented so far, $\mathrm{C}_{4}$ and CAM are both complex syndromes, involving multiple genes, complicated metabolite dynamics, biochemical adjustments, and sometimes anatomical specializations. This implies the existence of equally complex signaling routes, as stated by Freschi and Mercier (2012) for CAM signaling, and here extended to $\mathrm{C}_{4}$. Intricate transcriptional, translational, posttranslational, and metabolic regulatory changes are supposedly required to facilitate the establishment and functioning of either CCM in a given tissue (Fig. 33.2). This includes, but is not limited to, coarse changes in enzyme content/activity and the finetuned control of regulatory proteins (e.g., transcription factors, kinases, phosphatases) (Taybi et al., 2002).

Most of the studies regarding the establishment of the $\mathrm{C}_{4}$ machinery has been based on the developmental gradient in leaves (e.g., Li et al., 2010; Pick et al., 2011), since $C_{4}$ induction upon environmental variables is rare, with a few exceptions in the Eleocharis genus (Ueno, 2001) as previously mentioned.

In E. vivipara submerged plants, abscisic acid (ABA) was shown to induce Kranz anatomy formation and $\mathrm{C}_{4}$-like biochemical traits (Ueno, 1998) whereas in the $\mathrm{C}_{3}-\mathrm{C}_{4}$ intermediate $E$. baldwinii, full $\mathrm{C}_{4}$ was induced after ABA treatment causing auxin signaling and changes in the transcriptional profile of genes involved in the glycolytic pathway, ion and metabolite transporters, citrate metabolism, among other processes (Chen et al., 2014). Recent evidence also indicates that PEPC content and activity is increased in light-exposed Amaranthus hypochondriacus leaf disks upon ABA treatment (Aloor et al., 2017), suggesting that it may be advantageous for $\mathrm{C}_{4}$ plants to keep PEPC activated by ABA under drought stress conditions.

Although still fragmented, our current knowledge on the stress signaling cascades controlling CAM expression is relatively more detailed than for $\mathrm{C}_{4}$

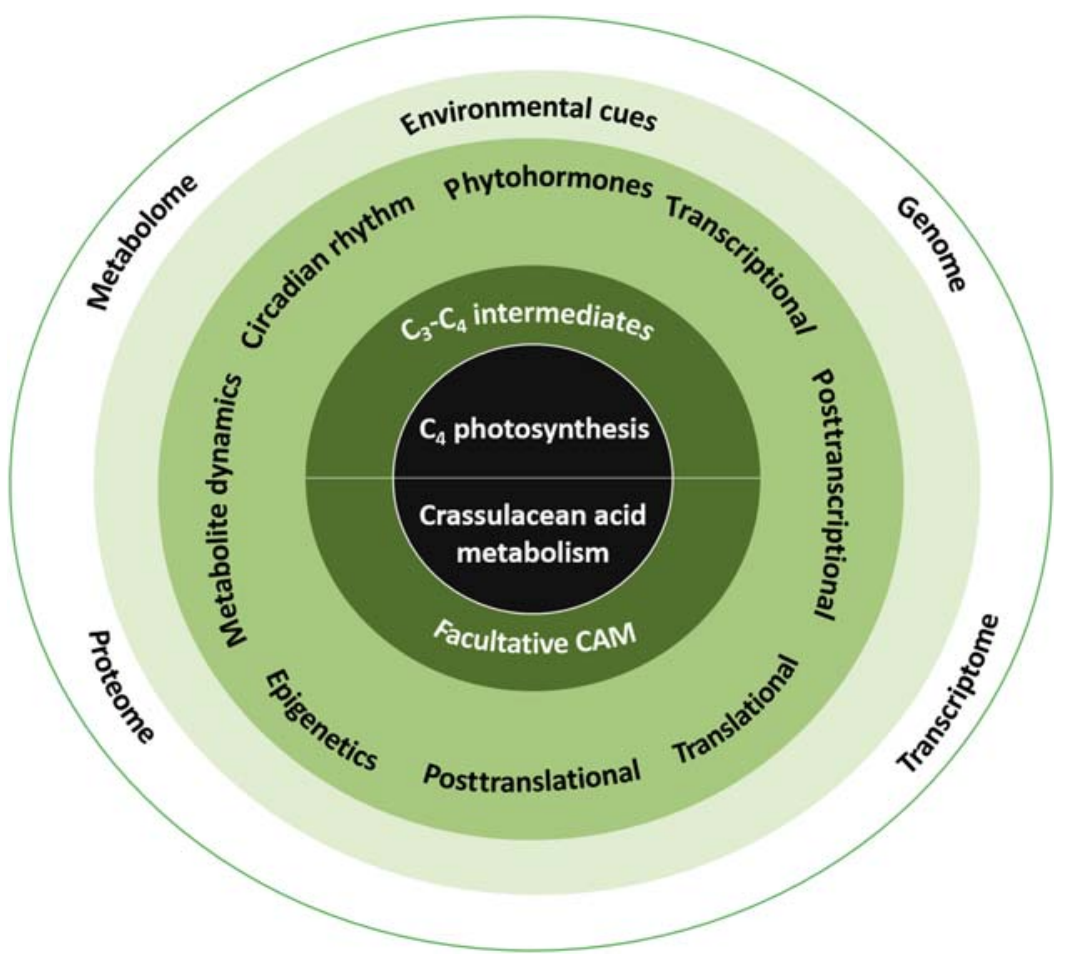

FIGURE 33.2 $\mathrm{C}_{4}$ and CAM are both complex syndromes that are regulated in many different levels. The study of intermediary and facultative systems for $\mathrm{C}_{4}$ and CAM, respectively, using omics technologies is very promising in understanding the regulatory processes. The two inner circles represent both photosynthetic metabolism types and their variations. The two outer circles represent the regulatory levels and omics fields that can be used to explore them. 
photosynthesis (Taybi et al., 2002; Freschi and Mercier, 2012). Most studies have been performed in the $C_{3^{-}}$ CAM halophyte $M$. crystallinum, but data is also available for a few other CAM species including pineapple (Ananas comosus) and Kalanchöe blossfeldiana. The signaling cascades controlling CAM expression in these species have been extensively reviewed first by Taybi et al. (2002) and later by Freschi and Mercier (2012); therefore, they are briefly discussed here.

Data indicate that ABA and cytokinins (Cks) play opposite roles in controlling CAM expression (Chu et al., 1990; Schmitt and Piepenbrock, 1992; Thomas and Bohnert, 1993; Thomas et al., 1992), which agrees with the contrasting impact of water availability on the biosynthesis of these two hormonal classes (Hare et al., 1997; Pospisilova et al., 2000, 2005; Tanaka et al., 2006). Two major lines of evidence support a promotive role of ABA on CAM expression. First, exogenous ABA has been shown to increase the activity and/or mRNA levels of CAM-related enzymes both in detached leaves and intact plants (Chu et al., 1990; Dai et al., 1994; Forsthoefel et al., 1995a,b, 2010; Taybi et al., 1995, 2002; Tsiantis et al., 1996). Second, stress-induced CAM expression is normally accompanied by an increment in endogenous ABA content (Freschi et al., 2010; Taybi et al., 1995, 2002). However, studies indicate that a parallel, non-ABA-dependent, signaling route is also involved in controlling CAM expression in response to environmental stresses (Freschi et al., 2010; Taybi et al., 2002).

In contrast, accumulating evidence implicates $\mathrm{Cks}$ as negative regulators of CAM expression in facultative CAM species as the stress-induced increase in CAM expression in facultative plants is usually accompanied by a reduction in endogenous $\mathrm{Ck}$ levels (Freschi et al., 2010; Peters et al., 1997), and exogenous Cks generally represses CAM induction in response to stress conditions (Freschi et al., 2010; Peters et al., 1997; Schmitt and Piepenbrock, 1992). Some studies also indicate that exogenous methyl jasmonate (MJA) can also limit CAM expression in droughted M. crystallinum plants (Dai et al., 1994; Schmitt and Piepenbrock, 1992), though the roles of endogenous MJA still remain elusive. More recently, gibberellin levels have been reported to decrease and not recover in Aptenia cordifolia plants exposed to successive drought stress, being thus connected to a drought stress memory response in CAM expression (Fleta-Soriano et al., 2015).

So far, only few second messengers have been demonstrated to control CAM expression in facultative plants (Freschi et al., 2010; Taybi et al., 2002). Among them, the influx of extracellular calcium $\left(\mathrm{Ca}^{2+}\right)$ has been identified as a key downstream event in both ABA-dependent and -independent signaling pathways leading to CAM expression (Freschi et al., 2010; Taybi et al., 2002). Data also indicate that inositol-1,4,5-triphosphate (IP3) and nitric oxide participate in the ABA-dependent signaling cascade promoting CAM expression (Taybi et al., 2002).

In addition to studies focused on specific signaling events, the generation of genomic and transcriptomic databases for both facultative and obligate CAM species has also recently opened a window of opportunities for more detailed characterization of the stress signaling cascades controlling CAM expression (Abraham et al., 2016; Brilhaus et al., 2016; Wai et al., 2017). In Talinum triangulare, ABA-responsive transcription factors were among the most upregulated transcription factors during the drought-triggered CAM induction (Brilhaus et al., 2016). In addition, studies also indicate that several families of miRNAs presumably target key CAM-related enzymes, and many of these miRNAs display a circadian pattern of expression in Ananas comosus (Wai et al., 2017). Cluster analysis of gene expression patterns leading to the generation of coexpression modules have also been increasingly employed to identify less obvious candidate genes potentially involved in controlling CAM expression. For example, CONSTANS-like genes and REVEILLE transcription factor-encoding genes have been grouped with PEPC and NADP-ME genes, thereby suggesting their potential involvement in controlling the CAM machinery in K. fedtschenkoi (Yang et al., 2017).

Metabolite fluctuation is also reported as an important layer of control in CAM plants (Borland and Taybi, 2004). In Agave americana, besides malic and fumaric acid nocturnal fluctuation, ascorbic acid was also shown to accumulate during the night, presumably providing antiredox power against reactive oxygen species in this CAM plant (Abraham et al., 2016). Glycolysis and carbohydrate metabolismrelated transcripts have been highlighted as a very important step for CAM regulation (Brilhaus et al., 2016; Yang et al., 2017). Moreover, the transcripts' abundance of core CAM and starch degradationrelated enzymes was drastically altered in a starch and CAM-deficient $M$. crystallinum mutant, thereby reinforcing the role of carbohydrates in CAM regulation (Taybi et al., 2017).

\section{5 $\mathrm{C}_{4} /$ CRASSULACEAN ACID METABOLISM COMPATIBILITY}

C4 and CAM have been considered incompatible syndromes for various reasons: (1) they show differential regulation of enzymes involved in the carboxylation and decarboxylation steps; (2) the metabolite transport dynamics of each syndrome is strikingly 
different; and (3) structural arrangements for each syndrome differ, favoring Kranz anatomy in $\mathrm{C}_{4}$ and succulence in CAM (Sage, 2002). In addition to this list, Sage (2002) also highlights that the evolutionary pathways selecting for each of these photosynthetic behaviors appear to be exclusive. Thus, the simultaneous evolution of $\mathrm{C}_{4}$ and CAM in a single organism would be unnecessary.

One of the most stunning cases, the order Caryophyllales, contains many $\mathrm{C}_{4}$ and/or CAM origins including its intermediates variants (Kellogg, 1999), being considered a hotbed for these CCMs (Edwards and Ogburn, 2012). To illustrate the photosynthetic diversity of Caryophyllales, the following representatives of the order are here listed: Borszczowia aralocaspica and Bienertia cycloptera (Chenopodiaceae), the two species known to perform single-cell $C_{4}$ in a family of many dual-cell $C_{4}$ (Edwards et al., 2004); cacti (Cactaceae), traditionally known to be obligate CAM plants (Ocampo and Columbus, 2012); and the "Portullugo" clade, as referred to by Edwards and Ogburn (2012), comprising the sister-groups Molluginaceae and Portulacaceae, that each evolved $\mathrm{C}_{4}$ independently (Christin et al., 2011, 2014). Both families contain $\mathrm{C}_{3}, \mathrm{C}_{4}$, and $\mathrm{C}_{3}-\mathrm{C}_{4}$ intermediates, and the latter, monogeneric, also includes $\mathrm{C}_{4}-\mathrm{CAM}$ species (Edwards and Ogburn, 2012). Recently, Christin et al. (2015) studying $\mathrm{C}_{4}$ evolution in Caryophyllales reinforced that genetic enablers were present in $C_{3}$ ancestors and genes were coopted into $\mathrm{C}_{4}$ when already expressed in a $\mathrm{C}_{4}$-manner. However, the identity of the coopted candidates varies in different lineages inside Caryophyllales, representing true independent evolution events of $\mathrm{C}_{4}$ photosynthesis.

The genus Portulaca is an exception to the $\mathrm{C}_{4}-\mathrm{CAM}$ incompatibility assumption. Portulaca comprises about 60 species of $\mathrm{C}_{4}$ annual weeds, of which at least six can undergo CAM induction upon environmental stimuli: $P$. oleracea, P. grandiflora, P. pilosa, P. australis, P. cyclophylla, and P. digyna (Guralnick and Jackson, 2001; Guralnick et al., 2002; Holtum et al., 2017; Koch and Kennedy, 1980, 1982; Ku et al., 1981; Winter and Holtum, 2017). This metabolic plasticity has been better studied in $P$. grandiflora and P. oleracea (Christin et al., 2014; Guralnick and Jackson, 2001; Guralnick et al., 2002; Mazen, 1996, 2000), revealing that both leaves and stems display the induction of CAM-like features under stress conditions (Guralnick et al., 2002; Koch and Kennedy, 1980, 1982; Mazen, 1996; Voznesenskaya et al., 2010).

The first discovery was carried out in P. oleracea, when leaves were shown to increase nocturnal acidity and perform a low level of $\mathrm{CO}_{2}$ assimilation in the dark after prolonged periods of water deprivation or under short-day conditions (Koch and Kennedy, 1982, 1980). Similar findings of a CAM-like behavior were made around the same time for $P$. grandiflora (Ku et al., 1981), and after a decade, the records of CAM in these two species plus $P$. pilosa (previously known as $P$. mundula) were confirmed (Kraybill and Martin, 1996). Moreover, leaf PEPC content was shown to increase in $P$. oleracea as the drought period was prolonged, associated with opposite diel changes in PEPC kinetic properties characterized by a peak in PEPC activity during the day and higher malate sensitivity at night in wellwatered plants, and the exact opposite pattern in drought-stressed individuals (Mazen, 1996, 2000).

In line with these findings, the comparison of the global transcriptional profile of well-watered and droughted $P$. oleracea plants led to the identification of two PEPC-encoding genes differentially regulated under each condition: while PoPPC1E1c was highly expressed during the night in droughted plants, PoPPC1E1 $a^{\prime}$ was predominantly expressed during the day and in well-watered samples (Christin et al., 2014). Therefore, PoPPC1E1c and PoPPC1E1a' were probably recruited by the CAM and $\mathrm{C}_{4}$ pathways, respectively, which may have facilitated the evolution of regulatory mechanisms to allow the expression of both CCM in leaves of this plant species. According to the evolutionary history of PEPC genes and considering the existence of other CAM plants with close phylogenetic proximity, $\mathrm{C}_{4}$ probably evolved on top of a CAM-performing organism (Christin et al., 2014), and the CAM pathway may have been lost in most species of the genus. In addition, the appearance of different types of $\mathrm{C}_{4}$ in different Portulaca species (e.g., P. oleracea is NAD-ME type and P. grandiflora is NADP-type) reinforces this hypothesis, where $\mathrm{C}_{4}$ would have evolved independently in each species (Voznesenskaya et al., 2010).

Based on immunoblot results, Lara et al. (2003, 2004) proposed a first hypothetical model of $\mathrm{C}_{4}-\mathrm{CAM}$ compatibility in P. oleracea. Most Portulaca leaves have three types of cells: water storage cells (WSC), whose contribution to overall carbon gain may be minimal due to their small number of chloroplasts, and photosynthetically active MC and BSC (Lara et al., 2003, 2004; Voznesenskaya et al., 2010). In the hypothetical model, malate generated from CAM at night in WSC and MC would be shuttled to BSC for decarboxylation during the day (Lara et al., 2004).

The compatibility between $\mathrm{C}_{4}$ and CAM observed in certain Portulaca species is much more than an evolutionary curiosity, as these species represent a particularly interesting model for exploring the molecular, biochemical, and signaling mechanisms responsible for allowing the occurrence of these distinct photosynthetic modes within a single individual (Yang et al., 2015). Among the six $C_{4}$-CAM facultative Portulaca species currently identified, $P$. oleracea display short 
size, rapid growth, flat leaves, and abundant production of long-lived seed (Zimmerman, 1976), making this species a particularly attractive target for future research on $\mathrm{C}_{4} / \mathrm{CAM}$ compatibility.

\subsection{ENGINEERING $\mathrm{C}_{4}$ AND CRASSULACEAN ACID METABOLISM: CHALLENGES AND POSSIBILITIES}

In the context of climate change, drier and warmer conditions are expected to occur in many important agricultural regions of the world (IPCC, 2014). Given the significant adaptive advantages of $\mathrm{C}_{4}$ and CAM pathways under hot and dry environments compared with $C_{3}$ photosynthesis, it is perhaps unsurprising that ambitious initiatives aiming to engineer either CCMs into $C_{3}$ crops have being initiated: the $C_{4}$ Rice project (von Caemmerer et al., 2012; von Caemmerer and Furbank, 2016; Furbank, 2016) and the CAM Biodesign project (Borland et al., 2014, 2015; Hartwell et al., 2016; Yang et al., 2015). Both initiatives are long-term endeavors (10 years or more) and agree on the intensive use of omics technologies to provide essential information on poorly understood aspects of both cycles. Moreover, since both syndromes involve hundreds or thousands of genes, considerable efforts have been done by both projects to develop new technologies to engineer plants for complex, multigene constructs (Czarnecki et al., 2016; Yang et al., 2015).

Though the engineering of functional $\mathrm{C}_{4}$ into rice or any other species has not been achieved yet, many important accomplishments have been obtained in recent years, such as massive advances in understanding the components and regulatory processes responsible for the $\mathrm{C}_{4}$ functioning (e.g., Wang et al., 2017), the successful increase of chloroplast, mitochondria, and plasmodesmata number in rice BSC (Wang et al., 2017), among others. Similarly, The CAM Biodesign initiative has also led to impressive advances in understanding CAM biology, including the development of an omics dataset for several key CAM model species (Abraham et al., 2016; Yang et al., 2017) and key functional genomics data (Boxall et al., 2017; Dever et al., 2015). In addition to engineering CAM in $\mathrm{C}_{3}$ crops, the CAM Biodesign initiative also aims to explore the photosynthetic plasticity of CAM plants for high biomass production in marginal lands and habitat restoration (Borland et al., 2011).

\subsubsection{Engineering Crassulacean Acid Metabolism Into $\mathrm{C}_{4}$}

In addition to the current efforts to increase drought tolerance in $C_{4}$ crops (Lopes et al., 2011), another elusive possibility would be to engineer inducible CAM into $\mathrm{C}_{4}$ crops. $\mathrm{C}_{4}$ and CAM have been considered incompatible to occur in the same cells (Sage, 2002), but as is the case for some Portulaca species, stress-induced CAM has been shown to occur in $\mathrm{C}_{4}{ }^{-}$ performing organisms (Guralnick and Jackson, 2001; Guralnick et al., 2002; Holtum et al., 2017; Koch and Kennedy, 1980, 1982; Ku et al., 1981; Winter and Holtum, 2017). Although an obligate CAM pathway would maximize WUE, a partial commitment to CAM, specifically when abiotic stress conditions challenge the functioning of $\mathrm{C}_{4}$, may prove to be beneficial by increasing the survival of the $\mathrm{C}_{4}$ crops until favorable environmental conditions are restored (Borland et al., 2014). Therefore, $C_{4}$ and CAM would not necessarily co-occur in a given tissue, providing that the stressinduced CAM is preceded by the downregulation of the $\mathrm{C}_{4}$ pathway.

A strong CAM response would require, most of all, a compact mesophyll for storing large amounts of nocturnal organic acid, and inverted stomatal control. On the other hand, CAM cycling seems to be more plausible to engineer into a $\mathrm{C}_{4}$ plant, as this mode of CAM does not require changes in the diel stomata pattern, nor much vacuolar space to accumulate the comparatively limited acids generated overnight (Borland et al., 2014). Although performing CAM cycling would only contribute with a small additional input in carbon supply, this could mean an increase in survival under dry seasons.

\subsubsection{Parts List for Crassulacean Acid Metabolism Into $\mathrm{C}_{4}$}

Engineering CAM or $\mathrm{C}_{4}$ in $\mathrm{C}_{3}$ plants involves transferring genes directly involved in either machinery as many of the modules required for either syndrome are absent in $\mathrm{C}_{3}$-performing species. In contrast, many genes and biochemical steps are common in both CCMs, so perhaps the most important and challenging task in engineering weak facultative CAM into $\mathrm{C}_{4}$ plants would be to coordinate the timing of expression of components of each CCM depending on the environmental conditions. Therefore, instead of transferring genes involved in CAM or $C_{4}$ to $C_{3}$ plants, perhaps one of the major challenges in engineering facultative CAM in a $\mathrm{C}_{4}$ species may rely in understanding the transcriptional regulation of genes involved in the $\mathrm{C}_{4}$ machinery aiming to the future adjustments in their expression patterns via genome editing technologies.

For example, the main players of the carboxylation module required for CAM functioning are already present in the $\mathrm{MC}$ of $\mathrm{C}_{4}$ plants, only functioning in a 
different moment of the diel cycle. Therefore, an important challenge to engineer the carboxylation module of CAM in a $\mathrm{C}_{4}$ species would be to invert the timing of PEPC expression and activation in the MC and guarantee available carbonic anhydrase (CA) activity. Adjustments to promote the nighttime accumulation of acids in the vacuoles and the subsequent transport of these molecules back to the cytosol during the day will also be necessary.

The decarboxylation module in CAM plants also take place in $\mathrm{MC}$, whereas in dual-cell $\mathrm{C}_{4}$ species this module is in the BSC. In P. oleracea plants under drought stress, it has been proposed that the acids accumulated overnight inside MC vacuoles are transferred to BSC during the day (Lara et al., 2004). Therefore, it seems tempting to propose that, with some adjustments, the acid transport mechanisms and decarboxylating enzymes already present in a $\mathrm{C}_{4}$ plant would be enough to decarboxylate the acids accumulated overnight in a $\mathrm{C}_{4}-\mathrm{CAM}$ engineered plant.

A hypothetical scheme of CAM cycling to be engineered into $\mathrm{C}_{4}$ as means to improve drought stress tolerance in crops is presented in Fig. 33.3, which is based on current knowledge available in $\mathrm{C}_{4}-\mathrm{CAM}$ facultative plants (Lara et al., 2004). Although accomplishing this

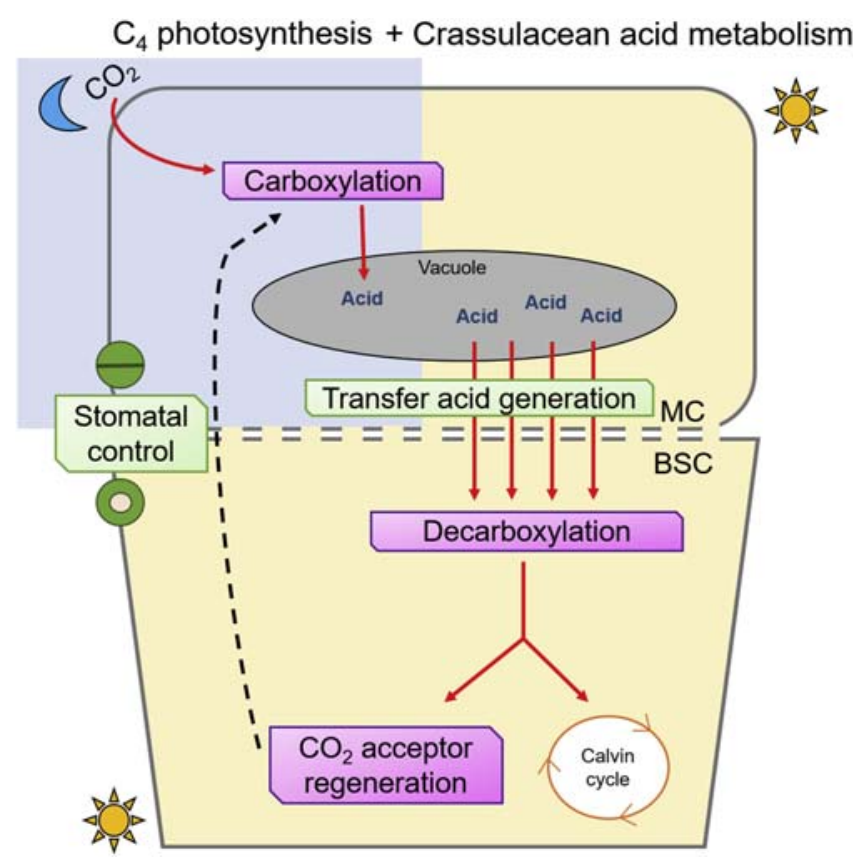

FIGURE 33.3 Hypothetical scheme of CAM cycling to be engineered into $\mathrm{C}_{4}$ as means to improve drought stress tolerance in crops, based on current knowledge available in $\mathrm{C}_{4}$-CAM facultative plants (Lara et al., 2004). Respiratory $\mathrm{CO}_{2}$ would be used in nocturnal acid formation on mesophyll cells. Acids stored overnight would then be transported to the $\mathrm{B}$ to be decarboxylated during the day. Red arrows indicate the carbon pathway inside the cells. BSC, Bundle sheath cell; $M C$, Mesophyll cell. idea would take place in a more distant future compared with the other bioengineering initiatives, technological progress achieved through other projects, such as the $\mathrm{C}_{4}$ rice and the CAM Biodesign projects, may shed light on this $\mathrm{C}_{4}-\mathrm{CAM}$ engineering endeavor and bring it closer to realization. Our discussion has not, by any means, exhausted such a rich and interesting research topic, which should be revisited as our current understanding on $\mathrm{C}_{4}$ and CAM functioning and compatibility is increased.

\subsection{CONCLUDING REMARKS}

In summary, there is still much room for improving our current knowledge on the complexity involving $C_{4}$ and CAM functioning and regulation, especially regarding metabolite transport, stomatal control, and molecular and hormonal events regulating the expression of these CCMs. Given the vast array of biochemical and anatomical variation found among $\mathrm{C}_{4}$ and CAM plants, it seems plausible to anticipate that equally diverse regulatory mechanisms have evolved to control the distinct $\mathrm{C}_{4}$ and CAM genotypes and physiotypes currently known. Therefore, generating and comparing data for different candidate species seems a valid approach to provide a broad and more complete overview of the regulatory mechanisms controlling $\mathrm{C}_{4}$ and CAM pathways. As both syndromes display higher WUE and lower crop water demand compared with $\mathrm{C}_{3}$ plants, $\mathrm{C}_{4}$ and CAM have been increasingly proposed as promising systems for bioengineering approaches in a context of climate change. A common initial step in these bioengineering initiatives is the use of omics approaches to unravel new candidate genes involved in both the core CCM pathway and the regulatory processes to produce a parts list for each syndrome. Besides exploring $\mathrm{C}_{4}$ and CAM in obligate organisms, further attention should be devoted to the few known examples of $\mathrm{C}_{4}-\mathrm{CAM}$ facultative plants, as they may help to explain how these syndromes interconnect, thereby holding a great value as a living blueprint for engineering CAM into $\mathrm{C}_{4}$ crops.

\section{Acknowledgments}

We thank the Royal Society (United Kingdom) through grant \#NA140007 and the Sao Paulo State Foundation for Research Support (FAPESP, Brazil) through grant \#2016/04755-4 for financial support.

\section{References}

Abraham, P.E., Yin, H., Borland, A.M., Weighill, D., Lim, S., Depaoli, H., et al., 2016. Transcript, protein, and metabolite temporal dynamics in the CAM plant Agave. Nat. Plants 2, 16178. 
Aloor, B.P., Avasthi, U.K., Raghavendra, A.S., 2017. Stimulation by abscisic acid of the activity of phosphoenolpyruvate carboxylase in leaf disks of Amaranthus hypochondriacus L., $\mathrm{C}_{4}$ plant: role of $\mathrm{pH}$ and protein levels. Protoplasma 254, 1973-1981. Available from: https:/ / doi.org/10.1007/s00709-017-1091-2.

Badger, M.R., Kaplan, A., Berry, J.A., 1980. Internal inorganic carbon pool of Chlamydomonas reinhardtii: evidence for a carbon dioxideconcentrating mechanism. Plant Physiol. 66, 407-413. Available from: https://doi.org/10.1104/pp.66.3.407.

Badger, M.R., Hanson, D., Price, G.D., 2002. Evolution and diversity of $\mathrm{CO}_{2}$-concentrating mechanisms in cyanobacteria. Funct. Plant Biol. 29, 161-173. Available from: https://doi.org/10.1071/ Pp01213.

Beerling, D.J., Royer, D.L., 2011. Convergent cenozoic $\mathrm{CO}_{2}$ history. Nat. Geosci. 4, 418-420. Available from: https://doi.org/ 10.1038 /ngeo1186.

Bellasio, C., Griffiths, H., 2014. Acclimation of $\mathrm{C}_{4}$ metabolism to low light in mature maize leaves could limit energetic losses during progressive shading in a crop canopy. J. Exp. Bot. 65, 3725-3736. Available from: https://doi.org/10.1093/jxb/eru052.

Bohnert, H.I., Ostrem, J.A., Cushman, J.C., Michalowski, C.B., Rickers, J., Meyer, G., et al., 1988. Mesembryanthemum crystallinum, a higher plant model for the study of environmentally induced changes in gene expression. Plant Mol. Biol. Report. 6, 10-28. Available from: https://doi.org/10.1007/BF02675305.

Borland, A.M., Taybi, T., 2004. Synchronization of metabolic processes in plants with Crassulacean acid metabolism. J. Exp. Bot. 55, 1255-1265. Available from: https://doi.org/10.1093/jxb/ erh105.

Borland, A.M., Zambrano, V.A.B., Ceusters, J., Shorrock, K., 2011. The photosynthetic plasticity of Crassulacean acid metabolism: an evolutionary innovation for sustainable productivity in a changing world. New Phytol. 191, 619-633.

Borland, A.M., Hartwell, J., Weston, D.J., Schlauch, K.A., Tschaplinski, T.J., Tuskan, G.A., et al., 2014. Engineering Crassulacean acid metabolism to improve water-use efficiency. Trends Plant Sci. 19, 327-338. Available from: https://doi.org/ 10.1016/j.tplants.2014.01.006.

Borland, A.M., Wullschleger, S.D., Weston, D.J., Hartwell, J., Tuskan, G.A., Yang, X., et al., 2015. Climate-resilient agroforestry: physiological responses to climate change and engineering of crassulacean acid metabolism (CAM) as a mitigation strategy. Plant, Cell Environ. 38, 1833-1849. Available from: https://doi.org/ 10.1111 / pce. 12479 .

Borland, A.M., Guo, H., Yang, X., Cushman, J.C., 2016. Orchestration of carbohydrate processing for crassulacean acid metabolism. Curr. Opin. Plant Biol. 31, 118-124.

Boxall, S.F., Dever, L.V., Kneřová, J., Gould, P.D., 2017. Phosphorylation of phosphoenol pyruvate Carboxylase is essential for maximal and sustained dark $\mathrm{CO}_{2}$ fixation and core circadian clock operation in the obligate Crassulacean acid metabolism species. Kalanchoë fedtschenkoi. Available from: https://doi.org/10.1105/tpc.17.00301.

Bräutigam, A., Schliesky, S., Külahoglu, C., Osborne, C.P., Weber, A.P.M., 2014. Towards an integrative model of $\mathrm{C}_{4}$ photosynthetic subtypes: insights from comparative transcriptome analysis of NAD-ME, NADP-ME, and PEP-CK $C_{4}$ species. J. Exp. Bot. 65, 3579-3593. Available from: https://doi.org/10.1093/jxb/ eru100.

Brilhaus, D., Bräutigam, A., Mettler-Altmann, T., Winter, K., Weber, A.P.M., 2016. Reversible burst of transcriptional changes during induction of Crassulacean acid metabolism in Talinum triangulare. Plant Phys. Available from: https://doi.org/10.1104/pp.15.01076.

Chen, T., Zhu, X.G., Lin, Y., 2014. Major alterations in transcript profiles between $\mathrm{C}_{3}-\mathrm{C}_{4}$ and $\mathrm{C}_{4}$ photosynthesis of an amphibious species Eleocharis baldwinii. Plant Mol. Biol. 86, 93-110. Available from: https://doi.org/10.1007/s11103-014-0215-8.

Chollet, R., Vidal, J., O'Leary, M.H., 1996. Phosphoenolpyruvate carboxylase: a ubiquitous, highly regulated enzyme in plants. Annu. Rev. Plant Physiol. Plant Mol. Biol. 47, 273-298. Available from: https://doi.org/10.1146/annurev.arplant.47.1.273.

Christin, P.A., Sage, T.L., Edwards, E.J., Ogburn, R.M., Khoshravesh, R., Sage, R.F., 2011. Complex evolutionary transitions and the significance of $\mathrm{C}_{3}-\mathrm{C}_{4}$ intermediate forms of photosynthesis in Molluginaceae. Evolution 65, 643-660. Available from: https:// doi.org/10.1111/j.1558-5646.2010.01168.x.

Christin, P.A., Boxall, S.F., Gregory, R., Edwards, E.J., Hartwell, J., Osborne, C.P., 2013. Parallel recruitment of multiple genes into $\mathrm{C}_{4}$ photosynthesis. Genome Biol. Evol. 5, 2174-2187. Available from: https:/ / doi.org/10.1093/gbe/evt168.

Christin, P.A., Arakaki, M., Osborne, C.P., Bräutigam, A., Sage, R.F., Hibberd, J.M., et al., 2014. Shared origins of a key enzyme during the evolution of $\mathrm{C}_{4}$ and CAM metabolism. J. Exp. Bot. 65, 3609-3621. Available from: https://doi.org/10.1093/jxb/eru087.

Christin, P.A., Arakaki, M., Osborne, C.P., Edwards, E.J., 2015. Genetic enablers underlying the clustered evolutionary origins of $\mathrm{C}_{4}$ photosynthesis in angiosperms. Mol. Biol. Evol. 32, 846-858. Available from: https://doi.org/10.1093/molbev/msu410.

Chu, C., Dai, Z.Y., Ku, M.S.B., Edwards, G.E., 1990. Induction of Crassulacean acid metabolism in the facultative halophyte Mesembryanthemum crystallinum by abscisic acid. Plant Physiol 93, 1253-1260. Available from: https:// doi.org/10.1104/pp.93.3.1253.

Cushman, J.C., 2001. Crassulacean acid metabolism. a plastic photosynthetic adaptation to arid environments. Plant Physiol. 127, 1439-1448. Available from: https://doi.org/10.1104/pp.010818.plants.

Cushman, J.C., Michalowski, C.B., Bohnert, H.J., 1990. Developmental control of Crassulacean acid metabolism inducibility by salt stress in the common ice plant. Plant Physiol. 94, 1137-1142. Available from: https:/ / doi.org/10.1104/pp.94.3.1137.

Cushman, J.C., Tillett, R.L., Wood, J.A., Branco, J.M., Schlauch, K.A., 2008. Large-scale mRNA expression profiling in the common ice plant, Mesembryanthemum crystallinum, performing $\mathrm{C}_{3}$ photosynthesis and Crassulacean acid metabolism (CAM). J. Exp. Bot. 59, 1875-1894. Available from: https://doi.org/10.1093/jxb/ern008.

Czarnecki, O., Bryan, A.C., Jawdy, S.S., Yang, X., Cheng, Z., Chen, J., et al., 2016. Simultaneous knockdown of six non-family genes using a single synthetic RNAi fragment in Arabidopsis thaliana. Plant Methods 12, 16. Available from: https://doi.org/10.1186/ s13007-016-0116-8.

Dai, Z., Ku, M.S.B., Zhang, D.Z., Edwards, G.E., 1994. Effects of growth regulators on the induction of Crassulacean acid metabolism in the facultative halophyte Mesembryanthemum crystallinum L. Planta 192, 287-294. Available from: https://doi.org/10.1007/ BF00198562.

Dengler, N.G., Nelson, T., 1999. Leaf structure and development in $\mathrm{C}_{4}$ plants. In: Sage, R., Monson, R. (Eds.), $\mathrm{C}_{4}$ Plant Biology. Academic Press, San Diego, CA, pp. 133-172.

Dever, L.V., Boxall, S.F., Kneřová, J., Hartwell, J., 2015. Transgenic perturbation of the decarboxylation phase of Crassulacean acid metabolism alters physiology and metabolism but has only a small effect on growth. Plant Physiol. 167, 44-59. Available from: https:/ / doi.org/10.1104/pp.114.251827.

Edwards, E.J., Ogburn, R.M., 2012. Angiosperm responses to a low$\mathrm{CO}_{2}$ world: $\mathrm{CAM}$ and $\mathrm{C}_{4}$ photosynthesis as parallel evolutionary trajectories. Int. J. Plant Sci. 173, 724-733. Available from: https:// doi.org/10.1086/666098.

Edwards, G.E., Franceschi, V.R., Voznesenskaya, E.V., 2004. Singlecell $\mathrm{C}_{4}$ photosynthesis versus the dual-cell (Kranz) paradigm. Annu. Rev. Plant Biol. 55, 173-196. Available from: https://doi. org/10.1146/annurev.arplant.55.031903.141725. 
Ehleringer, J.R., Monson, R.K., 1993. Evolutionary and ecological aspects of photosynthetic pathway variation. Annu. Rev. Ecol. Syst. 24, 411-439. Available from: https://doi.org/10.1146/ annurev.es.24.110193.002211.

Eisenhut, M., Ruth, W., Haimovich, M., Bauwe, H., Kaplan, A., Hagemann, M., 2008. The photorespiratory glycolate metabolism is essential for cyanobacteria and might have been conveyed endosymbiontically to plants. Proc. Natl. Acad. Sci. 105, 17199-17204. Available from: https://doi.org/10.1073/ pnas.0807043105.

Erb, T.J., Zarzycki, J., 2018. A short history of RubisCO: the rise and fall (?) of Nature's predominant $\mathrm{CO}_{2}$ fixing enzyme. Curr. Opin. Biotechnol. 49, 100-107. Available from: https://doi.org/ 10.1016/j.copbio.2017.07.017.

Fleta-Soriano, E., Pintó-Marijuan, M., Munné-Bosch, S., 2015. Evidence of drought stress memory in the facultative CAM, Aptenia cordifolia: possible role of phytohormones. PLoS One 10, 1-12. Available from: https://doi.org/10.1371/journal.pone.0135391.

Forsthoefel, N.R., Cushman, M.A.F., Cushman, J.C., 1995a. Posttranscriptional and post-translational control of enolase expression in the facultative Crassulacean acid metabolism plant Mesembryanthemum crystallinum L. Plant Physiol 108, 1185-1195. Available from: https://doi.org/10.1104/pp.108.3.1185.

Forsthoefel, N.R., Vernon, D.M., Cushman, J.C., 1995b. A salinityinduced gene from the halophyte $M$. crystallinum encodes a glycolytic enzyme, cofactor-independent phosphoglyceromutase. Plant Mol. Biol. 29, 213-226. Available from: https://doi.org/10.1007/ BF00043647.

Freschi, L., Mercier, H., 2012. Connecting environmental stimuli and Crassulacean acid metabolism expression: phytohormones and other signaling molecules. Prog. Bot. 73, 231-255. Available from: https: / / doi.org/10.1007/978-3-319-08807-5.

Freschi, L., Rodrigues, M.,A., Domingues, D.S., Purgatto, E., Van Sluys, M.A., Magalhaes, J.R., et al., 2010. Nitric oxide mediates the hormonal control of Crassulacean acid metabolism expression in young pineapple plants. Plant Physiol. 152 (4), 1971-1985. Available from: https://doi.org/10.1104/pp.109.151613.

Furbank, R.T., 2016. Walking the $C_{4}$ pathway: past, present, and future. J. Exp. Bot. 68, 4057-4066. Available from: https://doi. org /10.1093/jxb/erx006.

Ghannoum, O., Von Caemmerer, S., Conroy, J.P., 2002. The effect of drought on plant water use efficiency of nine NAD-ME and nine NADP-ME Australian $C_{4}$ grasses. Funct. Plant Biol. 29, 1337-1348. Available from: https://doi.org/10.1071/FP02056.

Giordano, M., Beardall, J., Raven, J.A., 2005. $\mathrm{CO}_{2}$ concentrating mechanisms in algae: mechanisms, environmental modulation, and evolution. Annu. Rev. Plant Biol. 56, 99-131. Available from: https:/ / doi.org/10.1146/annurev.arplant.56.032604.144052.

Guralnick, L.J., Jackson, M.D., 2001. The occurrence and phylogenetics of crassulacean acid metabolism in the Portulacaceae. Int. J. Plant Sci. 162, 257-262.

Guralnick, L.J., Edwards, G., Ku, M.S.B., Brandon, H., Franceschi, V. R., 2002. Photosynthetic and anatomical characteristics in the $\mathrm{C}_{4}$-crassulacean acid metabolism-cycling plant, Portulaca grandiflora. Funct. Plant Biol. 29, 763-773.

Hare, P.D., Cress, W.A., van Staden, J., 1997. The involvement of cytokinins in plant responses to environmental stress. Plant Growth. Regul. 23, 79-103.

Hartwell, J., 2005. The co-ordination of central plant metabolism by the circadian clock. Biochem. Soc. Trans. 33, 945-948. Available from: https://doi.org/10.1042/BST20050945.

Hartwell, J., Dever, L.V., Boxall, S.F., 2016. Emerging model systems for functional genomics analysis of Crassulacean acid metabolism. Curr. Opin. Plant Biol. 31, 100-108. Available from: https://doi. org/10.1016/j.pbi.2016.03.019.
Herppich, W., Herppich, M., Van Willert, D., 1992. The irreversible C3 to CAM shift in well-watered and salt-stressed plants of Mesembryanthemum crystallinum is under strict ontogenetic control. Botanica Acta 105, 34-40.

Herrera, A., 2009. Crassulacean acid metabolism and fitness under water deficit stress: if not for carbon gain, what is facultative CAM good for? Ann. Bot. 103, 645-653. Available from: https:// doi.org/10.1093/aob/mcn145.

Hibberd, J.M., Covshoff, S., 2010. The regulation of gene expression required for $\mathrm{C}_{4}$ photosynthesis. Annu. Rev. Plant Biol. 61, 181-207. Available from: https://doi.org/10.1146/annurevarplant-042809-112238.

Holtum, J.A.M., Winter, K., 1982. Activity of enzymes of carbon metabolism during the induction of Crassulacean acid metabolism in Mesembryanthemum crystallinum L. Planta 155, 8-16. Available from: https://doi.org/10.1007/BF00402925.

Holtum, J.A.M., Smith, J.A.C., Neuhaus, H.E., 2005. Intracellular transport and pathways of carbon flow in plants with Crassulacean acid metabolism. Funct. Plant Biol. 32, 429. Available from: https://doi.org/10.1071/FP04189.

Holtum, J.A.M., Hancock, L.P., Edwards, E.J., Winter, K., 2017. Optional use of CAM photosynthesis in two $\mathrm{C}_{4}$ species, Portulaca cyclophylla and Portulaca digyna. J. Plant Physiol. Available from: https:// doi.org/10.1016/j.jplph.2017.01.010.

IPCC, 2014. Climate change 2014: synthesis report. In: Pachauri, R.K., Meyer, L.A. (Eds.), Climate Change 2014: Synthesis Report Contribution of Working Groups I, II and III to the Fifth Assessment Report of the Intergovernmental Panel on Climate Change. IPCC, Geneva.

Izui, K., Matsumura, H., Furumoto, T., Kai, Y., 2004. Phosphoenolpyruvate carboxylate: a new era of structural biology. Annu. Rev. Plant Biol. 55, 69-84. Available from: https:// doi.org/10.1146/annurev.arplant.55.031903.141619.

Kanai, R., Edwards, G.E., 1999. The biochemistry of photosynthesis. In: Sage, R., Monson, R. (Eds.), $\mathrm{C}_{4}$ Plant Biology. Academic Press, San Diego, CA, pp. 49-87.

Keeley, J.E., Rundel, P.W., 2003. Evolution of CAM and $\mathrm{C}_{4}$ carbonconcentrating mechanisms. Int. J. Plant Sci. 164, S55-S77. Available from: https://doi.org/10.1086/374192.

Kellogg, E.A., 1999. Phylogenetic aspects of the evolution of $\mathrm{C}_{4}$ photosynthesis. In: Sage, R., Monson, R. (Eds.), $C_{4}$ Plant Biology. Academic Press, San Diego, CA, pp. 411-444.

Koch, K.E., Kennedy, R.A., 1980. Characteristics of Crassulacean acid metabolism in the succulent $\mathrm{C}_{4}$ dicot, Portulaca oleracea L. Plant Physiol. 65, 193-197. Available from: https://doi.org/10.1104/ pp.69.4.757.

Koch, K.E., Kennedy, R.A., 1982. Crassulacean acid metabolism in the succulent $\mathrm{C}_{4}$ dicot, Portulaca oleracea $\mathrm{L}$ under natural environmental conditions. Plant Physiol. 69, 757-761.

Kraybill, A.A., Martin, C.E., 1996. Crassulacean acid metabolism in three species of the $\mathrm{C}_{4}$ genus Portulaca. Int. J. Plant Sci. 157, 103-109.

Ku, S.B., Shieh, Y.J., Reger, B.J., Black, C.C., 1981. Photosynthetic characteristics of Portulaca grandiflora, a succulent $\mathrm{C}_{4}$ dicot. Plant Physiol. 68, 1073-1080. Available from: https://doi.org/10.1104/ pp.68.5.1073.

Lara, M., Disante, K.B., Podestá, F.P., Andreo, C.S., Drincovich, M.F., 2003. Induction of a Crassulacean acid like metabolism in the $\mathrm{C}_{4}$ succulent plant, Portulaca oleracea L.: physiological and morphological changes are accompanied by specific modifications in phosphoenolpyruvate carboxylase. Photosynth. Res. 77, 241-254. Available from: https://doi.org/10.1023/A:1025834120499.

Lara, M.V., Drincovich, M.F., Andreo, C.S., 2004. Induction of a Crassulacean acid-like metabolism in the $\mathrm{C}_{4}$ succulent plant, Portulaca oleracea L.: study of enzymes involved in carbon fixation 
and carbohydrate metabolism. Plant Cell Physiol. 45, 618-626. Available from: https://doi.org/10.1093/pcp/pch073.

Lepiniec, L., Vidal, J., Chollet, R., Gadal, P., Crétin, C., 1994. Phosphoenolpyruvate carboxylase: structure, regulation and evolution. Plant Sci. 99, 111-124. Available from: https://doi.org/ 10.1016/0168-9452(94)90168-6.

Li, P., Ponnala, L., Gandotra, N., Wang, L., Si, Y., Tausta, S.L., et al., 2010. The developmental dynamics of the maize leaf transcriptome. Nat. Gen. 42, 1060-1067. Available from: https://doi.org/ 10.1038/ng.703.

Long, S.P., 1999. Environmental responses. In: Sage, R., Monson, R. (Eds.), $C_{4}$ Plant Biology. Academic Press, San Diego, CA, pp. 215-249.

Lopes, M.S., Araus, J.L., Van Heerden, P.D.R., Foyer, C.H., 2011. Enhancing drought tolerance in $\mathrm{C}_{4}$ crops. J. Exp. Bot. 62, 3135-3153. Available from: https://doi.org/10.1093/jxb/err105.

Lüttge, U., 1996. Clusia: plasticity and diversity in a genus of $\mathrm{C}_{3}$ /CAM intermediate tropical trees. In: Winter, K., Smith, J. (Eds.), Crassulacean Acid Metabolism. Springer-Verlag, Berlin, pp. 296-311.

Lüttge, U., 2004. Ecophysiology of Crassulacean acid metabolism (CAM). Ann. Bot. 93, 629-652. Available from: https://doi.org/ 10.1093/aob/mch087.

Lüttge, U., 2006. Photosynthetic flexibility and ecophysiological plasticity: questions and lessons from Clusia, the only CAM tree, in the neotropics. New Phytol. 171, 7-25. Available from: https:// doi.org/10.1111/j.1469-8137.2006.01755.x.

Maberly, S.C., Madsen, T.V., 2002. Freshwater angiosperm carbon concentrating mechanisms: processes and patterns. Funct. Plant Biol. 29 (3), 393-405.

Mazen, A.M.A., 2000. Changes in properties of phosphoenolpyruvate carboxylase with induction of Crassulacean Acid Metabolism (CAM) in the $\mathrm{C}_{4}$ plant Portulaca oleracea. Photosynthetica. Available from: https://doi.org/10.1023/A:1010969419962.

Mazen, A.M.A., 1996. Changes in levels of phosphoenolpyruvate carboxylase with induction of Crassulacean acid metabolism (CAM)-like behavior in the $\mathrm{C}_{4}$ plant Portulaca oleracea. Physiol. Plant. 98, 111-116. Available from: https://doi.org/10.1111/j.1399-3054.1996.tb00681.x.

Meyer, M., Griffiths, H., 2013. Origins and diversity of eukaryotic $\mathrm{CO}_{2}$-concentrating mechanisms: lessons for the future. J. Exp. Bot. 64, 769-786. Available from: https://doi.org/10.1093/jxb/ers390S.

Miziorko, H.M., Lorimer, G.H., 1983. Ribulose-1,5-biphosphate carboxylase-oxygenase. Ann. Rev. Biochem. 52, 507-535.

Monson, R.K., 1999. The origins of $C_{4}$ genes and evolutionary pattern in the $\mathrm{C}_{4}$ metabolic phenotype. In: Sage, R., Monson, R. (Eds.), $\mathrm{C}_{4}$ Plant Biology. Academic Press, San Diego, CA, pp. 377-410. Available from: http://dx.doi.org/10.1016/B978-012614440-6/ 50012-4.

Moroney, J.V., Jungnick, N., DiMario, R.J., Longstreth, D.J., 2013. Photorespiration and carbon concentrating mechanisms: two adaptations to high $\mathrm{O}_{2}$, low $\mathrm{CO}_{2}$ conditions. Photosynth. Res. 117, 121-131. Available from: https://doi.org/10.1007/s11120-0139865-7.

Monson, R.K., Moore, B.D., 1989. On the significance of ${ }_{\mathrm{C}}^{3}{ }_{\mathrm{C}} \mathrm{C}$ intermediate photosynthesis to the evolution of $\mathrm{C}^{4}$ photosynthesis. Plant. Cell Environ. 12, 689-699. Available from: https://doi. org/10.1111/j.1365-3040.1989.tb01629.x.

Muhaidat, R.I.M., Sage, R.F., Denglres, N.G., 2007. Diversity of Kranz anatomy and biochemistry in $\mathrm{C}_{4}$ eudicots. Am. J. Bot. 94, 362-381.

Nelson, E.A., Sage, R.F., 2008. Functional constraints of CAM leaf anatomy: tight cell packing is associated with increased CAM function across a gradient of CAM expression. J. Exp. Bot. 59, 1841-1850. Available from: https://doi.org/10.1093/jxb/erm346.

Nelson, E.A., Sage, T.L., Sage, R.F., 2005. Functional leaf anatomy of plants with Crassulacean acid metabolism. Funct. Plant
Biol. 32, 409-419. Available from: https://doi.org/10.1071/ FP04195.

Nimmo, H.G., 2000. The regulation of phosphoenolpyruvate carboxylase. Trends Plant Sci. 5, 75-80.

Ocampo, G., Columbus, J.T., 2012. Molecular phylogenetics, historical biogeography, and chromosome number evolution of Portulaca (Portulacaceae). Mol. Phylogenet. Evol. 63, 97-112. Available from: https://doi.org/10.1016/j.ympev.2011.12.017.

Pospisilova, J., Synkova, H., Rulcova, J., 2000. Cytokinins and water stress. Biol. Plantarum 43, 321-328. Available from: https://doi. org/10.1023/A:1026754404857.

Pospisilova, J., Vagner, M., Malbeck, J., Travniakova, A., Batkova, P., 2005. Interactions between abscisic acid and cytokinins during water stress and subsequent rehydration. Biol. Plant. 49, 533-540. Available from: https://doi.org/10.1007/s10535-0050047-0.

Peters, W., Beck, E., Piepenbrock, M., Lenz, B., Schmitt, J.M., 1997. Cytokinin as a negative effector of phosphoenolpyruvate carboxylase induction in Mesembryanthemum crystallinum. J. Plant Physiol. 151, 362-367. Available from: https://doi.org/10.1016/ S0176-1617(97)80266-0.

Pengelly, J.J.L., Sirault, X.R.R., Tazoe, Y., Evans, J.R., Furbank, R.T., Von Caemmerer, S., 2010. Growth of the $\mathrm{C}_{4}$ dicot Flaveria bidentis: photosynthetic acclimation to low light through shifts in leaf anatomy and biochemistry. J. Exp. Bot. 61, 4109-4122. Available from: https://doi.org/10.1093/jxb/erq226.

Pick, T.R., Bräutigam, A., Schlüter, U., Denton, A.K., Colmsee, C., Scholz, U., et al., 2011. Systems Analysis of a maize leaf developmental gradient redefines the current $\mathrm{C}_{4}$ model and provides candidates for regulation. Plant Cell 23, 4208-4220. Available from: https:// doi.org/10.1105/tpc.111.090324.

Raven, J.A., Cockell, C.S., De La Rocha, C.L., 2008. The evolution of inorganic carbon concentrating mechanisms in photosynthesis. Philos. Trans. R. Soc. B Biol. Sci. 363, 2641-2650. Available from: https:/ / doi.org/10.1098/rstb.2008.0020.

Reeves, G., Grangé-guermente, M.J., Hibberd, J.M., 2016. Regulatory gateways for cell-specific gene expression in $\mathrm{C}_{4}$ leaves with Kranz anatomy. <https://doi.org/10.1093/jxb/erw438>.

Sage, R.F., 2002. Are crassulacean acid metabolism and $C_{4}$ photosynthesis incompatible? Funct. Plant Biol. 29, 775-785.

Sage, R.F., 2004. The evolution of $\mathrm{C}_{4}$ photosynthesis. New Phytol. 161, 341-370. Available from: https://doi.org/10.1046/j.14698137.2004.00974.x.

Sage, R.F., 2017. A portrait of the $C_{4}$ photosynthetic family on the 50th anniversary of its discovery: species number, evolutionary lineages, and Hall of Fame. J. Exp. Bot. 68, e11-e28. Available from: https://doi.org/10.1093/jxb/erx005.

Sage, R.F., McKown, A.D., 2006. Is $C_{4}$ photosynthesis less phenotypically plastic than $C_{3}$ photosynthesis? J. Exp. Bot. 57, 303-317. Available from: https://doi.org/10.1093/jxb/erj040.

Sage, R.F., Sage, T.L., Kocacinar, F., 2012. Photorespiration and the evolution of $C_{4}$ photosynthesis. Annu. Rev. Plant Biol. 63, $19-47$. Available from: https:// doi.org/10.1146/annurev-arplant-042811105511.

Schluter, U., Denton, A.K., Bräutigam, A., 2016. Understanding metabolite transport and metabolism in $\mathrm{C}_{4}$ plants through RNAseq 31. <https://doi.org/10.1016/j.pbi.2016.03.007>.

Schmitt, J.M., Piepenbrock, M., 1992. Regulation of phosphoenolpyruvate carboxylase and Crassulacean acid metabolism induction in Mesembryanthemum crystallinum L. by cytokinin. Modulation of leaf gene expression by roots? Plant Physiol. 99, 1664-1669. Available from: https://doi.org/10.1104/pp.99.4.1664.

Silvera, K., Neubig, K.M., Whitten, W.M., Williams, N.H., Winter, K., Cushman, J.C., 2010. Evolution along the Crassulacean acid metabolism continuum. Funct. Plant Biol. 37, 995-1010. Available from: https://doi.org/10.1071/FP10084. 
Tanaka, Y., Sano, T., Tamaoki, M., Nakajima, N., Kondo, N., Hasezawa, S., 2006. Cytokinin and auxin inhibit abscisic acidinduced stomatal closure by enhancing ethylene production in Arabidopsis. J. Exp. Bot. 57, 2259-2266.

Taybi, T., Sotta, B., Gehrig, H., Guclu, S., Kluge, M., Brulfert, J., 1995. Differential effects of abscisic acid on phosphoenolpyruvate carboxylase and CAM operation in Kalanchoë blossfeldiana. Bot. Acta 108, 240-246. Available from: https://doi.org/10.1111/j.14388677.1995.tb00856.x/abstract.

Taybi, T., Cushman, J.C., Borland, A.M., 2002. Environmental, hormonal and circadian regulation of Crassulacean acid metabolism expression. Funct. Plant Biol. 29, 669-678. Available from: https://doi.org/10.1071/PP01244.

Taybi, T., Cushman, J.C., Borland, A.M., 2017. Leaf carbohydrates influence transcriptional and post-transcriptional regulation of nocturnal carboxylation and starch degradation in the facultative CAM plant Mesembryanthemum crystallinum. J. Plant Physiol. 218, 144-154. Available from: https://doi.org/10.1016/j.jplph.2017.07.021.

Thomas, J.C., Bohnert, H.J., 1993. Salt stress perception and plant growth regulators in the halophyte Mesembryanthemum crystallinum. Plant Physiol. 103, 1299-1304. Available from: https://doi. org/10.1104/pp.103.4.1299.

Thomas, J.C., Mcelwain, E.F., Bohnert, H.J., 1992. Convergent induction of osmotic stress responses. Abscisic acid, cytokinin, and the effects of $\mathrm{NaCl}$. Plant Physiol. 100, 416-423. Available from: https:/ / doi.org/10.1104/pp.100.1.416.

Tsiantis, M.S., Bartholomew, D.M., Smith, J.A.C., 1996. Salt regulation of transcript levels for the c subunit of a leaf vacuolar $\mathrm{H}^{+}$-ATPase in the halophyte Mesembryanthemum crystallinum. Plant J. 9, 729-736. Available from: https://doi.org/10.1046/j.1365313X.1996.9050729.x/abstract.

Uchino, A., Sentoku, N., Nemoto, K., Ishii, R., Samejima, M., Matsuoka, M., 1998. C4-type gene expression is not directly dependent on Kranz anatomy in an amphibious sedge Eleocharis vivipara Link. Plant J. 14, 565-572. Available from: https://doi. org/10.1046/j.1365-313X.1998.00155.x.

Ueno, O., 1998. Induction of Kranz anatomy and $\mathrm{C}_{4}$-like biochemical characteristics in a submerged amphibious plant by abscisic acid. Plant Cell 10, 571-583. Available from: https://doi.org/10.1105/ tpc.10.4.571.

Ueno, O., 2001. Update on $\mathrm{C}_{4}$ photosynthesis environmental regulation of $\mathrm{C}_{3}$ and $\mathrm{C}_{4}$ differentiation in the amphibious sedge Eleocharis vivipara. Plant Physiol. 127, 1524-1532. Available from: https://doi.org/10.1104/pp.010704.1524.

Ueno, O., Samejima, M., Muto, S., Miyachi, S., 1988. Photosynthetic characteristics of an amphibious plant, Eleocharis vivipara: expression of $C_{4}$ and $C_{3}$ modes in contrasting environments. Proc. Natl. Acad. Sci. U. S. A. 85, 6733-6737. Available from: https://doi. org/10.1073/pnas.85.18.6733.

von Caemmerer, S., Furbank, R.T., 2016. Strategies for improving $C_{4}$ photosynthesis. Curr. Opin. Plant Biol. 31, 125-134.

von Caemmerer, S., Quick, W.P., Furbank, R.T., 2012. The development of $\mathrm{C}_{4}$ rice: current progress and future challenges. Science 336, 1671-1672. Available from: https://doi.org/10.1126/science.1220177.

Voznesenskaya, E.V., Franceschi, V.R., Kiirats, O., Freitag, H., Edwards, G.E., 2001. Kranz anatomy is not essential for terrestrial $\mathrm{C}_{4}$ plant photosynthesis. Nature 414, 543-546. Available from: https:/ / doi.org/10.1038/35107073.

Voznesenskaya, E.V., Franceschi, V.R., Kiirats, O., Artyusheva, E.G., Freitag, H., Edwards, G.E., 2002. Proof of $\mathrm{C}_{4}$ photosynthesis without Kranz anatomy in Bienertia cycloptera (Chenopodiaceae). Plant J. 31, 649-662. Available from: https://doi.org/10.1046/j.1365313X.2002.01385.x.

Voznesenskaya, E.V., Koteyeva, N.K., Edwards, G.E., Ocampo, G., 2010. Revealing diversity in structural and biochemical forms of
$\mathrm{C}_{4}$ photosynthesis and a $\mathrm{C}_{3}-\mathrm{C}_{4}$ intermediate in genus Portulaca $\mathrm{L}$. (Portulacaceae). J. Exp. Bot. 61, 3647-3662. Available from: https://doi.org/10.1093/jxb/erq178.

Wai, C.M., VanBuren, R., Zhang, J., Huang, L., Miao, W., Edger, P.P., et al., 2017. Temporal and spatial transcriptomic and microRNA dynamics of CAM photosynthesis in pineapple. Plant J. 92, 19-30. Available from: https://doi.org/10.1111/tpj.13630.

Wang, P., Khoshravesh, R., Karki, S., Furbank, R., Sage, T.L., Langdale, J.A., et al., 2017. Re-creation of a key step in the evolutionary switch from $\mathrm{C}_{3}$ to $\mathrm{C}_{4}$ leaf anatomy article re-creation of a key step in the. Ann. Bot. 93, 629-652. Available from: https:// doi.org/10.1093/aob/mch087.

Wang, Y., Bräutigam, A., Weber, A.P.M., Zhu, X.G., 2014. Three distinct biochemical subtypes of $\mathrm{C}_{4}$ photosynthesis? A modelling analysis. J. Exp. Bot. 65, 3567-3578. Available from: https://doi. org/10.1093/jxb/eru058.

Winter, K., Holtum, J.A.M., 2014. Facultative crassulacean acid metabolism (CAM) plants: powerful tools for unravelling the functional elements of CAM photosynthesis. J. Exp. Bot. 65, 3425-3441. Available from: https://doi.org/10.1093/jxb/eru063.

Winter, K., Holtum, J.A.M., 2017. Facultative crassulacean acid metabolism (CAM) in four small $\mathrm{C}_{3}$ and $\mathrm{C}_{4}$ leaf-succulents. Aust. J. Bot. Available from: https://doi.org/10.1071/BT16015.

Winter, K., Holtum, J.A.M., 2007. Environment or development? Lifetime net $\mathrm{CO}_{2}$ exchange and control of the expression of Crassulacean acid metabolism in Mesembryanthemum crystallinum. Plant Physiol. 143, 98-107. Available from: https://doi.org/ $10.1104 /$ pp.106.088922.

Winter, K., Smith, J.A.C., 1996. An introduction to Crassulacean acid metabolism. Biochemical principles and ecological diversity. In: Winter, K., Smith, J. (Eds.), Crassulacean Acid Metabolism. Springer-Verlag, Berlin, pp. 1-13.

Winter, K., Holtum, J.A.M., Smith, J.A.C., 2015. Crassulacean acid metabolism: a continuous or discrete trait? New Phytol. 208, 73-78. Available from: https://doi.org/10.1111/nph.13446.

Yang, X., Cushman, J.C., Borland, A.M., Edwards, E.J., Wullschleger, S.D., Tuskan, G.A., et al., 2015. A roadmap for research on Crassulacean acid metabolism (CAM) to enhance sustainable food and bioenergy production in a hotter, drier world. New Phytol. 207, 491-504. Available from: https://doi.org/10.1111/nph.13393.

Yang, X., Hu, R., Yin, H., Jenkins, J., Shu, S., Tang, H., et al., 2017. The Kalanchoë genome provides insights into convergent evolution and building blocks of Crassulacean acid metabolism. Nat. Commun. 8, 1899. Available from: https://doi.org/10.1038/ s41467-017-01491-7.

Zhang, Y.G., Pagani, M., Liu, Z., Bohaty, S.M., Deconto, R., 2013. A 40million-year history of atmospheric $\mathrm{CO}_{2} \mathrm{~A}$, P.T.R.S. Philos. Trans. R. Soc. Available from: https://doi.org/10.1098/rsta.2013.0096.

Zimmerman, C.A., 1976. Growth characteristics of weediness in Portulaca oleracea L. Ecology 57 (5), 964-974.

\section{Further Reading}

Cockburn, W., 1983. Stomatal mechanism as the basis of the evolution of CAM and $\mathrm{C}_{4}$ photosynthesis. Plant. Cell Environ. 6, 275-279. Available from: https://doi.org/10.1111/1365-3040.ep11611925.

McElwain, E.F., Bohnert, H.J., Thomas, J.C., 1992. Light moderates the induction of phosphoenolpyruvate carboxylase by $\mathrm{NaCl}$ and abscisic acid in Mesembryanthemum crystallinum. Plant Physiol. 99, $1261-1264$.

Winter, K., Garcia, M., Holtum, J.A.M., 2017. On the nature of facultative and constitutive CAM: environmental and developmental control of CAM expression during early growth of Clusia, Kalanchoe and Opuntia 59, 1829-1840. <https://doi.org/10.1093/ $\mathrm{jxb} / \mathrm{ern} 080>$. 


\title{
34
}

\section{Protein Kinases and Phosphatases in Stress Transduction: Role in Crop Improvement}

\author{
Thammineni Chakradhar ${ }^{1}$, Ramesha A. Reddy ${ }^{2}$ and \\ Thummala Chandrasekhar ${ }^{3}$ \\ ${ }^{1}$ Thakora Crop Sciences Pvt. Ltd., Anantapur, Andhra Pradesh, India \\ ${ }^{2}$ Seri-Biotech Research Laboratory, Central Silk Board, Bangalore, Karnataka, India \\ ${ }^{3}$ Department of Environmental Sciences, Yogi Vemana University, Kadapa, Andhra Pradesh, India
}

\section{O U T L I N E}

34.1 Introduction

34.2 Receptors Like Kinases

34.3 Mitogen Activated Protein Kinases 34.3.1 MAPKKKs

34.3.2 MAPKKs

34.3.3 MAPKs

34.4 Calcium Kinases

34.4.1 Calcium-Dependent Protein Kinases

34.4.2 Calcineurin B-Like Proteins and CBLInteracting Protein Kinases:

34.4.3 CDPK-Regulated Protein Kinases
34.5 Sucrose Nonfermenting 1-Related Protein Kinases

34.6 Diacylglycerol Kinases

34.7 Histidine Kinases

34.8 Genomics Efforts in Plant Protein Kinase Towards Crop Improvement

34.9 Conclusions

References

Further Reading

\subsection{INTRODUCTION}

Due to their sessile nature, plants are exposed to various kinds of adverse environmental cues. This has makes the plant cell evolve with different signaling enzymes/molecules that can perceive environmental stimuli, transduce across membranes and cytoplasm, interact with regulatory elements in the genome, and finally elicit appropriate adaptive response. The majority of these signaling enzymes belong to kinase group. Plant protein kinases are a class of enzymes that catalyze phosphorylation of functional proteins through which signal cascades transduce. Protein kinases are mainly constituted of a larger group of phosphoryltransferases that transfer the terminal phosphate from ATP to the substrate protein. Protein phosphorylation 
induces the conformational changes in the protein kinase structure to regulate its activity. A network of such signals stimulates the production of second messenger molecules such as phorbol ester, phorbol myristate acetate (PMA) and $\mathrm{Ca}^{2+}$ (Melissa et al., 2012). The substrate proteins interact with $\mathrm{Ca}^{2+}$ in presence of phorbol ester, PMA, and lowers their $\mathrm{Km}$ value. This promotes the phosphorylation of serine, threonine residues in kinase enzymes using terminal phosphate of ATP. $\mathrm{Ca}^{2+}$ is an important secondary messenger that acts as a catalyst in signal transmission (Batistic and Kudla, 2004). $\mathrm{Ca}^{2+}$ binds to the protein kinases like CDPKs, CIPKs, and MAPKs and makes them transmit stress signals to the transcriptional cascade, through phosphorylation. Plant protein kinases contain a highly conserved domain of 250-300 amino acids, which are responsible for phosphorylation transferase activity. The activity of protein kinases depends on the concentrations of cytoplasmic $\mathrm{Ca}^{2+}$, which in turn causes development and metabolic changes in plant cells. During experimenting on $\mathrm{Ca}^{2+}$ activated phosphorylation, Harmon et al. (2001) identified the calcium-dependent protein kinases in soybean, which are unique in nature. In short, difference in the concentrations of cytoplasmic $\mathrm{Ca}^{2+}$ is a cause for the promoting and inhibiting activity of protein kinases in plants. The changes of cytosolic $\mathrm{Ca}^{2+}$ level in response to various environmental stimuli such as drought, salinity, extreme temperature, light, pathogenic infection, and $\mathrm{pH}$ alter the protein phosphorylation in plant cells. CDPKs with N-terminal kinase catalytic domain contiguous with a terminal calmodulin-like Ca2 + -binding domain is the best example of calcium-dependent protein phosphorylation in plants (Ludwig et al., 2004). Moreover, another type of CDPK with the sequence of CB1 has also been recognized in plant that can change cytoplasm calcium levels and was found to be involved in protein phosphorylation (Watillon et al., 1993). Different environmental stress signals utilize common protein kinases to transduce the stimuli to nucleus. Apart from external signals, plant protein kinases are also involved in cellular morphogenesis and development such as cell division, selfincompatibility, initiation of mitosis, and elongation. Crosstalk of signaling molecules is a common phenomenon in plant signal transduction. Hormonal, reactive oxygen species (ROS), abiotic and biotic signaling pathways are interdependent through a complex network involving ROS molecules like $\mathrm{H}_{2} \mathrm{O}_{2}, \mathrm{Ca}^{2+}, \mathrm{ABA}$, inositol 1,4,5-triphosphate (IP3), etc. The continuous exposure to various environmental cues has led the plants to evolve with different kinds of kinases with special and functional specificity. Further evolutionary process has created duplications and divergence in basic kinase groups to various extent and led to the formation of different subfamilies. A detailed description of structural divergence of plant protein kinases has been elaborately reviewed by Lehti-Shiu et al. (2012). Protein kinases occupy a large group of functional genes in eukaryotes. Initial investigations proved that the plant genome contains Ser/Thr and His protein kinases whereas the animal genome contains Tyr protein kinase in addition to the abovementioned kinases. Based on phosphorylation activity between the conserved amino acids and substrates, protein kinases are broadly categorized into (1) serine-tyrosine kinases, (2) serine-threonine kinases, and (3) histidine kinases. Depending on functional diversity plant protein kinases have also been categorized into (1) RLKs, (2) MAPKs, (3) CDPKS, (4) CDKs, and other less significant kinases like SNF1/AMPK, PDK1, HKs, DGKs, etc. Each group of plant protein kinases is involved in specific environmental signal, or developmental response, forming a unique signal cascade. But often the signaling components in one cascade participate in other signaling pathways forming a complex network. Calmodulin-like domain protein kinases (CDPKs) and receptor-like kinases (RLKs) are unique to plants and are lacking in other eukaryotes. RLKs in plants perform as the receptor of Tyr kinase (RTKs) in an animal system. Histidine kinases (HKs) exist in bacteria and eukaryotes, with different modes of operation in both. Transgenic expression of different signaling kinase genes are described in Table 34.1.

Plants are equipped with many enzymatic and nonprotein components to cope with abiotic stress. As indicated above plant protein kinases are important signaling enzymes that perceive and transfer the external environmental stresses down to the nucleus to get the defense response. The role of kinases is more projected in the defense mechanism in biotic stress rather than in abiotic stress. Most of the abiotic stress responsive genes in plants are grouped as ABA dependent and ABA-independent. SnRK2 are plant specific kinases belonging to serine/threonine group and found to be expressed in all abiotic stress conditions (Kulik et al., 2011). In response to ABA hormone, SNF1 (sucrose nonfermenting 1) a SnRK2 kinase, binds to $\mathrm{ABRE} / \mathrm{ABF}$ transcription factor and activates $\mathrm{ABA}$ responsive physiological process (Umezawa et al., 2009). Different classes of plant protein kinases, including MAPKs and RLKs, play vital role in cellular metabolic signaling and physiological process during abiotic stress (Wankhede et al., 2013; Jaggi et al., 2013). The salt overly sensitive (SOS) pathway is a signaling pathway that plays a crucial role in salt stress adaptation. SnRK3, a SNF like protein kinase, interacts with SOS3 protein to activate plasma membrane bound $\mathrm{Na}^{+} / \mathrm{H}^{+}$ antiporter. The phosphorylation of $\mathrm{Na}^{+} / \mathrm{H}^{+}$antiporter, 
TABLE 34.1 Plant Protein Kinase Gene Used in Genetic Engineering for Abiotic Stress Tolerance

\begin{tabular}{|c|c|c|c|c|c|}
\hline Functional category & Gene & $\begin{array}{l}\text { Transgenic } \\
\text { crop/plant }\end{array}$ & Tolerance & Mechanism of action & References \\
\hline \multirow[t]{15}{*}{ RLK } & $\begin{array}{l}\text { (FLORAL ORGAN } \\
\text { NUMBER1) FON1 }\end{array}$ & Rice & Drought stress & $\begin{array}{l}\text { Through action of ABA responsive } \\
\text { genes }\end{array}$ & $\begin{array}{l}\text { Feng et al. } \\
\text { (2014) }\end{array}$ \\
\hline & (Leaf Panicle 2) LP2 & Rice & Drought stress & $\begin{array}{l}\text { Through interaction of aquaporin } \\
\text { and drought related TF (DST) }\end{array}$ & Wu et al. (2015) \\
\hline & OsSIK1 & Arabidopsis & Drought stress & ABA mediated signaling & $\begin{array}{l}\text { Ouyang et al. } \\
(2010)\end{array}$ \\
\hline & MsSIK1 & Arabidopsis & Drought stress & $\begin{array}{l}\text { Improved water use efficiency and } \\
\text { controlled stomatal movement }\end{array}$ & $\begin{array}{l}\text { Guo et al. } \\
\text { (2016) }\end{array}$ \\
\hline & TaPRK2697 & Wheat & Salt stress & Enhanced $\mathrm{Na}$ ? efflux & Ma et al. (2015) \\
\hline & $\begin{array}{l}\text { (Phloem intercalated } \\
\text { with xylem-like 1) } \\
\text { AtPXL1 }\end{array}$ & Arabidopsis & Cold stress & $\begin{array}{l}\text { Auto phosphorylation other } \\
\text { signaling components }\end{array}$ & $\begin{array}{l}\text { Chang et al. } \\
\text { (2015) }\end{array}$ \\
\hline & GsLRPK & Arabidopsis & Cold stress & $\begin{array}{l}\text { Activation of cold specific TFs like } \\
\text { KIN1 and COR15b }\end{array}$ & $\begin{array}{l}\text { Yang et al. } \\
\text { (2014) }\end{array}$ \\
\hline & LRR-RLK-VIII & Arabidopsis & Heavy metal stress & Ethylene-related pathways & Fu et al. (2014) \\
\hline & WAK1 & Arabidopsis & Heavy metal stress & $\begin{array}{l}\text { Efficient functioning of vacuole } \\
\text { membrane porters and long root } \\
\text { morphology }\end{array}$ & $\begin{array}{l}\text { Sivaguru et al. } \\
\text { (2003) }\end{array}$ \\
\hline & OsWAKL124 & Rice & Heavy metal stress & Efficient sequestration to vacuoles & \\
\hline & GsRLCK & Arabidopsis & Drought stress & Through ABA signaling molecules & Sun et al. (2016) \\
\hline & PnRLK-1 & Arabidopsis & Salt stress & Efficient ROS scavenging & $\begin{array}{l}\text { Zhang et al. } \\
\text { (2015) }\end{array}$ \\
\hline & PsLecRLK & Tobacco & Salt stress & $\begin{array}{l}\text { Efficient osmotic and ionic } \\
\text { management }\end{array}$ & $\begin{array}{l}\text { Vaid et al. } \\
\text { (2015) }\end{array}$ \\
\hline & OsSIK2 & Rice & Drought and salt stress & $\begin{array}{l}\text { Through activation of DREB } \\
\text { transcription factor (TF) }\end{array}$ & $\begin{array}{l}\text { Chen et al. } \\
(2013 a, b)\end{array}$ \\
\hline & AtLRK10L1.2 & Arabidopsis & Drought stress & Through ABA signaling & Lim et al. (2015) \\
\hline \multirow[t]{3}{*}{ MAPKKK } & $N t \mathrm{NPK} 1$ & & Drought stress & $\begin{array}{l}\text { Through higher photosynthesis } \\
\text { rates }\end{array}$ & $\begin{array}{l}\text { Shou et al. } \\
\text { (2004) }\end{array}$ \\
\hline & OsMKK6 & & Abiotic and biotic & $\begin{array}{l}\text { Efficient functioning of ROS } \\
\text { scavenging genes, DREB \& WRKY } \\
\text { TFs }\end{array}$ & $\begin{array}{l}\text { Kumar and } \\
\text { Sinha (2014) }\end{array}$ \\
\hline & OsDSM1 & & Dehydration stress & Through activation of DREB TFs & $\begin{array}{l}\text { Ning et al. } \\
\text { (2010) }\end{array}$ \\
\hline \multirow[t]{3}{*}{ MAPKK } & GhMKK1 & & Drought and salt stress & Efficient ROS regulation & Lu et al. (2015) \\
\hline & ZmMKK1 & & $\begin{array}{l}\text { Chilling and defense } \\
\text { responses }\end{array}$ & $\begin{array}{l}\text { Enhancing antioxidant enzyme } \\
\text { activity and increasing osmolytes } \\
\text { accumulation }\end{array}$ & Cai et al. (2014) \\
\hline & PtMAPKK4 & Tobacco & Salt tolerance & $\begin{array}{l}\text { Efficient free radical scavenging } \\
\text { and long root morphology }\end{array}$ & $\begin{array}{l}\text { Zhang et al. } \\
\text { (2014) }\end{array}$ \\
\hline \multirow[t]{2}{*}{ MAPK } & OsMPKK10.2 & & $\begin{array}{l}\text { Drought and bacterial } \\
\text { blight }\end{array}$ & Through activation of DREB TFs & Ma et al. (2018) \\
\hline & ZmSIMK1 & & $\begin{array}{l}\text { Drought and salt stress } \\
\text { Also improves seed } \\
\text { germination }\end{array}$ & Efficient ROS regulation & $\begin{array}{l}\text { Wang et al. } \\
\text { (2014) }\end{array}$ \\
\hline
\end{tabular}


TABLE 34.1 (Continued)

\begin{tabular}{|c|c|c|c|c|c|}
\hline Functional category & Gene & $\begin{array}{l}\text { Transgenic } \\
\text { crop/plant }\end{array}$ & Tolerance & Mechanism of action & References \\
\hline & ZmMPK5 & & $\begin{array}{l}\text { Salt and defense } \\
\text { tolerance }\end{array}$ & Improved ROS scavenging activity & $\begin{array}{l}\text { Zhang et al. } \\
\text { (2014) }\end{array}$ \\
\hline & OsMAPK33 & & Salt stress & Efficient ion transportation & \\
\hline & OsBWMK1 & & & & $\begin{array}{l}\text { Cheong et al. } \\
\text { (2003) }\end{array}$ \\
\hline \multirow[t]{3}{*}{ CDPK } & OsCPK4 & & Salt and drought stress & $\begin{array}{l}\text { Preventing membrane lipid } \\
\text { peroxidation }\end{array}$ & $\begin{array}{l}\text { Campo et al. } \\
\text { (2014) }\end{array}$ \\
\hline & OsCPK12 & & Salt and drought stress & ROS production and scavenging & $\begin{array}{l}\text { Asano et al. } \\
(2012)\end{array}$ \\
\hline & OsCDPK2 & & Salt and drought stress & Inhibits seeds development & $\begin{array}{l}\text { Morello et al. } \\
\text { (2000) }\end{array}$ \\
\hline \multirow[t]{4}{*}{ CIPK } & $\mathrm{CaCIPK} 25$ & Tobacco & $\begin{array}{l}\text { Dehydration tolerance, } \\
\text { improved germination }\end{array}$ & Long root morphology & $\begin{array}{l}\text { Meena et al. } \\
\text { (2015) }\end{array}$ \\
\hline & ТaCIPK29 & Tobacco & Salt stress & ROS scavenging & $\begin{array}{l}\text { Deng et al. } \\
\text { (2013) }\end{array}$ \\
\hline & MdSOS2L1 & Tomato & $\begin{array}{l}\text { ROS scavenging; } \\
\text { antioxidative } \\
\text { metabolism }\end{array}$ & Drought and oxidative stress & Hu et al. (2016) \\
\hline & ZmCIPK16 & Arabidopsis & $\begin{array}{l}\text { Efficient } \mathrm{K}^{+} / \mathrm{Na}^{+} \\
\text {homeostasis and ROS } \\
\text { scavenging }\end{array}$ & Salt stress & $\begin{array}{l}\text { Wang et al. } \\
\text { (2012) }\end{array}$ \\
\hline
\end{tabular}

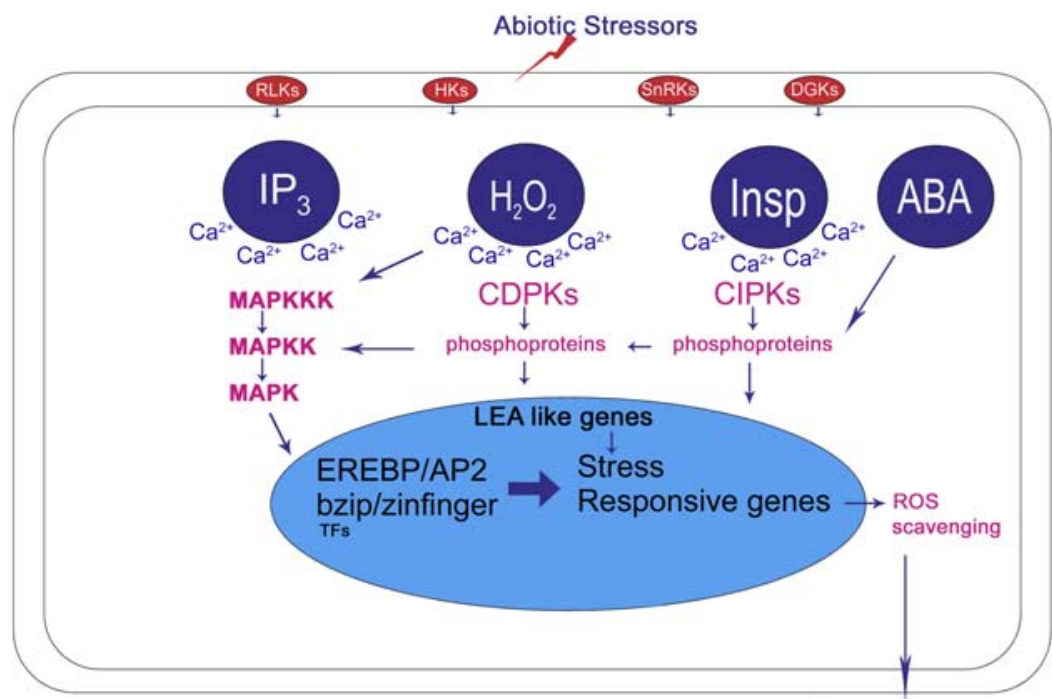

Stress tolerance
FIGURE 34.1 Schematic representation of abiotic stress signal transduction in plants. The external abiotic stress signals perceived by membrane bound receptor-like kinases (RLKs), histidine kinases (HKs), SNF1-related protein kinases (SnRKs), and diacylglycerol kinases (DGKs) are transduced to cytoplasm. The secondary messengers like inositol 1,4,5-triphosphate $\left(\mathrm{IP}_{3}\right)$, hydrogen peroxide $\left(\mathrm{H}_{2} \mathrm{O}_{2}\right)$, and abscisic acid (ABA) sense these signals through calcium ions $\left(\mathrm{Ca}^{2+}\right)$ and make conformational changes in the mitogen activated protein kinase (MAPK) cascade, which in turn regulates the master controls, that is, transcription factors in the nucleus, to get the right response. that is, SOS1 was mediated by MPK6, in response to $\mathrm{NaCl}$ during salt stress (Quintero et al., 2011). Another group of protein kinases that has a prominent role in abiotic stress adaptation are mitogen-activated protein kinases (MAPK). Many studies highlighted the MAPK role in different abiotic stress conditions (Kim et al., 2011). Another important study indicated the interplay of MAPK compounds with ROS signaling molecules
(Kim et al., 2011). Wei et al. (2014a,b) have demonstrated that RLKs have a major share in abiotic stress signaling followed by CDPKs and MAP kinases. MAP kinases can interact with ABA mediated signaling components and elicit stress response (Ara and Sinha, 2014). The hierarchy of various components in signal transduction and their interacting partners is depicted in Fig. 34.1. 


\subsection{RECEPTORS LIKE KINASES}

Cell surface RLKs are a major group of plant protein kinases. RLKs contain conserved serine/threonine catalytic domain that transduces external environmental signals through phosphorylation and dephosphorylation mechanisms. The processes controlled by RLKs include gas exchange (through stomatal control), disease reactions, and self-incompatibility. Arabidopsis is reported to have 610 RLK genes, whereas rice contains 1132 members of RLKs (Shiu et al., 2004). Plant RLKs are unique in nature, consisting of components resembling other eukaryotes. ZmPK1, a putative RLK, was the first cloned and characterized plant protein kinase and was reported to contain a transmembrane catalytic domain and a long highly conserved $\mathrm{N}$-terminal region (Walker and Zhang, 1990). Moreover leucine-rich repeat RLKs (LRR-RLKs) were the most studied surface RLKs and found to be involved in drought resistance (Morillo and Tax, 2006). A leucine-rich RLK, GHR1 (guard cell hydrogen peroxide resistant 1) was found as the initial component in $\mathrm{ABA}, \mathrm{H}_{2} \mathrm{O}_{2}$ mediated signaling pathway of stomatal movement in Arabidopsis (Hua et al., 2012). Another RLK from Arabidopsis, that is, RLK7 was found involved in seed germination and confers oxidative stress tolerance (Pitorre et al., 2010). Osakabe et al. (2013) have observed high water use efficiency (WUE) with drought tolerance in transgenic Arabidopsis plants overexpressing RPK1. The interaction of RLKs with aquaporins (plasma membrane intrinsic proteins) on the membrane could be a possible mechanism of ABA mediated drought stress tolerance in plants. FERONIA is also a group of RLKs identified from Catharanthus roseus (CrRLKs) that are found to activate transcription factors involved in mechanical and abiotic stress tolerance through ABA-independent signaling cascade (Chen et al., 2017). FON1, a LRR-RLK gene was initially isolated from rice, perceives drought signals and phosphorylates downstream ABA signaling molecules to confer drought tolerance (Feng et al., 2014). Several other RLKs were identified that are involved in ABAindependent mechanism and have been proposed to play an important role in the drought tolerance mechanism. OsSIKI1/OsSIKI2 are S-domain receptor kinases in plants and their overexpression leads to drought tolerance in many plant systems tested by the action of stomatal regulation and ROS detoxification (Ouyang et al., 2010; Chen et al., 2013a,b). MsSIKI1 is also a LRRRLK gene that promotes drought tolerance through improved water use efficiency (Guo et al., 2016). Another group of RLKs that are well studied in plants are the membrane bound lectin RLKs (Lec RLKs). A LecRLK from Pisum, that is, PsLecRLK, was found to be expressed efficiently under salinity (Vaid et al., 2015). PnRLK-1 is a cytoplasmic type of RLK found involved in salt tolerance mechanism through efficient ROS scavenging (Zhang et al., 2014).

Calcium regulated RLKs (CRLKs) are activated by $\mathrm{Ca}^{2+}$ signaling and play a vital role in cold stress tolerance in plants. CRLK1 is a membrane bound kinase molecule that was found to accumulate during cold stress and phosphorylate MAP kinase cascade to transmit cold stress signals to candidate genes in the genome (Furuya et al., 2013). AtPXL1 (phloem intercalated with xylem-like 1) is a LRR-RLK gene from Arabidopsis and found highly expressed by cold stress (Chang et al., 2015). GsLRPK is another LRR-RLK gene that autophosphorylates by cold stress, leading to acclimation (Yang et al., 2014).

\subsection{MITOGEN ACTIVATED PROTEIN KINASES}

MAPKs are the most studied plant protein kinases that connect environmental signals to the TFs/genes in the nucleus and make them to express accordingly. Three classes of MAPKs are recognized in plants that are activated through phosphorylation in sequential order starting from MAPK kinase kinase (MAPKKK) to MAPK kinase (MAPKK) to MAPK. MAPKs contain a conserved dual function kinase domain that has threonine/tyrosine/serine phosphorylation residues in their active domain site (T-loop). The stress stimuli are perceived by membrane bound receptors and transmitted to MAP cascade through common secondary messenger molecules like inositol phosphate $\left(\mathrm{IP}_{3}\right)$ and ROS molecules like hydrogen peroxide $\left(\mathrm{H}_{2} \mathrm{O}_{2}\right)$ as mentioned above. An interaction between $\mathrm{H}_{2} \mathrm{O}_{2}$ and MAPK molecule under stressful condition has been well explained in Arabidopsis (Tena et al., 2001). These secondary messenger molecules raise calcium concentrations in cytoplasm that trigger the initiation of protein phosphorylation in MAPK cascade to express the defense genes. Many attempts have been made to overexpress MAPKK derivative genes in different genetic backgrounds and the results are encouraging with superior performance compared with their nontransgenic counterparts. MAP kinase cascade is a circuit of various enzymes and each component has a prominent role and functions in a coordinate way with its interacting molecules. Transgenic overexpression of a single MAPK gene may not confer tolerance to complex traits to full extent as disproportionate concentrations of overexpressed enzymes could lead to limitation of interaction molecules or feedback inhibition. The concept of mutagen constructs with simultaneous expression of all MAPK genes involved in a signal cascade has been proposed by Moustafa et al. (2014). Multigene cloning and their transgenic 
overexpression was successfully demonstrated and found to be a successful biotechnology method to mitigate complex traits like drought, salt, and heat (Roy et al., 2011). A brief description of MAPK genes and their heterologous expression is presented in Table 34.1.

\subsubsection{MAPKKKs}

The whole genome-wide analysis of Arabidopsis has revealed the occurrence of approximately 80 MAP3Ks (Hashimoto et al., 2012). Wang et al. (2017) has identified 155 MAPKKK genes in wheat, and studied their phylogenetic relations with other crops. They also demonstrated tissue specific expression profile of selected MAPKKKs, under different simulated conditions using RNAseq analysis. Considering the phylogenetic relation and similarity among the catalytic domain, MAPKKKs are broadly categorized into two different families, that is, the Raf family that contains a highly conserved kinase domain at C-terminal and MKEE family with less conserved amino groups (Ichimura et al., 2002). Several individual MAP3K genes were isolated, characterized, and could establish their function in the stress tolerance mechanism. Functional characterization of cDNA clones from $N$. benthamiana has revealed the role of MAP3Ks in pathogen induced programmed cell death (PCD). OMTK1 is another MAPKKK gene, isolated from Medicago, and was found to be activated by $\mathrm{H}_{2} \mathrm{O}_{2}$, during stress and cell death defense mechanism (Nakagami et al., 2004). Transgenic overexpression of NPK1, a kinase domain of MAPKKK from tobacco, was reported to increase abiotic stress tolerance in maize (Shou et al., 2004). Most of the studies focused on MAP3Ks indicated their role in hypersensitive reactions through PCD. But Ning et al. (2010) has identified a putative MAP3K gene, that is, OsDSM1, whose overexpression leads to drought tolerance in rice.

The high occurrence of MAPKKK genes in cytoplasm compared with MAP2Ks and MAPKs indicates the interaction of more than one MAPKKK with single MAPKK and MAPK in the MAP cascade. The results obtained by Hashimoto et al. (2012) have supported this thought. Hashimoto et al. (2012) identified three novel MAPKKK genes and demonstrated a parallel, linear signaling pathway involving 3 MAPKKKs in its signaling cascade leading to programmed cell death (PCD). MEK1, a novel MAP3K gene in Arabidopsis, is activated by both biotic and abiotic stimuli, but the downstream signal module is different in both cases. This is due to the scaffolding mechanism of MAKKK proteins (Jalmi and Sinha, 2015). This indicates the high functional diversity of MAPKKKs, being the upstream proteins in MAP cascade.

\subsubsection{MAPKKs}

Mitogen activated protein kinase kinase (MAPKKs) are the central component in the MAPK cascade and are the junction point where the convergence and divergence of other signaling pathways takes place. The whole genome sequence analysis of Arabidopsis has revealed the occurrence of 10 MAPKK genes, which have been classified into four groups based on the similarity of their protein sequence (Sinha et al., 2011). Eight novel MAPKK genes were identified in rice and one among them, that is, OsMKK6 has been characterized and demonstrated its interaction with OsMPK3 (Kumar and Sinha, 2014). Compared with MAPKs fewer MAPKKs are present in cytoplasm indicating more than one MAPK interaction to each MAPKK. MAPKKs are reported to express under cold and pathogen stress. Transcriptome analysis in paddy has revealed that OsMKK4 and OsMKK6 are strongly regulated by salt and cold stresses (Kumar et al., 2008). Another MAPKK enzyme, OsMKK4, was found to be involved in heavy metal stress tolerance in rice (Rao et al., 2011). A MAP2K, identified as OsWNK1 (with no lysine kinase 1) in rice is involved in circadian rhythm and abiotic stress has been reported. Wankhede et al. (2013) have reported that OsMKK6EE is responsible for cellular protection under UV stress and also established MKK6EE's role in rice blast disease. In the same study, global gene expression analysis was performed for the overexpressed constitutive active OsMKK6 lines. Constitutive expression of $\mathrm{Nt} \mathrm{MeK2} \mathrm{(NtMAPKK2)} \mathrm{in}$ tobacco has efficiently expressed defense related genes when compared with controls, on artificial pathogen inoculation. Kumar and Sinha (2014) has studied the genes that upregulate in OsMKK6 overexpressed transgenic rice plants. $\mathrm{Xu}$ et al. (2008) have explained the role of MAPKK9 in transducing signals in salt stress and proposed ABA mediated defense pathway in Arabidopsis. They further identified the interacting molecule for MAPK3 in salt stress signal cascade. In another example, a cDNA clone of MAPKK from populous (PtMAPKK4) was overexpressed in tobacco and results showed increased water stress tolerance and salt tolerance up to $150 \mathrm{mM} \mathrm{NaCl}$ (Wang et al., 2017). The same group further studied tissue specific expression pattern of PtMAPKK4 and found more expression of transcript in leaves followed by roots.

\subsubsection{MAPKs}

Based on the amino acid composition in the activation loop the MAPKs are broadly categorized into two major groups, that is, TEY motif kinases and TDY motif kinases. TEY motif is further divided in to subgroups $\mathrm{A}, \mathrm{B}$, and $\mathrm{C}$ and TDY motif consists of only 
subgroup D (Opdenakker et al., 2012; Hamel et al., 2006). MAPKs are the last component in the MAP kinase cascade and are fewer in numbers, compared with MAP2Ks and MAP3Ks. Many genes encoding MAP kinase have been identified from different species and their role in gene expression related to biotic and abiotic stress has been studied. Twenty MAPKs are identified in Arabidopsis whereas 15 MAPKs are reported from rice. Since a lower number of MAPKs are available to their upstream counterparts, high functional diversity and crosstalk has been observed with MAPK genes. At MPK6 and At MAPK3 reported from Arabidopsis and their orthologous genes SIMK and SAMK from Medicago were found to share both biotic and abiotic stress signals and elicit the appropriate gene action (Cheong et al., 2003). BWMK1 was the first characterized MAPK isolated from indica rice and found involved in pathogen signaling and also in wound/ mechanical signaling cascade (He et al. 1999). A cDNA clone of MAPK (OsMAPK33) isolated from rice was found to be involved in salt stress tolerance. Overexpression and gene silencing studies of OsMAP33 indicated its negative regulation action in confirming salt tolerance in transgenic plants through efficient functioning of ion transport genes (Lee et al., 2011). Several independent MAPKs are found to be involved in plant developmental process (Wang et al., 2007). Studies showed that drought stress resulted in the activation of OsMSRMK2 and OsMAPK5 in rice plants, indicating the role of MAP kinase genes in abiotic stress (Agrawal et al., 2002). Cheong et al. (2003) have demonstrated the interaction of MAP kinase with the promoter region of a transcription factor EREBP1, that in turn activates stress related genes in rice.

\subsection{CALCIUM KINASES}

Calcium plays a pivotal role in plant adaptation to different biotic and abiotic stresses by modulating the signaling network that induces stress responsive genes. Perturbations from normal growth conditions result in elevated cellular calcium levels in the cytoplasm through influx from calcium stores such as endoplasmic reticulum, vacuole, mitochondria, and cell wall and then rapidly returns to basal level by $\mathrm{Ca}^{2+}$ efflux to generate calcium spikes or signatures (Yang and Poovaiah, 2003), that further act as secondary messengers. Consequent to spike in cellular calcium levels, calcium sensors, and calcium binding proteins are activated and phosphorylate downstream regulatory proteins including transcription factors. The activated regulatory proteins alter the gene expression pattern resulting in altered metabolism and physiology followed by phenotypic response to stress conditions.
The transient increase in cytosolic $\mathrm{Ca}^{2+}$ in response to stress signal is sensed by a plethora of calcium sensors or calcium binding proteins, which are estimated to be about 250 in Arabidopsis (Day et al., 2002). To date, the major classes of $\mathrm{Ca}^{2+}$ sensors identified and characterized include (1) calmodulin (CaMs) and calmodulinlike proteins, (2) calcium-dependent protein kinases (CDPKs/CPKs), and (3) calcineurin B like proteins (CBLs) (Kudla et al., 2010). Differential affinity of these families of proteins to $\mathrm{Ca}^{2+}$ coupled with their subcellular localization determines their activities. Calcium sensors and effectors transduce stress signals by changing their conformation upon binding to the $\mathrm{Ca}^{2+}$ ion, which triggers their association with target proteins and/or activation of kinase activity.

\subsubsection{Calcium-Dependent Protein Kinases}

Unlike CaM and CBLs, which acts as only $\mathrm{Ca}^{2+}$ sensors, CDPKs can sense, respond, and translate the $\mathrm{Ca}^{2+}$ signature generated by different environmental stresses into downstream effects. CDPKs have been identified in protozoans in addition to plants, but not in animals or the fungal genome so far (Valmonte et al., 2014). CDPKs represents a multigene family, estimated at about 34 CDPK genes in Arabidopsis (Cheng et al., 2002), 31 CDPK genes in rice (Ray et al., 2007), 20 CDPK genes in wheat (Li et al., 2008), and representing 29 CDPK genes in tomato (Wang et al., 2016a,b). CDPKs have three conserved domains, one at the $\mathrm{N}$-terminus variable domain that is fused to the serine/threonine protein kinase domain and another at the C-terminus, and one more CDPK activation domain (CAD) exists near the C-terminus. The CAD domain possesses an autoinhibitory region and four EF hands for binding $\mathrm{Ca}^{2+}$ ion (Singh et al., 2018).

Stress specific $\mathrm{Ca}^{2+}$ signatures generated are decoded by CDPKs through sensing and phosphorylation of downstream regulatory proteins. Several studies of genome-wide expression revealed that CDPK genes show differential expression in response to different stress conditions and also during developmental stages (Singh et al., 2018). CDPKs are often considered as positive regulators of abiotic stress tolerance; consequently, overexpression of CDPKs in plants imparts enhanced stress tolerance. During water deficit conditions, closure of stomata is a crucial adaptation to prevent water loss through transpiration. It has been shown that CDPKs regulate stomatal movement through phosphorylation of ion channels, as cpk3cpk6 double mutant shows impaired ABA activation through slow anion channel (SLAC) protein (Mori et al., 2006) interactions. In addition to CPK3 and CPK6, CPK21 and CPK23 were also reported to activate SLAC proteins. In Arabidopsis, 
CPK8 was found to regulate Catalase3 (CAT3) through phosphorylation by interaction and it is observed that absence of CPK8 activity leads to susceptibility to drought stress (Zou et al., 2015). In rice, CPK14 and CPK21 positively regulate drought, salinity, and ABA signaling through phosphorylation of Di19-4 transcription factor and 14-3-3 protein (Wang et al., 2016a,b; Chen et al., 2017). A few CDPKs were also enhanced by the accumulation of compatible solutes in response to drought stress through enhanced gene expression. Rice CPK9 was induced in response to drought, salinity, and ABA. Further, overexpression of CKP9 improves drought stress tolerance while mutant show increased sensitivity to stress (Wei et al., 2014a,b). Overexpression of AtCPK32 resulted in upregulation of ABA responsive genes such as RAB18, RD29A, and RD29B. This indicates that the AtCPK32 acts as a positive regulator of ABA-mediated stress signaling pathway. Further, overexpression of AtCPK4 and AtCPK11 also confers abiotic stress tolerance mediated by ABA-signaling transduction pathway (Zhu et al., 2007). The mutant of AtCPK23 results improved drought and salt tolerance but was not involved in ABA signaling pathway, which means AtCPK23 is the negative regulator for the ABAmediated signaling pathway (Ma and $\mathrm{Wu}, 2007$ ). Some of the CDPKs were also involved in ABA-independent signaling pathways under abiotic stress conditions. The AtCPK11 interacted with the zinc finger protein family of AtDi19-1 gene, and as a result induced drought tolerance (Rodriguez Milla et al., 2006). AtCPK1 also showed calcium sensing nature during stress conditions (Hwang et al., 2000). According to Wan et al. (2007), 17 OsCPK transcripts were identified in rice with response to cold, drought, salt, and heat stresses, which includes OsCPK1, 4, 6, 7, 8, 9, 10, 13, 14, 15, 16, 17, 19, 23, 24, 25, and 29. CDPKs in rice showed elevated expression when the crop is exposed to cold stress. Consequently, overexpressing of CDK13 along with a calreticulin interacting protein conferred cold tolerance in rice (Komatsu et al., 2007). Arabidopsis plants confer cold tolerance when Populus CPK10 and Vitis amurensis CPK20 were overexpressed (Chen et al., 2013a,b; Dubrovina et al., 2015). Tomato CPK2 showed higher expression when exposed to $42^{\circ} \mathrm{C}$ (Chang et al., 2009). Overexpression and silencing of CPK12 in rice conferred the role in salt stress tolerance through regulation of ROS scavenging enzymes (Asano et al., 2012).

\subsubsection{Calcineurin B-Like Proteins and CBL- Interacting Protein Kinases:}

CBLs are plant specific $\mathrm{Ca}^{2+}$ sensor proteins without kinase activity. CBLs were first identified in Arabidopsis and they are named after functional counterparts in yeast, calcineurin B. CBLs interact with CBL-interacting protein kinases (CIPKs), to relay perceived $\mathrm{Ca}^{2}+$ signal to induce downstream gene expression and response. CBLs contain four EF hands, helix-loop-helix structure for $\mathrm{Ca}^{2+}$ binding, and are responsible for interaction with CIPKs. The number of amino acids present between EF hands is highly conserved in CBLs; there are 22 amino acids between EF1 and EF2, 25 amino acids between EF2 and EF3, and 32 amino acids between EF3 and EF4 ( $\mathrm{Xi}$ et al., 2017). However, their first EF hand is unique and possesses distinguishing features in comparison with other $\mathrm{Ca}^{2+}$ sensor proteins, as $\mathrm{Ca}^{2+}$ binding loop as insertion of two amino acids in addition to the regular 12 amino acids (Nagae et al., 2003). CIPKs display a conserved domain architecture consisting of N-terminal, serine/ threonine protein kinase domain, junction domain and at the C-terminal, CIPK specific NAF/FISL motif is required for interaction with CBLs present. The NAF/ FISL domain, named for its highly conserved amino acids, aspargine $(\mathrm{N})$, alanine $(\mathrm{A})$, phenylalanine $(\mathrm{F})$, isoleucine (I), serine (S), and leucine (L), is required and sufficient to mediate binding with CBL proteins (Mao et al., 2016). In a few CIPKs, domain at the C-terminus can specifically interact with phosphatase 2C (Ohta et al., 2003). There are about 10 CBLs and 26 CIPKs genes in Arabidopsis, while the rice genome encodes 11CBLs and 34 CIPKs. The CBLs specifically interact with a subset of CIPKs in $\mathrm{Ca}^{2+}$ dependent manner for efficient transmission of signaling (Kudla et al., 2018). There are about 8 CBLs and 20 CIPKs genes identified in the grapevine genome ( $X_{i}$ et al., 2017).

In studies of gene expression and functional characterization, CBL-CIPK has been reported to play an important role in plant abiotic stress response. The physiological role of the CBL-CIPK complex was first identified while studying salt tolerance mechanism in Arabidopsis. Interaction of CBLs with CIPKs not only activate the kinase domain but is also important for localization of the resulting functional complex to different subcellular compartments that is driven by lipid modification of CBLs. This process determines the complex regulation of signaling, as the same CIPK can function in a different cellular compartment membrane by interacting with different CBLs. For example, CBL4 (SOS3) interacts with CIPK24 (SOS2) and recruits to the plasma membrane to activate the $\mathrm{Na}^{+} / \mathrm{H}^{+}$antiporter (SOS1) at the plasma membrane, and the same CIPK24 is targeted to the vacuole when it interacts with the vacuole targeted CBL10, which leads to salinity tolerance (Qiu et al., 2002; Kudla et al., 2018). Several studies highlighted that overexpression of CIPKs conferred tolerance to different abiotic stresses. A few examples include an overexpression of CIPK6 in cotton (He et al., 2013) and overexpression of 
CIPK24 to salt tolerance (Huertas et al., 2012). Over expression of CIPK3 in Arabidopsis increased tolerance to drought, salt, low temperature, and ABA (Kim et al., 2003). It has been shown that CIPK31 is involved in stress tolerance when rice plants are exposed to stress (Piao et al., 2010). Transgenic Brassica producing higher levels of CBL1-CIPK6 has enhanced tolerance to high $\mathrm{NaCl}$, mannitol, and $\mathrm{ABA}$ compared with wild type (Chen et al., 2012). The loss-of-function cipk21 mutant was hypersensitive to salt and osmotic stress conditions and CBL2 and CBL3 were found to interact with CIPK21 and target to tonoplast under salt stress (Pandey et al., 2015). It was found that the CBL-CIPK complex is more responsive to environmental stress than the developmental process (Xi et al., 2017). The CBL1/9-CIPK23 complex plays an important role in ABA mediated closure of stomata in Arabidopsis, where this complex phosphorylates and activates slow anion channel associated1 (SLAC1) and slow anion channel1 homolog3 (SLAH3) proteins and that resulting closure of stomata. The CBL9-CIPK3 complex was involved in salt induced ABA signaling as indicated from changes in $\mathrm{ABA}$ regulated gene expression in mutants (Mao et al., 2016).

\subsubsection{CDPK-Regulated Protein Kinases}

In addition to CDPKs and CIPKs some other classes of calcium regulated protein kinases are identified such as CDPK-regulated protein kinase (CRK) and $\mathrm{Ca} 2+/ \mathrm{CAM}$ kinase (CCaMK), which are yet to be explored in detail but play a major role in the abiotic stress signaling mechanism. The CRKs has $\mathrm{N}$-terminal myristoylation motif, which helps in plasma membrane associations. And also they have degenerated EF-hands, which are incapable of binding calcium ions (Harmon, 2003). A good potential substrate such as AtGLN1 (cytosolic glutamine synthetase involved in nitrogen assimilation) has been identified for AtCRK3 by using yeast two-hybrid approach that regulates mainly nitrogen mobilization during leaf senescence (Li et al., 2006).

Most of the CDPK-related protein kinase (CRKS) expression studies were reported in Arabidopsis and maize plants. All of the CRKs have kinase activity towards $\mathrm{Ca} 2+$ and CaM independently. The example is that AtCRK3 and ZmCRK exhibit autophosphorylation and substrate phosphorylation to $\mathrm{Ca} 2+$ and $\mathrm{CaM}$ independently (Furumoto et al., 1996; Du et al., 2004). With AtGLN1 (cytosolic glutamine synthetase take part in $\mathrm{N}$ assimilation) as a substrate for AtCRK3 gene using yeast two-hybrid approach, those interactions mainly induced nitrogen mobilization during leaf senescence ( $\mathrm{Li}$ et al., 2006). Moreover, the AtCRK1 has affinity to bind with CaM-isoforms such as AtCaM2, 4, 7, and 8, which stimulates kinase activity of AtCRK1 (Wang et al., 2004).

\subsection{SUCROSE NONFERMENTING 1-RELATED PROTEIN KINASES}

One of the well-characterized protein kinases involved in stress responses is the group of sucrose nonfermenting 1 (SNF1)-related protein kinases SnRKs (Halford and Hey, 2009). SnRKs are grouped into three subfamilies, that is, SnRK1, SnRK2, and SnRK3 (Halford and Hey, 2009). Recent studies have indicated the pivotal roles of plant-specific subgroups of SnRK2 and SnRK3 in the link between abiotic stress and abscisic acid (ABA) signaling to regulate metabolic pathways (Hrabak et al., 2003; Halford and Hey, 2009). Further evidence has shown that SnRK2 proteins function as positive regulators of $\mathrm{ABA}$ signaling for stress responses, as well as development, in plants (Umezawa et al., 2013). The SnRK2 family consists of 10 members, which includes SRK2A, SRK2J, and SnRK2.10 in Arabidopsis and in rice SAPK1-10 was reported (Hrabak et al., 2003; Kobayashi et al., 2004). They are further classified into three subclasses based on their domain structures (Kobayashi et al., 2004). Most SnRK2 proteins are activated by abiotic stresses, while the members of subclasses 2 and 3 are also activated by ABA (Boudsocq et al., 2004, 2007; Kobayashi et al., 2004). In the current model, ABA-induced activation is largely explained by the interaction between SnRK2s and protein phosphatase type 2C (PP2C) proteins in the ABA signaling pathway (Leung et al., 1994, 1997; Meyer et al., 1994; Saez et al., 2004; Nishimura et al., 2007; Umezawa et al., 2009; Cutler et al., 2010). In the absence of ABA, group A PP2Cs physically bind to SnRK2s to dephosphorylate SnRK2s, resulting in the inhibition of ABA signal transduction. But in the presence of ABA, SnRK2 will be released from such inhibitory regulation by $\mathrm{PP} 2 \mathrm{C}$, because the soluble $\mathrm{ABA}$ receptor PYR/PYL/RCAR inhibits PP2C activity (Umezawa et al., 2009). In Arabidopsis, detailed analyses of subclass 2 (SRK2F/SnRK2.7, SRK2C/SnRK2.8) and subclass 3 (SRK2D/SnRK2.2, SRK2I/SnRK2.3, SRK2E/ SnRK2.6) have revealed their redundant functions in ABA signaling for abiotic stress responses and developmental controls (Yoshida et al., 2002; Fujii et al., 2007, 2009; Fujii and Zhu, 2009; Nakashima et al., 2009; Mizoguchi et al., 2010). Overexpression of SnRK2 (AtSRK2C/SnRK2.8, TaSnRK2.3, TaSnRK2.4, TaSnRK2.7, and TaSnRK2.8) genes resulted in enhanced abiotic stress tolerance in Arabidopsis (Umezawa et al., 2004; Mao et al., 2010; Zhang et al., 2010). Overexpression of SnRK2 genes in Arabidopsis induced the upregulation of several 
important stress responsive genes, including RD29A and DREB1A/CBF3, and ABA biosynthetic genes, such as ABA1, under normal conditions (Umezawa et al., 2004). Early and quick expression of such key genes was noticed under abiotic stress conditions in the same study, indicating their role in stress tolerance. The rice SAPK4 overexpression exhibited enhanced salt tolerance and major aspects of its tolerance were explained by changes in the expression of genes related to ion homeostasis and oxidative stress responses (Diédhiou et al., 2008). In the above examples of SnRK2 overexpression studies, the results clearly indicated that SnRK2 can function in abiotic stress responses in plant cells, through the modulation of stress response related gene expression. Comparative genomics studies have demonstrated that the core components of ABA signaling, that is, PYR/ PYL/RCAR, SnRK2, and PP2C, are well-conserved in land plant species suggesting the evolutionary conservation of key molecular system (Umezawa et al., 2010). Indeed, the conserved molecular characteristics of SnRK2, such as transcriptional induction by abiotic stresses and activation by stress and/or ABA, have been reported for SnRK2 genes not only in Arabidopsis and rice, but also in other crop plants like maize (Huai et al., 2008) and wheat (Holappa and Walker-Simmons, 1995; Gómez-Cadenas et al., 1999; Mao et al., 2010; Zhang et al., 2010).

\subsection{DIACYLGLYCEROL KINASES}

Diacylglycerol kinases (DGKs) are important signaling kinases that exist in all higher plants. Unlike other plant kinases, DGKs participate indirectly in abiotic stress tolerance. DGKs play a crucial role in synthesis of phosphotidic acid (PA), an important secondary messenger molecule that functions during abiotic stress in plants. DAG (diaceylglycerol) is a wellcharacterized membrane that binds secondary messenger molecules in higher plants and functions during abiotic stress conditions. DAG (diaceylglycerol) is a precursor of PA phosphorylated by DGK giving rise to PA. The primary stress signals such as drought, cold, and salinity or stress respondent hormones like ABA, ethylene initiates the synthesis of PA through DGKs. Apart from stress tolerant molecules, PA acts as a precursor in the synthesis of triacylglycerols and phosphoglycerolipids, important components of cell wall synthesis. After its synthesis PA may be further metabolized to give PPI (Escobar-Sepúlveda et al., 2017). Phospholipase C (PLC) acts on PPI yielding two important secondary messenger molecules, that is, DAG and IP3. DAG and IP3 activate many kinases in cytoplasm in response to $\mathrm{Ca}^{2+}$ concentrations. Plant DGKs exist in many isoforms with conserved catalytic domain, containing ATP binding domain. Stress specific expression profile of DGK genes indicated their role in drought and salt tolerance (Zimmermann et al., 2014).

\subsection{HISTIDINE KINASES}

Plant HKs are membrane bound proteins with a highly conserved two-component system of operation. Like yeast HKs, plant HKs contain a CHASE domain, transmembrane domain, and receiver domain. The $\mathrm{N}$ terminal motifs are variable depending on the functional diversity of $\mathrm{HKs}$, whereas the C-terminal domain is highly conserved with kinase activity. The transfer of phosphoryl group to aspartate domain takes place at the C-terminal residue. Since their discovery many HKs have been identified and characterized in plant species like rice, maize, Arabidopsis, soybean, poplar, etc. (Pareek et al., 2006). Plant HKs mostly perceive ethylene and cytokinin stimuli and are involved in their signaling pathways. Based on their functional diversity plant HKs could be categorized into ethylene receptors, cytokinin receptors, osmosensors, and other less significant HKs. Arabidopsis AHK5/CK12 and CK11 are osmosensing type HKs, whereas AHK4 (CRE1/WOL), AHK3, and AHK2 belong to ethylene receptor HKs. Plant HKs exhibit cold, drought, and salt stress tolerance and evidence also confirms their role in some biotic stress tolerance (Nongpiur et al., 2012). Gene expression profile of AtHKs indicates abundance of AtHK1 and AtHK5 transcripts in root tissue (Iwama et al., 2007). This data supports the results of root phenotype in transgenic Arabidopsis overexpressing AtHK1.

Constitutive expression of AtHKI in Arabidopsis has conferred in water stress tolerance and improved resistance to changes in osmolality (Tran et al., 2007). Heterologous expression of a corn HK (ZmHK9) showed improved water stress tolerance in Arabidopsis, through control of stomatal openings indicating the role of HKs in the drought stress mechanism (Wang et al.,2012). AHK5/CK12 is another HK involved in gourd cell control through $\mathrm{H}_{2} \mathrm{O}_{2}$ regulation (Desikan et al., 2008).

\subsection{GENOMICS EFFORTS IN PLANT PROTEIN KINASE TOWARDS CROP IMPROVEMENT}

The innovations in sequencing chemistries have heavily reduced the cost per data point. The sequence information together with bioinformatics tools has created many genomic tools that help in improving crop productivity. Genomics based breeding tools have 
become an integral part of breeding programs in many institutes and industry. Genomic selection (GS) and genome-wide association studies (GWAS) for dissecting complex traits are a few innovative genomic tools that help in identification of rare QTLs and predicting superior breeding lines that have great impact on crop improvement. The precision in selection has increased with identification of SNP markers for complex traits.

The increased number of genome sequencings and improvements in trait association methods has led to the discovery of gene families using genome-wide markers. This helps in accurate classification of protein kinases and to establish phylogenetic relations among different crop species. It also helps in designing accurate markers for various kinase genes that participate in abiotic stress tolerance and helps in improved selections. Recently Guo et al. (2013) identified 18 CDPK and 7CRK genes in watermelon using whole genome sequencing and found structural polymorphism in one gene that is responsible for abiotic stress tolerance in this species. Rudrabhatla et al. (2006) has applied genome-wide study to identify tyrosine kinase genes in Arabidopsis using animal tyrosine kinase motifs. Recently genome-wide expression analysis of the MAPK gene family was performed in Cicer arietinum using informatics tools and great functional diversity among MAPK genes in different tissues under abiotic stress conditions was found (Singh et al., 2018). Similarly a comprehensive expression profile of MAPK genes in Brassica was studied using sequence similarities in the Brassica database (BRAD) using qRT-PCR (Lu et al., 2015).

Genomic selection is an important tool in recent molecular breeding, where the performance of genotypes can be predicated without phenotyping. During a recent study on identifying maize lines for drought tolerance, genomic selection method was employed and among the top 10 significant SNPs found contributing to drought tolerance, SnRK2 (SnSNF1-related protein kinase 2) was identified as one of the genes, indicating the role of plant protein kinases in abiotic stress tolerance (Umezawa et al.,2013). During genome-wide association study for salt tolerance using a core set of rice genotypes, many functional genes including MAP kinase genes were recognized as candidates responsible for salt tolerance (Patishtan et al., 2017). A genome-wide association study for dissection of the genomic region for leaf angle trait in Brassica has revealed a CDPK related gene (CRK5) is closely associated to the trait (Sun et al.,2016). Genome-wide transcriptome profiling in maize crop has revealed the profound role of protein kinases in drought tolerance. RNA-seq and analysis is an excellent method for global gene expression analysis in plants. Sequence analysis of drought and cold stress cDNAs in cassava showed 12 CDPKs and 4 MAPKs (Lu et al., 2015). Furthermore, the study revealed crosstalk between protein kinase genes like SnRK2 indicating the common mechanism in abiotic stress tolerance.

\subsection{CONCLUSIONS}

Protein kinases are the largest group of molecules involved in various biotic and abiotic stress regulation in plants and hence have attracted researchers all over the globe. Most of the work on plant protein kinases was focused on identification and structural characterization of various groups of kinase genes belonging to different species. But functional validation of plant kinase genes and their specific role in metabolic pathways needs to be elucidated. Most of the research works are focused on overexpression of single kinase genes and the transgenic plants showed a certain degree of tolerance to abiotic stress under study. Plant kinase genes act in a circuit fashion and functionally get activated through phosphorylation by their neighboring component in the cascade. Hence overexpression of a single gene in a signal cascade may not address the problem as a whole. The technological revolution in molecular biology has made multigene cloning and expression of whole pathway genes in single transfer. The recent advancements in genomic technologies has allowed scanning the genomic regions at low cost and faster rate. Identification of functional polymorphisms between the extreme genotypes will facilitate parental line selections and crop improvement programs.

\section{References}

Agrawal, G.K., Rakwal, R., Iwahashi, H., 2002. Isolation of novel rice (Oryza sativa L.) multiple stress responsive MAP kinase gene, OsMSRMK2, whose mRNA accumulates rapidly in response to environmental cues. Biochem. Biophys. Res. Commun. 294, 1009-1016.

Ara, H., Sinha, A.K., 2014. Conscientiousness of mitogen activated protein kinases in acquiring tolerance for abiotic stresses in plants. Proc. Ind. Natl. Sci. Acad. 2, 211-219.

Asano, T., Hayashi, N., Kobayashi, M., Aoki, N., Miyao, A., Mitsuhara, I., et al., 2012. A rice calcium-dependent protein kinase OsCPK12 oppositely modulates salt-stress tolerance and blast disease resistance. Plant J. 69, 26-36.

Batistic, O., Kudla, J., 2004. Integration and channeling of calcium signaling through the CBL calcium sensor/CIPK protein kinase network. Planta 219, 915-924.

Boudsocq, M., Barbier-Brygoo, H., Laurière, C., 2004. Identification of nine sucrose nonfermenting 1-related protein kinases 2 activated by hyperosmotic and saline stresses in Arabidopsis thaliana. J. Biol. Chem. 279 (40), 41758-41766.

Boudsocq, M., Droillard, M.J., Barbier-Brygoo, H., Laurière, C., 2007. Different phosphorylation mechanisms are involved in the activation of sucrose non-fermenting 1 related protein kinases 2 by osmotic stresses and abscisic acid. Plant Mol. Biol. 63 (4), 491-503. 
Cai, G., Wang, G., Wang, L., Pan, J., Liu, Y., Li, D., 2014. ZmMKK1, a novel group A mitogen-activated protein kinase kinase gene in maize, conferred chilling stress tolerance and was involved in pathogen defense in transgenic tobacco. Plant Sci. 214, 57-73.

Campo, S., Baldrich, P., Messeguer, J., Lalanne, E., Coca, M., San Segundo, B., 2014. Overexpression of a calcium-dependent protein kinase confers salt and drought tolerance in rice by preventing membrane lipid peroxidation. Plant Physiol. 165 (2), $688-704$.

Chang, G.J., Hwang, S.G., Yong, C.P., Hyeon, M.P., Dong, S.K., Duck, H.P., et al., 2015. Molecular characterization of the coldand heat-induced Arabidopsis PXL1 gene and its potential role in transduction pathways under temperature fluctuations. J. Plant Physiol. 176, 138-146.

Chang, W.J., Su, H.S., Li, W.J., Zhang, Z.L., 2009. Expression profiling of a novel calcium-dependent protein kinase gene, LeCPK2, from tomato (Solanum lycopersicum) under heat and pathogen-related hormones. Biosci. Biotechnol. Biochem. 73, 2427-2431.

Chen, J., Xue, B., Xia, X., Yin, W., 2013a. A novel calcium-dependent protein kinase gene from Populus euphratica, confers both drought and cold stress tolerance. Biochem. Biophys. Res. Commun. 441, $630-636$.

Chen, L., Ren, F., Zhou, L., Wang, Q.-Q., Zhong, H., Li, X.-B., 2012. The Brassica napus calcineurin B-Like 1/CBL-interacting protein kinase 6 (CBL1/CIPK6) component is involved in the plant response to abiotic stress and ABA signalling. J. Exp. Bot. 63, 6211-6222.

Chen, L.J., Wuriyanghan, H., Zhang, Y.Q., Duan, K.X., Chen, H.W., Li, Q.T., et al., 2013b. An S-domain receptor-like kinase, OsSIK2, confers abiotic stress tolerance and delays dark-induced leaf senescence in rice. Plant Physiol. 163 (4), 1752-1765.

Chen, Y., Zhou, X., Chang, S., Chu, Z., Wang, H., Han, S., et al., 2017. Calcium-dependent protein kinase 21 phosphorylates 14-3-3 proteins in response to $\mathrm{ABA}$ signaling and salt stress in rice. Biochem. Biophys. Res. Commun. 493, 1450-1456.

Cheng, S.-H., Willmann, M.R., Chen, H.-C., Sheen, J., 2002. Calcium signaling through protein kinases. The Arabidopsis calciumdependent protein kinase gene family. Plant Physiol. 129, 469-485.

Cheong, Y.H., Moon, B.C., Kim, J.K., Kim, C.Y., Kim, M.C., Kim, I.H., et al., 2003. BWMK1, a Rice mitogen-activated protein kinase, locates in the nucleus and mediates pathogenesis-related gene expression by activation of a transcription factor1. Plant Physiol. 132 (1961-1972).

Cutler, S.R., Rodriguez, P.L., Finkelstein, R.R., Abrams, S.R., 2010. Abscisic acid: emergence of a core signaling network. Ann. Rev. Plant Biol. 61, 651-679.

Day, I.S., Reddy, V.S., Shad Ali, G., Reddy, A.S.N., 2002. Analysis of EF-hand-containing proteins in Arabidopsis. Genome Biol. 3, 1-24.

Deng, X., Hu, W., Wei, S., Zhou, S., Zhang, F., Han, J., et al., 2013. TaCIPK29, a CBL-interacting protein kinase gene from wheat, confers salt stress tolerance in transgenic tobacco. PLoS ONE. 8, e69881.

Desikan, R., Horák, J., Chaban, C., Mira-Rodado, V., Witthöft, J., Elgass, K., et al., 2008. The histidine kinase AHK5 integrates endogenous and environmental signals in Arabidopsis guard cells. PLoS One 3 (6), 1-12.

Diédhiou, C.J., Popova, O.V., Dietz, K.J., Golldack, D., 2008. The SNF1-type serine-threonine protein kinase SAPK4 regulates stressresponsive gene expression in rice. BMC Plant Biol. 8, 49-53.

Du, W., Wang, Y., Liang, S., Lu, Y.T., 2004. Biochemical and expression analysis of an Arabidopsis calcium-dependent protein kinaserelated kinase. Plant Sci. 168, 1181-1192.

Dubrovina, A.S., Kiselev, K.V., Khristenko, V.S., Aleynova, O.A., 2015. VaCPK20, a calcium-dependent protein kinase gene of wild grapevine Vitis amurensis Rupr. mediates cold and drought stress tolerance. J. Plant Physiol. 185, 1-12.
Escobar-Sepúlveda, H.F., Trejo-Téllez, L.I., Pérez-Rodríguez, P., Hidalgo-Contreras, J.V., Gómez-Merino, F.C., 2017. Diacylglycerol kinases are widespread in higher plants and display inducible gene expression in response to beneficial elements, metal, and metalloid ions. Front. Plant Sci. 8, 129-137.

Feng, L., Gao, Z., Xiao, G., Huang, R., Zhang, H., 2014. Leucine-rich repeat receptor-like kinase FON1 regulates drought stress and seed germination by activating the expression of ABA-responsive genes in rice. Plant Mol. Biol. Rep. 32 (6), 1158-1168.

Fu, S.F., Chen, P.Y., Nguyen, Q.T.T., Huang, L.Y., Zeng, G.R., Huang, T.L., et al., 2014. Transcriptome profiling of genes and pathways associated with arsenic toxicity and tolerance in Arabidopsis. BMC Plant Biol. 14, 94.

Fujii, H., Zhu, J.K., 2009. Arabidopsis mutant deficient in 3 abscisic acidactivated protein kinases reveals critical roles in growth, reproduction, and stress. Proc. Natl. Acad. Sci. USA 106 (20), 8380-8385.

Fujii, H., Verslues, P.E., Zhu, J.K., 2007. Identification of two protein kinases required for abscisic acid regulation of seed germination, root growth, and gene expression in Arabidopsis. Plant Cell 19 (2), 485-494.

Fujita, Y., Nakashima, K., Yoshida, T., Katagiri, T., Kidokoro, S., Kanamori, N., et al., 2009. Three SnRK2 protein kinases are the main positive regulators of abscisic acid signaling in response to water stress in Arabidopsis. Plant Cell Physiol. 50 (12), 2123-2132.

Furumoto, T., Ogawa, N., Hata, S., Izui, K., 1996. Plant calciumdependent protein kinase-related kinases (CRKs) do not require calcium for their activities. FEBS Lett. 396, 147-151.

Furuya, T., Matsuoka, D., Nanmori, T., 2013. Phosphorylation of Arabidopsis thaliana MEKK1 via Ca $2+$ signaling as a part of the cold stress response. J. Plant Res. 126, 833-840.

Gómez-Cadenas, A., Verhey, S.D., Holappa, L.D., Shen, Q., Ho, T.H. D., Walker-Simmons, M.K., 1999. An abscisic acid-induced protein kinase, PKABA1, mediates abscisic acid-suppressed gene expression in barley aleurone layers. Proc. Natl. Acad. Sci. USA (4), $1767-1772$.

Guo, P., Wei, H., Zhang, W., Yang, B., Bao, Y., 2016. The dehydration-induced ERECTA gene, MsSIK1, from alfalfa improved water use efficiency in transgenic Arabidopsis. Acta Physiol. Planta. 38 (2), 46.

Guo, S., Zhang, J., Sun, H., Salse, J., Lucas, W.J., Zhang, H., 2013. The draft genome of watermelon (Citrullus lanatus) and resequencing of 20 diverse accessions. Nature Gen. 45, 51-58.

Halford, N.G., Hey, S.J., 2009. Snf1-related protein kinases (SnRKs) act within an intricate network that links metabolic and stress signalling in plants. Biochem. J. 419 (2), 247-259.

Hamel, L.P., Nicole, M.C., Sritubtim, S., Morency, M.J., Ellis, M., Ehlting, J., et al., 2006. Ancient signals: comparative genomics of plant MAPK and MAPKK gene families. Trends Plant Sci. 11 (4), 192-198.

Harmon, A.C., 2003. Calcium-regulated protein kinases of plants. Gravit. Space Biol. Bull. 16, 83-90.

Harmon, A.C., Gribskov, M., Gubrium, E., Harper, J.F., 2001. The CDPK superfamily of protein kinases. New Phytol. 151, 175-183.

Hashimoto, M., Komatsu, K., Maejima, K., Okano, Y., Shiraishi, T., Ishikawa, K., et al., 2012. Identification of three MAPKKKs forming a linear signalling pathway leading to programmed cell death in Nicotiana benthamiana. BMC Plant Biol. 12 (1), 103-113.

He, C., Fong, S.H.T., Yang, D., Wang, G.L., 1999. BWMK1, a novel MAP kinase induced by fungal infection and mechanical wounding in rice. Mol. Plant Microb. Int. 12 (12), 1064-1073.

He, L., Yang, X., Wang, L., Zhu, L., Zhou, T., Deng, J., et al., 2013. Molecular cloning and functional characterization of a novel cotton CBL-interacting protein kinase gene (GhCIPK6) reveals its involvement in multiple abiotic stress tolerance in transgenic plants. Biochem. Biophys. Res. Commun. 435, 209-215. 
Holappa, L.D., Walker-Simmons, M.K., 1995. The wheat abscisic acid-responsive protein kinase mRNA, PKABA1, is up-regulated by dehydration, cold temperature, and osmotic stress. Plant Physiol. 108 (3), 1203-1210.

Hrabak, E.M., Chan, C.W., Gribskov, M., Harper, J.F., Choi, J.H., Halford, N., et al., 2003. The Arabidopsis CDPK-SnRK super family of protein kinases. Plant Physiol. 132, 666-680.

Hu, D.G., Ma, Q.J., Sun, C.H., Sun, M.H., You, C.X., Hao, Y.J., 2016. Overexpression of MdSOS2L1, a CIPK protein kinase, increases the antioxidant metabolites to enhance salt tolerance in apple and tomato. Physiol. Plant 156 (2), 201-214.

Hua, D., Wang, C., He, J., Liao, H., Duan, Y., Zhu, Z., et al., 2012. A plasma membrane receptor kinase, GHR1, mediates abscisic acidand hydrogen peroxide-regulated stomatal movement in Arabidopsis. Plant Cell 24 (6), 2546-2561.

Huai, J., Wang, M., He, J., Zheng, J., Dong, Z., Lv, H., et al., 2008. Cloning and characterization of the SnRK2 gene family from Zea mays. Plant Cell Rep. 27 (12), 1861-1868.

Huertas, R., Olias, R., Eljakaoui, Z., Galvez, F.J., Li, J., De Morales, P. A., et al., 2012. Rodriguez-Rosales MP Overexpression of SISOS2 (SICIPK24) confers salt tolerance to transgenic tomato. Plant Cell Environ. 35, 1467-1482.

Hwang, I., Sze, H., Harper, J.F., 2000. A calcium-dependent protein kinase can inhibit a calmodulin-stimulated Ca2 + pump (ACA2) located in the endo-plasmic reticulum of Arabidopsis. Proc. Natl. Acad. Sci. USA 97 (11), 6224-6229.

Ichimura, K., Shinozaki, K., Tena, G., Sheen, J., Henry, Y., Champion, A., et al., 2002. Mitogen-activated protein kinase cascades in plants: a new nomenclature. Trends Plant Sci. 7 (7), 301-308.

Iwama, A., Yamashino, T., Tanaka, Y., Sakakibara, H., Kakimoto, T., Sato, S., et al., 2007. AHK5 histidine kinase regulates root elongation through an ETR1-dependent abscisic acid and ethylene signaling pathway in Arabidopsis thaliana. Plant Cell Physiol. 48 (2), 375-380.

Jaggi, M., Gupta, M., Tuteja, N., Sinha, A.K., 2013. Mitogen-activated protein kinases in abiotic stress tolerance in crop plants: "omics" approaches. Imp. Crop Product. Sustain. Agric. 107-132.

Jalmi, S.K., Sinha, A.K., 2015. ROS mediated MAPK signaling in abiotic and biotic stress- striking similarities and differences. Front. Plant Sci. 6, 769-772.

Kim, K.N., Cheong, Y.H., Grant, J.J., Pandey, G.K., Luan, S., 2003. CIPK3, a calcium sensor-associated protein kinase that regulates abscisic acid and cold signal transduction in Arabidopsis. Plant Cell 15, 411-423.

Kim, S.H., Woo, D.H., Kim, J.M., Lee, S.Y., Chung, W.S., Moon, Y.H., 2011. Arabidopsis MKK4 mediates osmotic-stress response via its regulation of MPK3 activity. Biochem. Biophys. Res. Comm. 412 (1), 150-154.

Kobayashi, Y., Yamamoto, S., Minami, H., Kagaya, Y., Hattori, T., 2004. Differential activation of the rice sucrosenonfermenting1-related protein kinase2 family by hyperosmotic stress and abscisic acid. Plant Cell 16 (5), 1163-1177.

Komatsu, S., Yang, G., Khan, M., Onodera, H., Toki, S., Yamaguchi, M., 2007. Over-expression of calcium-dependent protein kinase 13 and calreticulin interacting protein 1 confers cold tolerance on rice plants. Mol. Genet. Genomics 277 (6), 713-723.

Kudla, J., Batistič, O., Hashimoto, K., 2010. Calcium signals: the lead currency of plant information processing. Plant Cell 22, 541-563.

Kudla, J., Becker, D., Grill, E., Hedrich, R., Hippler, M., Kummer, U., et al., 2018. Advances and current challenges in calcium signaling. New Phytol. 218, 414-431.

Kulik, A., Wawer, I., Krzywińska, E., Bucholc, M., Dobrowolska, G., 2011. SnRK2 protein kinases-key regulators of plant response to abiotic stresses. Omics 15 (12), 859-872.

Kumar, K., Rao, K.P., Sharma, P., Sinha, A.K., 2008. Differential regulation of rice mitogen activated protein kinase kinase (MKK) by abiotic stress. Plant Physiol. Biochem. 46, 891-897.
Kumar, K., Sinha, A.K., 2014. Genome-wide transcriptome modulation in rice transgenic lines expressing engineered mitogen activated protein kinase kinase 6. Plant Signal. Behav. 9 (5), e28502.

Lee, S.K., Kim, B.G., Kwon, T.R., Jeong, M.J., Park, S.R., Lee, J.W., et al., 2011. Overexpression of the mitogen-activated protein kinase gene OsMAPK33 enhances sensitivity to salt stress in rice (Oryza sativa L.). J. Biosci. 36 (1), 139-151.

Lehti-Shiu, M.D., Zou, C., Shiu, S.H., 2012. Origin, diversity, expansion history, and functional evolution of the plant receptor-like kinase/pelle family. Receptor-Like Kinases inPlants. Springer, Berlin, Heidelberg, pp. 1-22.

Leung, J., Bouvier-Durand, M., Morris, P.C., Guerrier, D., Chefdor, F., Giraudat, J., 1994. Arabidopsis ABA response gene ABI1: features of a calcium-modulated protein phosphatase. Science 264 (5164), 1448-1452.

Leung, J., Merlot, S., Giraudat, J., 1997. The Arabidopsis Abscisic Acid-Insensitive 2 (ABI2) and ABI1 genes encode homologous protein phosphatases $2 \mathrm{C}$ involved in abscisic acid signal transduction. Plant Cell 9 (5), 759-771.

Li, A.L., Zhu, Y.F., Tan, X.M., Wang, X., Wei, B., Guo, H.Z., et al., 2008. Evolutionary and functional study of the CDPK gene family in wheat (Triticum aestivum L.). Plant Mol. Biol. 66, 429-443.

Li, R.J., Hua, W., Lu, Y.T., 2006. Arabidopsis cytosolic glutamine synthetase AtGLN1; 1 is a potential substrate of AtCRK3 involved in leaf senescence. Biochem. Biophys. Res. Commun. 342, 119-126.

Lim, C.W., Yang, S.H., Shin, K.H., Lee, S.C., Kim, S.H., 2015. The AtLRK10L1. 2, Arabidopsis ortholog of wheat LRK10, is involved in ABA-mediated signaling and drought resistance. Plant cell rep 34 (3), 447-455.

Lu, K., Guo, W., Lu, J., Yu, H., Qu, C., Tang, Z., et al., 2015. Genomewide survey and expression profile analysis of the mitogenactivated protein kinase (MAPK) gene family in Brassica rapa. PLoS One 10 (7), e0132051.

Ludwig, A.A., Romeis, T., Jones, J.D., 2004. CDPK-mediated signaling pathways: specificity and cross-talk. J. Exp. Bot. 395, 181-188.

Ma, S.Y., Wu, W.H., 2007. AtCPK23 functions in Arabidopsis responses to drought and salt stresses. Plant Mol. Biol. 65 (4), $511-518$

Ma, X.L., Cui, W.N., Zhao, Q., Zhao, J., Hou, X.N., Li, D.Y., et al., 2015. Functional study of a salt-inducible TaSR, gene in Triticum aestivum. Physiol. Plant 156, 40-53.

Ma, J., Chen, J., Wang, M., Ren, Y., Wang, S., Lei, C., et al., 2018. Disruption of OsSEC3A increases the content of salicylic acid and induces plant defense responses in rice. J. Exp. Bot. 69, 1051-1064.

Mao, H., Sun, S., Yao, J., Wang, C., Yu, S., Xu, C., et al., 2010. Linking differential domain functions of the GS3 protein to natural variation of grain size in rice. Proc. Natl. Acad. Sci. 107, 19579-19584.

Mao, J., Manik, S.M.N., Shi, S., Chao, J., Jin, Y., Wang, Q., et al., 2016. Mechanisms and physiological roles of the CBL-CIPK networking system in Arabidopsis thaliana. Genes. 7, 1-15.

Meena, M.K., Ghawana, S., Dwivedi, V., Roy, A., Chattopadhyay, D., 2015. Expression of chickpea CIPK25 enhances root growth and tolerance to dehydration and salt stress in transgenic tobacco. Front. Plant Sci. 6, 683.

Meyer, K., Leube, M.P., Grill, E., 1994. A protein phosphatase 2C involved in ABA signal transduction in Arabidopsis thaliana. Science 264 (5164), 1452-1455.

Mizoguchi, M., Umezawa, T., Nakashima, K., Kidokoro, S., Takasaki, H., Fujita, Y., et al., 2010. Two closely related subclass II SnRK2 protein kinases cooperatively regulate drought-inducible gene expression. Plant Cell Physiol. 51 (5), 842-847.

Morello, L., Frattini, M., Giani, S., Christou, P., Breviario, D., 2000. Overexpression of the calcium-dependent protein kinase OsCDPK2 in transgenic rice is repressed by light in leaves and disrupts seed development. Transgenic Res. 9 (6), 453-462. 
Mori, I.C., Murata, Y., Yang, Y., Munemasa, S., Wang, Y.-F., Andreoli, S., et al., 2006. CDPKs CPK6 and CPK3 function in ABA regulation of guard cell S-type anion- and $\mathrm{Ca}(2+)$-permeable channels and stomatal closure. PLoS. Biol. 4, e327.

Morillo, S.A., Tax, F.E., 2006. Functional analysis of receptor-like kinases in monocots and dicots. Current Opin. Plant Biol. 9, 460-469.

Moustafa, K., AbuQamar, S., Jarrar, M., Al-Rajab, A.J., TrémouillauxGuiller, J., 2014. MAPK cascades and major abiotic stresses. Plant Cell Rep. 33, 1217-1225.

Nagae, M., Nozawa, A., Koizumi, N., Sano, H., Hashimoto, H., Sato, M., et al., 2003. The crystal structure of the novel calcium-binding protein AtCBL2 from Arabidopsis thaliana. J. Biol. Chem. 278, $42240-42246$.

Nakagami, H., Kiegerl, S., Hirt, H., 2004. OMTK1, a novel MAPKKK, channels oxidative stress signaling through direct MAPK interaction. J. Biol. Chem. 279 (26), 26959-26966.

Nakashima, K., Ito, Y., Yamaguchi-Shinozaki, K., 2009. Transcriptional regulatory networks in response to abiotic stresses in Arabidopsis and grasses. Plant Physiol. 149 (1), 88-95.

Ning, J., Li, X., Hicks, L.M., Xiong, L., 2010. A Raf-like MAPKKK gene DSM1 mediates drought resistance through reactive oxygen species scavenging in rice. Plant Physiol. 152 (2), 876-890.

Nishimura, N., Yoshida, T., Kitahata, N., Asami, T., Shinozaki, K., Hirayama, T., 2007. ABA Hypersensitive Germination1 encodes a protein phosphatase $2 \mathrm{C}$, an essential component of abscisic acid signaling in Arabidopsis seed. Plant J. 50 (6), 935-949.

Nongpiur, R., Soni, P., Karan, R., Singla-Pareek, S.L., Pareek, A., 2012. Histidine kinases in plants: cross talk between hormone and stress responses. Plant Signal. Behav. 7 (10), 1230-1237.

Ohta, M., Guo, Y., Halfter, U., Zhu, J.K., 2003. A novel domain in the protein kinase SOS2 mediates interaction with the protein phosphatase 2C ABI2. Proc. Natl. Acad. Sci. 100, 11771-11776.

Opdenakker, K., Remans, T., Vangronsveld, J., Cuypers, A., 2012. Mitogen-activated protein (MAP) kinases in plant metal stress: regulation and responses in comparison to other biotic and abiotic stresses. Int. J. Mol. Sci. 13 (6), 7828-7853.

Osakabe, K., Yamaguchi-Shinozaki, K., Shinozaki, K., Tran, L.S., 2013. Sensing the environment: key roles of membrane-localized kinases in plant perception and response to abiotic stress. J. Exp. Bot. 64, 445-658.

Ouyang, S.Q., Liu, Y.F., Liu, P., Lei, G., He, S.J., Ma, B., et al., 2010. Receptor like kinase OsSIK1 improves drought and salt stress tolerance in rice (Oryza sativa) plants. Plant J. 62 (2), 316-329.

Pandey, G.K., Kanwar, P., Singh, A., Steinhorst, L., Pandey, A., Yadav, A.K., et al., 2015. Calcineurin B-Like Protein-Interacting Protein Kinase CIPK21 Regulates Osmotic and Salt Stress Responses in Arabidopsis. Plant Physiol. 169, 780-792.

Pareek, A., Singh, A., Kumar, M., Kushwaha, H.R., Lynn, A.M., Singla-Pareek, S.L., 2006. Whole-genome analysis of Oryza sativa reveals similar architecture of two-component signalling machinery with Arabidopsis. Plant physiol. 142 (2), 380-397.

Patishtan, J., Hartley, T.N., Fonseca de Carvalho, R., Maathuis, F.J., 2017. Genome-wide association studies to identify rice salt-tolerance markers. Plant Cell Environ. 41, 970-982.

Piao, H., Xuan, Y., Park, S.H., Je, B.L., Park, S.J., Park, S.H., et al., 2010. OsCIPK31, a CBL-interacting protein kinase is involved in germination and seedling growth under abiotic stress conditions in rice plants. Mol. Cells 30, 19-27.

Pitorre, D., Llauro, C., Jobet, E., Guilleminot, J., Brizard, J.P., Delseny, M., et al., 2010. RLK7, a leucine-rich repeat receptor-like kinase, is required for proper germination speed and tolerance to oxidative stress in Arabidopsis thaliana. Planta 232 (6), 1339-1353.

Qiu, Q.S., Guo, Y., Dietrich, M.A., Schumaker, K.S., Zhu, J.K., 2002. Regulation of SOS1, a plasma membrane $\mathrm{Na}+/ \mathrm{H}+$ exchanger in Arabidopsis thaliana, by SOS2 and SOS3. Proc. Natl. Acad. Sci. USA 99, 8436-8441.

Quintero, F.J., Martinez-Atienza, J., Villalta, I., Jiang, X., Kim, W.Y., Ali, Z., et al., 2011. Activation of the plasma membrane $\mathrm{Na} / \mathrm{H}$ antiporter SaltOverly-Sensitive 1 (SOS1) by phosphorylation of an auto-inhibitory Cterminal domain. Proc. Natl. Acad. Sci. USA 108 (6), 2611-2616.

Rao, K.P., Vani, G., Kumar, K., Wankhede, D.P., Misra, M., Gupta, M., et al., 2011. Arsenic stress activates MAP kinase in rice roots and leaves. Arch. Biochem. Biophys. 506, 73-82.

Ray, S., Agarwal, P., Arora, R., Kapoor, S., Tyagi, A.K., 2007. Expression analysis of calcium-dependent protein kinase gene family during reproductive development and abiotic stress conditions in rice (Oryza sativa L. ssp. indica). Mol. Genet. Genomics 278, 493-505.

Rodriguez Milla, M.A., Uno, Y., Chang, I.F., Townsend, J., Maher, E. A., Quilici, D., et al., 2006. A novel yeast two-hybrid approach to identify CDPK substrates: characterization of the interaction between AtCPK11 and AtDi19, a nuclear zinc- finger protein. FEBS Lett. 580, 904-911.

Roy, S.J., Tucker, E.J., Tester, M., 2011. Genetic analysis of abiotic stress tolerance in crops. Curr. Opi. Plant Biol. 14 (3), 232-239.

Rudrabhatla, P., Reddy, M.M., Rajasekharan, R., 2006. Genome-wide analysis and experimentation of plant serine/ threonine/ tyrosine-specific protein kinases. Plant Mol. Biol. 60, 293-319.

Saez, A., Apostolova, N., Guzman, M., Garcia, M.P., Nicolas, C., Lorenzo, O., et al., 2004. Gain of function and loss of function phenotypes of the protein phosphatase $2 \mathrm{C}$ HAB1 reveal its role as a negative regulator of abscisic acid signalling. Plant J. 37 (3), 354-369.

Shiu, S.-H., Karlowski, W.M., Pan, R., Tzeng, Y.-H., Mayer, K.F.X., Li, W.H., 2004. Comparative analysis of the receptor-like kinase family in Arabidopsis and rice. Plant Cell. 16, 1220-1234.

Shou, H., Bordallo, P., Wang, K., 2004. Expression of the Nicotiana protein kinase (NPK1) enhanced drought tolerance in transgenic maize. J. Exp. Bot. 55 (399), 1013-1019.

Singh, A., Sagar, S., Biswas, D.K., 2018. Calcium dependent protein kinase, a versatile player in plant stress management and development. CRC Crit. Rev. Plant Sci. 2689, 1-17.

Sinha, A.K., Jaggi, M., Raghuram, B., Tuteja, N., 2011. Mitogenactivated protein kinase signaling in plants under abiotic stress. Plant Signa. Behav. 6, 196-203.

Sivaguru, M., Ezaki, B., He, Z.H., Tong, H., Osawa, H., Baluska, F., et al., 2003. Aluminum-induced gene expression and protein localization of a cell wall-associated receptor kinase in Arabidopsis. Plant Physiol. 132 (4), 2256-2266.

Sun, X., Xu, L., Wang, Y., Luo, X., Zhu, X., Kinuthia, K.B., et al., 2016. Transcriptome-based gene expression profiling identifies differentially expressed genes critical for salt stress response in radish (Raphanus sativus L.). Plant Cell Rep. 35 (2), 329-346.

Tena, G., Asai, T., Chiu, W.L., Sheen, J., 2001. Plant mitogenactivated protein kinase signaling cascades. Curr. Opi. Plant Biol. 4 (5), 392-400.

Tran, L.S.P., Urao, T., Qin, F., Maruyama, K., Kakimoto, T., Shinozaki, K., et al., 2007. Functional analysis of AHK1/ATHK1 and cytokinin receptor histidine kinases in response to abscisic acid, drought, and salt stress in Arabidopsis. Proc. Natl. Acad. Sci. 104 (51), 20623-20628.

Umezawa, T., Nakashima, K., Miyakawa, T., Kuromori, T., Tanokura, M., Shinozaki, K., et al., 2010. Molecular basis of the core regulatory network in ABA responses: sensing, signaling and transport. Plant Cell Physiol. 51 (11), 1821-1839.

Umezawa, T., Sugiyama, N., Mizoguchi, M., Hayashi, S., Myouga, F., Yamaguchi-Shinozaki, K., et al., 2009. Type 2C protein phosphatases directly regulate abscisic acid-activated protein kinases in Arabidopsis. Proc. Natl. Acad. Sci. USA 106 (41), 17588-17593. 
Umezawa, T., Sugiyama, N., Takahashi, F., Anderson, J.C., Ishihama, Y., Peck, S.C., et al., 2013. Genetics and phosphoproteomics reveal a protein phosphorylation network in the abscisic acid signaling pathway in Arabidopsis thaliana. Sci. Signal 6 (270), 8-8.

Umezawa, T., Yoshida, R., Maruyama, K., Yamaguchi-Shinozaki, K., Shinozaki, K., 2004. SRK2C, a SNF1-related protein kinase 2, improves drought tolerance by controlling stress-responsive gene expression in Arabidopsis thaliana. Proc. Natl. Acad. Sci. USA 101 (49), 17306-17311.

Vaid, N., Pandey, P., Srivastava, V.K., Tuteja, N., 2015. Pea lectin receptor-like kinase functions in salinity adaptation without yield penalty, by alleviating osmotic and ionic stresses and upregulating stress-responsive genes. Plant Mol. Biol. 88 (1-2), 193-206.

Valmonte, G.R., Arthur, K., Higgins, C.M., MacDiarmid, R.M., 2014. Calcium-dependent protein kinases in plants: evolution, expression and function. Plant Cell Physiol. 55, 551-569.

Walker, J.C., Zhang, R., 1990. Relationship of a putative receptor protein kinase from maize to the S-locus glycoproteins of Brassica. Nature 345 (6277), 743.

Wan, B., Lin, Y., Mou, T., 2007. Expression of rice Ca (2+)-dependent protein kinases (CDPKs) genes under different environmental stresses. FEBS Lett. 581, 1179-1189.

Wang, B., Guo, B., Xie, X., Yao, Y., Peng, H., Xie, C., et al., 2012. A novel histidine kinase gene, $\mathrm{ZmHK}$, mediate drought tolerance through the regulation of stomatal development in Arabidopsis. Gene 501 (2), 171-179.

Wang, J.P., Xu, Y.P., Munyampundu, J.P., Liu, T.Y., Cai, X.Z., $2016 a$. Calcium-dependent protein kinase (CDPK) and CDPK-related kinase (CRK) gene families in tomato: genome-wide identification and functional analyses in disease resistance. Mol. Genet. Genom. 291, 661-676.

Wang, L., Yu, C., Xu, S., Zhu, Y., Huang, W., 2016b. OsDi19-4 acts downstream of OsCDPK14 to positively regulate ABA response in rice. Plant Cell Environ. 39, 2740-2753.

Wang, L., Hu, W., Tie, W., Ding, Z., Ding, X., Liu, Y., et al., 2017. The MAPKKK and MAPKK gene families in banana: identification, phylogeny and expression during development, ripening and abiotic stress. Sci. Rep. 7 (1), 1159.

Wang, M., Gu, D., Liu, T., Wang, Z., Guo, X., Hou, W., et al., 2007. Overexpression of a putative maize calcineurin B-like protein in Arabidopsis confers salt tolerance. Plant Mol. Biol. 65, 733-746.

Wang, Y., Liang, S., Xie, Q.G., Lu, Y.T., 2004. Characterization of a calmodulin- regulated $\mathrm{Ca} 2+-$ dependent-protein-kinase related protein kinase, AtCRK1, from Arabidopsis. Biochem. J. 383, 73-81.

Wang, L., Liu, Y., Cai, G., Jiang, S., Pan, J., Li, D., 2014. Ectopic expression of ZmSIMK1 leads to improved drought tolerance and activation of systematic acquired resistance in transgenic tobacco. J. Biotechnol. 172, 18-29.

Wankhede, D.P., Kumar, K., Singh, P., Sinha, A.K., 2013. Involvement of mitogen activated protein kinase kinase 6 in UV induced transcripts accumulation of genes in phytoalexin biosynthesis in rice. Rice 6 (1), 35.

Watillon, B., Kettmann, R., Boxus, P., Burny, A., 1993. A calcium/ calmodulin-binding serine/threonine protein kinase homologous to the mammalian type II calcium/calmodulin-dependent protein kinase is expressed in plant cells. Plant Physiol. 101, 1381-1384.

Wei, K., Wang, Y., Zhong, X., Pan, S., 2014a. Protein kinase structure, expression and regulation in maize drought signalling. Mol. Breed. 34 (2), 583-602.

Wei, S., Hu, W., Deng, X., Zhang, Y., Liu, X., Zhao, X., et al., 2014b. A rice calcium-dependent protein kinase OsCPK9 positively regulates drought stress tolerance and spikelet fertility. BMC Plant Biol. 14, 133 .
Wu, F., Sheng, P., Tan, J., Chen, X., Lu, G., Ma, W., et al., 2015. Plasma membrane receptor-like kinase leaf panicle 2 acts downstream of the drought and salt tolerance transcription factor to regulate drought sensitivity in rice. J. Exp. Bot. 66 (1), 271-281.

Xi, Y., Liu, J., Dong, C., Cheng, Z.M.M., 2017. The CBL and CIPK Gene Family in Grapevine (Vitis vinifera): genome-wide analysis and expression profiles in response to various abiotic stresses. Front. Plant Sci. 8, 978-980.

Xu, J., Li, Y., Wang, Y., Liu, H., Lei, L., Yang, H., et al., 2008. Activation of MAPK kinase 9 induces ethylene and camalexin biosynthesis and enhances sensitivity to salt stress in Arabidopsis. J. Bio. Chem. 283 (40), 26996-27006.

Yang, L., Wu, K.C., Gao, P., Liu, X.J., Li, G.P., Wu, Z.J., 2014. GsLRPK, a novel cold-activated leucine-rich repeat receptor-like protein kinase from Glycine soja, is a positive regulator to cold stress tolerance. Plant Sci. 215, 19-28.

Yang, T., Poovaiah, B.W., 2003. Calcium/calmodulin-mediated signal network in plants. Trends Plant Sci. 8, 505-512.

Yoshida, R., Hobo, T., Ichimura, K., Mizoguchi, T., Takahashi, F., Aronso, J., et al., 2002. ABA-activated SnRK2 protein kinase is required for dehydration stress signaling in Arabidopsis. Plant Cell Physiol. 43 (12), 1473-1483.

Zhang, D., Jiang, S., Pan, J., Kong, X., Zhou, Y., Liu, Y., et al., 2014. The overexpression of a maize mitogen-activated protein kinase gene (ZmMPK5) confers salt stress tolerance and induces defence responses in tobacco. Plant Biol. 16, 558-570.

Zhang, H., Mao, X., Wang, C., Jing, R., 2010. Overexpression of a common wheat gene TaSnRK2. 8 enhances tolerance to drought, salt and low temperature in Arabidopsis. PLoS One 5 (12), e16041.

Zhang, P., Zhang, Z., Wang, J., Cong, B., Chen, K., Liu, S., 2015. A novel receptor-like kinase (PnRLK-1) from the Antarctic Moss Pohlia nutans enhances salt and oxidative stress tolerance. Plant Mol. Biol. Report 33 (4), 1156-1170.

Zhu, S.Y., Yu, X.C., Wang, X.J., Zhao, R., Li, Y., Fan, R.C., et al., 2007. Two calcium dependent protein kinases, CPK4 and CPK11, regulate abscisic acid signal transduction in Arabidopsis. Plant Cell 19, 3019-3036.

Zimmermann, P., Bleuler, S., Laule, O., Martin, F., Ivanov, N., Campanoni, P., 2014. Expression Data-A public resource of high quality curated datasets representing gene expression across anatomy, development and experimental conditions. Bio. Data Min. 7, 18.

Zou, J.J., Li, X.D., Ratnasekera, D., Wang, C., Liu, W.X., Song, L.F., et al., 2015. Arabidopsis calcium-dependent protein kinase 8 and catalase 3 function in abscisic acid-mediated signaling and $\mathrm{H}_{2} \mathrm{O}_{2}$ homeostasis in stomatal guard cells under drought stress. Plant Cell 27, 1445-1460.

\section{Further Reading}

Botella, J.R., Arteca, J.M., Somodevilla, M., Arteca, R.N., 1996. Calcium- dependent protein kinase gene expression in response to physical and chemical stimuli in mungbean (Vigna radiata). Plant Mol. Biol. 30, 1129-1137.

Hwang, Y.S., Bethke, P.C., Cheong, Y.H., Chang, H.S., Zhu, T., Jones, R.L., 2005. A gibberellin-regulated calcineurin B in rice localizes to the tonoplast and is implicated in vacuole function. Plant Physiol. 138, 1347-1358.

Kudla, J., Xu, Q., Harter, K., Gruissem, W., Luan, S., 1999. Genes for calcineurin B-like proteins in Arabidopsis are differentially regulated by stress signals. Proc. Natl. Acad. Sci. 96 (8), 4718-4723.

Liu, G., Chen, J., Wang, X., 2006. VfCPK1, a gene encoding calciumdependent protein kinase from Vicia faba, is induced by drought and abscisic acid. Plant Cell Environ. 29, 2091-2099.

Rudd, J.J., Franklin-Tong, V.E., 2001. Unravelling response, specificity in $\mathrm{Ca}^{2+}$ signaling pathways in plant cells. New Phytol. 151, 7-33. 
This page intentionally left blank 


\title{
35
}

\section{Nanoparticles and Abiotic Stress Tolerance in Plants: Synthesis, Action, and Signaling Mechanisms}

\author{
Syed Uzma Jalil ${ }^{1}$ and Mohammad Israil Ansari ${ }^{2}$
}

${ }^{1}$ Amity Institute of Biotechnology, Amity University Uttar Pradesh, Lucknow Campus, Lucknow, Uttar Pradesh, India

${ }^{2}$ Department of Botany, University of Lucknow, Lucknow, Uttar Pradesh, India

\section{O U T L I N E}

\subsection{Introduction}

35.2 Plant and Abiotic Stress

35.3 Mode of Action of Nanomaterials Under Abiotic Stresses

35.3.1 Drought Stress

35.3.2 Salinity Stress

35.3.3 Chilling Stress

35.3.4 Heat Stress
549

551

552

552

553

554

554

\subsection{INTRODUCTION}

Abiotic stresses are the main constraints that adversely affect the crop productivity and plant growth. Realizing the increased incidences of diverse abiotic stresses due to both natural as well as anthropogenic activities, the scientific community has a major concern to mitigate their effect to increase the yield potential of crops. According to FAO reports, there is a major challenge among the scientific community to increase world food crop production by $70 \%$ (FAO, 2009). Therefore, in such changing environmental scenario there is a constant need to identify a new area of research to overcome the technological challenges in addressing the yield barrier, resource use efficiency, and development of environmentally accepted technology. In recent years, nanobiotechnology is gaining momentum to occupy the promising position of mitigating the constraints associated with abiotic and biotic stresses to obtain a sustainable and secure future of agriculture worldwide. Plants being sessile organisms are constantly exposed to environmental variations and multiple stress factors alone or in combination throughout their life. However, plants develop various 
mechanisms to respond to adverse conditions but their responses may vary considerably even in the same plant species. Therefore, identification of tolerant plant material or augmentation of stress tolerance in plants is always the prime concern in terms of sustainable agriculture and crop production. Nanotechnology, a novel approach that allows innovative study in various extents, and nanotechnological findings could open up new way in the field of biotechnology and agriculture (Torney et al., 2007; Perez-de-Luque and Diego, 2009).

Nanoparticles are small molecules of $1-100 \mathrm{~nm}$ dimensions (Roco, 2003) including very small size, nanoparticles may also acquire some other physiochemical properties, that is, improved reactivity, large surface area, malleable pore size, as well as diverse morphology (Nel et al., 2006). Nanoparticles retain a high surface dynamism and large surface to volume ratio that improves their reactivity as well as biochemical activity; these characteristics of nanoparticles may show diverse activities and impact (Dubchak et al., 2010). In the present circumstance, nanoparticles have potential to boost plant growth and development, used as herbicides, nanopesticide, and nanofertilizers, etc. that can proficiently release their content in required amounts to target cellular organelles in plants. There is an extensive scope of nanotechnology in the agriculture sector and the potential uses of nanoparticles are still unknown, particularly their role and mechanism in plant growth and development (Manzer et al., 2015). Application of fertilizers in agriculture is a common practice to increase the productivity and maintain growing food demand. Since fertilizers play a vital role in crop growth, development, and production, they must be applied in bulk quantity and most remains unutilized by plants, because of several inherent aspects (Singh et al., 2015). Therefore, there is a constant need to develop novel approaches with the support of nanotechnology and nanoparticles that not only increase the crop production and yield, but also minimize the nutrient losses of fertilizers and augment their effective availability to plants. Development of nanofertilizers or nanoencapsulated nutrients could be an effective tool in this direction towards sustainable production substantially by effective release of nutrients and availability. Application of nanofertilizers may provide a suitable alternative to increase resource use efficiency and help to reduce soil toxicity created due to accumulation of chemical fertilizers and pesticides in the soil. Most of the chemical fertilizer applied in the field remains unutilized by plants and gets accumulated in the soil leading to increased soil toxicity; therefore application of nanofertilizers could help to reduce such problems (DeRosa et al., 2010; Nair et al., 2010).

Plants cannot move from their growing place so they cannot escape from environmental stress conditions, that is, salinity, drought, chilling, heat, heavy metals, waterlogging, UV radiation, etc. These stresses produce reactive oxygen species (ROS) in plants and cause oxidative burst. Extreme generation of ROS degrades macromolecules and membrane lipids (Foyer and Noctor, 2000), prompts toxicity in cells (Shen et al., 2010; Yadav et al., 2014), as well as conquers growth of plant (Begum and Fugetsu, 2012; Khan et al., 2012a,b, 2013, 2014a,b, 2015, 2016). Plants have an antioxidant system to mitigate oxidative stress by scavenging ROS (Khan and Khan, 2014, 2017). While, plants counteract osmotic stress by increasing the production of trehalose and polyols, that is, inositol, sorbitol, glycerol, etc., as well as amino acids (betaine, glycine, proline, and taurine) that sustain the required osmotic level in the plant's cells. During hypoxia, roots are not able to provide required oxygen to the plants, which results in depletion of energy as well as adapts the plants with low vigor, though, to retain vigor level plants modify their metabolic rate and shift over from starch metabolism to fermentation (Banti et al., 2013). On responding to heavy metal stress, plants accumulate polyphosphates, metal-chelates, and organic acids that results in limiting as well as requisitioning of toxic metals in the plasma membrane. Furthermore, nanoparticles play an important role in the growth and development of plants, and are also involved in the protection of plants against different abiotic stress conditions (Khan et al., 2017). The nanoparticles imitate the activities of antioxidative enzymes and scavenge these ROS (Rico et al., 2013a,b; Wei and Wang, 2013). Small size and large surface area of nanoparticles are available to toxic metals for binding, thus condensed the accessibility and toxicity of heavy metals (Worms et al., 2012). Photosynthesis is an important process of plants; however, during abiotic stress conditions, nanoparticles improve photosynthesis rate by conquering oxidative and osmotic stress and defending the photosynthetic system (Fig. 35.1) (Haghighi and Pessarakli, 2013; Qi et al., 2013; Siddiqui et al., 2014). Therefore, the response of plants to nanoparticles varies from plant species and type or concentration of nanoparticles (Lin and Xing, 2007). Apart from their beneficial effects several nanoparticles show toxicity symptoms (Slomberg and Schoenfisch, 2012; Begum and Fugetsu, 2012). Exposure of some nanoparticles prompts oxidative stress and causes decline in germination rate, root and shoot length, loss of photosystem and crop yields (Barhoumi et al., 2015; Da Costa and Sharma, 2016; Wang et al., 2016), and nutritive value of crop plants (Peralta-Videa et al., 2014). The nanoparticles also alter expression of genes involved cell biosynthesis, cell organization, electron transport, and energy pathways in biotic and abiotic stress responses (Landa et al., 2012; Kaveh et al., 2013; 


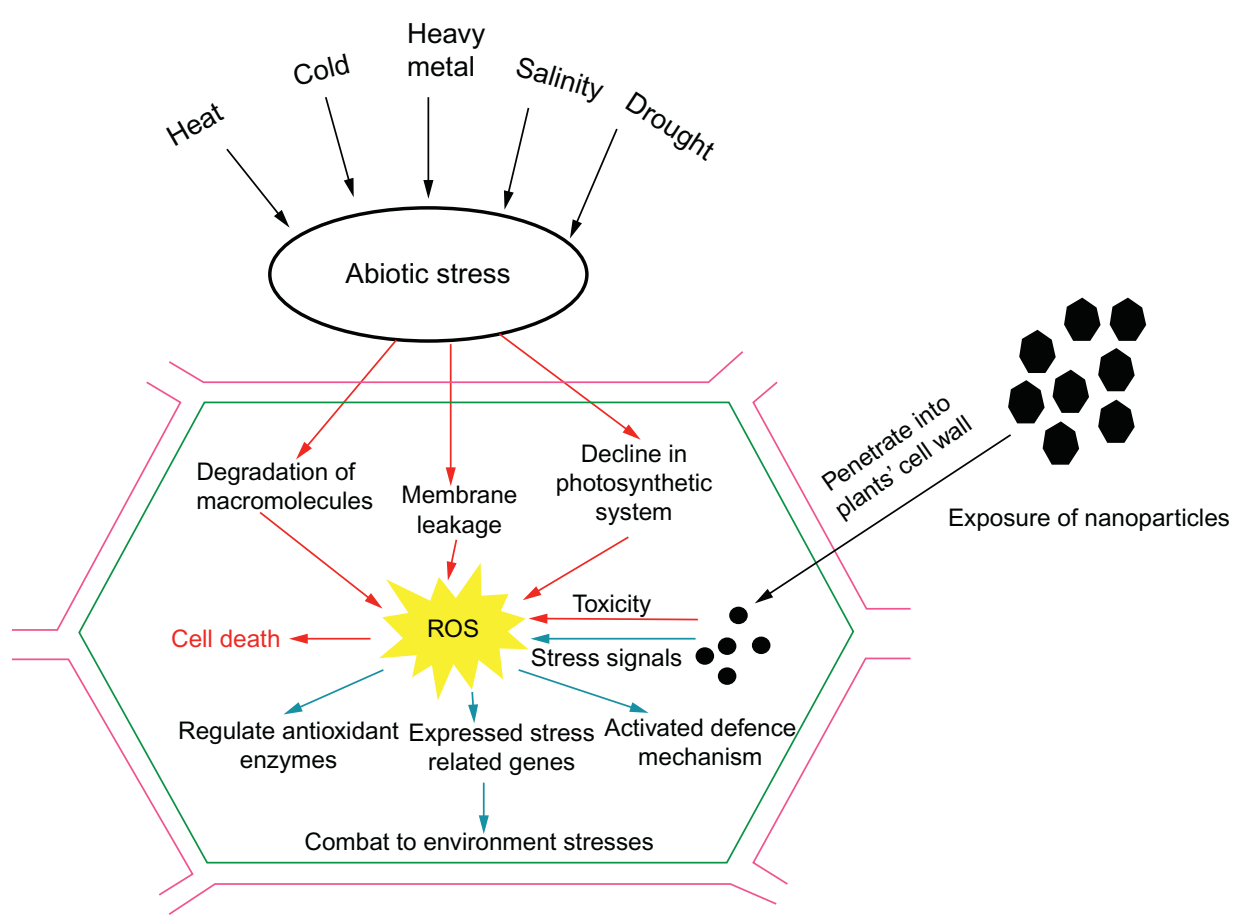

FIGURE 35.1 Mechanism of nanoparticles under abiotic stress condition in plant cell. The red arrows show the effect of abiotic stress and toxic effect of nanoparticles that leads to cell death. Blue arrows show the positive role of nanoparticles to act as stress signals that activate the defense mechanism in plants to mitigate abiotic stresses. ROS, reactive oxygen species.
Aken, 2015). Therefore, the present chapter is focused on the potential applications of nanoparticles in the agriculture sector, exploiting peculiar properties of different nanoparticles and their impact on crop plants especially toward growth and mitigation of abiotic stress tolerance in plants to achieve sustainable agriculture.

\subsection{PLANT AND ABIOTIC STRESS}

Plants are constantly affected by various adverse environmental conditions; among them abiotic stress is the prime cause of limiting crop production worldwide, reducing more than $50 \%$ average yields for most major crop plants (Bray et al., 2000; Wang et al., 2003). The effect of abiotic stresses on the plant is influenced by its extent or strength. The plant needs optimum abiotic environmental conditions for proper growth. Any alteration from such optimal environmental conditions, which is insufficient in the physical or chemical condition, is considered as abiotic stress, and also critically impacts the growth, development, as well as productivity of plant (Bray et al., 2000). Abiotic stresses includes temperature (heat, cold, and freezing), irradiation, drought, waterlogging, heavy metals, nutrients deficiency, and salinity.

Several abiotic conditions, that is, salinity, alkalinity of soils, toxicity by heavy metals, extremely high or low temperature, etc. (Bromham et al., 2013; Khan and Khan 2013; Pasala et al., 2016) have effects on the growth and development of plants that negatively impact on the production of agroeconomic crop plants (Emamverdian et al., 2015). Diverse acidic conditions in soil adversely influence nutrients in soil, which causes nutrient deficiency in plants causing them to lose their normal physiological ability of growth and development (Rorison, 1986). Primary and prolonged salinity treatment causes toxicity in the plant cell as well as disrupts the osmotic equilibrium. The effect of ionic followed with osmotic stresses leads to disturbing the growth and development of plants (Munns and Tester, 2008). Abiotic stress conditions are the main reason for the generation of ROS. Production and elimination of ROS is in balance under normal conditions; however during abiotic stress condition equilibrium of ROS is disturbed by increasing ROS production, which causes phytotoxicity by adversely impacting the structure and function of the proteins. The production of ROS occurs in the cell organelle, that is, mitochondria, peroxisomes, and chloroplasts in plants. Oxygen radicals and hydrogen peroxide are produced in mitochondria due to the overreduction of the electron transport chain. Chloroplasts are the main source of the production of $\mathrm{O}_{2}$ and $\mathrm{H}_{2} \mathrm{O}_{2}$ (Davletova et al., 2005). These superoxides are transformed to hydrogen peroxide either impulsively or by superoxide dismutase enzyme. In the peroxisomal matrix, the oxidation of xanthine and hypoxanthine to uric acid in the presence of the enzyme xanthine oxidase generates $\mathrm{O}_{2}{ }^{-}$radicals (Halliwell and Gutteridge 2000). They damage the biomolecules, that is, proteins, lipids, carbohydrates, and DNA, which results in the death of the cells (Foyer and Noctor 2005). 
Plants acclimatize to sudden variation and adverse abiotic conditions due to their inherent metabolism (Simontacchi et al., 2015). Fluctuation in the environmental conditions might disturb the metabolic equilibrium of the plant (Foyer and Noctor, 2005), and prompts the plant to harbor metabolic and genetic processes in the cell (Apel and Hirt, 2004; Gill and Tuteja, 2010). Plants retain defense mechanisms assimilated to combat the abiotic stress conditions (Yolcu et al., 2016). These mechanisms lead reprogramming of metabolic processes in the plant system (Heil and Bostock, 2002; Swarbrick et al., 2006; Shao et al., 2008; Bolton, 2009; Massad et al., 2012) to assist biophysicochemical progressions of the abiotic stress conditions (Mickelbart et al., 2015).

\subsection{MODE OF ACTION OF NANOMATERIALS UNDER ABIOTIC STRESSES}

Plants have ability to adapt or cope with adverse environment conditions, for example, chilling, salinity, drought, heat stress, etc. Cellular and molecular responses of plants to these abiotic stresses have been studied widely (Yoshioka and Shinozaki, 2009; Hirayama and Shinozaki, 2010; Duque et al., 2013). The preliminary response of plants against abiotic stresses comprises a transitory escalation of cytoplasmic $\mathrm{Ca}^{2+}$, raised intracellular secondary messengers (inositol, polyphosphate), ROS, abscisic acid, and increase in mitogen-activated protein kinase (MAPK) pathways (Hirt, 1997; Nakagami et al., 2005; Bailey-Serres and Mittler, 2006; Alcázar-Román and Wente, 2008; Gill and Tuteja, 2010; Baxter et al., 2013). The advanced level of stress response includes regulation of proteins involved in protection from cellular damage, and regulation of the expression of stress-specific genes (Xiong et al., 2002; Mahalingam and Fedoroff, 2003). Secondary metabolites play a key role in plants to combat abiotic stress conditions by stabilizing cell structure, protection of photosystem from ROS, signal transduction, and biosynthesis of polyamines (Dixon and Paiva, 1995; Dixon et al., 2002; Edreva et al., 2008; Oh et al., 2009). During abiotic and biotic stress conditions, the plant cell wall acts as a physical barrier to stress perception and plays a vigorous role in plant adaptation (Degenhardt and Gimmler, 2000). Extracellular peroxidases are involved in the modification of the cell wall and accumulate ROS and oxidative stress when encountering stresses (Passardi et al., 2004; Daudi et al., 2012; Roet et al., 2006). Oxidative stress activates generation of ROS, accumulation of phenylpropanoid, biosynthesis of enzymes, and regulation of gene expression in plant defense response (Fig. 35.1) (Minibayeva et al., 2009; Daudi et al., 2012).
There are several studies that indicated that nanoparticles' mediated effect on plants growth and development is concentration dependent. Nanoparticles upregulate the antioxidant enzyme activities (Laware and Raskar, 2014). Laware and Raskar (2014) conducted a study to assess the impact on onion seedlings when exposed to $\mathrm{TiO}_{2}$ nanoparticles; the results suggested that $\mathrm{TiO}_{2}$ nanoparticles elevate the activity of superoxide dismutase enzyme and it further enhanced with increasing nanoparticle concentration. However, seed germination and seedling growth in onion were improved at low concentration of $\mathrm{TiO}_{2}$ nanoparticles whereas the effect was reversed (suppressed) at higher concentrations. Beside superoxide dismutase, which showed concentration dependent increase, there was significant induction of hydrolytic enzyme (amylase) and catalase as well as peroxidase enzyme activities, although enzyme activity was higher at lower concentration $(10-30 \mu \mathrm{g} / \mathrm{mL})$ of $\mathrm{TiO}_{2}$ and decreased at higher concentration (40 and $50 \mu \mathrm{g} / \mathrm{mL}$ ) (Laware and Raskar, 2014). Some studies suggested that $\mathrm{TiO}_{2}$ and $\mathrm{SiO}_{2}$ nanoparticles have shown potential to enhance seed germination as well as growth of Glycine max seeds (Lu et al., 2002).

\subsubsection{Drought Stress}

Drought is among the most frequently occurring abiotic stresses, and has significantly contributed to limit crop production in arid regions (Martínez-Vilalta and Piñol, 2002). Studies indicated that application of different fractions of $\mathrm{Si}$ nanoparticles improves the plant tolerance toward drought stress, that is, hawthorns (Crataegus sp.) showed increased drought tolerance. The physiological and biochemical responses vary in seedlings of hawthorn to different doses of $\mathrm{Si}$ nanoparticles at different level of dehydration stress from temperate to severe stress. The results suggested the positive effect on photosynthesis parameters, malondialdehyde (MDA), water content, ion leakage of membrane, leaf pigments, proline, as well as carbohydrate contents by pretreatment of $\mathrm{Si}$ nanoparticles. Perhaps the involvement of $\mathrm{Si}$ nanoparticles in maintaining critical physiological and biochemical attributes induces drought tolerance in seedlings of hawthorn under drought stress (Ashkavand et al., 2015). Application of silicon on two sorghums (Sorghum bicolor (L.) Moench) cultivars having different drought susceptibility showed improved drought tolerance irrespective of their drought susceptibility by lowering shoot to root ratio, which perhaps suggested the improved root growth and the maintenance of the photosynthetic rate. These findings could be attributed to improve the drought tolerance of sorghum via the augmenting water uptake efficiency of plants 
(Hattori et al., 2005). Silicon can be potentially used to mitigate effects of drought stress impact to some extent. A study conducted by Pei et al. (2010), suggested that exposure of low concentration $(1.0 \mathrm{mM})$ of sodium silicate could moderately alleviate the harmful effects of drought stress in wheat. Although the exact mechanism is unclear silicon partially improves shoot growth, increases the leaf chlorophyll contents, and maintains leaf water potential in stressed plants. Moreover, it also reduces membrane lipid peroxidation in wheat (Pei et al., 2010). Application of Zn could increase the radicle growth in germinated seeds and high $\mathrm{Zn}$ content in grains can increase the seed viability and establishment especially in $\mathrm{Zn}$-deficient areas (Cakmak et al., 1996; Degenhardt and Gimmler, 2000). Sedghi et al (2013) demonstrated that $\mathrm{ZnO}$ nanoparticles have the potential to increase seed germination percentage and germination rate in soybean as compared with those subjected to water stress. It was further suggested that $\mathrm{ZnO}$ nanoparticle application under drought stress reduces fresh and dry weight of seeds, which shows that $\mathrm{ZnO}$ nanoparticles were effective for using germination and growth of seedlings and improved resistance to drought stress (Sedghi et al., 2013). Iron is an important micronutrient and plays a crucial role in plant growth and development; its deficiency leads to significant changes in plant metabolism and causes chlorosis.

Therefore, iron in plants under drought stress may play a pivotal role in drought tolerance. Several studies indicated that the application of micronutrients can be used to ameliorate the effects of drought and salinity stresses. A study revealed the significant effect of iron nanoparticles under drought stress in plants on traits like number of bolls per branch, number of seeds per boll, the thousand-seed weight, and yield at probability level. Foliar application of iron nanoparticles exhibited drought stress mitigating effects on biomass and oil content of safflower cultivars. Application of Fe nanoparticles also enhances biomass at two stages of flowering and granulation, although it was better at the flowering stage than seed formation in contrast to drought stress conditions without $\mathrm{Fe}$ nanoparticle application (Davar et al., 2014). The mitigation of the adverse effect of drought stress using titanium nanoparticle foliar application on wheat has also shown promising results on certain agronomic traits such as starch and gluten. The results suggested that application of $0.02 \% \mathrm{TiO}_{2}$ nanoparticles exhibited enhancement in various agronomic traits, that is, plant height, ear weight, ear number, seed number, thousand-seed weight, final yield, biomass, harvest index including gluten, and starch content under drought stress (Jaberzadeh et al., 2013). Advances in silver nanoparticles (AgNPs) application were also appreciated to reduce negative effects of drought stress on lentil (Lens culinaris Medic). A study suggested the significant effect of different concentrations of PEG and silver nanoparticles on germination rate and germination percentage, root length, root fresh, and dry weight in lentil seeds. Moreover, application of AgNPs could be attributed to mitigating water stress, mediating loss of plant growth and yield (Hojjat, 2016).

\subsubsection{Salinity Stress}

Salinity is a major abiotic stress factor. It limits food production and deteriorates the growing demand of food crops. Salinity is a major concern in the scientific community to attain sustainable crop production. Since, the majority of major crop plant species belong to the lycophyte category, they are susceptible to salt stress; hence it is the most critical environmental stress that can cripple crop productivity (Flowers, 2004; Munns and Tester, 2008). Salinity stress causes the negative impact on various biochemical and physiological processes that are associated with plant growth and yield. Lowering of soil osmotic potential, creation of nutritional imbalance, enhancing specific ionic toxicity (salt stress), or one or more combinations of these factors are some of the common implications of salinity stress experienced by plants (Ashraf, 1994). Some other vital processes like photosynthesis, protein synthesis, lipid metabolisms, etc. are severely affected by salinity stress within a plant (Parida and Das, 2005).

Nanotechnology is recently gaining the attention of researchers because of their wide application in diverse sectors including agriculture. Application of nanofertilizers is among the most promising methods that can potentially enhance plant resource use efficiency and reduce environmental toxicity due to accumulation of unused chemical fertilizers and pesticides in the soil. Plants utilize a much lower amount of chemical fertilizers and pesticides than the amount applied to the soil, therefore the rest of the chemicals remain unused and accumulate in soil to increase soil toxicity. The application of nanofertilizers could be a potential approach to address such issues of soil toxicity and other associated stress problems. It has been reported that $\mathrm{Si}$ nanoparticles and silicon fertilizer exhibited promising effects on physiological and morphological traits on vegetative features of basil under salinity stress. This was evident from results that indicated significant increase in growth and development indices, chlorophyll content, and proline level in basil (Ocimum basilicum) under salinity stress, when treated with Si nanoparticles and silicon fertilizer. Results suggested this could be due to tolerance induction in plants thereby mitigating the effect of salinity stress in 
basil (Ocimum basilicum) (Kalteh et al., 2014). Other studies also revealed the salinity stress mitigating capability of $\mathrm{SiO}_{2}$ nanoparticles. Application of $\mathrm{SiO}_{2}$ nanoparticles have shown potential increase in chlorophyll content, leaf fresh weight, leaf dry weight, proline accumulation, and upregulation of antioxidant enzyme activity under salinity stress. Such increases may be corroborated to enhancing the abiotic stress tolerance in plants (Haghighi et al., 2012; Kalteh et al., 2014). Application of Si nanoparticles on lentil (Lens culinaris Medik.) genotypes under salinity stress revealed significant increase in germination of seed and growth of seedling, whereas there was significant reduction in germination percent and seedling growth due to the salinity stress under without treatment of nanoparticles. Adding $\mathrm{SiO}_{2}$ nanoparticles not only enhances seed germination and early seedling growth, but also increases other related traits in lentil genotypes under salinity stress. Therefore, $\mathrm{SiO}_{2}$ nanoparticles ameliorate different defense mechanisms of plants against salt toxicity (Sabaghnia and Janmohammad, 2015). Stress mitigation effects of Si nanoparticle were also studied in tomato seeds and seedlings under salt stress. The results suggested the reduced salt toxicity impact on seed germination, and root length and plant dry weight in basil (Ocimum basilicum), exposed under salinity stress (Haghighi et al., 2012). Salinity stress reduces the crop growth and yield because of the $\mathrm{Na}^{+}$ion toxicity and nanoparticles $\left(\mathrm{SiO}_{2}\right)$ have been suggested to decrease the ionic toxicity leading to enhance crop growth and yield, thus helping crop improvement under adverse conditions (Savvasd et al., 2009). Other studies in maize suggested that fresh and dry weight of shoot and root increase under salinity stress after the application of $\mathrm{SiO}_{2}$ nanoparticles (Gao et al., 2006). One strategy by which silica nanoparticles are used to mitigate salinity stress in plants is to reduce $\mathrm{Na}^{+}$ion concentration, perhaps by reducing $\mathrm{Na}^{+}$ion absorption by plant tissues. Since the primary impact of salinity stress on plant growth is due to reduction of osmotic potential and toxicity of $\mathrm{Na}^{+}$ion, $\mathrm{Si}$ nanoparticles may help to improve plant growth under salinity stress following $\mathrm{Na}^{+}$ion toxicity (Raven, 1982). Multiwalled carbon nanotubes exposed to broccoli under salinity stress reported to induce water uptake and transportation by increasing the net assimilation of $\mathrm{CO}_{2}$ and aquaporin transduction, slightly changing the properties of salt stressed root plasma membrane to alleviate the stress and increasing growth (Martínez-Ballesta et al., 2016).

\subsubsection{Chilling Stress}

Chilling stress is produced by the very low temperatures that cause damage in the cells of the plant
(Hasanuzzaman et al., 2013). Distortion of permeability as well as ion leakage from the membrane are the discrete impacts of chilling stress, which negatively affect the plant by reducing germination and growth (Welti et al., 2002; Suzuki et al., 2008). Conversely, vulnerability to chilling stress differs among the species; the plants with greater tolerance ability express low damage of membrane as compared with sensitive plants (Maali Amiri et al., 2010; Heidarvand et al., 2011). However, $\mathrm{TiO}_{2}$ nanoparticles retain the ability to reduce the negative effect of chilling stress by decreasing the damage of plant membrane and ion leakage (Mohammadi et al., 2013). Photosynthesis is the integral mechanism of plant system that is much susceptible to chilling stress. Plants suffering from chilling stress express damage in the photosystem by reducing chlorophyll content, transpiration rate, $\mathrm{CO}_{2}$ assimilation, as well as photosystem enzyme (Rubisco) degradation (Yordanova and Popova, 2007; Liu et al., 2012). Effect of nanoparticles on the photosystem have been concluded by increasing production of Rubisco enzyme (Gao et al., 2006), light immersion ability of chloroplast (Ze et al., 2011), as well as inhibiting the production of ROS (Giraldo et al., 2014). Exposure to $\mathrm{TiO}_{2}$ nanoparticles increases the Rubisco and chlorophyll binding protein gene expression (Hasanpour et al., 2015), activities of antioxidant enzyme (Mohammadi et al., 2014), leaf pigments, and improves susceptibility to chilling stress. Plants that suffered with cold stress upregulated $\mathrm{MeCu}$ / $\mathrm{ZnSOD}$ and MeAPX2 genes and increased the monodehydroascorbate reductase, dehydroascorbate reductase, and glutathione reductase activities that scavenge ROS, which results in repressed oxidative stress, that is, lipid peroxidation, chlorophyll degradation, and $\mathrm{H}_{2} \mathrm{O}_{2}$ generation and finally ensured stress tolerance ( $\mathrm{Xu}$ et al., 2014). However, nanoparticle exposure along with chilling stress have shown increased growth and biochemical physiognomies of plants exposed to cold stress (Hawrylak-Nowak et al., 2010; Azimi et al., 2014; Haghighi et al., 2014; Kohan-Baghkheirati and GeislerLee, 2015).

\subsubsection{Heat Stress}

Heat stress involves high temperature at such severe level for a long enough time to result in irretrievable loss to development as well as growth of plants (Wahid, 2007). Heat stress increases the ROS generation and produces oxidative stress, which results in membrane lipid degeneration and membrane ion leakage, followed by degradation of protein (Moller et al., 2007; Savicka and Skute, 2010; Karuppanapandian et al., 2011) along with reduced rate of photosynthesis and chlorophyll content (Prasad et al., 2011). Application of 
Se nanoparticle in low concentration reduced the effect of heat stress by increasing hydration ability, chlorophyll content, and plant development (Haghighi et al., 2014). Low concentration of Se nanoparticles shows antioxidative properties to plants whereas high concentration of Se nanoparticles induces oxidative stress (Hartikainen et al., 2000; Hasanuzzaman et al., 2014). During heat stress, plant synthesized some heat shock proteins and molecular chaperones (Schulze et al., 2005). Heat shock proteins assist other proteins in sustaining their constancy in stress conditions (Wahid, 2007) and are involved in heat stress resistance. It has been reported that multiwall carbon nanotubes are involved in upregulating gene expression of heat shock proteins, for example, HSP90 (Khodakovskaya et al., 2011). Furthermore, exposure to $\mathrm{CeO}_{2}$ nanoparticle in maize causes excessive generation of $\mathrm{H}_{2} \mathrm{O}_{2}$ and upregulation of HSP70 (Zhao et al., 2012). Additionally, treatment with $\mathrm{TiO}_{2}$ nanoparticles reduced the effect of heat stress by stomatal opening regulation (Qi et al., 2013).

\subsubsection{Heavy-Metal Stress}

Heavy metal stress becomes a serious threat to crops worldwide by increasing toxicity and reducing growth of plants (Rahimi et al., 2012; Chibuike and Obiora, 2014). Under heavy metal stress, plants reduce their growth due to deficiency of essential nutrients by disturbance of uptake of vital supplements as well as suppression of enzyme activities (Capuana, 2011). Supplement of heavy metals increases extreme ROS production, which causes oxidative damage in the cell by altering cell structure, reduces membrane permeability, and degrades proteins (Rascio and Navari-Izzo, 2011; Sharma et al., 2012). Conversely, plants developed defense systems to combat heavy metal stress. Furthermore, plants produce metal-chelates, organic acids, and polyphosphates that limit the uptake of heavy metals and efflux of metal ions and activation of antioxidant enzymes that scavenge ROS production. However, the activation of these defense systems is important for resistance to heavy metal stress. It has been reported that synthesized nanoparticles are extremely active in lessening heavy metal-induced phytotoxicity (Gunjan et al., 2014; Tripathi et al., 2015). Nanoparticles are very small and have large surface area due to these properties; nanoparticles easily enter into plant cells and retain high affinity to the heavy metals. It has been studied that exposure of quantum dots reduces accessibility of $\mathrm{Cu}$ and $\mathrm{Pb}$ (Worms et al., 2012). However, if heavy metals cross the cell wall and the biophysical barriers and go into the plant cell, plants respond to the stress by accumulating biomolecules and nutrients, and by activating antioxidant enzymes to combat heavy metal stress. Although, exposure to $\mathrm{TiO}_{2}$ nanoparticle limits cadmium toxicity and increases photosynthesis rate as well as plant growth (Singh and Lee, 2016). In addition, hydroxyapatite nanoparticle treatment in Brassica juncea lessens cadmium toxicity ( $\mathrm{Li}$ and Huang 2014). It has been observed that the supplement of Si nanoparticle in growth media alleviates toxicity of chromium in pea (Tripathi et al., 2015). Furthermore, treatment of cowpea with gold ion stress incites the reduction of $\mathrm{Au}^{3+}$ to nontoxic gold nanoparticle by the phenolic compounds of germinating seeds (Shabnam et al., 2014).

\subsection{SIGNALING MECHANISM OF NANOPARTICLES DURING ABIOTIC STRESS CONDITIONS}

Plants have a network of defense systems; however, the exact recognition and transduction of stress to the defense system and precise sequence and stimulation of these defense systems in response to stress conditions before the stress-induced destruction are decisive for the fortification of the plant's cell. According to the existing data on nanoparticle-plant interactions during abiotic stress conditions, it was observed that production of ROS is a common response of plants to all stresses. ROS play a role as a stress signal to trigger the plant's defense system as well as aggravate cellular damage (Dat et al., 2000). Though, nanoparticles not only induce the ROS (Qi et al., 2013; Oukarroum et al., 2012; Van Hoecke et al., 2008; Ma et al., 2010; Simon et al., 2013), but also imitate antioxidant enzyme activities to scavenge ROS (Fig. 35.1) (Rico et al., 2013a,b; Wei and Wang, 2013). The dichotomy of actions ought to be deliberately broken down to the solution that nanoparticles give fortification against ROS whereas nanoparticles are also involved in the cause of oxidative stress (Fig. 35.1). This investigation might be handled by researching the part of nanoparticles in plant signaling. While, exhaustive mechanism of nanoparticles is not well known, conversely, via proteomic and genomic approaches it will be conceivable to understand the mechanism of nanoparticles in plants in abiotic stress conditions. It has been reported that treatment with $\mathrm{Ag}$ and $\mathrm{AgNO}_{3}$ nanoparticles altered the proteins involved in the regulation of redox as well as metabolism of sulfur in the roots of Eruca sativa due to its physiochemical properties (Vannini et al., 2013). Exposer of $\mathrm{Ag}$ and $\mathrm{Ag}^{+}$nanoparticles coated with polyvinylpyrrolidone (PVP) on Arabidopsis thaliana regulated the gene expression of stress related genes (Kaveh et al., 2013). MiRNAs have been involved in the regulation of biological processes in plants and animals, and in addition play significant roles in plant 
responses to abiotic and biotic stresses (Macovei et al., 2012; Frazier et al., 2014). Association of nanoparticles with miRNAs likewise reveals insight into the mechanism of nanoparticles under abiotic stress conditions. Exposure of $\mathrm{TiO}_{2}$ and $\mathrm{Al}_{2} \mathrm{O}_{3}$ nanoparticles on tobacco plants is involved in the upregulation of miRNA expression as well as response to metal stress, though enhanced concentration of these nanoparticles displayed wilting, reduced biomass, leaf sizes, and leaf counts, as well as decrease root growth (Frazier et al., 2014; Burklew et al., 2012). It has been studied that the exposure of zero valent iron nanoparticle on Arabidopsis upregulated the gene expression of $A H A 2$ (involved in stomatal opening process), which enhanced drought tolerance (Kim et al., 2015). Furthermore, the treatment of $\mathrm{TiO}_{2}$, multiwall carbon nanotubes, and Ag nanoparticles on Arabidopsis thaliana suppressed the expression of root-development as well as phosphate starvation genes under different stress conditions (García-Sanchez et al., 2015). In a recent study, it has been reported that exposure of $\mathrm{NiO}$ nanoparticles on barley (Hordeum vulgare) induces overproduction of ROS that favored oxidative stress as well as increased lipid peroxidation in the plant. Wherever, cotreatment with $\mathrm{SiO}_{2}$ nanoparticle on nano-NiO treated plant provoked proficient antioxidant response, reduced the levels of lipid peroxidation, and stimulated the redox pathway that mitigates the phytotoxicity of the $\mathrm{NiO}$ nanoparticles. This study revealed that $\mathrm{SiO}_{2}$ nanoparticles play a protective role in barley in response to $\mathrm{NiO}$ nanoparticle stress (Soares et al., 2018).

A signaling network activates the defense system in plants, which prompts the molecular mechanism in response to particular stress conditions. Calcium ions involved in signal transduction during various stress conditions act as a second messenger. Sensitivity of stress signals causes enlistment of calcium ions to the cytosol via calcium ion channels, which causes enhancement in calcium ion level in the cytosol, which is recognized through calcium ion-binding proteins that cause alterations in gene expression as well as plant adaptation to stress conditions (Tuteja and Mahajan, 2007; Khan et al., 2014a,b). It has been observed that nitric oxide (NO) prompts the enhancement of cytosolic calcium ions in plant cells during various biotic and abiotic stress conditions (Lamotte et al., 2006; Khan et al., 2012a,b) and therefore calcium ions persuade the synthesis of nitric oxide (Del Rio et al., 2004; Corpas et al., 2004). It has been reported that Ag nanoparticle treatment on Oryza sativa roots revealed the involvement of nanoparticle responsive proteins in regulation and signaling of calcium ions, protein degradation, cell wall synthesis, transcription, oxidative stress response pathway, cell division, and apoptosis (Mirzajani et al., 2014). In addition, it has been supposed that $\mathrm{Ag}$ nanoparticles bind with calcium ion channels or $\mathrm{Ca}^{+} / \mathrm{Na}^{+}$ion pumps via calcium ion receptors that affect cell metabolism (Goyer, 1995; Mirzajani et al., 2014). It has been also studied that that interaction of $\mathrm{C} 60$ nanocrystals instigated functional modulation of the $\mathrm{Ca}^{+}$/calmodulin-dependent protein kinase II (Miao et al., 2014). Furthermore, the application of cadmium sulfide QDs on Arabidopsis thaliana caused overexpression of calcium-binding protein CML45 as well as calcium-dependent protein kinase 23 (Marmiroli et al., 2015). These calcium-binding proteins have been revealed to control stress responses, and overexpression caused improved resistance in plants against several abiotic stress conditions (Delk et al., 2005; Xu et al., 2011; Boudsocq and Sheen, 2013).

Nanoparticles enhanced the activity of nitrate reductase enzyme in plants, which increased the concentration of nitric oxide to modulate immune response in plants (Carpenter et al., 2012; Shahrokh et al., 2014; Chandra et al., 2015). Conversely, it has been reported that nitric oxide causes nanoparticle-induced toxicity and activates the antioxidant genes expression as well as suppresses the lipid peroxidation and generation of ROS (Chen et al., 2015). Relating the mechanism of nanoparticles with calcium ions revealed that nanoparticles impersonate calcium ions and bind with calcium-binding proteins that stimulate the cascade of stress responsive genes (Mirzajani et al., 2014). Additionally, the treatment of nanoparticles enhances the expression of cell elongation, cell division, as well as stress responsive genes (Almutairi, 2016; Khodakovskaya et al., 2011, 2012). Multiwall carbon nanotubes penetrate the cell wall of the plant; considering this characteristics, carbon nanotubes can be sensed by plants as a stress stimulus like biotic stress. Therefore, the complete mechanism of signal transduction in plants induced by nanoparticles needs further study (Khodakovskaya et al., 2012). Additionally, studies on nanoparticle-induced phytotoxicity have shown enhanced generation of ROS that act as toxic compounds as well as signaling molecules in plant cells (Fig. 35.1). The different roles of ROS are precisely governed by their production as well as scavenging activity; disproportion in any of these processes will result in extreme production or diminished accessibility of ROS, which leads to oxidative stress or disruption of signaling respectively. Conversely, this symmetry is sustained by incessant production as well as scavenging of ROS. Moreover, it has been noticed that higher concentration of nanoparticles showed toxic effect, whereas its lower concentrations show beneficial or no influence on the plant's system. It concludes that lower concentration of nanoparticles sustains an active antioxidant defense system that regulates the generation of ROS 
into a precise concentration adequate for signaling but incapable of causing damage (Syu et al., 2014).

\subsection{CONCLUSION}

Nanoparticles lessen abiotic stress-induced damage by stimulating the defense mechanism of plants. The very small size of nanoparticles enables them to easily penetrate as well as control ion channels, which supports germination of seed and plant growth; furthermore, their large surface area assists high absorption as well as targeted delivery of molecules. On the other hand, nanoparticles are involved in the production of ROS and cause phytotoxicity. The elevated concentration of ROS by exposure of nanoparticles could be related to the intensification of stress signals, which trigger defense mechanisms in plants at proficient mode. Concerning the mechanism of nanoparticles, the available reports are emerging and are still too inconsistent to illustrate the complete mechanism. However, reported data illuminate that nanoparticles imitate calcium ions or a signaling substance in cytosol as recognized by calcium-binding proteins or by nanoparticle-specific proteins. Thus, initiation of the signaling substance promotes gene expression, which results in improved resistance to stress. It is concluded that the function of nanoparticles in plant systems requires additional research at the molecular and cellular level and it is greatly necessary to confirm whether nanoparticles are involved in stress promoters or stress inhibitors.

\section{References}

Aken, B.V., 2015. Gene expression changes in plants and microorganisms exposed to nanomaterials. Curr. Opin. Biotechnol. 33, 206-219.

Alcázar-Román, A., Wente, S., 2008. Inositol polyphosphates: a new frontier for regulating gene expression. Chromosoma 117, 1-13.

Almutairi, Z.M., 2016. Effect of nano-silicon application on the expression of salt tolerance genes in germinating tomato (Solanum lycopersicum L.) seedlings under salt stress. Plant Omics J. 9, 106-114.

Apel, K., Hirt, H., 2004. Reactive oxygen species: metabolism, oxidative stress, and signal transduction. Annu. Rev. Plant Biol. 55, 373-399.

Ashkavand, P., Tabari, M., Zarafshar, M., Tomášková, I., Struve, D., 2015. Effect of $\mathrm{SiO}_{2}$ nanoparticles on drought resistance in hawthorn seedlings. Leśne Prace Badawcze/Forest Research Papers Grudzień 76 (4), 350-359.

Ashraf, M., 1994. Organic substances responsible for salt tolerance in Eruca sativa. Biol. Plant 36, 255-259.

Azimi, R., Borzelabad, M.J., Feizi, H., Azimi, A., 2014. Interaction of $\mathrm{SiO}_{2}$ nanoparticles with seed prechilling on germination and early seedling growth of tall wheatgrass (Agropyron elongatum L.). Pol. J. Chem. Tech. 16, 25-29.
Bailey-Serres, J., Mittler, R., 2006. The roles of reactive oxygen species in plant cells. Plant Physiol. 141, 311.

Banti, V., Giuntoli, B., Gonzali, S., Loreti, E., Magneschi, L., Novi, G., et al., 2013. Low oxygen response mechanisms in green organisms. Int. J. Mol. Sci. 14, 4734-4761.

Barhoumi, L., Oukarroum, A., Taher, L.B., Smiri, L.S., Abdelmelek, H., Dewez, D., 2015. Effects of superparamagnetic iron oxide nanoparticles on photosynthesis and growth of the aquatic plant Lemna gibba. Arch. Environ. Contam. Toxicol. 68, 510-520.

Baxter, A., Mittler, R., Suzuki, N., 2013. Ros as key players in plant stress signalling. J. Exp. Botany 65, 1229-1240.

Begum, P., Fugetsu, B., 2012. Phytotoxicity of multi-walled carbon nanotubes on red spinach (Amaranthus tricolor L) and the role of ascorbic acid as an antioxidant. J. Hazard. Mater. 243, 212-222.

Bolton, M.V., 2009. Primary metabolism and plant defence-Fuel for the fire. Mol. Plant Microbe Interact. 22, 487-497.

Boudsocq, M., Sheen, J., 2013. CDPKs in immune and stress signalling. Trends Plant Sci. 18, 30-40.

Bray, A.B., Bailey-Serres, J., Weretilnyk, E., 2000. Responses to abiotic stress. In: Buchanan, B.B., Gruissem, W., Jones, R.L. (Eds.), Biochemistry \& Molecular Biology of Plants. American Society of Plant Physiol, Rockville, pp. 1158-1203.

Bromham, L., Saslis-Lagoudakis, C.H., Bennett, T.H., Flowers, T.J., 2013. Soil alkalinity and salt tolerance: adapting to multiple stresses. Biol. Lett. 9, 20130642.

Burklew, C.E., Ashlock, J., Winfrey, W.B., Zhang, B., 2012. Effects of aluminum oxide nanoparticles on the growth, development, and microRNA expression of tobacco (Nicotiana tabacum). PLoS One 7, 34783.

Cakmak, I., Yilmaz, A., Torun, B., Erenoglu, B., Broun, H.J., 1996. Zinc deficiency as a critical nutritional problem in wheat production in central Anatolia. Plant Soil. 180, 165-172.

Capuana, M., 2011. Heavy metals and woody plants biotechnologies for phytoremediation. J. Biogeo. Sci. For. 4, 7-15.

Carpenter, A.W., Worley, B.V., Slomberg, D.L., Schoenfisch, M.H., 2012. Dual action antimicrobials: nitric oxide release from quaternary ammonium-functionalized silica nanoparticles. Biomacromolecules 13, 3334-3342.

Chandra, S., Chakraborty, N., Dasgupta, A., Sarkar, J., Panda, K., Acharya, K., 2015. Chitosan nanoparticles: a positive modulator of innate immune responses in plants. Sci. Rep 5, 15195.

Chen, J., Liu, X., Wang, C., Yin, S.-S., Li, X.-L., Hu, W.-J., et al., 2015. Nitric oxide ameliorates zinc oxide nanoparticles-induced phytotoxicity in rice seedlings. J. Hazard. Mater. 297, 173-182.

Chibuike, G.U., Obiora, S.C., 2014. Heavy metal polluted soils: effect on plants and bioremediation methods. Appl. Environ. Soil Sci. 2014, 1-13.

Corpas, F.J., Barroso, J.B., Carreras, A., Quiros, M., Leon, A.M., Romero-Puertas, M.C., et al., 2004. Cellular and subcellular localization of endogenous nitric oxide in young and senescent pea plants. Plant Physiol. 136, 2722-2733.

Da Costa, M.V.J., Sharma, P.K., 2016. Effect of copper oxide nanoparticles on growth, morphology, photosynthesis, and antioxidant response in Oryza sativa. Photosynthetica 54, 110e119.

Dat, J., Vandenabeele, S., Vranov_a, E., Van Montagu, M., Inz_e, D., Van Breusegem, F., 2000. Dual action of the active oxygen species during plant stress responses. Cell. Mol. Life Sci. 57, 779-795.

Daudi, A., Cheng, Z., O'Brien, J.A., Mammarella, N., Khan, S., Ausubel, F.M., et al., 2012. The apoplastic oxidative burst peroxidase in Arabidopsis is a major component of pattern-triggered immunity. Plant Cell Online 24, 275-287.

Davar, F.Z., Arash, R., Amir, H., 2014. Evaluation the effect of water stress and foliar application of Fe nanoparticles on yield, yield components and oil percentage of safflower (Carthamus tinctorious L.). Int. J. Adv. Biol. Biom. Res. 2 (4), 1150-1159. 
Davletova, S., Rizhsky, L., Liang, H., Shengqiang, Z., Oliver, D.J., Coutu, J., et al., 2005. Cytosolic ascorbate peroxidase 1 is a central component of the reactive oxygen gene network of Arabidopsis. Plant Cell 17, 268-281.

Degenhardt, B., Gimmler, H., 2000. Cell wall adaptations to multiple environmental stresses in maize roots. J. Exp. Bot. 51, 595-603.

Delk, N.A., Johnson, K.A., Chowdhury, N.I., Braam, J., 2005. CML24, regulated in expression by diverse stimuli, encodes a potential $\mathrm{Ca} 2 \mathrm{p}$ sensor that functions in response to abscisic acid, day length, and ion stress. Plant Physiol. 139, 240-253.

Del Rio, L.A., Corpas, F.J., Barroso, J.B., 2004. Nitric oxide and nitric oxide synthase activity in plants. Phytochem. 65, 783-792.

DeRosa, M.C., Monreal, C., Schnitzer, M., Walsh, R., Sultan, Y., 2010. Nanotechnology in fertilizers. Nat. Nanotechnol. 5, 91.

Dixon, R.A., Paiva, N.L., 1995. Stress-induced phenylpropanoid metabolism. Plant Cell 7, 1085-1097.

Dixon, R.A., Achnine, L., Kota, P., Liu, C.J., Reddy, M., Wang, L., 2002. The phenylpropanoid pathway and plant defence-A genomics perspective. Mol. Plant Pathol. 3, 371-390

Dubchak, S., Ogar, A., Mietelski, J.W., Turnau, K., 2010. Influence of silver and titanium knanoparticles on arbuscular mycorrhiza colonization and accumulation of radiocaesium in Helianthus annuus. Span. J. Agric. Res. 8, S103-S108.

Duque, A.S., de Almeida, A.M., da Silva, A.B., da Silva, J.M., Farinha, A.P., Santos, D., et al., 2013. Abiotic stress responses in plants: unraveling the complexity of genes and networks to survive. In: Vahdati, K., Leslie, C. (Eds.), Abiotic Stress-Plant Responses and Applications in Agriculture. INTECH, Rijeka, Croatia.

Edreva, A., Velikova, V., Tsonev, T., Dagnon, S., Gürel, A., Aktaş, L., et al., 2008. Stress-protective role of secondary metabolites: diversity of functions and mechanisms. Gen. Appl. Plant Physiol. 34, $67-78$.

Emamverdian, A., Ding, Y., Mokhberdoran, F., Xie, Y., 2015. Heavy metal stress and some mechanisms of plant defence response. Sci. World J. 756120

FAO, 2009. High Level Expert Forum-How to Feed the World in 2050. Economic and Social Development, Food and Agricultural Organization of the United Nations, Rome, Italy.

Flowers, T.J., 2004. Improving crop salt tolerance. J. Exp. Bot. 55 (396), 307-319.

Foyer, C.H., Noctor, G., 2000. Oxygen processing in photosynthesis: regulation and signalling. New Phytol. 146, 359-388.

Foyer, C.H., Noctor, G., 2005. Oxidant and antioxidant signalling in plants: a re-evaluation of the concept of oxidative stress in a physiological context. Plant Cell Environ. 28, 1056-1071

Frazier, T.P., Burklew, C.E., Zhang, B., 2014. Titanium dioxide nanoparticles affect the growth and microRNA expression of tobacco (Nicotiana tabacum). Funct. Integr. Genom. 14, 75-83.

Gao, F.Q., Hong, F.S., Liu, C., Zheng, L., Su, M.Y., Wu, X., et al., 2006. Mechanism of nanoanatase $\mathrm{TiO}_{2}$ on promoting photosynthetic carbon reaction of spinach: inducing complex of RubiscoRubisco activase. Biol. Trace Elem. Res. 11, 239-254.

Gao, X., Zou, C.H., Wang, L., Zhang, F., 2006. Silicon decreases transpiration rate and conductance from stomata of maize plants. J. Plant Nutr. 29, 1637-1647

García-Sanchez, S., Bernales, I., Cristobal, S., 2015. Early response to nanoparticles in the Arabidopsis transcriptome compromises plant defence and root-hair development through salicylic acid signalling. BMC Genom. 16, 341.

Gill, S.S., Tuteja, N., 2010. Reactive oxygen species and antioxidant machinery in abiotic stress tolerance in crop plants. Plant Physiol. Biochem. 48, 909-930.

Giraldo, J.P., Landry, M.P., Faltermeier, S.M., McNicholas, T.P., Iverson, N.M., Boghossian, A.A., et al., 2014. Plant nanobionics approach to augment photosynthesis and biochemical sensing. Nat. Mater. 13

Goyer, R.A., 1995. Nutrition and metal toxicity. Am. J. Clin. Nutr. 61, 646Se650S

Gunjan, B., Zaidi, M.G.H., Sandeep, A., 2014. Impact of gold nanoparticles on physiological and biochemical characteristics of Brassica juncea. J. Plant Biochem. Physiol. 2, 133.

Haghighi, M., Afifipour, Z., Mozafarian, M., 2012. The effect of N-Si on tomato seed germination under salinity levels. Intern. J. Environ. Sci. 6, 87-90.

Haghighi, M., Pessarakli, M., 2013. Influence of silicon and nanosilicon on salinity tolerance of cherry tomatoes (Solanum lycopersicum L.) at early growth stage. Sci. Hortic. 161, 111-117.

Haghighi, M., Abolghasemi, R., Teixeira da Silva, J.A., 2014. Low and high temperature stress affect the growth characteristics of tomato in hydroponic culture with Se and nano-Se amendment. Sci. Hortic. 178, 231-240

Halliwell, B., Gutteridge, J.M.C., 2000. Free Radicals in Biology and Medicine, third ed. Oxford University Press, Oxford.

Hasanpour, H., Maali-Amiri, R., Zeinali, H., 2015. Effect of $\mathrm{TiO}_{2}$ nanoparticles on metabolic limitations to photosynthesis under cold in chickpea. Russ. J. Plant Physiol. 62, 779e787.

Hartikainen, H., Xue, T., Piironen, V., 2000. Selenium as an antioxidant and prooxidant in ryegrass. Plant Soil 225, 193e200.

Hasanuzzaman, M., Nahar, K., Fujita, M., 2013. Extreme temperature responses, oxidative stress and antioxidant defense in plants. In: Vahdati, K., Leslie, C. (Eds.), Abiotic Stress - Plant Responses and Applications in Agriculture. InTech Open Access Publisher.

Hasanuzzaman, M., Nahar, K., Fujita, M., 2014. Silicon and selenium: two vital trace elements that confer abiotic stress tolerance to plants. Emerging Technologies and Management of Crop Stress Tolerance. Elsevier, The Netherlands, pp. 377-422.

Hattori, T., Inanaga, S., Araki, H., An, P., Morita, S., Luxová, M., et al., 2005. Application of silicon enhanced drought tolerance in Sorghum bicolour. Physiol. Plant. 123, 459-466.

Hawrylak-Nowak, B., Matraszek, R., Szymanska, M., 2010. Selenium modifies the effect of short-term chilling stress on cucumber plants. Biol. Trace Elem. Res. 138, 307-315.

Heidarvand, L., Maali-Amiri, R., Naghavi, M.R., Farayedi, Y., Sadeghzadeh, B., Alizadeh, K.H., 2011. Physiological and morphological characteristics of chickpea accessions under low temperature stress. Russ. J. Plant Physiol. 58, 157-163.

Heil, M., Bostock, R.M., 2002. Induced systemic resistance (ISR) against pathogens in the context of induced plant defences. Ann. Bot. 89, 503-512.

Hirayama, T., Shinozaki, K., 2010. Research on plant abiotic stress responses in the post-genome era: past, present and future. Plant J. 61, 1041-1052.

Hirt, H., 1997. Multiple roles of map kinases in plant signal transduction. Trends Plant Sci. 2, 11-15.

Hojjat, 2016. The Effect of silver nanoparticle on lentil seed germination under drought stress. Intl. J. Farm \& Alli. Sci. 5 (3), 208-212.

Jaberzadeh, A., Payam, M., Hamid, R., Tohidi, M., Hossein, Z., 2013. Influence of bulk and nanoparticles titanium foliar application on some agronomic traits, seed gluten and starch contents of wheat subjected to water deficit stress. Not. Bot. Horti. Agrobo. 41 (1), 201-207.

Kalteh, M., Zarrin, T.A., Shahram, A., Maryam, M.A., Alireza, F.N., 2014. Effect of silica nanoparticles on Basil (Ocimum basilicum) Under Salinity. Stress. J. Chem.1 Health Risks 4 (3), 49-55.

Karuppanapandian, T., Wang, H.W., Prabakaran, N., Jeyalakshmi, K., Kwon, M., Manoharan, K., et al., 2011. 2,4-dichlorophenoxyacetic acid-induced leaf senescence in mung bean (Vigna radiata L. Wilczek) and senescence inhibition by co-treatment with silver nanoparticles. Plant Physiol. Biochem. 49, 168-217. 
Kaveh, R., Li, Y.-S., Ranjbar, S., Tehrani, R., Brueck, C.L., Aken, B.V., 2013. Changes in Arabidopsis thaliana gene expression in response to silver nanoparticles and silver ions. Environ. Sci. Technol. 47, $10637 \mathrm{e} 10644$.

Khan, M.I.R., Syeed, S., Nazar, R., Anjum, N.A., 2012a. An insight into the role of salicylic acid and jasmonic acid in salt stress tolerance. In: Khan, N.A., Nazar, R., Iqbal, N., Anjum, N.A. (Eds.), Phytohormones and Abiotic Stress Tolerance in Plants. SpringerVerlag, pp. 277-300.

Khan, M.I.R., Asgher, M., Khan, N.A., 2013. Rising temperature in the changing environment: a serious threat to plants. Clim. Change Environ. Sust. 1, 25-36.

Khan, M.I.R., Khan, N.A., 2013. Salicylic acid and jasmonates: approaches in abiotic stress tolerance. Plant Biochemistry \& Physiology 1, 4 .

Khan, M.I.R., Khan, N.A., 2014. Ethylene reverses photosynthetic inhibition by nickel and zinc in mustard through changes in PS II activity, photosynthetic-nitrogen use efficiency and antioxidant metabolism. Protoplasma 251, 1007-1019.

Khan, M.I.R., Khan, N., 2017. Reactive Oxygen Species and Antioxidant System in Plants: Role and Regulation Under Abiotic Stress. Springer Nature, New York978-981-10-5254-5.

Khan, M.I.R., Asgher, M., Khan, N.A., 2014a. Alleviation of saltinduced photosynthesis and growth inhibition by salicylic acid involves glycinebetaine and ethylene in mungbean (Vigna radiata L.). Plant Physiology and Biochemistry 80, 67-74.

Khan, M.I.R., Asgher, M., Fatma, M., Per, T.S., Khan, N.A., 2015. Drought stress vis a vis plant functions in the era of climate change. Clim. Chan. Environ. Sustain. 3, 13-25.

Khan, M.I.R., Khan, N.A., Masood, A., Per, T.S., Asgher, M., 2016. Hydrogen peroxide alleviates nickel-inhibited photosynthetic responses through increase in use-efficiency of nitrogen and sulfur, and glutathione production in mustard. Front. Plant Sci. 7, 44.

Khan, M.N., Siddiqui, M.H., Mohammad, F., Naeem, M., 2012b. Interactive role of nitric oxide and calcium chloride in the tolerance of plants to salt stress. Nitric Oxide 27, 210-218.

Khan, M.N., Mohammad, F., Mobin, M., Saqib, M.A., 2014b. Tolerance of plants to abiotic stress: a role of nitric oxide and calcium. In: Khan, M.N., Mobin, M., Mohammad, F., Corpas, F.J. (Eds.), Nitric Oxide in Plants: Metabolism and Role. Springer International Publishing Switzerland.

Khan, M.N., Mobin, M., Abbas, Z.K., AlMutairi, K.A., Siddiqui, Z.H., 2017. Role of nanomaterials in plants under challenging environments. Plant Physiol. Biochem. 110, 194-209.

Khodakovskaya, M.V., de Silva, K., Nedosekin, D.A., Dervishi, E., Biris, A.S., Shashkov, E.V., et al., 2011. Complex genetic, photothermal, and photoacoustic analysis of nanoparticle-plant interactions. Proc. Natl. Acad. Sci. U. S. A. 108, 1028-1033.

Khodakovskaya, M.V., de Silva, K., Biris, A.S., Dervishi, E., Villagarcia, H., 2012. Carbon nanotubes induce growth enhancement of tobacco cells. ACS Nano 6, 2128-2135.

Kim, J.H., Oh, Y., Yoon, H., Hwang, I., Chang, Y.-S., 2015. Iron nanoparticle-induced activation of plasma membrane Hp-ATPase promotes stomatal opening in Arabidopsis thaliana. Environ. Sci. Technol. 49, 1113-1119.

Kohan-Baghkheirati, E., Geisler-Lee, J., 2015. Gene expression, protein function and pathways of Arabidopsis thaliana responding to silver nanoparticles in comparison to silver ions, cold, salt, drought, and heat. Nanomaterials 5, 436-467.

Lamotte, O., Courtois, C., Dobrowolska, G., Besson, A., Pugin, A., Wendehenne, D., 2006. Mechanism of nitric-oxide-induced increase of free cytosolic Ca2p concentration in Nicotiana plumbaginifolia cells. Free Radic. Biol. Med. 40, 1369-1376.

Landa, P., Vankova, R., Andrlova, J., Hodekd, J., Marsik, P., Storchov, H., et al., 2012. Nanoparticle-specific changes in
Arabidopsis thaliana gene expression after exposure to $\mathrm{ZnO}, \mathrm{TiO}_{2}$, and fullerene soot. J. Hazard. Mater. 55-62. 241e242.

Laware, S.L., Raskar, S., 2014. Effect of titanium dioxide nanoparticles on hydrolytic and antioxidant enzymes during seed germination in onion. Int. J. Curr. Microbiol. App. Sci. 3 (7), 749-760.

Lin, D., Xing, B., 2007. Phytotoxicity of nanoparticles: inhibition of seed germination and root growth. Environ. Pollut. 150, 243-250.

Liu, Y.F., Qi, M.F., Li, T.L., 2012. Photosynthesis, photoinhibition, and antioxidant system in tomato leaves stressed by low night temperature and their subsequent recovery. Plant Sci. 196, 8-17.

Li, Z., Huang, J., 2014. Effects of nanoparticle hydroxyapatite on growth and antioxidant system in pakchoi (Brassica chinensis L.) from cadmium-contaminated soil. J. Nanomater. 2014, 1-7.

Lu, C.M., Zhang, C.Y., Wen, J.Q., Wu, G.R., Tao, M.X., 2002. Research of the effect of nanometer materials on germination and growth enhancement of Glycine max and its mechanism. Soybean Sci. 21, 168-172.

Maali Amiri, R., Yur'eva, N.O., Shimshilashvili, K.R., GoldenkovaPavlova, I.V., Pchelkin, V.P., Kuznitsova, E.I., et al., 2010. Expression of acyl-lipid D 12-desaturase gene in prokaryotic and eukaryotic cells and its effect on cold stress tolerance of potato. J. Integr. Plant Biol. 52, 289-297.

Macovei, A., Gill, S.S., Tuteja, N., 2012. MicroRNAs as promising tools for improving stress tolerance in rice. Plant Signal. Behav. 7, 1296-1301.

Mahalingam, R., Fedoroff, N., 2003. Stress response, cell death, and signalling: the many faces of reactive oxygen species. Physiol. Plant 119, 56-68.

Manzer, H., Siddiqui, Mohamed, H., Al-Whaibi, Firoz, M., AlKhaishany, M.Y., 2015. Role of nanoparticles in plants. Nanotechnol. Plant Sci. 19-35.

Marmiroli, M., Imperiale, D., Pagano, L., Villani, M., Zappettini, A., Marmiroli, N., 2015. The Proteomic response of Arabidopsis thaliana to cadmium sulphide quantum dots, and its correlation with the transcriptomic response. Front. Plant Sci. 6, 1104.

Martínez-Ballesta, M.C., Zapata, L., Chalbi, N., Carvajal, M., 2016. Multiwalled carbon nanotubes enter broccoli cells enhancing growth and water uptake of plants exposed to salinity. J. Nanobiotechnol. 14, 42.

Martínez-Vilalta, J., Piñol, J., 2002. Drought-induced mortality and hydraulic architecture in pine populations of the NE Iberian Peninsula. Forest Ecol. Manag. 161, 247-256.

Massad, T.J., Dyer, L.A., Vega, C.G., 2012. Cost of defence and a test of the carbon-nutrient balanceand growth-differentation balance hypotheses for two co-occurring classes of plant defence. PLoS One 7, 7554 .

Ma, X., Geiser-Lee, J., Deng, Y., Kolmakov, A., 2010. Interactions between engineered nanoparticles (ENPs) and plants: phytotoxicity, uptake and accumulation. Sci. Total Environ. 408, 3053-3061.

Miao, Y., Xu, J., Shen, Y., Chen, L., Bian, Y., Hu, Y., et al., 2014. Nanoparticle as signalling protein mimic: robust structural and functional modulation of CaMKII upon specific binding to fullerene C60 nanocrystals. ACS Nano 8, 6131-6144.

Mickelbart, M.V., Paul, M., Hasegawa, P.M., Bailey-Serres, J., 2015. Genetic mechanisms of abiotic stress tolerance that translate to crop yield stability. Nat. Rev. Genet. 16, 237-251.

Minibayeva, F., Kolesnikov, O., Chasov, A., Beckett, RP., et al., 2009. Wound-induced apoplastic peroxidase activities: their roles in the production and detoxification of reactive oxygen species. Plant Cell Environ. 32, 497-508.

Mirzajani, F., Askari, H., Hamzelou, S., Schober, Y., R€ompp, A., Ghassempour, A., et al., 2014. Proteomics study of silver nanoparticles toxicity on Oryza sativa L. Ecotoxicol. Environ. Saf. 108, $335-339$. 
Mohammadi, R., Maali-Amiri, R., Abbasi, A., 2013. Effect of $\mathrm{TiO}_{2}$ nanoparticles on chickpea response to cold stress. Biol. Trace Elem. Res. 152, 403-410.

Mohammadi, R., MaaliAmiri, R., Mantri, N., 2014. Effect of $\mathrm{TiO}_{2}$ nanoparticles on oxidative damage and antioxidant defence systems in chickpea seedlings during cold stress. Russ. J. Plant Physiol. 61, 768-775.

Moller, I.M., Jensen, P.E., Hansson, A., 2007. Oxidative modifications to cellular components in plants. Ann. Rev. Plant Biol. 58, 459-481.

Munns, R., Tester, M., 2008. Mechanisms of salinity tolerance. Annu. Rev. Plant Biol. 59, 651-681.

Nair, R., Varghese, S.H., Nair, B.G., Maekawa, T., Yoshida, Y., Kumar, D.S., 2010. Nanoparticulate material delivery to plants. Plant Sci. 179, 154-163.

Nakagami, H., Pitzschke, A., Hirt, H., 2005. Emerging map kinase pathways in plant stress signalling. Trends Plant Sci. 10, 339-346.

Nel, A., Xia, T., Madler, L., Li, N., 2006. Toxic potential of materials at the nanolevel. Science 311, 622-627.

Oh, M.-M., Trick, H.N., Rajashekar, C., 2009. Secondary metabolism and antioxidants are involved in environmental adaptation and stress tolerance in lettuce. J. Plant Physiol. 166, 180-191.

Oukarroum, A., Bras, S., Perreault, F., Popovic, R., 2012. Inhibitory effects of silver nanoparticles in two green algae, Chlorella vulgaris and Dunaliella tertiolecta. Ecotoxicol. Environ. Saf. 78, 80-85.

Pasala, R.K., Khan, M.I.R., Minhas, P.S., Farooq, M.A., Sultana, R., Per, T.S., et al., 2016. Can plant bio-regulators minimize crop productivity losses caused by drought, heat and salinity stress? An integrated review. J. Appl. Bot. Food. 89.

Passardi, F., Longet, D., Penel, C., Dunand, C., 2004. The plant peroxidase multigenic family in rice and its evolution in green plants. Phytochem. 65, 187-1893.

Parida, A.K., Das, A.B., 2005. Salt tolerance and salinity effect on plants: a review. Ecotoxicol. Environ. Saf. 60, 324-349.

Pei, Z.F., Ming, D.F., Liu, D., Wan, G.L., Geng, X.X., Gong, H.J., et al., 2010. Silicon improves the tolerance to water-deficit stress induced by polyethylene glycol in wheat (Triticum aestivum L.) seedlings. J. Plant Growth Regul. 29, 106-115.

Peralta-Videa, J.R., Hernandez-Viezcas, J.A., Zhao, L., Diaz, B.C., Ge, Y., Priester, J.H., et al., 2014. Cerium dioxide and zinc oxide nanoparticles alter the nutritional value of soil cultivated soybean plants. Plant Physiol. Biochem. 80, 128-135.

Perez-de-Luque, A., Diego, R., 2009. Nanotechnology for parasitic plant control. Pest Manag. Sci. 65, 540-545.

Prasad, P.V.V., Pisipati, S.R., Mom_cilovi_c, I., Ristic, Z., 2011. Independent and combined effects of high temperature and drought stress during grain filling on plant yield and chloroplast EF-Tu Expression in spring wheat. J. Agron. Crop Sci. 197, 430-441.

Qi, M., Liu, Y., Li, T., 2013. Nano-TiO 2 improves the photosynthesis of tomato leaves under mild heat stress. Biol. Trace Elem. Res. $156,323-328$

Rahimi, R., Mohammakhani, A., Roohi, V., Armand, N., 2012. Effects of salt stress and silicon nutrition on chlorophyll content, yield, and yield components in fennel (Foeniculum vulgare Mill.). Int. J. Agric. Crop. Sci. 4, 1591-1595.

Rascio, N., Navari-Izzo, F., 2011. Heavy metal hyper accumulating plants: how and why do they do it? and what makes them so interesting? Plant Sci. 180, 169-181.

Raven, J.A., 1982. Transport and function of silicon in plants. Biol. Rev. 58, 179-207.

Rico, C.M., Hong, J., Morales, M.I., Zhao, L., Barrios, A.C., Zhang, J. Y., et al., 2013a. Effect of cerium oxide nanoparticles on rice: a study involving the antioxidant defence system and in vivo fluorescence imaging. Environ. Sci. Technol. 47, 5635-5642.

Rico, C.M., Morales, M.I., McCreary, R., Castillo-Michel, H., Barrios, A.C., Hong, J., et al., 2013b. Cerium oxide nanoparticles modify the antioxidative stress enzyme activities and macromolecule composition in rice seedlings. Environ. Sci. Technol. 47, 14110-14118.

Roco, M.C., 2003. Broader societal issue of nanotechnology. J. Nanoparticle Res. 5, 181-189.

Rorison, I.H., 1986. The response of plants to acid soils. Experintia 42, 357-362.

Roet, M., Mathieu, Y., Barbier-Brygoo, H., Laurière, C., 2006. Characterization of active oxygen-producing proteins in response to hypo-osmolarity in tobacco and Arabidopsis cell suspensions: identification of a cell wall peroxidase. J. Exp. Bot. 57, 1323-1332.

Sabaghnia, N., Janmohammad, M., 2015. Effect of nano-silicon particles application on salinity tolerance in early growth of some lentil genotypes. Ann. UMCS, Biol. 69 (2), 39-55.

Savvasd, G., Giotes, D., Chatzieustratiou, E., Bakea, M., Patakioutad, G., 2009. Silicon supply in soilless cultivation of Zucchini alleviates stress induced by salinity and powdery mildew infection. Environ. Exp. Bot. 65, 11-17.

Savicka, M., Skute, N., 2010. Effects of high temperature on malondialdehyde content, superoxide production and growth changes in wheat seedlings (Triticum aestivum L.). Ekologija 56, 26-33.

Schulze, E.-D., Beck, E., Muller-Hohenstein, K., 2005. Plant Ecology. Springer, Berlin.

Sedghi, M., Hadi, M., Toluie, S.G., 2013. Effect of nano zinc oxide on the germination parameters of soybean seeds under drought stress. Ann. WUT-ser. Biol. 14, 73-78.

Shabnam, N., Pardha-Saradhi, P., Sharmila, P., 2014. Phenolics impart Au3b-stress tolerance to cowpea by generating nanoparticles. PLoS One 9, 85242.

Shahrokh, S., Hosseinkhani, B., Emtiazi, G., 2014. The impact of silver nanoparticles on bacterial aerobic nitrate reduction process. J. Bioprocess Biotech. 4, 152.

Shao, H.B., Chu, L.Y., Jaleel, C.A., Zhao, C.X., 2008. Water-deficit stress-Induced anatomical changes in higher plants. Crit. Rev. Biol. 331, 215-225.

Sharma, P., Jha, A.B., Dubey, R.S., Pessarakli, M., 2012. Reactive oxygen species, oxidative damage, and antioxidant defence mechanisms in plants under stressful conditions. J. Bot. 2012, 1-26.

Shen, C.X., Zhang, Q.F., Li, J., Bi, F.C., Yao, N., 2010. Induction of programmed cell death in Arabidopsis and rice by single-wall carbon nanotubes. Am. J. Bot. 97, 1-8.

Siddiqui, M.H., Al-Whaibi, Faisal, M., Alsahli, A.A., 2014. Nanosilicon dioxide mitigates the adverse effects of salt stress on Cucurbita pepo L. Environ. Toxicol. Chem. 33, 2429-2437.

Simon, D.F., Domingos, R.F., Hauser, C., Hutchins, C.M., Zerges, W., Wilkinson, K.J., 2013. Transcriptome sequencing (RNA-seq) analysis of the effects of metal nanoparticle exposure on the transcriptome of Chlamydomonas reinhardtii. Appl. Environ. Microbiol. 79, 4774-4785

Simontacchi, M., Galatro, A., Ramos-Artuso, F., Santa-Maria, G.E., 2015. Plant survival in a changing environment: the role of nitric oxide in plant responses to abiotic stress. Front. Plant Sci. 6, 977.

Singh, A., Singh, N.B., Hussain, I., Singh, H., Singh, S.C., 2015. Plantnanoparticle interaction: an approach to improve agricultural practices and plant productivity. Inter. J. Pharm. Sci. Inv. 4 (8), 25-40.

Singh, J., Lee, B.K., 2016. Influence of nano- $\mathrm{TiO}_{2}$ particles on the bioaccumulation of $\mathrm{Cd}$ in soybean plants (Glycine max): a possible mechanism for the removal of $\mathrm{Cd}$ from the contaminated soil. J. Environ. Manag. 170, 88-96.

Slomberg, D.L., Schoenfisch, M.H., 2012. Silica nanoparticle phytotoxicity to Arabidopsis thaliana. Environ. Sci. Technol. 46, 10247-10254.

Soares, C., Branco-Neves, S., Sousa, A., Azenha, M., Cunha, A., Pereira, R., et al., 2018. $\mathrm{SiO}_{2}$ nanomaterial as a tool to improve 
Hordeum vulgare L. tolerance to nano-NiO stress. Sci. Total Environ. 622-623, 517-525.

Suzuki, K., Nagasuga, K., Okada, M., 2008. The chilling injury induced by high root temperature in the leaves of rice seedlings. Plant Cell Physiol. 49, 433-442.

Syu, Y.Y., Hung, J.H., Chen, J.C., Chuang, H.W., 2014. Impact of size and shape of silver nanoparticles on Arabidopsis plant growth and gene expression. Plant Physiol. Biochem. 83, 57-64.

Swarbrick, P.J., Schulze-Lefert, P., Scholes, J.D., 2006. Metabolic consequences of susceptibility and resistance in barley leaves challenged with powdery mildew. Plant Cell Environ. 29, 1061-1076.

Torney, F., Trewyn, B.G., Lin, V.S.Y., Wang, K., 2007. Mesoporous silica nanoparticles deliver DNA and chemicals into plants. Nat. Nanotechnol. 2, 295-300.

Tripathi, D.K., Singh, V.P., Prasad, S.M., Chauhan, D.K., Dubey, N. K., 2015. Silicon nanoparticles (SiNp) alleviate chromium (VI) phytotoxicity in Pisum sativum (L.) seedlings. Plant Physiol. Biochem. 96, 189-198.

Tuteja, N., Mahajan, S., 2007. Calcium signalling network in plants. Plant Sig. Behav. 2, 79-85.

Van Hoecke, K., De Schamphelaere, K.A., Van der Meeren, P., Lucas, S., Janssen, C.R., 2008. Ecotoxicity of silica nanoparticles to the green alga Pseudokirchneriella subcapitata: importance of surface area. Environ. Toxicol. Chem. 9, 1948-1957.

Vannini, C., Domingo, G., Onelli, E., Prinsi, B., Marsoni, M., Espen, L., et al., 2013. Morphological and proteomic responses of Eruca sativa exposed to silver nanoparticles or silver nitrate. PLoS ONE 8, 68752.

Wahid, A., 2007. Physiological implications of metabolites biosynthesis in net assimilation and heat stress tolerance of sugarcane (Saccharum officinarum) sprouts. J. Plant Res. 120, 219-228.

Wang, W., Vinocur, B., Altman, A., 2003. Plant responses to drought, salinity and extreme temperatures: towards genetic engineering for stress tolerance. Planta 218, 1-14.

Wang, X., Yang, X., Chen, S., Li, Q., Wang, Hou, C., et al., 2016. Zinc oxide nanoparticles affect biomass accumulation and photosynthesis in Arabidopsis. Front. Plant Sci. 6, 1243.

Wei, H., Wang, E., 2013. Nanomaterials with enzyme-like characteristics (nanozymes): next-generation artificial enzymes. Chem. Soc. Rev. 42, 6060-6093.

Welti, R., Li, W., Li, M., Sang, Y., Biesiada, H., Zhou, H.E., et al., 2002. Profiling membrane lipids in plant stress responses: role of phospholipase $\mathrm{Da}$ in freezing induced lipid changes in Arabidopsis. J. Biol. Chem. 277, 31994-32002.

Worms, I.A.M., Boltzman, J., Garcia, M., Slaveykova, V.I., 2012. Cellwall-dependent effect of carboxyl-CdSe/ZnS quantum dots on lead and copper availability to green microalgae. Environ. Pollut. $167,27-33$.
Xiong, L., Schumaker, K.S., Zhu, J.-K., 2002. Cell signalling during cold, drought, and salt stress. Plant Cell Online 14, S165-S183.

Xu, G.Y., Rocha, P., Wang, M.L., Xu, M.L., Cui, Y.C., Li, L.Y., et al., 2011. A novel rice calmodulin-like gene, OsMSR2, enhances drought and salt tolerance and increases ABA sensitivity in Arabidopsis. Planta 234, 47-59.

Xu, J., Yang, J., Duan, X., Jiang, Y., Zhang, P., 2014. Increased expression of native cytosolic $\mathrm{Cu} / \mathrm{Zn}$ superoxide dismutase and ascorbate peroxidase improves tolerance to oxidative and chilling stresses in cassava (Manihot esculenta Crantz). BMC Plant Biol. 14, 208

Yadav, T., Mungray, A.A., Mungray, A.K., 2014. Fabricated nanoparticles: current status and potential phytotoxic threats. In: Whitacre, D.M. (Ed.), Reviews of Environmental Contamination and Toxicology. Springer International Publishing, Switzerland.

Yolcu, S., Ozdemir, F., Güler, A., Bor, M., 2016. Histone acetylation influences the transcriptional activation of POX in Beta vulgaris L. and Beta maritima L. under salt stress. Plant Physiol. Biochem. 100, 37-46.

Yordanova, R., Popova, L., 2007. Effect of exogenous treatment with salicylic acid on photosynthetic activity and antioxidant capacity of chilled wheat plants. Gen. Appl. Plant Physiol. 33, 155-170.

Yoshioka, K., Shinozaki, K., 2009. Signal Crosstalk in Plant Stress Responses. Wiley, Hoboken, Germany.

Ze, Y., Liu, C., Wang, L., Hong, M., Hong, F., 2011. The regulation of $\mathrm{TiO}_{2}$ nanoparticles on the expression of light-harvesting complex II and photosynthesis of chloroplasts of Arabidopsis thaliana. Biol. Trace Elem. Res. 143, 1131-1141.

Zhao, L., Peng, B., Hernandez-Viezcas, J.A., Rico, C., Sun, Y., PeraltaVidea, J.R., et al., 2012. Stress response and tolerance of Zea mays to $\mathrm{CeO}_{2}$ nanoparticles: cross talk among $\mathrm{H}_{2} \mathrm{O}_{2}$, heat shock protein and lipid peroxidation. ACS Nano 6, 9615-9622.

\section{Further Reading}

Corral-Diaz, B., Peralta-Videa, J.R., Alvarez-Parrilla, E., RodrigoGarcía, J., Morales, M.I., Osuna-Avila, P., et al., 2014. Cerium oxide nanoparticles alter the antioxidant capacity but do not impact tuber ionome in Raphanus sativus (L). Plant Physiol. Biochem. 84, 277-285.

Green, M., Howman, E., 2005. Semiconductor quantum dots and free radical induced DNA nicking. Chem. Commun. 1, 121-123.

Wang, S., Liang, D., Li, C., Hao, Y., Ma, F., Shu, H., 2012. Influence of drought stress on the cellular ultrastructure and antioxidant system in leaves of drought-tolerant and drought-sensitive apple rootstocks. Plant Physiol. Biochem. 51, 81-89. 
This page intentionally left blank 


\section{Index}

Note: Page numbers followed by " $f$ " and " $t$ " refer to figures and tables, respectively.

\section{A}

AA. See Amino acid (AA); Ascorbic acid (AA)

AAO. See Abscisic aldehyde oxidase (AAO)

AAO3 gene. See Aldehyde oxidase gene (AAO3 gene)

AAP. See 4-Aminoantipyrine (AAP)

ABA. See Abscisic acid (ABA)

ABA biosynthesis (aba), 214

aba3 mutants, 342-343

ABA insensitive 1-1 (ABI1-1), 335-336

ABA insensitive-1 (ABI1), 112-113, 241

ABA insensitive-2 (ABI2), 112-113

ABA insensitive-3 (ABI3), 322

ABA insensitive-5 (ABI5), 335-336

ABA receptors (ABAR), 344-345

ABA-glucosyltransferase (AOG), 343-344

ABA-insensitive (abi), 214

ABA-responsive elements (ABREs), 51, 112-113

ABA-responsive factors (ABFs), 53-54, 112-113

ABA/ABRE. See Abscisic acid/ABAresponsive element (ABA/ABRE)

ABA receptors (ABAR), 344-345

$\mathrm{ABC}$. See ATP-binding cassette (ABC)

ABCB. See ATP binding cassette type B (ABCB)

ABFs. See ABA-responsive factors (ABFs)

ABI1. See ABA insensitive-1 (ABI1)

Abiotic and biotic stress combinations

DNA damage of plants under concurrent of, 33-34

genomic and biochemical approaches for plants under, 34-36

Abiotic stress tolerance, 158-164

calcium, 278-280

improving by overexpressing glyoxalase genes, 227

mineral nutrients and stress tolerance, 270-276

phosphorous, 276-278

relating to MG in plants, 222-227, 223t

chilling tolerance, 226-227

drought tolerance, 224-225

heat tolerance, 226

heavy metal stress tolerance, 225

salt tolerance, 222-224

Abiotic stress-responsive Raf-like kinase (ARK), 45
Abiotic stresses, 20-21, 32-33, 43-44, 64, 125-126, 141-142, 183-184, 235-236, 248t, 290, 450, 459, 461f, 501, 508, $549-550$

antioxidants and signaling, 150-151

auxin signaling under, 320-325

drought stress, $320-322$

encompass auxin-mediated signaling response in plants, $321 t$

heavy metal stress, 325

nutrient deficiency stress, 323-325

salinity stress, 322

temperature stress, 322-323

conditions, 22

cross-talk signaling of jasmonate and ethylene biosynthesis, 67-68

genetic engineering approaches to modulating signaling process, $68-73$

genome editing approaches to modulating signaling processes, $74-75$

inducing Hsps-mediated signaling pathways, $504-508$

calcium and calcium-regulated proteins, 507-508

MAPKs, 506-507

ROS, 506

mode of action of nanomaterials under, 552-555

osmolytes regulation in response to, 461-471

photosynthetic machinery during, 424

and physiological changes in phenylpropanoid pathway, 184-185

plant and, 551-552

plant response in developing tolerance, 64-68

HyPRPs and abiotic stress responses, 66

LEA and abundant proteins, 65

methyglyoxyl production under abiotic stress, 66

protein kinases role in response, 66-67

regulation by plant hormones, $305-312$

responses, 66

signaling, ABA in, 347-349

mechanism of nanoparticles, 555-557

pathways during, $126 f$

trehalose as protectant against, 236-239

Abiotic stressors, 28

ABP1. See Auxin-binding protein 1 (ABP1)

ABREs. See ABA-responsive elements (ABREs)
Abscisic acid (ABA), 2, 8-9, 19-20, 25-27, $32-33,45,64-65,67-68,106$, 112-113, 133, 142-143, 195, 214, 227, $241,264-265,287-288,292,303-304$, $311,322,333-334,342,355-356,361$, 371-372, 407-408, 418-419, 419f, 438, $442-445,444 t, 524$

ABA- $8^{\prime}$ hydroxylase, 343-344

ABA-activated aquaporins, 11

ABA-dependent gene expression, 346-347

ABA-regulated abiotic stress response, 32-33

ABA-responsive transcription factors, 525

ABA-sensing mechanism of plants and downstream events, 418-419

ABA-signaling pathway, 487-488, $488 f$

ABA-dependent signaling pathways, 419-420

ABA-independent signaling pathways, 419-420

in abiotic stress signaling, 347-349

biosynthesis, 342-343, $343 f$

catabolism, 343-344

glycine betaine biosynthesis and modulation by, $422-423$

in osmolyte biosynthesis, 419-422

PP2C, 345-346

role, 305-306

signal transduction pathway in plants, $345 f$

signaling pathway, 51-53, 344

SNRK2, 346

Abscisic acid/ABA-responsive element (ABA/ABRE), 126

Abscisic aldehyde oxidase (AAO), 342-343

Absolute quantification method (AQUA method), 484

ABTS method. See 2,2'-Azino-bis (3ethylbenzthiazoline-6-sulfonic acid) diammonium salt (ABTS method)

ACC. See 1-Aminocyclopropane carboxylate (ACC)

Accumulation

accumulation stress, 423

nitrate, 175

of osmolytes, $461-471$

phenylpropanoids, 186-187

plant growth regulators, $19-20$

proline, 4, 419-420

ACD. See Alpha-crystallin domain (ACD)

Acetol monooxygenase (AMO), 221 
10-Acetyl-3,7-dihydroxyphenoxazine, 97

3'-O-Acetyl-6'-Opentafluorobenzenesulfonyl-2'-7'difluorofluorescein method (BES$\mathrm{H}_{2} \mathrm{O}_{2}$-Ac method), 97-98

$\mathrm{N}$-Acetyl-L-cysteine method (NAC method), 89

transcription factor, 106-107, 116-117, 129-130

Acetylated BESSo method (BESSo-AM method), 100

Acetylation, 150

ACO. See Aminocyclopropane-1-acid carboxylic oxidase (ACO)

Acquired thermotolerance, 504

ACS. See 1-Aminocyclopropane carboxylic acid synthase (ACS)

ACTIVATION-TAGGED bri1 SUPPRESSOR1/PACLOBUTRAZOLRESISTANCE (ATBS1/PRE), 409-410

ADAP. See ARIA-interacting double AP2 domain protein (ADAP)

$A D C$. See Arginine decarboxylase (ADC)

Adenosine 5'-phosphosulfate (APS), 288-289

Adenosine 5'-phosphosulfate reductase (APR), 293

APR1, 292

Adenosine triphosphate (ATP), 391-393

ADPglucose pyrophosphorylase (AGPase), 240

Adverse effects, 438, 462

Adverse environmental

conditions, 240

factors, 450

Adzuki bean (Vigna angularis), 343-344

Aequorin, 44

Aerobic respiration, 243

AFB proteins. See Auxin F-Box proteins (AFB proteins)

Affinity and reactive chemistry-based proteomics, 484

Agmatine iminohydrolase (AIH), 465

AGPase. See ADPglucose pyrophosphorylase (AGPase)

Agricultural production, 437

AHb1. See Arabidopsis hemoglobin 1 (AHb1)

AIFs/IBH1. See ATBS1-INTERACTING FACTORS/INCREASED LAMINA INCLINATION INTERACTING bHLH1 (AIFs/IBH1)

AIH. See Agmatine iminohydrolase (AIH)

AIP. See 2-Amino-indan-2-phosphonic acid (AIP)

Aizoaceae, 452

AKR. See Aldo-keto reductase (AKR)

AKT2/3. See Potassium transporter $2 / 3$ (AKT2/3)

Aldehyde oxidase gene (AAO3 gene), 305-306

Aldehyde reductase (ALR), 220-221

Aldo-keto reductase (AKR), 220-221

Allene oxide cyclase (AOC), 372-373, 375

ALMT. See Aluminum-activated malate transporter (ALMT)

$\alpha, \beta$-Dicarbonyl, 219-220
$\mathrm{N}$-a-acetyl-S-(1-hydroxy-2-oxo-prop-1-yl) cysteine (NASC), 89

$\alpha$-Amylases ( $\alpha \mathrm{AMY}), 264$

hydrolytic enzymes gene expression, 244

Alpha-crystallin domain (ACD), 501-502

$\alpha$-Linolenic acid, 372-373

$\alpha$-Tocopherol ( $\alpha$-toc), 109-110, 490

ALR. See Aldehyde reductase (ALR)

Alternative oxidases (AOX), 54

AOX1a, 395

Alternative pathway, 259

Aluminum (Al), 394

toxicity, 325

Aluminum-activated malate transporter (ALMT), 173

AMA. See Aminoacetone (AMA)

Amaranthaceae, 451

Amelioration of $\mathrm{NaCl}$-and metal-induced $\mathrm{K}+$ efflux under stress, 426

AMF. See Arabuscular mycorrhizal fungi (AMF); Arbuscular mycorrhizal fungi (AMF)

3-Amino,4-aminomethyl-2', , ${ }^{\prime}$ difluorescein, diacetate (DAF-FM DA method), 91

Amino acid (AA), 4, 356-357, 423, 533-534

and derivates under stress, 171-174

serine, 173

2-Amino-indan-2-phosphonic acid (AIP), 187

Aminoacetone (AMA), 221

4-Aminoantipyrine (AAP), 94

1-Aminocyclopropane carboxylate (ACC), 214, 291, 442-443

1-Aminocyclopropane carboxylic acid synthase (ACS), 113, 442-443

Aminocyclopropane-1-acid carboxylic oxidase (ACO), 113

Ammonium $\left(\mathrm{NH}_{4}{ }^{+}\right), 270-271$

AMO. See Acetol monooxygenase (AMO)

AMP-activated protein kinase (AMPK), 240, 259

Amplex UltraRed reagent method (AUR method), 97

Ampliflu Red (AR). See 10-Acetyl-3,7dihydroxyphenoxazine

Anatomy module, 521

Anhydrobiotic organisms, 428

Annexins, 488-489

Anthocyanidin

biosynthesis, 184

synthase transcriptional changes, 189

Anthocyanin, 161-162, 184, 187

Anthropogenic activities, 479-480

"Anti-ROS" enzymes, 7

Antioxidant

in abiotic stress, $150-151$

defense, 423

enzymes, 7, 106, 466

function, 188

Antisense cell wall invertase, 211

AOC. See Allene oxide cyclase (AOC)

AOG. See ABA-glucosyltransferase (AOG)

AOX. See Alternative oxidases (AOX)

AP2. See Apetala 2 (AP2)
AP2/ERF family. See APETALA2/ethylene response element-binding factors family (AP2/ERF family)

AP2/ERF transcription factor, 106-107

Apetala 2 (AP2), 115

APETALA2/ethylene response elementbinding factors family (AP2/ERF family), 115-116, 335

APK. See APS kinase (APK)

APR. See Adenosine 5'-phosphosulfate reductase (APR)

APS. See Adenosine 5'-phosphosulfate (APS)

APS kinase (APK), 288-289

APX. See Ascorbate peroxidase (APX)

AQUA method. See Absolute quantification method (AQUA method)

Aquaporin genes, 69-70

with abiotic stress tolerance, $68-70$

Aquastat, 307

Arabidopsis, 190, 260, 323-324

$A B A 2$ gene, $342-343$

ascorbate-deficient $v t c 1$ mutant, 110

bZIP63, 242

leaves T6P, 240

mechanosensitive ion channel proteins, 44

p5cdh mutant, 424-425

YUCCA6 gene, 133

Arabidopsis hemoglobin 1 (AHb1), 393

Arabidopsis overexpression of pyrroline-5carboxylate reductase gene (AtP5CR), 439-440

Arabidopsis P5CR gene (AtP5R gene), 107

Arabidopsis response regulator 2 (ARR2), 311-312

Arabidopsis revealed cellulose synthase like D6 (AtCSLD6), 45

Arabidopsis thaliana, 2-4, 22-23, 236, 258, 272-273, 293

Arabidopsis thaliana deficient for inositol-3phosphate synthase 1 (atips1), 396

Arabidopsis thaliana Drought induced F Box1 (AtDIF1), 347-348

Arabidopsis transcription activation factor 1 (ATAF1), 116-117, 242

Arabidopsis type-B response regulator 18 (ARR18), 422

Arabuscular mycorrhizal fungi (AMF), 35

Arachis hypogeae, 394-395

AhCuZnSOD gene, 109-110

Arbuscular mycorrhizal fungi (AMF), 277-278

ARE. See Auxin responsive elements (ARE)

AREB/ABF transcription factor, 106-107

ARFs. See Auxin response factors (ARFs)

Arginine, 483

Arginine decarboxylase (ADC), 34, 465

ARIA-interacting double AP2 domain protein (ADAP), 346-347

ARK. See Abiotic stress-responsive Raf-like kinase (ARK)

ARR18. See Arabidopsis type-B response regulator 18 (ARR18)

ARR2. See Arabidopsis response regulator 2 (ARR2)

Arsenic (Ar), 325, 394 
AsA. See Ascorbate (ASC)

AsA-GSH cycle. See Ascorbic acid-glutathione cycle (AsA-GSH cycle)

ASC. See Ascorbate (ASC)

Ascorbate (ASC), 109, 290

Ascorbate peroxidase (APX), 31, 54, 109, 126, $141-142,303-304,388,418,490$

Ascorbate-glutathione cycle, 170

Ascorbic acid (AA), 106, 141-142, 170, 490

in stress signaling, 170-171

Ascorbic acid-glutathione cycle (AsA-GSH cycle), 388

ASMT. See Hydroxyindole-Omethyltransferase (ASMT)

ATAF1. See Arabidopsis transcription activation factor 1 (ATAF1)

ATBS1-INTERACTING FACTORS/ INCREASED LAMINA INCLINATION INTERACTING bHLH1 (AIFs/IBH1), 409-410

ATBS1/PRE. See ACTIVATION-TAGGED bri1 SUPPRESSOR1/ PACLOBUTRAZOL-RESISTANCE (ATBS1/PRE)

AtCPK23, 539-540

AtCRK1, 541

AtCSLD6. See Arabidopsis revealed cellulose synthase like D6 (AtCSLD6)

AtDIF1. See Arabidopsis thaliana Drought induced F Box1 (AtDIF1)

AtERF15, 346-347

AtERF19, 346-347

AtGH3. 5 gene, 322

atips1. See Arabidopsis thaliana deficient for inositol-3-phosphate synthase 1 (atips1)

AtMPK3-6. See Mitogen-activated protein kinase 3 and /or 6 (AtMPK3-6)

AtMYB15 gene, 115

AtMYB21 gene, 115

AtMYB44 gene, 115

Atnoa1/rif1 mutant, 395

ATP. See Adenosine triphosphate (ATP)

ATP binding cassette type $\mathrm{B}$ (ABCB), 319

ATP-binding cassette (ABC), 51, 171

ATP-S. See ATP-sulfurylase (ATP-S)

AtP5CR. See Arabidopsis overexpression of pyrroline-5-carboxylate reductase gene (AtP5CR)

AtP5R gene. See Arabidopsis P5CR gene (AtP5R gene)

ATP-sulfurylase (ATP-S), 287-288

reductase, $272-273$

AtPXL1 gene, 537

AtWRKY18, 346-347

AtWRKY40, 346-347

AtWRKY46, 116

AtWRKY60, 346-347

AtWRKY63, 346-347

AtZEP gene, 342-343

AUR method. See Amplex UltraRed reagent method (AUR method)

Autoinhibitory N-terminal domain, 236

AUX. See Auxin (AUX)
Aux/IAAs protein. See Auxin/IAA protein (Aux/IAAs protein)

AUX1/LAX1. See Auxin Resistant 1/Like Aux1 (AUX1/LAX1)

Auxin (AUX), 67-68, 106, 111-112, 287-288, 291-292, 303-304, 311, 319-320, 322, 324-325, 355-356, 361, 440-441

crosstalk with hormones, 326

reduction, 323

role, 307

signaling in plants, 320

signaling under abiotic stresses, 320-325

transport inhibitors, 325

Auxin F-Box proteins (AFB proteins), 311, 320

Auxin Resistant 1/Like Aux1 (AUX1/LAX1), 319

Auxin response factors (ARFs), 111-112, $320,324,409-410$

ARF8, 323

Auxin responsive elements (ARE), 320

Auxin-binding protein 1 (ABP1), 320

Auxin/IAA protein (Aux/IAAs protein), 320

2,2'-Azino-bis (3-ethylbenzthiazoline-6sulfonic acid) diammonium salt (ABTS method), 93-94

\section{B}

b-glucuronidase gene (GUS gene), 66

BA 2-hydroxylase (BA2H), 356

BA2H. See BA 2-hydroxylase (BA2H)

Bacillus subtilis gene ( $\mathrm{SacB}$ ), 470

$B A D H$. See Betaine aldehyde dehydrogenase $(B A D H)$

BAK1. See BRI1 associated kinase 1 (BAK1)

Basic helix-loop-helix transcription factors (bHLH transcription factors), 490-491

Basic leucine zipper protein (bZIP), 52-53, $126,240,334-335,422,460$

transcription factor, 106-107, 130, 260

transcription family, 116

BCAAs. See Branched-chain amino acids (BCAAs)

BCNU. See 1,3-Bischloroethyl-nitrosourea (BCNU)

Bean, common (Phaseolus vulgaris), 20-21, $222,322-323$

Begonia semperflorens, 188

Benzoate method (BZ method), 101

Benzothiadiazole-S-methyl ester, 311

BES1-INTERACTING MYC-LIKE1 (BIM1), 409-410

BESSo-AM method. See Acetylated BESSo method (BESSo-AM method)

$\beta$-Cyclocitral, 170-171

$\beta$-Ionone, 170-171

Betaine aldehyde dehydrogenase $(B A D H)$, $108,422-423,453-454$

Betalains, 162

bHLH transcription factors. See Basic helix-loop-helix transcription factors (bHLH transcription factors)

BIL2. See BRZ-INSENSITIVELONG HYPOCOTYLS 2 (BIL2)
BIM1. See BES1-INTERACTING MYC-LIKE1 (BIM1)

BIN2. See BRASSINOSTEROID INSENSITIVE 2 (BIN2)

Bioactive molecules, 169-170

amino acids and derivates under stress, 171-174

ascorbic acid, carotenoids, and flavonoids in stress signaling, $170-171$

melatonin bioactive molecule in regulation of abiotic stress, $175-177$

nitrate accumulation and stress regulation, 175

poly-and oligosaccharides and plant responses, 174-175

Biomolecules, 207

Biosynthesis, 183-184, 235-236, 461-471

of glycine betaine, $453-454$

betaine derivatives, $454-455$

of proline, 452

derivatives, 452-453

of trehalose and sugar alcohols, 455-456

Biotic stress, 20-21, 183-184, 235-236, 290, 479-480. See also Abiotic stresses

Biotic stressors, 28

Biotin, 482-483

Biphasic response model, 460

1,3-Bischloroethyl-nitrosourea (BCNU), 228

BKI1. See BRI1-KINASE INHIBITOR 1 (BKI1)

BMV. See Brome mosaic virus (BMV)

Botrytis cinerea, 20-21

BR. See Brassinosteroid (BR)

BR signaling. See Brassinosteroid signaling (BR signaling)

BR-insensitive mutants (bri1 mutants), 407-408

Brachypodium distachyon, 491-493

BRAD. See Brassica database (BRAD)

Branched-chain amino acids (BCAAs), 172

Brassica database (BRAD), 543

Brassica juncea, 175, 358-359

Brassica napus, 411-412

seedlings, 113-114

Brassica rapa plants, 189

Brassinazole (Bz), 364, 413

Brassinosteroid (BR), 25-27, 303-304

BRASSINOSTEROID INSENSITIVE 2 (BIN2), 408-409

Brassinosteroid signaling (BR signaling), 502-503

Brassinosteroids (BRs), 113-114, 142-143, 287-288, 294, 341-342, 355-356, 364, 407-408, 408f, 420-421

brassinosteroid signaling in plants, 408-409

mediated defense signaling, 411-412, 412t

mediated ROS signaling and role in plant defense, $412-413$

RD26 in BR signaling, 410-411

responsive transcription factor, 408-409

role, 307

transcription factors involving in BR signalling, 409-410

BRI1 associated kinase 1 (BAK1), 407-408 
bri1 mutants. See BR-insensitive mutants (bri1 mutants)

BRI1-KINASE INHIBITOR 1 (BKI1), 408-409

BRI1-SUPPRESSOR 1 (BSU1), 408-409

Brome mosaic virus (BMV), 24

BRs. See Brassinosteroids (BRs)

BRZ. See Brassica rapa plants

BRZ-INSENSITIVELONG HYPOCOTYLS 2 (BIL2), 509-510

BSC. See Bundle sheath cells (BSC)

BSO. See Buthionine sulfoximine (BSO)

BSU1. See BRI1-SUPPRESSOR 1 (BSU1)

Bundle sheath cells (BSC), 518

1-Butanol, 489

Buthionine sulfoximine (BSO), 228

Bz. See Brassinazole (Bz)

BZ method. See Benzoate method (BZ method)

bZIP. See Basic leucine zipper protein (bZIP)

C

C-repeat binding factor (CBF), 246

CBF2, 337

C-repeat/dehydration-responsive element binding protein (CBF/DREB), 126

$\mathrm{C}_{4}$ and Crassulacean acid metabolism, 518-519

challenges and possibilities, $527-528$

engineering Crassulacean acid metabolism into $\mathrm{C}_{4}, 527$

parts list for Crassulacean acid

metabolism into $\mathrm{C}_{4}, 527-528$

compatibility, 525-527

core candidate genes for, $520 t$

environmental cues controlling, 522-524

modulation of $\mathrm{C}_{4}$ by environmental cues, 522-523

modulation of Crassulacean acid metabolism, 523-524

exclusive modules, 521-522

modules common to, $519-521$

similarities and differences, 518-522

stress signaling networks controlling, $524-525$

CA. See Carbonic anhydrase (CA); Citric acid (CA)

$\mathrm{Ca}^{2+} /$ Calmodulin signaling pathway (CaM signaling pathway), 488-489

$\mathrm{Ca}^{2+} / \mathrm{CaM}$ dependent kinase II (CaMK II), 507-508

$\mathrm{CAB}$ binding protein. See Chlorophyll $\mathrm{a} / \mathrm{b}$ binding protein (CAB binding protein)

CAD. See CDPK activation domain (CAD)

Cadmium (Cd), 35, 222, 293, 325, 394

cADPR. See Cyclic ADP-ribose (cADPR)

CaHSP genes. See Capsicum annuum heat shock protein genes (CaHSP genes)

Calcineurin B-like proteins (CBLs), 51-53, $279,485-486,488-489,507,539-541$

CBL10, 45

Calcium (Ca), 4-5, 28-29, 51-53, 84, $228-229,275,278-280,507-509$

kinases, 539-541

CBLs, 540-541
CDPKs, 539-540

CIPKs, 540-541

CRK, 541

messenger systems, 84

quantification

Fura-2 AM fluorescence method, 85

MTB method, 84-85

reporters, 44

sensors, $43-44$

and signaling, 53-54

signature, 278-279

Calcium and calmodulin-dependent protein kinases (CCaMKs), 55, 507, 541

Calcium regulated RLKs (CRLKs), 537

CRLK1, 537

Calcium-binding protein kinase proteins, 45

Calcium-dependent protein kinases (CDPKs), 31-33, 51-54, 64-65, $106-107,279,488-489,507-508$, $533-534,539-540$

CPK6, 53-54, 306

Calcium-regulated proteins, 507-508

Calmodulin (CaM), 32-33, 53, 143, 228, 279, $488-489,507,539$

CAM-idling, 523

cycling, 523

Calmodulin-binding transcription activator (CAMTA), 503-504

Calmodulin-like domain protein kinases (CDPKs), 67, 507, 533-534

Calmodulin-like protein (CML), 53

Calreticulin, 488-489

CaM. See Calmodulin (CaM)

CAM. See Crassulacean acid metabolism (CAM)

CaM signaling pathway. See $\mathrm{Ca}^{2+} /$ Calmodulin signaling pathway $(\mathrm{CaM}$ signaling pathway)

CaMK II. See $\mathrm{Ca}^{2+} / \mathrm{CaM}$ dependent kinase II (CaMK II)

CAMTA. See Calmodulin-binding transcription activator (CAMTA)

CaPO1 gene, 24-25

CaPR1 gene, 24-25

Capsicum annuum. See Pepper (Capsicum annuum)

Capsicum annuum heat shock protein genes (CaHSP genes), 24-25

CaHsp26 gene, $509-510$

N-Carbamoyl Put amidohydrolase, 465

Carbohydrate metabolite signal responsive elements (CMSRE), 264

Carbohydrate-binding motif (CBM), 241

Carbohydrates, 174

metabolism, 245, 262-263

Carbon $(\mathrm{C})$

metabolism, 244, 261

reserve during and after release of stress, 429

Carbon concentrating mechanisms (CCMs), 518

Carbon monoxide (CO) 418

Carbonic anhydrase (CA), 527-528

Carbonyl group (CO group), 147

Carbonylated proteins, 146
Carboxylation modules, 519-520

2-(4-Carboxyphenyl)-4,4,5,5tetramethylimidazoline-1-oxyl-3-oxide (c-PTIO), 388

CaRLK1. See Pepper receptor-like protein kinase 1 (CaRLK1)

Carotenoids in stress signaling, 170-171

Caryophyllales, 526

Cas9. See CRISPR associated protein (Cas9)

CaSAR82A gene, 24-25

Casein kinase II (CKII), 337

Catabolism of ABA, 343-344

Catalase (CAT), 7, 54, 93, 109, 141-142, 418, 490

CAT3, 539-540

Catharanthus roseus (CrRLKs), 537

CBF. See C-repeat binding factor (CBF)

CBF/DREB. See C-repeat/dehydrationresponsive element binding protein (CBF/DREB)

CBL-interacting protein kinases (CIPKs), 51-53, 106-107, 485-486, 540-541

CIPK26, 51-52

CBL1/9-CIPK23 complex, 540-541

CBLs. See Calcineurin B-like proteins (CBLs)

CBM. See Carbohydrate-binding motif (CBM)

CBS. See Cystathionine- $\beta$-synthase (CBS)

CCaMKs. See Calcium and calmodulindependent protein kinases (CCaMKs)

CCMs. See Carbon concentrating mechanisms (CCMs)

CDG1. See CONSTITUTIVE DIFFERENTIAL GROWTH 1 (CDG1)

$\mathrm{CDH}$. See Choline dehydrogenase $(\mathrm{CDH})$

CDPK activation domain (CAD), 539

CDPK-regulated protein kinase (CRK), 541

CRK45, 133

CDPKs. See Calcium-dependent protein kinases (CDPKs); Calmodulin-like

Cell domain protein kinases (CDPKs)

response mechanisms, $4-8$

sorting technique, 323

surface receptors, 213

surface RLKs, 537

wall, 143

Cellular metabolism, 219-220

Cellulose, 174

CFA. See Coronafacic acid (CFA)

cGMP. See Cyclic guanosine monophosphate (cGMP)

$\mathrm{CH}_{3} \mathrm{C}(\mathrm{O}) \mathrm{CHO}$. See Methylglyoxyl (MG)

Chalcone synthase (CHS), 158-160, 188

transcriptional induction, 188-189

Chaperones, 499-500

Chemical priming, 224

Cherry tomatoes, 186

Chickpea (Cicer arietinum), 441

plants, 425-426

Chilling

stress, 554

tolerance in plants, 393

tolerance, 226-227

Chilling-tolerance divergence 1 (COLD1), 44 Chitin, 174 
Chl. See Chlorophyll (Chl)

Chlamydomonas reinhardtii, 149, 426-427

Chlorella vulgaris, 427

Chloride channel family (CLC-c), 522

Chlorophyll (Chl), 224-225, 394

degradation, 187

fluorescence, 438

Chlorophyll $\mathrm{a} / \mathrm{b}$ binding protein (CAB binding protein), 195

Chloroplasts, 424-425, 551

Chlorosis, 245

Chlorpromazine (CPZ), 228

Choline dehydrogenase (CDH), 133-134, 424

Choline monooxygenase (CMO), 108, 451, 463-464

Choline oxidase (COD), 108, 424

Chorispora bungeana, 393

Chromium (Cr), 225

CHS. See Chalcone synthase (CHS)

Cicer arietinum. See Chickpea (Cicer arietinum)

cipk23 null mutants, 346

CIPKs. See CBL-interacting protein kinases (CIPKs)

9-Cis-epoxy-carotenoid dioxygenase (NCED), 342-343

NCED3, 112-113

Cis-jasmone, 371-372

Citric acid (CA), 225

CK. See Cytokinins (CK)

CKB1 overexpressing plants, 337

CKII. See Casein kinase II (CKII)

CKX1. See Cytokinin oxidase/ dehydrogenase1 (CKX1)

Classic pathway, 259

Classical plant hormones, 106

CLC-c. See Chloride channel family (CLC-c)

Clustered regularly interspaced short palindromic repeats (CRISPR-Cas9), $55,64,74$

genome editing, $74-75,77 f$

application in plant abiotic stress tolerance, 75

CML. See Calmodulin-like protein (CML)

CMO. See Choline monooxygenase (CMO)

CMSRE. See Carbohydrate metabolite signal responsive elements (CMSRE)

CMV. See Cucumber mosaic virus (CMV)

CNGCs. See Cyclic nucleotide-gated channels (CNGCs)

$\mathrm{CO}$ group. See Carbonyl group (CO group)

Cochliobolus sativus, 20-21

COD. See Choline oxidase (COD)

$\operatorname{codA}$. See Choline oxidase (COD)

COI 1. See Coronatine insensitive 1 (COI 1)

Cold, 245-247

stress, 186, 323

plant phenolics and role, 163

Cold-regulated proteins (COR proteins), 245-246

COLD1. See Chilling-tolerance divergence 1 (COLD1)

Compatible osmolytes, 172

CONSTANS-like genes, 525
CONSTITUTIVE DIFFERENTIAL GROWTH

1 (CDG1), 408-409

Constitutive triple response 1 (ctr1), 336

CONSTITUTIVE TRIPLE RESPONSE1 (CTR1), 491

Conventional breeding, 43-44, 64

Copper $(\mathrm{Cu}), 325,394$

COR proteins. See Cold-regulated proteins (COR proteins)

Coronafacic acid (CFA), 374-375

Coronatine insensitive 1 (COI 1), 306, 373-374

Cotton (Gossypium tomentosum), 68

CPK. See Calcium-dependent protein kinases (CDPKs)

CPZ. See Chlorpromazine (CPZ)

Crassulacean acid metabolism (CAM), 518

Crataegus monogyna, 186

CRISPR associated protein (Cas9), 74

CRISPR-Cas9. See Clustered regularly interspaced short palindromic repeats (CRISPR-Cas9)

CRISPR-Cpf1, 64

CRK. See CDPK-regulated protein kinase (CRK)

CRLKs. See Calcium regulated RLKs (CRLKs)

Crop improvement of cereals

engineering of osmoregulatory genes, 133-134

genetic engineering of kinases for drought and salinity tolerance, $130-132$

modulation of key genes involved in phytohormone signaling, 132-133

reactive oxygen species signaling during drought and salinity stress, 134

signaling pathways during abiotic stresses, $126 f$

TFs associating with signaling mechanism, $127-130$

CrRLKs. See Catharanthus roseus (CrRLKs)

CsNOA1, 393

CsSPDS. See Cucurbita ficifolia spermidine synthase gene (CsSPDS)

CstF64-like putative RNA processing factor, 212-213

ctr1. See Constitutive triple response 1 (ctr1)

CTR1. See CONSTITUTIVE TRIPLE RESPONSE1 (CTR1)

CUC. See Cup-shaped cotyledon (CUC)

Cucumber mosaic virus (CMV), 24

Cucurbita ficifolia spermidine synthase gene (CsSPDS), 466-467

Cup-shaped cotyledon (CUC), 116-117, 242

CycD. See CyclinD (CycD)

Cyclic ADP-ribose (cADPR), 387

Cyclic guanosine monophosphate (cGMP), 387

Cyclic nucleotide-gated channels (CNGCs), 44

CyclinD (CycD), 213-214

CycD3 expression, 266

Cycloheximide, 239-240

CYP79B2 gene, 324

CYP79B3 gene, 324
Cystathionine- $\beta$-synthase (CBS), 212-213

Cysteine (Cys), 147, 149, 287-288, 293-294

Cytapx, 109-110

Cytokinin oxidase/dehydrogenase1 (CKX1), 112

Cytokinins (CK), 25-27, 67-68, 106, 112, 266, 287-288, 291-292, 303-304, 311-312, 333-334, 341-342, 355-356, $440-441,525$

role, 307

Cytoplasm, 194

Cytoskeleton depolymerization, 245-246

Cytosol, 462, 556

Cytosolic acidification, 10

Cytosolic APX gene, 109-110

Cytosolic calcium $\left(\mathrm{Ca}^{2+}\right), 32-33$

Cytosolic glycine betaine synthesis, 453-454

Cytsod, 109-110

D

D. stramonium SPDS gene (DsSPDS), 466-467

DA NSCCs. See Depolarization-activated NSCCs (DA NSCCs)

DAB method. See 1,2-Diaminobenzene method (DAB method)

DAF-2 method. See 4,5-Diaminofluorescein method (DAF-2 method)

DAF-2DA. See 4,5-Diaminofluorescein diacetate (DAF-2DA)

DAF-FM DA method. See 3-Amino,4aminomethyl-2', $7^{\prime}$-difluorescein, diacetate (DAF-FM DA method)

DAG. See Diacylglycerol (DAG)

DAG kinase (DGK), 489

DAG-pyrophosphate (DAG-PP), 489

DAR-1 method. See 4,5-Diamino-rhodamine method (DAR-1 method)

Datura stramonium ADC gene (DsADC), 466-467

DCF method. See 2', 7'-Dichlorofluorescein method (DCF method)

$\mathrm{DCFH}_{2}$-DA method. See $2^{\prime}, 7^{\prime}$ Dichlorofluorescein diacetate method (DCFH ${ }_{2}$-DA method)

DCHBS method. See 3,5-Dichloro-2-hydroxybenzene sulfonic acid method (DCHBS method)

DCHBS-AAP method, 94-95

in vitro method, 95

in vivo method, 94-95

dcSAM. See Decarboxylated Sadenosylmethionine (dcSAM)

DDF1. See Dwarf and delayed flowering 1 (DDF1)

DDR method. See 2-Deoxy-D-ribose method (DDR method)

DEAD-box RNA helicases, 64-65

Decarboxylated $S$-adenosylmethionine (dcSAM), 465

Decarboxylation modules, 519-520

Deep proteome analysis, 481, 483

Deep proteomics. See Deep proteome analysis

Deetiolated 2 mutants (det2 mutants) $420-421$ 
Defense mechanism, 184, 407-408

Deficit, 305-307

DEGs. See Differentially expressed genes (DEGs)

Dehydration-responsive element (DRE), 113, 312

Dehydration-responsive element binding 1/ C-repeat binding factor (DREB1/CBF), 335

Dehydration-responsive element-binding proteins (DREB proteins), 53, 115, $128-129,312$

Dehydrin, 108-109, 114, 347-348

Dehydroascorbate (DHA), 150-151, 170

Dehydroascorbate reductase (DHAR), 54 , $109,126,303-304$

DHAR1, 238

Dehydroascorbic acid. See Dehydroascorbate (DHA)

DELLA mutants, 3

DELLA protein, 112, 142-143, 334-335

interaction and involvement with SA-GA crosstalk under stress, 363-364

Delta pyroline-5-carboylate synthetase, 133-134

$\delta$-Aminotransferase (OAT), 420-421

2-Deoxy-D-ribose method (DDR method), 101-102

3-Deoxyarabinoheptulosonate 7-phosphate synthase, 160-161

Depolarization-activated NSCCs (DA NSCCs), 5

$\operatorname{det} 2$ mutants. See Deetiolated 2 mutants (det 2 mutants)

DETA/NO. See DiethylenetriamineNONOate (DETA/NO)

DGK. See DAG kinase (DGK)

DGKs. See Diacylglycerol kinases (DGKs)

DHA. See Dehydroascorbate (DHA)

DHAP. See Dihydroxy-acetone phosphate (DHAP)

DHAR. See Dehydroascorbate reductase (DHAR)

DHBA. See 2,4-Dihydroxy-benzylamine (DHBA)

Diacylglycerol (DAG), 278-279, 489, 542

Diacylglycerol kinases (DGKs), 542

Diamine putrescine (Put), 35

4,5-Diamino-rhodamine method (DAR-1 method), 89-90

1,2-Diaminobenzene method (DAB method), $87-88,95$

4,5-Diaminofluorescein diacetate (DAF-2DA), 390

4,5-Diaminofluorescein method (DAF-2 method), 89-90

3,5-Dichloro-2-hydroxy-benzene sulfonic acid method (DCHBS method), 94-95

$2^{\prime}, 7^{\prime}$-Dichlorofluorescein diacetate method (DCFH ${ }_{2}-\mathrm{DA}$ method), 95-97

in vivo method, 96

2',7'-Dichlorofluorescein method (DCF method), 95-97

in vitro method, $96-97$

Diethylenetriamine-NONOate (DETA/NO), 391-393
Differentially expressed genes (DEGs), 337

Dihydroxy-acetone phosphate (DHAP), 66, $220-221$

2,4-Dihydroxy-benzylamine (DHBA), 228

2-Dimensional electrophoresis (2-DE), 480-481, 491

3'-o-(4,5-Dimethoxy-2-nitrobenzenesulfonyl)$2^{\prime}, 4^{\prime}, 5^{\prime}, 7^{\prime}$-tetrafluorofluorescein method (BESSo method), 100

$\mathrm{N}, \mathrm{N}$-Dimethyl-L-proline, $452-453$

$N, N$-Dimethylpphenylenediaminedihydrochloride (DMPD), 86

3-Dimethylsulfoniopropionate (DMSP), 450

Dimethylsulfoxide (DMSO), 85, 96

2,4-Dinitrophenylhydrazine method (DNP method), 88-89

Diospyros kaki, 108

Diphenylene iodonium (DPI), 143

Disaccharides, 207-208

Disulfide bonds (S-S bonds), 149

5,5'-Dithiobis(2-nitrobenzoic acid) (DTNB), 85-86

DMPD. See N,N-Dimethylpphenylenediaminedihydrochloride (DMPD)

DMSO. See Dimethylsulfoxide (DMSO)

DMSP. See 3-Dimethylsulfoniopropionate (DMSP)

DN. See Dominant negative (DN)

DNA, 146-147

damage of plants, 33-34

helicases, $71-73$

DNP method. See 2,4Dinitrophenylhydrazine method (DNP method)

DOLE. See Dry olive leaf extract (DOLE)

Dominant negative (DN), 509-510

Dopamine (DOPA), 147

Dormancy, 113

Double-strand break (DSB), 74

Downstream signaling processes, 64-65

DPI. See Diphenylene iodonium (DPI)

DRE. See Dehydration-responsive element (DRE)

DRE/CRT cis-acting motif, 346-347

DREB proteins. See Dehydration-responsive element-binding proteins (DREB proteins)

DREB1/CBF. See Dehydration-responsive element binding $1 / \mathrm{C}$-repeat binding factor (DREB1/CBF)

Drought, 185, 459

drought-induced pathogen resistance, 23-24

drought-responsive molecular mechanisms, 106-107

Drought stress, 106, 320-322, 552-553

molecular mechanism of regulatory elements in, 114-117

NO in drought stress tolerance, 390-391

osmoprotectant regulatory genes in, 107-109

plant phenolics and role in, 163

Drought tolerance, 224-225 in plants, 106

hormonal regulation, 111-114

redox regulatory machinery of plants, 109-111

response, $106-107$

Dry olive leaf extract (DOLE), 33-34

DsADC. See Datura stramonium ADC gene (DsADC)

DSB. See Double-strand break (DSB)

DsSPDS. See D. stramonium SPDS gene (DsSPDS)

DTNB. See 5,5'-Dithiobis(2-nitrobenzoic acid) (DTNB)

Dual-cell C 4 , 522-523

Dwarf and delayed flowering 1 (DDF1), 335

E

EBL. See Epibrassinolide (EBR)

EBR. See Epibrassinolide (EBR)

ECLB. See Extracellular ligand-binding domain (ECLB)

EDTA. See Ethylenediaminetetraacetic acid (EDTA)

Effector-triggered immunity, 210

EGL1. See ENHANCER OF GLABRA3 1 (EGL1)

EIN2. See Ethylene insensitive 2 (EIN2)

EIN3. See Ethylene insensitive 3 (EIN3)

EIN3/EILs. See Ethylene-insensitive-like (EIN3/EILs)

Electron transport chain (ETC), 143-144

ELISA. See Enzyme linked immunosorbent assay (ELISA)

Embryo development, 461-462

Endogenous NO, 10-11

Endogenous signal molecule, 441

Endogenous Suc levels, 247

Endoplasmic reticulum (ER), 44, 502-503

Endothelial NOS (eNOS), 386

Energy

homeostasis, 240

sources, 429

starvation, 244

Engineering signaling molecules

ABA signaling pathway, 51-53

calcium sensors and signaling, 53-54

osmotic stress sensors, 45-51

ROS signaling, 54-55

salt stress sensors, 45

stress signal sensors, $44-45$

ENHANCER OF GLABRA3 1 (EGL1), 374-375

eNOS. See Endothelial NOS (eNOS)

Environmental stresses, 44, 71, 193

conditions, 479-480

MG signaling triggering by, 221-222

Enzymatic/enzyme

antioxidants, $320-322$

condenses $p$-cumaryl-CoA, 184-185

pathways, 221

ROS regulation during drought, 109-110

sensors, 212-213

Enzyme linked immunosorbent assay (ELISA), 483-484

Epibrassinolide (EBR), 35, 113-114, 307, 413 
Epigenetic regulation of abiotic stress tolerance, 70-71

ER. See Endoplasmic reticulum (ER)

ERD10, 113-114

ERF-VII TFs. See Ethylene response factorVII TFs (ERF-VII TFs)

ERFs. See Ethylene-responsive factor (ERFs)

Erythose-4-phosphate, 158

Escherichia coli, 227

ETC. See Electron transport chain (ETC)

Ethylene (ET), 19-20, 67-68, 113, 287-288, 291, 293, 303-304, 306, 312, 341-342, $355-356,363,407-408,442-445,444 t$

ethylene-deficient mutants, 444-445

signaling pathways, 490-491

Ethylene insensitive 2 (EIN2), 491

Ethylene insensitive 3 (EIN3), 201, 312, 335

transcription factors, 293

Ethylene receptor (ETR), 491-493

Ethylene response factor-VII TFs (ERF-VII TFs), 149

Ethylene-insensitive-like (EIN3/EILs), 491

Ethylene-responsive factor (ERFs), 113

ERF1, 242, 312

ERF6, 112

Ethylenediaminetetraacetic acid (EDTA), 86

N-Ethymaleimide, 482-483

ETR. See Ethylene receptor (ETR)

Exclusive modules, 521-522

Exogenous application, 210

Extracellular ligand-binding domain (ECLB), 44-45

Extracellular peroxidases, 552

$\mathrm{F}$

F-6-P. See Fructose-6-phosphate (F-6-P)

F3H. See Flavanone 3-hydroxylase (F3H)

Fabaceae, 452

Fatty acid degradation, 219-220

FBPase. See Fru-1,6-bisphosphatase (FBPase)

FCR activity. See Ferric chelate reductase activity (FCR activity)

Fermentation, 245

Ferric chelate reductase activity (FCR activity), 324

Fertilizers, 550

FFAs. See Free fatty acids (FFAs)

FLAGELLIN SENSING 2 (FLS2), 411-412

Flavanone 3-hydroxylase (F3H), 188

Flavones, 161-162

Flavonoids, 161-162, 184

in stress signaling, 170-171

synthesis, 210

Flavonols, 161-162

Flooding, 305-307, 459

NO in flooding stress tolerance, 396-397

FLS2. See FLAGELLIN SENSING 2 (FLS2)

FON1 gene, 537

Free fatty acids (FFAs), 489

Free radical, 27-28

Fresh weight (FW), 226

Fru-1,6-bisphosphatase (FBPase), 149

Fructan 6-fructosyltransferase (6-SFT), 470

Fructans, 174-175, 208, 428, 470, 470f

Fructose (Fru), 199-200, 235-236
Fructose-6-phosphate (F-6-P), 455-456

Fura-2 AM fluorescence method, 85

FW. See Fresh weight (FW)

G

G-6-P. See Glucose-6-phosphate (G-6-P)

G6PDH. See Glucose-6-phosphate dehydrogenase (G6PDH)

GA-20-oxidase (GA20ox), 334

GA20ox. See GA-20-oxidase (GA20ox)

GABA. See Gamma-aminobutyric acid (GABA)

GAD. See Glutamate decarboxylase (GAD)

gai-t6. See Gibberellic acid insensitive-t6 (gait6)

Gamma-aminobutyric acid (GABA), 173, 224, 361,451

$\gamma$-glutamyl kinase (GK), 441

$\gamma$-glutamylcysteinyln-glycine, 149

GAP. See Glyceraldehydes-3-phosphate (GAP)

GAs. See Gibberellic acids (GAs); Gibberellins (GAs)

GB. See Glycine betaine (GB)

GbMYB5 gene, 115

GDH. See Glutamate dehydrogenase (GDH)

Gel-based targeted proteomics, 484

Gel-free proteomics approach, 481

Genes encoding protective proteins, 64-65

Genetic approaches

in plants, 260

of sugar regulation in plants, 261-264

Genetic engineering

approaches to modulating abiotic stress signaling process, $69 f$

transgenic approaches, $68-73$

of Hsps signaling molecules, 509-510

of kinases for drought and salinity

tolerance, $130-132$

protein kinases, 130-131

RLKs, 131-132

Genetically modified organisms (GMO), 35

Genome editing technologies, 55

to modulating abiotic stress signaling processes, $74-75$

comparison of mechanism, application, advantages, and disadvantages, $76 t$

CRISPR-Cas9 genome editing, 74-75

CRISPR-Cpf1, 75

TALENs, 74

ZFN, 74

Genome-wide analysis, 409-410

Genomics, 480, 543

Geranylgeranyl diphosphate (GGPP), 342-343

Germination, 244

GGPP. See Geranylgeranyl diphosphate (GGPP)

GhHyPRP4 proteins, 66

GHR1. See Guard cell hydrogen peroxide resistant 1 (GHR1)

Gibberellic acid insensitive-t6 (gai-t6), 336

Gibberellic acids (GAs), 292-293, 333-334, 355-356, 361-363, 440-441

metabolism in plants, $334-335$ in plant abiotic stress, 337

regulatory signaling during abiotic stresses, 335

signaling crosstalks between phytohormones and, 335-336

Gibberellins (GAs), 2-3, 25-27, 67-68, 106, 112, 142-143, 287-288, 303-304, $341-342,361-363,420-421$

GID1 nuclear receptor, 112, 334-335

gin. See Glucose insensitive (gin)

Gin2 mutant, 211

GK. See $\gamma$-glutamyl kinase (GK)

GL3. See GLABRA3 (GL3)

GLABRA3 (GL3), 374-375

GLOase. See L-gulono- $\gamma$-lactone oxidase (GLOase)

Global climatic changes, 43-44, 64

Global proteome analysis, 481-483. See also Targeted proteome analysis

deep proteome analysis, 483

2DGE, 481

3DGE, 482

ICAT, 482-483

iTRAQ, 483

MudPIT, 483

SILAC, 483

Global warming, 437

GLR. See Glutamate receptor-like (GLR)

Glucose (Glu), 199-202, 211, 235-236, 430

metabolism, 212

repression, 263-264

in salt stress responses, 195-201, $196 t$

sensing, transport, and signaling in plants, 195

Glucose insensitive (gin), 213

Glucose-6-phosphate (G-6-P), 455, 455f, 519

Glucose-6-phosphate dehydrogenase (G6PDH), 158

Glucosinolates (GS), 171, 290

Glucuronosyldiacylglycerol, 278

Glutamate, 107, 439-440

Glutamate decarboxylase (GAD), 173

Glutamate dehydrogenase (GDH), 270-271

Glutamate receptor-like (GLR), 44

Glutamate-semialdehyde (GSA), 439-440

Glutamatic- $\gamma$-glutamyl kinase, 462

Glutamine synthetase (GS), 270-271

Glutamyl-cysteinyl synthetase ( $\gamma$-ECS), $110-111,228$

Glutathione (GSH), 106, 110-111, 141-142, 149-151, 221, 287-288, 290, 294, 427, 466,490

Glutathione peroxidase (GPX), 54, 109, 223-224, 303-304

Glutathione reductase (GR), 54, 109, 126, 388, 490

Glutathione stransferase (GST), 54, 109, 223-224

Glutathione synthetase (GS), 110-111

Gly I. See Glyoxalase I (Gly I)

Glyceraldehydes-3-phosphate (GAP), 66

Glycine betaine (GB), 108, 133-134, 223-224, 360-361, 418, 453-454, 454f, 460, $463-465,465 f, 466 t, 491$ 
Glycine betaine (GB) (Continued)

biosynthesis and modulation by $\mathrm{ABA}$ $422-423$

derivatives, 454-455

Glycine max MAP kinase 1 (GMK1), 486-487

Glycine sarcosine methyltransferase (GSMT), 453-454

Glycine-rich proteins (GRPs), 66

Glycolysis, 219-220

Glycophytes, 1-2

Glyoxalase I (Gly I), 221

Glyoxalase I (Gly II), 221

Glyoxalase III (Gly III), 221

Glyoxalase system, 221, 423

GmbZIP1, 130

GMK1. See Glycine max MAP kinase 1 (GMK1)

GMO. See Genetically modified organisms (GMO)

GORK. See Guard cell outward-rectifying K ${ }^{+}$ channel (GORK)

GPX. See Glutathione peroxidase (GPX); Guaiacol peroxidase (GPX)

GR. See Glutathione reductase (GR)

Grapevine (Vitis vinifera L.), 246-247

Griess reagent method, 90-91

Groucho/Tup1-type corepressor, 373-374

GRPs. See Glycine-rich proteins (GRPs)

GS. See Glucosinolates (GS); Glutamine synthetase (GS); Glutathione synthetase (GS)

GSA. See Glutamate-semialdehyde (GSA)

GSH. See Glutathione (GSH)

GsLRPK gene, 537

GSMT. See Glycine sarcosine methyltransferase (GSMT)

GSNO. See Nitrosoglutathione (GSNO)

GSNOR. See S-nitrosoglutathione reductase (GSNOR)

GST. See Glutathione stransferase (GST)

Guaiacol peroxidase (GPX), 109

Guard cell hydrogen peroxide resistant 1 (GHR1), 537

Guard cell outward-rectifying $\mathrm{K}^{+}$channel (GORK), 306

GUS gene. See b-glucuronidase gene (GUS gene)

$\mathrm{H}$

H-type Trx (OsTRXh1), 55

HA NSCCs. See Hyperpolarization-activated NSCCs (HA NSCCs)

Halliwell-Asada cycle, 170

Halophytes, 1-2, 34-35

HAMK. See Heat stressed A. thaliana. MAP kinase (HAMK)

HATS. See High affinity transport system (HATS)

hba mutant. See High beta amylase mutant (hba mutant)

HCAs. See Hydroxycinnamic acids (HCAs) HDAC6. See Histone deacetylase6 (HDAC6) HDR. See Homology directed repair (HDR)

Heat shock (HS), 225

transcription factors, 503-504
Heat shock factor (Hsf), 503-504, 508-509

Heat shock response (HSR), 502

Heat stress, 322-323, 393, 437, 554-555

aftermaths of, 438-439, $439 f$

Heat stress elements (HSEs), 503-504

Heat stress transcription factors (HSFs), 70

Heat stressed $A$. thaliana. MAP kinase (HAMK), 507-508

Heat tolerance, 226

phytohormones in, 440-441

proline in, 439-440

salicyclic acid, ethylene, and abscisic acid for, $442-445$

salicyclic acid role in, $441-442$

Heat-shock proteins (HSPs), 64-65, 70, 359, $438,500-501$

abiotic stresses inducing Hsps-mediated signaling pathways, 504-508

complex signaling network of plant response, $500 f$

crosstalk between ROS, MAPKs cascades, $\mathrm{Ca}^{+2}$, and Hsf/Hsps, 508-509

expression of HSP families, 501t

general account on, 501-504

genetic engineering, 70

of Hsps signaling molecules, 509-510

Hsp10 protein, 502

Hsp60 protein, 502

Hsp70 protein, 500-502, 509

Hsp90 protein, $500-503$

Hsp100 protein, 502-503

induction phenomena in plants, 504, 505t

SA interaction with, 359

sHsps, 501-504

Heavy metal (HM), 303, 307

stress, 325, 555

plant phenolics and role in, 162

tolerance, 225, 394-395

toxicity, 162

Helicases, genetic engineering of, 71-73

Heme-regulated inhibitor (HRI), 502-503

Hemithioacetal (HTA), 221

Hexokinase (HXK), 29, 208, 212, 258

HXK1, 3-4, 195

Hexose kinases, 212-213

High affinity transport system (HATS), 270-271

High beta amylase mutant (hba mutant) 263-264

High leaf temperature 1 (HT1), 522

High osmolarity glycerol 1 (HOG1), 423

High sugar-response mutant (hsr mutant), 263-264

High temperature stress tolerance in plants, 393-394

High temperatures (HT), 239

High-sugar resistance, 210

HIOMT. See Hydroxymethyl O methyltransferase (HIOMT)

Histidine kinases (HKs), 134, 533-534, 542

Histochemical staining method, 99

Histone deacetylase gene, 70-71

Histone deacetylase6 (HDAC6), 347-348

Histones, 70-71

modification, $70-71$
HKs. See Histidine kinases (HKs)

HKT1, 5

HM. See Heavy metal (HM)

HOG1. See High osmolarity glycerol 1 (HOG1)

Homeostasis, 193

Homology directed repair (HDR), 74

Homostachydrine, 454-455

Hormonal regulation of drought tolerance, 111-114

ABA, 112-113

auxin, $111-112$

brassinosteroids, 113-114

CKs, 112

ethylene, 113

GAs, 112

JA, 114

SA, 114

Hormonal signaling, 32-33

Hormones

auxin crosstalk with, 326

biosynthesis, 214

control, 440-441

crosstalk, 311-312

effect in regulation

of metal stress, $310 t$

of salinity stress, $309 t$

of temperature stress, $308 t$

role in regulation of $P 5 C S$ and proline synthesis, 420-422

Horseradish peroxidase (HRP), 93-94, 97

13-HPOT. See 13(S)-hydroperoxy linolenic acid derivatives (13-HPOT)

HRI. See Heme-regulated inhibitor (HRI)

HRP. See Horseradish peroxidase (HRP)

HS. See Heat shock (HS)

HSEs. See Heat stress elements (HSEs)

Hsf. See Heat shock factor (Hsf)

HSFs. See Heat stress transcription factors (HSFs)

HSPs. See Heat-shock proteins (HSPs)

HSR. See Heat shock response (HSR)

hsr mutant. See High sugar-response mutant (hsr mutant)

HT. See High temperatures (HT)

HT1. See High leaf temperature 1 (HT1)

HTA. See Hemithioacetal (HTA)

Human anthropogenic activities, 64

HXK. See Hexokinase (HXK)

Hybrid Proline Rich Proteins (HyPRPs), 64, 66

Hydrogen peroxide $\left(\mathrm{H}_{2} \mathrm{O}_{2}\right), 7,35,54,84$, 143-144, 219-220, 307, 420-421, 489-490, 537-538

quantification

ABTS method, 93-94

$\mathrm{AR}$ and AUR fluorescence methods, 97

BES- $\mathrm{H}_{2} \mathrm{O}_{2}$-Ac and BES- $\mathrm{H}_{2} \mathrm{O}_{2}$

fluorescence method, $97-98$

DAB method, 95

$\mathrm{DCFH}_{2}-\mathrm{DA}$ and DCF fluorescence methods, 95-97

DCHBS-AAP method, 94-95

KI method, 91-92

$\mathrm{Ti}\left(\mathrm{SO}_{4}\right)_{2}$ method, 92 
XO method, 93

Hydrogen sulfide $\left(\mathrm{H}_{2} \mathrm{~S}\right), 84,228-229,292$, 418

quantification

DTNB method, 85-86

MB method, 86-87

WSP-1 fluorescence method, 87

9S-Hydroperoxy derivative of LA

hydroperoxy octadecadienoic acids (9S-HPODE), 372-373

Hydroxy-L-proline (Hyp), 452-453

2-Hydroxybenzoic acid, 355-356

Hydroxycinnamic acids (HCAs), 158

Hydroxyindole-O-methyltransferase (ASMT), 176

Hydroxyl pipecolate betaine, $455 f$

Hydroxyl radical $(\bullet \mathrm{OH}), 54,84,425-426$, 489-490

quantification

BZ method, 101

DDR method, 101-102

Hydroxylation, 343-344

Hydroxymethyl O methyltransferase (HIOMT). See Hydroxyindole-Omethyltransferase (ASMT)

Hydroxypipecolate, 454-455

Hydroxypipecolate betaine, 454-455

Hydroxyproline, 452-453

Hydroxyproline betaine, 452-453

Hyp. See Hydroxy-L-proline (Hyp)

Hypericum brasiliense plants, 186

Hyperpolarization-activated NSCCs (HA NSCCs), 5

Hypoxia, 243-245

HyPRPs. See Hybrid Proline Rich Proteins (HyPRPs)

I

IAA. See Indole acetic acid (IAA); Indole-3acetic acid (IAA)

IaaM-OX transgenic lines of Arabidopsis, 111-112

IAM pathway. See Indole-3-acetamide pathway (IAM pathway)

IAOX pathway. See Indole-3-acetaldoxime pathway (IAOX pathway)

ICAT. See Isotope-coded affinity tag (ICAT)

ICDH. See Isocitrate dehydrogenase (ICDH)

ICL. See Isocitratelyase (ICL)

ICS. See Isochorismate synthase (ICS)

Immobilized metal affinity chromatography (IMAC), 484

Immobilized $\mathrm{pH}$ gradient gels (IPG gels), 481

Immunoprecipitation method, 484

Impaired sucrose induction (isi), 213, 263-264

In vitro

conditions

phytohormones functions under, 266

phytohormones in regulation of growth and development under, 264-266

sugar role in plant growth and

development under, 258-259

methods, 84

$\mathrm{DCFH}_{2}$-DA method, 96

DCHBS-AAP method, 95
In vivo method

DCF method, 96-97

DCHBS-AAP method, 94-95

In-gel iso-electric focusing (In-gel IEF), 483

Indole acetic acid (IAA), 264-265, 291-292, 361

biosynthesis genes, 322

Indole-3 acetaldoxime, 291-292

Indole-3-acetaldoxime pathway (IAOX pathway), 319

Indole-3-acetamide pathway (IAM pathway), 319

Indole-3-acetic acid (IAA), 22, 311, 319, 325, 336

Indole-3-pyruvic acid pathway (IPA pathway), 319

Indolic glucosinolates, 291-292

Induced dormancy, 2-3

Induced thermotolerance, 504

Inducible NOS (iNOS), 386

Inocybe microfastigiata, 22

Inorganic phosphate $(\mathrm{Pi}), 276$

iNOS. See Inducible NOS (iNOS)

Inositol 1,4,5-trisphosphate (IP3), 45, 525, 533-534, 537-538

Inositol polyphosphates, 489

Intracellular ROS levels, 238

Inulin, 428

INV. See Invertase (INV)

Invertase (INV), 195

Iodine ion $\left(\mathrm{I}^{-}\right), 91$

Iodo-acetamide group, 482-483

Ion exclusion, 194-195

Ion transport genes, 50-51

Ionic stress, 485

IP3. See Inositol 1,4,5-trisphosphate (IP3)

IPA pathway. See Indole-3-pyruvic acid pathway (IPA pathway)

IPG gels. See Immobilized pH gradient gels (IPG gels)

IPL. See Isochorismate pyruvate lyase (IPL)

IPP. See Isopentenyl diphosphate (IPP)

IPT gene. See Isopentyl transferase gene (IPT gene)

Iris hexagona, 7

Iron, 324

isi. See Impaired sucrose induction (isi)

Isobaric tags for relative and absolute quantitation (iTRAQ), 481, 483

Isochorismate pyruvate lyase (IPL), 356

Isochorismate synthase (ICS), 356

Isocitrate dehydrogenase (ICDH), 391-393

Isocitratelyase (ICL), 363

Isopentenyl diphosphate (IPP), 342-343

Isopentyl transferase gene (IPT gene), 112, 133

Isoprene, 170-171

Isotope-coded affinity tag (ICAT), $481,482-483$

Isotopes, $482-483$

Isotopic lysine, 483

iTRAQ. See Isobaric tags for relative and absolute quantitation (iTRAQ)
J

J-protein. See LeCDJ1 DnaJ protein-coding gene

JA. See Jasmonic acid (JA)

JA carboxyl methyltransferase (JMT), 372-373

JA ZIM-domain protein (JAZ protein), 336, 490-491

JA-ACC. See Jasmonoyl ACC (JA-ACC)

JA-Ile. See Jasmonoyl isoleucine (JA-Ile)

JAR1. See Jasmonate resistant 1 (JAR1)

Jasminum grandiflorum, 371-372

Jasmonate resistant 1 (JAR1), 490-491

Jasmonate Zim domain (JAZ), 373-374

Jasmonates (JA). See Jasmonic acid (JA)

Jasmonic acid (JA), 5, 7, 19-20, 52, 67-68, $111,114,293,303-304,312,336$, $341-342,355-356,363-364,371-373$, $407-408,420-421$

biosynthesis, 306

counteract salinity stress, 376-379

role, 306

effect of salt stress on plants, 376-380

signaling, 373-375

signaling pathways, 490-491

Jasmonoyl ACC (JA-ACC), 371-372

Jasmonoyl isoleucine (JA-Ile), 371-374

JASMONOYL-L-ISOLEUCINE HYDROLASE 1 (JIH1), 374-375

Jasmonoylisoleucine synthetase, 373-374

JAZ. See Jasmonate Zim domain (JAZ)

JAZ protein. See JA ZIM-domain protein (JAZ protein)

JIH1. See JASMONOYL-L-ISOLEUCINE HYDROLASE 1 (JIH1)

JMT. See JA carboxyl methyltransferase (JMT)

$\mathrm{K}$

K acetyl transferases (KATs), 150

$\mathrm{K}$ deacetylases (KDACs), 150

$\mathrm{K}^{+}$channel in Arabidopsis thaliana 1 (KAT1), 51,344

$\mathrm{K}^{+}$efflux channel. See Guard cell outwardrectifying $\mathrm{K}^{+}$channel (GORK)

KI method. See Potassium iodide method (KI method)

Kinases as enzyme sensors, 212-213

KINK SUPPRESSED IN BZR1-1D (KIB1), 408-409

Kranz anatomy, 521

L

L-gulono- $\gamma$-lactone oxidase (GLOase), 110

L-NAME. See N(G)-Nitro-L-arginine methyl ester (L-NAME)

L-proline betaine, 452-453, $453 f$

LA. See Low affinity (LA)

Laccaria montana, 22

Lactarius salicis-herbaceae, 22

Late embryogenesis abundant proteins (LEA proteins), $64-65,106-107,311,320$, 347-348, 460

genetic engineering for biosynthesis, 68 
Lateral root meristem activation (LR meristem activation), 320-322

LATS. See Low affinity transport system (LATS)

lba mutant. See Low beta amylase mutant (lba mutant)

LEA proteins. See Late embryogenesis abundant proteins (LEA proteins)

Lead, 325

Leaf gaseous exchanges

photosynthesis, $11-12$

stomatal conductance and water relations, $8-11$

Leaf senescence, 358

LeCDJ1 DnaJ protein-coding gene, 509-510

Lectin RLKs (Lec RLKs), 537

Leucine zipper, 130

Leucine-rich repeat-receptor like kinase (LRR-RLK), 407-408

Life span plants, $499-500$

Light, 161-162

stress, 187

Ligustrum vulgare, 186

Linear trap quadrupole-orbitrap (LTQOrbitrap), 484

Lipids, 29, 145-146

metabolism, 66

Lipoxygenase (LOX), 224, 372-373

LOX2, 312

Lissamine Rhodamine B sulfonyl hydrazine (LRSH), 484

LOS6 gene. See Low expression ofosmotic stress-responsive gene (LOS6 gene)

Low affinity (LA), 270-271

Low affinity transport system (LATS), 270-271

Low beta amylase mutant (lba mutant), 263-264

Low expression ofosmotic stress-responsive gene (LOS6 gene), 305-306

LOX. See Lipoxygenase (LOX)

LR meristem activation. See Lateral root meristem activation (LR meristem activation)

LRK1, 131-132

LRR-RLK. See Leucine-rich repeat-receptor like kinase (LRR-RLK)

LRSH. See Lissamine Rhodamine B sulfonyl hydrazine (LRSH)

LTI29 gene, 114

LTI30 gene, 114

LTQ-Orbitrap. See Linear trap quadrupoleorbitrap (LTQ-Orbitrap)

LUC. See Luciferase (LUC)

Luciferase (LUC), 263-264

Lupines liteus, 7

Lycopene, 342-343

Lysophospholipids, 489

M

MA. See Maleic acid (MA)

Macronutrients, 292

Macrophomina phaseolina, 20-21

Maize seedlings, 189

Major facilitator superfamily (MFS), 277
Major intrinsic protein (MIP), 68-69

Malate, 519

Maleic acid (MA), 225

Malondialdehyde (MDA), 24, 109-110, 130, 145, 201, 223-224, 238, 393, 424, $552-553$

Mannitol, 470-471, 471f

Mannitol 1-phosphate dehydrogenase (mtlD), 108, 455-456

Mannose-1-phosphate phosphatase, 470-471

Mannose-6-phosphate isomerase, 470-471

Mannose-6-phosphate reductase, 470-471

MAP. See Mitogen activated protein (MAP)

MAPK. See Mitogen activated protein kinase (MAPK)

MAPK kinase (MAPKK), 144-145, 359, 461, 506-507, 537-538

MAPK kinase kinase (MAPKKK), 66-67, 144-145, 359, 461, 506-507, 537-538

MAPKK. See MAPK kinase (MAPKK)

MAPKKK. See MAPK kinase kinase (MAPKKK)

MAPMAN tool, 263-264

Mass spectrometry (MS), 481

media, 259

targeted proteomics, 484

MB method. See Methylene blue method (MB method)

MBF1c. See Multiprotein bridging factor1c (MBF1c)

MC. See Mesophyll cells (MC)

MDA. See Malondialdehyde (MDA)

MDHA. See Monodehydroascorbate (MDHA)

MDHAR. See Monodehydroascorbate reductase (MDHAR)

MDR. See MG reductase (MDR)

MDR-PGPs. See Multidrug resistant proteins or P-glycoproteins (MDR-PGPs)

Mean germination time (MGT), 357-358

Medicago sativa, 504

MeJA. See Methyl jasmonate (MeJA)

Melatonin, 35

bioactive molecule in regulation of abiotic stress, 175-177

melatonin biosynthesis and level under stressful conditions, 176-177

priming plants with melatonin against abiotic stresses, 177

Membrane

depolarization, 10, 347-348

and native protein structure stabilizations, 428

rigidification/fluidification, 245-246

MeSA. See Methyl SA (MeSA)

Mesophyll cells (MC), 518

Met. See Methionine (Met)

Metabolic enzymes, 210, 213

Metabolic processes, 219-220, 235-236

Metabolites, 290

fluctuation, 525

Metal chelation properties during metal stress, 426-427

Metal stress, 307

hormones effect in regulation, $310 t$
Metals, 459

Methionine (Met), 147, 287-288, 293

Methyl jasmonate (MeJA), 293, 306, 371-373, $420-421,525$

in alleviating salt stress in different crop species, $377 t$

multifunctional roles in abiotic stress tolerance, 375-376

Methyl SA (MeSA), 356-357

Methylation (MET), 356-357

Methylene blue method (MB method), 86-87

Methylerythritol phosphate pathway, 334

Methylglyoxal (MG), 28, 84, 219-220, $229 f$

abiotic stress tolerance

improving by overexpressing glyoxalase genes, 227

relating to MG in plants, 222-227

elimination in plants, 221

generation in plants, 220-221

priming-induced abiotic tolerance, 228

quantification

DAB method, $87-88$

DAF-2 and DAR-1 fluorescence methods, 89-90

DNP method, 88-89

NAC method, 89

signaling crosstalk between $\mathrm{MG}$ and $\mathrm{CA}^{2+}$, ROS, NO, AND $\mathrm{H}_{2} \mathrm{~S}, 228-229$

signaling triggered by environmental stress in plants, $221-222$

Methylglyoxyl (MG), 64, 66

production under abiotic stress, 66

Methylthymol blue method (MTB method), $84-85$

MFS. See Major facilitator superfamily (MFS)

MG. See Methylglyoxal (MG); Methylglyoxyl (MG)

MG dehydrogenase (MGDH), 220-221

MG detoxification system, 221

MG reductase (MDR), 220-221

MG synthase (MGS), 221

MGDH. See MG dehydrogenase (MGDH)

MGS. See MG synthase (MGS)

MGT. See Mean germination time (MGT)

Micronutrients, 291

MicroRNAs (miRNAs), 73, 190, 242, 555-556

Mineral nutrients, 291

nitrogen, $270-271$

potassium, 273-276

SA interaction with, 359-361

sulfur, 271-273

MIP. See Major intrinsic protein (MIP)

miR167a, 111-112

miR393, 111-112

miRNAs. See MicroRNAs (miRNAs)

Mitochondria, 424-425, 429

enzymes, 148-149

Mitogen activated protein (MAP), 66-67, 275

Mitogen activated protein kinase (MAPK), $5-7,27-28,43-44,64-65,106-107$, 130-131, 144-145, 242, 303-304, 355-356, 359, 387, 407-408, 411-412, $460,506-507,534-539,552$

crosstalk between ROS, MAPKs cascades, $\mathrm{Ca}^{+2}$, and Hsf/Hsps, 508-509 


\section{MAPKKKs, 538}

\section{MAPKKs, 538}

SA interaction with, 359

signaling pathway, 486-487

Mitogen-activated protein kinase 3 and/or 6 (AtMPK3-6), 113

MIZ1, 111-112

MKKK20 genes, 50-51

MKKs. See MAPK kinase (MAPKK)

MM. See Molecular mass (MM)

Modern genome editing system, 64

Molecular approaches

in plants, 260

of sugar regulation in plants, 261-264

Molecular biology tools and plant transformation, 185

Molecular changes of genes

anthocyanidin synthase transcriptional changes, 189

chalcone synthase transcriptional induction, 188-189

involvement in phenylpropanoid pathways, 188-189, 189t

phenylalanine ammonia-lyase, 188-189

Molecular chaperones, genetic engineering of, 70

Molecular mass (MM), 482

Monodehydroascorbate (MDHA), 150-151, 170

Monodehydroascorbate reductase (MDHAR), 54, 109, 126, 223-224, 303-304

Monodehydroascorbic acid. See Monodehydroascorbate (MDHA)

Monosaccharide transporters (MSTs), 195

Monosaccharides, 207-208

MPK6 activity, 5-7, 66-67

MRM. See Multiple reaction monitoring (MRM)

MS. See Mass spectrometry (MS)

MS/MS. See Tandem mass spectrometry (MS/MS)

MSL8 protein, 44

MsSIKI1 gene, 537

MSTs. See Monosaccharide transporters (MSTs)

MTB method. See Methylthymol blue method (MTB method)

$m t l D$. See Mannitol 1-phosphate dehydrogenase ( $m t l D)$

Multidimensional protein identification technology (MudPIT), 481, 483

Multidrug resistant proteins or Pglycoproteins (MDR-PGPs), 319

Multiple abiotic stresses, 71-73

Multiple biosynthetic pathways, 450

Multiple macronutrient transporters, 292

Multiple metabolic pathways, 220

Multiple reaction monitoring (MRM), 484

Multiple stress responses, 33

Multiprotein bridging factor1c (MBF1c), 509-510

Mung bean (Vigna radiata L.), 223-224 seedlings, 227

Mustard (Brassica campestris), 222
MYB 96, 346-347

MYB TFs, 148

MYB transcription factor, 106-107, 115, 129

MYB108, 346-347

MYB44, 346-347

MYB60 expression, 115

MYB61 gene, 115

MYC2, 373-374

Myosin activated protein kinase. See Mitogen activated protein kinase (MAPK)

Myriad functions, 418

Myristolylated $\mathrm{Ca}^{+}$binding protein, 485-486

$\mathbf{N}$

$\mathrm{N}(\mathrm{G})$-Nitro-L-arginine methyl ester (LNAME), 388

NA. See Nicotianamine (NA)

NAA. See Naphthalene acetic acid (NAA)

NAC method. See N-Acetyl-L-cysteine method (NAC method)

NADH. See Nicotinamide adenine dinucleotide (NADH)

NADPH. See Nicotinamide adenine dinucleotide phosphate (NADPH)

NADPH thioredoxin reductase (NTRC), 55

NAM. See No apical meristem (NAM)

Nanofertilizers, 553-554

Nanoparticles, 550

under abiotic stress condition in plant cell, $551 f$

signaling mechanism during abiotic stress conditions, 555-557

Nanotechnology, 553-554

Naphthalene acetic acid (NAA), 336

$\mathrm{N}$-Naphthylethylenediamine (NNED), 90

Naphthylphthalamic acid (NPA), 323-325

NASC. See N- $\alpha$-acetyl-S-(1-hydroxy-2-oxoprop-1-yl) cysteine (NASC)

NbPHAN, 115

NBT method, 98-99

histochemical staining method, 99

spectrophotometric method, 98-99

NCED. See 9-Cis-epoxy-carotenoid dioxygenase (NCED)

Necrosis, 245

Necrotrophic fungus, 23

Neural nitric oxide synthase (nNOS), 386, 388, 390-391

NF-YC. See Nuclear factor-Y C (NF-YC)

NF-YC-RGL2-ABI5 module, 335-336

NHEJ. See Nonhomologous end joining (NHEJ)

nia 1 nia $2,387-388$

Nickel (Ni), 395

Nicotiana benthamiana, 23

Nicotiana mutabilis, 188

Nicotiana tabacum. See Tobacco (Nicotiana tabacum)

Nicotianamine (NA), 293

Nicotinamide adenine dinucleotide (NADH), 220-221

Nicotinamide adenine dinucleotide phosphate (NADPH), 390

oxidase, 134,143

NINJA. See Novel Interactor of JAZ (NINJA)
Niotiana benthamiana, 413

NIP. See Nodulin 26-like intrinsic protein (NIP)

NiR. See Nitrite reductase (NiR)

NIT3 gene. See Nitrilase3 gene (NIT3 gene)

Nitrate $\left(\mathrm{NO}_{3}{ }^{-}\right), 270-271$

accumulation and stress regulation, 175

Nitrate reductase (NR), 175, 270-271, 386-387

Nitration, 294

Nitric oxide (NO), 7, 19-20, 84, 106 $142-143,148,175,219-220,228-229$, 287-288, 294, 303-304, 307, 322, 355-356, 364, 385-386, 418, 556 and chilling stress tolerance in plants, 393 in drought stress tolerance, 390-391 in flooding stress tolerance, 396-397 function in plants, 386

and heavy metal stress tolerance in plants, 394-395

and high temperature stress tolerance in plants, 393-394

modulation of endogenous nitric oxide levels in plants, $387-388$

modulators, $389 t$

in ozone stress tolerance, 395

and phytohormones crosstalk in abiotic stress tolerance, 397-398

in plant salt stress tolerance, 391-393

in plant stress responses, 388-397

quantification

DAF-FMDA fluorescence method, 91

Griess reagent method, 90-91

signaling in plants, 387

synthesis in plants, 386-387

in UV-B tolerance of plants, 395-396

in wounding stress tolerance, 396

Nitric oxide synthase (NOS), 386

Nitrilase3 gene (NIT3 gene), 324

Nitrite reductase (NiR), 270-271

Nitrogen (N), 175, 188, 261, 270-271, 287-288, 323

deficiency and phenylpropanoids, 188 metabolism, 271

Nitrosoglutathione (GSNO), 294

NNED. See N-Naphthylethylenediamine (NNED)

nNOS. See Neural nitric oxide synthase (nNOS)

No apical meristem (NAM), 116-117, 242

noa $1,387-388$

Nodulin 26-like intrinsic protein (NIP), 67

Nonenzymatic

antioxidants, 106

metabolites, 25-27

pathway, 220-221, $221 f$

ROS regulation during drought, 110-111

Nonexpression of pathogenesis-related genes (NPR1), 364

Nonglyoxalase system, 221

Nonhomologous end joining (NHEJ), 74

Nonmetabolizable sucrose analogs, 262-263

Nonradical forms, 27-28

Nonradical nitrous acid $\left(\mathrm{HNO}_{2}\right), 28$

Nonselective cation channel (NSCCs), 5 
NOS. See Nitric oxide synthase (NOS)

Novel Interactor of JAZ (NINJA), 373-374 nox1, 387-388

NPA. See Naphthylphthalamic acid (NPA)

NPR1. See Nonexpression of pathogenesisrelated genes (NPR1)

NR. See Nitrate reductase (NR)

NSCCs. See Nonselective cation channel (NSCCs)

NtHsp70-1 protein, 509-510

NTRC. See NADPH thioredoxin reductase (NTRC)

Nuclear factor-Y C (NF-YC), 335-336

NUE. See Nutrient use efficiency (NUE)

Nutrient, 269-270

availability, 258

deficiency, 188, 450

stress, 323-325

plant phenolics and role in nutrient stress, 163-164

uptake, 208

Nutrient use efficiency (NUE), 271

\section{$\mathrm{O}$}

O-acetylserine (OAS), 288-289

OAA. See Oxaloacetate (OAA)

OAS. See O-acetylserine (OAS)

Octadecatrienoic acid, 372-373

OD. See Optical density (OD)

ODC. See Ornithine decarboxylase (ODC)

OGs. See Oligogalacturonides (OGs)

Oidium neolycopersici, 23

Oligogalacturonides (OGs), 208, 211-212

Oligosaccharides, 174-175

"Oligosaccharin" signalling carbohydrates, 212

OLPs. See Osmotin-like proteins (OLPs)

"Omics", 480

OMTK1, 538

One-dimensional gel electrophoresis (1DGE), 481

12-OPDA. See 12-Oxophytodienoic acid (12OPDA)

OPDA reductase (OPR), 372-373

Open stomata 1 (OST1), 9, 346-348, 522

OPP pathway. See Oxidative pentose-5-P pathway (OPP pathway)

OPR. See OPDA reductase (OPR)

Optical density (OD), 87

Organelles, 5, 502

Organic osmolytes, 451

Ornithine decarboxylase (ODC), 465

Ornithine-delta-aminotransferase (OAT), $439-440$

Ornithine- $\delta$-aminotransferase, 107

Orthologs, 240

Oryza sativa L. Multistress-Responsive gene 2 (OsMSR2), 53, 488-489

OsABP, 71-73

OsbHLH062, 375

OsbHLH089, 375

OsbZIP12, 130

OSCA1 gene, 44

OsCDPK7, 131

OsCIPK12, 131
OsCPK4, 131

OsGH3 genes, 323

OsGMST1 knockdown rice seedlings, 200

OsJAZ9, 375

OsjDHN genes, 108-109

OsMKK6, 538

OsMKK6EE, 538

Osmolytes, 3, 106, 360-361, 450-452, 460

ABA in osmolyte biosynthesis

ABA-dependent and-independent signaling pathways, 419-420

role of hormones in regulation of P5CS and proline synthesis, $420-422$

during abiotic stress

accumulation and osmotic adjustment during stress, 423

in membrane and native protein structure stabilizations, 428

and metal chelation properties during metal stress, 426-427

and protection of photosynthetic machinery during abiotic stress, 424

as sources of energy and carbon reserve during and after release of stress, 429

accumulation, 418

and oxidative stress, $424-426$

and amelioration of $\mathrm{NaCl}$-and metalinduced $\mathrm{K}+$ efflux under stress, 426

diversity and distribution in different plant species

compatible, 450

osmoprotectants, 450

quaternary ammonium compounds, 451

sugars and sugar alcohols, 452

tertiary sulfonium compounds, 451-452

mediated abiotic stress responses, 460-461

regulation in response to abiotic stress

GB, 463-465

polyamines, $465-467$

proline, $461-463$

sugars and sugar alcohols, 468-471

and signaling processes

proline and signaling processes, 429

proline metabolism and signaling

pathways in plant senescence, 429-430

as sensing compounds and/or growth regulators, 430

synthesis, 423

Osmoprotectants, 133-134, 172, 450

genetic engineering for osmoprotectants biosynthesis, 68

regulatory genes in drought stress, 107-109

dehydrins, 108-109

GB, 108

osmotin, 108

polyols/sugars, 108

proline, 107

SA interaction with, 359-361

synthesis, 34

Osmoprotection, 210, 460-461

Osmoregulatory genes, engineering of 133-134

Osmosensing $\mathrm{Ca}^{2+}$ channels, 44
Osmosensors, 44

Osmotic adjustment during stress, 423

Osmotic stress, 5, 8, 485

sensors, $45-51$

Osmotin, 108

Osmotin-like proteins (OLPs), 108

OsMPK44 gene, 50-51

OsMPK5 gene, $50-51$

OsMSR2. See Oryza sativa L. MultistressResponsive gene 2 (OsMSR2)

OsNAC022, 346-347

OST1. See Open stomata 1 (OST1)

OsTPP7 gene, 244

OsTRXh1. See H-type Trx (OsTRXh1)

Overexpressing glyoxalase genes, 227

Oxaloacetate (OAA), 519

Oxidative damage, 461

Oxidative pentose-5-P pathway (OPP pathway), 241-242

Oxidative stress, $145,424-426,552$

12-Oxophytodienoic acid (12-OPDA), 114, 372-373

Oxygen $\left(\mathrm{O}_{2}\right), 90,385-386$

activation, 144

Oxylipins, 372-373, 489

Ozone $\left(\mathrm{O}_{3}\right), 395$

NO in ozone stress tolerance, 395

$P$

P starvation response transcription factors (PHR TFs), 276-277

P5C. See Pyrroline 5-carboxylate (P5C); Pyrroline-5-carboxylate (P5C)

P5C dehydrogenase (P5CDH), 107, 439-440

P5CDH. See P5C dehydrogenase (P5CDH)

P5CR. See Pyrroline 5-carboxylate reductase (P5CR)

P5CS. See Pyrroline-5-carboxylate synthetase (P5CS)

PA. See Perchloric acid (PA); Phosphatidic acid (PA); Phosphotidic acid (PA)

Paclobutrazol (PB), 337

PAL. See Phenylalanine ammonia lyase (PAL)

Palatinose, 262-263

PAMPs. See Pathogen associated molecular patterns (PAMPs)

PaNCED1 gene, $342-343$

PaNCED3 gene, $342-343$

PAPS. See 3-Phosphoadenosine 5phosphosulfate (PAPS)

Paracoccus denitrificans, 452-453

Parallel reaction monitoring (PRM), 484

Partial $\mathrm{CO} 2$ pressure $\left(\mathrm{pCO}_{2}\right), 522$

PAs. See Polyamines (PAs)

PAT. See Polar auxin transport (PAT)

Pathogen associated molecular patterns (PAMPs), 411-412

PAMPs-triggered immunity, 210

Pathogenesis-related proteins (PR proteins), 24-25

PB. See Paclobutrazol (PB)

PBL. See Peripheral blood lymphocytes (PBL)

PC. See Phytochelatin (PC); Protein carbonyl (PC) 
PCD. See Programmed cell death (PCD)

PCOs. See Plant Cys oxidases (PCOs)

PCP. See Pistil-expressed $\mathrm{Ca}^{2+}$ binding protein (PCP)

PCR cycle. See Photosynthetic carbon reductive cycle (PCR cycle)

PDH. See Proline dehydrogenase (PDH)

PDH45. See Pea DNA Helicase 45 (PDH45)

Pea DNA Helicase 45 (PDH45), 68

PeCBL1. See Populus euphratica CBL1 (PeCBL1)

Pectin, 174

PEG. See Polyethylene glycol (PEG)

6'-O-Pentafluorobenzenesulfonyl-2'-7'difluorofluorescein method (BES$\mathrm{H}_{2} \mathrm{O}_{2}$ method), 97-98

Pentose phosphate pathway (PPP), 158

PEP. See Phosphoenolpyruvate (PEP)

PEPC. See Phosphoenolpyruvate carboxylase (PEPC)

Pepper (Capsicum annuum), 22-23

Pepper receptor-like protein kinase 1 (CaRLK1), 54

Perchloric acid (PA), 93

Peripheral blood lymphocytes (PBL), 33-34

Peroxidase (POD), 7, 141-142, 229, 303-304, 363-364, 393, 490

Peroxiredoxin (PRX), 54, 149

Peroxisomes, 11-12

PFK. See Phosphofructokinase (PFK)

PFP. See Pyrophosphate-dependent phosphofructokinase (PFP)

PG. See Phosphoglycolate (PG)

PGA. See 3-Phosphoglyceric acid (PGA)

PGPR. See Plant growth-promoting rhizobacteria (PGPR)

PGRs. See Plant growth regulators (PGRs)

Phaseic acid, 343-344

Phenolic(s)

composition, 184

compounds, 158, 159f, 159t, 161-162

metabolism, 163

Phenols, 184

Phenylalanine ammonia lyase (PAL), 158-160, 174-175, 184, 188-189, 363-364, 395

3'-[1-[(Phenylamino)-carbonyl]-3,4tetrazolium] (4-methoxy-6-nitro) benzene sulfonic acid hydrate (XTT), 99

Phenylpropanoids pathway, 183-184, $185 f$ abiotic stress and physiological changes in, 184-185

classes of phenolic compounds in plants and carbon number, $184 t$

cold stress, 186

light stress, 187

molecular changes of genes involved in, 188-189

nitrogen deficiency and phenylpropanoids, 188

regulation and, 190

salinity stress and phenylpropanoids accumulation, $186-187$

senescence, $187-188$ water stress, 185-186

Phenylpropanoids pathway, 190

PHL1. See PHR1-LIKE1 (PHL1)

pho3 mutant. See Phosphorus-deficient3 mutant (pho3 mutant)

Phorbol myristate acetate (PMA), 533-534

Phosphate starvation response1 (PHR1), 419-420

Phosphate transporter 1 (PHT1), 276-277

Phosphate transporter B (PTB), 276-277

Phosphatidic acid (PA), 489

3-Phosphoadenosine 5-phosphosulfate (PAPS), 288-289

Phosphoenolpyruvate (PEP), 158, 160-161

Phosphoenolpyruvate carboxylase (PEPC), 519

Phosphofructokinase (PFK), 259

3-Phosphoglyceric acid (PGA), 518

Phosphoglycolate (PG), 518

Phosphoinositides (PIs), 489

Phospholipase, 64-65, 372-373

Phospholipase C pathway (PLC pathway), $278-279,542$

Phospholipase D (PLD), 488-489

Phospholipid(s), 186

signaling pathway, 489

Phosphoproteomic analyses, 243

Phosphoribulokinase, 426

Phosphorous use efficiency (PUE), 276-277

Phosphorus (P), 210-211, 276-278, 287-288, 323-324

Phosphorus-deficient3 mutant (pho3 mutant), 263-264

5-Phosphosulfate reductase, 272-273

Phosphotidic acid (PA), 542

PHOT2. See Phototropin 2 (PHOT2)

Photooxidative stress, 418

Photorespiration, 239

Photosynthates, $4-5$

Photosynthesis, 11-12, 185, 187, 194, 207-208, 219-220, 239, 258, 287-288 423-424, 459-460, 554

Photosynthetic carbon reductive cycle (PCR cycle), 518

Photosynthetic enzymes, 438

Photosynthetic machinery during abiotic stress, 424

Photosystem I (PS I), 229

Photosystem II (PSII), 239, 390

Phototropin 2 (PHOT2), 522

PHR TFs. See P starvation response transcription factors (PHR TFs)

PHR1-LIKE1 (PHL1), 419-420

PHR1. See Phosphate starvation response1 (PHR1)

PHT1. See Phosphate transporter 1 (PHT1)

Physiologic life cycle, 187

Physiological processes, $186-187$

Phytochelatin (PC), 225

Phytochrome interacting factors (PIFs), 336, 409-410

Phytohormone(s), 29, 67-68, 193, 214, 287-288, 291, 304-305, 333-334, 355-356, 371-372

application, 376 crosstalk between sulfur and, 290-294

ABA, 292

AU, 291-292

BRs, 294

CK, 292

ET, 293

GA, 292-293

JA, 293

$\mathrm{NO}, 294$

SA, 293-294

and functions in growth and development, $265 t$

functions under in vitro conditions, 266

in heat tolerance, $440-441$

importance of sugars interaction with, 264-266

levels, 438

modulation of key genes involved in phytohormone signaling, 132-133

under salt stress, 201-202

signaling crosstalks between GAs and, 335-336

Phytophthora capsici, 20-21

Phytoremediation, 34-35

Pi. See Inorganic phosphate (Pi)

PIFs. See Phytochrome interacting factors (PIFs)

PIN-LIKES (PILS), 319

PIP. See Plasma membrane intrinsic proteins (PIP)

Pipecolate betaine, $455 f$

Pipecolic acid, 454-455

PIs. See Phosphoinositides (PIs)

Pistil-expressed $\mathrm{Ca}^{2+}$ binding protein (PCP), 488-489

Pisum sativum seedlings, 325

PKABA1, 346

PKC. See Protein kinase catalytic domain (PKC)

Plant Cys oxidases (PCOs), 149

Plant growth regulators (PGRs), 303-304, 312f, 407-408

abiotic stresses regulation by plant hormones, 305-312

accumulation, 19-20

perception and transduction of signals, 304-305

signal transduction, $305 f$

Plant growth-promoting rhizobacteria (PGPR), 35, 277-278

Plant hormones, 25-27, 51, 64-65, 264-265, 304,319

abiotic stress regulation by crosstalk of different hormones, 311-312 metal stress, 307

salinity stress, 307

temperature stress, 307

UV radiation, $307-311,311 f$

water stress, 305-307

Plant(s), 43-44, 303-304, 407-408, 418, 460

and abiotic stress, 551-552

abiotic stress tolerance related to MG in, 222-227

adaptation strategy, 30

auxin signaling in, 320 
Plant(s) (Continued)

BRs signaling in, 408-409

CRISPR-Cas9 application in plant abiotic stress tolerance, 75

defense

BR mediated ROS signaling and role in, 412-413

mechanism, 33-34

development stages, 3

genetic engineering of plant transcription factors, 70

glucose sensing, transport, and signaling in, 195

GSTs, 110

Hsps induction phenomena in, 504, $505 t$

improvement technologies, 43-44

metabolism, 213-214

sulfur assimilation, 288-289

sulfur role and compounds in, 290

sulfur transport mechanism in plants, 289-290

MG elimination in, 221

glyoxalase system, 221

nonglyoxalase system, 221

MG generation in, 220-221

enzymatic pathways, 221

nonenzymatic pathway, 220-221

MG signaling triggered by environmental stress in, 221-222

molecular and genetic approaches of sugar

$\mathrm{NO}$ regulation in, 261-264

in plant salt stress tolerance, 391-393, $392 t$

in plant stress responses, $388-397$

nutrition, 22

phenolics, 158

and abiotic stress tolerance, 158-164

biosynthesis of polyphenols, 158

role in cold stress, 163

and role in drought stress, 163

and role in heavy metal stress, 162

role in nutrient stress, $163-164$

structure and classification, 158

as ultraviolet sunscreens, 161-162

physiological, molecular, and genetic approaches in, 260

physiological approach of sugar signaling in, 260-261

physiological processes, 491

protein kinases, 533-536, 535t

genomics efforts in, 542-543

responses, $174-175$

to abiotic stress in developing tolerance, 64-68

ionic stress, 485

osmotic stress, 485

to salinity, 2-4

salt stress effects on, 194-195

senescence, 358, 429-430

species, 1-2

osmolytes diversity and distribution in different, 450-452

sucrose, 259

metabolism in tissues, $262 f$ sugar role in plant growth, 258-259

sugar signaling systems in, 240

Tre in, 236

Plasma membrane (PM), 25-27, 44, 307

Plasma membrane intrinsic proteins (PIP), 68-69

Plastid proteins, 502

PLC pathway. See Phospholipase C pathway (PLC pathway)

PLD. See Phospholipase D (PLD)

Pleiotropic regulatory locus1 (prl1), 420-421

Plumbaginaceae, 451

PM. See Plasma membrane (PM)

PMA. See Phorbol myristate acetate (PMA)

PnRLK-1, 537

POD. See Peroxidase (POD)

Polar auxin transport (PAT), 319

Pollen sterility, 245

Polyamines (PAs), 35, 172-173, 293, 394, 460-461, 465-467, 467f, 467t

Polyethylene glycol (PEG), 109-110, 224

Polyhydroxysteroids, 113-114

Polyols, 3

polyols/sugars, 108

Polypeptides, 439

Polyphenol oxidase (PPO), 363-364

Polyphenols, 158

biosynthesis, 158

Polysaccharides, 174-175, 207-208, 413

Polyunsaturated fatty acids (PUFAs), 145

Polyvinylpyrrolidone (PVP), 555-556

PoPPC1E1a, 526

PoPPC1E1c, 526

Populus euphratica CBL1 (PeCBL1), 53

Porphyra yezoensis gene (PyTPS gene), $469-470$

Portulaca genus, 526

Posttranslational modification (PTM), 387, 482

Potassium (K), 4, 273-276, 287-288, 324

Potassium iodide method (KI method), 91-92

Potassium transporter 2/3 (AKT2/3), 522

POX. See Peroxidase (POD); Proline oxidase (POX)

PP2Cs. See Type 2C protein phosphatases (PP2Cs)

PPC-1, 519

PPC-2, 519

PPO. See Polyphenol oxidase (PPO)

PPP. See Pentose phosphate pathway (PPP)

PR proteins. See Pathogenesis-related proteins (PR proteins)

Predominantly salinity, 459

Priming plants with melatonin against abiotic stresses, 177

prl1. See Pleiotropic regulatory locus1 ( $p r l 1$ )

PRM. See Parallel reaction monitoring (PRM)

ProDH. See Proline dehydrogenase (PDH)

Programmed cell death (PCD), 67-68, 113, $398,412,538$

Proline (Pro), 107, 172, 223-224, 425-426, $461-463,462 f, 464 t$

accumulation, 4, 419-420

biosynthesis, 419-420, 452, 452f derivatives biosynthesis, 452-453

in heat tolerance, $439-440$

metabolism, 491

enzyme, 439-440

and signaling pathways in plant senescence, 429-430

and signaling processes, 429

synthesis, $420-422$

Proline dehydrogenase (PDH), 107, 419-420, $421 t, 439-440$

ProDH2, 419-420

regulation, 422-423

glycine betaine biosynthesis and modulation by ABA, 422-423

Proline oxidase (POX), 107, 439-440

Proline-rich proteins (PRPs), 66

Protein carbonyl (PC), 24

Protein kinase catalytic domain (PKC), $44-45$

Protein kinases, 64, 106-107, 114-115, 130-131, 195, 533-534

role in response to abiotic stress, 66-67

Protein phosphatase (PP), 114-115

PP2A, 408-409, 519

PP2C, 9, 112-113, 344-346, 361, 487

PP2CA, 241

Protein standard absolute quantification method (PSAQ method), 484

Protein(s), 4, 146, 222, 480-481

abundant, 65

accessory, 502-503

acetylation, 150

carbonylation, 148-149

degradation, 439

glutathionylation, 149

separation techniques, 483

synthesis, 439

Proteome analysis, 480

Proteomics, 480-481

approach in salt stress signaling pathways, $491-493,492 t$

plants response to salt stress, 485

salt stress

in plants, 485

signaling pathways, 485-491

technical advances in, 481-484

global proteome analysis, 481-483

targeted proteome analysis, $483-484$

Proton extrusion, 325

PRPs. See Proline-rich proteins (PRPs)

PRX. See Peroxiredoxin (PRX)

PS I. See Photosystem I (PS I)

PSAQ method. See Protein standard absolute quantification method (PSAQ method)

Psyscomitrella patens, 45

PTB. See Phosphate transporter B (PTB)

PTM. See Posttranslational modification (PTM)

PtPYL1 gene, $344-345$

PtPYL5 gene, $344-345$

PUE. See Phosphorous use efficiency (PUE)

PUFAs. See Polyunsaturated fatty acids (PUFAs)

Put. See Putrescine (Put)

Putrescine (Put), 223-224, 465 
Putricine (PUT), 394

PVP. See Polyvinylpyrrolidone (PVP)

PYL. See Pyrabactin resistance1-like (PYL)

Pyrabactin, 344-345

Pyrabactin resistance (PYR), 112-113, 418-419

Pyrabactin resistance/Pyrabactin resistance like/Regulatory component of ABA receptor (PYR/PYL/RCAR), 344

Pyrabactin resistance1-like (PYL), 51, 112-113, 418-419

Pyrophosphate-dependent phosphofructokinase (PFP), 259

Pyrroline 5-carboxylate (P5C), 462

Pyrroline 5-carboxylate reductase (P5CR), 107, 439-440, 462

Pyrroline-5-carboxylate (P5C), 422, 439-440

Pyrroline-5-carboxylate synthetase (P5CS), 107, 421-422, 439-440, 462

PyTPS gene. See Porphyra yezoensis gene (PyTPS gene)

Q

Quadrupole trap (Q-Trap), 484

Quantification concatamers method (QconCAT method), 484

Quaternary ammonium compounds, $450-451$

R

Raffinose family oligosaccharides (RFOs), 174

Ramosa3 mutants, 208

Rapeseed (Brassica napus), 224

RAV. See Related to ABI3/VP1 (RAV)

RBCS. See Rubisco small subunit (RBCS)

RBOHF. See Respiratory burst oxidase homolog F (RBOHF)

RBOHs. See Respiratory burst oxidase homologs (RBOHs)

RCAR. See Regulatory component of ABA receptor (RCAR)

RCARs. See Regulatory component of abscisic acid receptor proteins (RCARs)

RCS. See Reactive carboxyl species (RCS)

RD26 in BR signaling, 410-411

RD26. See Responsive to desiccation 26 (RD26)

RD29A genes, $113-114$

$R D 29 B$ genes, 113

Reactive carboxyl species (RCS), 84

Reactive nitrogen species (RNS), 8-9, 11-12, 28,84

Reactive oxygen species (ROS), 5-7, 19-20, $25-27,43-44,66,84,106,126$, $141-142,158-160,170,185,194$, 207-208, 219-220, 228-229, 238, 269-270, 287-288, 320-322, 325, 335, $376,386,407-408,418,438,459-460$, $480-481,500-501,506,550-551$, $555-556$

crosstalk between ROS, MAPKs cascades, $\mathrm{Ca}^{+2}$, and Hsf/Hsps, 508-509

in plant defense, $144-145$ production and intracellular protein oxidation, $147-150$

ROS-induced posttranslational modifications, 147-150

ROS-mediated damage to macromolecules, 145-147

SA interaction with, 359

signaling, 54-55, 106

during drought and salinity stress, 134

pathway, 489-490

in plants, $144-145$

as signaling molecules, $142-144$

Reactive sulfur species (RSS), 84

Really interesting new gene domain (RING domain), 277

Reaumuria trigyna RtWRKY gene, 347-348

Receptor protein kinase (RPK), 491-493

Receptor-like kinases (RLKs), 44-45, 131-132, 533-534, 537

Redox homeostasis, SA involvement in modulation of, 358-359

Redox regulatory machinery of plants during drought conditions, 109-111

enzymatic ROS regulation during drought, 109-110

nonenzymatic ROS regulation during drought, 110-111

transgenic intervention of antioxidant enzymes, $111 t$

Reduced sugar response mutant (rsr mutant), 263-264

Regulatory component of $\mathrm{ABA}$ receptor (RCAR), 51, 418-419

Regulatory component of abscisic acid receptor proteins (RCARs), 112-113

Related to ABI3/VP1 (RAV), 115

Relative water content (RWC), 224

Repressor of gibberellic acid (rga-24), 336

Respiration, 423

Respiratory burst oxidase, 134

Respiratory burst oxidase homolog $\mathrm{F}$ (RBOHF), 344

Respiratory burst oxidase homologs (RBOHs), 54, 141-142, 144, 407-408 $489-490$

Responsive to desiccation 26 (RD26), 410-411, $411 f$

REVEILLE transcription factor-encoding genes, 525

Reverse phase liquid chromatography-mass spectrometry (Reverse phase LC-MS), 483

RFOs. See Raffinose family oligosaccharides (RFOs)

rga-24. See Repressor of gibberellic acid (rga24)

RGA-like 3 (RGL3), 112, 336

Rhizoctonia solani, 20-21

Rhodobacter sphaeroides, 452-453

Ribulose-1,5-bisphosphate carboxylase/ oxygenase (RuBisCo), 390, 518

carboxylase, 393

Rice (Oryza sativa), 222

Rice salt sensitive3 (RSS3), 375
RING domain. See Really interesting new gene domain (RING domain)

RLK7 gene, 537

RLKs. See Receptor-like kinases (RLKs)

RNA helicases, 71-73

RNS. See Reactive nitrogen species (RNS)

ROS. See Reactive oxygen species (ROS)

RPK. See Receptor protein kinase (RPK)

rsr mutant. See Reduced sugar response mutant (rsr mutant)

RSS. See Reactive sulfur species (RSS)

RSS3. See Rice salt sensitive3 (RSS3)

RuBisCo. See Ribulose-1,5-bisphosphate carboxylase/oxygenase (RuBisCo)

Rubisco small subunit (RBCS), 195

RWC. See Relative water content (RWC)

\section{$\mathrm{S}$}

S-adenosyl methionine synthase (SAMS), 293

S-adenosyl-methionine (SAM), 287-288, 290, 293, 453-454, 465

S-D-lactoylglutahione (SLG), 221

S-domain receptor-like kinases (SRKs), $131-132$

$S$-nitroso- $N$-acetylpenicillamine (SNAP), 391-393

$S$-nitrosoglutathione, 291

$S$-nitrosoglutathione reductase (GSNOR), 294, 387-388

S-nitrosylation (SNO), 148, 394

S-use efficiency (SUE), 292-293

13(S)-hydroperoxy linolenic acid derivatives (13-HPOT), 372-373

S6P. See Sorbitol-6-phosphate (S6P)

S6PDH. See Sorbitol-6-phosphate dehydrogenase (S6PDH)

S6PP. See Sorbitol-6-pyrophosphatase (S6PP)

SA. See Salicylic acid (SA); Sulfanilamide (SA)

SA 2-O-3-D-glucoside (SAG), 356-357

SA methyl transferase 1 (SAMT1), 491

SA-binding protein 2 (SABP2), 491

SAA. See Systemic acquired acclimation (SAA)

SABP2. See SA-binding protein 2 (SABP2)

$\mathrm{SacB}$. See Bacillus subtilis gene (SacB)

SAG. See SA 2-O- $\beta$-D-glucoside (SAG)

SAGs. See Senescence-associated genes (SAGs)

Salicylate glucose ester (SGE), 356-357

Salicylhydroxamic acid (SHAM), 229

Salicylic acid (SA), 7, 19-20, 32-33, 67-68, 106, 114, 287-288, 293-294, 303-304, $311,341-342,355-356,407-408$, 420-421, 438, 442-445, 443t, 444t, 502. See also Abscisic acid (ABA)

and abscisic acid, 361

and auxins, 361

biosynthetic pathway and modifications, 356-357, $357 f$

and $\mathrm{BR}, 364$

crosstalk with other phytohormones under abiotic stress, 361-363

and ET, 363

and gibberellic acid, 361-363 
Salicylic acid (SA) (Continued)

interaction

with HSP, ROS and MAPK, 359

and involvement of DELLA with SA-GA crosstalk under stress, 363-364

with mineral nutrients and osmoprotectant, 359-361

involvement in modulation of redox homeostasis, 358-359

and JA, 363-364

and $\mathrm{NO}, 364$

in plant growth and development, 357-358

role in heat tolerance, $441-442$

SA-mediated defense mechanisms to abiotic stress, 358-361

signaling pathways, 490-491

Salicyloyl-aspartate, 356-357

Saline soils, $371-372$

Saline water, 187

Salinity, 479-480, 485

plant response to, $2-4$

salinity-like stress, $11-12$

stress, 307, 322, 553-554

hormones effect in regulation, $309 t$

and phenylpropanoids accumulation 186-187

Salt overly sensitive pathway (SOS pathway), 5, 45, 64-65, 485-486, 534-536

SOS3 pathway, $5,45,322$

Salt stress, 8, 450, 479-480. See also Water stress

effect on plants, $376-380$

jasmonates counteract salinity stress, 376-379

salt stress response mediated by JA signaling, 379-380

effects on plants, 194-195

glucose

and phytohormones under salt stress, 201-202

in salt stress responses, 195-201

sensing, transport, and signaling in plants, 195

in plants, 485

representation of glucose metabolism, transport, storage, $194 f$

sensors, $45,46 t$

signaling pathways, $485-491$

ABA-signaling pathway, 487-488

CaM, 488-489

JA, ET and SA signaling pathways, 490-491

MAPK, 486-487

phospholipid signaling pathway, 489

proteomics approach in, 491-493, 492t

ROS signaling pathway, 489-490

SOS, 485-486

Salt tolerance, 222-224

cultivars of Oryza sativa, 7

SAM. See S-adenosyl-methionine (SAM); Shoot apical meristem (SAM)

SAM decarboxylase (SAMDC), 465

SAMDC. See SAM decarboxylase (SAMDC)
SAMS. See S-adenosyl methionine synthase (SAMS)

SAMT1. See SA methyl transferase 1 (SAMT1)

SAR. See Systemic acquired resistance (SAR)

Sarcosine dimethylglycine methyltransferase (SDMT), 453-454

SAT. See Serine acetyl transferase (SAT)

SAURs. See Small auxin-upregulated RNAs (SAURs)

ScaBPs. See SOS3-like calcium-binding proteins (ScaBPs)

SCF ubiquitin ligase. See SKP, CULLIN, FBOX CONTAINING ubiquitin ligase (SCF ubiquitin ligase)

Sclerotinia sclerotiorum, 23

Sclerotium rolfsii, 20-21

Scrophularia striata seedlings, 185-186

SCX chromatography. See Strong cation exchange chromatography (SCX chromatography)

SDMT. See Sarcosine dimethylglycine methyltransferase (SDMT)

SDS. See Sodium dodecyl sulfate (SDS)

Secondary messengers, 32-33

Secondary metabolites, 160-161, 184, 552

Secondary signaling molecules, 504

Seed germination, 2-3, 357-358

Seedling, 244

Selected reaction monitoring (SRM), 484

Selenium, 226

Semicarbazide-sensitive amine oxidase (SSAO), 221

Senescence, 113, 187-188

Senescence-associated genes (SAGs), 358 SAG12, 177, 195

Sensor

protein kinases, 507

relays, $488-489,507$

responders, $488-489$

Sequential window acquisition of all theoretical spectra (SWATH), 484

Serine acetyl transferase (SAT), 287-288

SERK3. See Somatic embryogenesis receptorlike kinase 3 (SERK3)

Serotonin N-acetyltransferase (SNAT), 176

6-SFT. See Fructan 6-fructosyltransferase (6SFT)

SGE. See Salicylate glucose ester (SGE)

SHAM. See Salicylhydroxamic acid (SHAM)

Shoot apical meristem (SAM), 214

Shotgun approach, 481

Shotgun proteomics, 480-481

sHsps. See Small heat shock proteins (sHsps)

Signal transduction pathway, 25-27, 304, 499-500, 504

Signaling lipid molecules, 29

Signaling molecules, 84, 423, 500-501

measurement

$\mathrm{Ca}^{2+}$ quantification, $84-85$

$\mathrm{H}_{2} \mathrm{O}_{2}$ quantification, 91-98

$\mathrm{H}_{2} \mathrm{~S}$ quantification, 85-87

HO• quantification, 101-102

MG quantification, 87-90

NO quantification, 90-91 superoxide radical measurement 98-101

and plant responses under combined stress conditions, 29-33

ROS as, $142-144$

in salt-stressed plants

growth and development, 2-8

leaf gaseous exchanges, $8-12$

under stress conditions, 25-29, $26 t$

sugars as, 208-212, 209t

glucose, 211

OGs, 211-212

sucrose, 211

Signaling pathways, 1

Signals, perception and transduction of, 304-305

SILAC. See Stable isotope labeling by amino acids in cell culture (SILAC)

Silver nanoparticles (AgNPs), 553

Simultaneous stress, 20

Singlet oxygen (O), 54, 489-490

$\mathrm{SiO}_{2}$ nanoparticles, 553-554

SiR. See Sulfite reductase (SiR)

sis. See Sugar insensitive (sis)

Site-specific nuclease (SSN), 74

Site-specific recombinase (SSR), 74

SKP, CULLIN, F-BOX CONTAINING ubiquitin ligase (SCF ubiquitin ligase), 334-335

Skp/Cullin/F-box complex (SCF ${ }^{\mathrm{COI} 1}$ ), 373-374

SL. See Strigolactones (SL)

SLAC. See Slow anion channel (SLAC)

SLAH3. See Slow anion channel1 homolog3 (SLAH3)

Slender like-1 (SLR1), 335

SLG. See S-D-lactoylglutahione (SLG)

SLIM1. See Sulfate limitation 1 (SLIM1)

Slow anion channel (SLAC), 10, 51-52, 539-540

SLAC1 channel, 51, 344, 540-541

Slow anion channel1 homolog3 (SLAH3), 540-541

Slowing down energy-consuming processes, 241

SLR1. See Slender like-1 (SLR1)

Small auxin-upregulated RNAs (SAURs), 111-112

Small heat shock proteins (sHsps), 501-504 chaperonins, 502

heat shock transcription factors, 503-504

Hsp70, 502

Hsp90, 502-503

Hsp100, 503

Small noncoding RNAs, 70-71

Small RNAs, genetic engineering of, 73

Small signaling peptides, 291

SNAP. See S-nitroso-N-acetylpenicillamine (SNAP)

SNAT. See Serotonin N-acetyltransferase (SNAT)

Snf1-related kinases (SnRKs), 212, 258

SnRK1, 240, 259

SnRK2, 9, 45, 51, 342, 344, 346

SNF1. See Sucrose nonfermenting 1 (SNF1) 
SNO. See S-nitrosylation (SNO)

SNP. See Sodium nitroprusside (SNP)

SnRK. See Sucrose nonfermenting related protein kinases (SnRK)

SnRKs. See Snf1-related kinases (SnRKs)

SOD. See Superoxide dismutase (SOD)

Sodium chloride $(\mathrm{NaCl})$

cellular concentration, 1-2

$\mathrm{NaCl}$-and metal-induced $\mathrm{K}+$ efflux under stress, 426

Sodium dodecyl sulfate (SDS), 481

Sodium hydrosulfide, 35

Sodium hydrosulfide hydrate (NaHS), 86

Sodium nitroprusside (SNP), 28, 35, 224, 364, 388

Soil salinity, 391-393

Solanum tuberosum, 33

Soluble START domain proteins, 51

Somatic embryogenesis receptor-like kinase 3 (SERK3), 407-408

Sorbitol, 471

Sorbitol-6-phosphate (S6P), 455-456

Sorbitol-6-phosphate dehydrogenase (S6PDH), 455-456, 471

Sorbitol-6-phosphate-dehydrogenase (Stpd1), 108

Sorbitol-6-pyrophosphatase (S6PP), 471

SOS pathway. See Salt overly sensitive pathway (SOS pathway)

SOS3-like calcium-binding proteins (ScaBPs), 45

Soybean (Glycine max L.), 201

Spd. See Spermidine (Spd)

SPDS. See Spermidine synthase (SPDS)

Spectrophotometric method, 98-99

Spermidine (Spd), 35, 173, 223-224, 398, 465

Spermidine synthase (SPDS), 465

Spermine (Spm), 35, 173, 223-224, 465

Spermine synthase (SPMS), 465

Spm. See Spermine (Spm)

SPMS. See Spermine synthase (SPMS)

Spore germination, 429

SPS. See Sucrose phosphate synthases (SPS)

SRKs. See S-domain receptor-like kinases (SRKs)

SRM. See Selected reaction monitoring (SRM)

SRS. See Sugar-response sequence (SRS)

$\mathrm{S}-\mathrm{S}$ bonds. See Disulfide bonds (S-S bonds)

SSAO. See Semicarbazide-sensitive amine oxidase (SSAO)

SSN. See Site-specific nuclease (SSN)

SSR. See Site-specific recombinase (SSR)

1-SST. See Sucrose 1-fructosyltransferase (1SST)

Stable isotope labeling by amino acids in cell culture (SILAC), 483

Stachydrine, 452-453

Starvation-induced $\alpha$-amylase Ramy $3 D$ gene, 244

Stem cell-like Arabidopsis suspension culture, 208-210

Stimpy mutants, 208

Stomatal conductance and water relations, $8-11,9 f, 10 t$

Stomatal control, 521-522
STP. See Sugar transport protein (STP)

Stpd1. See Sorbitol-6-phosphatedehydrogenase (Stpd1)

Stress

agents, 23-24

hormone, 342

memory, $70-71$

osmotic adjustment during, 423

responses, $243-247$

responsive genes, $50-52,66-67,70-71$

signal sensors, $44-45$

signaling

molecules, 64

networks controlling $\mathrm{C}_{4}$ and crassulacean acid metabolism, $524-525$

stimuli, 537-538

stress-induced genes, $499-500$

tolerance, 24, 28-29, 35, 270-276

Stress response suppressor 1 (STRS1), 71-73

Stress responsive signaling molecules and genes

DNA damage of plants, 33-34

genomic and biochemical approaches for plants in combined stresses, 34-36

signaling molecules

and plant responses under combined stress conditions, 29-33

in stress conditions, 25-29

Strigolactones (SL), 132-133

Strong cation exchange chromatography (SCX chromatography), 484

STRS1. See Stress response suppressor 1 (STRS1)

Submergence 1A (Sub1A), 335-336

SUC2. See Sucrose transporter2 (SUC2)

Sucrose (Suc), 258

Sucrose, 208, 211, 260-261, 430

Sucrose 1-fructosyltransferase (1-SST), 470

Sucrose metabolic enzymes, 213-214

Sucrose metabolism, 211

Sucrose nonfermenting 1 (SNF1), 240, 259, 344

SNF1-related protein kinases, 541-542

Sucrose nonfermenting related protein kinases (SnRK), 45, 130-131

SnRK1 and SnRK2, 130-131

SnRK2s, 112-113, 306, 418-419

Sucrose phosphate synthases (SPS), 211, 263-264

Sucrose transporter2 (SUC2), 263-264

Sucrose transporters (SUTs), 195

SUT4, 211

Sucrose-responsive element (SURE), 264

SUE. See S-use efficiency (SUE)

Sugar insensitive (sis), 213

Sugar transport protein (STP), 195

Sugar-response sequence (SRS), 264

Sugar(s), 3, 195, 207, 235-236, 258, 266, 430

alcohols biosynthesis, 455-456

interaction with phytohormones, 264-266

kinases as enzyme sensors, 212-213

molecular and genetic approaches of sugar regulation in plants, 261-264

molecule, 260-261 physiological approach of sugar signaling in plants, 260-261

phytohormones functions under in vitro conditions, 266

role in plant growth and development, 258-259

signaling, 260, 260f

at gene level, 213

and phytohormones, 214

and plant metabolism, 213-214

in plants in response to stress, $208 \mathrm{f}$

systems in plants, 240

as signaling molecules, 208-212

and sugar alcohols, 452, 468-471, 468t

fructans, 470

mannitol, 470-471

sorbitol, 471

trehalose, 469-470

sugar-hormone cross-regulation networks, 208

Sulfanilamide (SA), 90

Sulfate assimilation, 288-289, $289 f$

Sulfate limitation 1 (SLIM1), 293

Sulfate transporters (SULTRs), 289-290

Sulfite reductase (SiR), 288-289

Sulfonylation, 149

Sulfur (S), 271-273, 287-288, 290f, 324

assimilation and role in plant metabolism 288-290

crosstalk between sulfur and phytohormones, 290-294

glutathionylation, 149

metabolism, 290

Sulfur responsive element (SURE), 273, 324

Sulfurase, 342-343

Sulhydryl oxidations of Met and Cys, 149

SULTRs. See Sulfate transporters (SULTRs)

Superoxide dismutase (SOD), 7, 54, 109, 126 141-142, 239, 303-304, 393, 418, 466, 490,552

Superoxide radical $\left(\mathrm{O}_{2} \bullet^{-}\right), 54,84,100$, 489-490

BESSo-AM and BESSo fluorescence methods, 100-101

NBT method, 98-99

XTT method, 99

SURE. See Sucrose-responsive element (SURE); Sulfur responsive element (SURE)

Sustainable crop production, 269-270

SUTs. See Sucrose transporters (SUTs)

SWATH. See Sequential window acquisition of all theoretical spectra (SWATH)

Sweet immunity, 174

SWEET15 gene, 113

Systemic acquired acclimation (SAA), 413

Systemic acquired resistance (SAR), 32-33, 311, 360-361

Systemin, 375

\section{$\mathrm{T}$}

T5H. See Tryptamine 5-hydroxylase (T5H)

T6P. See Trehalose-6-P (T6P)

TaAOC1 gene, 375 
TaAQP8 gene, 68-69

TaCBF14 gene, 129

TaCBF15 gene, 129

TaHDZip $1-5$ gene, 130

TALENs. See Transcription activator like effector nucleases (TALENs)

TAM pathway. See Tryptamine pathway (TAM pathway)

Tamarix albiflonum TaMnSOD, 109-110

TaNAC8 gene, $129-130$

Tandem mass spectrometry (MS/MS), 483

Tangut nitraria, 491-493

TaOPR1 enzyme, 134

Target of rapamycin (TOR), 172

kinase, 259, 271

pathway, 271

Targeted proteome analysis, 483-484. See also Global proteome analysis

affinity and reactive chemistry-based proteomics, 484

gel-based targeted proteomics, 484

mass spectrometry-based targeted proteomics, 484

TaWRKY1 gene, 346-347

TaWRKY33 gene, 346-347

TBARS. See Thiobarbituric acid reactive substances (TBARS)

TCA. See Trichloroacetic acid (TCA)

TCP cell factors. See Teosinite branched/ cycloidea/proliferating cell factors (TCP cell factors)

TDC. See Tryptophan decarboxylase (TDC)

Temperature stress, 307, 322-323

hormones effect in regulation, $308 t$

Temporal signature, $4-8$

Teosinite branched/cycloidea/proliferating cell factors (TCP cell factors), 73

Tertiary sulfonium compounds, 451-452

Tetramines Spm, 35

Tetrazolium compounds, 99

TFBP. See Transcription factor binding protein (TFBP)

TFP. See Trifluoperazine (TFP)

TFs. See Transcription factors (TFs)

Thermospermine ( $\mathrm{tSpm}$ ), 35

5-Thio-2-nitrobenzoic acid (TNB), 85-86

Thiobarbituric acid reactive substances (TBARS), 201

Thioredoxins (Trxs), 55, 149

Three-dimensional gel electrophoresis (3DGE), 482

$\mathrm{Ti}\left(\mathrm{SO}_{4}\right)_{2}$ method, 92

TIBA. See 2,3,5-Triiodobenzoic acid (TIBA)

TIP. See Tonoplast intrinsic protein (TIP)

TIR1. See Transport inhibitor resistant 1 (TIR1)

Tissue culture medium sucrose, 259

Tissue ratio, 275

Tissue tolerance, 194-195

Titanium dioxide $\left(\mathrm{TiO}_{2}\right), 484$

TM. See Transmembrane domain (TM)

TMV. See Tobacco mosaic virus (TMV)

TNB. See 5-Thio-2-nitrobenzoic acid (TNB)

Tobacco (Nicotiana tabacum), 22-23

Tobacco mosaic virus (TMV), 22-24
Tobacco NtC7, 45-50

Tomato (Solanum lycopersicum L.), 7, 200

Tomato plants (Lycopersicon esculentum Mill.), 20-21, 391-393

Tonoplast intrinsic protein (TIP), 68-69

TOPLESS (TPL), 373-375

TOR. See Target of rapamycin (TOR)

TP. See Triosephosphate (TP)

TPI. See Triose phosphate isomerize (TPI)

TPL. See TOPLESS (TPL)

TPL-related proteins (TPRs), 373-374

TPP. See Trehalose-6-phosphate phosphatase (TPP)

TPRs. See TPL-related proteins (TPRs)

TPS. See Trehalose-6-phosphate synthase (TPS)

TPSP. See Trehalose-6-phosphate synthase/ phosphatase (TPSP)

Trans-4-hydroxy-L-proline betaine or betonicine, 452-453

Transcription activator like effector nucleases (TALENs), 55, 74

Transcription factor binding protein (TFBP), 279

Transcription factors (TFs), 54, 64-65, 71-73, 106-107, 114-115, 126, 141-142, 271, $275,320,334-335,499-500$

associating with signaling mechanism, 127-130

involving in BR signalling, 409-410, $409 f$

Transfer acid generation, 521-522

Transgenic approaches, $43-44,68-73$

aquaporin genes with abiotic stress tolerance, $68-70$

epigenetic regulation of abiotic stress tolerance, $70-71$

functional role of ubiquitination encoding proteins in abiotic stress tolerance, 71

genetic engineering

for biosynthesis of osmoprotectants and LEA, 68

of helicases in plant abiotic stress tolerance, $71-73$

of molecular chaperones, HSPs, and plant transcription factors, 70

of small RNAs for abiotic stress responses, 73

Transgenic plants, 34, 52

Transmembrane domain (TM), 44-45

TRANSPARENT TESTA 8 (TT8), 374-375

Transpiration, 185

Transport inhibitor resistant 1 (TIR1), 311, 320

Transport inhibitor response 1. See Transport inhibitor resistant 1 (TIR1)

Trehalose (Tre), 108, 235-236, 237f, 469-470, $469 f$

biosynthesis, 455-456

metabolism, $248 t$

in plants, 236

as protectant against abiotic stresses, 236-239

T6P in plant metabolic and physiological activities, 239-240

trehalose/trehalose-6-P/SNRK1 system, 240-243 in specific stress responses, 243-247

Trehalose-6-P (T6P), 213, 235-236, 259

in plant metabolic and physiological activities, 239-240

signal, 259

Trehalose-6-phosphate phosphatase (TPP), 236, 469

Trehalose-6-phosphate synthase (TPS), 236 469

TPS1, 108

Trehalose-6-phosphate synthase/ phosphatase (TPSP), 108

Trehalose/trehalose-6-P/SNRK1 system, 240-243, 243f

in specific stress responses

cold, 245-247

hypoxia, $243-245$

Triamine Spd, 35

Trichloroacetic acid (TCA), 92, 221, 481

Trifluoperazine (TFP), 228

Triiodide $\left(\mathrm{I}_{3}{ }^{-}\right), 91$

2,3,5-Triiodobenzoic acid (TIBA), 323-325

N,N,N-Trimethylglycine, 360-361

Triose phosphate isomerize (TPI), 66

Triose-P positive effectors, 519

Triosephosphate (TP), 220-221

Tripeptide glutathione, 149

Triple quadrupole, 484

Trxs. See Thioredoxins (Trxs)

Tryptamine 5-hydroxylase (T5H), 176

Tryptamine pathway (TAM pathway), 319

Tryptophan decarboxylase (TDC), 176

Tryptophan-dependent IAA biosynthesis, 319

tSpm. See Thermospermine (tSpm)

TT8. See TRANSPARENT TESTA 8 (TT8)

Turanose, 262-263

Turnip mosaic virus (TuMV), 22-23

TWD1. See TWISTED DWARF 1 (TWD1)

TWISTED DWARF 1 (TWD1), 408-409

Two-dimensional gel electrophoresis (2DGE), 481

Two-dimensional-differential in gel electrophoresis (2DE-DIGE), 481

Type $2 \mathrm{C}$ protein phosphatases (PP2Cs), 51

Type I ACSs, 113

Type II ACS proteins, 113

$\mathrm{U}$

Ubiquitin (UQ), 236

Ubiquitination encoding proteins, $71,72 t$

UDPG. See Uridine diphosphate glucose (UDPG)

Ultraviolet (UV), 157

light, 161-162, 187

phenolics as UV sunscreens, 161-162

radiation, $238,307-311,311 f$

Ultraviolet light-B (UV-B), 27-28

NO in UV-B tolerance of plants, 395-396

radiation, 24,424

Unicellular organisms, 45

Upland cotton (Gossypium hirsutum), 65

UQ. See Ubiquitin (UQ) 
Uridine diphosphate glucose (UDPG), 236

UV. See Ultraviolet (UV)

UV-B. See Ultraviolet light-B (UV-B)

\section{$\mathrm{V}$}

V-ATPase. See Vacuolar type $\mathrm{H}^{+}$-ATPase (VATPase)

V-PPase. See Vacuolar pyrophosphatase (VPPase)

Vacuolar glucose transporter (VGT), 195

Vacuolar invertase, 211

Vacuolar phosphate transporter 1 (VPT1), 277

Vacuolar pyrophosphatase (V-PPase), 485-486

Vacuolar type $\mathrm{H}^{+}$-ATPase (V-ATPase), 485-486

Vacuole, 277

Vegetative storage protein (VSP), 312

Verticillium albo-atrum, 20-21

VGT. See Vacuolar glucose transporter (VGT)

Vicia faba, 12

Vigna aconitifolia pyrroline T carboxylate synthetase, 133-134

VIGS. See Virus-induced gene silencing (VIGS)

Virus-induced gene silencing (VIGS), 115
Volatile organic compound (VOC), 35

VPT1. See Vacuolar phosphate transporter 1 (VPT1)

VSP. See Vegetative storage protein (VSP)

\section{W}

Washington StateProbe-1 (WSP-1), 87 fluorescence method, 87

Water scarcity, 133-134, 305

Water storage cells (WSC), 526

Water stress, 185-186, 305-307, 450

ABA role, 305-306

analogously, 189

auxin and cytokinin role, 307

BR role, 307

ET role, 306

JA role, 306

Water use efficiency (WUE), 522, 537

Water-soluble sugars, 207-208

Wheat (Triticum aestivum), 20-21, 222, 358-359

aquaporins genes, $68-69$

Wounding stress tolerance, NO in, 396

WRKY transcription factors, 346-347

WRKY63, 346-347

WRKY70 transcription factor, 364

WSC. See Water storage cells (WSC)
WSP-1. See Washington StateProbe-1 (WSP-1)

WUE. See Water use efficiency (WUE)

\section{$\mathrm{X}$}

Xanthomonas campestris, 74

Xanthoxin, 342-343

xylenol orange (XO method), 93

Y

yис1уuс2yuc6 triple mutants, 111-112

YUCCA genes, 111-112, 320

\section{$\mathrm{Z}$}

ZAT10, 239

Zeaxanthin, 342-343

Zeaxanthin epoxidase (ZEP), 342-343 gene, 305-306

Zinc (Zn), 395

Zn-finger transcription factor family, 116

Zinc finger nucleases (ZFNs), 55, 74

Zinc-finger proteins (ZFPs), 116 ZFP252, 116

Zirconium dioxide $\left(\mathrm{ZrO}_{2}\right), 484$

Zmhdz10 gene, 130

ZmPK1 (RLK gene), 131-132 


\section{Plant Signaling Molecules Role and Regulation Under Stressful Environments}

Edited by

M. Iqbal R. Khan

Department of Botany, School of Chemical and Life Sciences, Jamia Hamdard, New Delhi, India

\section{Palakolanu Sudhakar Reddy}

Cell, Molecular Biology and Genetic Engineering Group, International Crops Research Institute for the Semi-Arid Tropics (ICRISAT), Telangana, India

\section{Antonio Ferrante}

Department of Agricultural and Environmental Sciences, University of Milano, Milano, Italy

\section{Nafees A. Khan}

\section{Department of Botany, Aligarh Muslim University, Aligarh, India}

Plant Signaling Molecules explores stress tolerance mechanisms mediated by different "signaling molecules" in plants for achieving sustainability under changing environmental conditions through mechanistic strategies. This book includes a wide range of signaling molecules, exploring the present status and future prospects of the role and regulation of such molecules at physiological, biochemical, molecular, and structural levels under abiotic stresses. Understanding how these mechanisms direct plant responses provides an important contribution to anticipating and addressing underlying mechanisms. This book is designed to enhance the mechanistic understanding of signaling molecules and will be an important resource for graduate students, researchers, professors, and plant scientists. This book will also assist in developing stress tolerant crop plants to achieve sustainability under changing environmental conditions.

\section{Key Features}

- Provides a compendium of knowledge related to plant physiology, plant biochemistry, and plant molecular responses for adaptation of plants under stressful environments.

- Focuses on plant signaling molecules under stressful environments and identifies treatments able to enhance tolerance to abiotic stresses.

- Illustrates specific physiological and molecular pathways that are considered key points for plant adaptation or tolerance to abiotic stresses.

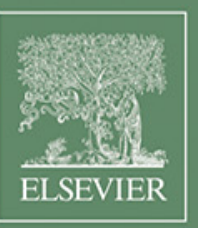

